\title{
Computational Studies of Chiral Hydroxyl Carboxylic Acids: The Allylboration of Aldehydes \\ Supporting Information
}

\author{
Elliot H. E. Farrar and Matthew N. Grayson* \\ Department of Chemistry, University of Bath, Claverton Down, Bath, BA2 7AY, United Kingdom \\ *Corresponding Author: M.N.Grayson@bath.ac.uk
}

\section{Contents}

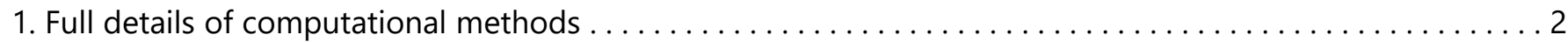

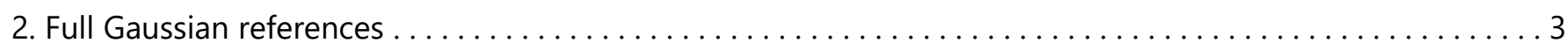

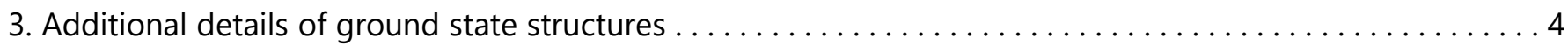

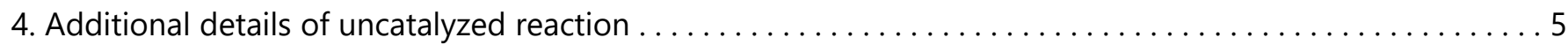

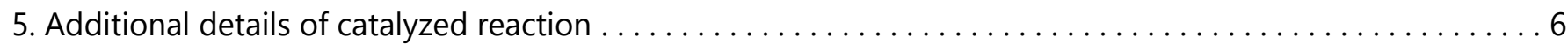

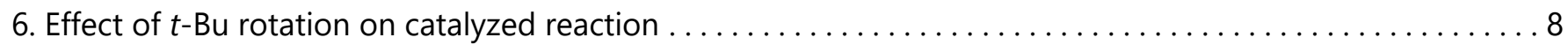

7. Additional details of alternative catalyzed reaction conditions $\ldots \ldots \ldots \ldots \ldots \ldots \ldots \ldots \ldots \ldots \ldots$

8. Energies, frequencies and molecular geometries of all computed structures $\ldots \ldots \ldots \ldots \ldots \ldots \ldots \ldots$

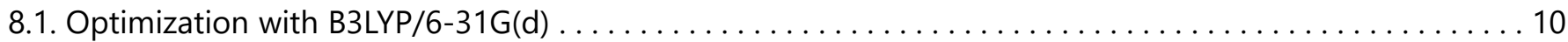

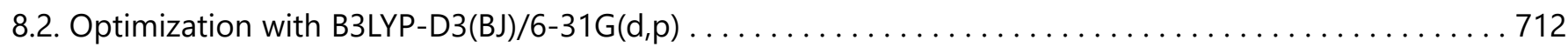

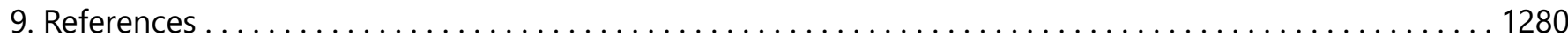




\section{Full computational methods}

Conformational searches were carried out using the conformational search tool within Schrödinger's MacroModel (version 11.3) ${ }^{1,2}$ with the OPLS 2005 force field. ${ }^{3}$ A mixed Monte Carlo Multiple Minimum (MCMM) ${ }^{4}$ and low-mode sampling approach ${ }^{5,6}$ was used to explore the possible conformations of the molecule or complex. Conformations provided by these searches were optimized by DFT ${ }^{7,8}$ calculations carried out using Gaussian09 (Revision D.01) ${ }^{9}$ with the B3LYP density functional ${ }^{10,11}$ and split-valence polarized $6-31 \mathrm{G}(\mathrm{d})$ basis set. ${ }^{12}$ In order to correct the Gibbs free energies derived from the B3LYP/6-31G(d) calculations, ${ }^{13}$ single point energy (SPE) calculations were performed using B3LYP, dispersion-corrected with the D3 version of Grimme's dispersion ${ }^{14}$ with Becke-Johnson damping (B3LYP$\mathrm{D} 3(\mathrm{BJ})){ }_{1}^{15-17}$ the polarized triple- $\zeta$ valence quality (def2-TZVPP) basis set, ${ }^{18}$ and the integral equation formalism version of the polarizable continuum model (IEF-PCM) ${ }^{19}$ (dichloromethane). All conformers within $8.0 \mathrm{kcal} \cdot \mathrm{mol}^{-1}$ of the lowest energy conformer for each species, as determined from the original B3LYP-D3(BJ)/def2-TZVPP/IEFPCM(dichloromethane)//B3LYP/6-31G(d) calculations, were subsequently reoptimized using Gaussian16 (Revision A.03) ${ }^{20}$ with B3LYP-D3(BJ) and the 6-31G(d,p) basis set, ${ }^{12}$ followed by SPE calculations at the same level of theory as above. Following conformational searching with MacroModel, conformations of the simple phenyl BHCA and BPA species, the alternative catalyst, and the alternative BHCA-catalyzed reaction transition state, were optimized immediately at the higher B3LYP-D3(BJ)/6-31G(d,p) level of theory. SPE calculations were performed with the same level of theory as above in order to correct the Gibbs free energies from these B3LYP-D3(BJ)/6-31G(d,p) calculations. ${ }^{13}$ All DFT calculations were performed using the pruned version of the $(99,590)$ integration grid. Temperature $(195.15 \mathrm{~K})$ and concentration-corrected $(1 \mathrm{~mol} / \mathrm{l})$ quasiharmonic (Grimme approximation ${ }^{21}$ ) free energies were calculated with GoodVibes $^{22}$ with a vibrational scaling factor of 0.977 .23 NBO analyses were performed using Gaussian 16 (Revision A.03) with the B3LYP-D3(BJ)/def2-TZVPP/IEF-PCM(dichloromethane) level of theory. 


\section{Full Gaussian references}

Gaussian 09, Revision D.01, Frisch, M. J.; Trucks, G. W.; Schlegel, H. B.; Scuseria, G. E.; Robb, M. A.; Cheeseman, J. R.; Scalmani, G.; Barone, V.; Mennucci, B.; Petersson, G. A.; Nakatsuji, H.; Caricato, M.; Li, X.; Hratchian, H. P.; Izmaylov, A. F.;Bloino, J.; Zheng, G.; Sonnenberg, J. L.; Hada, M.; Ehara, M.; Toyota, K.; Fukuda, R.; Hasegawa, J.; Ishida, M.; Nakajima, T.; Honda, Y.; Kitao, O.; Nakai, H.; Vreven, T.; Montgomery, J. A., Jr;; Peralta, J. E.; Ogliaro, F.; Bearpark, M. J.; Heyd, J. J.; Brothers, E. N.; Kudin, K. N.; Staroverov, V. N.; Kobayashi, R.; Normand, J.; Raghavachari, K.; Rendell, A. P.; Burant, J. C.; lyengar, S. S.; Tomasi, J.; Cossi, M.; Rega, N.; Millam, J. M.; Klene, M.; Knox, J. E.; Cross, J. B.; Bakken, V.; Adamo, C.; Jaramillo, J.; Gomperts, R.; Stratmann, R. E.; Yazyev, O.; Austin, A. J.; Cammi, R.; Pomelli, C.; Ochterski, J. W.; Martin, R. L.; Morokuma, K.; Zakrzewski, V. G.; Voth, G. A.; Salvador, P.; Dannenberg, J. J.; Dapprich, S.; Daniels, A. D.; Farkas, O.; Foresman, J. B.; Ortiz, J. V.; Cioslowski, J.; Fox, D. J. Gaussian, Inc., Wallingford CT, 2009.

Gaussian 16, Revision A.03, Frisch, M. J.; Trucks, G. W.; Schlegel, H. B.; Scuseria, G. E.; Robb, M. A.; Cheeseman, J. R.; Scalmani, G.; Barone, V.; Petersson, G. A.; Nakatsuji, H.; Li, X.; Caricato, M.; Marenich, A. V.; Bloino, J.; Janesko, B. G.; Gomperts, R.; Mennucci, B.; Hratchian, H. P.; Ortiz, J. V.; Izmaylov, A. F.; Sonnenberg, J. L.; Williams-Young, D.; Ding, F.; Lipparini, F.; Egidi, F.; Goings, J.; Peng, B.; Petrone, A.; Henderson, T.; Ranasinghe, D.; Zakrzewski, V. G.; Gao, J.; Rega, N.; Zheng, G.; Liang, W.; Hada, M.; Ehara, M.; Toyota, K.; Fukuda, R.; Hasegawa, J.; Ishida, M.; Nakajima, T.; Honda, Y.; Kitao, O.; Nakai, H.; Vreven, T.; Throssell, K.; Montgomery, J. A., J.; Peralta, J. E.; Ogliaro, F.; Bearpark, M. J.; Heyd, J. J.; Brothers, E. N.; Kudin, K. N.; Staroverov, V. N.; Keith, T. A.; Kobayashi, R.; Normand, J.; Raghavachari, K.; Rendell, A. P.; Burant, J. C.; lyengar, S. S.; Tomasi, J.; Cossi, M.; Millam, J. M.; Klene, M.; Adamo, C.; Cammi, R.; Ochterski, J. W.; Martin, R. L.; Morokuma, K.; Farkas, O.; Foresman, J. B.; Fox, D. J. Gaussian, Inc., Wallingford CT, 2016. 


\section{Additional details of ground state structures}

Conformational searching and DFT optimizations, as described above, yielded one, three and 82 unique conformations for benzaldehyde, the allyl boronate, and the BHCA catalyst, respectively. The lowest energy conformers for each are shown in Figure S1. In the lowest energy catalyst structure, BHCA-1, intramolecular hydrogen bonding occurrs between the carboxyl and alcohol groups, with the carboxyl group acting as the hydrogen bond donor. The lowest energy catalyst structure where the alcohol group acts as the hydrogen bond donor and the carboxyl group as the acceptor, BHCA-2, is $1.3 \mathrm{kcal}^{-\mathrm{mol}^{-1}}$ higher in energy than BHCA-1.

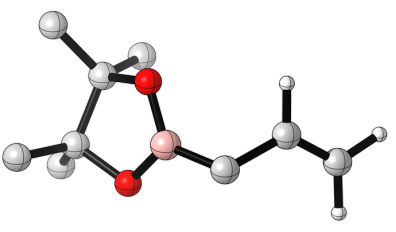

Allyl Boronate<smiles>COB1OOOOO1</smiles>

Benzaldehyde
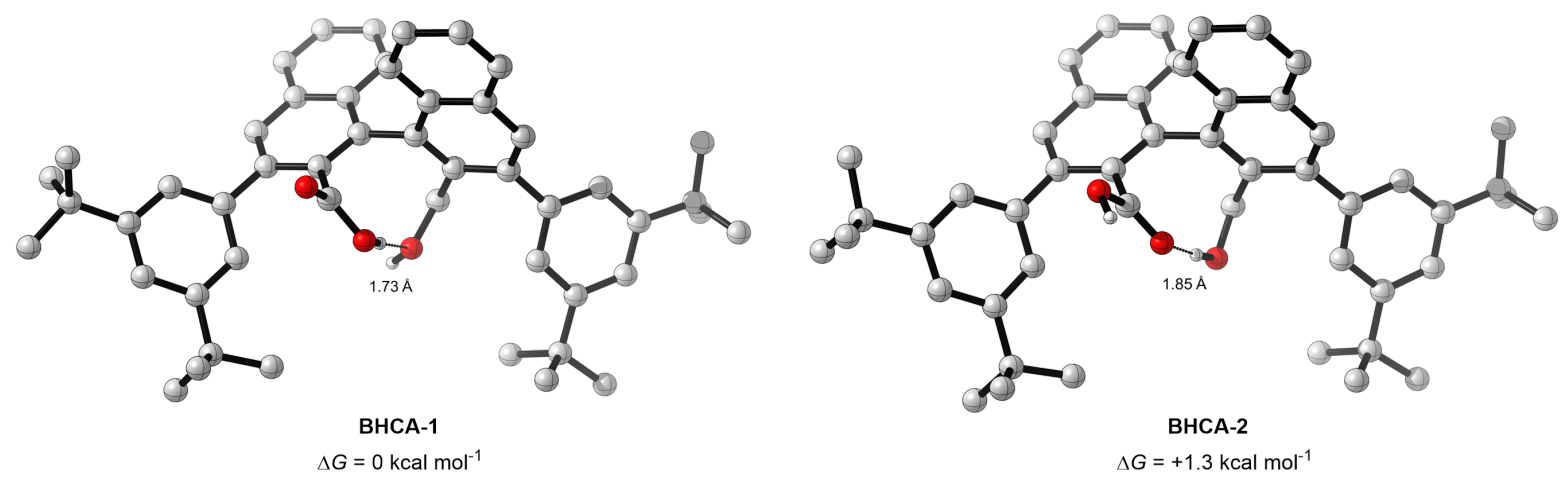

Figure S1. Lowest energy conformers for benzaldehyde, allyl boronate, and BHCA catalyst (B3LYP-D3(BJ)/def2-TZVPP/IEF-PCM(dichloromethane)//B3LYP-D3(BJ)/6-31G(d,p)). 


\section{Additional details of uncatalyzed reaction}

No conformational search was carried out for the uncatalyzed reaction, since the lowest energy TS geometries are well-documented for these types of reactions, ${ }^{24}$ however four unique TSs for the uncatalyzed reaction, corresponding to chair and boat conformations with the phenyl group of benzaldehyde positioned either pseudoequatorially or pseudoaxially, were investigated (Figure S2). The chair conformation with a pseudoequatorial phenyl group, TS$\mathbf{1}_{\text {chair,eq, }}$, was found to be the lowest energy with a $\Delta G \neq$ value of $17.2 \mathrm{kcal} \cdot \mathrm{mol}^{-1}$. The Boltzmann population ratios for each TS of the uncatalyzed reaction were calculated and indicate that $\mathbf{T S} \mathbf{-} \mathbf{1}_{\text {chair,eq }}$ is the only significantly populated $\mathrm{TS}$, and hence only TSs of this nature needed to be considered during investigation of the catalyzed reaction.

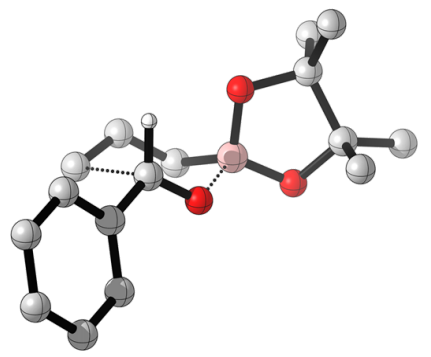

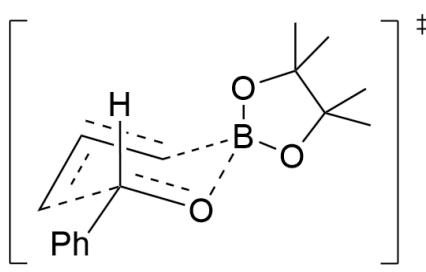

TS- $1_{\text {chair-eq }}$

$\Delta \Delta G^{\ddagger}=0 \mathrm{kcal} \mathrm{mol}^{-1}$

Ratio $=1$

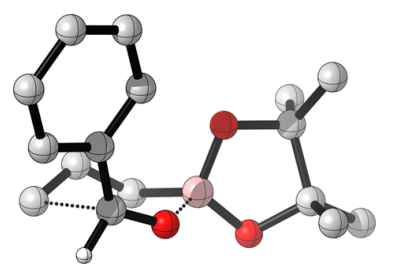<smiles></smiles>

TS-1 chair-ax $_{\text {}}$

$\Delta \Delta G^{\ddagger}=4.6 \mathrm{kcal} \mathrm{mol}^{-1}$

Ratio $=6.38 \times 10^{-6}$

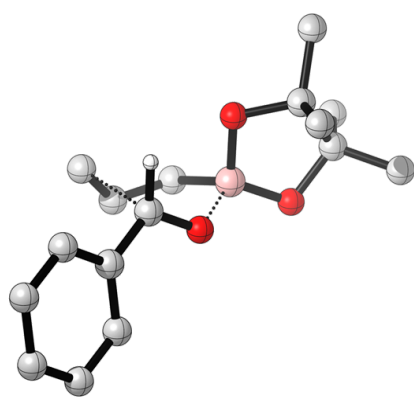<smiles>CC1(C)OB(C=Cc2ccccc2)OC1(C)C</smiles>

TS- $\boldsymbol{1}_{\text {boat-eq }}$

$\Delta \Delta G^{\ddagger}=6.5 \mathrm{kcal} \mathrm{mol}^{-1}$

Ratio $=5.42 \times 10^{-8}$

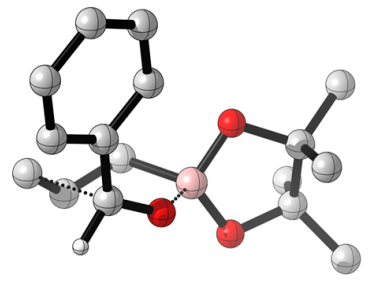<smiles>CC1(C)OB(C=Cc2ccccc2)OC1(C)C</smiles>

TS-1 $1_{\text {boat-ax }}$

$\Delta \Delta G^{\ddagger}=8.3 \mathrm{kcal} \mathrm{mol}^{-1}$

Ratio $=4.73 \times 10^{-10}$

Figure S2. Relative energy reaction barriers and Boltzmann ratios for the TSs of the uncatalyzed allylboration (B3LYP-D3(BJ)/def2-TZVPP/IEFPCM(dichloromethane)//B3LYP-D3(BJ)/6-31G(d,p)). 


\section{Additional details of catalyzed reaction}

The specific conditions chosen for computational analysis of the catalyzed reaction are summarized in Figure S3. ${ }^{25}$ Although a comparable ee and higher yield were achieved under the optimized conditions (Figure S4), the chosen conditions significantly reduced the computational complexity of the calculations due to the use of a truncated catalyst.
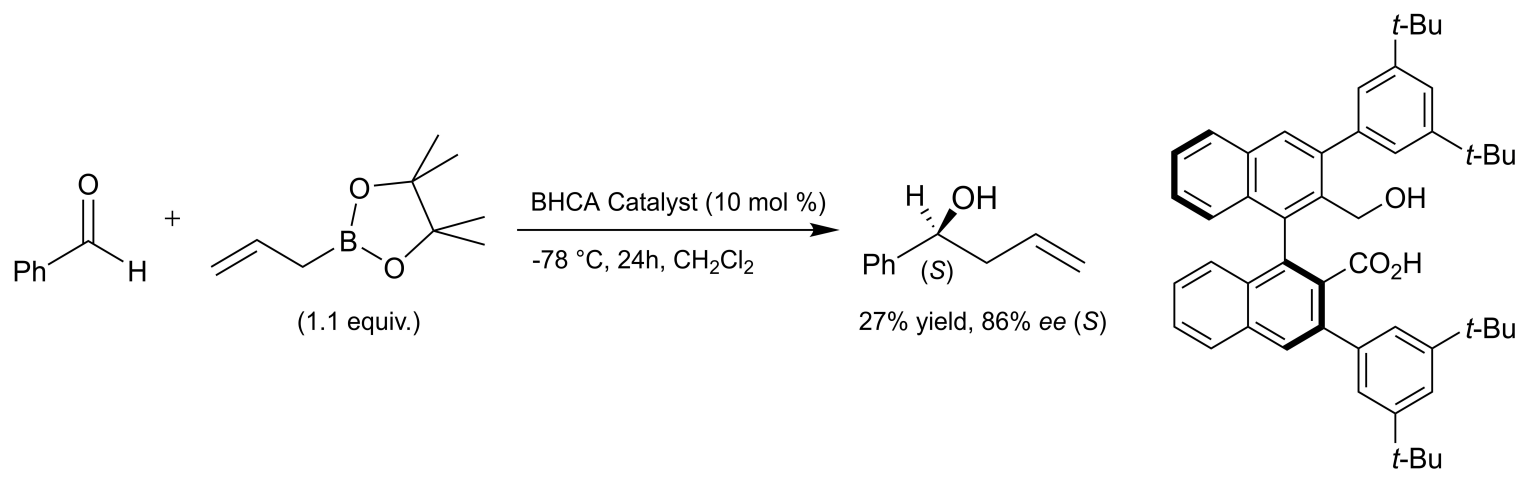

Figure S3. Chosen reaction conditions for computational analysis of BHCA-catalyzed allylboration.<smiles>C=CCB1OC(C)(C)C(C)(C)O1</smiles>

$\mathrm{Ph}(\mathrm{S})$ $73 \%$ yield, $85 \%$ ee $(S)$<smiles>OCc1c(-c2c(-c3cc([Al])cc([Al])c3)cc3ccccc3c2-c2cc([Al])cc([Al])c2)cc2ccccc2c1-c1cc([Al])cc([Al])c1</smiles><smiles>CC(C)(C)c1cc(C(C)(C)C)cc(C(C)(C)C)c1</smiles>

Figure S4. Optimized reaction conditions for BHCA-catalyzed allylboration.

Conformational searches were performed for the catalyzed reaction transition state with either the alcohol or carboxylic acid group constrained to protonate the boronate oxygen. Additional attempts were also made to locate modes in which both the aldehyde oxygen and one of the boronate oxygens were protonated by the catalyst carboxylic acid and alcohol groups, respectively, and vice versa. However, only one such mode was found (Figure S5) other attempts resulted in relaxation to previously-located modes, such as mode A TSs. The free energy of TS-2.6 is $10.9 \mathrm{kcal} \cdot \mathrm{mol}^{-1}$ higher than TS-2.1 (B3LYP-D3(BJ)/def2-TZVPP/IEFPCM(dichloromethane)//B3LYP-D3(BJ)/6-31G(d,p)). This difference is largely attributed to the substantially higher energy of the catalyst structure in TS-2.6, which is also $10.9 \mathrm{kcal} \cdot \mathrm{mol}^{-1}$ higher than the lowest energy conformation of the isolated catalyst - a significantly larger difference than that found for any of the TSs presented in the manuscript (Figure 8). Furthermore, the intramolecular hydrogen

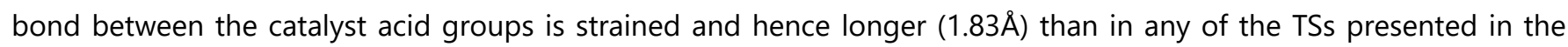

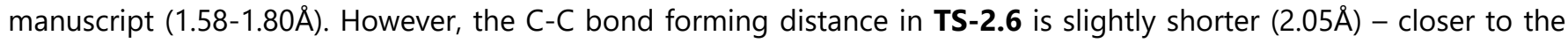
product structure - than in TS-2.1 $(2.11 \AA)$, indicating a later TS. Overall, these results suggest that it is not geometrically feasible for the substrate and catalyst to interact favourably in this manner, and hence TSs of this nature were not considered further. Additionally, modes in which the catalyst and substrate interact only via hydrogen bonding with the aldehyde oxygen (no interaction with boronate oxygens) were consistently found to be significantly higher in energy (between 9.0 and $14.4 \mathrm{kcal} \cdot \mathrm{mol}^{-1}$ ) than modes of type A (Figure 2) during our original work on BPAcatalyzed allylboration and propargylation reactions. ${ }^{26,27}$ 

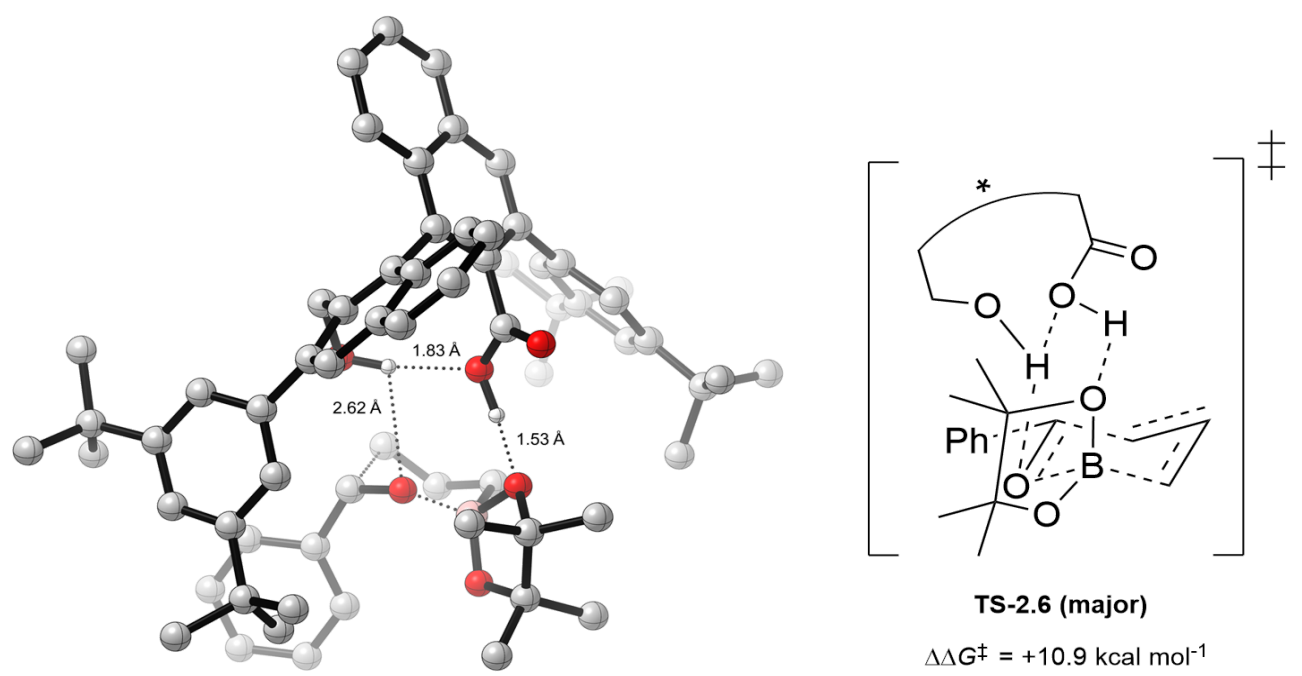

Figure S5. Lowest energy TS for BHCA-catalyzed allylboration in which the aldehyde oxygen is protonated by the catalyst alcohol group, and a boronate oxygen is protonated by the carboxylic acid. Energy relative to TS-2.1 (B3LYP-D3(BJ)/def2-TZVPP/IEF-PCM(dichloromethane)//B3LYP-D3(BJ)/6$31 G(d, p))$.

Overall, conformational searching and DFT optimizations, as described above, yielded 145 unique TSs for the catalyzed reaction. All major $((S)$-enantiomer) products resulted from si-face attack of benzaldehyde, whilst all minor $((R)$ enantiomer) products resulted from re-face attack of benzaldehyde. 


\section{Effect of $t$-Bu rotation on catalyzed reaction}

The $t$-Bu groups of the catalyst aryl groups in TS-2.1 are all orientated in the same way, with a methyl of the $t$-Bu eclipsing the ortho carbons of the aryl in each case. In order to investigate the effect of rotating these $t$-Bu groups, an alternative TS was located and optimized (B3LYP-D3(BJ)/def2-TZVPP/IEFPCM(dichloromethane)//B3LYP-D3(BJ)/6$31 \mathrm{G}(\mathrm{d}, \mathrm{p})$ ) in which the methyls instead eclipse the para carbons (rotation through $180^{\circ}$ ) (Figure S6). The most significant contribution to the change in free energy upon rotation of the catalyst $t$-Bu groups was found to come from the enthalpy, however, overall, rotation of the catalyst $t$-Bu groups was found not to have a significant effect on the quasiharmonic enthalpy, entropy, or free energy of TS-2.1 (Table S1).

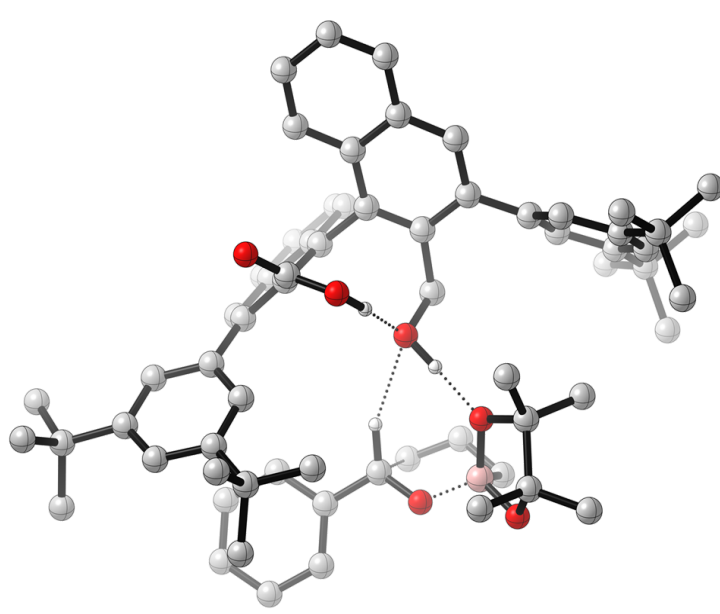

TS-2.1

$\Delta \Delta G^{\ddagger}=0 \mathrm{kcal} \mathrm{mol}^{-1}$

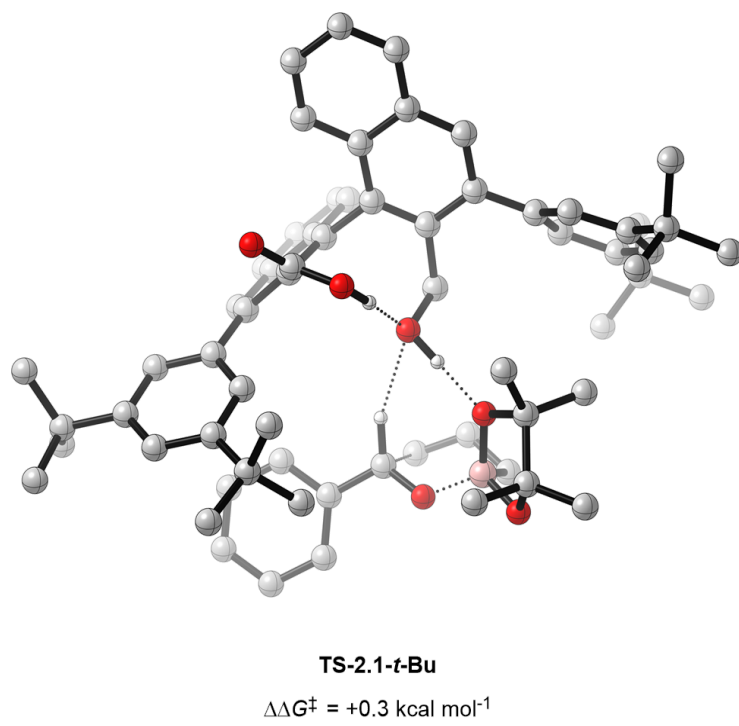

$\Delta \Delta G^{\ddagger}=+0.3 \mathrm{kcal} \mathrm{mol}^{-1}$

Figure S6. Relative energy reaction barriers for TS-2.1 and TS-2.1-t-Bu (B3LYP-D3(BJ)/def2TZVPP/IEFPCM(dichloromethane)//B3LYP-D3(BJ)/6-31G(d,p)).

Property
TS-2.1 / Hartrees
TS-2.1-t-Bu / Hartrees
Rel. difference / $\mathrm{kcal} \cdot \mathrm{mol}^{-1}$

\begin{tabular}{cccc}
\hline Quasiharmonic Free Energy (G) & -3039.301929 & -3039.301501 & +0.269 \\
\hline Quasiharmonic Enthalpy (H) & -3039.223798 & -3039.301501 & +0.248 \\
\hline Quasiharmonic Entropy (T.S) & 0.078131 & 0.078098 & -0.021
\end{tabular}

Table S1. Effect of rotating catalyst $t$-Bu groups on quasiharmonic free energy, enthalpy, and entropy. 


\section{Additional details of alternative catalyzed reaction conditions}

In order to assess the validity of the proposed reaction model, and the effect on the transition state of changing the catalyst aryl groups, an alternative set of conditions from the original experimental paper was analysed computationally (Figure S7). ${ }^{25}$ This was achieved by reoptimizing all TSs within $3.0 \mathrm{kcal} \cdot \mathrm{mol}^{-1}$ of the original TS-2.1. As a result, low-energy TSs analogous to TS-2.1, TS-2.2, and TS-2.3, were all identified (Figure S8), denoted as TS-2.1', TS-2.2', and TS-2.3', respectively. TS-2.3' was found to become significantly more energetically relevant than for TS2.3 in the original conditions, as a result of the reduced extent of catalyst distortion in the mode A TSs (Table 1). This is because the para-phenyl group of the new catalyst is less sterically demanding than the bulky meta- $t$-Bu substituents of the original catalyst, and in mode A TSs, such as TS-2.3', this para-phenyl group lies away from the substrate and hence reduces the potential for substrate-catalyst steric clashing.

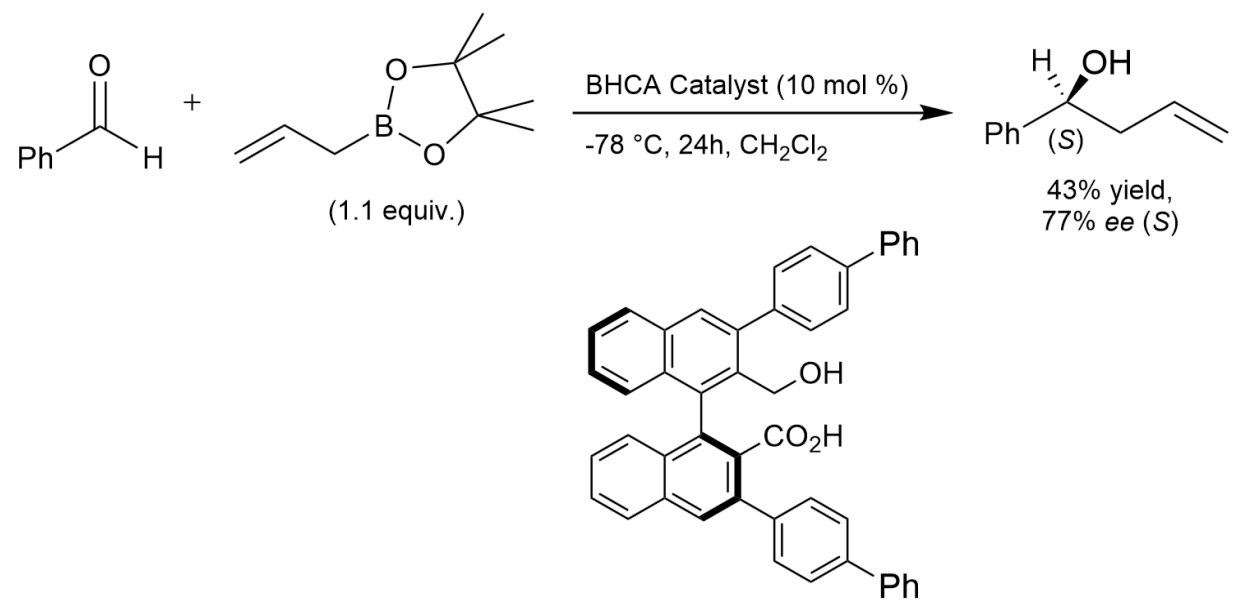

Figure S7. Alternative reaction conditions for computational analysis of BHCA-catalyzed allylboration.
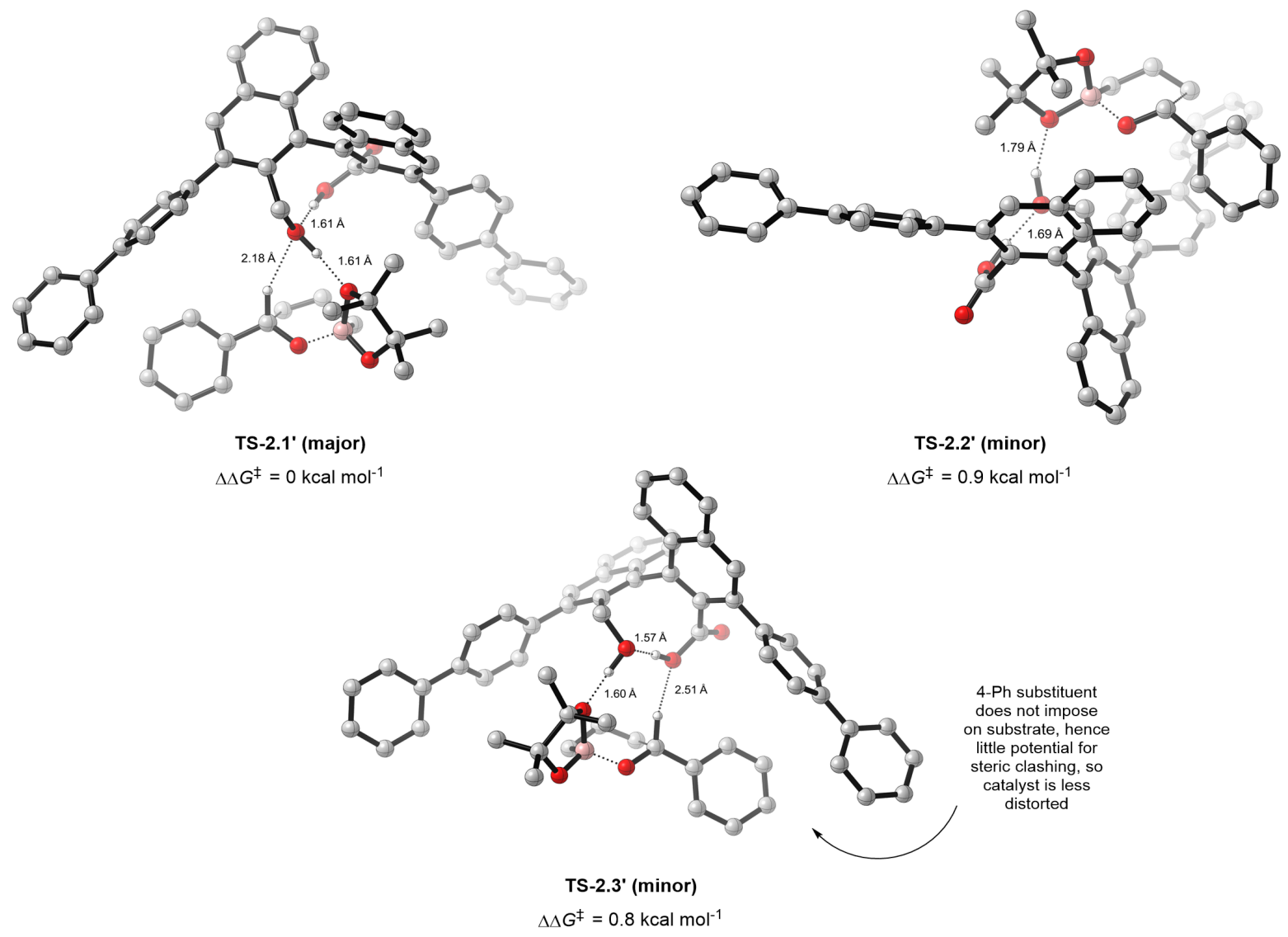

Figure S8. Relative energy reaction barriers for the TSs of the alternative BHCA-catalyzed allylboration (B3LYP-D3(BJ)/def2-TZVPP/IEFPCM(dichloromethane)//B3LYP-D3(BJ)/6-31G(d,p)). 


\section{Energies, frequencies and molecular geometries of all computed structures}

All energies in Hartrees, coordinates in $\AA$. Cartesian coordinates generated by EsiGen software. ${ }^{28}$ Structures ordered by quasiharmonic free energy (from lowest to highest). Catalyst $2 \mathrm{C}$ refers to the original catalyst $(3,5-t$-Bu-substituted), whilst catalyst $2 \mathrm{~A}$ refers to the alternative catalyst (4-Ph-substituted). Ar $=\mathrm{Ph}$ for simple BPA and simple BHCA. Key TSs indicated where appropriate.

\subsection{Optimization with B3LYP/6-31G(d).}

\section{Benzaldehyde}

\begin{tabular}{lr} 
Datum & Value \\
\hline B3LYP/6-31G(d) Energy & -345.573442 \\
\hline B3LYP-D3(BJ)/def2-TZVPP-IEF-PCM(DCM) Energy & -345.740369 \\
\hline B3LYP-D3(BJ)/def2-TZVPP-IEF-PCM(DCM)//B3LYP/6-31G(d) Free Energy (Quasiharmonic) & -345.649218 \\
\hline Number of Imaginary Frequencies & 0
\end{tabular}

Frequencies (Top 3 out of 36)

$\begin{array}{ll}\text { 1. } & 124.9931 \mathrm{~cm}^{-1} \\ \text { 2. } & 221.1956 \mathrm{~cm}^{-1} \\ \text { 3. } & 241.0274 \mathrm{~cm}^{-1}\end{array}$

B3LYP/6-31G(d) Molecular Geometry in Cartesian Coordinates 


\begin{tabular}{lc}
\hline B3LYP/6-31G(d) Energy & -528.591061 \\
\hline B3LYP-D3(BJ)/def2-TZVPP-IEF-PCM(DCM) Energy & -528.858533 \\
\hline B3LYP-D3(BJ)/def2-TZVPP-IEF-PCM(DCM)//B3LYP/6-31G(d) Free Energy (Quasiharmonic) & -528.631305 \\
\hline
\end{tabular}

Number of Imaginary Frequencies

Frequencies (Top 3 out of 81)

$\begin{array}{ll}\text { 1. } & 24.6237 \mathrm{~cm}^{-1} \\ \text { 2. } & 54.3619 \mathrm{~cm}^{-1} \\ \text { 3. } & 92.7322 \mathrm{~cm}^{-1}\end{array}$

\section{B3LYP/6-31G(d) Molecular Geometry in Cartesian Coordinates}

C

C

$\mathrm{C}$

$\mathrm{H}$

$\mathrm{H}$

C

C

C

$\mathrm{H}$

$\mathrm{H}$

$\mathrm{H}$

C

$\mathrm{H}$

$\mathrm{H}$

$\mathrm{H}$

C

$\mathrm{H}$

H

$\mathrm{H}$

C

$\mathrm{H}$

$\mathrm{H}$

$\mathrm{H}$

0

0

B

$\mathrm{H}$

$\mathrm{H}$

$\mathrm{H}$
$-4.237633$

$-2.299294$

$-3.266095$

$-2.376419$

$-2.555434$

1.073091

1.412532

2.017719

1.671064

3.033768

2. 054861

0.898978

1.854979

0.472889

0.214335

2. 165734

2. 320428

3.148897

1.609853

2.118953

3.152288

2.133709

1.591402

$-0.240952$

0.089204

$-0.797298$

$-3.121080$

$-4.417876$

$-4.891107$
0.165334

$-0.824921$

0.269140

$-1.023531$

$-1.761424$

0.848004

$-0.669538$

1.561873

2.587957

1.607590

1.065049

1.680052

1.849810

2.652245

1.194646

$-0.942006$

$-2.019618$

$-0.457582$

$-0.587367$

$-1.363117$

$-1.018191$

$-2.441923$

$-1.188762$

0.762638

$-1.271114$

$-0.440199$

1. 220334

$-0.760671$

1.001317
0.642161

$-0.639734$

$-0.266169$

$-1.722064$

$-0.130895$

$-0.080466$

0.197076

$-1.045587$

$-1.204969$

$-0.636951$

$-2.017592$

1.197094

1.703707

0.930775

1.899581

1.497813

1.613176

1. 488971

2. 368582

$-0.975546$

$-1.087166$

$-0.792213$

$-1.918749$

$-0.702712$

0.298029

$-0.340030$

$-0.779254$

1.185244

0.877580 


\section{Allylboronic acid pinacol ester 2}

Datum

\begin{tabular}{lr}
\hline B3LYP/6-31G(d) Energy & -528.591005 \\
\hline B3LYP-D3(BJ)/def2-TZVPP-IEF-PCM(DCM) Energy & -528.858408 \\
\hline B3LYP-D3(BJ)/def2-TZVPP-IEF-PCM(DCM)//B3LYP/6-31G(d) Free Energy (Quasiharmonic) & -528.631067 \\
\hline
\end{tabular}

Number of Imaginary Frequencies

Frequencies (Top 3 out of 81)

\section{B3LYP/6-31G(d) Molecular Geometry in Cartesian Coordinates}

C

C

C

$\mathrm{H}$

$\mathrm{H}$

C

C

C

$\mathrm{H}$

$\mathrm{H}$

$\mathrm{H}$

C

$\mathrm{H}$

$\mathrm{H}$

$\mathrm{H}$

C

$\mathrm{H}$

$\mathrm{H}$

$\mathrm{H}$

C

$\mathrm{H}$

$\mathrm{H}$

$\mathrm{H}$

0

0

B

$\mathrm{H}$

$\mathrm{H}$

$\mathrm{H}$
$-4.211012$

$-2.318751$

- 3.246594

$-2.604587$

$-2.405657$

1. 208945

1. 272467

1.725560

1.601785

2. 791416

1.178648

1.858341

2.949615

1.599379

1.494659

2.459398

2.388491

3.406146

2.481460

1.161968

2.069405

1.012078

0.308859

$-0.221252$

0.050963

$-0.808956$

$-3.076627$

$-4.415654$

$-4.834247$
0.264182

0.080585

$-0.439198$

1.094685

$-0.556361$

$-0.786647$

0.790061

$-1.396276$

$-2.483570$

$-1.180525$

$-1.019873$

$-1.485829$

$-1.396575$

$-2.548847$

$-1.073293$

1. 358104

2.450110

1.093490

0.994817

1.474473

1.333686

2. 547905

1.092007

$-1.044828$

1.115261

0.054384

$-1.471028$

1. 298856

$-0.167603$
0.654282

$-1.010344$

0.058393

$-1.311919$

$-1.906121$

0.122495

0.044504

1.424429

1. 394032

1.561863

2. 291701

$-1.079438$

$-1.058889$

$-1.053359$

$-2.025969$

$-0.731234$

$-0.760940$

$-0.246410$

$-1.761029$

1.413522

2.010176

1. 262103

1.982594

0.030370

$-0.680985$

$-0.547663$

0.367535

0.385187

1.433018 


\section{Allylboronic acid pinacol ester 3}

Datum

Value

\begin{tabular}{lc}
\hline B3LYP/6-31G(d) Energy & -528.588534 \\
\hline B3LYP-D3(BJ)/def2-TZVPP-IEF-PCM(DCM) Energy & -528.85632 \\
\hline B3LYP-D3(BJ)/def2-TZVPP-IEF-PCM(DCM)//B3LYP/6-31G(d) Free Energy (Quasiharmonic) & -528.629031 \\
\hline
\end{tabular}

Number of Imaginary Frequencies

Frequencies (Top 3 out of 81)

$\begin{array}{ll}\text { 1. } & 22.8300 \mathrm{~cm}^{-1} \\ \text { 2. } & 63.3228 \mathrm{~cm}^{-1} \\ \text { 3. } & 91.3240 \mathrm{~cm}^{-1}\end{array}$

\section{B3LYP/6-31G(d) Molecular Geometry in Cartesian Coordinates}

C

C

$\mathrm{C}$

$\mathrm{H}$

$\mathrm{H}$

C

C

C

$\mathrm{H}$

$\mathrm{H}$

$\mathrm{H}$

C

$\mathrm{H}$

$\mathrm{H}$

$\mathrm{H}$

C

$\mathrm{H}$

$\mathrm{H}$

$\mathrm{H}$

C

$\mathrm{H}$

$\mathrm{H}$

$\mathrm{H}$

0

0

B

$\mathrm{H}$
3. 315324

2. 346492

3.434238

2. 533178

2.444528

$-1.092974$

$-1.225877$

$-1.751656$

$-1.448833$

$-2.844498$

$-1.435148$

$-1.539904$

$-0.985596$

$-2.609837$

$-1.367152$

$-1.095663$

$-0.997343$

$-1.973895$

$-0.206261$

$-2.463246$

$-2.440786$

$-2.505725$

$-3.379947$

0.343658

$-0.047021$

0.865925

4.410652
$-0.652169$

0.597853

0.192759

0.086872

1.669613

$-0.798590$

0.715434

$-1.112911$

$-2.115445$

$-1.087451$

$-0.404745$

$-1.814723$

$-1.702720$

$-1.710410$

$-2.828587$

0.939934

2.012937

0.572368

0.444725

1.436906

2.484482

1.416984

0.983342

$-0.941427$

1. 314057

0.313070

0.644408
0.975431

$-1.025647$

$-0.050411$

$-1.984726$

$-1.244199$

$-0.152810$

0.274291

$-1.502833$

$-1.820571$

$-1.436127$

$-2.275175$

0.896445

1.830958

1.110346

0.521506

1.787085

1.978303

2.328384

2. 189022

$-0.257146$

0.059916

$-1.348397$

0.137034

$-0.349972$

$-0.335870$

$-0.561544$

$-0.235306$ 


\section{Catalyst 2C 1}

Datum

Value

\begin{tabular}{|c|c|}
\hline B3LYP/6-31G(d) Energy & -2164.800593 \\
\hline B3LYP-D3(BJ)/def2-TZVPP-IEF-PCM(DCM) Energy & -2165.9029 \\
\hline B3LYP-D3(BJ)/def2-TZVPP-IEF-PCM(DCM)//B3LYP/6-31G(d) Free Energy (Quasiharmonic) & -2165.024475 \\
\hline Number of Imaginary Frequencies & 0 \\
\hline \multicolumn{2}{|l|}{ Frequencies (Top 3 out of 321) } \\
\hline $10.4179 \mathrm{~cm}^{-1}$ & \\
\hline $12.6481 \mathrm{~cm}^{-1}$ & \\
\hline $14.0488 \mathrm{~cm}^{-1}$ & \\
\hline
\end{tabular}

\section{B3LYP/6-31G(d) Molecular Geometry in Cartesian Coordinates}

\begin{tabular}{|c|c|c|c|}
\hline C & 1.546860 & 0.000240 & -1.264130 \\
\hline $\mathrm{H}$ & 2.514210 & 0.239210 & -1.713680 \\
\hline $\mathrm{H}$ & 0.938330 & -0.536310 & -1.998130 \\
\hline C & -1.602670 & -0.027490 & 1.268190 \\
\hline 0 & -2.283390 & -0.309240 & 2.226820 \\
\hline 0 & -0.763580 & 1.021850 & 1.288350 \\
\hline $\mathrm{H}$ & -0.244140 & 1.088180 & 0.450270 \\
\hline 0 & 0.875570 & 1.237910 & -0.905410 \\
\hline $\mathrm{H}$ & 0.591220 & 1.678700 & -1.721950 \\
\hline C & -0.615620 & -1.679090 & -0.355160 \\
\hline C & -1.671790 & -0.845840 & -0.009630 \\
\hline C & -2.907640 & -0.869440 & -0.741840 \\
\hline C & -3.029950 & -1.749980 & -1.798090 \\
\hline $\mathrm{H}$ & -3.946640 & -1.753220 & -2.382250 \\
\hline C & 1.729380 & -0.840160 & -0.022610 \\
\hline C & 0.678710 & -1.646210 & 0.412230 \\
\hline C & 3.127960 & -1.655710 & 1.783730 \\
\hline C & 2.974540 & -0.823500 & 0.691170 \\
\hline $\mathrm{H}$ & 4.068250 & -1.646200 & 2.329070 \\
\hline C & 2.085890 & -2.492220 & 2.247930 \\
\hline C & 0.828170 & -2.482640 & 1.564290 \\
\hline C & -0.218130 & -3.311920 & 2.056470 \\
\hline C & -0.023420 & -4.112830 & 3.158630 \\
\hline C & 1.224240 & -4.126430 & 3.828190 \\
\hline
\end{tabular}




\begin{tabular}{|c|c|c|c|}
\hline C & 2.253840 & -3.330850 & 3.383160 \\
\hline $\mathrm{H}$ & -1.183280 & -3.295240 & 1.562670 \\
\hline $\mathrm{H}$ & -0.836240 & -4.734140 & 3.523920 \\
\hline $\mathrm{H}$ & 1.362040 & -4.762140 & 4.698640 \\
\hline $\mathrm{H}$ & 3.212850 & -3.327170 & 3.895660 \\
\hline C & -1.990870 & -2.639300 & -2.166120 \\
\hline C & -0.759120 & -2.610780 & -1.438440 \\
\hline C & 0.273280 & -3.511610 & -1.820690 \\
\hline C & 0.097520 & -4.386660 & -2.870490 \\
\hline C & -1.118650 & -4.406390 & -3.593560 \\
\hline C & -2.138490 & -3.549990 & -3.246830 \\
\hline $\mathrm{H}$ & 1.207250 & -3.507750 & -1.268770 \\
\hline $\mathrm{H}$ & 0.897670 & -5.068860 & -3.144510 \\
\hline $\mathrm{H}$ & -1.244780 & -5.100550 & -4.419980 \\
\hline $\mathrm{H}$ & -3.077950 & -3.558780 & -3.794450 \\
\hline C & 6.267180 & 1.695600 & -0.360950 \\
\hline C & 6.481770 & 0.309660 & -0.243950 \\
\hline C & 5.388260 & -0.493450 & 0.086420 \\
\hline C & 4.112700 & 0.058750 & 0.297910 \\
\hline C & 3.944460 & 1.440900 & 0.179380 \\
\hline C & 5.015680 & 2.283420 & -0.157180 \\
\hline $\mathrm{H}$ & 7.108490 & 2.327520 & -0.620450 \\
\hline $\mathrm{H}$ & 5.499930 & -1.568370 & 0.172550 \\
\hline $\mathrm{H}$ & 2.960310 & 1.859410 & 0.359760 \\
\hline C & 7.891030 & -0.264000 & -0.489790 \\
\hline C & 4.769120 & 3.799180 & -0.283580 \\
\hline C & 8.341360 & 0.071230 & -1.931700 \\
\hline $\mathrm{H}$ & 9.345660 & -0.327780 & -2.120420 \\
\hline $\mathrm{H}$ & 7.657740 & -0.368140 & -2.667290 \\
\hline $\mathrm{H}$ & 8.373640 & 1.151210 & -2.109370 \\
\hline C & 8.885740 & 0.362760 & 0.516130 \\
\hline $\mathrm{H}$ & 9.897010 & -0.026420 & 0.345250 \\
\hline H & 8.928510 & 1.452840 & 0.423370 \\
\hline $\mathrm{H}$ & 8.600410 & 0.126550 & 1.547680 \\
\hline C & 7.941150 & -1.794150 & -0.318720 \\
\hline $\mathrm{H}$ & 8.961340 & -2.151370 & -0.499350 \\
\hline H & 7.658100 & -2.101070 & 0.694460 \\
\hline $\mathrm{H}$ & 7.281780 & -2.306470 & -1.028500 \\
\hline C & 6.039110 & 4.574310 & -0.682220 \\
\hline $\mathrm{H}$ & 5.807570 & 5.642030 & -0.768110 \\
\hline H & 6.833820 & 4.469660 & 0.065320 \\
\hline $\mathrm{H}$ & 6.433120 & 4.243200 & -1.650040 \\
\hline C & 3.692490 & 4.051320 & -1.367120 \\
\hline H & 3.482380 & 5.124370 & -1.454570 \\
\hline $\mathrm{H}$ & 4.033290 & 3.692250 & -2.345640 \\
\hline $\mathrm{H}$ & 2.751960 & 3.542670 & -1.130920 \\
\hline C & 4.270130 & 4.353390 & 1.072090 \\
\hline $\mathrm{H}$ & 4.081840 & 5.431560 & 0.997960 \\
\hline H & 3.339710 & 3.872920 & 1.390920 \\
\hline $\mathrm{H}$ & 5.016380 & 4.192130 & 1.858400 \\
\hline C & -4.035170 & 0.049050 & -0.419990 \\
\hline C & -3.810000 & 1.421250 & -0.222180 \\
\hline C & -5.342460 & -0.438370 & -0.352600 \\
\hline C & -4.858910 & 2.297510 & 0.053990 \\
\hline
\end{tabular}




\begin{tabular}{|c|c|c|c|}
\hline $\mathrm{H}$ & -2.795510 & 1.793800 & -0.285800 \\
\hline C & -6.428960 & 0.408769 & -0.083800 \\
\hline $\mathrm{H}$ & -5.504810 & -1.504090 & -0.483560 \\
\hline C & -6.160010 & 1.766039 & 0.114290 \\
\hline $\mathrm{H}$ & -6.981110 & 2.439199 & 0.330950 \\
\hline C & -7.850690 & -0.180581 & -0.003780 \\
\hline C & -4.635030 & 3.801520 & 0.300910 \\
\hline C & -8.916850 & 0.888159 & 0.302720 \\
\hline $\mathrm{H}$ & -8.740600 & 1.374909 & 1.268560 \\
\hline $\mathrm{H}$ & -8.953080 & 1.663489 & -0.471100 \\
\hline $\mathrm{H}$ & -9.906180 & 0.418639 & 0.345890 \\
\hline C & -8.210370 & -0.842031 & -1.355540 \\
\hline $\mathrm{H}$ & -9.215820 & -1.278681 & -1.312010 \\
\hline H & -8.194380 & -0.106601 & -2.168320 \\
\hline $\mathrm{H}$ & -7.510630 & -1.643121 & -1.616120 \\
\hline C & -7.903760 & -1.244951 & 1.118100 \\
\hline $\mathrm{H}$ & -7.200360 & -2.064251 & 0.938100 \\
\hline $\mathrm{H}$ & -7.655830 & -0.802051 & 2.089200 \\
\hline $\mathrm{H}$ & -8.909850 & -1.677261 & 1.186630 \\
\hline C & -5.110580 & 4.158760 & 1.729700 \\
\hline $\mathrm{H}$ & -6.174270 & 3.940499 & 1.871710 \\
\hline $\mathrm{H}$ & -4.549140 & 3.592190 & 2.480630 \\
\hline $\mathrm{H}$ & -4.960370 & 5.227970 & 1.924490 \\
\hline C & -3.151420 & 4.200110 & 0.178800 \\
\hline $\mathrm{H}$ & -2.521360 & 3.660100 & 0.894000 \\
\hline $\mathrm{H}$ & -2.764270 & 4.013010 & -0.830080 \\
\hline $\mathrm{H}$ & -3.040660 & 5.271920 & 0.379470 \\
\hline C & -5.441880 & 4.625230 & -0.730290 \\
\hline $\mathrm{H}$ & -5.294320 & 5.698930 & -0.560490 \\
\hline $\mathrm{H}$ & -5.120470 & 4.395900 & -1.753020 \\
\hline $\mathrm{H}$ & -6.516300 & 4.423489 & -0.665430 \\
\hline
\end{tabular}

\section{Catalyst 2C 2}

Datum

Value

\begin{tabular}{lc}
\hline B3LYP/6-31G(d) Energy & -2164.800123 \\
\hline B3LYP-D3(BJ)/def2-TZVPP-IEF-PCM(DCM) Energy & -2165.902672 \\
\hline
\end{tabular}

B3LYP-D3(BJ)/def2-TZVPP-IEF-PCM(DCM)//B3LYP/6-31G(d) Free Energy (Quasiharmonic) $\quad$-2165.024394

Number of Imaginary Frequencies

Frequencies (Top 3 out of 321)

$\begin{array}{ll}\text { 1. } & 10.3214 \mathrm{~cm}^{-1} \\ \text { 2. } & 12.4575 \mathrm{~cm}^{-1} \\ \text { 3. } & 14.2375 \mathrm{~cm}^{-1}\end{array}$




\begin{tabular}{|c|c|c|c|}
\hline C & 1.539370 & 0.008460 & -1.271040 \\
\hline $\mathrm{H}$ & 2.507030 & 0.252510 & -1.717700 \\
\hline $\mathrm{H}$ & 0.937570 & -0.533740 & -2.006580 \\
\hline C & -1.609560 & -0.020211 & 1.264980 \\
\hline 0 & -2.289500 & -0.302011 & 2.224210 \\
\hline 0 & -0.771500 & 1.029789 & 1.283670 \\
\hline $\mathrm{H}$ & -0.252540 & 1.095649 & 0.445080 \\
\hline 0 & 0.859060 & 1.241330 & -0.915880 \\
\hline $\mathrm{H}$ & 0.567650 & 1.676260 & -1.732980 \\
\hline C & -0.621029 & -1.670911 & -0.358200 \\
\hline C & -1.678590 & -0.839581 & -0.012220 \\
\hline C & -2.914820 & -0.865531 & -0.743630 \\
\hline C & -3.036009 & -1.746331 & -1.799820 \\
\hline $\mathrm{H}$ & -3.952989 & -1.751361 & -2.383530 \\
\hline C & 1.722850 & -0.828400 & -0.027300 \\
\hline C & 0.673201 & -1.634770 & 0.409210 \\
\hline C & 3.119691 & -1.632240 & 1.785290 \\
\hline C & 2.966800 & -0.806050 & 0.688020 \\
\hline $\mathrm{H}$ & 4.058491 & -1.616990 & 2.333000 \\
\hline C & 2.079131 & -2.470080 & 2.250610 \\
\hline C & 0.822891 & -2.466960 & 1.564330 \\
\hline C & -0.221789 & -3.297791 & 2.057270 \\
\hline C & -0.026779 & -4.094241 & 3.162620 \\
\hline C & 1.219421 & -4.101320 & 3.834950 \\
\hline C & 2.247351 & -3.303900 & 3.389310 \\
\hline $\mathrm{H}$ & -1.185929 & -3.286041 & 1.561370 \\
\hline $\mathrm{H}$ & -0.838329 & -4.716991 & 3.528310 \\
\hline $\mathrm{H}$ & 1.357401 & -4.733450 & 4.707970 \\
\hline $\mathrm{H}$ & 3.205221 & -3.295160 & 3.903890 \\
\hline C & -1.995449 & -2.633751 & -2.168290 \\
\hline C & -0.763409 & -2.603041 & -1.441140 \\
\hline C & 0.270361 & -3.502190 & -1.823650 \\
\hline C & 0.095751 & -4.377581 & -2.873350 \\
\hline C & -1.120659 & -4.399411 & -3.596000 \\
\hline C & -2.141859 & -3.544781 & -3.248900 \\
\hline $\mathrm{H}$ & 1.204481 & -3.496650 & -1.271960 \\
\hline $\mathrm{H}$ & 0.896961 & -5.058440 & -3.147650 \\
\hline $\mathrm{H}$ & -1.245879 & -5.093791 & -4.422350 \\
\hline $\mathrm{H}$ & -3.081529 & -3.555281 & -3.796160 \\
\hline C & 6.265240 & 1.706600 & -0.361290 \\
\hline C & 6.478490 & 0.330140 & -0.244230 \\
\hline C & 5.374950 & -0.473470 & 0.085740 \\
\hline C & 4.105760 & 0.074220 & 0.291790 \\
\hline C & 3.935280 & 1.464730 & 0.171080 \\
\hline C & 5.005160 & 2.298770 & -0.157790 \\
\hline $\mathrm{H}$ & 7.099990 & 2.348180 & -0.618300 \\
\hline $\mathrm{H}$ & 5.492911 & -1.549790 & 0.173290 \\
\hline $\mathrm{H}$ & 2.950040 & 1.877040 & 0.348110 \\
\hline C & 7.856870 & -0.322199 & -0.466750 \\
\hline C & 4.845920 & 3.824430 & -0.305610 \\
\hline
\end{tabular}




\begin{tabular}{|c|c|c|c|}
\hline C & 8.948290 & 0.708191 & -0.813820 \\
\hline $\mathrm{H}$ & 9.906280 & 0.195711 & -0.957020 \\
\hline $\mathrm{H}$ & 8.721590 & 1.247711 & -1.740520 \\
\hline $\mathrm{H}$ & 9.085020 & 1.444391 & -0.013500 \\
\hline C & 8.289660 & -1.062649 & 0.821000 \\
\hline H & 9.266691 & -1.540069 & 0.676950 \\
\hline $\mathrm{H}$ & 8.372390 & -0.366739 & 1.663700 \\
\hline $\mathrm{H}$ & 7.575751 & -1.843940 & 1.101960 \\
\hline C & 7.766170 & -1.334309 & -1.633540 \\
\hline $\mathrm{H}$ & 8.739131 & -1.812569 & -1.800590 \\
\hline $\mathrm{H}$ & 7.036581 & -2.125790 & -1.432660 \\
\hline $\mathrm{H}$ & 7.469480 & -0.834550 & -2.562870 \\
\hline C & 5.205880 & 4.238390 & -1.752470 \\
\hline $\mathrm{H}$ & 5.106080 & 5.323830 & -1.875290 \\
\hline $\mathrm{H}$ & 6.234730 & 3.966870 & -2.011830 \\
\hline $\mathrm{H}$ & 4.540370 & 3.752550 & -2.475590 \\
\hline C & 3.406510 & 4.292110 & -0.016710 \\
\hline $\mathrm{H}$ & 3.336950 & 5.377610 & -0.150660 \\
\hline $\mathrm{H}$ & 2.680530 & 3.825360 & -0.691840 \\
\hline $\mathrm{H}$ & 3.102990 & 4.065630 & 1.011490 \\
\hline C & 5.791760 & 4.546230 & 0.683220 \\
\hline $\mathrm{H}$ & 5.685420 & 5.633800 & 0.587450 \\
\hline $\mathrm{H}$ & 5.558430 & 4.272320 & 1.718340 \\
\hline $\mathrm{H}$ & 6.842510 & 4.298010 & 0.500370 \\
\hline C & -4.043880 & 0.050709 & -0.420650 \\
\hline C & -3.820970 & 1.422999 & -0.220950 \\
\hline C & -5.350290 & -0.439041 & -0.353080 \\
\hline C & -4.871090 & 2.296979 & 0.057770 \\
\hline $\mathrm{H}$ & -2.807180 & 1.797409 & -0.284690 \\
\hline C & -6.438020 & 0.405809 & -0.082000 \\
\hline $\mathrm{H}$ & -5.510959 & -1.504841 & -0.485500 \\
\hline C & -6.171240 & 1.763189 & 0.118300 \\
\hline $\mathrm{H}$ & -6.993270 & 2.434529 & 0.337100 \\
\hline C & -7.858690 & -0.186072 & -0.001800 \\
\hline C & -4.649330 & 3.800739 & 0.308050 \\
\hline C & -7.909060 & -1.252652 & 1.118080 \\
\hline $\mathrm{H}$ & -7.204429 & -2.070431 & 0.935990 \\
\hline $\mathrm{H}$ & -7.661110 & -0.811172 & 2.089830 \\
\hline $\mathrm{H}$ & -8.914369 & -1.686792 & 1.186610 \\
\hline C & -8.926350 & 0.880298 & 0.307720 \\
\hline $\mathrm{H}$ & -9.914850 & 0.409038 & 0.350920 \\
\hline $\mathrm{H}$ & -8.750010 & 1.365478 & 1.274320 \\
\hline $\mathrm{H}$ & -8.964630 & 1.657068 & -0.464560 \\
\hline C & -8.218500 & -0.845532 & -1.354500 \\
\hline $\mathrm{H}$ & -8.204420 & -0.108522 & -2.165890 \\
\hline $\mathrm{H}$ & -7.517659 & -1.644972 & -1.617230 \\
\hline $\mathrm{H}$ & -9.223200 & -1.283902 & -1.310930 \\
\hline C & -5.121640 & 4.152939 & 1.739170 \\
\hline $\mathrm{H}$ & -6.184510 & 3.932069 & 1.883370 \\
\hline $\mathrm{H}$ & -4.557020 & 3.585259 & 2.486870 \\
\hline $\mathrm{H}$ & -4.973000 & 5.221869 & 1.936690 \\
\hline C & -3.166820 & 4.202479 & 0.182960 \\
\hline $\mathrm{H}$ & -2.533740 & 3.662329 & 0.895360 \\
\hline $\mathrm{H}$ & -2.781940 & 4.018229 & -0.827320 \\
\hline
\end{tabular}




\section{Catalyst 2C 3}

\section{Datum}

Value

\begin{tabular}{lc} 
B3LYP/6-31G(d) Energy & -2164.800037 \\
\hline B3LYP-D3(BJ)/def2-TZVPP-IEF-PCM(DCM) Energy & -2165.902499 \\
\hline B3LYP-D3(BJ)/def2-TZVPP-IEF-PCM(DCM)//B3LYP/6-31G(d) Free Energy (Quasiharmonic) & -2165.024149 \\
\hline
\end{tabular}

Frequencies (Top 3 out of 321)

$\begin{array}{ll}\text { 1. } & 10.7778 \mathrm{~cm}^{-1} \\ \text { 2. } & 13.9417 \mathrm{~cm}^{-1} \\ \text { 3. } & 15.0184 \mathrm{~cm}^{-1}\end{array}$

\section{B3LYP/6-31G(d) Molecular Geometry in Cartesian Coordinates}

C

$\mathrm{H}$

$\mathrm{H}$

C

0

0

$\mathrm{H}$

0

$\mathrm{H}$

C

C

C

C

$\mathrm{H}$

C

$\mathrm{C}$

C

C

$\mathrm{H}$

C

C

\subsection{0}

2.501580

0.929910

$-1.613080$

$-2.294100$

$-0.776680$

$-0.255900$

0.858220

0.565290

$-0.618850$

$-1.678600$

$-2.913640$

$-3.031270$

$-3.947000$

1.723530

0.675790

3.127910

2.970370

4.069600

2.088730

0.829680
$-0.001700$

0.242410

$-0.541520$

$-0.040310$

$-0.324010$

1.011040

1.078710

1. 231210

1.668980

$-1.688160$

$-0.858880$

$-0.887130$

$-1.769740$

$-1.777100$

$-0.841240$

$-1.648950$

$-1.649330$

$-0.820460$

$-1.636590$

$-2.487010$

$-2.482440$
$-1.254400$

$-1.704440$

$-1.988530$

1.281060

2.238980

1. 302320

0.464930

$-0.893370$

$-1.708440$

$-0.341090$

0.003210

$-0.730420$

$-1.785750$

$-2.371300$

$-0.013290$

0.425400

1.791490

0.696900

2.334320

2.260160

1.578960 


\begin{tabular}{|c|c|c|c|}
\hline C & -0.213350 & -3.313600 & 2.074860 \\
\hline C & -0.014301 & -4.111520 & 3.178420 \\
\hline C & 1.234639 & -4.120100 & 3.845670 \\
\hline C & 2.261150 & -3.322600 & 3.396950 \\
\hline $\mathrm{H}$ & -1.179460 & -3.300670 & 1.582810 \\
\hline $\mathrm{H}$ & -0.824661 & -4.734380 & 3.546570 \\
\hline $\mathrm{H}$ & 1.375869 & -4.753530 & 4.717230 \\
\hline $\mathrm{H}$ & 3.221160 & -3.315160 & 3.907570 \\
\hline C & -1.988950 & -2.655930 & -2.151620 \\
\hline C & -0.757850 & -2.621810 & -1.423270 \\
\hline C & 0.278139 & -3.519320 & -1.803680 \\
\hline C & 0.106259 & -4.396490 & -2.852360 \\
\hline C & -1.109431 & -4.421800 & -3.576100 \\
\hline C & -2.132611 & -3.568700 & -3.231150 \\
\hline $\mathrm{H}$ & 1.211759 & -3.511250 & -1.251250 \\
\hline $\mathrm{H}$ & 0.909089 & -5.076120 & -3.124940 \\
\hline $\mathrm{H}$ & -1.232541 & -5.117640 & -4.401550 \\
\hline $\mathrm{H}$ & -3.071801 & -3.581840 & -3.779170 \\
\hline C & 6.263060 & 1.688110 & -0.360280 \\
\hline C & 5.012540 & 2.284260 & -0.143540 \\
\hline C & 3.940350 & 1.448200 & 0.188190 \\
\hline C & 4.107920 & 0.060110 & 0.297210 \\
\hline C & 5.376080 & -0.495610 & 0.075090 \\
\hline C & 6.474660 & 0.305700 & -0.257930 \\
\hline $\mathrm{H}$ & 7.104000 & 2.325190 & -0.618230 \\
\hline $\mathrm{H}$ & 2.957950 & 1.862830 & 0.375480 \\
\hline $\mathrm{H}$ & 5.484040 & -1.571430 & 0.154420 \\
\hline C & 4.855350 & 3.811530 & -0.278890 \\
\hline C & 7.878980 & -0.274590 & -0.517710 \\
\hline C & 5.205910 & 4.235330 & -1.725140 \\
\hline $\mathrm{H}$ & 5.106960 & 5.321740 & -1.839720 \\
\hline $\mathrm{H}$ & 6.232520 & 3.964080 & -1.993610 \\
\hline $\mathrm{H}$ & 4.534880 & 3.755560 & -2.447190 \\
\hline C & 3.418840 & 4.279790 & 0.023310 \\
\hline $\mathrm{H}$ & 3.350600 & 5.366380 & -0.102150 \\
\hline $\mathrm{H}$ & 2.687460 & 3.819520 & -0.650420 \\
\hline $\mathrm{H}$ & 3.121750 & 4.046420 & 1.051860 \\
\hline C & 5.809590 & 4.523770 & 0.708610 \\
\hline H & 5.705940 & 5.612290 & 0.621140 \\
\hline $\mathrm{H}$ & 5.582450 & 4.243290 & 1.743330 \\
\hline H & 6.858390 & 4.273650 & 0.517090 \\
\hline C & 8.884100 & 0.338330 & 0.486230 \\
\hline $\mathrm{H}$ & 9.892010 & -0.054920 & 0.305260 \\
\hline H & 8.932200 & 1.428910 & 0.401960 \\
\hline $\mathrm{H}$ & 8.604550 & 0.095550 & 1.517810 \\
\hline C & 7.920730 & -1.806360 & -0.358930 \\
\hline $\mathrm{H}$ & 8.937230 & -2.168510 & -0.550180 \\
\hline $\mathrm{H}$ & 7.643600 & -2.119580 & 0.653960 \\
\hline H & 7.252750 & -2.308960 & -1.067610 \\
\hline C & 8.321390 & 0.069250 & -1.959950 \\
\hline $\mathrm{H}$ & 9.322000 & -0.334080 & -2.158680 \\
\hline $\mathrm{H}$ & 7.630190 & -0.360020 & -2.694390 \\
\hline $\mathrm{H}$ & 8.358730 & 1.150440 & -2.129210 \\
\hline C & -4.045000 & 0.028550 & -0.414210 \\
\hline
\end{tabular}




\begin{tabular}{|c|c|c|c|}
\hline C & -3.825820 & 1.396930 & -0.208610 \\
\hline C & -5.355190 & -0.467130 & -0.365410 \\
\hline C & -4.882610 & 2.272660 & 0.057640 \\
\hline $\mathrm{H}$ & -2.812010 & 1.773430 & -0.257940 \\
\hline C & -6.442760 & 0.375410 & -0.111210 \\
\hline $\mathrm{H}$ & -5.506450 & -1.531460 & -0.502000 \\
\hline C & -6.177430 & 1.736680 & 0.095630 \\
\hline $\mathrm{H}$ & -7.009870 & 2.403140 & 0.301880 \\
\hline C & -7.893990 & -0.139380 & -0.045460 \\
\hline C & -4.663780 & 3.776090 & 0.314180 \\
\hline C & -7.989840 & -1.658770 & -0.281570 \\
\hline $\mathrm{H}$ & -7.611500 & -1.942960 & -1.270240 \\
\hline $\mathrm{H}$ & -7.433390 & -2.224770 & 0.473830 \\
\hline $\mathrm{H}$ & -9.037760 & -1.974720 & -0.225960 \\
\hline C & -8.490110 & 0.165980 & 1.349240 \\
\hline $\mathrm{H}$ & -9.524490 & -0.194840 & 1.409080 \\
\hline $\mathrm{H}$ & -7.910960 & -0.326380 & 2.138440 \\
\hline $\mathrm{H}$ & -8.499720 & 1.239630 & 1.563730 \\
\hline C & -8.738990 & 0.568640 & -1.131220 \\
\hline $\mathrm{H}$ & -8.749620 & 1.655180 & -0.995820 \\
\hline $\mathrm{H}$ & -8.343310 & 0.361220 & -2.132250 \\
\hline $\mathrm{H}$ & -9.777850 & 0.217640 & -1.096900 \\
\hline C & -3.179190 & 4.177060 & 0.213970 \\
\hline $\mathrm{H}$ & -2.557830 & 3.634700 & 0.934920 \\
\hline $\mathrm{H}$ & -2.778220 & 3.995330 & -0.790470 \\
\hline $\mathrm{H}$ & -3.072770 & 5.248070 & 0.421170 \\
\hline $\mathrm{C}$ & -5.457690 & 4.603230 & -0.724150 \\
\hline $\mathrm{H}$ & -5.122060 & 4.379030 & -1.743430 \\
\hline H & -6.532570 & 4.399390 & -0.674970 \\
\hline $\mathrm{H}$ & -5.314360 & 5.676400 & -0.547510 \\
\hline $\mathrm{C}$ & -5.159090 & 4.126160 & 1.737950 \\
\hline H & -5.012850 & 5.194590 & 1.940000 \\
\hline H & -6.224360 & 3.905890 & 1.864410 \\
\hline $\mathrm{H}$ & -4.607440 & 3.556660 & 2.493890 \\
\hline
\end{tabular}

\section{Catalyst 2C 4}

Datum

Value

\begin{tabular}{lc}
\hline B3LYP/6-31G(d) Energy & -2164.800625 \\
\hline B3LYP-D3(BJ)/def2-TZVPP-IEF-PCM(DCM) Energy & -2165.902813 \\
\hline B3LYP-D3(BJ)/def2-TZVPP-IEF-PCM(DCM)//B3LYP/6-31G(d) Free Energy (Quasiharmonic) & -2165.024113 \\
\hline Number of Imaginary Frequencies & 0
\end{tabular}

Frequencies (Top 3 out of 321) 
1. $\quad 11.1271 \mathrm{~cm}^{-1}$

2. $13.8987 \mathrm{~cm}^{-1}$

3. $14.6624 \mathrm{~cm}^{-1}$

B3LYP/6-31G(d) Molecular Geometry in Cartesian Coordinates

C

$\mathrm{H}$

$\mathrm{H}$

C

0

0

$\mathrm{H}$

0

$\mathrm{H}$

C

C

C

C

$\mathrm{H}$

C

C

C

C

$\mathrm{H}$

C

C

C

C

C

C

$\mathrm{H}$

$\mathrm{H}$

$\mathrm{H}$

$\mathrm{H}$

C

C

C

C

C

C

$\mathrm{H}$

$\mathrm{H}$

H

$\mathrm{H}$

C

C

C

C

C
1.536110

2.500580

0.926810

$-1.603140$

$-2.274930$

$-0.772010$

$-0.255070$

0.861210

0.573260

$-0.615160$

$-1.673670$

$-2.909460$

$-3.028090$

$-3.943660$

1.727080

0.681100

3.136260

2.975530

4.079130

2.098780

0.838260

$-0.202970$

$-0.000899$

1. 249401

2. 274290

$-1.170090$

$-0.809919$

1.392971

3.235310

$-1.987510$

$-0.756100$

0.278080

0.104071

$-1.111899$

$-2.133270$

1.211860

0.905391

$-1.236609$

$-3.072620$

6.258110

6.474870

5.384610

4.110150

3.939790
$-0.002190$

0.240070

$-0.542200$

$-0.041540$

$-0.328500$

1.015260

1.082380

1.233570

1.671800

$-1.688020$

$-0.858270$

$-0.882700$

$-1.762580$

$-1.767370$

$-0.839750$

$-1.648710$

$-1.646920$

$-0.818000$

$-1.633610$

$-2.485440$

$-2.482020$

$-3.314160$

$-4.111790$

$-4.119130$

$-3.320770$

$-3.302130$

$-4.735360$

$-4.752300$

$-3.312410$

$-2.649740$

$-2.619580$

$-3.518300$

$-4.393130$

$-4.414540$

$-3.560060$

$-3.513140$

$-5.073920$

$-5.108550$

$-3.570290$

1.700580

0.315330

$-0.487360$

0.064530

1.446040
$-1.252510$

$-1.706360$

$-1.983250$

1.291890

2. 254930

1.306270

0.466530

$-0.893150$

$-1.709830$

$-0.333300$

0.013560

$-0.718850$

$-1.776580$

$-2.362320$

$-0.010420$

0.430510

1.791390

0.697370

2. 332200

2. 262270

1.583710

2.081740

3.184940

3.849660

3.398780

1.591650

3.554820

4.721020

3.907450

$-2.144750$

$-1.416800$

$-1.799190$

$-2.849440$

$-3.572920$

$-3.226100$

$-1.246960$

$-3.123530$

$-4.399660$

$-3.773890$

$-0.387260$

$-0.266140$

0.075800

0.294510

0.171740 


\begin{tabular}{|c|c|c|c|}
\hline C & 5.007640 & 2.288100 & -0.176460 \\
\hline H & 7.096810 & 2.332130 & -0.655860 \\
\hline $\mathrm{H}$ & 5.497810 & -1.561840 & 0.165260 \\
\hline $\mathrm{H}$ & 2.956580 & 1.864470 & 0.357460 \\
\hline C & 7.882670 & -0.258240 & -0.520600 \\
\hline C & 4.758550 & 3.803010 & -0.308020 \\
\hline C & 8.884570 & 0.373210 & 0.475190 \\
\hline H & 9.894820 & -0.015780 & 0.298010 \\
\hline H & 8.925680 & 1.462960 & 0.377890 \\
\hline H & 8.607420 & 0.140750 & 1.509810 \\
\hline C & 7.935240 & -1.787660 & -0.343960 \\
\hline H & 8.954270 & -2.144870 & -0.531050 \\
\hline $\mathrm{H}$ & 7.660260 & -2.090860 & 0.672560 \\
\hline $\mathrm{H}$ & 7.270790 & -2.303200 & -1.046630 \\
\hline C & 8.321680 & 0.071620 & -1.967240 \\
\hline $\mathrm{H}$ & 9.324840 & -0.327330 & -2.161960 \\
\hline $\mathrm{H}$ & 7.632870 & -0.371310 & -2.695820 \\
\hline H & 8.351700 & 1.150900 & -2.149510 \\
\hline C & 6.024460 & 4.577480 & -0.720700 \\
\hline H & 5.791110 & 5.644540 & -0.809880 \\
\hline $\mathrm{H}$ & 6.825320 & 4.477290 & 0.020860 \\
\hline $\mathrm{H}$ & 6.411060 & 4.242180 & -1.690080 \\
\hline C & 3.673030 & 4.048990 & -1.384120 \\
\hline $\mathrm{H}$ & 3.461730 & 5.121480 & -1.475460 \\
\hline $\mathrm{H}$ & 4.006140 & 3.685000 & -2.363470 \\
\hline $\mathrm{H}$ & 2.734670 & 3.541160 & -1.137730 \\
\hline C & 4.270000 & 4.363250 & 1.048930 \\
\hline H & 4.080210 & 5.440900 & 0.971160 \\
\hline $\mathrm{H}$ & 3.342580 & 3.883530 & 1.377590 \\
\hline $\mathrm{H}$ & 5.022680 & 4.206380 & 1.829980 \\
\hline C & -4.041050 & 0.032490 & -0.400930 \\
\hline C & -3.827990 & 1.393800 & -0.173910 \\
\hline C & -5.356180 & -0.462800 & -0.373820 \\
\hline c & -4.890070 & 2.271300 & 0.090140 \\
\hline $\mathrm{H}$ & -2.813810 & 1.777660 & -0.202410 \\
\hline C & -6.442980 & 0.375920 & -0.124130 \\
\hline $\mathrm{H}$ & -5.506160 & -1.525270 & -0.525390 \\
\hline C & -6.182400 & 1.739810 & 0.103520 \\
\hline $\mathrm{H}$ & -7.019640 & 2.398239 & 0.303850 \\
\hline C & -7.895580 & -0.136931 & -0.082880 \\
\hline c & -4.592030 & 3.758600 & 0.359680 \\
\hline C & -7.990940 & -1.653251 & -0.337730 \\
\hline $\mathrm{H}$ & -7.600370 & -1.926591 & -1.324720 \\
\hline $\mathrm{H}$ & -7.444990 & -2.228851 & 0.418090 \\
\hline $\mathrm{H}$ & -9.039910 & -1.968301 & -0.299300 \\
\hline C & -8.509420 & 0.153399 & 1.307500 \\
\hline $\mathrm{H}$ & -9.545100 & -0.206131 & 1.349140 \\
\hline $\mathrm{H}$ & -7.941720 & -0.349641 & 2.098310 \\
\hline $\mathrm{H}$ & -8.520050 & 1.224409 & 1.534590 \\
\hline C & -8.725610 & 0.585089 & -1.171090 \\
\hline $\mathrm{H}$ & -8.735500 & 1.670069 & -1.023950 \\
\hline $\mathrm{H}$ & -8.318210 & 0.387459 & -2.169420 \\
\hline $\mathrm{H}$ & -9.765590 & 0.236239 & -1.153170 \\
\hline & -3.859590 & 4.369740 & -0.858590 \\
\hline
\end{tabular}




\begin{tabular}{|lllr|}
$H$ & -2.909660 & 3.863650 & -1.059900 \\
$H$ & -4.474710 & 4.299790 & -1.763410 \\
$H$ & -3.638760 & 5.428960 & -0.678110 \\
$\mathrm{C}$ & -5.871120 & 4.580800 & 0.605440 \\
$\mathrm{H}$ & -6.544970 & 4.559209 & -0.259170 \\
$\mathrm{H}$ & -6.424170 & 4.221470 & 1.480550 \\
$\mathrm{H}$ & -5.605840 & 5.627720 & 0.791410 \\
$\mathrm{C}$ & -3.692100 & 3.876400 & 1.613620 \\
$\mathrm{H}$ & -3.448460 & 4.928290 & 1.809140 \\
$\mathrm{H}$ & -4.201830 & 3.475390 & 2.496720 \\
$\mathrm{H}$ & -2.752140 & 3.326780 & 1.501090 \\
\hline
\end{tabular}

\section{Catalyst 2C 5}

Datum

Value

\begin{tabular}{lc}
\hline B3LYP/6-31G(d) Energy & -2164.800393 \\
\hline B3LYP-D3(BJ)/def2-TZVPP-IEF-PCM(DCM) Energy & -2165.902619 \\
\hline B3LYP-D3(BJ)/def2-TZVPP-IEF-PCM(DCM)//B3LYP/6-31G(d) Free Energy (Quasiharmonic) & -2165.02411 \\
\hline Number of Imaginary Frequencies & 0
\end{tabular}

Frequencies (Top 3 out of 321)

$\begin{array}{ll}\text { 1. } & 11.0509 \mathrm{~cm}^{-1} \\ \text { 2. } & 12.8311 \mathrm{~cm}^{-1} \\ \text { 3. } & 14.6518 \mathrm{~cm}^{-1}\end{array}$

B3LYP/6-31G(d) Molecular Geometry in Cartesian Coordinates

$\begin{array}{lrrr}\mathrm{C} & 1.551500 & 0.019290 & -1.257751 \\ \mathrm{H} & 2.514790 & 0.269110 & -1.710081 \\ \mathrm{H} & 0.947130 & -0.523840 & -1.990281 \\ \mathrm{C} & -1.593380 & -0.043340 & 1.282189 \\ \mathrm{O} & -2.269570 & -0.332940 & 2.241659 \\ \mathrm{O} & -0.764600 & 1.014400 & 1.302379 \\ \mathrm{H} & -0.247310 & 1.086950 & 0.463459 \\ \mathrm{O} & 0.867370 & 1.249160 & -0.896511 \\ \mathrm{H} & 0.575640 & 1.687000 & -1.712031 \\ \mathrm{C} & -0.590280 & -1.681710 & -0.343461 \\ \mathrm{C} & -1.655540 & -0.860530 & 0.003359 \\ \mathrm{C} & -2.891020 & -0.895650 & -0.729331 \\ \mathrm{C} & -3.002810 & -1.776440 & -1.786891 \\ \mathrm{H} & -3.918630 & -1.788670 & -2.372191 \\ \mathrm{C} & 1.746360 & -0.818830 & -0.016561\end{array}$




\begin{tabular}{|c|c|c|c|}
\hline C & 0.704740 & -1.634480 & 0.422079 \\
\hline C & 3.157010 & -1.617060 & 1.788179 \\
\hline C & 2.993270 & -0.789470 & 0.693659 \\
\hline $\mathrm{H}$ & 4.098370 & -1.597030 & 2.331409 \\
\hline C & 2.124230 & -2.462940 & 2.256309 \\
\hline C & 0.865220 & -2.467650 & 1.574999 \\
\hline C & -0.171290 & -3.307370 & 2.070129 \\
\hline C & 0.034030 & -4.104790 & 3.172909 \\
\hline C & 1.282910 & -4.104090 & 3.840289 \\
\hline C & 2.303120 & -3.298030 & 3.392439 \\
\hline $\mathrm{H}$ & -1.137420 & -3.301610 & 1.577989 \\
\hline $\mathrm{H}$ & -0.771380 & -4.734430 & 3.540349 \\
\hline $\mathrm{H}$ & 1.429090 & -4.737200 & 4.711249 \\
\hline $\mathrm{H}$ & 3.262960 & -3.283420 & 3.903199 \\
\hline C & -1.954570 & -2.654420 & -2.155541 \\
\hline C & -0.723390 & -2.613790 & -1.427681 \\
\hline C & 0.318650 & -3.502990 & -1.811021 \\
\hline C & 0.152410 & -4.378360 & -2.862111 \\
\hline C & -1.063430 & -4.410100 & -3.585371 \\
\hline C & -2.092390 & -3.565170 & -3.237551 \\
\hline $\mathrm{H}$ & 1.252520 & -3.489840 & -1.259111 \\
\hline $\mathrm{H}$ & 0.959870 & -5.051510 & -3.137051 \\
\hline $\mathrm{H}$ & -1.182020 & -5.104390 & -4.412791 \\
\hline $\mathrm{H}$ & -3.031690 & -3.583300 & -3.785231 \\
\hline C & 6.265180 & 1.751150 & -0.389411 \\
\hline C & 5.004170 & 2.328830 & -0.182191 \\
\hline C & 3.943870 & 1.483020 & 0.164969 \\
\hline C & 4.123480 & 0.099450 & 0.292389 \\
\hline C & 5.399740 & -0.438300 & 0.079939 \\
\hline C & 6.489820 & 0.372370 & -0.261741 \\
\hline $\mathrm{H}$ & 7.093940 & 2.391570 & -0.658391 \\
\hline $\mathrm{H}$ & 2.956920 & 1.894130 & 0.347219 \\
\hline $\mathrm{H}$ & 5.527470 & -1.513140 & 0.171939 \\
\hline C & 4.746250 & 3.842370 & -0.317961 \\
\hline C & 7.873510 & -0.267290 & -0.491581 \\
\hline C & 6.007700 & 4.624060 & -0.731111 \\
\hline $\mathrm{H}$ & 5.767320 & 5.689380 & -0.822751 \\
\hline $\mathrm{H}$ & 6.808560 & 4.530810 & 0.011299 \\
\hline $\mathrm{H}$ & 6.397630 & 4.289370 & -1.699341 \\
\hline C & 3.660350 & 4.079970 & -1.395561 \\
\hline $\mathrm{H}$ & 3.443620 & 5.151130 & -1.490011 \\
\hline $\mathrm{H}$ & 3.996030 & 3.715060 & -2.373711 \\
\hline $\mathrm{H}$ & 2.724460 & 3.567970 & -1.148441 \\
\hline C & 4.252970 & 4.403060 & 1.037149 \\
\hline H & 4.057220 & 5.479500 & 0.956869 \\
\hline $\mathrm{H}$ & 3.327930 & 3.918640 & 1.365569 \\
\hline $\mathrm{H}$ & 5.005520 & 4.252150 & 1.819509 \\
\hline C & 8.953510 & 0.772220 & -0.847711 \\
\hline $\mathrm{H}$ & 9.915290 & 0.267880 & -0.994591 \\
\hline $\mathrm{H}$ & 8.717210 & 1.306940 & -1.774741 \\
\hline $\mathrm{H}$ & 9.087890 & 1.512160 & -0.050471 \\
\hline C & 8.322210 & -1.001080 & 0.794509 \\
\hline H & 9.303240 & -1.468540 & 0.644939 \\
\hline $\mathrm{H}$ & 8.403220 & -0.302980 & 1.635579 \\
\hline
\end{tabular}




\begin{tabular}{|c|c|c|c|}
\hline $\mathrm{H}$ & 7.618390 & -1.789310 & 1.081509 \\
\hline C & 7.785120 & -1.282580 & -1.655871 \\
\hline $\mathrm{H}$ & 8.761370 & -1.752190 & -1.828321 \\
\hline $\mathrm{H}$ & 7.063940 & -2.080160 & -1.448811 \\
\hline $\mathrm{H}$ & 7.477940 & -0.787370 & -2.584261 \\
\hline C & -4.029050 & 0.010660 & -0.410061 \\
\hline C & -5.335980 & -0.494300 & -0.366921 \\
\hline C & -3.819950 & 1.379320 & -0.195861 \\
\hline C & -6.429960 & 0.339160 & -0.110371 \\
\hline $\mathrm{H}$ & -5.479740 & -1.558830 & -0.509991 \\
\hline C & -4.883310 & 2.245990 & 0.073839 \\
\hline $\mathrm{H}$ & -2.808850 & 1.763470 & -0.241181 \\
\hline C & -6.174490 & 1.700910 & 0.105529 \\
\hline $\mathrm{H}$ & -7.011970 & 2.360410 & 0.313789 \\
\hline C & -4.675290 & 3.749070 & 0.341209 \\
\hline C & -7.877840 & -0.185680 & -0.051551 \\
\hline C & -3.193550 & 4.161300 & 0.245279 \\
\hline $\mathrm{H}$ & -2.568950 & 3.618590 & 0.963159 \\
\hline $\mathrm{H}$ & -2.790330 & 3.989330 & -0.759981 \\
\hline $\mathrm{H}$ & -3.095100 & 5.231610 & 0.459919 \\
\hline C & -5.474140 & 4.578290 & -0.691621 \\
\hline $\mathrm{H}$ & -5.339130 & 5.651070 & -0.506301 \\
\hline $\mathrm{H}$ & -5.135530 & 4.364720 & -1.712191 \\
\hline $\mathrm{H}$ & -6.547510 & 4.366010 & -0.645491 \\
\hline C & -5.174230 & 4.085040 & 1.767129 \\
\hline $\mathrm{H}$ & -6.237960 & 3.856010 & 1.891019 \\
\hline $\mathrm{H}$ & -4.619040 & 3.513930 & 2.519269 \\
\hline $\mathrm{H}$ & -5.035940 & 5.152980 & 1.977319 \\
\hline C & -8.724900 & 0.523970 & -1.134631 \\
\hline $\mathrm{H}$ & -8.743310 & 1.609440 & -0.991811 \\
\hline $\mathrm{H}$ & -8.325270 & 0.326160 & -2.136051 \\
\hline $\mathrm{H}$ & -9.761420 & 0.165670 & -1.105391 \\
\hline C & -7.963000 & -1.704080 & -0.298121 \\
\hline $\mathrm{H}$ & -7.579950 & -1.979100 & -1.287581 \\
\hline $\mathrm{H}$ & -7.405040 & -2.271530 & 0.455069 \\
\hline $\mathrm{H}$ & -9.008950 & -2.027290 & -0.247801 \\
\hline C & -8.479430 & 0.106380 & 1.343679 \\
\hline $\mathrm{H}$ & -9.511530 & -0.261640 & 1.398359 \\
\hline $\mathrm{H}$ & -7.899060 & -0.387510 & 2.131029 \\
\hline $\mathrm{H}$ & -8.496730 & 1.178470 & 1.565369 \\
\hline
\end{tabular}

\section{Catalyst 2C 6}

Datum

Value

\begin{tabular}{lr}
\hline B3LYP/6-31G(d) Energy & -2164.800158 \\
\hline B3LYP-D3(BJ)/def2-TZVPP-IEF-PCM(DCM) Energy & -2165.902584 \\
\hline B3LYP-D3(BJ)/def2-TZVPP-IEF-PCM(DCM)//B3LYP/6-31G(d) Free Energy (Quasiharmonic) & -2165.024026 \\
\hline
\end{tabular}


Frequencies (Top 3 out of 321)

$\begin{array}{ll}\text { 1. } & 11.0361 \mathrm{~cm}^{-1} \\ \text { 2. } & 13.9121 \mathrm{~cm}^{-1} \\ \text { 3. } & 14.5983 \mathrm{~cm}^{-1}\end{array}$

\section{B3LYP/6-31G(d) Molecular Geometry in Cartesian Coordinates}

C

$\mathrm{H}$

$\mathrm{H}$

C

0

0

$\mathrm{H}$

0

$\mathrm{H}$

C

C

C

C

$\mathrm{H}$

C

C

C

C

$\mathrm{H}$

C

C

C

C

C

C

$\mathrm{H}$

$\mathrm{H}$

$\mathrm{H}$

$\mathrm{H}$

C

C

C

C

C

C

$\mathrm{H}$

$\mathrm{H}$

\subsection{0}

2.492610

0.925360

$-1.609440$

$-2.280300$

$-0.779210$

$-0.263130$

0.843930

0.548020

$-0.620540$

$-1.680220$

$-2.916450$

$-3.034240$

$-3.950090$

1.720490

0.675850

3.128650

2.967920

4.070130

2.093060

0.833790

$-0.205470$

$-0.002560$

1. 246500

2.269390

$-1.171720$

$-0.810020$

1. 390690

3. 229420

$-1.992470$

$-0.760670$

0.274650

0.101440

$-1.114870$

$-2.137380$

1. 208660

0.903650
0.005230

0.252840

$-0.540400$

$-0.036000$

$-0.323380$

1.021340

1.088450

1.235960

1.668080

$-1.681000$

$-0.853110$

$-0.879260$

$-1.759060$

$-1.765150$

$-0.828960$

$-1.638590$

$-1.624590$

$-0.801350$

$-1.605430$

$-2.464890$

$-2.467990$

$-3.302070$

$-4.095590$

$-4.096350$

$-3.295770$

$-3.295020$

$-4.720890$

$-4.726230$

$-3.282330$

$-2.644510$

$-2.612630$

$-3.509750$

$-4.384590$

$-4.407630$

$-3.554820$

$-3.503250$

$-5.064100$
$-1.259270$

$-1.710480$

$-1.991470$

1. 289910

2. 253530

1.303170

0.462720

$-0.903050$

$-1.720060$

$-0.335930$

0.011820

$-0.719740$

$-1.777680$

$-2.362980$

$-0.015170$

0.427500

1. 792210

0.693820

2. 335170

2. 264200

1.583420

2.082270

3.188290

3.855350

3.403810

1.590390

3.558620

4.729000

3.914250

$-2.146610$

$-1.419370$

$-1.802420$

$-2.852810$

$-3.575680$

$-3.228110$

$-1.250570$

$-3.127500$ 


\begin{tabular}{|c|c|c|c|}
\hline $\mathrm{H}$ & -1.238950 & -5.101610 & -4.402540 \\
\hline H & -3.077000 & -3.566400 & -3.775410 \\
\hline C & 6.254900 & 1.712900 & -0.387410 \\
\hline C & 6.471190 & 0.337270 & -0.266270 \\
\hline C & 5.371370 & -0.466680 & 0.075080 \\
\hline C & 4.102850 & 0.079840 & 0.288140 \\
\hline C & 3.929320 & 1.469610 & 0.163080 \\
\hline C & 4.995380 & 2.303950 & -0.177270 \\
\hline $\mathrm{H}$ & 7.086690 & 2.354610 & -0.653460 \\
\hline $\mathrm{H}$ & 5.491630 & -1.542470 & 0.165710 \\
\hline $\mathrm{H}$ & 2.944640 & 1.881070 & 0.345250 \\
\hline C & 7.848670 & -0.314000 & -0.497260 \\
\hline C & 4.832480 & 3.828590 & -0.331320 \\
\hline C & 8.936360 & 0.716910 & -0.854510 \\
\hline $\mathrm{H}$ & 9.893980 & 0.205300 & -1.003200 \\
\hline $\mathrm{H}$ & 8.702540 & 1.253860 & -1.780930 \\
\hline $\mathrm{H}$ & 9.077770 & 1.455250 & -0.056970 \\
\hline C & 8.291850 & -1.051130 & 0.788830 \\
\hline $\mathrm{H}$ & 9.268660 & -1.527140 & 0.638710 \\
\hline $\mathrm{H}$ & 8.379470 & -0.353400 & 1.629520 \\
\hline $\mathrm{H}$ & 7.581440 & -1.833140 & 1.076560 \\
\hline C & 7.750530 & -1.328690 & -1.661220 \\
\hline $\mathrm{H}$ & 8.722670 & -1.806460 & -1.834240 \\
\hline $\mathrm{H}$ & 7.023000 & -2.120310 & -1.453470 \\
\hline $\mathrm{H}$ & 7.446780 & -0.831100 & -2.589450 \\
\hline C & 5.182420 & 4.236290 & -1.782430 \\
\hline $\mathrm{H}$ & 5.079910 & 5.320950 & -1.909690 \\
\hline $\mathrm{H}$ & 6.210050 & 3.965390 & -2.047230 \\
\hline $\mathrm{H}$ & 4.513150 & 3.745900 & -2.499000 \\
\hline C & 3.394080 & 4.295060 & -0.035650 \\
\hline $\mathrm{H}$ & 3.321600 & 5.379740 & -0.174620 \\
\hline $\mathrm{H}$ & 2.664870 & 3.823530 & -0.703950 \\
\hline $\mathrm{H}$ & 3.097460 & 4.073190 & 0.995560 \\
\hline C & 5.783270 & 4.556850 & 0.647960 \\
\hline $\mathrm{H}$ & 5.674800 & 5.643770 & 0.547160 \\
\hline $\mathrm{H}$ & 5.556630 & 4.288000 & 1.685890 \\
\hline $\mathrm{H}$ & 6.833250 & 4.309160 & 0.460010 \\
\hline C & -4.049240 & 0.034050 & -0.400620 \\
\hline C & -3.837900 & 1.395200 & -0.170940 \\
\hline C & -5.363780 & -0.462880 & -0.374390 \\
\hline C & -4.900960 & 2.270800 & 0.095340 \\
\hline $\mathrm{H}$ & -2.824230 & 1.780460 & -0.198680 \\
\hline C & -6.451580 & 0.374000 & -0.122810 \\
\hline $\mathrm{H}$ & -5.512540 & -1.525230 & -0.528010 \\
\hline C & -6.192630 & 1.737700 & 0.107800 \\
\hline $\mathrm{H}$ & -7.030620 & 2.394650 & 0.309780 \\
\hline C & -7.903570 & -0.140650 & -0.082270 \\
\hline C & -4.604630 & 3.757760 & 0.368710 \\
\hline C & -8.735080 & 0.583550 & -1.167890 \\
\hline $\mathrm{H}$ & -8.746340 & 1.668070 & -1.017480 \\
\hline $\mathrm{H}$ & -8.327930 & 0.389470 & -2.167030 \\
\hline $\mathrm{H}$ & -9.774600 & 0.233290 & -1.150480 \\
\hline C & -7.997310 & -1.656330 & -0.341480 \\
\hline $\mathrm{H}$ & -9.045900 & -1.972690 & -0.303630 \\
\hline
\end{tabular}




\begin{tabular}{|c|c|c|c|}
\hline $\mathrm{H}$ & -7.606750 & -1.926350 & -1.329400 \\
\hline $\mathrm{H}$ & -7.450460 & -2.233500 & 0.412490 \\
\hline C & -8.516940 & 0.144940 & 1.309300 \\
\hline $\mathrm{H}$ & -7.948120 & -0.359640 & 2.098340 \\
\hline $\mathrm{H}$ & -8.528760 & 1.215290 & 1.539460 \\
\hline $\mathrm{H}$ & -9.552140 & -0.215990 & 1.350550 \\
\hline C & -5.884640 & 4.577720 & 0.617180 \\
\hline $\mathrm{H}$ & -6.558880 & 4.557550 & -0.247170 \\
\hline $\mathrm{H}$ & -6.436810 & 4.215380 & 1.491610 \\
\hline $\mathrm{H}$ & -5.620590 & 5.624480 & 0.805800 \\
\hline C & -3.704390 & 3.873220 & 1.622660 \\
\hline $\mathrm{H}$ & -4.213250 & 3.468910 & 2.504760 \\
\hline $\mathrm{H}$ & -2.763650 & 3.325360 & 1.508210 \\
\hline $\mathrm{H}$ & -3.462230 & 4.924900 & 1.821130 \\
\hline C & -3.873330 & 4.373110 & -0.848130 \\
\hline $\mathrm{H}$ & -3.653810 & 5.432130 & -0.664850 \\
\hline $\mathrm{H}$ & -2.922790 & 3.868820 & -1.050970 \\
\hline H & -4.488640 & 4.304780 & -1.752970 \\
\hline
\end{tabular}

\section{Catalyst 2C 7}

\section{Datum}

Value

\begin{tabular}{lc}
\hline B3LYP/6-31G(d) Energy & -2164.79986 \\
\hline B3LYP-D3(BJ)/def2-TZVPP-IEF-PCM(DCM) Energy & -2165.902296 \\
\hline B3LYP-D3(BJ)/def2-TZVPP-IEF-PCM(DCM)//B3LYP/6-31G(d) Free Energy (Quasiharmonic) & -2165.023941 \\
\hline
\end{tabular}

Number of Imaginary Frequencies

Frequencies (Top 3 out of 321)

$\begin{array}{lr}\text { 1. } & 9.4086 \mathrm{~cm}^{-1} \\ \text { 2. } & 12.3440 \mathrm{~cm}^{-1} \\ \text { 3. } & 15.0268 \mathrm{~cm}^{-1}\end{array}$

\section{B3LYP/6-31G(d) Molecular Geometry in Cartesian Coordinates}

$\begin{array}{rrrr}\mathrm{C} & -1.521846 & -0.011367 & -1.262067 \\ \mathrm{H} & -0.914340 & -0.549729 & -1.995713 \\ \mathrm{H} & -2.489944 & 0.226106 & -1.711216 \\ \mathrm{C} & 1.619517 & -0.021636 & 1.280421 \\ \mathrm{O} & 2.295600 & -0.301933 & 2.242527 \\ \mathrm{O} & 0.778619 & 1.026939 & 1.295179 \\ \mathrm{H} & 0.260361 & 1.088955 & 0.455697 \\ \mathrm{O} & -0.850776 & 1.226637 & -0.906223\end{array}$




\begin{tabular}{|c|c|c|c|}
\hline H & -0.561580 & 1.662780 & -1.723500 \\
\hline C & 0.645613 & -1.678134 & -0.344197 \\
\hline C & 1.696570 & -0.838940 & 0.002735 \\
\hline C & 2.932997 & -0.853758 & -0.728467 \\
\hline C & 3.060945 & -1.732941 & -1.785393 \\
\hline H & 3.977483 & -1.730008 & -2.369747 \\
\hline C & -1.703379 & -0.849052 & -0.018698 \\
\hline C & -0.650365 & -1.649698 & 0.420509 \\
\hline C & -3.101405 & -1.663112 & 1.788303 \\
\hline C & -2.949731 & -0.834535 & 0.692613 \\
\hline $\mathrm{H}$ & -4.042447 & -1.654817 & 2.332328 \\
\hline C & -2.057116 & -2.494421 & 2.256873 \\
\hline C & -0.798534 & -2.483027 & 1.574910 \\
\hline C & 0.249842 & -3.307207 & 2.071197 \\
\hline C & 0.056352 & -4.104876 & 3.175908 \\
\hline C & -1.192077 & -4.120134 & 3.844015 \\
\hline C & -2.223735 & -3.329547 & 3.394883 \\
\hline $\mathrm{H}$ & 1.215546 & -3.289095 & 1.578525 \\
\hline $\mathrm{H}$ & 0.870732 & -4.722248 & 3.544400 \\
\hline $\mathrm{H}$ & -1.328838 & -4.753129 & 4.716602 \\
\hline $\mathrm{H}$ & -3.183398 & -3.327199 & 3.906186 \\
\hline C & 2.028106 & -2.629196 & -2.153945 \\
\hline C & 0.795762 & -2.609026 & -1.427000 \\
\hline C & -0.230274 & -3.517011 & -1.809444 \\
\hline C & -0.047869 & -4.391191 & -2.858803 \\
\hline C & 1.168798 & -4.402662 & -3.581318 \\
\hline C & 2.182500 & -3.539097 & -3.234416 \\
\hline H & -1.164407 & -3.519484 & -1.257755 \\
\hline $\mathrm{H}$ & -0.843034 & -5.079162 & -3.132915 \\
\hline $\mathrm{H}$ & 1.300156 & -5.096249 & -4.407385 \\
\hline $\mathrm{H}$ & 3.122287 & -3.541614 & -3.781549 \\
\hline C & -6.255975 & 1.657793 & -0.360803 \\
\hline C & -6.461886 & 0.274905 & -0.253243 \\
\hline C & -5.358987 & -0.521176 & 0.077987 \\
\hline C & -4.092195 & 0.040091 & 0.294014 \\
\hline C & -3.930580 & 1.428541 & 0.180549 \\
\hline C & -5.007062 & 2.259433 & -0.150181 \\
\hline H & -7.100218 & 2.290795 & -0.617998 \\
\hline $\mathrm{H}$ & -5.462589 & -1.597177 & 0.160671 \\
\hline $\mathrm{H}$ & -2.949375 & 1.847735 & 0.363812 \\
\hline C & -7.865003 & -0.311424 & -0.505564 \\
\hline C & -4.856102 & 3.786833 & -0.290977 \\
\hline C & -8.314351 & 0.025925 & -1.947178 \\
\hline $\mathrm{H}$ & -9.314333 & -0.381544 & -2.140614 \\
\hline $\mathrm{H}$ & -8.356161 & 1.106398 & -2.119984 \\
\hline $\mathrm{H}$ & -7.624562 & -0.403459 & -2.682881 \\
\hline C & -7.900515 & -1.842817 & -0.341474 \\
\hline $\mathrm{H}$ & -8.916467 & -2.209227 & -0.527440 \\
\hline $\mathrm{H}$ & -7.233584 & -2.345398 & -1.051147 \\
\hline $\mathrm{H}$ & -7.618155 & -2.151610 & 0.671334 \\
\hline C & -8.868396 & 0.301163 & 0.500318 \\
\hline $\mathrm{H}$ & -9.875391 & -0.097065 & 0.325227 \\
\hline $\mathrm{H}$ & -8.583397 & 0.063634 & 1.531633 \\
\hline H & -8.921514 & 1.391188 & 0.412009 \\
\hline
\end{tabular}




\begin{tabular}{|c|c|c|c|}
\hline C & -5.212415 & 4.204459 & -1.737625 \\
\hline $\mathrm{H}$ & -5.117884 & 5.290848 & -1.856056 \\
\hline H & -4.541706 & 3.724815 & -2.460057 \\
\hline $\mathrm{H}$ & -6.238785 & 3.928425 & -2.002083 \\
\hline C & -5.810199 & 4.498836 & 0.696825 \\
\hline $\mathrm{H}$ & -5.710221 & 5.587418 & 0.605964 \\
\hline H & -6.858673 & 4.244911 & 0.508568 \\
\hline H & -5.579669 & 4.222059 & 1.731793 \\
\hline C & -3.420499 & 4.261516 & 0.005646 \\
\hline H & -3.356697 & 5.347939 & -0.123618 \\
\hline H & -3.119670 & 4.032650 & 1.034117 \\
\hline $\mathrm{H}$ & -2.689268 & 3.801734 & -0.668603 \\
\hline C & 4.055398 & 0.071682 & -0.407430 \\
\hline C & 5.370531 & -0.407538 & -0.358991 \\
\hline C & 3.828011 & 1.436453 & -0.194080 \\
\hline C & 6.451313 & 0.443027 & -0.097884 \\
\hline H & 5.538014 & -1.471078 & -0.500740 \\
\hline C & 4.876879 & 2.322057 & 0.075469 \\
\hline H & 2.810497 & 1.810120 & -0.238328 \\
\hline C & 6.178731 & 1.802423 & 0.114207 \\
\hline H & 7.000999 & 2.473265 & 0.321969 \\
\hline C & 4.564744 & 3.810216 & 0.326004 \\
\hline C & 7.878806 & -0.136264 & -0.040048 \\
\hline C & 5.834393 & 4.645910 & 0.575901 \\
\hline $\mathrm{H}$ & 6.380194 & 4.303136 & 1.462096 \\
\hline H & 6.518677 & 4.618534 & -0.280262 \\
\hline H & 5.558337 & 5.692963 & 0.744856 \\
\hline C & 3.840331 & 4.402829 & -0.906230 \\
\hline H & 3.610630 & 5.462489 & -0.739817 \\
\hline H & 4.464741 & 4.327006 & -1.804208 \\
\hline H & 2.895849 & 3.888071 & -1.111245 \\
\hline C & 3.650677 & 3.934799 & 1.569037 \\
\hline $\mathrm{H}$ & 2.717004 & 3.375333 & 1.452890 \\
\hline H & 4.154431 & 3.548496 & 2.462088 \\
\hline H & 3.395845 & 4.986728 & 1.749772 \\
\hline C & 8.227070 & -0.783945 & -1.401434 \\
\hline $\mathrm{H}$ & 7.530661 & -1.588736 & -1.659559 \\
\hline H & 8.194664 & -0.042253 & -2.208033 \\
\hline H & 9.236633 & -1.212420 & -1.374240 \\
\hline C & 8.941265 & 0.937550 & 0.262277 \\
\hline $\mathrm{H}$ & 8.962885 & 1.719418 & -0.505431 \\
\hline H & 8.773605 & 1.415354 & 1.234053 \\
\hline H & 9.934189 & 0.474422 & 0.289553 \\
\hline C & 7.953892 & -1.209457 & 1.072187 \\
\hline H & 8.964312 & -1.633770 & 1.125703 \\
\hline H & 7.713418 & -0.776779 & 2.049775 \\
\hline $\mathrm{H}$ & 7.255335 & -2.033126 & 0.893252 \\
\hline
\end{tabular}


Frequencies (Top 3 out of 321)

$\begin{array}{lr}\text { 1. } & 9.7969 \mathrm{~cm}^{-1} \\ \text { 2. } & 11.9544 \mathrm{~cm}^{-1} \\ \text { 3. } & 14.0492 \mathrm{~cm}^{-1}\end{array}$

\section{B3LYP/6-31G(d) Molecular Geometry in Cartesian Coordinates}

\begin{tabular}{|c|c|c|c|}
\hline C & 1.539780 & 0.011490 & -1.267460 \\
\hline $\mathrm{H}$ & 2.505520 & 0.253910 & -1.718520 \\
\hline $\mathrm{H}$ & 0.933751 & -0.530480 & -1.999460 \\
\hline C & -1.599870 & -0.019960 & 1.278690 \\
\hline 0 & -2.270720 & -0.305420 & 2.242810 \\
\hline 0 & -0.766900 & 1.035370 & 1.291140 \\
\hline $\mathrm{H}$ & -0.251740 & 1.101210 & 0.450270 \\
\hline 0 & 0.862140 & 1.247020 & -0.912720 \\
\hline $\mathrm{H}$ & 0.574840 & 1.682700 & -1.730970 \\
\hline C & -0.616849 & -1.667540 & -0.348610 \\
\hline C & -1.673709 & -0.836660 & 0.000540 \\
\hline C & -2.910579 & -0.858850 & -0.729830 \\
\hline C & -3.031709 & -1.735650 & -1.789650 \\
\hline $\mathrm{H}$ & -3.948209 & -1.737851 & -2.374030 \\
\hline C & 1.726961 & -0.823820 & -0.023150 \\
\hline C & 0.679041 & -1.631170 & 0.416070 \\
\hline C & 3.128891 & -1.626690 & 1.786260 \\
\hline C & 2.972881 & -0.800870 & 0.689190 \\
\hline $\mathrm{H}$ & 4.069051 & -1.611040 & 2.331670 \\
\hline C & 2.089911 & -2.464830 & 2.254430 \\
\hline C & 0.832081 & -2.462970 & 1.570970 \\
\hline C & -0.210769 & -3.294610 & 2.066410 \\
\hline C & -0.012649 & -4.090410 & 3.171660 \\
\hline C & 1.234991 & -4.096050 & 3.841350 \\
\hline C & 2.261301 & -3.297990 & 3.393160 \\
\hline $\mathrm{H}$ & -1.175969 & -3.283920 & 1.572560 \\
\hline $\mathrm{H}$ & -0.822829 & -4.713730 & 3.539360 \\
\hline $\mathrm{H}$ & 1.375371 & -4.727620 & 4.714380 \\
\hline $\mathrm{H}$ & 3.220271 & -3.288230 & 3.905660 \\
\hline C & -1.992399 & -2.623170 & -2.160990 \\
\hline C & -0.760419 & -2.596740 & -1.433680 \\
\hline 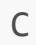 & 0.271961 & -3.496480 & -1.818540 \\
\hline
\end{tabular}




\begin{tabular}{|c|c|c|c|}
\hline C & 0.095941 & -4.368540 & -2.870740 \\
\hline C & -1.120369 & -4.386050 & -3.593750 \\
\hline C & -2.140159 & -3.530670 & -3.244420 \\
\hline $\mathrm{H}$ & 1.205971 & -3.494150 & -1.266630 \\
\hline $\mathrm{H}$ & 0.895931 & -5.050090 & -3.146820 \\
\hline $\mathrm{H}$ & -1.246629 & -5.077800 & -4.422140 \\
\hline $\mathrm{H}$ & -3.079729 & -3.537960 & -3.791880 \\
\hline C & 6.264140 & 1.716200 & -0.390770 \\
\hline C & 5.004740 & 2.301520 & -0.195510 \\
\hline C & 3.938160 & 1.463440 & 0.151120 \\
\hline C & 4.109740 & 0.079770 & 0.288580 \\
\hline C & 5.384621 & -0.465680 & 0.087450 \\
\hline C & 6.481010 & 0.337220 & -0.252220 \\
\hline $\mathrm{H}$ & 7.097760 & 2.350690 & -0.658840 \\
\hline $\mathrm{H}$ & 2.952480 & 1.881010 & 0.325220 \\
\hline $\mathrm{H}$ & 5.506491 & -1.540540 & 0.186840 \\
\hline C & 4.755110 & 3.815300 & -0.343400 \\
\hline C & 7.863120 & -0.310850 & -0.467300 \\
\hline C & 4.258210 & 4.388300 & 1.005210 \\
\hline $\mathrm{H}$ & 4.068110 & 5.465040 & 0.916040 \\
\hline $\mathrm{H}$ & 3.329300 & 3.910810 & 1.332920 \\
\hline $\mathrm{H}$ & 5.006340 & 4.239560 & 1.792210 \\
\hline C & 6.022430 & 4.587630 & -0.756240 \\
\hline $\mathrm{H}$ & 5.787760 & 5.653360 & -0.857400 \\
\hline $\mathrm{H}$ & 6.819090 & 4.496360 & -0.009090 \\
\hline $\mathrm{H}$ & 6.415580 & 4.243500 & -1.719850 \\
\hline C & 3.675550 & 4.050660 & -1.427860 \\
\hline $\mathrm{H}$ & 3.464700 & 5.122200 & -1.530930 \\
\hline $\mathrm{H}$ & 4.013880 & 3.677090 & -2.401810 \\
\hline $\mathrm{H}$ & 2.735910 & 3.545210 & -1.181460 \\
\hline C & 7.778201 & -1.335460 & -1.623600 \\
\hline $\mathrm{H}$ & 8.753481 & -1.810920 & -1.785120 \\
\hline $\mathrm{H}$ & 7.051941 & -2.128020 & -1.414980 \\
\hline $\mathrm{H}$ & 7.479881 & -0.846690 & -2.558250 \\
\hline C & 8.950610 & 0.720520 & -0.824370 \\
\hline $\mathrm{H}$ & 9.910970 & 0.210330 & -0.959990 \\
\hline $\mathrm{H}$ & 8.723670 & 1.248460 & -1.757600 \\
\hline $\mathrm{H}$ & 9.082930 & 1.466520 & -0.032460 \\
\hline C & 8.299151 & -1.035880 & 0.828140 \\
\hline $\mathrm{H}$ & 9.279051 & -1.509100 & 0.689620 \\
\hline $\mathrm{H}$ & 8.377260 & -0.331140 & 1.663920 \\
\hline $\mathrm{H}$ & 7.589541 & -1.818380 & 1.116540 \\
\hline C & -4.041280 & 0.054939 & -0.404430 \\
\hline C & -3.826780 & 1.419830 & -0.178720 \\
\hline C & -5.352309 & -0.436331 & -0.363150 \\
\hline C & -4.884140 & 2.293409 & 0.096950 \\
\hline $\mathrm{H}$ & -2.812750 & 1.803300 & -0.217650 \\
\hline C & -6.441320 & 0.402129 & -0.097240 \\
\hline $\mathrm{H}$ & -5.510149 & -1.500051 & -0.514350 \\
\hline C & -6.181380 & 1.761869 & 0.128050 \\
\hline $\mathrm{H}$ & -7.009980 & 2.423419 & 0.340460 \\
\hline C & -7.863790 & -0.190221 & -0.048920 \\
\hline C & -4.585800 & 3.781779 & 0.362890 \\
\hline c & -8.936320 & 0.871029 & 0.261880 \\
\hline
\end{tabular}




\begin{tabular}{|c|c|c|c|}
\hline $\mathrm{H}$ & -8.775000 & 1.340229 & 1.238910 \\
\hline $\mathrm{H}$ & -8.963280 & 1.660599 & -0.497730 \\
\hline $\mathrm{H}$ & -9.925170 & 0.398929 & 0.282160 \\
\hline C & -8.202809 & -0.826621 & -1.417940 \\
\hline $\mathrm{H}$ & -9.208639 & -1.264191 & -1.398060 \\
\hline $\mathrm{H}$ & -8.174730 & -0.076271 & -2.216660 \\
\hline H & -7.498599 & -1.622501 & -1.682470 \\
\hline C & -7.932439 & -1.275621 & 1.051780 \\
\hline $\mathrm{H}$ & -7.226079 & -2.091181 & 0.866330 \\
\hline $\mathrm{H}$ & -7.698729 & -0.851101 & 2.034570 \\
\hline $\mathrm{H}$ & -8.939169 & -1.709451 & 1.097860 \\
\hline C & -3.674880 & 3.901790 & 1.608700 \\
\hline $\mathrm{H}$ & -4.176380 & 3.501139 & 2.496680 \\
\hline $\mathrm{H}$ & -2.735670 & 3.352630 & 1.488210 \\
\hline $\mathrm{H}$ & -3.430450 & 4.954110 & 1.801140 \\
\hline C & -3.864910 & 4.393380 & -0.862090 \\
\hline $\mathrm{H}$ & -2.915830 & 3.888860 & -1.071080 \\
\hline $\mathrm{H}$ & -4.487500 & 4.321549 & -1.761660 \\
\hline $\mathrm{H}$ & -3.644550 & 5.453190 & -0.684370 \\
\hline C & -5.863370 & 4.603319 & 0.619430 \\
\hline $\mathrm{H}$ & -5.597020 & 5.650849 & 0.800550 \\
\hline $\mathrm{H}$ & -6.545720 & 4.579339 & -0.238380 \\
\hline $\mathrm{H}$ & -6.407650 & 4.245719 & 1.500670 \\
\hline
\end{tabular}

\section{Catalyst 2C 9}

Datum

Value

\begin{tabular}{lc}
\hline B3LYP/6-31G(d) Energy & -2164.804927 \\
\hline B3LYP-D3(BJ)/def2-TZVPP-IEF-PCM(DCM) Energy & -2165.900846 \\
\hline B3LYP-D3(BJ)/def2-TZVPP-IEF-PCM(DCM)//B3LYP/6-31G(d) Free Energy (Quasiharmonic) & -2165.022427 \\
\hline
\end{tabular}

Number of Imaginary Frequencies

Frequencies (Top 3 out of 321)
1. $9.3798 \mathrm{~cm}^{-1}$
2. $\quad 11.1275 \mathrm{~cm}^{-1}$
3. $13.1858 \mathrm{~cm}^{-1}$

\section{B3LYP/6-31G(d) Molecular Geometry in Cartesian Coordinates}

$\begin{array}{rrrr}C & 0.170876 & -4.207432 & 3.050381 \\ C & 0.329497 & -3.347999 & 1.985892 \\ C & -0.737431 & -2.510771 & 1.557897\end{array}$




\begin{tabular}{|c|c|c|c|}
\hline C & -1.984316 & -2.583457 & 2.257425 \\
\hline C & -2.114498 & -3.478728 & 3.353114 \\
\hline C & -1.061918 & -4.274762 & 3.742104 \\
\hline $\mathrm{H}$ & 0.998181 & -4.838552 & 3.362867 \\
\hline $\mathrm{H}$ & 1.277602 & -3.300205 & 1.461707 \\
\hline C & -0.608405 & -1.604253 & 0.452841 \\
\hline C & -3.056421 & -1.750923 & 1.852119 \\
\hline $\mathrm{H}$ & -3.064726 & -3.521751 & 3.879874 \\
\hline $\mathrm{H}$ & -1.173605 & -4.956162 & 4.581085 \\
\hline C & -2.944562 & -0.877244 & 0.790400 \\
\hline C & -1.698751 & -0.828770 & 0.081112 \\
\hline $\mathrm{H}$ & -3.984485 & -1.782843 & 2.416912 \\
\hline C & 1.196167 & -4.224482 & -3.626580 \\
\hline C & 2.217048 & -3.385753 & -3.243928 \\
\hline C & 2.058761 & -2.497866 & -2.145319 \\
\hline C & 0.818565 & -2.476428 & -1.432961 \\
\hline C & -0.216205 & -3.357967 & -1.854898 \\
\hline C & -0.031699 & -4.208398 & -2.922479 \\
\hline $\mathrm{H}$ & 4.036435 & -1.654743 & -2.285294 \\
\hline $\mathrm{H}$ & 1.328199 & -4.899522 & -4.467881 \\
\hline $\mathrm{H}$ & 3.164907 & -3.388281 & -3.777100 \\
\hline C & 3.099984 & -1.633763 & -1.733637 \\
\hline C & 0.678508 & -1.571468 & -0.328700 \\
\hline $\mathrm{H}$ & -1.164883 & -3.352958 & -1.329216 \\
\hline $\mathrm{H}$ & -0.836063 & -4.871961 & -3.228369 \\
\hline C & 1.715481 & -0.718451 & 0.043045 \\
\hline C & 2.960732 & -0.751532 & -0.678242 \\
\hline C & 1.514801 & 0.252665 & 1.196741 \\
\hline $\mathrm{H}$ & 0.797064 & -0.166274 & 1.913498 \\
\hline $\mathrm{H}$ & 2.458079 & 0.403728 & 1.726829 \\
\hline C & -1.583974 & 0.042416 & -1.134288 \\
\hline 0 & -0.870717 & 1.025795 & -1.245101 \\
\hline 0 & -2.364097 & -0.383010 & -2.142770 \\
\hline $\mathrm{H}$ & -2.238116 & 0.249369 & -2.876920 \\
\hline 0 & 1.094148 & 1.547540 & 0.771223 \\
\hline $\mathrm{H}$ & 0.365306 & 1.431399 & 0.129571 \\
\hline C & 4.135058 & 0.099489 & -0.318185 \\
\hline C & 4.026640 & 1.485606 & -0.181994 \\
\hline C & 5.396944 & -0.503574 & -0.158688 \\
\hline C & 5.144983 & 2.280881 & 0.114913 \\
\hline $\mathrm{H}$ & 3.049001 & 1.939432 & -0.294293 \\
\hline C & 6.533646 & 0.251838 & 0.133105 \\
\hline $\mathrm{H}$ & 5.463450 & -1.581908 & -0.248955 \\
\hline C & 6.380151 & 1.644677 & 0.263235 \\
\hline $\mathrm{H}$ & 7.255480 & 2.241097 & 0.493359 \\
\hline C & -4.083719 & 0.016394 & 0.440047 \\
\hline C & -5.368992 & -0.514757 & 0.268091 \\
\hline C & -3.888925 & 1.399331 & 0.318536 \\
\hline C & -6.460015 & 0.309246 & -0.029984 \\
\hline $\mathrm{H}$ & -5.495562 & -1.588110 & 0.350346 \\
\hline C & -4.952471 & 2.257701 & 0.017838 \\
\hline $\mathrm{H}$ & -2.895801 & 1.799679 & 0.482316 \\
\hline C & -6.221733 & 1.686416 & -0.149886 \\
\hline $\mathrm{H}$ & -7.057534 & 2.339912 & -0.382125 \\
\hline
\end{tabular}




\begin{tabular}{|c|c|c|c|}
\hline C & -4.767823 & 3.780667 & -0.129363 \\
\hline C & -7.884580 & -0.241191 & -0.237673 \\
\hline C & -5.181950 & 4.210360 & -1.557040 \\
\hline $\mathrm{H}$ & -4.559285 & 3.714993 & -2.310853 \\
\hline $\mathrm{H}$ & -6.227173 & 3.964103 & -1.772327 \\
\hline $\mathrm{H}$ & -5.062649 & 5.293880 & -1.678061 \\
\hline C & -3.307536 & 4.217590 & 0.097196 \\
\hline $\mathrm{H}$ & -3.223658 & 5.302491 & -0.032432 \\
\hline $\mathrm{H}$ & -2.962451 & 3.980363 & 1.109894 \\
\hline H & -2.621363 & 3.744957 & -0.614445 \\
\hline C & -5.656223 & 4.513350 & 0.903610 \\
\hline $\mathrm{H}$ & -5.383728 & 4.228933 & 1.926295 \\
\hline $\mathrm{H}$ & -5.533561 & 5.599250 & 0.809303 \\
\hline $\mathrm{H}$ & -6.718378 & 4.285272 & 0.765085 \\
\hline C & -8.842071 & 0.402293 & 0.793352 \\
\hline $\mathrm{H}$ & -8.533082 & 0.163662 & 1.817529 \\
\hline $\mathrm{H}$ & -8.871186 & 1.492761 & 0.699572 \\
\hline $\mathrm{H}$ & -9.863231 & 0.028031 & 0.650399 \\
\hline C & -7.954916 & -1.770321 & -0.064031 \\
\hline $\mathrm{H}$ & -7.649480 & -2.081380 & 0.941460 \\
\hline $\mathrm{H}$ & -8.985454 & -2.110544 & -0.216173 \\
\hline $\mathrm{H}$ & -7.324034 & -2.293760 & -0.791249 \\
\hline C & -8.365807 & 0.101071 & -1.667772 \\
\hline $\mathrm{H}$ & -8.385641 & 1.181545 & -1.844389 \\
\hline $\mathrm{H}$ & -7.707642 & -0.348406 & -2.420111 \\
\hline $\mathrm{H}$ & -9.380614 & -0.282197 & -1.831186 \\
\hline C & 7.925929 & -0.380148 & 0.325539 \\
\hline C & 4.960388 & 3.802818 & 0.267292 \\
\hline C & 3.955250 & 4.080963 & 1.411562 \\
\hline $\mathrm{H}$ & 2.987681 & 3.601378 & 1.233495 \\
\hline H & 4.342783 & 3.707723 & 2.367088 \\
\hline $\mathrm{H}$ & 3.786463 & 5.160412 & 1.514638 \\
\hline C & 4.405469 & 4.387337 & -1.053614 \\
\hline $\mathrm{H}$ & 5.097357 & 4.205252 & -1.884425 \\
\hline $\mathrm{H}$ & 3.439653 & 3.945617 & -1.318114 \\
\hline $\mathrm{H}$ & 4.263339 & 5.471248 & -0.960594 \\
\hline C & 6.278903 & 4.528309 & 0.595679 \\
\hline $\mathrm{H}$ & 6.713805 & 4.176890 & 1.538526 \\
\hline H & 7.026739 & 4.399372 & -0.195707 \\
\hline $\mathrm{H}$ & 6.091928 & 5.603207 & 0.700157 \\
\hline C & 7.907930 & -1.910382 & 0.146749 \\
\hline $\mathrm{H}$ & 7.252666 & -2.398754 & 0.876925 \\
\hline $\mathrm{H}$ & 7.576714 & -2.200046 & -0.857012 \\
\hline $\mathrm{H}$ & 8.918227 & -2.310425 & 0.290161 \\
\hline C & 8.441352 & -0.072147 & 1.751623 \\
\hline $\mathrm{H}$ & 9.435328 & -0.511546 & 1.903201 \\
\hline H & 8.522398 & 1.004625 & 1.932854 \\
\hline $\mathrm{H}$ & 7.766830 & -0.487808 & 2.509002 \\
\hline C & 8.909514 & 0.210704 & -0.712558 \\
\hline $\mathrm{H}$ & 9.004260 & 1.296919 & -0.612998 \\
\hline $\mathrm{H}$ & 9.908429 & -0.224427 & -0.583965 \\
\hline H & 8.574589 & -0.002461 & -1.734256 \\
\hline
\end{tabular}




\section{Catalyst 2C 10}

Datum

Value

\begin{tabular}{lc}
\hline B3LYP/6-31G(d) Energy & -2164.804954 \\
\hline B3LYP-D3(BJ)/def2-TZVPP-IEF-PCM(DCM) Energy & -2165.900892 \\
\hline B3LYP-D3(BJ)/def2-TZVPP-IEF-PCM(DCM)//B3LYP/6-31G(d) Free Energy (Quasiharmonic) & -2165.022347 \\
\hline
\end{tabular}

Number of Imaginary Frequencies

Frequencies (Top 3 out of 321)

$\begin{array}{lr}\text { 1. } & 9.6291 \mathrm{~cm}^{-1} \\ \text { 2. } & 10.8587 \mathrm{~cm}^{-1} \\ \text { 3. } & 14.1849 \mathrm{~cm}^{-1}\end{array}$

\section{B3LYP/6-31G(d) Molecular Geometry in Cartesian Coordinates}

C

C

C

C

C

C

$\mathrm{H}$

$\mathrm{H}$

C

C

$\mathrm{H}$

$\mathrm{H}$

C

C

$\mathrm{H}$

C

C

C

C

C

C

$\mathrm{H}$

$\mathrm{H}$

$\mathrm{H}$

C

C

$\mathrm{H}$

$\mathrm{H}$

C
$-0.149555$

$-0.311349$

0.751718

1.998086

2.131506

1.082613

$-0.973877$

$-1.258974$

0.619197

3.066654

3.081234

1.196767

2.952170

1.706278

3.994194

$-1.170043$

$-2.195245$

$-2.042125$

$-0.802604$

0.236671

0.057120

$-4.023726$

$-1.298121$

$-3.142636$

$-3.087863$

$-0.667709$

1.184890

0.864891

$-1.709132$
$-4.193589$

$-3.335993$

$-2.493585$

$-2.559133$

$-3.452423$

$-4.253626$

$-4.828871$

$-3.293720$

$-1.588365$

$-1.722099$

$-3.489869$

$-4.933558$

$-0.851306$

$-0.808444$

$-1.748507$

$-4.208787$

$-3.375921$

$-2.490046$

$-2.464386$

$-3.340022$

$-4.188658$

$-1.656336$

$-4.882294$

$-3.381650$

$-1.632089$

$-1.561108$

$-3.331839$

$-4.847615$

$-0.714074$
3.050670

1.985171

1.557778

2. 258891

3. 355777

3.744163

3.362600

1.459652

0.452080

1.853429

3.883863

4.584000

0.789656

0.080028

2.419352

$-3.634229$

$-3.250339$

$-2.149364$

$-1.435975$

$-1.859153$

$-2.929004$

$-2.288859$

$-4.477369$

$-3.784316$

$-1.736362$

$-0.329724$

$-1.332686$

$-3.235867$

0.043308 


\begin{tabular}{|c|c|c|c|}
\hline C & -2.953915 & -0.751812 & -0.678596 \\
\hline C & -1.513043 & 0.256404 & 1.198287 \\
\hline $\mathrm{H}$ & -2.456987 & 0.402747 & 1.728500 \\
\hline $\mathrm{H}$ & -0.793532 & -0.159978 & 1.914713 \\
\hline C & 1.586797 & 0.062150 & -1.135344 \\
\hline 0 & 0.867515 & 1.041131 & -1.245995 \\
\hline 0 & 2.370203 & -0.358062 & -2.143391 \\
\hline $\mathrm{H}$ & 2.241009 & 0.273780 & -2.877440 \\
\hline 0 & -1.098062 & 1.553569 & 0.774022 \\
\hline $\mathrm{H}$ & -0.369590 & 1.441194 & 0.131287 \\
\hline C & -4.133150 & 0.091656 & -0.316860 \\
\hline C & -5.392280 & -0.518693 & -0.163082 \\
\hline C & -4.032310 & 1.477614 & -0.173137 \\
\hline C & -6.533507 & 0.229219 & 0.130257 \\
\hline $\mathrm{H}$ & -5.453084 & -1.596868 & -0.258950 \\
\hline C & -5.155332 & 2.265418 & 0.126056 \\
\hline $\mathrm{H}$ & -3.056992 & 1.937376 & -0.281552 \\
\hline C & -6.387504 & 1.622082 & 0.268444 \\
\hline $\mathrm{H}$ & -7.266376 & 2.212649 & 0.500130 \\
\hline C & 4.089262 & 0.043991 & 0.436663 \\
\hline C & 3.890112 & 1.429212 & 0.306575 \\
\hline C & 5.373643 & -0.478353 & 0.269428 \\
\hline C & 4.948561 & 2.286341 & 0.001383 \\
\hline $\mathrm{H}$ & 2.895393 & 1.826509 & 0.467981 \\
\hline C & 6.466658 & 0.348823 & -0.033992 \\
\hline $\mathrm{H}$ & 5.510652 & -1.552164 & 0.357512 \\
\hline C & 6.225734 & 1.719992 & -0.164202 \\
\hline $\mathrm{H}$ & 7.053004 & 2.378672 & -0.401077 \\
\hline C & 7.864108 & -0.273739 & -0.219583 \\
\hline C & 4.760509 & 3.807747 & -0.154697 \\
\hline C & 8.279961 & -1.001579 & 1.080945 \\
\hline $\mathrm{H}$ & 7.574829 & -1.795909 & 1.347306 \\
\hline $\mathrm{H}$ & 8.329230 & -0.301771 & 1.923085 \\
\hline H & 9.269116 & -1.460439 & 0.961755 \\
\hline C & 8.941486 & 0.778883 & -0.542452 \\
\hline $\mathrm{H}$ & 9.913080 & 0.286163 & -0.660939 \\
\hline $\mathrm{H}$ & 9.043056 & 1.519443 & 0.259063 \\
\hline $\mathrm{H}$ & 8.726165 & 1.311257 & -1.475893 \\
\hline C & 7.823792 & -1.290612 & -1.385182 \\
\hline $\mathrm{H}$ & 7.106075 & -2.096542 & -1.200469 \\
\hline $\mathrm{H}$ & 8.810611 & -1.748440 & -1.526299 \\
\hline $\mathrm{H}$ & 7.539593 & -0.799804 & -2.323021 \\
\hline C & 5.173001 & 4.230159 & -1.585105 \\
\hline $\mathrm{H}$ & 6.218589 & 3.984964 & -1.799680 \\
\hline $\mathrm{H}$ & 4.550711 & 3.729348 & -2.335652 \\
\hline $\mathrm{H}$ & 5.051341 & 5.312742 & -1.712037 \\
\hline C & 5.647829 & 4.548655 & 0.873492 \\
\hline $\mathrm{H}$ & 6.710463 & 4.322412 & 0.735856 \\
\hline $\mathrm{H}$ & 5.522440 & 5.633667 & 0.772717 \\
\hline $\mathrm{H}$ & 5.376273 & 4.269720 & 1.897953 \\
\hline C & 3.299469 & 4.242860 & 0.070125 \\
\hline $\mathrm{H}$ & 2.613937 & 3.764612 & -0.638381 \\
\hline $\mathrm{H}$ & 2.955546 & 4.010551 & 1.084360 \\
\hline $\mathrm{H}$ & 3.213080 & 5.326821 & -0.065628 \\
\hline
\end{tabular}




\begin{tabular}{|rrrr|} 
C & -4.978961 & 3.787426 & 0.287206 \\
C & -7.922902 & -0.411064 & 0.315867 \\
H & -7.896532 & -1.940111 & 0.128163 \\
H & -7.561336 & -2.222102 & -0.876473 \\
H & -7.240464 & -2.429383 & 0.857022 \\
C & -8.905038 & -2.346353 & 0.266648 \\
H & -8.907143 & 0.180737 & -0.721083 \\
H & -9.008314 & 1.265748 & -0.614941 \\
H & -8.568392 & -0.024090 & -1.743225 \\
C & -9.903909 & -0.260826 & -0.597842 \\
H & -8.443226 & -0.114023 & 1.742516 \\
H & -7.768388 & -0.530695 & 2.499061 \\
H & -8.530183 & 0.961244 & 1.929855 \\
C & -9.435313 & -0.559307 & 1.889189 \\
H & -6.301601 & 4.503924 & 0.618766 \\
H & -7.048085 & 4.375694 & -0.174011 \\
H & -6.735503 & 4.144679 & 1.559144 \\
C & -6.120492 & 5.579181 & 0.729736 \\
H & -4.426212 & 4.382409 & -1.029918 \\
H & -4.290114 & 5.466530 & -0.930595 \\
H & -3.457753 & 3.947476 & -1.296105 \\
C & -5.116415 & 4.201267 & -1.862331 \\
H & -3.976120 & 4.064574 & 1.433728 \\
H & -3.005411 & 3.592419 & 1.252908 \\
H & -3.814217 & 5.144390 & 1.543782 \\
& -4.361610 & 3.682758 & 2.386688 \\
\hline & & & \\
\hline
\end{tabular}

\section{Catalyst 2C 11}

Datum

Value

\begin{tabular}{lr}
\hline B3LYP/6-31G(d) Energy & -2164.805066 \\
\hline B3LYP-D3(BJ)/def2-TZVPP-IEF-PCM(DCM) Energy & -2165.900835 \\
\hline B3LYP-D3(BJ)/def2-TZVPP-IEF-PCM(DCM)//B3LYP/6-31G(d) Free Energy (Quasiharmonic) & -2165.022231 \\
\hline
\end{tabular}

Number of Imaginary Frequencies

Frequencies (Top 3 out of 321)

$\begin{array}{lr}\text { 1. } & 9.8989 \mathrm{~cm}^{-1} \\ \text { 2. } & 11.4683 \mathrm{~cm}^{-1} \\ \text { 3. } & 13.6296 \mathrm{~cm}^{-1}\end{array}$




\begin{tabular}{|c|c|c|c|}
\hline C & 0.166875 & -4.191566 & 3.054322 \\
\hline C & 0.325684 & -3.332769 & 1.989368 \\
\hline C & -0.741583 & -2.496658 & 1.560060 \\
\hline C & -1.989169 & -2.570138 & 2.258284 \\
\hline C & -2.119493 & -3.464773 & 3.354522 \\
\hline C & -1.066485 & -4.259444 & 3.745058 \\
\hline $\mathrm{H}$ & 0.994445 & -4.821726 & 3.368028 \\
\hline H & 1.274196 & -3.284447 & 1.465931 \\
\hline C & -0.612321 & -1.590081 & 0.455288 \\
\hline C & -3.061963 & -1.739636 & 1.850873 \\
\hline $\mathrm{H}$ & -3.070169 & -3.508389 & 3.880417 \\
\hline $\mathrm{H}$ & -1.178243 & -4.940267 & 4.584491 \\
\hline C & -2.950216 & -0.866277 & 0.788711 \\
\hline C & -1.703068 & -0.816024 & 0.081926 \\
\hline $\mathrm{H}$ & -3.990774 & -1.773297 & 2.414320 \\
\hline C & 1.191948 & -4.190248 & -3.637124 \\
\hline C & 2.211453 & -3.350553 & -3.252932 \\
\hline C & 2.053448 & -2.468708 & -2.149443 \\
\hline C & 0.814872 & -2.453852 & -1.434177 \\
\hline C & -0.218521 & -3.336150 & -1.857874 \\
\hline C & -0.034170 & -4.181031 & -2.929871 \\
\hline $\mathrm{H}$ & 4.028683 & -1.620057 & -2.289682 \\
\hline H & 1.323753 & -4.860851 & -4.481994 \\
\hline $\mathrm{H}$ & 3.158024 & -3.347860 & -3.788378 \\
\hline C & 3.093765 & -1.604701 & -1.735308 \\
\hline C & 0.675016 & -1.554306 & -0.325432 \\
\hline $\mathrm{H}$ & -1.165903 & -3.336387 & -1.329869 \\
\hline $\mathrm{H}$ & -0.837374 & -4.845476 & -3.236873 \\
\hline C & 1.711577 & -0.702190 & 0.049670 \\
\hline C & 2.955836 & -0.729928 & -0.673547 \\
\hline C & 1.510677 & 0.263071 & 1.208255 \\
\hline $\mathrm{H}$ & 0.794472 & -0.160265 & 1.923887 \\
\hline $\mathrm{H}$ & 2.454194 & 0.413342 & 1.738149 \\
\hline C & -1.585457 & 0.058848 & -1.130609 \\
\hline 0 & -0.878544 & 1.048338 & -1.231055 \\
\hline 0 & -2.354553 & -0.367632 & -2.146291 \\
\hline $\mathrm{H}$ & -2.230194 & 0.270373 & -2.875940 \\
\hline 0 & 1.087288 & 1.559220 & 0.789078 \\
\hline $\mathrm{H}$ & 0.359025 & 1.444858 & 0.146514 \\
\hline C & 4.131271 & 0.117675 & -0.308834 \\
\hline C & 5.391592 & -0.480920 & -0.162640 \\
\hline C & 4.023603 & 1.505615 & -0.155110 \\
\hline C & 6.534208 & 0.272207 & 0.132576 \\
\hline $\mathrm{H}$ & 5.465235 & -1.559974 & -0.265177 \\
\hline C & 5.140442 & 2.294795 & 0.146383 \\
\hline $\mathrm{H}$ & 3.045229 & 1.959973 & -0.258855 \\
\hline C & 6.382617 & 1.658769 & 0.282087 \\
\hline $\mathrm{H}$ & 7.252831 & 2.257119 & 0.515648 \\
\hline C & -4.091557 & 0.023198 & 0.434819 \\
\hline C & -5.380617 & -0.512842 & 0.276086 \\
\hline C & -3.904618 & 1.400616 & 0.294209 \\
\hline C & -6.471439 & 0.303962 & -0.025256 \\
\hline $\mathrm{H}$ & -5.504260 & -1.585370 & 0.372174 \\
\hline
\end{tabular}




\begin{tabular}{|c|c|c|c|}
\hline C & -4.973395 & 2.257212 & -0.014060 \\
\hline $\mathrm{H}$ & -2.911846 & 1.812067 & 0.445447 \\
\hline C & -6.239361 & 1.685243 & -0.166164 \\
\hline $\mathrm{H}$ & -7.079802 & 2.326969 & -0.403325 \\
\hline C & -4.709827 & 3.766880 & -0.173046 \\
\hline C & -7.896487 & -0.249995 & -0.217352 \\
\hline C & -4.100604 & 4.327578 & 1.134225 \\
\hline $\mathrm{H}$ & -4.785080 & 4.185183 & 1.978458 \\
\hline $\mathrm{H}$ & -3.152515 & 3.842320 & 1.386282 \\
\hline $\mathrm{H}$ & -3.905907 & 5.402044 & 1.031835 \\
\hline C & -5.993389 & 4.560815 & -0.480947 \\
\hline $\mathrm{H}$ & -5.751863 & 5.624621 & -0.584213 \\
\hline $\mathrm{H}$ & -6.459197 & 4.236945 & -1.418684 \\
\hline $\mathrm{H}$ & -6.734640 & 4.467389 & 0.321139 \\
\hline C & -3.714905 & 3.987617 & -1.338434 \\
\hline $\mathrm{H}$ & -4.140644 & 3.633404 & -2.284948 \\
\hline $\mathrm{H}$ & -3.487724 & 5.054978 & -1.448966 \\
\hline $\mathrm{H}$ & -2.769350 & 3.459362 & -1.178046 \\
\hline C & -8.387555 & 0.074551 & -1.648364 \\
\hline $\mathrm{H}$ & -8.410100 & 1.152703 & -1.838181 \\
\hline $\mathrm{H}$ & -7.733901 & -0.383605 & -2.399435 \\
\hline $\mathrm{H}$ & -9.402977 & -0.311843 & -1.800342 \\
\hline C & -8.847920 & 0.405026 & 0.812169 \\
\hline $\mathrm{H}$ & -8.879923 & 1.494108 & 0.704521 \\
\hline $\mathrm{H}$ & -9.869310 & 0.026927 & 0.681404 \\
\hline $\mathrm{H}$ & -8.531090 & 0.180046 & 1.837058 \\
\hline C & -7.964480 & -1.776989 & -0.025100 \\
\hline $\mathrm{H}$ & -7.337422 & -2.308447 & -0.749816 \\
\hline $\mathrm{H}$ & -7.652634 & -2.075731 & 0.982164 \\
\hline $\mathrm{H}$ & -8.995592 & -2.119862 & -0.167003 \\
\hline C & 4.957246 & 3.815325 & 0.318211 \\
\hline C & 7.894372 & -0.436495 & 0.290100 \\
\hline C & 9.040376 & 0.545220 & 0.600789 \\
\hline $\mathrm{H}$ & 9.170047 & 1.287680 & -0.194909 \\
\hline $\mathrm{H}$ & 8.876836 & 1.079629 & 1.543449 \\
\hline $\mathrm{H}$ & 9.982805 & -0.006572 & 0.694115 \\
\hline C & 8.244313 & -1.178905 & -1.021566 \\
\hline $\mathrm{H}$ & 7.488916 & -1.928684 & -1.278782 \\
\hline $\mathrm{H}$ & 8.317865 & -0.477245 & -1.860431 \\
\hline $\mathrm{H}$ & 9.207385 & -1.695479 & -0.924432 \\
\hline C & 7.813344 & -1.458196 & 1.449178 \\
\hline $\mathrm{H}$ & 7.573865 & -0.958423 & 2.394773 \\
\hline $\mathrm{H}$ & 7.045417 & -2.218154 & 1.270297 \\
\hline $\mathrm{H}$ & 8.773156 & -1.975615 & 1.571240 \\
\hline C & 3.953603 & 4.080657 & 1.466853 \\
\hline $\mathrm{H}$ & 2.984692 & 3.606067 & 1.282824 \\
\hline $\mathrm{H}$ & 4.340634 & 3.693566 & 2.417069 \\
\hline $\mathrm{H}$ & 3.787598 & 5.159058 & 1.584635 \\
\hline C & 4.401256 & 4.416932 & -0.994577 \\
\hline $\mathrm{H}$ & 4.260104 & 5.499727 & -0.887838 \\
\hline $\mathrm{H}$ & 5.091938 & 4.244767 & -1.828515 \\
\hline $\mathrm{H}$ & 3.434832 & 3.979069 & -1.263365 \\
\hline C & 6.276401 & 4.536437 & 0.654230 \\
\hline $\mathrm{H}$ & 7.023234 & 4.418262 & -0.139731 \\
\hline
\end{tabular}




\section{Catalyst 2C 12}

Datum

Value

\begin{tabular}{lr}
\hline B3LYP/6-31G(d) Energy & -2164.804737 \\
\hline B3LYP-D3(BJ)/def2-TZVPP-IEF-PCM(DCM) Energy & -2165.900733 \\
\hline B3LYP-D3(BJ)/def2-TZVPP-IEF-PCM(DCM)//B3LYP/6-31G(d) Free Energy (Quasiharmonic) & -2165.022142 \\
\hline
\end{tabular}

Number of Imaginary Frequencies

Frequencies (Top 3 out of 321)

$\begin{array}{ll}\text { 1. } & 10.1678 \mathrm{~cm}^{-1} \\ \text { 2. } & 11.5871 \mathrm{~cm}^{-1} \\ \text { 3. } & 14.2812 \mathrm{~cm}^{-1}\end{array}$

\section{B3LYP/6-31G(d) Molecular Geometry in Cartesian Coordinates}

\begin{tabular}{|c|c|c|c|}
\hline C & -0.152681 & -4.214906 & 3.021200 \\
\hline C & -0.311502 & -3.349881 & 1.961289 \\
\hline C & 0.754450 & -2.508368 & 1.539374 \\
\hline C & 2.000676 & -2.582820 & 2.239889 \\
\hline C & 2.131046 & -3.483878 & 3.330843 \\
\hline C & 1.079368 & -4.283822 & 3.714146 \\
\hline $\mathrm{H}$ & -0.979219 & -4.849258 & 3. 329142 \\
\hline $\mathrm{H}$ & -1.258961 & -3.300893 & 1.436036 \\
\hline C & 0.625288 & -1.595835 & 0.439407 \\
\hline C & 3.071988 & -1.746656 & 1.840199 \\
\hline $\mathrm{H}$ & 3.080716 & -3.528227 & 3.858498 \\
\hline $\mathrm{H}$ & 1.191193 & -4.969628 & 4.549510 \\
\hline C & 2.960237 & -0.867461 & 0.782914 \\
\hline C & 1.714989 & -0.817174 & 0.072752 \\
\hline $\mathrm{H}$ & 3.999348 & -1.780598 & 2.406027 \\
\hline C & -1.172707 & -4.185704 & -3.662888 \\
\hline C & -2.194532 & -3.350408 & -3.275144 \\
\hline C & -2.038266 & -2.471273 & -2.169323 \\
\hline C & -0.799136 & -2.454829 & -1.454915 \\
\hline C & 0.236644 & -3.332495 & -1.882363 \\
\hline C & 0.054056 & -4.174612 & -2.956862 \\
\hline $\mathrm{H}$ & -4.016670 & -1.627471 & -2.304871 \\
\hline $\mathrm{H}$ & -1.303175 & -4.854113 & -4.509706 \\
\hline $\mathrm{H}$ & -3.141575 & -3.349027 & -3.809772 \\
\hline
\end{tabular}




\begin{tabular}{|c|c|c|c|}
\hline C & -3.080773 & -1.611064 & -1.752102 \\
\hline C & -0.660962 & -1.558813 & -0.342968 \\
\hline $\mathrm{H}$ & 1.184600 & -3.331174 & -1.355367 \\
\hline $\mathrm{H}$ & 0.859187 & -4.835370 & -3.266777 \\
\hline C & -1.699388 & -0.710262 & 0.034629 \\
\hline C & -2.943070 & -0.738493 & -0.688828 \\
\hline C & -1.503618 & 0.251977 & 1.196733 \\
\hline $\mathrm{H}$ & -2.449624 & 0.398089 & 1.723698 \\
\hline $\mathrm{H}$ & -0.789487 & -0.173396 & 1.913573 \\
\hline C & 1.599233 & 0.062319 & -1.136632 \\
\hline 0 & 0.889644 & 1.049810 & -1.236332 \\
\hline 0 & 2.373866 & -0.358116 & -2.150868 \\
\hline $\mathrm{H}$ & 2.250420 & 0.282347 & -2.878503 \\
\hline 0 & -1.081751 & 1.549828 & 0.783465 \\
\hline $\mathrm{H}$ & -0.352627 & 1.438353 & 0.141656 \\
\hline C & -4.118729 & 0.107143 & -0.319933 \\
\hline C & -5.368545 & -0.495759 & -0.141885 \\
\hline C & -4.012797 & 1.503112 & -0.196482 \\
\hline C & -6.513743 & 0.256953 & 0.162248 \\
\hline $\mathrm{H}$ & -5.437625 & -1.576745 & -0.225668 \\
\hline C & -5.127469 & 2.287272 & 0.105843 \\
\hline H & -3.037756 & 1.953406 & -0.324963 \\
\hline C & -6.365044 & 1.641815 & 0.281036 \\
\hline $\mathrm{H}$ & -7.232253 & 2.245739 & 0.521908 \\
\hline C & 4.098910 & 0.029124 & 0.438556 \\
\hline C & 3.906024 & 1.406644 & 0.307220 \\
\hline C & 5.390962 & -0.499835 & 0.280657 \\
\hline C & 4.972002 & 2.270457 & 0.009569 \\
\hline $\mathrm{H}$ & 2.910710 & 1.812213 & 0.457702 \\
\hline C & 6.479048 & 0.324215 & -0.010735 \\
\hline $\mathrm{H}$ & 5.519204 & -1.572446 & 0.369533 \\
\hline C & 6.241158 & 1.705461 & -0.142085 \\
\hline $\mathrm{H}$ & 7.079591 & 2.352799 & -0.371156 \\
\hline C & 7.907319 & -0.221807 & -0.201875 \\
\hline C & 4.702183 & 3.780097 & -0.138924 \\
\hline C & 7.981570 & -1.749864 & -0.020935 \\
\hline $\mathrm{H}$ & 7.667488 & -2.057632 & 0.982911 \\
\hline H & 7.359539 & -2.278656 & -0.751908 \\
\hline $\mathrm{H}$ & 9.014729 & -2.087042 & -0.161654 \\
\hline C & 8.852105 & 0.429653 & 0.836003 \\
\hline $\mathrm{H}$ & 9.875781 & 0.057629 & 0.705641 \\
\hline $\mathrm{H}$ & 8.879087 & 1.519716 & 0.737212 \\
\hline $\mathrm{H}$ & 8.533008 & 0.194877 & 1.857988 \\
\hline C & 8.401924 & 0.115895 & -1.628596 \\
\hline $\mathrm{H}$ & 8.420014 & 1.195572 & -1.810026 \\
\hline $\mathrm{H}$ & 9.419669 & -0.264593 & -1.779929 \\
\hline $\mathrm{H}$ & 7.753047 & -0.339467 & -2.385479 \\
\hline C & 5.983800 & 4.582509 & -0.432335 \\
\hline $\mathrm{H}$ & 6.720927 & 4.484899 & 0.373047 \\
\hline H & 6.456354 & 4.269439 & -1.370361 \\
\hline $\mathrm{H}$ & 5.737995 & 5.646108 & -0.527183 \\
\hline C & 4.082427 & 4. 327568 & 1.169011 \\
\hline $\mathrm{H}$ & 4.762291 & 4.181426 & 2.016333 \\
\hline H & 3.883630 & 5.401970 & 1.074029 \\
\hline
\end{tabular}




\begin{tabular}{|c|c|c|c|}
\hline $\mathrm{H}$ & 3.134878 & 3.836380 & 1.411414 \\
\hline C & 3.713311 & 4.005406 & -1.308545 \\
\hline $\mathrm{H}$ & 4.146614 & 3.661014 & -2.255248 \\
\hline $\mathrm{H}$ & 2.769486 & 3.471127 & -1.158209 \\
\hline $\mathrm{H}$ & 3.481418 & 5.072488 & -1.411801 \\
\hline C & -5.034035 & 3.817263 & 0.264906 \\
\hline C & -7.865442 & -0.457661 & 0.356652 \\
\hline C & -8.235634 & -1.219984 & -0.937880 \\
\hline $\mathrm{H}$ & -8.329680 & -0.530208 & -1.784501 \\
\hline $\mathrm{H}$ & -7.480573 & -1.968633 & -1.199374 \\
\hline $\mathrm{H}$ & -9.193366 & -1.740996 & -0.815259 \\
\hline C & -9.010693 & 0.521942 & 0.676200 \\
\hline $\mathrm{H}$ & -8.831101 & 1.070844 & 1.607607 \\
\hline $\mathrm{H}$ & -9.160551 & 1.251911 & -0.127491 \\
\hline $\mathrm{H}$ & -9.947924 & -0.033424 & 0.796469 \\
\hline C & -7.755215 & -1.462666 & 1.527812 \\
\hline $\mathrm{H}$ & -6.986475 & -2.220730 & 1.344430 \\
\hline $\mathrm{H}$ & -7.500378 & -0.948450 & 2.461561 \\
\hline $\mathrm{H}$ & -8.709260 & -1.983921 & 1.675603 \\
\hline C & -5.405209 & 4.200430 & 1.717449 \\
\hline $\mathrm{H}$ & -6.419482 & 3.878112 & 1.977454 \\
\hline $\mathrm{H}$ & -4.713305 & 3.738997 & 2.431062 \\
\hline $\mathrm{H}$ & -5.355297 & 5.288347 & 1.850432 \\
\hline C & -6.015086 & 4.507062 & -0.712256 \\
\hline $\mathrm{H}$ & -5.949446 & 5.597428 & -0.610826 \\
\hline $\mathrm{H}$ & -5.779031 & 4.248489 & -1.750963 \\
\hline $\mathrm{H}$ & -7.055045 & 4.219341 & -0.524254 \\
\hline C & -3.616570 & 4.348086 & -0.025897 \\
\hline $\mathrm{H}$ & -3.313253 & 4.142481 & -1.059036 \\
\hline $\mathrm{H}$ & -3.595301 & 5.435191 & 0.115091 \\
\hline $\mathrm{H}$ & -2.865819 & 3.906781 & 0.637712 \\
\hline
\end{tabular}

\section{Catalyst 2C 13}

Datum

Value

\begin{tabular}{lr}
\hline B3LYP/6-31G(d) Energy & -2164.804734 \\
\hline B3LYP-D3(BJ)/def2-TZVPP-IEF-PCM(DCM) Energy & -2165.900633 \\
\hline B3LYP-D3(BJ)/def2-TZVPP-IEF-PCM(DCM)//B3LYP/6-31G(d) Free Energy (Quasiharmonic) & -2165.022039 \\
\hline Number of Imaginary Frequencies & 0
\end{tabular}

Frequencies (Top 3 out of 321)

$\begin{array}{ll}\text { 1. } & 10.1756 \mathrm{~cm}^{-1} \\ \text { 2. } & 12.5061 \mathrm{~cm}^{-1} \\ \text { 3. } & 13.7551 \mathrm{~cm}^{-1}\end{array}$




\begin{tabular}{|c|c|c|c|}
\hline C & -0.190339 & -4.186245 & 3.067827 \\
\hline C & -0.346062 & -3.328728 & 2.001373 \\
\hline C & 0.724105 & -2.497053 & 1.570663 \\
\hline C & 1.971334 & -2.573573 & 2.269219 \\
\hline C & 2.098490 & -3.466826 & 3.366922 \\
\hline C & 1.042725 & -4.257245 & 3.758725 \\
\hline $\mathrm{H}$ & -1.020141 & -4.812944 & 3.382571 \\
\hline $\mathrm{H}$ & -1.294394 & -3.277977 & 1.477834 \\
\hline C & 0.597977 & -1.592154 & 0.464074 \\
\hline C & 3.046937 & -1.747131 & 1.860684 \\
\hline $\mathrm{H}$ & 3.048961 & -3.512798 & 3.892993 \\
\hline $\mathrm{H}$ & 1.152093 & -4.937033 & 4.599314 \\
\hline C & 2.937843 & -0.875057 & 0.797319 \\
\hline C & 1.691140 & -0.821753 & 0.090058 \\
\hline $\mathrm{H}$ & 3.975751 & -1.782685 & 2.424027 \\
\hline C & -1.207806 & -4.200418 & -3.622266 \\
\hline C & -2.225704 & -3.357017 & -3.241924 \\
\hline C & -2.066882 & -2.472123 & -2.141000 \\
\hline C & -0.829186 & -2.458146 & -1.424202 \\
\hline C & 0.202545 & -3.344285 & -1.843906 \\
\hline C & 0.017453 & -4.192024 & -2.913530 \\
\hline $\mathrm{H}$ & -4.039708 & -1.618813 & -2.286543 \\
\hline $\mathrm{H}$ & -1.340236 & -4.873310 & -4.465217 \\
\hline $\mathrm{H}$ & -3.171600 & -3.353642 & -3.778564 \\
\hline C & -3.105487 & -1.604110 & -1.730974 \\
\hline C & -0.688438 & -1.555433 & -0.318139 \\
\hline $\mathrm{H}$ & 1.149249 & -3.345236 & -1.314686 \\
\hline $\mathrm{H}$ & 0.819401 & -4.859390 & -3.217472 \\
\hline C & -1.723185 & -0.699408 & 0.052813 \\
\hline C & -2.966588 & -0.726213 & -0.671921 \\
\hline C & -1.520870 & 0.269257 & 1.208290 \\
\hline $\mathrm{H}$ & -2.464401 & 0.423456 & 1.737065 \\
\hline $\mathrm{H}$ & -0.806135 & -0.153469 & 1.925777 \\
\hline C & 1.577489 & 0.049669 & -1.125306 \\
\hline 0 & 0.868914 & 1.036707 & -1.233685 \\
\hline 0 & 2.352312 & -0.379745 & -2.136101 \\
\hline $\mathrm{H}$ & 2.227247 & 0.253123 & -2.869985 \\
\hline 0 & -1.094180 & 1.562904 & 0.785067 \\
\hline $\mathrm{H}$ & -0.365455 & 1.444829 & 0.143628 \\
\hline C & -4.140201 & 0.125840 & -0.311740 \\
\hline C & -4.028869 & 1.513960 & -0.162339 \\
\hline C & -5.402456 & -0.468736 & -0.165778 \\
\hline C & -5.144013 & 2.307251 & 0.134651 \\
\hline $\mathrm{H}$ & -3.048995 & 1.965134 & -0.265885 \\
\hline C & -6.543440 & 0.288544 & 0.125087 \\
\hline $\mathrm{H}$ & -5.478900 & -1.547899 & -0.265073 \\
\hline C & -6.388193 & 1.675162 & 0.270363 \\
\hline $\mathrm{H}$ & -7.257103 & 2.276712 & 0.500586 \\
\hline C & 4.081402 & 0.010833 & 0.441633 \\
\hline C & 3.894801 & 1.394538 & 0.316294 \\
\hline
\end{tabular}




\begin{tabular}{|c|c|c|c|}
\hline C & 5.362679 & -0.528892 & 0.266530 \\
\hline C & 4.962380 & 2.245160 & 0.007996 \\
\hline $\mathrm{H}$ & 2.904849 & 1.801673 & 0.482519 \\
\hline C & 6.457597 & 0.287204 & -0.038945 \\
\hline $\mathrm{H}$ & 5.482891 & -1.602743 & 0.351871 \\
\hline C & 6.227338 & 1.665364 & -0.163074 \\
\hline H & 7.066063 & 2.312784 & -0.401634 \\
\hline C & 7.877782 & -0.272877 & -0.250694 \\
\hline C & 4.786235 & 3.768547 & -0.145074 \\
\hline C & 7.939444 & -1.801722 & -0.071134 \\
\hline H & 7.636345 & -2.106835 & 0.936881 \\
\hline $\mathrm{H}$ & 7.302269 & -2.324162 & -0.793565 \\
\hline $\mathrm{H}$ & 8.967218 & -2.148866 & -0.226292 \\
\hline C & 8.843842 & 0.368836 & 0.773401 \\
\hline $\mathrm{H}$ & 9.861979 & -0.012447 & 0.627538 \\
\hline $\mathrm{H}$ & 8.879407 & 1.458685 & 0.674869 \\
\hline $\mathrm{H}$ & 8.537871 & 0.136481 & 1.799923 \\
\hline C & 8.354751 & 0.060489 & -1.684335 \\
\hline $\mathrm{H}$ & 8.380587 & 1.140068 & -1.865598 \\
\hline $\mathrm{H}$ & 9.366410 & -0.329804 & -1.850620 \\
\hline H & 7.690451 & -0.388024 & -2.431844 \\
\hline C & 3.330220 & 4.215520 & 0.089202 \\
\hline $\mathrm{H}$ & 2.636429 & 3.744091 & -0.615822 \\
\hline $\mathrm{H}$ & 2.990465 & 3.984732 & 1.105192 \\
\hline $\mathrm{H}$ & 3.252119 & 5.300344 & -0.044632 \\
\hline C & 5.193341 & 4.188704 & -1.577613 \\
\hline H & 4.562515 & 3.693593 & -2.324778 \\
\hline $\mathrm{H}$ & 5.079991 & 5.272348 & -1.703144 \\
\hline H & 6.235526 & 3.934761 & -1.798648 \\
\hline C & 5.686088 & 4.500862 & 0.878183 \\
\hline $\mathrm{H}$ & 5.419302 & 4.222765 & 1.904103 \\
\hline $\mathrm{H}$ & 6.745945 & 4.266221 & 0.733170 \\
\hline $\mathrm{H}$ & 5.568914 & 5.586989 & 0.779639 \\
\hline C & -7.905935 & -0.415719 & 0.282379 \\
\hline C & -4.956833 & 3.827843 & 0.301686 \\
\hline C & -3.954923 & 4.094254 & 1.451586 \\
\hline $\mathrm{H}$ & -4.345324 & 3.711850 & 2.402326 \\
\hline $\mathrm{H}$ & -2.987083 & 3.616003 & 1.271460 \\
\hline H & -3.785833 & 5.172557 & 1.565815 \\
\hline C & -6.274635 & 4.553885 & 0.632319 \\
\hline $\mathrm{H}$ & -7.020096 & 4.435037 & -0.162829 \\
\hline $\mathrm{H}$ & -6.714212 & 4.194862 & 1.570098 \\
\hline H & -6.084735 & 5.627181 & 0.747758 \\
\hline C & -4.396272 & 4.423343 & -1.011958 \\
\hline $\mathrm{H}$ & -3.430502 & 3.981853 & -1.277039 \\
\hline $\mathrm{H}$ & -5.085597 & 4.250205 & -1.846821 \\
\hline $\mathrm{H}$ & -4.252304 & 5.506107 & -0.908687 \\
\hline C & -9.049452 & 0.570157 & 0.588919 \\
\hline $\mathrm{H}$ & -8.885363 & 1.106832 & 1.530193 \\
\hline $\mathrm{H}$ & -9.176095 & 1.310658 & -0.209079 \\
\hline H & -9.993569 & 0.021351 & 0.682774 \\
\hline C & -7.829754 & -1.434769 & 1.444119 \\
\hline H & -8.791386 & -1.948800 & 1.566187 \\
\hline $\mathrm{H}$ & -7.064074 & -2.197662 & 1.268209 \\
\hline
\end{tabular}




\section{Catalyst 2C 14}

Datum

Value

\begin{tabular}{lr}
\hline B3LYP/6-31G(d) Energy & -2164.804403 \\
\hline B3LYP-D3(BJ)/def2-TZVPP-IEF-PCM(DCM) Energy & -2165.900532 \\
\hline B3LYP-D3(BJ)/def2-TZVPP-IEF-PCM(DCM)//B3LYP/6-31G(d) Free Energy (Quasiharmonic) & -2165.022 \\
\hline Number of Imaginary Frequencies & 0
\end{tabular}

Frequencies (Top 3 out of 321)
1. $10.3873 \mathrm{~cm}^{-1}$
2. $12.3128 \mathrm{~cm}^{-1}$
3. $14.2526 \mathrm{~cm}^{-1}$

\section{B3LYP/6-31G(d) Molecular Geometry in Cartesian Coordinates}

\begin{tabular}{|c|c|c|c|}
\hline $\mathrm{C}$ & 0.177112 & -4.207533 & 3.035155 \\
\hline C & 0.332605 & -3.344040 & 1.973490 \\
\hline C & -0.736398 & -2.507155 & 1.550095 \\
\hline C & -1.982160 & -2.584610 & 2.251105 \\
\hline C & -2.109121 & -3.484010 & 3.343790 \\
\hline C & -1.054538 & -4.279507 & 3.728446 \\
\hline $\mathrm{H}$ & 1.006000 & -4.838267 & 3.344194 \\
\hline $\mathrm{H}$ & 1.279816 & -3.292666 & 1.448011 \\
\hline C & -0.610623 & -1.596506 & 0.448104 \\
\hline C & -3.056470 & -1.752824 & 1.850110 \\
\hline $\mathrm{H}$ & -3.058523 & -3.530677 & 3.871735 \\
\hline $\mathrm{H}$ & -1.163780 & -4.964078 & 4.565159 \\
\hline C & -2.947696 & -0.875203 & 0.791318 \\
\hline C & -1.702902 & -0.821743 & 0.080657 \\
\hline $\mathrm{H}$ & -3.983781 & -1.788697 & 2.415909 \\
\hline C & 1.187970 & -4.194770 & -3.648334 \\
\hline C & 2.208446 & -3.356043 & -3.264432 \\
\hline C & 2.051645 & -2.473840 & -2.161139 \\
\hline C & 0.813411 & -2.457960 & -1.445174 \\
\hline C & -0.220961 & -3.339212 & -1.868609 \\
\hline C & -0.037907 & -4.184206 & -2.940784 \\
\hline
\end{tabular}




\begin{tabular}{|c|c|c|c|}
\hline $\mathrm{H}$ & 4.027933 & -1.625999 & -2.301927 \\
\hline $\mathrm{H}$ & 1.318844 & -4.865478 & -4.493266 \\
\hline $\mathrm{H}$ & 3.154803 & -3.354213 & -3.800276 \\
\hline C & 3.092719 & -1.609954 & -1.747992 \\
\hline C & 0.674615 & -1.558717 & -0.335918 \\
\hline H & -1.168230 & -3.338370 & -1.340374 \\
\hline $\mathrm{H}$ & -0.841982 & -4.847670 & -3.247637 \\
\hline C & 1.711457 & -0.706492 & 0.037489 \\
\hline C & 2.954327 & -0.734222 & -0.687403 \\
\hline C & 1.514377 & 0.259409 & 1.196306 \\
\hline $\mathrm{H}$ & 0.801578 & -0.165020 & 1.915047 \\
\hline $\mathrm{H}$ & 2.460371 & 0.409398 & 1.722222 \\
\hline C & -1.591131 & 0.054168 & -1.131656 \\
\hline 0 & -0.879681 & 1.039046 & -1.239434 \\
\hline 0 & -2.371738 & -0.368965 & -2.140898 \\
\hline $\mathrm{H}$ & -2.247635 & 0.266424 & -2.872748 \\
\hline 0 & 1.089478 & 1.554740 & 0.778564 \\
\hline $\mathrm{H}$ & 0.359784 & 1.439471 & 0.138002 \\
\hline C & 4.128524 & 0.115333 & -0.322894 \\
\hline C & 5.380158 & -0.483998 & -0.145440 \\
\hline C & 4.019408 & 1.511375 & -0.203184 \\
\hline C & 6.524056 & 0.272354 & 0.154516 \\
\hline $\mathrm{H}$ & 5.451667 & -1.565042 & -0.226385 \\
\hline C & 5.132725 & 2.299097 & 0.094844 \\
\hline $\mathrm{H}$ & 3.043018 & 1.958907 & -0.331136 \\
\hline C & 6.372183 & 1.657190 & 0.269708 \\
\hline $\mathrm{H}$ & 7.238326 & 2.263950 & 0.507342 \\
\hline C & -4.089170 & 0.017015 & 0.444907 \\
\hline C & -3.897602 & 1.400765 & 0.327659 \\
\hline C & -5.373244 & -0.516577 & 0.271611 \\
\hline C & -4.963203 & 2.257581 & 0.029842 \\
\hline $\mathrm{H}$ & -2.905321 & 1.802898 & 0.492209 \\
\hline C & -6.466213 & 0.305788 & -0.023863 \\
\hline $\mathrm{H}$ & -5.497282 & -1.590486 & 0.350470 \\
\hline C & -6.231149 & 1.683867 & -0.139550 \\
\hline $\mathrm{H}$ & -7.068469 & 2.336131 & -0.369778 \\
\hline C & -7.889419 & -0.247372 & -0.233647 \\
\hline C & -4.781996 & 3.781392 & -0.112872 \\
\hline C & -8.848994 & 0.391233 & 0.798453 \\
\hline $\mathrm{H}$ & -8.540127 & 0.150560 & 1.822183 \\
\hline $\mathrm{H}$ & -8.880501 & 1.481874 & 0.707562 \\
\hline $\mathrm{H}$ & -9.869223 & 0.015096 & 0.653834 \\
\hline C & -7.956333 & -1.777105 & -0.063968 \\
\hline $\mathrm{H}$ & -8.986071 & -2.119285 & -0.217146 \\
\hline $\mathrm{H}$ & -7.324172 & -2.297224 & -0.792447 \\
\hline $\mathrm{H}$ & -7.650308 & -2.090055 & 0.940754 \\
\hline C & -8.370618 & 0.097452 & -1.663138 \\
\hline $\mathrm{H}$ & -7.710988 & -0.348601 & -2.416224 \\
\hline $\mathrm{H}$ & -9.384461 & -0.287676 & -1.828134 \\
\hline $\mathrm{H}$ & -8.392740 & 1.178330 & -1.836975 \\
\hline C & -5.672756 & 4.509088 & 0.921579 \\
\hline $\mathrm{H}$ & -6.734309 & 4.279110 & 0.781615 \\
\hline $\mathrm{H}$ & -5.400380 & 4.222254 & 1.943617 \\
\hline $\mathrm{H}$ & -5.552388 & 5.595522 & 0.830566 \\
\hline
\end{tabular}




\begin{tabular}{|c|c|c|c|}
\hline C & -5.196182 & 4.214166 & -1.539603 \\
\hline $\mathrm{H}$ & -6.240667 & 3.966019 & -1.756308 \\
\hline $\mathrm{H}$ & -5.079374 & 5.298300 & -1.657498 \\
\hline $\mathrm{H}$ & -4.571807 & 3.722424 & -2.294366 \\
\hline C & -3.322871 & 4.221028 & 0.115897 \\
\hline $\mathrm{H}$ & -2.977973 & 3.981792 & 1.128184 \\
\hline $\mathrm{H}$ & -2.635066 & 3.751954 & -0.596504 \\
\hline $\mathrm{H}$ & -3.241349 & 5.306468 & -0.010676 \\
\hline C & 5.035661 & 3.829280 & 0.249932 \\
\hline C & 7.877922 & -0.438318 & 0.348309 \\
\hline C & 7.772270 & -1.441128 & 1.521759 \\
\hline $\mathrm{H}$ & 7.517340 & -0.925652 & 2.454781 \\
\hline $\mathrm{H}$ & 7.005460 & -2.201792 & 1.341158 \\
\hline $\mathrm{H}$ & 8.728003 & -1.959362 & 1.669267 \\
\hline C & 9.021095 & 0.544934 & 0.663971 \\
\hline $\mathrm{H}$ & 9.168064 & 1.273290 & -0.141704 \\
\hline $\mathrm{H}$ & 8.841221 & 1.095671 & 1.594234 \\
\hline $\mathrm{H}$ & 9.959895 & -0.007731 & 0.784401 \\
\hline C & 8.248013 & -1.202449 & -0.945185 \\
\hline $\mathrm{H}$ & 7.494286 & -1.953364 & -1.203997 \\
\hline $\mathrm{H}$ & 8.339186 & -0.514198 & -1.793353 \\
\hline $\mathrm{H}$ & 9.207140 & -1.720975 & -0.822924 \\
\hline C & 5.407543 & 4.217149 & 1.701040 \\
\hline $\mathrm{H}$ & 4.717552 & 3.755878 & 2.416593 \\
\hline $\mathrm{H}$ & 6.422893 & 3.897977 & 1.960741 \\
\hline $\mathrm{H}$ & 5.355111 & 5.305278 & 1.831278 \\
\hline C & 3.616542 & 4.355784 & -0.040625 \\
\hline $\mathrm{H}$ & 3.592657 & 5.443193 & 0.097557 \\
\hline $\mathrm{H}$ & 3.312587 & 4.146745 & -1.072888 \\
\hline $\mathrm{H}$ & 2.867596 & 3.914329 & 0.624902 \\
\hline C & 6.013838 & 4.519008 & -0.730137 \\
\hline $\mathrm{H}$ & 5.777196 & 4.257191 & -1.767894 \\
\hline $\mathrm{H}$ & 5.945599 & 5.609463 & -0.631439 \\
\hline $\mathrm{H}$ & 7.054723 & 4.234349 & -0.542652 \\
\hline
\end{tabular}

\section{Catalyst 2C 15}

Datum

Value

\begin{tabular}{lc}
\hline B3LYP/6-31G(d) Energy & -2164.804475 \\
\hline B3LYP-D3(BJ)/def2-TZVPP-IEF-PCM(DCM) Energy & -2165.900422 \\
\hline B3LYP-D3(BJ)/def2-TZVPP-IEF-PCM(DCM)//B3LYP/6-31G(d) Free Energy (Quasiharmonic) & -2165.021716 \\
\hline Number of Imaginary Frequencies & 0
\end{tabular}

Frequencies (Top 3 out of 321) 


$\begin{array}{lr}\text { 1. } & 9.5977 \mathrm{~cm}^{-1} \\ \text { 2. } & 13.2633 \mathrm{~cm}^{-1} \\ \text { 3. } & 14.8247 \mathrm{~cm}^{-1}\end{array}$

\section{B3LYP/6-31G(d) Molecular Geometry in Cartesian Coordinates}

C

C

C

C

C

C

$\mathrm{H}$

$\mathrm{H}$

C

C

$\mathrm{H}$

$\mathrm{H}$

C

C

$\mathrm{H}$

C

C

C

C

C

C

$\mathrm{H}$

$\mathrm{H}$

$\mathrm{H}$

C

C

$\mathrm{H}$

$\mathrm{H}$

C

C

C

$\mathrm{H}$

$\mathrm{H}$

C

0

0

$\mathrm{H}$

0

$\mathrm{H}$

C

C

C

C

$\mathrm{H}$
0.115838

0.279374

$-0.780217$

$-2.024877$

$-2.160120$

$-1.114630$

0.937453

1.225613

$-0.646030$

$-3.089806$

$-3.108518$

$-1.230169$

$-2.973818$

$-1.730197$

$-4.015338$

1.133569

2.162675

2. 012520

0.771991

$-0.271294$

$-0.094649$

3.999610

1.259378

3.110882

3.062228

0.639985

$-1.220421$

$-0.905574$

1.685003

2.929700

1.496096

0.776987

2.442611

$-1.610350$

$-0.890843$

$-2.394320$

$-2.267847$

1.086857

0.356539

4.111031

4.016525

5.356341

5.138685

3.046672
$-4.220159$

$-3.355426$

$-2.503993$

$-2.567877$

$-3.468607$

$-4.278554$

$-4.862446$

$-3.314575$

$-1.591534$

$-1.721739$

$-3.504742$

$-4.964201$

$-0.843975$

$-0.803848$

$-1.746757$

$-4.196302$

$-3.371039$

$-2.489965$

$-2.461401$

$-3.329134$

$-4.173058$

$-1.665911$

$-4.866052$

$-3.379097$

$-1.639387$

$-1.563849$

$-3.318379$

$-4.825813$

$-0.723667$

$-0.763490$

0.241703

$-0.176095$

0.379940

0.074699

1.054618

$-0.337257$

0.302094

1.542817

1.436736

0.073621

1.467511

$-0.545436$

2. 246096

1.926517
3.003538

1.944093

1.526104

2. 230359

3. 320945

3.700202

3.308193

1.416007

0.426535

1.834536

3.851505

4.535189

0.776631

0.062801

2.403803

$-3.674803$

$-3.284847$

$-2.179699$

$-1.468013$

$-1.897525$

$-2.971598$

$-2.310653$

$-4.521271$

$-3.817351$

$-1.760748$

$-0.356611$

$-1.372717$

$-3.283363$

0.021577

$-0.699561$

1.182192

1.898519

1.710297

$-1.146858$

$-1.249793$

$-2.157406$

$-2.885492$

0.766394

0.125048

$-0.329545$

$-0.215357$

$-0.139012$

0.090157

$-0.351889$ 


\begin{tabular}{|c|c|c|c|}
\hline C & 6.501398 & 0.197617 & 0.169070 \\
\hline $\mathrm{H}$ & 5.408749 & -1.625554 & -0.216712 \\
\hline C & 6.362745 & 1.588860 & 0.276857 \\
\hline $\mathrm{H}$ & 7.239602 & 2.181669 & 0.520810 \\
\hline C & -4.106192 & 0.062047 & 0.436019 \\
\hline C & -5.400810 & -0.449398 & 0.284444 \\
\hline C & -3.900987 & 1.441150 & 0.302296 \\
\hline C & -6.486825 & 0.385835 & -0.004354 \\
\hline $\mathrm{H}$ & -5.545524 & -1.522052 & 0.374396 \\
\hline C & -4.957613 & 2.311852 & 0.009159 \\
\hline $\mathrm{H}$ & -2.901001 & 1.836641 & 0.448647 \\
\hline C & -6.238414 & 1.760272 & -0.138795 \\
\hline $\mathrm{H}$ & -7.065345 & 2.419240 & -0.365318 \\
\hline C & -4.675865 & 3.819703 & -0.140170 \\
\hline C & -7.892699 & -0.223533 & -0.173590 \\
\hline C & -4.044229 & 4.361434 & 1.164522 \\
\hline $\mathrm{H}$ & -4.720250 & 4.220051 & 2.015730 \\
\hline $\mathrm{H}$ & -3.099199 & 3.862642 & 1.401199 \\
\hline $\mathrm{H}$ & -3.837559 & 5.434341 & 1.069172 \\
\hline C & -5.951927 & 4.633895 & -0.425654 \\
\hline $\mathrm{H}$ & -5.696988 & 5.695442 & -0.519630 \\
\hline $\mathrm{H}$ & -6.432294 & 4.327192 & -1.361784 \\
\hline H & -6.685868 & 4.541299 & 0.383175 \\
\hline C & -3.691555 & 4.037740 & -1.314993 \\
\hline $\mathrm{H}$ & -4.132537 & 3.697090 & -2.259516 \\
\hline $\mathrm{H}$ & -3.451756 & 5.103007 & -1.419242 \\
\hline $\mathrm{H}$ & -2.751307 & 3.495814 & -1.169611 \\
\hline C & -7.875662 & -1.240678 & -1.339619 \\
\hline $\mathrm{H}$ & -7.596648 & -0.752747 & -2.280525 \\
\hline $\mathrm{H}$ & -7.164148 & -2.053744 & -1.162589 \\
\hline $\mathrm{H}$ & -8.868579 & -1.688471 & -1.470075 \\
\hline C & -8.964555 & 0.838613 & -0.483907 \\
\hline $\mathrm{H}$ & -8.755550 & 1.369602 & -1.419530 \\
\hline $\mathrm{H}$ & -9.941508 & 0.353972 & -0.591397 \\
\hline $\mathrm{H}$ & -9.050721 & 1.579724 & 0.318822 \\
\hline C & -8.300444 & -0.947857 & 1.131517 \\
\hline $\mathrm{H}$ & -7.599522 & -1.748628 & 1.389564 \\
\hline $\mathrm{H}$ & -8.333506 & -0.247880 & 1.974319 \\
\hline $\mathrm{H}$ & -9.295141 & -1.397534 & 1.023732 \\
\hline C & 7.879025 & -0.454211 & 0.399550 \\
\hline C & 5.056811 & 3.777865 & 0.240028 \\
\hline C & 3.645857 & 4. 318618 & -0.064165 \\
\hline $\mathrm{H}$ & 3.348152 & 4.109423 & -1.098224 \\
\hline $\mathrm{H}$ & 2.886882 & 3.887551 & 0.596828 \\
\hline $\mathrm{H}$ & 3.632670 & 5.406677 & 0.070187 \\
\hline C & 6.050847 & 4.453705 & -0.733564 \\
\hline $\mathrm{H}$ & 7.086834 & 4.157710 & -0.536439 \\
\hline $\mathrm{H}$ & 5.820195 & 4.191555 & -1.772570 \\
\hline $\mathrm{H}$ & 5.994301 & 5.545129 & -0.638461 \\
\hline C & 5.420289 & 4.165770 & 1.693189 \\
\hline $\mathrm{H}$ & 4.720062 & 3.713054 & 2.404238 \\
\hline $\mathrm{H}$ & 6.430268 & 3.837235 & 1.962128 \\
\hline $\mathrm{H}$ & 5.377501 & 5.254678 & 1.820379 \\
\hline C & 8.894532 & 0.108470 & -0.623221 \\
\hline
\end{tabular}




$\begin{array}{rrrr}\text { H } & 8.578796 & -0.111589 & -1.649522 \\ \text { H } & 9.004255 & 1.194249 & -0.534438 \\ \text { H } & 9.883360 & -0.340565 & -0.467403 \\ \text { C } & 7.839894 & -1.985966 & 0.238043 \\ \text { H } & 8.839836 & -2.400539 & 0.409924 \\ \text { H } & 7.159924 & -2.454998 & 0.958245 \\ \text { H } & 7.527621 & -2.282376 & -0.769806 \\ \text { C } & 8.367709 & -0.137514 & 1.832992 \\ \text { H } & 7.670518 & -0.533760 & 2.580152 \\ \text { H } & 9.351263 & -0.590071 & 2.011282 \\ \text { H } & 8.461296 & 0.940010 & 2.003602\end{array}$

\section{Catalyst 2C 16}

Datum

Value

\begin{tabular}{lc}
\hline B3LYP/6-31G(d) Energy & -2164.799246 \\
\hline B3LYP-D3(BJ)/def2-TZVPP-IEF-PCM(DCM) Energy & -2165.899566 \\
\hline B3LYP-D3(BJ)/def2-TZVPP-IEF-PCM(DCM)//B3LYP/6-31G(d) Free Energy (Quasiharmonic) & -2165.021151 \\
\hline
\end{tabular}

Number of Imaginary Frequencies

Frequencies (Top 3 out of 321)

$\begin{array}{ll}\text { 1. } & 11.1322 \mathrm{~cm}^{-1} \\ \text { 2. } & 13.2365 \mathrm{~cm}^{-1} \\ \text { 3. } & 14.6553 \mathrm{~cm}^{-1}\end{array}$

\section{B3LYP/6-31G(d) Molecular Geometry in Cartesian Coordinates}

$\begin{array}{rrrr}\text { C } & -1.500271 & 0.301010 & 1.221340 \\ \text { H } & -2.453341 & 0.692340 & 1.587810 \\ \text { H } & -0.929710 & -0.086800 & 2.070560 \\ \text { C } & 1.537020 & -0.496470 & -1.248650 \\ \text { O } & 2.050500 & -1.103440 & -2.164550 \\ \text { O } & 0.861269 & 0.637290 & -1.459250 \\ \text { H } & 0.398309 & 0.940950 & -0.639910 \\ \text { O } & -0.755041 & 1.385140 & 0.605230 \\ \text { H } & -0.475181 & 1.996160 & 1.305320 \\ \text { C } & 0.555710 & -1.664970 & 0.738410 \\ \mathrm{C} & 1.636790 & -0.991270 & 0.184300 \\ \mathrm{C} & 2.862010 & -0.823070 & 0.915320 \\ \mathrm{C} & 2.946200 & -1.366490 & 2.183040 \\ \mathrm{H} & 3.868030 & -1.247240 & 2.746650 \\ \mathrm{C} & -1.737180 & -0.794900 & 0.207800\end{array}$




\begin{tabular}{|c|c|c|c|}
\hline C & -0.747460 & -1.753290 & -0.007800 \\
\hline C & -3.202360 & -1.934320 & -1.354280 \\
\hline C & -2.984170 & -0.870590 & -0.498760 \\
\hline $\mathrm{H}$ & -4.143270 & -1.994390 & -1.895250 \\
\hline C & -2.226230 & -2.931490 & -1.584790 \\
\hline C & -0.965030 & -2.838410 & -0.914890 \\
\hline C & 0.016330 & -3.835820 & -1.172590 \\
\hline C & -0.244250 & -4.873300 & -2.038340 \\
\hline C & -1.495590 & -4.966890 & -2.695020 \\
\hline C & -2.462600 & -4.014380 & -2.474760 \\
\hline $\mathrm{H}$ & 0.984050 & -3.762010 & -0.689120 \\
\hline $\mathrm{H}$ & 0.518920 & -5.623080 & -2.227250 \\
\hline $\mathrm{H}$ & -1.685290 & -5.790280 & -3.378300 \\
\hline $\mathrm{H}$ & -3.423350 & -4.072500 & -2.980690 \\
\hline C & 1.886710 & -2.102650 & 2.766990 \\
\hline C & 0.666060 & -2.261330 & 2.038180 \\
\hline C & -0.389860 & -3.002970 & 2.637490 \\
\hline C & -0.242730 & -3.557010 & 3.890330 \\
\hline C & 0.965560 & -3.396870 & 4.609920 \\
\hline C & 2.005790 & -2.685220 & 4.057780 \\
\hline $\mathrm{H}$ & -1.317090 & -3.135990 & 2.089890 \\
\hline $\mathrm{H}$ & -1.058660 & -4.125090 & 4.328850 \\
\hline $\mathrm{H}$ & 1.068990 & -3.839530 & 5.596880 \\
\hline $\mathrm{H}$ & 2.939240 & -2.559090 & 4.601210 \\
\hline C & -6.102231 & 2.030209 & -0.165450 \\
\hline C & -6.409511 & 0.681389 & 0.063430 \\
\hline C & -5.366220 & -0.246870 & -0.034400 \\
\hline C & -4.059471 & 0.153810 & -0.348650 \\
\hline c & -3.795621 & 1.513040 & -0.572060 \\
\hline C & -4.810321 & 2.472820 & -0.483510 \\
\hline $\mathrm{H}$ & -6.899901 & 2.763709 & -0.094240 \\
\hline $\mathrm{H}$ & -5.549060 & -1.300261 & 0.145140 \\
\hline $\mathrm{H}$ & -2.784221 & 1.804150 & -0.825590 \\
\hline C & -7.855601 & 0.274579 & 0.407940 \\
\hline C & -4.550041 & 3.972460 & -0.726160 \\
\hline C & -8.303561 & 0.995559 & 1.701570 \\
\hline H & -9.334871 & 0.720209 & 1.954510 \\
\hline $\mathrm{H}$ & -7.661621 & 0.719799 & 2.546150 \\
\hline $\mathrm{H}$ & -8.266921 & 2.084899 & 1.596450 \\
\hline C & -8.793561 & 0.678949 & -0.754420 \\
\hline $\mathrm{H}$ & -9.829421 & 0.401379 & -0.523620 \\
\hline $\mathrm{H}$ & -8.771711 & 1.757769 & -0.940830 \\
\hline $\mathrm{H}$ & -8.505071 & 0.174219 & -1.683500 \\
\hline C & -8.001870 & -1.242491 & 0.633240 \\
\hline H & -9.043980 & -1.480421 & 0.874850 \\
\hline $\mathrm{H}$ & -7.731330 & -1.816351 & -0.260360 \\
\hline $\mathrm{H}$ & -7.381790 & -1.593561 & 1.465970 \\
\hline C & -5.420791 & 4.464700 & -1.906370 \\
\hline $\mathrm{H}$ & -5.246551 & 5.531970 & -2.090510 \\
\hline $\mathrm{H}$ & -5.179081 & 3.917720 & -2.824670 \\
\hline $\mathrm{H}$ & -6.489731 & 4.330959 & -1.709340 \\
\hline C & -4.916461 & 4.770670 & 0.547830 \\
\hline H & -4.744171 & 5.842340 & 0.389500 \\
\hline $\mathrm{H}$ & -5.967921 & 4.639879 & 0.824540 \\
\hline
\end{tabular}




\begin{tabular}{|c|c|c|c|}
\hline $\mathrm{H}$ & -4.305371 & 4.451020 & 1.400040 \\
\hline C & -3.075481 & 4.262150 & -1.066440 \\
\hline $\mathrm{H}$ & -2.933941 & 5.339760 & -1.207230 \\
\hline $\mathrm{H}$ & -2.399201 & 3.937990 & -0.267580 \\
\hline $\mathrm{H}$ & -2.765211 & 3.764130 & -1.991670 \\
\hline C & 4.019410 & -0.031600 & 0.398530 \\
\hline C & 4.523639 & 1.018150 & 1.173560 \\
\hline C & 4.646240 & -0.343310 & -0.819260 \\
\hline C & 5.632259 & 1.772680 & 0.759240 \\
\hline $\mathrm{H}$ & 4.026469 & 1.254430 & 2.110390 \\
\hline C & 5.748059 & 0.386210 & -1.269090 \\
\hline $\mathrm{H}$ & 4.250450 & -1.159930 & -1.405570 \\
\hline C & 6.221809 & 1.436720 & -0.462760 \\
\hline $\mathrm{H}$ & 7.076349 & 2.005990 & -0.809360 \\
\hline C & 6.441459 & 0.079950 & -2.610680 \\
\hline C & 6.144509 & 2.926110 & 1.644120 \\
\hline C & 7.927540 & -0.269210 & -2.360720 \\
\hline $\mathrm{H}$ & 8.467159 & 0.553240 & -1.878710 \\
\hline $\mathrm{H}$ & 8.019380 & -1.152090 & -1.717540 \\
\hline $\mathrm{H}$ & 8.432580 & -0.487400 & -3.309810 \\
\hline C & 5.785340 & -1.102270 & -3.349710 \\
\hline $\mathrm{H}$ & 6.297070 & -1.267820 & -4.304900 \\
\hline $\mathrm{H}$ & 5.856950 & -2.031560 & -2.772790 \\
\hline $\mathrm{H}$ & 4.727280 & -0.916900 & -3.562340 \\
\hline C & 6.354519 & 1.325460 & -3.525210 \\
\hline $\mathrm{H}$ & 5.310349 & 1.589670 & -3.726040 \\
\hline $\mathrm{H}$ & 6.841469 & 2.198010 & -3.076190 \\
\hline $\mathrm{H}$ & 6.846269 & 1.127180 & -4.485540 \\
\hline C & 7.366949 & 3.638750 & 1.035520 \\
\hline $\mathrm{H}$ & 8.214259 & 2.956100 & 0.905010 \\
\hline $\mathrm{H}$ & 7.136229 & 4.087170 & 0.062590 \\
\hline $\mathrm{H}$ & 7.693009 & 4.445640 & 1.701760 \\
\hline C & 5.021269 & 3.974800 & 1.826180 \\
\hline $\mathrm{H}$ & 4.713259 & 4.389370 & 0.859520 \\
\hline $\mathrm{H}$ & 4.134519 & 3.543320 & 2.302550 \\
\hline $\mathrm{H}$ & 5.369159 & 4.802620 & 2.456570 \\
\hline C & 6.554779 & 2.371460 & 3.028990 \\
\hline $\mathrm{H}$ & 6.917539 & 3.181440 & 3.674020 \\
\hline $\mathrm{H}$ & 5.714759 & 1.889750 & 3.540370 \\
\hline $\mathrm{H}$ & 7.356179 & 1.630040 & 2.932220 \\
\hline
\end{tabular}

\section{Catalyst 2C 17}

Datum

Value

\begin{tabular}{lc}
\hline B3LYP/6-31G(d) Energy & -2164.799787 \\
\hline B3LYP-D3(BJ)/def2-TZVPP-IEF-PCM(DCM) Energy & -2165.899853 \\
\hline B3LYP-D3(BJ)/def2-TZVPP-IEF-PCM(DCM)//B3LYP/6-31G(d) Free Energy (Quasiharmonic) & -2165.02113 \\
\hline
\end{tabular}


Frequencies (Top 3 out of 321)

$\begin{array}{ll}\text { 1. } & 11.2531 \mathrm{~cm}^{-1} \\ \text { 2. } & 12.8881 \mathrm{~cm}^{-1} \\ \text { 3. } & 15.0718 \mathrm{~cm}^{-1}\end{array}$

\section{B3LYP/6-31G(d) Molecular Geometry in Cartesian Coordinates}

C

$\mathrm{H}$

$\mathrm{H}$

C

0

0

$\mathrm{H}$

0

$\mathrm{H}$

C

C

C

C

$\mathrm{H}$

C

C

C

C

$\mathrm{H}$

C

$\mathrm{C}$

$\mathrm{C}$

C

C

C

$\mathrm{H}$

$\mathrm{H}$

$\mathrm{H}$

$\mathrm{H}$

C

C

C

C

C

C

$\mathrm{H}$

$\mathrm{H}$
1.523870

0.954140

2.476210

$-1.517960$

$-2.027171$

$-0.851050$

$-0.388330$

0.775900

0.499560

$-0.526051$

$-1.612401$

$-2.836770$

$-2.913561$

$-3.834251$

1.761500

0.774899

3. 225549

3.006490

4.164849

2. 252509

0.993549

0.015539

0.277199

1. 526298

2.490029

$-0.950531$

$-0.483422$

1. 716898

3.449059

$-1.848051$

$-0.629041$

0.432719

0.292739

$-0.913881$

$-1.959651$

1. 358809

1.113119
0.314960

$-0.073250$

0.708179

$-0.498930$

$-1.109490$

0.640340

0.946200

1.399150

2.010460

$-1.659900$

$-0.993380$

$-0.830379$

$-1.369859$

$-1.254129$

$-0.781021$

$-1.743990$

$-1.918221$

$-0.853761$

$-1.976081$

$-2.919521$

$-2.830170$

$-3.832460$

$-4.870840$

$-4.960560$

$-4.003381$

$-3.761840$

$-5.624420$

$-5.784771$

$-4.058571$

$-2.097830$

$-2.252840$

$-2.986890$

$-3.537090$

$-3.380490$

$-2.676290$

$-3.117060$

$-4.099310$
1. 222200

2.071620

1.587920

$-1.236650$

$-2.152420$

$-1.447640$

$-0.629230$

0.607410

1.308750

0.749190

0.196690

0.930370

2.200320

2.766520

0.208740

$-0.001380$

$-1.356480$

$-0.502120$

$-1.900500$

$-1.581570$

$-0.906970$

$-1.158470$

$-2.022790$

$-2.684260$

$-2.470050$

$-0.671260$

$-2.206800$

$-3.366320$

$-2.979560$

2.783470

2.051090

2.649430

3.904770

4.627960

4.076800

2.099220

4.342570 


\begin{tabular}{|c|c|c|c|}
\hline $\mathrm{H}$ & -1.011581 & -3.819940 & 5.616940 \\
\hline $\mathrm{H}$ & -2.891851 & -2.552909 & 4.623000 \\
\hline C & 6.116350 & 2.062588 & -0.164940 \\
\hline C & 6.429500 & 0.706838 & 0.045040 \\
\hline C & 5.393420 & -0.223801 & -0.054280 \\
\hline C & 4.078840 & 0.173969 & -0.355990 \\
\hline C & 3.811940 & 1.530059 & -0.563140 \\
\hline C & 4.823410 & 2.498829 & -0.467310 \\
\hline $\mathrm{H}$ & 6.913490 & 2.792558 & -0.087670 \\
\hline $\mathrm{H}$ & 5.581759 & -1.278011 & 0.114400 \\
\hline $\mathrm{H}$ & 2.797950 & 1.825679 & -0.809510 \\
\hline C & 7.880430 & 0.305328 & 0.373900 \\
\hline C & 4.470841 & 3.980789 & -0.698640 \\
\hline C & 8.038319 & -1.213382 & 0.579140 \\
\hline $\mathrm{H}$ & 9.083999 & -1.447662 & 0.808650 \\
\hline $\mathrm{H}$ & 7.427809 & -1.578662 & 1.412820 \\
\hline $\mathrm{H}$ & 7.763439 & -1.777682 & -0.319210 \\
\hline C & 8.807160 & 0.729948 & -0.790400 \\
\hline $\mathrm{H}$ & 9.846580 & 0.457468 & -0.569800 \\
\hline $\mathrm{H}$ & 8.515930 & 0.233228 & -1.722950 \\
\hline $\mathrm{H}$ & 8.776130 & 1.810548 & -0.964600 \\
\hline C & 8.333820 & 1.013118 & 1.673030 \\
\hline $\mathrm{H}$ & 9.368640 & 0.740758 & 1.914690 \\
\hline $\mathrm{H}$ & 8.290140 & 2.103428 & 1.581810 \\
\hline $\mathrm{H}$ & 7.699970 & 0.722878 & 2.518890 \\
\hline C & 3.904461 & 4.158669 & -2.127510 \\
\hline $\mathrm{H}$ & 3.642761 & 5.208959 & -2.305790 \\
\hline $\mathrm{H}$ & 4.642321 & 3.859739 & -2.880760 \\
\hline $\mathrm{H}$ & 3.003171 & 3.558269 & -2.287180 \\
\hline C & 3.402291 & 4.416629 & 0.333150 \\
\hline $\mathrm{H}$ & 3.113991 & 5.461959 & 0.166900 \\
\hline $\mathrm{H}$ & 2.497921 & 3.803169 & 0.263430 \\
\hline $\mathrm{H}$ & 3.790551 & 4.330789 & 1.355150 \\
\hline C & 5.689991 & 4.909579 & -0.545870 \\
\hline $\mathrm{H}$ & 5.384731 & 5.948809 & -0.712490 \\
\hline $\mathrm{H}$ & 6.125641 & 4.850518 & 0.458230 \\
\hline $\mathrm{H}$ & 6.475101 & 4.676408 & -1.274190 \\
\hline C & -4.001350 & -0.049979 & 0.413140 \\
\hline C & -4.512710 & 0.999901 & 1.191900 \\
\hline C & -4.624960 & -0.369469 & -0.800120 \\
\hline C & -5.624890 & 1.740491 & 0.777050 \\
\hline $\mathrm{H}$ & -4.012870 & 1.236561 & 2.124560 \\
\hline C & -5.736650 & 0.350621 & -1.252090 \\
\hline $\mathrm{H}$ & -4.224061 & -1.184199 & -1.385570 \\
\hline C & -6.213700 & 1.394461 & -0.447600 \\
\hline $\mathrm{H}$ & -7.074960 & 1.961302 & -0.789180 \\
\hline C & -6.428620 & 0.033472 & -2.592200 \\
\hline C & -6.206210 & 2.905271 & 1.602620 \\
\hline C & -7.911330 & -0.327318 & -2.339280 \\
\hline $\mathrm{H}$ & -7.995191 & -1.208258 & -1.692390 \\
\hline $\mathrm{H}$ & -8.457250 & 0.492512 & -1.859880 \\
\hline $\mathrm{H}$ & -8.415600 & -0.553508 & -3.286900 \\
\hline C & -6.353060 & 1.276301 & -3.511170 \\
\hline $\mathrm{H}$ & -6.843420 & 1.070392 & -4.470600 \\
\hline
\end{tabular}




\begin{tabular}{|c|c|c|c|}
\hline $\mathrm{H}$ & -6.847530 & 2.146032 & -3.064850 \\
\hline $\mathrm{H}$ & -5.311400 & 1.549201 & -3.713350 \\
\hline C & -5.763241 & -1.145949 & -3.327460 \\
\hline $\mathrm{H}$ & -4.707091 & -0.952439 & -3.542350 \\
\hline $\mathrm{H}$ & -5.826211 & -2.073619 & -2.746950 \\
\hline $\mathrm{H}$ & -6.274861 & -1.319479 & -4.281270 \\
\hline C & -5.434120 & 3.139081 & 2.915070 \\
\hline $\mathrm{H}$ & -5.459720 & 2.258261 & 3.566780 \\
\hline $\mathrm{H}$ & -4.385159 & 3.397801 & 2.730660 \\
\hline $\mathrm{H}$ & -5.886829 & 3.970981 & 3.466540 \\
\hline C & -6.144329 & 4.209301 & 0.772420 \\
\hline $\mathrm{H}$ & -5.109839 & 4.456391 & 0.507890 \\
\hline $\mathrm{H}$ & -6.714709 & 4.127512 & -0.158580 \\
\hline $\mathrm{H}$ & -6.559529 & 5.047742 & 1.345410 \\
\hline C & -7.679150 & 2.598112 & 1.963010 \\
\hline $\mathrm{H}$ & -8.111029 & 3.423192 & 2.542990 \\
\hline $\mathrm{H}$ & -8.297360 & 2.459812 & 1.069990 \\
\hline $\mathrm{H}$ & -7.753360 & 1.684412 & 2.563930 \\
\hline
\end{tabular}

\section{Catalyst 2C 18}

\section{Datum}

Value

\begin{tabular}{lc}
\hline B3LYP/6-31G(d) Energy & -2164.800257 \\
\hline B3LYP-D3(BJ)/def2-TZVPP-IEF-PCM(DCM) Energy & -2165.900002 \\
\hline B3LYP-D3(BJ)/def2-TZVPP-IEF-PCM(DCM)//B3LYP/6-31G(d) Free Energy (Quasiharmonic) & -2165.020959 \\
\hline
\end{tabular}

Number of Imaginary Frequencies

Frequencies (Top 3 out of 321)

$\begin{array}{ll}\text { 1. } & 11.7340 \mathrm{~cm}^{-1} \\ \text { 2. } & 12.9244 \mathrm{~cm}^{-1} \\ \text { 3. } & 16.3077 \mathrm{~cm}^{-1}\end{array}$

\section{B3LYP/6-31G(d) Molecular Geometry in Cartesian Coordinates}

$\begin{array}{rrrr}C & -1.515270 & 0.326840 & 1.220840 \\ H & -2.467710 & 0.720380 & 1.585900 \\ H & -0.943060 & -0.054760 & 2.071560 \\ C & 1.523980 & -0.494880 & -1.236610 \\ 0 & 2.035320 & -1.110280 & -2.148640 \\ 0 & 0.852170 & 0.639260 & -1.455290 \\ H & 0.389700 & 0.949920 & -0.638340 \\ 0 & -0.770790 & 1.408710 & 0.597660\end{array}$




\begin{tabular}{|c|c|c|c|}
\hline $\mathrm{H}$ & -0.493210 & 2.024520 & 1.294570 \\
\hline C & 0.537860 & -1.646360 & 0.756830 \\
\hline C & 1.621950 & -0.980000 & 0.199590 \\
\hline C & 2.846960 & -0.809870 & 0.930850 \\
\hline C & 2.926360 & -1.343370 & 2.203260 \\
\hline $\mathrm{H}$ & 3.847420 & -1.223050 & 2.767890 \\
\hline C & -1.752340 & -0.775760 & 0.214480 \\
\hline C & -0.763880 & -1.737700 & 0.008400 \\
\hline C & -3.215900 & -1.924800 & -1.342430 \\
\hline C & -2.998220 & -0.855270 & -0.494070 \\
\hline H & -4.155800 & -1.987809 & -1.884840 \\
\hline C & -2.240851 & -2.925070 & -1.563440 \\
\hline C & -0.981311 & -2.829420 & -0.890780 \\
\hline C & -0.001561 & -3.831150 & -1.137820 \\
\hline C & -0.262031 & -4.874710 & -1.996240 \\
\hline C & -1.511611 & -4.970480 & -2.655980 \\
\hline C & -2.477081 & -4.014170 & -2.445830 \\
\hline $\mathrm{H}$ & 0.964799 & -3.756120 & -0.651820 \\
\hline $\mathrm{H}$ & 0.499839 & -5.627840 & -2.176860 \\
\hline $\mathrm{H}$ & -1.701221 & -5.798720 & -3.333400 \\
\hline $\mathrm{H}$ & -3.436551 & -4.074070 & -2.953960 \\
\hline C & 1.863540 & -2.071170 & 2.791290 \\
\hline C & 0.643810 & -2.232480 & 2.061530 \\
\hline C & -0.415481 & -2.965930 & 2.664930 \\
\hline C & -0.272381 & -3.509900 & 3.922610 \\
\hline C & 0.935049 & -3.347280 & 4.643160 \\
\hline C & 1.978419 & -2.643350 & 4.087150 \\
\hline $\mathrm{H}$ & -1.342121 & -3.100690 & 2.116750 \\
\hline $\mathrm{H}$ & -1.090851 & -4.071820 & 4.364340 \\
\hline $\mathrm{H}$ & 1.035239 & -3.781870 & 5.634020 \\
\hline H & 2.911129 & -2.515340 & 4.631400 \\
\hline C & -6.115480 & 2.054201 & -0.165850 \\
\hline C & -6.424520 & 0.698631 & 0.051180 \\
\hline C & -5.386020 & -0.229589 & -0.045500 \\
\hline C & -4.073090 & 0.170350 & -0.351480 \\
\hline C & -3.810340 & 1.526150 & -0.565740 \\
\hline C & -4.824320 & 2.492561 & -0.472710 \\
\hline $\mathrm{H}$ & -6.914480 & 2.782321 & -0.090530 \\
\hline $\mathrm{H}$ & -5.571170 & -1.283489 & 0.128600 \\
\hline $\mathrm{H}$ & -2.797650 & 1.823420 & -0.815410 \\
\hline C & -7.873730 & 0.294641 & 0.384590 \\
\hline C & -4.476390 & 3.974391 & -0.711830 \\
\hline C & -8.326870 & 1.007711 & 1.680920 \\
\hline $\mathrm{H}$ & -9.360560 & 0.733831 & 1.925660 \\
\hline $\mathrm{H}$ & -7.690870 & 0.723381 & 2.527160 \\
\hline $\mathrm{H}$ & -8.286230 & 2.097671 & 1.584190 \\
\hline C & -8.803670 & 0.710781 & -0.780220 \\
\hline $\mathrm{H}$ & -9.841880 & 0.436101 & -0.556650 \\
\hline $\mathrm{H}$ & -8.776360 & 1.790641 & -0.959570 \\
\hline $\mathrm{H}$ & -8.512360 & 0.210581 & -1.710880 \\
\hline C & -8.026880 & -1.223449 & 0.597800 \\
\hline $\mathrm{H}$ & -9.071470 & -1.459569 & 0.830380 \\
\hline $\mathrm{H}$ & -7.751980 & -1.791549 & -0.298150 \\
\hline H & -7.413850 & -1.582729 & 1.432230 \\
\hline
\end{tabular}




\begin{tabular}{|c|c|c|c|}
\hline C & -5.697870 & 4.900431 & -0.561010 \\
\hline $\mathrm{H}$ & -5.396020 & 5.939681 & -0.733620 \\
\hline $\mathrm{H}$ & -6.483930 & 4.661241 & -1.286360 \\
\hline $\mathrm{H}$ & -6.131100 & 4.845221 & 0.444350 \\
\hline C & -3.407020 & 4.418180 & 0.315730 \\
\hline $\mathrm{H}$ & -3.122630 & 5.463750 & 0.144410 \\
\hline $\mathrm{H}$ & -3.792750 & 4.335370 & 1.338940 \\
\hline $\mathrm{H}$ & -2.500740 & 3.807500 & 0.246610 \\
\hline C & -3.913380 & 4.147020 & -2.142670 \\
\hline $\mathrm{H}$ & -3.654840 & 5.197130 & -2.326450 \\
\hline $\mathrm{H}$ & -3.010810 & 3.548280 & -2.301330 \\
\hline $\mathrm{H}$ & -4.651960 & 3.842561 & -2.893000 \\
\hline C & 4.010040 & -0.030030 & 0.409370 \\
\hline C & 4.532900 & 1.015500 & 1.193220 \\
\hline C & 4.624050 & -0.338400 & -0.806180 \\
\hline C & 5.642390 & 1.752050 & 0.776210 \\
\hline $\mathrm{H}$ & 4.039880 & 1.247510 & 2.130640 \\
\hline C & 5.738900 & 0.381960 & -1.263950 \\
\hline $\mathrm{H}$ & 4.218920 & -1.146410 & -1.401390 \\
\hline C & 6.226390 & 1.414300 & -0.458750 \\
\hline $\mathrm{H}$ & 7.085890 & 1.982320 & -0.795180 \\
\hline C & 6.357720 & 0.016300 & -2.626640 \\
\hline C & 6.234870 & 2.908670 & 1.604970 \\
\hline C & 7.599540 & 0.864830 & -2.958480 \\
\hline $\mathrm{H}$ & 7.360930 & 1.932920 & -3.019070 \\
\hline $\mathrm{H}$ & 8.396250 & 0.732670 & -2.216830 \\
\hline $\mathrm{H}$ & 8.001160 & 0.563090 & -3.932430 \\
\hline C & 6.782610 & -1.471450 & -2.621610 \\
\hline $\mathrm{H}$ & 7.222810 & -1.743140 & -3.588900 \\
\hline $\mathrm{H}$ & 7.529230 & -1.664970 & -1.842300 \\
\hline $\mathrm{H}$ & 5.932870 & -2.138480 & -2.446090 \\
\hline C & 5.301290 & 0.244990 & -3.735260 \\
\hline $\mathrm{H}$ & 4.387780 & -0.330890 & -3.558160 \\
\hline $\mathrm{H}$ & 5.019190 & 1.302950 & -3.789290 \\
\hline $\mathrm{H}$ & 5.706480 & -0.048110 & -4.712040 \\
\hline C & 6.175970 & 4.217960 & 0.782680 \\
\hline $\mathrm{H}$ & 6.741060 & 4.138860 & -0.151750 \\
\hline $\mathrm{H}$ & 5.141430 & 4.472330 & 0.525260 \\
\hline $\mathrm{H}$ & 6.598760 & 5.050620 & 1.358550 \\
\hline C & 5.471570 & 3.139660 & 2.922940 \\
\hline $\mathrm{H}$ & 4.422950 & 3.404760 & 2.745720 \\
\hline $\mathrm{H}$ & 5.496200 & 2.255280 & 3.569840 \\
\hline $\mathrm{H}$ & 5.931690 & 3.966280 & 3.476240 \\
\hline C & 7.708080 & 2.591030 & 1.955370 \\
\hline $\mathrm{H}$ & 8.147910 & 3.410480 & 2.537370 \\
\hline $\mathrm{H}$ & 7.780090 & 1.673760 & 2.551130 \\
\hline $\mathrm{H}$ & 8.320530 & 2.453950 & 1.058230 \\
\hline
\end{tabular}




\begin{tabular}{lc}
\hline B3LYP/6-31G(d) Energy & -2164.800011 \\
\hline B3LYP-D3(BJ)/def2-TZVPP-IEF-PCM(DCM) Energy & -2165.899764 \\
\hline B3LYP-D3(BJ)/def2-TZVPP-IEF-PCM(DCM)//B3LYP/6-31G(d) Free Energy (Quasiharmonic) & -2165.020874 \\
\hline
\end{tabular}

Number of Imaginary Frequencies

Frequencies (Top 3 out of 321)

$\begin{array}{ll}\text { 1. } & 11.7978 \mathrm{~cm}^{-1} \\ \text { 2. } & 12.5321 \mathrm{~cm}^{-1} \\ \text { 3. } & 15.5166 \mathrm{~cm}^{-1}\end{array}$

\section{B3LYP/6-31G(d) Molecular Geometry in Cartesian Coordinates}

$\mathrm{H}$

$\mathrm{H}$

C

0

0

$\mathrm{H}$

0

$\mathrm{H}$

C

C

C

C

$\mathrm{H}$

C

$\mathrm{C}$

C

C

$\mathrm{H}$

C

C

C

C

C

C

$\mathrm{H}$

$\mathrm{H}$

$\mathrm{H}$

$\mathrm{H}$

C

C

C

\subsection{0}

0.931711

2.459380

$-1.531639$

$-2.044439$

$-0.855850$

$-0.393720$

0.766280

0.490460

$-0.550499$

$-1.632489$

$-2.858639$

$-2.941889$

$-3.864259$

1.742731

0.752281

3. 206001

2. 989571

4.146641

2. 228821

0.968431

$-0.013359$

0.245941

1.496341

2.463821

$-0.980339$

$-0.517499$

1.685001

3.423981

$-1.881079$

$-0.659929$

0. 397271
0.334980

$-0.039080$

0.728560

$-0.496500$

$-1.115920$

0.633910

0.948960

1.414220

2.036960

$-1.637030$

$-0.972450$

$-0.796600$

$-1.322860$

$-1.198430$

$-0.775190$

$-1.736080$

$-1.938070$

$-0.862430$

$-2.006980$

$-2.937330$

$-2.834210$

$-3.835150$

$-4.884860$

$-4.987970$

$-4.032690$

$-3.754700$

$-5.637260$

$-5.821020$

$-4.098210$

$-2.048350$

$-2.215120$

$-2.946120$
1.213930

2.066220

1.578110

$-1.242360$

$-2.150810$

$-1.467680$

$-0.652220$

0.581440

1.272870

0.760130

0.196730

0.924620

2.199690

2. 761250

0.215730

0.014440

$-1.331120$

$-0.490230$

$-1.871530$

$-1.547220$

$-0.877250$

$-1.119420$

$-1.970670$

$-2.627730$

$-2.422130$

$-0.635530$

$-2.147670$

$-3.299480$

$-2.928260$

2. 794320

2.068180

2.678180 


\begin{tabular}{|c|c|c|c|}
\hline C & 0.250871 & -3.482540 & 3.938720 \\
\hline C & -0.957969 & -3.314540 & 4.655630 \\
\hline C & -1.999439 & -2.612860 & 4.093190 \\
\hline H & 1.324961 & -3.085000 & 2.132840 \\
\hline $\mathrm{H}$ & 1.067821 & -4.042630 & 4.385550 \\
\hline $\mathrm{H}$ & -1.060809 & -3.743250 & 5.648780 \\
\hline $\mathrm{H}$ & -2.933249 & -2.480850 & 4.634600 \\
\hline c & 6.113860 & 2.041070 & -0.176400 \\
\hline C & 6.418870 & 0.686270 & 0.050870 \\
\hline c & 5.378081 & -0.239840 & -0.041290 \\
\hline c & 4.066820 & 0.161410 & -0.352670 \\
\hline C & 3.808080 & 1.516350 & -0.577070 \\
\hline C & 4.824490 & 2.480680 & -0.488950 \\
\hline H & 6.914630 & 2.767570 & -0.104670 \\
\hline $\mathrm{H}$ & 5.560101 & -1.292980 & 0.140530 \\
\hline $\mathrm{H}$ & 2.796680 & 1.814510 & -0.830890 \\
\hline C & 7.866370 & 0.280760 & 0.389870 \\
\hline c & 4.481130 & 3.961690 & -0.739540 \\
\hline C & 8.014781 & -1.236060 & 0.615060 \\
\hline H & 9.058221 & -1.473330 & 0.851570 \\
\hline $\mathrm{H}$ & 7.399061 & -1.587160 & 1.451010 \\
\hline $\mathrm{H}$ & 7.740061 & -1.810340 & -0.277000 \\
\hline C & 8.799410 & 0.685390 & -0.776500 \\
\hline $\mathrm{H}$ & 9.836410 & 0.409020 & -0.549370 \\
\hline $\mathrm{H}$ & 8.507880 & 0.179420 & -1.703970 \\
\hline $\mathrm{H}$ & 8.775850 & 1.764020 & -0.963650 \\
\hline C & 8.319340 & 1.002500 & 1.681450 \\
\hline H & 9.351850 & 0.727670 & 1.930020 \\
\hline $\mathrm{H}$ & 8.281850 & 2.091790 & 1.576320 \\
\hline $\mathrm{H}$ & 7.681150 & 0.726400 & 2.528780 \\
\hline C & 3.921380 & 4.125380 & -2.172700 \\
\hline $\mathrm{H}$ & 3.665820 & 5.174770 & -2.364580 \\
\hline $\mathrm{H}$ & 4.660650 & 3.813650 & -2.919350 \\
\hline $\mathrm{H}$ & 3.017620 & 3.527770 & -2.328750 \\
\hline C & 3.411010 & 4.415950 & 0.282670 \\
\hline $\mathrm{H}$ & 3.130560 & 5.461300 & 0.103750 \\
\hline $\mathrm{H}$ & 2.502800 & 3.807930 & 0.215600 \\
\hline $\mathrm{H}$ & 3.794220 & 4.338630 & 1.307250 \\
\hline C & 5.704900 & 4.885420 & -0.593080 \\
\hline $\mathrm{H}$ & 5.406420 & 5.924170 & -0.774300 \\
\hline $\mathrm{H}$ & 6.135770 & 4.836650 & 0.413630 \\
\hline $\mathrm{H}$ & 6.491860 & 4.638410 & -1.314810 \\
\hline C & -4.019239 & -0.017430 & 0.396460 \\
\hline C & -4.540550 & 1.033230 & 1.165610 \\
\hline C & -4.635359 & -0.334790 & -0.819740 \\
\hline C & -5.650710 & 1.773070 & 0.741650 \\
\hline $\mathrm{H}$ & -4.051450 & 1.277680 & 2.104570 \\
\hline C & -5.744670 & 0.383590 & -1.282570 \\
\hline $\mathrm{H}$ & -4.231239 & -1.148880 & -1.407300 \\
\hline C & -6.233910 & 1.428880 & -0.486740 \\
\hline $\mathrm{H}$ & -7.089840 & 1.991030 & -0.834080 \\
\hline C & -6.364860 & 0.009260 & -2.642880 \\
\hline $\mathrm{C}$ & -6.180590 & 2.925430 & 1.618530 \\
\hline & -6.791429 & -1.477970 & -2.628180 \\
\hline
\end{tabular}




\begin{tabular}{|c|c|c|c|}
\hline $\mathrm{H}$ & -5.942189 & -2.144810 & -2.449640 \\
\hline $\mathrm{H}$ & -7.537209 & -1.665870 & -1.846700 \\
\hline $\mathrm{H}$ & -7.233249 & -1.755220 & -3.593180 \\
\hline C & -7.606160 & 0.856380 & -2.980720 \\
\hline $\mathrm{H}$ & -8.008300 & 0.547180 & -3.952150 \\
\hline $\mathrm{H}$ & -8.402780 & 0.730850 & -2.237840 \\
\hline $\mathrm{H}$ & -7.367060 & 1.923810 & -3.049880 \\
\hline C & -5.308700 & 0.229380 & -3.753530 \\
\hline $\mathrm{H}$ & -5.024830 & 1.286530 & -3.814140 \\
\hline $\mathrm{H}$ & -4.396119 & -0.346900 & -3.573020 \\
\hline $\mathrm{H}$ & -5.714729 & -0.068920 & -4.728420 \\
\hline C & -5.068810 & 3.985560 & 1.804840 \\
\hline $\mathrm{H}$ & -4.181610 & 3.564380 & 2.289450 \\
\hline $\mathrm{H}$ & -4.757550 & 4.399350 & 0.838870 \\
\hline $\mathrm{H}$ & -5.429210 & 4.812550 & 2.429300 \\
\hline C & -7.405310 & 3.624850 & 0.999060 \\
\hline $\mathrm{H}$ & -7.172140 & 4.072210 & 0.026250 \\
\hline $\mathrm{H}$ & -8.245690 & 2.934420 & 0.864920 \\
\hline $\mathrm{H}$ & -7.742910 & 4.430970 & 1.660490 \\
\hline C & -6.595710 & 2.372500 & 3.002670 \\
\hline $\mathrm{H}$ & -6.972160 & 3.181330 & 3.641330 \\
\hline $\mathrm{H}$ & -7.388350 & 1.622070 & 2.903040 \\
\hline H & -5.754520 & 1.902050 & 3.522550 \\
\hline
\end{tabular}

\section{Catalyst 2C 20}

Datum

Value

\begin{tabular}{lc}
\hline B3LYP/6-31G(d) Energy & -2164.799792 \\
\hline B3LYP-D3(BJ)/def2-TZVPP-IEF-PCM(DCM) Energy & -2165.89972 \\
\hline B3LYP-D3(BJ)/def2-TZVPP-IEF-PCM(DCM)//B3LYP/6-31G(d) Free Energy (Quasiharmonic) & -2165.020723 \\
\hline
\end{tabular}

Number of Imaginary Frequencies

Frequencies (Top 3 out of 321)

$\begin{array}{ll}\text { 1. } & 12.0414 \mathrm{~cm}^{-1} \\ \text { 2. } & 13.3057 \mathrm{~cm}^{-1} \\ \text { 3. } & 16.4255 \mathrm{~cm}^{-1}\end{array}$

B3LYP/6-31G(d) Molecular Geometry in Cartesian Coordinates

$\begin{array}{rrrr}C & -1.507220 & 0.344830 & 1.211000 \\ H & -2.459650 & 0.741350 & 1.573490 \\ H & -0.938740 & -0.038000 & 2.063850\end{array}$




\begin{tabular}{|c|c|c|c|}
\hline C & 1.538140 & -0.481719 & -1.242720 \\
\hline 0 & 2.053340 & -1.095869 & -2.153550 \\
\hline 0 & 0.866090 & 0.651730 & -1.462750 \\
\hline $\mathrm{H}$ & 0.400310 & 0.961970 & -0.647290 \\
\hline 0 & -0.758730 & 1.422140 & 0.586910 \\
\hline $\mathrm{H}$ & -0.480330 & 2.039400 & 1.282130 \\
\hline C & 0.543290 & -1.632320 & 0.746690 \\
\hline C & 1.631150 & -0.968959 & 0.193110 \\
\hline C & 2.855070 & -0.804809 & 0.927640 \\
\hline C & 2.929380 & -1.341059 & 2.199240 \\
\hline $\mathrm{H}$ & 3.849560 & -1.225289 & 2.766240 \\
\hline C & -1.745090 & -0.757050 & 0.204300 \\
\hline C & -0.757010 & -1.718540 & -0.004800 \\
\hline C & -3.207620 & -1.897720 & -1.359380 \\
\hline C & -2.990580 & -0.833350 & -0.504450 \\
\hline $\mathrm{H}$ & -4.147260 & -1.957431 & -1.902560 \\
\hline C & -2.232549 & -2.897130 & -1.584690 \\
\hline C & -0.974149 & -2.806010 & -0.909320 \\
\hline C & 0.005011 & -3.807490 & -1.159550 \\
\hline C & -0.254869 & -4.846400 & -2.023810 \\
\hline C & -1.503219 & -4.937540 & -2.686440 \\
\hline C & -2.468179 & -3.981360 & -2.473160 \\
\hline $\mathrm{H}$ & 0.970571 & -3.735890 & -0.671460 \\
\hline $\mathrm{H}$ & 0.506621 & -5.599340 & -2.206820 \\
\hline $\mathrm{H}$ & -1.692319 & -5.762040 & -3.368550 \\
\hline $\mathrm{H}$ & -3.426769 & -4.037700 & -2.983380 \\
\hline C & 1.862570 & -2.066069 & 2.783400 \\
\hline C & 0.644000 & -2.221450 & 2.050470 \\
\hline c & -0.419159 & -2.952610 & 2.649860 \\
\hline C & -0.280849 & -3.499770 & 3.906700 \\
\hline C & 0.925431 & -3.342860 & 4.630430 \\
\hline C & 1.972481 & -2.641359 & 4.078310 \\
\hline $\mathrm{H}$ & -1.344989 & -3.083060 & 2.099300 \\
\hline H & -1.102269 & -4.059840 & 4.345280 \\
\hline $\mathrm{H}$ & 1.021811 & -3.779940 & 5.620570 \\
\hline $\mathrm{H}$ & 2.904431 & -2.517769 & 4.624890 \\
\hline C & -6.123990 & 2.057969 & -0.164180 \\
\hline C & -6.421010 & 0.711719 & 0.066570 \\
\hline C & -5.369090 & -0.213001 & -0.034500 \\
\hline C & -4.068770 & 0.187960 & -0.353140 \\
\hline C & -3.812120 & 1.551750 & -0.579760 \\
\hline C & -4.829230 & 2.503709 & -0.488760 \\
\hline $\mathrm{H}$ & -6.918690 & 2.791389 & -0.092160 \\
\hline $\mathrm{H}$ & -5.553150 & -1.267691 & 0.149020 \\
\hline $\mathrm{H}$ & -2.802630 & 1.846480 & -0.836950 \\
\hline C & -7.835730 & 0.217909 & 0.426810 \\
\hline C & -4.578211 & 4.004289 & -0.734210 \\
\hline C & -7.805900 & -0.458031 & 1.818390 \\
\hline $\mathrm{H}$ & -8.804770 & -0.823281 & 2.087180 \\
\hline $\mathrm{H}$ & -7.121330 & -1.312441 & 1.843150 \\
\hline $\mathrm{H}$ & -7.484370 & 0.250289 & 2.590640 \\
\hline C & -8.866180 & 1.361849 & 0.472980 \\
\hline $\mathrm{H}$ & -9.853510 & 0.958538 & 0.724880 \\
\hline $\mathrm{H}$ & -8.612540 & 2.110059 & 1.232520 \\
\hline
\end{tabular}




\begin{tabular}{|c|c|c|c|}
\hline $\mathrm{H}$ & -8.954740 & 1.870909 & -0.493550 \\
\hline C & -8.307700 & -0.808811 & -0.630290 \\
\hline $\mathrm{H}$ & -9.313260 & -1.172981 & -0.386360 \\
\hline $\mathrm{H}$ & -8.342790 & -0.356141 & -1.627830 \\
\hline $\mathrm{H}$ & -7.643290 & -1.677551 & -0.681280 \\
\hline C & -5.456911 & 4.490739 & -1.911050 \\
\hline $\mathrm{H}$ & -5.289161 & 5.558759 & -2.096790 \\
\hline $\mathrm{H}$ & -5.216111 & 3.944319 & -2.829940 \\
\hline $\mathrm{H}$ & -6.524261 & 4.351589 & -1.709300 \\
\hline C & -4.943071 & 4.802159 & 0.540540 \\
\hline $\mathrm{H}$ & -4.777391 & 5.874549 & 0.379910 \\
\hline $\mathrm{H}$ & -5.992441 & 4.666099 & 0.822480 \\
\hline $\mathrm{H}$ & -4.325951 & 4.487049 & 1.390110 \\
\hline C & -3.106871 & 4.301700 & -1.081510 \\
\hline $\mathrm{H}$ & -2.971901 & 5.379830 & -1.224900 \\
\hline $\mathrm{H}$ & -2.425341 & 3.982980 & -0.284900 \\
\hline $\mathrm{H}$ & -2.797851 & 3.803610 & -2.007110 \\
\hline C & 4.022760 & -0.028359 & 0.411310 \\
\hline C & 4.548190 & 1.012221 & 1.200060 \\
\hline C & 4.638990 & -0.335629 & -0.803390 \\
\hline C & 5.662420 & 1.744831 & 0.788860 \\
\hline $\mathrm{H}$ & 4.053470 & 1.243491 & 2.136770 \\
\hline C & 5.758460 & 0.381121 & -1.255540 \\
\hline $\mathrm{H}$ & 4.231960 & -1.139879 & -1.402330 \\
\hline C & 6.248410 & 1.408521 & -0.445550 \\
\hline $\mathrm{H}$ & 7.111500 & 1.973662 & -0.777640 \\
\hline C & 6.379520 & 0.016991 & -2.617620 \\
\hline C & 6.257880 & 2.895831 & 1.623270 \\
\hline C & 7.625450 & 0.861722 & -2.943710 \\
\hline $\mathrm{H}$ & 7.391100 & 1.930912 & -3.001370 \\
\hline H & 8.419860 & 0.724022 & -2.200600 \\
\hline $\mathrm{H}$ & 8.028220 & 0.561582 & -3.917680 \\
\hline C & 6.798770 & -1.472368 & -2.615590 \\
\hline $\mathrm{H}$ & 7.240300 & -1.743138 & -3.582540 \\
\hline $\mathrm{H}$ & 7.542750 & -1.670778 & -1.834990 \\
\hline $\mathrm{H}$ & 5.946100 & -2.136639 & -2.443870 \\
\hline C & 5.327000 & 0.252821 & -3.728440 \\
\hline $\mathrm{H}$ & 4.410720 & -0.319849 & -3.555300 \\
\hline H & 5.049240 & 1.312041 & -3.780450 \\
\hline $\mathrm{H}$ & 5.733650 & -0.039329 & -4.704900 \\
\hline C & 6.205679 & 4.208451 & 0.805860 \\
\hline $\mathrm{H}$ & 6.772259 & 4.130582 & -0.127770 \\
\hline $\mathrm{H}$ & 5.172629 & 4.467741 & 0.547400 \\
\hline $\mathrm{H}$ & 6.630559 & 5.037331 & 1.385640 \\
\hline C & 5.492890 & 3.124731 & 2.940650 \\
\hline $\mathrm{H}$ & 4.445749 & 3.394941 & 2.762410 \\
\hline H & 5.512470 & 2.237641 & 3.584000 \\
\hline $\mathrm{H}$ & 5.955359 & 3.947181 & 3.498190 \\
\hline C & 7.729130 & 2.571082 & 1.975380 \\
\hline H & 8.170899 & 3.386402 & 2.561700 \\
\hline $\mathrm{H}$ & 7.796400 & 1.651052 & 2.567420 \\
\hline $\mathrm{H}$ & 8.342930 & 2.435442 & 1.078950 \\
\hline
\end{tabular}




\section{Catalyst 2C 21}

Datum

Value

\begin{tabular}{lc}
\hline B3LYP/6-31G(d) Energy & -2164.799835 \\
\hline B3LYP-D3(BJ)/def2-TZVPP-IEF-PCM(DCM) Energy & -2165.899522 \\
\hline B3LYP-D3(BJ)/def2-TZVPP-IEF-PCM(DCM)//B3LYP/6-31G(d) Free Energy (Quasiharmonic) & -2165.020573 \\
\hline
\end{tabular}

Number of Imaginary Frequencies

Frequencies (Top 3 out of 321)

$\begin{array}{ll}\text { 1. } & 11.5843 \mathrm{~cm}^{-1} \\ \text { 2. } & 12.7071 \mathrm{~cm}^{-1} \\ \text { 3. } & 15.8086 \mathrm{~cm}^{-1}\end{array}$

\section{B3LYP/6-31G(d) Molecular Geometry in Cartesian Coordinates}

C

$\mathrm{H}$

$\mathrm{H}$

C

0

0

$\mathrm{H}$

0

$\mathrm{H}$

C

C

C

C

$\mathrm{H}$

C

C

C

C

C

$\mathrm{H}$

C

C

C

C

C

C

$\mathrm{H}$

$\mathrm{H}$

$\mathrm{H}$

$\mathrm{H}$

-1.513509
-2.465819
-0.939899
1.534101
2.048191
0.862711
0.397591
-0.771279
-0.499769
0.538581
1.627491
2.852821
2.927321
3.848951
-1.750479
-0.761649
-3.211899
-2.996109
-4.151629
-2.235699
-0.977619
0.002231
-0.256659
-1.504649
-2.470299
0.967541
0.505381
-1.692939
-3.428709

$-1.513509$

0.939899

534101

0.862711

$-0.499769$

38581

2.852821

3.848951

1.750479

761649

$$
\begin{array}{r}
0.375060 \\
0.773240 \\
0.007240 \\
-0.487490 \\
-1.113300 \\
0.643770 \\
0.965080 \\
1.447210 \\
2.078780 \\
-1.608750 \\
-0.956010 \\
-0.785610 \\
-1.303730 \\
-1.183350 \\
-0.742170 \\
-1.706090 \\
-1.908430 \\
-0.831070 \\
-1.977810 \\
-2.909910 \\
-2.807450 \\
-3.812120 \\
-4.864260 \\
-4.966350 \\
-4.007690 \\
-3.732710 \\
-5.619450 \\
-5.801320 \\
-4.072440
\end{array}
$$

1.195910

1.556310

2.051810

$-1.243871$

$-2.147201$

$-1.477931$

$-0.666631$

0.553930

1.239060

0.762080

0.198090

0.928770

2. 207720

2.771370

0.205940

0.011740

$-1.340241$

$-0.501561$

$-1.882151$

$-1.550571$

$-0.876211$

$-1.110741$

$-1.959121$

- 2.620831

$-2.422481$

$-0.623421$

$-2.130200$

$-3.290291$

$-2.932011$ 


\begin{tabular}{|c|c|c|c|}
\hline C & 1.858581 & -2.016080 & 2.803930 \\
\hline C & 0.638821 & -2.178610 & 2.074540 \\
\hline C & -0.425979 & -2.897400 & 2.685850 \\
\hline C & -0.288239 & -3.425740 & 3.950770 \\
\hline C & 0.919181 & -3.261690 & 4.670960 \\
\hline C & 1.967981 & -2.572080 & 4.107250 \\
\hline $\mathrm{H}$ & -1.352799 & -3.033190 & 2.138270 \\
\hline $\mathrm{H}$ & -1.111069 & -3.976350 & 4.398600 \\
\hline $\mathrm{H}$ & 1.015121 & -3.683890 & 5.667570 \\
\hline $\mathrm{H}$ & 2.900861 & -2.443280 & 4.651040 \\
\hline C & -6.134309 & 2.064310 & -0.183891 \\
\hline C & -4.842509 & 2.505890 & -0.506101 \\
\hline C & -3.824259 & 1.548820 & -0.595451 \\
\hline C & -4.075939 & 0.190170 & -0.363861 \\
\hline C & -5.380329 & -0.210090 & -0.045090 \\
\hline C & -6.429349 & 0.712940 & 0.049470 \\
\hline $\mathrm{H}$ & -6.931749 & 2.791300 & -0.112191 \\
\hline $\mathrm{H}$ & -2.815039 & 1.849470 & -0.855251 \\
\hline $\mathrm{H}$ & -5.563439 & -1.264030 & 0.143740 \\
\hline C & -4.508139 & 3.987980 & -0.765461 \\
\hline C & -7.845719 & 0.221720 & 0.408860 \\
\hline C & -3.958699 & 4.148320 & -2.203021 \\
\hline $\mathrm{H}$ & -3.709919 & 5.198170 & -2.401371 \\
\hline $\mathrm{H}$ & -3.052929 & 3.554600 & -2.362311 \\
\hline $\mathrm{H}$ & -4.701219 & 3.829370 & -2.943401 \\
\hline C & -5.735119 & 4.907040 & -0.615181 \\
\hline $\mathrm{H}$ & -5.442189 & 5.946190 & -0.803211 \\
\hline $\mathrm{H}$ & -6.525789 & 4.653410 & -1.330491 \\
\hline $\mathrm{H}$ & -6.159419 & 4.861260 & 0.394430 \\
\hline C & -3.433489 & 4.452520 & 0.247380 \\
\hline $\mathrm{H}$ & -3.160709 & 5.498930 & 0.062840 \\
\hline $\mathrm{H}$ & -3.808719 & 4.376470 & 1.275010 \\
\hline $\mathrm{H}$ & -2.522039 & 3.849840 & 0.175890 \\
\hline C & -7.820369 & -0.444840 & 1.805070 \\
\hline $\mathrm{H}$ & -8.820369 & -0.806960 & 2.074040 \\
\hline $\mathrm{H}$ & -7.136909 & -1.299900 & 1.837080 \\
\hline $\mathrm{H}$ & -7.499669 & 0.268180 & 2.573350 \\
\hline C & -8.876909 & 1.365550 & 0.444230 \\
\hline $\mathrm{H}$ & -9.864969 & 0.962810 & 0.694210 \\
\hline $\mathrm{H}$ & -8.627349 & 2.118380 & 1.200500 \\
\hline $\mathrm{H}$ & -8.961799 & 1.869240 & -0.525421 \\
\hline C & -8.315069 & -0.812080 & -0.642501 \\
\hline $\mathrm{H}$ & -9.321749 & -1.173780 & -0.399391 \\
\hline $\mathrm{H}$ & -8.346529 & -0.366420 & -1.643311 \\
\hline $\mathrm{H}$ & -7.651149 & -1.681640 & -0.685191 \\
\hline C & 4.022861 & -0.021410 & 0.399530 \\
\hline C & 4.552591 & 1.028361 & 1.164160 \\
\hline C & 4.640411 & -0.353179 & -0.812111 \\
\hline C & 5.672561 & 1.753201 & 0.740170 \\
\hline $\mathrm{H}$ & 4.062611 & 1.284140 & 2.099590 \\
\hline C & 5.759501 & 0.349981 & -1.274751 \\
\hline $\mathrm{H}$ & 4.230111 & -1.166660 & -1.396171 \\
\hline C & 6.257001 & 1.394911 & -0.483581 \\
\hline $\mathrm{H}$ & 7.120541 & 1.945351 & -0.830811 \\
\hline
\end{tabular}




\begin{tabular}{|rrrr|} 
C & 6.381661 & -0.040309 & -2.629701 \\
C & 6.211921 & 2.904601 & 1.612610 \\
H & 5.332411 & 0.181621 & -3.746481 \\
H & 4.413321 & -0.384040 & -3.565391 \\
H & 5.059361 & 1.241031 & -3.816511 \\
C & 5.739471 & -0.128279 & -4.717300 \\
H & 7.632481 & 0.792211 & -2.968501 \\
H & 8.035291 & 0.472381 & -3.936211 \\
H & 7.404101 & 1.861421 & -3.045851 \\
C & 8.424971 & 0.663961 & -2.221681 \\
H & 6.793921 & -1.531409 & -2.602041 \\
H & 7.534211 & -1.720569 & -1.815651 \\
H & 5.937561 & -2.188829 & -2.422591 \\
C & 7.237641 & -1.819999 & -3.562851 \\
H & 7.444061 & 3.589751 & 0.991920 \\
H & 8.278521 & 2.891211 & 0.862860 \\
H & 7.216651 & 4.034141 & 0.016380 \\
C & 7.787631 & 4.396181 & 1.649870 \\
H & 5.109991 & 3.975991 & 1.792720 \\
H & 4.803761 & 4.388371 & 0.824560 \\
H & 4.218301 & 3.565430 & 2.278210 \\
C & 5.477431 & 4.802301 & 2.413940 \\
H & 6.619691 & 2.353661 & 2.999720 \\
H & 7.003081 & 3.161501 & 3.635510 \\
H & 5.773151 & 1.893591 & 3.520230 \\
& 7.405101 & 1.595111 & 2.904400 \\
\hline & & & \\
\hline & & & \\
\hline & & & \\
\hline
\end{tabular}

\section{Catalyst 2C 22}

Datum

Value

\begin{tabular}{lr}
\hline B3LYP/6-31G(d) Energy & -2164.800394 \\
\hline B3LYP-D3(BJ)/def2-TZVPP-IEF-PCM(DCM) Energy & -2165.897491 \\
\hline B3LYP-D3(BJ)/def2-TZVPP-IEF-PCM(DCM)//B3LYP/6-31G(d) Free Energy (Quasiharmonic) & -2165.019426 \\
\hline
\end{tabular}

Number of Imaginary Frequencies

Frequencies (Top 3 out of 321)

$\begin{array}{ll}\text { 1. } & 10.2642 \mathrm{~cm}^{-1} \\ \text { 2. } & 11.0765 \mathrm{~cm}^{-1} \\ \text { 3. } & 11.5903 \mathrm{~cm}^{-1}\end{array}$




\begin{tabular}{|c|c|c|c|}
\hline C & 0.231736 & -4.015336 & 3.156493 \\
\hline C & 0.360465 & -3.189918 & 2.061354 \\
\hline C & -0.722756 & -2.376839 & 1.627257 \\
\hline C & -1.956125 & -2.442880 & 2.350922 \\
\hline C & -2.055135 & -3.301743 & 3.478492 \\
\hline C & -0.986298 & -4.072225 & 3.874477 \\
\hline $\mathrm{H}$ & 1.071387 & -4.628388 & 3.472104 \\
\hline $\mathrm{H}$ & 1.297414 & -3.152616 & 1.516610 \\
\hline C & -0.625293 & -1.504418 & 0.490840 \\
\hline C & -3.049059 & -1.644409 & 1.932788 \\
\hline $\mathrm{H}$ & -2.995501 & -3.338585 & 4.023117 \\
\hline $\mathrm{H}$ & -1.074381 & -4.726336 & 4.737627 \\
\hline C & -2.969127 & -0.810565 & 0.837247 \\
\hline C & -1.734155 & -0.759135 & 0.110098 \\
\hline $\mathrm{H}$ & -3.970080 & -1.676254 & 2.509076 \\
\hline C & 1.039268 & -4.085768 & -3.671317 \\
\hline C & 2.085995 & -3.276636 & -3.295848 \\
\hline C & 1.969461 & -2.406742 & -2.177710 \\
\hline C & 0.744654 & -2.370343 & -1.439731 \\
\hline C & -0.317433 & -3.221849 & -1.855322 \\
\hline C & -0.172970 & -4.056155 & -2.941130 \\
\hline $\mathrm{H}$ & 3.963841 & -1.610145 & -2.341510 \\
\hline $\mathrm{H}$ & 1.138389 & -4.747370 & -4.527646 \\
\hline $\mathrm{H}$ & 3.022639 & -3.290004 & -3.848303 \\
\hline C & 3.039905 & -1.577935 & -1.769741 \\
\hline C & 0.647763 & -1.484400 & -0.314862 \\
\hline$H$ & -1.256458 & -3.203402 & -1.313628 \\
\hline $\mathrm{H}$ & -0.998687 & -4.694418 & -3.243276 \\
\hline C & 1.717583 & -0.670784 & 0.057252 \\
\hline C & 2.947311 & -0.719620 & -0.689477 \\
\hline C & 1.573915 & 0.271498 & 1.242213 \\
\hline $\mathrm{H}$ & 0.861213 & -0.140791 & 1.965957 \\
\hline $\mathrm{H}$ & 2.533365 & 0.385597 & 1.750915 \\
\hline C & -1.705122 & 0.000059 & -1.185699 \\
\hline 0 & -2.300426 & -0.309859 & -2.188277 \\
\hline 0 & -0.916442 & 1.116684 & -1.134316 \\
\hline $\mathrm{H}$ & -0.923500 & 1.504118 & -2.031380 \\
\hline 0 & 1.181741 & 1.593663 & 0.862387 \\
\hline $\mathrm{H}$ & 0.400824 & 1.511711 & 0.287629 \\
\hline C & 4.155947 & 0.083762 & -0.332558 \\
\hline C & 4.098684 & 1.467396 & -0.146647 \\
\hline C & 5.401554 & -0.564174 & -0.229069 \\
\hline C & 5.250919 & 2.215391 & 0.144128 \\
\hline $\mathrm{H}$ & 3.135283 & 1.958361 & -0.217980 \\
\hline C & 6.570718 & 0.143218 & 0.054150 \\
\hline $\mathrm{H}$ & 5.429732 & -1.640229 & -0.357087 \\
\hline C & 6.467816 & 1.534864 & 0.235536 \\
\hline $\mathrm{H}$ & 7.368858 & 2.093929 & 0.459498 \\
\hline C & -4.136072 & 0.034067 & 0.458044 \\
\hline C & -3.995153 & 1.414788 & 0.302266 \\
\hline C & -5.402248 & -0.549209 & 0.287141 \\
\hline C & -5.087957 & 2.229140 & -0.033149 \\
\hline $\mathrm{H}$ & -3.019148 & 1.861688 & 0.464068 \\
\hline
\end{tabular}




\begin{tabular}{|c|c|c|c|}
\hline C & -6.515666 & 0.224156 & -0.044973 \\
\hline $\mathrm{H}$ & -5.488992 & -1.624179 & 0.395341 \\
\hline C & -6.330057 & 1.610691 & -0.199472 \\
\hline $\mathrm{H}$ & -7.188013 & 2.218812 & -0.460819 \\
\hline C & -7.915573 & -0.383010 & -0.258193 \\
\hline C & -4.878220 & 3.746817 & -0.195746 \\
\hline C & -7.931679 & -1.909607 & -0.051062 \\
\hline $\mathrm{H}$ & -7.270774 & -2.424209 & -0.757545 \\
\hline $\mathrm{H}$ & -7.629732 & -2.187039 & 0.965343 \\
\hline $\mathrm{H}$ & -8.946480 & -2.291471 & -0.210051 \\
\hline C & -8.389359 & -0.091295 & -1.702124 \\
\hline $\mathrm{H}$ & -9.386409 & -0.517184 & -1.869646 \\
\hline $\mathrm{H}$ & -8.448771 & 0.983285 & -1.903921 \\
\hline $\mathrm{H}$ & -7.703557 & -0.531356 & -2.434881 \\
\hline C & -8.910048 & 0.247423 & 0.745606 \\
\hline $\mathrm{H}$ & -8.979034 & 1.333522 & 0.625357 \\
\hline $\mathrm{H}$ & -9.914442 & -0.168467 & 0.599113 \\
\hline $\mathrm{H}$ & -8.605719 & 0.044524 & 1.778920 \\
\hline C & -3.818826 & 4.002207 & -1.294966 \\
\hline $\mathrm{H}$ & -2.857267 & 3.539349 & -1.048675 \\
\hline $\mathrm{H}$ & -4.149648 & 3.596820 & -2.258137 \\
\hline $\mathrm{H}$ & -3.648503 & 5.078680 & -1.418215 \\
\hline C & -4.381447 & 4.343246 & 1.142670 \\
\hline $\mathrm{H}$ & -3.436608 & 3.892852 & 1.464185 \\
\hline $\mathrm{H}$ & -4.219284 & 5.423487 & 1.042572 \\
\hline $\mathrm{H}$ & -5.116778 & 4.182250 & 1.939308 \\
\hline C & -6.170506 & 4.481798 & -0.599329 \\
\hline $\mathrm{H}$ & -6.565601 & 4.118309 & -1.554900 \\
\hline $\mathrm{H}$ & -6.955162 & 4.376516 & 0.158647 \\
\hline $\mathrm{H}$ & -5.965311 & 5.552285 & -0.712840 \\
\hline C & 7.945924 & -0.540751 & 0.181662 \\
\hline C & 5.123617 & 3.736767 & 0.352087 \\
\hline C & 4.166623 & 4.010328 & 1.537819 \\
\hline $\mathrm{H}$ & 3.175700 & 3.575434 & 1.373774 \\
\hline $\mathrm{H}$ & 4.568267 & 3.586800 & 2.466036 \\
\hline $\mathrm{H}$ & 4.043671 & 5.090834 & 1.685428 \\
\hline C & 4.547058 & 4.384862 & -0.929266 \\
\hline $\mathrm{H}$ & 5.203876 & 4.207755 & -1.789017 \\
\hline $\mathrm{H}$ & 3.557630 & 3.986307 & -1.175325 \\
\hline $\mathrm{H}$ & 4.446739 & 5.469127 & -0.795495 \\
\hline C & 6.476294 & 4.406626 & 0.659170 \\
\hline $\mathrm{H}$ & 6.928228 & 4.012541 & 1.576762 \\
\hline $\mathrm{H}$ & 7.193891 & 4.276066 & -0.159388 \\
\hline $\mathrm{H}$ & 6.330510 & 5.483616 & 0.801031 \\
\hline C & 7.869810 & -2.062095 & -0.048869 \\
\hline $\mathrm{H}$ & 7.220521 & -2.554064 & 0.684234 \\
\hline $\mathrm{H}$ & 7.498280 & -2.304679 & -1.051056 \\
\hline $\mathrm{H}$ & 8.869269 & -2.501369 & 0.047800 \\
\hline C & 8.514573 & -0.299707 & 1.600499 \\
\hline $\mathrm{H}$ & 9.497294 & -0.776184 & 1.705033 \\
\hline $\mathrm{H}$ & 8.637395 & 0.766876 & 1.815517 \\
\hline $\mathrm{H}$ & 7.850247 & -0.719650 & 2.364476 \\
\hline C & 8.917025 & 0.053721 & -0.866149 \\
\hline $\mathrm{H}$ & 9.053446 & 1.131684 & -0.731214 \\
\hline
\end{tabular}


$\mathrm{H}$

$\mathrm{H}$

\section{Catalyst 2C 23}

Datum

Value

\begin{tabular}{lc}
\hline B3LYP/6-31G(d) Energy & -2164.80016 \\
\hline B3LYP-D3(BJ)/def2-TZVPP-IEF-PCM(DCM) Energy & -2165.897345 \\
\hline B3LYP-D3(BJ)/def2-TZVPP-IEF-PCM(DCM)//B3LYP/6-31G(d) Free Energy (Quasiharmonic) & -2165.019327 \\
\hline
\end{tabular}

Number of Imaginary Frequencies

Frequencies (Top 3 out of 321)
1.
$9.9838 \mathrm{~cm}^{-1}$
2. $\quad 10.7764 \mathrm{~cm}^{-1}$
3. $\quad 12.1177 \mathrm{~cm}^{-1}$

\section{B3LYP/6-31G(d) Molecular Geometry in Cartesian Coordinates}

\begin{tabular}{|rrrr}
\hline C & -0.239373 & -4.001537 & 3.133420 \\
C & -0.367303 & -3.174681 & 2.039252 \\
C & 0.715601 & -2.359515 & 1.608218 \\
C & 1.947736 & -2.425435 & 2.333645 \\
C & 2.045918 & -3.285353 & 3.460470 \\
C & 0.977381 & -4.057715 & 3.853597 \\
H & -1.078736 & -4.616240 & 3.446568 \\
H & -1.303375 & -3.137930 & 1.493036 \\
C & 0.618861 & -1.484247 & 0.473828 \\
C & 3.040630 & -1.626090 & 1.917601 \\
H & 2.985428 & -3.321705 & 4.006605 \\
H & 1.064768 & -4.712821 & 4.716063 \\
C & 2.963531 & -0.792715 & 0.821255 \\
C & 1.728243 & -0.738582 & 0.094202 \\
H & 3.960166 & -1.656911 & 2.496189 \\
C & -1.041924 & -4.046179 & -3.702349 \\
C & -2.091282 & -3.243726 & -3.319943 \\
C & -1.976400 & -2.380375 & -2.196576 \\
C & -0.750363 & -2.343399 & -1.460538 \\
C & 0.314298 & -3.188318 & -1.883133 \\
C & 0.171382 & -4.016336 & -2.973956 \\
H & -3.974902 & -1.592148 & -2.351053 \\
H & -1.139840 & -4.702756 & -4.562673 \\
H & -3.028870 & -3.257524 & -3.870786 \\
\hline & & &
\end{tabular}




\begin{tabular}{|c|c|c|c|}
\hline C & -3.049763 & -1.559026 & -1.781283 \\
\hline C & -0.654986 & -1.463690 & -0.330702 \\
\hline $\mathrm{H}$ & 1.254089 & -3.169969 & -1.342740 \\
\hline $\mathrm{H}$ & 0.999110 & -4.649456 & -3.281367 \\
\hline C & -1.727417 & -0.656646 & 0.048048 \\
\hline C & -2.958660 & -0.706904 & -0.695997 \\
\hline C & -1.584296 & 0.281844 & 1.236071 \\
\hline $\mathrm{H}$ & -2.542565 & 0.390013 & 1.748255 \\
\hline $\mathrm{H}$ & -0.867433 & -0.129521 & 1.956162 \\
\hline C & 1.697507 & 0.024829 & -1.198671 \\
\hline 0 & 2.308760 & -0.268803 & -2.196781 \\
\hline 0 & 0.886780 & 1.125137 & -1.150359 \\
\hline $\mathrm{H}$ & 0.889957 & 1.513043 & -2.047143 \\
\hline 0 & -1.199267 & 1.606890 & 0.859070 \\
\hline $\mathrm{H}$ & -0.418679 & 1.529946 & 0.283184 \\
\hline C & -4.170458 & 0.087882 & -0.330567 \\
\hline C & -4.120167 & 1.470627 & -0.136220 \\
\hline C & -5.411998 & -0.567652 & -0.226330 \\
\hline C & -5.275379 & 2.210260 & 0.163977 \\
\hline $\mathrm{H}$ & -3.159762 & 1.967376 & -0.208117 \\
\hline C & -6.583935 & 0.131364 & 0.066114 \\
\hline $\mathrm{H}$ & -5.434607 & -1.643028 & -0.360977 \\
\hline C & -6.488121 & 1.522401 & 0.255891 \\
\hline $\mathrm{H}$ & -7.391348 & 2.074994 & 0.487166 \\
\hline C & 4.135078 & 0.046979 & 0.445509 \\
\hline C & 3.994711 & 1.434613 & 0.275205 \\
\hline C & 5.397104 & -0.532408 & 0.299717 \\
\hline C & 5.089588 & 2.238227 & -0.045369 \\
\hline $\mathrm{H}$ & 3.015891 & 1.876579 & 0.418849 \\
\hline C & 6.524919 & 0.238984 & -0.022181 \\
\hline $\mathrm{H}$ & 5.488919 & -1.608148 & 0.416944 \\
\hline C & 6.342614 & 1.614796 & -0.189533 \\
\hline $\mathrm{H}$ & 7.197517 & 2.231065 & -0.441950 \\
\hline C & 7.894300 & -0.447781 & -0.187085 \\
\hline C & 4.968506 & 3.761550 & -0.242061 \\
\hline C & 9.017842 & 0.549682 & -0.527633 \\
\hline $\mathrm{H}$ & 8.832330 & 1.066295 & -1.476165 \\
\hline $\mathrm{H}$ & 9.145755 & 1.305730 & 0.255618 \\
\hline $\mathrm{H}$ & 9.968355 & 0.013231 & -0.625913 \\
\hline C & 7.813464 & -1.487374 & -1.330214 \\
\hline $\mathrm{H}$ & 8.780495 & -1.989392 & -1.457599 \\
\hline $\mathrm{H}$ & 7.061505 & -2.257720 & -1.130090 \\
\hline $\mathrm{H}$ & 7.551684 & -1.005992 & -2.279172 \\
\hline C & 8.273068 & -1.163703 & 1.131276 \\
\hline $\mathrm{H}$ & 7.534213 & -1.922134 & 1.410398 \\
\hline $\mathrm{H}$ & 9.242685 & -1.666011 & 1.027664 \\
\hline $\mathrm{H}$ & 8.347481 & -0.448545 & 1.958626 \\
\hline C & 5.398265 & 4.127254 & -1.682963 \\
\hline $\mathrm{H}$ & 6.434150 & 3.836360 & -1.885890 \\
\hline $\mathrm{H}$ & 4.761066 & 3.626609 & -2.420802 \\
\hline $\mathrm{H}$ & 5.318414 & 5.209789 & -1.841396 \\
\hline C & 5.886422 & 4.491430 & 0.767111 \\
\hline $\mathrm{H}$ & 6.938305 & 4.217278 & 0.635364 \\
\hline $\mathrm{H}$ & 5.807116 & 5.577860 & 0.638052 \\
\hline
\end{tabular}




\begin{tabular}{|c|c|c|c|}
\hline $\mathrm{H}$ & 5.604658 & 4.250610 & 1.798501 \\
\hline C & 3.528675 & 4.266926 & -0.028487 \\
\hline $\mathrm{H}$ & 2.823672 & 3.802775 & -0.727577 \\
\hline $\mathrm{H}$ & 3.174210 & 4.072089 & 0.989969 \\
\hline $\mathrm{H}$ & 3.489833 & 5.350107 & -0.190120 \\
\hline C & -7.954606 & -0.561396 & 0.195488 \\
\hline C & -5.155686 & 3.730887 & 0.381699 \\
\hline C & -6.510589 & 4.390801 & 0.700270 \\
\hline $\mathrm{H}$ & -7.231738 & 4.261309 & -0.115328 \\
\hline $\mathrm{H}$ & -6.955468 & 3.988278 & 1.617673 \\
\hline $\mathrm{H}$ & -6.370327 & 5.467701 & 0.848275 \\
\hline C & -4.589487 & 4. 391073 & -0.898061 \\
\hline $\mathrm{H}$ & -3.599348 & 3.999443 & -1.152330 \\
\hline $\mathrm{H}$ & -5.250082 & 4.216699 & -1.755474 \\
\hline $\mathrm{H}$ & -4.494021 & 5.474860 & -0.757082 \\
\hline C & -4.194159 & 4.001930 & 1.564347 \\
\hline $\mathrm{H}$ & -4.587959 & 3.568885 & 2.491530 \\
\hline $\mathrm{H}$ & -3.201267 & 3.574898 & 1.391785 \\
\hline $\mathrm{H}$ & -4.077498 & 5.082096 & 1.719316 \\
\hline C & -8.518429 & -0.331919 & 1.618179 \\
\hline $\mathrm{H}$ & -7.848279 & -0.752282 & 2.376816 \\
\hline $\mathrm{H}$ & -8.646568 & 0.732667 & 1.839867 \\
\hline $\mathrm{H}$ & -9.497835 & -0.814827 & 1.724228 \\
\hline C & -7.870550 & -2.080864 & -0.044184 \\
\hline $\mathrm{H}$ & -8.866982 & -2.526638 & 0.054057 \\
\hline $\mathrm{H}$ & -7.501794 & -2.315495 & -1.049286 \\
\hline $\mathrm{H}$ & -7.215310 & -2.573192 & 0.683363 \\
\hline C & -8.933763 & 0.033437 & -0.844565 \\
\hline $\mathrm{H}$ & -8.564305 & -0.123562 & -1.864516 \\
\hline $\mathrm{H}$ & -9.917232 & -0.445646 & -0.761444 \\
\hline $\mathrm{H}$ & -9.075128 & 1.109948 & -0.703298 \\
\hline
\end{tabular}

\section{Catalyst 2C 24}

Datum

Value

\begin{tabular}{lc}
\hline B3LYP/6-31G(d) Energy & -2164.799612 \\
\hline B3LYP-D3(BJ)/def2-TZVPP-IEF-PCM(DCM) Energy & -2165.896959 \\
\hline
\end{tabular}

B3LYP-D3(BJ)/def2-TZVPP-IEF-PCM(DCM)//B3LYP/6-31G(d) Free Energy (Quasiharmonic) $\quad$-2165.019246

Number of Imaginary Frequencies

Frequencies (Top 3 out of 321)

$\begin{array}{lr}\text { 1. } & 7.9707 \mathrm{~cm}^{-1} \\ \text { 2. } & 9.4883 \mathrm{~cm}^{-1} \\ \text { 3. } & 12.8797 \mathrm{~cm}^{-1}\end{array}$




\begin{tabular}{|c|c|c|c|}
\hline C & 0.256007 & -4.037494 & 3.097534 \\
\hline C & 0.377841 & -3.202727 & 2.008688 \\
\hline C & -0.709083 & -2.388343 & 1.586360 \\
\hline C & -1.938711 & -2.463162 & 2.315214 \\
\hline C & -2.030686 & -3.331379 & 3.436199 \\
\hline C & -0.958443 & -4.102885 & 3.820802 \\
\hline $\mathrm{H}$ & 1.098416 & -4.651410 & 3.404008 \\
\hline $\mathrm{H}$ & 1.312135 & -3.159073 & 1.459966 \\
\hline C & -0.618938 & -1.506001 & 0.456805 \\
\hline C & -3.034992 & -1.663334 & 1.909048 \\
\hline $\mathrm{H}$ & -2.968433 & -3.374682 & 3.984861 \\
\hline $\mathrm{H}$ & -1.041106 & -4.764313 & 4.678908 \\
\hline C & -2.963092 & -0.820943 & 0.819336 \\
\hline C & -1.731555 & -0.760624 & 0.086612 \\
\hline $\mathrm{H}$ & -3.952412 & -1.700568 & 2.490662 \\
\hline C & 1.045840 & -4.061185 & -3.722350 \\
\hline C & 2.093703 & -3.256814 & -3.339666 \\
\hline C & 1.976572 & -2.392533 & -2.217344 \\
\hline C & 0.749950 & -2.357211 & -1.481980 \\
\hline C & -0.313118 & -3.203893 & -1.904962 \\
\hline C & -0.168161 & -4.032410 & -2.995174 \\
\hline $\mathrm{H}$ & 3.974752 & -1.600382 & -2.370066 \\
\hline $\mathrm{H}$ & 1.145498 & -4.718301 & -4.582072 \\
\hline $\mathrm{H}$ & 3.031705 & -3.269551 & -3.889842 \\
\hline C & 3.048211 & -1.568242 & -1.802357 \\
\hline C & 0.652159 & -1.478025 & -0.351754 \\
\hline $\mathrm{H}$ & -1.253451 & -3.186251 & -1.365460 \\
\hline $\mathrm{H}$ & -0.994802 & -4.666790 & -3.302946 \\
\hline C & 1.722164 & -0.667454 & 0.025581 \\
\hline C & 2.952547 & -0.714414 & -0.719305 \\
\hline C & 1.581171 & 0.270446 & 1.214545 \\
\hline $\mathrm{H}$ & 0.868971 & -0.144507 & 1.937601 \\
\hline $\mathrm{H}$ & 2.541899 & 0.379975 & 1.722120 \\
\hline C & -1.710390 & 0.008678 & -1.203013 \\
\hline 0 & -2.323618 & -0.285218 & -2.199916 \\
\hline 0 & -0.906552 & 1.113664 & -1.153693 \\
\hline $\mathrm{H}$ & -0.916055 & 1.505442 & -2.048748 \\
\hline 0 & 1.191733 & 1.594310 & 0.840637 \\
\hline $\mathrm{H}$ & 0.407901 & 1.515363 & 0.269669 \\
\hline C & 4.159547 & 0.086823 & -0.351772 \\
\hline C & 4.103234 & 1.479545 & -0.202386 \\
\hline C & 5.392534 & -0.566697 & -0.199553 \\
\hline C & 5.249864 & 2.222693 & 0.101134 \\
\hline $\mathrm{H}$ & 3.143748 & 1.966652 & -0.311314 \\
\hline C & 6.561455 & 0.139816 & 0.104577 \\
\hline $\mathrm{H}$ & 5.416434 & -1.645424 & -0.304217 \\
\hline C & 6.460073 & 1.530853 & 0.249351 \\
\hline $\mathrm{H}$ & 7.356057 & 2.094675 & 0.492124 \\
\hline C & -4.134975 & 0.024399 & 0.457492 \\
\hline C & -5.399761 & -0.557903 & 0.302809 \\
\hline
\end{tabular}




\begin{tabular}{|c|c|c|c|}
\hline C & -3.993867 & 1.410998 & 0.310999 \\
\hline C & -6.523057 & 0.218072 & -0.003996 \\
\hline $\mathrm{H}$ & -5.484417 & -1.633803 & 0.403016 \\
\hline C & -5.091598 & 2.223207 & 0.006371 \\
\hline $\mathrm{H}$ & -3.014789 & 1.850656 & 0.459981 \\
\hline C & -6.338811 & 1.601108 & -0.145420 \\
\hline $\mathrm{H}$ & -7.200095 & 2.217954 & -0.384409 \\
\hline C & -4.969156 & 3.749949 & -0.163166 \\
\hline C & -7.925197 & -0.390557 & -0.198804 \\
\hline C & -5.883813 & 4.462044 & 0.861398 \\
\hline $\mathrm{H}$ & -6.936244 & 4.191107 & 0.727144 \\
\hline $\mathrm{H}$ & -5.600031 & 4.201928 & 1.887522 \\
\hline $\mathrm{H}$ & -5.803872 & 5.550604 & 0.752343 \\
\hline C & -5.401946 & 4.141989 & -1.596086 \\
\hline $\mathrm{H}$ & -5.321294 & 5.227077 & -1.735345 \\
\hline $\mathrm{H}$ & -4.767211 & 3.654003 & -2.344439 \\
\hline $\mathrm{H}$ & -6.438656 & 3.855765 & -1.801744 \\
\hline C & -3.528089 & 4.249545 & 0.056095 \\
\hline $\mathrm{H}$ & -2.825129 & 3.796887 & -0.652502 \\
\hline $\mathrm{H}$ & -3.488098 & 5.335353 & -0.086463 \\
\hline $\mathrm{H}$ & -3.171556 & 4.036496 & 1.070185 \\
\hline C & -8.423995 & -0.086636 & -1.631620 \\
\hline $\mathrm{H}$ & -8.489176 & 0.989721 & -1.822028 \\
\hline $\mathrm{H}$ & -7.749583 & -0.517722 & -2.380092 \\
\hline $\mathrm{H}$ & -9.422748 & -0.513321 & -1.786612 \\
\hline C & -8.903697 & 0.228226 & 0.827587 \\
\hline $\mathrm{H}$ & -8.978218 & 1.315070 & 0.717553 \\
\hline $\mathrm{H}$ & -9.909250 & -0.189713 & 0.695589 \\
\hline $\mathrm{H}$ & -8.580426 & 0.018120 & 1.853667 \\
\hline C & -7.934221 & -1.919104 & -0.005431 \\
\hline $\mathrm{H}$ & -7.284001 & -2.425645 & -0.727478 \\
\hline $\mathrm{H}$ & -7.614787 & -2.205117 & 1.003231 \\
\hline $\mathrm{H}$ & -8.950632 & -2.302016 & -0.151002 \\
\hline C & 7.926091 & -0.552094 & 0.291011 \\
\hline C & 5.210427 & 3.751961 & 0.289379 \\
\hline C & 6.207728 & 4.426092 & -0.682127 \\
\hline $\mathrm{H}$ & 7.238575 & 4.100751 & -0.505892 \\
\hline $\mathrm{H}$ & 5.957503 & 4.193882 & -1.723731 \\
\hline $\mathrm{H}$ & 6.179375 & 5.516020 & -0.561084 \\
\hline C & 5.605064 & 4.093885 & 1.746014 \\
\hline $\mathrm{H}$ & 4.904215 & 3.641219 & 2.456481 \\
\hline $\mathrm{H}$ & 6.609786 & 3.732129 & 1.990002 \\
\hline $\mathrm{H}$ & 5.593047 & 5.179961 & 1.901213 \\
\hline C & 3.810329 & 4.337272 & 0.019536 \\
\hline $\mathrm{H}$ & 3.493387 & 4.160990 & -1.015067 \\
\hline $\mathrm{H}$ & 3.049680 & 3.911200 & 0.681978 \\
\hline $\mathrm{H}$ & 3.828845 & 5.421652 & 0.179900 \\
\hline C & 8.935185 & 0.010321 & -0.738318 \\
\hline $\mathrm{H}$ & 8.592664 & -0.173770 & -1.763097 \\
\hline $\mathrm{H}$ & 9.076692 & 1.089978 & -0.623477 \\
\hline $\mathrm{H}$ & 9.914241 & -0.469192 & -0.615311 \\
\hline C & 7.843970 & -2.077773 & 0.092671 \\
\hline $\mathrm{H}$ & 8.836027 & -2.522336 & 0.231831 \\
\hline $\mathrm{H}$ & 7.167444 & -2.547612 & 0.815594 \\
\hline
\end{tabular}




\section{Catalyst 2C 25}

\section{Datum}

Value

\begin{tabular}{lc}
\hline B3LYP/6-31G(d) Energy & -2164.801871 \\
\hline B3LYP-D3(BJ)/def2-TZVPP-IEF-PCM(DCM) Energy & -2165.897577 \\
\hline B3LYP-D3(BJ)/def2-TZVPP-IEF-PCM(DCM)//B3LYP/6-31G(d) Free Energy (Quasiharmonic) & -2165.019211 \\
\hline
\end{tabular}

Number of Imaginary Frequencies

Frequencies (Top 3 out of 321)
1. $10.2601 \mathrm{~cm}^{-1}$
2. $11.0591 \mathrm{~cm}^{-1}$
3. $12.9003 \mathrm{~cm}^{-1}$

\section{B3LYP/6-31G(d) Molecular Geometry in Cartesian Coordinates}

C

C

C

C

C

C

$\mathrm{H}$

$\mathrm{H}$

C

C

$\mathrm{H}$

$\mathrm{H}$

C

C

$\mathrm{H}$

C

C

C

C

C

C

\subsection{8}

0.364642

$-0.701385$

$-1.926319$

$-2.039904$

$-0.990271$

1.046614

1. 296457

$-0.589820$

$-2.997832$

$-2.974956$

$-1.088556$

$-2.909022$

$-1.680065$

$-3.925790$

1.411995

2.368057

2.145989

0.908793

$-0.058749$

0.187593
$-3.457187$

$-2.897201$

$-2.171412$

$-2.030011$

$-2.619132$

$-3.317543$

$-4.011121$

$-3.007209$

$-1.570066$

$-1.307961$

$-2.506438$

$-3.763380$

$-0.748565$

$-0.899444$

$-1.210494$

$-4.968221$

$-3.991145$

$-2.882910$

$-2.781309$

$-3.808695$

$-4.873874$

\subsection{4}

2. 700238

2.101206

2.828187

4.116405

4.667117

4.391293

2. 155991

0.804417

2. 246926

4.659801

5.653142

0.988194

0.268916

2.804567

$-2.548853$

$-2.396102$

$-1.534066$

$-0.825161$

$-1.007009$

$-1.844462$ 


\begin{tabular}{|c|c|c|c|}
\hline $\mathrm{H}$ & 4.056266 & -1.958274 & -1.903535 \\
\hline $\mathrm{H}$ & 1.592541 & -5.813056 & -3.208101 \\
\hline $\mathrm{H}$ & 3.312922 & -4.052181 & -2.931082 \\
\hline C & 3.122372 & -1.875748 & -1.353337 \\
\hline C & 0.703977 & -1.655091 & 0.039506 \\
\hline H & -1.004307 & -3.745280 & -0.479455 \\
\hline $\mathrm{H}$ & -0.564976 & -5.648016 & -1.968249 \\
\hline C & 1.684412 & -0.675132 & 0.198203 \\
\hline C & 2.928357 & -0.790603 & -0.518548 \\
\hline C & 1.453331 & 0.491836 & 1.152732 \\
\hline $\mathrm{H}$ & 0.757145 & 0.187745 & 1.943949 \\
\hline $\mathrm{H}$ & 2.398190 & 0.757037 & 1.633468 \\
\hline C & -1.515290 & -0.227281 & -1.065572 \\
\hline 0 & -1.146423 & 0.920970 & -1.223980 \\
\hline 0 & -1.800609 & -1.033782 & -2.106522 \\
\hline $\mathrm{H}$ & -1.628201 & -0.512782 & -2.915751 \\
\hline 0 & 1.000609 & 1.686255 & 0.521491 \\
\hline $\mathrm{H}$ & 0.175790 & 1.492813 & 0.035687 \\
\hline C & 4.052094 & 0.183882 & -0.372789 \\
\hline C & 3.862393 & 1.562419 & -0.542702 \\
\hline C & 5.343873 & -0.300205 & -0.110009 \\
\hline C & 4.933956 & 2.458047 & -0.446771 \\
\hline $\mathrm{H}$ & 2.859451 & 1.920128 & -0.731093 \\
\hline C & 6.441162 & 0.561855 & -0.009980 \\
\hline $\mathrm{H}$ & 5.471891 & -1.366923 & 0.034448 \\
\hline C & 6.206617 & 1.933653 & -0.181707 \\
\hline $\mathrm{H}$ & 7.045211 & 2.618857 & -0.098871 \\
\hline C & -4.068672 & 0.006498 & 0.430635 \\
\hline C & -4.652031 & -0.353152 & -0.790435 \\
\hline C & -4.613045 & 1.074202 & 1.155372 \\
\hline C & -5.758035 & 0.338544 & -1.300502 \\
\hline $\mathrm{H}$ & -4.243108 & -1.197352 & -1.334760 \\
\hline C & -5.716652 & 1.793233 & 0.680893 \\
\hline $\mathrm{H}$ & -4.143875 & 1.351864 & 2.094542 \\
\hline C & -6.269141 & 1.406260 & -0.548972 \\
\hline $\mathrm{H}$ & -7.120410 & 1.951728 & -0.931837 \\
\hline C & -6.270373 & 2.971070 & 1.507194 \\
\hline C & -6.368079 & -0.096709 & -2.647864 \\
\hline C & -6.710126 & 2.460256 & 2.899997 \\
\hline $\mathrm{H}$ & -7.496576 & 1.701952 & 2.809932 \\
\hline $\mathrm{H}$ & -5.876856 & 2.013936 & 3.452701 \\
\hline $\mathrm{H}$ & -7.103872 & 3.287735 & 3.502855 \\
\hline C & -7.485847 & 3.642131 & 0.840079 \\
\hline $\mathrm{H}$ & -7.840461 & 4.466986 & 1.468288 \\
\hline $\mathrm{H}$ & -7.234533 & 4.060591 & -0.141110 \\
\hline $\mathrm{H}$ & -8.320576 & 2.943535 & 0.711247 \\
\hline C & -5.166199 & 4.041574 & 1.679617 \\
\hline $\mathrm{H}$ & -4.838366 & 4.426260 & 0.707293 \\
\hline $\mathrm{H}$ & -5.543105 & 4.885653 & 2.270234 \\
\hline $\mathrm{H}$ & -4.285843 & 3.641974 & 2.193275 \\
\hline C & -5.292891 & 0.014797 & -3.755414 \\
\hline $\mathrm{H}$ & -4.942105 & 1.048451 & -3.856042 \\
\hline $\mathrm{H}$ & -4.423964 & -0.616333 & -3.541331 \\
\hline H & -5.705058 & -0.300225 & -4.721920 \\
\hline
\end{tabular}




\begin{tabular}{|c|c|c|c|}
\hline C & -7.571029 & 0.771311 & -3.062057 \\
\hline $\mathrm{H}$ & -7.294058 & 1.824997 & -3.181088 \\
\hline $\mathrm{H}$ & -7.962255 & 0.422488 & -4.024447 \\
\hline $\mathrm{H}$ & -8.387833 & 0.711955 & -2.333746 \\
\hline C & -6.847707 & -1.564446 & -2.546988 \\
\hline $\mathrm{H}$ & -6.026330 & -2.246474 & -2.304223 \\
\hline $\mathrm{H}$ & -7.613746 & -1.673568 & -1.770760 \\
\hline $\mathrm{H}$ & -7.281097 & -1.890598 & -3.500488 \\
\hline C & 7.869251 & 0.059510 & 0.281243 \\
\hline C & 4.746584 & 3.979726 & -0.606360 \\
\hline C & 3.293810 & 4.355042 & -0.957433 \\
\hline $\mathrm{H}$ & 2.987311 & 3.923922 & -1.917503 \\
\hline $\mathrm{H}$ & 2.583983 & 4.016606 & -0.195758 \\
\hline $\mathrm{H}$ & 3.206544 & 5.444712 & -1.040822 \\
\hline C & 5.665503 & 4.505736 & -1.734072 \\
\hline $\mathrm{H}$ & 6.724808 & 4.326251 & -1.520797 \\
\hline $\mathrm{H}$ & 5.429926 & 4.020877 & -2.688450 \\
\hline $\mathrm{H}$ & 5.531743 & 5.587179 & -1.860830 \\
\hline C & 5.117666 & 4.678087 & 0.723801 \\
\hline $\mathrm{H}$ & 4.467667 & 4.336829 & 1.537402 \\
\hline $\mathrm{H}$ & 6.154012 & 4.475434 & 1.015430 \\
\hline $\mathrm{H}$ & 5.002658 & 5.765275 & 0.630151 \\
\hline C & 8.801680 & 0.455470 & -0.888384 \\
\hline $\mathrm{H}$ & 8.463210 & 0.003874 & -1.828048 \\
\hline $\mathrm{H}$ & 8.835792 & 1.540183 & -1.033988 \\
\hline $\mathrm{H}$ & 9.825603 & 0.112159 & -0.694994 \\
\hline C & 7.932558 & -1.471320 & 0.443429 \\
\hline $\mathrm{H}$ & 8.965042 & -1.779296 & 0.644377 \\
\hline $\mathrm{H}$ & 7.315377 & -1.819061 & 1.279635 \\
\hline $\mathrm{H}$ & 7.603589 & -1.991535 & -0.463410 \\
\hline C & 8.389266 & 0.701071 & 1.589628 \\
\hline $\mathrm{H}$ & 7.752498 & 0.428755 & 2.439143 \\
\hline $\mathrm{H}$ & 9.409001 & 0.358186 & 1.804816 \\
\hline $\mathrm{H}$ & 8.411946 & 1.794051 & 1.527614 \\
\hline
\end{tabular}

\section{Catalyst 2C 26}

Datum

Value

\begin{tabular}{lc}
\hline B3LYP/6-31G(d) Energy & -2164.799955 \\
\hline B3LYP-D3(BJ)/def2-TZVPP-IEF-PCM(DCM) Energy & -2165.897219 \\
\hline B3LYP-D3(BJ)/def2-TZVPP-IEF-PCM(DCM)//B3LYP/6-31G(d) Free Energy (Quasiharmonic) & -2165.019195 \\
\hline Number of Imaginary Frequencies & 0
\end{tabular}

Frequencies (Top 3 out of 321) 


$\begin{array}{ll}\text { 1. } & 10.8674 \mathrm{~cm}^{-1} \\ \text { 2. } & 11.6066 \mathrm{~cm}^{-1} \\ \text { 3. } & 13.0855 \mathrm{~cm}^{-1}\end{array}$

\section{B3LYP/6-31G(d) Molecular Geometry in Cartesian Coordinates}

C

C

C

C

C

C

$\mathrm{H}$

$\mathrm{H}$

C

$\mathrm{C}$

$\mathrm{H}$

$\mathrm{H}$

C

C

$\mathrm{H}$

C

C

C

C

C

C

$\mathrm{H}$

$\mathrm{H}$

$\mathrm{H}$

C

C

$\mathrm{H}$

$\mathrm{H}$

C

C

C

$\mathrm{H}$

$\mathrm{H}$

C

0

0

$\mathrm{H}$

0

$\mathrm{H}$

C

C

C

C

$\mathrm{H}$
0.241363

0.366260

$-0.719274$

$-1.950840$

$-2.045918$

$-0.974964$

1.082746

1.301906

$-0.625954$

$-3.045873$

$-2.984996$

$-1.060060$

$-2.969754$

$-1.736963$

$-3.965279$

1.042207

2.087810

1.969688

0.744527

$-0.316306$

$-0.170380$

3.963820

1.142512

3.024642

3.038968

0.645706

$-1.255672$

$-0.995277$

1.713944

2.942990

1.571982

0.863882

2.533583

$-1.713269$

$-2.310945$

$-0.927011$

$-0.937537$

1.175087

0.393075

4.148458

4.086996

5.383527

5.229817

3. 124094
$-4.030779$

$-3.197678$

$-2.383525$

$-2.456462$

$-3.323169$

$-4.094619$

$-4.644554$

$-3.155107$

$-1.503642$

$-1.656607$

$-3.365284$

$-4.754795$

$-0.815370$

$-0.757879$

$-1.693308$

$-4.061420$

$-3.252654$

$-2.387815$

$-2.356327$

$-3.207203$

$-4.036433$

$-1.586886$

$-4.719001$

$-3.262246$

$-1.558875$

$-1.476051$

$-3.192152$

$-4.674178$

$-0.662147$

$-0.705428$

0.275198

$-0.143252$

0.389175

0.008630

$-0.297428$

1.126429

1.519287

1.597253

1.515032

0.099914

1.495576

$-0.542078$

2. 240847

1.977715
3.121633

2.031875

1.605660

2. 331698

3.453591

3.841904

3.431187

1.485303

0.474587

1.921756

4.000061

4.700729

0.831641

0.101269

2.500340

$-3.701554$

$-3.321959$

$-2.200192$

$-1.462382$

$-1.882358$

$-2.971894$

$-2.359001$

$-4.560841$

$-3.874183$

$-1.788382$

$-0.333037$

$-1.341133$

$-3.277372$

0.042258

$-0.705054$

1.231567

1.956641

1.736562

$-1.190370$

$-2.192789$

$-1.135140$

$-2.029797$

0.859044

0.286044

$-0.341391$

$-0.186814$

$-0.200524$

0.109249

$-0.287646$ 


\begin{tabular}{|c|c|c|c|}
\hline C & 6.556239 & 0.170419 & 0.095132 \\
\hline $\mathrm{H}$ & 5.419329 & -1.622570 & -0.307233 \\
\hline C & 6.451105 & 1.556111 & 0.245362 \\
\hline $\mathrm{H}$ & 7.340754 & 2.128561 & 0.480449 \\
\hline C & -4.138125 & 0.031602 & 0.462135 \\
\hline C & -3.997950 & 1.413645 & 0.317710 \\
\hline C & -5.404599 & -0.550610 & 0.289955 \\
\hline C & -5.092034 & 2.230542 & -0.007245 \\
\hline $\mathrm{H}$ & -3.021547 & 1.859329 & 0.480548 \\
\hline C & -6.519171 & 0.225247 & -0.032387 \\
\hline $\mathrm{H}$ & -5.490712 & -1.626481 & 0.389462 \\
\hline C & -6.334393 & 1.613120 & -0.175408 \\
\hline $\mathrm{H}$ & -7.193277 & 2.223228 & -0.428931 \\
\hline C & -7.919506 & -0.380477 & -0.246954 \\
\hline C & -4.883467 & 3.749762 & -0.156581 \\
\hline C & -7.934737 & -1.908698 & -0.052198 \\
\hline $\mathrm{H}$ & -7.275498 & -2.417356 & -0.764514 \\
\hline $\mathrm{H}$ & -7.630182 & -2.194294 & 0.961162 \\
\hline $\mathrm{H}$ & -8.949847 & -2.289518 & -0.211767 \\
\hline C & -8.396948 & -0.077116 & -1.687260 \\
\hline $\mathrm{H}$ & -9.394363 & -0.501775 & -1.855753 \\
\hline $\mathrm{H}$ & -8.456964 & 0.999075 & -1.880129 \\
\hline $\mathrm{H}$ & -7.712913 & -0.511059 & -2.425297 \\
\hline C & -8.911628 & 0.241479 & 0.764446 \\
\hline $\mathrm{H}$ & -8.980927 & 1.328558 & 0.653589 \\
\hline $\mathrm{H}$ & -9.916364 & -0.173166 & 0.616779 \\
\hline $\mathrm{H}$ & -8.604880 & 0.029829 & 1.795290 \\
\hline C & -3.828618 & 4.015730 & -1.257625 \\
\hline $\mathrm{H}$ & -2.866007 & 3.550769 & -1.019585 \\
\hline $\mathrm{H}$ & -4.163279 & 3.619349 & -2.223223 \\
\hline $\mathrm{H}$ & -3.658947 & 5.093342 & -1.371467 \\
\hline C & -4.381459 & 4.333780 & 1.185389 \\
\hline $\mathrm{H}$ & -3.435156 & 3.880750 & 1.498679 \\
\hline $\mathrm{H}$ & -4.220117 & 5.414992 & 1.094912 \\
\hline $\mathrm{H}$ & -5.113477 & 4.164865 & 1.983443 \\
\hline C & -6.177672 & 4.487968 & -0.547957 \\
\hline $\mathrm{H}$ & -6.576320 & 4.133379 & -1.505382 \\
\hline $\mathrm{H}$ & -6.959273 & 4.375040 & 0.212079 \\
\hline $\mathrm{H}$ & -5.973333 & 5.559571 & -0.652051 \\
\hline C & 7.889497 & -0.587882 & 0.247365 \\
\hline C & 5.185561 & 3.769062 & 0.303214 \\
\hline C & 3.780560 & 4.349389 & 0.048914 \\
\hline $\mathrm{H}$ & 3.454928 & 4.176156 & -0.983491 \\
\hline $\mathrm{H}$ & 3.027826 & 3.917021 & 0.716287 \\
\hline $\mathrm{H}$ & 3.795860 & 5.433127 & 0.213926 \\
\hline C & 6.170587 & 4.451433 & -0.675201 \\
\hline $\mathrm{H}$ & 7.204575 & 4.130430 & -0.509996 \\
\hline $\mathrm{H}$ & 5.911390 & 4.222023 & -1.715244 \\
\hline $\mathrm{H}$ & 6.138174 & 5.540761 & -0.549734 \\
\hline C & 5.592637 & 4.107611 & 1.757324 \\
\hline $\mathrm{H}$ & 4.900017 & 3.649953 & 2.472673 \\
\hline $\mathrm{H}$ & 6.600933 & 3.748979 & 1.990759 \\
\hline $\mathrm{H}$ & 5.577961 & 5.193122 & 1.916283 \\
\hline C & 7.773736 & -1.608507 & 1.404431 \\
\hline
\end{tabular}




\begin{tabular}{|lrrr|}
$H$ & 7.558078 & -1.102426 & 2.352392 \\
$H$ & 6.976357 & -2.337858 & 1.226920 \\
$H$ & 8.713359 & -2.162813 & 1.520660 \\
$\mathrm{C}$ & 9.071188 & 0.351054 & 0.556200 \\
$\mathrm{H}$ & 9.993223 & -0.234479 & 0.646871 \\
$\mathrm{H}$ & 9.225736 & 1.089145 & -0.239184 \\
$\mathrm{H}$ & 8.929353 & 0.889855 & 1.499951 \\
$\mathrm{C}$ & 8.207354 & -1.339695 & -1.067150 \\
$\mathrm{H}$ & 8.302305 & -0.639511 & -1.905072 \\
$\mathrm{H}$ & 9.151964 & -1.889939 & -0.974677 \\
$\mathrm{H}$ & 7.425327 & -2.062284 & -1.322543 \\
& & & \\
\hline
\end{tabular}

\section{Catalyst 2C 27}

Datum

Value

\begin{tabular}{lr}
\hline B3LYP/6-31G(d) Energy & -2164.801788 \\
\hline B3LYP-D3(BJ)/def2-TZVPP-IEF-PCM(DCM) Energy & -2165.897571 \\
\hline B3LYP-D3(BJ)/def2-TZVPP-IEF-PCM(DCM)//B3LYP/6-31G(d) Free Energy (Quasiharmonic) & -2165.019188 \\
\hline Number of Imaginary Frequencies & 0
\end{tabular}

Frequencies (Top 3 out of 321)
1. $9.6851 \mathrm{~cm}^{-1}$
2. $12.3075 \mathrm{~cm}^{-1}$
3. $12.9752 \mathrm{~cm}^{-1}$

\section{B3LYP/6-31G(d) Molecular Geometry in Cartesian Coordinates}

$\begin{array}{lrrl}\text { C } & 0.269978 & -3.486188 & 3.924630 \\ \text { C } & 0.404278 & -2.918084 & 2.677157 \\ \text { C } & -0.667269 & -2.192746 & 2.087446 \\ \text { C } & -1.889337 & -2.060617 & 2.820917 \\ \text { C } & -1.994621 & -2.657820 & 4.106130 \\ \text { C } & -0.939808 & -3.355484 & 4.647780 \\ \text { H } & 1.098337 & -4.039670 & 4.358188 \\ \text { H } & 1.333984 & -3.021322 & 2.127999 \\ \text { C } & -0.564115 & -1.582730 & 0.794027 \\ \text { C } & -2.966461 & -1.339691 & 2.248821 \\ \text { H } & -2.927571 & -2.551859 & 4.654470 \\ \text { H } & -1.031744 & -3.807593 & 5.631557 \\ \text { C } & -2.886225 & -0.772651 & 0.992827 \\ \text { C } & -1.659691 & -0.913703 & 0.267492 \\ \text { H } & -3.892160 & -1.249275 & 2.811354\end{array}$




\begin{tabular}{|c|c|c|c|}
\hline C & 1.440152 & -4.946621 & -2.592579 \\
\hline C & 2.393184 & -3.967434 & -2.434502 \\
\hline C & 2.169528 & -2.866935 & -1.563004 \\
\hline C & 0.933820 & -2.775339 & -0.850119 \\
\hline C & -0.030520 & -3.804751 & -1.037533 \\
\hline C & 0.217317 & -4.862270 & -1.884201 \\
\hline $\mathrm{H}$ & 4.076213 & -1.933445 & -1.928844 \\
\hline $\mathrm{H}$ & 1.621902 & -5.785508 & -3.259049 \\
\hline $\mathrm{H}$ & 3.336868 & -4.020883 & -2.972377 \\
\hline C & 3.143093 & -1.858148 & -1.376302 \\
\hline C & 0.727204 & -1.656691 & 0.023840 \\
\hline $\mathrm{H}$ & -0.974946 & -3.748910 & -0.507103 \\
\hline $\mathrm{H}$ & -0.532871 & -5.638017 & -2.012262 \\
\hline C & 1.704498 & -0.674427 & 0.187564 \\
\hline C & 2.947419 & -0.780371 & -0.532388 \\
\hline C & 1.470555 & 0.485514 & 1.149833 \\
\hline $\mathrm{H}$ & 0.776914 & 0.174015 & 1.940345 \\
\hline $\mathrm{H}$ & 2.415086 & 0.751872 & 1.630515 \\
\hline C & -1.503864 & -0.232835 & -1.063522 \\
\hline 0 & -1.139148 & 0.917581 & -1.216960 \\
\hline 0 & -1.792716 & -1.033540 & -2.107471 \\
\hline $\mathrm{H}$ & -1.623209 & -0.507942 & -2.914278 \\
\hline 0 & 1.011752 & 1.681567 & 0.525975 \\
\hline $\mathrm{H}$ & 0.186830 & 1.487114 & 0.040726 \\
\hline C & 4.068972 & 0.195573 & -0.379712 \\
\hline C & 3.876026 & 1.575192 & -0.536651 \\
\hline C & 5.362050 & -0.288009 & -0.122422 \\
\hline C & 4.945652 & 2.472328 & -0.433051 \\
\hline $\mathrm{H}$ & 2.872098 & 1.932342 & -0.720970 \\
\hline C & 6.457450 & 0.575548 & -0.015058 \\
\hline $\mathrm{H}$ & 5.492494 & -1.355723 & 0.012116 \\
\hline C & 6.219687 & 1.948348 & -0.173787 \\
\hline $\mathrm{H}$ & 7.056832 & 2.634573 & -0.084907 \\
\hline C & -4.052626 & -0.020275 & 0.445630 \\
\hline C & -4.644387 & -0.384747 & -0.771412 \\
\hline C & -4.591846 & 1.043995 & 1.181576 \\
\hline C & -5.760399 & 0.300691 & -1.266197 \\
\hline $\mathrm{H}$ & -4.233055 & -1.224616 & -1.316491 \\
\hline C & -5.703770 & 1.756827 & 0.719602 \\
\hline $\mathrm{H}$ & -4.111179 & 1.317159 & 2.113703 \\
\hline C & -6.264410 & 1.363651 & -0.504046 \\
\hline $\mathrm{H}$ & -7.127604 & 1.906518 & -0.877401 \\
\hline C & -6.312611 & 2.941563 & 1.495117 \\
\hline C & -6.441034 & -0.078096 & -2.596450 \\
\hline C & -7.789783 & 2.631050 & 1.834897 \\
\hline $\mathrm{H}$ & -8.390253 & 2.462122 & 0.934845 \\
\hline $\mathrm{H}$ & -7.868745 & 1.735100 & 2.461406 \\
\hline $\mathrm{H}$ & -8.238832 & 3.469041 & 2.381973 \\
\hline C & -6.244784 & 4.217794 & 0.622945 \\
\hline $\mathrm{H}$ & -6.678748 & 5.069819 & 1.160506 \\
\hline $\mathrm{H}$ & -5.207391 & 4.466103 & 0.372200 \\
\hline $\mathrm{H}$ & -6.796406 & 4.100829 & -0.315785 \\
\hline C & -5.568437 & 3.224727 & 2.813853 \\
\hline $\mathrm{H}$ & -4.518437 & 3.486628 & 2.642235 \\
\hline
\end{tabular}




\begin{tabular}{|c|c|c|c|}
\hline $\mathrm{H}$ & -6.039238 & 4.070072 & 3.328221 \\
\hline $\mathrm{H}$ & -5.600613 & 2.365863 & 3.494020 \\
\hline C & -5.754568 & -1.271501 & -3.287540 \\
\hline $\mathrm{H}$ & -4.703896 & -1.062093 & -3.518017 \\
\hline $\mathrm{H}$ & -5.792521 & -2.177149 & -2.671698 \\
\hline $\mathrm{H}$ & -6.263652 & -1.493341 & -4.232238 \\
\hline C & -6.390999 & 1.130272 & -3.561631 \\
\hline $\mathrm{H}$ & -5.355722 & 1.423744 & -3.769708 \\
\hline $\mathrm{H}$ & -6.871442 & 0.877701 & -4.514850 \\
\hline $\mathrm{H}$ & -6.907818 & 2.003555 & -3.150139 \\
\hline C & -7.916451 & -0.461377 & -2.332350 \\
\hline $\mathrm{H}$ & -7.982551 & -1.319114 & -1.653282 \\
\hline $\mathrm{H}$ & -8.478775 & 0.364073 & -1.883454 \\
\hline $\mathrm{H}$ & -8.414823 & -0.732119 & -3.271328 \\
\hline C & 7.886745 & 0.073830 & 0.271399 \\
\hline C & 4.754550 & 3.995107 & -0.577264 \\
\hline C & 3.301017 & 4.370365 & -0.925237 \\
\hline $\mathrm{H}$ & 2.995996 & 3.948070 & -1.889686 \\
\hline $\mathrm{H}$ & 2.591708 & 4.022700 & -0.167261 \\
\hline $\mathrm{H}$ & 3.211176 & 5.460601 & -0.997802 \\
\hline C & 5.672665 & 4.535281 & -1.698904 \\
\hline $\mathrm{H}$ & 6.732356 & 4.356766 & -1.486793 \\
\hline $\mathrm{H}$ & 5.439117 & 4.059632 & -2.658391 \\
\hline $\mathrm{H}$ & 5.535854 & 5.617594 & -1.814555 \\
\hline C & 5.123178 & 4.680619 & 0.760258 \\
\hline $\mathrm{H}$ & 4.473403 & 4.329632 & 1.569884 \\
\hline $\mathrm{H}$ & 6.159765 & 4.477210 & 1.050494 \\
\hline $\mathrm{H}$ & 5.005864 & 5.768460 & 0.677682 \\
\hline C & 8.818973 & 0.484926 & -0.893150 \\
\hline $\mathrm{H}$ & 8.482638 & 0.042343 & -1.837853 \\
\hline $\mathrm{H}$ & 8.850217 & 1.571184 & -1.027279 \\
\hline $\mathrm{H}$ & 9.843669 & 0.142453 & -0.702459 \\
\hline C & 7.954093 & -1.458503 & 0.416842 \\
\hline $\mathrm{H}$ & 8.987420 & -1.766009 & 0.614151 \\
\hline $\mathrm{H}$ & 7.338063 & -1.817012 & 1.249327 \\
\hline $\mathrm{H}$ & 7.626226 & -1.969567 & -0.495577 \\
\hline C & 8.404029 & 0.702507 & 1.587115 \\
\hline $\mathrm{H}$ & 7.767669 & 0.418667 & 2.433154 \\
\hline $\mathrm{H}$ & 9.424760 & 0.360592 & 1.799115 \\
\hline $\mathrm{H}$ & 8.423066 & 1.796168 & 1.537179 \\
\hline
\end{tabular}

\section{Catalyst 2C 28}

Datum

Value

\begin{tabular}{lc}
\hline B3LYP/6-31G(d) Energy & -2164.799674 \\
\hline B3LYP-D3(BJ)/def2-TZVPP-IEF-PCM(DCM) Energy & -2165.897012 \\
\hline B3LYP-D3(BJ)/def2-TZVPP-IEF-PCM(DCM)//B3LYP/6-31G(d) Free Energy (Quasiharmonic) & -2165.019153 \\
\hline
\end{tabular}


Frequencies (Top 3 out of 321)

$\begin{array}{lr}\text { 1. } & 9.6487 \mathrm{~cm}^{-1} \\ \text { 2. } & 10.8185 \mathrm{~cm}^{-1} \\ \text { 3. } & 13.0984 \mathrm{~cm}^{-1}\end{array}$

\section{B3LYP/6-31G(d) Molecular Geometry in Cartesian Coordinates}

C

C

C

C

C

C

$\mathrm{H}$

$\mathrm{H}$

C

C

$\mathrm{H}$

$\mathrm{H}$

C

C

$\mathrm{H}$

C

C

C

C

C

C

$\mathrm{H}$

$\mathrm{H}$

$\mathrm{H}$

C

C

$\mathrm{H}$

$\mathrm{H}$

C

C

C

$\mathrm{H}$

$\mathrm{H}$

C

0

0

$\mathrm{H}$

\section{$-0.265081$}

$-0.387346$

0.700804

1.932219

2.024624

0.951126

$-1.108485$

$-1.322968$

0.610173

3.029960

2.963711

1.034127

2. 957307

1.723787

3.949266

$-1.059647$

$-2.105035$

$-1.986398$

$-0.760889$

0.299663

0.153234

$-3.980721$

$-1.160389$

$-3.042155$

$-3.055550$

$-0.661595$

1. 239181

0.977931

$-1.729687$

$-2.958958$

$-1.587505$

$-2.548918$

$-0.878777$

1.700963

2.308450

0.902859

0.910451
$-4.027585$

$-3.192547$

$-2.381297$

$-2.459700$

$-3.328217$

$-4.096515$

$-4.638948$

$-3.146114$

$-1.498693$

$-1.663443$

$-3.374324$

$-4.758135$

$-0.820537$

$-0.756079$

$-1.704187$

$-4.045364$

$-3.237439$

$-2.375186$

$-2.345338$

$-3.195429$

$-4.022131$

$-1.574014$

$-4.700999$

$-3.245771$

$-1.547296$

$-1.467791$

$-3.182060$

$-4.659316$

$-0.654874$

$-0.696386$

0.279959

0.392233

$-0.139764$

0.014956

$-0.280390$

1.123815

1.517287

\section{113479}

2.024897

1. 599715

2. 325243

3. 445964

3.833478

3.422307

1.478633

0.470482

1.915852

3.992101

4.691403

0.826624

0.097776

2. 494262

$-3.711435$

$-3.329462$

$-2.205762$

$-1.468405$

$-1.890828$

$-2.982222$

$-2.361688$

$-4.572158$

$-3.881222$

$-1.791517$

$-0.336932$

$-1.349823$

- 3.289416

0.040712

$-0.706279$

1. 232018

1.737776

1.955757

$-1.190837$

$-2.190792$

$-1.136122$

$-2.030456$ 


\begin{tabular}{|c|c|c|c|}
\hline 0 & -1.191669 & 1.603022 & 0.862286 \\
\hline $\mathrm{H}$ & -0.407908 & 1.522516 & 0.291441 \\
\hline C & -4.164057 & 0.108594 & -0.340544 \\
\hline C & -4.102369 & 1.504202 & -0.185712 \\
\hline C & -5.398912 & -0.533472 & -0.198360 \\
\hline C & -5.244861 & 2.249438 & 0.111647 \\
\hline $\mathrm{H}$ & -3.139503 & 1.986233 & -0.287318 \\
\hline C & -6.571262 & 0.178967 & 0.098954 \\
\hline $\mathrm{H}$ & -5.434825 & -1.613934 & -0.305401 \\
\hline C & -6.465973 & 1.564650 & 0.249152 \\
\hline $\mathrm{H}$ & -7.355353 & 2.137081 & 0.485306 \\
\hline C & 4.130949 & 0.020134 & 0.459447 \\
\hline C & 3.995575 & 1.407759 & 0.317452 \\
\hline C & 5.391411 & -0.568208 & 0.293102 \\
\hline C & 5.094844 & 2.215135 & 0.005541 \\
\hline $\mathrm{H}$ & 3.019903 & 1.851938 & 0.475225 \\
\hline C & 6.515925 & 0.202754 & -0.021838 \\
\hline $\mathrm{H}$ & 5.471390 & -1.644746 & 0.390293 \\
\hline C & 6.337442 & 1.586997 & -0.158680 \\
\hline $\mathrm{H}$ & 7.199631 & 2.199952 & -0.404217 \\
\hline C & 7.913084 & -0.412642 & -0.230937 \\
\hline C & 4.978674 & 3.742811 & -0.159920 \\
\hline C & 7.916012 & -1.941611 & -0.040926 \\
\hline $\mathrm{H}$ & 7.604710 & -2.228212 & 0.970108 \\
\hline $\mathrm{H}$ & 7.256340 & -2.443171 & -0.757863 \\
\hline $\mathrm{H}$ & 8.928984 & -2.329479 & -0.197024 \\
\hline C & 8.904327 & 0.198890 & 0.787524 \\
\hline $\mathrm{H}$ & 9.906614 & -0.223266 & 0.644614 \\
\hline $\mathrm{H}$ & 8.982782 & 1.285676 & 0.679664 \\
\hline $\mathrm{H}$ & 8.590060 & -0.012515 & 1.816132 \\
\hline C & 8.400025 & -0.108208 & -1.667728 \\
\hline $\mathrm{H}$ & 8.468687 & 0.968206 & -1.856597 \\
\hline $\mathrm{H}$ & 9.395203 & -0.539436 & -1.832762 \\
\hline $\mathrm{H}$ & 7.716592 & -0.534475 & -2.410757 \\
\hline C & 3.542839 & 4.249592 & 0.076611 \\
\hline $\mathrm{H}$ & 2.829671 & 3.802670 & -0.625402 \\
\hline $\mathrm{H}$ & 3.196319 & 4.035660 & 1.094004 \\
\hline $\mathrm{H}$ & 3.507090 & 5.335982 & -0.062598 \\
\hline C & 5.397205 & 4.134904 & -1.597040 \\
\hline $\mathrm{H}$ & 4.750944 & 3.652065 & -2.338843 \\
\hline $\mathrm{H}$ & 5.321466 & 5.220692 & -1.733493 \\
\hline $\mathrm{H}$ & 6.429743 & 3.842776 & -1.815145 \\
\hline C & 5.908719 & 4.448364 & 0.855267 \\
\hline $\mathrm{H}$ & 5.635561 & 4.187750 & 1.884149 \\
\hline $\mathrm{H}$ & 6.958195 & 4.172560 & 0.708296 \\
\hline $\mathrm{H}$ & 5.832967 & 5.537494 & 0.749078 \\
\hline C & -7.904269 & -0.579406 & 0.253015 \\
\hline C & -5.200468 & 3.777687 & 0.305244 \\
\hline C & -3.795641 & 4.357920 & 0.049768 \\
\hline $\mathrm{H}$ & -3.042424 & 3.925610 & 0.716635 \\
\hline $\mathrm{H}$ & -3.470818 & 4.184517 & -0.982867 \\
\hline $\mathrm{H}$ & -3.810748 & 5.441689 & 0.214605 \\
\hline C & -5.606494 & 4.116714 & 1.759524 \\
\hline $\mathrm{H}$ & -6.614628 & 3.758186 & 1.993824 \\
\hline
\end{tabular}




\begin{tabular}{|lrrr|}
$H$ & -4.913346 & 3.659305 & 2.474518 \\
$H$ & -5.591694 & 5.202282 & 1.918085 \\
$\mathrm{C}$ & -6.186213 & 4.459704 & -0.672703 \\
$\mathrm{H}$ & -5.927725 & 4.229961 & -1.712852 \\
$\mathrm{H}$ & -7.220064 & 4.138667 & -0.506677 \\
$\mathrm{H}$ & -6.153780 & 5.549078 & -0.547621 \\
$\mathrm{C}$ & -8.223707 & -1.331681 & -1.060845 \\
$\mathrm{H}$ & -8.319706 & -0.631789 & -1.898894 \\
$\mathrm{H}$ & -7.441987 & -2.054349 & -1.316948 \\
$\mathrm{H}$ & -9.168191 & -1.881914 & -0.967022 \\
$\mathrm{C}$ & -9.085676 & 0.359507 & 0.563016 \\
$\mathrm{H}$ & -10.007566 & -0.226081 & 0.654828 \\
$\mathrm{H}$ & -8.942835 & 0.898488 & 1.506513 \\
$\mathrm{H}$ & -9.241162 & 1.097446 & -0.232328 \\
$\mathrm{C}$ & -7.786936 & -1.599637 & 1.410276 \\
$\mathrm{H}$ & -7.570175 & -1.093200 & 2.357797 \\
$\mathrm{H}$ & -8.726327 & -2.154055 & 1.527856 \\
$\mathrm{H}$ & -6.989641 & -2.328896 & 1.232001 \\
\hline
\end{tabular}

\section{Catalyst 2C 29}

\section{Datum}

Value

\begin{tabular}{lc}
\hline B3LYP/6-31G(d) Energy & -2164.801887 \\
\hline B3LYP-D3(BJ)/def2-TZVPP-IEF-PCM(DCM) Energy & -2165.897704 \\
\hline B3LYP-D3(BJ)/def2-TZVPP-IEF-PCM(DCM)//B3LYP/6-31G(d) Free Energy (Quasiharmonic) & -2165.01915 \\
\hline
\end{tabular}

Number of Imaginary Frequencies

Frequencies (Top 3 out of 321)
1. $9.9838 \mathrm{~cm}^{-1}$
2. $12.3933 \mathrm{~cm}^{-1}$
3. $12.7805 \mathrm{~cm}^{-1}$

\section{B3LYP/6-31G(d) Molecular Geometry in Cartesian Coordinates}

C

C

C

C

C

C

$\mathrm{H}$

$\mathrm{H}$
0.266570

0.402739

$-0.669375$

$-1.893869$

$-2.001121$

$-0.945720$

1.095399

1.334354
$-3.503194$

$-2.926066$

$-2.200073$

$-2.076681$

$-2.683160$

$-3.381363$

$-4.057062$

$-3.022711$

\subsection{3}

2.654671

2.066834

2.797728

4.078408

4.618258

4.329960

2.107570 


\begin{tabular}{|c|c|c|c|}
\hline C & -0.564715 & -1.581574 & 0.777533 \\
\hline C & -2.971416 & -1.354682 & 2. 227701 \\
\hline $\mathrm{H}$ & -2.936023 & -2.583932 & 4.624667 \\
\hline $\mathrm{H}$ & -1.039115 & -3.840715 & 5.598531 \\
\hline C & -2.889064 & -0.778238 & 0.976159 \\
\hline C & -1.660640 & -0.911975 & 0.252541 \\
\hline $\mathrm{H}$ & -3.899060 & -1.270930 & 2.788033 \\
\hline C & 1.463248 & -4.925445 & -2.614940 \\
\hline C & 2.412487 & -3.943734 & -2.449565 \\
\hline C & 2.181691 & -2.847118 & -1.575118 \\
\hline C & 0.942975 & -2.762155 & -0.866661 \\
\hline C & -0.017251 & -3.793976 & -1.061615 \\
\hline C & 0.237370 & -4.847584 & -1.911181 \\
\hline $\mathrm{H}$ & 4.086650 & -1.905009 & -1.930506 \\
\hline $\mathrm{H}$ & 1.650304 & -5.761250 & -3.283804 \\
\hline $\mathrm{H}$ & 3.358354 & -3.992046 & -2.984077 \\
\hline C & 3.150991 & -1.835334 & -1.381487 \\
\hline C & 0.728902 & -1.647328 & 0.010537 \\
\hline $\mathrm{H}$ & -0.964028 & -3.743015 & -0.534901 \\
\hline $\mathrm{H}$ & -0.509857 & -5.625170 & -2.045216 \\
\hline C & 1.701733 & -0.661810 & 0.180563 \\
\hline C & 2.947248 & -0.761124 & -0.535274 \\
\hline C & 1.461959 & 0.494973 & 1.145374 \\
\hline $\mathrm{H}$ & 0.767005 & 0.179332 & 1.933185 \\
\hline $\mathrm{H}$ & 2.404564 & 0.762167 & 1.629467 \\
\hline C & -1.503587 & -0.223462 & -1.074402 \\
\hline 0 & -1.148178 & 0.930745 & -1.220715 \\
\hline 0 & -1.780222 & -1.022030 & -2.123308 \\
\hline $\mathrm{H}$ & -1.610924 & -0.491614 & -2.926984 \\
\hline 0 & 1.002492 & 1.691597 & 0.523537 \\
\hline $\mathrm{H}$ & 0.176534 & 1.498063 & 0.039769 \\
\hline C & 4.063585 & 0.219854 & -0.376310 \\
\hline C & 3.866223 & 1.600791 & -0.547157 \\
\hline C & 5.352707 & -0.250786 & -0.102662 \\
\hline C & 4.929117 & 2.500149 & -0.443109 \\
\hline $\mathrm{H}$ & 2.861935 & 1.950987 & -0.742125 \\
\hline C & 6.448206 & 0.619654 & 0.006890 \\
\hline $\mathrm{H}$ & 5.493283 & -1.317860 & 0.044629 \\
\hline C & 6.209133 & 1.985745 & -0.167795 \\
\hline $\mathrm{H}$ & 7.036869 & 2.680025 & -0.082500 \\
\hline C & -4.055263 & -0.023432 & 0.432133 \\
\hline C & -4.641954 & -0.376876 & -0.794778 \\
\hline C & -4.602138 & 1.028203 & 1.171551 \\
\hline C & -5.754369 & 0.309676 & -1.285724 \\
\hline $\mathrm{H}$ & -4.225211 & -1.209946 & -1.346171 \\
\hline C & -5.717293 & 1.746222 & 0.711845 \\
\hline $\mathrm{H}$ & -4.130551 & 1.298522 & 2.111638 \\
\hline C & -6.269925 & 1.367285 & -0.514831 \\
\hline $\mathrm{H}$ & -7.131001 & 1.905952 & -0.892100 \\
\hline C & -6.274984 & 2.908594 & 1.556243 \\
\hline C & -6.427867 & -0.054791 & -2.623311 \\
\hline C & -5.177405 & 3.984310 & 1.737738 \\
\hline $\mathrm{H}$ & -4.291566 & 3.584081 & 2.241359 \\
\hline $\mathrm{H}$ & -4.857443 & 4.384743 & 0.769176 \\
\hline
\end{tabular}




\begin{tabular}{|c|c|c|c|}
\hline $\mathrm{H}$ & -5.557190 & 4.817348 & 2.341948 \\
\hline C & -6.704508 & 2.375946 & 2.944008 \\
\hline $\mathrm{H}$ & -7.100823 & 3.192571 & 3.559840 \\
\hline $\mathrm{H}$ & -7.486274 & 1.613638 & 2.847414 \\
\hline $\mathrm{H}$ & -5.865600 & 1.927955 & 3.486712 \\
\hline C & -7.498087 & 3.579276 & 0.903148 \\
\hline $\mathrm{H}$ & -8.328465 & 2.876305 & 0.769911 \\
\hline $\mathrm{H}$ & -7.855580 & 4.394066 & 1.542718 \\
\hline $\mathrm{H}$ & -7.254085 & 4.011064 & -0.074137 \\
\hline C & -6.375477 & 1.165089 & -3.573985 \\
\hline $\mathrm{H}$ & -6.895382 & 2.032755 & -3.154632 \\
\hline $\mathrm{H}$ & -5.339670 & 1.462271 & -3.774069 \\
\hline $\mathrm{H}$ & -6.851379 & 0.922928 & -4.532160 \\
\hline C & -7.903929 & -0.444054 & -2.371336 \\
\hline $\mathrm{H}$ & -8.470332 & 0.374545 & -1.915159 \\
\hline $\mathrm{H}$ & -8.396856 & -0.704033 & -3.316200 \\
\hline $\mathrm{H}$ & -7.971705 & -1.310482 & -1.703524 \\
\hline C & -5.735824 & -1.238581 & -3.325171 \\
\hline $\mathrm{H}$ & -4.684727 & -1.024062 & -3.548934 \\
\hline $\mathrm{H}$ & -5.774038 & -2.151202 & -2.719724 \\
\hline $\mathrm{H}$ & -6.240452 & -1.451001 & -4.274425 \\
\hline C & 7.845524 & 0.048272 & 0.318208 \\
\hline C & 4.734999 & 4.020580 & -0.604412 \\
\hline C & 3.280678 & 4.388852 & -0.956336 \\
\hline $\mathrm{H}$ & 2.976941 & 3.956744 & -1.916822 \\
\hline $\mathrm{H}$ & 2.572368 & 4.046179 & -0.195105 \\
\hline $\mathrm{H}$ & 3.187745 & 5.478104 & -1.039142 \\
\hline C & 5.652167 & 4.549333 & -1.732390 \\
\hline $\mathrm{H}$ & 6.712074 & 4.374002 & -1.518732 \\
\hline $\mathrm{H}$ & 5.418444 & 4.062839 & -2.686412 \\
\hline $\mathrm{H}$ & 5.514206 & 5.630163 & -1.860024 \\
\hline C & 5.102089 & 4.722784 & 0.724947 \\
\hline $\mathrm{H}$ & 4.453111 & 4.379739 & 1.538617 \\
\hline $\mathrm{H}$ & 6.139221 & 4.525988 & 1.017597 \\
\hline $\mathrm{H}$ & 4.981796 & 5.809217 & 0.629323 \\
\hline C & 8.247577 & -0.961058 & -0.783606 \\
\hline $\mathrm{H}$ & 7.543049 & -1.796834 & -0.848777 \\
\hline $\mathrm{H}$ & 8.281134 & -0.475372 & -1.765649 \\
\hline $\mathrm{H}$ & 9.240793 & -1.378557 & -0.576359 \\
\hline C & 7.813694 & -0.674762 & 1.685764 \\
\hline $\mathrm{H}$ & 8.799087 & -1.097318 & 1.918773 \\
\hline $\mathrm{H}$ & 7.543028 & 0.020065 & 2.489056 \\
\hline $\mathrm{H}$ & 7.087958 & -1.494794 & 1.696057 \\
\hline C & 8.929976 & 1.140627 & 0.380038 \\
\hline $\mathrm{H}$ & 8.727926 & 1.872745 & 1.170105 \\
\hline $\mathrm{H}$ & 9.902434 & 0.683246 & 0.595484 \\
\hline $\mathrm{H}$ & 9.021172 & 1.679283 & -0.570078 \\
\hline
\end{tabular}




\begin{tabular}{lc}
\hline B3LYP/6-31G(d) Energy & -2164.802431 \\
\hline B3LYP-D3(BJ)/def2-TZVPP-IEF-PCM(DCM) Energy & -2165.897999 \\
\hline B3LYP-D3(BJ)/def2-TZVPP-IEF-PCM(DCM)//B3LYP/6-31G(d) Free Energy (Quasiharmonic) & -2165.019125 \\
\hline
\end{tabular}

Number of Imaginary Frequencies

Frequencies (Top 3 out of 321)

$\begin{array}{ll}\text { 1. } & 11.0030 \mathrm{~cm}^{-1} \\ \text { 2. } & 12.4399 \mathrm{~cm}^{-1} \\ \text { 3. } & 13.8602 \mathrm{~cm}^{-1}\end{array}$

\section{B3LYP/6-31G(d) Molecular Geometry in Cartesian Coordinates}

C

C

C

C

C

C

$\mathrm{H}$

$\mathrm{H}$

C

C

$\mathrm{H}$

$\mathrm{H}$

C

C

$\mathrm{H}$

C

C

C

C

C

C

$\mathrm{H}$

$\mathrm{H}$

$\mathrm{H}$

C

C

$\mathrm{H}$

$\mathrm{H}$

C

C

C

$\mathrm{H}$

\subsection{2}

0.393666

$-0.675198$

$-1.902684$

$-2.015962$

$-0.963581$

1.078034

1.327464

$-0.564394$

$-2.977021$

$-2.953036$

$-1.061656$

$-2.888765$

$-1.657433$

$-3.906875$

1.467593

2.417560

2.186317

0.946281

$-0.014738$

0.240388

4.092016

1.655036

3. 364480

3.156157

0.732049

$-0.962437$

$-0.507384$

1. 705850

2.953441

1.462057

0.763689
$-3.480051$

$-2.912996$

$-2.190606$

$-2.060071$

$-2.656246$

$-3.351191$

$-4.031330$

$-3.014940$

$-1.582592$

$-1.341515$

$-2.551789$

$-3.802585$

$-0.775217$

$-0.916142$

$-1.252197$

$-4.953577$

$-3.971847$

$-2.868942$

$-2.777709$

$-3.809824$

$-4.869557$

$-1.932076$

$-5.794214$

$-4.024937$

$-1.857117$

$-1.656267$

$-3.754271$

$-5.647298$

$-0.670690$

$-0.776265$

0.492258

0.182519

\subsection{8}

2.690286

2. 092181

2. 816817

4.101803

4.651669

4.377444

2.147876

0.798424

2. 236460

4.643354

5.635176

0.980703

0.263437

2. 792261

$-2.563807$

$-2.403353$

$-1.536826$

$-0.831577$

$-1.021221$

$-1.862918$

$-1.896181$

$-3.226476$

$-2.935536$

$-1.348327$

0.036844

$-0.496644$

$-1.992944$

0.202449

$-0.509790$

1.158549

1.945395 


\begin{tabular}{|c|c|c|c|}
\hline $\mathrm{H}$ & 2.402147 & 0.764318 & 1.644399 \\
\hline C & -1.494174 & -0.238686 & -1.068397 \\
\hline 0 & -1.133662 & 0.913033 & -1.222702 \\
\hline 0 & -1.771310 & -1.043937 & -2.111800 \\
\hline $\mathrm{H}$ & -1.598122 & -0.520359 & -2.919143 \\
\hline 0 & 1.003385 & 1.684630 & 0.526428 \\
\hline $\mathrm{H}$ & 0.179436 & 1.487801 & 0.040207 \\
\hline C & 4.071103 & 0.204440 & -0.359631 \\
\hline C & 3.874885 & 1.580025 & -0.505031 \\
\hline C & 5.372998 & -0.274696 & -0.117963 \\
\hline C & 4.944096 & 2.485339 & -0.410424 \\
\hline $\mathrm{H}$ & 2.868238 & 1.941441 & -0.677376 \\
\hline C & 6.462759 & 0.591538 & -0.021932 \\
\hline H & 5.509207 & -1.342315 & 0.011413 \\
\hline C & 6.220690 & 1.969497 & -0.173113 \\
\hline $\mathrm{H}$ & 7.058945 & 2.652236 & -0.096594 \\
\hline C & -4.051497 & -0.022936 & 0.425746 \\
\hline C & -4.634776 & -0.386475 & -0.799850 \\
\hline C & -4.598282 & 1.036977 & 1.153302 \\
\hline C & -5.743525 & 0.298423 & -1.301298 \\
\hline $\mathrm{H}$ & -4.218278 & -1.225765 & -1.341897 \\
\hline C & -5.709783 & 1.753659 & 0.682790 \\
\hline $\mathrm{H}$ & -4.129364 & 1.314850 & 2.092510 \\
\hline C & -6.258859 & 1.364775 & -0.542357 \\
\hline $\mathrm{H}$ & -7.116862 & 1.902431 & -0.927963 \\
\hline C & -6.267242 & 2.925300 & 1.514486 \\
\hline C & -6.413439 & -0.076837 & -2.637722 \\
\hline C & -5.167201 & 3.999412 & 1.690467 \\
\hline $\mathrm{H}$ & -4.284483 & 3.601193 & 2.201113 \\
\hline $\mathrm{H}$ & -4.842379 & 4.390130 & 0.719557 \\
\hline $\mathrm{H}$ & -5.546816 & 4.838990 & 2.285669 \\
\hline C & -6.703514 & 2.406261 & 2.905309 \\
\hline $\mathrm{H}$ & -7.099553 & 3.229479 & 3.512457 \\
\hline H & -7.487289 & 1.645543 & 2.812489 \\
\hline $\mathrm{H}$ & -5.868009 & 1.960452 & 3.455037 \\
\hline C & -7.485909 & 3.593632 & 0.850751 \\
\hline $\mathrm{H}$ & -8.317971 & 2.892017 & 0.720925 \\
\hline $\mathrm{H}$ & -7.843186 & 4.415346 & 1.481509 \\
\hline $\mathrm{H}$ & -7.236964 & 4.015679 & -0.129540 \\
\hline C & -6.353844 & 1.133578 & -3.599975 \\
\hline $\mathrm{H}$ & -6.872476 & 2.006902 & -3.190920 \\
\hline $\mathrm{H}$ & -5.316406 & 1.425562 & -3.799285 \\
\hline $\mathrm{H}$ & -6.827143 & 0.883621 & -4.557451 \\
\hline C & -7.891611 & -0.458994 & -2.387170 \\
\hline $\mathrm{H}$ & -8.457100 & 0.365953 & -1.941405 \\
\hline $\mathrm{H}$ & -8.381954 & -0.727041 & -3.331123 \\
\hline $\mathrm{H}$ & -7.964420 & -1.318383 & -1.710852 \\
\hline C & -5.722680 & -1.269520 & -3.325652 \\
\hline $\mathrm{H}$ & -4.670032 & -1.060548 & -3.547373 \\
\hline $\mathrm{H}$ & -5.766293 & -2.176232 & -2.711757 \\
\hline $\mathrm{H}$ & -6.224401 & -1.489152 & -4.274795 \\
\hline C & 7.897855 & 0.096257 & 0.244901 \\
\hline C & 4.663831 & 3.992324 & -0.565875 \\
\hline$c$ & 3.672917 & 4.441816 & 0.535544 \\
\hline
\end{tabular}




\begin{tabular}{|c|c|c|c|}
\hline $\mathrm{H}$ & 2.733963 & 3.881118 & 0.496944 \\
\hline $\mathrm{H}$ & 4.108774 & 4.296380 & 1.531253 \\
\hline $\mathrm{H}$ & 3.438483 & 5.507994 & 0.422475 \\
\hline C & 4.038302 & 4.258358 & -1.955865 \\
\hline $\mathrm{H}$ & 4.717428 & 3.946608 & -2.758193 \\
\hline $\mathrm{H}$ & 3.094492 & 3.719568 & -2.086356 \\
\hline $\mathrm{H}$ & 3.831994 & 5.328523 & -2.081508 \\
\hline C & 5.939963 & 4.846451 & -0.444661 \\
\hline $\mathrm{H}$ & 6.419220 & 4.729347 & 0.534284 \\
\hline $\mathrm{H}$ & 6.675782 & 4.596284 & -1.218067 \\
\hline $\mathrm{H}$ & 5.686181 & 5.906300 & -0.561320 \\
\hline C & 7.975200 & -1.436157 & 0.383703 \\
\hline $\mathrm{H}$ & 7.371895 & -1.801058 & 1.222714 \\
\hline $\mathrm{H}$ & 7.638118 & -1.945440 & -0.526368 \\
\hline $\mathrm{H}$ & 9.012609 & -1.738996 & 0.566350 \\
\hline C & 8.427986 & 0.722911 & 1.556554 \\
\hline $\mathrm{H}$ & 9.452654 & 0.384787 & 1.755315 \\
\hline $\mathrm{H}$ & 8.441553 & 1.816777 & 1.510132 \\
\hline $\mathrm{H}$ & 7.802893 & 0.433153 & 2.408981 \\
\hline C & 8.813827 & 0.516352 & -0.929444 \\
\hline $\mathrm{H}$ & 8.837674 & 1.603135 & -1.060623 \\
\hline $\mathrm{H}$ & 9.842575 & 0.179051 & -0.751900 \\
\hline $\mathrm{H}$ & 8.468802 & 0.074480 & -1.871370 \\
\hline
\end{tabular}

\section{Catalyst 2C 31}

Datum

Value

\begin{tabular}{lc}
\hline B3LYP/6-31G(d) Energy & -2164.802753 \\
\hline B3LYP-D3(BJ)/def2-TZVPP-IEF-PCM(DCM) Energy & -2165.898174 \\
\hline B3LYP-D3(BJ)/def2-TZVPP-IEF-PCM(DCM)//B3LYP/6-31G(d) Free Energy (Quasiharmonic) & -2165.019121 \\
\hline
\end{tabular}

Number of Imaginary Frequencies

Frequencies (Top 3 out of 321)
1. $12.1887 \mathrm{~cm}^{-1}$
2. $\quad 13.1310 \mathrm{~cm}^{-1}$
3. $14.2356 \mathrm{~cm}^{-1}$

\section{B3LYP/6-31G(d) Molecular Geometry in Cartesian Coordinates}

$\begin{array}{rrrr}C & 0.254549 & -3.474563 & 3.941471 \\ C & 0.394988 & -2.906575 & 2.694639 \\ C & -0.674312 & -2.182861 & 2.098853\end{array}$




\begin{tabular}{|c|c|c|c|}
\hline C & -1.900544 & -2.052095 & 2.825598 \\
\hline C & -2.012161 & -2.649222 & 4.110329 \\
\hline C & -0.959423 & -3.345378 & 4.657879 \\
\hline $\mathrm{H}$ & 1.081237 & -4.026829 & 4.379727 \\
\hline H & 1.327850 & -3.008720 & 2.150670 \\
\hline C & -0.564964 & -1.573364 & 0.805715 \\
\hline C & -2.975308 & -1.332577 & 2.247515 \\
\hline $\mathrm{H}$ & -2.948256 & -2.544444 & 4.653499 \\
\hline $\mathrm{H}$ & -1.056183 & -3.797462 & 5.641198 \\
\hline C & -2.888965 & -0.765715 & 0.991818 \\
\hline C & -1.658411 & -0.905577 & 0.273008 \\
\hline $\mathrm{H}$ & -3.904109 & -1.243271 & 2.805092 \\
\hline C & 1.457008 & -4.942617 & -2.563973 \\
\hline C & 2.408852 & -3.962743 & -2.403305 \\
\hline C & 2.180582 & -2.860682 & -1.534925 \\
\hline C & 0.941551 & -2.768230 & -0.828092 \\
\hline C & -0.021449 & -3.798458 & -1.017977 \\
\hline C & 0.230834 & -4.857483 & -1.861424 \\
\hline $\mathrm{H}$ & 4.087679 & -1.926844 & -1.894899 \\
\hline $\mathrm{H}$ & 1.642206 & -5.782669 & -3.228014 \\
\hline $\mathrm{H}$ & 3.355043 & -4.016732 & -2.936690 \\
\hline C & 3.152533 & -1.850965 & -1.345957 \\
\hline C & 0.730321 & -1.647663 & 0.042229 \\
\hline $\mathrm{H}$ & -0.968314 & -3.742114 & -0.491972 \\
\hline $\mathrm{H}$ & -0.518347 & -5.633874 & -1.991461 \\
\hline C & 1.706167 & -0.664203 & 0.208274 \\
\hline C & 2.952761 & -0.771075 & -0.505500 \\
\hline C & 1.465827 & 0.497753 & 1.166456 \\
\hline $\mathrm{H}$ & 0.767523 & 0.188356 & 1.953485 \\
\hline $\mathrm{H}$ & 2.406990 & 0.766977 & 1.651807 \\
\hline C & -1.495647 & -0.224463 & -1.057148 \\
\hline 0 & -1.133739 & 0.927122 & -1.208097 \\
\hline 0 & -1.773426 & -1.026363 & -2.103612 \\
\hline $\mathrm{H}$ & -1.601664 & -0.499685 & -2.909300 \\
\hline 0 & 1.009197 & 1.692069 & 0.536535 \\
\hline $\mathrm{H}$ & 0.183568 & 1.497885 & 0.052143 \\
\hline C & 4.072500 & 0.207218 & -0.354996 \\
\hline C & 3.879018 & 1.583298 & -0.499286 \\
\hline C & 5.373497 & -0.274794 & -0.114311 \\
\hline C & 4.950166 & 2.486310 & -0.404656 \\
\hline $\mathrm{H}$ & 2.873021 & 1.946881 & -0.670848 \\
\hline C & 6.465086 & 0.589127 & -0.018090 \\
\hline $\mathrm{H}$ & 5.507557 & -1.342799 & 0.014141 \\
\hline C & 6.225805 & 1.967682 & -0.168258 \\
\hline $\mathrm{H}$ & 7.065493 & 2.648663 & -0.091687 \\
\hline C & -4.053362 & -0.015118 & 0.438336 \\
\hline C & -4.629979 & -0.366228 & -0.784399 \\
\hline C & -4.607624 & 1.042867 & 1.179156 \\
\hline C & -5.745127 & 0.321775 & -1.289984 \\
\hline $\mathrm{H}$ & -4.213218 & -1.200292 & -1.338291 \\
\hline C & -5.714574 & 1.752648 & 0.711649 \\
\hline $\mathrm{H}$ & -4.138106 & 1.309190 & 2.118909 \\
\hline C & -6.263231 & 1.371311 & -0.526826 \\
\hline H & -7.121614 & 1.917897 & -0.89878 \\
\hline
\end{tabular}




\begin{tabular}{|c|c|c|c|}
\hline C & -6.339231 & 2.926374 & 1.490842 \\
\hline C & -6.347620 & -0.104184 & -2.643177 \\
\hline C & -6.272184 & 4.210814 & 0.630620 \\
\hline $\mathrm{H}$ & -5.234201 & 4.468601 & 0.392118 \\
\hline $\mathrm{H}$ & -6.814203 & 4.098921 & -0.314276 \\
\hline $\mathrm{H}$ & -6.717115 & 5.054838 & 1.171786 \\
\hline C & -5.610204 & 3.203613 & 2.819221 \\
\hline $\mathrm{H}$ & -6.091704 & 4.041503 & 3.335900 \\
\hline $\mathrm{H}$ & -5.643246 & 2.338966 & 3.491973 \\
\hline $\mathrm{H}$ & -4.560333 & 3.473878 & 2.660129 \\
\hline C & -7.817459 & 2.602274 & 1.813292 \\
\hline $\mathrm{H}$ & -7.895747 & 1.700719 & 2.431835 \\
\hline $\mathrm{H}$ & -8.278045 & 3.432440 & 2.362710 \\
\hline $\mathrm{H}$ & -8.407693 & 2.436276 & 0.905974 \\
\hline C & -7.559619 & 0.755295 & -3.048259 \\
\hline $\mathrm{H}$ & -8.376110 & 0.678266 & -2.321205 \\
\hline H & -7.293860 & 1.813257 & -3.154479 \\
\hline $\mathrm{H}$ & -7.946466 & 0.413820 & -4.015009 \\
\hline C & -6.810193 & -1.578509 & -2.561041 \\
\hline $\mathrm{H}$ & -7.576477 & -1.706163 & -1.787912 \\
\hline $\mathrm{H}$ & -7.237716 & -1.897927 & -3.519425 \\
\hline $\mathrm{H}$ & -5.981411 & -2.253941 & -2.325090 \\
\hline C & -5.271581 & 0.033269 & -3.746984 \\
\hline $\mathrm{H}$ & -4.933092 & 1.072142 & -3.834800 \\
\hline $\mathrm{H}$ & -4.395601 & -0.589872 & -3.538338 \\
\hline $\mathrm{H}$ & -5.677997 & -0.275560 & -4.717882 \\
\hline C & 7.899232 & 0.090632 & 0.247874 \\
\hline C & 4.672967 & 3.993934 & -0.559242 \\
\hline C & 5.950931 & 4.845340 & -0.438205 \\
\hline $\mathrm{H}$ & 6.430325 & 4.726932 & 0.540519 \\
\hline H & 6.685916 & 4.593864 & -1.211982 \\
\hline $\mathrm{H}$ & 5.699355 & 5.905763 & -0.554428 \\
\hline C & 3.683458 & 4.444967 & 0.542813 \\
\hline $\mathrm{H}$ & 2.743330 & 3.886224 & 0.504370 \\
\hline $\mathrm{H}$ & 4.119423 & 4.298155 & 1.538268 \\
\hline $\mathrm{H}$ & 3.451199 & 5.511685 & 0.430329 \\
\hline C & 4.047433 & 4.261905 & -1.948852 \\
\hline $\mathrm{H}$ & 4.725604 & 3.949130 & -2.751591 \\
\hline H & 3.102463 & 3.725122 & -2.079255 \\
\hline $\mathrm{H}$ & 3.843291 & 5.332552 & -2.073921 \\
\hline C & 8.431143 & 0.715268 & 1.559768 \\
\hline $\mathrm{H}$ & 8.446973 & 1.809135 & 1.514099 \\
\hline $\mathrm{H}$ & 7.805748 & 0.426231 & 2.412218 \\
\hline H & 9.455167 & 0.374852 & 1.757925 \\
\hline C & 8.815656 & 0.509588 & -0.926516 \\
\hline $\mathrm{H}$ & 9.843780 & 0.170094 & -0.749543 \\
\hline H & 8.469409 & 0.069004 & -1.868601 \\
\hline $\mathrm{H}$ & 8.841647 & 1.596405 & -1.057025 \\
\hline C & 7.973345 & -1.442031 & 0.385626 \\
\hline $\mathrm{H}$ & 7.634889 & -1.949990 & -0.524675 \\
\hline $\mathrm{H}$ & 9.010156 & -1.747217 & 0.567741 \\
\hline H & 7.369525 & -1.806180 & 1.224594 \\
\hline
\end{tabular}




\section{Catalyst 2C 32}

Datum

Value

\begin{tabular}{lc}
\hline B3LYP/6-31G(d) Energy & -2164.802211 \\
\hline B3LYP-D3(BJ)/def2-TZVPP-IEF-PCM(DCM) Energy & -2165.897879 \\
\hline B3LYP-D3(BJ)/def2-TZVPP-IEF-PCM(DCM)//B3LYP/6-31G(d) Free Energy (Quasiharmonic) & -2165.019109
\end{tabular}

Number of Imaginary Frequencies

Frequencies (Top 3 out of 321)

$\begin{array}{ll}\text { 1. } & 10.8386 \mathrm{~cm}^{-1} \\ \text { 2. } & 12.5965 \mathrm{~cm}^{-1} \\ \text { 3. } & 14.0095 \mathrm{~cm}^{-1}\end{array}$

\section{B3LYP/6-31G(d) Molecular Geometry in Cartesian Coordinates}

C

C

$\mathrm{C}$

C

C

C

$\mathrm{H}$

$\mathrm{H}$

C

C

$\mathrm{H}$

$\mathrm{H}$

C

C

$\mathrm{H}$

C

C

C

C

C

C

$\mathrm{H}$

$\mathrm{H}$

$\mathrm{H}$

C

C

$\mathrm{H}$

$\mathrm{H}$

C
0.269604

0.404035

$-0.668461$

$-1.891538$

$-1.996950$

$-0.941247$

1.098701

1. 334571

$-0.565369$

$-2.969488$

$-2.930720$

$-1.033245$

$-2.889174$

$-1.661699$

$-3.895941$

1.451817

2.402946

2.175359

0.937807

$-0.024408$

0.227145

4.081597

1.636482

3. 347954

3.146793

0.726967

$-0.970220$

$-0.521526$

1. 701893
$-3.495956$

$-2.918664$

$-2.191487$

$-2.067182$

$-2.673799$

$-3.373099$

$-4.050728$

$-3.016050$

$-1.572300$

$-1.344398$

$-2.573726$

$-3.832509$

$-0.768250$

$-0.901552$

$-1.260091$

$-4.915877$

$-3.935937$

$-2.839824$

$-2.753383$

$-3.783435$

$-4.836656$

$-1.900838$

$-5.751338$

$-3.985330$

$-1.830065$

$-1.639105$

$-3.731509$

$-5.612953$

$-0.655661$
3.904068

2.660850

2.075109

2.808294

4.089096

4.626837

4.334550

2. 112054

0.786055

2. 240309

4.637129

5.607214

0.988450

0.263167

2.802552

$-2.612919$

$-2.447952$

$-1.572037$

$-0.861712$

$-1.056245$

$-1.907199$

$-1.928938$

$-3.282875$

$-2.983886$

$-1.378603$

0.016991

$-0.527887$

$-2.040711$

0.186863 


\begin{tabular}{|c|c|c|c|}
\hline C & 2.946235 & -0.756504 & -0.530823 \\
\hline C & 1.465724 & 0.500409 & 1.153417 \\
\hline $\mathrm{H}$ & 0.770972 & 0.185302 & 1.941618 \\
\hline $\mathrm{H}$ & 2.409469 & 0.764991 & 1.636705 \\
\hline C & -1.505394 & -0.210147 & -1.062465 \\
\hline 0 & -1.147500 & 0.943488 & -1.206146 \\
\hline 0 & -1.784322 & -1.005523 & -2.113824 \\
\hline $\mathrm{H}$ & -1.616554 & -0.472550 & -2.916209 \\
\hline 0 & 1.008066 & 1.698750 & 0.533530 \\
\hline $\mathrm{H}$ & 0.180666 & 1.507545 & 0.051347 \\
\hline C & 4.064685 & 0.222130 & -0.372212 \\
\hline C & 3.869868 & 1.603558 & -0.541819 \\
\hline C & 5.353162 & -0.251292 & -0.100264 \\
\hline C & 4.934643 & 2.500750 & -0.438149 \\
\hline $\mathrm{H}$ & 2.866081 & 1.955875 & -0.735524 \\
\hline C & 6.450485 & 0.616891 & 0.008826 \\
\hline $\mathrm{H}$ & 5.491740 & -1.318765 & 0.046072 \\
\hline C & 6.213903 & 1.983588 & -0.164575 \\
\hline $\mathrm{H}$ & 7.043091 & 2.676164 & -0.079609 \\
\hline C & -4.056921 & -0.015338 & 0.445367 \\
\hline C & -4.637776 & -0.358157 & -0.777687 \\
\hline C & -4.610162 & 1.035903 & 1.196482 \\
\hline C & -5.756430 & 0.331330 & -1.273393 \\
\hline $\mathrm{H}$ & -4.221407 & -1.186962 & -1.339731 \\
\hline C & -5.720278 & 1.747154 & 0.738825 \\
\hline $\mathrm{H}$ & -4.137345 & 1.295924 & 2.136357 \\
\hline C & -6.273419 & 1.374008 & -0.500180 \\
\hline $\mathrm{H}$ & -7.134440 & 1.921594 & -0.864466 \\
\hline C & -6.343742 & 2.914190 & 1.528972 \\
\hline C & -6.363689 & -0.085914 & -2.627169 \\
\hline C & -7.820315 & 2.585762 & 1.854570 \\
\hline $\mathrm{H}$ & -8.413701 & 2.425441 & 0.948296 \\
\hline $\mathrm{H}$ & -7.895066 & 1.679712 & 2.466955 \\
\hline $\mathrm{H}$ & -8.279998 & 3.411345 & 2.411605 \\
\hline C & -6.281516 & 4.204913 & 0.677860 \\
\hline $\mathrm{H}$ & -6.725232 & 5.044518 & 1.226839 \\
\hline $\mathrm{H}$ & -5.244764 & 4.465536 & 0.437118 \\
\hline $\mathrm{H}$ & -6.827130 & 4.099256 & -0.265691 \\
\hline C & -5.610171 & 3.182766 & 2.856620 \\
\hline $\mathrm{H}$ & -4.561311 & 3.455776 & 2.695594 \\
\hline $\mathrm{H}$ & -6.091015 & 4.016122 & 3.381186 \\
\hline $\mathrm{H}$ & -5.639428 & 2.313163 & 3.523129 \\
\hline C & -7.579379 & 0.773759 & -3.020704 \\
\hline $\mathrm{H}$ & -8.392581 & 0.689455 & -2.290781 \\
\hline $\mathrm{H}$ & -7.316475 & 1.833093 & -3.120156 \\
\hline $\mathrm{H}$ & -7.969495 & 0.438547 & -3.988322 \\
\hline C & -6.822468 & -1.561892 & -2.554020 \\
\hline $\mathrm{H}$ & -7.585155 & -1.697170 & -1.778634 \\
\hline $\mathrm{H}$ & -7.253239 & -1.875259 & -3.512949 \\
\hline $\mathrm{H}$ & -5.991076 & -2.237042 & -2.326573 \\
\hline C & -5.292620 & 0.062149 & -3.734437 \\
\hline $\mathrm{H}$ & -4.957037 & 1.102452 & -3.816179 \\
\hline $\mathrm{H}$ & -4.414250 & -0.560278 & -3.533878 \\
\hline $\mathrm{H}$ & -5.702338 & -0.240769 & -4.705823 \\
\hline
\end{tabular}




\begin{tabular}{|lrrr|} 
C & 7.846971 & 0.042598 & 0.318533 \\
C & 4.743359 & 4.021707 & -0.597877 \\
H & 5.112365 & 4.721765 & 0.732097 \\
H & 4.463069 & 4.379074 & 1.545662 \\
H & 6.149239 & 4.522609 & 1.024075 \\
C & 4.994158 & 5.808544 & 0.637731 \\
H & 3.289597 & 4.393123 & -0.948786 \\
H & 2.984597 & 3.962532 & -1.909555 \\
H & 2.580996 & 4.051087 & -0.187536 \\
C & 3.198755 & 5.482634 & -1.030495 \\
H & 5.661045 & 4.549936 & -1.725663 \\
H & 6.720711 & 4.372316 & -1.512695 \\
H & 5.425950 & 4.065006 & -2.680143 \\
C & 5.525140 & 5.631181 & -1.851979 \\
H & 7.814732 & -0.681719 & 1.685404 \\
H & 7.545883 & 0.012803 & 2.489571 \\
H & 7.087459 & -1.500396 & 1.695333 \\
C & 8.799491 & -1.106350 & 1.917325 \\
H & 8.933514 & 1.132866 & 0.380647 \\
H & 9.905272 & 0.673425 & 0.594863 \\
H & 9.024975 & 1.672325 & -0.568990 \\
C & 8.733487 & 1.864567 & 1.171622 \\
H & 8.246409 & -0.966403 & -0.784529 \\
H & 8.280112 & -0.479842 & -1.766133 \\
H & 9.239043 & -1.385841 & -0.578398 \\
& 7.540385 & -1.800904 & -0.849976 \\
\hline & & & \\
\hline & & & \\
\hline & & & \\
\hline
\end{tabular}

\section{Catalyst 2C 33}

Datum

Value

\begin{tabular}{lr}
\hline B3LYP/6-31G(d) Energy & -2164.802424 \\
\hline B3LYP-D3(BJ)/def2-TZVPP-IEF-PCM(DCM) Energy & -2165.897952 \\
\hline B3LYP-D3(BJ)/def2-TZVPP-IEF-PCM(DCM)//B3LYP/6-31G(d) Free Energy (Quasiharmonic) & -2165.019035 \\
\hline
\end{tabular}

Number of Imaginary Frequencies

Frequencies (Top 3 out of 321)

$\begin{array}{ll}\text { 1. } & 11.4255 \mathrm{~cm}^{-1} \\ \text { 2. } & 12.6218 \mathrm{~cm}^{-1} \\ \text { 3. } & 14.0571 \mathrm{~cm}^{-1}\end{array}$

\section{B3LYP/6-31G(d) Molecular Geometry in Cartesian Coordinates}




\begin{tabular}{|c|c|c|c|}
\hline C & -0.276314 & -3.498285 & 3.922611 \\
\hline C & -0.413789 & -2.925170 & 2.677783 \\
\hline C & 0.657931 & -2.201559 & 2.086247 \\
\hline C & 1.883428 & -2.076320 & 2.815167 \\
\hline C & 1.991949 & -2.678590 & 4.097749 \\
\hline C & 0.936933 & -3.374540 & 4.641187 \\
\hline $\mathrm{H}$ & -1.104817 & -4.050435 & 4.357580 \\
\hline $\mathrm{H}$ & -1.346123 & -3.023229 & 2.132156 \\
\hline C & 0.551873 & -1.586971 & 0.795229 \\
\hline C & 2.960600 & -1.356913 & 2.241334 \\
\hline $\mathrm{H}$ & 2.927534 & -2.577923 & 4.642577 \\
\hline $\mathrm{H}$ & 1.031340 & -3.830642 & 5.622881 \\
\hline C & 2.877377 & -0.785000 & 0.987697 \\
\hline C & 1.647664 & -0.919955 & 0.266533 \\
\hline $\mathrm{H}$ & 3.888789 & -1.271551 & 2.800523 \\
\hline C & -1.478440 & -4.936631 & -2.589346 \\
\hline C & -2.427459 & -3.954614 & -2.425080 \\
\hline C & -2.196239 & -2.856722 & -1.552200 \\
\hline C & -0.957195 & -2.770824 & -0.844535 \\
\hline C & 0.002825 & -3.803129 & -1.038185 \\
\hline C & -0.252263 & -4.857951 & -1.886039 \\
\hline $\mathrm{H}$ & -4.100544 & -1.915908 & -1.908878 \\
\hline H & -1.665862 & -5.773440 & -3.256853 \\
\hline $\mathrm{H}$ & -3.373607 & -4.003672 & -2.959023 \\
\hline C & -3.165262 & -1.844890 & -1.359508 \\
\hline C & -0.742903 & -1.654378 & 0.030274 \\
\hline $\mathrm{H}$ & 0.949735 & -3.751627 & -0.511766 \\
\hline $\mathrm{H}$ & 0.494729 & -5.635938 & -2.019067 \\
\hline C & -1.715715 & -0.668461 & 0.199596 \\
\hline C & -2.962455 & -0.768857 & -0.514830 \\
\hline C & -1.471750 & 0.489509 & 1.161639 \\
\hline $\mathrm{H}$ & -2.412096 & 0.760528 & 1.647562 \\
\hline $\mathrm{H}$ & -0.774750 & 0.175239 & 1.947881 \\
\hline C & 1.489096 & -0.235096 & -1.062060 \\
\hline 0 & 1.130668 & 0.917974 & -1.211152 \\
\hline 0 & 1.767544 & -1.035096 & -2.109224 \\
\hline $\mathrm{H}$ & 1.596639 & -0.506942 & -2.914071 \\
\hline 0 & -1.010799 & 1.684166 & 0.535468 \\
\hline H & -0.185968 & 1.488504 & 0.050270 \\
\hline C & -4.079369 & 0.212023 & -0.360334 \\
\hline C & -3.881993 & 1.588141 & -0.498898 \\
\hline C & -5.381642 & -0.267268 & -0.121117 \\
\hline C & -4.950483 & 2.493832 & -0.399917 \\
\hline $\mathrm{H}$ & -2.875003 & 1.949531 & -0.669315 \\
\hline C & -6.470683 & 0.599388 & -0.020668 \\
\hline $\mathrm{H}$ & -5.518680 & -1.335415 & 0.002933 \\
\hline C & -6.227502 & 1.977870 & -0.165096 \\
\hline $\mathrm{H}$ & -7.065200 & 2.660926 & -0.085114 \\
\hline C & 4.043853 & -0.033532 & 0.439582 \\
\hline C & 4.627911 & -0.390902 & -0.783306 \\
\hline C & 4.591133 & 1.022970 & 1.180812 \\
\hline C & 5.743977 & 0.294106 & -1.278620 \\
\hline $\mathrm{H}$ & 4.210749 & -1.225073 & -1.332706 \\
\hline
\end{tabular}




\begin{tabular}{|c|c|c|c|}
\hline C & 5.703693 & 1.734736 & 0.718846 \\
\hline $\mathrm{H}$ & 4.116188 & 1.291039 & 2.117334 \\
\hline C & 6.256375 & 1.348908 & -0.510744 \\
\hline H & 7.119867 & 1.891143 & -0.884332 \\
\hline C & 6.321626 & 2.910507 & 1.500776 \\
\hline C & 6.415666 & -0.076131 & -2.615820 \\
\hline C & 6.257056 & 4.193635 & 0.638577 \\
\hline $\mathrm{H}$ & 6.804704 & 4.081168 & -0.303028 \\
\hline $\mathrm{H}$ & 5.220035 & 4.449053 & 0.393506 \\
\hline $\mathrm{H}$ & 6.697236 & 5.039343 & 1.181007 \\
\hline C & 7.798458 & 2.589685 & 1.832347 \\
\hline H & 8.254115 & 3.421152 & 2.383891 \\
\hline $\mathrm{H}$ & 7.875110 & 1.688647 & 2.451815 \\
\hline $\mathrm{H}$ & 8.394424 & 2.424305 & 0.928648 \\
\hline C & 5.584086 & 3.187598 & 2.824527 \\
\hline $\mathrm{H}$ & 5.614591 & 2.323618 & 3.498258 \\
\hline $\mathrm{H}$ & 6.061144 & 4.026746 & 3.343261 \\
\hline $\mathrm{H}$ & 4.534749 & 3.456024 & 2.659015 \\
\hline C & 5.720978 & -1.261289 & -3.312856 \\
\hline $\mathrm{H}$ & 5.758709 & -2.172046 & -2.704587 \\
\hline $\mathrm{H}$ & 4.669910 & -1.046055 & -3.536068 \\
\hline $\mathrm{H}$ & 6.224178 & -1.477397 & -4.262022 \\
\hline C & 7.891228 & -0.466806 & -2.363636 \\
\hline $\mathrm{H}$ & 7.958360 & -1.330605 & -1.692395 \\
\hline $\mathrm{H}$ & 8.383102 & -0.731157 & -3.307836 \\
\hline $\mathrm{H}$ & 8.459114 & 0.352763 & -1.911002 \\
\hline C & 6.364304 & 1.140455 & -3.570585 \\
\hline $\mathrm{H}$ & 5.328867 & 1.439056 & -3.770400 \\
\hline $\mathrm{H}$ & 6.886324 & 2.008556 & -3.154708 \\
\hline $\mathrm{H}$ & 6.838601 & 0.894277 & -4.528535 \\
\hline C & -7.906078 & 0.103988 & 0.244319 \\
\hline C & -4.669012 & 4.001336 & -0.547860 \\
\hline C & -4.042970 & 4.273806 & -1.936363 \\
\hline $\mathrm{H}$ & -3.099614 & 3.734837 & -2.069446 \\
\hline $\mathrm{H}$ & -4.722221 & 3.966742 & -2.740394 \\
\hline $\mathrm{H}$ & -3.835682 & 5.344410 & -2.056546 \\
\hline C & -3.678026 & 4.444618 & 0.556016 \\
\hline $\mathrm{H}$ & -4.114318 & 4.294781 & 1.550880 \\
\hline $\mathrm{H}$ & -2.739568 & 3.883262 & 0.515000 \\
\hline $\mathrm{H}$ & -3.442603 & 5.511122 & 0.448156 \\
\hline C & -5.944487 & 4.855860 & -0.422740 \\
\hline H & -6.680296 & 4.610101 & -1.197568 \\
\hline $\mathrm{H}$ & -6.424108 & 4.734333 & 0.555491 \\
\hline $\mathrm{H}$ & -5.689803 & 5.916065 & -0.534080 \\
\hline C & -8.822555 & 0.531459 & -0.926959 \\
\hline $\mathrm{H}$ & -8.845520 & 1.618967 & -1.052112 \\
\hline $\mathrm{H}$ & -8.478721 & 0.094494 & -1.871608 \\
\hline $\mathrm{H}$ & -9.851466 & 0.194123 & -0.750443 \\
\hline C & -8.434578 & 0.723841 & 1.559861 \\
\hline $\mathrm{H}$ & -9.459520 & 0.385838 & 1.757426 \\
\hline $\mathrm{H}$ & -7.809238 & 0.428477 & 2.410183 \\
\hline $\mathrm{H}$ & -8.446795 & 1.817966 & 1.519618 \\
\hline C & -7.984789 & -1.429095 & 0.374645 \\
\hline $\mathrm{H}$ & -7.381518 & -1.799209 & 1.211392 \\
\hline
\end{tabular}




\section{Catalyst 2C 34}

Datum

Value

\begin{tabular}{lc}
\hline B3LYP/6-31G(d) Energy & -2164.799936 \\
\hline B3LYP-D3(BJ)/def2-TZVPP-IEF-PCM(DCM) Energy & -2165.897062 \\
\hline B3LYP-D3(BJ)/def2-TZVPP-IEF-PCM(DCM)//B3LYP/6-31G(d) Free Energy (Quasiharmonic) & -2165.019014 \\
\hline
\end{tabular}

Number of Imaginary Frequencies

Frequencies (Top 3 out of 321)

$\begin{array}{ll}\text { 1. } & 10.1730 \mathrm{~cm}^{-1} \\ \text { 2. } & 11.4246 \mathrm{~cm}^{-1} \\ \text { 3. } & 13.2758 \mathrm{~cm}^{-1}\end{array}$

\section{B3LYP/6-31G(d) Molecular Geometry in Cartesian Coordinates}

\begin{tabular}{|c|c|c|c|}
\hline C & 0.272639 & -4.003805 & 3.151683 \\
\hline C & 0.396718 & -3.175284 & 2.058347 \\
\hline C & -0.691680 & -2.368809 & 1.624774 \\
\hline C & -1.925344 & -2.445347 & 2.346727 \\
\hline C & -2.019587 & -3.307154 & 3.472459 \\
\hline C & -0.945750 & -4.070780 & 3.868182 \\
\hline $\mathrm{H}$ & 1.116289 & -4.611481 & 3.467019 \\
\hline $\mathrm{H}$ & 1.334045 & -3.130278 & 1.514858 \\
\hline C & -0.599072 & -1.492625 & 0.490811 \\
\hline C & -3.023588 & -1.654366 & 1.928542 \\
\hline $\mathrm{H}$ & -2.960345 & -3.351852 & 4.015822 \\
\hline $\mathrm{H}$ & -1.030147 & -4.727215 & 4.729936 \\
\hline C & -2.949020 & -0.817702 & 0.834624 \\
\hline C & -1.712822 & -0.754103 & 0.110321 \\
\hline $\mathrm{H}$ & -3.945103 & -1.694347 & 2.503474 \\
\hline C & 1.075430 & -4.052605 & -3.680239 \\
\hline C & 2.119131 & -3.240883 & -3.301880 \\
\hline C & 1.999414 & -2.375528 & -2.180610 \\
\hline C & 0.774510 & -2.346176 & -1.442415 \\
\hline C & -0.284337 & -3.200269 & -1.861054 \\
\hline C & -0.136794 & -4.030176 & -2.949835 \\
\hline $\mathrm{H}$ & 3.990875 & -1.570344 & -2.341722 \\
\hline $\mathrm{H}$ & 1.176977 & -4.710741 & -4.538947 \\
\hline $\mathrm{H}$ & 3.055775 & -3.248665 & -3.854442 \\
\hline
\end{tabular}




\begin{tabular}{|c|c|c|c|}
\hline C & 3.066912 & -1.544275 & -1.769678 \\
\hline C & 0.674250 & -1.464579 & -0.314301 \\
\hline $\mathrm{H}$ & -1.223320 & -3.187636 & -1.319131 \\
\hline $\mathrm{H}$ & -0.960100 & -4.670548 & -3.254092 \\
\hline C & 1.741221 & -0.648819 & 0.060881 \\
\hline C & 2.970906 & -0.690812 & -0.686044 \\
\hline C & 1.594826 & 0.289549 & 1.248725 \\
\hline $\mathrm{H}$ & 0.884173 & -0.127634 & 1.971728 \\
\hline $\mathrm{H}$ & 2.554215 & 0.405547 & 1.757177 \\
\hline C & -1.686272 & 0.011263 & -1.181581 \\
\hline 0 & -2.288522 & -0.289425 & -2.183038 \\
\hline 0 & -0.891059 & 1.122547 & -1.127927 \\
\hline $\mathrm{H}$ & -0.895784 & 1.511969 & -2.024060 \\
\hline 0 & 1.197771 & 1.611250 & 0.873040 \\
\hline $\mathrm{H}$ & 0.414785 & 1.528355 & 0.301254 \\
\hline C & 4.176420 & 0.116082 & -0.326215 \\
\hline C & 4.113972 & 1.503187 & -0.141541 \\
\hline C & 5.421631 & -0.521425 & -0.219547 \\
\hline C & 5.260276 & 2.253464 & 0.148872 \\
\hline $\mathrm{H}$ & 3.148169 & 1.989172 & -0.214480 \\
\hline C & 6.592360 & 0.191683 & 0.064293 \\
\hline $\mathrm{H}$ & 5.461122 & -1.599759 & -0.345110 \\
\hline C & 6.485738 & 1.578821 & 0.243247 \\
\hline $\mathrm{H}$ & 7.378752 & 2.146402 & 0.466524 \\
\hline C & -4.124079 & 0.016136 & 0.456489 \\
\hline C & -5.380843 & -0.578833 & 0.285882 \\
\hline C & -3.993966 & 1.403443 & 0.306843 \\
\hline C & -6.506686 & 0.185073 & -0.041190 \\
\hline $\mathrm{H}$ & -5.456650 & -1.655104 & 0.389105 \\
\hline C & -5.094761 & 2.203945 & -0.017256 \\
\hline $\mathrm{H}$ & -3.021263 & 1.852947 & 0.467913 \\
\hline C & -6.333443 & 1.569205 & -0.185642 \\
\hline $\mathrm{H}$ & -7.196679 & 2.176578 & -0.441075 \\
\hline C & -4.984219 & 3.730979 & -0.192102 \\
\hline C & -7.899672 & -0.437938 & -0.255547 \\
\hline C & -5.924728 & 4.439280 & 0.811455 \\
\hline $\mathrm{H}$ & -6.971945 & 4.158170 & 0.658513 \\
\hline $\mathrm{H}$ & -5.658162 & 4.186661 & 1.844050 \\
\hline $\mathrm{H}$ & -5.852750 & 5.527982 & 0.698517 \\
\hline C & -5.394003 & 4.111499 & -1.634855 \\
\hline $\mathrm{H}$ & -5.322197 & 5.196677 & -1.778094 \\
\hline $\mathrm{H}$ & -4.740221 & 3.626605 & -2.368683 \\
\hline $\mathrm{H}$ & -6.423593 & 3.813178 & -1.858412 \\
\hline C & -3.552373 & 4.245733 & 0.051340 \\
\hline $\mathrm{H}$ & -2.832368 & 3.797561 & -0.642869 \\
\hline $\mathrm{H}$ & -3.520484 & 5.331366 & -0.094540 \\
\hline $\mathrm{H}$ & -3.212097 & 4.039747 & 1.072474 \\
\hline C & -7.896704 & -1.965864 & -0.057307 \\
\hline $\mathrm{H}$ & -7.230109 & -2.468146 & -0.767308 \\
\hline $\mathrm{H}$ & -7.590533 & -2.245599 & 0.957209 \\
\hline $\mathrm{H}$ & -8.906822 & -2.359354 & -0.217733 \\
\hline C & -8.379283 & -0.143562 & -1.696907 \\
\hline H & -7.689492 & -0.570831 & -2.433455 \\
\hline H & -9.371498 & -0.580187 & -1.865486 \\
\hline
\end{tabular}




\begin{tabular}{|c|c|c|c|}
\hline $\mathrm{H}$ & -8.451697 & 0.931456 & -1.892172 \\
\hline C & -8.899871 & 0.174514 & 0.753551 \\
\hline H & -8.590886 & -0.029879 & 1.785167 \\
\hline $\mathrm{H}$ & -8.982557 & 1.260329 & 0.639287 \\
\hline $\mathrm{H}$ & -9.899345 & -0.252976 & 0.606884 \\
\hline C & 7.933495 & -0.560249 & 0.179051 \\
\hline C & 5.128073 & 3.775123 & 0.355133 \\
\hline C & 4.549745 & 4.420278 & -0.926932 \\
\hline $\mathrm{H}$ & 5.206963 & 4.243837 & -1.786536 \\
\hline $\mathrm{H}$ & 3.561426 & 4.018654 & -1.172412 \\
\hline $\mathrm{H}$ & 4.446545 & 5.504470 & -0.794587 \\
\hline C & 6.478190 & 4.450544 & 0.661941 \\
\hline $\mathrm{H}$ & 6.931560 & 4.059290 & 1.579995 \\
\hline $\mathrm{H}$ & 7.196724 & 4.322250 & -0.156118 \\
\hline $\mathrm{H}$ & 6.327846 & 5.527074 & 0.802721 \\
\hline C & 4.169935 & 4.047031 & 1.540363 \\
\hline $\mathrm{H}$ & 3.180726 & 3.608094 & 1.376805 \\
\hline $\mathrm{H}$ & 4.572970 & 3.626171 & 2.469208 \\
\hline $\mathrm{H}$ & 4.043023 & 5.127288 & 1.686617 \\
\hline C & 8.228993 & -1.295803 & -1.149814 \\
\hline $\mathrm{H}$ & 7.446386 & -2.020549 & -1.397191 \\
\hline $\mathrm{H}$ & 8.302611 & -0.586121 & -1.981882 \\
\hline $\mathrm{H}$ & 9.178611 & -1.841112 & -1.082593 \\
\hline C & 7.847989 & -1.594415 & 1.326753 \\
\hline $\mathrm{H}$ & 8.793322 & -2.143757 & 1.418099 \\
\hline $\mathrm{H}$ & 7.648158 & -1.100300 & 2.284454 \\
\hline $\mathrm{H}$ & 7.051726 & -2.326880 & 1.157119 \\
\hline C & 9.116400 & 0.381846 & 0.473838 \\
\hline $\mathrm{H}$ & 8.992042 & 0.908672 & 1.426707 \\
\hline $\mathrm{H}$ & 10.043332 & -0.199503 & 0.537701 \\
\hline H & 9.249825 & 1.130366 & -0.315600 \\
\hline
\end{tabular}

\section{Catalyst 2C 35}

Datum

Value

\begin{tabular}{lc}
\hline B3LYP/6-31G(d) Energy & -2164.794897 \\
\hline B3LYP-D3(BJ)/def2-TZVPP-IEF-PCM(DCM) Energy & -2165.895088 \\
\hline
\end{tabular}

B3LYP-D3(BJ)/def2-TZVPP-IEF-PCM(DCM)//B3LYP/6-31G(d) Free Energy (Quasiharmonic) $\quad$-2165.018988

Number of Imaginary Frequencies

Frequencies (Top 3 out of 321)

$\begin{array}{lr}\text { 1. } & 6.2396 \mathrm{~cm}^{-1} \\ \text { 2. } & 6.9827 \mathrm{~cm}^{-1} \\ \text { 3. } & 12.4421 \mathrm{~cm}^{-1}\end{array}$




\begin{tabular}{|c|c|c|c|}
\hline C & 0.091784 & -4.675878 & -2.600271 \\
\hline C & -0.146664 & -3.700986 & -1.657466 \\
\hline C & 0.864481 & -2.765603 & -1.305372 \\
\hline C & 2.135670 & -2.853244 & -1.957472 \\
\hline C & 2.349216 & -3.871130 & -2.925915 \\
\hline C & 1.351719 & -4.765287 & -3.239316 \\
\hline $\mathrm{H}$ & -0.694189 & -5.379374 & -2.860508 \\
\hline $\mathrm{H}$ & -1.118004 & -3.627964 & -1.181039 \\
\hline C & 0.655452 & -1.740115 & -0.326281 \\
\hline C & 3.144936 & -1.913636 & -1.634609 \\
\hline $\mathrm{H}$ & 3.317722 & -3.927813 & -3.417099 \\
\hline $\mathrm{H}$ & 1.526200 & -5.539507 & -3.981634 \\
\hline C & 2.946976 & -0.912550 & -0.704331 \\
\hline C & 1.678026 & -0.845380 & -0.040636 \\
\hline $\mathrm{H}$ & 4.093237 & -1.965424 & -2.163342 \\
\hline C & -1.135123 & -4.081720 & 3.929451 \\
\hline C & -2.160887 & -3.275566 & 3.491976 \\
\hline C & -2.009471 & -2.467786 & 2.333501 \\
\hline C & -0.774352 & -2.495243 & 1.612000 \\
\hline C & 0.267215 & -3.334857 & 2.096307 \\
\hline C & 0.090546 & -4.107472 & 3.223124 \\
\hline $\mathrm{H}$ & -3.975123 & -1.576581 & 2.446316 \\
\hline $\mathrm{H}$ & -1.261217 & -4.695800 & 4.817093 \\
\hline $\mathrm{H}$ & -3.105978 & -3.241667 & 4.029162 \\
\hline C & -3.047609 & -1.617219 & 1.879695 \\
\hline C & -0.641510 & -1.685185 & 0.432957 \\
\hline $\mathrm{H}$ & 1.214489 & -3.360520 & 1.568862 \\
\hline $\mathrm{H}$ & 0.900605 & -4.740976 & 3.574439 \\
\hline C & -1.681999 & -0.870015 & 0.007416 \\
\hline C & -2.900112 & -0.820740 & 0.764713 \\
\hline C & -1.576576 & -0.061869 & -1.278578 \\
\hline $\mathrm{H}$ & -1.660465 & 1.010591 & -1.059290 \\
\hline $\mathrm{H}$ & -0.601761 & -0.214276 & -1.740821 \\
\hline C & 1.442655 & 0.200489 & 1.012803 \\
\hline 0 & 0.680506 & 1.140913 & 0.916630 \\
\hline 0 & 2.183139 & -0.024495 & 2.120426 \\
\hline $\mathrm{H}$ & 1.981347 & 0.707940 & 2.734281 \\
\hline 0 & -2.533456 & -0.465598 & -2.256413 \\
\hline $\mathrm{H}$ & -3.407670 & -0.195835 & -1.931251 \\
\hline C & -4.026513 & 0.088594 & 0.381094 \\
\hline C & -5.255760 & -0.448634 & -0.040042 \\
\hline C & -3.888076 & 1.475921 & 0.479949 \\
\hline C & -6.334776 & 0.377792 & -0.367435 \\
\hline $\mathrm{H}$ & -5.342459 & -1.527093 & -0.109257 \\
\hline C & -4.947300 & 2.341889 & 0.162392 \\
\hline $\mathrm{H}$ & -2.940815 & 1.877159 & 0.828369 \\
\hline C & -6.151187 & 1.768180 & -0.258716 \\
\hline $\mathrm{H}$ & -6.980404 & 2.417638 & -0.512688 \\
\hline C & 4.029146 & 0.077769 & -0.443744 \\
\hline C & 5.332864 & -0.355296 & -0.190799 \\
\hline
\end{tabular}




\begin{tabular}{|c|c|c|c|}
\hline C & 3.764345 & 1.457278 & -0.490406 \\
\hline C & 6.379777 & 0.554796 & 0.024051 \\
\hline $\mathrm{H}$ & 5.522098 & -1.423559 & -0.140432 \\
\hline C & 4.775139 & 2.395822 & -0.277257 \\
\hline $\mathrm{H}$ & 2.755562 & 1.782832 & -0.712236 \\
\hline C & 6.073734 & 1.918129 & -0.022307 \\
\hline $\mathrm{H}$ & 6.864826 & 2.639646 & 0.143772 \\
\hline C & 4.512294 & 3.913739 & -0.315374 \\
\hline C & 7.800446 & 0.027033 & 0.304448 \\
\hline C & 4.878105 & 4.532295 & 1.055169 \\
\hline $\mathrm{H}$ & 4.266751 & 4.099589 & 1.855421 \\
\hline $\mathrm{H}$ & 5.929802 & 4.367137 & 1.311686 \\
\hline $\mathrm{H}$ & 4.703958 & 5.615324 & 1.043446 \\
\hline C & 3.036331 & 4.244814 & -0.609657 \\
\hline $\mathrm{H}$ & 2.896574 & 5.331824 & -0.607153 \\
\hline $\mathrm{H}$ & 2.724213 & 3.874811 & -1.592853 \\
\hline $\mathrm{H}$ & 2.361473 & 3.820102 & 0.141780 \\
\hline C & 5.381044 & 4.564638 & -1.417672 \\
\hline $\mathrm{H}$ & 5.140830 & 4.147688 & -2.402322 \\
\hline $\mathrm{H}$ & 5.202821 & 5.646510 & -1.454125 \\
\hline $\mathrm{H}$ & 6.450715 & 4.409841 & -1.240970 \\
\hline C & 8.819694 & 1.161417 & 0.523470 \\
\hline $\mathrm{H}$ & 8.905976 & 1.808719 & -0.356675 \\
\hline $\mathrm{H}$ & 8.556907 & 1.786950 & 1.384222 \\
\hline $\mathrm{H}$ & 9.810149 & 0.734146 & 0.716973 \\
\hline C & 8.278290 & -0.821657 & -0.897888 \\
\hline $\mathrm{H}$ & 8.310476 & -0.220390 & -1.813720 \\
\hline $\mathrm{H}$ & 9.285826 & -1.214071 & -0.712789 \\
\hline $\mathrm{H}$ & 7.616936 & -1.674873 & -1.081065 \\
\hline C & 7.782007 & -0.854102 & 1.576328 \\
\hline $\mathrm{H}$ & 7.449461 & -0.278174 & 2.447455 \\
\hline $\mathrm{H}$ & 7.110589 & -1.712027 & 1.468112 \\
\hline $\mathrm{H}$ & 8.786727 & -1.240857 & 1.787038 \\
\hline C & -4.750070 & 3.864560 & 0.295090 \\
\hline C & -7.693613 & -0.177083 & -0.836283 \\
\hline C & -8.801398 & 0.277679 & 0.143805 \\
\hline $\mathrm{H}$ & -8.604254 & -0.091700 & 1.156692 \\
\hline H & -8.878766 & 1.368726 & 0.195344 \\
\hline $\mathrm{H}$ & -9.776190 & -0.110691 & -0.175904 \\
\hline C & -7.709864 & -1.716597 & -0.895224 \\
\hline $\mathrm{H}$ & -6.966637 & -2.106726 & -1.599674 \\
\hline $\mathrm{H}$ & -7.519130 & -2.165306 & 0.086310 \\
\hline $\mathrm{H}$ & -8.694538 & -2.061762 & -1.229911 \\
\hline C & -8.010171 & 0.360517 & -2.252322 \\
\hline $\mathrm{H}$ & -8.060600 & 1.454164 & -2.272825 \\
\hline $\mathrm{H}$ & -7.243852 & 0.047576 & -2.970713 \\
\hline $\mathrm{H}$ & -8.977335 & -0.023729 & -2.599112 \\
\hline C & -4.405367 & 4.210139 & 1.763464 \\
\hline $\mathrm{H}$ & -5.215255 & 3.908956 & 2.437861 \\
\hline $\mathrm{H}$ & -3.490049 & 3.709701 & 2.095510 \\
\hline $\mathrm{H}$ & -4.254201 & 5.290865 & 1.875021 \\
\hline C & -6.008488 & 4.659931 & -0.101595 \\
\hline $\mathrm{H}$ & -5.814597 & 5.732849 & 0.008034 \\
\hline H & -6.292740 & 4.481256 & -1.144913 \\
\hline
\end{tabular}




\section{Catalyst 2C 36}

Datum

Value

\begin{tabular}{lc}
\hline B3LYP/6-31G(d) Energy & -2164.802512 \\
\hline B3LYP-D3(BJ)/def2-TZVPP-IEF-PCM(DCM) Energy & -2165.897943 \\
\hline B3LYP-D3(BJ)/def2-TZVPP-IEF-PCM(DCM)//B3LYP/6-31G(d) Free Energy (Quasiharmonic) & -2165.018976 \\
\hline
\end{tabular}

Number of Imaginary Frequencies

Frequencies (Top 3 out of 321)
1. $11.7149 \mathrm{~cm}^{-1}$
2. $12.7299 \mathrm{~cm}^{-1}$
3. $13.9878 \mathrm{~cm}^{-1}$

\section{B3LYP/6-31G(d) Molecular Geometry in Cartesian Coordinates}

\begin{tabular}{|c|c|c|c|}
\hline C & -0.229644 & -3.457248 & 3.956306 \\
\hline C & -0.374703 & -2.895218 & 2.707294 \\
\hline C & 0.691764 & -2.172856 & 2.104884 \\
\hline C & 1.920065 & -2.036993 & 2.827196 \\
\hline C & 2.036510 & -2.628129 & 4.114237 \\
\hline C & 0.986394 & -3.323175 & 4.668260 \\
\hline $\mathrm{H}$ & -1.054235 & -4.008543 & 4.399703 \\
\hline $\mathrm{H}$ & -1.309092 & -3.001070 & 2.166658 \\
\hline C & 0.577605 & -1.569881 & 0.809076 \\
\hline C & 2.991986 & -1.318361 & 2.242564 \\
\hline $\mathrm{H}$ & 2.974153 & -2.519722 & 4.654021 \\
\hline $\mathrm{H}$ & 1.086876 & -3.770642 & 5.653317 \\
\hline C & 2.900505 & -0.756993 & 0.984858 \\
\hline C & 1.668287 & -0.902737 & 0.270045 \\
\hline $\mathrm{H}$ & 3.922478 & -1.225159 & 2.796685 \\
\hline C & -1.446877 & -4.960245 & -2.537739 \\
\hline C & -2.399418 & -3.980413 & -2.380999 \\
\hline C & -2.170854 & -2.873336 & -1.519104 \\
\hline C & -0.930832 & -2.775812 & -0.814705 \\
\hline C & 0.032909 & -3.806090 & -1.000474 \\
\hline C & -0.219671 & -4.870022 & -1.837630 \\
\hline
\end{tabular}




\begin{tabular}{|c|c|c|c|}
\hline $\mathrm{H}$ & -4.079063 & -1.942977 & -1.881902 \\
\hline $\mathrm{H}$ & -1.632296 & -5.804128 & -3.196841 \\
\hline $\mathrm{H}$ & -3.346376 & -4.038243 & -2.912614 \\
\hline C & -3.143355 & -1.863359 & -1.334453 \\
\hline C & -0.719395 & -1.650232 & 0.049090 \\
\hline $\mathrm{H}$ & 0.980563 & -3.745885 & -0.476327 \\
\hline $\mathrm{H}$ & 0.530090 & -5.646362 & -1.964583 \\
\hline C & -1.695987 & -0.666873 & 0.211338 \\
\hline C & -2.943439 & -0.778656 & -0.500237 \\
\hline C & -1.455590 & 0.500013 & 1.163523 \\
\hline H & -2.396452 & 0.770490 & 1.648781 \\
\hline $\mathrm{H}$ & -0.756007 & 0.195007 & 1.951155 \\
\hline C & 1.500835 & -0.228949 & -1.063267 \\
\hline 0 & 1.137329 & 0.921439 & -1.219319 \\
\hline 0 & 1.776774 & -1.036194 & -2.106014 \\
\hline $\mathrm{H}$ & 1.602925 & -0.513980 & -2.914160 \\
\hline 0 & -1.001000 & 1.691936 & 0.527663 \\
\hline $\mathrm{H}$ & -0.176204 & 1.496481 & 0.042362 \\
\hline C & -4.063654 & 0.199766 & -0.354195 \\
\hline C & -3.870920 & 1.575230 & -0.505294 \\
\hline C & -5.364438 & -0.281696 & -0.111163 \\
\hline C & -4.942527 & 2.478154 & -0.415131 \\
\hline $\mathrm{H}$ & -2.865150 & 1.938542 & -0.678710 \\
\hline C & -6.456506 & 0.582101 & -0.019488 \\
\hline $\mathrm{H}$ & -5.498004 & -1.349105 & 0.022631 \\
\hline C & -6.217922 & 1.960032 & -0.176388 \\
\hline $\mathrm{H}$ & -7.057971 & 2.640930 & -0.103343 \\
\hline C & 4.060844 & -0.005182 & 0.424485 \\
\hline C & 4.636522 & -0.362049 & -0.801058 \\
\hline C & 4.614062 & 1.056504 & 1.151418 \\
\hline C & 5.743366 & 0.326657 & -1.313376 \\
\hline $\mathrm{H}$ & 4.220998 & -1.201777 & -1.347288 \\
\hline C & 5.719131 & 1.772027 & 0.675117 \\
\hline $\mathrm{H}$ & 4.150726 & 1.332198 & 2.094052 \\
\hline C & 6.263586 & 1.388148 & -0.559280 \\
\hline $\mathrm{H}$ & 7.115713 & 1.931162 & -0.943724 \\
\hline C & 6.283136 & 2.942767 & 1.504564 \\
\hline C & 6.344473 & -0.104774 & -2.665986 \\
\hline C & 6.727564 & 2.422423 & 2.892349 \\
\hline $\mathrm{H}$ & 5.894942 & 1.977390 & 3.447067 \\
\hline $\mathrm{H}$ & 7.509697 & 1.660670 & 2.794209 \\
\hline $\mathrm{H}$ & 7.128573 & 3.244738 & 3.497495 \\
\hline C & 5.185356 & 4.017930 & 1.688372 \\
\hline $\mathrm{H}$ & 5.569393 & 4.856690 & 2.281927 \\
\hline $\mathrm{H}$ & 4.854894 & 4.409834 & 0.719826 \\
\hline $\mathrm{H}$ & 4.305428 & 3.619948 & 2.203988 \\
\hline C & 7.498525 & 3.611106 & 0.834537 \\
\hline $\mathrm{H}$ & 7.244294 & 4.035555 & -0.143319 \\
\hline $\mathrm{H}$ & 7.860359 & 4.431133 & 1.464932 \\
\hline $\mathrm{H}$ & 8.329110 & 2.909029 & 0.698079 \\
\hline C & 6.816289 & -1.575715 & -2.575560 \\
\hline $\mathrm{H}$ & 7.585516 & -1.693530 & -1.803775 \\
\hline $\mathrm{H}$ & 5.992276 & -2.254485 & -2.332604 \\
\hline $\mathrm{H}$ & 7.243135 & -1.898959 & -3.532987 \\
\hline
\end{tabular}




\begin{tabular}{|c|c|c|c|}
\hline C & 7.550247 & 0.758757 & -3.081376 \\
\hline $\mathrm{H}$ & 8.370010 & 0.691377 & -2.357097 \\
\hline $\mathrm{H}$ & 7.935239 & 0.412648 & -4.047255 \\
\hline $\mathrm{H}$ & 7.278499 & 1.814514 & -3.193915 \\
\hline C & 5.264532 & 0.018742 & -3.767639 \\
\hline $\mathrm{H}$ & 4.393274 & -0.608863 & -3.552673 \\
\hline $\mathrm{H}$ & 4.918880 & 1.054803 & -3.860864 \\
\hline $\mathrm{H}$ & 5.670238 & -0.293231 & -4.737848 \\
\hline C & -7.890526 & 0.084160 & 0.248183 \\
\hline C & -4.666064 & 3.985143 & -0.577129 \\
\hline C & -4.040657 & 4.246532 & -1.968058 \\
\hline $\mathrm{H}$ & -3.095369 & 3.709655 & -2.095686 \\
\hline $\mathrm{H}$ & -4.718614 & 3.929316 & -2.769233 \\
\hline $\mathrm{H}$ & -3.837139 & 5.316657 & -2.098501 \\
\hline C & -3.676742 & 4.442057 & 0.522665 \\
\hline $\mathrm{H}$ & -4.112576 & 4.299833 & 1.518845 \\
\hline $\mathrm{H}$ & -2.736309 & 3.883650 & 0.486875 \\
\hline $\mathrm{H}$ & -3.445075 & 5.508344 & 0.404994 \\
\hline C & -5.944461 & 4.836490 & -0.460226 \\
\hline $\mathrm{H}$ & -6.679384 & 4.580836 & -1.232694 \\
\hline $\mathrm{H}$ & -6.423714 & 4.722636 & 0.519105 \\
\hline $\mathrm{H}$ & -5.693449 & 5.896458 & -0.581713 \\
\hline C & -8.806131 & 0.495819 & -0.929432 \\
\hline $\mathrm{H}$ & -8.832723 & 1.581877 & -1.066046 \\
\hline $\mathrm{H}$ & -8.458626 & 0.050196 & -1.868678 \\
\hline $\mathrm{H}$ & -9.834202 & 0.156613 & -0.751575 \\
\hline C & -8.424111 & 0.715907 & 1.555976 \\
\hline $\mathrm{H}$ & -9.447976 & 0.375660 & 1.755234 \\
\hline $\mathrm{H}$ & -7.799171 & 0.432463 & 2.410634 \\
\hline $\mathrm{H}$ & -8.441007 & 1.809479 & 1.503953 \\
\hline C & -7.963725 & -1.447746 & 0.394626 \\
\hline $\mathrm{H}$ & -7.360199 & -1.806710 & 1.236042 \\
\hline $\mathrm{H}$ & -9.000455 & -1.752553 & 0.577833 \\
\hline $\mathrm{H}$ & -7.624363 & -1.960682 & -0.512543 \\
\hline
\end{tabular}

\section{Catalyst 2C 37}

Datum

Value

\begin{tabular}{lr}
\hline B3LYP/6-31G(d) Energy & -2164.799693 \\
\hline B3LYP-D3(BJ)/def2-TZVPP-IEF-PCM(DCM) Energy & -2165.896927 \\
\hline B3LYP-D3(BJ)/def2-TZVPP-IEF-PCM(DCM)//B3LYP/6-31G(d) Free Energy (Quasiharmonic) & -2165.018793 \\
\hline Number of Imaginary Frequencies & 0
\end{tabular}

Frequencies (Top 3 out of 321) 

1. $\quad 9.8675 \mathrm{~cm}^{-1}$
2. $\quad 11.8854 \mathrm{~cm}^{-1}$
3. $\quad 13.8063 \mathrm{~cm}^{-1}$

\section{B3LYP/6-31G(d) Molecular Geometry in Cartesian Coordinates}

\begin{tabular}{|c|c|c|c|}
\hline C & -0.214609 & -4.021946 & 3.103973 \\
\hline C & -0.341126 & -3.190698 & 2.012973 \\
\hline C & 0.740001 & -2.368595 & 1.590860 \\
\hline C & 1.968821 & -2.431615 & 2.322296 \\
\hline C & 2.065537 & -3.296237 & 3.445643 \\
\hline C & 0.998841 & -4.075594 & 3.829883 \\
\hline $\mathrm{H}$ & -1.052516 & -4.642139 & 3.410138 \\
\hline $\mathrm{H}$ & -1.274574 & -3.155930 & 1.462160 \\
\hline C & 0.644811 & -1.489715 & 0.459089 \\
\hline C & 3.059766 & -1.624613 & 1.915713 \\
\hline $\mathrm{H}$ & 3.002457 & -3.330548 & 3.996344 \\
\hline $\mathrm{H}$ & 1.085164 & -4.734287 & 4.689722 \\
\hline C & 2.983243 & -0.786758 & 0.822948 \\
\hline C & 1.752370 & -0.737423 & 0.088398 \\
\hline $\mathrm{H}$ & 3.976547 & -1.653278 & 2.498847 \\
\hline C & -1.000603 & -4.048698 & -3.726081 \\
\hline C & -2.053014 & -3.250009 & -3.344027 \\
\hline C & -1.942249 & -2.387516 & -2.219662 \\
\hline C & -0.717289 & -2.347821 & -1.481841 \\
\hline C & 0.350561 & -3.188773 & -1.904121 \\
\hline C & 0.211703 & -4.015816 & -2.996230 \\
\hline $\mathrm{H}$ & -3.944008 & -1.605525 & -2.374446 \\
\hline $\mathrm{H}$ & -1.095335 & -4.704457 & -4.587392 \\
\hline $\mathrm{H}$ & -2.989793 & -3.266021 & -3.896192 \\
\hline C & -3.018777 & -1.569635 & -1.804873 \\
\hline C & -0.625813 & -1.469603 & -0.350399 \\
\hline $\mathrm{H}$ & 1.289754 & -3.167617 & -1.362778 \\
\hline $\mathrm{H}$ & 1.041932 & -4.645691 & -3.303576 \\
\hline C & -1.700662 & -0.665190 & 0.026728 \\
\hline C & -2.930099 & -0.717856 & -0.719469 \\
\hline C & -1.564552 & 0.272924 & 1.215979 \\
\hline $\mathrm{H}$ & -2.525762 & 0.378208 & 1.723495 \\
\hline $\mathrm{H}$ & -0.850545 & -0.138383 & 1.939233 \\
\hline C & 1.726452 & 0.028115 & -1.203691 \\
\hline 0 & 2.333053 & -0.271183 & -2.202786 \\
\hline 0 & 0.926560 & 1.136521 & -1.153155 \\
\hline $\mathrm{H}$ & 0.936536 & 1.528496 & -2.048201 \\
\hline 0 & -1.180976 & 1.598519 & 0.841575 \\
\hline $\mathrm{H}$ & -0.399209 & 1.522793 & 0.267325 \\
\hline C & -4.143574 & 0.072953 & -0.350741 \\
\hline C & -5.372928 & -0.590363 & -0.211153 \\
\hline C & -4.097727 & 1.464518 & -0.186890 \\
\hline C & -6.548209 & 0.104901 & 0.094007 \\
\hline $\mathrm{H}$ & -5.388876 & -1.668123 & -0.326436 \\
\hline
\end{tabular}




\begin{tabular}{|c|c|c|c|}
\hline C & -5.250926 & 2.196492 & 0.119290 \\
\hline $\mathrm{H}$ & -3.141337 & 1.959664 & -0.287164 \\
\hline C & -6.457115 & 1.494954 & 0.254141 \\
\hline $\mathrm{H}$ & -7.358185 & 2.049816 & 0.498673 \\
\hline C & 4.149777 & 0.063649 & 0.455852 \\
\hline C & 5.419486 & -0.506313 & 0.306474 \\
\hline C & 4.002829 & 1.446388 & 0.291773 \\
\hline C & 6.537471 & 0.273847 & -0.012703 \\
\hline $\mathrm{H}$ & 5.518690 & -1.581870 & 0.419585 \\
\hline C & 5.092475 & 2.263419 & -0.031514 \\
\hline $\mathrm{H}$ & 3.021777 & 1.887225 & 0.439019 \\
\hline C & 6.346813 & 1.653755 & -0.178397 \\
\hline $\mathrm{H}$ & 7.198383 & 2.270387 & -0.430978 \\
\hline C & 4.876948 & 3.779829 & -0.202657 \\
\hline C & 7.913272 & -0.400160 & -0.181216 \\
\hline C & 4.363500 & 4.378997 & 1.128312 \\
\hline $\mathrm{H}$ & 3.417774 & 3.924702 & 1.441548 \\
\hline $\mathrm{H}$ & 5.091289 & 4.224719 & 1.933193 \\
\hline $\mathrm{H}$ & 4.196935 & 5.458050 & 1.022505 \\
\hline C & 3.827502 & 4.026434 & -1.313364 \\
\hline $\mathrm{H}$ & 3.654281 & 5.101732 & -1.442929 \\
\hline $\mathrm{H}$ & 4.169072 & 3.618104 & -2.271515 \\
\hline $\mathrm{H}$ & 2.865349 & 3.561017 & -1.074368 \\
\hline C & 6.169382 & 4.520418 & -0.595911 \\
\hline $\mathrm{H}$ & 6.576777 & 4.155059 & -1.545548 \\
\hline $\mathrm{H}$ & 5.959003 & 5.589169 & -0.716358 \\
\hline $\mathrm{H}$ & 6.946703 & 4.423323 & 0.170601 \\
\hline C & 8.299053 & -1.119022 & 1.133534 \\
\hline $\mathrm{H}$ & 8.367715 & -0.407190 & 1.964261 \\
\hline $\mathrm{H}$ & 7.566921 & -1.885093 & 1.409463 \\
\hline $\mathrm{H}$ & 9.272994 & -1.612340 & 1.027094 \\
\hline C & 9.028567 & 0.608135 & -0.517421 \\
\hline $\mathrm{H}$ & 9.150265 & 1.361977 & 0.268898 \\
\hline $\mathrm{H}$ & 9.983368 & 0.079692 & -0.617703 \\
\hline $\mathrm{H}$ & 8.839224 & 1.127311 & -1.463751 \\
\hline C & 7.841482 & -1.435131 & -1.329197 \\
\hline $\mathrm{H}$ & 7.096794 & -2.213268 & -1.132157 \\
\hline H & 7.574484 & -0.951786 & -2.275715 \\
\hline $\mathrm{H}$ & 8.813050 & -1.927552 & -1.459748 \\
\hline C & -5.222764 & 3.723746 & 0.325452 \\
\hline C & -7.909021 & -0.598456 & 0.265433 \\
\hline C & -7.815047 & -2.121179 & 0.050761 \\
\hline $\mathrm{H}$ & -7.465636 & -2.370293 & -0.957744 \\
\hline $\mathrm{H}$ & -7.139752 & -2.594375 & 0.772637 \\
\hline $\mathrm{H}$ & -8.804767 & -2.574311 & 0.178495 \\
\hline C & -8.916367 & -0.031739 & -0.763250 \\
\hline $\mathrm{H}$ & -9.065651 & 1.045663 & -0.637649 \\
\hline $\mathrm{H}$ & -8.567283 & -0.202636 & -1.788101 \\
\hline $\mathrm{H}$ & -9.892843 & -0.518992 & -0.650557 \\
\hline C & -8.443835 & -0.349058 & 1.695814 \\
\hline $\mathrm{H}$ & -7.753174 & -0.749198 & 2.446926 \\
\hline $\mathrm{H}$ & -8.577515 & 0.718105 & 1.901440 \\
\hline $\mathrm{H}$ & -9.415985 & -0.839058 & 1.832148 \\
\hline C & -5.623431 & 4.045208 & 1.785171 \\
\hline
\end{tabular}




\begin{tabular}{|llll|}
$H$ & -6.626002 & 3.673056 & 2.022141 \\
$H$ & -4.921048 & 3.589048 & 2.491900 \\
$H$ & -5.619817 & 5.129379 & 1.953652 \\
C & -6.222518 & 4.402629 & -0.640189 \\
$H$ & -6.202598 & 5.491106 & -0.505177 \\
$H$ & -5.967816 & 4.185617 & -1.683975 \\
$H$ & -7.251402 & 4.067552 & -0.470933 \\
C & -3.826357 & 4.322348 & 0.066114 \\
$H$ & -3.505584 & 4.160340 & -0.969610 \\
$H$ & -3.853512 & 5.404600 & 0.239033 \\
$H$ & -3.064078 & 3.894437 & 0.725461 \\
\hline
\end{tabular}

\section{Catalyst 2C 38}

Datum

Value

\begin{tabular}{lc}
\hline B3LYP/6-31G(d) Energy & -2164.795277 \\
\hline B3LYP-D3(BJ)/def2-TZVPP-IEF-PCM(DCM) Energy & -2165.895294 \\
\hline B3LYP-D3(BJ)/def2-TZVPP-IEF-PCM(DCM)//B3LYP/6-31G(d) Free Energy (Quasiharmonic) & -2165.01879 \\
\hline
\end{tabular}

Number of Imaginary Frequencies

Frequencies (Top 3 out of 321)
1. $6.5580 \mathrm{~cm}^{-1}$
2. $7.1169 \mathrm{~cm}^{-1}$
3. $13.8781 \mathrm{~cm}^{-1}$

\section{B3LYP/6-31G(d) Molecular Geometry in Cartesian Coordinates}

$\begin{array}{lrrr}\text { C } & 0.098230 & -4.695881 & -2.594419 \\ \mathrm{C} & -0.140892 & -3.718271 & -1.654614 \\ \mathrm{C} & 0.869547 & -2.780954 & -1.305806 \\ \mathrm{C} & 2.140874 & -2.869488 & -1.957610 \\ \mathrm{C} & 2.355027 & -3.890307 & -2.922926 \\ \mathrm{C} & 1.358177 & -4.786155 & -3.233427 \\ \mathrm{H} & -0.687109 & -5.400960 & -2.852274 \\ \mathrm{H} & -1.112107 & -3.644690 & -1.177932 \\ \mathrm{C} & 0.660242 & -1.752715 & -0.329945 \\ \mathrm{C} & 3.149817 & -1.928818 & -1.637062 \\ \mathrm{H} & 3.323573 & -3.947933 & -3.413916 \\ \mathrm{H} & 1.533171 & -5.562565 & -3.973327 \\ \mathrm{C} & 2.951380 & -0.924386 & -0.710211 \\ \mathrm{C} & 1.682029 & -0.856448 & -0.047609 \\ \mathrm{H} & 4.098702 & -1.983282 & -2.164445\end{array}$




\begin{tabular}{|c|c|c|c|}
\hline C & -1.130763 & -4.074361 & 3.936692 \\
\hline C & -2.154791 & -3.266648 & 3.498042 \\
\hline C & -2.002930 & -2.463967 & 2.336077 \\
\hline C & -0.769292 & -2.498136 & 1.612476 \\
\hline C & 0.270584 & -3.338983 & 2.098106 \\
\hline C & 0.093505 & -4.106712 & 3.228194 \\
\hline $\mathrm{H}$ & -3.965189 & -1.565659 & 2.449420 \\
\hline $\mathrm{H}$ & -1.257108 & -4.684617 & 4.826928 \\
\hline $\mathrm{H}$ & -3.098763 & -3.227615 & 4.036839 \\
\hline C & -3.039116 & -1.611800 & 1.880870 \\
\hline C & -0.636207 & -1.693215 & 0.429871 \\
\hline $\mathrm{H}$ & 1.216822 & -3.369430 & 1.569099 \\
\hline $\mathrm{H}$ & 0.902204 & -4.741397 & 3.580497 \\
\hline C & -1.675188 & -0.876871 & 0.002518 \\
\hline C & -2.891671 & -0.820374 & 0.762213 \\
\hline C & -1.569963 & -0.076283 & -1.288461 \\
\hline $\mathrm{H}$ & -1.644488 & 0.998053 & -1.074961 \\
\hline $\mathrm{H}$ & -0.598488 & -0.238710 & -1.754324 \\
\hline C & 1.444214 & 0.193621 & 1.001514 \\
\hline 0 & 0.704707 & 1.150388 & 0.886541 \\
\hline 0 & 2.152985 & -0.046917 & 2.125577 \\
\hline $\mathrm{H}$ & 1.952964 & 0.689256 & 2.735678 \\
\hline 0 & -2.534143 & -0.478090 & -2.259623 \\
\hline $\mathrm{H}$ & -3.404897 & -0.200359 & -1.931974 \\
\hline C & -4.016813 & 0.089887 & 0.377427 \\
\hline C & -3.871649 & 1.477797 & 0.457887 \\
\hline C & -5.252408 & -0.446872 & -0.025453 \\
\hline C & -4.929918 & 2.344438 & 0.138888 \\
\hline $\mathrm{H}$ & -2.919893 & 1.879541 & 0.793231 \\
\hline C & -6.330934 & 0.380106 & -0.352696 \\
\hline $\mathrm{H}$ & -5.344318 & -1.525668 & -0.080844 \\
\hline C & -6.140242 & 1.770925 & -0.263571 \\
\hline $\mathrm{H}$ & -6.968983 & 2.420805 & -0.517973 \\
\hline C & 4.033808 & 0.065437 & -0.449091 \\
\hline C & 5.346113 & -0.375978 & -0.207636 \\
\hline C & 3.773983 & 1.437864 & -0.477535 \\
\hline C & 6.386768 & 0.528154 & 0.009602 \\
\hline $\mathrm{H}$ & 5.527440 & -1.443917 & -0.171163 \\
\hline C & 4.790105 & 2.380979 & -0.257081 \\
\hline $\mathrm{H}$ & 2.764542 & 1.774850 & -0.687935 \\
\hline C & 6.081028 & 1.901845 & -0.018510 \\
\hline $\mathrm{H}$ & 6.882040 & 2.611389 & 0.154206 \\
\hline C & 4.443737 & 3.882069 & -0.280691 \\
\hline C & 7.833771 & 0.077867 & 0.289154 \\
\hline C & 3.831208 & 4.252602 & -1.652294 \\
\hline $\mathrm{H}$ & 4.537197 & 4.047533 & -2.465307 \\
\hline $\mathrm{H}$ & 2.913465 & 3.691953 & -1.856965 \\
\hline $\mathrm{H}$ & 3.581243 & 5.320265 & -1.680463 \\
\hline C & 5.677254 & 4.775978 & -0.053265 \\
\hline $\mathrm{H}$ & 5.377932 & 5.829895 & -0.078932 \\
\hline $\mathrm{H}$ & 6.143595 & 4.590707 & 0.921214 \\
\hline $\mathrm{H}$ & 6.436710 & 4.629425 & -0.829985 \\
\hline C & 3.416369 & 4.182535 & 0.837781 \\
\hline $\mathrm{H}$ & 3.841762 & 3.962088 & 1.824196 \\
\hline
\end{tabular}




\begin{tabular}{|c|c|c|c|}
\hline $\mathrm{H}$ & 3.133419 & 5.242369 & 0.821432 \\
\hline $\mathrm{H}$ & 2.502771 & 3.589169 & 0.727003 \\
\hline C & 8.775736 & 0.666407 & -0.788110 \\
\hline $\mathrm{H}$ & 8.495174 & 0.316072 & -1.788029 \\
\hline $\mathrm{H}$ & 8.750991 & 1.760973 & -0.799040 \\
\hline $\mathrm{H}$ & 9.811386 & 0.358669 & -0.597973 \\
\hline C & 7.986451 & -1.454959 & 0.269722 \\
\hline $\mathrm{H}$ & 7.712275 & -1.879679 & -0.702723 \\
\hline $\mathrm{H}$ & 9.030674 & -1.724508 & 0.464524 \\
\hline $\mathrm{H}$ & 7.372249 & -1.936934 & 1.038708 \\
\hline C & 8.271260 & 0.585890 & 1.683823 \\
\hline $\mathrm{H}$ & 8.229884 & 1.678005 & 1.751479 \\
\hline $\mathrm{H}$ & 7.625026 & 0.177575 & 2.469218 \\
\hline $\mathrm{H}$ & 9.302184 & 0.277142 & 1.896417 \\
\hline C & -7.696965 & -0.174694 & -0.800374 \\
\hline C & -4.724514 & 3.867784 & 0.249523 \\
\hline C & -5.982731 & 4.663672 & -0.146791 \\
\hline $\mathrm{H}$ & -6.276735 & 4.472780 & -1.185226 \\
\hline $\mathrm{H}$ & -6.836045 & 4.429704 & 0.500112 \\
\hline $\mathrm{H}$ & -5.783230 & 5.737039 & -0.052793 \\
\hline C & -3.568875 & 4.300151 & -0.684326 \\
\hline $\mathrm{H}$ & -2.628071 & 3.802363 & -0.428008 \\
\hline $\mathrm{H}$ & -3.799163 & 4.063395 & -1.729450 \\
\hline $\mathrm{H}$ & -3.403766 & 5.381992 & -0.609816 \\
\hline C & -4.365165 & 4.231645 & 1.709976 \\
\hline $\mathrm{H}$ & -5.170637 & 3.944205 & 2.395565 \\
\hline $\mathrm{H}$ & -3.449530 & 3.731064 & 2.040904 \\
\hline $\mathrm{H}$ & -4.207414 & 5.312984 & 1.805096 \\
\hline C & -7.720344 & -1.714705 & -0.839417 \\
\hline $\mathrm{H}$ & -6.986148 & -2.117003 & -1.546483 \\
\hline $\mathrm{H}$ & -7.521164 & -2.151538 & 0.145790 \\
\hline $\mathrm{H}$ & -8.709886 & -2.060056 & -1.159221 \\
\hline C & -8.026411 & 0.346303 & -2.219695 \\
\hline $\mathrm{H}$ & -8.998708 & -0.038480 & -2.551095 \\
\hline $\mathrm{H}$ & -8.072851 & 1.439793 & -2.253588 \\
\hline $\mathrm{H}$ & -7.269064 & 0.021212 & -2.942189 \\
\hline C & -8.792145 & 0.297470 & 0.185647 \\
\hline $\mathrm{H}$ & -8.863208 & 1.389451 & 0.225134 \\
\hline $\mathrm{H}$ & -9.772255 & -0.089379 & -0.119264 \\
\hline $\mathrm{H}$ & -8.586310 & -0.061043 & 1.200711 \\
\hline
\end{tabular}

\section{Catalyst 2C 39}

Datum

Value

\begin{tabular}{lc}
\hline B3LYP/6-31G(d) Energy & -2164.800016 \\
\hline B3LYP-D3(BJ)/def2-TZVPP-IEF-PCM(DCM) Energy & -2165.897046 \\
\hline B3LYP-D3(BJ)/def2-TZVPP-IEF-PCM(DCM)//B3LYP/6-31G(d) Free Energy (Quasiharmonic) & -2165.018701 \\
\hline
\end{tabular}


Frequencies (Top 3 out of 321)

$\begin{array}{ll}\text { 1. } & 11.8304 \mathrm{~cm}^{-1} \\ \text { 2. } & 12.9344 \mathrm{~cm}^{-1} \\ \text { 3. } & 13.3609 \mathrm{~cm}^{-1}\end{array}$

\section{B3LYP/6-31G(d) Molecular Geometry in Cartesian Coordinates}

C

C

C

C

C

C

$\mathrm{H}$

$\mathrm{H}$

C

C

$\mathrm{H}$

$\mathrm{H}$

C

C

$\mathrm{H}$

C

C

C

C

C

C

$\mathrm{H}$

$\mathrm{H}$

$\mathrm{H}$

C

C

$\mathrm{H}$

$\mathrm{H}$

C

C

C

$\mathrm{H}$

$\mathrm{H}$

C

0

0

$\mathrm{H}$
0.227385

0.357662

$-0.724154$

$-1.957651$

$-2.058190$

$-0.990739$

1.065913

1.294713

$-0.625129$

$-3.049282$

$-2.998618$

$-1.080007$

$-2.968871$

$-1.733320$

$-3.969878$

1.047347

2.093387

1.974939

0.748844

$-0.312396$

$-0.166038$

3.969527

1. 147891

3.030878

3.044698

0.649665

$-1.252322$

$-0.991186$

1.718827

2. 950039

1.572192

0.858873

2. 530591

$-1.702099$

$-2.300863$

$-0.906960$

$-0.912731$
$-4.003908$

$-3.177117$

$-2.361662$

$-2.426613$

$-3.286606$

$-4.059568$

$-4.618937$

$-3.140700$

$-1.487582$

$-1.626042$

$-3.322466$

$-4.714693$

$-0.792579$

$-0.741439$

$-1.656098$

$-4.050343$

$-3.243033$

$-2.378959$

$-2.346357$

$-3.195963$

$-4.024687$

$-1.580849$

$-4.707406$

$-3.253334$

$-1.552122$

$-1.465595$

$-3.180433$

$-4.661471$

$-0.653872$

$-0.699666$

0.284728

$-0.130403$

0.398654

0.018388

$-0.287586$

1.130370

1.518407
3.143833

2.049901

1.616812

2. 340251

3.466803

3. 861735

3.458567

1.505289

0.481793

1.922808

4.011382

4.723987

0.826972

0.100501

2.499826

$-3.689682$

- 3.308351

$-2.185949$

$-1.449852$

$-1.871446$

$-2.961277$

$-2.342499$

$-4.549327$

$-3.859431$

$-1.771999$

$-0.321151$

$-1.331204$

$-3.268047$

0.056877

$-0.687384$

1. 244424

1.965901

1. 755152

$-1.194844$

$-2.196573$

$-1.143943$

$-2.040746$ 


\begin{tabular}{|c|c|c|c|}
\hline 0 & 1.178897 & 1.607316 & 0.867310 \\
\hline $\mathrm{H}$ & 0.401177 & 1.525834 & 0.288198 \\
\hline C & 4.158123 & 0.101492 & -0.323374 \\
\hline C & 5.401094 & -0.540842 & -0.218951 \\
\hline C & 4.100392 & 1.487803 & -0.131020 \\
\hline C & 6.573970 & 0.166629 & 0.070038 \\
\hline $\mathrm{H}$ & 5.437109 & -1.618612 & -0.350211 \\
\hline C & 5.248814 & 2.232310 & 0.165779 \\
\hline $\mathrm{H}$ & 3.136541 & 1.977842 & -0.202824 \\
\hline C & 6.471874 & 1.552994 & 0.257374 \\
\hline $\mathrm{H}$ & 7.366506 & 2.116197 & 0.485175 \\
\hline C & -4.136041 & 0.051658 & 0.447771 \\
\hline C & -5.402331 & -0.523743 & 0.290533 \\
\hline C & -3.993021 & 1.434258 & 0.279003 \\
\hline C & -6.520608 & 0.250874 & -0.040944 \\
\hline $\mathrm{H}$ & -5.498537 & -1.599189 & 0.407178 \\
\hline C & -5.082974 & 2.245793 & -0.056742 \\
\hline $\mathrm{H}$ & -3.014893 & 1.879499 & 0.432217 \\
\hline C & -6.333783 & 1.630765 & -0.211044 \\
\hline $\mathrm{H}$ & -7.185586 & 2.243087 & -0.473075 \\
\hline C & -4.871558 & 3.762145 & -0.233330 \\
\hline C & -7.892440 & -0.429029 & -0.217900 \\
\hline C & -4.371869 & 4.369778 & 1.098996 \\
\hline $\mathrm{H}$ & -5.105908 & 4.216543 & 1.898370 \\
\hline $\mathrm{H}$ & -3.427018 & 3.920926 & 1.422671 \\
\hline $\mathrm{H}$ & -4.208727 & 5.448964 & 0.989288 \\
\hline C & -6.163246 & 4.496010 & -0.641341 \\
\hline $\mathrm{H}$ & -5.955741 & 5.564923 & -0.765289 \\
\hline $\mathrm{H}$ & -6.561258 & 4.124565 & -1.592613 \\
\hline $\mathrm{H}$ & -6.946758 & 4.399864 & 0.118963 \\
\hline C & -3.813561 & 4.007284 & -1.336246 \\
\hline H & -4.145508 & 3.593103 & -2.295270 \\
\hline $\mathrm{H}$ & -3.643211 & 5.082576 & -1.469549 \\
\hline $\mathrm{H}$ & -2.851766 & 3.546598 & -1.086841 \\
\hline C & -9.009126 & 0.573999 & -0.565301 \\
\hline $\mathrm{H}$ & -9.139115 & 1.330021 & 0.217600 \\
\hline $\mathrm{H}$ & -8.815266 & 1.090718 & -1.512068 \\
\hline $\mathrm{H}$ & -9.961249 & 0.041671 & -0.670433 \\
\hline C & -8.285448 & -1.145632 & 1.095915 \\
\hline $\mathrm{H}$ & -8.362369 & -0.431847 & 1.924236 \\
\hline $\mathrm{H}$ & -9.257048 & -1.642263 & 0.983712 \\
\hline $\mathrm{H}$ & -7.553028 & -1.908735 & 1.379220 \\
\hline C & -7.807860 & -1.466889 & -1.362389 \\
\hline $\mathrm{H}$ & -7.535759 & -0.985042 & -2.308216 \\
\hline $\mathrm{H}$ & -7.061445 & -2.241382 & -1.157574 \\
\hline $\mathrm{H}$ & -8.776415 & -1.963614 & -1.498790 \\
\hline C & 5.121416 & 3.752936 & 0.382453 \\
\hline C & 7.912466 & -0.590513 & 0.181489 \\
\hline C & 7.822316 & -1.631630 & 1.322517 \\
\hline $\mathrm{H}$ & 7.623306 & -1.142919 & 2.283155 \\
\hline $\mathrm{H}$ & 7.023650 & -2.360180 & 1.147427 \\
\hline $\mathrm{H}$ & 8.765645 & -2.184831 & 1.411217 \\
\hline C & 9.098197 & 0.345824 & 0.483371 \\
\hline $\mathrm{H}$ & 9.234876 & 1.098822 & -0.301239 \\
\hline
\end{tabular}




\begin{tabular}{|rrrr|}
$H$ & 8.974605 & 0.867111 & 1.439388 \\
$H$ & 10.023145 & -0.238965 & 0.544540 \\
$\mathrm{C}$ & 8.206891 & -1.318611 & -1.151722 \\
$\mathrm{H}$ & 7.422268 & -2.039383 & -1.404296 \\
$\mathrm{H}$ & 8.283389 & -0.603972 & -1.979273 \\
$\mathrm{H}$ & 9.154768 & -1.867257 & -1.087122 \\
$\mathrm{C}$ & 6.473002 & 4.421238 & 0.698280 \\
$\mathrm{H}$ & 6.922154 & 4.021915 & 1.614954 \\
$\mathrm{H}$ & 7.193654 & 4.296193 & -0.118412 \\
$\mathrm{H}$ & 6.326089 & 5.497286 & 0.846200 \\
$\mathrm{C}$ & 4.160689 & 4.019856 & 1.566746 \\
$\mathrm{H}$ & 4.038654 & 5.099496 & 1.721433 \\
$\mathrm{H}$ & 3.169808 & 3.587377 & 1.396236 \\
$\mathrm{H}$ & 4.558443 & 3.589381 & 2.493457 \\
$\mathrm{C}$ & 4.549248 & 4.409433 & -0.896565 \\
$\mathrm{H}$ & 3.560636 & 4.012456 & -1.148434 \\
$\mathrm{H}$ & 4.448603 & 5.492865 & -0.756273 \\
$\mathrm{H}$ & 5.208883 & 4.237740 & -1.755272 \\
& & & \\
\hline
\end{tabular}

\section{Catalyst 2C 40}

\section{Datum}

Value

\begin{tabular}{lc}
\hline B3LYP/6-31G(d) Energy & -2164.802261 \\
\hline B3LYP-D3(BJ)/def2-TZVPP-IEF-PCM(DCM) Energy & -2165.897739 \\
\hline B3LYP-D3(BJ)/def2-TZVPP-IEF-PCM(DCM)//B3LYP/6-31G(d) Free Energy (Quasiharmonic) & -2165.018681 \\
\hline
\end{tabular}

Number of Imaginary Frequencies

Frequencies (Top 3 out of 321)

$\begin{array}{ll}\text { 1. } & 11.8029 \mathrm{~cm}^{-1} \\ \text { 2. } & 13.3264 \mathrm{~cm}^{-1} \\ \text { 3. } & 13.4828 \mathrm{~cm}^{-1}\end{array}$

\section{B3LYP/6-31G(d) Molecular Geometry in Cartesian Coordinates}

$\begin{array}{lrrr}C & 0.298536 & -3.513270 & 3.897493 \\ C & 0.432811 & -2.930716 & 2.656708 \\ C & -0.642712 & -2.208428 & 2.070467 \\ C & -1.868589 & -2.094493 & 2.800606 \\ C & -1.973773 & -2.706334 & 4.078933 \\ C & -0.915138 & -3.400798 & 4.617200 \\ H & 1.129946 & -4.064252 & 4.328379 \\ H & 1.365521 & -3.020289 & 2.110279\end{array}$




\begin{tabular}{|c|c|c|c|}
\hline C & -0.540301 & -1.584507 & 0.783638 \\
\hline C & -2.949498 & -1.376467 & 2.232150 \\
\hline $\mathrm{H}$ & -2.909736 & -2.614182 & 4.624606 \\
\hline $\mathrm{H}$ & -1.006972 & -3.864350 & 5.595640 \\
\hline C & -2.869578 & -0.795227 & 0.982567 \\
\hline C & -1.639529 & -0.919206 & 0.259951 \\
\hline $\mathrm{H}$ & -3.877958 & -1.299763 & 2.792125 \\
\hline C & 1.507003 & -4.904395 & -2.620058 \\
\hline C & 2.451519 & -3.919099 & -2.449393 \\
\hline C & 2.214730 & -2.827408 & -1.570305 \\
\hline C & 0.974868 & -2.751154 & -0.862973 \\
\hline C & 0.019661 & -3.786619 & -1.063305 \\
\hline C & 0.280049 & -4.835304 & -1.917168 \\
\hline $\mathrm{H}$ & 4.115298 & -1.875502 & -1.919825 \\
\hline $\mathrm{H}$ & 1.698589 & -5.736384 & -3.292389 \\
\hline $\mathrm{H}$ & 3.398171 & -3.960707 & -2.983078 \\
\hline C & 3.179096 & -1.812262 & -1.371042 \\
\hline C & 0.754619 & -1.640706 & 0.018098 \\
\hline $\mathrm{H}$ & -0.927870 & -3.742416 & -0.537347 \\
\hline $\mathrm{H}$ & -0.463395 & -5.615788 & -2.055339 \\
\hline C & 1.722567 & -0.651135 & 0.193314 \\
\hline C & 2.970105 & -0.742267 & -0.520478 \\
\hline C & 1.473517 & 0.501590 & 1.160428 \\
\hline $\mathrm{H}$ & 0.777411 & 0.181037 & 1.944988 \\
\hline $\mathrm{H}$ & 2.412576 & 0.774126 & 1.648054 \\
\hline C & -1.484746 & -0.224470 & -1.063941 \\
\hline 0 & -1.135739 & 0.932469 & -1.205060 \\
\hline 0 & -1.754986 & -1.020091 & -2.116630 \\
\hline $\mathrm{H}$ & -1.586822 & -0.485571 & -2.917844 \\
\hline 0 & 1.008555 & 1.696991 & 0.538946 \\
\hline $\mathrm{H}$ & 0.182440 & 1.501223 & 0.056054 \\
\hline C & 4.082377 & 0.242869 & -0.359163 \\
\hline C & 3.880263 & 1.621339 & -0.502314 \\
\hline C & 5.381718 & -0.225421 & -0.111545 \\
\hline C & 4.942461 & 2.528445 & -0.400991 \\
\hline $\mathrm{H}$ & 2.872159 & 1.977200 & -0.677674 \\
\hline C & 6.471483 & 0.646933 & -0.007785 \\
\hline $\mathrm{H}$ & 5.529282 & -1.293868 & 0.018025 \\
\hline C & 6.226274 & 2.019827 & -0.158253 \\
\hline $\mathrm{H}$ & 7.055082 & 2.710133 & -0.079823 \\
\hline C & -4.039996 & -0.045650 & 0.440464 \\
\hline C & -4.594421 & 1.000118 & 1.191575 \\
\hline C & -4.620767 & -0.394380 & -0.786483 \\
\hline C & -5.711006 & 1.709486 & 0.735750 \\
\hline $\mathrm{H}$ & -4.121849 & 1.261931 & 2.131065 \\
\hline C & -5.740436 & 0.288808 & -1.276142 \\
\hline $\mathrm{H}$ & -4.198205 & -1.220626 & -1.343701 \\
\hline C & -6.260149 & 1.332582 & -0.498200 \\
\hline $\mathrm{H}$ & -7.126597 & 1.873178 & -0.867281 \\
\hline C & -6.407909 & -0.071380 & -2.618182 \\
\hline C & -6.336945 & 2.873547 & 1.528759 \\
\hline C & -6.361019 & 1.155200 & -3.560352 \\
\hline $\mathrm{H}$ & -6.887824 & 2.016471 & -3.136379 \\
\hline $\mathrm{H}$ & -5.326726 & 1.460790 & -3.755462 \\
\hline
\end{tabular}




\begin{tabular}{|c|c|c|c|}
\hline $\mathrm{H}$ & -6.832675 & 0.916545 & -4.521494 \\
\hline C & -7.881904 & -0.472278 & -2.372980 \\
\hline $\mathrm{H}$ & -8.370565 & -0.729030 & -3.320932 \\
\hline $\mathrm{H}$ & -7.945976 & -1.343546 & -1.711155 \\
\hline $\mathrm{H}$ & -8.454937 & 0.339433 & -1.912761 \\
\hline C & -5.705827 & -1.245751 & -3.326001 \\
\hline $\mathrm{H}$ & -5.739284 & -2.162676 & -2.726815 \\
\hline $\mathrm{H}$ & -6.206623 & -1.455257 & -4.277919 \\
\hline $\mathrm{H}$ & -4.655675 & -1.022604 & -3.545730 \\
\hline C & -7.811923 & 2.540093 & 1.856038 \\
\hline $\mathrm{H}$ & -8.406171 & 2.379847 & 0.950287 \\
\hline $\mathrm{H}$ & -7.883233 & 1.632507 & 2.466527 \\
\hline $\mathrm{H}$ & -8.273278 & 3.363136 & 2.415430 \\
\hline C & -6.279823 & 4.165466 & 0.679292 \\
\hline H & -6.826596 & 4.059091 & -0.263531 \\
\hline H & -6.725122 & 5.003179 & 1.229864 \\
\hline $\mathrm{H}$ & -5.244287 & 4.429416 & 0.437013 \\
\hline C & -5.602088 & 3.142284 & 2.855721 \\
\hline $\mathrm{H}$ & -5.627678 & 2.271641 & 3.521021 \\
\hline $\mathrm{H}$ & -4.554324 & 3.418839 & 2.693639 \\
\hline $\mathrm{H}$ & -6.084741 & 3.973363 & 3.382213 \\
\hline C & 7.877354 & 0.079084 & 0.272327 \\
\hline C & 4.657055 & 4.035278 & -0.553231 \\
\hline C & 5.929345 & 4.894608 & -0.427057 \\
\hline $\mathrm{H}$ & 6.407225 & 4.777162 & 0.552479 \\
\hline $\mathrm{H}$ & 6.668026 & 4.650000 & -1.199476 \\
\hline $\mathrm{H}$ & 5.670837 & 5.953598 & -0.541370 \\
\hline C & 3.662357 & 4.478037 & 0.547574 \\
\hline $\mathrm{H}$ & 2.726246 & 3.912802 & 0.506362 \\
\hline $\mathrm{H}$ & 4.097281 & 4.332320 & 1.543657 \\
\hline $\mathrm{H}$ & 3.423146 & 5.543411 & 0.436637 \\
\hline C & 4.033257 & 4.303523 & -1.943573 \\
\hline $\mathrm{H}$ & 4.714757 & 3.996196 & -2.745610 \\
\hline $\mathrm{H}$ & 3.091412 & 3.762117 & -2.077410 \\
\hline $\mathrm{H}$ & 3.823873 & 5.373421 & -2.066581 \\
\hline C & 8.957540 & 1.176059 & 0.330081 \\
\hline $\mathrm{H}$ & 9.030204 & 1.727684 & -0.614161 \\
\hline $\mathrm{H}$ & 8.765679 & 1.896883 & 1.132920 \\
\hline $\mathrm{H}$ & 9.935645 & 0.720573 & 0.522861 \\
\hline C & 8.266559 & -0.913915 & -0.848786 \\
\hline $\mathrm{H}$ & 9.265594 & -1.328038 & -0.663791 \\
\hline $\mathrm{H}$ & 7.565879 & -1.753027 & -0.912630 \\
\hline $\mathrm{H}$ & 8.280532 & -0.415732 & -1.825038 \\
\hline C & 7.872861 & -0.661393 & 1.630903 \\
\hline $\mathrm{H}$ & 7.151880 & -1.485602 & 1.643000 \\
\hline $\mathrm{H}$ & 8.864429 & -1.081129 & 1.841945 \\
\hline $\mathrm{H}$ & 7.611905 & 0.021688 & 2.447386 \\
\hline
\end{tabular}


Frequencies (Top 3 out of 321)

$\begin{array}{lr}\text { 1. } & 5.6653 \mathrm{~cm}^{-1} \\ \text { 2. } & 7.3076 \mathrm{~cm}^{-1} \\ \text { 3. } & 13.3438 \mathrm{~cm}^{-1}\end{array}$

\section{B3LYP/6-31G(d) Molecular Geometry in Cartesian Coordinates}

C

C

$\mathrm{C}$

C

C

C

$\mathrm{H}$

$\mathrm{H}$

C

C

$\mathrm{H}$

$\mathrm{H}$

C

$\mathrm{C}$

$\mathrm{H}$

$\mathrm{C}$

C

C

C

C

C

$\mathrm{H}$

$H$

$\mathrm{H}$

C

$\mathrm{C}$

$\mathrm{H}$

$\mathrm{H}$

C

C

C

$\mathrm{H}$

\subsection{2}

$-0.139603$

0.871270

2. 144369

2. 359801

1. 362422

$-0.685168$

$-1.112203$

0.660389

3. 153816

3. 329778

1. 538441

2.953305

1.682467

4.104531

$-1.130277$

$-2.155312$

$-2.003624$

$-0.769219$

0.271699

0.094874

$-3.966874$

$-1.256528$

$-3.099957$

$-3.040502$

$-0.636470$

1.218668

0.904494

$-1.675976$

$-2.893124$

$-1.570958$

$-1.646082$
$-4.688394$

$-3.713570$

$-2.779072$

$-2.867633$

$-3.885647$

$-4.778784$

$-5.391147$

$-3.639880$

$-1.754015$

$-1.929452$

$-3.943250$

$-5.553022$

$-0.927552$

$-0.859484$

$-1.983403$

$-4.112804$

$-3.303257$

$-2.490157$

$-2.515810$

$-3.358796$

$-4.136568$

$-1.594981$

$-4.730935$

$-3.270626$

$-1.635246$

$-1.700366$

$-3.382968$

$-4.772572$

$-0.881345$

$-0.833047$

$-0.068907$

1.003257
$-2.622612$

$-1.680203$

$-1.325015$

$-1.973399$

$-2.941327$

$-3.257952$

$-2.885284$

$-1.206390$

$-0.345909$

$-1.646858$

$-3.429504$

$-3.999887$

$-0.717988$

$-0.058337$

$-2.171060$

3.899400

3.466489

2. 311793

1.589177

2.068880

3.192155

2.431924

4.784204

4.004551

1.863459

0.413636

1.540870

3.539950

$-0.006903$

0.752445

$-1.290326$

$-1.066429$ 


\begin{tabular}{|c|c|c|c|}
\hline $\mathrm{H}$ & -0.599323 & -0.226446 & -1.757576 \\
\hline C & 1.445302 & 0.187256 & 0.993957 \\
\hline 0 & 0.692135 & 1.134102 & 0.890893 \\
\hline 0 & 2.172325 & -0.045149 & 2.108928 \\
\hline $\mathrm{H}$ & 1.969793 & 0.688038 & 2.721679 \\
\hline 0 & -2.534859 & -0.462024 & -2.265510 \\
\hline $\mathrm{H}$ & -3.406304 & -0.192875 & -1.932471 \\
\hline C & -4.018159 & 0.081693 & 0.378286 \\
\hline C & -3.865451 & 1.472755 & 0.474202 \\
\hline C & -5.253716 & -0.447309 & -0.022020 \\
\hline C & -4.921949 & 2.340658 & 0.173899 \\
\hline $\mathrm{H}$ & -2.910610 & 1.861246 & 0.809073 \\
\hline C & -6.336047 & 0.386921 & -0.333831 \\
\hline $\mathrm{H}$ & -5.349287 & -1.525149 & -0.088907 \\
\hline C & -6.139371 & 1.770741 & -0.228309 \\
\hline $\mathrm{H}$ & -6.967358 & 2.431884 & -0.466522 \\
\hline C & 4.035351 & 0.061250 & -0.451188 \\
\hline C & 5.337862 & -0.380436 & -0.181465 \\
\hline C & 3.775224 & 1.437656 & -0.504868 \\
\hline C & 6.380141 & 0.525500 & 0.043520 \\
\hline $\mathrm{H}$ & 5.515841 & -1.448217 & -0.127741 \\
\hline C & 4.788367 & 2.376857 & -0.281650 \\
\hline $\mathrm{H}$ & 2.770147 & 1.766124 & -0.738815 \\
\hline C & 6.076270 & 1.893720 & -0.010335 \\
\hline $\mathrm{H}$ & 6.873958 & 2.609201 & 0.167042 \\
\hline C & 4.529068 & 3.895423 & -0.327354 \\
\hline C & 7.820487 & 0.074047 & 0.354833 \\
\hline C & 5.412901 & 4.540726 & -1.420784 \\
\hline $\mathrm{H}$ & 6.479914 & 4.384531 & -1.229589 \\
\hline $\mathrm{H}$ & 5.184937 & 4.120386 & -2.406882 \\
\hline $\mathrm{H}$ & 5.237226 & 5.622776 & -1.463699 \\
\hline C & 4.879332 & 4.517381 & 1.045607 \\
\hline $\mathrm{H}$ & 4.707379 & 5.600687 & 1.028497 \\
\hline $\mathrm{H}$ & 4.257705 & 4.088221 & 1.839799 \\
\hline $\mathrm{H}$ & 5.927583 & 4.351017 & 1.315167 \\
\hline C & 3.057462 & 4.228445 & -0.640771 \\
\hline $\mathrm{H}$ & 2.372576 & 3.807156 & 0.103460 \\
\hline $\mathrm{H}$ & 2.919801 & 5.315723 & -0.642966 \\
\hline $\mathrm{H}$ & 2.756961 & 3.856314 & -1.626776 \\
\hline C & 8.783359 & 0.645572 & -0.712970 \\
\hline $\mathrm{H}$ & 8.518291 & 0.284743 & -1.713356 \\
\hline $\mathrm{H}$ & 8.763550 & 1.740052 & -0.737350 \\
\hline $\mathrm{H}$ & 9.814289 & 0.335911 & -0.501591 \\
\hline C & 7.968091 & -1.459512 & 0.357881 \\
\hline $\mathrm{H}$ & 7.710511 & -1.895981 & -0.613891 \\
\hline $\mathrm{H}$ & 9.007596 & -1.729747 & 0.575591 \\
\hline $\mathrm{H}$ & 7.338170 & -1.929571 & 1.121500 \\
\hline C & 8.234749 & 0.598545 & 1.750398 \\
\hline $\mathrm{H}$ & 8.195301 & 1.691574 & 1.803360 \\
\hline $\mathrm{H}$ & 7.573573 & 0.202193 & 2.529441 \\
\hline $\mathrm{H}$ & 9.260889 & 0.289864 & 1.985190 \\
\hline C & -7.705376 & -0.161745 & -0.779888 \\
\hline C & -4.792210 & 3.873032 & 0.276912 \\
\hline C & -3.387821 & 4.314401 & 0.731113 \\
\hline
\end{tabular}




\begin{tabular}{|c|c|c|c|}
\hline $\mathrm{H}$ & -3.139382 & 3.925275 & 1.724773 \\
\hline $\mathrm{H}$ & -2.609115 & 3.988335 & 0.032627 \\
\hline $\mathrm{H}$ & -3.346189 & 5.408183 & 0.784891 \\
\hline C & -5.819029 & 4.409228 & 1.302808 \\
\hline $\mathrm{H}$ & -6.847742 & 4.164195 & 1.018431 \\
\hline $\mathrm{H}$ & -5.637217 & 3.985528 & 2.297129 \\
\hline $\mathrm{H}$ & -5.745747 & 5.500963 & 1.379900 \\
\hline C & -5.071405 & 4.508068 & -1.106350 \\
\hline $\mathrm{H}$ & -4.354171 & 4.151322 & -1.854274 \\
\hline $\mathrm{H}$ & -6.077389 & 4.272883 & -1.469240 \\
\hline $\mathrm{H}$ & -4.985677 & 5.600046 & -1.047422 \\
\hline C & -8.041432 & 0.374780 & -2.191727 \\
\hline $\mathrm{H}$ & -8.085778 & 1.468688 & -2.213417 \\
\hline $\mathrm{H}$ & -7.288429 & 0.056353 & -2.921661 \\
\hline $\mathrm{H}$ & -9.016185 & -0.004544 & -2.522241 \\
\hline C & -8.794717 & 0.300961 & 0.216862 \\
\hline $\mathrm{H}$ & -9.776749 & -0.082426 & -0.086250 \\
\hline $\mathrm{H}$ & -8.583362 & -0.067384 & 1.227239 \\
\hline $\mathrm{H}$ & -8.865044 & 1.392544 & 0.267533 \\
\hline C & -7.730730 & -1.701314 & -0.835238 \\
\hline $\mathrm{H}$ & -7.527249 & -2.149027 & 0.144191 \\
\hline $\mathrm{H}$ & -8.722303 & -2.041907 & -1.153839 \\
\hline $\mathrm{H}$ & -7.000680 & -2.096958 & -1.550298 \\
\hline
\end{tabular}

\section{Catalyst 2C 42}

Datum

Value

\begin{tabular}{lc}
\hline B3LYP/6-31G(d) Energy & -2164.794751 \\
\hline B3LYP-D3(BJ)/def2-TZVPP-IEF-PCM(DCM) Energy & -2165.894983 \\
\hline B3LYP-D3(BJ)/def2-TZVPP-IEF-PCM(DCM)//B3LYP/6-31G(d) Free Energy (Quasiharmonic) & -2165.01846 \\
\hline
\end{tabular}

Number of Imaginary Frequencies

Frequencies (Top 3 out of 321)
1. $6.9743 \mathrm{~cm}^{-1}$
2. $10.6143 \mathrm{~cm}^{-1}$
3. $13.4319 \mathrm{~cm}^{-1}$

\section{B3LYP/6-31G(d) Molecular Geometry in Cartesian Coordinates}

$\begin{array}{lrrr}C & 0.003113 & -4.645580 & -2.591859 \\ C & 0.218922 & -3.667907 & -1.646519 \\ C & -0.810923 & -2.751606 & -1.298496\end{array}$




\begin{tabular}{|c|c|c|c|}
\hline C & -2.076675 & -2.860890 & -1.957862 \\
\hline C & -2.266784 & -3.881416 & -2.928428 \\
\hline C & -1.251724 & -4.757114 & -3.237448 \\
\hline $\mathrm{H}$ & 0.803212 & -5.334011 & -2.849319 \\
\hline $\mathrm{H}$ & 1.186757 & -3.576882 & -1.166178 \\
\hline C & -0.626350 & -1.724462 & -0.316368 \\
\hline C & -3.104069 & -1.939444 & -1.640091 \\
\hline $\mathrm{H}$ & -3.231482 & -3.954852 & -3.424864 \\
\hline $\mathrm{H}$ & -1.408260 & -5.533466 & -3.981544 \\
\hline C & -2.928886 & -0.934935 & -0.708767 \\
\hline C & -1.665603 & -0.847283 & -0.036749 \\
\hline H & -4.048464 & -2.008129 & -2.173864 \\
\hline C & 1.150802 & -4.008925 & 3.976271 \\
\hline C & 2.173404 & -3.199013 & 3.538359 \\
\hline C & 2.024429 & -2.404313 & 2.370557 \\
\hline C & 0.794705 & -2.448410 & 1.640512 \\
\hline C & -0.243460 & -3.292144 & 2.125032 \\
\hline C & -0.069068 & -4.052034 & 3.260790 \\
\hline $\mathrm{H}$ & 3.985467 & -1.502596 & 2.484559 \\
\hline $\mathrm{H}$ & 1.275050 & -4.613024 & 4.870989 \\
\hline $\mathrm{H}$ & 3.114202 & -3.152315 & 4.082078 \\
\hline C & 3.060859 & -1.553008 & 1.913992 \\
\hline C & 0.663657 & -1.650403 & 0.453119 \\
\hline $\mathrm{H}$ & -1.186322 & -3.331060 & $1.59056 e$ \\
\hline $\mathrm{H}$ & -0.876438 & -4.688985 & 3.612056 \\
\hline C & 1.701026 & -0.831593 & 0.027276 \\
\hline C & 2.915596 & -0.770014 & 0.789299 \\
\hline C & 1.598942 & -0.036478 & -1.266471 \\
\hline $\mathrm{H}$ & 0.616597 & -0.172682 & -1.717527 \\
\hline $\mathrm{H}$ & 1.709742 & 1.036262 & -1.061203 \\
\hline C & -1.453045 & 0.201461 & 1.018653 \\
\hline 0 & -0.706858 & 1.154859 & 0.925906 \\
\hline 0 & -2.192915 & -0.037929 & 2.123797 \\
\hline $\mathrm{H}$ & -2.005245 & 0.696755 & 2.739447 \\
\hline 0 & 2.538193 & -0.473266 & -2.247782 \\
\hline $\mathrm{H}$ & 3.421339 & -0.222507 & -1.931385 \\
\hline C & 4.042334 & 0.134013 & 0.394043 \\
\hline C & 3.908098 & 1.525968 & 0.482368 \\
\hline C & 5.264019 & -0.404002 & -0.031870 \\
\hline C & 4.963277 & 2.384089 & 0.147424 \\
\hline $\mathrm{H}$ & 2.964407 & 1.931475 & 0.835493 \\
\hline C & 6.345430 & 0.418478 & -0.380052 \\
\hline $\mathrm{H}$ & 5.355397 & -1.484801 & -0.092252 \\
\hline C & 6.167305 & 1.805873 & -0.283398 \\
\hline $\mathrm{H}$ & 6.988977 & 2.455562 & -0.551337 \\
\hline C & -4.029425 & 0.036492 & -0.454465 \\
\hline C & -3.789013 & 1.416988 & -0.494255 \\
\hline C & -5.330293 & -0.425960 & -0.212722 \\
\hline C & -4.820125 & 2.339536 & -0.284323 \\
\hline H & -2.784893 & 1.762291 & -0.707180 \\
\hline C & -6.390277 & 0.462862 & -0.002957 \\
\hline $\mathrm{H}$ & -5.493447 & -1.496559 & -0.169266 \\
\hline C & -6.105871 & 1.835729 & -0.042000 \\
\hline H & -6.917389 & 2.538320 & 0.123673 \\
\hline
\end{tabular}




\begin{tabular}{|c|c|c|c|}
\hline C & -7.829633 & -0.012226 & 0.276873 \\
\hline C & -4.582423 & 3.862106 & -0.313558 \\
\hline C & -7.954462 & -1.547682 & 0.264465 \\
\hline $\mathrm{H}$ & -7.670109 & -1.972070 & -0.705193 \\
\hline $\mathrm{H}$ & -7.333833 & -2.014906 & 1.037364 \\
\hline $\mathrm{H}$ & -8.994191 & -1.835059 & 0.457854 \\
\hline C & -8.780328 & 0.554044 & -0.804544 \\
\hline $\mathrm{H}$ & -9.810639 & 0.229103 & -0.614219 \\
\hline $\mathrm{H}$ & -8.774919 & 1.648828 & -0.821057 \\
\hline $\mathrm{H}$ & -8.492223 & 0.203919 & -1.802362 \\
\hline C & -8.277895 & 0.494688 & 1.668378 \\
\hline $\mathrm{H}$ & -8.255132 & 1.587700 & 1.730603 \\
\hline $\mathrm{H}$ & -9.303688 & 0.169369 & 1.881103 \\
\hline $\mathrm{H}$ & -7.625838 & 0.101506 & 2.456646 \\
\hline C & -3.110016 & 4.219311 & -0.594833 \\
\hline $\mathrm{H}$ & -2.433783 & 3.802421 & 0.159733 \\
\hline $\mathrm{H}$ & -2.783968 & 3.859752 & -1.577401 \\
\hline $\mathrm{H}$ & -2.988624 & 5.308482 & -0.585980 \\
\hline C & -4.969132 & 4.467739 & 1.056896 \\
\hline $\mathrm{H}$ & -4.356329 & 4.042074 & 1.859786 \\
\hline $\mathrm{H}$ & -4.814265 & 5.553719 & 1.051591 \\
\hline $\mathrm{H}$ & -6.019642 & 4.282665 & 1.304765 \\
\hline C & -5.453516 & 4.503131 & -1.419627 \\
\hline $\mathrm{H}$ & -5.197892 & 4.095738 & -2.404383 \\
\hline $\mathrm{H}$ & -6.521610 & 4.327897 & -1.252668 \\
\hline $\mathrm{H}$ & -5.294630 & 5.588217 & -1.449081 \\
\hline C & 7.668461 & -0.219846 & -0.846439 \\
\hline C & 4.771468 & 3.908843 & 0.268968 \\
\hline C & 6.026195 & 4.698301 & -0.151089 \\
\hline $\mathrm{H}$ & 6.891899 & 4.456821 & 0.476241 \\
\hline $\mathrm{H}$ & 6.296839 & 4.509828 & -1.196274 \\
\hline $\mathrm{H}$ & 5.835296 & 5.772508 & -0.048684 \\
\hline C & 4.446592 & 4.268366 & 1.738595 \\
\hline $\mathrm{H}$ & 3.534275 & 3.773323 & 2.086799 \\
\hline $\mathrm{H}$ & 5.264165 & 3.970734 & 2.405276 \\
\hline $\mathrm{H}$ & 4.299949 & 5.350469 & 1.842662 \\
\hline C & 3.599039 & 4.354132 & -0.637486 \\
\hline $\mathrm{H}$ & 3.804225 & 4.119109 & -1.688213 \\
\hline $\mathrm{H}$ & 2.660134 & 3.863116 & -0.362013 \\
\hline $\mathrm{H}$ & 3.444613 & 5.437028 & -0.555861 \\
\hline C & 7.409723 & -1.087121 & -2.101443 \\
\hline $\mathrm{H}$ & 6.692295 & -1.890392 & -1.903778 \\
\hline $\mathrm{H}$ & 7.013618 & -0.479846 & -2.923482 \\
\hline $\mathrm{H}$ & 8.343080 & -1.551529 & -2.442418 \\
\hline C & 8.232811 & -1.112438 & 0.284612 \\
\hline H & 9.172978 & -1.580585 & -0.031778 \\
\hline $\mathrm{H}$ & 8.433498 & -0.522199 & 1.186085 \\
\hline $\mathrm{H}$ & 7.536980 & -1.912818 & 0.556865 \\
\hline C & 8.737050 & 0.830365 & -1.204950 \\
\hline $\mathrm{H}$ & 8.997152 & 1.460539 & -0.346765 \\
\hline $\mathrm{H}$ & 9.653253 & 0.325742 & -1.531820 \\
\hline $\mathrm{H}$ & 8.410934 & 1.483093 & -2.022725 \\
\hline
\end{tabular}




\section{Catalyst 2C 43}

Datum

Value

\begin{tabular}{lc}
\hline B3LYP/6-31G(d) Energy & -2164.795329 \\
\hline B3LYP-D3(BJ)/def2-TZVPP-IEF-PCM(DCM) Energy & -2165.895333 \\
\hline B3LYP-D3(BJ)/def2-TZVPP-IEF-PCM(DCM)//B3LYP/6-31G(d) Free Energy (Quasiharmonic) & -2165.018458
\end{tabular}

Number of Imaginary Frequencies

Frequencies (Top 3 out of 321)

$\begin{array}{lr}\text { 1. } & 6.9500 \mathrm{~cm}^{-1} \\ \text { 2. } & 10.2208 \mathrm{~cm}^{-1} \\ \text { 3. } & 14.1878 \mathrm{~cm}^{-1}\end{array}$

\section{B3LYP/6-31G(d) Molecular Geometry in Cartesian Coordinates}

C

$\mathrm{C}$

C

C

C

C

$\mathrm{H}$

$\mathrm{H}$

C

C

$\mathrm{H}$

$\mathrm{H}$

C

C

$\mathrm{H}$

C

C

C

C

C

C

$\mathrm{H}$

$\mathrm{H}$

$\mathrm{H}$

C

C

$\mathrm{H}$

$\mathrm{H}$

C
$-0.076702$

0.154859

$-0.861969$

$-2.131450$

$-2.337801$

$-1.335067$

0.713529

1.125141

$-0.660984$

$-3.146207$

$-3.305083$

$-1.504111$

$-2.955296$

$-1.688339$

$-4.093355$

1.125924

2.150002

1.999015

0.766013

$-0.273753$

$-0.097485$

3.962413

1.251669

3.093378

3.036030

0.633181

$-1.219352$

$-0.906171$

1.671738
$-4.676024$

$-3.697352$

$-2.766610$

$-2.862352$

$-3.884172$

$-4.773954$

$-5.375884$

$-3.617288$

$-1.738088$

$-1.927156$

$-3.947369$

$-5.551202$

$-0.921535$

$-0.847511$

$-1.986455$

$-4.047309$

$-3.240026$

$-2.440029$

$-2.476328$

$-3.316992$

$-4.082079$

$-1.543257$

$-4.655432$

$-3.199347$

$-1.589485$

$-1.673428$

$-3.349226$

$-4.716534$

$-0.856476$
$-2.584013$

$-1.643440$

$-1.295673$

$-1.950061$

$-2.915996$

$-3.224973$

$-2.841151$

$-1.166006$

$-0.318345$

$-1.631919$

$-3.408795$

$-3.965382$

$-0.704786$

$-0.038257$

$-2.161903$

3.956998

3.517623

2. 353723

1.628983

2.115166

3.247173

2.465029

4.848779

4.057347

1.897026

0.444949

1.585125

3.599917

0.018034 


\begin{tabular}{|c|c|c|c|}
\hline C & 2.889000 & -0.800696 & 0.776546 \\
\hline C & 1.568954 & -0.057778 & -1.273709 \\
\hline $\mathrm{H}$ & 0.587924 & -0.196340 & -1.726910 \\
\hline $\mathrm{H}$ & 1.675214 & 1.014740 & -1.064704 \\
\hline C & -1.458498 & 0.203619 & 1.011447 \\
\hline 0 & -0.721518 & 1.162504 & 0.898918 \\
\hline 0 & -2.171117 & -0.038719 & 2.132815 \\
\hline $\mathrm{H}$ & -1.975847 & 0.698475 & 2.743244 \\
\hline 0 & 2.511085 & -0.487950 & -2.255003 \\
\hline $\mathrm{H}$ & 3.393277 & -0.239245 & -1.934324 \\
\hline C & 4.015262 & 0.105667 & 0.385609 \\
\hline C & 5.238363 & -0.426695 & -0.031405 \\
\hline C & 3.871856 & 1.501569 & 0.477626 \\
\hline C & 6.323312 & 0.401704 & -0.369777 \\
\hline $\mathrm{H}$ & 5.334426 & -1.506936 & -0.094324 \\
\hline C & 4.925231 & 2.359455 & 0.155717 \\
\hline $\mathrm{H}$ & 2.924094 & 1.895809 & 0.825662 \\
\hline C & 6.137812 & 1.782855 & -0.268476 \\
\hline $\mathrm{H}$ & 6.958566 & 2.442552 & -0.524531 \\
\hline C & -4.042675 & 0.064093 & -0.448456 \\
\hline C & -5.354413 & -0.381990 & -0.212593 \\
\hline C & -3.787883 & 1.437457 & -0.477149 \\
\hline C & -6.399433 & 0.518484 & -0.001186 \\
\hline $\mathrm{H}$ & -5.531956 & -1.450568 & -0.176054 \\
\hline C & -4.808550 & 2.377004 & -0.262603 \\
\hline $\mathrm{H}$ & -2.778746 & 1.777982 & -0.683279 \\
\hline C & -6.098752 & 1.893297 & -0.029470 \\
\hline $\mathrm{H}$ & -6.903218 & 2.600059 & 0.138571 \\
\hline C & -4.467767 & 3.879380 & -0.286732 \\
\hline C & -7.846079 & 0.063057 & 0.271987 \\
\hline C & -3.850454 & 4.250349 & -1.656061 \\
\hline $\mathrm{H}$ & -2.929813 & 3.692696 & -1.855859 \\
\hline $\mathrm{H}$ & -4.552039 & 4.041721 & -2.471979 \\
\hline $\mathrm{H}$ & -3.604153 & 5.318862 & -1.684481 \\
\hline C & -3.446703 & 4.185409 & 0.835987 \\
\hline $\mathrm{H}$ & -3.167960 & 5.246356 & 0.819556 \\
\hline $\mathrm{H}$ & -3.875707 & 3.964510 & 1.820730 \\
\hline $\mathrm{H}$ & -2.530175 & 3.595689 & 0.730189 \\
\hline C & -5.705711 & 4.768891 & -0.066196 \\
\hline $\mathrm{H}$ & -6.175727 & 4.583311 & 0.906459 \\
\hline $\mathrm{H}$ & -5.410242 & 5.823886 & -0.092108 \\
\hline $\mathrm{H}$ & -6.461124 & 4.618271 & -0.846081 \\
\hline C & -8.291961 & 0.570832 & 1.664074 \\
\hline $\mathrm{H}$ & -7.647947 & 0.165575 & 2.452868 \\
\hline $\mathrm{H}$ & -8.254743 & 1.663151 & 1.730813 \\
\hline $\mathrm{H}$ & -9.322771 & 0.258623 & 1.872142 \\
\hline C & -7.992904 & -1.470330 & 0.253313 \\
\hline $\mathrm{H}$ & -7.380844 & -1.949253 & 1.025906 \\
\hline $\mathrm{H}$ & -9.037103 & -1.743604 & 0.443013 \\
\hline $\mathrm{H}$ & -7.712145 & -1.894963 & -0.717293 \\
\hline C & -8.785146 & 0.647024 & -0.810289 \\
\hline $\mathrm{H}$ & -8.764282 & 1.741658 & -0.822344 \\
\hline $\mathrm{H}$ & -8.498723 & 0.296604 & -1.808515 \\
\hline H & -9.820550 & 0.335776 & -0.624591 \\
\hline
\end{tabular}




\begin{tabular}{|rrrr|} 
C & 4.807158 & 3.892756 & 0.254574 \\
C & 7.650664 & -0.232366 & -0.828145 \\
H & 8.209146 & -1.125887 & 0.305041 \\
H & 8.403303 & -0.536759 & 1.208656 \\
H & 7.512859 & -1.927790 & 0.571629 \\
C & 9.151916 & -1.592156 & -0.006257 \\
H & 8.718441 & 0.821650 & -1.177168 \\
H & 8.395146 & 1.475493 & -1.995218 \\
H & 8.971343 & 1.450162 & -0.315574 \\
C & 9.638166 & 0.320744 & -1.499719 \\
H & 7.402395 & -1.097551 & -2.086666 \\
H & 6.685297 & -1.902741 & -1.895815 \\
H & 7.010992 & -0.489368 & -2.910268 \\
C & 8.339072 & -1.559456 & -2.421857 \\
H & 5.066271 & 4.520960 & -1.135805 \\
H & 6.064482 & 4.278787 & -1.515071 \\
H & 4.334099 & 4.165710 & -1.869846 \\
C & 4.987817 & 5.613606 & -1.079426 \\
H & 5.855107 & 4.426423 & 1.260395 \\
H & 5.790396 & 5.518974 & 1.333697 \\
H & 5.687167 & 4.008525 & 2.259616 \\
C & 6.877116 & 4.173165 & 0.959683 \\
H & 3.413876 & 4.345091 & 0.731668 \\
H & 3.180287 & 3.960829 & 1.730824 \\
H & 3.380517 & 5.439301 & 0.782503 \\
& 2.620916 & 4.021928 & 0.047998 \\
\hline & & & \\
\hline & & & \\
\hline & & & \\
\hline
\end{tabular}

\section{Catalyst 2C 44}

Datum

Value

\begin{tabular}{lc}
\hline B3LYP/6-31G(d) Energy & -2164.794991 \\
\hline B3LYP-D3(BJ)/def2-TZVPP-IEF-PCM(DCM) Energy & -2165.894883 \\
\hline B3LYP-D3(BJ)/def2-TZVPP-IEF-PCM(DCM)//B3LYP/6-31G(d) Free Energy (Quasiharmonic) & -2165.018257 \\
\hline
\end{tabular}

Number of Imaginary Frequencies

Frequencies (Top 3 out of 321)

$\begin{array}{lr}\text { 1. } & 7.0698 \mathrm{~cm}^{-1} \\ \text { 2. } & 7.4063 \mathrm{~cm}^{-1} \\ \text { 3. } & 13.9518 \mathrm{~cm}^{-1}\end{array}$




\begin{tabular}{|c|c|c|c|}
\hline C & 0.158685 & -4.688047 & 2.599870 \\
\hline C & -0.091189 & -3.714598 & 1.658529 \\
\hline C & 0.909852 & -2.768733 & 1.305795 \\
\hline C & 2.183089 & -2.844143 & 1.955542 \\
\hline C & 2.408375 & -3.860746 & 2.922727 \\
\hline C & 1.420497 & -4.765246 & 3.236852 \\
\hline $\mathrm{H}$ & -0.619613 & -5.399894 & 2.860459 \\
\hline $\mathrm{H}$ & -1.063839 & -3.651184 & 1.183330 \\
\hline C & 0.689088 & -1.744418 & 0.328216 \\
\hline C & 3.182573 & -1.894627 & 1.631399 \\
\hline $\mathrm{H}$ & 3.378222 & -3.908207 & 3.412245 \\
\hline $\mathrm{H}$ & 1.604059 & -5.538396 & 3.978082 \\
\hline C & 2.973549 & -0.894976 & 0.701830 \\
\hline C & 1.702481 & -0.840081 & 0.041199 \\
\hline $\mathrm{H}$ & 4.132264 & -1.937937 & 2.158366 \\
\hline C & -1.100304 & -4.099306 & -3.920753 \\
\hline C & -2.128991 & -3.297812 & -3.481531 \\
\hline C & -1.978720 & -2.488306 & -2.324129 \\
\hline C & -0.741854 & -2.509193 & -1.605586 \\
\hline C & 0.302767 & -3.343811 & -2.091714 \\
\hline C & 0.127213 & -4.118288 & -3.217457 \\
\hline $\mathrm{H}$ & -3.947777 & -1.604700 & -2.434605 \\
\hline $\mathrm{H}$ & -1.225514 & -4.714725 & -4.807590 \\
\hline $\mathrm{H}$ & -3.075482 & -3.268971 & -4.016549 \\
\hline C & -3.019407 & -1.641510 & -1.869167 \\
\hline C & -0.610359 & -1.697598 & -0.427349 \\
\hline$H$ & 1.251492 & -3.363988 & -1.566670 \\
\hline $\mathrm{H}$ & 0.939630 & -4.747893 & -3.570315 \\
\hline C & -1.653696 & -0.886813 & -0.000044 \\
\hline C & -2.873561 & -0.842921 & -0.755331 \\
\hline C & -1.550039 & -0.079257 & 1.286680 \\
\hline H & -0.576096 & -0.232458 & 1.750486 \\
\hline $\mathrm{H}$ & -1.632841 & 0.993377 & 1.067696 \\
\hline C & 1.453601 & 0.205242 & -1.010025 \\
\hline 0 & 0.702154 & 1.152940 & -0.897972 \\
\hline 0 & 2.168017 & -0.027922 & -2.132094 \\
\hline $\mathrm{H}$ & 1.960721 & 0.705310 & -2.743327 \\
\hline 0 & -2.508374 & -0.482850 & 2.262970 \\
\hline H & -3.382618 & -0.218148 & 1.933711 \\
\hline C & -4.002985 & 0.063135 & -0.373264 \\
\hline C & -5.233996 & -0.475370 & 0.028353 \\
\hline C & -3.858819 & 1.455602 & -0.461854 \\
\hline C & -6.320141 & 0.350685 & 0.348675 \\
\hline H & -5.322787 & -1.554120 & 0.089641 \\
\hline C & -4.919219 & 2.315570 & -0.152695 \\
\hline $\mathrm{H}$ & -2.907529 & 1.851717 & -0.797881 \\
\hline C & -6.131890 & 1.736212 & 0.250409 \\
\hline H & -6.962826 & 2.391135 & 0.495324 \\
\hline C & 4.046035 & 0.104947 & 0.437831 \\
\hline C & 3.770681 & 1.478022 & 0.462900 \\
\hline C & 5.360236 & -0.316772 & 0.199516 \\
\hline C & 4.774083 & 2.429109 & 0.242647 \\
\hline $\mathrm{H}$ & 2.757018 & 1.803285 & 0.671429 \\
\hline
\end{tabular}




\begin{tabular}{|c|c|c|c|}
\hline C & 6.395504 & 0.600206 & -0.018289 \\
\hline $\mathrm{H}$ & 5.561220 & -1.383500 & 0.164031 \\
\hline C & 6.076502 & 1.966210 & 0.006194 \\
\hline $\mathrm{H}$ & 6.863136 & 2.688414 & -0.163797 \\
\hline C & 7.824653 & 0.085681 & -0.282186 \\
\hline C & 4.412062 & 3.927024 & 0.263609 \\
\hline C & 8.303242 & -0.745598 & 0.931949 \\
\hline $\mathrm{H}$ & 8.320218 & -0.135471 & 1.842315 \\
\hline $\mathrm{H}$ & 7.651287 & -1.605470 & 1.117838 \\
\hline $\mathrm{H}$ & 9.317186 & -1.126875 & 0.758859 \\
\hline C & 8.833445 & 1.228901 & -0.504779 \\
\hline H & 9.829953 & 0.810129 & -0.685592 \\
\hline $\mathrm{H}$ & 8.571583 & 1.842749 & -1.374150 \\
\hline $\mathrm{H}$ & 8.905678 & 1.886400 & 0.369027 \\
\hline C & 7.826150 & -0.808139 & -1.545359 \\
\hline $\mathrm{H}$ & 7.492704 & -0.244964 & -2.424454 \\
\hline $\mathrm{H}$ & 8.836977 & -1.184575 & -1.745329 \\
\hline $\mathrm{H}$ & 7.164355 & -1.673039 & -1.433172 \\
\hline C & 3.382187 & 4.215295 & -0.855802 \\
\hline $\mathrm{H}$ & 3.809889 & 3.996700 & -1.841648 \\
\hline $\mathrm{H}$ & 2.474336 & 3.613389 & -0.743853 \\
\hline $\mathrm{H}$ & 3.089118 & 5.272429 & -0.842007 \\
\hline C & 5.635989 & 4.834120 & 0.035393 \\
\hline $\mathrm{H}$ & 6.105575 & 4.652070 & -0.938098 \\
\hline $\mathrm{H}$ & 5.324715 & 5.884668 & 0.058484 \\
\hline $\mathrm{H}$ & 6.396405 & 4.698153 & 0.813082 \\
\hline C & 3.794959 & 4.293586 & 1.634246 \\
\hline $\mathrm{H}$ & 2.882671 & 3.724096 & 1.838951 \\
\hline $\mathrm{H}$ & 4.502310 & 4.096480 & 2.448059 \\
\hline $\mathrm{H}$ & 3.534593 & 5.358823 & 1.660880 \\
\hline C & -4.798489 & 3.849261 & -0.246730 \\
\hline C & -7.684589 & -0.208619 & 0.796476 \\
\hline C & -7.700360 & -1.748565 & 0.843403 \\
\hline $\mathrm{H}$ & -7.497171 & -2.189560 & -0.139126 \\
\hline $\mathrm{H}$ & -6.965671 & -2.143692 & 1.553987 \\
\hline $\mathrm{H}$ & -8.688841 & -2.097014 & 1.163095 \\
\hline C & -8.780212 & 0.252987 & -0.193864 \\
\hline $\mathrm{H}$ & -8.857006 & 1.344400 & -0.238500 \\
\hline $\mathrm{H}$ & -8.570491 & -0.108848 & -1.206929 \\
\hline $\mathrm{H}$ & -9.758903 & -0.137628 & 0.110828 \\
\hline C & -8.018937 & 0.318110 & 2.212431 \\
\hline $\mathrm{H}$ & -7.261077 & 0.000919 & 2.937863 \\
\hline $\mathrm{H}$ & -8.070666 & 1.411559 & 2.240177 \\
\hline $\mathrm{H}$ & -8.989836 & -0.069627 & 2.544500 \\
\hline C & -5.831440 & 4.386019 & -1.266140 \\
\hline $\mathrm{H}$ & -5.649597 & 3.970468 & -2.263889 \\
\hline $\mathrm{H}$ & -6.857808 & 4.132467 & -0.980766 \\
\hline $\mathrm{H}$ & -5.765256 & 5.478709 & -1.335785 \\
\hline C & -3.398025 & 4.301520 & -0.702362 \\
\hline $\mathrm{H}$ & -3.362994 & 5.395825 & -0.749522 \\
\hline $\mathrm{H}$ & -2.615378 & 3.975760 & -0.008132 \\
\hline $\mathrm{H}$ & -3.150198 & 3.920004 & -1.699116 \\
\hline C & -5.076996 & 4.474209 & 1.141289 \\
\hline $\mathrm{H}$ & -4.355543 & 4.116855 & 1.884858 \\
\hline
\end{tabular}



H

\section{Catalyst 2C 45}

Datum

Value

\begin{tabular}{lc}
\hline B3LYP/6-31G(d) Energy & -2164.795109 \\
\hline B3LYP-D3(BJ)/def2-TZVPP-IEF-PCM(DCM) Energy & -2165.895083 \\
\hline B3LYP-D3(BJ)/def2-TZVPP-IEF-PCM(DCM)//B3LYP/6-31G(d) Free Energy (Quasiharmonic) & -2165.018209
\end{tabular}

Number of Imaginary Frequencies

Frequencies (Top 3 out of 321)

$\begin{array}{lr}\text { 1. } & 7.4058 \mathrm{~cm}^{-1} \\ \text { 2. } & 10.1723 \mathrm{~cm}^{-1} \\ \text { 3. } & 14.0736 \mathrm{~cm}^{-1}\end{array}$

\section{B3LYP/6-31G(d) Molecular Geometry in Cartesian Coordinates}

$\begin{array}{lrrr}\text { C } & 0.106060 & -4.668921 & -2.578157 \\ \mathrm{C} & -0.131128 & -3.692525 & -1.636614 \\ \mathrm{C} & 0.880338 & -2.756338 & -1.287836 \\ \mathrm{C} & 2.150276 & -2.844124 & -1.942403 \\ \mathrm{C} & 2.362452 & -3.863567 & -2.909550 \\ \mathrm{C} & 1.364882 & -4.758864 & -3.219354 \\ \mathrm{H} & -0.680112 & -5.373110 & -2.835908 \\ \mathrm{H} & -1.101793 & -3.618653 & -1.158990 \\ \mathrm{C} & 0.673249 & -1.729725 & -0.309700 \\ \mathrm{C} & 3.159431 & -1.903062 & -1.623699 \\ \mathrm{H} & 3.329975 & -3.920504 & -3.402639 \\ \mathrm{H} & 1.538352 & -5.534285 & -3.960649 \\ \mathrm{C} & 2.963202 & -0.900391 & -0.694581 \\ \mathrm{C} & 1.695894 & -0.834256 & -0.027674 \\ \mathrm{H} & 4.106148 & -1.954999 & -2.155210 \\ \mathrm{C} & -1.115346 & -4.051025 & 3.958814 \\ \mathrm{C} & -2.141612 & -3.247601 & 3.517458 \\ \mathrm{C} & -1.990817 & -2.445684 & 2.354857 \\ \mathrm{C} & -0.755649 & -2.476086 & 1.633485 \\ \mathrm{C} & 0.286325 & -3.312863 & 2.121664 \\ \mathrm{C} & 0.110187 & -4.079874 & 3.252386 \\ \mathrm{H} & -3.958124 & -1.556886 & 2.461923 \\ \mathrm{H} & -1.240999 & -4.660612 & 4.849606 \\ \mathrm{H} & -3.086626 & -3.211498 & 4.054637 \\ & & & \end{array}$




\begin{tabular}{|c|c|c|c|}
\hline C & -3.030057 & -1.598810 & 1.896340 \\
\hline C & -0.622923 & -1.671471 & 0.450692 \\
\hline $\mathrm{H}$ & 1.233493 & -3.340558 & 1.594140 \\
\hline $\mathrm{H}$ & 0.920569 & -4.711258 & 3.606740 \\
\hline C & -1.663696 & -0.858401 & 0.021784 \\
\hline C & -2.883099 & -0.808203 & 0.777146 \\
\hline C & -1.560866 & -0.057660 & -1.268681 \\
\hline $\mathrm{H}$ & -1.672952 & 1.014034 & -1.058539 \\
\hline $\mathrm{H}$ & -0.577893 & -0.190907 & -1.719231 \\
\hline C & 1.460320 & 0.213976 & 1.023573 \\
\hline 0 & 0.712414 & 1.164887 & 0.915564 \\
\hline 0 & 2.182150 & -0.020710 & 2.140680 \\
\hline $\mathrm{H}$ & 1.983016 & 0.714617 & 2.752096 \\
\hline 0 & -2.498042 & -0.491101 & -2.253298 \\
\hline $\mathrm{H}$ & -3.382325 & -0.246607 & -1.935140 \\
\hline C & -4.011196 & 0.095045 & 0.384204 \\
\hline C & -5.230369 & -0.440263 & -0.040400 \\
\hline C & -3.873010 & 1.491123 & 0.481237 \\
\hline C & -6.316422 & 0.385516 & -0.381771 \\
\hline $\mathrm{H}$ & -5.322454 & -1.520634 & -0.106982 \\
\hline C & -4.927663 & 2.346410 & 0.156677 \\
\hline $\mathrm{H}$ & -2.928241 & 1.887435 & 0.835008 \\
\hline C & -6.136079 & 1.766976 & -0.275483 \\
\hline $\mathrm{H}$ & -6.957689 & 2.424685 & -0.533877 \\
\hline C & 4.044599 & 0.092105 & -0.439184 \\
\hline C & 5.357747 & -0.338347 & -0.210904 \\
\hline C & 3.778558 & 1.467029 & -0.464021 \\
\hline C & 6.401076 & 0.571798 & -0.003090 \\
\hline $\mathrm{H}$ & 5.551671 & -1.406392 & -0.175659 \\
\hline C & 4.790232 & 2.411473 & -0.253362 \\
\hline $\mathrm{H}$ & 2.765546 & 1.799004 & -0.665051 \\
\hline C & 6.091343 & 1.939947 & -0.026984 \\
\hline $\mathrm{H}$ & 6.884317 & 2.656980 & 0.135337 \\
\hline C & 4.438414 & 3.911844 & -0.273537 \\
\hline C & 7.828859 & 0.047860 & 0.249551 \\
\hline C & 3.812937 & 4.280916 & -1.639684 \\
\hline $\mathrm{H}$ & 4.512559 & 4.078384 & -2.458824 \\
\hline $\mathrm{H}$ & 2.895423 & 3.717072 & -1.836522 \\
\hline $\mathrm{H}$ & 3.559199 & 5.347777 & -1.665456 \\
\hline C & 5.670327 & 4.810770 & -0.056244 \\
\hline $\mathrm{H}$ & 5.366040 & 5.863376 & -0.078422 \\
\hline $\mathrm{H}$ & 6.146288 & 4.626880 & 0.913805 \\
\hline $\mathrm{H}$ & 6.423699 & 4.668473 & -0.839635 \\
\hline C & 3.419599 & 4.208810 & 0.853680 \\
\hline $\mathrm{H}$ & 3.853514 & 3.988054 & 1.836317 \\
\hline $\mathrm{H}$ & 3.134209 & 5.268059 & 0.841090 \\
\hline $\mathrm{H}$ & 2.506424 & 3.613603 & 0.749579 \\
\hline C & 8.847336 & 1.184533 & 0.461286 \\
\hline $\mathrm{H}$ & 8.916500 & 1.839896 & -0.414375 \\
\hline $\mathrm{H}$ & 8.596903 & 1.801713 & 1.331651 \\
\hline $\mathrm{H}$ & 9.842485 & 0.759353 & 0.634417 \\
\hline C & 8.291210 & -0.789092 & -0.967022 \\
\hline $\mathrm{H}$ & 8.305084 & -0.180751 & -1.878636 \\
\hline $\mathrm{H}$ & 9.303697 & -1.177608 & -0.801678 \\
\hline
\end{tabular}




\begin{tabular}{|c|c|c|c|}
\hline $\mathrm{H}$ & 7.631334 & -1.644375 & -1.145811 \\
\hline C & 7.834891 & -0.843563 & 1.514399 \\
\hline $\mathrm{H}$ & 7.512618 & -0.276465 & 2.395130 \\
\hline $\mathrm{H}$ & 7.166296 & -1.704130 & 1.409407 \\
\hline $\mathrm{H}$ & 8.844764 & -1.226518 & 1.706651 \\
\hline C & -4.815209 & 3.879781 & 0.260570 \\
\hline C & -7.639366 & -0.251652 & -0.848491 \\
\hline C & -8.708853 & 0.799822 & -1.200007 \\
\hline $\mathrm{H}$ & -8.968070 & 1.425216 & -0.338022 \\
\hline $\mathrm{H}$ & -8.383684 & 1.456899 & -2.014718 \\
\hline $\mathrm{H}$ & -9.625339 & 0.296779 & -1.528416 \\
\hline C & -8.200488 & -1.149974 & 0.279565 \\
\hline $\mathrm{H}$ & -7.503017 & -1.950488 & 0.547218 \\
\hline $\mathrm{H}$ & -8.400664 & -0.563894 & 1.183846 \\
\hline $\mathrm{H}$ & -9.140370 & -1.618232 & -0.037431 \\
\hline C & -7.382010 & -1.112636 & -2.108076 \\
\hline $\mathrm{H}$ & -6.988161 & -0.501028 & -2.927967 \\
\hline $\mathrm{H}$ & -6.663516 & -1.916238 & -1.915739 \\
\hline $\mathrm{H}$ & -8.315561 & -1.576366 & -2.449368 \\
\hline C & -5.067537 & 4.510924 & -1.129722 \\
\hline $\mathrm{H}$ & -4.329547 & 4.160068 & -1.860036 \\
\hline $\mathrm{H}$ & -6.062512 & 4.266415 & -1.515943 \\
\hline $\mathrm{H}$ & -4.993061 & 5.603663 & -1.069927 \\
\hline C & -3.426442 & 4.335230 & 0.747877 \\
\hline $\mathrm{H}$ & -3.397118 & 5.429380 & 0.802418 \\
\hline $\mathrm{H}$ & -3.197864 & 3.948513 & 1.747253 \\
\hline $\mathrm{H}$ & -2.628041 & 4.016950 & 0.068264 \\
\hline C & -5.871166 & 4.407415 & 1.261185 \\
\hline $\mathrm{H}$ & -5.708724 & 3.986728 & 2.260140 \\
\hline $\mathrm{H}$ & -5.809767 & 5.499892 & 1.338428 \\
\hline $\mathrm{H}$ & -6.890516 & 4.152545 & 0.952935 \\
\hline
\end{tabular}

\section{Catalyst 2C 46}

Datum

Value

\begin{tabular}{lc}
\hline B3LYP/6-31G(d) Energy & -2164.797742 \\
\hline B3LYP-D3(BJ)/def2-TZVPP-IEF-PCM(DCM) Energy & -2165.895778 \\
\hline
\end{tabular}

B3LYP-D3(BJ)/def2-TZVPP-IEF-PCM(DCM)//B3LYP/6-31G(d) Free Energy (Quasiharmonic) $\quad$-2165.018177

Number of Imaginary Frequencies

Frequencies (Top 3 out of 321)

$\begin{array}{ll}\text { 1. } & 10.5411 \mathrm{~cm}^{-1} \\ \text { 2. } & 11.0681 \mathrm{~cm}^{-1} \\ \text { 3. } & 13.1996 \mathrm{~cm}^{-1}\end{array}$




\begin{tabular}{|c|c|c|c|}
\hline C & 0.072943 & -3.680696 & 3.678892 \\
\hline C & 0.241009 & -2.465445 & 3.050403 \\
\hline C & -0.794818 & -1.909065 & 2.246039 \\
\hline C & -2.017745 & -2.641182 & 2.106672 \\
\hline C & -2.159124 & -3.888363 & 2.773476 \\
\hline C & -1.137961 & -4.400191 & 3.540569 \\
\hline $\mathrm{H}$ & 0.874078 & -4.087346 & 4.289747 \\
\hline $\mathrm{H}$ & 1.167336 & -1.913599 & 3.171907 \\
\hline C & -0.653247 & -0.654656 & 1.565217 \\
\hline C & -3.058634 & -2.103599 & 1.311329 \\
\hline $\mathrm{H}$ & -3.092930 & -4.434111 & 2.661996 \\
\hline $\mathrm{H}$ & -1.257606 & -5.356485 & 4.042116 \\
\hline C & -2.932489 & -0.894348 & 0.656291 \\
\hline C & -1.703126 & -0.172975 & 0.795518 \\
\hline $\mathrm{H}$ & -3.992127 & -2.654672 & 1.232088 \\
\hline C & 1.252957 & 2.906212 & 4.809154 \\
\hline C & 2.244130 & 2.680009 & 3.882638 \\
\hline C & 2.055751 & 1.746210 & 2.827491 \\
\hline C & 0.818054 & 1.037040 & 2.731384 \\
\hline C & -0.186658 & 1.297010 & 3.704141 \\
\hline C & 0.025740 & 2.206285 & 4.716213 \\
\hline $\mathrm{H}$ & 4.012898 & 2.019721 & 1.967444 \\
\hline $\mathrm{H}$ & 1.407636 & 3.620983 & 5.612979 \\
\hline H & 3.191035 & 3.211389 & 3.943730 \\
\hline C & 3.067800 & 1.490862 & 1.872483 \\
\hline C & 0.646218 & 0.099005 & 1.661246 \\
\hline $\mathrm{H}$ & -1.133803 & 0.772463 & 3.638597 \\
\hline $\mathrm{H}$ & -0.754862 & 2.390202 & 5.449536 \\
\hline C & 1.662849 & -0.136792 & 0.737030 \\
\hline C & 2.910364 & 0.571717 & 0.852002 \\
\hline C & 1.450428 & -1.146940 & -0.380524 \\
\hline H & 0.406107 & -1.137775 & -0.708254 \\
\hline $\mathrm{H}$ & 2.065709 & -0.887674 & -1.242382 \\
\hline C & -1.470657 & 1.055796 & -0.042232 \\
\hline 0 & -1.235052 & 1.035777 & -1.229288 \\
\hline 0 & -1.532506 & 2.204241 & 0.674260 \\
\hline $\mathrm{H}$ & -1.319909 & 2.924883 & 0.049332 \\
\hline 0 & 1.852003 & -2.475742 & -0.016014 \\
\hline $\mathrm{H}$ & 1.265045 & -2.773630 & 0.697998 \\
\hline C & 4.050274 & 0.366190 & -0.090689 \\
\hline C & 4.553817 & -0.909616 & -0.358954 \\
\hline C & 4.668659 & 1.481650 & -0.684302 \\
\hline C & 5.653470 & -1.097725 & -1.211380 \\
\hline $\mathrm{H}$ & 4.068910 & -1.763148 & 0.101528 \\
\hline C & 5.767613 & 1.338096 & -1.532725 \\
\hline $\mathrm{H}$ & 4.256413 & 2.463273 & -0.480293 \\
\hline C & 6.240451 & 0.036063 & -1.779776 \\
\hline $\mathrm{H}$ & 7.090420 & -0.086282 & -2.441076 \\
\hline C & -4.063847 & -0.371484 & -0.159344 \\
\hline C & -4.547633 & 0.925197 & 0.029372 \\
\hline
\end{tabular}




\begin{tabular}{|c|c|c|c|}
\hline C & -4.681485 & -1.201037 & -1.110493 \\
\hline C & -5.627330 & 1.419007 & -0.719575 \\
\hline $\mathrm{H}$ & -4.084673 & 1.551596 & 0.784778 \\
\hline C & -5.758510 & -0.749882 & -1.874709 \\
\hline $\mathrm{H}$ & -4.281677 & -2.197800 & -1.256204 \\
\hline C & -6.210186 & 0.565592 & -1.660173 \\
\hline $\mathrm{H}$ & -7.041801 & 0.927268 & -2.253090 \\
\hline C & -6.442968 & -1.624368 & -2.943035 \\
\hline C & -6.121311 & 2.858047 & -0.475005 \\
\hline C & -5.851485 & -3.045451 & -3.005485 \\
\hline $\mathrm{H}$ & -4.788772 & -3.035253 & -3.271977 \\
\hline $\mathrm{H}$ & -5.960773 & -3.574737 & -2.051788 \\
\hline $\mathrm{H}$ & -6.376619 & -3.629853 & -3.769423 \\
\hline C & -6.264094 & -0.967116 & -4.332502 \\
\hline $\mathrm{H}$ & -6.749640 & -1.573183 & -5.107408 \\
\hline $\mathrm{H}$ & -6.703863 & 0.035094 & -4.367819 \\
\hline $\mathrm{H}$ & -5.202226 & -0.875241 & -4.587073 \\
\hline C & -7.952126 & -1.749146 & -2.625355 \\
\hline $\mathrm{H}$ & -8.452321 & -0.775119 & -2.616930 \\
\hline $\mathrm{H}$ & -8.449079 & -2.370232 & -3.380674 \\
\hline $\mathrm{H}$ & -8.110260 & -2.214665 & -1.645698 \\
\hline C & -4.962532 & 3.848525 & -0.742879 \\
\hline $\mathrm{H}$ & -4.105051 & 3.655977 & -0.089536 \\
\hline $\mathrm{H}$ & -4.617187 & 3.774661 & -1.780437 \\
\hline $\mathrm{H}$ & -5.292918 & 4.879505 & -0.565853 \\
\hline C & -6.586079 & 3.000605 & 0.993996 \\
\hline $\mathrm{H}$ & -5.776996 & 2.786936 & 1.700114 \\
\hline $\mathrm{H}$ & -6.935104 & 4.022691 & 1.186152 \\
\hline $\mathrm{H}$ & -7.411192 & 2.313052 & 1.212394 \\
\hline C & -7.299567 & 3.242464 & -1.389529 \\
\hline $\mathrm{H}$ & -7.028341 & 3.188739 & -2.450065 \\
\hline $\mathrm{H}$ & -8.171480 & 2.598822 & -1.225608 \\
\hline $\mathrm{H}$ & -7.607577 & 4.273014 & -1.179831 \\
\hline C & 6.465264 & 2.538795 & -2.201313 \\
\hline C & 6.156867 & -2.528173 & -1.484520 \\
\hline C & 5.016596 & -3.355777 & -2.126139 \\
\hline $\mathrm{H}$ & 4.126800 & -3.392350 & -1.489945 \\
\hline $\mathrm{H}$ & 4.720643 & -2.925873 & -3.090378 \\
\hline $\mathrm{H}$ & 5.349158 & -4.386347 & -2.304021 \\
\hline C & 6.580031 & -3.188747 & -0.150881 \\
\hline $\mathrm{H}$ & 7.389807 & -2.625215 & 0.327336 \\
\hline $\mathrm{H}$ & 5.746745 & -3.244153 & 0.556569 \\
\hline $\mathrm{H}$ & 6.936016 & -4.211149 & -0.328640 \\
\hline C & 7.366946 & -2.556627 & -2.437059 \\
\hline $\mathrm{H}$ & 7.125237 & -2.127564 & -3.416277 \\
\hline $\mathrm{H}$ & 8.225208 & -2.011694 & -2.026633 \\
\hline $\mathrm{H}$ & 7.682400 & -3.593339 & -2.600645 \\
\hline C & 5.812854 & 3.882695 & -1.825033 \\
\hline $\mathrm{H}$ & 4.765514 & 3.931071 & -2.144123 \\
\hline $\mathrm{H}$ & 5.850061 & 4.066832 & -0.745269 \\
\hline $\mathrm{H}$ & 6.346922 & 4.702886 & -2.318254 \\
\hline C & 6.396332 & 2.388206 & -3.739701 \\
\hline H & 6.891531 & 3.235814 & -4.229624 \\
\hline $\mathrm{H}$ & 6.888223 & 1.472007 & -4.082426 \\
\hline
\end{tabular}




\section{Catalyst 2C 47}

Datum

Value

\begin{tabular}{lr}
\hline B3LYP/6-31G(d) Energy & -2164.7975 \\
\hline B3LYP-D3(BJ)/def2-TZVPP-IEF-PCM(DCM) Energy & -2165.895526 \\
\hline B3LYP-D3(BJ)/def2-TZVPP-IEF-PCM(DCM)//B3LYP/6-31G(d) Free Energy (Quasiharmonic) & -2165.018127 \\
\hline
\end{tabular}

Number of Imaginary Frequencies

Frequencies (Top 3 out of 321)
1. $7.4389 \mathrm{~cm}^{-1}$
2. $11.2819 \mathrm{~cm}^{-1}$
3. $13.6866 \mathrm{~cm}^{-1}$

\section{B3LYP/6-31G(d) Molecular Geometry in Cartesian Coordinates}

$\begin{array}{lrrl}\text { C } & -0.108782 & -3.618205 & 3.720680 \\ \mathrm{C} & -0.268978 & -2.411365 & 3.074194 \\ \mathrm{C} & 0.769483 & -1.874794 & 2.259716 \\ \mathrm{C} & 1.986637 & -2.618338 & 2.129451 \\ \mathrm{C} & 2.119688 & -3.856619 & 2.814251 \\ \mathrm{C} & 1.096263 & -4.349043 & 3.590950 \\ \mathrm{H} & -0.911769 & -4.009421 & 4.339137 \\ \mathrm{H} & -1.191166 & -1.851202 & 3.188854 \\ \mathrm{C} & 0.636087 & -0.629581 & 1.560473 \\ \mathrm{C} & 3.030897 & -2.099929 & 1.325687 \\ \mathrm{H} & 3.049159 & -4.410950 & 2.709144 \\ \mathrm{H} & 1.209592 & -5.298551 & 4.106642 \\ \mathrm{C} & 2.913645 & -0.897909 & 0.656248 \\ \mathrm{C} & 1.689728 & -0.166002 & 0.785012 \\ \mathrm{H} & 3.960114 & -2.659046 & 1.252550 \\ \mathrm{C} & -1.244101 & 3.014989 & 4.727361 \\ \mathrm{C} & -2.237703 & 2.774656 & 3.806989 \\ \mathrm{C} & -2.056888 & 1.815741 & 2.773285 \\ \mathrm{C} & -0.823841 & 1.096407 & 2.692106 \\ \mathrm{C} & 0.183290 & 1.371125 & 3.658226 \\ \mathrm{C} & -0.021946 & 2.304458 & 4.649655\end{array}$




\begin{tabular}{|c|c|c|c|}
\hline H & -4.014588 & 2.080265 & 1.909970 \\
\hline $\mathrm{H}$ & -1.393180 & 3.748860 & 5.514872 \\
\hline $\mathrm{H}$ & -3.180997 & 3.313569 & 3.857063 \\
\hline C & -3.072454 & 1.544170 & 1.826242 \\
\hline C & -0.658764 & 0.133437 & 1.643196 \\
\hline H & 1.126447 & 0.838176 & 3.603950 \\
\hline H & 0.760380 & 2.499229 & 5.378320 \\
\hline C & -1.678496 & -0.117485 & 0.726675 \\
\hline C & -2.921605 & 0.599870 & 0.828310 \\
\hline C & -1.475071 & -1.152217 & -0.370020 \\
\hline $\mathrm{H}$ & -2.095279 & -0.909791 & -1.233290 \\
\hline H & -0.432567 & -1.151699 & -0.704246 \\
\hline C & 1.469787 & 1.053529 & -0.069347 \\
\hline 0 & 1.230019 & 1.019412 & -1.255232 \\
\hline 0 & 1.550199 & 2.211108 & 0.630145 \\
\hline $\mathrm{H}$ & 1.347306 & 2.925727 & -0.004855 \\
\hline 0 & -1.877170 & -2.471909 & 0.024818 \\
\hline $\mathrm{H}$ & -1.283991 & -2.757628 & 0.738616 \\
\hline C & -4.064528 & 0.373669 & -0.106039 \\
\hline C & -4.669294 & 1.465312 & -0.744311 \\
\hline C & -4.584806 & -0.908947 & -0.323031 \\
\hline C & -5.773237 & 1.301380 & -1.590293 \\
\hline $\mathrm{H}$ & -4.247791 & 2.453890 & -0.584673 \\
\hline C & -5.684381 & -1.117351 & -1.164408 \\
\hline $\mathrm{H}$ & -4.107189 & -1.748312 & 0.169929 \\
\hline C & -6.262324 & 0.000924 & -1.782712 \\
\hline $\mathrm{H}$ & -7.111689 & -0.145319 & -2.436030 \\
\hline C & 4.051425 & -0.390815 & -0.160882 \\
\hline C & 4.559866 & 0.894811 & 0.037824 \\
\hline C & 4.651764 & -1.225213 & -1.118700 \\
\hline C & 5.653482 & 1.370736 & -0.702594 \\
\hline $\mathrm{H}$ & 4.105606 & 1.525385 & 0.795104 \\
\hline C & 5.739691 & -0.790336 & -1.877036 \\
\hline $\mathrm{H}$ & 4.230588 & -2.211908 & -1.272876 \\
\hline C & 6.221870 & 0.511616 & -1.646748 \\
\hline $\mathrm{H}$ & 7.066133 & 0.857974 & -2.230857 \\
\hline C & 6.413534 & -1.671328 & -2.946754 \\
\hline C & 6.177155 & 2.797054 & -0.445595 \\
\hline C & 7.895509 & -1.900407 & -2.565292 \\
\hline $\mathrm{H}$ & 8.447949 & -0.958156 & -2.487588 \\
\hline $\mathrm{H}$ & 7.976143 & -2.415796 & -1.601239 \\
\hline H & 8.393593 & -2.517728 & -3.323105 \\
\hline C & 6.340709 & -0.962920 & -4.320644 \\
\hline $\mathrm{H}$ & 6.811220 & -1.582616 & -5.093893 \\
\hline $\mathrm{H}$ & 5.300186 & -0.784054 & -4.614085 \\
\hline $\mathrm{H}$ & 6.855692 & 0.003426 & -4.311036 \\
\hline C & 5.737003 & -3.048301 & -3.084587 \\
\hline $\mathrm{H}$ & 4.685602 & -2.958638 & -3.379917 \\
\hline $\mathrm{H}$ & 6.248456 & -3.634137 & -3.856644 \\
\hline $\mathrm{H}$ & 5.783644 & -3.621529 & -2.151622 \\
\hline C & 6.633524 & 2.921402 & 1.027645 \\
\hline $\mathrm{H}$ & 5.814873 & 2.720685 & 1.726481 \\
\hline $\mathrm{H}$ & 7.442481 & 2.215575 & 1.248252 \\
\hline H & 7.002286 & 3.934863 & 1.228364 \\
\hline
\end{tabular}




\begin{tabular}{|c|c|c|c|}
\hline C & 5.042198 & 3.813975 & -0.716622 \\
\hline $\mathrm{H}$ & 4.174721 & 3.635003 & -0.072670 \\
\hline $\mathrm{H}$ & 5.392815 & 4.836402 & -0.529093 \\
\hline $\mathrm{H}$ & 4.705118 & 3.754933 & -1.757873 \\
\hline C & 7.370531 & 3.160599 & -1.348948 \\
\hline $\mathrm{H}$ & 8.226407 & 2.496411 & -1.182623 \\
\hline $\mathrm{H}$ & 7.106068 & 3.119472 & -2.411742 \\
\hline $\mathrm{H}$ & 7.699964 & 4.182664 & -1.130464 \\
\hline C & -6.205279 & -2.551651 & -1.381152 \\
\hline C & -6.391993 & 2.531258 & -2.284413 \\
\hline C & -7.617389 & 2.170714 & -3.145555 \\
\hline $\mathrm{H}$ & -7.359633 & 1.476482 & -3.953262 \\
\hline $\mathrm{H}$ & -8.417871 & 1.718964 & -2.548502 \\
\hline $\mathrm{H}$ & -8.021841 & 3.078345 & -3.607803 \\
\hline C & -5.334722 & 3.185680 & -3.205304 \\
\hline $\mathrm{H}$ & -4.449536 & 3.504948 & -2.645535 \\
\hline $\mathrm{H}$ & -5.003765 & 2.484985 & -3.980322 \\
\hline $\mathrm{H}$ & -5.753536 & 4.069831 & -3.702113 \\
\hline C & -6.844761 & 3.556121 & -1.217289 \\
\hline $\mathrm{H}$ & -7.598890 & 3.120685 & -0.551601 \\
\hline $\mathrm{H}$ & -6.008830 & 3.896553 & -0.597554 \\
\hline $\mathrm{H}$ & -7.284051 & 4.439584 & -1.697148 \\
\hline C & -5.077180 & -3.415635 & -1.996095 \\
\hline $\mathrm{H}$ & -4.782228 & -3.026709 & -2.977882 \\
\hline $\mathrm{H}$ & -4.183964 & -3.436308 & -1.363934 \\
\hline $\mathrm{H}$ & -5.421436 & -4.448796 & -2.131943 \\
\hline C & -7.420316 & -2.603081 & -2.326545 \\
\hline $\mathrm{H}$ & -7.748207 & -3.641675 & -2.448341 \\
\hline $\mathrm{H}$ & -8.270191 & -2.032704 & -1.933652 \\
\hline $\mathrm{H}$ & -7.179136 & -2.215293 & -3.322942 \\
\hline C & -6.629886 & -3.157536 & -0.022203 \\
\hline $\mathrm{H}$ & -7.431092 & -2.567336 & 0.437932 \\
\hline $\mathrm{H}$ & -6.998309 & -4.181687 & -0.160274 \\
\hline $\mathrm{H}$ & -5.794455 & -3.196533 & 0.683840 \\
\hline
\end{tabular}

\section{Catalyst 2C 48}

Datum

Value

\begin{tabular}{lr}
\hline B3LYP/6-31G(d) Energy & -2164.798894 \\
\hline B3LYP-D3(BJ)/def2-TZVPP-IEF-PCM(DCM) Energy & -2165.896145 \\
\hline B3LYP-D3(BJ)/def2-TZVPP-IEF-PCM(DCM)//B3LYP/6-31G(d) Free Energy (Quasiharmonic) & -2165.018077 \\
\hline Number of Imaginary Frequencies & 0
\end{tabular}

Frequencies (Top 3 out of 321) 


$\begin{array}{ll}\text { 1. } & 10.6121 \mathrm{~cm}^{-1} \\ \text { 2. } & 12.4899 \mathrm{~cm}^{-1} \\ \text { 3. } & 13.7531 \mathrm{~cm}^{-1}\end{array}$

B3LYP/6-31G(d) Molecular Geometry in Cartesian Coordinates

C

C

C

C

C

C

$\mathrm{H}$

$\mathrm{H}$

C

$\mathrm{C}$

$\mathrm{H}$

$\mathrm{H}$

C

C

$\mathrm{H}$

C

C

C

C

C

C

$\mathrm{H}$

$\mathrm{H}$

$\mathrm{H}$

C

C

$\mathrm{H}$

$\mathrm{H}$

C

C

C

$\mathrm{H}$

$\mathrm{H}$

C

0

0

$\mathrm{H}$

0

$\mathrm{H}$

C

C

C

C

$\mathrm{H}$
0.043544

$-0.151188$

0.868627

2.109820

2. 276693

1.267803

$-0.746786$

$-1.087060$

0.700263

3.137877

3. 221312

1.408187

2. 986297

1.744237

4.063881

$-0.926122$

$-1.986599$

$-1.886242$

$-0.664205$

0.412800

0.284813

$-3.900899$

$-1.013874$

$-2.922238$

$-2.969574$

$-0.583645$

1.347915

1.120157

$-1.666207$

$-2.894862$

$-1.532196$

$-2.196406$

$-0.508090$

1.550747

0.828450

2. 279638

2. 105609

$-1.918286$

$-1.286663$

$-4.099694$

$-4.627590$

$-4.752039$

$-5.784969$

$-4.116989$
$-4.857938$

$-3.751206$

$-2.767386$

$-2.963926$

$-4.116746$

$-5.043103$

$-5.596634$

$-3.623178$

$-1.598047$

$-2.000528$

$-4.251992$

$-5.919440$

$-0.872730$

$-0.690147$

$-2.143204$

$-2.876025$

$-2.233695$

$-1.736963$

$-1.910154$

$-2.580055$

$-3.049409$

$-0.977873$

$-3.251778$

$-2.095779$

$-1.083970$

$-1.403388$

$-2.716227$

$-3.557431$

$-0.760670$

$-0.605847$

$-0.210669$

0.643915

0.138001

0.517871

1.458059

0.443750

1.270845

$-1.148983$

$-1.885962$

0.065842

$-0.309117$

1.066335

0.295982

$-1.078302$
$-1.722990$

$-0.924808$

$-0.776674$

$-1.463776$

$-2.278250$

$-2.408668$

$-1.823018$

$-0.390976$

0.036456

$-1.324380$

$-2.799492$

$-3.035295$

$-0.541587$

0.149122

$-1.875270$

4.782991

4.187622

2.859441

2.136030

2.782039

4.070283

2.778137

5.798883

4.724575

2. 227477

0.797234

2. 249745

4.544605

0.201370

0.932106

$-1.209659$

$-1.341521$

$-1.376601$

1.023463

0.765064

2.157256

2.647177

$-2.224820$

$-2.198650$

0.360599

$-0.877969$

1.104086

$-1.393501$

$-1.446528$ 


\begin{tabular}{|c|c|c|c|}
\hline C & -5.907791 & 1.688481 & 0.629806 \\
\hline $\mathrm{H}$ & -4.319983 & 1.360465 & 2.053641 \\
\hline C & -6.403983 & 1.284365 & -0.623393 \\
\hline $\mathrm{H}$ & -7.297428 & 1.765993 & -1.003591 \\
\hline C & 4.090537 & 0.124499 & -0.459208 \\
\hline C & 5.400365 & -0.302491 & -0.181304 \\
\hline C & 3.851702 & 1.481466 & -0.690946 \\
\hline C & 6.460536 & 0.603384 & -0.124870 \\
\hline $\mathrm{H}$ & 5.565048 & -1.356262 & 0.011810 \\
\hline C & 4.888371 & 2.426611 & -0.636530 \\
\hline $\mathrm{H}$ & 2.843411 & 1.802913 & -0.929304 \\
\hline C & 6.175833 & 1.962452 & -0.354116 \\
\hline $\mathrm{H}$ & 6.991981 & 2.673408 & -0.307367 \\
\hline C & 4.568086 & 3.913583 & -0.882447 \\
\hline C & 7.907663 & 0.172498 & 0.182419 \\
\hline C & 3.944143 & 4.083192 & -2.288174 \\
\hline $\mathrm{H}$ & 4.635923 & 3.744202 & -3.067908 \\
\hline $\mathrm{H}$ & 3.014946 & 3.514436 & -2.394232 \\
\hline $\mathrm{H}$ & 3.710871 & 5.138418 & -2.475557 \\
\hline C & 5.819926 & 4.807719 & -0.803181 \\
\hline $\mathrm{H}$ & 5.538583 & 5.851218 & -0.984152 \\
\hline $\mathrm{H}$ & 6.293742 & 4.761155 & 0.184081 \\
\hline $\mathrm{H}$ & 6.567910 & 4.532431 & -1.555707 \\
\hline C & 3.560194 & 4.399267 & 0.187677 \\
\hline $\mathrm{H}$ & 3.993197 & 4.320798 & 1.192127 \\
\hline $\mathrm{H}$ & 3.297825 & 5.450266 & 0.014514 \\
\hline $\mathrm{H}$ & 2.633719 & 3.816160 & 0.175628 \\
\hline C & 8.820921 & 0.554755 & -1.006952 \\
\hline $\mathrm{H}$ & 8.498524 & 0.051069 & -1.925478 \\
\hline $\mathrm{H}$ & 8.814132 & 1.632970 & -1.197891 \\
\hline $\mathrm{H}$ & 9.857323 & 0.259275 & -0.802554 \\
\hline C & 8.032816 & -1.345866 & 0.411492 \\
\hline $\mathrm{H}$ & 7.725309 & -1.918786 & -0.470838 \\
\hline $\mathrm{H}$ & 9.077113 & -1.601926 & 0.623168 \\
\hline $\mathrm{H}$ & 7.431589 & -1.681350 & 1.264133 \\
\hline C & 8.402774 & 0.891623 & 1.459922 \\
\hline $\mathrm{H}$ & 8.385646 & 1.980854 & 1.349034 \\
\hline $\mathrm{H}$ & 7.777005 & 0.633480 & 2.321863 \\
\hline $\mathrm{H}$ & 9.434478 & 0.596660 & 1.687834 \\
\hline C & -6.638219 & 2.794418 & 1.416489 \\
\hline C & -6.313942 & -0.144766 & -2.771830 \\
\hline C & -7.586669 & 0.616651 & -3.186933 \\
\hline $\mathrm{H}$ & -8.410146 & 0.453018 & -2.481897 \\
\hline $\mathrm{H}$ & -7.410331 & 1.695786 & -3.263009 \\
\hline $\mathrm{H}$ & -7.920943 & 0.267877 & -4.170759 \\
\hline C & -6.649106 & -1.654881 & -2.736280 \\
\hline $\mathrm{H}$ & -5.770252 & -2.260518 & -2.493436 \\
\hline $\mathrm{H}$ & -7.421342 & -1.868473 & -1.987925 \\
\hline $\mathrm{H}$ & -7.022408 & -1.985064 & -3.713719 \\
\hline C & -5.224998 & 0.112373 & -3.842020 \\
\hline $\mathrm{H}$ & -4.994800 & 1.182145 & -3.911479 \\
\hline $\mathrm{H}$ & -4.292800 & -0.414741 & -3.615852 \\
\hline $\mathrm{H}$ & -5.574310 & -0.222445 & -4.827022 \\
\hline C & -8.094792 & 2.353868 & 1.696506 \\
\hline
\end{tabular}




\begin{tabular}{|llll|}
$H$ & -8.650937 & 2.168187 & 0.771714 \\
$H$ & -8.118265 & 1.432581 & 2.289966 \\
$H$ & -8.628701 & 3.132267 & 2.255714 \\
$\mathrm{C}$ & -6.649068 & 4.098891 & 0.584459 \\
$\mathrm{H}$ & -7.170677 & 4.895887 & 1.128839 \\
$\mathrm{H}$ & -5.627893 & 4.439571 & 0.378882 \\
$\mathrm{H}$ & -7.155830 & 3.964882 & -0.376846 \\
$\mathrm{C}$ & -5.964578 & 3.097286 & 2.768633 \\
$\mathrm{H}$ & -4.935341 & 3.451447 & 2.642055 \\
$\mathrm{H}$ & -6.522216 & 3.883176 & 3.290878 \\
$\mathrm{H}$ & -5.944796 & 2.216865 & 3.421211 \\
\hline
\end{tabular}

\section{Catalyst 2C 49}

Datum

Value

\begin{tabular}{lc}
\hline B3LYP/6-31G(d) Energy & -2164.798456 \\
\hline B3LYP-D3(BJ)/def2-TZVPP-IEF-PCM(DCM) Energy & -2165.895895 \\
\hline B3LYP-D3(BJ)/def2-TZVPP-IEF-PCM(DCM)//B3LYP/6-31G(d) Free Energy (Quasiharmonic) & -2165.01807 \\
\hline
\end{tabular}

Number of Imaginary Frequencies

Frequencies (Top 3 out of 321)
1. $10.1204 \mathrm{~cm}^{-1}$
2. $12.1601 \mathrm{~cm}^{-1}$
3. $13.4364 \mathrm{~cm}^{-1}$

\section{B3LYP/6-31G(d) Molecular Geometry in Cartesian Coordinates}

$\begin{array}{lrrr}C & 0.041567 & -4.866318 & -1.709558 \\ C & -0.152711 & -3.756126 & -0.916126 \\ C & 0.867667 & -2.772351 & -0.771800 \\ C & 2.108841 & -2.972468 & -1.457898 \\ C & 2.275247 & -4.128787 & -2.267478 \\ C & 1.265876 & -5.055137 & -2.394123 \\ H & -0.749318 & -5.604768 & -1.806876 \\ H & -1.088852 & -3.625073 & -0.383551 \\ C & 0.699962 & -1.599658 & 0.036762 \\ C & 3.137415 & -2.009109 & -1.322289 \\ H & 3.219887 & -4.266731 & -2.787981 \\ H & 1.405879 & -5.934182 & -3.017034 \\ C & 2.986524 & -0.878179 & -0.543915 \\ C & 1.744565 & -0.692094 & 0.146098 \\ H & 4.063363 & -2.154528 & -1.872558\end{array}$




\begin{tabular}{|c|c|c|c|}
\hline C & -0.928013 & -2.863219 & 4.786876 \\
\hline c & -1.988665 & -2.223504 & 4.188910 \\
\hline C & -1.887948 & -1.730815 & 2.859322 \\
\hline C & -0.665371 & -1.905286 & 2.137038 \\
\hline C & 0.411679 & -2.572524 & 2.785686 \\
\hline C & 0.283372 & -3.037939 & 4.075349 \\
\hline $\mathrm{H}$ & -3.904176 & -0.974457 & 2.773148 \\
\hline $\mathrm{H}$ & -1.016076 & -3.235929 & 5.803862 \\
\hline $\mathrm{H}$ & -2.924750 & -2.084753 & 4.724883 \\
\hline C & -2.971711 & -1.080979 & 2.224445 \\
\hline C & -0.583946 & -1.402171 & 0.796784 \\
\hline $\mathrm{H}$ & 1.347183 & -2.709675 & 2.254327 \\
\hline $\mathrm{H}$ & 1.118844 & -3.543893 & 4.551654 \\
\hline C & -1.666271 & -0.761298 & 0.198802 \\
\hline C & -2.895445 & -0.606549 & 0.928056 \\
\hline C & -1.532453 & -0.211853 & -1.212737 \\
\hline $\mathrm{H}$ & -2.201610 & 0.638910 & -1.345758 \\
\hline $\mathrm{H}$ & -0.510017 & 0.143926 & -1.376124 \\
\hline C & 1.552435 & 0.518750 & 1.016788 \\
\hline 0 & 0.830484 & 1.458636 & 0.756319 \\
\hline 0 & 2.282251 & 0.447621 & 2.150231 \\
\hline $\mathrm{H}$ & 2.109345 & 1.276447 & 2.637612 \\
\hline 0 & -1.909980 & -1.151882 & -2.228553 \\
\hline $\mathrm{H}$ & -1.277740 & -1.887993 & -2.195776 \\
\hline C & -4.099860 & 0.061986 & 0.351772 \\
\hline C & -4.637448 & -0.341024 & -0.883224 \\
\hline C & -4.741258 & 1.074329 & 1.072184 \\
\hline C & -5.789754 & 0.255330 & -1.398272 \\
\hline $\mathrm{H}$ & -4.127686 & -1.121808 & -1.432510 \\
\hline C & -5.902495 & 1.698944 & 0.590167 \\
\hline $\mathrm{H}$ & -4.307454 & 1.388811 & 2.017070 \\
\hline C & -6.403144 & 1.271521 & -0.642855 \\
\hline $\mathrm{H}$ & -7.295039 & 1.742149 & -1.039633 \\
\hline C & 4.091555 & 0.118467 & -0.465350 \\
\hline C & 5.401157 & -0.308677 & -0.186599 \\
\hline C & 3.853779 & 1.474845 & -0.701565 \\
\hline C & 6.462158 & 0.596433 & -0.133844 \\
\hline $\mathrm{H}$ & 5.565020 & -1.361919 & 0.010050 \\
\hline C & 4.891294 & 2.419273 & -0.650812 \\
\hline $\mathrm{H}$ & 2.845659 & 1.796338 & -0.940614 \\
\hline C & 6.178513 & 1.954963 & -0.367539 \\
\hline $\mathrm{H}$ & 6.995298 & 2.665363 & -0.323641 \\
\hline C & 4.572184 & 3.905688 & -0.901594 \\
\hline C & 7.909077 & 0.165278 & 0.174074 \\
\hline C & 3.947430 & 4.071018 & -2.307470 \\
\hline $\mathrm{H}$ & 4.638401 & 3.728769 & -3.086497 \\
\hline $\mathrm{H}$ & 3.017695 & 3.502675 & -2.410908 \\
\hline $\mathrm{H}$ & 3.714901 & 5.125789 & -2.498323 \\
\hline C & 5.824886 & 4.798957 & -0.826277 \\
\hline $\mathrm{H}$ & 5.544371 & 5.842085 & -1.010631 \\
\hline $\mathrm{H}$ & 6.299361 & 4.755327 & 0.160800 \\
\hline $\mathrm{H}$ & 6.572080 & 4.520405 & -1.578388 \\
\hline C & 3.565447 & 4.395936 & 0.167532 \\
\hline $\mathrm{H}$ & 3.999005 & 4.320388 & 1.171967 \\
\hline
\end{tabular}




\begin{tabular}{|c|c|c|c|}
\hline $\mathrm{H}$ & 3.303986 & 5.446604 & -0.008968 \\
\hline $\mathrm{H}$ & 2.638398 & 3.813690 & 0.158020 \\
\hline C & 8.821941 & 0.542558 & -1.017182 \\
\hline $\mathrm{H}$ & 8.498346 & 0.036202 & -1.933815 \\
\hline $\mathrm{H}$ & 8.816190 & 1.620151 & -1.211658 \\
\hline $\mathrm{H}$ & 9.858168 & 0.246628 & -0.812531 \\
\hline C & 8.032982 & -1.352388 & 0.408406 \\
\hline $\mathrm{H}$ & 7.724508 & -1.928151 & -0.471733 \\
\hline $\mathrm{H}$ & 9.077158 & -1.608633 & 0.620445 \\
\hline $\mathrm{H}$ & 7.431902 & -1.684346 & 1.262529 \\
\hline C & 8.405627 & 0.888419 & 1.448738 \\
\hline $\mathrm{H}$ & 8.389541 & 1.977264 & 1.333977 \\
\hline $\mathrm{H}$ & 7.780051 & 0.633970 & 2.311916 \\
\hline $\mathrm{H}$ & 9.437151 & 0.593211 & 1.677145 \\
\hline C & -6.565634 & 2.816168 & 1.419563 \\
\hline C & -6.391629 & -0.154670 & -2.756449 \\
\hline C & -5.608969 & -1.305161 & -3.418817 \\
\hline $\mathrm{H}$ & -4.559887 & -1.044174 & -3.593295 \\
\hline $\mathrm{H}$ & -5.632620 & -2.214054 & -2.806513 \\
\hline $\mathrm{H}$ & -6.062251 & -1.547179 & -4.387186 \\
\hline C & -6.363684 & 1.060830 & -3.713826 \\
\hline $\mathrm{H}$ & -6.936616 & 1.906108 & -3.317370 \\
\hline $\mathrm{H}$ & -5.335734 & 1.401721 & -3.881165 \\
\hline $\mathrm{H}$ & -6.795107 & 0.791775 & -4.686065 \\
\hline C & -7.854522 & -0.618232 & -2.561448 \\
\hline $\mathrm{H}$ & -7.905057 & -1.480111 & -1.886081 \\
\hline $\mathrm{H}$ & -8.484264 & 0.172959 & -2.140812 \\
\hline $\mathrm{H}$ & -8.291658 & -0.914543 & -3.523085 \\
\hline C & -6.989479 & 2.251336 & 2.796253 \\
\hline $\mathrm{H}$ & -7.712155 & 1.435551 & 2.678987 \\
\hline $\mathrm{H}$ & -6.133568 & 1.861042 & 3.356742 \\
\hline $\mathrm{H}$ & -7.457065 & 3.035618 & 3.404620 \\
\hline C & -7.818734 & 3.395398 & 0.735531 \\
\hline $\mathrm{H}$ & -8.251306 & 4.181579 & 1.364743 \\
\hline $\mathrm{H}$ & -7.582648 & 3.843243 & -0.236497 \\
\hline $\mathrm{H}$ & -8.591080 & 2.633005 & 0.582265 \\
\hline C & -5.558042 & 3.971364 & 1.629544 \\
\hline $\mathrm{H}$ & -5.244644 & 4.396696 & 0.669368 \\
\hline $\mathrm{H}$ & -6.013608 & 4.772717 & 2.224503 \\
\hline $\mathrm{H}$ & -4.657708 & 3.637111 & 2.155162 \\
\hline
\end{tabular}

\section{Catalyst 2C 50}

Datum

Value

\begin{tabular}{lc}
\hline B3LYP/6-31G(d) Energy & -2164.797735 \\
\hline B3LYP-D3(BJ)/def2-TZVPP-IEF-PCM(DCM) Energy & -2165.895468 \\
\hline B3LYP-D3(BJ)/def2-TZVPP-IEF-PCM(DCM)//B3LYP/6-31G(d) Free Energy (Quasiharmonic) & -2165.018027 \\
\hline
\end{tabular}


Frequencies (Top 3 out of 321)

$\begin{array}{lr}\text { 1. } & 9.2191 \mathrm{~cm}^{-1} \\ \text { 2. } & 11.6003 \mathrm{~cm}^{-1} \\ \text { 3. } & 13.3104 \mathrm{~cm}^{-1}\end{array}$

\section{B3LYP/6-31G(d) Molecular Geometry in Cartesian Coordinates}

C

C

C

C

C

C

$\mathrm{H}$

$\mathrm{H}$

C

C

$\mathrm{H}$

$\mathrm{H}$

C

C

$\mathrm{H}$

C

C

C

C

C

C

$\mathrm{H}$

$\mathrm{H}$

$\mathrm{H}$

C

C

C

$\mathrm{H}$

$\mathrm{H}$

C

C

C

$\mathrm{H}$

$\mathrm{H}$

C

0

0

$\mathrm{H}$
$-0.114663$

$-0.269134$

0.772968

1.990053

2.116971

1.088627

$-0.920018$

$-1.188075$

0.645080

3.040815

3.046119

1.197418

2. 931694

1.705773

3.970273

$-1.193954$

$-2.200505$

$-2.031365$

$-0.798122$

0.223480

0.029719

$-3.997455$

$-1.332988$

$-3.144031$

$-3.057636$

$-0.648216$

1.169013

0.823554

$-1.679567$

$-2.919612$

$-1.490068$

$-2.101308$

$-0.445045$

1. 528621

1.575701

1. 295562

1. 204461
$-3.690131$

$-2.465477$

$-1.910987$

$-2.651910$

- 3.909191

$-4.420860$

$-4.094803$

$-1.903323$

$-0.647755$

$-2.112075$

$-4.462141$

$-5.384602$

$-0.889920$

$-0.162211$

$-2.669711$

2.993314

2. 751770

1.795836

1.079842

1. 357446

2. 288189

2.064793

3.726030

3. 288729

1. 526751

0.115903

0.831226

2. 487131

$-0.134984$

0.586561

$-1.171265$

$-0.915563$

$-1.191128$

1. 146852

2. 245835

0.957672

1.848895
3.620170

3.006309

2. 208517

2.065746

2. 716534

3.473973

4. 226780

3.137116

1.542128

1. 285307

2. 601628

3.963572

0.650734

0.790922

1.204986

4.731110

3. 825499

2. 787324

2. 687507

3.637033

4.632847

1.947384

5. 521518

3. 889505

1.851737

1.637734

3. 565265

5.347690

0.733770

0.847733

$-0.363479$

$-1.229818$

$-0.689680$

0.069091

0.576280

$-1.250626$

$-1.640646$ 


\begin{tabular}{|c|c|c|c|}
\hline 0 & -1.916689 & -2.486061 & 0.021143 \\
\hline $\mathrm{H}$ & -1.338584 & -2.782078 & 0.743290 \\
\hline C & -4.070725 & 0.376812 & -0.080743 \\
\hline C & -4.592070 & -0.896959 & -0.323907 \\
\hline C & -4.682303 & 1.489833 & -0.686588 \\
\hline C & -5.702464 & -1.085560 & -1.162358 \\
\hline $\mathrm{H}$ & -4.113072 & -1.748780 & 0.145605 \\
\hline C & -5.791778 & 1.345852 & -1.521162 \\
\hline $\mathrm{H}$ & -4.257351 & 2.469817 & -0.501462 \\
\hline C & -6.282221 & 0.045743 & -1.742861 \\
\hline $\mathrm{H}$ & -7.140698 & -0.076990 & -2.393057 \\
\hline C & 4.076020 & -0.365495 & -0.146317 \\
\hline C & 4.564332 & 0.935713 & 0.063390 \\
\hline C & 4.701554 & -1.183452 & -1.091043 \\
\hline C & 5.651505 & 1.424181 & -0.663271 \\
\hline $\mathrm{H}$ & 4.088065 & 1.554309 & 0.813135 \\
\hline C & 5.801633 & -0.733480 & -1.837573 \\
\hline $\mathrm{H}$ & 4.302647 & -2.179728 & -1.257770 \\
\hline C & 6.252624 & 0.569329 & -1.604825 \\
\hline $\mathrm{H}$ & 7.098105 & 0.942056 & -2.170744 \\
\hline C & 6.450814 & -1.668108 & -2.876860 \\
\hline C & 6.199378 & 2.851255 & -0.468951 \\
\hline C & 7.651739 & -1.016415 & -3.588094 \\
\hline $\mathrm{H}$ & 7.359847 & -0.116388 & -4.141010 \\
\hline $\mathrm{H}$ & 8.445477 & -0.743929 & -2.883107 \\
\hline $\mathrm{H}$ & 8.079068 & -1.721795 & -4.309655 \\
\hline C & 5.404065 & -2.046200 & -3.951897 \\
\hline $\mathrm{H}$ & 5.846899 & -2.720165 & -4.695686 \\
\hline $\mathrm{H}$ & 4.536284 & -2.553219 & -3.517475 \\
\hline $\mathrm{H}$ & 5.041212 & -1.154229 & -4.475307 \\
\hline C & 6.950311 & -2.953208 & -2.174433 \\
\hline $\mathrm{H}$ & 6.132584 & -3.499314 & -1.692549 \\
\hline $\mathrm{H}$ & 7.417328 & -3.628491 & -2.901817 \\
\hline $\mathrm{H}$ & 7.693947 & -2.715694 & -1.405067 \\
\hline C & 6.084008 & 3.627235 & -1.803071 \\
\hline $\mathrm{H}$ & 6.649862 & 3.145232 & -2.607399 \\
\hline $\mathrm{H}$ & 5.038781 & 3.698038 & -2.125181 \\
\hline $\mathrm{H}$ & 6.472722 & 4.646325 & -1.686254 \\
\hline C & 7.685038 & 2.785869 & -0.042603 \\
\hline $\mathrm{H}$ & 8.305193 & 2.281601 & -0.791296 \\
\hline $\mathrm{H}$ & 8.086415 & 3.797409 & 0.095061 \\
\hline $\mathrm{H}$ & 7.796938 & 2.244697 & 0.903844 \\
\hline C & 5.424554 & 3.634875 & 0.608249 \\
\hline $\mathrm{H}$ & 4.360114 & 3.726336 & 0.365820 \\
\hline $\mathrm{H}$ & 5.508059 & 3.162818 & 1.593916 \\
\hline $\mathrm{H}$ & 5.835499 & 4.647331 & 0.692763 \\
\hline C & -6.483386 & 2.544039 & -2.200536 \\
\hline C & -6.225695 & -2.514047 & -1.407215 \\
\hline C & -6.642055 & -3.148542 & -0.058833 \\
\hline $\mathrm{H}$ & -5.801971 & -3.203007 & 0.640593 \\
\hline $\mathrm{H}$ & -7.439770 & -2.567733 & 0.418914 \\
\hline $\mathrm{H}$ & -7.012156 & -4.169232 & -0.216547 \\
\hline C & -5.102407 & -3.365357 & -2.047723 \\
\hline $\mathrm{H}$ & -4.812984 & -2.955316 & -3.022607 \\
\hline
\end{tabular}




\begin{tabular}{|llll|}
$H$ & -4.205841 & -3.401105 & -1.421040 \\
$\mathrm{H}$ & -5.448547 & -4.394931 & -2.204177 \\
$\mathrm{C}$ & -7.446482 & -2.543190 & -2.345946 \\
$\mathrm{H}$ & -8.293736 & -1.982070 & -1.934478 \\
$\mathrm{H}$ & -7.210923 & -2.131966 & -3.334302 \\
$\mathrm{H}$ & -7.775603 & -3.578523 & -2.490086 \\
$\mathrm{C}$ & -5.812761 & 3.886238 & -1.851191 \\
$\mathrm{H}$ & -5.836754 & 4.086996 & -0.774117 \\
$\mathrm{H}$ & -4.768158 & 3.918316 & -2.181155 \\
$\mathrm{H}$ & -6.342658 & 4.704818 & -2.351528 \\
$\mathrm{C}$ & -7.959906 & 2.613162 & -1.742162 \\
$\mathrm{H}$ & -8.470002 & 3.457986 & -2.221560 \\
$\mathrm{H}$ & -8.510836 & 1.702388 & -1.998981 \\
$\mathrm{H}$ & -8.026453 & 2.747727 & -0.656378 \\
$\mathrm{C}$ & -6.432564 & 2.369794 & -3.737095 \\
$\mathrm{H}$ & -6.936676 & 1.453322 & -4.060863 \\
$\mathrm{H}$ & -6.924742 & 3.214757 & -4.234531 \\
$\mathrm{H}$ & -5.396336 & 2.323624 & -4.091193 \\
\hline
\end{tabular}

\section{Catalyst 2C 51}

\section{Datum}

Value

\begin{tabular}{lc}
\hline B3LYP/6-31G(d) Energy & -2164.797123 \\
\hline B3LYP-D3(BJ)/def2-TZVPP-IEF-PCM(DCM) Energy & -2165.895507 \\
\hline B3LYP-D3(BJ)/def2-TZVPP-IEF-PCM(DCM)//B3LYP/6-31G(d) Free Energy (Quasiharmonic) & -2165.017999 \\
\hline
\end{tabular}

Number of Imaginary Frequencies

Frequencies (Top 3 out of 321)
1. $9.6956 \mathrm{~cm}^{-1}$
2. $12.0034 \mathrm{~cm}^{-1}$
3. $13.6127 \mathrm{~cm}^{-1}$

\section{B3LYP/6-31G(d) Molecular Geometry in Cartesian Coordinates}

C

C

C

C

C

C

$\mathrm{H}$

$\mathrm{H}$
0.001539

$-0.175008$

0.852923

2.084962

2. 232693

1. 215134

$-0.794815$

$-1.101984$
$-4.848952$

$-3.734185$

$-2.755638$

$-2.969409$

$-4.128976$

$-5.048290$

$-5.583345$

$-3.597891$
$-1.668299$

$-0.876679$

$-0.749921$

$-1.447896$

$-2.256282$

$-2.368386$

$-1.750676$

$-0.329562$ 


\begin{tabular}{|c|c|c|c|}
\hline C & 0.703431 & -1.578591 & 0.057255 \\
\hline C & 3.124398 & -2.015974 & -1.323644 \\
\hline $\mathrm{H}$ & 3.170441 & -4.275917 & -2.786693 \\
\hline $\mathrm{H}$ & 1.341257 & -5.930302 & -2.990037 \\
\hline C & 2.993727 & -0.886898 & -0.540160 \\
\hline C & 1.760612 & -0.683941 & 0.160555 \\
\hline $\mathrm{H}$ & 4.043668 & -2.170141 & -1.882696 \\
\hline C & -0.894604 & -2.724659 & 4.848291 \\
\hline C & -1.963470 & -2.111326 & 4.237802 \\
\hline C & -1.870864 & -1.652070 & 2.895736 \\
\hline C & -0.648784 & -1.833699 & 2.174300 \\
\hline C & 0.437410 & -2.471374 & 2.836970 \\
\hline C & 0.317021 & -2.904038 & 4.138411 \\
\hline H & -3.892095 & -0.910943 & 2.799181 \\
\hline $\mathrm{H}$ & -0.975512 & -3.071170 & 5.875053 \\
\hline $\mathrm{H}$ & -2.899263 & -1.967720 & 4.772988 \\
\hline C & -2.961687 & -1.026485 & 2.248789 \\
\hline C & -0.577111 & -1.367920 & 0.819974 \\
\hline $\mathrm{H}$ & 1.374864 & -2.607369 & 2.309093 \\
\hline $\mathrm{H}$ & 1.160274 & -3.384884 & 4.626496 \\
\hline C & -1.669099 & -0.755713 & 0.207983 \\
\hline C & -2.895051 & -0.586137 & 0.939719 \\
\hline C & -1.550353 & -0.252046 & -1.222191 \\
\hline $\mathrm{H}$ & -2.212490 & 0.601968 & -1.371855 \\
\hline $\mathrm{H}$ & -0.525983 & 0.084184 & -1.414349 \\
\hline C & 1.658117 & 0.462853 & 1.129385 \\
\hline 0 & 2.253993 & 0.528293 & 2.179288 \\
\hline 0 & 0.810998 & 1.435788 & 0.705114 \\
\hline $\mathrm{H}$ & 0.785822 & 2.100677 & 1.420719 \\
\hline 0 & -1.952652 & -1.215951 & -2.204659 \\
\hline $\mathrm{H}$ & -1.326167 & -1.956677 & -2.161752 \\
\hline C & -4.105034 & 0.066466 & 0.355721 \\
\hline C & -4.654236 & -0.367002 & -0.863756 \\
\hline C & -4.740500 & 1.096816 & 1.056160 \\
\hline C & -5.810893 & 0.217076 & -1.383349 \\
\hline $\mathrm{H}$ & -4.150578 & -1.162268 & -1.397397 \\
\hline C & -5.905681 & 1.710131 & 0.569118 \\
\hline $\mathrm{H}$ & -4.300350 & 1.432339 & 1.990962 \\
\hline C & -6.416841 & 1.252789 & -0.648752 \\
\hline $\mathrm{H}$ & -7.312094 & 1.713796 & -1.049295 \\
\hline C & 4.108824 & 0.098537 & -0.469942 \\
\hline C & 3.884529 & 1.451389 & -0.735020 \\
\hline C & 5.411472 & -0.334896 & -0.172660 \\
\hline C & 4.929753 & 2.387253 & -0.697250 \\
\hline $\mathrm{H}$ & 2.880158 & 1.774369 & -0.990041 \\
\hline C & 6.479631 & 0.562342 & -0.127157 \\
\hline $\mathrm{H}$ & 5.563674 & -1.385447 & 0.046256 \\
\hline C & 6.210179 & 1.917680 & -0.392565 \\
\hline $\mathrm{H}$ & 7.032670 & 2.621774 & -0.355378 \\
\hline C & 7.918932 & 0.125663 & 0.207029 \\
\hline C & 4.629683 & 3.869357 & -0.992966 \\
\hline C & 8.848893 & 0.466434 & -0.981925 \\
\hline H & 8.530369 & -0.056789 & -1.890879 \\
\hline H & 8.856844 & 1.539522 & -1.200141 \\
\hline
\end{tabular}




\begin{tabular}{|c|c|c|c|}
\hline $\mathrm{H}$ & 9.879657 & 0.164528 & -0.758973 \\
\hline C & 8.025531 & -1.387022 & 0.479066 \\
\hline $\mathrm{H}$ & 9.064562 & -1.647232 & 0.710607 \\
\hline $\mathrm{H}$ & 7.410593 & -1.692403 & 1.333137 \\
\hline $\mathrm{H}$ & 7.722978 & -1.981343 & -0.390806 \\
\hline C & 8.406769 & 0.874324 & 1.470304 \\
\hline $\mathrm{H}$ & 7.767445 & 0.647345 & 2.330870 \\
\hline $\mathrm{H}$ & 9.432328 & 0.574414 & 1.718685 \\
\hline $\mathrm{H}$ & 8.403272 & 1.960184 & 1.329165 \\
\hline C & 3.579463 & 4.390160 & 0.017691 \\
\hline $\mathrm{H}$ & 2.642567 & 3.826593 & -0.043090 \\
\hline $\mathrm{H}$ & 3.953107 & 4.310087 & 1.044919 \\
\hline $\mathrm{H}$ & 3.348958 & 5.444093 & -0.180848 \\
\hline C & 4.068945 & 4.004330 & -2.428726 \\
\hline $\mathrm{H}$ & 3.147844 & 3.427529 & -2.562010 \\
\hline $\mathrm{H}$ & 3.841033 & 5.053788 & -2.652426 \\
\hline $\mathrm{H}$ & 4.795531 & 3.649078 & -3.168443 \\
\hline C & 5.882077 & 4.759186 & -0.880529 \\
\hline $\mathrm{H}$ & 6.315115 & 4.729014 & 0.125831 \\
\hline $\mathrm{H}$ & 6.658195 & 4.465482 & -1.596514 \\
\hline $\mathrm{H}$ & 5.614674 & 5.800265 & -1.093969 \\
\hline C & -6.563394 & 2.845569 & 1.377889 \\
\hline C & -6.426222 & -0.227898 & -2.724259 \\
\hline C & -6.409234 & 0.962426 & -3.712975 \\
\hline $\mathrm{H}$ & -6.977755 & 1.817757 & -3.332027 \\
\hline $\mathrm{H}$ & -5.383326 & 1.298780 & -3.901010 \\
\hline $\mathrm{H}$ & -6.851604 & 0.668476 & -4.672974 \\
\hline C & -7.886659 & -0.686892 & -2.501798 \\
\hline $\mathrm{H}$ & -7.929166 & -1.530270 & -1.802988 \\
\hline $\mathrm{H}$ & -8.512590 & 0.114987 & -2.095973 \\
\hline $\mathrm{H}$ & -8.333572 & -1.009152 & -3.450500 \\
\hline C & -5.649582 & -1.394915 & -3.364650 \\
\hline $\mathrm{H}$ & -4.601865 & -1.138971 & -3.554169 \\
\hline $\mathrm{H}$ & -5.668512 & -2.288498 & -2.730053 \\
\hline $\mathrm{H}$ & -6.111106 & -1.660129 & -4.322950 \\
\hline C & -6.983496 & 2.309013 & 2.766958 \\
\hline $\mathrm{H}$ & -7.708949 & 1.493338 & 2.667934 \\
\hline $\mathrm{H}$ & -6.126562 & 1.926774 & 3.331444 \\
\hline $\mathrm{H}$ & -7.446615 & 3.106067 & 3.361933 \\
\hline C & -7.817551 & 3.413619 & 0.686337 \\
\hline $\mathrm{H}$ & -8.245594 & 4.214258 & 1.300187 \\
\hline $\mathrm{H}$ & -7.584032 & 3.839647 & -0.296095 \\
\hline $\mathrm{H}$ & -8.592587 & 2.650387 & 0.552608 \\
\hline C & -5.553247 & 4.003027 & 1.561295 \\
\hline $\mathrm{H}$ & -5.240089 & 4.406570 & 0.591597 \\
\hline $\mathrm{H}$ & -6.006277 & 4.818262 & 2.138936 \\
\hline $\mathrm{H}$ & -4.653376 & 3.678512 & 2.094021 \\
\hline
\end{tabular}


Frequencies (Top 3 out of 321)

$\begin{array}{lr}\text { 1. } & 7.7406 \mathrm{~cm}^{-1} \\ \text { 2. } & 11.9810 \mathrm{~cm}^{-1} \\ \text { 3. } & 14.1121 \mathrm{~cm}^{-1}\end{array}$

\section{B3LYP/6-31G(d) Molecular Geometry in Cartesian Coordinates}

C

C

C

$\mathrm{C}$

C

C

$\mathrm{H}$

$\mathrm{H}$

C

C

$\mathrm{H}$

$\mathrm{H}$

C

C

$\mathrm{H}$

$\mathrm{C}$

C

C

C

C

C

$\mathrm{H}$

$\mathrm{H}$

$\mathrm{H}$

C

C

$\mathrm{H}$

$\mathrm{H}$

C

C

C

$\mathrm{H}$
$-0.057397$

$-0.228888$

0.805214

2.029885

2.175036

1.155433

$-0.857433$

$-1.156828$

0.660702

3.068861

3. 110352

1. 277803

2.939131

1.708697

4.004328

$-1.253356$

$-2.245623$

$-2.055443$

$-0.814795$

0.190922

$-0.023229$

$-4.016647$

$-1.409414$

$-3.194805$

$-3.068870$

$-0.640783$

1.140457

0.758323

$-1.658226$

$-2.909155$

$-1.443613$

$-2.066830$
$-3.673730$

$-2.458000$

$-1.897644$

$-2.626319$

$-3.873940$

$-4.389675$

$-4.083558$

$-1.909118$

$-0.641902$

$-2.084816$

$-4.416916$

$-5.346374$

$-0.875820$

$-0.156585$

$-2.632484$

2.896305

2.670177

1. 742482

1.039387

1. 299104

2. 202427

2.009859

3.606396

3. 196914

1.486714

0.107778

0.779566

2. 386387

$-0.127601$

0.573383

$-1.128765$

$-0.868397$

\subsection{2}

3.050660

2. 246861

2.106085

2. 771196

3. 537762

4. 287438

3. 172927

1.568777

1. 311044

2. 658765

4.037889

0.656061

0.798593

1.231955

4.832956

3.907566

2.847415

2. 744947

3. 716712

4.733736

1.993781

5. 640659

3.973441

1.893827

1.669468

3. 646199

5.466031

0.746093

0.868656

$-0.379398$

$-1.235383$ 


\begin{tabular}{|c|c|c|c|}
\hline $\mathrm{H}$ & -0.401386 & -1.106476 & -0.713736 \\
\hline C & 1.474279 & 1.075417 & -0.033435 \\
\hline 0 & 1.252767 & 1.062176 & -1.223466 \\
\hline 0 & 1.518072 & 2.218559 & 0.691933 \\
\hline $\mathrm{H}$ & 1.303511 & 2.941760 & 0.070762 \\
\hline 0 & -1.829966 & -2.463587 & -0.023038 \\
\hline $\mathrm{H}$ & -1.241882 & -2.757119 & 0.691774 \\
\hline C & -4.050590 & 0.364718 & -0.071550 \\
\hline C & -4.556167 & -0.918001 & -0.327394 \\
\hline C & -4.665843 & 1.474400 & -0.670273 \\
\hline C & -5.654459 & -1.108181 & -1.173534 \\
\hline $\mathrm{H}$ & -4.068589 & -1.763548 & 0.140108 \\
\hline C & -5.768560 & 1.326830 & -1.519218 \\
\hline $\mathrm{H}$ & -4.252728 & 2.457144 & -0.473437 \\
\hline C & -6.239349 & 0.026841 & -1.752511 \\
\hline $\mathrm{H}$ & -7.090644 & -0.106866 & -2.413790 \\
\hline C & 4.069912 & -0.353110 & -0.160529 \\
\hline C & 4.691433 & -1.178747 & -1.100493 \\
\hline C & 4.551425 & 0.953619 & 0.027674 \\
\hline C & 5.779905 & -0.730650 & -1.864941 \\
\hline $\mathrm{H}$ & 4.297030 & -2.179364 & -1.250559 \\
\hline C & 5.630395 & 1.438788 & -0.712892 \\
\hline $\mathrm{H}$ & 4.078776 & 1.577215 & 0.776462 \\
\hline C & 6.226385 & 0.576574 & -1.650987 \\
\hline $\mathrm{H}$ & 7.063682 & 0.947393 & -2.230060 \\
\hline C & 6.177744 & 2.868576 & -0.538796 \\
\hline C & 6.423418 & -1.673258 & -2.900210 \\
\hline C & 5.416268 & 3.660921 & 0.541196 \\
\hline $\mathrm{H}$ & 4.350205 & 3.754370 & 0.305558 \\
\hline $\mathrm{H}$ & 5.505030 & 3.194306 & 1.528898 \\
\hline $\mathrm{H}$ & 5.829527 & 4.673129 & 0.615759 \\
\hline C & 6.044918 & 3.632122 & -1.878351 \\
\hline $\mathrm{H}$ & 6.428445 & 4.654730 & -1.774922 \\
\hline $\mathrm{H}$ & 6.606747 & 3.145806 & -2.682658 \\
\hline $\mathrm{H}$ & 4.996802 & 3.691184 & -2.193094 \\
\hline C & 7.668106 & 2.810646 & -0.127741 \\
\hline $\mathrm{H}$ & 8.280202 & 2.299048 & -0.877792 \\
\hline $\mathrm{H}$ & 8.069670 & 3.824214 & -0.006028 \\
\hline $\mathrm{H}$ & 7.791574 & 2.279866 & 0.823173 \\
\hline C & 5.362663 & -2.087182 & -3.947898 \\
\hline $\mathrm{H}$ & 4.515452 & -2.607434 & -3.489231 \\
\hline $\mathrm{H}$ & 4.969067 & -1.210317 & -4.474163 \\
\hline $\mathrm{H}$ & 5.804869 & -2.761155 & -4.692074 \\
\hline C & 6.956760 & -2.936863 & -2.183818 \\
\hline $\mathrm{H}$ & 6.157359 & -3.484396 & -1.673635 \\
\hline $\mathrm{H}$ & 7.418252 & -3.619903 & -2.907502 \\
\hline $\mathrm{H}$ & 7.713101 & -2.673263 & -1.435491 \\
\hline C & 7.599600 & -1.015371 & -3.646358 \\
\hline $\mathrm{H}$ & 7. 282381 & -0.128450 & -4.206483 \\
\hline $\mathrm{H}$ & 8.404460 & -0.720398 & -2.963305 \\
\hline $\mathrm{H}$ & 8.021978 & -1.725713 & -4.365976 \\
\hline C & -6.464007 & 2.524745 & -2.195522 \\
\hline C & -6.215501 & -2.508600 & -1.489569 \\
\hline C & -7.712876 & -2.572494 & -1.106621 \\
\hline
\end{tabular}




\begin{tabular}{|c|c|c|c|}
\hline $\mathrm{H}$ & -7.851513 & -2.384467 & -0.035739 \\
\hline $\mathrm{H}$ & -8.309863 & -1.836045 & -1.655042 \\
\hline $\mathrm{H}$ & -8.120994 & -3.565305 & -1.332872 \\
\hline C & -5.476814 & -3.621370 & -0.720540 \\
\hline $\mathrm{H}$ & -4.406701 & -3.643362 & -0.951600 \\
\hline $\mathrm{H}$ & -5.584733 & -3.502251 & 0.363815 \\
\hline $\mathrm{H}$ & -5.899918 & -4.596212 & -0.989754 \\
\hline C & -6.062973 & -2.786093 & -3.004300 \\
\hline $\mathrm{H}$ & -6.597169 & -2.046239 & -3.610274 \\
\hline $\mathrm{H}$ & -5.008322 & -2.763059 & -3.300949 \\
\hline $\mathrm{H}$ & -6.465264 & -3.775928 & -3.253516 \\
\hline C & -5.807695 & 3.869557 & -1.829173 \\
\hline $\mathrm{H}$ & -5.843620 & 4.061547 & -0.750749 \\
\hline $\mathrm{H}$ & -4.760479 & 3.912875 & -2.149371 \\
\hline $\mathrm{H}$ & -6.339945 & 4.687594 & -2.327886 \\
\hline C & -7.945044 & 2.577681 & -1.750237 \\
\hline $\mathrm{H}$ & -8.458230 & 3.421680 & -2.227793 \\
\hline $\mathrm{H}$ & -8.485347 & 1.664021 & -2.019175 \\
\hline $\mathrm{H}$ & -8.022877 & 2.703032 & -0.664075 \\
\hline C & -6.396988 & 2.363151 & -3.732763 \\
\hline $\mathrm{H}$ & -6.891503 & 1.445683 & -4.068332 \\
\hline $\mathrm{H}$ & -6.890588 & 3.208421 & -4.228291 \\
\hline $\mathrm{H}$ & -5.357202 & 2.327026 & -4.077161 \\
\hline
\end{tabular}

\section{Catalyst 2C 53}

Datum

Value

\begin{tabular}{lc}
\hline B3LYP/6-31G(d) Energy & -2164.797563 \\
\hline B3LYP-D3(BJ)/def2-TZVPP-IEF-PCM(DCM) Energy & -2165.895753 \\
\hline B3LYP-D3(BJ)/def2-TZVPP-IEF-PCM(DCM)//B3LYP/6-31G(d) Free Energy (Quasiharmonic) & -2165.017954 \\
\hline Number of Imaginary Frequencies & 0
\end{tabular}

Frequencies (Top 3 out of 321)

$\begin{array}{ll}\text { 1. } & 10.0100 \mathrm{~cm}^{-1} \\ \text { 2. } & 12.5096 \mathrm{~cm}^{-1} \\ \text { 3. } & 13.8813 \mathrm{~cm}^{-1}\end{array}$

\section{B3LYP/6-31G(d) Molecular Geometry in Cartesian Coordinates}

$\begin{array}{lrrr}C & 0.003843 & -4.837466 & -1.689283 \\ C & -0.173303 & -3.727602 & -0.890897 \\ C & 0.854106 & -2.749253 & -0.758219\end{array}$




\begin{tabular}{|c|c|c|c|}
\hline C & 2.086343 & -2.958218 & -1.457308 \\
\hline C & 2.234679 & -4.112789 & -2.272699 \\
\hline C & 1.217573 & -5.031895 & -2.390537 \\
\hline $\mathrm{H}$ & -0.791990 & -5.571946 & -1.775919 \\
\hline $\mathrm{H}$ & -1.100163 & -3.595327 & -0.342558 \\
\hline C & 0.703830 & -1.577086 & 0.055788 \\
\hline C & 3.125242 & -2.004936 & -1.327374 \\
\hline $\mathrm{H}$ & 3.172547 & -4.256051 & -2.803900 \\
\hline $\mathrm{H}$ & 1.344206 & -5.910071 & -3.017486 \\
\hline C & 2.993790 & -0.880543 & -0.537323 \\
\hline C & 1.760528 & -0.682466 & 0.164499 \\
\hline $\mathrm{H}$ & 4.044585 & -2.155172 & -1.887373 \\
\hline C & -0.895472 & -2.748052 & 4.840136 \\
\hline C & -1.963880 & -2.130977 & 4.232729 \\
\hline C & -1.870794 & -1.664670 & 2.893060 \\
\hline C & -0.648699 & -1.843200 & 2.170964 \\
\hline C & 0.437109 & -2.484880 & 2.830433 \\
\hline C & 0.316238 & -2.924351 & 4.129524 \\
\hline $\mathrm{H}$ & -3.890623 & -0.921328 & 2.801390 \\
\hline $\mathrm{H}$ & -0.976715 & -3.099861 & 5.865064 \\
\hline $\mathrm{H}$ & -2.899645 & -1.989572 & 4.768542 \\
\hline C & -2.960874 & -1.034761 & 2.249514 \\
\hline C & -0.577076 & -1.370724 & 0.819117 \\
\hline $\mathrm{H}$ & 1.374550 & -2.618680 & 2.301967 \\
\hline $\mathrm{H}$ & 1.159131 & -3.408209 & 4.615240 \\
\hline C & -1.668924 & -0.755293 & 0.209788 \\
\hline C & -2.894979 & -0.587531 & 0.942458 \\
\hline C & -1.548612 & -0.247546 & -1.218546 \\
\hline $\mathrm{H}$ & -2.206112 & 0.610193 & -1.365987 \\
\hline $\mathrm{H}$ & -0.522693 & 0.082595 & -1.411879 \\
\hline C & 1.657203 & 0.458778 & 1.139786 \\
\hline 0 & 2.253839 & 0.519084 & 2.189559 \\
\hline 0 & 0.808498 & 1.432927 & 0.721575 \\
\hline H & 0.782505 & 2.093477 & 1.441160 \\
\hline 0 & -1.957523 & -1.207471 & -2.203211 \\
\hline $\mathrm{H}$ & -1.331555 & -1.949098 & -2.167627 \\
\hline c & -4.105237 & 0.069248 & 0.363939 \\
\hline C & -4.642177 & -0.331476 & -0.862621 \\
\hline C & -4.754117 & 1.084257 & 1.091189 \\
\hline C & -5.803772 & 0.262140 & -1.382258 \\
\hline $\mathrm{H}$ & -4.135867 & -1.112529 & -1.418379 \\
\hline C & -5.913996 & 1.695565 & 0.612830 \\
\hline $\mathrm{H}$ & -4.317522 & 1.396407 & 2.033019 \\
\hline C & -6.418133 & 1.265986 & -0.628679 \\
\hline $\mathrm{H}$ & -7.314779 & 1.738830 & -1.012387 \\
\hline C & 4.107834 & 0.105713 & -0.462010 \\
\hline C & 3.881856 & 1.459538 & -0.720641 \\
\hline C & 5.410996 & -0.327593 & -0.166872 \\
\hline C & 4.925960 & 2.396457 & -0.678681 \\
\hline $\mathrm{H}$ & 2.877050 & 1.782502 & -0.973979 \\
\hline C & 6.478043 & 0.570761 & -0.117097 \\
\hline $\mathrm{H}$ & 5.564504 & -1.379004 & 0.046968 \\
\hline C & 6.206944 & 1.926984 & -0.376198 \\
\hline $\mathrm{H}$ & 7.028611 & 2.631867 & -0.335812 \\
\hline
\end{tabular}




\begin{tabular}{|c|c|c|c|}
\hline C & 7.917934 & 0.134351 & 0.214866 \\
\hline C & 4.624169 & 3.879513 & -0.967781 \\
\hline C & 8.847274 & 0.482047 & -0.972578 \\
\hline $\mathrm{H}$ & 8.529506 & -0.037537 & -1.883882 \\
\hline $\mathrm{H}$ & 8.853501 & 1.556127 & -1.185911 \\
\hline $\mathrm{H}$ & 9.878520 & 0.180754 & -0.751071 \\
\hline C & 8.026516 & -1.379485 & 0.479594 \\
\hline $\mathrm{H}$ & 9.065873 & -1.639492 & 0.709904 \\
\hline $\mathrm{H}$ & 7.411940 & -1.689773 & 1.332157 \\
\hline $\mathrm{H}$ & 7.724752 & -1.969963 & -0.393162 \\
\hline C & 8.405013 & 0.877560 & 1.481650 \\
\hline $\mathrm{H}$ & 7.766134 & 0.645656 & 2.341229 \\
\hline $\mathrm{H}$ & 9.430990 & 0.577743 & 1.728402 \\
\hline $\mathrm{H}$ & 8.400164 & 1.964082 & 1.345754 \\
\hline C & 3.573604 & 4.394690 & 0.045383 \\
\hline $\mathrm{H}$ & 2.637393 & 3.830219 & -0.017512 \\
\hline $\mathrm{H}$ & 3.947663 & 4.310713 & 1.072147 \\
\hline $\mathrm{H}$ & 3.341728 & 5.449174 & -0.148593 \\
\hline C & 4.062994 & 4.020178 & -2.402828 \\
\hline $\mathrm{H}$ & 3.142353 & 3.443180 & -2.538452 \\
\hline $\mathrm{H}$ & 3.834149 & 5.070394 & -2.621950 \\
\hline $\mathrm{H}$ & 4.789736 & 3.668747 & -3.144211 \\
\hline C & 5.875613 & 4.770207 & -0.851708 \\
\hline $\mathrm{H}$ & 6.308890 & 4.736134 & 0.154424 \\
\hline $\mathrm{H}$ & 6.651894 & 4.480470 & -1.569126 \\
\hline $\mathrm{H}$ & 5.607030 & 5.811916 & -1.060533 \\
\hline C & -6.640760 & 2.815514 & 1.382866 \\
\hline C & -6.342544 & -0.208109 & -2.746958 \\
\hline C & -5.262201 & 0.028580 & -3.830499 \\
\hline $\mathrm{H}$ & -5.035036 & 1.097178 & -3.924358 \\
\hline $\mathrm{H}$ & -4.327327 & -0.491902 & -3.600088 \\
\hline $\mathrm{H}$ & -5.618052 & -0.327621 & -4.805547 \\
\hline C & -7.619876 & 0.542346 & -3.167868 \\
\hline $\mathrm{H}$ & -8.437479 & 0.392390 & -2.453027 \\
\hline $\mathrm{H}$ & -7.446122 & 1.619917 & -3.268330 \\
\hline $\mathrm{H}$ & -7.961369 & 0.172300 & -4.141378 \\
\hline C & -6.674436 & -1.717787 & -2.677339 \\
\hline $\mathrm{H}$ & -5.792206 & -2.316849 & -2.430469 \\
\hline $\mathrm{H}$ & -7.439203 & -1.917354 & -1.917558 \\
\hline $\mathrm{H}$ & -7.056231 & -2.068514 & -3.644250 \\
\hline C & -8.096833 & 2.381572 & 1.675649 \\
\hline $\mathrm{H}$ & -8.656836 & 2.181947 & 0.756156 \\
\hline $\mathrm{H}$ & -8.119379 & 1.470093 & 2.284012 \\
\hline $\mathrm{H}$ & -8.627402 & 3.169755 & 2.224150 \\
\hline C & -6.653551 & 4.105309 & 0.528188 \\
\hline $\mathrm{H}$ & -7.173932 & 4.911830 & 1.059526 \\
\hline $\mathrm{H}$ & -5.632763 & 4.442429 & 0.314458 \\
\hline $\mathrm{H}$ & -7.162111 & 3.954235 & -0.429588 \\
\hline C & -5.962310 & 3.140836 & 2.727354 \\
\hline $\mathrm{H}$ & -4.933683 & 3.493542 & 2.591341 \\
\hline $\mathrm{H}$ & -6.518164 & 3.934935 & 3.238885 \\
\hline $\mathrm{H}$ & -5.939515 & 2.271149 & 3.394001 \\
\hline
\end{tabular}




\section{Catalyst 2C 54}

Datum

Value

\begin{tabular}{lc}
\hline B3LYP/6-31G(d) Energy & -2164.797289 \\
\hline B3LYP-D3(BJ)/def2-TZVPP-IEF-PCM(DCM) Energy & -2165.895442 \\
\hline B3LYP-D3(BJ)/def2-TZVPP-IEF-PCM(DCM)//B3LYP/6-31G(d) Free Energy (Quasiharmonic) & -2165.017946
\end{tabular}

Number of Imaginary Frequencies

Frequencies (Top 3 out of 321)

$\begin{array}{ll}\text { 1. } & 10.1199 \mathrm{~cm}^{-1} \\ \text { 2. } & 11.6214 \mathrm{~cm}^{-1} \\ \text { 3. } & 12.9275 \mathrm{~cm}^{-1}\end{array}$

\section{B3LYP/6-31G(d) Molecular Geometry in Cartesian Coordinates}

C

C

C

C

C

C

$\mathrm{H}$

$\mathrm{H}$

C

C

$\mathrm{H}$

$\mathrm{H}$

C

C

$\mathrm{H}$

C

C

C

C

C

C

$\mathrm{H}$

$\mathrm{H}$

$\mathrm{H}$

C

C

$\mathrm{H}$

$\mathrm{H}$

C
$-0.064464$

$-0.231639$

0.803316

2.024319

2.164841

1.144606

$-0.865031$

$-1.156792$

0.662737

3.064240

3.097216

1. 263511

2. 939132

1.711895

3.996228

$-1.227894$

$-2.222359$

$-2.038892$

$-0.802546$

0.205527

$-0.002179$

$-3.999385$

$-1.378899$

- 3.168249

$-3.054851$

$-0.635065$

1.151555

0.780990

$-1.654862$
$-3.666079$

$-2.452659$

$-1.901059$

$-2.636277$

$-3.881485$

$-4.388540$

$-4.069103$

$-1.898886$

$-0.648451$

$-2.103654$

$-4.429552$

$-5.343389$

$-0.896152$

$-0.171396$

$-2.657141$

2.927850

2.699611

1.761358

1.049908

1. 311903

2. 225474

2.032760

3.646018

3. 232697

1. 503051

0.107944

0.785462

2.410867

$-0.129598$
3.695739

3.063544

2. 254769

2.114799

2. 785442

3.556908

4.309724

3. 185042

1.570282

1. 314900

2.673346

4.061379

0.656501

0.796741

1. 234761

4.806747

3.884238

2.832216

2.734874

3. 703591

4.712773

1.978099

5.608246

3.946312

1.882002

1.667422

3.637116

5.442983

0.747230 


\begin{tabular}{|c|c|c|c|}
\hline C & -2.901558 & 0.579541 & 0.864993 \\
\hline C & -1.446819 & -1.140690 & -0.370616 \\
\hline $\mathrm{H}$ & -2.069939 & -0.883744 & -1.227699 \\
\hline H & -0.404955 & -1.125992 & -0.706493 \\
\hline C & 1.481213 & 1.056388 & -0.042910 \\
\hline 0 & 1.242457 & 1.035084 & -1.229329 \\
\hline 0 & 1.549168 & 2.205973 & 0.671209 \\
\hline H & 1.337660 & 2.926182 & 0.045416 \\
\hline 0 & -1.839402 & -2.470749 & -0.003441 \\
\hline H & -1.250300 & -2.762623 & 0.711203 \\
\hline C & -4.044993 & 0.368463 & -0.072227 \\
\hline C & -4.558472 & -0.913799 & -0.314204 \\
\hline C & -4.653572 & 1.475470 & -0.682557 \\
\hline C & -5.658260 & -1.106302 & -1.157845 \\
\hline $\mathrm{H}$ & -4.075462 & -1.757287 & 0.161657 \\
\hline C & -5.757366 & 1.325519 & -1.529737 \\
\hline $\mathrm{H}$ & -4.234236 & 2.457698 & -0.496534 \\
\hline C & -6.236372 & 0.026042 & -1.748725 \\
\hline $\mathrm{H}$ & -7.088639 & -0.109596 & -2.408353 \\
\hline C & 4.069745 & -0.378824 & -0.163754 \\
\hline C & 4.559503 & 0.916097 & 0.021475 \\
\hline C & 4.680879 & -1.212455 & -1.115500 \\
\hline C & 5.639321 & 1.403988 & -0.731201 \\
\hline H & 4.101204 & 1.545579 & 0.777189 \\
\hline C & 5.757478 & -0.767084 & -1.883707 \\
\hline H & 4.276380 & -2.207765 & -1.258216 \\
\hline C & 6.215654 & 0.546642 & -1.672257 \\
\hline $\mathrm{H}$ & 7.047265 & 0.903700 & -2.267985 \\
\hline C & 6.434699 & -1.646032 & -2.952981 \\
\hline C & 6.140714 & 2.840994 & -0.489821 \\
\hline C & 5.836751 & -3.064572 & -3.011484 \\
\hline H & 5.946902 & -3.593052 & -2.057438 \\
\hline $\mathrm{H}$ & 4.773193 & -3.049976 & -3.274363 \\
\hline H & 6.356716 & -3.652328 & -3.776389 \\
\hline C & 7.944327 & -1.776941 & -2.640058 \\
\hline $\mathrm{H}$ & 8.436066 & -2.401382 & -3.396030 \\
\hline $\mathrm{H}$ & 8.448761 & -0.805081 & -2.634847 \\
\hline $\mathrm{H}$ & 8.103642 & -2.241612 & -1.660193 \\
\hline C & 6.254167 & -0.989952 & -4.342778 \\
\hline $\mathrm{H}$ & 6.698218 & 0.010266 & -4.380922 \\
\hline $\mathrm{H}$ & 6.734518 & -1.599218 & -5.118415 \\
\hline $\mathrm{H}$ & 5.191882 & -0.893776 & -4.593997 \\
\hline C & 6.609608 & 2.983380 & 0.977884 \\
\hline H & 5.801153 & 2.774537 & 1.686162 \\
\hline $\mathrm{H}$ & 7.431930 & 2.292196 & 1.195353 \\
\hline $\mathrm{H}$ & 6.963946 & 4.004059 & 1.167792 \\
\hline C & 4.986028 & 3.836620 & -0.756285 \\
\hline $\mathrm{H}$ & 4.129114 & 3.648831 & -0.100813 \\
\hline $\mathrm{H}$ & 5.321656 & 4.866239 & -0.581175 \\
\hline $\mathrm{H}$ & 4.638033 & 3.763179 & -1.792988 \\
\hline C & 7.318615 & 3.218435 & -1.407695 \\
\hline H & 8.187684 & 2.570634 & -1.245089 \\
\hline $\mathrm{H}$ & 7.044515 & 3.164736 & -2.467491 \\
\hline $\mathrm{H}$ & 7.632258 & 4.247702 & -1.200031 \\
\hline
\end{tabular}




\begin{tabular}{|llll|} 
C & -6.444837 & 2.520127 & -2.219841 \\
C & -6.227733 & -2.506585 & -1.458989 \\
H & -7.725473 & -2.557395 & -1.075261 \\
H & -7.862962 & -2.357175 & -0.006433 \\
H & -8.318054 & -1.823190 & -1.631443 \\
C & -8.139705 & -3.550023 & -1.290911 \\
H & -5.495559 & -3.615465 & -0.678220 \\
H & -4.425842 & -3.646960 & -0.910042 \\
H & -5.601593 & -3.483399 & 0.404810 \\
C & -5.925209 & -4.590519 & -0.936042 \\
H & -6.077208 & -2.801097 & -2.970678 \\
H & -6.607494 & -2.064828 & -3.584425 \\
H & -5.022538 & -2.787118 & -3.267796 \\
C & -6.485063 & -3.791324 & -3.209111 \\
H & -7.926180 & 2.586988 & -1.777393 \\
H & -8.471762 & 1.673733 & -2.036880 \\
H & -8.004932 & 2.725004 & -0.692830 \\
C & -8.433425 & 3.428694 & -2.265218 \\
H & -6.376764 & 2.341346 & -3.755133 \\
H & -6.864706 & 3.184038 & -4.260560 \\
H & -5.336778 & 2.295265 & -4.097740 \\
C & -6.876191 & 1.423144 & -4.081310 \\
H & -5.780825 & 3.864863 & -1.867302 \\
H & -4.733085 & 3.898323 & -2.186928 \\
H & -6.307591 & 4.680686 & -2.375383 \\
& -5.816577 & 4.068607 & -0.791041 \\
\hline & & & \\
\hline & & & \\
\hline
\end{tabular}

\section{Catalyst 2C 55}

Datum

Value

\begin{tabular}{lc}
\hline B3LYP/6-31G(d) Energy & -2164.79728 \\
\hline B3LYP-D3(BJ)/def2-TZVPP-IEF-PCM(DCM) Energy & -2165.895418 \\
\hline B3LYP-D3(BJ)/def2-TZVPP-IEF-PCM(DCM)//B3LYP/6-31G(d) Free Energy (Quasiharmonic) & -2165.017896 \\
\hline
\end{tabular}

Number of Imaginary Frequencies

Frequencies (Top 3 out of 321)

$\begin{array}{lr}\text { 1. } & 9.4756 \mathrm{~cm}^{-1} \\ \text { 2. } & 10.4896 \mathrm{~cm}^{-1} \\ \text { 3. } & 12.9484 \mathrm{~cm}^{-1}\end{array}$




\begin{tabular}{|c|c|c|c|}
\hline C & 0.088441 & -3.773617 & 3.572822 \\
\hline C & 0.256292 & -2.541410 & 2.978107 \\
\hline C & -0.780171 & -1.962616 & 2.190569 \\
\hline C & -2.003759 & -2.689666 & 2.032796 \\
\hline C & -2.144952 & -3.954777 & 2.664860 \\
\hline C & -1.122932 & -4.488416 & 3.415838 \\
\hline $\mathrm{H}$ & 0.890027 & -4.197385 & 4.171315 \\
\hline $\mathrm{H}$ & 1.182751 & -1.993163 & 3.114069 \\
\hline C & -0.638771 & -0.689651 & 1.544877 \\
\hline C & -3.045884 & -2.128824 & 1.255260 \\
\hline$H$ & -3.079317 & -4.496666 & 2.539925 \\
\hline $\mathrm{H}$ & -1.242484 & -5.458300 & 3.890606 \\
\hline C & -2.919727 & -0.902082 & 0.633679 \\
\hline C & -1.689684 & -0.185672 & 0.790907 \\
\hline $\mathrm{H}$ & -3.980713 & -2.675685 & 1.163629 \\
\hline C & 1.282335 & 2.783153 & 4.875998 \\
\hline C & 2.270596 & 2.579228 & 3.941220 \\
\hline C & 2.077683 & 1.673266 & 2.862970 \\
\hline C & 0.838511 & 0.968781 & 2.751890 \\
\hline C & -0.163141 & 1.205411 & 3.733784 \\
\hline C & 0.053681 & 2.087919 & 4.768347 \\
\hline $\mathrm{H}$ & 4.033208 & 1.965823 & 2.004310 \\
\hline $\mathrm{H}$ & 1.440415 & 3.476682 & 5.697567 \\
\hline $\mathrm{H}$ & 3.218627 & 3.107206 & 4.013285 \\
\hline C & 3.086857 & 1.441191 & 1.898840 \\
\hline C & 0.662226 & 0.058885 & 1.658376 \\
\hline$H$ & -1.111654 & 0.684801 & 3.657408 \\
\hline $\mathrm{H}$ & -0.724700 & 2.254307 & 5.508185 \\
\hline C & 1.676468 & -0.155145 & 0.726244 \\
\hline C & 2.924878 & 0.548574 & 0.856050 \\
\hline C & 1.462147 & -1.137923 & -0.415087 \\
\hline $\mathrm{H}$ & 0.417362 & -1.120038 & -0.741285 \\
\hline $\mathrm{H}$ & 2.076734 & -0.858450 & -1.271163 \\
\hline C & -1.460098 & 1.066286 & -0.012161 \\
\hline 0 & -1.239908 & 1.080609 & -1.202411 \\
\hline 0 & -1.507030 & 2.192374 & 0.738927 \\
\hline $\mathrm{H}$ & -1.296868 & 2.930252 & 0.133711 \\
\hline 0 & 1.863408 & -2.475124 & -0.082928 \\
\hline $\mathrm{H}$ & 1.278142 & -2.789141 & 0.625566 \\
\hline C & 4.061968 & 0.365275 & -0.094752 \\
\hline C & 4.571884 & -0.906431 & -0.387229 \\
\hline C & 4.673773 & 1.487418 & -0.670611 \\
\hline C & 5.668715 & -1.073527 & -1.241609 \\
\hline $\mathrm{H}$ & 4.089669 & -1.769249 & 0.058615 \\
\hline C & 5.775285 & 1.365039 & -1.526463 \\
\hline $\mathrm{H}$ & 4.260149 & 2.468055 & -0.452335 \\
\hline C & 6.254063 & 0.074295 & -1.795365 \\
\hline $\mathrm{H}$ & 7.101937 & -0.039588 & -2.457104 \\
\hline C & -4.054222 & -0.356171 & -0.162444 \\
\hline C & -4.544314 & 0.938679 & 0.078378 \\
\hline C & -4.670440 & -1.147866 & -1.134366 \\
\hline C & -5.628085 & 1.445137 & -0.640654 \\
\hline $\mathrm{H}$ & -4.074563 & 1.535188 & 0.850668 \\
\hline
\end{tabular}




\begin{tabular}{|c|c|c|c|}
\hline C & -5.763073 & -0.677126 & -1.879116 \\
\hline $\mathrm{H}$ & -4.268877 & -2.138662 & -1.325014 \\
\hline C & -6.219116 & 0.616904 & -1.611904 \\
\hline $\mathrm{H}$ & -7.060124 & 1.004478 & -2.174362 \\
\hline C & -6.397623 & -1.580233 & -2.954433 \\
\hline C & -6.186578 & 2.862273 & -0.407981 \\
\hline C & -6.896308 & -2.890995 & -2.300465 \\
\hline $\mathrm{H}$ & -6.080449 & -3.448018 & -1.828028 \\
\hline $\mathrm{H}$ & -7.650290 & -2.684451 & -1.532156 \\
\hline $\mathrm{H}$ & -7.350232 & -3.544320 & -3.055654 \\
\hline C & -5.337924 & -1.916116 & -4.030893 \\
\hline $\mathrm{H}$ & -5.769723 & -2.567372 & -4.800972 \\
\hline $\mathrm{H}$ & -4.974738 & -1.005215 & -4.520042 \\
\hline $\mathrm{H}$ & -4.471518 & -2.432166 & -3.604685 \\
\hline C & -7.595550 & -0.911863 & -3.655127 \\
\hline $\mathrm{H}$ & -7.303455 & 0.006946 & -4.176031 \\
\hline $\mathrm{H}$ & -8.012258 & -1.595695 & -4.403137 \\
\hline $\mathrm{H}$ & -8.397282 & -0.666532 & -2.949064 \\
\hline C & -7.675188 & 2.775397 & 0.004508 \\
\hline $\mathrm{H}$ & -8.285345 & 2.289959 & -0.764293 \\
\hline $\mathrm{H}$ & -7.791610 & 2.205137 & 0.933190 \\
\hline $\mathrm{H}$ & -8.084481 & 3.779818 & 0.168696 \\
\hline C & -6.064728 & 3.680472 & -1.715966 \\
\hline $\mathrm{H}$ & -6.626762 & 3.223617 & -2.537270 \\
\hline $\mathrm{H}$ & -6.455171 & 4.695113 & -1.568954 \\
\hline $\mathrm{H}$ & -5.018481 & 3.760101 & -2.032335 \\
\hline C & -5.428207 & 3.616968 & 0.700683 \\
\hline $\mathrm{H}$ & -5.510039 & 3.110243 & 1.669022 \\
\hline $\mathrm{H}$ & -4.363681 & 3.728689 & 0.465994 \\
\hline $\mathrm{H}$ & -5.849628 & 4.621812 & 0.817404 \\
\hline C & 6.404095 & 2.629163 & -2.145933 \\
\hline C & 6.179409 & -2.496793 & -1.540405 \\
\hline C & 5.044399 & -3.316669 & -2.200915 \\
\hline $\mathrm{H}$ & 4.152593 & -3.367177 & -1.568526 \\
\hline $\mathrm{H}$ & 4.750239 & -2.870255 & -3.158195 \\
\hline $\mathrm{H}$ & 5.380860 & -4.342804 & -2.396402 \\
\hline C & 6.601701 & -3.181128 & -0.218496 \\
\hline $\mathrm{H}$ & 7.407093 & -2.622985 & 0.273277 \\
\hline $\mathrm{H}$ & 5.766386 & -3.254269 & 0.484909 \\
\hline $\mathrm{H}$ & 6.963463 & -4.198129 & -0.414834 \\
\hline C & 7.392407 & -2.503151 & -2.489852 \\
\hline $\mathrm{H}$ & 7.151737 & -2.058782 & -3.462427 \\
\hline $\mathrm{H}$ & 8.247088 & -1.961424 & -2.067948 \\
\hline $\mathrm{H}$ & 7.712897 & -3.535629 & -2.669976 \\
\hline C & 6.859920 & 3.586755 & -1.019452 \\
\hline $\mathrm{H}$ & 6.023842 & 3.897920 & -0.384680 \\
\hline $\mathrm{H}$ & 7.607087 & 3.106732 & -0.377017 \\
\hline $\mathrm{H}$ & 7.309118 & 4.492649 & -1.445154 \\
\hline C & 5.354226 & 3.343050 & -3.030520 \\
\hline $\mathrm{H}$ & 5.779303 & 4.253662 & -3.471079 \\
\hline $\mathrm{H}$ & 5.023745 & 2.692347 & -3.848240 \\
\hline $\mathrm{H}$ & 4.467505 & 3.632009 & -2.456772 \\
\hline C & 7.630355 & 2.311146 & -3.022410 \\
\hline $\mathrm{H}$ & 7.371791 & 1.666682 & -3.870111 \\
\hline
\end{tabular}




\section{Catalyst 2C 56}

Datum

Value

\begin{tabular}{lr}
\hline B3LYP/6-31G(d) Energy & -2164.798508 \\
\hline B3LYP-D3(BJ)/def2-TZVPP-IEF-PCM(DCM) Energy & -2165.895917 \\
\hline B3LYP-D3(BJ)/def2-TZVPP-IEF-PCM(DCM)//B3LYP/6-31G(d) Free Energy (Quasiharmonic) & -2165.017855 \\
\hline
\end{tabular}

Number of Imaginary Frequencies

Frequencies (Top 3 out of 321)

$\begin{array}{ll}\text { 1. } & 11.2575 \mathrm{~cm}^{-1} \\ \text { 2. } & 13.2243 \mathrm{~cm}^{-1} \\ \text { 3. } & 13.5380 \mathrm{~cm}^{-1}\end{array}$

\section{B3LYP/6-31G(d) Molecular Geometry in Cartesian Coordinates}

C

C

C

C

C

C

$\mathrm{H}$

$\mathrm{H}$

C

C

$\mathrm{H}$

$\mathrm{H}$

C

C

$\mathrm{H}$

C

C

C

C

C

C

$\mathrm{H}$

$\mathrm{H}$

$\mathrm{H}$
$-0.029212$

0.165159

$-0.856824$

$-2.099819$

$-2.266395$

$-1.255350$

0.762837

1.102486

$-0.688664$

$-3.129215$

$-3.212368$

$-1.395443$

$-2.976713$

$-1.734050$

$-4.056115$

0.952762

2.008759

1.903780

0.681810

$-0.390602$

$-0.258173$

3.913364

1.044062

2.944347
$-4.841760$

$-3.749669$

$-2.771593$

$-2.958748$

$-4.096715$

$-5.017584$

$-5.576176$

$-3.629200$

$-1.617190$

$-1.999579$

$-4.224765$

$-5.882448$

$-0.885259$

$-0.713308$

$-2.133091$

$-2.978925$

$-2.319329$

$-1.797993$

$-1.964474$

$-2.652441$

$-3.145556$

$-1.024072$

$-3.373505$

$-2.186340$
$-1.808900$

$-0.990694$

$-0.820795$

$-1.507343$

$-2.342412$

$-2.493654$

$-1.925547$

$-0.457602$

0.013415

$-1.348614$

$-2.863015$

$-3.136097$

$-0.547218$

0.144963

$-1.900341$

4.731318

4.146939

2.828591

2.103500

2.738092

4.017003

2. 759868

5.739736

4.685225 


\begin{tabular}{|c|c|c|c|}
\hline C & 2.982280 & -1.126284 & 2.208041 \\
\hline C & 0.596541 & -1.433165 & 0.774593 \\
\hline $\mathrm{H}$ & -1.325595 & -2.783880 & 2.204359 \\
\hline $\mathrm{H}$ & -1.089939 & -3.667333 & 4.482610 \\
\hline C & 1.674858 & -0.773857 & 0.189323 \\
\hline C & 2.903066 & -0.624645 & 0.921888 \\
\hline C & 1.537102 & -0.200063 & -1.211759 \\
\hline $\mathrm{H}$ & 0.510675 & 0.144186 & -1.373205 \\
\hline $\mathrm{H}$ & 2.195344 & 0.661283 & -1.328608 \\
\hline C & -1.542565 & 0.476841 & 1.043883 \\
\hline 0 & -0.813050 & 1.418042 & 0.812839 \\
\hline 0 & -2.282371 & 0.382458 & 2.169949 \\
\hline $\mathrm{H}$ & -2.105422 & 1.196420 & 2.680243 \\
\hline 0 & 1.930001 & -1.118160 & -2.242734 \\
\hline $\mathrm{H}$ & 1.302802 & -1.859269 & -2.229914 \\
\hline C & 4.102296 & 0.067037 & 0.362551 \\
\hline C & 4.634019 & -0.281809 & -0.881991 \\
\hline C & 4.745068 & 1.060729 & 1.123330 \\
\hline C & 5.786240 & 0.342338 & -1.386264 \\
\hline $\mathrm{H}$ & 4.130545 & -1.045438 & -1.464205 \\
\hline C & 5.895243 & 1.701411 & 0.660313 \\
\hline $\mathrm{H}$ & 4.309833 & 1.335017 & 2.077324 \\
\hline C & 6.396073 & 1.322838 & -0.598994 \\
\hline $\mathrm{H}$ & 7.285523 & 1.818646 & -0.970132 \\
\hline C & -4.077714 & 0.114247 & -0.451239 \\
\hline C & -3.828899 & 1.473374 & -0.687543 \\
\hline C & -5.383375 & -0.305370 & -0.162241 \\
\hline C & -4.857898 & 2.419652 & -0.627018 \\
\hline $\mathrm{H}$ & -2.820806 & 1.781576 & -0.934797 \\
\hline C & -6.441220 & 0.608437 & -0.095970 \\
\hline $\mathrm{H}$ & -5.552744 & -1.358204 & 0.032144 \\
\hline C & -6.148605 & 1.959983 & -0.330152 \\
\hline $\mathrm{H}$ & -6.957661 & 2.682530 & -0.279175 \\
\hline C & -7.887465 & 0.184311 & 0.225703 \\
\hline C & -4.612542 & 3.920707 & -0.875910 \\
\hline C & -8.016883 & -1.333124 & 0.458864 \\
\hline $\mathrm{H}$ & -7.721024 & -1.909153 & -0.425415 \\
\hline $\mathrm{H}$ & -7.408535 & -1.669680 & 1.306003 \\
\hline $\mathrm{H}$ & -9.060113 & -1.584031 & 0.681646 \\
\hline C & -8.810191 & 0.568366 & -0.955608 \\
\hline $\mathrm{H}$ & -9.845846 & 0.277307 & -0.741283 \\
\hline $\mathrm{H}$ & -8.800792 & 1.646308 & -1.148106 \\
\hline $\mathrm{H}$ & -8.498310 & 0.062374 & -1.876473 \\
\hline C & -8.367116 & 0.907840 & 1.506501 \\
\hline $\mathrm{H}$ & -8.345372 & 1.996801 & 1.393573 \\
\hline $\mathrm{H}$ & -9.398140 & 0.618558 & 1.744564 \\
\hline $\mathrm{H}$ & -7.734653 & 0.648123 & 2.363054 \\
\hline C & -5.022743 & 4.722983 & 0.382169 \\
\hline $\mathrm{H}$ & -6.079638 & 4.581685 & 0.631812 \\
\hline $\mathrm{H}$ & -4.429903 & 4.417611 & 1.251981 \\
\hline $\mathrm{H}$ & -4.859075 & 5.795309 & 0.219659 \\
\hline C & -5.461003 & 4.390523 & -2.081142 \\
\hline $\mathrm{H}$ & -6.532338 & 4.239864 & -1.910609 \\
\hline $\mathrm{H}$ & -5.299932 & 5.459403 & -2.267621 \\
\hline
\end{tabular}




\begin{tabular}{|c|c|c|c|}
\hline $\mathrm{H}$ & -5.186653 & 3.842519 & -2.989755 \\
\hline C & -3.133502 & 4.229186 & -1.178941 \\
\hline $\mathrm{H}$ & -2.472692 & 3.924151 & -0.360025 \\
\hline $\mathrm{H}$ & -2.792612 & 3.729905 & -2.093022 \\
\hline $\mathrm{H}$ & -3.005293 & 5.307557 & -1.326128 \\
\hline C & 6.615342 & 2.800836 & 1.465592 \\
\hline C & 6.319684 & -0.069938 & -2.771774 \\
\hline C & 7.585866 & 0.709488 & -3.173116 \\
\hline $\mathrm{H}$ & 7.400410 & 1.788285 & -3.230073 \\
\hline $\mathrm{H}$ & 8.410612 & 0.540317 & -2.470897 \\
\hline $\mathrm{H}$ & 7.923070 & 0.380975 & -4.162880 \\
\hline C & 5.228919 & 0.196211 & -3.837900 \\
\hline $\mathrm{H}$ & 4.301489 & -0.343363 & -3.621730 \\
\hline $\mathrm{H}$ & 4.988920 & 1.264887 & -3.888513 \\
\hline $\mathrm{H}$ & 5.581774 & -0.117818 & -4.828482 \\
\hline C & 6.668040 & -1.577436 & -2.762179 \\
\hline $\mathrm{H}$ & 7.442539 & -1.796993 & -2.017904 \\
\hline $\mathrm{H}$ & 5.794702 & -2.194889 & -2.529447 \\
\hline $\mathrm{H}$ & 7.043716 & -1.887535 & -3.745261 \\
\hline C & 5.934215 & 3.080960 & 2.818808 \\
\hline $\mathrm{H}$ & 4.902984 & 3.429423 & 2.692543 \\
\hline $\mathrm{H}$ & 5.918155 & 2.192071 & 3.459870 \\
\hline $\mathrm{H}$ & 6.484129 & 3.863913 & 3.353506 \\
\hline C & 6.621717 & 4.117050 & 0.652238 \\
\hline $\mathrm{H}$ & 7.134505 & 4.909769 & 1.211048 \\
\hline $\mathrm{H}$ & 7.135119 & 4.000751 & -0.307857 \\
\hline $\mathrm{H}$ & 5.599409 & 4.453544 & 0.445457 \\
\hline C & 8.073345 & 2.365061 & 1.745563 \\
\hline $\mathrm{H}$ & 8.633919 & 2.194340 & 0.820556 \\
\hline $\mathrm{H}$ & 8.600722 & 3.139152 & 2.316850 \\
\hline $\mathrm{H}$ & 8.099697 & 1.436332 & 2.327202 \\
\hline
\end{tabular}

\section{Catalyst 2C 57}

Datum

Value

\begin{tabular}{lc}
\hline B3LYP/6-31G(d) Energy & -2164.800125 \\
\hline B3LYP-D3(BJ)/def2-TZVPP-IEF-PCM(DCM) Energy & -2165.896249 \\
\hline
\end{tabular}

B3LYP-D3(BJ)/def2-TZVPP-IEF-PCM(DCM)//B3LYP/6-31G(d) Free Energy (Quasiharmonic) $\quad$-2165.017848

Number of Imaginary Frequencies

Frequencies (Top 3 out of 321)

$\begin{array}{ll}\text { 1. } & 11.4884 \mathrm{~cm}^{-1} \\ \text { 2. } & 12.7601 \mathrm{~cm}^{-1} \\ \text { 3. } & 13.5621 \mathrm{~cm}^{-1}\end{array}$




\begin{tabular}{|c|c|c|c|}
\hline C & 0.294467 & -3.349897 & 3.972894 \\
\hline C & 0.415475 & -2.805320 & 2.713559 \\
\hline C & -0.664286 & -2.096611 & 2.118574 \\
\hline C & -1.881635 & -1.958267 & 2.858937 \\
\hline C & -1.973182 & -2.531025 & 4.156319 \\
\hline C & -0.910019 & -3.211612 & 4.703286 \\
\hline $\mathrm{H}$ & 1.129085 & -3.891225 & 4.409768 \\
\hline $\mathrm{H}$ & 1.340880 & -2.915553 & 2.158599 \\
\hline C & -0.574982 & -1.512056 & 0.811779 \\
\hline C & -2.969992 & -1.260379 & 2.280060 \\
\hline $\mathrm{H}$ & -2.902776 & -2.420643 & 4.709461 \\
\hline $\mathrm{H}$ & -0.991644 & -3.644996 & 5.696349 \\
\hline C & -2.903437 & -0.716534 & 1.012935 \\
\hline C & -1.678589 & -0.858229 & 0.282695 \\
\hline $\mathrm{H}$ & -3.895068 & -1.175792 & 2.844476 \\
\hline C & 1.350915 & -4.905749 & -2.585516 \\
\hline C & 2.326564 & -3.949636 & -2.425379 \\
\hline C & 2.126289 & -2.844429 & -1.554612 \\
\hline C & 0.892093 & -2.724606 & -0.843895 \\
\hline C & -0.097120 & -3.728400 & -1.036143 \\
\hline C & 0.127992 & -4.791349 & -1.881934 \\
\hline $\mathrm{H}$ & 4.052794 & -1.952751 & -1.921755 \\
\hline $\mathrm{H}$ & 1.513838 & -5.748180 & -3.252351 \\
\hline $\mathrm{H}$ & 3.269485 & -4.024546 & -2.961965 \\
\hline C & 3.122164 & -1.857570 & -1.368062 \\
\hline C & 0.710627 & -1.604060 & 0.033146 \\
\hline $\mathrm{H}$ & -1.043597 & -3.646336 & -0.513391 \\
\hline $\mathrm{H}$ & -0.641796 & -5.546533 & -2.015681 \\
\hline C & 1.711168 & -0.645703 & 0.198886 \\
\hline C & 2.950031 & -0.776159 & -0.524414 \\
\hline C & 1.511294 & 0.512743 & 1.168115 \\
\hline $\mathrm{H}$ & 0.828252 & 0.213615 & 1.971583 \\
\hline $\mathrm{H}$ & 2.466815 & 0.771981 & 1.628925 \\
\hline C & -1.573650 & -0.272936 & -1.098041 \\
\hline 0 & -1.678347 & -0.875421 & -2.140590 \\
\hline 0 & -1.337383 & 1.069159 & -1.059047 \\
\hline $\mathrm{H}$ & -1.285027 & 1.369990 & -1.987734 \\
\hline 0 & 1.052555 & 1.716885 & 0.550514 \\
\hline $\mathrm{H}$ & 0.188304 & 1.538185 & 0.140883 \\
\hline C & 4.089906 & 0.178875 & -0.374869 \\
\hline C & 3.922219 & 1.561670 & -0.533744 \\
\hline C & 5.374397 & -0.326569 & -0.117525 \\
\hline C & 5.007036 & 2.440406 & -0.431039 \\
\hline $\mathrm{H}$ & 2.925463 & 1.936650 & -0.721339 \\
\hline C & 6.485060 & 0.517640 & -0.011893 \\
\hline $\mathrm{H}$ & 5.485900 & -1.396290 & 0.017815 \\
\hline C & 6.271671 & 1.894347 & -0.171295 \\
\hline $\mathrm{H}$ & 7.120684 & 2.565861 & -0.082715 \\
\hline C & -4.085879 & -0.006370 & 0.447539 \\
\hline C & -4.607772 & -0.350991 & -0.801853 \\
\hline
\end{tabular}




\begin{tabular}{|c|c|c|c|}
\hline C & -4.712305 & 1.005327 & 1.196126 \\
\hline C & -5.735946 & 0.299545 & -1.327390 \\
\hline $\mathrm{H}$ & -4.134936 & -1.147414 & -1.365793 \\
\hline C & -5.837560 & 1.673511 & 0.711775 \\
\hline $\mathrm{H}$ & -4.284192 & 1.270886 & 2.155724 \\
\hline C & -6.327827 & 1.301697 & -0.554102 \\
\hline H & -7.198696 & 1.817994 & -0.940440 \\
\hline C & -6.542435 & 2.793647 & 1.501321 \\
\hline C & -6.265365 & -0.108920 & -2.715570 \\
\hline C & -5.867003 & 3.071125 & 2.857897 \\
\hline $\mathrm{H}$ & -5.876179 & 2.188494 & 3.507636 \\
\hline H & -4.827530 & 3.396585 & 2.737793 \\
\hline $\mathrm{H}$ & -6.404431 & 3.870475 & 3.380508 \\
\hline C & -8.011036 & 2.387845 & 1.771003 \\
\hline H & -8.528451 & 3.177664 & 2.329355 \\
\hline H & -8.566034 & 2.218738 & 0.842317 \\
\hline $\mathrm{H}$ & -8.060785 & 1.465940 & 2.361815 \\
\hline C & -6.515757 & 4.102866 & 0.677076 \\
\hline $\mathrm{H}$ & -7.024858 & 3.990307 & -0.285799 \\
\hline H & -7.016739 & 4.909393 & 1.226296 \\
\hline $\mathrm{H}$ & -5.485553 & 4.417898 & 0.475863 \\
\hline C & -5.165079 & 0.146749 & -3.774253 \\
\hline $\mathrm{H}$ & -4.915122 & 1.213268 & -3.824076 \\
\hline H & -4.244210 & -0.402146 & -3.552461 \\
\hline H & -5.512109 & -0.165409 & -4.767019 \\
\hline C & -7.520186 & 0.684410 & -3.125503 \\
\hline $\mathrm{H}$ & -7.322038 & 1.760635 & -3.187235 \\
\hline H & -7.857143 & 0.355216 & -4.114924 \\
\hline $\mathrm{H}$ & -8.349396 & 0.528069 & -2.425793 \\
\hline C & -6.630678 & -1.612489 & -2.709401 \\
\hline $\mathrm{H}$ & -5.766756 & -2.243059 & -2.476429 \\
\hline H & -7.410299 & -1.824145 & -1.968472 \\
\hline H & -7.006584 & -1.915534 & -3.694258 \\
\hline C & 7.905361 & -0.009373 & 0.274174 \\
\hline C & 4.843431 & 3.966240 & -0.576310 \\
\hline C & 3.397678 & 4.367464 & -0.927832 \\
\hline $\mathrm{H}$ & 3.087734 & 3.949012 & -1.892577 \\
\hline H & 2.681258 & 4.034376 & -0.169787 \\
\hline $\mathrm{H}$ & 3.327688 & 5.458967 & -1.002552 \\
\hline C & 5.773260 & 4.490085 & -1.696000 \\
\hline H & 6.828909 & 4.291737 & -1.481934 \\
\hline H & 5.533082 & 4.019307 & -2.656245 \\
\hline $\mathrm{H}$ & 5.657002 & 5.574822 & -1.811157 \\
\hline C & 5.220486 & 4.645602 & 0.762003 \\
\hline $\mathrm{H}$ & 4.562761 & 4.306103 & 1.570142 \\
\hline H & 6.252474 & 4.423696 & 1.054726 \\
\hline H & 5.122862 & 5.735378 & 0.679226 \\
\hline C & 8.844294 & 0.384873 & -0.890776 \\
\hline H & 8.499987 & -0.051932 & -1.835275 \\
\hline H & 8.894835 & 1.470392 & -1.025112 \\
\hline $\mathrm{H}$ & 9.862759 & 0.024297 & -0.700216 \\
\hline C & 7.945103 & -1.542578 & 0.419921 \\
\hline $\mathrm{H}$ & 8.972778 & -1.868555 & 0.616858 \\
\hline H & 7.323212 & -1.889686 & 1.25285 \\
\hline
\end{tabular}




\section{Catalyst 2C 58}

\section{Datum}

Value

\begin{tabular}{lr}
\hline B3LYP/6-31G(d) Energy & -2164.797108 \\
\hline B3LYP-D3(BJ)/def2-TZVPP-IEF-PCM(DCM) Energy & -2165.895279 \\
\hline B3LYP-D3(BJ)/def2-TZVPP-IEF-PCM(DCM)//B3LYP/6-31G(d) Free Energy (Quasiharmonic) & -2165.017834 \\
\hline
\end{tabular}

Number of Imaginary Frequencies

Frequencies (Top 3 out of 321)
1. $8.5168 \mathrm{~cm}^{-1}$
2. $11.8798 \mathrm{~cm}^{-1}$
3. $13.8813 \mathrm{~cm}^{-1}$

\section{B3LYP/6-31G(d) Molecular Geometry in Cartesian Coordinates}

$\begin{array}{lrrl}\text { C } & 0.069787 & -3.642473 & 3.698433 \\ \text { C } & 0.235825 & -2.431934 & 3.060397 \\ \text { C } & -0.798080 & -1.887134 & 2.245660 \\ \text { C } & -2.017028 & -2.625990 & 2.106426 \\ \text { C } & -2.156319 & -3.868128 & 2.782901 \\ \text { C } & -1.137007 & -4.368720 & 3.559823 \\ \text { H } & 0.869538 & -4.040231 & 4.316896 \\ \text { H } & 1.159336 & -1.875364 & 3.181665 \\ \text { C } & -0.658445 & -0.637780 & 1.554966 \\ \text { C } & -3.056959 & -2.099044 & 1.302576 \\ \text { H } & -3.087145 & -4.418902 & 2.671221 \\ \text { H } & -1.255004 & -5.321218 & 4.068918 \\ \text { C } & -2.933102 & -0.893945 & 0.639924 \\ \text { C } & -1.707367 & -0.166495 & 0.777707 \\ \text { H } & -3.988275 & -2.653928 & 1.224077 \\ \text { C } & 1.211500 & 2.978853 & 4.759387 \\ \text { C } & 2.209101 & 2.744668 & 3.841725 \\ \text { C } & 2.032195 & 1.793935 & 2.799888 \\ \text { C } & 0.798919 & 1.076374 & 2.707389 \\ \text { C } & -0.212372 & 1.344624 & 3.670992 \\ \text { C } & -0.010879 & 2.270087 & 4.670551\end{array}$




\begin{tabular}{|c|c|c|c|}
\hline H & 3.994448 & 2.062886 & 1.947326 \\
\hline $\mathrm{H}$ & 1.357616 & 3.706474 & 5.553228 \\
\hline $\mathrm{H}$ & 3.152645 & 3.282295 & 3.900240 \\
\hline C & 3.052010 & 1.528638 & 1.855479 \\
\hline C & 0.637411 & 0.122123 & 1.649842 \\
\hline H & -1.155851 & 0.813179 & 3.608275 \\
\hline H & -0.796417 & 2.459949 & 5.397055 \\
\hline C & 1.661073 & -0.122527 & 0.736082 \\
\hline C & 2.904470 & 0.592194 & 0.849876 \\
\hline C & 1.462158 & -1.146571 & -0.371673 \\
\hline $\mathrm{H}$ & 0.422270 & -1.137325 & -0.714373 \\
\hline H & 2.090412 & -0.897640 & -1.227424 \\
\hline C & -1.479774 & 1.057525 & -0.068204 \\
\hline 0 & -1.240402 & 1.030054 & -1.254353 \\
\hline 0 & -1.552795 & 2.210819 & 0.639164 \\
\hline $\mathrm{H}$ & -1.345274 & 2.928414 & 0.009051 \\
\hline 0 & 1.855215 & -2.471472 & 0.012524 \\
\hline $\mathrm{H}$ & 1.259764 & -2.758206 & 0.723955 \\
\hline C & 4.051271 & 0.370730 & -0.080895 \\
\hline C & 4.581163 & -0.915101 & -0.286276 \\
\hline C & 4.647693 & 1.460797 & -0.721578 \\
\hline C & 5.682556 & -1.117521 & -1.119294 \\
\hline $\mathrm{H}$ & 4.104158 & -1.749562 & 0.211030 \\
\hline C & 5.757539 & 1.300248 & -1.566780 \\
\hline $\mathrm{H}$ & 4.219657 & 2.447541 & -0.568122 \\
\hline C & 6.252941 & 0.005565 & -1.746032 \\
\hline $\mathrm{H}$ & 7.105961 & -0.146120 & -2.397122 \\
\hline C & -4.066344 & -0.378539 & -0.178177 \\
\hline C & -4.565581 & 0.914605 & 0.020053 \\
\hline C & -4.674554 & -1.202269 & -1.133267 \\
\hline C & -5.650547 & 1.398682 & -0.720597 \\
\hline $\mathrm{H}$ & -4.106916 & 1.540283 & 0.778709 \\
\hline C & -5.761812 & -0.758366 & -1.895428 \\
\hline $\mathrm{H}$ & -4.266669 & -2.196185 & -1.291994 \\
\hline C & -6.230196 & 0.544601 & -1.669637 \\
\hline $\mathrm{H}$ & -7.068156 & 0.904589 & -2.250229 \\
\hline C & -6.387570 & -1.694104 & -2.948337 \\
\hline C & -6.163073 & 2.829745 & -0.464685 \\
\hline C & -6.891724 & -2.984238 & -2.258499 \\
\hline $\mathrm{H}$ & -6.079540 & -3.526209 & -1.762826 \\
\hline $\mathrm{H}$ & -7.651380 & -2.753773 & -1.502651 \\
\hline H & -7.340432 & -3.660603 & -2.996348 \\
\hline C & -5.319785 & -2.062737 & -4.005909 \\
\hline $\mathrm{H}$ & -5.746920 & -2.733933 & -4.761381 \\
\hline $\mathrm{H}$ & -4.949524 & -1.166888 & -4.517036 \\
\hline $\mathrm{H}$ & -4.458601 & -2.569261 & -3.558098 \\
\hline C & -7.579967 & -1.048069 & -3.679096 \\
\hline $\mathrm{H}$ & -7.284249 & -0.144831 & -4.224586 \\
\hline $\mathrm{H}$ & -7.989486 & -1.754490 & -4.409949 \\
\hline $\mathrm{H}$ & -8.388337 & -0.782606 & -2.988123 \\
\hline C & -7.350300 & 3.204909 & -1.371618 \\
\hline $\mathrm{H}$ & -7.083317 & 3.161862 & -2.433683 \\
\hline $\mathrm{H}$ & -8.213155 & 2.549057 & -1.208426 \\
\hline H & -7.670509 & 4.229994 & -1.153434 \\
\hline
\end{tabular}




\begin{tabular}{|c|c|c|c|}
\hline C & -5.018798 & 3.837435 & -0.731023 \\
\hline H & -4.677521 & 3.775302 & -1.770728 \\
\hline $\mathrm{H}$ & -5.361903 & 4.862772 & -0.545395 \\
\hline $\mathrm{H}$ & -4.155715 & 3.651593 & -0.083130 \\
\hline C & -6.623559 & 2.957344 & 1.007044 \\
\hline $\mathrm{H}$ & -7.438616 & 2.257648 & 1.224737 \\
\hline $\mathrm{H}$ & -5.808764 & 2.750048 & 1.708459 \\
\hline $\mathrm{H}$ & -6.985310 & 3.973532 & 1.206902 \\
\hline C & 6.367705 & 2.530977 & -2.265901 \\
\hline C & 6.271588 & -2.517937 & -1.377572 \\
\hline C & 5.545586 & -3.614104 & -0.573619 \\
\hline $\mathrm{H}$ & 5.639412 & -3.450970 & 0.506273 \\
\hline $\mathrm{H}$ & 4.478652 & -3.664805 & -0.814901 \\
\hline $\mathrm{H}$ & 5.989388 & -4.590574 & -0.800204 \\
\hline C & 7.766250 & -2.538933 & -0.978437 \\
\hline $\mathrm{H}$ & 8.354714 & -1.812618 & -1.549185 \\
\hline $\mathrm{H}$ & 7.890732 & -2.307482 & 0.085727 \\
\hline $\mathrm{H}$ & 8.195488 & -3.531623 & -1.162183 \\
\hline C & 6.139626 & -2.856014 & -2.881853 \\
\hline $\mathrm{H}$ & 5.087830 & -2.862562 & -3.189278 \\
\hline $\mathrm{H}$ & 6.667940 & -2.131557 & -3.511137 \\
\hline $\mathrm{H}$ & 6.560750 & -3.847874 & -3.088413 \\
\hline C & 6.816168 & 3.561536 & -1.202446 \\
\hline $\mathrm{H}$ & 5.979433 & 3.898024 & -0.581609 \\
\hline $\mathrm{H}$ & 7.574784 & 3.132923 & -0.537437 \\
\hline $\mathrm{H}$ & 7.248320 & 4.446597 & -1.685783 \\
\hline $\mathrm{C}$ & 5.305180 & 3.176394 & -3.187035 \\
\hline $\mathrm{H}$ & 5.718170 & 4.061671 & -3.686613 \\
\hline H & 4.977515 & 2.471642 & -3.959742 \\
\hline $\mathrm{H}$ & 4.418808 & 3.492153 & -2.627183 \\
\hline $\mathrm{C}$ & 7.593417 & 2.173817 & -3.127744 \\
\hline $\mathrm{H}$ & 7.337779 & 1.474060 & -3.931404 \\
\hline H & 7.991641 & 3.081439 & -3.595325 \\
\hline $\mathrm{H}$ & 8.397746 & 1.729796 & -2.530032 \\
\hline
\end{tabular}

\section{Catalyst 2C 59}

Datum

Value

\begin{tabular}{lr}
\hline B3LYP/6-31G(d) Energy & -2164.799727 \\
\hline B3LYP-D3(BJ)/def2-TZVPP-IEF-PCM(DCM) Energy & -2165.896056 \\
\hline B3LYP-D3(BJ)/def2-TZVPP-IEF-PCM(DCM)//B3LYP/6-31G(d) Free Energy (Quasiharmonic) & -2165.017824 \\
\hline Number of Imaginary Frequencies & 0
\end{tabular}

Frequencies (Top 3 out of 321) 

1.
$10.6304 \mathrm{~cm}^{-1}$
2.
$12.5919 \mathrm{~cm}^{-1}$
3.
$12.9434 \mathrm{~cm}^{-1}$

\section{B3LYP/6-31G(d) Molecular Geometry in Cartesian Coordinates}

C

C

C

C

C

C

$\mathrm{H}$

$\mathrm{H}$

$\mathrm{C}$

C

$\mathrm{H}$

$\mathrm{H}$

C

C

$\mathrm{H}$

C

C

C

C

C

C

$\mathrm{H}$

$\mathrm{H}$

$\mathrm{H}$

C

C

$\mathrm{H}$

$\mathrm{H}$

C

C

C

$\mathrm{H}$

$\mathrm{H}$

C

0

0

$\mathrm{H}$

0

$\mathrm{H}$

C

C

C

C

$\mathrm{H}$
0.290297

0.412605

$-0.666371$

$-1.884450$

$-1.977418$

$-0.914859$

1.124419

1.338536

$-0.575318$

$-2.972375$

$-2.907612$

$-0.997563$

$-2.903545$

$-1.678015$

$-3.898752$

1.351743

2. 327767

2. 127235

0.892506

$-0.097149$

0.128158

4.054058

1.514836

3. 271140

3.123205

0.710946

$-1.044187$

$-0.642046$

1.711659

2. 950712

1.512208

0.829495

2.467985

$-1.570385$

$-1.665575$

$-1.342115$

$-1.284667$

1.053395

0.187488

4.090182

3.921750

5.374990

5.006180

2.924736
$-3.354576$

$-2.809969$

$-2.100920$

$-1.962544$

$-2.535418$

$-3.216157$

$-3.896031$

$-2.920317$

$-1.516060$

$-1.264847$

$-2.425095$

$-3.649596$

$-0.720357$

$-0.861509$

$-1.181012$

$-4.912898$

$-3.957181$

$-2.851084$

$-2.730056$

$-3.733350$

$-4.797134$

$-1.960035$

$-5.755992$

$-4.033039$

$-1.864243$

$-1.608849$

$-3.650208$

$-5.551836$

$-0.650712$

$-0.781917$

0.507952

0.208831

0.766997

$-0.271881$

$-0.869988$

1.072125

1. 376543

1.712260

1.534757

0.173800

1.556161

$-0.330440$

2.435646

1.930205
3.969811

2.710593

2.114609

2.853780

4.150951

4.698998

4.407476

2.156535

0.807955

2. 273537

4.703106

5.691950

1.007010

0.277918

2.835964

$-2.584982$

$-2.424738$

$-1.555175$

$-0.845625$

$-1.038109$

$-1.882796$

$-1.922230$

$-3.250939$

$-2.960400$

$-1.369015$

0.030558

$-0.516551$

$-2.016839$

0.196210

$-0.526652$

1.165312

1.969096

1.625712

$-1.100865$

$-2.146347$

$-1.054791$

$-1.981965$

0.548135

0.141423

$-0.378581$

$-0.540370$

$-0.120509$

$-0.440187$

$-0.728422$ 


\begin{tabular}{|c|c|c|c|}
\hline C & 6.485284 & 0.514549 & -0.017197 \\
\hline $\mathrm{H}$ & 5.487068 & -1.399817 & 0.017060 \\
\hline C & 6.271189 & 1.890785 & -0.179734 \\
\hline $\mathrm{H}$ & 7.119939 & 2.562895 & -0.093242 \\
\hline C & -4.084887 & -0.010691 & 0.438731 \\
\hline C & -4.612966 & -0.375463 & -0.811606 \\
\hline C & -4.702841 & 1.008002 & 1.168649 \\
\hline C & -5.734157 & 0.269501 & -1.337099 \\
\hline $\mathrm{H}$ & -4.141093 & -1.181821 & -1.358167 \\
\hline C & -5.832680 & 1.680512 & 0.677131 \\
\hline $\mathrm{H}$ & -4.275216 & 1.289599 & 2.126439 \\
\hline C & -6.323363 & 1.293589 & -0.573532 \\
\hline $\mathrm{H}$ & -7.192382 & 1.800322 & -0.976132 \\
\hline C & -6.471409 & 2.806620 & 1.513478 \\
\hline C & -6.335361 & -0.104272 & -2.706029 \\
\hline C & -6.922780 & 2.239537 & 2.880478 \\
\hline $\mathrm{H}$ & -7.665891 & 1.444515 & 2.749526 \\
\hline $\mathrm{H}$ & -6.083269 & 1.822425 & 3.446433 \\
\hline $\mathrm{H}$ & -7.374988 & 3.030729 & 3.491082 \\
\hline C & -7.701403 & 3.428697 & 0.825502 \\
\hline $\mathrm{H}$ & -8.118081 & 4.217961 & 1.461248 \\
\hline H & -7.444396 & 3.883473 & -0.137947 \\
\hline $\mathrm{H}$ & -8.492816 & 2.689739 & 0.655365 \\
\hline C & -5.431411 & 3.929037 & 1.743609 \\
\hline $\mathrm{H}$ & -5.100575 & 4.357599 & 0.790713 \\
\hline $\mathrm{H}$ & -5.867211 & 4.735476 & 2.346075 \\
\hline $\mathrm{H}$ & -4.544268 & 3.562283 & 2.269951 \\
\hline C & -5.559886 & -1.244074 & -3.394205 \\
\hline $\mathrm{H}$ & -4.509774 & -0.982723 & -3.565193 \\
\hline $\mathrm{H}$ & -5.587950 & -2.168349 & -2.805959 \\
\hline $\mathrm{H}$ & -6.012632 & -1.460707 & -4.368426 \\
\hline C & -6.298264 & 1.131474 & -3.636783 \\
\hline $\mathrm{H}$ & -5.268277 & 1.471018 & -3.795085 \\
\hline $\mathrm{H}$ & -6.727805 & 0.884155 & -4.615269 \\
\hline $\mathrm{H}$ & -6.868375 & 1.971567 & -3.226119 \\
\hline C & -7.801810 & -0.561154 & -2.521103 \\
\hline $\mathrm{H}$ & -7.858925 & -1.439295 & -1.867738 \\
\hline $\mathrm{H}$ & -8.423420 & 0.224922 & -2.079391 \\
\hline $\mathrm{H}$ & -8.242211 & -0.829317 & -3.489283 \\
\hline C & 7.905987 & -0.011159 & 0.269271 \\
\hline C & 4.841859 & 3.961015 & -0.589475 \\
\hline C & 3.395586 & 4.360710 & -0.940604 \\
\hline $\mathrm{H}$ & 3.084872 & 3.939617 & -1.903952 \\
\hline $\mathrm{H}$ & 2.680075 & 4.029251 & -0.180983 \\
\hline $\mathrm{H}$ & 3.325040 & 5.451984 & -1.018096 \\
\hline C & 5.770320 & 4.481940 & -1.711667 \\
\hline $\mathrm{H}$ & 6.826255 & 4.284541 & -1.498125 \\
\hline $\mathrm{H}$ & 5.529287 & 4.008255 & -2.670267 \\
\hline $\mathrm{H}$ & 5.653570 & 5.566296 & -1.829898 \\
\hline C & 5.220079 & 4.644330 & 0.746485 \\
\hline $\mathrm{H}$ & 4.563391 & 4.306826 & 1.556298 \\
\hline $\mathrm{H}$ & 6.252488 & 4.423733 & 1.038704 \\
\hline $\mathrm{H}$ & 5.121877 & 5.733824 & 0.660728 \\
\hline C & 8.843725 & 0.379808 & -0.897751 \\
\hline
\end{tabular}




\begin{tabular}{|lrrr|}
$H$ & 8.498613 & -0.059910 & -1.840602 \\
$H$ & 8.893830 & 1.464940 & -1.035370 \\
$H$ & 9.862474 & 0.020072 & -0.707104 \\
$\mathrm{C}$ & 7.946312 & -1.543902 & 0.419695 \\
$\mathrm{H}$ & 8.974237 & -1.868948 & 0.616878 \\
$\mathrm{H}$ & 7.325123 & -1.888642 & 1.254132 \\
$\mathrm{H}$ & 7.608410 & -2.052076 & -0.490631 \\
$\mathrm{C}$ & 8.435600 & 0.612793 & 1.582339 \\
$\mathrm{H}$ & 7.795623 & 0.342668 & 2.430143 \\
$\mathrm{H}$ & 9.450493 & 0.253927 & 1.794122 \\
$\mathrm{H}$ & 8.473496 & 1.705810 & 1.528868 \\
& & & \\
\hline
\end{tabular}

\section{Catalyst 2C 60}

Datum

Value

\begin{tabular}{lc}
\hline B3LYP/6-31G(d) Energy & -2164.797489 \\
\hline B3LYP-D3(BJ)/def2-TZVPP-IEF-PCM(DCM) Energy & -2165.895459 \\
\hline B3LYP-D3(BJ)/def2-TZVPP-IEF-PCM(DCM)//B3LYP/6-31G(d) Free Energy (Quasiharmonic) & -2165.017824 \\
\hline
\end{tabular}

Number of Imaginary Frequencies

Frequencies (Top 3 out of 321)
1. $11.4951 \mathrm{~cm}^{-1}$
2. $\quad 12.5552 \mathrm{~cm}^{-1}$
3. $13.2415 \mathrm{~cm}^{-1}$

\section{B3LYP/6-31G(d) Molecular Geometry in Cartesian Coordinates}

$\begin{array}{lrrl}\mathrm{C} & -0.059016 & -3.319778 & 3.971033 \\ \mathrm{C} & -0.230881 & -2.169369 & 3.231418 \\ \mathrm{C} & 0.803875 & -1.686169 & 2.379700 \\ \mathrm{C} & 2.029381 & -2.424276 & 2.310544 \\ \mathrm{C} & 2.174730 & -3.602937 & 3.091252 \\ \mathrm{C} & 1.154740 & -4.044421 & 3.902314 \\ \mathrm{H} & -0.859679 & -3.670691 & 4.616115 \\ \mathrm{H} & -1.160332 & -1.613771 & 3.299693 \\ \mathrm{C} & 0.659735 & -0.500169 & 1.585695 \\ \mathrm{C} & 3.069719 & -1.959295 & 1.470047 \\ \mathrm{H} & 3.110680 & -4.153098 & 3.031567 \\ \mathrm{H} & 1.277294 & -4.949011 & 4.491413 \\ \mathrm{C} & 2.940135 & -0.817413 & 0.704515 \\ \mathrm{C} & 1.708418 & -0.089930 & 0.774388 \\ \mathrm{H} & 4.005894 & -2.510829 & 1.444313\end{array}$




\begin{tabular}{|c|c|c|c|}
\hline C & -1.223442 & 3.340060 & 4.505006 \\
\hline C & -2.221112 & 3.032068 & 3.609572 \\
\hline C & -2.040401 & 2.006852 & 2.641506 \\
\hline C & -0.803308 & 1.291211 & 2.600866 \\
\hline C & 0.207730 & 1.636312 & 3.539849 \\
\hline C & 0.002656 & 2.633120 & 4.467498 \\
\hline $\mathrm{H}$ & -4.005317 & 2.199195 & 1.777281 \\
\hline $\mathrm{H}$ & -1.372503 & 4.124513 & 5.242141 \\
\hline $\mathrm{H}$ & -3.167606 & 3.567334 & 3.629542 \\
\hline C & -3.060122 & 1.664835 & 1.722424 \\
\hline C & -0.639130 & 0.259970 & 1.618568 \\
\hline $\mathrm{H}$ & 1.153331 & 1.105517 & 3.516370 \\
\hline $\mathrm{H}$ & 0.788027 & 2.880778 & 5.176543 \\
\hline C & -1.662667 & -0.059043 & 0.728101 \\
\hline C & -2.910244 & 0.656204 & 0.789113 \\
\hline C & -1.459104 & -1.166499 & -0.295333 \\
\hline $\mathrm{H}$ & -2.086964 & -0.989153 & -1.168769 \\
\hline $\mathrm{H}$ & -0.419267 & -1.183767 & -0.636874 \\
\hline C & 1.474368 & 1.054420 & -0.174945 \\
\hline 0 & 1.234427 & 0.921943 & -1.353873 \\
\hline 0 & 1.541422 & 2.265541 & 0.428534 \\
\hline $\mathrm{H}$ & 1.329459 & 2.924241 & -0.261581 \\
\hline 0 & -1.849053 & -2.458126 & 0.193895 \\
\hline $\mathrm{H}$ & -1.249679 & -2.687894 & 0.922514 \\
\hline C & -4.059497 & 0.361047 & -0.117677 \\
\hline C & -4.561244 & -0.935757 & -0.258486 \\
\hline C & -4.688847 & 1.413570 & -0.807021 \\
\hline C & -5.669130 & -1.207198 & -1.076766 \\
\hline $\mathrm{H}$ & -4.068469 & -1.741279 & 0.274321 \\
\hline C & -5.796810 & 1.186940 & -1.625280 \\
\hline $\mathrm{H}$ & -4.278086 & 2.411373 & -0.702372 \\
\hline C & -6.267594 & -0.133970 & -1.742396 \\
\hline $\mathrm{H}$ & -7.124286 & -0.321194 & -2.379505 \\
\hline C & 4.073835 & -0.367849 & -0.151012 \\
\hline C & 4.698905 & -1.272256 & -1.018279 \\
\hline C & 4.558094 & 0.943830 & -0.073321 \\
\hline C & 5.787725 & -0.891174 & -1.811406 \\
\hline $\mathrm{H}$ & 4.303561 & -2.281780 & -1.082525 \\
\hline C & 5.644345 & 1.366884 & -0.848892 \\
\hline $\mathrm{H}$ & 4.086766 & 1.633204 & 0.619726 \\
\hline C & 6.239942 & 0.432730 & -1.708406 \\
\hline $\mathrm{H}$ & 7.079012 & 0.744403 & -2.314978 \\
\hline C & 6.143708 & 2.819660 & -0.724267 \\
\hline C & 6.438695 & -1.919498 & -2.757160 \\
\hline C & 6.603226 & 3.082001 & 0.729924 \\
\hline $\mathrm{H}$ & 5.789343 & 2.932965 & 1.447050 \\
\hline $\mathrm{H}$ & 7.422304 & 2.409256 & 1.008988 \\
\hline $\mathrm{H}$ & 6.958821 & 4.114159 & 0.837887 \\
\hline C & 4.991536 & 3.789856 & -1.079628 \\
\hline $\mathrm{H}$ & 5.324123 & 4.830562 & -0.981061 \\
\hline $\mathrm{H}$ & 4.655462 & 3.636082 & -2.111534 \\
\hline $\mathrm{H}$ & 4.127172 & 3.651513 & -0.421745 \\
\hline C & 7.327692 & 3.121031 & -1.662553 \\
\hline $\mathrm{H}$ & 7.061480 & 2.975635 & -2.715660 \\
\hline
\end{tabular}




\begin{tabular}{|c|c|c|c|}
\hline $\mathrm{H}$ & 7.636494 & 4.165496 & -1.541381 \\
\hline $\mathrm{H}$ & 8.197567 & 2.492854 & -1.439140 \\
\hline C & 6.984942 & -3.104833 & -1.925996 \\
\hline $\mathrm{H}$ & 7.743536 & -2.765648 & -1.211033 \\
\hline $\mathrm{H}$ & 6.191943 & -3.604482 & -1.359705 \\
\hline $\mathrm{H}$ & 7.447128 & -3.852331 & -2.582584 \\
\hline C & 7.608011 & -1.323138 & -3.563764 \\
\hline $\mathrm{H}$ & 8.413620 & -0.963031 & -2.913694 \\
\hline $\mathrm{H}$ & 8.032650 & -2.092380 & -4.218670 \\
\hline $\mathrm{H}$ & 7.283452 & -0.491697 & -4.199759 \\
\hline C & 5.382030 & -2.441047 & -3.759957 \\
\hline $\mathrm{H}$ & 4.541206 & -2.925402 & -3.252980 \\
\hline $\mathrm{H}$ & 4.978286 & -1.621658 & -4.365184 \\
\hline $\mathrm{H}$ & 5.831706 & -3.176631 & -4.438173 \\
\hline C & -6.504612 & 2.316035 & -2.399596 \\
\hline C & -6.167461 & -2.659051 & -1.209542 \\
\hline C & -5.029712 & -3.537353 & -1.785431 \\
\hline $\mathrm{H}$ & -4.745309 & -3.195352 & -2.787580 \\
\hline $\mathrm{H}$ & -4.133159 & -3.513232 & -1.157953 \\
\hline $\mathrm{H}$ & -5.359917 & -4.580800 & -1.865906 \\
\hline C & -7.388819 & -2.781132 & -2.139844 \\
\hline $\mathrm{H}$ & -8.243294 & -2.201086 & -1.771885 \\
\hline $\mathrm{H}$ & -7.161283 & -2.446987 & -3.158701 \\
\hline $\mathrm{H}$ & -7.703073 & -3.829292 & -2.200207 \\
\hline C & -6.571262 & -3.194918 & 0.184616 \\
\hline $\mathrm{H}$ & -5.731101 & -3.177946 & 0.886037 \\
\hline $\mathrm{H}$ & -7.381254 & -2.595261 & 0.616261 \\
\hline $\mathrm{H}$ & -6.920164 & -4.232225 & 0.107613 \\
\hline C & -7.982988 & 2.400615 & -1.950102 \\
\hline $\mathrm{H}$ & -8.518882 & 1.462666 & -2.129290 \\
\hline $\mathrm{H}$ & -8.054613 & 2.625857 & -0.879748 \\
\hline $\mathrm{H}$ & -8.505003 & 3.193373 & -2.500291 \\
\hline C & -6.448153 & 2.017960 & -3.916781 \\
\hline $\mathrm{H}$ & -6.953169 & 2.811404 & -4.481641 \\
\hline $\mathrm{H}$ & -5.410853 & 1.959295 & -4.265606 \\
\hline $\mathrm{H}$ & -6.936863 & 1.070076 & -4.164806 \\
\hline C & -5.853904 & 3.691619 & -2.159677 \\
\hline $\mathrm{H}$ & -4.809147 & 3.711133 & -2.489773 \\
\hline $\mathrm{H}$ & -6.393692 & 4.459397 & -2.725743 \\
\hline $\mathrm{H}$ & -5.883979 & 3.978618 & -1.102379 \\
\hline
\end{tabular}

\section{Catalyst 2C 61}

Datum

Value

\begin{tabular}{lc}
\hline B3LYP/6-31G(d) Energy & -2164.798069 \\
\hline B3LYP-D3(BJ)/def2-TZVPP-IEF-PCM(DCM) Energy & -2165.895666 \\
\hline B3LYP-D3(BJ)/def2-TZVPP-IEF-PCM(DCM)//B3LYP/6-31G(d) Free Energy (Quasiharmonic) & -2165.017807 \\
\hline
\end{tabular}


Frequencies (Top 3 out of 321)

$\begin{array}{ll}\text { 1. } & 10.9354 \mathrm{~cm}^{-1} \\ \text { 2. } & 12.9406 \mathrm{~cm}^{-1} \\ \text { 3. } & 13.4065 \mathrm{~cm}^{-1}\end{array}$

\section{B3LYP/6-31G(d) Molecular Geometry in Cartesian Coordinates}

C

C

C

C

C

C

$\mathrm{H}$

$\mathrm{H}$

C

C

$\mathrm{H}$

$\mathrm{H}$

C

C

$\mathrm{H}$

C

C

C

C

C

C

$\mathrm{H}$

$\mathrm{H}$

$\mathrm{H}$

C

C

$\mathrm{H}$

$\mathrm{H}$

C

C

C

$\mathrm{H}$

$\mathrm{H}$

C

0

0

$\mathrm{H}$

$$
-0.031151
$$

0.164071

$-0.857583$

$-2.101045$

$-2.268520$

$-1.257837$

0.760796

1.102038

$-0.688696$

$-3.129977$

$-3.214881$

$-1.398603$

$-2.976743$

$-1.733852$

$-4.057098$

0.957057

2.013055

1.906993

0.683944

$-0.388310$

$-0.254867$

3.918124

1.049228

2. 949491

2.985604

0.597099

$-1.324088$

$-1.086629$

1.674936

2.904053

1.536676

0.511730

2.199598

$-1.542396$

$-0.812072$

$-2.283425$

$-2.106696$
$-4.849182$

$-3.755987$

$-2.777495$

$-2.965277$

$-4.104380$

$-5.025753$

$-5.583726$

$-3.634802$

$-1.622129$

$-2.005501$

$-4.232884$

$-5.891492$

$-0.890245$

$-0.717913$

$-2.139292$

$-2.985296$

$-2.326741$

$-1.805126$

$-1.970210$

$-2.657248$

$-3.150592$

$-1.033650$

$-3.380097$

$-2.194917$

$-1.134567$

$-1.438273$

$-2.787685$

$-3.671539$

$-0.779063$

$-0.632192$

$-0.201685$

0.149765

0.656415

0.472561

1.413270

0.379112

1.193349
$-1.808028$

$-0.991534$

$-0.822147$

$-1.507681$

$-2.340984$

$-2.491567$

$-1.924445$

$-0.459762$

0.010694

$-1.349796$

$-2.860776$

$-3.132677$

$-0.549886$

0.141828

$-1.901083$

4.726975

4.141316

2.823244

2.099561

2.735380

4.014128

2. 750943

5.735223

4.678429

2. 201251

0.770882

2. 202769

4.480665

0.184886

0.915776

$-1.214938$

$-1.371355$

$-1.330788$

1.040274

0.809762

2. 165688

2.675605 


\begin{tabular}{|c|c|c|c|}
\hline 0 & 1.920881 & -1.118484 & -2.249375 \\
\hline $\mathrm{H}$ & 1.293546 & -1.859302 & -2.231417 \\
\hline C & 4.102057 & 0.059498 & 0.353619 \\
\hline C & 4.642755 & -0.310851 & -0.890213 \\
\hline C & 4.733615 & 1.062471 & 1.095516 \\
\hline C & 5.788327 & 0.308646 & -1.392801 \\
\hline $\mathrm{H}$ & 4.140598 & -1.084441 & -1.456362 \\
\hline C & 5.888016 & 1.709285 & 0.626599 \\
\hline $\mathrm{H}$ & 4.297280 & 1.352586 & 2.046964 \\
\hline C & 6.392126 & 1.314146 & -0.615726 \\
\hline $\mathrm{H}$ & 7.278757 & 1.802343 & -1.002823 \\
\hline C & -4.077139 & 0.110088 & -0.455526 \\
\hline C & -3.827258 & 1.468665 & -0.693832 \\
\hline C & -5.383230 & -0.308199 & -0.166612 \\
\hline C & -4.855714 & 2.415691 & -0.635696 \\
\hline $\mathrm{H}$ & -2.818784 & 1.775790 & -0.940892 \\
\hline C & -6.440547 & 0.606387 & -0.102636 \\
\hline $\mathrm{H}$ & -5.553379 & -1.360613 & 0.029365 \\
\hline C & -6.146924 & 1.957334 & -0.339002 \\
\hline $\mathrm{H}$ & -6.955583 & 2.680468 & -0.290009 \\
\hline C & -7.887307 & 0.183720 & 0.218671 \\
\hline C & -4.609320 & 3.916150 & -0.887142 \\
\hline C & -8.017755 & -1.333157 & 0.454876 \\
\hline $\mathrm{H}$ & -7.721687 & -1.911200 & -0.428012 \\
\hline $\mathrm{H}$ & -7.410123 & -1.668310 & 1.303080 \\
\hline $\mathrm{H}$ & -9.061265 & -1.583004 & 0.677525 \\
\hline C & -8.808770 & 0.565860 & -0.964248 \\
\hline $\mathrm{H}$ & -9.844767 & 0.275729 & -0.750318 \\
\hline $\mathrm{H}$ & -8.798655 & 1.643420 & -1.158833 \\
\hline $\mathrm{H}$ & -8.496271 & 0.057914 & -1.883821 \\
\hline C & -8.367684 & 0.910157 & 1.497535 \\
\hline $\mathrm{H}$ & -8.345356 & 1.998862 & 1.382330 \\
\hline $\mathrm{H}$ & -9.399032 & 0.621843 & 1.735364 \\
\hline $\mathrm{H}$ & -7.736038 & 0.651949 & 2.355141 \\
\hline C & -5.020630 & 4.721042 & 0.368889 \\
\hline $\mathrm{H}$ & -6.077881 & 4.580710 & 0.617554 \\
\hline $\mathrm{H}$ & -4.428958 & 4.417088 & 1.239987 \\
\hline $\mathrm{H}$ & -4.856281 & 5.792971 & 0.204483 \\
\hline C & -5.456172 & 4.384042 & -2.094259 \\
\hline $\mathrm{H}$ & -6.527747 & 4.233974 & -1.924749 \\
\hline $\mathrm{H}$ & -5.294574 & 5.452540 & -2.282459 \\
\hline $\mathrm{H}$ & -5.180872 & 3.834325 & -3.001544 \\
\hline C & -3.129780 & 4.223358 & -1.189041 \\
\hline $\mathrm{H}$ & -2.470078 & 3.919473 & -0.368810 \\
\hline $\mathrm{H}$ & -2.788097 & 3.722264 & -2.101825 \\
\hline $\mathrm{H}$ & -3.000832 & 5.301392 & -1.338035 \\
\hline C & 6.539088 & 2.816439 & 1.478883 \\
\hline C & 6.392280 & -0.064055 & -2.760919 \\
\hline C & 7.861987 & -0.511571 & -2.580015 \\
\hline $\mathrm{H}$ & 8.482334 & 0.278791 & -2.144161 \\
\hline $\mathrm{H}$ & 7.926574 & -1.387256 & -1.923875 \\
\hline $\mathrm{H}$ & 8.300024 & -0.780832 & -3.549137 \\
\hline C & 6.345065 & 1.171490 & -3.691583 \\
\hline $\mathrm{H}$ & 5.312187 & 1.502390 & -3.848204 \\
\hline
\end{tabular}




\begin{tabular}{|lrrr|}
$H$ & 6.908308 & 2.015386 & -3.278594 \\
$H$ & 6.776714 & 0.929111 & -4.670710 \\
$\mathrm{C}$ & 5.623767 & -1.210574 & -3.446122 \\
$\mathrm{H}$ & 5.659800 & -2.131423 & -2.852560 \\
$\mathrm{H}$ & 4.571253 & -0.959565 & -3.614407 \\
$\mathrm{H}$ & 6.079004 & -1.427071 & -4.419618 \\
$\mathrm{C}$ & 5.520294 & 3.958329 & 1.707183 \\
$\mathrm{H}$ & 5.207445 & 4.399870 & 0.754163 \\
$\mathrm{H}$ & 4.620671 & 3.605635 & 2.221809 \\
$\mathrm{H}$ & 5.966079 & 4.751696 & 2.320008 \\
$\mathrm{C}$ & 7.788822 & 3.419571 & 0.809779 \\
$\mathrm{H}$ & 8.213192 & 4.196857 & 1.455424 \\
$\mathrm{H}$ & 8.567829 & 2.666690 & 0.643612 \\
$\mathrm{H}$ & 7.551469 & 3.884528 & -0.153850 \\
$\mathrm{C}$ & 6.964111 & 2.230364 & 2.846323 \\
$\mathrm{H}$ & 7.693364 & 1.422364 & 2.716623 \\
$\mathrm{H}$ & 7.424153 & 3.007458 & 3.469482 \\
$\mathrm{H}$ & 6.110052 & 1.823783 & 3.397981 \\
& & & \\
\hline
\end{tabular}

\section{Catalyst 2C 62}

\section{Datum}

Value

\begin{tabular}{lc}
\hline B3LYP/6-31G(d) Energy & -2164.797604 \\
\hline B3LYP-D3(BJ)/def2-TZVPP-IEF-PCM(DCM) Energy & -2165.895263 \\
\hline B3LYP-D3(BJ)/def2-TZVPP-IEF-PCM(DCM)//B3LYP/6-31G(d) Free Energy (Quasiharmonic) & -2165.017789 \\
\hline
\end{tabular}

Number of Imaginary Frequencies

Frequencies (Top 3 out of 321)

$\begin{array}{ll}\text { 1. } & 10.7236 \mathrm{~cm}^{-1} \\ \text { 2. } & 11.0179 \mathrm{~cm}^{-1} \\ \text { 3. } & 11.3974 \mathrm{~cm}^{-1}\end{array}$

\section{B3LYP/6-31G(d) Molecular Geometry in Cartesian Coordinates}

C

C

C

C

C

C

$\mathrm{H}$

$\mathrm{H}$

\subsection{5}

0.276391

$-0.767512$

$-1.975707$

$-2.091861$

$-1.062016$

0.938720

1.188646
$-3.645826$

$-2.425743$

$-1.890676$

$-2.646442$

- 3.898919

$-4.391566$

$-4.035381$

$-1.852583$

\section{642243}

3.016943

2.208220

2.067012

2. 729034

3. 496891

4.257063

3.146625 


\begin{tabular}{|c|c|c|c|}
\hline C & -0.650298 & -0.632621 & 1.530065 \\
\hline C & -3.029214 & -2.125349 & 1.277707 \\
\hline $\mathrm{H}$ & -3.014194 & -4.463236 & 2.614579 \\
\hline $\mathrm{H}$ & -1.162575 & -5.351634 & 3.995374 \\
\hline C & -2.932451 & -0.905993 & 0.635818 \\
\hline C & -1.714673 & -0.164449 & 0.773148 \\
\hline $\mathrm{H}$ & -3.951534 & -2.694742 & 1.197674 \\
\hline C & 1.132818 & 3.088942 & 4.659184 \\
\hline C & 2.147725 & 2.839079 & 3.765212 \\
\hline C & 1.995246 & 1.860256 & 2.745972 \\
\hline C & 0.769513 & 1.130331 & 2.652495 \\
\hline C & -0.260730 & 1.416538 & 3.590051 \\
\hline C & -0.082845 & 2.369231 & 4.567901 \\
\hline $\mathrm{H}$ & 3.966779 & 2.127244 & 1.916450 \\
\hline $\mathrm{H}$ & 1.259317 & 3.839076 & 5.435234 \\
\hline $\mathrm{H}$ & 3.085521 & 3.386528 & 3.824535 \\
\hline C & 3.031795 & 1.580056 & 1.824826 \\
\hline C & 0.635343 & 0.144494 & 1.620878 \\
\hline $\mathrm{H}$ & -1.200205 & 0.878981 & 3.523195 \\
\hline $\mathrm{H}$ & -0.883110 & 2.574205 & 5.273814 \\
\hline C & 1.675834 & -0.115289 & 0.730090 \\
\hline C & 2.909485 & 0.616819 & 0.841110 \\
\hline C & 1.503274 & -1.169226 & -0.353511 \\
\hline $\mathrm{H}$ & 0.462305 & -1.194569 & -0.692927 \\
\hline $\mathrm{H}$ & 2.125358 & -0.923032 & -1.215083 \\
\hline C & -1.554559 & 1.143112 & 0.044852 \\
\hline 0 & -1.633609 & 2.243644 & 0.545657 \\
\hline 0 & -1.300476 & 0.952377 & -1.270066 \\
\hline $\mathrm{H}$ & -1.226259 & 1.843308 & -1.664460 \\
\hline 0 & 1.927178 & -2.477381 & 0.053162 \\
\hline $\mathrm{H}$ & 1.342473 & -2.763276 & 0.773954 \\
\hline C & 4.071411 & 0.388946 & -0.069515 \\
\hline C & 4.667468 & 1.480869 & -0.718579 \\
\hline C & 4.615048 & -0.891471 & -0.247047 \\
\hline C & 5.787921 & 1.317017 & -1.541210 \\
\hline $\mathrm{H}$ & 4.226224 & 2.461625 & -0.581239 \\
\hline C & 5.733229 & -1.097423 & -1.062858 \\
\hline $\mathrm{H}$ & 4.140475 & -1.723340 & 0.256574 \\
\hline C & 6.297781 & 0.019750 & -1.694179 \\
\hline $\mathrm{H}$ & 7.164081 & -0.126720 & -2.332935 \\
\hline C & -4.083052 & -0.398019 & -0.163163 \\
\hline C & -4.588274 & 0.888770 & 0.039275 \\
\hline C & -4.700729 & -1.235634 & -1.108276 \\
\hline C & -5.693275 & 1.362715 & -0.685953 \\
\hline $\mathrm{H}$ & -4.120759 & 1.524504 & 0.783113 \\
\hline C & -5.803498 & -0.804588 & -1.846775 \\
\hline $\mathrm{H}$ & -4.283103 & -2.223192 & -1.266858 \\
\hline C & -6.280236 & 0.499295 & -1.614591 \\
\hline $\mathrm{H}$ & -7.135759 & 0.843090 & -2.183769 \\
\hline C & -6.502119 & -1.692713 & -2.894632 \\
\hline C & -6.201265 & 2.795638 & -0.434807 \\
\hline C & -6.471468 & -0.990935 & -4.273428 \\
\hline $\mathrm{H}$ & -6.989838 & -0.026572 & -4.254372 \\
\hline $\mathrm{H}$ & -5.440422 & -0.809866 & -4.597864 \\
\hline
\end{tabular}




\begin{tabular}{|c|c|c|c|}
\hline $\mathrm{H}$ & -6.961165 & -1.616272 & -5.030002 \\
\hline C & -7.971829 & -1.926032 & -2.470476 \\
\hline $\mathrm{H}$ & -8.488909 & -2.549151 & -3.210638 \\
\hline $\mathrm{H}$ & -8.022668 & -2.436708 & -1.501925 \\
\hline $\mathrm{H}$ & -8.525434 & -0.985514 & -2.381141 \\
\hline C & -5.824098 & -3.067564 & -3.045817 \\
\hline $\mathrm{H}$ & -5.843638 & -3.638099 & -2.110311 \\
\hline $\mathrm{H}$ & -6.353250 & -3.657963 & -3.802310 \\
\hline $\mathrm{H}$ & -4.781037 & -2.974907 & -3.368900 \\
\hline C & -5.074960 & 3.799682 & -0.781486 \\
\hline $\mathrm{H}$ & -4.167906 & 3.619952 & -0.195334 \\
\hline $\mathrm{H}$ & -4.806765 & 3.730199 & -1.842480 \\
\hline $\mathrm{H}$ & -5.405822 & 4.826800 & -0.583764 \\
\hline C & -6.589895 & 2.954582 & 1.054394 \\
\hline $\mathrm{H}$ & -5.740743 & 2.772802 & 1.720764 \\
\hline $\mathrm{H}$ & -6.950179 & 3.972641 & 1.246206 \\
\hline $\mathrm{H}$ & -7.387658 & 2.254475 & 1.328346 \\
\hline C & -7.434946 & 3.143301 & -1.288910 \\
\hline $\mathrm{H}$ & -7.219540 & 3.084926 & -2.361974 \\
\hline $\mathrm{H}$ & -8.281894 & 2.481882 & -1.072446 \\
\hline $\mathrm{H}$ & -7.754120 & 4.169187 & -1.073418 \\
\hline C & 6.338762 & -2.496548 & -1.289318 \\
\hline C & 6.461153 & 2.493844 & -2.274624 \\
\hline C & 7.934035 & 2.612674 & -1.815251 \\
\hline $\mathrm{H}$ & 7.994037 & 2.801417 & -0.737216 \\
\hline $\mathrm{H}$ & 8.502220 & 1.700505 & -2.025240 \\
\hline $\mathrm{H}$ & 8.430621 & 3.442031 & -2.334134 \\
\hline C & 5.763933 & 3.838548 & -1.993145 \\
\hline $\mathrm{H}$ & 4.720076 & 3.834128 & -2.326701 \\
\hline $\mathrm{H}$ & 5.780775 & 4.092229 & -0.927149 \\
\hline $\mathrm{H}$ & 6.279507 & 4.641686 & -2.532031 \\
\hline C & 6.420496 & 2.244363 & -3.800987 \\
\hline $\mathrm{H}$ & 6.941721 & 1.322118 & -4.077910 \\
\hline $\mathrm{H}$ & 5.387058 & 2.162379 & -4.156761 \\
\hline $\mathrm{H}$ & 6.900306 & 3.072614 & -4.337078 \\
\hline C & 5.613426 & -3.585656 & -0.475186 \\
\hline $\mathrm{H}$ & 5.691724 & -3.402315 & 0.602683 \\
\hline $\mathrm{H}$ & 4.550246 & -3.651702 & -0.728852 \\
\hline $\mathrm{H}$ & 6.070033 & -4.561355 & -0.678468 \\
\hline C & 7.828334 & -2.495917 & -0.871331 \\
\hline $\mathrm{H}$ & 8.269414 & -3.487702 & -1.030388 \\
\hline $\mathrm{H}$ & 8.417215 & -1.775034 & -1.448535 \\
\hline $\mathrm{H}$ & 7.937402 & -2.242834 & 0.189577 \\
\hline C & 6.228987 & -2.861521 & -2.788966 \\
\hline $\mathrm{H}$ & 6.758194 & -2.142380 & -3.423640 \\
\hline $\mathrm{H}$ & 6.661788 & -3.852633 & -2.973896 \\
\hline $\mathrm{H}$ & 5.181243 & -2.883343 & -3.109427 \\
\hline
\end{tabular}




\begin{tabular}{lc}
\hline B3LYP/6-31G(d) Energy & -2164.799834 \\
\hline B3LYP-D3(BJ)/def2-TZVPP-IEF-PCM(DCM) Energy & -2165.896104 \\
\hline B3LYP-D3(BJ)/def2-TZVPP-IEF-PCM(DCM)//B3LYP/6-31G(d) Free Energy (Quasiharmonic) & -2165.017698 \\
\hline
\end{tabular}

Number of Imaginary Frequencies

Frequencies (Top 3 out of 321)

$\begin{array}{ll}\text { 1. } & 10.9706 \mathrm{~cm}^{-1} \\ \text { 2. } & 12.5946 \mathrm{~cm}^{-1} \\ \text { 3. } & 12.8876 \mathrm{~cm}^{-1}\end{array}$

\section{B3LYP/6-31G(d) Molecular Geometry in Cartesian Coordinates}

C

C

C

C

C

C

$\mathrm{H}$

$\mathrm{H}$

C

C

$\mathrm{H}$

$\mathrm{H}$

C

C

$\mathrm{H}$

C

$\mathrm{C}$

C

C

C

C

$\mathrm{H}$

$\mathrm{H}$

$\mathrm{H}$

C

C

$\mathrm{H}$

$\mathrm{H}$

C

C

C

$\mathrm{H}$
0.331419

0.447583

$-0.636577$

$-1.853199$

$-1.939716$

$-0.872345$

1.169397

1.372516

$-0.552236$

$-2.946129$

$-2.868901$

$-0.950073$

$-2.884237$

$-1.659939$

$-3.870976$

1. 392637

2. 363919

2.157224

0.921386

$-0.063213$

0.167908

4.079903

1.560316

3. 307991

3.147964

0.733297

$-1.011179$

$-0.598596$

1.728439

2.968069

1.523549

0.844038
$-3.418403$

$-2.850596$

$-2.136400$

$-2.017460$

$-2.613894$

$-3.299151$

$-3.963562$

$-2.946525$

$-1.527675$

$-1.314932$

$-2.517918$

$-3.750852$

$-0.748115$

$-0.869888$

$-1.245396$

$-4.854593$

$-3.897030$

$-2.806892$

$-2.704038$

$-3.708674$

$-4.756707$

$-1.899183$

$-5.685353$

$-3.959085$

$-1.817970$

$-1.598981$

$-3.638772$

$-5.512600$

$-0.638049$

$-0.750688$

0.504312

0.188480

\subsection{8}

2.662747

2.082457

2.827372

4.114368

4.647146

4.337789

2.104373

0.786357

2. 262790

4.670862

5.632328

1.005611

0.270835

2.829623

$-2.665555$

$-2.488172$

$-1.600147$

$-0.889611$

$-1.100037$

$-1.962683$

$-1.950688$

$-3.345727$

$-3.024381$

$-1.396917$

0.005564

$-0.578213$

$-2.110526$

0.187033

$-0.537372$

1.174318

1.974489 


\begin{tabular}{|c|c|c|c|}
\hline $\mathrm{H}$ & 2.478533 & 0.761973 & 1.637215 \\
\hline C & -1.558833 & -0.255907 & -1.097769 \\
\hline 0 & -1.651579 & -0.836955 & -2.153108 \\
\hline 0 & -1.339008 & 1.088558 & -1.029428 \\
\hline $\mathrm{H}$ & -1.284463 & 1.408829 & -1.951420 \\
\hline 0 & 1.056797 & 1.714996 & 0.576174 \\
\hline $\mathrm{H}$ & 0.190295 & 1.539037 & 0.170096 \\
\hline C & 4.100604 & 0.210846 & -0.374458 \\
\hline C & 3.922864 & 1.596230 & -0.531087 \\
\hline c & 5.384162 & -0.280112 & -0.111238 \\
\hline C & 4.998208 & 2.480000 & -0.422733 \\
\hline $\mathrm{H}$ & 2.923462 & 1.962730 & -0.721089 \\
\hline C & 6.492423 & 0.573833 & 0.001422 \\
\hline H & 5.510116 & -1.350489 & 0.024696 \\
\hline C & 6.271946 & 1.944818 & -0.158360 \\
\hline $\mathrm{H}$ & 7.109668 & 2.626462 & -0.069899 \\
\hline C & -4.071321 & -0.035094 & 0.453885 \\
\hline c & -4.598047 & -0.375385 & -0.799899 \\
\hline C & -4.694322 & 0.965873 & 1.212674 \\
\hline C & -5.727769 & 0.274435 & -1.310750 \\
\hline $\mathrm{H}$ & -4.122901 & -1.166278 & -1.365842 \\
\hline C & -5.826870 & 1.637091 & 0.739067 \\
\hline $\mathrm{H}$ & -4.262475 & 1.225244 & 2.172298 \\
\hline C & -6.318370 & 1.272124 & -0.522853 \\
\hline $\mathrm{H}$ & -7.194314 & 1.786358 & -0.907000 \\
\hline c & -6.529672 & 2.749241 & 1.542193 \\
\hline C & -6.326562 & -0.072050 & -2.688202 \\
\hline C & -6.510214 & 4.065186 & 0.728724 \\
\hline $\mathrm{H}$ & -5.481857 & 4.383322 & 0.523092 \\
\hline $\mathrm{H}$ & -7.025550 & 3.959752 & -0.231670 \\
\hline $\mathrm{H}$ & -7.008630 & 4.866549 & 1.287743 \\
\hline C & -5.847026 & 3.016809 & 2.897170 \\
\hline $\mathrm{H}$ & -6.383195 & 3.810706 & 3.429273 \\
\hline $\mathrm{H}$ & -5.850763 & 2.128901 & 3.539714 \\
\hline $\mathrm{H}$ & -4.808946 & 3.345515 & 2.773973 \\
\hline C & -7.995751 & 2.338042 & 1.816789 \\
\hline $\mathrm{H}$ & -8.040340 & 1.410779 & 2.399542 \\
\hline $\mathrm{H}$ & -8.511646 & 3.121754 & 2.385062 \\
\hline $\mathrm{H}$ & -8.555683 & 2.175946 & 0.889787 \\
\hline C & -6.292269 & 1.183859 & -3.591610 \\
\hline $\mathrm{H}$ & -6.865368 & 2.012902 & -3.162970 \\
\hline $\mathrm{H}$ & -5.263237 & 1.530180 & -3.741123 \\
\hline $\mathrm{H}$ & -6.719944 & 0.956713 & -4.575784 \\
\hline C & -7.791653 & -0.537654 & -2.515308 \\
\hline $\mathrm{H}$ & -8.416660 & 0.236580 & -2.057729 \\
\hline $\mathrm{H}$ & -8.229762 & -0.786258 & -3.489705 \\
\hline $\mathrm{H}$ & -7.847090 & -1.429833 & -1.881132 \\
\hline C & -5.546639 & -1.194182 & -3.400016 \\
\hline $\mathrm{H}$ & -4.497532 & -0.925480 & -3.565594 \\
\hline $\mathrm{H}$ & -5.571196 & -2.130691 & -2.831263 \\
\hline $\mathrm{H}$ & -5.998587 & -1.392180 & -4.378570 \\
\hline C & 7.882979 & -0.019693 & 0.301104 \\
\hline c & 4.825425 & 4.004710 & -0.566731 \\
\hline & 5.203509 & 4.686410 & 0.770203 \\
\hline
\end{tabular}




\begin{tabular}{|c|c|c|c|}
\hline $\mathrm{H}$ & 4.550605 & 4.343196 & 1.580686 \\
\hline $\mathrm{H}$ & 6.237853 & 4.471011 & 1.059299 \\
\hline $\mathrm{H}$ & 5.099101 & 5.775558 & 0.687493 \\
\hline C & 3.376075 & 4. 397708 & -0.912354 \\
\hline $\mathrm{H}$ & 3.065399 & 3.979741 & -1.877071 \\
\hline $\mathrm{H}$ & 2.664748 & 4.057878 & -0.152461 \\
\hline $\mathrm{H}$ & 3.298738 & 5.488928 & -0.984023 \\
\hline C & 5.748118 & 4.533998 & -1.689882 \\
\hline $\mathrm{H}$ & 6.805585 & 4.340894 & -1.480072 \\
\hline $\mathrm{H}$ & 5.506330 & 4.062445 & -2.649367 \\
\hline $\mathrm{H}$ & 5.625740 & 5.618194 & -1.804053 \\
\hline C & 8.982648 & 1.057035 & 0.369291 \\
\hline $\mathrm{H}$ & 9.077944 & 1.603324 & -0.576058 \\
\hline $\mathrm{H}$ & 8.793837 & 1.784339 & 1.167104 \\
\hline $\mathrm{H}$ & 9.949372 & 0.584151 & 0.576730 \\
\hline C & 8.266713 & -1.023416 & -0.812313 \\
\hline $\mathrm{H}$ & 9.254941 & -1.456289 & -0.613094 \\
\hline $\mathrm{H}$ & 7.550784 & -1.849010 & -0.882975 \\
\hline $\mathrm{H}$ & 8.303149 & -0.528511 & -1.789628 \\
\hline C & 7.846378 & -0.755825 & 1.661561 \\
\hline $\mathrm{H}$ & 7.108970 & -1.565376 & 1.666530 \\
\hline $\mathrm{H}$ & 8.826523 & -1.194811 & 1.885990 \\
\hline $\mathrm{H}$ & 7.589109 & -0.065365 & 2.473005 \\
\hline
\end{tabular}

\section{Catalyst 2C 64}

Datum

Value

\begin{tabular}{lc}
\hline B3LYP/6-31G(d) Energy & -2164.796903 \\
\hline B3LYP-D3(BJ)/def2-TZVPP-IEF-PCM(DCM) Energy & -2165.895354 \\
\hline B3LYP-D3(BJ)/def2-TZVPP-IEF-PCM(DCM)//B3LYP/6-31G(d) Free Energy (Quasiharmonic) & -2165.017687 \\
\hline
\end{tabular}

Number of Imaginary Frequencies

Frequencies (Top 3 out of 321)
1. $11.0547 \mathrm{~cm}^{-1}$
2. $12.7891 \mathrm{~cm}^{-1}$
3. $13.6059 \mathrm{~cm}^{-1}$

\section{B3LYP/6-31G(d) Molecular Geometry in Cartesian Coordinates}

$\begin{array}{lrrr}C & -0.006245 & -4.842910 & -1.627306 \\ C & -0.181915 & -3.721222 & -0.845287 \\ C & 0.845269 & -2.740448 & -0.730039\end{array}$




\begin{tabular}{|c|c|c|c|}
\hline C & 2.075753 & -2.959553 & -1.428875 \\
\hline C & 2.222507 & -4.125867 & -2.227655 \\
\hline C & 1.205600 & -5.047162 & -2.328967 \\
\hline $\mathrm{H}$ & -0.802009 & -5.578868 & -1.700857 \\
\hline $\mathrm{H}$ & -1.107662 & -3.581033 & -0.297129 \\
\hline C & 0.696367 & -1.555676 & 0.066082 \\
\hline C & 3.115259 & -2.005110 & -1.314022 \\
\hline $\mathrm{H}$ & 3.159064 & -4.276560 & -2.759124 \\
\hline $\mathrm{H}$ & 1.330975 & -5.934480 & -2.943170 \\
\hline C & 2.987149 & -0.870624 & -0.537851 \\
\hline C & 1.753899 & -0.660161 & 0.160868 \\
\hline $\mathrm{H}$ & 4.033310 & -2.163363 & -1.873849 \\
\hline C & -0.888032 & -2.650544 & 4.873477 \\
\hline C & -1.960865 & -2.049887 & 4.257368 \\
\hline C & -1.872692 & -1.606342 & 2.909719 \\
\hline C & -0.650868 & -1.790625 & 2.188440 \\
\hline C & 0.439443 & -2.415109 & 2.857004 \\
\hline C & 0.323321 & -2.832535 & 4.163802 \\
\hline $\mathrm{H}$ & -3.897754 & -0.876645 & 2.807593 \\
\hline $\mathrm{H}$ & -0.965613 & -2.985035 & 5.904469 \\
\hline $\mathrm{H}$ & -2.896510 & -1.904431 & 4. 792321 \\
\hline C & -2.967583 & -0.993883 & 2.257177 \\
\hline C & -0.583593 & -1.340513 & 0.828614 \\
\hline $\mathrm{H}$ & 1.376748 & -2.553046 & 2.329349 \\
\hline $\mathrm{H}$ & 1.169737 & -3.303289 & 4.656222 \\
\hline C & -1.679286 & -0.740380 & 0.211427 \\
\hline C & -2.905014 & -0.568392 & 0.942971 \\
\hline C & -1.564046 & -0.250552 & -1.223846 \\
\hline $\mathrm{H}$ & -2.229634 & 0.599425 & -1.381049 \\
\hline $\mathrm{H}$ & -0.541215 & 0.087898 & -1.420183 \\
\hline C & 1.651521 & 0.496885 & 1.117044 \\
\hline 0 & 2.263890 & 0.584595 & 2.156011 \\
\hline 0 & 0.784736 & 1.452066 & 0.693959 \\
\hline $\mathrm{H}$ & 0.758924 & 2.124481 & 1.402366 \\
\hline 0 & -1.963449 & -1.225385 & -2.196700 \\
\hline $\mathrm{H}$ & -1.333244 & -1.962589 & -2.148276 \\
\hline C & -4.119067 & 0.071446 & 0.353302 \\
\hline C & -4.666955 & -0.377791 & -0.861047 \\
\hline C & -4.759590 & 1.105583 & 1.043522 \\
\hline C & -5.827239 & 0.194477 & -1.385672 \\
\hline $\mathrm{H}$ & -4.159294 & -1.175738 & -1.386849 \\
\hline C & -5.928682 & 1.707220 & 0.551330 \\
\hline $\mathrm{H}$ & -4.320316 & 1.453199 & 1.974326 \\
\hline C & -6.438324 & 1.234303 & -0.661230 \\
\hline $\mathrm{H}$ & -7.336632 & 1.686001 & -1.065611 \\
\hline C & 4.106926 & 0.109937 & -0.473447 \\
\hline C & 5.401531 & -0.323546 & -0.180830 \\
\hline C & 3.885395 & 1.470373 & -0.745724 \\
\hline C & 6.482922 & 0.570648 & -0.141586 \\
\hline $\mathrm{H}$ & 5.556221 & -1.374654 & 0.043999 \\
\hline C & 4.932420 & 2.392476 & -0.718396 \\
\hline $\mathrm{H}$ & 2.881304 & 1.790787 & -0.996216 \\
\hline C & 6.220691 & 1.916635 & -0.412517 \\
\hline $\mathrm{H}$ & 7.038633 & 2.627121 & -0.385269 \\
\hline
\end{tabular}




\begin{tabular}{|c|c|c|c|}
\hline C & 4.722151 & 3.891125 & -1.008241 \\
\hline C & 7.890439 & 0.046534 & 0.202627 \\
\hline C & 5.583203 & 4. 310981 & -2.223189 \\
\hline $\mathrm{H}$ & 6.649991 & 4.137720 & -2.047154 \\
\hline H & 5.296367 & 3.748817 & -3.119280 \\
\hline $\mathrm{H}$ & 5.449668 & 5.379004 & -2.434765 \\
\hline C & 5.146103 & 4.716805 & 0.230071 \\
\hline $\mathrm{H}$ & 5.002142 & 5.787803 & 0.041330 \\
\hline $\mathrm{H}$ & 4.549664 & 4.441609 & 1.107332 \\
\hline H & 6.200263 & 4.562202 & 0.482587 \\
\hline C & 3.252281 & 4.225650 & -1.326441 \\
\hline H & 2.584431 & 3.966676 & -0.497067 \\
\hline H & 3.149837 & 5.301010 & -1.510898 \\
\hline $\mathrm{H}$ & 2.899148 & 3.701949 & -2.221978 \\
\hline C & 8.307017 & -1.026270 & -0.831590 \\
\hline $\mathrm{H}$ & 7.616356 & -1.876020 & -0.839380 \\
\hline H & 8.332268 & -0.606090 & -1.843632 \\
\hline $\mathrm{H}$ & 9.306863 & -1.412847 & -0.598928 \\
\hline C & 7.872547 & -0.583620 & 1.615740 \\
\hline H & 7.169070 & -1.420097 & 1.681473 \\
\hline H & 8.868174 & -0.963444 & 1.876406 \\
\hline $\mathrm{H}$ & 7.581409 & 0.155508 & 2.370519 \\
\hline C & 8.954808 & 1.159873 & 0.188919 \\
\hline $\mathrm{H}$ & 9.037936 & 1.634913 & -0.795418 \\
\hline H & 8.740603 & 1.939498 & 0.928735 \\
\hline H & 9.934811 & 0.734716 & 0.433283 \\
\hline C & -6.592459 & 2.846914 & 1.348958 \\
\hline C & -6.441144 & -0.267760 & -2.721286 \\
\hline C & -5.657835 & -1.436432 & -3.350582 \\
\hline $\mathrm{H}$ & -4.611688 & -1.176227 & -3.542891 \\
\hline H & -5.671308 & -2.323882 & -2.707287 \\
\hline $\mathrm{H}$ & -6.118072 & -1.713643 & -4.306103 \\
\hline C & -6.432553 & 0.912508 & -3.722089 \\
\hline $\mathrm{H}$ & -7.005534 & 1.768329 & -3.349030 \\
\hline H & -5.408932 & 1.253000 & -3.915065 \\
\hline $\mathrm{H}$ & -6.874537 & 0.606195 & -4.678394 \\
\hline C & -7.898445 & -0.733544 & -2.492507 \\
\hline H & -7.934838 & -1.570124 & -1.785242 \\
\hline H & -8.528780 & 0.068458 & -2.093824 \\
\hline H & -8.344513 & -1.067981 & -3.437390 \\
\hline C & -7.849246 & 3.401802 & 0.651429 \\
\hline H & -7.617477 & 3.819489 & -0.334995 \\
\hline H & -8.620307 & 2.633362 & 0.524821 \\
\hline H & -8.281698 & 4.206108 & 1.257348 \\
\hline C & -5.588491 & 4.011338 & 1.521751 \\
\hline H & -6.046419 & 4.830056 & 2.090538 \\
\hline H & -4.687558 & 3.696978 & 2.058790 \\
\hline H & -5.276206 & 4.406598 & 0.548372 \\
\hline C & -7.010409 & 2.321262 & 2.742842 \\
\hline H & -6.151809 & 1.948184 & 3.310905 \\
\hline $\mathrm{H}$ & -7.477420 & 3.121515 & 3.330423 \\
\hline $\mathrm{H}$ & -7.732031 & 1.501357 & 2.651046 \\
\hline
\end{tabular}




\section{Catalyst 2C 65}

Datum

Value

\begin{tabular}{lc}
\hline B3LYP/6-31G(d) Energy & -2164.798475 \\
\hline B3LYP-D3(BJ)/def2-TZVPP-IEF-PCM(DCM) Energy & -2165.895662 \\
\hline B3LYP-D3(BJ)/def2-TZVPP-IEF-PCM(DCM)//B3LYP/6-31G(d) Free Energy (Quasiharmonic) & -2165.017668
\end{tabular}

Number of Imaginary Frequencies

Frequencies (Top 3 out of 321)

$\begin{array}{ll}\text { 1. } & 10.7272 \mathrm{~cm}^{-1} \\ \text { 2. } & 12.8111 \mathrm{~cm}^{-1} \\ \text { 3. } & 14.0720 \mathrm{~cm}^{-1}\end{array}$

\section{B3LYP/6-31G(d) Molecular Geometry in Cartesian Coordinates}

C

C

C

C

C

C

$\mathrm{H}$

$\mathrm{H}$

C

C

$\mathrm{H}$

$\mathrm{H}$

C

C

$\mathrm{H}$

C

C

C

C

C

C

$\mathrm{H}$

$\mathrm{H}$

$\mathrm{H}$

C

C

$\mathrm{H}$

$\mathrm{H}$

C
$-0.038157$

0.155333

$-0.864100$

$-2.103524$

$-2.268984$

$-1.260530$

0.751841

1.089958

$-0.697041$

$-3.132210$

$-3.212272$

$-1.399854$

$-2.982964$

$-1.741282$

$-4.057673$

0.915061

1.980275

1.884259

0.661710

$-0.420117$

$-0.296368$

3.905070

0.999465

2. 916381

2.972695

0.585478

$-1.355790$

$-1.135523$

1.672072
$-4.857989$

$-3.736284$

$-2.749066$

$-2.957940$

$-4.125736$

$-5.055230$

$-5.599068$

$-3.598629$

$-1.564390$

$-1.992728$

$-4.270174$

$-5.943214$

$-0.851817$

$-0.654749$

$-2.145983$

$-2.756177$

$-2.132449$

$-1.660655$

$-1.839665$

$-2.490118$

$-2.935246$

$-0.919160$

$-3.112853$

$-1.990461$

$-1.027675$

$-1.357906$

$-2.630147$

$-3.428355$

$-0.732119$
$-1.645423$

$-0.868117$

$-0.741550$

$-1.428125$

$-2.221272$

$-2.331055$

$-1.728979$

$-0.334587$

0.049668

$-1.307504$

$-2.742464$

$-2.941302$

$-0.543539$

0.143798

$-1.856442$

4.823405

4.216789

2.879247

2.158071

2.815777

4.112994

2. 784062

5.846428

4.751862

2.235650

0.809737

2. 285511

4.596237

0.203479 


\begin{tabular}{|c|c|c|c|}
\hline C & 2.901454 & -0.572792 & 0.931844 \\
\hline C & 1.541563 & -0.205150 & -1.216779 \\
\hline $\mathrm{H}$ & 0.520556 & 0.150797 & -1.387752 \\
\hline $\mathrm{H}$ & 2.213632 & 0.640791 & -1.363905 \\
\hline C & -1.548772 & 0.570479 & 0.994106 \\
\hline 0 & -0.828422 & 1.506443 & 0.715913 \\
\hline 0 & -2.276523 & 0.517785 & 2.129752 \\
\hline $\mathrm{H}$ & -2.104069 & 1.354942 & 2.602867 \\
\hline 0 & 1.917049 & -1.163881 & -2.216585 \\
\hline $\mathrm{H}$ & 1.278199 & -1.894016 & -2.177910 \\
\hline C & 4.111836 & 0.077368 & 0.347234 \\
\hline C & 4.637036 & -0.329050 & -0.886477 \\
\hline C & 4.774708 & 1.083218 & 1.064533 \\
\hline C & 5.797248 & 0.252232 & -1.412600 \\
\hline $\mathrm{H}$ & 4.117360 & -1.104539 & -1.438045 \\
\hline C & 5.939979 & 1.687292 & 0.576992 \\
\hline $\mathrm{H}$ & 4.350124 & 1.404613 & 2.011311 \\
\hline C & 6.430920 & 1.254228 & -0.663527 \\
\hline $\mathrm{H}$ & 7.327013 & 1.712947 & -1.058623 \\
\hline C & -4.092797 & 0.140168 & -0.472369 \\
\hline C & -3.865160 & 1.498936 & -0.724617 \\
\hline C & -5.394820 & -0.286834 & -0.182966 \\
\hline C & -4.906354 & 2.434171 & -0.677576 \\
\hline $\mathrm{H}$ & -2.860146 & 1.823598 & -0.972469 \\
\hline C & -6.465833 & 0.613155 & -0.132230 \\
\hline $\mathrm{H}$ & -5.557494 & -1.339880 & 0.027037 \\
\hline C & -6.194946 & 1.967081 & -0.380662 \\
\hline $\mathrm{H}$ & -7.010019 & 2.676496 & -0.340662 \\
\hline C & -7.879553 & 0.096030 & 0.199798 \\
\hline C & -4.599196 & 3.920459 & -0.946498 \\
\hline C & -8.300786 & -0.959877 & -0.849878 \\
\hline $\mathrm{H}$ & -8.316820 & -0.526745 & -1.856576 \\
\hline $\mathrm{H}$ & -7.617834 & -1.815684 & -0.865134 \\
\hline $\mathrm{H}$ & -9.305368 & -1.340089 & -0.627277 \\
\hline C & -8.935605 & 1.217504 & 0.196224 \\
\hline $\mathrm{H}$ & -9.920030 & 0.796181 & 0.429255 \\
\hline $\mathrm{H}$ & -8.720084 & 1.985373 & 0.947846 \\
\hline $\mathrm{H}$ & -9.009675 & 1.706973 & -0.781624 \\
\hline C & -7.873602 & -0.553067 & 1.604400 \\
\hline $\mathrm{H}$ & -7.582920 & 0.174525 & 2.370734 \\
\hline $\mathrm{H}$ & -8.872820 & -0.930247 & 1.854965 \\
\hline $\mathrm{H}$ & -7.175520 & -1.394692 & 1.662697 \\
\hline C & -3.589083 & 4.429504 & 0.110634 \\
\hline $\mathrm{H}$ & -4.014944 & 4.360411 & 1.118828 \\
\hline $\mathrm{H}$ & -2.657492 & 3.854535 & 0.100220 \\
\hline $\mathrm{H}$ & -3.337342 & 5.480450 & -0.077983 \\
\hline C & -5.857242 & 4.806597 & -0.873061 \\
\hline $\mathrm{H}$ & -6.607590 & 4.515013 & -1.617012 \\
\hline $\mathrm{H}$ & -5.584401 & 5.849286 & -1.071066 \\
\hline $\mathrm{H}$ & -6.325318 & 4.771374 & 0.117370 \\
\hline C & -3.984442 & 4.075205 & -2.358018 \\
\hline $\mathrm{H}$ & -3.051432 & 3.512209 & -2.461199 \\
\hline $\mathrm{H}$ & -4.677773 & 3.719784 & -3.129017 \\
\hline $\mathrm{H}$ & -3.760556 & 5.129476 & -2.561680 \\
\hline
\end{tabular}




\begin{tabular}{|rrrr|} 
C & 6.627412 & 2.793103 & 1.402600 \\
C & 6.322803 & -0.222354 & -2.781373 \\
H & 6.640830 & -1.735181 & -2.715082 \\
H & 7.408753 & -1.942586 & -1.960551 \\
H & 5.754529 & -2.325739 & -2.462329 \\
C & 7.012690 & -2.089124 & -3.684747 \\
H & 7.604675 & 0.515768 & -3.210883 \\
H & 7.440975 & 1.594966 & -3.310189 \\
H & 8.425801 & 0.357991 & -2.501799 \\
C & 7.935723 & 0.142331 & -4.186762 \\
H & 5.237998 & 0.025241 & -3.858032 \\
H & 4.299644 & -0.486373 & -3.621948 \\
H & 5.020069 & 1.095931 & -3.949537 \\
C & 5.584255 & -0.333545 & -4.835665 \\
H & 7.050712 & 2.220897 & 2.776479 \\
$H$ & 6.191935 & 1.844955 & 3.342353 \\
H & 7.758581 & 1.392929 & 2.654292 \\
C & 7.535581 & 2.996885 & 3.381994 \\
H & 5.641450 & 3.965519 & 1.619674 \\
H & 6.115152 & 4.759193 & 2.210844 \\
H & 5.328029 & 4.395928 & 0.661765 \\
C & 4.739480 & 3.646767 & 2.152137 \\
H & 7.886098 & 3.351516 & 0.711107 \\
H & 7.652164 & 3.803697 & -0.259396 \\
H & 8.335340 & 4.130031 & 1.338222 \\
& 8.644816 & 2.576584 & 0.552982 \\
\hline & & & \\
\hline & & & \\
\hline & & & \\
\hline
\end{tabular}

\section{Catalyst 2C 66}

Datum

Value

\begin{tabular}{lr}
\hline B3LYP/6-31G(d) Energy & -2164.800262 \\
\hline B3LYP-D3(BJ)/def2-TZVPP-IEF-PCM(DCM) Energy & -2165.896378 \\
\hline B3LYP-D3(BJ)/def2-TZVPP-IEF-PCM(DCM)//B3LYP/6-31G(d) Free Energy (Quasiharmonic) & -2165.017665 \\
\hline
\end{tabular}

Number of Imaginary Frequencies

Frequencies (Top 3 out of 321)

$\begin{array}{ll}\text { 1. } & 12.1782 \mathrm{~cm}^{-1} \\ \text { 2. } & 13.0949 \mathrm{~cm}^{-1} \\ \text { 3. } & 13.8144 \mathrm{~cm}^{-1}\end{array}$




\begin{tabular}{|c|c|c|c|}
\hline C & -0.295752 & -3.370803 & 3.965121 \\
\hline C & -0.421278 & -2.819930 & 2.708951 \\
\hline C & 0.657911 & -2.112218 & 2.111746 \\
\hline C & 1.879446 & -1.981593 & 2.846571 \\
\hline C & 1.975698 & -2.560808 & 4.140683 \\
\hline C & 0.912924 & -3.240241 & 4.689922 \\
\hline $\mathrm{H}$ & -1.130039 & -3.911232 & 4.403726 \\
\hline H & -1.349884 & -2.924408 & 2.158260 \\
\hline C & 0.563927 & -1.521647 & 0.807870 \\
\hline C & 2.967374 & -1.284879 & 2.265217 \\
\hline H & 2.908567 & -2.456438 & 4.689464 \\
\hline H & 0.998134 & -3.678682 & 5.680458 \\
\hline C & 2.895540 & -0.734311 & 1.001454 \\
\hline C & 1.666875 & -0.868723 & 0.276240 \\
\hline H & 3.896156 & -1.206638 & 2.824455 \\
\hline C & -1.390898 & -4.901410 & -2.585746 \\
\hline C & -2.362773 & -3.942777 & -2.418134 \\
\hline C & -2.154199 & -2.839979 & -1.546209 \\
\hline C & -0.915793 & -2.725448 & -0.842125 \\
\hline C & 0.069378 & -3.731716 & -1.042034 \\
\hline C & -0.163652 & -4.792116 & -1.888858 \\
\hline $\mathrm{H}$ & -4.078823 & -1.941091 & -1.902647 \\
\hline $\mathrm{H}$ & -1.560014 & -5.741892 & -3.253496 \\
\hline H & -3.308861 & -4.013646 & -2.949663 \\
\hline C & -3.145531 & -1.850064 & -1.352830 \\
\hline C & -0.726027 & -1.607034 & 0.035724 \\
\hline H & 1.019147 & -3.653489 & -0.524697 \\
\hline H & 0.603227 & -5.549119 & -2.028830 \\
\hline C & -1.722108 & -0.645084 & 0.207960 \\
\hline C & -2.965467 & -0.770056 & -0.508831 \\
\hline C & -1.511710 & 0.511598 & 1.176898 \\
\hline H & -2.463039 & 0.775540 & 1.643373 \\
\hline $\mathrm{H}$ & -0.824902 & 0.209801 & 1.975975 \\
\hline C & 1.556365 & -0.274392 & -1.100231 \\
\hline 0 & 1.642603 & -0.870079 & -2.147829 \\
\hline 0 & 1.337098 & 1.071147 & -1.049683 \\
\hline H & 1.277973 & 1.378103 & -1.975917 \\
\hline 0 & -1.051048 & 1.714221 & 0.556534 \\
\hline H & -0.184973 & 1.535622 & 0.150452 \\
\hline C & -4.100463 & 0.190474 & -0.357734 \\
\hline C & -3.928193 & 1.569715 & -0.499968 \\
\hline C & -5.394372 & -0.310382 & -0.118271 \\
\hline C & -5.012233 & 2.457061 & -0.404438 \\
\hline H & -2.928341 & 1.948904 & -0.673576 \\
\hline C & -6.498814 & 0.537134 & -0.021568 \\
\hline $\mathrm{H}$ & -5.512630 & -1.380481 & 0.007942 \\
\hline C & -6.280148 & 1.919373 & -0.169115 \\
\hline H & -7.129787 & 2.587804 & -0.091764 \\
\hline C & 4.076722 & -0.024585 & 0.432947 \\
\hline C & 4.701160 & 0.986415 & 1.168078 \\
\hline C & 4.598169 & -0.381066 & -0.822578 \\
\hline C & 5.831125 & 1.659056 & 0.677085 \\
\hline H & 4.278545 & 1.261981 & 2.129831 \\
\hline
\end{tabular}




\begin{tabular}{|c|c|c|c|}
\hline C & 5.718773 & 0.264927 & -1.348119 \\
\hline $\mathrm{H}$ & 4.121807 & -1.181931 & -1.373300 \\
\hline C & 6.314812 & 1.280804 & -0.578938 \\
\hline $\mathrm{H}$ & 7.183567 & 1.788069 & -0.981427 \\
\hline C & 6.311915 & -0.098745 & -2.723286 \\
\hline C & 6.477298 & 2.776233 & 1.519632 \\
\hline C & 5.530413 & -1.231201 & -3.416731 \\
\hline $\mathrm{H}$ & 5.559498 & -2.160298 & -2.836190 \\
\hline $\mathrm{H}$ & 4.479990 & -0.966292 & -3.580160 \\
\hline $\mathrm{H}$ & 5.977597 & -1.440897 & -4.395035 \\
\hline C & 7.778439 & -0.560049 & -2.550343 \\
\hline $\mathrm{H}$ & 8.212916 & -0.820710 & -3.523224 \\
\hline $\mathrm{H}$ & 8.404095 & 0.220952 & -2.105367 \\
\hline $\mathrm{H}$ & 7.837449 & -1.443907 & -1.904910 \\
\hline C & 6.272247 & 1.144860 & -3.643446 \\
\hline $\mathrm{H}$ & 6.846309 & 1.980334 & -3.228889 \\
\hline $\mathrm{H}$ & 6.695934 & 0.904812 & -4.626283 \\
\hline $\mathrm{H}$ & 5.242110 & 1.487801 & -3.793243 \\
\hline C & 5.442686 & 3.901102 & 1.761693 \\
\hline $\mathrm{H}$ & 5.110964 & 4.338658 & 0.813201 \\
\hline $\mathrm{H}$ & 4.555504 & 3.533844 & 2.287607 \\
\hline $\mathrm{H}$ & 5.883569 & 4.700875 & 2.369337 \\
\hline C & 7.707528 & 3.398365 & 0.832136 \\
\hline $\mathrm{H}$ & 7.449128 & 3.861868 & -0.126764 \\
\hline $\mathrm{H}$ & 8.129943 & 4.180759 & 1.472568 \\
\hline $\mathrm{H}$ & 8.495033 & 2.657265 & 0.653375 \\
\hline C & 6.930999 & 2.197304 & 2.880914 \\
\hline $\mathrm{H}$ & 6.091673 & 1.779961 & 3.446969 \\
\hline $\mathrm{H}$ & 7.670052 & 1.399922 & 2.741665 \\
\hline $\mathrm{H}$ & 7.388865 & 2.982152 & 3.495469 \\
\hline C & -7.925518 & 0.016602 & 0.242515 \\
\hline C & -4.758007 & 3.968940 & -0.557834 \\
\hline C & -6.048535 & 4.800927 & -0.434785 \\
\hline $\mathrm{H}$ & -6.780493 & 4.538307 & -1.207626 \\
\hline $\mathrm{H}$ & -6.524715 & 4.674952 & 0.544498 \\
\hline $\mathrm{H}$ & -5.813572 & 5.865156 & -0.551041 \\
\hline C & -4.139101 & 4.247908 & -1.948191 \\
\hline $\mathrm{H}$ & -3.187140 & 3.724059 & -2.081605 \\
\hline $\mathrm{H}$ & -4.814103 & 3.926520 & -2.750055 \\
\hline $\mathrm{H}$ & -3.950072 & 5.321439 & -2.072427 \\
\hline C & -3.774567 & 4.434722 & 0.543433 \\
\hline $\mathrm{H}$ & -4.206782 & 4.279928 & 1.539213 \\
\hline $\mathrm{H}$ & -2.825727 & 3.890837 & 0.504064 \\
\hline $\mathrm{H}$ & -3.559245 & 5.505101 & 0.431968 \\
\hline C & -8.467020 & 0.630317 & 1.555649 \\
\hline $\mathrm{H}$ & -7.838164 & 0.348038 & 2.407825 \\
\hline $\mathrm{H}$ & -8.498443 & 1.723970 & 1.512717 \\
\hline $\mathrm{H}$ & -9.486141 & 0.274745 & 1.752236 \\
\hline C & -7.976473 & -1.517273 & 0.376688 \\
\hline $\mathrm{H}$ & -9.008623 & -1.838443 & 0.557555 \\
\hline $\mathrm{H}$ & -7.629957 & -2.017979 & -0.534556 \\
\hline $\mathrm{H}$ & -7.367787 & -1.874140 & 1.215243 \\
\hline C & -8.847469 & 0.424590 & -0.931386 \\
\hline $\mathrm{H}$ & -8.494454 & -0.008799 & -1.874273 \\
\hline
\end{tabular}




$\begin{array}{llll}H & -9.870480 & 0.069535 & -0.755530 \\ H & -8.889549 & 1.511203 & -1.059498\end{array}$

\section{Catalyst 2C 67}

Datum

Value

\begin{tabular}{lc}
\hline B3LYP/6-31G(d) Energy & -2164.797369 \\
\hline B3LYP-D3(BJ)/def2-TZVPP-IEF-PCM(DCM) Energy & -2165.895497 \\
\hline B3LYP-D3(BJ)/def2-TZVPP-IEF-PCM(DCM)//B3LYP/6-31G(d) Free Energy (Quasiharmonic) & -2165.017631 \\
\hline
\end{tabular}

Number of Imaginary Frequencies

Frequencies (Top 3 out of 321)

$\begin{array}{ll}\text { 1. } & 10.5703 \mathrm{~cm}^{-1} \\ \text { 2. } & 13.0323 \mathrm{~cm}^{-1} \\ \text { 3. } & 14.1274 \mathrm{~cm}^{-1}\end{array}$

\section{B3LYP/6-31G(d) Molecular Geometry in Cartesian Coordinates}

\begin{tabular}{|c|c|c|c|}
\hline C & -0.009997 & -4.832577 & -1.625270 \\
\hline C & 0.166034 & -3.711060 & -0.843067 \\
\hline C & -0.859420 & -2.728117 & -0.730681 \\
\hline C & -2.088636 & -2.944839 & -1.432651 \\
\hline C & -2.235700 & -4.111053 & -2.231550 \\
\hline C & -1.220446 & -5.034459 & -2.330052 \\
\hline $\mathrm{H}$ & 0.784265 & -5.570411 & -1.696349 \\
\hline $\mathrm{H}$ & 1.090467 & -3.572815 & -0.292173 \\
\hline C & -0.710241 & -1.543819 & 0.066014 \\
\hline C & -3.126942 & -1.988789 & -1.319844 \\
\hline $\mathrm{H}$ & -3.171226 & -4.260011 & -2.765312 \\
\hline $\mathrm{H}$ & -1.346111 & -5.921702 & -2.944297 \\
\hline C & -2.997912 & -0.854456 & -0.543721 \\
\hline C & -1.766069 & -0.646284 & 0.157652 \\
\hline $\mathrm{H}$ & -4.044744 & -2.146240 & -1.880359 \\
\hline C & 0.858360 & -2.636976 & 4.878759 \\
\hline C & 1.933929 & -2.038794 & 4.265148 \\
\hline C & 1.850207 & -1.596261 & 2.916805 \\
\hline C & 0.630057 & -1.778950 & 2.192446 \\
\hline C & -0.463229 & -2.400912 & 2.858457 \\
\hline C & -0.351392 & -2.817428 & 4.165880 \\
\hline $\mathrm{H}$ & 3.876509 & -0.871548 & 2.820364 \\
\hline $\mathrm{H}$ & 0.932413 & -2.970589 & 5.910295 \\
\hline $\mathrm{H}$ & 2.868304 & -1.894379 & 4.802574 \\
\hline
\end{tabular}




\begin{tabular}{|c|c|c|c|}
\hline C & 2.947984 & -0.986714 & 2.266811 \\
\hline C & 0.567753 & -1.329802 & 0.832159 \\
\hline $\mathrm{H}$ & -1.399457 & -2.537207 & 2.328524 \\
\hline $\mathrm{H}$ & -1.200037 & -3.286091 & 4.656452 \\
\hline C & 1.666400 & -0.732212 & 0.217325 \\
\hline C & 2.891072 & -0.562093 & 0.951798 \\
\hline C & 1.554259 & -0.245089 & -1.218888 \\
\hline $\mathrm{H}$ & 0.531730 & 0.091417 & -1.419343 \\
\hline $\mathrm{H}$ & 2.219640 & 0.604612 & -1.377189 \\
\hline C & -1.663136 & 0.510775 & 1.114142 \\
\hline 0 & -2.261667 & 0.589898 & 2.161557 \\
\hline 0 & -0.812604 & 1.476437 & 0.680382 \\
\hline $\mathrm{H}$ & -0.787100 & 2.149663 & 1.388145 \\
\hline 0 & 1.956779 & -1.222667 & -2.188622 \\
\hline $\mathrm{H}$ & 1.323599 & -1.957670 & -2.144718 \\
\hline C & 4.109997 & 0.071704 & 0.365941 \\
\hline C & 4.646071 & -0.356639 & -0.851599 \\
\hline C & 4.768628 & 1.091376 & 1.077685 \\
\hline C & 5.816316 & 0.213923 & -1.377531 \\
\hline $\mathrm{H}$ & 4.132284 & -1.141338 & -1.395217 \\
\hline C & 5.937294 & 1.680473 & 0.592840 \\
\hline H & 4.332831 & 1.424502 & 2.012721 \\
\hline C & 6.440046 & 1.223511 & -0.639486 \\
\hline $\mathrm{H}$ & 7.343493 & 1.678863 & -1.028456 \\
\hline C & -4.116501 & 0.127343 & -0.477241 \\
\hline C & -3.899421 & 1.484051 & -0.746756 \\
\hline C & -5.413336 & -0.306111 & -0.177720 \\
\hline C & -4.946847 & 2.412025 & -0.708486 \\
\hline $\mathrm{H}$ & -2.896861 & 1.810545 & -1.004603 \\
\hline C & -6.490167 & 0.587298 & -0.130681 \\
\hline $\mathrm{H}$ & -5.566792 & -1.357731 & 0.045648 \\
\hline C & -6.230308 & 1.939456 & -0.398265 \\
\hline $\mathrm{H}$ & -7.050052 & 2.643566 & -0.361648 \\
\hline C & -7.897401 & 0.065193 & 0.219746 \\
\hline C & -4.655536 & 3.895335 & -1.009387 \\
\hline C & -7.874528 & -0.564001 & 1.633254 \\
\hline $\mathrm{H}$ & -7.171954 & -1.401454 & 1.696373 \\
\hline $\mathrm{H}$ & -7.578855 & 0.175185 & 2.386228 \\
\hline $\mathrm{H}$ & -8.869617 & -0.941997 & 1.898678 \\
\hline C & -8.319997 & -1.007883 & -0.811777 \\
\hline $\mathrm{H}$ & -9.319640 & -1.392439 & -0.574872 \\
\hline $\mathrm{H}$ & -8.348468 & -0.588677 & -1.824152 \\
\hline $\mathrm{H}$ & -7.630886 & -1.858847 & -0.821465 \\
\hline C & -8.961183 & 1.179339 & 0.209720 \\
\hline $\mathrm{H}$ & -9.047213 & 1.654899 & -0.774092 \\
\hline $\mathrm{H}$ & -9.940562 & 0.754348 & 0.456962 \\
\hline $\mathrm{H}$ & -8.744724 & 1.958721 & 0.949081 \\
\hline C & -5.911391 & 4.780034 & -0.893278 \\
\hline $\mathrm{H}$ & -6.689568 & 4.481674 & -1.605039 \\
\hline $\mathrm{H}$ & -6.340024 & 4.750451 & 0.114960 \\
\hline $\mathrm{H}$ & -5.649180 & 5.821731 & -1.110263 \\
\hline C & -4.102491 & 4.029642 & -2.448240 \\
\hline $\mathrm{H}$ & -4.830789 & 3.668781 & -3.183559 \\
\hline $\mathrm{H}$ & -3.881072 & 5.079683 & -2.675795 \\
\hline
\end{tabular}




\begin{tabular}{|c|c|c|c|}
\hline $\mathrm{H}$ & -3.179161 & 3.457076 & -2.584463 \\
\hline C & -3.602942 & 4.424366 & -0.005450 \\
\hline $\mathrm{H}$ & -3.970201 & 4.343643 & 1.024018 \\
\hline $\mathrm{H}$ & -2.662934 & 3.866473 & -0.070571 \\
\hline $\mathrm{H}$ & -3.380016 & 5.479378 & -0.206975 \\
\hline C & 6.674827 & 2.804632 & 1.346114 \\
\hline C & 6.353958 & -0.287396 & -2.731493 \\
\hline C & 6.667052 & -1.799508 & -2.633108 \\
\hline $\mathrm{H}$ & 7.426365 & -1.994452 & -1.866682 \\
\hline $\mathrm{H}$ & 5.776566 & -2.383066 & -2.378778 \\
\hline $\mathrm{H}$ & 7.048131 & -2.172586 & -3.591888 \\
\hline C & 7.641908 & 0.439805 & -3.161073 \\
\hline $\mathrm{H}$ & 7.481648 & 1.517404 & -3.281592 \\
\hline $\mathrm{H}$ & 8.455155 & 0.292930 & -2.440627 \\
\hline $\mathrm{H}$ & 7.982303 & 0.048190 & -4.126506 \\
\hline C & 5.280318 & -0.057305 & -3.823061 \\
\hline $\mathrm{H}$ & 4.338271 & -0.561841 & -3.586445 \\
\hline $\mathrm{H}$ & 5.066750 & 1.012114 & -3.937270 \\
\hline $\mathrm{H}$ & 5.634936 & -0.435702 & -4.790154 \\
\hline C & 8.126887 & 2.361688 & 1.645381 \\
\hline $\mathrm{H}$ & 8.141168 & 1.458714 & 2.266522 \\
\hline $\mathrm{H}$ & 8.685358 & 2.144310 & 0.729001 \\
\hline $\mathrm{H}$ & 8.664385 & 3.152718 & 2.182913 \\
\hline C & 5.999601 & 3.155927 & 2.685714 \\
\hline $\mathrm{H}$ & 6.563569 & 3.951360 & 3.186155 \\
\hline $\mathrm{H}$ & 4.974628 & 3.517154 & 2.544692 \\
\hline $\mathrm{H}$ & 5.968007 & 2.295775 & 3.364276 \\
\hline C & 6.699421 & 4.081225 & 0.472031 \\
\hline $\mathrm{H}$ & 5.681668 & 4.425040 & 0.254411 \\
\hline $\mathrm{H}$ & 7.228373 & 4.890606 & 0.990451 \\
\hline $\mathrm{H}$ & 7.205101 & 3.910359 & -0.483948 \\
\hline
\end{tabular}

\section{Catalyst 2C 68}

Datum

Value

\begin{tabular}{lc}
\hline B3LYP/6-31G(d) Energy & -2164.797341 \\
\hline B3LYP-D3(BJ)/def2-TZVPP-IEF-PCM(DCM) Energy & -2165.895598 \\
\hline B3LYP-D3(BJ)/def2-TZVPP-IEF-PCM(DCM)//B3LYP/6-31G(d) Free Energy (Quasiharmonic) & -2165.017629 \\
\hline Number of Imaginary Frequencies & 0
\end{tabular}

Frequencies (Top 3 out of 321)

$\begin{array}{ll}\text { 1. } & 11.5917 \mathrm{~cm}^{-1} \\ \text { 2. } & 12.9682 \mathrm{~cm}^{-1} \\ \text { 3. } & 13.7828 \mathrm{~cm}^{-1}\end{array}$




\begin{tabular}{|c|c|c|c|}
\hline C & -0.002620 & -4.830073 & -1.656669 \\
\hline C & -0.179368 & -3.714613 & -0.865991 \\
\hline C & 0.847084 & -2.734000 & -0.742786 \\
\hline C & 2.078025 & -2.946840 & -1.442759 \\
\hline C & 2.225897 & -4.106838 & -2.250477 \\
\hline C & 1.209646 & -5.027996 & -2.359435 \\
\hline $\mathrm{H}$ & -0.797700 & -5.566185 & -1.736027 \\
\hline $\mathrm{H}$ & -1.105196 & -3.579560 & -0.316643 \\
\hline C & 0.696900 & -1.555443 & 0.062167 \\
\hline C & 3.116709 & -1.992393 & -1.320401 \\
\hline $\mathrm{H}$ & 3.162773 & -4.252771 & -2.782704 \\
\hline $\mathrm{H}$ & 1.335903 & -5.910448 & -2.980430 \\
\hline C & 2.987189 & -0.863764 & -0.535992 \\
\hline C & 1.753603 & -0.659695 & 0.163954 \\
\hline $\mathrm{H}$ & 4.035066 & -2.145577 & -1.881140 \\
\hline C & -0.889727 & -2.685446 & 4.860408 \\
\hline C & -1.961980 & -2.079714 & 4.248375 \\
\hline C & -1.873038 & -1.626215 & 2.904021 \\
\hline C & -0.651059 & -1.805879 & 2.181945 \\
\hline C & 0.438736 & -2.435804 & 2.846269 \\
\hline C & 0.321856 & -2.862844 & 4.149868 \\
\hline $\mathrm{H}$ & -3.896610 & -0.893841 & 2.807928 \\
\hline $\mathrm{H}$ & -0.967844 & -3.027443 & 5.888894 \\
\hline $\mathrm{H}$ & -2.897705 & -1.937595 & 4.784071 \\
\hline C & -2.967029 & -1.007924 & 2.255920 \\
\hline C & -0.583590 & -1.346202 & 0.825486 \\
\hline $\mathrm{H}$ & 1.376133 & -2.570440 & 2.317922 \\
\hline $\mathrm{H}$ & 1.167811 & -3.337685 & 4.639148 \\
\hline C & -1.679011 & -0.741502 & 0.211958 \\
\hline C & -2.904940 & -0.572680 & 0.944602 \\
\hline C & -1.561967 & -0.244926 & -1.220577 \\
\hline $\mathrm{H}$ & -2.222616 & 0.609318 & -1.374152 \\
\hline $\mathrm{H}$ & -0.537438 & 0.087420 & -1.417407 \\
\hline C & 1.649845 & 0.490484 & 1.128224 \\
\hline 0 & 2.262660 & 0.571840 & 2.167434 \\
\hline 0 & 0.781510 & 1.447318 & 0.712152 \\
\hline $\mathrm{H}$ & 0.754652 & 2.114504 & 1.425453 \\
\hline 0 & -1.968298 & -1.213983 & -2.197373 \\
\hline $\mathrm{H}$ & -1.338916 & -1.952478 & -2.157461 \\
\hline C & -4.118988 & 0.073122 & 0.361682 \\
\hline C & -4.654710 & -0.340075 & -0.861251 \\
\hline C & -4.772680 & 1.090483 & 1.081320 \\
\hline C & -5.819602 & 0.243531 & -1.384838 \\
\hline $\mathrm{H}$ & -4.144754 & -1.122943 & -1.411081 \\
\hline C & -5.936025 & 1.692016 & 0.599035 \\
\hline $\mathrm{H}$ & -4.337039 & 1.412193 & 2.020388 \\
\hline C & -6.438589 & 1.250182 & -0.638830 \\
\hline $\mathrm{H}$ & -7.337812 & 1.715456 & -1.025794 \\
\hline C & 4.105436 & 0.118094 & -0.464958 \\
\hline C & 5.400491 & -0.314758 & -0.173544 \\
\hline
\end{tabular}




\begin{tabular}{|c|c|c|c|}
\hline C & 3.881793 & 1.479521 & -0.730474 \\
\hline C & 6.480345 & 0.581073 & -0.128968 \\
\hline $\mathrm{H}$ & 5.556792 & -1.366755 & 0.045963 \\
\hline C & 4.927268 & 2.403210 & -0.697930 \\
\hline $\mathrm{H}$ & 2.877299 & 1.799472 & -0.979961 \\
\hline C & 6.216101 & 1.927947 & -0.393493 \\
\hline $\mathrm{H}$ & 7.032897 & 2.639586 & -0.362283 \\
\hline C & 4.714775 & 3.902824 & -0.981161 \\
\hline C & 7.888440 & 0.057653 & 0.213939 \\
\hline C & 5.575586 & 4.329198 & -2.194003 \\
\hline $\mathrm{H}$ & 6.642590 & 4.156914 & -2.018286 \\
\hline $\mathrm{H}$ & 5.289987 & 3.770298 & -3.092528 \\
\hline $\mathrm{H}$ & 5.440378 & 5.397874 & -2.401186 \\
\hline C & 5.137079 & 4.723764 & 0.260847 \\
\hline $\mathrm{H}$ & 4.991695 & 5.795361 & 0.076634 \\
\hline $\mathrm{H}$ & 4.540664 & 4.443978 & 1.136672 \\
\hline $\mathrm{H}$ & 6.191356 & 4.569582 & 0.513137 \\
\hline C & 3.244494 & 4.236509 & -1.298372 \\
\hline $\mathrm{H}$ & 2.576765 & 3.972748 & -0.470409 \\
\hline $\mathrm{H}$ & 3.140468 & 5.312534 & -1.477998 \\
\hline $\mathrm{H}$ & 2.892483 & 3.716338 & -2.196404 \\
\hline C & 8.307174 & -1.010605 & -0.824105 \\
\hline $\mathrm{H}$ & 7.617897 & -1.861429 & -0.835449 \\
\hline $\mathrm{H}$ & 8.332272 & -0.586608 & -1.834560 \\
\hline $\mathrm{H}$ & 9.307507 & -1.396475 & -0.592380 \\
\hline C & 7.870592 & -0.577941 & 1.624620 \\
\hline $\mathrm{H}$ & 7.167929 & -1.415385 & 1.686819 \\
\hline $\mathrm{H}$ & 8.866504 & -0.957709 & 1.884291 \\
\hline $\mathrm{H}$ & 7.578331 & 0.157995 & 2.382081 \\
\hline C & 8.951221 & 1.172556 & 0.205035 \\
\hline $\mathrm{H}$ & 9.033902 & 1.651655 & -0.777371 \\
\hline $\mathrm{H}$ & 8.735683 & 1.948890 & 0.947912 \\
\hline $\mathrm{H}$ & 9.931772 & 0.747851 & 0.447982 \\
\hline C & -6.667957 & 2.814135 & 1.360877 \\
\hline C & -6.356689 & -0.240323 & -2.745398 \\
\hline C & -7.638058 & 0.500323 & -3.171528 \\
\hline $\mathrm{H}$ & -8.454492 & 0.352127 & -2.454974 \\
\hline $\mathrm{H}$ & -7.469734 & 1.577890 & -3.280844 \\
\hline $\mathrm{H}$ & -7.978309 & 0.120735 & -4.141799 \\
\hline C & -6.680849 & -1.751062 & -2.663449 \\
\hline $\mathrm{H}$ & -5.795385 & -2.343581 & -2.412368 \\
\hline $\mathrm{H}$ & -7.444047 & -1.948404 & -1.901510 \\
\hline $\mathrm{H}$ & -7.061495 & -2.111521 & -3.627226 \\
\hline C & -5.278122 & -0.006833 & -3.831394 \\
\hline $\mathrm{H}$ & -5.056476 & 1.062126 & -3.933940 \\
\hline $\mathrm{H}$ & -4.340480 & -0.520649 & -3.597286 \\
\hline $\mathrm{H}$ & -5.632616 & -0.372667 & -4.803363 \\
\hline C & -6.684838 & 4.098074 & 0.497472 \\
\hline $\mathrm{H}$ & -5.665097 & 4.437891 & 0.282969 \\
\hline $\mathrm{H}$ & -7.191272 & 3.938263 & -0.460015 \\
\hline $\mathrm{H}$ & -7.209314 & 4.906071 & 1.022531 \\
\hline C & -5.992062 & 3.150878 & 2.703863 \\
\hline $\mathrm{H}$ & -6.551838 & 3.945687 & 3.209983 \\
\hline H & -5.966080 & 2.285379 & 3.375830 \\
\hline
\end{tabular}




\section{Catalyst 2C 69}

\section{Datum}

Value

\begin{tabular}{lc}
\hline B3LYP/6-31G(d) Energy & -2164.799877 \\
\hline B3LYP-D3(BJ)/def2-TZVPP-IEF-PCM(DCM) Energy & -2165.896016 \\
\hline B3LYP-D3(BJ)/def2-TZVPP-IEF-PCM(DCM)//B3LYP/6-31G(d) Free Energy (Quasiharmonic) & -2165.017628 \\
\hline
\end{tabular}

Number of Imaginary Frequencies

Frequencies (Top 3 out of 321)
1. $11.1149 \mathrm{~cm}^{-1}$
2. $12.5742 \mathrm{~cm}^{-1}$
3. $13.9395 \mathrm{~cm}^{-1}$

\section{B3LYP/6-31G(d) Molecular Geometry in Cartesian Coordinates}

\begin{tabular}{|c|c|c|c|}
\hline C & -0.268307 & -3.345697 & 3.978789 \\
\hline C & -0.394756 & -2.803340 & 2.719026 \\
\hline C & 0.681937 & -2.094477 & 2.118725 \\
\hline C & 1.901970 & -1.953499 & 2.854170 \\
\hline C & 1.999216 & -2.524168 & 4.152026 \\
\hline C & 0.938884 & -3.205008 & 4.704211 \\
\hline $\mathrm{H}$ & -1.100664 & -3.887136 & 4.419814 \\
\hline H & -1.322161 & -2.915434 & 2.167787 \\
\hline C & 0.586993 & -1.512657 & 0.811086 \\
\hline C & 2.987171 & -1.254899 & 2.270088 \\
\hline $\mathrm{H}$ & 2.930839 & -2.412014 & 4.701390 \\
\hline $\mathrm{H}$ & 1.024868 & -3.636742 & 5.697623 \\
\hline C & 2.914526 & -0.712487 & 1.002778 \\
\hline C & 1.687536 & -0.857862 & 0.276882 \\
\hline $\mathrm{H}$ & 3.914494 & -1.168345 & 2.830526 \\
\hline C & -1.346231 & -4.921010 & -2.567097 \\
\hline C & -2.321852 & -3.964619 & -2.408448 \\
\hline C & -2.119861 & -2.855790 & -1.542707 \\
\hline C & -0.884001 & -2.732654 & -0.835471 \\
\hline C & 0.105169 & -3.736781 & -1.026152 \\
\hline C & -0.121608 & -4.803264 & -1.867041 \\
\hline
\end{tabular}




\begin{tabular}{|c|c|c|c|}
\hline $\mathrm{H}$ & -4.047157 & -1.965721 & -1.909604 \\
\hline $\mathrm{H}$ & -1.510471 & -5.766204 & -3.230099 \\
\hline $\mathrm{H}$ & -3.266040 & -4.042043 & -2.942442 \\
\hline C & -3.115465 & -1.868284 & -1.358095 \\
\hline C & -0.700899 & -1.608577 & 0.036709 \\
\hline $\mathrm{H}$ & 1.052904 & -3.652254 & -0.506074 \\
\hline $\mathrm{H}$ & 0.648167 & -5.558660 & -1.999644 \\
\hline C & -1.701411 & -0.649969 & 0.201105 \\
\hline C & -2.941661 & -0.783385 & -0.519300 \\
\hline C & -1.500453 & 0.511477 & 1.166577 \\
\hline $\mathrm{H}$ & -2.455393 & 0.771262 & 1.628313 \\
\hline $\mathrm{H}$ & -0.815884 & 0.214861 & 1.969718 \\
\hline C & 1.577051 & -0.276050 & -1.104900 \\
\hline 0 & 1.676525 & -0.881453 & -2.146238 \\
\hline 0 & 1.342786 & 1.066423 & -1.068239 \\
\hline $\mathrm{H}$ & 1.287056 & 1.365165 & -1.997404 \\
\hline 0 & -1.043622 & 1.714384 & 0.545279 \\
\hline $\mathrm{H}$ & -0.179694 & 1.535702 & 0.134991 \\
\hline C & -4.080865 & 0.172845 & -0.372311 \\
\hline C & -3.912545 & 1.554715 & -0.538360 \\
\hline C & -5.365333 & -0.330507 & -0.110800 \\
\hline C & -4.996689 & 2.434633 & -0.438943 \\
\hline $\mathrm{H}$ & -2.915808 & 1.928116 & -0.729047 \\
\hline C & -6.475358 & 0.514928 & -0.008180 \\
\hline $\mathrm{H}$ & -5.477354 & -1.399461 & 0.030068 \\
\hline C & -6.261336 & 1.890676 & -0.174917 \\
\hline $\mathrm{H}$ & -7.109854 & 2.563145 & -0.088961 \\
\hline C & 4.092708 & 0.000807 & 0.432355 \\
\hline C & 4.612614 & -0.345201 & -0.821388 \\
\hline C & 4.718470 & 1.013745 & 1.170641 \\
\hline C & 5.732679 & 0.307667 & -1.351268 \\
\hline $\mathrm{H}$ & 4.140050 & -1.145039 & -1.380741 \\
\hline C & 5.842267 & 1.689031 & 0.679862 \\
\hline $\mathrm{H}$ & 4.297017 & 1.284413 & 2.134300 \\
\hline C & 6.327817 & 1.318677 & -0.583216 \\
\hline $\mathrm{H}$ & 7.192490 & 1.832628 & -0.979746 \\
\hline C & 6.488408 & 2.805842 & 1.523787 \\
\hline C & 6.259392 & -0.101223 & -2.740852 \\
\hline C & 6.944547 & 2.225757 & 2.883789 \\
\hline $\mathrm{H}$ & 6.106340 & 1.807182 & 3.450585 \\
\hline $\mathrm{H}$ & 7.683955 & 1.429027 & 2.742639 \\
\hline $\mathrm{H}$ & 7.402799 & 3.010223 & 3.498586 \\
\hline C & 5.452761 & 3.929153 & 1.768880 \\
\hline $\mathrm{H}$ & 5.893326 & 4.728739 & 2.377068 \\
\hline $\mathrm{H}$ & 5.119178 & 4.367626 & 0.821446 \\
\hline $\mathrm{H}$ & 4.566743 & 3.559752 & 2.295267 \\
\hline C & 7.717356 & 3.431105 & 0.836545 \\
\hline $\mathrm{H}$ & 7.457821 & 3.897143 & -0.120775 \\
\hline $\mathrm{H}$ & 8.139507 & 4.212102 & 1.478904 \\
\hline $\mathrm{H}$ & 8.505461 & 2.691411 & 0.654787 \\
\hline C & 7.510937 & 0.694626 & -3.156347 \\
\hline $\mathrm{H}$ & 7.310058 & 1.770195 & -3.220147 \\
\hline $\mathrm{H}$ & 8.342817 & 0.542320 & -2.458959 \\
\hline $\mathrm{H}$ & 7.845589 & 0.363840 & -4.146050 \\
\hline
\end{tabular}




\begin{tabular}{|c|c|c|c|}
\hline C & 5.155341 & 0.150069 & -3.796717 \\
\hline $\mathrm{H}$ & 4.901844 & 1.215763 & -3.846704 \\
\hline $\mathrm{H}$ & 5.500449 & -0.161744 & -4.790287 \\
\hline $\mathrm{H}$ & 4.236913 & -0.401545 & -3.571596 \\
\hline C & 6.628889 & -1.603795 & -2.734344 \\
\hline $\mathrm{H}$ & 7.410995 & -1.812681 & -1.995237 \\
\hline $\mathrm{H}$ & 5.767296 & -2.236472 & -2.498517 \\
\hline $\mathrm{H}$ & 7.003119 & -1.906770 & -3.719881 \\
\hline C & -7.895717 & -0.009761 & 0.281779 \\
\hline C & -4.832422 & 3.959546 & -0.592828 \\
\hline C & -5.208648 & 4.646705 & 0.741732 \\
\hline $\mathrm{H}$ & -6.240695 & 4.427146 & 1.035991 \\
\hline $\mathrm{H}$ & -4.550900 & 4.311358 & 1.551581 \\
\hline $\mathrm{H}$ & -5.110333 & 5.735929 & 0.652745 \\
\hline C & -5.762464 & 4.477361 & -1.715148 \\
\hline $\mathrm{H}$ & -5.522974 & 4.000814 & -2.672718 \\
\hline $\mathrm{H}$ & -6.818122 & 4.280831 & -1.499439 \\
\hline $\mathrm{H}$ & -5.645654 & 5.561336 & -1.836738 \\
\hline C & -3.386616 & 4.358097 & -0.947194 \\
\hline $\mathrm{H}$ & -2.670042 & 4.028933 & -0.187579 \\
\hline $\mathrm{H}$ & -3.077300 & 3.934051 & -1.909693 \\
\hline $\mathrm{H}$ & -3.316128 & 5.449126 & -1.028118 \\
\hline C & -8.834979 & 0.377981 & -0.885095 \\
\hline $\mathrm{H}$ & -8.885312 & 1.462740 & -1.025560 \\
\hline $\mathrm{H}$ & -8.491051 & -0.064249 & -1.827201 \\
\hline $\mathrm{H}$ & -9.853468 & 0.018712 & -0.692172 \\
\hline C & -8.423519 & 0.618039 & 1.593742 \\
\hline $\mathrm{H}$ & -9.438151 & 0.259859 & 1.807909 \\
\hline $\mathrm{H}$ & -7.782439 & 0.350346 & 2.441484 \\
\hline $\mathrm{H}$ & -8.461447 & 1.710900 & 1.537160 \\
\hline C & -7.936019 & -1.542060 & 0.436695 \\
\hline $\mathrm{H}$ & -7.313580 & -1.884466 & 1.271164 \\
\hline $\mathrm{H}$ & -8.963682 & -1.866382 & 0.636411 \\
\hline $\mathrm{H}$ & -7.599598 & -2.052922 & -0.472675 \\
\hline
\end{tabular}

\section{Catalyst 2C 70}

Datum

Value

\begin{tabular}{lc}
\hline B3LYP/6-31G(d) Energy & -2164.798083 \\
\hline B3LYP-D3(BJ)/def2-TZVPP-IEF-PCM(DCM) Energy & -2165.895613 \\
\hline B3LYP-D3(BJ)/def2-TZVPP-IEF-PCM(DCM)//B3LYP/6-31G(d) Free Energy (Quasiharmonic) & -2165.017626 \\
\hline Number of Imaginary Frequencies & 0
\end{tabular}

Frequencies (Top 3 out of 321) 
1. $10.8798 \mathrm{~cm}^{-1}$

2. $\quad 12.5156 \mathrm{~cm}^{-1}$

3. $13.2983 \mathrm{~cm}^{-1}$

B3LYP/6-31G(d) Molecular Geometry in Cartesian Coordinates

C

C

C

C

$\mathrm{C}$

C

$\mathrm{H}$

$\mathrm{H}$

C

C

$\mathrm{H}$

$\mathrm{H}$

C

C

$\mathrm{H}$

C

C

C

C

C

C

$\mathrm{H}$

$\mathrm{H}$

$\mathrm{H}$

C

C

$\mathrm{H}$

$\mathrm{H}$

C

C

C

$\mathrm{H}$

$\mathrm{H}$

C

0

0

$\mathrm{H}$

0

$\mathrm{H}$

C

C

C

C

$\mathrm{H}$
0.042892

$-0.149500$

0.870509

2.109616

2. 274125

1. 264919

$-0.747762$

$-1.083939$

0.704283

3.138400

3. 217125

1. 403372

2.988974

1. 748651

4.063202

$-0.894280$

$-1.959937$

$-1.868165$

$-0.649262$

0.433048

0.313329

$-3.887103$

$-0.975502$

$-2.893314$

$-2.957518$

$-0.576729$

1. 365762

1.152763

$-1.664357$

$-2.890575$

$-1.538290$

$-2.210030$

$-0.517404$

1.558663

0.827632

2. 302227

2. 126233

$-1.918041$

$-1.283202$

$-4.101766$

$-4.639394$

$-4.747808$

$-5.801210$

$-4.126865$
$-4.821140$

$-3.724981$

$-2.742777$

$-2.930554$

$-4.072440$

$-4.997164$

$-5.558471$

$-3.604118$

$-1.583834$

$-1.969335$

$-4.200567$

$-5.865157$

$-0.852530$

$-0.677362$

$-2.104384$

$-2.914151$

$-2.269811$

$-1.760293$

$-1.922189$

$-2.595040$

$-3.076996$

$-1.009933$

$-3.299719$

$-2.140220$

$-1.106342$

$-1.401185$

$-2.723386$

$-3.586904$

$-0.756239$

$-0.615902$

$-0.185526$

0.665561

0.175081

0.518799

1.457512

0.433758

1. 251311

$-1.110614$

$-1.844988$

0.052744

$-0.333230$

1.051055

0.263637

$-1.101098$
$-1.787988$

$-0.974794$

$-0.817172$

$-1.510473$

$-2.340537$

$-2.479965$

$-1.895220$

$-0.436843$

0.011415

$-1.361001$

$-2.866501$

$-3.118527$

$-0.562561$

0.132847

$-1.915841$

4.752996

4.169126

2.845212

2. 113866

2. 747854

4.032272

2. 779946

5.765751

4. 712074

2. 224538

0.779975

2. 209451

4.497270

0.196074

0.933457

$-1.207654$

$-1.324585$

$-1.370092$

1.023998

0.787811

2. 148262

2.653127

$-2.236369$

$-2.218458$

0.371884

$-0.864453$

1.116599

$-1.367330$

$-1.429105$ 


\begin{tabular}{|c|c|c|c|}
\hline C & -5.912381 & 1.670651 & 0.649802 \\
\hline $\mathrm{H}$ & -4.308745 & 1.349544 & 2.061588 \\
\hline C & -6.414871 & 1.258020 & -0.592546 \\
\hline $\mathrm{H}$ & -7.313936 & 1.733974 & -0.973186 \\
\hline C & 4.094832 & 0.141900 & -0.468365 \\
\hline C & 5.394096 & -0.280713 & -0.178410 \\
\hline C & 3.855583 & 1.505939 & -0.707151 \\
\hline C & 6.462722 & 0.627095 & -0.111335 \\
\hline $\mathrm{H}$ & 5.562822 & -1.335215 & 0.019244 \\
\hline C & 4.889277 & 2.442026 & -0.647216 \\
\hline $\mathrm{H}$ & 2.849476 & 1.819919 & -0.955378 \\
\hline C & 6.182676 & 1.976488 & -0.347039 \\
\hline $\mathrm{H}$ & 6.990685 & 2.697164 & -0.297772 \\
\hline C & 4.656068 & 3.944225 & -0.899702 \\
\hline C & 7.876662 & 0.113245 & 0.222708 \\
\hline C & 5.070475 & 4.746312 & 0.357214 \\
\hline $\mathrm{H}$ & 4.473640 & 4.447664 & 1.226654 \\
\hline $\mathrm{H}$ & 6.125802 & 4.597575 & 0.609020 \\
\hline $\mathrm{H}$ & 4.915333 & 5.819467 & 0.191781 \\
\hline C & 3.180099 & 4.263820 & -1.205947 \\
\hline $\mathrm{H}$ & 3.060650 & 5.342781 & -1.356188 \\
\hline $\mathrm{H}$ & 2.836671 & 3.764707 & -2.119161 \\
\hline $\mathrm{H}$ & 2.515592 & 3.966284 & -0.387235 \\
\hline C & 5.510147 & 4.404952 & -2.104612 \\
\hline $\mathrm{H}$ & 5.232313 & 3.857702 & -3.012637 \\
\hline $\mathrm{H}$ & 5.358038 & 5.474796 & -2.293057 \\
\hline $\mathrm{H}$ & 6.579973 & 4.245928 & -1.932410 \\
\hline C & 8.307945 & -0.930447 & -0.835072 \\
\hline $\mathrm{H}$ & 7.628854 & -1.788976 & -0.862634 \\
\hline $\mathrm{H}$ & 8.327756 & -0.487355 & -1.837358 \\
\hline $\mathrm{H}$ & 9.312821 & -1.308651 & -0.610512 \\
\hline C & 7.866093 & -0.549559 & 1.620848 \\
\hline $\mathrm{H}$ & 7.170008 & -1.393553 & 1.667959 \\
\hline $\mathrm{H}$ & 8.865207 & -0.926519 & 1.872081 \\
\hline $\mathrm{H}$ & 7.570125 & 0.169857 & 2.392844 \\
\hline C & 8.926729 & 1.240033 & 0.235335 \\
\hline $\mathrm{H}$ & 9.002657 & 1.739170 & -0.737496 \\
\hline $\mathrm{H}$ & 8.702902 & 1.999180 & 0.993375 \\
\hline $\mathrm{H}$ & 9.912364 & 0.822063 & 0.469192 \\
\hline C & -6.636564 & 2.779148 & 1.439159 \\
\hline C & -6.403028 & -0.128651 & -2.731086 \\
\hline C & -6.379392 & 1.101077 & -3.670121 \\
\hline $\mathrm{H}$ & -6.954938 & 1.938360 & -3.260595 \\
\hline $\mathrm{H}$ & -5.352661 & 1.447954 & -3.832468 \\
\hline $\mathrm{H}$ & -6.810192 & 0.845266 & -4.646203 \\
\hline C & -7.864199 & -0.599766 & -2.542212 \\
\hline $\mathrm{H}$ & -7.911942 & -1.471602 & -1.879566 \\
\hline $\mathrm{H}$ & -8.496429 & 0.183048 & -2.109776 \\
\hline $\mathrm{H}$ & -8.300824 & -0.883244 & -3.507923 \\
\hline C & -5.617197 & -1.266788 & -3.410829 \\
\hline $\mathrm{H}$ & -4.569477 & -0.999530 & -3.583867 \\
\hline $\mathrm{H}$ & -5.636207 & -2.184147 & -2.811098 \\
\hline $\mathrm{H}$ & -6.071568 & -1.497196 & -4.381523 \\
\hline C & -6.652017 & 4.081222 & 0.603594 \\
\hline
\end{tabular}




\begin{tabular}{|lllr|}
$H$ & -5.632032 & 4.420145 & 0.389469 \\
$H$ & -7.165832 & 3.944697 & -0.353629 \\
$H$ & -7.168817 & 4.880447 & 1.149293 \\
$\mathrm{C}$ & -5.952897 & 3.084817 & 2.785661 \\
$\mathrm{H}$ & -6.506682 & 3.871874 & 3.310231 \\
$\mathrm{H}$ & -5.928400 & 2.205782 & 3.439938 \\
$\mathrm{H}$ & -4.924641 & 3.438744 & 2.650881 \\
$\mathrm{C}$ & -8.091406 & 2.340771 & 1.730715 \\
$\mathrm{H}$ & -8.111802 & 1.420433 & 2.325715 \\
$\mathrm{H}$ & -8.620270 & 3.120737 & 2.292508 \\
$\mathrm{H}$ & -8.654594 & 2.154286 & 0.810343 \\
\hline
\end{tabular}

\section{Catalyst 2C 71}

Datum

Value

\begin{tabular}{lc}
\hline B3LYP/6-31G(d) Energy & -2164.797167 \\
\hline B3LYP-D3(BJ)/def2-TZVPP-IEF-PCM(DCM) Energy & -2165.895286 \\
\hline B3LYP-D3(BJ)/def2-TZVPP-IEF-PCM(DCM)//B3LYP/6-31G(d) Free Energy (Quasiharmonic) & -2165.017565 \\
\hline
\end{tabular}

Number of Imaginary Frequencies

Frequencies (Top 3 out of 321)
1. $10.2014 \mathrm{~cm}^{-1}$
2. $13.3602 \mathrm{~cm}^{-1}$
3. $14.0473 \mathrm{~cm}^{-1}$

\section{B3LYP/6-31G(d) Molecular Geometry in Cartesian Coordinates}

$\begin{array}{lrrr}\text { C } & -0.003208 & -4.848221 & -1.574818 \\ \text { C } & -0.178472 & -3.717481 & -0.805835 \\ \text { C } & 0.848501 & -2.735066 & -0.703232 \\ \text { C } & 2.078245 & -2.961755 & -1.401101 \\ \text { C } & 2.224453 & -4.137296 & -2.186396 \\ \text { C } & 1.207846 & -5.060153 & -2.275585 \\ \text { H } & -0.798629 & -5.585466 & -1.638586 \\ \text { H } & -1.103541 & -3.571307 & -0.258083 \\ \text { C } & 0.700291 & -1.541507 & 0.079777 \\ \text { C } & 3.117908 & -2.006133 & -1.297924 \\ \text { H } & 3.160416 & -4.293879 & -2.717201 \\ \text { H } & 1.332867 & -5.954580 & -2.879451 \\ \text { C } & 2.989952 & -0.862902 & -0.534727 \\ \text { C } & 1.757558 & -0.644858 & 0.162797 \\ \text { H } & 4.036026 & -2.171401 & -1.855656\end{array}$




\begin{tabular}{|c|c|c|c|}
\hline C & -0.882442 & -2.582755 & 4.899577 \\
\hline C & -1.955709 & -1.989708 & 4.276953 \\
\hline C & -1.867651 & -1.560610 & 2.924579 \\
\hline C & -0.645578 & -1.751694 & 2.205588 \\
\hline C & 0.445255 & -2.367816 & 2.880950 \\
\hline C & 0.329265 & -2.771182 & 4.192127 \\
\hline $\mathrm{H}$ & -3.893410 & -0.835391 & 2.814431 \\
\hline $\mathrm{H}$ & -0.959838 & -2.906162 & 5.934111 \\
\hline $\mathrm{H}$ & -2.891526 & -1.839228 & 4.810192 \\
\hline C & -2.963011 & -0.956482 & 2.265289 \\
\hline C & -0.578839 & -1.316618 & 0.840930 \\
\hline $\mathrm{H}$ & 1.382942 & -2.509934 & 2.355138 \\
\hline $\mathrm{H}$ & 1.176088 & -3.235579 & 4.689845 \\
\hline C & -1.674884 & -0.723643 & 0.217225 \\
\hline C & -2.901233 & -0.544736 & 0.946549 \\
\hline C & -1.558702 & -0.251605 & -1.223806 \\
\hline $\mathrm{H}$ & -2.224337 & 0.595735 & -1.393346 \\
\hline $\mathrm{H}$ & -0.535904 & 0.083973 & -1.424515 \\
\hline C & 1.655149 & 0.522567 & 1.106712 \\
\hline 0 & 2.251489 & 0.611462 & 2.154589 \\
\hline 0 & 0.807513 & 1.485274 & 0.660912 \\
\hline $\mathrm{H}$ & 0.782072 & 2.166313 & 1.361163 \\
\hline 0 & -1.957142 & -1.239906 & -2.184198 \\
\hline $\mathrm{H}$ & -1.323175 & -1.973596 & -2.130820 \\
\hline C & -4.117355 & 0.084462 & 0.349863 \\
\hline C & -4.776136 & 1.112090 & 1.040168 \\
\hline C & -4.652471 & -0.360857 & -0.866011 \\
\hline C & -5.945629 & 1.700109 & 0.543087 \\
\hline $\mathrm{H}$ & -4.346422 & 1.461236 & 1.974879 \\
\hline C & -5.817158 & 0.203066 & -1.401208 \\
\hline $\mathrm{H}$ & -4.137209 & -1.153595 & -1.396417 \\
\hline C & -6.445477 & 1.228234 & -0.679637 \\
\hline $\mathrm{H}$ & -7.345099 & 1.673573 & -1.082045 \\
\hline C & 4.110319 & 0.117429 & -0.477616 \\
\hline C & 3.895688 & 1.472248 & -0.758388 \\
\hline C & 5.406571 & -0.315871 & -0.175203 \\
\hline C & 4.944758 & 2.398660 & -0.727846 \\
\hline $\mathrm{H}$ & 2.893743 & 1.798473 & -1.018878 \\
\hline C & 6.485074 & 0.575878 & -0.136141 \\
\hline $\mathrm{H}$ & 5.558223 & -1.365870 & 0.056853 \\
\hline C & 6.227497 & 1.926332 & -0.414361 \\
\hline $\mathrm{H}$ & 7.048483 & 2.629264 & -0.383597 \\
\hline C & 7.891673 & 0.053963 & 0.217127 \\
\hline C & 4.655853 & 3.880069 & -1.040229 \\
\hline C & 8.312256 & -1.026003 & -0.808017 \\
\hline $\mathrm{H}$ & 7.621678 & -1.875838 & -0.812359 \\
\hline $\mathrm{H}$ & 8.341102 & -0.612915 & -1.822889 \\
\hline $\mathrm{H}$ & 9.311340 & -1.410779 & -0.569113 \\
\hline C & 7.868590 & -0.566474 & 1.634482 \\
\hline $\mathrm{H}$ & 8.863403 & -0.943756 & 1.901925 \\
\hline $\mathrm{H}$ & 7.573843 & 0.177602 & 2.382987 \\
\hline $\mathrm{H}$ & 7.165326 & -1.402904 & 1.702995 \\
\hline C & 8.957047 & 1.166509 & 0.199918 \\
\hline $\mathrm{H}$ & 8.741642 & 1.950998 & 0.934175 \\
\hline
\end{tabular}




\begin{tabular}{|c|c|c|c|}
\hline $\mathrm{H}$ & 9.935780 & 0.741694 & 0.450000 \\
\hline $\mathrm{H}$ & 9.043926 & 1.635543 & -0.786946 \\
\hline C & 5.913062 & 4.763687 & -0.930645 \\
\hline $\mathrm{H}$ & 6.341476 & 4.741094 & 0.077862 \\
\hline $\mathrm{H}$ & 6.690904 & 4.458756 & -1.639988 \\
\hline $\mathrm{H}$ & 5.652479 & 5.804094 & -1.155624 \\
\hline C & 3.603870 & 4.418456 & -0.040617 \\
\hline $\mathrm{H}$ & 3.970620 & 4.344832 & 0.989562 \\
\hline $\mathrm{H}$ & 3.382833 & 5.472297 & -0.250146 \\
\hline $\mathrm{H}$ & 2.662916 & 3.861722 & -0.101892 \\
\hline C & 4.103262 & 4.004173 & -2.480170 \\
\hline $\mathrm{H}$ & 4.831243 & 3.636879 & -3.212611 \\
\hline $\mathrm{H}$ & 3.179270 & 3.431716 & -2.612163 \\
\hline $\mathrm{H}$ & 3.883147 & 5.052729 & -2.715700 \\
\hline C & -6.353176 & -0.315832 & -2.749667 \\
\hline C & -6.629929 & 2.828429 & 1.340341 \\
\hline C & -7.050374 & 2.292811 & 2.729733 \\
\hline $\mathrm{H}$ & -7.760096 & 1.463437 & 2.630440 \\
\hline $\mathrm{H}$ & -6.190660 & 1.929553 & 3.302437 \\
\hline $\mathrm{H}$ & -7.532248 & 3.084746 & 3.316636 \\
\hline C & -7.889597 & 3.369502 & 0.636815 \\
\hline $\mathrm{H}$ & -7.657624 & 3.793540 & -0.346809 \\
\hline $\mathrm{H}$ & -8.650090 & 2.591869 & 0.502879 \\
\hline $\mathrm{H}$ & -8.335855 & 4.166101 & 1.242966 \\
\hline C & -5.642983 & 4.005692 & 1.524064 \\
\hline $\mathrm{H}$ & -4.741858 & 3.701657 & 2.066734 \\
\hline $\mathrm{H}$ & -5.328798 & 4.408100 & 0.554218 \\
\hline $\mathrm{H}$ & -6.116017 & 4.816573 & 2.091803 \\
\hline C & -5.278262 & -0.098999 & -3.842744 \\
\hline $\mathrm{H}$ & -5.064782 & 0.969009 & -3.969760 \\
\hline $\mathrm{H}$ & -4.336465 & -0.600288 & -3.598395 \\
\hline $\mathrm{H}$ & -5.631423 & -0.489230 & -4.805678 \\
\hline C & -7.641064 & 0.404851 & -3.190896 \\
\hline $\mathrm{H}$ & -7.979592 & -0.000272 & -4.151425 \\
\hline $\mathrm{H}$ & -8.455458 & 0.267528 & -2.469887 \\
\hline $\mathrm{H}$ & -7.481552 & 1.480788 & -3.326046 \\
\hline C & -6.665701 & -1.826768 & -2.632878 \\
\hline $\mathrm{H}$ & -7.425209 & -2.012632 & -1.864376 \\
\hline $\mathrm{H}$ & -7.046224 & -2.211954 & -3.587091 \\
\hline $\mathrm{H}$ & -5.775042 & -2.406653 & -2.370886 \\
\hline
\end{tabular}

\section{Catalyst 2C 72}

Datum

Value

\begin{tabular}{lc}
\hline B3LYP/6-31G(d) Energy & -2164.797253 \\
\hline B3LYP-D3(BJ)/def2-TZVPP-IEF-PCM(DCM) Energy & -2165.895063 \\
\hline B3LYP-D3(BJ)/def2-TZVPP-IEF-PCM(DCM)//B3LYP/6-31G(d) Free Energy (Quasiharmonic) & -2165.017562 \\
\hline
\end{tabular}


Frequencies (Top 3 out of 321)

$\begin{array}{ll}\text { 1. } & 10.9371 \mathrm{~cm}^{-1} \\ \text { 2. } & 11.5329 \mathrm{~cm}^{-1} \\ \text { 3. } & 11.9692 \mathrm{~cm}^{-1}\end{array}$

\section{B3LYP/6-31G(d) Molecular Geometry in Cartesian Coordinates}

C

C

C

C

C

C

$\mathrm{H}$

$\mathrm{H}$

C

C

$\mathrm{H}$

$\mathrm{H}$

C

C

$\mathrm{H}$

C

C

C

C

C

C

$\mathrm{H}$

$\mathrm{H}$

$\mathrm{H}$

C

C

$\mathrm{H}$

$\mathrm{H}$

C

C

C

$\mathrm{H}$

$\mathrm{H}$

C

0

0

$\mathrm{H}$

$$
-0.144218
$$

$-0.287962$

0.757460

1.966565

2.082268

1.050963

$-0.951985$

$-1.200946$

0.640515

3.021191

3.005336

1. 151184

2.924112

1.705670

3.944346

$-1.163341$

$-2.174219$

$-2.015157$

$-0.787236$

0. 238871

0.054746

$-3.983542$

$-1.294763$

$-3.113673$

$-3.046961$

$-0.646611$

1.180342

0.851996

$-1.682398$

$-2.918129$

$-1.501926$

$-2.119976$

$-0.459199$

1. 543335

1.608597

1. 301770

1. 221214
$-3.713956$

$-2.482588$

$-1.931786$

$-2.683822$

$-3.948140$

$-4.455814$

$-4.115577$

$-1.912533$

$-0.661527$

$-2.147466$

$-4.509562$

$-5.424921$

$-0.917175$

$-0.178892$

$-2.714276$

2.987500

2. 754576

1.798847

1.074203

1. 342729

2. 273415

2.080163

3. 720112

3. 298213

1.537195

0.111717

0.809767

2.465388

$-0.130014$

0.597113

$-1.159564$

$-0.896152$

$-1.175366$

1.139521

2.232692

0.966891

1.863212
3.589814

2.986903

2.190824

2.038349

2.677484

3. 433543

4. 195232

3. 124878

1.535642

1. 260789

2.554905

3.914306

0.640243

0.788877

1.172273

4. 737609

3.834539

2. 794627

2.690017

3.637324

4.635004

1.962686

5.529421

3.902099

1.862801

1.637566

3. 562037

5.347953

0.736151

0.857686

$-0.369325$

$-1.228696$

$-0.703813$

0.080781

0.598440

$-1.239686$

$-1.620243$ 


\begin{tabular}{|c|c|c|c|}
\hline 0 & -1.925144 & -2.477102 & 0.006856 \\
\hline $\mathrm{H}$ & -1.345606 & -2.776121 & 0.726526 \\
\hline C & -4.074858 & 0.389852 & -0.064395 \\
\hline C & -4.612455 & -0.886991 & -0.281564 \\
\hline C & -4.672344 & 1.497587 & -0.684827 \\
\hline C & -5.725136 & -1.073731 & -1.109494 \\
\hline $\mathrm{H}$ & -4.137856 & -1.731181 & 0.201119 \\
\hline C & -5.788181 & 1.353355 & -1.517228 \\
\hline $\mathrm{H}$ & -4.235803 & 2.475624 & -0.516872 \\
\hline C & -6.291197 & 0.058926 & -1.711239 \\
\hline $\mathrm{H}$ & -7.153381 & -0.072497 & -2.358811 \\
\hline C & 4.074287 & -0.394542 & -0.149620 \\
\hline C & 4.582621 & 0.890530 & 0.085895 \\
\hline C & 4.684034 & -1.208908 & -1.114487 \\
\hline C & 5.681535 & 1.376584 & -0.631680 \\
\hline $\mathrm{H}$ & 4.116957 & 1.502443 & 0.847703 \\
\hline C & 5.787434 & -0.762163 & -1.849542 \\
\hline $\mathrm{H}$ & 4.263787 & -2.191385 & -1.295807 \\
\hline C & 6.263005 & 0.530815 & -1.586350 \\
\hline $\mathrm{H}$ & 7.117612 & 0.894241 & -2.149134 \\
\hline C & 6.478461 & -1.627353 & -2.921738 \\
\hline C & 6.253580 & 2.789771 & -0.406434 \\
\hline C & 5.796389 & -2.996846 & -3.101332 \\
\hline $\mathrm{H}$ & 5.819244 & -3.589286 & -2.179625 \\
\hline $\mathrm{H}$ & 4.751950 & -2.894486 & -3.416910 \\
\hline $\mathrm{H}$ & 6.320346 & -3.570486 & -3.874146 \\
\hline C & 7.949825 & -1.873597 & -2.511304 \\
\hline $\mathrm{H}$ & 8.461279 & -2.480967 & -3.268261 \\
\hline $\mathrm{H}$ & 8.506076 & -0.936492 & -2.403903 \\
\hline $\mathrm{H}$ & 8.005229 & -2.405893 & -1.554729 \\
\hline C & 6.441760 & -0.893636 & -4.283506 \\
\hline H & 6.962551 & 0.068872 & -4.244898 \\
\hline $\mathrm{H}$ & 6.925708 & -1.502145 & -5.057289 \\
\hline $\mathrm{H}$ & 5.409439 & -0.702450 & -4.597827 \\
\hline C & 5.490372 & 3.563374 & 0.686229 \\
\hline $\mathrm{H}$ & 4.428339 & 3.679314 & 0.443704 \\
\hline $\mathrm{H}$ & 5.563070 & 3.068418 & 1.661455 \\
\hline $\mathrm{H}$ & 5.919330 & 4.566115 & 0.793838 \\
\hline C & 6.154021 & 3.595943 & -1.723702 \\
\hline $\mathrm{H}$ & 5.110859 & 3.691794 & -2.045908 \\
\hline $\mathrm{H}$ & 6.560273 & 4.605325 & -1.584348 \\
\hline $\mathrm{H}$ & 6.712905 & 3.121370 & -2.537306 \\
\hline C & 7.737099 & 2.689567 & 0.020484 \\
\hline $\mathrm{H}$ & 7.838386 & 2.125998 & 0.954976 \\
\hline $\mathrm{H}$ & 8.349682 & 2.191076 & -0.738287 \\
\hline $\mathrm{H}$ & 8.155586 & 3.690770 & 0.180697 \\
\hline C & -6.464226 & 2.548896 & -2.217037 \\
\hline C & -6.322301 & -2.468523 & -1.380994 \\
\hline C & -5.598782 & -3.577407 & -0.592243 \\
\hline $\mathrm{H}$ & -4.533088 & -3.631260 & -0.838049 \\
\hline $\mathrm{H}$ & -5.687390 & -3.425709 & 0.489752 \\
\hline $\mathrm{H}$ & -6.048830 & -4.548857 & -0.827913 \\
\hline C & -6.197751 & -2.789507 & -2.889520 \\
\hline $\mathrm{H}$ & -6.723910 & -2.054025 & -3.507775 \\
\hline
\end{tabular}




\begin{tabular}{|lrrr|}
$H$ & -5.147115 & -2.798432 & -3.200974 \\
$H$ & -6.625393 & -3.776297 & -3.106694 \\
$\mathrm{C}$ & -7.815406 & -2.487076 & -0.976352 \\
$\mathrm{H}$ & -7.934821 & -2.266613 & 0.090681 \\
$\mathrm{H}$ & -8.402822 & -1.751939 & -1.536810 \\
$\mathrm{H}$ & -8.249988 & -3.475693 & -1.169295 \\
$\mathrm{C}$ & -6.416427 & 2.346972 & -3.750199 \\
$\mathrm{H}$ & -5.381180 & 2.280249 & -4.103868 \\
$\mathrm{H}$ & -6.932897 & 1.431697 & -4.057566 \\
$\mathrm{H}$ & -6.897388 & 3.189517 & -4.262475 \\
$\mathrm{C}$ & -5.774980 & 3.887706 & -1.891323 \\
$\mathrm{H}$ & -6.293443 & 4.704550 & -2.406254 \\
$\mathrm{H}$ & -5.796167 & 4.107794 & -0.817984 \\
$\mathrm{H}$ & -4.730095 & 3.899410 & -2.221651 \\
$\mathrm{C}$ & -7.939387 & 2.646174 & -1.759759 \\
$\mathrm{H}$ & -8.004064 & 2.800356 & -0.676494 \\
$\mathrm{H}$ & -8.438065 & 3.489334 & -2.253839 \\
$\mathrm{H}$ & -8.502620 & 1.738560 & -2.000602 \\
\hline
\end{tabular}

\section{Catalyst 2C 73}

\section{Datum}

Value

\begin{tabular}{lc}
\hline B3LYP/6-31G(d) Energy & -2164.798222 \\
\hline B3LYP-D3(BJ)/def2-TZVPP-IEF-PCM(DCM) Energy & -2165.895554 \\
\hline B3LYP-D3(BJ)/def2-TZVPP-IEF-PCM(DCM)//B3LYP/6-31G(d) Free Energy (Quasiharmonic) & -2165.017548 \\
\hline
\end{tabular}

Number of Imaginary Frequencies

Frequencies (Top 3 out of 321)

$\begin{array}{ll}\text { 1. } & 10.6791 \mathrm{~cm}^{-1} \\ \text { 2. } & 11.9187 \mathrm{~cm}^{-1} \\ \text { 3. } & 13.6931 \mathrm{~cm}^{-1}\end{array}$

\section{B3LYP/6-31G(d) Molecular Geometry in Cartesian Coordinates}

C

C

C

C

C

C

$\mathrm{H}$

$\mathrm{H}$

$$
\begin{array}{r}
-0.055692 \\
0.136555 \\
-0.881735 \\
-2.118908 \\
-2.283170 \\
-1.275721 \\
0.733557 \\
1.069466
\end{array}
$$

$-4.839630$

$-3.727236$

$-2.739779$

$-2.939018$

$-4.097376$

$-5.027114$

$-5.580854$

$-3.597137$
$-1.694764$

$-0.903910$

$-0.770070$

$-1.463549$

$-2.270628$

$-2.387267$

$-1.783892$

$-0.365528$ 


\begin{tabular}{|c|c|c|c|}
\hline C & -0.715845 & -1.564351 & 0.035082 \\
\hline C & -3.146795 & -1.973958 & -1.335387 \\
\hline $\mathrm{H}$ & -3.224718 & -4.234333 & -2.796964 \\
\hline $\mathrm{H}$ & -1.414042 & -5.907826 & -3.008190 \\
\hline C & -2.998745 & -0.842074 & -0.557920 \\
\hline C & -1.759110 & -0.654234 & 0.135589 \\
\hline $\mathrm{H}$ & -4.070642 & -2.119780 & -1.889052 \\
\hline C & 0.865984 & -2.799852 & 4.807842 \\
\hline C & 1.936095 & -2.173629 & 4.212544 \\
\hline C & 1.849390 & -1.691743 & 2.877969 \\
\hline C & 0.630924 & -1.862524 & 2.147931 \\
\hline C & -0.455998 & -2.516295 & 2.793867 \\
\hline C & -0.341159 & -2.971547 & 4.088407 \\
\hline $\mathrm{H}$ & 3.872997 & -0.955568 & 2.800125 \\
\hline $\mathrm{H}$ & 0.943301 & -3.164298 & 5.828689 \\
\hline $\mathrm{H}$ & 2.869149 & -2.037489 & 4.754431 \\
\hline C & 2.943548 & -1.057446 & 2.245495 \\
\hline C & 0.563529 & -1.368993 & 0.803312 \\
\hline $\mathrm{H}$ & -1.388523 & -2.650872 & 2.256705 \\
\hline $\mathrm{H}$ & -1.184152 & -3.466984 & 4.562508 \\
\hline C & 1.655349 & -0.741357 & 0.208414 \\
\hline C & 2.881544 & -0.593459 & 0.944411 \\
\hline C & 1.533442 & -0.197000 & -1.206175 \\
\hline $\mathrm{H}$ & 0.514916 & 0.167316 & -1.375424 \\
\hline $\mathrm{H}$ & 2.210824 & 0.647258 & -1.339067 \\
\hline C & -1.567714 & 0.561629 & 0.999439 \\
\hline 0 & -0.844852 & 1.499269 & 0.733604 \\
\hline 0 & -2.299914 & 0.498619 & 2.131710 \\
\hline $\mathrm{H}$ & -2.127473 & 1.330426 & 2.614156 \\
\hline 0 & 1.907102 & -1.143747 & -2.217189 \\
\hline $\mathrm{H}$ & 1.267575 & -1.873569 & -2.185700 \\
\hline C & 4.098398 & 0.053920 & 0.370326 \\
\hline C & 4.633570 & -0.361834 & -0.857383 \\
\hline C & 4.752847 & 1.061141 & 1.095383 \\
\hline C & 5.801135 & 0.214264 & -1.371068 \\
\hline $\mathrm{H}$ & 4.114780 & -1.136627 & -1.406702 \\
\hline C & 5.923430 & 1.660678 & 0.617541 \\
\hline $\mathrm{H}$ & 4.315748 & 1.382047 & 2.033962 \\
\hline C & 6.422986 & 1.218679 & -0.615891 \\
\hline $\mathrm{H}$ & 7.326658 & 1.678802 & -1.005136 \\
\hline C & -4.108089 & 0.149858 & -0.478826 \\
\hline C & -3.879217 & 1.511088 & -0.716123 \\
\hline C & -5.411035 & -0.279578 & -0.197300 \\
\hline C & -4.920250 & 2.446139 & -0.662384 \\
\hline $\mathrm{H}$ & -2.873356 & 1.837915 & -0.957666 \\
\hline C & -6.481850 & 0.620241 & -0.140017 \\
\hline $\mathrm{H}$ & -5.574703 & -1.334689 & 0.001243 \\
\hline C & -6.209837 & 1.976535 & -0.373819 \\
\hline $\mathrm{H}$ & -7.024754 & 2.685839 & -0.328852 \\
\hline C & -7.896562 & 0.100139 & 0.183011 \\
\hline C & -4.611893 & 3.934929 & -0.915587 \\
\hline C & -8.314836 & -0.945914 & -0.877663 \\
\hline $\mathrm{H}$ & -8.327887 & -0.503500 & -1.880361 \\
\hline $\mathrm{H}$ & -7.631961 & -1.801647 & -0.898906 \\
\hline
\end{tabular}




\begin{tabular}{|c|c|c|c|}
\hline $\mathrm{H}$ & -9.320100 & -1.328037 & -0.661508 \\
\hline C & -8.952507 & 1.221699 & 0.186826 \\
\hline $\mathrm{H}$ & -9.937713 & 0.798378 & 0.412831 \\
\hline $\mathrm{H}$ & -8.739163 & 1.982357 & 0.946354 \\
\hline $\mathrm{H}$ & -9.023438 & 1.720415 & -0.786569 \\
\hline C & -7.894701 & -0.562106 & 1.581477 \\
\hline $\mathrm{H}$ & -7.606923 & 0.158472 & 2.355497 \\
\hline $\mathrm{H}$ & -8.894486 & -0.942214 & 1.825293 \\
\hline $\mathrm{H}$ & -7.196238 & -1.403800 & 1.634110 \\
\hline C & -3.604004 & 4.432975 & 0.148866 \\
\hline $\mathrm{H}$ & -4.032221 & 4.354083 & 1.155342 \\
\hline $\mathrm{H}$ & -2.672657 & 3.857695 & 0.134902 \\
\hline $\mathrm{H}$ & -3.351340 & 5.485628 & -0.028718 \\
\hline C & -5.869742 & 4.820824 & -0.836054 \\
\hline $\mathrm{H}$ & -6.618406 & 4.537259 & -1.584778 \\
\hline $\mathrm{H}$ & -5.595982 & 5.865385 & -1.022637 \\
\hline $\mathrm{H}$ & -6.340248 & 4.775609 & 0.152825 \\
\hline C & -3.993931 & 4.103534 & -2.324117 \\
\hline $\mathrm{H}$ & -3.060728 & 3.541463 & -2.430755 \\
\hline $\mathrm{H}$ & -4.685548 & 3.755732 & -3.100110 \\
\hline $\mathrm{H}$ & -3.769580 & 5.159746 & -2.516901 \\
\hline C & 6.656676 & 2.777970 & 1.385627 \\
\hline C & 6.401103 & -0.212016 & -2.725250 \\
\hline C & 7.855389 & -0.698495 & -2.522719 \\
\hline $\mathrm{H}$ & 8.496425 & 0.084349 & -2.103505 \\
\hline $\mathrm{H}$ & 7.889563 & -1.557629 & -1.842882 \\
\hline $\mathrm{H}$ & 8.291161 & -1.006529 & -3.481294 \\
\hline C & 6.396120 & 0.999166 & -3.688327 \\
\hline $\mathrm{H}$ & 5.374430 & 1.355816 & -3.861030 \\
\hline $\mathrm{H}$ & 6.980948 & 1.837009 & -3.293432 \\
\hline H & 6.826662 & 0.718898 & -4.657787 \\
\hline C & 5.601984 & -1.352890 & -3.384855 \\
\hline H & 5.609101 & -2.259246 & -2.768368 \\
\hline $\mathrm{H}$ & 4.557757 & -1.076112 & -3.563976 \\
\hline $\mathrm{H}$ & 6.054463 & -1.606489 & -4.350622 \\
\hline C & 8.109861 & 2.336329 & 1.680621 \\
\hline $\mathrm{H}$ & 8.126905 & 1.425632 & 2.290356 \\
\hline $\mathrm{H}$ & 8.669827 & 2.132158 & 0.762057 \\
\hline $\mathrm{H}$ & 8.644308 & 3.122394 & 2.228424 \\
\hline C & 5.978697 & 3.110313 & 2.728741 \\
\hline $\mathrm{H}$ & 6.539301 & 3.901849 & 3.239123 \\
\hline $\mathrm{H}$ & 4.952529 & 3.469097 & 2.591043 \\
\hline $\mathrm{H}$ & 5.949986 & 2.241979 & 3.397010 \\
\hline C & 6.677186 & 4.065262 & 0.527487 \\
\hline $\mathrm{H}$ & 5.658466 & 4.407046 & 0.311789 \\
\hline $\mathrm{H}$ & 7.201494 & 4.870510 & 1.056967 \\
\hline $\mathrm{H}$ & 7.185824 & 3.908351 & -0.429378 \\
\hline
\end{tabular}




\begin{tabular}{ll}
\hline B3LYP/6-31G(d) Energy & -2164.796798 \\
\hline B3LYP-D3(BJ)/def2-TZVPP-IEF-PCM(DCM) Energy & -2165.895254 \\
\hline
\end{tabular}

B3LYP-D3(BJ)/def2-TZVPP-IEF-PCM(DCM)//B3LYP/6-31G(d) Free Energy (Quasiharmonic) - 2165.017537

Number of Imaginary Frequencies

Frequencies (Top 3 out of 321)

$\begin{array}{ll}\text { 1. } & 10.8254 \mathrm{~cm}^{-1} \\ \text { 2. } & 12.2018 \mathrm{~cm}^{-1} \\ \text { 3. } & 13.2546 \mathrm{~cm}^{-1}\end{array}$

\section{B3LYP/6-31G(d) Molecular Geometry in Cartesian Coordinates}

C

C

C

$\mathrm{C}$

$\mathrm{C}$

C

$\mathrm{H}$

$\mathrm{H}$

C

C

$\mathrm{H}$

$\mathrm{H}$

C

C

$\mathrm{H}$

C

C

C

c

C

C

$\mathrm{H}$

$\mathrm{H}$

$\mathrm{H}$

C

C

$\mathrm{H}$

$\mathrm{H}$

C

C

C

$\mathrm{H}$
0.001562

0.178234

$-0.849849$

$-2.082321$

$-2.230267$

$-1.212442$

0.798065

1.105439

$-0.699816$

- 3.121465

$-3.168331$

$-1.338692$

$-2.990843$

$-1.757284$

$-4.040205$

0.901539

1.969096

1.875768

0.654171

$-0.430681$

$-0.309530$

3. 894542

0.983078

2.904474

2.965104

0.581783

$-1.367512$

$-1.151661$

1.672984

2.898346

1.553226

0.527227
$-4.833971$

$-3.731758$

$-2.755519$

$-2.958854$

$-4.105591$

$-5.022640$

$-5.566762$

$-3.603993$

$-1.590686$

$-2.007127$

$-4.244426$

$-5.894782$

$-0.890100$

$-0.697801$

$-2.151734$

$-2.798893$

$-2.175407$

$-1.699227$

$-1.874253$

$-2.522932$

$-2.972063$

$-0.950855$

$-3.158331$

$-2.036557$

$-1.062683$

$-1.391263$

$-2.655005$

$-3.461349$

$-0.770167$

$-0.606523$

$-0.249091$

0.083385
$-1.728573$

$-0.919556$

$-0.776903$

$-1.477089$

$-2.303491$

$-2.430896$

$-1.822988$

$-0.370745$

0.047639

$-1.338254$

$-2.835521$

$-3.066300$

$-0.537615$

0.165739

$-1.900674$

4.822238

4.219826

2.883693

2.159777

2.814034

4.109829

2.797978

5.844496

4.756996

2. 245199

0.811641

2. 284004

4.591421

0.207168

0.941415

$-1.216556$

$-1.406188$ 


\begin{tabular}{|c|c|c|c|}
\hline $\mathrm{H}$ & 2.210674 & 0.610493 & -1.354399 \\
\hline C & -1.655155 & 0.434458 & 1.151131 \\
\hline 0 & -2.269299 & 0.495709 & 2.190983 \\
\hline 0 & -0.786659 & 1.399674 & 0.755034 \\
\hline $\mathrm{H}$ & -0.761151 & 2.052444 & 1.481591 \\
\hline 0 & 1.962562 & -1.198249 & -2.210589 \\
\hline $\mathrm{H}$ & 1.339957 & -1.942784 & -2.178089 \\
\hline C & 4.106581 & 0.058029 & 0.367404 \\
\hline C & 4.656015 & -0.352625 & -0.855714 \\
\hline C & 4.737574 & 1.082475 & 1.089771 \\
\hline C & 5.813282 & 0.245681 & -1.367639 \\
\hline $\mathrm{H}$ & 4.156695 & -1.141297 & -1.402982 \\
\hline C & 5.897086 & 1.704456 & 0.613597 \\
\hline $\mathrm{H}$ & 4.291828 & 1.397532 & 2.026338 \\
\hline C & 6.410526 & 1.267314 & -0.615773 \\
\hline $\mathrm{H}$ & 7.305768 & 1.744475 & -1.003880 \\
\hline C & -4.104451 & 0.096283 & -0.458045 \\
\hline C & -5.406513 & -0.336560 & -0.174900 \\
\hline C & -3.870965 & 1.455199 & -0.708758 \\
\hline C & -6.476383 & 0.564320 & -0.127569 \\
\hline $\mathrm{H}$ & -5.563550 & -1.388770 & 0.032282 \\
\hline C & -4.912651 & 2.388204 & -0.672131 \\
\hline $\mathrm{H}$ & -2.863676 & 1.771805 & -0.951039 \\
\hline C & -6.199664 & 1.915709 & -0.379306 \\
\hline $\mathrm{H}$ & -7.018118 & 2.628473 & -0.343934 \\
\hline C & -4.688031 & 3.889111 & -0.940003 \\
\hline C & -7.918504 & 0.126021 & 0.193255 \\
\hline C & -5.539785 & 4.332799 & -2.152885 \\
\hline $\mathrm{H}$ & -5.254465 & 3.779546 & -3.054964 \\
\hline $\mathrm{H}$ & -6.608866 & 4.166950 & -1.983521 \\
\hline $\mathrm{H}$ & -5.395589 & 5.402187 & -2.350143 \\
\hline C & -3.213773 & 4.214987 & -1.246838 \\
\hline $\mathrm{H}$ & -3.101023 & 5.292010 & -1.414775 \\
\hline H & -2.551918 & 3.937633 & -0.418634 \\
\hline $\mathrm{H}$ & -2.861377 & 3.701600 & -2.148614 \\
\hline C & -5.110638 & 4.701047 & 0.307675 \\
\hline H & -4.520694 & 4.408733 & 1.183775 \\
\hline $\mathrm{H}$ & -4.957082 & 5.773363 & 0.134592 \\
\hline $\mathrm{H}$ & -6.167189 & 4.551565 & 0.553310 \\
\hline C & -8.028382 & -1.389060 & 0.450641 \\
\hline $\mathrm{H}$ & -7.721204 & -1.975672 & -0.422829 \\
\hline $\mathrm{H}$ & -7.419093 & -1.703123 & 1.305610 \\
\hline $\mathrm{H}$ & -9.069111 & -1.650199 & 0.673349 \\
\hline C & -8.840206 & 0.478676 & -0.998545 \\
\hline $\mathrm{H}$ & -8.516494 & -0.036744 & -1.910107 \\
\hline $\mathrm{H}$ & -9.872845 & 0.176375 & -0.785094 \\
\hline $\mathrm{H}$ & -8.845051 & 1.553691 & -1.207243 \\
\hline C & -8.413590 & 0.863626 & 1.460080 \\
\hline $\mathrm{H}$ & -7.779569 & 0.628993 & 2.322485 \\
\hline $\mathrm{H}$ & -8.408619 & 1.950697 & 1.328426 \\
\hline $\mathrm{H}$ & -9.440792 & 0.562174 & 1.699644 \\
\hline C & 6.604377 & 2.840083 & 1.379364 \\
\hline c & 6.428239 & -0.174723 & -2.717038 \\
\hline$c$ & 5.658528 & -1.337690 & -3.372900 \\
\hline
\end{tabular}




\begin{tabular}{|rrrr|} 
H & 5.684131 & -2.239965 & -2.750976 \\
H & 4.608949 & -1.086176 & -3.557917 \\
H & 6.120799 & -1.586403 & -4.335254 \\
$\mathrm{C}$ & 7.892687 & -0.625891 & -2.506021 \\
$\mathrm{H}$ & 8.513931 & 0.174554 & -2.090238 \\
$\mathrm{H}$ & 7.944197 & -1.479305 & -1.820140 \\
$\mathrm{H}$ & 8.338913 & -0.930390 & -3.460870 \\
$\mathrm{C}$ & 6.399206 & 1.030491 & -3.687176 \\
$\mathrm{H}$ & 5.370376 & 1.362966 & -3.865772 \\
$\mathrm{H}$ & 6.963617 & 1.883559 & -3.295099 \\
$\mathrm{H}$ & 6.839596 & 0.754467 & -4.653385 \\
$\mathrm{C}$ & 8.063652 & 2.427387 & 1.685987 \\
$\mathrm{H}$ & 8.093821 & 1.521546 & 2.302350 \\
$\mathrm{H}$ & 8.632432 & 2.226953 & 0.772063 \\
$\mathrm{H}$ & 8.580338 & 3.227082 & 2.231047 \\
$\mathrm{C}$ & 5.912106 & 3.168139 & 2.716131 \\
$\mathrm{H}$ & 6.454624 & 3.973010 & 3.225132 \\
$\mathrm{H}$ & 4.880430 & 3.507489 & 2.569672 \\
$\mathrm{H}$ & 5.894714 & 2.303768 & 3.389791 \\
$\mathrm{C}$ & 6.606813 & 4.122522 & 0.513684 \\
$\mathrm{H}$ & 5.583449 & 4.444394 & 0.289139 \\
$\mathrm{H}$ & 7.112610 & 4.940358 & 1.041830 \\
$\mathrm{H}$ & 7.124598 & 3.969758 & -0.438896 \\
& & & \\
\hline & & & \\
\hline & & &
\end{tabular}

\section{Catalyst 2C 75}

Datum

Value

\begin{tabular}{lc}
\hline B3LYP/6-31G(d) Energy & -2164.79998 \\
\hline B3LYP-D3(BJ)/def2-TZVPP-IEF-PCM(DCM) Energy & -2165.896108 \\
\hline B3LYP-D3(BJ)/def2-TZVPP-IEF-PCM(DCM)//B3LYP/6-31G(d) Free Energy (Quasiharmonic) & -2165.017536 \\
\hline
\end{tabular}

Number of Imaginary Frequencies

Frequencies (Top 3 out of 321)
1. $10.9617 \mathrm{~cm}^{-1}$
2. $13.0533 \mathrm{~cm}^{-1}$
3. $14.1074 \mathrm{~cm}^{-1}$

\section{B3LYP/6-31G(d) Molecular Geometry in Cartesian Coordinates}

$\begin{array}{lrrr}C & 0.287402 & -3.393822 & 3.932577 \\ C & 0.411682 & -2.834098 & 2.680220 \\ C & -0.667643 & -2.121008 & 2.089697\end{array}$




\begin{tabular}{|c|c|c|c|}
\hline C & -1.887855 & -1.994062 & 2.827385 \\
\hline C & -1.982815 & -2.582432 & 4.117487 \\
\hline C & -0.920046 & -3.267101 & 4.660150 \\
\hline $\mathrm{H}$ & 1.121722 & -3.938342 & 4.366037 \\
\hline $\mathrm{H}$ & 1.339300 & -2.935754 & 2.127338 \\
\hline C & -0.575294 & -1.521774 & 0.789775 \\
\hline C & -2.975444 & -1.291005 & 2.253228 \\
\hline $\mathrm{H}$ & -2.914674 & -2.480762 & 4.668475 \\
\hline $\mathrm{H}$ & -1.004250 & -3.712577 & 5.647628 \\
\hline C & -2.905197 & -0.731587 & 0.993174 \\
\hline C & -1.678357 & -0.863957 & 0.264502 \\
\hline $\mathrm{H}$ & -3.902660 & -1.214552 & 2.815297 \\
\hline C & 1.380624 & -4.882456 & -2.623498 \\
\hline C & 2.351759 & -3.923769 & -2.451447 \\
\hline C & 2.142507 & -2.825438 & -1.574196 \\
\hline C & 0.904185 & -2.715469 & -0.869033 \\
\hline C & -0.080156 & -3.721563 & -1.073678 \\
\hline C & 0.153504 & -4.777606 & -1.925817 \\
\hline $\mathrm{H}$ & 4.066825 & -1.921798 & -1.926428 \\
\hline $\mathrm{H}$ & 1.550282 & -5.719471 & -3.295455 \\
\hline $\mathrm{H}$ & 3.297668 & -3.991162 & -2.983755 \\
\hline C & 3.133040 & -1.835132 & -1.376604 \\
\hline C & 0.713470 & -1.602051 & 0.015167 \\
\hline $\mathrm{H}$ & -1.029836 & -3.646596 & -0.555668 \\
\hline $\mathrm{H}$ & -0.612772 & -5.534576 & -2.069277 \\
\hline C & 1.708599 & -0.640123 & 0.191519 \\
\hline C & 2.950684 & -0.759957 & -0.527510 \\
\hline C & 1.501343 & 0.510905 & 1.168240 \\
\hline $\mathrm{H}$ & 0.818062 & 0.202769 & 1.968235 \\
\hline $\mathrm{H}$ & 2.454859 & 0.770826 & 1.632930 \\
\hline C & -1.570885 & -0.265368 & -1.110317 \\
\hline 0 & -1.665335 & -0.859774 & -2.158459 \\
\hline 0 & -1.345860 & 1.078137 & -1.058241 \\
\hline $\mathrm{H}$ & -1.291517 & 1.387916 & -1.983867 \\
\hline 0 & 1.039124 & 1.717419 & 0.558406 \\
\hline $\mathrm{H}$ & 0.174891 & 1.539412 & 0.148526 \\
\hline C & 4.082928 & 0.202773 & -0.369465 \\
\hline C & 3.906223 & 1.586530 & -0.540817 \\
\hline C & 5.365184 & -0.285771 & -0.095735 \\
\hline C & 4.981217 & 2.471183 & -0.436478 \\
\hline $\mathrm{H}$ & 2.907789 & 1.951279 & -0.738851 \\
\hline C & 6.473079 & 0.569112 & 0.013539 \\
\hline $\mathrm{H}$ & 5.490337 & -1.354756 & 0.051352 \\
\hline C & 6.253600 & 1.938492 & -0.160795 \\
\hline $\mathrm{H}$ & 7.091016 & 2.620882 & -0.075117 \\
\hline C & -4.085628 & -0.013327 & 0.433786 \\
\hline C & -4.605645 & -0.341863 & -0.824603 \\
\hline C & -4.713498 & 0.986820 & 1.187586 \\
\hline C & -5.727932 & 0.315890 & -1.343704 \\
\hline $\mathrm{H}$ & -4.131744 & -1.132141 & -1.396306 \\
\hline C & -5.839748 & 1.666091 & 0.708085 \\
\hline $\mathrm{H}$ & -4.291886 & 1.244557 & 2.154701 \\
\hline C & -6.325430 & 1.313374 & -0.559992 \\
\hline $\mathrm{H}$ & -7.192046 & 1.830546 & -0.947946 \\
\hline
\end{tabular}




\begin{tabular}{|c|c|c|c|}
\hline C & -6.488156 & 2.768498 & 1.569021 \\
\hline C & -6.254781 & -0.073810 & -2.738805 \\
\hline C & -7.720767 & 3.398905 & 0.893229 \\
\hline H & -7.464917 & 3.879650 & -0.057778 \\
\hline $\mathrm{H}$ & -8.506385 & 2.658835 & 0.702412 \\
\hline $\mathrm{H}$ & -8.144616 & 4.168981 & 1.547549 \\
\hline C & -5.456031 & 3.891994 & 1.827766 \\
\hline $\mathrm{H}$ & -5.898037 & 4.681043 & 2.448569 \\
\hline $\mathrm{H}$ & -4.567284 & 3.518374 & 2.346518 \\
\hline $\mathrm{H}$ & -5.126486 & 4.345391 & 0.885956 \\
\hline C & -6.939415 & 2.168004 & 2.921811 \\
\hline $\mathrm{H}$ & -6.098435 & 1.745586 & 3.481600 \\
\hline $\mathrm{H}$ & -7.399961 & 2.942183 & 3.547829 \\
\hline $\mathrm{H}$ & -7.675597 & 1.370024 & 2.771228 \\
\hline C & -6.623038 & -1.576614 & -2.753106 \\
\hline $\mathrm{H}$ & -5.761035 & -2.211740 & -2.525492 \\
\hline H & -7.405286 & -1.796173 & -2.017257 \\
\hline $\mathrm{H}$ & -6.996594 & -1.866392 & -3.742853 \\
\hline C & -5.151417 & 0.193210 & -3.791506 \\
\hline $\mathrm{H}$ & -4.232841 & -0.361778 & -3.575390 \\
\hline $\mathrm{H}$ & -5.497180 & -0.103758 & -4.789389 \\
\hline $\mathrm{H}$ & -4.897886 & 1.259518 & -3.825845 \\
\hline C & -7.507149 & 0.726722 & -3.142691 \\
\hline $\mathrm{H}$ & -8.339068 & 0.562808 & -2.448007 \\
\hline $\mathrm{H}$ & -7.307559 & 1.803384 & -3.190082 \\
\hline $\mathrm{H}$ & -7.841029 & 0.410501 & -4.137386 \\
\hline C & 7.862173 & -0.021724 & 0.325087 \\
\hline C & 4.809495 & 3.994321 & -0.597234 \\
\hline C & 5.736606 & 4.511169 & -1.722547 \\
\hline $\mathrm{H}$ & 6.793202 & 4.319990 & -1.506638 \\
\hline H & 5.498288 & 4.029323 & -2.677782 \\
\hline $\mathrm{H}$ & 5.615028 & 5.594093 & -1.848921 \\
\hline C & 5.182843 & 4.690177 & 0.733734 \\
\hline $\mathrm{H}$ & 4.527154 & 4.355448 & 1.545517 \\
\hline $\mathrm{H}$ & 6.216208 & 4.478057 & 1.028655 \\
\hline $\mathrm{H}$ & 5.078543 & 5.778362 & 0.639097 \\
\hline C & 3.361568 & 4.384072 & -0.952448 \\
\hline H & 3.054363 & 3.955797 & -1.913741 \\
\hline H & 2.647297 & 4.052713 & -0.191566 \\
\hline $\mathrm{H}$ & 3.284877 & 5.474478 & -1.036214 \\
\hline C & 8.961486 & 1.055592 & 0.389470 \\
\hline $\mathrm{H}$ & 9.061652 & 1.593668 & -0.560077 \\
\hline $\mathrm{H}$ & 8.768729 & 1.789818 & 1.179968 \\
\hline $\mathrm{H}$ & 9.927121 & 0.584495 & 0.605889 \\
\hline C & 8.251217 & -1.034808 & -0.777954 \\
\hline $\mathrm{H}$ & 9.238498 & -1.465935 & -0.570423 \\
\hline $\mathrm{H}$ & 7.535571 & -1.860953 & -0.844966 \\
\hline $\mathrm{H}$ & 8.292277 & -0.548190 & -1.759237 \\
\hline C & 7.819053 & -0.746339 & 1.691522 \\
\hline $\mathrm{H}$ & 7.082246 & -1.556422 & 1.699584 \\
\hline $\mathrm{H}$ & 8.798338 & -1.182669 & 1.924690 \\
\hline H & 7.557142 & -0.049262 & 2.495792 \\
\hline
\end{tabular}




\section{Catalyst 2C 76}

Datum

Value

\begin{tabular}{lc}
\hline B3LYP/6-31G(d) Energy & -2164.797078 \\
\hline B3LYP-D3(BJ)/def2-TZVPP-IEF-PCM(DCM) Energy & -2165.895361 \\
\hline B3LYP-D3(BJ)/def2-TZVPP-IEF-PCM(DCM)//B3LYP/6-31G(d) Free Energy (Quasiharmonic) & -2165.017514 \\
\hline Number of Imaginary Frequencies & 0
\end{tabular}

Frequencies (Top 3 out of 321)

$\begin{array}{ll}\text { 1. } & 11.2662 \mathrm{~cm}^{-1} \\ \text { 2. } & 12.5285 \mathrm{~cm}^{-1} \\ \text { 3. } & 14.3075 \mathrm{~cm}^{-1}\end{array}$

\section{B3LYP/6-31G(d) Molecular Geometry in Cartesian Coordinates}

C

C

C

C

C

C

$\mathrm{H}$

$\mathrm{H}$

C

C

$\mathrm{H}$

$\mathrm{H}$

C

C

$\mathrm{H}$

C

C

C

C

C

C

$\mathrm{H}$

$\mathrm{H}$

$\mathrm{H}$

C

C

$\mathrm{H}$

$\mathrm{H}$

C
$-0.017128$

$-0.195922$

0.833049

2.068342

2. 218427

1.199882

$-0.814221$

$-1.125399$

0.680957

3.107875

3.158696

1. 327833

2.975000

1.738834

4.028716

$-0.953153$

$-2.015657$

$-1.912253$

$-0.686012$

0.393549

0. 262771

$-3.928419$

$-1.042347$

$-2.954522$

$-2.995627$

$-0.604124$

1.333933

1.100928

$-1.689803$
$-4.849833$

$-3.739656$

$-2.763744$

$-2.975266$

$-4.130128$

$-5.046951$

$-5.582407$

$-3.605317$

$-1.590998$

$-2.023246$

$-4.275377$

$-5.925326$

$-0.898210$

$-0.698157$

$-2.174155$

$-2.769849$

$-2.147612$

$-1.677365$

$-1.857742$

$-2.504512$

$-2.947533$

$-0.924611$

$-3.124684$

$-2.005058$

$-1.040718$

$-1.382457$

$-2.639859$

$-3.435478$

$-0.761661$
$-1.689430$

$-0.891831$

$-0.753544$

$-1.446277$

$-2.260918$

$-2.384199$

$-1.780501$

$-0.348441$

0.059292

$-1.312346$

$-2.787313$

$-3.010606$

$-0.523329$

0.173297

$-1.869647$

4.829962

4.217391

2.879896

2.165248

2.830009

4.126957

2. 777653

5.853183

4.747453

2.231470

0.815001

2. 307135

4.616766

0.200402 


\begin{tabular}{|c|c|c|c|}
\hline C & -2.918923 & -0.589261 & 0.926574 \\
\hline C & -1.560253 & -0.253886 & -1.227139 \\
\hline $\mathrm{H}$ & -2.212287 & 0.607494 & -1.377612 \\
\hline $\mathrm{H}$ & -0.531797 & 0.070607 & -1.416153 \\
\hline C & 1.633460 & 0.443430 & 1.147631 \\
\hline 0 & 2.240571 & 0.512238 & 2.191107 \\
\hline 0 & 0.770413 & 1.407310 & 0.736765 \\
\hline $\mathrm{H}$ & 0.742047 & 2.067331 & 1.456642 \\
\hline 0 & -1.970305 & -1.211936 & -2.213214 \\
\hline $\mathrm{H}$ & -1.348406 & -1.956851 & -2.174842 \\
\hline C & -4.120355 & 0.079907 & 0.343694 \\
\hline C & -4.656417 & -0.313814 & -0.889482 \\
\hline C & -4.762732 & 1.097533 & 1.063716 \\
\hline C & -5.805500 & 0.292055 & -1.412667 \\
\hline $\mathrm{H}$ & -4.154196 & -1.098964 & -1.443195 \\
\hline C & -5.916480 & 1.725805 & 0.579500 \\
\hline $\mathrm{H}$ & -4.331994 & 1.407758 & 2.011519 \\
\hline C & -6.417734 & 1.305509 & -0.661232 \\
\hline $\mathrm{H}$ & -7.304840 & 1.783076 & -1.054190 \\
\hline C & 4.088778 & 0.088418 & -0.449356 \\
\hline C & 3.856506 & 1.445047 & -0.713356 \\
\hline C & 5.389820 & -0.342152 & -0.158103 \\
\hline C & 4.898348 & 2.378069 & -0.681962 \\
\hline $\mathrm{H}$ & 2.850172 & 1.759750 & -0.961918 \\
\hline C & 6.459847 & 0.558807 & -0.115944 \\
\hline $\mathrm{H}$ & 5.545875 & -1.392431 & 0.059396 \\
\hline C & 6.184315 & 1.907896 & -0.381012 \\
\hline H & 7.002845 & 2.620770 & -0.349838 \\
\hline C & 7.900835 & 0.123120 & 0.213391 \\
\hline C & 4.674968 & 3.876679 & -0.963330 \\
\hline C & 8.009338 & -1.389413 & 0.485837 \\
\hline $\mathrm{H}$ & 7.397152 & -1.694954 & 1.341822 \\
\hline $\mathrm{H}$ & 7.704897 & -1.984477 & -0.382863 \\
\hline $\mathrm{H}$ & 9.049250 & -1.648603 & 0.714513 \\
\hline C & 8.392322 & 0.872774 & 1.474527 \\
\hline $\mathrm{H}$ & 9.418762 & 0.573527 & 1.720038 \\
\hline $\mathrm{H}$ & 8.387865 & 1.958523 & 1.332417 \\
\hline H & 7.755706 & 0.646477 & 2.337251 \\
\hline C & 8.826258 & 0.463947 & -0.978960 \\
\hline $\mathrm{H}$ & 8.832720 & 1.537016 & -1.197408 \\
\hline $\mathrm{H}$ & 9.858015 & 0.162673 & -0.759807 \\
\hline $\mathrm{H}$ & 8.504664 & -0.059442 & -1.886717 \\
\hline C & 3.201631 & 4.200444 & -1.276767 \\
\hline $\mathrm{H}$ & 2.851327 & 3.679475 & -2.175002 \\
\hline $\mathrm{H}$ & 2.537536 & 3.930482 & -0.447918 \\
\hline $\mathrm{H}$ & 3.089653 & 5.276015 & -1.454271 \\
\hline C & 5.530109 & 4.309882 & -2.177630 \\
\hline $\mathrm{H}$ & 5.246738 & 3.749561 & -3.075953 \\
\hline $\mathrm{H}$ & 5.387130 & 5.377729 & -2.383870 \\
\hline H & 6.598648 & 4.144765 & -2.004198 \\
\hline C & 5.094620 & 4.698961 & 0.278579 \\
\hline $\mathrm{H}$ & 4.502178 & 4.414355 & 1.155533 \\
\hline $\mathrm{H}$ & 6.150436 & 4.551080 & 0.528329 \\
\hline H & 4.942008 & 5.769820 & 0.095995 \\
\hline
\end{tabular}




\begin{tabular}{|rrrr|} 
C & -6.580505 & 2.843370 & 1.408774 \\
C & -6.342589 & -0.168728 & -2.781812 \\
H & -7.610731 & 0.594888 & -3.207194 \\
H & -8.433393 & 0.451297 & -2.496904 \\
H & -7.426588 & 1.670996 & -3.304518 \\
C & -7.950694 & 0.230058 & -4.183229 \\
H & -6.689259 & -1.675406 & -2.719405 \\
H & -5.813898 & -2.283746 & -2.470838 \\
H & -7.459324 & -1.870535 & -1.963820 \\
C & -7.070076 & -2.018921 & -3.689312 \\
H & -5.254966 & 0.061241 & -3.859571 \\
H & -5.016503 & 1.127854 & -3.948038 \\
H & -4.326570 & -0.469638 & -3.626762 \\
C & -5.609857 & -0.287339 & -4.837751 \\
H & -7.012789 & 2.276920 & 2.782201 \\
H & -7.736723 & 1.463102 & 2.659585 \\
H & -6.160778 & 1.883202 & 3.346246 \\
C & -7.481669 & 3.060998 & 3.389830 \\
H & -7.828692 & 3.428755 & 0.720709 \\
H & -8.261467 & 4.214353 & 1.350522 \\
H & -7.587312 & 3.878701 & -0.249015 \\
C & -8.602785 & 2.669410 & 0.561779 \\
H & -5.570911 & 3.995374 & 1.626780 \\
H & -5.250958 & 4.421820 & 0.669160 \\
H & -6.027158 & 4.797013 & 2.220789 \\
& -4.674922 & 3.656978 & 2.157366 \\
\hline & & & \\
\hline
\end{tabular}

\section{Catalyst 2C 77}

Datum

Value

\begin{tabular}{lr}
\hline B3LYP/6-31G(d) Energy & -2164.800501 \\
\hline B3LYP-D3(BJ)/def2-TZVPP-IEF-PCM(DCM) Energy & -2165.896369 \\
\hline B3LYP-D3(BJ)/def2-TZVPP-IEF-PCM(DCM)//B3LYP/6-31G(d) Free Energy (Quasiharmonic) & -2165.017456 \\
\hline
\end{tabular}

Number of Imaginary Frequencies

Frequencies (Top 3 out of 321)

$\begin{array}{ll}\text { 1. } & 12.5391 \mathrm{~cm}^{-1} \\ \text { 2. } & 13.0592 \mathrm{~cm}^{-1} \\ \text { 3. } & 14.4276 \mathrm{~cm}^{-1}\end{array}$




\begin{tabular}{|c|c|c|c|}
\hline C & 0.318161 & -3.400261 & 3.933102 \\
\hline C & 0.440431 & -2.835627 & 2.682763 \\
\hline C & -0.642102 & -2.125910 & 2.094000 \\
\hline C & -1.863337 & -2.007534 & 2.831404 \\
\hline C & -1.956086 & -2.600644 & 4.119525 \\
\hline C & -0.890299 & -3.281948 & 4.660440 \\
\hline $\mathrm{H}$ & 1.154880 & -3.942221 & 4.365139 \\
\hline $\mathrm{H}$ & 1.368877 & -2.930937 & 2.130156 \\
\hline C & -0.552163 & -1.521849 & 0.796170 \\
\hline C & -2.954164 & -1.308343 & 2.258887 \\
\hline$H$ & -2.888737 & -2.505345 & 4.670298 \\
\hline $\mathrm{H}$ & -0.972843 & -3.731223 & 5.646333 \\
\hline C & -2.886742 & -0.745065 & 1.000278 \\
\hline C & -1.658502 & -0.868374 & 0.272228 \\
\hline $\mathrm{H}$ & -3.881790 & -1.238290 & 2.821075 \\
\hline C & 1.420688 & -4.858966 & -2.630001 \\
\hline C & 2.387991 & -3.897517 & -2.452167 \\
\hline C & 2.173541 & -2.804288 & -1.569723 \\
\hline C & 0.933963 & -2.702316 & -0.865676 \\
\hline C & -0.046263 & -3.711248 & -1.076135 \\
\hline C & 0.192403 & -4.762215 & -1.933123 \\
\hline $\mathrm{H}$ & 4.094553 & -1.892488 & -1.915401 \\
\hline $\mathrm{H}$ & 1.594234 & -5.692024 & -3.305867 \\
\hline $\mathrm{H}$ & 3.334768 & -3.958745 & -2.983666 \\
\hline C & 3.160109 & -1.811549 & -1.365921 \\
\hline C & 0.737839 & -1.593307 & 0.022716 \\
\hline$H$ & -0.996810 & -3.642441 & -0.558877 \\
\hline $\mathrm{H}$ & -0.570789 & -5.521419 & -2.081132 \\
\hline C & 1.728978 & -0.628092 & 0.204531 \\
\hline C & 2.973327 & -0.740649 & -0.512064 \\
\hline C & 1.512787 & 0.519653 & 1.182855 \\
\hline $\mathrm{H}$ & 0.827495 & 0.207861 & 1.979455 \\
\hline $\mathrm{H}$ & 2.462748 & 0.784665 & 1.651571 \\
\hline C & -1.553220 & -0.264879 & -1.100553 \\
\hline 0 & -1.644616 & -0.856058 & -2.150776 \\
\hline 0 & -1.333803 & 1.079618 & -1.044445 \\
\hline $\mathrm{H}$ & -1.280861 & 1.391977 & -1.969295 \\
\hline 0 & 1.046334 & 1.724626 & 0.571756 \\
\hline $\mathrm{H}$ & 0.181904 & 1.544474 & 0.162997 \\
\hline C & 4.102790 & 0.224841 & -0.351008 \\
\hline C & 3.923532 & 1.606651 & -0.493692 \\
\hline C & 5.394658 & -0.263703 & -0.104200 \\
\hline C & 4.999707 & 2.497039 & -0.392820 \\
\hline $\mathrm{H}$ & 2.921845 & 1.979158 & -0.671115 \\
\hline C & 6.498400 & 0.591184 & -0.000999 \\
\hline $\mathrm{H}$ & 5.525130 & -1.334446 & 0.024425 \\
\hline C & 6.275240 & 1.967867 & -0.150742 \\
\hline $\mathrm{H}$ & 7.114896 & 2.644848 & -0.072755 \\
\hline C & -4.071456 & -0.032633 & 0.442582 \\
\hline C & -4.707716 & 0.960527 & 1.207614 \\
\hline C & -4.585877 & -0.356531 & -0.815448 \\
\hline C & -5.835510 & 1.630285 & 0.731536 \\
\hline $\mathrm{H}$ & -4.285355 & 1.210699 & 2.173859 \\
\hline
\end{tabular}




\begin{tabular}{|c|c|c|c|}
\hline C & -5.715686 & 0.297205 & -1.333569 \\
\hline $\mathrm{H}$ & -4.106265 & -1.139512 & -1.392363 \\
\hline C & -6.317706 & 1.280046 & -0.543557 \\
\hline $\mathrm{H}$ & -7.190285 & 1.798004 & -0.923691 \\
\hline C & -6.236141 & -0.087453 & -2.731938 \\
\hline C & -6.552307 & 2.729178 & 1.539974 \\
\hline C & -5.131346 & 0.191658 & -3.779938 \\
\hline $\mathrm{H}$ & -4.883780 & 1.259551 & -3.807634 \\
\hline $\mathrm{H}$ & -4.210200 & -0.359424 & -3.564732 \\
\hline $\mathrm{H}$ & -5.472762 & -0.101677 & -4.780358 \\
\hline C & -7.491632 & 0.708879 & -3.134058 \\
\hline H & -7.820952 & 0.398063 & -4.131935 \\
\hline $\mathrm{H}$ & -8.324602 & 0.535298 & -2.442955 \\
\hline $\mathrm{H}$ & -7.297522 & 1.786909 & $-3.17306 e$ \\
\hline C & -6.596003 & -1.592133 & -2.755189 \\
\hline $\mathrm{H}$ & -7.379669 & -1.819661 & -2.023300 \\
\hline $\mathrm{H}$ & -6.964364 & -1.878812 & -3.747755 \\
\hline $\mathrm{H}$ & -5.731346 & -2.223709 & -2.527709 \\
\hline C & -6.536465 & 4.053672 & 0.740265 \\
\hline $\mathrm{H}$ & -5.508932 & 4.381313 & 0.545647 \\
\hline $\mathrm{H}$ & -7.044217 & 3.955071 & -0.224859 \\
\hline H & -7.044583 & 4.845466 & 1.304157 \\
\hline C & -5.882350 & 2.988143 & 2.902908 \\
\hline $\mathrm{H}$ & -4.845466 & 3.324701 & 2.791196 \\
\hline $\mathrm{H}$ & -6.427999 & 3.773357 & 3.438243 \\
\hline $\mathrm{H}$ & -5.885422 & 2.094094 & 3.536885 \\
\hline C & -8.017420 & 2.304178 & 1.798961 \\
\hline H & -8.568700 & 2.146157 & 0.866111 \\
\hline $\mathrm{H}$ & -8.059058 & 1.371363 & 2.373048 \\
\hline $\mathrm{H}$ & -8.543907 & 3.078572 & 2.370294 \\
\hline C & 7.895239 & 0.001143 & 0.278106 \\
\hline C & 4.739145 & 4.008399 & -0.545502 \\
\hline C & 3.751777 & 4.468523 & 0.554688 \\
\hline $\mathrm{H}$ & 2.805967 & 3.919532 & 0.513613 \\
\hline $\mathrm{H}$ & 4.183307 & 4. 315001 & 1.550976 \\
\hline $\mathrm{H}$ & 3.531142 & 5.537927 & 0.443959 \\
\hline C & 4.121385 & 4.286786 & -1.936476 \\
\hline H & 4.798773 & 3.968863 & -2.737720 \\
\hline $\mathrm{H}$ & 3.171640 & 3.759475 & -2.072018 \\
\hline $\mathrm{H}$ & 3.928538 & 5.359763 & -2.059828 \\
\hline C & 6.025310 & 4.846863 & -0.419171 \\
\hline $\mathrm{H}$ & 6.500333 & 4.722686 & 0.560863 \\
\hline $\mathrm{H}$ & 6.760329 & 4.588941 & -1.190648 \\
\hline $\mathrm{H}$ & 5.784749 & 5.909918 & -0.534949 \\
\hline C & 7.878769 & -0.742305 & 1.634993 \\
\hline $\mathrm{H}$ & 7.628219 & -0.057312 & $2.45315 e$ \\
\hline $\mathrm{H}$ & 7.145156 & -1.555276 & 1.644716 \\
\hline $\mathrm{H}$ & 8.863617 & -1.177869 & 1.845182 \\
\hline C & 8.992283 & 1.081200 & $0.33856 e$ \\
\hline H & 9.962910 & 0.610128 & 0.531493 \\
\hline $\mathrm{H}$ & 9.074555 & 1.632975 & -0.604810 \\
\hline $\mathrm{H}$ & 8.811204 & 1.803779 & 1.142393 \\
\hline C & 8.269248 & -0.995208 & -0.845212 \\
\hline H & 8.291062 & -0.495151 & -1.820348 \\
\hline
\end{tabular}




\section{Catalyst 2C 78}

Datum

Value

\begin{tabular}{lr}
\hline B3LYP/6-31G(d) Energy & -2164.797366 \\
\hline B3LYP-D3(BJ)/def2-TZVPP-IEF-PCM(DCM) Energy & -2165.895221 \\
\hline B3LYP-D3(BJ)/def2-TZVPP-IEF-PCM(DCM)//B3LYP/6-31G(d) Free Energy (Quasiharmonic) & -2165.017454 \\
\hline
\end{tabular}

Number of Imaginary Frequencies

Frequencies (Top 3 out of 321)

$\begin{array}{ll}\text { 1. } & 11.0782 \mathrm{~cm}^{-1} \\ \text { 2. } & 11.8188 \mathrm{~cm}^{-1} \\ \text { 3. } & 13.3563 \mathrm{~cm}^{-1}\end{array}$

\section{B3LYP/6-31G(d) Molecular Geometry in Cartesian Coordinates}

\begin{tabular}{|rrrl}
\hline & & & \\
C & -0.117732 & -3.887702 & 3.429972 \\
C & -0.270921 & -2.631644 & 2.882902 \\
C & 0.769610 & -2.038313 & 2.111505 \\
C & 1.983741 & -2.774018 & 1.923553 \\
C & 2.109335 & -4.064591 & 2.505708 \\
C & 1.082649 & -4.612940 & 3.239418 \\
H & -0.921849 & -4.322020 & 4.017391 \\
H & -1.187710 & -2.074664 & 3.046745 \\
$\mathrm{C}$ & 0.642828 & -0.740417 & 1.515098 \\
$\mathrm{C}$ & 3.032928 & -2.196071 & 1.168538 \\
$\mathrm{H}$ & 3.036104 & -4.613610 & 2.357401 \\
$\mathrm{H}$ & 1.190604 & -5.601863 & 3.676141 \\
$\mathrm{C}$ & 2.924966 & -0.940782 & 0.602524 \\
$\mathrm{C}$ & 1.702431 & -0.217854 & 0.787583 \\
$\mathrm{H}$ & 3.960031 & -2.751555 & 1.053196 \\
$\mathrm{C}$ & -1.182486 & 2.743499 & 4.886094 \\
$\mathrm{C}$ & -2.190005 & 2.550960 & 3.969809 \\
$\mathrm{C}$ & -2.024988 & 1.646499 & 2.886043 \\
$\mathrm{C}$ & -0.794749 & 0.930845 & 2.750765 \\
$\mathrm{C}$ & 0.227947 & 1.157211 & 3.712758 \\
$\mathrm{C}$ & 0.038052 & 2.038754 & 4.753085 \\
$\mathrm{H}$ & -3.990251 & 1.965442 & 2.059064 \\
$\mathrm{H}$ & -1.318396 & 3.437127 & 5.711521 \\
$\mathrm{H}$ & 3.131207 & 3.088231 & 4.060012 \\
& & &
\end{tabular}




\begin{tabular}{|c|c|c|c|}
\hline C & -3.052540 & 1.428724 & 1.938160 \\
\hline C & -0.648320 & 0.020199 & 1.653855 \\
\hline $\mathrm{H}$ & 1.171528 & 0.631559 & 3.615255 \\
\hline $\mathrm{H}$ & 0.832757 & 2.198942 & 5.476651 \\
\hline C & -1.680911 & -0.180970 & 0.738888 \\
\hline C & -2.917544 & 0.538552 & 0.889688 \\
\hline C & -1.497878 & -1.160591 & -0.410570 \\
\hline $\mathrm{H}$ & -2.113777 & -0.859907 & -1.259176 \\
\hline $\mathrm{H}$ & -0.454445 & -1.162359 & -0.743242 \\
\hline C & 1.529335 & 1.129465 & 0.139028 \\
\hline 0 & 1.583904 & 2.198848 & 0.705218 \\
\hline 0 & 1.291581 & 1.013497 & -1.188258 \\
\hline $\mathrm{H}$ & 1.203994 & 1.924951 & -1.529234 \\
\hline 0 & -1.923122 & -2.492548 & -0.091990 \\
\hline $\mathrm{H}$ & -1.345872 & -2.822231 & 0.616087 \\
\hline C & -4.067555 & 0.378838 & -0.050206 \\
\hline C & -4.613420 & -0.886514 & -0.328042 \\
\hline C & -4.651077 & 1.510359 & -0.628519 \\
\hline C & -5.716632 & -1.027935 & -1.171481 \\
\hline $\mathrm{H}$ & -4.147929 & -1.753449 & 0.122183 \\
\hline C & -5.763112 & 1.411921 & -1.480124 \\
\hline $\mathrm{H}$ & -4.211601 & 2.481463 & -0.418256 \\
\hline C & -6.273527 & 0.135515 & -1.733527 \\
\hline $\mathrm{H}$ & -7.128324 & 0.031741 & -2.391949 \\
\hline C & 4.067428 & -0.375487 & -0.168994 \\
\hline C & 4.570709 & 0.903568 & 0.124393 \\
\hline C & 4.675517 & -1.132970 & -1.173408 \\
\hline C & 5.656916 & 1.429618 & -0.577058 \\
\hline $\mathrm{H}$ & 4.106796 & 1.473960 & 0.918795 \\
\hline C & 5.772428 & -0.642917 & -1.899128 \\
\hline $\mathrm{H}$ & 4.265287 & -2.111983 & -1.403003 \\
\hline C & 6.239372 & 0.636181 & -1.582052 \\
\hline $\mathrm{H}$ & 7.082831 & 1.038839 & -2.130214 \\
\hline C & 6.400261 & -1.510210 & -3.007775 \\
\hline C & 6.225049 & 2.832518 & -0.287236 \\
\hline C & 7.604272 & -0.826359 & -3.682621 \\
\hline $\mathrm{H}$ & 7.320774 & 0.113957 & -4.168816 \\
\hline $\mathrm{H}$ & 8.407407 & -0.614347 & -2.967518 \\
\hline $\mathrm{H}$ & 8.015642 & -1.485269 & -4.455543 \\
\hline C & 5.338973 & -1.797650 & -4.096628 \\
\hline $\mathrm{H}$ & 5.765220 & -2.425300 & -4.888981 \\
\hline $\mathrm{H}$ & 4.467256 & -2.320239 & -3.689607 \\
\hline $\mathrm{H}$ & 4.985825 & -0.866367 & -4.554055 \\
\hline C & 6.887513 & -2.848573 & -2.403005 \\
\hline $\mathrm{H}$ & 6.067001 & -3.415175 & -1.950425 \\
\hline $\mathrm{H}$ & 7.335288 & -3.477870 & -3.181956 \\
\hline $\mathrm{H}$ & 7.643476 & -2.677133 & -1.628107 \\
\hline C & 5.465628 & 3.549047 & 0.845946 \\
\hline $\mathrm{H}$ & 4.401692 & 3.672028 & 0.615477 \\
\hline $\mathrm{H}$ & 5.546273 & 3.007413 & 1.795346 \\
\hline $\mathrm{H}$ & 5.891252 & 4.547147 & 1.000071 \\
\hline C & 6.115785 & 3.701845 & -1.562945 \\
\hline $\mathrm{H}$ & 5.070758 & 3.808975 & -1.875346 \\
\hline $\mathrm{H}$ & 6.518483 & 4.704777 & -1.375294 \\
\hline
\end{tabular}




\begin{tabular}{|c|c|c|c|}
\hline $\mathrm{H}$ & 6.673327 & 3.271063 & -2.401468 \\
\hline C & 7.711484 & 2.716882 & 0.125812 \\
\hline $\mathrm{H}$ & 7.820096 & 2.108904 & 1.031225 \\
\hline $\mathrm{H}$ & 8.320965 & 2.258071 & -0.659999 \\
\hline $\mathrm{H}$ & 8.127619 & 3.710595 & 0.332152 \\
\hline C & -6.360645 & 2.688673 & -2.103534 \\
\hline C & -6.321167 & -2.404592 & -1.509812 \\
\hline C & -5.610307 & -3.553218 & -0.767827 \\
\hline $\mathrm{H}$ & -4.542550 & -3.600230 & -1.006034 \\
\hline $\mathrm{H}$ & -5.708726 & -3.453108 & 0.319339 \\
\hline $\mathrm{H}$ & -6.062130 & -4.510159 & -1.053972 \\
\hline C & -6.187850 & -2.657932 & -3.030565 \\
\hline $\mathrm{H}$ & -6.702829 & -1.890663 & -3.618726 \\
\hline $\mathrm{H}$ & -5.134860 & -2.662267 & -3.334169 \\
\hline $\mathrm{H}$ & -6.622404 & -3.629928 & -3.295384 \\
\hline C & -7.817112 & -2.431332 & -1.116458 \\
\hline $\mathrm{H}$ & -7.941921 & -2.260011 & -0.041055 \\
\hline $\mathrm{H}$ & -8.395775 & -1.666886 & -1.645897 \\
\hline $\mathrm{H}$ & -8.256698 & -3.406948 & -1.358229 \\
\hline C & -6.795335 & 3.658886 & -0.979523 \\
\hline $\mathrm{H}$ & -7.555971 & 3.198495 & -0.338576 \\
\hline $\mathrm{H}$ & -5.953497 & 3.949863 & -0.342774 \\
\hline $\mathrm{H}$ & -7.220536 & 4.575055 & -1.407740 \\
\hline C & -7.592234 & 2.396595 & -2.981335 \\
\hline $\mathrm{H}$ & -7.981508 & 3.334306 & -3.393854 \\
\hline $\mathrm{H}$ & -7.347288 & 1.742183 & -3.825595 \\
\hline $\mathrm{H}$ & -8.399749 & 1.927483 & -2.407609 \\
\hline C & -5.292994 & 3.375977 & -2.987798 \\
\hline $\mathrm{H}$ & -4.976589 & 2.715819 & -3.803617 \\
\hline $\mathrm{H}$ & -5.696381 & 4.295003 & -3.431077 \\
\hline $\mathrm{H}$ & -4.401108 & 3.646472 & -2.413030 \\
\hline
\end{tabular}

\section{Catalyst 2C 79}

Datum

Value

\begin{tabular}{lr}
\hline B3LYP/6-31G(d) Energy & -2164.797577 \\
\hline B3LYP-D3(BJ)/def2-TZVPP-IEF-PCM(DCM) Energy & -2165.89521 \\
\hline B3LYP-D3(BJ)/def2-TZVPP-IEF-PCM(DCM)//B3LYP/6-31G(d) Free Energy (Quasiharmonic) & -2165.017413 \\
\hline Number of Imaginary Frequencies & 0
\end{tabular}

Frequencies (Top 3 out of 321)

$\begin{array}{ll}\text { 1. } & 10.4376 \mathrm{~cm}^{-1} \\ \text { 2. } & 11.7400 \mathrm{~cm}^{-1} \\ \text { 3. } & 12.4116 \mathrm{~cm}^{-1}\end{array}$




\begin{tabular}{|c|c|c|c|}
\hline C & 0.153179 & -3.910312 & 3.390077 \\
\hline C & 0.302015 & -2.651350 & 2.848440 \\
\hline C & -0.743749 & -2.054930 & 2.086536 \\
\hline C & -1.958548 & -2.790504 & 1.902552 \\
\hline C & -2.079474 & -4.084189 & 2.478831 \\
\hline C & -1.047821 & -4.635531 & 3.203246 \\
\hline $\mathrm{H}$ & 0.961147 & -4.346887 & 3.970508 \\
\hline $\mathrm{H}$ & 1.219279 & -2.094370 & 3.009663 \\
\hline C & -0.621761 & -0.754076 & 1.495681 \\
\hline C & -3.013268 & -2.209087 & 1.158129 \\
\hline $\mathrm{H}$ & -3.006766 & -4.633132 & 2.333572 \\
\hline $\mathrm{H}$ & -1.152333 & -5.626741 & 3.635595 \\
\hline C & -2.910357 & -0.950578 & 0.598034 \\
\hline C & -1.686292 & -0.228705 & 0.777404 \\
\hline $\mathrm{H}$ & -3.940846 & -2.764569 & 1.046825 \\
\hline C & 1.212098 & 2.715817 & 4.875491 \\
\hline C & 2.216700 & 2.528043 & 3.955082 \\
\hline C & 2.048352 & 1.628828 & 2.867420 \\
\hline C & 0.818018 & 0.913386 & 2.732602 \\
\hline C & -0.201649 & 1.134719 & 3.699017 \\
\hline C & -0.008650 & 2.011297 & 4.742950 \\
\hline $\mathrm{H}$ & 4.010652 & 1.952287 & 2.036738 \\
\hline $\mathrm{H}$ & 1.350427 & 3.405483 & 5.703826 \\
\hline $\mathrm{H}$ & 3.158036 & 3.065147 & 4.044805 \\
\hline C & 3.072760 & 1.416098 & 1.915296 \\
\hline C & 0.668999 & 0.007604 & 1.632045 \\
\hline $\mathrm{H}$ & -1.145364 & 0.609216 & 3.602198 \\
\hline $\mathrm{H}$ & -0.801068 & 2.167705 & 5.469842 \\
\hline C & 1.698738 & -0.188721 & 0.712684 \\
\hline C & 2.935397 & 0.531346 & 0.862297 \\
\hline C & 1.512145 & -1.165144 & -0.438667 \\
\hline $\mathrm{H}$ & 0.466568 & -1.171986 & -0.763889 \\
\hline $\mathrm{H}$ & 2.120580 & -0.859954 & -1.290897 \\
\hline C & -1.516558 & 1.120674 & 0.132164 \\
\hline 0 & -1.570441 & 2.188646 & 0.701103 \\
\hline 0 & -1.281572 & 1.008246 & -1.195932 \\
\hline $\mathrm{H}$ & -1.194701 & 1.920616 & -1.534656 \\
\hline 0 & 1.946188 & -2.496352 & -0.126017 \\
\hline $\mathrm{H}$ & 1.370616 & -2.833982 & 0.579764 \\
\hline C & 4.084622 & 0.378289 & -0.079621 \\
\hline C & 4.684595 & 1.519028 & -0.631633 \\
\hline C & 4.618791 & -0.879997 & -0.386612 \\
\hline C & 5.797669 & 1.428386 & -1.476262 \\
\hline $\mathrm{H}$ & 4.253549 & 2.489333 & -0.401376 \\
\hline C & 5.728369 & -1.015458 & -1.230293 \\
\hline $\mathrm{H}$ & 4.146385 & -1.757658 & 0.040092 \\
\hline C & 6.301025 & 0.150078 & -1.759600 \\
\hline $\mathrm{H}$ & 7.158900 & 0.060581 & -2.412128 \\
\hline C & -4.060033 & -0.381117 & -0.159444 \\
\hline C & -4.556150 & 0.895123 & 0.141274 \\
\hline
\end{tabular}




\begin{tabular}{|c|c|c|c|}
\hline C & -4.681309 & -1.141919 & -1.159965 \\
\hline C & -5.653215 & 1.425620 & -0.547078 \\
\hline $\mathrm{H}$ & -4.082337 & 1.464529 & 0.930478 \\
\hline C & -5.783241 & -0.649808 & -1.867634 \\
\hline $\mathrm{H}$ & -4.271339 & -2.118271 & -1.390770 \\
\hline C & -6.245115 & 0.633538 & -1.540661 \\
\hline $\mathrm{H}$ & -7.097362 & 1.032360 & -2.082800 \\
\hline C & -6.486115 & -1.456644 & -2.977016 \\
\hline C & -6.213767 & 2.830031 & -0.248561 \\
\hline C & -6.437198 & -0.664650 & -4.305200 \\
\hline $\mathrm{H}$ & -6.939746 & 0.304728 & -4.222815 \\
\hline $\mathrm{H}$ & -5.401895 & -0.479099 & -4.612964 \\
\hline $\mathrm{H}$ & -6.933436 & -1.229304 & -5.103987 \\
\hline C & -7.961471 & -1.698823 & -2.578633 \\
\hline $\mathrm{H}$ & -8.480171 & -2.266441 & -3.361031 \\
\hline $\mathrm{H}$ & -8.026262 & -2.269796 & -1.645242 \\
\hline $\mathrm{H}$ & -8.504695 & -0.759282 & -2.432712 \\
\hline C & -5.824469 & -2.827243 & -3.216326 \\
\hline $\mathrm{H}$ & -5.854164 & -3.458170 & -2.320658 \\
\hline $\mathrm{H}$ & -6.357892 & -3.359839 & -4.011739 \\
\hline $\mathrm{H}$ & -4.779185 & -2.726926 & -3.529762 \\
\hline C & -6.115772 & 3.700739 & -1.524134 \\
\hline $\mathrm{H}$ & -5.073940 & 3.805004 & -1.847912 \\
\hline $\mathrm{H}$ & -6.683624 & 3.272505 & -2.357062 \\
\hline $\mathrm{H}$ & -6.513373 & 4.704651 & -1.330926 \\
\hline C & -5.439269 & 3.542167 & 0.877220 \\
\hline $\mathrm{H}$ & -4.377737 & 3.662838 & 0.634829 \\
\hline $\mathrm{H}$ & -5.860416 & 4.541080 & 1.038200 \\
\hline $\mathrm{H}$ & -5.510446 & 2.998866 & 1.826415 \\
\hline C & -7.695722 & 2.718361 & 0.180900 \\
\hline $\mathrm{H}$ & -8.315385 & 2.262874 & -0.598897 \\
\hline $\mathrm{H}$ & -7.796339 & 2.108949 & 1.086258 \\
\hline $\mathrm{H}$ & -8.106482 & 3.712938 & 0.393691 \\
\hline C & 6.266706 & -2.425493 & -1.543337 \\
\hline C & 6.414327 & 2.712775 & -2.065512 \\
\hline C & 6.852589 & 3.651199 & -0.916176 \\
\hline $\mathrm{H}$ & 6.009591 & 3.937833 & -0.278930 \\
\hline $\mathrm{H}$ & 7.602600 & 3.166508 & -0.280649 \\
\hline $\mathrm{H}$ & 7.292467 & 4.571561 & -1.319868 \\
\hline C & 5.361289 & 3.433680 & -2.940629 \\
\hline $\mathrm{H}$ & 5.042200 & 2.796614 & -3.773619 \\
\hline $\mathrm{H}$ & 4.468869 & 3.701966 & -2.365612 \\
\hline $\mathrm{H}$ & 5.778970 & 4.357590 & -3.359943 \\
\hline C & 7.648803 & 2.427764 & -2.941895 \\
\hline $\mathrm{H}$ & 8.447433 & 1.936744 & -2.374158 \\
\hline $\mathrm{H}$ & 7.403043 & 1.795905 & -3.802899 \\
\hline $\mathrm{H}$ & 8.050872 & 3.370637 & -3.329714 \\
\hline C & 7.491640 & -2.398094 & -2.477020 \\
\hline $\mathrm{H}$ & 7.256193 & -1.944062 & -3.446419 \\
\hline $\mathrm{H}$ & 8.331619 & -1.848368 & -2.036399 \\
\hline $\mathrm{H}$ & 7.831638 & -3.422524 & -2.667328 \\
\hline C & 5.153960 & -3.254226 & -2.229950 \\
\hline $\mathrm{H}$ & 5.509324 & -4.272087 & -2.434970 \\
\hline $\mathrm{H}$ & 4.255062 & -3.327315 & -1.609926 \\
\hline
\end{tabular}




\section{Catalyst 2C 80}

\section{Datum}

Value

\begin{tabular}{lc}
\hline B3LYP/6-31G(d) Energy & -2164.800108 \\
\hline B3LYP-D3(BJ)/def2-TZVPP-IEF-PCM(DCM) Energy & -2165.896135 \\
\hline B3LYP-D3(BJ)/def2-TZVPP-IEF-PCM(DCM)//B3LYP/6-31G(d) Free Energy (Quasiharmonic) & -2165.017347 \\
\hline
\end{tabular}

Number of Imaginary Frequencies

Frequencies (Top 3 out of 321)
1. $12.2751 \mathrm{~cm}^{-1}$
2. $12.8058 \mathrm{~cm}^{-1}$
3. $13.6346 \mathrm{~cm}^{-1}$

\section{B3LYP/6-31G(d) Molecular Geometry in Cartesian Coordinates}

$\begin{array}{lrrr}\text { C } & -0.337907 & -3.422710 & 3.915238 \\ \text { C } & -0.456914 & -2.851536 & 2.667542 \\ \text { C } & 0.627720 & -2.140282 & 2.084512 \\ \text { C } & 1.847744 & -2.027735 & 2.824828 \\ \text { C } & 1.937152 & -2.627527 & 4.110064 \\ \text { C } & 0.869282 & -3.309909 & 4.645522 \\ \text { H } & -1.176274 & -3.965615 & 4.342875 \\ \text { H } & -1.384456 & -2.942664 & 2.112722 \\ \text { C } & 0.540798 & -1.528774 & 0.789890 \\ \text { C } & 2.941055 & -1.328057 & 2.257532 \\ \text { H } & 2.868946 & -2.536484 & 4.663006 \\ \text { H } & 0.949236 & -3.764297 & 5.629285 \\ \text { C } & 2.876497 & -0.758031 & 1.001900 \\ \text { C } & 1.649003 & -0.874064 & 0.271415 \\ \text { H } & 3.868333 & -1.263327 & 2.820935 \\ \text { C } & -1.428621 & -4.845452 & -2.657368 \\ \text { C } & -2.396018 & -3.884910 & -2.475180 \\ \text { C } & -2.182258 & -2.796510 & -1.586621 \\ \text { C } & -0.943384 & -2.698550 & -0.880811 \\ \text { C } & 0.037021 & -3.706286 & -1.096009 \\ \text { C } & -0.200908 & -4.752514 & -1.958977\end{array}$




\begin{tabular}{|c|c|c|c|}
\hline $\mathrm{H}$ & -4.102617 & -1.882252 & -1.929525 \\
\hline $\mathrm{H}$ & -1.601614 & -5.674812 & -3.337911 \\
\hline $\mathrm{H}$ & -3.342314 & -3.943159 & -3.007874 \\
\hline C & -3.168762 & -1.804595 & -1.378567 \\
\hline C & -0.748073 & -1.594648 & 0.014102 \\
\hline $\mathrm{H}$ & 0.987204 & -3.640217 & -0.577740 \\
\hline $\mathrm{H}$ & 0.562480 & -5.510794 & -2.110638 \\
\hline C & -1.739083 & -0.630138 & 0.200019 \\
\hline C & -2.982496 & -0.738308 & -0.518866 \\
\hline C & -1.523998 & 0.511961 & 1.185189 \\
\hline $\mathrm{H}$ & -2.474726 & 0.775081 & 1.653408 \\
\hline $\mathrm{H}$ & -0.840624 & 0.195093 & 1.981442 \\
\hline C & 1.545242 & -0.258081 & -1.096029 \\
\hline 0 & 1.629998 & -0.838435 & -2.152382 \\
\hline 0 & 1.333207 & 1.087722 & -1.025671 \\
\hline $\mathrm{H}$ & 1.277164 & 1.408808 & -1.947299 \\
\hline 0 & -1.055314 & 1.719979 & 0.581832 \\
\hline $\mathrm{H}$ & -0.188617 & 1.542220 & 0.176753 \\
\hline C & -4.111517 & 0.227133 & -0.354671 \\
\hline C & -3.931060 & 1.609414 & -0.491102 \\
\hline C & -5.404070 & -0.261583 & -0.111909 \\
\hline C & -5.006811 & 2.500086 & -0.388302 \\
\hline $\mathrm{H}$ & -2.928810 & 1.981921 & -0.665364 \\
\hline C & -6.507404 & 0.593610 & -0.006858 \\
\hline $\mathrm{H}$ & -5.535435 & -1.332795 & 0.011862 \\
\hline C & -6.283097 & 1.970752 & -0.150531 \\
\hline $\mathrm{H}$ & -7.122449 & 2.647960 & -0.071189 \\
\hline C & 4.063978 & -0.047400 & 0.447981 \\
\hline C & 4.694998 & 0.946616 & 1.209339 \\
\hline C & 4.583240 & -0.382775 & -0.810255 \\
\hline C & 5.828259 & 1.615491 & 0.734216 \\
\hline $\mathrm{H}$ & 4.268895 & 1.202511 & 2.172452 \\
\hline C & 5.712913 & 0.265561 & -1.323143 \\
\hline $\mathrm{H}$ & 4.102418 & -1.168748 & -1.378252 \\
\hline C & 6.311769 & 1.255983 & -0.532346 \\
\hline $\mathrm{H}$ & 7.187879 & 1.768838 & -0.917931 \\
\hline C & 6.302732 & -0.074484 & -2.706060 \\
\hline C & 6.540441 & 2.719102 & 1.540803 \\
\hline C & 7.767390 & -0.545545 & -2.544685 \\
\hline $\mathrm{H}$ & 8.397506 & 0.223950 & -2.086127 \\
\hline $\mathrm{H}$ & 7.823862 & -1.441766 & -1.916332 \\
\hline $\mathrm{H}$ & 8.198936 & -0.789477 & -3.523170 \\
\hline C & 6.266969 & 1.187046 & -3.601575 \\
\hline $\mathrm{H}$ & 6.688297 & 0.964627 & -4.589562 \\
\hline $\mathrm{H}$ & 5.238133 & 1.537334 & -3.743007 \\
\hline $\mathrm{H}$ & 6.844978 & 2.011759 & -3.171177 \\
\hline C & 5.515238 & -1.189904 & -3.420089 \\
\hline $\mathrm{H}$ & 4.466050 & -0.916997 & -3.578095 \\
\hline $\mathrm{H}$ & 5.960946 & -1.383503 & -4.402379 \\
\hline $\mathrm{H}$ & 5.540062 & -2.129848 & -2.857045 \\
\hline C & 8.005163 & 2.297753 & 1.807230 \\
\hline $\mathrm{H}$ & 8.046481 & 1.366947 & 2.384550 \\
\hline $\mathrm{H}$ & 8.560434 & 2.137557 & 0.877096 \\
\hline $\mathrm{H}$ & 8.527961 & 3.075116 & 2.377907 \\
\hline
\end{tabular}




\begin{tabular}{|c|c|c|c|}
\hline C & 5.864698 & 2.982789 & 2.900023 \\
\hline $\mathrm{H}$ & 5.866229 & 2.091340 & 3.537655 \\
\hline $\mathrm{H}$ & 6.407355 & 3.770786 & 3.434298 \\
\hline $\mathrm{H}$ & 4.827918 & 3.317788 & 2.782918 \\
\hline C & 6.525388 & 4.039839 & 0.735075 \\
\hline $\mathrm{H}$ & 7.036765 & 3.937280 & -0.227743 \\
\hline $\mathrm{H}$ & 5.498103 & 4.364904 & 0.534991 \\
\hline $\mathrm{H}$ & 7.030207 & 4.835143 & 1.296981 \\
\hline C & -7.905054 & 0.003360 & 0.267714 \\
\hline C & -4.745025 & 4.011887 & -0.534317 \\
\hline C & -3.759310 & 4.466840 & 0.569500 \\
\hline $\mathrm{H}$ & -4.192788 & 4.309702 & 1.564377 \\
\hline $\mathrm{H}$ & -2.813879 & 3.917231 & 0.527996 \\
\hline $\mathrm{H}$ & -3.537608 & 5.536497 & 0.463434 \\
\hline C & -6.030855 & 4.850684 & -0.406848 \\
\hline $\mathrm{H}$ & -6.764637 & 4.596435 & -1.180717 \\
\hline $\mathrm{H}$ & -6.507744 & 4.722799 & 0.571801 \\
\hline $\mathrm{H}$ & -5.789357 & 5.914041 & -0.517805 \\
\hline C & -4.124642 & 4.295613 & -1.923045 \\
\hline $\mathrm{H}$ & -3.175055 & 3.768163 & -2.059156 \\
\hline $\mathrm{H}$ & -4.800877 & 3.981545 & -2.726780 \\
\hline $\mathrm{H}$ & -3.930789 & 5.368953 & -2.041578 \\
\hline C & -7.891422 & -0.744345 & 1.622294 \\
\hline $\mathrm{H}$ & -7.158021 & -1.557518 & 1.630870 \\
\hline $\mathrm{H}$ & -7.642253 & -0.061972 & 2.443058 \\
\hline $\mathrm{H}$ & -8.876792 & -1.180291 & 1.829230 \\
\hline $\mathrm{C}$ & -8.277503 & -0.989276 & -0.859406 \\
\hline $\mathrm{H}$ & -9.270612 & -1.418955 & -0.678237 \\
\hline $\mathrm{H}$ & -8.297316 & -0.486151 & -1.833006 \\
\hline $\mathrm{H}$ & -7.564566 & -1.817754 & -0.926594 \\
\hline C & -9.001686 & 1.083758 & 0.329516 \\
\hline $\mathrm{H}$ & -9.081794 & 1.638614 & -0.612231 \\
\hline $\mathrm{H}$ & -9.972932 & 0.612571 & 0.519015 \\
\hline $\mathrm{H}$ & -8.821812 & 1.803632 & 1.136039 \\
\hline
\end{tabular}

\section{Catalyst 2C 81}

Datum

Value

\begin{tabular}{lc}
\hline B3LYP/6-31G(d) Energy & -2164.797739 \\
\hline B3LYP-D3(BJ)/def2-TZVPP-IEF-PCM(DCM) Energy & -2165.895209 \\
\hline B3LYP-D3(BJ)/def2-TZVPP-IEF-PCM(DCM)//B3LYP/6-31G(d) Free Energy (Quasiharmonic) & -2165.017275 \\
\hline Number of Imaginary Frequencies & 0
\end{tabular}

Frequencies (Top 3 out of 321) 


$\begin{array}{ll}\text { 1. } & 10.8830 \mathrm{~cm}^{-1} \\ \text { 2. } & 11.9836 \mathrm{~cm}^{-1} \\ \text { 3. } & 12.9828 \mathrm{~cm}^{-1}\end{array}$

\section{B3LYP/6-31G(d) Molecular Geometry in Cartesian Coordinates}

C

C

C

C

C

C

$\mathrm{H}$

$\mathrm{H}$

C

$\mathrm{C}$

$\mathrm{H}$

$\mathrm{H}$

C

C

$\mathrm{H}$

C

C

C

C

C

C

$\mathrm{H}$

$\mathrm{H}$

$\mathrm{H}$

C

C

$\mathrm{H}$

$\mathrm{H}$

C

C

C

$\mathrm{H}$

$\mathrm{H}$

C

0

0

$\mathrm{H}$

0

$\mathrm{H}$

C

C

C

C

$\mathrm{H}$
0.108746

0.264644

$-0.774706$

$-1.990788$

$-2.119054$

$-1.093317$

0.911798

1.182317

$-0.645086$

$-3.039255$

$-3.047196$

$-1.203413$

$-2.929012$

$-1.703959$

$-3.967774$

1.180710

2.187750

2.022793

0.793121

$-0.229095$

$-0.039233$

3.986547

1. 316529

3.128504

3.049681

0.647052

$-1.172111$

$-0.833441$

1.679822

2.915447

1.498089

0.453478

2.108846

$-1.528095$

$-1.589645$

$-1.280938$

$-1.195119$

1.933177

1.354600

4.065889

4.657532

4.607705

5.769807

4.219895
$-3.853874$

$-2.604192$

$-2.017171$

$-2.751822$

$-4.035755$

$-4.578463$

$-4.283402$

$-2.046989$

$-0.726574$

$-2.179459$

$-4.584157$

$-5.562264$

$-0.930402$

$-0.209346$

$-2.734037$

2.802511

2.598269

1.678619

0.960124

1.198765

2.094746

1.988857

3.507638

3.137604

1.448921

0.033988

0.670662

2.264025

$-0.180058$

0.543641

$-1.178862$

$-1.193607$

$-0.889505$

1.131387

2. 205953

1.003603

1.911925

$-2.502931$

$-2.828351$

0.373401

1.502783

$-0.889299$

1.396333

2.476032

\subsection{7}

2.895705

2.117926

1.938640

2.534838

3.274033

4.048886

3.054022

1.506966

1.178398

2. 393054

3.721639

0.598913

0.774666

1.070372

4.829846

3.915617

2. 844642

2.720105

3.679650

4.707568

2. 011741

5.645485

3.997707

1.899047

1.636204

3.590321

5.429610

0.724315

0.863326

$-0.408372$

$-0.736537$

$-1.264441$

0.113292

0.669753

$-1.210463$

$-1.560312$

$-0.068211$

0.640831

$-0.074163$

$-0.657403$

$-0.347618$

$-1.501408$

$-0.453221$ 


\begin{tabular}{|c|c|c|c|}
\hline C & 5.717323 & -1.040402 & -1.188545 \\
\hline $\mathrm{H}$ & 4.140399 & -1.758045 & 0.102329 \\
\hline C & 6.281577 & 0.114186 & -1.750005 \\
\hline $\mathrm{H}$ & 7.138949 & 0.012576 & -2.401441 \\
\hline C & -4.071304 & -0.369785 & -0.176345 \\
\hline C & -4.561006 & 0.915480 & 0.088471 \\
\hline C & -4.696041 & -1.143860 & -1.162802 \\
\hline C & -5.649073 & 1.441260 & -0.618973 \\
\hline $\mathrm{H}$ & -4.089462 & 1.504980 & 0.866936 \\
\hline C & -5.790432 & -0.659282 & -1.888857 \\
\hline $\mathrm{H}$ & -4.296543 & -2.131444 & -1.373916 \\
\hline C & -6.246321 & 0.635623 & -1.598972 \\
\hline $\mathrm{H}$ & -7.088069 & 1.027926 & -2.152654 \\
\hline C & -6.435770 & -1.543171 & -2.974737 \\
\hline C & -6.137198 & 2.868393 & -0.301517 \\
\hline C & -5.386920 & -1.861145 & -4.067149 \\
\hline $\mathrm{H}$ & -5.029970 & -0.942133 & -4.545979 \\
\hline $\mathrm{H}$ & -4.515964 & -2.382961 & -3.657479 \\
\hline $\mathrm{H}$ & -5.825393 & -2.500598 & -4.843306 \\
\hline C & -7.640313 & -0.863095 & -3.652787 \\
\hline $\mathrm{H}$ & -8.064492 & -1.534835 & -4.407564 \\
\hline $\mathrm{H}$ & -8.434829 & -0.628932 & -2.935023 \\
\hline $\mathrm{H}$ & -7.353696 & 0.064151 & -4.161625 \\
\hline C & -6.929000 & -2.865363 & -2.339823 \\
\hline $\mathrm{H}$ & -7.675064 & -2.672284 & -1.560440 \\
\hline $\mathrm{H}$ & -7.390840 & -3.504873 & -3.102062 \\
\hline $\mathrm{H}$ & -6.108989 & -3.431104 & -1.885267 \\
\hline C & -4.993287 & 3.871005 & -0.590363 \\
\hline $\mathrm{H}$ & -4.094133 & 3.649734 & -0.006299 \\
\hline $\mathrm{H}$ & -4.717001 & 3.848832 & -1.651347 \\
\hline $\mathrm{H}$ & -5.311062 & 4.892218 & -0.345926 \\
\hline C & -6.535047 & 2.961368 & 1.190867 \\
\hline $\mathrm{H}$ & -5.693952 & 2.734868 & 1.853742 \\
\hline $\mathrm{H}$ & -6.881337 & 3.974543 & 1.428854 \\
\hline $\mathrm{H}$ & -7.345516 & 2.261537 & 1.425297 \\
\hline C & -7.358796 & 3.277598 & -1.145888 \\
\hline $\mathrm{H}$ & -7.136404 & 3.269901 & -2.219106 \\
\hline $\mathrm{H}$ & -8.217543 & 2.619517 & -0.969357 \\
\hline $\mathrm{H}$ & -7.664129 & 4.296236 & -0.881324 \\
\hline C & 6.264585 & -2.454684 & -1.464642 \\
\hline C & 6.374940 & 2.667606 & -2.129891 \\
\hline C & 7.611693 & 2.367276 & -2.997849 \\
\hline $\mathrm{H}$ & 8.414716 & 1.900093 & -2.416337 \\
\hline $\mathrm{H}$ & 7.371074 & 1.708214 & -3.839692 \\
\hline $\mathrm{H}$ & 8.005394 & 3.301750 & -3.413535 \\
\hline C & 6.804696 & 3.645255 & -1.010440 \\
\hline $\mathrm{H}$ & 5.959382 & 3.943384 & -0.381660 \\
\hline $\mathrm{H}$ & 7.559668 & 3.187776 & -0.360741 \\
\hline $\mathrm{H}$ & 7.235372 & 4.557024 & -1.442635 \\
\hline C & 5.314856 & 3.351006 & -3.026238 \\
\hline $\mathrm{H}$ & 5.001805 & 2.685304 & -3.838847 \\
\hline $\mathrm{H}$ & 4.420180 & 3.628109 & -2.459030 \\
\hline $\mathrm{H}$ & 5.723394 & 4.265509 & -3.474211 \\
\hline C & 6.686571 & -3.113984 & -0.129958 \\
\hline
\end{tabular}




$\begin{array}{|llll|}H & 7.472402 & -2.531248 & 0.364854 \\ H & 5.844771 & -3.199045 & 0.564288 \\ H & 7.074691 & -4.124218 & -0.310258 \\ \mathrm{C} & 7.489000 & -2.443739 & -2.399304 \\ \mathrm{H} & 7.834948 & -3.470559 & -2.564088 \\ \mathrm{H} & 7.250474 & -2.015508 & -3.379635 \\ \mathrm{H} & 8.325857 & -1.878138 & -1.973026 \\ \mathrm{C} & 5.157042 & -3.308298 & -2.128922 \\ \mathrm{H} & 4.866462 & -2.881667 & -3.096329 \\ \mathrm{H} & 5.518440 & -4.329367 & -2.305507 \\ \mathrm{H} & 4.258015 & -3.369402 & -1.507801 \\ & & \end{array}$

\section{Catalyst 2C 82}

Datum

Value

\begin{tabular}{lr}
\hline B3LYP/6-31G(d) Energy & -2164.800258 \\
\hline B3LYP-D3(BJ)/def2-TZVPP-IEF-PCM(DCM) Energy & -2165.896132 \\
\hline B3LYP-D3(BJ)/def2-TZVPP-IEF-PCM(DCM)//B3LYP/6-31G(d) Free Energy (Quasiharmonic) & -2165.017181 \\
\hline
\end{tabular}

Number of Imaginary Frequencies

Frequencies (Top 3 out of 321)
1. $12.6101 \mathrm{~cm}^{-1}$
2. $\quad 13.5529 \mathrm{~cm}^{-1}$
3. $14.5112 \mathrm{~cm}^{-1}$

\section{B3LYP/6-31G(d) Molecular Geometry in Cartesian Coordinates}

$\begin{array}{lrrl}\text { C } & -0.294968 & -3.386717 & 3.944304 \\ \text { C } & -0.421630 & -2.827165 & 2.692110 \\ \text { C } & 0.658457 & -2.118925 & 2.097144 \\ \text { C } & 1.881894 & -1.996541 & 2.830235 \\ \text { C } & 1.979258 & -2.584605 & 4.120296 \\ \text { C } & 0.915714 & -3.264618 & 4.667274 \\ \text { H } & -1.129899 & -3.927556 & 4.381171 \\ \text { H } & -1.351725 & -2.925352 & 2.142788 \\ \text { C } & 0.563950 & -1.520501 & 0.796998 \\ \text { C } & 2.970166 & -1.298191 & 2.251695 \\ \text { H } & 2.913582 & -2.486509 & 4.667738 \\ \text { H } & 1.001781 & -3.709966 & 5.654644 \\ \text { C } & 2.897699 & -0.739224 & 0.991546 \\ \text { C } & 1.667808 & -0.867585 & 0.267210 \\ \text { H } & 3.899645 & -1.225032 & 2.810445\end{array}$




\begin{tabular}{|c|c|c|c|}
\hline C & -1.415226 & -4.877784 & -2.605653 \\
\hline C & -2.382361 & -3.915504 & -2.431423 \\
\hline C & -2.166401 & -2.817216 & -1.555667 \\
\hline C & -0.925502 & -2.711039 & -0.854586 \\
\hline C & 0.054539 & -3.720932 & -1.061255 \\
\hline C & -0.185600 & -4.776828 & -1.911749 \\
\hline H & -4.088041 & -1.907472 & -1.903225 \\
\hline $\mathrm{H}$ & -1.589929 & -5.714713 & -3.276416 \\
\hline $\mathrm{H}$ & -3.330147 & -3.979908 & -2.960740 \\
\hline C & -3.152686 & -1.823392 & -1.355771 \\
\hline C & -0.727929 & -1.596989 & 0.027156 \\
\hline H & 1.006079 & -3.649043 & -0.546240 \\
\hline $\mathrm{H}$ & 0.577457 & -5.536712 & -2.056920 \\
\hline C & -1.718949 & -0.631004 & 0.205517 \\
\hline C & -2.964516 & -0.747666 & -0.508328 \\
\hline C & -1.501609 & 0.521764 & 1.177716 \\
\hline $\mathrm{H}$ & -2.450928 & 0.788356 & 1.646858 \\
\hline $\mathrm{H}$ & -0.814722 & 0.214230 & 1.974620 \\
\hline C & 1.558253 & -0.270698 & -1.108135 \\
\hline 0 & 1.646041 & -0.867101 & -2.155674 \\
\hline 0 & 1.340101 & 1.074194 & -1.057765 \\
\hline H & 1.284784 & 1.382324 & -1.983891 \\
\hline 0 & -1.037100 & 1.724164 & 0.560187 \\
\hline $\mathrm{H}$ & -0.173067 & 1.543038 & 0.151038 \\
\hline C & -4.093710 & 0.218765 & -0.350957 \\
\hline C & -3.914678 & 1.599719 & -0.501997 \\
\hline C & -5.385192 & -0.268290 & -0.099265 \\
\hline C & -4.990673 & 2.490711 & -0.404611 \\
\hline $\mathrm{H}$ & -2.913301 & 1.971221 & -0.683160 \\
\hline C & -6.488737 & 0.587218 & 0.000836 \\
\hline $\mathrm{H}$ & -5.515506 & -1.338260 & 0.035725 \\
\hline C & -6.265804 & 1.963001 & -0.157285 \\
\hline $\mathrm{H}$ & -7.105324 & 2.640427 & -0.081791 \\
\hline C & 4.078704 & -0.024993 & 0.428208 \\
\hline C & 4.592519 & -0.353509 & -0.832744 \\
\hline C & 4.713292 & 0.971704 & 1.180980 \\
\hline C & 5.714789 & 0.301346 & -1.355584 \\
\hline H & 4.113783 & -1.141478 & -1.403601 \\
\hline C & 5.840148 & 1.647531 & 0.698086 \\
\hline $\mathrm{H}$ & 4.296474 & 1.229485 & 2.150159 \\
\hline C & 6.319127 & 1.295344 & -0.572681 \\
\hline $\mathrm{H}$ & 7.185892 & 1.810181 & -0.963420 \\
\hline C & 6.496384 & 2.745731 & 1.558437 \\
\hline C & 6.234176 & -0.087447 & -2.753707 \\
\hline C & 7.729323 & 3.371840 & 0.879204 \\
\hline $\mathrm{H}$ & 8.510907 & 2.628591 & 0.684232 \\
\hline $\mathrm{H}$ & 7.472174 & 3.855423 & -0.070010 \\
\hline $\mathrm{H}$ & 8.159007 & 4.138839 & 1.533327 \\
\hline C & 6.949935 & 2.141045 & 2.908611 \\
\hline H & 7.416054 & 2.912209 & 3.534209 \\
\hline $\mathrm{H}$ & 6.109168 & 1.721350 & 3.470768 \\
\hline $\mathrm{H}$ & 7.682092 & 1.340131 & 2.754069 \\
\hline C & 5.470094 & 3.873237 & 1.822795 \\
\hline H & 4.581534 & 3.502524 & 2.343939 \\
\hline
\end{tabular}




\begin{tabular}{|c|c|c|c|}
\hline $\mathrm{H}$ & 5.917662 & 4.659208 & 2.443520 \\
\hline $\mathrm{H}$ & 5.139258 & 4.329809 & 0.882974 \\
\hline C & 6.598924 & -1.591054 & -2.771997 \\
\hline $\mathrm{H}$ & 7.384263 & -1.813433 & -2.040307 \\
\hline $\mathrm{H}$ & 5.736595 & -2.224533 & -2.541042 \\
\hline $\mathrm{H}$ & 6.966966 & -1.880233 & -3.763979 \\
\hline C & 7.486436 & 0.710914 & -3.162275 \\
\hline $\mathrm{H}$ & 8.321346 & 0.543931 & -2.471927 \\
\hline $\mathrm{H}$ & 7.814791 & 0.395655 & -4.159108 \\
\hline $\mathrm{H}$ & 7.289080 & 1.788108 & -3.206861 \\
\hline C & 5.126570 & 0.183738 & -3.800880 \\
\hline $\mathrm{H}$ & 4.207830 & -0.369753 & -3.581630 \\
\hline $\mathrm{H}$ & 4.875152 & 1.250646 & -3.832205 \\
\hline $\mathrm{H}$ & 5.467143 & -0.112163 & -4.800857 \\
\hline C & -7.885145 & -0.001196 & 0.285454 \\
\hline C & -4.730330 & 4.001124 & -0.566821 \\
\hline C & -6.016341 & 4.840328 & -0.443762 \\
\hline $\mathrm{H}$ & -6.752507 & 4.577615 & -1.212525 \\
\hline $\mathrm{H}$ & -6.489884 & 4.722172 & 0.537726 \\
\hline $\mathrm{H}$ & -5.776023 & 5.902661 & -0.566476 \\
\hline C & -4.114521 & 4.271014 & -1.960341 \\
\hline $\mathrm{H}$ & -3.164857 & 3.743056 & -2.093873 \\
\hline $\mathrm{H}$ & -4.792916 & 3.947972 & -2.758677 \\
\hline $\mathrm{H}$ & -3.922067 & 5.343240 & -2.090614 \\
\hline C & -3.741383 & 4.467868 & 0.529151 \\
\hline $\mathrm{H}$ & -4.171584 & 4.320550 & 1.526950 \\
\hline $\mathrm{H}$ & -2.795736 & 3.918441 & 0.490167 \\
\hline $\mathrm{H}$ & -3.520724 & 5.536530 & 0.411542 \\
\hline C & -8.981997 & 1.079257 & 0.342154 \\
\hline $\mathrm{H}$ & -8.799365 & 1.806181 & 1.141709 \\
\hline $\mathrm{H}$ & -9.066090 & 1.625940 & -0.604017 \\
\hline $\mathrm{H}$ & -9.952280 & 0.609292 & 0.539481 \\
\hline C & -7.866473 & -0.737586 & 1.646152 \\
\hline $\mathrm{H}$ & -8.851144 & -1.171543 & 1.860447 \\
\hline $\mathrm{H}$ & -7.133288 & -1.550908 & 1.658745 \\
\hline $\mathrm{H}$ & -7.614048 & -0.048492 & 2.460279 \\
\hline C & -8.261107 & -1.003344 & -0.832035 \\
\hline $\mathrm{H}$ & -7.547980 & -1.832018 & -0.894727 \\
\hline $\mathrm{H}$ & -9.253404 & -1.431893 & -0.643871 \\
\hline $\mathrm{H}$ & -8.284497 & -0.508366 & -1.809719 \\
\hline
\end{tabular}

\section{Uncatalyzed reaction TS 1 (Chair, Equatorial)}

Datum

Value

\begin{tabular}{lc}
\hline B3LYP/6-31G(d) Energy & -874.148983 \\
\hline B3LYP-D3(BJ)/def2-TZVPP-IEF-PCM(DCM) Energy & -874.588116 \\
\hline B3LYP-D3(BJ)/def2-TZVPP-IEF-PCM(DCM)//B3LYP/6-31G(d) Free Energy (Quasiharmonic) & -874.25358 \\
\hline
\end{tabular}


Frequencies (Top 3 out of 123)

$\begin{array}{rr}\text { 1. } & -327.5603 \mathrm{~cm}^{-1} \\ \text { 2. } & 38.0843 \mathrm{~cm}^{-1} \\ \text { 3. } & 46.5121 \mathrm{~cm}^{-1}\end{array}$

\section{B3LYP/6-31G(d) Molecular Geometry in Cartesian Coordinates}

C

C

C

C

$\mathrm{H}$

$\mathrm{H}$

$\mathrm{H}$

C

C

C

$\mathrm{H}$

$\mathrm{H}$

$\mathrm{H}$

C

$\mathrm{H}$

$\mathrm{H}$

$\mathrm{H}$

C

$\mathrm{H}$

$\mathrm{H}$

$\mathrm{H}$

C

$\mathrm{H}$

$\mathrm{H}$

$\mathrm{H}$

0

0

B

0

c

C

C

$\mathrm{C}$

$\mathrm{H}$

C

$\mathrm{H}$

C

\section{$-1.550560$}

$-1.265612$

0.716481

$-0.193847$

$-0.901129$

1.711341

0.321874

2. 822468

2. 767433

3.884074

3.720836

4.902176

3.807860

2.992712

3.940080

2.997884

2.174596

2. 106514

1.915938

2. 746184

1.147254

4.109340

4.827304

3.963448

4. 542015

1.527022

1.909077

1.017742

$-0.400407$

$-2.659784$

$-3.533975$

$-3.116449$

$-4.846697$

$-3.179108$

$-4.429335$

$-2.428981$

$-5.297605$
2.447838

0.333506

2.088688

2.689499

0.589680

2.535807

1.920083

$-0.077638$

$-0.994172$

1.030897

1.752442

0.636385

1.563456

$-0.826913$

$-1.378686$

$-0.111577$

$-1.531119$

$-2.358258$

$-2.847510$

$-3.015317$

$-2.242754$

$-1.204505$

$-1.711385$

$-1.828935$

$-0.255847$

0.551152

$-0.244217$

0.495842

$-0.079670$

$-0.132745$

0.028529

$-0.766459$

$-0.430753$

0.512179

$-1.223108$

$-0.902758$

$-1.055341$
$-0.275502$

0.321258

$-1.205761$

$-0.277154$

1. 317379

$-1.231576$

$-2.209871$

0.832072

$-0.452911$

0.729369

1.537009

0.822475

$-0.223591$

2. 155597

2. 181038

2.985506

2. 325013

$-0.186837$

$-1.147527$

0.413413

0.326245

$-1.156372$

$-0.500319$

$-2.044299$

$-1.482473$

0.825388

$-1.332172$

$-0.515744$

$-0.542050$

0.206417

1. 292078

$-0.960236$

1. 212347

2. 199437

$-1.036407$

$-1.788802$

0.046827 


\begin{tabular}{|lrrr|}
$H$ & -5.516499 & -0.305408 & 2.058497 \\
$H$ & -4.777193 & -1.716059 & -1.940059 \\
$H$ & -6.321077 & -1.414793 & -0.015909 \\
$H$ & 0.240734 & 3.143653 & 0.613817 \\
$H$ & -2.051395 & 2.150583 & -1.192281 \\
$H$ & -2.189348 & 2.866150 & 0.497557 \\
\hline
\end{tabular}

\section{Uncatalyzed reaction TS 2 (Chair, Axial)}

\section{Datum}

Value

\begin{tabular}{lc}
\hline B3LYP/6-31G(d) Energy & -874.140136 \\
\hline B3LYP-D3(BJ)/def2-TZVPP-IEF-PCM(DCM) Energy & -874.580081 \\
\hline B3LYP-D3(BJ)/def2-TZVPP-IEF-PCM(DCM)//B3LYP/6-31G(d) Free Energy (Quasiharmonic) & -874.245748 \\
\hline
\end{tabular}

Number of Imaginary Frequencies

Frequencies (Top 3 out of 123)

$\begin{array}{lr}\text { 1. } & -316.8927 \mathrm{~cm}^{-1} \\ \text { 2. } & 37.8174 \mathrm{~cm}^{-1} \\ \text { 3. } & 46.9917 \mathrm{~cm}^{-1}\end{array}$

\section{B3LYP/6-31G(d) Molecular Geometry in Cartesian Coordinates}

C

C

C

C

$\mathrm{H}$

$\mathrm{H}$

C

C

C

$\mathrm{H}$

$\mathrm{H}$

$\mathrm{H}$

C

$\mathrm{H}$

$\mathrm{H}$

$\mathrm{H}$

C

$\mathrm{H}$

$\mathrm{H}$

$\mathrm{H}$
$-1.808150$

$-1.518625$

0.558579

$-0.833034$

1.231525

0.873879

2.275206

2.838576

3.029175

2. 454545

4.024130

3.146301

2. 185522

3.177602

1.764725

1.543755

2.444997

2. 681449

2.988820

1. 371591

\section{741951}

1.090333

2. 253809

2. 240489

2.082546

3.108836

$-0.921991$

$-0.200372$

$-0.534934$

$-0.880023$

$-0.991827$

0.550121

$-2.446154$

$-2.891055$

$-2.854157$

$-2.757012$

$-0.912046$

$-0.253374$

$-1.853867$

$-1.121894$
0.504601

$-0.952835$

1.000986

1.335297

1.842947

0.398421

0.586540

$-0.696552$

1.869842

2.735886

1.913773

1.948614

0.486589

0.344351

1.412481

$-0.341442$

$-2.003074$

$-2.844869$

$-2.138863$

$-2.033143$ 


\begin{tabular}{|lrrr|} 
C & 4.346057 & 0.058537 & -0.686316 \\
H & 4.909515 & -0.881170 & -0.639844 \\
H & 4.634517 & 0.579926 & -1.605277 \\
H & 4.639659 & 0.685256 & 0.159216 \\
O & 0.939906 & -0.386457 & 0.678619 \\
O & 2.158035 & 1.065275 & -0.655516 \\
B & 0.905496 & 0.865335 & -0.020586 \\
O & -0.240091 & 0.980597 & -1.052504 \\
H & -1.147374 & 1.602561 & 2.162210 \\
H & -1.550798 & 3.506314 & -0.224555 \\
H & -2.859394 & 2.659188 & 0.762550 \\
C & -2.447050 & 0.042209 & -0.465544 \\
C & -3.807189 & 0.186788 & -0.791437 \\
C & -2.029926 & -1.092443 & 0.248829 \\
C & -4.737630 & -0.772618 & -0.403041 \\
$H$ & -4.133564 & 1.058805 & -1.354918 \\
C & -2.967212 & -2.049363 & 0.635601 \\
$H$ & -0.979622 & -1.208525 & 0.495855 \\
C & -4.318279 & -1.895492 & 0.315154 \\
H & -5.785273 & -0.648738 & -0.663276 \\
H & -2.638138 & -2.925607 & 1.187941 \\
H & -5.040751 & -2.648597 & 0.618266 \\
H & -1.963489 & 1.748070 & -1.706016 \\
& & & \\
\hline & & & \\
\hline
\end{tabular}

\section{Uncatalyzed reaction TS 3 (Boat, Equatorial)}

\section{Datum}

Value

\begin{tabular}{lc}
\hline B3LYP/6-31G(d) Energy & -874.138217 \\
\hline B3LYP-D3(BJ)/def2-TZVPP-IEF-PCM(DCM) Energy & -874.577238 \\
\hline B3LYP-D3(BJ)/def2-TZVPP-IEF-PCM(DCM)//B3LYP/6-31G(d) Free Energy (Quasiharmonic) & -874.243489 \\
\hline
\end{tabular}

Number of Imaginary Frequencies

Frequencies (Top 3 out of 123)

$$
\begin{array}{lr}
\text { 1. } & -389.2354 \mathrm{~cm}^{-1} \\
\text { 2. } & 29.5130 \mathrm{~cm}^{-1} \\
\text { 3. } & 42.5563 \mathrm{~cm}^{-1}
\end{array}
$$

\section{B3LYP/6-31G(d) Molecular Geometry in Cartesian Coordinates}

$\begin{array}{rrrr}\text { C } & 1.357813 & 2.188113 & 1.064476 \\ \text { C } & 1.280901 & 0.068285 & 0.462298 \\ \text { C } & -0.678715 & 2.344930 & -0.332539\end{array}$




$\begin{array}{lrrr}\text { C } & 0.704987 & 2.568089 & -0.092689 \\ \text { H } & -1.055470 & 2.703246 & -1.289511 \\ \text { H } & -1.339905 & 2.588651 & 0.500203 \\ \text { C } & -3.092229 & -0.319666 & -0.695373 \\ \text { C } & -2.629087 & -0.707720 & 0.762629 \\ \text { C } & -4.073546 & 0.864577 & -0.716039 \\ \text { H } & -4.178285 & 1.216234 & -1.747553 \\ \text { H } & -5.065847 & 0.582525 & -0.346504 \\ \text { H } & -3.704248 & 1.697757 & -0.110229 \\ \text { C } & -3.667176 & -1.473494 & -1.520020 \\ \text { H } & -4.567257 & -1.888125 & -1.050137 \\ \text { H } & -3.943170 & -1.110278 & -2.515781 \\ \text { H } & -2.937270 & -2.276169 & -1.648609 \\ \text { C } & -2.067489 & -2.137537 & 0.847145 \\ \text { H } & -1.583152 & -2.269008 & 1.820754 \\ \text { H } & -2.854296 & -2.894321 & 0.752744 \\ \text { H } & -1.319452 & -2.315243 & 0.068627 \\ \text { C } & -3.678792 & -0.500810 & 1.855462 \\ \text { H } & -4.557101 & -1.135036 & 1.685955 \\ \text { H } & -3.253991 & -0.767398 & 2.829446 \\ \text { H } & -4.004663 & 0.540965 & 1.906106 \\ \text { O } & -1.863475 & 0.106526 & -1.311967 \\ \text { O } & -1.542869 & 0.213690 & 0.993398 \\ \text { B } & -0.981123 & 0.521058 & -0.288556 \\ \text { O } & 0.398845 & -0.027001 & -0.487923 \\ \text { H } & 1.337189 & 2.780833 & -0.957265 \\ \text { H } & 0.790004 & 2.068258 & 1.984340 \\ \text { H } & 2.422866 & 2.362001 & 1.177931 \\ \text { C } & 2.682972 & -0.247887 & 0.141128 \\ \text { C } & 3.592106 & -0.499223 & 1.181010 \\ \text { C } & 3.121543 & -0.328821 & -1.190094 \\ \text { C } & 4.917463 & -0.819034 & 0.896868 \\ \text { H } & 3.254096 & -0.445009 & 2.213582 \\ \text { C } & 4.447900 & -0.647236 & -1.470369 \\ \text { H } & 2.409752 & -0.151649 & -1.989754 \\ \text { C } & 5.349371 & -0.891414 & -0.430143 \\ \text { H } & 5.612703 & -1.015267 & 1.708423 \\ \text { H } & 4.780129 & -0.710373 & -2.503054 \\ \text { H } & 6.383026 & -1.141680 & -0.652812 \\ & 0.931812 & -0.138836 & 1.475317\end{array}$

\section{Uncatalyzed reaction TS 4 (Boat, Axial)}

Datum

Value

\begin{tabular}{lc}
\hline B3LYP/6-31G(d) Energy & -874.134081 \\
\hline B3LYP-D3(BJ)/def2-TZVPP-IEF-PCM(DCM) Energy & -874.574043 \\
\hline B3LYP-D3(BJ)/def2-TZVPP-IEF-PCM(DCM)//B3LYP/6-31G(d) Free Energy (Quasiharmonic) & -874.240229 \\
\hline
\end{tabular}


Frequencies (Top 3 out of 123)

$\begin{array}{lr}\text { 1. } & -364.1575 \mathrm{~cm}^{-1} \\ \text { 2. } & 34.0653 \mathrm{~cm}^{-1} \\ \text { 3. } & 37.3671 \mathrm{~cm}^{-1}\end{array}$

\section{B3LYP/6-31G(d) Molecular Geometry in Cartesian Coordinates}

C

C

C

C

$\mathrm{H}$

$\mathrm{H}$

C

C

C

$\mathrm{H}$

$\mathrm{H}$

$\mathrm{H}$

C

$\mathrm{H}$

$\mathrm{H}$

$\mathrm{H}$

C

$\mathrm{H}$

$\mathrm{H}$

$\mathrm{H}$

C

$\mathrm{H}$

$\mathrm{H}$

$\mathrm{H}$

0

0

B

0

$\mathrm{H}$

$\mathrm{H}$

$\mathrm{H}$

C

C

C

C

$\mathrm{H}$

C

\section{$-1.828383$}

$-1.540376$

0.427738

$-0.473751$

1.406895

$-0.004169$

3.077884

2. 204524

3.934785

4.332933

4. 778991

3.339800

3.960903

4.696857

4.506584

3. 365020

1.738890

0.986061

2.566920

1. 284052

2.834636

3. 761524

2. 140571

3.056577

2.080852

1.049066

0.882763

$-0.284514$

$-0.044484$

$-2.316914$

$-2.475282$

$-2.553456$

$-3.899746$

$-2.193567$

$-4.875886$

$-4.177393$

$-3.177030$
2.638882

1.018403

1.944452

2.875686

2. 346808

1. 349280

0.025038

$-1.197620$

0.622320

1.586733

$-0.025472$

0.791530

$-0.247125$

$-1.032653$

0.664091

$-0.546236$

$-2.084197$

$-2.790936$

$-2.659977$

$-1.485579$

$-2.066639$

$-2.534540$

$-2.866383$

$-1.485691$

0.991524

$-0.537627$

0.698962

0.733010

3.673826

1.995703

3. 342545

0.059097

0.252210

$-1.049606$

$-0.642349$

1.107135

$-1.938583$
$-0.535434$

0.941585

$-1.234341$

$-0.640846$

$-1.495283$

$-2.042394$

0.305099

$-0.173728$

$-0.824701$

$-0.492164$

$-1.086667$

$-1.727518$

1. 525135

1. 315273

1. 793217

2. 390510

0.993892

0.628561

1.422380

1. 788620

$-1.263132$

$-0.910261$

$-1.544192$

$-2.161598$

0.673443

$-0.730341$

$-0.029461$

0.959231

$-0.032548$

$-1.259816$

$-0.018113$

0.451377

0.802790

$-0.333169$

0.373294

1.416090

$-0.763259$ 


\begin{tabular}{|lrrr|}
$H$ & -1.149911 & -1.202274 & -0.593494 \\
$\mathrm{C}$ & -4.515658 & -1.739765 & -0.414185 \\
$\mathrm{H}$ & -5.914355 & -0.488306 & 0.653391 \\
$\mathrm{H}$ & -2.896803 & -2.795589 & -1.369867 \\
$\mathrm{H}$ & -5.275922 & -2.439842 & -0.750120 \\
$\mathrm{H}$ & -1.873626 & 1.684536 & 1.742372 \\
\hline
\end{tabular}

\section{C-Catalyzed reaction TS 1 (TS-2.1)}

\section{Datum}

Value

\begin{tabular}{lc}
\hline B3LYP/6-31G(d) Energy & -3038.966021 \\
\hline B3LYP-D3(BJ)/def2-TZVPP-IEF-PCM(DCM) Energy & -3040.523001 \\
\hline B3LYP-D3(BJ)/def2-TZVPP-IEF-PCM(DCM)//B3LYP/6-31G(d) Free Energy (Quasiharmonic) & -3039.295156 \\
\hline
\end{tabular}

Number of Imaginary Frequencies

Frequencies (Top 3 out of 450)
1. $-340.8829 \mathrm{~cm}^{-1}$
2. $\quad 3.7694 \mathrm{~cm}^{-1}$
3. $8.8329 \mathrm{~cm}^{-1}$

B3LYP/6-31G(d) Molecular Geometry in Cartesian Coordinates

\begin{tabular}{|rrrr|}
\hline C & 1.477428 & 0.633840 & 0.829812 \\
H & 2.445179 & 0.137507 & 0.912434 \\
H & 1.043897 & 0.701572 & 1.835862 \\
C & -1.622177 & 1.915335 & -1.489792 \\
O & -2.278895 & 2.635268 & -2.207458 \\
O & -0.726394 & 1.049573 & -1.986315 \\
H & -0.239374 & 0.573666 & -1.260550 \\
O & 0.610424 & -0.175920 & 0.012550 \\
H & 1.000862 & -1.082455 & -0.073160 \\
C & -0.200595 & -2.691044 & 3.424682 \\
C & -0.971445 & -3.011577 & 1.431866 \\
C & 1.823003 & -3.722651 & 2.437813 \\
C & 1.107644 & -2.612131 & 2.991654 \\
H & -0.739394 & -2.001175 & 1.089820 \\
H & 2.897744 & -3.569295 & 2.327953 \\
H & 1.597366 & -4.692451 & 2.886561 \\
C & 2.437227 & -3.169217 & -1.132857 \\
C & 2.077328 & -4.701040 & -1.200094 \\
C & 2.101044 & -2.364123 & -2.388383 \\
H & 2.363631 & -1.310854 & -2.241485 \\
& & &
\end{tabular}




\begin{tabular}{|c|c|c|c|}
\hline H & 2.669796 & -2.731561 & -3.250283 \\
\hline $\mathrm{H}$ & 1.036463 & -2.415638 & -2.629703 \\
\hline C & 3.896818 & -2.920783 & -0.725523 \\
\hline $\mathrm{H}$ & 4.588730 & -3.182071 & -1.533724 \\
\hline $\mathrm{H}$ & 4.038023 & -1.861131 & -0.491512 \\
\hline H & 4.165835 & -3.508706 & 0.157325 \\
\hline C & 3.213556 & -5.607330 & -1.677355 \\
\hline $\mathrm{H}$ & 3.522934 & -5.353593 & -2.698168 \\
\hline $\mathrm{H}$ & 4.083101 & -5.539596 & -1.019409 \\
\hline $\mathrm{H}$ & 2.873415 & -6.648147 & -1.676557 \\
\hline C & 0.811596 & -4.982800 & -2.028736 \\
\hline H & 0.987347 & -4.850544 & -3.102082 \\
\hline $\mathrm{H}$ & 0.505840 & -6.019368 & -1.855164 \\
\hline $\mathrm{H}$ & -0.017603 & -4.335306 & -1.729736 \\
\hline 0 & 1.588928 & -2.707994 & -0.045385 \\
\hline 0 & 1.800592 & -5.013893 & 0.175949 \\
\hline B & 1.249052 & -3.859806 & 0.783713 \\
\hline 0 & -0.240233 & -3.990023 & 0.992607 \\
\hline C & -2.384591 & -3.297514 & 1.747582 \\
\hline C & -2.838570 & -4.617575 & 1.901594 \\
\hline C & -3.292631 & -2.233984 & 1.854787 \\
\hline C & -4.182975 & -4.863331 & 2.164218 \\
\hline $\mathrm{H}$ & -2.132258 & -5.434900 & 1.797292 \\
\hline C & -4.638982 & -2.485776 & 2.111506 \\
\hline $\mathrm{H}$ & -2.946942 & -1.212058 & 1.723995 \\
\hline C & -5.085544 & -3.799492 & 2.270141 \\
\hline $\mathrm{H}$ & -4.532315 & -5.885815 & 2.278868 \\
\hline H & -5.338951 & -1.657603 & 2.172421 \\
\hline $\mathrm{H}$ & -6.135626 & -3.996328 & 2.468674 \\
\hline $\mathrm{H}$ & 1.525709 & -1.617679 & 2.831575 \\
\hline $\mathrm{H}$ & -0.596660 & -3.638920 & 3.777277 \\
\hline $\mathrm{H}$ & -0.722146 & -1.803154 & 3.770988 \\
\hline C & -0.768517 & 2.467186 & 0.808927 \\
\hline C & -1.786524 & 1.948934 & 0.019655 \\
\hline C & -3.051629 & 1.575516 & 0.587075 \\
\hline C & -3.236591 & 1.747106 & 1.946273 \\
\hline $\mathrm{H}$ & -4.178906 & 1.444782 & 2.396519 \\
\hline C & 1.645128 & 2.027192 & 0.246523 \\
\hline C & 0.543808 & 2.881148 & 0.199928 \\
\hline C & 2.989374 & 3.696448 & -0.899593 \\
\hline C & 2.900774 & 2.451245 & -0.305013 \\
\hline H & 3.944128 & 4.022396 & -1.304587 \\
\hline C & 1.889048 & 4.580112 & -0.972330 \\
\hline C & 0.638260 & 4.174795 & -0.408594 \\
\hline C & -0.457648 & 5.080621 & -0.476916 \\
\hline C & -0.316564 & 6.316111 & -1.066508 \\
\hline C & 0.924378 & 6.714845 & -1.619741 \\
\hline C & 2.002573 & 5.863061 & -1.573596 \\
\hline $\mathrm{H}$ & -1.418747 & 4.781786 & -0.074676 \\
\hline $\mathrm{H}$ & -1.167618 & 6.989604 & -1.114967 \\
\hline $\mathrm{H}$ & 1.019052 & 7.692827 & -2.083886 \\
\hline $\mathrm{H}$ & 2.960181 & 6.156592 & -1.997135 \\
\hline C & -2.235743 & 2.295692 & 2.784932 \\
\hline C & -0.977866 & 2.668915 & 2.214332 \\
\hline
\end{tabular}




\begin{tabular}{|c|c|c|c|}
\hline C & 0.009411 & 3.234601 & 3.068344 \\
\hline C & -0.230715 & 3.406733 & 4.414203 \\
\hline C & -1.471187 & 3.023846 & 4.977531 \\
\hline C & -2.450382 & 2.480910 & 4.177487 \\
\hline $\mathrm{H}$ & 0.960671 & 3.537684 & 2.643696 \\
\hline $\mathrm{H}$ & 0.536105 & 3.843627 & 5.048231 \\
\hline $\mathrm{H}$ & -1.648854 & 3.164777 & 6.040240 \\
\hline $\mathrm{H}$ & -3.409793 & 2.189924 & 4.599033 \\
\hline C & 6.555504 & 0.218148 & -0.195070 \\
\hline C & 6.037871 & 0.618113 & -1.435729 \\
\hline C & 4.824105 & 1.318688 & -1.441889 \\
\hline C & 4.145122 & 1.624196 & -0.255434 \\
\hline C & 4.704964 & 1.217405 & 0.963439 \\
\hline C & 5.910559 & 0.506113 & 1.017452 \\
\hline $\mathrm{H}$ & 7.487885 & -0.328753 & -0.171514 \\
\hline $\mathrm{H}$ & 4.383354 & 1.640812 & -2.380796 \\
\hline $\mathrm{H}$ & 4.196414 & 1.492035 & 1.883666 \\
\hline C & 6.760662 & 0.333628 & -2.767339 \\
\hline C & 6.489602 & 0.089656 & 2.384420 \\
\hline C & 8.053079 & -0.482509 & -2.575209 \\
\hline $\mathrm{H}$ & 8.522160 & -0.662521 & -3.549093 \\
\hline $\mathrm{H}$ & 8.783328 & 0.046234 & -1.952120 \\
\hline $\mathrm{H}$ & 7.855174 & -1.458609 & -2.117782 \\
\hline C & 5.828104 & -0.461961 & -3.710844 \\
\hline $\mathrm{H}$ & 6.334585 & -0.666106 & -4.662081 \\
\hline $\mathrm{H}$ & 5.545096 & -1.421626 & -3.263826 \\
\hline $\mathrm{H}$ & 4.907390 & 0.085552 & -3.936544 \\
\hline C & 7.135376 & 1.677911 & -3.436781 \\
\hline $\mathrm{H}$ & 7.647201 & 1.500106 & -4.390557 \\
\hline $\mathrm{H}$ & 6.250263 & 2.289088 & -3.641181 \\
\hline $\mathrm{H}$ & 7.805103 & 2.262237 & -2.795338 \\
\hline C & 7.798443 & -0.712250 & 2.252319 \\
\hline $\mathrm{H}$ & 8.160545 & -0.991740 & 3.248151 \\
\hline $\mathrm{H}$ & 7.654518 & -1.636221 & 1.680915 \\
\hline $\mathrm{H}$ & 8.588904 & -0.128850 & 1.766933 \\
\hline C & 6.783494 & 1.355696 & 3.224340 \\
\hline $\mathrm{H}$ & 7.193102 & 1.078235 & 4.203488 \\
\hline $\mathrm{H}$ & 7.513653 & 1.999117 & 2.720386 \\
\hline $\mathrm{H}$ & 5.879556 & 1.949097 & 3.396595 \\
\hline C & 5.463634 & -0.792876 & 3.134952 \\
\hline $\mathrm{H}$ & 5.862891 & -1.097766 & 4.109954 \\
\hline $\mathrm{H}$ & 4.522635 & -0.261993 & 3.314594 \\
\hline $\mathrm{H}$ & 5.234603 & -1.699705 & 2.563157 \\
\hline C & -4.148426 & 0.982660 & -0.229318 \\
\hline C & -3.894694 & -0.029184 & -1.157904 \\
\hline C & -5.473448 & 1.412493 & -0.038195 \\
\hline C & -4.924928 & -0.614246 & -1.909197 \\
\hline $\mathrm{H}$ & -2.873591 & -0.366196 & -1.301102 \\
\hline C & -6.529645 & 0.852029 & -0.757875 \\
\hline $\mathrm{H}$ & -5.654075 & 2.218884 & 0.662761 \\
\hline C & -6.228015 & -0.161445 & -1.686419 \\
\hline $\mathrm{H}$ & -7.040999 & -0.597075 & -2.255249 \\
\hline C & -7.989922 & 1.313161 & -0.584494 \\
\hline C & -4.580155 & -1.705401 & -2.940901 \\
\hline
\end{tabular}




\begin{tabular}{|llll|} 
C & -8.522296 & 1.858526 & -1.931269 \\
$H$ & -9.562303 & 2.191363 & -1.824569 \\
$H$ & -7.924243 & 2.711713 & -2.270412 \\
$H$ & -8.494988 & 1.098238 & -2.718613 \\
C & -8.860521 & 0.114683 & -0.137477 \\
$H$ & -9.907014 & 0.423557 & -0.023633 \\
$H$ & -8.832342 & -0.704324 & -0.863648 \\
$H$ & -8.517155 & -0.281162 & 0.825651 \\
C & -8.134556 & 2.427004 & 0.470049 \\
$H$ & -7.795741 & 2.099767 & 1.459909 \\
$H$ & -7.570275 & 3.324932 & 0.194691 \\
$H$ & -9.188374 & 2.713973 & 0.560619 \\
C & -3.902894 & -2.899007 & -2.226655 \\
$H$ & -2.979820 & -2.600635 & -1.719178 \\
$H$ & -4.567977 & -3.338652 & -1.474685 \\
$H$ & -3.645608 & -3.679358 & -2.953464 \\
C & -5.825545 & -2.234397 & -3.676641 \\
$H$ & -6.544643 & -2.693782 & -2.988229 \\
$H$ & -6.339395 & -1.440947 & -4.231126 \\
$H$ & -5.526126 & -3.000786 & -4.400587 \\
C & -3.610203 & -1.117745 & -3.994725 \\
$H$ & -3.340750 & -1.885705 & -4.730742 \\
$H$ & -4.075108 & -0.281718 & -4.529054 \\
$H$ & -2.686081 & -0.743640 & -3.543113 \\
& & & \\
\hline
\end{tabular}

\section{C-Catalyzed reaction TS 2}

\section{Datum}

Value

\begin{tabular}{lc}
\hline B3LYP/6-31G(d) Energy & -3038.965802 \\
\hline B3LYP-D3(BJ)/def2-TZVPP-IEF-PCM(DCM) Energy & -3040.522698 \\
\hline B3LYP-D3(BJ)/def2-TZVPP-IEF-PCM(DCM)//B3LYP/6-31G(d) Free Energy (Quasiharmonic) & -3039.295086 \\
\hline
\end{tabular}

Number of Imaginary Frequencies

Frequencies (Top 3 out of 450)

$\begin{array}{lr}\text { 1. } & -340.9302 \mathrm{~cm}^{-1} \\ \text { 2. } & 3.8126 \mathrm{~cm}^{-1} \\ \text { 3. } & 9.3552 \mathrm{~cm}^{-1}\end{array}$

\section{B3LYP/6-31G(d) Molecular Geometry in Cartesian Coordinates}

$\begin{array}{llll}\mathrm{C} & -1.471420 & 0.633465 & 0.827380 \\ \mathrm{H} & -2.440337 & 0.139782 & 0.912397\end{array}$




\begin{tabular}{|c|c|c|c|}
\hline $\mathrm{H}$ & -1.033938 & 0.697516 & 1.832001 \\
\hline C & 1.622909 & 1.912504 & -1.499728 \\
\hline 0 & 2.280912 & 2.631359 & -2.217317 \\
\hline 0 & 0.721334 & 1.052659 & -1.995866 \\
\hline $\mathrm{H}$ & 0.235754 & 0.575363 & -1.270048 \\
\hline 0 & -0.609981 & -0.176246 & 0.004368 \\
\hline H & -0.999580 & -1.083569 & -0.076516 \\
\hline C & 0.213853 & -2.686761 & 3.420498 \\
\hline C & 0.975518 & -3.017318 & 1.426030 \\
\hline C & -1.817820 & -3.713558 & 2.445217 \\
\hline C & -1.095870 & -2.604130 & 2.992607 \\
\hline $\mathrm{H}$ & 0.746054 & -2.007348 & 1.080922 \\
\hline $\mathrm{H}$ & -2.892334 & -3.556164 & 2.338909 \\
\hline $\mathrm{H}$ & -1.594455 & -4.682775 & 2.896355 \\
\hline C & -2.442274 & -3.170506 & -1.125798 \\
\hline C & -2.089831 & -4.704372 & -1.187967 \\
\hline C & -2.105721 & -2.372172 & -2.385549 \\
\hline $\mathrm{H}$ & -2.362473 & -1.317009 & -2.242023 \\
\hline $\mathrm{H}$ & -2.678785 & -2.740112 & -3.244374 \\
\hline $\mathrm{H}$ & -1.042110 & -2.430120 & -2.629665 \\
\hline C & -3.899445 & -2.913330 & -0.715320 \\
\hline $\mathrm{H}$ & -4.595053 & -3.175235 & -1.520142 \\
\hline $\mathrm{H}$ & -4.035037 & -1.851926 & -0.485911 \\
\hline $\mathrm{H}$ & -4.168506 & -3.495738 & 0.171179 \\
\hline C & -3.232117 & -5.607192 & -1.657254 \\
\hline $\mathrm{H}$ & -3.544314 & -5.356030 & -2.677839 \\
\hline $\mathrm{H}$ & -4.098731 & -5.532771 & -0.996172 \\
\hline $\mathrm{H}$ & -2.896871 & -6.649591 & -1.653650 \\
\hline C & -0.828568 & -4.995690 & -2.020117 \\
\hline $\mathrm{H}$ & -1.007657 & -4.866998 & -3.093347 \\
\hline $\mathrm{H}$ & -0.527309 & -6.033051 & -1.843440 \\
\hline $\mathrm{H}$ & 0.005012 & -4.351171 & -1.726900 \\
\hline 0 & -1.588715 & -2.708884 & -0.042652 \\
\hline 0 & -1.809439 & -5.012974 & 0.188266 \\
\hline B & -1.250686 & -3.858975 & 0.789515 \\
\hline 0 & 0.238850 & -3.994552 & 0.993223 \\
\hline C & 2.388738 & -3.307543 & 1.737549 \\
\hline C & 2.838161 & -4.628806 & 1.894517 \\
\hline C & 3.301201 & -2.247175 & 1.838236 \\
\hline C & 4.182446 & -4.878816 & 2.153718 \\
\hline $\mathrm{H}$ & 2.128402 & -5.443772 & 1.795292 \\
\hline C & 4.647379 & -2.503176 & 2.091710 \\
\hline $\mathrm{H}$ & 2.958916 & -1.224386 & 1.705345 \\
\hline C & 5.089353 & -3.818082 & 2.253368 \\
\hline $\mathrm{H}$ & 4.528228 & -5.902244 & 2.270721 \\
\hline $\mathrm{H}$ & 5.350633 & -1.677445 & 2.147978 \\
\hline H & 6.139240 & -4.018312 & 2.449582 \\
\hline $\mathrm{H}$ & -1.510717 & -1.608558 & 2.831210 \\
\hline $\mathrm{H}$ & 0.607585 & -3.635004 & 3.774705 \\
\hline $\mathrm{H}$ & 0.740172 & -1.799766 & 3.761880 \\
\hline C & 0.779404 & 2.460347 & 0.803695 \\
\hline C & 1.792691 & 1.940817 & 0.009196 \\
\hline C & 3.058237 & 1.560743 & 0.571096 \\
\hline C & 3.248287 & 1.726403 & 1.930280 \\
\hline
\end{tabular}




\begin{tabular}{|c|c|c|c|}
\hline $\mathrm{H}$ & 4.190475 & 1.417688 & 2.376439 \\
\hline C & -1.637205 & 2.028720 & 0.248262 \\
\hline C & -0.533843 & 2.879972 & 0.200581 \\
\hline C & -2.980446 & 3.704059 & -0.890047 \\
\hline C & -2.893229 & 2.457156 & -0.298883 \\
\hline H & -3.935423 & 4.033178 & -1.291952 \\
\hline C & -1.878180 & 4.585281 & -0.963400 \\
\hline C & -0.626801 & 4.175425 & -0.404259 \\
\hline C & 0.471216 & 5.078626 & -0.473493 \\
\hline C & 0.331597 & 6.315918 & -1.059642 \\
\hline C & -0.909921 & 6.719149 & -1.608310 \\
\hline C & -1.990151 & 5.869997 & -1.561158 \\
\hline $\mathrm{H}$ & 1.432705 & 4.776357 & -0.074738 \\
\hline $\mathrm{H}$ & 1.184224 & 6.987356 & -1.108930 \\
\hline $\mathrm{H}$ & -1.003430 & 7.698500 & -2.069796 \\
\hline $\mathrm{H}$ & -2.948201 & 6.166991 & -1.981264 \\
\hline C & 2.252648 & 2.276611 & 2.774110 \\
\hline C & 0.994428 & 2.656888 & 2.208944 \\
\hline C & 0.012278 & 3.223715 & 3.068091 \\
\hline C & 0.257633 & 3.390511 & 4.413669 \\
\hline C & 1.498400 & 3.000707 & 4.971588 \\
\hline C & 2.472684 & 2.456304 & 4.166539 \\
\hline $\mathrm{H}$ & -0.939183 & 3.531942 & 2.647620 \\
\hline $\mathrm{H}$ & -0.505261 & 3.828446 & 5.051700 \\
\hline $\mathrm{H}$ & 1.680157 & 3.137383 & 6.034159 \\
\hline $\mathrm{H}$ & 3.432195 & 2.159815 & 4.584006 \\
\hline C & -6.551584 & 0.230194 & -0.187250 \\
\hline C & -5.905058 & 0.516138 & 1.024909 \\
\hline C & -4.698319 & 1.225460 & 0.970333 \\
\hline C & -4.139088 & 1.632391 & -0.248759 \\
\hline C & -4.819630 & 1.328995 & -1.434835 \\
\hline C & -6.034440 & 0.630221 & -1.428088 \\
\hline $\mathrm{H}$ & -7.484828 & -0.315220 & -0.163252 \\
\hline $\mathrm{H}$ & -4.188243 & 1.498313 & 1.890257 \\
\hline $\mathrm{H}$ & -4.379176 & 1.651168 & -2.373867 \\
\hline C & -6.483510 & 0.099451 & 2.392054 \\
\hline C & -6.758626 & 0.347385 & -2.759284 \\
\hline C & -5.458522 & -0.785921 & 3.140578 \\
\hline $\mathrm{H}$ & -5.857263 & -1.090853 & 4.115780 \\
\hline $\mathrm{H}$ & -4.516208 & -0.257166 & 3.319564 \\
\hline $\mathrm{H}$ & -5.232072 & -1.692708 & 2.567691 \\
\hline C & -7.794106 & -0.699671 & 2.260527 \\
\hline $\mathrm{H}$ & -8.155810 & -0.979271 & 3.256469 \\
\hline $\mathrm{H}$ & -7.652592 & -1.623451 & 1.688213 \\
\hline $\mathrm{H}$ & -8.583848 & -0.114272 & 1.776378 \\
\hline C & -6.773981 & 1.365206 & 3.233589 \\
\hline $\mathrm{H}$ & -7.183049 & 1.087550 & 4.212907 \\
\hline $\mathrm{H}$ & -7.503436 & 2.010576 & 2.731105 \\
\hline $\mathrm{H}$ & -5.868711 & 1.956684 & 3.405457 \\
\hline C & -5.827964 & -0.449626 & -3.703475 \\
\hline $\mathrm{H}$ & -6.335409 & -0.652979 & -4.654368 \\
\hline $\mathrm{H}$ & -5.546067 & -1.409727 & -3.256675 \\
\hline $\mathrm{H}$ & -4.906601 & 0.096534 & -3.929796 \\
\hline C & -7.131408 & 1.692440 & -3.428246 \\
\hline
\end{tabular}




\begin{tabular}{lrrr}
$\mathrm{H}$ & -7.644089 & 1.515696 & -4.381757 \\
$\mathrm{H}$ & -6.245372 & 2.302134 & -3.633059 \\
$\mathrm{H}$ & -7.799761 & 2.277795 & -2.786309 \\
$\mathrm{C}$ & -8.052331 & -0.466545 & -2.566523 \\
$\mathrm{H}$ & -8.522420 & -0.645385 & -3.540135 \\
$\mathrm{H}$ & -8.781200 & 0.063252 & -1.942712 \\
$\mathrm{H}$ & -7.855814 & -1.443154 & -2.109587 \\
$\mathrm{C}$ & 4.150045 & 0.966427 & -0.251069 \\
$\mathrm{C}$ & 3.887733 & -0.041860 & -1.186503 \\
$\mathrm{C}$ & 5.474855 & 1.383084 & -0.064784 \\
$\mathrm{C}$ & 4.909974 & -0.627097 & -1.941552 \\
$\mathrm{H}$ & 2.863985 & -0.371412 & -1.327947 \\
$\mathrm{C}$ & 6.530141 & 0.819237 & -0.791936 \\
$\mathrm{H}$ & 5.670163 & 2.186140 & -0.639778 \\
$\mathrm{C}$ & 6.222252 & -0.183919 & -1.722677 \\
$\mathrm{H}$ & 7.024164 & -0.625181 & -2.298398 \\
$\mathrm{C}$ & 7.968194 & 1.324541 & -0.559857 \\
$\mathrm{C}$ & 4.557017 & -1.711670 & -2.978184 \\
$\mathrm{C}$ & 8.042520 & 2.835895 & -0.883556 \\
$\mathrm{H}$ & 9.059799 & 3.211594 & -0.717427 \\
$\mathrm{H}$ & 7.363302 & 3.421906 & -0.255999 \\
$\mathrm{H}$ & 7.774786 & 3.025473 & -1.928947 \\
$\mathrm{C}$ & 9.000268 & 0.596492 & -1.441725 \\
$\mathrm{H}$ & 10.002506 & 0.989993 & -1.237146 \\
$\mathrm{H}$ & 8.800183 & 0.743008 & -2.508968 \\
$\mathrm{H}$ & 9.021320 & -0.481072 & -1.242202 \\
$\mathrm{C}$ & 8.361044 & 1.099417 & 0.919877 \\
$\mathrm{H}$ & 8.331203 & 0.033503 & 1.175193 \\
$\mathrm{H}$ & 7.688810 & 1.627107 & 1.604658 \\
$\mathrm{H}$ & 9.378876 & 1.464148 & 1.105750 \\
$\mathrm{C}$ & 3.591027 & -1.112514 & -4.029256 \\
$\mathrm{H}$ & 4.061410 & -0.277009 & -4.559628 \\
$\mathrm{H}$ & 2.669604 & -0.734396 & -3.575469 \\
$\mathrm{H}$ & 3.316363 & -1.874998 & -4.769068 \\
$\mathrm{C}$ & 3.871028 & -2.903587 & -2.269437 \\
$\mathrm{H}$ & 2.949654 & -2.600769 & -1.761507 \\
$\mathrm{H}$ & 4.532535 & -3.351006 & -1.518876 \\
$\mathrm{H}$ & 3.608832 & -3.679125 & -2.999658 \\
$\mathrm{H}$ & 5.797969 & -2.247192 & -3.716914 \\
$\mathrm{H}$ & 5.492084 & -3.008760 & -4.443282 \\
& 6.514325 & -2.714409 & -3.030951 \\
\hline & & -1.455885 & -4.269140
\end{tabular}

\section{C-Catalyzed reaction TS 3}

\section{Datum}

Value 
Frequencies (Top 3 out of 450)

$\begin{array}{lr}\text { 1. } & -340.9335 \mathrm{~cm}^{-1} \\ \text { 2. } & 3.8889 \mathrm{~cm}^{-1} \\ \text { 3. } & 9.3634 \mathrm{~cm}^{-1}\end{array}$

\section{B3LYP/6-31G(d) Molecular Geometry in Cartesian Coordinates}

C

$\mathrm{H}$

$\mathrm{H}$

C

0

0

$\mathrm{H}$

0

$\mathrm{H}$

C

C

C

C

$\mathrm{H}$

$\mathrm{H}$

$\mathrm{H}$

C

C

C

$\mathrm{H}$

$\mathrm{H}$

$\mathrm{H}$

C

$\mathrm{H}$

$\mathrm{H}$

$\mathrm{H}$

C

$\mathrm{H}$

$\mathrm{H}$

$\mathrm{H}$

C

$\mathrm{H}$

$\mathrm{H}$

$\mathrm{H}$

0

0
1.471512

2.440463

1.033949

$-1.622807$

$-2.280810$

$-0.721218$

$-0.235629$

0.610201

0.999602

$-0.214278$

$-0.975680$

1.817695

1.095471

$-0.746304$

2.892181

1.594521

2. 442099

2.090088

2. 105233

2. 361623

2. 678389

1.041632

3. 899214

4.594864

4.034530

4.168467

3. 232646

3. 544818

4. 099209

2. 897691

0.828933

1.008008

0.527987

$-0.004852$

1.588465

1.809739
0.633407

0.139782

0.697221

1.912902

2.631926

1.053191

0.575710

$-0.176154$

$-1.083578$

$-2.686367$

$-3.017529$

$-3.712977$

$-2.603578$

$-2.007625$

$-3.555371$

$-4.682136$

$-3.170726$

$-4.704721$

$-2.372872$

$-1.317580$

$-2.740859$

$-2.431255$

$-2.912999$

$-3.175013$

$-1.851474$

$-3.495001$

$-5.607360$

$-5.356415$

$-5.532500$

$-6.649853$

$-4.996667$

$-4.868245$

$-6.034066$

$-4.352316$

$-2.709016$

$-5.012984$
0.827324

0.912311

1.831927

$-1.499515$

$-2.216934$

$-1.995860$

$-1.270174$

0.004044

$-0.076632$

3.420366

1.425920

2.445493

2.992581

1.080556

2. 339215

2.896849

$-1.125718$

$-1.187414$

$-2.385691$

$-2.242465$

- 3.244434

$-2.629743$

$-0.715401$

$-1.520151$

$-0.486392$

0.171308

$-1.656386$

$-2.677033$

$-0.995288$

$-1.652486$

$-2.019508$

$-3.092774$

$-1.842526$

$-1.726504$

$-0.042673$

0.188902 


\begin{tabular}{|c|c|c|c|}
\hline B & 1.250687 & -3.858951 & 0.789803 \\
\hline 0 & -0.238838 & -3.994784 & 0.993458 \\
\hline C & -2.388902 & -3.307838 & 1.737352 \\
\hline C & -2.838193 & -4.629111 & 1.894602 \\
\hline C & -3.301496 & -2.247547 & 1.837659 \\
\hline C & -4.182482 & -4.879206 & 2.153702 \\
\hline $\mathrm{H}$ & -2.128331 & -5.444023 & 1.795672 \\
\hline C & -4.647677 & -2.503634 & 2.091031 \\
\hline $\mathrm{H}$ & -2.959307 & -1.224753 & 1.704562 \\
\hline C & -5.089522 & -3.818549 & 2.252968 \\
\hline $\mathrm{H}$ & -4.528163 & -5.902643 & 2.270923 \\
\hline $\mathrm{H}$ & -5.351027 & -1.677965 & 2.147004 \\
\hline $\mathrm{H}$ & -6.139410 & -4.018850 & 2.449103 \\
\hline $\mathrm{H}$ & 1.510137 & -1.607958 & 2.831024 \\
\hline $\mathrm{H}$ & -0.607862 & -3.634607 & 3.774746 \\
\hline $\mathrm{H}$ & -0.740790 & -1.799395 & 3.761511 \\
\hline C & -0.779375 & 2.460209 & 0.804067 \\
\hline C & -1.792622 & 1.940837 & 0.009412 \\
\hline C & -3.058172 & 1.560588 & 0.571183 \\
\hline C & -3.248262 & 1.725896 & 1.930405 \\
\hline $\mathrm{H}$ & -4.190448 & 1.417033 & 2.376464 \\
\hline C & 1.637243 & 2.028795 & 0.248522 \\
\hline C & 0.533862 & 2.880034 & 0.201073 \\
\hline C & 2.980414 & 3.704412 & -0.889456 \\
\hline C & 2.893233 & 2.457370 & -0.298585 \\
\hline $\mathrm{H}$ & 3.935366 & 4.033625 & -1.291345 \\
\hline C & 1.878136 & 4.585644 & -0.962541 \\
\hline C & 0.626783 & 4.175637 & -0.403451 \\
\hline C & -0.471249 & 5.078840 & -0.472428 \\
\hline C & -0.331668 & 6.316275 & -1.058285 \\
\hline C & 0.909824 & 6.719653 & -1.606903 \\
\hline C & 1.990067 & 5.870504 & -1.559995 \\
\hline $\mathrm{H}$ & -1.432718 & 4.776464 & -0.073707 \\
\hline $\mathrm{H}$ & -1.184305 & 6.987714 & -1.107379 \\
\hline $\mathrm{H}$ & 1.003303 & 7.699115 & -2.068158 \\
\hline $\mathrm{H}$ & 2.948097 & 6.167611 & -1.980068 \\
\hline C & -2.252657 & 2.275913 & 2.774401 \\
\hline C & -0.994436 & 2.656378 & 2.209361 \\
\hline C & -0.012321 & 3.223011 & 3.068675 \\
\hline C & -0.257706 & 3.389442 & 4.414293 \\
\hline C & -1.498472 & 2.999451 & 4.972085 \\
\hline C & -2.472725 & 2.455232 & 4.166873 \\
\hline $\mathrm{H}$ & 0.939137 & 3.531377 & 2.648302 \\
\hline $\mathrm{H}$ & 0.505162 & 3.827230 & 5.052454 \\
\hline $\mathrm{H}$ & -1.680253 & 3.135839 & 6.034688 \\
\hline $\mathrm{H}$ & -3.432235 & 2.158603 & 4.584245 \\
\hline C & 6.551516 & 0.230253 & -0.187804 \\
\hline C & 6.034180 & 0.630434 & -1.428513 \\
\hline C & 4.819409 & 1.329279 & -1.434978 \\
\hline C & 4.139082 & 1.632571 & -0.248755 \\
\hline C & 4.698492 & 1.225476 & 0.970199 \\
\hline C & 5.905207 & 0.516093 & 1.024495 \\
\hline $\mathrm{H}$ & 7.484733 & -0.315216 & -0.164028 \\
\hline $\mathrm{H}$ & 4.378814 & 1.651583 & -2.373898 \\
\hline
\end{tabular}




\begin{tabular}{|c|c|c|c|}
\hline $\mathrm{H}$ & 4.188573 & 1.498241 & 1.890236 \\
\hline C & 6.758112 & 0.347685 & -2.759864 \\
\hline C & 6.483875 & 0.099235 & 2.391497 \\
\hline C & 7.130945 & 1.692784 & -3.428709 \\
\hline $\mathrm{H}$ & 7.643436 & 1.516107 & -4.382335 \\
\hline H & 6.244947 & 2.302615 & -3.633280 \\
\hline $\mathrm{H}$ & 7.799483 & 2.277968 & -2.786809 \\
\hline C & 8.051751 & -0.466427 & -2.567420 \\
\hline $\mathrm{H}$ & 8.521648 & -0.645215 & -3.541134 \\
\hline $\mathrm{H}$ & 8.780796 & 0.063207 & -1.943677 \\
\hline $\mathrm{H}$ & 7.855190 & -1.443064 & -2.110561 \\
\hline C & 5.827204 & -0.449094 & -3.704008 \\
\hline $\mathrm{H}$ & 6.334475 & -0.652385 & -4.655007 \\
\hline $\mathrm{H}$ & 5.545267 & -1.409220 & -3.257285 \\
\hline $\mathrm{H}$ & 4.905867 & 0.097198 & -3.930116 \\
\hline C & 6.774680 & 1.364901 & 3.233051 \\
\hline $\mathrm{H}$ & 7.183930 & 1.087133 & 4.212261 \\
\hline $\mathrm{H}$ & 7.504099 & 2.010219 & 2.730448 \\
\hline $\mathrm{H}$ & 5.869513 & 1.956469 & 3.405152 \\
\hline C & 5.458924 & -0.786049 & 3.140173 \\
\hline $\mathrm{H}$ & 5.857838 & -1.091120 & 4.115260 \\
\hline $\mathrm{H}$ & 4.516724 & -0.257177 & 3.319416 \\
\hline $\mathrm{H}$ & 5.232216 & -1.692756 & 2.567261 \\
\hline C & 7.794335 & -0.700057 & 2.259650 \\
\hline $\mathrm{H}$ & 8.156167 & -0.979839 & 3.255496 \\
\hline $\mathrm{H}$ & 7.652592 & -1.623740 & 1.687237 \\
\hline $\mathrm{H}$ & 8.584082 & -0.114709 & 1.775447 \\
\hline C & -4.149943 & 0.966449 & -0.251162 \\
\hline C & -3.887580 & -0.041614 & -1.186824 \\
\hline C & -5.474768 & 1.383032 & -0.064819 \\
\hline C & -4.909785 & -0.626704 & -1.942035 \\
\hline $\mathrm{H}$ & -2.863819 & -0.371105 & -1.328316 \\
\hline C & -6.530018 & 0.819338 & -0.792143 \\
\hline $\mathrm{H}$ & -5.670117 & 2.185914 & 0.639930 \\
\hline C & -6.222078 & -0.183600 & -1.723104 \\
\hline $\mathrm{H}$ & -7.023963 & -0.624748 & -2.298949 \\
\hline C & -7.968088 & 1.324565 & -0.560003 \\
\hline C & -4.556773 & -1.711043 & -2.978892 \\
\hline C & -8.042425 & 2.835990 & -0.883374 \\
\hline $\mathrm{H}$ & -9.059712 & 3.211640 & -0.717186 \\
\hline $\mathrm{H}$ & -7.363227 & 3.421873 & -0.255674 \\
\hline $\mathrm{H}$ & -7.774671 & 3.025799 & -1.928718 \\
\hline C & -9.000118 & 0.596696 & -1.442070 \\
\hline $\mathrm{H}$ & -10.002371 & 0.990129 & -1.237432 \\
\hline $\mathrm{H}$ & -8.800003 & 0.743461 & -2.509274 \\
\hline $\mathrm{H}$ & -9.021154 & -0.480915 & -1.242795 \\
\hline C & -8.360988 & 1.099112 & 0.919667 \\
\hline $\mathrm{H}$ & -8.331133 & 0.033144 & 1.174753 \\
\hline $\mathrm{H}$ & -7.688790 & 1.626669 & 1.604586 \\
\hline $\mathrm{H}$ & -9.378834 & 1.463781 & 1.105583 \\
\hline C & -3.590835 & -1.111609 & -4.029853 \\
\hline $\mathrm{H}$ & -4.061268 & -0.276010 & -4.560031 \\
\hline $\mathrm{H}$ & -2.669421 & -0.733549 & -3.575998 \\
\hline $\mathrm{H}$ & -3.316150 & -1.873917 & -4.769839 \\
\hline
\end{tabular}




\begin{tabular}{|llll|}
$\mathrm{C}$ & -3.870697 & -2.903062 & -2.270401 \\
$\mathrm{H}$ & -2.949328 & -2.600292 & -1.762434 \\
$\mathrm{H}$ & -4.532161 & -3.350675 & -1.519917 \\
$\mathrm{H}$ & -3.608469 & -3.678433 & -3.000787 \\
$\mathrm{C}$ & -5.797701 & -2.246496 & -3.717712 \\
$\mathrm{H}$ & -5.491783 & -3.007929 & -4.444208 \\
$\mathrm{H}$ & -6.514036 & -2.713859 & -3.031827 \\
$\mathrm{H}$ & -6.317223 & -1.455122 & -4.269808 \\
\hline
\end{tabular}

\section{C-Catalyzed reaction TS 4}

Datum

Value

\begin{tabular}{lc}
\hline B3LYP/6-31G(d) Energy & -3038.970007 \\
\hline B3LYP-D3(BJ)/def2-TZVPP-IEF-PCM(DCM) Energy & -3040.523642 \\
\hline B3LYP-D3(BJ)/def2-TZVPP-IEF-PCM(DCM)//B3LYP/6-31G(d) Free Energy (Quasiharmonic) & -3039.294315 \\
\hline
\end{tabular}

Number of Imaginary Frequencies

Frequencies (Top 3 out of 450)

$\begin{array}{rr}\text { 1. } & -324.6697 \mathrm{~cm}^{-1} \\ \text { 2. } & 9.2187 \mathrm{~cm}^{-1} \\ \text { 3. } & 10.1996 \mathrm{~cm}^{-1}\end{array}$

\section{B3LYP/6-31G(d) Molecular Geometry in Cartesian Coordinates}

C

$\mathrm{H}$

$\mathrm{H}$

C

0

0

$\mathrm{H}$

0

$\mathrm{H}$

C

C

C

C

$\mathrm{H}$

$\mathrm{H}$

$\mathrm{H}$

C

C

-1.451930
-2.425659
-0.911769
1.735262
2.437364
0.931625
0.377498
-0.707254
-0.332916
1.827117
-0.193172
2.213042
2.395533
-0.040902
2.161870
2.887894
-0.407328
0.258233

-0.329987
0.168346
0.045984
-2.475095
-3.434945
-1.949818
-1.201224
-0.010091
0.901446
3.557950
3.871417
2.893170
3.775080
4.926858
1.831716
3.074094
3.431673
4.827624

0.122736

0.085473

0.998351

$-0.996352$

$-1.224274$

$-1.927511$

$-1.568835$

$-1.067650$

$-0.969765$

3. 174080

2.411995

0.818423

1.935968

2.181980

1.069746

$-0.020218$

$-1.873688$

$-1.567988$ 


\begin{tabular}{|c|c|c|c|}
\hline C & -0.172815 & 2.892942 & -3.284661 \\
\hline $\mathrm{H}$ & -0.626830 & 1.901174 & -3.381665 \\
\hline $\mathrm{H}$ & -0.631130 & 3.548197 & -4.034435 \\
\hline $\mathrm{H}$ & 0.891601 & 2.796671 & -3.510045 \\
\hline C & -1.908248 & 3.396547 & -1.542760 \\
\hline $\mathrm{H}$ & -2.492978 & 3.987821 & -2.255638 \\
\hline $\mathrm{H}$ & -2.258989 & 2.360745 & -1.591060 \\
\hline $\mathrm{H}$ & -2.104112 & 3.773846 & -0.534958 \\
\hline C & -0.659220 & 6.032974 & -1.786758 \\
\hline $\mathrm{H}$ & -0.976836 & 6.105680 & -2.833610 \\
\hline $\mathrm{H}$ & -1.548928 & 5.980745 & -1.154742 \\
\hline $\mathrm{H}$ & -0.120710 & 6.952608 & -1.534019 \\
\hline C & 1.577343 & 5.044016 & -2.328027 \\
\hline $\mathrm{H}$ & 1.412430 & 5.213980 & -3.397667 \\
\hline $\mathrm{H}$ & 2.078750 & 5.925451 & -1.915113 \\
\hline $\mathrm{H}$ & 2.248766 & 4.187522 & -2.215503 \\
\hline 0 & 0.278487 & 2.558469 & -0.934919 \\
\hline 0 & 0.566340 & 4.737050 & -0.165727 \\
\hline B & 0.646272 & 3.356656 & 0.205376 \\
\hline 0 & -0.239933 & 3.054202 & 1.405953 \\
\hline C & -0.967216 & 3.560609 & 3.627112 \\
\hline C & -1.283883 & 4.601909 & 4.513558 \\
\hline C & -1.416435 & 2.259383 & 3.899950 \\
\hline C & -2.041600 & 4.348328 & 5.654578 \\
\hline $\mathrm{H}$ & -0.940051 & 5.611635 & 4.300644 \\
\hline C & -2.170775 & 2.009927 & 5.042693 \\
\hline $\mathrm{H}$ & -1.169627 & 1.454075 & 3.216356 \\
\hline C & -2.485858 & 3.051074 & 5.921306 \\
\hline $\mathrm{H}$ & -2.288059 & 5.160544 & 6.332608 \\
\hline $\mathrm{H}$ & -2.512565 & 0.999800 & 5.248553 \\
\hline $\mathrm{H}$ & -3.077163 & 2.851859 & 6.810886 \\
\hline $\mathrm{H}$ & 2.776255 & 4.773936 & 1.720795 \\
\hline $\mathrm{H}$ & 1.593433 & 2.546508 & 3.494903 \\
\hline $\mathrm{H}$ & 1.933875 & 4.293025 & 3.967260 \\
\hline C & 0.664700 & -1.973442 & 1.227150 \\
\hline C & 1.750714 & -1.821233 & 0.376184 \\
\hline C & 2.971209 & -1.215421 & 0.831299 \\
\hline C & 3.051478 & -0.797263 & 2.145085 \\
\hline $\mathrm{H}$ & 3.959886 & -0.312264 & 2.493450 \\
\hline C & -1.637888 & -1.829238 & 0.232776 \\
\hline C & -0.609597 & -2.614086 & 0.750237 \\
\hline C & -3.031978 & -3.803002 & -0.008304 \\
\hline C & -2.868710 & -2.440720 & -0.174910 \\
\hline H & -3.967143 & -4.265555 & -0.314073 \\
\hline C & -2.007189 & -4.627811 & 0.511330 \\
\hline C & -0.761869 & -4.031667 & 0.886626 \\
\hline C & 0.269043 & -4.875038 & 1.387854 \\
\hline C & 0.070358 & -6.230712 & 1.518923 \\
\hline C & -1.166700 & -6.816317 & 1.155769 \\
\hline C & -2.180755 & -6.030695 & 0.660165 \\
\hline $\mathrm{H}$ & 1.227454 & -4.440547 & 1.649416 \\
\hline $\mathrm{H}$ & 0.872506 & -6.859109 & 1.895857 \\
\hline $\mathrm{H}$ & -1.308166 & -7.888467 & 1.262815 \\
\hline $\mathrm{H}$ & -3.131511 & -6.471255 & 0.369232 \\
\hline
\end{tabular}




\begin{tabular}{|c|c|c|c|}
\hline C & 1.979791 & -0.963358 & 3.056936 \\
\hline C & 0.762453 & -1.559434 & 2.598643 \\
\hline C & -0.299032 & -1.729839 & 3.530583 \\
\hline C & -0.163684 & -1.326999 & 4.842099 \\
\hline C & 1.036632 & -0.724895 & 5.288992 \\
\hline C & 2.083443 & -0.547786 & 4.412560 \\
\hline $\mathrm{H}$ & -1.220463 & -2.195976 & 3.198117 \\
\hline $\mathrm{H}$ & -0.981392 & -1.480274 & 5.541515 \\
\hline $\mathrm{H}$ & 1.131108 & -0.410631 & 6.324840 \\
\hline $\mathrm{H}$ & 3.014359 & -0.096273 & 4.747974 \\
\hline C & -6.176346 & -0.397746 & -1.940586 \\
\hline C & -6.361620 & -1.043741 & -0.710728 \\
\hline C & -5.254211 & -1.686468 & -0.144976 \\
\hline C & -4.000993 & -1.669319 & -0.772545 \\
\hline C & -3.852773 & -0.998180 & -1.994785 \\
\hline C & -4.942220 & -0.364832 & -2.606281 \\
\hline $\mathrm{H}$ & -7.027398 & 0.095310 & -2.401359 \\
\hline $\mathrm{H}$ & -5.343568 & -2.208705 & 0.800920 \\
\hline $\mathrm{H}$ & -2.876090 & -0.996639 & -2.463740 \\
\hline C & -7.748717 & -1.029659 & -0.039013 \\
\hline C & -4.835624 & 0.337124 & -3.974649 \\
\hline C & -8.785144 & -1.691780 & -0.977749 \\
\hline $\mathrm{H}$ & -9.779232 & -1.686547 & -0.513736 \\
\hline $\mathrm{H}$ & -8.862358 & -1.167097 & -1.935661 \\
\hline $\mathrm{H}$ & -8.513687 & -2.732481 & -1.188698 \\
\hline C & -7.761520 & -1.793588 & 1.298881 \\
\hline $\mathrm{H}$ & -8.765858 & -1.754859 & 1.736034 \\
\hline $\mathrm{H}$ & -7.498756 & -2.849535 & 1.168925 \\
\hline $\mathrm{H}$ & -7.067764 & -1.355876 & 2.025647 \\
\hline C & -8.171583 & 0.432020 & 0.240729 \\
\hline $\mathrm{H}$ & -9.160955 & 0.459777 & 0.714097 \\
\hline $\mathrm{H}$ & -7.459246 & 0.923510 & 0.913403 \\
\hline $\mathrm{H}$ & -8.225092 & 1.024692 & -0.678491 \\
\hline C & -5.826828 & -0.316593 & -4.967273 \\
\hline $\mathrm{H}$ & -5.756957 & 0.167527 & -5.949214 \\
\hline $\mathrm{H}$ & -5.604370 & -1.381736 & -5.097275 \\
\hline $\mathrm{H}$ & -6.864451 & -0.230867 & -4.627763 \\
\hline C & -5.185786 & 1.835518 & -3.816704 \\
\hline $\mathrm{H}$ & -5.120802 & 2.346875 & -4.785260 \\
\hline $\mathrm{H}$ & -6.201227 & 1.979485 & -3.432124 \\
\hline $\mathrm{H}$ & -4.493756 & 2.328705 & -3.124942 \\
\hline C & -3.422731 & 0.231388 & -4.578775 \\
\hline $\mathrm{H}$ & -3.396156 & 0.746910 & -5.545624 \\
\hline $\mathrm{H}$ & -2.666553 & 0.693231 & -3.935242 \\
\hline $\mathrm{H}$ & -3.129908 & -0.810298 & -4.750565 \\
\hline C & 4.129304 & -0.995364 & -0.078714 \\
\hline C & 5.429071 & -1.321736 & 0.343639 \\
\hline C & 3.949258 & -0.428099 & -1.341752 \\
\hline C & 6.535348 & -1.095441 & -0.476482 \\
\hline $\mathrm{H}$ & 5.550547 & -1.784208 & 1.316235 \\
\hline C & 5.032281 & -0.190234 & -2.200631 \\
\hline $\mathrm{H}$ & 2.945111 & -0.169911 & -1.660741 \\
\hline C & 6.309191 & -0.527385 & -1.743836 \\
\hline $\mathrm{H}$ & 7.161681 & -0.354417 & -2.390264 \\
\hline
\end{tabular}




\begin{tabular}{|c|c|c|c|}
\hline C & 4.770761 & 0.410841 & -3.594270 \\
\hline C & 7.972370 & -1.455301 & -0.052380 \\
\hline C & 6.066607 & 0.617497 & -4.400720 \\
\hline $\mathrm{H}$ & 6.756284 & 1.306699 & -3.899288 \\
\hline H & 6.592938 & -0.327769 & -4.575160 \\
\hline $\mathrm{H}$ & 5.826419 & 1.045384 & -5.380736 \\
\hline C & 3.850511 & -0.543313 & -4.393862 \\
\hline $\mathrm{H}$ & 3.639987 & -0.124990 & -5.386331 \\
\hline $\mathrm{H}$ & 4.328822 & -1.519587 & -4.530267 \\
\hline H & 2.894760 & -0.716093 & -3.889701 \\
\hline C & 4.078411 & 1.786044 & -3.440621 \\
\hline $\mathrm{H}$ & 3.127414 & 1.705667 & -2.903357 \\
\hline $\mathrm{H}$ & 4.717580 & 2.487208 & -2.890388 \\
\hline $\mathrm{H}$ & 3.870267 & 2.219878 & -4.426648 \\
\hline C & 8.563591 & -2.482038 & -1.047703 \\
\hline $\mathrm{H}$ & 7.963934 & -3.398930 & -1.065669 \\
\hline $\mathrm{H}$ & 8.598979 & -2.087356 & -2.068419 \\
\hline H & 9.587558 & -2.748673 & -0.757592 \\
\hline C & 8.846505 & -0.178431 & -0.054743 \\
\hline $\mathrm{H}$ & 8.886278 & 0.287334 & -1.044943 \\
\hline H & 8.455350 & 0.565730 & 0.648739 \\
\hline $\mathrm{H}$ & 9.874878 & -0.419402 & 0.241828 \\
\hline C & 8.031321 & -2.071772 & 1.358267 \\
\hline $\mathrm{H}$ & 9.070458 & -2.307347 & 1.615273 \\
\hline $\mathrm{H}$ & 7.650726 & -1.382909 & 2.121271 \\
\hline H & 7.456603 & -3.002581 & 1.420154 \\
\hline
\end{tabular}

\section{C-Catalyzed reaction TS 5}

Datum

Value

\begin{tabular}{lc}
\hline B3LYP/6-31G(d) Energy & -3038.965408 \\
\hline B3LYP-D3(BJ)/def2-TZVPP-IEF-PCM(DCM) Energy & -3040.522101 \\
\hline B3LYP-D3(BJ)/def2-TZVPP-IEF-PCM(DCM)//B3LYP/6-31G(d) Free Energy (Quasiharmonic) & -3039.293647 \\
\hline
\end{tabular}

Number of Imaginary Frequencies

Frequencies (Top 3 out of 450)

$$
\begin{array}{lr}
\text { 1. } & -329.0569 \mathrm{~cm}^{-1} \\
\text { 2. } & 6.5483 \mathrm{~cm}^{-1} \\
\text { 3. } & 8.2466 \mathrm{~cm}^{-1}
\end{array}
$$

\section{B3LYP/6-31G(d) Molecular Geometry in Cartesian Coordinates}




\begin{tabular}{|c|c|c|c|}
\hline C & -1.862888 & 0.479141 & -0.756796 \\
\hline $\mathrm{H}$ & -2.817374 & -0.031082 & -0.917483 \\
\hline $\mathrm{H}$ & -1.309355 & 0.465955 & -1.704207 \\
\hline C & 1.051563 & 2.248739 & 1.399922 \\
\hline 0 & 1.613105 & 3.113090 & 2.034262 \\
\hline 0 & 0.227563 & 1.367600 & 1.983381 \\
\hline $\mathrm{H}$ & -0.195597 & 0.757805 & 1.320577 \\
\hline 0 & -1.112551 & -0.206733 & 0.256597 \\
\hline $\mathrm{H}$ & -0.900878 & -1.124132 & -0.047028 \\
\hline C & 1.879315 & -1.918384 & -3.254846 \\
\hline C & 2.163618 & -2.919614 & -1.357543 \\
\hline C & -0.356951 & -2.970491 & -3.074033 \\
\hline C & 0.515161 & -1.831460 & -3.074056 \\
\hline $\mathrm{H}$ & 1.884531 & -2.052571 & -0.755055 \\
\hline $\mathrm{H}$ & -1.420970 & -2.733164 & -3.129104 \\
\hline $\mathrm{H}$ & -0.085442 & -3.758749 & -3.779863 \\
\hline C & -1.684681 & -3.633823 & 0.307872 \\
\hline C & -1.358524 & -5.116170 & -0.116517 \\
\hline C & -1.542686 & -3.335611 & 1.801266 \\
\hline $\mathrm{H}$ & -1.758485 & -2.278558 & 1.989267 \\
\hline $\mathrm{H}$ & -2.247639 & -3.936039 & 2.388303 \\
\hline $\mathrm{H}$ & -0.530732 & -3.541668 & 2.158007 \\
\hline C & -3.062132 & -3.171639 & -0.189491 \\
\hline $\mathrm{H}$ & -3.872824 & -3.687000 & 0.335830 \\
\hline $\mathrm{H}$ & -3.178646 & -2.099947 & -0.004895 \\
\hline $\mathrm{H}$ & -3.177095 & -3.358022 & -1.261675 \\
\hline C & -2.570337 & -6.047985 & -0.184622 \\
\hline $\mathrm{H}$ & -3.053691 & -6.147617 & 0.794510 \\
\hline $\mathrm{H}$ & -3.308822 & -5.692562 & -0.906922 \\
\hline $\mathrm{H}$ & -2.243972 & -7.043770 & -0.502512 \\
\hline C & -0.266110 & -5.764024 & 0.753463 \\
\hline $\mathrm{H}$ & -0.630237 & -5.991280 & 1.761652 \\
\hline $\mathrm{H}$ & 0.045886 & -6.700821 & 0.280995 \\
\hline $\mathrm{H}$ & 0.615458 & -5.122864 & 0.839182 \\
\hline 0 & -0.669885 & -2.873077 & -0.409974 \\
\hline 0 & -0.848246 & -4.949987 & -1.449653 \\
\hline B & -0.174345 & -3.705573 & -1.499717 \\
\hline 0 & 1.328998 & -3.906755 & -1.426310 \\
\hline C & 3.605319 & -3.225994 & -1.454414 \\
\hline C & 4.043677 & -4.468285 & -1.940906 \\
\hline C & 4.545396 & -2.277174 & -1.025291 \\
\hline C & 5.405300 & -4.750356 & -2.000186 \\
\hline $\mathrm{H}$ & 3.308284 & -5.202541 & -2.253624 \\
\hline C & 5.907788 & -2.566973 & -1.083103 \\
\hline $\mathrm{H}$ & 4.210835 & -1.319121 & -0.636704 \\
\hline C & 6.340038 & -3.801351 & -1.573243 \\
\hline $\mathrm{H}$ & 5.740683 & -5.714022 & -2.373691 \\
\hline $\mathrm{H}$ & 6.626929 & -1.829936 & -0.737603 \\
\hline $\mathrm{H}$ & 7.402074 & -4.027132 & -1.617452 \\
\hline $\mathrm{H}$ & 0.127287 & -0.896110 & -2.669909 \\
\hline $\mathrm{H}$ & 2.300863 & -2.750043 & -3.812028 \\
\hline $\mathrm{H}$ & 2.498946 & -1.029978 & -3.180167 \\
\hline C & 0.257260 & 2.463180 & -0.977126 \\
\hline
\end{tabular}




\begin{tabular}{|c|c|c|c|}
\hline C & 1.271513 & 2.109824 & -0.097015 \\
\hline C & 2.582869 & 1.768033 & -0.572254 \\
\hline C & 2.816292 & 1.794560 & -1.934174 \\
\hline $\mathrm{H}$ & 3.797534 & 1.515825 & -2.310815 \\
\hline C & -2.112991 & 1.913466 & -0.341077 \\
\hline C & -1.097599 & 2.859472 & -0.457988 \\
\hline C & -3.626423 & 3.617546 & 0.494664 \\
\hline C & -3.393454 & 2.296602 & 0.170563 \\
\hline $\mathrm{H}$ & -4.603063 & 3.907754 & 0.874376 \\
\hline C & -2.624030 & 4.608792 & 0.368914 \\
\hline C & -1.326381 & 4.227410 & -0.099057 \\
\hline C & -0.320654 & 5.230042 & -0.194625 \\
\hline C & -0.592072 & 6.536744 & 0.141196 \\
\hline C & -1.880172 & 6.912418 & 0.593353 \\
\hline C & -2.872059 & 5.966749 & 0.706664 \\
\hline $\mathrm{H}$ & 0.674642 & 4.951649 & -0.522353 \\
\hline $\mathrm{H}$ & 0.191036 & 7.286114 & 0.066587 \\
\hline $\mathrm{H}$ & -2.078865 & 7.947755 & 0.856908 \\
\hline $\mathrm{H}$ & -3.862541 & 6.242034 & 1.061207 \\
\hline C & 1.816172 & 2.165130 & -2.867157 \\
\hline C & 0.511775 & 2.508864 & -2.388481 \\
\hline C & -0.478256 & 2.888447 & -3.336679 \\
\hline C & -0.195012 & 2.916205 & -4.684749 \\
\hline C & 1.093195 & 2.567516 & -5.155780 \\
\hline C & 2.075243 & 2.200398 & -4.264359 \\
\hline $\mathrm{H}$ & -1.466646 & 3.162373 & -2.982950 \\
\hline $\mathrm{H}$ & -0.965076 & 3.210358 & -5.392653 \\
\hline $\mathrm{H}$ & 1.304272 & 2.593592 & -6.221320 \\
\hline $\mathrm{H}$ & 3.069974 & 1.936547 & -4.616272 \\
\hline C & -6.614871 & -0.414626 & 0.889370 \\
\hline C & -5.620301 & -0.277553 & 1.868610 \\
\hline C & -4.551883 & 0.585448 & 1.591359 \\
\hline C & -4.496822 & 1.306149 & 0.391711 \\
\hline C & -5.513571 & 1.147597 & -0.557668 \\
\hline C & -6.586950 & 0.276339 & -0.330299 \\
\hline $\mathrm{H}$ & -7.450004 & -1.080280 & 1.087292 \\
\hline $\mathrm{H}$ & -3.753953 & 0.729353 & 2.310758 \\
\hline $\mathrm{H}$ & -5.453202 & 1.721694 & -1.475705 \\
\hline C & -5.753348 & -1.031463 & 3.206628 \\
\hline C & -7.719703 & 0.066028 & -1.353768 \\
\hline C & -5.880634 & -2.551189 & 2.949612 \\
\hline $\mathrm{H}$ & -5.987619 & -3.089235 & 3.899435 \\
\hline $\mathrm{H}$ & -6.752200 & -2.792569 & 2.332200 \\
\hline $\mathrm{H}$ & -4.991587 & -2.937472 & 2.439082 \\
\hline C & -4.542353 & -0.802279 & 4.131161 \\
\hline $\mathrm{H}$ & -4.677562 & -1.365831 & 5.061298 \\
\hline $\mathrm{H}$ & -3.607066 & -1.140581 & 3.671534 \\
\hline $\mathrm{H}$ & -4.427002 & 0.253406 & 4.400272 \\
\hline C & -7.020842 & -0.532532 & 3.941935 \\
\hline $\mathrm{H}$ & -7.136859 & -1.053697 & 4.900310 \\
\hline $\mathrm{H}$ & -6.957163 & 0.542428 & 4.145614 \\
\hline $\mathrm{H}$ & -7.927926 & -0.707930 & 3.353735 \\
\hline C & -9.071129 & 0.475683 & -0.721479 \\
\hline $\mathrm{H}$ & -9.889009 & 0.326604 & -1.437234 \\
\hline
\end{tabular}




\begin{tabular}{|c|c|c|c|}
\hline H & -9.299933 & -0.114117 & 0.172327 \\
\hline H & -9.062802 & 1.532374 & -0.430602 \\
\hline C & -7.518584 & 0.902071 & -2.632083 \\
\hline $\mathrm{H}$ & -8.342927 & 0.714085 & -3.329273 \\
\hline $\mathrm{H}$ & -7.505239 & 1.977063 & -2.419418 \\
\hline $\mathrm{H}$ & -6.585913 & 0.643641 & -3.146397 \\
\hline C & -7.775517 & -1.425866 & -1.760331 \\
\hline $\mathrm{H}$ & -8.583017 & -1.595171 & -2.483285 \\
\hline $\mathrm{H}$ & -6.833234 & -1.740994 & -2.223119 \\
\hline H & -7.957598 & -2.077251 & -0.899196 \\
\hline C & 3.682033 & 1.368767 & 0.351703 \\
\hline C & 3.457450 & 0.453941 & 1.390436 \\
\hline C & 4.973033 & 1.884407 & 0.166379 \\
\hline C & 4.487660 & 0.057419 & 2.249790 \\
\hline $\mathrm{H}$ & 2.459325 & 0.057945 & 1.528791 \\
\hline C & 6.033994 & 1.509177 & 0.997967 \\
\hline $\mathrm{H}$ & 5.125559 & 2.613697 & -0.620370 \\
\hline C & 5.763232 & 0.595459 & 2.027394 \\
\hline $\mathrm{H}$ & 6.572474 & 0.301985 & 2.689557 \\
\hline C & 7.458166 & 2.075192 & 0.832257 \\
\hline C & 4.253383 & -0.914608 & 3.421929 \\
\hline C & 7.877530 & 2.805918 & 2.130085 \\
\hline $\mathrm{H}$ & 8.888521 & 3.218566 & 2.025322 \\
\hline $\mathrm{H}$ & 7.192806 & 3.631940 & 2.351814 \\
\hline $\mathrm{H}$ & 7.880051 & 2.134265 & 2.994718 \\
\hline C & 8.445444 & 0.915994 & 0.556855 \\
\hline $\mathrm{H}$ & 9.468406 & 1.299203 & 0.456330 \\
\hline $\mathrm{H}$ & 8.444220 & 0.178766 & 1.366390 \\
\hline $\mathrm{H}$ & 8.188427 & 0.395165 & -0.373660 \\
\hline C & 7.560847 & 3.077260 & -0.333561 \\
\hline $\mathrm{H}$ & 7.301550 & 2.615995 & -1.293548 \\
\hline $\mathrm{H}$ & 6.909027 & 3.945005 & -0.183410 \\
\hline $\mathrm{H}$ & 8.589706 & 3.446350 & -0.412513 \\
\hline C & 4.532280 & -0.173559 & 4.751653 \\
\hline $\mathrm{H}$ & 5.561980 & 0.196550 & 4.801961 \\
\hline $\mathrm{H}$ & 3.861562 & 0.684721 & 4.867835 \\
\hline $\mathrm{H}$ & 4.376832 & -0.847572 & 5.603132 \\
\hline C & 2.806228 & -1.443514 & 3.462758 \\
\hline H & 2.075178 & -0.635700 & 3.575196 \\
\hline $\mathrm{H}$ & 2.554997 & -2.009493 & 2.557615 \\
\hline $\mathrm{H}$ & 2.687616 & -2.120347 & 4.316410 \\
\hline C & 5.206286 & -2.126899 & 3.298448 \\
\hline $\mathrm{H}$ & 5.038402 & -2.827644 & 4.125419 \\
\hline $\mathrm{H}$ & 5.039675 & -2.664706 & 2.358086 \\
\hline $\mathrm{H}$ & 6.259224 & -1.826715 & 3.329362 \\
\hline
\end{tabular}

\section{C-Catalyzed reaction TS 6}




\begin{tabular}{lc}
\hline B3LYP/6-31G(d) Energy & -3038.966949 \\
\hline B3LYP-D3(BJ)/def2-TZVPP-IEF-PCM(DCM) Energy & -3040.522711 \\
\hline B3LYP-D3(BJ)/def2-TZVPP-IEF-PCM(DCM)//B3LYP/6-31G(d) Free Energy (Quasiharmonic) & -3039.29272 \\
\hline
\end{tabular}

Number of Imaginary Frequencies

Frequencies (Top 3 out of 450)

$\begin{array}{rr}\text { 1. } & -292.7495 \mathrm{~cm}^{-1} \\ \text { 2. } & 11.0820 \mathrm{~cm}^{-1} \\ \text { 3. } & 12.7296 \mathrm{~cm}^{-1}\end{array}$

\section{B3LYP/6-31G(d) Molecular Geometry in Cartesian Coordinates}

C

$\mathrm{H}$

$\mathrm{H}$

C

0

0

$\mathrm{H}$

0

$\mathrm{H}$

C

C

C

C

$\mathrm{H}$

$\mathrm{H}$

$\mathrm{H}$

C

C

C

$\mathrm{H}$

$\mathrm{H}$

$\mathrm{H}$

C

$\mathrm{H}$

$\mathrm{H}$

$\mathrm{H}$

C

$\mathrm{H}$

$\mathrm{H}$

$\mathrm{H}$

C

$\mathrm{H}$
$-1.005271$

$-2.043202$

$-0.433711$

2. 359042

3.146673

1. 352435

0.751362

$-0.474796$

$-0.306499$

$-2.478618$

$-2.908800$

$-0.290682$

$-1.138566$

$-2.636519$

0.777669

$-0.523468$

0.431943

0.981938

$-0.694631$

$-1.164760$

$-0.312700$

$-1.463688$

1.495929

2.027751

1.032263

2.228673

2. 377628

3. 161432

2.421385

2.594748

0.990679

1.638262
$-0.839626$

$-0.580143$

$-0.794693$

$-1.527428$

$-2.105521$

$-0.798397$

$-0.451018$

0.112267

0.972686

3.049852

3. 129174

3.868123

2.857372

2.083064

3.663312

4.889027

2.944729

4.395622

2.917036

1.927350

3.102676

3.661943

1.907889

2.197333

0.933271

1.782902

4. 392495

4.106164

3. 707622

5.401564

5.313636

4.916459
0.792172

1.017634

1. 725844

$-1.403187$

$-2.117464$

$-1.907057$

$-1.192712$

$-0.148845$

0.313479

$-2.233361$

$-0.062709$

$-1.410621$

$-1.986120$

0.088020

$-1.502012$

$-1.723717$

2. 206369

1.934907

3. 251890

3. 247120

4.261474

3.029086

2. 561907

3.475673

2. 750114

1.761624

1.287639

1.997528

0.435252

0.923223

3. 159955

3.950860 


\begin{tabular}{|c|c|c|c|}
\hline $\mathrm{H}$ & 1.373613 & 6.298633 & 2.873192 \\
\hline $\mathrm{H}$ & -0.014928 & 5.451861 & 3.564226 \\
\hline 0 & -0.142338 & 2.603769 & 0.915236 \\
\hline 0 & 0.046800 & 4.922225 & 0.982762 \\
\hline B & -0.582454 & 3.849180 & 0.300178 \\
\hline 0 & -2.095272 & 4.038506 & 0.358261 \\
\hline C & -4.345327 & 3.454176 & -0.125224 \\
\hline C & -4.783626 & 4.784745 & -0.028625 \\
\hline C & -5.286522 & 2.420467 & -0.248664 \\
\hline C & -6.144783 & 5.073943 & -0.063729 \\
\hline $\mathrm{H}$ & -4.048383 & 5.574756 & 0.084414 \\
\hline C & -6.648215 & 2.716878 & -0.281584 \\
\hline $\mathrm{H}$ & -4.954392 & 1.387411 & -0.317269 \\
\hline C & -7.080044 & 4.042468 & -0.192163 \\
\hline $\mathrm{H}$ & -6.479375 & 6.104723 & 0.013643 \\
\hline $\mathrm{H}$ & -7.372096 & 1.912252 & -0.374656 \\
\hline $\mathrm{H}$ & -8.141917 & 4.271362 & -0.218150 \\
\hline H & -0.768799 & 1.832295 & -1.999830 \\
\hline $\mathrm{H}$ & -2.870388 & 4.051897 & -2.383972 \\
\hline $\mathrm{H}$ & -3.097134 & 2.235563 & -2.600056 \\
\hline c & 1.517827 & -2.275747 & 0.843565 \\
\hline C & 2.474064 & -1.584950 & 0.109534 \\
\hline C & 3.651600 & -1.051146 & 0.736174 \\
\hline C & 3.805313 & -1.227959 & 2.097973 \\
\hline $\mathrm{H}$ & 4.671441 & -0.797761 & 2.593840 \\
\hline C & -0.917573 & -2.230971 & 0.197044 \\
\hline C & 0.315432 & -2.882053 & 0.170862 \\
\hline C & -1.916093 & -4.062642 & -1.048171 \\
\hline c & -2.060725 & -2.841894 & -0.416486 \\
\hline $\mathrm{H}$ & -2.786105 & -4.529555 & -1.503183 \\
\hline C & -0.679667 & -4.745914 & -1.097547 \\
\hline C & 0.462493 & -4.155364 & -0.470239 \\
\hline C & 1.696998 & -4.862792 & -0.514229 \\
\hline C & 1.788831 & -6.084439 & -1.141181 \\
\hline C & 0.655205 & -6.666371 & -1.758332 \\
\hline C & -0.552212 & -6.009113 & -1.736327 \\
\hline H & 2.577583 & -4.420768 & -0.062750 \\
\hline $\mathrm{H}$ & 2.742353 & -6.604213 & -1.169629 \\
\hline $\mathrm{H}$ & 0.744493 & -7.630414 & -2.251773 \\
\hline $\mathrm{H}$ & -1.429849 & -6.444665 & -2.208160 \\
\hline C & 2.866049 & -1.940178 & 2.882051 \\
\hline C & 1.702678 & -2.484816 & 2.251919 \\
\hline C & 0.782625 & -3.217760 & 3.052086 \\
\hline C & 0.994773 & -3.386826 & 4.402973 \\
\hline c & 2.138932 & -2.833817 & 5.025680 \\
\hline C & 3.052971 & -2.127094 & 4.278539 \\
\hline $\mathrm{H}$ & -0.094078 & -3.651042 & 2.582628 \\
\hline $\mathrm{H}$ & 0.280233 & -3.952158 & 4.995236 \\
\hline $\mathrm{H}$ & 2.294668 & -2.973245 & 6.092031 \\
\hline $\mathrm{H}$ & 3.938931 & -1.703083 & 4.745338 \\
\hline C & -6.075894 & -1.351697 & -0.393977 \\
\hline C & -5.419563 & -1.523094 & -1.622330 \\
\hline C & -4.091064 & -1.968338 & -1.595978 \\
\hline$c$ & -3.432411 & -2.245982 & -0.392014 \\
\hline
\end{tabular}




\begin{tabular}{|c|c|c|c|}
\hline C & -4.123768 & -2.059247 & 0.812611 \\
\hline C & -5.450409 & -1.609647 & 0.836401 \\
\hline $\mathrm{H}$ & -7.105756 & -1.021461 & -0.395079 \\
\hline $\mathrm{H}$ & -3.544784 & -2.112486 & -2.523314 \\
\hline $\mathrm{H}$ & -3.619029 & -2.305097 & 1.742873 \\
\hline C & -6.105062 & -1.257105 & -2.977556 \\
\hline C & -6.173780 & -1.444072 & 2.187909 \\
\hline C & -6.090604 & -2.553444 & -3.823287 \\
\hline $\mathrm{H}$ & -6.572538 & -2.380102 & -4.793040 \\
\hline $\mathrm{H}$ & -5.071882 & -2.904207 & -4.016929 \\
\hline $\mathrm{H}$ & -6.631476 & -3.359348 & -3.314110 \\
\hline C & -7.570847 & -0.808165 & -2.823991 \\
\hline $\mathrm{H}$ & -8.010924 & -0.645672 & -3.814223 \\
\hline $\mathrm{H}$ & -8.179203 & -1.562799 & -2.312583 \\
\hline $\mathrm{H}$ & -7.655570 & 0.134266 & -2.270642 \\
\hline C & -5.339164 & -0.145935 & -3.734662 \\
\hline $\mathrm{H}$ & -5.806676 & 0.045476 & -4.708224 \\
\hline $\mathrm{H}$ & -5.348545 & 0.791757 & -3.166219 \\
\hline $\mathrm{H}$ & -4.294972 & -0.421513 & -3.915343 \\
\hline C & -7.619587 & -0.935658 & 2.028849 \\
\hline $\mathrm{H}$ & -8.083584 & -0.834028 & 3.016273 \\
\hline $\mathrm{H}$ & -7.656425 & 0.048092 & 1.546817 \\
\hline $\mathrm{H}$ & -8.236618 & -1.628338 & 1.445347 \\
\hline C & -6.225090 & -2.810114 & 2.913641 \\
\hline $\mathrm{H}$ & -6.733539 & -2.709153 & 3.880306 \\
\hline $\mathrm{H}$ & -6.771549 & -3.550190 & 2.317957 \\
\hline $\mathrm{H}$ & -5.223725 & -3.209610 & 3.104479 \\
\hline C & -5.404643 & -0.427486 & 3.065196 \\
\hline $\mathrm{H}$ & -5.903372 & -0.306347 & 4.034503 \\
\hline $\mathrm{H}$ & -4.376944 & -0.751593 & 3.259880 \\
\hline $\mathrm{H}$ & -5.361718 & 0.555430 & 2.581866 \\
\hline C & 4.699428 & -0.309336 & -0.020453 \\
\hline C & 4.367094 & 0.677285 & -0.958539 \\
\hline C & 6.052016 & -0.580421 & 0.231743 \\
\hline C & 5.355165 & 1.381108 & -1.655041 \\
\hline $\mathrm{H}$ & 3.321202 & 0.885872 & -1.146876 \\
\hline C & 7.071510 & 0.110131 & -0.431450 \\
\hline $\mathrm{H}$ & 6.293937 & -1.366600 & 0.937124 \\
\hline C & 6.694589 & 1.083194 & -1.367478 \\
\hline $\mathrm{H}$ & 7.472747 & 1.621447 & -1.900918 \\
\hline C & 8.565352 & -0.174336 & -0.180832 \\
\hline C & 5.019457 & 2.439809 & -2.723040 \\
\hline C & 9.260408 & 1.116793 & 0.312538 \\
\hline $\mathrm{H}$ & 10.327873 & 0.933406 & 0.487308 \\
\hline $\mathrm{H}$ & 9.177380 & 1.929398 & -0.416583 \\
\hline $\mathrm{H}$ & 8.817280 & 1.465579 & 1.252537 \\
\hline C & 8.785484 & -1.268943 & 0.880736 \\
\hline $\mathrm{H}$ & 9.859604 & -1.429513 & 1.027345 \\
\hline $\mathrm{H}$ & 8.358398 & -0.988822 & 1.850644 \\
\hline $\mathrm{H}$ & 8.347101 & -2.226178 & 0.577016 \\
\hline C & 9.229506 & -0.644071 & -1.496829 \\
\hline $\mathrm{H}$ & 8.758431 & -1.561863 & -1.866225 \\
\hline $\mathrm{H}$ & 9.150747 & 0.111317 & -2.285479 \\
\hline H & 10.295418 & -0.848132 & -1.335512 \\
\hline
\end{tabular}




$\begin{array}{|llll|}\mathrm{C} & 3.501374 & 2.632858 & -2.897430 \\ \mathrm{H} & 3.000841 & 1.705516 & -3.196394 \\ \mathrm{H} & 3.033696 & 2.992077 & -1.972969 \\ \mathrm{H} & 3.311553 & 3.382083 & -3.674413 \\ \mathrm{C} & 5.636224 & 3.800915 & -2.323236 \\ \mathrm{H} & 5.232435 & 4.148827 & -1.365273 \\ \mathrm{H} & 6.725539 & 3.744030 & -2.225036 \\ \mathrm{H} & 5.410134 & 4.559868 & -3.082432 \\ \mathrm{C} & 5.603010 & 1.990246 & -4.084082 \\ \mathrm{H} & 5.375302 & 2.732191 & -4.859546 \\ \mathrm{H} & 6.691283 & 1.874596 & -4.042835 \\ \mathrm{H} & 5.175376 & 1.030045 & -4.392695 \\ & & \end{array}$

\section{C-Catalyzed reaction TS 7}

\section{Datum}

Value

\begin{tabular}{lc}
\hline B3LYP/6-31G(d) Energy & -3038.966861 \\
\hline B3LYP-D3(BJ)/def2-TZVPP-IEF-PCM(DCM) Energy & -3040.522007 \\
\hline B3LYP-D3(BJ)/def2-TZVPP-IEF-PCM(DCM)//B3LYP/6-31G(d) Free Energy (Quasiharmonic) & -3039.292382 \\
\hline
\end{tabular}

Number of Imaginary Frequencies

Frequencies (Top 3 out of 450)

$\begin{array}{lr}\text { 1. } & -305.4167 \mathrm{~cm}^{-1} \\ \text { 2. } & 8.8467 \mathrm{~cm}^{-1} \\ \text { 3. } & 10.3817 \mathrm{~cm}^{-1}\end{array}$

\section{B3LYP/6-31G(d) Molecular Geometry in Cartesian Coordinates}

$\begin{array}{lrrr}\mathrm{C} & 1.007884 & 0.804349 & 0.846917 \\ \mathrm{H} & 2.052904 & 0.554462 & 1.047115 \\ \mathrm{H} & 0.457464 & 0.735496 & 1.791363 \\ \mathrm{C} & -2.349087 & 1.512604 & -1.364585 \\ \mathrm{O} & -3.116245 & 2.115581 & -2.079705 \\ \mathrm{O} & -1.337707 & 0.786002 & -1.866326 \\ \mathrm{H} & -0.748282 & 0.426845 & -1.148173 \\ \mathrm{O} & 0.471200 & -0.142624 & -0.096178 \\ \mathrm{H} & 0.325556 & -1.010434 & 0.362918 \\ \mathrm{C} & 2.334942 & -3.007989 & -2.320634 \\ \mathrm{C} & 2.890832 & -3.080513 & -0.203056 \\ \mathrm{C} & 0.205045 & -3.863162 & -1.389459 \\ \mathrm{C} & 1.003618 & -2.839507 & -2.008514 \\ \mathrm{H} & 2.612918 & -2.040451 & -0.022679\end{array}$




\begin{tabular}{|c|c|c|c|}
\hline $\mathrm{H}$ & -0.869856 & -3.673534 & -1.412389 \\
\hline $\mathrm{H}$ & 0.431893 & -4.880541 & -1.717940 \\
\hline C & -0.700606 & -2.977275 & 2.059159 \\
\hline C & -0.380708 & -4.503218 & 2.284346 \\
\hline C & -0.441274 & -2.081050 & 3.270719 \\
\hline $\mathrm{H}$ & -0.720003 & -1.045628 & 3.045371 \\
\hline $\mathrm{H}$ & -1.046058 & -2.402881 & 4.126455 \\
\hline $\mathrm{H}$ & 0.611005 & -2.097194 & 3.564897 \\
\hline C & -2.120561 & -2.738522 & 1.528146 \\
\hline $\mathrm{H}$ & -2.876402 & -2.905721 & 2.302964 \\
\hline $\mathrm{H}$ & -2.212995 & -1.701485 & 1.191112 \\
\hline $\mathrm{H}$ & -2.343506 & -3.396991 & 0.683886 \\
\hline C & -1.571401 & -5.343437 & 2.749669 \\
\hline $\mathrm{H}$ & -1.947879 & -4.997781 & 3.719857 \\
\hline $\mathrm{H}$ & -2.388539 & -5.314072 & 2.025376 \\
\hline $\mathrm{H}$ & -1.259000 & -6.387082 & 2.860701 \\
\hline C & 0.811184 & -4.731392 & 3.231780 \\
\hline $\mathrm{H}$ & 0.559865 & -4.490695 & 4.270860 \\
\hline $\mathrm{H}$ & 1.098086 & -5.786663 & 3.182929 \\
\hline $\mathrm{H}$ & 1.679056 & -4.135045 & 2.936308 \\
\hline 0 & 0.235144 & -2.624520 & 1.004092 \\
\hline 0 & -0.008122 & -4.939518 & 0.967546 \\
\hline B & 0.607311 & -3.850541 & 0.302487 \\
\hline 0 & 2.120796 & -4.009664 & 0.260342 \\
\hline C & 4.329213 & -3.379633 & -0.340215 \\
\hline C & 4.792385 & -4.704403 & -0.294879 \\
\hline C & 5.247421 & -2.328013 & -0.480209 \\
\hline C & 6.154931 & -4.970209 & -0.396507 \\
\hline $\mathrm{H}$ & 4.075630 & -5.509147 & -0.167599 \\
\hline C & 6.611176 & -2.600874 & -0.578584 \\
\hline $\mathrm{H}$ & 4.897228 & -1.299106 & -0.507363 \\
\hline C & 7.067461 & -3.920502 & -0.540335 \\
\hline $\mathrm{H}$ & 6.508559 & -5.996844 & -0.358531 \\
\hline $\mathrm{H}$ & 7. 317344 & -1.781945 & -0.681562 \\
\hline $\mathrm{H}$ & 8.130690 & -4.130798 & -0.617593 \\
\hline $\mathrm{H}$ & 0.615820 & -1.821216 & -2.005420 \\
\hline $\mathrm{H}$ & 2.732695 & -4.003069 & -2.498962 \\
\hline $\mathrm{H}$ & 2.916451 & -2.183684 & -2.724042 \\
\hline C & -1.548582 & 2.185074 & 0.920183 \\
\hline C & -2.492859 & 1.521205 & 0.146345 \\
\hline C & -3.675990 & 0.957271 & 0.734500 \\
\hline C & -3.839756 & 1.064582 & 2.102820 \\
\hline $\mathrm{H}$ & -4.709434 & 0.609566 & 2.569448 \\
\hline C & 0.891628 & 2.206761 & 0.282398 \\
\hline C & -0.352581 & 2.837141 & 0.279952 \\
\hline C & 1.853299 & 4.098303 & -0.902059 \\
\hline C & 2.021414 & 2.857929 & -0.315278 \\
\hline $\mathrm{H}$ & 2.713152 & 4.595202 & -1.344330 \\
\hline C & 0.606841 & 4.763897 & -0.919967 \\
\hline C & -0.523050 & 4.131429 & -0.312130 \\
\hline C & -1.768000 & 4.821665 & -0.322923 \\
\hline C & -1.880967 & 6.065777 & -0.900173 \\
\hline C & -0.759435 & 6.688469 & -1.499409 \\
\hline C & 0.457540 & 6.048985 & -1.508658 \\
\hline
\end{tabular}




\begin{tabular}{|c|c|c|c|}
\hline $\mathrm{H}$ & -2.639602 & 4.350434 & 0.116037 \\
\hline $\mathrm{H}$ & -2.842045 & 6.572246 & -0.902906 \\
\hline $\mathrm{H}$ & -0.865561 & 7.669778 & -1.953940 \\
\hline $\mathrm{H}$ & 1.326392 & 6.515856 & -1.966584 \\
\hline C & -2.908644 & 1.739120 & 2.928764 \\
\hline C & -1.746337 & 2.325388 & 2.334655 \\
\hline C & -0.839717 & 3.028232 & 3.176181 \\
\hline C & -1.062033 & 3.127379 & 4.532253 \\
\hline C & -2.203558 & 2.531167 & 5.119190 \\
\hline C & -3.105753 & 1.853656 & 4.331812 \\
\hline $\mathrm{H}$ & 0.035043 & 3.493753 & 2.734486 \\
\hline $\mathrm{H}$ & -0.357532 & 3.670152 & 5.156733 \\
\hline $\mathrm{H}$ & -2.366913 & 2.615092 & 6.190182 \\
\hline $\mathrm{H}$ & -3.990445 & 1.398513 & 4.770841 \\
\hline C & 6.062432 & 1.440769 & -0.391675 \\
\hline C & 5.394936 & 1.665498 & -1.605369 \\
\hline C & 4.058279 & 2.082366 & -1.547216 \\
\hline C & 3.402886 & 2.284976 & -0.326334 \\
\hline C & 4.106297 & 2.048485 & 0.862385 \\
\hline C & 5.440363 & 1.621047 & 0.854041 \\
\hline $\mathrm{H}$ & 7.097932 & 1.129666 & -0.415637 \\
\hline $\mathrm{H}$ & 3.502656 & 2.264917 & -2.462250 \\
\hline $\mathrm{H}$ & 3.605149 & 2.236606 & 1.807753 \\
\hline C & 6.076829 & 1.488983 & -2.976752 \\
\hline C & 6.174148 & 1.392625 & 2.190734 \\
\hline C & 5.328283 & 0.407611 & -3.792333 \\
\hline $\mathrm{H}$ & 5.792675 & 0.283526 & -4.778209 \\
\hline $\mathrm{H}$ & 5.359518 & -0.561532 & -3.280362 \\
\hline $\mathrm{H}$ & 4.277625 & 0.672086 & -3.950528 \\
\hline C & 6.032038 & 2.830479 & -3.747804 \\
\hline $\mathrm{H}$ & 6.514324 & 2.722974 & -4.727046 \\
\hline $\mathrm{H}$ & 5.005444 & 3.169681 & -3.918986 \\
\hline $\mathrm{H}$ & 6.557371 & 3.617669 & -3.194912 \\
\hline C & 7.552359 & 1.062490 & -2.856342 \\
\hline $\mathrm{H}$ & 7.988461 & 0.963088 & -3.856653 \\
\hline $\mathrm{H}$ & 8.149047 & 1.800594 & -2.308355 \\
\hline $\mathrm{H}$ & 7.660077 & 0.093719 & -2.355046 \\
\hline C & 6.206311 & 2.716010 & 2.992515 \\
\hline $\mathrm{H}$ & 6.720847 & 2.568966 & 3.949921 \\
\hline $\mathrm{H}$ & 6.737332 & 3.497869 & 2.437700 \\
\hline $\mathrm{H}$ & 5.199237 & 3.086839 & 3.209588 \\
\hline C & 5.426540 & 0.314844 & 3.011787 \\
\hline $\mathrm{H}$ & 5.935541 & 0.142647 & 3.967962 \\
\hline $\mathrm{H}$ & 4.396349 & 0.612337 & 3.233895 \\
\hline $\mathrm{H}$ & 5.392296 & -0.637438 & 2.470046 \\
\hline C & 7.627087 & 0.918194 & 1.996023 \\
\hline $\mathrm{H}$ & 8.099091 & 0.770242 & 2.973757 \\
\hline $\mathrm{H}$ & 7.677016 & -0.036881 & 1.460466 \\
\hline $\mathrm{H}$ & 8.228854 & 1.652161 & 1.448102 \\
\hline C & -4.722986 & 0.262342 & -0.067669 \\
\hline C & -4.398816 & -0.675986 & -1.049926 \\
\hline C & -6.078337 & 0.533715 & 0.189805 \\
\hline C & -5.390647 & -1.339253 & -1.788374 \\
\hline $\mathrm{H}$ & -3.354011 & -0.891193 & -1.247275 \\
\hline
\end{tabular}




\begin{tabular}{|c|c|c|c|}
\hline C & -7.096733 & -0.111743 & -0.511890 \\
\hline $\mathrm{H}$ & -6.316747 & 1.284660 & 0.933732 \\
\hline C & -6.725370 & -1.044935 & -1.496702 \\
\hline $\mathrm{H}$ & -7.508413 & -1.545612 & -2.054098 \\
\hline C & -8.589761 & 0.169951 & -0.254604 \\
\hline C & -4.976629 & -2.343975 & -2.880741 \\
\hline C & -8.807911 & 1.226940 & 0.844743 \\
\hline $\mathrm{H}$ & -9.881676 & 1.387691 & 0.993844 \\
\hline $\mathrm{H}$ & -8.385290 & 0.910545 & 1.805345 \\
\hline $\mathrm{H}$ & -8.363658 & 2.192075 & 0.576362 \\
\hline C & -9.251265 & 0.688686 & -1.553363 \\
\hline $\mathrm{H}$ & -10.316485 & 0.890333 & -1.384890 \\
\hline $\mathrm{H}$ & -8.776921 & 1.617804 & -1.888663 \\
\hline $\mathrm{H}$ & -9.175039 & -0.037691 & -2.368986 \\
\hline C & -9.289640 & -1.136053 & 0.191388 \\
\hline $\mathrm{H}$ & -9.207279 & -1.922204 & -0.566273 \\
\hline $\mathrm{H}$ & -8.849250 & -1.519471 & 1.119152 \\
\hline $\mathrm{H}$ & -10.357010 & -0.956208 & 0.370727 \\
\hline C & -6.188096 & -2.965009 & -3.601140 \\
\hline $\mathrm{H}$ & -6.833270 & -3.522805 & -2.912201 \\
\hline $\mathrm{H}$ & -6.797481 & -2.204628 & -4.102420 \\
\hline $\mathrm{H}$ & -5.840311 & -3.666944 & -4.367587 \\
\hline C & -4.106587 & -1.619848 & -3.936761 \\
\hline $\mathrm{H}$ & -4.670800 & -0.814764 & -4.420447 \\
\hline $\mathrm{H}$ & -3.209833 & -1.171075 & -3.498144 \\
\hline $\mathrm{H}$ & -3.786203 & -2.326408 & -4.712765 \\
\hline C & -4.161096 & -3.492633 & -2.242069 \\
\hline $\mathrm{H}$ & -4.756742 & -4.030666 & -1.495302 \\
\hline $\mathrm{H}$ & -3.846483 & -4.211376 & -3.008713 \\
\hline $\mathrm{H}$ & -3.259693 & -3.120080 & -1.744455 \\
\hline
\end{tabular}

\section{C-Catalyzed reaction TS 8 (TS-2.2)}

\section{Datum}

Value

\begin{tabular}{lc}
\hline B3LYP/6-31G(d) Energy & -3038.968576 \\
\hline B3LYP-D3(BJ)/def2-TZVPP-IEF-PCM(DCM) Energy & -3040.521233 \\
\hline B3LYP-D3(BJ)/def2-TZVPP-IEF-PCM(DCM)//B3LYP/6-31G(d) Free Energy (Quasiharmonic) & -3039.292341 \\
\hline
\end{tabular}

Number of Imaginary Frequencies

Frequencies (Top 3 out of 450)

$\begin{array}{lr}\text { 1. } & -327.4231 \mathrm{~cm}^{-1} \\ \text { 2. } & 9.2249 \mathrm{~cm}^{-1} \\ \text { 3. } & 10.9013 \mathrm{~cm}^{-1}\end{array}$




\begin{tabular}{|c|c|c|c|}
\hline C & 1.029575 & 0.420713 & -0.252030 \\
\hline $\mathrm{H}$ & 0.630457 & 0.005762 & -1.183287 \\
\hline $\mathrm{H}$ & 2.074954 & 0.106122 & -0.165744 \\
\hline C & -2.457516 & 2.058536 & 0.821728 \\
\hline 0 & -3.245156 & 2.942787 & 1.071519 \\
\hline 0 & -1.628578 & 1.568247 & 1.754808 \\
\hline $\mathrm{H}$ & -0.996155 & 0.895770 & 1.379663 \\
\hline 0 & 0.286437 & -0.110232 & 0.860246 \\
\hline $\mathrm{H}$ & 0.132996 & -1.068031 & 0.670418 \\
\hline C & 4.552407 & -3.561513 & 0.475712 \\
\hline C & 3.350173 & -3.500010 & -1.351156 \\
\hline C & 2.310555 & -3.347338 & 1.505433 \\
\hline C & 3.508189 & -4.080524 & 1.212080 \\
\hline $\mathrm{H}$ & 3.265537 & -4.585231 & -1.426239 \\
\hline $\mathrm{H}$ & 2.447149 & -2.275850 & 1.668014 \\
\hline $\mathrm{H}$ & 1.677713 & -3.794357 & 2.273798 \\
\hline C & -0.966801 & -3.697484 & 0.002895 \\
\hline C & -0.292294 & -4.910709 & -0.741887 \\
\hline C & -1.419027 & -4.049551 & 1.428318 \\
\hline $\mathrm{H}$ & -1.659309 & -3.126249 & 1.965906 \\
\hline $\mathrm{H}$ & -2.313292 & -4.681930 & 1.424090 \\
\hline $\mathrm{H}$ & -0.631748 & -4.570582 & 1.981174 \\
\hline C & -2.112327 & -3.030266 & -0.756512 \\
\hline $\mathrm{H}$ & -2.922846 & -3.744679 & -0.942992 \\
\hline $\mathrm{H}$ & -2.528672 & -2.207113 & -0.167430 \\
\hline $\mathrm{H}$ & -1.778692 & -2.621759 & -1.712820 \\
\hline C & -0.252623 & -4.721252 & -2.268174 \\
\hline $\mathrm{H}$ & -1.245479 & -4.825334 & -2.719103 \\
\hline $\mathrm{H}$ & 0.147828 & -3.739778 & -2.538020 \\
\hline $\mathrm{H}$ & 0.399356 & -5.487437 & -2.701172 \\
\hline C & -0.883462 & -6.280293 & -0.406538 \\
\hline $\mathrm{H}$ & -1.939185 & -6.338155 & -0.696404 \\
\hline $\mathrm{H}$ & -0.340738 & -7.057994 & -0.954587 \\
\hline $\mathrm{H}$ & -0.800692 & -6.502311 & 0.659955 \\
\hline 0 & 0.133871 & -2.756908 & 0.100759 \\
\hline 0 & 1.059304 & -4.866390 & -0.238131 \\
\hline B & 1.365563 & -3.500034 & 0.044906 \\
\hline 0 & 2.294003 & -2.852198 & -0.973865 \\
\hline C & 4.313137 & -2.838467 & -2.249789 \\
\hline C & 4.294209 & -1.448695 & -2.446333 \\
\hline C & 5.250539 & -3.621792 & -2.941097 \\
\hline C & 5.205364 & -0.857026 & -3.317023 \\
\hline $\mathrm{H}$ & 3.559367 & -0.847914 & -1.921076 \\
\hline C & 6.159687 & -3.026058 & -3.811934 \\
\hline $\mathrm{H}$ & 5.261394 & -4.699612 & -2.795100 \\
\hline C & 6.140158 & -1.641805 & -3.999529 \\
\hline $\mathrm{H}$ & 5.185799 & 0.218617 & -3.466861 \\
\hline $\mathrm{H}$ & 6.879872 & -3.639569 & -4.345777 \\
\hline $\mathrm{H}$ & 6.848597 & -1.175527 & -4.678581 \\
\hline $\mathrm{H}$ & 3.474973 & -5.160722 & 1.357505 \\
\hline
\end{tabular}




\begin{tabular}{|c|c|c|c|}
\hline $\mathrm{H}$ & 4.719695 & -2.488435 & 0.452837 \\
\hline $\mathrm{H}$ & 5.407722 & -4.179328 & 0.216612 \\
\hline C & -1.299389 & 1.735072 & -1.385187 \\
\hline C & -2.381590 & 1.443106 & -0.565243 \\
\hline C & -3.508340 & 0.699668 & -1.056638 \\
\hline C & -3.485412 & 0.266037 & -2.368432 \\
\hline H & -4.313401 & -0.329021 & -2.744880 \\
\hline C & 0.953546 & 1.932404 & -0.286543 \\
\hline C & -0.151079 & 2.554554 & -0.862505 \\
\hline C & 1.992961 & 4.096022 & 0.084340 \\
\hline C & 2.032224 & 2.722548 & 0.231574 \\
\hline H & 2.810040 & 4.695564 & 0.477951 \\
\hline C & 0.898084 & 4.757415 & -0.519273 \\
\hline C & -0.210881 & 3.980996 & -0.982996 \\
\hline C & -1.321775 & 4.662317 & -1.554343 \\
\hline C & -1.324550 & 6.033664 & -1.672466 \\
\hline C & -0.220924 & 6.798126 & -1.222497 \\
\hline C & 0.863973 & 6.171843 & -0.655178 \\
\hline H & -2.180067 & 4.088673 & -1.885731 \\
\hline H & -2.184744 & 6.535582 & -2.106730 \\
\hline H & -0.238599 & 7.880377 & -1.319617 \\
\hline H & 1.712310 & 6.749734 & -0.295875 \\
\hline C & -2.408040 & 0.549978 & -3.242387 \\
\hline C & -1.293892 & 1.302249 & -2.752730 \\
\hline C & -0.225956 & 1.589453 & -3.646951 \\
\hline C & -0.254969 & 1.146540 & -4.951314 \\
\hline C & -1.353468 & 0.393514 & -5.429992 \\
\hline C & -2.405352 & 0.103229 & -4.591567 \\
\hline H & 0.617857 & 2.169611 & -3.288229 \\
\hline H & 0.570453 & 1.377821 & -5.619291 \\
\hline H & -1.364395 & 0.049010 & -6.460588 \\
\hline H & -3.256242 & -0.471118 & -4.950626 \\
\hline C & 5.373195 & 1.193615 & 2.416656 \\
\hline C & 5.613640 & 1.921421 & 1.247990 \\
\hline C & 4.498861 & 2.390951 & 0.533808 \\
\hline C & 3.193944 & 2.126349 & 0.956968 \\
\hline C & 2.993611 & 1.373819 & 2.128109 \\
\hline C & 4.074646 & 0.912082 & 2.882455 \\
\hline H & 6.216095 & 0.837754 & 2.997493 \\
\hline H & 4.638043 & 2.973854 & -0.372619 \\
\hline H & 1.975607 & 1.180491 & 2.443031 \\
\hline C & 7.033199 & 2.235712 & 0.736482 \\
\hline C & 3.892139 & 0.160920 & 4.216637 \\
\hline C & 7.197976 & 1.695436 & -0.704060 \\
\hline H & 8.207001 & 1.909092 & -1.077868 \\
\hline H & 7.044356 & 0.610786 & -0.739335 \\
\hline H & 6.484221 & 2.155951 & -1.395261 \\
\hline C & 7.247898 & 3.768033 & 0.732598 \\
\hline H & 8.249611 & 4.013835 & 0.358559 \\
\hline H & 6.518493 & 4.278095 & 0.094712 \\
\hline $\mathrm{H}$ & 7.151821 & 4.178446 & 1.744230 \\
\hline C & 8.130717 & 1.600613 & 1.611114 \\
\hline $\mathrm{H}$ & 9.116868 & 1.840237 & 1.197245 \\
\hline H & 8.105024 & 1.979938 & 2.638850 \\
\hline
\end{tabular}




\begin{tabular}{|c|c|c|c|}
\hline $\mathrm{H}$ & 8.042880 & 0.508683 & 1.647025 \\
\hline C & 4.478397 & 1.022564 & 5.361386 \\
\hline $\mathrm{H}$ & 4.359234 & 0.510848 & 6.324504 \\
\hline $\mathrm{H}$ & 5.546326 & 1.218764 & 5.216338 \\
\hline $\mathrm{H}$ & 3.966497 & 1.989420 & 5.424419 \\
\hline C & 2.409957 & -0.114749 & 4.534717 \\
\hline $\mathrm{H}$ & 2.333494 & -0.677214 & 5.472377 \\
\hline $\mathrm{H}$ & 1.839937 & 0.812235 & 4.659759 \\
\hline $\mathrm{H}$ & 1.923805 & -0.704230 & 3.749673 \\
\hline C & 4.636717 & -1.193964 & 4.173003 \\
\hline $\mathrm{H}$ & 4.518378 & -1.721115 & 5.127709 \\
\hline $\mathrm{H}$ & 4.240214 & -1.838692 & 3.381209 \\
\hline $\mathrm{H}$ & 5.710380 & -1.068917 & 3.997265 \\
\hline C & -4.681122 & 0.365376 & -0.199945 \\
\hline C & -5.978482 & 0.495504 & -0.716522 \\
\hline C & -4.515752 & -0.113181 & 1.106948 \\
\hline C & -7.103829 & 0.161211 & 0.044146 \\
\hline $\mathrm{H}$ & -6.094071 & 0.893274 & -1.717824 \\
\hline C & -5.613688 & -0.446594 & 1.906302 \\
\hline $\mathrm{H}$ & -3.513069 & -0.216137 & 1.501415 \\
\hline C & -6.892130 & -0.305452 & 1.349300 \\
\hline $\mathrm{H}$ & -7.754931 & -0.557701 & 1.958758 \\
\hline C & -5.458198 & -0.934462 & 3.359626 \\
\hline C & -8.541401 & 0.301390 & -0.494102 \\
\hline C & -6.117876 & -2.324235 & 3.520311 \\
\hline $\mathrm{H}$ & -7.185920 & -2.302280 & 3.279033 \\
\hline $\mathrm{H}$ & -5.642235 & -3.062357 & 2.863972 \\
\hline $\mathrm{H}$ & -6.018388 & -2.675711 & 4.554701 \\
\hline C & -3.981276 & -1.052153 & 3.783521 \\
\hline $\mathrm{H}$ & -3.923394 & -1.383616 & 4.826656 \\
\hline $\mathrm{H}$ & -3.443316 & -1.788712 & 3.174623 \\
\hline $\mathrm{H}$ & -3.452854 & -0.095758 & 3.707951 \\
\hline C & -6.148953 & 0.074863 & 4.307974 \\
\hline $\mathrm{H}$ & -5.692968 & 1.067000 & 4.219746 \\
\hline $\mathrm{H}$ & -7.216757 & 0.176213 & 4.086533 \\
\hline $\mathrm{H}$ & -6.053475 & -0.254400 & 5.350238 \\
\hline C & -9.235836 & -1.081137 & -0.471909 \\
\hline $\mathrm{H}$ & -8.700409 & -1.798498 & -1.104650 \\
\hline $\mathrm{H}$ & -9.281510 & -1.498244 & 0.539413 \\
\hline $\mathrm{H}$ & -10.264098 & -0.999244 & -0.845850 \\
\hline C & -9.334053 & 1.287209 & 0.396823 \\
\hline $\mathrm{H}$ & -9.392087 & 0.942862 & 1.434630 \\
\hline $\mathrm{H}$ & -8.863425 & 2.276723 & 0.400033 \\
\hline $\mathrm{H}$ & -10.360139 & 1.398126 & 0.024447 \\
\hline C & -8.578732 & 0.830174 & -1.940497 \\
\hline $\mathrm{H}$ & -9.618591 & 0.905225 & -2.278641 \\
\hline $\mathrm{H}$ & -8.131476 & 1.827102 & -2.022045 \\
\hline $\mathrm{H}$ & -8.053948 & 0.161897 & -2.632898 \\
\hline
\end{tabular}


Frequencies (Top 3 out of 450)

$\begin{array}{lr}\text { 1. } & -327.1527 \mathrm{~cm}^{-1} \\ \text { 2. } & 6.5338 \mathrm{~cm}^{-1} \\ \text { 3. } & 8.5035 \mathrm{~cm}^{-1}\end{array}$

\section{B3LYP/6-31G(d) Molecular Geometry in Cartesian Coordinates}

C

$\mathrm{H}$

$\mathrm{H}$

C

0

0

$\mathrm{H}$

0

$\mathrm{H}$

C

C

C

C

$\mathrm{H}$

$\mathrm{H}$

$\mathrm{H}$

C

C

C

$\mathrm{H}$

$\mathrm{H}$

$\mathrm{H}$

C

$\mathrm{H}$

$\mathrm{H}$

$\mathrm{H}$

C

$\mathrm{H}$

$\mathrm{H}$

$\mathrm{H}$

C

$\mathrm{H}$

\section{$-1.228015$}

$-0.823017$

$-2.262960$

2. 239012

2.986805

1.467171

0.850616

$-0.458562$

$-0.365408$

$-4.408890$

$-2.618375$

$-2.813032$

$-3.666621$

$-2.344534$

$-3.173397$

$-2.469104$

0.811680

0.642987

0.717631

0.607757

1.617239

$-0.147608$

2.064614

2.971193

2.096541

2.088734

1.176325

2. 271184

0.801897

0.838139

1. 227205

2. 309701
$-0.310441$

0.377574

$-0.014706$

$-2.283553$

$-3.230580$

$-2.093541$

$-1.319662$

$-0.194718$

0.772096

4.156239

3.956927

3. 442155

4.414434

5.008831

2.412205

3.705134

3.317930

4.676164

3. 485597

2.498688

3.956353

4.092386

2.520584

3.090269

1.596465

2.247869

4.606148

4.586610

3. 722676

5.495278

5.899667

5.796517
$-0.095086$

$-0.843958$

0.107331

0.245023

0.157112

1. 326622

1. 219354

1.115494

1. 295762

0.153247

$-1.090428$

1.905363

1.286350

$-0.997772$

1.860868

2.906920

1. 787751

1.008803

3. 311906

3. 773189

3. 722874

3.594623

1.430825

1.664997

2.017451

0.373580

$-0.432502$

$-0.462342$

$-0.957656$

$-0.975382$

1. 716013

1.855285 


\begin{tabular}{|c|c|c|c|}
\hline H & 1.049672 & 6.794027 & 1.109083 \\
\hline $\mathrm{H}$ & 0.762428 & 6.059635 & 2.691810 \\
\hline 0 & -0.351802 & 2.569838 & 1.349067 \\
\hline 0 & -0.791734 & 4.822919 & 0.962048 \\
\hline B & -1.359363 & 3.511587 & 0.934671 \\
\hline 0 & -1.903452 & 3.103494 & -0.428019 \\
\hline C & -3.250855 & 3.534667 & -2.353044 \\
\hline C & -3.375064 & 2.178134 & -2.691325 \\
\hline C & -3.715789 & 4.514614 & -3.243983 \\
\hline C & -3.963205 & 1.813493 & -3.899530 \\
\hline $\mathrm{H}$ & -3.000087 & 1.424125 & -2.007686 \\
\hline C & -4.300913 & 4.145781 & -4.452817 \\
\hline $\mathrm{H}$ & -3.613886 & 5.566366 & -2.986034 \\
\hline C & -4.428495 & 2.793799 & -4.781328 \\
\hline $\mathrm{H}$ & -4.057338 & 0.762138 & -4.156632 \\
\hline $\mathrm{H}$ & -4.655090 & 4.910231 & -5.138562 \\
\hline $\mathrm{H}$ & -4.886492 & 2.504566 & -5.723198 \\
\hline $\mathrm{H}$ & -3.518368 & 5.456222 & 1.571684 \\
\hline $\mathrm{H}$ & -4.724679 & 3.140965 & -0.068887 \\
\hline $\mathrm{H}$ & -4.983984 & 4.944497 & -0.324568 \\
\hline C & 1.028000 & -1.213636 & -1.674682 \\
\hline C & 2.148287 & -1.240137 & -0.854341 \\
\hline C & 3.282071 & -0.395680 & -1.112736 \\
\hline C & 3.214686 & 0.477082 & -2.183058 \\
\hline $\mathrm{H}$ & 4.045945 & 1.151926 & -2.369714 \\
\hline C & -1.197752 & -1.727838 & -0.625763 \\
\hline C & -0.126210 & -2.142710 & -1.412332 \\
\hline C & -2.282936 & -3.876184 & -0.949734 \\
\hline C & -2.283675 & -2.626988 & -0.358587 \\
\hline $\mathrm{H}$ & -3.103511 & -4.559944 & -0.746817 \\
\hline C & -1.222589 & -4.318964 & -1.774524 \\
\hline C & -0.107452 & -3.450229 & -1.997785 \\
\hline C & 0.967189 & -3.921776 & -2.802338 \\
\hline C & 0.930512 & -5.177860 & -3.364047 \\
\hline C & -0.178352 & -6.031620 & -3.148725 \\
\hline C & -1.229071 & -5.610695 & -2.367765 \\
\hline $\mathrm{H}$ & 1.829671 & -3.283696 & -2.958949 \\
\hline H & 1.763685 & -5.521596 & -3.970780 \\
\hline $\mathrm{H}$ & -0.191655 & -7.022036 & -3.595835 \\
\hline $\mathrm{H}$ & -2.081101 & -6.261883 & -2.186823 \\
\hline C & 2.094625 & 0.534819 & -3.047252 \\
\hline C & 0.983028 & -0.332324 & -2.805075 \\
\hline C & -0.124254 & -0.277777 & -3.695675 \\
\hline C & -0.136382 & 0.598300 & -4.759040 \\
\hline C & 0.959941 & 1.462950 & -4.989713 \\
\hline C & 2.050167 & 1.429443 & -4.150832 \\
\hline $\mathrm{H}$ & -0.965266 & -0.942898 & -3.528677 \\
\hline $\mathrm{H}$ & -0.993550 & 0.627736 & -5.426078 \\
\hline $\mathrm{H}$ & 0.938555 & 2.149746 & -5.831579 \\
\hline $\mathrm{H}$ & 2.900000 & 2.086049 & -4.322422 \\
\hline C & -5.511826 & -1.761724 & 2.308379 \\
\hline C & -4.201291 & -1.622723 & 2.774902 \\
\hline C & -3.155489 & -1.880333 & 1.874656 \\
\hline C & -3.408057 & -2.276370 & 0.558532 \\
\hline
\end{tabular}




\begin{tabular}{|c|c|c|c|}
\hline C & -4.740058 & -2.409497 & 0.129131 \\
\hline C & -5.809460 & -2.151044 & 0.989339 \\
\hline $\mathrm{H}$ & -6.334780 & -1.565163 & 2.985661 \\
\hline $\mathrm{H}$ & -2.124055 & -1.784550 & 2.195643 \\
\hline $\mathrm{H}$ & -4.918173 & -2.710507 & -0.897241 \\
\hline C & -3.865776 & -1.218438 & 4.223479 \\
\hline C & -7.281240 & -2.270478 & 0.547160 \\
\hline C & -3.030694 & -2.339789 & 4.887098 \\
\hline $\mathrm{H}$ & -2.786243 & -2.069928 & 5.921967 \\
\hline $\mathrm{H}$ & -3.587046 & -3.284034 & 4.904359 \\
\hline $\mathrm{H}$ & -2.089293 & -2.515962 & 4.357497 \\
\hline C & -3.043990 & 0.092572 & 4.214966 \\
\hline $\mathrm{H}$ & -2.749670 & 0.364007 & 5.236520 \\
\hline $\mathrm{H}$ & -2.133904 & 0.002384 & 3.613164 \\
\hline $\mathrm{H}$ & -3.635901 & 0.919337 & 3.804671 \\
\hline C & -5.125481 & -0.990643 & 5.080134 \\
\hline $\mathrm{H}$ & -4.833527 & -0.702965 & 6.096578 \\
\hline $\mathrm{H}$ & -5.753891 & -0.187335 & 4.678116 \\
\hline $\mathrm{H}$ & -5.736986 & -1.897156 & 5.157500 \\
\hline C & -7.975918 & -0.895653 & 0.693877 \\
\hline $\mathrm{H}$ & -9.027993 & -0.963038 & 0.390252 \\
\hline $\mathrm{H}$ & -7.949811 & -0.534114 & 1.727205 \\
\hline $\mathrm{H}$ & -7.488344 & -0.142315 & 0.064191 \\
\hline C & -7.420086 & -2.720993 & -0.919567 \\
\hline $\mathrm{H}$ & -8.480992 & -2.793787 & -1.184692 \\
\hline $\mathrm{H}$ & -6.955165 & -2.009280 & -1.611370 \\
\hline $\mathrm{H}$ & -6.969294 & -3.705503 & -1.088526 \\
\hline C & -8.008336 & -3.307655 & 1.435862 \\
\hline $\mathrm{H}$ & -9.059506 & -3.398119 & 1.135076 \\
\hline $\mathrm{H}$ & -7.542767 & -4.295645 & 1.345067 \\
\hline $\mathrm{H}$ & -7.988414 & -3.025909 & 2.493656 \\
\hline C & 4.516308 & -0.425752 & -0.277000 \\
\hline C & 5.771797 & -0.380838 & -0.901709 \\
\hline C & 4.457707 & -0.473379 & 1.122343 \\
\hline C & 6.957509 & -0.382509 & -0.159937 \\
\hline $\mathrm{H}$ & 5.805923 & -0.376116 & -1.984745 \\
\hline C & 5.618593 & -0.491291 & 1.901798 \\
\hline $\mathrm{H}$ & 3.489148 & -0.504901 & 1.603168 \\
\hline C & 6.851458 & -0.438867 & 1.236902 \\
\hline $\mathrm{H}$ & 7.763114 & -0.455621 & 1.827045 \\
\hline C & 5.577834 & -0.594578 & 3.438912 \\
\hline C & 8.350290 & -0.343463 & -0.819079 \\
\hline C & 6.339839 & 0.594542 & 4.069198 \\
\hline $\mathrm{H}$ & 7.390051 & 0.621030 & 3.760050 \\
\hline $\mathrm{H}$ & 5.883270 & 1.549466 & 3.783516 \\
\hline $\mathrm{H}$ & 6.318711 & 0.522475 & 5.163680 \\
\hline C & 4.138691 & -0.586409 & 3.990256 \\
\hline $\mathrm{H}$ & 4.164493 & -0.672733 & 5.082614 \\
\hline $\mathrm{H}$ & 3.616786 & 0.346792 & 3.746606 \\
\hline $\mathrm{H}$ & 3.543698 & -1.420875 & 3.604263 \\
\hline C & 6.248193 & -1.921295 & 3.869964 \\
\hline $\mathrm{H}$ & 5.720802 & -2.780237 & 3.440973 \\
\hline H & 7.292833 & -1.973024 & 3.544651 \\
\hline $\mathrm{H}$ & 6.232439 & -2.020214 & 4.962667 \\
\hline
\end{tabular}




\begin{tabular}{|rrrr|} 
C & 9.117546 & 0.907736 & -0.329425 \\
$H$ & 8.586728 & 1.826205 & -0.605796 \\
$H$ & 9.242954 & 0.910073 & 0.758291 \\
$H$ & 10.116989 & 0.943883 & -0.780495 \\
$\mathrm{C}$ & 9.142005 & -1.614610 & -0.429901 \\
$\mathrm{H}$ & 9.276796 & -1.695144 & 0.653706 \\
$\mathrm{H}$ & 8.622417 & -2.517854 & -0.768839 \\
$\mathrm{H}$ & 10.137941 & -1.600431 & -0.890000 \\
$\mathrm{C}$ & 8.272116 & -0.282459 & -2.356414 \\
$\mathrm{H}$ & 9.283818 & -0.245747 & -2.776419 \\
$\mathrm{H}$ & 7.772460 & -1.163282 & -2.774941 \\
$\mathrm{H}$ & 7.738564 & 0.610408 & -2.702230 \\
& & & \\
\hline
\end{tabular}

\section{C-Catalyzed reaction TS 10 (TS-2.3)}

\section{Datum}

Value

\begin{tabular}{lc}
\hline B3LYP/6-31G(d) Energy & -3038.970341 \\
\hline B3LYP-D3(BJ)/def2-TZVPP-IEF-PCM(DCM) Energy & -3040.52006 \\
\hline B3LYP-D3(BJ)/def2-TZVPP-IEF-PCM(DCM)//B3LYP/6-31G(d) Free Energy (Quasiharmonic) & -3039.291097 \\
\hline
\end{tabular}

Number of Imaginary Frequencies

Frequencies (Top 3 out of 450)

$\begin{array}{lr}\text { 1. } & -309.8415 \mathrm{~cm}^{-1} \\ \text { 2. } & 7.0425 \mathrm{~cm}^{-1} \\ \text { 3. } & 10.6542 \mathrm{~cm}^{-1}\end{array}$

\section{B3LYP/6-31G(d) Molecular Geometry in Cartesian Coordinates}

$\begin{array}{lrrr}\mathrm{C} & 1.480137 & -1.146099 & 1.069729 \\ \mathrm{H} & 1.176112 & -1.724267 & 1.948083 \\ \mathrm{H} & 2.454722 & -0.705595 & 1.278651 \\ \mathrm{C} & -1.939350 & -1.312179 & -1.118754 \\ \mathrm{O} & -2.646868 & -1.583865 & -2.064593 \\ \mathrm{O} & -1.156112 & -0.222941 & -1.122805 \\ \mathrm{H} & -0.568526 & -0.197043 & -0.308230 \\ \mathrm{O} & 0.519262 & -0.084437 & 0.905678 \\ \mathrm{H} & 0.970173 & 0.798108 & 0.898519 \\ \mathrm{C} & 0.141386 & 2.394738 & -2.733257 \\ \mathrm{C} & -0.699351 & 3.219415 & -0.908565 \\ \mathrm{C} & 2.252521 & 2.939435 & -1.546619 \\ \mathrm{C} & 1.288349 & 2.011079 & -2.073344 \\ \mathrm{H} & -0.787319 & 2.248213 & -0.424065\end{array}$




\begin{tabular}{|c|c|c|c|}
\hline $\mathrm{H}$ & 3.201316 & 2.485832 & -1.253009 \\
\hline $\mathrm{H}$ & 2.405869 & 3.835924 & -2.152516 \\
\hline C & 1.778998 & 3.037372 & 2.228918 \\
\hline C & 2.777401 & 4.189544 & 1.832437 \\
\hline C & 0.454640 & 3.557960 & 2.811275 \\
\hline H & -0.261333 & 2.730177 & 2.857752 \\
\hline $\mathrm{H}$ & 0.586528 & 3.951481 & 3.825065 \\
\hline $\mathrm{H}$ & 0.026751 & 4.345934 & 2.185769 \\
\hline C & 2.358412 & 1.964865 & 3.150944 \\
\hline $\mathrm{H}$ & 3.226382 & 1.473726 & 2.703598 \\
\hline $\mathrm{H}$ & 2.663574 & 2.400818 & 4.109133 \\
\hline $\mathrm{H}$ & 1.601132 & 1.200606 & 3.358020 \\
\hline C & 4.231743 & 3.701760 & 1.708776 \\
\hline $\mathrm{H}$ & 4.680795 & 3.491613 & 2.686421 \\
\hline $\mathrm{H}$ & 4.301391 & 2.797830 & 1.095683 \\
\hline $\mathrm{H}$ & 4.821140 & 4.486015 & 1.222929 \\
\hline C & 2.725753 & 5.416477 & 2.746764 \\
\hline $\mathrm{H}$ & 2.999104 & 5.158185 & 3.777053 \\
\hline $\mathrm{H}$ & 3.435632 & 6.168749 & 2.387108 \\
\hline $\mathrm{H}$ & 1.732087 & 5.870314 & 2.749463 \\
\hline 0 & 1.502390 & 2.439398 & 0.934542 \\
\hline 0 & 2.324127 & 4.577577 & 0.528383 \\
\hline B & 1.581323 & 3.514055 & -0.051968 \\
\hline 0 & 0.222757 & 4.029534 & -0.490168 \\
\hline C & -1.945791 & 3.793512 & -1.449032 \\
\hline C & -3.071005 & 2.967337 & -1.600905 \\
\hline C & -2.032243 & 5.156311 & -1.774534 \\
\hline C & -4.268566 & 3.501218 & -2.072901 \\
\hline $\mathrm{H}$ & -3.000189 & 1.910443 & -1.357138 \\
\hline C & -3.230977 & 5.682599 & -2.248011 \\
\hline $\mathrm{H}$ & -1.160431 & 5.788087 & -1.638116 \\
\hline C & -4.350104 & 4.857612 & -2.398521 \\
\hline $\mathrm{H}$ & -5.136398 & 2.857685 & -2.184108 \\
\hline $\mathrm{H}$ & -3.296455 & 6.738647 & -2.495593 \\
\hline $\mathrm{H}$ & -5.284586 & 5.272567 & -2.766854 \\
\hline $\mathrm{H}$ & 1.333389 & 0.981495 & -1.718269 \\
\hline $\mathrm{H}$ & 0.102954 & 3.351155 & -3.247448 \\
\hline H & -0.592100 & 1.650730 & -3.026159 \\
\hline C & -0.748776 & -2.894933 & 0.423235 \\
\hline C & -1.888036 & -2.151348 & 0.141252 \\
\hline C & -3.020875 & -2.157187 & 1.020787 \\
\hline C & -2.961595 & -2.931462 & 2.161776 \\
\hline $\mathrm{H}$ & -3.809139 & -2.935089 & 2.842603 \\
\hline C & 1.554688 & -2.052325 & -0.152588 \\
\hline C & 0.452620 & -2.843838 & -0.483210 \\
\hline C & 2.703611 & -2.816042 & -2.154678 \\
\hline C & 2.717474 & -2.059645 & -0.996274 \\
\hline $\mathrm{H}$ & 3.588210 & -2.832357 & -2.786374 \\
\hline C & 1.595191 & -3.608371 & -2.528527 \\
\hline C & 0.448156 & -3.641287 & -1.673737 \\
\hline C & -0.645789 & -4.472808 & -2.045857 \\
\hline C & -0.603396 & -5.222636 & -3.198947 \\
\hline C & 0.532297 & -5.183977 & -4.044000 \\
\hline C & 1.607436 & -4.393548 & -3.713626 \\
\hline
\end{tabular}




\begin{tabular}{|c|c|c|c|}
\hline $\mathrm{H}$ & -1.527194 & -4.501405 & -1.415657 \\
\hline $\mathrm{H}$ & -1.451485 & -5.846505 & -3.467083 \\
\hline $\mathrm{H}$ & 0.549534 & -5.779473 & -4.952797 \\
\hline $\mathrm{H}$ & 2.486372 & -4.357082 & -4.352937 \\
\hline C & -1.842638 & -3.745069 & 2.465207 \\
\hline C & -0.716824 & -3.740548 & 1.581847 \\
\hline C & 0.389505 & -4.579182 & 1.894114 \\
\hline C & 0.384281 & -5.369267 & 3.022531 \\
\hline C & -0.726819 & -5.363554 & 3.899162 \\
\hline C & -1.815343 & -4.568896 & 3.622793 \\
\hline $\mathrm{H}$ & 1.242989 & -4.595293 & 1.224570 \\
\hline $\mathrm{H}$ & 1.237761 & -6.005812 & 3.240040 \\
\hline $\mathrm{H}$ & -0.718475 & -5.990875 & 4.786375 \\
\hline $\mathrm{H}$ & -2.676864 & -4.561339 & 4.286313 \\
\hline C & 6.424307 & -0.082905 & -0.173291 \\
\hline C & 5.903168 & -0.948412 & 0.793331 \\
\hline C & 4.672256 & -1.566208 & 0.518754 \\
\hline C & 3.979978 & -1.326077 & -0.672142 \\
\hline C & 4.544504 & -0.453662 & -1.619293 \\
\hline C & 5.771072 & 0.176220 & -1.392116 \\
\hline $\mathrm{H}$ & 7.375346 & 0.401009 & 0.014564 \\
\hline $\mathrm{H}$ & 4.253524 & -2.274107 & 1.228723 \\
\hline $\mathrm{H}$ & 4.000519 & -0.282715 & -2.541323 \\
\hline C & 6.637167 & -1.265119 & 2.111096 \\
\hline C & 6.431809 & 1.110543 & -2.425109 \\
\hline C & 5.730946 & -0.903531 & 3.311581 \\
\hline $\mathrm{H}$ & 6.239201 & -1.133582 & 4. 255971 \\
\hline $\mathrm{H}$ & 5.486563 & 0.165056 & 3.310259 \\
\hline $\mathrm{H}$ & 4.789674 & -1.462802 & 3.298202 \\
\hline C & 6.970398 & -2.775688 & 2.159976 \\
\hline $\mathrm{H}$ & 7.488350 & -3.022280 & 3.094944 \\
\hline $\mathrm{H}$ & 6.068446 & -3.394093 & 2.105541 \\
\hline $\mathrm{H}$ & 7.621234 & -3.060355 & 1.325419 \\
\hline C & 7.954538 & -0.479645 & 2.256738 \\
\hline $\mathrm{H}$ & 8.430151 & -0.732831 & 3.210973 \\
\hline $\mathrm{H}$ & 8.666765 & -0.723080 & 1.460206 \\
\hline $\mathrm{H}$ & 7.787264 & 0.603442 & 2.248273 \\
\hline C & 5.579458 & 1.274836 & -3.698154 \\
\hline $\mathrm{H}$ & 5.426525 & 0.319369 & -4.212984 \\
\hline $\mathrm{H}$ & 4.597106 & 1.707963 & -3.480684 \\
\hline $\mathrm{H}$ & 6.089315 & 1.947206 & -4.397379 \\
\hline C & 6.646483 & 2.511427 & -1.804539 \\
\hline $\mathrm{H}$ & 7.133207 & 3.176268 & -2.528532 \\
\hline $\mathrm{H}$ & 5.692374 & 2.966978 & -1.516765 \\
\hline $\mathrm{H}$ & 7.279990 & 2.471340 & -0.912290 \\
\hline C & 7.802278 & 0.521997 & -2.839274 \\
\hline $\mathrm{H}$ & 8.287697 & 1.171867 & -3.577642 \\
\hline $\mathrm{H}$ & 8.480196 & 0.424900 & -1.984804 \\
\hline $\mathrm{H}$ & 7.683496 & -0.471351 & -3.286984 \\
\hline C & -4.228166 & -1.305757 & 0.788109 \\
\hline C & -5.067765 & -1.513783 & -0.317933 \\
\hline C & -4.552515 & -0.311283 & 1.714436 \\
\hline C & -6.213881 & -0.740120 & -0.506815 \\
\hline $\mathrm{H}$ & -4.795746 & -2.280551 & -1.029295 \\
\hline
\end{tabular}




\begin{tabular}{|c|c|c|c|}
\hline C & -5.691793 & 0.495035 & 1.556495 \\
\hline $\mathrm{H}$ & -3.890032 & -0.157730 & 2.561455 \\
\hline C & -6.502067 & 0.257983 & 0.442606 \\
\hline $\mathrm{H}$ & -7.387765 & 0.865739 & 0.298489 \\
\hline C & -5.991187 & 1.598950 & 2.589450 \\
\hline C & -7.153538 & -0.943538 & -1.711472 \\
\hline C & -6.157766 & 0.964432 & 3.990607 \\
\hline $\mathrm{H}$ & -6.366410 & 1.738834 & 4.739121 \\
\hline $\mathrm{H}$ & -6.989010 & 0.249937 & 4.000420 \\
\hline $\mathrm{H}$ & -5.255138 & 0.431122 & 4.306718 \\
\hline C & -4.814962 & 2.604357 & 2.617770 \\
\hline $\mathrm{H}$ & -5.011859 & 3.404606 & 3.342081 \\
\hline $\mathrm{H}$ & -3.874203 & 2.121950 & 2.903147 \\
\hline $\mathrm{H}$ & -4.668902 & 3.063876 & 1.633605 \\
\hline C & -7.278023 & 2.380077 & 2.263575 \\
\hline $\mathrm{H}$ & -7.211405 & 2.889800 & 1.295690 \\
\hline $\mathrm{H}$ & -8.159484 & 1.728801 & 2.248762 \\
\hline $\mathrm{H}$ & -7.446950 & 3.146791 & 3.028218 \\
\hline C & -8.573466 & -1.296823 & -1.209088 \\
\hline $\mathrm{H}$ & -8.993913 & -0.506826 & -0.577629 \\
\hline $\mathrm{H}$ & -8.561338 & -2.222628 & -0.622528 \\
\hline $\mathrm{H}$ & -9.252933 & -1.441567 & -2.058207 \\
\hline C & -7.216299 & 0.359829 & -2.543269 \\
\hline $\mathrm{H}$ & -6.225103 & 0.619947 & -2.932703 \\
\hline $\mathrm{H}$ & -7.582338 & 1.205867 & -1.951172 \\
\hline $\mathrm{H}$ & -7.892877 & 0.234797 & -3.397787 \\
\hline C & -6.675032 & -2.075671 & -2.640313 \\
\hline $\mathrm{H}$ & -7.362212 & -2.170008 & -3.489103 \\
\hline $\mathrm{H}$ & -6.654351 & -3.042277 & -2.124132 \\
\hline $\mathrm{H}$ & -5.673607 & -1.880289 & -3.038348 \\
\hline
\end{tabular}

\section{C-Catalyzed reaction TS 11}

\section{Datum}

Value

\begin{tabular}{lc}
\hline B3LYP/6-31G(d) Energy & -3038.970341 \\
\hline B3LYP-D3(BJ)/def2-TZVPP-IEF-PCM(DCM) Energy & -3040.520059 \\
\hline B3LYP-D3(BJ)/def2-TZVPP-IEF-PCM(DCM)//B3LYP/6-31G(d) Free Energy (Quasiharmonic) & -3039.291096 \\
\hline
\end{tabular}

Number of Imaginary Frequencies

Frequencies (Top 3 out of 450)

$$
\begin{array}{lr}
\text { 1. } & -309.8428 \mathrm{~cm}^{-1} \\
\text { 2. } & 7.0424 \mathrm{~cm}^{-1} \\
\text { 3. } & 10.6546 \mathrm{~cm}^{-1}
\end{array}
$$




\begin{tabular}{|c|c|c|c|}
\hline C & 1.480135 & -1.146052 & 1.069791 \\
\hline $\mathrm{H}$ & 1.176117 & -1.724183 & 1.948171 \\
\hline $\mathrm{H}$ & 2.454722 & -0.705539 & 1.278688 \\
\hline C & -1.939373 & -1.312209 & -1.118660 \\
\hline 0 & -2.646904 & -1.583923 & -2.064482 \\
\hline 0 & -1.156128 & -0.222976 & -1.122758 \\
\hline H & -0.568537 & -0.197052 & -0.308187 \\
\hline 0 & 0.519259 & -0.084396 & 0.905706 \\
\hline $\mathrm{H}$ & 0.970172 & 0.798147 & 0.898483 \\
\hline C & 0.141425 & 2.394579 & -2.733404 \\
\hline C & -0.699320 & 3.219371 & -0.908774 \\
\hline C & 2.252554 & 2.939319 & -1.546776 \\
\hline C & 1.288377 & 2.010944 & -2.073457 \\
\hline $\mathrm{H}$ & -0.787303 & 2.248199 & -0.424216 \\
\hline $\mathrm{H}$ & 3.201340 & 2.485721 & -1.253127 \\
\hline $\mathrm{H}$ & 2.405920 & 3.835770 & -2.152723 \\
\hline C & 1.778978 & 3.037491 & 2.228750 \\
\hline C & 2.777404 & 4.189624 & 1.832216 \\
\hline C & 0.454615 & 3.558133 & 2.811051 \\
\hline $\mathrm{H}$ & -0.261370 & 2.730363 & 2.857561 \\
\hline $\mathrm{H}$ & 0.586488 & 3.951710 & 3.824821 \\
\hline $\mathrm{H}$ & 0.026751 & 4.346076 & 2.185490 \\
\hline C & 2.358361 & 1.965033 & 3.150853 \\
\hline $\mathrm{H}$ & 3.226336 & 1.473860 & 2.703553 \\
\hline $\mathrm{H}$ & 2.663507 & 2.401038 & 4.109023 \\
\hline $\mathrm{H}$ & 1.601068 & 1.200793 & 3.357956 \\
\hline C & 4.231741 & 3.701813 & 1.708609 \\
\hline $\mathrm{H}$ & 4.680774 & 3.491721 & 2.686275 \\
\hline $\mathrm{H}$ & 4.301386 & 2.797843 & 1.095574 \\
\hline $\mathrm{H}$ & 4.821156 & 4.486029 & 1.222722 \\
\hline C & 2.725756 & 5.416614 & 2.746467 \\
\hline $\mathrm{H}$ & 2.999081 & 5.158381 & 3.776777 \\
\hline $\mathrm{H}$ & 3.435654 & 6.168854 & 2.386780 \\
\hline $\mathrm{H}$ & 1.732097 & 5.870467 & 2.749117 \\
\hline 0 & 1.502385 & 2.439440 & 0.934407 \\
\hline 0 & 2.324157 & 4.577584 & 0.528129 \\
\hline B & 1.581345 & 3.514037 & -0.052166 \\
\hline 0 & 0.222792 & 4.029506 & -0.490415 \\
\hline C & -1.945750 & 3.793449 & -1.449286 \\
\hline C & -3.070970 & 2.967274 & -1.601124 \\
\hline C & -2.032187 & 5.156230 & -1.774863 \\
\hline C & -4.268521 & 3.501139 & -2.073160 \\
\hline $\mathrm{H}$ & -3.000163 & 1.910393 & -1.357298 \\
\hline C & -3.230913 & 5.682502 & -2.248382 \\
\hline $\mathrm{H}$ & -1.160372 & 5.788007 & -1.638472 \\
\hline C & -4.350045 & 4.857516 & -2.398856 \\
\hline $\mathrm{H}$ & -5.136358 & 2.857607 & -2.184340 \\
\hline $\mathrm{H}$ & -3.296380 & 6.738537 & -2.496022 \\
\hline $\mathrm{H}$ & -5.284520 & 5.272457 & -2.767221 \\
\hline $\mathrm{H}$ & 1.333399 & 0.981380 & -1.718322 \\
\hline
\end{tabular}




\begin{tabular}{|c|c|c|c|}
\hline $\mathrm{H}$ & 0.103011 & 3.350966 & -3.247652 \\
\hline $\mathrm{H}$ & -0.592067 & 1.650563 & -3.026273 \\
\hline C & -0.748786 & -2.894907 & 0.423379 \\
\hline C & -1.888049 & -2.151334 & 0.141375 \\
\hline C & -3.020884 & -2.157143 & 1.020917 \\
\hline C & -2.961596 & -2.931375 & 2.161934 \\
\hline $\mathrm{H}$ & -3.809136 & -2.934977 & 2.842766 \\
\hline C & 1.554678 & -2.052328 & -0.152489 \\
\hline C & 0.452604 & -2.843849 & -0.483075 \\
\hline C & 2.703588 & -2.816133 & -2.154553 \\
\hline C & 2.717461 & -2.059689 & -0.996179 \\
\hline $\mathrm{H}$ & 3.588186 & -2.832480 & -2.786251 \\
\hline C & 1.595162 & -3.608468 & -2.528367 \\
\hline C & 0.448130 & -3.641344 & -1.673572 \\
\hline C & -0.645821 & -4.472872 & -2.045656 \\
\hline C & -0.603438 & -5.222745 & -3.198717 \\
\hline C & 0.532252 & -5.184126 & -4.043776 \\
\hline C & 1.607397 & -4.393691 & -3.713436 \\
\hline $\mathrm{H}$ & -1.527224 & -4.501441 & -1.415451 \\
\hline $\mathrm{H}$ & -1.451532 & -5.846619 & -3.466826 \\
\hline $\mathrm{H}$ & 0.549482 & -5.779657 & -4.952551 \\
\hline $\mathrm{H}$ & 2.486331 & -4.357255 & -4.352752 \\
\hline C & -1.842636 & -3.744969 & 2.465389 \\
\hline C & -0.716827 & -3.740479 & 1.582023 \\
\hline C & 0.389505 & -4.579100 & 1.894315 \\
\hline C & 0.384289 & -5.369141 & 3.022763 \\
\hline C & -0.726806 & -5.363397 & 3.899400 \\
\hline C & -1.815332 & -4.568752 & 3.623007 \\
\hline $\mathrm{H}$ & 1.242985 & -4.595234 & 1.224767 \\
\hline $\mathrm{H}$ & 1.237772 & -6.005677 & 3.240291 \\
\hline $\mathrm{H}$ & -0.718455 & -5.990685 & 4.786636 \\
\hline $\mathrm{H}$ & -2.676850 & -4.561171 & 4.286532 \\
\hline C & 6.424310 & -0.082940 & -0.173291 \\
\hline C & 5.903162 & -0.948393 & 0.793375 \\
\hline C & 4.672245 & -1.566193 & 0.518828 \\
\hline C & 3.979971 & -1.326117 & -0.672081 \\
\hline C & 4.544506 & -0.453756 & -1.619276 \\
\hline C & 5.771079 & 0.176128 & -1.392130 \\
\hline $\mathrm{H}$ & 7.375352 & 0.400977 & 0.014542 \\
\hline $\mathrm{H}$ & 4.253507 & -2.274052 & 1.228833 \\
\hline $\mathrm{H}$ & 4.000524 & -0.282850 & -2.541315 \\
\hline C & 6.637153 & -1.265033 & 2.111160 \\
\hline C & 6.431824 & 1.110394 & -2.425168 \\
\hline C & 5.730937 & -0.903349 & 3.311620 \\
\hline $\mathrm{H}$ & 6.239185 & -1.133348 & 4.256026 \\
\hline $\mathrm{H}$ & 5.486575 & 0.165243 & 3.310227 \\
\hline $\mathrm{H}$ & 4.789655 & -1.462604 & 3.298275 \\
\hline C & 6.970353 & -2.775606 & 2.160138 \\
\hline $\mathrm{H}$ & 7.488303 & -3.022146 & 3.095121 \\
\hline $\mathrm{H}$ & 6.068388 & -3.393995 & 2.105746 \\
\hline $\mathrm{H}$ & 7.621181 & -3.060341 & 1.325598 \\
\hline C & 7.954541 & -0.479577 & 2.256754 \\
\hline $\mathrm{H}$ & 8.430150 & -0.732717 & 3.211003 \\
\hline $\mathrm{H}$ & 8.666761 & -0.723074 & 1.460235 \\
\hline
\end{tabular}




\begin{tabular}{|c|c|c|c|}
\hline $\mathrm{H}$ & 7.787290 & 0.603513 & 2.248226 \\
\hline C & 5.579481 & 1.274623 & -3.698227 \\
\hline $\mathrm{H}$ & 5.426550 & 0.319129 & -4.213008 \\
\hline $\mathrm{H}$ & 4.597128 & 1.707761 & -3.480785 \\
\hline $\mathrm{H}$ & 6.089343 & 1.946956 & -4.397484 \\
\hline C & 6.646496 & 2.511310 & -1.804670 \\
\hline $\mathrm{H}$ & 7.133226 & 3.176114 & -2.528694 \\
\hline $\mathrm{H}$ & 5.692386 & 2.966878 & -1.516927 \\
\hline $\mathrm{H}$ & 7.279996 & 2.471269 & -0.912413 \\
\hline C & 7.802295 & 0.521826 & -2.839294 \\
\hline $\mathrm{H}$ & 8.287719 & 1.171657 & -3.577693 \\
\hline $\mathrm{H}$ & 8.480208 & 0.424773 & -1.984815 \\
\hline $\mathrm{H}$ & 7.683514 & -0.471545 & -3.286953 \\
\hline C & -4.228176 & -1.305721 & 0.788214 \\
\hline C & -5.067787 & -1.513797 & -0.317809 \\
\hline C & -4.552510 & -0.311201 & 1.714496 \\
\hline C & -6.213901 & -0.740138 & -0.506716 \\
\hline $\mathrm{H}$ & -4.795779 & -2.280600 & -1.029137 \\
\hline C & -5.691785 & 0.495116 & 1.556527 \\
\hline $\mathrm{H}$ & -3.890017 & -0.157610 & 2.561501 \\
\hline C & -6.502072 & 0.258013 & 0.442659 \\
\hline $\mathrm{H}$ & -7.387768 & 0.865768 & 0.298521 \\
\hline C & -5.991160 & 1.599086 & 2.589429 \\
\hline C & -7.153571 & -0.943606 & -1.711355 \\
\hline C & -6.157721 & 0.964642 & 3.990622 \\
\hline $\mathrm{H}$ & -6.366348 & 1.739086 & 4.739099 \\
\hline $\mathrm{H}$ & -6.988971 & 0.250154 & 4.000486 \\
\hline H & -5.255093 & 0.431342 & 4.306747 \\
\hline C & -4.814929 & 2.604488 & 2.617680 \\
\hline $\mathrm{H}$ & -5.011811 & 3.404775 & 3.341953 \\
\hline $\mathrm{H}$ & -3.874167 & 2.122091 & 2.903068 \\
\hline $\mathrm{H}$ & -4.668881 & 3.063957 & 1.633489 \\
\hline C & -7.277996 & 2.380202 & 2.263532 \\
\hline H & -7.211388 & 2.889875 & 1.295619 \\
\hline $\mathrm{H}$ & -8.159460 & 1.728929 & 2.248764 \\
\hline H & -7.446908 & 3.146957 & 3.028137 \\
\hline C & -8.573497 & -1.296855 & -1.208943 \\
\hline $\mathrm{H}$ & -8.993932 & -0.506827 & -0.577516 \\
\hline $\mathrm{H}$ & -8.561372 & -2.222634 & -0.622341 \\
\hline $\mathrm{H}$ & -9.252973 & -1.441632 & -2.058049 \\
\hline C & -7.216328 & 0.359723 & -2.543213 \\
\hline $\mathrm{H}$ & -6.225134 & 0.619812 & -2.932670 \\
\hline $\mathrm{H}$ & -7.582350 & 1.205793 & -1.951152 \\
\hline $\mathrm{H}$ & -7.892916 & 0.234658 & -3.397718 \\
\hline C & -6.675082 & -2.075786 & -2.640147 \\
\hline $\mathrm{H}$ & -7.362270 & -2.170158 & -3.488926 \\
\hline $\mathrm{H}$ & -6.654402 & -3.042367 & -2.123920 \\
\hline $\mathrm{H}$ & -5.673659 & -1.880429 & -3.038201 \\
\hline
\end{tabular}




\begin{tabular}{lc}
\hline B3LYP/6-31G(d) Energy & -3038.970322 \\
\hline B3LYP-D3(BJ)/def2-TZVPP-IEF-PCM(DCM) Energy & -3040.520217 \\
\hline B3LYP-D3(BJ)/def2-TZVPP-IEF-PCM(DCM)//B3LYP/6-31G(d) Free Energy (Quasiharmonic) & -3039.290916 \\
\hline
\end{tabular}

Number of Imaginary Frequencies

Frequencies (Top 3 out of 450)

$\begin{array}{lr}\text { 1. } & -347.7330 \mathrm{~cm}^{-1} \\ \text { 2. } & 8.0127 \mathrm{~cm}^{-1} \\ \text { 3. } & 11.3127 \mathrm{~cm}^{-1}\end{array}$

\section{B3LYP/6-31G(d) Molecular Geometry in Cartesian Coordinates}

C

$\mathrm{H}$

$\mathrm{H}$

C

0

0

$\mathrm{H}$

0

$\mathrm{H}$

C

C

C

C

$\mathrm{H}$

$\mathrm{H}$

$\mathrm{H}$

C

C

C

$\mathrm{H}$

$\mathrm{H}$

$\mathrm{H}$

C

$\mathrm{H}$

$\mathrm{H}$

$\mathrm{H}$

C

$\mathrm{H}$

$\mathrm{H}$

$\mathrm{H}$

C

$\mathrm{H}$

\subsection{6}

3.020806

1.601327

$-0.982516$

$-1.472180$

$-0.392228$

0.097042

1. 226674

0.956143

$-0.720568$

$-1.967135$

0.853970

$-0.021168$

$-2.482092$

1.416470

1.488263

$-0.109917$

$-0.665823$

$-1.218766$

$-0.794595$

$-2.005790$

$-1.671116$

0.966939

1. 289275

0.579004

1.844793

$-1.920746$

$-1.727501$

$-2.746886$

$-2.240512$

0.398974

0.633005
0.390988

$-0.137936$

0.597766

1.960982

2. 750853

0.842327

0.355674

$-0.440938$

$-1.228959$

$-5.070604$

$-3.723016$

$-4.624779$

$-5.484660$

$-4.514626$

$-3.909004$

$-5.129335$

$-2.618246$

$-4.082940$

$-1.556364$

$-0.602252$

$-1.832001$

$-1.403557$

$-2.208882$

$-1.179521$

$-2.248394$

$-2.855682$

$-4.185886$

$-3.835437$

$-3.606100$

$-5.231868$

$-5.065456$

$-4.898173$
0.919531

1.115655

1.882397

$-1.110434$

$-1.892381$

$-1.540341$

$-0.815424$

0.118535

0.653548

$-2.027331$

$-0.900857$

$-0.172610$

$-0.910737$

$-0.353715$

$-0.774544$

0.557790

2. 885841

3.069385

2. 844421

2. 516258

2. 136789

3.830279

3.890686

3. 699701

4.915174

3.824130

3.939036

4.959798

3.520653

3.997099

3. 587545

4.644674 


\begin{tabular}{|c|c|c|c|}
\hline $\mathrm{H}$ & 0.016348 & -6.085504 & 3.478864 \\
\hline $\mathrm{H}$ & 1.327578 & -4.987536 & 3.013949 \\
\hline 0 & 0.492972 & -2.690777 & 1.567380 \\
\hline 0 & -1.009343 & -4.466776 & 1.726719 \\
\hline B & -0.245007 & -3.674088 & 0.814028 \\
\hline 0 & -1.101388 & -3.016209 & -0.237837 \\
\hline C & -2.708019 & -3.075631 & -1.999454 \\
\hline C & -2.325670 & -1.815305 & -2.484279 \\
\hline C & -3.827989 & -3.721087 & -2.549488 \\
\hline C & -3.054178 & -1.213922 & -3.507339 \\
\hline H & -1.478787 & -1.298261 & -2.048346 \\
\hline C & -4.550001 & -3.117943 & -3.576186 \\
\hline $\mathrm{H}$ & -4.128754 & -4.695615 & -2.170403 \\
\hline C & -4.161806 & -1.864297 & -4.058570 \\
\hline $\mathrm{H}$ & -2.757754 & -0.231074 & -3.861399 \\
\hline H & -5.415853 & -3.621419 & -3.997165 \\
\hline $\mathrm{H}$ & -4.726786 & -1.392961 & -4.858342 \\
\hline $\mathrm{H}$ & -0.346147 & -6.402348 & -0.419302 \\
\hline $\mathrm{H}$ & -0.327423 & -4.268465 & -2.645196 \\
\hline H & -1.424560 & -5.738437 & -2.515995 \\
\hline C & 0.155616 & 2.579640 & 1.033478 \\
\hline C & -1.014356 & 2.190787 & 0.390473 \\
\hline C & -2.252459 & 2.063673 & 1.112464 \\
\hline C & -2.242425 & 2.318788 & 2.471227 \\
\hline $\mathrm{H}$ & -3.170860 & 2.229228 & 3.029129 \\
\hline C & 2.366851 & 1.697603 & 0.206124 \\
\hline C & 1.441009 & 2.737346 & 0.266484 \\
\hline C & 3.878793 & 3.106030 & -1.072604 \\
\hline C & 3.601098 & 1.878984 & -0.501882 \\
\hline $\mathrm{H}$ & 4.815917 & 3.242234 & -1.606692 \\
\hline C & 2.964458 & 4.183651 & -1.023491 \\
\hline C & 1.711498 & 3.999273 & -0.357755 \\
\hline C & 0.793115 & 5.086072 & -0.334644 \\
\hline C & 1.104065 & 6.287296 & -0.930401 \\
\hline C & 2.347430 & 6.467804 & -1.583276 \\
\hline C & 3.255241 & 5.436064 & -1.629558 \\
\hline $\mathrm{H}$ & -0.168842 & 4.954747 & 0.147684 \\
\hline $\mathrm{H}$ & 0.386407 & 7.102722 & -0.906063 \\
\hline $\mathrm{H}$ & 2.578055 & 7.421030 & -2.051423 \\
\hline $\mathrm{H}$ & 4.210309 & 5.560193 & -2.134622 \\
\hline C & -1.078513 & 2.731226 & 3.162688 \\
\hline C & 0.144215 & 2.879226 & 2.436429 \\
\hline C & 1.300304 & 3.317508 & 3.140912 \\
\hline C & 1.247440 & 3.585676 & 4.491064 \\
\hline C & 0.036578 & 3.432507 & 5.208042 \\
\hline C & -1.100326 & 3.015500 & 4.555140 \\
\hline $\mathrm{H}$ & 2.231650 & 3.442616 & 2.598966 \\
\hline $\mathrm{H}$ & 2.141061 & 3.921602 & 5.010162 \\
\hline $\mathrm{H}$ & 0.008095 & 3.648661 & 6.272626 \\
\hline $\mathrm{H}$ & -2.037282 & 2.899532 & 5.094898 \\
\hline C & 6.523991 & -1.175858 & -1.094421 \\
\hline C & 6.900513 & -0.011032 & -0.410979 \\
\hline C & 5.920223 & 0.968708 & -0.212625 \\
\hline C & 4.605794 & 0.785659 & -0.662920 \\
\hline
\end{tabular}




\begin{tabular}{|c|c|c|c|}
\hline C & 4.267348 & -0.396936 & -1.334168 \\
\hline C & 5.223210 & -1.391132 & -1.572530 \\
\hline $\mathrm{H}$ & 7.272894 & -1.944165 & -1.263225 \\
\hline $\mathrm{H}$ & 6.158373 & 1.890429 & 0.306017 \\
\hline $\mathrm{H}$ & 3.246000 & -0.516273 & -1.673680 \\
\hline C & 8.349604 & 0.149977 & 0.088816 \\
\hline C & 4.889854 & -2.688493 & -2.334175 \\
\hline C & 9.323318 & 0.076487 & -1.111367 \\
\hline $\mathrm{H}$ & 10.359919 & 0.185068 & -0.769095 \\
\hline $\mathrm{H}$ & 9.246403 & -0.878006 & -1.642311 \\
\hline $\mathrm{H}$ & 9.117533 & 0.876195 & -1.832053 \\
\hline C & 8.577619 & 1.494491 & 0.806244 \\
\hline $\mathrm{H}$ & 9.618365 & 1.560795 & 1.143276 \\
\hline H & 8.390176 & 2.347839 & 0.144626 \\
\hline $\mathrm{H}$ & 7.937283 & 1.599632 & 1.689345 \\
\hline C & 8.679698 & -0.986275 & 1.085849 \\
\hline $\mathrm{H}$ & 9.710325 & -0.889190 & 1.449170 \\
\hline $\mathrm{H}$ & 8.009325 & -0.953220 & 1.952372 \\
\hline $\mathrm{H}$ & 8.580678 & -1.974683 & 0.625091 \\
\hline C & 5.059705 & -3.897627 & -1.384289 \\
\hline $\mathrm{H}$ & 4.839287 & -4.834137 & -1.911655 \\
\hline $\mathrm{H}$ & 6.081035 & -3.965966 & -0.994183 \\
\hline $\mathrm{H}$ & 4.379885 & -3.821062 & -0.528071 \\
\hline C & 3.446183 & -2.689554 & -2.871886 \\
\hline $\mathrm{H}$ & 3.246329 & -3.632902 & -3.393074 \\
\hline $\mathrm{H}$ & 2.710808 & -2.588360 & -2.066676 \\
\hline $\mathrm{H}$ & 3.278116 & -1.873142 & -3.583264 \\
\hline C & 5.844674 & -2.848079 & -3.541000 \\
\hline $\mathrm{H}$ & 5.607009 & -3.764366 & -4.095516 \\
\hline $\mathrm{H}$ & 5.750333 & -2.000754 & -4.229674 \\
\hline $\mathrm{H}$ & 6.893126 & -2.911811 & -3.231793 \\
\hline C & -3.547973 & 1.646303 & 0.492263 \\
\hline C & -4.275558 & 0.590411 & 1.073536 \\
\hline C & -4.101935 & 2.332694 & -0.590522 \\
\hline C & -5.529168 & 0.216218 & 0.587561 \\
\hline $\mathrm{H}$ & -3.831292 & 0.062981 & 1.909709 \\
\hline C & -5.356392 & 1.981574 & -1.114060 \\
\hline $\mathrm{H}$ & -3.538998 & 3.145736 & -1.029260 \\
\hline C & -6.046699 & 0.928458 & -0.509554 \\
\hline $\mathrm{H}$ & -7.017944 & 0.643688 & -0.897204 \\
\hline C & -5.905913 & 2.758670 & -2.325745 \\
\hline C & -6.354570 & -0.931260 & 1.202488 \\
\hline C & -5.995043 & 4.264235 & -1.980845 \\
\hline $\mathrm{H}$ & -6.664717 & 4.432472 & -1.129215 \\
\hline $\mathrm{H}$ & -5.016412 & 4.683066 & -1.726921 \\
\hline $\mathrm{H}$ & -6.385707 & 4.827827 & -2.837189 \\
\hline C & -4.946570 & 2.563448 & -3.524770 \\
\hline $\mathrm{H}$ & -5.295570 & 3.139717 & -4.391074 \\
\hline $\mathrm{H}$ & -3.926650 & 2.882906 & -3.289640 \\
\hline $\mathrm{H}$ & -4.904444 & 1.506630 & -3.814288 \\
\hline C & -7.309318 & 2.283396 & -2.746332 \\
\hline $\mathrm{H}$ & -7.312615 & 1.227338 & -3.040139 \\
\hline $\mathrm{H}$ & -8.044436 & 2.417634 & -1.944050 \\
\hline $\mathrm{H}$ & -7.651879 & 2.865796 & -3.609308 \\
\hline
\end{tabular}




\begin{tabular}{|lrrr|} 
C & -5.637098 & -1.605214 & 2.387253 \\
$H$ & -4.681855 & -2.049719 & 2.085260 \\
$H$ & -5.442992 & -0.898805 & 3.202414 \\
$H$ & -6.263923 & -2.409495 & 2.789157 \\
C & -7.704761 & -0.376120 & 1.716011 \\
$H$ & -7.546705 & 0.386957 & 2.486826 \\
$H$ & -8.290741 & 0.079893 & 0.911242 \\
$H$ & -8.308426 & -1.181514 & 2.152945 \\
C & -6.621871 & -2.011688 & 0.127924 \\
H & -7.212444 & -2.833933 & 0.551536 \\
H & -7.176416 & -1.608826 & -0.725804 \\
H & -5.681909 & -2.425623 & -0.253933 \\
\hline
\end{tabular}

\section{C-Catalyzed reaction TS 13}

\section{Datum}

Value

\begin{tabular}{lc}
\hline B3LYP/6-31G(d) Energy & -3038.969042 \\
\hline B3LYP-D3(BJ)/def2-TZVPP-IEF-PCM(DCM) Energy & -3040.519452 \\
\hline B3LYP-D3(BJ)/def2-TZVPP-IEF-PCM(DCM)//B3LYP/6-31G(d) Free Energy (Quasiharmonic) & -3039.290596 \\
\hline
\end{tabular}

Number of Imaginary Frequencies

Frequencies (Top 3 out of 450)

$$
\begin{array}{lr}
\text { 1. } & -315.0382 \mathrm{~cm}^{-1} \\
\text { 2. } & 6.8966 \mathrm{~cm}^{-1} \\
\text { 3. } & 10.3882 \mathrm{~cm}^{-1}
\end{array}
$$

\section{B3LYP/6-31G(d) Molecular Geometry in Cartesian Coordinates}

C

$\mathrm{H}$

$\mathrm{H}$

C

0

0

$\mathrm{H}$

0

$\mathrm{H}$

C

C

C

C

$\mathrm{H}$

\section{511242}

1.172509

2.487647

$-1.877420$

$-2.552745$

$-1.134088$

$-0.543000$

0.570969

1.008739

$-0.087174$

$-0.856766$

2.063399

1.126949

$-0.854130$
$-1.064755$

$-1.585423$

$-0.628362$

$-1.424830$

$-1.786755$

$-0.310981$

$-0.217075$

$-0.005286$

0.883374

2.386920

3. 163682

3.072955

2.080897

2.199303

\subsection{2}

1.951912

1. 261397

$-1.140289$

$-2.079979$

$-1.204345$

$-0.398728$

0.785818

0.804473

$-2.744988$

$-0.885895$

$-1.713727$

$-2.167532$

$-0.379253$ 


\begin{tabular}{|c|c|c|c|}
\hline $\mathrm{H}$ & 3.054957 & 2.682925 & -1.475026 \\
\hline $\mathrm{H}$ & 2.123261 & 3.969524 & -2.335677 \\
\hline C & 2.250239 & 3.055545 & 1.966170 \\
\hline C & 2.245991 & 4.616044 & 1.739411 \\
\hline C & 1.585685 & 2.586896 & 3.261605 \\
\hline $\mathrm{H}$ & 1.613486 & 1.493395 & 3.326342 \\
\hline $\mathrm{H}$ & 2.109898 & 2.986851 & 4.137385 \\
\hline $\mathrm{H}$ & 0.539658 & 2.897416 & 3.309693 \\
\hline C & 3.656486 & 2.450078 & 1.846995 \\
\hline $\mathrm{H}$ & 4. 284212 & 2.715549 & 2.705023 \\
\hline $\mathrm{H}$ & 3.587044 & 1.358347 & 1.807651 \\
\hline $\mathrm{H}$ & 4.156761 & 2.788341 & 0.935352 \\
\hline C & 3.501461 & 5.339197 & 2.232248 \\
\hline $\mathrm{H}$ & 3.631878 & 5.220355 & 3.314547 \\
\hline $\mathrm{H}$ & 4.398722 & 4.973844 & 1.727500 \\
\hline $\mathrm{H}$ & 3.410595 & 6.409384 & 2.018838 \\
\hline C & 0.999464 & 5.304316 & 2.325317 \\
\hline $\mathrm{H}$ & 1.023488 & 5.328828 & 3.420692 \\
\hline $\mathrm{H}$ & 0.967473 & 6.335306 & 1.958883 \\
\hline $\mathrm{H}$ & 0.077951 & 4.809018 & 2.007771 \\
\hline 0 & 1.462292 & 2.578214 & 0.844620 \\
\hline 0 & 2.201157 & 4.719955 & 0.308207 \\
\hline B & 1.450296 & 3.620961 & -0.183240 \\
\hline 0 & 0.036214 & 4.038263 & -0.537292 \\
\hline C & -2.173693 & 3.651673 & -1.339646 \\
\hline C & -2.363522 & 4.994970 & -1.700522 \\
\hline C & -3.259677 & 2.762948 & -1.366807 \\
\hline C & -3.625319 & 5.439023 & -2.086135 \\
\hline $\mathrm{H}$ & -1.520538 & 5.677377 & -1.659890 \\
\hline C & -4.521645 & 3.214972 & -1.748359 \\
\hline $\mathrm{H}$ & -3.111261 & 1.721652 & -1.094233 \\
\hline C & -4.706115 & 4.551630 & -2.110758 \\
\hline $\mathrm{H}$ & -3.770072 & 6.480033 & -2.362099 \\
\hline $\mathrm{H}$ & -5.357748 & 2.521913 & -1.755260 \\
\hline $\mathrm{H}$ & -5.690211 & 4.903196 & -2.409144 \\
\hline $\mathrm{H}$ & 1.261501 & 1.059539 & -1.811297 \\
\hline $\mathrm{H}$ & -0.218435 & 3.334089 & -3.261117 \\
\hline $\mathrm{H}$ & -0.786592 & 1.594207 & -2.989207 \\
\hline C & -0.677975 & -2.894630 & 0.494590 \\
\hline C & -1.824725 & -2.180375 & 0.171258 \\
\hline C & -2.961023 & -2.153063 & 1.047285 \\
\hline C & -2.895162 & -2.870803 & 2.225089 \\
\hline $\mathrm{H}$ & -3.745455 & -2.853262 & 2.902156 \\
\hline C & 1.610192 & -2.039236 & -0.114956 \\
\hline C & 0.526484 & -2.869183 & -0.408392 \\
\hline C & 2.784529 & -2.877866 & -2.071619 \\
\hline C & 2.777059 & -2.067380 & -0.951159 \\
\hline $\mathrm{H}$ & 3.672505 & -2.906401 & -2.698266 \\
\hline C & 1.693765 & -3.710641 & -2.409390 \\
\hline C & 0.544979 & -3.726544 & -1.556470 \\
\hline C & -0.529455 & -4.599114 & -1.889015 \\
\hline C & -0.466756 & -5.404680 & -3.002944 \\
\hline C & 0.670548 & -5.383045 & -3.846333 \\
\hline C & 1.726979 & -4.553369 & -3.553718 \\
\hline
\end{tabular}




\begin{tabular}{|c|c|c|c|}
\hline H & -1.412616 & -4.614317 & -1.260720 \\
\hline $\mathrm{H}$ & -1.300141 & -6.059554 & -3.241608 \\
\hline $\mathrm{H}$ & 0.703863 & -6.022633 & -4.724191 \\
\hline $\mathrm{H}$ & 2.606940 & -4.529628 & -4.192205 \\
\hline C & -1.768385 & -3.655110 & 2.572297 \\
\hline C & -0.638453 & -3.679437 & 1.694781 \\
\hline C & 0.477761 & -4.485763 & 2.052751 \\
\hline C & 0.477294 & -5.219470 & 3.218620 \\
\hline C & -0.638453 & -5.186041 & 4.088783 \\
\hline C & -1.736075 & -4.420630 & 3.769192 \\
\hline $\mathrm{H}$ & 1.334879 & -4.522490 & 1.388707 \\
\hline H & 1.338091 & -5.832777 & 3.471187 \\
\hline $\mathrm{H}$ & -0.626235 & -5.768873 & 5.005782 \\
\hline $\mathrm{H}$ & -2.600714 & -4.391815 & 4.428043 \\
\hline C & 6.453141 & -0.017297 & -0.179309 \\
\hline C & 5.963960 & -0.897258 & 0.791146 \\
\hline C & 4.739568 & -1.534723 & 0.532950 \\
\hline C & 4.026777 & -1.303276 & -0.647467 \\
\hline C & 4.558579 & -0.416484 & -1.598936 \\
\hline C & 5.775570 & 0.236854 & -1.385723 \\
\hline $\mathrm{H}$ & 7.397504 & 0.484050 & -0.003824 \\
\hline H & 4.342403 & -2.251903 & 1.246254 \\
\hline $\mathrm{H}$ & 3.998518 & -0.254624 & -2.513085 \\
\hline C & 6.730029 & -1.214586 & 2.090611 \\
\hline C & 6.398183 & 1.195353 & -2.420008 \\
\hline C & 8.016485 & -0.379485 & 2.235547 \\
\hline $\mathrm{H}$ & 8.513457 & -0.629282 & 3.179764 \\
\hline H & 8.728914 & -0.580001 & 1.427413 \\
\hline $\mathrm{H}$ & 7.804644 & 0.695757 & 2.247617 \\
\hline C & 5.832104 & -0.921963 & 3.315803 \\
\hline $\mathrm{H}$ & 6.368069 & -1.155288 & 4.243838 \\
\hline $\mathrm{H}$ & 5.542761 & 0.134613 & 3.347848 \\
\hline $\mathrm{H}$ & 4.915150 & -1.520435 & $3.30593 e$ \\
\hline C & 7.123909 & -2.711475 & 2.093207 \\
\hline $\mathrm{H}$ & 7.665526 & -2.962793 & 3.013470 \\
\hline $\mathrm{H}$ & 6.245954 & -3.363323 & 2.033492 \\
\hline $\mathrm{H}$ & 7.772647 & -2.946772 & 1.241745 \\
\hline C & 7.786260 & 0.657828 & -2.844510 \\
\hline $\mathrm{H}$ & 8.242186 & 1.326087 & -3.585164 \\
\hline $\mathrm{H}$ & 8.473941 & 0.584360 & -1.995545 \\
\hline $\mathrm{H}$ & 7.700804 & -0.338562 & -3.293022 \\
\hline C & 5.533801 & 1.333669 & -3.688033 \\
\hline $\mathrm{H}$ & 6.018771 & 2.021069 & -4.390307 \\
\hline $\mathrm{H}$ & 5.406382 & 0.373792 & -4.201573 \\
\hline $\mathrm{H}$ & 4.539988 & 1.737278 & -3.465650 \\
\hline C & 6.566331 & 2.600780 & -1.795192 \\
\hline $\mathrm{H}$ & 7.029544 & 3.284747 & -2.516784 \\
\hline $\mathrm{H}$ & 5.597101 & 3.023229 & -1.506208 \\
\hline $\mathrm{H}$ & 7.201033 & 2.578640 & -0.903118 \\
\hline C & -4.178598 & -1.328050 & 0.777499 \\
\hline C & -4.565553 & -0.356416 & 1.711184 \\
\hline C & -4.973845 & -1.533043 & -0.356183 \\
\hline C & -5.718798 & 0.417171 & 1.529650 \\
\hline H & -3.936426 & -0.200065 & 2.582681 \\
\hline
\end{tabular}




\begin{tabular}{|c|c|c|c|}
\hline C & -6.136846 & -0.783963 & -0.574498 \\
\hline $\mathrm{H}$ & -4.666057 & -2.282008 & -1.074611 \\
\hline C & -6.487543 & 0.182984 & 0.379609 \\
\hline $\mathrm{H}$ & -7.383945 & 0.767732 & 0.224108 \\
\hline C & -6.971696 & -1.044405 & -1.843950 \\
\hline C & -6.091235 & 1.485449 & 2.577243 \\
\hline C & -6.109484 & -0.732571 & -3.091524 \\
\hline $\mathrm{H}$ & -6.665210 & -0.968861 & -4.007748 \\
\hline $\mathrm{H}$ & -5.842409 & 0.330636 & -3.125491 \\
\hline $\mathrm{H}$ & -5.176489 & -1.303979 & -3.100217 \\
\hline C & -7.405854 & -2.529437 & -1.880725 \\
\hline $\mathrm{H}$ & -8.005088 & -2.727757 & -2.777929 \\
\hline $\mathrm{H}$ & -6.545789 & -3.206060 & -1.900150 \\
\hline $\mathrm{H}$ & -8.012992 & -2.782732 & -1.003709 \\
\hline C & -8.243551 & -0.178066 & -1.905399 \\
\hline $\mathrm{H}$ & -8.910381 & -0.368675 & -1.056404 \\
\hline $\mathrm{H}$ & -8.010763 & 0.893069 & -1.922828 \\
\hline $\mathrm{H}$ & -8.800527 & -0.406002 & -2.821323 \\
\hline C & -4.944073 & 2.518381 & 2.687801 \\
\hline $\mathrm{H}$ & -4.005603 & 2.051945 & 3.005367 \\
\hline $\mathrm{H}$ & -4.764181 & 3.008130 & 1.724167 \\
\hline $\mathrm{H}$ & -5.196422 & 3.292604 & 3.423050 \\
\hline C & -7.381840 & 2.244522 & 2.215020 \\
\hline $\mathrm{H}$ & -7.286348 & 2.780507 & 1.263931 \\
\hline $\mathrm{H}$ & -8.246211 & 1.574214 & 2.145961 \\
\hline $\mathrm{H}$ & -7.601494 & 2.987014 & 2.990577 \\
\hline C & -6.304237 & 0.807297 & 3.951476 \\
\hline $\mathrm{H}$ & -6.564807 & 1.554987 & 4.710886 \\
\hline $\mathrm{H}$ & -7.117148 & 0.073410 & 3.904419 \\
\hline $\mathrm{H}$ & -5.403326 & 0.286605 & 4.292706 \\
\hline
\end{tabular}

\section{C-Catalyzed reaction TS 14}

\section{Datum}

Value

\begin{tabular}{lc}
\hline B3LYP/6-31G(d) Energy & -3038.969568 \\
\hline B3LYP-D3(BJ)/def2-TZVPP-IEF-PCM(DCM) Energy & -3040.519085 \\
\hline B3LYP-D3(BJ)/def2-TZVPP-IEF-PCM(DCM)//B3LYP/6-31G(d) Free Energy (Quasiharmonic) & -3039.290048 \\
\hline
\end{tabular}

Number of Imaginary Frequencies

Frequencies (Top 3 out of 450)

$\begin{array}{lr}\text { 1. } & -343.4134 \mathrm{~cm}^{-1} \\ \text { 2. } & 5.4421 \mathrm{~cm}^{-1} \\ \text { 3. } & 9.3271 \mathrm{~cm}^{-1}\end{array}$




\begin{tabular}{|c|c|c|c|}
\hline C & -2.119105 & -0.348524 & 0.899196 \\
\hline $\mathrm{H}$ & -3.069842 & 0.163521 & 1.079200 \\
\hline $\mathrm{H}$ & -1.630170 & -0.508604 & 1.866229 \\
\hline C & 1.006399 & -1.914941 & -1.079076 \\
\hline 0 & 1.540756 & -2.697036 & -1.838519 \\
\hline 0 & 0.363499 & -0.838572 & -1.543788 \\
\hline $\mathrm{H}$ & -0.143158 & -0.345059 & -0.835620 \\
\hline 0 & -1.284216 & 0.475687 & 0.067593 \\
\hline $\mathrm{H}$ & -0.998109 & 1.264429 & 0.590582 \\
\hline C & 0.803710 & 5.037190 & -2.211783 \\
\hline C & 1.995698 & 3.704837 & -0.996868 \\
\hline C & -0.823630 & 4.687497 & -0.383812 \\
\hline C & 0.088966 & 5.504177 & -1.127080 \\
\hline $\mathrm{H}$ & 2.508660 & 4.513739 & -0.473645 \\
\hline $\mathrm{H}$ & -1.380933 & 3.961512 & -0.978492 \\
\hline $\mathrm{H}$ & -1.472312 & 5.231092 & 0.304665 \\
\hline C & -0.395778 & 3.098323 & 2.834778 \\
\hline C & 0.929955 & 3.950379 & 2.868068 \\
\hline C & -0.347259 & 1.804196 & 3.648299 \\
\hline $\mathrm{H}$ & -1.311079 & 1.287118 & 3.587371 \\
\hline $\mathrm{H}$ & 0.425816 & 1.124071 & 3.283021 \\
\hline $\mathrm{H}$ & -0.153243 & 2.015106 & 4.706450 \\
\hline C & -1.641057 & 3.909558 & 3.225767 \\
\hline $\mathrm{H}$ & -2.536015 & 3.338427 & 2.957868 \\
\hline $\mathrm{H}$ & -1.673109 & 4.110891 & 4.302128 \\
\hline $\mathrm{H}$ & -1.675752 & 4.865862 & 2.695866 \\
\hline C & 2.193105 & 3.075964 & 2.946879 \\
\hline $\mathrm{H}$ & 2.306276 & 2.605931 & 3.930082 \\
\hline $\mathrm{H}$ & 2.178517 & 2.290485 & 2.185121 \\
\hline $\mathrm{H}$ & 3.069297 & 3.707920 & 2.768787 \\
\hline C & 0.978289 & 5.022174 & 3.958179 \\
\hline $\mathrm{H}$ & 0.921967 & 4.575106 & 4.957738 \\
\hline $\mathrm{H}$ & 1.922517 & 5.572723 & 3.888379 \\
\hline $\mathrm{H}$ & 0.163034 & 5.741511 & 3.850039 \\
\hline 0 & -0.506591 & 2.763875 & 1.430309 \\
\hline 0 & 0.907427 & 4.608782 & 1.587198 \\
\hline B & 0.219215 & 3.753501 & 0.670482 \\
\hline 0 & 1.102990 & 3.042384 & -0.325429 \\
\hline C & 2.748425 & 2.998502 & -2.049537 \\
\hline C & 2.363818 & 1.717425 & -2.473298 \\
\hline C & 3.884887 & 3.603608 & -2.611445 \\
\hline C & 3.108366 & 1.052112 & -3.444063 \\
\hline $\mathrm{H}$ & 1.502122 & 1.233359 & -2.029369 \\
\hline C & 4.621794 & 2.938017 & -3.587772 \\
\hline $\mathrm{H}$ & 4.187560 & 4.594933 & -2.280599 \\
\hline C & 4.233594 & 1.661184 & -4.006184 \\
\hline $\mathrm{H}$ & 2.809620 & 0.052402 & -3.745836 \\
\hline $\mathrm{H}$ & 5.500050 & 3.410529 & -4.019001 \\
\hline $\mathrm{H}$ & 4.812775 & 1.140217 & -4.763707 \\
\hline $\mathrm{H}$ & 0.419049 & 6.433016 & -0.660459 \\
\hline
\end{tabular}




\begin{tabular}{|c|c|c|c|}
\hline $\mathrm{H}$ & 0.409459 & 4.219278 & -2.807833 \\
\hline $\mathrm{H}$ & 1.535295 & 5.670408 & -2.705800 \\
\hline C & -0.132646 & -2.469498 & 1.083077 \\
\hline C & 1.036048 & -2.095043 & 0.428415 \\
\hline C & 2.268971 & -1.922683 & 1.150362 \\
\hline C & 2.249101 & -2.093701 & 2.522153 \\
\hline $\mathrm{H}$ & 3.173329 & -1.967493 & 3.079929 \\
\hline C & -2.369432 & -1.684006 & 0.228573 \\
\hline C & -1.410140 & -2.690738 & 0.318000 \\
\hline C & -3.832271 & -3.174184 & -1.012606 \\
\hline C & -3.597378 & -1.924079 & -0.472888 \\
\hline $\mathrm{H}$ & -4.763181 & -3.355500 & -1.544107 \\
\hline C & -2.882753 & -4.219044 & -0.935188 \\
\hline C & -1.638323 & -3.977467 & -0.271451 \\
\hline C & -0.686824 & -5.034258 & -0.216101 \\
\hline C & -0.957119 & -6.259608 & -0.781725 \\
\hline C & -2.191092 & -6.495901 & -1.434594 \\
\hline C & -3.131046 & -5.495173 & -1.509995 \\
\hline $\mathrm{H}$ & 0.267824 & -4.860742 & 0.267309 \\
\hline $\mathrm{H}$ & -0.214421 & -7.051183 & -0.733349 \\
\hline $\mathrm{H}$ & -2.389386 & -7.467549 & -1.879082 \\
\hline $\mathrm{H}$ & -4.079813 & -5.662557 & -2.014463 \\
\hline C & 1.084718 & -2.481527 & 3.227125 \\
\hline C & -0.126807 & -2.697818 & 2.499379 \\
\hline C & -1.278125 & -3.127957 & 3.216676 \\
\hline C & -1.232092 & -3.321228 & 4.579816 \\
\hline C & -0.033200 & -3.097172 & 5.298271 \\
\hline C & 1.099540 & -2.687831 & 4.633401 \\
\hline $\mathrm{H}$ & -2.200318 & -3.307017 & 2.674302 \\
\hline $\mathrm{H}$ & -2.121907 & -3.652514 & 5.108365 \\
\hline $\mathrm{H}$ & -0.010169 & -3.254141 & 6.373304 \\
\hline $\mathrm{H}$ & 2.028339 & -2.520191 & 5.173656 \\
\hline C & -6.625638 & 1.017375 & -1.112232 \\
\hline C & -6.968256 & -0.164016 & -0.439565 \\
\hline C & -5.954132 & -1.105401 & -0.226744 \\
\hline C & -4.640411 & -0.871308 & -0.655802 \\
\hline C & -4.337746 & 0.325067 & -1.320049 \\
\hline C & -5.326428 & 1.284153 & -1.567967 \\
\hline $\mathrm{H}$ & -7.400129 & 1.758026 & -1.288876 \\
\hline $\mathrm{H}$ & -6.164848 & -2.035472 & 0.288812 \\
\hline $\mathrm{H}$ & -3.317058 & 0.484128 & -1.644892 \\
\hline C & -8.417960 & -0.381690 & 0.036546 \\
\hline C & -5.029441 & 2.599990 & -2.312496 \\
\hline C & -8.803833 & 0.733641 & 1.037285 \\
\hline $\mathrm{H}$ & -9.835749 & 0.596877 & 1.383910 \\
\hline $\mathrm{H}$ & -8.145988 & 0.717269 & 1.913838 \\
\hline $\mathrm{H}$ & -8.733148 & 1.728852 & 0.585935 \\
\hline C & -9.375422 & -0.333172 & -1.177813 \\
\hline $\mathrm{H}$ & -10.412428 & -0.481849 & -0.852313 \\
\hline $\mathrm{H}$ & -9.324975 & 0.627855 & -1.700077 \\
\hline $\mathrm{H}$ & -9.130048 & -1.118929 & -1.901406 \\
\hline C & -8.607896 & -1.739668 & 0.739578 \\
\hline $\mathrm{H}$ & -9.651199 & -1.847814 & 1.057332 \\
\hline $\mathrm{H}$ & -8.376627 & -2.579548 & 0.074629 \\
\hline
\end{tabular}




\begin{tabular}{|c|c|c|c|}
\hline H & -7.979897 & -1.828473 & 1.633300 \\
\hline C & -5.966725 & 2.733753 & -3.535938 \\
\hline $\mathrm{H}$ & -5.753841 & 3.663185 & -4.078467 \\
\hline $\mathrm{H}$ & -5.826882 & 1.896539 & -4.229170 \\
\hline $\mathrm{H}$ & -7.022391 & 2.754926 & -3.246015 \\
\hline C & -5.264652 & 3.792210 & -1.354975 \\
\hline $\mathrm{H}$ & -5.071824 & 4.741766 & -1.869670 \\
\hline $\mathrm{H}$ & -6.294867 & 3.816463 & -0.983309 \\
\hline $\mathrm{H}$ & -4.598072 & 3.734320 & -0.486854 \\
\hline C & -3.576677 & 2.662988 & -2.820876 \\
\hline $\mathrm{H}$ & -3.403938 & 3.618978 & -3.328620 \\
\hline $\mathrm{H}$ & -2.853868 & 2.581865 & -2.002056 \\
\hline $\mathrm{H}$ & -3.362876 & 1.861803 & -3.537373 \\
\hline C & 3.578527 & -1.572027 & 0.517219 \\
\hline C & 4.137935 & -2.378043 & -0.488819 \\
\hline C & 4.319687 & -0.493368 & 1.012271 \\
\hline C & 5.407270 & -2.112346 & -1.003600 \\
\hline H & 3.556215 & -3.208533 & -0.859901 \\
\hline C & 5.607439 & -0.203070 & 0.532654 \\
\hline $\mathrm{H}$ & 3.875546 & 0.127492 & 1.785344 \\
\hline C & 6.122469 & -1.023901 & -0.474150 \\
\hline $\mathrm{H}$ & 7.111752 & -0.818759 & -0.865925 \\
\hline C & 6.395655 & 0.985998 & 1.117367 \\
\hline C & 6.036388 & -2.966754 & -2.121210 \\
\hline C & 6.522672 & 0.822643 & 2.650754 \\
\hline H & 5.544946 & 0.800958 & 3.143247 \\
\hline $\mathrm{H}$ & 7.045762 & -0.106708 & 2.904153 \\
\hline $\mathrm{H}$ & 7.089700 & 1.659241 & 3.077810 \\
\hline C & 7.817983 & 1.094476 & 0.535198 \\
\hline $\mathrm{H}$ & 8.340053 & 1.941665 & 0.994608 \\
\hline $\mathrm{H}$ & 8.409568 & 0.193339 & 0.733428 \\
\hline $\mathrm{H}$ & 7.805053 & 1.263935 & -0.547376 \\
\hline C & 5.647006 & 2.301862 & 0.802978 \\
\hline H & 5.558181 & 2.450831 & -0.278788 \\
\hline $\mathrm{H}$ & 4.636502 & 2.300950 & 1.223459 \\
\hline $\mathrm{H}$ & 6.186136 & 3.159795 & 1.225156 \\
\hline C & 7.368409 & -3.577430 & -1.625594 \\
\hline $\mathrm{H}$ & 8.092811 & -2.806796 & -1.340292 \\
\hline $\mathrm{H}$ & 7.203153 & -4.219465 & -0.752727 \\
\hline $\mathrm{H}$ & 7.825388 & -4.187842 & -2.414471 \\
\hline C & 5.113801 & -4.117804 & -2.566154 \\
\hline $\mathrm{H}$ & 4.921431 & -4.822534 & -1.749012 \\
\hline $\mathrm{H}$ & 4.148296 & -3.751381 & -2.930085 \\
\hline $\mathrm{H}$ & 5.590904 & -4.677798 & -3.378888 \\
\hline C & 6.312468 & -2.071168 & -3.352648 \\
\hline $\mathrm{H}$ & 6.763591 & -2.660997 & -4.160394 \\
\hline $\mathrm{H}$ & 5.381663 & -1.632311 & -3.729375 \\
\hline $\mathrm{H}$ & 6.997607 & -1.249861 & -3.115964 \\
\hline
\end{tabular}




\begin{tabular}{lc}
\hline B3LYP/6-31G(d) Energy & -3038.970208 \\
\hline B3LYP-D3(BJ)/def2-TZVPP-IEF-PCM(DCM) Energy & -3040.519776 \\
\hline B3LYP-D3(BJ)/def2-TZVPP-IEF-PCM(DCM)//B3LYP/6-31G(d) Free Energy (Quasiharmonic) & -3039.289787 \\
\hline
\end{tabular}

Number of Imaginary Frequencies

Frequencies (Top 3 out of 450)

$\begin{array}{lr}\text { 1. } & -288.4255 \mathrm{~cm}^{-1} \\ \text { 2. } & 7.6046 \mathrm{~cm}^{-1} \\ \text { 3. } & 8.6270 \mathrm{~cm}^{-1}\end{array}$

\section{B3LYP/6-31G(d) Molecular Geometry in Cartesian Coordinates}

C

$\mathrm{H}$

$\mathrm{H}$

C

0

0

$\mathrm{H}$

0

$\mathrm{H}$

C

C

C

C

$\mathrm{H}$

$\mathrm{H}$

$\mathrm{H}$

C

C

C

$\mathrm{H}$

$\mathrm{H}$

$\mathrm{H}$

C

$\mathrm{H}$

$\mathrm{H}$

$\mathrm{H}$

C

$\mathrm{H}$

$\mathrm{H}$

$\mathrm{H}$

C

$\mathrm{H}$
$-1.566495$

$-2.546135$

$-1.017510$

1.575495

2.100423

0.879167

0.346679

$-0.841186$

$-0.638127$

2. 352257

0.205953

2. 198610

2.355570

$-0.108125$

2.475087

2.652990

$-1.200843$

$-0.512296$

$-2.510971$

$-2.377715$

$-2.828928$

$-3.314647$

$-1.424872$

$-2.059939$

$-1.933149$

$-0.480619$

0.499110

0.004975

1.177704

1.098931

$-1.484697$

$-2.117369$
$-0.797309$

$-0.412630$

$-1.085551$

$-1.702096$

$-2.306417$

$-0.578719$

$-0.296321$

0.238747

0.990484

2. 532623

3.069127

3. 342637

2. 302388

2.052742

3.050661

4. 298382

3.048158

4. 385096

3. 288811

4.040170

2. 354480

3.619747

2.020682

2.436066

1.134164

1.698729

4.168885

3.964635

3. 339380

5.077779

5.501873

5.204750
1.081624

1.382035

1.984665

$-1.225817$

$-2.136588$

$-1.452799$

$-0.655843$

0.390974

1.006542

$-1.218413$

$-1.156999$

1.118655

0.136932

$-0.926820$

2.133875

0.845818

2. 729251

3. 195591

1.964304

1.180676

1.489161

2. 631245

3.839698

4.630772

3. 443945

4.285378

4.335824

5.292270

4.115891

4.446061

3.584520

4.429636 


\begin{tabular}{|c|c|c|c|}
\hline $\mathrm{H}$ & -0.917809 & 6.390045 & 3.882830 \\
\hline $\mathrm{H}$ & -2.126173 & 5.781978 & 2.745990 \\
\hline 0 & -0.221103 & 2.519537 & 1.796473 \\
\hline 0 & 0.203812 & 4.798204 & 2.026171 \\
\hline B & 0.496157 & 3.660215 & 1.226749 \\
\hline 0 & 0.140032 & 3.961138 & -0.221728 \\
\hline C & 0.055540 & 3.494889 & -2.559404 \\
\hline C & 0.052948 & 4.854057 & -2.909862 \\
\hline C & -0.111925 & 2.516868 & -3.554174 \\
\hline C & -0.115771 & 5.226796 & -4.240873 \\
\hline $\mathrm{H}$ & 0.172164 & 5.600421 & -2.131061 \\
\hline C & -0.278769 & 2.897073 & -4.883578 \\
\hline $\mathrm{H}$ & -0.092072 & 1.464057 & -3.281019 \\
\hline C & -0.280728 & 4.251414 & -5.229081 \\
\hline $\mathrm{H}$ & -0.122062 & 6.279572 & -4.509849 \\
\hline $\mathrm{H}$ & -0.405326 & 2.137792 & -5.650052 \\
\hline $\mathrm{H}$ & -0.411511 & 4.546713 & -6.266658 \\
\hline $\mathrm{H}$ & 2.231806 & 1.272223 & 0.469150 \\
\hline $\mathrm{H}$ & 2.627413 & 3.509001 & -1.607122 \\
\hline $\mathrm{H}$ & 2.384211 & 1.702863 & -1.916983 \\
\hline C & 0.571729 & -2.765792 & 0.803520 \\
\hline C & 1.677030 & -2.158665 & 0.218783 \\
\hline C & 2.913269 & -2.009066 & 0.940151 \\
\hline C & 2.969391 & -2.487308 & 2.236358 \\
\hline $\mathrm{H}$ & 3.897904 & -2.388814 & 2.792510 \\
\hline C & -1.744728 & -1.997157 & 0.174125 \\
\hline C & -0.713670 & -2.926056 & 0.038014 \\
\hline C & -3.144093 & -3.324635 & -1.301459 \\
\hline C & -2.982687 & -2.194572 & -0.522399 \\
\hline $\mathrm{H}$ & -4.083576 & -3.474405 & -1.827456 \\
\hline C & -2.118146 & -4.283959 & -1.463562 \\
\hline C & -0.871575 & -4.084724 & -0.790960 \\
\hline C & 0.153132 & -5.056886 & -0.966438 \\
\hline C & -0.051082 & -6.163170 & -1.759286 \\
\hline C & -1.287424 & -6.357976 & -2.421469 \\
\hline C & -2.297042 & -5.435863 & -2.276859 \\
\hline $\mathrm{H}$ & 1.109797 & -4.911945 & -0.477629 \\
\hline $\mathrm{H}$ & 0.745923 & -6.890885 & -1.884291 \\
\hline $\mathrm{H}$ & -1.432700 & -7.234969 & -3.046465 \\
\hline $\mathrm{H}$ & -3.249291 & -5.571688 & -2.784113 \\
\hline C & 1.876761 & -3.132822 & 2.861834 \\
\hline C & 0.653518 & -3.286291 & 2.137751 \\
\hline C & -0.430504 & -3.952783 & 2.774145 \\
\hline C & -0.308783 & -4.434096 & 4.059135 \\
\hline C & 0.902106 & -4.276162 & 4.774956 \\
\hline C & 1.970494 & -3.640321 & 4.186109 \\
\hline $\mathrm{H}$ & -1.360782 & -4.083506 & 2.231732 \\
\hline $\mathrm{H}$ & -1.147482 & -4.942670 & 4.526779 \\
\hline $\mathrm{H}$ & 0.985030 & -4.661004 & 5.787732 \\
\hline $\mathrm{H}$ & 2.906832 & -3.517477 & 4.725259 \\
\hline C & -6.380741 & 0.397922 & -0.470372 \\
\hline C & -5.164591 & 0.902207 & -0.968058 \\
\hline C & -4.043448 & 0.067970 & -0.927820 \\
\hline C & -4.136824 & -1.246480 & -0.437654 \\
\hline
\end{tabular}




\begin{tabular}{|c|c|c|c|}
\hline C & -5.364602 & -1.707696 & 0.044296 \\
\hline C & -6.508104 & -0.893038 & 0.047856 \\
\hline $\mathrm{H}$ & -7.253029 & 1.039996 & -0.499760 \\
\hline $\mathrm{H}$ & -3.083954 & 0.409036 & -1.297920 \\
\hline $\mathrm{H}$ & -5.418610 & -2.724618 & 0.422586 \\
\hline C & -5.130110 & 2.317208 & -1.579375 \\
\hline C & -7.836645 & -1.444835 & 0.600200 \\
\hline C & -6.042051 & 2.345173 & -2.830163 \\
\hline $\mathrm{H}$ & -6.035288 & 3.343417 & -3.285004 \\
\hline $\mathrm{H}$ & -5.696715 & 1.626578 & -3.582186 \\
\hline $\mathrm{H}$ & -7.079649 & 2.097006 & -2.582326 \\
\hline C & -5.646444 & 3.353657 & -0.553543 \\
\hline $\mathrm{H}$ & -5.627203 & 4.359541 & -0.990302 \\
\hline $\mathrm{H}$ & -6.676585 & 3.148768 & -0.243619 \\
\hline $\mathrm{H}$ & -5.021492 & 3.363524 & 0.345964 \\
\hline C & -3.712554 & 2.734263 & -2.010870 \\
\hline $\mathrm{H}$ & -3.732601 & 3.750163 & -2.420857 \\
\hline $\mathrm{H}$ & -3.013874 & 2.732582 & -1.167569 \\
\hline $\mathrm{H}$ & -3.309856 & 2.076605 & -2.788762 \\
\hline C & -7.651101 & -1.841600 & 2.084275 \\
\hline $\mathrm{H}$ & -8.585953 & -2.246440 & 2.491126 \\
\hline $\mathrm{H}$ & -6.875781 & -2.604714 & 2.208786 \\
\hline $\mathrm{H}$ & -7.365472 & -0.973176 & 2.688987 \\
\hline C & -8.980951 & -0.416454 & 0.520296 \\
\hline $\mathrm{H}$ & -9.898607 & -0.853415 & 0.930159 \\
\hline $\mathrm{H}$ & -8.758730 & 0.487494 & 1.098723 \\
\hline $\mathrm{H}$ & -9.191077 & -0.118989 & -0.513406 \\
\hline C & -8.255359 & -2.692121 & -0.213965 \\
\hline $\mathrm{H}$ & -9.197892 & -3.100959 & 0.170626 \\
\hline $\mathrm{H}$ & -8.400817 & -2.439231 & -1.270493 \\
\hline $\mathrm{H}$ & -7.502651 & -3.485604 & -0.161060 \\
\hline C & 4.126189 & -1.326970 & 0.392911 \\
\hline C & 4.702800 & -1.693663 & -0.825638 \\
\hline C & 4.749245 & -0.327968 & 1.166623 \\
\hline C & 5.870471 & -1.072826 & -1.298023 \\
\hline $\mathrm{H}$ & 4.229967 & -2.471109 & -1.410657 \\
\hline C & 5.917301 & 0.304202 & 0.737880 \\
\hline $\mathrm{H}$ & 4.290408 & -0.048599 & 2.108212 \\
\hline C & 6.455081 & -0.084533 & -0.502622 \\
\hline $\mathrm{H}$ & 7.356697 & 0.406345 & -0.849769 \\
\hline C & 6.619795 & 1.403346 & 1.558958 \\
\hline C & 6.444306 & -1.495883 & -2.663958 \\
\hline C & 8.071809 & 0.968269 & 1.869728 \\
\hline $\mathrm{H}$ & 8.585485 & 1.743031 & 2.452286 \\
\hline $\mathrm{H}$ & 8.087190 & 0.039445 & 2.451644 \\
\hline $\mathrm{H}$ & 8.652773 & 0.799058 & 0.957202 \\
\hline C & 6.641839 & 2.718638 & 0.744309 \\
\hline $\mathrm{H}$ & 7.135199 & 3.514682 & 1.315646 \\
\hline $\mathrm{H}$ & 7.182602 & 2.602573 & -0.200764 \\
\hline $\mathrm{H}$ & 5.623689 & 3.047793 & 0.508622 \\
\hline C & 5.911474 & 1.682576 & 2.898110 \\
\hline $\mathrm{H}$ & 4.886006 & 2.039459 & 2.750810 \\
\hline $\mathrm{H}$ & 5.876600 & 0.791431 & 3.535460 \\
\hline $\mathrm{H}$ & 6.454266 & 2.460388 & 3.447154 \\
\hline
\end{tabular}




$\begin{array}{llll}\mathrm{C} & 6.756932 & -3.011082 & -2.648940 \\ \mathrm{H} & 7.495294 & -3.252213 & -1.875144 \\ \mathrm{H} & 5.861870 & -3.611333 & -2.458224 \\ \mathrm{H} & 7.165923 & -3.323338 & -3.617759 \\ \mathrm{C} & 5.396383 & -1.198764 & -3.764347 \\ \mathrm{H} & 4.443364 & -1.700649 & -3.570804 \\ \mathrm{H} & 5.196986 & -0.122431 & -3.828532 \\ \mathrm{H} & 5.766395 & -1.532948 & -4.741883 \\ \mathrm{C} & 7.742387 & -0.746017 & -3.016878 \\ \mathrm{H} & 8.108445 & -1.082922 & -3.993416 \\ \mathrm{H} & 7.584650 & 0.336741 & -3.081352 \\ \mathrm{H} & 8.535886 & -0.933930 & -2.283962\end{array}$

\section{C-Catalyzed reaction TS 16}

\section{Datum}

Value

\begin{tabular}{lc}
\hline B3LYP/6-31G(d) Energy & -3038.970635 \\
\hline B3LYP-D3(BJ)/def2-TZVPP-IEF-PCM(DCM) Energy & -3040.518901 \\
\hline B3LYP-D3(BJ)/def2-TZVPP-IEF-PCM(DCM)//B3LYP/6-31G(d) Free Energy (Quasiharmonic) & -3039.289671 \\
\hline
\end{tabular}

Number of Imaginary Frequencies

Frequencies (Top 3 out of 450)

$\begin{array}{lr}\text { 1. } & -319.3917 \mathrm{~cm}^{-1} \\ \text { 2. } & 5.7616 \mathrm{~cm}^{-1} \\ \text { 3. } & 9.6426 \mathrm{~cm}^{-1}\end{array}$

\section{B3LYP/6-31G(d) Molecular Geometry in Cartesian Coordinates}

$\begin{array}{rrrr}\mathrm{C} & -1.784661 & 0.828177 & 0.970511 \\ \mathrm{H} & -1.279600 & 1.057692 & 1.914846 \\ \mathrm{H} & -2.760773 & 0.394108 & 1.200878 \\ \mathrm{C} & 1.523373 & 1.886448 & -1.034310 \\ \mathrm{O} & 2.173478 & 2.541671 & -1.821122 \\ \mathrm{O} & 0.840722 & 0.804457 & -1.433529 \\ \mathrm{H} & 0.228175 & 0.461825 & -0.721890 \\ \mathrm{O} & -1.013054 & -0.122783 & 0.218763 \\ \mathrm{H} & -0.945863 & -0.985394 & 0.703430 \\ \mathrm{C} & -0.542728 & -2.715946 & -2.699760 \\ \mathrm{C} & 0.998162 & -2.935413 & -1.173517 \\ \mathrm{C} & -1.916614 & -3.768103 & -0.926856 \\ \mathrm{C} & -1.542773 & -2.652239 & -1.752610 \\ \mathrm{H} & 0.865189 & -1.923516 & -0.791377\end{array}$




\begin{tabular}{|c|c|c|c|}
\hline H & -2.861734 & -3.643563 & -0.394296 \\
\hline $\mathrm{H}$ & -1.859973 & -4.744320 & -1.414602 \\
\hline C & -0.547211 & -3.079871 & 2.553556 \\
\hline C & -1.121528 & -4.546169 & 2.520909 \\
\hline C & 0.968428 & -3.032575 & 2.805159 \\
\hline H & 1.329865 & -2.017872 & 2.604439 \\
\hline H & 1.214982 & -3.282449 & 3.842721 \\
\hline $\mathrm{H}$ & 1.501588 & -3.722468 & 2.145105 \\
\hline C & -1.260232 & -2.125266 & 3.511392 \\
\hline $\mathrm{H}$ & -2.317716 & -2.016399 & 3.257772 \\
\hline $\mathrm{H}$ & -1.187083 & -2.482963 & 4.544808 \\
\hline H & -0.794633 & -1.133599 & 3.472735 \\
\hline C & -2.643225 & -4.594437 & 2.746172 \\
\hline $\mathrm{H}$ & -2.913217 & -4.383252 & 3.787112 \\
\hline $\mathrm{H}$ & -3.165210 & -3.879330 & 2.102621 \\
\hline $\mathrm{H}$ & -3.000921 & -5.598119 & 2.495559 \\
\hline C & -0.432169 & -5.521329 & 3.478828 \\
\hline $\mathrm{H}$ & -0.543195 & -5.202556 & 4.522293 \\
\hline $\mathrm{H}$ & -0.886501 & -6.512562 & 3.378211 \\
\hline $\mathrm{H}$ & 0.632008 & -5.618013 & 3.251736 \\
\hline 0 & -0.794360 & -2.642524 & 1.192966 \\
\hline 0 & -0.852363 & -4.967652 & 1.176973 \\
\hline B & -0.722571 & -3.826536 & 0.340658 \\
\hline 0 & 0.567304 & -3.922027 & -0.451467 \\
\hline C & 2.167245 & -3.159880 & -2.043459 \\
\hline C & 2.831668 & -2.053946 & -2.598152 \\
\hline C & 2.636382 & -4.458391 & -2.300807 \\
\hline C & 3.950401 & -2.249289 & -3.405259 \\
\hline $\mathrm{H}$ & 2.467036 & -1.049204 & -2.398753 \\
\hline C & 3.755778 & -4.644821 & -3.107053 \\
\hline $\mathrm{H}$ & 2.122455 & -5.303933 & -1.854995 \\
\hline C & 4.413066 & -3.542212 & -3.662336 \\
\hline $\mathrm{H}$ & 4.460821 & -1.390815 & -3.832139 \\
\hline $\mathrm{H}$ & 4.120068 & -5.649996 & -3.301112 \\
\hline $\mathrm{H}$ & 5.286442 & -3.691612 & -4.291580 \\
\hline $\mathrm{H}$ & -1.872482 & -1.662849 & -1.437233 \\
\hline $\mathrm{H}$ & -0.286140 & -3.665381 & -3.161320 \\
\hline $\mathrm{H}$ & -0.235115 & -1.823484 & -3.237137 \\
\hline C & 0.314281 & 2.821262 & 0.961257 \\
\hline C & 1.461112 & 2.232387 & 0.442244 \\
\hline C & 2.610178 & 1.985452 & 1.269181 \\
\hline C & 2.547145 & 2.345815 & 2.601150 \\
\hline $\mathrm{H}$ & 3.407440 & 2.157891 & 3.238512 \\
\hline C & -1.938803 & 2.082683 & 0.135704 \\
\hline C & -0.905160 & 3.017076 & 0.101395 \\
\hline C & -3.174223 & 3.367386 & -1.513487 \\
\hline C & -3.094380 & 2.258975 & -0.694184 \\
\hline $\mathrm{H}$ & -4.053945 & 3.504011 & -2.137726 \\
\hline C & -2.153053 & 4.346513 & -1.556600 \\
\hline C & -0.992330 & 4.175515 & -0.737007 \\
\hline C & 0.025774 & 5.167904 & -0.789845 \\
\hline C & -0.104436 & 6.270424 & -1.603274 \\
\hline C & -1.256047 & 6.438985 & -2.409555 \\
\hline C & -2.256302 & 5.495407 & -2.386692 \\
\hline
\end{tabular}




\begin{tabular}{|c|c|c|c|}
\hline $\mathrm{H}$ & 0.917836 & 5.040637 & -0.186897 \\
\hline $\mathrm{H}$ & 0.685402 & 7.015890 & -1.631835 \\
\hline $\mathrm{H}$ & -1.343951 & 7.313459 & -3.048635 \\
\hline $\mathrm{H}$ & -3.143460 & 5.613094 & -3.004494 \\
\hline C & 1.415828 & 2.986377 & 3.162195 \\
\hline C & 0.278814 & 3.241236 & 2.332604 \\
\hline C & -0.844129 & 3.899141 & 2.907494 \\
\hline C & -0.841299 & 4.277858 & 4.231950 \\
\hline C & 0.282902 & 4.018170 & 5.051849 \\
\hline C & 1.386225 & 3.386880 & 4.525428 \\
\hline $\mathrm{H}$ & -1.708433 & 4.106072 & 2.285394 \\
\hline $\mathrm{H}$ & -1.707134 & 4.783504 & 4.650872 \\
\hline $\mathrm{H}$ & 0.272307 & 4.321846 & 6.095125 \\
\hline $\mathrm{H}$ & 2.257181 & 3.186682 & 5.145005 \\
\hline C & -6.360744 & -0.496206 & -0.807260 \\
\hline C & -5.531339 & -0.387840 & -1.931877 \\
\hline C & -4.460132 & 0.513058 & -1.864200 \\
\hline C & -4.223512 & 1.276478 & -0.714123 \\
\hline C & -5.081647 & 1.142973 & 0.386059 \\
\hline C & -6.163104 & 0.254374 & 0.361117 \\
\hline $\mathrm{H}$ & -7.194200 & -1.191396 & -0.842871 \\
\hline $\mathrm{H}$ & -3.785738 & 0.631829 & -2.704344 \\
\hline $\mathrm{H}$ & -4.897871 & 1.759147 & 1.259469 \\
\hline C & -5.823375 & -1.239087 & -3.183579 \\
\hline C & -7.124476 & 0.080131 & 1.553476 \\
\hline C & -7.215335 & -0.860184 & -3.743676 \\
\hline $\mathrm{H}$ & -7.444470 & -1.457411 & -4.634870 \\
\hline $\mathrm{H}$ & -8.010741 & -1.035430 & -3.011498 \\
\hline $\mathrm{H}$ & -7.249250 & 0.198270 & -4.026284 \\
\hline C & -4.782150 & -1.022653 & -4.298572 \\
\hline $\mathrm{H}$ & -5.033028 & -1.649160 & -5.162014 \\
\hline $\mathrm{H}$ & -4.759812 & 0.017518 & -4.642632 \\
\hline $\mathrm{H}$ & -3.772425 & -1.298532 & -3.973552 \\
\hline C & -5.813859 & -2.739880 & -2.808181 \\
\hline $\mathrm{H}$ & -6.029128 & -3.353881 & -3.691241 \\
\hline $\mathrm{H}$ & -4.835090 & -3.040261 & -2.417569 \\
\hline $\mathrm{H}$ & -6.565351 & -2.978199 & -2.048277 \\
\hline C & -6.762581 & 0.994419 & 2.739315 \\
\hline $\mathrm{H}$ & -5.760064 & 0.782135 & 3.128126 \\
\hline $\mathrm{H}$ & -6.806137 & 2.054717 & 2.465644 \\
\hline $\mathrm{H}$ & -7.473638 & 0.835415 & 3.557864 \\
\hline C & -8.567460 & 0.420145 & 1.110597 \\
\hline $\mathrm{H}$ & -9.263186 & 0.294487 & 1.949285 \\
\hline $\mathrm{H}$ & -8.636612 & 1.457551 & 0.763930 \\
\hline $\mathrm{H}$ & -8.908631 & -0.227135 & 0.296148 \\
\hline C & -7.071328 & -1.386148 & 2.044556 \\
\hline $\mathrm{H}$ & -7.750953 & -1.529806 & 2.893536 \\
\hline $\mathrm{H}$ & -7.365205 & -2.090694 & 1.259662 \\
\hline $\mathrm{H}$ & -6.059501 & -1.653483 & 2.370590 \\
\hline C & 3.850242 & 1.312418 & 0.773473 \\
\hline C & 4.295527 & 0.139943 & 1.410640 \\
\hline C & 4.614295 & 1.855050 & -0.261631 \\
\hline C & 5.477484 & -0.493722 & 1.022166 \\
\hline $\mathrm{H}$ & 3.687993 & -0.270294 & 2.209541 \\
\hline
\end{tabular}




\begin{tabular}{|c|c|c|c|}
\hline C & 5.809259 & 1.249503 & -0.680879 \\
\hline $\mathrm{H}$ & 4.262366 & 2.755700 & -0.747821 \\
\hline C & 6.215991 & 0.083587 & -0.027018 \\
\hline $\mathrm{H}$ & 7.134003 & -0.401365 & -0.337756 \\
\hline C & 6.603765 & 1.882550 & -1.839527 \\
\hline C & 5.987423 & -1.787888 & 1.686112 \\
\hline C & 5.735049 & 1.854114 & -3.120404 \\
\hline $\mathrm{H}$ & 6.258684 & 2.348200 & -3.948480 \\
\hline $\mathrm{H}$ & 5.528153 & 0.820028 & -3.421735 \\
\hline $\mathrm{H}$ & 4.772188 & 2.353403 & -2.976004 \\
\hline C & 6.952341 & 3.349226 & -1.490774 \\
\hline $\mathrm{H}$ & 7.520658 & 3.808988 & -2.308668 \\
\hline $\mathrm{H}$ & 6.055492 & 3.955361 & -1.329317 \\
\hline $\mathrm{H}$ & 7.562100 & 3.402675 & -0.581230 \\
\hline C & 7.920249 & 1.138212 & -2.130691 \\
\hline $\mathrm{H}$ & 8.590636 & 1.137700 & -1.263163 \\
\hline $\mathrm{H}$ & 7.745271 & 0.097847 & -2.428032 \\
\hline $\mathrm{H}$ & 8.447287 & 1.630704 & -2.955868 \\
\hline C & 5.069118 & -2.264949 & 2.826783 \\
\hline $\mathrm{H}$ & 4.060080 & -2.496848 & 2.468456 \\
\hline $\mathrm{H}$ & 4.986561 & -1.517382 & 3.624158 \\
\hline $\mathrm{H}$ & 5.478554 & -3.178765 & 3.272313 \\
\hline C & 6.062068 & -2.914265 & 0.627614 \\
\hline $\mathrm{H}$ & 6.744315 & -2.660042 & -0.190337 \\
\hline $\mathrm{H}$ & 5.076938 & -3.108422 & 0.188974 \\
\hline $\mathrm{H}$ & 6.421774 & -3.844352 & 1.085337 \\
\hline $\mathrm{C}$ & 7.396426 & -1.546849 & 2.278378 \\
\hline $\mathrm{H}$ & 7.774868 & -2.463976 & 2.746604 \\
\hline $\mathrm{H}$ & 7.371395 & -0.760993 & 3.042224 \\
\hline $\mathrm{H}$ & 8.117116 & -1.243521 & 1.511944 \\
\hline
\end{tabular}

\section{C-Catalyzed reaction TS 17}

\section{Datum}

Value

\begin{tabular}{lc}
\hline B3LYP/6-31G(d) Energy & -3038.968729 \\
\hline B3LYP-D3(BJ)/def2-TZVPP-IEF-PCM(DCM) Energy & -3040.518166 \\
\hline B3LYP-D3(BJ)/def2-TZVPP-IEF-PCM(DCM)//B3LYP/6-31G(d) Free Energy (Quasiharmonic) & -3039.289641 \\
\hline
\end{tabular}

Number of Imaginary Frequencies

Frequencies (Top 3 out of 450)

$$
\begin{array}{lr}
\text { 1. } & -333.9529 \mathrm{~cm}^{-1} \\
\text { 2. } & 6.0166 \mathrm{~cm}^{-1} \\
\text { 3. } & 11.1367 \mathrm{~cm}^{-1}
\end{array}
$$




\begin{tabular}{|c|c|c|c|}
\hline C & -1.406612 & -1.173370 & 0.842462 \\
\hline $\mathrm{H}$ & -2.411899 & -0.914665 & 1.182399 \\
\hline $\mathrm{H}$ & -0.846612 & -1.553787 & 1.704639 \\
\hline C & 1.878547 & -1.246822 & -1.541612 \\
\hline 0 & 2.609124 & -1.566484 & -2.452146 \\
\hline 0 & 0.957246 & -0.281373 & -1.681658 \\
\hline $\mathrm{H}$ & 0.391811 & -0.183997 & -0.863234 \\
\hline 0 & -0.768767 & 0.005078 & 0.324638 \\
\hline $\mathrm{H}$ & -0.670911 & 0.651129 & 1.066446 \\
\hline C & -2.888516 & 4.466726 & -0.624316 \\
\hline C & -0.748638 & 4.298158 & -0.291865 \\
\hline C & -2.963431 & 3.007643 & 1.371568 \\
\hline C & -3.193273 & 4.254965 & 0.704848 \\
\hline $\mathrm{H}$ & -0.679459 & 5.236272 & 0.262244 \\
\hline $\mathrm{H}$ & -3.101846 & 2.116520 & 0.755199 \\
\hline $\mathrm{H}$ & -3.445541 & 2.911205 & 2.345928 \\
\hline C & 0.121143 & 2.148119 & 3.400262 \\
\hline C & -0.359088 & 3.583157 & 3.848155 \\
\hline C & 1.520934 & 2.154325 & 2.767872 \\
\hline $\mathrm{H}$ & 2.301958 & 2.330852 & 3.515185 \\
\hline $\mathrm{H}$ & 1.712526 & 1.180479 & 2.304961 \\
\hline $\mathrm{H}$ & 1.604962 & 2.920346 & 1.992220 \\
\hline C & 0.045034 & 1.069223 & 4.480439 \\
\hline $\mathrm{H}$ & 0.387317 & 0.111123 & 4.074152 \\
\hline $\mathrm{H}$ & 0.690213 & 1.319750 & 5.330601 \\
\hline $\mathrm{H}$ & -0.976326 & 0.935969 & 4.844499 \\
\hline C & 0.755474 & 4.502144 & 4.352642 \\
\hline $\mathrm{H}$ & 1.241881 & 4.084770 & 5.242274 \\
\hline $\mathrm{H}$ & 1.514898 & 4.670600 & 3.585502 \\
\hline $\mathrm{H}$ & 0.332192 & 5.474850 & 4.625478 \\
\hline C & -1.492953 & 3.532693 & 4.886537 \\
\hline $\mathrm{H}$ & -1.135038 & 3.207428 & 5.869684 \\
\hline $\mathrm{H}$ & -1.918874 & 4.535836 & 4.991606 \\
\hline $\mathrm{H}$ & -2.292138 & 2.855104 & 4.570463 \\
\hline 0 & -0.843818 & 1.825067 & 2.366524 \\
\hline 0 & -0.893998 & 4.139231 & 2.632419 \\
\hline B & -1.255131 & 3.066119 & 1.760977 \\
\hline 0 & -0.646354 & 3.197820 & 0.384839 \\
\hline C & -0.277763 & 4.296518 & -1.688896 \\
\hline C & -0.049473 & 3.087692 & -2.366953 \\
\hline C & -0.027740 & 5.515753 & -2.337361 \\
\hline C & 0.424147 & 3.105181 & -3.675844 \\
\hline $\mathrm{H}$ & -0.228949 & 2.145198 & -1.861476 \\
\hline C & 0.443672 & 5.527724 & -3.648143 \\
\hline $\mathrm{H}$ & -0.197906 & 6.451868 & -1.809747 \\
\hline C & 0.668946 & 4.322604 & -4.319311 \\
\hline $\mathrm{H}$ & 0.611995 & 2.165085 & -4.185941 \\
\hline $\mathrm{H}$ & 0.639868 & 6.474139 & -4.144355 \\
\hline $\mathrm{H}$ & 1.039854 & 4.331919 & -5.340610 \\
\hline $\mathrm{H}$ & -3.364432 & 5.129150 & 1.334129 \\
\hline
\end{tabular}




\begin{tabular}{|c|c|c|c|}
\hline H & -2.887442 & 3.631514 & -1.318120 \\
\hline $\mathrm{H}$ & -3.009889 & 5.450100 & -1.070312 \\
\hline C & 0.951011 & -2.744646 & 0.252212 \\
\hline C & 1.971864 & -1.904660 & -0.176373 \\
\hline C & 3.177438 & -1.746967 & 0.589293 \\
\hline C & 3.288967 & -2.435774 & 1.782323 \\
\hline H & 4.175602 & -2.294371 & 2.394712 \\
\hline C & -1.457374 & -2.221254 & -0.250129 \\
\hline C & -0.299039 & -2.929269 & -0.566541 \\
\hline C & -2.634360 & -3.312198 & -2.073685 \\
\hline C & -2.651446 & -2.419890 & -1.018960 \\
\hline H & -3.543118 & -3.470297 & -2.649338 \\
\hline C & -1.478095 & -4.050961 & -2.417176 \\
\hline C & -0.283314 & -3.864861 & -1.651711 \\
\hline C & 0.869464 & -4.621344 & -2.004544 \\
\hline C & 0.835111 & -5.514645 & -3.051048 \\
\hline C & -0.350376 & -5.699958 & -3.802631 \\
\hline C & -1.480766 & -4.981286 & -3.491685 \\
\hline $\mathrm{H}$ & 1.788617 & -4.475395 & -1.448649 \\
\hline $\mathrm{H}$ & 1.727928 & -6.078008 & -3.307702 \\
\hline $\mathrm{H}$ & -0.361557 & -6.407637 & -4.627293 \\
\hline H & -2.396401 & -5.113063 & -4.063507 \\
\hline C & 2.283841 & -3.316683 & 2.247935 \\
\hline C & 1.095796 & -3.490155 & 1.470540 \\
\hline C & 0.107797 & -4.398607 & 1.942463 \\
\hline C & 0.280819 & -5.083539 & 3.125334 \\
\hline C & 1.450794 & -4.897668 & 3.899514 \\
\hline C & 2.429323 & -4.032532 & 3.467171 \\
\hline $\mathrm{H}$ & -0.791138 & -4.550444 & 1.354716 \\
\hline $\mathrm{H}$ & -0.485879 & -5.774104 & 3.466312 \\
\hline $\mathrm{H}$ & 1.574741 & -5.442726 & 4.831407 \\
\hline $\mathrm{H}$ & 3.335403 & -3.886063 & 4.050520 \\
\hline C & -6.378211 & -0.460390 & -0.256294 \\
\hline C & -5.731130 & -0.227355 & -1.472566 \\
\hline C & -4.497836 & -0.866382 & -1.686903 \\
\hline C & -3.933409 & -1.707201 & -0.726023 \\
\hline C & -4.621902 & -1.921648 & 0.480710 \\
\hline C & -5.847003 & -1.303557 & 0.737654 \\
\hline $\mathrm{H}$ & -7.329577 & 0.022354 & -0.065897 \\
\hline $\mathrm{H}$ & -3.953853 & -0.710066 & -2.613968 \\
\hline $\mathrm{H}$ & -4.185712 & -2.599250 & 1.206358 \\
\hline C & -6.318348 & 0.683953 & -2.567489 \\
\hline C & -6.627592 & -1.518102 & 2.048971 \\
\hline C & -5.329973 & 1.835554 & -2.867902 \\
\hline $\mathrm{H}$ & -5.729372 & 2.486417 & -3.655569 \\
\hline $\mathrm{H}$ & -5.161951 & 2.448273 & -1.974586 \\
\hline $\mathrm{H}$ & -4.358481 & 1.461007 & -3.206562 \\
\hline C & -6.544510 & -0.144153 & -3.855049 \\
\hline $\mathrm{H}$ & -6.959342 & 0.488914 & -4.649174 \\
\hline $\mathrm{H}$ & -5.611675 & -0.579002 & -4.228436 \\
\hline $\mathrm{H}$ & -7.247116 & -0.965725 & -3.673840 \\
\hline C & -7.665906 & 1.307519 & -2.156700 \\
\hline $\mathrm{H}$ & -8.038474 & 1.945366 & -2.966236 \\
\hline H & -8.428015 & 0.544414 & -1.961762 \\
\hline
\end{tabular}




\begin{tabular}{|c|c|c|c|}
\hline $\mathrm{H}$ & -7.569964 & 1.932392 & -1.261339 \\
\hline C & -6.828302 & -0.157455 & 2.758294 \\
\hline $\mathrm{H}$ & -7.383957 & -0.293650 & 3.694247 \\
\hline $\mathrm{H}$ & -5.863923 & 0.304349 & 2.999410 \\
\hline $\mathrm{H}$ & -7.390346 & 0.548380 & 2.137909 \\
\hline C & -8.009060 & -2.139157 & 1.733656 \\
\hline $\mathrm{H}$ & -8.579205 & -2.291723 & 2.658487 \\
\hline $\mathrm{H}$ & -8.605212 & -1.496709 & 1.077332 \\
\hline $\mathrm{H}$ & -7.897438 & -3.110648 & 1.238822 \\
\hline C & -5.892466 & -2.458627 & 3.022916 \\
\hline $\mathrm{H}$ & -6.480752 & -2.572383 & 3.940508 \\
\hline $\mathrm{H}$ & -5.750146 & -3.458209 & 2.596793 \\
\hline $\mathrm{H}$ & -4.909979 & -2.064917 & 3.307284 \\
\hline C & 4.302122 & -0.866125 & 0.161262 \\
\hline C & 5.623370 & -1.331657 & 0.286855 \\
\hline C & 4.086857 & 0.425488 & -0.325458 \\
\hline C & 6.713932 & -0.532901 & -0.057503 \\
\hline $\mathrm{H}$ & 5.776411 & -2.346106 & 0.635470 \\
\hline C & 5.154752 & 1.258573 & -0.693762 \\
\hline $\mathrm{H}$ & 3.069138 & 0.788369 & -0.423840 \\
\hline C & 6.451549 & 0.760489 & -0.543192 \\
\hline $\mathrm{H}$ & 7.290752 & 1.387665 & -0.819422 \\
\hline C & 4.864104 & 2.655866 & -1.274298 \\
\hline C & 8.171594 & -1.019422 & 0.059473 \\
\hline C & 4.118105 & 2.487532 & -2.619625 \\
\hline $\mathrm{H}$ & 4.737107 & 1.942025 & -3.341001 \\
\hline H & 3.183947 & 1.930836 & -2.499034 \\
\hline $\mathrm{H}$ & 3.874428 & 3.468006 & -3.047664 \\
\hline C & 3.980249 & 3.463227 & -0.294945 \\
\hline H & 3.779419 & 4.462024 & -0.701698 \\
\hline H & 3.014341 & 2.979430 & -0.121414 \\
\hline H & 4.477004 & 3.583818 & 0.675050 \\
\hline C & 6.148188 & 3.467632 & -1.530917 \\
\hline $\mathrm{H}$ & 6.725627 & 3.622144 & -0.611719 \\
\hline $\mathrm{H}$ & 6.799227 & 2.982567 & -2.266861 \\
\hline H & 5.885618 & 4.455057 & -1.927603 \\
\hline C & 8.270349 & -2.456816 & 0.604331 \\
\hline H & 7.838088 & -2.543424 & 1.607866 \\
\hline H & 7.764734 & -3.176468 & -0.049174 \\
\hline $\mathrm{H}$ & 9.323192 & -2.753996 & 0.671187 \\
\hline C & 8.839002 & -0.990349 & -1.336282 \\
\hline H & 8.308858 & -1.645893 & -2.036248 \\
\hline H & 8.845634 & 0.017556 & -1.763921 \\
\hline H & 9.879698 & -1.331778 & -1.270172 \\
\hline C & 8.951050 & -0.089125 & 1.019285 \\
\hline H & 9.993835 & -0.418613 & 1.107534 \\
\hline H & 8.958587 & 0.947778 & 0.667352 \\
\hline H & 8.505995 & -0.098204 & 2.021020 \\
\hline
\end{tabular}




\begin{tabular}{lc}
\hline B3LYP/6-31G(d) Energy & -3038.970588 \\
\hline B3LYP-D3(BJ)/def2-TZVPP-IEF-PCM(DCM) Energy & -3040.520204 \\
\hline B3LYP-D3(BJ)/def2-TZVPP-IEF-PCM(DCM)//B3LYP/6-31G(d) Free Energy (Quasiharmonic) & -3039.289629 \\
\hline
\end{tabular}

Number of Imaginary Frequencies

Frequencies (Top 3 out of 450)

$\begin{array}{lr}\text { 1. } & -289.8384 \mathrm{~cm}^{-1} \\ \text { 2. } & 8.6552 \mathrm{~cm}^{-1} \\ \text { 3. } & 11.7425 \mathrm{~cm}^{-1}\end{array}$

\section{B3LYP/6-31G(d) Molecular Geometry in Cartesian Coordinates}

C

$\mathrm{H}$

$\mathrm{H}$

C

0

0

$\mathrm{H}$

0

$\mathrm{H}$

C

C

C

C

$\mathrm{H}$

$\mathrm{H}$

$\mathrm{H}$

C

C

C

$\mathrm{H}$

$\mathrm{H}$

$\mathrm{H}$

C

$\mathrm{H}$

$\mathrm{H}$

$\mathrm{H}$

C

$\mathrm{H}$

$\mathrm{H}$

$\mathrm{H}$

C

$\mathrm{H}$
$-1.567581$

$-1.002666$

$-2.544523$

1.556931

2.075459

0.850866

0.323113

$-0.859701$

$-0.650657$

2. 214150

0.095533

2. 250837

2. 321952

$-0.216348$

2. 588519

2.702285

$-0.683057$

$-0.678091$

0.311860

0.427770

$-0.038136$

1.295034

$-2.060407$

$-2.500327$

$-1.975961$

$-2.747011$

$-1.953772$

$-2.826320$

$-2.176343$

$-1.792605$

$-0.413536$

$-1.192266$
0.818256

1.096225

0.438764

1.711247

2. 320737

0.594559

0.310360

$-0.221271$

$-0.972854$

$-2.566530$

$-3.115641$

$-3.289980$

$-2.286977$

$-2.098862$

$-2.953450$

$-4.249652$

$-2.895129$

$-4.469041$

$-2.347415$

$-1.268364$

$-2.512828$

$-2.814591$

$-2.272195$

$-2.640495$

$-1.182783$

$-2.497565$

$-5.039699$

$-4.945107$

$-4.545769$

$-6.102809$

$-5.178685$

$-4.947950$
1.084887

1.981556

1. 397464

$-1.246177$

$-2.157189$

$-1.475861$

$-0.676613$

0.379244

0.991959

$-1.402376$

$-1.162266$

0.968350

$-0.059972$

$-0.928236$

1.950606

0.704158

3.059274

2.973847

4.094990

3.946133

5.119738

3.988632

3.291392

4. 225440

3.378303

2.472277

2. 330086

2.986552

1.380393

2. 124885

4.303527

5.040185 


\begin{tabular}{|c|c|c|c|}
\hline H & -0.410327 & -6.261886 & 4.143503 \\
\hline $\mathrm{H}$ & 0.557358 & -4.899567 & 4.719411 \\
\hline 0 & -0.207209 & -2.516625 & 1.742584 \\
\hline 0 & 0.425508 & -4.729998 & 2.095411 \\
\hline B & 0.566361 & -3.636231 & 1.203037 \\
\hline 0 & 0.118831 & -3.989527 & -0.207201 \\
\hline C & -0.168862 & -3.572828 & -2.538575 \\
\hline C & -0.422201 & -2.618263 & -3.537506 \\
\hline C & -0.198444 & -4.939211 & -2.856826 \\
\hline C & -0.701508 & -3.028058 & -4.839037 \\
\hline $\mathrm{H}$ & -0.381095 & -1.559414 & -3.291188 \\
\hline C & -0.480409 & -5.342334 & -4.159679 \\
\hline $\mathrm{H}$ & -0.011616 & -5.667563 & -2.074313 \\
\hline C & -0.731303 & -4.389960 & -5.152180 \\
\hline H & -0.894606 & -2.286358 & -5.608786 \\
\hline $\mathrm{H}$ & -0.508077 & -6.401019 & -4.402829 \\
\hline H & -0.950525 & -4.708687 & -6.167632 \\
\hline $\mathrm{H}$ & 2.215211 & -1.246067 & 0.242183 \\
\hline $\mathrm{H}$ & 2.468903 & -3.554123 & -1.776475 \\
\hline H & 2.190200 & -1.760539 & -2.128824 \\
\hline C & 0.585782 & 2.765040 & 0.804549 \\
\hline C & 1.678837 & 2.153720 & 0.201457 \\
\hline C & 2.922386 & 1.988052 & 0.906977 \\
\hline C & 2.996780 & 2.452143 & 2.207398 \\
\hline $\mathrm{H}$ & 3.931040 & 2.341810 & 2.751517 \\
\hline C & -1.745056 & 2.027964 & 0.189568 \\
\hline C & -0.705766 & 2.947858 & 0.054449 \\
\hline C & -3.144484 & 3.390716 & -1.254259 \\
\hline C & -2.988864 & 2.248028 & -0.491969 \\
\hline $\mathrm{H}$ & -4.085995 & 3.556688 & -1.771626 \\
\hline C & -2.110299 & 4.341020 & -1.413887 \\
\hline C & -0.859176 & 4.119455 & -0.757016 \\
\hline C & 0.174086 & 5.082880 & -0.929901 \\
\hline C & -0.026091 & 6.201928 & -1.705716 \\
\hline C & -1.266764 & 6.418619 & -2.352852 \\
\hline C & -2.284725 & 5.505479 & -2.210290 \\
\hline $\mathrm{H}$ & 1.133878 & 4.921362 & -0.452593 \\
\hline $\mathrm{H}$ & 0.777381 & 6.922794 & -1.828930 \\
\hline H & -1.408850 & 7.305392 & -2.964643 \\
\hline $\mathrm{H}$ & -3.240501 & 5.658288 & -2.705957 \\
\hline C & 1.916333 & 3.099186 & 2.852180 \\
\hline C & 0.686669 & 3.270829 & 2.143194 \\
\hline C & -0.384695 & 3.939484 & 2.798545 \\
\hline C & -0.244971 & 4.405803 & 4.087226 \\
\hline C & 0.972313 & 4.230106 & 4.787940 \\
\hline C & 2.028818 & 3.591690 & 4.180670 \\
\hline $\mathrm{H}$ & -1.319715 & 4.083830 & 2.267880 \\
\hline $\mathrm{H}$ & -1.074200 & 4.916294 & 4.569437 \\
\hline $\mathrm{H}$ & 1.069585 & 4.603361 & 5.803775 \\
\hline H & 2.969893 & 3.455292 & 4.708196 \\
\hline C & -6.381788 & -0.352105 & -0.419020 \\
\hline C & -6.531191 & 0.977380 & 0.015206 \\
\hline C & -5.399675 & 1.794497 & 0.004197 \\
\hline C & -4.147448 & 1.307791 & -0.411213 \\
\hline
\end{tabular}




\begin{tabular}{|c|c|c|c|}
\hline C & -4.037764 & -0.024251 & -0.818026 \\
\hline C & -5.156009 & -0.874693 & -0.841013 \\
\hline $\mathrm{H}$ & -7.255779 & -0.992705 & -0.426657 \\
\hline $\mathrm{H}$ & -5.462789 & 2.827691 & 0.326208 \\
\hline $\mathrm{H}$ & -3.066268 & -0.388369 & -1.136349 \\
\hline C & -7.913651 & 1.476497 & 0.477770 \\
\hline C & -4.997053 & -2.325158 & -1.335327 \\
\hline C & -7.890179 & 2.954638 & 0.912041 \\
\hline $\mathrm{H}$ & -8.893015 & 3.261238 & 1.230506 \\
\hline $\mathrm{H}$ & -7.210515 & 3.122257 & 1.755275 \\
\hline $\mathrm{H}$ & -7.587862 & 3.616390 & 0.092428 \\
\hline C & -8.928981 & 1.334641 & -0.681257 \\
\hline $\mathrm{H}$ & -9.921068 & 1.678430 & -0.363622 \\
\hline $\mathrm{H}$ & -8.622049 & 1.934449 & -1.545726 \\
\hline $\mathrm{H}$ & -9.027542 & 0.296213 & -1.014013 \\
\hline C & -8.390398 & 0.630686 & 1.682595 \\
\hline $\mathrm{H}$ & -9.375895 & 0.971821 & 2.023135 \\
\hline $\mathrm{H}$ & -8.475197 & -0.430994 & 1.428258 \\
\hline $\mathrm{H}$ & -7.691902 & 0.717994 & 2.522795 \\
\hline C & -6.329151 & -3.099155 & -1.340527 \\
\hline $\mathrm{H}$ & -6.158817 & -4.119761 & -1.701449 \\
\hline $\mathrm{H}$ & -6.763442 & -3.173410 & -0.336979 \\
\hline $\mathrm{H}$ & -7.069587 & -2.634326 & -2.001671 \\
\hline C & -4.442937 & -2.320254 & -2.779790 \\
\hline $\mathrm{H}$ & -4.328708 & -3.347161 & -3.147529 \\
\hline $\mathrm{H}$ & -5.121918 & -1.788856 & -3.456710 \\
\hline $\mathrm{H}$ & -3.462776 & -1.838064 & -2.841700 \\
\hline C & -4.012057 & -3.074423 & -0.408366 \\
\hline $\mathrm{H}$ & -3.835829 & -4.092424 & -0.776800 \\
\hline $\mathrm{H}$ & -3.043529 & -2.568230 & -0.343898 \\
\hline $\mathrm{H}$ & -4.417775 & -3.148302 & 0.607615 \\
\hline C & 4.126145 & 1.307454 & 0.337809 \\
\hline C & 4.757253 & 0.299711 & 1.093585 \\
\hline C & 4.689003 & 1.686669 & -0.883346 \\
\hline C & 5.920469 & -0.327814 & 0.645125 \\
\hline $\mathrm{H}$ & 4.309391 & 0.011112 & 2.037698 \\
\hline C & 5.850106 & 1.069611 & -1.376233 \\
\hline $\mathrm{H}$ & 4.210366 & 2.471068 & -1.454156 \\
\hline C & 6.443339 & 0.072842 & -0.597988 \\
\hline $\mathrm{H}$ & 7.340531 & -0.414884 & -0.960775 \\
\hline C & 6.408292 & 1.506456 & -2.744202 \\
\hline C & 6.635056 & -1.431642 & 1.449247 \\
\hline C & 6.724082 & 3.020833 & -2.716400 \\
\hline $\mathrm{H}$ & 5.832476 & 3.620492 & -2.508367 \\
\hline $\mathrm{H}$ & 7.472136 & 3.252278 & -1.948971 \\
\hline $\mathrm{H}$ & 7.121952 & 3.342920 & -3.686634 \\
\hline C & 7.700432 & 0.757893 & -3.120851 \\
\hline $\mathrm{H}$ & 8.055855 & 1.104990 & -4.097765 \\
\hline $\mathrm{H}$ & 8.503001 & 0.935716 & -2.395307 \\
\hline $\mathrm{H}$ & 7.539480 & -0.323727 & -3.195693 \\
\hline C & 5.346656 & 1.223440 & -3.835155 \\
\hline $\mathrm{H}$ & 5.143678 & 0.148365 & -3.907962 \\
\hline $\mathrm{H}$ & 4.397261 & 1.725633 & -3.625314 \\
\hline $\mathrm{H}$ & 5.706019 & 1.566832 & -4.813468 \\
\hline
\end{tabular}




\begin{tabular}{|llll|} 
C & 8.089776 & -0.995172 & 1.745482 \\
H & 8.659498 & -0.818796 & 0.827249 \\
$H$ & 8.109945 & -0.070012 & 2.333053 \\
H & 8.612032 & -1.772406 & 2.317005 \\
C & 5.944140 & -1.721439 & 2.795260 \\
$H$ & 5.916326 & -0.834932 & 3.439394 \\
$H$ & 4.917188 & -2.078173 & 2.658521 \\
$H$ & 6.494503 & -2.502653 & 3.331776 \\
C & 6.650838 & -2.741251 & 0.625398 \\
$H$ & 7.155868 & -3.538882 & 1.184152 \\
$H$ & 5.631011 & -3.073934 & 0.402240 \\
$H$ & 7.177336 & -2.616334 & -0.326568 \\
\hline
\end{tabular}

\section{C-Catalyzed reaction TS 19}

\section{Datum}

Value

\begin{tabular}{lc}
\hline B3LYP/6-31G(d) Energy & -3038.970392 \\
\hline B3LYP-D3(BJ)/def2-TZVPP-IEF-PCM(DCM) Energy & -3040.518586 \\
\hline B3LYP-D3(BJ)/def2-TZVPP-IEF-PCM(DCM)//B3LYP/6-31G(d) Free Energy (Quasiharmonic) & -3039.289568 \\
\hline
\end{tabular}

Number of Imaginary Frequencies

Frequencies (Top 3 out of 450)

$\begin{array}{lr}\text { 1. } & -317.9565 \mathrm{~cm}^{-1} \\ \text { 2. } & 6.5706 \mathrm{~cm}^{-1} \\ \text { 3. } & 8.6925 \mathrm{~cm}^{-1}\end{array}$

\section{B3LYP/6-31G(d) Molecular Geometry in Cartesian Coordinates}

\section{C}

$\mathrm{H}$

$\mathrm{H}$

C

0

0

$\mathrm{H}$

0

$\mathrm{H}$

C

C

C

C

$\mathrm{H}$
$-1.765199$

$-1.262568$

$-2.747100$

1.556051

2. 209149

0.861470

0.248534

$-1.000282$

$-0.959543$

$-0.561532$

0.955516

$-1.977939$

$-1.571844$

0.837873
0.834926

1.054703

0.410629

1.864244

2.515780

0.789884

0.451252

$-0.121281$

$-0.991290$

$-2.686773$

$-2.956014$

$-3.734921$

$-2.616097$

$-1.945524$
0.977774

1.925817

1.201480

$-1.027300$

$-1.814524$

$-1.427260$

$-0.714388$

0.226207

0.700514

$-2.713466$

$-1.170702$

$-0.971509$

$-1.777835$

$-0.779951$ 


\begin{tabular}{|c|c|c|c|}
\hline $\mathrm{H}$ & -2.927385 & -3.598743 & -0.449620 \\
\hline $\mathrm{H}$ & -1.933321 & -4.705846 & -1.470903 \\
\hline C & -0.643728 & -3.112479 & 2.534873 \\
\hline C & -1.240110 & -4.569338 & 2.478089 \\
\hline C & 0.869204 & -3.090817 & 2.806433 \\
\hline $\mathrm{H}$ & 1.247525 & -2.079021 & 2.622129 \\
\hline $\mathrm{H}$ & 1.098886 & -3.356290 & 3.844049 \\
\hline $\mathrm{H}$ & 1.400944 & -3.780034 & 2.144717 \\
\hline C & -1.354868 & -2.157907 & 3.494119 \\
\hline $\mathrm{H}$ & -2.407634 & -2.031640 & 3.229339 \\
\hline $\mathrm{H}$ & -1.298789 & -2.527062 & 4.524570 \\
\hline $\mathrm{H}$ & -0.875244 & -1.172461 & 3.471063 \\
\hline C & -2.764845 & -4.597297 & 2.686352 \\
\hline $\mathrm{H}$ & -3.043250 & -4.394824 & 3.726830 \\
\hline $\mathrm{H}$ & -3.269228 & -3.866827 & 2.045990 \\
\hline $\mathrm{H}$ & -3.134585 & -5.592384 & 2.419477 \\
\hline C & -0.576223 & -5.566112 & 3.431793 \\
\hline $\mathrm{H}$ & -0.693479 & -5.257689 & 4.477692 \\
\hline $\mathrm{H}$ & -1.044796 & -6.548868 & 3.314836 \\
\hline $\mathrm{H}$ & 0.488655 & -5.676921 & 3.214769 \\
\hline 0 & -0.865996 & -2.656049 & 1.176396 \\
\hline 0 & -0.963224 & -4.979308 & 1.132288 \\
\hline B & -0.802635 & -3.831183 & 0.311217 \\
\hline 0 & 0.495705 & -3.941351 & -0.465249 \\
\hline C & 2.130811 & -3.194749 & -2.028426 \\
\hline C & 2.573222 & -4.499809 & -2.299295 \\
\hline C & 2.829350 & -2.096502 & -2.555730 \\
\hline C & 3.699838 & -4.700263 & -3.091946 \\
\hline $\mathrm{H}$ & 2.033371 & -5.339646 & -1.873829 \\
\hline C & 3.955661 & -2.305621 & -3.348899 \\
\hline $\mathrm{H}$ & 2.485331 & -1.086558 & -2.346566 \\
\hline C & 4. 391491 & -3.605082 & -3.619710 \\
\hline $\mathrm{H}$ & 4.043066 & -5.710708 & -3.296801 \\
\hline $\mathrm{H}$ & 4.492450 & -1.452862 & -3.754481 \\
\hline $\mathrm{H}$ & 5.270368 & -3.765452 & -4.238544 \\
\hline $\mathrm{H}$ & -1.886468 & -1.624616 & -1.453838 \\
\hline $\mathrm{H}$ & -0.317382 & -3.634770 & -3.184637 \\
\hline $\mathrm{H}$ & -0.229823 & -1.793241 & -3.234502 \\
\hline C & 0.362009 & 2.799128 & 0.977207 \\
\hline C & 1.502435 & 2.204277 & 0.450921 \\
\hline C & 2.652496 & 1.944376 & 1.272294 \\
\hline C & 2.595990 & 2.294595 & 2.607209 \\
\hline $\mathrm{H}$ & 3.457421 & 2.097066 & 3.240099 \\
\hline C & -1.901537 & 2.095031 & 0.148128 \\
\hline C & -0.855623 & 3.015844 & 0.119890 \\
\hline C & -3.117466 & 3.404427 & -1.496135 \\
\hline C & -3.053359 & 2.290656 & -0.682642 \\
\hline $\mathrm{H}$ & -3.994350 & 3.555681 & -2.120985 \\
\hline C & -2.084033 & 4.370794 & -1.532035 \\
\hline C & -0.926826 & 4.180261 & -0.711848 \\
\hline C & 0.103479 & 5.160392 & -0.757153 \\
\hline C & -0.011820 & 6.269364 & -1.564031 \\
\hline C & -1.159854 & 6.457007 & -2.371173 \\
\hline C & -2.171710 & 5.525762 & -2.355499 \\
\hline
\end{tabular}




\begin{tabular}{|c|c|c|c|}
\hline $\mathrm{H}$ & 0.992910 & 5.018977 & -0.153486 \\
\hline $\mathrm{H}$ & 0.787156 & 7.005233 & -1.586719 \\
\hline $\mathrm{H}$ & -1.235872 & 7.336306 & -3.005135 \\
\hline $\mathrm{H}$ & -3.056378 & 5.658108 & -2.973907 \\
\hline C & 1.470481 & 2.938262 & 3.176510 \\
\hline C & 0.333622 & 3.209010 & 2.351721 \\
\hline C & -0.782259 & 3.872076 & 2.934373 \\
\hline C & -0.773252 & 4.240068 & 4.261810 \\
\hline C & 0.350535 & 3.964275 & 5.077003 \\
\hline C & 1.447343 & 3.328019 & 4.542966 \\
\hline $\mathrm{H}$ & -1.646128 & 4.091293 & 2.315866 \\
\hline $\mathrm{H}$ & -1.633794 & 4.749702 & 4.686764 \\
\hline $\mathrm{H}$ & 0.344873 & 4.259679 & 6.122686 \\
\hline $\mathrm{H}$ & 2.318058 & 3.115742 & 5.158843 \\
\hline C & -6.360255 & -0.414580 & -0.819633 \\
\hline C & -5.528086 & -0.310096 & -1.942574 \\
\hline C & -4.442981 & 0.573366 & -1.866620 \\
\hline C & -4.195713 & 1.323805 & -0.710260 \\
\hline C & -5.056531 & 1.194233 & 0.388291 \\
\hline C & -6.151763 & 0.322929 & 0.355184 \\
\hline $\mathrm{H}$ & -7.204912 & -1.095700 & -0.862003 \\
\hline $\mathrm{H}$ & -3.766181 & 0.688702 & -2.705322 \\
\hline $\mathrm{H}$ & -4.864146 & 1.800444 & 1.266807 \\
\hline C & -5.832602 & -1.145573 & -3.201906 \\
\hline C & -7.116876 & 0.154238 & 1.545296 \\
\hline C & -4.786528 & -0.936541 & -4.313793 \\
\hline $\mathrm{H}$ & -5.046128 & -1.551801 & -5.182710 \\
\hline $\mathrm{H}$ & -4.747220 & 0.105939 & -4.649236 \\
\hline $\mathrm{H}$ & -3.781742 & -1.231014 & -3.989771 \\
\hline C & -5.848225 & -2.649593 & -2.839717 \\
\hline $\mathrm{H}$ & -6.072101 & -3.252232 & -3.728439 \\
\hline $\mathrm{H}$ & -4.875134 & -2.969447 & -2.450322 \\
\hline $\mathrm{H}$ & -6.604814 & -2.882288 & -2.083130 \\
\hline C & -7.217432 & -0.738716 & -3.759985 \\
\hline $\mathrm{H}$ & -7.455404 & -1.323878 & -4.656854 \\
\hline $\mathrm{H}$ & -8.016497 & -0.907355 & -3.030232 \\
\hline $\mathrm{H}$ & -7.233538 & 0.322696 & -4.032912 \\
\hline C & -7.088751 & -1.317034 & 2.023388 \\
\hline $\mathrm{H}$ & -7.393644 & -2.009802 & 1.232240 \\
\hline $\mathrm{H}$ & -6.081754 & -1.603915 & 2.347825 \\
\hline $\mathrm{H}$ & -7.771354 & -1.456736 & 2.870644 \\
\hline C & -6.740308 & 1.051543 & 2.739550 \\
\hline $\mathrm{H}$ & -7.454330 & 0.897081 & 3.556390 \\
\hline $\mathrm{H}$ & -5.741713 & 0.818790 & 3.126714 \\
\hline $\mathrm{H}$ & -6.765912 & 2.114840 & 2.475445 \\
\hline C & -8.553478 & 0.522648 & 1.104428 \\
\hline $\mathrm{H}$ & -9.252083 & 0.401477 & 1.941373 \\
\hline $\mathrm{H}$ & -8.604709 & 1.564069 & 0.766771 \\
\hline $\mathrm{H}$ & -8.904704 & -0.111539 & 0.283971 \\
\hline C & 3.889568 & 1.273532 & 0.766011 \\
\hline C & 4.654766 & 1.831574 & -0.264895 \\
\hline C & 4.336853 & 0.096162 & 1.381708 \\
\hline C & 5.846881 & 1.234220 & -0.691084 \\
\hline $\mathrm{H}$ & 4.300236 & 2.737687 & -0.738881 \\
\hline
\end{tabular}




\begin{tabular}{|c|c|c|c|}
\hline C & 5.523160 & -0.533824 & 0.984500 \\
\hline $\mathrm{H}$ & 3.732671 & -0.332692 & 2.176406 \\
\hline C & 6.260191 & 0.056526 & -0.052350 \\
\hline $\mathrm{H}$ & 7.179161 & -0.414947 & -0.372465 \\
\hline C & 5.968051 & -1.835594 & 1.680615 \\
\hline C & 6.639743 & 1.880185 & -1.844278 \\
\hline C & 6.150187 & -1.576581 & 3.195063 \\
\hline $\mathrm{H}$ & 6.459817 & -2.496854 & 3.705803 \\
\hline $\mathrm{H}$ & 6.918152 & -0.814829 & 3.372879 \\
\hline $\mathrm{H}$ & 5.222948 & -1.232099 & 3.664768 \\
\hline C & 4.887960 & -2.923930 & 1.472727 \\
\hline $\mathrm{H}$ & 5.191365 & -3.861019 & 1.956107 \\
\hline $\mathrm{H}$ & 3.924283 & -2.627011 & 1.899543 \\
\hline $\mathrm{H}$ & 4.732946 & -3.124802 & 0.406674 \\
\hline C & 7.300671 & -2.376059 & 1.127995 \\
\hline $\mathrm{H}$ & 7.231379 & -2.620938 & 0.062140 \\
\hline $\mathrm{H}$ & 8.120080 & -1.660720 & 1.262919 \\
\hline $\mathrm{H}$ & 7.573272 & -3.294524 & 1.660111 \\
\hline C & 6.979265 & 3.346789 & -1.486532 \\
\hline $\mathrm{H}$ & 7.586738 & 3.398560 & -0.575346 \\
\hline $\mathrm{H}$ & 6.078793 & 3.947114 & -1.323687 \\
\hline $\mathrm{H}$ & 7.546851 & 3.814357 & -2.300510 \\
\hline C & 7.961477 & 1.146381 & -2.139129 \\
\hline $\mathrm{H}$ & 7.794014 & 0.107600 & -2.446047 \\
\hline $\mathrm{H}$ & 8.630262 & 1.142294 & -1.270388 \\
\hline $\mathrm{H}$ & 8.486912 & 1.649402 & -2.958993 \\
\hline C & 5.772576 & 1.854333 & -3.126384 \\
\hline $\mathrm{H}$ & 6.294140 & 2.355985 & -3.951259 \\
\hline $\mathrm{H}$ & 4.806918 & 2.347460 & -2.979465 \\
\hline $\mathrm{H}$ & 5.571422 & 0.820921 & -3.434261 \\
\hline
\end{tabular}

\section{C-Catalyzed reaction TS 20}

\section{Datum}

Value

\begin{tabular}{lc}
\hline B3LYP/6-31G(d) Energy & -3038.969768 \\
\hline B3LYP-D3(BJ)/def2-TZVPP-IEF-PCM(DCM) Energy & -3040.518815 \\
\hline B3LYP-D3(BJ)/def2-TZVPP-IEF-PCM(DCM)//B3LYP/6-31G(d) Free Energy (Quasiharmonic) & -3039.289562 \\
\hline
\end{tabular}

Number of Imaginary Frequencies

Frequencies (Top 3 out of 450)

$\begin{array}{lr}\text { 1. } & -321.5110 \mathrm{~cm}^{-1} \\ \text { 2. } & 7.4535 \mathrm{~cm}^{-1} \\ \text { 3. } & 9.7526 \mathrm{~cm}^{-1}\end{array}$




\begin{tabular}{|c|c|c|c|}
\hline C & 1.598645 & -1.125984 & -1.011600 \\
\hline $\mathrm{H}$ & 2.554696 & -0.677780 & -1.281361 \\
\hline $\mathrm{H}$ & 1.285368 & -1.773975 & -1.835916 \\
\hline C & -1.777284 & -1.238994 & 1.240630 \\
\hline 0 & -2.481730 & -1.445766 & 2.207903 \\
\hline 0 & -0.994915 & -0.155865 & 1.180470 \\
\hline $\mathrm{H}$ & -0.403062 & -0.166807 & 0.367845 \\
\hline 0 & 0.605664 & -0.083727 & -0.899049 \\
\hline $\mathrm{H}$ & 1.001547 & 0.822113 & -0.946298 \\
\hline C & -2.714134 & 2.438298 & -0.552525 \\
\hline C & -1.140072 & 3.153046 & 0.757345 \\
\hline C & -1.093625 & 3.156069 & -2.281203 \\
\hline C & -1.814897 & 2.147139 & -1.556578 \\
\hline $\mathrm{H}$ & -0.675889 & 2.175221 & 0.867005 \\
\hline $\mathrm{H}$ & -0.589435 & 2.798098 & -3.180997 \\
\hline $\mathrm{H}$ & -1.657209 & 4.071064 & -2.478755 \\
\hline C & 2.497081 & 3.259185 & -1.041115 \\
\hline C & 2.247214 & 4.489562 & -1.990931 \\
\hline C & 2.810117 & 3.673722 & 0.405434 \\
\hline $\mathrm{H}$ & 2.768707 & 2.786435 & 1.046445 \\
\hline $\mathrm{H}$ & 3.810607 & 4.111606 & 0.493078 \\
\hline $\mathrm{H}$ & 2.080773 & 4.398484 & 0.777433 \\
\hline C & 3.549499 & 2.270552 & -1.539649 \\
\hline $\mathrm{H}$ & 4.519572 & 2.765588 & -1.663205 \\
\hline $\mathrm{H}$ & 3.688385 & 1.461704 & -0.815113 \\
\hline $\mathrm{H}$ & 3.263298 & 1.832779 & -2.499907 \\
\hline C & 2.390754 & 4.131601 & -3.481266 \\
\hline $\mathrm{H}$ & 3.436379 & 3.980026 & -3.772099 \\
\hline $\mathrm{H}$ & 1.828868 & 3.225815 & -3.729629 \\
\hline $\mathrm{H}$ & 1.984476 & 4.955164 & -4.077004 \\
\hline C & 3.102427 & 5.720838 & -1.678620 \\
\hline $\mathrm{H}$ & 4.172012 & 5.503368 & -1.786548 \\
\hline $\mathrm{H}$ & 2.849660 & 6.525653 & -2.376993 \\
\hline $\mathrm{H}$ & 2.917792 & 6.088083 & -0.666422 \\
\hline 0 & 1.197794 & 2.610420 & -1.051379 \\
\hline 0 & 0.876732 & 4.815760 & -1.738038 \\
\hline B & 0.203516 & 3.668090 & -1.235389 \\
\hline 0 & -0.535085 & 4.043018 & 0.031809 \\
\hline C & -1.965598 & 3.612785 & 1.890647 \\
\hline C & -2.314606 & 4.965737 & 2.027442 \\
\hline C & -2.382500 & 2.682414 & 2.857493 \\
\hline C & -3.073827 & 5.380858 & 3.118112 \\
\hline $\mathrm{H}$ & -1.974356 & 5.676969 & 1.281563 \\
\hline C & -3.143021 & 3.106291 & 3.945735 \\
\hline $\mathrm{H}$ & -2.114656 & 1.634227 & 2.750710 \\
\hline C & -3.490876 & 4.453228 & 4.077763 \\
\hline $\mathrm{H}$ & -3.338279 & 6.429571 & 3.224136 \\
\hline $\mathrm{H}$ & -3.462071 & 2.383883 & 4.691655 \\
\hline $\mathrm{H}$ & -4.083152 & 4.780869 & 4.928090 \\
\hline $\mathrm{H}$ & -1.450557 & 1.122476 & -1.628835 \\
\hline
\end{tabular}




\begin{tabular}{|c|c|c|c|}
\hline H & -3.221090 & 3.399272 & -0.541557 \\
\hline $\mathrm{H}$ & -3.185281 & 1.642475 & 0.015000 \\
\hline C & -0.558444 & -2.897525 & -0.178272 \\
\hline C & -1.724030 & -2.173340 & 0.048011 \\
\hline C & -2.868632 & -2.316284 & -0.810079 \\
\hline C & -2.789303 & -3.219794 & -1.853033 \\
\hline $\mathrm{H}$ & -3.646128 & -3.338156 & -2.511041 \\
\hline C & 1.726740 & -1.933782 & 0.275310 \\
\hline C & 0.652594 & -2.719527 & 0.698717 \\
\hline C & 2.934931 & -2.496786 & 2.309547 \\
\hline C & 2.908756 & -1.850720 & 1.086773 \\
\hline $\mathrm{H}$ & 3.835431 & -2.442626 & 2.916001 \\
\hline C & 1.850956 & -3.270359 & 2.780742 \\
\hline C & 0.690471 & -3.407269 & 1.955496 \\
\hline C & -0.374859 & -4.227909 & 2.423003 \\
\hline C & -0.293107 & -4.867556 & 3.638772 \\
\hline C & 0.855475 & -4.724603 & 4.454559 \\
\hline C & 1.904234 & -3.942577 & 4.032169 \\
\hline $\mathrm{H}$ & -1.266534 & -4.335633 & 1.816290 \\
\hline $\mathrm{H}$ & -1.120137 & -5.484556 & 3.978905 \\
\hline $\mathrm{H}$ & 0.903590 & -5.233971 & 5.413213 \\
\hline $\mathrm{H}$ & 2.793583 & -3.827045 & 4.647137 \\
\hline C & -1.644425 & -4.017395 & -2.087125 \\
\hline C & -0.503945 & -3.862435 & -1.238356 \\
\hline C & 0.633957 & -4.681914 & -1.481120 \\
\hline C & 0.642197 & -5.595660 & -2.511949 \\
\hline C & -0.485830 & -5.740542 & -3.354572 \\
\hline C & -1.604028 & -4.967812 & -3.143127 \\
\hline $\mathrm{H}$ & 1.500553 & -4.585750 & -0.835888 \\
\hline $\mathrm{H}$ & 1.519786 & -6.214829 & -2.677266 \\
\hline $\mathrm{H}$ & -0.466241 & -6.465151 & -4.164040 \\
\hline $\mathrm{H}$ & -2.478470 & -5.073294 & -3.780799 \\
\hline C & 6.617110 & -0.028759 & -0.004522 \\
\hline C & 6.052838 & -0.969178 & -0.878850 \\
\hline C & 4.819237 & -1.526919 & -0.523638 \\
\hline C & 4.161389 & -1.156469 & 0.658950 \\
\hline C & 4.758030 & -0.205540 & 1.499505 \\
\hline C & 5.996467 & 0.370419 & 1.187527 \\
\hline $\mathrm{H}$ & 7.579796 & 0.404314 & -0.258689 \\
\hline $\mathrm{H}$ & 4.365386 & -2.288380 & -1.147929 \\
\hline $\mathrm{H}$ & 4.235389 & 0.073563 & 2.407080 \\
\hline C & 6.803398 & -1.366092 & -2.165500 \\
\hline C & 6.696689 & 1.386301 & 2.112305 \\
\hline C & 6.026950 & -2.399142 & -3.004323 \\
\hline $\mathrm{H}$ & 6.595684 & -2.640927 & -3.909191 \\
\hline $\mathrm{H}$ & 5.868185 & -3.334744 & -2.456201 \\
\hline $\mathrm{H}$ & 5.049650 & -2.017424 & -3.321190 \\
\hline C & 7.027454 & -0.109642 & -3.040228 \\
\hline $\mathrm{H}$ & 7.561264 & -0.376303 & -3.960652 \\
\hline $\mathrm{H}$ & 6.072284 & 0.348244 & -3.321721 \\
\hline $\mathrm{H}$ & 7.620971 & 0.649753 & -2.520540 \\
\hline C & 8.173542 & -1.982165 & -1.794596 \\
\hline $\mathrm{H}$ & 8.721839 & -2.266238 & -2.701136 \\
\hline $\mathrm{H}$ & 8.798774 & -1.279537 & -1.233902 \\
\hline
\end{tabular}




\begin{tabular}{|c|c|c|c|}
\hline $\mathrm{H}$ & 8.045597 & -2.879999 & -1.179240 \\
\hline C & 7.017532 & 2.681508 & 1.330065 \\
\hline $\mathrm{H}$ & 7.522378 & 3.403612 & 1.983068 \\
\hline $\mathrm{H}$ & 7.675053 & 2.494517 & 0.474781 \\
\hline $\mathrm{H}$ & 6.101239 & 3.148976 & 0.952664 \\
\hline C & 5.831198 & 1.767867 & 3.328340 \\
\hline $\mathrm{H}$ & 6.365841 & 2.497757 & 3.946489 \\
\hline $\mathrm{H}$ & 4.881286 & 2.222759 & 3.025633 \\
\hline $\mathrm{H}$ & 5.609445 & 0.901763 & 3.961853 \\
\hline C & 8.015722 & 0.766503 & 2.633676 \\
\hline $\mathrm{H}$ & 8.535643 & 1.473194 & 3.292105 \\
\hline $\mathrm{H}$ & 7.820219 & -0.148573 & 3.204434 \\
\hline $\mathrm{H}$ & 8.696259 & 0.509500 & 1.814849 \\
\hline C & -4.107678 & -1.485859 & -0.697683 \\
\hline C & -4.550829 & -0.788003 & -1.837890 \\
\hline C & -4.869996 & -1.423673 & 0.471136 \\
\hline C & -5.725264 & -0.034261 & -1.821863 \\
\hline $\mathrm{H}$ & -3.943396 & -0.838137 & -2.734322 \\
\hline C & -6.053466 & -0.669661 & 0.531185 \\
\hline $\mathrm{H}$ & -4.526449 & -1.965132 & 1.342319 \\
\hline C & -6.458230 & 0.008600 & -0.621636 \\
\hline $\mathrm{H}$ & -7.369977 & 0.594112 & -0.594338 \\
\hline C & -6.840213 & -0.615940 & 1.854734 \\
\hline C & -6.232481 & 0.741174 & -3.053546 \\
\hline C & -5.942575 & 0.008267 & 2.951056 \\
\hline $\mathrm{H}$ & -5.010004 & -0.549079 & 3.082754 \\
\hline $\mathrm{H}$ & -5.677008 & 1.041160 & 2.696216 \\
\hline H & -6.471962 & 0.021799 & 3.912331 \\
\hline C & -8.125269 & 0.226115 & 1.744739 \\
\hline $\mathrm{H}$ & -8.646782 & 0.228856 & 2.708601 \\
\hline $\mathrm{H}$ & -7.909611 & 1.268913 & 1.485218 \\
\hline $\mathrm{H}$ & -8.816979 & -0.177908 & 0.996214 \\
\hline C & -7.241675 & -2.049638 & 2.276323 \\
\hline H & -7.878839 & -2.518154 & 1.517010 \\
\hline $\mathrm{H}$ & -6.367254 & -2.691298 & 2.423178 \\
\hline H & -7.799215 & -2.026398 & 3.220800 \\
\hline C & -7.625447 & 0.204238 & -3.460868 \\
\hline $\mathrm{H}$ & -7.574677 & -0.859201 & -3.721754 \\
\hline $\mathrm{H}$ & -8.357251 & 0.314508 & -2.653769 \\
\hline $\mathrm{H}$ & -8.005354 & 0.750824 & -4.332925 \\
\hline C & -6.344353 & 2.245582 & -2.710787 \\
\hline H & -7.033482 & 2.425143 & -1.879076 \\
\hline $\mathrm{H}$ & -5.366961 & 2.654280 & -2.430923 \\
\hline H & -6.714113 & 2.807796 & -3.577245 \\
\hline C & -5.290681 & 0.599477 & -4.264491 \\
\hline $\mathrm{H}$ & -5.690584 & 1.168090 & -5.111842 \\
\hline $\mathrm{H}$ & -4.289718 & 0.989200 & -4.047690 \\
\hline $\mathrm{H}$ & -5.190758 & -0.443764 & -4.585152 \\
\hline
\end{tabular}




\begin{tabular}{lc}
\hline B3LYP/6-31G(d) Energy & -3038.969264 \\
\hline B3LYP-D3(BJ)/def2-TZVPP-IEF-PCM(DCM) Energy & -3040.517463 \\
\hline B3LYP-D3(BJ)/def2-TZVPP-IEF-PCM(DCM)//B3LYP/6-31G(d) Free Energy (Quasiharmonic) & -3039.289543 \\
\hline
\end{tabular}

Number of Imaginary Frequencies

Frequencies (Top 3 out of 450)

$\begin{array}{lr}\text { 1. } & -338.1967 \mathrm{~cm}^{-1} \\ \text { 2. } & 2.4033 \mathrm{~cm}^{-1} \\ \text { 3. } & 9.6437 \mathrm{~cm}^{-1}\end{array}$

\section{B3LYP/6-31G(d) Molecular Geometry in Cartesian Coordinates}

C

$\mathrm{H}$

$\mathrm{H}$

C

0

0

$\mathrm{H}$

0

$\mathrm{H}$

C

C

C

C

$\mathrm{H}$

$\mathrm{H}$

$\mathrm{H}$

C

C

C

$\mathrm{H}$

$\mathrm{H}$

$\mathrm{H}$

C

$\mathrm{H}$

$\mathrm{H}$

$\mathrm{H}$

C

$\mathrm{H}$

$\mathrm{H}$

$\mathrm{H}$

C

$\mathrm{H}$
1.358286

2.354499

0.748832

$-2.033837$

$-2.859524$

$-1.100445$

$-0.470229$

0.772611

0.838604

2. 300293

0.440872

2.897074

3.038726

0.729604

2.670142

3. 685874

1.194821

1.079923

0.074263

0.229187

$-0.904497$

0.062682

2. 560136

2. 655178

2.669645

3. 381200

$-0.378059$

$-0.880735$

$-0.955478$

$-0.381668$

1.843701

1.462299
$-1.283734$

$-1.085959$

$-1.731635$

$-1.083454$

$-1.228662$

$-0.128002$

$-0.128774$

$-0.051064$

0.653189

3.915069

4.252379

2. 774089

3.838425

5. 244581

1. 792544

2. 722882

2. 789452

4.337728

2.206415

1.129967

2. 349771

2.668306

2. 387422

1. 296699

2. 706631

2.818063

4.817560

4.821440

4.191837

5.841286

5.228930

5.112722
$-0.920823$

$-1.323173$

$-1.714730$

1.297096

2. 172278

1. 371514

0.594576

$-0.479228$

$-1.168649$

2.074600

1.006734

$-0.038208$

0.911044

0.654218

0.382593

$-0.790687$

$-3.240101$

$-2.954128$

$-4.102345$

$-4.236448$

$-3.638919$

$-5.096524$

$-3.820720$

$-3.786950$

$-4.862949$

$-3.240188$

$-2.848946$

$-3.822503$

$-2.162390$

$-2.459963$

$-3.935100$

$-4.956469$ 


\begin{tabular}{|c|c|c|c|}
\hline $\mathrm{H}$ & 1.721352 & 6.278618 & -3.647445 \\
\hline $\mathrm{H}$ & 2.912887 & 5.004232 & -3.932726 \\
\hline 0 & 1.090254 & 2.228051 & -1.913271 \\
\hline 0 & 1.695167 & 4.460889 & -1.657961 \\
\hline B & 1.478185 & 3.235072 & -0.954133 \\
\hline 0 & 0.428203 & 3.294854 & 0.131741 \\
\hline C & -0.504871 & 4.186969 & 2.135849 \\
\hline C & -1.111657 & 2.974214 & 2.500428 \\
\hline C & -0.818725 & 5.359924 & 2.840297 \\
\hline C & -2.024833 & 2.941384 & 3.551317 \\
\hline $\mathrm{H}$ & -0.877765 & 2.061487 & 1.964138 \\
\hline C & -1.732368 & 5.322014 & 3.891513 \\
\hline $\mathrm{H}$ & -0.351538 & 6.300878 & 2.557127 \\
\hline C & -2.336824 & 4.113068 & 4.248406 \\
\hline $\mathrm{H}$ & -2.489512 & 1.995563 & 3.815866 \\
\hline $\mathrm{H}$ & -1.976269 & 6.234079 & 4.429328 \\
\hline $\mathrm{H}$ & -3.050086 & 4.085153 & 5.067821 \\
\hline $\mathrm{H}$ & 3.552308 & 4.739530 & 0.573563 \\
\hline $\mathrm{H}$ & 1.920686 & 3.008023 & 2.535527 \\
\hline $\mathrm{H}$ & 2.386790 & 4.777238 & 2.730091 \\
\hline C & -0.969071 & -2.848428 & -0.166252 \\
\hline C & -2.025262 & -1.975744 & 0.069633 \\
\hline C & -3.208940 & -2.010091 & -0.742332 \\
\hline C & -3.286246 & -2.933926 & -1.763804 \\
\hline $\mathrm{H}$ & -4.166956 & -2.946711 & -2.400910 \\
\hline C & 1.423281 & -2.202461 & 0.284037 \\
\hline C & 0.264511 & -2.857342 & 0.697283 \\
\hline C & 2.590420 & -2.995479 & 2.260415 \\
\hline C & 2.617284 & -2.285853 & 1.075198 \\
\hline $\mathrm{H}$ & 3.498650 & -3.072255 & 2.853182 \\
\hline C & 1.426590 & -3.656291 & 2.718480 \\
\hline C & 0.238923 & -3.601305 & 1.922224 \\
\hline C & -0.918649 & -4.286038 & 2.387734 \\
\hline C & -0.896764 & -4.985943 & 3.572917 \\
\hline C & 0.281501 & -5.041796 & 4.355574 \\
\hline C & 1.417272 & -4.389798 & 3.935658 \\
\hline $\mathrm{H}$ & -1.832507 & -4.237027 & 1.806615 \\
\hline $\mathrm{H}$ & -1.793613 & -5.495526 & 3.913977 \\
\hline $\mathrm{H}$ & 0.283607 & -5.597729 & 5.289346 \\
\hline $\mathrm{H}$ & 2.327827 & -4.424521 & 4.529316 \\
\hline C & -2.245822 & -3.857896 & -2.025861 \\
\hline C & -1.065484 & -3.823357 & -1.218391 \\
\hline C & -0.037897 & -4.769820 & -1.489119 \\
\hline C & -0.167770 & -5.687626 & -2.508578 \\
\hline C & -1.331723 & -5.709584 & -3.312554 \\
\hline C & -2.347204 & -4.812527 & -3.073276 \\
\hline $\mathrm{H}$ & 0.857337 & -4.765706 & -0.877102 \\
\hline $\mathrm{H}$ & 0.629183 & -6.402330 & -2.695777 \\
\hline $\mathrm{H}$ & -1.421790 & -6.437362 & -4.114513 \\
\hline $\mathrm{H}$ & -3.248526 & -4.822089 & -3.681687 \\
\hline C & 6.406500 & -0.635174 & -0.014047 \\
\hline C & 5.799307 & -1.547497 & -0.889480 \\
\hline C & 4.547984 & -2.058819 & -0.527695 \\
\hline C & 3.914462 & -1.668967 & 0.661463 \\
\hline
\end{tabular}




\begin{tabular}{|c|c|c|c|}
\hline C & 4.556121 & -0.753547 & 1.506411 \\
\hline C & 5.813053 & -0.223302 & 1.186934 \\
\hline $\mathrm{H}$ & 7.381459 & -0.234630 & -0.275164 \\
\hline $\mathrm{H}$ & 4.054762 & -2.792305 & -1.155769 \\
\hline $\mathrm{H}$ & 4.050861 & -0.460445 & 2.419367 \\
\hline C & 6.519993 & -1.956823 & -2.189195 \\
\hline C & 6.558715 & 0.761568 & 2.109720 \\
\hline C & 7.880385 & -2.608549 & -1.845073 \\
\hline $\mathrm{H}$ & 8.406261 & -2.901298 & -2.762197 \\
\hline $\mathrm{H}$ & 8.532456 & -1.925126 & -1.291375 \\
\hline $\mathrm{H}$ & 7.740154 & -3.506291 & -1.232253 \\
\hline C & 5.705556 & -2.966019 & -3.020908 \\
\hline $\mathrm{H}$ & 6.257547 & -3.222798 & -3.932095 \\
\hline $\mathrm{H}$ & 5.525300 & -3.896905 & -2.471474 \\
\hline $\mathrm{H}$ & 4.736232 & -2.556244 & -3.326978 \\
\hline C & 6.759677 & -0.700672 & -3.060320 \\
\hline $\mathrm{H}$ & 7.271142 & -0.973539 & -3.991721 \\
\hline $\mathrm{H}$ & 5.810874 & -0.218248 & -3.322032 \\
\hline $\mathrm{H}$ & 7.379474 & 0.040935 & -2.545518 \\
\hline C & 7.867654 & 0.099586 & 2.603228 \\
\hline $\mathrm{H}$ & 8.419189 & 0.784517 & 3.259173 \\
\hline $\mathrm{H}$ & 7.655046 & -0.814815 & 3.168955 \\
\hline $\mathrm{H}$ & 8.526632 & -0.169527 & 1.770789 \\
\hline C & 6.901816 & 2.054631 & 1.333141 \\
\hline $\mathrm{H}$ & 7.437160 & 2.757270 & 1.983509 \\
\hline $\mathrm{H}$ & 7.539783 & 1.856822 & 0.465446 \\
\hline $\mathrm{H}$ & 5.991538 & 2.548327 & 0.975287 \\
\hline C & 5.725697 & 1.157305 & 3.343831 \\
\hline $\mathrm{H}$ & 6.297655 & 1.856043 & 3.964971 \\
\hline $\mathrm{H}$ & 4.790703 & 1.654131 & 3.061365 \\
\hline $\mathrm{H}$ & 5.479146 & 0.290552 & 3.967227 \\
\hline C & -4.343179 & -1.062203 & -0.545824 \\
\hline C & -5.642876 & -1.553826 & -0.412171 \\
\hline C & -4.133733 & 0.326457 & -0.561582 \\
\hline C & -6.743300 & -0.692538 & -0.282302 \\
\hline $\mathrm{H}$ & -5.786911 & -2.629911 & -0.385064 \\
\hline C & -5.201986 & 1.218212 & -0.451945 \\
\hline $\mathrm{H}$ & -3.120367 & 0.695007 & -0.673261 \\
\hline C & -6.494907 & 0.682104 & -0.308222 \\
\hline $\mathrm{H}$ & -7.327749 & 1.368501 & -0.212608 \\
\hline C & -5.015055 & 2.747411 & -0.483034 \\
\hline C & -8.155919 & -1.283340 & -0.109799 \\
\hline C & -3.550930 & 3.152947 & -0.734960 \\
\hline $\mathrm{H}$ & -2.880696 & 2.793806 & 0.052395 \\
\hline $\mathrm{H}$ & -3.185979 & 2.771803 & -1.696123 \\
\hline $\mathrm{H}$ & -3.471691 & 4. 245943 & -0.760638 \\
\hline C & -5.877296 & 3.356928 & -1.614213 \\
\hline $\mathrm{H}$ & -5.745292 & 4.445598 & -1.646693 \\
\hline $\mathrm{H}$ & -5.587573 & 2.950635 & -2.590266 \\
\hline $\mathrm{H}$ & -6.944616 & 3.157488 & -1.473214 \\
\hline C & -5.455515 & 3.344313 & 0.874852 \\
\hline $\mathrm{H}$ & -6.501137 & 3.108937 & 1.101255 \\
\hline $\mathrm{H}$ & -4.838122 & 2.954397 & 1.691353 \\
\hline $\mathrm{H}$ & -5.352738 & 4.436650 & 0.863214 \\
\hline
\end{tabular}




\begin{tabular}{|lrrr|} 
C & -8.202108 & -2.127699 & 1.186034 \\
$H$ & -7.476637 & -2.947344 & 1.163635 \\
$H$ & -7.976916 & -1.510119 & 2.062702 \\
$H$ & -9.199251 & -2.564823 & 1.322260 \\
C & -9.243100 & -0.196253 & -0.012609 \\
$H$ & -9.088678 & 0.459253 & 0.851921 \\
$H$ & -9.278837 & 0.427222 & -0.913439 \\
$H$ & -10.226019 & -0.667242 & 0.102554 \\
C & -8.490825 & -2.185885 & -1.321242 \\
H & -9.493136 & -2.617986 & -1.210334 \\
H & -8.469562 & -1.612159 & -2.255109 \\
H & -7.782088 & -3.014465 & -1.421903 \\
\hline
\end{tabular}

\section{C-Catalyzed reaction TS 22}

\section{Datum}

Value

\begin{tabular}{lc}
\hline B3LYP/6-31G(d) Energy & -3038.968643 \\
\hline B3LYP-D3(BJ)/def2-TZVPP-IEF-PCM(DCM) Energy & -3040.518185 \\
\hline B3LYP-D3(BJ)/def2-TZVPP-IEF-PCM(DCM)//B3LYP/6-31G(d) Free Energy (Quasiharmonic) & -3039.289168 \\
\hline
\end{tabular}

Number of Imaginary Frequencies

Frequencies (Top 3 out of 450)

$\begin{array}{lr}\text { 1. } & -326.5675 \mathrm{~cm}^{-1} \\ \text { 2. } & 5.4179 \mathrm{~cm}^{-1} \\ \text { 3. } & 7.7438 \mathrm{~cm}^{-1}\end{array}$

\section{B3LYP/6-31G(d) Molecular Geometry in Cartesian Coordinates}

$\begin{array}{lrrr}\mathrm{C} & 0.835451 & 0.776531 & 0.457624 \\ \mathrm{H} & 1.893832 & 0.498032 & 0.499907 \\ \mathrm{H} & 0.361505 & 0.459220 & 1.393731 \\ \mathrm{C} & -2.685251 & 2.001756 & -0.853998 \\ \mathrm{O} & -3.434427 & 2.879213 & -1.235734 \\ \mathrm{O} & -1.901231 & 1.339814 & -1.702800 \\ \mathrm{H} & -1.218503 & 0.784077 & -1.234850 \\ \mathrm{O} & 0.214027 & 0.107311 & -0.651617 \\ \mathrm{H} & 0.315972 & -0.869849 & -0.532630 \\ \mathrm{C} & 2.980295 & -3.520151 & 3.270176 \\ \mathrm{C} & 3.535021 & -3.787736 & 1.167562 \\ \mathrm{C} & 0.813179 & -2.931219 & 2.231385 \\ \mathrm{C} & 1.652426 & -3.789948 & 3.015752 \\ \mathrm{H} & 3.326320 & -4.858536 & 1.174197\end{array}$




\begin{tabular}{|c|c|c|c|}
\hline $\mathrm{H}$ & 1.022753 & -1.864196 & 2.335593 \\
\hline $\mathrm{H}$ & -0.254958 & -3.139860 & 2.307246 \\
\hline C & -0.367181 & -3.491676 & -1.138925 \\
\hline C & 0.356882 & -4.879554 & -0.963234 \\
\hline C & -1.751493 & -3.445930 & -0.474695 \\
\hline $\mathrm{H}$ & -2.095679 & -2.407539 & -0.430165 \\
\hline $\mathrm{H}$ & -2.489425 & -4.022012 & -1.043407 \\
\hline $\mathrm{H}$ & -1.718812 & -3.841297 & 0.544950 \\
\hline C & -0.472491 & -2.993401 & -2.580238 \\
\hline $\mathrm{H}$ & -1.068439 & -3.685846 & -3.186078 \\
\hline H & -0.969225 & -2.017447 & -2.606045 \\
\hline $\mathrm{H}$ & 0.510976 & -2.884493 & -3.041340 \\
\hline C & 1.496852 & -5.088417 & -1.974546 \\
\hline $\mathrm{H}$ & 1.117364 & -5.267815 & -2.986356 \\
\hline $\mathrm{H}$ & 2.167634 & -4.224746 & -2.004657 \\
\hline $\mathrm{H}$ & 2.080848 & -5.963849 & -1.670869 \\
\hline C & -0.572764 & -6.093487 & -0.974989 \\
\hline $\mathrm{H}$ & -1.100522 & -6.178766 & -1.932073 \\
\hline $\mathrm{H}$ & 0.014319 & -7.006951 & -0.831688 \\
\hline $\mathrm{H}$ & -1.311215 & -6.040094 & -0.171681 \\
\hline 0 & 0.513558 & -2.602600 & -0.402998 \\
\hline 0 & 0.930274 & -4.761476 & 0.355731 \\
\hline B & 1.213882 & -3.379994 & 0.586159 \\
\hline 0 & 2.691755 & -3.027083 & 0.543658 \\
\hline C & 4.947123 & -3.373640 & 1.244086 \\
\hline C & 5.918784 & -4.320894 & 1.603479 \\
\hline C & 5.339565 & -2.059316 & 0.944368 \\
\hline C & 7.262491 & -3.960606 & 1.667379 \\
\hline $\mathrm{H}$ & 5.616570 & -5.340938 & 1.830039 \\
\hline C & 6.684394 & -1.704408 & 1.007799 \\
\hline $\mathrm{H}$ & 4.590483 & -1.331480 & 0.650470 \\
\hline C & 7.647433 & -2.650320 & 1.371799 \\
\hline $\mathrm{H}$ & 8.008841 & -4.700088 & 1.943403 \\
\hline $\mathrm{H}$ & 6.981211 & -0.687885 & 0.766045 \\
\hline $\mathrm{H}$ & 8.695605 & -2.368334 & 1.419963 \\
\hline $\mathrm{H}$ & 1.295789 & -4.805354 & 3.190099 \\
\hline $\mathrm{H}$ & 3.334609 & -2.493457 & 3.274912 \\
\hline $\mathrm{H}$ & 3.599877 & -4.235352 & 3.803952 \\
\hline C & -1.562668 & 2.112951 & 1.379483 \\
\hline C & -2.611340 & 1.616791 & 0.614979 \\
\hline C & -3.653850 & 0.823017 & 1.208909 \\
\hline C & -3.586677 & 0.571525 & 2.566647 \\
\hline $\mathrm{H}$ & -4.371547 & -0.019351 & 3.031729 \\
\hline C & 0.699604 & 2.276530 & 0.297018 \\
\hline C & -0.459984 & 2.910970 & 0.737750 \\
\hline C & 1.626517 & 4.418642 & -0.373413 \\
\hline C & 1.761133 & 3.045305 & -0.289028 \\
\hline $\mathrm{H}$ & 2.425251 & 5.002775 & -0.823526 \\
\hline C & 0.464417 & 5.093305 & 0.067319 \\
\hline C & -0.610343 & 4.331133 & 0.624062 \\
\hline C & -1.781114 & 5.019897 & 1.046513 \\
\hline C & -1.875254 & 6.388286 & 0.931190 \\
\hline C & -0.807234 & 7.140805 & 0.385353 \\
\hline C & 0.335635 & 6.504875 & -0.039577 \\
\hline
\end{tabular}




\begin{tabular}{|c|c|c|c|}
\hline H & -2.612413 & 4.451007 & 1.447307 \\
\hline $\mathrm{H}$ & -2.780344 & 6.895581 & 1.253596 \\
\hline $\mathrm{H}$ & -0.897338 & 8.220237 & 0.297446 \\
\hline $\mathrm{H}$ & 1.158758 & 7.071745 & -0.468351 \\
\hline C & -2.554136 & 1.084035 & 3.387989 \\
\hline C & -1.520993 & 1.873805 & 2.793046 \\
\hline C & -0.494526 & 2.388687 & 3.632771 \\
\hline C & -0.493011 & 2.134011 & 4.986805 \\
\hline C & -1.515968 & 1.350337 & 5.572408 \\
\hline C & -2.523421 & 0.838346 & 4.787517 \\
\hline $\mathrm{H}$ & 0.290884 & 2.994846 & 3.193717 \\
\hline $\mathrm{H}$ & 0.297345 & 2.541056 & 5.611864 \\
\hline $\mathrm{H}$ & -1.504027 & 1.157782 & 6.641861 \\
\hline H & -3.316376 & 0.237895 & 5.227320 \\
\hline C & 5.374441 & 1.398494 & -1.882081 \\
\hline C & 5.460010 & 2.420965 & -0.932242 \\
\hline C & 4.258370 & 2.924422 & -0.408429 \\
\hline C & 3.015649 & 2.428685 & -0.814248 \\
\hline C & 2.972854 & 1.399284 & -1.771459 \\
\hline C & 4.143991 & 0.873310 & -2.320499 \\
\hline $\mathrm{H}$ & 6.286426 & 0.996084 & -2.307621 \\
\hline $\mathrm{H}$ & 4. 279701 & 3.714491 & 0.336945 \\
\hline H & 2.002967 & 1.029156 & -2.078649 \\
\hline C & 6.802516 & 3.015271 & -0.461904 \\
\hline C & 4.125467 & -0.233695 & -3.393243 \\
\hline C & 6.938428 & 2.850051 & 1.070500 \\
\hline $\mathrm{H}$ & 7.884818 & 3.281153 & 1.419675 \\
\hline $\mathrm{H}$ & 6.126650 & 3.349451 & 1.609083 \\
\hline $\mathrm{H}$ & 6.923070 & 1.791417 & 1.356657 \\
\hline C & 8.015128 & 2.335588 & -1.125858 \\
\hline H & 8.941604 & 2.787044 & -0.753279 \\
\hline $\mathrm{H}$ & 8.057552 & 1.262944 & -0.902665 \\
\hline $\mathrm{H}$ & 8.004987 & 2.455401 & -2.215002 \\
\hline C & 6.846561 & 4.521236 & -0.815408 \\
\hline $\mathrm{H}$ & 7.794192 & 4.964757 & -0.485324 \\
\hline $\mathrm{H}$ & 6.758995 & 4.670620 & -1.897502 \\
\hline $\mathrm{H}$ & 6.033496 & 5.075296 & -0.334982 \\
\hline C & 4.819563 & 0.280834 & -4.676796 \\
\hline H & 4.807359 & -0.494431 & -5.452846 \\
\hline $\mathrm{H}$ & 4.305468 & 1.164444 & -5.071678 \\
\hline $\mathrm{H}$ & 5.864667 & 0.554403 & -4.496499 \\
\hline C & 4.877007 & -1.480273 & -2.869839 \\
\hline $\mathrm{H}$ & 4.900723 & -2.260984 & -3.640488 \\
\hline H & 5.912917 & -1.251690 & -2.597007 \\
\hline $\mathrm{H}$ & 4.380917 & -1.893868 & -1.984491 \\
\hline C & 2.692414 & -0.661533 & -3.760728 \\
\hline $\mathrm{H}$ & 2.728964 & -1.459305 & -4.511964 \\
\hline $\mathrm{H}$ & 2.146925 & -1.041796 & -2.890741 \\
\hline $\mathrm{H}$ & 2.115545 & 0.167099 & -4.186226 \\
\hline C & -4.779737 & 0.217973 & 0.433378 \\
\hline C & -5.065241 & -1.151130 & 0.601232 \\
\hline C & -5.598008 & 0.979639 & -0.403975 \\
\hline C & -6.137659 & -1.757669 & -0.054688 \\
\hline H & -4.419471 & -1.731841 & 1.250192 \\
\hline
\end{tabular}




\begin{tabular}{|c|c|c|c|}
\hline C & -6.672691 & 0.402534 & -1.099174 \\
\hline $\mathrm{H}$ & -5.385388 & 2.034393 & -0.516268 \\
\hline C & -6.921224 & -0.958512 & -0.907462 \\
\hline $\mathrm{H}$ & -7.747545 & -1.421456 & -1.434367 \\
\hline C & -7.514353 & 1.285029 & -2.040205 \\
\hline C & -6.480676 & -3.251106 & 0.112054 \\
\hline C & -6.597487 & 1.858150 & -3.148628 \\
\hline $\mathrm{H}$ & -5.758372 & 2.427123 & -2.736962 \\
\hline $\mathrm{H}$ & -6.179526 & 1.050760 & -3.761266 \\
\hline $\mathrm{H}$ & -7.169752 & 2.522605 & -3.808385 \\
\hline C & -8.658276 & 0.505194 & -2.715475 \\
\hline $\mathrm{H}$ & -9.222384 & 1.176263 & -3.373181 \\
\hline $\mathrm{H}$ & -8.281651 & -0.319066 & -3.332217 \\
\hline $\mathrm{H}$ & -9.362120 & 0.091950 & -1.983272 \\
\hline C & -8.138303 & 2.450641 & -1.236666 \\
\hline $\mathrm{H}$ & -8.799311 & 2.073951 & -0.447029 \\
\hline $\mathrm{H}$ & -7.373810 & 3.075943 & -0.765358 \\
\hline $\mathrm{H}$ & -8.731491 & 3.093166 & -1.899227 \\
\hline C & -5.555944 & -3.959448 & 1.120260 \\
\hline $\mathrm{H}$ & -4.508034 & -3.937901 & 0.801288 \\
\hline $\mathrm{H}$ & -5.619166 & -3.507013 & 2.116615 \\
\hline $\mathrm{H}$ & -5.850285 & -5.011066 & 1.215208 \\
\hline C & -7.934608 & -3.396175 & 0.621769 \\
\hline $\mathrm{H}$ & -8.058946 & -2.906527 & 1.594486 \\
\hline $\mathrm{H}$ & -8.657843 & -2.951954 & -0.069524 \\
\hline $\mathrm{H}$ & -8.193547 & -4.455895 & 0.738685 \\
\hline C & -6.344639 & -3.966798 & -1.252933 \\
\hline $\mathrm{H}$ & -6.589527 & -5.031910 & -1.154980 \\
\hline $\mathrm{H}$ & -7.014569 & -3.537797 & -2.005357 \\
\hline $\mathrm{H}$ & -5.320536 & -3.886408 & -1.635482 \\
\hline
\end{tabular}

\section{C-Catalyzed reaction TS 23}

\section{Datum}

Value

\begin{tabular}{lc}
\hline B3LYP/6-31G(d) Energy & -3038.966046 \\
\hline B3LYP-D3(BJ)/def2-TZVPP-IEF-PCM(DCM) Energy & -3040.518521 \\
\hline B3LYP-D3(BJ)/def2-TZVPP-IEF-PCM(DCM)//B3LYP/6-31G(d) Free Energy (Quasiharmonic) & -3039.28879 \\
\hline
\end{tabular}

Number of Imaginary Frequencies

Frequencies (Top 3 out of 450)

$$
\begin{array}{lr}
\text { 1. } & -329.3809 \mathrm{~cm}^{-1} \\
\text { 2. } & 7.4981 \mathrm{~cm}^{-1} \\
\text { 3. } & 13.1833 \mathrm{~cm}^{-1}
\end{array}
$$




\begin{tabular}{|c|c|c|c|}
\hline C & 1.549604 & 0.357246 & 0.410624 \\
\hline $\mathrm{H}$ & 2.499910 & -0.171826 & 0.535822 \\
\hline $\mathrm{H}$ & 0.922009 & 0.148780 & 1.285693 \\
\hline C & -1.492132 & 2.448517 & -1.075159 \\
\hline 0 & -2.011256 & 3.467322 & -1.484278 \\
\hline 0 & -0.803289 & 1.648098 & -1.889481 \\
\hline H & -0.289930 & 0.948388 & -1.401474 \\
\hline 0 & 0.894352 & -0.097256 & -0.783010 \\
\hline $\mathrm{H}$ & 0.738026 & -1.074059 & -0.768180 \\
\hline C & 2.317943 & -3.425205 & 2.153555 \\
\hline C & 0.149780 & -3.204388 & 1.845319 \\
\hline C & 2.163301 & -4.409523 & -0.112852 \\
\hline C & 2.585492 & -3.389246 & 0.801642 \\
\hline $\mathrm{H}$ & 0.228137 & -2.172579 & 1.498694 \\
\hline $\mathrm{H}$ & 2.628036 & -4.365510 & -1.099210 \\
\hline $\mathrm{H}$ & 2.170579 & -5.424901 & 0.289133 \\
\hline C & -0.762082 & -2.954928 & -2.075923 \\
\hline C & -0.597399 & -4.473979 & -2.456744 \\
\hline C & -2.095632 & -2.650208 & -1.377629 \\
\hline $\mathrm{H}$ & -2.067306 & -1.633285 & -0.972767 \\
\hline $\mathrm{H}$ & -2.935667 & -2.704413 & -2.078069 \\
\hline $\mathrm{H}$ & -2.283466 & -3.346421 & -0.554733 \\
\hline C & -0.552053 & -1.980861 & -3.234997 \\
\hline $\mathrm{H}$ & -1.267723 & -2.189673 & -4.038715 \\
\hline $\mathrm{H}$ & -0.717602 & -0.948078 & -2.913464 \\
\hline $\mathrm{H}$ & 0.457259 & -2.052731 & -3.645637 \\
\hline C & 0.471929 & -4.708549 & -3.537422 \\
\hline $\mathrm{H}$ & 0.135370 & -4.381876 & -4.527383 \\
\hline $\mathrm{H}$ & 1.402113 & -4.183103 & -3.300816 \\
\hline $\mathrm{H}$ & 0.689498 & -5.780337 & -3.584721 \\
\hline C & -1.896892 & -5.172662 & -2.863951 \\
\hline $\mathrm{H}$ & -2.337288 & -4.708259 & -3.754284 \\
\hline $\mathrm{H}$ & -1.687607 & -6.221606 & -3.098655 \\
\hline $\mathrm{H}$ & -2.631888 & -5.151766 & -2.056047 \\
\hline 0 & 0.308544 & -2.783311 & -1.096838 \\
\hline 0 & -0.132958 & -5.066746 & -1.233348 \\
\hline B & 0.475854 & -4.068314 & -0.436829 \\
\hline 0 & -0.104926 & -4.117549 & 0.968111 \\
\hline C & -0.313803 & -3.408044 & 3.229236 \\
\hline C & -0.728365 & -4.675434 & 3.670280 \\
\hline C & -0.372493 & -2.314449 & 4.106259 \\
\hline C & -1.186731 & -4.842200 & 4.973631 \\
\hline $\mathrm{H}$ & -0.697313 & -5.511152 & 2.978690 \\
\hline C & -0.833912 & -2.486809 & 5.409365 \\
\hline $\mathrm{H}$ & -0.065378 & -1.327901 & 3.767923 \\
\hline C & -1.238485 & -3.750268 & 5.846311 \\
\hline $\mathrm{H}$ & -1.510165 & -5.823246 & 5.310314 \\
\hline $\mathrm{H}$ & -0.883750 & -1.631449 & 6.076340 \\
\hline $\mathrm{H}$ & -1.598957 & -3.884205 & 6.862608 \\
\hline $\mathrm{H}$ & 2.916461 & -2.442415 & 0.372959 \\
\hline
\end{tabular}




\begin{tabular}{|c|c|c|c|}
\hline $\mathrm{H}$ & 2.171192 & -4.378899 & 2.651741 \\
\hline $\mathrm{H}$ & 2.591218 & -2.593417 & 2.796870 \\
\hline C & -0.503370 & 2.244136 & 1.214920 \\
\hline C & -1.601249 & 2.044127 & 0.386220 \\
\hline C & -2.847745 & 1.549899 & 0.909932 \\
\hline C & -2.925798 & 1.289606 & 2.265666 \\
\hline H & -3.863419 & 0.925997 & 2.677473 \\
\hline C & 1.805732 & 1.845400 & 0.301171 \\
\hline C & 0.804217 & 2.743984 & 0.662308 \\
\hline C & 3.286592 & 3.692344 & -0.232207 \\
\hline C & 3.073642 & 2.327963 & -0.168000 \\
\hline H & 4.244006 & 4.061929 & -0.590471 \\
\hline C & 2.289543 & 4.631723 & 0.118930 \\
\hline C & 1.017007 & 4.157703 & 0.568897 \\
\hline C & 0.020181 & 5.113822 & 0.909938 \\
\hline C & 0.275182 & 6.463116 & 0.815888 \\
\hline C & 1.537435 & 6.929250 & 0.375119 \\
\hline C & 2.520852 & 6.031461 & 0.031941 \\
\hline H & -0.956606 & 4.768382 & 1.229209 \\
\hline H & -0.501397 & 7.177707 & 1.074016 \\
\hline H & 1.723066 & 7.997538 & 0.303333 \\
\hline H & 3.491468 & 6.377980 & -0.314985 \\
\hline C & -1.844666 & 1.513899 & 3.150750 \\
\hline C & -0.606499 & 1.998488 & 2.624970 \\
\hline C & 0.470431 & 2.222337 & 3.528343 \\
\hline C & 0.324907 & 1.984968 & 4.877990 \\
\hline C & -0.905082 & 1.511861 & 5.396171 \\
\hline C & -1.964355 & 1.279570 & 4.547957 \\
\hline H & 1.413164 & 2.594464 & 3.142015 \\
\hline H & 1.156799 & 2.171036 & 5.551906 \\
\hline H & -1.011749 & 1.342476 & 6.464559 \\
\hline H & -2.914482 & 0.918765 & 4.934581 \\
\hline C & 6.350446 & -0.134942 & -1.373150 \\
\hline C & 5.105958 & -0.342609 & -1.985212 \\
\hline C & 4.029009 & 0.448154 & -1.564340 \\
\hline C & 4.193297 & 1.423800 & -0.569409 \\
\hline C & 5.455892 & 1.597558 & 0.017128 \\
\hline C & 6.554596 & 0.821805 & -0.369532 \\
\hline H & 7.193866 & -0.739520 & -1.693164 \\
\hline H & 3.048448 & 0.329226 & -2.008852 \\
\hline H & 5.559144 & 2.347862 & 0.792707 \\
\hline C & 4.973050 & -1.397271 & -3.101256 \\
\hline C & 7.952605 & 0.985364 & 0.258322 \\
\hline C & 3.527608 & -1.520652 & -3.619712 \\
\hline H & 3.479634 & -2.298888 & -4.390314 \\
\hline H & 3.175225 & -0.587313 & -4.072688 \\
\hline H & 2.826532 & -1.793977 & -2.823277 \\
\hline C & 5.878780 & -0.997860 & -4.290739 \\
\hline H & 5.796840 & -1.735837 & -5.098309 \\
\hline H & 5.587318 & -0.020831 & -4.692234 \\
\hline $\mathrm{H}$ & 6.932562 & -0.938278 & -3.998291 \\
\hline C & 5.412854 & -2.782072 & -2.570182 \\
\hline $\mathrm{H}$ & 5.332020 & -3.536146 & -3.362548 \\
\hline H & 6.450753 & -2.779465 & -2.221108 \\
\hline
\end{tabular}




\begin{tabular}{|c|c|c|c|}
\hline $\mathrm{H}$ & 4.781756 & -3.102274 & -1.732885 \\
\hline C & 7.982960 & 2.072179 & 1.349960 \\
\hline $\mathrm{H}$ & 8.993671 & 2.148021 & 1.766870 \\
\hline $\mathrm{H}$ & 7.715454 & 3.057847 & 0.952637 \\
\hline $\mathrm{H}$ & 7.301609 & 1.840714 & 2.176659 \\
\hline C & 8.391251 & -0.351926 & 0.901319 \\
\hline $\mathrm{H}$ & 9.388559 & -0.252060 & 1.347150 \\
\hline $\mathrm{H}$ & 7.694558 & -0.654034 & 1.691817 \\
\hline $\mathrm{H}$ & 8.434560 & -1.163439 & 0.167367 \\
\hline C & 8.967388 & 1.381280 & -0.840548 \\
\hline $\mathrm{H}$ & 9.969787 & 1.497690 & -0.410404 \\
\hline $\mathrm{H}$ & 9.029579 & 0.625669 & -1.630720 \\
\hline $\mathrm{H}$ & 8.686187 & 2.331231 & -1.309171 \\
\hline C & -4.052234 & 1.256487 & 0.073521 \\
\hline C & -4.617866 & 2.206819 & -0.779422 \\
\hline C & -4.682705 & 0.004458 & 0.211505 \\
\hline C & -5.783300 & 1.930003 & -1.511755 \\
\hline $\mathrm{H}$ & -4.137501 & 3.171509 & -0.871645 \\
\hline C & -5.852087 & -0.303193 & -0.485428 \\
\hline $\mathrm{H}$ & -4.229491 & -0.726504 & 0.871338 \\
\hline C & -6.378823 & 0.677372 & -1.346301 \\
\hline $\mathrm{H}$ & -7.282202 & 0.446978 & -1.899262 \\
\hline C & -6.577065 & -1.655495 & -0.339610 \\
\hline C & -6.341874 & 3.007004 & -2.460747 \\
\hline C & -5.859581 & -2.605996 & 0.637648 \\
\hline $\mathrm{H}$ & -6.415092 & -3.547946 & 0.711144 \\
\hline $\mathrm{H}$ & -4.844268 & -2.846675 & 0.303305 \\
\hline $\mathrm{H}$ & -5.795731 & -2.181492 & 1.646137 \\
\hline C & -8.008826 & -1.413175 & 0.195835 \\
\hline $\mathrm{H}$ & -8.543521 & -2.365193 & 0.302985 \\
\hline $\mathrm{H}$ & -7.981982 & -0.926399 & 1.177606 \\
\hline $\mathrm{H}$ & -8.593440 & -0.776223 & -0.475902 \\
\hline C & -6.659499 & -2.355513 & -1.716621 \\
\hline $\mathrm{H}$ & -7.201484 & -1.750590 & -2.450893 \\
\hline $\mathrm{H}$ & -5.658013 & -2.548696 & -2.117959 \\
\hline $\mathrm{H}$ & -7.180922 & -3.316604 & -1.626633 \\
\hline C & -7.638547 & 2.560906 & -3.162179 \\
\hline $\mathrm{H}$ & -7.483727 & 1.668827 & -3.779978 \\
\hline $\mathrm{H}$ & -8.439483 & 2.346054 & -2.444698 \\
\hline $\mathrm{H}$ & -7.993253 & 3.360039 & -3.822989 \\
\hline C & -6.649988 & 4.294272 & -1.659030 \\
\hline $\mathrm{H}$ & -7.395374 & 4.102174 & -0.878169 \\
\hline $\mathrm{H}$ & -5.754591 & 4.698663 & -1.176878 \\
\hline $\mathrm{H}$ & -7.047644 & 5.069902 & -2.325045 \\
\hline C & -5.282478 & 3.319238 & -3.545788 \\
\hline $\mathrm{H}$ & -5.642935 & 4.113008 & -4.212395 \\
\hline $\mathrm{H}$ & -4.332296 & 3.644518 & -3.111054 \\
\hline $\mathrm{H}$ & -5.079817 & 2.431241 & -4.155998 \\
\hline
\end{tabular}




\begin{tabular}{lc}
\hline B3LYP/6-31G(d) Energy & -3038.964964 \\
\hline B3LYP-D3(BJ)/def2-TZVPP-IEF-PCM(DCM) Energy & -3040.518086 \\
\hline B3LYP-D3(BJ)/def2-TZVPP-IEF-PCM(DCM)//B3LYP/6-31G(d) Free Energy (Quasiharmonic) & -3039.288403 \\
\hline
\end{tabular}

Number of Imaginary Frequencies

Frequencies (Top 3 out of 450)

$\begin{array}{lr}\text { 1. } & -310.1522 \mathrm{~cm}^{-1} \\ \text { 2. } & 6.7847 \mathrm{~cm}^{-1} \\ \text { 3. } & 10.4365 \mathrm{~cm}^{-1}\end{array}$

\section{B3LYP/6-31G(d) Molecular Geometry in Cartesian Coordinates}

C

$\mathrm{H}$

$\mathrm{H}$

C

0

0

$\mathrm{H}$

0

$\mathrm{H}$

C

C

C

C

$\mathrm{H}$

$\mathrm{H}$

$\mathrm{H}$

C

C

C

$\mathrm{H}$

$\mathrm{H}$

$\mathrm{H}$

C

$\mathrm{H}$

$\mathrm{H}$

$\mathrm{H}$

C

$\mathrm{H}$

$\mathrm{H}$

$\mathrm{H}$

C

$\mathrm{H}$
$-1.082934$

$-2.153796$

$-0.658836$

2. 364598

3. 024125

1. 577441

0.952895

$-0.448485$

$-0.442429$

$-4.166500$

$-3.302746$

$-2.134803$

$-2.928059$

$-2.793995$

$-1.295855$

$-2.701807$

0.840317

0.538840

1.468258

1.624988

2.444493

0.830340

1.664256

2. 698142

1.696147

1. 225351

1.639004

2. 580656

1.816071

1. 336221

0.183645

1.056882
$-0.484922$

$-0.247791$

$-0.015768$

$-2.030995$

$-3.015262$

$-1.459912$

$-0.792548$

0.054150

1.041862

2. 229234

3.082029

3. 251664

2. 118367

2.159805

3.015615

4. 109342

3. 377502

4.923578

2.910677

1.827770

3. 384548

3.143209

2.868037

3. 226073

1.773717

3.190694

5.755499

5.685751

5.437894

6.807609

5.541485

5.610488
0.616552

0.584184

1.511450

$-0.536131$

$-0.801458$

$-1.450772$

$-1.058419$

$-0.555122$

$-0.468014$

$-1.307878$

0.520256

$-2.291266$

$-1.902935$

0.806414

$-2.948990$

$-2.660724$

$-0.128817$

$-0.209797$

1.183577

1.161143

1.340470

2.039504

$-1.321132$

$-1.281184$

$-1.311709$

$-2.270066$

$-0.871886$

$-0.314522$

$-1.901902$

$-0.893436$

1.154819

1.813112 


\begin{tabular}{|c|c|c|c|}
\hline $\mathrm{H}$ & -0.201445 & 6.552480 & 0.988189 \\
\hline $\mathrm{H}$ & -0.594159 & 4.968773 & 1.667692 \\
\hline 0 & -0.490107 & 2.795755 & -0.229994 \\
\hline 0 & -0.627324 & 4.973058 & -1.048051 \\
\hline B & -1.372754 & 3.791559 & -0.827873 \\
\hline 0 & -2.583247 & 4.054052 & 0.066548 \\
\hline C & -4.548279 & 3.402040 & 1.242826 \\
\hline C & -5.097030 & 4.693294 & 1.198484 \\
\hline C & -5.176803 & 2.408344 & 2.008920 \\
\hline C & -6.260269 & 4.979278 & 1.908122 \\
\hline $\mathrm{H}$ & -4.595903 & 5.459725 & 0.616188 \\
\hline C & -6.340689 & 2.698929 & 2.717230 \\
\hline $\mathrm{H}$ & -4.751071 & 1.408270 & 2.046888 \\
\hline C & -6.885416 & 3.984646 & 2.666398 \\
\hline $\mathrm{H}$ & -6.679522 & 5.981030 & 1.874795 \\
\hline $\mathrm{H}$ & -6.820225 & 1.925330 & 3.310107 \\
\hline $\mathrm{H}$ & -7.792412 & 4.212388 & 3.219580 \\
\hline $\mathrm{H}$ & -2.427466 & 1.151393 & -1.848929 \\
\hline $\mathrm{H}$ & -4.772704 & 3.115509 & -1.473306 \\
\hline $\mathrm{H}$ & -4.698229 & 1.341276 & -0.977992 \\
\hline C & 1.362138 & -1.666668 & 1.725830 \\
\hline C & 2.403987 & -1.396687 & 0.845326 \\
\hline C & 3.534433 & -0.604328 & 1.251312 \\
\hline C & 3.545416 & -0.104738 & 2.541433 \\
\hline $\mathrm{H}$ & 4.396822 & 0.486064 & 2.868797 \\
\hline C & -0.919776 & -1.988699 & 0.704997 \\
\hline C & 0.219127 & -2.540184 & 1.287075 \\
\hline C & -1.882823 & -4.205757 & 0.461089 \\
\hline C & -1.969675 & -2.845826 & 0.240909 \\
\hline $\mathrm{H}$ & -2.684799 & -4.852299 & 0.113401 \\
\hline C & -0.759264 & -4.794635 & 1.087765 \\
\hline C & 0.329637 & -3.956058 & 1.483986 \\
\hline C & 1.471867 & -4.566217 & 2.073658 \\
\hline C & 1.521997 & -5.927163 & 2.272947 \\
\hline C & 0.437545 & -6.752550 & 1.889255 \\
\hline C & -0.676753 & -6.196398 & 1.306076 \\
\hline $\mathrm{H}$ & 2.314234 & -3.946174 & 2.358512 \\
\hline $\mathrm{H}$ & 2.404604 & -6.374027 & 2.722127 \\
\hline $\mathrm{H}$ & 0.492746 & -7.825819 & 2.050254 \\
\hline $\mathrm{H}$ & -1.511303 & -6.821374 & 0.997110 \\
\hline C & 2.507494 & -0.358059 & 3.469785 \\
\hline C & 1.394647 & -1.160083 & 3.065716 \\
\hline C & 0.367834 & -1.422735 & 4.014542 \\
\hline C & 0.437527 & -0.911527 & 5.291807 \\
\hline C & 1.538115 & -0.114007 & 5.687927 \\
\hline C & 2.550150 & 0.153753 & 4.795202 \\
\hline $\mathrm{H}$ & -0.475182 & -2.039256 & 3.720117 \\
\hline $\mathrm{H}$ & -0.355431 & -1.125080 & 6.003541 \\
\hline $\mathrm{H}$ & 1.581642 & 0.282153 & 6.698832 \\
\hline $\mathrm{H}$ & 3.401884 & 0.761795 & 5.090803 \\
\hline C & -5.354309 & -1.708863 & -2.104410 \\
\hline C & -4.071464 & -1.634927 & -2.681166 \\
\hline C & -2.973834 & -1.933659 & -1.868742 \\
\hline C & -3.147537 & -2.345025 & -0.535620 \\
\hline
\end{tabular}




\begin{tabular}{|c|c|c|c|}
\hline C & -4.436211 & -2.410789 & -0.002864 \\
\hline C & -5.564962 & -2.080368 & -0.772782 \\
\hline H & -6.211567 & -1.485340 & -2.727987 \\
\hline $\mathrm{H}$ & -1.964420 & -1.893711 & -2.260963 \\
\hline $\mathrm{H}$ & -4.554841 & -2.741434 & 1.025334 \\
\hline C & -3.926050 & -1.324403 & -4.185058 \\
\hline C & -6.969740 & -2.173067 & -0.145347 \\
\hline C & -2.457822 & -1.104452 & -4.598826 \\
\hline $\mathrm{H}$ & -2.409604 & -0.855825 & -5.664968 \\
\hline $\mathrm{H}$ & -1.995445 & -0.280571 & -4.043765 \\
\hline $\mathrm{H}$ & -1.847236 & -2.000378 & -4.444907 \\
\hline C & -4.479141 & -2.529579 & -4.985246 \\
\hline $\mathrm{H}$ & -4.391791 & -2.345535 & -6.063261 \\
\hline $\mathrm{H}$ & -3.923336 & -3.444255 & -4.750002 \\
\hline $\mathrm{H}$ & -5.535502 & -2.711891 & -4.759050 \\
\hline C & -4.726131 & -0.058006 & -4.568680 \\
\hline $\mathrm{H}$ & -4.618353 & 0.141316 & -5.641551 \\
\hline $\mathrm{H}$ & -5.796428 & -0.164443 & -4.363151 \\
\hline $\mathrm{H}$ & -4.366060 & 0.820617 & -4.022976 \\
\hline C & -7.241336 & -3.633055 & 0.290422 \\
\hline $\mathrm{H}$ & -8.235921 & -3.717225 & 0.745569 \\
\hline $\mathrm{H}$ & -6.507838 & -3.982545 & 1.024471 \\
\hline $\mathrm{H}$ & -7.202589 & -4.311178 & -0.569607 \\
\hline C & -7.047185 & -1.250510 & 1.094445 \\
\hline $\mathrm{H}$ & -8.038102 & -1.319969 & 1.559905 \\
\hline $\mathrm{H}$ & -6.876905 & -0.203318 & 0.817503 \\
\hline $\mathrm{H}$ & -6.304973 & -1.523546 & 1.852300 \\
\hline C & -8.083272 & -1.750628 & -1.122566 \\
\hline $\mathrm{H}$ & -9.056344 & -1.816101 & -0.622776 \\
\hline $\mathrm{H}$ & -8.123012 & -2.400493 & -2.003984 \\
\hline $\mathrm{H}$ & -7.955893 & -0.717271 & -1.464931 \\
\hline C & 4.682626 & -0.265940 & 0.355022 \\
\hline C & 5.114427 & 1.072230 & 0.271933 \\
\hline C & 5.388847 & -1.246256 & -0.346214 \\
\hline C & 6.221146 & 1.434640 & -0.497082 \\
\hline $\mathrm{H}$ & 4.555183 & 1.824758 & 0.815456 \\
\hline C & 6.495185 & -0.920106 & -1.146616 \\
\hline $\mathrm{H}$ & 5.065011 & -2.275189 & -0.267583 \\
\hline C & 6.890464 & 0.418099 & -1.203180 \\
\hline $\mathrm{H}$ & 7.744562 & 0.689262 & -1.812709 \\
\hline C & 7.208506 & -2.040059 & -1.927557 \\
\hline C & 6.725430 & 2.887450 & -0.600888 \\
\hline C & 7.716937 & -3.118369 & -0.941033 \\
\hline $\mathrm{H}$ & 8.222919 & -3.923231 & -1.488204 \\
\hline $\mathrm{H}$ & 8.430751 & -2.692519 & -0.225906 \\
\hline $\mathrm{H}$ & 6.898258 & -3.568857 & -0.371325 \\
\hline C & 6.204754 & -2.681923 & -2.916349 \\
\hline $\mathrm{H}$ & 6.683204 & -3.505588 & -3.461304 \\
\hline $\mathrm{H}$ & 5.321068 & -3.077596 & -2.406481 \\
\hline $\mathrm{H}$ & 5.860232 & -1.944885 & -3.651121 \\
\hline C & 8.416100 & -1.522035 & -2.731427 \\
\hline $\mathrm{H}$ & 8.120083 & -0.778684 & -3.480678 \\
\hline $\mathrm{H}$ & 9.177302 & -1.070936 & -2.083709 \\
\hline $\mathrm{H}$ & 8.887949 & -2.355052 & -3.265001 \\
\hline
\end{tabular}




\begin{tabular}{|lllr|} 
C & 6.655601 & 3.357122 & -2.073332 \\
H & 7.267931 & 2.730511 & -2.729941 \\
H & 5.625644 & 3.322745 & -2.446688 \\
H & 7.016739 & 4.389169 & -2.164600 \\
C & 5.893060 & 3.863384 & 0.252335 \\
$H$ & 4.845450 & 3.894690 & -0.068774 \\
$H$ & 5.917065 & 3.598752 & 1.315739 \\
$H$ & 6.297544 & 4.877273 & 0.154441 \\
C & 8.191188 & 2.960923 & -0.109594 \\
H & 8.566972 & 3.989155 & -0.180835 \\
H & 8.269962 & 2.641868 & 0.936111 \\
H & 8.853596 & 2.322799 & -0.703277 \\
\hline
\end{tabular}

\section{C-Catalyzed reaction TS 25 (TS-2.4)}

\section{Datum}

Value

\begin{tabular}{lc}
\hline B3LYP/6-31G(d) Energy & -3038.967392 \\
\hline B3LYP-D3(BJ)/def2-TZVPP-IEF-PCM(DCM) Energy & -3040.518162 \\
\hline B3LYP-D3(BJ)/def2-TZVPP-IEF-PCM(DCM)//B3LYP/6-31G(d) Free Energy (Quasiharmonic) & -3039.288021 \\
\hline
\end{tabular}

Number of Imaginary Frequencies

Frequencies (Top 3 out of 450)

$\begin{array}{lr}\text { 1. } & -331.4551 \mathrm{~cm}^{-1} \\ \text { 2. } & 9.7198 \mathrm{~cm}^{-1} \\ \text { 3. } & 12.7538 \mathrm{~cm}^{-1}\end{array}$

\section{B3LYP/6-31G(d) Molecular Geometry in Cartesian Coordinates}

C

$\mathrm{H}$

$\mathrm{H}$

C

0

0

$\mathrm{H}$

0

$\mathrm{H}$

C

C

C

C

$\mathrm{H}$

$$
\begin{array}{r}
2.949110 \\
3.944330 \\
2.220620 \\
-0.184990 \\
0.818770 \\
-1.154600 \\
-1.870500 \\
2.896190 \\
2.161300 \\
-5.328651 \\
-4.355171 \\
-4.819271 \\
-5.734171 \\
-5.285771
\end{array}
$$

\subsection{0}

0.632030

0.654180

1.278130

1.887610

1.068030

0.442470

2.009090

2.012580

$-2.490059$

$-3.529609$

$-0.533819$

$-1.454499$

$-3.733209$
$-1.254280$

$-1.697620$

$-2.072620$

0.889130

1.221970

1.775320

1.483890

$-0.648140$

0.001830

$-1.787250$

$-0.142120$

$-0.361120$

$-0.971100$

0.389670 


\begin{tabular}{|c|c|c|c|}
\hline $\mathrm{H}$ & -5.282310 & 0.352461 & 0.075150 \\
\hline $\mathrm{H}$ & -3.943271 & -0.284429 & -0.962920 \\
\hline C & -3.757581 & -0.481559 & 3.136370 \\
\hline C & -4.842851 & -1.618269 & 3.256400 \\
\hline C & -2.603781 & -0.601489 & 4.134220 \\
\hline $\mathrm{H}$ & -1.904461 & 0.229320 & 4.008240 \\
\hline $\mathrm{H}$ & -2.984001 & -0.573469 & 5.162370 \\
\hline $\mathrm{H}$ & -2.047191 & -1.530480 & 3.991310 \\
\hline C & -4.357330 & 0.931641 & 3.195660 \\
\hline $\mathrm{H}$ & -4.690360 & 1.184301 & 4.208180 \\
\hline $\mathrm{H}$ & -5.210660 & 1.033401 & 2.518000 \\
\hline $\mathrm{H}$ & -3.594930 & 1.658201 & 2.898840 \\
\hline C & -6.038551 & -1.269179 & 4.144320 \\
\hline $\mathrm{H}$ & -5.724011 & -1.081339 & 5.177400 \\
\hline $\mathrm{H}$ & -6.567781 & -0.388399 & 3.773790 \\
\hline $\mathrm{H}$ & -6.744081 & -2.106929 & 4.153730 \\
\hline C & -4.251251 & -2.967319 & 3.701440 \\
\hline $\mathrm{H}$ & -3.953961 & -2.955119 & 4.755470 \\
\hline $\mathrm{H}$ & -5.011341 & -3.745089 & 3.570760 \\
\hline $\mathrm{H}$ & -3.377211 & -3.238409 & 3.102560 \\
\hline 0 & -3.240251 & -0.686539 & 1.787410 \\
\hline 0 & -5.312231 & -1.748379 & 1.900660 \\
\hline B & -4.231701 & -1.408099 & 1.030900 \\
\hline 0 & -3.583141 & -2.616469 & 0.361150 \\
\hline C & -3.754151 & -4.649979 & -0.888290 \\
\hline C & -2.450551 & -4.575779 & -1.403630 \\
\hline C & -4.501961 & -5.825189 & -1.063890 \\
\hline C & -1.909401 & -5.663750 & -2.082770 \\
\hline $\mathrm{H}$ & -1.868001 & -3.671930 & -1.264010 \\
\hline C & -3.955502 & -6.911689 & -1.742750 \\
\hline $\mathrm{H}$ & -5.510581 & -5.885459 & -0.661030 \\
\hline C & -2.658002 & -6.831869 & -2.254880 \\
\hline $\mathrm{H}$ & -0.899951 & -5.597550 & -2.477710 \\
\hline $\mathrm{H}$ & -4.538192 & -7.819749 & -1.869510 \\
\hline $\mathrm{H}$ & -2.230402 & -7.678949 & -2.784290 \\
\hline $\mathrm{H}$ & -6.746111 & -1.489779 & -0.566870 \\
\hline $\mathrm{H}$ & -4.399271 & -2.409409 & -2.344100 \\
\hline $\mathrm{H}$ & -6.048151 & -3.208549 & -2.169940 \\
\hline C & 0.296789 & -0.379720 & -0.917770 \\
\hline C & -0.419690 & 0.734240 & -0.501100 \\
\hline C & -1.397220 & 1.365220 & -1.346180 \\
\hline C & -1.706240 & 0.760190 & -2.548400 \\
\hline $\mathrm{H}$ & -2.416070 & 1.240031 & -3.217290 \\
\hline C & 2.719229 & -0.423730 & -0.274560 \\
\hline C & 1.432509 & -0.932410 & -0.095920 \\
\hline C & 3.572389 & -2.031490 & 1.337350 \\
\hline C & 3.817309 & -0.983940 & 0.468190 \\
\hline $\mathrm{H}$ & 4.398129 & -2.450790 & 1.906430 \\
\hline C & 2.279299 & -2.566170 & 1.538560 \\
\hline C & 1.178389 & -2.006610 & 0.816720 \\
\hline C & -0.117471 & -2.551170 & 1.033590 \\
\hline C & -0.306741 & -3.603550 & 1.902540 \\
\hline C & 0.785399 & -4.158740 & 2.611570 \\
\hline C & 2.049069 & -3.644460 & 2.435680 \\
\hline
\end{tabular}




\begin{tabular}{|c|c|c|c|}
\hline $\mathrm{H}$ & -0.972901 & -2.126830 & 0.520610 \\
\hline $\mathrm{H}$ & -1.304801 & -4.009690 & 2.039820 \\
\hline $\mathrm{H}$ & 0.621469 & -4.986730 & 3.296170 \\
\hline H & 2.895699 & -4.056820 & 2.979710 \\
\hline C & -1.075981 & -0.436230 & -2.973370 \\
\hline C & -0.037221 & -1.003140 & -2.167510 \\
\hline C & 0.629919 & -2.166190 & -2.642300 \\
\hline C & 0.276889 & -2.745470 & -3.842250 \\
\hline C & -0.757441 & -2.188840 & -4.631880 \\
\hline C & -1.414481 & -1.056670 & -4.206710 \\
\hline $\mathrm{H}$ & 1.428609 & -2.591940 & -2.044930 \\
\hline H & 0.803179 & -3.630070 & -4.190960 \\
\hline $\mathrm{H}$ & -1.024561 & -2.652780 & -5.577430 \\
\hline $\mathrm{H}$ & -2.199841 & -0.612739 & -4.813980 \\
\hline C & 7.888349 & 0.317569 & 0.160370 \\
\hline C & 7.599159 & -1.051821 & 0.064470 \\
\hline C & 6.257499 & -1.442971 & 0.147860 \\
\hline C & 5.226459 & -0.508361 & 0.326160 \\
\hline C & 5.558470 & 0.849279 & 0.419230 \\
\hline C & 6.886710 & 1.283409 & 0.332360 \\
\hline $\mathrm{H}$ & 8.918590 & 0.640449 & 0.094440 \\
\hline $\mathrm{H}$ & 5.992549 & -2.492621 & 0.056070 \\
\hline H & 4.756090 & 1.566830 & 0.544300 \\
\hline C & 8.693099 & -2.119961 & -0.134520 \\
\hline C & 7.187150 & 2.792269 & 0.423090 \\
\hline C & 8.652559 & -3.124921 & 1.041230 \\
\hline $\mathrm{H}$ & 9.421509 & -3.897071 & 0.912760 \\
\hline H & 8.836009 & -2.617621 & 1.995380 \\
\hline $\mathrm{H}$ & 7.683229 & -3.629191 & 1.112990 \\
\hline C & 8.442249 & -2.875371 & -1.461350 \\
\hline H & 9.207839 & -3.646411 & -1.614460 \\
\hline $\mathrm{H}$ & 7.464969 & -3.369161 & -1.470730 \\
\hline $\mathrm{H}$ & 8.476499 & -2.187811 & -2.314280 \\
\hline C & 10.108679 & -1.514491 & -0.192200 \\
\hline $\mathrm{H}$ & 10.845999 & -2.314161 & -0.327880 \\
\hline H & 10.221259 & -0.816611 & -1.029640 \\
\hline $\mathrm{H}$ & 10.363569 & -0.983451 & 0.731960 \\
\hline C & 8.691000 & 3.104679 & 0.305880 \\
\hline H & 8.847920 & 4.187509 & 0.370650 \\
\hline $\mathrm{H}$ & 9.270830 & 2.638829 & 1.111410 \\
\hline H & 9.104980 & 2.769369 & -0.652240 \\
\hline C & 6.448590 & 3.527299 & -0.722160 \\
\hline $\mathrm{H}$ & 6.630830 & 4.607669 & -0.658840 \\
\hline $\mathrm{H}$ & 6.807620 & 3.181599 & -1.699070 \\
\hline $\mathrm{H}$ & 5.367180 & 3.362629 & -0.685490 \\
\hline C & 6.688060 & 3.335439 & 1.783330 \\
\hline $\mathrm{H}$ & 6.888400 & 4.411419 & 1.860360 \\
\hline $\mathrm{H}$ & 5.611030 & 3.187129 & 1.909050 \\
\hline $\mathrm{H}$ & 7.195740 & 2.834789 & 2.616250 \\
\hline C & -1.979780 & 2.697570 & -1.013760 \\
\hline C & -1.137990 & 3.735010 & -0.590480 \\
\hline C & -3.342390 & 2.967341 & -1.203710 \\
\hline C & -1.630070 & 5.019710 & -0.334610 \\
\hline $\mathrm{H}$ & -0.078420 & 3.536220 & -0.482500 \\
\hline
\end{tabular}




\begin{tabular}{|c|c|c|c|}
\hline C & -3.873480 & 4.242401 & -0.971780 \\
\hline $\mathrm{H}$ & -3.992180 & 2.160871 & -1.529350 \\
\hline C & -2.998780 & 5.247741 & -0.532380 \\
\hline $\mathrm{H}$ & -3.393290 & 6.236671 & -0.344340 \\
\hline C & -5.376100 & 4.500071 & -1.204430 \\
\hline C & -0.660020 & 6.113810 & 0.151010 \\
\hline C & -6.210960 & 3.581301 & -0.281500 \\
\hline $\mathrm{H}$ & -6.017370 & 2.522351 & -0.481180 \\
\hline $\mathrm{H}$ & -5.981620 & 3.771401 & 0.773230 \\
\hline $\mathrm{H}$ & -7.282940 & 3.758011 & -0.434530 \\
\hline C & -5.778570 & 5.958391 & -0.912000 \\
\hline $\mathrm{H}$ & -6.850740 & 6.088181 & -1.098080 \\
\hline $\mathrm{H}$ & -5.590030 & 6.233831 & 0.131800 \\
\hline $\mathrm{H}$ & -5.244160 & 6.666851 & -1.554940 \\
\hline C & -5.728090 & 4.195441 & -2.680200 \\
\hline $\mathrm{H}$ & -5.159370 & 4.840611 & -3.359580 \\
\hline $\mathrm{H}$ & -5.509670 & 3.155611 & -2.945900 \\
\hline $\mathrm{H}$ & -6.796130 & 4.368151 & -2.861500 \\
\hline C & 0.488490 & 6.283430 & -0.872300 \\
\hline $\mathrm{H}$ & 1.067730 & 5.363530 & -0.998710 \\
\hline $\mathrm{H}$ & 0.099290 & 6.574610 & -1.855060 \\
\hline $\mathrm{H}$ & 1.180550 & 7.065170 & -0.536230 \\
\hline C & -1.350100 & 7.480030 & 0.323490 \\
\hline $\mathrm{H}$ & -1.775989 & 7.846190 & -0.618200 \\
\hline $\mathrm{H}$ & -2.150740 & 7.443791 & 1.071260 \\
\hline $\mathrm{H}$ & -0.617329 & 8.220210 & 0.664180 \\
\hline C & -0.070180 & 5.691470 & 1.518840 \\
\hline $\mathrm{H}$ & 0.651050 & 6.440670 & 1.868490 \\
\hline $\mathrm{H}$ & -0.860910 & 5.604080 & 2.273130 \\
\hline $\mathrm{H}$ & 0.443600 & 4.726660 & 1.464930 \\
\hline
\end{tabular}

\section{C-Catalyzed reaction TS 26}

\section{Datum}

Value

B3LYP/6-31G(d) Energy

$-3038.963287$

B3LYP-D3(BJ)/def2-TZVPP-IEF-PCM(DCM) Energy

$-3040.517761$

B3LYP-D3(BJ)/def2-TZVPP-IEF-PCM(DCM)//B3LYP/6-31G(d) Free Energy (Quasiharmonic)

$-3039.287635$

Number of Imaginary Frequencies

Frequencies (Top 3 out of 450)
1. $-306.0782 \mathrm{~cm}^{-1}$
2. $9.5826 \mathrm{~cm}^{-1}$
3. $11.7265 \mathrm{~cm}^{-1}$ 


\begin{tabular}{|c|c|c|c|}
\hline C & -2.001402 & -0.266033 & -0.824550 \\
\hline $\mathrm{H}$ & -1.471428 & -0.102466 & -1.770134 \\
\hline $\mathrm{H}$ & -2.995970 & 0.178016 & -0.911956 \\
\hline C & 0.963849 & -2.231936 & 1.041933 \\
\hline 0 & 1.435547 & -3.208595 & 1.575496 \\
\hline 0 & 0.179774 & -1.370220 & 1.717951 \\
\hline $\mathrm{H}$ & -0.227380 & -0.688999 & 1.121046 \\
\hline 0 & -1.280636 & 0.393446 & 0.235437 \\
\hline $\mathrm{H}$ & -1.140679 & 1.329541 & -0.045984 \\
\hline C & 2.323776 & 2.851589 & 1.615963 \\
\hline C & 2.098445 & 3.220640 & -0.529856 \\
\hline C & 0.090319 & 3.916402 & 1.654536 \\
\hline C & 0.960065 & 2.780074 & 1.794853 \\
\hline $\mathrm{H}$ & 1.681941 & 2.232366 & -0.734906 \\
\hline $\mathrm{H}$ & -0.919754 & 3.765901 & 2.041050 \\
\hline $\mathrm{H}$ & 0.514415 & 4.858189 & 2.011029 \\
\hline C & -1.732790 & 3.737678 & -1.688119 \\
\hline C & -1.969835 & 5.169237 & -1.074286 \\
\hline C & -0.914166 & 3.768542 & -2.989110 \\
\hline $\mathrm{H}$ & -1.500952 & 4.164686 & -3.824985 \\
\hline $\mathrm{H}$ & -0.012149 & 4.376498 & -2.880137 \\
\hline $\mathrm{H}$ & -0.610485 & 2.747150 & -3.242834 \\
\hline C & -3.012039 & 2.932581 & -1.907661 \\
\hline $\mathrm{H}$ & -2.789687 & 1.989843 & -2.417151 \\
\hline $\mathrm{H}$ & -3.513658 & 2.705155 & -0.963930 \\
\hline $\mathrm{H}$ & -3.709017 & 3.490814 & -2.543360 \\
\hline C & -3.146029 & 5.209938 & -0.083833 \\
\hline $\mathrm{H}$ & -4.116783 & 5.133692 & -0.586509 \\
\hline H & -3.071294 & 4.402893 & 0.650622 \\
\hline $\mathrm{H}$ & -3.113190 & 6.161596 & 0.456152 \\
\hline C & -2.133914 & 6.285026 & -2.110218 \\
\hline $\mathrm{H}$ & -3.010054 & 6.113349 & -2.747150 \\
\hline $\mathrm{H}$ & -2.273857 & 7.240980 & -1.594756 \\
\hline $\mathrm{H}$ & -1.249123 & 6.374179 & -2.744777 \\
\hline 0 & -0.920166 & 3.101023 & -0.659163 \\
\hline 0 & -0.765035 & 5.408190 & -0.337182 \\
\hline B & -0.136764 & 4.167942 & -0.051637 \\
\hline 0 & 1.300779 & 4.235356 & -0.558590 \\
\hline C & 3.516767 & 3.438129 & -0.874623 \\
\hline C & 4.358521 & 2.334576 & -1.080289 \\
\hline C & 4.027664 & 4.737988 & -1.019663 \\
\hline C & 5.694447 & 2.527818 & -1.423316 \\
\hline $\mathrm{H}$ & 3.968119 & 1.328350 & -0.956400 \\
\hline C & 5.363112 & 4.925101 & -1.366000 \\
\hline H & 3.365793 & 5.584706 & -0.870301 \\
\hline C & 6.199421 & 3.822622 & -1.566752 \\
\hline $\mathrm{H}$ & 6.340586 & 1.667721 & -1.574654 \\
\hline $\mathrm{H}$ & 5.753893 & 5.932211 & -1.482504 \\
\hline $\mathrm{H}$ & 7.241665 & 3.973069 & -1.834857 \\
\hline $\mathrm{H}$ & 0.502874 & 1.789703 & 1.810866 \\
\hline
\end{tabular}




\begin{tabular}{|c|c|c|c|}
\hline $\mathrm{H}$ & 2.840786 & 3.796170 & 1.759398 \\
\hline $\mathrm{H}$ & 2.940572 & 1.959285 & 1.652951 \\
\hline C & 0.237779 & -2.063437 & -1.341464 \\
\hline C & 1.245290 & -1.872449 & -0.404444 \\
\hline C & 2.562532 & -1.448518 & -0.795016 \\
\hline C & 2.771968 & -1.156353 & -2.132599 \\
\hline $\mathrm{H}$ & 3.744547 & -0.792573 & -2.452742 \\
\hline C & -2.142554 & -1.754381 & -0.582030 \\
\hline C & -1.091653 & -2.610896 & -0.899011 \\
\hline C & -3.547580 & -3.662175 & -0.047369 \\
\hline C & -3.384778 & -2.291262 & -0.104804 \\
\hline $\mathrm{H}$ & -4.488237 & -4.069259 & 0.314947 \\
\hline C & -2.512793 & -4.559769 & -0.397991 \\
\hline C & -1.246907 & -4.032804 & -0.806928 \\
\hline C & -0.203187 & -4.948199 & -1.119177 \\
\hline C & -0.408916 & -6.306935 & -1.043671 \\
\hline C & -1.665527 & -6.824955 & -0.647027 \\
\hline C & -2.692408 & -5.968048 & -0.327945 \\
\hline $\mathrm{H}$ & 0.768702 & -4.564626 & -1.408271 \\
\hline $\mathrm{H}$ & 0.402408 & -6.988955 & -1.282458 \\
\hline $\mathrm{H}$ & -1.812096 & -7.900126 & -0.588889 \\
\hline $\mathrm{H}$ & -3.658601 & -6.354067 & -0.011751 \\
\hline C & 1.769570 & -1.320391 & -3.118811 \\
\hline C & 0.483409 & -1.809194 & -2.729619 \\
\hline C & -0.499223 & -2.014023 & -3.736864 \\
\hline C & -0.227372 & -1.730032 & -5.057327 \\
\hline C & 1.040960 & -1.229821 & -5.438241 \\
\hline C & 2.016617 & -1.031335 & -4.488456 \\
\hline $\mathrm{H}$ & -1.471879 & -2.401329 & -3.451285 \\
\hline $\mathrm{H}$ & -0.990576 & -1.892529 & -5.813479 \\
\hline $\mathrm{H}$ & 1.242238 & -1.008157 & -6.482745 \\
\hline H & 2.996500 & -0.655342 & -4.773277 \\
\hline C & -6.627519 & 0.056160 & 1.388381 \\
\hline C & -5.355539 & 0.243170 & 1.937356 \\
\hline C & -4.296132 & -0.507647 & 1.403696 \\
\hline C & -4.503791 & -1.427431 & 0.372839 \\
\hline C & -5.798418 & -1.590337 & -0.149078 \\
\hline C & -6.876472 & -0.850939 & 0.341849 \\
\hline $\mathrm{H}$ & -7.459579 & 0.627547 & 1.782772 \\
\hline H & -3.292918 & -0.397568 & 1.802069 \\
\hline $\mathrm{H}$ & -5.938342 & -2.302566 & -0.954269 \\
\hline C & -5.079562 & 1.213131 & 3.101765 \\
\hline C & -8.306187 & -0.994163 & -0.215461 \\
\hline C & -6.347150 & 1.950984 & 3.571916 \\
\hline $\mathrm{H}$ & -6.093831 & 2.630965 & 4. 393121 \\
\hline H & -6.790184 & 2.553381 & 2.770413 \\
\hline H & -7.111061 & 1.257300 & 3.941522 \\
\hline C & -4.510158 & 0.421057 & 4.303337 \\
\hline H & -4.315521 & 1.095805 & 5.146135 \\
\hline H & -5.218828 & -0.345069 & 4.638331 \\
\hline $\mathrm{H}$ & -3.569779 & -0.080192 & 4.053429 \\
\hline C & -4.046799 & 2.272513 & 2.650488 \\
\hline $\mathrm{H}$ & -3.793215 & 2.939188 & 3.484258 \\
\hline H & -3.121121 & 1.813128 & 2.288426 \\
\hline
\end{tabular}




\begin{tabular}{|c|c|c|c|}
\hline H & -4.455037 & 2.886768 & 1.839646 \\
\hline C & -8.779862 & 0.367704 & -0.777315 \\
\hline $\mathrm{H}$ & -9.799218 & 0.283917 & -1.174190 \\
\hline $\mathrm{H}$ & -8.785005 & 1.147086 & -0.008203 \\
\hline $\mathrm{H}$ & -8.125708 & 0.704502 & -1.589793 \\
\hline C & -8.393700 & -2.033434 & -1.349508 \\
\hline $\mathrm{H}$ & -9.425738 & -2.096839 & -1.712689 \\
\hline $\mathrm{H}$ & -7.760167 & -1.762461 & -2.201730 \\
\hline $\mathrm{H}$ & -8.101223 & -3.033702 & -1.010453 \\
\hline C & -9.261295 & -1.440386 & 0.917179 \\
\hline $\mathrm{H}$ & -10.284893 & -1.541026 & 0.535651 \\
\hline $\mathrm{H}$ & -8.954931 & -2.409099 & 1.328239 \\
\hline $\mathrm{H}$ & -9.283103 & -0.720497 & 1.742010 \\
\hline C & 3.707007 & -1.355365 & 0.160756 \\
\hline C & 3.557842 & -0.900935 & 1.480669 \\
\hline C & 4.983512 & -1.753415 & -0.271436 \\
\hline C & 4.637692 & -0.868896 & 2.370860 \\
\hline $\mathrm{H}$ & 2.580335 & -0.589319 & 1.823710 \\
\hline C & 6.097840 & -1.694845 & 0.571563 \\
\hline $\mathrm{H}$ & 5.085369 & -2.150856 & -1.273755 \\
\hline C & 5.895727 & -1.250893 & 1.885338 \\
\hline $\mathrm{H}$ & 6.744309 & -1.221931 & 2.562183 \\
\hline C & 7.505240 & -2.130152 & 0.118452 \\
\hline C & 4.479369 & -0.476909 & 3.853762 \\
\hline C & 7.980549 & -3.320721 & 0.985136 \\
\hline H & 8.981986 & -3.643792 & 0.674805 \\
\hline $\mathrm{H}$ & 8.029080 & -3.057934 & 2.046913 \\
\hline H & 7.300525 & -4.173943 & 0.883749 \\
\hline C & 7.539404 & -2.571232 & -1.357462 \\
\hline $\mathrm{H}$ & 8.561288 & -2.854918 & -1.633423 \\
\hline H & 6.896211 & -3.439229 & -1.540283 \\
\hline $\mathrm{H}$ & 7.226900 & -1.765872 & -2.032556 \\
\hline C & 8.490140 & -0.949097 & 0.287460 \\
\hline H & 8.184705 & -0.092325 & -0.325025 \\
\hline H & 8.548650 & -0.609849 & 1.326661 \\
\hline H & 9.499230 & -1.245971 & -0.023698 \\
\hline C & 5.340669 & 0.769747 & 4.163519 \\
\hline $\mathrm{H}$ & 6.401664 & 0.596197 & 3.953145 \\
\hline H & 5.018380 & 1.631017 & 3.566757 \\
\hline H & 5.251234 & 1.040854 & 5.222599 \\
\hline C & 3.017293 & -0.168370 & 4.231213 \\
\hline H & 2.629026 & 0.706125 & 3.697647 \\
\hline H & 2.353255 & -1.015066 & 4.027356 \\
\hline H & 2.956661 & 0.053882 & 5.302727 \\
\hline C & 4.951328 & -1.658001 & 4.737027 \\
\hline H & 4.834614 & -1.405874 & 5.798093 \\
\hline H & 4.359614 & -2.556990 & 4.532888 \\
\hline H & 6.005087 & -1.903486 & 4.568433 \\
\hline
\end{tabular}


Frequencies (Top 3 out of 450)

$\begin{array}{rr}\text { 1. } & -326.8930 \mathrm{~cm}^{-1} \\ \text { 2. } & 5.0997 \mathrm{~cm}^{-1} \\ \text { 3. } & 7.8726 \mathrm{~cm}^{-1}\end{array}$

\section{B3LYP/6-31G(d) Molecular Geometry in Cartesian Coordinates}

C

$\mathrm{H}$

$\mathrm{H}$

C

0

0

$\mathrm{H}$

0

$\mathrm{H}$

C

C

C

C

$\mathrm{H}$

$\mathrm{H}$

$\mathrm{H}$

C

C

C

$\mathrm{H}$

$\mathrm{H}$

$\mathrm{H}$

C

$\mathrm{H}$

$\mathrm{H}$

$\mathrm{H}$

C

$\mathrm{H}$

$\mathrm{H}$

$\mathrm{H}$

C

$\mathrm{H}$

\subsection{6}

1.846735

0.321186

$-2.777862$

$-3.533904$

$-1.962999$

$-1.276564$

0.186482

0.390828

3. 367015

3. 880032

1.164188

2.039637

3. 695289

1.353399

0.102798

$-0.081958$

0.687628

$-1.446653$

$-1.818508$

$-2.184379$

$-1.372765$

$-0.243576$

$-0.838015$

$-0.767883$

0.722348

1.806447

1.406185

2.449251

2.424576

$-0.205154$

$-0.752618$

\subsection{5}

0.551603

0.438305

1.870422

2.733930

1. 223561

0.685717

0.073394

$-0.889975$

$-3.370812$

$-3.666651$

$-2.849439$

$-3.673707$

$-4.741386$

$-1.776356$

$-3.078953$

$-3.531946$

$-4.892704$

$-3.508701$

$-2.479129$

$-4.118399$

$-3.876884$

$-3.077829$

$-3.802991$

$-2.116822$

$-2.955579$

$-5.094013$

$-5.310958$

$-4.211603$

$-5.943514$

$-6.133252$

$-6.260637$
0.492397

0.532654

1.430922

$-0.867364$

$-1.266127$

$-1.699783$

$-1.218792$

$-0.612976$

$-0.518471$

3. 211130

1. 103155

2. 212150

2.993822

1.133626

2. 289104

2. 317036

$-1.109755$

$-0.918764$

$-0.405673$

$-0.375910$

$-0.937068$

0.621979

$-2.560342$

$-3.128216$

$-2.598306$

$-3.054255$

$-1.954637$

$-2.950951$

$-2.024394$

$-1.644977$

$-0.874429$

$-1.815572$ 


\begin{tabular}{|c|c|c|c|}
\hline $\mathrm{H}$ & 0.412289 & -7.024759 & -0.720635 \\
\hline $\mathrm{H}$ & -0.926056 & -6.079099 & -0.055353 \\
\hline 0 & 0.793689 & -2.596920 & -0.424718 \\
\hline 0 & 1.290707 & -4.722429 & 0.380859 \\
\hline B & 1.539057 & -3.327322 & 0.567959 \\
\hline 0 & 3.005751 & -2.936921 & 0.484321 \\
\hline C & 5.284204 & -3.221440 & 1.141057 \\
\hline C & 6.284137 & -4.147358 & 1.477604 \\
\hline C & 5.641266 & -1.899899 & 0.829071 \\
\hline C & 7.621498 & -3.759551 & 1.505498 \\
\hline $\mathrm{H}$ & 6.009061 & -5.172844 & 1.713916 \\
\hline C & 6.979696 & -1.517344 & 0.857693 \\
\hline $\mathrm{H}$ & 4.869808 & -1.188225 & 0.554173 \\
\hline C & 7.971419 & -2.442451 & 1.197759 \\
\hline $\mathrm{H}$ & 8.389825 & -4.482952 & 1.763232 \\
\hline $\mathrm{H}$ & 7.249882 & -0.494995 & 0.609227 \\
\hline $\mathrm{H}$ & 9.014373 & -2.138620 & 1.218376 \\
\hline $\mathrm{H}$ & 1.709161 & -4.692644 & 3.196499 \\
\hline $\mathrm{H}$ & 3.698850 & -2.336901 & 3.187953 \\
\hline $\mathrm{H}$ & 4.014006 & -4.061810 & 3.744278 \\
\hline C & -1.699816 & 1.997964 & 1.385214 \\
\hline C & -2.725163 & 1.482303 & 0.601689 \\
\hline C & -3.758662 & 0.661432 & 1.174670 \\
\hline C & -3.698102 & 0.392686 & 2.529705 \\
\hline $\mathrm{H}$ & -4.477397 & -0.216381 & 2.980316 \\
\hline C & 0.568404 & 2.268468 & 0.332403 \\
\hline C & -0.621647 & 2.847290 & 0.767784 \\
\hline C & 1.407329 & 4.454866 & -0.311309 \\
\hline C & 1.598532 & 3.087770 & -0.241060 \\
\hline $\mathrm{H}$ & 2.183159 & 5.076718 & -0.750851 \\
\hline C & 0.215379 & 5.074873 & 0.130185 \\
\hline C & -0.830938 & 4.261368 & 0.668383 \\
\hline C & -2.032429 & 4.896010 & 1.089498 \\
\hline C & -2.182090 & 6.260770 & 0.991173 \\
\hline C & -1.142117 & 7.063935 & 0.463802 \\
\hline C & 0.029184 & 6.481138 & 0.040158 \\
\hline $\mathrm{H}$ & -2.842677 & 4.288686 & 1.476668 \\
\hline $\mathrm{H}$ & -3.109716 & 6.725987 & 1.313034 \\
\hline $\mathrm{H}$ & -1.276061 & 8.139804 & 0.389155 \\
\hline $\mathrm{H}$ & 0.831567 & 7.086871 & -0.374503 \\
\hline C & -2.683717 & 0.913943 & 3.367760 \\
\hline C & -1.666441 & 1.739959 & 2.795409 \\
\hline C & -0.663089 & 2.270111 & 3.653425 \\
\hline C & -0.667085 & 1.993526 & 5.003106 \\
\hline C & -1.673598 & 1.172836 & 5.566169 \\
\hline C & -2.659672 & 0.646940 & 4.763582 \\
\hline $\mathrm{H}$ & 0.109312 & 2.904708 & 3.231727 \\
\hline $\mathrm{H}$ & 0.105947 & 2.411774 & 5.642271 \\
\hline $\mathrm{H}$ & -1.666297 & 0.963341 & 6.632472 \\
\hline $\mathrm{H}$ & -3.440599 & 0.019000 & 5.186200 \\
\hline C & 5.269555 & 1.600175 & -1.858205 \\
\hline C & 5.318894 & 2.606147 & -0.888155 \\
\hline C & 4.100321 & 3.057586 & -0.355735 \\
\hline C & 2.876162 & 2.527675 & -0.773657 \\
\hline
\end{tabular}




\begin{tabular}{|c|c|c|c|}
\hline C & 2.869509 & 1.517937 & -1.752169 \\
\hline C & 4.058059 & 1.042895 & -2.309942 \\
\hline $\mathrm{H}$ & 6.195483 & 1.237233 & -2.289323 \\
\hline $\mathrm{H}$ & 4.093888 & 3.832648 & 0.405608 \\
\hline $\mathrm{H}$ & 1.912944 & 1.122124 & -2.068519 \\
\hline C & 6.639599 & 3.234238 & -0.400558 \\
\hline C & 4.076525 & -0.044295 & -3.402618 \\
\hline C & 6.625778 & 4.753128 & -0.696325 \\
\hline $\mathrm{H}$ & 7.557355 & 5.218743 & -0.351413 \\
\hline $\mathrm{H}$ & 6.528974 & 4.940756 & -1.771678 \\
\hline $\mathrm{H}$ & 5.794819 & 5.258194 & -0.193326 \\
\hline C & 6.786944 & 3.015328 & 1.124062 \\
\hline $\mathrm{H}$ & 7.718122 & 3.466837 & 1.488156 \\
\hline H & 5.958738 & 3.463827 & 1.682174 \\
\hline $\mathrm{H}$ & 6.810373 & 1.946485 & 1.368465 \\
\hline C & 7.874714 & 2.628101 & -1.093410 \\
\hline $\mathrm{H}$ & 8.784164 & 3.106572 & -0.712576 \\
\hline $\mathrm{H}$ & 7.964889 & 1.551675 & -0.905011 \\
\hline $\mathrm{H}$ & 7.850636 & 2.781373 & -2.178065 \\
\hline C & 4.774599 & 0.506047 & -4.668879 \\
\hline $\mathrm{H}$ & 4.786226 & -0.255900 & -5.457984 \\
\hline H & 4.247266 & 1.385934 & -5.054549 \\
\hline $\mathrm{H}$ & 5.812195 & 0.796839 & -4.472845 \\
\hline C & 4.847965 & -1.283070 & -2.889525 \\
\hline $\mathrm{H}$ & 4.898534 & -2.050592 & -3.672169 \\
\hline $\mathrm{H}$ & 5.875178 & -1.036534 & -2.599640 \\
\hline $\mathrm{H}$ & 4.348853 & -1.720967 & -2.017654 \\
\hline C & 2.657522 & -0.496187 & -3.794477 \\
\hline $\mathrm{H}$ & 2.719664 & -1.277980 & -4.560677 \\
\hline $\mathrm{H}$ & 2.112084 & -0.906277 & -2.938330 \\
\hline $\mathrm{H}$ & 2.067018 & 0.327935 & -4.210102 \\
\hline C & -4.879670 & 0.061209 & 0.387785 \\
\hline C & -5.178146 & -1.301606 & 0.545148 \\
\hline C & -5.696098 & 0.835370 & -0.445995 \\
\hline C & -6.263671 & -1.897274 & -0.108502 \\
\hline $\mathrm{H}$ & -4.538769 & -1.898304 & 1.189864 \\
\hline C & -6.775446 & 0.272729 & -1.138277 \\
\hline $\mathrm{H}$ & -5.474720 & 1.888848 & -0.552221 \\
\hline C & -7.041766 & -1.090386 & -0.951861 \\
\hline $\mathrm{H}$ & -7.877125 & -1.534938 & -1.475475 \\
\hline C & -7.613637 & 1.167212 & -2.071928 \\
\hline C & -6.573415 & -3.390355 & 0.122907 \\
\hline C & -6.696721 & 1.737459 & -3.181781 \\
\hline $\mathrm{H}$ & -5.850767 & 2.296770 & -2.770893 \\
\hline $\mathrm{H}$ & -6.288911 & 0.929317 & -3.800288 \\
\hline $\mathrm{H}$ & -7.265846 & 2.410314 & -3.835778 \\
\hline C & -8.767567 & 0.401288 & -2.746289 \\
\hline $\mathrm{H}$ & -9.326869 & 1.080702 & -3.399553 \\
\hline $\mathrm{H}$ & -8.401431 & -0.424415 & -3.367344 \\
\hline $\mathrm{H}$ & -9.473386 & -0.007589 & -2.013576 \\
\hline C & -8.223910 & 2.334803 & -1.260730 \\
\hline $\mathrm{H}$ & -8.884271 & 1.960422 & -0.469434 \\
\hline $\mathrm{H}$ & -7.451745 & 2.951441 & -0.790539 \\
\hline $\mathrm{H}$ & -8.814873 & 2.985225 & -1.917625 \\
\hline
\end{tabular}




\begin{tabular}{|lrrr|} 
C & -5.357331 & -4.249487 & -0.294438 \\
H & -5.124394 & -4.106783 & -1.355994 \\
H & -4.462081 & -3.994110 & 0.281380 \\
H & -5.566893 & -5.314178 & -0.131294 \\
C & -6.870171 & -3.623959 & 1.623531 \\
$H$ & -6.020440 & -3.341765 & 2.254034 \\
$H$ & -7.736102 & -3.035181 & 1.947284 \\
$H$ & -7.089087 & -4.682615 & 1.811615 \\
C & -7.794583 & -3.872354 & -0.683489 \\
H & -7.974646 & -4.934301 & -0.480202 \\
H & -8.704613 & -3.325596 & -0.412147 \\
H & -7.639692 & -3.764041 & -1.762958 \\
\hline
\end{tabular}

\section{C-Catalyzed reaction TS 28}

\section{Datum}

Value

\begin{tabular}{lr}
\hline B3LYP/6-31G(d) Energy & -3038.966285 \\
\hline B3LYP-D3(BJ)/def2-TZVPP-IEF-PCM(DCM) Energy & -3040.517145 \\
\hline B3LYP-D3(BJ)/def2-TZVPP-IEF-PCM(DCM)//B3LYP/6-31G(d) Free Energy (Quasiharmonic) & -3039.287435 \\
\hline
\end{tabular}

Number of Imaginary Frequencies

Frequencies (Top 3 out of 450)

$\begin{array}{lr}\text { 1. } & -312.0631 \mathrm{~cm}^{-1} \\ \text { 2. } & 9.4527 \mathrm{~cm}^{-1} \\ \text { 3. } & 10.4863 \mathrm{~cm}^{-1}\end{array}$

\section{B3LYP/6-31G(d) Molecular Geometry in Cartesian Coordinates}

C

$\mathrm{H}$

$\mathrm{H}$

C

0

0

$\mathrm{H}$

0

$\mathrm{H}$

C

C

C

C

$\mathrm{H}$

\subsection{6}

3.620109

1.931619

$-0.707508$

0.159678

$-1.637276$

$-2.279156$

2. 316216

1.525414

$-2.175954$

$-1.560972$

$-4.316688$

$-3.138370$

$-1.165617$
$-1.966595$

$-2.299063$

$-2.361548$

$-0.625876$

$-1.013743$

0.213384

0.614275

$-2.541308$

$-2.085623$

2.669697

3.451566

2.390250

1.868392

2.496279

\subsection{3}

1.257377

1.766988

$-0.461361$

$-1.228347$

$-0.922170$

$-0.274843$

$-0.268867$

$-0.625006$

$-3.703734$

$-1.760215$

$-2.494458$

$-3.129722$

$-1.415962$ 


\begin{tabular}{|c|c|c|c|}
\hline $\mathrm{H}$ & -5.084888 & 1.643223 & -2.286079 \\
\hline $\mathrm{H}$ & -4.745048 & 3.271724 & -2.978339 \\
\hline C & -4.464654 & 1.947473 & 1.104748 \\
\hline C & -4.988494 & 3.429232 & 1.039938 \\
\hline C & -5.553506 & 0.922665 & 0.750571 \\
\hline $\mathrm{H}$ & -5.085577 & -0.048304 & 0.561308 \\
\hline $\mathrm{H}$ & -6.276114 & 0.803573 & 1.565851 \\
\hline $\mathrm{H}$ & -6.099734 & 1.220166 & -0.149192 \\
\hline C & -3.798329 & 1.560233 & 2.424117 \\
\hline $\mathrm{H}$ & -4.512543 & 1.633372 & 3.252691 \\
\hline $\mathrm{H}$ & -2.946468 & 2.204985 & 2.651466 \\
\hline $\mathrm{H}$ & -3.442793 & 0.524820 & 2.384655 \\
\hline C & -4.074066 & 4.420082 & 1.782274 \\
\hline $\mathrm{H}$ & -4.145780 & 4.312870 & 2.870625 \\
\hline H & -3.029902 & 4.290675 & 1.483221 \\
\hline $\mathrm{H}$ & -4.374917 & 5.438285 & 1.516216 \\
\hline C & -6.433917 & 3.619930 & 1.503955 \\
\hline $\mathrm{H}$ & -6.555207 & 3.340433 & 2.557368 \\
\hline $\mathrm{H}$ & -6.713088 & 4.673762 & 1.400371 \\
\hline $\mathrm{H}$ & -7.129286 & 3.030595 & 0.901763 \\
\hline 0 & -3.472540 & 1.929981 & 0.044311 \\
\hline 0 & -4.920489 & 3.713077 & -0.362770 \\
\hline B & -3.842669 & 2.973433 & -0.920480 \\
\hline 0 & -2.674088 & 3.878893 & -1.252813 \\
\hline C & -0.578653 & 4.450341 & -2.223788 \\
\hline C & -0.944223 & 5.792892 & -2.410162 \\
\hline C & 0.745241 & 4.052589 & -2.464458 \\
\hline C & 0.002641 & 6.719002 & -2.838713 \\
\hline $\mathrm{H}$ & -1.966946 & 6.093772 & -2.206682 \\
\hline C & 1.688967 & 4.982971 & -2.894271 \\
\hline H & 1.033015 & 3.015872 & -2.307955 \\
\hline C & 1.319242 & 6.316599 & -3.084964 \\
\hline H & -0.283675 & 7.757723 & -2.979654 \\
\hline $\mathrm{H}$ & 2.712487 & 4.668197 & -3.077279 \\
\hline $\mathrm{H}$ & 2.055337 & 7.041965 & -3.420994 \\
\hline $\mathrm{H}$ & -2.873350 & 0.831742 & -2.930291 \\
\hline $\mathrm{H}$ & -2.435853 & 3.659241 & -4.069021 \\
\hline H & -1.266893 & 2.235944 & -4.110366 \\
\hline C & 0.196694 & -0.596937 & 1.861117 \\
\hline C & -0.786419 & -1.047008 & 0.985116 \\
\hline C & -1.854205 & -1.906180 & 1.424342 \\
\hline C & -1.935418 & -2.200841 & 2.772135 \\
\hline $\mathrm{H}$ & -2.708764 & -2.879865 & 3.120620 \\
\hline C & 2.554294 & -0.445004 & 1.001548 \\
\hline C & 1.381022 & 0.205404 & 1.383436 \\
\hline C & 3.622739 & 1.707097 & 0.627270 \\
\hline C & 3.703933 & 0.326726 & 0.608230 \\
\hline $\mathrm{H}$ & 4.487150 & 2. 292291 & 0.323942 \\
\hline C & 2.449154 & 2.394252 & 1.013067 \\
\hline C & 1.299184 & 1.636207 & 1.400256 \\
\hline C & 0.132238 & 2.343796 & 1.801980 \\
\hline C & 0.107690 & 3.721384 & 1.821353 \\
\hline C & 1.245930 & 4.467199 & 1.432377 \\
\hline C & 2.389380 & 3.814312 & 1.035219 \\
\hline
\end{tabular}




\begin{tabular}{|c|c|c|c|}
\hline H & -0.748034 & 1.785002 & 2.099458 \\
\hline $\mathrm{H}$ & -0.791607 & 4.240813 & 2.139510 \\
\hline $\mathrm{H}$ & 1.210969 & 5.552896 & 1.445982 \\
\hline $\mathrm{H}$ & 3.270389 & 4.375939 & 0.733607 \\
\hline C & -1.009932 & -1.695207 & 3.716268 \\
\hline C & 0.085013 & -0.894784 & 3.261022 \\
\hline C & 1.026139 & -0.427590 & 4.219712 \\
\hline C & 0.885089 & -0.731498 & 5.555682 \\
\hline C & -0.199312 & -1.523524 & 6.002601 \\
\hline C & -1.123677 & -1.996747 & 5.100345 \\
\hline $\mathrm{H}$ & 1.861415 & 0.177420 & 3.885496 \\
\hline H & 1.612862 & -0.362320 & 6.272944 \\
\hline $\mathrm{H}$ & -0.297231 & -1.758177 & 7.059002 \\
\hline $\mathrm{H}$ & -1.957264 & -2.610852 & 5.432495 \\
\hline C & 7.476789 & -1.313914 & -0.574481 \\
\hline C & 6.317928 & -1.818754 & -1.170034 \\
\hline C & 5.083494 & -1.286169 & -0.764947 \\
\hline C & 5.006351 & -0.287904 & 0.209510 \\
\hline C & 6.196755 & 0.189044 & 0.790404 \\
\hline C & 7.443556 & -0.310422 & 0.411882 \\
\hline $\mathrm{H}$ & 8.439812 & -1.710989 & -0.874629 \\
\hline H & 4.161122 & -1.658345 & -1.195213 \\
\hline $\mathrm{H}$ & 6.121205 & 0.947994 & 1.560581 \\
\hline C & 6.338412 & -2.929437 & -2.237842 \\
\hline C & 8.763108 & 0.188799 & 1.033129 \\
\hline C & 7.765691 & -3.402604 & -2.570250 \\
\hline $\mathrm{H}$ & 7.724671 & -4.193480 & -3.327910 \\
\hline H & 8.382714 & -2.590912 & -2.973569 \\
\hline $\mathrm{H}$ & 8.274248 & -3.814795 & -1.691128 \\
\hline C & 5.530605 & -4.145317 & -1.721423 \\
\hline $\mathrm{H}$ & 5.509037 & -4.936922 & -2.481262 \\
\hline $\mathrm{H}$ & 5.989689 & -4.560198 & -0.816023 \\
\hline $\mathrm{H}$ & 4.497056 & -3.878270 & -1.479597 \\
\hline C & 5.691021 & -2.402814 & -3.540637 \\
\hline $\mathrm{H}$ & 5.693629 & -3.184966 & -4.309995 \\
\hline $\mathrm{H}$ & 4.653013 & -2.094603 & -3.381864 \\
\hline $\mathrm{H}$ & 6.242897 & -1.539831 & -3.931641 \\
\hline C & 9.659259 & 0.788400 & -0.076870 \\
\hline $\mathrm{H}$ & 10.610463 & 1.136910 & 0.344683 \\
\hline $\mathrm{H}$ & 9.888984 & 0.053961 & -0.855588 \\
\hline $\mathrm{H}$ & 9.167851 & 1.641726 & -0.558492 \\
\hline C & 8.537473 & 1.275114 & 2.102105 \\
\hline $\mathrm{H}$ & 9.502725 & 1.598387 & 2.508334 \\
\hline $\mathrm{H}$ & 8.040600 & 2.159685 & 1.687579 \\
\hline $\mathrm{H}$ & 7.934108 & 0.904718 & 2.938641 \\
\hline C & 9.501764 & -0.993904 & 1.703344 \\
\hline $\mathrm{H}$ & 10.446216 & -0.653843 & 2.146234 \\
\hline $\mathrm{H}$ & 8.891259 & -1.433981 & 2.500082 \\
\hline $\mathrm{H}$ & 9.736461 & -1.787049 & 0.986247 \\
\hline C & -2.806392 & -2.555186 & 0.477237 \\
\hline C & -4.164711 & -2.697232 & 0.820070 \\
\hline C & -2.355201 & -3.107025 & -0.725350 \\
\hline C & -5.060403 & -3.365297 & -0.016420 \\
\hline H & -4.507928 & -2.266628 & 1.753570 \\
\hline
\end{tabular}




\begin{tabular}{|c|c|c|c|}
\hline C & -3.227194 & -3.764419 & -1.607268 \\
\hline $\mathrm{H}$ & -1.301825 & -3.044110 & -0.970584 \\
\hline C & -4.567207 & -3.879405 & -1.230268 \\
\hline $\mathrm{H}$ & -5.258241 & -4.387611 & -1.892236 \\
\hline C & -2.676120 & -4.325612 & -2.931611 \\
\hline C & -6.548859 & -3.554840 & 0.336128 \\
\hline C & -3.765516 & -5.007287 & -3.780705 \\
\hline $\mathrm{H}$ & -4.210963 & -5.866129 & -3.265223 \\
\hline $\mathrm{H}$ & -4.569612 & -4.311902 & -4.047611 \\
\hline $\mathrm{H}$ & -3.326251 & -5.376070 & -4.714373 \\
\hline C & -2.071926 & -3.167369 & -3.762541 \\
\hline $\mathrm{H}$ & -1.653583 & -3.552746 & -4.700523 \\
\hline $\mathrm{H}$ & -2.840662 & -2.427063 & -4.014381 \\
\hline $\mathrm{H}$ & -1.271212 & -2.647312 & -3.227085 \\
\hline C & -1.575025 & -5.370892 & -2.632384 \\
\hline $\mathrm{H}$ & -0.739044 & -4.938723 & -2.073450 \\
\hline $\mathrm{H}$ & -1.975715 & -6.205590 & -2.045507 \\
\hline $\mathrm{H}$ & -1.173231 & -5.775780 & -3.569284 \\
\hline C & -7.426433 & -2.857639 & -0.730558 \\
\hline $\mathrm{H}$ & -7.215425 & -1.782982 & -0.770003 \\
\hline $\mathrm{H}$ & -7.255866 & -3.268788 & -1.731060 \\
\hline $\mathrm{H}$ & -8.489771 & -2.986750 & -0.493954 \\
\hline C & -6.881336 & -5.066058 & 0.364792 \\
\hline $\mathrm{H}$ & -6.695198 & -5.545888 & -0.601490 \\
\hline $\mathrm{H}$ & -6.277448 & -5.585918 & 1.117359 \\
\hline $\mathrm{H}$ & -7.938849 & -5.217613 & 0.613498 \\
\hline C & -6.908639 & -2.965883 & 1.713675 \\
\hline $\mathrm{H}$ & -7.970034 & -3.141300 & 1.922585 \\
\hline $\mathrm{H}$ & -6.332551 & -3.434461 & 2.520001 \\
\hline $\mathrm{H}$ & -6.740028 & -1.884194 & 1.754955 \\
\hline
\end{tabular}

\section{C-Catalyzed reaction TS 29}

\section{Datum}

Value

\begin{tabular}{lc}
\hline B3LYP/6-31G(d) Energy & -3038.966335 \\
\hline B3LYP-D3(BJ)/def2-TZVPP-IEF-PCM(DCM) Energy & -3040.516282 \\
\hline B3LYP-D3(BJ)/def2-TZVPP-IEF-PCM(DCM)//B3LYP/6-31G(d) Free Energy (Quasiharmonic) & -3039.287431 \\
\hline
\end{tabular}

Number of Imaginary Frequencies

Frequencies (Top 3 out of 450)

$\begin{array}{lr}\text { 1. } & -326.9510 \mathrm{~cm}^{-1} \\ \text { 2. } & 7.2706 \mathrm{~cm}^{-1} \\ \text { 3. } & 8.7997 \mathrm{~cm}^{-1}\end{array}$




\begin{tabular}{|c|c|c|c|}
\hline C & 0.995120 & -0.345000 & 0.229828 \\
\hline $\mathrm{H}$ & 0.669232 & 0.250472 & 1.089445 \\
\hline $\mathrm{H}$ & 2.046152 & -0.105373 & 0.038033 \\
\hline C & -2.494014 & -2.077192 & -0.345676 \\
\hline 0 & -3.144955 & -3.101120 & -0.378577 \\
\hline 0 & -1.816627 & -1.648375 & -1.413120 \\
\hline $\mathrm{H}$ & -1.198038 & -0.900911 & -1.192037 \\
\hline 0 & 0.218750 & 0.022107 & -0.923345 \\
\hline $\mathrm{H}$ & 0.299808 & 1.002762 & -1.003887 \\
\hline C & 5.067912 & 3.590919 & -0.759429 \\
\hline C & 3.699335 & 3.472262 & 0.939342 \\
\hline C & 2.944011 & 3.344402 & -2.005561 \\
\hline C & 4.091314 & 4.098460 & -1.590710 \\
\hline $\mathrm{H}$ & 3.597690 & 4.554712 & 1.029827 \\
\hline $\mathrm{H}$ & 2.382485 & 3.780249 & -2.833599 \\
\hline $\mathrm{H}$ & 3.119358 & 2.277004 & -2.152378 \\
\hline C & -0.468518 & 3.705561 & -0.779733 \\
\hline C & 0.158336 & 4.915563 & 0.012046 \\
\hline C & -0.830398 & 4.057911 & -2.230420 \\
\hline $\mathrm{H}$ & -1.043422 & 3.135049 & -2.779685 \\
\hline $\mathrm{H}$ & -1.718080 & 4.697460 & -2.283299 \\
\hline $\mathrm{H}$ & -0.005445 & 4.571226 & -2.733460 \\
\hline C & -1.659170 & 3.035915 & -0.094359 \\
\hline $\mathrm{H}$ & -2.020099 & 2.198591 & -0.700621 \\
\hline $\mathrm{H}$ & -1.394634 & 2.645380 & 0.890712 \\
\hline $\mathrm{H}$ & -2.487281 & 3.745054 & 0.023186 \\
\hline C & 0.076161 & 4.736254 & 1.538011 \\
\hline $\mathrm{H}$ & 0.443883 & 3.753409 & 1.846604 \\
\hline $\mathrm{H}$ & 0.699338 & 5.499879 & 2.015623 \\
\hline $\mathrm{H}$ & -0.947911 & 4.853910 & 1.908280 \\
\hline C & -0.390797 & 6.289519 & -0.374481 \\
\hline $\mathrm{H}$ & -1.464822 & 6.360639 & -0.166424 \\
\hline $\mathrm{H}$ & 0.117064 & 7.064556 & 0.209531 \\
\hline $\mathrm{H}$ & -0.224642 & 6.504108 & -1.432692 \\
\hline 0 & 0.629592 & 2.759010 & -0.814162 \\
\hline 0 & 1.543690 & 4.855780 & -0.383671 \\
\hline B & 1.860446 & 3.486376 & -0.643952 \\
\hline 0 & 2.687834 & 2.825955 & 0.451623 \\
\hline C & 4.576587 & 2.796579 & 1.912443 \\
\hline C & 4.574275 & 1.399781 & 2.053918 \\
\hline C & 5.409989 & 3.572970 & 2.732636 \\
\hline C & 5.395426 & 0.795637 & 3.001874 \\
\hline $\mathrm{H}$ & 3.925134 & 0.801171 & 1.423455 \\
\hline C & 6.229618 & 2.964284 & 3.680289 \\
\hline $\mathrm{H}$ & 5.408585 & 4.655677 & 2.628245 \\
\hline C & 6.224678 & 1.573998 & 3.815539 \\
\hline $\mathrm{H}$ & 5.387838 & -0.285228 & 3.109248 \\
\hline $\mathrm{H}$ & 6.868270 & 3.572613 & 4.314437 \\
\hline $\mathrm{H}$ & 6.863107 & 1.097704 & 4.554444 \\
\hline $\mathrm{H}$ & 4.049685 & 5.179275 & -1.728007 \\
\hline
\end{tabular}




\begin{tabular}{|c|c|c|c|}
\hline H & 5.256295 & 2.521425 & -0.733969 \\
\hline $\mathrm{H}$ & 5.880287 & 4.221596 & -0.409012 \\
\hline C & -1.302710 & -1.338784 & 1.727634 \\
\hline C & -2.416710 & -1.217789 & 0.905853 \\
\hline C & -3.504317 & -0.343952 & 1.249585 \\
\hline C & -3.403089 & 0.395459 & 2.413349 \\
\hline H & -4.220837 & 1.056034 & 2.690203 \\
\hline C & 0.869462 & -1.818986 & 0.556312 \\
\hline C & -0.190468 & -2.273044 & 1.336647 \\
\hline C & 1.851446 & -4.041857 & 0.576800 \\
\hline C & 1.885493 & -2.736394 & 0.127084 \\
\hline H & 2.622067 & -4.736599 & 0.251779 \\
\hline C & 0.812205 & -4.523505 & 1.407391 \\
\hline C & -0.249668 & -3.636954 & 1.772697 \\
\hline C & -1.313018 & -4.147012 & 2.568152 \\
\hline C & -1.312941 & -5.456458 & 2.992389 \\
\hline C & -0.254677 & -6.328115 & 2.638940 \\
\hline C & 0.782006 & -5.870538 & 1.859649 \\
\hline $\mathrm{H}$ & -2.136348 & -3.492047 & 2.830132 \\
\hline $\mathrm{H}$ & -2.136033 & -5.827814 & 3.596688 \\
\hline $\mathrm{H}$ & -0.269553 & -7.360454 & 2.978175 \\
\hline H & 1.593630 & -6.534619 & 1.571568 \\
\hline C & -2.290845 & 0.295865 & 3.284381 \\
\hline C & -1.222484 & -0.594985 & 2.949386 \\
\hline C & -0.122467 & -0.701707 & 3.844883 \\
\hline C & -0.081336 & 0.037219 & 5.006708 \\
\hline C & -1.137848 & 0.920711 & 5.334297 \\
\hline C & -2.217561 & 1.044644 & 4.490371 \\
\hline $\mathrm{H}$ & 0.688235 & -1.381043 & 3.602135 \\
\hline $\mathrm{H}$ & 0.766457 & -0.060110 & 5.679571 \\
\hline $\mathrm{H}$ & -1.094016 & 1.497272 & 6.254394 \\
\hline $\mathrm{H}$ & -3.036718 & 1.716677 & 4.735578 \\
\hline C & 4.937977 & -1.807817 & -2.722135 \\
\hline C & 3.594501 & -1.612444 & -3.077325 \\
\hline C & 2.615988 & -1.874291 & -2.110629 \\
\hline C & 2.957201 & -2.346817 & -0.836683 \\
\hline C & 4.308645 & -2.539521 & -0.523796 \\
\hline C & 5.321511 & -2.266149 & -1.452933 \\
\hline $\mathrm{H}$ & 5.705324 & -1.606659 & -3.457112 \\
\hline $\mathrm{H}$ & 1.565376 & -1.739007 & -2.343144 \\
\hline $\mathrm{H}$ & 4.559505 & -2.910047 & 0.466546 \\
\hline C & 3.160975 & -1.166316 & -4.487813 \\
\hline C & 6.796057 & -2.495183 & -1.064924 \\
\hline C & 4.357697 & -0.930545 & -5.428903 \\
\hline $\mathrm{H}$ & 3.994580 & -0.607216 & -6.411141 \\
\hline $\mathrm{H}$ & 5.028560 & -0.149415 & -5.052392 \\
\hline $\mathrm{H}$ & 4.945934 & -1.843081 & -5.579594 \\
\hline C & 2.269622 & -2.266609 & -5.113729 \\
\hline $\mathrm{H}$ & 1.961364 & -1.975642 & -6.125567 \\
\hline $\mathrm{H}$ & 2.811632 & -3.216782 & -5.183387 \\
\hline $\mathrm{H}$ & 1.362833 & -2.440499 & -4.525992 \\
\hline C & 2.354171 & 0.150028 & -4.395965 \\
\hline $\mathrm{H}$ & 1.966864 & 0.429036 & -5.383890 \\
\hline H & 1.504886 & 0.064527 & -3.711032 \\
\hline
\end{tabular}




\begin{tabular}{|c|c|c|c|}
\hline $\mathrm{H}$ & 2.988765 & 0.970430 & -4.041473 \\
\hline C & 7.772980 & -2.088151 & -2.184567 \\
\hline $\mathrm{H}$ & 8.804025 & -2.257876 & -1.853930 \\
\hline $\mathrm{H}$ & 7.619309 & -2.676769 & -3.095878 \\
\hline $\mathrm{H}$ & 7.678535 & -1.027177 & -2.442988 \\
\hline C & 7.139638 & -1.662193 & 0.192612 \\
\hline $\mathrm{H}$ & 8.189392 & -1.810470 & 0.474582 \\
\hline $\mathrm{H}$ & 6.984244 & -0.592436 & 0.011329 \\
\hline $\mathrm{H}$ & 6.522741 & -1.947369 & 1.051225 \\
\hline C & 7.016331 & -3.995672 & -0.757398 \\
\hline $\mathrm{H}$ & 8.059977 & -4.180828 & -0.473742 \\
\hline $\mathrm{H}$ & 6.380413 & -4.339040 & 0.065379 \\
\hline $\mathrm{H}$ & 6.788234 & -4.611774 & -1.634580 \\
\hline C & -4.725227 & -0.180234 & 0.403070 \\
\hline C & -5.143145 & 1.102723 & 0.033189 \\
\hline C & -5.504524 & -1.285961 & 0.022765 \\
\hline C & -6.313157 & 1.308872 & -0.714933 \\
\hline $\mathrm{H}$ & -4.531411 & 1.950617 & 0.326644 \\
\hline C & -6.668468 & -1.124088 & -0.730000 \\
\hline $\mathrm{H}$ & -5.176079 & -2.270892 & 0.320759 \\
\hline C & -7.052016 & 0.181589 & -1.084732 \\
\hline $\mathrm{H}$ & -7.954650 & 0.312066 & -1.670394 \\
\hline C & -7.521213 & -2.322749 & -1.188713 \\
\hline C & -6.729633 & 2.741716 & -1.101837 \\
\hline C & -8.966132 & -2.173499 & -0.657303 \\
\hline $\mathrm{H}$ & -9.581028 & -3.021107 & -0.984447 \\
\hline $\mathrm{H}$ & -8.979987 & -2.148057 & 0.438552 \\
\hline $\mathrm{H}$ & -9.445175 & -1.257245 & -1.018993 \\
\hline C & -7.545110 & -2.363738 & -2.735673 \\
\hline $\mathrm{H}$ & -8.153170 & -3.207343 & -3.085528 \\
\hline $\mathrm{H}$ & -7.968379 & -1.447852 & -3.162338 \\
\hline $\mathrm{H}$ & -6.532796 & -2.484714 & -3.136962 \\
\hline C & -6.958149 & -3.665497 & -0.684047 \\
\hline $\mathrm{H}$ & -5.934732 & -3.838212 & -1.032592 \\
\hline $\mathrm{H}$ & -6.956509 & -3.717428 & 0.410897 \\
\hline $\mathrm{H}$ & -7.583106 & -4.487399 & -1.052437 \\
\hline C & -6.920136 & 3.588345 & 0.179075 \\
\hline $\mathrm{H}$ & -7.706332 & 3.164676 & 0.814498 \\
\hline $\mathrm{H}$ & -6.002701 & 3.640543 & 0.774823 \\
\hline $\mathrm{H}$ & -7.208161 & 4.615014 & -0.079437 \\
\hline C & -5.626569 & 3.380786 & -1.978424 \\
\hline $\mathrm{H}$ & -4.665614 & 3.424213 & -1.455293 \\
\hline $\mathrm{H}$ & -5.478213 & 2.806297 & -2.899830 \\
\hline $\mathrm{H}$ & -5.903839 & 4.405433 & -2.256617 \\
\hline C & -8.049277 & 2.780201 & -1.895711 \\
\hline $\mathrm{H}$ & -8.303889 & 3.818644 & -2.136779 \\
\hline $\mathrm{H}$ & -7.973932 & 2.231943 & -2.841613 \\
\hline $\mathrm{H}$ & -8.883251 & 2.359890 & -1.322206 \\
\hline
\end{tabular}




\begin{tabular}{lc}
\hline B3LYP/6-31G(d) Energy & -3038.961536 \\
\hline B3LYP-D3(BJ)/def2-TZVPP-IEF-PCM(DCM) Energy & -3040.515705 \\
\hline B3LYP-D3(BJ)/def2-TZVPP-IEF-PCM(DCM)//B3LYP/6-31G(d) Free Energy (Quasiharmonic) & -3039.286656 \\
\hline
\end{tabular}

Number of Imaginary Frequencies

Frequencies (Top 3 out of 450)

$\begin{array}{lr}\text { 1. } & -285.6655 \mathrm{~cm}^{-1} \\ \text { 2. } & 5.5265 \mathrm{~cm}^{-1} \\ \text { 3. } & 8.8279 \mathrm{~cm}^{-1}\end{array}$

\section{B3LYP/6-31G(d) Molecular Geometry in Cartesian Coordinates}

C

$\mathrm{H}$

$\mathrm{H}$

C

0

0

$\mathrm{H}$

0

$\mathrm{H}$

C

C

C

C

$\mathrm{H}$

$\mathrm{H}$

$\mathrm{H}$

C

C

C

$\mathrm{H}$

$\mathrm{H}$

$\mathrm{H}$

C

$\mathrm{H}$

$\mathrm{H}$

$\mathrm{H}$

$\mathrm{H}$

C

$\mathrm{H}$

$\mathrm{H}$

$\mathrm{H}$

C

$\mathrm{H}$

\section{$-2.069658$}

$-1.452091$

$-2.932304$

0.523516

0.956449

$-0.159554$

$-0.498272$

$-1.305938$

$-0.842672$

3.453015

3. 391295

1.157660

2. 537761

3.739373

0.495660

0.762480

$-0.968425$

0.173464

$-1.937515$

$-2.563221$

$-2.596955$

$-1.399011$

$-1.758252$

$-2.504355$

$-1.109023$

$-2.285034$

0.842546

1.142931

1.741867

0.180452

$-0.241060$

$-1.031883$

\subsection{8}

0.284160

$-0.417276$

2. 388368

3. 324341

1. 396648

0.729506

$-0.371712$

$-1.165318$

$-1.184183$

$-2.015694$

$-2.089243$

$-2.104944$

$-1.046328$

$-2.670806$

$-1.085792$

$-3.868592$

$-4.950051$

$-3.957910$

$-3.059603$

$-4.828926$

$-4.019454$

$-3.828137$

$-3.028267$

$-3.636072$

$-4.774984$

$-5.176156$

$-4.229320$

$-5.785250$

$-5.704064$

$-6.293253$

$-6.765554$

\subsection{1}

1.616112

0.938850

$-1.417022$

$-2.052089$

$-1.999132$

$-1.338610$

$-0.351103$

0.014281

2.947056

0.890145

2.995164

3. 396141

0.526233

3. 640288

2.817567

0.682990

0.737320

1.871888

1.891750

1.792629

2.822138

$-0.624888$

$-0.583883$

$-1.482342$

$-0.791125$

$-0.629032$

$-1.087141$

$-0.490983$

$-1.323985$

1. 339156

0. 744671 


\begin{tabular}{|c|c|c|c|}
\hline $\mathrm{H}$ & 0.618555 & -6.972038 & 1.353255 \\
\hline $\mathrm{H}$ & -0.595674 & -6.179837 & 2.366279 \\
\hline 0 & -0.219925 & -2.635917 & 0.807411 \\
\hline 0 & 1.128139 & -4.332196 & 1.623648 \\
\hline B & 1.032854 & -2.913051 & 1.470887 \\
\hline 0 & 2.135469 & -2.220772 & 0.662361 \\
\hline C & 4.451752 & -3.049488 & 0.935426 \\
\hline C & 4.221238 & -4.409664 & 1.200743 \\
\hline C & 5.761743 & -2.614970 & 0.663289 \\
\hline C & 5.288056 & -5.305921 & 1.197622 \\
\hline $\mathrm{H}$ & 3.212055 & -4.746920 & 1.412476 \\
\hline C & 6.822716 & -3.517521 & 0.658295 \\
\hline $\mathrm{H}$ & 5.944002 & -1.564501 & 0.446996 \\
\hline C & 6.587688 & -4.867323 & 0.927875 \\
\hline $\mathrm{H}$ & 5.103718 & -6.356724 & 1.404884 \\
\hline $\mathrm{H}$ & 7.828547 & -3.169101 & 0.441125 \\
\hline $\mathrm{H}$ & 7.412436 & -5.574878 & 0.924570 \\
\hline $\mathrm{H}$ & 2.910972 & -3.005789 & 3.884642 \\
\hline H & 3.113294 & -0.210201 & 2.606715 \\
\hline $\mathrm{H}$ & 4.503117 & -1.266829 & 3.210189 \\
\hline C & -0.290890 & 2.515833 & 0.963727 \\
\hline C & 0.759903 & 2.299611 & 0.082382 \\
\hline C & 2.111872 & 2.184006 & 0.555104 \\
\hline C & 2.349715 & 2.314310 & 1.909611 \\
\hline $\mathrm{H}$ & 3.367635 & 2.225253 & 2.281989 \\
\hline C & -2.546906 & 1.602054 & 0.319972 \\
\hline C & -1.694521 & 2.695082 & 0.454215 \\
\hline C & -4.332408 & 3.073489 & -0.419403 \\
\hline C & -3.883527 & 1.793150 & -0.160215 \\
\hline $\mathrm{H}$ & -5.348818 & 3.216532 & -0.777748 \\
\hline C & -3.497758 & 4.206759 & -0.277567 \\
\hline C & -2.143582 & 4.020369 & 0.144731 \\
\hline C & -1.304832 & 5.165348 & 0.248908 \\
\hline C & -1.787132 & 6.422704 & -0.035676 \\
\hline C & -3.131260 & 6.604605 & -0.441691 \\
\hline C & -3.965635 & 5.518175 & -0.562632 \\
\hline $\mathrm{H}$ & -0.268652 & 5.037809 & 0.541442 \\
\hline $\mathrm{H}$ & -1.128563 & 7.283183 & 0.044017 \\
\hline $\mathrm{H}$ & -3.497382 & 7.603170 & -0.664778 \\
\hline $\mathrm{H}$ & -4.996815 & 5.644029 & -0.884252 \\
\hline C & 1.309394 & 2.546600 & 2.843399 \\
\hline C & -0.037458 & 2.643229 & 2.371536 \\
\hline C & -1.070988 & 2.874712 & 3.320554 \\
\hline C & -0.786264 & 2.996810 & 4.663270 \\
\hline C & 0.546636 & 2.896361 & 5.127074 \\
\hline C & 1.570362 & 2.675920 & 4.234157 \\
\hline $\mathrm{H}$ & -2.095045 & 2.957736 & 2.972458 \\
\hline $\mathrm{H}$ & -1.590726 & 3.173593 & 5.372021 \\
\hline $\mathrm{H}$ & 0.758613 & 2.995299 & 6.188232 \\
\hline $\mathrm{H}$ & 2.598412 & 2.598411 & 4.580574 \\
\hline C & -6.688058 & -1.298115 & -1.075405 \\
\hline C & -5.487750 & -1.235833 & -1.808246 \\
\hline C & -4.553323 & -0.260168 & -1.452770 \\
\hline C & -4.822253 & 0.659393 & -0.423881 \\
\hline
\end{tabular}




\begin{tabular}{|c|c|c|c|}
\hline C & -6.028917 & 0.569855 & 0.273062 \\
\hline C & -6.980245 & -0.418576 & -0.029823 \\
\hline $\mathrm{H}$ & -7.415576 & -2.054924 & -1.343744 \\
\hline $\mathrm{H}$ & -3.612602 & -0.173205 & -1.982713 \\
\hline $\mathrm{H}$ & -6.222326 & 1.287552 & 1.065442 \\
\hline C & -5.272646 & -2.198206 & -2.993653 \\
\hline C & -8.291595 & -0.485689 & 0.777063 \\
\hline C & -5.375343 & -3.664237 & -2.511496 \\
\hline $\mathrm{H}$ & -5.226176 & -4.351852 & -3.353160 \\
\hline $\mathrm{H}$ & -4.613757 & -3.883581 & -1.755447 \\
\hline $\mathrm{H}$ & -6.354595 & -3.885391 & -2.073812 \\
\hline C & -6.365741 & -1.937967 & -4.058652 \\
\hline $\mathrm{H}$ & -6.231344 & -2.610506 & -4.914968 \\
\hline $\mathrm{H}$ & -7.372031 & -2.102728 & -3.658809 \\
\hline $\mathrm{H}$ & -6.315925 & -0.906547 & -4.425575 \\
\hline C & -3.899542 & -2.004335 & -3.664838 \\
\hline $\mathrm{H}$ & -3.789807 & -2.717659 & -4.489811 \\
\hline $\mathrm{H}$ & -3.790004 & -0.997958 & -4.083555 \\
\hline $\mathrm{H}$ & -3.072300 & -2.171478 & -2.966871 \\
\hline C & -9.060992 & 0.847720 & 0.621509 \\
\hline $\mathrm{H}$ & -9.997617 & 0.818255 & 1.192112 \\
\hline $\mathrm{H}$ & -8.477344 & 1.700169 & 0.984258 \\
\hline $\mathrm{H}$ & -9.309199 & 1.035636 & -0.429378 \\
\hline C & -9.215634 & -1.626523 & 0.310972 \\
\hline $\mathrm{H}$ & -10.127091 & -1.634411 & 0.919491 \\
\hline $\mathrm{H}$ & -9.519004 & -1.505494 & -0.735191 \\
\hline $\mathrm{H}$ & -8.738937 & -2.607631 & 0.418066 \\
\hline C & -7.964268 & -0.715669 & 2.271844 \\
\hline $\mathrm{H}$ & -8.887225 & -0.757273 & 2.863382 \\
\hline $\mathrm{H}$ & -7.427072 & -1.660520 & 2.413724 \\
\hline $\mathrm{H}$ & -7.341864 & 0.086813 & 2.681141 \\
\hline C & 3.248503 & 1.902701 & -0.366217 \\
\hline C & 3.141041 & 0.913011 & -1.354100 \\
\hline C & 4.455734 & 2.603186 & -0.232545 \\
\hline C & 4.201608 & 0.629378 & -2.221784 \\
\hline $\mathrm{H}$ & 2.210656 & 0.367085 & -1.443036 \\
\hline C & 5.547030 & 2.339644 & -1.067936 \\
\hline $\mathrm{H}$ & 4.515812 & 3.383754 & 0.516886 \\
\hline C & 5.391614 & 1.351790 & -2.051931 \\
\hline $\mathrm{H}$ & 6.221834 & 1.149984 & -2.722315 \\
\hline C & 6.879824 & 3.105684 & -0.957272 \\
\hline C & 4.077810 & -0.402507 & -3.360036 \\
\hline C & 7.153509 & 3.856526 & -2.282277 \\
\hline $\mathrm{H}$ & 8.098665 & 4.410064 & -2.219709 \\
\hline $\mathrm{H}$ & 7.224619 & 3.170241 & -3.132469 \\
\hline $\mathrm{H}$ & 6.352335 & 4.572095 & -2.497817 \\
\hline C & 6.869586 & 4.140430 & 0.184264 \\
\hline $\mathrm{H}$ & 7.838381 & 4.650932 & 0.228098 \\
\hline $\mathrm{H}$ & 6.100121 & 4.905947 & 0.034813 \\
\hline $\mathrm{H}$ & 6.699836 & 3.670606 & 1.160059 \\
\hline C & 8.030388 & 2.107377 & -0.687006 \\
\hline $\mathrm{H}$ & 7.870993 & 1.572030 & 0.256679 \\
\hline $\mathrm{H}$ & 8.121100 & 1.361533 & -1.483728 \\
\hline $\mathrm{H}$ & 8.987971 & 2.637923 & -0.617211 \\
\hline
\end{tabular}




$\begin{array}{|llll|}\mathrm{C} & 5.206909 & -1.453433 & -3.255086 \\ \mathrm{H} & 6.201041 & -0.997455 & -3.315000 \\ \mathrm{H} & 5.147852 & -2.006649 & -2.310708 \\ \mathrm{H} & 5.127226 & -2.177980 & -4.074673 \\ \mathrm{C} & 2.725694 & -1.142583 & -3.331962 \\ \mathrm{H} & 2.582473 & -1.695958 & -2.396229 \\ \mathrm{H} & 1.880369 & -0.456813 & -3.454234 \\ \mathrm{H} & 2.687918 & -1.866484 & -4.154061 \\ \mathrm{C} & 4.193490 & 0.338682 & -4.714283 \\ \mathrm{H} & 4.105926 & -0.371622 & -5.545845 \\ \mathrm{H} & 3.400183 & 1.086758 & -4.818230 \\ \mathrm{H} & 5.155316 & 0.853654 & -4.813636 \\ & & \end{array}$

\section{C-Catalyzed reaction TS 31}

\section{Datum}

Value

\begin{tabular}{lc}
\hline B3LYP/6-31G(d) Energy & -3038.96688 \\
\hline B3LYP-D3(BJ)/def2-TZVPP-IEF-PCM(DCM) Energy & -3040.515641 \\
\hline B3LYP-D3(BJ)/def2-TZVPP-IEF-PCM(DCM)//B3LYP/6-31G(d) Free Energy (Quasiharmonic) & -3039.285956 \\
\hline
\end{tabular}

Number of Imaginary Frequencies

Frequencies (Top 3 out of 450)

$\begin{array}{lr}\text { 1. } & -317.1497 \mathrm{~cm}^{-1} \\ \text { 2. } & 8.0300 \mathrm{~cm}^{-1} \\ \text { 3. } & 10.9556 \mathrm{~cm}^{-1}\end{array}$

\section{B3LYP/6-31G(d) Molecular Geometry in Cartesian Coordinates}

C

$\mathrm{H}$

$\mathrm{H}$

C

0

0

$\mathrm{H}$

0

$\mathrm{H}$

C

$\mathrm{C}$

C

C

$\mathrm{H}$

\subsection{1}

3. 765691

2.087311

$-0.626680$

0. 238041

$-1.596410$

$-2.229040$

2.443411

1.639411

$-3.361110$

$-2.590990$

$-4.287350$

$-4.431330$

$-3.419230$
$-1.921440$

$-2.240790$

$-2.364560$

$-0.623330$

$-0.947480$

0.200100

0.489140

$-2.447300$

$-1.991380$

3. 510140

4.306190

1.798190

2.903190

5.006970
0.877890

1.104810

1.636120

$-0.479240$

$-1.279470$

$-0.865970$

$-0.157090$

$-0.411260$

$-0.739130$

$-3.691710$

$-1.798460$

$-2.166730$

$-3.070410$

$-1.677540$ 


\begin{tabular}{|c|c|c|c|}
\hline $\mathrm{H}$ & -3.478230 & 1.105800 & -2.406710 \\
\hline $\mathrm{H}$ & -5.216990 & 1.274459 & -1.937020 \\
\hline C & -4.523390 & 1.739120 & 1.435640 \\
\hline C & -4.986930 & 3.233950 & 1.247090 \\
\hline C & -5.654400 & 0.732829 & 1.172920 \\
\hline $\mathrm{H}$ & -5.219710 & -0.264661 & 1.060270 \\
\hline H & -6.374650 & 0.708399 & 1.998250 \\
\hline $\mathrm{H}$ & -6.195070 & 0.972659 & 0.252660 \\
\hline C & -3.858270 & 1.431020 & 2.777040 \\
\hline $\mathrm{H}$ & -2.979960 & 2.058400 & 2.945860 \\
\hline $\mathrm{H}$ & -3.536390 & 0.384390 & 2.803170 \\
\hline $\mathrm{H}$ & -4.558840 & 1.587330 & 3.605750 \\
\hline C & -4.037750 & 4.241290 & 1.918330 \\
\hline $\mathrm{H}$ & -4.298990 & 5.249520 & 1.580040 \\
\hline $\mathrm{H}$ & -4.117180 & 4.217350 & 3.010850 \\
\hline $\mathrm{H}$ & -2.998420 & 4.047590 & 1.638170 \\
\hline C & -6.426580 & 3.518059 & 1.678960 \\
\hline $\mathrm{H}$ & -6.566470 & 3.315639 & 2.747420 \\
\hline $\mathrm{H}$ & -6.661640 & 4.573139 & 1.502320 \\
\hline $\mathrm{H}$ & -7.141390 & 2.916839 & 1.112240 \\
\hline 0 & -3.539330 & 1.593670 & 0.384440 \\
\hline 0 & -4.897080 & 3.404150 & -0.179840 \\
\hline B & -3.848740 & 2.553210 & -0.653620 \\
\hline 0 & -2.571310 & 3.266440 & -1.029640 \\
\hline C & -1.303770 & 4.845860 & -2.270050 \\
\hline C & -0.128030 & 4.084780 & -2.167300 \\
\hline C & -1.249460 & 6.141940 & -2.805540 \\
\hline C & 1.082480 & 4.618870 & -2.600090 \\
\hline $\mathrm{H}$ & -0.177470 & 3.087520 & -1.742420 \\
\hline C & -0.035720 & 6.670780 & -3.238030 \\
\hline H & -2.159870 & 6.732970 & -2.879260 \\
\hline C & 1.131510 & 5.908730 & -3.137810 \\
\hline H & 1.989910 & 4.028610 & -2.512870 \\
\hline $\mathrm{H}$ & 0.001650 & 7.675560 & -3.649620 \\
\hline $\mathrm{H}$ & 2.078610 & 6.321070 & -3.475190 \\
\hline $\mathrm{H}$ & -5.384720 & 3.432889 & -3.061840 \\
\hline $\mathrm{H}$ & -2.448980 & 2.948560 & -3.870460 \\
\hline H & -3.504010 & 4.391670 & -4.310740 \\
\hline C & 0.315890 & -0.651540 & 1.830170 \\
\hline C & -0.660719 & -1.107930 & 0.951560 \\
\hline C & -1.693849 & -2.015480 & 1.376610 \\
\hline C & -1.748759 & -2.354510 & 2.715250 \\
\hline $\mathrm{H}$ & -2.493639 & -3.070770 & 3.051150 \\
\hline C & 2.653010 & -0.402970 & 0.944130 \\
\hline C & 1.468650 & 0.201770 & 1.364150 \\
\hline C & 3.666040 & 1.787860 & 0.650530 \\
\hline C & 3.779270 & 0.412160 & 0.571840 \\
\hline $\mathrm{H}$ & 4.513730 & 2.406170 & 0.365910 \\
\hline C & 2.478560 & 2.429300 & 1.071660 \\
\hline C & 1.349680 & 1.628350 & 1.434300 \\
\hline C & 0.165820 & 2.290080 & 1.863630 \\
\hline C & 0.108700 & 3.664420 & 1.937910 \\
\hline C & 1.227640 & 4.453090 & 1.579400 \\
\hline C & 2.385020 & 3.845320 & 1.151910 \\
\hline
\end{tabular}




\begin{tabular}{|c|c|c|c|}
\hline $\mathrm{H}$ & -0.704300 & 1.699440 & 2.129240 \\
\hline $\mathrm{H}$ & -0.802850 & 4.148910 & 2.275460 \\
\hline $\mathrm{H}$ & 1.167350 & 5.536300 & 1.639890 \\
\hline $\mathrm{H}$ & 3.251810 & 4.439810 & 0.872200 \\
\hline C & -0.828519 & -1.847580 & 3.664150 \\
\hline C & 0.233271 & -0.997220 & 3.221360 \\
\hline C & 1.171330 & -0.530210 & 4.183050 \\
\hline C & 1.058401 & -0.880730 & 5.510350 \\
\hline C & 0.006471 & -1.721690 & 5.945040 \\
\hline C & -0.913879 & -2.196140 & 5.039230 \\
\hline $\mathrm{H}$ & 1.982250 & 0.111840 & 3.858040 \\
\hline $\mathrm{H}$ & 1.783850 & -0.511140 & 6.229840 \\
\hline $\mathrm{H}$ & -0.069519 & -1.993230 & 6.994450 \\
\hline $\mathrm{H}$ & -1.722379 & -2.847990 & 5.361570 \\
\hline C & 7.577211 & -1.110459 & -0.702120 \\
\hline C & 6.416851 & -1.609279 & -1.311010 \\
\hline C & 5.179581 & -1.114959 & -0.880090 \\
\hline C & 5.091060 & -0.156129 & 0.137050 \\
\hline C & 6.275150 & 0.314521 & 0.724100 \\
\hline C & 7.532320 & -0.149429 & 0.318920 \\
\hline $\mathrm{H}$ & 8.539341 & -1.484199 & -1.025440 \\
\hline $\mathrm{H}$ & 4.259851 & -1.485330 & -1.317610 \\
\hline $\mathrm{H}$ & 6.199040 & 1.042571 & 1.526890 \\
\hline C & 6.449261 & -2.679549 & -2.419780 \\
\hline C & 8.801910 & 0.392001 & 1.006560 \\
\hline C & 5.781271 & -2.118509 & -3.697570 \\
\hline $\mathrm{H}$ & 5.792221 & -2.871979 & -4.495070 \\
\hline $\mathrm{H}$ & 4.739081 & -1.836039 & -3.519730 \\
\hline $\mathrm{H}$ & 6.313601 & -1.231449 & -4.060880 \\
\hline C & 7.882061 & -3.113809 & -2.780920 \\
\hline $\mathrm{H}$ & 7.848081 & -3.878249 & -3.565660 \\
\hline $\mathrm{H}$ & 8.480381 & -2.277129 & -3.160610 \\
\hline $\mathrm{H}$ & 8.406461 & -3.547279 & -1.921570 \\
\hline C & 5.668531 & -3.928559 & -1.941950 \\
\hline $\mathrm{H}$ & 5.654871 & -4.692239 & -2.730120 \\
\hline $\mathrm{H}$ & 6.143351 & -4.367519 & -1.056180 \\
\hline $\mathrm{H}$ & 4.632561 & -3.689610 & -1.681910 \\
\hline C & 10.093170 & -0.223149 & 0.433940 \\
\hline $\mathrm{H}$ & 10.962980 & 0.196851 & 0.952160 \\
\hline $\mathrm{H}$ & 10.122091 & -1.310229 & 0.568780 \\
\hline $\mathrm{H}$ & 10.209290 & -0.008029 & -0.634390 \\
\hline C & 8.878800 & 1.925701 & 0.815560 \\
\hline $\mathrm{H}$ & 9.772550 & 2.329061 & 1.307930 \\
\hline $\mathrm{H}$ & 8.930250 & 2.185461 & -0.248130 \\
\hline $\mathrm{H}$ & 8.006690 & 2.433471 & 1.240820 \\
\hline C & 8.747860 & 0.068421 & 2.518490 \\
\hline $\mathrm{H}$ & 9.642330 & 0.452461 & 3.024820 \\
\hline $\mathrm{H}$ & 7.873100 & 0.517461 & 3.000100 \\
\hline $\mathrm{H}$ & 8.700891 & -1.013839 & 2.684880 \\
\hline C & -2.633869 & -2.667280 & 0.419330 \\
\hline C & -3.980209 & -2.879770 & 0.773570 \\
\hline C & -2.177909 & -3.154170 & -0.808980 \\
\hline C & -4.858639 & -3.554100 & -0.076060 \\
\hline $\mathrm{H}$ & -4.327829 & -2.499770 & 1.727340 \\
\hline
\end{tabular}




\begin{tabular}{|c|c|c|c|}
\hline C & -3.034119 & -3.812660 & -1.705040 \\
\hline $\mathrm{H}$ & -1.131919 & -3.037790 & -1.065300 \\
\hline C & -4.362379 & -3.999190 & -1.315840 \\
\hline $\mathrm{H}$ & -5.040659 & -4.510040 & -1.988970 \\
\hline C & -2.478699 & -4.296880 & -3.057690 \\
\hline C & -6.332089 & -3.823531 & 0.288310 \\
\hline C & -3.549699 & -4.993520 & -3.917940 \\
\hline $\mathrm{H}$ & -3.950809 & -5.888900 & -3.428780 \\
\hline $\mathrm{H}$ & -4.386369 & -4.324160 & -4.149050 \\
\hline $\mathrm{H}$ & -3.108049 & -5.308770 & -4.869960 \\
\hline C & -1.939229 & -3.081010 & -3.850040 \\
\hline $\mathrm{H}$ & -1.153699 & -2.547070 & -3.306040 \\
\hline $\mathrm{H}$ & -2.742759 & -2.365270 & -4.060100 \\
\hline $\mathrm{H}$ & -1.520049 & -3.410570 & -4.808800 \\
\hline C & -1.328489 & -5.303090 & -2.814830 \\
\hline $\mathrm{H}$ & -0.504139 & -4.856760 & -2.249940 \\
\hline $\mathrm{H}$ & -1.683669 & -6.177450 & -2.256970 \\
\hline $\mathrm{H}$ & -0.923089 & -5.652500 & -3.772240 \\
\hline C & -6.600759 & -5.347341 & 0.252390 \\
\hline $\mathrm{H}$ & -6.410999 & -5.773771 & -0.737650 \\
\hline $\mathrm{H}$ & -5.962539 & -5.874411 & 0.970780 \\
\hline $\mathrm{H}$ & -7.646609 & -5.555251 & 0.509910 \\
\hline C & -6.695189 & -3.314631 & 1.696580 \\
\hline $\mathrm{H}$ & -6.085189 & -3.792501 & 2.471880 \\
\hline $\mathrm{H}$ & -6.574679 & -2.229551 & 1.785760 \\
\hline $\mathrm{H}$ & -7.744109 & -3.547451 & 1.912380 \\
\hline C & -7.254939 & -3.117391 & -0.733250 \\
\hline $\mathrm{H}$ & -8.308349 & -3.306241 & -0.491850 \\
\hline $\mathrm{H}$ & -7.092659 & -2.033581 & -0.723490 \\
\hline $\mathrm{H}$ & -7.078339 & -3.472091 & -1.754090 \\
\hline
\end{tabular}

\section{C-Catalyzed reaction TS 32}

\section{Datum}

Value

\begin{tabular}{lc}
\hline B3LYP/6-31G(d) Energy & -3038.966728 \\
\hline B3LYP-D3(BJ)/def2-TZVPP-IEF-PCM(DCM) Energy & -3040.515605 \\
\hline B3LYP-D3(BJ)/def2-TZVPP-IEF-PCM(DCM)//B3LYP/6-31G(d) Free Energy (Quasiharmonic) & -3039.285855 \\
\hline
\end{tabular}

Number of Imaginary Frequencies

Frequencies (Top 3 out of 450)

$$
\begin{array}{lr}
\text { 1. } & -317.3739 \mathrm{~cm}^{-1} \\
\text { 2. } & 7.7186 \mathrm{~cm}^{-1} \\
\text { 3. } & 10.8709 \mathrm{~cm}^{-1}
\end{array}
$$




\begin{tabular}{|c|c|c|c|}
\hline C & 2.734019 & -1.925749 & 0.910677 \\
\hline $\mathrm{H}$ & 3.752911 & -2.246419 & 1.142100 \\
\hline $\mathrm{H}$ & 2.072631 & -2.363490 & 1.669249 \\
\hline C & -0.628963 & -0.623141 & -0.461641 \\
\hline 0 & 0.241691 & -0.948050 & -1.254438 \\
\hline 0 & -1.599358 & 0.195093 & -0.859692 \\
\hline H & -2.232960 & 0.492064 & -0.155519 \\
\hline 0 & 2.434336 & -2.457430 & -0.376484 \\
\hline $\mathrm{H}$ & 1.632694 & -2.000702 & -0.709193 \\
\hline C & -3.291278 & 3.492314 & -3.725068 \\
\hline C & -2.528616 & 4.295312 & -1.832565 \\
\hline C & -4.260931 & 1.810819 & -2.193135 \\
\hline C & -4.377567 & 2.908756 & -3.109189 \\
\hline $\mathrm{H}$ & -3.347272 & 5.009692 & -1.726834 \\
\hline $\mathrm{H}$ & -3.461017 & 1.102863 & -2.418039 \\
\hline $\mathrm{H}$ & -5.201345 & 1.304818 & -1.967634 \\
\hline C & -4.528451 & 1.789763 & 1.408106 \\
\hline C & -4.966646 & 3.289770 & 1.201427 \\
\hline C & -5.673197 & 0.798815 & 1.146400 \\
\hline $\mathrm{H}$ & -5.252915 & -0.206250 & 1.046883 \\
\hline $\mathrm{H}$ & -6.400203 & 0.793321 & 1.966155 \\
\hline $\mathrm{H}$ & -6.202804 & 1.037781 & 0.219514 \\
\hline C & -3.878358 & 1.483913 & 2.757423 \\
\hline $\mathrm{H}$ & -2.991651 & 2.099127 & 2.927198 \\
\hline $\mathrm{H}$ & -3.572724 & 0.432736 & 2.795355 \\
\hline $\mathrm{H}$ & -4.582545 & 1.658495 & 3.579400 \\
\hline C & -4.006767 & 4.288614 & 1.870149 \\
\hline $\mathrm{H}$ & -4.250505 & 5.297581 & 1.521114 \\
\hline $\mathrm{H}$ & -4.094015 & 4.275850 & 2.962272 \\
\hline $\mathrm{H}$ & -2.968680 & 4.076830 & 1.598812 \\
\hline C & -6.404881 & 3.601619 & 1.618732 \\
\hline $\mathrm{H}$ & -6.556431 & 3.413794 & 2.688285 \\
\hline $\mathrm{H}$ & -6.621220 & 4.658288 & 1.428317 \\
\hline $\mathrm{H}$ & -7.124919 & 3.005707 & 1.053031 \\
\hline 0 & -3.538983 & 1.618160 & 0.366207 \\
\hline 0 & -4.863145 & 3.444393 & -0.226384 \\
\hline B & -3.823981 & 2.572933 & -0.682925 \\
\hline 0 & -2.532380 & 3.262945 & -1.053624 \\
\hline C & -1.228719 & 4.811209 & -2.296047 \\
\hline C & -0.065339 & 4.034251 & -2.173598 \\
\hline C & -1.150005 & 6.100857 & -2.843882 \\
\hline C & 1.157183 & 4.546328 & -2.599243 \\
\hline $\mathrm{H}$ & -0.133605 & 3.042138 & -1.739361 \\
\hline C & 0.075627 & 6.607651 & -3.269119 \\
\hline $\mathrm{H}$ & -2.050876 & 6.704253 & -2.933025 \\
\hline C & 1.230520 & 5.829825 & -3.149320 \\
\hline $\mathrm{H}$ & 2.054919 & 3.943828 & -2.496689 \\
\hline $\mathrm{H}$ & 0.131786 & 7.607567 & -3.690328 \\
\hline $\mathrm{H}$ & 2.186913 & 6.224929 & -3.481078 \\
\hline $\mathrm{H}$ & -5.322402 & 3.453638 & -3.116341 \\
\hline
\end{tabular}




\begin{tabular}{|c|c|c|c|}
\hline $\mathrm{H}$ & -2.386329 & 2.914565 & -3.888323 \\
\hline $\mathrm{H}$ & -3.413667 & 4.369506 & -4.354539 \\
\hline C & 0.304717 & -0.644869 & 1.852130 \\
\hline C & -0.671525 & -1.099100 & 0.971802 \\
\hline C & -1.713627 & -1.995754 & 1.398166 \\
\hline C & -1.777156 & -2.326470 & 2.738447 \\
\hline $\mathrm{H}$ & -2.529365 & -3.034788 & 3.074956 \\
\hline C & 2.645645 & -0.406776 & 0.970634 \\
\hline C & 1.462569 & 0.202444 & 1.387760 \\
\hline C & 3.665601 & 1.780464 & 0.674823 \\
\hline C & 3.774792 & 0.404212 & 0.597461 \\
\hline $\mathrm{H}$ & 4.515309 & 2.395955 & 0.390188 \\
\hline C & 2.480317 & 2.426287 & 1.094990 \\
\hline C & 1.348339 & 1.629493 & 1.456941 \\
\hline C & 0.166812 & 2.295731 & 1.885818 \\
\hline C & 0.115023 & 3.670258 & 1.960665 \\
\hline C & 1.236931 & 4.454795 & 1.602356 \\
\hline C & 2.392018 & 3.842695 & 1.175032 \\
\hline $\mathrm{H}$ & -0.705391 & 1.708360 & 2.151741 \\
\hline $\mathrm{H}$ & -0.794581 & 4.158087 & 2.298626 \\
\hline $\mathrm{H}$ & 1.180797 & 5.538220 & 1.663242 \\
\hline $\mathrm{H}$ & 3.261157 & 4.433877 & 0.895658 \\
\hline C & -0.856414 & -1.822013 & 3.688135 \\
\hline C & 0.214068 & -0.982908 & 3.244780 \\
\hline C & 1.152506 & -0.518823 & 4.207553 \\
\hline C & 1.031858 & -0.861494 & 5.536224 \\
\hline C & -0.028773 & -1.691191 & 5.971421 \\
\hline C & -0.949774 & -2.162592 & 5.064685 \\
\hline $\mathrm{H}$ & 1.970021 & 0.114600 & 3.882170 \\
\hline $\mathrm{H}$ & 1.757845 & -0.494350 & 6.256419 \\
\hline $\mathrm{H}$ & -0.110903 & -1.956567 & 7.021946 \\
\hline $\mathrm{H}$ & -1.764906 & -2.805949 & 5.387395 \\
\hline C & 7.564666 & -1.117922 & -0.686083 \\
\hline C & 6.408455 & -1.623001 & -1.286546 \\
\hline C & 5.169293 & -1.127838 & -0.849878 \\
\hline C & 5.084621 & -0.167154 & 0.160998 \\
\hline C & 6.272710 & 0.311627 & 0.745133 \\
\hline C & 7.524064 & -0.150159 & 0.335068 \\
\hline $\mathrm{H}$ & 8.531464 & -1.486608 & -1.009585 \\
\hline $\mathrm{H}$ & 4.248811 & -1.501835 & -1.282701 \\
\hline $\mathrm{H}$ & 6.192005 & 1.040249 & 1.543589 \\
\hline C & 6.436267 & -2.696912 & -2.391227 \\
\hline C & 8.841260 & 0.350463 & 0.960098 \\
\hline C & 5.762409 & -2.140263 & -3.667845 \\
\hline $\mathrm{H}$ & 5.769431 & -2.896714 & -4.462529 \\
\hline $\mathrm{H}$ & 4.721094 & -1.856929 & -3.486239 \\
\hline $\mathrm{H}$ & 6.293306 & -1.254733 & -4.036932 \\
\hline C & 7.867832 & -3.131322 & -2.756422 \\
\hline $\mathrm{H}$ & 7.831759 & -3.898714 & -3.538139 \\
\hline $\mathrm{H}$ & 8.463702 & -2.295336 & -3.141536 \\
\hline $\mathrm{H}$ & 8.395751 & -3.560929 & -1.897238 \\
\hline C & 5.657953 & -3.944326 & -1.905454 \\
\hline $\mathrm{H}$ & 5.641184 & -4.710699 & -2.690911 \\
\hline $\mathrm{H}$ & 6.136796 & -4.379962 & -1.020215 \\
\hline
\end{tabular}




\begin{tabular}{|c|c|c|c|}
\hline $\mathrm{H}$ & 4.622939 & -3.704934 & -1.641994 \\
\hline C & 9.718827 & 0.997709 & -0.137846 \\
\hline $\mathrm{H}$ & 10.667743 & 1.348454 & 0.287044 \\
\hline H & 9.953857 & 0.290951 & -0.940256 \\
\hline H & 9.209908 & 1.857149 & -0.589401 \\
\hline C & 8.608603 & 1.399900 & 2.063837 \\
\hline H & 9.572309 & 1.725915 & 2.471684 \\
\hline $\mathrm{H}$ & 8.094445 & 2.288645 & 1.680371 \\
\hline H & 8.018151 & 0.995270 & 2.893657 \\
\hline C & 9.603749 & -0.840861 & 1.587166 \\
\hline H & 10.547523 & -0.500563 & 2.031480 \\
\hline H & 9.007693 & -1.314423 & 2.375656 \\
\hline $\mathrm{H}$ & 9.842895 & -1.608316 & 0.844012 \\
\hline C & -2.654366 & -2.645970 & 0.440687 \\
\hline C & -4.002994 & -2.835750 & 0.784175 \\
\hline C & -2.187332 & -3.154692 & -0.780014 \\
\hline C & -4.885167 & -3.509831 & -0.067802 \\
\hline H & -4.354929 & -2.440393 & 1.730162 \\
\hline C & -3.041744 & -3.811884 & -1.671540 \\
\hline H & -1.138084 & -3.052446 & -1.020697 \\
\hline C & -4.380686 & -3.973407 & -1.291707 \\
\hline H & -5.055415 & -4.483960 & -1.972340 \\
\hline C & -2.555938 & -4.349268 & -3.031277 \\
\hline C & -6.365976 & -3.753829 & 0.285571 \\
\hline C & -2.818977 & -5.871057 & -3.117049 \\
\hline H & -2.280875 & -6.406819 & -2.326730 \\
\hline H & -3.883107 & -6.111800 & -3.019751 \\
\hline H & -2.477782 & -6.261048 & -4.083794 \\
\hline C & -3.328258 & -3.629986 & -4.163182 \\
\hline H & -3.147592 & -2.549563 & -4.133117 \\
\hline H & -4.409096 & -3.791666 & -4.087694 \\
\hline H & -3.002283 & -4.002513 & -5.142119 \\
\hline C & -1.049435 & -4.109791 & -3.251699 \\
\hline H & -0.790682 & -3.045924 & -3.219816 \\
\hline H & -0.440040 & -4.629110 & -2.503338 \\
\hline H & -0.757848 & -4.493670 & -4.235973 \\
\hline C & -6.732809 & -3.231182 & 1.687905 \\
\hline H & -6.135311 & -3.712461 & 2.470801 \\
\hline H & -6.598719 & -2.147310 & 1.771661 \\
\hline H & -7.786434 & -3.448589 & 1.896799 \\
\hline C & -7.267824 & -3.038586 & -0.748190 \\
\hline H & -7.085697 & -1.957920 & -0.745061 \\
\hline H & -7.089268 & -3.403996 & -1.764958 \\
\hline H & -8.326438 & -3.206302 & -0.514055 \\
\hline C & -6.659123 & -5.273118 & 0.256167 \\
\hline H & -7.710189 & -5.462432 & 0.506606 \\
\hline H & -6.468727 & -5.708527 & -0.729858 \\
\hline H & -6.035220 & -5.806469 & 0.982429 \\
\hline
\end{tabular}

\section{C-Catalyzed reaction TS 33 (TS-2.5)}


Frequencies (Top 3 out of 450)

$\begin{array}{rr}\text { 1. } & -300.2626 \mathrm{~cm}^{-1} \\ \text { 2. } & 7.9649 \mathrm{~cm}^{-1} \\ \text { 3. } & 11.7809 \mathrm{~cm}^{-1}\end{array}$

\section{B3LYP/6-31G(d) Molecular Geometry in Cartesian Coordinates}

C

$\mathrm{H}$

$\mathrm{H}$

C

0

0

$\mathrm{H}$

0

$\mathrm{H}$

C

$\mathrm{C}$

C

C

$\mathrm{H}$

$\mathrm{H}$

$\mathrm{H}$

C

C

C

$\mathrm{H}$

$\mathrm{H}$

$\mathrm{H}$

C

$\mathrm{H}$

$\mathrm{H}$

$\mathrm{H}$

C

$\mathrm{H}$

$\mathrm{H}$

$\mathrm{H}$

C

$\mathrm{H}$

\subsection{2}

4.208858

2.604592

$-0.372337$

0.381246

$-1.373404$

$-1.900991$

2.742490

1.881776

$-1.803538$

$-3.484061$

$-2.222364$

$-1.431715$

$-2.832258$

$-2.731533$

$-1.693268$

$-4.071941$

$-4.621021$

$-5.133026$

$-4.648139$

$-5.891670$

$-5.635494$

$-3.413216$

$-3.031956$

$-4.137758$

$-2.577344$

$-3.758867$

$-3.880930$

$-2.698362$

$-4.060148$

$-6.086971$

$-6.240421$

\subsection{8}

0.895738

0.613671

0.406834

1.227660

$-0.074836$

$-0.841116$

1.942980

1.764054

$-1.057652$

$-0.354766$

$-3.117700$

$-1.966195$

0.079800

$-3.585287$

$-3.856620$

$-2.612423$

$-3.906690$

$-1.506858$

$-0.573532$

$-1.757319$

$-1.336489$

$-2.855284$

$-1.916183$

$-3.264041$

$-3.554739$

$-5.151952$

$-5.526851$

$-4.940632$

$-5.942272$

$-4.226567$

$-4.410436$
$-1.548188$

$-1.807636$

$-2.486792$

$-0.055451$

0.442601

0.683113

0.326145

$-0.893579$

$-0.461717$

4. 315918

3.097770

3.008532

3. 349923

2. 341245

3.855060

2.404502

$-0.282034$

0.425748

$-0.401311$

$-0.705552$

$-1.151353$

0.554873

$-1.638551$

$-2.052352$

$-2.352687$

$-1.561823$

0.152942

$-0.869520$

0.320287

0.847738

0.119593

$-0.950640$ 


\begin{tabular}{|c|c|c|c|}
\hline $\mathrm{H}$ & -6.383473 & -5.129670 & 0.663411 \\
\hline $\mathrm{H}$ & -6.746915 & -3.415370 & 0.435593 \\
\hline 0 & -3.057896 & -2.167191 & 0.660230 \\
\hline 0 & -4.508214 & -3.581300 & 1.814748 \\
\hline B & -3.518372 & -2.574720 & 1.991074 \\
\hline 0 & -4.121253 & -1.446015 & 2.814360 \\
\hline C & -4.097971 & 0.579618 & 4.060396 \\
\hline C & -5.176466 & 0.187039 & 4.868535 \\
\hline C & -3.612271 & 1.893989 & 4.142891 \\
\hline C & -5.753731 & 1.098333 & 5.748692 \\
\hline H & -5.555357 & -0.826565 & 4.785758 \\
\hline C & -4.192926 & 2.802118 & 5.025381 \\
\hline $\mathrm{H}$ & -2.779715 & 2.198818 & 3.513000 \\
\hline C & -5.263323 & 2.405360 & 5.831055 \\
\hline $\mathrm{H}$ & -6.590786 & 0.792150 & 6.370200 \\
\hline H & -3.813463 & 3.818425 & 5.082926 \\
\hline $\mathrm{H}$ & -5.717252 & 3.113614 & 6.518882 \\
\hline $\mathrm{H}$ & -0.641393 & -1.675421 & 2.659453 \\
\hline H & -2.462549 & -1.360395 & 5.124782 \\
\hline $\mathrm{H}$ & -1.212628 & -0.163396 & 4.491660 \\
\hline C & 0.803236 & -0.938747 & -1.799758 \\
\hline C & -0.247572 & -0.087060 & -1.476112 \\
\hline C & -1.205366 & 0.340352 & -2.461114 \\
\hline C & -1.111349 & -0.195140 & -3.731341 \\
\hline $\mathrm{H}$ & -1.801490 & 0.140455 & -4.500655 \\
\hline C & 3.014124 & -0.501140 & -0.688735 \\
\hline C & 1.871548 & -1.293964 & -0.797243 \\
\hline C & 3.915498 & -2.031596 & 0.972072 \\
\hline C & 4.060658 & -0.877491 & 0.224918 \\
\hline $\mathrm{H}$ & 4.701541 & -2.314218 & 1.667674 \\
\hline C & 2.774466 & -2.860761 & 0.875506 \\
\hline C & 1.724568 & -2.492715 & -0.024317 \\
\hline C & 0.595481 & -3.352888 & -0.127425 \\
\hline C & 0.518962 & -4.514461 & 0.610348 \\
\hline C & 1.556347 & -4.870439 & 1.505404 \\
\hline C & 2.656536 & -4.055618 & 1.636183 \\
\hline $\mathrm{H}$ & -0.212803 & -3.089630 & -0.801275 \\
\hline $\mathrm{H}$ & -0.343256 & -5.166544 & 0.501064 \\
\hline $\mathrm{H}$ & 1.480044 & -5.786671 & 2.084455 \\
\hline H & 3.460997 & -4.317186 & 2.319572 \\
\hline C & -0.109706 & -1.129909 & -4.087567 \\
\hline C & 0.877299 & -1.498580 & -3.119785 \\
\hline C & 1.899745 & -2.407709 & -3.508462 \\
\hline C & 1.935712 & -2.928713 & -4.783029 \\
\hline C & 0.956478 & -2.563590 & -5.737296 \\
\hline C & -0.041801 & -1.680785 & -5.395733 \\
\hline $\mathrm{H}$ & 2.655115 & -2.691131 & -2.784163 \\
\hline $\mathrm{H}$ & 2.723023 & -3.624423 & -5.059696 \\
\hline $\mathrm{H}$ & 0.997788 & -2.980350 & -6.739919 \\
\hline $\mathrm{H}$ & -0.795365 & -1.388923 & -6.123249 \\
\hline C & 7.720679 & 1.269016 & 0.746651 \\
\hline C & 7.771968 & -0.107773 & 0.485592 \\
\hline C & 6.557347 & -0.778274 & 0.304227 \\
\hline C & 5.328982 & -0.102064 & 0.377764 \\
\hline
\end{tabular}




\begin{tabular}{|c|c|c|c|}
\hline C & 5.322582 & 1.274627 & 0.642133 \\
\hline C & 6.516236 & 1.981377 & 0.827834 \\
\hline $\mathrm{H}$ & 8.653821 & 1.807413 & 0.884532 \\
\hline $\mathrm{H}$ & 6.545840 & -1.839100 & 0.081379 \\
\hline $\mathrm{H}$ & 4.368674 & 1.782953 & 0.678430 \\
\hline C & 9.136351 & -0.820572 & 0.401642 \\
\hline C & 6.537496 & 3.498622 & 1.097722 \\
\hline C & 9.877331 & -0.674172 & 1.751947 \\
\hline $\mathrm{H}$ & 10.854618 & -1.171238 & 1.708831 \\
\hline $\mathrm{H}$ & 10.048567 & 0.375847 & 2.010946 \\
\hline $\mathrm{H}$ & 9.300475 & -1.128432 & 2.565791 \\
\hline C & 8.997295 & -2.323919 & 0.095198 \\
\hline $\mathrm{H}$ & 9.990889 & -2.784428 & 0.049482 \\
\hline $\mathrm{H}$ & 8.426473 & -2.847722 & 0.870434 \\
\hline $\mathrm{H}$ & 8.506320 & -2.500307 & -0.868577 \\
\hline C & 9.987880 & -0.181206 & -0.720915 \\
\hline $\mathrm{H}$ & 10.964181 & -0.677040 & -0.790136 \\
\hline $\mathrm{H}$ & 9.489021 & -0.275403 & -1.692255 \\
\hline $\mathrm{H}$ & 10.167794 & 0.883798 & -0.541020 \\
\hline C & 7.276876 & 3.783968 & 2.426185 \\
\hline $\mathrm{H}$ & 7.297170 & 4.862413 & 2.626299 \\
\hline $\mathrm{H}$ & 6.774172 & 3.292525 & 3.267125 \\
\hline $\mathrm{H}$ & 8.313723 & 3.431394 & 2.404603 \\
\hline C & 7.276683 & 4.209180 & -0.061427 \\
\hline $\mathrm{H}$ & 7.307807 & 5.292172 & 0.111819 \\
\hline $\mathrm{H}$ & 8.309151 & 3.857336 & -0.163196 \\
\hline $\mathrm{H}$ & 6.766719 & 4.032230 & -1.015124 \\
\hline C & 5.119352 & 4.093313 & 1.199992 \\
\hline $\mathrm{H}$ & 5.186307 & 5.174017 & 1.372264 \\
\hline $\mathrm{H}$ & 4.536367 & 3.931960 & 0.287434 \\
\hline $\mathrm{H}$ & 4.559581 & 3.659193 & 2.036443 \\
\hline C & -2.228130 & 1.391578 & -2.187432 \\
\hline C & -1.886147 & 2.561484 & -1.503061 \\
\hline C & -3.535474 & 1.259627 & -2.692706 \\
\hline C & -2.819422 & 3.587692 & -1.288123 \\
\hline $\mathrm{H}$ & -0.868796 & 2.685840 & -1.152633 \\
\hline C & -4.488722 & 2.264328 & -2.517668 \\
\hline $\mathrm{H}$ & -3.793180 & 0.349015 & -3.220728 \\
\hline C & -4.106425 & 3.415081 & -1.803002 \\
\hline $\mathrm{H}$ & -4.842059 & 4.196511 & -1.653429 \\
\hline C & -5.922829 & 2.157376 & -3.072350 \\
\hline C & -2.387456 & 4.845669 & -0.510531 \\
\hline C & -6.158244 & 0.847654 & -3.849134 \\
\hline $\mathrm{H}$ & -5.484629 & 0.756850 & -4.709124 \\
\hline $\mathrm{H}$ & -6.025415 & -0.035370 & -3.214274 \\
\hline $\mathrm{H}$ & -7.184956 & 0.826358 & -4.231731 \\
\hline C & -6.936197 & 2.214370 & -1.904576 \\
\hline $\mathrm{H}$ & -7.962365 & 2.150537 & -2.286936 \\
\hline $\mathrm{H}$ & -6.780312 & 1.382382 & -1.208615 \\
\hline $\mathrm{H}$ & -6.850374 & 3.146064 & -1.335641 \\
\hline C & -6.188600 & 3.337074 & -4.038312 \\
\hline $\mathrm{H}$ & -6.088164 & 4.306268 & -3.539148 \\
\hline $\mathrm{H}$ & -5.486826 & 3.320521 & -4.880117 \\
\hline H & -7.206297 & 3.275254 & -4.442810 \\
\hline
\end{tabular}




\begin{tabular}{|llll|} 
C & -3.522998 & 5.878865 & -0.384016 \\
$H$ & -3.864352 & 6.233234 & -1.363531 \\
$H$ & -4.387333 & 5.474182 & 0.155110 \\
$H$ & -3.165268 & 6.750763 & 0.175224 \\
C & -1.945968 & 4.434697 & 0.915603 \\
$H$ & -2.781538 & 3.983194 & 1.464354 \\
$H$ & -1.125357 & 3.710364 & 0.899790 \\
$H$ & -1.607965 & 5.315246 & 1.475948 \\
C & -1.201417 & 5.522736 & -1.238101 \\
$H$ & -0.885707 & 6.420218 & -0.692498 \\
$H$ & -0.333848 & 4.860038 & -1.314869 \\
$H$ & -1.484754 & 5.824163 & -2.253172 \\
\hline
\end{tabular}

\section{C-Catalyzed reaction TS 34}

\section{Datum}

Value

\begin{tabular}{lc}
\hline B3LYP/6-31G(d) Energy & -3038.96643 \\
\hline B3LYP-D3(BJ)/def2-TZVPP-IEF-PCM(DCM) Energy & -3040.515481 \\
\hline B3LYP-D3(BJ)/def2-TZVPP-IEF-PCM(DCM)//B3LYP/6-31G(d) Free Energy (Quasiharmonic) & -3039.285732 \\
\hline
\end{tabular}

Number of Imaginary Frequencies

Frequencies (Top 3 out of 450)
1. $-300.2633 \mathrm{~cm}^{-1}$
2. $7.9658 \mathrm{~cm}^{-1}$
3. $11.7814 \mathrm{~cm}^{-1}$

\section{B3LYP/6-31G(d) Molecular Geometry in Cartesian Coordinates}

\section{C}

$\mathrm{H}$

$\mathrm{H}$

C

0

0

$\mathrm{H}$

0

$\mathrm{H}$

C

C

c

C

$\mathrm{H}$
$-3.157417$

$-2.604690$

$-4.208940$

0.372257

$-0.381325$

1. 373363

1.900955

$-2.742537$

$-1.881863$

1.803737

3.484298

2. 222354

1.431811

2.832519
0.748278

0.613320

0.895482

0.406781

1.227620

$-0.074822$

$-0.841089$

1.942835

1.763934

$-1.056960$

$-0.354395$

$-3.117222$

$-1.965607$

0.080087
$-1.548176$

$-2.486776$

$-1.807613$

$-0.055437$

0.442593

0.683118

0.326136

$-0.893733$

$-0.461783$

4.315862

3.097586

3.008741

3. 350002

2. 340992 


\begin{tabular}{|c|c|c|c|}
\hline $\mathrm{H}$ & 1.693188 & -3.856162 & 2.404799 \\
\hline $\mathrm{H}$ & 2.731489 & -3.584758 & 3.855317 \\
\hline C & 4.072000 & -2.612450 & -0.281884 \\
\hline C & 4.620976 & -3.906699 & 0.426021 \\
\hline C & 3.413249 & -2.855365 & -1.638379 \\
\hline H & 3.031866 & -1.916296 & -2.052132 \\
\hline H & 4.137806 & -3.264016 & -2.352560 \\
\hline $\mathrm{H}$ & 2.577459 & -3.554916 & -1.561638 \\
\hline C & 5.133169 & -1.506973 & -0.401255 \\
\hline H & 5.891829 & -1.757575 & -1.151235 \\
\hline $\mathrm{H}$ & 4.648350 & -0.573653 & -0.705629 \\
\hline H & 5.635607 & -1.336517 & 0.554928 \\
\hline C & 6.086915 & -4.226695 & 0.119936 \\
\hline $\mathrm{H}$ & 6.383331 & -5.129801 & 0.663795 \\
\hline $\mathrm{H}$ & 6.240397 & -4.410609 & -0.950285 \\
\hline H & 6.746902 & -3.415533 & 0.435939 \\
\hline C & 3.758749 & -5.151928 & 0.153294 \\
\hline H & 3.880867 & -5.526959 & -0.869113 \\
\hline $\mathrm{H}$ & 4.059916 & -5.942186 & 0.848211 \\
\hline H & 2.698247 & -4.940505 & 0.320530 \\
\hline 0 & 3.057989 & -2.167057 & 0.660331 \\
\hline 0 & 4.508147 & -3.581201 & 1.814998 \\
\hline B & 3.518413 & -2.574497 & 1.991214 \\
\hline 0 & 4.121410 & -1.445738 & 2.814354 \\
\hline C & 4.098292 & 0.580116 & 4.060036 \\
\hline C & 3.612664 & 1.894526 & 4.142332 \\
\hline C & 5.176801 & 0.187616 & 4.868193 \\
\hline C & 4.193406 & 2.802772 & 5.024645 \\
\hline $\mathrm{H}$ & 2.780095 & 2.199295 & 3.512429 \\
\hline C & 5.754154 & 1.099027 & 5.748171 \\
\hline H & 5.555637 & -0.826021 & 4.785566 \\
\hline C & 5.263819 & 2.406094 & 5.830337 \\
\hline H & 3.813998 & 3.819108 & 5.082037 \\
\hline H & 6.591222 & 0.792904 & 6.369691 \\
\hline $\mathrm{H}$ & 5.717817 & 3.114439 & 6.518024 \\
\hline H & 0.641478 & -1.674868 & 2.659526 \\
\hline $\mathrm{H}$ & 2.462740 & -1.359648 & 5.124753 \\
\hline $\mathrm{H}$ & 1.212902 & -0.162633 & 4.491498 \\
\hline C & -0.803320 & -0.938987 & -1.799580 \\
\hline C & 0.247459 & -0.087222 & -1.476054 \\
\hline C & 1.205224 & 0.340114 & -2.461120 \\
\hline C & 1.111198 & -0.195526 & -3.731284 \\
\hline $\mathrm{H}$ & 1.801316 & 0.139999 & -4.500649 \\
\hline C & -3.014182 & -0.501260 & -0.688568 \\
\hline C & -1.871607 & -1.294095 & -0.796999 \\
\hline C & -3.915526 & -2.031514 & 0.972441 \\
\hline C & -4.060700 & -0.877502 & 0.225148 \\
\hline H & -4.701559 & -2.314055 & 1.668087 \\
\hline C & -2.774483 & -2.860678 & 0.875972 \\
\hline C & -1.724597 & -2.492737 & -0.023910 \\
\hline C & -0.595489 & -3.352896 & -0.126898 \\
\hline C & -0.518943 & -4.514364 & 0.611038 \\
\hline C & -1.556321 & -4.870243 & 1.506141 \\
\hline C & -2.656528 & -4.055430 & 1.636809 \\
\hline
\end{tabular}




\begin{tabular}{|c|c|c|c|}
\hline $\mathrm{H}$ & 0.212789 & -3.089710 & -0.800786 \\
\hline $\mathrm{H}$ & 0.343291 & -5.166443 & 0.501844 \\
\hline $\mathrm{H}$ & -1.479996 & -5.786394 & 2.085318 \\
\hline $\mathrm{H}$ & -3.460981 & -4.316921 & 2.320236 \\
\hline C & 0.109581 & -1.130372 & -4.087384 \\
\hline C & -0.877391 & -1.498974 & -3.119540 \\
\hline C & -1.899807 & -2.408189 & -3.508089 \\
\hline C & -1.935778 & -2.929342 & -4.782596 \\
\hline C & -0.956579 & -2.564287 & -5.736925 \\
\hline C & 0.041671 & -1.681403 & -5.395484 \\
\hline $\mathrm{H}$ & -2.655149 & -2.691559 & -2.783740 \\
\hline H & -2.723066 & -3.625117 & -5.059167 \\
\hline $\mathrm{H}$ & -0.997893 & -2.981164 & -6.739499 \\
\hline $\mathrm{H}$ & 0.795209 & -1.389596 & -6.123049 \\
\hline C & -7.720704 & 1.269114 & 0.746552 \\
\hline C & -6.516252 & 1.981464 & 0.827696 \\
\hline C & -5.322605 & 1.274671 & 0.642121 \\
\hline C & -5.329018 & -0.102049 & 0.377905 \\
\hline C & -6.557391 & -0.778249 & 0.304415 \\
\hline C & -7.772007 & -0.107707 & 0.485659 \\
\hline $\mathrm{H}$ & -8.653841 & 1.807543 & 0.884339 \\
\hline H & -4.368691 & 1.782988 & 0.678383 \\
\hline $\mathrm{H}$ & -6.545893 & -1.839101 & 0.081689 \\
\hline C & -6.537494 & 3.498743 & 1.097393 \\
\hline C & -9.136399 & -0.820493 & 0.401739 \\
\hline C & -5.119341 & 4.093414 & 1.199650 \\
\hline $\mathrm{H}$ & -5.186278 & 5.174141 & 1.371790 \\
\hline H & -4.559620 & 3.659383 & 2.036181 \\
\hline $\mathrm{H}$ & -4.536323 & 3.931933 & 0.287137 \\
\hline C & -7.276617 & 4.209168 & -0.061878 \\
\hline $\mathrm{H}$ & -7.307668 & 5.292190 & 0.111192 \\
\hline $\mathrm{H}$ & -6.766655 & 4.032028 & -1.015539 \\
\hline $\mathrm{H}$ & -8.309110 & 3.857380 & -0.163604 \\
\hline C & -7.276914 & 3.784280 & 2.425794 \\
\hline $\mathrm{H}$ & -7.297251 & 4.862758 & 2.625727 \\
\hline $\mathrm{H}$ & -8.313748 & 3.431667 & 2.404258 \\
\hline $\mathrm{H}$ & -6.774206 & 3.292998 & 3.266826 \\
\hline C & -9.877440 & -0.673904 & 1.751990 \\
\hline $\mathrm{H}$ & -10.854715 & -1.170996 & 1.708907 \\
\hline $\mathrm{H}$ & -9.300610 & -1.128027 & 2.565929 \\
\hline $\mathrm{H}$ & -10.048711 & 0.376151 & 2.010821 \\
\hline C & -9.987862 & -0.181258 & -0.720940 \\
\hline $\mathrm{H}$ & -10.964177 & -0.677068 & -0.790128 \\
\hline $\mathrm{H}$ & -10.167746 & 0.883778 & -0.541203 \\
\hline $\mathrm{H}$ & -9.488969 & -0.275610 & -1.692248 \\
\hline C & -8.997356 & -2.323882 & 0.095497 \\
\hline $\mathrm{H}$ & -9.990960 & -2.784369 & 0.049760 \\
\hline $\mathrm{H}$ & -8.506308 & -2.500406 & -0.868216 \\
\hline $\mathrm{H}$ & -8.426611 & -2.847603 & 0.870845 \\
\hline C & 2.227988 & 1.391377 & -2.187566 \\
\hline C & 3.535339 & 1.259340 & -2.692804 \\
\hline C & 1.886019 & 2.561368 & -1.503334 \\
\hline C & 4.488611 & 2.264031 & -2.517844 \\
\hline $\mathrm{H}$ & 3.793030 & 0.348670 & -3.220732 \\
\hline
\end{tabular}




\begin{tabular}{|c|c|c|c|}
\hline C & 2.819320 & 3.587572 & -1.288479 \\
\hline $\mathrm{H}$ & 0.868667 & 2.685787 & -1.152937 \\
\hline C & 4.106331 & 3.414865 & -1.803302 \\
\hline $\mathrm{H}$ & 4.841990 & 4.196282 & -1.653786 \\
\hline C & 2.387383 & 4.845630 & -0.511000 \\
\hline C & 5.922740 & 2.156969 & -3.072446 \\
\hline C & 1.201231 & 5.522564 & -1.238509 \\
\hline $\mathrm{H}$ & 1.484443 & 5.823912 & -2.253639 \\
\hline $\mathrm{H}$ & 0.333681 & 4.859823 & -1.315121 \\
\hline $\mathrm{H}$ & 0.885537 & 6.420081 & -0.692955 \\
\hline C & 1.946074 & 4.434801 & 0.915230 \\
\hline $\mathrm{H}$ & 1.607992 & 5.315381 & 1.475479 \\
\hline $\mathrm{H}$ & 1.125564 & 3.710349 & 0.899584 \\
\hline $\mathrm{H}$ & 2.781754 & 3.983490 & 1.463972 \\
\hline C & 3.522891 & 5.878894 & -0.384734 \\
\hline $\mathrm{H}$ & 4.387298 & 5.474328 & 0.154364 \\
\hline $\mathrm{H}$ & 3.864131 & 6.233149 & -1.364330 \\
\hline $\mathrm{H}$ & 3.165174 & 6.750847 & 0.174428 \\
\hline C & 6.936044 & 2.213921 & -1.904616 \\
\hline $\mathrm{H}$ & 6.850249 & 3.145634 & -1.335706 \\
\hline $\mathrm{H}$ & 6.780062 & 1.381956 & -1.208650 \\
\hline $\mathrm{H}$ & 7.962230 & 2.150010 & -2.286915 \\
\hline C & 6.158106 & 0.847209 & -3.849179 \\
\hline $\mathrm{H}$ & 6.025252 & -0.035783 & -3.214278 \\
\hline $\mathrm{H}$ & 5.484480 & 0.756390 & -4.709160 \\
\hline $\mathrm{H}$ & 7.184814 & 0.825859 & -4.231785 \\
\hline $\mathrm{C}$ & 6.188651 & 3.336624 & -4.038424 \\
\hline $\mathrm{H}$ & 7.206359 & 3.274708 & -4.442879 \\
\hline $\mathrm{H}$ & 5.486911 & 3.320115 & -4.880257 \\
\hline $\mathrm{H}$ & 6.088280 & 4.305839 & -3.539288 \\
\hline
\end{tabular}

\section{C-Catalyzed reaction TS 35}

\section{Datum}

Value

B3LYP/6-31G(d) Energy

$-3038.9611$

B3LYP-D3(BJ)/def2-TZVPP-IEF-PCM(DCM) Energy

$-3040.514169$

B3LYP-D3(BJ)/def2-TZVPP-IEF-PCM(DCM)//B3LYP/6-31G(d) Free Energy (Quasiharmonic)

$-3039.285633$

Number of Imaginary Frequencies

Frequencies (Top 3 out of 450)
1. $\quad-295.9890 \mathrm{~cm}^{-1}$
2. $\quad 7.6643 \mathrm{~cm}^{-1}$
3. $9.6850 \mathrm{~cm}^{-1}$ 


\begin{tabular}{|c|c|c|c|}
\hline C & 1.962146 & -0.456132 & 0.703488 \\
\hline $\mathrm{H}$ & 1.325373 & -0.479993 & 1.595783 \\
\hline $\mathrm{H}$ & 2.837974 & 0.158070 & 0.930113 \\
\hline C & -0.837383 & -2.482591 & -1.340020 \\
\hline 0 & -1.389240 & -3.380710 & -1.937087 \\
\hline 0 & -0.090294 & -1.570801 & -1.970611 \\
\hline $\mathrm{H}$ & 0.337072 & -0.925142 & -1.339208 \\
\hline 0 & 1.246619 & 0.126594 & -0.396176 \\
\hline $\mathrm{H}$ & 0.916612 & 1.014470 & -0.108300 \\
\hline C & -2.964788 & 1.717755 & 2.900510 \\
\hline C & -3.037055 & 2.327523 & 0.788189 \\
\hline C & -0.650736 & 2.550281 & 2.706225 \\
\hline C & -2.001267 & 2.657937 & 3.180656 \\
\hline $\mathrm{H}$ & -3.473865 & 1.350937 & 0.566707 \\
\hline $\mathrm{H}$ & 0.060416 & 3.201697 & 3.218112 \\
\hline H & -0.283812 & 1.521778 & 2.653334 \\
\hline C & 1.160843 & 3.679869 & -0.399022 \\
\hline C & 0.665941 & 4.974041 & 0.348881 \\
\hline C & 2.677523 & 3.500784 & -0.469928 \\
\hline $\mathrm{H}$ & 3.131908 & 3.495326 & 0.524047 \\
\hline $\mathrm{H}$ & 2.913227 & 2.546166 & -0.952120 \\
\hline $\mathrm{H}$ & 3.138341 & 4.302311 & -1.059126 \\
\hline C & 0.550750 & 3.520683 & -1.800539 \\
\hline $\mathrm{H}$ & 0.775322 & 2.516792 & -2.175109 \\
\hline $\mathrm{H}$ & -0.536431 & 3.638620 & -1.777417 \\
\hline $\mathrm{H}$ & 0.965732 & 4.247294 & -2.507410 \\
\hline C & 0.403246 & 6.176849 & -0.559443 \\
\hline $\mathrm{H}$ & 1.320825 & 6.495222 & -1.068125 \\
\hline $\mathrm{H}$ & 0.042985 & 7.019372 & 0.040949 \\
\hline $\mathrm{H}$ & -0.353755 & 5.952342 & -1.314608 \\
\hline C & 1.595486 & 5.393585 & 1.499776 \\
\hline $\mathrm{H}$ & 1.839782 & 4.544983 & 2.145919 \\
\hline $\mathrm{H}$ & 1.085013 & 6.148388 & 2.106791 \\
\hline $\mathrm{H}$ & 2.532687 & 5.825594 & 1.132072 \\
\hline 0 & 0.620735 & 2.635068 & 0.447607 \\
\hline 0 & -0.586423 & 4.549211 & 0.921339 \\
\hline B & -0.587993 & 3.125492 & 1.060414 \\
\hline 0 & -1.793778 & 2.419174 & 0.440267 \\
\hline C & -4.019482 & 3.437101 & 0.779779 \\
\hline C & -3.666134 & 4.795346 & 0.726863 \\
\hline C & -5.380386 & 3.080214 & 0.781241 \\
\hline C & -4.663457 & 5.768679 & 0.694127 \\
\hline $\mathrm{H}$ & -2.618025 & 5.075525 & 0.711453 \\
\hline C & -6.370844 & 4.058196 & 0.746933 \\
\hline $\mathrm{H}$ & -5.658732 & 2.028687 & 0.802718 \\
\hline C & -6.013301 & 5.408025 & 0.706652 \\
\hline $\mathrm{H}$ & -4.383796 & 6.818029 & 0.652691 \\
\hline $\mathrm{H}$ & -7.417907 & 3.768439 & 0.745478 \\
\hline $\mathrm{H}$ & -6.783219 & 6.174356 & 0.677817 \\
\hline $\mathrm{H}$ & -2.325740 & 3.621959 & 3.573986 \\
\hline
\end{tabular}




\begin{tabular}{|c|c|c|c|}
\hline $\mathrm{H}$ & -2.670493 & 0.699432 & 2.658654 \\
\hline $\mathrm{H}$ & -3.991203 & 1.859501 & 3.224727 \\
\hline C & 0.088894 & -2.644641 & 0.994757 \\
\hline C & -0.987360 & -2.353743 & 0.167153 \\
\hline C & -2.294117 & -2.101697 & 0.709444 \\
\hline C & -2.462485 & -2.167424 & 2.079236 \\
\hline $\mathrm{H}$ & -3.441274 & -1.960369 & 2.505623 \\
\hline C & 2.377978 & -1.861486 & 0.323757 \\
\hline C & 1.455797 & -2.900919 & 0.419139 \\
\hline C & 4.008710 & -3.377613 & -0.645699 \\
\hline C & 3.679263 & -2.105719 & -0.223017 \\
\hline H & 4.999206 & -3.562674 & -1.054342 \\
\hline C & 3.098635 & -4.457972 & -0.554920 \\
\hline C & 1.794524 & -4.223750 & -0.013502 \\
\hline C & 0.888501 & -5.318249 & 0.065757 \\
\hline C & 1.259103 & -6.573946 & -0.358844 \\
\hline C & 2.552630 & -6.803814 & -0.886457 \\
\hline C & 3.450488 & -5.766529 & -0.982946 \\
\hline $\mathrm{H}$ & -0.111360 & -5.150314 & 0.450163 \\
\hline H & 0.550365 & -7.395031 & -0.296191 \\
\hline $\mathrm{H}$ & 2.830212 & -7.800210 & -1.219804 \\
\hline $\mathrm{H}$ & 4.444847 & -5.930336 & -1.391703 \\
\hline C & -1.399019 & -2.489300 & 2.958718 \\
\hline C & -0.097821 & -2.731955 & 2.415705 \\
\hline C & 0.958125 & -3.056058 & 3.311572 \\
\hline C & 0.738686 & -3.129256 & 4.669924 \\
\hline C & -0.547839 & -2.881503 & 5.204223 \\
\hline C & -1.591971 & -2.568317 & 4.364276 \\
\hline $\mathrm{H}$ & 1.946957 & -3.249832 & 2.909952 \\
\hline $\mathrm{H}$ & 1.558927 & -3.380902 & 5.336782 \\
\hline H & -0.708507 & -2.942129 & 6.277230 \\
\hline $\mathrm{H}$ & -2.585553 & -2.379804 & 4.764581 \\
\hline C & 6.656471 & 0.939501 & -0.633391 \\
\hline C & 6.018030 & 0.464109 & -1.782064 \\
\hline C & 5.035518 & -0.527601 & -1.618335 \\
\hline C & 4.702387 & -1.021097 & -0.357008 \\
\hline C & 5.369697 & -0.519980 & 0.773046 \\
\hline C & 6.353157 & 0.464540 & 0.656457 \\
\hline $\mathrm{H}$ & 7.418565 & 1.703545 & -0.733779 \\
\hline $\mathrm{H}$ & 4.507524 & -0.923097 & -2.480817 \\
\hline $\mathrm{H}$ & 5.109394 & -0.927619 & 1.743694 \\
\hline C & 6.349943 & 0.978320 & -3.195962 \\
\hline C & 7.108433 & 1.033035 & 1.873423 \\
\hline C & 7.430505 & 2.076102 & -3.186208 \\
\hline $\mathrm{H}$ & 7.626724 & 2.409336 & -4.211635 \\
\hline $\mathrm{H}$ & 7.115722 & 2.953193 & -2.609027 \\
\hline H & 8.377994 & 1.714483 & -2.770585 \\
\hline C & 6.866680 & -0.196946 & -4.060270 \\
\hline $\mathrm{H}$ & 7.105749 & 0.150588 & -5.072937 \\
\hline $\mathrm{H}$ & 7.774752 & -0.632380 & -3.627326 \\
\hline $\mathrm{H}$ & 6.123039 & -0.995665 & -4.149686 \\
\hline C & 5.074118 & 1.566498 & -3.844038 \\
\hline $\mathrm{H}$ & 5.293843 & 1.931922 & -4.854768 \\
\hline $\mathrm{H}$ & 4.275534 & 0.822283 & -3.925022 \\
\hline
\end{tabular}




\begin{tabular}{|c|c|c|c|}
\hline H & 4.688656 & 2.406542 & -3.254813 \\
\hline C & 6.871168 & 2.559693 & 1.960825 \\
\hline $\mathrm{H}$ & 7.405662 & 2.980995 & 2.821287 \\
\hline $\mathrm{H}$ & 7.222646 & 3.079612 & 1.063580 \\
\hline $\mathrm{H}$ & 5.804651 & 2.783323 & 2.080408 \\
\hline C & 6.644015 & 0.402457 & 3.200120 \\
\hline $\mathrm{H}$ & 6.813632 & -0.679995 & 3.219897 \\
\hline $\mathrm{H}$ & 5.579639 & 0.584804 & 3.387612 \\
\hline $\mathrm{H}$ & 7.205709 & 0.840091 & 4.033175 \\
\hline C & 8.623283 & 0.759217 & 1.716500 \\
\hline $\mathrm{H}$ & 9.175181 & 1.162055 & 2.574813 \\
\hline $\mathrm{H}$ & 8.822167 & -0.317048 & 1.659154 \\
\hline $\mathrm{H}$ & 9.030510 & 1.222280 & 0.811664 \\
\hline C & -3.453573 & -1.746632 & -0.156599 \\
\hline C & -3.316951 & -0.814135 & -1.195147 \\
\hline C & -4.708634 & -2.327450 & 0.075460 \\
\hline C & -4.396123 & -0.475389 & -2.019402 \\
\hline $\mathrm{H}$ & -2.347821 & -0.362205 & -1.363343 \\
\hline C & -5.819400 & -1.999776 & -0.709884 \\
\hline $\mathrm{H}$ & -4.793398 & -3.068372 & 0.861731 \\
\hline C & -5.634213 & -1.075399 & -1.748685 \\
\hline $\mathrm{H}$ & -6.481482 & -0.826314 & -2.380745 \\
\hline C & -7.208600 & -2.628526 & -0.484821 \\
\hline C & -4.245348 & 0.482670 & -3.217127 \\
\hline C & -7.644749 & -3.390273 & -1.758820 \\
\hline H & -8.632014 & -3.845587 & -1.611983 \\
\hline $\mathrm{H}$ & -7.709571 & -2.728340 & -2.628480 \\
\hline H & -6.933225 & -4.187911 & -1.999396 \\
\hline C & -7.219747 & -3.623198 & 0.691856 \\
\hline $\mathrm{H}$ & -8.227165 & -4.036512 & 0.816112 \\
\hline H & -6.536834 & -4.462967 & 0.521963 \\
\hline $\mathrm{H}$ & -6.941610 & -3.142088 & 1.636778 \\
\hline C & -8.235392 & -1.512122 & -0.178905 \\
\hline H & -7.960387 & -0.965214 & 0.730916 \\
\hline H & -8.305038 & -0.787187 & -0.996690 \\
\hline H & -9.233451 & -1.941357 & -0.027167 \\
\hline C & -5.270823 & 1.635348 & -3.114345 \\
\hline H & -6.303326 & 1.269514 & -3.107350 \\
\hline H & -5.117073 & 2.222605 & -2.201865 \\
\hline H & -5.167463 & 2.311600 & -3.971743 \\
\hline C & -2.834569 & 1.098829 & -3.298536 \\
\hline H & -2.592011 & 1.685117 & -2.404547 \\
\hline H & -2.059649 & 0.335032 & -3.424369 \\
\hline H & -2.779456 & 1.772709 & -4.161210 \\
\hline C & -4.498414 & -0.313029 & -4.520829 \\
\hline $\mathrm{H}$ & -4.391690 & 0.342969 & -5.393807 \\
\hline H & -3.781607 & -1.135254 & -4.620910 \\
\hline H & -5.506244 & -0.741910 & -4.545510 \\
\hline
\end{tabular}


Frequencies (Top 3 out of 450)

$\begin{array}{lr}\text { 1. } & -324.8548 \mathrm{~cm}^{-1} \\ \text { 2. } & 6.7248 \mathrm{~cm}^{-1} \\ \text { 3. } & 10.8247 \mathrm{~cm}^{-1}\end{array}$

\section{B3LYP/6-31G(d) Molecular Geometry in Cartesian Coordinates}

C

$\mathrm{H}$

$\mathrm{H}$

C

0

0

$\mathrm{H}$

0

$\mathrm{H}$

C

C

C

C

$\mathrm{H}$

$\mathrm{H}$

$\mathrm{H}$

C

C

C

$\mathrm{H}$

$\mathrm{H}$

$\mathrm{H}$

C

$\mathrm{H}$

$\mathrm{H}$

$\mathrm{H}$

C

$\mathrm{H}$

$\mathrm{H}$

$\mathrm{H}$

C

$\mathrm{H}$

\section{$-3.165532$}

$-2.610718$

$-4.218266$

0.361080

$-0.403644$

1.366027

1.891959

$-2.761019$

$-1.897054$

2. 602591

4.188337

1.874369

2.402340

4.896053

1.131055

1.551520

4.005473

4. 564699

3.467232

3.070907

4.263115

2.661690

5.012861

5.832881

4.501750

5.438944

6.064498

6.312407

6.654311

6.364185

3. 791799

4.014372
0.927804

0.902997

1.094606

0.450095

1. 211343

$-0.091699$

$-0.792599$

2.043698

1.824001

$-2.271303$

$-1.394832$

$-3.108505$

$-3.303919$

$-2.203104$

$-2.313928$

$-4.020000$

$-2.673753$

$-3.916659$

$-2.983164$

$-2.071442$

$-3.368413$

$-3.720585$

$-1.515485$

$-1.737768$

$-0.614235$

$-1.296842$

$-4.164402$

$-4.370217$

$-3.309053$

$-5.034602$

$-5.212281$

$-5.603652$
$-1.447729$

$-2.394845$

$-1.690928$

$-0.005658$

0.568192

0.674369

0.202029

$-0.662955$

$-0.255153$

4.682971

3. 475544

2.472412

3. 791043

3. 670466

2. 382152

1.968039

$-0.649426$

0.143503

$-2.045330$

$-2.505022$

$-2.693550$

$-2.012853$

$-0.719027$

$-1.411242$

$-1.070645$

0.263762

$-0.033135$

$-1.081195$

0.304643

0.560731

$-0.156018$

$-1.154835$ 


\begin{tabular}{|c|c|c|c|}
\hline $\mathrm{H}$ & 4.077492 & -5.969850 & 0.580914 \\
\hline $\mathrm{H}$ & 2.711887 & -5.053485 & -0.079519 \\
\hline 0 & 2.895575 & -2.263953 & 0.192364 \\
\hline 0 & 4.317381 & -3.558981 & 1.514196 \\
\hline B & 3.262920 & -2.591729 & 1.553596 \\
\hline 0 & 3.658961 & -1.334486 & 2.296249 \\
\hline C & 4.380631 & -0.130878 & 4.210889 \\
\hline C & 5.287283 & -0.082302 & 5.280047 \\
\hline C & 3.678818 & 1.027762 & 3.838417 \\
\hline C & 5.492517 & 1.109615 & 5.971940 \\
\hline $\mathrm{H}$ & 5.833150 & -0.979334 & 5.564405 \\
\hline C & 3.885276 & 2.214483 & 4.536349 \\
\hline $\mathrm{H}$ & 2.984198 & 0.982643 & 3.004851 \\
\hline C & 4.790058 & 2.259001 & 5.602198 \\
\hline $\mathrm{H}$ & 6.199536 & 1.142771 & 6.796158 \\
\hline $\mathrm{H}$ & 3.339584 & 3.108594 & 4.248292 \\
\hline $\mathrm{H}$ & 4.947727 & 3.188523 & 6.142454 \\
\hline $\mathrm{H}$ & 2.896927 & -4.254881 & 3.992482 \\
\hline $\mathrm{H}$ & 1.995356 & -1.373710 & 4.613941 \\
\hline $\mathrm{H}$ & 3.070948 & -2.452886 & 5.646573 \\
\hline C & -0.802368 & -0.710890 & -1.896463 \\
\hline C & 0.241024 & 0.106148 & -1.473864 \\
\hline C & 1.201627 & 0.639846 & -2.403602 \\
\hline C & 1.117984 & 0.244694 & -3.725002 \\
\hline $\mathrm{H}$ & 1.809496 & 0.666921 & -4.449097 \\
\hline C & -3.015751 & -0.413986 & -0.741228 \\
\hline C & -1.873501 & -1.187154 & -0.948552 \\
\hline C & -3.928147 & -2.152215 & 0.694959 \\
\hline C & -4.064689 & -0.905333 & 0.113662 \\
\hline $\mathrm{H}$ & -4.717962 & -2.523526 & 1.343045 \\
\hline C & -2.792616 & -2.967756 & 0.484992 \\
\hline C & -1.735238 & -2.481814 & -0.347157 \\
\hline C & -0.610024 & -3.324468 & -0.566003 \\
\hline C & -0.549010 & -4.583478 & -0.009243 \\
\hline C & -1.595597 & -5.059489 & 0.816304 \\
\hline C & -2.688648 & -4.262016 & 1.063160 \\
\hline $\mathrm{H}$ & 0.210975 & -2.967020 & -1.177594 \\
\hline $\mathrm{H}$ & 0.310135 & -5.218946 & -0.207239 \\
\hline $\mathrm{H}$ & -1.532013 & -6.052977 & 1.252213 \\
\hline $\mathrm{H}$ & -3.499005 & -4.613874 & 1.697439 \\
\hline C & 0.120789 & -0.646299 & -4.188177 \\
\hline C & -0.868961 & -1.121550 & -3.271385 \\
\hline C & -1.888783 & -1.981346 & -3.765526 \\
\hline C & -1.919525 & -2.354898 & -5.091028 \\
\hline C & -0.936903 & -1.884563 & -5.994331 \\
\hline C & 0.058926 & -1.045745 & -5.550562 \\
\hline $\mathrm{H}$ & -2.648015 & -2.344804 & -3.082285 \\
\hline $\mathrm{H}$ & -2.706075 & -3.014377 & -5.447401 \\
\hline $\mathrm{H}$ & -0.974132 & -2.185104 & -7.037907 \\
\hline $\mathrm{H}$ & 0.815081 & -0.672491 & -6.237087 \\
\hline C & -7.705644 & 1.182112 & 0.930108 \\
\hline C & -6.494509 & 1.868151 & 1.096049 \\
\hline C & -5.307946 & 1.181662 & 0.813843 \\
\hline c & -5.326880 & -0.148583 & 0.372337 \\
\hline
\end{tabular}




\begin{tabular}{|c|c|c|c|}
\hline C & -6.561454 & -0.799604 & 0.219097 \\
\hline C & -7.769259 & -0.149066 & 0.493533 \\
\hline $\mathrm{H}$ & -8.634172 & 1.704717 & 1.142168 \\
\hline $\mathrm{H}$ & -4.349644 & 1.673236 & 0.909931 \\
\hline $\mathrm{H}$ & -6.559734 & -1.822953 & -0.138655 \\
\hline C & -6.498727 & 3.338528 & 1.558605 \\
\hline C & -9.139652 & -0.834233 & 0.325238 \\
\hline C & -7.223595 & 4.201532 & 0.498108 \\
\hline $\mathrm{H}$ & -7.246317 & 5.252963 & 0.811119 \\
\hline $\mathrm{H}$ & -6.709224 & 4.145919 & -0.467886 \\
\hline $\mathrm{H}$ & -8.258608 & 3.875920 & 0.345714 \\
\hline C & -7.240326 & 3.462506 & 2.910409 \\
\hline $\mathrm{H}$ & -7.242886 & 4.506430 & 3.248199 \\
\hline $\mathrm{H}$ & -8.283136 & 3.134819 & 2.840915 \\
\hline $\mathrm{H}$ & -6.750537 & 2.857780 & 3.682289 \\
\hline C & -5.073621 & 3.896217 & 1.741326 \\
\hline $\mathrm{H}$ & -5.128230 & 4.946193 & 2.052571 \\
\hline $\mathrm{H}$ & -4.523440 & 3.349532 & 2.515893 \\
\hline $\mathrm{H}$ & -4.489284 & 3.846296 & 0.816979 \\
\hline C & -9.980883 & -0.059897 & -0.717175 \\
\hline $\mathrm{H}$ & -10.962577 & -0.532994 & -0.845141 \\
\hline H & -10.148347 & 0.978991 & -0.414686 \\
\hline $\mathrm{H}$ & -9.479919 & -0.046574 & -1.691944 \\
\hline C & -9.014115 & -2.293264 & -0.153841 \\
\hline $\mathrm{H}$ & -10.011932 & -2.735598 & -0.254433 \\
\hline $\mathrm{H}$ & -8.522548 & -2.361312 & -1.130923 \\
\hline $\mathrm{H}$ & -8.449580 & -2.908946 & 0.555723 \\
\hline C & -9.884829 & -0.839439 & 1.681285 \\
\hline $\mathrm{H}$ & -10.865702 & -1.320131 & 1.577382 \\
\hline $\mathrm{H}$ & -9.314873 & -1.389776 & 2.438686 \\
\hline $\mathrm{H}$ & -10.049527 & 0.174349 & 2.060627 \\
\hline C & 2.214605 & 1.666324 & -2.021324 \\
\hline C & 1.860795 & 2.758701 & -1.224518 \\
\hline C & 3.521142 & 1.602287 & -2.541929 \\
\hline C & 2.780873 & 3.772611 & -0.915876 \\
\hline $\mathrm{H}$ & 0.844554 & 2.832620 & -0.856998 \\
\hline C & 4.462272 & 2.596799 & -2.271404 \\
\hline $\mathrm{H}$ & 3.786680 & 0.753282 & -3.161116 \\
\hline C & 4.067756 & 3.668321 & -1.448735 \\
\hline $\mathrm{H}$ & 4.793882 & 4.441422 & -1.226742 \\
\hline C & 5.897219 & 2.562807 & -2.832811 \\
\hline C & 2.335753 & 4.943065 & -0.018812 \\
\hline C & 6.908516 & 2.509410 & -1.663226 \\
\hline $\mathrm{H}$ & 6.815030 & 3.378169 & -1.003096 \\
\hline $\mathrm{H}$ & 6.756003 & 1.610425 & -1.055377 \\
\hline $\mathrm{H}$ & 7.935993 & 2.491233 & -2.047317 \\
\hline C & 6.147852 & 1.342361 & -3.738885 \\
\hline $\mathrm{H}$ & 7.174309 & 1.372755 & -4.121736 \\
\hline $\mathrm{H}$ & 6.026375 & 0.397833 & -3.197126 \\
\hline H & 5.474276 & 1.330887 & -4.603614 \\
\hline C & 6.151656 & 3.837924 & -3.672246 \\
\hline $\mathrm{H}$ & 5.451497 & 3.900073 & -4.513276 \\
\hline $\mathrm{H}$ & 6.038785 & 4.749684 & -3.076808 \\
\hline H & 7.170766 & 3.829606 & -4.078221 \\
\hline
\end{tabular}




$\begin{array}{llll}\mathrm{C} & 1.912155 & 4.387436 & 1.362835 \\ \mathrm{H} & 1.100803 & 3.656981 & 1.283837 \\ \mathrm{H} & 2.759027 & 3.893100 & 1.854242 \\ \mathrm{H} & 1.569216 & 5.203261 & 2.011534 \\ \mathrm{C} & 3.455814 & 5.977622 & 0.200131 \\ \mathrm{H} & 4.330702 & 5.536927 & 0.691993 \\ \mathrm{H} & 3.784101 & 6.430588 & -0.742684 \\ \mathrm{H} & 3.088840 & 6.785706 & 0.842954 \\ \mathrm{C} & 1.134145 & 5.667923 & -0.670432 \\ \mathrm{H} & 0.806772 & 6.502422 & -0.038354 \\ \mathrm{H} & 1.405946 & 6.071908 & -1.652575 \\ \mathrm{H} & 0.277236 & 5.001098 & -0.807345\end{array}$

\section{C-Catalyzed reaction TS 37}

Datum

Value

\begin{tabular}{lc}
\hline B3LYP/6-31G(d) Energy & -3038.963786 \\
\hline B3LYP-D3(BJ)/def2-TZVPP-IEF-PCM(DCM) Energy & -3040.514298 \\
\hline B3LYP-D3(BJ)/def2-TZVPP-IEF-PCM(DCM)//B3LYP/6-31G(d) Free Energy (Quasiharmonic) & -3039.284758 \\
\hline
\end{tabular}

Number of Imaginary Frequencies

Frequencies (Top 3 out of 450)

$\begin{array}{lr}\text { 1. } & -292.8328 \mathrm{~cm}^{-1} \\ \text { 2. } & 9.8223 \mathrm{~cm}^{-1} \\ \text { 3. } & 10.8399 \mathrm{~cm}^{-1}\end{array}$

\section{B3LYP/6-31G(d) Molecular Geometry in Cartesian Coordinates}

$\begin{array}{lrrr}C & 3.155953 & 0.595237 & 1.306472 \\ H & 2.598789 & 0.397498 & 2.230481 \\ H & 4.183551 & 0.836126 & 1.590308 \\ \mathrm{C} & -0.269694 & 0.096983 & -0.318957 \\ \mathrm{O} & 0.499176 & 0.841729 & -0.903246 \\ \mathrm{O} & -1.343257 & -0.362239 & -0.970775 \\ \mathrm{H} & -1.867383 & -1.087737 & -0.536546 \\ \mathrm{O} & 2.635261 & 1.757064 & 0.657764 \\ \mathrm{H} & 1.895797 & 1.473608 & 0.080102 \\ \mathrm{C} & -3.398751 & -0.175790 & -4.094238 \\ \mathrm{C} & -4.473203 & -0.057842 & -2.177546 \\ \mathrm{C} & -3.198283 & -2.539328 & -3.373270 \\ \mathrm{C} & -2.646197 & -1.236347 & -3.641630 \\ \mathrm{H} & -3.583357 & 0.210512 & -1.607400\end{array}$




\begin{tabular}{|c|c|c|c|}
\hline $\mathrm{H}$ & -2.448548 & -3.321483 & -3.234554 \\
\hline $\mathrm{H}$ & -3.969103 & -2.857111 & -4.080256 \\
\hline C & -3.775081 & -3.164195 & 0.348557 \\
\hline C & -4.459186 & -4.303726 & -0.488981 \\
\hline C & -2.694447 & -3.638061 & 1.316762 \\
\hline $\mathrm{H}$ & -2.245593 & -2.784694 & 1.835811 \\
\hline $\mathrm{H}$ & -3.125172 & -4.297432 & 2.079505 \\
\hline $\mathrm{H}$ & -1.901403 & -4.186244 & 0.804412 \\
\hline C & -4.790855 & -2.288833 & 1.099103 \\
\hline $\mathrm{H}$ & -5.213427 & -2.810500 & 1.964794 \\
\hline $\mathrm{H}$ & -4.280131 & -1.389924 & 1.457081 \\
\hline $\mathrm{H}$ & -5.609943 & -1.976103 & 0.446130 \\
\hline C & -5.695930 & -4.923043 & 0.166908 \\
\hline $\mathrm{H}$ & -5.446034 & -5.393505 & 1.125461 \\
\hline $\mathrm{H}$ & -6.475448 & -4.176064 & 0.333798 \\
\hline $\mathrm{H}$ & -6.108689 & -5.695251 & -0.490782 \\
\hline C & -3.477418 & -5.418568 & -0.892639 \\
\hline $\mathrm{H}$ & -3.212058 & -6.063861 & -0.047451 \\
\hline $\mathrm{H}$ & -3.950910 & -6.037461 & -1.661319 \\
\hline $\mathrm{H}$ & -2.557654 & -4.999607 & -1.313238 \\
\hline 0 & -3.166142 & -2.356783 & -0.699046 \\
\hline 0 & -4.869108 & -3.617210 & -1.676349 \\
\hline B & -4.048120 & -2.470035 & -1.863459 \\
\hline 0 & -4.948348 & -1.253838 & -2.054395 \\
\hline C & -5.406929 & 1.037237 & -2.500149 \\
\hline C & -5.014332 & 2.364014 & -2.263721 \\
\hline C & -6.690028 & 0.772637 & -3.005549 \\
\hline C & -5.889728 & 3.412858 & -2.539688 \\
\hline $\mathrm{H}$ & -4.027447 & 2.567912 & -1.855238 \\
\hline C & -7.559557 & 1.823997 & -3.281216 \\
\hline $\mathrm{H}$ & -6.991105 & -0.257571 & -3.166568 \\
\hline C & -7.161582 & 3.145179 & -3.052200 \\
\hline $\mathrm{H}$ & -5.580291 & 4.437757 & -2.354666 \\
\hline $\mathrm{H}$ & -8.551996 & 1.615400 & -3.671199 \\
\hline $\mathrm{H}$ & -7.843380 & 3.963197 & -3.268273 \\
\hline $\mathrm{H}$ & -1.657910 & -1.014755 & -3.244798 \\
\hline $\mathrm{H}$ & -4.323038 & -0.349924 & -4.638137 \\
\hline $\mathrm{H}$ & -2.951222 & 0.806346 & -4.217405 \\
\hline C & 0.926645 & -1.227561 & 1.430161 \\
\hline C & -0.109141 & -0.359451 & 1.108952 \\
\hline C & -1.051083 & 0.093878 & 2.096955 \\
\hline C & -1.009577 & -0.483175 & 3.349534 \\
\hline $\mathrm{H}$ & -1.688037 & -0.129548 & 4.121768 \\
\hline C & 3.139559 & -0.643440 & 0.421386 \\
\hline C & 2.043314 & -1.506109 & 0.457495 \\
\hline C & 4.185330 & -2.048400 & -1.265292 \\
\hline C & 4.239241 & -0.923720 & -0.462191 \\
\hline $\mathrm{H}$ & 5.010417 & -2.255566 & -1.942005 \\
\hline C & 3.081055 & -2.931169 & -1.261202 \\
\hline C & 1.984539 & -2.663279 & -0.382841 \\
\hline C & 0.883544 & -3.562778 & -0.385425 \\
\hline C & 0.868193 & -4.665092 & -1.210940 \\
\hline C & 1.954199 & -4.925961 & -2.081028 \\
\hline C & 3.034760 & -4.075013 & -2.103863 \\
\hline
\end{tabular}




\begin{tabular}{|c|c|c|c|}
\hline $\mathrm{H}$ & 0.045296 & -3.369605 & 0.274043 \\
\hline $\mathrm{H}$ & 0.017491 & -5.341264 & -1.194676 \\
\hline $\mathrm{H}$ & 1.929888 & -5.797926 & -2.729015 \\
\hline $\mathrm{H}$ & 3.873966 & -4.264352 & -2.769093 \\
\hline C & -0.051544 & -1.472630 & 3.688598 \\
\hline C & 0.957597 & -1.825849 & 2.735454 \\
\hline C & 1.955736 & -2.762192 & 3.120554 \\
\hline C & 1.945107 & -3.331666 & 4.374972 \\
\hline C & 0.942762 & -2.986654 & 5.311967 \\
\hline C & -0.030531 & -2.073671 & 4.975547 \\
\hline $\mathrm{H}$ & 2.727409 & -3.030223 & 2.407479 \\
\hline $\mathrm{H}$ & 2.713088 & -4.049789 & 4.648619 \\
\hline $\mathrm{H}$ & 0.946993 & -3.441138 & 6.298928 \\
\hline $\mathrm{H}$ & -0.798451 & -1.794630 & 5.693010 \\
\hline C & 7.791228 & 1.446515 & -0.745499 \\
\hline C & 6.545558 & 2.090577 & -0.857066 \\
\hline C & 5.390618 & 1.313811 & -0.748591 \\
\hline C & 5.465633 & -0.072970 & -0.531623 \\
\hline C & 6.724180 & -0.674999 & -0.428232 \\
\hline C & 7.909219 & 0.070253 & -0.531349 \\
\hline $\mathrm{H}$ & 8.690661 & 2.046030 & -0.824180 \\
\hline $\mathrm{H}$ & 4.410964 & 1.768591 & -0.813219 \\
\hline $\mathrm{H}$ & 6.771224 & -1.744763 & -0.245097 \\
\hline C & 6.492619 & 3.614537 & -1.078781 \\
\hline C & 9.270535 & -0.640589 & -0.398093 \\
\hline C & 7.131105 & 4.326826 & 0.137711 \\
\hline $\mathrm{H}$ & 7.117261 & 5.414564 & -0.005368 \\
\hline $\mathrm{H}$ & 6.579693 & 4.098176 & 1.056795 \\
\hline $\mathrm{H}$ & 8.172855 & 4.022692 & 0.287628 \\
\hline C & 7.278916 & 3.985007 & -2.358559 \\
\hline $\mathrm{H}$ & 7.245897 & 5.068825 & -2.525051 \\
\hline $\mathrm{H}$ & 8.332332 & 3.691761 & -2.295049 \\
\hline $\mathrm{H}$ & 6.847557 & 3.493333 & -3.238119 \\
\hline C & 5.049642 & 4.131927 & -1.237339 \\
\hline $\mathrm{H}$ & 5.062713 & 5.219782 & -1.373579 \\
\hline $\mathrm{H}$ & 4.559027 & 3.694607 & -2.114524 \\
\hline $\mathrm{H}$ & 4.431406 & 3.909315 & -0.361531 \\
\hline C & 10.459095 & 0.326323 & -0.557022 \\
\hline $\mathrm{H}$ & 11.400278 & -0.227673 & -0.464754 \\
\hline $\mathrm{H}$ & 10.458162 & 0.815650 & -1.537671 \\
\hline $\mathrm{H}$ & 10.457802 & 1.104857 & 0.214185 \\
\hline C & 9.372629 & -1.300600 & 0.997663 \\
\hline $\mathrm{H}$ & 10.333372 & -1.819244 & 1.106669 \\
\hline $\mathrm{H}$ & 9.300416 & -0.548377 & 1.791546 \\
\hline $\mathrm{H}$ & 8.576954 & -2.034937 & 1.161054 \\
\hline C & 9.392882 & -1.731112 & -1.489010 \\
\hline $\mathrm{H}$ & 10.354223 & -2.252710 & -1.402571 \\
\hline $\mathrm{H}$ & 8.599420 & -2.481172 & -1.407151 \\
\hline $\mathrm{H}$ & 9.333897 & -1.290637 & -2.490945 \\
\hline C & -1.924413 & 1.275033 & 1.839120 \\
\hline C & -3.262804 & 1.321493 & 2.252601 \\
\hline C & -1.356505 & 2.416033 & 1.255018 \\
\hline C & -4.042072 & 2.472996 & 2.073834 \\
\hline $\mathrm{H}$ & -3.694435 & 0.441542 & 2.720251 \\
\hline
\end{tabular}




\begin{tabular}{|c|c|c|c|}
\hline C & -2.098398 & 3.584895 & 1.054850 \\
\hline $\mathrm{H}$ & -0.306514 & 2.395333 & 0.988699 \\
\hline C & -3.438692 & 3.586186 & 1.467394 \\
\hline $\mathrm{H}$ & -4.025641 & 4.483242 & 1.325988 \\
\hline C & -1.418515 & 4.802680 & 0.399458 \\
\hline C & -5.507973 & 2.491651 & 2.553904 \\
\hline C & -1.040127 & 4.443356 & -1.058201 \\
\hline $\mathrm{H}$ & -0.393620 & 3.561642 & -1.108375 \\
\hline $\mathrm{H}$ & -1.937381 & 4.233680 & -1.654151 \\
\hline $\mathrm{H}$ & -0.511252 & 5.279498 & -1.532167 \\
\hline C & -2.331946 & 6.042603 & 0.371959 \\
\hline $\mathrm{H}$ & -1.799417 & 6.881238 & -0.090379 \\
\hline $\mathrm{H}$ & -3.242001 & 5.870243 & -0.214630 \\
\hline $\mathrm{H}$ & -2.628805 & 6.353779 & 1.380362 \\
\hline C & -0.134174 & 5.170445 & 1.181749 \\
\hline $\mathrm{H}$ & -0.365547 & 5.406217 & 2.227004 \\
\hline $\mathrm{H}$ & 0.603932 & 4.362364 & 1.173070 \\
\hline $\mathrm{H}$ & 0.339047 & 6.051947 & 0.732411 \\
\hline C & -6.304394 & 1.366587 & 1.852502 \\
\hline $\mathrm{H}$ & -6.295532 & 1.498136 & 0.764721 \\
\hline $\mathrm{H}$ & -5.895475 & 0.375915 & 2.073324 \\
\hline $\mathrm{H}$ & -7.349023 & 1.376137 & 2.187115 \\
\hline C & -5.545866 & 2.266688 & 4.084556 \\
\hline $\mathrm{H}$ & -5.100143 & 1.306456 & 4.364956 \\
\hline $\mathrm{H}$ & -4.998166 & 3.056331 & 4.611631 \\
\hline $\mathrm{H}$ & -6.581619 & 2.271328 & 4.445679 \\
\hline C & -6.211720 & 3.828193 & 2.249103 \\
\hline $\mathrm{H}$ & -7.246593 & 3.786913 & 2.606947 \\
\hline $\mathrm{H}$ & -5.724477 & 4.673198 & 2.748644 \\
\hline $\mathrm{H}$ & -6.242756 & 4.034545 & 1.173180 \\
\hline
\end{tabular}

\section{C-Catalyzed reaction TS 38}

\section{Datum}

Value

\begin{tabular}{lc}
\hline B3LYP/6-31G(d) Energy & -3038.959212 \\
\hline B3LYP-D3(BJ)/def2-TZVPP-IEF-PCM(DCM) Energy & -3040.513056 \\
\hline B3LYP-D3(BJ)/def2-TZVPP-IEF-PCM(DCM)//B3LYP/6-31G(d) Free Energy (Quasiharmonic) & -3039.284616 \\
\hline
\end{tabular}

Number of Imaginary Frequencies

Frequencies (Top 3 out of 450)

$\begin{array}{lr}\text { 1. } & -288.9657 \mathrm{~cm}^{-1} \\ \text { 2. } & 7.5314 \mathrm{~cm}^{-1} \\ \text { 3. } & 9.3607 \mathrm{~cm}^{-1}\end{array}$




\begin{tabular}{|c|c|c|c|}
\hline C & -0.763134 & 0.948540 & 0.659736 \\
\hline $\mathrm{H}$ & -0.271135 & 0.786696 & 1.626227 \\
\hline $\mathrm{H}$ & -1.809133 & 0.651671 & 0.763052 \\
\hline C & 2.687504 & 1.795204 & -1.278003 \\
\hline 0 & 3.574604 & 2.403399 & -1.835149 \\
\hline 0 & 1.675114 & 1.242124 & -1.954793 \\
\hline H & 1.021262 & 0.808032 & -1.338441 \\
\hline 0 & -0.141988 & 0.129503 & -0.343968 \\
\hline $\mathrm{H}$ & 0.005040 & -0.771403 & 0.035137 \\
\hline C & -3.394597 & -2.364986 & -2.311277 \\
\hline C & -3.589511 & -2.276130 & -0.107565 \\
\hline C & -1.109732 & -3.085184 & -1.729617 \\
\hline C & -2.432408 & -3.342376 & -2.224414 \\
\hline $\mathrm{H}$ & -4.016852 & -1.279631 & -0.246339 \\
\hline $\mathrm{H}$ & -0.377386 & -3.855788 & -1.979338 \\
\hline $\mathrm{H}$ & -0.740383 & -2.084175 & -1.966403 \\
\hline C & 0.509708 & -3.164517 & 1.672212 \\
\hline C & 0.052381 & -4.636039 & 1.345182 \\
\hline C & 2.019653 & -2.960623 & 1.788324 \\
\hline $\mathrm{H}$ & 2.240692 & -1.907323 & 1.990872 \\
\hline $\mathrm{H}$ & 2.431114 & -3.553701 & 2.613529 \\
\hline $\mathrm{H}$ & 2.537980 & -3.238016 & 0.867758 \\
\hline C & -0.193713 & -2.580082 & 2.907582 \\
\hline $\mathrm{H}$ & 0.023015 & -1.508472 & 2.970642 \\
\hline $\mathrm{H}$ & -1.278682 & -2.704517 & 2.845805 \\
\hline $\mathrm{H}$ & 0.159549 & -3.048575 & 3.832537 \\
\hline C & -0.270508 & -5.493051 & 2.570797 \\
\hline $\mathrm{H}$ & 0.610351 & -5.608940 & 3.213009 \\
\hline $\mathrm{H}$ & -1.078852 & -5.057983 & 3.163413 \\
\hline $\mathrm{H}$ & -0.585419 & -6.491822 & 2.249474 \\
\hline C & 1.047382 & -5.384518 & 0.443101 \\
\hline $\mathrm{H}$ & 1.958115 & -5.664352 & 0.983807 \\
\hline $\mathrm{H}$ & 0.570924 & -6.299850 & 0.077146 \\
\hline $\mathrm{H}$ & 1.335214 & -4.781085 & -0.423030 \\
\hline 0 & 0.037503 & -2.454808 & 0.501877 \\
\hline 0 & -1.162012 & -4.430867 & 0.594951 \\
\hline B & -1.137219 & -3.127333 & 0.009200 \\
\hline 0 & -2.371081 & -2.262608 & 0.319045 \\
\hline C & -4.592030 & -3.322259 & 0.199746 \\
\hline C & -4.262634 & -4.567913 & 0.758169 \\
\hline C & -5.942771 & -3.009822 & -0.038324 \\
\hline C & -5.273521 & -5.480250 & 1.057316 \\
\hline $\mathrm{H}$ & -3.221831 & -4.809327 & 0.948205 \\
\hline C & -6.946726 & -3.925469 & 0.263480 \\
\hline $\mathrm{H}$ & -6.200570 & -2.043003 & -0.465975 \\
\hline C & -6.612689 & -5.166511 & 0.812146 \\
\hline $\mathrm{H}$ & -5.013363 & -6.442579 & 1.489964 \\
\hline $\mathrm{H}$ & -7.986365 & -3.672072 & 0.075410 \\
\hline $\mathrm{H}$ & -7.393603 & -5.883239 & 1.051545 \\
\hline H & -2.747895 & -4.381923 & -2.320973 \\
\hline
\end{tabular}




\begin{tabular}{|c|c|c|c|}
\hline H & -3.098680 & -1.320903 & -2.375444 \\
\hline $\mathrm{H}$ & -4.403922 & -2.602407 & -2.632721 \\
\hline C & 1.752799 & 2.316001 & 1.001438 \\
\hline C & 2.690442 & 1.638666 & 0.233686 \\
\hline C & 3.770748 & 0.917515 & 0.846052 \\
\hline C & 3.882128 & 0.938802 & 2.222266 \\
\hline H & 4.676987 & 0.371396 & 2.699903 \\
\hline C & -0.642478 & 2.403729 & 0.253540 \\
\hline C & 0.596962 & 3.034534 & 0.358439 \\
\hline C & -1.570708 & 4.387611 & -0.797696 \\
\hline C & -1.753366 & 3.101702 & -0.324262 \\
\hline H & -2.415914 & 4.920648 & -1.226354 \\
\hline C & -0.324716 & 5.051147 & -0.715107 \\
\hline C & 0.784082 & 4.371334 & -0.118000 \\
\hline C & 2.029123 & 5.055415 & -0.029791 \\
\hline C & 2.162645 & 6.341021 & -0.502370 \\
\hline C & 1.062014 & 7.012377 & -1.088195 \\
\hline C & -0.154066 & 6.379053 & -1.192345 \\
\hline $\mathrm{H}$ & 2.884341 & 4.544867 & 0.398350 \\
\hline $\mathrm{H}$ & 3.123412 & 6.843416 & -0.432895 \\
\hline $\mathrm{H}$ & 1.183960 & 8.026659 & -1.458782 \\
\hline H & -1.005406 & 6.883928 & -1.642937 \\
\hline C & 2.978254 & 1.658754 & 3.041674 \\
\hline C & 1.888581 & 2.357036 & 2.430431 \\
\hline C & 0.989973 & 3.071947 & 3.270046 \\
\hline C & 1.156786 & 3.088513 & 4.637748 \\
\hline C & 2.230149 & 2.389646 & 5.239250 \\
\hline C & 3.119255 & 1.690755 & 4.455256 \\
\hline $\mathrm{H}$ & 0.167587 & 3.615876 & 2.817478 \\
\hline $\mathrm{H}$ & 0.460922 & 3.644914 & 5.260031 \\
\hline $\mathrm{H}$ & 2.351116 & 2.409696 & 6.319011 \\
\hline $\mathrm{H}$ & 3.949588 & 1.152550 & 4.906558 \\
\hline C & -5.755953 & 1.607816 & -0.601250 \\
\hline C & -5.067053 & 1.934158 & -1.784836 \\
\hline C & -3.749064 & 2.384809 & -1.669144 \\
\hline C & -3.127964 & 2.518948 & -0.416116 \\
\hline C & -3.850598 & 2.190187 & 0.734232 \\
\hline C & -5.176246 & 1.730578 & 0.666849 \\
\hline $\mathrm{H}$ & -6.782915 & 1.269729 & -0.678777 \\
\hline $\mathrm{H}$ & -3.174494 & 2.646263 & -2.550170 \\
\hline $\mathrm{H}$ & -3.375237 & 2.328401 & 1.701195 \\
\hline C & -5.790132 & 1.825032 & -3.142394 \\
\hline C & -5.946000 & 1.435356 & 1.970099 \\
\hline C & -6.308911 & 0.383647 & -3.354669 \\
\hline $\mathrm{H}$ & -6.843961 & 0.308158 & -4.309076 \\
\hline $\mathrm{H}$ & -5.478806 & -0.331767 & -3.378084 \\
\hline $\mathrm{H}$ & -7.000674 & 0.074749 & -2.563581 \\
\hline C & -6.989540 & 2.803493 & -3.156211 \\
\hline $\mathrm{H}$ & -7.518600 & 2.745381 & -4.115339 \\
\hline H & -7.709490 & 2.576623 & -2.362671 \\
\hline $\mathrm{H}$ & -6.652238 & 3.836638 & -3.015931 \\
\hline C & -4.871066 & 2.177024 & -4.328081 \\
\hline $\mathrm{H}$ & -5.425948 & 2.073815 & -5.267448 \\
\hline H & -4.508734 & 3.209384 & -4.272548 \\
\hline
\end{tabular}




\begin{tabular}{|c|c|c|c|}
\hline H & -4.000772 & 1.513279 & -4.383133 \\
\hline $\mathrm{C}$ & -5.183238 & 0.383849 & 2.810063 \\
\hline $\mathrm{H}$ & -5.713291 & 0.193817 & 3.751262 \\
\hline $\mathrm{H}$ & -5.102051 & -0.568333 & 2.273282 \\
\hline $\mathrm{H}$ & -4.170387 & 0.713821 & 3.062128 \\
\hline C & -6.072684 & 2.744299 & 2.786835 \\
\hline H & -6.616513 & 2.560314 & 3.721509 \\
\hline $\mathrm{H}$ & -5.092495 & 3.157713 & 3.046010 \\
\hline $\mathrm{H}$ & -6.617646 & 3.508109 & 2.220524 \\
\hline C & -7.365293 & 0.895898 & 1.709657 \\
\hline $\mathrm{H}$ & -7.347699 & -0.043262 & 1.144302 \\
\hline $\mathrm{H}$ & -7.984396 & 1.615531 & 1.162241 \\
\hline $\mathrm{H}$ & -7.862988 & 0.694376 & 2.664866 \\
\hline C & 4.728748 & 0.107001 & 0.044286 \\
\hline C & 4.263095 & -0.761078 & -0.952126 \\
\hline C & 6.103488 & 0.168573 & 0.308493 \\
\hline C & 5.141925 & -1.550506 & -1.699153 \\
\hline H & 3.197992 & -0.811996 & -1.138331 \\
\hline C & 7.016982 & -0.612384 & -0.408458 \\
\hline $\mathrm{H}$ & 6.449958 & 0.861156 & 1.066758 \\
\hline C & 6.509511 & -1.459212 & -1.404315 \\
\hline $\mathrm{H}$ & 7.205960 & -2.064594 & -1.977140 \\
\hline C & 8.536255 & -0.556464 & -0.154425 \\
\hline C & 4.654560 & -2.486603 & -2.821433 \\
\hline C & 8.905103 & 0.405383 & 0.991187 \\
\hline H & 9.991008 & 0.403013 & 1.139776 \\
\hline $\mathrm{H}$ & 8.604025 & 1.435694 & 0.771435 \\
\hline $\mathrm{H}$ & 8.441121 & 0.107921 & 1.938680 \\
\hline C & 9.051885 & -1.966305 & 0.220123 \\
\hline $\mathrm{H}$ & 10.134157 & -1.941672 & 0.398069 \\
\hline $\mathrm{H}$ & 8.565697 & -2.331993 & 1.132002 \\
\hline $\mathrm{H}$ & 8.862090 & -2.695907 & -0.574005 \\
\hline C & 9.254449 & -0.072247 & -1.436612 \\
\hline H & 9.072961 & -0.743294 & -2.282689 \\
\hline $\mathrm{H}$ & 8.909076 & 0.927043 & -1.724308 \\
\hline $\mathrm{H}$ & 10.338583 & -0.025353 & -1.273892 \\
\hline C & 5.292390 & -2.048358 & -4.161611 \\
\hline $\mathrm{H}$ & 5.004645 & -1.021500 & -4.412423 \\
\hline $\mathrm{H}$ & 6.386232 & -2.089664 & -4.125368 \\
\hline $\mathrm{H}$ & 4.960200 & -2.705493 & -4.975076 \\
\hline C & 5.072020 & -3.941986 & -2.505741 \\
\hline $\mathrm{H}$ & 6.158624 & -4.045990 & -2.416192 \\
\hline $\mathrm{H}$ & 4.625433 & -4.282421 & -1.564058 \\
\hline $\mathrm{H}$ & 4.737705 & -4.617165 & -3.303310 \\
\hline C & 3.122783 & -2.450007 & -2.989230 \\
\hline $\mathrm{H}$ & 2.827117 & -3.120253 & -3.804749 \\
\hline $\mathrm{H}$ & 2.604661 & -2.784179 & -2.082142 \\
\hline $\mathrm{H}$ & 2.761019 & -1.445513 & -3.234553 \\
\hline
\end{tabular}


Frequencies (Top 3 out of 450)

$\begin{array}{rr}\text { 1. } & -321.4324 \mathrm{~cm}^{-1} \\ \text { 2. } & 4.3345 \mathrm{~cm}^{-1} \\ \text { 3. } & 7.7036 \mathrm{~cm}^{-1}\end{array}$

\section{B3LYP/6-31G(d) Molecular Geometry in Cartesian Coordinates}

C

$\mathrm{H}$

$\mathrm{H}$

C

0

0

$\mathrm{H}$

0

$\mathrm{H}$

C

C

C

C

$\mathrm{H}$

$\mathrm{H}$

$\mathrm{H}$

C

C

C

$\mathrm{H}$

$\mathrm{H}$

$\mathrm{H}$

C

$\mathrm{H}$

$\mathrm{H}$

$\mathrm{H}$

C

$\mathrm{H}$

$\mathrm{H}$

$\mathrm{H}$

C

$\mathrm{H}$
2.939122

3. 972415

2. 319238

$-0.483491$

0.387833

$-1.525316$

$-2.192627$

2.586785

1.802471

$-3.841443$

$-3.409394$

$-4.443859$

$-4.779836$

$-4.389340$

$-3.491132$

$-5.246272$

$-4.503738$

$-5.863128$

$-4.638892$

$-3.648360$

$-5.217903$

$-5.127702$

$-3.665655$

$-4.118748$

$-3.539539$

$-2.673524$

$-6.505935$

$-6.757553$

$-5.846967$

$-7.432318$

$-6.897656$

$-7.325491$
$-1.693304$

$-1.998930$

$-2.148645$

$-0.456420$

$-0.677374$

0.301675

0.466803

$-2.218091$

$-1.724126$

2. 840892

3.939416

1.073603

2.046567

4.419305

0.564040

0.373963

1.658568

2. 188401

0.492266

0.171151

0.786658

$-0.364503$

2. 775886

3.139142

3. 620695

2. 383398

3. 335974

3.030244

4.206224

3. 642422

1.069572

0.718771
0.890694

1.072929

1.673281

$-0.382357$

$-1.208880$

$-0.720430$

$-0.005350$

$-0.388040$

$-0.709125$

$-3.693062$

$-1.849916$

$-2.069897$

$-3.069457$

$-1.816523$

$-2.222732$

$-1.828450$

1.676671

1.077453

2.653510

2.992899

3.536712

2. 185522

2. 317325

3. 246014

1.634702

2.559164

1.859986

2.882493

1.906591

1.362530

0.865809

1.811637 


\begin{tabular}{|c|c|c|c|}
\hline $\mathrm{H}$ & -7.711858 & 1.459092 & 0.246072 \\
\hline $\mathrm{H}$ & -6.455448 & 0.213379 & 0.347158 \\
\hline 0 & -3.818135 & 1.190739 & 0.487107 \\
\hline 0 & -5.471976 & 2.681831 & -0.214046 \\
\hline B & -4.267077 & 2.016837 & -0.609635 \\
\hline 0 & -3.178415 & 2.990190 & -1.002363 \\
\hline C & -2.267975 & 4.754720 & -2.302499 \\
\hline C & -2.501424 & 5.977162 & -2.950729 \\
\hline C & -0.949819 & 4.335773 & -2.058805 \\
\hline C & -1.430987 & 6.771075 & -3.355427 \\
\hline $\mathrm{H}$ & -3.523090 & 6.303460 & -3.133008 \\
\hline C & 0.116354 & 5.135121 & -2.462241 \\
\hline $\mathrm{H}$ & -0.778932 & 3.393961 & -1.547644 \\
\hline C & -0.120637 & 6.350295 & -3.112300 \\
\hline $\mathrm{H}$ & -1.616808 & 7.717634 & -3.855284 \\
\hline $\mathrm{H}$ & 1.133479 & 4.811533 & -2.261780 \\
\hline $\mathrm{H}$ & 0.714677 & 6.970488 & -3.426125 \\
\hline $\mathrm{H}$ & -5.827717 & 2.338734 & -3.149754 \\
\hline $\mathrm{H}$ & -2.813843 & 2.499546 & -3.777548 \\
\hline $\mathrm{H}$ & -4.145045 & 3.611852 & -4.396069 \\
\hline C & 0.517875 & -0.477204 & 1.901892 \\
\hline C & -0.450813 & -0.971940 & 1.035837 \\
\hline C & -1.431410 & -1.931286 & 1.466663 \\
\hline C & -1.468994 & -2.265881 & 2.806000 \\
\hline $\mathrm{H}$ & -2.177869 & -3.016027 & 3.146440 \\
\hline C & 2.824580 & -0.177393 & 0.962304 \\
\hline C & 1.639001 & 0.403536 & 1.411406 \\
\hline C & 3.770623 & 2.033706 & 0.603082 \\
\hline C & 3.918874 & 0.659853 & 0.547169 \\
\hline $\mathrm{H}$ & 4.591727 & 2.668501 & 0.279685 \\
\hline C & 2.575343 & 2.650618 & 1.037822 \\
\hline C & 1.483695 & 1.826312 & 1.455891 \\
\hline C & 0.290476 & 2.462179 & 1.895983 \\
\hline C & 0.179463 & 3.834693 & 1.913534 \\
\hline C & 1.260540 & 4.647325 & 1.494890 \\
\hline C & 2.431879 & 4.064742 & 1.068574 \\
\hline $\mathrm{H}$ & -0.543062 & 1.847428 & 2.218237 \\
\hline $\mathrm{H}$ & -0.744778 & 4.297199 & 2.248178 \\
\hline $\mathrm{H}$ & 1.161462 & 5.729281 & 1.512889 \\
\hline $\mathrm{H}$ & 3.270972 & 4.678739 & 0.749052 \\
\hline C & -0.568439 & -1.710169 & 3.748021 \\
\hline C & 0.459261 & -0.822988 & 3.293962 \\
\hline C & 1.385613 & -0.316045 & 4.246558 \\
\hline C & 1.292421 & -0.660051 & 5.577105 \\
\hline C & 0.273730 & -1.535007 & 6.023533 \\
\hline C & -0.633220 & -2.050308 & 5.126263 \\
\hline $\mathrm{H}$ & 2.170839 & 0.352767 & 3.911876 \\
\hline $\mathrm{H}$ & 2.007679 & -0.258965 & 6.289893 \\
\hline $\mathrm{H}$ & 0.212804 & -1.800856 & 7.075376 \\
\hline $\mathrm{H}$ & -1.414876 & -2.729447 & 5.458205 \\
\hline C & 7.709485 & -0.785697 & -0.818910 \\
\hline C & 7.672274 & 0.191277 & 0.193583 \\
\hline C & 6.421274 & 0.629422 & 0.630150 \\
\hline C & 5.230706 & 0.117676 & 0.080694 \\
\hline
\end{tabular}




\begin{tabular}{|c|c|c|c|}
\hline C & 5.311896 & -0.849570 & -0.924188 \\
\hline C & 6.550619 & -1.321132 & -1.387136 \\
\hline $\mathrm{H}$ & 8.676068 & -1.136577 & -1.162278 \\
\hline $\mathrm{H}$ & 6.343449 & 1.364717 & 1.422780 \\
\hline $\mathrm{H}$ & 4.389787 & -1.246340 & -1.332892 \\
\hline C & 8.992989 & 0.726633 & 0.781192 \\
\hline C & 6.575396 & -2.401286 & -2.485785 \\
\hline C & 9.832242 & 1.376339 & -0.344798 \\
\hline $\mathrm{H}$ & 10.782417 & 1.753037 & 0.054188 \\
\hline $\mathrm{H}$ & 10.064855 & 0.663483 & -1.142427 \\
\hline $\mathrm{H}$ & 9.295257 & 2.218343 & -0.796627 \\
\hline C & 8.764365 & 1.786207 & 1.876062 \\
\hline $\mathrm{H}$ & 9.730355 & 2.136825 & 2.257211 \\
\hline $\mathrm{H}$ & 8.223790 & 2.659117 & 1.492532 \\
\hline $\mathrm{H}$ & 8.201274 & 1.381498 & 2.724663 \\
\hline C & 9.792950 & -0.441146 & 1.405975 \\
\hline $\mathrm{H}$ & 10.740862 & -0.077170 & 1.821815 \\
\hline $\mathrm{H}$ & 9.225660 & -0.914321 & 2.215664 \\
\hline $\mathrm{H}$ & 10.027852 & -1.214771 & 0.667882 \\
\hline C & 5.869355 & -1.861978 & -3.752539 \\
\hline $\mathrm{H}$ & 5.871642 & -2.623058 & -4.542844 \\
\hline $\mathrm{H}$ & 4.827890 & -1.591805 & -3.552309 \\
\hline $\mathrm{H}$ & 6.380205 & -0.971304 & -4.137150 \\
\hline C & 8.006835 & -2.814965 & -2.874688 \\
\hline $\mathrm{H}$ & 7.968817 & -3.586917 & -3.651800 \\
\hline $\mathrm{H}$ & 8.556779 & -3.231522 & -2.022862 \\
\hline $\mathrm{H}$ & 8.582323 & -1.971810 & -3.274722 \\
\hline C & 5.826418 & -3.658517 & -1.979954 \\
\hline $\mathrm{H}$ & 5.810259 & -4.429952 & -2.760407 \\
\hline $\mathrm{H}$ & 6.326745 & -4.080284 & -1.099930 \\
\hline $\mathrm{H}$ & 4.791592 & -3.435589 & -1.701726 \\
\hline C & -2.308296 & -2.647451 & 0.496727 \\
\hline C & -3.656259 & -2.914364 & 0.797758 \\
\hline C & -1.771218 & -3.142411 & -0.695407 \\
\hline C & -4.460035 & -3.653112 & -0.072041 \\
\hline $\mathrm{H}$ & -4.063507 & -2.522816 & 1.722255 \\
\hline C & -2.550407 & -3.869113 & -1.608242 \\
\hline $\mathrm{H}$ & -0.720747 & -2.977942 & -0.904845 \\
\hline C & -3.885129 & -4.108953 & -1.273690 \\
\hline $\mathrm{H}$ & -4.505394 & -4.672907 & -1.960601 \\
\hline C & -1.909292 & -4.361869 & -2.919275 \\
\hline C & -5.935179 & -3.982586 & 0.230375 \\
\hline C & -1.444279 & -3.137057 & -3.744429 \\
\hline $\mathrm{H}$ & -2.296912 & -2.502030 & -4.012702 \\
\hline $\mathrm{H}$ & -0.729028 & -2.517470 & -3.194405 \\
\hline $\mathrm{H}$ & -0.961599 & -3.466505 & -4.672945 \\
\hline C & -0.688138 & -5.256326 & -2.597437 \\
\hline $\mathrm{H}$ & -0.226162 & -5.613098 & -3.525981 \\
\hline $\mathrm{H}$ & 0.079894 & -4.717753 & -2.033423 \\
\hline $\mathrm{H}$ & -0.986642 & -6.131553 & -2.008637 \\
\hline C & -2.888095 & -5.181218 & -3.781156 \\
\hline $\mathrm{H}$ & -3.239597 & -6.078904 & -3.259060 \\
\hline $\mathrm{H}$ & -3.763362 & -4.592453 & -4.079316 \\
\hline $\mathrm{H}$ & -2.385283 & -5.508159 & -4.698249 \\
\hline
\end{tabular}




\begin{tabular}{|lllr|} 
C & -6.839754 & -3.353493 & -0.855659 \\
$H$ & -6.736214 & -2.262113 & -0.866218 \\
$H$ & -6.591449 & -3.721559 & -1.856504 \\
$H$ & -7.892974 & -3.592761 & -0.662831 \\
C & -6.130460 & -5.518027 & 0.232604 \\
$H$ & -5.869466 & -5.966241 & -0.731320 \\
$H$ & -5.506451 & -5.990644 & 0.999753 \\
$H$ & -7.177422 & -5.768494 & 0.443584 \\
C & -6.391001 & -3.448193 & 1.601183 \\
H & -7.436487 & -3.726318 & 1.775891 \\
H & -5.796002 & -3.867142 & 2.420749 \\
$H$ & -6.329079 & -2.355688 & 1.655995 \\
\hline
\end{tabular}

\section{C-Catalyzed reaction TS 40}

\section{Datum}

Value

\begin{tabular}{lc}
\hline B3LYP/6-31G(d) Energy & -3038.966899 \\
\hline B3LYP-D3(BJ)/def2-TZVPP-IEF-PCM(DCM) Energy & -3040.514055 \\
\hline B3LYP-D3(BJ)/def2-TZVPP-IEF-PCM(DCM)//B3LYP/6-31G(d) Free Energy (Quasiharmonic) & -3039.284016 \\
\hline
\end{tabular}

Number of Imaginary Frequencies

Frequencies (Top 3 out of 450)

$$
\begin{array}{rr}
\text { 1. } & -322.6821 \mathrm{~cm}^{-1} \\
\text { 2. } & 7.7690 \mathrm{~cm}^{-1} \\
\text { 3. } & 11.8749 \mathrm{~cm}^{-1}
\end{array}
$$

\section{B3LYP/6-31G(d) Molecular Geometry in Cartesian Coordinates}

$\begin{array}{lrrr}\mathrm{C} & -3.138540 & 0.824790 & -1.470530 \\ \mathrm{H} & -2.572220 & 0.772300 & -2.409030 \\ \mathrm{H} & -4.185120 & 1.002610 & -1.730090 \\ \mathrm{C} & 0.372210 & 0.367220 & 0.025370 \\ \mathrm{O} & -0.396780 & 1.115750 & 0.609640 \\ \mathrm{O} & 1.387000 & -0.170230 & 0.696560 \\ \mathrm{H} & 1.929250 & -0.842090 & 0.208390 \\ \mathrm{O} & -2.724230 & 1.952420 & -0.704160 \\ \mathrm{H} & -1.882580 & 1.720380 & -0.257660 \\ \mathrm{C} & 2.863220 & -2.418060 & 4.719710 \\ \mathrm{C} & 4.157660 & -1.181210 & 3.475170 \\ \mathrm{C} & 2.309020 & -3.410720 & 2.523100 \\ \mathrm{C} & 2.900310 & -3.469240 & 3.828050 \\ \mathrm{H} & 5.040380 & -1.798810 & 3.652540\end{array}$




\begin{tabular}{|c|c|c|c|}
\hline $\mathrm{H}$ & 1.390980 & -2.824190 & 2.451520 \\
\hline $\mathrm{H}$ & 2.209970 & -4.375300 & 2.023200 \\
\hline C & 3.954200 & -2.985100 & -0.690630 \\
\hline C & 5.260250 & -3.143130 & 0.178530 \\
\hline C & 3.320120 & -4.336450 & -1.055640 \\
\hline H & 2.326500 & -4.165240 & -1.481710 \\
\hline $\mathrm{H}$ & 3.917290 & -4.875500 & -1.799050 \\
\hline $\mathrm{H}$ & 3.212130 & -4.974710 & -0.173730 \\
\hline C & 4.122900 & -2.139770 & -1.951370 \\
\hline $\mathrm{H}$ & 4.858880 & -2.591100 & -2.627510 \\
\hline $\mathrm{H}$ & 3.171110 & -2.072290 & -2.489160 \\
\hline $\mathrm{H}$ & 4.444910 & -1.125030 & -1.709200 \\
\hline C & 6.144400 & -1.883560 & 0.155590 \\
\hline $\mathrm{H}$ & 6.653330 & -1.758790 & -0.806740 \\
\hline $\mathrm{H}$ & 5.559870 & -0.981420 & 0.359180 \\
\hline $\mathrm{H}$ & 6.908820 & -1.977470 & 0.934180 \\
\hline C & 6.108130 & -4.372280 & -0.152510 \\
\hline $\mathrm{H}$ & 6.472080 & -4.337810 & -1.186150 \\
\hline $\mathrm{H}$ & 6.979460 & -4.405860 & 0.510350 \\
\hline $\mathrm{H}$ & 5.544340 & -5.297270 & -0.010410 \\
\hline 0 & 3.058810 & -2.296980 & 0.218130 \\
\hline 0 & 4.725970 & -3.307000 & 1.504590 \\
\hline B & 3.502850 & -2.568240 & 1.570380 \\
\hline 0 & 3.603970 & -1.253110 & 2.308130 \\
\hline C & 4.056540 & 0.091230 & 4.212700 \\
\hline C & 4.934880 & 0.344010 & 5.277190 \\
\hline C & 3.104210 & 1.058100 & 3.848270 \\
\hline C & 4.863860 & 1.547960 & 5.974530 \\
\hline $\mathrm{H}$ & 5.675180 & -0.403820 & 5.553430 \\
\hline C & 3.036070 & 2.257520 & 4.552540 \\
\hline $\mathrm{H}$ & 2.435730 & 0.860770 & 3.015380 \\
\hline C & 3.912120 & 2.505120 & 5.614460 \\
\hline $\mathrm{H}$ & 5.549350 & 1.740430 & 6.795130 \\
\hline $\mathrm{H}$ & 2.298050 & 3.003050 & 4.270880 \\
\hline $\mathrm{H}$ & 3.853820 & 3.443660 & 6.159060 \\
\hline $\mathrm{H}$ & 3.616500 & -4.270720 & 4.013100 \\
\hline $\mathrm{H}$ & 2.053650 & -1.695900 & 4.671400 \\
\hline $\mathrm{H}$ & 3.387750 & -2.477210 & 5.669480 \\
\hline C & -0.785830 & -0.826440 & -1.839530 \\
\hline C & 0.245890 & 0.014890 & -1.438440 \\
\hline C & 1.193620 & 0.548550 & -2.380070 \\
\hline C & 1.118960 & 0.119010 & -3.690870 \\
\hline $\mathrm{H}$ & 1.801260 & 0.537510 & -4.425870 \\
\hline C & -3.011000 & -0.496730 & -0.724930 \\
\hline C & -1.861910 & -1.271700 & -0.882120 \\
\hline C & -3.932580 & -2.173970 & 0.775310 \\
\hline C & -4.071770 & -0.957450 & 0.132560 \\
\hline $\mathrm{H}$ & -4.729180 & -2.522310 & 1.427770 \\
\hline C & -2.781440 & -2.981870 & 0.632530 \\
\hline C & -1.716450 & -2.527470 & -0.207430 \\
\hline C & -0.568470 & -3.355380 & -0.346350 \\
\hline C & -0.488630 & -4.571110 & 0.296410 \\
\hline C & -1.545550 & -5.018890 & 1.124680 \\
\hline C & -2.663630 & -4.235530 & 1.292000 \\
\hline
\end{tabular}




\begin{tabular}{|c|c|c|c|}
\hline H & 0.257570 & -3.016350 & -0.961930 \\
\hline $\mathrm{H}$ & 0.394940 & -5.189950 & 0.168030 \\
\hline $\mathrm{H}$ & -1.468820 & -5.978910 & 1.628160 \\
\hline $\mathrm{H}$ & -3.480700 & -4.565710 & 1.929330 \\
\hline C & 0.143490 & -0.808840 & -4.129110 \\
\hline C & -0.840110 & -1.277990 & -3.201660 \\
\hline C & -1.838560 & -2.175350 & -3.671630 \\
\hline C & -1.855130 & -2.591220 & -4.984720 \\
\hline C & -0.879980 & -2.126340 & -5.898970 \\
\hline C & 0.095070 & -1.251650 & -5.478620 \\
\hline $\mathrm{H}$ & -2.590680 & -2.535490 & -2.978710 \\
\hline H & -2.624250 & -3.280200 & -5.322810 \\
\hline $\mathrm{H}$ & -0.906220 & -2.459960 & -6.932780 \\
\hline $\mathrm{H}$ & 0.845030 & -0.883040 & -6.174400 \\
\hline C & -7.751690 & 1.132320 & 0.782140 \\
\hline C & -6.554820 & 1.843410 & 0.900010 \\
\hline C & -5.353440 & 1.151990 & 0.676750 \\
\hline C & -5.345470 & -0.202430 & 0.333670 \\
\hline C & -6.573540 & -0.881610 & 0.219320 \\
\hline C & -7.788810 & -0.232550 & 0.441280 \\
\hline $\mathrm{H}$ & -8.690300 & 1.647430 & 0.951200 \\
\hline H & -4.404800 & 1.671040 & 0.748200 \\
\hline $\mathrm{H}$ & -6.554970 & -1.926610 & -0.068220 \\
\hline C & -6.500380 & 3.343140 & 1.248970 \\
\hline C & -9.148740 & -0.946710 & 0.314510 \\
\hline C & -5.811170 & 4.107300 & 0.092020 \\
\hline $\mathrm{H}$ & -5.739560 & 5.175840 & 0.332190 \\
\hline H & -4.800720 & 3.733670 & -0.101550 \\
\hline $\mathrm{H}$ & -6.388410 & 4.008140 & -0.835110 \\
\hline C & -7.899060 & 3.950670 & 1.465050 \\
\hline $\mathrm{H}$ & -7.805020 & 5.015560 & 1.706910 \\
\hline $\mathrm{H}$ & -8.522230 & 3.870990 & 0.566800 \\
\hline $\mathrm{H}$ & -8.430990 & 3.469970 & 2.294620 \\
\hline C & -5.684960 & 3.541150 & 2.548880 \\
\hline $\mathrm{H}$ & -5.634020 & 4.606320 & 2.807430 \\
\hline $\mathrm{H}$ & -6.149010 & 3.009900 & 3.388300 \\
\hline $\mathrm{H}$ & -4.659310 & 3.173980 & 2.444630 \\
\hline C & -9.901990 & -0.866560 & 1.663710 \\
\hline $\mathrm{H}$ & -10.876070 & -1.366260 & 1.588900 \\
\hline $\mathrm{H}$ & -9.329570 & -1.354990 & 2.460710 \\
\hline $\mathrm{H}$ & -10.082040 & 0.169160 & 1.969730 \\
\hline C & -9.994210 & -0.259430 & -0.784220 \\
\hline $\mathrm{H}$ & -10.968060 & -0.754970 & -0.884250 \\
\hline $\mathrm{H}$ & -10.178960 & 0.795510 & -0.556480 \\
\hline $\mathrm{H}$ & -9.487000 & -0.307040 & -1.754670 \\
\hline C & -9.001020 & -2.433910 & -0.059630 \\
\hline $\mathrm{H}$ & -9.992190 & -2.896620 & -0.130030 \\
\hline $\mathrm{H}$ & -8.505790 & -2.564010 & -1.028550 \\
\hline $\mathrm{H}$ & -8.429860 & -2.989290 & 0.693100 \\
\hline C & 2.176810 & 1.609130 & -2.014810 \\
\hline C & 1.784510 & 2.708010 & -1.245030 \\
\hline C & 3.490490 & 1.568050 & -2.518230 \\
\hline C & 2.674690 & 3.750730 & -0.944520 \\
\hline H & 0.760790 & 2.764080 & -0.894900 \\
\hline
\end{tabular}




\begin{tabular}{|c|c|c|c|}
\hline C & 4.404040 & 2.588280 & -2.250410 \\
\hline $\mathrm{H}$ & 3.783810 & 0.716340 & -3.119660 \\
\hline C & 3.972540 & 3.664930 & -1.453340 \\
\hline $\mathrm{H}$ & 4.677620 & 4.458010 & -1.234130 \\
\hline C & 5.848950 & 2.576620 & -2.786930 \\
\hline C & 2.185520 & 4.933630 & -0.087380 \\
\hline C & 6.840220 & 2.558040 & -1.599280 \\
\hline $\mathrm{H}$ & 6.713980 & 3.430670 & -0.949960 \\
\hline $\mathrm{H}$ & 6.697830 & 1.661540 & -0.985050 \\
\hline $\mathrm{H}$ & 7.874690 & 2.559910 & -1.964540 \\
\hline C & 6.140300 & 1.350830 & -3.673450 \\
\hline $\mathrm{H}$ & 7.171720 & 1.398880 & -4.040850 \\
\hline $\mathrm{H}$ & 6.031430 & 0.409900 & -3.122360 \\
\hline $\mathrm{H}$ & 5.480380 & 1.313260 & -4.547850 \\
\hline C & 6.093290 & 3.846060 & -3.637980 \\
\hline $\mathrm{H}$ & 5.405200 & 3.885390 & -4.490240 \\
\hline $\mathrm{H}$ & 5.954860 & 4.762680 & -3.055610 \\
\hline $\mathrm{H}$ & 7.118540 & 3.851640 & -4.028250 \\
\hline C & 3.280390 & 5.994300 & 0.133390 \\
\hline $\mathrm{H}$ & 4.150840 & 5.581390 & 0.656160 \\
\hline $\mathrm{H}$ & 3.622760 & 6.432010 & -0.811620 \\
\hline $\mathrm{H}$ & 2.883260 & 6.809690 & 0.748480 \\
\hline C & 0.987740 & 5.618200 & -0.788100 \\
\hline $\mathrm{H}$ & 1.276850 & 6.001350 & -1.773650 \\
\hline $\mathrm{H}$ & 0.147150 & 4.931510 & -0.928400 \\
\hline $\mathrm{H}$ & 0.629600 & 6.462600 & -0.186630 \\
\hline C & 1.736760 & 4.406170 & 1.297110 \\
\hline $\mathrm{H}$ & 1.355090 & 5.231890 & 1.910940 \\
\hline $\mathrm{H}$ & 0.945510 & 3.654110 & 1.215590 \\
\hline $\mathrm{H}$ & 2.579360 & 3.947350 & 1.827870 \\
\hline
\end{tabular}

\section{C-Catalyzed reaction TS 41}

\section{Datum}

Value

\begin{tabular}{lc}
\hline B3LYP/6-31G(d) Energy & -3038.966137 \\
\hline B3LYP-D3(BJ)/def2-TZVPP-IEF-PCM(DCM) Energy & -3040.51359 \\
\hline B3LYP-D3(BJ)/def2-TZVPP-IEF-PCM(DCM)//B3LYP/6-31G(d) Free Energy (Quasiharmonic) & -3039.283676 \\
\hline
\end{tabular}

Number of Imaginary Frequencies

Frequencies (Top 3 out of 450)

$$
\begin{array}{rrr}
\text { 1. } & -321.6208 \mathrm{~cm}^{-1} \\
\text { 2. } & 7.9717 \mathrm{~cm}^{-1} \\
\text { 3. } & 10.4273 \mathrm{~cm}^{-1}
\end{array}
$$




\begin{tabular}{|c|c|c|c|}
\hline C & -3.125302 & 0.860993 & -1.444598 \\
\hline $\mathrm{H}$ & -2.549950 & 0.821053 & -2.378213 \\
\hline $\mathrm{H}$ & -4.168598 & 1.046762 & -1.711570 \\
\hline C & 0.379118 & 0.360823 & 0.057312 \\
\hline 0 & -0.390166 & 1.102207 & 0.649562 \\
\hline 0 & 1.391646 & -0.186806 & 0.724214 \\
\hline H & 1.927267 & -0.861774 & 0.232881 \\
\hline 0 & -2.715068 & 1.974434 & -0.655945 \\
\hline $\mathrm{H}$ & -1.873347 & 1.735743 & -0.213237 \\
\hline C & 2.829372 & -2.435363 & 4.739973 \\
\hline C & 4.125750 & -1.205701 & 3.486809 \\
\hline C & 2.275521 & -3.442313 & 2.549674 \\
\hline C & 2.866663 & -3.492180 & 3.855194 \\
\hline $\mathrm{H}$ & 5.009077 & -1.819256 & 3.675245 \\
\hline $\mathrm{H}$ & 1.358499 & -2.854716 & 2.473886 \\
\hline $\mathrm{H}$ & 2.174726 & -4.410485 & 2.057044 \\
\hline C & 3.917498 & -3.031192 & -0.671751 \\
\hline C & 5.223683 & -3.194255 & 0.197089 \\
\hline C & 3.279901 & -4.380483 & -1.038022 \\
\hline $\mathrm{H}$ & 2.286998 & -4.206530 & -1.464554 \\
\hline $\mathrm{H}$ & 3.876087 & -4.920653 & -1.781415 \\
\hline $\mathrm{H}$ & 3.169855 & -5.018982 & -0.156510 \\
\hline C & 4.086317 & -2.183991 & -1.931601 \\
\hline $\mathrm{H}$ & 4.823048 & -2.633737 & -2.608045 \\
\hline $\mathrm{H}$ & 3.134622 & -2.116760 & -2.469694 \\
\hline $\mathrm{H}$ & 4.407546 & -1.169453 & -1.687739 \\
\hline C & 6.113527 & -1.939318 & 0.172977 \\
\hline $\mathrm{H}$ & 6.621779 & -1.816201 & -0.789640 \\
\hline $\mathrm{H}$ & 5.533875 & -1.034202 & 0.376393 \\
\hline $\mathrm{H}$ & 6.877995 & -2.036979 & 0.951150 \\
\hline C & 6.065615 & -4.427735 & -0.133079 \\
\hline $\mathrm{H}$ & 6.429543 & -4.395785 & -1.166817 \\
\hline $\mathrm{H}$ & 6.936961 & -4.464581 & 0.529585 \\
\hline $\mathrm{H}$ & 5.497766 & -5.350093 & 0.009950 \\
\hline 0 & 3.023949 & -2.341732 & 0.237624 \\
\hline 0 & 4.689467 & -3.354582 & 1.523959 \\
\hline B & 3.470310 & -2.610360 & 1.589782 \\
\hline 0 & 3.577120 & -1.290558 & 2.318849 \\
\hline C & 4.016768 & 0.075132 & 4.208832 \\
\hline C & 4.890326 & 0.346759 & 5.272449 \\
\hline C & 3.060841 & 1.032343 & 3.827929 \\
\hline C & 4.811948 & 1.560530 & 5.952008 \\
\hline $\mathrm{H}$ & 5.633001 & -0.393713 & 5.561848 \\
\hline C & 2.985459 & 2.241524 & 4.514542 \\
\hline $\mathrm{H}$ & 2.395095 & 0.819353 & 2.996708 \\
\hline C & 3.857686 & 2.508556 & 5.575059 \\
\hline $\mathrm{H}$ & 5.493983 & 1.767758 & 6.771882 \\
\hline $\mathrm{H}$ & 2.244121 & 2.979534 & 4.221440 \\
\hline $\mathrm{H}$ & 3.794043 & 3.454768 & 6.105524 \\
\hline $\mathrm{H}$ & 3.582374 & -4.292812 & 4.045772 \\
\hline
\end{tabular}




\begin{tabular}{|c|c|c|c|}
\hline $\mathrm{H}$ & 2.020175 & -1.713144 & 4.686607 \\
\hline $\mathrm{H}$ & 3.353846 & -2.488184 & 5.690173 \\
\hline C & -0.780406 & -0.799421 & -1.824233 \\
\hline C & 0.257648 & 0.028759 & -1.411270 \\
\hline C & 1.211204 & 0.567147 & -2.345263 \\
\hline C & 1.128166 & 0.158872 & -3.662762 \\
\hline H & 1.809735 & 0.585430 & -4.393598 \\
\hline C & -3.009509 & -0.471558 & -0.717339 \\
\hline C & -1.862335 & -1.249168 & -0.875160 \\
\hline C & -3.949216 & -2.162312 & 0.756223 \\
\hline C & -4.079086 & -0.938578 & 0.125522 \\
\hline $\mathrm{H}$ & -4.752813 & -2.515522 & 1.397408 \\
\hline C & -2.799231 & -2.972014 & 0.614546 \\
\hline C & -1.726459 & -2.512606 & -0.212819 \\
\hline C & -0.580522 & -3.343258 & -0.351803 \\
\hline C & -0.509560 & -4.565646 & 0.279327 \\
\hline C & -1.574081 & -5.018193 & 1.095116 \\
\hline C & -2.690698 & -4.232706 & 1.262035 \\
\hline $\mathrm{H}$ & 0.251383 & -3.001004 & -0.957509 \\
\hline H & 0.372869 & -5.186079 & 0.151325 \\
\hline $\mathrm{H}$ & -1.504334 & -5.983542 & 1.589355 \\
\hline $\mathrm{H}$ & -3.513732 & -4.566473 & 1.889755 \\
\hline C & 0.146594 & -0.755927 & -4.113593 \\
\hline C & -0.838151 & -1.233128 & -3.191927 \\
\hline C & -1.842024 & -2.118125 & -3.673694 \\
\hline C & -1.862549 & -2.514288 & -4.992820 \\
\hline C & -0.886507 & -2.040719 & -5.901662 \\
\hline C & 0.093699 & -1.177564 & -5.469746 \\
\hline $\mathrm{H}$ & -2.595265 & -2.484568 & -2.985359 \\
\hline $\mathrm{H}$ & -2.635695 & -3.194222 & -5.339935 \\
\hline $\mathrm{H}$ & -0.916161 & -2.358558 & -6.940344 \\
\hline $\mathrm{H}$ & 0.844564 & -0.802360 & -6.161008 \\
\hline C & -7.763497 & 1.149421 & 0.754547 \\
\hline C & -6.567125 & 1.856476 & 0.899253 \\
\hline C & -5.363857 & 1.166013 & 0.683092 \\
\hline C & -5.353605 & -0.183420 & 0.321083 \\
\hline C & -6.581072 & -0.858600 & 0.180145 \\
\hline C & -7.798246 & -0.210414 & 0.394015 \\
\hline $\mathrm{H}$ & -8.703513 & 1.663946 & 0.917520 \\
\hline $\mathrm{H}$ & -4.415258 & 1.681971 & 0.774998 \\
\hline $\mathrm{H}$ & -6.560137 & -1.899566 & -0.121524 \\
\hline C & -6.515057 & 3.351191 & 1.269413 \\
\hline C & -9.157363 & -0.920114 & 0.237675 \\
\hline C & -5.808967 & 4.129709 & 0.132359 \\
\hline $\mathrm{H}$ & -5.739366 & 5.194859 & 0.387696 \\
\hline $\mathrm{H}$ & -4.796385 & 3.757256 & -0.052183 \\
\hline $\mathrm{H}$ & -6.373393 & 4.043659 & -0.803923 \\
\hline C & -7.915532 & 3.958240 & 1.474843 \\
\hline H & -7.822979 & 5.019366 & 1.733314 \\
\hline H & -8.526283 & 3.892688 & 0.566977 \\
\hline $\mathrm{H}$ & -8.459770 & 3.466666 & 2.289935 \\
\hline C & -5.716828 & 3.530172 & 2.582693 \\
\hline $\mathrm{H}$ & -5.667334 & 4.591692 & 2.856104 \\
\hline $\mathrm{H}$ & -6.193064 & 2.988750 & 3.408685 \\
\hline
\end{tabular}




\begin{tabular}{|c|c|c|c|}
\hline $\mathrm{H}$ & -4.690560 & 3.162359 & 2.487250 \\
\hline C & -9.006671 & -2.402373 & -0.154754 \\
\hline $\mathrm{H}$ & -9.997445 & -2.862163 & -0.246493 \\
\hline $\mathrm{H}$ & -8.497236 & -2.520020 & -1.117881 \\
\hline $\mathrm{H}$ & -8.447765 & -2.969271 & 0.598586 \\
\hline C & -9.930454 & -0.857122 & 1.576558 \\
\hline $\mathrm{H}$ & -10.903972 & -1.354178 & 1.480330 \\
\hline $\mathrm{H}$ & -9.370658 & -1.357300 & 2.375228 \\
\hline $\mathrm{H}$ & -10.113719 & 0.174563 & 1.894073 \\
\hline C & -9.985482 & -0.216304 & -0.863831 \\
\hline $\mathrm{H}$ & -10.958191 & -0.709235 & -0.985529 \\
\hline $\mathrm{H}$ & -10.172626 & 0.835490 & -0.623874 \\
\hline $\mathrm{H}$ & -9.463787 & -0.250734 & -1.827139 \\
\hline C & 2.208738 & 1.612799 & -1.972834 \\
\hline C & 1.835630 & 2.700112 & -1.162697 \\
\hline C & 3.502025 & 1.584879 & -2.508277 \\
\hline C & 2.732192 & 3.728193 & -0.868532 \\
\hline $\mathrm{H}$ & 0.822915 & 2.744579 & -0.786439 \\
\hline C & 4.426477 & 2.610857 & -2.257063 \\
\hline $\mathrm{H}$ & 3.786907 & 0.744260 & -3.133128 \\
\hline C & 4.019646 & 3.661315 & -1.429146 \\
\hline $\mathrm{H}$ & 4.717418 & 4.461499 & -1.213068 \\
\hline C & 5.825973 & 2.557570 & -2.902573 \\
\hline C & 2.350906 & 4.922344 & 0.027388 \\
\hline C & 6.700180 & 3.765916 & -2.516243 \\
\hline $\mathrm{H}$ & 6.252975 & 4.713321 & -2.837745 \\
\hline $\mathrm{H}$ & 6.872078 & 3.817148 & -1.435081 \\
\hline $\mathrm{H}$ & 7.678766 & 3.682110 & -3.002373 \\
\hline C & 6.563154 & 1.273695 & -2.455825 \\
\hline $\mathrm{H}$ & 7.563707 & 1.233095 & -2.904010 \\
\hline $\mathrm{H}$ & 6.677794 & 1.245535 & -1.366289 \\
\hline $\mathrm{H}$ & 6.027387 & 0.368738 & -2.759792 \\
\hline C & 5.676032 & 2.550131 & -4.442807 \\
\hline $\mathrm{H}$ & 5.103181 & 1.684555 & -4.791976 \\
\hline $\mathrm{H}$ & 5.162043 & 3.453531 & -4.790444 \\
\hline $\mathrm{H}$ & 6.661467 & 2.513172 & -4.923540 \\
\hline C & 3.311815 & 4.979036 & 1.239030 \\
\hline $\mathrm{H}$ & 3.247398 & 4.061778 & 1.835242 \\
\hline $\mathrm{H}$ & 4.355333 & 5.102192 & 0.930132 \\
\hline $\mathrm{H}$ & 3.054970 & 5.826657 & 1.886440 \\
\hline C & 2.470509 & 6.236146 & -0.781179 \\
\hline $\mathrm{H}$ & 3.487968 & 6.397496 & -1.153409 \\
\hline $\mathrm{H}$ & 1.795531 & 6.227257 & -1.644577 \\
\hline $\mathrm{H}$ & 2.205755 & 7.095260 & -0.152518 \\
\hline C & 0.909729 & 4.816964 & 0.562749 \\
\hline $\mathrm{H}$ & 0.697771 & 5.671276 & 1.215932 \\
\hline $\mathrm{H}$ & 0.170393 & 4.831995 & -0.245837 \\
\hline$H$ & 0.752105 & 3.902647 & 1.145237 \\
\hline
\end{tabular}




\begin{tabular}{lc}
\hline B3LYP/6-31G(d) Energy & -3038.959027 \\
\hline B3LYP-D3(BJ)/def2-TZVPP-IEF-PCM(DCM) Energy & -3040.512014 \\
\hline B3LYP-D3(BJ)/def2-TZVPP-IEF-PCM(DCM)//B3LYP/6-31G(d) Free Energy (Quasiharmonic) & -3039.28337 \\
\hline
\end{tabular}

Number of Imaginary Frequencies

Frequencies (Top 3 out of 450)

$\begin{array}{lr}\text { 1. } & -281.7231 \mathrm{~cm}^{-1} \\ \text { 2. } & 8.3802 \mathrm{~cm}^{-1} \\ \text { 3. } & 8.6827 \mathrm{~cm}^{-1}\end{array}$

\section{B3LYP/6-31G(d) Molecular Geometry in Cartesian Coordinates}

C

$\mathrm{H}$

$\mathrm{H}$

C

0

0

$\mathrm{H}$

0

$\mathrm{H}$

C

C

C

C

$\mathrm{H}$

$\mathrm{H}$

$\mathrm{H}$

C

C

C

$\mathrm{H}$

$\mathrm{H}$

$\mathrm{H}$

C

$\mathrm{H}$

$\mathrm{H}$

$\mathrm{H}$

$\mathrm{H}$

C

$\mathrm{H}$

$\mathrm{H}$

$\mathrm{H}$

C

H
$-0.699047$

$-0.262286$

$-1.760073$

2.800770

3.685613

1.774259

1.116972

$-0.066854$

$-0.011657$

$-3.364123$

$-3.811509$

$-1.223795$

$-2.493033$

$-4.152420$

$-0.520413$

$-0.764192$

0.305316

$-0.860371$

1.486772

2.156299

2.065112

1.147193

0.819254

1.602285

0.022222

1.253826

$-1.793961$

$-1.303600$

$-2.136174$

$-2.674657$

$-0.407920$

0.220345
0.883531

0.674259

0.630401

1.662196

2. 281255

1.139037

0.706269

0.055927

$-0.855665$

$-2.283775$

$-2.082520$

$-3.122983$

$-3.317351$

$-1.067011$

$-3.947987$

$-2.158710$

$-3.382590$

$-4.405384$

$-4.007095$

$-3.210522$

$-4.683804$

$-4.566746$

$-2.628275$

$-1.922377$

$-2.062985$

$-3.320187$

$-3.941255$

$-3.994479$

$-2.914910$

$-4.591476$

$-5.842490$

$-5.904139$
0.690369

1.674054

0.735792

$-1.230214$

$-1.777717$

$-1.913290$

$-1.300326$

$-0.300612$

0.074891

$-2.547959$

$-0.379530$

$-1.658811$

$-2.303425$

$-0.597301$

$-1.791365$

$-1.889776$

1.601537

1.883493

0.844647

0.504067

1.483008

$-0.031928$

2.828770

2.531616

3. 317154

3.559802

3.014056

3. 992101

2.852206

3.041976

2.142951

3.039296 


\begin{tabular}{|c|c|c|c|}
\hline H & -1.283742 & -6.480775 & 2.302422 \\
\hline $\mathrm{H}$ & 0.150882 & -6.245785 & 1.295320 \\
\hline 0 & -0.344181 & -2.438691 & 0.720522 \\
\hline 0 & -1.594696 & -4.381565 & 0.640534 \\
\hline B & -1.454013 & -3.082813 & 0.063270 \\
\hline 0 & -2.654925 & -2.128790 & 0.189723 \\
\hline C & -4.911823 & -3.053177 & -0.175660 \\
\hline C & -6.210357 & -2.621575 & -0.500286 \\
\hline C & -4.727433 & -4.343074 & 0.348884 \\
\hline C & -7.305577 & -3.460442 & -0.311791 \\
\hline $\mathrm{H}$ & -6.355563 & -1.620991 & -0.902329 \\
\hline C & -5.828072 & -5.178028 & 0.533392 \\
\hline $\mathrm{H}$ & -3.726126 & -4.678715 & 0.598966 \\
\hline C & -7.115539 & -4.743959 & 0.205916 \\
\hline $\mathrm{H}$ & -8.303839 & -3.114314 & -0.564842 \\
\hline $\mathrm{H}$ & -5.680161 & -6.175425 & 0.938746 \\
\hline $\mathrm{H}$ & -7.967413 & -5.401683 & 0.355920 \\
\hline $\mathrm{H}$ & -2.866738 & -4.337515 & -2.398222 \\
\hline $\mathrm{H}$ & -2.989011 & -1.265764 & -2.614148 \\
\hline $\mathrm{H}$ & -4.342772 & -2.467961 & -2.980637 \\
\hline C & 1.891344 & 2.101411 & 1.071754 \\
\hline C & 2.813556 & 1.439576 & 0.271850 \\
\hline C & 3.872327 & 0.657895 & 0.845295 \\
\hline C & 3.964084 & 0.586052 & 2.221892 \\
\hline $\mathrm{H}$ & 4.739263 & -0.029508 & 2.671220 \\
\hline C & -0.497598 & 2.342064 & 0.331275 \\
\hline C & 0.772687 & 2.903246 & 0.461404 \\
\hline C & -1.320619 & 4.414277 & -0.635020 \\
\hline C & -1.569772 & 3.120606 & -0.216606 \\
\hline $\mathrm{H}$ & -2.136697 & 5.007625 & -1.040295 \\
\hline C & -0.042256 & 5.008467 & -0.525932 \\
\hline C & 1.029627 & 4.247541 & 0.040254 \\
\hline C & 2.308076 & 4.862923 & 0.154716 \\
\hline C & 2.507815 & 6.158424 & -0.264259 \\
\hline C & 1.443662 & 6.909570 & -0.819748 \\
\hline C & 0.196652 & 6.344601 & -0.947576 \\
\hline $\mathrm{H}$ & 3.136146 & 4.291617 & 0.558554 \\
\hline $\mathrm{H}$ & 3.493326 & 6.607032 & -0.175826 \\
\hline $\mathrm{H}$ & 1.617892 & 7.930696 & -1.148252 \\
\hline $\mathrm{H}$ & -0.627572 & 6.910738 & -1.375188 \\
\hline C & 3.070848 & 1.277001 & 3.076582 \\
\hline C & 2.013775 & 2.051480 & 2.500938 \\
\hline C & 1.132153 & 2.744858 & 3.376093 \\
\hline C & 1.282526 & 2.667214 & 4.743405 \\
\hline C & 2.322538 & 1.891993 & 5.309353 \\
\hline C & 3.195504 & 1.212889 & 4.490923 \\
\hline $\mathrm{H}$ & 0.335236 & 3.346221 & 2.951430 \\
\hline $\mathrm{H}$ & 0.599879 & 3.208059 & 5.393442 \\
\hline $\mathrm{H}$ & 2.430821 & 1.838459 & 6.389318 \\
\hline $\mathrm{H}$ & 4.000949 & 0.617497 & 4.914684 \\
\hline C & -5.637561 & 1.818302 & -0.568513 \\
\hline C & -5.050251 & 1.852731 & 0.707002 \\
\hline C & -3.711430 & 2.253961 & 0.797617 \\
\hline C & -2.970738 & 2.611038 & -0.337095 \\
\hline
\end{tabular}




\begin{tabular}{|c|c|c|c|}
\hline C & -3.596004 & 2.568458 & -1.588931 \\
\hline C & -4.935367 & 2.178077 & -1.729329 \\
\hline $\mathrm{H}$ & -6.674575 & 1.523236 & -0.656259 \\
\hline $\mathrm{H}$ & -3.230705 & 2.321975 & 1.769492 \\
\hline $\mathrm{H}$ & -3.013866 & 2.851794 & -2.461024 \\
\hline C & -5.833113 & 1.515603 & 1.992617 \\
\hline C & -5.589775 & 2.192736 & -3.125694 \\
\hline C & -7.266905 & 1.031392 & 1.703458 \\
\hline $\mathrm{H}$ & -7.772748 & 0.798054 & 2.647115 \\
\hline $\mathrm{H}$ & -7.863617 & 1.795132 & 1.192053 \\
\hline $\mathrm{H}$ & -7.275230 & 0.121107 & 1.092707 \\
\hline C & -5.103238 & 0.401920 & 2.779982 \\
\hline $\mathrm{H}$ & -5.646551 & 0.173073 & 3.704892 \\
\hline $\mathrm{H}$ & -5.036562 & -0.520579 & 2.192217 \\
\hline $\mathrm{H}$ & -4.085837 & 0.694893 & 3.057930 \\
\hline C & -5.923055 & 2.785391 & 2.873524 \\
\hline $\mathrm{H}$ & -6.475012 & 2.572025 & 3.797199 \\
\hline $\mathrm{H}$ & -4.931140 & 3.154763 & 3.154023 \\
\hline $\mathrm{H}$ & -6.442635 & 3.592945 & 2.345284 \\
\hline C & -4.794895 & 1.283511 & -4.091960 \\
\hline $\mathrm{H}$ & -5.235669 & 1.316109 & -5.095756 \\
\hline $\mathrm{H}$ & -3.748264 & 1.591566 & -4.179206 \\
\hline $\mathrm{H}$ & -4.809907 & 0.241222 & -3.752345 \\
\hline C & -7.049606 & 1.701274 & -3.102028 \\
\hline $\mathrm{H}$ & -7.462279 & 1.724156 & -4.116880 \\
\hline $\mathrm{H}$ & -7.128222 & 0.669783 & -2.738241 \\
\hline $\mathrm{H}$ & -7.686403 & 2.334423 & -2.474070 \\
\hline C & -5.581010 & 3.641712 & -3.670818 \\
\hline $\mathrm{H}$ & -6.040691 & 3.678538 & -4.666106 \\
\hline $\mathrm{H}$ & -6.143921 & 4.311574 & -3.010831 \\
\hline $\mathrm{H}$ & -4.563291 & 4.035631 & -3.757721 \\
\hline C & 4.845757 & -0.097812 & 0.007478 \\
\hline C & 4.423676 & -0.869881 & -1.077329 \\
\hline C & 6.214118 & -0.066563 & 0.326863 \\
\hline C & 5.333263 & -1.593361 & -1.863355 \\
\hline $\mathrm{H}$ & 3.366716 & -0.899057 & -1.319909 \\
\hline C & 7.151315 & -0.781804 & -0.419329 \\
\hline $\mathrm{H}$ & 6.531385 & 0.556312 & 1.154907 \\
\hline C & 6.684392 & -1.537113 & -1.510727 \\
\hline $\mathrm{H}$ & 7.406399 & -2.086319 & -2.103631 \\
\hline C & 8.659578 & -0.755972 & -0.103827 \\
\hline C & 4.817169 & -2.392274 & -3.075838 \\
\hline C & 8.989490 & 0.115452 & 1.123130 \\
\hline $\mathrm{H}$ & 10.069598 & 0.097090 & 1.308480 \\
\hline $\mathrm{H}$ & 8.698071 & 1.160743 & 0.971487 \\
\hline $\mathrm{H}$ & 8.492680 & -0.250466 & 2.029158 \\
\hline C & 9.151958 & -2.194124 & 0.184997 \\
\hline $\mathrm{H}$ & 10.227218 & -2.192971 & 0.402904 \\
\hline $\mathrm{H}$ & 8.630266 & -2.620311 & 1.049801 \\
\hline $\mathrm{H}$ & 8.985519 & -2.861823 & -0.666703 \\
\hline C & 9.430111 & -0.184228 & -1.317661 \\
\hline $\mathrm{H}$ & 9.277217 & -0.787664 & -2.218366 \\
\hline $\mathrm{H}$ & 9.102951 & 0.837376 & -1.541333 \\
\hline $\mathrm{H}$ & 10.507329 & -0.160405 & -1.110442 \\
\hline
\end{tabular}




$\begin{array}{llll}\mathrm{C} & 4.136536 & -1.420822 & -4.070859 \\ \mathrm{H} & 3.307181 & -0.873369 & -3.612422 \\ \mathrm{H} & 4.852308 & -0.678953 & -4.442216 \\ \mathrm{H} & 3.742415 & -1.975366 & -4.931992 \\ \mathrm{C} & 5.946525 & -3.129599 & -3.819545 \\ \mathrm{H} & 6.697236 & -2.434613 & -4.212348 \\ \mathrm{H} & 6.454907 & -3.856233 & -3.174998 \\ \mathrm{H} & 5.528839 & -3.678375 & -4.671337 \\ \mathrm{C} & 3.788864 & -3.447194 & -2.604044 \\ \mathrm{H} & 3.410997 & -4.020066 & -3.460118 \\ \mathrm{H} & 4.244401 & -4.151966 & -1.898276 \\ \mathrm{H} & 2.928584 & -2.985667 & -2.108587\end{array}$

\section{C-Catalyzed reaction TS 43}

Datum

Value

\begin{tabular}{lc}
\hline B3LYP/6-31G(d) Energy & -3038.963766 \\
\hline B3LYP-D3(BJ)/def2-TZVPP-IEF-PCM(DCM) Energy & -3040.513438 \\
\hline B3LYP-D3(BJ)/def2-TZVPP-IEF-PCM(DCM)//B3LYP/6-31G(d) Free Energy (Quasiharmonic) & -3039.282971 \\
\hline
\end{tabular}

Number of Imaginary Frequencies

Frequencies (Top 3 out of 450)

$\begin{array}{lr}\text { 1. } & -283.4360 \mathrm{~cm}^{-1} \\ \text { 2. } & 11.6969 \mathrm{~cm}^{-1} \\ \text { 3. } & 12.2832 \mathrm{~cm}^{-1}\end{array}$

\section{B3LYP/6-31G(d) Molecular Geometry in Cartesian Coordinates}

C

$\mathrm{H}$

$\mathrm{H}$

$\mathrm{C}$

0

0

$\mathrm{H}$

0

$\mathrm{H}$

C

C

C

C

$\mathrm{H}$

$$
\begin{array}{r}
-2.217203 \\
-3.099112 \\
-1.677566 \\
0.758054 \\
1.184990 \\
0.323041 \\
-0.183429 \\
-1.369039 \\
-1.003565 \\
1.567929 \\
2.658956 \\
0.091723 \\
1.081229 \\
2.713401
\end{array}
$$

$-0.249153$

0.332370

$-0.523844$

$-1.967506$

$-2.762644$

$-0.762250$

$-0.278957$

0.549850

1. 321865

2.068262

1.773095

3. 380741

3. 275772

0.712115
0.896069

1.182123

1.808668

$-1.130124$

$-1.942803$

$-1.511594$

$-0.794686$

0.051002

0.551744

$-3.077184$

$-1.203939$

$-1.595623$

$-2.633891$

$-1.455806$ 


\begin{tabular}{|c|c|c|c|}
\hline $\mathrm{H}$ & -0.389583 & 4.359337 & -1.533513 \\
\hline $\mathrm{H}$ & -0.646045 & 2.575418 & -1.619786 \\
\hline C & -0.191161 & 3.768138 & 2.006701 \\
\hline C & 1.189718 & 4.510931 & 1.856664 \\
\hline C & -1.394077 & 4.676431 & 1.709022 \\
\hline $\mathrm{H}$ & -2.294863 & 4.059911 & 1.619328 \\
\hline $\mathrm{H}$ & -1.561511 & 5.406446 & 2.508565 \\
\hline $\mathrm{H}$ & -1.258230 & 5.218730 & 0.768790 \\
\hline C & -0.401261 & 3.055001 & 3.343897 \\
\hline $\mathrm{H}$ & -0.380457 & 3.767730 & 4.176622 \\
\hline H & -1.380105 & 2.562011 & 3.354126 \\
\hline $\mathrm{H}$ & 0.361839 & 2.292823 & 3.517202 \\
\hline C & 2.332385 & 3.789579 & 2.592217 \\
\hline $\mathrm{H}$ & 2.238830 & 3.882008 & 3.679894 \\
\hline $\mathrm{H}$ & 2.363341 & 2.727373 & 2.333176 \\
\hline $\mathrm{H}$ & 3.286616 & 4.236480 & 2.294941 \\
\hline C & 1.174574 & 5.988611 & 2.250579 \\
\hline $\mathrm{H}$ & 0.916974 & 6.115034 & 3.308820 \\
\hline $\mathrm{H}$ & 2.169021 & 6.420213 & 2.093801 \\
\hline $\mathrm{H}$ & 0.463898 & 6.557408 & 1.646312 \\
\hline 0 & -0.107647 & 2.775073 & 0.963146 \\
\hline 0 & 1.425480 & 4.425196 & 0.437995 \\
\hline B & 0.846305 & 3.206524 & -0.040045 \\
\hline 0 & 1.792727 & 2.031756 & -0.278161 \\
\hline C & 3.923794 & 2.515005 & -1.431214 \\
\hline C & 4.159192 & 3.822076 & -0.972914 \\
\hline C & 4.940790 & 1.838823 & -2.126827 \\
\hline C & 5.387223 & 4.433529 & -1.221111 \\
\hline $\mathrm{H}$ & 3.380775 & 4.346181 & -0.427866 \\
\hline C & 6.165714 & 2.455786 & -2.371548 \\
\hline $\mathrm{H}$ & 4.770807 & 0.819767 & -2.465758 \\
\hline C & 6.391617 & 3.758131 & -1.919875 \\
\hline $\mathrm{H}$ & 5.562437 & 5.444752 & -0.863406 \\
\hline $\mathrm{H}$ & 6.942234 & 1.920953 & -2.911645 \\
\hline $\mathrm{H}$ & 7.345975 & 4.242487 & -2.108507 \\
\hline $\mathrm{H}$ & 1.618840 & 4.180804 & -2.919371 \\
\hline $\mathrm{H}$ & 0.989808 & 1.157860 & -2.940064 \\
\hline $\mathrm{H}$ & 2.356931 & 2.032407 & -3.822601 \\
\hline C & -0.514027 & -2.600733 & 0.928050 \\
\hline C & 0.710627 & -2.280307 & 0.354627 \\
\hline C & 1.924245 & -2.304973 & 1.128959 \\
\hline C & 1.832679 & -2.644776 & 2.466036 \\
\hline $\mathrm{H}$ & 2.740249 & -2.680094 & 3.062497 \\
\hline C & -2.631016 & -1.491341 & 0.133905 \\
\hline C & -1.780225 & -2.595433 & 0.114728 \\
\hline C & -4.217613 & -2.685028 & -1.263204 \\
\hline C & -3.881569 & -1.535647 & -0.570812 \\
\hline $\mathrm{H}$ & -5.159886 & -2.719244 & -1.803974 \\
\hline C & -3.369398 & -3.814409 & -1.317983 \\
\hline C & -2.123540 & -3.779280 & -0.616501 \\
\hline C & -1.284635 & -4.927449 & -0.672401 \\
\hline C & -1.661668 & -6.043977 & -1.383558 \\
\hline C & -2.895470 & -6.074796 & -2.077504 \\
\hline C & -3.728910 & -4.981671 & -2.045439 \\
\hline
\end{tabular}




\begin{tabular}{|c|c|c|c|}
\hline $\mathrm{H}$ & -0.329505 & -4.911316 & -0.159927 \\
\hline $\mathrm{H}$ & -1.003530 & -6.907761 & -1.418876 \\
\hline $\mathrm{H}$ & -3.178186 & -6.962316 & -2.637267 \\
\hline $\mathrm{H}$ & -4.677603 & -4.991739 & -2.576956 \\
\hline C & 0.610202 & -2.994159 & 3.086160 \\
\hline C & -0.589000 & -2.985849 & 2.308696 \\
\hline C & -1.806432 & -3.362496 & 2.941588 \\
\hline C & -1.831637 & -3.719976 & 4.271806 \\
\hline C & -0.642752 & -3.721664 & 5.039730 \\
\hline C & 0.551534 & -3.367067 & 4.456239 \\
\hline $\mathrm{H}$ & -2.722967 & -3.368436 & 2.361795 \\
\hline $\mathrm{H}$ & -2.771614 & -4.006797 & 4.735507 \\
\hline $\mathrm{H}$ & -0.676700 & -4.006426 & 6.087847 \\
\hline $\mathrm{H}$ & 1.472538 & -3.368620 & 5.034431 \\
\hline C & -6.812161 & 1.575218 & -0.638636 \\
\hline C & -5.496454 & 1.901427 & -0.996571 \\
\hline C & -4.532237 & 0.885930 & -0.949452 \\
\hline C & -4.873830 & -0.418141 & -0.561668 \\
\hline C & -6.203252 & -0.699803 & -0.210347 \\
\hline C & -7.194347 & 0.286922 & -0.241034 \\
\hline $\mathrm{H}$ & -7.567589 & 2.354920 & -0.669893 \\
\hline $\mathrm{H}$ & -3.502887 & 1.086974 & -1.220962 \\
\hline $\mathrm{H}$ & -6.445255 & -1.709469 & 0.101032 \\
\hline C & -5.164795 & 3.342303 & -1.432195 \\
\hline C & -8.660838 & 0.005816 & 0.141499 \\
\hline C & -3.677896 & 3.514817 & -1.790173 \\
\hline $\mathrm{H}$ & -3.485909 & 4.554491 & -2.079664 \\
\hline $\mathrm{H}$ & -3.024461 & 3.278730 & -0.943998 \\
\hline $\mathrm{H}$ & -3.384943 & 2.878078 & -2.632380 \\
\hline C & -6.005194 & 3.713170 & -2.677284 \\
\hline $\mathrm{H}$ & -5.780404 & 4.738019 & -2.997739 \\
\hline $\mathrm{H}$ & -5.783463 & 3.039704 & -3.512944 \\
\hline $\mathrm{H}$ & -7.080441 & 3.655293 & -2.477870 \\
\hline C & -5.499224 & 4.320205 & -0.280731 \\
\hline $\mathrm{H}$ & -5.272861 & 5.351706 & -0.577793 \\
\hline $\mathrm{H}$ & -6.557635 & 4.279097 & -0.002464 \\
\hline $\mathrm{H}$ & -4.910066 & 4.088330 & 0.614181 \\
\hline C & -8.890747 & -1.462223 & 0.548083 \\
\hline $\mathrm{H}$ & -9.944889 & -1.612952 & 0.807253 \\
\hline $\mathrm{H}$ & -8.649263 & -2.153406 & -0.267312 \\
\hline $\mathrm{H}$ & -8.292631 & -1.742520 & 1.422513 \\
\hline C & -9.065229 & 0.902887 & 1.335468 \\
\hline $\mathrm{H}$ & -10.110688 & 0.721041 & 1.613710 \\
\hline $\mathrm{H}$ & -8.439567 & 0.694870 & 2.211079 \\
\hline $\mathrm{H}$ & -8.963892 & 1.967219 & 1.099022 \\
\hline C & -9.576539 & 0.317178 & -1.066190 \\
\hline $\mathrm{H}$ & -10.626129 & 0.128698 & -0.808701 \\
\hline $\mathrm{H}$ & -9.494036 & 1.362144 & -1.382873 \\
\hline $\mathrm{H}$ & -9.318821 & -0.312733 & -1.925278 \\
\hline C & 3.281174 & -1.974079 & 0.593245 \\
\hline C & 3.797000 & -2.587113 & -0.556075 \\
\hline C & 4.111889 & -1.106480 & 1.321511 \\
\hline C & 5.104284 & -2.337698 & -0.993513 \\
\hline $\mathrm{H}$ & 3.163570 & -3.270968 & -1.104322 \\
\hline
\end{tabular}




\begin{tabular}{|c|c|c|c|}
\hline C & 5.433135 & -0.852130 & 0.935206 \\
\hline $\mathrm{H}$ & 3.706825 & -0.628608 & 2.208550 \\
\hline C & 5.903050 & -1.472970 & -0.232602 \\
\hline $\mathrm{H}$ & 6.918802 & -1.283068 & -0.551201 \\
\hline C & 6.327993 & 0.049167 & 1.810802 \\
\hline C & 5.607817 & -3.030594 & -2.275294 \\
\hline C & 5.661736 & 1.428337 & 2.019746 \\
\hline $\mathrm{H}$ & 6.291329 & 2.058817 & 2.659986 \\
\hline $\mathrm{H}$ & 5.520284 & 1.946781 & 1.066028 \\
\hline $\mathrm{H}$ & 4.682738 & 1.343700 & 2.502313 \\
\hline C & 6.527242 & -0.632175 & 3.186562 \\
\hline $\mathrm{H}$ & 7.164372 & -0.014505 & 3.831861 \\
\hline $\mathrm{H}$ & 5.573459 & -0.782511 & 3.703902 \\
\hline $\mathrm{H}$ & 7.004669 & -1.612466 & 3.074509 \\
\hline C & 7.717163 & 0.285626 & 1.187267 \\
\hline $\mathrm{H}$ & 8.277409 & -0.648314 & 1.064695 \\
\hline $\mathrm{H}$ & 7.644473 & 0.775154 & 0.209478 \\
\hline $\mathrm{H}$ & 8.307046 & 0.937721 & 1.841514 \\
\hline C & 5.517419 & -4.565822 & -2.102607 \\
\hline $\mathrm{H}$ & 6.129112 & -4.902196 & -1.257356 \\
\hline $\mathrm{H}$ & 4.489099 & -4.895908 & -1.926049 \\
\hline $\mathrm{H}$ & 5.878564 & -5.071672 & -3.006613 \\
\hline C & 4.721253 & -2.600561 & -3.469324 \\
\hline $\mathrm{H}$ & 3.662465 & -2.814485 & -3.294218 \\
\hline $\mathrm{H}$ & 4.816819 & -1.523459 & -3.656299 \\
\hline $\mathrm{H}$ & 5.029931 & -3.125793 & -4.381933 \\
\hline C & 7.069668 & -2.674786 & -2.605239 \\
\hline $\mathrm{H}$ & 7.374981 & -3.186626 & -3.524941 \\
\hline $\mathrm{H}$ & 7.202855 & -1.598780 & -2.768228 \\
\hline $\mathrm{H}$ & 7.756990 & -2.986331 & -1.810067 \\
\hline
\end{tabular}

\section{C-Catalyzed reaction TS 44}

\section{Datum}

Value

\begin{tabular}{lc}
\hline B3LYP/6-31G(d) Energy & -3038.963861 \\
\hline B3LYP-D3(BJ)/def2-TZVPP-IEF-PCM(DCM) Energy & -3040.513486 \\
\hline B3LYP-D3(BJ)/def2-TZVPP-IEF-PCM(DCM)//B3LYP/6-31G(d) Free Energy (Quasiharmonic) & -3039.282968 \\
\hline
\end{tabular}

Number of Imaginary Frequencies

Frequencies (Top 3 out of 450)

$\begin{array}{lr}\text { 1. } & -282.8167 \mathrm{~cm}^{-1} \\ \text { 2. } & 11.5601 \mathrm{~cm}^{-1} \\ \text { 3. } & 12.9928 \mathrm{~cm}^{-1}\end{array}$




\begin{tabular}{|c|c|c|c|}
\hline C & -2.220717 & -0.234948 & 0.885339 \\
\hline $\mathrm{H}$ & -1.684598 & -0.513350 & 1.799000 \\
\hline $\mathrm{H}$ & -3.099569 & 0.351977 & 1.170027 \\
\hline C & 0.747881 & -1.965308 & -1.139877 \\
\hline 0 & 1.171033 & -2.761319 & -1.953737 \\
\hline 0 & 0.321992 & -0.756134 & -1.518767 \\
\hline $\mathrm{H}$ & -0.183969 & -0.272269 & -0.801701 \\
\hline 0 & -1.366480 & 0.559190 & 0.042218 \\
\hline $\mathrm{H}$ & -0.998865 & 1.329459 & 0.544244 \\
\hline C & 1.593576 & 2.074451 & -3.067893 \\
\hline C & 2.675398 & 1.771506 & -1.189776 \\
\hline C & 0.115362 & 3.388006 & -1.589392 \\
\hline C & 1.109207 & 3.282419 & -2.623477 \\
\hline $\mathrm{H}$ & 2.727854 & 0.711111 & -1.444592 \\
\hline $\mathrm{H}$ & -0.624921 & 2.585191 & -1.619274 \\
\hline $\mathrm{H}$ & -0.363038 & 4.368021 & -1.527207 \\
\hline C & -0.188595 & 3.765875 & 2.010484 \\
\hline C & 1.195150 & 4.505477 & 1.872129 \\
\hline C & -1.387269 & 4.677288 & 1.705042 \\
\hline $\mathrm{H}$ & -2.288288 & 4.062437 & 1.606301 \\
\hline $\mathrm{H}$ & -1.560017 & 5.405625 & 2.504985 \\
\hline $\mathrm{H}$ & -1.242729 & 5.221715 & 0.767345 \\
\hline C & -0.410483 & 3.050939 & 3.344794 \\
\hline $\mathrm{H}$ & -0.393428 & 3.762061 & 4.178978 \\
\hline $\mathrm{H}$ & -1.390832 & 2.560854 & 3.347228 \\
\hline $\mathrm{H}$ & 0.349163 & 2.286216 & 3.521973 \\
\hline C & 2.330666 & 3.779377 & 2.614101 \\
\hline $\mathrm{H}$ & 2.229494 & 3.869361 & 3.701297 \\
\hline $\mathrm{H}$ & 2.360766 & 2.717711 & 2.352695 \\
\hline $\mathrm{H}$ & 3.288161 & 4.224595 & 2.324889 \\
\hline C & 1.180792 & 5.982214 & 2.269509 \\
\hline $\mathrm{H}$ & 0.915205 & 6.106697 & 3.326009 \\
\hline $\mathrm{H}$ & 2.177531 & 6.411665 & 2.121629 \\
\hline $\mathrm{H}$ & 0.476328 & 6.554340 & 1.661118 \\
\hline 0 & -0.099246 & 2.774512 & 0.965948 \\
\hline 0 & 1.440844 & 4.422447 & 0.454924 \\
\hline B & 0.861274 & 3.206866 & -0.030494 \\
\hline 0 & 1.805692 & 2.030230 & -0.267383 \\
\hline C & 3.943105 & 2.510806 & -1.409068 \\
\hline C & 4.960779 & 1.835484 & -2.104542 \\
\hline C & 4.180438 & 3.814731 & -0.942854 \\
\hline C & 6.188321 & 2.450298 & -2.341363 \\
\hline $\mathrm{H}$ & 4.789175 & 0.818730 & -2.449532 \\
\hline C & 5.411148 & 4.423996 & -1.183084 \\
\hline $\mathrm{H}$ & 3.401406 & 4.338264 & -0.398125 \\
\hline C & 6.416230 & 3.749519 & -1.881704 \\
\hline $\mathrm{H}$ & 6.965341 & 1.916245 & -2.881511 \\
\hline $\mathrm{H}$ & 5.587890 & 5.432751 & -0.819215 \\
\hline $\mathrm{H}$ & 7.372686 & 4.232138 & -2.064062 \\
\hline $\mathrm{H}$ & 1.651177 & 4.186347 & -2.904148 \\
\hline
\end{tabular}




\begin{tabular}{|c|c|c|c|}
\hline $\mathrm{H}$ & 1.011729 & 1.165690 & -2.935582 \\
\hline $\mathrm{H}$ & 2.385877 & 2.037744 & -3.809760 \\
\hline C & -0.531341 & -2.598339 & 0.914240 \\
\hline C & 0.695823 & -2.282075 & 0.343917 \\
\hline C & 1.908019 & -2.315404 & 1.120289 \\
\hline C & 1.812690 & -2.660893 & 2.455626 \\
\hline $\mathrm{H}$ & 2.719111 & -2.703324 & 3.053347 \\
\hline C & -2.641278 & -1.474296 & 0.122304 \\
\hline C & -1.797070 & -2.583247 & 0.100271 \\
\hline C & -4.238748 & -2.656758 & -1.272258 \\
\hline C & -3.893204 & -1.510560 & -0.579746 \\
\hline $\mathrm{H}$ & -5.182846 & -2.684353 & -1.810288 \\
\hline C & -3.396808 & -3.790875 & -1.330884 \\
\hline C & -2.148856 & -3.763657 & -0.632664 \\
\hline C & -1.316101 & -4.916053 & -0.693101 \\
\hline C & -1.700865 & -6.029187 & -1.405467 \\
\hline C & -2.936768 & -6.052265 & -2.095900 \\
\hline C & -3.764481 & -4.954882 & -2.059379 \\
\hline $\mathrm{H}$ & -0.359384 & -4.905641 & -0.183443 \\
\hline $\mathrm{H}$ & -1.047230 & -6.896223 & -1.444520 \\
\hline $\mathrm{H}$ & -3.225625 & -6.937165 & -2.656675 \\
\hline $\mathrm{H}$ & -4.714606 & -4.958939 & -2.588421 \\
\hline C & 0.587575 & -3.006953 & 3.072360 \\
\hline C & -0.610457 & -2.988561 & 2.293288 \\
\hline C & -1.830787 & -3.361223 & 2.922957 \\
\hline C & -1.859728 & -3.724779 & 4.251447 \\
\hline C & -0.671885 & -3.736763 & 5.020873 \\
\hline C & 0.525079 & -3.385962 & 4.440589 \\
\hline $\mathrm{H}$ & -2.746636 & -3.359397 & 2.342089 \\
\hline $\mathrm{H}$ & -2.801915 & -4.008454 & 4.712587 \\
\hline $\mathrm{H}$ & -0.708769 & -4.026307 & 6.067579 \\
\hline $\mathrm{H}$ & 1.445304 & -3.395220 & 5.019948 \\
\hline C & -6.801390 & 1.626611 & -0.651527 \\
\hline C & -5.481490 & 1.933609 & -1.029723 \\
\hline C & -4.527329 & 0.913960 & -0.978154 \\
\hline C & -4.876611 & -0.385147 & -0.568384 \\
\hline C & -6.200021 & -0.645705 & -0.198221 \\
\hline C & -7.186801 & 0.351913 & -0.229626 \\
\hline $\mathrm{H}$ & -7.544170 & 2.414518 & -0.690470 \\
\hline $\mathrm{H}$ & -3.498714 & 1.101029 & -1.262042 \\
\hline $\mathrm{H}$ & -6.453278 & -1.649125 & 0.131825 \\
\hline C & -5.144747 & 3.364354 & -1.493195 \\
\hline C & -8.628380 & 0.013016 & 0.197178 \\
\hline C & -5.989699 & 3.715893 & -2.740911 \\
\hline $\mathrm{H}$ & -5.761603 & 4.733485 & -3.081566 \\
\hline $\mathrm{H}$ & -5.775099 & 3.025822 & -3.564808 \\
\hline $\mathrm{H}$ & -7.064167 & 3.666914 & -2.534999 \\
\hline C & -5.467959 & 4.366288 & -0.359189 \\
\hline $\mathrm{H}$ & -5.236325 & 5.390310 & -0.677372 \\
\hline $\mathrm{H}$ & -6.525250 & 4.337941 & -0.075393 \\
\hline $\mathrm{H}$ & -4.875869 & 4.148169 & 0.537263 \\
\hline C & -3.658786 & 3.522508 & -1.861312 \\
\hline $\mathrm{H}$ & -3.462456 & 4.555948 & -2.169551 \\
\hline H & -3.003170 & 3.297283 & -1.013854 \\
\hline
\end{tabular}




\begin{tabular}{|c|c|c|c|}
\hline $\mathrm{H}$ & -3.373106 & 2.869634 & -2.693598 \\
\hline C & -8.628962 & -0.471053 & 1.666789 \\
\hline $\mathrm{H}$ & -9.647248 & -0.726739 & 1.985057 \\
\hline $\mathrm{H}$ & -8.003930 & -1.359690 & 1.802461 \\
\hline H & -8.249563 & 0.309503 & 2.336279 \\
\hline C & -9.575512 & 1.223442 & 0.092458 \\
\hline H & -10.583894 & 0.930113 & 0.405803 \\
\hline $\mathrm{H}$ & -9.256640 & 2.049020 & 0.738993 \\
\hline $\mathrm{H}$ & -9.645219 & 1.599775 & -0.934484 \\
\hline C & -9.184543 & -1.109770 & -0.710780 \\
\hline $\mathrm{H}$ & -10.209304 & -1.367334 & -0.415847 \\
\hline H & -9.202339 & -0.792108 & -1.759624 \\
\hline H & -8.581407 & -2.021350 & -0.647885 \\
\hline C & 3.267272 & -1.987122 & 0.588753 \\
\hline C & 4.100119 & -1.127021 & 1.323544 \\
\hline C & 3.782836 & -2.595361 & -0.563228 \\
\hline C & 5.423091 & -0.875680 & 0.941257 \\
\hline $\mathrm{H}$ & 3.695396 & -0.652839 & 2.212708 \\
\hline C & 5.091830 & -2.348302 & -0.996949 \\
\hline H & 3.147969 & -3.273876 & -1.116426 \\
\hline C & 5.892673 & -1.491354 & -0.229447 \\
\hline $\mathrm{H}$ & 6.909767 & -1.303671 & -0.545080 \\
\hline C & 5.594967 & -3.035791 & -2.281787 \\
\hline C & 6.320086 & 0.016215 & 1.824317 \\
\hline C & 5.500083 & -4.571632 & -2.117160 \\
\hline H & 6.109780 & -4.914067 & -1.272905 \\
\hline $\mathrm{H}$ & 4.470607 & -4.899687 & -1.943570 \\
\hline H & 5.860928 & -5.073790 & -3.023341 \\
\hline C & 4.711097 & -2.597251 & -3.474724 \\
\hline $\mathrm{H}$ & 5.019522 & -3.118750 & -4.389559 \\
\hline H & 3.651500 & -2.809209 & -3.302134 \\
\hline $\mathrm{H}$ & 4.809800 & -1.519500 & -3.656212 \\
\hline C & 7.058193 & -2.682303 & -2.608174 \\
\hline H & 7.194599 & -1.605796 & -2.765133 \\
\hline $\mathrm{H}$ & 7.743820 & -3.000135 & -1.814034 \\
\hline H & 7.363059 & -3.189959 & -3.530342 \\
\hline C & 6.514662 & -0.675561 & 3.195537 \\
\hline $\mathrm{H}$ & 7.153841 & -0.065313 & 3.845854 \\
\hline H & 5.559574 & -0.825136 & 3.710687 \\
\hline H & 6.987790 & -1.657194 & 3.077203 \\
\hline C & 7.711052 & 0.251630 & 1.204381 \\
\hline H & 8.268012 & -0.683560 & 1.076459 \\
\hline H & 7.641612 & 0.747860 & 0.229739 \\
\hline H & 8.302373 & 0.897165 & 1.863813 \\
\hline C & 5.658938 & 1.396495 & 2.042071 \\
\hline H & 6.289689 & 2.019577 & 2.688370 \\
\hline H & 5.521838 & 1.922679 & 1.091976 \\
\hline H & 4.678504 & 1.312350 & 2.521820 \\
\hline
\end{tabular}




\begin{tabular}{lc}
\hline B3LYP/6-31G(d) Energy & -3038.963661 \\
\hline B3LYP-D3(BJ)/def2-TZVPP-IEF-PCM(DCM) Energy & -3040.512913 \\
\hline B3LYP-D3(BJ)/def2-TZVPP-IEF-PCM(DCM)//B3LYP/6-31G(d) Free Energy (Quasiharmonic) & -3039.282867 \\
\hline
\end{tabular}

Number of Imaginary Frequencies

Frequencies (Top 3 out of 450)

$\begin{array}{lr}\text { 1. } & -285.1900 \mathrm{~cm}^{-1} \\ \text { 2. } & 10.7041 \mathrm{~cm}^{-1} \\ \text { 3. } & 14.0768 \mathrm{~cm}^{-1}\end{array}$

\section{B3LYP/6-31G(d) Molecular Geometry in Cartesian Coordinates}

C

$\mathrm{H}$

$\mathrm{H}$

C

0

0

$\mathrm{H}$

0

$\mathrm{H}$

C

C

C

C

$\mathrm{H}$

$\mathrm{H}$

$\mathrm{H}$

C

C

C

$\mathrm{H}$

$\mathrm{H}$

$\mathrm{H}$

C

$\mathrm{H}$

$\mathrm{H}$

$\mathrm{H}$

C

$\mathrm{H}$

$\mathrm{H}$

$\mathrm{H}$

C

$\mathrm{H}$

\section{$-2.227475$}

$-3.102328$

$-1.695387$

0.758034

1. 216276

0.310675

$-0.197906$

$-1.364152$

$-0.967405$

1.466613

2.650890

0.076844

1.011434

2.688355

$-0.668868$

$-0.390135$

$-0.042827$

1. 332659

$-1.254351$

$-2.159448$

$-1.389970$

$-1.152955$

$-0.202154$

$-0.143105$

$-1.182595$

0.562651

2. 502045

2.452783

2.518354

3.444606

1.339410

1.129283
$-0.256800$

0.329738

$-0.561518$

$-1.959814$

$-2.736120$

$-0.757263$

$-0.281794$

0.550639

1.290633

2.087989

1.748220

3. 375113

3. 288079

0.693599

2.577047

4. 356294

3.664896

4.408157

4. 581088

3.968077

5.293396

5.143599

2. 923081

3. 616638

2.434753

2.153150

3.665333

3. 728655

2. 610191

4.118931

5.875537

5.976248
0.896182

1.193148

1.803422

$-1.140008$

$-1.954064$

$-1.514840$

$-0.794330$

0.074067

0.598890

$-3.100796$

$-1.298915$

$-1.517149$

$-2.605875$

$-1.579282$

$-1.522309$

$-1.406327$

2.096295

1.908156

1.864884

1.795836

2.686106

0.932259

3.424896

4.271850

3. 464221

3.549609

2. 578141

3. 670797

2. 289586

2. 254592

2. 339126

3.410546 


\begin{tabular}{|c|c|c|c|}
\hline $\mathrm{H}$ & 2.327215 & 6.308902 & 2.148915 \\
\hline $\mathrm{H}$ & 0.604534 & 6.460592 & 1.781110 \\
\hline 0 & -0.001281 & 2.694568 & 1.029415 \\
\hline 0 & 1.510419 & 4.357135 & 0.479793 \\
\hline B & 0.906396 & 3.152809 & -0.005249 \\
\hline 0 & 1.836897 & 1.985637 & -0.320835 \\
\hline C & 3.902759 & 2.495140 & -1.575943 \\
\hline C & 4.177811 & 3.775993 & -1.068910 \\
\hline C & 4.861588 & 1.855622 & -2.380381 \\
\hline C & 5.387000 & 4.398424 & -1.376675 \\
\hline $\mathrm{H}$ & 3.441849 & 4.274011 & -0.446057 \\
\hline C & 6.066269 & 2.483756 & -2.685757 \\
\hline $\mathrm{H}$ & 4.658270 & 0.857959 & -2.762508 \\
\hline C & 6.331911 & 3.760280 & -2.184535 \\
\hline $\mathrm{H}$ & 5.593368 & 5.389638 & -0.981487 \\
\hline $\mathrm{H}$ & 6.797838 & 1.977157 & -3.309313 \\
\hline $\mathrm{H}$ & 7.271526 & 4.253189 & -2.419342 \\
\hline $\mathrm{H}$ & 1.541410 & 4.195817 & -2.896980 \\
\hline $\mathrm{H}$ & 0.890449 & 1.177744 & -2.955277 \\
\hline $\mathrm{H}$ & 2.215848 & 2.065300 & -3.886603 \\
\hline C & -0.556321 & -2.625663 & 0.881721 \\
\hline C & 0.679759 & -2.297918 & 0.337406 \\
\hline C & 1.878367 & -2.340829 & 1.133902 \\
\hline C & 1.761689 & -2.711332 & 2.460766 \\
\hline $\mathrm{H}$ & 2.657609 & -2.757459 & 3.073855 \\
\hline C & -2.652351 & -1.473003 & 0.099039 \\
\hline C & -1.811738 & -2.584280 & 0.052804 \\
\hline C & -4.238158 & -2.607499 & -1.346805 \\
\hline C & -3.898470 & -1.484114 & -0.614438 \\
\hline $\mathrm{H}$ & -5.176957 & -2.616696 & -1.894586 \\
\hline C & -3.398192 & -3.741119 & -1.432860 \\
\hline C & -2.158620 & -3.740348 & -0.719365 \\
\hline C & -1.328814 & -4.893322 & -0.805276 \\
\hline C & -1.708303 & -5.982151 & -1.556884 \\
\hline C & -2.935730 & -5.978993 & -2.262797 \\
\hline C & -3.760499 & -4.880496 & -2.201949 \\
\hline $\mathrm{H}$ & -0.378435 & -4.901863 & -0.283785 \\
\hline $\mathrm{H}$ & -1.057141 & -6.849956 & -1.615144 \\
\hline $\mathrm{H}$ & -3.220528 & -6.844930 & -2.854417 \\
\hline $\mathrm{H}$ & -4.704280 & -4.864927 & -2.742038 \\
\hline C & 0.528263 & -3.077148 & 3.048918 \\
\hline C & -0.656735 & -3.046284 & 2.250504 \\
\hline C & -1.885947 & -3.436714 & 2.851619 \\
\hline C & -1.935463 & -3.829680 & 4.171074 \\
\hline C & -0.760516 & -3.853971 & 4.959789 \\
\hline C & 0.444446 & -3.485846 & 4.407449 \\
\hline $\mathrm{H}$ & -2.792071 & -3.425775 & 2.255824 \\
\hline $\mathrm{H}$ & -2.883988 & -4.127104 & 4.610034 \\
\hline $\mathrm{H}$ & -0.813588 & -4.166371 & 5.999212 \\
\hline $\mathrm{H}$ & 1.354841 & -3.503881 & 5.001940 \\
\hline C & -6.812837 & 1.642921 & -0.578891 \\
\hline C & -7.202832 & 0.342574 & -0.231711 \\
\hline C & -6.216597 & -0.649426 & -0.234258 \\
\hline C & -4.884546 & -0.361708 & -0.570760 \\
\hline
\end{tabular}




\begin{tabular}{|c|c|c|c|}
\hline C & -4.535497 & 0.954208 & -0.909167 \\
\hline C & -5.494417 & 1.975836 & -0.919967 \\
\hline $\mathrm{H}$ & -7.564321 & 2.427013 & -0.583211 \\
\hline $\mathrm{H}$ & -6.464476 & -1.668695 & 0.038819 \\
\hline $\mathrm{H}$ & -3.504621 & 1.160435 & -1.171062 \\
\hline C & -8.672153 & 0.055419 & 0.134950 \\
\hline C & -5.154764 & 3.431494 & -1.295948 \\
\hline C & -9.583128 & 0.427435 & -1.059138 \\
\hline $\mathrm{H}$ & -10.634385 & 0.234484 & -0.812111 \\
\hline $\mathrm{H}$ & -9.493230 & 1.485161 & -1.327775 \\
\hline $\mathrm{H}$ & -9.327921 & -0.164352 & -1.945651 \\
\hline C & -8.913670 & -1.427437 & 0.475306 \\
\hline $\mathrm{H}$ & -9.970082 & -1.582593 & 0.722394 \\
\hline $\mathrm{H}$ & -8.672189 & -2.083831 & -0.368384 \\
\hline $\mathrm{H}$ & -8.322197 & -1.749991 & 1.339647 \\
\hline C & -9.072195 & 0.901988 & 1.366739 \\
\hline $\mathrm{H}$ & -10.119788 & 0.716667 & 1.634532 \\
\hline $\mathrm{H}$ & -8.450269 & 0.650158 & 2.233430 \\
\hline $\mathrm{H}$ & -8.961479 & 1.974796 & 1.177399 \\
\hline C & -3.666815 & 3.611379 & -1.645381 \\
\hline $\mathrm{H}$ & -3.470236 & 4.660461 & -1.895182 \\
\hline $\mathrm{H}$ & -3.015718 & 3.341234 & -0.807591 \\
\hline $\mathrm{H}$ & -3.374714 & 3.005913 & -2.510590 \\
\hline C & -5.992135 & 3.858409 & -2.524989 \\
\hline $\mathrm{H}$ & -5.763018 & 4.895090 & -2.801290 \\
\hline $\mathrm{H}$ & -5.772081 & 3.220228 & -3.388364 \\
\hline $\mathrm{H}$ & -7.067841 & 3.796244 & -2.329555 \\
\hline C & -5.484329 & 4.362028 & -0.104393 \\
\hline $\mathrm{H}$ & -5.252158 & 5.403794 & -0.357876 \\
\hline $\mathrm{H}$ & -6.543028 & 4.315022 & 0.171928 \\
\hline $\mathrm{H}$ & -4.896475 & 4.089010 & 0.779713 \\
\hline C & 3.244313 & -1.990553 & 0.634804 \\
\hline C & 4.045286 & -1.118877 & 1.398901 \\
\hline C & 3.795188 & -2.584899 & -0.503561 \\
\hline C & 5.369533 & -0.848824 & 1.051167 \\
\hline $\mathrm{H}$ & 3.604850 & -0.657194 & 2.274862 \\
\hline c & 5.114701 & -2.317570 & -0.903788 \\
\hline $\mathrm{H}$ & 3.183953 & -3.269120 & -1.076229 \\
\hline C & 5.877790 & -1.454093 & -0.113817 \\
\hline $\mathrm{H}$ & 6.902626 & -1.244849 & -0.398293 \\
\hline C & 5.659727 & -2.997483 & -2.174827 \\
\hline C & 6.286300 & 0.052747 & 1.901988 \\
\hline C & 5.584748 & -4.534415 & -2.009710 \\
\hline $\mathrm{H}$ & 6.180582 & -4.866026 & -1.151372 \\
\hline H & 4.556798 & -4.877883 & -1.858132 \\
\hline $\mathrm{H}$ & 5.973262 & -5.032535 & -2.906631 \\
\hline C & 4.799071 & -2.574682 & -3.390013 \\
\hline $\mathrm{H}$ & 5.136816 & -3.094159 & -4.295570 \\
\hline $\mathrm{H}$ & 3.738444 & -2.800312 & -3.242914 \\
\hline $\mathrm{H}$ & 4.888029 & -1.496188 & -3.571987 \\
\hline C & 7.124748 & -2.620918 & -2.464884 \\
\hline $\mathrm{H}$ & 7.247756 & -1.542331 & -2.618267 \\
\hline $\mathrm{H}$ & 7.794901 & -2.928213 & -1.653559 \\
\hline $\mathrm{H}$ & 7.461007 & -3.123840 & -3.378746 \\
\hline
\end{tabular}




\begin{tabular}{|llll|} 
C & 7.505520 & -0.772321 & 2.380826 \\
$\mathrm{H}$ & 7.186855 & -1.627856 & 2.987373 \\
$\mathrm{H}$ & 8.091870 & -1.159162 & 1.540813 \\
$\mathrm{H}$ & 8.170503 & -0.149859 & 2.992468 \\
$\mathrm{C}$ & 6.781555 & 1.250566 & 1.059194 \\
$\mathrm{H}$ & 7.323920 & 0.925015 & 0.165784 \\
$\mathrm{H}$ & 5.944156 & 1.873994 & 0.728977 \\
$\mathrm{H}$ & 7.460218 & 1.876798 & 1.651988 \\
$\mathrm{C}$ & 5.571761 & 0.608026 & 3.148794 \\
$\mathrm{H}$ & 6.264253 & 1.236615 & 3.720266 \\
$\mathrm{H}$ & 4.707496 & 1.227315 & 2.883793 \\
$\mathrm{H}$ & 5.228844 & -0.193513 & 3.813229 \\
& & & \\
\hline
\end{tabular}

\section{C-Catalyzed reaction TS 46}

\section{Datum}

Value

\begin{tabular}{lr}
\hline B3LYP/6-31G(d) Energy & -3038.955418 \\
\hline B3LYP-D3(BJ)/def2-TZVPP-IEF-PCM(DCM) Energy & -3040.510621 \\
\hline B3LYP-D3(BJ)/def2-TZVPP-IEF-PCM(DCM)//B3LYP/6-31G(d) Free Energy (Quasiharmonic) & -3039.28279 \\
\hline
\end{tabular}

Number of Imaginary Frequencies

Frequencies (Top 3 out of 450)

$\begin{array}{lr}\text { 1. } & -337.8366 \mathrm{~cm}^{-1} \\ \text { 2. } & 3.6696 \mathrm{~cm}^{-1} \\ \text { 3. } & 8.4581 \mathrm{~cm}^{-1}\end{array}$

\section{B3LYP/6-31G(d) Molecular Geometry in Cartesian Coordinates}

C

$\mathrm{H}$

$\mathrm{H}$

C

0

0

$\mathrm{H}$

0

$\mathrm{H}$

C

C

C

C

$\mathrm{H}$

\subsection{6}

2.909440

1. 398886

$-0.662094$

$-1.150420$

0.061347

0.442110

1. 304144

0.881781

$-2.956783$

$-3.173028$

$-0.800170$

$-1.581313$

$-3.398969$
0.270110

$-0.336185$

0.333883

2. 296414

3. 192647

1. 324998

0.691554

$-0.356586$

$-1.178392$

$-1.354534$

$-1.907932$

$-2.519207$

$-1.329532$

$-0.879028$
0.637893

0.888278

1.531077

$-1.533846$

$-2.186189$

$-2.098819$

$-1.427861$

$-0.429089$

$-0.074536$

3. 307440

1. 207178

3. 077475

3. 224215

0.922316 


\begin{tabular}{|c|c|c|c|}
\hline $\mathrm{H}$ & 0.266960 & -2.392085 & 3.267214 \\
\hline $\mathrm{H}$ & -1.204436 & -3.383457 & 3.610841 \\
\hline C & 1.055689 & -3.914041 & 0.282232 \\
\hline C & -0.075036 & -5.007783 & 0.354877 \\
\hline C & 1.681380 & -3.721805 & -1.098741 \\
\hline $\mathrm{H}$ & 2.417606 & -2.912918 & -1.060434 \\
\hline $\mathrm{H}$ & 2.193321 & -4.633901 & -1.426524 \\
\hline $\mathrm{H}$ & 0.931857 & -3.454443 & -1.847507 \\
\hline C & 2.159480 & -4.123796 & 1.330269 \\
\hline $\mathrm{H}$ & 2.797610 & -4.978127 & 1.080002 \\
\hline $\mathrm{H}$ & 2.791276 & -3.230127 & 1.370270 \\
\hline $\mathrm{H}$ & 1.736138 & -4.289922 & 2.325357 \\
\hline C & 0.403469 & -6.402644 & 0.760670 \\
\hline $\mathrm{H}$ & 1.117048 & -6.804667 & 0.031784 \\
\hline $\mathrm{H}$ & 0.876280 & -6.393738 & 1.745611 \\
\hline $\mathrm{H}$ & -0.451947 & -7.085253 & 0.805040 \\
\hline C & -0.900478 & -5.099054 & -0.939324 \\
\hline $\mathrm{H}$ & -0.326840 & -5.547845 & -1.757623 \\
\hline $\mathrm{H}$ & -1.778360 & -5.727509 & -0.756361 \\
\hline $\mathrm{H}$ & -1.250859 & -4.113990 & -1.260580 \\
\hline 0 & 0.330482 & -2.709041 & 0.628752 \\
\hline 0 & -0.920577 & -4.496791 & 1.402606 \\
\hline B & -0.840360 & -3.069714 & 1.394617 \\
\hline 0 & -2.023739 & -2.338830 & 0.790189 \\
\hline C & -4.344519 & -2.802931 & 1.322790 \\
\hline C & -4.207675 & -4.197946 & 1.424746 \\
\hline C & -5.627298 & -2.229571 & 1.305434 \\
\hline C & -5.344938 & -4.996838 & 1.521537 \\
\hline $\mathrm{H}$ & -3.214754 & -4.638242 & 1.424932 \\
\hline C & -6.759122 & -3.036019 & 1.395698 \\
\hline $\mathrm{H}$ & -5.731584 & -1.151132 & 1.209181 \\
\hline C & -6.619327 & -4.421762 & 1.508458 \\
\hline $\mathrm{H}$ & -5.238248 & -6.075375 & 1.600701 \\
\hline $\mathrm{H}$ & -7.748035 & -2.586682 & 1.373560 \\
\hline $\mathrm{H}$ & -7.501464 & -5.052432 & 1.579092 \\
\hline $\mathrm{H}$ & -1.104176 & -0.371486 & 3.015325 \\
\hline $\mathrm{H}$ & -3.453439 & -2.239338 & 3.692370 \\
\hline $\mathrm{H}$ & -3.522722 & -0.429246 & 3.364653 \\
\hline C & 0.181874 & 2.516401 & 0.830690 \\
\hline C & -0.872629 & 2.241401 & -0.029135 \\
\hline C & -2.213559 & 2.094734 & 0.466100 \\
\hline C & -2.439263 & 2.251246 & 1.819369 \\
\hline $\mathrm{H}$ & -3.448076 & 2.129838 & 2.207402 \\
\hline C & 2.469646 & 1.658869 & 0.220760 \\
\hline C & 1.575399 & 2.723409 & 0.303571 \\
\hline C & 4.204006 & 3.164349 & -0.569655 \\
\hline C & 3.804366 & 1.882161 & -0.248650 \\
\hline $\mathrm{H}$ & 5.220447 & 3.331802 & -0.917257 \\
\hline C & 3.321663 & 4.267820 & -0.495324 \\
\hline C & 1.974243 & 4.049081 & -0.066687 \\
\hline C & 1.090555 & 5.163817 & -0.020553 \\
\hline C & 1.523178 & 6.423042 & -0.369207 \\
\hline C & 2.860195 & 6.637407 & -0.782766 \\
\hline$\sigma$ & 3.737864 & 5.580629 & -0.846214 \\
\hline
\end{tabular}




\begin{tabular}{|c|c|c|c|}
\hline $\mathrm{H}$ & 0.059057 & 5.010099 & 0.275859 \\
\hline $\mathrm{H}$ & 0.830507 & 7.259380 & -0.334866 \\
\hline $\mathrm{H}$ & 3.186776 & 7.637014 & -1.056713 \\
\hline $\mathrm{H}$ & 4.764492 & 5.731255 & -1.171685 \\
\hline C & -1.396305 & 2.551064 & 2.731001 \\
\hline C & -0.059118 & 2.679068 & 2.237717 \\
\hline C & 0.977240 & 2.973564 & 3.166384 \\
\hline C & 0.705224 & 3.126769 & 4.508662 \\
\hline C & -0.617540 & 2.996426 & 4.993364 \\
\hline C & -1.644106 & 2.714491 & 4.121024 \\
\hline $\mathrm{H}$ & 1.993783 & 3.079532 & 2.803104 \\
\hline $\mathrm{H}$ & 1.512049 & 3.351919 & 5.200760 \\
\hline $\mathrm{H}$ & -0.819831 & 3.121840 & 6.053573 \\
\hline $\mathrm{H}$ & -2.665075 & 2.616207 & 4.483027 \\
\hline C & 6.766652 & -1.141783 & -0.834632 \\
\hline C & 5.653397 & -1.113835 & -1.695706 \\
\hline C & 4.664505 & -0.156299 & -1.455481 \\
\hline C & 4.800689 & 0.777407 & -0.413835 \\
\hline C & 5.927117 & 0.725026 & 0.409215 \\
\hline C & 6.926800 & -0.244725 & 0.224426 \\
\hline $\mathrm{H}$ & 7.533771 & -1.886628 & -1.011211 \\
\hline $\mathrm{H}$ & 3.782636 & -0.097147 & -2.082616 \\
\hline $\mathrm{H}$ & 6.016359 & 1.457403 & 1.206911 \\
\hline C & 5.587284 & -2.101957 & -2.876844 \\
\hline C & 8.142613 & -0.278371 & 1.170255 \\
\hline C & 5.621611 & -3.555104 & -2.347486 \\
\hline $\mathrm{H}$ & 5.579074 & -4.265662 & -3.182187 \\
\hline $\mathrm{H}$ & 6.535969 & -3.762716 & -1.781609 \\
\hline $\mathrm{H}$ & 4.768757 & -3.753224 & -1.689298 \\
\hline C & 4.310989 & -1.922395 & -3.721019 \\
\hline $\mathrm{H}$ & 4.308588 & -2.646390 & -4.543946 \\
\hline $\mathrm{H}$ & 3.401541 & -2.086113 & -3.133471 \\
\hline $\mathrm{H}$ & 4.253060 & -0.921247 & -4.162272 \\
\hline C & 6.806130 & -1.870885 & -3.802949 \\
\hline $\mathrm{H}$ & 6.776835 & -2.565845 & -4.651315 \\
\hline $\mathrm{H}$ & 6.807046 & -0.849690 & -4.200749 \\
\hline $\mathrm{H}$ & 7.754707 & -2.025805 & -3.278007 \\
\hline C & 8.893223 & 1.072537 & 1.095858 \\
\hline $\mathrm{H}$ & 9.757083 & 1.067964 & 1.772040 \\
\hline $\mathrm{H}$ & 9.258160 & 1.261915 & 0.079830 \\
\hline $\mathrm{H}$ & 8.251578 & 1.912274 & 1.381958 \\
\hline C & 7.657013 & -0.514550 & 2.620248 \\
\hline $\mathrm{H}$ & 8.508921 & -0.534322 & 3.311205 \\
\hline $\mathrm{H}$ & 6.974579 & 0.273882 & 2.954345 \\
\hline $\mathrm{H}$ & 7.128805 & -1.471289 & 2.704876 \\
\hline C & 9.138810 & -1.397604 & 0.811711 \\
\hline $\mathrm{H}$ & 9.980476 & -1.380834 & 1.513466 \\
\hline $\mathrm{H}$ & 8.677886 & -2.390038 & 0.873198 \\
\hline $\mathrm{H}$ & 9.548421 & -1.272110 & -0.197049 \\
\hline C & -3.351827 & 1.746808 & -0.429950 \\
\hline C & -3.229045 & 0.710547 & -1.371811 \\
\hline C & -4.572996 & 2.416518 & -0.315424 \\
\hline C & -4.293361 & 0.350943 & -2.199131 \\
\hline $\mathrm{H}$ & -2.283043 & 0.189581 & -1.448046 \\
\hline
\end{tabular}




\begin{tabular}{|c|c|c|c|}
\hline C & -5.671779 & 2.082810 & -1.123210 \\
\hline $\mathrm{H}$ & -4.649706 & 3.234777 & 0.394416 \\
\hline C & -5.505099 & 1.050613 & -2.051772 \\
\hline $\mathrm{H}$ & -6.334959 & 0.781112 & -2.694240 \\
\hline C & -6.990751 & 2.866345 & -0.976710 \\
\hline C & -4.172044 & -0.756237 & -3.263077 \\
\hline C & -6.737316 & 4.360824 & -1.287455 \\
\hline $\mathrm{H}$ & -7.665985 & 4.935072 & -1.180407 \\
\hline $\mathrm{H}$ & -5.994118 & 4.797747 & -0.612639 \\
\hline $\mathrm{H}$ & -6.371740 & 4.490363 & -2.312110 \\
\hline C & -8.087812 & 2.358547 & -1.931769 \\
\hline $\mathrm{H}$ & -9.003870 & 2.941563 & -1.783586 \\
\hline $\mathrm{H}$ & -7.795526 & 2.465949 & -2.982258 \\
\hline $\mathrm{H}$ & -8.333181 & 1.305668 & -1.750488 \\
\hline C & -7.516712 & 2.728065 & 0.471944 \\
\hline $\mathrm{H}$ & -7.718508 & 1.678457 & 0.717362 \\
\hline $\mathrm{H}$ & -6.799848 & 3.112050 & 1.205307 \\
\hline $\mathrm{H}$ & -8.450756 & 3.289886 & 0.594977 \\
\hline C & -4.382110 & -0.132592 & -4.663815 \\
\hline $\mathrm{H}$ & -5.368195 & 0.335142 & -4.757482 \\
\hline $\mathrm{H}$ & -3.626610 & 0.634409 & -4.866225 \\
\hline $\mathrm{H}$ & -4.303192 & -0.904403 & -5.439458 \\
\hline C & -2.788459 & -1.435728 & -3.242577 \\
\hline $\mathrm{H}$ & -1.979431 & -0.724977 & -3.443557 \\
\hline $\mathrm{H}$ & -2.587345 & -1.919541 & -2.279366 \\
\hline $\mathrm{H}$ & -2.750184 & -2.210707 & -4.016783 \\
\hline C & -5.246513 & -1.841635 & -3.017537 \\
\hline $\mathrm{H}$ & -5.168025 & -2.630328 & -3.775732 \\
\hline $\mathrm{H}$ & -5.121446 & -2.305049 & -2.032262 \\
\hline $\mathrm{H}$ & -6.261658 & -1.433545 & -3.069110 \\
\hline
\end{tabular}

\section{C-Catalyzed reaction TS 47}

\section{Datum}

Value

\begin{tabular}{lc}
\hline B3LYP/6-31G(d) Energy & -3038.958696 \\
\hline B3LYP-D3(BJ)/def2-TZVPP-IEF-PCM(DCM) Energy & -3040.511357 \\
\hline B3LYP-D3(BJ)/def2-TZVPP-IEF-PCM(DCM)//B3LYP/6-31G(d) Free Energy (Quasiharmonic) & -3039.282732 \\
\hline
\end{tabular}

Number of Imaginary Frequencies

Frequencies (Top 3 out of 450)

$\begin{array}{lr}\text { 1. } & -306.5222 \mathrm{~cm}^{-1} \\ \text { 2. } & 7.0884 \mathrm{~cm}^{-1} \\ \text { 3. } & 11.6558 \mathrm{~cm}^{-1}\end{array}$




\begin{tabular}{|c|c|c|c|}
\hline C & -2.646089 & -2.099412 & -0.473113 \\
\hline $\mathrm{H}$ & -1.684493 & -2.455737 & -0.093511 \\
\hline $\mathrm{H}$ & -3.374094 & -2.200688 & 0.332008 \\
\hline C & 0.644968 & -0.521169 & 0.636797 \\
\hline 0 & -0.244669 & -0.726657 & 1.436212 \\
\hline 0 & 1.556586 & 0.432665 & 0.886598 \\
\hline $\mathrm{H}$ & 2.229998 & 0.628341 & 0.184034 \\
\hline 0 & -3.124831 & -2.951202 & -1.525185 \\
\hline $\mathrm{H}$ & -2.459257 & -2.944909 & -2.232124 \\
\hline C & 2.114606 & 3.506669 & 2.941464 \\
\hline C & 1.582213 & 3.762869 & 0.833991 \\
\hline C & 4.291077 & 2.909816 & 1.926552 \\
\hline C & 3.082098 & 2.577660 & 2.628673 \\
\hline $\mathrm{H}$ & 1.183249 & 2.753116 & 0.741774 \\
\hline $\mathrm{H}$ & 5.054976 & 2.130280 & 1.950316 \\
\hline $\mathrm{H}$ & 4.714366 & 3.884982 & 2.180450 \\
\hline C & 4.534640 & 1.514378 & -1.411325 \\
\hline C & 5.089933 & 2.950826 & -1.732094 \\
\hline C & 5.591806 & 0.607991 & -0.761463 \\
\hline $\mathrm{H}$ & 5.099088 & -0.273150 & -0.339329 \\
\hline $\mathrm{H}$ & 6.336400 & 0.270357 & -1.491282 \\
\hline $\mathrm{H}$ & 6.116265 & 1.126868 & 0.046055 \\
\hline C & 3.895782 & 0.793739 & -2.597825 \\
\hline $\mathrm{H}$ & 4.632927 & 0.625089 & -3.391827 \\
\hline $\mathrm{H}$ & 3.513198 & -0.185322 & -2.288790 \\
\hline $\mathrm{H}$ & 3.066871 & 1.367595 & -3.017760 \\
\hline C & 4.220731 & 3.717005 & -2.745405 \\
\hline $\mathrm{H}$ & 4.320606 & 3.317227 & -3.761079 \\
\hline $\mathrm{H}$ & 3.165564 & 3.691482 & -2.458178 \\
\hline $\mathrm{H}$ & 4.539644 & 4.764091 & -2.755497 \\
\hline C & 6.552362 & 2.988315 & -2.180825 \\
\hline $\mathrm{H}$ & 6.698389 & 2.424306 & -3.109805 \\
\hline $\mathrm{H}$ & 6.850894 & 4.025713 & -2.364957 \\
\hline $\mathrm{H}$ & 7.216625 & 2.581202 & -1.414909 \\
\hline 0 & 3.514374 & 1.797035 & -0.419289 \\
\hline 0 & 4.987597 & 3.605456 & -0.461456 \\
\hline B & 3.879058 & 3.055582 & 0.238440 \\
\hline 0 & 2.719887 & 4.032443 & 0.276619 \\
\hline C & 0.603959 & 4.857698 & 0.981741 \\
\hline C & 0.985431 & 6.197542 & 0.808032 \\
\hline C & -0.731993 & 4.550503 & 1.281332 \\
\hline C & 0.041704 & 7.212502 & 0.939704 \\
\hline $\mathrm{H}$ & 2.017843 & 6.424022 & 0.561689 \\
\hline C & -1.672211 & 5.569901 & 1.414004 \\
\hline $\mathrm{H}$ & -1.030156 & 3.512089 & 1.403141 \\
\hline C & -1.287058 & 6.902330 & 1.246089 \\
\hline $\mathrm{H}$ & 0.340078 & 8.248236 & 0.801755 \\
\hline $\mathrm{H}$ & -2.705148 & 5.325340 & 1.645369 \\
\hline $\mathrm{H}$ & -2.020634 & 7.697352 & 1.349861 \\
\hline $\mathrm{H}$ & 2.804965 & 1.526591 & 2.688389 \\
\hline
\end{tabular}




\begin{tabular}{|c|c|c|c|}
\hline $\mathrm{H}$ & 2.377888 & 4.555170 & 3.048778 \\
\hline $\mathrm{H}$ & 1.183411 & 3.198927 & 3.408330 \\
\hline C & -0.180766 & -1.114588 & -1.636116 \\
\hline C & 0.779447 & -1.307445 & -0.647406 \\
\hline C & 1.822532 & -2.286530 & -0.792312 \\
\hline C & 1.900866 & -2.980813 & -1.986299 \\
\hline $\mathrm{H}$ & 2.659289 & -3.750033 & -2.103123 \\
\hline C & -2.531950 & -0.643376 & -0.894746 \\
\hline C & -1.339089 & -0.170553 & -1.439024 \\
\hline C & -3.508771 & 1.572740 & -1.108418 \\
\hline C & -3.647260 & 0.249222 & -0.732862 \\
\hline $\mathrm{H}$ & -4.354970 & 2.246274 & -0.997890 \\
\hline C & -2.316837 & 2.077939 & -1.676275 \\
\hline C & -1.205757 & 1.193607 & -1.854996 \\
\hline C & -0.024511 & 1.711110 & -2.455606 \\
\hline C & 0.051031 & 3.027246 & -2.855786 \\
\hline C & -1.047532 & 3.900546 & -2.669976 \\
\hline C & -2.204540 & 3.432578 & -2.092485 \\
\hline $\mathrm{H}$ & 0.823243 & 1.052069 & -2.605889 \\
\hline $\mathrm{H}$ & 0.959500 & 3.398850 & -3.321070 \\
\hline $\mathrm{H}$ & -0.972162 & 4.937775 & -2.984060 \\
\hline $\mathrm{H}$ & -3.056131 & 4.092942 & -1.947043 \\
\hline C & 0.988220 & -2.771780 & -3.046747 \\
\hline C & -0.081268 & -1.836422 & -2.871846 \\
\hline C & -1.011442 & -1.665819 & -3.938006 \\
\hline C & -0.887033 & -2.384024 & -5.107749 \\
\hline C & 0.171544 & -3.308537 & -5.275494 \\
\hline C & 1.086974 & -3.496105 & -4.265899 \\
\hline $\mathrm{H}$ & -1.820219 & -0.952037 & -3.823154 \\
\hline $\mathrm{H}$ & -1.605619 & -2.237335 & -5.909257 \\
\hline $\mathrm{H}$ & 0.257293 & -3.868735 & -6.202396 \\
\hline $\mathrm{H}$ & 1.901730 & -4.206448 & -4.383644 \\
\hline C & -7.452224 & -0.891657 & 0.856800 \\
\hline C & -6.795142 & 0.230592 & 1.394130 \\
\hline C & -5.547080 & 0.568209 & 0.868501 \\
\hline C & -4.958812 & -0.183584 & -0.164715 \\
\hline C & -5.645979 & -1.289638 & -0.672268 \\
\hline C & -6.901174 & -1.665679 & -0.168023 \\
\hline $\mathrm{H}$ & -8.419819 & -1.164635 & 1.261496 \\
\hline $\mathrm{H}$ & -4.993918 & 1.412048 & 1.264408 \\
\hline $\mathrm{H}$ & -5.183634 & -1.865752 & -1.465858 \\
\hline C & -7.461659 & 1.029551 & 2.531314 \\
\hline C & -7.607365 & -2.902222 & -0.756761 \\
\hline C & -8.821329 & 1.584249 & 2.043557 \\
\hline $\mathrm{H}$ & -9.312172 & 2.152054 & 2.843794 \\
\hline $\mathrm{H}$ & -8.685957 & 2.252976 & 1.185615 \\
\hline $\mathrm{H}$ & -9.502991 & 0.784022 & 1.737040 \\
\hline C & -7.693607 & 0.103614 & 3.748976 \\
\hline $\mathrm{H}$ & -8.169377 & 0.659426 & 4.566624 \\
\hline $\mathrm{H}$ & -8.342030 & -0.743132 & 3.500799 \\
\hline $\mathrm{H}$ & -6.744584 & -0.300409 & 4.119317 \\
\hline C & -6.601242 & 2.219657 & 2.996467 \\
\hline $\mathrm{H}$ & -7.116074 & 2.754729 & 3.802668 \\
\hline $\mathrm{H}$ & -5.629587 & 1.893567 & 3.384323 \\
\hline
\end{tabular}




\begin{tabular}{|c|c|c|c|}
\hline $\mathrm{H}$ & -6.423419 & 2.935709 & 2.185803 \\
\hline C & -7.854306 & -2.680793 & -2.268161 \\
\hline $\mathrm{H}$ & -8.354268 & -3.554371 & -2.704721 \\
\hline $\mathrm{H}$ & -8.491834 & -1.804933 & -2.436840 \\
\hline $\mathrm{H}$ & -6.918507 & -2.524790 & -2.814194 \\
\hline C & -6.707123 & -4.146492 & -0.561672 \\
\hline $\mathrm{H}$ & -7.183686 & -5.031854 & -1.001349 \\
\hline $\mathrm{H}$ & -5.725487 & -4.022405 & -1.029597 \\
\hline $\mathrm{H}$ & -6.545373 & -4.345367 & 0.504398 \\
\hline C & -8.965823 & -3.181854 & -0.086956 \\
\hline $\mathrm{H}$ & -9.421577 & -4.071751 & -0.535950 \\
\hline $\mathrm{H}$ & -8.859339 & -3.373390 & 0.987039 \\
\hline $\mathrm{H}$ & -9.667717 & -2.350006 & -0.218678 \\
\hline C & 2.763456 & -2.636782 & 0.311998 \\
\hline C & 4.119266 & -2.893099 & 0.030818 \\
\hline C & 2.306344 & -2.799970 & 1.622951 \\
\hline C & 5.007282 & -3.294462 & 1.030318 \\
\hline $\mathrm{H}$ & 4.468097 & -2.761943 & -0.986932 \\
\hline C & 3.170665 & -3.179701 & 2.661655 \\
\hline $\mathrm{H}$ & 1.255737 & -2.645496 & 1.836753 \\
\hline C & 4.508585 & -3.420358 & 2.340340 \\
\hline $\mathrm{H}$ & 5.192911 & -3.719093 & 3.125728 \\
\hline C & 2.611244 & -3.319121 & 4.090168 \\
\hline C & 6.493496 & -3.594970 & 0.753639 \\
\hline C & 2.010506 & -1.963860 & 4.537293 \\
\hline $\mathrm{H}$ & 2.782401 & -1.185373 & 4.560194 \\
\hline $\mathrm{H}$ & 1.213040 & -1.623415 & 3.869063 \\
\hline $\mathrm{H}$ & 1.589266 & -2.051792 & 5.546429 \\
\hline C & 1.504172 & -4.400100 & 4.106883 \\
\hline $\mathrm{H}$ & 1.091372 & -4.504816 & 5.117736 \\
\hline $\mathrm{H}$ & 0.676797 & -4.148501 & 3.435755 \\
\hline $\mathrm{H}$ & 1.902032 & -5.374321 & 3.799749 \\
\hline C & 3.692351 & -3.725627 & 5.109042 \\
\hline $\mathrm{H}$ & 4.136136 & -4.699029 & 4.869633 \\
\hline $\mathrm{H}$ & 4.498937 & -2.985599 & 5.166850 \\
\hline $\mathrm{H}$ & 3.245815 & -3.804262 & 6.106618 \\
\hline C & 6.861133 & -3.433415 & -0.733859 \\
\hline $\mathrm{H}$ & 6.282405 & -4.110744 & -1.372527 \\
\hline $\mathrm{H}$ & 6.701276 & -2.408617 & -1.086453 \\
\hline $\mathrm{H}$ & 7.921335 & -3.669956 & -0.878690 \\
\hline C & 7.378209 & -2.628419 & 1.576613 \\
\hline $\mathrm{H}$ & 7.182300 & -1.586253 & 1.300227 \\
\hline $\mathrm{H}$ & 7.198946 & -2.728151 & 2.652249 \\
\hline $\mathrm{H}$ & 8.440143 & -2.834947 & 1.395061 \\
\hline C & 6.808821 & -5.052952 & 1.166062 \\
\hline $\mathrm{H}$ & 7.865679 & -5.280125 & 0.979956 \\
\hline $\mathrm{H}$ & 6.613224 & -5.229816 & 2.228612 \\
\hline $\mathrm{H}$ & 6.202329 & -5.762837 & 0.591936 \\
\hline
\end{tabular}


Frequencies (Top 3 out of 450)

$\begin{array}{lr}\text { 1. } & -311.5147 \mathrm{~cm}^{-1} \\ \text { 2. } & 5.9082 \mathrm{~cm}^{-1} \\ \text { 3. } & 9.6594 \mathrm{~cm}^{-1}\end{array}$

\section{B3LYP/6-31G(d) Molecular Geometry in Cartesian Coordinates}

C

$\mathrm{H}$

$\mathrm{H}$

C

0

0

$\mathrm{H}$

0

$\mathrm{H}$

C

C

C

C

$\mathrm{H}$

$\mathrm{H}$

$\mathrm{H}$

C

C

C

$\mathrm{H}$

$\mathrm{H}$

$\mathrm{H}$

C

$\mathrm{H}$

$\mathrm{H}$

$\mathrm{H}$

C

$\mathrm{H}$

$\mathrm{H}$

$\mathrm{H}$

C

$\mathrm{H}$

\subsection{1}

2.571672

4.177493

$-0.382914$

0.387257

$-1.407002$

$-1.963175$

2.697779

1.859727

$-1.678721$

$-3.328681$

$-2.406824$

$-1.471721$

$-2.677426$

$-2.007413$

$-2.927028$

$-4.031857$

$-5.298026$

$-3.216854$

$-2.259914$

$-3.737343$

$-3.017432$

$-4.316120$

$-5.002936$

$-3.387627$

$-4.753170$

$-6.353662$

$-6.875890$

$-5.906566$

$-7.092511$

$-5.969520$

$-6.336012$

\subsection{8}

0.634801

0.923872

0.299892

1.064038

$-0.195599$

$-0.911978$

1.908285

1.686335

$-1.324987$

$-0.413342$

$-3.311805$

$-2.262779$

$-0.014800$

$-4.104600$

$-3.719397$

$-2.939815$

$-3.334520$

$-4.166676$

$-3.837169$

$-4.741373$

$-4.830484$

$-2.055336$

$-2.554943$

$-1.849390$

$-1.100240$

$-2.214168$

$-2.097145$

$-1.254339$

$-2.469201$

$-4.645677$

$-4.599208$

\subsection{6}

2.492246

1.818582

0.049013

$-0.509167$

$-0.650391$

$-0.245872$

0.853509

0.397320

$-4.224854$

$-3.139217$

$-2.943022$

$-3.236275$

$-2.363933$

$-2.308537$

$-3.813305$

0.318678

$-0.526199$

0.755610

1.171678

1.529437

$-0.090579$

1.530268

2. 224020

2.074777

1. 233302

$-0.580034$

0.376310

$-0.853151$

$-1.346337$

$-0.111233$

0.921285 


\begin{tabular}{|c|c|c|c|}
\hline $\mathrm{H}$ & -6.827128 & -4.836074 & -0.764976 \\
\hline $\mathrm{H}$ & -5.285937 & -5.492811 & -0.204348 \\
\hline 0 & -3.244203 & -2.183589 & -0.639033 \\
\hline 0 & -4.743153 & -3.509303 & -1.836165 \\
\hline B & -3.668456 & -2.591901 & -1.983614 \\
\hline 0 & -4.093368 & -1.419978 & -2.850953 \\
\hline C & -3.798776 & 0.541840 & -4.162316 \\
\hline C & -3.177342 & 1.796482 & -4.262302 \\
\hline C & -4.872061 & 0.230415 & -5.011127 \\
\hline C & -3.620412 & 2.725886 & -5.200992 \\
\hline $\mathrm{H}$ & -2.347347 & 2.037611 & -3.601885 \\
\hline C & -5.311314 & 1.162335 & -5.947810 \\
\hline $\mathrm{H}$ & -5.355958 & -0.736319 & -4.915473 \\
\hline C & -4.686945 & 2.409677 & -6.046364 \\
\hline $\mathrm{H}$ & -3.136656 & 3.696017 & -5.271719 \\
\hline H & -6.145085 & 0.919287 & -6.600869 \\
\hline $\mathrm{H}$ & -5.033463 & 3.134550 & -6.777986 \\
\hline $\mathrm{H}$ & -0.699640 & -2.057566 & -2.496091 \\
\hline $\mathrm{H}$ & -2.307419 & -1.563663 & -5.078097 \\
\hline H & -0.978107 & -0.506728 & -4.364456 \\
\hline C & 0.801950 & -0.958589 & 1.842564 \\
\hline C & -0.252992 & -0.122157 & 1.490647 \\
\hline C & -1.205598 & 0.347584 & 2.462824 \\
\hline C & -1.111110 & -0.149456 & 3.748908 \\
\hline $\mathrm{H}$ & -1.791264 & 0.220068 & 4.511329 \\
\hline C & 3.016333 & -0.531122 & 0.734997 \\
\hline C & 1.879977 & -1.331020 & 0.855343 \\
\hline C & 3.932473 & -2.081905 & -0.899159 \\
\hline C & 4.070431 & -0.917280 & -0.166334 \\
\hline H & 4.720950 & -2.369166 & -1.589992 \\
\hline C & 2.792998 & -2.912217 & -0.798756 \\
\hline C & 1.742811 & -2.539797 & 0.098260 \\
\hline C & 0.615092 & -3.399386 & 0.205928 \\
\hline C & 0.530223 & -4.559187 & -0.533008 \\
\hline C & 1.566874 & -4.918110 & -1.428014 \\
\hline C & 2.671909 & -4.109008 & -1.556132 \\
\hline H & -0.184715 & -3.134043 & 0.888289 \\
\hline H & -0.337318 & -5.204116 & -0.425166 \\
\hline $\mathrm{H}$ & 1.486903 & -5.832978 & -2.008710 \\
\hline H & 3.476146 & -4.375099 & -2.238033 \\
\hline C & -0.115642 & -1.080266 & 4.132418 \\
\hline C & 0.873593 & -1.478753 & 3.178488 \\
\hline C & 1.894111 & -2.377187 & 3.595789 \\
\hline C & 1.925702 & -2.861627 & 4.884805 \\
\hline C & 0.944594 & -2.467279 & 5.825484 \\
\hline C & -0.051255 & -1.592839 & 5.456248 \\
\hline $\mathrm{H}$ & 2.650600 & -2.682408 & 2.881529 \\
\hline $\mathrm{H}$ & 2.710929 & -3.550517 & 5.183520 \\
\hline $\mathrm{H}$ & 0.982709 & -2.854856 & 6.839861 \\
\hline H & -0.805478 & -1.278437 & 6.173585 \\
\hline $\mathrm{C}$ & 7.728978 & 1.235438 & -0.718835 \\
\hline C & 6.526919 & 1.944812 & -0.780385 \\
\hline C & 5.332029 & 1.233734 & -0.585557 \\
\hline C & 5.336217 & -0.139401 & -0.327659 \\
\hline
\end{tabular}




\begin{tabular}{|c|c|c|c|}
\hline C & 6.569155 & -0.816732 & -0.268412 \\
\hline C & 7.778226 & -0.147323 & -0.462022 \\
\hline $\mathrm{H}$ & 8.662406 & 1.765687 & -0.867312 \\
\hline $\mathrm{H}$ & 4.379500 & 1.749785 & -0.614937 \\
\hline $\mathrm{H}$ & 6.559658 & -1.877882 & -0.047174 \\
\hline C & 6.460027 & 3.461380 & -1.043384 \\
\hline C & 9.143576 & -0.859956 & -0.396913 \\
\hline C & 5.772190 & 4.155183 & 0.157834 \\
\hline $\mathrm{H}$ & 5.693301 & 5.234916 & -0.022201 \\
\hline $\mathrm{H}$ & 4.764721 & 3.765725 & 0.334666 \\
\hline $\mathrm{H}$ & 6.354315 & 4.008074 & 1.075448 \\
\hline C & 7.853081 & 4.090468 & -1.232575 \\
\hline $\mathrm{H}$ & 7.750321 & 5.166798 & -1.412137 \\
\hline $\mathrm{H}$ & 8.482668 & 3.963719 & -0.344256 \\
\hline $\mathrm{H}$ & 8.382777 & 3.662260 & -2.091721 \\
\hline C & 5.635496 & 3.725365 & -2.325855 \\
\hline $\mathrm{H}$ & 5.576109 & 4.802803 & -2.524650 \\
\hline $\mathrm{H}$ & 6.097905 & 3.244353 & -3.195884 \\
\hline $\mathrm{H}$ & 4.612862 & 3.345936 & -2.236150 \\
\hline C & 10.007785 & -0.225204 & 0.718652 \\
\hline $\mathrm{H}$ & 10.985153 & -0.720682 & 0.774099 \\
\hline $\mathrm{H}$ & 10.184811 & 0.840765 & 0.541776 \\
\hline $\mathrm{H}$ & 9.520306 & -0.324646 & 1.695255 \\
\hline C & 9.008662 & -2.364637 & -0.095734 \\
\hline $\mathrm{H}$ & 10.002760 & -2.825279 & -0.064288 \\
\hline $\mathrm{H}$ & 8.529580 & -2.545573 & 0.873179 \\
\hline $\mathrm{H}$ & 8.428334 & -2.884845 & -0.866330 \\
\hline C & 9.869078 & -0.707270 & -1.755013 \\
\hline $\mathrm{H}$ & 10.847146 & -1.203690 & -1.724810 \\
\hline $\mathrm{H}$ & 9.283423 & -1.158965 & -2.564007 \\
\hline $\mathrm{H}$ & 10.036661 & 0.343874 & -2.011727 \\
\hline C & -2.203707 & 1.416570 & 2.163927 \\
\hline C & -3.489706 & 1.369675 & 2.715709 \\
\hline C & -1.836549 & 2.540318 & 1.401757 \\
\hline C & -4.413029 & 2.409433 & 2.522991 \\
\hline $\mathrm{H}$ & -3.772788 & 0.501193 & 3.302302 \\
\hline C & -2.734163 & 3.581241 & 1.160721 \\
\hline $\mathrm{H}$ & -0.826580 & 2.604654 & 1.021502 \\
\hline C & -4.014915 & 3.492173 & 1.732869 \\
\hline $\mathrm{H}$ & -4.714362 & 4.300945 & 1.557992 \\
\hline C & -2.361484 & 4.808300 & 0.306396 \\
\hline C & -5.800687 & 2.338380 & 3.192098 \\
\hline C & -3.322343 & 4.895896 & -0.903686 \\
\hline $\mathrm{H}$ & -3.243880 & 4.002680 & -1.534233 \\
\hline $\mathrm{H}$ & -4.367737 & 4.991387 & -0.591528 \\
\hline $\mathrm{H}$ & -3.077722 & 5.769968 & -1.519762 \\
\hline C & -2.492163 & 6.094318 & 1.156568 \\
\hline $\mathrm{H}$ & -2.231167 & 6.974789 & 0.556720 \\
\hline $\mathrm{H}$ & -3.511469 & 6.237706 & 1.530677 \\
\hline $\mathrm{H}$ & -1.819473 & 6.061859 & 2.021141 \\
\hline C & -0.919133 & 4.732947 & -0.231048 \\
\hline $\mathrm{H}$ & -0.180600 & 4.720287 & 0.578397 \\
\hline $\mathrm{H}$ & -0.755323 & 3.844099 & -0.850158 \\
\hline $\mathrm{H}$ & -0.712189 & 5.613600 & -0.849782 \\
\hline
\end{tabular}




\begin{tabular}{|llll|} 
C & -6.548422 & 1.066754 & 2.728824 \\
H & -6.682607 & 1.063628 & 1.641011 \\
H & -6.011461 & 0.153342 & 3.003090 \\
H & -7.541291 & 1.019387 & 3.192794 \\
C & -5.619063 & 2.291969 & 4.728307 \\
$H$ & -5.037769 & 1.418609 & 5.042514 \\
$H$ & -5.099124 & 3.186974 & 5.088594 \\
$H$ & -6.594406 & 2.240861 & 5.227789 \\
C & -6.681262 & 3.556445 & 2.853390 \\
$H$ & -7.651259 & 3.458846 & 3.353804 \\
H & -6.229393 & 4.495374 & 3.192633 \\
$H$ & -6.871751 & 3.636333 & 1.777049 \\
\hline
\end{tabular}

\section{C-Catalyzed reaction TS 49}

\section{Datum}

Value

\begin{tabular}{lc}
\hline B3LYP/6-31G(d) Energy & -3038.964977 \\
\hline B3LYP-D3(BJ)/def2-TZVPP-IEF-PCM(DCM) Energy & -3040.512229 \\
\hline B3LYP-D3(BJ)/def2-TZVPP-IEF-PCM(DCM)//B3LYP/6-31G(d) Free Energy (Quasiharmonic) & -3039.282515 \\
\hline
\end{tabular}

Number of Imaginary Frequencies

Frequencies (Top 3 out of 450)
1. $-322.5714 \mathrm{~cm}^{-1}$
2. $7.1224 \mathrm{~cm}^{-1}$
3. $8.7162 \mathrm{~cm}^{-1}$

\section{B3LYP/6-31G(d) Molecular Geometry in Cartesian Coordinates}

C

$\mathrm{H}$

$\mathrm{H}$

C

0

0

$\mathrm{H}$

0

$\mathrm{H}$

C

C

C

C

$\mathrm{H}$

\subsection{0}

3.891053

2.235950

$-0.534130$

0.358528

$-1.612745$

$-2.284105$

2. 504178

1.749530

$-3.883601$

$-3.423187$

$-4.531165$

$-4.841929$

$-4.390122$
$-1.591691$

$-1.894674$

$-2.012681$

$-0.430458$

$-0.630676$

0.254614

0.431837

$-2.165980$

$-1.653141$

2.805526

3.904229

1.065123

2.039354

4.410020
0.963533

1.159886

1. 762071

$-0.399295$

$-1.208068$

$-0.777327$

$-0.069314$

$-0.294104$

$-0.655393$

$-3.783506$

$-1.947563$

$-2.149015$

$-3.155111$

$-1.916707$ 


\begin{tabular}{|c|c|c|c|}
\hline $\mathrm{H}$ & -3.591143 & 0.530848 & -2.296734 \\
\hline $\mathrm{H}$ & -5.351118 & 0.388033 & -1.902374 \\
\hline C & -4.593567 & 1.662897 & 1.591307 \\
\hline C & -5.931202 & 2.240364 & 0.984219 \\
\hline C & -4.774233 & 0.493786 & 2.558132 \\
\hline $\mathrm{H}$ & -3.796774 & 0.140357 & 2.903206 \\
\hline $\mathrm{H}$ & -5.351924 & 0.799245 & 3.438434 \\
\hline $\mathrm{H}$ & -5.285886 & -0.343778 & 2.079076 \\
\hline C & -3.726869 & 2.748368 & 2.247963 \\
\hline $\mathrm{H}$ & -3.561303 & 3.589048 & 1.568448 \\
\hline $\mathrm{H}$ & -2.753281 & 2.320806 & 2.505465 \\
\hline $\mathrm{H}$ & -4.181238 & 3.126376 & 3.170081 \\
\hline C & -6.534733 & 3.413444 & 1.761016 \\
\hline $\mathrm{H}$ & -7.446857 & 3.752136 & 1.258135 \\
\hline $\mathrm{H}$ & -6.802605 & 3.120070 & 2.783056 \\
\hline $\mathrm{H}$ & -5.844901 & 4.259398 & 1.808681 \\
\hline C & -7.006372 & 1.161363 & 0.771494 \\
\hline $\mathrm{H}$ & -7.445933 & 0.824960 & 1.717191 \\
\hline H & -7.806389 & 1.582358 & 0.153778 \\
\hline $\mathrm{H}$ & -6.598892 & 0.289871 & 0.251032 \\
\hline 0 & -3.909888 & 1.181509 & 0.406407 \\
\hline 0 & -5.517980 & 2.716166 & -0.306947 \\
\hline B & -4.332084 & 2.014766 & -0.694904 \\
\hline 0 & -3.216731 & 2.955159 & -1.093187 \\
\hline C & -2.262078 & 4.685981 & -2.408882 \\
\hline C & -0.954445 & 4.231061 & -2.173590 \\
\hline C & -2.466127 & 5.912315 & -3.059774 \\
\hline C & 0.130583 & 4.998930 & -2.587863 \\
\hline $\mathrm{H}$ & -0.805975 & 3.286248 & -1.660924 \\
\hline C & -1.377064 & 6.674735 & -3.475120 \\
\hline $\mathrm{H}$ & -3.479671 & 6.266242 & -3.235741 \\
\hline C & -0.077230 & 6.218122 & -3.240336 \\
\hline $\mathrm{H}$ & 1.139737 & 4.647826 & -2.393918 \\
\hline $\mathrm{H}$ & -1.540150 & 7.624433 & -3.976972 \\
\hline $\mathrm{H}$ & 0.772775 & 6.813511 & -3.562669 \\
\hline $\mathrm{H}$ & -5.882009 & 2.357661 & -3.237211 \\
\hline $\mathrm{H}$ & -2.865069 & 2.437585 & -3.865799 \\
\hline $\mathrm{H}$ & -4.167428 & 3.579558 & -4.491357 \\
\hline C & 0.438454 & -0.329771 & 1.900329 \\
\hline C & -0.498695 & -0.888312 & 1.038672 \\
\hline C & -1.455536 & -1.860722 & 1.492996 \\
\hline C & -1.526098 & -2.123785 & 2.846141 \\
\hline $\mathrm{H}$ & -2.216559 & -2.882737 & 3.204725 \\
\hline C & 2.752822 & -0.073234 & 0.976589 \\
\hline C & 1.565709 & 0.530953 & 1.390474 \\
\hline C & 3.719296 & 2.118421 & 0.556342 \\
\hline C & 3.857461 & 0.742274 & 0.546731 \\
\hline $\mathrm{H}$ & 4.549292 & 2.736204 & 0.222845 \\
\hline C & 2.523240 & 2.757739 & 0.955289 \\
\hline C & 1.419708 & 1.954935 & 1.383757 \\
\hline C & 0.224000 & 2.611863 & 1.783683 \\
\hline C & 0.122466 & 3.984883 & 1.756943 \\
\hline C & 1.216174 & 4.776551 & 1.330829 \\
\hline C & 2.389442 & 4.173149 & 0.940074 \\
\hline
\end{tabular}




\begin{tabular}{|c|c|c|c|}
\hline $\mathrm{H}$ & -0.619243 & 2.012779 & 2.109225 \\
\hline $\mathrm{H}$ & -0.803770 & 4.463635 & 2.062142 \\
\hline $\mathrm{H}$ & 1.124826 & 5.859183 & 1.313653 \\
\hline $\mathrm{H}$ & 3.237257 & 4.771417 & 0.613829 \\
\hline C & -0.671924 & -1.488272 & 3.781734 \\
\hline C & 0.349859 & -0.604491 & 3.307204 \\
\hline C & 1.240272 & -0.029156 & 4.255208 \\
\hline C & 1.115509 & -0.301123 & 5.599898 \\
\hline C & 0.100926 & -1.170100 & 6.066298 \\
\hline C & -0.769523 & -1.753212 & 5.174237 \\
\hline H & 2.022489 & 0.635953 & 3.906414 \\
\hline $\mathrm{H}$ & 1.802853 & 0.152798 & 6.308446 \\
\hline $\mathrm{H}$ & 0.014802 & -1.378625 & 7.129239 \\
\hline $\mathrm{H}$ & -1.546269 & -2.430231 & 5.521605 \\
\hline C & 7.646583 & -0.773060 & -0.744628 \\
\hline C & 6.487700 & -1.315934 & -1.305803 \\
\hline C & 5.249512 & -0.822474 & -0.864916 \\
\hline C & 5.168818 & 0.174948 & 0.110062 \\
\hline C & 6.359191 & 0.692718 & 0.653960 \\
\hline C & 7.609810 & 0.232044 & 0.239976 \\
\hline $\mathrm{H}$ & 8.612792 & -1.140144 & -1.071686 \\
\hline $\mathrm{H}$ & 4.327515 & -1.224693 & -1.268506 \\
\hline $\mathrm{H}$ & 6.281293 & 1.451938 & 1.423683 \\
\hline C & 6.512005 & -2.425080 & -2.375115 \\
\hline C & 8.930469 & 0.774169 & 0.821506 \\
\hline C & 5.820787 & -1.913008 & -3.661251 \\
\hline $\mathrm{H}$ & 5.823685 & -2.694062 & -4.431801 \\
\hline $\mathrm{H}$ & 4.779875 & -1.629811 & -3.476900 \\
\hline $\mathrm{H}$ & 6.341824 & -1.036429 & -4.064130 \\
\hline C & 7.942951 & -2.860458 & -2.741584 \\
\hline $\mathrm{H}$ & 7.904631 & -3.651369 & -3.499375 \\
\hline $\mathrm{H}$ & 8.528862 & -2.032525 & -3.157999 \\
\hline $\mathrm{H}$ & 8.482473 & -3.259930 & -1.875068 \\
\hline C & 5.748481 & -3.662500 & -1.843005 \\
\hline $\mathrm{H}$ & 5.730431 & -4.453034 & -2.604041 \\
\hline $\mathrm{H}$ & 6.239184 & -4.066538 & -0.949362 \\
\hline $\mathrm{H}$ & 4.713867 & -3.423807 & -1.577210 \\
\hline C & 9.777526 & 1.393855 & -0.315600 \\
\hline $\mathrm{H}$ & 10.728362 & 1.774319 & 0.078259 \\
\hline $\mathrm{H}$ & 10.008973 & 0.661892 & -1.096093 \\
\hline $\mathrm{H}$ & 9.247066 & 2.228402 & -0.788556 \\
\hline C & 8.702064 & 1.859519 & 1.890894 \\
\hline $\mathrm{H}$ & 9.668043 & 2.213466 & 2.268969 \\
\hline $\mathrm{H}$ & 8.168052 & 2.726461 & 1.485365 \\
\hline $\mathrm{H}$ & 8.132719 & 1.476685 & 2.745491 \\
\hline C & 9.722162 & -0.382460 & 1.476624 \\
\hline $\mathrm{H}$ & 10.669262 & -0.012860 & 1.889258 \\
\hline $\mathrm{H}$ & 9.148417 & -0.834669 & 2.293664 \\
\hline $\mathrm{H}$ & 9.957903 & -1.173448 & 0.757487 \\
\hline C & -2.243138 & -2.683341 & 0.531253 \\
\hline C & -3.586918 & -3.004976 & 0.765610 \\
\hline C & -1.600255 & -3.235196 & -0.585282 \\
\hline C & -4.291189 & -3.861382 & -0.090274 \\
\hline $\mathrm{H}$ & -4.081911 & -2.570532 & 1.628974 \\
\hline
\end{tabular}




\begin{tabular}{|c|c|c|c|}
\hline C & -2.273609 & -4.074781 & -1.478983 \\
\hline $\mathrm{H}$ & -0.548067 & -3.024389 & -0.737439 \\
\hline C & -3.615816 & -4.374529 & -1.208348 \\
\hline $\mathrm{H}$ & -4.148580 & -5.030311 & -1.882859 \\
\hline C & -1.523369 & -4.623362 & -2.707809 \\
\hline C & -5.760617 & -4.214773 & 0.217354 \\
\hline C & -0.257671 & -5.388278 & -2.251916 \\
\hline $\mathrm{H}$ & 0.441976 & -4.745392 & -1.708575 \\
\hline $\mathrm{H}$ & -0.520081 & -6.228559 & -1.598454 \\
\hline $\mathrm{H}$ & 0.273536 & -5.789598 & -3.123719 \\
\hline C & -2.387822 & -5.586144 & -3.543624 \\
\hline $\mathrm{H}$ & -3.284779 & -5.094664 & -3.937766 \\
\hline $\mathrm{H}$ & -2.703423 & -6.461465 & -2.963696 \\
\hline $\mathrm{H}$ & -1.809184 & -5.948448 & -4.400734 \\
\hline C & -1.106844 & -3.436701 & -3.611103 \\
\hline $\mathrm{H}$ & -1.988685 & -2.895609 & -3.973903 \\
\hline H & -0.472917 & -2.719586 & -3.080157 \\
\hline $\mathrm{H}$ & -0.549645 & -3.800793 & -4.483281 \\
\hline C & -5.847592 & -4.881817 & 1.611190 \\
\hline $\mathrm{H}$ & -5.255434 & -5.803512 & 1.642086 \\
\hline $\mathrm{H}$ & -5.478885 & -4.221338 & 2.403026 \\
\hline $\mathrm{H}$ & -6.887665 & -5.137705 & 1.848665 \\
\hline C & -6.617031 & -2.927389 & 0.214760 \\
\hline $\mathrm{H}$ & -6.277106 & -2.212363 & 0.970570 \\
\hline $\mathrm{H}$ & -6.572464 & -2.429882 & -0.760922 \\
\hline $\mathrm{H}$ & -7.666555 & -3.164549 & 0.428995 \\
\hline C & -6.364503 & -5.188529 & -0.812817 \\
\hline $\mathrm{H}$ & -7.402586 & -5.412316 & -0.541961 \\
\hline $\mathrm{H}$ & -6.371982 & -4.763311 & -1.822823 \\
\hline $\mathrm{H}$ & -5.819942 & -6.138906 & -0.845702 \\
\hline
\end{tabular}

\section{C-Catalyzed reaction TS 50}

\section{Datum}

Value

\begin{tabular}{lc}
\hline B3LYP/6-31G(d) Energy & -3038.958763 \\
\hline B3LYP-D3(BJ)/def2-TZVPP-IEF-PCM(DCM) Energy & -3040.511333 \\
\hline B3LYP-D3(BJ)/def2-TZVPP-IEF-PCM(DCM)//B3LYP/6-31G(d) Free Energy (Quasiharmonic) & -3039.282498 \\
\hline
\end{tabular}

Number of Imaginary Frequencies

Frequencies (Top 3 out of 450)

$$
\begin{array}{lr}
\text { 1. } & -291.1268 \mathrm{~cm}^{-1} \\
\text { 2. } & 9.3704 \mathrm{~cm}^{-1} \\
\text { 3. } & 10.6330 \mathrm{~cm}^{-1}
\end{array}
$$




\begin{tabular}{|c|c|c|c|}
\hline C & -0.783530 & 0.956001 & 0.676035 \\
\hline $\mathrm{H}$ & -0.315956 & 0.769608 & 1.650364 \\
\hline $\mathrm{H}$ & -1.839829 & 0.688242 & 0.755229 \\
\hline C & 2.686640 & 1.764953 & -1.264162 \\
\hline 0 & 3.569756 & 2.376847 & -1.822845 \\
\hline 0 & 1.663208 & 1.225358 & -1.937290 \\
\hline $\mathrm{H}$ & 1.009367 & 0.793924 & -1.319014 \\
\hline 0 & -0.164899 & 0.128798 & -0.322873 \\
\hline $\mathrm{H}$ & -0.035158 & -0.773727 & 0.058700 \\
\hline C & -3.419932 & -2.451572 & -2.326800 \\
\hline C & -3.658500 & -2.391554 & -0.128333 \\
\hline C & -1.122407 & -3.096642 & -1.709792 \\
\hline C & -2.425726 & -3.395981 & -2.230460 \\
\hline $\mathrm{H}$ & -4.123013 & -1.410913 & -0.263591 \\
\hline $\mathrm{H}$ & -0.783956 & -2.081425 & -1.932073 \\
\hline $\mathrm{H}$ & -0.359027 & -3.839621 & -1.949923 \\
\hline C & 0.437800 & -3.158031 & 1.719806 \\
\hline C & 0.024636 & -4.639515 & 1.377968 \\
\hline C & 1.940245 & -2.916737 & 1.858242 \\
\hline $\mathrm{H}$ & 2.354819 & -3.504968 & 2.685388 \\
\hline $\mathrm{H}$ & 2.477984 & -3.174956 & 0.943319 \\
\hline $\mathrm{H}$ & 2.131569 & -1.859869 & 2.071607 \\
\hline C & -0.298365 & -2.599034 & 2.947812 \\
\hline $\mathrm{H}$ & -1.378925 & -2.749474 & 2.868320 \\
\hline $\mathrm{H}$ & 0.052028 & -3.064603 & 3.875314 \\
\hline $\mathrm{H}$ & -0.109210 & -1.522812 & 3.020354 \\
\hline C & -0.302772 & -5.508749 & 2.593930 \\
\hline $\mathrm{H}$ & 0.566778 & -5.605024 & 3.254509 \\
\hline $\mathrm{H}$ & -1.134457 & -5.096536 & 3.170339 \\
\hline $\mathrm{H}$ & -0.585303 & -6.513885 & 2.262515 \\
\hline C & 1.056199 & -5.360773 & 0.495239 \\
\hline $\mathrm{H}$ & 1.961955 & -5.619353 & 1.054562 \\
\hline $\mathrm{H}$ & 0.609961 & -6.286889 & 0.118337 \\
\hline $\mathrm{H}$ & 1.347837 & -4.749097 & -0.363491 \\
\hline 0 & -0.036708 & -2.452167 & 0.547752 \\
\hline 0 & -1.178418 & -4.462775 & 0.602965 \\
\hline B & -1.181994 & -3.154178 & 0.029045 \\
\hline 0 & -2.448987 & -2.332319 & 0.320201 \\
\hline C & -4.625878 & -3.479674 & 0.146865 \\
\hline C & -5.982414 & -3.215649 & -0.114668 \\
\hline C & -4.261092 & -4.720978 & 0.692970 \\
\hline C & -6.956534 & -4.173754 & 0.150147 \\
\hline $\mathrm{H}$ & -6.269804 & -2.251184 & -0.527803 \\
\hline C & -5.242079 & -5.676275 & 0.955224 \\
\hline $\mathrm{H}$ & -3.216275 & -4.925859 & 0.901831 \\
\hline C & -6.586812 & -5.410362 & 0.685522 \\
\hline $\mathrm{H}$ & -8.000957 & -3.956429 & -0.055656 \\
\hline $\mathrm{H}$ & -4.953926 & -6.634931 & 1.378202 \\
\hline $\mathrm{H}$ & -7.344366 & -6.160470 & 0.896015 \\
\hline $\mathrm{H}$ & -2.702979 & -4.445080 & -2.339585 \\
\hline
\end{tabular}




\begin{tabular}{|c|c|c|c|}
\hline $\mathrm{H}$ & -3.158071 & -1.397999 & -2.381006 \\
\hline $\mathrm{H}$ & -4.413924 & -2.721202 & -2.670325 \\
\hline C & 1.777877 & 2.247564 & 1.032266 \\
\hline C & 2.704206 & 1.578884 & 0.243401 \\
\hline $\mathrm{C}$ & 3.780774 & 0.832741 & 0.830938 \\
\hline C & 3.894731 & 0.812970 & 2.207132 \\
\hline $\mathrm{H}$ & 4.685732 & 0.225296 & 2.666272 \\
\hline C & -0.617642 & 2.413028 & 0.291636 \\
\hline C & 0.638368 & 3.007875 & 0.408732 \\
\hline C & -1.490715 & 4.441732 & -0.721143 \\
\hline C & -1.708082 & 3.152293 & -0.273225 \\
\hline H & -2.321183 & 5.004949 & -1.139815 \\
\hline C & -0.227506 & 5.069774 & -0.625304 \\
\hline C & 0.861987 & 4.348600 & -0.041492 \\
\hline C & 2.124776 & 4.997663 & 0.060119 \\
\hline C & 2.292889 & 6.288175 & -0.387291 \\
\hline C & 1.211182 & 7.000084 & -0.960237 \\
\hline C & -0.021269 & 6.401592 & -1.076674 \\
\hline $\mathrm{H}$ & 2.966213 & 4.456647 & 0.477888 \\
\hline H & 3.266771 & 6.763102 & -0.307892 \\
\hline $\mathrm{H}$ & 1.360507 & 8.017721 & -1.311074 \\
\hline $\mathrm{H}$ & -0.858675 & 6.937450 & -1.517457 \\
\hline C & 2.998927 & 1.516573 & 3.049310 \\
\hline C & 1.916821 & 2.245973 & 2.461167 \\
\hline C & 1.028435 & 2.946785 & 3.323329 \\
\hline C & 1.196961 & 2.919741 & 4.690574 \\
\hline C & 2.262543 & 2.190069 & 5.269083 \\
\hline C & 3.142194 & 1.504461 & 4.463088 \\
\hline $\mathrm{H}$ & 0.211918 & 3.513771 & 2.888706 \\
\hline $\mathrm{H}$ & 0.508554 & 3.465280 & 5.330494 \\
\hline $\mathrm{H}$ & 2.384960 & 2.176073 & 6.348768 \\
\hline $\mathrm{H}$ & 3.966616 & 0.943091 & 4.896670 \\
\hline C & -5.753329 & 1.789748 & -0.579058 \\
\hline C & -5.053239 & 2.088268 & -1.751970 \\
\hline C & -3.714518 & 2.498297 & -1.625567 \\
\hline C & -3.098103 & 2.609867 & -0.377753 \\
\hline C & -3.838718 & 2.302177 & 0.777059 \\
\hline C & -5.171778 & 1.893666 & 0.699654 \\
\hline $\mathrm{H}$ & -6.791094 & 1.484984 & -0.648598 \\
\hline $\mathrm{H}$ & -3.131776 & 2.743209 & -2.508882 \\
\hline $\mathrm{H}$ & -3.356683 & 2.421368 & 1.740879 \\
\hline C & -5.698311 & 2.016602 & -3.150153 \\
\hline C & -6.025735 & 1.606725 & 1.950973 \\
\hline C & -4.898918 & 1.043617 & -4.048113 \\
\hline $\mathrm{H}$ & -5.330372 & 1.012680 & -5.056015 \\
\hline $\mathrm{H}$ & -3.850236 & 1.341900 & -4.144762 \\
\hline $\mathrm{H}$ & -4.921709 & 0.025866 & -3.641185 \\
\hline C & -7.159634 & 1.531382 & -3.103883 \\
\hline $\mathrm{H}$ & -7.565531 & 1.488482 & -4.120834 \\
\hline $\mathrm{H}$ & -7.243109 & 0.526836 & -2.672640 \\
\hline $\mathrm{H}$ & -7.798115 & 2.206876 & -2.523380 \\
\hline C & -5.681447 & 3.426552 & -3.788729 \\
\hline $\mathrm{H}$ & -6.136445 & 3.399348 & -4.786528 \\
\hline $\mathrm{H}$ & -6.245320 & 4.139882 & -3.176843 \\
\hline
\end{tabular}




\begin{tabular}{|c|c|c|c|}
\hline H & -4.662287 & 3.811180 & -3.897042 \\
\hline C & -5.216020 & 1.730263 & 3.256006 \\
\hline $\mathrm{H}$ & -5.861415 & 1.505078 & 4.112530 \\
\hline $\mathrm{H}$ & -4.374180 & 1.028964 & 3.282607 \\
\hline $\mathrm{H}$ & -4.822558 & 2.742894 & 3.398431 \\
\hline C & -7.193428 & 2.620860 & 2.012501 \\
\hline $\mathrm{H}$ & -7.814315 & 2.434250 & 2.897321 \\
\hline $\mathrm{H}$ & -6.816484 & 3.648134 & 2.071983 \\
\hline $\mathrm{H}$ & -7.839094 & 2.553063 & 1.130458 \\
\hline C & -6.601374 & 0.173050 & 1.882777 \\
\hline $\mathrm{H}$ & -7.225057 & -0.028981 & 2.762040 \\
\hline $\mathrm{H}$ & -7.225363 & 0.022861 & 0.995301 \\
\hline $\mathrm{H}$ & -5.799759 & -0.574049 & 1.861814 \\
\hline C & 4.737202 & 0.044551 & 0.004663 \\
\hline C & 6.110616 & 0.089938 & 0.273459 \\
\hline C & 4.279250 & -0.786153 & -1.024994 \\
\hline C & 7.026975 & -0.668906 & -0.464559 \\
\hline $\mathrm{H}$ & 6.461909 & 0.756500 & 1.055570 \\
\hline C & 5.159699 & -1.552817 & -1.795267 \\
\hline $\mathrm{H}$ & 3.214470 & -0.826588 & -1.227451 \\
\hline C & 6.527156 & -1.482153 & -1.492305 \\
\hline $\mathrm{H}$ & 7.221406 & -2.069752 & -2.077167 \\
\hline C & 4.601541 & -2.414978 & -2.944177 \\
\hline C & 8.531471 & -0.568746 & -0.143654 \\
\hline C & 3.916768 & -1.491275 & -3.981105 \\
\hline $\mathrm{H}$ & 3.105466 & -0.905120 & -3.538448 \\
\hline $\mathrm{H}$ & 4.637006 & -0.783739 & -4.406937 \\
\hline H & 3.497738 & -2.086710 & -4.802375 \\
\hline C & 5.699450 & -3.219007 & -3.666170 \\
\hline $\mathrm{H}$ & 6.451480 & -2.564628 & -4.121363 \\
\hline H & 6.212430 & -3.911844 & -2.988843 \\
\hline $\mathrm{H}$ & 5.251765 & -3.813059 & -4.471102 \\
\hline C & 3.563969 & -3.418990 & -2.387505 \\
\hline H & 4.024480 & -4.092725 & -1.654967 \\
\hline H & 2.725684 & -2.911541 & -1.898436 \\
\hline H & 3.153967 & -4.031106 & -3.200496 \\
\hline C & 9.006398 & 0.886685 & -0.368502 \\
\hline H & 8.464041 & 1.594359 & 0.266905 \\
\hline H & 8.852869 & 1.191164 & -1.409844 \\
\hline H & 10.075331 & 0.980439 & -0.138721 \\
\hline C & 8.774776 & -0.963915 & 1.332401 \\
\hline H & 8.452407 & -1.994682 & 1.520215 \\
\hline H & 8.231273 & -0.312275 & 2.024543 \\
\hline H & 9.841938 & -0.891409 & 1.576753 \\
\hline C & 9.388437 & -1.493050 & -1.029638 \\
\hline H & 10.444566 & -1.390177 & -0.755230 \\
\hline H & 9.298520 & -1.239570 & -2.091922 \\
\hline$H$ & 9.115387 & -2.547295 & -0.904915 \\
\hline
\end{tabular}




\begin{tabular}{lc}
\hline B3LYP/6-31G(d) Energy & -3038.961001 \\
\hline B3LYP-D3(BJ)/def2-TZVPP-IEF-PCM(DCM) Energy & -3040.512084 \\
\hline B3LYP-D3(BJ)/def2-TZVPP-IEF-PCM(DCM)//B3LYP/6-31G(d) Free Energy (Quasiharmonic) & -3039.282491 \\
\hline
\end{tabular}

Number of Imaginary Frequencies

Frequencies (Top 3 out of 450)

$\begin{array}{lr}\text { 1. } & -320.5348 \mathrm{~cm}^{-1} \\ \text { 2. } & 8.5216 \mathrm{~cm}^{-1} \\ \text { 3. } & 11.8239 \mathrm{~cm}^{-1}\end{array}$

\section{B3LYP/6-31G(d) Molecular Geometry in Cartesian Coordinates}

C

$\mathrm{H}$

$\mathrm{H}$

C

0

0

$\mathrm{H}$

0

$\mathrm{H}$

C

C

C

C

$\mathrm{H}$

$\mathrm{H}$

$\mathrm{H}$

C

C

C

$\mathrm{H}$

$\mathrm{H}$

$\mathrm{H}$

C

$\mathrm{H}$

$\mathrm{H}$

$\mathrm{H}$

C

$\mathrm{H}$

$\mathrm{H}$

$\mathrm{H}$

C

$\mathrm{H}$
$-1.457499$

$-2.185069$

$-0.463729$

1.506991

1. 349411

1. 446501

1.528651

$-1.642899$

$-0.998119$

0.572480

$-0.903550$

2. 129050

1.475140

$-1.144190$

2. 341540

2.989080

1.554390

0.576050

3.028980

3.657500

3. 271480

3. 278490

1. 226551

1. 318911

0. 217731

1.937901

$-0.879980$

$-1.023700$

$-1.202850$

$-1.525980$

0.998190

1.029620
0.606810

$-0.180460$

0.174329

0.887399

1.369369

$-0.440321$

$-0.734441$

0.946510

1.639049

$-4.963341$

$-3.860991$

$-3.869611$

$-5.025851$

$-4.733240$

$-3.076071$

$-4.084201$

$-2.216811$

$-3.425011$

$-2.589411$

$-1.813171$

$-2.661781$

$-3.543141$

$-0.940171$

$-1.114301$

$-0.575251$

$-0.151431$

$-2.978551$

$-2.485431$

$-2.289500$

$-3.862520$

$-4.356111$

$-3.822151$
1.092630

0.893450

0.945220

$-1.242890$

$-2.344370$

$-1.061230$

$-0.118210$

2.477700

2.698310

$-2.094770$

$-0.879970$

$-0.517620$

$-1.056010$

$-0.269130$

$-1.237200$

0.118660

2.621690

2.881200

2.845750

2. 398740

3.911620

2. 372000

3. 394990

4.473930

3.190630

3.125070

3.089600

4.056830

2.304620

3.056990

4.018770

4.975800 


\begin{tabular}{|c|c|c|c|}
\hline $\mathrm{H}$ & 0.273530 & -5.172071 & 4.112670 \\
\hline $\mathrm{H}$ & 1.979260 & -4.799681 & 3.832830 \\
\hline 0 & 1.367230 & -1.978861 & 1.203370 \\
\hline 0 & 0.659160 & -4.157761 & 1.641680 \\
\hline B & 0.944420 & -3.222501 & 0.601500 \\
\hline 0 & -0.203320 & -2.921861 & -0.335630 \\
\hline C & -1.837880 & -3.494120 & -1.958400 \\
\hline C & -2.867470 & -4.378540 & -2.314020 \\
\hline C & -1.705980 & -2.268810 & -2.633660 \\
\hline C & -3.751880 & -4.049070 & -3.338780 \\
\hline $\mathrm{H}$ & -2.971390 & -5.323930 & -1.785980 \\
\hline C & -2.591070 & -1.946390 & -3.658590 \\
\hline $\mathrm{H}$ & -0.908460 & -1.588001 & -2.351290 \\
\hline C & -3.611260 & -2.834540 & -4.016110 \\
\hline $\mathrm{H}$ & -4.547020 & -4.737470 & -3.610990 \\
\hline $\mathrm{H}$ & -2.483139 & -1.001020 & -4.182670 \\
\hline $\mathrm{H}$ & -4.294850 & -2.581080 & -4.821760 \\
\hline $\mathrm{H}$ & 1.502190 & -5.935291 & -0.454730 \\
\hline $\mathrm{H}$ & 0.632940 & -4.154971 & -2.816990 \\
\hline $\mathrm{H}$ & 0.035180 & -5.850021 & -2.420190 \\
\hline C & 0.727731 & 2.474509 & 0.513830 \\
\hline C & 1.770541 & 1.715489 & -0.003650 \\
\hline C & 3.070811 & 1.702029 & 0.609500 \\
\hline C & 3.245971 & 2.414289 & 1.781490 \\
\hline $\mathrm{H}$ & 4.229651 & 2.446529 & 2.242050 \\
\hline C & -1.672469 & 1.768520 & 0.139290 \\
\hline C & -0.602329 & 2.599649 & -0.185690 \\
\hline C & -3.110619 & 3.028070 & -1.361370 \\
\hline C & -2.967079 & 2.001920 & -0.446410 \\
\hline $\mathrm{H}$ & -4.089849 & 3.221630 & -1.791770 \\
\hline C & -2.036979 & 3.870250 & -1.732630 \\
\hline C & -0.756449 & 3.667289 & -1.131050 \\
\hline C & 0.306931 & 4.535829 & -1.501260 \\
\hline C & 0.109991 & 5.549059 & -2.412200 \\
\hline C & -1.160529 & 5.749410 & -3.003260 \\
\hline C & -2.209609 & 4.925380 & -2.669410 \\
\hline $\mathrm{H}$ & 1.288421 & 4.384769 & -1.066710 \\
\hline $\mathrm{H}$ & 0.937791 & 6.198359 & -2.684190 \\
\hline $\mathrm{H}$ & -1.302089 & 6.553060 & -3.721010 \\
\hline $\mathrm{H}$ & -3.190609 & 5.068050 & -3.116740 \\
\hline C & 2.198741 & 3.152929 & 2.381650 \\
\hline C & 0.922661 & 3.207359 & 1.734330 \\
\hline C & -0.103779 & 3.993629 & 2.333840 \\
\hline C & 0.118701 & 4.670199 & 3.514190 \\
\hline C & 1.379741 & 4.607729 & 4.152930 \\
\hline C & 2.396481 & 3.868789 & 3.593580 \\
\hline $\mathrm{H}$ & -1.069449 & 4.062189 & 1.844750 \\
\hline $\mathrm{H}$ & -0.678869 & 5.262759 & 3.953690 \\
\hline $\mathrm{H}$ & 1.542441 & 5.147439 & 5.081730 \\
\hline $\mathrm{H}$ & 3.371571 & 3.819399 & 4.072350 \\
\hline C & -6.531389 & -0.158290 & 0.529340 \\
\hline C & -5.711879 & 0.280020 & 1.572590 \\
\hline C & -4.536749 & 0.971510 & 1.238020 \\
\hline C & -4.179949 & 1.205060 & -0.093580 \\
\hline
\end{tabular}




\begin{tabular}{|c|c|c|c|}
\hline C & -5.029029 & 0.738390 & -1.114240 \\
\hline C & -6.213349 & 0.059420 & -0.823950 \\
\hline $\mathrm{H}$ & -7.446259 & -0.689280 & 0.765210 \\
\hline $\mathrm{H}$ & -3.880979 & 1.335160 & 2.021120 \\
\hline $\mathrm{H}$ & -4.731599 & 0.908060 & -2.142510 \\
\hline C & -6.043649 & 0.037270 & 3.057600 \\
\hline C & -7.169129 & -0.452520 & -1.919300 \\
\hline C & -4.901849 & -0.782060 & 3.707040 \\
\hline $\mathrm{H}$ & -5.111069 & -0.948310 & 4.771480 \\
\hline $\mathrm{H}$ & -4.804760 & -1.762230 & 3.225180 \\
\hline H & -3.936079 & -0.273490 & 3.627030 \\
\hline C & -6.174909 & 1.396310 & 3.785460 \\
\hline $\mathrm{H}$ & -6.408139 & 1.239730 & 4.846020 \\
\hline $\mathrm{H}$ & -5.248899 & 1.977350 & 3.730000 \\
\hline $\mathrm{H}$ & -6.977289 & 2.000670 & 3.346310 \\
\hline C & -7.361339 & -0.737290 & 3.249100 \\
\hline $\mathrm{H}$ & -7.547699 & -0.889290 & 4.318440 \\
\hline $\mathrm{H}$ & -8.220199 & -0.193220 & 2.838930 \\
\hline $\mathrm{H}$ & -7.325440 & -1.725870 & 2.776940 \\
\hline C & -8.553889 & 0.215990 & -1.746630 \\
\hline $\mathrm{H}$ & -9.250099 & -0.141650 & -2.515560 \\
\hline $\mathrm{H}$ & -8.994619 & -0.005330 & -0.769130 \\
\hline $\mathrm{H}$ & -8.476099 & 1.305410 & -1.838660 \\
\hline C & -6.660199 & -0.133750 & -3.338030 \\
\hline $\mathrm{H}$ & -7.372909 & -0.512970 & -4.079670 \\
\hline $\mathrm{H}$ & -5.690929 & -0.604520 & -3.536490 \\
\hline $\mathrm{H}$ & -6.556679 & 0.945000 & -3.501700 \\
\hline C & -7.323230 & -1.987570 & -1.799440 \\
\hline $\mathrm{H}$ & -8.005730 & -2.364050 & -2.571930 \\
\hline $\mathrm{H}$ & -6.356490 & -2.488320 & -1.923960 \\
\hline $\mathrm{H}$ & -7.729880 & -2.281630 & -0.826110 \\
\hline C & 4.240971 & 1.013809 & -0.008240 \\
\hline C & 4.480871 & 1.104099 & -1.382290 \\
\hline C & 5.167071 & 0.325219 & 0.797820 \\
\hline C & 5.603041 & 0.503969 & -1.973930 \\
\hline $\mathrm{H}$ & 3.787651 & 1.661619 & -2.000760 \\
\hline C & 6.305491 & -0.270001 & 0.252300 \\
\hline $\mathrm{H}$ & 4.972061 & 0.256579 & 1.861670 \\
\hline C & 6.495041 & -0.173141 & -1.139110 \\
\hline $\mathrm{H}$ & 7.369361 & -0.642851 & -1.574280 \\
\hline C & 7.342351 & -1.025231 & 1.106990 \\
\hline C & 5.798241 & 0.617239 & -3.497730 \\
\hline C & 8.738461 & -0.386962 & 0.911140 \\
\hline $\mathrm{H}$ & 9.065101 & -0.424812 & -0.132990 \\
\hline $\mathrm{H}$ & 8.735711 & 0.663978 & 1.222300 \\
\hline $\mathrm{H}$ & 9.486891 & -0.917722 & 1.512320 \\
\hline C & 7.011371 & -0.982681 & 2.610850 \\
\hline $\mathrm{H}$ & 7.787050 & -1.513711 & 3.174210 \\
\hline $\mathrm{H}$ & 6.973101 & 0.045179 & 2.989790 \\
\hline $\mathrm{H}$ & 6.054240 & -1.466051 & 2.835320 \\
\hline C & 7.391370 & -2.507401 & 0.665320 \\
\hline $\mathrm{H}$ & 6.416630 & -2.989201 & 0.802580 \\
\hline $\mathrm{H}$ & 7.667160 & -2.608571 & -0.389640 \\
\hline $\mathrm{H}$ & 8.130880 & -3.059302 & 1.258760 \\
\hline
\end{tabular}




$\begin{array}{lrrr}\mathrm{C} & 4.596091 & -0.046651 & -4.212820 \\ \mathrm{H} & 4.543171 & -1.115031 & -3.972660 \\ \mathrm{H} & 3.642561 & 0.406149 & -3.923560 \\ \mathrm{H} & 4.701231 & 0.051209 & -5.300580 \\ \mathrm{C} & 5.873241 & 2.109629 & -3.900180 \\ \mathrm{H} & 4.958851 & 2.650869 & -3.637310 \\ \mathrm{H} & 6.714391 & 2.608679 & -3.404750 \\ \mathrm{H} & 6.013271 & 2.202899 & -4.984090 \\ \mathrm{C} & 7.088471 & -0.072481 & -3.979180 \\ \mathrm{H} & 7.179591 & 0.035369 & -5.065960 \\ \mathrm{H} & 7.983471 & 0.371118 & -3.527330 \\ \mathrm{H} & 7.086661 & -1.145311 & -3.754620\end{array}$

\section{C-Catalyzed reaction TS 52}

Datum

Value

\begin{tabular}{lc}
\hline B3LYP/6-31G(d) Energy & -3038.959669 \\
\hline B3LYP-D3(BJ)/def2-TZVPP-IEF-PCM(DCM) Energy & -3040.511513 \\
\hline B3LYP-D3(BJ)/def2-TZVPP-IEF-PCM(DCM)//B3LYP/6-31G(d) Free Energy (Quasiharmonic) & -3039.282462 \\
\hline
\end{tabular}

Number of Imaginary Frequencies

Frequencies (Top 3 out of 450)

$\begin{array}{lr}\text { 1. } & -304.6400 \mathrm{~cm}^{-1} \\ \text { 2. } & 7.4561 \mathrm{~cm}^{-1} \\ \text { 3. } & 9.8913 \mathrm{~cm}^{-1}\end{array}$

\section{B3LYP/6-31G(d) Molecular Geometry in Cartesian Coordinates}

C

$\mathrm{H}$

$\mathrm{H}$

C

0

0

$\mathrm{H}$

0

$\mathrm{H}$

C

C

C

C

$\mathrm{H}$

\subsection{0}

2.615400

0.983900

$-1.127660$

$-1.157040$

$-1.109050$

$-0.997870$

2.459030

1.954250

0.244490

$-1.598420$

1.242160

0.910880

$-1.738730$
$-0.844600$

0.079900

$-0.566870$

$-0.965050$

$-1.152180$

0.288970

0.394790

$-1.526550$

$-2.354390$

3.710420

3.491340

3.103630

2.820130

2.455100
0.842010

0.830130

0.924720

$-1.314870$

$-2.511510$

$-0.827900$

0.154140

2.032970

2.092990

$-1.655930$

$-0.482030$

0.525950

$-0.843570$

$-0.788410$ 


\begin{tabular}{|c|c|c|c|}
\hline $\mathrm{H}$ & 1.514100 & 4.143950 & 0.720700 \\
\hline $\mathrm{H}$ & 1.967120 & 2.418940 & 0.969790 \\
\hline C & -1.065070 & 1.259330 & 2.933970 \\
\hline C & -0.207970 & 2.309310 & 3.732790 \\
\hline C & -2.568660 & 1.577980 & 2.956800 \\
\hline H & -3.079490 & 0.951740 & 2.217940 \\
\hline $\mathrm{H}$ & -3.008650 & 1.374820 & 3.939670 \\
\hline $\mathrm{H}$ & -2.755650 & 2.625340 & 2.703550 \\
\hline C & -0.830350 & -0.194250 & 3.342910 \\
\hline $\mathrm{H}$ & -1.460760 & -0.864380 & 2.748710 \\
\hline $\mathrm{H}$ & -1.097620 & -0.339430 & 4.396500 \\
\hline $\mathrm{H}$ & 0.211460 & -0.495540 & 3.208810 \\
\hline C & 1.186470 & 1.782700 & 4.115740 \\
\hline $\mathrm{H}$ & 1.139520 & 1.050850 & 4.929880 \\
\hline $\mathrm{H}$ & 1.692680 & 1.312110 & 3.267960 \\
\hline $\mathrm{H}$ & 1.795520 & 2.628870 & 4.450170 \\
\hline C & -0.895550 & 2.874070 & 4.979500 \\
\hline $\mathrm{H}$ & -1.115550 & 2.083760 & 5.707390 \\
\hline $\mathrm{H}$ & -0.233140 & 3.601100 & 5.461000 \\
\hline $\mathrm{H}$ & -1.825760 & 3.387360 & 4.725160 \\
\hline 0 & -0.588190 & 1.458980 & 1.571880 \\
\hline 0 & -0.046840 & 3.373870 & 2.787780 \\
\hline B & -0.199720 & 2.867130 & 1.470060 \\
\hline 0 & -1.207840 & 3.734750 & 0.727440 \\
\hline C & -2.428930 & 4.503340 & -1.162470 \\
\hline C & -2.557190 & 5.803170 & -0.648250 \\
\hline C & -3.127050 & 4.146740 & -2.326780 \\
\hline C & -3.368680 & 6.730640 & -1.296120 \\
\hline $\mathrm{H}$ & -2.026630 & 6.064460 & 0.261730 \\
\hline C & -3.937450 & 5.078380 & -2.971720 \\
\hline $\mathrm{H}$ & -3.031240 & 3.138060 & -2.722160 \\
\hline C & -4.058330 & 6.372390 & -2.458650 \\
\hline $\mathrm{H}$ & -3.468270 & 7.734810 & -0.893090 \\
\hline $\mathrm{H}$ & -4.476410 & 4.795190 & -3.871510 \\
\hline $\mathrm{H}$ & -4.691320 & 7.099020 & -2.960770 \\
\hline $\mathrm{H}$ & 0.966150 & 1.784790 & -1.176300 \\
\hline $\mathrm{H}$ & 0.296320 & 4.777050 & -1.456320 \\
\hline $\mathrm{H}$ & -0.051060 & 3.427020 & -2.661930 \\
\hline C & 0.063710 & -2.787350 & -0.100930 \\
\hline C & -1.121080 & -2.089590 & -0.303520 \\
\hline C & -2.328050 & -2.430250 & 0.398490 \\
\hline C & -2.268880 & -3.440300 & 1.340920 \\
\hline $\mathrm{H}$ & -3.176160 & -3.732230 & 1.862900 \\
\hline C & 2.276260 & -1.618940 & -0.441990 \\
\hline C & 1.302760 & -2.499810 & -0.909570 \\
\hline C & 3.652340 & -2.099420 & -2.386760 \\
\hline C & 3.487170 & -1.422030 & -1.193790 \\
\hline $\mathrm{H}$ & 4.571420 & -1.962900 & -2.951170 \\
\hline C & 2.680070 & -2.994850 & -2.890480 \\
\hline C & 1.480720 & -3.210770 & -2.142480 \\
\hline C & 0.518260 & -4.121020 & -2.659850 \\
\hline C & 0.735970 & -4.783020 & -3.847100 \\
\hline C & 1.926220 & -4.569970 & -4.583010 \\
\hline$\sigma$ & 2.875440 & -3.692910 & -4.113170 \\
\hline
\end{tabular}




\begin{tabular}{|c|c|c|c|}
\hline $\mathrm{H}$ & -0.404150 & -4.282700 & -2.113720 \\
\hline $\mathrm{H}$ & -0.014340 & -5.471640 & -4.226040 \\
\hline $\mathrm{H}$ & 2.084750 & -5.098650 & -5.519130 \\
\hline $\mathrm{H}$ & 3.792960 & -3.518690 & -4.670370 \\
\hline C & -1.074920 & -4.146130 & 1.620690 \\
\hline C & 0.110070 & -3.834920 & 0.879850 \\
\hline C & 1.290680 & -4.586690 & 1.147820 \\
\hline C & 1.298290 & -5.575530 & 2.108270 \\
\hline C & 0.126660 & -5.873340 & 2.843890 \\
\hline C & -1.033740 & -5.175910 & 2.599500 \\
\hline $\mathrm{H}$ & 2.189970 & -4.376940 & 0.578680 \\
\hline $\mathrm{H}$ & 2.209730 & -6.135750 & 2.297290 \\
\hline $\mathrm{H}$ & 0.146270 & -6.655720 & 3.597410 \\
\hline $\mathrm{H}$ & -1.941230 & -5.401350 & 3.154410 \\
\hline C & 6.659740 & 1.161670 & 0.039760 \\
\hline C & 6.144910 & 1.294400 & -1.257820 \\
\hline C & 5.097750 & 0.443920 & -1.630550 \\
\hline C & 4.578000 & -0.508430 & -0.741120 \\
\hline C & 5.126150 & -0.612200 & 0.545670 \\
\hline C & 6.171700 & 0.221450 & 0.958500 \\
\hline $\mathrm{H}$ & 7.466430 & 1.820660 & 0.347600 \\
\hline $\mathrm{H}$ & 4.652830 & 0.519980 & -2.616170 \\
\hline $\mathrm{H}$ & 4.712050 & -1.350090 & 1.220920 \\
\hline C & 6.724320 & 2.363110 & -2.205480 \\
\hline C & 6.775550 & 0.142150 & 2.374270 \\
\hline C & 8.241480 & 2.124619 & -2.392190 \\
\hline $\mathrm{H}$ & 8.667260 & 2.882479 & -3.061400 \\
\hline $\mathrm{H}$ & 8.783750 & 2.178019 & -1.442470 \\
\hline $\mathrm{H}$ & 8.432790 & 1.138299 & -2.830300 \\
\hline C & 6.063170 & 2.335220 & -3.597000 \\
\hline $\mathrm{H}$ & 6.515330 & 3.102900 & -4.235310 \\
\hline $\mathrm{H}$ & 6.200140 & 1.368470 & -4.095010 \\
\hline $\mathrm{H}$ & 4.988540 & 2.542530 & -3.543410 \\
\hline C & 6.498440 & 3.767060 & -1.595450 \\
\hline $\mathrm{H}$ & 6.909930 & 4.541840 & -2.254340 \\
\hline $\mathrm{H}$ & 5.429350 & 3.967270 & -1.461040 \\
\hline $\mathrm{H}$ & 6.981920 & 3.868640 & -0.618200 \\
\hline C & 6.541800 & 1.487190 & 3.102840 \\
\hline $\mathrm{H}$ & 6.971850 & 1.455070 & 4.111600 \\
\hline $\mathrm{H}$ & 7.003430 & 2.325290 & 2.569670 \\
\hline $\mathrm{H}$ & 5.470860 & 1.699480 & 3.197070 \\
\hline C & 6.138310 & -0.978720 & 3.218580 \\
\hline $\mathrm{H}$ & 6.587660 & -0.985290 & 4.218460 \\
\hline $\mathrm{H}$ & 5.057930 & -0.844130 & 3.336360 \\
\hline $\mathrm{H}$ & 6.308770 & -1.965800 & 2.773320 \\
\hline C & 8.295050 & -0.131261 & 2.280410 \\
\hline $\mathrm{H}$ & 8.734720 & -0.187061 & 3.284000 \\
\hline $\mathrm{H}$ & 8.491060 & -1.081791 & 1.770970 \\
\hline $\mathrm{H}$ & 8.821430 & 0.656649 & 1.731280 \\
\hline C & -3.638640 & -1.775590 & 0.115160 \\
\hline C & -4.070750 & -1.566730 & -1.197640 \\
\hline C & -4.495350 & -1.424580 & 1.175930 \\
\hline C & -5.321430 & -0.993250 & -1.476040 \\
\hline $\mathrm{H}$ & -3.427200 & -1.866570 & -2.015550 \\
\hline
\end{tabular}




\begin{tabular}{|c|c|c|c|}
\hline C & -5.753940 & -0.868370 & 0.941840 \\
\hline $\mathrm{H}$ & -4.149960 & -1.585010 & 2.190660 \\
\hline C & -6.139430 & -0.656340 & -0.395060 \\
\hline $\mathrm{H}$ & -7.110540 & -0.215690 & -0.587050 \\
\hline C & -6.718150 & -0.480730 & 2.080140 \\
\hline C & -5.728930 & -0.756510 & -2.942660 \\
\hline C & -8.043760 & -1.262870 & 1.919570 \\
\hline $\mathrm{H}$ & -8.534000 & -1.047760 & 0.964430 \\
\hline $\mathrm{H}$ & -7.869560 & -2.343880 & 1.969270 \\
\hline $\mathrm{H}$ & -8.743430 & -0.994270 & 2.720540 \\
\hline C & -6.144800 & -0.801910 & 3.473610 \\
\hline $\mathrm{H}$ & -6.870370 & -0.517840 & 4.244200 \\
\hline $\mathrm{H}$ & -5.939740 & -1.872050 & 3.593320 \\
\hline $\mathrm{H}$ & -5.219380 & -0.250680 & 3.674100 \\
\hline C & -7.008630 & 1.037710 & 2.017660 \\
\hline $\mathrm{H}$ & -6.086840 & 1.618300 & 2.136290 \\
\hline $\mathrm{H}$ & -7.465240 & 1.327100 & 1.065340 \\
\hline $\mathrm{H}$ & -7.698910 & 1.327110 & 2.819580 \\
\hline C & -7.137780 & -0.147790 & -3.073250 \\
\hline $\mathrm{H}$ & -7.908200 & -0.803180 & -2.650470 \\
\hline $\mathrm{H}$ & -7.209120 & 0.828100 & -2.579440 \\
\hline $\mathrm{H}$ & -7.376790 & 0.001110 & -4.132320 \\
\hline C & -4.717330 & 0.217110 & -3.595830 \\
\hline $\mathrm{H}$ & -4.737720 & 1.189740 & -3.088940 \\
\hline $\mathrm{H}$ & -3.691630 & -0.162870 & -3.550440 \\
\hline $\mathrm{H}$ & -4.972310 & 0.379000 & -4.650640 \\
\hline C & -5.714290 & -2.100110 & -3.710190 \\
\hline $\mathrm{H}$ & -6.005420 & -1.942410 & -4.755960 \\
\hline $\mathrm{H}$ & -4.721730 & -2.561090 & -3.709360 \\
\hline $\mathrm{H}$ & -6.417860 & -2.813570 & -3.265610 \\
\hline
\end{tabular}

\section{C-Catalyzed reaction TS 53}

\section{Datum}

Value

\begin{tabular}{lc}
\hline B3LYP/6-31G(d) Energy & -3038.954218 \\
\hline B3LYP-D3(BJ)/def2-TZVPP-IEF-PCM(DCM) Energy & -3040.509913 \\
\hline B3LYP-D3(BJ)/def2-TZVPP-IEF-PCM(DCM)//B3LYP/6-31G(d) Free Energy (Quasiharmonic) & -3039.282406 \\
\hline
\end{tabular}

Number of Imaginary Frequencies

Frequencies (Top 3 out of 450)

$\begin{array}{lr}\text { 1. } & -311.6938 \mathrm{~cm}^{-1} \\ \text { 2. } & 7.4220 \mathrm{~cm}^{-1} \\ \text { 3. } & 9.0202 \mathrm{~cm}^{-1}\end{array}$




\begin{tabular}{|c|c|c|c|}
\hline C & 1.688133 & 0.809582 & -1.028369 \\
\hline $\mathrm{H}$ & 1.573206 & -0.212934 & -0.639493 \\
\hline H & 0.730572 & 1.079403 & -1.470050 \\
\hline C & -1.177337 & 1.019531 & 1.198962 \\
\hline 0 & -1.024341 & 1.254899 & 2.378189 \\
\hline 0 & -1.172727 & -0.249776 & 0.756993 \\
\hline H & -1.259098 & -0.356636 & -0.221779 \\
\hline 0 & 2.619573 & 0.843823 & -2.105859 \\
\hline $\mathrm{H}$ & 3.444745 & 0.444997 & -1.787109 \\
\hline C & -2.493074 & -4.638950 & 1.363975 \\
\hline C & -0.463483 & -4.153216 & 0.622215 \\
\hline C & -3.110151 & -3.048448 & -0.424358 \\
\hline C & -3.062371 & -4.365648 & 0.140642 \\
\hline $\mathrm{H}$ & -0.411729 & -5.050786 & 0.002489 \\
\hline H & -3.214606 & -2.235676 & 0.297151 \\
\hline $\mathrm{H}$ & -3.799502 & -2.936345 & -1.262750 \\
\hline C & -1.234653 & -1.649930 & -3.146807 \\
\hline C & -0.696093 & -3.121460 & -3.307677 \\
\hline C & -2.638641 & -1.464125 & -3.745471 \\
\hline $\mathrm{H}$ & -3.047677 & -0.512535 & -3.391468 \\
\hline $\mathrm{H}$ & -2.611603 & -1.439924 & -4.840345 \\
\hline $\mathrm{H}$ & -3.317220 & -2.264604 & -3.436975 \\
\hline C & -0.309607 & -0.561540 & -3.689130 \\
\hline $\mathrm{H}$ & -0.192616 & -0.668843 & -4.774296 \\
\hline $\mathrm{H}$ & 0.682925 & -0.580062 & -3.236136 \\
\hline $\mathrm{H}$ & -0.748160 & 0.425387 & -3.502969 \\
\hline C & 0.827022 & -3.216798 & -3.115626 \\
\hline $\mathrm{H}$ & 1.374029 & -2.794290 & -3.965397 \\
\hline $\mathrm{H}$ & 1.144984 & -2.695229 & -2.207789 \\
\hline $\mathrm{H}$ & 1.103727 & -4.272176 & -3.019693 \\
\hline C & -1.095934 & -3.814978 & -4.611030 \\
\hline $\mathrm{H}$ & -0.697035 & -3.282225 & -5.482308 \\
\hline $\mathrm{H}$ & -0.691580 & -4.832942 & -4.624256 \\
\hline $\mathrm{H}$ & -2.181868 & -3.885419 & -4.710210 \\
\hline 0 & -1.340370 & -1.527250 & -1.705701 \\
\hline 0 & -1.340511 & -3.805783 & -2.214998 \\
\hline B & -1.538230 & -2.852617 & -1.169138 \\
\hline 0 & -0.623547 & -3.019708 & 0.027507 \\
\hline C & 0.245884 & -4.165092 & 1.913825 \\
\hline C & 0.393745 & -2.983512 & 2.661798 \\
\hline C & 0.789268 & -5.366521 & 2.393538 \\
\hline C & 1.078950 & -3.016955 & 3.873806 \\
\hline H & -0.030293 & -2.055926 & 2.287624 \\
\hline C & 1.475943 & -5.391516 & 3.605716 \\
\hline $\mathrm{H}$ & 0.674352 & -6.279085 & 1.812516 \\
\hline C & 1.620607 & -4.216741 & 4.347929 \\
\hline $\mathrm{H}$ & 1.190523 & -2.103297 & 4.450853 \\
\hline $\mathrm{H}$ & 1.898244 & -6.323830 & 3.970237 \\
\hline $\mathrm{H}$ & 2.153920 & -4.235366 & 5.294571 \\
\hline $\mathrm{H}$ & -3.254434 & -5.201788 & -0.532850 \\
\hline
\end{tabular}




\begin{tabular}{|c|c|c|c|}
\hline $\mathrm{H}$ & -2.438501 & -3.863842 & 2.122338 \\
\hline $\mathrm{H}$ & -2.402700 & -5.660778 & 1.722382 \\
\hline C & -0.251700 & 2.858058 & -0.194118 \\
\hline C & -1.357277 & 2.089374 & 0.147061 \\
\hline C & -2.636056 & 2.287752 & -0.475951 \\
\hline C & -2.732654 & 3.240841 & -1.472360 \\
\hline $\mathrm{H}$ & -3.698938 & 3.433347 & -1.931251 \\
\hline C & 2.006351 & 1.763992 & 0.113729 \\
\hline C & 1.063984 & 2.700780 & 0.523623 \\
\hline C & 3.564015 & 2.584060 & 1.793265 \\
\hline C & 3.284663 & 1.704532 & 0.768799 \\
\hline $\mathrm{H}$ & 4.534651 & 2.540141 & 2.281889 \\
\hline C & 2.616633 & 3.531733 & 2.252006 \\
\hline C & 1.338968 & 3.593109 & 1.615466 \\
\hline C & 0.394973 & 4.541649 & 2.094433 \\
\hline C & 0.704968 & 5.386912 & 3.137036 \\
\hline C & 1.974273 & 5.328315 & 3.758783 \\
\hline C & 2.908202 & 4.415961 & 3.324400 \\
\hline $\mathrm{H}$ & -0.586691 & 4.586763 & 1.636635 \\
\hline $\mathrm{H}$ & -0.034287 & 6.100914 & 3.490026 \\
\hline $\mathrm{H}$ & 2.205626 & 5.999951 & 4.581224 \\
\hline $\mathrm{H}$ & 3.885426 & 4.355060 & 3.798019 \\
\hline C & -1.623249 & 4.015264 & -1.888951 \\
\hline C & -0.360437 & 3.834567 & -1.239432 \\
\hline C & 0.739917 & 4.628013 & -1.666001 \\
\hline C & 0.594795 & 5.554846 & -2.674231 \\
\hline C & -0.657180 & 5.738121 & -3.308506 \\
\hline C & -1.740861 & 4.983769 & -2.922694 \\
\hline $\mathrm{H}$ & 1.704412 & 4.487912 & -1.191143 \\
\hline $\mathrm{H}$ & 1.448505 & 6.149478 & -2.987538 \\
\hline $\mathrm{H}$ & -0.758086 & 6.473904 & -4.101835 \\
\hline $\mathrm{H}$ & -2.706694 & 5.115449 & -3.404865 \\
\hline C & 6.450015 & -0.944704 & -0.362515 \\
\hline C & 5.240104 & -1.509007 & 0.066288 \\
\hline C & 4.202953 & -0.636317 & 0.424406 \\
\hline C & 4.365520 & 0.756116 & 0.349884 \\
\hline C & 5.595257 & 1.275492 & -0.084134 \\
\hline C & 6.657470 & 0.439111 & -0.446101 \\
\hline $\mathrm{H}$ & 7.262057 & -1.609534 & -0.641279 \\
\hline $\mathrm{H}$ & 3.261856 & -1.028289 & 0.792104 \\
\hline $\mathrm{H}$ & 5.697300 & 2.352672 & -0.144265 \\
\hline C & 5.100183 & -3.042349 & 0.136515 \\
\hline C & 8.016497 & 0.982797 & -0.927877 \\
\hline C & 6.139270 & -3.608029 & 1.133939 \\
\hline $\mathrm{H}$ & 6.062022 & -4.701071 & 1.185249 \\
\hline $\mathrm{H}$ & 7.164851 & -3.359782 & 0.840980 \\
\hline $\mathrm{H}$ & 5.972794 & -3.208368 & 2.140756 \\
\hline C & 3.701100 & -3.484682 & 0.601088 \\
\hline $\mathrm{H}$ & 3.650628 & -4.578939 & 0.625314 \\
\hline $\mathrm{H}$ & 3.467680 & -3.125167 & 1.608617 \\
\hline $\mathrm{H}$ & 2.914131 & -3.134026 & -0.076174 \\
\hline C & 5.353450 & -3.648477 & -1.264841 \\
\hline $\mathrm{H}$ & 5.257947 & -4.740644 & -1.228278 \\
\hline $\mathrm{H}$ & 4.627679 & -3.269364 & -1.993461 \\
\hline
\end{tabular}




\begin{tabular}{|c|c|c|c|}
\hline $\mathrm{H}$ & 6.355758 & -3.415524 & -1.638942 \\
\hline C & 8.302563 & 0.465416 & -2.357766 \\
\hline $\mathrm{H}$ & 9.269291 & 0.842267 & -2.713850 \\
\hline $\mathrm{H}$ & 8.337541 & -0.628349 & -2.397843 \\
\hline $\mathrm{H}$ & 7.529008 & 0.800967 & -3.057903 \\
\hline C & 8.054427 & 2.522677 & -0.960939 \\
\hline $\mathrm{H}$ & 9.038761 & 2.859249 & -1.305372 \\
\hline $\mathrm{H}$ & 7.885606 & 2.957200 & 0.030895 \\
\hline $\mathrm{H}$ & 7.305822 & 2.935062 & -1.646671 \\
\hline C & 9.132311 & 0.494887 & 0.026662 \\
\hline $\mathrm{H}$ & 10.108238 & 0.871886 & -0.303032 \\
\hline $\mathrm{H}$ & 8.957671 & 0.851543 & 1.048194 \\
\hline $\mathrm{H}$ & 9.191944 & -0.598014 & 0.060457 \\
\hline C & -3.858969 & 1.552944 & -0.042024 \\
\hline C & -4.769687 & 1.069737 & -0.994566 \\
\hline C & -4.151582 & 1.394352 & 1.320572 \\
\hline C & -5.957838 & 0.436960 & -0.612428 \\
\hline $\mathrm{H}$ & -4.527192 & 1.188044 & -2.044305 \\
\hline C & -5.316704 & 0.745803 & 1.744179 \\
\hline $\mathrm{H}$ & -3.462506 & 1.796988 & 2.050589 \\
\hline C & -6.200397 & 0.281787 & 0.760406 \\
\hline $\mathrm{H}$ & -7.112067 & -0.217998 & 1.074500 \\
\hline C & -5.641070 & 0.530893 & 3.235431 \\
\hline C & -6.993725 & -0.078312 & -1.631413 \\
\hline C & -4.569849 & 1.139314 & 4.161438 \\
\hline $\mathrm{H}$ & -3.576255 & 0.715522 & 3.980564 \\
\hline $\mathrm{H}$ & -4.499650 & 2.226434 & 4.040781 \\
\hline $\mathrm{H}$ & -4.833584 & 0.940822 & 5.206748 \\
\hline C & -7.000314 & 1.186117 & 3.575849 \\
\hline $\mathrm{H}$ & -7.239564 & 1.037062 & 4.635974 \\
\hline $\mathrm{H}$ & -6.974164 & 2.264703 & 3.382857 \\
\hline $\mathrm{H}$ & -7.820803 & 0.761247 & 2.987799 \\
\hline C & -5.719501 & -0.987848 & 3.522341 \\
\hline $\mathrm{H}$ & -6.488912 & -1.479080 & 2.916863 \\
\hline $\mathrm{H}$ & -4.761996 & -1.475443 & 3.306758 \\
\hline $\mathrm{H}$ & -5.961155 & -1.164150 & 4.577858 \\
\hline C & -7.201662 & -1.599337 & -1.442179 \\
\hline $\mathrm{H}$ & -6.264767 & -2.145630 & -1.600236 \\
\hline $\mathrm{H}$ & -7.561009 & -1.840850 & -0.436473 \\
\hline $\mathrm{H}$ & -7.941178 & -1.975961 & -2.159658 \\
\hline C & -8.338518 & 0.653218 & -1.403679 \\
\hline $\mathrm{H}$ & -8.728531 & 0.484963 & -0.394551 \\
\hline $\mathrm{H}$ & -8.223158 & 1.734527 & -1.540443 \\
\hline $\mathrm{H}$ & -9.092596 & 0.298368 & -2.117167 \\
\hline C & -6.559940 & 0.167223 & -3.088989 \\
\hline $\mathrm{H}$ & -7.330634 & -0.209189 & -3.771276 \\
\hline $\mathrm{H}$ & -6.425738 & 1.234215 & -3.300610 \\
\hline H & -5.624531 & -0.349797 & -3.329942 \\
\hline
\end{tabular}




\begin{tabular}{lc}
\hline B3LYP/6-31G(d) Energy & -3038.954294 \\
\hline B3LYP-D3(BJ)/def2-TZVPP-IEF-PCM(DCM) Energy & -3040.509583 \\
\hline B3LYP-D3(BJ)/def2-TZVPP-IEF-PCM(DCM)//B3LYP/6-31G(d) Free Energy (Quasiharmonic) & -3039.282353 \\
\hline
\end{tabular}

Number of Imaginary Frequencies

Frequencies (Top 3 out of 450)

$\begin{array}{lr}\text { 1. } & -317.0149 \mathrm{~cm}^{-1} \\ \text { 2. } & 4.8171 \mathrm{~cm}^{-1} \\ \text { 3. } & 9.3403 \mathrm{~cm}^{-1}\end{array}$

\section{B3LYP/6-31G(d) Molecular Geometry in Cartesian Coordinates}

C

$\mathrm{H}$

$\mathrm{H}$

C

0

0

$\mathrm{H}$

0

$\mathrm{H}$

C

C

C

C

$\mathrm{H}$

$\mathrm{H}$

$\mathrm{H}$

C

C

C

$\mathrm{H}$

$\mathrm{H}$

$\mathrm{H}$

C

$\mathrm{H}$

$\mathrm{H}$

$\mathrm{H}$

C

$\mathrm{H}$

$\mathrm{H}$

$\mathrm{H}$

C

$\mathrm{H}$

\section{$-2.126970$}

$-1.078019$

$-2.449766$

1.058677

1.044450

1.073219

0.960817

$-2.811896$

$-3.749320$

$-0.100236$

1.677427

$-1.217129$

$-0.837100$

1.796362

$-1.983498$

$-1.447088$

0.926821

0.069485

0.634702

1.263954

0.863970

$-0.409800$

2.436500

2.830425

2.956852

2.668650

0.717276

0.876343

1.675116

0.058384

$-1.357240$

$-1.370025$
$-0.690098$

$-0.637274$

0.330996

$-0.962667$

$-1.147222$

0.295510

0.393189

$-1.167727$

$-0.941922$

3.765744

3.483883

3. 202450

2.903401

2.443722

2. 547534

4.253733

1. 239087

2. 303045

$-0.212156$

$-0.892748$

$-0.379828$

$-0.482870$

1. 510301

1. 274111

0.881809

2. 555144

2.824567

2.015547

3. 309117

3. 566536

1.815983

1.082141
0.719856

1.000886

0.482662

$-1.265913$

$-2.462447$

$-0.778324$

0.202533

1.882371

1.768423

$-1.593959$

$-0.343690$

0.540841

$-0.810905$

$-0.646370$

0.958842

0.730240

3.011927

3. 790584

3. 389599

2.806404

4.448807

3. 219981

3. 100507

4.095180

2. 370542

2.875542

5.076550

5.799609

4.873603

5.540013

4.100104

4.913710 


\begin{tabular}{|c|c|c|c|}
\hline H & -1.957941 & 2.678753 & 4.406456 \\
\hline $\mathrm{H}$ & -1.833005 & 1.357289 & 3.228462 \\
\hline 0 & 0.513367 & 1.475136 & 1.633571 \\
\hline 0 & -0.012319 & 3.389368 & 2.857816 \\
\hline B & 0.181819 & 2.894709 & 1.542146 \\
\hline 0 & 1.242917 & 3.737559 & 0.848783 \\
\hline C & 2.571121 & 4.468762 & -0.982831 \\
\hline C & 3.308323 & 4.091584 & -2.116034 \\
\hline C & 2.718773 & 5.762894 & -0.459508 \\
\hline C & 4.176538 & 4.997095 & -2.721654 \\
\hline $\mathrm{H}$ & 3.197655 & 3.086865 & -2.517695 \\
\hline C & 3.587764 & 6.664493 & -1.068090 \\
\hline $\mathrm{H}$ & 2.157400 & 6.039830 & 0.427070 \\
\hline C & 4.316224 & 6.285764 & -2.200089 \\
\hline $\mathrm{H}$ & 4.746071 & 4.697450 & -3.596898 \\
\hline $\mathrm{H}$ & 3.702125 & 7.664186 & -0.657921 \\
\hline $\mathrm{H}$ & 4.994189 & 6.992070 & -2.671561 \\
\hline $\mathrm{H}$ & -0.907004 & 1.867993 & -1.140614 \\
\hline $\mathrm{H}$ & -0.124086 & 4.834639 & -1.401231 \\
\hline $\mathrm{H}$ & 0.231613 & 3.466412 & -2.583838 \\
\hline C & -0.128293 & -2.758577 & -0.017129 \\
\hline C & 1.061191 & -2.075736 & -0.243934 \\
\hline C & 2.271123 & -2.416612 & 0.453681 \\
\hline C & 2.207479 & -3.411147 & 1.412448 \\
\hline $\mathrm{H}$ & 3.118605 & -3.716669 & 1.919916 \\
\hline C & -2.308681 & -1.568402 & -0.502954 \\
\hline C & -1.350304 & -2.508975 & -0.862473 \\
\hline C & -3.670419 & -2.183120 & -2.424469 \\
\hline C & -3.494012 & -1.414293 & -1.296156 \\
\hline $\mathrm{H}$ & -4.570694 & -2.061619 & -3.022495 \\
\hline C & -2.707638 & -3.142066 & -2.827310 \\
\hline C & -1.526912 & -3.315525 & -2.038269 \\
\hline C & -0.575312 & -4.284816 & -2.459645 \\
\hline C & -0.785078 & -5.040674 & -3.591282 \\
\hline C & -1.955972 & -4.867936 & -4.366429 \\
\hline C & -2.894941 & -3.935679 & -3.990360 \\
\hline $\mathrm{H}$ & 0.331399 & -4.420563 & -1.881401 \\
\hline $\mathrm{H}$ & -0.042462 & -5.773504 & -3.895115 \\
\hline $\mathrm{H}$ & -2.108321 & -5.470143 & -5.258106 \\
\hline $\mathrm{H}$ & -3.797828 & -3.790074 & -4.578919 \\
\hline C & 1.003692 & -4.084777 & 1.728808 \\
\hline C & -0.187138 & -3.765596 & 1.002293 \\
\hline C & -1.383635 & -4.463551 & 1.323339 \\
\hline C & -1.396048 & -5.430056 & 2.303917 \\
\hline C & -0.214414 & -5.751140 & 3.013978 \\
\hline C & 0.958787 & -5.091205 & 2.731415 \\
\hline $\mathrm{H}$ & -2.295922 & -4.212566 & 0.795035 \\
\hline $\mathrm{H}$ & -2.321399 & -5.948999 & 2.538434 \\
\hline $\mathrm{H}$ & -0.238636 & -6.517141 & 3.784404 \\
\hline $\mathrm{H}$ & 1.871817 & -5.327747 & 3.272671 \\
\hline C & -6.541769 & 1.385351 & -0.198300 \\
\hline C & -6.525615 & 0.136920 & 0.429303 \\
\hline C & -5.512168 & -0.764307 & 0.056185 \\
\hline C & -4.555129 & -0.429722 & -0.906512 \\
\hline
\end{tabular}




\begin{tabular}{|c|c|c|c|}
\hline C & -4.613075 & 0.831838 & -1.519262 \\
\hline C & -5.601792 & 1.758103 & -1.177000 \\
\hline $\mathrm{H}$ & -7.310695 & 2.098963 & 0.072072 \\
\hline $\mathrm{H}$ & -5.478638 & -1.759093 & 0.493803 \\
\hline $\mathrm{H}$ & -3.866743 & 1.071219 & -2.268013 \\
\hline C & -7.568399 & -0.280099 & 1.484711 \\
\hline C & -5.695964 & 3.151785 & -1.827813 \\
\hline C & -8.574813 & 0.843997 & 1.795274 \\
\hline $\mathrm{H}$ & -9.286006 & 0.501221 & 2.555125 \\
\hline $\mathrm{H}$ & -8.079036 & 1.739496 & 2.186953 \\
\hline $\mathrm{H}$ & -9.153044 & 1.132011 & 0.909953 \\
\hline C & -8.358808 & -1.505235 & 0.965877 \\
\hline $\mathrm{H}$ & -9.104418 & -1.819656 & 1.706420 \\
\hline $\mathrm{H}$ & -8.884533 & -1.266499 & 0.034364 \\
\hline $\mathrm{H}$ & -7.702782 & -2.359833 & 0.769918 \\
\hline C & -6.851660 & -0.656724 & 2.803497 \\
\hline $\mathrm{H}$ & -7.584744 & -0.946219 & 3.566132 \\
\hline $\mathrm{H}$ & -6.166369 & -1.501335 & 2.672637 \\
\hline $\mathrm{H}$ & -6.275389 & 0.189915 & 3.194407 \\
\hline C & -7.058963 & 3.290774 & -2.547677 \\
\hline $\mathrm{H}$ & -7.145990 & 4. 281995 & -3.009248 \\
\hline H & -7.163486 & 2.538268 & -3.337735 \\
\hline $\mathrm{H}$ & -7.900759 & 3.170170 & -1.857732 \\
\hline C & -5.579511 & 4.241333 & -0.735079 \\
\hline $\mathrm{H}$ & -5.646955 & 5.239303 & -1.185215 \\
\hline $\mathrm{H}$ & -6.375834 & 4.160365 & 0.012196 \\
\hline $\mathrm{H}$ & -4.619718 & 4.169313 & -0.211136 \\
\hline C & -4.581797 & 3.394600 & -2.863713 \\
\hline $\mathrm{H}$ & -4.696637 & 4.394143 & -3.298042 \\
\hline $\mathrm{H}$ & -3.584257 & 3.344435 & -2.412823 \\
\hline $\mathrm{H}$ & -4.623865 & 2.671884 & -3.686457 \\
\hline C & 3.594086 & -1.805460 & 0.130239 \\
\hline C & 4.495790 & -1.484218 & 1.152546 \\
\hline C & 3.996111 & -1.630066 & -1.206136 \\
\hline C & 5.784248 & -0.999801 & 0.874484 \\
\hline $\mathrm{H}$ & 4.180469 & -1.616929 & 2.183458 \\
\hline C & 5.257555 & -1.124867 & -1.524353 \\
\hline $\mathrm{H}$ & 3.313406 & -1.913468 & -1.995205 \\
\hline C & 6.133216 & -0.820541 & -0.467451 \\
\hline $\mathrm{H}$ & 7.118324 & -0.438564 & -0.707750 \\
\hline C & 5.708809 & -0.900301 & -2.980635 \\
\hline C & 6.761607 & -0.709598 & 2.031195 \\
\hline C & 6.981769 & -1.730857 & $-3.26864 e$ \\
\hline $\mathrm{H}$ & 7.812602 & -1.451474 & -2.612141 \\
\hline $\mathrm{H}$ & 6.789970 & -2.800838 & -3.129069 \\
\hline $\mathrm{H}$ & 7.310167 & -1.577437 & -4.303993 \\
\hline C & 4.627173 & -1.311258 & -3.998526 \\
\hline $\mathrm{H}$ & 4.984398 & -1.110499 & -5.015183 \\
\hline $\mathrm{H}$ & 4.397340 & -2.380996 & -3.936448 \\
\hline H & 3.692259 & -0.758909 & -3.855924 \\
\hline C & 6.021308 & 0.601053 & -3.187838 \\
\hline $\mathrm{H}$ & 5.134704 & 1.214693 & $-2.98986 e$ \\
\hline $\mathrm{H}$ & 6.821008 & 0.948451 & -2.524929 \\
\hline H & 6.339722 & 0.785527 & -4.221345 \\
\hline
\end{tabular}




\begin{tabular}{|c|c|c|c|}
\hline C & 6.153499 & 0.342740 & 2.986895 \\
\hline $\mathrm{H}$ & 5.956763 & 1.284289 & 2.461827 \\
\hline $\mathrm{H}$ & 5.210077 & 0.002139 & 3.424386 \\
\hline $\mathrm{H}$ & 6.845997 & 0.551601 & 3.811636 \\
\hline C & 7.022282 & -2.018623 & 2.814866 \\
\hline $\mathrm{H}$ & 6.098798 & -2.432852 & 3.232980 \\
\hline $\mathrm{H}$ & 7.467973 & -2.781729 & 2.166479 \\
\hline $\mathrm{H}$ & 7.712029 & -1.834515 & 3.647947 \\
\hline C & 8.118700 & -0.173367 & 1.536304 \\
\hline $\mathrm{H}$ & 8.773192 & 0.017069 & 2.394304 \\
\hline $\mathrm{H}$ & 8.630272 & -0.891392 & 0.885348 \\
\hline H & 8.009783 & 0.769635 & 0.988539 \\
\hline
\end{tabular}

\section{C-Catalyzed reaction TS 55}

\section{Datum}

Value

\begin{tabular}{lc}
\hline B3LYP/6-31G(d) Energy & -3038.963789 \\
\hline B3LYP-D3(BJ)/def2-TZVPP-IEF-PCM(DCM) Energy & -3040.512338 \\
\hline B3LYP-D3(BJ)/def2-TZVPP-IEF-PCM(DCM)//B3LYP/6-31G(d) Free Energy (Quasiharmonic) & -3039.282321 \\
\hline
\end{tabular}

Number of Imaginary Frequencies

Frequencies (Top 3 out of 450)

$\begin{array}{lr}\text { 1. } & -289.9175 \mathrm{~cm}^{-1} \\ \text { 2. } & 9.0262 \mathrm{~cm}^{-1} \\ \text { 3. } & 14.2652 \mathrm{~cm}^{-1}\end{array}$

\section{B3LYP/6-31G(d) Molecular Geometry in Cartesian Coordinates}

C

$\mathrm{H}$

$\mathrm{H}$

C

0

0

$\mathrm{H}$

0

$\mathrm{H}$

C

C

C

C

$\mathrm{H}$

-2.153495
-3.024268
-1.605582
0.890554
1.415027
0.426174
-0.114328
-1.307890
-0.981843
1.527620
2.604135
-0.012852
0.992232
2.686540

$-2.153495$

$-3.024268$

$-1.605582$

1.415027

2.686540
$-0.360521$

0.217550

$-0.682238$

$-1.996257$

$-2.738398$

$-0.798418$

$-0.342031$

0.460515

1. 256982

2.015632

1.815585

3. 320167

3. 218647

0.749398
0.846103

1.169148

1.738110

$-1.165477$

$-1.970534$

$-1.536907$

$-0.827157$

0.020190

0.509774

$-3.149531$

$-1.265368$

$-1.728895$

$-2.750684$

$-1.485708$ 


\begin{tabular}{|c|c|c|c|}
\hline $\mathrm{H}$ & -0.721304 & 2.488355 & -1.731352 \\
\hline $\mathrm{H}$ & -0.528104 & 4.282635 & -1.702743 \\
\hline C & 0.009722 & 3.545931 & 2.041609 \\
\hline C & 0.634414 & 4.883826 & 1.495201 \\
\hline C & -1.300599 & 3.708687 & 2.812192 \\
\hline $\mathrm{H}$ & -1.661611 & 2.729841 & 3.147743 \\
\hline $\mathrm{H}$ & -1.156286 & 4.332111 & 3.702208 \\
\hline $\mathrm{H}$ & -2.078233 & 4.161409 & 2.192393 \\
\hline C & 1.003557 & 2.716452 & 2.871078 \\
\hline $\mathrm{H}$ & 1.198610 & 3.175409 & 3.846423 \\
\hline $\mathrm{H}$ & 0.583418 & 1.719630 & 3.042621 \\
\hline $\mathrm{H}$ & 1.954802 & 2.594613 & 2.345284 \\
\hline C & 1.649114 & 5.546805 & 2.429494 \\
\hline $\mathrm{H}$ & 1.184454 & 5.832524 & 3.380643 \\
\hline $\mathrm{H}$ & 2.494393 & 4.886598 & 2.638175 \\
\hline $\mathrm{H}$ & 2.039594 & 6.456501 & 1.960550 \\
\hline C & -0.431044 & 5.913128 & 1.080764 \\
\hline $\mathrm{H}$ & -0.926619 & 6.362395 & 1.948694 \\
\hline $\mathrm{H}$ & 0.054857 & 6.712590 & 0.512020 \\
\hline $\mathrm{H}$ & -1.196882 & 5.460159 & 0.443963 \\
\hline 0 & -0.261597 & 2.835265 & 0.813140 \\
\hline 0 & 1.320782 & 4.447610 & 0.308735 \\
\hline B & 0.737547 & 3.226772 & -0.163033 \\
\hline 0 & 1.724870 & 2.079625 & -0.351001 \\
\hline C & 3.851248 & 2.584456 & -1.500251 \\
\hline C & 4.086525 & 3.865555 & -0.974908 \\
\hline C & 4.851096 & 1.963918 & -2.269594 \\
\hline C & 5.295323 & 4.510121 & -1.236641 \\
\hline $\mathrm{H}$ & 3.322834 & 4.346248 & -0.372432 \\
\hline C & 6.053986 & 2.614425 & -2.529768 \\
\hline $\mathrm{H}$ & 4.681890 & 0.963373 & -2.660378 \\
\hline C & 6.278454 & 3.893689 & -2.014676 \\
\hline $\mathrm{H}$ & 5.471318 & 5.501339 & -0.827019 \\
\hline $\mathrm{H}$ & 6.816502 & 2.122327 & -3.127182 \\
\hline $\mathrm{H}$ & 7.217043 & 4.404127 & -2.213494 \\
\hline $\mathrm{H}$ & 1.502671 & 4.132914 & -3.056099 \\
\hline $\mathrm{H}$ & 0.978392 & 1.090465 & -2.994813 \\
\hline $\mathrm{H}$ & 2.325669 & 1.986630 & -3.885439 \\
\hline C & -0.496116 & -2.731995 & 0.791165 \\
\hline C & 0.752091 & -2.372626 & 0.297375 \\
\hline C & 1.922223 & -2.418641 & 1.133333 \\
\hline C & 1.771400 & -2.829400 & 2.444054 \\
\hline $\mathrm{H}$ & 2.647462 & -2.870377 & 3.085775 \\
\hline C & -2.581537 & -1.558002 & 0.023228 \\
\hline C & -1.734414 & -2.661889 & -0.061232 \\
\hline C & -4.156021 & -2.644451 & -1.470297 \\
\hline C & -3.827863 & -1.550336 & -0.690035 \\
\hline $\mathrm{H}$ & -5.095143 & -2.640478 & -2.017662 \\
\hline C & -3.303358 & -3.764168 & -1.605359 \\
\hline C & -2.067400 & -3.785121 & -0.885548 \\
\hline C & -1.225833 & -4.924823 & -1.018967 \\
\hline C & -1.590473 & -5.981361 & -1.822357 \\
\hline C & -2.814527 & -5.957460 & -2.533666 \\
\hline C & -3.650619 & -4.870910 & -2.427133 \\
\hline
\end{tabular}




\begin{tabular}{|c|c|c|c|}
\hline $\mathrm{H}$ & -0.277469 & -4.946651 & -0.494074 \\
\hline $\mathrm{H}$ & -0.930188 & -6.838917 & -1.917652 \\
\hline $\mathrm{H}$ & -3.087908 & -6.798064 & -3.165833 \\
\hline H & -4.591830 & -4.840074 & -2.971068 \\
\hline C & 0.527019 & -3.239957 & 2.977700 \\
\hline C & -0.631714 & -3.201613 & 2.141787 \\
\hline C & -1.872323 & -3.633304 & 2.689027 \\
\hline C & -1.957013 & -4.073824 & 3.991583 \\
\hline C & -0.808102 & -4.105241 & 4.817316 \\
\hline C & 0.406948 & -3.696641 & 4. 317997 \\
\hline $\mathrm{H}$ & -2.758954 & -3.617227 & 2.064958 \\
\hline $\mathrm{H}$ & -2.913562 & -4.403160 & 4.388323 \\
\hline $\mathrm{H}$ & -0.888839 & -4.454823 & 5.843035 \\
\hline $\mathrm{H}$ & 1.297817 & -3.719400 & 4.941245 \\
\hline C & -6.773338 & 1.538825 & -0.446346 \\
\hline C & -7.147829 & 0.215417 & -0.178171 \\
\hline C & -6.152618 & -0.764881 & -0.252875 \\
\hline C & -4.827035 & -0.443719 & -0.584762 \\
\hline C & -4.494510 & 0.894050 & -0.845625 \\
\hline C & -5.461603 & 1.905335 & -0.778492 \\
\hline $\mathrm{H}$ & -7.531609 & 2.314564 & -0.393470 \\
\hline $\mathrm{H}$ & -6.387190 & -1.801163 & -0.038385 \\
\hline $\mathrm{H}$ & -3.468956 & 1.126514 & -1.106124 \\
\hline C & -8.610138 & -0.107573 & 0.186404 \\
\hline C & -5.136560 & 3.386128 & -1.055666 \\
\hline C & -8.833523 & -1.609649 & 0.444883 \\
\hline $\mathrm{H}$ & -9.885635 & -1.788876 & 0.694066 \\
\hline $\mathrm{H}$ & -8.595500 & -2.215163 & -0.436988 \\
\hline $\mathrm{H}$ & -8.229346 & -1.974370 & 1.283315 \\
\hline C & -9.006200 & 0.663543 & 1.468073 \\
\hline $\mathrm{H}$ & -10.048013 & 0.449071 & 1.736825 \\
\hline $\mathrm{H}$ & -8.370622 & 0.372296 & 2.312204 \\
\hline $\mathrm{H}$ & -8.912202 & 1.746774 & 1.338199 \\
\hline C & -9.537059 & 0.321613 & -0.975749 \\
\hline $\mathrm{H}$ & -10.584182 & 0.107899 & -0.728131 \\
\hline $\mathrm{H}$ & -9.456943 & 1.392891 & -1.187786 \\
\hline $\mathrm{H}$ & -9.287770 & -0.219779 & -1.895534 \\
\hline C & -5.460708 & 4.228154 & 0.201620 \\
\hline $\mathrm{H}$ & -5.240041 & 5.287113 & 0.018895 \\
\hline $\mathrm{H}$ & -6.515686 & 4.152451 & 0.485698 \\
\hline $\mathrm{H}$ & -4.860417 & 3.898500 & 1.057688 \\
\hline C & -3.653591 & 3.603942 & -1.405957 \\
\hline $\mathrm{H}$ & -3.472737 & 4.669713 & -1.589803 \\
\hline H & -2.986629 & 3.287554 & -0.596732 \\
\hline $\mathrm{H}$ & -3.365795 & 3.061314 & -2.313321 \\
\hline C & -5.989789 & 3.890111 & -2.243800 \\
\hline $\mathrm{H}$ & -5.772530 & 4.945648 & -2.449278 \\
\hline $\mathrm{H}$ & -5.772716 & 3.315619 & -3.151542 \\
\hline $\mathrm{H}$ & -7.062899 & 3.803935 & -2.043269 \\
\hline C & 3.293864 & -2.014684 & 0.693685 \\
\hline C & 4.000668 & -1.079127 & 1.466353 \\
\hline C & 3.933594 & -2.623099 & -0.395380 \\
\hline C & 5.331064 & -0.750603 & 1.182725 \\
\hline $\mathrm{H}$ & 3.486403 & -0.611892 & 2.298172 \\
\hline
\end{tabular}




\begin{tabular}{|c|c|c|c|}
\hline C & 5.258727 & -2.310327 & -0.721294 \\
\hline $\mathrm{H}$ & 3.379484 & -3.345543 & -0.976461 \\
\hline C & 5.932212 & -1.379294 & 0.082545 \\
\hline $\mathrm{H}$ & 6.965421 & -1.139685 & -0.152347 \\
\hline C & 5.985012 & -2.963529 & -1.913946 \\
\hline C & 6.147904 & 0.225295 & 2.053121 \\
\hline C & 5.089603 & -3.968939 & -2.664145 \\
\hline H & 4.798938 & -4.809671 & -2.024092 \\
\hline $\mathrm{H}$ & 4.174434 & -3.501214 & -3.042083 \\
\hline $\mathrm{H}$ & 5.636781 & -4.380279 & -3.520327 \\
\hline C & 6.416112 & -1.867155 & -2.916999 \\
\hline $\mathrm{H}$ & 6.963570 & -2.311042 & -3.757755 \\
\hline $\mathrm{H}$ & 5.540768 & -1.346898 & -3.323762 \\
\hline $\mathrm{H}$ & 7.067567 & -1.119492 & -2.451411 \\
\hline C & 7.239878 & -3.715510 & -1.411454 \\
\hline $\mathrm{H}$ & 7.948189 & -3.045692 & -0.912167 \\
\hline H & 6.964400 & -4.501180 & -0.698768 \\
\hline $\mathrm{H}$ & 7.763867 & -4.187603 & -2.251887 \\
\hline C & 7.293220 & -0.556711 & 2.740683 \\
\hline $\mathrm{H}$ & 6.895834 & -1.351012 & 3.383083 \\
\hline $\mathrm{H}$ & 7.960584 & -1.023749 & 2.008505 \\
\hline $\mathrm{H}$ & 7.896309 & 0.115921 & 3.363423 \\
\hline C & 6.751897 & 1.348933 & 1.180045 \\
\hline H & 7.414730 & 0.954566 & 0.403293 \\
\hline $\mathrm{H}$ & 5.967594 & 1.930125 & 0.684700 \\
\hline $\mathrm{H}$ & 7.342409 & 2.033480 & 1.801770 \\
\hline C & 5.292448 & 0.887844 & 3.150166 \\
\hline $\mathrm{H}$ & 5.910036 & 1.583749 & 3.729383 \\
\hline $\mathrm{H}$ & 4.458733 & 1.458552 & 2.724954 \\
\hline $\mathrm{H}$ & 4.881029 & 0.152657 & 3.850894 \\
\hline
\end{tabular}

\section{C-Catalyzed reaction TS 56}

\section{Datum}

Value

\begin{tabular}{lc}
\hline B3LYP/6-31G(d) Energy & -3038.957179 \\
\hline B3LYP-D3(BJ)/def2-TZVPP-IEF-PCM(DCM) Energy & -3040.510337 \\
\hline B3LYP-D3(BJ)/def2-TZVPP-IEF-PCM(DCM)//B3LYP/6-31G(d) Free Energy (Quasiharmonic) & -3039.282227 \\
\hline
\end{tabular}

Number of Imaginary Frequencies

Frequencies (Top 3 out of 450)

$\begin{array}{lr}\text { 1. } & -301.7378 \mathrm{~cm}^{-1} \\ \text { 2. } & 8.3335 \mathrm{~cm}^{-1} \\ \text { 3. } & 10.7148 \mathrm{~cm}^{-1}\end{array}$




\begin{tabular}{|c|c|c|c|}
\hline C & -3.029762 & -0.686808 & -1.851801 \\
\hline $\mathrm{H}$ & -3.031298 & 0.408084 & -1.905018 \\
\hline $\mathrm{H}$ & -2.212701 & -1.032126 & -2.483837 \\
\hline C & 0.247775 & 0.126184 & -0.559762 \\
\hline 0 & -0.548380 & 0.989704 & -0.871108 \\
\hline 0 & 1.088181 & 0.337879 & 0.463174 \\
\hline $\mathrm{H}$ & 1.664075 & -0.418044 & 0.753779 \\
\hline 0 & -4.215557 & -1.238210 & -2.421801 \\
\hline $\mathrm{H}$ & -4.970131 & -0.810934 & -1.985561 \\
\hline C & 0.844462 & 1.821872 & 3.921248 \\
\hline C & 2.720386 & 1.868585 & 2.785300 \\
\hline C & 1.576632 & -0.515121 & 4.289417 \\
\hline C & 0.703876 & 0.468169 & 3.709080 \\
\hline $\mathrm{H}$ & 2.224791 & 1.641819 & 1.842343 \\
\hline $\mathrm{H}$ & 1.203615 & -1.538638 & 4.222588 \\
\hline $\mathrm{H}$ & 1.920187 & -0.279353 & 5.299796 \\
\hline C & 3.988132 & -1.860063 & 1.673424 \\
\hline C & 4.440267 & -2.363820 & 3.094544 \\
\hline C & 3.589172 & -2.966900 & 0.699057 \\
\hline $\mathrm{H}$ & 3.267806 & -2.536681 & -0.254831 \\
\hline $\mathrm{H}$ & 4.438389 & -3.630042 & 0.495709 \\
\hline $\mathrm{H}$ & 2.767866 & -3.571288 & 1.090821 \\
\hline C & 5.018479 & -0.927993 & 1.015306 \\
\hline $\mathrm{H}$ & 5.907799 & -1.477972 & 0.687042 \\
\hline $\mathrm{H}$ & 5.334236 & -0.138821 & 1.703464 \\
\hline $\mathrm{H}$ & 4.566555 & -0.456510 & 0.136346 \\
\hline C & 5.946471 & -2.599531 & 3.238017 \\
\hline $\mathrm{H}$ & 6.297138 & -3.374406 & 2.545536 \\
\hline $\mathrm{H}$ & 6.512992 & -1.683155 & 3.057456 \\
\hline $\mathrm{H}$ & 6.166957 & -2.933008 & 4.257586 \\
\hline C & 3.680400 & -3.620501 & 3.555426 \\
\hline $\mathrm{H}$ & 3.992717 & -4.518077 & 3.009627 \\
\hline $\mathrm{H}$ & 3.882272 & -3.778011 & 4.619795 \\
\hline $\mathrm{H}$ & 2.600782 & -3.495007 & 3.430756 \\
\hline 0 & 2.810613 & -1.074141 & 1.998189 \\
\hline 0 & 4.067751 & -1.279212 & 3.951002 \\
\hline B & 3.030134 & -0.524887 & 3.336103 \\
\hline 0 & 3.428741 & 0.943988 & 3.350651 \\
\hline C & 3.099377 & 3.275644 & 3.016480 \\
\hline C & 3.995664 & 3.629546 & 4.037112 \\
\hline C & 2.575894 & 4.271574 & 2.177191 \\
\hline C & 4.354296 & 4.962823 & 4.216428 \\
\hline $\mathrm{H}$ & 4.408160 & 2.849857 & 4.669351 \\
\hline C & 2.937906 & 5.604325 & 2.360508 \\
\hline $\mathrm{H}$ & 1.886838 & 3.995112 & 1.382457 \\
\hline C & 3.825691 & 5.952513 & 3.381702 \\
\hline $\mathrm{H}$ & 5.050740 & 5.232911 & 5.005574 \\
\hline $\mathrm{H}$ & 2.531125 & 6.370193 & 1.706094 \\
\hline $\mathrm{H}$ & 4.108887 & 6.991869 & 3.524471 \\
\hline $\mathrm{H}$ & 0.067566 & 0.151612 & 2.884133 \\
\hline
\end{tabular}




\begin{tabular}{|c|c|c|c|}
\hline $\mathrm{H}$ & 1.330869 & 2.186177 & 4.821811 \\
\hline $\mathrm{H}$ & 0.206711 & 2.532285 & 3.403243 \\
\hline C & -0.610339 & -2.166303 & -1.048738 \\
\hline C & 0.353572 & -1.193225 & -1.292608 \\
\hline C & 1.396301 & -1.392573 & -2.261406 \\
\hline C & 1.452075 & -2.611310 & -2.912449 \\
\hline $\mathrm{H}$ & 2.217350 & -2.773723 & -3.666842 \\
\hline C & -2.817037 & -1.151182 & -0.414871 \\
\hline C & -1.710470 & -1.911411 & -0.054456 \\
\hline C & -3.729548 & -1.391292 & 1.830299 \\
\hline C & -3.824917 & -0.856000 & 0.564139 \\
\hline $\mathrm{H}$ & -4.497637 & -1.163917 & 2.565953 \\
\hline C & -2.641409 & -2.213001 & 2.214285 \\
\hline C & -1.598135 & -2.464479 & 1.267549 \\
\hline C & -0.506947 & -3.280679 & 1.675950 \\
\hline C & -0.462999 & -3.830115 & 2.939717 \\
\hline C & -1.496660 & -3.576756 & 3.872169 \\
\hline C & -2.559437 & -2.779180 & 3.514429 \\
\hline $\mathrm{H}$ & 0.293596 & -3.482337 & 0.972931 \\
\hline $\mathrm{H}$ & 0.371926 & -4.465435 & 3.222163 \\
\hline $\mathrm{H}$ & -1.447477 & -4.012140 & 4.866459 \\
\hline $\mathrm{H}$ & -3.359133 & -2.573008 & 4.222098 \\
\hline C & 0.510720 & -3.641731 & -2.677484 \\
\hline C & -0.551067 & -3.417904 & -1.744586 \\
\hline C & -1.517438 & -4.444423 & -1.558044 \\
\hline C & -1.425967 & -5.633779 & -2.245929 \\
\hline C & -0.367068 & -5.858141 & -3.158332 \\
\hline C & 0.577355 & -4.880910 & -3.370646 \\
\hline $\mathrm{H}$ & -2.338496 & -4.275825 & -0.870066 \\
\hline $\mathrm{H}$ & -2.175643 & -6.405029 & -2.092269 \\
\hline $\mathrm{H}$ & -0.307400 & -6.801354 & -3.694573 \\
\hline $\mathrm{H}$ & 1.388577 & -5.039136 & -4.077274 \\
\hline C & -7.128820 & 1.742565 & -0.235458 \\
\hline C & -7.391558 & 0.403707 & 0.065940 \\
\hline C & -6.290798 & -0.435462 & 0.313490 \\
\hline C & -4.979445 & 0.044621 & 0.254502 \\
\hline C & -4.758480 & 1.400323 & -0.048805 \\
\hline C & -5.823903 & 2.268192 & -0.295491 \\
\hline $\mathrm{H}$ & -7.960333 & 2.409046 & -0.431383 \\
\hline $\mathrm{H}$ & -6.444995 & -1.484734 & 0.548793 \\
\hline $\mathrm{H}$ & -3.735543 & 1.758815 & -0.068808 \\
\hline C & -8.818827 & -0.173053 & 0.134042 \\
\hline C & -5.619821 & 3.760042 & -0.623344 \\
\hline C & -9.080441 & -0.728490 & 1.554395 \\
\hline $\mathrm{H}$ & -10.088688 & -1.155914 & 1.616896 \\
\hline $\mathrm{H}$ & -8.368017 & -1.515867 & 1.821876 \\
\hline $\mathrm{H}$ & -9.000577 & 0.064970 & 2.306381 \\
\hline C & -9.897477 & 0.882803 & -0.173143 \\
\hline $\mathrm{H}$ & -10.890186 & 0.422971 & -0.111596 \\
\hline $\mathrm{H}$ & -9.873433 & 1.712368 & 0.542685 \\
\hline $\mathrm{H}$ & -9.788023 & 1.296717 & -1.182008 \\
\hline C & -8.961617 & -1.318062 & -0.897220 \\
\hline $\mathrm{H}$ & -9.971814 & -1.743516 & -0.856612 \\
\hline $\mathrm{H}$ & -8.788590 & -0.952260 & -1.915871 \\
\hline
\end{tabular}




\begin{tabular}{|c|c|c|c|}
\hline $\mathrm{H}$ & -8.251485 & -2.129744 & -0.708915 \\
\hline C & -4.132803 & 4.161829 & -0.625818 \\
\hline $\mathrm{H}$ & -4.040765 & 5.227889 & -0.862896 \\
\hline $\mathrm{H}$ & -3.664120 & 3.998653 & 0.351126 \\
\hline $\mathrm{H}$ & -3.558417 & 3.606131 & -1.375141 \\
\hline C & -6.201436 & 4.061333 & -2.025405 \\
\hline $\mathrm{H}$ & -6.071050 & 5.122219 & -2.272084 \\
\hline $\mathrm{H}$ & -7.271620 & 3.835743 & -2.081986 \\
\hline $\mathrm{H}$ & -5.693605 & 3.469228 & -2.795253 \\
\hline C & -6.349420 & 4.625774 & 0.431313 \\
\hline $\mathrm{H}$ & -6.218969 & 5.691167 & 0.205204 \\
\hline $\mathrm{H}$ & -7.424906 & 4.421228 & 0.457290 \\
\hline $\mathrm{H}$ & -5.949344 & 4.440002 & 1.434673 \\
\hline C & 2.382701 & -0.330906 & -2.619799 \\
\hline C & 3.741949 & -0.659003 & -2.786031 \\
\hline C & 1.973497 & 0.982372 & -2.868150 \\
\hline C & 4.679759 & 0.296866 & -3.180517 \\
\hline $\mathrm{H}$ & 4.052749 & -1.678855 & -2.591257 \\
\hline C & 2.887768 & 1.978654 & -3.245269 \\
\hline $\mathrm{H}$ & 0.922593 & 1.228646 & -2.779689 \\
\hline C & 4.227142 & 1.612055 & -3.395214 \\
\hline $\mathrm{H}$ & 4.949538 & 2.363947 & -3.690749 \\
\hline C & 2.380081 & 3.414789 & -3.476356 \\
\hline C & 6.170438 & -0.034217 & -3.389741 \\
\hline C & 1.323081 & 3.414254 & -4.606286 \\
\hline $\mathrm{H}$ & 1.754572 & 3.051784 & -5.546542 \\
\hline $\mathrm{H}$ & 0.465609 & 2.778638 & -4.363969 \\
\hline $\mathrm{H}$ & 0.946522 & 4.430716 & -4.775088 \\
\hline C & 1.734492 & 3.941505 & -2.170935 \\
\hline $\mathrm{H}$ & 1.342336 & 4.954787 & -2.324652 \\
\hline $\mathrm{H}$ & 0.908353 & 3.307142 & -1.834057 \\
\hline $\mathrm{H}$ & 2.475237 & 3.983387 & -1.363155 \\
\hline C & 3.508953 & 4.382200 & -3.878324 \\
\hline $\mathrm{H}$ & 4.281169 & 4.453514 & -3.103720 \\
\hline $\mathrm{H}$ & 3.990519 & 4.081926 & -4.816148 \\
\hline $\mathrm{H}$ & 3.096926 & 5.386746 & -4.026728 \\
\hline C & 6.558837 & 0.266847 & -4.857314 \\
\hline $\mathrm{H}$ & 7.619963 & 0.044330 & -5.023950 \\
\hline $\mathrm{H}$ & 6.394431 & 1.318055 & -5.116037 \\
\hline $\mathrm{H}$ & 5.970078 & -0.343803 & -5.551476 \\
\hline C & 7.035486 & 0.833141 & -2.444585 \\
\hline $\mathrm{H}$ & 6.885779 & 1.903323 & -2.621108 \\
\hline $\mathrm{H}$ & 6.794341 & 0.630844 & -1.394952 \\
\hline $\mathrm{H}$ & 8.100024 & 0.616674 & -2.597173 \\
\hline C & 6.490687 & -1.514481 & -3.107565 \\
\hline $\mathrm{H}$ & 7.558144 & -1.698484 & -3.273548 \\
\hline $\mathrm{H}$ & 6.265420 & -1.791393 & -2.071579 \\
\hline$H$ & 5.933747 & -2.186379 & -3.770735 \\
\hline
\end{tabular}


Frequencies (Top 3 out of 450)

$\begin{array}{lr}\text { 1. } & -389.1308 \mathrm{~cm}^{-1} \\ \text { 2. } & 6.5424 \mathrm{~cm}^{-1} \\ \text { 3. } & 10.8058 \mathrm{~cm}^{-1}\end{array}$

\section{B3LYP/6-31G(d) Molecular Geometry in Cartesian Coordinates}

C

$\mathrm{H}$

$\mathrm{H}$

C

0

0

$\mathrm{H}$

0

$\mathrm{H}$

C

C

C

C

$\mathrm{H}$

$\mathrm{H}$

$\mathrm{H}$

C

C

C

$\mathrm{H}$

$\mathrm{H}$

$\mathrm{H}$

C

$\mathrm{H}$

$\mathrm{H}$

$\mathrm{H}$

C

$\mathrm{H}$

$\mathrm{H}$

$\mathrm{H}$

C

$\mathrm{H}$

\section{$-2.141804$}

$-1.654725$

$-3.073633$

0.986349

1.533383

0.375494

$-0.169710$

$-1.274630$

$-0.869450$

0.128155

1.829457

$-0.322879$

0.111038

1. 368377

$-0.272884$

$-1.271584$

$-0.234078$

0.917256

$-0.187245$

$-1.049657$

$-0.226630$

0.719282

$-1.633426$

$-2.368544$

$-1.925627$

$-1.681207$

0.576220

0.362987

$-0.284560$

1. 429279

2. 243231

2. 211403

\subsection{0}

0.295432

$-0.389160$

1.906929

2. 724910

0.837057

0.331695

$-0.606632$

$-1.352427$

$-2.779856$

$-2.376898$

$-4.556312$

$-4.111557$

$-1.428781$

$-5.632571$

$-4.132779$

$-3.179934$

$-4.230343$

$-1.978411$

$-1.324291$

$-2.306379$

$-1.386921$

- 3.815296

$-3.097217$

$-4.088122$

$-4.713592$

$-5.369495$

$-4.990706$

$-5.945480$

$-6.052297$

$-3.576244$

$-3.212582$
0.861010

1.831211

1.017637

$-0.999073$

$-1.707064$

$-1.533470$

$-0.865572$

$-0.002213$

0.511050

$-2.412089$

$-1.195464$

$-0.753559$

$-2.034629$

$-0.917353$

$-0.591931$

$-0.420932$

2.563646

2.781963

3.508503

3. 340694

4.553800

3. 362413

2. 598432

2. 218465

3. 618339

1.976453

3. 744439

4. 751081

3. 396447

3.814724

3. 204506

4.237799 


\begin{tabular}{|c|c|c|c|}
\hline $\mathrm{H}$ & 3.041501 & -4.320844 & 3.126236 \\
\hline $\mathrm{H}$ & 2.499275 & -2.739949 & 2.548122 \\
\hline 0 & 0.030208 & -2.726685 & 1.209687 \\
\hline 0 & 1.084986 & -4.783993 & 1.467303 \\
\hline B & 0.741819 & -3.796874 & 0.514573 \\
\hline 0 & 1.903280 & -3.299028 & -0.268156 \\
\hline C & 2.933580 & -2.304734 & -2.174403 \\
\hline C & 3.790980 & -3.397445 & -2.378621 \\
\hline C & 3.118548 & -1.128633 & -2.918940 \\
\hline C & 4.812679 & -3.315736 & -3.322028 \\
\hline $\mathrm{H}$ & 3.649586 & -4.297888 & -1.789162 \\
\hline C & 4.144817 & -1.052499 & -3.858602 \\
\hline $\mathrm{H}$ & 2.457654 & -0.280195 & -2.761992 \\
\hline C & 4.990928 & -2.144732 & -4.065390 \\
\hline $\mathrm{H}$ & 5.473049 & -4.164766 & -3.477265 \\
\hline $\mathrm{H}$ & 4.282218 & -0.138739 & -4.429897 \\
\hline $\mathrm{H}$ & 5.788718 & -2.083990 & -4.800808 \\
\hline $\mathrm{H}$ & 0.722520 & -4.794369 & -2.627642 \\
\hline $\mathrm{H}$ & -0.553290 & -2.077275 & -1.938153 \\
\hline $\mathrm{H}$ & 0.488079 & -2.494872 & -3.394872 \\
\hline C & -0.220986 & 2.315745 & 1.158100 \\
\hline C & 0.971491 & 2.007568 & 0.513092 \\
\hline C & 2.191735 & 1.818612 & 1.250207 \\
\hline C & 2.138978 & 1.911464 & 2.628172 \\
\hline $\mathrm{H}$ & 3.052532 & 1.767234 & 3.199077 \\
\hline C & -2.427886 & 1.514183 & 0.240909 \\
\hline C & -1.487519 & 2.536029 & 0.374588 \\
\hline C & -3.891744 & 3.016315 & -0.981789 \\
\hline C & -3.657874 & 1.757445 & -0.458462 \\
\hline $\mathrm{H}$ & -4.817722 & 3.200708 & -1.520168 \\
\hline C & -2.956635 & 4.069781 & -0.871895 \\
\hline C & -1.724837 & 3.834165 & -0.183845 \\
\hline C & -0.794843 & 4.906434 & -0.077900 \\
\hline C & -1.072649 & 6.139014 & -0.623474 \\
\hline C & -2.292519 & 6.367797 & -1.305307 \\
\hline C & -3.212418 & 5.353371 & -1.426934 \\
\hline H & 0.149006 & 4.740175 & 0.428594 \\
\hline $\mathrm{H}$ & -0.346863 & 6.942793 & -0.536478 \\
\hline $\mathrm{H}$ & -2.496111 & 7.345404 & -1.733879 \\
\hline $\mathrm{H}$ & -4.151176 & 5.515803 & -1.951191 \\
\hline C & 0.951686 & 2.240019 & 3.325660 \\
\hline C & -0.249550 & 2.469985 & 2.584115 \\
\hline C & -1.424321 & 2.838384 & 3.297741 \\
\hline C & -1.410310 & 2.960191 & 4.669802 \\
\hline C & -0.222155 & 2.721841 & 5.401350 \\
\hline C & 0.932507 & 2.371000 & 4.740826 \\
\hline $\mathrm{H}$ & -2.338739 & 3.028328 & 2.745954 \\
\hline $\mathrm{H}$ & -2.317498 & 3.245456 & 5.195549 \\
\hline $\mathrm{H}$ & -0.224785 & 2.821319 & 6.483390 \\
\hline $\mathrm{H}$ & 1.852942 & 2.192834 & 5.291725 \\
\hline C & -6.782140 & -1.098523 & -1.077989 \\
\hline C & -7.094338 & 0.144394 & -0.497896 \\
\hline C & -6.048139 & 1.043310 & -0.286380 \\
\hline C & -4.722947 & 0.725813 & -0.636577 \\
\hline
\end{tabular}




\begin{tabular}{|c|c|c|c|}
\hline C & -4.454880 & -0.522823 & -1.203512 \\
\hline C & -5.479448 & -1.455538 & -1.436036 \\
\hline $\mathrm{H}$ & -7.586890 & -1.803239 & -1.250851 \\
\hline $\mathrm{H}$ & -6.237073 & 2.009247 & 0.167409 \\
\hline $\mathrm{H}$ & -3.431600 & -0.757921 & -1.477964 \\
\hline C & -8.554128 & 0.463317 & -0.120509 \\
\hline C & -5.132426 & -2.819482 & -2.062198 \\
\hline C & -9.440598 & 0.397554 & -1.387174 \\
\hline $\mathrm{H}$ & -10.484642 & 0.619508 & -1.133973 \\
\hline $\mathrm{H}$ & -9.109803 & 1.127078 & -2.135225 \\
\hline $\mathrm{H}$ & -9.414661 & -0.592809 & -1.853251 \\
\hline C & -9.059687 & -0.573143 & 0.911310 \\
\hline $\mathrm{H}$ & -10.100062 & -0.361018 & 1.187141 \\
\hline $\mathrm{H}$ & -9.021859 & -1.593917 & 0.516808 \\
\hline $\mathrm{H}$ & -8.453626 & -0.544910 & 1.824109 \\
\hline C & -8.708255 & 1.865995 & 0.497723 \\
\hline $\mathrm{H}$ & -9.760404 & 2.046280 & 0.745696 \\
\hline $\mathrm{H}$ & -8.128483 & 1.970591 & 1.421790 \\
\hline $\mathrm{H}$ & -8.392551 & 2.654455 & -0.194857 \\
\hline C & -4.444244 & -2.604268 & -3.430997 \\
\hline $\mathrm{H}$ & -4.194313 & -3.570217 & -3.886731 \\
\hline $\mathrm{H}$ & -5.103340 & -2.063683 & -4.119947 \\
\hline $\mathrm{H}$ & -3.516240 & -2.031421 & -3.337320 \\
\hline C & -4.168106 & -3.572389 & -1.115809 \\
\hline $\mathrm{H}$ & -3.870940 & -4.533839 & -1.552324 \\
\hline $\mathrm{H}$ & -3.259167 & -2.992161 & -0.927404 \\
\hline $\mathrm{H}$ & -4.645717 & -3.770696 & -0.148996 \\
\hline C & -6.373593 & -3.703677 & -2.286526 \\
\hline $\mathrm{H}$ & -6.069700 & -4.657435 & -2.732845 \\
\hline $\mathrm{H}$ & -6.891172 & -3.930420 & -1.347465 \\
\hline $\mathrm{H}$ & -7.091335 & -3.233944 & -2.968937 \\
\hline C & 3.516962 & 1.523097 & 0.621750 \\
\hline C & 4.245790 & 0.394559 & 1.039352 \\
\hline C & 4.098003 & 2.406092 & -0.291599 \\
\hline C & 5.535849 & 0.145303 & 0.564042 \\
\hline $\mathrm{H}$ & 3.778265 & -0.285764 & 1.742453 \\
\hline C & 5.389056 & 2.189188 & -0.796188 \\
\hline $\mathrm{H}$ & 3.528190 & 3.268878 & -0.610456 \\
\hline C & 6.084820 & 1.062951 & -0.349401 \\
\hline $\mathrm{H}$ & 7.087858 & 0.885081 & -0.717919 \\
\hline C & 5.975523 & 3.186739 & -1.813215 \\
\hline C & 6.377688 & -1.057296 & 1.034781 \\
\hline C & 7.414620 & 2.829386 & -2.230779 \\
\hline $\mathrm{H}$ & 8.101232 & 2.827697 & -1.376000 \\
\hline $\mathrm{H}$ & 7.468416 & 1.847180 & -2.714215 \\
\hline $\mathrm{H}$ & 7.783796 & 3.569833 & -2.949573 \\
\hline C & 5.089017 & 3.191014 & -3.082192 \\
\hline $\mathrm{H}$ & 5.461905 & 3.926212 & -3.806551 \\
\hline $\mathrm{H}$ & 5.101955 & 2.205297 & -3.562419 \\
\hline $\mathrm{H}$ & 4.046453 & 3.432863 & -2.854460 \\
\hline C & 5.996020 & 4.606078 & -1.197440 \\
\hline $\mathrm{H}$ & 4.992344 & 4.948416 & -0.926734 \\
\hline $\mathrm{H}$ & 6.615192 & 4.632990 & -0.293033 \\
\hline $\mathrm{H}$ & 6.410347 & 5.325473 & -1.914575 \\
\hline
\end{tabular}




$\begin{array}{|llll|}\text { C } & 6.960513 & -1.818234 & -0.179151 \\ \mathrm{H} & 6.163954 & -2.185939 & -0.834019 \\ \mathrm{H} & 7.626299 & -1.190805 & -0.780623 \\ \mathrm{H} & 7.545535 & -2.680137 & 0.164983 \\ \mathrm{C} & 7.540767 & -0.536199 & 1.913031 \\ \mathrm{H} & 8.180513 & 0.160431 & 1.360323 \\ \mathrm{H} & 7.159883 & -0.010226 & 2.796198 \\ \mathrm{H} & 8.166861 & -1.369590 & 2.256284 \\ \mathrm{C} & 5.551975 & -2.060143 & 1.862393 \\ \mathrm{H} & 6.181662 & -2.911434 & 2.145733 \\ \mathrm{H} & 5.171156 & -1.615689 & 2.788890 \\ \mathrm{H} & 4.701093 & -2.450031 & 1.292992 \\ & & \end{array}$

\section{C-Catalyzed reaction TS 58}

\section{Datum}

Value

\begin{tabular}{lr}
\hline B3LYP/6-31G(d) Energy & -3038.960546 \\
\hline B3LYP-D3(BJ)/def2-TZVPP-IEF-PCM(DCM) Energy & -3040.511521 \\
\hline B3LYP-D3(BJ)/def2-TZVPP-IEF-PCM(DCM)//B3LYP/6-31G(d) Free Energy (Quasiharmonic) & -3039.282178 \\
\hline
\end{tabular}

Number of Imaginary Frequencies

Frequencies (Top 3 out of 450)

$\begin{array}{lr}\text { 1. } & -321.1306 \mathrm{~cm}^{-1} \\ \text { 2. } & 8.6105 \mathrm{~cm}^{-1} \\ \text { 3. } & 11.6989 \mathrm{~cm}^{-1}\end{array}$

\section{B3LYP/6-31G(d) Molecular Geometry in Cartesian Coordinates}

C

$\mathrm{H}$

$\mathrm{H}$

C

0

0

$\mathrm{H}$

0

$\mathrm{H}$

C

C

C

C

$\mathrm{H}$
$-1.456115$

$-2.188548$

$-0.465054$

1. 513651

1. 357057

1.453898

1.532916

$-1.636029$

$-0.991902$

0.582339

$-0.897433$

2.131083

1.474068

$-1.153468$

0.619539
-0.164426
0.181024
0.880643
1.354901
-0.445952
-0.733803
0.964851
1.659989
-4.980052
-3.853936
-3.882290
-5.038857
-4.719074

1.083064

0.887953

0.933953

$-1.242908$

$-2.347887$

$-1.052579$

$-0.107433$

2.466922

2.680942

$-2.057238$

$-0.868876$

$-0.475373$

$-1.008857$

$-0.254148$ 


\begin{tabular}{|c|c|c|c|}
\hline $\mathrm{H}$ & 2.355348 & -3.096796 & -1.200170 \\
\hline $\mathrm{H}$ & 2.983722 & -4.096620 & 0.170766 \\
\hline C & 1.542375 & -2.199472 & 2.643661 \\
\hline C & 0.553149 & -3.398296 & 2.905857 \\
\hline C & 3.012601 & -2.581175 & 2.880912 \\
\hline $\mathrm{H}$ & 3.649740 & -1.813030 & 2.432086 \\
\hline $\mathrm{H}$ & 3.247238 & -2.646536 & 3.948974 \\
\hline $\mathrm{H}$ & 3.258561 & -3.540425 & 2.416612 \\
\hline C & 1.219156 & -0.914234 & 3.404495 \\
\hline $\mathrm{H}$ & 1.303865 & -1.080260 & 4.485357 \\
\hline $\mathrm{H}$ & 0.214344 & -0.543533 & 3.191072 \\
\hline $\mathrm{H}$ & 1.937954 & -0.132981 & 3.132520 \\
\hline C & -0.900833 & -2.939013 & 3.100945 \\
\hline $\mathrm{H}$ & -1.046360 & -2.436151 & 4.062909 \\
\hline $\mathrm{H}$ & -1.213083 & -2.254181 & 2.307963 \\
\hline $\mathrm{H}$ & -1.553428 & -3.818279 & 3.071887 \\
\hline C & 0.960335 & -4.323748 & 4.053477 \\
\hline $\mathrm{H}$ & 0.988779 & -3.782870 & 5.006699 \\
\hline $\mathrm{H}$ & 0.229218 & -5.133841 & 4.148229 \\
\hline $\mathrm{H}$ & 1.939478 & -4.775744 & 3.877951 \\
\hline 0 & 1.366770 & -1.971561 & 1.222185 \\
\hline 0 & 0.638969 & -4.141391 & 1.672783 \\
\hline B & 0.940438 & -3.217205 & 0.627300 \\
\hline 0 & -0.195710 & -2.916494 & -0.323494 \\
\hline C & -1.814796 & -3.487116 & -1.961848 \\
\hline C & -2.847512 & -4.365035 & -2.324463 \\
\hline C & -1.662557 & -2.268381 & -2.644708 \\
\hline C & -3.714842 & -4.035655 & -3.363769 \\
\hline $\mathrm{H}$ & -2.967055 & -5.305358 & -1.790732 \\
\hline C & -2.530755 & -1.946007 & -3.684047 \\
\hline $\mathrm{H}$ & -0.862769 & -1.592521 & -2.356889 \\
\hline C & -3.553965 & -2.827795 & -4.048559 \\
\hline $\mathrm{H}$ & -4.512345 & -4.719064 & -3.641618 \\
\hline $\mathrm{H}$ & -2.407090 & -1.005767 & -4.213840 \\
\hline $\mathrm{H}$ & -4.224265 & -2.574645 & -4.865415 \\
\hline $\mathrm{H}$ & 1.488283 & -5.942346 & -0.398249 \\
\hline $\mathrm{H}$ & 0.656552 & -4.179796 & -2.787176 \\
\hline $\mathrm{H}$ & 0.041457 & -5.865886 & -2.379029 \\
\hline C & 0.733177 & 2.482677 & 0.499877 \\
\hline C & 1.775442 & 1.717157 & -0.009098 \\
\hline C & 3.073938 & 1.704733 & 0.607811 \\
\hline C & 3.248035 & 2.425396 & 1.774759 \\
\hline $\mathrm{H}$ & 4.230606 & 2.458368 & 2.237626 \\
\hline C & -1.667140 & 1.778023 & 0.124741 \\
\hline C & -0.595227 & 2.605509 & -0.203258 \\
\hline C & -3.101160 & 3.029568 & -1.386750 \\
\hline C & -2.960357 & 2.010174 & -0.464080 \\
\hline $\mathrm{H}$ & -4.079501 & 3.221079 & -1.820202 \\
\hline C & -2.025343 & 3.867521 & -1.761825 \\
\hline C & -0.746381 & 3.667225 & -1.155806 \\
\hline C & 0.318820 & 4.532073 & -1.529358 \\
\hline C & 0.125271 & 5.539285 & -2.447690 \\
\hline C & -1.143561 & 5.736926 & -3.043233 \\
\hline C & -2.194388 & 4.916338 & -2.706282 \\
\hline
\end{tabular}




\begin{tabular}{|c|c|c|c|}
\hline $\mathrm{H}$ & 1.299042 & 4.382940 & -1.091281 \\
\hline $\mathrm{H}$ & 0.954447 & 6.185842 & -2.722024 \\
\hline $\mathrm{H}$ & -1.282507 & 6.535798 & -3.766812 \\
\hline $\mathrm{H}$ & -3.174147 & 5.057119 & -3.156926 \\
\hline C & 2.201278 & 3.171604 & 2.366328 \\
\hline C & 0.926954 & 3.224247 & 1.715338 \\
\hline C & -0.099041 & 4.017438 & 2.306386 \\
\hline C & 0.122322 & 4.702818 & 3.481813 \\
\hline C & 1.381734 & 4.642521 & 4.123946 \\
\hline C & 2.397945 & 3.896578 & 3.572997 \\
\hline H & -1.063590 & 4.083984 & 1.814868 \\
\hline $\mathrm{H}$ & -0.674967 & 5.300423 & 3.914921 \\
\hline $\mathrm{H}$ & 1.543551 & 5.189203 & 5.048818 \\
\hline $\mathrm{H}$ & 3.371742 & 3.848479 & 4.054512 \\
\hline C & -6.516046 & -0.145459 & 0.530085 \\
\hline C & -5.707991 & 0.326370 & 1.573857 \\
\hline C & -4.537784 & 1.014576 & 1.234594 \\
\hline C & -4.173798 & 1.216956 & -0.105126 \\
\hline C & -5.010321 & 0.725309 & -1.119045 \\
\hline C & -6.194965 & 0.042126 & -0.821862 \\
\hline $\mathrm{H}$ & -7.427327 & -0.681355 & 0.779079 \\
\hline $\mathrm{H}$ & -3.886322 & 1.401703 & 2.007069 \\
\hline $\mathrm{H}$ & -4.707603 & 0.874790 & -2.148930 \\
\hline C & -6.115964 & 0.069782 & 3.037904 \\
\hline C & -7.138252 & -0.501146 & -1.913195 \\
\hline C & -6.133632 & -1.455499 & 3.298390 \\
\hline $\mathrm{H}$ & -6.434271 & -1.663347 & 4.332943 \\
\hline $\mathrm{H}$ & -6.835634 & -1.973268 & 2.635703 \\
\hline $\mathrm{H}$ & -5.140088 & -1.889614 & 3.138848 \\
\hline C & -5.140147 & 0.717125 & 4.039842 \\
\hline $\mathrm{H}$ & -5.463317 & 0.493292 & 5.063274 \\
\hline $\mathrm{H}$ & -4.117286 & 0.345019 & 3.920675 \\
\hline $\mathrm{H}$ & -5.115813 & 1.807717 & 3.931840 \\
\hline C & -7.526385 & 0.648813 & 3.299518 \\
\hline $\mathrm{H}$ & -7.825341 & 0.468490 & 4.339589 \\
\hline $\mathrm{H}$ & -7.544134 & 1.730657 & 3.124338 \\
\hline $\mathrm{H}$ & -8.284922 & 0.193783 & 2.653691 \\
\hline C & -7.270841 & -2.035958 & -1.768234 \\
\hline $\mathrm{H}$ & -7.945578 & -2.435069 & -2.536205 \\
\hline $\mathrm{H}$ & -6.296858 & -2.525179 & -1.881648 \\
\hline $\mathrm{H}$ & -7.676189 & -2.319132 & -0.791058 \\
\hline C & -8.533331 & 0.149945 & -1.758823 \\
\hline $\mathrm{H}$ & -9.219435 & -0.229470 & -2.526355 \\
\hline $\mathrm{H}$ & -8.470814 & 1.238852 & -1.866932 \\
\hline $\mathrm{H}$ & -8.977240 & -0.062742 & -0.780844 \\
\hline C & -6.626901 & -0.198270 & -3.334603 \\
\hline $\mathrm{H}$ & -7.331911 & -0.597718 & -4.072992 \\
\hline $\mathrm{H}$ & -6.535963 & 0.879043 & -3.514477 \\
\hline $\mathrm{H}$ & -5.651346 & -0.660235 & -3.522188 \\
\hline C & 4.243905 & 1.009003 & -0.001832 \\
\hline C & 4.488456 & 1.090545 & -1.375604 \\
\hline C & 5.165140 & 0.322197 & 0.811285 \\
\hline C & 5.610718 & 0.483675 & -1.960107 \\
\hline $\mathrm{H}$ & 3.798809 & 1.646515 & -1.999529 \\
\hline
\end{tabular}




\begin{tabular}{|c|c|c|c|}
\hline C & 6.303570 & -0.279496 & 0.272885 \\
\hline $\mathrm{H}$ & 4.966302 & 0.260170 & 1.874858 \\
\hline C & 6.497997 & -0.191121 & -1.118409 \\
\hline $\mathrm{H}$ & 7.372523 & -0.665675 & -1.547908 \\
\hline C & 7.335696 & -1.032652 & 1.135133 \\
\hline C & 5.811467 & 0.587444 & -3.483836 \\
\hline C & 8.733671 & -0.397741 & 0.941629 \\
\hline $\mathrm{H}$ & 9.063931 & -0.440643 & -0.101169 \\
\hline $\mathrm{H}$ & 8.731695 & 0.654529 & 1.248269 \\
\hline $\mathrm{H}$ & 9.478973 & -0.927249 & 1.547776 \\
\hline C & 6.998982 & -0.982511 & 2.637461 \\
\hline $\mathrm{H}$ & 7.771287 & -1.512604 & 3.206318 \\
\hline $\mathrm{H}$ & 6.961398 & 0.047128 & 3.011614 \\
\hline $\mathrm{H}$ & 6.039937 & -1.462831 & 2.860258 \\
\hline C & 7.383888 & -2.516974 & 0.700672 \\
\hline $\mathrm{H}$ & 6.407886 & -2.996580 & 0.836571 \\
\hline $\mathrm{H}$ & 7.663473 & -2.623656 & -0.352744 \\
\hline $\mathrm{H}$ & 8.120271 & -3.067211 & 1.299516 \\
\hline C & 5.893300 & 2.077240 & -3.894490 \\
\hline $\mathrm{H}$ & 4.980141 & 2.623412 & -3.637518 \\
\hline $\mathrm{H}$ & 6.734797 & 2.575907 & -3.399259 \\
\hline $\mathrm{H}$ & 6.036993 & 2.163968 & -4.978469 \\
\hline C & 7.100794 & -0.109680 & -3.956873 \\
\hline $\mathrm{H}$ & 7.995822 & 0.333315 & -3.504485 \\
\hline $\mathrm{H}$ & 7.094374 & -1.181179 & -3.726069 \\
\hline $\mathrm{H}$ & 7.196062 & -0.008535 & -5.043937 \\
\hline C & 4.609388 & -0.076177 & -4.199254 \\
\hline $\mathrm{H}$ & 4.719060 & 0.014309 & -5.287207 \\
\hline $\mathrm{H}$ & 4.551240 & -1.142764 & -3.952449 \\
\hline $\mathrm{H}$ & 3.656561 & 0.382210 & -3.916529 \\
\hline
\end{tabular}

\section{C-Catalyzed reaction TS 59}

\section{Datum}

Value

\begin{tabular}{lc}
\hline B3LYP/6-31G(d) Energy & -3038.958064 \\
\hline B3LYP-D3(BJ)/def2-TZVPP-IEF-PCM(DCM) Energy & -3040.511583 \\
\hline B3LYP-D3(BJ)/def2-TZVPP-IEF-PCM(DCM)//B3LYP/6-31G(d) Free Energy (Quasiharmonic) & -3039.282149 \\
\hline
\end{tabular}

Number of Imaginary Frequencies

Frequencies (Top 3 out of 450)

$\begin{array}{lr}\text { 1. } & -320.4478 \mathrm{~cm}^{-1} \\ \text { 2. } & 7.0895 \mathrm{~cm}^{-1} \\ \text { 3. } & 10.6540 \mathrm{~cm}^{-1}\end{array}$




\begin{tabular}{|c|c|c|c|}
\hline C & 3.181772 & 0.318748 & -1.273081 \\
\hline $\mathrm{H}$ & 4.166156 & 0.383559 & -1.743702 \\
\hline $\mathrm{H}$ & 2.443962 & 0.183073 & -2.073812 \\
\hline C & 0.081651 & 0.469129 & 0.942612 \\
\hline 0 & 1.121738 & 0.948128 & 1.364199 \\
\hline 0 & -0.951055 & 0.352787 & 1.779396 \\
\hline H & -1.826717 & 0.064685 & 1.414685 \\
\hline 0 & 2.961726 & 1.567600 & -0.613717 \\
\hline $\mathrm{H}$ & 2.344149 & 1.401020 & 0.130237 \\
\hline C & -3.796438 & -3.279825 & -0.761306 \\
\hline C & -5.308201 & -1.810444 & -0.183653 \\
\hline C & -3.026495 & -2.813184 & 1.552056 \\
\hline C & -2.889012 & -2.741813 & 0.124546 \\
\hline $\mathrm{H}$ & -4.752575 & -0.958463 & -0.580917 \\
\hline $\mathrm{H}$ & -2.126669 & -2.551136 & 2.111805 \\
\hline $\mathrm{H}$ & -3.473023 & -3.737304 & 1.926093 \\
\hline C & -4.251987 & 0.617457 & 2.738119 \\
\hline C & -4.301625 & -0.366359 & 3.964811 \\
\hline C & -3.400535 & 1.869543 & 2.940439 \\
\hline $\mathrm{H}$ & -3.383257 & 2.464633 & 2.020799 \\
\hline $\mathrm{H}$ & -3.836904 & 2.489286 & 3.732667 \\
\hline $\mathrm{H}$ & -2.370501 & 1.636473 & 3.209492 \\
\hline C & -5.652074 & 1.033551 & 2.255924 \\
\hline $\mathrm{H}$ & -5.556315 & 1.523411 & 1.281544 \\
\hline $\mathrm{H}$ & -6.115726 & 1.743580 & 2.949099 \\
\hline $\mathrm{H}$ & -6.318821 & 0.174741 & 2.146007 \\
\hline C & -5.431433 & -0.089534 & 4.960615 \\
\hline $\mathrm{H}$ & -5.327383 & 0.903137 & 5.414805 \\
\hline $\mathrm{H}$ & -6.412294 & -0.158311 & 4.484225 \\
\hline $\mathrm{H}$ & -5.396885 & -0.833010 & 5.763881 \\
\hline C & -2.963482 & -0.454571 & 4.716285 \\
\hline $\mathrm{H}$ & -2.773731 & 0.442113 & 5.316708 \\
\hline $\mathrm{H}$ & -2.999056 & -1.316548 & 5.390378 \\
\hline $\mathrm{H}$ & -2.121473 & -0.589868 & 4.031715 \\
\hline 0 & -3.656162 & -0.235452 & 1.704630 \\
\hline 0 & -4.555158 & -1.638918 & 3.342159 \\
\hline B & -4.165989 & -1.570546 & 1.983469 \\
\hline 0 & -5.360661 & -1.946048 & 1.098889 \\
\hline C & -6.438571 & -2.307427 & -0.992155 \\
\hline C & -6.581216 & -1.876607 & -2.319749 \\
\hline C & -7.391159 & -3.179050 & -0.441139 \\
\hline C & -7.656775 & -2.314494 & -3.089200 \\
\hline $\mathrm{H}$ & -5.848460 & -1.194518 & -2.745168 \\
\hline C & -8.463965 & -3.615015 & -1.213728 \\
\hline $\mathrm{H}$ & -7.282917 & -3.493069 & 0.591965 \\
\hline C & -8.598674 & -3.186694 & -2.538075 \\
\hline $\mathrm{H}$ & -7.762912 & -1.973795 & -4.115251 \\
\hline $\mathrm{H}$ & -9.201085 & -4.286668 & -0.782540 \\
\hline $\mathrm{H}$ & -9.438393 & -3.528244 & -3.136993 \\
\hline H & -2.174360 & -2.024077 & -0.277009 \\
\hline
\end{tabular}




\begin{tabular}{|c|c|c|c|}
\hline $\mathrm{H}$ & -4.425225 & -4.113664 & -0.462375 \\
\hline $\mathrm{H}$ & -3.669462 & -3.138824 & -1.830825 \\
\hline C & 0.712239 & -1.114786 & -0.889181 \\
\hline C & -0.096910 & -0.064142 & -0.459990 \\
\hline $\mathrm{C}$ & -1.111881 & 0.495366 & -1.313690 \\
\hline C & -1.395027 & -0.146592 & -2.503018 \\
\hline $\mathrm{H}$ & -2.126697 & 0.286748 & -3.180045 \\
\hline C & 3.138378 & -0.872309 & -0.326560 \\
\hline C & 1.934731 & -1.547534 & -0.119219 \\
\hline C & 4.245050 & -2.369491 & 1.236636 \\
\hline C & 4.324017 & -1.295022 & 0.369338 \\
\hline H & 5.134993 & -2.678613 & 1.778696 \\
\hline C & 3.041054 & -3.073155 & 1.465027 \\
\hline C & 1.859703 & -2.668889 & 0.767248 \\
\hline C & 0.665575 & -3.409021 & 0.985501 \\
\hline C & 0.635861 & -4.474822 & 1.856954 \\
\hline C & 1.802686 & -4.859560 & 2.560080 \\
\hline C & 2.978228 & -4.173197 & 2.363407 \\
\hline $\mathrm{H}$ & -0.231820 & -3.133721 & 0.444690 \\
\hline $\mathrm{H}$ & -0.290079 & -5.023486 & 2.006124 \\
\hline $\mathrm{H}$ & 1.766571 & -5.698450 & 3.249752 \\
\hline $\mathrm{H}$ & 3.882999 & -4.462819 & 2.892668 \\
\hline C & -0.693011 & -1.305162 & -2.918707 \\
\hline C & 0.404306 & -1.775438 & -2.127914 \\
\hline C & 1.152436 & -2.887164 & -2.604891 \\
\hline C & 0.819844 & -3.510778 & -3.787286 \\
\hline C & -0.270736 & -3.049047 & -4.561439 \\
\hline C & -1.006579 & -1.966366 & -4.137031 \\
\hline $\mathrm{H}$ & 1.990908 & -3.246408 & -2.019247 \\
\hline $\mathrm{H}$ & 1.401045 & -4.361998 & -4.130589 \\
\hline $\mathrm{H}$ & -0.519922 & -3.546918 & -5.494572 \\
\hline $\mathrm{H}$ & -1.835361 & -1.592775 & -4.734006 \\
\hline C & 8.185714 & 0.498596 & -0.072451 \\
\hline C & 8.059474 & -0.897405 & -0.196244 \\
\hline C & 6.784406 & -1.449664 & -0.065372 \\
\hline C & 5.656565 & -0.645085 & 0.183980 \\
\hline C & 5.826360 & 0.736664 & 0.301432 \\
\hline C & 7.090995 & 1.332230 & 0.170536 \\
\hline $\mathrm{H}$ & 9.170741 & 0.938756 & -0.175553 \\
\hline $\mathrm{H}$ & 6.635753 & -2.518060 & -0.172818 \\
\hline $\mathrm{H}$ & 4.952538 & 1.351698 & 0.483150 \\
\hline C & 9.311406 & -1.752678 & -0.473697 \\
\hline C & 7.212968 & 2.862958 & 0.295273 \\
\hline C & 10.329466 & -1.564267 & 0.676303 \\
\hline $\mathrm{H}$ & 11.228861 & -2.165630 & 0.494021 \\
\hline $\mathrm{H}$ & 10.642250 & -0.519796 & 0.777176 \\
\hline $\mathrm{H}$ & 9.899438 & -1.877458 & 1.634564 \\
\hline C & 8.985298 & -3.254546 & -0.580474 \\
\hline $\mathrm{H}$ & 9.904233 & -3.819853 & -0.773414 \\
\hline $\mathrm{H}$ & 8.546361 & -3.643402 & 0.345415 \\
\hline $\mathrm{H}$ & 8.290390 & -3.463554 & -1.401749 \\
\hline C & 9.958448 & -1.307806 & -1.806954 \\
\hline $\mathrm{H}$ & 10.854700 & -1.905206 & -2.016349 \\
\hline $\mathrm{H}$ & 9.260904 & -1.437208 & -2.642453 \\
\hline
\end{tabular}




\begin{tabular}{|c|c|c|c|}
\hline $\mathrm{H}$ & 10.258399 & -0.254957 & -1.783732 \\
\hline C & 6.339413 & 3.532852 & -0.793391 \\
\hline $\mathrm{H}$ & 6.394317 & 4.625378 & -0.705084 \\
\hline $\mathrm{H}$ & 6.689806 & 3.257768 & -1.795447 \\
\hline $\mathrm{H}$ & 5.288272 & 3.238299 & -0.714076 \\
\hline C & 6.716965 & 3.306415 & 1.692288 \\
\hline $\mathrm{H}$ & 6.794285 & 4.396041 & 1.794997 \\
\hline $\mathrm{H}$ & 5.671523 & 3.029033 & 1.859176 \\
\hline $\mathrm{H}$ & 7.317281 & 2.846851 & 2.486256 \\
\hline C & 8.661965 & 3.355871 & 0.122681 \\
\hline $\mathrm{H}$ & 8.694002 & 4.447435 & 0.216665 \\
\hline $\mathrm{H}$ & 9.330264 & 2.939692 & 0.885707 \\
\hline $\mathrm{H}$ & 9.065729 & 3.098431 & -0.863371 \\
\hline C & -1.711761 & 1.830077 & -1.034003 \\
\hline C & -3.058300 & 2.111167 & -1.326893 \\
\hline C & -0.884046 & 2.866388 & -0.592733 \\
\hline C & -3.573250 & 3.402344 & -1.195907 \\
\hline $\mathrm{H}$ & -3.692773 & 1.300317 & -1.666021 \\
\hline C & -1.367713 & 4.171127 & -0.417428 \\
\hline $\mathrm{H}$ & 0.167089 & 2.661884 & -0.426912 \\
\hline C & -2.708651 & 4.411799 & -0.728400 \\
\hline $\mathrm{H}$ & -3.099809 & 5.416502 & -0.620127 \\
\hline C & -0.407491 & 5.261786 & 0.092211 \\
\hline C & -5.021863 & 3.765210 & -1.580202 \\
\hline C & 0.818132 & 5.359601 & -0.848708 \\
\hline $\mathrm{H}$ & 1.388626 & 4.426040 & -0.889172 \\
\hline $\mathrm{H}$ & 0.509949 & 5.610946 & -1.870420 \\
\hline $\mathrm{H}$ & 1.498001 & 6.145325 & -0.497567 \\
\hline C & -1.075244 & 6.648160 & 0.155794 \\
\hline $\mathrm{H}$ & -0.353106 & 7.387368 & 0.519895 \\
\hline $\mathrm{H}$ & -1.419832 & 6.981560 & -0.830270 \\
\hline $\mathrm{H}$ & -1.930818 & 6.660851 & 0.841017 \\
\hline C & 0.069360 & 4.884704 & 1.516142 \\
\hline $\mathrm{H}$ & -0.777138 & 4.834339 & 2.210948 \\
\hline $\mathrm{H}$ & 0.573627 & 3.913356 & 1.536714 \\
\hline $\mathrm{H}$ & 0.773128 & 5.637588 & 1.891850 \\
\hline C & -5.842448 & 2.535442 & -2.014765 \\
\hline $\mathrm{H}$ & -5.414329 & 2.052628 & -2.901190 \\
\hline $\mathrm{H}$ & -5.915002 & 1.787755 & -1.216563 \\
\hline $\mathrm{H}$ & -6.862167 & 2.844212 & -2.270692 \\
\hline C & -5.744675 & 4.426967 & -0.383675 \\
\hline $\mathrm{H}$ & -5.790832 & 3.748151 & 0.474618 \\
\hline $\mathrm{H}$ & -5.242169 & 5.343277 & -0.058149 \\
\hline $\mathrm{H}$ & -6.771853 & 4.693478 & -0.661056 \\
\hline C & -4.989875 & 4.759953 & -2.766434 \\
\hline $\mathrm{H}$ & -6.009978 & 5.032422 & -3.064310 \\
\hline $\mathrm{H}$ & -4.459344 & 5.682241 & -2.508338 \\
\hline $\mathrm{H}$ & -4.487742 & 4.318689 & -3.635064 \\
\hline
\end{tabular}


Frequencies (Top 3 out of 450)

$\begin{array}{lr}\text { 1. } & -384.8991 \mathrm{~cm}^{-1} \\ \text { 2. } & 8.8354 \mathrm{~cm}^{-1} \\ \text { 3. } & 12.6508 \mathrm{~cm}^{-1}\end{array}$

\section{B3LYP/6-31G(d) Molecular Geometry in Cartesian Coordinates}

C

$\mathrm{H}$

$\mathrm{H}$

C

0

0

$\mathrm{H}$

0

$\mathrm{H}$

C

C

C

C

$\mathrm{H}$

$\mathrm{H}$

$\mathrm{H}$

C

C

C

$\mathrm{H}$

$\mathrm{H}$

$\mathrm{H}$

C

$\mathrm{H}$

$\mathrm{H}$

$\mathrm{H}$

C

$\mathrm{H}$

$\mathrm{H}$

$\mathrm{H}$

C

$\mathrm{H}$

\subsection{0}

3.037630

1.592570

$-1.055700$

$-1.666400$

$-0.423240$

0.147000

1.275490

0.910160

$-0.175820$

$-1.854490$

0.350730

$-0.127870$

$-1.406310$

0.324471

1. 303410

$-0.076860$

$-0.364360$

1.190570

1.303580

1.138370

2.084600

$-1.270540$

$-1.418320$

$-1.087730$

$-2.192070$

$-1.273059$

$-0.824219$

$-2.252210$

$-1.428129$

0.920591

1.447581
0.223010

$-0.321350$

0.385600

1.904329

2.675109

0.844419

0.360649

$-0.564900$

$-1.350490$

$-2.603801$

$-2.321721$

$-4.474681$

$-3.956191$

$-1.378241$

$-5.560211$

$-4.057820$

$-3.085441$

$-4.634081$

$-2.667690$

$-1.578050$

$-2.971510$

$-3.112140$

$-2.251181$

$-2.348711$

$-1.194301$

$-2.552171$

$-5.107441$

$-4.905941$

$-4.625171$

$-6.188121$

$-5.481140$

$-5.447750$
0.861050

1.066350

1.810080

$-0.990030$

$-1.699340$

$-1.517860$

$-0.854760$

$-0.014110$

0.468760

$-2.562330$

$-1.277780$

$-1.036740$

$-2.274170$

$-0.966290$

$-0.947950$

$-0.707050$

2.458850

2.468430

3. 204980

3.186690

4.256800

2.760530

2.946240

4.027380

2.723920

2.440530

3.605480

4.585520

3.560610

3.520950

2.451550

3.411510 


\begin{tabular}{|c|c|c|c|}
\hline $\mathrm{H}$ & 0.649971 & -6.521571 & 2.245090 \\
\hline $\mathrm{H}$ & 1.608951 & -5.149270 & 1.668170 \\
\hline 0 & 0.102620 & -2.827011 & 1.040440 \\
\hline 0 & -1.054540 & -4.835991 & 1.228170 \\
\hline B & -0.684280 & -3.821931 & 0.313710 \\
\hline 0 & -1.876280 & -3.291901 & -0.398690 \\
\hline C & -2.989230 & -2.231231 & -2.219490 \\
\hline C & -3.833580 & -3.331361 & -2.438700 \\
\hline C & -3.216930 & -1.032591 & -2.915020 \\
\hline C & -4.883700 & -3.235401 & -3.348950 \\
\hline $\mathrm{H}$ & -3.659340 & -4.249131 & -1.886010 \\
\hline C & -4.269630 & -0.943681 & -3.823760 \\
\hline $\mathrm{H}$ & -2.569020 & -0.176441 & -2.746410 \\
\hline C & -5.102410 & -2.043171 & -4.045930 \\
\hline $\mathrm{H}$ & -5.533610 & -4.090411 & -3.515160 \\
\hline $\mathrm{H}$ & -4.437620 & -0.013951 & -4.359840 \\
\hline $\mathrm{H}$ & -5.920930 & -1.971421 & -4.757210 \\
\hline $\mathrm{H}$ & -0.743770 & -4.609821 & -2.894890 \\
\hline $\mathrm{H}$ & 0.504650 & -1.921981 & -2.057750 \\
\hline $\mathrm{H}$ & -0.567370 & -2.258561 & -3.513060 \\
\hline C & 0.222230 & 2.400719 & 1.111660 \\
\hline C & -0.986260 & 2.058119 & 0.515360 \\
\hline C & -2.180790 & 1.885019 & 1.297020 \\
\hline C & -2.089620 & 2.035519 & 2.667470 \\
\hline $\mathrm{H}$ & -2.983060 & 1.897849 & 3.270920 \\
\hline C & 2.409120 & 1.551210 & 0.196450 \\
\hline C & 1.471380 & 2.579760 & 0.290370 \\
\hline C & 3.851970 & 2.982600 & -1.130340 \\
\hline C & 3.631510 & 1.755850 & -0.529710 \\
\hline $\mathrm{H}$ & 4.768340 & 3.138130 & -1.693600 \\
\hline C & 2.915990 & 4.039310 & -1.067290 \\
\hline C & 1.697920 & 3.844710 & -0.342920 \\
\hline C & 0.768720 & 4.920910 & -0.281460 \\
\hline C & 1.033519 & 6.119020 & -0.904990 \\
\hline C & 2.239389 & 6.307270 & -1.623090 \\
\hline C & 3.158560 & 5.287870 & -1.702350 \\
\hline $\mathrm{H}$ & -0.164710 & 4.784269 & 0.252620 \\
\hline $\mathrm{H}$ & 0.308289 & 6.926209 & -0.851570 \\
\hline $\mathrm{H}$ & 2.432919 & 7.257710 & -2.113010 \\
\hline $\mathrm{H}$ & 4.086640 & 5.419710 & -2.253520 \\
\hline C & -0.887450 & 2.410989 & 3.314500 \\
\hline C & 0.289110 & 2.619569 & 2.528440 \\
\hline C & 1.479910 & 3.030490 & 3.190670 \\
\hline C & 1.503620 & 3.213980 & 4.555710 \\
\hline C & 0.339810 & 2.996649 & 5.331480 \\
\hline C & -0.829220 & 2.604719 & 4.721200 \\
\hline $\mathrm{H}$ & 2.376350 & 3.203620 & 2.605070 \\
\hline $\mathrm{H}$ & 2.422260 & 3.531470 & 5.041530 \\
\hline $\mathrm{H}$ & 0.372350 & 3.144529 & 6.407480 \\
\hline $\mathrm{H}$ & -1.731240 & 2.441269 & 5.306010 \\
\hline C & 6.779580 & -1.120350 & -0.945430 \\
\hline C & 7.087960 & 0.177430 & -0.514210 \\
\hline C & 6.031090 & 1.083320 & -0.368120 \\
\hline C & 4.704320 & 0.723180 & -0.645600 \\
\hline
\end{tabular}




\begin{tabular}{|c|c|c|c|}
\hline C & 4.438890 & -0.585290 & -1.071650 \\
\hline C & 5.466430 & -1.525400 & -1.224080 \\
\hline $\mathrm{H}$ & 7.582210 & -1.834800 & -1.064020 \\
\hline $\mathrm{H}$ & 6.227680 & 2.092120 & -0.017250 \\
\hline $\mathrm{H}$ & 3.412240 & -0.859500 & -1.290890 \\
\hline C & 8.526790 & 0.626670 & -0.190570 \\
\hline C & 5.120840 & -2.962540 & -1.659530 \\
\hline C & 8.607790 & 1.061890 & 1.292120 \\
\hline $\mathrm{H}$ & 9.624630 & 1.391660 & 1.538580 \\
\hline $\mathrm{H}$ & 7.926180 & 1.890240 & 1.511510 \\
\hline $\mathrm{H}$ & 8.349160 & 0.230910 & 1.958440 \\
\hline C & 9.561480 & -0.492460 & -0.416370 \\
\hline $\mathrm{H}$ & 10.564130 & -0.119500 & -0.178170 \\
\hline $\mathrm{H}$ & 9.372480 & -1.360540 & 0.225380 \\
\hline $\mathrm{H}$ & 9.575080 & -0.832970 & -1.457980 \\
\hline C & 8.910250 & 1.821920 & -1.095370 \\
\hline $\mathrm{H}$ & 9.929600 & 2.159560 & -0.870960 \\
\hline $\mathrm{H}$ & 8.870860 & 1.540600 & -2.153990 \\
\hline $\mathrm{H}$ & 8.238490 & 2.674640 & -0.951300 \\
\hline C & 4.321380 & -2.938450 & -2.983320 \\
\hline $\mathrm{H}$ & 4.074640 & -3.960540 & -3.295340 \\
\hline $\mathrm{H}$ & 3.380790 & -2.386860 & -2.887990 \\
\hline $\mathrm{H}$ & 4.904330 & -2.469870 & -3.784480 \\
\hline C & 6.372510 & -3.834290 & -1.877540 \\
\hline $\mathrm{H}$ & 6.071280 & -4.837410 & -2.200180 \\
\hline $\mathrm{H}$ & 7.027310 & -3.419550 & -2.652600 \\
\hline $\mathrm{H}$ & 6.959700 & -3.948950 & -0.959320 \\
\hline C & 4.263040 & -3.619970 & -0.551580 \\
\hline $\mathrm{H}$ & 3.968900 & -4.635910 & -0.843350 \\
\hline $\mathrm{H}$ & 4.824300 & -3.685540 & 0.388070 \\
\hline $\mathrm{H}$ & 3.351740 & -3.044990 & -0.355630 \\
\hline C & -3.517450 & 1.534409 & 0.722150 \\
\hline C & -4.169850 & 2.390839 & -0.167420 \\
\hline C & -4.179990 & 0.377449 & 1.169420 \\
\hline C & -5.471530 & 2.121269 & -0.616180 \\
\hline $\mathrm{H}$ & -3.647710 & 3.274249 & -0.511390 \\
\hline C & -5.475860 & 0.072289 & 0.744940 \\
\hline $\mathrm{H}$ & -3.656300 & -0.280011 & 1.853930 \\
\hline C & -6.100320 & 0.966319 & -0.142910 \\
\hline $\mathrm{H}$ & -7.109170 & 0.746199 & -0.470450 \\
\hline C & -6.243860 & -1.166581 & 1.247880 \\
\hline C & -6.142620 & 3.096969 & -1.601840 \\
\hline C & -7.381960 & -0.698041 & 2.186420 \\
\hline $\mathrm{H}$ & -7.956490 & -1.557871 & 2.553590 \\
\hline $\mathrm{H}$ & -6.979330 & -0.162101 & 3.053830 \\
\hline $\mathrm{H}$ & -8.075490 & -0.024401 & 1.671110 \\
\hline C & -6.854070 & -1.945561 & 0.059330 \\
\hline $\mathrm{H}$ & -7.385810 & -2.832231 & 0.426140 \\
\hline $\mathrm{H}$ & -7.573650 & -1.343831 & -0.505190 \\
\hline $\mathrm{H}$ & -6.076230 & -2.277301 & -0.636140 \\
\hline C & -5.337720 & -2.139371 & 2.027340 \\
\hline $\mathrm{H}$ & -4.503820 & -2.495131 & 1.411450 \\
\hline $\mathrm{H}$ & -4.926810 & -1.682101 & 2.934520 \\
\hline $\mathrm{H}$ & -5.918450 & -3.015171 & 2.338860 \\
\hline
\end{tabular}




\begin{tabular}{|llll|} 
C & -6.197800 & 4.510989 & -0.975860 \\
$H$ & -6.779170 & 4.506779 & -0.046340 \\
$H$ & -5.198990 & 4.894239 & -0.744700 \\
$H$ & -6.671870 & 5.215699 & -1.670280 \\
C & -5.312980 & 3.147249 & -2.907410 \\
$H$ & -4.272840 & 3.432239 & -2.722720 \\
$H$ & -5.304140 & 2.165259 & -3.394710 \\
$H$ & -5.747910 & 3.870519 & -3.608780 \\
C & -7.581330 & 2.679899 & -1.960480 \\
$H$ & -8.011410 & 3.405309 & -2.660570 \\
$H$ & -7.613370 & 1.697049 & -2.444540 \\
$H$ & -8.231020 & 2.647559 & -1.077950 \\
\hline
\end{tabular}

\section{C-Catalyzed reaction TS 61}

\section{Datum}

Value

\begin{tabular}{lc}
\hline B3LYP/6-31G(d) Energy & -3038.955696 \\
\hline B3LYP-D3(BJ)/def2-TZVPP-IEF-PCM(DCM) Energy & -3040.50981 \\
\hline B3LYP-D3(BJ)/def2-TZVPP-IEF-PCM(DCM)//B3LYP/6-31G(d) Free Energy (Quasiharmonic) & -3039.281859 \\
\hline
\end{tabular}

Number of Imaginary Frequencies

Frequencies (Top 3 out of 450)
1. $-325.8135 \mathrm{~cm}^{-1}$
2. $\quad 6.8031 \mathrm{~cm}^{-1}$
3. $9.7667 \mathrm{~cm}^{-1}$

\section{B3LYP/6-31G(d) Molecular Geometry in Cartesian Coordinates}

\begin{tabular}{|c|c|c|c|}
\hline C & -2.183948 & -0.605049 & 0.716756 \\
\hline $\mathrm{H}$ & -1.138460 & -0.605997 & 1.014650 \\
\hline $\mathrm{H}$ & -2.429428 & 0.417921 & 0.406773 \\
\hline C & 0.983504 & -1.130363 & -1.273046 \\
\hline 0 & 0.933137 & -1.405351 & -2.453219 \\
\hline 0 & 1.094858 & 0.147257 & -0.873922 \\
\hline $\mathrm{H}$ & 1.043563 & 0.282122 & 0.106469 \\
\hline 0 & -2.919969 & -0.955246 & 1.895045 \\
\hline $\mathrm{H}$ & -3.843652 & -0.701975 & 1.734493 \\
\hline $\mathrm{C}$ & 0.202159 & 4.831685 & -1.162455 \\
\hline C & 1.903557 & 4. 324172 & 0.119950 \\
\hline C & -0.802012 & 2.915061 & 0.037025 \\
\hline C & -0.707788 & 4.312889 & -0.265896 \\
\hline $\mathrm{H}$ & 1.651903 & 5.065679 & 0.880695 \\
\hline
\end{tabular}




\begin{tabular}{|c|c|c|c|}
\hline $\mathrm{H}$ & -0.591711 & 2.238779 & -0.794066 \\
\hline $\mathrm{H}$ & -1.699253 & 2.627543 & 0.587283 \\
\hline C & 0.902412 & 1.234559 & 2.992928 \\
\hline C & 0.193546 & 2.541051 & 3.516269 \\
\hline C & 0.372294 & -0.069326 & 3.587765 \\
\hline $\mathrm{H}$ & 0.918950 & -0.921373 & 3.169401 \\
\hline $\mathrm{H}$ & 0.520065 & -0.081361 & 4.674400 \\
\hline $\mathrm{H}$ & -0.690045 & -0.215983 & 3.379863 \\
\hline C & 2.431730 & 1.293002 & 3.133873 \\
\hline $\mathrm{H}$ & 2.743604 & 1.172862 & 4.177474 \\
\hline H & 2.873887 & 0.482815 & 2.546377 \\
\hline $\mathrm{H}$ & 2.835432 & 2.237263 & 2.757552 \\
\hline C & 0.865596 & 3.190738 & 4.727951 \\
\hline $\mathrm{H}$ & 0.870243 & 2.513121 & 5.589857 \\
\hline $\mathrm{H}$ & 1.894878 & 3.483225 & 4.506937 \\
\hline $\mathrm{H}$ & 0.313090 & 4.092906 & 5.012247 \\
\hline C & -1.301634 & 2.329743 & 3.810449 \\
\hline $\mathrm{H}$ & -1.457082 & 1.764202 & 4.735837 \\
\hline $\mathrm{H}$ & -1.777465 & 3.309651 & 3.923653 \\
\hline $\mathrm{H}$ & -1.803866 & 1.791944 & 3.001004 \\
\hline 0 & 0.581740 & 1.273424 & 1.575176 \\
\hline 0 & 0.311019 & 3.439577 & 2.396042 \\
\hline B & 0.478146 & 2.663170 & 1.208649 \\
\hline 0 & 1.690151 & 3.080834 & 0.402426 \\
\hline C & 3.003598 & 4.645211 & -0.807717 \\
\hline C & 3.514453 & 5.950937 & -0.851000 \\
\hline C & 3.558944 & 3.651898 & -1.631221 \\
\hline C & 4.567282 & 6.264170 & -1.707944 \\
\hline $\mathrm{H}$ & 3.086852 & 6.717488 & -0.208405 \\
\hline C & 4.608916 & 3.971749 & -2.487309 \\
\hline $\mathrm{H}$ & 3.161956 & 2.642199 & -1.584502 \\
\hline C & 5.114563 & 5.275141 & -2.528856 \\
\hline $\mathrm{H}$ & 4.961956 & 7.276024 & -1.734235 \\
\hline $\mathrm{H}$ & 5.036896 & 3.202389 & -3.123976 \\
\hline $\mathrm{H}$ & 5.935029 & 5.518430 & -3.198477 \\
\hline $\mathrm{H}$ & -1.191227 & 5.003475 & 0.426025 \\
\hline $\mathrm{H}$ & 0.577712 & 4.212885 & -1.971979 \\
\hline $\mathrm{H}$ & 0.289343 & 5.905120 & -1.307148 \\
\hline C & -0.281388 & -2.796252 & 0.089045 \\
\hline C & 0.933217 & -2.175353 & -0.178155 \\
\hline C & 2.126603 & -2.519805 & 0.545221 \\
\hline C & 2.026524 & -3.449833 & 1.563400 \\
\hline $\mathrm{H}$ & 2.925020 & -3.755078 & 2.093319 \\
\hline C & -2.412873 & -1.546317 & -0.450111 \\
\hline C & -1.498593 & -2.545212 & -0.762151 \\
\hline C & -3.824779 & -2.220929 & -2.315272 \\
\hline C & -3.599596 & -1.390737 & -1.240719 \\
\hline $\mathrm{H}$ & -4.727110 & -2.098705 & -2.910125 \\
\hline C & -2.907025 & -3.241649 & -2.668992 \\
\hline C & -1.722866 & -3.412941 & -1.885060 \\
\hline C & -0.815120 & -4.442271 & -2.257575 \\
\hline C & -1.070091 & -5.257444 & -3.337593 \\
\hline C & -2.244928 & -5.087047 & -4.107252 \\
\hline C & -3.141750 & -4.097180 & -3.778211 \\
\hline
\end{tabular}




\begin{tabular}{|c|c|c|c|}
\hline $\mathrm{H}$ & 0.094464 & -4.575908 & -1.683426 \\
\hline $\mathrm{H}$ & -0.360187 & -6.035506 & -3.605110 \\
\hline $\mathrm{H}$ & -2.433251 & -5.736168 & -4.958285 \\
\hline $\mathrm{H}$ & -4.046640 & -3.952301 & -4.363982 \\
\hline C & 0.798627 & -4.060361 & 1.913640 \\
\hline C & -0.378491 & -3.739180 & 1.165762 \\
\hline C & -1.600473 & -4.372149 & 1.524215 \\
\hline C & -1.649925 & -5.280164 & 2.558086 \\
\hline C & -0.481734 & -5.604242 & 3.288704 \\
\hline C & 0.715259 & -5.005334 & 2.972142 \\
\hline $\mathrm{H}$ & -2.502412 & -4.118904 & 0.979448 \\
\hline $\mathrm{H}$ & -2.594320 & -5.750128 & 2.818778 \\
\hline $\mathrm{H}$ & -0.534977 & -6.323725 & 4.101443 \\
\hline $\mathrm{H}$ & 1.618601 & -5.244265 & 3.528563 \\
\hline C & -6.521028 & 1.596176 & -0.300511 \\
\hline C & -6.589986 & 0.364524 & 0.355935 \\
\hline C & -5.615469 & -0.597898 & 0.035361 \\
\hline C & -4.613571 & -0.338432 & -0.904161 \\
\hline C & -4.583488 & 0.910195 & -1.545013 \\
\hline C & -5.531816 & 1.895006 & -1.256338 \\
\hline $\mathrm{H}$ & -7.260255 & 2.354630 & -0.071091 \\
\hline $\mathrm{H}$ & -5.645857 & -1.580926 & 0.498699 \\
\hline $\mathrm{H}$ & -3.801317 & 1.089950 & -2.273654 \\
\hline C & -7.682537 & 0.027064 & 1.389034 \\
\hline C & -5.534026 & 3.273231 & -1.945836 \\
\hline C & -8.637604 & 1.208261 & 1.645192 \\
\hline $\mathrm{H}$ & -9.385251 & 0.920892 & 2.392846 \\
\hline $\mathrm{H}$ & -8.106815 & 2.086623 & 2.029823 \\
\hline $\mathrm{H}$ & -9.176481 & 1.503444 & 0.737704 \\
\hline C & -8.520739 & -1.167690 & 0.874160 \\
\hline $\mathrm{H}$ & -9.301094 & -1.427162 & 1.600052 \\
\hline $\mathrm{H}$ & -9.008155 & -0.923255 & -0.076519 \\
\hline $\mathrm{H}$ & -7.904325 & -2.058438 & 0.714286 \\
\hline C & -7.023174 & -0.354817 & 2.735940 \\
\hline $\mathrm{H}$ & -7.790573 & -0.598360 & 3.480721 \\
\hline $\mathrm{H}$ & -6.370228 & -1.229042 & 2.640946 \\
\hline $\mathrm{H}$ & -6.422760 & 0.473873 & 3.129168 \\
\hline C & -4.367065 & 3.435189 & -2.938496 \\
\hline $\mathrm{H}$ & -4.413673 & 4.427259 & -3.401833 \\
\hline $\mathrm{H}$ & -3.393781 & 3.346982 & -2.442713 \\
\hline $\mathrm{H}$ & -4.409784 & 2.693604 & -3.743890 \\
\hline C & -6.858707 & 3.452872 & -2.725613 \\
\hline $\mathrm{H}$ & -6.881562 & 4.432732 & -3.218242 \\
\hline $\mathrm{H}$ & -6.966027 & 2.682134 & -3.497373 \\
\hline $\mathrm{H}$ & -7.731807 & 3.390241 & -2.067525 \\
\hline C & -5.412716 & 4.387769 & -0.879402 \\
\hline $\mathrm{H}$ & -5.420062 & 5.374484 & -1.358592 \\
\hline $\mathrm{H}$ & -6.238724 & 4.361419 & -0.161231 \\
\hline $\mathrm{H}$ & -4.476920 & 4.290483 & -0.317024 \\
\hline C & 3.467747 & -1.969709 & 0.192353 \\
\hline C & 4.371094 & -1.601460 & 1.207323 \\
\hline C & 3.880276 & -1.877466 & -1.140006 \\
\hline C & 5.652442 & -1.135650 & 0.909204 \\
\hline $\mathrm{H}$ & 4.043616 & -1.676824 & 2.237677 \\
\hline
\end{tabular}




\begin{tabular}{|c|c|c|c|}
\hline C & 5.154360 & -1.400070 & -1.483707 \\
\hline $\mathrm{H}$ & 3.200551 & -2.192743 & -1.922385 \\
\hline C & 6.016556 & -1.036229 & -0.446601 \\
\hline $\mathrm{H}$ & 7.005436 & -0.664561 & -0.689020 \\
\hline C & 5.540671 & -1.300427 & -2.971660 \\
\hline C & 6.661854 & -0.720440 & 1.997688 \\
\hline C & 5.458820 & -2.702183 & -3.622004 \\
\hline $\mathrm{H}$ & 6.148702 & -3.401138 & -3.134809 \\
\hline $\mathrm{H}$ & 4.450924 & -3.123905 & -3.556828 \\
\hline $\mathrm{H}$ & 5.726655 & -2.644534 & -4.684208 \\
\hline C & 4.556150 & -0.345283 & -3.689181 \\
\hline $\mathrm{H}$ & 4.800363 & -0.279126 & -4.756879 \\
\hline $\mathrm{H}$ & 3.516593 & -0.675570 & -3.599764 \\
\hline $\mathrm{H}$ & 4.619783 & 0.663747 & -3.263454 \\
\hline C & 6.969534 & -0.762550 & -3.175831 \\
\hline $\mathrm{H}$ & 7.090680 & 0.245518 & -2.762028 \\
\hline $\mathrm{H}$ & 7.722392 & -1.413218 & -2.715803 \\
\hline $\mathrm{H}$ & 7.192183 & -0.707217 & -4.247449 \\
\hline C & 7.960329 & -1.547925 & 1.844458 \\
\hline $\mathrm{H}$ & 7.757582 & -2.619327 & 1.955955 \\
\hline $\mathrm{H}$ & 8.431116 & -1.398022 & 0.867422 \\
\hline $\mathrm{H}$ & 8.688670 & -1.258337 & 2.611991 \\
\hline C & 6.991372 & 0.783592 & 1.844718 \\
\hline $\mathrm{H}$ & 7.415719 & 1.008696 & 0.860654 \\
\hline $\mathrm{H}$ & 6.090164 & 1.395088 & 1.966975 \\
\hline $\mathrm{H}$ & 7.720321 & 1.095074 & 2.603317 \\
\hline $\mathrm{C}$ & 6.121238 & -0.950215 & 3.421843 \\
\hline $\mathrm{H}$ & 6.880091 & -0.655974 & 4.155924 \\
\hline $\mathrm{H}$ & 5.223289 & -0.354938 & 3.620474 \\
\hline $\mathrm{H}$ & 5.880336 & -2.004540 & 3.600239 \\
\hline
\end{tabular}

\section{C-Catalyzed reaction TS 62}

\section{Datum}

Value

\begin{tabular}{lc}
\hline B3LYP/6-31G(d) Energy & -3038.959161 \\
\hline B3LYP-D3(BJ)/def2-TZVPP-IEF-PCM(DCM) Energy & -3040.51018 \\
\hline B3LYP-D3(BJ)/def2-TZVPP-IEF-PCM(DCM)//B3LYP/6-31G(d) Free Energy (Quasiharmonic) & -3039.281826 \\
\hline
\end{tabular}

Number of Imaginary Frequencies

Frequencies (Top 3 out of 450)

$$
\begin{array}{lr}
\text { 1. } & -305.6632 \mathrm{~cm}^{-1} \\
\text { 2. } & 5.7177 \mathrm{~cm}^{-1} \\
\text { 3. } & 11.1349 \mathrm{~cm}^{-1}
\end{array}
$$




\begin{tabular}{|c|c|c|c|}
\hline C & 3.158665 & -1.567872 & 0.653211 \\
\hline $\mathrm{H}$ & 3.801183 & -1.074277 & 1.382460 \\
\hline $\mathrm{H}$ & 2.195041 & -1.752336 & 1.135487 \\
\hline C & -0.332030 & -0.148506 & 0.487254 \\
\hline 0 & 0.423041 & 0.217779 & 1.363346 \\
\hline 0 & -1.275503 & 0.687224 & 0.027069 \\
\hline $\mathrm{H}$ & -1.819211 & 0.395753 & -0.751300 \\
\hline 0 & 3.818729 & -2.804630 & 0.342225 \\
\hline $\mathrm{H}$ & 3.232194 & -3.309147 & -0.244364 \\
\hline C & -1.538552 & 4.352089 & -0.796280 \\
\hline C & -3.263110 & 3.207447 & -0.077065 \\
\hline C & -2.149929 & 3.210122 & -2.905334 \\
\hline C & -1.279814 & 3.425110 & -1.781834 \\
\hline $\mathrm{H}$ & -2.642648 & 2.388223 & 0.283247 \\
\hline $\mathrm{H}$ & -1.702641 & 2.626359 & -3.711289 \\
\hline $\mathrm{H}$ & -2.632959 & 4.113447 & -3.286287 \\
\hline C & -4.120670 & -0.013013 & -2.451189 \\
\hline C & -4.702430 & 0.775654 & -3.682798 \\
\hline C & -3.536277 & -1.384296 & -2.786414 \\
\hline $\mathrm{H}$ & -3.134727 & -1.858548 & -1.884930 \\
\hline $\mathrm{H}$ & -4.310173 & -2.045519 & -3.194124 \\
\hline $\mathrm{H}$ & -2.728391 & -1.311725 & -3.518579 \\
\hline C & -5.128731 & -0.139317 & -1.297417 \\
\hline $\mathrm{H}$ & -5.930476 & -0.846667 & -1.538250 \\
\hline $\mathrm{H}$ & -5.581020 & 0.827741 & -1.060460 \\
\hline $\mathrm{H}$ & -4.610417 & -0.504161 & -0.404925 \\
\hline C & -6.194022 & 0.544676 & -3.940511 \\
\hline $\mathrm{H}$ & -6.401891 & -0.509063 & -4.162615 \\
\hline $\mathrm{H}$ & -6.798963 & 0.850045 & -3.083695 \\
\hline $\mathrm{H}$ & -6.511398 & 1.140474 & -4.802851 \\
\hline C & -3.915283 & 0.524969 & -4.981308 \\
\hline $\mathrm{H}$ & -4.099384 & -0.473972 & -5.392542 \\
\hline $\mathrm{H}$ & -4.225481 & 1.265899 & -5.724955 \\
\hline $\mathrm{H}$ & -2.839490 & 0.640032 & -4.817655 \\
\hline 0 & -3.045668 & 0.863218 & -2.018950 \\
\hline 0 & -4.513329 & 2.142373 & -3.302479 \\
\hline B & -3.465911 & 2.227498 & -2.342809 \\
\hline 0 & -3.967755 & 3.025714 & -1.148475 \\
\hline C & -3.768886 & 4.153366 & 0.936082 \\
\hline C & -4.813357 & 5.043850 & 0.643130 \\
\hline C & -3.213358 & 4.136420 & 2.225151 \\
\hline C & -5.288296 & 5.906902 & 1.627120 \\
\hline $\mathrm{H}$ & -5.246902 & 5.037834 & -0.351745 \\
\hline C & -3.691835 & 5.001931 & 3.206428 \\
\hline $\mathrm{H}$ & -2.405953 & 3.443607 & 2.451486 \\
\hline C & -4.728677 & 5.889929 & 2.908525 \\
\hline $\mathrm{H}$ & -6.099526 & 6.592479 & 1.397768 \\
\hline $\mathrm{H}$ & -3.259009 & 4.982461 & 4.202654 \\
\hline $\mathrm{H}$ & -5.102722 & 6.564581 & 3.673851 \\
\hline $\mathrm{H}$ & -0.526435 & 2.665876 & -1.576211 \\
\hline
\end{tabular}




\begin{tabular}{|c|c|c|c|}
\hline $\mathrm{H}$ & -2.152302 & 5.222093 & -1.012559 \\
\hline $\mathrm{H}$ & -0.893260 & 4.428704 & 0.074099 \\
\hline C & 0.777116 & -1.825664 & -0.984088 \\
\hline C & -0.266992 & -1.535711 & -0.112040 \\
\hline C & -1.214321 & -2.540106 & 0.289019 \\
\hline C & -1.102761 & -3.798967 & -0.273404 \\
\hline $\mathrm{H}$ & -1.789441 & -4.580883 & 0.039128 \\
\hline C & 2.970145 & -0.676810 & -0.564290 \\
\hline C & 1.817168 & -0.794336 & -1.338515 \\
\hline C & 3.776533 & 1.086847 & -2.030832 \\
\hline C & 3.979332 & 0.281534 & -0.925688 \\
\hline $\mathrm{H}$ & 4.543309 & 1.804511 & -2.311362 \\
\hline C & 2.626666 & 0.978081 & -2.846003 \\
\hline C & 1.623651 & 0.015348 & -2.505302 \\
\hline C & 0.491278 & -0.106560 & -3.358655 \\
\hline C & 0.366701 & 0.673294 & -4.487836 \\
\hline C & 1.357568 & 1.629728 & -4.817016 \\
\hline C & 2.460154 & 1.778838 & -4.008679 \\
\hline $\mathrm{H}$ & -0.278309 & -0.831756 & -3.117133 \\
\hline $\mathrm{H}$ & -0.496318 & 0.549188 & -5.136084 \\
\hline $\mathrm{H}$ & 1.244315 & 2.240272 & -5.708636 \\
\hline $\mathrm{H}$ & 3.230219 & 2.507191 & -4.251721 \\
\hline C & -0.088684 & -4.128449 & -1.202952 \\
\hline C & 0.879169 & -3.136125 & -1.559493 \\
\hline C & 1.912271 & -3.495933 & -2.472653 \\
\hline C & 1.979794 & -4.767856 & -2.999257 \\
\hline C & 1.020163 & -5.745736 & -2.643985 \\
\hline C & 0.009877 & -5.430336 & -1.765101 \\
\hline $\mathrm{H}$ & 2.646275 & -2.750250 & -2.758748 \\
\hline $\mathrm{H}$ & 2.774408 & -5.021565 & -3.695333 \\
\hline $\mathrm{H}$ & 1.085472 & -6.744607 & -3.066299 \\
\hline $\mathrm{H}$ & -0.729904 & -6.175606 & -1.482881 \\
\hline C & 7.675136 & 0.819370 & 1.159949 \\
\hline C & 6.867154 & 1.942402 & 0.902924 \\
\hline C & 5.656356 & 1.735935 & 0.239586 \\
\hline C & 5.251003 & 0.449823 & -0.160939 \\
\hline C & 6.082741 & -0.639001 & 0.114166 \\
\hline C & 7.306728 & -0.474406 & 0.781570 \\
\hline $\mathrm{H}$ & 8.616642 & 0.969429 & 1.675146 \\
\hline $\mathrm{H}$ & 4.991893 & 2.567773 & 0.035779 \\
\hline $\mathrm{H}$ & 5.759714 & -1.626991 & -0.194144 \\
\hline C & 7.339228 & 3.336933 & 1.358757 \\
\hline C & 8.177753 & -1.712648 & 1.067677 \\
\hline C & 8.678638 & 3.678831 & 0.663339 \\
\hline $\mathrm{H}$ & 9.033575 & 4.667489 & 0.980289 \\
\hline $\mathrm{H}$ & 9.460892 & 2.951479 & 0.904104 \\
\hline $\mathrm{H}$ & 8.560993 & 3.693275 & -0.426342 \\
\hline C & 6.325342 & 4.443543 & 1.011480 \\
\hline $\mathrm{H}$ & 6.704465 & 5.412665 & 1.355576 \\
\hline $\mathrm{H}$ & 6.157913 & 4.519346 & -0.068982 \\
\hline $\mathrm{H}$ & 5.356926 & 4.276125 & 1.496366 \\
\hline C & 7.543509 & 3.340736 & 2.892567 \\
\hline $\mathrm{H}$ & 7.883341 & 4. 327791 & 3.230094 \\
\hline $\mathrm{H}$ & 6.607223 & 3.107078 & 3.412072 \\
\hline
\end{tabular}




\begin{tabular}{|c|c|c|c|}
\hline $\mathrm{H}$ & 8.291971 & 2.606049 & 3.207184 \\
\hline C & 8.543804 & -2.404577 & -0.266984 \\
\hline $\mathrm{H}$ & 9.164146 & -3.289797 & -0.078952 \\
\hline $\mathrm{H}$ & 7.653174 & -2.731958 & -0.812562 \\
\hline $\mathrm{H}$ & 9.107480 & -1.727069 & -0.919075 \\
\hline C & 9.487872 & -1.358497 & 1.796372 \\
\hline H & 10.067461 & -2.271312 & 1.975663 \\
\hline $\mathrm{H}$ & 9.300637 & -0.891307 & 2.770048 \\
\hline $\mathrm{H}$ & 10.114876 & -0.680241 & 1.205815 \\
\hline C & 7.381468 & -2.700989 & 1.954294 \\
\hline $\mathrm{H}$ & 7.975455 & -3.603865 & 2.144906 \\
\hline $\mathrm{H}$ & 7.139473 & -2.245837 & 2.922053 \\
\hline $\mathrm{H}$ & 6.440855 & -3.006417 & 1.485257 \\
\hline C & -2.268433 & -2.301675 & 1.319013 \\
\hline C & -3.562354 & -2.813713 & 1.127482 \\
\hline C & -1.970190 & -1.642869 & 2.520882 \\
\hline C & -4.555659 & -2.675597 & 2.103396 \\
\hline H & -3.783834 & -3.317207 & 0.193868 \\
\hline C & -2.940896 & -1.465834 & 3.513323 \\
\hline $\mathrm{H}$ & -0.963049 & -1.285207 & 2.683221 \\
\hline C & -4.218774 & -1.991236 & 3.280063 \\
\hline $\mathrm{H}$ & -4.979935 & -1.865825 & 4.044168 \\
\hline C & -2.645006 & -0.720037 & 4.828994 \\
\hline C & -5.978261 & -3.243583 & 1.930171 \\
\hline C & -1.182820 & -0.241078 & 4.915394 \\
\hline H & -0.477929 & -1.080170 & 4.903559 \\
\hline $\mathrm{H}$ & -0.917449 & 0.431672 & 4.092666 \\
\hline H & -1.030378 & 0.302561 & 5.854880 \\
\hline C & -3.566102 & 0.519727 & 4.925687 \\
\hline $\mathrm{H}$ & -3.373657 & 1.065145 & 5.857919 \\
\hline $\mathrm{H}$ & -3.391795 & 1.205010 & 4.088183 \\
\hline $\mathrm{H}$ & -4.625911 & 0.243578 & 4.914430 \\
\hline C & -2.918114 & -1.654350 & 6.031441 \\
\hline $\mathrm{H}$ & -3.959456 & -1.992668 & 6.061114 \\
\hline $\mathrm{H}$ & -2.278952 & -2.543680 & 5.989737 \\
\hline $\mathrm{H}$ & -2.711662 & -1.132221 & 6.973717 \\
\hline C & -6.164114 & -3.966910 & 0.582304 \\
\hline $\mathrm{H}$ & -7.185845 & -4.356793 & 0.511912 \\
\hline $\mathrm{H}$ & -5.479941 & -4.816910 & 0.477363 \\
\hline $\mathrm{H}$ & -6.008893 & -3.294615 & -0.268924 \\
\hline C & -6.262992 & -4.261747 & 3.060363 \\
\hline $\mathrm{H}$ & -6.194082 & -3.801956 & 4.051623 \\
\hline $\mathrm{H}$ & -7.273333 & -4.675525 & 2.954516 \\
\hline H & -5.549815 & -5.093404 & 3.027797 \\
\hline C & -7.011131 & -2.094095 & 2.006357 \\
\hline $\mathrm{H}$ & -8.028599 & -2.488298 & 1.893801 \\
\hline $\mathrm{H}$ & -6.963772 & -1.565718 & 2.964340 \\
\hline H & -6.841188 & -1.359080 & 1.211653 \\
\hline
\end{tabular}




\begin{tabular}{lc}
\hline B3LYP/6-31G(d) Energy & -3038.960021 \\
\hline B3LYP-D3(BJ)/def2-TZVPP-IEF-PCM(DCM) Energy & -3040.510557 \\
\hline B3LYP-D3(BJ)/def2-TZVPP-IEF-PCM(DCM)//B3LYP/6-31G(d) Free Energy (Quasiharmonic) & -3039.281787 \\
\hline
\end{tabular}

Number of Imaginary Frequencies

Frequencies (Top 3 out of 450)

$\begin{array}{rrr}\text { 1. } & -284.1642 \mathrm{~cm}^{-1} \\ \text { 2. } & 6.5031 \mathrm{~cm}^{-1} \\ \text { 3. } & 8.5887 \mathrm{~cm}^{-1}\end{array}$

\section{B3LYP/6-31G(d) Molecular Geometry in Cartesian Coordinates}

C

$\mathrm{H}$

$\mathrm{H}$

C

0

0

$\mathrm{H}$

0

$\mathrm{H}$

C

C

C

C

$\mathrm{H}$

$\mathrm{H}$

$\mathrm{H}$

C

C

C

$\mathrm{H}$

$\mathrm{H}$

$\mathrm{H}$

C

$\mathrm{H}$

$\mathrm{H}$

$\mathrm{H}$

C

$\mathrm{H}$

$\mathrm{H}$

$\mathrm{H}$

C

$\mathrm{H}$

\section{$-1.331062$}

$-2.358594$

$-0.886226$

2.098704

2. 774762

1. 365874

0.730775

$-0.584825$

$-0.332354$

$-0.748950$

$-2.197664$

0.861280

0.142316

$-2.651810$

1.117870

1.728426

0.708928

$-0.123652$

2. 224483

2.720180

2.626096

2.473906

0.403729

0.659962

1.001709

$-0.649148$

$-1.554479$

$-1.566654$

$-2.054449$

$-2.135320$

0.540269

0.691244
$-0.615786$

$-0.255169$

$-0.115086$

$-2.451871$

$-3.453511$

$-1.972219$

$-1.259440$

$-0.272788$

0.682784

3. 232143

3. 114927

3. 316283

3.922973

2. 268085

2. 267682

3.888694

3.033559

4.366182

3. 264239

2. 303737

3. 698483

3.932917

2.155494

2. 677237

1.238778

1.869761

4. 210592

4.104556

3.341573

5.102066

5.587473

5.437864
0.261423

0.155469

1.127048

$-0.569862$

$-0.701659$

$-1.571678$

$-1.276014$

$-0.921601$

$-0.872703$

3.423293

1.730083

1.552487

2.638205

2.251676

1. 722259

1.216416

$-2.030940$

$-1.937461$

$-1.934141$

$-1.761340$

$-2.856207$

$-1.104759$

- 3.244591

$-4.173901$

$-3.201137$

$-3.286817$

$-2.477318$

$-3.567443$

$-2.040019$

$-2.218186$

$-2.574022$

$-3.649499$ 


\begin{tabular}{|c|c|c|c|}
\hline $\mathrm{H}$ & -0.101055 & 6.465976 & -2.444242 \\
\hline $\mathrm{H}$ & 1.505895 & 5.806389 & -2.112268 \\
\hline 0 & 0.289230 & 2.337805 & -0.832412 \\
\hline 0 & -0.190643 & 4.579444 & -0.510029 \\
\hline B & -0.152845 & 3.307493 & 0.137631 \\
\hline 0 & -1.475709 & 2.768880 & 0.717886 \\
\hline C & -2.964342 & 4.373931 & 1.859385 \\
\hline C & -2.706358 & 5.525320 & 1.097167 \\
\hline C & -4.028166 & 4.381906 & 2.778786 \\
\hline C & -3.498536 & 6.659020 & 1.267743 \\
\hline $\mathrm{H}$ & -1.886087 & 5.520613 & 0.386864 \\
\hline C & -4.817033 & 5.517088 & 2.942519 \\
\hline $\mathrm{H}$ & -4.237143 & 3.489507 & 3.365280 \\
\hline C & -4.551825 & 6.661323 & 2.186314 \\
\hline H & -3.294383 & 7.547370 & 0.676101 \\
\hline $\mathrm{H}$ & -5.638067 & 5.508630 & 3.653887 \\
\hline $\mathrm{H}$ & -5.165793 & 7.549302 & 2.309844 \\
\hline $\mathrm{H}$ & 0.135739 & 5.012155 & 2.694721 \\
\hline $\mathrm{H}$ & -0.664514 & 2.152442 & 3.518880 \\
\hline $\mathrm{H}$ & -1.323499 & 3.740101 & 4.191967 \\
\hline C & 0.934173 & -1.869563 & 1.560892 \\
\hline C & 2.051684 & -1.700976 & 0.752003 \\
\hline C & 3.168187 & -0.900407 & 1.182169 \\
\hline C & 3.093190 & -0.299124 & 2.425794 \\
\hline $\mathrm{H}$ & 3.932143 & 0.298259 & 2.772493 \\
\hline C & -1.321568 & -2.116628 & 0.474596 \\
\hline C & -0.225677 & -2.710068 & 1.099122 \\
\hline C & -2.413886 & -4.281292 & 0.330889 \\
\hline C & -2.435951 & -2.924040 & 0.065219 \\
\hline $\mathrm{H}$ & -3.252739 & -4.895865 & 0.014349 \\
\hline C & -1.320048 & -4.911560 & 0.966233 \\
\hline C & -0.193244 & -4.119549 & 1.352309 \\
\hline C & 0.906571 & -4.768869 & 1.979632 \\
\hline C & 0.884827 & -6.124528 & 2.216054 \\
\hline C & -0.234076 & -6.905070 & 1.836256 \\
\hline C & -1.310720 & -6.309964 & 1.222206 \\
\hline $\mathrm{H}$ & 1.776915 & -4.185159 & 2.257137 \\
\hline $\mathrm{H}$ & 1.737288 & -6.602675 & 2.690590 \\
\hline H & -0.235160 & -7.975071 & 2.026199 \\
\hline $\mathrm{H}$ & -2.172524 & -6.900062 & 0.919372 \\
\hline C & 1.983774 & -0.461687 & 3.289737 \\
\hline C & 0.881950 & -1.265521 & 2.860495 \\
\hline C & -0.220612 & -1.430292 & 3.744697 \\
\hline C & -0.230232 & -0.830068 & 4.985408 \\
\hline C & 0.862349 & -0.033910 & 5.407437 \\
\hline C & 1.944142 & 0.144208 & 4.575432 \\
\hline $\mathrm{H}$ & -1.057533 & -2.045746 & 3.432104 \\
\hline $\mathrm{H}$ & -1.078047 & -0.976181 & 5.649575 \\
\hline $\mathrm{H}$ & 0.845757 & 0.430161 & 6.389948 \\
\hline $\mathrm{H}$ & 2.790215 & 0.750236 & 4.890802 \\
\hline C & -5.899041 & -1.483645 & -1.991484 \\
\hline C & -6.067389 & -2.244359 & -0.820031 \\
\hline C & -4.918946 & -2.679203 & -0.156858 \\
\hline C & -3.632494 & -2.371130 & -0.635639 \\
\hline
\end{tabular}




\begin{tabular}{|c|c|c|c|}
\hline C & -3.508845 & -1.607900 & -1.799620 \\
\hline C & -4.639336 & -1.152100 & -2.497716 \\
\hline $\mathrm{H}$ & -6.783803 & -1.143091 & -2.516679 \\
\hline $\mathrm{H}$ & -4.997295 & -3.259202 & 0.755564 \\
\hline $\mathrm{H}$ & -2.512814 & -1.378852 & -2.164710 \\
\hline C & -7.489411 & -2.562695 & -0.317801 \\
\hline C & -4.448747 & -0.315398 & -3.777134 \\
\hline C & -7.483725 & -3.401464 & 0.974260 \\
\hline $\mathrm{H}$ & -8.514415 & -3.602138 & 1.288057 \\
\hline $\mathrm{H}$ & -6.988291 & -4.368386 & 0.831118 \\
\hline $\mathrm{H}$ & -6.983200 & -2.879704 & 1.797895 \\
\hline C & -8.243379 & -1.242927 & -0.027808 \\
\hline $\mathrm{H}$ & -9.261011 & -1.452810 & 0.324518 \\
\hline $\mathrm{H}$ & -7.729297 & -0.661146 & 0.746071 \\
\hline $\mathrm{H}$ & -8.323295 & -0.613786 & -0.920315 \\
\hline C & -8.252960 & -3.359828 & -1.402115 \\
\hline $\mathrm{H}$ & -9.271885 & -3.585879 & -1.063997 \\
\hline $\mathrm{H}$ & -8.329795 & -2.801787 & -2.341012 \\
\hline $\mathrm{H}$ & -7.748051 & -4.308280 & -1.618263 \\
\hline C & -3.670976 & 0.973740 & -3.422078 \\
\hline $\mathrm{H}$ & -3.484515 & 1.569936 & -4.324142 \\
\hline $\mathrm{H}$ & -4.241340 & 1.592380 & -2.718956 \\
\hline $\mathrm{H}$ & -2.705763 & 0.745408 & -2.959031 \\
\hline C & -3.641003 & -1.132293 & -4.813619 \\
\hline $\mathrm{H}$ & -3.492103 & -0.545938 & -5.728849 \\
\hline $\mathrm{H}$ & -2.653972 & -1.413055 & -4.433180 \\
\hline $\mathrm{H}$ & -4.170129 & -2.053559 & -5.083221 \\
\hline C & -5.785156 & 0.091824 & -4.424838 \\
\hline $\mathrm{H}$ & -5.591876 & 0.688300 & -5.323764 \\
\hline $\mathrm{H}$ & -6.374482 & -0.781087 & -4.728339 \\
\hline $\mathrm{H}$ & -6.397725 & 0.701478 & -3.750590 \\
\hline C & 4.389687 & -0.649484 & 0.356869 \\
\hline C & 4.869142 & 0.669691 & 0.231391 \\
\hline C & 5.117656 & -1.687992 & -0.228633 \\
\hline C & 6.045600 & 0.955478 & -0.463198 \\
\hline $\mathrm{H}$ & 4.293047 & 1.468091 & 0.685022 \\
\hline C & 6.289913 & -1.439099 & -0.960211 \\
\hline $\mathrm{H}$ & 4.758695 & -2.701463 & -0.113182 \\
\hline C & 6.731414 & -0.118125 & -1.060881 \\
\hline $\mathrm{H}$ & 7.636412 & 0.093794 & -1.618274 \\
\hline C & 7.026318 & -2.623087 & -1.615142 \\
\hline C & 6.608635 & 2.383884 & -0.599590 \\
\hline C & 8.296832 & -2.185285 & -2.367675 \\
\hline $\mathrm{H}$ & 8.069296 & -1.484651 & -3.179430 \\
\hline $\mathrm{H}$ & 9.027061 & -1.711831 & -1.700625 \\
\hline $\mathrm{H}$ & 8.778833 & -3.061628 & -2.815889 \\
\hline C & 7.440842 & -3.639662 & -0.524741 \\
\hline $\mathrm{H}$ & 7.966945 & -4.489461 & -0.977202 \\
\hline $\mathrm{H}$ & 8.111217 & -3.177138 & 0.209495 \\
\hline $\mathrm{H}$ & 6.574019 & -4.034867 & 0.013840 \\
\hline C & 6.076681 & -3.312634 & -2.625245 \\
\hline $\mathrm{H}$ & 5.147423 & -3.648174 & -2.154776 \\
\hline $\mathrm{H}$ & 5.807625 & -2.624676 & -3.435388 \\
\hline $\mathrm{H}$ & 6.568279 & -4.185997 & -3.072303 \\
\hline
\end{tabular}




\begin{tabular}{|lllr|} 
C & 5.778293 & 3.421720 & 0.179233 \\
H & 4.747940 & 3.480330 & -0.187483 \\
H & 5.748007 & 3.195032 & 1.251317 \\
H & 6.226774 & 4.415151 & 0.063249 \\
C & 8.053509 & 2.426577 & -0.046626 \\
H & 8.075808 & 2.142402 & 1.011782 \\
$H$ & 8.721086 & 1.748426 & -0.587630 \\
$H$ & 8.465603 & 3.439448 & -0.135721 \\
C & 6.621293 & 2.794194 & -2.091366 \\
$H$ & 7.032145 & 3.804916 & -2.208723 \\
H & 7.231900 & 2.113306 & -2.693656 \\
$H$ & 5.607347 & 2.788820 & -2.507393 \\
\hline
\end{tabular}

\section{C-Catalyzed reaction TS 64}

\section{Datum}

Value

\begin{tabular}{lc}
\hline B3LYP/6-31G(d) Energy & -3038.955074 \\
\hline B3LYP-D3(BJ)/def2-TZVPP-IEF-PCM(DCM) Energy & -3040.512165 \\
\hline B3LYP-D3(BJ)/def2-TZVPP-IEF-PCM(DCM)//B3LYP/6-31G(d) Free Energy (Quasiharmonic) & -3039.281773 \\
\hline
\end{tabular}

Number of Imaginary Frequencies

Frequencies (Top 3 out of 450)

$\begin{array}{lr}\text { 1. } & -314.1238 \mathrm{~cm}^{-1} \\ \text { 2. } & 11.9869 \mathrm{~cm}^{-1} \\ \text { 3. } & 14.9165 \mathrm{~cm}^{-1}\end{array}$

\section{B3LYP/6-31G(d) Molecular Geometry in Cartesian Coordinates}

\section{C}

$\mathrm{H}$

$\mathrm{H}$

C

0

0

$\mathrm{H}$

0

$\mathrm{H}$

C

C

c

C

$\mathrm{H}$

$$
\begin{array}{r}
-1.489757 \\
-0.981325 \\
-2.465915 \\
1.706491 \\
2.297268 \\
0.982891 \\
0.441522 \\
-0.714823 \\
-0.391910 \\
0.059440 \\
-0.947271 \\
1.587013 \\
0.561150 \\
-0.938150
\end{array}
$$

$$
\begin{array}{r}
0.316913 \\
0.085489 \\
-0.174224 \\
2.337871 \\
3.329106 \\
1.601711 \\
0.905504 \\
-0.192189 \\
-1.102752 \\
-3.359861 \\
-4.508394 \\
-2.784113 \\
-2.453079 \\
-5.348080
\end{array}
$$
0.400767
1. 342962
0.416828
$-0.922563$
$-1.300562$
$-1.769204$
$-1.305643$
$-0.702385$
$-0.495737$
3. 772237
2. 208874
1.921535
2.866577
2.911098 


\begin{tabular}{|c|c|c|c|}
\hline $\mathrm{H}$ & 2.056546 & -1.918515 & 1.450548 \\
\hline $\mathrm{H}$ & 2.330655 & -3.489313 & 2.301643 \\
\hline C & 1.045732 & -3.044537 & -1.708561 \\
\hline C & 1.672081 & -4.463494 & -1.458849 \\
\hline C & 0.054461 & -2.966665 & -2.867782 \\
\hline $\mathrm{H}$ & -0.789410 & -3.645415 & -2.722931 \\
\hline $\mathrm{H}$ & -0.335398 & -1.947617 & -2.956716 \\
\hline $\mathrm{H}$ & 0.546006 & -3.219073 & -3.814616 \\
\hline C & 2.115557 & -1.955245 & -1.860801 \\
\hline $\mathrm{H}$ & 1.656659 & -0.963634 & -1.855224 \\
\hline $\mathrm{H}$ & 2.847080 & -1.995904 & -1.049687 \\
\hline $\mathrm{H}$ & 2.651908 & -2.058340 & -2.809974 \\
\hline C & 2.988643 & -4.727903 & -2.190156 \\
\hline $\mathrm{H}$ & 2.854996 & -4.685104 & -3.277469 \\
\hline $\mathrm{H}$ & 3.759010 & -4.008790 & -1.903907 \\
\hline $\mathrm{H}$ & 3.351800 & -5.728641 & -1.933751 \\
\hline C & 0.690493 & -5.614265 & -1.746503 \\
\hline $\mathrm{H}$ & 0.524235 & -5.746058 & -2.821434 \\
\hline $\mathrm{H}$ & 1.113357 & -6.541638 & -1.347385 \\
\hline $\mathrm{H}$ & -0.276749 & -5.451890 & -1.262926 \\
\hline 0 & 0.320850 & -2.812068 & -0.458597 \\
\hline 0 & 1.936013 & -4.425937 & -0.046317 \\
\hline B & 0.916618 & -3.655098 & 0.566642 \\
\hline 0 & -0.079807 & -4.608051 & 1.261473 \\
\hline C & -2.278248 & -3.875162 & 2.060015 \\
\hline C & -2.645797 & -3.153216 & 0.914859 \\
\hline C & -3.226039 & -4.082539 & 3.076916 \\
\hline C & -3.935536 & -2.631280 & 0.807035 \\
\hline $\mathrm{H}$ & -1.922403 & -3.015586 & 0.117871 \\
\hline C & -4.510700 & -3.559797 & 2.965951 \\
\hline $\mathrm{H}$ & -2.949746 & -4.658592 & 3.957824 \\
\hline C & -4.866797 & -2.828886 & 1.829286 \\
\hline $\mathrm{H}$ & -4.217762 & -2.069587 & -0.078526 \\
\hline $\mathrm{H}$ & -5.234330 & -3.726283 & 3.759129 \\
\hline $\mathrm{H}$ & -5.869654 & -2.421739 & 1.734427 \\
\hline $\mathrm{H}$ & 0.009461 & -1.524302 & 2.721954 \\
\hline $\mathrm{H}$ & 0.672823 & -4.203231 & 4.079741 \\
\hline $\mathrm{H}$ & -0.748636 & -3.085443 & 4.443275 \\
\hline C & 0.610268 & 2.104978 & 1.309030 \\
\hline C & 1.744250 & 1.897675 & 0.531400 \\
\hline C & 2.949637 & 1.356116 & 1.103708 \\
\hline C & 2.943881 & 1.046064 & 2.451835 \\
\hline H & 3.847612 & 0.643689 & 2.901474 \\
\hline C & -1.664075 & 1.815719 & 0.272484 \\
\hline C & -0.647935 & 2.666970 & 0.703913 \\
\hline C & -3.034698 & 3.734173 & -0.307415 \\
\hline C & -2.878304 & 2.361392 & -0.260871 \\
\hline $\mathrm{H}$ & -3.954415 & 4.148880 & -0.712364 \\
\hline C & -2.023853 & 4.624858 & 0.121237 \\
\hline C & -0.797909 & 4.089504 & 0.628469 \\
\hline C & 0.214905 & 4.997554 & 1.046647 \\
\hline C & 0.018584 & 6.357577 & 0.970819 \\
\hline C & -1.197844 & 6.884011 & 0.472375 \\
\hline C & -2.195038 & 6.034439 & 0.054868 \\
\hline
\end{tabular}




\begin{tabular}{|c|c|c|c|}
\hline $\mathrm{H}$ & 1.157199 & 4.606343 & 1.413121 \\
\hline $\mathrm{H}$ & 0.806716 & 7.034445 & 1.288722 \\
\hline $\mathrm{H}$ & -1.336977 & 7.960265 & 0.415879 \\
\hline H & -3.130533 & 6.427254 & -0.336421 \\
\hline C & 1.820474 & 1.264079 & 3.284348 \\
\hline C & 0.629210 & 1.810210 & 2.712895 \\
\hline C & -0.487751 & 2.036083 & 3.565295 \\
\hline C & -0.427289 & 1.731165 & 4.907654 \\
\hline C & 0.752372 & 1.186313 & 5.469160 \\
\hline C & 1.851359 & 0.959893 & 4.672610 \\
\hline $\mathrm{H}$ & -1.393936 & 2.459409 & 3.145407 \\
\hline H & -1.290006 & 1.915161 & 5.542302 \\
\hline $\mathrm{H}$ & 0.788112 & 0.952424 & 6.529765 \\
\hline $\mathrm{H}$ & 2.764591 & 0.546490 & 5.094016 \\
\hline C & -6.179567 & 0.110676 & -1.785624 \\
\hline C & -6.390236 & 0.986379 & -0.705030 \\
\hline C & -5.287622 & 1.687380 & -0.213640 \\
\hline C & -4.005116 & 1.517960 & -0.764360 \\
\hline C & -3.834439 & 0.627484 & -1.828274 \\
\hline C & -4.922739 & -0.079724 & -2.369422 \\
\hline $\mathrm{H}$ & -7.030805 & -0.427504 & -2.185397 \\
\hline $\mathrm{H}$ & -5.398016 & 2.379229 & 0.613504 \\
\hline H & -2.840637 & 0.508699 & -2.248246 \\
\hline C & -7.806702 & 1.145419 & -0.118747 \\
\hline C & -4.706505 & -0.995751 & -3.590693 \\
\hline C & -8.297127 & -0.217994 & 0.424219 \\
\hline $\mathrm{H}$ & -9.312373 & -0.127004 & 0.829716 \\
\hline H & -8.317228 & -0.985378 & -0.356680 \\
\hline $\mathrm{H}$ & -7.643013 & -0.573924 & 1.229368 \\
\hline C & -7.853758 & 2.163574 & 1.036567 \\
\hline H & -8.877932 & 2.241193 & 1.418678 \\
\hline $\mathrm{H}$ & -7.211744 & 1.863700 & 1.872618 \\
\hline $\mathrm{H}$ & -7.545870 & 3.163655 & 0.711345 \\
\hline C & -8.773989 & 1.631932 & -1.224285 \\
\hline $\mathrm{H}$ & -9.786845 & 1.749503 & -0.819826 \\
\hline H & -8.453607 & 2.600225 & -1.625224 \\
\hline $\mathrm{H}$ & -8.830034 & 0.927189 & -2.060427 \\
\hline C & -6.010278 & -1.660888 & -4.070890 \\
\hline H & -5.800092 & -2.298501 & -4.936889 \\
\hline $\mathrm{H}$ & -6.455941 & -2.295360 & -3.295979 \\
\hline $\mathrm{H}$ & -6.757490 & -0.921080 & -4.380199 \\
\hline C & -4.138319 & -0.155989 & -4.760247 \\
\hline $\mathrm{H}$ & -3.984076 & -0.789004 & -5.642694 \\
\hline H & -4.829181 & 0.648964 & -5.035982 \\
\hline $\mathrm{H}$ & -3.176816 & 0.301010 & -4.505920 \\
\hline C & -3.700574 & -2.112853 & -3.231007 \\
\hline H & -3.486416 & -2.732684 & -4.110382 \\
\hline $\mathrm{H}$ & -2.752304 & -1.703865 & -2.870777 \\
\hline $\mathrm{H}$ & -4.103647 & -2.768321 & -2.449687 \\
\hline C & 4.195046 & 1.060257 & 0.329775 \\
\hline C & 4.825474 & 2.018450 & -0.467011 \\
\hline C & 4.797254 & -0.205508 & 0.474730 \\
\hline C & 6.027068 & 1.736557 & -1.136974 \\
\hline $\mathrm{H}$ & 4.366470 & 2.992844 & -0.565030 \\
\hline
\end{tabular}




\begin{tabular}{|c|c|c|c|}
\hline C & 5.998580 & -0.519977 & -0.161556 \\
\hline $\mathrm{H}$ & 4.298931 & -0.943598 & 1.092606 \\
\hline C & 6.591070 & 0.470053 & -0.966989 \\
\hline $\mathrm{H}$ & 7.521113 & 0.235217 & -1.471732 \\
\hline C & 6.690231 & -1.888580 & -0.005043 \\
\hline C & 6.659152 & 2.822967 & -2.027482 \\
\hline C & 5.896988 & -2.854674 & 0.896662 \\
\hline $\mathrm{H}$ & 6.438712 & -3.803575 & 0.983124 \\
\hline H & 5.773604 & -2.454873 & 1.909984 \\
\hline $\mathrm{H}$ & 4.902934 & -3.082587 & 0.495230 \\
\hline C & 6.856519 & -2.551879 & -1.392649 \\
\hline $\mathrm{H}$ & 7.348587 & -3.527287 & -1.293655 \\
\hline H & 5.883138 & -2.709881 & -1.870661 \\
\hline $\mathrm{H}$ & 7.463515 & -1.940257 & -2.068324 \\
\hline C & 8.086845 & -1.682508 & 0.629866 \\
\hline $\mathrm{H}$ & 8.722311 & -1.035576 & 0.016106 \\
\hline $\mathrm{H}$ & 8.001972 & -1.223024 & 1.621429 \\
\hline $\mathrm{H}$ & 8.600772 & -2.645054 & 0.744133 \\
\hline C & 7.986417 & 2.366942 & -2.662129 \\
\hline $\mathrm{H}$ & 8.740751 & 2.123000 & -1.904631 \\
\hline H & 7.851655 & 1.490006 & -3.305720 \\
\hline $\mathrm{H}$ & 8.392456 & 3.172179 & -3.284942 \\
\hline C & 5.671752 & 3.178206 & -3.165879 \\
\hline $\mathrm{H}$ & 5.491916 & 2.307969 & -3.807973 \\
\hline H & 4.702857 & 3.510076 & -2.780596 \\
\hline $\mathrm{H}$ & 6.084674 & 3.980455 & -3.790455 \\
\hline C & 6.942557 & 4.087073 & -1.181386 \\
\hline $\mathrm{H}$ & 7.393190 & 4.868353 & -1.805890 \\
\hline $\mathrm{H}$ & 6.027657 & 4.498783 & -0.744219 \\
\hline H & 7.636729 & 3.864780 & -0.362249 \\
\hline
\end{tabular}

\section{C-Catalyzed reaction TS 65}

\section{Datum}

Value

\begin{tabular}{lc}
\hline B3LYP/6-31G(d) Energy & -3038.957329 \\
\hline B3LYP-D3(BJ)/def2-TZVPP-IEF-PCM(DCM) Energy & -3040.509227 \\
\hline B3LYP-D3(BJ)/def2-TZVPP-IEF-PCM(DCM)//B3LYP/6-31G(d) Free Energy (Quasiharmonic) & -3039.281644 \\
\hline
\end{tabular}

Number of Imaginary Frequencies

Frequencies (Top 3 out of 450)

$\begin{array}{lr}\text { 1. } & -320.0902 \mathrm{~cm}^{-1} \\ \text { 2. } & 6.6363 \mathrm{~cm}^{-1} \\ \text { 3. } & 10.5243 \mathrm{~cm}^{-1}\end{array}$




\begin{tabular}{|c|c|c|c|}
\hline C & -2.946554 & -0.594309 & -1.985808 \\
\hline $\mathrm{H}$ & -2.961568 & 0.500205 & -1.948225 \\
\hline $\mathrm{H}$ & -2.119801 & -0.876689 & -2.636127 \\
\hline C & 0.261425 & 0.197206 & -0.619359 \\
\hline 0 & -0.555500 & 1.050813 & -0.911742 \\
\hline 0 & 1.069159 & 0.374533 & 0.427930 \\
\hline $\mathrm{H}$ & 1.654449 & -0.395695 & 0.657584 \\
\hline 0 & -4.122059 & -1.110347 & -2.610020 \\
\hline $\mathrm{H}$ & -4.883975 & -0.716575 & -2.155647 \\
\hline C & 1.154062 & 1.285237 & 5.073700 \\
\hline C & 2.984821 & 1.529912 & 3.903677 \\
\hline C & 1.076031 & -0.852886 & 3.831899 \\
\hline C & 1.235467 & -0.090594 & 5.035609 \\
\hline $\mathrm{H}$ & 3.635864 & 1.175232 & 4.705432 \\
\hline $\mathrm{H}$ & 0.360024 & -0.444923 & 3.115547 \\
\hline $\mathrm{H}$ & 0.929820 & -1.923489 & 3.980404 \\
\hline C & 3.920454 & -2.025949 & 1.605019 \\
\hline C & 4.300809 & -2.386915 & 3.093074 \\
\hline C & 3.754642 & -3.222482 & 0.669001 \\
\hline $\mathrm{H}$ & 3.456543 & -2.878742 & -0.327278 \\
\hline $\mathrm{H}$ & 4.697009 & -3.773706 & 0.567377 \\
\hline $\mathrm{H}$ & 2.987472 & -3.912087 & 1.028723 \\
\hline C & 4.877958 & -0.999288 & 0.979071 \\
\hline $\mathrm{H}$ & 5.853966 & -1.444779 & 0.755279 \\
\hline $\mathrm{H}$ & 5.031353 & -0.142311 & 1.640544 \\
\hline $\mathrm{H}$ & 4.447996 & -0.629223 & 0.043128 \\
\hline C & 5.803521 & -2.401591 & 3.382131 \\
\hline $\mathrm{H}$ & 6.317817 & -3.157133 & 2.776311 \\
\hline $\mathrm{H}$ & 6.257192 & -1.427447 & 3.185741 \\
\hline $\mathrm{H}$ & 5.970492 & -2.644701 & 4.436988 \\
\hline C & 3.673324 & -3.709356 & 3.565229 \\
\hline $\mathrm{H}$ & 4.152679 & -4.580006 & 3.103854 \\
\hline $\mathrm{H}$ & 3.794682 & -3.786953 & 4.650719 \\
\hline $\mathrm{H}$ & 2.603362 & -3.741559 & 3.339173 \\
\hline 0 & 2.625462 & -1.395473 & 1.777213 \\
\hline 0 & 3.701617 & -1.318142 & 3.846158 \\
\hline B & 2.641960 & -0.750541 & 3.070258 \\
\hline 0 & 2.790971 & 0.745033 & 2.895426 \\
\hline C & 2.927868 & 2.983602 & 3.661815 \\
\hline C & 2.337743 & 3.488186 & 2.490609 \\
\hline C & 3.489351 & 3.866609 & 4.595862 \\
\hline C & 2.311692 & 4.862333 & 2.268372 \\
\hline $\mathrm{H}$ & 1.913206 & 2.795863 & 1.769304 \\
\hline C & 3.462209 & 5.240560 & 4.365883 \\
\hline $\mathrm{H}$ & 3.950464 & 3.473273 & 5.499301 \\
\hline C & 2.871683 & 5.740086 & 3.202572 \\
\hline $\mathrm{H}$ & 1.855436 & 5.250575 & 1.362063 \\
\hline $\mathrm{H}$ & 3.902556 & 5.920085 & 5.090272 \\
\hline $\mathrm{H}$ & 2.849549 & 6.811547 & 3.022691 \\
\hline $\mathrm{H}$ & 1.687722 & -0.597992 & 5.888724 \\
\hline
\end{tabular}




\begin{tabular}{|c|c|c|c|}
\hline $\mathrm{H}$ & 0.562126 & 1.811863 & 4.331057 \\
\hline $\mathrm{H}$ & 1.352427 & 1.828777 & 5.993583 \\
\hline C & -0.507063 & -2.095148 & -1.274081 \\
\hline C & 0.427209 & -1.075431 & -1.427543 \\
\hline C & 1.502533 & -1.182237 & -2.375295 \\
\hline C & 1.617248 & -2.353033 & -3.102352 \\
\hline H & 2.409747 & -2.441657 & -3.840785 \\
\hline C & -2.742597 & -1.178479 & -0.591739 \\
\hline C & -1.631249 & -1.954285 & -0.284423 \\
\hline C & -3.696893 & -1.652972 & 1.598080 \\
\hline C & -3.769523 & -0.988089 & 0.393728 \\
\hline $\mathrm{H}$ & -4.481446 & -1.507425 & 2.337179 \\
\hline C & -2.610735 & -2.505114 & 1.916439 \\
\hline C & -1.539993 & -2.639702 & 0.976557 \\
\hline C & -0.446865 & -3.480642 & 1.324480 \\
\hline C & -0.433058 & -4.172439 & 2.516888 \\
\hline C & -1.497709 & -4.041357 & 3.439503 \\
\hline C & -2.558219 & -3.215050 & 3.145420 \\
\hline $\mathrm{H}$ & 0.382973 & -3.582627 & 0.634733 \\
\hline $\mathrm{H}$ & 0.403229 & -4.826129 & 2.750386 \\
\hline $\mathrm{H}$ & -1.473630 & -4.590782 & 4.376849 \\
\hline $\mathrm{H}$ & -3.380283 & -3.098564 & 3.847986 \\
\hline C & 0.700532 & -3.422633 & -2.968210 \\
\hline C & -0.392372 & -3.292789 & -2.054482 \\
\hline C & -1.334433 & -4.355060 & -1.970345 \\
\hline C & -1.190320 & -5.489936 & -2.736779 \\
\hline C & -0.099500 & -5.621969 & -3.629382 \\
\hline C & 0.822558 & -4.607724 & -3.743703 \\
\hline $\mathrm{H}$ & -2.179128 & -4.256231 & -1.297906 \\
\hline $\mathrm{H}$ & -1.922914 & -6.288789 & -2.661238 \\
\hline $\mathrm{H}$ & 0.002000 & -6.522994 & -4.228370 \\
\hline $\mathrm{H}$ & 1.657858 & -4.693724 & -4.434769 \\
\hline C & -7.042574 & 1.705582 & -0.185559 \\
\hline C & -7.319594 & 0.337152 & -0.013539 \\
\hline C & -6.236995 & -0.530014 & 0.160173 \\
\hline C & -4.914261 & -0.053650 & 0.159260 \\
\hline C & -4.682481 & 1.314792 & -0.010831 \\
\hline C & -5.741707 & 2.219706 & -0.185939 \\
\hline $\mathrm{H}$ & -7.875655 & 2.385248 & -0.322922 \\
\hline $\mathrm{H}$ & -6.394927 & -1.594493 & 0.291700 \\
\hline $\mathrm{H}$ & -3.656255 & 1.670129 & 0.011464 \\
\hline C & -8.781310 & -0.151148 & -0.023896 \\
\hline C & -5.436662 & 3.720143 & -0.359122 \\
\hline C & -9.437333 & 0.206172 & -1.378992 \\
\hline $\mathrm{H}$ & -10.481045 & -0.131005 & -1.397231 \\
\hline $\mathrm{H}$ & -9.433121 & 1.285396 & -1.563735 \\
\hline H & -8.910048 & -0.277093 & -2.209421 \\
\hline C & -8.892571 & -1.675110 & 0.172430 \\
\hline $\mathrm{H}$ & -9.947165 & -1.972633 & 0.154828 \\
\hline $\mathrm{H}$ & -8.380452 & -2.228123 & -0.622995 \\
\hline $\mathrm{H}$ & -8.475345 & -1.994519 & 1.134160 \\
\hline C & -9.562062 & 0.538286 & 1.120419 \\
\hline $\mathrm{H}$ & -10.607427 & 0.205705 & 1.123477 \\
\hline $\mathrm{H}$ & -9.124761 & 0.293677 & 2.095239 \\
\hline
\end{tabular}




\begin{tabular}{|c|c|c|c|}
\hline H & -9.559635 & 1.628424 & 1.017698 \\
\hline C & -4.532635 & 3.922798 & -1.598440 \\
\hline $\mathrm{H}$ & -4.298953 & 4.986776 & -1.728362 \\
\hline $\mathrm{H}$ & -3.584351 & 3.383592 & -1.506271 \\
\hline $\mathrm{H}$ & -5.031400 & 3.571840 & -2.509257 \\
\hline C & -6.711093 & 4.563210 & -0.552944 \\
\hline H & -6.438847 & 5.617804 & -0.673387 \\
\hline $\mathrm{H}$ & -7.383946 & 4.493481 & 0.309575 \\
\hline $\mathrm{H}$ & -7.268367 & 4.262727 & -1.447734 \\
\hline C & -4.702385 & 4.241446 & 0.899332 \\
\hline $\mathrm{H}$ & -4.470720 & 5.308109 & 0.789746 \\
\hline $\mathrm{H}$ & -5.323094 & 4.118732 & 1.794512 \\
\hline $\mathrm{H}$ & -3.759685 & 3.711748 & 1.069984 \\
\hline C & 2.466612 & -0.073110 & -2.637987 \\
\hline C & 3.839375 & -0.353245 & -2.778587 \\
\hline C & 2.026166 & 1.240026 & -2.823758 \\
\hline C & 4.760044 & 0.648878 & -3.089480 \\
\hline H & 4.172690 & -1.374047 & -2.630687 \\
\hline C & 2.921511 & 2.280386 & -3.117467 \\
\hline $\mathrm{H}$ & 0.966610 & 1.451503 & -2.749792 \\
\hline C & 4.274964 & 1.960428 & -3.246567 \\
\hline $\mathrm{H}$ & 4.982826 & 2.747598 & -3.478018 \\
\hline C & 2.377833 & 3.712184 & -3.283535 \\
\hline C & 6.265837 & 0.371952 & -3.267140 \\
\hline C & 3.486908 & 4.731190 & -3.605749 \\
\hline H & 4.238517 & 4.781850 & -2.809573 \\
\hline $\mathrm{H}$ & 3.998779 & 4.496000 & -4.546218 \\
\hline H & 3.049238 & 5.730546 & -3.709982 \\
\hline C & 1.347760 & 3.743616 & -4.437791 \\
\hline $\mathrm{H}$ & 0.949310 & 4.757954 & -4.564057 \\
\hline $\mathrm{H}$ & 1.810358 & 3.440220 & -5.384232 \\
\hline H & 0.501520 & 3.075799 & -4.249126 \\
\hline C & 1.687248 & 4.148112 & -1.967763 \\
\hline H & 0.876990 & 3.469637 & -1.682290 \\
\hline $\mathrm{H}$ & 2.409060 & 4.170389 & -1.142284 \\
\hline $\mathrm{H}$ & 1.264510 & 5.154916 & -2.078499 \\
\hline C & 6.621037 & -1.112455 & -3.056914 \\
\hline $\mathrm{H}$ & 6.371443 & -1.453966 & -2.046053 \\
\hline $\mathrm{H}$ & 6.105387 & -1.761065 & -3.774332 \\
\hline H & 7.698285 & -1.257065 & -3.197189 \\
\hline C & 6.692115 & 0.766043 & -4.701743 \\
\hline H & 6.142188 & 0.181055 & -5.447846 \\
\hline $\mathrm{H}$ & 6.506801 & 1.825625 & -4.906340 \\
\hline $\mathrm{H}$ & 7.763687 & 0.580720 & -4.846034 \\
\hline C & 7.073837 & 1.207598 & -2.245950 \\
\hline $\mathrm{H}$ & 8.148697 & 1.026622 & -2.370574 \\
\hline H & 6.901271 & 2.281747 & -2.369399 \\
\hline $\mathrm{H}$ & 6.800923 & 0.941886 & -1.218369 \\
\hline
\end{tabular}


Frequencies (Top 3 out of 450)

$\begin{array}{rr}\text { 1. } & -302.9763 \mathrm{~cm}^{-1} \\ \text { 2. } & 7.7140 \mathrm{~cm}^{-1} \\ \text { 3. } & 9.7506 \mathrm{~cm}^{-1}\end{array}$

\section{B3LYP/6-31G(d) Molecular Geometry in Cartesian Coordinates}

C

$\mathrm{H}$

$\mathrm{H}$

C

0

0

$\mathrm{H}$

0

$\mathrm{H}$

C

C

C

C

$\mathrm{H}$

$\mathrm{H}$

$\mathrm{H}$

C

C

C

$\mathrm{H}$

$\mathrm{H}$

$\mathrm{H}$

C

$\mathrm{H}$

$\mathrm{H}$

$\mathrm{H}$

C

$\mathrm{H}$

$\mathrm{H}$

$\mathrm{H}$

C

$\mathrm{H}$

\subsection{3}

1.065889

2.627731

$-0.960295$

$-1.290745$

$-0.302582$

0.040599

1.036943

0.744737

0.966281

$-0.945444$

1.634640

1. 293317

$-0.956748$

2. 124361

2. 178387

0.047463

$-0.957414$

1.339997

2.078670

1.172614

1.761179

$-0.541724$

0.222732

$-1.380138$

$-0.888261$

$-2.394095$

$-2.420738$

$-3.000230$

$-2.857496$

$-0.961518$

$-1.262345$
$-0.088196$

0.373534

0.510427

$-2.897850$

$-4.066432$

$-2.327893$

$-1.421147$

$-0.082522$

0.846055

3.774902

3.169437

3.955350

4.531159

2. 321211

4.643812

3.010411

3. 180176

4. 313499

3.731950

2. 927087

4.117116

4.536736

2.096978

1. 347950

1. 583484

2. 525745

3. 795469

2.907488

4.573105

3. 545888

5.545796

5. 284072
0. 324767

1.078783

0.247957

$-0.498416$

$-0.535174$

$-1.505695$

$-1.274039$

$-0.942895$

$-1.116321$

2.928323

2.026795

0.557331

1.826624

2. 714217

$-0.134031$

0.631819

$-2.606203$

$-2.179807$

- 3.226157

$-3.287378$

$-4.237818$

$-2.616219$

$-3.510912$

$-3.744507$

$-3.034567$

$-4.458336$

$-2.001568$

$-1.362937$

$-1.525351$

$-2.961952$

$-3.084872$

$-4.106008$ 


\begin{tabular}{|c|c|c|c|}
\hline $\mathrm{H}$ & -1.677192 & 6.281703 & -2.702670 \\
\hline $\mathrm{H}$ & 0.021376 & 6.021284 & -3.120252 \\
\hline 0 & 0.383335 & 2.584526 & -1.330204 \\
\hline 0 & -0.442649 & 4.700585 & -0.888497 \\
\hline B & 0.155006 & 3.551662 & -0.284433 \\
\hline 0 & -0.633461 & 2.839113 & 0.815228 \\
\hline C & -1.866667 & 4.262302 & 2.422507 \\
\hline C & -2.195597 & 5.354542 & 1.602854 \\
\hline C & -2.456893 & 4.156949 & 3.694687 \\
\hline C & -3.091985 & 6.318736 & 2.060415 \\
\hline $\mathrm{H}$ & -1.743178 & 5.438982 & 0.620542 \\
\hline C & -3.353328 & 5.122811 & 4.144509 \\
\hline $\mathrm{H}$ & -2.212398 & 3.306754 & 4.327316 \\
\hline C & -3.672589 & 6.209166 & 3.326873 \\
\hline $\mathrm{H}$ & -3.341708 & 7.161639 & 1.421503 \\
\hline $\mathrm{H}$ & -3.804737 & 5.026919 & 5.128180 \\
\hline $\mathrm{H}$ & -4.372566 & 6.964646 & 3.673485 \\
\hline $\mathrm{H}$ & 1.058904 & 5.595839 & 1.852146 \\
\hline $\mathrm{H}$ & 1.320609 & 2.750393 & 3.010009 \\
\hline $\mathrm{H}$ & 0.654164 & 4.246854 & 3.854725 \\
\hline C & -0.279053 & -1.761636 & 1.624408 \\
\hline C & -1.285471 & -2.025425 & 0.703405 \\
\hline C & -2.627792 & -1.555012 & 0.911791 \\
\hline C & -2.893929 & -0.828599 & 2.057401 \\
\hline $\mathrm{H}$ & -3.907134 & -0.477087 & 2.233991 \\
\hline C & 2.066544 & -1.499741 & 0.748066 \\
\hline C & 1.113186 & -2.283334 & 1.394296 \\
\hline C & 3.708773 & -3.270018 & 1.014475 \\
\hline C & 3.388574 & -2.015629 & 0.529908 \\
\hline $\mathrm{H}$ & 4.707718 & -3.663627 & 0.844656 \\
\hline C & 2.769093 & -4.082852 & 1.689314 \\
\hline C & 1.436977 & -3.595220 & 1.872106 \\
\hline C & 0.495203 & -4.433593 & 2.531312 \\
\hline C & 0.858449 & -5.678852 & 2.991595 \\
\hline C & 2.179813 & -6.155782 & 2.814732 \\
\hline C & 3.112294 & -5.374299 & 2.173997 \\
\hline $\mathrm{H}$ & -0.524292 & -4.087090 & 2.656877 \\
\hline $\mathrm{H}$ & 0.122761 & -6.305885 & 3.487701 \\
\hline $\mathrm{H}$ & 2.451000 & -7.142614 & 3.180162 \\
\hline $\mathrm{H}$ & 4.127713 & -5.733392 & 2.023697 \\
\hline C & -1.905056 & -0.558268 & 3.033381 \\
\hline C & -0.570858 & -1.028068 & 2.820762 \\
\hline C & 0.411600 & -0.749334 & 3.812176 \\
\hline C & 0.088927 & -0.050252 & 4.955336 \\
\hline C & -1.236250 & 0.401981 & 5.170047 \\
\hline C & -2.209338 & 0.152757 & 4.227269 \\
\hline $\mathrm{H}$ & 1.425339 & -1.105373 & 3.660810 \\
\hline $\mathrm{H}$ & 0.851842 & 0.143362 & 5.704601 \\
\hline $\mathrm{H}$ & -1.483721 & 0.933972 & 6.085154 \\
\hline $\mathrm{H}$ & -3.231813 & 0.488275 & 4.385360 \\
\hline C & 6.479810 & 0.001652 & -1.627782 \\
\hline C & 5.223569 & -0.158467 & -2.241209 \\
\hline C & 4.212626 & -0.788746 & -1.513231 \\
\hline C & 4.443153 & -1.260385 & -0.209134 \\
\hline
\end{tabular}




\begin{tabular}{|c|c|c|c|}
\hline C & 5.705905 & -1.082596 & 0.363666 \\
\hline C & 6.747058 & -0.446353 & -0.331219 \\
\hline $\mathrm{H}$ & 7.267885 & 0.491175 & -2.188124 \\
\hline $\mathrm{H}$ & 3.229962 & -0.936592 & -1.943103 \\
\hline $\mathrm{H}$ & 5.868424 & -1.442461 & 1.375867 \\
\hline C & 5.009324 & 0.349688 & -3.679946 \\
\hline C & 8.119195 & -0.267726 & 0.347351 \\
\hline C & 3.582081 & 0.070248 & -4.188269 \\
\hline $\mathrm{H}$ & 3.464915 & 0.472345 & -5.201263 \\
\hline $\mathrm{H}$ & 3.369166 & -1.003590 & -4.231295 \\
\hline $\mathrm{H}$ & 2.822625 & 0.536032 & -3.550606 \\
\hline C & 5.252699 & 1.876834 & -3.728877 \\
\hline $\mathrm{H}$ & 5.102945 & 2.254647 & -4.747876 \\
\hline $\mathrm{H}$ & 4.559990 & 2.405912 & -3.064445 \\
\hline $\mathrm{H}$ & 6.271495 & 2.137827 & -3.423367 \\
\hline C & 6.003275 & -0.354931 & -4.633665 \\
\hline $\mathrm{H}$ & 5.860302 & -0.000872 & -5.662183 \\
\hline $\mathrm{H}$ & 7.044884 & -0.160302 & -4.356807 \\
\hline $\mathrm{H}$ & 5.851457 & -1.440172 & -4.624166 \\
\hline C & 9.141401 & 0.437882 & -0.563992 \\
\hline $\mathrm{H}$ & 10.098154 & 0.535003 & -0.038361 \\
\hline $\mathrm{H}$ & 9.326466 & -0.127312 & -1.484511 \\
\hline $\mathrm{H}$ & 8.813790 & 1.446297 & -0.841252 \\
\hline C & 7.953123 & 0.582702 & 1.629062 \\
\hline $\mathrm{H}$ & 7.266911 & 0.114503 & 2.342421 \\
\hline $\mathrm{H}$ & 7.559419 & 1.577363 & 1.389999 \\
\hline $\mathrm{H}$ & 8.920188 & 0.711280 & 2.131109 \\
\hline C & 8.692465 & -1.654497 & 0.724976 \\
\hline $\mathrm{H}$ & 9.667734 & -1.544522 & 1.215061 \\
\hline $\mathrm{H}$ & 8.033637 & -2.194766 & 1.412767 \\
\hline $\mathrm{H}$ & 8.828169 & -2.277925 & -0.166186 \\
\hline C & -3.747302 & -1.785537 & -0.051650 \\
\hline C & -4.513033 & -0.693812 & -0.487650 \\
\hline C & -4.103987 & -3.072339 & -0.472634 \\
\hline C & -5.617171 & -0.863273 & -1.331997 \\
\hline $\mathrm{H}$ & -4.221888 & 0.301816 & -0.164268 \\
\hline C & -5.193186 & -3.283249 & -1.326815 \\
\hline $\mathrm{H}$ & -3.511710 & -3.910281 & -0.129264 \\
\hline C & -5.935009 & -2.167665 & -1.738354 \\
\hline $\mathrm{H}$ & -6.780933 & -2.317659 & -2.395406 \\
\hline C & -5.518635 & -4.717462 & -1.786644 \\
\hline C & -6.426531 & 0.366254 & -1.791264 \\
\hline C & -4.318207 & -5.269392 & -2.593913 \\
\hline $\mathrm{H}$ & -4.505851 & -6.308756 & -2.892077 \\
\hline $\mathrm{H}$ & -4.160049 & -4.679544 & -3.504427 \\
\hline $\mathrm{H}$ & -3.388557 & -5.241291 & -2.017481 \\
\hline C & -5.763379 & -5.616918 & -0.551697 \\
\hline $\mathrm{H}$ & -5.993582 & -6.641742 & -0.868427 \\
\hline $\mathrm{H}$ & -4.886227 & -5.661631 & 0.101038 \\
\hline $\mathrm{H}$ & -6.607431 & -5.247544 & 0.042938 \\
\hline C & -6.772916 & -4.783026 & -2.678149 \\
\hline $\mathrm{H}$ & -7.666826 & -4.421040 & -2.156297 \\
\hline $\mathrm{H}$ & -6.652074 & -4.198251 & -3.597218 \\
\hline $\mathrm{H}$ & -6.960198 & -5.821847 & -2.972807 \\
\hline
\end{tabular}




\begin{tabular}{|lrrr|} 
C & -7.632294 & -0.014316 & -2.671545 \\
$H$ & -7.322071 & -0.518735 & -3.593530 \\
$H$ & -8.333701 & -0.669359 & -2.142327 \\
$H$ & -8.177932 & 0.891501 & -2.959567 \\
C & -6.961813 & 1.127848 & -0.555272 \\
$H$ & -7.623827 & 0.489327 & 0.041079 \\
$H$ & -6.151616 & 1.470248 & 0.096823 \\
$H$ & -7.532439 & 2.011267 & -0.867819 \\
C & -5.511420 & 1.304362 & -2.612708 \\
H & -6.066964 & 2.191964 & -2.941053 \\
H & -4.651887 & 1.645822 & -2.027244 \\
$H$ & -5.128135 & 0.793356 & -3.503393 \\
\hline
\end{tabular}

\section{C-Catalyzed reaction TS 67}

\section{Datum}

Value

\begin{tabular}{lc}
\hline B3LYP/6-31G(d) Energy & -3038.955493 \\
\hline B3LYP-D3(BJ)/def2-TZVPP-IEF-PCM(DCM) Energy & -3040.510047 \\
\hline B3LYP-D3(BJ)/def2-TZVPP-IEF-PCM(DCM)//B3LYP/6-31G(d) Free Energy (Quasiharmonic) & -3039.281558 \\
\hline
\end{tabular}

Number of Imaginary Frequencies

Frequencies (Top 3 out of 450)

$\begin{array}{lr}\text { 1. } & -318.5944 \mathrm{~cm}^{-1} \\ \text { 2. } & 6.6404 \mathrm{~cm}^{-1} \\ \text { 3. } & 8.0130 \mathrm{~cm}^{-1}\end{array}$

\section{B3LYP/6-31G(d) Molecular Geometry in Cartesian Coordinates}

C

$\mathrm{H}$

$\mathrm{H}$

C

0

0

$\mathrm{H}$

0

$\mathrm{H}$

C

C

C

C

$\mathrm{H}$

\subsection{1}

0.863133

2.421314

$-1.768298$

$-2.484609$

$-0.897236$

$-0.342648$

0.754997

0.510436

$-2.475321$

$-0.335292$

$-1.942499$

$-2.553572$

$-0.562316$
$-0.355891$

0.215427

0.087408

$-2.604205$

$-3.577981$

$-2.287822$

$-1.491250$

$-0.272404$

0.670781

3.497438

3.003873

3. 757371

3.054316

2.083895
0.308647

1.055767

0.224036

$-0.561034$

$-0.638661$

$-1.522538$

$-1.286119$

$-0.960635$

$-1.132733$

1.879100

2.004649

$-0.510251$

0.577497

2.546685 


\begin{tabular}{|c|c|c|c|}
\hline $\mathrm{H}$ & -2.293109 & 3.454983 & -1.498443 \\
\hline $\mathrm{H}$ & -1.950789 & 4.844412 & -0.394050 \\
\hline C & 0.866494 & 3.009013 & -2.626251 \\
\hline C & 1.503375 & 4.247756 & -1.897863 \\
\hline C & 1.866190 & 2.005769 & -3.199202 \\
\hline $\mathrm{H}$ & 1.329912 & 1.172894 & -3.665829 \\
\hline H & 2.488951 & 2.476804 & -3.968335 \\
\hline $\mathrm{H}$ & 2.517212 & 1.591093 & -2.426305 \\
\hline C & -0.138282 & 3.412825 & -3.717119 \\
\hline H & -0.710287 & 2.529993 & -4.020532 \\
\hline $\mathrm{H}$ & -0.840782 & 4.169359 & -3.355446 \\
\hline $\mathrm{H}$ & 0.368339 & 3.811406 & -4.602860 \\
\hline C & 1.751382 & 5.465378 & -2.788905 \\
\hline $\mathrm{H}$ & 0.823875 & 5.829868 & -3.236347 \\
\hline $\mathrm{H}$ & 2.177331 & 6.278133 & -2.190755 \\
\hline $\mathrm{H}$ & 2.460499 & 5.231105 & -3.591582 \\
\hline C & 2.789492 & 3.890822 & -1.134306 \\
\hline H & 2.646581 & 3.010703 & -0.500599 \\
\hline $\mathrm{H}$ & 3.064779 & 4.731268 & -0.488639 \\
\hline $\mathrm{H}$ & 3.626548 & 3.697082 & -1.814093 \\
\hline 0 & 0.134325 & 2.375064 & -1.548322 \\
\hline 0 & 0.480098 & 4.586298 & -0.941121 \\
\hline B & -0.216622 & 3.391198 & -0.580039 \\
\hline 0 & 0.101449 & 2.826965 & 0.797801 \\
\hline C & 0.114951 & 4.137921 & 2.837104 \\
\hline C & 0.719212 & 5.274736 & 2.273716 \\
\hline C & -0.045868 & 4.053604 & 4.230696 \\
\hline C & 1.143402 & 6.311718 & 3.102016 \\
\hline $\mathrm{H}$ & 0.849695 & 5.331812 & 1.196943 \\
\hline C & 0.385141 & 5.092306 & 5.051195 \\
\hline $\mathrm{H}$ & -0.504804 & 3.167482 & 4.663738 \\
\hline C & 0.978459 & 6.225433 & 4.487435 \\
\hline $\mathrm{H}$ & 1.610880 & 7.190271 & 2.665653 \\
\hline $\mathrm{H}$ & 0.263489 & 5.018895 & 6.128409 \\
\hline $\mathrm{H}$ & 1.315913 & 7.036557 & 5.127071 \\
\hline $\mathrm{H}$ & -2.871872 & 2.025321 & 0.405424 \\
\hline $\mathrm{H}$ & -2.335585 & 4.555096 & 2.077653 \\
\hline $\mathrm{H}$ & -2.892019 & 2.910842 & 2.692388 \\
\hline C & -0.827570 & -1.691767 & 1.592988 \\
\hline C & -1.851488 & -1.696066 & 0.656738 \\
\hline C & -3.091736 & -1.015578 & 0.910160 \\
\hline C & -3.265185 & -0.384618 & 2.125740 \\
\hline $\mathrm{H}$ & -4.195636 & 0.142642 & 2.322909 \\
\hline C & 1.539279 & -1.802809 & 0.741519 \\
\hline C & 0.454816 & -2.437229 & 1.343250 \\
\hline C & 2.877101 & -3.811125 & 1.018183 \\
\hline C & 2.768173 & -2.514773 & 0.554209 \\
\hline H & 3.814373 & -4.345494 & 0.883843 \\
\hline C & 1.794644 & -4.484746 & 1.631049 \\
\hline C & 0.549241 & -3.797445 & 1.784126 \\
\hline C & -0.540329 & -4.496319 & 2.375900 \\
\hline C & -0.396630 & -5.796663 & 2.803533 \\
\hline C & 0.841290 & -6.469282 & 2.662485 \\
\hline C & 1.911237 & -5.826345 & 2.085477 \\
\hline
\end{tabular}




\begin{tabular}{|c|c|c|c|}
\hline $\mathrm{H}$ & -1.499575 & -3.999650 & 2.469838 \\
\hline $\mathrm{H}$ & -1.243020 & -6.315356 & 3.245129 \\
\hline $\mathrm{H}$ & 0.939398 & -7.496420 & 3.003498 \\
\hline $\mathrm{H}$ & 2.863604 & -6.336667 & 1.962111 \\
\hline C & -2.258273 & -0.380621 & 3.123586 \\
\hline C & -1.009971 & -1.026917 & 2.854034 \\
\hline C & -0.003371 & -0.996598 & 3.858606 \\
\hline C & -0.219902 & -0.364555 & 5.064319 \\
\hline C & -1.457826 & 0.267992 & 5.330804 \\
\hline C & -2.452783 & 0.260382 & 4.377623 \\
\hline $\mathrm{H}$ & 0.945064 & -1.486107 & 3.665514 \\
\hline $\mathrm{H}$ & 0.561880 & -0.357784 & 5.818830 \\
\hline $\mathrm{H}$ & -1.624626 & 0.748372 & 6.291456 \\
\hline $\mathrm{H}$ & -3.411473 & 0.734668 & 4.576411 \\
\hline C & 6.246147 & -1.019648 & -1.418267 \\
\hline C & 5.090509 & -1.243278 & -2.181300 \\
\hline C & 3.939357 & -1.675529 & -1.510162 \\
\hline C & 3.957883 & -1.917855 & -0.129728 \\
\hline C & 5.135473 & -1.692021 & 0.594627 \\
\hline C & 6.297342 & -1.225949 & -0.032930 \\
\hline $\mathrm{H}$ & 7.144622 & -0.679765 & -1.925156 \\
\hline $\mathrm{H}$ & 3.017321 & -1.856113 & -2.049743 \\
\hline H & 5.124714 & -1.887063 & 1.661110 \\
\hline C & 5.139015 & -1.048001 & -3.709926 \\
\hline C & 7.606618 & -0.957087 & 0.734277 \\
\hline C & 3.785848 & -1.346784 & -4.382781 \\
\hline $\mathrm{H}$ & 3.870106 & -1.188684 & -5.464136 \\
\hline $\mathrm{H}$ & 3.473635 & -2.384910 & -4.224417 \\
\hline $\mathrm{H}$ & 2.989455 & -0.693079 & -4.012186 \\
\hline C & 5.536704 & 0.409891 & -4.040141 \\
\hline H & 5.578651 & 0.556947 & -5.126499 \\
\hline $\mathrm{H}$ & 4.808679 & 1.118104 & -3.629399 \\
\hline $\mathrm{H}$ & 6.520009 & 0.666940 & -3.632413 \\
\hline C & 6.192229 & -2.009993 & -4.311299 \\
\hline $\mathrm{H}$ & 6.239378 & -1.890547 & -5.400789 \\
\hline $\mathrm{H}$ & 7.194232 & -1.821427 & -3.911510 \\
\hline $\mathrm{H}$ & 5.936882 & -3.053560 & -4.095056 \\
\hline C & 8.030310 & 0.517688 & 0.535830 \\
\hline H & 8.964953 & 0.721934 & 1.072744 \\
\hline $\mathrm{H}$ & 8.194510 & 0.757672 & -0.519776 \\
\hline $\mathrm{H}$ & 7.262728 & 1.200541 & 0.917769 \\
\hline C & 7.463851 & -1.211415 & 2.247192 \\
\hline $\mathrm{H}$ & 7.204656 & -2.253854 & 2.464282 \\
\hline H & 6.699761 & -0.568262 & 2.698484 \\
\hline $\mathrm{H}$ & 8.414601 & -0.997570 & 2.748594 \\
\hline C & 8.719622 & -1.886774 & 0.194690 \\
\hline $\mathrm{H}$ & 9.661719 & -1.705425 & 0.726915 \\
\hline $\mathrm{H}$ & 8.449150 & -2.940040 & 0.331610 \\
\hline $\mathrm{H}$ & 8.903214 & -1.725965 & -0.872734 \\
\hline C & -4.167214 & -0.939103 & -0.116834 \\
\hline C & -5.499590 & -1.173680 & 0.229971 \\
\hline C & -3.858210 & -0.585521 & -1.441092 \\
\hline C & -6.531984 & -1.068970 & -0.715253 \\
\hline H & -5.725281 & -1.472991 & 1.249237 \\
\hline
\end{tabular}




\begin{tabular}{|c|c|c|c|}
\hline C & -4.852052 & -0.480214 & -2.413803 \\
\hline $\mathrm{H}$ & -2.823082 & -0.400005 & -1.699158 \\
\hline C & -6.181783 & -0.721432 & -2.023253 \\
\hline $\mathrm{H}$ & -6.960332 & -0.642388 & -2.772719 \\
\hline C & -4.536296 & -0.127123 & -3.879414 \\
\hline C & -7.985145 & -1.353214 & -0.287949 \\
\hline C & -5.272341 & 1.175648 & -4.271335 \\
\hline $\mathrm{H}$ & -5.062432 & 1.433058 & -5.316906 \\
\hline $\mathrm{H}$ & -6.358169 & 1.080173 & -4.163895 \\
\hline $\mathrm{H}$ & -4.947272 & 2.013937 & -3.643906 \\
\hline C & -3.027656 & 0.078192 & -4.117906 \\
\hline $\mathrm{H}$ & -2.851506 & 0.309401 & -5.174877 \\
\hline $\mathrm{H}$ & -2.631055 & 0.912634 & -3.527170 \\
\hline $\mathrm{H}$ & -2.449139 & -0.818651 & -3.870792 \\
\hline C & -5.010373 & -1.281142 & -4.795157 \\
\hline $\mathrm{H}$ & -4.494903 & -2.214420 & -4.543508 \\
\hline $\mathrm{H}$ & -6.087361 & -1.458400 & -4.705787 \\
\hline $\mathrm{H}$ & -4.797281 & -1.044915 & -5.845055 \\
\hline C & -8.092872 & -2.807386 & 0.229567 \\
\hline $\mathrm{H}$ & -7.817160 & -3.522436 & -0.553500 \\
\hline $\mathrm{H}$ & -7.435516 & -2.985053 & 1.086770 \\
\hline $\mathrm{H}$ & -9.120970 & -3.024993 & 0.544840 \\
\hline C & -8.390606 & -0.375845 & 0.841061 \\
\hline $\mathrm{H}$ & -7.743547 & -0.475612 & 1.719005 \\
\hline $\mathrm{H}$ & -8.332708 & 0.664003 & 0.498839 \\
\hline $\mathrm{H}$ & -9.420893 & -0.570483 & 1.163789 \\
\hline C & -8.986015 & -1.183009 & -1.446731 \\
\hline $\mathrm{H}$ & -10.001110 & -1.389715 & -1.088959 \\
\hline $\mathrm{H}$ & -8.978317 & -0.162740 & -1.847165 \\
\hline $\mathrm{H}$ & -8.779802 & -1.876367 & -2.269859 \\
\hline
\end{tabular}

\section{C-Catalyzed reaction TS 68}

\section{Datum}

Value

\begin{tabular}{lc}
\hline B3LYP/6-31G(d) Energy & -3038.960793 \\
\hline B3LYP-D3(BJ)/def2-TZVPP-IEF-PCM(DCM) Energy & -3040.511074 \\
\hline B3LYP-D3(BJ)/def2-TZVPP-IEF-PCM(DCM)//B3LYP/6-31G(d) Free Energy (Quasiharmonic) & -3039.28153 \\
\hline
\end{tabular}

Number of Imaginary Frequencies

Frequencies (Top 3 out of 450)

$\begin{array}{lr}\text { 1. } & -319.6333 \mathrm{~cm}^{-1} \\ \text { 2. } & 7.4889 \mathrm{~cm}^{-1} \\ \text { 3. } & 11.0200 \mathrm{~cm}^{-1}\end{array}$




\begin{tabular}{|c|c|c|c|}
\hline C & -2.066202 & -0.865298 & 0.868226 \\
\hline $\mathrm{H}$ & -1.011553 & -0.603854 & 0.978546 \\
\hline H & -2.629568 & 0.067105 & 0.831756 \\
\hline C & 1.130641 & -0.990895 & -1.323800 \\
\hline 0 & 1.127660 & -1.227758 & -2.513475 \\
\hline 0 & 1.155618 & 0.273154 & -0.879203 \\
\hline $\mathrm{H}$ & 1.083920 & 0.378636 & 0.104902 \\
\hline 0 & -2.532319 & -1.530394 & 2.055288 \\
\hline $\mathrm{H}$ & -2.022259 & -2.351739 & 2.150299 \\
\hline C & -0.441578 & 4.779017 & -1.023080 \\
\hline C & 1.424793 & 4.448361 & 0.079021 \\
\hline C & -1.115335 & 2.763421 & 0.243585 \\
\hline C & -1.197855 & 4.164930 & -0.048097 \\
\hline $\mathrm{H}$ & 1.176050 & 5.163045 & 0.865967 \\
\hline $\mathrm{H}$ & -0.923445 & 2.118920 & -0.616925 \\
\hline $\mathrm{H}$ & -1.924774 & 2.382875 & 0.868592 \\
\hline C & 1.012961 & 1.231452 & 2.985794 \\
\hline C & 0.225627 & 2.451732 & 3.598748 \\
\hline C & 0.658961 & -0.131500 & 3.580354 \\
\hline $\mathrm{H}$ & 1.253408 & -0.914279 & 3.096361 \\
\hline $\mathrm{H}$ & 0.886173 & -0.156218 & 4.652894 \\
\hline $\mathrm{H}$ & -0.397384 & -0.375329 & 3.442070 \\
\hline C & 2.535090 & 1.440638 & 3.007366 \\
\hline $\mathrm{H}$ & 2.939430 & 1.345145 & 4.021390 \\
\hline $\mathrm{H}$ & 3.010552 & 0.685346 & 2.374358 \\
\hline $\mathrm{H}$ & 2.807287 & 2.424886 & 2.615892 \\
\hline C & 0.929934 & 3.143121 & 4.768358 \\
\hline $\mathrm{H}$ & 1.073623 & 2.454092 & 5.609032 \\
\hline $\mathrm{H}$ & 1.903007 & 3.541766 & 4.471874 \\
\hline $\mathrm{H}$ & 0.316984 & 3.979860 & 5.120346 \\
\hline C & -1.211949 & 2.089093 & 4.007750 \\
\hline $\mathrm{H}$ & -1.235971 & 1.479899 & 4.917947 \\
\hline $\mathrm{H}$ & -1.765041 & 3.014252 & 4.200901 \\
\hline $\mathrm{H}$ & -1.730761 & 1.537818 & 3.218392 \\
\hline 0 & 0.575773 & 1.262560 & 1.600398 \\
\hline 0 & 0.161981 & 3.375955 & 2.496496 \\
\hline B & 0.289425 & 2.641019 & 1.277627 \\
\hline 0 & 1.369397 & 3.190445 & 0.371438 \\
\hline C & 2.387378 & 4.875601 & -0.952548 \\
\hline C & 2.765201 & 6.224073 & -1.033074 \\
\hline C & 2.944423 & 3.940843 & -1.841302 \\
\hline C & 3.688764 & 6.637472 & -1.990873 \\
\hline $\mathrm{H}$ & 2.336555 & 6.945535 & -0.340851 \\
\hline C & 3.864050 & 4.360835 & -2.798190 \\
\hline $\mathrm{H}$ & 2.650440 & 2.898025 & -1.766666 \\
\hline C & 4.237746 & 5.706540 & -2.876005 \\
\hline $\mathrm{H}$ & 3.981407 & 7.682282 & -2.046000 \\
\hline $\mathrm{H}$ & 4.291893 & 3.636938 & -3.486140 \\
\hline $\mathrm{H}$ & 4.956670 & 6.028224 & -3.624638 \\
\hline $\mathrm{H}$ & -1.681637 & 4.798364 & 0.696257 \\
\hline
\end{tabular}




\begin{tabular}{|c|c|c|c|}
\hline $\mathrm{H}$ & -0.086189 & 4.205756 & -1.874173 \\
\hline $\mathrm{H}$ & -0.480957 & 5.856195 & -1.160947 \\
\hline C & -0.058567 & -2.781822 & -0.049690 \\
\hline C & 1.123820 & -2.085854 & -0.274984 \\
\hline C & 2.330842 & -2.406245 & 0.437984 \\
\hline C & 2.272301 & -3.383822 & 1.414527 \\
\hline $\mathrm{H}$ & 3.180563 & -3.661261 & 1.942599 \\
\hline C & -2.285906 & -1.650555 & -0.412352 \\
\hline C & -1.300125 & -2.525370 & -0.864707 \\
\hline C & -3.648131 & -2.175903 & -2.355810 \\
\hline C & -3.493234 & -1.474905 & -1.175256 \\
\hline $\mathrm{H}$ & -4.564928 & -2.055697 & -2.927640 \\
\hline C & -2.668120 & -3.074673 & -2.837701 \\
\hline C & -1.468089 & -3.262374 & -2.083795 \\
\hline C & -0.497199 & -4.175572 & -2.579647 \\
\hline C & -0.708136 & -4.867064 & -3.751163 \\
\hline C & -1.899116 & -4.681279 & -4.493221 \\
\hline C & -2.856119 & -3.801642 & -4.044573 \\
\hline $\mathrm{H}$ & 0.425979 & -4.316664 & -2.029315 \\
\hline $\mathrm{H}$ & 0.048554 & -5.557834 & -4.113254 \\
\hline $\mathrm{H}$ & -2.051908 & -5.232651 & -5.417179 \\
\hline $\mathrm{H}$ & -3.774405 & -3.647524 & -4.606492 \\
\hline C & 1.079698 & -4.082193 & 1.716948 \\
\hline C & -0.103541 & -3.798795 & 0.962983 \\
\hline C & -1.281015 & -4.548973 & 1.249621 \\
\hline C & -1.287759 & -5.507764 & 2.240316 \\
\hline C & -0.117880 & -5.776248 & 2.989917 \\
\hline C & 1.039845 & -5.080932 & 2.727593 \\
\hline $\mathrm{H}$ & -2.178499 & -4.363536 & 0.669326 \\
\hline $\mathrm{H}$ & -2.196855 & -6.067531 & 2.441908 \\
\hline $\mathrm{H}$ & -0.136452 & -6.534963 & 3.767361 \\
\hline $\mathrm{H}$ & 1.946778 & -5.284852 & 3.291832 \\
\hline C & -6.703690 & 1.117193 & -0.040917 \\
\hline C & -6.212140 & 0.214890 & 0.906635 \\
\hline C & -5.150450 & -0.621929 & 0.527061 \\
\hline C & -4.592762 & -0.556823 & -0.753016 \\
\hline C & -5.116231 & 0.365909 & -1.677027 \\
\hline C & -6.173195 & 1.213088 & -1.340674 \\
\hline $\mathrm{H}$ & -7.521498 & 1.774412 & 0.231647 \\
\hline $\mathrm{H}$ & -4.737257 & -1.332838 & 1.234192 \\
\hline $\mathrm{H}$ & -4.661943 & 0.416440 & -2.659832 \\
\hline C & -6.779719 & 0.109178 & 2.335115 \\
\hline C & -6.757118 & 2.244358 & -2.326133 \\
\hline C & -7.304176 & -1.326308 & 2.576347 \\
\hline $\mathrm{H}$ & -7.709568 & -1.418172 & 3.591683 \\
\hline $\mathrm{H}$ & -8.102104 & -1.576817 & 1.867563 \\
\hline $\mathrm{H}$ & -6.510793 & -2.072139 & 2.465574 \\
\hline C & -5.656673 & 0.420851 & 3.354182 \\
\hline $\mathrm{H}$ & -6.034919 & 0.321652 & 4.379605 \\
\hline $\mathrm{H}$ & -4.802052 & -0.253574 & 3.241321 \\
\hline $\mathrm{H}$ & -5.291660 & 1.447192 & 3.227720 \\
\hline C & -7.941471 & 1.089697 & 2.582629 \\
\hline $\mathrm{H}$ & -8.307000 & 0.976620 & 3.609685 \\
\hline $\mathrm{H}$ & -7.629084 & 2.132887 & 2.457434 \\
\hline
\end{tabular}




\begin{tabular}{|c|c|c|c|}
\hline $\mathrm{H}$ & -8.785705 & 0.902174 & 1.908876 \\
\hline C & -6.078099 & 2.187369 & -3.708137 \\
\hline $\mathrm{H}$ & -6.532999 & 2.930194 & -4.373474 \\
\hline $\mathrm{H}$ & -5.007144 & 2.410942 & -3.647254 \\
\hline $\mathrm{H}$ & -6.195044 & 1.205049 & -4.179954 \\
\hline C & -8.267943 & 1.976110 & -2.524426 \\
\hline H & -8.697289 & 2.706660 & -3.221267 \\
\hline $\mathrm{H}$ & -8.437337 & 0.974260 & -2.935423 \\
\hline $\mathrm{H}$ & -8.823113 & 2.048333 & -1.583447 \\
\hline C & -6.561573 & 3.669157 & -1.755027 \\
\hline $\mathrm{H}$ & -6.978297 & 4.417586 & -2.440617 \\
\hline $\mathrm{H}$ & -7.057353 & 3.791153 & -0.786251 \\
\hline $\mathrm{H}$ & -5.497184 & 3.890684 & -1.615908 \\
\hline C & 3.646315 & -1.775444 & 0.124876 \\
\hline C & 4.525229 & -1.419928 & 1.165807 \\
\hline C & 4.063460 & -1.599124 & -1.197367 \\
\hline C & 5.788328 & -0.887741 & 0.903455 \\
\hline H & 4.193599 & -1.558195 & 2.188307 \\
\hline C & 5.319036 & -1.052157 & -1.504507 \\
\hline H & 3.403151 & -1.902588 & -2.000437 \\
\hline C & 6.157999 & -0.707158 & -0.442394 \\
\hline $\mathrm{H}$ & 7.132626 & -0.284577 & -0.656719 \\
\hline C & 5.708707 & -0.852672 & -2.981376 \\
\hline C & 6.772869 & -0.489433 & 2.020513 \\
\hline C & 4.697607 & 0.115693 & -3.642697 \\
\hline H & 3.668914 & -0.252605 & -3.578108 \\
\hline $\mathrm{H}$ & 4.730920 & 1.097196 & -3.153963 \\
\hline H & 4.942101 & 0.254410 & -4.703397 \\
\hline C & 7.121682 & -0.261879 & -3.144824 \\
\hline $\mathrm{H}$ & 7.347159 & -0.138897 & -4.210165 \\
\hline $\mathrm{H}$ & 7.210051 & 0.723854 & -2.673594 \\
\hline $\mathrm{H}$ & 7.891414 & -0.915746 & -2.718323 \\
\hline C & 5.670363 & -2.213625 & -3.716787 \\
\hline $\mathrm{H}$ & 6.375715 & -2.922289 & -3.267179 \\
\hline H & 4.674205 & -2.665764 & -3.687729 \\
\hline $\mathrm{H}$ & 5.944640 & -2.083523 & -4.770957 \\
\hline C & 8.092531 & -1.279628 & 1.851696 \\
\hline $\mathrm{H}$ & 7.914604 & -2.358909 & 1.921664 \\
\hline H & 8.568365 & -1.082375 & 0.885568 \\
\hline $\mathrm{H}$ & 8.806160 & -1.001306 & 2.636978 \\
\hline C & 7.068116 & 1.026670 & 1.930232 \\
\hline H & 7.508395 & 1.299213 & 0.965359 \\
\hline $\mathrm{H}$ & 6.149933 & 1.611746 & 2.055063 \\
\hline H & 7.773132 & 1.326213 & 2.715622 \\
\hline C & 6.220560 & -0.786901 & 3.427660 \\
\hline $\mathrm{H}$ & 6.961103 & -0.498260 & 4.182277 \\
\hline $\mathrm{H}$ & 5.302892 & -0.225687 & 3.635896 \\
\hline H & 6.008887 & -1.853614 & 3.564993 \\
\hline
\end{tabular}




\begin{tabular}{lc}
\hline B3LYP/6-31G(d) Energy & -3038.958472 \\
\hline B3LYP-D3(BJ)/def2-TZVPP-IEF-PCM(DCM) Energy & -3040.511115 \\
\hline B3LYP-D3(BJ)/def2-TZVPP-IEF-PCM(DCM)//B3LYP/6-31G(d) Free Energy (Quasiharmonic) & -3039.281526 \\
\hline
\end{tabular}

Number of Imaginary Frequencies

Frequencies (Top 3 out of 450)

$\begin{array}{lr}\text { 1. } & -305.1055 \mathrm{~cm}^{-1} \\ \text { 2. } & 8.6373 \mathrm{~cm}^{-1} \\ \text { 3. } & 12.2914 \mathrm{~cm}^{-1}\end{array}$

\section{B3LYP/6-31G(d) Molecular Geometry in Cartesian Coordinates}

C

$\mathrm{H}$

$\mathrm{H}$

C

0

0

$\mathrm{H}$

0

$\mathrm{H}$

C

C

C

C

$\mathrm{H}$

$\mathrm{H}$

$\mathrm{H}$

C

C

C

$\mathrm{H}$

$\mathrm{H}$

$\mathrm{H}$

C

$\mathrm{H}$

$\mathrm{H}$

$\mathrm{H}$

C

$\mathrm{H}$

$\mathrm{H}$

$\mathrm{H}$

C

$\mathrm{H}$

\section{$-1.486439$}

$-2.225879$

$-0.497796$

1.499161

1. 291723

1.508043

1.566827

$-1.655734$

$-1.002966$

0.947270

$-0.562459$

1.995048

1.939448

$-0.124750$

2.949762

1.652952

1.571236

0.672582

3.072610

3.636937

3.402369

3. 315137

1. 258255

1.918851

1.439014

0.223333

$-0.787497$

$-0.894540$

$-1.190024$

$-1.392541$

1. 207476

1. 283347
0.642258

$-0.142926$

0.192613

0.811002

1. 228894

$-0.516989$

$-0.812642$

1.037659

1.731844

- 3.893083

$-2.946004$

$-4.327187$

$-3.661397$

$-1.957865$

$-4.230687$

$-5.364956$

$-2.180044$

$-3.365292$

$-2.478263$

$-1.760614$

$-2.388692$

$-3.485436$

$-0.823040$

$-0.053257$

$-0.859515$

$-0.514010$

$-2.945508$

$-2.326623$

-2. 389191

$-3.848592$

$-4.095582$

$-3.419614$
1.075209

0.914497

0.937009

$-1.232364$

$-2.349781$

$-1.001475$

$-0.054778$

2. 445407

2.634789

$-2.468058$

$-1.180077$

$-0.271893$

$-1.542371$

$-1.313279$

0.247509

$-0.283303$

2. 582336

3.087493

2. 726914

2. 123297

3.768016

2. 377339

3. 210060

2. 795044

4. 291255

3.050511

3. 328780

4. 226320

2.477894

3.458580

4. 321383

5. 181465 


\begin{tabular}{|c|c|c|c|}
\hline $\mathrm{H}$ & 0.522934 & -4.907134 & 4.589836 \\
\hline $\mathrm{H}$ & 2.189384 & -4.536671 & 4.133229 \\
\hline 0 & 1.255419 & -2.151540 & 1.161530 \\
\hline 0 & 0.711884 & -4.269913 & 1.973872 \\
\hline B & 0.859941 & -3.512026 & 0.782631 \\
\hline 0 & -0.410930 & -3.546384 & -0.043056 \\
\hline C & -1.759834 & -3.261252 & -1.981514 \\
\hline C & -2.565315 & -4.370117 & -1.678645 \\
\hline C & -2.101596 & -2.427760 & -3.058139 \\
\hline C & -3.690130 & -4.644704 & -2.451709 \\
\hline $\mathrm{H}$ & -2.301214 & -4.998384 & -0.834075 \\
\hline C & -3.225857 & -2.708854 & -3.831190 \\
\hline $\mathrm{H}$ & -1.481253 & -1.563845 & -3.286013 \\
\hline C & -4.020485 & -3.819276 & -3.531486 \\
\hline H & -4.312315 & -5.503187 & -2.214007 \\
\hline $\mathrm{H}$ & -3.482360 & -2.064008 & -4.667085 \\
\hline $\mathrm{H}$ & -4.895864 & -4.039728 & -4.136221 \\
\hline $\mathrm{H}$ & 2.549015 & -2.767856 & -1.671951 \\
\hline $\mathrm{H}$ & 0.416935 & -4.841148 & -2.474391 \\
\hline H & 0.896248 & -3.312094 & -3.384275 \\
\hline C & 0.707974 & 2.484542 & 0.433272 \\
\hline C & 1.755149 & 1.701706 & -0.039971 \\
\hline C & 3.056811 & 1.730709 & 0.570644 \\
\hline C & 3.228780 & 2.508031 & 1.700737 \\
\hline $\mathrm{H}$ & 4.213369 & 2.573177 & 2.155674 \\
\hline C & -1.693023 & 1.765756 & 0.075492 \\
\hline C & -0.618724 & 2.579178 & -0.278335 \\
\hline C & -3.119623 & 2.961092 & -1.486546 \\
\hline C & -2.984272 & 1.976776 & -0.525709 \\
\hline $\mathrm{H}$ & -4.095801 & 3.137782 & -1.930895 \\
\hline C & -2.041557 & 3.783784 & -1.887768 \\
\hline C & -0.765206 & 3.606247 & -1.269111 \\
\hline C & 0.301092 & 4.457974 & -1.668948 \\
\hline C & 0.111444 & 5.430494 & -2.624736 \\
\hline C & -1.154455 & 5.604933 & -3.233578 \\
\hline C & -2.206420 & 4.796788 & -2.871341 \\
\hline $\mathrm{H}$ & 1.279258 & 4.327856 & -1.220218 \\
\hline $\mathrm{H}$ & 0.941438 & 6.067511 & -2.918156 \\
\hline $\mathrm{H}$ & -1.290334 & 6.376273 & -3.986930 \\
\hline H & -3.184110 & 4.920308 & -3.331330 \\
\hline C & 2.176392 & 3.269112 & 2.262387 \\
\hline C & 0.899127 & 3.279701 & 1.614819 \\
\hline C & -0.132464 & 4.088981 & 2.173479 \\
\hline C & 0.086918 & 4.829602 & 3.315110 \\
\hline C & 1.349429 & 4.811200 & 3.953730 \\
\hline C & 2.370880 & 4.050599 & 3.433470 \\
\hline $\mathrm{H}$ & -1.099782 & 4.123273 & 1.684355 \\
\hline $\mathrm{H}$ & -0.714567 & 5.438372 & 3.724116 \\
\hline $\mathrm{H}$ & 1.509331 & 5.401285 & 4.851805 \\
\hline H & 3.346831 & 4.033856 & 3.912541 \\
\hline C & -6.555838 & -0.123669 & 0.546608 \\
\hline C & -5.738409 & 0.365828 & 1.568523 \\
\hline C & -4.560956 & 1.036813 & 1.202010 \\
\hline C & -4.200643 & 1.201475 & -0.138898 \\
\hline
\end{tabular}




\begin{tabular}{|c|c|c|c|}
\hline C & -5.048061 & 0.685755 & -1.136900 \\
\hline C & -6.233975 & 0.023562 & -0.815287 \\
\hline $\mathrm{H}$ & -7.471895 & -0.640730 & 0.807180 \\
\hline $\mathrm{H}$ & -3.906853 & 1.438900 & 1.967520 \\
\hline $\mathrm{H}$ & -4.748590 & 0.803080 & -2.171927 \\
\hline C & -6.074840 & 0.201225 & 3.063255 \\
\hline C & -7.188774 & -0.540869 & -1.885343 \\
\hline C & -7.395172 & -0.558742 & 3.291139 \\
\hline $\mathrm{H}$ & -7.584442 & -0.653406 & 4.366526 \\
\hline $\mathrm{H}$ & -8.251555 & -0.034815 & 2.850643 \\
\hline $\mathrm{H}$ & -7.360214 & -1.570963 & 2.872232 \\
\hline C & -4.937235 & -0.586074 & 3.758193 \\
\hline $\mathrm{H}$ & -5.148061 & -0.690912 & 4.830039 \\
\hline $\mathrm{H}$ & -4.844503 & -1.592174 & 3.332528 \\
\hline $\mathrm{H}$ & -3.968533 & -0.088092 & 3.651165 \\
\hline C & -6.204576 & 1.596970 & 3.718372 \\
\hline $\mathrm{H}$ & -6.440955 & 1.496917 & 4.785060 \\
\hline $\mathrm{H}$ & -5.276844 & 2.171761 & 3.635028 \\
\hline $\mathrm{H}$ & -7.004218 & 2.179648 & 3.246120 \\
\hline C & -7.361488 & -2.064673 & -1.679942 \\
\hline $\mathrm{H}$ & -8.045835 & -2.475192 & -2.433226 \\
\hline $\mathrm{H}$ & -6.400795 & -2.583588 & -1.772034 \\
\hline $\mathrm{H}$ & -7.775402 & -2.299819 & -0.693817 \\
\hline C & -8.566919 & 0.151531 & -1.759008 \\
\hline $\mathrm{H}$ & -9.262862 & -0.242003 & -2.510417 \\
\hline $\mathrm{H}$ & -9.015375 & -0.009190 & -0.773059 \\
\hline $\mathrm{H}$ & -8.477159 & 1.233165 & -1.912009 \\
\hline C & -6.668567 & -0.309165 & -3.316895 \\
\hline $\mathrm{H}$ & -7.380418 & -0.724286 & -4.039789 \\
\hline $\mathrm{H}$ & -6.554750 & 0.757364 & -3.542053 \\
\hline $\mathrm{H}$ & -5.702625 & -0.799450 & -3.482141 \\
\hline C & 4.230802 & 1.018897 & -0.012453 \\
\hline C & 4.470786 & 1.050287 & -1.394085 \\
\hline C & 5.152795 & 0.369292 & 0.823858 \\
\hline C & 5.588461 & 0.421968 & -1.953724 \\
\hline $\mathrm{H}$ & 3.779736 & 1.586296 & -2.030797 \\
\hline C & 6.292776 & -0.256133 & 0.306174 \\
\hline $\mathrm{H}$ & 4.958469 & 0.350160 & 1.889893 \\
\hline C & 6.477890 & -0.223322 & -1.083740 \\
\hline $\mathrm{H}$ & 7.349844 & -0.716573 & -1.502921 \\
\hline C & 7.327558 & -0.971955 & 1.196993 \\
\hline C & 5.856055 & 0.420866 & -3.471340 \\
\hline C & 8.722485 & -0.338716 & 0.978288 \\
\hline $\mathrm{H}$ & 9.051913 & -0.419585 & -0.062555 \\
\hline $\mathrm{H}$ & 8.716522 & 0.724369 & 1.245090 \\
\hline $\mathrm{H}$ & 9.470290 & -0.841855 & 1.603365 \\
\hline C & 6.989548 & -0.865135 & 2.696169 \\
\hline $\mathrm{H}$ & 7.762099 & -1.372565 & 3.284837 \\
\hline $\mathrm{H}$ & 6.951427 & 0.177788 & 3.031517 \\
\hline $\mathrm{H}$ & 6.030879 & -1.337860 & 2.935753 \\
\hline C & 7.381807 & -2.471633 & 0.819948 \\
\hline $\mathrm{H}$ & 6.408309 & -2.950557 & 0.974533 \\
\hline $\mathrm{H}$ & 7.662969 & -2.617783 & -0.228365 \\
\hline $\mathrm{H}$ & 8.120270 & -2.994959 & 1.439684 \\
\hline
\end{tabular}




$\begin{array}{|llll|}\mathrm{C} & 7.236245 & 1.057619 & -3.758679 \\ \mathrm{H} & 7.271676 & 2.093019 & -3.400870 \\ \mathrm{H} & 8.051364 & 0.508433 & -3.275432 \\ \mathrm{H} & 7.434602 & 1.064666 & -4.837359 \\ \mathrm{C} & 5.848260 & -1.038600 & -3.985535 \\ \mathrm{H} & 6.609786 & -1.650514 & -3.490181 \\ \mathrm{H} & 4.873992 & -1.509247 & -3.810832 \\ \mathrm{H} & 6.048770 & -1.063919 & -5.063671 \\ \mathrm{C} & 4.789619 & 1.211035 & -4.254427 \\ \mathrm{H} & 5.011708 & 1.162681 & -5.326579 \\ \mathrm{H} & 3.782331 & 0.808200 & -4.103679 \\ \mathrm{H} & 4.776625 & 2.269191 & -3.968905 \\ & & \end{array}$

\section{C-Catalyzed reaction TS 70}

\section{Datum}

Value

\begin{tabular}{lr}
\hline B3LYP/6-31G(d) Energy & -3038.958534 \\
\hline B3LYP-D3(BJ)/def2-TZVPP-IEF-PCM(DCM) Energy & -3040.510408 \\
\hline B3LYP-D3(BJ)/def2-TZVPP-IEF-PCM(DCM)//B3LYP/6-31G(d) Free Energy (Quasiharmonic) & -3039.281298 \\
\hline
\end{tabular}

Number of Imaginary Frequencies

Frequencies (Top 3 out of 450)

$\begin{array}{lr}\text { 1. } & -288.0761 \mathrm{~cm}^{-1} \\ \text { 2. } & 8.9948 \mathrm{~cm}^{-1} \\ \text { 3. } & 12.0093 \mathrm{~cm}^{-1}\end{array}$

\section{B3LYP/6-31G(d) Molecular Geometry in Cartesian Coordinates}

\section{C}

$\mathrm{H}$

$\mathrm{H}$

C

0

0

$\mathrm{H}$

0

$\mathrm{H}$

C

C

c

C

$\mathrm{H}$

-3.242323
-4.252230
-2.544408
0.043665
-0.968122
1.102694
1.875170
-3.186188
-2.417116
3.850251
3.291198
4.486053
4.807221
2.323895

$-3.242323$

$-4.252230$

$-2.544408$

$-0.968122$

694

2.323895
0.730735

0.669554

0.763895

1.163945

1.660193

0.953450

0.512616

1.949077

1.900667

$-2.847651$

$-3.547038$

$-0.827801$

$-2.010506$

$-3.829180$
1.143550

1.556538

1.989971

$-0.868883$

$-1.335922$

$-1.649552$

$-1.206001$

0.405187

$-0.201412$

2.470283

0.430399

1.199742

1.949648

0.854253 


\begin{tabular}{|c|c|c|c|}
\hline $\mathrm{H}$ & 5.310701 & -0.117123 & 1.113352 \\
\hline $\mathrm{H}$ & 3.563322 & -0.338415 & 1.519538 \\
\hline C & 4.134331 & -0.116742 & -2.488903 \\
\hline C & 5.595640 & -0.637358 & -2.229558 \\
\hline C & 3.330371 & -1.026811 & -3.430383 \\
\hline $\mathrm{H}$ & 2.277822 & -0.730357 & -3.403565 \\
\hline H & 3.681894 & -0.943933 & -4.464645 \\
\hline $\mathrm{H}$ & 3. 395971 & -2.075210 & -3.124297 \\
\hline C & 4.050510 & 1.334833 & -2.963523 \\
\hline $\mathrm{H}$ & 3.010052 & 1.612928 & -3.152272 \\
\hline $\mathrm{H}$ & 4.611147 & 1.465362 & -3.896540 \\
\hline $\mathrm{H}$ & 4.451422 & 2.023497 & -2.215684 \\
\hline C & 6.540270 & 0.457300 & -1.705092 \\
\hline $\mathrm{H}$ & 6.811908 & 1.172510 & -2.488874 \\
\hline $\mathrm{H}$ & 6.087020 & 1.011918 & -0.878117 \\
\hline $\mathrm{H}$ & 7.457711 & -0.014596 & -1.338169 \\
\hline C & 6.245353 & -1.340005 & -3.423385 \\
\hline $\mathrm{H}$ & 5.664700 & -2.208034 & -3.744080 \\
\hline $\mathrm{H}$ & 6.349509 & -0.656628 & -4.274264 \\
\hline $\mathrm{H}$ & 7.247471 & -1.685160 & -3.146096 \\
\hline 0 & 3.546229 & -0.214513 & -1.159192 \\
\hline 0 & 5.404162 & -1.601350 & -1.176886 \\
\hline B & 4.212282 & -1.281540 & -0.456795 \\
\hline 0 & 3.243288 & -2.466971 & -0.276576 \\
\hline C & 4.196087 & -4.694966 & 0.197873 \\
\hline C & 3.838001 & -5.919217 & 0.790813 \\
\hline C & 5.341173 & -4.633366 & -0.613222 \\
\hline C & 4.611201 & -7.058638 & 0.589608 \\
\hline $\mathrm{H}$ & 2.945245 & -5.974354 & 1.410281 \\
\hline C & 6.111218 & -5.778443 & -0.809774 \\
\hline $\mathrm{H}$ & 5.616053 & -3.691025 & -1.075137 \\
\hline C & 5.753573 & -6.989662 & -0.212004 \\
\hline $\mathrm{H}$ & 4.321864 & -7.998597 & 1.051154 \\
\hline $\mathrm{H}$ & 6.995270 & -5.725548 & -1.439485 \\
\hline $\mathrm{H}$ & 6.358497 & -7.877685 & -0.374169 \\
\hline $\mathrm{H}$ & 5.835361 & -2.371899 & 1.906469 \\
\hline $\mathrm{H}$ & 2.853606 & -2.467627 & 2.679524 \\
\hline $\mathrm{H}$ & 4.129553 & -3.769099 & 2.972008 \\
\hline C & -0.564903 & -0.345481 & 1.019387 \\
\hline C & 0.182365 & 0.736986 & 0.572431 \\
\hline C & 1.101320 & 1.431027 & 1.432642 \\
\hline C & 1.308117 & 0.931630 & 2.703547 \\
\hline $\mathrm{H}$ & 1.968369 & 1.463968 & 3.383367 \\
\hline C & -2.954464 & -0.490231 & 0.282766 \\
\hline C & -1.648236 & -0.965816 & 0.173826 \\
\hline C & -3.710139 & -2.216418 & -1.253482 \\
\hline C & -4.012413 & -1.132309 & -0.450436 \\
\hline $\mathrm{H}$ & -4.507040 & -2.698884 & -1.813673 \\
\hline C & -2.390680 & -2.699885 & -1.408343 \\
\hline C & -1.330761 & -2.064973 & -0.687235 \\
\hline C & -0.005794 & -2.546381 & -0.873088 \\
\hline C & 0.252786 & -3.600269 & -1.722306 \\
\hline C & -0.799991 & -4.236089 & -2.423036 \\
\hline C & -2.092513 & -3.791991 & -2.267849 \\
\hline
\end{tabular}




\begin{tabular}{|c|c|c|c|}
\hline $\mathrm{H}$ & 0.817610 & -2.059780 & -0.362445 \\
\hline $\mathrm{H}$ & 1.276074 & -3.935492 & -1.865332 \\
\hline $\mathrm{H}$ & -0.582477 & -5.066925 & -3.088987 \\
\hline $\mathrm{H}$ & -2.909248 & -4.264864 & -2.808356 \\
\hline C & 0.647206 & -0.231337 & 3.171714 \\
\hline C & -0.318133 & -0.874071 & 2.331696 \\
\hline C & -0.998639 & -2.017315 & 2.834685 \\
\hline C & -0.731089 & -2.504068 & 4.095624 \\
\hline C & 0.223762 & -1.867208 & 4.923736 \\
\hline C & 0.893635 & -0.752990 & 4.471238 \\
\hline $\mathrm{H}$ & -1.735808 & -2.506236 & 2.207549 \\
\hline $\mathrm{H}$ & -1.260620 & -3.380096 & 4.460101 \\
\hline $\mathrm{H}$ & 0.421408 & -2.256361 & 5.918919 \\
\hline $\mathrm{H}$ & 1.622154 & -0.251222 & 5.103681 \\
\hline C & -8.129809 & 0.021503 & -0.268741 \\
\hline C & -7.789910 & -1.315631 & -0.044248 \\
\hline C & -6.429553 & -1.657931 & -0.096170 \\
\hline C & -5.441193 & -0.703927 & -0.360760 \\
\hline C & -5.825213 & 0.629710 & -0.583976 \\
\hline C & -7.167226 & 1.011414 & -0.538576 \\
\hline $\mathrm{H}$ & -9.172921 & 0.313211 & -0.229318 \\
\hline $\mathrm{H}$ & -6.121380 & -2.682614 & 0.092153 \\
\hline $\mathrm{H}$ & -5.048165 & 1.358066 & -0.774926 \\
\hline C & -8.836792 & -2.403125 & 0.267348 \\
\hline C & -7.611381 & 2.471047 & -0.755308 \\
\hline C & -8.555710 & -3.000548 & 1.666716 \\
\hline $\mathrm{H}$ & -9.289560 & -3.780684 & 1.905149 \\
\hline $\mathrm{H}$ & -7.559053 & -3.450462 & 1.724060 \\
\hline H & -8.617350 & -2.227385 & 2.441138 \\
\hline C & -10.277205 & -1.856392 & 0.262586 \\
\hline $\mathrm{H}$ & -10.980241 & -2.668522 & 0.480992 \\
\hline $\mathrm{H}$ & -10.423125 & -1.080925 & 1.023117 \\
\hline $\mathrm{H}$ & -10.550796 & -1.435641 & -0.711712 \\
\hline C & -8.748975 & -3.525333 & -0.794096 \\
\hline $\mathrm{H}$ & -9.481599 & -4.313629 & -0.580127 \\
\hline $\mathrm{H}$ & -8.955247 & -3.132526 & -1.796349 \\
\hline H & -7.757547 & -3.989703 & -0.815492 \\
\hline C & -8.629537 & 2.543972 & -1.917731 \\
\hline $\mathrm{H}$ & -8.950745 & 3.580889 & -2.076869 \\
\hline $\mathrm{H}$ & -8.183888 & 2.180550 & -2.850864 \\
\hline $\mathrm{H}$ & -9.525842 & 1.946317 & -1.720337 \\
\hline C & -8.276151 & 2.995702 & 0.540157 \\
\hline $\mathrm{H}$ & -8.607471 & 4.033197 & 0.406804 \\
\hline $\mathrm{H}$ & -9.151337 & 2.399404 & 0.821062 \\
\hline $\mathrm{H}$ & -7.570259 & 2.969709 & 1.378053 \\
\hline C & -6.427912 & 3.396856 & -1.097958 \\
\hline $\mathrm{H}$ & -6.790435 & 4.423799 & -1.224474 \\
\hline $\mathrm{H}$ & -5.664956 & 3.402346 & -0.312835 \\
\hline $\mathrm{H}$ & -5.941930 & 3.099591 & -2.034232 \\
\hline C & 1.748180 & 2.708867 & 1.019277 \\
\hline C & 3.102004 & 2.952070 & 1.295645 \\
\hline C & 0.984633 & 3.711803 & 0.404127 \\
\hline C & 3.703179 & 4.170862 & 0.961201 \\
\hline $\mathrm{H}$ & 3.683032 & 2.166690 & 1.764398 \\
\hline
\end{tabular}




\begin{tabular}{|c|c|c|c|}
\hline C & 1.553026 & 4.935195 & 0.034782 \\
\hline $\mathrm{H}$ & -0.068245 & 3.531459 & 0.231722 \\
\hline C & 2.909198 & 5.136362 & 0.324812 \\
\hline $\mathrm{H}$ & 3.363816 & 6.082755 & 0.047041 \\
\hline C & 0.742097 & 6.040543 & -0.668272 \\
\hline C & 5.183444 & 4.480428 & 1.261325 \\
\hline C & 0.757655 & 7.325284 & 0.193167 \\
\hline $\mathrm{H}$ & 0.303104 & 7.144816 & 1.174016 \\
\hline $\mathrm{H}$ & 1.774913 & 7.696415 & 0.358832 \\
\hline $\mathrm{H}$ & 0.189646 & 8.121905 & -0.302598 \\
\hline C & 1.380271 & 6.339709 & -2.046159 \\
\hline $\mathrm{H}$ & 0.818690 & 7.128864 & -2.560985 \\
\hline $\mathrm{H}$ & 2.418308 & 6.677001 & -1.952721 \\
\hline $\mathrm{H}$ & 1.373561 & 5.447463 & -2.682107 \\
\hline C & -0.725258 & 5.631437 & -0.900218 \\
\hline $\mathrm{H}$ & -0.807586 & 4.729301 & -1.516035 \\
\hline $\mathrm{H}$ & -1.253087 & 5.447439 & 0.042404 \\
\hline $\mathrm{H}$ & -1.253439 & 6.438854 & -1.420036 \\
\hline C & 5.937230 & 4.747034 & -0.063241 \\
\hline $\mathrm{H}$ & 5.907133 & 3.867231 & -0.716545 \\
\hline $\mathrm{H}$ & 5.503753 & 5.586955 & -0.615687 \\
\hline $\mathrm{H}$ & 6.989305 & 4.985454 & 0.136333 \\
\hline C & 5.273757 & 5.736207 & 2.161266 \\
\hline $\mathrm{H}$ & 4.831231 & 6.615340 & 1.681839 \\
\hline $\mathrm{H}$ & 4.751162 & 5.575694 & 3.111175 \\
\hline $\mathrm{H}$ & 6.321744 & 5.970975 & 2.384708 \\
\hline $\mathrm{C}$ & 5.891219 & 3.323339 & 1.991091 \\
\hline $\mathrm{H}$ & 6.934185 & 3.594788 & 2.189867 \\
\hline $\mathrm{H}$ & 5.418422 & 3.098359 & 2.953936 \\
\hline $\mathrm{H}$ & 5.898645 & 2.406034 & 1.391367 \\
\hline
\end{tabular}

\section{C-Catalyzed reaction TS 71}

\section{Datum}

Value

B3LYP/6-31G(d) Energy

$-3038.950831$

B3LYP-D3(BJ)/def2-TZVPP-IEF-PCM(DCM) Energy

$-3040.50909$

B3LYP-D3(BJ)/def2-TZVPP-IEF-PCM(DCM)//B3LYP/6-31G(d) Free Energy (Quasiharmonic)

$-3039.281205$

Number of Imaginary Frequencies

Frequencies (Top 3 out of 450)
1. $\quad-305.7485 \mathrm{~cm}^{-1}$
2. $\quad 8.1959 \mathrm{~cm}^{-1}$
3. $9.8985 \mathrm{~cm}^{-1}$ 


\begin{tabular}{|c|c|c|c|}
\hline C & 2.493505 & 0.135875 & 0.847835 \\
\hline $\mathrm{H}$ & 1.520911 & 0.248948 & 1.324534 \\
\hline $\mathrm{H}$ & 2.460626 & -0.793941 & 0.263883 \\
\hline C & -0.505460 & 1.082788 & -0.927402 \\
\hline 0 & -0.377941 & 1.489767 & -2.059019 \\
\hline 0 & -0.623075 & -0.247381 & -0.697853 \\
\hline $\mathrm{H}$ & -0.679855 & -0.525949 & 0.250298 \\
\hline 0 & 3.431462 & 0.027499 & 1.918472 \\
\hline $\mathrm{H}$ & 4.276441 & -0.255988 & 1.531907 \\
\hline C & -1.523495 & -3.571518 & -2.159270 \\
\hline C & -2.900967 & -2.654645 & -0.710343 \\
\hline C & -0.305521 & -4.135670 & -0.079406 \\
\hline C & -0.453710 & -3.406617 & -1.308185 \\
\hline $\mathrm{H}$ & -2.465199 & -1.663879 & -0.839546 \\
\hline $\mathrm{H}$ & 0.690771 & -4.080572 & 0.363530 \\
\hline $\mathrm{H}$ & -0.672061 & -5.164688 & -0.109206 \\
\hline C & -0.547186 & -2.066424 & 2.914350 \\
\hline C & -1.453181 & -3.258908 & 3.396733 \\
\hline C & 0.945372 & -2.379491 & 3.100957 \\
\hline $\mathrm{H}$ & 1.569061 & -1.621579 & 2.622580 \\
\hline $\mathrm{H}$ & 1.212442 & -2.393319 & 4.163390 \\
\hline $\mathrm{H}$ & 1.195757 & -3.356147 & 2.676034 \\
\hline C & -0.879559 & -0.713077 & 3.539976 \\
\hline $\mathrm{H}$ & -0.774829 & -0.754677 & 4.630481 \\
\hline $\mathrm{H}$ & -0.193034 & 0.058325 & 3.175467 \\
\hline $\mathrm{H}$ & -1.898725 & -0.399807 & 3.300913 \\
\hline C & -2.911800 & -2.838163 & 3.647735 \\
\hline $\mathrm{H}$ & -3.012084 & -2.234801 & 4.557324 \\
\hline $\mathrm{H}$ & -3.312656 & -2.269652 & 2.804717 \\
\hline $\mathrm{H}$ & -3.522139 & -3.739457 & 3.764372 \\
\hline C & -0.929921 & -4.022815 & 4.615345 \\
\hline $\mathrm{H}$ & -0.842302 & -3.367433 & 5.490154 \\
\hline $\mathrm{H}$ & -1.628321 & -4.828531 & 4.865594 \\
\hline $\mathrm{H}$ & 0.044202 & -4.474975 & 4.416534 \\
\hline 0 & -0.847637 & -2.012598 & 1.488293 \\
\hline 0 & -1.416541 & -4.139434 & 2.264578 \\
\hline B & -1.316751 & -3.338868 & 1.096610 \\
\hline 0 & -2.660365 & -3.306915 & 0.378712 \\
\hline C & -4.178365 & -2.921044 & -1.401415 \\
\hline C & -4.639186 & -2.019124 & -2.371893 \\
\hline C & -4.952720 & -4.047580 & -1.079079 \\
\hline C & -5.852870 & -2.244339 & -3.018774 \\
\hline $\mathrm{H}$ & -4.047354 & -1.138802 & -2.608053 \\
\hline C & -6.165078 & -4.267769 & -1.727111 \\
\hline $\mathrm{H}$ & -4.596268 & -4.730819 & -0.315023 \\
\hline C & -6.616953 & -3.369793 & -2.699487 \\
\hline $\mathrm{H}$ & -6.202684 & -1.542805 & -3.771231 \\
\hline $\mathrm{H}$ & -6.762335 & -5.139182 & -1.472903 \\
\hline $\mathrm{H}$ & -7.563194 & -3.545503 & -3.204066 \\
\hline $\mathrm{H}$ & 0.158869 & -2.516414 & -1.443499 \\
\hline
\end{tabular}




\begin{tabular}{|c|c|c|c|}
\hline $\mathrm{H}$ & -2.076033 & -4.506880 & -2.167224 \\
\hline $\mathrm{H}$ & -1.631794 & -2.945141 & -3.040073 \\
\hline C & 0.675326 & 2.486766 & 0.758682 \\
\hline C & -0.524679 & 1.954905 & 0.304628 \\
\hline C & -1.764146 & 2.197455 & 0.986518 \\
\hline C & -1.724995 & 2.875443 & 2.188974 \\
\hline $\mathrm{H}$ & -2.655688 & 3.099470 & 2.704200 \\
\hline C & 2.768879 & 1.318314 & -0.060886 \\
\hline C & 1.919945 & 2.415907 & -0.086004 \\
\hline C & 4.259047 & 2.400266 & -1.650361 \\
\hline C & 3.955426 & 1.309311 & -0.868323 \\
\hline $\mathrm{H}$ & 5.162584 & 2.391372 & -2.255648 \\
\hline C & 3.410863 & 3.534958 & -1.707355 \\
\hline C & 2.214517 & 3.547001 & -0.924028 \\
\hline C & 1.369976 & 4.687741 & -1.009703 \\
\hline C & 1.696855 & 5.755650 & -1.816275 \\
\hline C & 2.884575 & 5.741517 & -2.583728 \\
\hline C & 3.721260 & 4.650507 & -2.529242 \\
\hline $\mathrm{H}$ & 0.451780 & 4.711937 & -0.433698 \\
\hline $\mathrm{H}$ & 1.034060 & 6.615354 & -1.866630 \\
\hline $\mathrm{H}$ & 3.130469 & 6.589712 & -3.216950 \\
\hline $\mathrm{H}$ & 4.634582 & 4.623291 & -3.119048 \\
\hline C & -0.509969 & 3.341913 & 2.749851 \\
\hline C & 0.709744 & 3.172468 & 2.017997 \\
\hline C & 1.914817 & 3.675434 & 2.580001 \\
\hline C & 1.912030 & 4.314719 & 3.799797 \\
\hline C & 0.704696 & 4.489349 & 4.517361 \\
\hline C & -0.478831 & 4.013226 & 4.001938 \\
\hline $\mathrm{H}$ & 2.845054 & 3.530301 & 2.042111 \\
\hline $\mathrm{H}$ & 2.844146 & 4.685052 & 4.217434 \\
\hline $\mathrm{H}$ & 0.716445 & 4.997513 & 5.477805 \\
\hline $\mathrm{H}$ & -1.410921 & 4.141801 & 4.547060 \\
\hline C & 6.661767 & -2.013582 & -0.894572 \\
\hline C & 7.027312 & -0.850677 & -0.202004 \\
\hline C & 6.120181 & 0.219673 & -0.199996 \\
\hline C & 4.889040 & 0.136669 & -0.864022 \\
\hline C & 4.564631 & -1.041917 & -1.547123 \\
\hline C & 5.441243 & -2.134574 & -1.576445 \\
\hline $\mathrm{H}$ & 7.347765 & -2.849245 & -0.905371 \\
\hline $\mathrm{H}$ & 6.362427 & 1.143534 & 0.317819 \\
\hline $\mathrm{H}$ & 3.614964 & -1.087708 & -2.072781 \\
\hline C & 8.370988 & -0.711540 & 0.539750 \\
\hline C & 5.046355 & -3.407317 & -2.351056 \\
\hline C & 8.106843 & -0.423644 & 2.037213 \\
\hline $\mathrm{H}$ & 9.055110 & -0.318196 & 2.578231 \\
\hline $\mathrm{H}$ & 7.537731 & 0.500408 & 2.182224 \\
\hline $\mathrm{H}$ & 7.542304 & -1.241269 & 2.500229 \\
\hline C & 9.233583 & -1.984511 & 0.444032 \\
\hline $\mathrm{H}$ & 10.173460 & -1.832558 & 0.986432 \\
\hline $\mathrm{H}$ & 8.732930 & -2.851996 & 0.889037 \\
\hline $\mathrm{H}$ & 9.488009 & -2.230148 & -0.593230 \\
\hline C & 9.174236 & 0.461137 & -0.072254 \\
\hline $\mathrm{H}$ & 10.131330 & 0.581565 & 0.449889 \\
\hline $\mathrm{H}$ & 9.386005 & 0.280224 & -1.132327 \\
\hline
\end{tabular}




\begin{tabular}{|c|c|c|c|}
\hline $\mathrm{H}$ & 8.632170 & 1.409582 & 0.002802 \\
\hline C & 4.848638 & -3.055812 & -3.845016 \\
\hline $\mathrm{H}$ & 4.555835 & -3.948644 & -4.410954 \\
\hline $\mathrm{H}$ & 4.068576 & -2.300809 & -3.986690 \\
\hline $\mathrm{H}$ & 5.774965 & -2.665216 & -4.281719 \\
\hline C & 6.114945 & -4.513120 & -2.256433 \\
\hline $\mathrm{H}$ & 5.783216 & -5.392988 & -2.819007 \\
\hline $\mathrm{H}$ & 7.073884 & -4.194003 & -2.680282 \\
\hline $\mathrm{H}$ & 6.285784 & -4.829633 & -1.221068 \\
\hline C & 3.723880 & -3.970456 & -1.778631 \\
\hline $\mathrm{H}$ & 3.428329 & -4.875866 & -2.322261 \\
\hline $\mathrm{H}$ & 3.834006 & -4.231227 & -0.719712 \\
\hline $\mathrm{H}$ & 2.902737 & -3.251093 & -1.863270 \\
\hline C & -3.077390 & 1.864798 & 0.361675 \\
\hline C & -4.112762 & 1.272071 & 1.097605 \\
\hline C & -3.323649 & 2.249104 & -0.964349 \\
\hline C & -5.377287 & 1.053884 & 0.534114 \\
\hline $\mathrm{H}$ & -3.916353 & 0.978333 & 2.124724 \\
\hline C & -4.572897 & 2.053699 & -1.564176 \\
\hline $\mathrm{H}$ & -2.528688 & 2.731557 & -1.521249 \\
\hline C & -5.579645 & 1.451077 & -0.796718 \\
\hline $\mathrm{H}$ & -6.550673 & 1.291307 & -1.243818 \\
\hline C & -4.798518 & 2.536385 & -3.011094 \\
\hline C & -6.501472 & 0.425849 & 1.382798 \\
\hline C & -6.223443 & 2.240751 & -3.516062 \\
\hline $\mathrm{H}$ & -6.446488 & 1.167366 & -3.504172 \\
\hline $\mathrm{H}$ & -6.985813 & 2.753132 & -2.917963 \\
\hline $\mathrm{H}$ & -6.327406 & 2.588337 & -4.550265 \\
\hline C & -4.576520 & 4.067159 & -3.075548 \\
\hline $\mathrm{H}$ & -4.748081 & 4.432757 & -4.095471 \\
\hline $\mathrm{H}$ & -5.266338 & 4.592222 & -2.404767 \\
\hline $\mathrm{H}$ & -3.556186 & 4.342237 & -2.790367 \\
\hline C & -3.794576 & 1.839140 & -3.960302 \\
\hline $\mathrm{H}$ & -2.756272 & 1.978280 & -3.644375 \\
\hline $\mathrm{H}$ & -3.984795 & 0.759976 & -4.006429 \\
\hline $\mathrm{H}$ & -3.895475 & 2.237533 & -4.977555 \\
\hline C & -6.029217 & -0.920874 & 1.976432 \\
\hline $\mathrm{H}$ & -5.756834 & -1.628463 & 1.185589 \\
\hline $\mathrm{H}$ & -5.160330 & -0.798485 & 2.630052 \\
\hline $\mathrm{H}$ & -6.829576 & -1.372418 & 2.575366 \\
\hline C & -6.859350 & 1.392705 & 2.537335 \\
\hline $\mathrm{H}$ & -5.995968 & 1.587546 & 3.182696 \\
\hline $\mathrm{H}$ & -7.210791 & 2.355503 & 2.148829 \\
\hline $\mathrm{H}$ & -7.654475 & 0.966588 & 3.161656 \\
\hline C & -7.779478 & 0.158515 & 0.563816 \\
\hline $\mathrm{H}$ & -8.535325 & -0.307813 & 1.205843 \\
\hline $\mathrm{H}$ & -8.214245 & 1.082141 & 0.165118 \\
\hline $\mathrm{H}$ & -7.589685 & -0.522422 & -0.273743 \\
\hline
\end{tabular}


Frequencies (Top 3 out of 450)

$\begin{array}{rr}\text { 1. } & -307.7750 \mathrm{~cm}^{-1} \\ \text { 2. } & 7.7924 \mathrm{~cm}^{-1} \\ \text { 3. } & 10.7381 \mathrm{~cm}^{-1}\end{array}$

\section{B3LYP/6-31G(d) Molecular Geometry in Cartesian Coordinates}

C

$\mathrm{H}$

$\mathrm{H}$

C

0

0

$\mathrm{H}$

0

$\mathrm{H}$

C

C

C

C

$\mathrm{H}$

$\mathrm{H}$

$\mathrm{H}$

C

C

C

$\mathrm{H}$

$\mathrm{H}$

$\mathrm{H}$

C

$\mathrm{H}$

$\mathrm{H}$

$\mathrm{H}$

C

$\mathrm{H}$

$\mathrm{H}$

$\mathrm{H}$

C

$\mathrm{H}$

\section{$-2.000005$}

$-2.167407$

$-1.000624$

1.189775

0.986474

1.305174

1.428951

$-2.888583$

$-3.792037$

2.443424

0.520188

3. 258030

3.121904

0.535613

4.007485

3. 362814

1. 512640

1.105298

2.934190

3. 252944

2. 976923

3. 642821

0.538540

0.460756

$-0.460661$

0.894433

$-0.415468$

$-0.940228$

$-0.835183$

$-0.606944$

1.639385

1.254834
$-1.073622$

0.005788

$-1.201805$

$-0.927279$

$-1.152535$

0.349598

0.517387

$-1.617971$

$-1.410925$

3. 625621

3. 393739

3.642308

3.033528

2. 310295

3. 176742

4. 729978

1.816679

3. 264667

1.438472

0.550963

1.210027

2.245130

0.714148

0.649360

0.885330

$-0.257807$

3. 499511

2.970389

3. 181289

4.571574

3. 684502

3.033155
$-1.258611$

$-1.140769$

$-1.671635$

1.150043

2. 323173

0.733936

$-0.232875$

$-2.234142$

$-1.942771$

2.004020

0.983173

$-0.331708$

0.961272

1.086002

$-0.974363$

$-0.320428$

$-2.929541$

$-3.383874$

$-3.374317$

$-2.819794$

$-4.445091$

- 3.165664

$-3.338468$

$-4.430006$

$-2.933548$

$-2.977300$

$-3.331093$

$-4.134491$

$-2.372011$

$-3.441504$

$-4.755001$

$-5.548962$ 


\begin{tabular}{|c|c|c|c|}
\hline $\mathrm{H}$ & 1.317659 & 4.708351 & -4.972890 \\
\hline $\mathrm{H}$ & 2.731491 & 3.665814 & -4.782917 \\
\hline 0 & 1.512400 & 1.941160 & -1.483368 \\
\hline 0 & 1.730748 & 4.081651 & -2.384246 \\
\hline B & 1.752282 & 3.349316 & -1.166811 \\
\hline 0 & 0.738098 & 3.910386 & -0.184960 \\
\hline C & -0.336240 & 4.136598 & 1.927472 \\
\hline C & -0.853339 & 3.465939 & 3.047367 \\
\hline C & -0.664199 & 5.484430 & 1.713098 \\
\hline C & -1.682376 & 4.136444 & 3.944610 \\
\hline $\mathrm{H}$ & -0.601134 & 2.420170 & 3.209053 \\
\hline C & -1.492959 & 6.149926 & 2.612813 \\
\hline $\mathrm{H}$ & -0.271059 & 5.989342 & 0.836450 \\
\hline C & -2.001623 & 5.479932 & 3.730058 \\
\hline $\mathrm{H}$ & -2.079794 & 3.611906 & 4.809022 \\
\hline $\mathrm{H}$ & -1.746330 & 7.192959 & 2.443254 \\
\hline $\mathrm{H}$ & -2.647778 & 6.003204 & 4.429703 \\
\hline $\mathrm{H}$ & 3.341060 & 1.969771 & 1.043225 \\
\hline $\mathrm{H}$ & 2.345732 & 4.706785 & 2.047409 \\
\hline $\mathrm{H}$ & 2.298233 & 3.095036 & 2.940609 \\
\hline C & 0.200756 & -2.763139 & -0.215512 \\
\hline C & 1.324123 & -2.004827 & 0.100807 \\
\hline C & 2.597658 & -2.251099 & -0.519805 \\
\hline C & 2.664658 & -3.218806 & -1.504616 \\
\hline $\mathrm{H}$ & 3.625265 & -3.450108 & -1.957034 \\
\hline C & -2.111190 & -1.783259 & 0.080533 \\
\hline C & -1.090380 & -2.602421 & 0.545426 \\
\hline C & -3.511162 & -2.422735 & 1.965154 \\
\hline C & -3.340741 & -1.681086 & 0.817162 \\
\hline $\mathrm{H}$ & -4.444632 & -2.345332 & 2.517978 \\
\hline C & -2.497463 & -3.281900 & 2.457704 \\
\hline C & -1.260548 & -3.373515 & 1.746941 \\
\hline C & -0.258935 & -4.244382 & 2.255466 \\
\hline C & -0.473455 & -4.984373 & 3. 396991 \\
\hline C & -1.698258 & -4.888744 & 4.098087 \\
\hline C & -2.687039 & -4.050900 & 3.636620 \\
\hline $\mathrm{H}$ & 0.689433 & -4.319409 & 1.735961 \\
\hline $\mathrm{H}$ & 0.307546 & -5.643458 & 3.766506 \\
\hline $\mathrm{H}$ & -1.853192 & -5.475104 & 4.999832 \\
\hline $\mathrm{H}$ & -3.632705 & -3.964931 & 4.166912 \\
\hline C & 1.533635 & -3.966675 & -1.910095 \\
\hline C & 0.280198 & -3.750408 & -1.253311 \\
\hline C & -0.841342 & -4.523186 & -1.662458 \\
\hline C & -0.723284 & -5.464549 & -2.660569 \\
\hline C & 0.519903 & -5.684817 & -3.300074 \\
\hline C & 1.623062 & -4.950033 & -2.932216 \\
\hline $\mathrm{H}$ & -1.802156 & -4.344869 & -1.194189 \\
\hline $\mathrm{H}$ & -1.593213 & -6.040310 & -2.963925 \\
\hline $\mathrm{H}$ & 0.598389 & -6.432363 & -4.084860 \\
\hline $\mathrm{H}$ & 2.582186 & -5.109029 & -3.419358 \\
\hline C & -6.613124 & 0.763470 & -0.469635 \\
\hline C & -6.775867 & -0.623808 & -0.451455 \\
\hline C & -5.680450 & -1.400994 & -0.035349 \\
\hline C & -4.469577 & -0.815596 & 0.347934 \\
\hline
\end{tabular}




\begin{tabular}{|c|c|c|c|}
\hline C & -4.351123 & 0.585499 & 0.318435 \\
\hline C & -5.413055 & 1.394590 & -0.090683 \\
\hline $\mathrm{H}$ & -7.441546 & 1.384898 & -0.787867 \\
\hline $\mathrm{H}$ & -5.758818 & -2.484129 & -0.007300 \\
\hline $\mathrm{H}$ & -3.416058 & 1.028797 & 0.641019 \\
\hline C & -8.086074 & -1.317865 & -0.870203 \\
\hline C & -5.318688 & 2.932238 & -0.131399 \\
\hline C & -8.631849 & -2.147566 & 0.316507 \\
\hline $\mathrm{H}$ & -9.563155 & -2.652186 & 0.031725 \\
\hline $\mathrm{H}$ & -7.921982 & -2.916726 & 0.638174 \\
\hline $\mathrm{H}$ & -8.843216 & -1.505619 & 1.179359 \\
\hline C & -9.175948 & -0.314033 & -1.292013 \\
\hline $\mathrm{H}$ & -10.083546 & -0.855924 & -1.580920 \\
\hline $\mathrm{H}$ & -9.443866 & 0.365506 & -0.474908 \\
\hline $\mathrm{H}$ & -8.865436 & 0.289911 & -2.152242 \\
\hline C & -7.809506 & -2.258404 & -2.067394 \\
\hline $\mathrm{H}$ & -8.732218 & -2.765726 & -2.374553 \\
\hline $\mathrm{H}$ & -7.429090 & -1.696450 & -2.928194 \\
\hline $\mathrm{H}$ & -7.072772 & -3.029678 & -1.819535 \\
\hline C & -5.614011 & 3.430378 & -1.566523 \\
\hline $\mathrm{H}$ & -5.549622 & 4.524443 & -1.608924 \\
\hline $\mathrm{H}$ & -4.889902 & 3.021084 & -2.280140 \\
\hline $\mathrm{H}$ & -6.615479 & 3.144155 & -1.904682 \\
\hline C & -6.357169 & 3.535506 & 0.844384 \\
\hline $\mathrm{H}$ & -6.312449 & 4.631121 & 0.818335 \\
\hline $\mathrm{H}$ & -7.379629 & 3.237196 & 0.589110 \\
\hline $\mathrm{H}$ & -6.161004 & 3.212245 & 1.873126 \\
\hline C & -3.924548 & 3.446858 & 0.271976 \\
\hline $\mathrm{H}$ & -3.904623 & 4.540780 & 0.219470 \\
\hline $\mathrm{H}$ & -3.660195 & 3.165823 & 1.297169 \\
\hline $\mathrm{H}$ & -3.142240 & 3.072842 & -0.398608 \\
\hline C & 3.851815 & -1.573442 & -0.078007 \\
\hline C & 4.810312 & -1.165060 & -1.024506 \\
\hline C & 4.136415 & -1.407261 & 1.281284 \\
\hline C & 6.022446 & -0.593122 & -0.634476 \\
\hline $\mathrm{H}$ & 4.583722 & -1.297598 & -2.075912 \\
\hline C & 5.336046 & -0.821473 & 1.716254 \\
\hline $\mathrm{H}$ & 3.419523 & -1.758663 & 2.014253 \\
\hline C & 6.256361 & -0.423448 & 0.743009 \\
\hline $\mathrm{H}$ & 7.190750 & 0.028044 & 1.055330 \\
\hline C & 5.590731 & -0.660766 & 3.227680 \\
\hline C & 7.099657 & -0.151298 & -1.644627 \\
\hline C & 6.949046 & -0.000735 & 3.529444 \\
\hline $\mathrm{H}$ & 7.787072 & -0.597833 & 3.151494 \\
\hline $\mathrm{H}$ & 7.020780 & 1.003851 & 3.096989 \\
\hline $\mathrm{H}$ & 7.077364 & 0.097763 & 4.613211 \\
\hline C & 4.476931 & 0.218936 & 3.845848 \\
\hline $\mathrm{H}$ & 4.626414 & 0.314043 & 4.928551 \\
\hline $\mathrm{H}$ & 4.493506 & 1.227041 & 3.414197 \\
\hline $\mathrm{H}$ & 3.479418 & -0.200468 & 3.679858 \\
\hline C & 5.574444 & -2.053927 & 3.901363 \\
\hline $\mathrm{H}$ & 4.612303 & -2.559396 & 3.771161 \\
\hline $\mathrm{H}$ & 6.354015 & -2.700941 & 3.482597 \\
\hline $\mathrm{H}$ & 5.755587 & -1.956715 & 4.978818 \\
\hline
\end{tabular}




$\begin{array}{llll}\mathrm{C} & 8.414456 & -0.916585 & -1.359128 \\ \mathrm{H} & 8.790473 & -0.723017 & -0.349341 \\ \mathrm{H} & 8.267110 & -1.998052 & -1.459294 \\ \mathrm{H} & 9.193724 & -0.612349 & -2.068672 \\ \mathrm{C} & 6.693400 & -0.435714 & -3.103068 \\ \mathrm{H} & 6.522033 & -1.504276 & -3.277347 \\ \mathrm{H} & 5.786770 & 0.108163 & -3.389932 \\ \mathrm{H} & 7.495986 & -0.116643 & -3.777544 \\ \mathrm{C} & 7.351940 & 1.368429 & -1.502281 \\ \mathrm{H} & 8.125538 & 1.694849 & -2.208181 \\ \mathrm{H} & 6.439708 & 1.938927 & -1.711693 \\ \mathrm{H} & 7.687440 & 1.633751 & -0.494295\end{array}$

\section{C-Catalyzed reaction TS 73}

Datum

Value

\begin{tabular}{lc}
\hline B3LYP/6-31G(d) Energy & -3038.95796 \\
\hline B3LYP-D3(BJ)/def2-TZVPP-IEF-PCM(DCM) Energy & -3040.508709 \\
\hline B3LYP-D3(BJ)/def2-TZVPP-IEF-PCM(DCM)//B3LYP/6-31G(d) Free Energy (Quasiharmonic) & -3039.280701 \\
\hline
\end{tabular}

Number of Imaginary Frequencies

Frequencies (Top 3 out of 450)

$\begin{array}{lr}\text { 1. } & -384.6848 \mathrm{~cm}^{-1} \\ \text { 2. } & 6.6585 \mathrm{~cm}^{-1} \\ \text { 3. } & 10.6041 \mathrm{~cm}^{-1}\end{array}$

\section{B3LYP/6-31G(d) Molecular Geometry in Cartesian Coordinates}

C

$\mathrm{H}$

$\mathrm{H}$

C

0

0

$\mathrm{H}$

0

$\mathrm{H}$

C

C

C

C

$\mathrm{H}$
$-1.320269$

$-0.762963$

$-2.321958$

1.881981

2. 591995

0.941578

0.414966

$-0.665857$

$-0.476798$

$-3.107762$

$-0.950233$

$-2.884003$

$-3.237936$

$-0.903316$
1.125146

1.479879

0.842108

1.324388

1.698203

0.383308

0.231491

$-0.027985$

$-0.653482$

$-4.334831$

$-4.200666$

$-2.893744$

$-3.109815$

$-5.204825$
0.874624

1.748635

1. 207293

$-1.596103$

$-2.501594$

$-1.776615$

$-0.942247$

0.317969

1.059424

$-0.218007$

$-0.253147$

1. 773771

0.412331

0.176543 


\begin{tabular}{|c|c|c|c|}
\hline $\mathrm{H}$ & -3.139611 & -1.911349 & 2.170642 \\
\hline H & -3.158927 & -3.704360 & 2.451339 \\
\hline C & 0.429347 & -2.091464 & 3.453834 \\
\hline C & -0.113718 & -3.474392 & 3.986434 \\
\hline C & 1.800480 & -2.209480 & 2.772503 \\
\hline $\mathrm{H}$ & 2.030791 & -1.271430 & 2.256579 \\
\hline H & 2.598252 & -2.397707 & 3.499172 \\
\hline $\mathrm{H}$ & 1.808500 & -3.015003 & 2.032969 \\
\hline C & 0.451846 & -0.960647 & 4.481601 \\
\hline $\mathrm{H}$ & -0.546541 & -0.755421 & 4.875057 \\
\hline $\mathrm{H}$ & 1.114010 & -1.206390 & 5.320049 \\
\hline $\mathrm{H}$ & 0.829211 & -0.042679 & 4.017775 \\
\hline C & -1.215056 & -3.312974 & 5.048109 \\
\hline $\mathrm{H}$ & -0.813152 & -2.964337 & 6.005857 \\
\hline $\mathrm{H}$ & -1.984912 & -2.607598 & 4.720592 \\
\hline $\mathrm{H}$ & -1.691361 & -4.285331 & 5.210952 \\
\hline C & 0.964226 & -4.427232 & 4.506117 \\
\hline $\mathrm{H}$ & 1.495333 & -3.995074 & 5.362469 \\
\hline $\mathrm{H}$ & 0.498960 & -5.362750 & 4.834649 \\
\hline $\mathrm{H}$ & 1.691648 & -4.669281 & 3.728092 \\
\hline 0 & -0.555445 & -1.766501 & 2.439105 \\
\hline 0 & -0.703754 & -4.058530 & 2.809611 \\
\hline B & -1.070081 & -3.007079 & 1.921468 \\
\hline 0 & -0.602696 & -3.213357 & 0.522288 \\
\hline C & -0.652961 & -4.066825 & -1.690785 \\
\hline C & -0.435640 & -2.801929 & -2.260520 \\
\hline C & -0.563722 & -5.213712 & -2.494805 \\
\hline C & -0.129566 & -2.689709 & -3.613846 \\
\hline $\mathrm{H}$ & -0.503492 & -1.917372 & -1.637623 \\
\hline C & -0.260524 & -5.096952 & -3.849904 \\
\hline $\mathrm{H}$ & -0.725157 & -6.195615 & -2.054743 \\
\hline C & -0.043105 & -3.835575 & -4.411331 \\
\hline $\mathrm{H}$ & 0.053907 & -1.706125 & -4.035958 \\
\hline $\mathrm{H}$ & -0.187294 & -5.988721 & -4.466344 \\
\hline $\mathrm{H}$ & 0.198710 & -3.746095 & -5.466924 \\
\hline $\mathrm{H}$ & -3.344471 & -2.229587 & -0.223595 \\
\hline $\mathrm{H}$ & -3.161075 & -5.246453 & 0.373464 \\
\hline $\mathrm{H}$ & -3.356657 & -4.439206 & -1.268879 \\
\hline C & 1.020755 & 2.722575 & 0.304793 \\
\hline C & 2.014924 & 1.889290 & -0.192844 \\
\hline C & 3.226798 & 1.652776 & 0.540494 \\
\hline C & 3.381303 & 2.274577 & 1.765165 \\
\hline $\mathrm{H}$ & 4.273941 & 2.075377 & 2.352368 \\
\hline C & -1.392231 & 2.213089 & -0.176402 \\
\hline C & -0.247934 & 2.952716 & -0.472006 \\
\hline C & -2.604099 & 3.366139 & -1.937650 \\
\hline C & -2.594902 & 2.423700 & -0.927315 \\
\hline H & -3.519229 & 3.530876 & -2.501245 \\
\hline C & -1.465337 & 4.143963 & -2.252428 \\
\hline C & -0.259302 & 3.939903 & -1.509467 \\
\hline C & 0.878512 & 4.729613 & -1.836988 \\
\hline C & 0.818961 & 5.672683 & -2.837537 \\
\hline C & -0.377853 & 5.876212 & -3.566423 \\
\hline C & -1.493909 & 5.125767 & -3.279772 \\
\hline
\end{tabular}




\begin{tabular}{|c|c|c|c|}
\hline H & 1.805539 & 4.570130 & -1.297946 \\
\hline $\mathrm{H}$ & 1.700179 & 6.261746 & -3.075819 \\
\hline $\mathrm{H}$ & -0.408789 & 6.623177 & -4.355124 \\
\hline $\mathrm{H}$ & -2.417363 & 5.270807 & -3.835547 \\
\hline C & 2.409003 & 3.155961 & 2.296674 \\
\hline C & 1.207765 & 3.396414 & 1.557590 \\
\hline C & 0.249197 & 4.297122 & 2.099441 \\
\hline C & 0.463065 & 4.915255 & 3.312026 \\
\hline C & 1.646134 & 4.664817 & 4.047282 \\
\hline C & 2.596069 & 3.803615 & 3.548086 \\
\hline $\mathrm{H}$ & -0.659232 & 4.496920 & 1.540874 \\
\hline H & -0.280759 & 5.602053 & 3.706943 \\
\hline $\mathrm{H}$ & 1.802750 & 5.157219 & 5.003256 \\
\hline $\mathrm{H}$ & 3.510934 & 3.608429 & 4.102760 \\
\hline C & -6.278963 & 0.354170 & -0.275629 \\
\hline C & -5.593481 & 0.147820 & -1.480832 \\
\hline C & -4.374156 & 0.816764 & -1.656755 \\
\hline C & -3.858611 & 1.664765 & -0.667936 \\
\hline C & -4.576812 & 1.842311 & 0.522781 \\
\hline C & -5.795829 & 1.189817 & 0.742322 \\
\hline $\mathrm{H}$ & -7.228824 & -0.149909 & -0.125402 \\
\hline H & -3.801841 & 0.691231 & -2.568730 \\
\hline $\mathrm{H}$ & -4.175379 & 2.518935 & 1.269187 \\
\hline C & -6.205069 & -0.760196 & -2.566658 \\
\hline C & -6.612505 & 1.368464 & 2.037365 \\
\hline C & -6.541719 & -2.148756 & -1.974498 \\
\hline $\mathrm{H}$ & -6.975549 & -2.794653 & -2.747483 \\
\hline H & -5.643697 & -2.642461 & -1.586038 \\
\hline $\mathrm{H}$ & -7.265606 & -2.084015 & -1.155664 \\
\hline C & -7.504955 & -0.105970 & -3.094410 \\
\hline $\mathrm{H}$ & -7.964326 & -0.734590 & -3.867346 \\
\hline $\mathrm{H}$ & -8.240545 & 0.034750 & -2.295162 \\
\hline $\mathrm{H}$ & -7.297984 & 0.876375 & -3.534043 \\
\hline C & -5.252351 & -0.970556 & -3.759531 \\
\hline $\mathrm{H}$ & -5.718958 & -1.640879 & -4.490304 \\
\hline $\mathrm{H}$ & -5.026464 & -0.030783 & -4.275197 \\
\hline $\mathrm{H}$ & -4.304063 & -1.424985 & -3.450077 \\
\hline C & -6.806566 & -0.007112 & 2.719012 \\
\hline $\mathrm{H}$ & -7.341895 & -0.711671 & 2.074049 \\
\hline $\mathrm{H}$ & -5.840810 & -0.457929 & 2.975584 \\
\hline $\mathrm{H}$ & -7.385981 & 0.103886 & 3.643743 \\
\hline C & -5.918530 & 2.305240 & 3.044409 \\
\hline $\mathrm{H}$ & -6.531390 & 2.393697 & 3.948523 \\
\hline $\mathrm{H}$ & -4.936671 & 1.923677 & 3.346994 \\
\hline $\mathrm{H}$ & -5.783198 & 3.313936 & 2.638091 \\
\hline C & -7.996365 & 1.970448 & 1.696868 \\
\hline $\mathrm{H}$ & -8.592546 & 2.097247 & 2.609018 \\
\hline $\mathrm{H}$ & -7.889832 & 2.951901 & 1.220971 \\
\hline $\mathrm{H}$ & -8.563999 & 1.328685 & 1.014984 \\
\hline C & 4.301021 & 0.745367 & 0.045830 \\
\hline C & 5.646902 & 1.134297 & 0.165541 \\
\hline C & 4.007436 & -0.505474 & -0.502478 \\
\hline C & 6.685409 & 0.299077 & -0.246766 \\
\hline H & 5.860966 & 2.119014 & 0.564044 \\
\hline
\end{tabular}




\begin{tabular}{|c|c|c|c|}
\hline C & 5.020077 & -1.370698 & -0.944345 \\
\hline $\mathrm{H}$ & 2.970410 & -0.810360 & -0.591844 \\
\hline C & 6.344479 & -0.949778 & -0.797629 \\
\hline $\mathrm{H}$ & 7.143628 & -1.603100 & -1.128097 \\
\hline C & 4.637426 & -2.715386 & -1.592058 \\
\hline C & 8.169184 & 0.699595 & -0.132682 \\
\hline C & 5.869150 & -3.562065 & -1.964501 \\
\hline $\mathrm{H}$ & 5.543107 & -4.506431 & -2.415108 \\
\hline $\mathrm{H}$ & 6.508392 & -3.053847 & -2.695243 \\
\hline $\mathrm{H}$ & 6.478651 & -3.806565 & -1.086485 \\
\hline C & 3.767183 & -3.542710 & -0.616912 \\
\hline $\mathrm{H}$ & 3.494582 & -4.502048 & -1.073849 \\
\hline $\mathrm{H}$ & 4.308523 & -3.750390 & 0.313768 \\
\hline $\mathrm{H}$ & 2.837332 & -3.027024 & -0.358150 \\
\hline C & 3.833355 & -2.434694 & -2.884752 \\
\hline $\mathrm{H}$ & 2.930615 & -1.849243 & -2.686247 \\
\hline $\mathrm{H}$ & 4.439727 & -1.873019 & -3.604450 \\
\hline $\mathrm{H}$ & 3.526628 & -3.377097 & -3.355652 \\
\hline C & 8.907277 & -0.315335 & 0.772472 \\
\hline $\mathrm{H}$ & 8.843916 & -1.334925 & 0.378279 \\
\hline $\mathrm{H}$ & 8.480989 & -0.321176 & 1.782388 \\
\hline $\mathrm{H}$ & 9.969764 & -0.053870 & 0.853229 \\
\hline C & 8.816097 & 0.697968 & -1.538314 \\
\hline $\mathrm{H}$ & 8.315406 & 1.414339 & -2.199333 \\
\hline $\mathrm{H}$ & 8.761040 & -0.287408 & -2.012314 \\
\hline $\mathrm{H}$ & 9.875100 & 0.977276 & -1.471814 \\
\hline C & 8.355646 & 2.103276 & 0.474111 \\
\hline $\mathrm{H}$ & 9.424269 & 2.338624 & 0.535870 \\
\hline $\mathrm{H}$ & 7.944497 & 2.167851 & 1.488112 \\
\hline $\mathrm{H}$ & 7.881080 & 2.878662 & -0.137713 \\
\hline
\end{tabular}

\section{C-Catalyzed reaction TS 74}

\section{Datum}

Value

\begin{tabular}{lc}
\hline B3LYP/6-31G(d) Energy & -3038.959032 \\
\hline B3LYP-D3(BJ)/def2-TZVPP-IEF-PCM(DCM) Energy & -3040.509453 \\
\hline B3LYP-D3(BJ)/def2-TZVPP-IEF-PCM(DCM)//B3LYP/6-31G(d) Free Energy (Quasiharmonic) & -3039.28067 \\
\hline
\end{tabular}

Number of Imaginary Frequencies

Frequencies (Top 3 out of 450)

$\begin{array}{lr}\text { 1. } & -312.7641 \mathrm{~cm}^{-1} \\ \text { 2. } & 8.5237 \mathrm{~cm}^{-1} \\ \text { 3. } & 10.5443 \mathrm{~cm}^{-1}\end{array}$




\begin{tabular}{|c|c|c|c|}
\hline C & -2.750834 & -2.097765 & -0.247636 \\
\hline $\mathrm{H}$ & -1.793760 & -2.429934 & 0.163421 \\
\hline $\mathrm{H}$ & -3.473656 & -2.092269 & 0.568376 \\
\hline C & 0.583141 & -0.526995 & 0.644308 \\
\hline 0 & -0.284763 & -0.673299 & 1.481852 \\
\hline 0 & 1.487424 & 0.448721 & 0.770794 \\
\hline $\mathrm{H}$ & 2.146372 & 0.524980 & 0.035208 \\
\hline 0 & -3.257379 & -3.050649 & -1.195551 \\
\hline $\mathrm{H}$ & -2.594511 & -3.143198 & -1.898965 \\
\hline C & 3.046127 & 4.394924 & 2.667805 \\
\hline C & 2.408524 & 4.614622 & 0.573893 \\
\hline C & 4.105201 & 2.361271 & 1.739066 \\
\hline C & 4.166102 & 3.676539 & 2.309728 \\
\hline $\mathrm{H}$ & 3.235360 & 5.282584 & 0.324922 \\
\hline $\mathrm{H}$ & 3.289735 & 1.734131 & 2.104541 \\
\hline $\mathrm{H}$ & 5.056926 & 1.826823 & 1.727846 \\
\hline C & 4.586723 & 1.317401 & -1.689716 \\
\hline C & 5.018336 & 2.820255 & -1.892651 \\
\hline C & 5.710858 & 0.452479 & -1.098143 \\
\hline $\mathrm{H}$ & 5.281114 & -0.489706 & -0.744882 \\
\hline $\mathrm{H}$ & 6.484407 & 0.226509 & -1.840826 \\
\hline $\mathrm{H}$ & 6.187141 & 0.946930 & -0.246380 \\
\hline C & 4.011161 & 0.635478 & -2.930935 \\
\hline $\mathrm{H}$ & 4.758376 & 0.585955 & -3.731581 \\
\hline $\mathrm{H}$ & 3.710780 & -0.389853 & -2.688348 \\
\hline $\mathrm{H}$ & 3.133574 & 1.164194 & -3.309688 \\
\hline C & 4.099350 & 3.577023 & -2.867452 \\
\hline $\mathrm{H}$ & 4.248438 & 3.256375 & -3.904763 \\
\hline $\mathrm{H}$ & 3.046366 & 3.438807 & -2.605674 \\
\hline $\mathrm{H}$ & 4.327313 & 4.646472 & -2.806229 \\
\hline C & 6.478201 & 3.021238 & -2.302624 \\
\hline $\mathrm{H}$ & 6.688749 & 2.549216 & -3.269708 \\
\hline $\mathrm{H}$ & 6.686961 & 4.092176 & -2.398624 \\
\hline $\mathrm{H}$ & 7.164118 & 2.612878 & -1.556804 \\
\hline 0 & 3.541933 & 1.434564 & -0.697510 \\
\hline 0 & 4.837884 & 3.369778 & -0.574371 \\
\hline B & 3.769252 & 2.651246 & 0.049920 \\
\hline 0 & 2.460060 & 3.403971 & 0.126313 \\
\hline C & 1.081776 & 5.218700 & 0.787205 \\
\hline C & -0.071061 & 4.417105 & 0.814170 \\
\hline C & 0.966808 & 6.609365 & 0.936099 \\
\hline C & -1.319373 & 5.006428 & 0.993216 \\
\hline $\mathrm{H}$ & 0.027094 & 3.343944 & 0.686412 \\
\hline C & -0.284971 & 7.193370 & 1.114869 \\
\hline $\mathrm{H}$ & 1.859963 & 7.229856 & 0.908051 \\
\hline C & -1.429491 & 6.392070 & 1.146124 \\
\hline $\mathrm{H}$ & -2.208348 & 4.382625 & 1.008191 \\
\hline $\mathrm{H}$ & -0.369397 & 8.270751 & 1.226819 \\
\hline $\mathrm{H}$ & -2.406160 & 6.847609 & 1.285967 \\
\hline $\mathrm{H}$ & 5.110563 & 4.214869 & 2.220036 \\
\hline
\end{tabular}




\begin{tabular}{|c|c|c|c|}
\hline $\mathrm{H}$ & 2.129576 & 3.875095 & 2.930487 \\
\hline $\mathrm{H}$ & 3.131636 & 5.417094 & 3.026387 \\
\hline C & -0.282906 & -1.308149 & -1.556179 \\
\hline C & 0.690326 & -1.426591 & -0.569545 \\
\hline C & 1.725042 & -2.422187 & -0.650300 \\
\hline C & 1.770055 & -3.223315 & -1.777405 \\
\hline $\mathrm{H}$ & 2.521075 & -4.005849 & -1.841725 \\
\hline C & -2.609171 & -0.699981 & -0.828688 \\
\hline C & -1.414053 & -0.319581 & -1.435768 \\
\hline C & -3.538611 & 1.502097 & -1.275858 \\
\hline C & -3.701351 & 0.231901 & -0.754620 \\
\hline $\mathrm{H}$ & -4.369359 & 2.202318 & -1.234713 \\
\hline C & -2.342201 & 1.913273 & -1.907510 \\
\hline C & -1.253566 & 0.988495 & -1.997633 \\
\hline C & -0.066224 & 1.409544 & -2.658759 \\
\hline C & 0.032469 & 2.669618 & -3.205873 \\
\hline C & -1.045823 & 3.581950 & -3.113402 \\
\hline C & -2.205600 & 3.209502 & -2.475024 \\
\hline $\mathrm{H}$ & 0.769539 & 0.722172 & -2.732254 \\
\hline $\mathrm{H}$ & 0.944880 & 2.967382 & -3.714815 \\
\hline $\mathrm{H}$ & -0.953324 & 4.573931 & -3.547149 \\
\hline $\mathrm{H}$ & -3.042015 & 3.900528 & -2.399450 \\
\hline C & 0.832596 & -3.106222 & -2.830140 \\
\hline C & -0.221057 & -2.143626 & -2.720917 \\
\hline C & -1.173639 & -2.060908 & -3.777523 \\
\hline C & -1.086538 & -2.888807 & -4.875998 \\
\hline C & -0.044104 & -3.840804 & -4.978464 \\
\hline C & 0.892979 & -3.944655 & -3.976604 \\
\hline H & -1.970275 & -1.327627 & -3.711939 \\
\hline $\mathrm{H}$ & -1.822577 & -2.808828 & -5.671145 \\
\hline H & 0.012181 & -4.488320 & -5.849111 \\
\hline H & 1.695801 & -4.674989 & -4.044339 \\
\hline C & -7.523046 & -0.649059 & 0.956105 \\
\hline C & -6.835422 & 0.504088 & 1.377722 \\
\hline C & -5.582010 & 0.756368 & 0.817842 \\
\hline C & -5.017945 & -0.108585 & -0.137359 \\
\hline C & -5.733531 & -1.243574 & -0.527917 \\
\hline C & -6.994706 & -1.536444 & 0.014634 \\
\hline H & -8.496230 & -0.855533 & 1.386518 \\
\hline H & -5.005273 & 1.620369 & 1.127437 \\
\hline H & -5.286896 & -1.909253 & -1.257848 \\
\hline C & -7.477824 & 1.427827 & 2.431215 \\
\hline C & -7.726844 & -2.814336 & -0.438023 \\
\hline C & -8.815033 & 1.981009 & 1.883372 \\
\hline H & -9.292750 & 2.632246 & 2.626013 \\
\hline H & -8.651704 & 2.567347 & 0.971660 \\
\hline H & -9.519913 & 1.178877 & 1.640942 \\
\hline C & -7.748611 & 0.628010 & 3.727822 \\
\hline H & -8.212153 & 1.272796 & 4.484920 \\
\hline H & -8.422716 & -0.216949 & 3.553395 \\
\hline $\mathrm{H}$ & -6.816214 & 0.230045 & 4.144068 \\
\hline C & -6.577764 & 2.625228 & 2.791012 \\
\hline H & -7.077113 & 3.250620 & 3.539906 \\
\hline H & -5.620285 & 2.302091 & 3.214812 \\
\hline
\end{tabular}




\begin{tabular}{|c|c|c|c|}
\hline $\mathrm{H}$ & -6.370935 & 3.256179 & 1.918951 \\
\hline C & -6.860127 & -4.047122 & -0.082419 \\
\hline $\mathrm{H}$ & -7.354496 & -4.968304 & -0.416300 \\
\hline $\mathrm{H}$ & -5.872056 & -4.003273 & -0.551257 \\
\hline $\mathrm{H}$ & -6.712750 & -4.117061 & 1.001838 \\
\hline C & -9.100976 & -2.979661 & 0.237804 \\
\hline $\mathrm{H}$ & -9.577747 & -3.900001 & -0.118300 \\
\hline $\mathrm{H}$ & -9.014013 & -3.054847 & 1.327868 \\
\hline $\mathrm{H}$ & -9.775007 & -2.146987 & 0.004504 \\
\hline C & -7.947731 & -2.769674 & -1.968730 \\
\hline $\mathrm{H}$ & -8.462867 & -3.678012 & -2.305371 \\
\hline $\mathrm{H}$ & -8.561043 & -1.906323 & -2.252418 \\
\hline $\mathrm{H}$ & -7.001054 & -2.702960 & -2.514113 \\
\hline C & 2.698803 & -2.671185 & 0.453094 \\
\hline C & 4.041937 & -2.973784 & 0.156329 \\
\hline C & 2.286050 & -2.689547 & 1.788193 \\
\hline C & 4.958626 & -3.283941 & 1.162212 \\
\hline $\mathrm{H}$ & 4.357131 & -2.952813 & -0.880530 \\
\hline C & 3.181761 & -2.967974 & 2.832181 \\
\hline $\mathrm{H}$ & 1.245808 & -2.497354 & 2.019747 \\
\hline C & 4.504257 & -3.263345 & 2.494326 \\
\hline $\mathrm{H}$ & 5.211038 & -3.488280 & 3.284330 \\
\hline C & 2.671639 & -2.935891 & 4.285358 \\
\hline C & 6.429362 & -3.641157 & 0.869055 \\
\hline C & 2.116088 & -1.524506 & 4.596764 \\
\hline $\mathrm{H}$ & 2.904009 & -0.767719 & 4.504611 \\
\hline $\mathrm{H}$ & 1.302735 & -1.241395 & 3.921065 \\
\hline $\mathrm{H}$ & 1.729872 & -1.488451 & 5.623074 \\
\hline C & 1.543426 & -3.980471 & 4.460441 \\
\hline $\mathrm{H}$ & 1.170674 & -3.966235 & 5.491939 \\
\hline $\mathrm{H}$ & 0.694805 & -3.779902 & 3.798996 \\
\hline $\mathrm{H}$ & 1.907187 & -4.991899 & 4. 244471 \\
\hline C & 3.780660 & -3.254040 & 5.305813 \\
\hline $\mathrm{H}$ & 4.194194 & -4.259219 & 5.162269 \\
\hline $\mathrm{H}$ & 4.604477 & -2.532959 & 5.251595 \\
\hline $\mathrm{H}$ & 3.370303 & -3.209000 & 6.320887 \\
\hline C & 7.359594 & -2.604500 & 1.542743 \\
\hline $\mathrm{H}$ & 7.168495 & -1.596906 & 1.156432 \\
\hline $\mathrm{H}$ & 7.219946 & -2.577752 & 2.628489 \\
\hline $\mathrm{H}$ & 8.410839 & -2.850617 & 1.347709 \\
\hline C & 6.738651 & -5.048327 & 1.434465 \\
\hline $\mathrm{H}$ & 6.580572 & -5.099559 & 2.516508 \\
\hline $\mathrm{H}$ & 6.099969 & -5.806983 & 0.967631 \\
\hline $\mathrm{H}$ & 7.784134 & -5.316376 & 1.237782 \\
\hline C & 6.743049 & -3.660796 & -0.639145 \\
\hline $\mathrm{H}$ & 7.791896 & -3.938974 & -0.792745 \\
\hline $\mathrm{H}$ & 6.126208 & -4.392823 & -1.173094 \\
\hline $\mathrm{H}$ & 6.591521 & -2.680426 & -1.103879 \\
\hline
\end{tabular}




\begin{tabular}{lc}
\hline B3LYP/6-31G(d) Energy & -3038.958972 \\
\hline B3LYP-D3(BJ)/def2-TZVPP-IEF-PCM(DCM) Energy & -3040.509206 \\
\hline B3LYP-D3(BJ)/def2-TZVPP-IEF-PCM(DCM)//B3LYP/6-31G(d) Free Energy (Quasiharmonic) & -3039.280653 \\
\hline
\end{tabular}

Number of Imaginary Frequencies

Frequencies (Top 3 out of 450)

$\begin{array}{lr}\text { 1. } & -317.2502 \mathrm{~cm}^{-1} \\ \text { 2. } & 7.2108 \mathrm{~cm}^{-1} \\ \text { 3. } & 10.1372 \mathrm{~cm}^{-1}\end{array}$

\section{B3LYP/6-31G(d) Molecular Geometry in Cartesian Coordinates}

C

$\mathrm{H}$

$\mathrm{H}$

C

0

0

$\mathrm{H}$

0

$\mathrm{H}$

C

C

C

C

$\mathrm{H}$

$\mathrm{H}$

$\mathrm{H}$

C

C

C

$\mathrm{H}$

$\mathrm{H}$

$\mathrm{H}$

C

$\mathrm{H}$

$\mathrm{H}$

$\mathrm{H}$

C

$\mathrm{H}$

$\mathrm{H}$

$\mathrm{H}$

C

$\mathrm{H}$

-2.164492
-1.107056
-2.719903
1.009638
0.919293
1.124894
1.080793
-2.633367
-2.131259
0.068941
1.799651
-0.864710
-0.815254
1.529216
-0.647145
-1.751991
0.922530
0.194700
0.429935
0.992471
0.591756
-0.631502
2.451643
2.784557
2.897141
2.832103
0.879702
0.915080
1.898488
0.316215
-1.288039
-1.412225

\section{$-2.164492$}

$-0.610557$

$-0.344655$

0.308203

$-1.086873$

$-1.433974$

0.212210

0.406409

$-1.065379$

$-1.865610$

4.828168

4.439428

2.951298

4.333223

5.210610

2.239680

2.668907

1.488610

2.808565

0.209373

$-0.651128$

0.260943

0.029660

1.582838

1.531623

0.745663

2.507779

3.541739

2.917502

3.838876

4.449356

2.584050

2.054752
0.888031

0.952672

0.696545

$-1.202494$

$-2.359962$

$-0.882571$

0.088796

2.169977

2.396739

$-1.412033$

$-0.121529$

$-0.096495$

$-0.477579$

0.602108

$-0.895572$

0.472959

2.922480

3. 385001

3. 597735

3. 218783

4.681169

3.411943

3.032953

4.075572

2.487600

2.591095

4.540685

5.441366

4.280599

4.782766

3. 726602

4.677728 


\begin{tabular}{|c|c|c|c|}
\hline $\mathrm{H}$ & -1.779074 & 3.559252 & 3.810268 \\
\hline $\mathrm{H}$ & -1.798994 & 2.007437 & 2.950233 \\
\hline 0 & 0.579486 & 1.438694 & 1.510712 \\
\hline 0 & 0.264417 & 3.641249 & 2.212709 \\
\hline B & 0.430502 & 2.803676 & 1.066471 \\
\hline 0 & 1.625982 & 3.206507 & 0.225851 \\
\hline C & 2.884183 & 4.745252 & -1.071970 \\
\hline C & 3.476755 & 3.724572 & -1.833616 \\
\hline C & 3.346787 & 6.063793 & -1.195931 \\
\hline C & 4.516320 & 4.028775 & -2.707985 \\
\hline H & 3.116181 & 2.706249 & -1.724056 \\
\hline C & 4.387866 & 6.362129 & -2.072249 \\
\hline $\mathrm{H}$ & 2.890982 & 6.852224 & -0.600959 \\
\hline C & 4.972933 & 5.345074 & -2.830627 \\
\hline $\mathrm{H}$ & 4.975369 & 3.237055 & -3.293450 \\
\hline $\mathrm{H}$ & 4.744676 & 7.384378 & -2.161698 \\
\hline $\mathrm{H}$ & 5.785135 & 5.576853 & -3.514270 \\
\hline $\mathrm{H}$ & -1.314743 & 5.046517 & 0.178947 \\
\hline $\mathrm{H}$ & 0.455867 & 4.177110 & -2.190309 \\
\hline $\mathrm{H}$ & 0.122088 & 5.894141 & -1.616371 \\
\hline C & -0.177957 & -2.691703 & 0.300844 \\
\hline C & 1.012608 & -2.060615 & -0.041403 \\
\hline C & 2.233788 & -2.320203 & 0.672962 \\
\hline C & 2.179798 & -3.149245 & 1.777986 \\
\hline $\mathrm{H}$ & 3.097472 & -3.386286 & 2.309650 \\
\hline C & -2.396132 & -1.598943 & -0.239940 \\
\hline C & -1.423513 & -2.550175 & -0.536846 \\
\hline C & -3.770156 & -2.424897 & -2.067268 \\
\hline C & -3.603559 & -1.539359 & -1.020452 \\
\hline $\mathrm{H}$ & -4.687406 & -2.390823 & -2.649862 \\
\hline C & -2.801580 & -3.403194 & -2.392746 \\
\hline C & -1.603540 & -3.478536 & -1.616369 \\
\hline C & -0.646029 & -4.474721 & -1.952889 \\
\hline C & -0.866431 & -5.347398 & -2.994637 \\
\hline C & -2.054958 & -5.271130 & -3.759251 \\
\hline C & -3.000092 & -4.317172 & -3.462895 \\
\hline $\mathrm{H}$ & 0.273760 & -4.538901 & -1.382852 \\
\hline H & -0.119449 & -6.098943 & -3.235622 \\
\hline $\mathrm{H}$ & -2.215637 & -5.965111 & -4.579993 \\
\hline H & -3.916872 & -4.245816 & -4.043593 \\
\hline C & 0.976118 & -3.755182 & 2.210957 \\
\hline C & -0.219829 & -3.551061 & 1.450749 \\
\hline C & -1.407445 & -4.217034 & 1.871222 \\
\hline C & -1.412172 & -5.019141 & 2.992558 \\
\hline C & -0.229543 & -5.209703 & 3.745724 \\
\hline C & 0.938265 & -4.594752 & 3.357144 \\
\hline $\mathrm{H}$ & -2.315457 & -4.091934 & 1.290872 \\
\hline H & -2.329748 & -5.515615 & 3.295867 \\
\hline $\mathrm{H}$ & -0.246256 & -5.845916 & 4.626285 \\
\hline H & 1.854810 & -4.741679 & 3.923605 \\
\hline C & -6.791988 & 1.226557 & -0.315207 \\
\hline C & -6.309378 & 0.466678 & 0.766236 \\
\hline C & -5.260428 & -0.422797 & 0.527751 \\
\hline C & -4.692865 & -0.553700 & -0.752138 \\
\hline
\end{tabular}




\begin{tabular}{|c|c|c|c|}
\hline C & -5.201560 & 0.220582 & -1.799614 \\
\hline C & -6.258900 & 1.123198 & -1.603274 \\
\hline $\mathrm{H}$ & -7.605908 & 1.919422 & -0.136061 \\
\hline $\mathrm{H}$ & -4.855683 & -1.025062 & 1.330610 \\
\hline $\mathrm{H}$ & -4.746247 & 0.123413 & -2.781030 \\
\hline C & -6.933029 & 0.645936 & 2.164160 \\
\hline C & -6.779325 & 1.955374 & -2.791555 \\
\hline C & -6.319539 & -0.310228 & 3.205546 \\
\hline $\mathrm{H}$ & -6.785209 & -0.136305 & 4.182671 \\
\hline $\mathrm{H}$ & -6.490974 & -1.359793 & 2.939575 \\
\hline $\mathrm{H}$ & -5.240157 & -0.162835 & 3.316112 \\
\hline C & -6.694345 & 2.097845 & 2.643506 \\
\hline $\mathrm{H}$ & -7.141027 & 2.253190 & 3.633654 \\
\hline $\mathrm{H}$ & -5.622085 & 2.312242 & 2.717717 \\
\hline $\mathrm{H}$ & -7.135957 & 2.830241 & 1.959059 \\
\hline C & -8.454166 & 0.371902 & 2.099615 \\
\hline $\mathrm{H}$ & -8.906099 & 0.493832 & 3.091842 \\
\hline $\mathrm{H}$ & -8.967618 & 1.055890 & 1.415632 \\
\hline $\mathrm{H}$ & -8.652969 & -0.651228 & 1.760271 \\
\hline C & -5.632989 & 2.832765 & -3.348598 \\
\hline $\mathrm{H}$ & -5.985523 & 3.426471 & -4.201266 \\
\hline $\mathrm{H}$ & -5.261819 & 3.523991 & -2.583140 \\
\hline $\mathrm{H}$ & -4.786164 & 2.229150 & -3.690823 \\
\hline C & -7.281296 & 1.005356 & -3.905037 \\
\hline $\mathrm{H}$ & -7.651465 & 1.582524 & -4.761477 \\
\hline $\mathrm{H}$ & -6.485542 & 0.346027 & -4.266907 \\
\hline $\mathrm{H}$ & -8.099724 & 0.373435 & -3.541320 \\
\hline C & -7.943041 & 2.884422 & -2.397090 \\
\hline $\mathrm{H}$ & -8.277307 & 3.450646 & -3.274006 \\
\hline $\mathrm{H}$ & -8.804706 & 2.322127 & -2.019434 \\
\hline $\mathrm{H}$ & -7.645149 & 3.608929 & -1.630589 \\
\hline C & 3.558255 & -1.810113 & 0.210770 \\
\hline C & 4.511563 & -1.354399 & 1.128788 \\
\hline C & 3.904591 & -1.871719 & -1.150768 \\
\hline C & 5.794263 & -0.953554 & 0.721502 \\
\hline $\mathrm{H}$ & 4.241189 & -1.308979 & 2.179611 \\
\hline C & 5.161266 & -1.464530 & -1.599504 \\
\hline $\mathrm{H}$ & 3.179378 & -2.258381 & -1.853308 \\
\hline C & 6.087450 & -1.009653 & -0.644508 \\
\hline $\mathrm{H}$ & 7.066640 & -0.695380 & -0.985741 \\
\hline C & 5.551449 & -1.499720 & -3.089703 \\
\hline C & 6.822211 & -0.484498 & 1.771010 \\
\hline C & 5.858080 & -0.059245 & -3.565917 \\
\hline $\mathrm{H}$ & 4.971370 & 0.577371 & -3.463845 \\
\hline $\mathrm{H}$ & 6.669959 & 0.397360 & -2.989261 \\
\hline $\mathrm{H}$ & 6.155913 & -0.061902 & -4.621737 \\
\hline C & 6.808709 & -2.380050 & -3.282359 \\
\hline $\mathrm{H}$ & 7.092169 & -2.411301 & -4.341602 \\
\hline $\mathrm{H}$ & 7.668501 & -2.000352 & -2.720076 \\
\hline $\mathrm{H}$ & 6.620275 & -3.407923 & -2.951763 \\
\hline C & 4.426328 & -2.067510 & -3.976618 \\
\hline $\mathrm{H}$ & 4.193464 & -3.107043 & -3.719129 \\
\hline $\mathrm{H}$ & 3.502171 & -1.485433 & -3.897273 \\
\hline $\mathrm{H}$ & 4.743473 & -2.051752 & -5.025769 \\
\hline
\end{tabular}




\begin{tabular}{|llll|} 
C & 8.177585 & -0.110110 & 1.141161 \\
$H$ & 8.633583 & -0.957787 & 0.617135 \\
$H$ & 8.083398 & 0.720625 & 0.432608 \\
H & 8.873639 & 0.204941 & 1.926787 \\
C & 6.281403 & 0.758864 & 2.514602 \\
$H$ & 6.093537 & 1.582376 & 1.816375 \\
$H$ & 5.344219 & 0.546406 & 3.038242 \\
$H$ & 7.008871 & 1.103605 & 3.260083 \\
C & 7.068224 & -1.622173 & 2.790928 \\
$H$ & 7.798976 & -1.306658 & 3.545750 \\
$H$ & 6.149507 & -1.904588 & 3.315849 \\
$H$ & 7.457978 & -2.517742 & 2.293652 \\
\hline
\end{tabular}

\section{C-Catalyzed reaction TS 76}

\section{Datum}

Value

\begin{tabular}{lc}
\hline B3LYP/6-31G(d) Energy & -3038.955493 \\
\hline B3LYP-D3(BJ)/def2-TZVPP-IEF-PCM(DCM) Energy & -3040.508247 \\
\hline B3LYP-D3(BJ)/def2-TZVPP-IEF-PCM(DCM)//B3LYP/6-31G(d) Free Energy (Quasiharmonic) & -3039.280635 \\
\hline
\end{tabular}

Number of Imaginary Frequencies

Frequencies (Top 3 out of 450)

$\begin{array}{lr}\text { 1. } & -336.4499 \mathrm{~cm}^{-1} \\ \text { 2. } & 7.2810 \mathrm{~cm}^{-1} \\ \text { 3. } & 10.2273 \mathrm{~cm}^{-1}\end{array}$

\section{B3LYP/6-31G(d) Molecular Geometry in Cartesian Coordinates}

\begin{tabular}{|c|c|c|c|}
\hline C & -2.155679 & -0.834760 & 0.818320 \\
\hline $\mathrm{H}$ & -1.115249 & -0.800910 & 1.134180 \\
\hline $\mathrm{H}$ & -2.475470 & 0.201189 & 0.651890 \\
\hline C & 1.077951 & -0.969789 & -1.246160 \\
\hline 0 & 1.121221 & -1.153719 & -2.444040 \\
\hline 0 & 1.016100 & 0.279111 & -0.755030 \\
\hline $\mathrm{H}$ & 0.956190 & 0.341551 & 0.229110 \\
\hline 0 & -2.877379 & -1.408091 & 1.915910 \\
\hline $\mathrm{H}$ & -3.809799 & -1.168941 & 1.789300 \\
\hline C & -0.469111 & 4.960920 & -0.619820 \\
\hline C & 1.495409 & 4. 242991 & 0.013220 \\
\hline C & -1.215341 & 3.100300 & 0.827590 \\
\hline C & -1.087051 & 4.493500 & 0.521710 \\
\hline $\mathrm{H}$ & 1.583879 & 4.978351 & 0.815330 \\
\hline
\end{tabular}




\begin{tabular}{|c|c|c|c|}
\hline $\mathrm{H}$ & -1.345870 & 2.442460 & -0.034280 \\
\hline H & -1.912541 & 2.864440 & 1.632830 \\
\hline C & 0.627200 & 1.161471 & 3.254780 \\
\hline C & 1.250760 & 2.557591 & 3.636370 \\
\hline C & -0.760150 & 0.947590 & 3.880460 \\
\hline $\mathrm{H}$ & -1.250680 & 0.080980 & 3.427290 \\
\hline H & -0.683020 & 0.768020 & 4.958640 \\
\hline $\mathrm{H}$ & -1.401560 & 1.820970 & 3.728000 \\
\hline C & 1.517601 & -0.043609 & 3.548670 \\
\hline $\mathrm{H}$ & 1.735871 & -0.112059 & 4.621030 \\
\hline $\mathrm{H}$ & 1.010821 & -0.967519 & 3.251750 \\
\hline $\mathrm{H}$ & 2.461570 & 0.015121 & 3.002420 \\
\hline C & 2.770320 & 2.614771 & 3.399070 \\
\hline $\mathrm{H}$ & 3.322010 & 2.032301 & 4.145580 \\
\hline $\mathrm{H}$ & 3.032370 & 2.241451 & 2.404550 \\
\hline $\mathrm{H}$ & 3.098859 & 3.657061 & 3.471130 \\
\hline C & 0.930919 & 3.043911 & 5.050370 \\
\hline $\mathrm{H}$ & 1.328650 & 2.356581 & 5.806360 \\
\hline $\mathrm{H}$ & 1.388919 & 4.025661 & 5.213470 \\
\hline $\mathrm{H}$ & -0.145781 & 3.146500 & 5.203240 \\
\hline 0 & 0.473530 & 1.285260 & 1.815140 \\
\hline 0 & 0.606689 & 3.442781 & 2.697930 \\
\hline B & 0.351870 & 2.690010 & 1.510270 \\
\hline 0 & 1.263499 & 3.016751 & 0.352920 \\
\hline C & 2.251609 & 4.489051 & -1.227560 \\
\hline C & 2.828478 & 5.749701 & -1.444080 \\
\hline C & 2.413789 & 3.472771 & -2.184470 \\
\hline C & 3.556148 & 5.998292 & -2.605530 \\
\hline $\mathrm{H}$ & 2.707058 & 6.532391 & -0.698270 \\
\hline C & 3.137799 & 3.730571 & -3.346040 \\
\hline $\mathrm{H}$ & 1.980270 & 2.493411 & -2.002770 \\
\hline C & 3.708569 & 4.989462 & -3.559910 \\
\hline $\mathrm{H}$ & 4.004478 & 6.974892 & -2.765840 \\
\hline $\mathrm{H}$ & 3.259829 & 2.944971 & -4.085990 \\
\hline $\mathrm{H}$ & 4.273749 & 5.182512 & -4.467760 \\
\hline $\mathrm{H}$ & -1.248081 & 5.196530 & 1.339690 \\
\hline $\mathrm{H}$ & -0.447731 & 4.345970 & -1.514700 \\
\hline $\mathrm{H}$ & -0.324692 & 6.026460 & -0.775860 \\
\hline C & -0.101909 & -2.791290 & -0.019190 \\
\hline C & 1.080991 & -2.092919 & -0.231190 \\
\hline C & 2.285631 & -2.423119 & 0.477670 \\
\hline C & 2.226352 & -3.419829 & 1.433530 \\
\hline $\mathrm{H}$ & 3.134902 & -3.707289 & 1.956380 \\
\hline C & -2.298009 & -1.625460 & -0.467570 \\
\hline C & -1.320179 & -2.529630 & -0.865370 \\
\hline C & -3.614989 & -2.140581 & -2.448290 \\
\hline C & -3.469079 & -1.437121 & -1.273640 \\
\hline $\mathrm{H}$ & -4.504339 & -1.992521 & -3.056720 \\
\hline C & -2.635968 & -3.067321 & -2.885460 \\
\hline C & -1.467628 & -3.272540 & -2.085910 \\
\hline C & -0.499718 & -4.209860 & -2.540770 \\
\hline C & -0.682458 & -4.906040 & -3.714550 \\
\hline C & -1.840478 & -4.701010 & -4.501200 \\
\hline C & -2.794598 & -3.798051 & -4.093190 \\
\hline
\end{tabular}




\begin{tabular}{|c|c|c|c|}
\hline $\mathrm{H}$ & 0.397792 & -4.367890 & -1.953900 \\
\hline $\mathrm{H}$ & 0.071942 & -5.615820 & -4.043190 \\
\hline $\mathrm{H}$ & -1.971058 & -5.255520 & -5.426690 \\
\hline $\mathrm{H}$ & -3.688078 & -3.628561 & -4.689780 \\
\hline C & 1.030032 & -4.116879 & 1.727510 \\
\hline C & -0.156668 & -3.810830 & 0.987710 \\
\hline C & -1.345098 & -4.532670 & 1.285550 \\
\hline C & -1.354268 & -5.508820 & 2.256500 \\
\hline C & -0.177417 & -5.815550 & 2.980830 \\
\hline C & 0.987782 & -5.132379 & 2.721310 \\
\hline $\mathrm{H}$ & -2.254678 & -4.290050 & 0.748830 \\
\hline $\mathrm{H}$ & -2.273657 & -6.046030 & 2.472850 \\
\hline $\mathrm{H}$ & -0.199057 & -6.589030 & 3.743930 \\
\hline $\mathrm{H}$ & 1.897162 & -5.357999 & 3.273410 \\
\hline C & -6.581990 & 1.252728 & -0.102110 \\
\hline C & -6.559069 & -0.029642 & 0.463790 \\
\hline C & -5.524529 & -0.891602 & 0.072570 \\
\hline C & -4.550029 & -0.487861 & -0.852320 \\
\hline C & -4.615720 & 0.799079 & -1.399340 \\
\hline C & -5.630870 & 1.692838 & -1.033730 \\
\hline $\mathrm{H}$ & -7.375910 & 1.934048 & 0.188640 \\
\hline $\mathrm{H}$ & -5.478159 & -1.906522 & 0.454240 \\
\hline $\mathrm{H}$ & -3.856030 & 1.088909 & -2.116090 \\
\hline C & -7.652599 & -0.442962 & 1.468480 \\
\hline C & -5.732711 & 3.116168 & -1.616030 \\
\hline C & -7.621280 & 0.510668 & 2.686500 \\
\hline $\mathrm{H}$ & -8.398540 & 0.231987 & 3.408430 \\
\hline $\mathrm{H}$ & -6.652290 & 0.463598 & 3.196730 \\
\hline $\mathrm{H}$ & -7.795180 & 1.551688 & 2.395300 \\
\hline C & -9.038229 & -0.356083 & 0.785110 \\
\hline $\mathrm{H}$ & -9.828949 & -0.642643 & 1.489270 \\
\hline $\mathrm{H}$ & -9.257340 & 0.657327 & 0.432890 \\
\hline H & -9.092249 & -1.028593 & -0.078580 \\
\hline C & -7.464609 & -1.882442 & 1.984020 \\
\hline $\mathrm{H}$ & -8.263949 & -2.126793 & 2.692590 \\
\hline $\mathrm{H}$ & -7.507779 & -2.617302 & 1.172370 \\
\hline $\mathrm{H}$ & -6.511309 & -2.009292 & 2.510040 \\
\hline C & -4.601801 & 3.425039 & -2.615700 \\
\hline $\mathrm{H}$ & -4.722881 & 4.442579 & -3.004190 \\
\hline $\mathrm{H}$ & -3.613941 & 3.367649 & -2.145030 \\
\hline $\mathrm{H}$ & -4.613740 & 2.741779 & -3.472300 \\
\hline C & -7.082821 & 3.273848 & -2.355620 \\
\hline $\mathrm{H}$ & -7.174221 & 4.285278 & -2.770230 \\
\hline $\mathrm{H}$ & -7.161830 & 2.559468 & -3.183110 \\
\hline $\mathrm{H}$ & -7.936291 & 3.108948 & -1.689620 \\
\hline C & -5.651551 & 4.151168 & -0.468800 \\
\hline $\mathrm{H}$ & -5.729741 & 5.169348 & -0.869380 \\
\hline $\mathrm{H}$ & -6.456721 & 4.018908 & 0.261720 \\
\hline $\mathrm{H}$ & -4.697841 & 4.068569 & 0.064910 \\
\hline C & 3.598031 & -1.781918 & 0.173990 \\
\hline C & 4.455381 & -1.391368 & 1.219530 \\
\hline C & 4.032221 & -1.630468 & -1.145900 \\
\hline C & 5.714871 & -0.847488 & 0.963510 \\
\hline $\mathrm{H}$ & 4.109471 & -1.515738 & 2.238820 \\
\hline
\end{tabular}




\begin{tabular}{|c|c|c|c|}
\hline C & 5.289201 & -1.082968 & -1.446700 \\
\hline $\mathrm{H}$ & 3.383471 & -1.956949 & -1.949800 \\
\hline C & 6.105971 & -0.699698 & -0.380280 \\
\hline $\mathrm{H}$ & 7.080081 & -0.273187 & -0.589540 \\
\hline C & 5.708681 & -0.932798 & -2.921280 \\
\hline C & 6.673241 & -0.402307 & 2.085460 \\
\hline C & 7.119501 & -0.334857 & -3.076890 \\
\hline $\mathrm{H}$ & 7.188740 & 0.668153 & -2.640300 \\
\hline $\mathrm{H}$ & 7.885771 & -0.965517 & -2.611000 \\
\hline $\mathrm{H}$ & 7. 366191 & -0.248367 & -4.141210 \\
\hline C & 5.698751 & -2.320918 & -3.605070 \\
\hline $\mathrm{H}$ & 5.993151 & -2.228128 & -4.657860 \\
\hline $\mathrm{H}$ & 6.401482 & -3.004787 & -3.114740 \\
\hline $\mathrm{H}$ & 4.706581 & -2.781908 & -3.577700 \\
\hline C & 4.704800 & 0.000402 & -3.640700 \\
\hline $\mathrm{H}$ & 3.677301 & -0.371688 & -3.576860 \\
\hline $\mathrm{H}$ & 4.724550 & 1.002272 & -3.196020 \\
\hline $\mathrm{H}$ & 4.967480 & 0.092852 & -4.702370 \\
\hline C & 6.102051 & -0.672238 & 3.490460 \\
\hline $\mathrm{H}$ & 5.170701 & -0.122598 & 3.667500 \\
\hline $\mathrm{H}$ & 5.905761 & -1.738308 & 3.652530 \\
\hline $\mathrm{H}$ & 6.823561 & -0.350147 & 4.249990 \\
\hline C & 8.010251 & -1.171387 & 1.961440 \\
\hline $\mathrm{H}$ & 7.851021 & -2.251677 & 2.056410 \\
\hline $\mathrm{H}$ & 8.500531 & -0.990327 & 0.999330 \\
\hline $\mathrm{H}$ & 8.704031 & -0.859537 & 2.752000 \\
\hline C & 6.941820 & 1.116213 & 1.959060 \\
\hline $\mathrm{H}$ & 7.628520 & 1.450663 & 2.746670 \\
\hline $\mathrm{H}$ & 7.391250 & 1.371313 & 0.993630 \\
\hline $\mathrm{H}$ & 6.010580 & 1.686362 & 2.054170 \\
\hline
\end{tabular}

\section{C-Catalyzed reaction TS 77}

\section{Datum}

Value

\begin{tabular}{lc}
\hline B3LYP/6-31G(d) Energy & -3038.958949 \\
\hline B3LYP-D3(BJ)/def2-TZVPP-IEF-PCM(DCM) Energy & -3040.509396 \\
\hline B3LYP-D3(BJ)/def2-TZVPP-IEF-PCM(DCM)//B3LYP/6-31G(d) Free Energy (Quasiharmonic) & -3039.280547 \\
\hline
\end{tabular}

Number of Imaginary Frequencies

Frequencies (Top 3 out of 450)

$\begin{array}{lr}\text { 1. } & -290.5132 \mathrm{~cm}^{-1} \\ \text { 2. } & 5.0534 \mathrm{~cm}^{-1} \\ \text { 3. } & 7.2401 \mathrm{~cm}^{-1}\end{array}$




\begin{tabular}{|c|c|c|c|}
\hline C & -3.229246 & 0.600038 & 1.257646 \\
\hline $\mathrm{H}$ & -4.224200 & 0.461661 & 1.688169 \\
\hline H & -2.517512 & 0.655968 & 2.091367 \\
\hline C & -0.054744 & 1.247839 & -0.827140 \\
\hline 0 & -1.086408 & 1.775257 & -1.211175 \\
\hline 0 & 0.944468 & 1.050324 & -1.682778 \\
\hline H & 1.699211 & 0.500758 & -1.343212 \\
\hline 0 & -3.270475 & 1.840954 & 0.557346 \\
\hline $\mathrm{H}$ & -2.500695 & 1.873851 & -0.049495 \\
\hline C & 4.470167 & -2.003506 & 2.465027 \\
\hline C & 3.697954 & -3.249559 & 0.795554 \\
\hline C & 4.634490 & -0.332277 & 0.656116 \\
\hline C & 5.219780 & -1.246582 & 1.596687 \\
\hline $\mathrm{H}$ & 2.851276 & -3.503389 & 1.439218 \\
\hline $\mathrm{H}$ & 5.331191 & 0.403008 & 0.248070 \\
\hline $\mathrm{H}$ & 3.714966 & 0.136181 & 1.012645 \\
\hline C & 3.800168 & -0.232880 & -2.875231 \\
\hline C & 4.887800 & -1.367030 & -2.967751 \\
\hline C & 2.710738 & -0.317760 & -3.946818 \\
\hline $\mathrm{H}$ & 2.010486 & 0.515445 & -3.847404 \\
\hline $\mathrm{H}$ & 3.156550 & -0.270064 & -4.947409 \\
\hline $\mathrm{H}$ & 2.139487 & -1.244927 & -3.862194 \\
\hline C & 4.398493 & 1.182317 & -2.851687 \\
\hline $\mathrm{H}$ & 4.780412 & 1.474713 & -3.835820 \\
\hline $\mathrm{H}$ & 5.216433 & 1.261262 & -2.128560 \\
\hline $\mathrm{H}$ & 3.617946 & 1.893748 & -2.564330 \\
\hline C & 6.133223 & -0.995041 & -3.773309 \\
\hline $\mathrm{H}$ & 5.877125 & -0.774263 & -4.815948 \\
\hline $\mathrm{H}$ & 6.641318 & -0.127370 & -3.346439 \\
\hline $\mathrm{H}$ & 6.838052 & -1.833575 & -3.770567 \\
\hline C & 4.316418 & -2.698889 & -3.483217 \\
\hline $\mathrm{H}$ & 4.070711 & -2.648990 & -4.549202 \\
\hline $\mathrm{H}$ & 5.063347 & -3.487631 & -3.344730 \\
\hline $\mathrm{H}$ & 3.413670 & -2.984054 & -2.935775 \\
\hline 0 & 3.210814 & -0.483541 & -1.568654 \\
\hline 0 & 5.276126 & -1.535875 & -1.588640 \\
\hline B & 4.158803 & -1.212862 & -0.763683 \\
\hline 0 & 3.400227 & -2.400128 & -0.130151 \\
\hline C & 4.709655 & -4.325827 & 0.692745 \\
\hline C & 4.560089 & -5.424441 & 1.557255 \\
\hline C & 5.767367 & -4.318017 & -0.231519 \\
\hline C & 5.449327 & -6.494474 & 1.506174 \\
\hline $\mathrm{H}$ & 3.737974 & -5.438815 & 2.269779 \\
\hline C & 6.655268 & -5.391025 & -0.275723 \\
\hline $\mathrm{H}$ & 5.883540 & -3.469484 & -0.897621 \\
\hline C & 6.501735 & -6.479247 & 0.587883 \\
\hline $\mathrm{H}$ & 5.319853 & -7.339163 & 2.176979 \\
\hline $\mathrm{H}$ & 7.472038 & -5.379691 & -0.992429 \\
\hline $\mathrm{H}$ & 7.197220 & -7.312965 & 0.543618 \\
\hline $\mathrm{H}$ & 6.269063 & -1.510754 & 1.460520 \\
\hline
\end{tabular}




\begin{tabular}{|c|c|c|c|}
\hline $\mathrm{H}$ & 3.474445 & -1.674718 & 2.751500 \\
\hline $\mathrm{H}$ & 4.940810 & -2.734843 & 3.114995 \\
\hline C & -0.489226 & -0.322665 & 1.067210 \\
\hline C & 0.177249 & 0.801802 & 0.597462 \\
\hline C & 1.093337 & 1.541204 & 1.423613 \\
\hline C & 1.384427 & 1.043420 & 2.678702 \\
\hline $\mathrm{H}$ & 2.046582 & 1.605091 & 3.332552 \\
\hline C & -2.883755 & -0.583913 & 0.365190 \\
\hline C & -1.557952 & -1.001722 & 0.249063 \\
\hline C & -3.566500 & -2.337576 & -1.174875 \\
\hline C & -3.914786 & -1.269512 & -0.368656 \\
\hline $\mathrm{H}$ & -4.342371 & -2.850616 & -1.737321 \\
\hline C & -2.230604 & -2.775853 & -1.320197 \\
\hline C & -1.197563 & -2.099706 & -0.597794 \\
\hline C & 0.141209 & -2.552713 & -0.752910 \\
\hline C & 0.437412 & -3.619263 & -1.573353 \\
\hline C & -0.587068 & -4.287223 & -2.286052 \\
\hline C & -1.891905 & -3.870075 & -2.161790 \\
\hline $\mathrm{H}$ & 0.945117 & -2.047451 & -0.230584 \\
\hline $\mathrm{H}$ & 1.468576 & -3.945789 & -1.672920 \\
\hline $\mathrm{H}$ & -0.339277 & -5.125400 & -2.932021 \\
\hline $\mathrm{H}$ & -2.688291 & -4.371498 & -2.706905 \\
\hline C & 0.802949 & -0.152744 & 3.168239 \\
\hline C & -0.163679 & -0.840385 & 2.366350 \\
\hline C & -0.766570 & -2.015937 & 2.892794 \\
\hline C & -0.423404 & -2.491540 & 4.139735 \\
\hline C & 0.533287 & -1.810967 & 4.929876 \\
\hline C & 1.128638 & -0.664320 & 4.454459 \\
\hline H & -1.504814 & -2.539155 & 2.295169 \\
\hline $\mathrm{H}$ & -0.894987 & -3.392463 & 4.522607 \\
\hline $\mathrm{H}$ & 0.789886 & -2.191354 & 5.914969 \\
\hline $\mathrm{H}$ & 1.854725 & -0.126456 & 5.059621 \\
\hline C & -8.081808 & -0.302755 & -0.204565 \\
\hline C & -7.679242 & -1.637161 & -0.010354 \\
\hline C & -6.312808 & -1.917679 & -0.052368 \\
\hline C & -5.361268 & -0.905495 & -0.280441 \\
\hline C & -5.803422 & 0.406216 & -0.469895 \\
\hline C & -7.169001 & 0.730514 & -0.431564 \\
\hline H & -9.140734 & -0.073053 & -0.171288 \\
\hline $\mathrm{H}$ & -5.955240 & -2.928129 & 0.110567 \\
\hline $\mathrm{H}$ & -5.063226 & 1.181041 & -0.632917 \\
\hline C & -8.743716 & -2.722114 & 0.245284 \\
\hline C & -7.594919 & 2.196552 & -0.638607 \\
\hline C & -8.125311 & -4.119460 & 0.441354 \\
\hline H & -8.919618 & -4.853164 & 0.620720 \\
\hline $\mathrm{H}$ & -7.567037 & -4.446361 & -0.443319 \\
\hline $\mathrm{H}$ & -7.447988 & -4.148544 & 1.302444 \\
\hline C & -9.545484 & -2.371279 & 1.521243 \\
\hline $\mathrm{H}$ & -10.311333 & -3.133025 & 1.714079 \\
\hline $\mathrm{H}$ & -8.886597 & -2.321954 & 2.395765 \\
\hline $\mathrm{H}$ & -10.052247 & -1.404924 & 1.431380 \\
\hline C & -9.708895 & -2.791900 & -0.962192 \\
\hline $\mathrm{H}$ & -10.480005 & -3.554162 & -0.793513 \\
\hline $\mathrm{H}$ & -10.216635 & -1.837242 & -1.134258 \\
\hline
\end{tabular}




\begin{tabular}{|c|c|c|c|}
\hline H & -9.168406 & -3.052047 & -1.879604 \\
\hline C & -9.121184 & 2.385204 & -0.556899 \\
\hline $\mathrm{H}$ & -9.370751 & 3.442275 & -0.703868 \\
\hline $\mathrm{H}$ & -9.647191 & 1.811786 & -1.329348 \\
\hline $\mathrm{H}$ & -9.517668 & 2.086780 & 0.420628 \\
\hline C & -6.937724 & 3.077583 & 0.451693 \\
\hline $\mathrm{H}$ & -7.208492 & 4.130614 & 0.302161 \\
\hline $\mathrm{H}$ & -7.280961 & 2.779826 & 1.449803 \\
\hline $\mathrm{H}$ & -5.845986 & 3.002324 & 0.437278 \\
\hline C & -7.124491 & 2.674263 & -2.033227 \\
\hline $\mathrm{H}$ & -7.415091 & 3.719638 & -2.195832 \\
\hline $\mathrm{H}$ & -6.037016 & 2.609917 & -2.138288 \\
\hline $\mathrm{H}$ & -7.575211 & 2.068513 & -2.828239 \\
\hline C & 1.652527 & 2.858077 & 1.000799 \\
\hline C & 0.816580 & 3.830492 & 0.444168 \\
\hline C & 3.006071 & 3.170078 & 1.223155 \\
\hline C & 1.302743 & 5.094947 & 0.077747 \\
\hline H & -0.234475 & 3.603277 & 0.310986 \\
\hline C & 3.526788 & 4.419913 & 0.884111 \\
\hline $\mathrm{H}$ & 3.646632 & 2.410841 & 1.655476 \\
\hline C & 2.654924 & 5.361337 & 0.306040 \\
\hline H & 3.052667 & 6.331372 & 0.031832 \\
\hline C & 5.005152 & 4.794231 & 1.107102 \\
\hline C & 0.340290 & 6.117861 & -0.554266 \\
\hline C & 5.801328 & 3.667914 & 1.792681 \\
\hline H & 5.388089 & 3.417886 & 2.776647 \\
\hline $\mathrm{H}$ & 5.821176 & 2.753607 & 1.189021 \\
\hline $\mathrm{H}$ & 6.839181 & 3.987086 & 1.941102 \\
\hline C & 5.670550 & 5.092592 & -0.257802 \\
\hline $\mathrm{H}$ & 6.724867 & 5.362558 & -0.119218 \\
\hline $\mathrm{H}$ & 5.626809 & 4.215976 & -0.914399 \\
\hline $\mathrm{H}$ & 5.179546 & 5.922126 & -0.777234 \\
\hline C & 5.092227 & 6.051867 & 2.004540 \\
\hline H & 4.584954 & 6.911831 & 1.555568 \\
\hline $\mathrm{H}$ & 4.633857 & 5.867351 & 2.982882 \\
\hline $\mathrm{H}$ & 6.140165 & 6.332044 & 2.167472 \\
\hline C & 1.026920 & 7.462892 & -0.857682 \\
\hline $\mathrm{H}$ & 1.422080 & 7.935427 & 0.049344 \\
\hline $\mathrm{H}$ & 1.850197 & 7.350143 & -1.572609 \\
\hline $\mathrm{H}$ & 0.300724 & 8.153920 & -1.300130 \\
\hline C & -0.207345 & 5.542290 & -1.883134 \\
\hline H & 0.605707 & 5.375105 & -2.599188 \\
\hline $\mathrm{H}$ & -0.721615 & 4.587203 & -1.738198 \\
\hline $\mathrm{H}$ & -0.918815 & 6.244266 & -2.335543 \\
\hline C & -0.837194 & 6.388456 & 0.412655 \\
\hline $\mathrm{H}$ & -1.529194 & 7.115785 & -0.029029 \\
\hline $\mathrm{H}$ & -1.408199 & 5.480547 & 0.630286 \\
\hline $\mathrm{H}$ & -0.477634 & 6.796781 & 1.364514 \\
\hline
\end{tabular}




\begin{tabular}{lc}
\hline B3LYP/6-31G(d) Energy & -3038.958364 \\
\hline B3LYP-D3(BJ)/def2-TZVPP-IEF-PCM(DCM) Energy & -3040.508939 \\
\hline B3LYP-D3(BJ)/def2-TZVPP-IEF-PCM(DCM)//B3LYP/6-31G(d) Free Energy (Quasiharmonic) & -3039.280365 \\
\hline
\end{tabular}

Number of Imaginary Frequencies

Frequencies (Top 3 out of 450)

$\begin{array}{lr}\text { 1. } & -313.3911 \mathrm{~cm}^{-1} \\ \text { 2. } & 9.1269 \mathrm{~cm}^{-1} \\ \text { 3. } & 10.9972 \mathrm{~cm}^{-1}\end{array}$

\section{B3LYP/6-31G(d) Molecular Geometry in Cartesian Coordinates}

C

$\mathrm{H}$

$\mathrm{H}$

C

0

0

$\mathrm{H}$

0

$\mathrm{H}$

C

C

C

C

$\mathrm{H}$

$\mathrm{H}$

$\mathrm{H}$

C

C

C

$\mathrm{H}$

$\mathrm{H}$

$\mathrm{H}$

C

$\mathrm{H}$

$\mathrm{H}$

$\mathrm{H}$

C

$\mathrm{H}$

$\mathrm{H}$

$\mathrm{H}$

C

$\mathrm{H}$

-2.752104
-1.792176
-3.473335
0.573959
-0.301680
1.486975
2.144182
-3.253633
-2.591981
3.016034
2.376730
4.084656
4.139379
3.200698
3.272094
5.038838
4.569694
4.992254
5.699527
5.275068
6.473306
6.174299
3.996123
4.742264
3.701927
3.114823
4.067505
4.217532
3.015685
4.288844
6.450364
6.662295

$-2.106321$

$-2.434889$

$-2.112351$

$-0.527922$

$-0.673354$

0.438316

0.522804

$-3.055296$

$-3.136698$

4.393233

4.621774

2. 370184

3. 682266

5. 294495

1.737096

1.840248

1.350498

2.857016

0.488284

$-0.458200$

0.270674

0.980092

0.673136

0.632946

$-0.355345$

1.199126

3.614602

3.301950

3.467976

4.685013

3.068991

2.603902
-0.261919
0.145128
0.555498
0.636801
1.465702
0.779373
0.043424

$-1.216423$

$-1.922340$

2.690433

0.598352

1. 749532

2. 327993

0. 352756

2.111146

1. 735031

$-1.686621$

$-1.880491$

$-1.101989$

$-0.753890$

$-1.846945$

$-0.247848$

$-2.931270$

$-3.733433$

$-2.694590$

- 3.305191

$-2.849171$

$-3.888777$

$-2.587255$

$-2.780876$

$-2.291130$

$-3.261258$ 


\begin{tabular}{|c|c|c|c|}
\hline $\mathrm{H}$ & 6.652838 & 4.141657 & -2.381019 \\
\hline H & 7.139568 & 2.660122 & -1.548624 \\
\hline 0 & 3.525694 & 1.454965 & -0.692219 \\
\hline 0 & 4.810381 & 3.396951 & -0.558418 \\
\hline B & 3.746287 & 2.668452 & 0.062156 \\
\hline 0 & 2.433174 & 3.413804 & 0.144028 \\
\hline C & 1.047575 & 5.219311 & 0.815203 \\
\hline C & -0.102277 & 4.413296 & 0.835466 \\
\hline C & 0.927275 & 6.608466 & 0.973760 \\
\hline C & -1.352944 & 4.996755 & 1.017259 \\
\hline $\mathrm{H}$ & -0.000090 & 3.341472 & 0.700035 \\
\hline C & -0.326811 & 7.186548 & 1.155484 \\
\hline $\mathrm{H}$ & 1.818134 & 7.232459 & 0.950893 \\
\hline C & -1.468408 & 6.380835 & 1.179844 \\
\hline $\mathrm{H}$ & -2.239575 & 4.369523 & 1.026394 \\
\hline $\mathrm{H}$ & -0.415347 & 8.262785 & 1.274976 \\
\hline $\mathrm{H}$ & -2.446903 & 6.831814 & 1.321691 \\
\hline $\mathrm{H}$ & 5.081258 & 4.225568 & 2.241340 \\
\hline $\mathrm{H}$ & 2.102015 & 3.867570 & 2.950313 \\
\hline H & 3.096790 & 5.413634 & 3.055097 \\
\hline C & -0.292710 & -1.291660 & -1.569907 \\
\hline C & 0.682401 & -1.415682 & -0.585540 \\
\hline C & 1.721029 & -2.406246 & -0.678138 \\
\hline C & 1.768582 & -3.195035 & -1.813839 \\
\hline $\mathrm{H}$ & 2.522363 & -3.974197 & -1.886494 \\
\hline C & -2.620967 & -0.703314 & -0.832882 \\
\hline C & -1.429392 & -0.310741 & -1.439079 \\
\hline C & -3.566593 & 1.494866 & -1.265312 \\
\hline C & -3.719192 & 0.220724 & -0.750713 \\
\hline $\mathrm{H}$ & -4.402428 & 2.188688 & -1.219396 \\
\hline C & -2.373593 & 1.918637 & -1.895164 \\
\hline C & -1.278308 & 1.002386 & -1.991675 \\
\hline C & -0.094002 & 1.436622 & -2.649768 \\
\hline C & -0.004325 & 2.701265 & -3.187867 \\
\hline C & -1.089336 & 3.604967 & -3.089431 \\
\hline C & -2.246534 & 3.219589 & -2.454006 \\
\hline $\mathrm{H}$ & 0.746812 & 0.755927 & -2.727771 \\
\hline $\mathrm{H}$ & 0.906107 & 3.009283 & -3.694268 \\
\hline $\mathrm{H}$ & -1.004036 & 4.600583 & -3.516270 \\
\hline $\mathrm{H}$ & -3.088058 & 3.903887 & -2.373917 \\
\hline C & 0.830123 & -3.070367 & -2.864750 \\
\hline C & -0.228012 & -2.114155 & -2.743792 \\
\hline C & -1.182282 & -2.024846 & -3.798393 \\
\hline C & -1.092227 & -2.840129 & -4.906010 \\
\hline C & -0.045095 & -3.785596 & -5.020161 \\
\hline C & 0.893522 & -3.895813 & -4.020426 \\
\hline $\mathrm{H}$ & -1.982732 & -1.296610 & -3.723982 \\
\hline $\mathrm{H}$ & -1.829647 & -2.755169 & -5.699360 \\
\hline H & 0.013511 & -4.423134 & -5.897993 \\
\hline $\mathrm{H}$ & 1.699889 & -4.621357 & -4.096959 \\
\hline C & -7.528166 & -0.698600 & 0.980292 \\
\hline C & -6.991798 & -1.579970 & 0.030496 \\
\hline C & -5.740486 & -1.275048 & -0.518650 \\
\hline C & -5.030753 & -0.131571 & -0.129274 \\
\hline
\end{tabular}




\begin{tabular}{|c|c|c|c|}
\hline C & -5.599603 & 0.721733 & 0.826468 \\
\hline C & -6.851497 & 0.457815 & 1.395276 \\
\hline $\mathrm{H}$ & -8.494306 & -0.921562 & 1.412127 \\
\hline $\mathrm{H}$ & -5.291454 & -1.934310 & -1.252928 \\
\hline $\mathrm{H}$ & -5.033914 & 1.594724 & 1.139328 \\
\hline C & -7.715658 & -2.864178 & -0.419382 \\
\hline C & -7.428757 & 1.424635 & 2.448436 \\
\hline C & -9.083091 & -3.044817 & 0.266399 \\
\hline $\mathrm{H}$ & -9.552781 & -3.969360 & -0.088323 \\
\hline $\mathrm{H}$ & -8.987575 & -3.121429 & 1.355626 \\
\hline $\mathrm{H}$ & -9.767601 & -2.218862 & 0.039972 \\
\hline C & -7.949294 & -2.818187 & -1.948187 \\
\hline $\mathrm{H}$ & -8.458831 & -3.730401 & -2.282971 \\
\hline $\mathrm{H}$ & -8.572810 & -1.959847 & -2.224789 \\
\hline $\mathrm{H}$ & -7.007632 & -2.741350 & -2.500833 \\
\hline C & -6.835123 & -4.089997 & -0.073817 \\
\hline H & -7.324393 & -5.014892 & -0.405024 \\
\hline $\mathrm{H}$ & -5.851686 & -4.036561 & -0.551326 \\
\hline $\mathrm{H}$ & -6.677482 & -4.160751 & 1.008966 \\
\hline C & -7.565937 & 2.837178 & 1.831686 \\
\hline $\mathrm{H}$ & -7.969439 & 3.540331 & 2.570980 \\
\hline $\mathrm{H}$ & -6.601187 & 3.228562 & 1.492187 \\
\hline $\mathrm{H}$ & -8.243153 & 2.824161 & 0.969894 \\
\hline C & -8.818146 & 0.990533 & 2.953083 \\
\hline $\mathrm{H}$ & -9.183649 & 1.712545 & 3.692317 \\
\hline $\mathrm{H}$ & -9.553035 & 0.948376 & 2.141020 \\
\hline $\mathrm{H}$ & -8.788388 & 0.008664 & 3.438796 \\
\hline C & -6.476139 & 1.489307 & 3.666128 \\
\hline $\mathrm{H}$ & -6.866868 & 2.182626 & 4.421561 \\
\hline $\mathrm{H}$ & -6.370280 & 0.503093 & 4.132270 \\
\hline $\mathrm{H}$ & -5.475233 & 1.831659 & 3.383962 \\
\hline C & 2.695602 & -2.665404 & 0.422171 \\
\hline C & 4.037735 & -2.952409 & 0.122823 \\
\hline C & 2.274877 & -2.710562 & 1.759150 \\
\hline C & 4.957619 & -3.275156 & 1.126776 \\
\hline $\mathrm{H}$ & 4.355235 & -2.911266 & -0.912755 \\
\hline C & 3.168126 & -3.004771 & 2.795035 \\
\hline H & 1.233130 & -2.529195 & 1.984189 \\
\hline C & 4.497826 & -3.282600 & 2.451884 \\
\hline $\mathrm{H}$ & 5.201225 & -3.517257 & 3.245237 \\
\hline C & 2.732453 & -3.020511 & 4.273164 \\
\hline C & 6.431846 & -3.614901 & 0.829413 \\
\hline C & 1.224527 & -2.751112 & 4.442284 \\
\hline $\mathrm{H}$ & 0.928104 & -1.779225 & 4.033855 \\
\hline $\mathrm{H}$ & 0.617331 & -3.521713 & 3.953536 \\
\hline $\mathrm{H}$ & 0.968671 & -2.757767 & 5.508097 \\
\hline C & 3.047667 & -4.398944 & 4.900408 \\
\hline $\mathrm{H}$ & 2.738706 & -4.415781 & 5.952742 \\
\hline $\mathrm{H}$ & 2.511897 & -5.199281 & 4.377244 \\
\hline $\mathrm{H}$ & 4.116823 & -4.633909 & 4.865751 \\
\hline C & 3.508136 & -1.920240 & 5.036784 \\
\hline $\mathrm{H}$ & 4.591426 & -2.075721 & 4.986975 \\
\hline $\mathrm{H}$ & 3.291832 & -0.930123 & 4.620113 \\
\hline $\mathrm{H}$ & 3.219306 & -1.914946 & 6.095055 \\
\hline
\end{tabular}




\begin{tabular}{|llll|} 
C & 6.755203 & -5.026031 & 1.376673 \\
H & 6.598982 & -5.092875 & 2.458128 \\
H & 6.123546 & -5.785061 & 0.901016 \\
H & 7.803050 & -5.281199 & 1.175654 \\
C & 6.745802 & -3.612407 & -0.678959 \\
$H$ & 6.134590 & -4.342129 & -1.222465 \\
$H$ & 6.586918 & -2.627291 & -1.130867 \\
$H$ & 7.796758 & -3.880547 & -0.835890 \\
C & 7.351260 & -2.577674 & 1.516720 \\
H & 8.405118 & -2.810500 & 1.319460 \\
$H$ & 7.150096 & -1.567196 & 1.143336 \\
$H$ & 7.210266 & -2.566291 & 2.602608 \\
\hline
\end{tabular}

\section{C-Catalyzed reaction TS 79}

\section{Datum}

Value

\begin{tabular}{lc}
\hline B3LYP/6-31G(d) Energy & -3038.959352 \\
\hline B3LYP-D3(BJ)/def2-TZVPP-IEF-PCM(DCM) Energy & -3040.508323 \\
\hline B3LYP-D3(BJ)/def2-TZVPP-IEF-PCM(DCM)//B3LYP/6-31G(d) Free Energy (Quasiharmonic) & -3039.280284 \\
\hline
\end{tabular}

Number of Imaginary Frequencies

Frequencies (Top 3 out of 450)

$$
\begin{array}{lr}
\text { 1. } & -321.1928 \mathrm{~cm}^{-1} \\
\text { 2. } & 5.8399 \mathrm{~cm}^{-1} \\
\text { 3. } & 11.3722 \mathrm{~cm}^{-1}
\end{array}
$$

\section{B3LYP/6-31G(d) Molecular Geometry in Cartesian Coordinates}

C

$\mathrm{H}$

$\mathrm{H}$

C

0

0

$\mathrm{H}$

0

$\mathrm{H}$

C

C

C

C

$\mathrm{H}$

\subsection{8}

3. 718959

2.117793

$-0.342797$

0.432980

$-1.249945$

$-1.807829$

3. 745258

3.159303

$-2.036952$

$-3.699204$

$-1.642003$

$-2.016359$

$-4.428936$

\section{$-1.709438$}

$-1.286773$

$-1.947952$

$-0.294342$

$-0.099502$

0.630101

0.437104

$-2.903685$

$-3.347339$

5. 195756

3.953791

3. 246088

4.612160

4.439948
0.419180

1.192587

0.883345

0.501004

1.416467

0.178831

$-0.620106$

$-0.018616$

$-0.653071$

$-0.813264$

$-0.143930$

$-2.283122$

$-2.062532$

$-0.794797$ 


\begin{tabular}{|c|c|c|c|}
\hline $\mathrm{H}$ & -0.864408 & 2.863311 & -1.619009 \\
\hline $\mathrm{H}$ & -1.445281 & 2.985440 & -3.323713 \\
\hline C & -4.087925 & 0.345982 & -2.504727 \\
\hline C & -4.635694 & 1.502831 & -3.425986 \\
\hline C & -3.731186 & -0.950553 & -3.231001 \\
\hline $\mathrm{H}$ & -3.330011 & -1.679157 & -2.518163 \\
\hline $\mathrm{H}$ & -4.617936 & -1.392701 & -3.700254 \\
\hline $\mathrm{H}$ & -2.976811 & -0.785678 & -4.003981 \\
\hline C & -5.018590 & 0.042935 & -1.319680 \\
\hline $\mathrm{H}$ & -5.929773 & -0.470759 & -1.646799 \\
\hline $\mathrm{H}$ & -5.305959 & 0.957468 & -0.793919 \\
\hline $\mathrm{H}$ & -4.497504 & -0.604091 & -0.607633 \\
\hline C & -6.159095 & 1.542997 & -3.566736 \\
\hline $\mathrm{H}$ & -6.540624 & 0.624114 & -4.027728 \\
\hline $\mathrm{H}$ & -6.647533 & 1.678476 & -2.598966 \\
\hline H & -6.444485 & 2.384210 & -4.207567 \\
\hline C & -3.990798 & 1.508003 & -4.822621 \\
\hline $\mathrm{H}$ & -4.346923 & 0.678874 & -5.444153 \\
\hline $\mathrm{H}$ & -4.246602 & 2.446882 & -5.324499 \\
\hline $\mathrm{H}$ & -2.900613 & 1.446204 & -4.753195 \\
\hline 0 & -2.866766 & 0.933804 & -1.988234 \\
\hline 0 & -4.208735 & 2.690122 & -2.736192 \\
\hline B & -3.094782 & 2.358746 & -1.900459 \\
\hline 0 & -3.316395 & 2.759362 & -0.458971 \\
\hline C & -3.711674 & 4.318079 & 1.285235 \\
\hline C & -4.466488 & 5.419855 & 1.713919 \\
\hline C & -2.990541 & 3.558986 & 2.222312 \\
\hline C & -4.502051 & 5.763004 & 3.063898 \\
\hline $\mathrm{H}$ & -5.028223 & 6.003294 & 0.987578 \\
\hline C & -3.027466 & 3.909501 & 3.569232 \\
\hline $\mathrm{H}$ & -2.414495 & 2.703143 & 1.882704 \\
\hline C & -3.780777 & 5.009348 & 3.992962 \\
\hline $\mathrm{H}$ & -5.092151 & 6.614528 & 3.391148 \\
\hline $\mathrm{H}$ & -2.467958 & 3.322119 & 4.291699 \\
\hline $\mathrm{H}$ & -3.806460 & 5.277206 & 5.045784 \\
\hline $\mathrm{H}$ & -2.544807 & 5.119537 & -2.870621 \\
\hline $\mathrm{H}$ & -1.385318 & 4.818618 & -0.030712 \\
\hline $\mathrm{H}$ & -2.395862 & 6.212915 & -0.680860 \\
\hline C & 0.676355 & -1.797458 & -1.219981 \\
\hline C & -0.336392 & -1.588076 & -0.289901 \\
\hline C & -1.310596 & -2.605749 & -0.002512 \\
\hline C & -1.262354 & -3.781087 & -0.729670 \\
\hline $\mathrm{H}$ & -1.975223 & -4.570579 & -0.507213 \\
\hline C & 2.882256 & -0.706279 & -0.706139 \\
\hline C & 1.729220 & -0.753803 & -1.487232 \\
\hline C & 3.703451 & 1.161192 & -2.029940 \\
\hline C & 3.894358 & 0.276171 & -0.985714 \\
\hline $\mathrm{H}$ & 4.474341 & 1.895491 & -2.250117 \\
\hline C & 2.560861 & 1.114792 & -2.861733 \\
\hline C & 1.547550 & 0.140799 & -2.593337 \\
\hline C & 0.420620 & 0.086530 & -3.460289 \\
\hline C & 0.316249 & 0.934539 & -4.541247 \\
\hline C & 1.319853 & 1.898421 & -4.802032 \\
\hline C & 2.413463 & 1.988939 & -3.972762 \\
\hline
\end{tabular}




\begin{tabular}{|c|c|c|c|}
\hline $\mathrm{H}$ & -0.363672 & -0.636599 & -3.266626 \\
\hline $\mathrm{H}$ & -0.542890 & 0.860777 & -5.202740 \\
\hline $\mathrm{H}$ & 1.223639 & 2.562185 & -5.657165 \\
\hline H & 3.192370 & 2.723979 & -4.161841 \\
\hline C & -0.272072 & -4.025848 & -1.709881 \\
\hline C & 0.727754 & -3.030873 & -1.951990 \\
\hline C & 1.743892 & -3.314223 & -2.909946 \\
\hline C & 1.763315 & -4.513708 & -3.588538 \\
\hline C & 0.768484 & -5.491743 & -3.350222 \\
\hline C & -0.225287 & -5.250504 & -2.429952 \\
\hline $\mathrm{H}$ & 2.505736 & -2.567707 & -3.107978 \\
\hline H & 2.547493 & -4.709351 & -4.314665 \\
\hline $\mathrm{H}$ & 0.794973 & -6.432681 & -3.892835 \\
\hline $\mathrm{H}$ & -0.990205 & -5.998074 & -2.233603 \\
\hline C & 7.534489 & 0.680415 & 1.216327 \\
\hline C & 7.204542 & -0.581758 & 0.702496 \\
\hline C & 6.003424 & -0.706433 & -0.004908 \\
\hline C & 5.153104 & 0.393100 & -0.190063 \\
\hline C & 5.521312 & 1.639395 & 0.338071 \\
\hline C & 6.714405 & 1.805534 & 1.050597 \\
\hline $\mathrm{H}$ & 8.460980 & 0.790423 & 1.772497 \\
\hline $\mathrm{H}$ & 5.702693 & -1.661660 & -0.415457 \\
\hline H & 4.843963 & 2.473965 & 0.198487 \\
\hline C & 8.145926 & -1.778677 & 0.940944 \\
\hline C & 7.138790 & 3.157976 & 1.656472 \\
\hline C & 8.246984 & -2.049689 & 2.461104 \\
\hline $\mathrm{H}$ & 8.919859 & -2.894367 & 2.655161 \\
\hline H & 8.635338 & -1.181385 & 3.004399 \\
\hline $\mathrm{H}$ & 7.264388 & -2.294366 & 2.879982 \\
\hline C & 7.642676 & -3.064053 & 0.255854 \\
\hline H & 8.334125 & -3.887806 & 0.468477 \\
\hline $\mathrm{H}$ & 6.649032 & -3.358806 & 0.609041 \\
\hline $\mathrm{H}$ & 7.591880 & -2.947932 & -0.832998 \\
\hline C & 9.553211 & -1.457794 & 0.384633 \\
\hline $\mathrm{H}$ & 10.231011 & -2.304330 & 0.551080 \\
\hline H & 9.512380 & -1.262755 & -0.693230 \\
\hline $\mathrm{H}$ & 9.996225 & -0.579507 & 0.866231 \\
\hline C & 6.114772 & 4.274609 & 1.377019 \\
\hline H & 6.462179 & 5.213634 & 1.822945 \\
\hline $\mathrm{H}$ & 5.982519 & 4.447969 & 0.302962 \\
\hline $\mathrm{H}$ & 5.134021 & 4.046554 & 1.809365 \\
\hline C & 7.282504 & 3.015291 & 3.190437 \\
\hline $\mathrm{H}$ & 7.584590 & 3.971044 & 3.636797 \\
\hline H & 8.035511 & 2.268122 & 3.462084 \\
\hline $\mathrm{H}$ & 6.332472 & 2.712572 & 3.645214 \\
\hline C & 8.496374 & 3.591685 & 1.053947 \\
\hline H & 8.816159 & 4.550101 & 1.481761 \\
\hline $\mathrm{H}$ & 9.284736 & 2.858617 & 1.254000 \\
\hline $\mathrm{H}$ & 8.421947 & 3.712751 & -0.032922 \\
\hline C & -2.324091 & -2.476040 & 1.085939 \\
\hline C & -3.652686 & -2.886899 & 0.866684 \\
\hline C & -1.957797 & -2.032714 & 2.359627 \\
\hline C & -4.604217 & -2.849908 & 1.887484 \\
\hline $\mathrm{H}$ & -3.928792 & -3.228421 & -0.124428 \\
\hline
\end{tabular}




\begin{tabular}{|c|c|c|c|}
\hline C & -2.887164 & -1.966181 & 3.409316 \\
\hline $\mathrm{H}$ & -0.928535 & -1.747540 & 2.538835 \\
\hline C & -4.196224 & -2.378165 & 3.149027 \\
\hline $\mathrm{H}$ & -4.929111 & -2.337535 & 3.946529 \\
\hline C & -2.427064 & -1.453082 & 4.786817 \\
\hline C & -6.063649 & -3.301074 & 1.682184 \\
\hline C & -3.567359 & -1.442028 & 5.821920 \\
\hline $\mathrm{H}$ & -4.393991 & -0.792486 & 5.511542 \\
\hline $\mathrm{H}$ & -3.969243 & -2.446411 & 5.999735 \\
\hline $\mathrm{H}$ & -3.192284 & -1.063736 & 6.779604 \\
\hline C & -1.297186 & -2.363124 & 5.325286 \\
\hline $\mathrm{H}$ & -0.960290 & -2.009315 & 6.307433 \\
\hline $\mathrm{H}$ & -1.645523 & -3.396506 & 5.437263 \\
\hline $\mathrm{H}$ & -0.428070 & -2.371757 & 4.660089 \\
\hline C & -1.892288 & -0.007439 & 4.638807 \\
\hline H & -1.063387 & 0.055702 & 3.926605 \\
\hline $\mathrm{H}$ & -2.685527 & 0.663694 & 4.288159 \\
\hline $\mathrm{H}$ & -1.534720 & 0.363637 & 5.607770 \\
\hline C & -6.327555 & -3.809602 & 0.251921 \\
\hline $\mathrm{H}$ & -6.153130 & -3.029758 & -0.497701 \\
\hline $\mathrm{H}$ & -5.698069 & -4.671628 & 0.002810 \\
\hline $\mathrm{H}$ & -7.372556 & -4.126930 & 0.160818 \\
\hline C & -6.389707 & -4.451539 & 2.664504 \\
\hline $\mathrm{H}$ & -5.734672 & -5.312958 & 2.490475 \\
\hline $\mathrm{H}$ & -6.267179 & -4.145760 & 3.708595 \\
\hline $\mathrm{H}$ & -7.427621 & -4.781864 & 2.533783 \\
\hline C & -7.016181 & -2.112174 & 1.952597 \\
\hline $\mathrm{H}$ & -8.060435 & -2.421200 & 1.819410 \\
\hline $\mathrm{H}$ & -6.909959 & -1.728985 & 2.972787 \\
\hline $\mathrm{H}$ & -6.815410 & -1.284717 & 1.262705 \\
\hline
\end{tabular}

\section{C-Catalyzed reaction TS 80}

\section{Datum}

Value

\begin{tabular}{lc}
\hline B3LYP/6-31G(d) Energy & -3038.955113 \\
\hline B3LYP-D3(BJ)/def2-TZVPP-IEF-PCM(DCM) Energy & -3040.508192 \\
\hline B3LYP-D3(BJ)/def2-TZVPP-IEF-PCM(DCM)//B3LYP/6-31G(d) Free Energy (Quasiharmonic) & -3039.280256 \\
\hline
\end{tabular}

Number of Imaginary Frequencies

Frequencies (Top 3 out of 450)

$$
\begin{array}{lr}
\text { 1. } & -315.4461 \mathrm{~cm}^{-1} \\
\text { 2. } & 8.2786 \mathrm{~cm}^{-1} \\
\text { 3. } & 10.7875 \mathrm{~cm}^{-1}
\end{array}
$$




\begin{tabular}{|c|c|c|c|}
\hline C & -1.579223 & 0.666747 & 0.795906 \\
\hline $\mathrm{H}$ & -2.073345 & -0.245039 & 0.436069 \\
\hline $\mathrm{H}$ & -0.523082 & 0.429772 & 0.909809 \\
\hline C & 1.414615 & 0.733992 & -1.310975 \\
\hline 0 & 1.199625 & 0.990011 & -2.476403 \\
\hline 0 & 1.427006 & -0.540064 & -0.881367 \\
\hline $\mathrm{H}$ & 1.521811 & -0.632521 & 0.099402 \\
\hline 0 & -2.042985 & 0.998417 & 2.111618 \\
\hline $\mathrm{H}$ & -2.996410 & 0.821400 & 2.123898 \\
\hline C & 1.241256 & -5.267099 & -0.922494 \\
\hline C & -0.463537 & -4.082747 & -0.138030 \\
\hline C & 2.495303 & -3.689782 & 0.507835 \\
\hline C & 2.012200 & -5.000276 & 0.186941 \\
\hline $\mathrm{H}$ & -0.702532 & -4.825836 & 0.625288 \\
\hline $\mathrm{H}$ & 2.720183 & -3.052093 & -0.349531 \\
\hline $\mathrm{H}$ & 3.290243 & -3.663312 & 1.254691 \\
\hline C & 1.428352 & -1.499000 & 3.143332 \\
\hline C & 0.525695 & -2.720930 & 3.561969 \\
\hline C & 2.892396 & -1.667406 & 3.580917 \\
\hline $\mathrm{H}$ & 3.502054 & -0.928471 & 3.052250 \\
\hline $\mathrm{H}$ & 3.012886 & -1.507468 & 4.657859 \\
\hline $\mathrm{H}$ & 3.274673 & -2.662807 & 3.337242 \\
\hline C & 0.915842 & -0.132206 & 3.593887 \\
\hline $\mathrm{H}$ & 0.913646 & -0.071942 & 4.689275 \\
\hline $\mathrm{H}$ & -0.093693 & 0.079095 & 3.236487 \\
\hline $\mathrm{H}$ & 1.581184 & 0.654418 & 3.221607 \\
\hline C & -0.975349 & -2.387664 & 3.539865 \\
\hline $\mathrm{H}$ & -1.260460 & -1.740098 & 4.375960 \\
\hline $\mathrm{H}$ & -1.263276 & -1.886711 & 2.611873 \\
\hline $\mathrm{H}$ & -1.542258 & -3.321594 & 3.619939 \\
\hline C & 0.889447 & -3.358738 & 4.904328 \\
\hline $\mathrm{H}$ & 0.778268 & -2.642580 & 5.726931 \\
\hline $\mathrm{H}$ & 0.221042 & -4.203970 & 5.101179 \\
\hline $\mathrm{H}$ & 1.914835 & -3.736314 & 4.904906 \\
\hline 0 & 1.383766 & -1.577809 & 1.693507 \\
\hline 0 & 0.800720 & -3.680196 & 2.520136 \\
\hline B & 1.136365 & -2.952883 & 1.339123 \\
\hline 0 & 0.088164 & -2.981807 & 0.246861 \\
\hline C & -1.282729 & -4.057896 & -1.362200 \\
\hline C & -1.160897 & -3.003937 & -2.284341 \\
\hline C & -2.191732 & -5.098023 & -1.608535 \\
\hline C & -1.934796 & -3.007812 & -3.441930 \\
\hline $\mathrm{H}$ & -0.458846 & -2.198898 & -2.087002 \\
\hline C & -2.966502 & -5.093277 & -2.766439 \\
\hline $\mathrm{H}$ & -2.287221 & -5.910558 & -0.891521 \\
\hline C & -2.834787 & -4.050713 & -3.687752 \\
\hline $\mathrm{H}$ & -1.833033 & -2.196223 & -4.156962 \\
\hline $\mathrm{H}$ & -3.668732 & -5.901195 & -2.952053 \\
\hline $\mathrm{H}$ & -3.432170 & -4.050156 & -4.595385 \\
\hline $\mathrm{H}$ & 2.043551 & -5.747236 & 0.980853 \\
\hline
\end{tabular}




\begin{tabular}{|c|c|c|c|}
\hline $\mathrm{H}$ & 1.317244 & -4.633203 & -1.800598 \\
\hline $\mathrm{H}$ & 0.822220 & -6.256265 & -1.086094 \\
\hline C & 0.607063 & 2.587159 & 0.144224 \\
\hline C & 1.665551 & 1.776523 & -0.246352 \\
\hline C & 2.965357 & 1.888549 & 0.356674 \\
\hline C & 3.127858 & 2.791523 & 1.389911 \\
\hline $\mathrm{H}$ & 4.112882 & 2.925644 & 1.829455 \\
\hline C & -1.765299 & 1.768527 & -0.232702 \\
\hline C & -0.707944 & 2.596970 & -0.592664 \\
\hline C & -3.188709 & 2.828890 & -1.900599 \\
\hline C & -3.039709 & 1.915935 & -0.879526 \\
\hline $\mathrm{H}$ & -4.156104 & 2.936490 & -2.385848 \\
\hline C & -2.119705 & 3.657190 & -2.321672 \\
\hline C & -0.860099 & 3.556308 & -1.652227 \\
\hline C & 0.194884 & 4.409112 & -2.079240 \\
\hline C & 0.010687 & 5.309263 & -3.104999 \\
\hline C & -1.238596 & 5.407425 & -3.761625 \\
\hline C & -2.279966 & 4.595665 & -3.375962 \\
\hline $\mathrm{H}$ & 1.161278 & 4.337591 & -1.593745 \\
\hline $\mathrm{H}$ & 0.833530 & 5.947113 & -3.416380 \\
\hline $\mathrm{H}$ & -1.370728 & 6.121484 & -4.570165 \\
\hline $\mathrm{H}$ & -3.245344 & 4.659323 & -3.872940 \\
\hline C & 2.061067 & 3.587745 & 1.872115 \\
\hline C & 0.779306 & 3.497080 & 1.240975 \\
\hline C & -0.273900 & 4.317192 & 1.731452 \\
\hline C & -0.067032 & 5.182657 & 2.782239 \\
\hline C & 1.203936 & 5.277216 & 3.397617 \\
\hline C & 2.243626 & 4.495637 & 2.950440 \\
\hline $\mathrm{H}$ & -1.253569 & 4.243071 & 1.274067 \\
\hline $\mathrm{H}$ & -0.886721 & 5.797151 & 3.144465 \\
\hline $\mathrm{H}$ & 1.354125 & 5.965775 & 4.224853 \\
\hline $\mathrm{H}$ & 3.223939 & 4.558187 & 3.417077 \\
\hline C & -6.525598 & -0.253718 & 0.322279 \\
\hline C & -5.993095 & 0.699815 & 1.201583 \\
\hline C & -4.843859 & 1.388097 & 0.791485 \\
\hline C & -4.237487 & 1.127604 & -0.448295 \\
\hline C & -4.813972 & 0.180001 & -1.302683 \\
\hline C & -5.969995 & -0.523152 & -0.936324 \\
\hline $\mathrm{H}$ & -7.414791 & -0.798420 & 0.625041 \\
\hline $\mathrm{H}$ & -4.423966 & 2.173694 & 1.410027 \\
\hline $\mathrm{H}$ & -4.338225 & 0.000728 & -2.259954 \\
\hline C & -6.679958 & 0.963743 & 2.555997 \\
\hline C & -6.631987 & -1.569078 & -1.854950 \\
\hline C & -6.683562 & -0.338077 & 3.392523 \\
\hline $\mathrm{H}$ & -7.181900 & -0.171421 & 4.355226 \\
\hline $\mathrm{H}$ & -7.209262 & -1.150969 & 2.880879 \\
\hline $\mathrm{H}$ & -5.661085 & -0.677481 & 3.595179 \\
\hline C & -5.966796 & 2.056826 & 3.375335 \\
\hline $\mathrm{H}$ & -6.493497 & 2.206862 & 4.324424 \\
\hline $\mathrm{H}$ & -4.932307 & 1.785388 & 3.615994 \\
\hline $\mathrm{H}$ & -5.953366 & 3.018483 & 2.850233 \\
\hline C & -8.138072 & 1.422712 & 2.315836 \\
\hline $\mathrm{H}$ & -8.640988 & 1.610305 & 3.272418 \\
\hline $\mathrm{H}$ & -8.166024 & 2.348996 & 1.730689 \\
\hline
\end{tabular}




\begin{tabular}{|c|c|c|c|}
\hline H & -8.720570 & 0.669224 & 1.775769 \\
\hline C & -8.122344 & -1.210971 & -2.066851 \\
\hline $\mathrm{H}$ & -8.597191 & -1.945485 & -2.728752 \\
\hline $\mathrm{H}$ & -8.683553 & -1.203167 & -1.126964 \\
\hline $\mathrm{H}$ & -8.226463 & -0.222016 & -2.527565 \\
\hline C & -5.962298 & -1.637291 & -3.240445 \\
\hline H & -6.482305 & -2.374459 & -3.863232 \\
\hline $\mathrm{H}$ & -4.913406 & -1.944254 & -3.173730 \\
\hline $\mathrm{H}$ & -6.007652 & -0.673460 & -3.760486 \\
\hline C & -6.529044 & -2.965163 & -1.195527 \\
\hline $\mathrm{H}$ & -7.016659 & -3.721083 & -1.823866 \\
\hline H & -5.481952 & -3.259500 & -1.062348 \\
\hline $\mathrm{H}$ & -7.012334 & -2.984813 & -0.212752 \\
\hline C & 4.143175 & 1.124176 & -0.146305 \\
\hline C & 4.374931 & 0.987894 & -1.518187 \\
\hline C & 5.074828 & 0.576664 & 0.755449 \\
\hline C & 5.489514 & 0.292286 & -2.010981 \\
\hline H & 3.677435 & 1.437220 & -2.215238 \\
\hline C & 6.208996 & -0.102029 & 0.307221 \\
\hline $\mathrm{H}$ & 4.885532 & 0.682644 & 1.817344 \\
\hline C & 6.387199 & -0.239349 & -1.082141 \\
\hline $\mathrm{H}$ & 7.256001 & -0.779577 & -1.439516 \\
\hline C & 7.248776 & -0.714031 & 1.266548 \\
\hline C & 5.669676 & 0.143408 & -3.533680 \\
\hline C & 8.650773 & -0.141603 & 0.947954 \\
\hline H & 8.963790 & -0.364843 & -0.077112 \\
\hline $\mathrm{H}$ & 8.666763 & 0.947293 & 1.072238 \\
\hline H & 9.399463 & -0.571898 & 1.624537 \\
\hline C & 6.941071 & -0.408490 & 2.744646 \\
\hline $\mathrm{H}$ & 7.719700 & -0.844824 & 3.380723 \\
\hline H & 6.919715 & 0.669727 & 2.940924 \\
\hline $\mathrm{H}$ & 5.982723 & -0.833938 & 3.061537 \\
\hline C & 7.270210 & -2.250625 & 1.085597 \\
\hline H & 6.290339 & -2.683973 & 1.316683 \\
\hline $\mathrm{H}$ & 7.526810 & -2.535274 & 0.059817 \\
\hline H & 8.010659 & -2.705882 & 1.755182 \\
\hline C & 4.451540 & -0.614547 & -4.116119 \\
\hline $\mathrm{H}$ & 4.383419 & -1.622875 & -3.691221 \\
\hline $\mathrm{H}$ & 3.506705 & -0.102991 & -3.907282 \\
\hline H & 4.550835 & -0.711211 & -5.204679 \\
\hline C & 5.760585 & 1.544500 & -4.183739 \\
\hline H & 4.858796 & 2.137468 & -4.002272 \\
\hline $\mathrm{H}$ & 6.617047 & 2.104990 & -3.790954 \\
\hline $\mathrm{H}$ & 5.883777 & 1.451926 & -5.269855 \\
\hline C & 6.944356 & -0.638245 & -3.903179 \\
\hline $\mathrm{H}$ & 7.023131 & -0.718314 & -4.993289 \\
\hline H & 7.851285 & -0.138670 & -3.542766 \\
\hline $\mathrm{H}$ & 6.930362 & -1.657136 & -3.499130 \\
\hline
\end{tabular}




\begin{tabular}{lc}
\hline B3LYP/6-31G(d) Energy & -3038.952376 \\
\hline B3LYP-D3(BJ)/def2-TZVPP-IEF-PCM(DCM) Energy & -3040.507438 \\
\hline B3LYP-D3(BJ)/def2-TZVPP-IEF-PCM(DCM)//B3LYP/6-31G(d) Free Energy (Quasiharmonic) & -3039.280108 \\
\hline
\end{tabular}

Number of Imaginary Frequencies

Frequencies (Top 3 out of 450)

$\begin{array}{lr}\text { 1. } & -335.8199 \mathrm{~cm}^{-1} \\ \text { 2. } & 3.8779 \mathrm{~cm}^{-1} \\ \text { 3. } & 8.2006 \mathrm{~cm}^{-1}\end{array}$

\section{B3LYP/6-31G(d) Molecular Geometry in Cartesian Coordinates}

C

$\mathrm{H}$

$\mathrm{H}$

C

0

0

$\mathrm{H}$

0

$\mathrm{H}$

C

C

C

C

$\mathrm{H}$

$\mathrm{H}$

$\mathrm{H}$

C

C

C

$\mathrm{H}$

$\mathrm{H}$

$\mathrm{H}$

C

$\mathrm{H}$

$\mathrm{H}$

$\mathrm{H}$

C

$\mathrm{H}$

$\mathrm{H}$

$\mathrm{H}$

C

$\mathrm{H}$

\section{$-1.731618$}

$-1.737987$

$-0.793441$

1. 296231

1.316048

1.033109

1.111019

$-2.757868$

$-3.602823$

0.295993

$-0.796951$

2. 229331

1.333473

$-1.057644$

2.478018

3.114098

1.684482

1.821623

0.732411

0.690849

1.078422

$-0.280164$

3.048038

2.930367

3. 491769

3. 746675

2.989276

2.889629

3.947973

3.004867

0.527023

0.332344
0.385592

$-0.166681$

0.154917

1.720587

2.693425

0.505100

$-0.266555$

$-0.099580$

$-0.002448$

$-2.429667$

$-3.210688$

$-2.837736$

$-1.971351$

$-2.229553$

$-3.768790$

$-2.348789$

$-2.688008$

$-4.249397$

$-2.254679$

$-1.163582$

$-2.632369$

$-2.629120$

$-2.003784$

$-0.918777$

$-2.248959$

$-2.309176$

$-4.882649$

$-4.733494$

$-4.471289$

$-5.960733$

$-5.012336$

$-5.025635$
0.514055

$-0.434822$

1.018104

$-0.889466$

$-1.609800$

$-1.408809$

$-0.799042$

1. 387061

0.915976

3.138296

1.450009

1.645906

2. 352309

1.053730

2.159993

1. 235921

$-2.021187$

$-1.847052$

$-3.137900$

$-3.194952$

$-4.107671$

$-2.964839$

$-2.197124$

$-2.148463$

$-3.168220$

$-1.412689$

$-2.607558$

$-3.689262$

-2. 283691

$-2.415141$

$-2.185566$

$-3.263742$ 


\begin{tabular}{|c|c|c|c|}
\hline $\mathrm{H}$ & 0.632672 & -6.046341 & -1.841859 \\
\hline $\mathrm{H}$ & -0.340964 & -4.580153 & -1.680667 \\
\hline 0 & 1.127116 & -2.269491 & -0.735217 \\
\hline 0 & 2.078882 & -4.381581 & -0.441447 \\
\hline B & 1.364095 & -3.347357 & 0.214112 \\
\hline 0 & 0.089103 & -3.914241 & 0.820385 \\
\hline C & -1.867114 & -3.936779 & 2.167947 \\
\hline C & -1.764850 & -5.317693 & 2.399514 \\
\hline C & -3.006345 & -3.239361 & 2.599109 \\
\hline C & -2.790509 & -5.989800 & 3.058721 \\
\hline H & -0.886548 & -5.848431 & 2.046583 \\
\hline C & -4.028307 & -3.919480 & 3.259384 \\
\hline $\mathrm{H}$ & -3.077329 & -2.171029 & 2.409552 \\
\hline C & -3.922913 & -5.292991 & 3.491978 \\
\hline $\mathrm{H}$ & -2.710667 & -7.059573 & 3.232280 \\
\hline $\mathrm{H}$ & -4.909795 & -3.377540 & 3.591542 \\
\hline $\mathrm{H}$ & -4.721719 & -5.820830 & 4.006281 \\
\hline $\mathrm{H}$ & 1.322272 & -0.920505 & 2.064047 \\
\hline $\mathrm{H}$ & 0.364146 & -3.407716 & 3.605755 \\
\hline $\mathrm{H}$ & -0.397783 & -1.731562 & 3.597888 \\
\hline C & 0.414909 & 2.251165 & 1.362804 \\
\hline C & 1.494817 & 1.802062 & 0.609284 \\
\hline C & 2.753846 & 1.474360 & 1.218972 \\
\hline C & 2.842671 & 1.551516 & 2.598267 \\
\hline $\mathrm{H}$ & 3.794807 & 1.345116 & 3.079473 \\
\hline C & -1.832071 & 1.878570 & 0.262631 \\
\hline C & -0.834859 & 2.746940 & 0.685276 \\
\hline C & -3.158249 & 3.751785 & -0.541627 \\
\hline C & -3.006033 & 2.392767 & -0.384830 \\
\hline $\mathrm{H}$ & -4.052475 & 4.137373 & -1.025713 \\
\hline C & -2.162572 & 4.668079 & -0.119232 \\
\hline C & -0.969485 & 4.164759 & 0.486718 \\
\hline C & 0.033457 & 5.096704 & 0.868941 \\
\hline C & -0.144496 & 6.449316 & 0.677772 \\
\hline C & -1.332412 & 6.943512 & 0.090609 \\
\hline C & -2.318210 & 6.067569 & -0.302106 \\
\hline $\mathrm{H}$ & 0.954636 & 4.732261 & 1.309133 \\
\hline $\mathrm{H}$ & 0.637450 & 7.142810 & 0.974975 \\
\hline $\mathrm{H}$ & -1.459713 & 8.012744 & -0.055483 \\
\hline $\mathrm{H}$ & -3.231760 & 6.433203 & -0.765148 \\
\hline C & 1.747586 & 1.928920 & 3.411753 \\
\hline C & 0.510471 & 2.295167 & 2.791837 \\
\hline C & -0.577796 & 2.681342 & 3.622021 \\
\hline C & -0.444576 & 2.708851 & 4.992332 \\
\hline C & 0.783159 & 2.356308 & 5.603369 \\
\hline C & 1.854210 & 1.975642 & 4.828797 \\
\hline $\mathrm{H}$ & -1.522991 & 2.944631 & 3.160158 \\
\hline $\mathrm{H}$ & -1.288032 & 3.002341 & 5.611188 \\
\hline $\mathrm{H}$ & 0.874914 & 2.385102 & 6.685720 \\
\hline $\mathrm{H}$ & 2.800153 & 1.700596 & 5.289146 \\
\hline C & -6.110721 & -0.152869 & -1.870078 \\
\hline C & -6.376466 & 0.643132 & -0.753078 \\
\hline C & -5.337427 & 1.463299 & -0.277337 \\
\hline C & -4.081938 & 1.482313 & -0.892512 \\
\hline
\end{tabular}




\begin{tabular}{|c|c|c|c|}
\hline C & -3.857248 & 0.667162 & -2.016118 \\
\hline C & -4.863951 & -0.156538 & -2.524653 \\
\hline $\mathrm{H}$ & -6.894473 & -0.794155 & -2.255385 \\
\hline $\mathrm{H}$ & -5.496699 & 2.103456 & 0.585868 \\
\hline $\mathrm{H}$ & -2.885260 & 0.710973 & -2.494211 \\
\hline C & -7.741994 & 0.655241 & -0.039148 \\
\hline C & -4.659469 & -1.049430 & -3.764019 \\
\hline C & -8.328991 & 2.086554 & -0.076717 \\
\hline $\mathrm{H}$ & -9.300401 & 2.112680 & 0.431920 \\
\hline $\mathrm{H}$ & -7.674389 & 2.810438 & 0.419690 \\
\hline $\mathrm{H}$ & -8.476887 & 2.422335 & -1.109423 \\
\hline C & -8.760016 & -0.296126 & -0.695940 \\
\hline $\mathrm{H}$ & -9.710724 & -0.248671 & -0.153182 \\
\hline $\mathrm{H}$ & -8.961673 & -0.023033 & -1.737922 \\
\hline $\mathrm{H}$ & -8.420015 & -1.337808 & -0.673895 \\
\hline C & -7.556739 & 0.216886 & 1.433319 \\
\hline $\mathrm{H}$ & -8.519986 & 0.227018 & 1.957774 \\
\hline $\mathrm{H}$ & -7.150090 & -0.799516 & 1.490773 \\
\hline $\mathrm{H}$ & -6.876541 & 0.881724 & 1.975825 \\
\hline C & -3.251182 & -0.899403 & -4.369815 \\
\hline $\mathrm{H}$ & -3.158900 & -1.547052 & -5.248829 \\
\hline $\mathrm{H}$ & -3.052835 & 0.128152 & -4.694670 \\
\hline $\mathrm{H}$ & -2.465939 & -1.191009 & -3.663484 \\
\hline C & -4.858391 & -2.531851 & -3.367365 \\
\hline $\mathrm{H}$ & -4.720794 & -3.180805 & -4.240806 \\
\hline $\mathrm{H}$ & -4.132864 & -2.834415 & -2.603686 \\
\hline $\mathrm{H}$ & -5.861385 & -2.718507 & -2.969009 \\
\hline C & -5.691639 & -0.666281 & -4.851361 \\
\hline $\mathrm{H}$ & -5.556932 & -1.294561 & -5.740147 \\
\hline $\mathrm{H}$ & -6.722069 & -0.797866 & -4.504786 \\
\hline $\mathrm{H}$ & -5.571236 & 0.380058 & -5.154312 \\
\hline C & 3.968410 & 1.119021 & 0.429151 \\
\hline C & 4.837306 & 0.109101 & 0.886411 \\
\hline C & 4.308450 & 1.827046 & -0.727518 \\
\hline C & 6.017061 & -0.199121 & 0.207895 \\
\hline $\mathrm{H}$ & 4.565863 & -0.436619 & 1.782616 \\
\hline C & 5.474943 & 1.533329 & -1.452182 \\
\hline $\mathrm{H}$ & 3.664108 & 2.632139 & -1.058541 \\
\hline C & 6.306873 & 0.522396 & -0.965661 \\
\hline $\mathrm{H}$ & 7.215277 & 0.283228 & -1.506108 \\
\hline C & 5.785444 & 2.334512 & -2.730635 \\
\hline C & 7.002139 & -1.279394 & 0.696389 \\
\hline C & 5.914097 & 3.835930 & -2.378602 \\
\hline $\mathrm{H}$ & 4.991838 & 4.233489 & -1.943338 \\
\hline $\mathrm{H}$ & 6.725266 & 4.003053 & -1.660166 \\
\hline $\mathrm{H}$ & 6.133692 & 4.419681 & -3.280906 \\
\hline C & 7.097323 & 1.885990 & -3.401856 \\
\hline $\mathrm{H}$ & 7.269445 & 2.480372 & -4.306273 \\
\hline $\mathrm{H}$ & 7.963714 & 2.027238 & -2.745049 \\
\hline $\mathrm{H}$ & 7.062899 & 0.832479 & -3.702499 \\
\hline C & 4.631470 & 2.142124 & -3.744495 \\
\hline $\mathrm{H}$ & 4.539760 & 1.088395 & -4.032808 \\
\hline $\mathrm{H}$ & 3.666658 & 2.460759 & -3.337732 \\
\hline $\mathrm{H}$ & 4.823942 & 2.726527 & -4.652859 \\
\hline
\end{tabular}




\begin{tabular}{|llll|} 
C & 6.539113 & -1.953199 & 2.002345 \\
$H$ & 6.440091 & -1.231316 & 2.821275 \\
$H$ & 5.579805 & -2.468719 & 1.882185 \\
$H$ & 7.276952 & -2.702266 & 2.311293 \\
C & 7.153306 & -2.376238 & -0.384263 \\
$H$ & 6.194146 & -2.868062 & -0.581912 \\
$H$ & 7.521386 & -1.967759 & -1.331223 \\
$H$ & 7.864978 & -3.142044 & -0.052178 \\
C & 8.381822 & -0.628987 & 0.959561 \\
$H$ & 9.099105 & -1.383924 & 1.304325 \\
$H$ & 8.794559 & -0.165303 & 0.057657 \\
$H$ & 8.308418 & 0.147174 & 1.730073 \\
\hline
\end{tabular}

\section{C-Catalyzed reaction TS 82}

\section{Datum}

Value

\begin{tabular}{lc}
\hline B3LYP/6-31G(d) Energy & -3038.952758 \\
\hline B3LYP-D3(BJ)/def2-TZVPP-IEF-PCM(DCM) Energy & -3040.508124 \\
\hline B3LYP-D3(BJ)/def2-TZVPP-IEF-PCM(DCM)//B3LYP/6-31G(d) Free Energy (Quasiharmonic) & -3039.28002 \\
\hline
\end{tabular}

Number of Imaginary Frequencies

Frequencies (Top 3 out of 450)

$\begin{array}{lr}\text { 1. } & -301.9892 \mathrm{~cm}^{-1} \\ \text { 2. } & 6.8001 \mathrm{~cm}^{-1} \\ \text { 3. } & 8.4072 \mathrm{~cm}^{-1}\end{array}$

\section{B3LYP/6-31G(d) Molecular Geometry in Cartesian Coordinates}

C

$\mathrm{H}$

$\mathrm{H}$

C

0

0

$\mathrm{H}$

0

$\mathrm{H}$

C

C

C

C

$\mathrm{H}$

\section{$-1.712275$}

$-2.197912$

$-0.656111$

1.392703

1. 216811

1.446838

1.505304

$-2.202396$

$-3.170736$

1.852248

0.009502

2.644592

2.637385

0.244404
$-0.893187$

0.082378

$-0.704317$

$-0.672345$

$-0.786672$

0.557966

0.618080

$-1.534112$

$-1.464682$

4.120281

3. 350279

3.961490

3. 533787

2. 322156
$-0.828752$

$-0.691847$

$-1.013308$

1.332966

2. 526376

0.783375

$-0.204142$

$-2.009599$

$-1.981329$

1.564175

0.627956

$-0.772881$

0.597530

0.897058 


\begin{tabular}{|c|c|c|c|}
\hline $\mathrm{H}$ & 3.481174 & 3.579552 & -1.360104 \\
\hline $\mathrm{H}$ & 2.514677 & 5.036621 & -0.919339 \\
\hline C & 1.419980 & 1.486185 & -3.075787 \\
\hline C & 0.705933 & 2.726341 & -3.722275 \\
\hline C & 2.916175 & 1.421795 & -3.425171 \\
\hline $\mathrm{H}$ & 3.409702 & 0.726840 & -2.738985 \\
\hline $\mathrm{H}$ & 3.076656 & 1.067981 & -4.449742 \\
\hline $\mathrm{H}$ & 3.394133 & 2.400212 & -3.323640 \\
\hline C & 0.770998 & 0.137292 & -3.378765 \\
\hline $\mathrm{H}$ & 0.822962 & -0.073049 & -4.453698 \\
\hline $\mathrm{H}$ & -0.277313 & 0.093152 & -3.079455 \\
\hline $\mathrm{H}$ & 1.308303 & -0.667330 & -2.863719 \\
\hline C & -0.827761 & 2.599666 & -3.715331 \\
\hline $\mathrm{H}$ & -1.183694 & 1.867519 & -4.448681 \\
\hline $\mathrm{H}$ & -1.197795 & 2.309177 & -2.727769 \\
\hline $\mathrm{H}$ & -1.258994 & 3.574689 & -3.964238 \\
\hline C & 1.185829 & 3.084222 & -5.130164 \\
\hline $\mathrm{H}$ & 0.995041 & 2.265940 & -5.834636 \\
\hline $\mathrm{H}$ & 0.646150 & 3.968484 & -5.485378 \\
\hline $\mathrm{H}$ & 2.253211 & 3.317492 & -5.142744 \\
\hline 0 & 1.303600 & 1.784801 & -1.656346 \\
\hline 0 & 1.080603 & 3.787920 & -2.831695 \\
\hline B & 1.240199 & 3.242257 & -1.530133 \\
\hline 0 & 0.126082 & 3.711724 & -0.609270 \\
\hline C & -0.968250 & 4.056454 & 1.477415 \\
\hline C & -1.339953 & 3.484996 & 2.705273 \\
\hline C & -1.536156 & 5.277024 & 1.079697 \\
\hline C & -2.259471 & 4.131617 & 3.529045 \\
\hline $\mathrm{H}$ & -0.899746 & 2.538025 & 3.010034 \\
\hline C & -2.452559 & 5.920139 & 1.907757 \\
\hline $\mathrm{H}$ & -1.252823 & 5.702768 & 0.122392 \\
\hline C & -2.813618 & 5.352224 & 3.133818 \\
\hline $\mathrm{H}$ & -2.541305 & 3.686121 & 4.479036 \\
\hline $\mathrm{H}$ & -2.888705 & 6.865966 & 1.597921 \\
\hline $\mathrm{H}$ & -3.527190 & 5.858702 & 3.778137 \\
\hline $\mathrm{H}$ & 3.073412 & 2.562085 & 0.825349 \\
\hline $\mathrm{H}$ & 1.529008 & 5.151446 & 1.453589 \\
\hline $\mathrm{H}$ & 1.815341 & 3.713059 & 2.570427 \\
\hline C & 0.452728 & -2.657509 & 0.167921 \\
\hline C & 1.554127 & -1.838272 & 0.390565 \\
\hline C & 2.819326 & -2.082286 & -0.247189 \\
\hline C & 2.901962 & -3.131289 & -1.143452 \\
\hline $\mathrm{H}$ & 3.858928 & -3.362985 & -1.603233 \\
\hline C & -1.880148 & -1.718246 & 0.433092 \\
\hline C & -0.836372 & -2.489649 & 0.931155 \\
\hline C & -3.299468 & -2.387594 & 2.292727 \\
\hline C & -3.143096 & -1.695650 & 1.112579 \\
\hline $\mathrm{H}$ & -4.259415 & -2.368900 & 2.803736 \\
\hline C & -2.243216 & -3.150366 & 2.851299 \\
\hline C & -0.993994 & -3.220596 & 2.157422 \\
\hline C & 0.043370 & -4.012423 & 2.722282 \\
\hline C & -0.146447 & -4.694308 & 3.903290 \\
\hline C & -1.383493 & -4.621616 & 4.586228 \\
\hline C & -2.408006 & -3.863370 & 4.068853 \\
\hline
\end{tabular}




\begin{tabular}{|c|c|c|c|}
\hline $\mathrm{H}$ & 1.000532 & -4.066530 & 2.216304 \\
\hline $\mathrm{H}$ & 0.662354 & -5.289937 & 4.317926 \\
\hline $\mathrm{H}$ & -1.519615 & -5.163567 & 5.518302 \\
\hline $\mathrm{H}$ & -3.363822 & -3.798622 & 4.583740 \\
\hline C & 1.792060 & -3.953118 & -1.452019 \\
\hline C & 0.545582 & -3.725008 & -0.786503 \\
\hline C & -0.552434 & -4.572314 & -1.100553 \\
\hline C & -0.419627 & -5.592087 & -2.015814 \\
\hline C & 0.816667 & -5.820337 & -2.666013 \\
\hline C & 1.897767 & -5.016821 & -2.388695 \\
\hline $\mathrm{H}$ & -1.506436 & -4.395837 & -0.618323 \\
\hline $\mathrm{H}$ & -1.271876 & -6.226063 & -2.244567 \\
\hline $\mathrm{H}$ & 0.907213 & -6.629552 & -3.385634 \\
\hline $\mathrm{H}$ & 2.851960 & -5.181347 & -2.883584 \\
\hline C & -6.561043 & 0.253762 & -0.576970 \\
\hline C & -6.207648 & -1.026424 & -1.040464 \\
\hline C & -5.078807 & -1.630365 & -0.479039 \\
\hline C & -4.322901 & -0.986077 & 0.518236 \\
\hline C & -4.711045 & 0.281158 & 0.952286 \\
\hline C & -5.837912 & 0.926713 & 0.411764 \\
\hline $\mathrm{H}$ & -7.434255 & 0.731702 & -1.004625 \\
\hline $\mathrm{H}$ & -4.780560 & -2.629929 & -0.777999 \\
\hline $\mathrm{H}$ & -4.118140 & 0.768895 & 1.720580 \\
\hline C & -7.067832 & -1.710528 & -2.120481 \\
\hline C & -6.218779 & 2.335355 & 0.905329 \\
\hline C & -6.529934 & -3.101945 & -2.505110 \\
\hline $\mathrm{H}$ & -7.172356 & -3.545790 & -3.273802 \\
\hline $\mathrm{H}$ & -5.514775 & -3.051118 & -2.915467 \\
\hline $\mathrm{H}$ & -6.518468 & -3.786684 & -1.649552 \\
\hline C & -8.512127 & -1.884011 & -1.592577 \\
\hline $\mathrm{H}$ & -9.140085 & -2.365723 & -2.352000 \\
\hline $\mathrm{H}$ & -8.527962 & -2.508926 & -0.692349 \\
\hline $\mathrm{H}$ & -8.972765 & -0.923275 & -1.340222 \\
\hline C & -7.090445 & -0.834088 & -3.395541 \\
\hline $\mathrm{H}$ & -7.705002 & -1.306613 & -4.171424 \\
\hline $\mathrm{H}$ & -7.506277 & 0.160020 & -3.201336 \\
\hline $\mathrm{H}$ & -6.079842 & -0.700581 & -3.798448 \\
\hline C & -5.082595 & 3.319784 & 0.541378 \\
\hline $\mathrm{H}$ & -5.324707 & 4.330337 & 0.891440 \\
\hline $\mathrm{H}$ & -4.130689 & 3.031466 & 0.997575 \\
\hline $\mathrm{H}$ & -4.935231 & 3.362385 & -0.543867 \\
\hline C & -7.524695 & 2.851582 & 0.271118 \\
\hline $\mathrm{H}$ & -7.754635 & 3.847354 & 0.666428 \\
\hline $\mathrm{H}$ & -7.444102 & 2.943116 & -0.817929 \\
\hline $\mathrm{H}$ & -8.376370 & 2.200202 & 0.499239 \\
\hline C & -6.410197 & 2.315519 & 2.440443 \\
\hline $\mathrm{H}$ & -6.685405 & 3.314245 & 2.800682 \\
\hline $\mathrm{H}$ & -7.206512 & 1.619405 & 2.728241 \\
\hline $\mathrm{H}$ & -5.496804 & 2.016125 & 2.963957 \\
\hline C & 4.046957 & -1.299011 & 0.077290 \\
\hline C & 4.953399 & -0.950284 & -0.942049 \\
\hline C & 4.355659 & -0.953825 & 1.396667 \\
\hline C & 6.136489 & -0.264254 & -0.662704 \\
\hline $\mathrm{H}$ & 4.707022 & -1.218923 & -1.962585 \\
\hline
\end{tabular}




$\begin{array}{lrrr}\text { C } & 5.522783 & -0.243680 & 1.718637 \\ \text { H } & 3.682719 & -1.256529 & 2.190411 \\ \text { C } & 6.391365 & 0.088197 & 0.675756 \\ \text { H } & 7.299730 & 0.634313 & 0.901005 \\ \text { C } & 5.795575 & 0.127737 & 3.188821 \\ \text { C } & 7.152056 & 0.123753 & -1.755574 \\ \text { C } & 5.878591 & -1.162386 & 4.039315 \\ \text { H } & 4.947357 & -1.736621 & 4.001937 \\ \text { H } & 6.689024 & -1.812089 & 3.688852 \\ \text { H } & 6.072997 & -0.912265 & 5.089432 \\ \text { C } & 7.115118 & 0.902921 & 3.365118 \\ \text { H } & 7.261102 & 1.145380 & 4.423807 \\ \text { H } & 7.981728 & 0.316059 & 3.039131 \\ \text { H } & 7.112181 & 1.847493 & 2.808948 \\ \text { C } & 4.639933 & 1.013881 & 3.714358 \\ \text { H } & 4.583353 & 1.952557 & 3.149904 \\ \text { H } & 3.668216 & 0.516258 & 3.633916 \\ \text { H } & 4.804090 & 1.264899 & 4.769716 \\ \text { C } & 8.540559 & -0.462158 & -1.403879 \\ \text { H } & 8.916651 & -0.086895 & -0.446597 \\ \text { H } & 8.499874 & -1.555637 & -1.340746 \\ \text { H } & 9.272116 & -0.193319 & -2.175671 \\ \text { C } & 6.755108 & -0.408894 & -3.145534 \\ \text { H } & 6.674981 & -1.502042 & -3.153463 \\ \text { H } & 5.802046 & 0.007532 & -3.489412 \\ \text { H } & 7.519422 & -0.128137 & -3.878966 \\ \text { C } & 7.253249 & 1.665446 & -1.838911 \\ \text { H } & 7.983987 & 1.960657 & -2.602080 \\ \text { H } & 6.286206 & 2.106653 & -2.106266 \\ \text { H } & 7.569037 & 2.104331 & -0.886526\end{array}$

\section{C-Catalyzed reaction TS 83}

Datum

Value

\begin{tabular}{lc}
\hline B3LYP/6-31G(d) Energy & -3038.951515 \\
\hline B3LYP-D3(BJ)/def2-TZVPP-IEF-PCM(DCM) Energy & -3040.509451 \\
\hline B3LYP-D3(BJ)/def2-TZVPP-IEF-PCM(DCM)//B3LYP/6-31G(d) Free Energy (Quasiharmonic) & -3039.279872 \\
\hline
\end{tabular}

Number of Imaginary Frequencies

Frequencies (Top 3 out of 450)

$\begin{array}{lr}\text { 1. } & -305.1002 \mathrm{~cm}^{-1} \\ \text { 2. } & 11.6842 \mathrm{~cm}^{-1} \\ \text { 3. } & 13.3637 \mathrm{~cm}^{-1}\end{array}$




\begin{tabular}{|c|c|c|c|}
\hline C & 1.356114 & -0.755401 & 0.991778 \\
\hline $\mathrm{H}$ & 2.205689 & -0.156677 & 0.660356 \\
\hline $\mathrm{H}$ & 0.467252 & -0.123913 & 0.922403 \\
\hline C & -1.907025 & -0.663964 & -1.241537 \\
\hline 0 & -2.204924 & -1.192426 & -2.283862 \\
\hline 0 & -1.246600 & 0.520851 & -1.238882 \\
\hline $\mathrm{H}$ & -1.082918 & 0.925475 & -0.351110 \\
\hline 0 & 1.638843 & -1.055762 & 2.366783 \\
\hline $\mathrm{H}$ & 0.875080 & -1.535033 & 2.728914 \\
\hline C & 1.986915 & 3.163252 & -2.400211 \\
\hline C & 2.418794 & 3.034663 & -0.265029 \\
\hline C & -0.247076 & 3.765923 & -1.511726 \\
\hline C & 0.657458 & 2.872135 & -2.186218 \\
\hline $\mathrm{H}$ & 2.205912 & 1.966758 & -0.196623 \\
\hline $\mathrm{H}$ & -1.299215 & 3.500568 & -1.626440 \\
\hline $\mathrm{H}$ & -0.080542 & 4.825380 & -1.722478 \\
\hline C & -0.756845 & 2.640804 & 2.128028 \\
\hline C & -1.341447 & 4.099598 & 1.998305 \\
\hline C & -1.763181 & 1.593672 & 2.595875 \\
\hline $\mathrm{H}$ & -2.132403 & 1.840332 & 3.598231 \\
\hline $\mathrm{H}$ & -1.286798 & 0.609655 & 2.655659 \\
\hline $\mathrm{H}$ & -2.622847 & 1.517886 & 1.927324 \\
\hline C & 0.496201 & 2.566218 & 3.017126 \\
\hline $\mathrm{H}$ & 0.242098 & 2.731022 & 4.070022 \\
\hline $\mathrm{H}$ & 1.243313 & 3.308611 & 2.725133 \\
\hline $\mathrm{H}$ & 0.945378 & 1.571028 & 2.931675 \\
\hline C & -1.157491 & 4.972120 & 3.243954 \\
\hline $\mathrm{H}$ & -1.679163 & 4.548827 & 4.110897 \\
\hline $\mathrm{H}$ & -0.100921 & 5.092313 & 3.493515 \\
\hline $\mathrm{H}$ & -1.572055 & 5.967777 & 3.053368 \\
\hline C & -2.820051 & 4.119647 & 1.575832 \\
\hline $\mathrm{H}$ & -3.488845 & 3.803177 & 2.384169 \\
\hline $\mathrm{H}$ & -3.085632 & 5.142334 & 1.289396 \\
\hline $\mathrm{H}$ & -2.993412 & 3.471185 & 0.712003 \\
\hline 0 & -0.366561 & 2.350994 & 0.751137 \\
\hline 0 & -0.572490 & 4.668401 & 0.931021 \\
\hline B & 0.053668 & 3.632464 & 0.188465 \\
\hline 0 & 1.568240 & 3.857518 & 0.250212 \\
\hline C & 3.841496 & 3.429591 & -0.288523 \\
\hline C & 4.215693 & 4.770523 & -0.105462 \\
\hline C & 4.831087 & 2.449561 & -0.456469 \\
\hline C & 5.561999 & 5.123613 & -0.101187 \\
\hline $\mathrm{H}$ & 3.442631 & 5.517380 & 0.043955 \\
\hline C & 6.178253 & 2.808797 & -0.445812 \\
\hline $\mathrm{H}$ & 4.550958 & 1.407457 & -0.588555 \\
\hline C & 6.546217 & 4.145030 & -0.272748 \\
\hline $\mathrm{H}$ & 5.846949 & 6.162225 & 0.042626 \\
\hline $\mathrm{H}$ & 6.937193 & 2.041356 & -0.565834 \\
\hline $\mathrm{H}$ & 7.596614 & 4.423326 & -0.265294 \\
\hline $\mathrm{H}$ & 0.327882 & 1.842738 & -2.320545 \\
\hline
\end{tabular}




\begin{tabular}{|c|c|c|c|}
\hline $\mathrm{H}$ & 2.321447 & 4.195944 & -2.442704 \\
\hline $\mathrm{H}$ & 2.647692 & 2.432700 & -2.859000 \\
\hline C & -1.258031 & -2.082487 & 0.697101 \\
\hline C & -2.210890 & -1.249507 & 0.118984 \\
\hline C & -3.484034 & -1.013971 & 0.742856 \\
\hline C & -3.675560 & -1.516274 & 2.018595 \\
\hline $\mathrm{H}$ & -4.611129 & -1.313199 & 2.531881 \\
\hline C & 1.206658 & -1.974044 & 0.099602 \\
\hline C & -0.049251 & -2.551616 & -0.073695 \\
\hline C & 2.169824 & -3.601092 & -1.427374 \\
\hline C & 2.348055 & -2.516594 & -0.589206 \\
\hline $\mathrm{H}$ & 3.032183 & -4.028300 & -1.932491 \\
\hline C & 0.908939 & -4.207183 & -1.628054 \\
\hline C & -0.229745 & -3.683564 & -0.940151 \\
\hline C & -1.486397 & -4.318610 & -1.144400 \\
\hline C & -1.603395 & -5.406880 & -1.979419 \\
\hline C & -0.472881 & -5.922415 & -2.656809 \\
\hline C & 0.756525 & -5.331924 & -2.483518 \\
\hline $\mathrm{H}$ & -2.365781 & -3.930531 & -0.644547 \\
\hline $\mathrm{H}$ & -2.574769 & -5.871664 & -2.124021 \\
\hline $\mathrm{H}$ & -0.580876 & -6.781398 & -3.313531 \\
\hline $\mathrm{H}$ & 1.634118 & -5.716040 & -2.998198 \\
\hline C & -2.703032 & -2.296509 & 2.685959 \\
\hline C & -1.490174 & -2.630651 & 2.003316 \\
\hline C & -0.566188 & -3.497765 & 2.655810 \\
\hline C & -0.811564 & -3.965910 & 3.929353 \\
\hline C & -1.997261 & -3.605690 & 4.612919 \\
\hline C & -2.923187 & -2.793997 & 3.999492 \\
\hline $\mathrm{H}$ & 0.337588 & -3.796437 & 2.135299 \\
\hline $\mathrm{H}$ & -0.091533 & -4.622614 & 4.409649 \\
\hline $\mathrm{H}$ & -2.176918 & -3.979568 & 5.617011 \\
\hline $\mathrm{H}$ & -3.845074 & -2.523892 & 4.508917 \\
\hline C & 6.390229 & -1.142218 & -0.220722 \\
\hline C & 5.855857 & -1.361964 & -1.500181 \\
\hline C & 4.519240 & -1.770524 & -1.586681 \\
\hline C & 3.733402 & -1.977315 & -0.442392 \\
\hline C & 4.306207 & -1.745239 & 0.816064 \\
\hline C & 5.633966 & -1.318200 & 0.948140 \\
\hline $\mathrm{H}$ & 7.421907 & -0.829318 & -0.132869 \\
\hline $\mathrm{H}$ & 4.064353 & -1.933782 & -2.559203 \\
\hline $\mathrm{H}$ & 3.696540 & -1.906934 & 1.698282 \\
\hline C & 6.680809 & -1.181144 & -2.790788 \\
\hline C & 6.206447 & -1.067306 & 2.357245 \\
\hline C & 8.128142 & -0.733260 & -2.510923 \\
\hline $\mathrm{H}$ & 8.670892 & -0.627629 & -3.456996 \\
\hline $\mathrm{H}$ & 8.167151 & 0.236824 & -2.001696 \\
\hline H & 8.670954 & -1.462043 & -1.898548 \\
\hline C & 6.738507 & -2.526978 & -3.553251 \\
\hline $\mathrm{H}$ & 7.321329 & -2.418408 & -4.476168 \\
\hline $\mathrm{H}$ & 7.211495 & -3.303966 & -2.941864 \\
\hline $\mathrm{H}$ & 5.740072 & -2.881072 & -3.830003 \\
\hline C & 6.013337 & -0.114924 & -3.691992 \\
\hline $\mathrm{H}$ & 6.583929 & 0.011927 & -4.620241 \\
\hline $\mathrm{H}$ & 4.991424 & -0.396113 & -3.966987 \\
\hline
\end{tabular}




\begin{tabular}{|c|c|c|c|}
\hline $\mathrm{H}$ & 5.969303 & 0.856572 & -3.186164 \\
\hline C & 7.672375 & -0.595304 & 2.324548 \\
\hline $\mathrm{H}$ & 8.028851 & -0.431144 & 3.347751 \\
\hline $\mathrm{H}$ & 8.332581 & -1.337603 & 1.860999 \\
\hline $\mathrm{H}$ & 7.784485 & 0.350636 & 1.782213 \\
\hline C & 5.362702 & 0.022130 & 3.063039 \\
\hline $\mathrm{H}$ & 5.746939 & 0.203790 & 4.074651 \\
\hline $\mathrm{H}$ & 5.407751 & 0.967814 & 2.510165 \\
\hline $\mathrm{H}$ & 4.310251 & -0.266242 & 3.148756 \\
\hline C & 6.142570 & -2.376513 & 3.179726 \\
\hline $\mathrm{H}$ & 6.542386 & -2.211388 & 4.187889 \\
\hline $\mathrm{H}$ & 5.115731 & -2.740853 & 3.284288 \\
\hline $\mathrm{H}$ & 6.733891 & -3.168444 & 2.705238 \\
\hline C & -4.635435 & -0.359013 & 0.050272 \\
\hline C & -4.518161 & 0.793222 & -0.741342 \\
\hline C & -5.902808 & -0.949647 & 0.196820 \\
\hline C & -5.631625 & 1.350359 & -1.384491 \\
\hline $\mathrm{H}$ & -3.549306 & 1.259523 & -0.858624 \\
\hline C & -7.042073 & -0.411127 & -0.406425 \\
\hline $\mathrm{H}$ & -5.977238 & -1.864798 & 0.771160 \\
\hline C & -6.876655 & 0.738664 & -1.189730 \\
\hline $\mathrm{H}$ & -7.747440 & 1.165666 & -1.678053 \\
\hline C & -8.440401 & -1.040810 & -0.254071 \\
\hline C & -5.533742 & 2.586223 & -2.300580 \\
\hline C & -8.424691 & -2.304188 & 0.627373 \\
\hline $\mathrm{H}$ & -7.792138 & -3.091577 & 0.202016 \\
\hline $\mathrm{H}$ & -8.069745 & -2.089676 & 1.641977 \\
\hline $\mathrm{H}$ & -9.440340 & -2.706800 & 0.711894 \\
\hline C & -9.395852 & -0.013215 & 0.398040 \\
\hline $\mathrm{H}$ & -10.401915 & -0.438400 & 0.500364 \\
\hline $\mathrm{H}$ & -9.043762 & 0.270949 & 1.396387 \\
\hline $\mathrm{H}$ & -9.479898 & 0.901136 & -0.198427 \\
\hline C & -8.985402 & -1.435778 & -1.647228 \\
\hline $\mathrm{H}$ & -9.069354 & -0.570963 & -2.313382 \\
\hline $\mathrm{H}$ & -8.328372 & -2.167021 & -2.131510 \\
\hline $\mathrm{H}$ & -9.983035 & -1.882437 & -1.554275 \\
\hline C & -6.402081 & 3.730190 & -1.725771 \\
\hline $\mathrm{H}$ & -7.455388 & 3.441879 & -1.642427 \\
\hline $\mathrm{H}$ & -6.054868 & 4.022251 & -0.728050 \\
\hline $\mathrm{H}$ & -6.348423 & 4.612464 & -2.375343 \\
\hline C & -4.090449 & 3.102651 & -2.441894 \\
\hline $\mathrm{H}$ & -3.689151 & 3.451182 & -1.483944 \\
\hline $\mathrm{H}$ & -3.417793 & 2.334817 & -2.839302 \\
\hline $\mathrm{H}$ & -4.070181 & 3.953944 & -3.131753 \\
\hline C & -6.043002 & 2.213641 & -3.714180 \\
\hline $\mathrm{H}$ & -5.978501 & 3.082063 & -4.381200 \\
\hline $\mathrm{H}$ & -5.440007 & 1.407048 & -4.145802 \\
\hline $\mathrm{H}$ & -7.086535 & 1.881944 & -3.701578 \\
\hline
\end{tabular}




\begin{tabular}{lc}
\hline B3LYP/6-31G(d) Energy & -3038.957121 \\
\hline B3LYP-D3(BJ)/def2-TZVPP-IEF-PCM(DCM) Energy & -3040.508244 \\
\hline B3LYP-D3(BJ)/def2-TZVPP-IEF-PCM(DCM)//B3LYP/6-31G(d) Free Energy (Quasiharmonic) & -3039.279816 \\
\hline
\end{tabular}

Number of Imaginary Frequencies

Frequencies (Top 3 out of 450)

$\begin{array}{lr}\text { 1. } & -387.0901 \mathrm{~cm}^{-1} \\ \text { 2. } & 8.1821 \mathrm{~cm}^{-1} \\ \text { 3. } & 10.2364 \mathrm{~cm}^{-1}\end{array}$

\section{B3LYP/6-31G(d) Molecular Geometry in Cartesian Coordinates}

C

$\mathrm{H}$

$\mathrm{H}$

C

0

0

$\mathrm{H}$

0

$\mathrm{H}$

C

C

C

C

$\mathrm{H}$

$\mathrm{H}$

$\mathrm{H}$

C

C

C

$\mathrm{H}$

$\mathrm{H}$

$\mathrm{H}$

C

$\mathrm{H}$

$\mathrm{H}$

$\mathrm{H}$

C

$\mathrm{H}$

$\mathrm{H}$

$\mathrm{H}$

C

$\mathrm{H}$

\subsection{5}

2. 216667

0.772727

$-2.217853$

$-2.870463$

$-1.548518$

$-0.925523$

0.412480

0.240182

1.079420

2. 317604

$-0.642049$

0.284783

2.163857

$-1.231499$

$-1.248819$

$-0.781398$

$-0.180811$

$-2.286714$

$-2.591744$

$-2.875978$

$-2.527435$

$-0.489125$

$-0.938804$

0.583640

$-0.919887$

1.174629

1.066800

1.862330

1.623944

$-1.119088$

$-1.374368$
0.442454

0.050729

$-0.007081$

2. 328730

3. 342291

1.778508

1.058715

0.068485

$-0.904340$

$-4.050804$

$-3.943564$

- 3.095921

$-2.997668$

$-5.017628$

$-2.198796$

$-4.003340$

$-3.443362$

$-4.869793$

$-3.353666$

$-2.302327$

$-3.878533$

$-3.780717$

$-2.892690$

$-3.530309$

$-2.820971$

$-1.890867$

$-5.098186$

$-5.219980$

$-4.268596$

$-6.012419$

$-6.038519$

$-6.075625$
0.098190

$-0.023889$

0.999791

$-0.665853$

$-0.815576$

$-1.677393$

$-1.379453$

$-1.045098$

$-0.992098$

3. 304898

1.535825

1.808611

2.885358

1.414275

1.621540

1.810227

$-1.791833$

$-1.494518$

$-1.502594$

$-1.500220$

$-2.262359$

$-0.524455$

$-3.187330$

$-3.957273$

$-3.377710$

- 3.292523

$-2.183937$

$-3.267312$

$-1.994659$

$-1.782022$

$-1.796947$

$-2.862453$ 


\begin{tabular}{|c|c|c|c|}
\hline $\mathrm{H}$ & -0.625988 & -6.980916 & -1.535803 \\
\hline $\mathrm{H}$ & -2.043314 & -5.972139 & -1.218106 \\
\hline 0 & -0.091167 & -2.622760 & -0.814257 \\
\hline 0 & 0.038359 & -4.821133 & -0.068681 \\
\hline B & 0.297538 & -3.461809 & 0.283105 \\
\hline 0 & 1.710371 & -3.177058 & 0.670519 \\
\hline C & 3.627595 & -3.505256 & 2.049548 \\
\hline C & 4.472903 & -4.441083 & 2.666823 \\
\hline C & 4.058554 & -2.177127 & 1.908713 \\
\hline C & 5.724664 & -4.055179 & 3.139891 \\
\hline $\mathrm{H}$ & 4.146392 & -5.473521 & 2.771081 \\
\hline C & 5.310754 & -1.794312 & 2.382519 \\
\hline $\mathrm{H}$ & 3.409938 & -1.459733 & 1.417779 \\
\hline C & 6.145668 & -2.729973 & 3.000356 \\
\hline $\mathrm{H}$ & 6.373475 & -4.787136 & 3.612750 \\
\hline $\mathrm{H}$ & 5.638616 & -0.765402 & 2.263187 \\
\hline $\mathrm{H}$ & 7.122985 & -2.428729 & 3.367289 \\
\hline $\mathrm{H}$ & 0.582857 & -1.997660 & 3.204929 \\
\hline $\mathrm{H}$ & 0.738755 & -5.071202 & 3.143474 \\
\hline $\mathrm{H}$ & 1.788751 & -3.910784 & 4.114094 \\
\hline C & -0.970032 & 1.813739 & 1.436513 \\
\hline C & -2.126700 & 1.638826 & 0.686462 \\
\hline C & -3.240613 & 0.885885 & 1.200731 \\
\hline C & -3.123491 & 0.334550 & 2.464076 \\
\hline $\mathrm{H}$ & -3.959629 & -0.227165 & 2.872005 \\
\hline C & 1.239788 & 1.950146 & 0.242566 \\
\hline C & 0.187724 & 2.600906 & 0.884299 \\
\hline C & 2.377325 & 4.074932 & -0.056636 \\
\hline C & 2.356200 & 2.706012 & -0.252032 \\
\hline $\mathrm{H}$ & 3.216111 & 4.651207 & -0.438637 \\
\hline C & 1.327228 & 4.764052 & 0.591757 \\
\hline C & 0.200957 & 4.021612 & 1.067700 \\
\hline C & -0.853469 & 4.729148 & 1.709453 \\
\hline C & -0.789219 & 6.093928 & 1.875415 \\
\hline C & 0.328920 & 6.825514 & 1.406629 \\
\hline C & 1.362145 & 6.173209 & 0.776150 \\
\hline $\mathrm{H}$ & -1.723556 & 4.182329 & 2.054670 \\
\hline $\mathrm{H}$ & -1.607641 & 6.616821 & 2.362464 \\
\hline $\mathrm{H}$ & 0.363664 & 7.903282 & 1.541414 \\
\hline $\mathrm{H}$ & 2.222575 & 6.725364 & 0.405423 \\
\hline C & -1.971732 & 0.504802 & 3.269418 \\
\hline C & -0.872270 & 1.262102 & 2.756884 \\
\hline C & 0.275407 & 1.430957 & 3.580853 \\
\hline C & 0.330337 & 0.878139 & 4.842305 \\
\hline C & -0.760259 & 0.129043 & 5.346927 \\
\hline C & -1.885491 & -0.051192 & 4.575152 \\
\hline $\mathrm{H}$ & 1.112138 & 2.009806 & 3.204367 \\
\hline $\mathrm{H}$ & 1.213732 & 1.024326 & 5.458154 \\
\hline $\mathrm{H}$ & -0.707198 & -0.298083 & 6.344746 \\
\hline $\mathrm{H}$ & -2.730072 & -0.621895 & 4.954007 \\
\hline C & 5.707639 & 1.024027 & -2.321171 \\
\hline C & 5.936279 & 1.864756 & -1.222346 \\
\hline C & 4.820146 & 2.382423 & -0.553896 \\
\hline C & 3.512350 & 2.080984 & -0.960024 \\
\hline
\end{tabular}




\begin{tabular}{|c|c|c|c|}
\hline C & 3.327066 & 1.235750 & -2.062044 \\
\hline C & 4.416024 & 0.693561 & -2.756238 \\
\hline $\mathrm{H}$ & 6.556846 & 0.612919 & -2.849540 \\
\hline $\mathrm{H}$ & 4.954395 & 3.025796 & 0.311257 \\
\hline $\mathrm{H}$ & 2.312609 & 1.008034 & -2.372488 \\
\hline C & 7.353171 & 2.219793 & -0.728835 \\
\hline C & 4.157375 & -0.244398 & -3.950872 \\
\hline C & 8.458387 & 1.583705 & -1.593375 \\
\hline $\mathrm{H}$ & 9.441783 & 1.870494 & -1.203590 \\
\hline $\mathrm{H}$ & 8.406672 & 0.489082 & -1.584009 \\
\hline $\mathrm{H}$ & 8.405507 & 1.919609 & -2.635168 \\
\hline C & 7.544318 & 3.754715 & -0.762697 \\
\hline $\mathrm{H}$ & 8.546396 & 4.023418 & -0.406154 \\
\hline $\mathrm{H}$ & 7.431369 & 4.139584 & -1.782575 \\
\hline $\mathrm{H}$ & 6.816066 & 4.270665 & -0.128539 \\
\hline C & 7.532401 & 1.715603 & 0.723311 \\
\hline $\mathrm{H}$ & 8.533079 & 1.967104 & 1.096401 \\
\hline $\mathrm{H}$ & 6.800648 & 2.165526 & 1.402719 \\
\hline $\mathrm{H}$ & 7.415211 & 0.626423 & 0.774348 \\
\hline C & 3.312356 & 0.491214 & -5.017812 \\
\hline $\mathrm{H}$ & 3.116574 & -0.170790 & -5.870405 \\
\hline $\mathrm{H}$ & 2.346069 & 0.817606 & -4.621117 \\
\hline $\mathrm{H}$ & 3.838276 & 1.377828 & -5.390299 \\
\hline C & 5.458336 & -0.725822 & -4.620307 \\
\hline $\mathrm{H}$ & 5.217405 & -1.386568 & -5.460822 \\
\hline $\mathrm{H}$ & 6.048058 & 0.109154 & -5.016195 \\
\hline $\mathrm{H}$ & 6.090092 & -1.292947 & -3.926993 \\
\hline C & 3.383097 & -1.487903 & -3.451017 \\
\hline $\mathrm{H}$ & 3.159349 & -2.160780 & -4.288716 \\
\hline $\mathrm{H}$ & 3.975321 & -2.046512 & -2.716388 \\
\hline $\mathrm{H}$ & 2.436925 & -1.211169 & -2.975194 \\
\hline C & -4.501548 & 0.632874 & 0.438128 \\
\hline C & -5.000078 & -0.678531 & 0.361234 \\
\hline C & -5.240341 & 1.676295 & -0.136501 \\
\hline C & -6.208961 & -0.964316 & -0.283050 \\
\hline $\mathrm{H}$ & -4.417219 & -1.475680 & 0.808572 \\
\hline C & -6.442436 & 1.428261 & -0.808826 \\
\hline $\mathrm{H}$ & -4.857760 & 2.682430 & -0.053374 \\
\hline C & -6.900564 & 0.105579 & -0.868768 \\
\hline $\mathrm{H}$ & -7.830791 & -0.099894 & -1.390367 \\
\hline C & -7.251252 & 2.554333 & -1.482093 \\
\hline C & -6.788637 & -2.389734 & -0.375860 \\
\hline C & -6.615011 & 3.940770 & -1.263687 \\
\hline $\mathrm{H}$ & -6.579766 & 4.207007 & -0.200921 \\
\hline $\mathrm{H}$ & -5.595173 & 3.992410 & -1.658430 \\
\hline $\mathrm{H}$ & -7.213773 & 4.703450 & -1.775221 \\
\hline C & -7.315022 & 2.288474 & -3.005408 \\
\hline $\mathrm{H}$ & -7.894049 & 3.074745 & -3.505605 \\
\hline $\mathrm{H}$ & -6.309960 & 2.276668 & -3.441525 \\
\hline $\mathrm{H}$ & -7.790562 & 1.327946 & -3.232869 \\
\hline C & -8.686911 & 2.585339 & -0.907497 \\
\hline $\mathrm{H}$ & -9.220171 & 1.643725 & -1.077830 \\
\hline $\mathrm{H}$ & -8.672334 & 2.770154 & 0.172824 \\
\hline $\mathrm{H}$ & -9.268826 & 3.385913 & -1.380700 \\
\hline
\end{tabular}




\begin{tabular}{|lrrr|} 
C & -6.845427 & -2.825894 & -1.859313 \\
$H$ & -7.465018 & -2.148949 & -2.456889 \\
$H$ & -5.842846 & -2.837345 & -2.302052 \\
$H$ & -7.268851 & -3.834295 & -1.948009 \\
C & -5.944931 & -3.420647 & 0.397666 \\
$H$ & -4.927437 & -3.498443 & -0.000044 \\
$H$ & -5.877977 & -3.172984 & 1.463413 \\
$H$ & -6.407050 & -4.411185 & 0.316057 \\
C & -8.217475 & -2.410053 & 0.217786 \\
H & -8.639801 & -3.420931 & 0.158965 \\
H & -8.207803 & -2.106213 & 1.270854 \\
H & -8.895037 & -1.736636 & -0.316693 \\
& & & \\
\hline
\end{tabular}

\section{C-Catalyzed reaction TS 85}

\section{Datum}

Value

\begin{tabular}{lr}
\hline B3LYP/6-31G(d) Energy & -3038.958325 \\
\hline B3LYP-D3(BJ)/def2-TZVPP-IEF-PCM(DCM) Energy & -3040.508652 \\
\hline B3LYP-D3(BJ)/def2-TZVPP-IEF-PCM(DCM)//B3LYP/6-31G(d) Free Energy (Quasiharmonic) & -3039.279781 \\
\hline
\end{tabular}

Number of Imaginary Frequencies

Frequencies (Top 3 out of 450)

$$
\begin{array}{lr}
\text { 1. } & -305.3239 \mathrm{~cm}^{-1} \\
\text { 2. } & 7.9854 \mathrm{~cm}^{-1} \\
\text { 3. } & 11.5453 \mathrm{~cm}^{-1}
\end{array}
$$

\section{B3LYP/6-31G(d) Molecular Geometry in Cartesian Coordinates}

C

$\mathrm{H}$

$\mathrm{H}$

C

0

0

$\mathrm{H}$

0

$\mathrm{H}$

C

C

C

C

$\mathrm{H}$

\subsection{7}

3.681051

2.080665

$-0.356042$

0.408090

$-1.225939$

$-1.785796$

3. 711391

3.127809

$-1.074682$

$-2.896970$

$-1.831840$

$-0.931091$

$-2.363843$
$-1.737573$

$-1.253804$

$-1.937822$

$-0.267248$

0.004598

0.654056

0.445106

$-2.962595$

$-3.457130$

4.358342

3. 397708

3. 316878

3.421032

2. 512121
0.548917

1. 288305

1.026879

0.481374

1. 385244

0.048575

$-0.746154$

0.211873

$-0.386079$

$-0.716035$

0.017378

$-2.829906$

$-1.715511$

0.360322 


\begin{tabular}{|c|c|c|c|}
\hline $\mathrm{H}$ & -1.461981 & 2.696720 & -3.647760 \\
\hline $\mathrm{H}$ & -2.219304 & 4.271887 & -3.193414 \\
\hline C & -4.139079 & 0.321007 & -2.368984 \\
\hline C & -4.662381 & 1.182926 & -3.577785 \\
\hline C & -3.715998 & -1.102119 & -2.730608 \\
\hline $\mathrm{H}$ & -3.349848 & -1.626409 & -1.841607 \\
\hline $\mathrm{H}$ & -4.565948 & -1.670790 & -3.125855 \\
\hline $\mathrm{H}$ & -2.921063 & -1.108314 & -3.480252 \\
\hline C & -5.120434 & 0.291851 & -1.187115 \\
\hline $\mathrm{H}$ & -4.621051 & -0.146485 & -0.316649 \\
\hline $\mathrm{H}$ & -6.004081 & -0.315222 & -1.410601 \\
\hline $\mathrm{H}$ & -5.450094 & 1.300261 & -0.922362 \\
\hline C & -6.177908 & 1.129282 & -3.790953 \\
\hline $\mathrm{H}$ & -6.515263 & 0.110271 & -4.016393 \\
\hline $\mathrm{H}$ & -6.717214 & 1.492298 & -2.913069 \\
\hline $\mathrm{H}$ & -6.448470 & 1.769246 & -4.637471 \\
\hline C & -3.948215 & 0.855130 & -4.901008 \\
\hline $\mathrm{H}$ & -4.256385 & -0.113657 & -5.310364 \\
\hline $\mathrm{H}$ & -4.195648 & 1.631627 & -5.631827 \\
\hline $\mathrm{H}$ & -2.861966 & 0.847686 & -4.769941 \\
\hline 0 & -2.961989 & 1.068306 & -1.961313 \\
\hline 0 & -4.306212 & 2.516144 & -3.197383 \\
\hline B & -3.239074 & 2.474577 & -2.257431 \\
\hline 0 & -3.632766 & 3.305077 & -1.044906 \\
\hline C & -3.287126 & 4.378961 & 1.048420 \\
\hline C & -4.226077 & 5.386352 & 0.776746 \\
\hline C & -2.730244 & 4.278470 & 2.332961 \\
\hline C & -4.594646 & 6.281839 & 1.777084 \\
\hline $\mathrm{H}$ & -4.663484 & 5.445312 & -0.214746 \\
\hline C & -3.102518 & 5.176710 & 3.330734 \\
\hline $\mathrm{H}$ & -2.007863 & 3.493488 & 2.544597 \\
\hline C & -4.033299 & 6.181311 & 3.053984 \\
\hline $\mathrm{H}$ & -5.324268 & 7.058386 & 1.564062 \\
\hline $\mathrm{H}$ & -2.669744 & 5.091796 & 4.323577 \\
\hline $\mathrm{H}$ & -4.324246 & 6.881802 & 3.832119 \\
\hline $\mathrm{H}$ & -0.263994 & 2.581020 & -1.527605 \\
\hline $\mathrm{H}$ & -1.592314 & 5.292537 & -0.915121 \\
\hline $\mathrm{H}$ & -0.413368 & 4.354305 & 0.145618 \\
\hline C & 0.638152 & -1.941141 & -1.073738 \\
\hline C & -0.375174 & -1.632552 & -0.171710 \\
\hline C & -1.355360 & -2.608561 & 0.222000 \\
\hline C & -1.304482 & -3.855235 & -0.375660 \\
\hline $\mathrm{H}$ & -2.016356 & -4.617318 & -0.070930 \\
\hline C & 2.852894 & -0.828619 & -0.655074 \\
\hline C & 1.701179 & -0.935263 & -1.432627 \\
\hline C & 3.680526 & 0.927055 & -2.120083 \\
\hline C & 3.867629 & 0.126883 & -1.008834 \\
\hline $\mathrm{H}$ & 4.452295 & 1.641930 & -2.394009 \\
\hline C & 2.539061 & 0.818397 & -2.947680 \\
\hline C & 1.524086 & -0.131574 & -2.607147 \\
\hline C & 0.398600 & -0.251087 & -3.470060 \\
\hline C & 0.292942 & 0.517308 & -4.609090 \\
\hline C & 1.295500 & 1.461661 & -4.937632 \\
\hline C & 2.391044 & 1.609541 & -4.119345 \\
\hline
\end{tabular}




\begin{tabular}{|c|c|c|c|}
\hline $\mathrm{H}$ & -0.378558 & -0.968715 & -3.230167 \\
\hline $\mathrm{H}$ & -0.564917 & 0.394724 & -5.264439 \\
\hline $\mathrm{H}$ & 1.196982 & 2.063857 & -5.836643 \\
\hline $\mathrm{H}$ & 3.169966 & 2.328581 & -4.361840 \\
\hline C & -0.317373 & -4.203300 & -1.327065 \\
\hline C & 0.683220 & -3.241936 & -1.676603 \\
\hline C & 1.693110 & -3.622874 & -2.606695 \\
\hline C & 1.706630 & -4.885955 & -3.158034 \\
\hline C & 0.712703 & -5.832576 & -2.812259 \\
\hline C & -0.275629 & -5.496258 & -1.916338 \\
\hline H & 2.452703 & -2.900272 & -2.885461 \\
\hline $\mathrm{H}$ & 2.484739 & -5.156919 & -3.866160 \\
\hline $\mathrm{H}$ & 0.735003 & -6.824608 & -3.254691 \\
\hline $\mathrm{H}$ & -1.040301 & -6.218348 & -1.640250 \\
\hline C & 7.490216 & 0.720881 & 1.187587 \\
\hline C & 7.162388 & -0.583662 & 0.774683 \\
\hline C & 5.972174 & -0.770206 & 0.069560 \\
\hline C & 5.120125 & 0.310957 & -0.216076 \\
\hline C & 5.484622 & 1.592289 & 0.208248 \\
\hline C & 6.674070 & 1.822939 & 0.917269 \\
\hline $\mathrm{H}$ & 8.410596 & 0.870517 & 1.740050 \\
\hline $\mathrm{H}$ & 5.676516 & -1.756341 & -0.264463 \\
\hline $\mathrm{H}$ & 4.811575 & 2.417518 & -0.005326 \\
\hline C & 8.105450 & -1.752553 & 1.120224 \\
\hline C & 7.027168 & 3.254503 & 1.366002 \\
\hline C & 7.616322 & -3.091211 & 0.534191 \\
\hline $\mathrm{H}$ & 8.308308 & -3.892047 & 0.819940 \\
\hline $\mathrm{H}$ & 6.619833 & -3.361671 & 0.898601 \\
\hline $\mathrm{H}$ & 7.578471 & -3.062238 & -0.560966 \\
\hline C & 9.518574 & -1.470075 & 0.557699 \\
\hline $\mathrm{H}$ & 10.196645 & -2.298180 & 0.798556 \\
\hline $\mathrm{H}$ & 9.490710 & -1.360724 & -0.532617 \\
\hline $\mathrm{H}$ & 9.953080 & -0.554898 & 0.973788 \\
\hline C & 8.187273 & -1.900230 & 2.658579 \\
\hline $\mathrm{H}$ & 8.866270 & -2.718653 & 2.928634 \\
\hline $\mathrm{H}$ & 8.557735 & -0.986909 & 3.136609 \\
\hline $\mathrm{H}$ & 7.201281 & -2.123187 & 3.081756 \\
\hline C & 5.913079 & 3.796833 & 2.292479 \\
\hline $\mathrm{H}$ & 6.148559 & 4.818695 & 2.615192 \\
\hline $\mathrm{H}$ & 4.940040 & 3.821657 & 1.791131 \\
\hline $\mathrm{H}$ & 5.810639 & 3.172597 & 3.187436 \\
\hline C & 8.360674 & 3.320528 & 2.134324 \\
\hline $\mathrm{H}$ & 8.567891 & 4.356849 & 2.424624 \\
\hline $\mathrm{H}$ & 9.201824 & 2.974905 & 1.522609 \\
\hline $\mathrm{H}$ & 8.334851 & 2.720500 & 3.051155 \\
\hline C & 7.147120 & 4.166978 & 0.122010 \\
\hline $\mathrm{H}$ & 7.389642 & 5.194080 & 0.421978 \\
\hline $\mathrm{H}$ & 7.939013 & 3.812880 & -0.548075 \\
\hline $\mathrm{H}$ & 6.214403 & 4.199309 & -0.450921 \\
\hline C & -2.377375 & -2.364235 & 1.283898 \\
\hline C & -3.689510 & -2.837863 & 1.123628 \\
\hline C & -2.029768 & -1.753242 & 2.496213 \\
\hline C & -4.644076 & -2.720675 & 2.141765 \\
\hline $\mathrm{H}$ & -3.959757 & -3.307884 & 0.182511 \\
\hline
\end{tabular}




\begin{tabular}{|c|c|c|c|}
\hline C & -2.963370 & -1.582150 & 3.525390 \\
\hline $\mathrm{H}$ & -1.008522 & -1.426057 & 2.643676 \\
\hline C & -4.259491 & -2.076745 & 3.327672 \\
\hline $\mathrm{H}$ & -4.987101 & -1.964026 & 4.119532 \\
\hline C & -2.525980 & -0.879636 & 4.825532 \\
\hline C & -6.053513 & -3.315459 & 1.943850 \\
\hline C & -3.676265 & -0.750212 & 5.841974 \\
\hline $\mathrm{H}$ & -4.513620 & -0.168005 & 5.440363 \\
\hline $\mathrm{H}$ & -4.058142 & -1.728593 & 6.155802 \\
\hline $\mathrm{H}$ & -3.316671 & -0.234411 & 6.739578 \\
\hline C & -1.385934 & -1.688294 & 5.489526 \\
\hline $\mathrm{H}$ & -1.062750 & -1.198313 & 6.416157 \\
\hline $\mathrm{H}$ & -1.718332 & -2.702510 & 5.739586 \\
\hline $\mathrm{H}$ & -0.512154 & -1.772874 & 4.835860 \\
\hline C & -2.014908 & 0.543282 & 4.490702 \\
\hline $\mathrm{H}$ & -1.176321 & 0.524301 & 3.787307 \\
\hline $\mathrm{H}$ & -2.813749 & 1.148084 & 4.044783 \\
\hline H & -1.677852 & 1.048068 & 5.404821 \\
\hline C & -5.931410 & -4.842867 & 1.723901 \\
\hline $\mathrm{H}$ & -5.330683 & -5.079155 & 0.839081 \\
\hline $\mathrm{H}$ & -5.460515 & -5.327452 & 2.586822 \\
\hline $\mathrm{H}$ & -6.923613 & -5.288865 & 1.582290 \\
\hline C & -6.969473 & -3.081029 & 3.160642 \\
\hline $\mathrm{H}$ & -6.571232 & -3.545497 & 4.069741 \\
\hline $\mathrm{H}$ & -7.119883 & -2.013686 & 3.358717 \\
\hline $\mathrm{H}$ & -7.953909 & -3.522938 & 2.969486 \\
\hline C & -6.730837 & -2.677420 & 0.709238 \\
\hline $\mathrm{H}$ & -7.728008 & -3.108757 & 0.557466 \\
\hline $\mathrm{H}$ & -6.844253 & -1.595511 & 0.839153 \\
\hline $\mathrm{H}$ & -6.153851 & -2.844487 & -0.205976 \\
\hline
\end{tabular}

\section{C-Catalyzed reaction TS 86}

\section{Datum}

Value

B3LYP/6-31G(d) Energy

$-3038.956658$

B3LYP-D3(BJ)/def2-TZVPP-IEF-PCM(DCM) Energy

$-3040.508196$

B3LYP-D3(BJ)/def2-TZVPP-IEF-PCM(DCM)//B3LYP/6-31G(d) Free Energy (Quasiharmonic)

$-3039.27955$

Number of Imaginary Frequencies

Frequencies (Top 3 out of 450)

$\begin{array}{lr}\text { 1. } & -309.8107 \mathrm{~cm}^{-1} \\ \text { 2. } & 4.8832 \mathrm{~cm}^{-1} \\ \text { 3. } & 10.6378 \mathrm{~cm}^{-1}\end{array}$




\begin{tabular}{|c|c|c|c|}
\hline C & -1.564070 & 0.671970 & 1.061630 \\
\hline $\mathrm{H}$ & -2.317010 & -0.100210 & 0.899870 \\
\hline $\mathrm{H}$ & -0.582750 & 0.201020 & 0.940670 \\
\hline C & 1.423570 & 0.730400 & -1.209120 \\
\hline 0 & 1.189480 & 1.102250 & -2.337820 \\
\hline 0 & 1.433430 & -0.586060 & -0.921750 \\
\hline $\mathrm{H}$ & 1.514960 & -0.841260 & 0.035760 \\
\hline 0 & -1.743710 & 1.087700 & 2.423890 \\
\hline $\mathrm{H}$ & -1.074520 & 1.765510 & 2.615060 \\
\hline C & 1.190860 & -4.081090 & -2.209400 \\
\hline C & -0.417660 & -3.184780 & -1.017010 \\
\hline C & 2.210950 & -4.306570 & 0.030280 \\
\hline C & 2.137560 & -3.719580 & -1.277270 \\
\hline $\mathrm{H}$ & -0.052480 & -2.174020 & -1.192000 \\
\hline $\mathrm{H}$ & 3.139680 & -4.103180 & 0.565570 \\
\hline $\mathrm{H}$ & 1.952910 & -5.367650 & 0.071560 \\
\hline C & 1.539940 & -2.046010 & 2.744300 \\
\hline C & 0.721190 & -3.267790 & 3.295790 \\
\hline C & 3.054910 & -2.215200 & 2.939380 \\
\hline $\mathrm{H}$ & 3.576060 & -1.489910 & 2.307260 \\
\hline $\mathrm{H}$ & 3.350200 & -2.039640 & 3.979880 \\
\hline $\mathrm{H}$ & 3.388120 & -3.216960 & 2.655150 \\
\hline C & 1.108410 & -0.685930 & 3.288290 \\
\hline $\mathrm{H}$ & 1.719380 & 0.109050 & 2.845590 \\
\hline $\mathrm{H}$ & 1.264670 & -0.650300 & 4.373330 \\
\hline $\mathrm{H}$ & 0.057590 & -0.466510 & 3.091520 \\
\hline C & -0.773660 & -2.949920 & 3.474320 \\
\hline $\mathrm{H}$ & -0.954970 & -2.292900 & 4.332050 \\
\hline $\mathrm{H}$ & -1.189100 & -2.473480 & 2.581830 \\
\hline $\mathrm{H}$ & -1.312470 & -3.888630 & 3.638660 \\
\hline C & 1.275270 & -3.881390 & 4.582970 \\
\hline $\mathrm{H}$ & 1.265440 & -3.155860 & 5.405020 \\
\hline $\mathrm{H}$ & 0.653610 & -4.733430 & 4.877870 \\
\hline $\mathrm{H}$ & 2.296610 & -4.245250 & 4.447180 \\
\hline 0 & 1.261990 & -2.121550 & 1.317170 \\
\hline 0 & 0.862410 & -4.227640 & 2.238230 \\
\hline B & 0.983770 & -3.528400 & 1.008620 \\
\hline 0 & -0.255830 & -3.709900 & 0.156100 \\
\hline C & -1.565040 & -3.630720 & -1.829640 \\
\hline C & -2.301210 & -4.774970 & -1.484930 \\
\hline C & -1.930920 & -2.884640 & -2.961020 \\
\hline C & -3.381850 & -5.168930 & -2.269630 \\
\hline $\mathrm{H}$ & -2.020180 & -5.335280 & -0.599030 \\
\hline C & -3.009990 & -3.285320 & -3.745130 \\
\hline $\mathrm{H}$ & -1.365080 & -1.993060 & -3.221830 \\
\hline C & -3.735830 & -4.429530 & -3.402550 \\
\hline $\mathrm{H}$ & -3.951610 & -6.053490 & -1.998270 \\
\hline $\mathrm{H}$ & -3.287030 & -2.705680 & -4.621120 \\
\hline $\mathrm{H}$ & -4.577540 & -4.741990 & -4.014540 \\
\hline $\mathrm{H}$ & 2.679620 & -2.789570 & -1.445450 \\
\hline
\end{tabular}




\begin{tabular}{|c|c|c|c|}
\hline $\mathrm{H}$ & 0.737130 & -5.067450 & -2.171590 \\
\hline H & 1.119900 & -3.559670 & -3.159450 \\
\hline C & 0.680010 & 2.465670 & 0.411810 \\
\hline C & 1.713650 & 1.662570 & -0.057450 \\
\hline C & 3.028880 & 1.703210 & 0.524870 \\
\hline C & 3.220640 & 2.501030 & 1.637520 \\
\hline H & 4.214220 & 2.580720 & 2.069710 \\
\hline C & -1.736900 & 1.788710 & 0.048300 \\
\hline C & -0.644360 & 2.578210 & -0.301930 \\
\hline C & -3.127030 & 2.999520 & -1.535050 \\
\hline C & -3.018010 & 2.019470 & -0.567030 \\
\hline H & -4.095810 & 3.190430 & -1.989570 \\
\hline C & -2.030170 & 3.799080 & -1.932220 \\
\hline C & -0.762740 & 3.601270 & -1.301610 \\
\hline C & 0.322330 & 4.430460 & -1.698890 \\
\hline C & 0.158910 & 5.399120 & -2.663600 \\
\hline C & -1.098100 & 5.592780 & -3.284540 \\
\hline C & -2.167950 & 4.807410 & -2.924550 \\
\hline $\mathrm{H}$ & 1.294200 & 4.288650 & -1.240040 \\
\hline H & 1.003151 & 6.018600 & -2.953990 \\
\hline H & -1.213219 & 6.360760 & -4.044730 \\
\hline H & -3.139470 & 4.945670 & -3.393320 \\
\hline C & 2.178010 & 3.270170 & 2.206950 \\
\hline C & 0.891930 & 3.278800 & 1.577240 \\
\hline C & -0.126970 & 4.103040 & 2.136720 \\
\hline C & 0.111770 & 4.858110 & 3.264950 \\
\hline C & 1.382570 & 4.841050 & 3.886880 \\
\hline C & 2.392800 & 4.067430 & 3.363740 \\
\hline H & -1.100400 & 4.136350 & 1.659630 \\
\hline $\mathrm{H}$ & -0.680640 & 5.477250 & 3.676050 \\
\hline H & 1.557950 & 5.442810 & 4.774250 \\
\hline $\mathrm{H}$ & 3.375250 & 4.052720 & 3.829430 \\
\hline C & -6.628520 & -0.033110 & 0.466680 \\
\hline C & -5.824540 & 0.477640 & 1.501800 \\
\hline C & -4.640130 & 1.128410 & 1.152740 \\
\hline C & -4.250650 & 1.264010 & -0.192050 \\
\hline C & -5.079920 & 0.742170 & -1.190260 \\
\hline C & -6.283670 & 0.088470 & -0.882410 \\
\hline H & -7.547990 & -0.541979 & 0.731710 \\
\hline H & -3.994540 & 1.540180 & 1.917410 \\
\hline $\mathrm{H}$ & -4.765870 & 0.840740 & -2.225440 \\
\hline C & -6.263980 & 0.299010 & 2.968080 \\
\hline C & -7.165640 & -0.461350 & -2.020760 \\
\hline C & -7.667850 & 0.915701 & 3.173550 \\
\hline H & -7.988300 & 0.793631 & 4.215540 \\
\hline H & -7.661870 & 1.987511 & 2.944020 \\
\hline H & -8.423130 & 0.442201 & 2.537390 \\
\hline C & -6.315270 & -1.211300 & 3.301460 \\
\hline H & -6.636400 & -1.363140 & 4.339580 \\
\hline H & -7.016490 & -1.747770 & 2.653030 \\
\hline $\mathrm{H}$ & -5.328180 & -1.671660 & 3.180590 \\
\hline C & -5.295330 & 0.977190 & 3.956430 \\
\hline H & -5.643990 & 0.812510 & 4.982660 \\
\hline H & -4.278420 & 0.578660 & 3.878860 \\
\hline
\end{tabular}




\begin{tabular}{|c|c|c|c|}
\hline H & -5.245840 & 2.060180 & 3.793800 \\
\hline C & -6.365540 & -1.501040 & -2.839540 \\
\hline $\mathrm{H}$ & -6.977930 & -1.890180 & -3.662950 \\
\hline H & -5.458320 & -1.068720 & -3.273700 \\
\hline $\mathrm{H}$ & -6.059540 & -2.345740 & -2.212570 \\
\hline C & -8.443210 & -1.146099 & -1.499840 \\
\hline H & -9.033540 & -1.517059 & -2.345540 \\
\hline $\mathrm{H}$ & -8.213340 & -2.002939 & -0.856420 \\
\hline $\mathrm{H}$ & -9.076920 & -0.453609 & -0.934160 \\
\hline C & -7.590440 & 0.702161 & -2.948250 \\
\hline $\mathrm{H}$ & -8.213690 & 0.328311 & -3.770160 \\
\hline $\mathrm{H}$ & -8.169130 & 1.451661 & -2.396210 \\
\hline $\mathrm{H}$ & -6.724970 & 1.207310 & -3.389630 \\
\hline C & 4.199560 & 1.005640 & -0.084310 \\
\hline C & 4.376070 & 0.991210 & -1.474860 \\
\hline C & 5.194900 & 0.432780 & 0.723250 \\
\hline C & 5.496850 & 0.398740 & -2.067720 \\
\hline H & 3.633350 & 1.466800 & -2.103800 \\
\hline C & 6.346470 & -0.144270 & 0.173190 \\
\hline H & 5.057920 & 0.438840 & 1.800420 \\
\hline C & 6.468130 & -0.157520 & -1.224620 \\
\hline $\mathrm{H}$ & 7.345180 & -0.608580 & -1.667430 \\
\hline C & 7.432850 & -0.727300 & 1.099770 \\
\hline C & 5.616670 & 0.386170 & -3.603830 \\
\hline C & 6.835830 & -1.866480 & 1.958080 \\
\hline H & 6.017490 & -1.514860 & 2.594010 \\
\hline $\mathrm{H}$ & 6.444020 & -2.671080 & 1.325350 \\
\hline H & 7.605630 & -2.293550 & 2.612660 \\
\hline C & 8.630560 & -1.299891 & 0.317040 \\
\hline $\mathrm{H}$ & 9.374510 & -1.692301 & 1.019440 \\
\hline $\mathrm{H}$ & 8.333440 & -2.124181 & -0.341280 \\
\hline H & 9.124680 & -0.535531 & -0.293370 \\
\hline C & 7.962790 & 0.389310 & 2.031470 \\
\hline H & 8.396730 & 1.211449 & 1.450920 \\
\hline $\mathrm{H}$ & 7.169120 & 0.806560 & 2.660260 \\
\hline $\mathrm{H}$ & 8.740720 & -0.005181 & 2.696560 \\
\hline C & 6.912940 & -0.290400 & -4.088670 \\
\hline $\mathrm{H}$ & 7.807300 & 0.230270 & -3.727020 \\
\hline $\mathrm{H}$ & 6.972960 & -1.337060 & -3.768850 \\
\hline H & 6.945750 & -0.279150 & -5.183930 \\
\hline C & 4.414480 & -0.387160 & -4.198990 \\
\hline $\mathrm{H}$ & 4.415090 & -1.428820 & -3.855950 \\
\hline $\mathrm{H}$ & 3.455920 & 0.058110 & -3.913980 \\
\hline $\mathrm{H}$ & 4.471690 & -0.392600 & -5.294650 \\
\hline C & 5.605350 & 1.838800 & -4.137180 \\
\hline $\mathrm{H}$ & 5.690410 & 1.841510 & -5.230700 \\
\hline H & 4.680860 & 2.363770 & -3.876200 \\
\hline $\mathrm{H}$ & 6.445430 & 2.413320 & -3.729710 \\
\hline
\end{tabular}




\begin{tabular}{lc}
\hline B3LYP/6-31G(d) Energy & -3038.958911 \\
\hline B3LYP-D3(BJ)/def2-TZVPP-IEF-PCM(DCM) Energy & -3040.507908 \\
\hline B3LYP-D3(BJ)/def2-TZVPP-IEF-PCM(DCM)//B3LYP/6-31G(d) Free Energy (Quasiharmonic) & -3039.279518 \\
\hline
\end{tabular}

Number of Imaginary Frequencies

Frequencies (Top 3 out of 450)

$\begin{array}{lr}\text { 1. } & -324.5421 \mathrm{~cm}^{-1} \\ \text { 2. } & 7.5642 \mathrm{~cm}^{-1} \\ \text { 3. } & 10.9882 \mathrm{~cm}^{-1}\end{array}$

\section{B3LYP/6-31G(d) Molecular Geometry in Cartesian Coordinates}

C

$\mathrm{H}$

$\mathrm{H}$

C

0

0

$\mathrm{H}$

0

$\mathrm{H}$

C

C

C

C

$\mathrm{H}$

$\mathrm{H}$

$\mathrm{H}$

C

C

C

$\mathrm{H}$

$\mathrm{H}$

$\mathrm{H}$

C

$\mathrm{H}$

$\mathrm{H}$

$\mathrm{H}$

$\mathrm{H}$

C

$\mathrm{H}$

$\mathrm{H}$

$\mathrm{H}$

C

$\mathrm{H}$

\subsection{9}

3. 784810

2.162167

$-0.357512$

0.417802

$-1.315593$

$-1.878920$

3. 754005

3.155959

$-2.672480$

$-3.941404$

$-2.300077$

$-2.826567$

$-4.854353$

$-1.349889$

$-2.293006$

$-4.070688$

$-5.340835$

$-3.548808$

$-2.570527$

$-4.219349$

$-3.435053$

$-4.242493$

$-4.487667$

$-3.312753$

$-5.034869$

$-6.137694$

$-6.683740$

$-5.485051$

$-6.867867$

$-6.286443$

$-6.696513$
$-1.623965$

$-1.238858$

$-1.847901$

$-0.156192$

0.108058

0.712148

0.457286

$-2.826214$

$-3.234990$

5. 266375

3. 780525

3. 395153

4.686446

4. 144409

3. 142072

3. 163819

0.236431

1.154090

0.222121

$-0.268547$

$-0.330809$

1.237353

$-1.197235$

$-1.221498$

$-1.758293$

$-1.711756$

0.823995

$-0.121515$

0.763039

1.621525

1.186472

0.191583

\subsection{5}

1.143598

0.834491

0.434210

1. 331475

0.094105

$-0.678385$

$-0.104097$

$-0.750619$

$-0.996071$

$-0.028213$

$-2.569000$

$-2.237790$

$-0.504002$

$-2.094881$

$-3.635079$

$-2.870796$

$-2.692741$

$-4.316112$

$-4.339696$

$-4.982816$

$-4.707373$

$-2.373457$

$-1.310119$

$-2.517936$

$-2.930367$

$-1.417868$

$-1.511244$

$-0.541972$

$-1.244099$

$-3.894460$

$-4.104669$ 


\begin{tabular}{|c|c|c|c|}
\hline $\mathrm{H}$ & -7.125132 & 1.858515 & -3.682953 \\
\hline $\mathrm{H}$ & -5.781584 & 1.554312 & -4.790799 \\
\hline 0 & -3.088195 & 0.902282 & -2.040837 \\
\hline 0 & -4.753839 & 2.459850 & -2.544806 \\
\hline B & -3.481925 & 2.289132 & -1.912396 \\
\hline 0 & -3.456025 & 2.657714 & -0.448292 \\
\hline C & -3.712979 & 4.148587 & 1.381059 \\
\hline C & -4.503792 & 5.145753 & 1.971681 \\
\hline C & -2.725847 & 3.497902 & 2.140763 \\
\hline C & -4.311108 & 5.494894 & 3.306458 \\
\hline $\mathrm{H}$ & -5.271341 & 5.643206 & 1.382598 \\
\hline C & -2.536553 & 3.854890 & 3.473595 \\
\hline $\mathrm{H}$ & -2.126638 & 2.716781 & 1.681776 \\
\hline C & -3.324813 & 4.851422 & 4.058232 \\
\hline $\mathrm{H}$ & -4.929046 & 6.264787 & 3.760134 \\
\hline H & -1.772727 & 3.351531 & 4.059238 \\
\hline $\mathrm{H}$ & -3.172167 & 5.123972 & 5.099063 \\
\hline $\mathrm{H}$ & -3.585366 & 5.099479 & -2.903496 \\
\hline $\mathrm{H}$ & -1.819259 & 5.002807 & -0.378078 \\
\hline $\mathrm{H}$ & -3.149942 & 6.214242 & -0.762976 \\
\hline C & 0.725078 & -1.634364 & -1.259524 \\
\hline C & -0.296494 & -1.460565 & -0.332156 \\
\hline C & -1.235568 & -2.510103 & -0.042462 \\
\hline C & -1.156995 & -3.676512 & -0.781003 \\
\hline $\mathrm{H}$ & -1.839673 & -4.491980 & -0.557658 \\
\hline C & 2.941467 & -0.583240 & -0.726178 \\
\hline C & 1.773918 & -0.576484 & -1.487804 \\
\hline C & 3.766835 & 1.334532 & -1.971605 \\
\hline C & 3.967088 & 0.392074 & -0.980119 \\
\hline $\mathrm{H}$ & 4.546945 & 2.063516 & -2.176037 \\
\hline C & 2.597648 & 1.361768 & -2.765250 \\
\hline C & 1.575739 & 0.388714 & -2.527889 \\
\hline C & 0.415133 & 0.418435 & -3.349613 \\
\hline C & 0.282083 & 1.349084 & -4.356247 \\
\hline C & 1.296554 & 2.308428 & -4.589772 \\
\hline C & 2.426537 & 2.314721 & -3.805958 \\
\hline $\mathrm{H}$ & -0.376448 & -0.301685 & -3.173904 \\
\hline $\mathrm{H}$ & -0.610468 & 1.347777 & -4.975335 \\
\hline $\mathrm{H}$ & 1.178252 & 3.037501 & -5.386943 \\
\hline $\mathrm{H}$ & 3.213020 & 3.046821 & -3.974063 \\
\hline C & -0.174252 & -3.876568 & -1.779110 \\
\hline C & 0.798828 & -2.852910 & -2.014146 \\
\hline C & 1.807637 & -3.092038 & -2.991820 \\
\hline C & 1.845538 & -4.276419 & -3.695813 \\
\hline C & 0.879188 & -5.283786 & -3.462411 \\
\hline C & -0.106531 & -5.085747 & -2.523356 \\
\hline $\mathrm{H}$ & 2.546993 & -2.322362 & -3.186208 \\
\hline $\mathrm{H}$ & 2.622205 & -4.437194 & -4.438362 \\
\hline $\mathrm{H}$ & 0.920834 & -6.212828 & -4.024210 \\
\hline $\mathrm{H}$ & -0.849742 & -5.855977 & -2.331423 \\
\hline C & 7.688491 & 0.600847 & 1.122084 \\
\hline C & 7.284692 & -0.633617 & 0.606013 \\
\hline C & 6.055832 & -0.692277 & -0.070590 \\
\hline C & 5.250511 & 0.440405 & -0.218106 \\
\hline
\end{tabular}




\begin{tabular}{|c|c|c|c|}
\hline C & 5.692100 & 1.663723 & 0.318027 \\
\hline C & 6.910064 & 1.765918 & 0.991979 \\
\hline $\mathrm{H}$ & 8.633361 & 0.667860 & 1.649143 \\
\hline $\mathrm{H}$ & 5.706553 & -1.631577 & -0.484584 \\
\hline H & 5.048433 & 2.529377 & 0.211624 \\
\hline C & 8.122594 & -1.918321 & 0.754250 \\
\hline C & 7.414473 & 3.090038 & 1.598343 \\
\hline C & 7.297201 & -2.979310 & 1.522238 \\
\hline $\mathrm{H}$ & 7.868612 & -3.911856 & 1.613541 \\
\hline $\mathrm{H}$ & 7.060077 & -2.628429 & 2.533613 \\
\hline $\mathrm{H}$ & 6.352932 & -3.208866 & 1.018801 \\
\hline C & 8.478847 & -2.465887 & -0.648459 \\
\hline $\mathrm{H}$ & 9.070911 & -3.385315 & -0.559346 \\
\hline $\mathrm{H}$ & 7.582947 & -2.701315 & -1.231459 \\
\hline H & 9.067494 & -1.736229 & -1.216892 \\
\hline C & 9.436015 & -1.681153 & 1.522723 \\
\hline $\mathrm{H}$ & 9.990055 & -2.623171 & 1.606115 \\
\hline $\mathrm{H}$ & 10.085565 & -0.960560 & 1.011957 \\
\hline H & 9.252570 & -1.315724 & 2.539674 \\
\hline C & 7.587018 & 2.925732 & 3.127188 \\
\hline H & 7.947958 & 3.860284 & 3.574710 \\
\hline $\mathrm{H}$ & 6.633627 & 2.667326 & 3.601940 \\
\hline $\mathrm{H}$ & 8.306756 & 2.138221 & 3.373686 \\
\hline C & 8.777133 & 3.461782 & 0.966463 \\
\hline $\mathrm{H}$ & 9.154631 & 4.399239 & 1.393849 \\
\hline $\mathrm{H}$ & 8.682094 & 3.596696 & -0.117220 \\
\hline $\mathrm{H}$ & 9.532778 & 2.688812 & 1.140718 \\
\hline C & 6.440506 & 4.258072 & 1.352105 \\
\hline H & 6.845063 & 5.175467 & 1.794837 \\
\hline $\mathrm{H}$ & 6.290093 & 4.445574 & 0.282790 \\
\hline $\mathrm{H}$ & 5.460805 & 4.075142 & 1.807617 \\
\hline C & -2.230909 & -2.421754 & 1.066519 \\
\hline C & -3.543502 & -2.895705 & 0.882092 \\
\hline C & -1.854633 & -1.956889 & 2.329892 \\
\hline C & -4.471445 & -2.893692 & 1.924581 \\
\hline $\mathrm{H}$ & -3.826409 & -3.257387 & -0.099112 \\
\hline C & -2.760262 & -1.927113 & 3.401868 \\
\hline $\mathrm{H}$ & -0.834362 & -1.628464 & 2.485115 \\
\hline C & -4.056297 & -2.394927 & 3.173462 \\
\hline $\mathrm{H}$ & -4.772270 & -2.379906 & 3.987067 \\
\hline C & -2.287272 & -1.399998 & 4.769709 \\
\hline C & -5.913894 & -3.410289 & 1.756076 \\
\hline C & -3.406596 & -1.419776 & 5.827307 \\
\hline $\mathrm{H}$ & -4.257703 & -0.795323 & 5.531896 \\
\hline $\mathrm{H}$ & -3.775197 & -2.434916 & 6.015751 \\
\hline $\mathrm{H}$ & -3.023635 & -1.027875 & 6.776327 \\
\hline C & -1.123519 & -2.281315 & 5.283785 \\
\hline $\mathrm{H}$ & -0.775223 & -1.919890 & 6.259223 \\
\hline $\mathrm{H}$ & -1.442882 & -3.323402 & 5.401515 \\
\hline $\mathrm{H}$ & -0.268514 & -2.267404 & 4.600366 \\
\hline C & -1.793940 & 0.059074 & 4.614451 \\
\hline $\mathrm{H}$ & -0.981498 & 0.144452 & 3.885774 \\
\hline H & -2.609496 & 0.712085 & 4.282625 \\
\hline $\mathrm{H}$ & -1.425693 & 0.436281 & 5.577063 \\
\hline
\end{tabular}




\begin{tabular}{|llll|} 
C & -6.910416 & -2.256429 & 2.020191 \\
$H$ & -6.800568 & -1.847060 & 3.029748 \\
$H$ & -6.757763 & -1.436177 & 1.309328 \\
$H$ & -7.943305 & -2.610915 & 1.914061 \\
C & -6.182043 & -3.960618 & 0.342223 \\
$H$ & -6.058986 & -3.191101 & -0.428065 \\
$H$ & -5.518010 & -4.797789 & 0.098200 \\
$H$ & -7.212822 & -4.327398 & 0.279293 \\
C & -6.174738 & -4.552657 & 2.766983 \\
$H$ & -7.200471 & -4.927241 & 2.662141 \\
$H$ & -5.488077 & -5.389936 & 2.597303 \\
$H$ & -6.046431 & -4.221391 & 3.802499 \\
\hline
\end{tabular}

\section{C-Catalyzed reaction TS 88}

\section{Datum}

Value

\begin{tabular}{lr}
\hline B3LYP/6-31G(d) Energy & -3038.957443 \\
\hline B3LYP-D3(BJ)/def2-TZVPP-IEF-PCM(DCM) Energy & -3040.508655 \\
\hline B3LYP-D3(BJ)/def2-TZVPP-IEF-PCM(DCM)//B3LYP/6-31G(d) Free Energy (Quasiharmonic) & -3039.279454 \\
\hline
\end{tabular}

Number of Imaginary Frequencies

Frequencies (Top 3 out of 450)

$\begin{array}{lr}\text { 1. } & -282.6386 \mathrm{~cm}^{-1} \\ \text { 2. } & 7.2895 \mathrm{~cm}^{-1} \\ \text { 3. } & 9.3469 \mathrm{~cm}^{-1}\end{array}$

\section{B3LYP/6-31G(d) Molecular Geometry in Cartesian Coordinates}

\begin{tabular}{|c|c|c|c|}
\hline C & -0.666129 & 0.726130 & 0.808228 \\
\hline $\mathrm{H}$ & -0.305777 & 0.390654 & 1.788559 \\
\hline $\mathrm{H}$ & -1.733037 & 0.502581 & 0.752356 \\
\hline C & 2.849526 & 1.746938 & -0.923654 \\
\hline 0 & 3.635752 & 2.554398 & -1.378002 \\
\hline 0 & 1.892441 & 1.205484 & -1.675194 \\
\hline $\mathrm{H}$ & 1.258165 & 0.669139 & -1.123447 \\
\hline 0 & 0.011142 & -0.005838 & -0.225630 \\
\hline $\mathrm{H}$ & -0.041636 & -0.959888 & 0.020656 \\
\hline C & -3.155082 & -1.892996 & -3.101772 \\
\hline C & -3.845216 & -1.804034 & -0.995601 \\
\hline C & -1.238277 & -3.031163 & -2.049150 \\
\hline C & -2.433810 & -3.034056 & -2.846405 \\
\hline $\mathrm{H}$ & -4.067181 & -0.747288 & -1.164435 \\
\hline
\end{tabular}




\begin{tabular}{|c|c|c|c|}
\hline $\mathrm{H}$ & -0.649264 & -2.116839 & -2.156541 \\
\hline $\mathrm{H}$ & -0.619554 & -3.923794 & -2.163429 \\
\hline C & -0.128505 & -3.619860 & 1.334224 \\
\hline C & -1.411564 & -4.528416 & 1.425064 \\
\hline C & 1.062224 & -4.326319 & 0.668279 \\
\hline $\mathrm{H}$ & 1.827660 & -3.580582 & 0.429362 \\
\hline $\mathrm{H}$ & 1.509765 & -5.077450 & 1.328103 \\
\hline $\mathrm{H}$ & 0.764823 & -4.817987 & -0.262497 \\
\hline C & 0.316857 & -2.988496 & 2.653794 \\
\hline $\mathrm{H}$ & 0.590790 & -3.759411 & 3.383655 \\
\hline $\mathrm{H}$ & 1.196681 & -2.357035 & 2.488611 \\
\hline H & -0.468407 & -2.364230 & 3.086200 \\
\hline C & -2.422833 & -4.024616 & 2.469203 \\
\hline $\mathrm{H}$ & -2.063781 & -4.181487 & 3.492162 \\
\hline H & -2.636855 & -2.960251 & 2.335233 \\
\hline $\mathrm{H}$ & -3.361585 & -4.576114 & 2.352235 \\
\hline C & -1.133477 & -6.015111 & 1.649580 \\
\hline $\mathrm{H}$ & -0.615138 & -6.180740 & 2.601291 \\
\hline $\mathrm{H}$ & -2.079592 & -6.566084 & 1.682245 \\
\hline H & -0.528207 & -6.435963 & 0.843254 \\
\hline 0 & -0.577500 & -2.570426 & 0.449456 \\
\hline 0 & -1.988498 & -4.369943 & 0.111476 \\
\hline B & -1.662497 & -3.062018 & -0.364025 \\
\hline 0 & -2.773871 & -2.001974 & -0.303956 \\
\hline C & -5.042647 & -2.677148 & -0.995075 \\
\hline C & -5.036336 & -4.008428 & -0.547321 \\
\hline C & -6.249700 & -2.106951 & -1.437632 \\
\hline C & -6.218269 & -4.747372 & -0.556381 \\
\hline $\mathrm{H}$ & -4.107000 & -4.450649 & -0.202963 \\
\hline C & -7.427170 & -2.850210 & -1.442018 \\
\hline H & -6.259512 & -1.073063 & -1.775869 \\
\hline C & -7.413194 & -4.176000 & -1.001806 \\
\hline $\mathrm{H}$ & -6.206947 & -5.777226 & -0.209613 \\
\hline $\mathrm{H}$ & -8.353029 & -2.396957 & -1.785169 \\
\hline $\mathrm{H}$ & -8.329965 & -4.759351 & -1.002836 \\
\hline H & -2.899223 & -3.995240 & -3.067352 \\
\hline $\mathrm{H}$ & -2.668867 & -0.922902 & -3.038336 \\
\hline $\mathrm{H}$ & -4.085088 & -1.932846 & -3.660603 \\
\hline C & 1.952464 & 1.822463 & 1.409591 \\
\hline C & 2.900943 & 1.312022 & 0.531263 \\
\hline C & 3.930634 & 0.418592 & 0.984052 \\
\hline C & 3.953375 & 0.075236 & 2.323281 \\
\hline $\mathrm{H}$ & 4.728040 & -0.598248 & 2.681013 \\
\hline C & -0.415774 & 2.213915 & 0.653095 \\
\hline C & 0.855971 & 2.726372 & 0.912510 \\
\hline C & -1.177816 & 4.430351 & 0.005543 \\
\hline C & -1.456756 & 3.090093 & 0.198930 \\
\hline $\mathrm{H}$ & -1.971402 & 5.094262 & -0.328541 \\
\hline C & 0.098647 & 4.979945 & 0.262586 \\
\hline C & 1.140290 & 4.118814 & 0.731974 \\
\hline C & 2.414899 & 4.689810 & 1.006161 \\
\hline C & 2.639019 & 6.035766 & 0.828766 \\
\hline C & 1.605026 & 6.884798 & 0.364492 \\
\hline C & 0.362832 & 6.365589 & 0.086328 \\
\hline
\end{tabular}




\begin{tabular}{|c|c|c|c|}
\hline $\mathrm{H}$ & 3.219250 & 4.046980 & 1.344488 \\
\hline $\mathrm{H}$ & 3.620732 & 6.450741 & 1.039630 \\
\hline $\mathrm{H}$ & 1.798535 & 7.945162 & 0.226134 \\
\hline $\mathrm{H}$ & -0.439143 & 7.006949 & -0.271761 \\
\hline C & 3.031888 & 0.602426 & 3.259835 \\
\hline C & 2.016941 & 1.502604 & 2.805693 \\
\hline C & 1.116337 & 2.044398 & 3.765075 \\
\hline C & 1.210872 & 1.706134 & 5.097028 \\
\hline C & 2.210760 & 0.808062 & 5.541701 \\
\hline C & 3.100778 & 0.270807 & 4.640680 \\
\hline $\mathrm{H}$ & 0.351274 & 2.739054 & 3.434371 \\
\hline H & 0.516049 & 2.135356 & 5.814017 \\
\hline $\mathrm{H}$ & 2.275328 & 0.549480 & 6.595212 \\
\hline $\mathrm{H}$ & 3.877898 & -0.414085 & 4.972100 \\
\hline C & -5.529851 & 1.968997 & -0.526945 \\
\hline C & -4.767911 & 2.491977 & -1.583299 \\
\hline C & -3.427808 & 2.813796 & -1.325865 \\
\hline C & -2.859593 & 2.638014 & -0.058392 \\
\hline C & -3.659548 & 2.124138 & 0.971321 \\
\hline C & -4.999789 & 1.777603 & 0.759642 \\
\hline $\mathrm{H}$ & -6.567800 & 1.721311 & -0.703548 \\
\hline $\mathrm{H}$ & -2.800646 & 3.216372 & -2.115802 \\
\hline $\mathrm{H}$ & -3.225203 & 2.022567 & 1.961730 \\
\hline C & -5.355584 & 2.749866 & -2.985569 \\
\hline C & -5.844509 & 1.247815 & 1.936447 \\
\hline C & -6.832770 & 2.326627 & -3.097618 \\
\hline $\mathrm{H}$ & -7.198426 & 2.533170 & -4.109698 \\
\hline H & -6.968625 & 1.254207 & -2.912642 \\
\hline $\mathrm{H}$ & -7.471572 & 2.876939 & -2.397816 \\
\hline C & -5.268215 & 4.262825 & -3.300989 \\
\hline $\mathrm{H}$ & -5.680763 & 4.469900 & -4.296075 \\
\hline $\mathrm{H}$ & -5.835721 & 4.848587 & -2.568866 \\
\hline $\mathrm{H}$ & -4.234015 & 4.621265 & -3.287043 \\
\hline C & -4.550155 & 1.963195 & -4.046228 \\
\hline $\mathrm{H}$ & -4.949430 & 2.155556 & -5.049518 \\
\hline $\mathrm{H}$ & -3.492243 & 2.243988 & -4.050012 \\
\hline $\mathrm{H}$ & -4.609397 & 0.883754 & -3.862391 \\
\hline C & -5.169760 & -0.002443 & 2.549628 \\
\hline $\mathrm{H}$ & -5.759628 & -0.372503 & 3.397001 \\
\hline $\mathrm{H}$ & -5.091579 & -0.811260 & 1.814453 \\
\hline $\mathrm{H}$ & -4.161561 & 0.213487 & 2.917685 \\
\hline C & -5.951806 & 2.349975 & 3.017988 \\
\hline $\mathrm{H}$ & -6.541121 & 1.991219 & 3.870886 \\
\hline $\mathrm{H}$ & -4.967608 & 2.648482 & 3.393687 \\
\hline $\mathrm{H}$ & -6.442080 & 3.244979 & 2.618092 \\
\hline C & -7.272247 & 0.854100 & 1.511479 \\
\hline $\mathrm{H}$ & -7.822413 & 0.476664 & 2.380772 \\
\hline $\mathrm{H}$ & -7.833181 & 1.707870 & 1.114668 \\
\hline $\mathrm{H}$ & -7.268738 & 0.060964 & 0.754974 \\
\hline C & 4.951673 & -0.191928 & 0.078653 \\
\hline C & 5.135925 & -1.582642 & 0.087379 \\
\hline C & 5.778933 & 0.592150 & -0.734422 \\
\hline C & 6.117145 & -2.198522 & -0.699163 \\
\hline $\mathrm{H}$ & 4.484789 & -2.185543 & 0.714381 \\
\hline
\end{tabular}




$\begin{array}{llrl}\mathrm{C} & 6.766316 & 0.015552 & -1.542007 \\ \mathrm{H} & 5.632041 & 1.663897 & -0.738294 \\ \mathrm{C} & 6.918523 & -1.377326 & -1.505882 \\ \mathrm{H} & 7.678185 & -1.835032 & -2.124706 \\ \mathrm{C} & 7.619498 & 0.925088 & -2.446946 \\ \mathrm{C} & 6.275270 & -3.731993 & -0.664936 \\ \mathrm{C} & 6.693189 & 1.606372 & -3.484017 \\ \mathrm{H} & 6.230485 & 0.858870 & -4.139159 \\ \mathrm{H} & 5.885807 & 2.169088 & -3.005307 \\ \mathrm{H} & 7.268369 & 2.298320 & -4.112636 \\ \mathrm{C} & 8.316710 & 2.010166 & -1.592191 \\ \mathrm{H} & 8.926941 & 2.660453 & -2.231135 \\ \mathrm{H} & 7.595323 & 2.644903 & -1.068486 \\ \mathrm{H} & 8.974621 & 1.557291 & -0.841018 \\ \mathrm{C} & 8.710916 & 0.147696 & -3.206808 \\ \mathrm{H} & 9.407768 & -0.352445 & -2.523536 \\ \mathrm{H} & 8.285023 & -0.608856 & -3.875608 \\ \mathrm{H} & 9.293361 & 0.840640 & -3.824748 \\ \mathrm{C} & 4.962687 & -4.395966 & -1.144107 \\ \mathrm{H} & 4.113858 & -4.125128 & -0.507809 \\ \mathrm{H} & 4.721812 & -4.090560 & -2.168702 \\ \mathrm{H} & 5.057072 & -5.489161 & -1.127569 \\ \mathrm{C} & 7.417547 & -4.229424 & -1.571495 \\ \mathrm{H} & 7.245488 & -3.974508 & -2.623269 \\ \mathrm{H} & 8.386684 & -3.813749 & -1.273136 \\ \mathrm{H} & 7.491321 & -5.320962 & -1.505037 \\ \mathrm{C} & 6.581894 & -4.189636 & 0.780904 \\ \mathrm{H} & 6.688735 & -5.280838 & 0.823048 \\ \mathrm{H} & 7.514935 & -3.742772 & 1.143203 \\ \mathrm{H} & 5.784654 & -3.906547 & 1.476186\end{array}$

\section{C-Catalyzed reaction TS 89}

Datum

Value

\begin{tabular}{lc}
\hline B3LYP/6-31G(d) Energy & -3038.954187 \\
\hline B3LYP-D3(BJ)/def2-TZVPP-IEF-PCM(DCM) Energy & -3040.507865 \\
\hline B3LYP-D3(BJ)/def2-TZVPP-IEF-PCM(DCM)//B3LYP/6-31G(d) Free Energy (Quasiharmonic) & -3039.279426 \\
\hline
\end{tabular}

Number of Imaginary Frequencies

Frequencies (Top 3 out of 450)

$\begin{array}{lr}\text { 1. } & -339.4344 \mathrm{~cm}^{-1} \\ \text { 2. } & 7.1241 \mathrm{~cm}^{-1} \\ \text { 3. } & 10.3322 \mathrm{~cm}^{-1}\end{array}$




\begin{tabular}{|c|c|c|c|}
\hline C & 1.026484 & -0.550362 & -0.508134 \\
\hline $\mathrm{H}$ & 2.097662 & -0.337717 & -0.421991 \\
\hline $\mathrm{H}$ & 0.647085 & -0.001519 & -1.376113 \\
\hline C & -2.501302 & -2.084504 & 0.537704 \\
\hline 0 & -3.324931 & -2.951860 & 0.720774 \\
\hline 0 & -1.602037 & -1.756281 & 1.477360 \\
\hline $\mathrm{H}$ & -0.952846 & -1.073746 & 1.152368 \\
\hline 0 & 0.358670 & -0.086817 & 0.678826 \\
\hline $\mathrm{H}$ & 0.216213 & 0.887287 & 0.575583 \\
\hline C & 4.515198 & 1.962401 & 1.014756 \\
\hline C & 3.641345 & 2.367663 & -0.958244 \\
\hline C & 2.433300 & 3.033965 & 1.779690 \\
\hline C & 3.274951 & 1.890383 & 1.614082 \\
\hline $\mathrm{H}$ & 3.851377 & 1.338716 & -1.260599 \\
\hline $\mathrm{H}$ & 1.629865 & 2.912620 & 2.508240 \\
\hline $\mathrm{H}$ & 2.974348 & 3.972500 & 1.923403 \\
\hline C & -0.734293 & 3.563161 & -0.239460 \\
\hline C & -0.099392 & 4.962255 & 0.107953 \\
\hline C & -0.800392 & 3.295290 & -1.750452 \\
\hline $\mathrm{H}$ & -1.070882 & 2.248602 & -1.919635 \\
\hline $\mathrm{H}$ & -1.557582 & 3.920003 & -2.236667 \\
\hline $\mathrm{H}$ & 0.164729 & 3.480646 & -2.230491 \\
\hline C & -2.095500 & 3.297964 & 0.399476 \\
\hline $\mathrm{H}$ & -2.831854 & 4.039132 & 0.067562 \\
\hline $\mathrm{H}$ & -2.466269 & 2.311148 & 0.105283 \\
\hline $\mathrm{H}$ & -2.039789 & 3.329948 & 1.490491 \\
\hline C & -0.438364 & 5.438857 & 1.530282 \\
\hline $\mathrm{H}$ & -1.482467 & 5.758490 & 1.617707 \\
\hline $\mathrm{H}$ & -0.256460 & 4.651799 & 2.268379 \\
\hline $\mathrm{H}$ & 0.203293 & 6.290606 & 1.778018 \\
\hline C & -0.412305 & 6.075314 & -0.894028 \\
\hline $\mathrm{H}$ & -1.490019 & 6.269864 & -0.945728 \\
\hline $\mathrm{H}$ & 0.081876 & 7.001004 & -0.579904 \\
\hline $\mathrm{H}$ & -0.053678 & 5.825555 & -1.895279 \\
\hline 0 & 0.238835 & 2.655509 & 0.340290 \\
\hline 0 & 1.312999 & 4.687644 & 0.055905 \\
\hline B & 1.528471 & 3.297764 & 0.288435 \\
\hline 0 & 2.388596 & 2.638631 & -0.787836 \\
\hline C & 4.587404 & 3.386607 & -1.458779 \\
\hline C & 4.247785 & 4.749906 & -1.476239 \\
\hline C & 5.835065 & 2.974699 & -1.956689 \\
\hline C & 5.157732 & 5.680657 & -1.974190 \\
\hline $\mathrm{H}$ & 3.274470 & 5.063281 & -1.109645 \\
\hline C & 6.736997 & 3.909777 & -2.455594 \\
\hline $\mathrm{H}$ & 6.093465 & 1.917642 & -1.951200 \\
\hline C & 6.400204 & 5.266746 & -2.462006 \\
\hline $\mathrm{H}$ & 4.893358 & 6.734499 & -1.988811 \\
\hline $\mathrm{H}$ & 7.698462 & 3.583769 & -2.842484 \\
\hline $\mathrm{H}$ & 7.102697 & 5.998144 & -2.852398 \\
\hline $\mathrm{H}$ & 2.816523 & 0.907508 & 1.726547 \\
\hline
\end{tabular}




\begin{tabular}{|c|c|c|c|}
\hline $\mathrm{H}$ & 5.077014 & 2.890113 & 1.054792 \\
\hline $\mathrm{H}$ & 5.095032 & 1.058011 & 0.852105 \\
\hline C & -1.440640 & -1.565010 & -1.681219 \\
\hline C & -2.455182 & -1.308435 & -0.768082 \\
\hline C & -3.553257 & -0.443897 & -1.100362 \\
\hline C & -3.569824 & 0.142807 & -2.351838 \\
\hline $\mathrm{H}$ & -4.375686 & 0.826398 & -2.606175 \\
\hline C & 0.831147 & -2.037977 & -0.716903 \\
\hline C & -0.327743 & -2.511499 & -1.325623 \\
\hline C & 1.740172 & -4.290396 & -0.665638 \\
\hline C & 1.863790 & -2.955041 & -0.336790 \\
\hline $\mathrm{H}$ & 2.527476 & -4.983853 & -0.380120 \\
\hline C & 0.592127 & -4.798200 & -1.318910 \\
\hline C & -0.480449 & -3.903365 & -1.629705 \\
\hline C & -1.649354 & -4.435361 & -2.242351 \\
\hline C & -1.740751 & -5.775140 & -2.544229 \\
\hline C & -0.672392 & -6.655499 & -2.247018 \\
\hline C & 0.466825 & -6.175958 & -1.644011 \\
\hline $\mathrm{H}$ & -2.479808 & -3.773010 & -2.459579 \\
\hline $\mathrm{H}$ & -2.643697 & -6.163053 & -3.007508 \\
\hline $\mathrm{H}$ & -0.759940 & -7.711267 & -2.489152 \\
\hline $\mathrm{H}$ & 1.288456 & -6.845307 & -1.399898 \\
\hline C & -2.562707 & -0.100672 & -3.317519 \\
\hline C & -1.477003 & -0.971935 & -2.986446 \\
\hline C & -0.478812 & -1.214354 & -3.970002 \\
\hline C & -0.547533 & -0.619136 & -5.210639 \\
\hline C & -1.618235 & 0.248486 & -5.532944 \\
\hline C & -2.602356 & 0.500671 & -4.604804 \\
\hline $\mathrm{H}$ & 0.342800 & -1.881871 & -3.731746 \\
\hline $\mathrm{H}$ & 0.224502 & -0.818157 & -5.949055 \\
\hline $\mathrm{H}$ & -1.661173 & 0.711822 & -6.514921 \\
\hline $\mathrm{H}$ & -3.430864 & 1.163280 & -4.843855 \\
\hline C & 5.263947 & -1.999958 & 2.070775 \\
\hline C & 3.976757 & -1.873932 & 2.629089 \\
\hline C & 2.879734 & -2.128663 & 1.801787 \\
\hline C & 3.053723 & -2.537523 & 0.467522 \\
\hline C & 4.346221 & -2.655404 & -0.046723 \\
\hline C & 5.476990 & -2.378650 & 0.741475 \\
\hline $\mathrm{H}$ & 6.120709 & -1.810695 & 2.706515 \\
\hline $\mathrm{H}$ & 1.868056 & -2.047642 & 2.180998 \\
\hline $\mathrm{H}$ & 4.464696 & -2.977501 & -1.077691 \\
\hline C & 3.821645 & -1.543830 & 4.127603 \\
\hline C & 6.885177 & -2.520498 & 0.130820 \\
\hline C & 2.352519 & -1.297554 & 4.523228 \\
\hline $\mathrm{H}$ & 2.297303 & -1.030944 & 5.584726 \\
\hline $\mathrm{H}$ & 1.904515 & -0.478461 & 3.949403 \\
\hline $\mathrm{H}$ & 1.732694 & -2.188560 & 4.377291 \\
\hline C & 4.350043 & -2.743925 & 4.951301 \\
\hline $\mathrm{H}$ & 4.250841 & -2.543769 & 6.025399 \\
\hline $\mathrm{H}$ & 3.786075 & -3.654925 & 4.721513 \\
\hline $\mathrm{H}$ & 5.406944 & -2.942479 & 4.742190 \\
\hline C & 4.636479 & -0.282883 & 4.498943 \\
\hline $\mathrm{H}$ & 4.526905 & -0.067628 & 5.568573 \\
\hline $\mathrm{H}$ & 5.705925 & -0.405948 & 4.298346 \\
\hline
\end{tabular}




\begin{tabular}{|c|c|c|c|}
\hline $\mathrm{H}$ & 4.291117 & 0.594222 & 3.940886 \\
\hline C & 7.093396 & -3.974833 & -0.355264 \\
\hline $\mathrm{H}$ & 8.089903 & -4.090093 & -0.799105 \\
\hline $\mathrm{H}$ & 7.008794 & -4.681165 & 0.478329 \\
\hline $\mathrm{H}$ & 6.356329 & -4.261882 & -1.112257 \\
\hline C & 7.025172 & -1.558263 & -1.073128 \\
\hline $\mathrm{H}$ & 8.016103 & -1.660265 & -1.532400 \\
\hline $\mathrm{H}$ & 6.276934 & -1.760165 & -1.846657 \\
\hline $\mathrm{H}$ & 6.907776 & -0.515075 & -0.753966 \\
\hline C & 8.003371 & -2.189148 & 1.137199 \\
\hline $\mathrm{H}$ & 8.979898 & -2.297717 & 0.651908 \\
\hline $\mathrm{H}$ & 7.930430 & -1.159564 & 1.506075 \\
\hline $\mathrm{H}$ & 7.988213 & -2.863152 & 2.001048 \\
\hline C & -4.656955 & -0.150701 & -0.142724 \\
\hline C & -5.985468 & -0.154626 & -0.591735 \\
\hline C & -4.393021 & 0.164006 & 1.197125 \\
\hline C & -7.046592 & 0.147931 & 0.268117 \\
\hline $\mathrm{H}$ & -6.177155 & -0.428804 & -1.622394 \\
\hline C & -5.424500 & 0.464367 & 2.092409 \\
\hline $\mathrm{H}$ & -3.366228 & 0.167144 & 1.538932 \\
\hline C & -6.737183 & 0.454500 & 1.600877 \\
\hline $\mathrm{H}$ & -7.550046 & 0.683381 & 2.283671 \\
\hline C & -5.160567 & 0.778296 & 3.577728 \\
\hline C & -8.517484 & 0.138465 & -0.192722 \\
\hline C & -5.856928 & -0.292146 & 4.451869 \\
\hline $\mathrm{H}$ & -5.683046 & -0.087948 & 5.515802 \\
\hline $\mathrm{H}$ & -6.939820 & -0.309706 & 4.288264 \\
\hline $\mathrm{H}$ & -5.467898 & -1.291273 & 4.227194 \\
\hline C & -3.658112 & 0.771389 & 3.920103 \\
\hline $\mathrm{H}$ & -3.524126 & 0.986945 & 4.986444 \\
\hline $\mathrm{H}$ & -3.193283 & -0.198710 & 3.714181 \\
\hline $\mathrm{H}$ & -3.111248 & 1.537699 & 3.357889 \\
\hline C & -5.726633 & 2.174749 & 3.926974 \\
\hline $\mathrm{H}$ & -5.245664 & 2.954414 & 3.324736 \\
\hline $\mathrm{H}$ & -6.805984 & 2.236308 & 3.751984 \\
\hline $\mathrm{H}$ & -5.548231 & 2.404540 & 4.984809 \\
\hline C & -8.663626 & -0.220835 & -1.683728 \\
\hline $\mathrm{H}$ & -8.143338 & 0.496450 & -2.328955 \\
\hline $\mathrm{H}$ & -8.275292 & -1.221905 & -1.902111 \\
\hline $\mathrm{H}$ & -9.723007 & -0.209116 & -1.964472 \\
\hline C & -9.309869 & -0.904409 & 0.631108 \\
\hline $\mathrm{H}$ & -8.892457 & -1.908465 & 0.495943 \\
\hline $\mathrm{H}$ & -9.291222 & -0.677626 & 1.702192 \\
\hline $\mathrm{H}$ & -10.359637 & -0.925018 & 0.312852 \\
\hline C & -9.135880 & 1.539969 & 0.025013 \\
\hline $\mathrm{H}$ & -10.186215 & 1.547027 & -0.291620 \\
\hline $\mathrm{H}$ & -9.103594 & 1.841918 & 1.077075 \\
\hline $\mathrm{H}$ & -8.600367 & 2.299317 & -0.556592 \\
\hline
\end{tabular}




\begin{tabular}{lc}
\hline B3LYP/6-31G(d) Energy & -3038.955958 \\
\hline B3LYP-D3(BJ)/def2-TZVPP-IEF-PCM(DCM) Energy & -3040.507083 \\
\hline B3LYP-D3(BJ)/def2-TZVPP-IEF-PCM(DCM)//B3LYP/6-31G(d) Free Energy (Quasiharmonic) & -3039.278876 \\
\hline
\end{tabular}

Number of Imaginary Frequencies

Frequencies (Top 3 out of 450)

$\begin{array}{lr}\text { 1. } & -298.1071 \mathrm{~cm}^{-1} \\ \text { 2. } & 8.4216 \mathrm{~cm}^{-1} \\ \text { 3. } & 11.1936 \mathrm{~cm}^{-1}\end{array}$

\section{B3LYP/6-31G(d) Molecular Geometry in Cartesian Coordinates}

C

$\mathrm{H}$

$\mathrm{H}$

C

0

0

$\mathrm{H}$

0

$\mathrm{H}$

C

C

C

C

$\mathrm{H}$

$\mathrm{H}$

$\mathrm{H}$

C

C

C

$\mathrm{H}$

$\mathrm{H}$

$\mathrm{H}$

C

$\mathrm{H}$

$\mathrm{H}$

$\mathrm{H}$

C

$\mathrm{H}$

$\mathrm{H}$

$\mathrm{H}$

C

$\mathrm{H}$

-2.852741
-1.895516
-3.568224
0.528282
-0.375041
1.518932
2.240617
-3.377325
-2.726075
2.452839
2.199893
4.525670
3.212857
1.629725
5.085223
5.134769
4.508631
5.913528
4.524851
3.512652
5.151276
4.902877
3.779251
4.234942
2.740172
3.779173
6.662906
6.876579
6.095122
7.617838
6.835233
7.226442

$-1.953214$

$-2.326018$

$-2.001028$

$-0.396054$

$-0.455480$

0.494511

0.535033

$-2.828804$

$-2.870481$

3.468989

3. 828542

2. 397687

2. 347400

2.913156

1.462709

3.246275

1.407487

1.999731

$-0.051897$

$-0.383947$

$-0.178302$

$-0.704202$

2.267004

2. 172125

1.933364

3. 322581

2.675722

1.966734

3. 515255

3. 065871

0.965762

0.234039

$$
\begin{array}{r}
-0.410498 \\
-0.034780 \\
0.410587 \\
0.555138 \\
1.362696 \\
0.733577 \\
0.057472 \\
-1.419799 \\
-2.138767 \\
2.809262 \\
0.654375 \\
1.977130 \\
2.563171 \\
0.494142 \\
2.048999 \\
2.298854 \\
-1.695751 \\
-1.310521 \\
-2.144129 \\
-2.401577 \\
-3.035089 \\
-1.354426 \\
-2.739499 \\
-3.731135 \\
-2.809602 \\
-2.453780 \\
-2.462262 \\
-3.271298 \\
-2.869806 \\
-2.094502 \\
-0.639766 \\
-1.355717
\end{array}
$$




\begin{tabular}{|c|c|c|c|}
\hline H & 7.682786 & 1.494409 & -0.191807 \\
\hline $\mathrm{H}$ & 6.314320 & 0.424037 & 0.155439 \\
\hline 0 & 3.789218 & 1.501309 & -0.437111 \\
\hline 0 & 5.576371 & 2.998313 & -0.342202 \\
\hline B & 4.335487 & 2.659438 & 0.267578 \\
\hline 0 & 3.421552 & 3.875031 & 0.230958 \\
\hline C & 1.436107 & 5.088378 & 0.722597 \\
\hline C & 2.083210 & 6.332755 & 0.656124 \\
\hline C & 0.037596 & 5.038520 & 0.825676 \\
\hline C & 1.338193 & 7.507574 & 0.702235 \\
\hline $\mathrm{H}$ & 3.163807 & 6.360704 & 0.558790 \\
\hline C & -0.703486 & 6.217546 & 0.870937 \\
\hline $\mathrm{H}$ & -0.465133 & 4.075216 & 0.861297 \\
\hline C & -0.055078 & 7.453493 & 0.812831 \\
\hline $\mathrm{H}$ & 1.842165 & 8.468756 & 0.648568 \\
\hline $\mathrm{H}$ & -1.786178 & 6.171914 & 0.947604 \\
\hline $\mathrm{H}$ & -0.633287 & 8.372903 & 0.848682 \\
\hline $\mathrm{H}$ & 2.705280 & 1.385349 & 2.582038 \\
\hline $\mathrm{H}$ & 2.929223 & 4.435076 & 2.951289 \\
\hline $\mathrm{H}$ & 1.438278 & 3.377259 & 3.186176 \\
\hline C & -0.382926 & -1.093799 & -1.649473 \\
\hline C & 0.588412 & -1.279975 & -0.668962 \\
\hline C & 1.567756 & -2.329159 & -0.768040 \\
\hline C & 1.585068 & -3.091215 & -1.922615 \\
\hline $\mathrm{H}$ & 2.288148 & -3.915365 & -2.003707 \\
\hline C & -2.700338 & -0.517205 & -0.886164 \\
\hline C & -1.504381 & -0.102111 & -1.469863 \\
\hline C & -3.601194 & 1.727514 & -1.128285 \\
\hline C & -3.780327 & 0.417542 & -0.724190 \\
\hline $\mathrm{H}$ & -4.418512 & 2.434814 & -1.012124 \\
\hline C & -2.404078 & 2.175869 & -1.730984 \\
\hline C & -1.333784 & 1.245135 & -1.923141 \\
\hline C & -0.152960 & 1.704318 & -2.569906 \\
\hline C & -0.033613 & 3.010674 & -2.990464 \\
\hline C & -1.088754 & 3.931723 & -2.784370 \\
\hline C & -2.248109 & 3.518925 & -2.170237 \\
\hline $\mathrm{H}$ & 0.657848 & 1.003773 & -2.741994 \\
\hline $\mathrm{H}$ & 0.875402 & 3.338213 & -3.486523 \\
\hline $\mathrm{H}$ & -0.979889 & 4.960468 & -3.116076 \\
\hline $\mathrm{H}$ & -3.070309 & 4.214040 & -2.016253 \\
\hline C & 0.673878 & -2.882916 & -2.984286 \\
\hline C & -0.341443 & -1.883031 & -2.846049 \\
\hline C & -1.277064 & -1.716939 & -3.908218 \\
\hline C & -1.208416 & -2.499648 & -5.040567 \\
\hline C & -0.203835 & -3.488289 & -5.172047 \\
\hline C & 0.714799 & -3.673668 & -4.164987 \\
\hline $\mathrm{H}$ & -2.043951 & -0.954561 & -3.820813 \\
\hline $\mathrm{H}$ & -1.929208 & -2.355009 & -5.840449 \\
\hline $\mathrm{H}$ & -0.162150 & -4.099405 & -6.069324 \\
\hline $\mathrm{H}$ & 1.487473 & -4.433347 & -4.255057 \\
\hline C & -7.595722 & -0.557330 & 0.947742 \\
\hline C & -6.894886 & 0.555851 & 1.447584 \\
\hline C & -5.644065 & 0.839092 & 0.897115 \\
\hline C & -5.095936 & 0.042978 & -0.125157 \\
\hline
\end{tabular}




\begin{tabular}{|c|c|c|c|}
\hline C & -5.825427 & -1.052797 & -0.594356 \\
\hline C & -7.084470 & -1.375227 & -0.063408 \\
\hline $\mathrm{H}$ & -8.566105 & -0.787719 & 1.371906 \\
\hline $\mathrm{H}$ & -5.057449 & 1.672753 & 1.265568 \\
\hline $\mathrm{H}$ & -5.392572 & -1.664057 & -1.378146 \\
\hline C & -7.520145 & 1.402555 & 2.573608 \\
\hline C & -7.835530 & -2.605723 & -0.607595 \\
\hline C & -8.866460 & 1.991030 & 2.088428 \\
\hline $\mathrm{H}$ & -9.328440 & 2.593112 & 2.880787 \\
\hline $\mathrm{H}$ & -8.719319 & 2.635021 & 1.213678 \\
\hline $\mathrm{H}$ & -9.577907 & 1.207142 & 1.808312 \\
\hline C & -7.768465 & 0.512397 & 3.814564 \\
\hline $\mathrm{H}$ & -8.218729 & 1.101355 & 4.623388 \\
\hline $\mathrm{H}$ & -8.445204 & -0.319258 & 3.592346 \\
\hline $\mathrm{H}$ & -6.828751 & 0.087635 & 4.185438 \\
\hline C & -6.615009 & 2.573139 & 3.002086 \\
\hline $\mathrm{H}$ & -7.102399 & 3.142457 & 3.801787 \\
\hline $\mathrm{H}$ & -5.650214 & 2.223398 & 3.386255 \\
\hline $\mathrm{H}$ & -6.422959 & 3.265073 & 2.173968 \\
\hline C & -9.197194 & -2.818919 & 0.079813 \\
\hline $\mathrm{H}$ & -9.686216 & -3.705633 & -0.339362 \\
\hline $\mathrm{H}$ & -9.089179 & -2.981656 & 1.158385 \\
\hline $\mathrm{H}$ & -9.870904 & -1.967515 & -0.072525 \\
\hline C & -8.087122 & -2.426386 & -2.123761 \\
\hline $\mathrm{H}$ & -8.616995 & -3.298072 & -2.527630 \\
\hline $\mathrm{H}$ & -8.698947 & -1.537408 & -2.317081 \\
\hline $\mathrm{H}$ & -7.151407 & -2.317719 & -2.681228 \\
\hline C & -6.973778 & -3.871077 & -0.377263 \\
\hline $\mathrm{H}$ & -7.481977 & -4.755154 & -0.782762 \\
\hline $\mathrm{H}$ & -5.992762 & -3.794335 & -0.856595 \\
\hline $\mathrm{H}$ & -6.809362 & -4.038461 & 0.693833 \\
\hline C & 2.483335 & -2.691711 & 0.354226 \\
\hline C & 3.795881 & -3.118737 & 0.097938 \\
\hline C & 2.020926 & -2.711864 & 1.677241 \\
\hline C & 4.639095 & -3.561310 & 1.124529 \\
\hline $\mathrm{H}$ & 4.156756 & -3.101349 & -0.925809 \\
\hline C & 2.842691 & -3.109909 & 2.738068 \\
\hline $\mathrm{H}$ & 0.993782 & -2.435230 & 1.879846 \\
\hline C & 4.143991 & -3.532930 & 2.437225 \\
\hline $\mathrm{H}$ & 4.786490 & -3.856234 & 3.244389 \\
\hline C & 2.282967 & -3.083633 & 4.173700 \\
\hline C & 6.051936 & -4.081646 & 0.787957 \\
\hline C & 1.823972 & -1.645621 & 4.518011 \\
\hline $\mathrm{H}$ & 2.668456 & -0.947229 & 4.477378 \\
\hline $\mathrm{H}$ & 1.054383 & -1.281201 & 3.830064 \\
\hline $\mathrm{H}$ & 1.408720 & -1.613947 & 5.532953 \\
\hline C & 1.072676 & -4.043104 & 4.272014 \\
\hline $\mathrm{H}$ & 0.659144 & -4.030044 & 5.287863 \\
\hline $\mathrm{H}$ & 0.270210 & -3.759390 & 3.583557 \\
\hline $\mathrm{H}$ & 1.367790 & -5.072713 & 4.038507 \\
\hline C & 3.325696 & -3.518665 & 5.220664 \\
\hline $\mathrm{H}$ & 3.661505 & -4.550015 & 5.061868 \\
\hline $\mathrm{H}$ & 4.206945 & -2.866788 & 5.215411 \\
\hline $\mathrm{H}$ & 2.883866 & -3.467903 & 6.222109 \\
\hline
\end{tabular}




$\begin{array}{lllr}\mathrm{C} & 6.833656 & -4.520886 & 2.041122 \\ \mathrm{H} & 6.979841 & -3.692102 & 2.743039 \\ \mathrm{H} & 6.330617 & -5.336200 & 2.573170 \\ \mathrm{H} & 7.825329 & -4.882495 & 1.746683 \\ \mathrm{C} & 5.929717 & -5.306149 & -0.151167 \\ \mathrm{H} & 5.357586 & -6.110469 & 0.325174 \\ \mathrm{H} & 5.427720 & -5.049793 & -1.090126 \\ \mathrm{H} & 6.923955 & -5.697124 & -0.400702 \\ \mathrm{C} & 6.869820 & -2.977878 & 0.079286 \\ \mathrm{H} & 7.871534 & -3.347868 & -0.171776 \\ \mathrm{H} & 6.395363 & -2.650938 & -0.851603 \\ \mathrm{H} & 6.985508 & -2.098831 & 0.723019\end{array}$

\section{C-Catalyzed reaction TS 91}

\section{Datum}

Value

\begin{tabular}{lc} 
B3LYP/6-31G(d) Energy & -3038.956833 \\
\hline B3LYP-D3(BJ)/def2-TZVPP-IEF-PCM(DCM) Energy & -3040.507297 \\
\hline B3LYP-D3(BJ)/def2-TZVPP-IEF-PCM(DCM)//B3LYP/6-31G(d) Free Energy (Quasiharmonic) & -3039.27886 \\
\hline
\end{tabular}

Number of Imaginary Frequencies

Frequencies (Top 3 out of 450)

$\begin{array}{lr}\text { 1. } & -313.1043 \mathrm{~cm}^{-1} \\ \text { 2. } & 6.0843 \mathrm{~cm}^{-1} \\ \text { 3. } & 8.3399 \mathrm{~cm}^{-1}\end{array}$

\section{B3LYP/6-31G(d) Molecular Geometry in Cartesian Coordinates}

$\begin{array}{rrrr}C & -2.110440 & -0.924568 & 0.913353 \\ H & -1.049805 & -0.679632 & 1.025628 \\ H & -2.665986 & 0.012144 & 0.967256 \\ \mathrm{C} & 1.003940 & -0.883978 & -1.254560 \\ \mathrm{O} & 0.994426 & -0.962820 & -2.463035 \\ \mathrm{O} & 0.965582 & 0.324651 & -0.662067 \\ \mathrm{H} & 0.960120 & 0.359641 & 0.328781 \\ \mathrm{O} & -2.562386 & -1.689528 & 2.039665 \\ \mathrm{H} & -2.072979 & -2.528779 & 2.042966 \\ \mathrm{C} & -0.543806 & 3.776753 & -1.237029 \\ \mathrm{C} & 1.471343 & 3.426804 & -0.493324 \\ \mathrm{C} & -1.115228 & 3.282830 & 1.118259 \\ \mathrm{C} & -1.094839 & 2.953694 & -0.278889 \\ \mathrm{H} & 1.478127 & 2.389874 & -0.824092\end{array}$




\begin{tabular}{|c|c|c|c|}
\hline H & -1.779238 & 2.662438 & 1.722953 \\
\hline $\mathrm{H}$ & -1.255186 & 4.344629 & 1.335069 \\
\hline C & 0.984190 & 1.155441 & 3.213052 \\
\hline C & 1.399850 & 2.545556 & 3.820513 \\
\hline C & -0.333482 & 0.635302 & 3.806237 \\
\hline H & -0.696888 & -0.228060 & 3.242380 \\
\hline H & -0.199807 & 0.319305 & 4.846851 \\
\hline $\mathrm{H}$ & -1.109740 & 1.405623 & 3.782987 \\
\hline C & 2.056346 & 0.072376 & 3.303637 \\
\hline $\mathrm{H}$ & 2.323315 & -0.122379 & 4.349355 \\
\hline $\mathrm{H}$ & 1.685167 & -0.866170 & 2.877487 \\
\hline H & 2.958594 & 0.358126 & 2.758679 \\
\hline C & 2.889122 & 2.871851 & 3.601060 \\
\hline $\mathrm{H}$ & 3.539203 & 2.265330 & 4.242174 \\
\hline $\mathrm{H}$ & 3.183780 & 2.717051 & 2.559037 \\
\hline $\mathrm{H}$ & 3.054834 & 3.926398 & 3.843268 \\
\hline C & 1.048617 & 2.737418 & 5.297270 \\
\hline $\mathrm{H}$ & 1.560175 & 2.001116 & 5.928713 \\
\hline $\mathrm{H}$ & 1.364819 & 3.735409 & 5.619055 \\
\hline $\mathrm{H}$ & -0.027684 & 2.657917 & 5.466055 \\
\hline 0 & 0.771197 & 1.486690 & 1.811089 \\
\hline 0 & 0.609992 & 3.458573 & 3.047278 \\
\hline B & 0.474019 & 2.920310 & 1.740013 \\
\hline 0 & 1.372303 & 3.675369 & 0.774805 \\
\hline C & 2.209769 & 4.393757 & -1.330390 \\
\hline C & 2.535533 & 5.671972 & -0.851347 \\
\hline C & 2.619347 & 4.010678 & -2.617112 \\
\hline C & 3.256971 & 6.552465 & -1.653315 \\
\hline $\mathrm{H}$ & 2.227840 & 5.952720 & 0.150792 \\
\hline C & 3.340897 & 4.895281 & -3.416039 \\
\hline $\mathrm{H}$ & 2.371923 & 3.017751 & -2.985454 \\
\hline C & 3.659345 & 6.168266 & -2.936316 \\
\hline $\mathrm{H}$ & 3.510747 & 7.539965 & -1.277492 \\
\hline $\mathrm{H}$ & 3.656978 & 4.591167 & -4.409954 \\
\hline $\mathrm{H}$ & 4.222857 & 6.858310 & -3.558534 \\
\hline $\mathrm{H}$ & -1.289832 & 1.919005 & -0.559035 \\
\hline $\mathrm{H}$ & -0.483051 & 4.847428 & -1.063132 \\
\hline H & -0.488329 & 3.458845 & -2.274101 \\
\hline C & -0.142227 & -2.801970 & -0.166511 \\
\hline C & 1.037649 & -2.088334 & -0.341705 \\
\hline C & 2.261703 & -2.479124 & 0.301454 \\
\hline C & 2.219731 & -3.547524 & 1.178555 \\
\hline $\mathrm{H}$ & 3.137892 & -3.876927 & 1.657440 \\
\hline C & -2.350467 & -1.600362 & -0.424028 \\
\hline C & -1.388852 & -2.464018 & -0.943155 \\
\hline C & -3.730572 & -1.942009 & -2.394294 \\
\hline C & -3.556431 & -1.338762 & -1.163657 \\
\hline $\mathrm{H}$ & -4.646218 & -1.757635 & -2.950569 \\
\hline C & -2.773035 & -2.823758 & -2.948251 \\
\hline C & -1.577334 & -3.100522 & -2.214794 \\
\hline C & -0.629420 & -3.995022 & -2.783859 \\
\hline C & -0.857684 & -4.585631 & -4.006342 \\
\hline C & -2.044256 & -4.312251 & -4.727976 \\
\hline C & -2.979382 & -3.448133 & -4.208339 \\
\hline
\end{tabular}




\begin{tabular}{|c|c|c|c|}
\hline $\mathrm{H}$ & 0.289281 & -4.204798 & -2.247753 \\
\hline $\mathrm{H}$ & -0.118895 & -5.264384 & -4.423737 \\
\hline $\mathrm{H}$ & -2.211397 & -4.784573 & -5.692286 \\
\hline $\mathrm{H}$ & -3.894081 & -3.228334 & -4.753922 \\
\hline C & 1.029532 & -4.267527 & 1.439479 \\
\hline C & -0.169259 & -3.912848 & 0.741192 \\
\hline C & -1.344282 & -4.682739 & 0.980149 \\
\hline C & -1.334910 & -5.729401 & 1.877206 \\
\hline C & -0.150518 & -6.069443 & 2.573246 \\
\hline C & 1.005340 & -5.356600 & 2.352621 \\
\hline $\mathrm{H}$ & -2.252322 & -4.441076 & 0.437682 \\
\hline $\mathrm{H}$ & -2.242085 & -6.302881 & 2.045843 \\
\hline $\mathrm{H}$ & -0.156527 & -6.897166 & 3.276887 \\
\hline $\mathrm{H}$ & 1.922388 & -5.615728 & 2.876246 \\
\hline C & -6.704319 & 1.217024 & 0.213827 \\
\hline C & -6.227253 & 0.217169 & 1.073983 \\
\hline C & -5.190260 & -0.604508 & 0.615721 \\
\hline C & -4.633548 & -0.436125 & -0.659134 \\
\hline C & -5.138474 & 0.575403 & -1.488049 \\
\hline C & -6.176930 & 1.417075 & -1.070462 \\
\hline $\mathrm{H}$ & -7.504099 & 1.860040 & 0.554939 \\
\hline $\mathrm{H}$ & -4.791742 & -1.386756 & 1.252448 \\
\hline $\mathrm{H}$ & -4.690160 & 0.708736 & -2.468589 \\
\hline C & -6.791537 & -0.002008 & 2.491390 \\
\hline C & -6.689042 & 2.522484 & -2.015121 \\
\hline C & -7.342421 & -1.442937 & 2.610798 \\
\hline $\mathrm{H}$ & -7.743977 & -1.614750 & 3.617233 \\
\hline $\mathrm{H}$ & -8.149092 & -1.616500 & 1.888985 \\
\hline $\mathrm{H}$ & -6.564356 & -2.191458 & 2.430536 \\
\hline C & -5.658431 & 0.198750 & 3.527283 \\
\hline $\mathrm{H}$ & -6.036356 & 0.022970 & 4.542468 \\
\hline $\mathrm{H}$ & -4.819289 & -0.482861 & 3.355877 \\
\hline $\mathrm{H}$ & -5.271008 & 1.223591 & 3.484699 \\
\hline C & -7.933047 & 0.975041 & 2.830587 \\
\hline $\mathrm{H}$ & -8.298945 & 0.775884 & 3.844325 \\
\hline $\mathrm{H}$ & -7.600461 & 2.018964 & 2.800501 \\
\hline $\mathrm{H}$ & -8.782249 & 0.866249 & 2.145872 \\
\hline C & -7.218892 & 1.881836 & -3.320062 \\
\hline $\mathrm{H}$ & -7.577816 & 2.657255 & -4.008283 \\
\hline $\mathrm{H}$ & -6.441488 & 1.310963 & -3.838396 \\
\hline $\mathrm{H}$ & -8.051668 & 1.200384 & -3.111387 \\
\hline C & -7.830946 & 3.349059 & -1.393211 \\
\hline $\mathrm{H}$ & -8.158657 & 4.116824 & -2.103401 \\
\hline $\mathrm{H}$ & -8.701721 & 2.728122 & -1.153927 \\
\hline $\mathrm{H}$ & -7.513576 & 3.860936 & -0.477658 \\
\hline C & -5.528995 & 3.488330 & -2.355486 \\
\hline $\mathrm{H}$ & -5.877996 & 4.282741 & -3.026869 \\
\hline $\mathrm{H}$ & -5.131585 & 3.958831 & -1.448956 \\
\hline $\mathrm{H}$ & -4.701349 & 2.971806 & -2.852475 \\
\hline C & 3.568210 & -1.819919 & 0.010146 \\
\hline C & 4.475049 & -1.549987 & 1.052515 \\
\hline C & 3.945532 & -1.527771 & -1.304051 \\
\hline C & 5.726543 & -0.986451 & 0.800535 \\
\hline $\mathrm{H}$ & 4.173315 & -1.776591 & 2.067828 \\
\hline
\end{tabular}




\begin{tabular}{|c|c|c|c|}
\hline C & 5.189647 & -0.948245 & -1.599215 \\
\hline $\mathrm{H}$ & 3.265443 & -1.770069 & -2.111383 \\
\hline C & 6.055578 & -0.687551 & -0.534935 \\
\hline $\mathrm{H}$ & 7.021068 & -0.240603 & -0.739693 \\
\hline C & 5.542627 & -0.630910 & -3.064956 \\
\hline C & 6.741926 & -0.678050 & 1.918336 \\
\hline C & 5.503099 & -1.933562 & -3.899547 \\
\hline $\mathrm{H}$ & 6.222955 & -2.666731 & -3.517474 \\
\hline $\mathrm{H}$ & 4.511726 & -2.397183 & -3.886350 \\
\hline $\mathrm{H}$ & 5.756430 & -1.721606 & -4.945564 \\
\hline C & 4.507567 & 0.371150 & -3.632320 \\
\hline $\mathrm{H}$ & 4.726576 & 0.585099 & -4.686046 \\
\hline $\mathrm{H}$ & 3.485036 & -0.015465 & -3.572399 \\
\hline $\mathrm{H}$ & 4.541668 & 1.317919 & -3.080052 \\
\hline C & 6.944763 & -0.010709 & -3.212623 \\
\hline $\mathrm{H}$ & 7.031835 & 0.936256 & -2.667749 \\
\hline $\mathrm{H}$ & 7.731172 & -0.685807 & -2.855443 \\
\hline $\mathrm{H}$ & 7.145026 & 0.198040 & -4.269626 \\
\hline C & 6.230911 & -1.093941 & 3.311015 \\
\hline $\mathrm{H}$ & 5.317268 & -0.557640 & 3.590425 \\
\hline $\mathrm{H}$ & 6.027214 & -2.169584 & 3.366436 \\
\hline $\mathrm{H}$ & 6.991422 & -0.863330 & 4.065582 \\
\hline C & 8.057470 & -1.446174 & 1.645136 \\
\hline $\mathrm{H}$ & 7.883572 & -2.528264 & 1.630148 \\
\hline $\mathrm{H}$ & 8.502448 & -1.166178 & 0.684926 \\
\hline $\mathrm{H}$ & 8.794101 & -1.230810 & 2.428819 \\
\hline C & 7.033305 & 0.841334 & 1.946494 \\
\hline $\mathrm{H}$ & 7.758073 & 1.076253 & 2.735771 \\
\hline $\mathrm{H}$ & 7.449372 & 1.194246 & 0.997049 \\
\hline $\mathrm{H}$ & 6.119260 & 1.413032 & 2.143492 \\
\hline
\end{tabular}

\section{C-Catalyzed reaction TS 92}

\section{Datum}

Value

\begin{tabular}{lc}
\hline B3LYP/6-31G(d) Energy & -3038.948856 \\
\hline B3LYP-D3(BJ)/def2-TZVPP-IEF-PCM(DCM) Energy & -3040.506506 \\
\hline B3LYP-D3(BJ)/def2-TZVPP-IEF-PCM(DCM)//B3LYP/6-31G(d) Free Energy (Quasiharmonic) & -3039.278579 \\
\hline
\end{tabular}

Number of Imaginary Frequencies

Frequencies (Top 3 out of 450)

$$
\begin{array}{lr}
\text { 1. } & -301.9686 \mathrm{~cm}^{-1} \\
\text { 2. } & 9.1912 \mathrm{~cm}^{-1} \\
\text { 3. } & 11.2316 \mathrm{~cm}^{-1}
\end{array}
$$




\begin{tabular}{|c|c|c|c|}
\hline C & 1.564989 & -0.675610 & 0.792800 \\
\hline $\mathrm{H}$ & 2.187850 & 0.208740 & 0.602130 \\
\hline $\mathrm{H}$ & 0.561629 & -0.315350 & 1.013450 \\
\hline C & -1.872001 & -0.249089 & -1.300900 \\
\hline 0 & -2.183401 & -0.414859 & -2.455870 \\
\hline 0 & -1.382990 & 0.941240 & -0.883650 \\
\hline H & -1.233570 & 0.991550 & 0.091850 \\
\hline 0 & 2.002439 & -1.347720 & 1.978070 \\
\hline $\mathrm{H}$ & 2.969419 & -1.414461 & 1.922570 \\
\hline C & -0.044599 & 5.530150 & -0.901210 \\
\hline C & 1.364191 & 3.942750 & -0.212940 \\
\hline C & -1.546119 & 4.291371 & 0.617050 \\
\hline C & -0.772949 & 5.446390 & 0.262450 \\
\hline $\mathrm{H}$ & 1.831401 & 4.607900 & 0.515840 \\
\hline $\mathrm{H}$ & -1.973779 & 3.751441 & -0.229730 \\
\hline $\mathrm{H}$ & -2.281929 & 4.462781 & 1.404160 \\
\hline C & -0.979580 & 1.816330 & 3.151960 \\
\hline C & 0.182380 & 2.793900 & 3.582500 \\
\hline C & -2.364680 & 2.286251 & 3.625800 \\
\hline $\mathrm{H}$ & -3.133090 & 1.696201 & 3.115920 \\
\hline $\mathrm{H}$ & -2.490070 & 2.148411 & 4.705100 \\
\hline $\mathrm{H}$ & -2.534800 & 3.341741 & 3.395140 \\
\hline C & -0.779460 & 0.356780 & 3.561250 \\
\hline $\mathrm{H}$ & -0.804010 & 0.265020 & 4.653920 \\
\hline $\mathrm{H}$ & 0.164960 & -0.060870 & 3.208410 \\
\hline $\mathrm{H}$ & -1.594951 & -0.254879 & 3.160360 \\
\hline C & 1.567850 & 2.129220 & 3.557100 \\
\hline $\mathrm{H}$ & 1.698300 & 1.421170 & 4.382410 \\
\hline $\mathrm{H}$ & 1.738660 & 1.592280 & 2.620750 \\
\hline $\mathrm{H}$ & 2.330670 & 2.909749 & 3.650220 \\
\hline C & -0.029880 & 3.481830 & 4.932780 \\
\hline $\mathrm{H}$ & -0.087580 & 2.748380 & 5.745550 \\
\hline $\mathrm{H}$ & 0.813731 & 4.149000 & 5.140510 \\
\hline $\mathrm{H}$ & -0.941639 & 4.083930 & 4.938640 \\
\hline 0 & -0.946970 & 1.924660 & 1.702910 \\
\hline 0 & 0.144331 & 3.804050 & 2.553000 \\
\hline B & -0.391260 & 3.215210 & 1.371380 \\
\hline 0 & 0.590180 & 3.011280 & 0.228240 \\
\hline C & 2.052121 & 3.724430 & -1.497990 \\
\hline C & 1.664640 & 2.670310 & -2.343360 \\
\hline C & 3.097431 & 4.577899 & -1.884550 \\
\hline C & 2.318790 & 2.482329 & -3.558880 \\
\hline $\mathrm{H}$ & 0.851700 & 2.016610 & -2.042110 \\
\hline C & 3.744371 & 4.387219 & -3.103460 \\
\hline $\mathrm{H}$ & 3.397741 & 5.392799 & -1.229440 \\
\hline C & 3.355730 & 3.339369 & -3.943060 \\
\hline $\mathrm{H}$ & 2.014310 & 1.668000 & -4.210160 \\
\hline $\mathrm{H}$ & 4.551441 & 5.052179 & -3.397800 \\
\hline $\mathrm{H}$ & 3.857280 & 3.193239 & -4.895880 \\
\hline H & -0.563109 & 6.166310 & 1.054330 \\
\hline
\end{tabular}




\begin{tabular}{|c|c|c|c|}
\hline $\mathrm{H}$ & -0.333909 & 4.942180 & -1.766810 \\
\hline $\mathrm{H}$ & 0.599041 & 6.382980 & -1.099070 \\
\hline C & -0.874821 & -2.154590 & -0.030750 \\
\hline C & -1.970401 & -1.323109 & -0.240120 \\
\hline C & -3.208541 & -1.521939 & 0.458870 \\
\hline C & -3.255751 & -2.501649 & 1.432580 \\
\hline $\mathrm{H}$ & -4.179281 & -2.652999 & 1.985430 \\
\hline C & 1.539469 & -1.530330 & -0.460120 \\
\hline C & 0.353649 & -2.105740 & -0.906530 \\
\hline C & 2.712809 & -2.335571 & -2.431960 \\
\hline C & 2.749989 & -1.699091 & -1.210810 \\
\hline H & 3.632349 & -2.465811 & -2.998200 \\
\hline C & 1.509549 & -2.872630 & -2.953190 \\
\hline C & 0.312669 & -2.790290 & -2.170750 \\
\hline C & -0.867451 & -3.392220 & -2.689290 \\
\hline C & -0.863632 & -4.026730 & -3.911140 \\
\hline C & 0.319618 & -4.099240 & -4.683080 \\
\hline C & 1.480629 & -3.532890 & -4.210820 \\
\hline $\mathrm{H}$ & -1.786971 & -3.328299 & -2.120570 \\
\hline H & -1.780572 & -4.470319 & -4.289240 \\
\hline $\mathrm{H}$ & 0.307648 & -4.601950 & -5.646530 \\
\hline $\mathrm{H}$ & 2.399059 & -3.585731 & -4.791120 \\
\hline C & -2.148741 & -3.331159 & 1.728560 \\
\hline C & -0.943031 & -3.182450 & 0.972100 \\
\hline C & 0.131908 & -4.073290 & 1.245090 \\
\hline C & 0.023178 & -5.040030 & 2.219410 \\
\hline C & -1.164882 & -5.171560 & 2.976560 \\
\hline C & -2.227092 & -4.332979 & 2.733850 \\
\hline $\mathrm{H}$ & 1.051078 & -3.978470 & 0.680710 \\
\hline $\mathrm{H}$ & 0.859548 & -5.706500 & 2.412210 \\
\hline H & -1.235262 & -5.936780 & 3.745010 \\
\hline $\mathrm{H}$ & -3.149322 & -4.427079 & 3.302480 \\
\hline C & 6.605349 & -0.757982 & 0.396390 \\
\hline C & 5.892669 & -1.853131 & 0.915440 \\
\hline C & 4.627849 & -2.112541 & 0.382190 \\
\hline C & 4.078879 & -1.308671 & -0.636050 \\
\hline C & 4.819339 & -0.230071 & -1.121600 \\
\hline C & 6.100420 & 0.062348 & -0.616360 \\
\hline H & 7.589459 & -0.550282 & 0.798690 \\
\hline H & 4.054979 & -2.970041 & 0.717660 \\
\hline $\mathrm{H}$ & 4.382440 & 0.387459 & -1.900530 \\
\hline C & 6.523089 & -2.718022 & 2.024050 \\
\hline C & 6.886900 & 1.274368 & -1.153360 \\
\hline C & 6.808259 & -1.837002 & 3.263830 \\
\hline $\mathrm{H}$ & 7.267269 & -2.436482 & 4.059440 \\
\hline $\mathrm{H}$ & 7.490959 & -1.012772 & 3.032170 \\
\hline $\mathrm{H}$ & 5.882429 & -1.402681 & 3.658550 \\
\hline C & 5.604938 & -3.875451 & 2.461420 \\
\hline $\mathrm{H}$ & 6.094828 & -4.454172 & 3.252430 \\
\hline H & 4.650399 & -3.515431 & 2.862250 \\
\hline $\mathrm{H}$ & 5.390828 & -4.562511 & 1.634930 \\
\hline C & 7.850669 & -3.325982 & 1.511980 \\
\hline $\mathrm{H}$ & 8.312868 & -3.942612 & 2.292520 \\
\hline $\mathrm{H}$ & 7.678568 & -3.960272 & 0.634950 \\
\hline
\end{tabular}




\begin{tabular}{|c|c|c|c|}
\hline $\mathrm{H}$ & 8.572259 & -2.552762 & 1.228130 \\
\hline C & 8.350560 & 1.288328 & -0.669510 \\
\hline $\mathrm{H}$ & 8.875900 & 2.141128 & -1.114150 \\
\hline $\mathrm{H}$ & 8.425150 & 1.393658 & 0.418530 \\
\hline $\mathrm{H}$ & 8.885120 & 0.377488 & -0.963160 \\
\hline C & 6.903090 & 1.260728 & -2.699680 \\
\hline $\mathrm{H}$ & 7.484810 & 2.110818 & -3.076350 \\
\hline $\mathrm{H}$ & 5.898030 & 1.338919 & -3.124970 \\
\hline $\mathrm{H}$ & 7.362490 & 0.341248 & -3.080300 \\
\hline C & 6.199790 & 2.568608 & -0.658160 \\
\hline $\mathrm{H}$ & 6.730910 & 3.452528 & -1.033840 \\
\hline $\mathrm{H}$ & 5.162380 & 2.629529 & -0.999880 \\
\hline $\mathrm{H}$ & 6.197790 & 2.613888 & 0.437220 \\
\hline C & -4.472191 & -0.806389 & 0.106440 \\
\hline C & -4.568710 & 0.586601 & 0.004420 \\
\hline C & -5.622311 & -1.577998 & -0.120220 \\
\hline C & -5.780130 & 1.210562 & -0.326640 \\
\hline $\mathrm{H}$ & -3.687810 & 1.189791 & 0.193200 \\
\hline C & -6.852791 & -0.995348 & -0.439940 \\
\hline $\mathrm{H}$ & -5.532921 & -2.658688 & -0.066570 \\
\hline C & -6.904690 & 0.401862 & -0.538600 \\
\hline $\mathrm{H}$ & -7.844100 & 0.872992 & -0.792680 \\
\hline C & -8.082171 & -1.892988 & -0.683310 \\
\hline C & -5.835700 & 2.744982 & -0.456970 \\
\hline C & -8.366341 & -2.732058 & 0.585410 \\
\hline $\mathrm{H}$ & -7.518181 & -3.372748 & 0.848230 \\
\hline $\mathrm{H}$ & -8.575221 & -2.084288 & 1.444690 \\
\hline $\mathrm{H}$ & -9.237601 & -3.380007 & 0.428360 \\
\hline C & -9.349981 & -1.081517 & -1.011160 \\
\hline $\mathrm{H}$ & -10.192721 & -1.763807 & -1.170820 \\
\hline $\mathrm{H}$ & -9.623861 & -0.403497 & -0.194760 \\
\hline $\mathrm{H}$ & -9.229701 & -0.487317 & -1.924040 \\
\hline C & -7.799291 & -2.842608 & -1.871650 \\
\hline $\mathrm{H}$ & -7.602921 & -2.274898 & -2.788220 \\
\hline $\mathrm{H}$ & -6.930401 & -3.481518 & -1.683730 \\
\hline $\mathrm{H}$ & -8.662151 & -3.495628 & -2.053130 \\
\hline C & -4.867900 & 3.190901 & -1.579190 \\
\hline $\mathrm{H}$ & -3.843990 & 2.851661 & -1.396270 \\
\hline $\mathrm{H}$ & -5.179390 & 2.779501 & -2.546050 \\
\hline $\mathrm{H}$ & -4.855919 & 4.285031 & -1.661720 \\
\hline C & -7.243410 & 3.262502 & -0.810170 \\
\hline $\mathrm{H}$ & -7.596120 & 2.869522 & -1.770440 \\
\hline $\mathrm{H}$ & -7.980690 & 3.003232 & -0.041480 \\
\hline $\mathrm{H}$ & -7.221569 & 4.355292 & -0.891010 \\
\hline C & -5.417490 & 3.392542 & 0.883630 \\
\hline $\mathrm{H}$ & -5.433179 & 4.486582 & 0.800840 \\
\hline $\mathrm{H}$ & -6.105130 & 3.103422 & 1.687190 \\
\hline $\mathrm{H}$ & -4.408940 & 3.094871 & 1.184530 \\
\hline
\end{tabular}




\begin{tabular}{lc}
\hline B3LYP/6-31G(d) Energy & -3038.952479 \\
\hline B3LYP-D3(BJ)/def2-TZVPP-IEF-PCM(DCM) Energy & -3040.505876 \\
\hline B3LYP-D3(BJ)/def2-TZVPP-IEF-PCM(DCM)//B3LYP/6-31G(d) Free Energy (Quasiharmonic) & -3039.278571 \\
\hline
\end{tabular}

Number of Imaginary Frequencies

Frequencies (Top 3 out of 450)

$\begin{array}{lr}\text { 1. } & -320.0358 \mathrm{~cm}^{-1} \\ \text { 2. } & 7.0658 \mathrm{~cm}^{-1} \\ \text { 3. } & 9.8033 \mathrm{~cm}^{-1}\end{array}$

\section{B3LYP/6-31G(d) Molecular Geometry in Cartesian Coordinates}

C

$\mathrm{H}$

$\mathrm{H}$

C

0

0

$\mathrm{H}$

0

$\mathrm{H}$

C

C

C

C

$\mathrm{H}$

$\mathrm{H}$

$\mathrm{H}$

C

C

C

$\mathrm{H}$

$\mathrm{H}$

$\mathrm{H}$

C

$\mathrm{H}$

$\mathrm{H}$

$\mathrm{H}$

C

$\mathrm{H}$

$\mathrm{H}$

$\mathrm{H}$

C

$\mathrm{H}$

\section{$-2.233093$}

$-1.177859$

$-2.539058$

0.950090

0.964335

0.940556

0.923720

$-2.914621$

$-3.836866$

$-0.110961$

1.821809

$-0.842752$

$-0.791708$

1.737623

$-1.596378$

$-0.868230$

0.853841

1.422669

$-0.560019$

$-1.008826$

$-0.537309$

$-1.210702$

1.751428

1.928179

1.275000

2. 714941

2.951685

3.479135

3. 276289

3. 246795

1.025763

1.392315
$-0.727930$

$-0.720287$

0.316930

$-0.959746$

$-1.070718$

0.269673

0.322481

$-1.300065$

$-1.002971$

3.807414

3. 292532

3.505957

3.101238

2. 248926

2.992968

4.586232

1.230780

2.573239

0.927919

0.100662

0.639859

1.801800

0.011871

$-0.170553$

$-0.884121$

0.143721

2. 687791

2.028341

2. 450188

3.720135

2.869961

2.093195
0.807100

1.075800

0.667095

$-1.217853$

$-2.423528$

$-0.660716$

0. 327315

1.927697

1.873185

$-1.396797$

$-0.532945$

0.946721

$-0.428339$

$-0.831328$

1.546849

1.108061

3. 216148

3.803088

3. 736157

3. 180148

4.793119

3.636897

3.414082

4.480807

3.001483

2.916991

3.657639

4.356560

2. 640339

3.871007

5.250541

5.932196 


\begin{tabular}{|c|c|c|c|}
\hline $\mathrm{H}$ & 1.464724 & 3.824617 & 5.559387 \\
\hline $\mathrm{H}$ & -0.058165 & 2.950099 & 5.359518 \\
\hline 0 & 0.779471 & 1.532246 & 1.792745 \\
\hline 0 & 0.805704 & 3.554050 & 2.956631 \\
\hline B & 0.667142 & 2.984602 & 1.662981 \\
\hline 0 & 1.686696 & 3.595714 & 0.719480 \\
\hline C & 2.691146 & 4.153781 & -1.359641 \\
\hline C & 3.105966 & 3.698852 & -2.620986 \\
\hline C & 3.131500 & 5.402506 & -0.894539 \\
\hline C & 3.945681 & 4.483688 & -3.408583 \\
\hline $\mathrm{H}$ & 2.768749 & 2.728996 & -2.978989 \\
\hline C & 3.970768 & 6.183303 & -1.685140 \\
\hline $\mathrm{H}$ & 2.818646 & 5.738176 & 0.088932 \\
\hline C & 4.378158 & 5.728074 & -2.942915 \\
\hline $\mathrm{H}$ & 4.264572 & 4.123934 & -4.382834 \\
\hline $\mathrm{H}$ & 4.312706 & 7.147817 & -1.319585 \\
\hline $\mathrm{H}$ & 5.033944 & 6.340035 & -3.556365 \\
\hline $\mathrm{H}$ & -1.075586 & 2.075828 & -0.663129 \\
\hline $\mathrm{H}$ & 0.053774 & 4.874463 & -1.276702 \\
\hline $\mathrm{H}$ & -0.036390 & 3.424927 & -2.410643 \\
\hline C & -0.298682 & -2.782164 & -0.080361 \\
\hline C & 0.914918 & -2.129404 & -0.263384 \\
\hline C & 2.107471 & -2.545180 & 0.420216 \\
\hline C & 2.004905 & -3.581454 & 1.329531 \\
\hline $\mathrm{H}$ & 2.900811 & -3.935805 & 1.832606 \\
\hline C & -2.437205 & -1.494400 & -0.485658 \\
\hline C & -1.505159 & -2.433286 & -0.912885 \\
\hline C & -3.784499 & -1.889228 & -2.472591 \\
\hline C & -3.607156 & -1.234180 & -1.274068 \\
\hline $\mathrm{H}$ & -4.671749 & -1.687552 & -3.068395 \\
\hline C & -2.847736 & -2.841626 & -2.945290 \\
\hline C & -1.690783 & -3.130827 & -2.154855 \\
\hline C & -0.769960 & -4.098831 & -2.642201 \\
\hline C & -0.985497 & -4.744102 & -3.839332 \\
\hline C & -2.131425 & -4.455279 & -4.617376 \\
\hline C & -3.040804 & -3.521752 & -4.177380 \\
\hline $\mathrm{H}$ & 0.117804 & -4.322399 & -2.061673 \\
\hline $\mathrm{H}$ & -0.266925 & -5.478710 & -4.192627 \\
\hline $\mathrm{H}$ & -2.288395 & -4.970451 & -5.561255 \\
\hline $\mathrm{H}$ & -3.925023 & -3.289656 & -4.766528 \\
\hline C & 0.778106 & -4.234049 & 1.599135 \\
\hline C & -0.396150 & -3.842177 & 0.880165 \\
\hline C & -1.615424 & -4.521050 & 1.152447 \\
\hline C & -1.666098 & -5.536938 & 2.080224 \\
\hline C & -0.501902 & -5.928581 & 2.783822 \\
\hline C & 0.692718 & -5.289257 & 2.547685 \\
\hline $\mathrm{H}$ & -2.514385 & -4.214854 & 0.630543 \\
\hline $\mathrm{H}$ & -2.608323 & -6.040488 & 2.278236 \\
\hline $\mathrm{H}$ & -0.556454 & -6.732718 & 3.512724 \\
\hline $\mathrm{H}$ & 1.592500 & -5.580512 & 3.084405 \\
\hline C & -6.647974 & 1.477605 & 0.042170 \\
\hline C & -6.567988 & 0.207726 & 0.642987 \\
\hline C & -5.560961 & -0.656710 & 0.203570 \\
\hline C & -4.657675 & -0.274581 & -0.805519 \\
\hline
\end{tabular}




\begin{tabular}{|c|c|c|c|}
\hline C & -4.781835 & 0.988714 & -1.384119 \\
\hline C & -5.778305 & 1.890735 & -0.971015 \\
\hline $\mathrm{H}$ & -7.422831 & 2.156759 & 0.377312 \\
\hline $\mathrm{H}$ & -5.481959 & -1.661802 & 0.604971 \\
\hline $\mathrm{H}$ & -4.083195 & 1.267421 & -2.167712 \\
\hline C & -7.579888 & -0.181953 & 1.737968 \\
\hline C & -5.874444 & 3.276296 & -1.638311 \\
\hline C & -7.345694 & -1.606713 & 2.275265 \\
\hline $\mathrm{H}$ & -8.090975 & -1.836787 & 3.044752 \\
\hline $\mathrm{H}$ & -7.441217 & -2.362357 & 1.487501 \\
\hline $\mathrm{H}$ & -6.356840 & -1.715974 & 2.735911 \\
\hline C & -7.460216 & 0.804339 & 2.924331 \\
\hline $\mathrm{H}$ & -8.176697 & 0.541595 & 3.712097 \\
\hline $\mathrm{H}$ & -6.454139 & 0.776078 & 3.358500 \\
\hline $\mathrm{H}$ & -7.663628 & 1.836261 & 2.620232 \\
\hline C & -9.014215 & -0.117936 & 1.160838 \\
\hline $\mathrm{H}$ & -9.747080 & -0.387441 & 1.931142 \\
\hline $\mathrm{H}$ & -9.264814 & 0.884738 & 0.799394 \\
\hline $\mathrm{H}$ & -9.131051 & -0.814217 & 0.322544 \\
\hline C & -4.552641 & 4.048514 & -1.414078 \\
\hline $\mathrm{H}$ & -4.603945 & 5.036145 & -1.888692 \\
\hline $\mathrm{H}$ & -4.359799 & 4.195532 & -0.345335 \\
\hline $\mathrm{H}$ & -3.691266 & 3.521689 & -1.837068 \\
\hline C & -6.112362 & 3.098457 & -3.156978 \\
\hline $\mathrm{H}$ & -6.172082 & 4.076693 & -3.649532 \\
\hline $\mathrm{H}$ & -5.303744 & 2.535104 & -3.633958 \\
\hline $\mathrm{H}$ & -7.050198 & 2.564036 & -3.347259 \\
\hline C & -7.026666 & 4.126533 & -1.070084 \\
\hline $\mathrm{H}$ & -7.053485 & 5.096142 & -1.579766 \\
\hline $\mathrm{H}$ & -8.001332 & 3.648254 & -1.220269 \\
\hline $\mathrm{H}$ & -6.901237 & 4.321748 & 0.001052 \\
\hline C & 3.445635 & -1.951325 & 0.133012 \\
\hline C & 4.333770 & -1.660468 & 1.185490 \\
\hline C & 3.869867 & -1.745377 & -1.183144 \\
\hline C & 5.613061 & -1.159202 & 0.940991 \\
\hline $\mathrm{H}$ & 3.995608 & -1.823973 & 2.201632 \\
\hline C & 5.145623 & -1.235548 & -1.472154 \\
\hline $\mathrm{H}$ & 3.200096 & -2.002745 & -1.994843 \\
\hline C & 5.991520 & -0.948752 & -0.398231 \\
\hline $\mathrm{H}$ & 6.979782 & -0.551654 & -0.598130 \\
\hline C & 5.554092 & -1.020975 & -2.942125 \\
\hline C & 6.605950 & -0.823710 & 2.071124 \\
\hline C & 5.478692 & -2.367971 & -3.700178 \\
\hline $\mathrm{H}$ & 6.153640 & -3.107485 & -3.253785 \\
\hline H & 4.467398 & -2.786402 & -3.689431 \\
\hline $\mathrm{H}$ & 5.770164 & -2.230928 & -4.748895 \\
\hline C & 4.583166 & -0.009385 & -3.598176 \\
\hline $\mathrm{H}$ & 4.835403 & 0.126142 & -4.657539 \\
\hline $\mathrm{H}$ & 3.541224 & -0.340347 & -3.538878 \\
\hline $\mathrm{H}$ & 4.654007 & 0.967548 & -3.105292 \\
\hline C & 6.986660 & -0.473221 & -3.083712 \\
\hline $\mathrm{H}$ & 7.102591 & 0.499910 & -2.592885 \\
\hline H & 7.731566 & -1.159751 & -2.664706 \\
\hline $\mathrm{H}$ & 7.224281 & -0.337009 & -4.144826 \\
\hline
\end{tabular}




$\begin{array}{|llll|}\mathrm{C} & 6.045535 & -1.156065 & 3.467222 \\ \mathrm{H} & 5.141956 & -0.580022 & 3.695828 \\ \mathrm{H} & 5.806973 & -2.221156 & 3.567886 \\ \mathrm{H} & 6.792458 & -0.911642 & 4.231085 \\ \mathrm{C} & 7.906919 & -1.638628 & 1.876421 \\ \mathrm{H} & 7.703325 & -2.715171 & 1.908188 \\ \mathrm{H} & 8.389887 & -1.418764 & 0.918771 \\ \mathrm{H} & 8.624894 & -1.404696 & 2.672058 \\ \mathrm{C} & 6.937242 & 0.687181 & 2.032651 \\ \mathrm{H} & 7.649988 & 0.942452 & 2.826646 \\ \mathrm{H} & 7.382993 & 0.982163 & 1.076895 \\ \mathrm{H} & 6.033959 & 1.290181 & 2.180082 \\ & & \end{array}$

\section{C-Catalyzed reaction TS 94}

\section{Datum}

Value

\begin{tabular}{lc}
\hline B3LYP/6-31G(d) Energy & -3038.954233 \\
\hline B3LYP-D3(BJ)/def2-TZVPP-IEF-PCM(DCM) Energy & -3040.506827 \\
\hline B3LYP-D3(BJ)/def2-TZVPP-IEF-PCM(DCM)//B3LYP/6-31G(d) Free Energy (Quasiharmonic) & -3039.278504 \\
\hline
\end{tabular}

Number of Imaginary Frequencies

Frequencies (Top 3 out of 450)

$\begin{array}{lr}\text { 1. } & -341.9038 \mathrm{~cm}^{-1} \\ \text { 2. } & 6.7305 \mathrm{~cm}^{-1} \\ \text { 3. } & 10.4096 \mathrm{~cm}^{-1}\end{array}$

\section{B3LYP/6-31G(d) Molecular Geometry in Cartesian Coordinates}

C

$\mathrm{H}$

$\mathrm{H}$

C

0

0

$\mathrm{H}$

0

$\mathrm{H}$

C

C

C

C

$\mathrm{H}$

-0.762195
-0.292350
-1.837254
2.704263
3.443031
1.842774
1.189906
-0.225513
-0.306417
-4.305451
-4.068933
-2.141490
-2.944302
-4.259248

$-0.762195$

\subsection{0}

0.509410

0.615786

1.811569

2.659010

1.146992

0.615697

0.013971

$-0.928233$

$-1.468083$

$-1.820026$

$-2.643402$

$-1.465913$

$-0.767560$
0.611730

1.555667

0.692641

$-0.948382$

$-1.408819$

$-1.717680$

$-1.184012$

$-0.456694$

$-0.165874$

2.715668

0.562805

2.821324

2.935287

0.342327 


\begin{tabular}{|c|c|c|c|}
\hline H & -1.145123 & -2.562958 & 3.258609 \\
\hline $\mathrm{H}$ & -2.649399 & -3.560473 & 3.130356 \\
\hline C & 0.365986 & -3.597936 & 0.386874 \\
\hline C & -0.674100 & -4.737714 & 0.072098 \\
\hline C & 1.289273 & -3.226167 & -0.774062 \\
\hline H & 1.968349 & -2.420499 & -0.474989 \\
\hline H & 1.901993 & -4.084306 & -1.073697 \\
\hline $\mathrm{H}$ & 0.725858 & -2.885397 & -1.646189 \\
\hline C & 1.200187 & -3.876146 & 1.647069 \\
\hline $\mathrm{H}$ & 1.949411 & -4.655950 & 1.472984 \\
\hline $\mathrm{H}$ & 1.721753 & -2.957984 & 1.936919 \\
\hline H & 0.568242 & -4.189053 & 2.483659 \\
\hline C & -0.210557 & -6.149880 & 0.431318 \\
\hline $\mathrm{H}$ & 0.679314 & -6.432440 & -0.143421 \\
\hline $\mathrm{H}$ & 0.017637 & -6.238272 & 1.496065 \\
\hline $\mathrm{H}$ & -1.003875 & -6.867812 & 0.197275 \\
\hline C & -1.168558 & -4.706361 & -1.383565 \\
\hline $\mathrm{H}$ & -0.393398 & -5.036037 & -2.084031 \\
\hline $\mathrm{H}$ & -2.024202 & -5.382503 & -1.482501 \\
\hline $\mathrm{H}$ & -1.494947 & -3.703550 & -1.674363 \\
\hline 0 & -0.501505 & -2.473399 & 0.669382 \\
\hline 0 & -1.775713 & -4.388860 & 0.935314 \\
\hline B & -1.781256 & -2.973324 & 1.113271 \\
\hline 0 & -2.848390 & -2.200433 & 0.363128 \\
\hline C & -5.207267 & -2.735534 & 0.338608 \\
\hline C & -5.031633 & -4.128711 & 0.281111 \\
\hline C & -6.487508 & -2.188075 & 0.150046 \\
\hline C & -6.130861 & -4.952951 & 0.049250 \\
\hline $\mathrm{H}$ & -4.038447 & -4.548202 & 0.413680 \\
\hline C & -7.579985 & -3.018174 & -0.086529 \\
\hline $\mathrm{H}$ & -6.618729 & -1.108702 & 0.183945 \\
\hline C & -7.403229 & -4.403807 & -0.134282 \\
\hline $\mathrm{H}$ & -5.994137 & -6.029981 & 0.004430 \\
\hline $\mathrm{H}$ & -8.566055 & -2.587715 & -0.237219 \\
\hline $\mathrm{H}$ & -8.254433 & -5.053306 & -0.319662 \\
\hline $\mathrm{H}$ & -2.430763 & -0.503518 & 2.950221 \\
\hline $\mathrm{H}$ & -4.872450 & -2.377480 & 2.885949 \\
\hline $\mathrm{H}$ & -4.873926 & -0.542602 & 2.750444 \\
\hline C & 1.760382 & 2.026618 & 1.356491 \\
\hline C & 2.725479 & 1.463482 & 0.531454 \\
\hline C & 3.761513 & 0.621636 & 1.065082 \\
\hline C & 3.766063 & 0.378888 & 2.426403 \\
\hline $\mathrm{H}$ & 4.546862 & -0.249928 & 2.846541 \\
\hline C & -0.533575 & 2.285767 & 0.375172 \\
\hline C & 0.665977 & 2.873928 & 0.767554 \\
\hline C & -1.393715 & 4.454669 & -0.299955 \\
\hline C & -1.571850 & 3.088480 & -0.202316 \\
\hline $\mathrm{H}$ & -2.181013 & 5.064022 & -0.737101 \\
\hline C & -0.195481 & 5.086833 & 0.109461 \\
\hline C & 0.868106 & 4.285506 & 0.633574 \\
\hline C & 2.078827 & 4.929437 & 1.011360 \\
\hline C & 2.220970 & 6.293013 & 0.888966 \\
\hline C & 1.163740 & 7.084670 & 0.378628 \\
\hline C & -0.016456 & 6.492105 & -0.005456 \\
\hline
\end{tabular}




\begin{tabular}{|c|c|c|c|}
\hline $\mathrm{H}$ & 2.900235 & 4.328981 & 1.386085 \\
\hline $\mathrm{H}$ & 3.155149 & 6.766271 & 1.178510 \\
\hline $\mathrm{H}$ & 1.291563 & 8.159768 & 0.284895 \\
\hline $\mathrm{H}$ & -0.831005 & 7.089522 & -0.408274 \\
\hline C & 2.815143 & 0.950186 & 3.306085 \\
\hline C & 1.794864 & 1.797746 & 2.771061 \\
\hline C & 0.852393 & 2.372773 & 3.668277 \\
\hline C & 0.917706 & 2.120573 & 5.021208 \\
\hline C & 1.927722 & 1.280080 & 5.547876 \\
\hline C & 2.854933 & 0.709583 & 4.706396 \\
\hline H & 0.078735 & 3.023148 & 3.273266 \\
\hline $\mathrm{H}$ & 0.191740 & 2.573744 & 5.691080 \\
\hline $\mathrm{H}$ & 1.969401 & 1.090096 & 6.617006 \\
\hline $\mathrm{H}$ & 3.636836 & 0.064896 & 5.101015 \\
\hline C & -5.198910 & 1.488583 & -1.818002 \\
\hline C & -3.971654 & 1.043073 & -2.335806 \\
\hline C & -2.797623 & 1.550745 & -1.765512 \\
\hline C & -2.836529 & 2.493113 & -0.729201 \\
\hline C & -4.080201 & 2.920917 & -0.247611 \\
\hline C & -5.280217 & 2.424637 & -0.775212 \\
\hline $\mathrm{H}$ & -6.114259 & 1.106889 & -2.249765 \\
\hline $\mathrm{H}$ & -1.828235 & 1.232618 & -2.132823 \\
\hline $\mathrm{H}$ & -4.097512 & 3.652065 & 0.556045 \\
\hline C & -3.874371 & 0.072850 & -3.530469 \\
\hline C & -6.624939 & 2.925531 & -0.210533 \\
\hline C & -3.008071 & -1.152609 & -3.155315 \\
\hline $\mathrm{H}$ & -2.871963 & -1.797035 & -4.032517 \\
\hline $\mathrm{H}$ & -2.017730 & -0.863005 & -2.791290 \\
\hline $\mathrm{H}$ & -3.486410 & -1.748615 & -2.370873 \\
\hline C & -5.252241 & -0.440552 & -3.990150 \\
\hline $\mathrm{H}$ & -5.124108 & -1.136357 & -4.827076 \\
\hline $\mathrm{H}$ & -5.775379 & -0.979382 & -3.191336 \\
\hline $\mathrm{H}$ & -5.899093 & 0.373222 & -4.337884 \\
\hline C & -3.214777 & 0.815926 & -4.718117 \\
\hline $\mathrm{H}$ & -3.136543 & 0.151092 & -5.587250 \\
\hline $\mathrm{H}$ & -3.806370 & 1.690966 & -5.011462 \\
\hline $\mathrm{H}$ & -2.206365 & 1.161660 & -4.469060 \\
\hline C & -6.709212 & 2.595141 & 1.298546 \\
\hline $\mathrm{H}$ & -7.655694 & 2.956651 & 1.718995 \\
\hline $\mathrm{H}$ & -6.658801 & 1.511916 & 1.465845 \\
\hline $\mathrm{H}$ & -5.894291 & 3.058663 & 1.864026 \\
\hline C & -6.723658 & 4.458437 & -0.400971 \\
\hline $\mathrm{H}$ & -7.673291 & 4.834197 & -0.000338 \\
\hline $\mathrm{H}$ & -5.913296 & 4.985789 & 0.112692 \\
\hline $\mathrm{H}$ & -6.674010 & 4.724185 & -1.463003 \\
\hline C & -7.838020 & 2.281867 & -0.908716 \\
\hline $\mathrm{H}$ & -8.764721 & 2.671451 & -0.472447 \\
\hline $\mathrm{H}$ & -7.858426 & 2.505520 & -1.981024 \\
\hline $\mathrm{H}$ & -7.850699 & 1.191983 & -0.788721 \\
\hline C & 4.810416 & -0.028539 & 0.222167 \\
\hline C & 5.050627 & -1.408765 & 0.365413 \\
\hline C & 5.604524 & 0.706942 & -0.660728 \\
\hline C & 6.055226 & -2.051568 & -0.360035 \\
\hline $\mathrm{H}$ & 4.424395 & -1.968806 & 1.050907 \\
\hline
\end{tabular}




$\begin{array}{lrrr}\text { C } & 6.615417 & 0.095493 & -1.418779 \\ \text { H } & 5.423480 & 1.769009 & -0.758859 \\ \text { C } & 6.821063 & -1.275654 & -1.249631 \\ \text { H } & 7.598298 & -1.765544 & -1.824870 \\ \text { C } & 7.433639 & 0.952781 & -2.402682 \\ \text { C } & 6.346293 & -3.559258 & -0.227411 \\ \text { C } & 6.477583 & 1.546823 & -3.466029 \\ \text { H } & 7.032945 & 2.193214 & -4.157361 \\ \text { H } & 6.007114 & 0.748955 & -4.052487 \\ \text { H } & 5.676211 & 2.139613 & -3.014443 \\ \text { C } & 8.127360 & 2.103903 & -1.636164 \\ \text { H } & 8.706823 & 2.725328 & -2.330038 \\ \text { H } & 7.404526 & 2.754371 & -1.134012 \\ \text { H } & 8.813685 & 1.712940 & -0.875630 \\ \text { C } & 8.522169 & 0.140708 & -3.129524 \\ \text { H } & 9.248029 & -0.290853 & -2.429965 \\ \text { H } & 8.094860 & -0.673856 & -3.725640 \\ \text { H } & 9.072907 & 0.794629 & -3.815184 \\ \text { C } & 7.807923 & -3.763662 & 0.238153 \\ \text { H } & 8.525080 & -3.332474 & -0.467741 \\ \text { H } & 7.977229 & -3.294180 & 1.214053 \\ \text { H } & 8.033424 & -4.833313 & 0.331538 \\ \text { C } & 5.422838 & -4.249528 & 0.794000 \\ \text { H } & 5.534411 & -3.824101 & 1.797852 \\ \text { H } & 4.368240 & -4.172682 & 0.506536 \\ \text { H } & 5.672569 & -5.314948 & 0.857141 \\ \text { C } & 6.145341 & -4.248710 & -1.597797 \\ \text { H } & 6.353481 & -5.323214 & -1.520111 \\ \text { H } & 5.114664 & -4.125410 & -1.949851 \\ \text { H } & 6.809563 & -3.835286 & -2.363676\end{array}$

\section{C-Catalyzed reaction TS 95}

\section{Datum}

Value

\begin{tabular}{lc}
\hline B3LYP/6-31G(d) Energy & -3038.956578 \\
\hline B3LYP-D3(BJ)/def2-TZVPP-IEF-PCM(DCM) Energy & -3040.506612 \\
\hline B3LYP-D3(BJ)/def2-TZVPP-IEF-PCM(DCM)//B3LYP/6-31G(d) Free Energy (Quasiharmonic) & -3039.278355 \\
\hline
\end{tabular}

Number of Imaginary Frequencies

Frequencies (Top 3 out of 450)

$$
\begin{array}{lr}
\text { 1. } & -306.7521 \mathrm{~cm}^{-1} \\
\text { 2. } & 7.0194 \mathrm{~cm}^{-1} \\
\text { 3. } & 12.5487 \mathrm{~cm}^{-1}
\end{array}
$$




\begin{tabular}{|c|c|c|c|}
\hline C & 3.193660 & -1.579250 & 0.650429 \\
\hline $\mathrm{H}$ & 3.828270 & -1.045800 & 1.358249 \\
\hline $\mathrm{H}$ & 2.229580 & -1.750980 & 1.137419 \\
\hline C & -0.322751 & -0.132880 & 0.363159 \\
\hline 0 & 0.452639 & 0.338160 & 1.167839 \\
\hline 0 & -1.301521 & 0.635690 & -0.143191 \\
\hline $\mathrm{H}$ & -1.885921 & 0.254390 & -0.844791 \\
\hline 0 & 3.866090 & -2.822980 & 0.401259 \\
\hline $\mathrm{H}$ & 3.289780 & -3.357630 & -0.168731 \\
\hline C & -1.533601 & 4.229900 & -1.220961 \\
\hline C & -3.163161 & 3.181820 & -0.222721 \\
\hline C & -2.440151 & 3.018710 & -3.179101 \\
\hline C & -1.427821 & 3.253880 & -2.187941 \\
\hline $\mathrm{H}$ & -2.517921 & 2.370580 & 0.108679 \\
\hline $\mathrm{H}$ & -2.118381 & 2.384330 & -4.006851 \\
\hline $\mathrm{H}$ & -2.949241 & 3.916710 & -3.537901 \\
\hline C & -4.138841 & -0.186660 & -2.759211 \\
\hline C & -5.404121 & 0.698559 & -3.057771 \\
\hline C & -3.408761 & -0.614110 & -4.041961 \\
\hline $\mathrm{H}$ & -2.435950 & -1.039660 & -3.777041 \\
\hline $\mathrm{H}$ & -3.970110 & -1.376310 & -4.593351 \\
\hline $\mathrm{H}$ & -3.244161 & 0.239980 & -4.705021 \\
\hline C & -4.401860 & -1.411591 & -1.886641 \\
\hline $\mathrm{H}$ & -5.133880 & -2.077141 & -2.359981 \\
\hline $\mathrm{H}$ & -4.772060 & -1.126961 & -0.899721 \\
\hline $\mathrm{H}$ & -3.476570 & -1.982280 & -1.748381 \\
\hline C & -6.385671 & 0.754379 & -1.872231 \\
\hline $\mathrm{H}$ & -6.924771 & -0.191101 & -1.742691 \\
\hline $\mathrm{H}$ & -5.871401 & 0.994959 & -0.937551 \\
\hline $\mathrm{H}$ & -7.118851 & 1.544349 & -2.063651 \\
\hline C & -6.165761 & 0.327719 & -4.332221 \\
\hline $\mathrm{H}$ & -6.557321 & -0.695381 & -4.281611 \\
\hline $\mathrm{H}$ & -7.014851 & 1.007719 & -4.458001 \\
\hline $\mathrm{H}$ & -5.532731 & 0.418599 & -5.217991 \\
\hline 0 & -3.283321 & 0.731370 & -2.031791 \\
\hline 0 & -4.825951 & 1.997199 & -3.239831 \\
\hline B & -3.688141 & 2.092890 & -2.392571 \\
\hline 0 & -4.004001 & 2.954510 & -1.182701 \\
\hline C & -3.523501 & 4.196310 & 0.787549 \\
\hline C & -4.580541 & 5.094359 & 0.573819 \\
\hline C & -2.813231 & 4.236860 & 1.997709 \\
\hline C & -4.915871 & 6.021069 & 1.557419 \\
\hline $\mathrm{H}$ & -5.133971 & 5.043879 & -0.358371 \\
\hline C & -3.152761 & 5.165740 & 2.979179 \\
\hline $\mathrm{H}$ & -1.995671 & 3.538720 & 2.162819 \\
\hline C & -4.203261 & 6.060500 & 2.759989 \\
\hline $\mathrm{H}$ & -5.737471 & 6.712249 & 1.389999 \\
\hline $\mathrm{H}$ & -2.600561 & 5.190650 & 3.914429 \\
\hline $\mathrm{H}$ & -4.468921 & 6.784429 & 3.525609 \\
\hline $\mathrm{H}$ & -0.676441 & 2.478540 & -2.044691 \\
\hline
\end{tabular}




\begin{tabular}{|c|c|c|c|}
\hline $\mathrm{H}$ & -2.144451 & 5.110230 & -1.400271 \\
\hline $\mathrm{H}$ & -0.777471 & 4.324660 & -0.446961 \\
\hline C & 0.804390 & -1.912430 & -0.958301 \\
\hline C & -0.242170 & -1.566140 & -0.109381 \\
\hline C & -1.174840 & -2.545990 & 0.378389 \\
\hline C & -1.056470 & -3.841760 & -0.091821 \\
\hline $\mathrm{H}$ & -1.728530 & -4.606260 & 0.288119 \\
\hline C & 3.000669 & -0.748060 & -0.607781 \\
\hline C & 1.845850 & -0.904010 & -1.372051 \\
\hline C & 3.791289 & 0.959610 & -2.148011 \\
\hline C & 4.002389 & 0.200950 & -1.011741 \\
\hline $\mathrm{H}$ & 4.551459 & 1.671980 & -2.458431 \\
\hline C & 2.636139 & 0.815490 & -2.949971 \\
\hline C & 1.642189 & -0.140070 & -2.566611 \\
\hline C & 0.500799 & -0.294100 & -3.401221 \\
\hline C & 0.350339 & 0.455760 & -4.547131 \\
\hline C & 1.331859 & 1.406740 & -4.917691 \\
\hline C & 2.448759 & 1.579910 & -4.133791 \\
\hline $\mathrm{H}$ & -0.254080 & -1.023940 & -3.129201 \\
\hline $\mathrm{H}$ & -0.526021 & 0.313320 & -5.172961 \\
\hline $\mathrm{H}$ & 1.200649 & 1.994090 & -5.822321 \\
\hline $\mathrm{H}$ & 3.211869 & 2.303640 & -4.410371 \\
\hline C & -0.050070 & -4.228650 & -1.007661 \\
\hline C & 0.909440 & -3.258750 & -1.441531 \\
\hline C & 1.935980 & -3.674930 & -2.337901 \\
\hline C & 2.006760 & -4.980030 & -2.775341 \\
\hline C & 1.057300 & -5.936350 & -2.341981 \\
\hline C & 0.052900 & -5.566280 & -1.477791 \\
\hline $\mathrm{H}$ & 2.660500 & -2.945240 & -2.683521 \\
\hline $\mathrm{H}$ & 2.795500 & -5.277040 & -3.460871 \\
\hline $\mathrm{H}$ & 1.125440 & -6.962010 & -2.693681 \\
\hline $\mathrm{H}$ & -0.679030 & -6.294680 & -1.137231 \\
\hline C & 7.670469 & 0.881131 & 1.091489 \\
\hline C & 6.854409 & 1.976261 & 0.771539 \\
\hline C & 5.652879 & 1.720711 & 0.099709 \\
\hline C & 5.267269 & 0.418591 & -0.248241 \\
\hline C & 6.111889 & -0.647579 & 0.087429 \\
\hline C & 7. 319619 & -0.436419 & 0.763589 \\
\hline $\mathrm{H}$ & 8.601159 & 1.058591 & 1.612779 \\
\hline $\mathrm{H}$ & 4.983159 & 2.539540 & -0.146571 \\
\hline $\mathrm{H}$ & 5.805160 & -1.652529 & -0.179871 \\
\hline C & 7.229319 & 3.426371 & 1.136819 \\
\hline C & 8.200860 & -1.648719 & 1.122029 \\
\hline C & 7.328359 & 4.273421 & -0.154291 \\
\hline $\mathrm{H}$ & 7.586679 & 5.311751 & 0.088149 \\
\hline $\mathrm{H}$ & 8.101219 & 3.878151 & -0.823561 \\
\hline $\mathrm{H}$ & 6.382779 & 4.285931 & -0.706551 \\
\hline C & 6.140419 & 4.025411 & 2.058499 \\
\hline $\mathrm{H}$ & 6.392449 & 5.059271 & 2.325869 \\
\hline $\mathrm{H}$ & 5.157499 & 4.035961 & 1.576329 \\
\hline $\mathrm{H}$ & 6.050699 & 3.446761 & 2.984949 \\
\hline C & 8.580039 & 3.520561 & 1.872069 \\
\hline $\mathrm{H}$ & 8.801049 & 4.568731 & 2.104099 \\
\hline $\mathrm{H}$ & 8.569949 & 2.968761 & 2.818919 \\
\hline
\end{tabular}




\begin{tabular}{|c|c|c|c|}
\hline $\mathrm{H}$ & 9.405499 & 3.136891 & 1.261659 \\
\hline C & 7.402010 & -2.604039 & 2.042009 \\
\hline $\mathrm{H}$ & 8.005020 & -3.487269 & 2.288349 \\
\hline H & 7.135420 & -2.105139 & 2.981339 \\
\hline $\mathrm{H}$ & 6.474570 & -2.946309 & 1.572059 \\
\hline C & 8.597150 & -2.398689 & -0.172151 \\
\hline $\mathrm{H}$ & 9.225450 & -3.265819 & 0.066659 \\
\hline $\mathrm{H}$ & 9.162360 & -1.745469 & -0.847291 \\
\hline $\mathrm{H}$ & 7.719430 & -2.763409 & -0.715171 \\
\hline C & 9.494940 & -1.244579 & 1.853899 \\
\hline $\mathrm{H}$ & 10.081770 & -2.140779 & 2.085649 \\
\hline $\mathrm{H}$ & 10.123859 & -0.587409 & 1.241949 \\
\hline $\mathrm{H}$ & 9.287319 & -0.734139 & 2.801449 \\
\hline C & -2.204740 & -2.242430 & 1.415699 \\
\hline C & -3.490660 & -2.797780 & 1.314649 \\
\hline C & -1.885240 & -1.472520 & 2.543979 \\
\hline C & -4.457460 & -2.588721 & 2.303679 \\
\hline $\mathrm{H}$ & -3.729630 & -3.386600 & 0.437209 \\
\hline C & -2.828850 & -1.225390 & 3.547499 \\
\hline H & -0.881660 & -1.082880 & 2.643049 \\
\hline C & -4.101730 & -1.792350 & 3.402009 \\
\hline $\mathrm{H}$ & -4.843640 & -1.609521 & 4.173619 \\
\hline C & -2.506911 & -0.363740 & 4.784159 \\
\hline C & -5.875100 & -3.188671 & 2.222959 \\
\hline C & -1.057421 & 0.159420 & 4.772119 \\
\hline $\mathrm{H}$ & -0.328781 & -0.658610 & 4.801269 \\
\hline $\mathrm{H}$ & -0.843671 & 0.767380 & 3.886339 \\
\hline $\mathrm{H}$ & -0.885811 & 0.783530 & 5.656649 \\
\hline C & -3.459451 & 0.855290 & 4.817979 \\
\hline $\mathrm{H}$ & -3.252291 & 1.473890 & 5.699889 \\
\hline $\mathrm{H}$ & -3.331461 & 1.480190 & 3.926789 \\
\hline $\mathrm{H}$ & -4.510881 & 0.552299 & 4.864029 \\
\hline C & -2.705240 & -1.202520 & 6.069039 \\
\hline $\mathrm{H}$ & -3.732710 & -1.569320 & 6.165509 \\
\hline $\mathrm{H}$ & -2.038010 & -2.072010 & 6.077019 \\
\hline $\mathrm{H}$ & -2.483821 & -0.597010 & 6.956519 \\
\hline C & -6.076550 & -4.059331 & 0.967979 \\
\hline $\mathrm{H}$ & -7.091600 & -4.472431 & 0.966159 \\
\hline $\mathrm{H}$ & -5.377500 & -4.903201 & 0.939869 \\
\hline $\mathrm{H}$ & -5.954550 & -3.482591 & 0.044419 \\
\hline C & -6.128740 & -4.077491 & 3.464209 \\
\hline $\mathrm{H}$ & -5.407050 & -4.901141 & 3.511329 \\
\hline $\mathrm{H}$ & -6.048460 & -3.510541 & 4. 397379 \\
\hline $\mathrm{H}$ & -7.135970 & -4.510021 & 3.423369 \\
\hline C & -6.918550 & -2.047021 & 2.189129 \\
\hline $\mathrm{H}$ & -7.934120 & -2.459021 & 2.141689 \\
\hline $\mathrm{H}$ & -6.858020 & -1.412201 & 3.079369 \\
\hline $\mathrm{H}$ & -6.770840 & -1.406281 & 1.312259 \\
\hline
\end{tabular}


Frequencies (Top 3 out of 450)

$\begin{array}{lr}\text { 1. } & -404.0190 \mathrm{~cm}^{-1} \\ \text { 2. } & 5.9492 \mathrm{~cm}^{-1} \\ \text { 3. } & 10.7301 \mathrm{~cm}^{-1}\end{array}$

\section{B3LYP/6-31G(d) Molecular Geometry in Cartesian Coordinates}

C

$\mathrm{H}$

$\mathrm{H}$

C

0

0

$\mathrm{H}$

0

$\mathrm{H}$

C

C

C

C

$\mathrm{H}$

$\mathrm{H}$

$\mathrm{H}$

C

C

C

$\mathrm{H}$

$\mathrm{H}$

$\mathrm{H}$

C

$\mathrm{H}$

$\mathrm{H}$

$\mathrm{H}$

C

$\mathrm{H}$

$\mathrm{H}$

$\mathrm{H}$

C

$\mathrm{H}$

\subsection{3}

0.646104

2.148555

$-2.018864$

$-2.698669$

$-1.123212$

$-0.624570$

0.396767

0.983710

1. 284126

$-0.034073$

3. 042966

2. 353967

$-0.283967$

3.894320

3. 262738

1. 616112

2.622365

2.036699

2. 982955

1.271514

2. 146133

0.181757

$-0.494717$

$-0.146519$

0.099065

2. 347705

1. 356422

3.086494

2. 422181

4.090754

4.303128
$-0.804185$

$-1.062484$

$-0.389659$

$-1.457569$

$-1.941633$

$-0.487098$

$-0.243374$

0.233569

0.866934

2.824813

3.544036

3. 303558

3.573181

2. 503368

3.949685

2. 250598

2. 504281

3.706661

1. 210648

0.832099

0.440264

1. 370197

2. 874132

2.062644

3. 793942

3.011335

4.638133

5.091214

5.446548

4.100996

3. 252385

2. 548381
0.980323

1.899275

1. 257154

$-1.491242$

$-2.366238$

$-1.741899$

$-0.912861$

0.350293

$-0.138421$

2.801674

1. 270891

1. 124837

2. 338915

1.053579

0.915297

0.937417

$-2.329792$

$-2.468761$

- 3.025493

$-2.630661$

$-2.882159$

$-4.104512$

$-2.736847$

$-2.451511$

$-2.244248$

$-3.820310$

$-3.650835$

$-3.579176$

$-3.659157$

$-4.603874$

$-2.498068$

$-1.687598$ 


\begin{tabular}{|c|c|c|c|}
\hline $\mathrm{H}$ & 4.732509 & 4.129573 & -2.365724 \\
\hline $\mathrm{H}$ & 4.353724 & 2.775517 & -3.448981 \\
\hline 0 & 1.641284 & 2.283943 & -0.889194 \\
\hline 0 & 2.408228 & 4.446271 & -1.252837 \\
\hline B & 1.878176 & 3.578100 & -0.273727 \\
\hline 0 & 0.684851 & 4.176301 & 0.382351 \\
\hline C & -1.033344 & 4.330800 & 2.018641 \\
\hline C & -0.974027 & 5.732557 & 2.057261 \\
\hline C & -2.076209 & 3.665828 & 2.682823 \\
\hline C & -1.938465 & 6.452381 & 2.757589 \\
\hline $\mathrm{H}$ & -0.177354 & 6.241312 & 1.524305 \\
\hline C & -3.040733 & 4.390046 & 3.380125 \\
\hline $\mathrm{H}$ & -2.130797 & 2.580176 & 2.646150 \\
\hline C & -2.972035 & 5.784846 & 3.421770 \\
\hline $\mathrm{H}$ & -1.889039 & 7.537620 & 2.781766 \\
\hline $\mathrm{H}$ & -3.847398 & 3.867407 & 3.886440 \\
\hline $\mathrm{H}$ & -3.724416 & 6.350393 & 3.964690 \\
\hline $\mathrm{H}$ & 2.477002 & 4.567350 & 2.773054 \\
\hline $\mathrm{H}$ & 1.197842 & 1.778451 & 2.520568 \\
\hline $\mathrm{H}$ & 0.781639 & 3.108270 & 3.720688 \\
\hline C & -1.074179 & -2.584515 & 0.544384 \\
\hline C & -2.130949 & -1.903297 & -0.046291 \\
\hline C & -3.378066 & -1.717981 & 0.640483 \\
\hline C & -3.510145 & -2.240141 & 1.911851 \\
\hline $\mathrm{H}$ & -4.435492 & -2.077709 & 2.458580 \\
\hline C & 1.346012 & -2.034622 & 0.100346 \\
\hline C & 0.225053 & -2.817119 & -0.182578 \\
\hline C & 2.683633 & -3.403849 & -1.395884 \\
\hline c & 2.616224 & -2.363036 & -0.486855 \\
\hline $\mathrm{H}$ & 3.645525 & -3.667669 & -1.827900 \\
\hline C & 1.555405 & -4.178437 & -1.746994 \\
\hline C & 0.301497 & -3.902784 & -1.114757 \\
\hline C & -0.813766 & -4.724435 & -1.442970 \\
\hline C & -0.691036 & -5.752410 & -2.349802 \\
\hline C & 0.550847 & -6.019835 & -2.975036 \\
\hline C & 1.649526 & -5.248779 & -2.677585 \\
\hline $\mathrm{H}$ & -1.775845 & -4.520278 & -0.987867 \\
\hline $\mathrm{H}$ & -1.557555 & -6.360559 & -2.593718 \\
\hline $\mathrm{H}$ & 0.630912 & -6.833950 & -3.690322 \\
\hline $\mathrm{H}$ & 2.609630 & -5.445623 & -3.148779 \\
\hline C & -2.471792 & -2.965757 & 2.543875 \\
\hline C & -1.230998 & -3.151328 & 1.855635 \\
\hline C & -0.208094 & -3.899676 & 2.502529 \\
\hline C & -0.397676 & -4.419232 & 3.764610 \\
\hline C & -1.620716 & -4.220421 & 4.447654 \\
\hline C & -2.633947 & -3.509565 & 3.846764 \\
\hline $\mathrm{H}$ & 0.730803 & -4.065320 & 1.985443 \\
\hline $\mathrm{H}$ & 0.396592 & -4.990060 & 4.238136 \\
\hline $\mathrm{H}$ & -1.758081 & -4.634324 & 5.442967 \\
\hline $\mathrm{H}$ & -3.580663 & -3.355956 & 4. 359461 \\
\hline C & 6.399016 & -0.650351 & 0.557649 \\
\hline C & 6.047042 & -0.744063 & -0.801116 \\
\hline C & 4.786763 & -1.259645 & -1.114006 \\
\hline$C$ & 3.900718 & -1.696194 & -0.112203 \\
\hline
\end{tabular}




\begin{tabular}{|c|c|c|c|}
\hline C & 4.301434 & -1.599120 & 1.224793 \\
\hline C & 5.548792 & -1.065101 & 1.586299 \\
\hline $\mathrm{H}$ & 7.373285 & -0.249681 & 0.810770 \\
\hline $\mathrm{H}$ & 4.466412 & -1.339720 & -2.146422 \\
\hline $\mathrm{H}$ & 3.637643 & -1.980745 & 1.995592 \\
\hline C & 7.064864 & -0.318734 & -1.878269 \\
\hline C & 5.935035 & -0.973336 & 3.075610 \\
\hline C & 8.293311 & -1.258132 & -1.801624 \\
\hline $\mathrm{H}$ & 9.037788 & -0.975962 & -2.556319 \\
\hline $\mathrm{H}$ & 8.777601 & -1.213019 & -0.820366 \\
\hline $\mathrm{H}$ & 8.002344 & -2.299068 & -1.983530 \\
\hline C & 6.484767 & -0.404218 & -3.302683 \\
\hline $\mathrm{H}$ & 7.240544 & -0.082967 & -4.028207 \\
\hline $\mathrm{H}$ & 6.192130 & -1.426994 & -3.565515 \\
\hline $\mathrm{H}$ & 5.610244 & 0.244257 & -3.426565 \\
\hline C & 7.524601 & 1.137762 & -1.633906 \\
\hline $\mathrm{H}$ & 8.253143 & 1.437051 & -2.397173 \\
\hline $\mathrm{H}$ & 6.679027 & 1.832440 & -1.682130 \\
\hline $\mathrm{H}$ & 8.002923 & 1.259403 & -0.656643 \\
\hline C & 4.904454 & -0.089901 & 3.818637 \\
\hline $\mathrm{H}$ & 5.162874 & -0.013316 & 4.881930 \\
\hline $\mathrm{H}$ & 4.880833 & 0.923153 & 3.400611 \\
\hline $\mathrm{H}$ & 3.891896 & -0.501908 & 3.752885 \\
\hline C & 5.937745 & -2.390162 & 3.697582 \\
\hline $\mathrm{H}$ & 6.668236 & -3.035981 & 3.197104 \\
\hline $\mathrm{H}$ & 4.957971 & -2.872879 & 3.620857 \\
\hline $\mathrm{H}$ & 6.201361 & -2.339116 & 4.761153 \\
\hline C & 7.331140 & -0.357393 & 3.287005 \\
\hline $\mathrm{H}$ & 7.552514 & -0.308545 & 4.359044 \\
\hline $\mathrm{H}$ & 8.118350 & -0.956644 & 2.815558 \\
\hline $\mathrm{H}$ & 7.392367 & 0.662561 & 2.890719 \\
\hline C & -4.514061 & -0.957432 & 0.047408 \\
\hline C & -4.317670 & 0.290557 & -0.555639 \\
\hline C & -5.815518 & -1.468385 & 0.131391 \\
\hline C & -5.386024 & 1.027530 & -1.078811 \\
\hline $\mathrm{H}$ & -3.310284 & 0.689394 & -0.615999 \\
\hline C & -6.914436 & -0.763017 & -0.372838 \\
\hline $\mathrm{H}$ & -5.956534 & -2.449042 & 0.576118 \\
\hline C & -6.673619 & 0.482490 & -0.970596 \\
\hline $\mathrm{H}$ & -7.509776 & 1.040114 & -1.369385 \\
\hline C & -8.324953 & -1.376469 & -0.272063 \\
\hline C & -5.111017 & 2.381629 & -1.761144 \\
\hline C & -9.412711 & -0.456794 & -0.858981 \\
\hline $\mathrm{H}$ & -10.393947 & -0.933802 & -0.754913 \\
\hline $\mathrm{H}$ & -9.457156 & 0.507138 & -0.339094 \\
\hline $\mathrm{H}$ & -9.252212 & -0.263072 & -1.925536 \\
\hline C & -8.358649 & -2.714886 & -1.047972 \\
\hline $\mathrm{H}$ & -9.356529 & -3.167115 & -0.986808 \\
\hline $\mathrm{H}$ & -8.118299 & -2.559663 & -2.105693 \\
\hline $\mathrm{H}$ & -7.639610 & -3.436489 & -0.646805 \\
\hline C & -8.671163 & -1.638388 & 1.213104 \\
\hline $\mathrm{H}$ & -7.959167 & -2.325382 & 1.682428 \\
\hline $\mathrm{H}$ & -8.664358 & -0.705051 & 1.788010 \\
\hline H & -9.669795 & -2.084208 & 1.299895 \\
\hline
\end{tabular}




\begin{tabular}{|llll|} 
C & -4.383524 & 3.326209 & -0.775643 \\
$H$ & -4.992515 & 3.510588 & 0.117040 \\
$H$ & -3.423856 & 2.916372 & -0.445004 \\
$H$ & -4.181273 & 4.292321 & -1.253917 \\
$\mathrm{C}$ & -4.217804 & 2.145627 & -3.003082 \\
$\mathrm{H}$ & -3.270766 & 1.663656 & -2.740732 \\
$\mathrm{H}$ & -4.723763 & 1.499387 & -3.729153 \\
$\mathrm{H}$ & -3.988751 & 3.099233 & -3.495373 \\
$\mathrm{C}$ & -6.401622 & 3.083879 & -2.224068 \\
$\mathrm{H}$ & -6.149974 & 4.042618 & -2.691664 \\
$\mathrm{H}$ & -6.947180 & 2.490696 & -2.966655 \\
$\mathrm{H}$ & -7.078062 & 3.291680 & -1.386640 \\
\hline
\end{tabular}

\section{C-Catalyzed reaction TS 97}

\section{Datum}

Value

\begin{tabular}{lc}
\hline B3LYP/6-31G(d) Energy & -3038.956332 \\
\hline B3LYP-D3(BJ)/def2-TZVPP-IEF-PCM(DCM) Energy & -3040.506795 \\
\hline B3LYP-D3(BJ)/def2-TZVPP-IEF-PCM(DCM)//B3LYP/6-31G(d) Free Energy (Quasiharmonic) & -3039.277833 \\
\hline
\end{tabular}

Number of Imaginary Frequencies

Frequencies (Top 3 out of 450)

$\begin{array}{lr}\text { 1. } & -380.0220 \mathrm{~cm}^{-1} \\ \text { 2. } & 8.3023 \mathrm{~cm}^{-1} \\ \text { 3. } & 11.1895 \mathrm{~cm}^{-1}\end{array}$

\section{B3LYP/6-31G(d) Molecular Geometry in Cartesian Coordinates}

C

$\mathrm{H}$

$\mathrm{H}$

C

0

0

$\mathrm{H}$

0

$\mathrm{H}$

C

C

C

C

$\mathrm{H}$

\section{570647}

3. 579181

1.893656

$-0.735497$

0.118214

$-1.656210$

$-2.263050$

2. 265371

1.476427

$-1.755283$

$-1.332451$

$-4.014541$

$-3.097817$

$-0.853735$
$-1.996893$

$-2.335944$

$-2.388635$

$-0.631009$

$-1.027818$

0.226389

0.651705

$-2.567282$

$-2.106238$

2.379658

3.495766

2. 226031

2. 715721

2.605762
0.982386

1. 232721

1. 752491

$-0.465574$

$-1.242835$

$-0.908730$

$-0.239766$

$-0.285981$

$-0.639223$

$-3.453121$

$-1.678057$

$-2.455562$

$-3.430695$

$-1.267738$ 


\begin{tabular}{|c|c|c|c|}
\hline $\mathrm{H}$ & -3.881109 & 1.171579 & -2.205643 \\
\hline $\mathrm{H}$ & -5.058100 & 2.485295 & -2.631943 \\
\hline C & -4.446553 & 1.976962 & 1.103296 \\
\hline C & -4.965976 & 3.457278 & 0.982637 \\
\hline C & -5.487610 & 0.948510 & 0.635547 \\
\hline $\mathrm{H}$ & -6.297362 & 0.833590 & 1.364927 \\
\hline $\mathrm{H}$ & -5.002335 & -0.023891 & 0.506670 \\
\hline $\mathrm{H}$ & -5.927213 & 1.239509 & -0.322906 \\
\hline C & -3.911424 & 1.589468 & 2.481131 \\
\hline $\mathrm{H}$ & -4.703181 & 1.656692 & 3.236607 \\
\hline $\mathrm{H}$ & -3.086850 & 2.235741 & 2.790821 \\
\hline H & -3.548110 & 0.555957 & 2.471727 \\
\hline C & -4.131459 & 4.452029 & 1.808187 \\
\hline $\mathrm{H}$ & -4.305157 & 4.342121 & 2.884592 \\
\hline H & -3.063074 & 4.327677 & 1.608799 \\
\hline H & -4.411059 & 5.469227 & 1.516338 \\
\hline C & -6.450571 & 3.643010 & 1.300046 \\
\hline $\mathrm{H}$ & -6.672798 & 3.359170 & 2.335549 \\
\hline $\mathrm{H}$ & -6.722752 & 4.696045 & 1.172701 \\
\hline H & -7.080753 & 3.052308 & 0.631109 \\
\hline 0 & -3.349404 & 1.971761 & 0.153656 \\
\hline 0 & -4.757001 & 3.738243 & -0.408676 \\
\hline B & -3.633603 & 2.996895 & -0.855746 \\
\hline 0 & -2.460366 & 3.868202 & -1.134080 \\
\hline C & -0.437586 & 4.561711 & -2.172074 \\
\hline C & -0.911376 & 5.864745 & -2.388618 \\
\hline C & 0.912980 & 4.268610 & -2.416640 \\
\hline C & -0.046897 & 6.853316 & -2.852395 \\
\hline $\mathrm{H}$ & -1.952530 & 6.087967 & -2.179212 \\
\hline C & 1.774139 & 5.260225 & -2.880864 \\
\hline $\mathrm{H}$ & 1.286270 & 3.263767 & -2.234778 \\
\hline C & 1.295723 & 6.554044 & -3.102868 \\
\hline $\mathrm{H}$ & -0.418819 & 7.861093 & -3.016860 \\
\hline $\mathrm{H}$ & 2.818532 & 5.024933 & -3.066161 \\
\hline $\mathrm{H}$ & 1.967269 & 7.327694 & -3.465396 \\
\hline H & -3.399689 & 3.593453 & -4.005641 \\
\hline $\mathrm{H}$ & -1.436967 & 1.425016 & -3.043764 \\
\hline $\mathrm{H}$ & -1.101982 & 2.825522 & -4.196237 \\
\hline C & 0.170312 & -0.612616 & 1.858528 \\
\hline C & -0.812842 & -1.057572 & 0.980543 \\
\hline C & -1.886470 & -1.909941 & 1.418890 \\
\hline C & -1.973856 & -2.201097 & 2.767005 \\
\hline $\mathrm{H}$ & -2.752522 & -2.874841 & 3.114038 \\
\hline C & 2.524540 & -0.474484 & 0.990009 \\
\hline C & 1.359144 & 0.183265 & 1.382614 \\
\hline C & 3.613092 & 1.670839 & 0.633737 \\
\hline C & 3.679343 & 0.290296 & 0.598831 \\
\hline $\mathrm{H}$ & 4.482756 & 2.250088 & 0.333789 \\
\hline C & 2.447954 & 2.365543 & 1.033230 \\
\hline C & 1.291071 & 1.614769 & 1.415043 \\
\hline C & 0.131715 & 2.329379 & 1.827435 \\
\hline C & 0.122108 & 3.706941 & 1.863300 \\
\hline C & 1.267550 & 4.445426 & 1.481995 \\
\hline C & 2.403355 & 3.785640 & 1.073798 \\
\hline
\end{tabular}




\begin{tabular}{|c|c|c|c|}
\hline $\mathrm{H}$ & -0.756579 & 1.777819 & 2.114971 \\
\hline $\mathrm{H}$ & -0.771890 & 4.232006 & 2.186997 \\
\hline $\mathrm{H}$ & 1.243917 & 5.531169 & 1.508675 \\
\hline H & 3.289230 & 4.341937 & 0.776570 \\
\hline C & -1.046831 & -1.700997 & 3.712695 \\
\hline C & 0.055135 & -0.909838 & 3.258462 \\
\hline C & 0.999299 & -0.450874 & 4.218081 \\
\hline C & 0.854111 & -0.753033 & 5.554055 \\
\hline C & -0.237851 & -1.535047 & 6.000062 \\
\hline C & -1.165110 & -2.000654 & 5.096776 \\
\hline $\mathrm{H}$ & 1.840491 & 0.146349 & 3.884699 \\
\hline H & 1.584526 & -0.390300 & 6.271938 \\
\hline $\mathrm{H}$ & -0.339114 & -1.768397 & 7.056449 \\
\hline $\mathrm{H}$ & -2.004233 & -2.607681 & 5.428057 \\
\hline C & 7.423603 & -1.404571 & -0.597404 \\
\hline C & 7.409625 & -0.428818 & 0.403748 \\
\hline C & 6.164186 & 0.095457 & 0.783829 \\
\hline C & 4.972880 & -0.336866 & 0.190694 \\
\hline C & 5.030819 & -1.317861 & -0.814085 \\
\hline C & 6.249252 & -1.865698 & -1.219525 \\
\hline $\mathrm{H}$ & 8.372018 & -1.830005 & -0.904242 \\
\hline $\mathrm{H}$ & 6.108968 & 0.841749 & 1.571509 \\
\hline $\mathrm{H}$ & 4.100611 & -1.653766 & -1.251844 \\
\hline C & 8.690869 & 0.073456 & 1.098482 \\
\hline C & 6.334080 & -2.960474 & -2.300879 \\
\hline C & 9.962052 & -0.608595 & 0.558266 \\
\hline $\mathrm{H}$ & 10.841842 & -0.217151 & 1.081777 \\
\hline H & 9.941092 & -1.693067 & 0.714177 \\
\hline $\mathrm{H}$ & 10.103418 & -0.419447 & -0.511983 \\
\hline C & 8.834836 & 1.597814 & 0.874752 \\
\hline $\mathrm{H}$ & 9.737698 & 1.974421 & 1.371529 \\
\hline $\mathrm{H}$ & 8.912338 & 1.830685 & -0.193526 \\
\hline $\mathrm{H}$ & 7.978930 & 2.150616 & 1.275973 \\
\hline C & 8.601876 & -0.213301 & 2.616259 \\
\hline $\mathrm{H}$ & 9.504730 & 0.144638 & 3.126669 \\
\hline H & 7.740624 & 0.283069 & 3.075229 \\
\hline $\mathrm{H}$ & 8.507259 & -1.288570 & 2.806120 \\
\hline C & 7.269875 & -2.500251 & -3.443415 \\
\hline H & 7.331079 & -3.273096 & -4.219609 \\
\hline H & 6.895105 & -1.581480 & -3.909161 \\
\hline $\mathrm{H}$ & 8.287994 & -2.304803 & -3.090025 \\
\hline C & 6.899495 & -4.254511 & -1.667384 \\
\hline $\mathrm{H}$ & 6.978229 & -5.047234 & -2.421911 \\
\hline H & 7.896867 & -4.097688 & -1.242012 \\
\hline $\mathrm{H}$ & 6.245439 & -4.613526 & -0.864746 \\
\hline C & 4.956638 & -3.284322 & -2.911263 \\
\hline $\mathrm{H}$ & 5.063593 & -4.079770 & -3.658136 \\
\hline $\mathrm{H}$ & 4.238993 & -3.623268 & -2.157211 \\
\hline $\mathrm{H}$ & 4.525484 & -2.412079 & -3.416183 \\
\hline C & -2.837919 & -2.557474 & 0.470444 \\
\hline C & -2.374624 & -3.130894 & -0.722877 \\
\hline C & -4.197462 & -2.679288 & 0.800757 \\
\hline C & -3.242895 & -3.790186 & -1.599760 \\
\hline $\mathrm{H}$ & -1.318819 & -3.078978 & -0.951043 \\
\hline
\end{tabular}




\begin{tabular}{|c|c|c|c|}
\hline C & -5.095249 & -3.347870 & -0.039059 \\
\hline $\mathrm{H}$ & -4.545524 & -2.234642 & 1.725965 \\
\hline C & -4.592703 & -3.881306 & -1.234764 \\
\hline $\mathrm{H}$ & -5.278671 & -4.391702 & -1.904072 \\
\hline C & -6.590125 & -3.512142 & 0.299459 \\
\hline C & -2.760703 & -4.406161 & -2.927403 \\
\hline C & -7.445304 & -2.810577 & -0.782329 \\
\hline $\mathrm{H}$ & -7.218445 & -1.739394 & -0.827659 \\
\hline $\mathrm{H}$ & -7.268440 & -3.231613 & -1.777637 \\
\hline $\mathrm{H}$ & -8.513123 & -2.922859 & -0.557447 \\
\hline C & -6.946393 & -5.017648 & 0.336979 \\
\hline $\mathrm{H}$ & -8.008630 & -5.150964 & 0.575858 \\
\hline $\mathrm{H}$ & -6.758005 & -5.508339 & -0.623450 \\
\hline $\mathrm{H}$ & -6.358307 & -5.540804 & 1.099691 \\
\hline C & -6.955054 & -2.906313 & 1.668307 \\
\hline $\mathrm{H}$ & -6.393874 & -3.376223 & 2.484309 \\
\hline $\mathrm{H}$ & -6.770918 & -1.826873 & 1.702276 \\
\hline $\mathrm{H}$ & -8.020976 & -3.064289 & 1.867936 \\
\hline C & -3.497222 & -3.717359 & -4.101302 \\
\hline $\mathrm{H}$ & -4.583278 & -3.843233 & -4.033499 \\
\hline $\mathrm{H}$ & -3.284551 & -2.642488 & -4.118832 \\
\hline $\mathrm{H}$ & -3.170980 & -4.144188 & -5.057571 \\
\hline C & -1.245069 & -4.225586 & -3.140368 \\
\hline $\mathrm{H}$ & -0.953244 & -3.169943 & -3.154188 \\
\hline $\mathrm{H}$ & -0.660287 & -4.728545 & -2.361822 \\
\hline $\mathrm{H}$ & -0.955709 & -4.663600 & -4.102393 \\
\hline C & -3.073041 & -5.920986 & -2.943348 \\
\hline $\mathrm{H}$ & -2.735934 & -6.368006 & -3.886529 \\
\hline $\mathrm{H}$ & -2.560468 & -6.435370 & -2.122473 \\
\hline $\mathrm{H}$ & -4.145511 & -6.120673 & -2.845854 \\
\hline
\end{tabular}

\section{C-Catalyzed reaction TS 98}

\section{Datum}

Value

\begin{tabular}{lc}
\hline B3LYP/6-31G(d) Energy & -3038.957101 \\
\hline B3LYP-D3(BJ)/def2-TZVPP-IEF-PCM(DCM) Energy & -3040.507133 \\
\hline B3LYP-D3(BJ)/def2-TZVPP-IEF-PCM(DCM)//B3LYP/6-31G(d) Free Energy (Quasiharmonic) & -3039.277645 \\
\hline
\end{tabular}

Number of Imaginary Frequencies

Frequencies (Top 3 out of 450)

$$
\begin{array}{lr}
\text { 1. } & -379.4424 \mathrm{~cm}^{-1} \\
\text { 2. } & 8.5332 \mathrm{~cm}^{-1} \\
\text { 3. } & 11.6494 \mathrm{~cm}^{-1}
\end{array}
$$




\begin{tabular}{|c|c|c|c|}
\hline C & 2.565433 & -2.033163 & 0.966654 \\
\hline $\mathrm{H}$ & 3.571835 & -2.378106 & 1.216775 \\
\hline $\mathrm{H}$ & 1.883722 & -2.433567 & 1.727773 \\
\hline C & -0.735296 & -0.639289 & -0.468315 \\
\hline 0 & 0.112594 & -1.044638 & -1.248449 \\
\hline 0 & -1.639438 & 0.237472 & -0.904756 \\
\hline $\mathrm{H}$ & -2.247953 & 0.660377 & -0.235260 \\
\hline 0 & 2.264690 & -2.586909 & -0.310850 \\
\hline $\mathrm{H}$ & 1.472251 & -2.126340 & -0.657211 \\
\hline C & -1.719146 & 2.405337 & -3.438449 \\
\hline C & -1.297064 & 3.505316 & -1.652811 \\
\hline C & -3.982667 & 2.255669 & -2.450372 \\
\hline C & -3.059788 & 2.748708 & -3.418102 \\
\hline $\mathrm{H}$ & -0.822992 & 2.609949 & -1.248792 \\
\hline $\mathrm{H}$ & -3.855233 & 1.198489 & -2.208936 \\
\hline $\mathrm{H}$ & -5.024260 & 2.521378 & -2.628781 \\
\hline C & -4.434921 & 1.982139 & 1.104030 \\
\hline C & -4.943340 & 3.466967 & 0.992098 \\
\hline C & -5.480952 & 0.964788 & 0.623355 \\
\hline $\mathrm{H}$ & -6.294955 & 0.850379 & 1.348076 \\
\hline $\mathrm{H}$ & -5.002580 & -0.010337 & 0.489443 \\
\hline $\mathrm{H}$ & -5.913983 & 1.266467 & -0.334781 \\
\hline C & -3.909514 & 1.580321 & 2.481511 \\
\hline $\mathrm{H}$ & -4.704507 & 1.647996 & 3.233520 \\
\hline $\mathrm{H}$ & -3.081591 & 2.217943 & 2.800064 \\
\hline $\mathrm{H}$ & -3.554117 & 0.544077 & 2.466230 \\
\hline C & -4.105587 & 4.449551 & 1.828846 \\
\hline $\mathrm{H}$ & -4.284348 & 4.332374 & 2.903663 \\
\hline $\mathrm{H}$ & -3.037330 & 4.319525 & 1.632550 \\
\hline $\mathrm{H}$ & -4.377115 & 5.470897 & 1.543957 \\
\hline C & -6.428059 & 3.661039 & 1.303925 \\
\hline $\mathrm{H}$ & -6.657352 & 3.370828 & 2.336120 \\
\hline $\mathrm{H}$ & -6.691976 & 4.716992 & 1.183513 \\
\hline $\mathrm{H}$ & -7.059340 & 3.080216 & 0.627412 \\
\hline 0 & -3.332918 & 1.976940 & 0.159892 \\
\hline 0 & -4.725845 & 3.756794 & -0.396082 \\
\hline B & -3.605117 & 3.011502 & -0.843372 \\
\hline 0 & -2.424911 & 3.877844 & -1.108875 \\
\hline C & -0.396384 & 4.571567 & -2.135591 \\
\hline C & -0.864001 & 5.878357 & -2.342622 \\
\hline C & 0.953566 & 4.274704 & -2.379129 \\
\hline C & 0.005923 & 6.866965 & -2.796063 \\
\hline $\mathrm{H}$ & -1.904741 & 6.104348 & -2.134096 \\
\hline C & 1.820166 & 5.266330 & -2.833073 \\
\hline $\mathrm{H}$ & 1.322091 & 3.266764 & -2.204757 \\
\hline C & 1.347878 & 6.563993 & -3.045621 \\
\hline $\mathrm{H}$ & -0.361258 & 7.877643 & -2.953205 \\
\hline $\mathrm{H}$ & 2.863967 & 5.028008 & -3.017850 \\
\hline $\mathrm{H}$ & 2.023641 & 7.337671 & -3.400155 \\
\hline $\mathrm{H}$ & -3.354904 & 3.632725 & -3.986889 \\
\hline
\end{tabular}




\begin{tabular}{|c|c|c|c|}
\hline $\mathrm{H}$ & -1.407813 & 1.445460 & -3.036012 \\
\hline $\mathrm{H}$ & -1.060538 & 2.853656 & -4.175373 \\
\hline C & 0.166092 & -0.654361 & 1.857047 \\
\hline C & -0.820152 & -1.080126 & 0.973049 \\
\hline C & -1.901178 & -1.929084 & 1.399656 \\
\hline C & -1.990200 & -2.238183 & 2.743756 \\
\hline $\mathrm{H}$ & -2.774783 & -2.909277 & 3.082346 \\
\hline C & 2.522394 & -0.511010 & 0.991874 \\
\hline C & 1.357678 & 0.143816 & 1.391771 \\
\hline C & 3.611310 & 1.637102 & 0.655315 \\
\hline C & 3.678204 & 0.256756 & 0.608722 \\
\hline $\mathrm{H}$ & 4.480344 & 2.219290 & 0.359434 \\
\hline C & 2.447198 & 2.328610 & 1.062493 \\
\hline C & 1.290861 & 1.574957 & 1.439878 \\
\hline C & 0.133447 & 2.286391 & 1.863113 \\
\hline C & 0.125070 & 3.663517 & 1.912247 \\
\hline C & 1.269534 & 4.404948 & 1.533672 \\
\hline C & 2.403647 & 3.748331 & 1.115957 \\
\hline $\mathrm{H}$ & -0.754032 & 1.732861 & 2.149226 \\
\hline $\mathrm{H}$ & -0.767199 & 4.186054 & 2.244577 \\
\hline $\mathrm{H}$ & 1.246589 & 5.490404 & 1.570389 \\
\hline $\mathrm{H}$ & 3.289020 & 4.306739 & 0.821259 \\
\hline C & -1.058125 & -1.760188 & 3.695739 \\
\hline C & 0.049635 & -0.970962 & 3.252458 \\
\hline C & 0.998065 & -0.533525 & 4.217904 \\
\hline C & 0.851640 & -0.854602 & 5.549300 \\
\hline C & -0.246148 & -1.634589 & 5.984568 \\
\hline C & -1.177815 & -2.079299 & 5.075359 \\
\hline $\mathrm{H}$ & 1.843662 & 0.061897 & 3.892510 \\
\hline $\mathrm{H}$ & 1.585498 & -0.508502 & 6.271858 \\
\hline $\mathrm{H}$ & -0.348361 & -1.882876 & 7.037446 \\
\hline $\mathrm{H}$ & -2.021459 & -2.684503 & 5.398373 \\
\hline C & 7.434485 & -1.411512 & -0.604250 \\
\hline C & 7.412864 & -0.425672 & 0.393541 \\
\hline C & 6.167953 & 0.085181 & 0.779448 \\
\hline C & 4.973466 & -0.364047 & 0.196382 \\
\hline C & 5.038666 & -1.350122 & -0.796356 \\
\hline C & 6.263241 & -1.890529 & -1.208124 \\
\hline $\mathrm{H}$ & 8.386852 & -1.820780 & -0.912946 \\
\hline $\mathrm{H}$ & 6.109722 & 0.834930 & 1.563576 \\
\hline $\mathrm{H}$ & 4.111019 & -1.704103 & -1.230596 \\
\hline C & 8.694369 & 0.094473 & 1.075499 \\
\hline C & 6.271303 & -2.986461 & -2.291948 \\
\hline C & 8.621991 & -0.188948 & 2.594787 \\
\hline $\mathrm{H}$ & 9.525192 & 0.180252 & 3.096551 \\
\hline $\mathrm{H}$ & 7.759121 & 0.299047 & 3.059682 \\
\hline $\mathrm{H}$ & 8.540846 & -1.264661 & 2.788363 \\
\hline C & 9.969524 & -0.573442 & 0.526597 \\
\hline $\mathrm{H}$ & 10.848599 & -0.168520 & 1.041047 \\
\hline $\mathrm{H}$ & 9.964126 & -1.657453 & 0.686655 \\
\hline $\mathrm{H}$ & 10.099281 & -0.386783 & -0.545535 \\
\hline C & 8.818528 & 1.619825 & 0.846527 \\
\hline $\mathrm{H}$ & 9.721402 & 2.008386 & 1.334030 \\
\hline $\mathrm{H}$ & 8.883633 & 1.850832 & -0.223004 \\
\hline
\end{tabular}




\begin{tabular}{|c|c|c|c|}
\hline $\mathrm{H}$ & 7.959881 & 2.163781 & 1.253973 \\
\hline C & 5.626976 & -2.436018 & -3.586404 \\
\hline $\mathrm{H}$ & 5.621477 & -3.207329 & -4.366659 \\
\hline $\mathrm{H}$ & 4.592040 & -2.120625 & -3.421850 \\
\hline $\mathrm{H}$ & 6.186127 & -1.572665 & -3.966126 \\
\hline C & 7.693182 & -3.469314 & -2.633896 \\
\hline $\mathrm{H}$ & 7.642414 & -4.249118 & -3.402402 \\
\hline $\mathrm{H}$ & 8.317792 & -2.658523 & -3.027163 \\
\hline $\mathrm{H}$ & 8.199505 & -3.899005 & -1.761923 \\
\hline c & 5.452557 & -4.201573 & -1.791022 \\
\hline $\mathrm{H}$ & 5.422521 & -4.982814 & -2.561279 \\
\hline $\mathrm{H}$ & 5.908696 & -4.632899 & -0.891811 \\
\hline $\mathrm{H}$ & 4.422046 & -3.927597 & -1.544153 \\
\hline C & -2.861272 & -2.553579 & 0.444185 \\
\hline C & -2.416183 & -3.103571 & -0.761451 \\
\hline C & -4.222723 & -2.675165 & 0.782669 \\
\hline C & -3.296525 & -3.741872 & -1.649170 \\
\hline $\mathrm{H}$ & -1.361655 & -3.054279 & -1.004549 \\
\hline c & -5.127262 & -3.321905 & -0.060883 \\
\hline $\mathrm{H}$ & -4.561088 & -2.245604 & 1.718462 \\
\hline C & -4.639166 & -3.837340 & -1.276149 \\
\hline $\mathrm{H}$ & -5.336660 & -4.330339 & -1.942779 \\
\hline C & -6.619808 & -3.487476 & 0.286087 \\
\hline C & -2.751340 & -4.304283 & -2.975378 \\
\hline C & -6.975067 & -2.892933 & 1.662373 \\
\hline $\mathrm{H}$ & -6.410984 & -3.371895 & 2.471064 \\
\hline $\mathrm{H}$ & -6.786964 & -1.814410 & 1.704736 \\
\hline $\mathrm{H}$ & -8.040335 & -3.049276 & 1.866724 \\
\hline C & -7.482699 & -2.776166 & -0.783289 \\
\hline $\mathrm{H}$ & -8.548657 & -2.887495 & -0.549353 \\
\hline $\mathrm{H}$ & -7.253596 & -1.705224 & -0.822928 \\
\hline $\mathrm{H}$ & -7.316593 & -3.190524 & -1.783183 \\
\hline C & -6.976444 & -4.993210 & 0.312903 \\
\hline $\mathrm{H}$ & -6.793182 & -5.475567 & -0.652723 \\
\hline $\mathrm{H}$ & -6.384300 & -5.522717 & 1.068079 \\
\hline $\mathrm{H}$ & -8.037398 & -5.128321 & 0.556515 \\
\hline C & -2.130520 & -3.150367 & -3.799824 \\
\hline $\mathrm{H}$ & -2.887556 & -2.396748 & -4.046460 \\
\hline $\mathrm{H}$ & -1.322390 & -2.645346 & -3.261253 \\
\hline $\mathrm{H}$ & -1.717910 & -3.536837 & -4.739928 \\
\hline c & -1.664849 & -5.365755 & -2.679527 \\
\hline $\mathrm{H}$ & -0.823174 & -4.946860 & -2.119025 \\
\hline $\mathrm{H}$ & -2.076715 & -6.197251 & -2.095788 \\
\hline $\mathrm{H}$ & -1.268293 & -5.772442 & -3.617876 \\
\hline c & -3.848861 & -4.967408 & -3.828768 \\
\hline $\mathrm{H}$ & -3.413075 & -5.339553 & -4.762750 \\
\hline $\mathrm{H}$ & -4.308099 & -5.821305 & -3.317059 \\
\hline & -4.642080 & -4.259456 & -4.095191 \\
\hline
\end{tabular}




\begin{tabular}{lc}
\hline B3LYP/6-31G(d) Energy & -3038.956473 \\
\hline B3LYP-D3(BJ)/def2-TZVPP-IEF-PCM(DCM) Energy & -3040.506544 \\
\hline B3LYP-D3(BJ)/def2-TZVPP-IEF-PCM(DCM)//B3LYP/6-31G(d) Free Energy (Quasiharmonic) & -3039.277573 \\
\hline
\end{tabular}

Number of Imaginary Frequencies

Frequencies (Top 3 out of 450)

$\begin{array}{lr}\text { 1. } & -391.4920 \mathrm{~cm}^{-1} \\ \text { 2. } & 8.2210 \mathrm{~cm}^{-1} \\ \text { 3. } & 10.0057 \mathrm{~cm}^{-1}\end{array}$

\section{B3LYP/6-31G(d) Molecular Geometry in Cartesian Coordinates}

C

$\mathrm{H}$

$\mathrm{H}$

C

0

0

$\mathrm{H}$

0

$\mathrm{H}$

C

C

C

C

$\mathrm{H}$

$\mathrm{H}$

$\mathrm{H}$

C

C

C

$\mathrm{H}$

$\mathrm{H}$

$\mathrm{H}$

C

$\mathrm{H}$

$\mathrm{H}$

$\mathrm{H}$

C

$\mathrm{H}$

$\mathrm{H}$

$\mathrm{H}$

C

$\mathrm{H}$

\subsection{3}

2. 231871

0.727391

$-2.255412$

$-2.929649$

$-1.497938$

$-0.854395$

0.495163

0.271688

1.962364

2.664844

$-0.051655$

1.146461

2.424731

$-0.610443$

$-0.692954$

$-0.827113$

$-1.013151$

$-2.110860$

$-1.886295$

$-2.584064$

$-2.829357$

0.222567

$-0.136520$

0.438767

1.156517

$-0.841902$

$-1.589155$

0.153785

$-0.973374$

$-2.346395$

$-3.195430$
0.518401

0.168111

0.137500

2.169070

3. 130854

1.538688

0.900406

0.005089

$-0.945095$

$-4.407410$

$-4.169589$

$-3.253503$

$-3.293059$

$-5.222434$

$-2.319172$

$-4.129881$

$-3.300604$

$-4.751621$

$-2.603550$

$-1.592051$

$-3.149289$

$-2.513770$

- 3.240948

$-3.722714$

$-2.193353$

$-3.722900$

$-5.882472$

$-5.811881$

$-5.869742$

$-6.847746$

$-4.927514$

$-4.970263$
0.354688

0.334658

1.269089

$-0.805932$

$-1.114576$

$-1.704607$

$-1.286778$

$-0.791936$

$-0.625535$

3.067050

1.028335

2. 208909

2. 975131

0.869057

2. 260807

2. 314826

$-1.514280$

$-0.922140$

$-1.962474$

$-2.317813$

$-2.787121$

$-1.145010$

$-2.633484$

$-3.549185$

$-2.865455$

$-2.329527$

$-1.938094$

$-2.737032$

$-2.387459$

$-1.437592$

$-0.177574$

$-0.868128$ 


\begin{tabular}{|c|c|c|c|}
\hline $\mathrm{H}$ & -2.315565 & -5.866735 & 0.384517 \\
\hline $\mathrm{H}$ & -2.519918 & -4.111729 & 0.530659 \\
\hline 0 & -0.298914 & -2.581667 & -0.364699 \\
\hline 0 & 0.055818 & -4.843376 & 0.040318 \\
\hline B & 0.406394 & -3.523957 & 0.450524 \\
\hline 0 & 1.879170 & -3.305589 & 0.443785 \\
\hline C & 4.086979 & -3.815776 & 1.178932 \\
\hline C & 4.535976 & -2.504710 & 0.957086 \\
\hline C & 5.015289 & -4.813216 & 1.518637 \\
\hline C & 5.889337 & -2.198965 & 1.079445 \\
\hline $\mathrm{H}$ & 3.821594 & -1.739549 & 0.672666 \\
\hline C & 6.367055 & -4.503518 & 1.644070 \\
\hline $\mathrm{H}$ & 4.672614 & -5.832597 & 1.682701 \\
\hline C & 6.806695 & -3.194678 & 1.426474 \\
\hline $\mathrm{H}$ & 6.227495 & -1.183978 & 0.892488 \\
\hline $\mathrm{H}$ & 7.078367 & -5.282007 & 1.905420 \\
\hline $\mathrm{H}$ & 7.861942 & -2.953312 & 1.520038 \\
\hline $\mathrm{H}$ & 1.589861 & -2.337864 & 3.262594 \\
\hline $\mathrm{H}$ & 1.530492 & -5.396261 & 2.929826 \\
\hline $\mathrm{H}$ & 2.878680 & -4.366696 & 3.646743 \\
\hline C & -1.124488 & 1.903547 & 1.407263 \\
\hline C & -2.234153 & 1.629411 & 0.615852 \\
\hline C & -3.361880 & 0.904812 & 1.142254 \\
\hline C & -3.293648 & 0.461420 & 2.450610 \\
\hline $\mathrm{H}$ & -4.139334 & -0.079746 & 2.866466 \\
\hline C & 1.159322 & 2.032756 & 0.353531 \\
\hline C & 0.035855 & 2.687695 & 0.855282 \\
\hline C & 2.219638 & 4.173226 & -0.079804 \\
\hline C & 2.273490 & 2.792294 & -0.139019 \\
\hline $\mathrm{H}$ & 3.056486 & 4.752209 & -0.461686 \\
\hline C & 1.098259 & 4.867594 & 0.427611 \\
\hline C & -0.025167 & 4.118580 & 0.899617 \\
\hline C & -1.149452 & 4.831781 & 1.401938 \\
\hline C & -1.154229 & 6.207550 & 1.438788 \\
\hline C & -0.039293 & 6.945689 & 0.972711 \\
\hline C & 1.060849 & 6.287875 & 0.475496 \\
\hline $\mathrm{H}$ & -2.016530 & 4.279051 & 1.744920 \\
\hline $\mathrm{H}$ & -2.024798 & 6.733250 & 1.821156 \\
\hline $\mathrm{H}$ & -0.059435 & 8.031759 & 1.004482 \\
\hline $\mathrm{H}$ & 1.920727 & 6.843650 & 0.108902 \\
\hline C & -2.185544 & 0.721511 & 3.291339 \\
\hline C & -1.083249 & 1.471271 & 2.774342 \\
\hline C & 0.009649 & 1.751238 & 3.641601 \\
\hline C & 0.013228 & 1.303188 & 4.944629 \\
\hline C & -1.076396 & 0.554566 & 5.450935 \\
\hline C & -2.151937 & 0.273460 & 4.640173 \\
\hline $\mathrm{H}$ & 0.846283 & 2.329839 & 3.264510 \\
\hline $\mathrm{H}$ & 0.856054 & 1.531372 & 5.591690 \\
\hline $\mathrm{H}$ & -1.062404 & 0.208455 & 6.480955 \\
\hline $\mathrm{H}$ & -2.997811 & -0.294545 & 5.020356 \\
\hline C & 5.847916 & 1.173608 & -1.833455 \\
\hline C & 5.951743 & 2.102002 & -0.787790 \\
\hline C & 4.761806 & 2.588639 & -0.235120 \\
\hline C & 3.507600 & 2.170590 & -0.706343 \\
\hline
\end{tabular}




\begin{tabular}{|c|c|c|c|}
\hline C & 3.448115 & 1.236163 & -1.751460 \\
\hline C & 4.615900 & 0.726706 & -2.333731 \\
\hline $\mathrm{H}$ & 6.759864 & 0.789704 & -2.281471 \\
\hline $\mathrm{H}$ & 4.788114 & 3.301792 & 0.580871 \\
\hline $\mathrm{H}$ & 2.473541 & 0.926029 & -2.108765 \\
\hline C & 7.341333 & 2.559542 & -0.301174 \\
\hline C & 4.588397 & -0.279739 & -3.501249 \\
\hline C & 8.144217 & 1.340656 & 0.211903 \\
\hline $\mathrm{H}$ & 9.146269 & 1.649735 & 0.534322 \\
\hline $\mathrm{H}$ & 7.643806 & 0.877015 & 1.070773 \\
\hline $\mathrm{H}$ & 8.265358 & 0.574608 & -0.561471 \\
\hline C & 8.106514 & 3.215490 & -1.475231 \\
\hline $\mathrm{H}$ & 9.097545 & 3.551240 & -1.145685 \\
\hline $\mathrm{H}$ & 8.250388 & 2.520010 & -2.308424 \\
\hline $\mathrm{H}$ & 7.562028 & 4.086164 & -1.857615 \\
\hline C & 7.254573 & 3.586906 & 0.843592 \\
\hline $\mathrm{H}$ & 8.263795 & 3.878681 & 1.155616 \\
\hline $\mathrm{H}$ & 6.728674 & 4.497322 & 0.534863 \\
\hline $\mathrm{H}$ & 6.742341 & 3.178272 & 1.722060 \\
\hline C & 5.308357 & -1.582219 & -3.078351 \\
\hline $\mathrm{H}$ & 5.302614 & -2.305227 & -3.903453 \\
\hline $\mathrm{H}$ & 6.352934 & -1.401209 & -2.802934 \\
\hline $\mathrm{H}$ & 4.812676 & -2.045197 & -2.217728 \\
\hline C & 3.152661 & -0.640921 & -3.925069 \\
\hline $\mathrm{H}$ & 3.182343 & -1.373390 & -4.739906 \\
\hline $\mathrm{H}$ & 2.584375 & -1.081725 & -3.099592 \\
\hline $\mathrm{H}$ & 2.601742 & 0.234621 & -4.286084 \\
\hline C & 5.311821 & 0.328868 & -4.726171 \\
\hline $\mathrm{H}$ & 5.297908 & -0.377249 & -5.565561 \\
\hline $\mathrm{H}$ & 4.820211 & 1.252671 & -5.051446 \\
\hline $\mathrm{H}$ & 6.358779 & 0.565902 & -4.508575 \\
\hline C & -4.596477 & 0.583198 & 0.362049 \\
\hline C & -5.313058 & 1.573257 & -0.324283 \\
\hline C & -5.105552 & -0.726323 & 0.394322 \\
\hline C & -6.501111 & 1.273072 & -1.000137 \\
\hline $\mathrm{H}$ & -4.923728 & 2.580036 & -0.324244 \\
\hline C & -6.303117 & -1.060997 & -0.247886 \\
\hline $\mathrm{H}$ & -4.543099 & -1.482409 & 0.930422 \\
\hline C & -6.971062 & -0.045516 & -0.946861 \\
\hline $\mathrm{H}$ & -7.892334 & -0.291510 & -1.466653 \\
\hline C & -6.899399 & -2.482406 & -0.215444 \\
\hline C & -7.279949 & 2.336724 & -1.798318 \\
\hline C & -6.079223 & -3.444684 & 0.663964 \\
\hline $\mathrm{H}$ & -5.058365 & -3.574473 & 0.289087 \\
\hline $\mathrm{H}$ & -6.019911 & -3.095723 & 1.701399 \\
\hline $\mathrm{H}$ & -6.553786 & -4.432671 & 0.673449 \\
\hline C & -8.336099 & -2.429262 & 0.357118 \\
\hline $\mathrm{H}$ & -8.773625 & -3.435057 & 0.382253 \\
\hline $\mathrm{H}$ & -8.335571 & -2.032755 & 1.378902 \\
\hline $\mathrm{H}$ & -8.995124 & -1.795962 & -0.245334 \\
\hline C & -6.943784 & -3.056282 & -1.651536 \\
\hline $\mathrm{H}$ & -7.555285 & -2.437401 & -2.316631 \\
\hline $\mathrm{H}$ & -5.937369 & -3.111976 & -2.082023 \\
\hline $\mathrm{H}$ & -7.370775 & -4.067109 & -1.648361 \\
\hline
\end{tabular}




\begin{tabular}{|llll|} 
C & -8.736957 & 2.419882 & -1.286081 \\
$H$ & -9.268131 & 1.468181 & -1.395822 \\
$H$ & -8.765136 & 2.698531 & -0.226306 \\
$H$ & -9.295098 & 3.177056 & -1.850495 \\
$\mathrm{C}$ & -6.646864 & 3.736386 & -1.677411 \\
$\mathrm{H}$ & -6.647274 & 4.091848 & -0.640388 \\
$\mathrm{H}$ & -5.614097 & 3.753860 & -2.039847 \\
$\mathrm{H}$ & -7.225350 & 4.452962 & -2.272095 \\
$\mathrm{C}$ & -7.285142 & 1.937374 & -3.293685 \\
$\mathrm{H}$ & -7.841423 & 2.675858 & -3.884503 \\
$\mathrm{H}$ & -6.263456 & 1.887362 & -3.686310 \\
$\mathrm{H}$ & -7.754069 & 0.959963 & -3.452918 \\
\hline
\end{tabular}

\section{C-Catalyzed reaction TS 100}

\section{Datum}

Value

\begin{tabular}{lr}
\hline B3LYP/6-31G(d) Energy & -3038.955006 \\
\hline B3LYP-D3(BJ)/def2-TZVPP-IEF-PCM(DCM) Energy & -3040.505636 \\
\hline B3LYP-D3(BJ)/def2-TZVPP-IEF-PCM(DCM)//B3LYP/6-31G(d) Free Energy (Quasiharmonic) & -3039.276739 \\
\hline
\end{tabular}

Number of Imaginary Frequencies

Frequencies (Top 3 out of 450)
1. $-364.8400 \mathrm{~cm}^{-1}$
2. $9.2617 \mathrm{~cm}^{-1}$
3. $11.6317 \mathrm{~cm}^{-1}$

\section{B3LYP/6-31G(d) Molecular Geometry in Cartesian Coordinates}

C

$\mathrm{H}$

$\mathrm{H}$

C

0

0

$\mathrm{H}$

0

$\mathrm{H}$

C

C

C

C

$\mathrm{H}$

\section{226250}

2.694710

4.248870

$-0.182790$

0.591220

$-1.257720$

$-1.854930$

2.645450

1.921960

$-2.865370$

$-4.249650$

$-3.152380$

$-3.349160$

$-3.401370$
0.354020

0.016200

0.598860

$-0.055380$

0.753940

$-0.412730$

$-1.115120$

1.565980

1.324890

0.637710

0.656990

$-1.811700$

$-0.567720$

0.841080
1.422670

2. 321100

1.721070

$-0.262990$

$-0.747190$

$-0.972160$

$-0.596690$

0.938760

0.322160

$-3.711200$

$-2.064240$

$-3.516350$

$-4.185930$

$-1.402570$ 


\begin{tabular}{|c|c|c|c|}
\hline H & -2.155540 & -1.933650 & -3.087720 \\
\hline $\mathrm{H}$ & -3.477360 & -2.689950 & -4.073760 \\
\hline C & -3.683610 & -3.410510 & -0.330600 \\
\hline C & -5.159970 & -3.578210 & -0.848320 \\
\hline C & -2.725170 & -4.436410 & -0.953950 \\
\hline H & -1.694130 & -4.121680 & -0.769910 \\
\hline H & -2.862560 & -5.433400 & -0.521150 \\
\hline $\mathrm{H}$ & -2.867830 & -4.506620 & -2.036080 \\
\hline C & -3.531380 & -3.408410 & 1.189120 \\
\hline $\mathrm{H}$ & -3.873350 & -4.359440 & 1.614190 \\
\hline $\mathrm{H}$ & -2.480620 & -3.277510 & 1.468930 \\
\hline H & -4.105320 & -2.598980 & 1.645640 \\
\hline C & -6.192300 & -2.872480 & 0.048520 \\
\hline $\mathrm{H}$ & -6.336800 & -3.395830 & 1.000470 \\
\hline $\mathrm{H}$ & -5.895130 & -1.840490 & 0.256650 \\
\hline $\mathrm{H}$ & -7.151610 & -2.845580 & -0.477820 \\
\hline C & -5.595670 & -5.024520 & -1.091230 \\
\hline $\mathrm{H}$ & -5.563210 & -5.610310 & -0.164930 \\
\hline $\mathrm{H}$ & -6.625630 & -5.037430 & -1.462880 \\
\hline $\mathrm{H}$ & -4.965240 & -5.513220 & -1.837770 \\
\hline 0 & -3.336900 & -2.096910 & -0.840600 \\
\hline 0 & -5.115900 & -2.897640 & -2.110370 \\
\hline B & -4.165710 & -1.848120 & -2.020670 \\
\hline 0 & -4.828240 & -0.509220 & -1.985090 \\
\hline C & -5.113900 & 1.812160 & -2.381920 \\
\hline C & -6.389320 & 1.630720 & -2.939040 \\
\hline C & -4.662160 & 3.111090 & -2.103540 \\
\hline C & -7.191550 & 2.733830 & -3.220220 \\
\hline $\mathrm{H}$ & -6.739220 & 0.622330 & -3.134800 \\
\hline C & -5.467800 & 4.212220 & -2.385370 \\
\hline $\mathrm{H}$ & -3.680580 & 3.251300 & -1.658460 \\
\hline C & -6.733840 & 4.026320 & -2.946760 \\
\hline $\mathrm{H}$ & -8.178710 & 2.586830 & -3.650020 \\
\hline $\mathrm{H}$ & -5.109340 & 5.214470 & -2.166750 \\
\hline $\mathrm{H}$ & -7.362730 & 4.884690 & -3.166850 \\
\hline $\mathrm{H}$ & -4.129830 & -0.526340 & -4.948120 \\
\hline $\mathrm{H}$ & -1.977270 & 0.646820 & -3.085560 \\
\hline $\mathrm{H}$ & -3.090640 & 1.556840 & -4.242620 \\
\hline C & 1.058210 & -1.550270 & 1.328160 \\
\hline C & -0.016090 & -0.698500 & 1.092070 \\
\hline C & -0.996680 & -0.427960 & 2.107120 \\
\hline C & -0.948360 & -1.153990 & 3.278700 \\
\hline $\mathrm{H}$ & -1.665190 & -0.939510 & 4.067160 \\
\hline C & 3.246270 & -0.766410 & 0.391780 \\
\hline C & 2.191560 & -1.678560 & 0.343990 \\
\hline C & 4.328060 & -1.927810 & -1.450380 \\
\hline C & 4.342690 & -0.894240 & -0.531290 \\
\hline $\mathrm{H}$ & 5.145540 & -2.015140 & -2.161390 \\
\hline C & 3.278460 & -2.872210 & -1.514620 \\
\hline C & 2.188990 & -2.758980 & -0.595130 \\
\hline C & 1.159200 & -3.738190 & -0.648000 \\
\hline C & 1.197450 & -4.762200 & -1.568010 \\
\hline C & 2.267280 & -4.859080 & -2.490170 \\
\hline C & 3.284210 & -3.933460 & -2.460550 \\
\hline
\end{tabular}




\begin{tabular}{|c|c|c|c|}
\hline $\mathrm{H}$ & 0.340450 & -3.677000 & 0.061050 \\
\hline $\mathrm{H}$ & 0.401520 & -5.501590 & -1.586990 \\
\hline $\mathrm{H}$ & 2.283630 & -5.667970 & -3.215540 \\
\hline $\mathrm{H}$ & 4.114730 & -4.001250 & -3.159330 \\
\hline C & 0.058770 & -2.121760 & 3.519240 \\
\hline C & 1.101050 & -2.299400 & 2.553140 \\
\hline C & 2.145300 & -3.219100 & 2.847290 \\
\hline C & 2.147400 & -3.935850 & 4.023610 \\
\hline C & 1.110740 & -3.765050 & 4.971420 \\
\hline C & 0.092230 & -2.873450 & 4.724300 \\
\hline $\mathrm{H}$ & 2.944480 & -3.355780 & 2.127270 \\
\hline $\mathrm{H}$ & 2.952240 & -4.636770 & 4.227010 \\
\hline $\mathrm{H}$ & 1.124890 & -4.335580 & 5.896050 \\
\hline $\mathrm{H}$ & -0.702750 & -2.726330 & 5.451370 \\
\hline C & 7.753320 & 1.690730 & -0.626130 \\
\hline C & 7.949640 & 0.297310 & -0.605570 \\
\hline C & 6.818650 & -0.518850 & -0.557600 \\
\hline C & 5.520400 & 0.024930 & -0.531460 \\
\hline C & 5.370110 & 1.413650 & -0.555120 \\
\hline C & 6.481960 & 2.269660 & -0.597770 \\
\hline $\mathrm{H}$ & 8.624780 & 2.334510 & -0.659380 \\
\hline $\mathrm{H}$ & 6.922320 & -1.597250 & -0.519140 \\
\hline $\mathrm{H}$ & 4.368260 & 1.825480 & -0.522920 \\
\hline C & 9.383160 & -0.268170 & -0.626160 \\
\hline C & 6.251590 & 3.792890 & -0.606310 \\
\hline C & 10.158330 & 0.239100 & 0.613220 \\
\hline $\mathrm{H}$ & 11.182920 & -0.153550 & 0.610810 \\
\hline $\mathrm{H}$ & 10.220580 & 1.332010 & 0.636450 \\
\hline $\mathrm{H}$ & 9.671480 & -0.086950 & 1.539490 \\
\hline C & 9.409110 & -1.808710 & -0.605680 \\
\hline $\mathrm{H}$ & 10.447150 & -2.159860 & -0.627720 \\
\hline $\mathrm{H}$ & 8.942510 & -2.213320 & 0.299610 \\
\hline $\mathrm{H}$ & 8.896650 & -2.236800 & -1.474750 \\
\hline C & 10.106860 & 0.203860 & -1.909810 \\
\hline $\mathrm{H}$ & 11.131920 & -0.186500 & -1.936130 \\
\hline $\mathrm{H}$ & 9.585050 & -0.150600 & -2.806190 \\
\hline $\mathrm{H}$ & 10.164910 & 1.295630 & -1.968080 \\
\hline C & 7.569140 & 4.589320 & -0.653040 \\
\hline $\mathrm{H}$ & 7.351260 & 5.663530 & -0.652380 \\
\hline $\mathrm{H}$ & 8.202530 & 4.381950 & 0.217240 \\
\hline $\mathrm{H}$ & 8.148510 & 4.371560 & -1.558070 \\
\hline C & 5.412270 & 4.178460 & -1.847850 \\
\hline $\mathrm{H}$ & 5.238030 & 5.261530 & -1.867880 \\
\hline $\mathrm{H}$ & 5.929830 & 3.900310 & -2.773490 \\
\hline $\mathrm{H}$ & 4.436150 & 3.683400 & -1.847650 \\
\hline C & 5.483790 & 4.198380 & 0.675480 \\
\hline $\mathrm{H}$ & 5.285230 & 5.277750 & 0.673010 \\
\hline $\mathrm{H}$ & 4.525460 & 3.676610 & 0.761820 \\
\hline $\mathrm{H}$ & 6.073840 & 3.966420 & 1.570210 \\
\hline C & -1.939340 & 0.719140 & 1.978240 \\
\hline C & -1.433960 & 1.972870 & 1.618820 \\
\hline C & -3.299200 & 0.596180 & 2.308620 \\
\hline C & -2.259200 & 3.106210 & 1.561630 \\
\hline $\mathrm{H}$ & -0.371690 & 2.068720 & 1.420900 \\
\hline
\end{tabular}




\begin{tabular}{|c|c|c|c|}
\hline C & -4.153920 & 1.700080 & 2.270530 \\
\hline $\mathrm{H}$ & -3.672450 & -0.380980 & 2.591320 \\
\hline C & -3.610220 & 2.939750 & 1.881420 \\
\hline $\mathrm{H}$ & -4.269240 & 3.798850 & 1.842340 \\
\hline C & -5.649400 & 1.608000 & 2.632070 \\
\hline C & -1.639360 & 4.468950 & 1.195880 \\
\hline C & -5.976770 & 2.622320 & 3.754310 \\
\hline $\mathrm{H}$ & -5.385510 & 2.416990 & 4.654180 \\
\hline $\mathrm{H}$ & -5.774630 & 3.654350 & 3.450240 \\
\hline $\mathrm{H}$ & -7.038160 & 2.559150 & 4.023570 \\
\hline C & -6.501380 & 1.932710 & 1.382360 \\
\hline $\mathrm{H}$ & -7.569780 & 1.890550 & 1.628020 \\
\hline $\mathrm{H}$ & -6.286850 & 2.932690 & 0.991070 \\
\hline $\mathrm{H}$ & -6.309480 & 1.214220 & 0.577840 \\
\hline C & -6.046920 & 0.207340 & 3.135700 \\
\hline $\mathrm{H}$ & -5.898480 & -0.562210 & 2.370290 \\
\hline $\mathrm{H}$ & -5.479960 & -0.080970 & 4.028590 \\
\hline $\mathrm{H}$ & -7.109750 & 0.200590 & 3.402530 \\
\hline C & -2.683490 & 5.601030 & 1.164600 \\
\hline $\mathrm{H}$ & -3.476970 & 5.408080 & 0.432590 \\
\hline $\mathrm{H}$ & -3.153220 & 5.752140 & 2.143320 \\
\hline $\mathrm{H}$ & -2.197400 & 6.542210 & 0.884010 \\
\hline C & -0.563660 & 4.833900 & 2.248240 \\
\hline $\mathrm{H}$ & -0.999710 & 4.885700 & 3.252560 \\
\hline $\mathrm{H}$ & 0.250350 & 4.102260 & 2.270300 \\
\hline $\mathrm{H}$ & -0.124710 & 5.812200 & 2.017130 \\
\hline C & -0.970970 & 4.385110 & -0.197240 \\
\hline $\mathrm{H}$ & -0.475240 & 5.333650 & -0.436920 \\
\hline $\mathrm{H}$ & -0.219710 & 3.591420 & -0.249740 \\
\hline $\mathrm{H}$ & -1.712740 & 4.189570 & -0.980980 \\
\hline
\end{tabular}

\section{C-Catalyzed reaction TS 101}

\section{Datum}

Value

\begin{tabular}{lc}
\hline B3LYP/6-31G(d) Energy & -3038.956669 \\
\hline B3LYP-D3(BJ)/def2-TZVPP-IEF-PCM(DCM) Energy & -3040.504732 \\
\hline B3LYP-D3(BJ)/def2-TZVPP-IEF-PCM(DCM)//B3LYP/6-31G(d) Free Energy (Quasiharmonic) & -3039.27647 \\
\hline
\end{tabular}

Number of Imaginary Frequencies

Frequencies (Top 3 out of 450)

$$
\begin{array}{lr}
\text { 1. } & -371.2038 \mathrm{~cm}^{-1} \\
\text { 2. } & 6.9297 \mathrm{~cm}^{-1} \\
\text { 3. } & 10.1192 \mathrm{~cm}^{-1}
\end{array}
$$




\begin{tabular}{|c|c|c|c|}
\hline C & 3.116160 & 0.627229 & 1.381570 \\
\hline $\mathrm{H}$ & 2.562910 & 0.459849 & 2.313960 \\
\hline $\mathrm{H}$ & 4.153161 & 0.841998 & 1.651540 \\
\hline C & -0.339890 & 0.215139 & -0.199750 \\
\hline 0 & 0.443091 & 0.939109 & -0.793390 \\
\hline 0 & -1.425870 & -0.232770 & -0.832250 \\
\hline H & -1.941780 & -0.921950 & -0.336890 \\
\hline 0 & 2.623971 & 1.794059 & 0.721460 \\
\hline $\mathrm{H}$ & 1.858511 & 1.528979 & 0.167810 \\
\hline C & -3.555390 & -1.269140 & -4.641760 \\
\hline C & -4.856990 & -0.782850 & -2.989630 \\
\hline C & -2.435040 & -2.668640 & -2.939190 \\
\hline C & -2.488230 & -1.535200 & -3.801060 \\
\hline $\mathrm{H}$ & -5.568570 & -1.525340 & -3.360580 \\
\hline $\mathrm{H}$ & -1.481880 & -2.821870 & -2.433540 \\
\hline $\mathrm{H}$ & -2.831620 & -3.588370 & -3.374890 \\
\hline C & -3.823540 & -3.170080 & 0.579310 \\
\hline C & -4.494800 & -4.209090 & -0.398130 \\
\hline C & -2.954640 & -3.778490 & 1.678690 \\
\hline $\mathrm{H}$ & -2.509080 & -2.984260 & 2.287070 \\
\hline $\mathrm{H}$ & -3.555541 & -4.410860 & 2.342840 \\
\hline $\mathrm{H}$ & -2.143711 & -4.385200 & 1.268610 \\
\hline C & -4.839430 & -2.197770 & 1.198160 \\
\hline $\mathrm{H}$ & -5.458780 & -2.688470 & 1.956760 \\
\hline $\mathrm{H}$ & -4.294880 & -1.378750 & 1.676910 \\
\hline $\mathrm{H}$ & -5.493830 & -1.766700 & 0.435600 \\
\hline C & -5.890481 & -4.673810 & 0.024010 \\
\hline $\mathrm{H}$ & -5.862441 & -5.187140 & 0.992480 \\
\hline $\mathrm{H}$ & -6.588030 & -3.835749 & 0.091470 \\
\hline $\mathrm{H}$ & -6.281781 & -5.376989 & -0.718880 \\
\hline C & -3.605031 & -5.433730 & -0.670370 \\
\hline $\mathrm{H}$ & -3.546711 & -6.101810 & 0.196080 \\
\hline $\mathrm{H}$ & -4.028821 & -5.995790 & -1.508870 \\
\hline $\mathrm{H}$ & -2.590161 & -5.129870 & -0.944090 \\
\hline 0 & -2.975970 & -2.420720 & -0.330610 \\
\hline 0 & -4.615830 & -3.462110 & -1.620690 \\
\hline B & -3.639310 & -2.420030 & -1.614010 \\
\hline 0 & -4.231380 & -1.075210 & -1.885720 \\
\hline C & -5.184720 & 0.634450 & -3.230370 \\
\hline C & -6.186729 & 0.965101 & -4.155780 \\
\hline C & -4.526059 & 1.655740 & -2.526600 \\
\hline C & -6.525479 & 2.297561 & -4.379650 \\
\hline $\mathrm{H}$ & -6.702300 & 0.174001 & -4.696110 \\
\hline C & -4.869709 & 2.986030 & -2.754110 \\
\hline $\mathrm{H}$ & -3.753419 & 1.395380 & -1.810330 \\
\hline C & -5.866239 & 3.311080 & -3.679460 \\
\hline $\mathrm{H}$ & -7.304629 & 2.545431 & -5.095120 \\
\hline $\mathrm{H}$ & -4.360409 & 3.771810 & -2.203380 \\
\hline $\mathrm{H}$ & -6.130309 & 4.350870 & -3.852280 \\
\hline $\mathrm{H}$ & -1.808920 & -0.712140 & -3.576730 \\
\hline
\end{tabular}




\begin{tabular}{|c|c|c|c|}
\hline H & -4.156120 & -2.093890 & -5.019420 \\
\hline $\mathrm{H}$ & -3.561580 & -0.367640 & -5.245710 \\
\hline C & 0.834110 & -1.124351 & 1.559220 \\
\hline C & -0.180190 & -0.233521 & 1.233210 \\
\hline C & -1.116500 & 0.237110 & 2.217450 \\
\hline C & -1.085680 & -0.331291 & 3.474170 \\
\hline H & -1.763230 & 0.035090 & 4.241290 \\
\hline C & 3.055030 & -0.626451 & 0.519270 \\
\hline C & 1.933350 & -1.454051 & 0.583490 \\
\hline C & 4.054800 & -2.116062 & -1.121080 \\
\hline C & 4.141310 & -0.963072 & -0.361960 \\
\hline H & 4.870820 & -2.370762 & -1.792530 \\
\hline C & 2.927280 & -2.967861 & -1.082770 \\
\hline C & 1.835370 & -2.633941 & -0.221160 \\
\hline C & 0.702530 & -3.494641 & -0.201730 \\
\hline C & 0.663229 & -4.629761 & -0.982160 \\
\hline C & 1.749199 & -4.961671 & -1.827940 \\
\hline C & 2.853530 & -4.143611 & -1.878270 \\
\hline $\mathrm{H}$ & -0.145340 & -3.242761 & 0.426160 \\
\hline $\mathrm{H}$ & -0.207921 & -5.278341 & -0.948060 \\
\hline $\mathrm{H}$ & 1.703969 & -5.859791 & -2.438030 \\
\hline H & 3.690959 & -4.383501 & -2.529320 \\
\hline C & -0.142340 & -1.331021 & 3.822390 \\
\hline C & 0.857370 & -1.711511 & 2.870050 \\
\hline C & 1.838050 & -2.663541 & 3.262060 \\
\hline C & 1.820400 & -3.220541 & 4.521990 \\
\hline C & 0.827390 & -2.847841 & 5.458360 \\
\hline C & -0.129390 & -1.920211 & 5.115170 \\
\hline $\mathrm{H}$ & 2.603060 & -2.953011 & 2.550280 \\
\hline $\mathrm{H}$ & 2.575860 & -3.950051 & 4.800480 \\
\hline $\mathrm{H}$ & 0.825860 & -3.292631 & 6.449780 \\
\hline $\mathrm{H}$ & -0.890300 & -1.619811 & 5.831540 \\
\hline C & 7.724171 & 1.336358 & -0.801760 \\
\hline C & 7.823420 & -0.048512 & -0.572480 \\
\hline C & 6.638540 & -0.768772 & -0.414230 \\
\hline C & 5.381510 & -0.138812 & -0.480900 \\
\hline C & 5.327771 & 1.238658 & -0.709050 \\
\hline C & 6.496451 & 2.000058 & -0.868820 \\
\hline $\mathrm{H}$ & 8.638461 & 1.905228 & -0.923790 \\
\hline $\mathrm{H}$ & 6.666830 & -1.834222 & -0.216300 \\
\hline $\mathrm{H}$ & 4.356281 & 1.718208 & -0.744110 \\
\hline C & 9.214630 & -0.708183 & -0.499580 \\
\hline C & 6.374851 & 3.517988 & -1.102500 \\
\hline C & 9.134760 & -2.221843 & -0.224480 \\
\hline $\mathrm{H}$ & 10.145680 & -2.643253 & -0.181440 \\
\hline $\mathrm{H}$ & 8.646150 & -2.437032 & 0.732610 \\
\hline $\mathrm{H}$ & 8.589520 & -2.752482 & -1.013360 \\
\hline C & 9.951430 & -0.504193 & -1.844910 \\
\hline $\mathrm{H}$ & 10.947160 & -0.963833 & -1.810160 \\
\hline H & 9.393570 & -0.962903 & -2.669430 \\
\hline $\mathrm{H}$ & 10.082220 & 0.556967 & -2.081090 \\
\hline C & 10.038910 & -0.059723 & 0.638120 \\
\hline $\mathrm{H}$ & 11.035600 & -0.514903 & 0.695570 \\
\hline H & 10.173071 & 1.015977 & 0.484050 \\
\hline
\end{tabular}




\begin{tabular}{|c|c|c|c|}
\hline $\mathrm{H}$ & 9.545240 & -0.198813 & 1.606750 \\
\hline C & 7.745351 & 4.204088 & -1.253900 \\
\hline $\mathrm{H}$ & 7.603791 & 5.279088 & -1.413870 \\
\hline $\mathrm{H}$ & 8.363441 & 4.083298 & -0.356710 \\
\hline $\mathrm{H}$ & 8.306441 & 3.814108 & -2.111300 \\
\hline C & 5.562301 & 3.776338 & -2.393750 \\
\hline $\mathrm{H}$ & 5.464061 & 4.854568 & -2.571970 \\
\hline $\mathrm{H}$ & 6.057571 & 3.331618 & -3.264960 \\
\hline $\mathrm{H}$ & 4.553971 & 3.355718 & -2.329910 \\
\hline C & 5.640711 & 4.160148 & 0.100000 \\
\hline $\mathrm{H}$ & 5.521241 & 5.239208 & -0.060980 \\
\hline $\mathrm{H}$ & 4.647121 & 3.727548 & 0.252900 \\
\hline $\mathrm{H}$ & 6.213771 & 4.018608 & 1.024190 \\
\hline C & -1.992329 & 1.411710 & 1.944950 \\
\hline C & -1.435759 & 2.553090 & 1.359820 \\
\hline C & -3.340819 & 1.434720 & 2.340060 \\
\hline C & -2.197469 & 3.709940 & 1.137290 \\
\hline $\mathrm{H}$ & -0.382719 & 2.545569 & 1.102410 \\
\hline C & -4.134589 & 2.565110 & 2.135670 \\
\hline $\mathrm{H}$ & -3.758630 & 0.547880 & 2.802050 \\
\hline C & -3.539289 & 3.686970 & 1.527620 \\
\hline $\mathrm{H}$ & -4.148899 & 4.568610 & 1.366740 \\
\hline C & -5.620859 & 2.623570 & 2.539350 \\
\hline C & -1.528649 & 4.935030 & 0.485620 \\
\hline C & -6.485969 & 2.850411 & 1.276610 \\
\hline $\mathrm{H}$ & -6.234329 & 3.790311 & 0.773910 \\
\hline $\mathrm{H}$ & -6.345349 & 2.039621 & 0.553100 \\
\hline $\mathrm{H}$ & -7.549159 & 2.889641 & 1.544370 \\
\hline C & -6.098529 & 1.326310 & 3.219220 \\
\hline $\mathrm{H}$ & -7.154219 & 1.423611 & 3.496900 \\
\hline $\mathrm{H}$ & -6.011540 & 0.459620 & 2.554700 \\
\hline $\mathrm{H}$ & -5.535329 & 1.114120 & 4.135460 \\
\hline C & -5.844409 & 3.790700 & 3.530370 \\
\hline $\mathrm{H}$ & -5.250579 & 3.651310 & 4.441030 \\
\hline $\mathrm{H}$ & -5.566909 & 4.756540 & 3.095940 \\
\hline $\mathrm{H}$ & -6.901139 & 3.847481 & 3.819120 \\
\hline C & -0.299349 & 5.362449 & 1.323640 \\
\hline $\mathrm{H}$ & -0.593119 & 5.618729 & 2.348230 \\
\hline $\mathrm{H}$ & 0.458321 & 4.574449 & 1.376590 \\
\hline $\mathrm{H}$ & 0.173742 & 6.244689 & 0.875460 \\
\hline C & -1.065889 & 4.560129 & -0.943120 \\
\hline $\mathrm{H}$ & -0.384759 & 3.703809 & -0.943180 \\
\hline $\mathrm{H}$ & -1.922599 & 4.300240 & -1.576710 \\
\hline $\mathrm{H}$ & -0.546449 & 5.406549 & -1.409180 \\
\hline C & -2.480468 & 6.141790 & 0.385470 \\
\hline $\mathrm{H}$ & -1.956478 & 6.985920 & -0.076700 \\
\hline $\mathrm{H}$ & -3.358458 & 5.922890 & -0.234000 \\
\hline $\mathrm{H}$ & -2.831408 & 6.469720 & 1.370950 \\
\hline
\end{tabular}




\begin{tabular}{lc}
\hline B3LYP/6-31G(d) Energy & -3038.956687 \\
\hline B3LYP-D3(BJ)/def2-TZVPP-IEF-PCM(DCM) Energy & -3040.505643 \\
\hline B3LYP-D3(BJ)/def2-TZVPP-IEF-PCM(DCM)//B3LYP/6-31G(d) Free Energy (Quasiharmonic) & -3039.276273 \\
\hline
\end{tabular}

Number of Imaginary Frequencies

Frequencies (Top 3 out of 450)

$\begin{array}{lr}\text { 1. } & -381.9597 \mathrm{~cm}^{-1} \\ \text { 2. } & 7.0822 \mathrm{~cm}^{-1} \\ \text { 3. } & 10.4759 \mathrm{~cm}^{-1}\end{array}$

\section{B3LYP/6-31G(d) Molecular Geometry in Cartesian Coordinates}

C

$\mathrm{H}$

$\mathrm{H}$

C

0

0

$\mathrm{H}$

0

$\mathrm{H}$

C

C

C

C

$\mathrm{H}$

$\mathrm{H}$

$\mathrm{H}$

C

C

C

$\mathrm{H}$

$\mathrm{H}$

$\mathrm{H}$

C

$\mathrm{H}$

$\mathrm{H}$

$\mathrm{H}$

C

$\mathrm{H}$

$\mathrm{H}$

$\mathrm{H}$

C

$\mathrm{H}$

\section{638313}

3. 649722

1.963405

$-0.695935$

0.161068

$-1.632780$

$-2.241675$

2. 341729

1.540475

$-3.009442$

$-2.201634$

$-4.164623$

$-3.181800$

$-2.948722$

$-4.270361$

$-5.125021$

$-4.514860$

$-4.912564$

$-5.652001$

$-5.246612$

$-6.442423$

$-6.099123$

$-3.970380$

$-4.729858$

$-3.087836$

$-3.686405$

$-3.979059$

$-4.137254$

$-2.929774$

$-4.180811$

$-6.369214$

$-6.594925$
$-1.971063$

$-2.309799$

$-2.401740$

$-0.599270$

$-0.965952$

0.252937

0.597419

$-2.492218$

$-2.033581$

3. 339984

4.202643

1.850159

2.102478

4.997802

0.805282

2.341440

2.028324

3.524003

1.043547

0.027529

1.085930

1.249923

1.764820

1.980623

2. 373209

0.711274

4.536516

4.574722

4. 297122

5.531665

3. 876951

3. 750334
0.950110

1.188442

1.700867

$-0.433499$

$-1.223380$

$-0.841718$

$-0.136010$

$-0.341945$

$-0.671822$

$-3.850097$

$-2.050768$

$-2.256388$

$-3.253896$

$-2.121573$

$-1.966144$

$-2.426577$

1. 328404

1.031679

1.015859

0.994625

1.773415

0.038969

2. 731855

3. 492454

2.942874

2.831035

1. 716804

2.800439

1.522329

1. 307122

1.336538

2. 402071 


\begin{tabular}{|c|c|c|c|}
\hline $\mathrm{H}$ & -6.552605 & 4.924781 & 1.076149 \\
\hline $\mathrm{H}$ & -7.061292 & 3.262399 & 0.756063 \\
\hline 0 & -3.451187 & 1.811151 & 0.370778 \\
\hline 0 & -4.712257 & 3.615473 & -0.391495 \\
\hline B & -3.658232 & 2.715793 & -0.737326 \\
\hline 0 & -2.355788 & 3. 345271 & -1.081416 \\
\hline C & -0.822712 & 4.539837 & -2.448393 \\
\hline C & 0.246754 & 3.690091 & -2.125267 \\
\hline C & -0.575439 & 5.730466 & -3.149346 \\
\hline C & 1.542548 & 4.029750 & -2.504990 \\
\hline $\mathrm{H}$ & 0.049565 & 2.774451 & -1.577877 \\
\hline C & 0.722886 & 6.066769 & -3.525560 \\
\hline $\mathrm{H}$ & -1.403143 & 6.392426 & -3.395385 \\
\hline C & 1.783896 & 5.215286 & -3.205731 \\
\hline $\mathrm{H}$ & 2.365261 & 3.368607 & -2.248810 \\
\hline H & 0.908070 & 6.991811 & -4.064637 \\
\hline $\mathrm{H}$ & 2.796844 & 5.476458 & -3.500135 \\
\hline $\mathrm{H}$ & -2.361250 & 1.388455 & -3.333025 \\
\hline H & -3.859411 & 4.014277 & -3.932347 \\
\hline $\mathrm{H}$ & -2.217963 & 3.489451 & -4.576972 \\
\hline C & 0.222742 & -0.650375 & 1.887382 \\
\hline C & -0.764101 & -1.067808 & 1.000776 \\
\hline C & -1.842294 & -1.924630 & 1.419650 \\
\hline C & -1.922557 & -2.259048 & 2.757976 \\
\hline $\mathrm{H}$ & -2.703667 & -2.937678 & 3.089553 \\
\hline C & 2.575132 & -0.451040 & 1.019332 \\
\hline C & 1.401214 & 0.175506 & 1.436738 \\
\hline C & 3.638410 & 1.720391 & 0.760962 \\
\hline C & 3.721468 & 0.343428 & 0.662320 \\
\hline $\mathrm{H}$ & 4.501966 & 2.323489 & 0.491841 \\
\hline C & 2.464544 & 2.383335 & 1.187146 \\
\hline C & 1.313910 & 1.603785 & 1.527065 \\
\hline C & 0.142652 & 2.286924 & 1.958082 \\
\hline C & 0.119559 & 3.660577 & 2.058050 \\
\hline C & 1.261299 & 4.427555 & 1.725716 \\
\hline C & 2.406346 & 3.799418 & 1.295006 \\
\hline $\mathrm{H}$ & -0.744876 & 1.714987 & 2.205632 \\
\hline $\mathrm{H}$ & -0.783464 & 4.161424 & 2.394729 \\
\hline $\mathrm{H}$ & 1.227791 & 5.510513 & 1.807541 \\
\hline H & 3.289698 & 4.377072 & 1.032812 \\
\hline C & -0.984661 & -1.797621 & 3.712413 \\
\hline C & 0.116464 & -0.994707 & 3.277433 \\
\hline C & 1.068814 & -0.571965 & 4.245581 \\
\hline C & 0.933365 & -0.920968 & 5.571147 \\
\hline C & -0.157576 & -1.714995 & 5.997962 \\
\hline C & -1.093442 & -2.145167 & 5.086078 \\
\hline $\mathrm{H}$ & 1.909329 & 0.033997 & 3.926706 \\
\hline $\mathrm{H}$ & 1.670814 & -0.586246 & 6.295453 \\
\hline $\mathrm{H}$ & -0.251275 & -1.985498 & 7.046189 \\
\hline $\mathrm{H}$ & -1.932143 & -2.760834 & 5.402297 \\
\hline C & 7.490563 & -1.222649 & -0.630048 \\
\hline C & 6.327873 & -1.708591 & -1.233753 \\
\hline C & 5.095152 & -1.199921 & -0.794107 \\
\hline C & 5.022836 & -0.245107 & 0.223410 \\
\hline
\end{tabular}




\begin{tabular}{|c|c|c|c|}
\hline C & 6.217300 & 0.212937 & 0.811554 \\
\hline C & 7.462529 & -0.261748 & 0.397951 \\
\hline $\mathrm{H}$ & 8.452548 & -1.601261 & -0.956383 \\
\hline $\mathrm{H}$ & 4.170101 & -1.558297 & -1.230389 \\
\hline $\mathrm{H}$ & 6.146425 & 0.936138 & 1.615826 \\
\hline C & 6.342276 & -2.775829 & -2.345165 \\
\hline C & 8.786017 & 0.218797 & 1.025401 \\
\hline C & 5.549062 & -4.016608 & -1.866544 \\
\hline $\mathrm{H}$ & 5.523478 & -4.778422 & -2.656178 \\
\hline $\mathrm{H}$ & 6.022254 & -4.462756 & -0.983526 \\
\hline $\mathrm{H}$ & 4.516877 & -3.766228 & -1.602182 \\
\hline C & 5.674749 & -2.203527 & -3.618182 \\
\hline $\mathrm{H}$ & 5.672612 & -2.955220 & -4.417391 \\
\hline $\mathrm{H}$ & 4.636879 & -1.908965 & -3.434610 \\
\hline $\mathrm{H}$ & 6.216084 & -1.322203 & -3.982121 \\
\hline C & 7.768345 & -3.225280 & -2.713586 \\
\hline $\mathrm{H}$ & 7.722807 & -3.987765 & -3.499582 \\
\hline $\mathrm{H}$ & 8.373962 & -2.394313 & -3.094333 \\
\hline $\mathrm{H}$ & 8.291571 & -3.665989 & -1.857169 \\
\hline C & 8.566651 & 1.262755 & 2.136968 \\
\hline $\mathrm{H}$ & 9.534396 & 1.574463 & 2.546338 \\
\hline $\mathrm{H}$ & 8.062824 & 2.160386 & 1.760524 \\
\hline $\mathrm{H}$ & 7.972129 & 0.859037 & 2.964319 \\
\hline C & 9.535470 & -0.985700 & 1.642937 \\
\hline $\mathrm{H}$ & 10.483820 & -0.659474 & 2.088026 \\
\hline $\mathrm{H}$ & 8.935206 & -1.457671 & 2.429190 \\
\hline $\mathrm{H}$ & 9.764798 & -1.750850 & 0.894276 \\
\hline C & 9.669769 & 0.863856 & -0.068881 \\
\hline $\mathrm{H}$ & 10.622968 & 1.200829 & 0.357523 \\
\hline $\mathrm{H}$ & 9.896038 & 0.160128 & -0.876460 \\
\hline $\mathrm{H}$ & 9.170188 & 1.732185 & -0.513812 \\
\hline C & -2.809896 & -2.529345 & 0.458848 \\
\hline C & -4.167118 & -2.668229 & 0.807830 \\
\hline C & -2.375753 & -3.043008 & -0.766608 \\
\hline C & -5.076935 & -3.297683 & -0.043273 \\
\hline $\mathrm{H}$ & -4.497704 & -2.267356 & 1.759072 \\
\hline c & -3.262354 & -3.658270 & -1.663916 \\
\hline $\mathrm{H}$ & -1.324408 & -2.981028 & -1.020272 \\
\hline C & -4.600093 & -3.773324 & -1.279208 \\
\hline $\mathrm{H}$ & -5.301956 & -4.250735 & -1.952601 \\
\hline C & -2.728653 & -4.174259 & -3.013448 \\
\hline C & -6.563699 & -3.488012 & 0.316534 \\
\hline C & -3.830204 & -4.822898 & -3.872806 \\
\hline $\mathrm{H}$ & -4.274028 & -5.696533 & -3.381211 \\
\hline H & -4.634187 & -4.115882 & -4.107762 \\
\hline $\mathrm{H}$ & -3.402870 & -5.162168 & -4.823119 \\
\hline C & -2.130916 & -2.988392 & -3.809457 \\
\hline $\mathrm{H}$ & -1.727697 & -3.340457 & -4.767019 \\
\hline $\mathrm{H}$ & -2.899493 & -2.235806 & -4.022132 \\
\hline $\mathrm{H}$ & -1.320672 & -2.492092 & -3.265892 \\
\hline C & -1.626977 & -5.232225 & -2.765269 \\
\hline $\mathrm{H}$ & -0.782343 & -4.821947 & -2.202946 \\
\hline H & -2.022399 & -6.085942 & -2.202638 \\
\hline $\mathrm{H}$ & -1.238784 & -5.604934 & -3.720999 \\
\hline
\end{tabular}




\begin{tabular}{|llll|} 
C & -6.907757 & -2.941900 & 1.715621 \\
$H$ & -6.327119 & -3.439306 & 2.501094 \\
$H$ & -6.733074 & -1.863035 & 1.790837 \\
$H$ & -7.968245 & -3.118469 & 1.928412 \\
C & -7.446096 & -2.751432 & -0.719195 \\
$H$ & -7.224643 & -1.678190 & -0.729677 \\
$H$ & -7.290344 & -3.134544 & -1.733136 \\
$H$ & -8.508125 & -2.877405 & -0.474927 \\
C & -6.906713 & -4.997101 & 0.299780 \\
$H$ & -7.963154 & -5.149714 & 0.552647 \\
$H$ & -6.731670 & -5.446424 & -0.682988 \\
$H$ & -6.299926 & -5.544679 & 1.029987 \\
\hline
\end{tabular}

\section{C-Catalyzed reaction TS 103}

\section{Datum}

Value

\begin{tabular}{lc}
\hline B3LYP/6-31G(d) Energy & -3038.950807 \\
\hline B3LYP-D3(BJ)/def2-TZVPP-IEF-PCM(DCM) Energy & -3040.504539 \\
\hline B3LYP-D3(BJ)/def2-TZVPP-IEF-PCM(DCM)//B3LYP/6-31G(d) Free Energy (Quasiharmonic) & -3039.276171 \\
\hline
\end{tabular}

Number of Imaginary Frequencies

Frequencies (Top 3 out of 450)

$\begin{array}{lr}\text { 1. } & -305.6044 \mathrm{~cm}^{-1} \\ \text { 2. } & 7.0721 \mathrm{~cm}^{-1} \\ \text { 3. } & 10.7607 \mathrm{~cm}^{-1}\end{array}$

\section{B3LYP/6-31G(d) Molecular Geometry in Cartesian Coordinates}

C

$\mathrm{H}$

$\mathrm{H}$

C

0

0

$\mathrm{H}$

0

$\mathrm{H}$

C

C

C

C

$\mathrm{H}$

\subsection{0}

2.427149

0.741178

$-1.427572$

$-1.389242$

$-1.287282$

$-1.228528$

2.130404

2. 354287

$-2.141865$

$-0.062230$

$-2.561919$

$-2.610317$

$-0.056345$
0.049123

0.116845

0.272758

$-1.890681$

$-3.097028$

$-1.125617$

$-0.154090$

1.005264

1.834436

3.702941

3. 420309

3.374493

2.950375

2. 361445
0.392700

$-0.464006$

0.032092

$-0.286569$

$-0.377546$

$-1.386949$

$-1.232971$

1.403886

0.951913

1.014240

0.340172

$-1.408100$

$-0.038056$

0.600389 


\begin{tabular}{|c|c|c|c|}
\hline $\mathrm{H}$ & -2.691944 & 4.447656 & -1.565541 \\
\hline $\mathrm{H}$ & -3.187784 & 2.790781 & -2.085942 \\
\hline C & -0.301539 & 1.301877 & -3.422181 \\
\hline C & 0.105804 & 2.715827 & -3.981710 \\
\hline C & 0.817169 & 0.259877 & -3.461540 \\
\hline $\mathrm{H}$ & 0.462599 & -0.693297 & -3.060837 \\
\hline $\mathrm{H}$ & 1.145227 & 0.092170 & -4.494372 \\
\hline $\mathrm{H}$ & 1.682993 & 0.578626 & -2.875705 \\
\hline C & -1.562238 & 0.743960 & -4.101513 \\
\hline $\mathrm{H}$ & -1.911353 & -0.138214 & -3.558576 \\
\hline $\mathrm{H}$ & -1.358230 & 0.443962 & -5.135118 \\
\hline $\mathrm{H}$ & -2.364975 & 1.487615 & -4.113028 \\
\hline C & -0.179619 & 2.924789 & -5.470535 \\
\hline $\mathrm{H}$ & 0.379315 & 2.211085 & -6.087268 \\
\hline $\mathrm{H}$ & -1.244297 & 2.822439 & -5.693027 \\
\hline $\mathrm{H}$ & 0.126989 & 3.935180 & -5.761448 \\
\hline C & 1.573115 & 3.079894 & -3.688916 \\
\hline $\mathrm{H}$ & 2.269788 & 2.497282 & -4.301723 \\
\hline $\mathrm{H}$ & 1.720612 & 4.141056 & -3.913542 \\
\hline $\mathrm{H}$ & 1.823136 & 2.923214 & -2.635534 \\
\hline 0 & -0.636351 & 1.600433 & -2.029089 \\
\hline 0 & -0.739937 & 3.600682 & -3.232352 \\
\hline B & -0.947850 & 3.021795 & -1.957213 \\
\hline 0 & -0.106462 & 3.752688 & -0.906073 \\
\hline C & 0.609880 & 4.336528 & 1.283532 \\
\hline C & 0.895823 & 5.661896 & 0.915284 \\
\hline C & 0.991571 & 3.869081 & 2.551919 \\
\hline C & 1.547480 & 6.506758 & 1.808569 \\
\hline $\mathrm{H}$ & 0.610643 & 6.007969 & -0.073003 \\
\hline C & 1.645262 & 4.721391 & 3.440461 \\
\hline $\mathrm{H}$ & 0.792069 & 2.838521 & 2.831950 \\
\hline C & 1.921221 & 6.040324 & 3.073426 \\
\hline $\mathrm{H}$ & 1.769255 & 7.530322 & 1.519206 \\
\hline $\mathrm{H}$ & 1.941358 & 4.352748 & 4.418464 \\
\hline $\mathrm{H}$ & 2.430733 & 6.702941 & 3.767805 \\
\hline $\mathrm{H}$ & -2.794025 & 1.893800 & 0.147115 \\
\hline $\mathrm{H}$ & -2.080611 & 4.784203 & 0.929258 \\
\hline $\mathrm{H}$ & -2.130371 & 3.299677 & 2.022758 \\
\hline C & -0.548501 & -1.071443 & 1.893211 \\
\hline C & -1.628145 & -1.156226 & 1.020431 \\
\hline C & -2.913839 & -0.600854 & 1.349837 \\
\hline C & -3.028272 & 0.103873 & 2.534901 \\
\hline $\mathrm{H}$ & -3.999358 & 0.494007 & 2.828072 \\
\hline C & 1.807171 & -1.342647 & 0.974407 \\
\hline C & 0.710628 & -1.865320 & 1.652274 \\
\hline C & 3.025167 & -3.418821 & 1.308819 \\
\hline C & 2.997836 & -2.132171 & 0.810530 \\
\hline $\mathrm{H}$ & 3.926490 & -4.015118 & 1.190726 \\
\hline C & 1.928449 & -3.982205 & 2.001792 \\
\hline C & 0.747345 & -3.198331 & 2.187380 \\
\hline C & -0.340347 & -3.782868 & 2.893695 \\
\hline C & -0.258232 & -5.064426 & 3.390183 \\
\hline C & 0.914021 & -5.835216 & 3.205996 \\
\hline C & 1.982815 & -5.302552 & 2.524401 \\
\hline
\end{tabular}




\begin{tabular}{|c|c|c|c|}
\hline $\mathrm{H}$ & -1.249807 & -3.210909 & 3.036311 \\
\hline $\mathrm{H}$ & -1.103159 & -5.490038 & 3.924703 \\
\hline $\mathrm{H}$ & 0.964717 & -6.846149 & 3.601385 \\
\hline $\mathrm{H}$ & 2.889361 & -5.883884 & 2.373312 \\
\hline C & -1.934211 & 0.293952 & 3.413513 \\
\hline C & -0.674022 & -0.311180 & 3.103321 \\
\hline C & 0.403062 & -0.138768 & 4.016184 \\
\hline C & 0.240407 & 0.594293 & 5.171155 \\
\hline C & -1.007897 & 1.188508 & 5.478004 \\
\hline C & -2.070750 & 1.039451 & 4.617359 \\
\hline $\mathrm{H}$ & 1.364847 & -0.578593 & 3.780819 \\
\hline $\mathrm{H}$ & 1.076158 & 0.715125 & 5.855014 \\
\hline $\mathrm{H}$ & -1.123803 & 1.759666 & 6.395189 \\
\hline $\mathrm{H}$ & -3.035453 & 1.486281 & 4.846359 \\
\hline C & 6.550787 & -0.759122 & -1.139642 \\
\hline C & 6.032935 & 0.001463 & -0.087483 \\
\hline C & 4.857339 & -0.451479 & 0.532352 \\
\hline C & 4.217672 & -1.622103 & 0.114909 \\
\hline C & 4.773363 & -2.361253 & -0.944283 \\
\hline C & 5.941000 & -1.945328 & -1.587359 \\
\hline $\mathrm{H}$ & 7.455396 & -0.426754 & -1.635172 \\
\hline $\mathrm{H}$ & 4.423016 & 0.105419 & 1.355444 \\
\hline $\mathrm{H}$ & 4.259342 & -3.260587 & -1.263237 \\
\hline C & 6.700384 & 1.297076 & 0.414312 \\
\hline C & 6.566542 & -2.725108 & -2.760754 \\
\hline C & 7.962676 & 1.662849 & -0.388440 \\
\hline $\mathrm{H}$ & 8.391951 & 2.592252 & 0.002675 \\
\hline $\mathrm{H}$ & 8.733025 & 0.886976 & -0.313077 \\
\hline $\mathrm{H}$ & 7.740344 & 1.822740 & -1.449716 \\
\hline C & 5.698978 & 2.470823 & 0.293568 \\
\hline $\mathrm{H}$ & 6.152698 & 3.401754 & 0.655268 \\
\hline $\mathrm{H}$ & 5.398996 & 2.623413 & -0.749812 \\
\hline $\mathrm{H}$ & 4.794216 & 2.291282 & 0.883855 \\
\hline C & 7.106337 & 1.124196 & 1.897375 \\
\hline $\mathrm{H}$ & 7.581061 & 2.039216 & 2.273195 \\
\hline $\mathrm{H}$ & 6.240819 & 0.910846 & 2.532979 \\
\hline $\mathrm{H}$ & 7.818377 & 0.299237 & 2.014297 \\
\hline C & 6.583164 & -1.829283 & -4.022488 \\
\hline $\mathrm{H}$ & 7.032881 & -2.366514 & -4.866667 \\
\hline $\mathrm{H}$ & 5.565902 & -1.538203 & -4.308238 \\
\hline $\mathrm{H}$ & 7.161199 & -0.912837 & -3.864108 \\
\hline C & 8.016523 & -3.128103 & -2.400807 \\
\hline $\mathrm{H}$ & 8.474353 & -3.683144 & -3.228850 \\
\hline $\mathrm{H}$ & 8.646137 & -2.255408 & -2.198316 \\
\hline $\mathrm{H}$ & 8.036147 & -3.767933 & -1.511133 \\
\hline C & 5.784988 & -4.009205 & -3.096940 \\
\hline $\mathrm{H}$ & 6.271818 & -4.529720 & -3.929500 \\
\hline $\mathrm{H}$ & 5.753253 & -4.701358 & -2.247804 \\
\hline $\mathrm{H}$ & 4.754552 & -3.793497 & -3.400595 \\
\hline C & -4.128262 & -0.820677 & 0.508090 \\
\hline C & -5.058115 & 0.219579 & 0.315686 \\
\hline C & -4.403444 & -2.075676 & -0.043882 \\
\hline C & -6.223043 & 0.029144 & -0.428145 \\
\hline $\mathrm{H}$ & -4.848732 & 1.189183 & 0.750858 \\
\hline
\end{tabular}




\begin{tabular}{|c|c|c|c|}
\hline C & -5.558224 & -2.306841 & -0.807729 \\
\hline $\mathrm{H}$ & -3.713661 & -2.890784 & 0.134147 \\
\hline C & -6.445990 & -1.243401 & -0.985844 \\
\hline $\mathrm{H}$ & -7.343117 & -1.398734 & -1.573671 \\
\hline C & -5.789650 & -3.703996 & -1.414062 \\
\hline C & -7.251083 & 1.152240 & -0.665860 \\
\hline C & -4.622765 & -4.038960 & -2.375295 \\
\hline $\mathrm{H}$ & -4.583117 & -3.322134 & -3.203838 \\
\hline $\mathrm{H}$ & -3.651800 & -4.016701 & -1.870634 \\
\hline $\mathrm{H}$ & -4.759419 & -5.040986 & -2.800828 \\
\hline C & -5.839976 & -4.757890 & -0.282391 \\
\hline $\mathrm{H}$ & -6.001563 & -5.757764 & -0.703329 \\
\hline $\mathrm{H}$ & -4.907922 & -4.787448 & 0.290660 \\
\hline $\mathrm{H}$ & -6.658806 & -4.546516 & 0.415335 \\
\hline C & -7.107974 & -3.791206 & -2.205619 \\
\hline $\mathrm{H}$ & -7.980363 & -3.590689 & -1.572442 \\
\hline $\mathrm{H}$ & -7.127087 & -3.090331 & -3.048158 \\
\hline $\mathrm{H}$ & -7.223492 & -4.800634 & -2.615980 \\
\hline C & -6.855223 & 2.471912 & 0.024010 \\
\hline $\mathrm{H}$ & -6.777935 & 2.357465 & 1.111533 \\
\hline $\mathrm{H}$ & -5.900639 & 2.861170 & -0.347829 \\
\hline $\mathrm{H}$ & -7.619044 & 3.232769 & -0.172576 \\
\hline C & -7.375557 & 1.423601 & -2.184068 \\
\hline $\mathrm{H}$ & -6.415066 & 1.741551 & -2.605442 \\
\hline $\mathrm{H}$ & -7.701329 & 0.533345 & -2.731853 \\
\hline $\mathrm{H}$ & -8.108712 & 2.217796 & -2.371283 \\
\hline C & -8.628045 & 0.717870 & -0.109073 \\
\hline $\mathrm{H}$ & -9.372546 & 1.505781 & -0.276955 \\
\hline H & -8.995065 & -0.194395 & -0.590515 \\
\hline $\mathrm{H}$ & -8.572089 & 0.527426 & 0.968888 \\
\hline
\end{tabular}

\section{C-Catalyzed reaction TS 104}

\section{Datum}

Value

\begin{tabular}{lc}
\hline B3LYP/6-31G(d) Energy & -3038.956188 \\
\hline B3LYP-D3(BJ)/def2-TZVPP-IEF-PCM(DCM) Energy & -3040.504649 \\
\hline B3LYP-D3(BJ)/def2-TZVPP-IEF-PCM(DCM)//B3LYP/6-31G(d) Free Energy (Quasiharmonic) & -3039.275692 \\
\hline
\end{tabular}

Number of Imaginary Frequencies

Frequencies (Top 3 out of 450)

$\begin{array}{lr}\text { 1. } & -371.7222 \mathrm{~cm}^{-1} \\ \text { 2. } & 10.0521 \mathrm{~cm}^{-1} \\ \text { 3. } & 13.5309 \mathrm{~cm}^{-1}\end{array}$




\begin{tabular}{|c|c|c|c|}
\hline C & 3.105115 & 0.628279 & 1.342111 \\
\hline $\mathrm{H}$ & 2.544897 & 0.474233 & 2.272488 \\
\hline $\mathrm{H}$ & 4.140300 & 0.845737 & 1.616693 \\
\hline C & -0.338570 & 0.215702 & -0.258175 \\
\hline 0 & 0.443032 & 0.925085 & -0.868775 \\
\hline 0 & -1.430715 & -0.244647 & -0.875265 \\
\hline H & -1.937079 & -0.921881 & -0.356471 \\
\hline 0 & 2.619003 & 1.787132 & 0.663003 \\
\hline $\mathrm{H}$ & 1.860427 & 1.517542 & 0.101946 \\
\hline C & -3.711698 & -1.349227 & -4.573240 \\
\hline C & -4.938637 & -0.838329 & -2.870772 \\
\hline C & -2.514228 & -2.723341 & -2.902633 \\
\hline C & -2.607709 & -1.602638 & -3.777298 \\
\hline $\mathrm{H}$ & -5.665399 & -1.586415 & -3.198234 \\
\hline $\mathrm{H}$ & -1.540501 & -2.865597 & -2.434359 \\
\hline $\mathrm{H}$ & -2.924923 & -3.650570 & -3.308364 \\
\hline C & -3.764306 & -3.213870 & 0.665719 \\
\hline C & -4.486267 & -4.244886 & -0.284799 \\
\hline C & -2.850707 & -3.836568 & 1.720390 \\
\hline $\mathrm{H}$ & -2.373094 & -3.050701 & 2.314878 \\
\hline $\mathrm{H}$ & -3.426845 & -4.469801 & 2.405398 \\
\hline $\mathrm{H}$ & -2.063327 & -4.446590 & 1.271295 \\
\hline C & -4.743501 & -2.241350 & 1.339947 \\
\hline $\mathrm{H}$ & -5.335721 & -2.736433 & 2.117147 \\
\hline $\mathrm{H}$ & -4.169039 & -1.436481 & 1.808138 \\
\hline $\mathrm{H}$ & -5.424540 & -1.791909 & 0.612108 \\
\hline C & -5.867167 & -4.696693 & 0.195876 \\
\hline $\mathrm{H}$ & -5.802781 & -5.213180 & 1.160905 \\
\hline $\mathrm{H}$ & -6.552041 & -3.851376 & 0.295448 \\
\hline $\mathrm{H}$ & -6.297452 & -5.393370 & -0.531411 \\
\hline C & -3.620682 & -5.477295 & -0.596284 \\
\hline $\mathrm{H}$ & -3.533213 & -6.148558 & 0.265135 \\
\hline $\mathrm{H}$ & -4.084078 & -6.032650 & -1.418151 \\
\hline $\mathrm{H}$ & -2.614887 & -5.182778 & -0.910762 \\
\hline 0 & -2.956501 & -2.460609 & -0.276980 \\
\hline 0 & -4.652294 & -3.495441 & -1.500161 \\
\hline B & -3.668997 & -2.460843 & -1.532896 \\
\hline 0 & -4.263106 & -1.115344 & -1.792269 \\
\hline C & -5.278047 & 0.575045 & -3.117076 \\
\hline C & -4.589152 & 1.607201 & -2.459463 \\
\hline C & -6.321747 & 0.891275 & -4.000436 \\
\hline C & -4.944878 & 2.934125 & -2.689650 \\
\hline $\mathrm{H}$ & -3.783311 & 1.357751 & -1.776675 \\
\hline C & -6.672637 & 2.220042 & -4.227643 \\
\hline $\mathrm{H}$ & -6.859967 & 0.091734 & -4.505071 \\
\hline C & -5.983841 & 3.244161 & -3.572827 \\
\hline $\mathrm{H}$ & -4.409836 & 3.727952 & -2.176673 \\
\hline $\mathrm{H}$ & -7.484057 & 2.456759 & -4.910293 \\
\hline $\mathrm{H}$ & -6.257117 & 4.281119 & -3.748559 \\
\hline $\mathrm{H}$ & -1.921176 & -0.775243 & -3.594915 \\
\hline
\end{tabular}




\begin{tabular}{|c|c|c|c|}
\hline $\mathrm{H}$ & -4.327664 & -2.179582 & -4.912166 \\
\hline $\mathrm{H}$ & -3.745888 & -0.456305 & -5.188862 \\
\hline C & 0.817964 & -1.110204 & 1.522759 \\
\hline C & -0.181165 & -0.208715 & 1.181698 \\
\hline C & -1.114280 & 0.289946 & 2.154672 \\
\hline C & -1.100878 & -0.263683 & 3.417782 \\
\hline $\mathrm{H}$ & -1.775862 & 0.124078 & 4.176699 \\
\hline C & 3.048394 & -0.635863 & 0.495525 \\
\hline C & 1.922452 & -1.457965 & 0.558772 \\
\hline C & 4.058167 & -2.148524 & -1.116975 \\
\hline C & 4.141924 & -0.986749 & -0.371102 \\
\hline $\mathrm{H}$ & 4.879727 & -2.414836 & -1.777061 \\
\hline C & 2.925554 & -2.993568 & -1.081825 \\
\hline C & 1.825716 & -2.644202 & -0.236490 \\
\hline C & 0.685166 & -3.494562 & -0.225832 \\
\hline C & 0.647052 & -4.636122 & -0.996867 \\
\hline C & 1.742070 & -4.985306 & -1.823761 \\
\hline C & 2.853395 & -4.176405 & -1.866890 \\
\hline $\mathrm{H}$ & -0.170783 & -3.227974 & 0.384436 \\
\hline $\mathrm{H}$ & -0.230741 & -5.276089 & -0.970707 \\
\hline $\mathrm{H}$ & 1.697800 & -5.888637 & -2.426195 \\
\hline $\mathrm{H}$ & 3.697222 & -4.428458 & -2.504951 \\
\hline C & -0.176676 & -1.276339 & 3.781899 \\
\hline C & 0.823673 & -1.682721 & 2.840416 \\
\hline C & 1.786673 & -2.645392 & 3.249285 \\
\hline C & 1.751339 & -3.189295 & 4.514623 \\
\hline C & 0.757870 & -2.791498 & 5.439982 \\
\hline C & -0.181699 & -1.852321 & 5.080577 \\
\hline $\mathrm{H}$ & 2.551768 & -2.954215 & 2.545737 \\
\hline $\mathrm{H}$ & 2.493208 & -3.927762 & 4.805801 \\
\hline $\mathrm{H}$ & 0.742369 & -3.225970 & 6.435847 \\
\hline $\mathrm{H}$ & -0.942398 & -1.532413 & 5.788675 \\
\hline C & 7.735593 & 1.296701 & -0.807639 \\
\hline C & 6.510598 & 1.963855 & -0.888366 \\
\hline C & 5.338366 & 1.207688 & -0.729813 \\
\hline C & 5.385839 & -0.167911 & -0.488964 \\
\hline C & 6.640340 & -0.801345 & -0.408555 \\
\hline C & 7.828608 & -0.086495 & -0.565901 \\
\hline $\mathrm{H}$ & 8.652596 & 1.861373 & -0.928735 \\
\hline $\mathrm{H}$ & 4.368820 & 1.690218 & -0.776370 \\
\hline $\mathrm{H}$ & 6.663898 & -1.865029 & -0.200817 \\
\hline C & 6.395579 & 3.480102 & -1.136018 \\
\hline C & 9.217029 & -0.750308 & -0.479137 \\
\hline C & 5.654243 & 4.134927 & 0.055176 \\
\hline $\mathrm{H}$ & 5.541650 & 5.213326 & -0.114912 \\
\hline $\mathrm{H}$ & 4.657055 & 3.708652 & 0.202374 \\
\hline $\mathrm{H}$ & 6.218532 & 3.997444 & 0.985358 \\
\hline C & 7.769384 & 4.160607 & -1.282706 \\
\hline $\mathrm{H}$ & 7.632473 & 5.234643 & -1.452902 \\
\hline $\mathrm{H}$ & 8.380262 & 4.045514 & -0.379842 \\
\hline $\mathrm{H}$ & 8.335771 & 3.761682 & -2.132472 \\
\hline C & 5.593713 & 3.729762 & -2.435627 \\
\hline $\mathrm{H}$ & 5.500209 & 4.806700 & -2.623888 \\
\hline $\mathrm{H}$ & 6.094219 & 3.276032 & -3.299165 \\
\hline
\end{tabular}




\begin{tabular}{|c|c|c|c|}
\hline $\mathrm{H}$ & 4.583628 & 3.312809 & -2.375757 \\
\hline C & 9.962795 & -0.559572 & -1.821465 \\
\hline $\mathrm{H}$ & 10.956613 & -1.022573 & -1.777053 \\
\hline $\mathrm{H}$ & 9.408270 & -1.022683 & -2.645774 \\
\hline H & 10.099011 & 0.499206 & -2.065159 \\
\hline C & 10.036616 & -0.095746 & 0.658467 \\
\hline $\mathrm{H}$ & 11.031314 & -0.553934 & 0.725597 \\
\hline $\mathrm{H}$ & 10.175519 & 0.978226 & 0.496738 \\
\hline $\mathrm{H}$ & 9.536557 & -0.225434 & 1.625127 \\
\hline C & 9.130287 & -2.261493 & -0.192764 \\
\hline $\mathrm{H}$ & 10.139523 & -2.685919 & -0.140624 \\
\hline $\mathrm{H}$ & 8.635325 & -2.467610 & 0.763051 \\
\hline $\mathrm{H}$ & 8.587891 & -2.796330 & -0.980772 \\
\hline C & -1.959211 & 1.483170 & 1.862388 \\
\hline C & -1.347541 & 2.620469 & 1.304489 \\
\hline C & -3.312006 & 1.537486 & 2.206309 \\
\hline C & -2.069213 & 3.791263 & 1.072935 \\
\hline $\mathrm{H}$ & -0.288203 & 2.580443 & 1.086108 \\
\hline C & -4.074912 & 2.697333 & 1.991073 \\
\hline $\mathrm{H}$ & -3.775952 & 0.656621 & 2.639870 \\
\hline C & -3.431863 & 3.801991 & 1.422884 \\
\hline $\mathrm{H}$ & -3.999775 & 4.709011 & 1.252646 \\
\hline C & -5.566868 & 2.713237 & 2.378913 \\
\hline C & -1.413578 & 5.054916 & 0.483320 \\
\hline C & -6.237056 & 4.070387 & 2.091238 \\
\hline H & -5.765819 & 4.886805 & 2.650452 \\
\hline $\mathrm{H}$ & -6.207348 & 4.321933 & 1.025071 \\
\hline $\mathrm{H}$ & -7.290357 & 4.030238 & 2.390855 \\
\hline C & -6.319580 & 1.627703 & 1.573466 \\
\hline $\mathrm{H}$ & -7.384515 & 1.626825 & 1.837434 \\
\hline $\mathrm{H}$ & -6.234703 & 1.809661 & 0.496215 \\
\hline $\mathrm{H}$ & -5.925633 & 0.626145 & 1.772811 \\
\hline C & -5.709015 & 2.421477 & 3.891684 \\
\hline $\mathrm{H}$ & -5.291567 & 1.445390 & 4.159679 \\
\hline $\mathrm{H}$ & -5.191661 & 3.181719 & 4.488178 \\
\hline $\mathrm{H}$ & -6.766621 & 2.422215 & 4.182975 \\
\hline C & -1.474830 & 6.195130 & 1.527390 \\
\hline $\mathrm{H}$ & -2.506454 & 6.439884 & 1.803613 \\
\hline $\mathrm{H}$ & -0.940303 & 5.915358 & 2.442200 \\
\hline $\mathrm{H}$ & -1.010021 & 7.104876 & 1.127687 \\
\hline C & 0.061161 & 4.823210 & 0.099509 \\
\hline $\mathrm{H}$ & 0.680758 & 4.568285 & 0.966011 \\
\hline $\mathrm{H}$ & 0.168798 & 4.020206 & -0.637805 \\
\hline H & 0.473043 & 5.738649 & -0.340512 \\
\hline C & -2.173253 & 5.489566 & -0.792281 \\
\hline $\mathrm{H}$ & -1.720819 & 6.394936 & -1.215011 \\
\hline $\mathrm{H}$ & -2.131343 & 4.704622 & -1.556441 \\
\hline H & -3.226648 & 5.712007 & -0.588876 \\
\hline
\end{tabular}




\begin{tabular}{lc}
\hline B3LYP/6-31G(d) Energy & -3038.957435 \\
\hline B3LYP-D3(BJ)/def2-TZVPP-IEF-PCM(DCM) Energy & -3040.503084 \\
\hline B3LYP-D3(BJ)/def2-TZVPP-IEF-PCM(DCM)//B3LYP/6-31G(d) Free Energy (Quasiharmonic) & -3039.275537 \\
\hline
\end{tabular}

Number of Imaginary Frequencies

Frequencies (Top 3 out of 450)

$\begin{array}{lr}\text { 1. } & -276.8362 \mathrm{~cm}^{-1} \\ \text { 2. } & 7.5483 \mathrm{~cm}^{-1} \\ \text { 3. } & 10.7387 \mathrm{~cm}^{-1}\end{array}$

\section{B3LYP/6-31G(d) Molecular Geometry in Cartesian Coordinates}

C

C

0

$\mathrm{H}$

0

$\mathrm{H}$

C

C

C

C

$\mathrm{H}$

$\mathrm{H}$

$\mathrm{H}$

C

C

C

$\mathrm{H}$

$\mathrm{H}$

$\mathrm{H}$

C

$\mathrm{H}$

$\mathrm{H}$

$\mathrm{H}$

C

$\mathrm{H}$

$\mathrm{H}$

$\mathrm{H}$

C

$\mathrm{H}$

$\mathrm{H}$

$\mathrm{H}$

0
1.939297

$-1.123057$

$-0.488515$

$-0.780554$

2.074643

1.675116

$-0.828178$

$-2.135810$

0.670785

$-0.230918$

$-2.322569$

1. 264305

1.302900

$-0.103416$

0.359429

$-1.144109$

$-1.423512$

$-0.743827$

$-2.049969$

1.072004

1.528188

0.703272

1.847985

1.619674

1.458594

2.471808

1.871730

$-0.753182$

$-0.978737$

$-0.414825$

$-1.673778$

$-0.710964$

\subsection{8}

1.740514

0.461765

$-0.119158$

$-0.556599$

$-1.327026$

$-2.482468$

$-2.247267$

$-3.469948$

$-3.577657$

$-1.242153$

$-4.369808$

$-2.579395$

$-4.621562$

$-3.159267$

$-5.212155$

$-6.217019$

$-5.296440$

$-4.602040$

$-5.600169$

$-5.848281$

$-6.526025$

$-5.187423$

$-3.055083$

$-3.502817$

$-3.551649$

$-1.998981$

$-2.292207$

$-2.591764$

$-1.251639$

$-2.352129$

$-4.456766$
0.723104

$-1.155249$

$-1.270876$

$-0.547763$

$-0.021702$

0.472427

$-3.249790$

$-1.498454$

$-1.557190$

$-2.673998$

$-1.882371$

$-1.378379$

$-1.604578$

1.835873

2.193144

2.788575

2.453206

3.805789

2.821060

1.681390

2.646115

1. 227739

1.029548

3.052010

4.039606

2.580308

3.192306

2.803742

3.833231

2.809857

2. 214890

0.536580 


\begin{tabular}{|c|c|c|c|}
\hline 0 & 0.661239 & -2.635107 & 0.878664 \\
\hline B & -0.203689 & -3.269646 & -0.076921 \\
\hline 0 & -1.319962 & -2.266641 & -0.494503 \\
\hline C & -3.243192 & -3.203957 & -1.727195 \\
\hline C & -3.375440 & -4.418798 & -1.034612 \\
\hline C & -4.225672 & -2.836133 & -2.664037 \\
\hline C & -4.465427 & -5.248483 & -1.295710 \\
\hline $\mathrm{H}$ & -2.625070 & -4.701019 & -0.303107 \\
\hline C & -5.310003 & -3.669929 & -2.920717 \\
\hline $\mathrm{H}$ & -4.135534 & -1.888230 & -3.190306 \\
\hline C & -5.430921 & -4.882608 & -2.236747 \\
\hline $\mathrm{H}$ & -4.563009 & -6.186750 & -0.756158 \\
\hline $\mathrm{H}$ & -6.061715 & -3.373456 & -3.646933 \\
\hline $\mathrm{H}$ & -6.277373 & -5.535411 & -2.432372 \\
\hline $\mathrm{H}$ & -0.611841 & -4.565825 & -2.934756 \\
\hline $\mathrm{H}$ & -0.401578 & -1.492216 & -3.107904 \\
\hline $\mathrm{H}$ & -1.536492 & -2.600746 & -4.064533 \\
\hline C & 0.101161 & 2.796905 & 0.780392 \\
\hline C & -1.094909 & 2.315863 & 0.250846 \\
\hline C & -2.282261 & 2.311409 & 1.061458 \\
\hline C & -2.220561 & 2.785118 & 2.357429 \\
\hline $\mathrm{H}$ & -3.122747 & 2.789955 & 2.964228 \\
\hline C & 2.281859 & 1.797475 & -0.034661 \\
\hline C & 1.358899 & 2.838916 & -0.046788 \\
\hline C & 3.740728 & 3.008691 & -1.542141 \\
\hline C & 3.512663 & 1.889318 & -0.767092 \\
\hline $\mathrm{H}$ & 4.681134 & 3.099152 & -2.079856 \\
\hline C & 2.810071 & 4.071656 & -1.622155 \\
\hline C & 1.607890 & 4.003266 & -0.848559 \\
\hline C & 0.703273 & 5.100450 & -0.912429 \\
\hline C & 0.970527 & 6.196812 & -1.702226 \\
\hline C & 2.158687 & 6.258000 & -2.468843 \\
\hline C & 3.057851 & 5.217728 & -2.425211 \\
\hline $\mathrm{H}$ & -0.207743 & 5.065129 & -0.324989 \\
\hline $\mathrm{H}$ & 0.266058 & 7.023650 & -1.734541 \\
\hline $\mathrm{H}$ & 2.358727 & 7.128655 & -3.087667 \\
\hline $\mathrm{H}$ & 3.976635 & 5.256082 & -3.005788 \\
\hline C & -1.027477 & 3.302038 & 2.913464 \\
\hline C & 0.157521 & 3.316728 & 2.114905 \\
\hline C & 1.345898 & 3.850444 & 2.686089 \\
\hline C & 1.355178 & 4.346958 & 3.969679 \\
\hline C & 0.178156 & 4.332049 & 4.756619 \\
\hline C & -0.986553 & 3.817959 & 4.237386 \\
\hline $\mathrm{H}$ & 2.256926 & 3.855815 & 2.098437 \\
\hline $\mathrm{H}$ & 2.274791 & 4.749331 & 4. 386107 \\
\hline $\mathrm{H}$ & 0.200014 & 4.724952 & 5.769686 \\
\hline $\mathrm{H}$ & -1.897513 & 3.798999 & 4.831278 \\
\hline C & 6.653799 & -0.982185 & -0.448846 \\
\hline C & 6.247833 & -0.570614 & -1.726076 \\
\hline C & 5.205313 & 0.359600 & -1.808202 \\
\hline C & 4.584681 & 0.859943 & -0.656707 \\
\hline C & 5.024315 & 0.426783 & 0.601441 \\
\hline C & 6.061797 & -0.501893 & 0.728428 \\
\hline $\mathrm{H}$ & 7.459187 & -1.706380 & -0.367363 \\
\hline
\end{tabular}




\begin{tabular}{|c|c|c|c|}
\hline $\mathrm{H}$ & 4.839742 & 0.692822 & -2.772591 \\
\hline $\mathrm{H}$ & 4.544617 & 0.833151 & 1.484350 \\
\hline C & 6.941813 & -1.152366 & -2.973443 \\
\hline C & 6.558116 & -1.005208 & 2.097957 \\
\hline C & 6.756629 & -2.688288 & -2.998062 \\
\hline H & 7.248256 & -3.119060 & -3.879341 \\
\hline $\mathrm{H}$ & 7.186053 & -3.164983 & -2.110696 \\
\hline $\mathrm{H}$ & 5.693832 & -2.952780 & -3.037325 \\
\hline C & 6.367952 & -0.582362 & -4.284732 \\
\hline $\mathrm{H}$ & 6.894511 & -1.021852 & -5.139671 \\
\hline $\mathrm{H}$ & 5.302732 & -0.812827 & -4.398294 \\
\hline $\mathrm{H}$ & 6.490574 & 0.505096 & -4.344853 \\
\hline C & 8.452060 & -0.819477 & -2.927683 \\
\hline $\mathrm{H}$ & 8.961582 & -1.232527 & -3.807194 \\
\hline $\mathrm{H}$ & 8.613834 & 0.264575 & -2.919080 \\
\hline $\mathrm{H}$ & 8.935220 & -1.235262 & -2.037394 \\
\hline C & 8.064832 & -0.691392 & 2.254235 \\
\hline $\mathrm{H}$ & 8.430265 & -1.048287 & 3.225155 \\
\hline $\mathrm{H}$ & 8.666652 & -1.172056 & 1.475733 \\
\hline $\mathrm{H}$ & 8.247418 & 0.387930 & 2.198648 \\
\hline C & 5.808468 & -0.346081 & 3.271766 \\
\hline $\mathrm{H}$ & 6.191728 & -0.739241 & 4.220414 \\
\hline $\mathrm{H}$ & 5.947977 & 0.740909 & 3.284752 \\
\hline $\mathrm{H}$ & 4.732615 & -0.550200 & 3.238591 \\
\hline C & 6.341344 & -2.534657 & 2.188450 \\
\hline $\mathrm{H}$ & 6.694740 & -2.914396 & 3.155244 \\
\hline $\mathrm{H}$ & 5.278261 & -2.783681 & 2.092777 \\
\hline $\mathrm{H}$ & 6.882207 & -3.070750 & 1.401296 \\
\hline C & -3.612813 & 1.829128 & 0.572411 \\
\hline C & -4.237825 & 0.754329 & 1.219019 \\
\hline C & -4.290570 & 2.487979 & -0.463882 \\
\hline C & -5.521573 & 0.327697 & 0.854051 \\
\hline $\mathrm{H}$ & -3.698619 & 0.258560 & 2.018059 \\
\hline C & -5.569755 & 2.088335 & -0.867689 \\
\hline $\mathrm{H}$ & -3.808315 & 3.337312 & -0.934666 \\
\hline C & -6.158296 & 1.008590 & -0.192498 \\
\hline $\mathrm{H}$ & -7.154167 & 0.691803 & -0.488122 \\
\hline C & -6.341730 & 2.793545 & -2.000428 \\
\hline C & -6.248073 & -0.827768 & 1.570781 \\
\hline C & -6.651809 & 1.775027 & -3.123268 \\
\hline $\mathrm{H}$ & -7.209553 & 2.258317 & -3.934910 \\
\hline $\mathrm{H}$ & -5.726682 & 1.365067 & -3.545290 \\
\hline $\mathrm{H}$ & -7.253644 & 0.935995 & -2.758697 \\
\hline C & -7.669158 & 3.363598 & -1.446419 \\
\hline $\mathrm{H}$ & -8.231719 & 3.865333 & -2.243451 \\
\hline $\mathrm{H}$ & -8.309308 & 2.578602 & -1.030592 \\
\hline $\mathrm{H}$ & -7.479311 & 4.094738 & -0.652431 \\
\hline C & -5.544917 & 3.957538 & -2.620019 \\
\hline $\mathrm{H}$ & -5.319811 & 4.736337 & -1.882743 \\
\hline $\mathrm{H}$ & -4.599471 & 3.618312 & -3.058383 \\
\hline $\mathrm{H}$ & -6.132189 & 4.421363 & -3.420637 \\
\hline C & -7.530298 & -0.280464 & 2.242875 \\
\hline $\mathrm{H}$ & -7.287026 & 0.492283 & 2.981117 \\
\hline H & -8.216216 & 0.160810 & 1.511925 \\
\hline
\end{tabular}




$\begin{array}{|lrrr|}H & -8.065289 & -1.087473 & 2.758697 \\ \mathrm{C} & -6.634141 & -1.922332 & 0.548813 \\ \mathrm{H} & -7.292008 & -1.535812 & -0.236735 \\ \mathrm{H} & -5.745849 & -2.341633 & 0.064899 \\ \mathrm{H} & -7.164551 & -2.740184 & 1.052035 \\ \mathrm{C} & -5.377815 & -1.481262 & 2.661355 \\ \mathrm{H} & -5.933767 & -2.298345 & 3.135044 \\ \mathrm{H} & -4.455675 & -1.904927 & 2.247557 \\ \mathrm{H} & -5.104070 & -0.769776 & 3.448473 \\ \mathrm{H} & -0.570180 & 2.389851 & -1.839570 \\ \mathrm{H} & -2.154683 & 1.682561 & -1.516351 \\ \mathrm{O} & 1.555417 & 0.530042 & 1.881441 \\ & & \end{array}$

\section{C-Catalyzed reaction TS 106}

\section{Datum}

Value

\begin{tabular}{lr}
\hline B3LYP/6-31G(d) Energy & -3038.953719 \\
\hline B3LYP-D3(BJ)/def2-TZVPP-IEF-PCM(DCM) Energy & -3040.504248 \\
\hline B3LYP-D3(BJ)/def2-TZVPP-IEF-PCM(DCM)//B3LYP/6-31G(d) Free Energy (Quasiharmonic) & -3039.275338 \\
\hline Number of Imaginary Frequencies & 1
\end{tabular}

Frequencies (Top 3 out of 450)

$$
\begin{array}{lr}
\text { 1. } & -333.7591 \mathrm{~cm}^{-1} \\
\text { 2. } & 8.1365 \mathrm{~cm}^{-1} \\
\text { 3. } & 10.1324 \mathrm{~cm}^{-1}
\end{array}
$$

\section{B3LYP/6-31G(d) Molecular Geometry in Cartesian Coordinates}

\begin{tabular}{|rrrr|}
\hline C & 1.278743 & -0.658956 & 0.279559 \\
$H$ & 0.840454 & -0.181477 & 1.162405 \\
H & 2.322497 & -0.338003 & 0.205466 \\
C & -2.228055 & -2.304446 & -0.651045 \\
O & -2.939409 & -3.269667 & -0.846039 \\
O & -1.473999 & -1.791550 & -1.621811 \\
H & -0.804557 & -1.131201 & -1.284327 \\
O & 0.566996 & -0.234761 & -0.895530 \\
$H$ & 0.384695 & 0.736441 & -0.822688 \\
C & 1.404166 & 3.155021 & 3.406217 \\
C & 2.638873 & 2.969061 & 1.595522 \\
C & -0.395742 & 3.478149 & 1.755733 \\
$C$ & 0.275619 & 2.705142 & 2.755796
\end{tabular}




\begin{tabular}{|c|c|c|c|}
\hline $\mathrm{H}$ & 3.059334 & 2.025948 & 1.953516 \\
\hline $\mathrm{H}$ & -0.381613 & 4.554844 & 1.943857 \\
\hline $\mathrm{H}$ & -1.396764 & 3.122775 & 1.505927 \\
\hline C & -0.675980 & 3.111128 & -1.842291 \\
\hline C & 0.151303 & 4.450497 & -1.832955 \\
\hline C & -0.510669 & 2.248105 & -3.093873 \\
\hline $\mathrm{H}$ & -1.097104 & 1.328209 & -2.994256 \\
\hline $\mathrm{H}$ & -0.872643 & 2.779484 & -3.981621 \\
\hline $\mathrm{H}$ & 0.531780 & 1.967440 & -3.259601 \\
\hline C & -2.170101 & 3.327704 & -1.560434 \\
\hline $\mathrm{H}$ & -2.638820 & 2.360388 & -1.353715 \\
\hline $\mathrm{H}$ & -2.681974 & 3.778984 & -2.417418 \\
\hline $\mathrm{H}$ & -2.319770 & 3.975706 & -0.691410 \\
\hline C & -0.598021 & 5.675008 & -2.359881 \\
\hline $\mathrm{H}$ & -0.879329 & 5.543454 & -3.411304 \\
\hline $\mathrm{H}$ & -1.501061 & 5.875910 & -1.778732 \\
\hline $\mathrm{H}$ & 0.047507 & 6.557251 & -2.292323 \\
\hline C & 1.503350 & 4.320994 & -2.552745 \\
\hline $\mathrm{H}$ & 1.378642 & 4.235459 & -3.637770 \\
\hline $\mathrm{H}$ & 2.101382 & 5.215697 & -2.349888 \\
\hline $\mathrm{H}$ & 2.064225 & 3.451478 & -2.198504 \\
\hline 0 & -0.109672 & 2.405116 & -0.710529 \\
\hline 0 & 0.401193 & 4.643226 & -0.424875 \\
\hline B & 0.453076 & 3.364659 & 0.205746 \\
\hline 0 & 1.839203 & 2.852434 & 0.585983 \\
\hline C & 3.501658 & 4.154422 & 1.778069 \\
\hline C & 4.620365 & 4.051437 & 2.621651 \\
\hline C & 3.244335 & 5.363892 & 1.110449 \\
\hline C & 5.469366 & 5.139588 & 2.802096 \\
\hline $\mathrm{H}$ & 4.823405 & 3.112475 & 3.132452 \\
\hline C & 4.096225 & 6.450183 & 1.299714 \\
\hline $\mathrm{H}$ & 2.383057 & 5.438663 & 0.452914 \\
\hline C & 5.206633 & 6.343287 & 2.141942 \\
\hline $\mathrm{H}$ & 6.335688 & 5.050043 & 3.451495 \\
\hline $\mathrm{H}$ & 3.896680 & 7.384721 & 0.782492 \\
\hline $\mathrm{H}$ & 5.868607 & 7.193807 & 2.280231 \\
\hline $\mathrm{H}$ & 0.029311 & 1.645734 & 2.832872 \\
\hline $\mathrm{H}$ & 1.572427 & 4.221793 & 3.512616 \\
\hline $\mathrm{H}$ & 1.917822 & 2.520167 & 4.123019 \\
\hline C & -1.048903 & -1.879059 & 1.511183 \\
\hline C & -2.159766 & -1.633492 & 0.712596 \\
\hline C & -3.253359 & -0.825269 & 1.187081 \\
\hline C & -3.158744 & -0.287490 & 2.457882 \\
\hline $\mathrm{H}$ & -3.980866 & 0.313845 & 2.836546 \\
\hline C & 1.204383 & -2.165774 & 0.423922 \\
\hline C & 0.080111 & -2.741398 & 1.013211 \\
\hline C & 2.210146 & -4.363281 & 0.184824 \\
\hline C & 2.287648 & -2.997673 & -0.018888 \\
\hline $\mathrm{H}$ & 3.025399 & -4.996363 & -0.155973 \\
\hline C & 1.087630 & -4.976961 & 0.785792 \\
\hline C & -0.010332 & -4.159125 & 1.200444 \\
\hline C & -1.139902 & -4.792407 & 1.790179 \\
\hline C & -1.172889 & -6.157123 & 1.965650 \\
\hline & -0.082098 & -6.963159 & 1.558745 \\
\hline
\end{tabular}




\begin{tabular}{|c|c|c|c|}
\hline C & 1.021722 & -6.383858 & 0.978748 \\
\hline $\mathrm{H}$ & -1.989337 & -4.188357 & 2.088121 \\
\hline $\mathrm{H}$ & -2.047319 & -6.622062 & 2.412495 \\
\hline $\mathrm{H}$ & -0.124057 & -8.039768 & 1.700552 \\
\hline $\mathrm{H}$ & 1.862265 & -6.993255 & 0.655134 \\
\hline C & -2.052546 & -0.522599 & 3.308601 \\
\hline C & -0.976833 & -1.339216 & 2.838427 \\
\hline C & 0.121492 & -1.578504 & 3.711206 \\
\hline C & 0.153188 & -1.034333 & 4.977138 \\
\hline C & -0.912783 & -0.224927 & 5.438939 \\
\hline C & -1.991523 & 0.022359 & 4.620630 \\
\hline $\mathrm{H}$ & 0.938448 & -2.204471 & 3.368284 \\
\hline $\mathrm{H}$ & 0.998392 & -1.235112 & 5.630212 \\
\hline $\mathrm{H}$ & -0.878703 & 0.194709 & 6.440733 \\
\hline $\mathrm{H}$ & -2.818339 & 0.638188 & 4.966717 \\
\hline C & 5.804599 & -1.586110 & -2.002810 \\
\hline C & 4.558174 & -1.185810 & -2.492126 \\
\hline C & 3.411657 & -1.635969 & -1.817396 \\
\hline C & 3.506175 & -2.458910 & -0.692096 \\
\hline C & 4.779870 & -2.835874 & -0.229029 \\
\hline C & 5.943908 & -2.409577 & -0.870416 \\
\hline $\mathrm{H}$ & 6.701700 & -1.251239 & -2.510517 \\
\hline $\mathrm{H}$ & 2.425162 & -1.353413 & -2.169877 \\
\hline $\mathrm{H}$ & 4.836281 & -3.462239 & 0.653845 \\
\hline C & 4.396943 & -0.281698 & -3.729126 \\
\hline C & 7.353020 & -2.803507 & -0.385651 \\
\hline C & 3.658833 & 1.013176 & -3.313564 \\
\hline $\mathrm{H}$ & 3.495817 & 1.657331 & -4.186667 \\
\hline $\mathrm{H}$ & 2.684742 & 0.795716 & -2.863775 \\
\hline $\mathrm{H}$ & 4.246019 & 1.579049 & -2.580454 \\
\hline C & 5.747339 & 0.113861 & -4.354736 \\
\hline $\mathrm{H}$ & 5.575323 & 0.758173 & -5.224491 \\
\hline H & 6.376703 & 0.671276 & -3.651395 \\
\hline $\mathrm{H}$ & 6.309962 & -0.761622 & -4.698887 \\
\hline C & 3.566811 & -1.021841 & -4.804793 \\
\hline $\mathrm{H}$ & 3.436653 & -0.386350 & -5.689521 \\
\hline H & 4.068706 & -1.943884 & -5.119977 \\
\hline $\mathrm{H}$ & 2.571418 & -1.291687 & -4.438582 \\
\hline C & 7.316194 & -3.696600 & 0.869183 \\
\hline $\mathrm{H}$ & 8.338871 & -3.948584 & 1.172193 \\
\hline H & 6.835676 & -3.192638 & 1.715576 \\
\hline $\mathrm{H}$ & 6.785116 & -4.637274 & 0.685325 \\
\hline C & 8.083711 & -3.582258 & -1.505497 \\
\hline H & 9.093479 & -3.862303 & -1.180521 \\
\hline $\mathrm{H}$ & 7.541913 & -4.500136 & -1.760837 \\
\hline $\mathrm{H}$ & 8.180859 & -2.987694 & -2.419786 \\
\hline C & 8.157776 & -1.527658 & -0.041234 \\
\hline $\mathrm{H}$ & 9.166176 & -1.791486 & 0.301221 \\
\hline H & 8.262580 & -0.865820 & -0.906947 \\
\hline $\mathrm{H}$ & 7.665930 & -0.959107 & 0.756561 \\
\hline C & -4.474944 & -0.506093 & 0.385484 \\
\hline C & -4.916968 & 0.826355 & 0.317671 \\
\hline C & -5.241587 & -1.507912 & -0.225712 \\
\hline c & -6.097277 & 1.172455 & -0.349807 \\
\hline
\end{tabular}




\begin{tabular}{|c|c|c|c|}
\hline $\mathrm{H}$ & -4.314772 & 1.592015 & 0.793471 \\
\hline C & -6.414606 & -1.199058 & -0.923801 \\
\hline H & -4.904442 & -2.530561 & -0.149025 \\
\hline C & -6.817393 & 0.141918 & -0.970517 \\
\hline $\mathrm{H}$ & -7.726616 & 0.393936 & -1.508338 \\
\hline C & -7.251728 & -2.279361 & -1.636130 \\
\hline C & -6.619336 & 2.621190 & -0.426133 \\
\hline C & -6.669895 & -3.692662 & -1.439579 \\
\hline $\mathrm{H}$ & -5.644345 & -3.772304 & -1.814506 \\
\hline $\mathrm{H}$ & -6.666420 & -3.985775 & -0.383391 \\
\hline H & -7.284829 & -4.420615 & -1.981518 \\
\hline C & -8.697001 & -2.271147 & -1.085388 \\
\hline $\mathrm{H}$ & -9.299852 & -3.037941 & -1.587512 \\
\hline $\mathrm{H}$ & -8.707209 & -2.482899 & -0.009951 \\
\hline $\mathrm{H}$ & -9.192416 & -1.306445 & -1.240412 \\
\hline C & -7.280186 & -1.977138 & -3.153667 \\
\hline $\mathrm{H}$ & -7.719635 & -0.996362 & -3.366844 \\
\hline $\mathrm{H}$ & -6.267838 & -1.989374 & -3.572578 \\
\hline $\mathrm{H}$ & -7.876353 & -2.731866 & -3.681502 \\
\hline C & -5.748974 & 3.605030 & 0.378845 \\
\hline $\mathrm{H}$ & -5.710584 & 3.337359 & 1.441203 \\
\hline $\mathrm{H}$ & -4.722417 & 3.649758 & -0.000112 \\
\hline $\mathrm{H}$ & -6.170635 & 4.614164 & 0.306936 \\
\hline C & -6.637645 & 3.085682 & -1.901881 \\
\hline $\mathrm{H}$ & -5.629963 & 3.062641 & -2.332207 \\
\hline $\mathrm{H}$ & -7.277808 & 2.446998 & -2.519611 \\
\hline $\mathrm{H}$ & -7.016768 & 4.112611 & -1.977055 \\
\hline C & -8.055344 & 2.685755 & 0.146885 \\
\hline $\mathrm{H}$ & -8.437811 & 3.712974 & 0.100492 \\
\hline $\mathrm{H}$ & -8.749431 & 2.048302 & -0.409974 \\
\hline H & -8.073600 & 2.363100 & 1.194221 \\
\hline
\end{tabular}

\section{C-Catalyzed reaction TS 107}

Datum

Value

\begin{tabular}{lr}
\hline B3LYP/6-31G(d) Energy & -3038.945908 \\
\hline B3LYP-D3(BJ)/def2-TZVPP-IEF-PCM(DCM) Energy & -3040.501423 \\
\hline B3LYP-D3(BJ)/def2-TZVPP-IEF-PCM(DCM)//B3LYP/6-31G(d) Free Energy (Quasiharmonic) & -3039.274166 \\
\hline Number of Imaginary Frequencies & 1
\end{tabular}

Frequencies (Top 3 out of 450)

$\begin{array}{lr}\text { 1. } & -263.4838 \mathrm{~cm}^{-1} \\ \text { 2. } & 6.1875 \mathrm{~cm}^{-1} \\ \text { 3. } & 10.9655 \mathrm{~cm}^{-1}\end{array}$




\begin{tabular}{|c|c|c|c|}
\hline C & 3.506180 & -0.027010 & 1.632780 \\
\hline $\mathrm{H}$ & 3.392690 & 1.020830 & 1.333370 \\
\hline $\mathrm{H}$ & 2.718710 & -0.236800 & 2.355610 \\
\hline C & 0.250720 & 0.113690 & 0.099670 \\
\hline 0 & 1.042460 & 1.035350 & 0.052330 \\
\hline 0 & -0.639770 & -0.056580 & -0.882920 \\
\hline H & -1.249040 & -0.831030 & -0.778410 \\
\hline 0 & 4.732530 & -0.240931 & 2.330490 \\
\hline $\mathrm{H}$ & 5.444810 & 0.108509 & 1.771420 \\
\hline C & -2.919590 & 2.181051 & -3.280150 \\
\hline C & -4.207940 & 1.516711 & -1.587680 \\
\hline C & -2.633440 & -0.254289 & -3.543110 \\
\hline C & -3.188040 & 1.010571 & -3.945970 \\
\hline $\mathrm{H}$ & -3.723450 & 2.294361 & -0.992100 \\
\hline $\mathrm{H}$ & -1.612220 & -0.172750 & -3.165640 \\
\hline $\mathrm{H}$ & -2.721210 & -1.037519 & -4.299330 \\
\hline C & -3.214600 & -3.117389 & -1.441230 \\
\hline C & -4.731290 & -2.846509 & -1.767670 \\
\hline C & -2.490581 & -3.878580 & -2.562800 \\
\hline $\mathrm{H}$ & -1.411011 & -3.817630 & -2.395980 \\
\hline $\mathrm{H}$ & -2.777441 & -4.936059 & -2.586120 \\
\hline $\mathrm{H}$ & -2.706470 & -3.442789 & -3.542810 \\
\hline C & -2.956461 & -3.800689 & -0.098030 \\
\hline $\mathrm{H}$ & -3.429151 & -4.789219 & -0.063420 \\
\hline $\mathrm{H}$ & -3.331980 & -3.203009 & 0.735470 \\
\hline $\mathrm{H}$ & -1.880301 & -3.939470 & 0.049370 \\
\hline C & -5.552780 & -2.505759 & -0.513000 \\
\hline $\mathrm{H}$ & -5.701570 & -3.382699 & 0.126790 \\
\hline $\mathrm{H}$ & -5.063700 & -1.722929 & 0.074090 \\
\hline $\mathrm{H}$ & -6.537960 & -2.137509 & -0.818160 \\
\hline C & -5.429361 & -3.952699 & -2.559500 \\
\hline $\mathrm{H}$ & -5.439931 & -4.894919 & -1.998770 \\
\hline $\mathrm{H}$ & -6.468690 & -3.666539 & -2.754340 \\
\hline $\mathrm{H}$ & -4.942701 & -4.124059 & -3.522370 \\
\hline 0 & -2.680430 & -1.777709 & -1.389430 \\
\hline 0 & -4.654530 & -1.671899 & -2.599550 \\
\hline B & -3.513130 & -0.910339 & -2.192410 \\
\hline 0 & -3.784050 & 0.329211 & -1.332840 \\
\hline C & -5.578070 & 1.857711 & -2.035790 \\
\hline C & -6.476870 & 0.910151 & -2.553410 \\
\hline C & -5.993919 & 3.192421 & -1.888930 \\
\hline C & -7.761380 & 1.305771 & -2.923390 \\
\hline $\mathrm{H}$ & -6.160120 & -0.122569 & -2.662890 \\
\hline C & -7.278749 & 3.580231 & -2.259280 \\
\hline $\mathrm{H}$ & -5.302689 & 3.926831 & -1.480650 \\
\hline C & -8.166619 & 2.635371 & -2.779710 \\
\hline $\mathrm{H}$ & -8.453010 & 0.569221 & -3.323650 \\
\hline $\mathrm{H}$ & -7.588689 & 4.614641 & -2.139140 \\
\hline $\mathrm{H}$ & -9.170939 & 2.933682 & -3.068260 \\
\hline $\mathrm{H}$ & -4.007910 & 1.008541 & -4.665240 \\
\hline
\end{tabular}




\begin{tabular}{|c|c|c|c|}
\hline $\mathrm{H}$ & -2.033450 & 2.250650 & -2.653990 \\
\hline H & -3.384229 & 3.115751 & -3.579250 \\
\hline C & 1.209420 & -1.844360 & 1.331920 \\
\hline C & 0.206700 & -0.882330 & 1.241130 \\
\hline C & -0.843230 & -0.799270 & 2.218530 \\
\hline C & -0.878800 & -1.752550 & 3.219310 \\
\hline H & -1.648890 & -1.690390 & 3.983670 \\
\hline C & 3.367990 & -0.948080 & 0.424710 \\
\hline C & 2.330370 & -1.867960 & 0.327850 \\
\hline C & 4.363490 & -1.831951 & -1.613850 \\
\hline C & 4.374410 & -0.902271 & -0.597260 \\
\hline $\mathrm{H}$ & 5.128900 & -1.790641 & -2.385480 \\
\hline C & 3.358490 & -2.825810 & -1.704270 \\
\hline C & 2.306220 & -2.834970 & -0.734700 \\
\hline C & 1.295589 & -3.828200 & -0.856650 \\
\hline C & 1.333479 & -4.764290 & -1.867830 \\
\hline C & 2.375139 & -4.748640 & -2.824970 \\
\hline C & 3.362830 & -3.794430 & -2.743100 \\
\hline $\mathrm{H}$ & 0.493819 & -3.855090 & -0.126770 \\
\hline $\mathrm{H}$ & 0.556789 & -5.521690 & -1.930080 \\
\hline $\mathrm{H}$ & 2.391069 & -5.488870 & -3.620300 \\
\hline $\mathrm{H}$ & 4.168170 & -3.767991 & -3.473620 \\
\hline C & 0.095240 & -2.773090 & 3.329040 \\
\hline C & 1.174280 & -2.811480 & 2.389940 \\
\hline C & 2.179449 & -3.804440 & 2.553790 \\
\hline C & 2.106909 & -4.721430 & 3.578600 \\
\hline C & 1.029589 & -4.691210 & 4.496610 \\
\hline C & 0.049140 & -3.734200 & 4.375350 \\
\hline $\mathrm{H}$ & 3.013609 & -3.826970 & 1.861380 \\
\hline $\mathrm{H}$ & 2.885929 & -5.470890 & 3.688130 \\
\hline $\mathrm{H}$ & 0.984949 & -5.420740 & 5.300790 \\
\hline $\mathrm{H}$ & -0.775690 & -3.694100 & 5.082900 \\
\hline C & 7.344670 & 2.172749 & -0.747700 \\
\hline C & 7.765990 & 0.840709 & -0.581810 \\
\hline C & 6.782560 & -0.151231 & -0.521200 \\
\hline C & 5.417540 & 0.170359 & -0.617890 \\
\hline C & 5.041010 & 1.506239 & -0.787360 \\
\hline C & 5.997131 & 2.532199 & -0.855670 \\
\hline $\mathrm{H}$ & 8.099761 & 2.949029 & -0.793310 \\
\hline $\mathrm{H}$ & 7.052720 & -1.192871 & -0.388810 \\
\hline $\mathrm{H}$ & 3.983170 & 1.733789 & -0.882250 \\
\hline C & 9.270560 & 0.526669 & -0.471020 \\
\hline C & 5.533961 & 3.988239 & -1.054270 \\
\hline C & 9.865290 & 1.270119 & 0.748790 \\
\hline $\mathrm{H}$ & 10.938380 & 1.060308 & 0.837300 \\
\hline $\mathrm{H}$ & 9.744140 & 2.355119 & 0.664950 \\
\hline H & 9.379550 & 0.950389 & 1.677830 \\
\hline C & 9.544220 & -0.978941 & -0.291670 \\
\hline $\mathrm{H}$ & 10.623990 & -1.150172 & -0.216390 \\
\hline H & 9.083100 & -1.371941 & 0.621350 \\
\hline $\mathrm{H}$ & 9.175310 & -1.565211 & -1.140920 \\
\hline C & 9.991370 & 0.995158 & -1.757460 \\
\hline $\mathrm{H}$ & 11.065020 & 0.778938 & -1.693470 \\
\hline H & 9.593170 & 0.480139 & -2.639230 \\
\hline
\end{tabular}




\begin{tabular}{|c|c|c|c|}
\hline $\mathrm{H}$ & 9.877960 & 2.071968 & -1.920330 \\
\hline C & 4.587491 & 4.390519 & 0.102040 \\
\hline $\mathrm{H}$ & 4.246281 & 5.424859 & -0.029140 \\
\hline $\mathrm{H}$ & 3.700081 & 3.751229 & 0.146250 \\
\hline $\mathrm{H}$ & 5.098911 & 4.322519 & 1.069230 \\
\hline C & 6.709371 & 4.983119 & -1.079550 \\
\hline $\mathrm{H}$ & 6.327091 & 6.000759 & -1.217770 \\
\hline $\mathrm{H}$ & 7.401791 & 4.776889 & -1.903730 \\
\hline $\mathrm{H}$ & 7.277241 & 4.968989 & -0.142110 \\
\hline C & 4.776291 & 4.104029 & -2.398600 \\
\hline $\mathrm{H}$ & 4.429961 & 5.133509 & -2.552850 \\
\hline $\mathrm{H}$ & 5.426751 & 3.835059 & -3.238990 \\
\hline $\mathrm{H}$ & 3.899741 & 3.449049 & -2.430280 \\
\hline C & -1.838610 & 0.312360 & 2.244390 \\
\hline C & -3.187200 & 0.050111 & 2.531460 \\
\hline C & -1.424300 & 1.642980 & 2.085620 \\
\hline C & -4.121190 & 1.083481 & 2.662090 \\
\hline $\mathrm{H}$ & -3.495940 & -0.981979 & 2.649980 \\
\hline C & -2.331089 & 2.704240 & 2.182340 \\
\hline $\mathrm{H}$ & -0.377020 & 1.842230 & 1.905370 \\
\hline C & -3.668989 & 2.397511 & 2.467430 \\
\hline $\mathrm{H}$ & -4.382819 & 3.211631 & 2.554630 \\
\hline C & -1.896809 & 4.173240 & 2.007230 \\
\hline C & -5.599760 & 0.830281 & 3.019700 \\
\hline C & -2.651749 & 4.792531 & 0.806930 \\
\hline $\mathrm{H}$ & -2.398509 & 4.268760 & -0.122960 \\
\hline $\mathrm{H}$ & -3.738409 & 4.745521 & 0.940530 \\
\hline $\mathrm{H}$ & -2.376049 & 5.847060 & 0.682860 \\
\hline C & -2.235839 & 4.972730 & 3.287730 \\
\hline $\mathrm{H}$ & -1.926269 & 6.019270 & 3.177240 \\
\hline $\mathrm{H}$ & -3.309189 & 4.964791 & 3.505670 \\
\hline $\mathrm{H}$ & -1.715329 & 4.555370 & 4.157020 \\
\hline C & -0.384799 & 4.309490 & 1.741670 \\
\hline $\mathrm{H}$ & 0.208871 & 3.941810 & 2.586130 \\
\hline $\mathrm{H}$ & -0.071279 & 3.761830 & 0.846360 \\
\hline $\mathrm{H}$ & -0.130929 & 5.365830 & 1.595820 \\
\hline C & -5.953590 & 1.601111 & 4.314600 \\
\hline $\mathrm{H}$ & -7.002500 & 1.428011 & 4.585110 \\
\hline $\mathrm{H}$ & -5.814459 & 2.681141 & 4.200990 \\
\hline $\mathrm{H}$ & -5.327710 & 1.270531 & 5.151430 \\
\hline C & -6.506480 & 1.323151 & 1.868060 \\
\hline $\mathrm{H}$ & -6.370479 & 2.392001 & 1.671580 \\
\hline $\mathrm{H}$ & -6.292080 & 0.783751 & 0.939110 \\
\hline $\mathrm{H}$ & -7.562650 & 1.162721 & 2.118000 \\
\hline C & -5.899730 & -0.660909 & 3.263760 \\
\hline $\mathrm{H}$ & -5.712150 & -1.268829 & 2.372200 \\
\hline $\mathrm{H}$ & -6.955770 & -0.785259 & 3.529200 \\
\hline H & -5.301910 & -1.066729 & 4.088030 \\
\hline
\end{tabular}




\begin{tabular}{lc}
\hline B3LYP/6-31G(d) Energy & -3038.954739 \\
\hline B3LYP-D3(BJ)/def2-TZVPP-IEF-PCM(DCM) Energy & -3040.502803 \\
\hline B3LYP-D3(BJ)/def2-TZVPP-IEF-PCM(DCM)//B3LYP/6-31G(d) Free Energy (Quasiharmonic) & -3039.27394 \\
\hline
\end{tabular}

Number of Imaginary Frequencies

Frequencies (Top 3 out of 450)

$\begin{array}{lr}\text { 1. } & -381.3769 \mathrm{~cm}^{-1} \\ \text { 2. } & 10.3557 \mathrm{~cm}^{-1} \\ \text { 3. } & 11.9087 \mathrm{~cm}^{-1}\end{array}$

\section{B3LYP/6-31G(d) Molecular Geometry in Cartesian Coordinates}

C

$\mathrm{H}$

$\mathrm{H}$

C

0

0

$\mathrm{H}$

0

$\mathrm{H}$

C

C

C

C

$\mathrm{H}$

$\mathrm{H}$

$\mathrm{H}$

C

C

C

$\mathrm{H}$

$\mathrm{H}$

$\mathrm{H}$

C

$\mathrm{H}$

$\mathrm{H}$

$\mathrm{H}$

C

$\mathrm{H}$

$\mathrm{H}$

$\mathrm{H}$

C

$\mathrm{H}$

\subsection{6}

2.588283

4.168908

$-0.334012$

0.453293

$-1.457513$

$-1.992399$

2.617049

1.872680

$-3.691285$

$-4.937572$

$-2.714554$

$-2.652333$

$-5.704050$

$-1.776669$

$-3.218839$

$-3.838733$

$-5.216566$

$-2.931182$

$-1.938251$

$-3.320885$

$-2.826654$

$-3.956663$

$-4.473877$

$-2.960089$

$-4.502889$

$-6.308830$

$-6.661445$

$-5.953022$

$-7.161374$

$-5.756861$

$-5.948926$
0.501968

0.278028

0.722279

0.144561

0.863525

$-0.244520$

$-0.905885$

1.689882

1.432934

$-0.976695$

$-0.458643$

$-2.559235$

$-1.390344$

$-1.134195$

$-2.837308$

$-3.410628$

$-3.361497$

$-3.563919$

$-4.596278$

$-4.350290$

$-5.433599$

$-4.923195$

$-2.928386$

$-3.691702$

$-2.785795$

$-1.986922$

$-2.595895$

$-2.851071$

$-1.561907$

$-2.656175$

$-4.995090$

$-5.332299$
1. 341595

2.265915

1.619246

$-0.248864$

$-0.841175$

$-0.859476$

$-0.350968$

0.739457

0.154411

$-4.610934$

$-2.944971$

$-2.981518$

$-3.793091$

$-3.334405$

$-2.500583$

$-3.444712$

0.431545

$-0.313749$

0.329994

0.718130

0.918860

$-0.709018$

1.892657

2. 485964

2. 323003

1.985566

0.174656

1. 180249

0.181107

$-0.509994$

$-0.291546$

0.733897 


\begin{tabular}{|c|c|c|c|}
\hline H & -6.702568 & -5.035708 & -0.842353 \\
\hline $\mathrm{H}$ & -5.063234 & -5.692853 & -0.766446 \\
\hline 0 & -3.231837 & -2.285660 & -0.326919 \\
\hline 0 & -4.883735 & -3.230873 & -1.673910 \\
\hline B & -3.862298 & -2.234360 & -1.624420 \\
\hline 0 & -4.339032 & -0.833848 & -1.850521 \\
\hline C & -5.166631 & 0.987182 & -3.128933 \\
\hline C & -4.450584 & 1.930420 & -2.374144 \\
\hline C & -6.134712 & 1.423853 & -4.046273 \\
\hline C & -4.706252 & 3.289343 & -2.541902 \\
\hline $\mathrm{H}$ & -3.703235 & 1.587187 & -1.666031 \\
\hline C & -6.384273 & 2.784331 & -4.211390 \\
\hline $\mathrm{H}$ & -6.694630 & 0.693427 & -4.626477 \\
\hline C & -5.669674 & 3.720085 & -3.459202 \\
\hline H & -4.153719 & 4.014418 & -1.950999 \\
\hline $\mathrm{H}$ & -7.137626 & 3.114474 & -4.921264 \\
\hline $\mathrm{H}$ & -5.864757 & 4.781586 & -3.585550 \\
\hline $\mathrm{H}$ & -1.890188 & -0.652898 & -3.539686 \\
\hline $\mathrm{H}$ & -4.367833 & -1.719157 & -5.028745 \\
\hline $\mathrm{H}$ & -3.603636 & -0.052670 & -5.173222 \\
\hline C & 0.874298 & -1.280671 & 1.418388 \\
\hline C & -0.145790 & -0.376942 & 1.153769 \\
\hline C & -1.072214 & 0.035702 & 2.170519 \\
\hline C & -1.035510 & -0.604138 & 3.391025 \\
\hline $\mathrm{H}$ & -1.707591 & -0.282396 & 4.182666 \\
\hline C & 3.090434 & -0.703657 & 0.413681 \\
\hline C & 1.972659 & -1.538014 & 0.420954 \\
\hline C & 4.097071 & -2.072932 & -1.325568 \\
\hline C & 4.179626 & -0.978273 & -0.484107 \\
\hline $\mathrm{H}$ & 4.916426 & -2.277759 & -2.009914 \\
\hline C & 2.966778 & -2.921762 & -1.355894 \\
\hline C & 1.876396 & -2.652873 & -0.470538 \\
\hline C & 0.739465 & -3.505144 & -0.521111 \\
\hline C & 0.688155 & -4.570043 & -1.393481 \\
\hline C & 1.773273 & -4.838890 & -2.262758 \\
\hline C & 2.885049 & -4.029127 & -2.243713 \\
\hline $\mathrm{H}$ & -0.102707 & -3.297415 & 0.129667 \\
\hline H & -0.190782 & -5.208489 & -1.414183 \\
\hline $\mathrm{H}$ & 1.721833 & -5.682444 & -2.945864 \\
\hline $\mathrm{H}$ & 3.721179 & -4.222046 & -2.911832 \\
\hline C & -0.091460 & -1.625638 & 3.670307 \\
\hline C & 0.904381 & -1.945782 & 2.691337 \\
\hline C & 1.888117 & -2.918579 & 3.019520 \\
\hline C & 1.876822 & -3.552680 & 4.242569 \\
\hline C & 0.887503 & -3.240269 & 5.204381 \\
\hline C & -0.071662 & -2.293981 & 4.923782 \\
\hline $\mathrm{H}$ & 2.650317 & -3.162367 & 2.287734 \\
\hline $\mathrm{H}$ & 2.634270 & -4.297075 & 4.471991 \\
\hline $\mathrm{H}$ & 0.890715 & -3.745819 & 6.166236 \\
\hline $\mathrm{H}$ & -0.829236 & -2.039127 & 5.661037 \\
\hline C & 7.776034 & 1.330387 & -0.700417 \\
\hline C & 6.542388 & 2.001332 & -0.785066 \\
\hline C & 5.373466 & 1.243084 & -0.698651 \\
\hline C & 5.423057 & -0.151561 & -0.529631 \\
\hline
\end{tabular}




\begin{tabular}{|c|c|c|c|}
\hline C & 6.670026 & -0.780590 & -0.452311 \\
\hline C & 7.868514 & -0.054418 & -0.534548 \\
\hline $\mathrm{H}$ & 8.686537 & 1.914989 & -0.761511 \\
\hline $\mathrm{H}$ & 4.402138 & 1.717906 & -0.744336 \\
\hline $\mathrm{H}$ & 6.697789 & -1.856684 & -0.306070 \\
\hline C & 6.517304 & 3.532907 & -0.953462 \\
\hline C & 9.216341 & -0.795673 & -0.433573 \\
\hline C & 5.083931 & 4.082367 & -1.090022 \\
\hline $\mathrm{H}$ & 5.117463 & 5.173696 & -1.190186 \\
\hline $\mathrm{H}$ & 4.582926 & 3.683677 & -1.979614 \\
\hline $\mathrm{H}$ & 4.463531 & 3.842734 & -0.220241 \\
\hline C & 7.171453 & 4.190290 & 0.285424 \\
\hline $\mathrm{H}$ & 7.175587 & 5.282543 & 0.181295 \\
\hline $\mathrm{H}$ & 6.618756 & 3.938251 & 1.197570 \\
\hline $\mathrm{H}$ & 8.208267 & 3.863396 & 0.420727 \\
\hline C & 7.307813 & 3.933123 & -2.221627 \\
\hline $\mathrm{H}$ & 7.293139 & 5.022388 & -2.351230 \\
\hline $\mathrm{H}$ & 8.356194 & 3.620004 & -2.170331 \\
\hline $\mathrm{H}$ & 6.866689 & 3.479055 & -3.116419 \\
\hline C & 10.422979 & 0.154000 & -0.556386 \\
\hline $\mathrm{H}$ & 11.353580 & -0.420944 & -0.487541 \\
\hline $\mathrm{H}$ & 10.430586 & 0.681712 & -1.516893 \\
\hline $\mathrm{H}$ & 10.437269 & 0.901446 & 0.244914 \\
\hline C & 9.309146 & -1.514122 & 0.933637 \\
\hline $\mathrm{H}$ & 10.260293 & -2.054527 & 1.018433 \\
\hline $\mathrm{H}$ & 9.252732 & -0.793976 & 1.757960 \\
\hline $\mathrm{H}$ & 8.500260 & -2.239654 & 1.069207 \\
\hline C & 9.314882 & -1.842804 & -1.568528 \\
\hline $\mathrm{H}$ & 10.266665 & -2.385222 & -1.507685 \\
\hline $\mathrm{H}$ & 8.507639 & -2.580500 & -1.513529 \\
\hline $\mathrm{H}$ & 9.260361 & -1.360674 & -2.551364 \\
\hline C & -1.926885 & 1.239273 & 1.966597 \\
\hline C & -1.321383 & 2.414671 & 1.500534 \\
\hline C & -3.286367 & 1.258413 & 2.299075 \\
\hline C & -2.046536 & 3.601220 & 1.351157 \\
\hline $\mathrm{H}$ & -0.259205 & 2.399584 & 1.281931 \\
\hline C & -4.049542 & 2.426220 & 2.164863 \\
\hline $\mathrm{H}$ & -3.748141 & 0.340772 & 2.652121 \\
\hline C & -3.407725 & 3.580513 & 1.688924 \\
\hline $\mathrm{H}$ & -3.983531 & 4.490121 & 1.585813 \\
\hline C & -5.546338 & 2.408145 & 2.534327 \\
\hline C & -1.329573 & 4.858703 & 0.822592 \\
\hline C & -5.704882 & 2.014214 & 4.022286 \\
\hline $\mathrm{H}$ & -5.281394 & 1.025954 & 4.229792 \\
\hline $\mathrm{H}$ & -5.201931 & 2.737189 & 4.674821 \\
\hline $\mathrm{H}$ & -6.765944 & 1.986549 & 4.299519 \\
\hline C & -6.223366 & 3.776820 & 2.329617 \\
\hline $\mathrm{H}$ & -7.280456 & 3.709127 & 2.610291 \\
\hline $\mathrm{H}$ & -5.766636 & 4.556584 & 2.949740 \\
\hline $\mathrm{H}$ & -6.180445 & 4.100534 & 1.283543 \\
\hline C & -6.281961 & 1.374384 & 1.649415 \\
\hline $\mathrm{H}$ & -6.182556 & 1.624841 & 0.587196 \\
\hline $\mathrm{H}$ & -5.884758 & 0.364106 & 1.790694 \\
\hline H & -7.350395 & 1.349873 & 1.897540 \\
\hline
\end{tabular}




\begin{tabular}{|lrlr|} 
C & -0.872149 & 4.600919 & -0.633735 \\
$H$ & -0.223650 & 3.722590 & -0.709441 \\
$H$ & -1.733774 & 4.432357 & -1.291180 \\
$H$ & -0.318552 & 5.466468 & -1.018497 \\
$\mathrm{C}$ & -2.237620 & 6.102740 & 0.834313 \\
$\mathrm{H}$ & -3.114609 & 5.977995 & 0.188278 \\
$\mathrm{H}$ & -2.589221 & 6.342545 & 1.844647 \\
$\mathrm{H}$ & -1.679313 & 6.969267 & 0.462577 \\
$\mathrm{C}$ & -0.088256 & 5.162604 & 1.696536 \\
$\mathrm{H}$ & 0.408824 & 6.071999 & 1.337674 \\
$\mathrm{H}$ & -0.373243 & 5.322180 & 2.743025 \\
$\mathrm{H}$ & 0.647592 & 4.352987 & 1.665806 \\
& & & \\
\hline
\end{tabular}

\section{C-Catalyzed reaction TS 109}

\section{Datum}

Value

\begin{tabular}{lc}
\hline B3LYP/6-31G(d) Energy & -3038.950623 \\
\hline B3LYP-D3(BJ)/def2-TZVPP-IEF-PCM(DCM) Energy & -3040.502222 \\
\hline B3LYP-D3(BJ)/def2-TZVPP-IEF-PCM(DCM)//B3LYP/6-31G(d) Free Energy (Quasiharmonic) & -3039.273648 \\
\hline
\end{tabular}

Number of Imaginary Frequencies

Frequencies (Top 3 out of 450)
1. $-376.5853 \mathrm{~cm}^{-1}$
2. $8.3084 \mathrm{~cm}^{-1}$
3. $11.3314 \mathrm{~cm}^{-1}$

\section{B3LYP/6-31G(d) Molecular Geometry in Cartesian Coordinates}

\begin{tabular}{|c|c|c|c|}
\hline C & -1.453950 & 0.596472 & 1.113934 \\
\hline $\mathrm{H}$ & -2.175489 & -0.201603 & 0.937970 \\
\hline $\mathrm{H}$ & -0.457775 & 0.165877 & 0.980820 \\
\hline C & 1.520634 & 0.836822 & -1.232368 \\
\hline 0 & 1.362518 & 1.289069 & -2.346411 \\
\hline 0 & 1.485790 & -0.487893 & -1.021207 \\
\hline $\mathrm{H}$ & 1.529337 & -0.754317 & -0.067240 \\
\hline 0 & -1.642819 & 0.977067 & 2.488272 \\
\hline $\mathrm{H}$ & -0.997399 & 1.674470 & 2.690448 \\
\hline C & 0.501948 & -5.043672 & -1.922981 \\
\hline C & -0.962512 & -3.761381 & -0.951297 \\
\hline C & 2.087492 & -3.881995 & -0.429632 \\
\hline C & 1.505727 & -4.118823 & -1.707381 \\
\hline $\mathrm{H}$ & -1.313245 & -4.641640 & -0.406387 \\
\hline
\end{tabular}




\begin{tabular}{|c|c|c|c|}
\hline $\mathrm{H}$ & 2.916043 & -3.174513 & -0.423792 \\
\hline $\mathrm{H}$ & 2.297965 & -4.782955 & 0.149693 \\
\hline C & 1.485886 & -2.205715 & 2.679332 \\
\hline C & 0.504953 & -3.410311 & 2.947015 \\
\hline C & 2.963680 & -2.595216 & 2.845057 \\
\hline $\mathrm{H}$ & 3.585196 & -1.808704 & 2.405891 \\
\hline $\mathrm{H}$ & 3.237558 & -2.703475 & 3.900203 \\
\hline $\mathrm{H}$ & 3.192667 & -3.534183 & 2.332376 \\
\hline C & 1.193971 & -0.939396 & 3.483421 \\
\hline $\mathrm{H}$ & 1.307723 & -1.133726 & 4.556748 \\
\hline $\mathrm{H}$ & 0.186278 & -0.557516 & 3.305314 \\
\hline $\mathrm{H}$ & 1.909672 & -0.155169 & 3.211394 \\
\hline C & -0.938976 & -2.955680 & 3.213099 \\
\hline $\mathrm{H}$ & -1.044946 & -2.481783 & 4.194844 \\
\hline $\mathrm{H}$ & -1.281870 & -2.248202 & 2.453513 \\
\hline $\mathrm{H}$ & -1.593363 & -3.833350 & 3.183747 \\
\hline C & 0.957004 & -4.368546 & 4.049846 \\
\hline $\mathrm{H}$ & 1.026473 & -3.854375 & 5.015732 \\
\hline $\mathrm{H}$ & 0.227943 & -5.179427 & 4.152131 \\
\hline $\mathrm{H}$ & 1.926776 & -4.816894 & 3.821039 \\
\hline 0 & 1.251138 & -1.940707 & 1.272942 \\
\hline 0 & 0.534648 & -4.117607 & 1.689866 \\
\hline B & 0.807712 & -3.168591 & 0.662594 \\
\hline 0 & -0.316492 & -2.864964 & -0.264936 \\
\hline C & -1.774912 & -3.295138 & -2.089700 \\
\hline C & -2.812511 & -4.105350 & -2.576222 \\
\hline C & -1.517645 & -2.055425 & -2.698410 \\
\hline C & -3.578944 & -3.689178 & -3.662677 \\
\hline $\mathrm{H}$ & -3.015623 & -5.062874 & -2.101353 \\
\hline C & -2.285206 & -1.645592 & -3.785920 \\
\hline $\mathrm{H}$ & -0.715516 & -1.429373 & -2.319749 \\
\hline C & -3.312622 & -2.460058 & -4.273021 \\
\hline H & -4.380114 & -4.321759 & -4.034720 \\
\hline $\mathrm{H}$ & -2.077866 & -0.688537 & -4.256216 \\
\hline $\mathrm{H}$ & -3.903232 & -2.138985 & -5.126805 \\
\hline $\mathrm{H}$ & 1.648960 & -3.354259 & -2.472399 \\
\hline H & 0.402831 & -5.891093 & -1.247673 \\
\hline $\mathrm{H}$ & 0.072372 & -5.170310 & -2.911053 \\
\hline C & 0.715908 & 2.462735 & 0.479469 \\
\hline C & 1.766045 & 1.696221 & -0.011412 \\
\hline C & 3.060901 & 1.701204 & 0.613240 \\
\hline C & 3.226103 & 2.445397 & 1.766400 \\
\hline $\mathrm{H}$ & 4.205970 & 2.490799 & 2.233934 \\
\hline C & -1.677155 & 1.726910 & 0.125289 \\
\hline C & -0.613670 & 2.555954 & -0.225952 \\
\hline C & -3.124420 & 2.924119 & -1.417260 \\
\hline C & -2.973102 & 1.930323 & -0.468582 \\
\hline $\mathrm{H}$ & -4.104640 & 3.094444 & -1.855316 \\
\hline C & -2.057540 & 3.763311 & -1.814435 \\
\hline C & -0.776052 & 3.591633 & -1.204855 \\
\hline C & 0.279730 & 4.458148 & -1.600652 \\
\hline C & 0.075143 & 5.440041 & -2.543677 \\
\hline C & -1.195984 & 5.609152 & -3.143158 \\
\hline C & -2.237929 & 4.786254 & -2.784924 \\
\hline
\end{tabular}




\begin{tabular}{|c|c|c|c|}
\hline H & 1.261611 & 4.330560 & -1.159437 \\
\hline $\mathrm{H}$ & 0.897229 & 6.088453 & -2.834474 \\
\hline $\mathrm{H}$ & -1.343778 & 6.388101 & -3.886440 \\
\hline $\mathrm{H}$ & -3.219387 & 4.905601 & -3.238069 \\
\hline C & 2.173165 & 3.199388 & 2.336838 \\
\hline C & 0.900851 & 3.229934 & 1.680357 \\
\hline C & -0.132234 & 4.028877 & 2.251004 \\
\hline C & 0.081229 & 4.741995 & 3.411261 \\
\hline C & 1.338943 & 4.704124 & 4.058437 \\
\hline C & 2.361423 & 3.952118 & 3.527729 \\
\hline $\mathrm{H}$ & -1.095741 & 4.077942 & 1.755551 \\
\hline H & -0.721118 & 5.344181 & 3.828374 \\
\hline $\mathrm{H}$ & 1.494402 & 5.272536 & 4.971206 \\
\hline $\mathrm{H}$ & 3.333852 & 3.920611 & 4.013329 \\
\hline C & -6.508005 & -0.240826 & 0.586685 \\
\hline C & -5.697957 & 0.252960 & 1.612411 \\
\hline C & -4.533561 & 0.949035 & 1.251370 \\
\hline C & -4.178155 & 1.134365 & -0.088122 \\
\hline C & -5.019412 & 0.616861 & -1.090344 \\
\hline C & -6.192854 & -0.069628 & -0.773976 \\
\hline $\mathrm{H}$ & -7.412354 & -0.780254 & 0.842910 \\
\hline H & -3.884204 & 1.353018 & 2.019746 \\
\hline $\mathrm{H}$ & -4.723219 & 0.749352 & -2.124384 \\
\hline C & -6.029359 & 0.067403 & 3.105813 \\
\hline C & -7.133958 & -0.648285 & -1.848563 \\
\hline C & -7.334685 & -0.719853 & 3.327593 \\
\hline $\mathrm{H}$ & -7.521472 & -0.828518 & 4.402164 \\
\hline H & -8.201177 & -0.208283 & 2.892459 \\
\hline $\mathrm{H}$ & -7.280948 & -1.727181 & 2.898780 \\
\hline C & -4.876352 & -0.704244 & 3.792751 \\
\hline $\mathrm{H}$ & -5.085045 & -0.824800 & 4.863437 \\
\hline $\mathrm{H}$ & -4.763968 & -1.703802 & 3.356230 \\
\hline $\mathrm{H}$ & -3.917916 & -0.185894 & 3.690658 \\
\hline C & -6.185094 & 1.454124 & 3.774343 \\
\hline $\mathrm{H}$ & -6.418771 & 1.339733 & 4.840226 \\
\hline $\mathrm{H}$ & -5.268564 & 2.047308 & 3.695604 \\
\hline $\mathrm{H}$ & -6.996019 & 2.025724 & 3.307883 \\
\hline C & -6.660453 & -0.327273 & -3.278983 \\
\hline $\mathrm{H}$ & -7.368815 & -0.745347 & -4.003820 \\
\hline $\mathrm{H}$ & -6.604570 & 0.752922 & -3.456577 \\
\hline $\mathrm{H}$ & -5.676620 & -0.760883 & -3.488453 \\
\hline C & -7.200730 & -2.187205 & -1.699255 \\
\hline $\mathrm{H}$ & -7.874936 & -2.615693 & -2.451789 \\
\hline $\mathrm{H}$ & -7.571591 & -2.483818 & -0.712390 \\
\hline $\mathrm{H}$ & -6.209610 & -2.635032 & -1.834327 \\
\hline C & -8.551642 & -0.054124 & -1.671631 \\
\hline $\mathrm{H}$ & -9.232922 & -0.460771 & -2.429369 \\
\hline $\mathrm{H}$ & -8.973320 & -0.285142 & -0.688139 \\
\hline $\mathrm{H}$ & -8.534873 & 1.036499 & -1.779917 \\
\hline C & 4.235495 & 0.993868 & 0.026133 \\
\hline C & 4.494113 & 1.052237 & -1.346241 \\
\hline C & 5.144221 & 0.314586 & 0.859451 \\
\hline C & 5.618155 & 0.428920 & -1.909694 \\
\hline H & 3.813213 & 1.601380 & -1.985873 \\
\hline
\end{tabular}




$\begin{array}{lrrr}\text { C } & 6.284275 & -0.302682 & 0.342134 \\ \text { H } & 4.934227 & 0.271006 & 1.921844 \\ \text { C } & 6.492949 & -0.237564 & -1.048346 \\ \text { H } & 7.369033 & -0.723901 & -1.461227 \\ \text { C } & 7.303015 & -1.048415 & 1.226447 \\ \text { C } & 5.834084 & 0.504109 & -3.433002 \\ \text { C } & 7.350754 & -2.538533 & 0.812141 \\ \text { H } & 6.371868 & -3.013482 & 0.943816 \\ \text { H } & 7.641831 & -2.660238 & -0.236527 \\ \text { H } & 8.078584 & -3.083059 & 1.426365 \\ \text { C } & 8.705490 & -0.421076 & 1.041011 \\ \text { H } & 9.441810 & -0.945794 & 1.662058 \\ \text { H } & 9.047122 & -0.478131 & 0.002554 \\ \text { H } & 8.704138 & 0.634914 & 1.334602 \\ \text { C } & 6.948244 & -0.977433 & 2.723773 \\ \text { H } & 6.911908 & 0.056960 & 3.084681 \\ \text { H } & 5.983840 & -1.449806 & 2.940329 \\ \text { H } & 7.710517 & -1.504511 & 3.308704 \\ \text { C } & 5.927219 & 1.985553 & -3.870387 \\ \text { H } & 5.014951 & 2.541338 & -3.631455 \\ \text { H } & 6.767217 & 2.488802 & -3.377326 \\ \text { H } & 6.080492 & 2.051633 & -4.954495 \\ \text { C } & 7.124289 & -0.207805 & -3.880824 \\ \text { H } & 8.016908 & 0.238611 & -3.427121 \\ \text { H } & 7.110188 & -1.275077 & -3.631367 \\ \text { H } & 7.231041 & -0.126457 & -4.968445 \\ \text { C } & 4.635627 & -0.166971 & -4.147615 \\ \text { H } & 4.756778 & -0.098681 & -5.235934 \\ \text { H } & 4.569745 & -1.228305 & -3.880489 \\ \text { H } & 3.682409 & 0.301768 & -3.883653\end{array}$

\section{C-Catalyzed reaction TS 110}

\section{Datum}

Value

\begin{tabular}{lc}
\hline B3LYP/6-31G(d) Energy & -3038.950959 \\
\hline B3LYP-D3(BJ)/def2-TZVPP-IEF-PCM(DCM) Energy & -3040.501124 \\
\hline B3LYP-D3(BJ)/def2-TZVPP-IEF-PCM(DCM)//B3LYP/6-31G(d) Free Energy (Quasiharmonic) & -3039.273247 \\
\hline
\end{tabular}

Number of Imaginary Frequencies

Frequencies (Top 3 out of 450)

$\begin{array}{lr}\text { 1. } & -287.3262 \mathrm{~cm}^{-1} \\ \text { 2. } & 7.7072 \mathrm{~cm}^{-1} \\ \text { 3. } & 9.3178 \mathrm{~cm}^{-1}\end{array}$




\begin{tabular}{|c|c|c|c|}
\hline C & -1.932964 & -0.741938 & 0.937844 \\
\hline $\mathrm{H}$ & -0.873735 & -0.476684 & 0.903566 \\
\hline $\mathrm{H}$ & -2.500584 & 0.156397 & 0.693556 \\
\hline C & 1.099069 & -1.440622 & -1.267512 \\
\hline 0 & 0.972525 & -1.931569 & -2.369103 \\
\hline 0 & 1.192511 & -0.110905 & -1.118256 \\
\hline $\mathrm{H}$ & 1.218525 & 0.206627 & -0.179374 \\
\hline 0 & -2.312007 & -1.064086 & 2.287369 \\
\hline $\mathrm{H}$ & -1.805803 & -1.849562 & 2.553352 \\
\hline C & 0.075686 & 3.695497 & -2.636547 \\
\hline C & 1.905796 & 3.891893 & -1.405771 \\
\hline C & -0.768173 & 2.618197 & -0.586673 \\
\hline C & -0.716620 & 3.713692 & -1.513293 \\
\hline $\mathrm{H}$ & 2.455839 & 3.496022 & -2.264275 \\
\hline $\mathrm{H}$ & -0.654902 & 1.641848 & -1.062526 \\
\hline $\mathrm{H}$ & -1.623633 & 2.644645 & 0.091595 \\
\hline C & 1.225077 & 1.676761 & 2.488955 \\
\hline C & 0.424745 & 2.988387 & 2.823637 \\
\hline C & 0.852559 & 0.459147 & 3.333599 \\
\hline $\mathrm{H}$ & 1.448966 & -0.405847 & 3.023127 \\
\hline $\mathrm{H}$ & 1.067687 & 0.647446 & 4.392335 \\
\hline $\mathrm{H}$ & -0.203549 & 0.197188 & 3.234284 \\
\hline C & 2.747071 & 1.889613 & 2.512042 \\
\hline $\mathrm{H}$ & 3.123871 & 1.990444 & 3.536092 \\
\hline $\mathrm{H}$ & 3.233426 & 1.026506 & 2.047682 \\
\hline $\mathrm{H}$ & 3.035462 & 2.779760 & 1.945734 \\
\hline C & 1.082408 & 3.893578 & 3.867645 \\
\hline $\mathrm{H}$ & 1.171492 & 3.383026 & 4.833841 \\
\hline $\mathrm{H}$ & 2.076605 & 4.217995 & 3.550955 \\
\hline $\mathrm{H}$ & 0.466839 & 4.787370 & 4.016960 \\
\hline C & -1.033549 & 2.714351 & 3.227958 \\
\hline H & -1.105216 & 2.299793 & 4.239402 \\
\hline $\mathrm{H}$ & -1.586227 & 3.659439 & 3.204994 \\
\hline $\mathrm{H}$ & -1.518880 & 2.014728 & 2.541786 \\
\hline 0 & 0.828018 & 1.440979 & 1.113296 \\
\hline 0 & 0.419381 & 3.679551 & 1.558881 \\
\hline B & 0.584707 & 2.729268 & 0.503870 \\
\hline 0 & 1.741050 & 3.035128 & -0.457473 \\
\hline C & 2.052757 & 5.354887 & -1.222784 \\
\hline C & 1.723309 & 6.018845 & -0.030002 \\
\hline C & 2.598034 & 6.088539 & -2.290776 \\
\hline C & 1.926590 & 7.394080 & 0.074301 \\
\hline $\mathrm{H}$ & 1.310847 & 5.453033 & 0.799200 \\
\hline C & 2.799296 & 7.461352 & -2.179661 \\
\hline $\mathrm{H}$ & 2.865612 & 5.575275 & -3.212256 \\
\hline C & 2.461023 & 8.118792 & -0.994032 \\
\hline $\mathrm{H}$ & 1.670264 & 7.903095 & 0.999625 \\
\hline $\mathrm{H}$ & 3.222855 & 8.016491 & -3.012047 \\
\hline $\mathrm{H}$ & 2.618782 & 9.190042 & -0.902059 \\
\hline $\mathrm{H}$ & -1.143174 & 4.668144 & -1.202568 \\
\hline
\end{tabular}




\begin{tabular}{|c|c|c|c|}
\hline $\mathrm{H}$ & 0.388365 & 2.742309 & -3.055700 \\
\hline H & 0.134365 & 4.558121 & -3.293295 \\
\hline C & 0.005325 & -2.880840 & 0.452393 \\
\hline C & 1.168440 & -2.270396 & -0.002053 \\
\hline C & 2.419452 & -2.425772 & 0.689444 \\
\hline C & 2.427571 & -3.147228 & 1.869044 \\
\hline H & 3.368729 & -3.304521 & 2.389191 \\
\hline C & -2.236245 & -1.836732 & -0.068418 \\
\hline C & -1.283575 & -2.819347 & -0.326866 \\
\hline C & -3.719372 & -2.833716 & -1.716130 \\
\hline C & -3.490092 & -1.848895 & -0.774761 \\
\hline H & -4.670692 & -2.853841 & -2.242010 \\
\hline C & -2.771296 & -3.844076 & -2.001580 \\
\hline C & -1.527680 & -3.847823 & -1.296520 \\
\hline C & -0.588886 & -4.874076 & -1.592219 \\
\hline C & -0.870814 & -5.844839 & -2.526794 \\
\hline C & -2.105421 & -5.841169 & -3.219095 \\
\hline C & -3.033034 & -4.859143 & -2.961518 \\
\hline $\mathrm{H}$ & 0.366052 & -4.880829 & -1.079165 \\
\hline H & -0.137526 & -6.618086 & -2.739444 \\
\hline H & -2.314818 & -6.612798 & -3.955171 \\
\hline H & -3.983912 & -4.842830 & -3.489117 \\
\hline C & 1.258132 & -3.739471 & 2.402155 \\
\hline C & 0.027455 & -3.626737 & 1.679183 \\
\hline C & -1.128439 & -4.268573 & 2.210949 \\
\hline C & -1.069547 & -4.965319 & 3.399052 \\
\hline C & 0.147702 & -5.067289 & 4.113479 \\
\hline C & 1.285346 & -4.470997 & 3.620475 \\
\hline H & -2.062366 & -4.211780 & 1.662034 \\
\hline $\mathrm{H}$ & -1.963290 & -5.446724 & 3.786197 \\
\hline H & 0.181158 & -5.620819 & 5.047788 \\
\hline H & 2.227350 & -4.549545 & 4.158029 \\
\hline C & -6.618309 & 0.995514 & -0.146189 \\
\hline C & -6.070993 & 0.349494 & 0.971361 \\
\hline C & -5.039935 & -0.571459 & 0.754088 \\
\hline C & -4.561421 & -0.836098 & -0.537454 \\
\hline C & -5.138071 & -0.168803 & -1.628739 \\
\hline C & -6.174329 & 0.755686 & -1.454501 \\
\hline H & -7.417090 & 1.715028 & 0.008313 \\
\hline H & -4.584475 & -1.092448 & 1.586096 \\
\hline H & -4.745485 & -0.373800 & -2.618108 \\
\hline C & -6.599518 & 0.673287 & 2.382681 \\
\hline C & -6.819866 & 1.512162 & -2.632164 \\
\hline C & -6.334512 & 2.165833 & 2.694627 \\
\hline H & -6.714082 & 2.421111 & 3.691877 \\
\hline H & -5.260728 & 2.384209 & 2.674034 \\
\hline H & -6.824720 & 2.826654 & 1.971433 \\
\hline C & -8.120667 & 0.400399 & 2.448797 \\
\hline H & -8.504621 & 0.628212 & 3.450919 \\
\hline H & -8.680347 & 1.011583 & 1.732721 \\
\hline $\mathrm{H}$ & -8.339414 & -0.651530 & 2.232342 \\
\hline C & -5.913185 & -0.173284 & 3.472381 \\
\hline H & -6.309286 & 0.104079 & 4.456280 \\
\hline H & -6.101035 & -1.243805 & 3.330038 \\
\hline
\end{tabular}




\begin{tabular}{|c|c|c|c|}
\hline $\mathrm{H}$ & -4.828843 & -0.021680 & 3.490977 \\
\hline C & -8.337516 & 1.213474 & -2.669700 \\
\hline $\mathrm{H}$ & -8.837666 & 1.521449 & -1.745646 \\
\hline $\mathrm{H}$ & -8.812332 & 1.750310 & -3.500391 \\
\hline $\mathrm{H}$ & -8.521881 & 0.141941 & -2.807931 \\
\hline C & -6.602481 & 3.033250 & -2.449300 \\
\hline H & -7.061882 & 3.588191 & -3.276896 \\
\hline $\mathrm{H}$ & -7.044543 & 3.398921 & -1.516497 \\
\hline $\mathrm{H}$ & -5.533640 & 3.275431 & -2.429711 \\
\hline C & -6.221499 & 1.104371 & -3.992022 \\
\hline $\mathrm{H}$ & -6.718780 & 1.660305 & -4.795078 \\
\hline $\mathrm{H}$ & -5.149982 & 1.325872 & -4.051356 \\
\hline $\mathrm{H}$ & -6.360514 & 0.036250 & -4.194302 \\
\hline C & 3.705605 & -1.897140 & 0.149077 \\
\hline C & 4.652253 & -1.314270 & 1.012789 \\
\hline C & 4.023882 & -2.032266 & -1.205055 \\
\hline C & 5.885800 & -0.861860 & 0.542005 \\
\hline H & 4.397412 & -1.211743 & 2.061177 \\
\hline C & 5.245119 & -1.574162 & -1.722711 \\
\hline H & 3.312403 & -2.511421 & -1.866305 \\
\hline C & 6.153972 & -0.996586 & -0.833077 \\
\hline H & 7.104062 & -0.637315 & -1.210534 \\
\hline C & 5.524356 & -1.726493 & -3.229988 \\
\hline C & 6.943404 & -0.218865 & 1.460628 \\
\hline C & 6.904256 & -1.172609 & -3.632180 \\
\hline H & 6.994932 & -0.102597 & -3.412027 \\
\hline $\mathrm{H}$ & 7.721530 & -1.697671 & -3.123859 \\
\hline H & 7.051318 & -1.300350 & -4.710679 \\
\hline C & 5.476907 & -3.224305 & -3.615274 \\
\hline $\mathrm{H}$ & 5.673558 & -3.346527 & -4.687493 \\
\hline $\mathrm{H}$ & 6.232316 & -3.796131 & -3.063641 \\
\hline $\mathrm{H}$ & 4.498852 & -3.668150 & -3.404800 \\
\hline C & 4.442369 & -0.957743 & -4.027389 \\
\hline $\mathrm{H}$ & 3.431396 & -1.312145 & -3.803038 \\
\hline $\mathrm{H}$ & 4.477524 & 0.113064 & -3.794673 \\
\hline $\mathrm{H}$ & 4.611104 & -1.076461 & -5.105077 \\
\hline C & 8.263624 & -1.021241 & 1.370342 \\
\hline $\mathrm{H}$ & 8.115879 & -2.057468 & 1.695814 \\
\hline H & 8.660374 & -1.045638 & 0.350286 \\
\hline $\mathrm{H}$ & 9.028253 & -0.568848 & 2.013757 \\
\hline C & 7.198540 & 1.239044 & 1.009154 \\
\hline $\mathrm{H}$ & 7.559812 & 1.287656 & -0.023450 \\
\hline $\mathrm{H}$ & 6.279729 & 1.833060 & 1.070527 \\
\hline H & 7.953604 & 1.711279 & 1.649895 \\
\hline C & 6.506229 & -0.193675 & 2.937784 \\
\hline $\mathrm{H}$ & 7.298283 & 0.255370 & 3.547930 \\
\hline $\mathrm{H}$ & 5.598740 & 0.401596 & 3.087295 \\
\hline H & 6.323235 & -1.202456 & 3.325677 \\
\hline
\end{tabular}




\begin{tabular}{lc}
\hline B3LYP/6-31G(d) Energy & -3038.94498 \\
\hline B3LYP-D3(BJ)/def2-TZVPP-IEF-PCM(DCM) Energy & -3040.499985 \\
\hline B3LYP-D3(BJ)/def2-TZVPP-IEF-PCM(DCM)//B3LYP/6-31G(d) Free Energy (Quasiharmonic) & -3039.273172 \\
\hline
\end{tabular}

Number of Imaginary Frequencies

Frequencies (Top 3 out of 450)

$\begin{array}{lr}\text { 1. } & -367.2506 \mathrm{~cm}^{-1} \\ \text { 2. } & 5.9156 \mathrm{~cm}^{-1} \\ \text { 3. } & 7.9301 \mathrm{~cm}^{-1}\end{array}$

\section{B3LYP/6-31G(d) Molecular Geometry in Cartesian Coordinates}

C

$\mathrm{H}$

$\mathrm{H}$

C

0

0

$\mathrm{H}$

0

$\mathrm{H}$

C

C

C

C

$\mathrm{H}$

$\mathrm{H}$

$\mathrm{H}$

C

C

C

$\mathrm{H}$

$\mathrm{H}$

$\mathrm{H}$

C

$\mathrm{H}$

$\mathrm{H}$

$\mathrm{H}$

C

$\mathrm{H}$

$\mathrm{H}$

$\mathrm{H}$

C

$\mathrm{H}$
1.637580

2.054270

0.579200

$-1.445180$

$-1.323380$

$-1.443300$

$-1.445600$

2.205410

3.166290

$-1.555849$

0.274721

$-2.609009$

$-2.306449$

0.500041

$-3.293429$

$-2.868849$

$-1.233000$

$-0.451459$

$-2.711680$

$-3.246400$

$-2.830090$

$-3.178309$

$-0.606510$

$-0.602390$

0.417500

$-1.200030$

1.074081

1.424331

1. 398251

1. 552651

$-0.872079$

$-0.688539$
0.830880

$-0.171930$

0.702880

0.652839

0.769179

$-0.564791$

$-0.556021$

1.425300

1.297170

$-5.149621$

$-4.094750$

$-3.449741$

$-4.002411$

$-4.926090$

$-2.602051$

$-4.186721$

$-1.341541$

$-2.641291$

$-1.379701$

$-0.582731$

$-1.221571$

$-2.333891$

$-0.025491$

0.031839

0.105420

0.817169

$-2.450610$

$-1.824820$

$-1.992230$

$-3.432000$

$-3.234511$

$-2.528731$
$-0.839800$

$-0.676030$

$-1.058330$

1.347910

2.549040

0.780270

$-0.209630$

$-2.011960$

$-1.952900$

0.736860

0.183420

$-0.709990$

0.567480

$-0.489780$

$-0.693990$

$-1.472670$

$-3.254120$

$-3.678970$

$-3.672540$

$-3.146560$

$-4.750000$

$-3.410490$

$-3.712290$

$-4.807570$

$-3.357320$

$-3.340320$

$-3.648060$

$-4.476180$

$-2.709420$

$-3.732880$

$-5.024300$

$-5.842970$ 


\begin{tabular}{|c|c|c|c|}
\hline H & -0.289839 & -4.140611 & -5.223940 \\
\hline $\mathrm{H}$ & -1.929649 & -3.509201 & -5.028790 \\
\hline 0 & -1.173720 & -1.426811 & -1.806690 \\
\hline 0 & -0.814939 & -3.573521 & -2.639450 \\
\hline B & -1.069719 & -2.820471 & -1.456130 \\
\hline 0 & -0.060149 & -2.966970 & -0.366420 \\
\hline C & 0.999581 & -4.041430 & 1.466690 \\
\hline C & 1.742011 & -5.155030 & 1.890190 \\
\hline C & 0.956141 & -2.889210 & 2.270460 \\
\hline C & 2.432811 & -5.121400 & 3.099590 \\
\hline $\mathrm{H}$ & 1.776962 & -6.046770 & 1.267850 \\
\hline C & 1.649441 & -2.861920 & 3.478890 \\
\hline $\mathrm{H}$ & 0.370021 & -2.034140 & 1.948150 \\
\hline C & 2.387971 & -3.974240 & 3.896480 \\
\hline $\mathrm{H}$ & 3.006842 & -5.986700 & 3.419470 \\
\hline $\mathrm{H}$ & 1.605391 & -1.971350 & 4.099660 \\
\hline $\mathrm{H}$ & 2.923631 & -3.948290 & 4.841620 \\
\hline $\mathrm{H}$ & -2.425259 & -3.351301 & 1.434630 \\
\hline $\mathrm{H}$ & -1.519428 & -5.887591 & -0.061890 \\
\hline $\mathrm{H}$ & -1.323278 & -5.514441 & 1.731720 \\
\hline C & -0.498121 & 2.630249 & 0.167140 \\
\hline C & -1.601390 & 1.818649 & 0.400120 \\
\hline C & -2.862030 & 2.052489 & -0.248740 \\
\hline C & -2.944131 & 3.093839 & -1.153470 \\
\hline $\mathrm{H}$ & -3.898451 & 3.314779 & -1.624330 \\
\hline C & 1.822120 & 1.659080 & 0.418680 \\
\hline C & 0.793589 & 2.448340 & 0.921450 \\
\hline C & 3.257179 & 2.304670 & 2.275960 \\
\hline C & 3.085470 & 1.614060 & 1.097120 \\
\hline $\mathrm{H}$ & 4.218030 & 2.268841 & 2.784460 \\
\hline C & 2.216289 & 3.085630 & 2.837990 \\
\hline C & 0.966429 & 3.175470 & 2.148150 \\
\hline C & -0.057091 & 3.982380 & 2.716930 \\
\hline C & 0.147179 & 4.661090 & 3.897470 \\
\hline C & 1.385419 & 4.569780 & 4.575870 \\
\hline C & 2.396439 & 3.795890 & 4.054890 \\
\hline $\mathrm{H}$ & -1.015341 & 4.049959 & 2.214680 \\
\hline $\mathrm{H}$ & -0.651321 & 5.268289 & 4.315300 \\
\hline $\mathrm{H}$ & 1.533079 & 5.109510 & 5.507520 \\
\hline $\mathrm{H}$ & 3.352929 & 3.716370 & 4.566560 \\
\hline C & -1.835061 & 3.917969 & -1.460770 \\
\hline C & -0.589211 & 3.692949 & -0.792640 \\
\hline C & 0.510229 & 4.536610 & -1.111190 \\
\hline C & 0.379389 & 5.551790 & -2.032040 \\
\hline C & -0.856371 & 5.778109 & -2.683900 \\
\hline C & -1.938471 & 4.976929 & -2.403170 \\
\hline $\mathrm{H}$ & 1.463759 & 4.361300 & -0.627100 \\
\hline $\mathrm{H}$ & 1.232618 & 6.183470 & -2.263690 \\
\hline $\mathrm{H}$ & -0.945742 & 6.583759 & -3.407720 \\
\hline $\mathrm{H}$ & -2.892051 & 5.139779 & -2.899970 \\
\hline C & 6.495660 & -0.378979 & -0.557800 \\
\hline C & 6.168440 & 0.909291 & -1.017770 \\
\hline C & 5.039630 & 1.525711 & -0.470150 \\
\hline C & 4.256030 & 0.884261 & 0.507610 \\
\hline
\end{tabular}




\begin{tabular}{|c|c|c|c|}
\hline C & 4.615250 & -0.393849 & 0.935460 \\
\hline C & 5.744170 & -1.049679 & 0.411180 \\
\hline $\mathrm{H}$ & 7.370870 & -0.864959 & -0.972020 \\
\hline $\mathrm{H}$ & 4.760349 & 2.531221 & -0.766810 \\
\hline $\mathrm{H}$ & 3.998970 & -0.880639 & 1.685700 \\
\hline C & 7.056150 & 1.588751 & -2.078350 \\
\hline C & 6.100071 & -2.466359 & 0.901310 \\
\hline C & 8.492320 & 1.743322 & -1.523200 \\
\hline $\mathrm{H}$ & 9.139780 & 2.220932 & -2.268770 \\
\hline $\mathrm{H}$ & 8.498629 & 2.364172 & -0.620060 \\
\hline $\mathrm{H}$ & 8.937000 & 0.776162 & -1.266630 \\
\hline C & 7.092750 & 0.718071 & -3.357040 \\
\hline $\mathrm{H}$ & 7.726160 & 1.187742 & -4.119430 \\
\hline $\mathrm{H}$ & 7.494610 & -0.281299 & -3.160300 \\
\hline $\mathrm{H}$ & 6.088170 & 0.597321 & -3.778620 \\
\hline C & 6.540679 & 2.987581 & -2.466630 \\
\hline $\mathrm{H}$ & 7.200649 & 3.426631 & -3.223190 \\
\hline $\mathrm{H}$ & 5.531629 & 2.949601 & -2.892960 \\
\hline $\mathrm{H}$ & 6.523309 & 3.669491 & -1.608950 \\
\hline C & 6.249951 & -2.463419 & 2.441190 \\
\hline $\mathrm{H}$ & 6.518421 & -3.465559 & 2.797210 \\
\hline $\mathrm{H}$ & 7.036591 & -1.768789 & 2.757750 \\
\hline $\mathrm{H}$ & 5.321461 & -2.173889 & 2.942840 \\
\hline C & 4.965491 & -3.437159 & 0.498100 \\
\hline $\mathrm{H}$ & 5.190081 & -4.452819 & 0.846370 \\
\hline $\mathrm{H}$ & 4.848551 & -3.469159 & -0.591450 \\
\hline $\mathrm{H}$ & 4.005031 & -3.142139 & 0.930780 \\
\hline C & 7.418211 & -2.988029 & 0.297600 \\
\hline $\mathrm{H}$ & 7.629661 & -3.988888 & 0.690500 \\
\hline $\mathrm{H}$ & 7.366451 & -3.069829 & -0.794010 \\
\hline $\mathrm{H}$ & 8.268521 & -2.345398 & 0.553870 \\
\hline C & -4.081180 & 1.251288 & 0.062090 \\
\hline C & -4.411670 & 0.923528 & 1.380220 \\
\hline C & -4.952850 & 0.859778 & -0.971690 \\
\hline C & -5.567340 & 0.189708 & 1.688620 \\
\hline $\mathrm{H}$ & -3.760440 & 1.252569 & 2.181480 \\
\hline C & -6.124610 & 0.148978 & -0.706260 \\
\hline $\mathrm{H}$ & -4.687660 & 1.115478 & -1.991030 \\
\hline C & -6.402480 & -0.183852 & 0.632890 \\
\hline $\mathrm{H}$ & -7.301660 & -0.749432 & 0.847580 \\
\hline C & -7.105120 & -0.285392 & -1.813650 \\
\hline C & -5.859540 & -0.168862 & 3.158030 \\
\hline C & -8.514560 & 0.274917 & -1.506020 \\
\hline $\mathrm{H}$ & -8.903170 & -0.088693 & -0.549260 \\
\hline $\mathrm{H}$ & -8.500280 & 1.370107 & -1.465370 \\
\hline $\mathrm{H}$ & -9.222250 & -0.027093 & -2.287860 \\
\hline C & -6.688140 & 0.227378 & -3.205020 \\
\hline $\mathrm{H}$ & -7.427820 & -0.086832 & -3.950160 \\
\hline $\mathrm{H}$ & -6.632740 & 1.321638 & -3.234550 \\
\hline $\mathrm{H}$ & -5.717510 & -0.173632 & -3.516560 \\
\hline C & -7.169409 & -1.830402 & -1.867120 \\
\hline $\mathrm{H}$ & -6.186749 & -2.254532 & -2.103450 \\
\hline $\mathrm{H}$ & -7.495839 & -2.256892 & -0.912730 \\
\hline $\mathrm{H}$ & -7.876029 & -2.158572 & -2.639620 \\
\hline
\end{tabular}




\begin{tabular}{|llll|} 
C & -5.977740 & 1.128068 & 3.993590 \\
H & -5.055770 & 1.717538 & 3.965860 \\
$H$ & -6.792970 & 1.760758 & 3.623450 \\
H & -6.185140 & 0.885128 & 5.042940 \\
C & -7.169210 & -0.963282 & 3.321040 \\
$H$ & -8.038990 & -0.395743 & 2.969990 \\
$H$ & -7.140199 & -1.915512 & 2.778730 \\
$H$ & -7.331240 & -1.193392 & 4.380210 \\
C & -4.697910 & -1.030592 & 3.710420 \\
H & -4.873790 & -1.273762 & 4.765830 \\
H & -4.618439 & -1.973372 & 3.155740 \\
$H$ & -3.732920 & -0.519181 & 3.637640 \\
\hline
\end{tabular}

\section{C-Catalyzed reaction TS 112}

\section{Datum}

Value

\begin{tabular}{lc}
\hline B3LYP/6-31G(d) Energy & -3038.951899 \\
\hline B3LYP-D3(BJ)/def2-TZVPP-IEF-PCM(DCM) Energy & -3040.501856 \\
\hline B3LYP-D3(BJ)/def2-TZVPP-IEF-PCM(DCM)//B3LYP/6-31G(d) Free Energy (Quasiharmonic) & -3039.273165 \\
\hline
\end{tabular}

Number of Imaginary Frequencies

Frequencies (Top 3 out of 450)

$$
\begin{array}{lr}
\text { 1. } & -320.3033 \mathrm{~cm}^{-1} \\
\text { 2. } & 7.4679 \mathrm{~cm}^{-1} \\
\text { 3. } & 11.5930 \mathrm{~cm}^{-1}
\end{array}
$$

\section{B3LYP/6-31G(d) Molecular Geometry in Cartesian Coordinates}

\section{C}

$\mathrm{H}$

$\mathrm{H}$

C

0

0

$\mathrm{H}$

0

$\mathrm{H}$

C

C

c

C

$\mathrm{H}$

-3.199567
-4.206708
-2.499240
0.075379
-0.938948
1.111947
1.888521
-3.167171
-2.388940
4.319378
3.337861
4.950244
4.184253
2.392932

\section{$-3.199567$}

$$
\begin{array}{r}
0.738886 \\
0.663621 \\
0.788686 \\
1.197062 \\
1.709870 \\
0.979162 \\
0.506680 \\
1.955446 \\
1.925431 \\
-2.537833 \\
-3.394320 \\
-0.773069 \\
-1.262370 \\
-3.483379
\end{array}
$$

1.184874

1.601808

2.028308

$-0.833961$

$-1.278515$

$-1.640065$

$-1.239458$

0.441601

$-0.154326$

2.410953

0.610761

0.807005

1.913406

1.151659 


\begin{tabular}{|c|c|c|c|}
\hline $\mathrm{H}$ & 5.970136 & -1.163197 & 0.768573 \\
\hline $\mathrm{H}$ & 4.925627 & 0.309988 & 0.678450 \\
\hline C & 3.920274 & -0.317520 & -2.810811 \\
\hline C & 5.384326 & -0.889715 & -2.735861 \\
\hline C & 2.953273 & -1.240653 & -3.566551 \\
\hline $\mathrm{H}$ & 1.928091 & -0.893305 & -3.410688 \\
\hline $\mathrm{H}$ & 3.155037 & -1.237098 & -4.643315 \\
\hline $\mathrm{H}$ & 3.022020 & -2.270268 & -3.203498 \\
\hline C & 3.826670 & 1.106026 & -3.361905 \\
\hline $\mathrm{H}$ & 2.783820 & 1.431127 & -3.402466 \\
\hline $\mathrm{H}$ & 4.234513 & 1.147890 & -4.378795 \\
\hline $\mathrm{H}$ & 4.377696 & 1.813698 & -2.737536 \\
\hline C & 6.441637 & 0.186773 & -2.435199 \\
\hline $\mathrm{H}$ & 6.616771 & 0.840519 & -3.296413 \\
\hline $\mathrm{H}$ & 6.149867 & 0.811096 & -1.585244 \\
\hline $\mathrm{H}$ & 7.385347 & -0.308467 & -2.184040 \\
\hline C & 5.817499 & -1.700184 & -3.959467 \\
\hline $\mathrm{H}$ & 5.810911 & -1.083134 & -4.865477 \\
\hline $\mathrm{H}$ & 6.837802 & -2.070332 & -3.811588 \\
\hline $\mathrm{H}$ & 5.165390 & -2.561851 & -4.119473 \\
\hline 0 & 3.529882 & -0.312619 & -1.404910 \\
\hline 0 & 5.317612 & -1.775488 & -1.604638 \\
\hline B & 4.276391 & -1.344835 & -0.730070 \\
\hline 0 & 3.346556 & -2.483219 & -0.306955 \\
\hline C & 4.119229 & -4.641524 & 0.493772 \\
\hline C & 5.162234 & -4.779481 & -0.438102 \\
\hline C & 3.775212 & -5.727752 & 1.317030 \\
\hline C & 5.850368 & -5.987966 & -0.527476 \\
\hline $\mathrm{H}$ & 5.417648 & -3.943393 & -1.082433 \\
\hline C & 4.464960 & -6.932299 & 1.218915 \\
\hline $\mathrm{H}$ & 2.961821 & -5.622556 & 2.031806 \\
\hline C & 5.507270 & -7.063470 & 0.296479 \\
\hline $\mathrm{H}$ & 6.655350 & -6.094010 & -1.249612 \\
\hline $\mathrm{H}$ & 4.189447 & -7.768793 & 1.855018 \\
\hline $\mathrm{H}$ & 6.046088 & -8.003746 & 0.216683 \\
\hline $\mathrm{H}$ & 3.301181 & -0.695511 & 2.208389 \\
\hline $\mathrm{H}$ & 5.251303 & -3.077273 & 2.273792 \\
\hline $\mathrm{H}$ & 3.677636 & -2.888902 & 3.214381 \\
\hline C & -0.507551 & -0.307728 & 1.067247 \\
\hline C & 0.231823 & 0.775326 & 0.607265 \\
\hline C & 1.136824 & 1.492930 & 1.463157 \\
\hline C & 1.330466 & 1.021956 & 2.747295 \\
\hline $\mathrm{H}$ & 1.972653 & 1.577876 & 3.425775 \\
\hline C & -2.896296 & -0.483951 & 0.331282 \\
\hline C & -1.585101 & -0.948643 & 0.229119 \\
\hline C & -3.634533 & -2.242692 & -1.176352 \\
\hline C & -3.947840 & -1.146421 & -0.394058 \\
\hline $\mathrm{H}$ & -4.425985 & -2.741909 & -1.729423 \\
\hline C & -2.311849 & -2.720209 & -1.318280 \\
\hline C & -1.257535 & -2.061182 & -0.610794 \\
\hline C & 0.070370 & -2.538802 & -0.783136 \\
\hline C & 0.337161 & -3.615065 & -1.601099 \\
\hline C & -0.710430 & -4.274313 & -2.287679 \\
\hline C & -2.005344 & -3.831664 & -2.149596 \\
\hline
\end{tabular}




\begin{tabular}{|c|c|c|c|}
\hline $\mathrm{H}$ & 0.890360 & -2.031364 & -0.287701 \\
\hline $\mathrm{H}$ & 1.362214 & -3.950550 & -1.727308 \\
\hline $\mathrm{H}$ & -0.486853 & -5.122326 & -2.929471 \\
\hline $\mathrm{H}$ & -2.818053 & -4.322329 & -2.680189 \\
\hline C & 0.681098 & -0.142103 & 3.229394 \\
\hline C & -0.262300 & -0.814872 & 2.388473 \\
\hline C & -0.926140 & -1.963743 & 2.900913 \\
\hline C & -0.662120 & -2.427257 & 4.171307 \\
\hline C & 0.270101 & -1.759394 & 5.000786 \\
\hline C & 0.922875 & -0.638881 & 4.539419 \\
\hline $\mathrm{H}$ & -1.646858 & -2.476170 & 2.273508 \\
\hline $\mathrm{H}$ & -1.178078 & -3.308499 & 4.542547 \\
\hline $\mathrm{H}$ & 0.463311 & -2.129648 & 6.004018 \\
\hline $\mathrm{H}$ & 1.633326 & -0.112855 & 5.172845 \\
\hline C & -8.073273 & -0.012137 & -0.277066 \\
\hline C & -7.118740 & 0.978201 & -0.523191 \\
\hline C & -5.767529 & 0.597964 & -0.547083 \\
\hline C & -5.379677 & -0.725398 & -0.322703 \\
\hline C & -6.371787 & -1.692866 & -0.075202 \\
\hline C & -7.726120 & -1.356711 & -0.048860 \\
\hline $\mathrm{H}$ & -9.122126 & 0.260995 & -0.255816 \\
\hline $\mathrm{H}$ & -4.995388 & 1.337065 & -0.727016 \\
\hline $\mathrm{H}$ & -6.055981 & -2.712389 & 0.115329 \\
\hline C & -7.483664 & 2.455186 & -0.764330 \\
\hline C & -8.835474 & -2.391581 & 0.223301 \\
\hline C & -6.985138 & 2.885298 & -2.164722 \\
\hline $\mathrm{H}$ & -7.232712 & 3.937963 & -2.349718 \\
\hline $\mathrm{H}$ & -5.900433 & 2.776018 & -2.260852 \\
\hline $\mathrm{H}$ & -7.454609 & 2.282436 & -2.951018 \\
\hline C & -9.001828 & 2.706554 & -0.698259 \\
\hline $\mathrm{H}$ & -9.207754 & 3.768812 & -0.872480 \\
\hline $\mathrm{H}$ & -9.545044 & 2.135928 & -1.460755 \\
\hline $\mathrm{H}$ & -9.416362 & 2.447730 & 0.283049 \\
\hline C & -6.798697 & 3.331336 & 0.312786 \\
\hline $\mathrm{H}$ & -7.029454 & 4.390759 & 0.142908 \\
\hline $\mathrm{H}$ & -7.156605 & 3.064708 & 1.314535 \\
\hline $\mathrm{H}$ & -5.710348 & 3.215775 & 0.304268 \\
\hline C & -9.636970 & -1.976337 & 1.479986 \\
\hline $\mathrm{H}$ & -10.431080 & -2.705121 & 1.685104 \\
\hline $\mathrm{H}$ & -8.985911 & -1.925457 & 2.360226 \\
\hline $\mathrm{H}$ & -10.108549 & -0.995862 & 1.357586 \\
\hline C & -9.789424 & -2.455154 & -0.993376 \\
\hline $\mathrm{H}$ & -10.592843 & -3.180564 & -0.813465 \\
\hline $\mathrm{H}$ & -10.255497 & -1.485609 & -1.197185 \\
\hline $\mathrm{H}$ & -9.250105 & -2.762162 & -1.896876 \\
\hline C & -8.274828 & -3.806631 & 0.462535 \\
\hline $\mathrm{H}$ & -9.099606 & -4.503750 & 0.650083 \\
\hline $\mathrm{H}$ & -7.720089 & -4.177860 & -0.406704 \\
\hline $\mathrm{H}$ & -7.608914 & -3.840309 & 1.332355 \\
\hline C & 1.781494 & 2.769324 & 1.040105 \\
\hline C & 3.127926 & 3.028082 & 1.334467 \\
\hline C & 1.025075 & 3.764924 & 0.407582 \\
\hline C & 3.726777 & 4.250517 & 1.005568 \\
\hline $\mathrm{H}$ & 3.712015 & 2.252635 & 1.821923 \\
\hline
\end{tabular}




\begin{tabular}{|c|c|c|c|}
\hline C & 1.588978 & 4.993282 & 0.044556 \\
\hline $\mathrm{H}$ & -0.025390 & 3.580907 & 0.215003 \\
\hline C & 2.938156 & 5.211606 & 0.355167 \\
\hline $\mathrm{H}$ & 3.386106 & 6.158365 & 0.087460 \\
\hline C & 0.714952 & 6.043612 & -0.667708 \\
\hline C & 5.204570 & 4.504381 & 1.366328 \\
\hline C & 1.489607 & 7.336433 & -0.985796 \\
\hline $\mathrm{H}$ & 1.862200 & 7.826185 & -0.078450 \\
\hline $\mathrm{H}$ & 2.341779 & 7.149875 & -1.649559 \\
\hline $\mathrm{H}$ & 0.826916 & 8.045558 & -1.494262 \\
\hline C & 0.194637 & 5.451812 & -2.000257 \\
\hline $\mathrm{H}$ & -0.451272 & 6.177476 & -2.509899 \\
\hline $\mathrm{H}$ & 1.027399 & 5.209620 & -2.670707 \\
\hline $\mathrm{H}$ & -0.383997 & 4.535435 & -1.847895 \\
\hline C & -0.487000 & 6.413246 & 0.234242 \\
\hline $\mathrm{H}$ & -1.116309 & 5.545557 & 0.455791 \\
\hline $\mathrm{H}$ & -0.147572 & 6.835235 & 1.187478 \\
\hline $\mathrm{H}$ & -1.117174 & 7.160672 & -0.262906 \\
\hline C & 5.383207 & 4.395026 & 2.899650 \\
\hline $\mathrm{H}$ & 4.767496 & 5.138038 & 3.419269 \\
\hline $\mathrm{H}$ & 5.099794 & 3.405426 & 3.273649 \\
\hline $\mathrm{H}$ & 6.430583 & 4.567522 & 3.176699 \\
\hline C & 6.100829 & 3.451760 & 0.672801 \\
\hline $\mathrm{H}$ & 5.858043 & 2.433961 & 0.995219 \\
\hline $\mathrm{H}$ & 5.986864 & 3.495648 & -0.416453 \\
\hline $\mathrm{H}$ & 7.156344 & 3.631305 & 0.911788 \\
\hline $\mathrm{C}$ & 5.688530 & 5.900560 & 0.929754 \\
\hline $\mathrm{H}$ & 6.738973 & 6.030361 & 1.213592 \\
\hline $\mathrm{H}$ & 5.621299 & 6.035896 & -0.155680 \\
\hline $\mathrm{H}$ & 5.115638 & 6.701389 & 1.410642 \\
\hline
\end{tabular}

\section{C-Catalyzed reaction TS 113}

\section{Datum}

Value

\begin{tabular}{lr}
\hline B3LYP/6-31G(d) Energy & -3038.95122 \\
\hline B3LYP-D3(BJ)/def2-TZVPP-IEF-PCM(DCM) Energy & -3040.50262 \\
\hline B3LYP-D3(BJ)/def2-TZVPP-IEF-PCM(DCM)//B3LYP/6-31G(d) Free Energy (Quasiharmonic) & -3039.273119 \\
\hline
\end{tabular}

Number of Imaginary Frequencies

Frequencies (Top 3 out of 450)
1. $-277.1205 \mathrm{~cm}^{-1}$
2. $\quad 8.0918 \mathrm{~cm}^{-1}$
3. $11.7830 \mathrm{~cm}^{-1}$ 


\begin{tabular}{|c|c|c|c|}
\hline C & 1.267823 & -0.790061 & 1.038379 \\
\hline $\mathrm{H}$ & 2.068109 & -0.117605 & 0.730702 \\
\hline $\mathrm{H}$ & 0.326996 & -0.258922 & 0.879049 \\
\hline C & -1.782326 & -0.984216 & -1.232232 \\
\hline 0 & -1.768321 & -1.622755 & -2.263078 \\
\hline 0 & -1.510104 & 0.328274 & -1.234450 \\
\hline H & -1.464695 & 0.748377 & -0.336949 \\
\hline 0 & 1.476822 & -1.002546 & 2.446429 \\
\hline $\mathrm{H}$ & 0.761699 & -1.577857 & 2.765025 \\
\hline C & 0.538903 & 3.334404 & -3.196848 \\
\hline C & 1.851370 & 2.640104 & -1.538259 \\
\hline C & -1.040877 & 3.649552 & -1.329418 \\
\hline C & -0.135984 & 4.154811 & -2.326504 \\
\hline $\mathrm{H}$ & 2.120178 & 1.802654 & -2.187654 \\
\hline $\mathrm{H}$ & -1.607989 & 2.779630 & -1.667006 \\
\hline $\mathrm{H}$ & -1.692204 & 4.410636 & -0.894674 \\
\hline C & -1.204537 & 2.567299 & 2.100658 \\
\hline C & -0.045822 & 3.610492 & 2.314634 \\
\hline C & -2.594784 & 3.218538 & 2.054389 \\
\hline $\mathrm{H}$ & -3.312314 & 2.492687 & 1.660482 \\
\hline $\mathrm{H}$ & -2.930422 & 3.530260 & 3.049770 \\
\hline $\mathrm{H}$ & -2.606981 & 4.094335 & 1.398802 \\
\hline C & -1.212679 & 1.401772 & 3.090938 \\
\hline $\mathrm{H}$ & -2.039529 & 0.722280 & 2.856593 \\
\hline $\mathrm{H}$ & -1.363271 & 1.767450 & 4.113880 \\
\hline $\mathrm{H}$ & -0.281991 & 0.830419 & 3.059996 \\
\hline C & 1.253389 & 2.964967 & 2.824243 \\
\hline $\mathrm{H}$ & 1.166882 & 2.653633 & 3.870905 \\
\hline $\mathrm{H}$ & 1.532966 & 2.089391 & 2.232887 \\
\hline $\mathrm{H}$ & 2.064954 & 3.697342 & 2.755884 \\
\hline C & -0.410075 & 4.800907 & 3.203795 \\
\hline $\mathrm{H}$ & -0.668074 & 4.473249 & 4.217720 \\
\hline $\mathrm{H}$ & 0.447403 & 5.478345 & 3.278386 \\
\hline $\mathrm{H}$ & -1.249837 & 5.366572 & 2.793567 \\
\hline 0 & -0.909379 & 2.061101 & 0.776742 \\
\hline 0 & 0.171204 & 4.101544 & 0.975235 \\
\hline B & -0.154101 & 3.061537 & 0.051907 \\
\hline 0 & 1.020824 & 2.325838 & -0.605325 \\
\hline C & 2.890197 & 3.691879 & -1.450821 \\
\hline C & 2.894838 & 4.694517 & -0.467490 \\
\hline C & 3.936286 & 3.643558 & -2.388887 \\
\hline C & 3.927664 & 5.630467 & -0.439072 \\
\hline $\mathrm{H}$ & 2.088975 & 4.732580 & 0.258359 \\
\hline C & 4.964895 & 4.580948 & -2.354476 \\
\hline $\mathrm{H}$ & 3.940733 & 2.864175 & -3.147769 \\
\hline C & 4.961923 & 5.579515 & -1.377078 \\
\hline $\mathrm{H}$ & 3.926690 & 6.403765 & 0.324485 \\
\hline $\mathrm{H}$ & 5.767947 & 4.531168 & -3.084557 \\
\hline $\mathrm{H}$ & 5.764109 & 6.311898 & -1.345115 \\
\hline $\mathrm{H}$ & 0.186736 & 5.193742 & -2.249373 \\
\hline
\end{tabular}




\begin{tabular}{|c|c|c|c|}
\hline $\mathrm{H}$ & 0.160888 & 2.334475 & -3.395421 \\
\hline $\mathrm{H}$ & 1.251693 & 3.738106 & -3.909467 \\
\hline C & -1.150875 & -2.408820 & 0.713827 \\
\hline C & -2.106225 & -1.596624 & 0.114595 \\
\hline C & -3.378305 & -1.343364 & 0.737015 \\
\hline C & -3.603882 & -1.874197 & 1.993934 \\
\hline $\mathrm{H}$ & -4.571125 & -1.727329 & 2.466740 \\
\hline C & 1.303476 & -2.063511 & 0.212946 \\
\hline C & 0.130088 & -2.787297 & 0.014159 \\
\hline C & 2.519380 & -3.650652 & -1.170109 \\
\hline C & 2.533828 & -2.514689 & -0.383173 \\
\hline $\mathrm{H}$ & 3.449753 & -4.006640 & -1.605448 \\
\hline C & 1.341134 & -4.397652 & -1.402585 \\
\hline C & 0.118575 & -3.969797 & -0.798006 \\
\hline C & -1.053329 & -4.740997 & -1.030600 \\
\hline C & -1.013149 & -5.873042 & -1.812970 \\
\hline C & 0.200171 & -6.295877 & -2.406747 \\
\hline C & 1.351117 & -5.570807 & -2.205039 \\
\hline $\mathrm{H}$ & -1.991936 & -4.421966 & -0.592269 \\
\hline $\mathrm{H}$ & -1.921639 & -6.445207 & -1.980550 \\
\hline $\mathrm{H}$ & 0.217569 & -7.191087 & -3.022643 \\
\hline $\mathrm{H}$ & 2.289694 & -5.883750 & -2.656672 \\
\hline C & -2.638294 & -2.655551 & 2.671819 \\
\hline C & -1.398073 & -2.951279 & 2.020441 \\
\hline C & -0.459902 & -3.780152 & 2.701426 \\
\hline C & -0.729500 & -4.269448 & 3.961733 \\
\hline C & -1.953598 & -3.967598 & 4.604230 \\
\hline C & -2.887353 & -3.182504 & 3.968177 \\
\hline $\mathrm{H}$ & 0.474353 & -4.032583 & 2.211503 \\
\hline $\mathrm{H}$ & 0.000908 & -4.898593 & 4.462813 \\
\hline $\mathrm{H}$ & -2.154420 & -4.361120 & 5.596824 \\
\hline $\mathrm{H}$ & -3.834504 & -2.950723 & 4.449464 \\
\hline C & 6.345883 & -0.639527 & 0.180853 \\
\hline C & 5.541805 & -0.880266 & 1.304166 \\
\hline C & 4.292624 & -1.479934 & 1.103769 \\
\hline C & 3.840103 & -1.820650 & -0.178210 \\
\hline C & 4.672720 & -1.557517 & -1.275814 \\
\hline C & 5.935379 & -0.972508 & -1.118374 \\
\hline $\mathrm{H}$ & 7.315175 & -0.181216 & 0.321458 \\
\hline $\mathrm{H}$ & 3.648153 & -1.687358 & 1.950424 \\
\hline $\mathrm{H}$ & 4.311643 & -1.811132 & -2.268543 \\
\hline C & 5.979044 & -0.516441 & 2.736613 \\
\hline C & 6.818014 & -0.718607 & -2.357123 \\
\hline C & 4.953574 & 0.467081 & 3.351436 \\
\hline $\mathrm{H}$ & 5.242590 & 0.722406 & 4.378882 \\
\hline $\mathrm{H}$ & 4.910926 & 1.396961 & 2.771944 \\
\hline $\mathrm{H}$ & 3.944156 & 0.044982 & 3.379061 \\
\hline C & 6.034878 & -1.800330 & 3.598639 \\
\hline $\mathrm{H}$ & 6.339863 & -1.557569 & 4.624170 \\
\hline $\mathrm{H}$ & 5.060916 & -2.297498 & 3.649165 \\
\hline $\mathrm{H}$ & 6.757013 & -2.516749 & 3.189788 \\
\hline C & 7.367897 & 0.148556 & 2.779902 \\
\hline $\mathrm{H}$ & 7.626867 & 0.393710 & 3.816347 \\
\hline $\mathrm{H}$ & 8.151812 & -0.512663 & 2.392436 \\
\hline
\end{tabular}




\begin{tabular}{|c|c|c|c|}
\hline $\mathrm{H}$ & 7.391943 & 1.081696 & 2.205327 \\
\hline C & 7.099588 & -2.061432 & -3.072515 \\
\hline $\mathrm{H}$ & 7.723047 & -1.899031 & -3.960694 \\
\hline $\mathrm{H}$ & 7.628269 & -2.754563 & -2.408199 \\
\hline $\mathrm{H}$ & 6.175814 & -2.550512 & -3.398343 \\
\hline C & 6.082902 & 0.232689 & -3.331060 \\
\hline $\mathrm{H}$ & 6.695347 & 0.416986 & -4.222643 \\
\hline $\mathrm{H}$ & 5.127189 & -0.185923 & -3.664299 \\
\hline $\mathrm{H}$ & 5.879360 & 1.197126 & -2.851144 \\
\hline C & 8.172343 & -0.077696 & -1.998445 \\
\hline $\mathrm{H}$ & 8.760741 & 0.074047 & -2.910753 \\
\hline $\mathrm{H}$ & 8.048102 & 0.899566 & -1.518401 \\
\hline $\mathrm{H}$ & 8.760858 & -0.715057 & -1.328961 \\
\hline C & -4.480641 & -0.606360 & 0.051360 \\
\hline C & -5.307764 & 0.272013 & 0.769448 \\
\hline C & -4.768229 & -0.843539 & -1.299511 \\
\hline C & -6.401774 & 0.907574 & 0.168700 \\
\hline $\mathrm{H}$ & -5.083326 & 0.458021 & 1.815869 \\
\hline C & -5.833198 & -0.206117 & -1.946289 \\
\hline $\mathrm{H}$ & -4.156845 & -1.548364 & -1.849211 \\
\hline C & -6.635724 & 0.660588 & -1.192550 \\
\hline $\mathrm{H}$ & -7.467973 & 1.152778 & -1.676302 \\
\hline C & -6.078913 & -0.484803 & -3.441720 \\
\hline C & -7.315997 & 1.823112 & 1.008443 \\
\hline C & -6.333309 & -1.996630 & -3.651569 \\
\hline $\mathrm{H}$ & -5.480051 & -2.602059 & -3.330043 \\
\hline $\mathrm{H}$ & -7.213765 & -2.329437 & -3.089341 \\
\hline $\mathrm{H}$ & -6.508559 & -2.207460 & -4.713663 \\
\hline C & -7.293975 & 0.285309 & -3.992470 \\
\hline $\mathrm{H}$ & -7.424404 & 0.054573 & -5.055844 \\
\hline $\mathrm{H}$ & -8.222187 & 0.008289 & -3.478982 \\
\hline $\mathrm{H}$ & -7.164327 & 1.370200 & -3.904838 \\
\hline C & -4.827880 & -0.059840 & -4.249267 \\
\hline $\mathrm{H}$ & -4.642478 & 1.015189 & -4.140737 \\
\hline $\mathrm{H}$ & -3.926104 & -0.586910 & -3.921924 \\
\hline $\mathrm{H}$ & -4.975855 & -0.272545 & -5.315525 \\
\hline C & -8.434554 & 2.470800 & 0.169140 \\
\hline $\mathrm{H}$ & -8.030987 & 3.099615 & -0.632691 \\
\hline $\mathrm{H}$ & -9.094889 & 1.722170 & -0.282665 \\
\hline $\mathrm{H}$ & -9.052181 & 3.109172 & 0.811136 \\
\hline C & -7.981422 & 0.984648 & 2.126518 \\
\hline $\mathrm{H}$ & -8.589345 & 0.177473 & 1.702015 \\
\hline $\mathrm{H}$ & -7.237481 & 0.529143 & 2.788788 \\
\hline $\mathrm{H}$ & -8.634850 & 1.615877 & 2.741598 \\
\hline C & -6.486902 & 2.958725 & 1.650996 \\
\hline $\mathrm{H}$ & -7.137809 & 3.619703 & 2.236716 \\
\hline $\mathrm{H}$ & -5.715784 & 2.572493 & 2.324623 \\
\hline H & -5.988514 & 3.563804 & 0.885230 \\
\hline
\end{tabular}


Frequencies (Top 3 out of 450)

$\begin{array}{lr}\text { 1. } & -377.2184 \mathrm{~cm}^{-1} \\ \text { 2. } & 9.0488 \mathrm{~cm}^{-1} \\ \text { 3. } & 12.4966 \mathrm{~cm}^{-1}\end{array}$

\section{B3LYP/6-31G(d) Molecular Geometry in Cartesian Coordinates}

C

$\mathrm{H}$

$\mathrm{H}$

C

0

0

$\mathrm{H}$

0

$\mathrm{H}$

C

C

C

C

$\mathrm{H}$

$\mathrm{H}$

$\mathrm{H}$

C

C

C

$\mathrm{H}$

$\mathrm{H}$

$\mathrm{H}$

C

$\mathrm{H}$

$\mathrm{H}$

$\mathrm{H}$

C

$\mathrm{H}$

$\mathrm{H}$

$\mathrm{H}$

C

$\mathrm{H}$
$-1.457360$

$-2.178630$

$-0.460850$

1.517970

1.340960

1.498590

1.541430

$-1.654170$

$-1.014130$

0.493119

$-0.966010$

2.076650

1.505960

$-1.340331$

2. 910940

2. 273379

1.457210

0.459340

2.928560

3.563020

3.189870

3.153080

1.171760

1.274740

0.169510

1.897740

$-0.981470$

$-1.089830$

$-1.308910$

$-1.646040$

0.889619

0.955840
0.602810

$-0.192340$

0.172600

0.851270

1.305830

$-0.475620$

$-0.743890$

0.966890

1.666460

$-5.044970$

$-3.731560$

$-3.887710$

$-4.130430$

$-4.603190$

$-3.187071$

$-4.785540$

$-2.203820$

$-3.396960$

$-2.610261$

$-1.829591$

$-2.723031$

$-3.550671$

$-0.934370$

$-1.129580$

$-0.542450$

$-0.157730$

$-2.925410$

$-2.452050$

$-2.211980$

$-3.795140$

$-4.362080$

$-3.850140$
1.114870

0.924470

0.982390

$-1.210290$

$-2.319980$

$-1.004080$

$-0.051100$

2.492230

2.703740

$-1.898230$

$-0.960670$

$-0.399730$

$-1.681210$

$-0.417640$

$-0.390900$

0.189100

2.699750

2.956890

2.881140

2.450150

3.938950

2. 369180

3.501120

4.575400

3. 314510

3. 234740

3. 211100

4.192880

2.450270

3.174280

4.062560

5.029870 


\begin{tabular}{|c|c|c|c|}
\hline $\mathrm{H}$ & 0.150239 & -5.164580 & 4.156650 \\
\hline $\mathrm{H}$ & 1.856239 & -4.821510 & 3.842480 \\
\hline 0 & 1.241140 & -1.935670 & 1.290970 \\
\hline 0 & 0.491550 & -4.103120 & 1.699290 \\
\hline B & 0.790020 & -3.157700 & 0.675710 \\
\hline 0 & -0.316830 & -2.842340 & -0.267900 \\
\hline C & -1.750330 & -3.257650 & -2.115500 \\
\hline C & -2.787810 & -4.056210 & -2.621260 \\
\hline C & -1.465490 & -2.022610 & -2.721500 \\
\hline C & -3.526530 & -3.633580 & -3.724260 \\
\hline $\mathrm{H}$ & -3.012311 & -5.010050 & -2.148540 \\
\hline C & -2.205820 & -1.606110 & -3.825310 \\
\hline $\mathrm{H}$ & -0.663720 & -1.405190 & -2.328120 \\
\hline C & -3.232790 & -2.409220 & -4.331740 \\
\hline $\mathrm{H}$ & -4.327521 & -4.257349 & -4.111250 \\
\hline $\mathrm{H}$ & -1.977200 & -0.652650 & -4.293030 \\
\hline $\mathrm{H}$ & -3.801820 & -2.083069 & -5.198170 \\
\hline $\mathrm{H}$ & 1.666790 & -3.376280 & -2.453100 \\
\hline $\mathrm{H}$ & 0.375049 & -5.882740 & -1.214010 \\
\hline H & 0.073329 & -5.177590 & -2.889700 \\
\hline C & 0.719521 & 2.472260 & 0.509210 \\
\hline C & 1.767260 & 1.705540 & 0.013420 \\
\hline C & 3.061400 & 1.701089 & 0.639680 \\
\hline C & 3.227861 & 2.432889 & 1.800400 \\
\hline $\mathrm{H}$ & 4.207531 & 2.470489 & 2.269210 \\
\hline C & -1.673930 & 1.745130 & 0.138580 \\
\hline C & -0.608259 & 2.577280 & -0.197850 \\
\hline C & -3.113509 & 2.961830 & -1.396130 \\
\hline C & -2.966540 & 1.956090 & -0.459630 \\
\hline $\mathrm{H}$ & -4.091509 & 3.136811 & -1.837340 \\
\hline C & -2.044429 & 3.805780 & -1.777310 \\
\hline C & -0.765759 & 3.625210 & -1.164460 \\
\hline C & 0.292661 & 4.495120 & -1.545370 \\
\hline C & 0.093151 & 5.488810 & -2.477090 \\
\hline C & -1.175319 & 5.666910 & -3.079530 \\
\hline C & -2.219669 & 4.840870 & -2.735720 \\
\hline $\mathrm{H}$ & 1.272571 & 4.361020 & -1.101640 \\
\hline $\mathrm{H}$ & 0.917221 & 6.139660 & -2.756640 \\
\hline H & -1.319169 & 6.455160 & -3.813730 \\
\hline H & -3.199079 & 4.966810 & -3.191510 \\
\hline C & 2.176111 & 3.184030 & 2.377120 \\
\hline C & 0.905381 & 3.226580 & 1.718090 \\
\hline C & -0.126059 & 4.023040 & 2.295040 \\
\hline C & 0.087071 & 4.722200 & 3.463830 \\
\hline C & 1.343091 & 4.672470 & 4.113450 \\
\hline C & 2.364191 & 3.922830 & 3.576700 \\
\hline $\mathrm{H}$ & -1.088159 & 4.081080 & 1.797750 \\
\hline $\mathrm{H}$ & -0.714159 & 5.322380 & 3.885910 \\
\hline $\mathrm{H}$ & 1.498391 & 5.229950 & 5.032970 \\
\hline H & 3.335331 & 3.882449 & 4.064230 \\
\hline C & -6.492740 & -0.245509 & 0.538230 \\
\hline C & -5.702690 & 0.257281 & 1.581290 \\
\hline C & -4.542770 & 0.962131 & 1.241110 \\
\hline C & -4.171650 & 1.151231 & -0.098590 \\
\hline
\end{tabular}




\begin{tabular}{|c|c|c|c|}
\hline C & -4.991400 & 0.631051 & -1.111900 \\
\hline C & -6.165290 & -0.070179 & -0.813820 \\
\hline $\mathrm{H}$ & -7.394350 & -0.797069 & 0.788080 \\
\hline $\mathrm{H}$ & -3.904010 & 1.371311 & 2.012770 \\
\hline $\mathrm{H}$ & -4.682570 & 0.769581 & -2.141470 \\
\hline C & -6.119190 & 0.016411 & 3.045590 \\
\hline C & -7.085000 & -0.654219 & -1.904210 \\
\hline C & -6.118310 & -1.504689 & 3.329750 \\
\hline $\mathrm{H}$ & -6.423100 & -1.700229 & 4.365500 \\
\hline $\mathrm{H}$ & -6.809150 & -2.042269 & 2.671110 \\
\hline $\mathrm{H}$ & -5.118110 & -1.928029 & 3.183530 \\
\hline C & -5.161140 & 0.692611 & 4.045610 \\
\hline $\mathrm{H}$ & -5.489490 & 0.479221 & 5.069630 \\
\hline $\mathrm{H}$ & -4.132050 & 0.333771 & 3.940090 \\
\hline $\mathrm{H}$ & -5.151660 & 1.781741 & 3.921640 \\
\hline C & -7.539740 & 0.579821 & 3.286110 \\
\hline $\mathrm{H}$ & -7.844970 & 0.412651 & 4.326560 \\
\hline $\mathrm{H}$ & -7.571110 & 1.658241 & 3.092620 \\
\hline $\mathrm{H}$ & -8.286260 & 0.103561 & 2.641550 \\
\hline C & -6.595420 & -0.320389 & -3.326330 \\
\hline $\mathrm{H}$ & -7.289320 & -0.742279 & -4.062850 \\
\hline $\mathrm{H}$ & -6.549080 & 0.761291 & -3.497500 \\
\hline $\mathrm{H}$ & -5.604240 & -0.742099 & -3.524960 \\
\hline C & -7.136220 & -2.194399 & -1.763220 \\
\hline $\mathrm{H}$ & -7.795660 & -2.626769 & -2.526540 \\
\hline $\mathrm{H}$ & -7.516570 & -2.499829 & -0.782640 \\
\hline $\mathrm{H}$ & -6.138490 & -2.630469 & -1.887380 \\
\hline C & -8.511570 & -0.077049 & -1.744050 \\
\hline $\mathrm{H}$ & -9.177210 & -0.486069 & -2.514300 \\
\hline $\mathrm{H}$ & -8.944820 & -0.319419 & -0.768320 \\
\hline $\mathrm{H}$ & -8.505700 & 1.014411 & -1.844680 \\
\hline C & 4.233060 & 0.996669 & 0.043750 \\
\hline C & 4.492750 & 1.084979 & -1.331580 \\
\hline C & 5.130810 & 0.293549 & 0.862510 \\
\hline C & 5.606140 & 0.461159 & -1.904030 \\
\hline $\mathrm{H}$ & 3.817330 & 1.658199 & -1.952310 \\
\hline C & 6.267270 & -0.328161 & 0.332590 \\
\hline $\mathrm{H}$ & 4.919710 & 0.230589 & 1.923740 \\
\hline C & 6.472230 & -0.237571 & -1.052110 \\
\hline $\mathrm{H}$ & 7.340120 & -0.728831 & -1.481990 \\
\hline C & 7.277920 & -1.100431 & 1.203750 \\
\hline C & 5.891390 & 0.518359 & -3.417260 \\
\hline C & 7.307810 & -2.584051 & 0.765700 \\
\hline $\mathrm{H}$ & 6.323440 & -3.049541 & 0.889950 \\
\hline $\mathrm{H}$ & 7.597190 & -2.692281 & -0.284950 \\
\hline $\mathrm{H}$ & 8.029340 & -3.147011 & 1.370690 \\
\hline C & 8.687350 & -0.486511 & 1.026710 \\
\hline $\mathrm{H}$ & 9.418210 & -1.029112 & 1.638770 \\
\hline $\mathrm{H}$ & 9.027440 & -0.531251 & -0.012880 \\
\hline $\mathrm{H}$ & 8.698380 & 0.564809 & 1.336330 \\
\hline C & 6.925720 & -1.048731 & 2.702530 \\
\hline $\mathrm{H}$ & 6.900540 & -0.019661 & 3.079230 \\
\hline $\mathrm{H}$ & 5.956910 & -1.514451 & 2.913630 \\
\hline H & 7.683340 & -1.592551 & 3.278080 \\
\hline
\end{tabular}




$\begin{array}{|llrl|}\mathrm{C} & 7.288170 & 1.135779 & -3.663270 \\ \mathrm{H} & 7.342170 & 2.154109 & -3.261430 \\ \mathrm{H} & 8.085990 & 0.548849 & -3.195600 \\ \mathrm{H} & 7.498510 & 1.183889 & -4.738670 \\ \mathrm{C} & 5.857810 & -0.917871 & -3.992610 \\ \mathrm{H} & 6.600500 & -1.566311 & -3.515210 \\ \mathrm{H} & 4.871640 & -1.373221 & -3.847740 \\ \mathrm{H} & 6.070270 & -0.901861 & -5.068670 \\ \mathrm{C} & 4.850960 & 1.363879 & -4.177330 \\ \mathrm{H} & 5.083640 & 1.354919 & -5.248380 \\ \mathrm{H} & 3.833560 & 0.977989 & -4.053100 \\ \mathrm{H} & 4.858421 & 2.409339 & -3.847990 \\ & & \end{array}$

\section{C-Catalyzed reaction TS 115}

\section{Datum}

Value

\begin{tabular}{lc}
\hline B3LYP/6-31G(d) Energy & -3038.944205 \\
\hline B3LYP-D3(BJ)/def2-TZVPP-IEF-PCM(DCM) Energy & -3040.499656 \\
\hline B3LYP-D3(BJ)/def2-TZVPP-IEF-PCM(DCM)//B3LYP/6-31G(d) Free Energy (Quasiharmonic) & -3039.272595 \\
\hline
\end{tabular}

Number of Imaginary Frequencies

Frequencies (Top 3 out of 450)

$\begin{array}{lr}\text { 1. } & -359.8567 \mathrm{~cm}^{-1} \\ \text { 2. } & 8.4950 \mathrm{~cm}^{-1} \\ \text { 3. } & 10.4299 \mathrm{~cm}^{-1}\end{array}$

\section{B3LYP/6-31G(d) Molecular Geometry in Cartesian Coordinates}

C

$\mathrm{H}$

$\mathrm{H}$

C

0

0

$\mathrm{H}$

0

$\mathrm{H}$

C

C

C

C

$\mathrm{H}$

$$
\begin{array}{r}
3.257963 \\
3.145355 \\
2.441004 \\
0.024163 \\
0.837369 \\
-0.944390 \\
-1.572036 \\
4.452870 \\
5.186537 \\
-2.401657 \\
-3.918054 \\
-2.724703 \\
-2.865401 \\
-3.120565
\end{array}
$$

-0.026533
1.006601
-0.219765
-0.015981
0.876696
-0.167920
-0.927872
-0.192719
0.142731
1.483286
1.136897
-0.927888
0.447330
1.158450

\subsection{3}

1.265891

2. 307698

0.007259

$-0.120929$

$-0.912403$

$-0.787883$

2. 375524

1.835571

$-3.462444$

$-1.962340$

$-3.899776$

$-4.251134$

$-1.217256$ 


\begin{tabular}{|c|c|c|c|}
\hline $\mathrm{H}$ & -1.755860 & -1.176728 & -3.461887 \\
\hline $\mathrm{H}$ & -3.026970 & -1.634243 & -4.672629 \\
\hline C & -3.377522 & -3.222648 & -1.219799 \\
\hline C & -4.830426 & -3.269536 & -1.819097 \\
\hline C & -2.380842 & -4.053208 & -2.041698 \\
\hline H & -1.360609 & -3.784697 & -1.754611 \\
\hline $\mathrm{H}$ & -2.513709 & -5.127502 & -1.870520 \\
\hline $\mathrm{H}$ & -2.492072 & -3.859796 & -3.112489 \\
\hline C & -3.274888 & -3.591344 & 0.258938 \\
\hline H & -3.608985 & -4.621973 & 0.426740 \\
\hline $\mathrm{H}$ & -3.878786 & -2.925053 & 0.878923 \\
\hline $\mathrm{H}$ & -2.236539 & -3.517769 & 0.599511 \\
\hline C & -5.903912 & -2.800052 & -0.821828 \\
\hline $\mathrm{H}$ & -6.077158 & -3.534742 & -0.027199 \\
\hline $\mathrm{H}$ & -5.625313 & -1.846223 & -0.364298 \\
\hline $\mathrm{H}$ & -6.843598 & -2.651205 & -1.363168 \\
\hline C & -5.238336 & -4.617391 & -2.416212 \\
\hline H & -5.237884 & -5.405702 & -1.654049 \\
\hline $\mathrm{H}$ & -6.251798 & -4.543427 & -2.824562 \\
\hline $\mathrm{H}$ & -4.571733 & -4.915035 & -3.228963 \\
\hline 0 & -3.038801 & -1.820593 & -1.376436 \\
\hline 0 & -4.746911 & -2.306946 & -2.880737 \\
\hline B & -3.811740 & -1.308393 & -2.504481 \\
\hline 0 & -4.487524 & -0.010804 & -2.199490 \\
\hline C & -4.768429 & 2.339687 & -2.074979 \\
\hline C & -6.005007 & 2.291629 & -2.737388 \\
\hline C & -4.345051 & 3.544857 & -1.494694 \\
\hline C & -6.796406 & 3.434585 & -2.821937 \\
\hline $\mathrm{H}$ & -6.334920 & 1.352153 & -3.168657 \\
\hline C & -5.138885 & 4.686390 & -1.581619 \\
\hline $\mathrm{H}$ & -3.393699 & 3.578623 & -0.970915 \\
\hline C & -6.366364 & 4.634360 & -2.247470 \\
\hline $\mathrm{H}$ & -7.753726 & 3.390444 & -3.334250 \\
\hline $\mathrm{H}$ & -4.801486 & 5.615288 & -1.129762 \\
\hline $\mathrm{H}$ & -6.986704 & 5.523919 & -2.315660 \\
\hline $\mathrm{H}$ & -3.592954 & 0.697806 & -5.025699 \\
\hline $\mathrm{H}$ & -1.565976 & 1.309543 & -2.789904 \\
\hline $\mathrm{H}$ & -2.577511 & 2.511826 & -3.761001 \\
\hline C & 1.030376 & -1.926632 & 1.300173 \\
\hline C & 0.013920 & -0.982511 & 1.171297 \\
\hline C & -1.038885 & -0.878496 & 2.142827 \\
\hline C & -1.093652 & -1.814359 & 3.156632 \\
\hline $\mathrm{H}$ & -1.872774 & -1.735685 & 3.910564 \\
\hline C & 3.190678 & -0.995281 & 0.437457 \\
\hline C & 2.184204 & -1.949343 & 0.333679 \\
\hline C & 4.292190 & -1.917719 & -1.527998 \\
\hline C & 4.237443 & -0.956433 & -0.543065 \\
\hline $\mathrm{H}$ & 5.087399 & -1.880394 & -2.269010 \\
\hline C & 3.320190 & -2.943123 & -1.623013 \\
\hline C & 2.233083 & -2.954365 & -0.692352 \\
\hline C & 1.262702 & -3.986823 & -0.815523 \\
\hline C & 1.366701 & -4.952056 & -1.794160 \\
\hline C & 2.440741 & -4.932297 & -2.714059 \\
\hline C & 3.393999 & -3.943964 & -2.627987 \\
\hline
\end{tabular}




\begin{tabular}{|c|c|c|c|}
\hline $\mathrm{H}$ & 0.436575 & -4.021406 & -0.113646 \\
\hline $\mathrm{H}$ & 0.615177 & -5.733987 & -1.860539 \\
\hline $\mathrm{H}$ & 2.509010 & -5.696035 & -3.483928 \\
\hline $\mathrm{H}$ & 4.224819 & -3.914360 & -3.329184 \\
\hline C & -0.119161 & -2.832384 & 3.293322 \\
\hline C & 0.981789 & -2.872322 & 2.378685 \\
\hline C & 1.995167 & -3.849315 & 2.584123 \\
\hline C & 1.908440 & -4.752055 & 3.620333 \\
\hline C & 0.808106 & -4.722916 & 4.510292 \\
\hline C & -0.179951 & -3.779299 & 4.351498 \\
\hline $\mathrm{H}$ & 2.846630 & -3.872647 & 1.913422 \\
\hline $\mathrm{H}$ & 2.693973 & -5.489489 & 3.760275 \\
\hline $\mathrm{H}$ & 0.752003 & -5.441580 & 5.323411 \\
\hline $\mathrm{H}$ & -1.021569 & -3.738056 & 5.038788 \\
\hline C & 7.156110 & 2.168923 & -0.668877 \\
\hline C & 7.589954 & 0.854613 & -0.416751 \\
\hline C & 6.621612 & -0.152705 & -0.365919 \\
\hline C & 5.259007 & 0.137326 & -0.553833 \\
\hline C & 4.869825 & 1.456388 & -0.804900 \\
\hline C & 5.810877 & 2.496576 & -0.868274 \\
\hline $\mathrm{H}$ & 7.899426 & 2.956781 & -0.709246 \\
\hline $\mathrm{H}$ & 6.901616 & -1.182043 & -0.172072 \\
\hline $\mathrm{H}$ & 3.815015 & 1.659417 & -0.966412 \\
\hline C & 9.090337 & 0.575258 & -0.202904 \\
\hline C & 5.335046 & 3.933170 & -1.158109 \\
\hline C & 9.378878 & -0.916654 & 0.051057 \\
\hline $\mathrm{H}$ & 10.455232 & -1.064419 & 0.194199 \\
\hline $\mathrm{H}$ & 8.873005 & -1.283159 & 0.951348 \\
\hline $\mathrm{H}$ & 9.069355 & -1.542222 & -0.793875 \\
\hline C & 9.881540 & 1.009699 & -1.459701 \\
\hline $\mathrm{H}$ & 10.952561 & 0.817626 & -1.320567 \\
\hline $\mathrm{H}$ & 9.548700 & 0.454108 & -2.343914 \\
\hline $\mathrm{H}$ & 9.759950 & 2.077149 & -1.670922 \\
\hline C & 9.593658 & 1.376332 & 1.021504 \\
\hline $\mathrm{H}$ & 10.662870 & 1.192892 & 1.184500 \\
\hline $\mathrm{H}$ & 9.456914 & 2.454523 & 0.888104 \\
\hline $\mathrm{H}$ & 9.056604 & 1.080974 & 1.930145 \\
\hline C & 4.322400 & 4.369004 & -0.072223 \\
\hline $\mathrm{H}$ & 3.974184 & 5.391051 & -0.266202 \\
\hline $\mathrm{H}$ & 3.442855 & 3.717749 & -0.047681 \\
\hline $\mathrm{H}$ & 4.781947 & 4.350505 & 0.922777 \\
\hline C & 6.493595 & 4.947900 & -1.166878 \\
\hline $\mathrm{H}$ & 6.102445 & 5.951135 & -1.370152 \\
\hline $\mathrm{H}$ & 7.232189 & 4.719098 & -1.943662 \\
\hline $\mathrm{H}$ & 7.011258 & 4.985614 & -0.201429 \\
\hline C & 4.647528 & 3.974626 & -2.543775 \\
\hline $\mathrm{H}$ & 4.294608 & 4.989899 & -2.763391 \\
\hline $\mathrm{H}$ & 5.344908 & 3.678680 & -3.336014 \\
\hline $\mathrm{H}$ & 3.783312 & 3.304237 & -2.590410 \\
\hline C & -1.987622 & 0.272980 & 2.160304 \\
\hline C & -3.366491 & 0.076316 & 2.348760 \\
\hline C & -1.490831 & 1.578525 & 2.099348 \\
\hline C & -4.243638 & 1.157095 & 2.464647 \\
\hline $\mathrm{H}$ & -3.737746 & -0.940615 & 2.399046 \\
\hline
\end{tabular}




\begin{tabular}{|c|c|c|c|}
\hline C & -2.336730 & 2.692643 & 2.208560 \\
\hline $\mathrm{H}$ & -0.421737 & 1.724128 & 1.994489 \\
\hline C & -3.703656 & 2.454979 & 2.381005 \\
\hline $\mathrm{H}$ & -4.378129 & 3.298876 & 2.466396 \\
\hline C & -1.722796 & 4.106646 & 2.192924 \\
\hline C & -5.759654 & 0.977549 & 2.679764 \\
\hline C & -0.801589 & 4.263949 & 3.427454 \\
\hline $\mathrm{H}$ & -1.365492 & 4.125237 & 4.357025 \\
\hline $\mathrm{H}$ & 0.014946 & 3.534628 & 3.419132 \\
\hline $\mathrm{H}$ & -0.355797 & 5.266163 & 3.445532 \\
\hline C & -0.883843 & 4.309939 & 0.908731 \\
\hline $\mathrm{H}$ & -0.385568 & 5.286857 & 0.934496 \\
\hline $\mathrm{H}$ & -0.114326 & 3.542092 & 0.784857 \\
\hline $\mathrm{H}$ & -1.517721 & 4.284705 & 0.014396 \\
\hline C & -2.791661 & 5.214453 & 2.249095 \\
\hline $\mathrm{H}$ & -3.492385 & 5.149606 & 1.408262 \\
\hline $\mathrm{H}$ & -3.371835 & 5.178520 & 3.178242 \\
\hline $\mathrm{H}$ & -2.307372 & 6.196516 & 2.202978 \\
\hline C & -6.523651 & 1.545417 & 1.460365 \\
\hline $\mathrm{H}$ & -6.304271 & 2.606010 & 1.298872 \\
\hline $\mathrm{H}$ & -6.255734 & 1.008810 & 0.543544 \\
\hline $\mathrm{H}$ & -7.606166 & 1.445548 & 1.608423 \\
\hline C & -6.157694 & -0.500685 & 2.851588 \\
\hline $\mathrm{H}$ & -5.927604 & -1.093985 & 1.959982 \\
\hline $\mathrm{H}$ & -5.655218 & -0.960847 & 3.710398 \\
\hline $\mathrm{H}$ & -7.237751 & -0.573306 & 3.022015 \\
\hline C & -6.195954 & 1.735033 & 3.956846 \\
\hline $\mathrm{H}$ & -7.272999 & 1.609830 & 4.122272 \\
\hline $\mathrm{H}$ & -5.669928 & 1.352641 & 4.839221 \\
\hline $\mathrm{H}$ & -5.995138 & 2.809003 & 3.887956 \\
\hline
\end{tabular}

\section{C-Catalyzed reaction TS 116}

\section{Datum}

Value

\begin{tabular}{lc}
\hline B3LYP/6-31G(d) Energy & -3038.945328 \\
\hline B3LYP-D3(BJ)/def2-TZVPP-IEF-PCM(DCM) Energy & -3040.499838 \\
\hline B3LYP-D3(BJ)/def2-TZVPP-IEF-PCM(DCM)//B3LYP/6-31G(d) Free Energy (Quasiharmonic) & -3039.27258 \\
\hline
\end{tabular}

Number of Imaginary Frequencies

Frequencies (Top 3 out of 450)

$\begin{array}{lr}\text { 1. } & -334.4033 \mathrm{~cm}^{-1} \\ \text { 2. } & 7.6694 \mathrm{~cm}^{-1} \\ \text { 3. } & 9.8363 \mathrm{~cm}^{-1}\end{array}$




\begin{tabular}{|c|c|c|c|}
\hline C & 1.759100 & -0.434720 & 0.562040 \\
\hline $\mathrm{H}$ & 1.850660 & 0.119220 & -0.379490 \\
\hline $\mathrm{H}$ & 0.803920 & -0.153870 & 1.001430 \\
\hline C & -1.345360 & -1.774700 & -0.909350 \\
\hline 0 & -1.330390 & -2.789200 & -1.573220 \\
\hline 0 & -1.139480 & -0.579480 & -1.481720 \\
\hline $\mathrm{H}$ & -1.252850 & 0.211360 & -0.890370 \\
\hline 0 & 2.753890 & 0.003450 & 1.500480 \\
\hline $\mathrm{H}$ & 3.619340 & -0.133010 & 1.078820 \\
\hline C & 0.436290 & 2.796670 & 3.351080 \\
\hline C & 1.437560 & 2.972690 & 1.419320 \\
\hline C & -1.594620 & 2.748140 & 1.956670 \\
\hline C & -0.633890 & 2.117810 & 2.808920 \\
\hline $\mathrm{H}$ & 2.088250 & 2.127050 & 1.651370 \\
\hline $\mathrm{H}$ & -1.817140 & 3.782420 & 2.230290 \\
\hline $\mathrm{H}$ & -2.501950 & 2.167910 & 1.782130 \\
\hline C & -1.796120 & 2.651490 & -1.840630 \\
\hline C & -2.114310 & 4.107370 & -1.337700 \\
\hline C & -0.548870 & 2.585870 & -2.736800 \\
\hline $\mathrm{H}$ & -0.272300 & 1.537140 & -2.881690 \\
\hline $\mathrm{H}$ & -0.736940 & 3.027600 & -3.721530 \\
\hline $\mathrm{H}$ & 0.297480 & 3.104710 & -2.276770 \\
\hline C & -2.966690 & 1.939830 & -2.520090 \\
\hline $\mathrm{H}$ & -2.661500 & 0.942670 & -2.849370 \\
\hline $\mathrm{H}$ & -3.295950 & 2.502870 & -3.401620 \\
\hline $\mathrm{H}$ & -3.815600 & 1.823000 & -1.842510 \\
\hline C & -3.563170 & 4.271710 & -0.849420 \\
\hline $\mathrm{H}$ & -4.279240 & 4.276320 & -1.678620 \\
\hline $\mathrm{H}$ & -3.839020 & 3.468960 & -0.158980 \\
\hline $\mathrm{H}$ & -3.648940 & 5.224390 & -0.316480 \\
\hline C & -1.782950 & 5.216870 & -2.338390 \\
\hline $\mathrm{H}$ & -2.370180 & 5.108950 & -3.257950 \\
\hline $\mathrm{H}$ & -2.022020 & 6.191320 & -1.898680 \\
\hline $\mathrm{H}$ & -0.722130 & 5.216050 & -2.599840 \\
\hline 0 & -1.485090 & 1.972690 & -0.591230 \\
\hline 0 & -1.247470 & 4.244610 & -0.200140 \\
\hline B & -0.930160 & 2.949180 & 0.317970 \\
\hline 0 & 0.555590 & 2.725700 & 0.498150 \\
\hline C & 2.041380 & 4.314130 & 1.580350 \\
\hline C & 3.231170 & 4.423090 & 2.320790 \\
\hline C & 1.488320 & 5.461350 & 0.987220 \\
\hline C & 3.855300 & 5.657450 & 2.476480 \\
\hline $\mathrm{H}$ & 3.663690 & 3.532370 & 2.771320 \\
\hline C & 2.117670 & 6.694490 & 1.149480 \\
\hline $\mathrm{H}$ & 0.576280 & 5.373390 & 0.404360 \\
\hline C & 3.297050 & 6.797960 & 1.891920 \\
\hline $\mathrm{H}$ & 4.776700 & 5.731550 & 3.047680 \\
\hline $\mathrm{H}$ & 1.688520 & 7.580060 & 0.688270 \\
\hline $\mathrm{H}$ & 3.783490 & 7.762580 & 2.010320 \\
\hline H & -0.608070 & 1.027750 & 2.830790 \\
\hline
\end{tabular}




\begin{tabular}{|c|c|c|c|}
\hline $\mathrm{H}$ & 0.361980 & 3.866830 & 3.516680 \\
\hline H & 1.177150 & 2.272140 & 3.947480 \\
\hline C & -0.468910 & -2.206330 & 1.372010 \\
\hline C & -1.538950 & -1.779780 & 0.593670 \\
\hline C & -2.784980 & -1.378420 & 1.185280 \\
\hline C & -2.871060 & -1.359320 & 2.566110 \\
\hline $\mathrm{H}$ & -3.816270 & -1.099080 & 3.035010 \\
\hline C & 1.813480 & -1.931510 & 0.323080 \\
\hline C & 0.775420 & -2.759060 & 0.729140 \\
\hline C & 3.095260 & -3.863270 & -0.413930 \\
\hline C & 2.987750 & -2.496880 & -0.280850 \\
\hline $\mathrm{H}$ & 3.988230 & -4.287850 & -0.866850 \\
\hline C & 2.057290 & -4.737610 & -0.005280 \\
\hline C & 0.867510 & -4.183460 & 0.561060 \\
\hline C & -0.173680 & -5.073750 & 0.939660 \\
\hline C & -0.036910 & -6.435000 & 0.779140 \\
\hline C & 1.145950 & -6.979620 & 0.227090 \\
\hline C & 2.169270 & -6.144860 & -0.159280 \\
\hline $\mathrm{H}$ & -1.090830 & -4.669240 & 1.352510 \\
\hline H & -0.847410 & -7.096490 & 1.072970 \\
\hline H & 1.240270 & -8.055190 & 0.103800 \\
\hline $\mathrm{H}$ & 3.080280 & -6.549790 & -0.593860 \\
\hline C & -1.784440 & -1.717180 & 3.400230 \\
\hline C & -0.561370 & -2.159510 & 2.801650 \\
\hline C & 0.517320 & -2.526200 & 3.653130 \\
\hline C & 0.388150 & -2.465360 & 5.022920 \\
\hline C & -0.825930 & -2.038420 & 5.613120 \\
\hline C & -1.887260 & -1.672920 & 4.817820 \\
\hline H & 1.453040 & -2.844500 & 3.207160 \\
\hline $\mathrm{H}$ & 1.224450 & -2.746470 & 5.657140 \\
\hline H & -0.915280 & -1.998420 & 6.695400 \\
\hline $\mathrm{H}$ & -2.823140 & -1.341640 & 5.261650 \\
\hline C & 6.249590 & -0.105020 & -1.683500 \\
\hline C & 5.013930 & 0.009840 & -2.328710 \\
\hline C & 3.951320 & -0.771290 & -1.845720 \\
\hline C & 4.115970 & -1.636610 & -0.759920 \\
\hline C & 5.376840 & -1.725550 & -0.142380 \\
\hline C & 6.461030 & -0.965250 & -0.591200 \\
\hline H & 7.082860 & 0.489890 & -2.037710 \\
\hline H & 2.980630 & -0.728890 & -2.330790 \\
\hline H & 5.485500 & -2.405420 & 0.695190 \\
\hline C & 4.786050 & 0.944390 & -3.532590 \\
\hline C & 7.854810 & -1.037290 & 0.062290 \\
\hline C & 4.323900 & 0.111440 & -4.751950 \\
\hline H & 4.155900 & 0.765620 & -5.616140 \\
\hline $\mathrm{H}$ & 5.080910 & -0.630920 & -5.029750 \\
\hline H & 3.388960 & -0.422150 & -4.553450 \\
\hline C & 3.693170 & 1.980860 & -3.177190 \\
\hline H & 3.514660 & 2.651600 & -4.026530 \\
\hline H & 2.740380 & 1.502770 & -2.927490 \\
\hline H & 3.995170 & 2.593400 & -2.319750 \\
\hline C & 6.060510 & 1.711570 & -3.933140 \\
\hline H & 5.844740 & 2.359910 & -4.789670 \\
\hline H & 6.425920 & 2.350450 & -3.120910 \\
\hline
\end{tabular}




\begin{tabular}{|c|c|c|c|}
\hline $\mathrm{H}$ & 6.870240 & 1.034260 & -4.228110 \\
\hline C & 8.898460 & -1.474390 & -0.993190 \\
\hline $\mathrm{H}$ & 9.896290 & -1.529850 & -0.541050 \\
\hline $\mathrm{H}$ & 8.654300 & -2.462550 & -1.399410 \\
\hline $\mathrm{H}$ & 8.952610 & -0.772920 & -1.832240 \\
\hline C & 8.237790 & 0.356650 & 0.614310 \\
\hline $\mathrm{H}$ & 9.233490 & 0.325100 & 1.073410 \\
\hline $\mathrm{H}$ & 8.258210 & 1.116640 & -0.173670 \\
\hline $\mathrm{H}$ & 7.522860 & 0.685660 & 1.377420 \\
\hline C & 7.906100 & -2.043500 & 1.227830 \\
\hline $\mathrm{H}$ & 8.914640 & -2.059850 & 1.656000 \\
\hline $\mathrm{H}$ & 7.211690 & -1.774950 & 2.032070 \\
\hline $\mathrm{H}$ & 7.670730 & -3.061920 & 0.898740 \\
\hline C & -3.999060 & -1.069220 & 0.376050 \\
\hline C & -4.866750 & -0.030100 & 0.763820 \\
\hline C & -4.345460 & -1.859230 & -0.723860 \\
\hline C & -6.058690 & 0.216410 & 0.080500 \\
\hline $\mathrm{H}$ & -4.586880 & 0.584570 & 1.611840 \\
\hline C & -5.519350 & -1.625740 & -1.457290 \\
\hline $\mathrm{H}$ & -3.697910 & -2.681170 & -1.003530 \\
\hline C & -6.357020 & -0.591790 & -1.033370 \\
\hline $\mathrm{H}$ & -7.276340 & -0.404110 & -1.575700 \\
\hline C & -5.828780 & -2.513700 & -2.677220 \\
\hline C & -7.059210 & 1.306730 & 0.513250 \\
\hline C & -5.929550 & -3.991560 & -2.229560 \\
\hline $\mathrm{H}$ & -4.998450 & -4.344830 & -1.775470 \\
\hline $\mathrm{H}$ & -6.734480 & -4.126640 & -1.497490 \\
\hline $\mathrm{H}$ & -6.142580 & -4.634840 & -3.092140 \\
\hline C & -7.153530 & -2.130830 & -3.363680 \\
\hline $\mathrm{H}$ & -7.322760 & -2.785050 & -4.226320 \\
\hline $\mathrm{H}$ & -8.011710 & -2.243989 & -2.690730 \\
\hline $\mathrm{H}$ & -7.139920 & -1.098340 & -3.731550 \\
\hline C & -4.685850 & -2.368970 & -3.711580 \\
\hline $\mathrm{H}$ & -4.611750 & -1.334030 & -4.065870 \\
\hline $\mathrm{H}$ & -3.713260 & -2.648050 & -3.294200 \\
\hline $\mathrm{H}$ & -4.877380 & -3.011730 & -4.579920 \\
\hline C & -8.394250 & 0.633641 & 0.914670 \\
\hline $\mathrm{H}$ & -8.248120 & -0.056639 & 1.753420 \\
\hline $\mathrm{H}$ & -8.827830 & 0.064201 & 0.085940 \\
\hline $\mathrm{H}$ & -9.127140 & 1.390801 & 1.220160 \\
\hline C & -6.556500 & 2.122900 & 1.719290 \\
\hline $\mathrm{H}$ & -5.617240 & 2.642550 & 1.498630 \\
\hline $\mathrm{H}$ & -6.400510 & 1.492800 & 2.602330 \\
\hline $\mathrm{H}$ & -7.299450 & 2.883390 & 1.985040 \\
\hline C & -7.316610 & 2.287650 & -0.654760 \\
\hline $\mathrm{H}$ & -8.041860 & 3.053601 & -0.353900 \\
\hline $\mathrm{H}$ & -7.718900 & 1.779190 & -1.536760 \\
\hline $\mathrm{H}$ & -6.392510 & 2.794750 & -0.953590 \\
\hline
\end{tabular}




\begin{tabular}{lc}
\hline B3LYP/6-31G(d) Energy & -3038.948873 \\
\hline B3LYP-D3(BJ)/def2-TZVPP-IEF-PCM(DCM) Energy & -3040.499947 \\
\hline B3LYP-D3(BJ)/def2-TZVPP-IEF-PCM(DCM)//B3LYP/6-31G(d) Free Energy (Quasiharmonic) & -3039.272276 \\
\hline
\end{tabular}

Number of Imaginary Frequencies

Frequencies (Top 3 out of 450)

$\begin{array}{lr}\text { 1. } & -381.3043 \mathrm{~cm}^{-1} \\ \text { 2. } & 6.2645 \mathrm{~cm}^{-1} \\ \text { 3. } & 10.4241 \mathrm{~cm}^{-1}\end{array}$

\section{B3LYP/6-31G(d) Molecular Geometry in Cartesian Coordinates}

C

$\mathrm{H}$

$\mathrm{H}$

C

0

0

$\mathrm{H}$

0

$\mathrm{H}$

C

C

C

C

$\mathrm{H}$

$\mathrm{H}$

$\mathrm{H}$

C

C

C

$\mathrm{H}$

$\mathrm{H}$

$\mathrm{H}$

C

$\mathrm{H}$

$\mathrm{H}$

$\mathrm{H}$

C

$\mathrm{H}$

$\mathrm{H}$

$\mathrm{H}$

C

$\mathrm{H}$
$-1.520323$

$-2.232328$

$-0.518597$

1.461465

1. 273894

1.484019

1.535446

$-1.708343$

$-1.076810$

0.880425

$-0.688340$

2. 327713

1.834562

$-1.040471$

3.122102

2.541323

1.479286

0.526905

2.958760

3.575704

3.182262

3. 242710

1.107478

1.171240

0.099673

1.813806

$-0.944385$

$-1.120451$

$-1.267009$

$-1.566654$

0.945371

0.929973
0.713289

$-0.108371$

0.283008

0.750534

1.063997

$-0.541882$

$-0.697905$

1. 241241

1.970126

$-5.254461$

$-3.933237$

$-3.842660$

$-4.265786$

$-4.756565$

$-3.097975$

$-4.651605$

$-1.856621$

$-3.061192$

$-2.168919$

$-1.412257$

$-2.150029$

$-3.149007$

$-0.522328$

$-0.597643$

$-0.194829$

0.252694

$-2.636486$

$-2.049945$

$-2.042618$

$-3.536505$

$-3.869886$

$-3.251739$
1.038882

0.956621

0.946974

$-1.297815$

$-2.453911$

$-0.936721$

0.040353

2. 362703

2.479829

$-1.332035$

$-0.616667$

0.084861

$-1.181118$

0.010119

0.046256

0.785798

2.935437

3. 289367

3. 212236

2. 717461

4. 284529

2. 818113

3. 580344

4.672621

3. 315716

3. 260988

3. 422549

4.330621

2. 563081

3.469300

4.518180

5.423592 


\begin{tabular}{|c|c|c|c|}
\hline $\mathrm{H}$ & 0.244902 & -4.698909 & 4.664856 \\
\hline $\mathrm{H}$ & 1.945390 & -4.293815 & 4.399687 \\
\hline 0 & 1.305964 & -1.759010 & 1.498063 \\
\hline 0 & 0.653599 & -3.905595 & 2.126651 \\
\hline B & 0.950472 & -3.068591 & 1.011927 \\
\hline 0 & -0.122202 & -2.932650 & -0.007123 \\
\hline C & -1.444184 & -3.640675 & -1.848218 \\
\hline C & -2.407691 & -4.554233 & -2.303538 \\
\hline C & -1.205845 & -2.462239 & -2.574962 \\
\hline C & -3.120425 & -4.299378 & -3.472924 \\
\hline $\mathrm{H}$ & -2.595908 & -5.464613 & -1.738223 \\
\hline C & -1.920376 & -2.213861 & -3.744417 \\
\hline $\mathrm{H}$ & -0.460739 & -1.756225 & -2.221483 \\
\hline C & -2.874929 & -3.129796 & -4.197794 \\
\hline $\mathrm{H}$ & -3.866201 & -5.009871 & -3.818558 \\
\hline $\mathrm{H}$ & -1.728882 & -1.302458 & -4.303791 \\
\hline $\mathrm{H}$ & -3.426657 & -2.933161 & -5.113167 \\
\hline $\mathrm{H}$ & 1.994814 & -3.599658 & -2.030362 \\
\hline $\mathrm{H}$ & 0.773827 & -6.015220 & -0.561398 \\
\hline $\mathrm{H}$ & 0.517680 & -5.523307 & -2.318440 \\
\hline C & 0.609928 & 2.548533 & 0.205646 \\
\hline C & 1.680027 & 1.752811 & -0.186060 \\
\hline C & 2.969101 & 1.858146 & 0.442414 \\
\hline C & 3.109702 & 2.738069 & 1.499091 \\
\hline $\mathrm{H}$ & 4.084784 & 2.860362 & 1.962980 \\
\hline C & -1.762906 & 1.722058 & -0.070105 \\
\hline C & -0.716712 & 2.529233 & -0.511319 \\
\hline C & -3.229630 & 2.698781 & -1.743823 \\
\hline C & -3.060247 & 1.827397 & -0.684888 \\
\hline $\mathrm{H}$ & -4.212021 & 2.795027 & -2.199292 \\
\hline C & -2.178776 & 3.508533 & -2.234829 \\
\hline C & -0.897074 & 3.439358 & -1.605207 \\
\hline C & 0.141325 & 4.276551 & -2.098334 \\
\hline C & -0.079711 & 5.134454 & -3.152085 \\
\hline C & -1.350918 & 5.202632 & -3.770928 \\
\hline C & -2.376332 & 4.404740 & -3.320274 \\
\hline $\mathrm{H}$ & 1.123764 & 4.224183 & -1.643173 \\
\hline $\mathrm{H}$ & 0.729717 & 5.761547 & -3.515951 \\
\hline $\mathrm{H}$ & -1.511801 & 5.883918 & -4.602205 \\
\hline $\mathrm{H}$ & -3.357624 & 4.446828 & -3.787330 \\
\hline C & 2.036598 & 3.530230 & 1.971447 \\
\hline C & 0.768375 & 3.453697 & 1.310798 \\
\hline C & -0.286941 & 4.288170 & 1.781230 \\
\hline C & -0.097921 & 5.136086 & 2.851492 \\
\hline C & 1.156188 & 5.204418 & 3.502985 \\
\hline C & 2.199555 & 4.420901 & 3.067007 \\
\hline $\mathrm{H}$ & -1.248772 & 4.254719 & 1.281413 \\
\hline $\mathrm{H}$ & -0.917289 & 5.762252 & 3.193366 \\
\hline $\mathrm{H}$ & 1.292309 & 5.878251 & 4.344314 \\
\hline $\mathrm{H}$ & 3.169194 & 4.469276 & 3.556790 \\
\hline C & -6.554891 & -0.257882 & 0.639297 \\
\hline C & -5.759306 & 0.400152 & 1.594443 \\
\hline C & -4.612377 & 1.059913 & 1.150578 \\
\hline C & -4.250047 & 1.060283 & -0.208610 \\
\hline
\end{tabular}




\begin{tabular}{|c|c|c|c|}
\hline C & -5.066569 & 0.389660 & -1.125060 \\
\hline C & -6.234866 & -0.276503 & -0.721166 \\
\hline $\mathrm{H}$ & -7.448355 & -0.770050 & 0.976882 \\
\hline $\mathrm{H}$ & -3.972998 & 1.580827 & 1.851066 \\
\hline $\mathrm{H}$ & -4.770335 & 0.379731 & -2.170110 \\
\hline C & -6.165991 & 0.366456 & 3.080641 \\
\hline C & -7.108013 & -0.991124 & -1.771140 \\
\hline C & -6.144323 & -1.098395 & 3.579411 \\
\hline $\mathrm{H}$ & -6.441794 & -1.148029 & 4.634393 \\
\hline $\mathrm{H}$ & -6.830908 & -1.733040 & 3.008617 \\
\hline $\mathrm{H}$ & -5.139318 & -1.526251 & 3.489770 \\
\hline C & -5.211313 & 1.189512 & 3.967206 \\
\hline $\mathrm{H}$ & -5.532527 & 1.122809 & 5.013308 \\
\hline $\mathrm{H}$ & -4.179081 & 0.829215 & 3.907839 \\
\hline $\mathrm{H}$ & -5.214747 & 2.249422 & 3.687471 \\
\hline C & -7.591987 & 0.942214 & 3.247906 \\
\hline $\mathrm{H}$ & -7.888864 & 0.923396 & 4.303874 \\
\hline $\mathrm{H}$ & -7.637244 & 1.981160 & 2.901468 \\
\hline $\mathrm{H}$ & -8.336335 & 0.369675 & 2.684439 \\
\hline C & -6.276557 & -2.088527 & -2.475249 \\
\hline $\mathrm{H}$ & -6.883197 & -2.598824 & -3.234298 \\
\hline $\mathrm{H}$ & -5.393029 & -1.678174 & -2.973579 \\
\hline $\mathrm{H}$ & -5.929011 & -2.839249 & -1.756573 \\
\hline C & -8.350306 & -1.658894 & -1.151346 \\
\hline $\mathrm{H}$ & -8.936923 & -2.147539 & -1.937692 \\
\hline $\mathrm{H}$ & -9.003216 & -0.930537 & -0.657106 \\
\hline $\mathrm{H}$ & -8.077158 & -2.426585 & -0.418345 \\
\hline C & -7.592325 & 0.036899 & -2.821541 \\
\hline $\mathrm{H}$ & -8.211207 & -0.455393 & -3.582121 \\
\hline $\mathrm{H}$ & -8.194042 & 0.823221 & -2.351191 \\
\hline $\mathrm{H}$ & -6.754840 & 0.520017 & -3.335483 \\
\hline C & 4.161214 & 1.105523 & -0.044367 \\
\hline C & 4.429672 & 1.000081 & -1.416861 \\
\hline C & 5.068258 & 0.547826 & 0.871039 \\
\hline C & 5.560543 & 0.324692 & -1.887789 \\
\hline $\mathrm{H}$ & 3.748026 & 1.461697 & -2.118432 \\
\hline C & 6.223046 & -0.115990 & 0.442015 \\
\hline $\mathrm{H}$ & 4.850306 & 0.631729 & 1.929470 \\
\hline C & 6.436392 & -0.221881 & -0.940207 \\
\hline $\mathrm{H}$ & 7.318867 & -0.748659 & -1.290920 \\
\hline C & 7.241901 & -0.734644 & 1.419914 \\
\hline C & 5.853779 & 0.164483 & -3.392185 \\
\hline C & 8.647295 & -0.148180 & 1.144636 \\
\hline $\mathrm{H}$ & 8.987687 & -0.355220 & 0.125009 \\
\hline $\mathrm{H}$ & 8.652627 & 0.938953 & 1.284278 \\
\hline $\mathrm{H}$ & 9.381552 & -0.583017 & 1.833818 \\
\hline C & 6.894543 & -0.450599 & 2.893700 \\
\hline $\mathrm{H}$ & 7.661010 & -0.888706 & 3.543026 \\
\hline $\mathrm{H}$ & 6.859138 & 0.624740 & 3.103525 \\
\hline $\mathrm{H}$ & 5.932841 & -0.888794 & 3.181700 \\
\hline C & 7.278970 & -2.268333 & 1.218744 \\
\hline $\mathrm{H}$ & 6.298012 & -2.712834 & 1.421758 \\
\hline $\mathrm{H}$ & 7.562046 & -2.536374 & 0.195389 \\
\hline $\mathrm{H}$ & 8.007431 & -2.727007 & 1.898888 \\
\hline
\end{tabular}




$\begin{array}{llll}\mathrm{C} & 4.800552 & 0.866721 & -4.271091 \\ \mathrm{H} & 3.790910 & 0.479622 & -4.096844 \\ \mathrm{H} & 4.782413 & 1.948490 & -4.095702 \\ \mathrm{H} & 5.041633 & 0.709289 & -5.328641 \\ \mathrm{C} & 7.238839 & 0.768250 & -3.723463 \\ \mathrm{H} & 7.268852 & 1.836772 & -3.481182 \\ \mathrm{H} & 8.045257 & 0.276980 & -3.168570 \\ \mathrm{H} & 7.456014 & 0.656958 & -4.792847 \\ \mathrm{C} & 5.852836 & -1.341331 & -3.749301 \\ \mathrm{H} & 6.063587 & -1.479616 & -4.816943 \\ \mathrm{H} & 6.610701 & -1.895925 & -3.185313 \\ \mathrm{H} & 4.877580 & -1.792152 & -3.533702\end{array}$

\section{C-Catalyzed reaction TS 118}

\section{Datum}

Value

\begin{tabular}{lc}
\hline B3LYP/6-31G(d) Energy & -3038.949531 \\
\hline B3LYP-D3(BJ)/def2-TZVPP-IEF-PCM(DCM) Energy & -3040.500525 \\
\hline B3LYP-D3(BJ)/def2-TZVPP-IEF-PCM(DCM)//B3LYP/6-31G(d) Free Energy (Quasiharmonic) & -3039.272182 \\
\hline
\end{tabular}

Number of Imaginary Frequencies

Frequencies (Top 3 out of 450)

$$
\begin{array}{lr}
\text { 1. } & -382.5292 \mathrm{~cm}^{-1} \\
\text { 2. } & 7.8915 \mathrm{~cm}^{-1} \\
\text { 3. } & 10.9922 \mathrm{~cm}^{-1}
\end{array}
$$

\section{B3LYP/6-31G(d) Molecular Geometry in Cartesian Coordinates}

$\begin{array}{rrrr}C & -1.516980 & 0.749650 & 1.049540 \\ H & -2.221820 & -0.079500 & 0.984680 \\ H & -0.511530 & 0.326160 & 0.970010 \\ \mathrm{C} & 1.445890 & 0.744581 & -1.310570 \\ \mathrm{O} & 1.264840 & 1.043081 & -2.472110 \\ \mathrm{O} & 1.455660 & -0.541689 & -0.930570 \\ \mathrm{H} & 1.514320 & -0.684289 & 0.048260 \\ \mathrm{O} & -1.712410 & 1.305970 & 2.361110 \\ \mathrm{H} & -1.080640 & 2.036410 & 2.467240 \\ \mathrm{C} & 0.926321 & -5.267660 & -1.257230 \\ \mathrm{C} & -0.658359 & -3.959950 & -0.554940 \\ \mathrm{C} & 2.357981 & -3.815689 & 0.134680 \\ \mathrm{C} & 1.866971 & -4.263499 & -1.123560 \\ \mathrm{H} & -0.994099 & -4.777530 & 0.088100\end{array}$




\begin{tabular}{|c|c|c|c|}
\hline $\mathrm{H}$ & 3.141401 & -3.060229 & 0.083350 \\
\hline $\mathrm{H}$ & 2.584681 & -4.611249 & 0.846790 \\
\hline C & 1.486151 & -1.804579 & 2.960310 \\
\hline C & 0.545941 & -3.013640 & 3.329440 \\
\hline C & 2.968451 & -2.099459 & 3.240980 \\
\hline $\mathrm{H}$ & 3.578661 & -1.344189 & 2.735960 \\
\hline $\mathrm{H}$ & 3.191511 & -2.064059 & 4.312970 \\
\hline $\mathrm{H}$ & 3.261421 & -3.082269 & 2.860460 \\
\hline C & 1.102330 & -0.465839 & 3.588630 \\
\hline $\mathrm{H}$ & 1.167890 & -0.526949 & 4.681650 \\
\hline $\mathrm{H}$ & 0.091260 & -0.150580 & 3.321830 \\
\hline $\mathrm{H}$ & 1.801260 & 0.311541 & 3.258840 \\
\hline C & -0.930209 & -2.602780 & 3.451210 \\
\hline $\mathrm{H}$ & -1.116209 & -2.003940 & 4.349170 \\
\hline $\mathrm{H}$ & -1.256239 & -2.026530 & 2.581120 \\
\hline $\mathrm{H}$ & -1.543079 & -3.508490 & 3.510110 \\
\hline C & 0.968651 & -3.799570 & 4.571480 \\
\hline $\mathrm{H}$ & 0.943511 & -3.168090 & 5.467440 \\
\hline $\mathrm{H}$ & 0.276311 & -4.633560 & 4.728160 \\
\hline $\mathrm{H}$ & 1.973441 & -4.214749 & 4.462690 \\
\hline 0 & 1.311111 & -1.727349 & 1.521620 \\
\hline 0 & 0.686041 & -3.873580 & 2.179520 \\
\hline B & 0.971551 & -3.047789 & 1.053390 \\
\hline 0 & -0.104419 & -2.940490 & 0.034730 \\
\hline C & -1.426159 & -3.699790 & -1.786280 \\
\hline C & -2.379589 & -4.634790 & -2.218720 \\
\hline C & -1.209509 & -2.531150 & -2.535160 \\
\hline C & -3.103859 & -4.410570 & -3.387300 \\
\hline $\mathrm{H}$ & -2.550968 & -5.537560 & -1.636080 \\
\hline C & -1.935389 & -2.313520 & -3.703730 \\
\hline $\mathrm{H}$ & -0.472079 & -1.808860 & -2.198890 \\
\hline C & -2.879969 & -3.250620 & -4.134220 \\
\hline $\mathrm{H}$ & -3.841809 & -5.137471 & -3.715180 \\
\hline $\mathrm{H}$ & -1.760609 & -1.409700 & -4.280610 \\
\hline $\mathrm{H}$ & -3.440839 & -3.077880 & -5.048830 \\
\hline $\mathrm{H}$ & 2.014821 & -3.606829 & -1.982370 \\
\hline $\mathrm{H}$ & 0.833742 & -6.019140 & -0.475750 \\
\hline $\mathrm{H}$ & 0.564512 & -5.555570 & -2.238580 \\
\hline C & 0.606830 & 2.567130 & 0.169640 \\
\hline C & 1.672590 & 1.762351 & -0.214740 \\
\hline C & 2.965560 & 1.873541 & 0.404530 \\
\hline C & 3.114610 & 2.770681 & 1.445450 \\
\hline $\mathrm{H}$ & 4.092170 & 2.897051 & 1.903010 \\
\hline C & -1.765970 & 1.732980 & -0.080310 \\
\hline C & -0.723900 & 2.535770 & -0.539210 \\
\hline C & -3.241140 & 2.675050 & -1.766260 \\
\hline C & -3.065180 & 1.821020 & -0.694270 \\
\hline $\mathrm{H}$ & -4.224780 & 2.758799 & -2.221470 \\
\hline C & -2.195580 & 3.482090 & -2.272650 \\
\hline C & -0.911200 & 3.427160 & -1.647140 \\
\hline C & 0.122589 & 4.259630 & -2.157640 \\
\hline C & -0.105561 & 5.100320 & -3.223670 \\
\hline C & -1.379541 & 5.154790 & -3.838220 \\
\hline C & -2.400481 & 4.360640 & -3.371110 \\
\hline
\end{tabular}




\begin{tabular}{|c|c|c|c|}
\hline $\mathrm{H}$ & 1.106949 & 4.217601 & -1.705540 \\
\hline $\mathrm{H}$ & 0.700269 & 5.724330 & -3.600580 \\
\hline $\mathrm{H}$ & -1.546021 & 5.822560 & -4.679290 \\
\hline $\mathrm{H}$ & -3.383691 & 4.392050 & -3.834930 \\
\hline C & 2.046650 & 3.574821 & 1.909510 \\
\hline C & 0.774330 & 3.490990 & 1.257550 \\
\hline C & -0.275761 & 4.336960 & 1.718880 \\
\hline C & -0.078151 & 5.202810 & 2.773130 \\
\hline C & 1.179749 & 5.278101 & 3.416600 \\
\hline C & 2.218269 & 4.483771 & 2.988620 \\
\hline $\mathrm{H}$ & -1.240011 & 4.298580 & 1.223980 \\
\hline $\mathrm{H}$ & -0.893401 & 5.837970 & 3.108210 \\
\hline $\mathrm{H}$ & 1.322669 & 5.966011 & 4.245310 \\
\hline $\mathrm{H}$ & 3.190699 & 4.537631 & 3.472260 \\
\hline C & -6.542980 & -0.284411 & 0.658170 \\
\hline C & -5.748210 & 0.379469 & 1.603250 \\
\hline C & -4.606870 & 1.050509 & 1.148030 \\
\hline C & -4.249010 & 1.051259 & -0.207300 \\
\hline C & -5.067330 & 0.368949 & -1.119110 \\
\hline C & -6.225760 & -0.302001 & -0.707940 \\
\hline $\mathrm{H}$ & -7.430250 & -0.803141 & 0.994460 \\
\hline $\mathrm{H}$ & -3.972470 & 1.579799 & 1.849990 \\
\hline $\mathrm{H}$ & -4.774740 & 0.356539 & -2.165140 \\
\hline C & -6.082260 & 0.396859 & 3.107910 \\
\hline C & -7.099659 & -1.027001 & -1.750900 \\
\hline C & -7.363150 & -0.392241 & 3.439100 \\
\hline $\mathrm{H}$ & -7.550730 & -0.353101 & 4.518220 \\
\hline $\mathrm{H}$ & -8.243540 & 0.024588 & 2.936050 \\
\hline $\mathrm{H}$ & -7.276819 & -1.448241 & 3.158230 \\
\hline C & -4.909590 & -0.232351 & 3.898860 \\
\hline $\mathrm{H}$ & -5.120530 & -0.206421 & 4.975580 \\
\hline $\mathrm{H}$ & -4.764089 & -1.279771 & 3.608860 \\
\hline $\mathrm{H}$ & -3.966650 & 0.295409 & 3.725340 \\
\hline C & -6.286800 & 1.858269 & 3.573530 \\
\hline $\mathrm{H}$ & -6.520240 & 1.888199 & 4.645190 \\
\hline $\mathrm{H}$ & -5.390410 & 2.464789 & 3.410540 \\
\hline $\mathrm{H}$ & -7.114890 & 2.330309 & 3.031900 \\
\hline C & -7.596640 & -0.006561 & -2.802820 \\
\hline $\mathrm{H}$ & -8.216690 & -0.505832 & -3.557870 \\
\hline $\mathrm{H}$ & -8.200660 & 0.777898 & -2.332300 \\
\hline $\mathrm{H}$ & -6.765110 & 0.479769 & -3.323380 \\
\hline C & -6.264539 & -2.121501 & -2.455310 \\
\hline $\mathrm{H}$ & -6.871669 & -2.639051 & -3.209060 \\
\hline $\mathrm{H}$ & -5.907729 & -2.866951 & -1.735720 \\
\hline $\mathrm{H}$ & -5.386609 & -1.706861 & -2.960010 \\
\hline C & -8.334339 & -1.701332 & -1.122740 \\
\hline $\mathrm{H}$ & -8.921079 & -2.196832 & -1.904720 \\
\hline $\mathrm{H}$ & -8.052679 & -2.464602 & -0.388380 \\
\hline $\mathrm{H}$ & -8.990219 & -0.976132 & -0.627830 \\
\hline C & 4.150950 & 1.105541 & -0.075130 \\
\hline C & 4.408820 & 0.962631 & -1.441470 \\
\hline C & 5.065600 & 0.563111 & 0.847480 \\
\hline C & 5.531950 & 0.263082 & -1.908860 \\
\hline $\mathrm{H}$ & 3.726720 & 1.409861 & -2.154640 \\
\hline
\end{tabular}




$\begin{array}{lrrr}\text { C } & 6.208730 & -0.117288 & 0.424870 \\ \text { H } & 4.857350 & 0.674591 & 1.905290 \\ \text { C } & 6.411650 & -0.263798 & -0.960260 \\ \text { H } & 7.286390 & -0.806578 & -1.298320 \\ \text { C } & 7.233420 & -0.718048 & 1.407360 \\ \text { C } & 5.740780 & 0.106132 & -3.427100 \\ \text { C } & 7.254611 & -2.257018 & 1.248230 \\ \text { H } & 6.272491 & -2.686518 & 1.476540 \\ \text { H } & 7.521481 & -2.556288 & 0.229190 \\ \text { H } & 7.987991 & -2.703108 & 1.931450 \\ \text { C } & 8.640830 & -0.152298 & 1.100300 \\ \text { H } & 9.378360 & -0.573088 & 1.794690 \\ \text { H } & 8.969280 & -0.392388 & 0.083960 \\ \text { H } & 8.657020 & 0.938382 & 1.207660 \\ \text { C } & 6.906270 & -0.390238 & 2.876670 \\ \text { H } & 6.883160 & 0.690882 & 3.056470 \\ \text { H } & 5.944210 & -0.811088 & 3.188390 \\ \text { H } & 7.676450 & -0.817158 & 3.529060 \\ \text { C } & 5.850110 & 1.503942 & -4.081430 \\ \text { H } & 4.947170 & 2.101151 & -3.920750 \\ \text { H } & 6.700780 & 2.062832 & -3.674170 \\ \text { H } & 5.994650 & 1.405872 & -5.164330 \\ \text { C } & 7.018740 & -0.683068 & -3.768170 \\ \text { H } & 7.921010 & -0.185238 & -3.394020 \\ \text { H } & 6.992821 & -1.699548 & -3.358690 \\ \text { H } & 7.117370 & -0.769608 & -4.856120 \\ \text { C } & 4.530600 & -0.649059 & -4.029630 \\ \text { H } & 4.651160 & -0.751989 & -5.115320 \\ \text { H } & 4.449881 & -1.655199 & -3.601360 \\ \text { H } & 3.584200 & -0.131819 & -3.842890\end{array}$

\section{C-Catalyzed reaction TS 119}

\section{Datum}

Value

\begin{tabular}{lc}
\hline B3LYP/6-31G(d) Energy & -3038.948625 \\
\hline B3LYP-D3(BJ)/def2-TZVPP-IEF-PCM(DCM) Energy & -3040.500484 \\
\hline B3LYP-D3(BJ)/def2-TZVPP-IEF-PCM(DCM)//B3LYP/6-31G(d) Free Energy (Quasiharmonic) & -3039.271997 \\
\hline
\end{tabular}

Number of Imaginary Frequencies

Frequencies (Top 3 out of 450)

$$
\begin{array}{lr}
\text { 1. } & -289.5081 \mathrm{~cm}^{-1} \\
\text { 2. } & 10.1465 \mathrm{~cm}^{-1} \\
\text { 3. } & 13.4175 \mathrm{~cm}^{-1}
\end{array}
$$




\begin{tabular}{|c|c|c|c|}
\hline C & -1.518920 & 0.671511 & 1.134670 \\
\hline $\mathrm{H}$ & -2.183420 & -0.055129 & 0.666180 \\
\hline $\mathrm{H}$ & -0.501570 & 0.274140 & 1.077340 \\
\hline C & 1.531930 & 1.562750 & -1.162770 \\
\hline 0 & 1.630110 & 2.392640 & -2.035950 \\
\hline 0 & 1.197440 & 0.288470 & -1.468770 \\
\hline $\mathrm{H}$ & 1.067510 & -0.274980 & -0.678410 \\
\hline 0 & -1.933940 & 0.726731 & 2.510700 \\
\hline $\mathrm{H}$ & -1.306390 & 1.301960 & 2.979820 \\
\hline C & 1.450250 & -2.994800 & -2.825970 \\
\hline C & -0.373460 & -2.343850 & -1.718820 \\
\hline C & 2.279460 & -3.339480 & -0.523570 \\
\hline C & 1.807450 & -3.825850 & -1.792510 \\
\hline $\mathrm{H}$ & -0.368030 & -1.467170 & -2.369500 \\
\hline $\mathrm{H}$ & 2.869660 & -2.422040 & -0.594510 \\
\hline $\mathrm{H}$ & 2.791160 & -4.093900 & 0.078740 \\
\hline C & 1.034350 & -2.638770 & 2.799010 \\
\hline C & -0.044030 & -3.731560 & 2.435800 \\
\hline C & 2.356270 & -3.249220 & 3.294720 \\
\hline $\mathrm{H}$ & 3.115760 & -2.461700 & 3.333270 \\
\hline $\mathrm{H}$ & 2.258930 & -3.677720 & 4.298370 \\
\hline $\mathrm{H}$ & 2.713800 & -4.033600 & 2.620200 \\
\hline C & 0.563310 & -1.574510 & 3.792380 \\
\hline $\mathrm{H}$ & 0.319380 & -2.027810 & 4.761010 \\
\hline $\mathrm{H}$ & -0.315090 & -1.035390 & 3.430490 \\
\hline $\mathrm{H}$ & 1.365290 & -0.846080 & 3.955870 \\
\hline C & -1.479530 & -3.183319 & 2.449920 \\
\hline $\mathrm{H}$ & -1.826240 & -2.980309 & 3.468700 \\
\hline $\mathrm{H}$ & -1.562700 & -2.259149 & 1.872970 \\
\hline $\mathrm{H}$ & -2.151230 & -3.925719 & 2.005530 \\
\hline C & 0.024569 & -5.011800 & 3.270240 \\
\hline $\mathrm{H}$ & -0.149371 & -4.800150 & 4.331770 \\
\hline $\mathrm{H}$ & -0.749531 & -5.711610 & 2.936610 \\
\hline $\mathrm{H}$ & 0.992209 & -5.508620 & 3.166690 \\
\hline 0 & 1.287430 & -2.010060 & 1.526010 \\
\hline 0 & 0.309600 & -4.064040 & 1.074150 \\
\hline B & 0.928830 & -2.920710 & 0.489710 \\
\hline 0 & 0.059560 & -2.094320 & -0.525200 \\
\hline C & -1.384760 & -3.360339 & -2.079190 \\
\hline C & -1.714081 & -4.462209 & -1.272520 \\
\hline C & -2.068480 & -3.167739 & -3.293010 \\
\hline C & -2.705511 & -5.349919 & -1.686230 \\
\hline $\mathrm{H}$ & -1.187001 & -4.611690 & -0.335830 \\
\hline C & -3.059760 & -4.057149 & -3.698970 \\
\hline $\mathrm{H}$ & -1.821420 & -2.311939 & -3.917630 \\
\hline C & -3.380171 & -5.153389 & -2.894250 \\
\hline $\mathrm{H}$ & -2.956521 & -6.200589 & -1.058260 \\
\hline $\mathrm{H}$ & -3.581660 & -3.895429 & -4.637990 \\
\hline $\mathrm{H}$ & -4.154111 & -5.849429 & -3.206330 \\
\hline $\mathrm{H}$ & 1.519119 & -4.875690 & -1.857300 \\
\hline
\end{tabular}




\begin{tabular}{|c|c|c|c|}
\hline H & 1.835870 & -1.979210 & -2.866840 \\
\hline $\mathrm{H}$ & 1.040450 & -3.395960 & -3.747990 \\
\hline C & 0.775940 & 2.465030 & 1.037830 \\
\hline C & 1.776620 & 1.842390 & 0.303220 \\
\hline C & 3.022120 & 1.453090 & 0.898500 \\
\hline C & 3.195490 & 1.676070 & 2.250750 \\
\hline H & 4.138190 & 1.401150 & 2.716500 \\
\hline C & -1.623210 & 2.011581 & 0.427870 \\
\hline C & -0.522279 & 2.865350 & 0.388960 \\
\hline C & -2.926589 & 3.633511 & -0.828180 \\
\hline C & -2.856460 & 2.404411 & -0.200750 \\
\hline H & -3.860719 & 3.937641 & -1.293880 \\
\hline C & -1.833689 & 4.531151 & -0.863130 \\
\hline C & -0.601579 & 4.147280 & -0.248000 \\
\hline C & 0.488301 & 5.059530 & -0.292720 \\
\hline C & 0.359761 & 6.286150 & -0.904390 \\
\hline C & -0.862979 & 6.665000 & -1.508740 \\
\hline C & -1.934439 & 5.803001 & -1.489350 \\
\hline $\mathrm{H}$ & 1.433941 & 4.775680 & 0.155410 \\
\hline $\mathrm{H}$ & 1.205291 & 6.968230 & -0.928610 \\
\hline $\mathrm{H}$ & -0.949929 & 7.635710 & -1.989510 \\
\hline H & -2.878019 & 6.081121 & -1.953010 \\
\hline C & 2.201480 & 2.294830 & 3.046540 \\
\hline C & 0.977671 & 2.717240 & 2.434490 \\
\hline C & 0.006371 & 3.373880 & 3.244130 \\
\hline C & 0.228721 & 3.582160 & 4.588860 \\
\hline C & 1.434451 & 3.152230 & 5.192610 \\
\hline C & 2.398750 & 2.528160 & 4.435040 \\
\hline $\mathrm{H}$ & -0.911969 & 3.725180 & 2.784390 \\
\hline $\mathrm{H}$ & -0.523349 & 4.087750 & 5.188230 \\
\hline $\mathrm{H}$ & 1.597491 & 3.322090 & 6.253230 \\
\hline $\mathrm{H}$ & 3.332110 & 2.203410 & 4.888650 \\
\hline C & -6.379300 & -0.022689 & -0.330420 \\
\hline C & -5.785320 & 0.218471 & 0.911370 \\
\hline C & -4.623170 & 1.005741 & 0.941380 \\
\hline C & -4.068290 & 1.530891 & -0.228640 \\
\hline C & -4.696880 & 1.264891 & -1.458920 \\
\hline C & -5.853860 & 0.487261 & -1.531980 \\
\hline $\mathrm{H}$ & -7.275540 & -0.630689 & -0.376430 \\
\hline $\mathrm{H}$ & -4.129520 & 1.211591 & 1.884280 \\
\hline $\mathrm{H}$ & -4.245880 & 1.666631 & -2.359140 \\
\hline C & -6.346700 & -0.342639 & 2.231880 \\
\hline C & -6.554450 & 0.162561 & -2.865820 \\
\hline C & -7.618370 & -1.185869 & 2.021190 \\
\hline $\mathrm{H}$ & -7.974880 & -1.564209 & 2.986110 \\
\hline $\mathrm{H}$ & -8.430400 & -0.598508 & 1.576720 \\
\hline $\mathrm{H}$ & -7.430820 & -2.052789 & 1.377060 \\
\hline C & -5.276940 & -1.239589 & 2.901050 \\
\hline $\mathrm{H}$ & -5.650140 & -1.625769 & 3.858130 \\
\hline H & -5.034440 & -2.097269 & 2.262480 \\
\hline $\mathrm{H}$ & -4.347270 & -0.695319 & 3.093840 \\
\hline C & -6.698200 & 0.828081 & 3.180480 \\
\hline $\mathrm{H}$ & -7.095470 & 0.444131 & 4.128240 \\
\hline H & -5.821530 & 1.441991 & 3.410680 \\
\hline
\end{tabular}




\begin{tabular}{|c|c|c|c|}
\hline H & -7.456820 & 1.481231 & 2.733530 \\
\hline C & -5.843120 & 0.800721 & -4.074260 \\
\hline $\mathrm{H}$ & -6.380040 & 0.546371 & -4.995330 \\
\hline $\mathrm{H}$ & -5.813590 & 1.893731 & -4.000420 \\
\hline $\mathrm{H}$ & -4.814310 & 0.438421 & -4.180330 \\
\hline C & -6.576640 & -1.370219 & -3.077670 \\
\hline $\mathrm{H}$ & -7.072840 & -1.617919 & -4.024650 \\
\hline H & -5.559490 & -1.777679 & -3.108420 \\
\hline $\mathrm{H}$ & -7.115870 & -1.884439 & -2.275360 \\
\hline C & -8.006800 & 0.695001 & -2.829880 \\
\hline $\mathrm{H}$ & -8.522990 & 0.464392 & -3.770180 \\
\hline $\mathrm{H}$ & -8.585390 & 0.248432 & -2.014490 \\
\hline $\mathrm{H}$ & -8.021560 & 1.782332 & -2.692250 \\
\hline C & 4.133790 & 0.859100 & 0.101440 \\
\hline C & 4.808130 & -0.282820 & 0.571780 \\
\hline C & 4.562840 & 1.462980 & -1.083640 \\
\hline C & 5.897240 & -0.815411 & -0.121870 \\
\hline H & 4.444680 & -0.754210 & 1.477360 \\
\hline C & 5.641240 & 0.947299 & -1.819390 \\
\hline $\mathrm{H}$ & 4.052820 & 2.353250 & -1.432200 \\
\hline C & 6.289250 & -0.183681 & -1.317010 \\
\hline $\mathrm{H}$ & 7.128320 & -0.595501 & -1.865170 \\
\hline C & 6.056000 & 1.639959 & -3.131740 \\
\hline C & 6.674080 & -2.053901 & 0.367240 \\
\hline C & 7.275050 & 0.970259 & -3.792960 \\
\hline H & 8.159640 & 0.999469 & -3.145930 \\
\hline $\mathrm{H}$ & 7.075470 & -0.075311 & -4.054410 \\
\hline $\mathrm{H}$ & 7.526560 & 1.498049 & -4.719820 \\
\hline C & 4.872280 & 1.585310 & -4.128100 \\
\hline $\mathrm{H}$ & 5.135650 & 2.102160 & -5.059430 \\
\hline $\mathrm{H}$ & 4.622280 & 0.547190 & -4.376800 \\
\hline $\mathrm{H}$ & 3.970140 & 2.055040 & -3.723810 \\
\hline C & 6.418051 & 3.116869 & -2.845660 \\
\hline H & 5.572511 & 3.671429 & -2.426600 \\
\hline $\mathrm{H}$ & 7.251301 & 3.186449 & -2.136610 \\
\hline $\mathrm{H}$ & 6.717741 & 3.620509 & -3.772870 \\
\hline C & 6.580430 & -3.177751 & -0.692250 \\
\hline $\mathrm{H}$ & 5.538360 & -3.473711 & -0.857810 \\
\hline $\mathrm{H}$ & 6.996170 & -2.866231 & -1.656200 \\
\hline $\mathrm{H}$ & 7.137140 & -4.062561 & -0.359440 \\
\hline C & 8.159710 & -1.677341 & 0.582820 \\
\hline $\mathrm{H}$ & 8.630460 & -1.319421 & -0.338600 \\
\hline $\mathrm{H}$ & 8.258560 & -0.887711 & 1.336570 \\
\hline $\mathrm{H}$ & 8.726710 & -2.550461 & 0.928820 \\
\hline C & 6.126870 & -2.603811 & 1.697310 \\
\hline $\mathrm{H}$ & 6.716450 & -3.474401 & 2.006710 \\
\hline $\mathrm{H}$ & 6.188970 & -1.861061 & 2.501070 \\
\hline $\mathrm{H}$ & 5.083660 & -2.924990 & 1.609390 \\
\hline
\end{tabular}




\begin{tabular}{ll}
\hline B3LYP/6-31G(d) Energy & -3038.944479 \\
\hline B3LYP-D3(BJ)/def2-TZVPP-IEF-PCM(DCM) Energy & -3040.499689 \\
\hline B3LYP-D3(BJ)/def2-TZVPP-IEF-PCM(DCM)//B3LYP/6-31G(d) Free Energy (Quasiharmonic) & -3039.271987 \\
\hline
\end{tabular}

Number of Imaginary Frequencies

Frequencies (Top 3 out of 450)

$\begin{array}{lr}\text { 1. } & -387.1924 \mathrm{~cm}^{-1} \\ \text { 2. } & 6.9903 \mathrm{~cm}^{-1} \\ \text { 3. } & 8.4942 \mathrm{~cm}^{-1}\end{array}$

\section{B3LYP/6-31G(d) Molecular Geometry in Cartesian Coordinates}

C

$\mathrm{H}$

$\mathrm{H}$

C

0

0

$\mathrm{H}$

0

$\mathrm{H}$

C

C

C

C

$\mathrm{H}$

$\mathrm{H}$

$\mathrm{H}$

C

C

C

$\mathrm{H}$

$\mathrm{H}$

$\mathrm{H}$

C

$\mathrm{H}$

$\mathrm{H}$

$\mathrm{H}$

$\mathrm{H}$

C

$\mathrm{H}$

$\mathrm{H}$

$\mathrm{H}$

C

$\mathrm{H}$

\section{324110}

1.288510

2.470010

$-0.688580$

$-0.560850$

$-0.841610$

$-0.845690$

3. 105170

3.995320

$-1.413060$

$-2.756070$

0.259340

$-0.505320$

$-2.679330$

0.623280

1.025860

$-0.783540$

$-0.120700$

$-0.049900$

$-0.556700$

$-0.061390$

0.990370

$-2.265550$

$-2.384690$

$-2.712890$

$-2.816650$

$-0.633100$

$-0.428210$

$-1.706960$

$-0.125960$

1.415820

1.783740

\subsection{0}

0.281100

$-0.681480$

1.165140

1.527760

$-0.146220$

$-0.373690$

0.262080

$-0.042790$

$-3.121190$

$-2.884190$

$-3.319450$

$-3.859760$

$-1.802060$

$-2.305530$

$-3.979610$

$-1.618150$

$-2.999970$

$-0.393890$

0.522500

$-0.406490$

$-0.346450$

$-1.551490$

$-1.474160$

$-0.663330$

$-2.428120$

$-3.631900$

$-2.987980$

$-3.827230$

$-4.588690$

$-2.937560$

$-2.420080$
0.699600

1.029090

0.151460

$-1.074180$

$-2.221610$

$-0.786840$

0.180230

1.898570

1.657480

$-2.117640$

$-0.461320$

$-0.302460$

$-1.376090$

$-0.584440$

$-0.477040$

0.102170

2.962580

3. 322280

3.505090

3.184030

4.601810

3.177980

3. 363690

4.449790

2.906610

3.012900

4.619430

5.483070

4.572210

4.784050

3.355280

4. 248090 


\begin{tabular}{|c|c|c|c|}
\hline $\mathrm{H}$ & 1.804520 & -3.961340 & 3.366840 \\
\hline $\mathrm{H}$ & 1.820510 & -2.423760 & 2.478850 \\
\hline 0 & -0.713830 & -1.631560 & 1.505600 \\
\hline 0 & -0.518280 & -3.838820 & 2.226380 \\
\hline B & -0.842170 & -3.030570 & 1.110730 \\
\hline 0 & -2.201180 & -3.400820 & 0.599730 \\
\hline C & -3.996320 & -3.515970 & -0.953270 \\
\hline C & -4.866160 & -2.776240 & -1.769750 \\
\hline C & -4.335030 & -4.830570 & -0.596120 \\
\hline C & -6.051800 & -3.345930 & -2.230560 \\
\hline $\mathrm{H}$ & -4.614480 & -1.750910 & -2.030420 \\
\hline C & -5.518660 & -5.396680 & -1.061990 \\
\hline $\mathrm{H}$ & -3.667760 & -5.388120 & 0.053210 \\
\hline C & -6.378620 & -4.658470 & -1.881340 \\
\hline $\mathrm{H}$ & -6.721210 & -2.765850 & -2.859990 \\
\hline $\mathrm{H}$ & -5.776280 & -6.414550 & -0.781920 \\
\hline $\mathrm{H}$ & -7.302550 & -5.103230 & -2.241010 \\
\hline $\mathrm{H}$ & -0.583520 & -4.947370 & -1.434490 \\
\hline $\mathrm{H}$ & -1.252620 & -2.054340 & -2.244290 \\
\hline $\mathrm{H}$ & -2.005570 & -3.613330 & -2.882730 \\
\hline C & 0.515860 & 2.630650 & 0.542150 \\
\hline C & -0.689460 & 2.081390 & 0.125100 \\
\hline C & -1.919160 & 2.330200 & 0.824930 \\
\hline C & -1.865300 & 3.068400 & 1.991360 \\
\hline $\mathrm{H}$ & -2.787760 & 3.303460 & 2.516280 \\
\hline C & 2.617540 & 1.429610 & -0.218660 \\
\hline C & 1.758090 & 2.518290 & -0.302900 \\
\hline C & 4.094070 & 2.433760 & -1.873630 \\
\hline C & 3.809720 & 1.395550 & -1.016460 \\
\hline $\mathrm{H}$ & 4.999550 & 2.401640 & -2.475210 \\
\hline C & 3.232680 & 3.552930 & -1.995470 \\
\hline C & 2.043930 & 3.603600 & -1.201760 \\
\hline C & 1.191530 & 4.733200 & -1.345420 \\
\hline C & 1.502930 & 5.753110 & -2.217050 \\
\hline C & 2.682580 & 5.700290 & -2.995610 \\
\hline C & 3.526980 & 4.619720 & -2.885610 \\
\hline $\mathrm{H}$ & 0.279130 & 4.785960 & -0.762370 \\
\hline $\mathrm{H}$ & 0.834360 & 6.604660 & -2.310810 \\
\hline $\mathrm{H}$ & 2.916290 & 6.511080 & -3.680400 \\
\hline $\mathrm{H}$ & 4.434990 & 4.563510 & -3.481580 \\
\hline C & -0.646510 & 3.577150 & 2.505030 \\
\hline C & 0.564560 & 3.373900 & 1.768110 \\
\hline C & 1.773750 & 3.909150 & 2.290060 \\
\hline C & 1.782220 & 4.612350 & 3.474050 \\
\hline C & 0.582600 & 4.821380 & 4.195290 \\
\hline C & -0.604410 & 4.314380 & 3.719330 \\
\hline $\mathrm{H}$ & 2.698410 & 3.740790 & 1.749700 \\
\hline $\mathrm{H}$ & 2.717540 & 5.007970 & 3.860270 \\
\hline $\mathrm{H}$ & 0.603150 & 5.380450 & 5.126890 \\
\hline $\mathrm{H}$ & -1.530960 & 4.467780 & 4.267540 \\
\hline C & 6.555260 & -1.880820 & -0.754780 \\
\hline C & 5.573950 & -1.908570 & -1.750110 \\
\hline C & 4.681230 & -0.824830 & -1.817800 \\
\hline C & 4.763160 & 0.241950 & -0.922700 \\
\hline
\end{tabular}




\begin{tabular}{|c|c|c|c|}
\hline C & 5.766640 & 0.234640 & 0.062990 \\
\hline C & 6.678140 & -0.821550 & 0.162730 \\
\hline $\mathrm{H}$ & 7.255300 & -2.704690 & -0.684160 \\
\hline $\mathrm{H}$ & 3.903300 & -0.804750 & -2.575370 \\
\hline $\mathrm{H}$ & 5.832280 & 1.086980 & 0.730760 \\
\hline C & 5.435600 & -3.069270 & -2.754230 \\
\hline C & 7.795660 & -0.857600 & 1.223450 \\
\hline C & 4.043230 & -3.724490 & -2.588710 \\
\hline $\mathrm{H}$ & 3.925780 & -4.552490 & -3.298630 \\
\hline $\mathrm{H}$ & 3.916850 & -4.125100 & -1.576320 \\
\hline $\mathrm{H}$ & 3.230840 & -3.013150 & -2.769330 \\
\hline C & 5.577190 & -2.523830 & -4.195390 \\
\hline $\mathrm{H}$ & 5.474260 & -3.338930 & -4.922020 \\
\hline $\mathrm{H}$ & 4.813160 & -1.774570 & -4.426490 \\
\hline $\mathrm{H}$ & 6.558410 & -2.058490 & -4.344070 \\
\hline C & 6.504630 & -4.159090 & -2.549060 \\
\hline $\mathrm{H}$ & 6.363720 & -4.955750 & -3.288090 \\
\hline $\mathrm{H}$ & 7.519480 & -3.765700 & -2.678260 \\
\hline $\mathrm{H}$ & 6.437340 & -4.616840 & -1.555540 \\
\hline C & 7.627930 & -2.116140 & 2.108240 \\
\hline $\mathrm{H}$ & 8.422090 & -2.158240 & 2.863540 \\
\hline $\mathrm{H}$ & 6.664230 & -2.103820 & 2.630490 \\
\hline $\mathrm{H}$ & 7.677690 & -3.039330 & 1.521620 \\
\hline C & 9.172210 & -0.906940 & 0.518210 \\
\hline $\mathrm{H}$ & 9.979280 & -0.938040 & 1.260440 \\
\hline $\mathrm{H}$ & 9.272740 & -1.790740 & -0.120400 \\
\hline $\mathrm{H}$ & 9.322540 & -0.021580 & -0.110080 \\
\hline C & 7.774580 & 0.378120 & 2.143210 \\
\hline $\mathrm{H}$ & 8.584410 & 0.303560 & 2.877580 \\
\hline $\mathrm{H}$ & 7.922790 & 1.308000 & 1.582660 \\
\hline $\mathrm{H}$ & 6.833830 & 0.460210 & 2.699680 \\
\hline C & -3.238640 & 1.906550 & 0.270690 \\
\hline C & -3.531470 & 2.120070 & -1.083910 \\
\hline C & -4.233720 & 1.363900 & 1.097180 \\
\hline C & -4.778580 & 1.789120 & -1.626500 \\
\hline $\mathrm{H}$ & -2.773540 & 2.565960 & -1.716870 \\
\hline C & -5.499230 & 1.028360 & 0.597710 \\
\hline $\mathrm{H}$ & -4.004330 & 1.197890 & 2.145720 \\
\hline C & -5.743850 & 1.245460 & -0.767140 \\
\hline $\mathrm{H}$ & -6.715540 & 0.992490 & -1.168260 \\
\hline C & -6.569060 & 0.444190 & 1.542330 \\
\hline C & -5.038820 & 2.038880 & -3.125150 \\
\hline C & -7.898510 & 0.151140 & 0.820280 \\
\hline $\mathrm{H}$ & -8.336060 & 1.055180 & 0.381400 \\
\hline $\mathrm{H}$ & -7.775110 & -0.592850 & 0.024980 \\
\hline $\mathrm{H}$ & -8.623250 & -0.251010 & 1.537110 \\
\hline C & -6.057600 & -0.882250 & 2.151530 \\
\hline $\mathrm{H}$ & -6.815760 & -1.312280 & 2.817740 \\
\hline $\mathrm{H}$ & -5.838840 & -1.616350 & 1.367810 \\
\hline $\mathrm{H}$ & -5.144880 & -0.737330 & 2.737350 \\
\hline C & -6.855320 & 1.453480 & 2.679760 \\
\hline $\mathrm{H}$ & -5.956760 & 1.672640 & 3.265990 \\
\hline $\mathrm{H}$ & -7.232510 & 2.401000 & 2.277930 \\
\hline $\mathrm{H}$ & -7.609940 & 1.050770 & 3.366610 \\
\hline
\end{tabular}




\begin{tabular}{|llll|} 
C & -4.819310 & 3.536930 & -3.446410 \\
$H$ & -5.500280 & 4.167630 & -2.863110 \\
$H$ & -3.795480 & 3.856530 & -3.228560 \\
$H$ & -5.006450 & 3.727560 & -4.510170 \\
C & -4.053070 & 1.188430 & -3.962920 \\
$H$ & -3.008630 & 1.400730 & -3.714100 \\
$H$ & -4.223340 & 0.117470 & -3.796220 \\
$H$ & -4.194490 & 1.386500 & -5.032770 \\
C & -6.473980 & 1.669520 & -3.545210 \\
$H$ & -6.605910 & 1.865940 & -4.615110 \\
$H$ & -6.690230 & 0.607790 & -3.378110 \\
$H$ & -7.223740 & 2.259760 & -3.005650 \\
\hline
\end{tabular}

\section{C-Catalyzed reaction TS 121}

\section{Datum}

Value

\begin{tabular}{lr}
\hline B3LYP/6-31G(d) Energy & -3038.953158 \\
\hline B3LYP-D3(BJ)/def2-TZVPP-IEF-PCM(DCM) Energy & -3040.500622 \\
\hline B3LYP-D3(BJ)/def2-TZVPP-IEF-PCM(DCM)//B3LYP/6-31G(d) Free Energy (Quasiharmonic) & -3039.27191 \\
\hline
\end{tabular}

Number of Imaginary Frequencies

Frequencies (Top 3 out of 450)
1. $-299.3317 \mathrm{~cm}^{-1}$
2. $7.7173 \mathrm{~cm}^{-1}$
3. $9.3734 \mathrm{~cm}^{-1}$

\section{B3LYP/6-31G(d) Molecular Geometry in Cartesian Coordinates}

C

$\mathrm{H}$

$\mathrm{H}$

C

0

0

$\mathrm{H}$

0

$\mathrm{H}$

C

C

C

C

$\mathrm{H}$

\subsection{0}

3.934120

2. 262240

$-0.553130$

0.353780

$-1.620010$

$-2.249060$

2.671710

1.840570

$-2.017000$

$-1.800509$

$-3.729500$

$-2.444000$

$-0.727700$
$-1.706311$

$-1.917661$

$-2.166780$

$-0.831760$

$-1.095500$

$-0.177860$

0.186210

$-2.326371$

$-1.960030$

3.335150

4.115630

1.930060

2.112100

3.909400
0.876610

1.139560

1.646680

$-0.578140$

$-1.353750$

$-1.028310$

$-0.343460$

$-0.386160$

$-0.755210$

$-3.544670$

$-1.495270$

$-2.466730$

$-3.079960$

$-1.503100$ 


\begin{tabular}{|c|c|c|c|}
\hline $\mathrm{H}$ & -4.051450 & 0.888250 & -2.410920 \\
\hline $\mathrm{H}$ & -4.511970 & 2.568670 & -2.886250 \\
\hline C & -4.639150 & 1.153380 & 0.954900 \\
\hline C & -5.302040 & 2.578080 & 0.878990 \\
\hline C & -5.490850 & 0.068170 & 0.280300 \\
\hline $\mathrm{H}$ & -4.884450 & -0.832590 & 0.143030 \\
\hline $\mathrm{H}$ & -6.363430 & -0.198499 & 0.886450 \\
\hline $\mathrm{H}$ & -5.844170 & 0.395391 & -0.702010 \\
\hline C & -4.232040 & 0.703880 & 2.357820 \\
\hline $\mathrm{H}$ & -3.525470 & 1.400680 & 2.814980 \\
\hline $\mathrm{H}$ & -3.752460 & -0.279660 & 2.314590 \\
\hline $\mathrm{H}$ & -5.109470 & 0.621360 & 3.010250 \\
\hline C & -4.706010 & 3.567730 & 1.894990 \\
\hline $\mathrm{H}$ & -5.060319 & 4.575940 & 1.656500 \\
\hline $\mathrm{H}$ & -5.010710 & 3.333910 & 2.921030 \\
\hline $\mathrm{H}$ & -3.613320 & 3.573330 & 1.847780 \\
\hline C & -6.827740 & 2.585581 & 0.990180 \\
\hline $\mathrm{H}$ & -7.156770 & 2.184091 & 1.955890 \\
\hline $\mathrm{H}$ & -7.195360 & 3.614171 & 0.910210 \\
\hline $\mathrm{H}$ & -7.291240 & 2.002031 & 0.191370 \\
\hline 0 & -3.442170 & 1.344410 & 0.162220 \\
\hline 0 & -4.940550 & 3.007360 & -0.444820 \\
\hline B & -3.682030 & 2.422540 & -0.787030 \\
\hline 0 & -2.500500 & 3.373490 & -0.706450 \\
\hline C & -2.190509 & 5.519600 & -1.747870 \\
\hline C & -3.490539 & 5.971820 & -1.465510 \\
\hline C & -1.235849 & 6.417790 & -2.250970 \\
\hline C & -3.823469 & 7.304410 & -1.698150 \\
\hline $\mathrm{H}$ & -4.220999 & 5.274890 & -1.064300 \\
\hline C & -1.574229 & 7.749660 & -2.476650 \\
\hline $\mathrm{H}$ & -0.227529 & 6.067380 & -2.460700 \\
\hline C & -2.870629 & 8.194410 & -2.202950 \\
\hline $\mathrm{H}$ & -4.829069 & 7.653590 & -1.479850 \\
\hline $\mathrm{H}$ & -0.829749 & 8.440860 & -2.861900 \\
\hline $\mathrm{H}$ & -3.136089 & 9.233480 & -2.378270 \\
\hline $\mathrm{H}$ & -1.709980 & 1.318580 & -2.944880 \\
\hline $\mathrm{H}$ & -2.744119 & 4.082250 & -3.846740 \\
\hline $\mathrm{H}$ & -1.016460 & 3.456720 & -3.951030 \\
\hline C & 0.350910 & -0.607860 & 1.742110 \\
\hline C & -0.550000 & -1.224690 & 0.880440 \\
\hline C & -1.476980 & -2.223050 & 1.348010 \\
\hline C & -1.530490 & -2.473560 & 2.705560 \\
\hline $\mathrm{H}$ & -2.190190 & -3.253620 & 3.075580 \\
\hline C & 2.665710 & -0.200471 & 0.862190 \\
\hline C & 1.428500 & 0.320420 & 1.241640 \\
\hline C & 3.499900 & 2.053489 & 0.491980 \\
\hline C & 3.726060 & 0.689939 & 0.469100 \\
\hline $\mathrm{H}$ & 4.298900 & 2.727719 & 0.193920 \\
\hline C & 2.258880 & 2.610710 & 0.876770 \\
\hline C & 1.193020 & 1.734360 & 1.256000 \\
\hline C & -0.046040 & 2.312460 & 1.648270 \\
\hline C & -0.212300 & 3.679660 & 1.679570 \\
\hline C & 0.845251 & 4.543650 & 1.306940 \\
\hline C & 2.052160 & 4.016500 & 0.907400 \\
\hline
\end{tabular}




\begin{tabular}{|c|c|c|c|}
\hline $\mathrm{H}$ & -0.876070 & 1.665910 & 1.911600 \\
\hline $\mathrm{H}$ & -1.167349 & 4.098970 & 1.981440 \\
\hline $\mathrm{H}$ & 0.699341 & 5.620300 & 1.336340 \\
\hline H & 2.873021 & 4.668369 & 0.616850 \\
\hline $\mathrm{C}$ & -0.703620 & -1.797330 & 3.634640 \\
\hline C & 0.271630 & -0.868260 & 3.152710 \\
\hline C & 1.131370 & -0.241900 & 4.096940 \\
\hline C & 1.020680 & -0.511360 & 5.443350 \\
\hline C & 0.051240 & -1.427010 & 5.916630 \\
\hline C & -0.788840 & -2.058330 & 5.028750 \\
\hline $\mathrm{H}$ & 1.879880 & 0.458820 & 3.744660 \\
\hline H & 1.684730 & -0.018800 & 6.148300 \\
\hline $\mathrm{H}$ & -0.024030 & -1.632340 & 6.981000 \\
\hline $\mathrm{H}$ & -1.531630 & -2.770390 & 5.380300 \\
\hline C & 7.652010 & -0.549671 & -0.680820 \\
\hline C & 6.554590 & -1.157501 & -1.306190 \\
\hline C & 5.271910 & -0.757761 & -0.913880 \\
\hline C & 5.088140 & 0.216559 & 0.077260 \\
\hline C & 6.214380 & 0.799999 & 0.679590 \\
\hline C & 7.512840 & 0.428709 & 0.313880 \\
\hline $\mathrm{H}$ & 8.652580 & -0.854371 & -0.973630 \\
\hline $\mathrm{H}$ & 4.393510 & -1.209551 & -1.354890 \\
\hline $\mathrm{H}$ & 6.054300 & 1.538459 & 1.456940 \\
\hline C & 6.784640 & -2.238991 & -2.379570 \\
\hline C & 8.768740 & 1.042079 & 0.963710 \\
\hline C & 7.529610 & -3.436661 & -1.743630 \\
\hline $\mathrm{H}$ & 7.711460 & -4.215821 & -2.494370 \\
\hline $\mathrm{H}$ & 8.499060 & -3.140821 & -1.327980 \\
\hline $\mathrm{H}$ & 6.937910 & -3.877151 & -0.933280 \\
\hline C & 5.462450 & -2.758811 & -2.977710 \\
\hline $\mathrm{H}$ & 5.676640 & -3.540311 & -3.716250 \\
\hline $\mathrm{H}$ & 4.803250 & -3.185591 & -2.214790 \\
\hline $\mathrm{H}$ & 4.910050 & -1.962611 & -3.489860 \\
\hline C & 7.638770 & -1.658341 & -3.531340 \\
\hline $\mathrm{H}$ & 7.804910 & -2.419351 & -4.304040 \\
\hline H & 7.135540 & -0.804511 & -3.999600 \\
\hline $\mathrm{H}$ & 8.620770 & -1.318191 & -3.185140 \\
\hline C & 8.422320 & 2.096749 & 2.032250 \\
\hline $\mathrm{H}$ & 9.344780 & 2.505479 & 2.460820 \\
\hline $\mathrm{H}$ & 7.854770 & 2.933949 & 1.610090 \\
\hline $\mathrm{H}$ & 7.837740 & 1.668559 & 2.854410 \\
\hline C & 9.597350 & -0.073761 & 1.643310 \\
\hline $\mathrm{H}$ & 10.495910 & 0.347259 & 2.111520 \\
\hline $\mathrm{H}$ & 9.011940 & -0.576681 & 2.421490 \\
\hline $\mathrm{H}$ & 9.920770 & -0.835211 & 0.926040 \\
\hline C & 9.629790 & 1.727919 & -0.123650 \\
\hline $\mathrm{H}$ & 10.530830 & 2.167599 & 0.322070 \\
\hline $\mathrm{H}$ & 9.951130 & 1.020999 & -0.895660 \\
\hline $\mathrm{H}$ & 9.069570 & 2.529419 & -0.618840 \\
\hline C & -2.279400 & -3.075110 & 0.421970 \\
\hline C & -3.588320 & -3.462090 & 0.750150 \\
\hline C & -1.698130 & -3.599320 & -0.740320 \\
\hline C & -4.312480 & -4.355740 & -0.049320 \\
\hline $\mathrm{H}$ & -4.039770 & -3.057530 & 1.651500 \\
\hline
\end{tabular}




\begin{tabular}{|c|c|c|c|}
\hline C & -2.398021 & -4.467910 & -1.585580 \\
\hline H & -0.671510 & -3.344500 & -0.973290 \\
\hline C & -3.699901 & -4.833450 & -1.217920 \\
\hline $\mathrm{H}$ & -4.248701 & -5.514430 & -1.853430 \\
\hline C & -1.710681 & -4.989800 & -2.862040 \\
\hline C & -5.723871 & -4.804050 & 0.383010 \\
\hline C & -2.614941 & -5.937360 & -3.672860 \\
\hline $\mathrm{H}$ & -2.891941 & -6.830520 & -3.100660 \\
\hline $\mathrm{H}$ & -3.535641 & -5.441990 & -4.002230 \\
\hline $\mathrm{H}$ & -2.083251 & -6.273230 & -4.570210 \\
\hline C & -1.336640 & -3.788950 & -3.764740 \\
\hline $\mathrm{H}$ & -0.828200 & -4.140790 & -4.670970 \\
\hline $\mathrm{H}$ & -2.233670 & -3.238400 & -4.071610 \\
\hline $\mathrm{H}$ & -0.670800 & -3.083400 & -3.258030 \\
\hline C & -0.425851 & -5.762970 & -2.479010 \\
\hline $\mathrm{H}$ & 0.291519 & -5.130560 & -1.946580 \\
\hline $\mathrm{H}$ & -0.659731 & -6.620080 & -1.836680 \\
\hline $\mathrm{H}$ & 0.071379 & -6.140370 & -3.380890 \\
\hline C & -5.629581 & -5.525870 & 1.749110 \\
\hline $\mathrm{H}$ & -4.982851 & -6.408100 & 1.682380 \\
\hline $\mathrm{H}$ & -5.222591 & -4.871460 & 2.527290 \\
\hline $\mathrm{H}$ & -6.623261 & -5.855929 & 2.076580 \\
\hline C & -6.652110 & -3.575539 & 0.521780 \\
\hline $\mathrm{H}$ & -6.283350 & -2.866879 & 1.269860 \\
\hline $\mathrm{H}$ & -6.741040 & -3.040089 & -0.430290 \\
\hline $\mathrm{H}$ & -7.657000 & -3.890409 & 0.829260 \\
\hline C & -6.370551 & -5.775099 & -0.623710 \\
\hline $\mathrm{H}$ & -7.368301 & -6.058789 & -0.270190 \\
\hline H & -6.488551 & -5.319809 & -1.613580 \\
\hline $\mathrm{H}$ & -5.787911 & -6.696160 & -0.737470 \\
\hline
\end{tabular}

\section{C-Catalyzed reaction TS 122}

\section{Datum}

Value

\begin{tabular}{lc}
\hline B3LYP/6-31G(d) Energy & -3038.945497 \\
\hline B3LYP-D3(BJ)/def2-TZVPP-IEF-PCM(DCM) Energy & -3040.499168 \\
\hline B3LYP-D3(BJ)/def2-TZVPP-IEF-PCM(DCM)//B3LYP/6-31G(d) Free Energy (Quasiharmonic) & -3039.271786 \\
\hline
\end{tabular}

Number of Imaginary Frequencies

Frequencies (Top 3 out of 450)

$\begin{array}{lr}\text { 1. } & -387.6611 \mathrm{~cm}^{-1} \\ \text { 2. } & 7.4641 \mathrm{~cm}^{-1} \\ \text { 3. } & 9.8218 \mathrm{~cm}^{-1}\end{array}$




\begin{tabular}{|c|c|c|c|}
\hline C & -2.214364 & -0.652701 & 0.757614 \\
\hline $\mathrm{H}$ & -1.166430 & -0.650981 & 1.049840 \\
\hline $\mathrm{H}$ & -2.485863 & 0.380962 & 0.510608 \\
\hline C & 0.955258 & -1.078583 & -1.239833 \\
\hline 0 & 0.925439 & -1.290619 & -2.432232 \\
\hline 0 & 1.028969 & 0.186866 & -0.781559 \\
\hline $\mathrm{H}$ & 0.976343 & 0.305280 & 0.203875 \\
\hline 0 & -2.934752 & -1.088761 & 1.915014 \\
\hline $\mathrm{H}$ & -3.862742 & -0.838093 & 1.776418 \\
\hline C & 0.478631 & 3.355840 & -1.853413 \\
\hline C & 2.127376 & 3.273783 & -0.487803 \\
\hline C & -0.841795 & 3.053293 & 0.212231 \\
\hline C & -0.394224 & 3.834459 & -0.890669 \\
\hline $\mathrm{H}$ & 2.268467 & 2.231140 & -0.778722 \\
\hline $\mathrm{H}$ & -1.044905 & 2.010482 & -0.034518 \\
\hline $\mathrm{H}$ & -1.626179 & 3.504269 & 0.819300 \\
\hline C & 0.976970 & 1.230186 & 3.026536 \\
\hline C & 0.172448 & 2.430322 & 3.649493 \\
\hline C & 0.517355 & -0.154789 & 3.477839 \\
\hline $\mathrm{H}$ & 1.104319 & -0.931268 & 2.975707 \\
\hline $\mathrm{H}$ & 0.671002 & -0.271934 & 4.557369 \\
\hline $\mathrm{H}$ & -0.538496 & -0.332742 & 3.262523 \\
\hline C & 2.495900 & 1.369356 & 3.218247 \\
\hline $\mathrm{H}$ & 2.790884 & 1.173877 & 4.255290 \\
\hline $\mathrm{H}$ & 3.002669 & 0.643344 & 2.574251 \\
\hline $\mathrm{H}$ & 2.842810 & 2.369032 & 2.941478 \\
\hline C & 0.760648 & 2.985365 & 4.948984 \\
\hline $\mathrm{H}$ & 0.771225 & 2.223535 & 5.737565 \\
\hline $\mathrm{H}$ & 1.778210 & 3.354535 & 4.801684 \\
\hline $\mathrm{H}$ & 0.147488 & 3.823261 & 5.297503 \\
\hline C & -1.317829 & 2.108987 & 3.855904 \\
\hline $\mathrm{H}$ & -1.477651 & 1.438431 & 4.707418 \\
\hline $\mathrm{H}$ & -1.851020 & 3.045237 & 4.051845 \\
\hline $\mathrm{H}$ & -1.758260 & 1.640834 & 2.970746 \\
\hline 0 & 0.692375 & 1.396439 & 1.608216 \\
\hline 0 & 0.282841 & 3.442887 & 2.636924 \\
\hline B & 0.531005 & 2.829382 & 1.385491 \\
\hline 0 & 1.686371 & 3.501799 & 0.719115 \\
\hline C & 3.072337 & 4.257585 & -1.054395 \\
\hline C & 3.843658 & 3.909005 & -2.174123 \\
\hline C & 3.229493 & 5.527204 & -0.477633 \\
\hline C & 4.752720 & 4.816760 & -2.712586 \\
\hline $\mathrm{H}$ & 3.725797 & 2.924540 & -2.621429 \\
\hline C & 4.138820 & 6.432269 & -1.019402 \\
\hline $\mathrm{H}$ & 2.642947 & 5.782539 & 0.398894 \\
\hline C & 4.901053 & 6.081630 & -2.137677 \\
\hline $\mathrm{H}$ & 5.347490 & 4.537230 & -3.577827 \\
\hline $\mathrm{H}$ & 4.257886 & 7.412964 & -0.566654 \\
\hline $\mathrm{H}$ & 5.610908 & 6.789700 & -2.556683 \\
\hline $\mathrm{H}$ & -0.521478 & 4.916857 & -0.826122 \\
\hline
\end{tabular}




\begin{tabular}{|c|c|c|c|}
\hline $\mathrm{H}$ & 0.499755 & 2. 291551 & -2.072512 \\
\hline $\mathrm{H}$ & 0.823838 & 4.015416 & -2.643212 \\
\hline C & -0.302054 & -2.805016 & 0.044064 \\
\hline C & 0.910624 & -2.167982 & -0.192508 \\
\hline C & 2.102993 & -2.530621 & 0.522365 \\
\hline C & 2.003554 & -3.497780 & 1.505316 \\
\hline $\mathrm{H}$ & 2.901223 & -3.815290 & 2.029396 \\
\hline C & -2.427570 & -1.531994 & -0.459471 \\
\hline C & -1.514060 & -2.521192 & -0.803870 \\
\hline C & -3.815585 & -2.111443 & -2.372768 \\
\hline C & -3.602217 & -1.332533 & -1.257936 \\
\hline $\mathrm{H}$ & -4.707810 & -1.955796 & -2.974925 \\
\hline C & -2.900386 & -3.123009 & -2.757696 \\
\hline C & -1.728700 & -3.338275 & -1.965679 \\
\hline C & -0.824032 & -4.358602 & -2.369415 \\
\hline C & -1.070193 & -5.124171 & -3.487144 \\
\hline C & -2.232131 & -4.909872 & -4.265449 \\
\hline C & -3.125563 & -3.927433 & -3.906465 \\
\hline $\mathrm{H}$ & 0.075342 & -4.526457 & -1.788015 \\
\hline $\mathrm{H}$ & -0.363516 & -5.896920 & -3.777548 \\
\hline $\mathrm{H}$ & -2.413468 & -5.520074 & -5.146218 \\
\hline $\mathrm{H}$ & -4.020797 & -3.749916 & -4.497967 \\
\hline C & 0.777598 & -4.128321 & 1.825958 \\
\hline C & -0.397895 & -3.788133 & 1.083787 \\
\hline C & -1.617839 & -4.441176 & 1.411328 \\
\hline C & -1.666813 & -5.386166 & 2.411475 \\
\hline C & -0.500479 & -5.728527 & 3.136773 \\
\hline C & 0.694496 & -5.111152 & 2.849335 \\
\hline $\mathrm{H}$ & -2.518348 & -4.173499 & 0.871090 \\
\hline $\mathrm{H}$ & -2.609384 & -5.871242 & 2.649871 \\
\hline H & -0.553642 & -6.476940 & 3.922893 \\
\hline H & 1.595988 & -5.364159 & 3.402377 \\
\hline C & -6.502812 & 1.634780 & -0.222350 \\
\hline C & -5.518298 & 1.960449 & -1.166657 \\
\hline C & -4.576582 & 0.975375 & -1.490818 \\
\hline C & -4.611971 & -0.287838 & -0.888537 \\
\hline C & -5.615022 & -0.575369 & 0.048762 \\
\hline C & -6.581678 & 0.379274 & 0.396058 \\
\hline $\mathrm{H}$ & -7.240665 & 2.387796 & 0.037927 \\
\hline H & -3.796005 & 1.174313 & -2.216093 \\
\hline $\mathrm{H}$ & -5.645795 & -1.572099 & 0.476802 \\
\hline C & -5.504391 & 3.363514 & -1.804105 \\
\hline C & -7.709648 & 0.091569 & 1.406075 \\
\hline C & -4.357740 & 3.536480 & -2.818552 \\
\hline $\mathrm{H}$ & -4.392717 & 4.546210 & -3.243114 \\
\hline $\mathrm{H}$ & -3.374519 & 3.407928 & -2.351996 \\
\hline $\mathrm{H}$ & -4.437610 & 2.826941 & -3.649799 \\
\hline C & -6.841397 & 3.606414 & -2.544349 \\
\hline H & -6.848674 & 4.604082 & -3.000193 \\
\hline H & -6.987554 & 2.868118 & -3.341052 \\
\hline $\mathrm{H}$ & -7.701094 & 3.543343 & -1.868948 \\
\hline C & -5.330096 & 4.429770 & -0.696376 \\
\hline H & -5.327115 & 5.435898 & -1.133393 \\
\hline $\mathrm{H}$ & -6.138453 & 4.389948 & 0.041113 \\
\hline
\end{tabular}




\begin{tabular}{|c|c|c|c|}
\hline $\mathrm{H}$ & -4.383332 & 4.288085 & -0.162615 \\
\hline C & -7.620717 & 1.093906 & 2.581477 \\
\hline $\mathrm{H}$ & -8.420050 & 0.901804 & 3.307583 \\
\hline $\mathrm{H}$ & -6.660823 & 1.003563 & 3.102842 \\
\hline $\mathrm{H}$ & -7.720589 & 2.130698 & 2.243864 \\
\hline C & -9.079258 & 0.246984 & 0.702551 \\
\hline $\mathrm{H}$ & -9.895249 & 0.049761 & 1.408514 \\
\hline $\mathrm{H}$ & -9.220565 & 1.256783 & 0.303359 \\
\hline $\mathrm{H}$ & -9.173687 & -0.457947 & -0.131339 \\
\hline C & -7.630689 & -1.333617 & 1.986431 \\
\hline $\mathrm{H}$ & -8.453406 & -1.489185 & 2.693173 \\
\hline $\mathrm{H}$ & -7.717105 & -2.098735 & 1.206729 \\
\hline $\mathrm{H}$ & -6.694838 & -1.504398 & 2.531122 \\
\hline C & 3.441874 & -1.960762 & 0.193719 \\
\hline C & 3.864353 & -1.840849 & -1.133447 \\
\hline C & 4.333974 & -1.607807 & 1.223954 \\
\hline C & 5.141453 & -1.356454 & -1.456852 \\
\hline $\mathrm{H}$ & 3.192055 & -2.145266 & -1.926674 \\
\hline C & 5.616727 & -1.132896 & 0.945657 \\
\hline $\mathrm{H}$ & 3.997510 & -1.704271 & 2.249776 \\
\hline C & 5.992534 & -1.008799 & -0.405064 \\
\hline $\mathrm{H}$ & 6.983106 & -0.631918 & -0.631530 \\
\hline C & 6.616485 & -0.736492 & 2.049929 \\
\hline C & 5.545310 & -1.235685 & -2.938732 \\
\hline C & 6.943008 & 0.771077 & 1.928702 \\
\hline $\mathrm{H}$ & 7.375595 & 1.016381 & 0.953029 \\
\hline $\mathrm{H}$ & 6.039603 & 1.378337 & 2.055554 \\
\hline $\mathrm{H}$ & 7.664554 & 1.069401 & 2.699387 \\
\hline C & 6.066023 & -0.996384 & 3.465152 \\
\hline $\mathrm{H}$ & 6.819096 & -0.715092 & 4.210073 \\
\hline $\mathrm{H}$ & 5.165501 & -0.407412 & 3.670342 \\
\hline $\mathrm{H}$ & 5.827092 & -2.054804 & 3.620720 \\
\hline C & 7.918295 & -1.557612 & 1.890220 \\
\hline $\mathrm{H}$ & 7.717762 & -2.631570 & 1.978810 \\
\hline $\mathrm{H}$ & 8.396990 & -1.386836 & 0.920483 \\
\hline $\mathrm{H}$ & 8.639319 & -1.281339 & 2.669341 \\
\hline C & 5.456421 & -2.625468 & -3.613412 \\
\hline $\mathrm{H}$ & 6.131188 & -3.340242 & -3.128188 \\
\hline $\mathrm{H}$ & 4.443103 & -3.036834 & -3.569833 \\
\hline $\mathrm{H}$ & 5.740242 & -2.553495 & -4.670528 \\
\hline C & 4.578766 & -0.258478 & -3.650943 \\
\hline $\mathrm{H}$ & 3.534360 & -0.576179 & -3.570732 \\
\hline $\mathrm{H}$ & 4.658195 & 0.744711 & -3.214191 \\
\hline $\mathrm{H}$ & 4.829546 & -0.183706 & -4.716444 \\
\hline C & 6.981583 & -0.709125 & -3.118812 \\
\hline $\mathrm{H}$ & 7.216684 & -0.640840 & -4.186972 \\
\hline $\mathrm{H}$ & 7.108188 & 0.291696 & -2.689769 \\
\hline $\mathrm{H}$ & 7.722304 & -1.374324 & -2.659991 \\
\hline
\end{tabular}


Frequencies (Top 3 out of 450)

$\begin{array}{lr}\text { 1. } & -301.8491 \mathrm{~cm}^{-1} \\ \text { 2. } & 5.1604 \mathrm{~cm}^{-1} \\ \text { 3. } & 6.6605 \mathrm{~cm}^{-1}\end{array}$

\section{B3LYP/6-31G(d) Molecular Geometry in Cartesian Coordinates}

C

$\mathrm{H}$

$\mathrm{H}$

C

0

0

$\mathrm{H}$

0

$\mathrm{H}$

C

C

C

C

$\mathrm{H}$

$\mathrm{H}$

$\mathrm{H}$

C

C

C

$\mathrm{H}$

$\mathrm{H}$

$\mathrm{H}$

C

$\mathrm{H}$

$\mathrm{H}$

$\mathrm{H}$

C

$\mathrm{H}$

$\mathrm{H}$

$\mathrm{H}$

C

$\mathrm{H}$

\subsection{2}

2. 544282

4.183214

$-0.401415$

0.495074

$-1.529562$

$-2.164403$

2.898883

2.051533

$-2.422665$

$-2.508662$

$-3.770180$

$-2.546214$

$-1.419638$

$-3.819998$

$-4.666525$

$-4.486067$

$-5.799752$

$-4.544348$

$-3.589242$

$-5.326798$

$-4.744070$

$-4.016135$

$-4.682773$

$-3.967204$

$-3.016194$

$-6.792002$

$-7.149163$

$-6.347063$

$-7.661380$

$-6.526791$

$-7.027820$
$-1.473270$

$-1.971900$

$-1.626420$

$-0.814029$

$-0.987879$

$-0.225545$

0.042752

$-2.101627$

$-1.761754$

3. 327976

4.189830

1. 560483

2.048746

4. 276538

0.472828

1.969983

1.093881

1.549094

$-0.298805$

$-0.537446$

$-0.349228$

$-1.062161$

2. 112924

2. 130126

3. 121412

1.837317

2. 323103

1. 710761

3. 237183

2. 608390

0.387889

$-0.274157$
0.840893

1.626248

1.070978

$-0.546784$

$-1.357596$

$-0.941424$

$-0.224464$

$-0.419155$

$-0.777120$

$-3.385773$

$-1.351451$

$-2.324200$

$-2.893885$

$-1.321112$

$-2.245431$

$-2.797917$

1. 383748

0.646668

2.004709

2.484758

2.771069

1. 250001

2.433810

3. 302857

2.013364

2. 783631

1.517944

2. 354594

1.918409

0.915787

$-0.052692$

0.662262 


\begin{tabular}{|c|c|c|c|}
\hline $\mathrm{H}$ & -7.285083 & 0.802875 & -0.724532 \\
\hline $\mathrm{H}$ & -5.835116 & -0.212080 & -0.651907 \\
\hline 0 & -3.528999 & 1.082732 & 0.294533 \\
\hline 0 & -5.294497 & 2.433962 & -0.365135 \\
\hline B & -3.936962 & 2.094296 & -0.662795 \\
\hline 0 & -3.008449 & 3.300272 & -0.562460 \\
\hline C & -3.245674 & 5.433372 & -1.662607 \\
\hline C & -2.546752 & 6.526364 & -2.200602 \\
\hline C & -4.620550 & 5.549938 & -1.396882 \\
\hline C & -3.210397 & 7.718227 & -2.478214 \\
\hline $\mathrm{H}$ & -1.480812 & 6.436787 & -2.399087 \\
\hline C & -5.278881 & 6.744702 & -1.680802 \\
\hline $\mathrm{H}$ & -5.153852 & 4.707117 & -0.966035 \\
\hline C & -4.579990 & 7.828173 & -2.220953 \\
\hline $\mathrm{H}$ & -2.662768 & 8.561011 & -2.890839 \\
\hline $\mathrm{H}$ & -6.341882 & 6.834396 & -1.473946 \\
\hline $\mathrm{H}$ & -5.099488 & 8.758062 & -2.436126 \\
\hline $\mathrm{H}$ & -1.643994 & 1.466963 & -2.709392 \\
\hline $\mathrm{H}$ & -3.302430 & 3.857838 & -3.736836 \\
\hline $\mathrm{H}$ & -1.469146 & 3.690410 & -3.760562 \\
\hline C & 0.541698 & -0.542652 & 1.747099 \\
\hline C & -0.323515 & -1.227841 & 0.901933 \\
\hline C & -1.155855 & -2.300320 & 1.378573 \\
\hline C & -1.168269 & -2.557472 & 2.735370 \\
\hline $\mathrm{H}$ & -1.757931 & -3.388550 & 3.112994 \\
\hline C & 2.807240 & 0.015314 & 0.830915 \\
\hline C & 1.542065 & 0.454912 & 1.220248 \\
\hline C & 3.476976 & 2.315187 & 0.413679 \\
\hline C & 3.799037 & 0.970689 & 0.412027 \\
\hline $\mathrm{H}$ & 4.223106 & 3.038681 & 0.095025 \\
\hline C & 2.199376 & 2.789229 & 0.790240 \\
\hline C & 1.202676 & 1.846483 & 1.197037 \\
\hline C & -0.080201 & 2.338875 & 1.561859 \\
\hline C & -0.355690 & 3.688449 & 1.542942 \\
\hline C & 0.636963 & 4.619243 & 1.151489 \\
\hline C & 1.884863 & 4.175057 & 0.777682 \\
\hline $\mathrm{H}$ & -0.858315 & 1.637627 & 1.842169 \\
\hline $\mathrm{H}$ & -1.345687 & 4.037240 & 1.819986 \\
\hline $\mathrm{H}$ & 0.409894 & 5.682314 & 1.147782 \\
\hline $\mathrm{H}$ & 2.654526 & 4.879214 & 0.469779 \\
\hline C & -0.385634 & -1.814233 & 3.652659 \\
\hline C & 0.504967 & -0.808630 & 3.157901 \\
\hline C & 1.322884 & -0.111905 & 4.089568 \\
\hline C & 1.253043 & -0.387495 & 5.437458 \\
\hline C & 0.369327 & -1.380046 & 5.923662 \\
\hline C & -0.428640 & -2.079504 & 5.047954 \\
\hline $\mathrm{H}$ & 2.004154 & 0.648922 & 3.725437 \\
\hline $\mathrm{H}$ & 1.882833 & 0.159644 & 6.133520 \\
\hline $\mathrm{H}$ & 0.326210 & -1.589870 & 6.988952 \\
\hline $\mathrm{H}$ & -1.105007 & -2.849865 & 5.410485 \\
\hline C & 7.788760 & -0.032120 & -0.769264 \\
\hline C & 6.728510 & -0.730500 & -1.352759 \\
\hline C & 5.423975 & -0.401295 & -0.951516 \\
\hline C & 5.184970 & 0.584709 & 0.009009 \\
\hline
\end{tabular}




\begin{tabular}{|c|c|c|c|}
\hline C & 6.280660 & 1.263619 & 0.574962 \\
\hline C & 7.592257 & 0.970558 & 0.198307 \\
\hline $\mathrm{H}$ & 8.803706 & -0.271163 & -1.065219 \\
\hline $\mathrm{H}$ & 4.574633 & -0.927043 & -1.372302 \\
\hline $\mathrm{H}$ & 6.081872 & 2.013477 & 1.332374 \\
\hline C & 6.930325 & -1.838264 & -2.403873 \\
\hline C & 8.812479 & 1.692052 & 0.803045 \\
\hline C & 6.319364 & -3.159203 & -1.876301 \\
\hline $\mathrm{H}$ & 6.432521 & -3.954085 & -2.624442 \\
\hline $\mathrm{H}$ & 6.829035 & -3.482462 & -0.960796 \\
\hline $\mathrm{H}$ & 5.253988 & -3.057458 & -1.647623 \\
\hline C & 6.219005 & -1.434825 & -3.717628 \\
\hline $\mathrm{H}$ & 6.346698 & -2.217835 & -4.475537 \\
\hline $\mathrm{H}$ & 5.144796 & -1.287068 & -3.568685 \\
\hline $\mathrm{H}$ & 6.634120 & -0.502639 & -4.118921 \\
\hline C & 8.416649 & -2.088148 & -2.721986 \\
\hline $\mathrm{H}$ & 8.504505 & -2.886634 & -3.467651 \\
\hline $\mathrm{H}$ & 8.904133 & -1.197186 & -3.135158 \\
\hline $\mathrm{H}$ & 8.976695 & -2.404444 & -1.834209 \\
\hline C & 8.410329 & 2.760540 & 1.837578 \\
\hline $\mathrm{H}$ & 9.308288 & 3.247838 & 2.234737 \\
\hline $\mathrm{H}$ & 7.780211 & 3.540475 & 1.394779 \\
\hline $\mathrm{H}$ & 7.868822 & 2.324983 & 2.684955 \\
\hline C & 9.726056 & 0.662438 & 1.510148 \\
\hline $\mathrm{H}$ & 10.601637 & 1.161804 & 1.943574 \\
\hline $\mathrm{H}$ & 9.189413 & 0.153923 & 2.319337 \\
\hline $\mathrm{H}$ & 10.089259 & -0.104750 & 0.818250 \\
\hline C & 9.608786 & 2.392814 & -0.323168 \\
\hline $\mathrm{H}$ & 10.483054 & 2.909958 & 0.091591 \\
\hline $\mathrm{H}$ & 9.967990 & 1.680648 & -1.073276 \\
\hline $\mathrm{H}$ & 8.987264 & 3.134357 & -0.838123 \\
\hline C & -1.894811 & -3.205307 & 0.450991 \\
\hline C & -3.180542 & -3.669925 & 0.764589 \\
\hline C & -1.270066 & -3.686379 & -0.707678 \\
\hline C & -3.846613 & -4.591007 & -0.053398 \\
\hline $\mathrm{H}$ & -3.661940 & -3.298052 & 1.663730 \\
\hline C & -1.908680 & -4.589934 & -1.564857 \\
\hline $\mathrm{H}$ & -0.257946 & -3.367739 & -0.926403 \\
\hline C & -3.193910 & -5.027024 & -1.216407 \\
\hline $\mathrm{H}$ & -3.697588 & -5.731478 & -1.863632 \\
\hline C & -1.179328 & -5.066999 & -2.835721 \\
\hline C & -5.248643 & -5.095945 & 0.344612 \\
\hline C & -2.016336 & -6.067804 & -3.654648 \\
\hline $\mathrm{H}$ & -2.247057 & -6.974325 & -3.082954 \\
\hline $\mathrm{H}$ & -2.960571 & -5.628345 & -3.996465 \\
\hline $\mathrm{H}$ & -1.454877 & -6.373565 & -4.544596 \\
\hline C & -0.872902 & -3.844200 & -3.734263 \\
\hline $\mathrm{H}$ & -0.332049 & -4.161752 & -4.634429 \\
\hline $\mathrm{H}$ & -1.799822 & -3.352205 & -4.051509 \\
\hline $\mathrm{H}$ & -0.260699 & -3.096994 & -3.219636 \\
\hline C & 0.147370 & -5.759961 & -2.442309 \\
\hline $\mathrm{H}$ & 0.821234 & -5.084386 & -1.906133 \\
\hline $\mathrm{H}$ & -0.038887 & -6.628817 & -1.800162 \\
\hline $\mathrm{H}$ & 0.672913 & -6.108034 & -3.339943 \\
\hline
\end{tabular}




\begin{tabular}{|llll|} 
C & -5.817371 & -6.117725 & -0.658696 \\
H & -5.932930 & -5.688107 & -1.660221 \\
H & -5.184137 & -7.008498 & -0.739150 \\
H & -6.808172 & -6.446534 & -0.325308 \\
C & -5.172528 & -5.780621 & 1.730452 \\
$H$ & -4.484365 & -6.633287 & 1.708969 \\
$H$ & -4.825856 & -5.090919 & 2.507258 \\
$H$ & -6.161655 & -6.148794 & 2.029734 \\
C & -6.231045 & -3.903582 & 0.419226 \\
H & -7.234834 & -4.251184 & 0.693492 \\
H & -5.919731 & -3.166625 & 1.166390 \\
$H$ & -6.301954 & -3.392223 & -0.547616 \\
\hline
\end{tabular}

\section{C-Catalyzed reaction TS 124}

\section{Datum}

Value

\begin{tabular}{lc}
\hline B3LYP/6-31G(d) Energy & -3038.948708 \\
\hline B3LYP-D3(BJ)/def2-TZVPP-IEF-PCM(DCM) Energy & -3040.49977 \\
\hline B3LYP-D3(BJ)/def2-TZVPP-IEF-PCM(DCM)//B3LYP/6-31G(d) Free Energy (Quasiharmonic) & -3039.271265 \\
\hline
\end{tabular}

Number of Imaginary Frequencies

Frequencies (Top 3 out of 450)

$$
\begin{array}{lr}
\text { 1. } & -370.9807 \mathrm{~cm}^{-1} \\
\text { 2. } & 6.2870 \mathrm{~cm}^{-1} \\
\text { 3. } & 11.3861 \mathrm{~cm}^{-1}
\end{array}
$$

\section{B3LYP/6-31G(d) Molecular Geometry in Cartesian Coordinates}

$\begin{array}{rrrr}\mathrm{C} & -1.506538 & 0.792497 & 1.034022 \\ \mathrm{H} & -2.248263 & -0.002351 & 0.949871 \\ \mathrm{H} & -0.519966 & 0.326484 & 0.948728 \\ \mathrm{C} & 1.476977 & 0.719392 & -1.302491 \\ \mathrm{O} & 1.297031 & 1.025407 & -2.460880 \\ \mathrm{O} & 1.446134 & -0.573851 & -0.931478 \\ \mathrm{H} & 1.501760 & -0.760405 & 0.045146 \\ \mathrm{O} & -1.681940 & 1.326841 & 2.356119 \\ \mathrm{H} & -1.017349 & 2.024147 & 2.482698 \\ \mathrm{C} & 1.128884 & -3.728496 & -2.147150 \\ \mathrm{C} & -0.561238 & -3.079835 & -0.970251 \\ \mathrm{C} & 2.186747 & -3.996831 & 0.067663 \\ \mathrm{C} & 1.615862 & -4.523441 & -1.126987 \\ \mathrm{H} & -0.309927 & -2.061284 & -1.268621\end{array}$




\begin{tabular}{|c|c|c|c|}
\hline $\mathrm{H}$ & 2.821289 & -3.122190 & -0.090102 \\
\hline $\mathrm{H}$ & 2.647400 & -4.734341 & 0.724314 \\
\hline C & 1.562948 & -1.817759 & 2.815809 \\
\hline C & 0.721521 & -3.012078 & 3.394083 \\
\hline C & 3.076654 & -2.053678 & 2.939312 \\
\hline $\mathrm{H}$ & 3.603099 & -1.337915 & 2.300949 \\
\hline $\mathrm{H}$ & 3.421438 & -1.914362 & 3.969921 \\
\hline $\mathrm{H}$ & 3.350699 & -3.062765 & 2.618651 \\
\hline C & 1.207709 & -0.442857 & 3.378701 \\
\hline $\mathrm{H}$ & 1.835907 & 0.326704 & 2.915466 \\
\hline $\mathrm{H}$ & 1.399694 & -0.416266 & 4.458204 \\
\hline H & 0.161161 & -0.179627 & 3.211345 \\
\hline C & -0.750035 & -2.638764 & 3.639854 \\
\hline $\mathrm{H}$ & -0.867039 & -1.984332 & 4.510589 \\
\hline $\mathrm{H}$ & -1.184852 & -2.136856 & 2.771057 \\
\hline $\mathrm{H}$ & -1.317901 & -3.557171 & 3.820188 \\
\hline C & 1.307061 & -3.661045 & 4.649298 \\
\hline $\mathrm{H}$ & 1.366065 & -2.943597 & 5.476339 \\
\hline $\mathrm{H}$ & 0.663528 & -4.488509 & 4.966382 \\
\hline $\mathrm{H}$ & 2.304747 & -4.066387 & 4.464003 \\
\hline 0 & 1.210869 & -1.871566 & 1.405532 \\
\hline 0 & 0.778003 & -3.966182 & 2.320904 \\
\hline B & 0.877794 & -3.262109 & 1.095084 \\
\hline 0 & -0.357477 & -3.409579 & 0.274303 \\
\hline C & -1.698722 & -3.720781 & -1.659788 \\
\hline C & -2.324431 & -4.856032 & -1.122384 \\
\hline C & -2.169296 & -3.179557 & -2.866674 \\
\hline C & -3.396253 & -5.443737 & -1.789669 \\
\hline $\mathrm{H}$ & -1.966469 & -5.257334 & -0.179856 \\
\hline C & -3.240103 & -3.771830 & -3.532161 \\
\hline $\mathrm{H}$ & -1.690006 & -2.295651 & -3.281653 \\
\hline C & -3.854906 & -4.906917 & -2.996254 \\
\hline $\mathrm{H}$ & -3.878307 & -6.321429 & -1.367555 \\
\hline $\mathrm{H}$ & -3.597244 & -3.347921 & -4.466504 \\
\hline $\mathrm{H}$ & -4.690438 & -5.368677 & -3.515270 \\
\hline $\mathrm{H}$ & 1.310482 & -5.571544 & -1.121303 \\
\hline $\mathrm{H}$ & 1.528078 & -2.726935 & -2.282939 \\
\hline $\mathrm{H}$ & 0.663863 & -4.182771 & -3.016028 \\
\hline C & 0.701145 & 2.546708 & 0.202270 \\
\hline C & 1.744179 & 1.722233 & -0.203791 \\
\hline C & 3.051994 & 1.809378 & 0.388407 \\
\hline C & 3.232744 & 2.692342 & 1.436785 \\
\hline $\mathrm{H}$ & 4.221089 & 2.802489 & 1.874590 \\
\hline C & -1.706076 & 1.810007 & -0.075781 \\
\hline C & -0.627742 & 2.577721 & -0.510178 \\
\hline C & -3.127704 & 2.855141 & -1.747056 \\
\hline C & -2.996584 & 1.968358 & -0.695087 \\
\hline $\mathrm{H}$ & -4.103765 & 2.993849 & -2.204901 \\
\hline C & -2.045425 & 3.626998 & -2.229419 \\
\hline C & -0.768830 & 3.502006 & -1.598711 \\
\hline C & 0.302473 & 4.301555 & -2.084349 \\
\hline C & 0.117022 & 5.175124 & -3.132121 \\
\hline C & -1.149354 & 5.298009 & -3.752277 \\
\hline C & -2.206092 & 4.538224 & -3.308508 \\
\hline
\end{tabular}




\begin{tabular}{|c|c|c|c|}
\hline $\mathrm{H}$ & 1.281369 & 4.209768 & -1.627935 \\
\hline $\mathrm{H}$ & 0.951067 & 5.772845 & -3.489724 \\
\hline $\mathrm{H}$ & -1.281826 & 5.991109 & -4.578720 \\
\hline $\mathrm{H}$ & -3.184254 & 4.622333 & -3.776325 \\
\hline C & 2.184585 & 3.502288 & 1.934218 \\
\hline C & 0.901148 & 3.450519 & 1.301311 \\
\hline C & -0.126506 & 4.307808 & 1.791310 \\
\hline C & 0.102060 & 5.151740 & 2.856954 \\
\hline C & 1.370526 & 5.194330 & 3.482439 \\
\hline C & 2.388665 & 4.390085 & 3.025199 \\
\hline $\mathrm{H}$ & -1.098734 & 4.294832 & 1.310895 \\
\hline $\mathrm{H}$ & -0.696875 & 5.794877 & 3.215207 \\
\hline $\mathrm{H}$ & 1.537672 & 5.865371 & 4.320346 \\
\hline $\mathrm{H}$ & 3.369201 & 4.419362 & 3.494254 \\
\hline C & -6.580524 & -0.011009 & 0.568707 \\
\hline C & -5.754713 & 0.570203 & 1.541243 \\
\hline C & -4.578958 & 1.200154 & 1.115656 \\
\hline C & -4.217370 & 1.239340 & -0.238123 \\
\hline C & -5.068477 & 0.640029 & -1.178149 \\
\hline C & -6.261481 & 0.013849 & -0.796931 \\
\hline $\mathrm{H}$ & -7.493851 & -0.497396 & 0.882754 \\
\hline $\mathrm{H}$ & -3.922977 & 1.669942 & 1.839748 \\
\hline $\mathrm{H}$ & -4.776292 & 0.659253 & -2.224346 \\
\hline C & -6.092181 & 0.543589 & 3.045089 \\
\hline C & -7.169414 & -0.617560 & -1.871313 \\
\hline C & -7.410930 & -0.195082 & 3.342004 \\
\hline $\mathrm{H}$ & -7.600750 & -0.189654 & 4.421380 \\
\hline $\mathrm{H}$ & -8.268214 & 0.283301 & 2.854091 \\
\hline $\mathrm{H}$ & -7.373781 & -1.241996 & 3.019557 \\
\hline C & -4.954190 & -0.172577 & 3.812505 \\
\hline $\mathrm{H}$ & -5.168140 & -0.178866 & 4.888860 \\
\hline $\mathrm{H}$ & -4.857672 & -1.213437 & 3.481817 \\
\hline $\mathrm{H}$ & -3.986227 & 0.315983 & 3.663132 \\
\hline C & -6.226779 & 1.993741 & 3.567988 \\
\hline $\mathrm{H}$ & -6.462240 & 1.992423 & 4.639602 \\
\hline $\mathrm{H}$ & -5.301215 & 2.561655 & 3.430830 \\
\hline $\mathrm{H}$ & -7.028914 & 2.527175 & 3.044556 \\
\hline C & -6.389546 & -1.718131 & -2.627764 \\
\hline $\mathrm{H}$ & -7.020758 & -2.165450 & -3.406156 \\
\hline $\mathrm{H}$ & -5.491820 & -1.321781 & -3.112785 \\
\hline $\mathrm{H}$ & -6.071116 & -2.515145 & -1.947002 \\
\hline C & -8.436692 & -1.258056 & -1.273661 \\
\hline $\mathrm{H}$ & -9.045236 & -1.688243 & -2.077373 \\
\hline $\mathrm{H}$ & -8.194229 & -2.066051 & -0.574176 \\
\hline $\mathrm{H}$ & -9.057177 & -0.523926 & -0.747298 \\
\hline C & -7.614143 & 0.473741 & -2.874255 \\
\hline $\mathrm{H}$ & -8.253636 & 0.039779 & -3.653246 \\
\hline $\mathrm{H}$ & -8.182792 & 1.262487 & -2.368223 \\
\hline $\mathrm{H}$ & -6.758781 & 0.944518 & -3.369900 \\
\hline C & 4.220424 & 1.038901 & -0.128037 \\
\hline C & 5.160184 & 0.493587 & 0.767567 \\
\hline C & 4.441760 & 0.899405 & -1.501364 \\
\hline C & 6.287513 & -0.192367 & 0.313333 \\
\hline H & 4.983420 & 0.605843 & 1.830706 \\
\hline
\end{tabular}




$\begin{array}{lrrr}\text { C } & 5.551177 & 0.199077 & -2.000201 \\ \text { H } & 3.744945 & 1.353084 & -2.195733 \\ \text { C } & 6.453144 & -0.335013 & -1.076955 \\ \text { H } & 7.316428 & -0.880278 & -1.439652 \\ \text { C } & 5.723350 & 0.050897 & -3.523892 \\ \text { C } & 7.331136 & -0.805232 & 1.267785 \\ \text { C } & 4.491640 & -0.685657 & -4.105303 \\ \text { H } & 4.404823 & -1.692435 & -3.679837 \\ \text { H } & 3.556800 & -0.154831 & -3.899061 \\ \text { H } & 4.589451 & -0.785235 & -5.193551 \\ \text { C } & 5.834236 & 1.452697 & -4.169398 \\ \text { H } & 5.950844 & 1.361917 & -5.256280 \\ \text { H } & 4.943152 & 2.059799 & -3.981448 \\ \text { H } & 6.701761 & 1.997333 & -3.778748 \\ \text { C } & 6.984202 & -0.750126 & -3.899432 \\ \text { H } & 7.900428 & -0.264392 & -3.543840 \\ \text { H } & 6.956671 & -1.768792 & -3.495530 \\ \text { H } & 7.056478 & -0.830881 & -4.989824 \\ \text { C } & 7.344030 & -2.342616 & 1.092333 \\ \text { H } & 6.365329 & -2.771550 & 1.335653 \\ \text { H } & 7.589783 & -2.632512 & 0.065341 \\ \text { H } & 8.089477 & -2.797621 & 1.756259 \\ \text { C } & 8.733109 & -0.239930 & 0.936852 \\ \text { H } & 9.038654 & -0.469863 & -0.089016 \\ \text { H } & 8.755080 & 0.849465 & 1.055743 \\ \text { H } & 9.484068 & -0.670370 & 1.610593 \\ \text { C } & 7.034389 & -0.492681 & 2.746917 \\ \text { H } & 7.814684 & -0.931474 & 3.379009 \\ \text { H } & 7.021557 & 0.586375 & 2.939813 \\ \text { H } & 6.075859 & -0.911683 & 3.071961\end{array}$

\section{C-Catalyzed reaction TS 125}

\section{Datum}

Value

\begin{tabular}{lc}
\hline B3LYP/6-31G(d) Energy & -3038.949751 \\
\hline B3LYP-D3(BJ)/def2-TZVPP-IEF-PCM(DCM) Energy & -3040.499927 \\
\hline B3LYP-D3(BJ)/def2-TZVPP-IEF-PCM(DCM)//B3LYP/6-31G(d) Free Energy (Quasiharmonic) & -3039.271051 \\
\hline
\end{tabular}

Number of Imaginary Frequencies

Frequencies (Top 3 out of 450)

$\begin{array}{lr}\text { 1. } & -378.1044 \mathrm{~cm}^{-1} \\ \text { 2. } & 9.4195 \mathrm{~cm}^{-1} \\ \text { 3. } & 10.5530 \mathrm{~cm}^{-1}\end{array}$




\begin{tabular}{|c|c|c|c|}
\hline C & -2.125667 & -0.761278 & 0.856608 \\
\hline $\mathrm{H}$ & -1.062672 & -0.518920 & 0.939545 \\
\hline $\mathrm{H}$ & -2.671883 & 0.181499 & 0.815038 \\
\hline C & 1.044728 & -1.026340 & -1.281612 \\
\hline 0 & 1.033734 & -1.240697 & -2.474069 \\
\hline 0 & 1.103760 & 0.235964 & -0.821668 \\
\hline $\mathrm{H}$ & 1.037158 & 0.360864 & 0.163987 \\
\hline 0 & -2.576120 & -1.396734 & 2.062845 \\
\hline $\mathrm{H}$ & -2.095001 & -2.236269 & 2.149458 \\
\hline C & 0.362495 & 3.348713 & -1.938720 \\
\hline C & 2.028780 & 3.354355 & -0.598413 \\
\hline C & -0.912565 & 2.987089 & 0.147694 \\
\hline C & -0.518891 & 3.785629 & -0.964736 \\
\hline $\mathrm{H}$ & 2.215936 & 2.318640 & -0.887293 \\
\hline $\mathrm{H}$ & -1.073493 & 1.935960 & -0.097996 \\
\hline $\mathrm{H}$ & -1.715623 & 3.401835 & 0.756184 \\
\hline C & 1.026936 & 1.300392 & 2.961496 \\
\hline C & 0.175519 & 2.471196 & 3.578549 \\
\hline C & 0.640954 & -0.097984 & 3.441601 \\
\hline $\mathrm{H}$ & 1.264973 & -0.851904 & 2.948923 \\
\hline $\mathrm{H}$ & 0.807784 & -0.187215 & 4.521842 \\
\hline $\mathrm{H}$ & -0.406226 & -0.333003 & 3.236866 \\
\hline C & 2.539978 & 1.514553 & 3.128611 \\
\hline $\mathrm{H}$ & 2.857705 & 1.350441 & 4.164405 \\
\hline $\mathrm{H}$ & 3.072580 & 0.803153 & 2.489004 \\
\hline $\mathrm{H}$ & 2.834132 & 2.524957 & 2.831004 \\
\hline C & 0.756484 & 3.073585 & 4.860242 \\
\hline $\mathrm{H}$ & 0.814653 & 2.325986 & 5.660410 \\
\hline $\mathrm{H}$ & 1.753149 & 3.488039 & 4.691801 \\
\hline $\mathrm{H}$ & 0.109826 & 3.887231 & 5.205524 \\
\hline C & -1.294472 & 2.083605 & 3.812394 \\
\hline $\mathrm{H}$ & -1.411643 & 1.410839 & 4.668983 \\
\hline $\mathrm{H}$ & -1.866819 & 2.995571 & 4.011739 \\
\hline $\mathrm{H}$ & -1.725901 & 1.593980 & 2.934649 \\
\hline 0 & 0.713043 & 1.428779 & 1.546559 \\
\hline 0 & 0.221748 & 3.470388 & 2.549141 \\
\hline B & 0.477116 & 2.851389 & 1.300698 \\
\hline 0 & 1.597989 & 3.565861 & 0.615439 \\
\hline C & 2.919431 & 4.378133 & -1.182765 \\
\hline C & 3.029806 & 5.654810 & -0.610948 \\
\hline C & 3.687242 & 4.060940 & -2.314222 \\
\hline C & 3.889823 & 6.597523 & -1.168976 \\
\hline $\mathrm{H}$ & 2.446679 & 5.886198 & 0.274430 \\
\hline C & 4.546750 & 5.006337 & -2.869073 \\
\hline $\mathrm{H}$ & 3.605381 & 3.071208 & -2.758058 \\
\hline C & 4.648655 & 6.277896 & -2.298755 \\
\hline $\mathrm{H}$ & 3.972894 & 7.583664 & -0.720047 \\
\hline $\mathrm{H}$ & 5.139027 & 4.751225 & -3.743520 \\
\hline $\mathrm{H}$ & 5.319838 & 7.015453 & -2.730424 \\
\hline $\mathrm{H}$ & -0.700626 & 4.860345 & -0.901813 \\
\hline
\end{tabular}




\begin{tabular}{|c|c|c|c|}
\hline $\mathrm{H}$ & 0.431743 & 2.286396 & -2.157083 \\
\hline $\mathrm{H}$ & 0.662217 & 4.022414 & -2.735145 \\
\hline C & -0.202988 & -2.781523 & -0.022744 \\
\hline C & 1.001872 & -2.123723 & -0.241200 \\
\hline C & 2.194509 & -2.474694 & 0.479715 \\
\hline C & 2.102611 & -3.448284 & 1.457051 \\
\hline $\mathrm{H}$ & 2.999142 & -3.748308 & 1.992884 \\
\hline C & -2.381292 & -1.564535 & -0.406193 \\
\hline C & -1.431531 & -2.485301 & -0.844153 \\
\hline C & -3.765380 & -2.065196 & -2.339857 \\
\hline C & -3.584296 & -1.356941 & -1.167413 \\
\hline $\mathrm{H}$ & -4.679016 & -1.920456 & -2.911002 \\
\hline C & -2.817736 & -3.002874 & -2.812721 \\
\hline C & -1.626821 & -3.229382 & -2.054647 \\
\hline C & -0.690567 & -4.183751 & -2.539450 \\
\hline C & -0.925113 & -4.877158 & -3.705385 \\
\hline C & -2.106752 & -4.652780 & -4.451724 \\
\hline C & -3.030758 & -3.733622 & -4.013105 \\
\hline $\mathrm{H}$ & 0.224973 & -4.355619 & -1.984897 \\
\hline $\mathrm{H}$ & -0.194805 & -5.599759 & -4.059372 \\
\hline $\mathrm{H}$ & -2.278696 & -5.206392 & -5.370914 \\
\hline $\mathrm{H}$ & -3.941613 & -3.550630 & -4.578368 \\
\hline C & 0.887761 & -4.110671 & 1.753121 \\
\hline C & -0.283363 & -3.793583 & 0.992886 \\
\hline C & -1.485385 & -4.504383 & 1.277403 \\
\hline C & -1.525685 & -5.459865 & 2.270298 \\
\hline C & -0.367399 & -5.763471 & 3.024392 \\
\hline C & 0.812656 & -5.105295 & 2.765679 \\
\hline $\mathrm{H}$ & -2.375063 & -4.290458 & 0.694926 \\
\hline $\mathrm{H}$ & -2.452922 & -5.989263 & 2.470952 \\
\hline $\mathrm{H}$ & -0.412797 & -6.519233 & 3.803547 \\
\hline $\mathrm{H}$ & 1.710173 & -5.335820 & 3.334577 \\
\hline C & -6.701309 & 1.333015 & -0.022354 \\
\hline C & -6.237259 & 0.409335 & 0.925035 \\
\hline C & -5.208531 & -0.458372 & 0.541307 \\
\hline C & -4.652839 & -0.403338 & -0.745221 \\
\hline C & -5.147920 & 0.534410 & -1.663816 \\
\hline C & -6.178388 & 1.416931 & -1.320782 \\
\hline $\mathrm{H}$ & -7.495367 & 2.017142 & 0.262945 \\
\hline $\mathrm{H}$ & -4.814178 & -1.185931 & 1.239036 \\
\hline $\mathrm{H}$ & -4.696994 & 0.573032 & -2.648748 \\
\hline C & -6.848575 & 0.384949 & 2.339740 \\
\hline C & -6.735505 & 2.465721 & -2.303517 \\
\hline C & -6.576809 & 1.741322 & 3.033329 \\
\hline $\mathrm{H}$ & -7.011003 & 1.749166 & 4.040800 \\
\hline $\mathrm{H}$ & -5.500176 & 1.923530 & 3.126075 \\
\hline $\mathrm{H}$ & -7.011194 & 2.578190 & 2.475803 \\
\hline C & -8.375291 & 0.154764 & 2.246943 \\
\hline $\mathrm{H}$ & -8.819775 & 0.136982 & 3.249810 \\
\hline H & -8.876453 & 0.943956 & 1.676469 \\
\hline $\mathrm{H}$ & -8.598151 & -0.801846 & 1.760432 \\
\hline C & -6.248259 & -0.731776 & 3.215764 \\
\hline $\mathrm{H}$ & -6.702833 & -0.700605 & 4.212849 \\
\hline $\mathrm{H}$ & -6.444738 & -1.724370 & 2.794156 \\
\hline
\end{tabular}




\begin{tabular}{|c|c|c|c|}
\hline H & -5.165103 & -0.625111 & 3.336324 \\
\hline C & -6.056111 & 2.397200 & -3.684863 \\
\hline $\mathrm{H}$ & -6.491571 & 3.153770 & -4.347632 \\
\hline $\mathrm{H}$ & -4.980034 & 2.594151 & -3.621619 \\
\hline $\mathrm{H}$ & -6.196674 & 1.420245 & -4.161381 \\
\hline C & -8.251921 & 2.233035 & -2.503840 \\
\hline H & -8.664403 & 2.976866 & -3.196745 \\
\hline $\mathrm{H}$ & -8.444217 & 1.237431 & -2.919960 \\
\hline $\mathrm{H}$ & -8.805092 & 2.312656 & -1.562263 \\
\hline C & -6.507513 & 3.883724 & -1.727975 \\
\hline $\mathrm{H}$ & -6.904232 & 4.643501 & -2.412850 \\
\hline H & -7.003058 & 4.015695 & -0.760440 \\
\hline $\mathrm{H}$ & -5.438783 & 4.079850 & -1.584692 \\
\hline C & 3.525951 & -1.876371 & 0.171731 \\
\hline C & 4.397055 & -1.512914 & 1.216122 \\
\hline C & 3.961959 & -1.739030 & -1.149180 \\
\hline C & 5.673452 & -1.010676 & 0.957554 \\
\hline H & 4.049340 & -1.621300 & 2.237016 \\
\hline C & 5.232989 & -1.226447 & -1.452758 \\
\hline $\mathrm{H}$ & 3.305597 & -2.049929 & -1.953066 \\
\hline C & 6.063572 & -0.870426 & -0.387519 \\
\hline $\mathrm{H}$ & 7.049179 & -0.472439 & -0.598644 \\
\hline C & 5.651134 & -1.081075 & -2.928372 \\
\hline C & 6.651013 & -0.602204 & 2.077089 \\
\hline C & 7.081177 & -0.531144 & -3.085943 \\
\hline H & 7.187548 & 0.466624 & -2.644672 \\
\hline $\mathrm{H}$ & 7.826714 & -1.190373 & -2.626249 \\
\hline H & 7.326895 & -0.447470 & -4.150554 \\
\hline C & 5.590104 & -2.462916 & -3.622176 \\
\hline $\mathrm{H}$ & 5.885553 & -2.372731 & -4.674680 \\
\hline $\mathrm{H}$ & 6.269218 & -3.174618 & -3.138482 \\
\hline H & 4.582363 & -2.889133 & -3.596918 \\
\hline C & 4.677241 & -0.108609 & -3.637446 \\
\hline H & 3.637122 & -0.443584 & -3.572275 \\
\hline $\mathrm{H}$ & 4.736938 & 0.889747 & -3.186782 \\
\hline $\mathrm{H}$ & 4.937640 & -0.016413 & -4.699226 \\
\hline C & 6.956197 & 0.910570 & 1.965092 \\
\hline $\mathrm{H}$ & 7.402144 & 1.165399 & 0.997917 \\
\hline H & 6.041638 & 1.503703 & 2.077715 \\
\hline H & 7.659691 & 1.217490 & 2.748860 \\
\hline C & 6.084456 & -0.875128 & 3.483501 \\
\hline H & 5.171608 & -0.301432 & 3.677513 \\
\hline $\mathrm{H}$ & 5.860943 & -1.937821 & 3.633069 \\
\hline H & 6.822050 & -0.583852 & 4.239874 \\
\hline C & 7.967257 & -1.403010 & 1.932977 \\
\hline $\mathrm{H}$ & 8.673113 & -1.118284 & 2.722816 \\
\hline H & 7.782154 & -2.480214 & 2.015603 \\
\hline $\mathrm{H}$ & 8.456652 & -1.221715 & 0.970489 \\
\hline
\end{tabular}




\begin{tabular}{lc}
\hline B3LYP/6-31G(d) Energy & -3038.946512 \\
\hline B3LYP-D3(BJ)/def2-TZVPP-IEF-PCM(DCM) Energy & -3040.499702 \\
\hline B3LYP-D3(BJ)/def2-TZVPP-IEF-PCM(DCM)//B3LYP/6-31G(d) Free Energy (Quasiharmonic) & -3039.271018 \\
\hline
\end{tabular}

Number of Imaginary Frequencies

Frequencies (Top 3 out of 450)

$\begin{array}{lr}\text { 1. } & -333.2637 \mathrm{~cm}^{-1} \\ \text { 2. } & 9.0445 \mathrm{~cm}^{-1} \\ \text { 3. } & 10.8770 \mathrm{~cm}^{-1}\end{array}$

\section{B3LYP/6-31G(d) Molecular Geometry in Cartesian Coordinates}

C

$\mathrm{H}$

$\mathrm{H}$

C

0

0

$\mathrm{H}$

0

$\mathrm{H}$

C

C

C

C

$\mathrm{H}$

$\mathrm{H}$

$\mathrm{H}$

C

C

C

$\mathrm{H}$

$\mathrm{H}$

$\mathrm{H}$

C

$\mathrm{H}$

$\mathrm{H}$

$\mathrm{H}$

C

$\mathrm{H}$

$\mathrm{H}$

$\mathrm{H}$

C

$\mathrm{H}$

\subsection{7}

1.885998

0.173045

$-1.840491$

$-1.859264$

$-1.458220$

$-1.386961$

1.475466

0.795650

2.175077

2. 727684

$-0.046874$

0.866601

3.197280

$-1.094964$

0.111180

$-1.053090$

$-0.976656$

$-2.432179$

$-2.767104$

$-2.395186$

$-3.173638$

$-0.001455$

$-0.266727$

0.989404

0.070011

$-0.614643$

$-1.371033$

0.356602

$-0.564311$

$-2.251377$

$-3.087785$
$-0.793679$

$-0.135278$

$-0.294782$

$-1.185857$

$-1.957852$

0.089450

0.641486

$-0.877712$

$-1.418754$

2. 237223

2. 327247

2.835348

1.906047

1. 342324

2. 542372

3.871626

2. 865854

4.343763

2.424478

3.044716

1. 386127

2. 487474

2.570373

3.047126

2.922597

1.491421

5.389915

5.430051

5.182113

6.379714

4. 780302

4.951903
1.246457

0.810929

1.127732

$-1.052184$

$-1.986200$

$-1.231072$

$-0.410710$

2. 641592

3.076235

$-2.933075$

$-0.820405$

$-2.055694$

$-2.649005$

$-0.879028$

$-2.131620$

$-2.365115$

1.634437

1. 103407

2.114727

2.954770

2.461387

1. 315314

2. 716390

3. 666816

2.417374

2.882760

2.159584

2.952364

2. 614255

1.692899

0.361106

1.048220 


\begin{tabular}{|c|c|c|c|}
\hline $\mathrm{H}$ & -2.047100 & 5.716160 & -0.169128 \\
\hline $\mathrm{H}$ & -2.557308 & 4.032134 & -0.376622 \\
\hline 0 & -0.711271 & 2.116152 & 0.438525 \\
\hline 0 & 0.093570 & 4.270501 & 0.145541 \\
\hline B & 0.226122 & 2.922142 & -0.316369 \\
\hline 0 & 1.631619 & 2.363909 & -0.134214 \\
\hline C & 3.662062 & 3.474418 & -0.845992 \\
\hline C & 3.259167 & 4.764089 & -0.459850 \\
\hline C & 4.993513 & 3.249728 & -1.233716 \\
\hline C & 4.180145 & 5.809653 & -0.483357 \\
\hline $\mathrm{H}$ & 2.235140 & 4.930811 & -0.138140 \\
\hline C & 5.908577 & 4.299225 & -1.250572 \\
\hline $\mathrm{H}$ & 5.308093 & 2.247027 & -1.511833 \\
\hline C & 5.501653 & 5.583501 & -0.878996 \\
\hline $\mathrm{H}$ & 3.867533 & 6.806288 & -0.183437 \\
\hline $\mathrm{H}$ & 6.937397 & 4.116612 & -1.548674 \\
\hline $\mathrm{H}$ & 6.214343 & 6.403860 & -0.890665 \\
\hline H & 0.579384 & 0.854586 & -2.640175 \\
\hline H & 2.433358 & 3.272108 & -3.133991 \\
\hline $\mathrm{H}$ & 2.859708 & 1.493506 & -3.332260 \\
\hline C & -1.329844 & -2.341064 & 1.094451 \\
\hline C & -2.230207 & -1.581760 & 0.356427 \\
\hline C & -3.499468 & -1.181198 & 0.898082 \\
\hline C & -3.774109 & -1.501098 & 2.215381 \\
\hline $\mathrm{H}$ & -4.739175 & -1.232417 & 2.636657 \\
\hline C & 1.129968 & -2.141973 & 0.551834 \\
\hline C & -0.058293 & -2.865326 & 0.478723 \\
\hline C & 2.284963 & -3.926331 & -0.626296 \\
\hline C & 2.333690 & -2.680704 & -0.028182 \\
\hline $\mathrm{H}$ & 3.195131 & -4.351583 & -1.041161 \\
\hline C & 1.097303 & -4.689954 & -0.701558 \\
\hline C & -0.106623 & -4.153815 & -0.149834 \\
\hline C & -1.295361 & -4.928149 & -0.247258 \\
\hline C & -1.286012 & -6.167502 & -0.847117 \\
\hline C & -0.089342 & -6.699241 & -1.384350 \\
\hline C & 1.076124 & -5.972721 & -1.313095 \\
\hline $\mathrm{H}$ & -2.221771 & -4.528968 & 0.149853 \\
\hline $\mathrm{H}$ & -2.206326 & -6.741461 & -0.913187 \\
\hline $\mathrm{H}$ & -0.096569 & -7.678545 & -1.855352 \\
\hline $\mathrm{H}$ & 2.001427 & -6.366936 & -1.726798 \\
\hline C & -2.860103 & -2.213426 & 3.028112 \\
\hline C & -1.626532 & -2.665760 & 2.459451 \\
\hline C & -0.740104 & -3.421165 & 3.280136 \\
\hline C & -1.050165 & -3.692881 & 4.595678 \\
\hline C & -2.266589 & -3.235751 & 5.156375 \\
\hline C & -3.152102 & -2.518362 & 4.385533 \\
\hline $\mathrm{H}$ & 0.184779 & -3.794485 & 2.852402 \\
\hline $\mathrm{H}$ & -0.359638 & -4.269788 & 5.204610 \\
\hline $\mathrm{H}$ & -2.500074 & -3.457888 & 6.193931 \\
\hline $\mathrm{H}$ & -4.093018 & -2.170472 & 4.805209 \\
\hline C & 6.146538 & -0.726976 & -0.119676 \\
\hline C & 5.657255 & -1.339610 & -1.289217 \\
\hline C & 4.394698 & -1.932373 & -1.232612 \\
\hline C & 3.640149 & -1.955631 & -0.042551 \\
\hline
\end{tabular}




\begin{tabular}{|c|c|c|c|}
\hline C & 4.172045 & -1.348700 & 1.099872 \\
\hline C & 5.424180 & -0.712301 & 1.077489 \\
\hline $\mathrm{H}$ & 7.123658 & -0.258988 & -0.151113 \\
\hline $\mathrm{H}$ & 3.965802 & -2.389885 & -2.116386 \\
\hline $\mathrm{H}$ & 3.598286 & -1.380921 & 2.019250 \\
\hline C & 6.527593 & -1.362471 & -2.562146 \\
\hline C & 5.946870 & -0.040995 & 2.362561 \\
\hline C & 6.883770 & 0.079428 & -2.993577 \\
\hline $\mathrm{H}$ & 7.521951 & 0.065526 & -3.885582 \\
\hline $\mathrm{H}$ & 5.979837 & 0.649806 & -3.238822 \\
\hline $\mathrm{H}$ & 7.423756 & 0.621312 & -2.209754 \\
\hline C & 7.833172 & -2.139430 & -2.265885 \\
\hline $\mathrm{H}$ & 8.469894 & -2.173908 & -3.158688 \\
\hline $\mathrm{H}$ & 8.410229 & -1.672449 & -1.460945 \\
\hline $\mathrm{H}$ & 7.613782 & -3.169862 & -1.964326 \\
\hline C & 5.821315 & -2.049089 & -3.747114 \\
\hline $\mathrm{H}$ & 6.475489 & -2.033475 & -4.626232 \\
\hline $\mathrm{H}$ & 5.585428 & -3.097011 & -3.531141 \\
\hline $\mathrm{H}$ & 4.889919 & -1.539481 & -4.019455 \\
\hline C & 7.318285 & 0.630852 & 2.161737 \\
\hline $\mathrm{H}$ & 7.639271 & 1.099578 & 3.098848 \\
\hline $\mathrm{H}$ & 8.091424 & -0.091267 & 1.873951 \\
\hline $\mathrm{H}$ & 7.279509 & 1.415962 & 1.397598 \\
\hline C & 4.940758 & 1.043526 & 2.819877 \\
\hline $\mathrm{H}$ & 5.291128 & 1.517453 & 3.745417 \\
\hline $\mathrm{H}$ & 4.838427 & 1.826921 & 2.059551 \\
\hline $\mathrm{H}$ & 3.946439 & 0.627651 & 3.011177 \\
\hline C & 6.089727 & -1.105789 & 3.476145 \\
\hline $\mathrm{H}$ & 6.457249 & -0.642112 & 4. 399947 \\
\hline $\mathrm{H}$ & 5.132943 & -1.586747 & 3.702593 \\
\hline $\mathrm{H}$ & 6.799188 & -1.888356 & 3.182682 \\
\hline C & -4.539574 & -0.495282 & 0.077688 \\
\hline C & -5.298620 & 0.555299 & 0.627101 \\
\hline C & -4.827384 & -0.926666 & -1.220449 \\
\hline C & -6.312823 & 1.178573 & -0.101156 \\
\hline $\mathrm{H}$ & -5.067206 & 0.885684 & 1.632444 \\
\hline C & -5.830092 & -0.317982 & -1.991513 \\
\hline $\mathrm{H}$ & -4.268732 & -1.757374 & -1.634010 \\
\hline C & -6.553712 & 0.726794 & -1.412242 \\
\hline $\mathrm{H}$ & -7.332027 & 1.211011 & -1.990039 \\
\hline C & -6.081421 & -0.821403 & -3.425731 \\
\hline C & -7.155571 & 2.337158 & 0.467657 \\
\hline C & -6.456663 & -2.322150 & -3.388366 \\
\hline $\mathrm{H}$ & -5.659584 & -2.931558 & -2.950944 \\
\hline $\mathrm{H}$ & -7.367223 & -2.485089 & -2.799905 \\
\hline $\mathrm{H}$ & -6.637355 & -2.693072 & -4.404733 \\
\hline C & -7.223376 & -0.061325 & -4.125960 \\
\hline $\mathrm{H}$ & -7.357564 & -0.452934 & -5.140608 \\
\hline $\mathrm{H}$ & -8.177600 & -0.178000 & -3.598701 \\
\hline $\mathrm{H}$ & -7.008278 & 1.009912 & -4.213418 \\
\hline C & -4.791066 & -0.636026 & -4.261153 \\
\hline $\mathrm{H}$ & -4.520575 & 0.424230 & -4.328055 \\
\hline $\mathrm{H}$ & -3.938231 & -1.168637 & -3.828733 \\
\hline $\mathrm{H}$ & -4.943091 & -1.011794 & -5.280775 \\
\hline
\end{tabular}




\begin{tabular}{|llll|} 
C & -6.932451 & 3.605152 & -0.390671 \\
$H$ & -5.879597 & 3.909698 & -0.373072 \\
$H$ & -7.214896 & 3.444355 & -1.436314 \\
$H$ & -7.534018 & 4.438131 & -0.006053 \\
C & -8.654396 & 1.953305 & 0.433956 \\
$H$ & -9.002565 & 1.744906 & -0.582905 \\
$H$ & -8.844067 & 1.060955 & 1.041505 \\
$H$ & -9.265514 & 2.772347 & 0.832869 \\
C & -6.787326 & 2.674900 & 1.924863 \\
$H$ & -7.421213 & 3.493408 & 2.284514 \\
$H$ & -6.942000 & 1.819798 & 2.592964 \\
$H$ & -5.745226 & 2.999779 & 2.019989 \\
\hline
\end{tabular}

\section{C-Catalyzed reaction TS 127}

\section{Datum}

Value

\begin{tabular}{lr}
\hline B3LYP/6-31G(d) Energy & -3038.948159 \\
\hline B3LYP-D3(BJ)/def2-TZVPP-IEF-PCM(DCM) Energy & -3040.499791 \\
\hline B3LYP-D3(BJ)/def2-TZVPP-IEF-PCM(DCM)//B3LYP/6-31G(d) Free Energy (Quasiharmonic) & -3039.271004 \\
\hline
\end{tabular}

Number of Imaginary Frequencies

Frequencies (Top 3 out of 450)

$$
\begin{array}{lr}
\text { 1. } & -376.8493 \mathrm{~cm}^{-1} \\
\text { 2. } & 7.4006 \mathrm{~cm}^{-1} \\
\text { 3. } & 13.3231 \mathrm{~cm}^{-1}
\end{array}
$$

\section{B3LYP/6-31G(d) Molecular Geometry in Cartesian Coordinates}

C

$\mathrm{H}$

$\mathrm{H}$

C

0

0

$\mathrm{H}$

0

$\mathrm{H}$

C

C

C

C

$\mathrm{H}$
$-1.445472$

$-2.207051$

$-0.470799$

1.585033

1.421455

1.536891

1.533792

$-1.626325$

$-0.950464$

0.876841

$-0.747939$

1.840396

1. 245185

$-0.349819$
0.725669

$-0.044668$

0.236692

0.669463

1.035034

$-0.641956$

$-0.873494$

1. 211141

1.890344

$-3.655231$

$-2.866638$

$-4.161043$

$-4.552739$

$-1.874591$
1.080089

0.953571

0.989220

$-1.241969$

$-2.384908$

$-0.938057$

0.029791

2.420002

2.580474

$-2.337423$

$-1.152862$

$-0.119523$

$-1.352963$

$-1.367000$ 


\begin{tabular}{|c|c|c|c|}
\hline $\mathrm{H}$ & 2.572525 & -3.356214 & -0.211896 \\
\hline $\mathrm{H}$ & 2.197297 & -4.980794 & 0.503072 \\
\hline C & 1.385054 & -2.083247 & 2.738215 \\
\hline C & 0.419727 & -3.220838 & 3.232063 \\
\hline C & 2.867547 & -2.466123 & 2.866049 \\
\hline $\mathrm{H}$ & 3.468680 & -1.757081 & 2.288469 \\
\hline $\mathrm{H}$ & 3.203925 & -2.432088 & 3.908179 \\
\hline $\mathrm{H}$ & 3.059790 & -3.469811 & 2.476678 \\
\hline C & 1.152317 & -0.716893 & 3.380840 \\
\hline $\mathrm{H}$ & 1.853184 & 0.017155 & 2.966964 \\
\hline $\mathrm{H}$ & 1.336629 & -0.773304 & 4.460580 \\
\hline $\mathrm{H}$ & 0.136327 & -0.348352 & 3.225807 \\
\hline C & -1.009504 & -2.718726 & 3.498559 \\
\hline $\mathrm{H}$ & -1.068703 & -2.111816 & 4.408640 \\
\hline $\mathrm{H}$ & -1.387192 & -2.123082 & 2.662989 \\
\hline $\mathrm{H}$ & -1.665561 & -3.586724 & 3.619812 \\
\hline C & 0.922869 & -4.007584 & 4.444072 \\
\hline $\mathrm{H}$ & 1.042265 & -3.356805 & 5.318425 \\
\hline $\mathrm{H}$ & 0.196252 & -4.785891 & 4.699836 \\
\hline $\mathrm{H}$ & 1.877852 & -4.496911 & 4.237564 \\
\hline 0 & 1.059709 & -2.018253 & 1.321741 \\
\hline 0 & 0.386859 & -4.105303 & 2.100371 \\
\hline B & 0.589450 & -3.344898 & 0.922858 \\
\hline 0 & -0.632001 & -3.309488 & 0.068137 \\
\hline C & -1.934496 & -3.292911 & -1.920362 \\
\hline C & -2.731631 & -4.365739 & -1.492885 \\
\hline C & -2.275560 & -2.604173 & -3.095283 \\
\hline C & -3.842993 & -4.749867 & -2.239615 \\
\hline $\mathrm{H}$ & -2.473105 & -4.879722 & -0.572805 \\
\hline C & -3.386016 & -2.993099 & -3.840782 \\
\hline $\mathrm{H}$ & -1.664002 & -1.766681 & -3.423341 \\
\hline C & -4.170860 & -4.069445 & -3.416369 \\
\hline $\mathrm{H}$ & -4.456995 & -5.581162 & -1.903814 \\
\hline $\mathrm{H}$ & -3.639590 & -2.458923 & -4.752288 \\
\hline $\mathrm{H}$ & -5.036273 & -4.374350 & -3.998354 \\
\hline $\mathrm{H}$ & 0.821701 & -5.557325 & -1.410570 \\
\hline $\mathrm{H}$ & 1.392306 & -2.701758 & -2.414092 \\
\hline $\mathrm{H}$ & 0.384274 & -4.009614 & -3.237066 \\
\hline C & 0.825564 & 2.436765 & 0.344431 \\
\hline C & 1.854017 & 1.607244 & -0.088333 \\
\hline C & 3.152577 & 1.625435 & 0.530428 \\
\hline C & 3.335439 & 2.436483 & 1.634593 \\
\hline $\mathrm{H}$ & 4.318431 & 2.493904 & 2.094040 \\
\hline C & -1.599040 & 1.789873 & 0.008400 \\
\hline C & -0.491632 & 2.539105 & -0.383865 \\
\hline C & -2.965373 & 2.932781 & -1.644266 \\
\hline C & -2.875388 & 2.008101 & -0.621083 \\
\hline $\mathrm{H}$ & -3.929835 & 3.113943 & -2.111890 \\
\hline C & -1.854344 & 3.690323 & -2.082453 \\
\hline C & -0.591334 & 3.507526 & -1.437987 \\
\hline C & 0.507880 & 4.296310 & -1.876411 \\
\hline C & 0.362261 & 5.212963 & -2.893228 \\
\hline C & -0.889972 & 5.391589 & -3.528389 \\
\hline C & -1.973198 & 4.644267 & -3.129633 \\
\hline
\end{tabular}




\begin{tabular}{|c|c|c|c|}
\hline $\mathrm{H}$ & 1.476224 & 4.162541 & -1.407932 \\
\hline $\mathrm{H}$ & 1.217056 & 5.801752 & -3.214849 \\
\hline $\mathrm{H}$ & -0.990790 & 6.117883 & -4.330458 \\
\hline $\mathrm{H}$ & -2.941095 & 4.771778 & -3.608873 \\
\hline C & 2.300493 & 3.248591 & 2.156404 \\
\hline C & 1.031166 & 3.274577 & 1.493791 \\
\hline C & 0.022674 & 4.142127 & 2.005164 \\
\hline C & 0.255124 & 4.922269 & 3.117683 \\
\hline C & 1.508718 & 4.886854 & 3.772710 \\
\hline C & 2.508699 & 4.070553 & 3.296969 \\
\hline $\mathrm{H}$ & -0.937458 & 4.189618 & 1.503073 \\
\hline $\mathrm{H}$ & -0.529005 & 5.575349 & 3.490366 \\
\hline $\mathrm{H}$ & 1.679198 & 5.508159 & 4.647479 \\
\hline $\mathrm{H}$ & 3.478513 & 4.041178 & 3. 787791 \\
\hline C & -6.518461 & 0.093382 & 0.535115 \\
\hline C & -5.706977 & 0.644120 & 1.536453 \\
\hline C & -4.508491 & 1.251612 & 1.145954 \\
\hline C & -4.121834 & 1.300057 & -0.201854 \\
\hline C & -4.963976 & 0.735695 & -1.172438 \\
\hline C & -6.175455 & 0.127792 & -0.823962 \\
\hline $\mathrm{H}$ & -7.450807 & -0.382593 & 0.824834 \\
\hline $\mathrm{H}$ & -3.853093 & 1.693397 & 1.884900 \\
\hline $\mathrm{H}$ & -4.645522 & 0.767856 & -2.208100 \\
\hline C & -6.142162 & 0.557397 & 3.012543 \\
\hline C & -7.127152 & -0.491455 & -1.866380 \\
\hline C & -5.162970 & 1.283914 & 3.955211 \\
\hline $\mathrm{H}$ & -5.509985 & 1.186050 & 4.990524 \\
\hline $\mathrm{H}$ & -4.150657 & 0.870398 & 3.899461 \\
\hline $\mathrm{H}$ & -5.102799 & 2.354410 & 3.726597 \\
\hline C & -7.539315 & 1.197820 & 3.187176 \\
\hline $\mathrm{H}$ & -7.855240 & 1.141472 & 4.236182 \\
\hline $\mathrm{H}$ & -7.525357 & 2.253726 & 2.893412 \\
\hline $\mathrm{H}$ & -8.302255 & 0.693786 & 2.584500 \\
\hline C & -6.205020 & -0.930418 & 3.433906 \\
\hline $\mathrm{H}$ & -6.521480 & -1.019292 & 4.480735 \\
\hline $\mathrm{H}$ & -6.914438 & -1.496784 & 2.820679 \\
\hline $\mathrm{H}$ & -5.222924 & -1.406396 & 3.334716 \\
\hline C & -6.588590 & -0.360601 & -3.303696 \\
\hline $\mathrm{H}$ & -7.300581 & -0.809251 & -4.006147 \\
\hline $\mathrm{H}$ & -6.454412 & 0.687464 & -3.595008 \\
\hline $\mathrm{H}$ & -5.630551 & -0.877603 & -3.426626 \\
\hline C & -7.323451 & -1.995856 & -1.563466 \\
\hline $\mathrm{H}$ & -8.014017 & -2.443776 & -2.289334 \\
\hline $\mathrm{H}$ & -6.371023 & -2.534317 & -1.621679 \\
\hline $\mathrm{H}$ & -7.740416 & -2.159742 & -0.564316 \\
\hline C & -8.496467 & 0.226519 & -1.801352 \\
\hline $\mathrm{H}$ & -9.187737 & -0.202210 & -2.537665 \\
\hline $\mathrm{H}$ & -8.961171 & 0.131786 & -0.814310 \\
\hline $\mathrm{H}$ & -8.389294 & 1.295713 & -2.017644 \\
\hline C & 4.319624 & 0.883202 & -0.030266 \\
\hline C & 5.240979 & 0.252748 & 0.814570 \\
\hline C & 4.562170 & 0.886425 & -1.415575 \\
\hline C & 6.393763 & -0.371635 & 0.310869 \\
\hline $\mathrm{H}$ & 5.050087 & 0.249339 & 1.883806 \\
\hline
\end{tabular}




\begin{tabular}{|c|c|c|c|}
\hline C & 5.680736 & 0.254005 & -1.958979 \\
\hline $\mathrm{H}$ & 3.869767 & 1.406876 & -2.063217 \\
\hline C & 6.581513 & -0.364966 & -1.074636 \\
\hline $\mathrm{H}$ & 7.456965 & -0.851004 & -1.488071 \\
\hline C & 5.947545 & 0.216038 & -3.476009 \\
\hline C & 7.403063 & -1.019260 & 1.280342 \\
\hline C & 7.324391 & 0.851127 & -3.782462 \\
\hline $\mathrm{H}$ & 7.355646 & 1.896012 & -3.452790 \\
\hline $\mathrm{H}$ & 8.143154 & 0.318812 & -3.286678 \\
\hline $\mathrm{H}$ & 7.520714 & 0.830274 & -4.861367 \\
\hline C & 5.944910 & -1.256701 & -3.951443 \\
\hline $\mathrm{H}$ & 6.142048 & -1.309542 & -5.029302 \\
\hline $\mathrm{H}$ & 6.711186 & -1.851505 & -3.442683 \\
\hline $\mathrm{H}$ & 4.973633 & -1.726757 & -3.760175 \\
\hline C & 4.876418 & 0.980589 & -4.277726 \\
\hline $\mathrm{H}$ & 3.871431 & 0.576888 & -4.114907 \\
\hline $\mathrm{H}$ & 4.858600 & 2.045615 & -4.019229 \\
\hline $\mathrm{H}$ & 5.097459 & 0.905984 & -5.348617 \\
\hline C & 7.944939 & 0.059980 & 2.248550 \\
\hline $\mathrm{H}$ & 8.455260 & 0.859317 & 1.699161 \\
\hline $\mathrm{H}$ & 7.143542 & 0.517998 & 2.837916 \\
\hline $\mathrm{H}$ & 8.662294 & -0.382708 & 2.950450 \\
\hline C & 6.707629 & -2.132462 & 2.097487 \\
\hline $\mathrm{H}$ & 5.877437 & -1.743238 & 2.694760 \\
\hline $\mathrm{H}$ & 6.307770 & -2.912118 & 1.439230 \\
\hline $\mathrm{H}$ & 7.421325 & -2.601618 & 2.785935 \\
\hline C & 8.604800 & -1.646954 & 0.547845 \\
\hline $\mathrm{H}$ & 9.291854 & -2.086912 & 1.279415 \\
\hline $\mathrm{H}$ & 8.295740 & -2.446606 & -0.135001 \\
\hline $\mathrm{H}$ & 9.167486 & -0.903355 & -0.027822 \\
\hline
\end{tabular}

\section{C-Catalyzed reaction TS 128}

\section{Datum}

Value

B3LYP/6-31G(d) Energy

$-3038.944262$

B3LYP-D3(BJ)/def2-TZVPP-IEF-PCM(DCM) Energy

$-3040.498627$

B3LYP-D3(BJ)/def2-TZVPP-IEF-PCM(DCM)//B3LYP/6-31G(d) Free Energy (Quasiharmonic)

$-3039.270973$

Number of Imaginary Frequencies

Frequencies (Top 3 out of 450)

$\begin{array}{lr}\text { 1. } & -342.4000 \mathrm{~cm}^{-1} \\ \text { 2. } & 8.5483 \mathrm{~cm}^{-1} \\ \text { 3. } & 10.8771 \mathrm{~cm}^{-1}\end{array}$




\begin{tabular}{|c|c|c|c|}
\hline C & 1.671440 & -0.505878 & 0.545682 \\
\hline $\mathrm{H}$ & 1.866682 & 0.033071 & -0.388570 \\
\hline H & 0.699532 & -0.174515 & 0.905033 \\
\hline C & -1.594481 & -1.902253 & -0.862286 \\
\hline 0 & -1.755162 & -2.962588 & -1.429292 \\
\hline 0 & -1.303433 & -0.797440 & -1.560740 \\
\hline $\mathrm{H}$ & -1.193341 & 0.042198 & -1.045552 \\
\hline 0 & 2.608549 & -0.108866 & 1.559690 \\
\hline $\mathrm{H}$ & 3.497347 & -0.238893 & 1.187797 \\
\hline C & -0.025779 & 2.813094 & 3.227113 \\
\hline C & 1.397741 & 2.921277 & 1.592019 \\
\hline C & -1.675014 & 2.790620 & 1.397690 \\
\hline C & -0.966206 & 2.153573 & 2.463523 \\
\hline $\mathrm{H}$ & 1.961726 & 2.065388 & 1.968763 \\
\hline $\mathrm{H}$ & -1.902654 & 3.843972 & 1.580302 \\
\hline $\mathrm{H}$ & -2.544501 & 2.242822 & 1.031665 \\
\hline C & -1.417891 & 2.481571 & -2.235307 \\
\hline C & -0.884955 & 3.956312 & -2.095072 \\
\hline C & -0.885142 & 1.729910 & -3.457401 \\
\hline $\mathrm{H}$ & -1.297473 & 0.718299 & -3.490273 \\
\hline $\mathrm{H}$ & -1.172150 & 2.247610 & -4.380539 \\
\hline $\mathrm{H}$ & 0.204348 & 1.648385 & -3.430883 \\
\hline C & -2.950663 & 2.391400 & -2.196269 \\
\hline $\mathrm{H}$ & -3.247324 & 1.344879 & -2.076618 \\
\hline $\mathrm{H}$ & -3.400627 & 2.768896 & -3.121086 \\
\hline $\mathrm{H}$ & -3.358849 & 2.961706 & -1.356145 \\
\hline C & -1.775215 & 5.025216 & -2.731087 \\
\hline $\mathrm{H}$ & -1.863122 & 4.875519 & -3.813603 \\
\hline $\mathrm{H}$ & -2.776985 & 5.023862 & -2.295223 \\
\hline $\mathrm{H}$ & -1.336837 & 6.014894 & -2.562774 \\
\hline C & 0.559040 & 4.119515 & -2.600872 \\
\hline $\mathrm{H}$ & 0.619521 & 4.042471 & -3.691881 \\
\hline $\mathrm{H}$ & 0.927341 & 5.109005 & -2.309708 \\
\hline $\mathrm{H}$ & 1.222363 & 3.369061 & -2.161557 \\
\hline 0 & -0.902911 & 1.858300 & -1.027053 \\
\hline 0 & -0.893278 & 4.150063 & -0.669451 \\
\hline B & -0.646156 & 2.887955 & -0.045238 \\
\hline 0 & 0.756714 & 2.685432 & 0.488286 \\
\hline C & 1.973646 & 4.256035 & 1.873725 \\
\hline C & 1.548027 & 5.408089 & 1.191486 \\
\hline C & 2.994411 & 4.357138 & 2.833491 \\
\hline C & 2.135611 & 6.638489 & 1.479778 \\
\hline H & 0.763882 & 5.325713 & 0.444303 \\
\hline C & 3.579883 & 5.589029 & 3.113644 \\
\hline $\mathrm{H}$ & 3.328249 & 3.463036 & 3.355620 \\
\hline C & 3.149146 & 6.733960 & 2.437734 \\
\hline $\mathrm{H}$ & 1.805034 & 7.528347 & 0.950627 \\
\hline $\mathrm{H}$ & 4.372437 & 5.657311 & 3.853869 \\
\hline $\mathrm{H}$ & 3.605199 & 7.696405 & 2.654012 \\
\hline $\mathrm{H}$ & -0.988428 & 1.064749 & 2.512572 \\
\hline
\end{tabular}




\begin{tabular}{|c|c|c|c|}
\hline $\mathrm{H}$ & -0.103639 & 3.887775 & 3.356867 \\
\hline $\mathrm{H}$ & 0.533190 & 2.282199 & 3.992451 \\
\hline C & -0.601029 & -2.197264 & 1.397039 \\
\hline C & -1.690208 & -1.773552 & 0.644645 \\
\hline C & -2.890639 & -1.289547 & 1.268318 \\
\hline C & -2.921282 & -1.217458 & 2.649683 \\
\hline $\mathrm{H}$ & -3.832836 & -0.889750 & 3.142467 \\
\hline C & 1.673511 & -2.005557 & 0.319790 \\
\hline C & 0.608882 & -2.797392 & 0.731427 \\
\hline C & 2.887666 & -3.980912 & -0.417173 \\
\hline C & 2.828789 & -2.612672 & -0.276786 \\
\hline $\mathrm{H}$ & 3.768193 & -4.436558 & -0.864362 \\
\hline C & 1.818564 & -4.818305 & -0.010518 \\
\hline C & 0.652007 & -4.223579 & 0.564046 \\
\hline C & -0.415926 & -5.077584 & 0.952497 \\
\hline C & -0.327134 & -6.442348 & 0.791000 \\
\hline C & 0.831595 & -7.027202 & 0.228394 \\
\hline C & 1.880182 & -6.228570 & -0.166093 \\
\hline $\mathrm{H}$ & -1.315455 & -4.640763 & 1.370967 \\
\hline $\mathrm{H}$ & -1.157261 & -7.075830 & 1.091589 \\
\hline $\mathrm{H}$ & 0.887476 & -8.105349 & 0.104126 \\
\hline $\mathrm{H}$ & 2.773539 & -6.664824 & -0.606987 \\
\hline C & -1.819737 & -1.595005 & 3.455212 \\
\hline C & -0.634732 & -2.094877 & 2.826571 \\
\hline C & 0.463760 & -2.468135 & 3.649422 \\
\hline C & 0.387179 & -2.361959 & 5.020267 \\
\hline C & -0.790190 & -1.878650 & 5.640696 \\
\hline C & -1.868156 & -1.502523 & 4.873282 \\
\hline $\mathrm{H}$ & 1.372813 & -2.827260 & 3.180444 \\
\hline $\mathrm{H}$ & 1.237455 & -2.650332 & 5.632277 \\
\hline $\mathrm{H}$ & -0.837919 & -1.803542 & 6.723771 \\
\hline $\mathrm{H}$ & -2.775883 & -1.126820 & 5.339622 \\
\hline C & 6.181003 & -0.303476 & -1.603436 \\
\hline C & 6.285426 & -1.012629 & -0.393554 \\
\hline C & 5.174720 & -1.753330 & 0.024357 \\
\hline C & 3.991463 & -1.784936 & -0.735284 \\
\hline C & 3.929232 & -1.063153 & -1.929151 \\
\hline C & 5.023779 & -0.311909 & -2.388802 \\
\hline $\mathrm{H}$ & 7.037362 & 0.269946 & -1.937786 \\
\hline $\mathrm{H}$ & 5.205482 & -2.328682 & 0.943270 \\
\hline $\mathrm{H}$ & 3.012871 & -1.106462 & -2.510689 \\
\hline C & 7.597679 & -0.953417 & 0.412334 \\
\hline C & 4.913903 & 0.456743 & -3.719742 \\
\hline C & 7.523769 & -1.770513 & 1.716383 \\
\hline $\mathrm{H}$ & 8.474666 & -1.690534 & 2.254991 \\
\hline $\mathrm{H}$ & 6.734800 & -1.405338 & 2.383773 \\
\hline $\mathrm{H}$ & 7.342999 & -2.833916 & 1.523284 \\
\hline C & 8.753985 & -1.520696 & -0.445302 \\
\hline $\mathrm{H}$ & 9.697727 & -1.480304 & 0.112347 \\
\hline $\mathrm{H}$ & 8.564246 & -2.565432 & -0.716690 \\
\hline $\mathrm{H}$ & 8.889963 & -0.954448 & -1.372569 \\
\hline C & 7.909693 & 0.515618 & 0.785194 \\
\hline $\mathrm{H}$ & 8.845349 & 0.573357 & 1.354692 \\
\hline $\mathrm{H}$ & 8.020730 & 1.149409 & -0.100766 \\
\hline
\end{tabular}




\begin{tabular}{|c|c|c|c|}
\hline $\mathrm{H}$ & 7.110337 & 0.941761 & 1.402551 \\
\hline C & 4.620967 & -0.541114 & -4.865634 \\
\hline $\mathrm{H}$ & 4.535210 & -0.008574 & -5.820665 \\
\hline $\mathrm{H}$ & 5.426352 & -1.278703 & -4.959023 \\
\hline $\mathrm{H}$ & 3.685522 & -1.086175 & -4.703883 \\
\hline C & 3.759111 & 1.482443 & -3.626362 \\
\hline $\mathrm{H}$ & 3.662686 & 2.033781 & -4.569594 \\
\hline $\mathrm{H}$ & 2.797986 & 0.997889 & -3.425336 \\
\hline $\mathrm{H}$ & 3.942232 & 2.208647 & -2.826038 \\
\hline C & 6.205008 & 1.220925 & -4.068695 \\
\hline $\mathrm{H}$ & 6.073428 & 1.749595 & -5.019363 \\
\hline $\mathrm{H}$ & 6.455781 & 1.969192 & -3.308070 \\
\hline $\mathrm{H}$ & 7.061353 & 0.546408 & -4.183029 \\
\hline C & -4.107568 & -0.906762 & 0.494481 \\
\hline C & -4.836361 & 0.242994 & 0.855115 \\
\hline C & -4.585044 & -1.707338 & -0.547146 \\
\hline C & -6.012211 & 0.598018 & 0.192666 \\
\hline $\mathrm{H}$ & -4.455981 & 0.860177 & 1.660868 \\
\hline C & -5.753138 & -1.375496 & -1.251806 \\
\hline $\mathrm{H}$ & -4.046123 & -2.611079 & -0.803357 \\
\hline C & -6.444993 & -0.225559 & -0.863617 \\
\hline $\mathrm{H}$ & -7.351984 & 0.046265 & -1.391051 \\
\hline C & -6.217033 & -2.285196 & -2.404925 \\
\hline C & -6.839899 & 1.840351 & 0.575860 \\
\hline C & -7.507733 & -1.775640 & -3.073608 \\
\hline $\mathrm{H}$ & -8.348069 & -1.739778 & -2.370310 \\
\hline $\mathrm{H}$ & -7.377603 & -0.775761 & -3.503589 \\
\hline $\mathrm{H}$ & -7.790264 & -2.450242 & -3.889731 \\
\hline C & -5.105607 & -2.349831 & -3.480935 \\
\hline $\mathrm{H}$ & -5.409218 & -3.015015 & -4.298998 \\
\hline $\mathrm{H}$ & -4.917123 & -1.356605 & -3.905095 \\
\hline $\mathrm{H}$ & -4.159426 & -2.722826 & -3.076452 \\
\hline C & -6.486714 & -3.708518 & -1.860771 \\
\hline $\mathrm{H}$ & -5.588885 & -4.153022 & -1.419883 \\
\hline $\mathrm{H}$ & -7.267963 & -3.693654 & -1.091690 \\
\hline $\mathrm{H}$ & -6.820215 & -4.368031 & -2.671335 \\
\hline C & -6.226098 & 2.616257 & 1.756923 \\
\hline $\mathrm{H}$ & -6.159830 & 1.998686 & 2.660077 \\
\hline $\mathrm{H}$ & -5.222828 & 2.991494 & 1.525434 \\
\hline $\mathrm{H}$ & -6.853928 & 3.482261 & 1.995779 \\
\hline C & -6.930210 & 2.799826 & -0.634360 \\
\hline $\mathrm{H}$ & -5.934115 & 3.139407 & -0.939924 \\
\hline $\mathrm{H}$ & -7.399065 & 2.321250 & -1.500339 \\
\hline $\mathrm{H}$ & -7.527612 & 3.683502 & -0.377659 \\
\hline C & -8.266027 & 1.399646 & 0.985277 \\
\hline $\mathrm{H}$ & -8.872424 & 2.273514 & 1.253628 \\
\hline $\mathrm{H}$ & -8.780035 & 0.874042 & 0.174003 \\
\hline $\mathrm{H}$ & -8.235276 & 0.728001 & 1.850906 \\
\hline
\end{tabular}




\begin{tabular}{lc}
\hline B3LYP/6-31G(d) Energy & -3038.946051 \\
\hline B3LYP-D3(BJ)/def2-TZVPP-IEF-PCM(DCM) Energy & -3040.500331 \\
\hline B3LYP-D3(BJ)/def2-TZVPP-IEF-PCM(DCM)//B3LYP/6-31G(d) Free Energy (Quasiharmonic) & -3039.270945 \\
\hline
\end{tabular}

Number of Imaginary Frequencies

Frequencies (Top 3 out of 450)

$\begin{array}{lr}\text { 1. } & -329.3343 \mathrm{~cm}^{-1} \\ \text { 2. } & 12.0917 \mathrm{~cm}^{-1} \\ \text { 3. } & 13.1108 \mathrm{~cm}^{-1}\end{array}$

\section{B3LYP/6-31G(d) Molecular Geometry in Cartesian Coordinates}

C

$\mathrm{H}$

$\mathrm{H}$

C

0

0

$\mathrm{H}$

0

$\mathrm{H}$

C

C

C

C

$\mathrm{H}$

$\mathrm{H}$

$\mathrm{H}$

C

C

C

$\mathrm{H}$

$\mathrm{H}$

$\mathrm{H}$

C

$\mathrm{H}$

$\mathrm{H}$

$\mathrm{H}$

$\mathrm{H}$

C

$\mathrm{H}$

$\mathrm{H}$

$\mathrm{H}$

C

$\mathrm{H}$

\section{$-1.104558$}

$-1.858067$

$-0.143653$

1.837670

1.848601

1.445501

1.360566

$-1.432417$

$-0.744587$

$-2.229238$

$-2.800779$

$-0.034424$

$-0.915318$

$-3.238554$

1.022538

$-0.221759$

0.963180

0.851837

2. 353196

2. 336835

2.680745

3.089488

$-0.077669$

0.177833

$-1.077801$

$-0.121277$

0.467992

1.224401

$-0.497823$

0.394899

2.114728

2.949082
0.826614

0.158392

0.321771

1.149452

1.907322

$-0.125929$

$-0.662383$

0.950057

1.496518

$-2.178939$

$-2.235842$

$-2.835291$

$-1.883823$

$-1.237223$

$-2.575285$

$-3.868955$

$-2.847147$

$-4.328286$

$-2.428781$

$-1.387289$

$-3.048770$

$-2.512341$

$-2.519714$

$-2.999448$

$-2.848379$

$-1.438884$

$-5.355609$

$-5.405001$

$-5.121752$

$-6.348501$

$-4.801284$

$-4.981860$

\subsection{3}

0.862584

1.161902

$-1.072265$

$-2.017535$

$-1.228614$

$-0.399289$

2.669796

3.084621

$-2.927436$

$-0.814884$

$-2.023418$

$-2.631637$

$-0.885459$

$-2.096586$

$-2.325478$

1.665837

1.149588

2.136415

2.475472

2.979545

1.334250

2.748864

3.700503

2.453727

2. 912051

2. 216488

3.008736

2.670097

1.759482

0.409838

1.096874 


\begin{tabular}{|c|c|c|c|}
\hline $\mathrm{H}$ & 1.888885 & -5.738876 & -0.108552 \\
\hline $\mathrm{H}$ & 2.435664 & -4.069214 & -0.337511 \\
\hline 0 & 0.634547 & -2.101569 & 0.463922 \\
\hline 0 & -0.219075 & -4.239890 & 0.193616 \\
\hline B & -0.317468 & -2.895248 & -0.286407 \\
\hline 0 & -1.712885 & -2.304275 & -0.118524 \\
\hline C & -3.768494 & -3.354896 & -0.843308 \\
\hline C & -3.405580 & -4.653131 & -0.446444 \\
\hline C & -5.089857 & -3.095226 & -1.243921 \\
\hline C & -4.355084 & -5.672842 & -0.472902 \\
\hline $\mathrm{H}$ & -2.389566 & -4.846514 & -0.114006 \\
\hline C & -6.033803 & -4.118795 & -1.263222 \\
\hline $\mathrm{H}$ & -5.373580 & -2.084139 & -1.526397 \\
\hline C & -5.666114 & -5.412040 & -0.881675 \\
\hline $\mathrm{H}$ & -4.073180 & -6.676127 & -0.164866 \\
\hline $\mathrm{H}$ & -7.054817 & -3.909434 & -1.570591 \\
\hline $\mathrm{H}$ & -6.401404 & -6.212152 & -0.895510 \\
\hline $\mathrm{H}$ & -0.597595 & -0.841202 & -2.627152 \\
\hline $\mathrm{H}$ & -2.515667 & -3.207690 & -3.121760 \\
\hline $\mathrm{H}$ & -2.889225 & -1.418744 & -3.336628 \\
\hline C & 1.367758 & 2.352013 & 1.056392 \\
\hline C & 2.249387 & 1.564426 & 0.324848 \\
\hline C & 3.519290 & 1.158087 & 0.861948 \\
\hline C & 3.813303 & 1.506894 & 2.167863 \\
\hline $\mathrm{H}$ & 4.780789 & 1.238304 & 2.583425 \\
\hline C & -1.097937 & 2.156651 & 0.546499 \\
\hline C & 0.092334 & 2.873043 & 0.445664 \\
\hline C & -2.258778 & 3.918995 & -0.658932 \\
\hline C & -2.304480 & 2.686170 & -0.035220 \\
\hline $\mathrm{H}$ & -3.171448 & 4.337247 & -1.075661 \\
\hline C & -1.069506 & 4.677737 & -0.759342 \\
\hline C & 0.138316 & 4.148230 & -0.209374 \\
\hline C & 1.328695 & 4.915956 & -0.334193 \\
\hline C & 1.317346 & 6.143589 & -0.957679 \\
\hline C & 0.117025 & 6.669148 & -1.492764 \\
\hline C & -1.050182 & 5.948317 & -1.395760 \\
\hline $\mathrm{H}$ & 2.257776 & 4.521037 & 0.061007 \\
\hline $\mathrm{H}$ & 2.238880 & 6.712809 & -1.044440 \\
\hline $\mathrm{H}$ & 0.122768 & 7.639164 & -1.982634 \\
\hline $\mathrm{H}$ & -1.978259 & 6.337886 & -1.807656 \\
\hline C & 2.918587 & 2.249678 & 2.974471 \\
\hline C & 1.683768 & 2.703547 & 2.410291 \\
\hline C & 0.816205 & 3.488144 & 3.223751 \\
\hline C & 1.145960 & 3.787207 & 4.528533 \\
\hline C & 2.364176 & 3.329379 & 5.084797 \\
\hline C & 3.231452 & 2.583363 & 4.320416 \\
\hline $\mathrm{H}$ & -0.109702 & 3.861968 & 2.798675 \\
\hline $\mathrm{H}$ & 0.469760 & 4.386280 & 5.132118 \\
\hline $\mathrm{H}$ & 2.613389 & 3.573402 & 6.113740 \\
\hline $\mathrm{H}$ & 4.173509 & 2.234380 & 4.736631 \\
\hline C & -6.105351 & 0.698693 & -0.077857 \\
\hline C & -5.382724 & 0.730939 & 1.124466 \\
\hline C & -4.140490 & 1.378783 & 1.131932 \\
\hline C & -3.608844 & 1.957225 & -0.029592 \\
\hline
\end{tabular}




\begin{tabular}{|c|c|c|c|}
\hline C & -4.359423 & 1.890352 & -1.214532 \\
\hline C & -5.618892 & 1.279622 & -1.259369 \\
\hline $\mathrm{H}$ & -7.072901 & 0.215588 & -0.092857 \\
\hline $\mathrm{H}$ & -3.567188 & 1.442968 & 2.050026 \\
\hline $\mathrm{H}$ & -3.938389 & 2.328245 & -2.114771 \\
\hline C & -5.904796 & 0.091577 & 2.426494 \\
\hline C & -6.424015 & 1.292096 & -2.575232 \\
\hline C & -7.269859 & -0.597640 & 2.240174 \\
\hline $\mathrm{H}$ & -7.589801 & -1.042027 & 3.189453 \\
\hline $\mathrm{H}$ & -8.048167 & 0.109349 & 1.929646 \\
\hline $\mathrm{H}$ & -7.221921 & -1.403843 & 1.498990 \\
\hline C & -4.891223 & -0.970890 & 2.917577 \\
\hline $\mathrm{H}$ & -5.240875 & -1.422360 & 3.854588 \\
\hline $\mathrm{H}$ & -4.778935 & -1.773695 & 2.179329 \\
\hline $\mathrm{H}$ & -3.901285 & -0.541012 & 3.100310 \\
\hline C & -6.061195 & 1.186199 & 3.508887 \\
\hline $\mathrm{H}$ & -6.429994 & 0.746136 & 4.443684 \\
\hline $\mathrm{H}$ & -5.109084 & 1.680542 & 3.725980 \\
\hline $\mathrm{H}$ & -6.775034 & 1.954699 & 3.189916 \\
\hline C & -7.773297 & 0.558460 & -2.452197 \\
\hline $\mathrm{H}$ & -8.304032 & 0.600384 & -3.410064 \\
\hline $\mathrm{H}$ & -7.645175 & -0.499164 & -2.193105 \\
\hline $\mathrm{H}$ & -8.420407 & 1.017708 & -1.696684 \\
\hline C & -6.715123 & 2.759176 & -2.974582 \\
\hline $\mathrm{H}$ & -7.287662 & 2.795758 & -3.909706 \\
\hline $\mathrm{H}$ & -7.298764 & 3.266544 & -2.198056 \\
\hline $\mathrm{H}$ & -5.792811 & 3.329820 & -3.125351 \\
\hline C & -5.611422 & 0.613890 & -3.703470 \\
\hline $\mathrm{H}$ & -4.649965 & 1.109990 & -3.871694 \\
\hline $\mathrm{H}$ & -6.169467 & 0.643674 & -4.647338 \\
\hline $\mathrm{H}$ & -5.410595 & -0.438248 & -3.467346 \\
\hline C & 4.545345 & 0.441487 & 0.049268 \\
\hline C & 4.818465 & 0.840604 & -1.271207 \\
\hline C & 5.309299 & -0.586628 & 0.614809 \\
\hline C & 5.808536 & 0.213369 & -2.028745 \\
\hline $\mathrm{H}$ & 4.253889 & 1.660023 & -1.693836 \\
\hline c & 6.329193 & -1.228320 & -0.105428 \\
\hline $\mathrm{H}$ & 5.093746 & -0.894708 & 1.633351 \\
\hline C & 6.549194 & -0.816740 & -1.423514 \\
\hline $\mathrm{H}$ & 7.321870 & -1.304451 & -2.005543 \\
\hline C & 7.170629 & -2.330111 & 0.570068 \\
\hline C & 6.101158 & 0.610408 & -3.488539 \\
\hline C & 7.931039 & -1.720326 & 1.772245 \\
\hline $\mathrm{H}$ & 8.603307 & -0.919066 & 1.444467 \\
\hline H & 7.245204 & -1.297819 & 2.514368 \\
\hline $\mathrm{H}$ & 8.534874 & -2.487162 & 2.273277 \\
\hline C & 6.250647 & -3.466737 & 1.073337 \\
\hline $\mathrm{H}$ & 6.847266 & -4.256982 & 1.545573 \\
\hline $\mathrm{H}$ & 5.527125 & -3.111783 & 1.814184 \\
\hline $\mathrm{H}$ & 5.689773 & -3.914354 & 0.244967 \\
\hline C & 8.207693 & -2.949273 & -0.386262 \\
\hline $\mathrm{H}$ & 7.731945 & -3.419168 & -1.254610 \\
\hline H & 8.925718 & -2.205488 & -0.749507 \\
\hline $\mathrm{H}$ & 8.775757 & -3.724983 & 0.139647 \\
\hline
\end{tabular}




$\begin{array}{|llll|}\mathrm{C} & 5.224504 & 1.785618 & -3.963109 \\ \mathrm{H} & 4.155256 & 1.558178 & -3.898186 \\ \mathrm{H} & 5.411996 & 2.693784 & -3.378546 \\ \mathrm{H} & 5.454893 & 2.014770 & -5.009912 \\ \mathrm{C} & 7.583957 & 1.027642 & -3.631550 \\ \mathrm{H} & 7.813133 & 1.885137 & -2.988454 \\ \mathrm{H} & 8.268711 & 0.215849 & -3.363816 \\ \mathrm{H} & 7.799280 & 1.314279 & -4.668201 \\ \mathrm{C} & 5.819014 & -0.603180 & -4.406581 \\ \mathrm{H} & 6.028559 & -0.346783 & -5.452400 \\ \mathrm{H} & 6.439526 & -1.466817 & -4.143747 \\ \mathrm{H} & 4.769420 & -0.909723 & -4.335662 \\ & & \end{array}$

\section{C-Catalyzed reaction TS 130}

\section{Datum}

Value

\begin{tabular}{lc}
\hline B3LYP/6-31G(d) Energy & -3038.947844 \\
\hline B3LYP-D3(BJ)/def2-TZVPP-IEF-PCM(DCM) Energy & -3040.499654 \\
\hline B3LYP-D3(BJ)/def2-TZVPP-IEF-PCM(DCM)//B3LYP/6-31G(d) Free Energy (Quasiharmonic) & -3039.270763 \\
\hline
\end{tabular}

Number of Imaginary Frequencies

Frequencies (Top 3 out of 450)

$\begin{array}{lr}\text { 1. } & -306.0679 \mathrm{~cm}^{-1} \\ \text { 2. } & 10.4624 \mathrm{~cm}^{-1} \\ \text { 3. } & 11.2346 \mathrm{~cm}^{-1}\end{array}$

\section{B3LYP/6-31G(d) Molecular Geometry in Cartesian Coordinates}

C

$\mathrm{H}$

$\mathrm{H}$

C

0

0

$\mathrm{H}$

0

$\mathrm{H}$

C

C

C

C

$\mathrm{H}$

$$
\begin{array}{r}
-3.105748 \\
-4.106326 \\
-2.400891 \\
0.103247 \\
-0.815084 \\
0.972095 \\
1.744479 \\
-3.130924 \\
-2.288374 \\
4.767811 \\
3.505065 \\
5.193143 \\
4.592956 \\
2.652934
\end{array}
$$

0.743970

0.649219

0.844052

1.107514

1.809915

0.623273

0.117822

1.933150

1.995211

$-1.436233$

$-2.776633$

$-0.370231$

$-0.395704$

$-2.604248$
1.014730

1.445000

1.850549

$-0.951533$

$-1.328330$

$-1.845362$

$-1.480203$

0.236976

$-0.259668$

2.503283

1.250710

0. 319851

1.621059

1.912180 


\begin{tabular}{|c|c|c|c|}
\hline $\mathrm{H}$ & 6.181153 & -0.835955 & 0.285437 \\
\hline $\mathrm{H}$ & 5.197468 & 0.613666 & -0.152311 \\
\hline C & 3.626681 & -1.072855 & -3.062703 \\
\hline C & 5.081900 & -1.668228 & -2.996386 \\
\hline C & 2.559851 & -2.142284 & -3.331606 \\
\hline $\mathrm{H}$ & 1.565687 & -1.714927 & -3.174217 \\
\hline $\mathrm{H}$ & 2.612245 & -2.506529 & -4.363377 \\
\hline $\mathrm{H}$ & 2.672203 & -2.994116 & -2.656273 \\
\hline C & 3.451043 & 0.087839 & -4.041214 \\
\hline $\mathrm{H}$ & 2.420272 & 0.452532 & -4.007999 \\
\hline $\mathrm{H}$ & 3.659545 & -0.241963 & -5.065860 \\
\hline $\mathrm{H}$ & 4.115317 & 0.922186 & -3.805948 \\
\hline C & 6.180346 & -0.614705 & -3.218663 \\
\hline $\mathrm{H}$ & 6.227502 & -0.288217 & -4.263125 \\
\hline $\mathrm{H}$ & 6.025726 & 0.267203 & -2.590175 \\
\hline $\mathrm{H}$ & 7.147643 & -1.053821 & -2.953360 \\
\hline C & 5.328896 & -2.859810 & -3.924653 \\
\hline $\mathrm{H}$ & 5.200070 & -2.576237 & -4.975688 \\
\hline $\mathrm{H}$ & 6.356539 & -3.216386 & -3.795916 \\
\hline $\mathrm{H}$ & 4.653421 & -3.688672 & -3.701607 \\
\hline 0 & 3.443646 & -0.578451 & -1.699826 \\
\hline 0 & 5.169918 & -2.127099 & -1.636418 \\
\hline B & 4.279537 & -1.365795 & -0.821450 \\
\hline 0 & 3.401273 & -2.231741 & 0.083017 \\
\hline C & 4.229444 & -4.043752 & 1.468195 \\
\hline C & 5.119391 & -4.563564 & 0.512427 \\
\hline C & 3.982462 & -4.761670 & 2.651178 \\
\hline C & 5.756554 & -5.779031 & 0.754274 \\
\hline $\mathrm{H}$ & 5.297637 & -4.014576 & -0.407584 \\
\hline C & 4.620088 & -5.976594 & 2.883088 \\
\hline $\mathrm{H}$ & 3.284753 & -4.363068 & 3.384556 \\
\hline C & 5.511872 & -6.486289 & 1.934737 \\
\hline $\mathrm{H}$ & 6.443288 & -6.180822 & 0.014256 \\
\hline $\mathrm{H}$ & 4.420462 & -6.528517 & 3.797307 \\
\hline $\mathrm{H}$ & 6.009562 & -7.435609 & 2.113376 \\
\hline $\mathrm{H}$ & 3.784761 & 0.307938 & 1.819193 \\
\hline $\mathrm{H}$ & 5.651482 & -2.062669 & 2.434272 \\
\hline $\mathrm{H}$ & 4.244531 & -1.451478 & 3.455362 \\
\hline C & -0.405573 & -0.348701 & 1.013333 \\
\hline C & 0.312440 & 0.724982 & 0.497178 \\
\hline C & 1.157203 & 1.533239 & 1.333222 \\
\hline C & 1.345998 & 1.136199 & 2.644061 \\
\hline $\mathrm{H}$ & 1.979393 & 1.737789 & 3.291363 \\
\hline C & -2.781890 & -0.521274 & 0.225774 \\
\hline C & -1.484383 & -1.036437 & 0.214130 \\
\hline C & -3.536614 & -2.404209 & -1.119325 \\
\hline C & -3.831540 & -1.220882 & -0.468625 \\
\hline $\mathrm{H}$ & -4.328993 & -2.930773 & -1.645258 \\
\hline C & -2.236779 & -2.959633 & -1.131242 \\
\hline C & -1.182719 & -2.265113 & -0.459643 \\
\hline C & 0.114241 & -2.845406 & -0.463166 \\
\hline C & 0.349454 & -4.055950 & -1.077555 \\
\hline C & -0.696028 & -4.741296 & -1.740829 \\
\hline & -1.959202 & -4.197112 & -1.772860 \\
\hline
\end{tabular}




\begin{tabular}{|c|c|c|c|}
\hline $\mathrm{H}$ & 0.932424 & -2.319058 & 0.013763 \\
\hline $\mathrm{H}$ & 1.348508 & -4.482258 & -1.057366 \\
\hline $\mathrm{H}$ & -0.496892 & -5.692935 & -2.226356 \\
\hline $\mathrm{H}$ & -2.770519 & -4.710477 & -2.283736 \\
\hline C & 0.737119 & -0.024126 & 3.181255 \\
\hline C & -0.173824 & -0.769332 & 2.367693 \\
\hline C & -0.820886 & -1.899385 & 2.940993 \\
\hline C & -0.557403 & -2.284917 & 4.237419 \\
\hline C & 0.354797 & -1.553118 & 5.034851 \\
\hline C & 0.983525 & -0.443533 & 4.517228 \\
\hline $\mathrm{H}$ & -1.524152 & -2.464133 & 2.339527 \\
\hline $\mathrm{H}$ & -1.057870 & -3.154996 & 4.653424 \\
\hline $\mathrm{H}$ & 0.548138 & -1.863522 & 6.058191 \\
\hline $\mathrm{H}$ & 1.672720 & 0.136820 & 5.126503 \\
\hline C & -7.930512 & 0.010374 & -0.551524 \\
\hline C & -6.942698 & 0.944816 & -0.912384 \\
\hline C & -5.607021 & 0.540713 & -0.871456 \\
\hline C & -5.253341 & -0.759915 & -0.474564 \\
\hline C & -6.266269 & -1.659234 & -0.123109 \\
\hline C & -7.620955 & -1.293404 & -0.153270 \\
\hline $\mathrm{H}$ & -8.968973 & 0.319853 & -0.579159 \\
\hline $\mathrm{H}$ & -4.812569 & 1.229788 & -1.122084 \\
\hline $\mathrm{H}$ & -5.982432 & -2.657787 & 0.197148 \\
\hline C & -7.353467 & 2.372464 & -1.322461 \\
\hline C & -8.696017 & -2.321302 & 0.251863 \\
\hline C & -8.070014 & 3.056861 & -0.133649 \\
\hline $\mathrm{H}$ & -8.382386 & 4.071972 & -0.409137 \\
\hline $\mathrm{H}$ & -8.964081 & 2.505302 & 0.176941 \\
\hline $\mathrm{H}$ & -7.403248 & 3.130557 & 0.732916 \\
\hline C & -6.140625 & 3.238246 & -1.715106 \\
\hline $\mathrm{H}$ & -6.479636 & 4.248364 & -1.973645 \\
\hline $\mathrm{H}$ & -5.413817 & 3.321926 & -0.900742 \\
\hline $\mathrm{H}$ & -5.620253 & 2.830484 & -2.589256 \\
\hline C & -8.315102 & 2.313058 & -2.532861 \\
\hline $\mathrm{H}$ & -8.610952 & 3.325773 & -2.833980 \\
\hline $\mathrm{H}$ & -7.832827 & 1.831773 & -3.391477 \\
\hline $\mathrm{H}$ & -9.229463 & 1.754196 & -2.306297 \\
\hline C & -10.122293 & -1.744542 & 0.176707 \\
\hline $\mathrm{H}$ & -10.846924 & -2.510879 & 0.475121 \\
\hline $\mathrm{H}$ & -10.251320 & -0.888229 & 0.848362 \\
\hline $\mathrm{H}$ & -10.380420 & -1.424077 & -0.839038 \\
\hline C & -8.622366 & -3.542445 & -0.695706 \\
\hline $\mathrm{H}$ & -9.375980 & -4.290023 & -0.417887 \\
\hline $\mathrm{H}$ & -8.806858 & -3.243035 & -1.733820 \\
\hline $\mathrm{H}$ & -7.641437 & -4.027740 & -0.657334 \\
\hline C & -8.444987 & -2.788229 & 1.705462 \\
\hline $\mathrm{H}$ & -9.201211 & -3.523870 & 2.006999 \\
\hline $\mathrm{H}$ & -7.461568 & -3.256022 & 1.819248 \\
\hline $\mathrm{H}$ & -8.495167 & -1.943667 & 2.402216 \\
\hline C & 1.729366 & 2.843460 & 0.895675 \\
\hline C & 2.502975 & 3.000428 & -0.256844 \\
\hline C & 1.478276 & 3.967204 & 1.706202 \\
\hline C & 3.023149 & 4.254869 & -0.620675 \\
\hline $\mathrm{H}$ & 2.710569 & 2.137952 & -0.878500 \\
\hline
\end{tabular}




\begin{tabular}{|c|c|c|c|}
\hline C & 1.984713 & 5.226119 & 1.385730 \\
\hline $\mathrm{H}$ & 0.851881 & 3.834663 & 2.580286 \\
\hline C & 2.755770 & 5.341120 & 0.215310 \\
\hline $\mathrm{H}$ & 3.149405 & 6.315606 & -0.047771 \\
\hline C & 1.721439 & 6.473776 & 2.250567 \\
\hline C & 3.833775 & 4.392876 & -1.923404 \\
\hline C & 0.991285 & 7.544415 & 1.404914 \\
\hline $\mathrm{H}$ & 1.581907 & 7.852410 & 0.535869 \\
\hline $\mathrm{H}$ & 0.030552 & 7.165469 & 1.038880 \\
\hline $\mathrm{H}$ & 0.797034 & 8.439298 & 2.008947 \\
\hline C & 0.848739 & 6.162394 & 3.481162 \\
\hline $\mathrm{H}$ & 0.693452 & 7.077550 & 4.063588 \\
\hline $\mathrm{H}$ & -0.138153 & 5.781103 & 3.196201 \\
\hline $\mathrm{H}$ & 1.322352 & 5.427693 & 4.142576 \\
\hline C & 3.069338 & 7.046106 & 2.749958 \\
\hline $\mathrm{H}$ & 3.603424 & 6.311881 & 3.364225 \\
\hline $\mathrm{H}$ & 3.726574 & 7.328803 & 1.920883 \\
\hline $\mathrm{H}$ & 2.900130 & 7.941310 & 3.360944 \\
\hline C & 4.400495 & 5.811740 & -2.120828 \\
\hline $\mathrm{H}$ & 3.606429 & 6.563135 & -2.194148 \\
\hline $\mathrm{H}$ & 5.073719 & 6.101319 & -1.305375 \\
\hline $\mathrm{H}$ & 4.974374 & 5.851064 & -3.053549 \\
\hline C & 5.023384 & 3.404495 & -1.908852 \\
\hline $\mathrm{H}$ & 5.691194 & 3.606387 & -1.062895 \\
\hline $\mathrm{H}$ & 4.688879 & 2.364802 & -1.836984 \\
\hline $\mathrm{H}$ & 5.608170 & 3.501789 & -2.831811 \\
\hline C & 2.907922 & 4.070805 & -3.121599 \\
\hline $\mathrm{H}$ & 3.466056 & 4.135707 & -4.064205 \\
\hline $\mathrm{H}$ & 2.480373 & 3.066283 & -3.048389 \\
\hline $\mathrm{H}$ & 2.074891 & 4.781219 & -3.170862 \\
\hline
\end{tabular}

\section{C-Catalyzed reaction TS 131}

\section{Datum}

Value

\begin{tabular}{lc}
\hline B3LYP/6-31G(d) Energy & -3038.945396 \\
\hline B3LYP-D3(BJ)/def2-TZVPP-IEF-PCM(DCM) Energy & -3040.498784 \\
\hline B3LYP-D3(BJ)/def2-TZVPP-IEF-PCM(DCM)//B3LYP/6-31G(d) Free Energy (Quasiharmonic) & -3039.270747 \\
\hline
\end{tabular}

Number of Imaginary Frequencies

Frequencies (Top 3 out of 450)

$$
\begin{array}{lr}
\text { 1. } & -335.9217 \mathrm{~cm}^{-1} \\
\text { 2. } & 8.6548 \mathrm{~cm}^{-1} \\
\text { 3. } & 10.8694 \mathrm{~cm}^{-1}
\end{array}
$$




\begin{tabular}{|c|c|c|c|}
\hline C & -1.763760 & -0.419390 & 0.580410 \\
\hline $\mathrm{H}$ & -2.748650 & 0.037160 & 0.745710 \\
\hline $\mathrm{H}$ & -1.167990 & -0.270130 & 1.490650 \\
\hline C & 1.411990 & -1.960840 & -0.941310 \\
\hline 0 & 1.368620 & -3.020860 & -1.529280 \\
\hline 0 & 1.470120 & -0.805230 & -1.613640 \\
\hline $\mathrm{H}$ & 1.355240 & -0.010240 & -1.046060 \\
\hline 0 & -1.108940 & 0.176860 & -0.540860 \\
\hline $\mathrm{H}$ & -0.848339 & 1.083870 & -0.294700 \\
\hline C & 0.108521 & 2.958570 & 3.330070 \\
\hline C & -1.158509 & 3.132040 & 1.522680 \\
\hline C & 1.912991 & 2.815740 & 1.657490 \\
\hline C & 1.060471 & 2.232070 & 2.650270 \\
\hline $\mathrm{H}$ & -1.804659 & 2.332470 & 1.890900 \\
\hline $\mathrm{H}$ & 2.774441 & 2.204509 & 1.383670 \\
\hline $\mathrm{H}$ & 2.200361 & 3.846839 & 1.878440 \\
\hline C & 1.624831 & 2.605230 & -2.123130 \\
\hline C & 2.025581 & 4.073080 & -1.719230 \\
\hline C & 2.697651 & 1.850639 & -2.909260 \\
\hline $\mathrm{H}$ & 2.341021 & 0.852769 & -3.175840 \\
\hline $\mathrm{H}$ & 2.938171 & 2.386739 & -3.835190 \\
\hline $\mathrm{H}$ & 3.614041 & 1.732609 & -2.325960 \\
\hline C & 0.280481 & 2.524900 & -2.861890 \\
\hline $\mathrm{H}$ & 0.360061 & 2.909770 & -3.884490 \\
\hline $\mathrm{H}$ & -0.033269 & 1.477820 & -2.912230 \\
\hline $\mathrm{H}$ & -0.498079 & 3.090030 & -2.339860 \\
\hline C & 1.569951 & 5.155340 & -2.699860 \\
\hline $\mathrm{H}$ & 2.028191 & 5.011930 & -3.685240 \\
\hline $\mathrm{H}$ & 0.483931 & 5.158810 & -2.818340 \\
\hline $\mathrm{H}$ & 1.873832 & 6.139950 & -2.328000 \\
\hline C & 3.529151 & 4.232799 & -1.440460 \\
\hline $\mathrm{H}$ & 4.121451 & 4.191699 & -2.360780 \\
\hline $\mathrm{H}$ & 3.699521 & 5.204379 & -0.965130 \\
\hline $\mathrm{H}$ & 3.892151 & 3.453179 & -0.763680 \\
\hline 0 & 1.453161 & 1.974580 & -0.820060 \\
\hline 0 & 1.325871 & 4.261150 & -0.473130 \\
\hline B & 1.077241 & 2.989810 & 0.115140 \\
\hline 0 & -0.426549 & 2.804640 & 0.501950 \\
\hline C & -1.678429 & 4.497890 & 1.720900 \\
\hline C & -1.162749 & 5.601310 & 1.019530 \\
\hline C & -2.749969 & 4.680430 & 2.613040 \\
\hline C & -1.713058 & 6.864980 & 1.224540 \\
\hline $\mathrm{H}$ & -0.344019 & 5.455700 & 0.321340 \\
\hline C & -3.296238 & 5.944881 & 2.808500 \\
\hline $\mathrm{H}$ & -3.154879 & 3.824641 & 3.149050 \\
\hline C & -2.775368 & 7.041600 & 2.115280 \\
\hline $\mathrm{H}$ & -1.315308 & 7.716900 & 0.679940 \\
\hline $\mathrm{H}$ & -4.128148 & 6.076551 & 3.494570 \\
\hline $\mathrm{H}$ & -3.201538 & 8.029901 & 2.264920 \\
\hline $\mathrm{H}$ & 1.006851 & 1.143730 & 2.699080 \\
\hline
\end{tabular}




\begin{tabular}{|c|c|c|c|}
\hline H & 0.233401 & 4.030060 & 3.448880 \\
\hline $\mathrm{H}$ & -0.554259 & 2.475590 & 4.042860 \\
\hline C & 0.363860 & -2.257010 & 1.315740 \\
\hline C & 1.473800 & -1.877110 & 0.571000 \\
\hline C & 2.699980 & -1.474881 & 1.208420 \\
\hline C & 2.727180 & -1.401571 & 2.588930 \\
\hline $\mathrm{H}$ & 3.654700 & -1.132641 & 3.087710 \\
\hline C & -1.926330 & -1.902140 & 0.325390 \\
\hline C & -0.889530 & -2.768710 & 0.656890 \\
\hline C & -3.268510 & -3.779769 & -0.428620 \\
\hline C & -3.140200 & -2.415569 & -0.247950 \\
\hline H & -4.181050 & -4.173229 & -0.869220 \\
\hline C & -2.237700 & -4.687920 & -0.093040 \\
\hline C & -1.017200 & -4.181620 & 0.452580 \\
\hline C & 0.013649 & -5.106970 & 0.773380 \\
\hline C & -0.160761 & -6.457920 & 0.575210 \\
\hline C & -1.372601 & -6.956700 & 0.039470 \\
\hline C & -2.386161 & -6.087920 & -0.290270 \\
\hline $\mathrm{H}$ & 0.952969 & -4.734820 & 1.166200 \\
\hline H & 0.641389 & -7.147780 & 0.823200 \\
\hline H & -1.495461 & -8.025360 & -0.115440 \\
\hline H & -3.318911 & -6.457869 & -0.709700 \\
\hline C & 1.604330 & -1.728740 & 3.386970 \\
\hline C & 0.406460 & -2.184360 & 2.749330 \\
\hline C & -0.698740 & -2.544750 & 3.570390 \\
\hline C & -0.623180 & -2.452760 & 4.942840 \\
\hline C & 0.562090 & -1.998520 & 5.569500 \\
\hline C & 1.651180 & -1.647000 & 4.805840 \\
\hline H & -1.609230 & -2.898700 & 3.099400 \\
\hline H & -1.478200 & -2.736330 & 5.550650 \\
\hline $\mathrm{H}$ & 0.610020 & -1.933460 & 6.653200 \\
\hline $\mathrm{H}$ & 2.569530 & -1.303921 & 5.276700 \\
\hline C & -6.461440 & 0.016411 & -1.420950 \\
\hline C & -5.183490 & 0.380591 & -1.854020 \\
\hline C & -4.100620 & -0.419749 & -1.455910 \\
\hline C & -4.284300 & -1.540709 & -0.641300 \\
\hline C & -5.587120 & -1.872089 & -0.225410 \\
\hline C & -6.691140 & -1.108019 & -0.606690 \\
\hline H & -7.310849 & 0.620451 & -1.718930 \\
\hline $\mathrm{H}$ & -3.093540 & -0.175460 & -1.775720 \\
\hline $\mathrm{H}$ & -5.712870 & -2.735509 & 0.418010 \\
\hline C & -4.920339 & 1.614051 & -2.739210 \\
\hline C & -8.128590 & -1.446139 & -0.164790 \\
\hline C & -6.211109 & 2.377871 & -3.089130 \\
\hline H & -5.969589 & 3.243571 & -3.716420 \\
\hline H & -6.917139 & 1.752651 & -3.647840 \\
\hline H & -6.720169 & 2.751951 & -2.193180 \\
\hline C & -3.974389 & 2.583361 & -1.989590 \\
\hline $\mathrm{H}$ & -3.745799 & 3.455221 & -2.615410 \\
\hline H & -4.440289 & 2.945091 & -1.064910 \\
\hline $\mathrm{H}$ & -3.028159 & 2.101540 & -1.723640 \\
\hline C & -4.251419 & 1.167261 & -4.060890 \\
\hline $\mathrm{H}$ & -4.052029 & 2.036291 & -4.700220 \\
\hline H & -3.299369 & 0.657651 & -3.883380 \\
\hline
\end{tabular}




\begin{tabular}{|c|c|c|c|}
\hline $\mathrm{H}$ & -4.900950 & 0.479841 & -4.615050 \\
\hline C & -9.010340 & -1.682598 & -1.414160 \\
\hline H & -10.039810 & -1.918568 & -1.116910 \\
\hline H & -9.045190 & -0.801388 & -2.062950 \\
\hline H & -8.628000 & -2.519678 & -2.009450 \\
\hline C & -8.193630 & -2.713069 & 0.709060 \\
\hline H & -9.233680 & -2.914498 & 0.989920 \\
\hline $\mathrm{H}$ & -7.818070 & -3.594699 & 0.177450 \\
\hline H & -7.617370 & -2.601139 & 1.634520 \\
\hline C & -8.708490 & -0.269098 & 0.655090 \\
\hline H & -9.737410 & -0.488758 & 0.966470 \\
\hline H & -8.111830 & -0.089999 & 1.557110 \\
\hline H & -8.726439 & 0.660562 & 0.076860 \\
\hline C & 3.958320 & -1.215391 & 0.448620 \\
\hline C & 4.368780 & -2.079081 & -0.570130 \\
\hline C & 4.796500 & -0.142391 & 0.805850 \\
\hline C & 5.577100 & -1.886031 & -1.256700 \\
\hline $\mathrm{H}$ & 3.739640 & -2.922741 & -0.827120 \\
\hline C & 6.017650 & 0.072329 & 0.164300 \\
\hline H & 4.470550 & 0.523899 & 1.596410 \\
\hline C & 6.381150 & -0.811541 & -0.869180 \\
\hline H & 7.324030 & -0.649911 & -1.378390 \\
\hline C & 6.976581 & 1.215009 & 0.552620 \\
\hline C & 5.955700 & -2.854121 & -2.393060 \\
\hline C & 6.408991 & 2.108139 & 1.672280 \\
\hline H & 5.470441 & 2.590089 & 1.375470 \\
\hline H & 6.227201 & 1.543049 & 2.593600 \\
\hline H & 7.126141 & 2.902109 & 1.909490 \\
\hline C & 8.309521 & 0.609458 & 1.055090 \\
\hline $\mathrm{H}$ & 9.010431 & 1.405798 & 1.335100 \\
\hline H & 8.144740 & -0.023312 & 1.934770 \\
\hline H & 8.791490 & -0.005232 & 0.287930 \\
\hline C & 7.256251 & 2.109479 & -0.678220 \\
\hline $\mathrm{H}$ & 7.708431 & 1.544998 & -1.500020 \\
\hline H & 6.331381 & 2.562029 & -1.053460 \\
\hline H & 7.946811 & 2.919228 & -0.412140 \\
\hline C & 7.313150 & -2.507541 & -3.033220 \\
\hline H & 7.312750 & -1.504291 & -3.474820 \\
\hline $\mathrm{H}$ & 8.134190 & -2.563132 & -2.308540 \\
\hline H & 7.533740 & -3.220102 & -3.835970 \\
\hline C & 6.041680 & -4.296011 & -1.837910 \\
\hline H & 6.806140 & -4.371201 & -1.055520 \\
\hline H & 5.089530 & -4.626851 & -1.411450 \\
\hline H & 6.306279 & -4.995661 & -2.640200 \\
\hline C & 4.868160 & -2.791611 & -3.493430 \\
\hline H & 5.108920 & -3.490961 & -4.303940 \\
\hline H & 3.875640 & -3.048421 & -3.109920 \\
\hline H & 4.806680 & -1.784361 & -3.921690 \\
\hline
\end{tabular}


Frequencies (Top 3 out of 450)

$\begin{array}{rr}\text { 1. } & -398.0002 \mathrm{~cm}^{-1} \\ \text { 2. } & 7.9409 \mathrm{~cm}^{-1} \\ \text { 3. } & 12.8421 \mathrm{~cm}^{-1}\end{array}$

\section{B3LYP/6-31G(d) Molecular Geometry in Cartesian Coordinates}

C

$\mathrm{H}$

$\mathrm{H}$

C

0

0

$\mathrm{H}$

0

$\mathrm{H}$

C

C

C

C

$\mathrm{H}$

$\mathrm{H}$

$\mathrm{H}$

C

C

C

$\mathrm{H}$

$\mathrm{H}$

$\mathrm{H}$

C

$\mathrm{H}$

$\mathrm{H}$

$\mathrm{H}$

C

$\mathrm{H}$

$\mathrm{H}$

$\mathrm{H}$

C

$\mathrm{H}$

\subsection{0}

2. 155540

3.877340

$-0.115270$

0.862220

$-0.996960$

$-1.700780$

3. 383540

2.617780

$-4.989330$

$-3.611000$

$-5.178760$

$-5.717160$

$-2.805340$

$-5.910070$

$-4.430990$

$-3.121070$

$-4.163470$

$-1.740130$

$-1.078840$

$-1.825440$

$-1.273260$

$-3.634750$

$-2.961470$

$-3.665060$

$-4.639670$

$-5.038540$

$-4.431500$

$-5.627610$

$-5.735200$

$-3.519869$

$-2.936129$

\subsection{0}

1.422860

1.370760

1. 267690

1.959110

1.036350

0.364110

0.344890

$-0.124570$

$-1.365840$

$-2.617120$

$-0.279400$

$-1.017870$

$-1.944340$

0.103360

0.468300

$-0.631690$

$-1.753180$

$-0.827020$

0.001490

$-0.856810$

$-1.754420$

0.786290

1. 514890

0.985940

0.940070

$-1.436210$

$-1.308740$

$-0.529510$

$-2.263070$

$-3.139290$

$-3.205620$
$-1.308410$

$-1.329290$

$-0.967520$

0.735900

0.937160

1.712930

1.524570

$-2.634790$

$-3.002690$

$-1.588040$

$-0.476120$

0.627990

$-0.463310$

$-0.781820$

1.338880

0.353480

3.580590

3.950070

4.209140

3.943380

5.301970

3.870520

3.873430

3.412340

4.950040

3.468000

5.164310

6.068280

5.007680

5.339040

4.128650

5.053230 


\begin{tabular}{|c|c|c|c|}
\hline $\mathrm{H}$ & -4.315589 & -3.890140 & 4.168630 \\
\hline $\mathrm{H}$ & -2.865009 & -3.383820 & 3.287890 \\
\hline 0 & -3.017760 & -0.776380 & 2.129080 \\
\hline 0 & -5.003310 & -1.798160 & 2.785020 \\
\hline B & -4.230750 & -1.416790 & 1.663720 \\
\hline 0 & -3.959370 & -2.606910 & 0.773690 \\
\hline C & -3.710779 & -3.894420 & -1.206710 \\
\hline C & -3.007769 & -4.061290 & -2.410370 \\
\hline C & -4.478269 & -4.955010 & -0.699000 \\
\hline C & -3.077419 & -5.269960 & -3.099370 \\
\hline $\mathrm{H}$ & -2.404479 & -3.246720 & -2.803880 \\
\hline C & -4.546369 & -6.159300 & -1.393750 \\
\hline $\mathrm{H}$ & -5.003399 & -4.822020 & 0.241280 \\
\hline C & -3.848629 & -6.320060 & -2.595080 \\
\hline H & -2.525509 & -5.393350 & -4.026910 \\
\hline H & -5.139969 & -6.978100 & -0.996450 \\
\hline $\mathrm{H}$ & -3.901559 & -7.262940 & -3.132550 \\
\hline $\mathrm{H}$ & -6.648810 & -1.557670 & -0.284450 \\
\hline $\mathrm{H}$ & -4.189910 & -0.717760 & -1.937200 \\
\hline H & -5.432310 & -2.008860 & -2.341990 \\
\hline C & 0.389210 & -0.390960 & -1.057390 \\
\hline C & -0.403970 & 0.665980 & -0.623460 \\
\hline C & -1.411420 & 1.251260 & -1.468950 \\
\hline C & -1.629520 & 0.679540 & -2.710320 \\
\hline $\mathrm{H}$ & -2.354130 & 1.131740 & -3.382310 \\
\hline C & 2.798360 & -0.388780 & -0.354370 \\
\hline C & 1.520580 & -0.928970 & -0.218020 \\
\hline C & 3.645670 & -1.980120 & 1.277300 \\
\hline C & 3.889070 & -0.932730 & 0.408340 \\
\hline $\mathrm{H}$ & 4.472190 & -2.400589 & 1.844470 \\
\hline C & 2.363990 & -2.556630 & 1.427720 \\
\hline C & 1.273110 & -2.026990 & 0.668220 \\
\hline C & -0.004160 & -2.631750 & 0.820040 \\
\hline C & -0.190089 & -3.708040 & 1.658710 \\
\hline C & 0.892931 & -4.230590 & 2.406010 \\
\hline C & 2.139861 & -3.660590 & 2.294680 \\
\hline $\mathrm{H}$ & -0.846220 & -2.230690 & 0.269320 \\
\hline $\mathrm{H}$ & -1.175079 & -4.157930 & 1.743340 \\
\hline $\mathrm{H}$ & 0.734561 & -5.078770 & 3.066450 \\
\hline H & 2.979471 & -4.050350 & 2.865430 \\
\hline C & -0.890610 & -0.436550 & -3.171940 \\
\hline C & 0.145130 & -0.978540 & -2.344440 \\
\hline C & 0.901170 & -2.079710 & -2.841160 \\
\hline C & 0.645950 & -2.610560 & -4.087530 \\
\hline C & -0.377320 & -2.070250 & -4.903490 \\
\hline C & -1.127300 & -1.007120 & -4.453260 \\
\hline $\mathrm{H}$ & 1.682230 & -2.503730 & -2.219480 \\
\hline $\mathrm{H}$ & 1.234691 & -3.449840 & -4.446960 \\
\hline $\mathrm{H}$ & -0.563920 & -2.492760 & -5.887010 \\
\hline H & -1.909580 & -0.579870 & -5.076260 \\
\hline C & 7.934940 & 0.438331 & 0.181940 \\
\hline C & 7.268170 & 0.136561 & -1.008440 \\
\hline C & 5.935650 & -0.297129 & -0.922330 \\
\hline C & 5.287090 & -0.416619 & 0.309770 \\
\hline
\end{tabular}




\begin{tabular}{|c|c|c|c|}
\hline C & 5.991800 & -0.098349 & 1.484900 \\
\hline C & 7.319160 & 0.331291 & 1.442650 \\
\hline $\mathrm{H}$ & 8.964800 & 0.773191 & 0.138890 \\
\hline $\mathrm{H}$ & 5.383130 & -0.540679 & -1.823030 \\
\hline $\mathrm{H}$ & 5.468780 & -0.176709 & 2.431000 \\
\hline C & 7.928400 & 0.264361 & -2.394590 \\
\hline C & 8.117850 & 0.689861 & 2.711080 \\
\hline C & 7.915810 & -1.112629 & -3.100240 \\
\hline $\mathrm{H}$ & 8.380350 & -1.037269 & -4.091460 \\
\hline $\mathrm{H}$ & 8.473760 & -1.856319 & -2.519190 \\
\hline $\mathrm{H}$ & 6.897380 & -1.489569 & -3.237410 \\
\hline C & 7.131810 & 1.282751 & -3.246150 \\
\hline $\mathrm{H}$ & 7.569430 & 1.363481 & -4.249330 \\
\hline $\mathrm{H}$ & 6.081970 & 0.993551 & -3.356180 \\
\hline $\mathrm{H}$ & 7.157580 & 2.277531 & -2.785750 \\
\hline C & 9.389690 & 0.744381 & -2.311990 \\
\hline $\mathrm{H}$ & 9.808970 & 0.822671 & -3.321650 \\
\hline $\mathrm{H}$ & 9.468640 & 1.732801 & -1.845030 \\
\hline $\mathrm{H}$ & 10.019180 & 0.047291 & -1.746470 \\
\hline C & 7.283670 & 0.528501 & 3.996230 \\
\hline $\mathrm{H}$ & 7.893920 & 0.791991 & 4.867730 \\
\hline $\mathrm{H}$ & 6.404560 & 1.182741 & 3.998130 \\
\hline $\mathrm{H}$ & 6.941030 & -0.503509 & 4.133460 \\
\hline C & 9.350850 & -0.238359 & 2.821190 \\
\hline $\mathrm{H}$ & 9.934990 & 0.005611 & 3.717350 \\
\hline $\mathrm{H}$ & 9.044530 & -1.288499 & 2.890730 \\
\hline $\mathrm{H}$ & 10.013880 & -0.141389 & 1.955040 \\
\hline C & 8.590440 & 2.160941 & 2.629430 \\
\hline $\mathrm{H}$ & 9.162040 & 2.428881 & 3.526750 \\
\hline $\mathrm{H}$ & 9.233880 & 2.336101 & 1.760860 \\
\hline $\mathrm{H}$ & 7.735620 & 2.842641 & 2.554530 \\
\hline C & -2.171100 & 2.481220 & -1.093980 \\
\hline C & -3.540880 & 2.590580 & -1.403610 \\
\hline C & -1.527120 & 3.573720 & -0.506010 \\
\hline C & -4.261240 & 3.752870 & -1.123090 \\
\hline $\mathrm{H}$ & -4.037120 & 1.744090 & -1.863490 \\
\hline C & -2.219530 & 4.751260 & -0.184900 \\
\hline $\mathrm{H}$ & -0.465030 & 3.514000 & -0.306120 \\
\hline C & -3.578600 & 4.814170 & -0.500210 \\
\hline $\mathrm{H}$ & -4.133190 & 5.713900 & -0.261260 \\
\hline C & -1.457250 & 5.906780 & 0.490850 \\
\hline C & -5.757980 & 3.907480 & -1.456650 \\
\hline C & -0.872440 & 5.411000 & 1.836110 \\
\hline $\mathrm{H}$ & -1.673080 & 5.102790 & 2.518560 \\
\hline $\mathrm{H}$ & -0.200790 & 4.556500 & 1.706130 \\
\hline $\mathrm{H}$ & -0.305340 & 6.215600 & 2.320380 \\
\hline C & -0.303140 & 6.370820 & -0.429620 \\
\hline $\mathrm{H}$ & 0.247020 & 7.195010 & 0.040360 \\
\hline $\mathrm{H}$ & 0.411860 & 5.565760 & -0.626550 \\
\hline $\mathrm{H}$ & -0.686480 & 6.724370 & -1.393970 \\
\hline C & -2.360730 & 7.121630 & 0.774010 \\
\hline $\mathrm{H}$ & -2.783130 & 7.541980 & -0.146270 \\
\hline $\mathrm{H}$ & -3.187920 & 6.868780 & 1.447310 \\
\hline $\mathrm{H}$ & -1.773680 & 7.910730 & 1.257230 \\
\hline
\end{tabular}




\begin{tabular}{|llll|} 
C & -6.560210 & 4.077770 & -0.144740 \\
$H$ & -6.443910 & 3.199930 & 0.500950 \\
$H$ & -6.230320 & 4.954820 & 0.422150 \\
$H$ & -7.628190 & 4.201879 & -0.362670 \\
C & -5.964400 & 5.154820 & -2.349320 \\
$H$ & -5.635770 & 6.074780 & -1.855490 \\
$H$ & -5.406070 & 5.061710 & -3.287980 \\
$H$ & -7.026580 & 5.272769 & -2.596390 \\
C & -6.323690 & 2.690390 & -2.213260 \\
$H$ & -7.380550 & 2.862479 & -2.446900 \\
$H$ & -5.799940 & 2.522530 & -3.161760 \\
$H$ & -6.263980 & 1.770610 & -1.621610 \\
\hline
\end{tabular}

\section{C-Catalyzed reaction TS 133}

\section{Datum}

Value

\begin{tabular}{lc}
\hline B3LYP/6-31G(d) Energy & -3038.947619 \\
\hline B3LYP-D3(BJ)/def2-TZVPP-IEF-PCM(DCM) Energy & -3040.498957 \\
\hline B3LYP-D3(BJ)/def2-TZVPP-IEF-PCM(DCM)//B3LYP/6-31G(d) Free Energy (Quasiharmonic) & -3039.270132 \\
\hline
\end{tabular}

Number of Imaginary Frequencies

Frequencies (Top 3 out of 450)
1. $-345.6006 \mathrm{~cm}^{-1}$
2. $8.8288 \mathrm{~cm}^{-1}$
3. $11.1343 \mathrm{~cm}^{-1}$

\section{B3LYP/6-31G(d) Molecular Geometry in Cartesian Coordinates}

C

$\mathrm{H}$

$\mathrm{H}$

C

0

0

$\mathrm{H}$

0

$\mathrm{H}$

C

C

C

C

$\mathrm{H}$

\section{772231}

2.421695

0.748875

$-1.406632$

$-1.395125$

$-1.277405$

$-1.374344$

2.200346

1.647111

$-0.122397$

1.113240

$-1.969088$

$-1.135333$

1.698368
$-0.315156$

0.132177

$-0.000658$

$-1.955320$

$-3.077483$

$-0.887970$

0.002411

0.259250

$-0.126668$

3.469642

3. 207235

3.112584

2.685193

2.430787
0.625476

$-0.126730$

0.403554

$-0.692188$

$-1.151444$

$-1.489815$

$-1.059368$

1.878554

2.578435

2. 725764

0.949949

1.138002

2.215936

1.445877 


\begin{tabular}{|c|c|c|c|}
\hline $\mathrm{H}$ & -2.198733 & 4.180524 & 1.147422 \\
\hline $\mathrm{H}$ & -2.856658 & 2.502378 & 0.968811 \\
\hline C & -1.744423 & 2.196724 & -2.539351 \\
\hline C & -2.068117 & 3.729205 & -2.393520 \\
\hline C & -0.421701 & 1.929334 & -3.274393 \\
\hline $\mathrm{H}$ & -0.159185 & 0.873440 & -3.161397 \\
\hline $\mathrm{H}$ & -0.505555 & 2.146437 & -4.344923 \\
\hline $\mathrm{H}$ & 0.392227 & 2.531283 & -2.859299 \\
\hline C & -2.868685 & 1.366431 & -3.161195 \\
\hline $\mathrm{H}$ & -2.560937 & 0.321373 & -3.252420 \\
\hline $\mathrm{H}$ & -3.107866 & 1.739205 & -4.164338 \\
\hline $\mathrm{H}$ & -3.775230 & 1.397670 & -2.551759 \\
\hline C & -3.552793 & 4.005968 & -2.102501 \\
\hline $\mathrm{H}$ & -4.182372 & 3.834584 & -2.982481 \\
\hline $\mathrm{H}$ & -3.920549 & 3.374515 & -1.288033 \\
\hline $\mathrm{H}$ & -3.663752 & 5.052326 & -1.799435 \\
\hline C & -1.608486 & 4.595319 & -3.568603 \\
\hline $\mathrm{H}$ & -2.111235 & 4.301685 & -4.497590 \\
\hline $\mathrm{H}$ & -1.855290 & 5.643868 & -3.369437 \\
\hline $\mathrm{H}$ & -0.528614 & 4.525568 & -3.718972 \\
\hline 0 & -1.570059 & 1.799765 & -1.148172 \\
\hline 0 & -1.311257 & 4.101900 & -1.230476 \\
\hline B & -1.094457 & 2.948880 & -0.414424 \\
\hline 0 & 0.343975 & 2.762937 & 0.001481 \\
\hline C & 1.734591 & 4.548264 & 0.887750 \\
\hline C & 1.290986 & 5.538052 & -0.005124 \\
\hline C & 2.827167 & 4.815804 & 1.730795 \\
\hline C & 1.929680 & 6.776558 & -0.036273 \\
\hline $\mathrm{H}$ & 0.455752 & 5.325283 & -0.665459 \\
\hline C & 3.461767 & 6.054114 & 1.691833 \\
\hline $\mathrm{H}$ & 3.178039 & 4.045011 & 2.413159 \\
\hline C & 3.011357 & 7.039429 & 0.808555 \\
\hline $\mathrm{H}$ & 1.585522 & 7.539796 & -0.729169 \\
\hline $\mathrm{H}$ & 4.308707 & 6.250162 & 2.343619 \\
\hline $\mathrm{H}$ & 3.506641 & 8.006205 & 0.774738 \\
\hline $\mathrm{H}$ & -1.133320 & 1.623722 & 2.466603 \\
\hline $\mathrm{H}$ & -0.191173 & 4.549786 & 2.644221 \\
\hline $\mathrm{H}$ & 0.526943 & 3.094648 & 3.511737 \\
\hline C & -0.431079 & -1.974569 & 1.594815 \\
\hline C & -1.531224 & -1.687463 & 0.794009 \\
\hline C & -2.754538 & -1.177170 & 1.349417 \\
\hline C & -2.790671 & -0.910023 & 2.706509 \\
\hline $\mathrm{H}$ & -3.715646 & -0.556864 & 3.153999 \\
\hline C & 1.882129 & -1.826647 & 0.591882 \\
\hline C & 0.813260 & -2.607351 & 1.026293 \\
\hline C & 3.136311 & -3.835518 & 0.048614 \\
\hline C & 3.076536 & -2.456565 & 0.097907 \\
\hline $\mathrm{H}$ & 4.041936 & -4.314144 & -0.315606 \\
\hline C & 2.070446 & -4.655500 & 0.488043 \\
\hline C & 0.883233 & -4.039271 & 0.992274 \\
\hline C & -0.175755 & -4.878637 & 1.434967 \\
\hline C & -0.060174 & -6.249480 & 1.387333 \\
\hline C & 1.118497 & -6.856284 & 0.891165 \\
\hline C & 2.159310 & -6.073443 & 0.449684 \\
\hline
\end{tabular}




\begin{tabular}{|c|c|c|c|}
\hline $\mathrm{H}$ & -1.089900 & -4.427341 & 1.803628 \\
\hline $\mathrm{H}$ & -0.882989 & -6.872311 & 1.727652 \\
\hline $\mathrm{H}$ & 1.195453 & -7.939737 & 0.857215 \\
\hline $\mathrm{H}$ & 3.068790 & -6.526965 & 0.062684 \\
\hline C & -1.678955 & -1.133775 & 3.553598 \\
\hline C & -0.482789 & -1.694903 & 3.001110 \\
\hline C & 0.608418 & -1.957594 & 3.879878 \\
\hline C & 0.524788 & -1.659715 & 5.223530 \\
\hline C & -0.655612 & -1.094932 & 5.762813 \\
\hline C & -1.733283 & -0.844456 & 4.944816 \\
\hline $\mathrm{H}$ & 1.508593 & -2.413182 & 3.480283 \\
\hline $\mathrm{H}$ & 1.367838 & -1.870121 & 5.875797 \\
\hline $\mathrm{H}$ & -0.709734 & -0.867179 & 6.823736 \\
\hline $\mathrm{H}$ & -2.648248 & -0.420347 & 5.351580 \\
\hline C & 6.559982 & -0.348758 & -1.229684 \\
\hline C & 6.043469 & -0.058702 & 0.041557 \\
\hline C & 4.891797 & -0.739354 & 0.455135 \\
\hline C & 4. 266201 & -1.683845 & -0.369161 \\
\hline C & 4.813345 & -1.944839 & -1.632734 \\
\hline C & 5.962761 & -1.285332 & -2.085944 \\
\hline $\mathrm{H}$ & 7.448573 & 0.169964 & -1.562442 \\
\hline $\mathrm{H}$ & 4.461004 & -0.540819 & 1.430255 \\
\hline $\mathrm{H}$ & 4.312702 & -2.667180 & -2.270941 \\
\hline C & 6.695632 & 0.966467 & 0.990140 \\
\hline C & 6.519296 & -1.602273 & -3.488533 \\
\hline C & 7.942173 & 1.632001 & 0.377433 \\
\hline $\mathrm{H}$ & 8.361969 & 2.353424 & 1.087865 \\
\hline $\mathrm{H}$ & 8.725901 & 0.900419 & 0.149137 \\
\hline $\mathrm{H}$ & 7.703571 & 2.176454 & -0.543330 \\
\hline C & 5.672058 & 2.077936 & 1.325545 \\
\hline $\mathrm{H}$ & 6.112250 & 2.801825 & 2.023487 \\
\hline $\mathrm{H}$ & 5.373610 & 2.620176 & 0.420821 \\
\hline $\mathrm{H}$ & 4.765420 & 1.670826 & 1.784660 \\
\hline C & 7.124327 & 0.256564 & 2.296636 \\
\hline $\mathrm{H}$ & 7.590800 & 0.972160 & 2.985336 \\
\hline $\mathrm{H}$ & 6.270425 & -0.195113 & 2.811889 \\
\hline $\mathrm{H}$ & 7.849615 & -0.539110 & 2.090797 \\
\hline C & 7.785419 & -0.789746 & -3.820173 \\
\hline $\mathrm{H}$ & 8.139537 & -1.053352 & -4.823260 \\
\hline $\mathrm{H}$ & 7.592767 & 0.289093 & -3.811537 \\
\hline $\mathrm{H}$ & 8.600236 & -0.998000 & -3.117394 \\
\hline C & 6.880512 & -3.104532 & -3.571837 \\
\hline $\mathrm{H}$ & 7.268887 & -3.349653 & -4.568121 \\
\hline $\mathrm{H}$ & 7.648865 & -3.364091 & -2.834345 \\
\hline $\mathrm{H}$ & 6.010948 & -3.743786 & -3.387432 \\
\hline C & 5.446528 & -1.274566 & -4.554431 \\
\hline $\mathrm{H}$ & 5.824872 & -1.500179 & -5.559260 \\
\hline $\mathrm{H}$ & 4.531054 & -1.856018 & -4.404744 \\
\hline $\mathrm{H}$ & 5.175542 & -0.213033 & -4.523586 \\
\hline C & -3.992955 & -0.994426 & 0.538066 \\
\hline C & -4.384746 & -1.955578 & -0.398090 \\
\hline C & -4.833353 & 0.112120 & 0.765861 \\
\hline C & -5.576223 & -1.826931 & -1.128991 \\
\hline $\mathrm{H}$ & -3.759897 & -2.826942 & -0.550765 \\
\hline
\end{tabular}




\begin{tabular}{|c|c|c|c|}
\hline C & -6.039865 & 0.263570 & 0.081045 \\
\hline $\mathrm{H}$ & -4.519841 & 0.856891 & 1.487966 \\
\hline C & -6.383708 & -0.717392 & -0.868653 \\
\hline $\mathrm{H}$ & -7.314220 & -0.604787 & -1.412229 \\
\hline C & -7.004923 & 1.437489 & 0.340739 \\
\hline C & -5.938459 & -2.906113 & -2.167089 \\
\hline C & -8.347336 & 0.878101 & 0.871713 \\
\hline $\mathrm{H}$ & -8.816693 & 0.197613 & 0.153711 \\
\hline $\mathrm{H}$ & -8.199290 & 0.326133 & 1.807023 \\
\hline $\mathrm{H}$ & -9.051660 & 1.696116 & 1.067148 \\
\hline C & -6.458727 & 2.431714 & 1.382822 \\
\hline $\mathrm{H}$ & -7.181008 & 3.242159 & 1.532214 \\
\hline $\mathrm{H}$ & -6.294036 & 1.955055 & 2.355995 \\
\hline $\mathrm{H}$ & -5.515315 & 2.887080 & 1.060733 \\
\hline C & -7.261263 & 2.212517 & -0.973020 \\
\hline $\mathrm{H}$ & -6.328865 & 2.624240 & -1.374782 \\
\hline $\mathrm{H}$ & -7.705842 & 1.575805 & -1.744811 \\
\hline $\mathrm{H}$ & -7.950810 & 3.046767 & -0.793958 \\
\hline C & -6.072719 & -4.276431 & -1.460958 \\
\hline $\mathrm{H}$ & -5.140273 & -4.577284 & -0.973010 \\
\hline $\mathrm{H}$ & -6.859648 & -4.250038 & -0.697987 \\
\hline $\mathrm{H}$ & -6.331770 & -5.054331 & -2.189622 \\
\hline C & -7.266110 & -2.607750 & -2.888319 \\
\hline $\mathrm{H}$ & -8.113092 & -2.575580 & -2.192906 \\
\hline $\mathrm{H}$ & -7.231017 & -1.656234 & -3.431334 \\
\hline $\mathrm{H}$ & -7.471515 & -3.396769 & -3.620432 \\
\hline C & -4.816349 & -2.985319 & -3.231148 \\
\hline $\mathrm{H}$ & -5.049373 & -3.763729 & -3.968331 \\
\hline $\mathrm{H}$ & -4.718287 & -2.032550 & -3.764471 \\
\hline $\mathrm{H}$ & -3.842616 & -3.220600 & -2.790224 \\
\hline
\end{tabular}

\section{C-Catalyzed reaction TS 134}

\section{Datum}

Value

\begin{tabular}{lc}
\hline B3LYP/6-31G(d) Energy & -3038.944399 \\
\hline B3LYP-D3(BJ)/def2-TZVPP-IEF-PCM(DCM) Energy & -3040.497438 \\
\hline B3LYP-D3(BJ)/def2-TZVPP-IEF-PCM(DCM)//B3LYP/6-31G(d) Free Energy (Quasiharmonic) & -3039.270094 \\
\hline
\end{tabular}

Number of Imaginary Frequencies

Frequencies (Top 3 out of 450)
1. $-374.0484 \mathrm{~cm}^{-1}$
2. $6.7291 \mathrm{~cm}^{-1}$
3. $8.8478 \mathrm{~cm}^{-1}$ 


\begin{tabular}{|c|c|c|c|}
\hline C & -1.585446 & 0.626956 & 0.813458 \\
\hline $\mathrm{H}$ & -2.049784 & -0.294677 & 0.439659 \\
\hline $\mathrm{H}$ & -0.533478 & 0.404737 & 0.979746 \\
\hline C & 1.455029 & 0.631005 & -1.267922 \\
\hline 0 & 1.265818 & 0.869624 & -2.441000 \\
\hline 0 & 1.448419 & -0.636824 & -0.815828 \\
\hline H & 1.489718 & -0.702613 & 0.170327 \\
\hline 0 & -2.116015 & 0.964473 & 2.102867 \\
\hline $\mathrm{H}$ & -3.063307 & 0.758579 & 2.080075 \\
\hline C & 1.005761 & -5.436686 & -0.520215 \\
\hline C & -0.633035 & -4.048708 & -0.116845 \\
\hline C & 2.353536 & -3.737339 & 0.659430 \\
\hline C & 1.915383 & -4.397448 & -0.523984 \\
\hline $\mathrm{H}$ & -1.000085 & -4.777861 & 0.609659 \\
\hline $\mathrm{H}$ & 3.102027 & -2.960567 & 0.505660 \\
\hline $\mathrm{H}$ & 2.601357 & -4.403530 & 1.487668 \\
\hline C & 1.277823 & -1.496627 & 3.243516 \\
\hline C & 0.361720 & -2.704163 & 3.678206 \\
\hline C & 2.748754 & -1.692663 & 3.642313 \\
\hline $\mathrm{H}$ & 3.354070 & -0.940232 & 3.127491 \\
\hline $\mathrm{H}$ & 2.893753 & -1.569143 & 4.720921 \\
\hline $\mathrm{H}$ & 3.118937 & -2.681379 & 3.355666 \\
\hline C & 0.800207 & -0.122919 & 3.711408 \\
\hline $\mathrm{H}$ & 0.811838 & -0.071566 & 4.807171 \\
\hline $\mathrm{H}$ & -0.208109 & 0.110954 & 3.364411 \\
\hline $\mathrm{H}$ & 1.477915 & 0.652164 & 3.337411 \\
\hline C & -1.131211 & -2.337674 & 3.711727 \\
\hline $\mathrm{H}$ & -1.371942 & -1.696671 & 4.566804 \\
\hline $\mathrm{H}$ & -1.439899 & -1.815965 & 2.802139 \\
\hline $\mathrm{H}$ & -1.715431 & -3.260121 & 3.798377 \\
\hline C & 0.756857 & -3.365753 & 4.999999 \\
\hline $\mathrm{H}$ & 0.689102 & -2.656880 & 5.833626 \\
\hline $\mathrm{H}$ & 0.076728 & -4.198310 & 5.209817 \\
\hline $\mathrm{H}$ & 1.773124 & -3.765393 & 4.961506 \\
\hline 0 & 1.195268 & -1.566957 & 1.794262 \\
\hline 0 & 0.574181 & -3.657730 & 2.616320 \\
\hline B & 0.907795 & -2.928345 & 1.439206 \\
\hline 0 & -0.117404 & -2.953895 & 0.357468 \\
\hline C & -1.316146 & -3.971670 & -1.420818 \\
\hline C & -2.231871 & -4.971776 & -1.783913 \\
\hline C & -1.053051 & -2.914292 & -2.308211 \\
\hline C & -2.870758 & -4.924378 & -3.021014 \\
\hline $\mathrm{H}$ & -2.438281 & -5.788063 & -1.094831 \\
\hline C & -1.692759 & -2.874273 & -3.545126 \\
\hline $\mathrm{H}$ & -0.346193 & -2.140144 & -2.024538 \\
\hline C & -2.598146 & -3.877579 & -3.906317 \\
\hline $\mathrm{H}$ & -3.578493 & -5.701748 & -3.295484 \\
\hline $\mathrm{H}$ & -1.480655 & -2.058452 & -4.230525 \\
\hline $\mathrm{H}$ & -3.090485 & -3.843426 & -4.874457 \\
\hline $\mathrm{H}$ & 2.075810 & -3.886082 & -1.474407 \\
\hline
\end{tabular}




\begin{tabular}{|c|c|c|c|}
\hline $\mathrm{H}$ & 0.890447 & -6.041887 & 0.376591 \\
\hline H & 0.685316 & -5.894162 & -1.450286 \\
\hline C & 0.645265 & 2.497420 & 0.174544 \\
\hline C & 1.700380 & 1.679421 & -0.208280 \\
\hline C & 2.994912 & 1.776799 & 0.407616 \\
\hline C & 3.153827 & 2.664036 & 1.454236 \\
\hline $\mathrm{H}$ & 4.135685 & 2.785184 & 1.904721 \\
\hline C & -1.735347 & 1.717530 & -0.232382 \\
\hline C & -0.658087 & 2.524350 & -0.582243 \\
\hline C & -3.108307 & 2.778295 & -1.941443 \\
\hline C & -2.993600 & 1.875635 & -0.907066 \\
\hline H & -4.063581 & 2.893269 & -2.448554 \\
\hline C & -2.019312 & 3.587044 & -2.349418 \\
\hline C & -0.774784 & 3.475157 & -1.654109 \\
\hline C & 0.299982 & 4.309778 & -2.067552 \\
\hline C & 0.148741 & 5.202439 & -3.105258 \\
\hline C & -1.085402 & 5.310668 & -3.788265 \\
\hline C & -2.145326 & 4.516845 & -3.415914 \\
\hline H & 1.255150 & 4.231124 & -1.561314 \\
\hline $\mathrm{H}$ & 0.986109 & 5.826644 & -3.405413 \\
\hline H & -1.191450 & 6.018432 & -4.606119 \\
\hline H & -3.099592 & 4.588724 & -3.932812 \\
\hline C & 2.087805 & 3.465234 & 1.931641 \\
\hline C & 0.814386 & 3.397335 & 1.280199 \\
\hline C & -0.235058 & 4.227570 & 1.761284 \\
\hline C & -0.033126 & 5.080356 & 2.823447 \\
\hline C & 1.228864 & 5.151362 & 3.459841 \\
\hline C & 2.265137 & 4.360070 & 3.021513 \\
\hline H & -1.208210 & 4.170806 & 1.287705 \\
\hline $\mathrm{H}$ & -0.849905 & 5.702913 & 3.178357 \\
\hline H & 1.375189 & 5.829960 & 4.295936 \\
\hline $\mathrm{H}$ & 3.238823 & 4.405609 & 3.503711 \\
\hline C & -6.551253 & -0.211339 & 0.243428 \\
\hline C & -6.035753 & 0.759911 & 1.106148 \\
\hline C & -4.857718 & 1.417749 & 0.713018 \\
\hline C & -4.213224 & 1.112125 & -0.491141 \\
\hline C & -4.773359 & 0.140261 & -1.335159 \\
\hline C & -5.948844 & -0.531855 & -0.986904 \\
\hline H & -7.457330 & -0.734635 & 0.524482 \\
\hline H & -4.446135 & 2.213073 & 1.327990 \\
\hline H & -4.265996 & -0.077960 & -2.267810 \\
\hline C & -6.713367 & 1.142683 & 2.436638 \\
\hline C & -6.594491 & -1.600950 & -1.889935 \\
\hline C & -7.128956 & 2.632510 & 2.388576 \\
\hline H & -7.608380 & 2.925671 & 3.330644 \\
\hline $\mathrm{H}$ & -6.267628 & 3.290591 & 2.234102 \\
\hline H & -7.839328 & 2.814817 & 1.574140 \\
\hline C & -7.973187 & 0.302187 & 2.719847 \\
\hline $\mathrm{H}$ & -8.410679 & 0.607265 & 3.677013 \\
\hline H & -8.739341 & 0.440694 & 1.948669 \\
\hline H & -7.743876 & -0.767481 & 2.787473 \\
\hline C & -5.722680 & 0.927202 & 3.605837 \\
\hline H & -6.195003 & 1.190642 & 4.559983 \\
\hline H & -5.406441 & -0.120943 & 3.665022 \\
\hline
\end{tabular}




\begin{tabular}{|c|c|c|c|}
\hline H & -4.824937 & 1.546801 & 3.505115 \\
\hline C & -6.555009 & -2.969275 & -1.168257 \\
\hline $\mathrm{H}$ & -7.026535 & -3.741817 & -1.788521 \\
\hline $\mathrm{H}$ & -5.522117 & -3.277103 & -0.970018 \\
\hline $\mathrm{H}$ & -7.086878 & -2.938965 & -0.211052 \\
\hline C & -8.064668 & -1.218152 & -2.184184 \\
\hline H & -8.529912 & -1.973047 & -2.829809 \\
\hline $\mathrm{H}$ & -8.122266 & -0.251361 & -2.697413 \\
\hline $\mathrm{H}$ & -8.665774 & -1.148487 & -1.271895 \\
\hline C & -5.866307 & -1.744925 & -3.239269 \\
\hline $\mathrm{H}$ & -6.375457 & -2.497645 & -3.852301 \\
\hline H & -5.866638 & -0.804348 & -3.802074 \\
\hline $\mathrm{H}$ & -4.828716 & -2.070654 & -3.113769 \\
\hline C & 4.176362 & 1.028971 & -0.112629 \\
\hline C & 4.422878 & 0.967120 & -1.491010 \\
\hline C & 5.101373 & 0.441571 & 0.762987 \\
\hline C & 5.551811 & 0.320695 & -2.006476 \\
\hline H & 3.722536 & 1.441689 & -2.168366 \\
\hline C & 6.255419 & -0.196695 & 0.290492 \\
\hline $\mathrm{H}$ & 4.904394 & 0.483752 & 1.830343 \\
\hline C & 6.454289 & -0.248178 & -1.097358 \\
\hline $\mathrm{H}$ & 7.336539 & -0.742752 & -1.479527 \\
\hline C & 7.252591 & -0.817630 & 1.289641 \\
\hline C & 5.753121 & 0.258319 & -3.532936 \\
\hline C & 8.482125 & -1.433317 & 0.593727 \\
\hline H & 8.204435 & -2.250852 & -0.081226 \\
\hline $\mathrm{H}$ & 9.041209 & -0.688138 & 0.016495 \\
\hline H & 9.161945 & -1.846277 & 1.347645 \\
\hline C & 7.755834 & 0.273805 & 2.264567 \\
\hline $\mathrm{H}$ & 8.461060 & -0.155684 & 2.986839 \\
\hline H & 8.270117 & 1.075950 & 1.722865 \\
\hline $\mathrm{H}$ & 6.935480 & 0.725924 & 2.831516 \\
\hline C & 6.549309 & -1.935494 & 2.094636 \\
\hline H & 5.693201 & -1.552957 & 2.658770 \\
\hline $\mathrm{H}$ & 6.183447 & -2.726249 & 1.429527 \\
\hline $\mathrm{H}$ & 7.246718 & -2.388555 & 2.810338 \\
\hline C & 5.802189 & 1.692918 & -4.110581 \\
\hline $\mathrm{H}$ & 4.877568 & 2.244897 & -3.914505 \\
\hline $\mathrm{H}$ & 6.632763 & 2.262822 & -3.677776 \\
\hline H & 5.943436 & 1.659109 & -5.197866 \\
\hline C & 7.058158 & -0.458439 & -3.927288 \\
\hline H & 7.942544 & 0.050979 & -3.526991 \\
\hline $\mathrm{H}$ & 7.075311 & -1.498064 & -3.580351 \\
\hline $\mathrm{H}$ & 7.152916 & -0.475553 & -5.018957 \\
\hline C & 4.567469 & -0.509186 & -4.167461 \\
\hline $\mathrm{H}$ & 4.679703 & -0.545828 & -5.258427 \\
\hline H & 4.530253 & -1.540102 & -3.796071 \\
\hline $\mathrm{H}$ & 3.604212 & -0.040938 & -3.941634 \\
\hline
\end{tabular}


Frequencies (Top 3 out of 450)

$\begin{array}{rr}\text { 1. } & -312.4081 \mathrm{~cm}^{-1} \\ \text { 2. } & 8.3730 \mathrm{~cm}^{-1} \\ \text { 3. } & 10.1029 \mathrm{~cm}^{-1}\end{array}$

\section{B3LYP/6-31G(d) Molecular Geometry in Cartesian Coordinates}

C

$\mathrm{H}$

$\mathrm{H}$

C

0

0

$\mathrm{H}$

0

$\mathrm{H}$

C

C

C

C

$\mathrm{H}$

$\mathrm{H}$

$\mathrm{H}$

C

C

C

$\mathrm{H}$

$\mathrm{H}$

$\mathrm{H}$

C

$\mathrm{H}$

$\mathrm{H}$

$\mathrm{H}$

C

$\mathrm{H}$

$\mathrm{H}$

$\mathrm{H}$

C

$\mathrm{H}$

\section{$-3.018469$}

$-4.078097$

$-2.445852$

0.431084

$-0.383190$

1.401538

2.082504

$-2.828946$

$-1.932409$

2.576340

2. 311689

4.137322

2.895773

1. 229409

4. 386706

4.978279

4.602492

5.860348

4.888575

3.963918

5.309328

5.592356

3.850661

4.431847

3. 612527

2.914302

6.574403

6.973858

5.905640

7.414676

6.885197

7.419948

1.376292
1.482640
1.834909
0.796221
1.202204
-0.033383
-0.263090
2.100468
1.903719
-3.350574
-3.913555
-1.716646
-2.056232
-3.795009
-0.654671
-2.336627
-0.857491
-1.660116
0.582341
1.069433
1.171274
0.602791
-1.584578
-1.601910
-2.614198
-1.055995
-2.481243
-1.837428
-3.217871
-3.020306
-0.776880
-0.112614

0.858390

1.105593

1.674572

$-0.556410$

$-1.363476$

$-0.960375$

$-0.273589$

$-0.351258$

$-0.695447$

$-3.337112$

$-1.228142$

$-2.359921$

$-2.993616$

$-1.308966$

$-2.400301$

$-2.681819$

1. 317198

0.806730

1.740814

2.066902

0.923335

2.581345

2.441939

3. 369968

2.159978

2. 646511

1.883491

2.676089

2. 334703

1.432961

0.075684

0.763365 


\begin{tabular}{|c|c|c|c|}
\hline $\mathrm{H}$ & 7.618632 & -1.424796 & -0.415247 \\
\hline $\mathrm{H}$ & 6.407743 & -0.164005 & -0.694122 \\
\hline 0 & 3.758350 & -0.846244 & 0.134973 \\
\hline 0 & 5.290215 & -2.566881 & -0.149843 \\
\hline B & 4.055126 & -2.041199 & -0.635265 \\
\hline 0 & 2.906455 & -3.029895 & -0.497622 \\
\hline C & 2.814373 & -5.302789 & -1.294612 \\
\hline C & 4.110493 & -5.628119 & -0.860896 \\
\hline C & 1.964007 & -6.316816 & -1.765205 \\
\hline C & 4.544588 & -6.951140 & -0.914944 \\
\hline $\mathrm{H}$ & 4.757834 & -4.843253 & -0.479705 \\
\hline C & 2.403399 & -7.637114 & -1.812755 \\
\hline $\mathrm{H}$ & 0.956642 & -6.065099 & -2.089682 \\
\hline C & 3.697085 & -7.956105 & -1.390198 \\
\hline $\mathrm{H}$ & 5.546964 & -7.201615 & -0.578328 \\
\hline $\mathrm{H}$ & 1.739190 & -8.417214 & -2.174375 \\
\hline $\mathrm{H}$ & 4.040979 & -8.986319 & -1.425762 \\
\hline $\mathrm{H}$ & 2.096291 & -1.316787 & -2.965207 \\
\hline $\mathrm{H}$ & 3.367780 & -4.067931 & -3.529910 \\
\hline $\mathrm{H}$ & 1.606236 & -3.588185 & -3.765103 \\
\hline C & -0.425101 & 0.416579 & 1.753705 \\
\hline C & 0.412115 & 1.143570 & 0.913676 \\
\hline C & 1.198600 & 2.244505 & 1.399660 \\
\hline C & 1.215664 & 2.472127 & 2.763212 \\
\hline $\mathrm{H}$ & 1.811466 & 3.292932 & 3.153800 \\
\hline C & -2.673076 & -0.106893 & 0.767143 \\
\hline C & -1.426833 & -0.566402 & 1.198275 \\
\hline C & -3.337466 & -2.391378 & 0.257683 \\
\hline C & -3.650231 & -1.044955 & 0.276918 \\
\hline $\mathrm{H}$ & -4.070555 & -3.099607 & -0.119944 \\
\hline C & -2.092729 & -2.888297 & 0.706247 \\
\hline C & -1.111721 & -1.964954 & 1.187359 \\
\hline C & 0.125153 & -2.484685 & 1.657035 \\
\hline C & 0.373997 & -3.839535 & 1.658948 \\
\hline C & -0.596720 & -4.749780 & 1.175324 \\
\hline C & -1.801539 & -4.279671 & 0.704651 \\
\hline $\mathrm{H}$ & 0.881147 & -1.799435 & 2.021883 \\
\hline $\mathrm{H}$ & 1.326226 & -4.211796 & 2.025662 \\
\hline $\mathrm{H}$ & -0.386911 & -5.816135 & 1.179707 \\
\hline $\mathrm{H}$ & -2.558248 & -4.967502 & 0.334003 \\
\hline C & 0.469198 & 1.685793 & 3.674294 \\
\hline C & -0.392810 & 0.661934 & 3.167909 \\
\hline C & -1.187879 & -0.071245 & 4.091378 \\
\hline C & -1.113708 & 0.180061 & 5.443752 \\
\hline C & -0.249296 & 1.183444 & 5.942854 \\
\hline C & 0.522063 & 1.921086 & 5.074766 \\
\hline $\mathrm{H}$ & -1.852457 & -0.842165 & 3.717575 \\
\hline $\mathrm{H}$ & -1.723982 & -0.395963 & 6.133914 \\
\hline $\mathrm{H}$ & -0.201260 & 1.371976 & 7.011904 \\
\hline $\mathrm{H}$ & 1.181791 & 2.701311 & 5.446803 \\
\hline C & -7.578784 & -0.012170 & -1.074607 \\
\hline C & -6.492631 & 0.705444 & -1.581838 \\
\hline C & -5.209256 & 0.369039 & -1.122523 \\
\hline C & -5.015424 & -0.643625 & -0.180111 \\
\hline
\end{tabular}




\begin{tabular}{|c|c|c|c|}
\hline C & -6.136766 & -1.340494 & 0.309804 \\
\hline C & -7.428361 & -1.039888 & -0.124982 \\
\hline $\mathrm{H}$ & -8.577863 & 0.232163 & -1.416851 \\
\hline $\mathrm{H}$ & -4.342678 & 0.912373 & -1.479101 \\
\hline $\mathrm{H}$ & -5.974817 & -2.110780 & 1.055300 \\
\hline C & -6.644389 & 1.847052 & -2.605020 \\
\hline C & -8.675832 & -1.778207 & 0.398965 \\
\hline C & -8.108648 & 2.072312 & -3.026490 \\
\hline $\mathrm{H}$ & -8.161034 & 2.891635 & -3.752591 \\
\hline $\mathrm{H}$ & -8.539779 & 1.182775 & -3.500993 \\
\hline $\mathrm{H}$ & -8.741904 & 2.347166 & -2.175006 \\
\hline C & -6.110199 & 3.159396 & -1.980430 \\
\hline $\mathrm{H}$ & -6.180496 & 3.980718 & -2.705100 \\
\hline $\mathrm{H}$ & -6.700194 & 3.436607 & -1.098489 \\
\hline $\mathrm{H}$ & -5.065339 & 3.068754 & -1.667674 \\
\hline C & -5.825375 & 1.515038 & -3.874987 \\
\hline $\mathrm{H}$ & -5.920923 & 2.323105 & -4.610918 \\
\hline $\mathrm{H}$ & -4.760998 & 1.392675 & -3.652139 \\
\hline $\mathrm{H}$ & -6.181215 & 0.587983 & -4.339913 \\
\hline C & -8.323843 & -2.873893 & 1.423329 \\
\hline $\mathrm{H}$ & -9.240107 & -3.370708 & 1.762696 \\
\hline $\mathrm{H}$ & -7.672224 & -3.642216 & 0.991495 \\
\hline $\mathrm{H}$ & -7.824895 & -2.461048 & 2.307340 \\
\hline C & -9.624761 & -0.767986 & 1.087091 \\
\hline $\mathrm{H}$ & -10.522173 & -1.278143 & 1.459005 \\
\hline $\mathrm{H}$ & -9.130973 & -0.284508 & 1.937827 \\
\hline $\mathrm{H}$ & -9.950267 & 0.019819 & 0.399662 \\
\hline C & -9.414926 & -2.448883 & -0.783326 \\
\hline $\mathrm{H}$ & -10.307416 & -2.977613 & -0.425891 \\
\hline $\mathrm{H}$ & -9.738131 & -1.716433 & -1.530025 \\
\hline $\mathrm{H}$ & -8.767492 & -3.175640 & -1.287326 \\
\hline C & 1.859173 & 3.233520 & 0.492518 \\
\hline C & 2.712422 & 2.877764 & -0.554761 \\
\hline C & 1.585086 & 4.598223 & 0.708455 \\
\hline C & 3.277234 & 3.847565 & -1.400882 \\
\hline $\mathrm{H}$ & 2.950704 & 1.834035 & -0.714289 \\
\hline C & 2.139284 & 5.592404 & -0.097140 \\
\hline $\mathrm{H}$ & 0.900177 & 4.859903 & 1.505978 \\
\hline C & 2.980050 & 5.188449 & -1.149495 \\
\hline $\mathrm{H}$ & 3.403192 & 5.951137 & -1.792008 \\
\hline C & 1.844391 & 7.090555 & 0.108722 \\
\hline C & 4.172949 & 3.401045 & -2.572456 \\
\hline C & 1.147897 & 7.655299 & -1.152691 \\
\hline $\mathrm{H}$ & 1.772323 & 7.548840 & -2.046076 \\
\hline $\mathrm{H}$ & 0.201875 & 7.136077 & -1.342861 \\
\hline $\mathrm{H}$ & 0.930865 & 8.722790 & -1.022761 \\
\hline C & 0.925257 & 7.347762 & 1.317984 \\
\hline $\mathrm{H}$ & 0.753761 & 8.424572 & 1.428044 \\
\hline $\mathrm{H}$ & -0.053104 & 6.869326 & 1.197875 \\
\hline $\mathrm{H}$ & 1.370908 & 6.986893 & 2.252183 \\
\hline C & 3.170728 & 7.851312 & 0.345721 \\
\hline $\mathrm{H}$ & 3.682170 & 7.477832 & 1.240381 \\
\hline $\mathrm{H}$ & 3.859584 & 7.749292 & -0.499473 \\
\hline $\mathrm{H}$ & 2.975092 & 8.921243 & 0.487700 \\
\hline
\end{tabular}




\begin{tabular}{|llll|}
$\mathrm{C}$ & 4.730219 & 4.589520 & -3.378889 \\
$\mathrm{H}$ & 3.930881 & 5.188100 & -3.830048 \\
$\mathrm{H}$ & 5.347411 & 5.251310 & -2.760082 \\
$\mathrm{H}$ & 5.360933 & 4.216434 & -4.193781 \\
$\mathrm{C}$ & 5.371916 & 2.588197 & -2.031141 \\
$\mathrm{H}$ & 5.977880 & 3.190628 & -1.344050 \\
$\mathrm{H}$ & 5.043860 & 1.693265 & -1.493174 \\
$\mathrm{H}$ & 6.017524 & 2.264726 & -2.857215 \\
$\mathrm{C}$ & 3.342557 & 2.514829 & -3.531969 \\
$\mathrm{H}$ & 3.967279 & 2.161668 & -4.362114 \\
$\mathrm{H}$ & 2.927573 & 1.639537 & -3.022198 \\
$\mathrm{H}$ & 2.502532 & 3.078043 & -3.953737 \\
\hline
\end{tabular}

\section{C-Catalyzed reaction TS 136}

\section{Datum}

Value

\begin{tabular}{lc}
\hline B3LYP/6-31G(d) Energy & -3038.943391 \\
\hline B3LYP-D3(BJ)/def2-TZVPP-IEF-PCM(DCM) Energy & -3040.496382 \\
\hline B3LYP-D3(BJ)/def2-TZVPP-IEF-PCM(DCM)//B3LYP/6-31G(d) Free Energy (Quasiharmonic) & -3039.269719 \\
\hline
\end{tabular}

Number of Imaginary Frequencies

Frequencies (Top 3 out of 450)

$\begin{array}{lr}\text { 1. } & -381.0124 \mathrm{~cm}^{-1} \\ \text { 2. } & 7.0547 \mathrm{~cm}^{-1} \\ \text { 3. } & 8.3764 \mathrm{~cm}^{-1}\end{array}$

\section{B3LYP/6-31G(d) Molecular Geometry in Cartesian Coordinates}

C

$\mathrm{H}$

$\mathrm{H}$

C

0

0

$\mathrm{H}$

0

$\mathrm{H}$

C

C

C

C

$\mathrm{H}$

\section{$-2.255350$}

$-1.214389$

$-2.462158$

0.830863

0.803494

0.842766

0.867923

$-3.023872$

$-3.921679$

0.017019

1.958591

$-0.736542$

$-0.466425$

1.897209
$-0.720489$

$-0.793997$

0.339954

$-1.030490$

$-1.145189$

0.199075

0.245308

$-1.183417$

$-0.831733$

3.464668

3. 126621

3. 362195

4.072288

2. 109304
0.800519

1.110165

0.606426

$-1.205120$

$-2.410212$

$-0.648762$

0.340899

1.914901

1.801017

$-1.490803$

$-0.636511$

0.858660

$-0.345826$

$-1.023406$ 


\begin{tabular}{|c|c|c|c|}
\hline $\mathrm{H}$ & -1.202691 & 2.386359 & 0.710421 \\
\hline $\mathrm{H}$ & -1.217816 & 3.941201 & 1.646282 \\
\hline C & 0.780544 & 1.140998 & 3.256383 \\
\hline C & 1.470582 & 2.445762 & 3.800180 \\
\hline C & -0.677197 & 1.017651 & 3.726913 \\
\hline $\mathrm{H}$ & -1.189618 & 0.210729 & 3.196278 \\
\hline $\mathrm{H}$ & -0.725577 & 0.789841 & 4.797554 \\
\hline $\mathrm{H}$ & -1.228151 & 1.947090 & 3.553300 \\
\hline C & 1.533025 & -0.155074 & 3.548098 \\
\hline $\mathrm{H}$ & 1.632366 & -0.310613 & 4.628931 \\
\hline $\mathrm{H}$ & 0.987813 & -1.012377 & 3.139176 \\
\hline $\mathrm{H}$ & 2.530023 & -0.142515 & 3.102693 \\
\hline C & 3.006459 & 2.391037 & 3.709737 \\
\hline $\mathrm{H}$ & 3.435605 & 1.711613 & 4.455096 \\
\hline $\mathrm{H}$ & 3.335377 & 2.073305 & 2.715828 \\
\hline $\mathrm{H}$ & 3.403429 & 3.394901 & 3.891594 \\
\hline C & 1.054941 & 2.858467 & 5.212761 \\
\hline $\mathrm{H}$ & 1.317395 & 2.086512 & 5.945866 \\
\hline $\mathrm{H}$ & 1.576479 & 3.780302 & 5.491573 \\
\hline $\mathrm{H}$ & -0.018803 & 3.049339 & 5.273783 \\
\hline 0 & 0.798864 & 1.377400 & 1.820088 \\
\hline 0 & 0.996250 & 3.440014 & 2.878745 \\
\hline B & 0.828300 & 2.820207 & 1.614762 \\
\hline 0 & 1.873345 & 3.284275 & 0.654453 \\
\hline C & 2.824397 & 4.073625 & -1.370167 \\
\hline C & 3.277825 & 5.257882 & -0.769593 \\
\hline C & 3.220418 & 3.770863 & -2.682557 \\
\hline C & 4.106913 & 6.125799 & -1.476326 \\
\hline $\mathrm{H}$ & 2.983270 & 5.475231 & 0.251980 \\
\hline C & 4.050712 & 4.640965 & -3.385800 \\
\hline $\mathrm{H}$ & 2.874073 & 2.851249 & -3.148488 \\
\hline C & 4.494045 & 5.822030 & -2.784848 \\
\hline $\mathrm{H}$ & 4.456868 & 7.040309 & -1.004937 \\
\hline $\mathrm{H}$ & 4.354119 & 4.397039 & -4.400151 \\
\hline $\mathrm{H}$ & 5.142412 & 6.500847 & -3.332299 \\
\hline $\mathrm{H}$ & -0.378079 & 5.158568 & -0.283552 \\
\hline $\mathrm{H}$ & -0.183993 & 2.410509 & -1.664990 \\
\hline $\mathrm{H}$ & 0.239620 & 4.060470 & -2.370052 \\
\hline C & -0.384775 & -2.857280 & -0.040495 \\
\hline C & 0.820965 & -2.196621 & -0.245005 \\
\hline C & 2.024073 & -2.595461 & 0.429295 \\
\hline C & 1.940948 & -3.622158 & 1.351353 \\
\hline $\mathrm{H}$ & 2.845658 & -3.963818 & 1.847424 \\
\hline C & -2.489630 & -1.524246 & -0.463835 \\
\hline C & -1.602557 & -2.517842 & -0.858820 \\
\hline C & -3.890139 & -1.964116 & -2.403926 \\
\hline C & -3.657379 & -1.250424 & -1.250192 \\
\hline $\mathrm{H}$ & -4.776080 & -1.752141 & -2.998131 \\
\hline C & -3.003571 & -2.980901 & -2.839644 \\
\hline C & -1.839112 & -3.269300 & -2.060164 \\
\hline C & -0.962655 & -4.292109 & -2.516150 \\
\hline C & -1.228453 & -4.991824 & -3.672118 \\
\hline C & -2.382859 & -4.705239 & -4.438074 \\
\hline C & -3.249272 & -3.718315 & -4.028368 \\
\hline
\end{tabular}




\begin{tabular}{|c|c|c|c|}
\hline H & -0.069589 & -4.516575 & -1.943843 \\
\hline $\mathrm{H}$ & -0.543295 & -5.768132 & -4.001921 \\
\hline $\mathrm{H}$ & -2.579966 & -5.263601 & -5.349305 \\
\hline $\mathrm{H}$ & -4.138460 & -3.486095 & -4.609947 \\
\hline C & 0.721866 & -4.279425 & 1.644656 \\
\hline C & -0.463596 & -3.904413 & 0.935046 \\
\hline C & -1.676856 & -4.582787 & 1.234349 \\
\hline C & -1.711149 & -5.584340 & 2.178450 \\
\hline C & -0.535592 & -5.961164 & 2.871349 \\
\hline C & 0.653355 & -5.321043 & 2.609488 \\
\hline $\mathrm{H}$ & -2.583827 & -4.288109 & 0.719056 \\
\hline $\mathrm{H}$ & -2.648686 & -6.087906 & 2.397513 \\
\hline $\mathrm{H}$ & -0.577046 & -6.754350 & 3.613000 \\
\hline $\mathrm{H}$ & 1.561202 & -5.600647 & 3.138783 \\
\hline C & -6.457925 & 1.756917 & -0.048519 \\
\hline C & -6.570312 & 0.480244 & 0.508710 \\
\hline C & -5.636747 & -0.490250 & 0.102224 \\
\hline C & -4.635016 & -0.195633 & -0.827248 \\
\hline C & -4.564249 & 1.096382 & -1.371322 \\
\hline C & -5.467593 & 2.092193 & -0.990655 \\
\hline $\mathrm{H}$ & -7.160944 & 2.524649 & 0.252065 \\
\hline H & -5.700110 & -1.504297 & 0.488884 \\
\hline $\mathrm{H}$ & -3.783362 & 1.303860 & -2.093953 \\
\hline C & -7.663905 & 0.106457 & 1.528144 \\
\hline C & -5.409974 & 3.525402 & -1.553850 \\
\hline C & -7.004069 & -0.391314 & 2.836326 \\
\hline $\mathrm{H}$ & -7.771631 & -0.659575 & 3.572409 \\
\hline H & -6.383925 & -1.279289 & 2.672316 \\
\hline $\mathrm{H}$ & -6.370633 & 0.386756 & 3.278191 \\
\hline C & -8.574421 & 1.298384 & 1.879204 \\
\hline $\mathrm{H}$ & -9.326125 & 0.983806 & 2.611668 \\
\hline $\mathrm{H}$ & -8.009356 & 2.126713 & 2.321669 \\
\hline $\mathrm{H}$ & -9.108906 & 1.677978 & 1.001081 \\
\hline C & -8.548321 & -1.019809 & 0.941260 \\
\hline $\mathrm{H}$ & -9.330777 & -1.301743 & 1.656386 \\
\hline $\mathrm{H}$ & -9.035667 & -0.692874 & 0.015644 \\
\hline $\mathrm{H}$ & -7.966289 & -1.918784 & 0.712946 \\
\hline C & -4.284544 & 3.705557 & -2.590421 \\
\hline $\mathrm{H}$ & -4.293546 & 4.735210 & -2.965652 \\
\hline $\mathrm{H}$ & -3.294731 & 3.522150 & -2.157472 \\
\hline $\mathrm{H}$ & -4.411962 & 3.039691 & -3.451498 \\
\hline C & -6.753563 & 3.867172 & -2.240974 \\
\hline $\mathrm{H}$ & -6.725943 & 4.887419 & -2.642700 \\
\hline $\mathrm{H}$ & -6.954240 & 3.180717 & -3.071467 \\
\hline $\mathrm{H}$ & -7.597923 & 3.807013 & -1.546383 \\
\hline C & -5.157978 & 4.519754 & -0.394782 \\
\hline $\mathrm{H}$ & -5.121547 & 5.547838 & -0.775805 \\
\hline $\mathrm{H}$ & -5.947968 & 4.473282 & 0.362043 \\
\hline $\mathrm{H}$ & -4.204384 & 4.307452 & 0.102002 \\
\hline C & 3.352920 & -1.994330 & 0.116836 \\
\hline C & 4.254636 & -1.685209 & 1.152316 \\
\hline C & 3.754894 & -1.799938 & -1.208089 \\
\hline C & 5.527172 & -1.179897 & 0.882164 \\
\hline H & 3.933011 & -1.839648 & 2.175394 \\
\hline
\end{tabular}




\begin{tabular}{|c|c|c|c|}
\hline C & 5.023086 & -1.286605 & -1.522750 \\
\hline $\mathrm{H}$ & 3.073756 & -2.070517 & -2.006199 \\
\hline C & 5.883713 & -0.983285 & -0.465136 \\
\hline $\mathrm{H}$ & 6.867045 & -0.584272 & -0.684702 \\
\hline C & 5.409365 & -1.089413 & -3.001157 \\
\hline C & 6.537602 & -0.829555 & 1.992042 \\
\hline C & 5.330293 & -2.447017 & -3.739691 \\
\hline $\mathrm{H}$ & 6.016834 & -3.176203 & -3.293979 \\
\hline $\mathrm{H}$ & 4.321959 & -2.871447 & -3.707227 \\
\hline $\mathrm{H}$ & 5.604317 & -2.322591 & -4.794646 \\
\hline C & 4.424271 & -0.091687 & -3.657141 \\
\hline $\mathrm{H}$ & 4.660535 & 0.030222 & -4.721782 \\
\hline $\mathrm{H}$ & 3.384725 & -0.425948 & -3.578279 \\
\hline $\mathrm{H}$ & 4.498428 & 0.892417 & -3.179216 \\
\hline C & 6.837093 & -0.536752 & -3.170413 \\
\hline $\mathrm{H}$ & 6.955641 & 0.443314 & -2.694176 \\
\hline $\mathrm{H}$ & 7.591102 & -1.214395 & -2.753224 \\
\hline $\mathrm{H}$ & 7.058865 & -0.413424 & -4.236504 \\
\hline C & 5.997031 & -1.138438 & 3.401181 \\
\hline $\mathrm{H}$ & 5.097912 & -0.556630 & 3.632868 \\
\hline $\mathrm{H}$ & 5.758294 & -2.201302 & 3.522676 \\
\hline $\mathrm{H}$ & 6.755236 & -0.883020 & 4.150195 \\
\hline C & 7.832686 & -1.652060 & 1.789796 \\
\hline $\mathrm{H}$ & 7.626294 & -2.727258 & 1.842703 \\
\hline $\mathrm{H}$ & 8.300026 & -1.449659 & 0.820610 \\
\hline $\mathrm{H}$ & 8.564437 & -1.407434 & 2.569532 \\
\hline C & 6.873327 & 0.679309 & 1.925774 \\
\hline $\mathrm{H}$ & 7.599396 & 0.943555 & 2.704616 \\
\hline $\mathrm{H}$ & 7.304907 & 0.958729 & 0.958964 \\
\hline $\mathrm{H}$ & 5.974604 & 1.287655 & 2.078523 \\
\hline
\end{tabular}

\section{C-Catalyzed reaction TS 137}

\section{Datum}

Value

\begin{tabular}{lc}
\hline B3LYP/6-31G(d) Energy & -3038.943112 \\
\hline B3LYP-D3(BJ)/def2-TZVPP-IEF-PCM(DCM) Energy & -3040.497149 \\
\hline B3LYP-D3(BJ)/def2-TZVPP-IEF-PCM(DCM)//B3LYP/6-31G(d) Free Energy (Quasiharmonic) & -3039.26951 \\
\hline
\end{tabular}

Number of Imaginary Frequencies

Frequencies (Top 3 out of 450)

$\begin{array}{lr}\text { 1. } & -350.6398 \mathrm{~cm}^{-1} \\ \text { 2. } & 8.3619 \mathrm{~cm}^{-1} \\ \text { 3. } & 10.0433 \mathrm{~cm}^{-1}\end{array}$




\begin{tabular}{|c|c|c|c|}
\hline C & 1.650740 & -0.501950 & 0.595390 \\
\hline $\mathrm{H}$ & 2.269580 & 0.090120 & -0.076210 \\
\hline $\mathrm{H}$ & 0.627110 & -0.129120 & 0.502660 \\
\hline C & -1.608770 & -1.905120 & -0.957160 \\
\hline 0 & -1.747230 & -2.953670 & -1.551380 \\
\hline 0 & -1.391190 & -0.768190 & -1.626710 \\
\hline $\mathrm{H}$ & -1.280390 & 0.059440 & -1.091640 \\
\hline 0 & 2.169290 & -0.231080 & 1.910440 \\
\hline $\mathrm{H}$ & 1.631130 & -0.736400 & 2.542170 \\
\hline C & -0.220100 & 2.803750 & 3.246170 \\
\hline C & 1.241820 & 2.887260 & 1.657700 \\
\hline C & -1.829020 & 2.785390 & 1.383170 \\
\hline C & -1.146720 & 2.146340 & 2.463080 \\
\hline $\mathrm{H}$ & 1.773420 & 2.018240 & 2.051350 \\
\hline $\mathrm{H}$ & -2.060141 & 3.838800 & 1.559500 \\
\hline $\mathrm{H}$ & -2.685740 & 2.238070 & 0.988650 \\
\hline C & -1.486350 & 2.512180 & -2.243930 \\
\hline C & -0.941931 & 3.979680 & -2.076340 \\
\hline C & -0.936290 & 1.770870 & -3.464690 \\
\hline $\mathrm{H}$ & -1.362440 & 0.766410 & -3.524430 \\
\hline $\mathrm{H}$ & -1.194050 & 2.307760 & -4.385490 \\
\hline $\mathrm{H}$ & 0.150740 & 1.671180 & -3.414870 \\
\hline C & -3.020510 & 2.435280 & -2.236380 \\
\hline $\mathrm{H}$ & -3.327720 & 1.389530 & -2.137120 \\
\hline $\mathrm{H}$ & -3.448520 & 2.828680 & -3.164900 \\
\hline $\mathrm{H}$ & -3.441090 & 2.998020 & -1.397200 \\
\hline C & -1.808241 & 5.063890 & -2.719220 \\
\hline $\mathrm{H}$ & -1.875391 & 4.925880 & -3.804750 \\
\hline $\mathrm{H}$ & -2.818761 & 5.068270 & -2.303930 \\
\hline $\mathrm{H}$ & -1.363541 & 6.047280 & -2.531990 \\
\hline C & 0.513979 & 4.132690 & -2.549910 \\
\hline $\mathrm{H}$ & 0.597469 & 4.061540 & -3.639650 \\
\hline $\mathrm{H}$ & 0.885729 & 5.116390 & -2.244110 \\
\hline $\mathrm{H}$ & 1.160190 & 3.373110 & -2.100500 \\
\hline 0 & -1.001910 & 1.870740 & -1.031580 \\
\hline 0 & -0.977571 & 4.158360 & -0.648890 \\
\hline B & -0.755700 & 2.887700 & -0.034180 \\
\hline 0 & 0.625910 & 2.664470 & 0.535490 \\
\hline C & 1.838809 & 4.209370 & 1.953360 \\
\hline C & 2.846609 & 4.285851 & 2.928830 \\
\hline C & 1.445679 & 5.372630 & 1.270520 \\
\hline C & 3.450999 & 5.505081 & 3.224150 \\
\hline $\mathrm{H}$ & 3.158230 & 3.382251 & 3.448100 \\
\hline C & 2.051709 & 6.590140 & 1.574330 \\
\hline $\mathrm{H}$ & 0.672549 & 5.309070 & 0.510170 \\
\hline C & 3.052049 & 6.661351 & 2.548250 \\
\hline $\mathrm{H}$ & 4.234599 & 5.554161 & 3.975300 \\
\hline $\mathrm{H}$ & 1.746669 & 7.488720 & 1.044580 \\
\hline $\mathrm{H}$ & 3.523239 & 7.613761 & 2.776150 \\
\hline $\mathrm{H}$ & -1.169360 & 1.056900 & 2.506190 \\
\hline
\end{tabular}




\begin{tabular}{|c|c|c|c|}
\hline $\mathrm{H}$ & -0.297901 & 3.878880 & 3.372570 \\
\hline H & 0.317960 & 2.274430 & 4.027530 \\
\hline C & -0.566460 & -2.283870 & 1.270440 \\
\hline C & -1.670210 & -1.833820 & 0.555460 \\
\hline C & -2.860450 & -1.375820 & 1.218060 \\
\hline C & -2.863890 & -1.344040 & 2.601390 \\
\hline H & -3.763700 & -1.027160 & 3.121800 \\
\hline C & 1.715220 & -1.976090 & 0.241580 \\
\hline C & 0.657090 & -2.819170 & 0.572170 \\
\hline C & 2.994400 & -3.880189 & -0.562110 \\
\hline C & 2.905290 & -2.520279 & -0.350000 \\
\hline $\mathrm{H}$ & 3.896891 & -4.290209 & -1.009180 \\
\hline C & 1.934961 & -4.763520 & -0.240730 \\
\hline C & 0.735141 & -4.230250 & 0.325450 \\
\hline C & -0.325719 & -5.127960 & 0.626180 \\
\hline C & -0.199029 & -6.478270 & 0.389170 \\
\hline C & 0.992821 & -7.003670 & -0.164400 \\
\hline C & 2.034911 & -6.161280 & -0.475120 \\
\hline $\mathrm{H}$ & -1.250519 & -4.735210 & 1.033270 \\
\hline $\mathrm{H}$ & -1.023839 & -7.146400 & 0.621580 \\
\hline $\mathrm{H}$ & 1.078491 & -8.071320 & -0.348150 \\
\hline $\mathrm{H}$ & 2.952711 & -6.551499 & -0.908720 \\
\hline C & -1.752190 & -1.759760 & 3.372730 \\
\hline C & -0.586470 & -2.254790 & 2.704810 \\
\hline C & 0.507360 & -2.705300 & 3.500000 \\
\hline C & 0.454000 & -2.649020 & 4.876520 \\
\hline C & -0.696650 & -2.149120 & 5.531950 \\
\hline C & -1.775510 & -1.719630 & 4.793940 \\
\hline $\mathrm{H}$ & 1.384910 & -3.111470 & 3.007620 \\
\hline $\mathrm{H}$ & 1.298050 & -3.000410 & 5.463520 \\
\hline $\mathrm{H}$ & -0.726880 & -2.112200 & 6.617370 \\
\hline $\mathrm{H}$ & -2.667670 & -1.342470 & 5.288190 \\
\hline C & 6.265820 & -0.146469 & -1.501200 \\
\hline C & 6.408840 & -1.044259 & -0.434810 \\
\hline C & 5.288070 & -1.800189 & -0.068910 \\
\hline C & 4.067750 & -1.663219 & -0.740830 \\
\hline C & 3.968340 & -0.759689 & -1.807880 \\
\hline C & 5.063820 & 0.015701 & -2.205680 \\
\hline $\mathrm{H}$ & 7.123510 & 0.450561 & -1.796750 \\
\hline $\mathrm{H}$ & 5.340660 & -2.502819 & 0.754780 \\
\hline $\mathrm{H}$ & 3.021350 & -0.684309 & -2.330940 \\
\hline C & 7.764670 & -1.164069 & 0.288000 \\
\hline C & 4.993110 & 1.021391 & -3.371270 \\
\hline C & 8.853160 & -1.595999 & -0.723320 \\
\hline $\mathrm{H}$ & 9.825740 & -1.684349 & -0.223320 \\
\hline $\mathrm{H}$ & 8.610490 & -2.567699 & -1.168540 \\
\hline $\mathrm{H}$ & 8.963330 & -0.874009 & -1.539200 \\
\hline C & 8.147240 & 0.205531 & 0.897760 \\
\hline $\mathrm{H}$ & 9.115520 & 0.139031 & 1.409390 \\
\hline H & 8.227230 & 0.984591 & 0.132400 \\
\hline $\mathrm{H}$ & 7.397570 & 0.531351 & 1.627780 \\
\hline C & 7.733320 & -2.201659 & 1.426240 \\
\hline $\mathrm{H}$ & 8.717280 & -2.252119 & 1.906410 \\
\hline H & 7.001540 & -1.938539 & 2.198280 \\
\hline
\end{tabular}




\begin{tabular}{|c|c|c|c|}
\hline $\mathrm{H}$ & 7.494250 & -3.205249 & 1.056530 \\
\hline C & 3.602300 & 1.057601 & -4.033010 \\
\hline $\mathrm{H}$ & 3.602500 & 1.787291 & -4.850710 \\
\hline $\mathrm{H}$ & 3.324410 & 0.086141 & -4.457030 \\
\hline $\mathrm{H}$ & 2.821420 & 1.356881 & -3.324540 \\
\hline C & 5.309640 & 2.441061 & -2.843320 \\
\hline $\mathrm{H}$ & 5.269330 & 3.170441 & -3.661740 \\
\hline $\mathrm{H}$ & 4.584030 & 2.745701 & -2.080590 \\
\hline $\mathrm{H}$ & 6.307610 & 2.497291 & -2.395980 \\
\hline C & 6.028370 & 0.634391 & -4.454100 \\
\hline $\mathrm{H}$ & 5.993190 & 1.347071 & -5.287340 \\
\hline $\mathrm{H}$ & 7.050460 & 0.632331 & -4.060970 \\
\hline $\mathrm{H}$ & 5.822250 & -0.365229 & -4.853490 \\
\hline C & -4.095110 & -0.977830 & 0.480580 \\
\hline C & -4.829810 & 0.150030 & 0.894470 \\
\hline C & -4.583570 & -1.748980 & -0.577950 \\
\hline C & -6.022100 & 0.513019 & 0.266590 \\
\hline $\mathrm{H}$ & -4.442010 & 0.744490 & 1.713470 \\
\hline C & -5.768880 & -1.407851 & -1.248490 \\
\hline $\mathrm{H}$ & -4.040060 & -2.636830 & -0.876380 \\
\hline C & -6.465860 & -0.279951 & -0.808390 \\
\hline $\mathrm{H}$ & -7.385540 & -0.001091 & -1.309280 \\
\hline C & -6.243400 & -2.282331 & -2.424400 \\
\hline C & -6.856470 & 1.731939 & 0.706780 \\
\hline C & -6.479690 & -3.729131 & -1.928400 \\
\hline $\mathrm{H}$ & -5.566269 & -4.176161 & -1.523460 \\
\hline $\mathrm{H}$ & -7.244900 & -3.755731 & -1.143690 \\
\hline $\mathrm{H}$ & -6.819979 & -4.362581 & -2.756640 \\
\hline C & -7.555640 & -1.769631 & -3.046760 \\
\hline $\mathrm{H}$ & -7.845460 & -2.418751 & -3.880740 \\
\hline $\mathrm{H}$ & -8.380980 & -1.772441 & -2.325010 \\
\hline $\mathrm{H}$ & -7.449490 & -0.753261 & -3.443220 \\
\hline C & -5.154480 & -2.289040 & -3.525060 \\
\hline $\mathrm{H}$ & -4.993920 & -1.278760 & -3.919450 \\
\hline $\mathrm{H}$ & -4.192800 & -2.657020 & -3.154000 \\
\hline $\mathrm{H}$ & -5.463610 & -2.932081 & -4.358530 \\
\hline C & -8.270750 & 1.261109 & 1.123780 \\
\hline $\mathrm{H}$ & -8.794420 & 0.761839 & 0.302160 \\
\hline $\mathrm{H}$ & -8.218430 & 0.557379 & 1.962490 \\
\hline $\mathrm{H}$ & -8.880490 & 2.117749 & 1.436220 \\
\hline C & -6.231710 & 2.473199 & 1.903980 \\
\hline $\mathrm{H}$ & -6.142210 & 1.825049 & 2.783430 \\
\hline $\mathrm{H}$ & -5.237640 & 2.870040 & 1.669160 \\
\hline $\mathrm{H}$ & -6.866340 & 3.321599 & 2.184200 \\
\hline C & -6.977100 & 2.732059 & -0.467500 \\
\hline $\mathrm{H}$ & -7.575710 & 3.601399 & -0.168300 \\
\hline $\mathrm{H}$ & -5.989710 & 3.090309 & -0.780000 \\
\hline $\mathrm{H}$ & -7.459370 & 2.280479 & -1.340580 \\
\hline
\end{tabular}


Frequencies (Top 3 out of 450)

$\begin{array}{rr}\text { 1. } & -352.0108 \mathrm{~cm}^{-1} \\ \text { 2. } & 7.9889 \mathrm{~cm}^{-1} \\ \text { 3. } & 9.0584 \mathrm{~cm}^{-1}\end{array}$

\section{B3LYP/6-31G(d) Molecular Geometry in Cartesian Coordinates}

C

$\mathrm{H}$

$\mathrm{H}$

C

0

0

$\mathrm{H}$

0

$\mathrm{H}$

C

C

C

C

$\mathrm{H}$

$\mathrm{H}$

$\mathrm{H}$

C

C

C

$\mathrm{H}$

$\mathrm{H}$

$\mathrm{H}$

C

$\mathrm{H}$

$\mathrm{H}$

$\mathrm{H}$

C

$\mathrm{H}$

$\mathrm{H}$

$\mathrm{H}$

C

$\mathrm{H}$

\subsection{7}

2.403157

0.728681

$-1.448346$

$-1.439456$

$-1.313041$

$-1.379020$

2.183011

1.623755

$-0.250293$

1.106538

$-1.981061$

$-1.252950$

1.640842

$-2.160103$

$-2.879057$

$-1.489005$

$-1.758107$

$-0.121524$

0.076700

$-0.099600$

0.682233

$-2.590759$

$-2.330770$

$-2.713310$

$-3.548719$

$-3.249515$

$-3.796551$

$-3.725309$

$-3.339867$

$-1.145630$

$-1.561243$
$-0.330893$

0.109457

0.000109

$-1.945031$

$-3.072641$

$-0.890197$

0.009455

0.232107

$-0.148637$

3. 482895

3. 184299

3. 194016

2. 738239

2. 398282

4.271511

2. 623156

2. 191852

3.740896

1.847425

0.782252

2.048203

2.416663

1. 393807

0.332345

1.738112

1.494921

4.089868

3.921270

3.501406

5.148700

4.554489

4. 252004
0.653790

$-0.096543$

0.432743

$-0.688344$

$-1.133050$

$-1.502083$

$-1.087765$

1.909562

2.607518

2.691986

1.017721

0.965401

2.105515

1.554186

0.949550

0.726865

$-2.667957$

$-2.588563$

- 3.278891

$-3.127067$

$-4.355694$

$-2.802335$

- 3.367401

$-3.391778$

$-4.401229$

$-2.851401$

$-2.454728$

$-3.388754$

$-1.665266$

$-2.190635$

$-3.731224$

$-4.699581$ 


\begin{tabular}{|c|c|c|c|}
\hline $\mathrm{H}$ & -1.369789 & 5.616929 & -3.585982 \\
\hline $\mathrm{H}$ & -0.059800 & 4.440514 & -3.766660 \\
\hline 0 & -1.452579 & 1.824581 & -1.258674 \\
\hline 0 & -1.105496 & 4.114101 & -1.363703 \\
\hline B & -0.994521 & 2.972118 & -0.513164 \\
\hline 0 & 0.399894 & 2.749017 & 0.017362 \\
\hline C & 1.761890 & 4.510774 & 0.992936 \\
\hline C & 1.402683 & 5.507311 & 0.070174 \\
\hline C & 2.800684 & 4.756195 & 1.907625 \\
\hline C & 2.070171 & 6.730947 & 0.080426 \\
\hline $\mathrm{H}$ & 0.610696 & 5.310604 & -0.646080 \\
\hline C & 3.464890 & 5.979558 & 1.909553 \\
\hline $\mathrm{H}$ & 3.087137 & 3.979495 & 2.613015 \\
\hline C & 3.097783 & 6.972014 & 0.996323 \\
\hline $\mathrm{H}$ & 1.791519 & 7.499429 & -0.635731 \\
\hline $\mathrm{H}$ & 4.270389 & 6.158350 & 2.616523 \\
\hline $\mathrm{H}$ & 3.616201 & 7.927192 & 0.994513 \\
\hline $\mathrm{H}$ & -1.314195 & 1.678573 & 2.356899 \\
\hline $\mathrm{H}$ & -0.272676 & 4.564961 & 2.610919 \\
\hline $\mathrm{H}$ & 0.318540 & 3.083474 & 3.526956 \\
\hline C & -0.487752 & -1.956843 & 1.604300 \\
\hline C & -1.579107 & -1.660036 & 0.794313 \\
\hline C & -2.800809 & -1.134876 & 1.340742 \\
\hline C & -2.841617 & -0.862349 & 2.696938 \\
\hline $\mathrm{H}$ & -3.766649 & -0.501579 & 3.138102 \\
\hline C & 1.834751 & -1.843612 & 0.618387 \\
\hline C & 0.752613 & -2.608411 & 1.048050 \\
\hline C & 3.065662 & -3.870897 & 0.091083 \\
\hline C & 3.023416 & -2.491031 & 0.132787 \\
\hline $\mathrm{H}$ & 3.966818 & -4.362954 & -0.266242 \\
\hline C & 1.987412 & -4.675036 & 0.529581 \\
\hline C & 0.804656 & -4.041289 & 1.022349 \\
\hline C & -0.267116 & -4.864945 & 1.463690 \\
\hline C & -0.167664 & -6.237349 & 1.425947 \\
\hline C & 1.006613 & -6.861544 & 0.941083 \\
\hline C & 2.059405 & -6.094160 & 0.500875 \\
\hline $\mathrm{H}$ & -1.178103 & -4.400478 & 1.823649 \\
\hline $\mathrm{H}$ & -1.000012 & -6.847968 & 1.765215 \\
\hline $\mathrm{H}$ & 1.070729 & -7.946046 & 0.914734 \\
\hline $\mathrm{H}$ & 2.965728 & -6.561073 & 0.122491 \\
\hline C & -1.737325 & -1.092839 & 3.551609 \\
\hline C & -0.544714 & -1.670191 & 3.008925 \\
\hline C & 0.537504 & -1.942715 & 3.895832 \\
\hline C & 0.449182 & -1.637712 & 5.237600 \\
\hline C & -0.727635 & -1.056293 & 5.767008 \\
\hline C & -1.797224 & -0.797006 & 4.941215 \\
\hline $\mathrm{H}$ & 1.434216 & -2.411856 & 3.504172 \\
\hline $\mathrm{H}$ & 1.285419 & -1.855684 & 5.896120 \\
\hline $\mathrm{H}$ & -0.785643 & -0.823325 & 6.826595 \\
\hline $\mathrm{H}$ & -2.709862 & -0.360882 & 5.340465 \\
\hline C & 6.532118 & -0.431730 & -1.205398 \\
\hline C & 6.014514 & -0.122496 & 0.060829 \\
\hline C & 4.856112 & -0.788674 & 0.479271 \\
\hline C & 4.223700 & -1.736160 & -0.336348 \\
\hline
\end{tabular}




\begin{tabular}{|c|c|c|c|}
\hline C & 4.772378 & -2.016608 & -1.595193 \\
\hline C & 5.929351 & -1.373302 & -2.052268 \\
\hline $\mathrm{H}$ & 7.425783 & 0.075840 & -1.541702 \\
\hline $\mathrm{H}$ & 4.425112 & -0.575332 & 1.451125 \\
\hline $\mathrm{H}$ & 4.266721 & -2.741169 & -2.226890 \\
\hline C & 6.671802 & 0.909116 & 0.998909 \\
\hline C & 6.487932 & -1.712302 & -3.448878 \\
\hline C & 7.093857 & 0.211140 & 2.313971 \\
\hline $\mathrm{H}$ & 7.562791 & 0.931755 & 2.995753 \\
\hline $\mathrm{H}$ & 6.236324 & -0.230076 & 2.832252 \\
\hline $\mathrm{H}$ & 7.815141 & -0.590823 & 2.118604 \\
\hline C & 7.923642 & 1.559541 & 0.380745 \\
\hline $\mathrm{H}$ & 8.346952 & 2.286171 & 1.083730 \\
\hline $\mathrm{H}$ & 8.703091 & 0.820550 & 0.161718 \\
\hline $\mathrm{H}$ & 7.689891 & 2.095253 & -0.546382 \\
\hline C & 5.655058 & 2.030939 & 1.320314 \\
\hline $\mathrm{H}$ & 6.099606 & 2.761103 & 2.008766 \\
\hline $\mathrm{H}$ & 5.359285 & 2.563542 & 0.409074 \\
\hline $\mathrm{H}$ & 4.746070 & 1.635641 & 1.784867 \\
\hline C & 5.421599 & -1.389841 & -4.522780 \\
\hline $\mathrm{H}$ & 5.801818 & -1.631145 & -5.523251 \\
\hline $\mathrm{H}$ & 4.501083 & -1.962264 & -4.369223 \\
\hline $\mathrm{H}$ & 5.158775 & -0.325940 & -4.506366 \\
\hline C & 7.761652 & -0.914195 & -3.786222 \\
\hline $\mathrm{H}$ & 8.117241 & -1.193267 & -4.784609 \\
\hline $\mathrm{H}$ & 7.577702 & 0.166185 & -3.791776 \\
\hline $\mathrm{H}$ & 8.572232 & -1.120106 & -3.077881 \\
\hline C & 6.837519 & -3.218298 & -3.511840 \\
\hline $\mathrm{H}$ & 7.227990 & -3.479014 & -4.503353 \\
\hline $\mathrm{H}$ & 7.600787 & -3.474645 & -2.767974 \\
\hline $\mathrm{H}$ & 5.962120 & -3.848233 & -3.323021 \\
\hline C & -4.036823 & -0.946509 & 0.526135 \\
\hline C & -4.874200 & 0.153199 & 0.750425 \\
\hline C & -4.430537 & -1.916793 & -0.412429 \\
\hline C & -6.090863 & 0.304994 & 0.067180 \\
\hline $\mathrm{H}$ & -4.566397 & 0.904125 & 1.472226 \\
\hline C & -5.616094 & -1.786369 & -1.137057 \\
\hline $\mathrm{H}$ & -3.805006 & -2.786648 & -0.556702 \\
\hline C & -6.428805 & -0.669076 & -0.877675 \\
\hline $\mathrm{H}$ & -7.354820 & -0.567592 & -1.430727 \\
\hline C & -6.047860 & -2.817847 & -2.197230 \\
\hline C & -7.009347 & 1.500943 & 0.390775 \\
\hline C & -7.442220 & -3.385049 & -1.840727 \\
\hline $\mathrm{H}$ & -7.425106 & -3.881952 & -0.863885 \\
\hline $\mathrm{H}$ & -8.208790 & -2.603587 & -1.805537 \\
\hline $\mathrm{H}$ & -7.754696 & -4.122170 & -2.590442 \\
\hline C & -6.115235 & -2.121317 & -3.577703 \\
\hline $\mathrm{H}$ & -6.427589 & -2.835582 & -4.349488 \\
\hline $\mathrm{H}$ & -6.830286 & -1.291333 & -3.581699 \\
\hline $\mathrm{H}$ & -5.134965 & -1.722028 & -3.861024 \\
\hline C & -5.062989 & -3.998246 & -2.302183 \\
\hline $\mathrm{H}$ & -4.050308 & -3.673129 & -2.562813 \\
\hline $\mathrm{H}$ & -5.004670 & -4.563091 & -1.364541 \\
\hline $\mathrm{H}$ & -5.402519 & -4.689586 & -3.081928 \\
\hline
\end{tabular}




\begin{tabular}{|llll|} 
C & -7.419738 & 1.434775 & 1.881935 \\
$H$ & -7.953951 & 0.503019 & 2.100234 \\
$H$ & -6.550281 & 1.485484 & 2.546114 \\
H & -8.080726 & 2.273284 & 2.133988 \\
C & -6.263171 & 2.828904 & 0.127333 \\
$H$ & -5.358637 & 2.918989 & 0.737549 \\
$H$ & -5.968186 & 2.910288 & -0.924682 \\
$H$ & -6.908909 & 3.683288 & 0.364914 \\
C & -8.296109 & 1.502755 & -0.456375 \\
$H$ & -8.911431 & 2.368956 & -0.188199 \\
H & -8.079424 & 1.571640 & -1.528455 \\
$H$ & -8.899600 & 0.604239 & -0.285359 \\
\hline
\end{tabular}

\section{C-Catalyzed reaction TS 139}

\section{Datum}

Value

\begin{tabular}{lr}
\hline B3LYP/6-31G(d) Energy & -3038.946466 \\
\hline B3LYP-D3(BJ)/def2-TZVPP-IEF-PCM(DCM) Energy & -3040.495765 \\
\hline B3LYP-D3(BJ)/def2-TZVPP-IEF-PCM(DCM)//B3LYP/6-31G(d) Free Energy (Quasiharmonic) & -3039.268355 \\
\hline
\end{tabular}

Number of Imaginary Frequencies

Frequencies (Top 3 out of 450)

$\begin{array}{lr}\text { 1. } & -371.1448 \mathrm{~cm}^{-1} \\ \text { 2. } & 6.0827 \mathrm{~cm}^{-1} \\ \text { 3. } & 8.6193 \mathrm{~cm}^{-1}\end{array}$

\section{B3LYP/6-31G(d) Molecular Geometry in Cartesian Coordinates}

C

$\mathrm{H}$

$\mathrm{H}$

C

0

0

$\mathrm{H}$

0

$\mathrm{H}$

C

C

C

C

$\mathrm{H}$

\section{$-2.228452$}

$-1.158244$

$-2.751791$

0.852425

0.770115

0.929725

0.980523

$-2.683902$

$-2.221352$

0.040065

1.992317

$-0.680271$

$-0.448826$

1.944674
$-0.785693$

$-0.572945$

0.170839

$-0.930877$

$-1.014242$

0.279056

0.305298

$-1.484779$

$-2.338573$

3.542564

3. 208154

3. 297281

4.076695

2. 203311
0.894028

0.978374

0.914221

$-1.246224$

$-2.451257$

$-0.659414$

0.332046

2.061440

2.091705

$-1.471112$

$-0.652326$

0.877323

$-0.293192$

$-1.072091$ 


\begin{tabular}{|c|c|c|c|}
\hline $\mathrm{H}$ & -1.105843 & 2.311813 & 0.677210 \\
\hline $\mathrm{H}$ & -1.193626 & 3.815766 & 1.686682 \\
\hline C & 0.967442 & 1.128068 & 3.248510 \\
\hline C & 1.601744 & 2.464687 & 3.783705 \\
\hline C & -0.468541 & 0.918955 & 3.752815 \\
\hline H & -0.939728 & 0.077070 & 3.237538 \\
\hline $\mathrm{H}$ & -0.480923 & 0.696995 & 4.825731 \\
\hline $\mathrm{H}$ & -1.080490 & 1.809961 & 3.583359 \\
\hline C & 1.796454 & -0.126717 & 3.513003 \\
\hline $\mathrm{H}$ & 1.929795 & -0.283773 & 4.589950 \\
\hline $\mathrm{H}$ & 1.287869 & -1.008939 & 3.109289 \\
\hline $\mathrm{H}$ & 2.780148 & -0.058947 & 3.043965 \\
\hline C & 3.136902 & 2.486896 & 3.665166 \\
\hline $\mathrm{H}$ & 3.613766 & 1.827225 & 4.399258 \\
\hline $\mathrm{H}$ & 3.463348 & 2.191793 & 2.663518 \\
\hline $\mathrm{H}$ & 3.485757 & 3.508820 & 3.844920 \\
\hline C & 1.192449 & 2.851884 & 5.205560 \\
\hline $\mathrm{H}$ & 1.506774 & 2.092802 & 5.931753 \\
\hline $\mathrm{H}$ & 1.672015 & 3.798201 & 5.477383 \\
\hline $\mathrm{H}$ & 0.111735 & 2.988582 & 5.286551 \\
\hline 0 & 0.934594 & 1.371277 & 1.814746 \\
\hline 0 & 1.061463 & 3.436922 & 2.877059 \\
\hline B & 0.899110 & 2.817967 & 1.610842 \\
\hline 0 & 1.927409 & 3.319121 & 0.644711 \\
\hline C & 2.832231 & 4.192200 & -1.368021 \\
\hline C & 3.218787 & 3.934784 & -2.692845 \\
\hline C & 3.270046 & 5.367191 & -0.738677 \\
\hline C & 4.023546 & 4.840842 & -3.380274 \\
\hline $\mathrm{H}$ & 2.883138 & 3.022928 & -3.181936 \\
\hline C & 4.073662 & 6.271144 & -1.429397 \\
\hline $\mathrm{H}$ & 2.982699 & 5.549239 & 0.291848 \\
\hline C & 4.450807 & 6.012755 & -2.750540 \\
\hline $\mathrm{H}$ & 4.319416 & 4.632412 & -4.404700 \\
\hline $\mathrm{H}$ & 4.411332 & 7.178598 & -0.935953 \\
\hline $\mathrm{H}$ & 5.079000 & 6.719744 & -3.285677 \\
\hline $\mathrm{H}$ & -0.400955 & 5.161183 & -0.178049 \\
\hline $\mathrm{H}$ & -0.139205 & 2.494524 & -1.697016 \\
\hline H & 0.233372 & 4.188548 & -2.321447 \\
\hline C & -0.354125 & -2.791649 & -0.124676 \\
\hline C & 0.850270 & -2.125638 & -0.320530 \\
\hline C & 2.061344 & -2.546800 & 0.327688 \\
\hline C & 1.985645 & -3.596129 & 1.225265 \\
\hline $\mathrm{H}$ & 2.894103 & -3.951417 & 1.704081 \\
\hline C & -2.514453 & -1.511357 & -0.408015 \\
\hline C & -1.593088 & -2.430640 & -0.902951 \\
\hline C & -3.932475 & -1.879265 & -2.346358 \\
\hline C & -3.720089 & -1.235621 & -1.142665 \\
\hline $\mathrm{H}$ & -4.848698 & -1.683812 & -2.897886 \\
\hline C & -3.013684 & -2.814600 & -2.877777 \\
\hline C & -1.820081 & -3.107293 & -2.147282 \\
\hline C & -0.911884 & -4.055922 & -2.692809 \\
\hline C & -1.175665 & -4.682360 & -3.890106 \\
\hline C & -2.360287 & -4.393027 & -4.608652 \\
\hline$\sigma$ & -3.257559 & -3.477145 & -4.111299 \\
\hline
\end{tabular}




\begin{tabular}{|c|c|c|c|}
\hline $\mathrm{H}$ & 0.005223 & -4.279000 & -2.159232 \\
\hline $\mathrm{H}$ & -0.466501 & -5.402056 & -4.290022 \\
\hline $\mathrm{H}$ & -2.555798 & -4.894040 & -5.552963 \\
\hline $\mathrm{H}$ & -4.170356 & -3.244486 & -4.654764 \\
\hline C & 0.770455 & -4.262992 & 1.510292 \\
\hline C & -0.418756 & -3.876753 & 0.812359 \\
\hline C & -1.622484 & -4.590404 & 1.081084 \\
\hline C & -1.647743 & -5.614904 & 2.002956 \\
\hline C & -0.471494 & -5.988341 & 2.695680 \\
\hline C & 0.710462 & -5.328927 & 2.448790 \\
\hline $\mathrm{H}$ & -2.525470 & -4.322222 & 0.542944 \\
\hline $\mathrm{H}$ & -2.576571 & -6.144981 & 2.194245 \\
\hline $\mathrm{H}$ & -0.504825 & -6.798689 & 3.418564 \\
\hline $\mathrm{H}$ & 1.620977 & -5.613006 & 2.970894 \\
\hline C & -6.757975 & 1.475814 & 0.168737 \\
\hline C & -6.312082 & 0.483648 & 1.061451 \\
\hline C & -5.311230 & -0.387094 & 0.627044 \\
\hline C & -4.758324 & -0.276803 & -0.661070 \\
\hline C & -5.231164 & 0.721797 & -1.517803 \\
\hline C & -6.237422 & 1.616862 & -1.120843 \\
\hline $\mathrm{H}$ & -7.531482 & 2.158511 & 0.500623 \\
\hline $\mathrm{H}$ & -4.933258 & -1.162972 & 1.280504 \\
\hline $\mathrm{H}$ & -4.785930 & 0.807110 & -2.504964 \\
\hline C & -6.921406 & 0.396822 & 2.474176 \\
\hline C & -6.714009 & 2.707675 & -2.099928 \\
\hline C & -6.620910 & 1.707804 & 3.239610 \\
\hline $\mathrm{H}$ & -7.055514 & 1.670785 & 4.246296 \\
\hline $\mathrm{H}$ & -5.540554 & 1.860922 & 3.341700 \\
\hline $\mathrm{H}$ & -7.036260 & 2.583043 & 2.728586 \\
\hline C & -8.452854 & 0.203478 & 2.372517 \\
\hline $\mathrm{H}$ & -8.896259 & 0.139745 & 3.374001 \\
\hline $\mathrm{H}$ & -8.938311 & 1.032875 & 1.847252 \\
\hline $\mathrm{H}$ & -8.695688 & -0.720326 & 1.834842 \\
\hline C & -6.343170 & -0.777211 & 3.287983 \\
\hline $\mathrm{H}$ & -6.793035 & -0.787687 & 4.287625 \\
\hline $\mathrm{H}$ & -6.563265 & -1.741934 & 2.816392 \\
\hline $\mathrm{H}$ & -5.257437 & -0.700711 & 3.408560 \\
\hline C & -7.276885 & 2.042559 & -3.378486 \\
\hline $\mathrm{H}$ & -7.611120 & 2.806841 & -4.091132 \\
\hline $\mathrm{H}$ & -6.525261 & 1.425018 & -3.880991 \\
\hline $\mathrm{H}$ & -8.133079 & 1.400576 & -3.141420 \\
\hline C & -7.818888 & 3.598314 & -1.500296 \\
\hline $\mathrm{H}$ & -8.122297 & 4.353335 & -2.234473 \\
\hline $\mathrm{H}$ & -8.710904 & 3.019879 & -1.234089 \\
\hline $\mathrm{H}$ & -7.475066 & 4.128531 & -0.604898 \\
\hline C & -5.520013 & 3.615367 & -2.480657 \\
\hline $\mathrm{H}$ & -5.840569 & 4.393098 & -3.184918 \\
\hline $\mathrm{H}$ & -5.105062 & 4.108555 & -1.594240 \\
\hline $\mathrm{H}$ & -4.712528 & 3.048440 & -2.955201 \\
\hline C & 3.389727 & -1.943179 & 0.017810 \\
\hline C & 3.767265 & -1.688183 & -1.309115 \\
\hline C & 4.311601 & -1.695800 & 1.047602 \\
\hline C & 5.030802 & -1.172580 & -1.619070 \\
\hline $\mathrm{H}$ & 3.068231 & -1.914765 & -2.102572 \\
\hline
\end{tabular}




\begin{tabular}{|c|c|c|c|}
\hline C & 5.588867 & -1.192465 & 0.778684 \\
\hline $\mathrm{H}$ & 4.007454 & -1.891745 & 2.068661 \\
\hline C & 5.917058 & -0.934769 & -0.560271 \\
\hline $\mathrm{H}$ & 6.901276 & -0.535755 & -0.786414 \\
\hline C & 6.623814 & -0.909857 & 1.885417 \\
\hline C & 5.465392 & -0.873650 & -3.067069 \\
\hline C & 6.977308 & 0.596413 & 1.887478 \\
\hline $\mathrm{H}$ & 7.399768 & 0.916635 & 0.929296 \\
\hline $\mathrm{H}$ & 6.088782 & 1.207917 & 2.081479 \\
\hline $\mathrm{H}$ & 7.717134 & 0.814049 & 2.667717 \\
\hline C & 6.105570 & -1.282708 & 3.287635 \\
\hline $\mathrm{H}$ & 6.881605 & -1.075382 & 4.033107 \\
\hline $\mathrm{H}$ & 5.219412 & -0.701805 & 3.566594 \\
\hline $\mathrm{H}$ & 5.854485 & -2.347236 & 3.360579 \\
\hline C & 7.904417 & -1.736109 & 1.616951 \\
\hline $\mathrm{H}$ & 7.686064 & -2.810217 & 1.620172 \\
\hline $\mathrm{H}$ & 8.354781 & -1.490252 & 0.649876 \\
\hline $\mathrm{H}$ & 8.654132 & -1.538899 & 2.392932 \\
\hline C & 5.834110 & 0.623881 & -3.192672 \\
\hline $\mathrm{H}$ & 4.980082 & 1.263333 & -2.942674 \\
\hline $\mathrm{H}$ & 6.660117 & 0.900616 & -2.528838 \\
\hline $\mathrm{H}$ & 6.142498 & 0.853556 & -4.220069 \\
\hline C & 6.701192 & -1.733268 & -3.424351 \\
\hline $\mathrm{H}$ & 7.548705 & -1.526031 & -2.762185 \\
\hline $\mathrm{H}$ & 6.470468 & -2.801976 & -3.347965 \\
\hline $\mathrm{H}$ & 7.023697 & -1.528257 & -4.452595 \\
\hline C & 4.352608 & -1.182785 & -4.087574 \\
\hline $\mathrm{H}$ & 4.697214 & -0.925305 & -5.095738 \\
\hline $\mathrm{H}$ & 4.089680 & -2.246691 & -4.092901 \\
\hline $\mathrm{H}$ & 3.438243 & -0.612945 & -3.889610 \\
\hline
\end{tabular}

\section{C-Catalyzed reaction TS 140}

\section{Datum}

Value

\begin{tabular}{lc}
\hline B3LYP/6-31G(d) Energy & -3038.947418 \\
\hline B3LYP-D3(BJ)/def2-TZVPP-IEF-PCM(DCM) Energy & -3040.496294 \\
\hline B3LYP-D3(BJ)/def2-TZVPP-IEF-PCM(DCM)//B3LYP/6-31G(d) Free Energy (Quasiharmonic) & -3039.268148 \\
\hline
\end{tabular}

Number of Imaginary Frequencies

Frequencies (Top 3 out of 450)

$\begin{array}{lr}\text { 1. } & -370.4367 \mathrm{~cm}^{-1} \\ \text { 2. } & 6.7064 \mathrm{~cm}^{-1} \\ \text { 3. } & 10.4383 \mathrm{~cm}^{-1}\end{array}$




\begin{tabular}{|c|c|c|c|}
\hline C & -2.231566 & -0.747560 & 0.909189 \\
\hline $\mathrm{H}$ & -1.161031 & -0.539899 & 0.998162 \\
\hline $\mathrm{H}$ & -2.749200 & 0.212081 & 0.904229 \\
\hline C & 0.861532 & -0.946784 & -1.227573 \\
\hline 0 & 0.796762 & -1.061990 & -2.431401 \\
\hline 0 & 0.923375 & 0.277129 & -0.669527 \\
\hline $\mathrm{H}$ & 0.970685 & 0.323988 & 0.321670 \\
\hline 0 & -2.700965 & -1.418088 & 2.088565 \\
\hline $\mathrm{H}$ & -2.236555 & -2.269461 & 2.147534 \\
\hline C & 0.084283 & 3.555679 & -1.526035 \\
\hline C & 2.027649 & 3.201932 & -0.695965 \\
\hline C & -0.652213 & 3.335071 & 0.819539 \\
\hline C & -0.403906 & 4.104109 & -0.354454 \\
\hline $\mathrm{H}$ & 1.972194 & 2.196519 & -1.113912 \\
\hline $\mathrm{H}$ & -1.087787 & 2.353276 & 0.622734 \\
\hline $\mathrm{H}$ & -1.165296 & 3.864364 & 1.622069 \\
\hline C & 0.957385 & 1.179382 & 3.231290 \\
\hline C & 1.608215 & 2.515795 & 3.747473 \\
\hline C & -0.481427 & 0.996988 & 3.737409 \\
\hline $\mathrm{H}$ & -0.962548 & 0.151589 & 3.237510 \\
\hline $\mathrm{H}$ & -0.497028 & 0.794808 & 4.814154 \\
\hline $\mathrm{H}$ & -1.082748 & 1.891960 & 3.551625 \\
\hline C & 1.769726 & -0.081736 & 3.516079 \\
\hline $\mathrm{H}$ & 1.897993 & -0.224924 & 4.595570 \\
\hline $\mathrm{H}$ & 1.251615 & -0.963295 & 3.123388 \\
\hline $\mathrm{H}$ & 2.755559 & -0.032549 & 3.049298 \\
\hline C & 3.143752 & 2.516447 & 3.631546 \\
\hline $\mathrm{H}$ & 3.610705 & 1.861649 & 4.376305 \\
\hline $\mathrm{H}$ & 3.467986 & 2.202029 & 2.635084 \\
\hline $\mathrm{H}$ & 3.505460 & 3.536349 & 3.796818 \\
\hline C & 1.201526 & 2.929453 & 5.162563 \\
\hline $\mathrm{H}$ & 1.505585 & 2.177822 & 5.900789 \\
\hline $\mathrm{H}$ & 1.692147 & 3.873944 & 5.420775 \\
\hline $\mathrm{H}$ & 0.122454 & 3.080425 & 5.239683 \\
\hline 0 & 0.929473 & 1.401551 & 1.793674 \\
\hline 0 & 1.081864 & 3.480919 & 2.825302 \\
\hline B & 0.915697 & 2.845240 & 1.567809 \\
\hline 0 & 1.956662 & 3.316893 & 0.600241 \\
\hline C & 2.883426 & 4.175686 & -1.407578 \\
\hline C & 3.275496 & 3.913392 & -2.729737 \\
\hline C & 3.331566 & 5.345220 & -0.775216 \\
\hline C & 4.096274 & 4.809328 & -3.411490 \\
\hline $\mathrm{H}$ & 2.933225 & 3.005201 & -3.220595 \\
\hline C & 4.151166 & 6.239017 & -1.460359 \\
\hline $\mathrm{H}$ & 3.039879 & 5.530797 & 0.253453 \\
\hline C & 4.534087 & 5.975796 & -2.778891 \\
\hline $\mathrm{H}$ & 4.396515 & 4.597035 & -4.433889 \\
\hline $\mathrm{H}$ & 4.496810 & 7.142170 & -0.964542 \\
\hline $\mathrm{H}$ & 5.174852 & 6.674764 & -3.309673 \\
\hline $\mathrm{H}$ & -0.342392 & 5.188580 & -0.245871 \\
\hline
\end{tabular}




\begin{tabular}{|c|c|c|c|}
\hline $\mathrm{H}$ & -0.108115 & 2.508612 & -1.745760 \\
\hline $\mathrm{H}$ & 0.290985 & 4.193041 & -2.379703 \\
\hline C & -0.351632 & -2.773605 & -0.055072 \\
\hline C & 0.854518 & -2.117477 & -0.271438 \\
\hline C & 2.065550 & -2.524464 & 0.385143 \\
\hline C & 1.988549 & -3.543797 & 1.316111 \\
\hline $\mathrm{H}$ & 2.896805 & -3.886088 & 1.804798 \\
\hline C & -2.512002 & -1.501417 & -0.377523 \\
\hline C & -1.588409 & -2.432574 & -0.846004 \\
\hline C & -3.918930 & -1.913268 & -2.314746 \\
\hline C & -3.713806 & -1.241801 & -1.124786 \\
\hline $\mathrm{H}$ & -4.831566 & -1.730502 & -2.876422 \\
\hline C & -2.998357 & -2.861515 & -2.818862 \\
\hline C & -1.809238 & -3.138538 & -2.075104 \\
\hline C & -0.899899 & -4.101639 & -2.592761 \\
\hline C & -1.158095 & -4.755709 & -3.776429 \\
\hline C & -2.337938 & -4.481138 & -4.508558 \\
\hline C & -3.236372 & -3.552468 & -4.037916 \\
\hline $\mathrm{H}$ & 0.013305 & -4.314339 & -2.048386 \\
\hline $\mathrm{H}$ & -0.448248 & -5.486231 & -4.154944 \\
\hline $\mathrm{H}$ & -2.528908 & -5.003863 & -5.441957 \\
\hline $\mathrm{H}$ & -4.145673 & -3.331204 & -4.591877 \\
\hline C & 0.771170 & -4.197076 & 1.623492 \\
\hline C & -0.417613 & -3.829938 & 0.914202 \\
\hline C & -1.622677 & -4.532741 & 1.204756 \\
\hline C & -1.650075 & -5.527805 & 2.158349 \\
\hline C & -0.474673 & -5.881471 & 2.862748 \\
\hline C & 0.708690 & -5.232676 & 2.595207 \\
\hline $\mathrm{H}$ & -2.525002 & -4.279887 & 0.658055 \\
\hline $\mathrm{H}$ & -2.579887 & -6.050032 & 2.365809 \\
\hline $\mathrm{H}$ & -0.509695 & -6.668535 & 3.610829 \\
\hline $\mathrm{H}$ & 1.618641 & -5.502272 & 3.125883 \\
\hline C & -6.771779 & 1.488271 & 0.099744 \\
\hline C & -6.312710 & 0.546305 & 1.024640 \\
\hline C & -5.300006 & -0.335395 & 0.614396 \\
\hline C & -4.757005 & -0.274241 & -0.672234 \\
\hline C & -5.246188 & 0.689741 & -1.572548 \\
\hline C & -6.255610 & 1.581244 & -1.206031 \\
\hline $\mathrm{H}$ & -7.552125 & 2.180105 & 0.395379 \\
\hline $\mathrm{H}$ & -4.915725 & -1.080695 & 1.302165 \\
\hline $\mathrm{H}$ & -4.803795 & 0.735667 & -2.561054 \\
\hline C & -6.865763 & 0.442953 & 2.458963 \\
\hline C & -6.806047 & 2.654165 & -2.165785 \\
\hline C & -5.714044 & 0.673905 & 3.467579 \\
\hline $\mathrm{H}$ & -6.084312 & 0.575609 & 4.495950 \\
\hline $\mathrm{H}$ & -4.898437 & -0.043197 & 3.331630 \\
\hline $\mathrm{H}$ & -5.295691 & 1.681107 & 3.354734 \\
\hline C & -7.969231 & 1.479475 & 2.742005 \\
\hline $\mathrm{H}$ & -8.326427 & 1.365738 & 3.771877 \\
\hline $\mathrm{H}$ & -7.602522 & 2.506580 & 2.632373 \\
\hline $\mathrm{H}$ & -8.831542 & 1.350970 & 2.077358 \\
\hline C & -7.463579 & -0.966868 & 2.680216 \\
\hline $\mathrm{H}$ & -7.856320 & -1.058031 & 3.700518 \\
\hline $\mathrm{H}$ & -8.285896 & -1.158782 & 1.981074 \\
\hline
\end{tabular}




\begin{tabular}{|c|c|c|c|}
\hline H & -6.714303 & -1.752426 & 2.540329 \\
\hline C & -6.139857 & 2.599424 & -3.554047 \\
\hline $\mathrm{H}$ & -6.569407 & 3.373773 & -4.199920 \\
\hline $\mathrm{H}$ & -5.060425 & 2.779123 & -3.496710 \\
\hline $\mathrm{H}$ & -6.299233 & 1.633120 & -4.046110 \\
\hline C & -8.327424 & 2.446179 & -2.356306 \\
\hline $\mathrm{H}$ & -8.735394 & 3.207797 & -3.032360 \\
\hline $\mathrm{H}$ & -8.537281 & 1.460820 & -2.788105 \\
\hline $\mathrm{H}$ & -8.871157 & 2.517075 & -1.408556 \\
\hline C & -6.553277 & 4.059240 & -1.569058 \\
\hline $\mathrm{H}$ & -6.945878 & 4.835362 & -2.237794 \\
\hline $\mathrm{H}$ & -7.038254 & 4.182066 & -0.595018 \\
\hline $\mathrm{H}$ & -5.480702 & 4.238658 & -1.432739 \\
\hline C & 3.394541 & -1.938295 & 0.046080 \\
\hline C & 3.767062 & -1.740581 & -1.286994 \\
\hline C & 4.325328 & -1.649885 & 1.061734 \\
\hline C & 5.033416 & -1.240421 & -1.628805 \\
\hline H & 3.064714 & -1.997264 & -2.071035 \\
\hline C & 5.597600 & -1.159983 & 0.764028 \\
\hline $\mathrm{H}$ & 4.026967 & -1.805311 & 2.091535 \\
\hline C & 5.923157 & -0.956814 & -0.590162 \\
\hline $\mathrm{H}$ & 6.905605 & -0.567911 & -0.830658 \\
\hline C & 6.639884 & -0.830938 & 1.850604 \\
\hline C & 5.385823 & -1.034641 & -3.114471 \\
\hline C & 7.921454 & -1.663168 & 1.606060 \\
\hline H & 7.706844 & -2.736987 & 1.654320 \\
\hline $\mathrm{H}$ & 8.364690 & -1.455333 & 0.626852 \\
\hline $\mathrm{H}$ & 8.675678 & -1.432430 & 2.368306 \\
\hline C & 6.987827 & 0.675469 & 1.790340 \\
\hline $\mathrm{H}$ & 7.736557 & 0.925292 & 2.552239 \\
\hline $\mathrm{H}$ & 7.396537 & 0.960229 & 0.815213 \\
\hline $\mathrm{H}$ & 6.099294 & 1.290676 & 1.972432 \\
\hline C & 6.133413 & -1.149360 & 3.270278 \\
\hline $\mathrm{H}$ & 5.248067 & -0.559639 & 3.532812 \\
\hline $\mathrm{H}$ & 5.885459 & -2.210758 & 3.386614 \\
\hline $\mathrm{H}$ & 6.914535 & -0.911294 & 4.001145 \\
\hline C & 6.814835 & -0.495130 & -3.312896 \\
\hline $\mathrm{H}$ & 6.955146 & 0.479504 & -2.831400 \\
\hline $\mathrm{H}$ & 7.571298 & -1.184301 & -2.919731 \\
\hline $\mathrm{H}$ & 7.012640 & -0.364615 & -4.382780 \\
\hline C & 5.274857 & -2.384979 & -3.862021 \\
\hline $\mathrm{H}$ & 5.965928 & -3.124354 & -3.440735 \\
\hline $\mathrm{H}$ & 4.263699 & -2.800492 & -3.807150 \\
\hline $\mathrm{H}$ & 5.522449 & -2.253809 & -4.922579 \\
\hline C & 4.395530 & -0.021236 & -3.738023 \\
\hline $\mathrm{H}$ & 4.609647 & 0.109516 & -4.806209 \\
\hline $\mathrm{H}$ & 3.354927 & -0.347004 & -3.640233 \\
\hline $\mathrm{H}$ & 4.488950 & 0.957222 & -3.251870 \\
\hline
\end{tabular}


Frequencies (Top 3 out of 450)

$\begin{array}{lr}\text { 1. } & -371.9321 \mathrm{~cm}^{-1} \\ \text { 2. } & 6.1655 \mathrm{~cm}^{-1} \\ \text { 3. } & 9.7348 \mathrm{~cm}^{-1}\end{array}$

\section{B3LYP/6-31G(d) Molecular Geometry in Cartesian Coordinates}

C

$\mathrm{H}$

$\mathrm{H}$

C

0

0

$\mathrm{H}$

0

$\mathrm{H}$

C

C

C

C

$\mathrm{H}$

$\mathrm{H}$

$\mathrm{H}$

C

C

C

$\mathrm{H}$

$\mathrm{H}$

$\mathrm{H}$

C

$\mathrm{H}$

$\mathrm{H}$

$\mathrm{H}$

C

$\mathrm{H}$

$\mathrm{H}$

$\mathrm{H}$

C

$\mathrm{H}$

\section{$-2.224826$}

$-1.153527$

$-2.741710$

0.857035

0.775492

0.935545

0.986984

$-2.687605$

$-2.227850$

0.045702

1.997853

$-0.674836$

$-0.444576$

1.950638

$-1.097109$

$-1.189895$

0.978827

1.609131

$-0.456320$

$-0.924541$

$-0.468025$

$-1.071749$

1.812000

1.946417

1. 305981

2. 795193

3.144266

3. 622883

3.471949

3.490037

1.198194

1.514665
$-0.779230$

$-0.574314$

0.180623

$-0.927236$

$-1.011540$

0.283047

0.310368

$-1.476651$

$-2.331915$

3. 545054

3. 212703

3. 299481

4.078741

2. 206714

2. 312515

3. 816915

1.138825

2.478334

0.925857

0.081496

0.705630

1.814104

$-0.112820$

$-0.267434$

$-0.997416$

$-0.042868$

2.504823

1.847823

2.209076

3. 528078

2.867111

2.110490
0.891045

0.978732

0.911344

$-1.250788$

$-2.455809$

$-0.664986$

0.326412

2.057342

2.090607

$-1.483125$

$-0.663865$

0.865229

$-0.305480$

$-1.080922$

0.665635

1.674149

3. 240870

3.773635

3. 745766

3. 231882

4.819030

3.574667

3. 507264

4.584428

3. 105482

3.037498

3. 655583

4. 390924

2.654527

3.833714

5.194590

5.922420 


\begin{tabular}{|c|c|c|c|}
\hline $\mathrm{H}$ & 1.674769 & 3.815450 & 5.464607 \\
\hline $\mathrm{H}$ & 0.117039 & 3.000626 & 5.274999 \\
\hline 0 & 0.944754 & 1.379311 & 1.806682 \\
\hline 0 & 1.066469 & 3.447325 & 2.864935 \\
\hline B & 0.905985 & 2.825452 & 1.599985 \\
\hline 0 & 1.933062 & 3.327017 & 0.632936 \\
\hline C & 2.837383 & 4.195127 & -1.382194 \\
\hline C & 3.224230 & 3.934072 & -2.706223 \\
\hline C & 3.274549 & 5.372133 & -0.756169 \\
\hline C & 4.028641 & 4.838532 & -3.396159 \\
\hline $\mathrm{H}$ & 2.889098 & 3.020632 & -3.192707 \\
\hline C & 4.077807 & 6.274477 & -1.449399 \\
\hline $\mathrm{H}$ & 2.986966 & 5.557021 & 0.273783 \\
\hline C & 4.455249 & 6.012458 & -2.769747 \\
\hline $\mathrm{H}$ & 4.324746 & 4.627292 & -4.419942 \\
\hline $\mathrm{H}$ & 4.414971 & 7.183518 & -0.958537 \\
\hline $\mathrm{H}$ & 5.083173 & 6.718209 & -3.306830 \\
\hline $\mathrm{H}$ & -0.398674 & 5.163352 & -0.190631 \\
\hline $\mathrm{H}$ & -0.132383 & 2.496828 & -1.709107 \\
\hline $\mathrm{H}$ & 0.238341 & 4.191225 & -2.333479 \\
\hline C & -0.353669 & -2.784727 & -0.128605 \\
\hline C & 0.852199 & -2.121305 & -0.324183 \\
\hline C & 2.062028 & -2.544417 & 0.325124 \\
\hline C & 1.983587 & -3.592927 & 1.223461 \\
\hline $\mathrm{H}$ & 2.890979 & -3.949719 & 1.703166 \\
\hline C & -2.512970 & -1.502468 & -0.411659 \\
\hline C & -1.591772 & -2.421723 & -0.907405 \\
\hline C & -3.930913 & -1.869707 & -2.349824 \\
\hline C & -3.719289 & -1.225991 & -1.145698 \\
\hline $\mathrm{H}$ & -4.847200 & -1.675023 & -2.901439 \\
\hline C & -3.012354 & -2.804552 & -2.881927 \\
\hline C & -1.818585 & -3.097668 & -2.151989 \\
\hline C & -0.910551 & -4.046112 & -2.698133 \\
\hline C & -1.174746 & -4.672033 & -3.895578 \\
\hline C & -2.359622 & -4.382322 & -4.613655 \\
\hline C & -3.256671 & -3.466615 & -4.115704 \\
\hline $\mathrm{H}$ & 0.006761 & -4.269336 & -2.164986 \\
\hline $\mathrm{H}$ & -0.465735 & -5.391563 & -4.296058 \\
\hline $\mathrm{H}$ & -2.555400 & -4.882899 & -5.558141 \\
\hline H & -4.169558 & -3.233583 & -4.658847 \\
\hline C & 0.766880 & -4.257138 & 1.508153 \\
\hline C & -0.421050 & -3.869104 & 0.809074 \\
\hline C & -1.626300 & -4.580416 & 1.077303 \\
\hline C & -1.654224 & -5.604188 & 1.999949 \\
\hline C & -0.479254 & -5.979207 & 2.693994 \\
\hline C & 0.704124 & -5.322213 & 2.447462 \\
\hline $\mathrm{H}$ & -2.528193 & -4.311391 & 0.537744 \\
\hline H & -2.584106 & -6.132651 & 2.190625 \\
\hline H & -0.514658 & -6.788941 & 3.417463 \\
\hline H & 1.613704 & -5.607628 & 2.970462 \\
\hline C & -6.768791 & 1.475366 & 0.160875 \\
\hline C & -6.264077 & 1.595377 & -1.147181 \\
\hline C & -5.257217 & 0.712361 & -1.540471 \\
\hline C & -4.760213 & -0.269946 & -0.66455 \\
\hline
\end{tabular}




\begin{tabular}{|c|c|c|c|}
\hline C & -5.292973 & -0.358499 & 0.624790 \\
\hline C & -6.301941 & 0.514431 & 1.062061 \\
\hline $\mathrm{H}$ & -7.546661 & 2.160698 & 0.477425 \\
\hline $\mathrm{H}$ & -4.822690 & 0.779231 & -2.531305 \\
\hline $\mathrm{H}$ & -4.903220 & -1.118372 & 1.293274 \\
\hline C & -6.822950 & 2.687599 & -2.079836 \\
\hline C & -6.843012 & 0.380890 & 2.498462 \\
\hline C & -6.558833 & 4.080469 & -1.459923 \\
\hline $\mathrm{H}$ & -6.957550 & 4.870147 & -2.108908 \\
\hline $\mathrm{H}$ & -7.031417 & 4.185771 & -0.477767 \\
\hline $\mathrm{H}$ & -5.484142 & 4.254474 & -1.333589 \\
\hline C & -6.173733 & 2.656638 & -3.476771 \\
\hline $\mathrm{H}$ & -6.608866 & 3.443692 & -4.103248 \\
\hline $\mathrm{H}$ & -5.093202 & 2.832467 & -3.429247 \\
\hline H & -6.341754 & 1.699827 & -3.984263 \\
\hline C & -8.347097 & 2.487539 & -2.256164 \\
\hline $\mathrm{H}$ & -8.760334 & 3.262140 & -2.914040 \\
\hline $\mathrm{H}$ & -8.565085 & 1.510545 & -2.702691 \\
\hline $\mathrm{H}$ & -8.879917 & 2.543584 & -1.301239 \\
\hline C & -7.941471 & 1.413566 & 2.813368 \\
\hline $\mathrm{H}$ & -8.291377 & 1.277297 & 3.843031 \\
\hline H & -7.572672 & 2.441923 & 2.724505 \\
\hline $\mathrm{H}$ & -8.809119 & 1.302509 & 2.152555 \\
\hline C & -7.442350 & -1.032133 & 2.693684 \\
\hline $\mathrm{H}$ & -7.825320 & -1.145507 & 3.715492 \\
\hline $\mathrm{H}$ & -8.271998 & -1.206153 & 1.998515 \\
\hline $\mathrm{H}$ & -6.696520 & -1.816062 & 2.528462 \\
\hline C & -5.682545 & 0.587080 & 3.502397 \\
\hline $\mathrm{H}$ & -6.045363 & 0.468899 & 4.531331 \\
\hline $\mathrm{H}$ & -4.870313 & -0.129684 & 3.345751 \\
\hline $\mathrm{H}$ & -5.261647 & 1.595021 & 3.407024 \\
\hline C & 3.391807 & -1.943557 & 0.015923 \\
\hline C & 4.313727 & -1.698411 & 1.046216 \\
\hline C & 3.770504 & -1.688947 & -1.310744 \\
\hline C & 5.592136 & -1.197606 & 0.778072 \\
\hline $\mathrm{H}$ & 4.008682 & -1.893946 & 2.067076 \\
\hline C & 5.035162 & -1.175633 & -1.619915 \\
\hline H & 3.071493 & -1.914012 & -2.104637 \\
\hline C & 5.921389 & -0.939908 & -0.560627 \\
\hline $\mathrm{H}$ & 6.906417 & -0.542564 & -0.786180 \\
\hline C & 5.470928 & -0.876780 & -3.067579 \\
\hline C & 6.627130 & -0.917527 & 1.885403 \\
\hline C & 4.358417 & -1.184371 & -4.088849 \\
\hline $\mathrm{H}$ & 4.094155 & -2.247947 & -4.094490 \\
\hline $\mathrm{H}$ & 3.444633 & -0.613405 & -3.891459 \\
\hline $\mathrm{H}$ & 4.704025 & -0.927185 & -5.096744 \\
\hline C & 5.841324 & 0.620407 & -3.192406 \\
\hline $\mathrm{H}$ & 6.150758 & 0.850108 & -4.219481 \\
\hline $\mathrm{H}$ & 4.987763 & 1.260670 & -2.942878 \\
\hline $\mathrm{H}$ & 6.667094 & 0.896023 & -2.527810 \\
\hline C & 6.705983 & -1.737626 & -3.424462 \\
\hline $\mathrm{H}$ & 7.553384 & -1.531561 & -2.761791 \\
\hline $\mathrm{H}$ & 6.474049 & -2.806109 & -3.348584 \\
\hline H & 7.029267 & -1.532611 & -4.452459 \\
\hline
\end{tabular}




\begin{tabular}{|llll|}
$\mathrm{C}$ & 7.906784 & -1.745097 & 1.616481 \\
$\mathrm{H}$ & 7.687059 & -2.818928 & 1.618355 \\
$\mathrm{H}$ & 8.357919 & -1.498697 & 0.649908 \\
$\mathrm{H}$ & 8.656410 & -1.549756 & 2.393019 \\
$\mathrm{C}$ & 6.982478 & 0.588304 & 1.889134 \\
$\mathrm{H}$ & 7.405488 & 0.908992 & 0.931348 \\
$\mathrm{H}$ & 6.094654 & 1.200687 & 2.083595 \\
$\mathrm{H}$ & 7.722416 & 0.804238 & 2.669740 \\
$\mathrm{C}$ & 6.108040 & -1.291282 & 3.287079 \\
$\mathrm{H}$ & 6.884244 & -1.086024 & 4.032947 \\
$\mathrm{H}$ & 5.222700 & -0.709423 & 3.566628 \\
$\mathrm{H}$ & 5.855342 & -2.355523 & 3.358661 \\
& & & \\
\hline
\end{tabular}

\section{C-Catalyzed reaction TS 142}

\section{Datum}

Value

\begin{tabular}{lr}
\hline B3LYP/6-31G(d) Energy & -3038.947339 \\
\hline B3LYP-D3(BJ)/def2-TZVPP-IEF-PCM(DCM) Energy & -3040.495702 \\
\hline B3LYP-D3(BJ)/def2-TZVPP-IEF-PCM(DCM)//B3LYP/6-31G(d) Free Energy (Quasiharmonic) & -3039.267805 \\
\hline
\end{tabular}

Number of Imaginary Frequencies

Frequencies (Top 3 out of 450)

$\begin{array}{lr}\text { 1. } & -372.8214 \mathrm{~cm}^{-1} \\ \text { 2. } & 8.0291 \mathrm{~cm}^{-1} \\ \text { 3. } & 11.9121 \mathrm{~cm}^{-1}\end{array}$

\section{B3LYP/6-31G(d) Molecular Geometry in Cartesian Coordinates}

C

$\mathrm{H}$

$\mathrm{H}$

C

0

0

$\mathrm{H}$

0

$\mathrm{H}$

C

C

C

C

$\mathrm{H}$

\section{233258}

3.855970

2.265439

$-0.289801$

0.485581

$-1.286338$

$-1.895189$

3. 917675

3. 352879

$-1.682756$

$-3.336597$

$-2.533422$

$-2.300983$

$-2.627821$
$-1.436829$

$-0.825869$

$-1.564837$

$-0.052937$

0.522314

0.630369

0.138941

$-2.694409$

$-3.290536$

3.894664

3.012294

2.598348

3.824312

2. 337649

\subsection{6}

1. 391563

1. 230820

0.281899

1.016809

$-0.302725$

$-0.912896$

0.629495

0.111379

$-1.307806$

$-0.290214$

$-3.231288$

$-2.543853$

0.190357 


\begin{tabular}{|c|c|c|c|}
\hline $\mathrm{H}$ & -1.706122 & 1.887434 & -3.173763 \\
\hline $\mathrm{H}$ & -2.921968 & 2.703373 & -4.244181 \\
\hline C & -4.110025 & -0.630369 & -2.799417 \\
\hline C & -5.438679 & 0.169137 & -3.064919 \\
\hline C & -3.353366 & -0.958389 & -4.095562 \\
\hline $\mathrm{H}$ & -2.355293 & -1.331019 & -3.845696 \\
\hline $\mathrm{H}$ & -3.865518 & -1.733040 & -4.676548 \\
\hline $\mathrm{H}$ & -3.242668 & -0.070294 & -4.724578 \\
\hline C & -4.279263 & -1.898548 & -1.965635 \\
\hline $\mathrm{H}$ & -4.952404 & -2.605875 & -2.464790 \\
\hline $\mathrm{H}$ & -4.679309 & -1.669709 & -0.976088 \\
\hline $\mathrm{H}$ & -3.311988 & -2.396223 & -1.832572 \\
\hline C & -6.411189 & 0.119874 & -1.872412 \\
\hline $\mathrm{H}$ & -6.880973 & -0.864787 & -1.767614 \\
\hline $\mathrm{H}$ & -5.905788 & 0.368929 & -0.934752 \\
\hline $\mathrm{H}$ & -7.200144 & 0.861034 & -2.035108 \\
\hline C & -6.180276 & -0.215185 & -4.346677 \\
\hline $\mathrm{H}$ & -6.503824 & -1.262651 & -4.322816 \\
\hline $\mathrm{H}$ & -7.072539 & 0.410989 & -4.451164 \\
\hline $\mathrm{H}$ & -5.558371 & -0.059802 & -5.231476 \\
\hline 0 & -3.330479 & 0.326885 & -2.039416 \\
\hline 0 & -4.956740 & 1.512148 & -3.206928 \\
\hline B & -3.820194 & 1.664887 & -2.369346 \\
\hline 0 & -4.148157 & 2.495999 & -1.173391 \\
\hline C & -3.864052 & 4.121753 & 0.532755 \\
\hline C & -3.196151 & 4.480073 & 1.714279 \\
\hline C & -5.033956 & 4.805167 & 0.168192 \\
\hline C & -3.686751 & 5.508573 & 2.515923 \\
\hline $\mathrm{H}$ & -2.291543 & 3.948967 & 2.001229 \\
\hline C & -5.520572 & 5.833702 & 0.971545 \\
\hline $\mathrm{H}$ & -5.554264 & 4.509977 & -0.737194 \\
\hline C & -4.849255 & 6.189251 & 2.145219 \\
\hline $\mathrm{H}$ & -3.164913 & 5.777076 & 3.430295 \\
\hline $\mathrm{H}$ & -6.428678 & 6.357594 & 0.685376 \\
\hline $\mathrm{H}$ & -5.233241 & 6.990804 & 2.770465 \\
\hline $\mathrm{H}$ & -2.866352 & 4.700069 & -2.868391 \\
\hline $\mathrm{H}$ & -0.974897 & 3.121571 & -1.020358 \\
\hline $\mathrm{H}$ & -1.566591 & 4.854818 & -0.815305 \\
\hline C & 0.859477 & -1.952676 & -0.840777 \\
\hline C & -0.187088 & -1.528418 & -0.028212 \\
\hline C & -1.109059 & -2.459640 & 0.562314 \\
\hline C & -0.990038 & -3.794913 & 0.221816 \\
\hline $\mathrm{H}$ & -1.656024 & -4.520087 & 0.681467 \\
\hline C & 3.046349 & -0.744522 & -0.602116 \\
\hline C & 1.896554 & -0.985504 & -1.352095 \\
\hline C & 3.836456 & 0.799955 & -2.305631 \\
\hline C & 4.045376 & 0.163715 & -1.096081 \\
\hline H & 4.593918 & 1.481706 & -2.684131 \\
\hline C & 2.684168 & 0.570776 & -3.091548 \\
\hline C & 1.692505 & -0.344501 & -2.616249 \\
\hline C & 0.550281 & -0.580483 & -3.429995 \\
\hline C & 0.396385 & 0.055678 & -4.642179 \\
\hline C & 1.377776 & 0.964823 & -5.106261 \\
\hline C & 2.495949 & 1.213776 & -4.345402 \\
\hline
\end{tabular}




\begin{tabular}{|c|c|c|c|}
\hline $\mathrm{H}$ & -0.204361 & -1.280158 & -3.086308 \\
\hline $\mathrm{H}$ & -0.482642 & -0.143671 & -5.248409 \\
\hline $\mathrm{H}$ & 1.244583 & 1.460957 & -6.063763 \\
\hline $\mathrm{H}$ & 3.258235 & 1.907368 & -4.692601 \\
\hline C & 0.013742 & -4.266304 & -0.656852 \\
\hline C & 0.969548 & -3.340447 & -1.185974 \\
\hline C & 1.997214 & -3.840461 & -2.036917 \\
\hline C & 2.071950 & -5.182204 & -2.343252 \\
\hline C & 1.125519 & -6.094020 & -1.817276 \\
\hline C & 0.120674 & -5.643419 & -0.992791 \\
\hline $\mathrm{H}$ & 2.719267 & -3.146458 & -2.454005 \\
\hline $\mathrm{H}$ & 2.861685 & -5.542977 & -2.996314 \\
\hline $\mathrm{H}$ & 1.196711 & -7.149193 & -2.066048 \\
\hline $\mathrm{H}$ & -0.608416 & -6.336862 & -0.580697 \\
\hline C & 7.669711 & 1.086727 & 0.982671 \\
\hline C & 7.340534 & -0.257041 & 0.784711 \\
\hline C & 6.145549 & -0.546958 & 0.107265 \\
\hline C & 5.300885 & 0.469149 & -0.347429 \\
\hline C & 5.668629 & 1.809214 & -0.129092 \\
\hline C & 6.851076 & 2.140132 & 0.534718 \\
\hline $\mathrm{H}$ & 8.586119 & 1.332623 & 1.506933 \\
\hline $\mathrm{H}$ & 5.851965 & -1.576906 & -0.061472 \\
\hline $\mathrm{H}$ & 4.994488 & 2.585760 & -0.472056 \\
\hline C & 8.220208 & -1.419006 & 1.285116 \\
\hline C & 7.273452 & 3.597978 & 0.802703 \\
\hline C & 7.406969 & -2.279343 & 2.282903 \\
\hline $\mathrm{H}$ & 8.009003 & -3.128652 & 2.630251 \\
\hline $\mathrm{H}$ & 7.120433 & -1.687844 & 3.160575 \\
\hline $\mathrm{H}$ & 6.490139 & -2.674438 & 1.834063 \\
\hline C & 8.647829 & -2.295847 & 0.084109 \\
\hline $\mathrm{H}$ & 9.271104 & -3.131670 & 0.425391 \\
\hline $\mathrm{H}$ & 7.783381 & -2.716887 & -0.438953 \\
\hline $\mathrm{H}$ & 9.227932 & -1.712855 & -0.640806 \\
\hline C & 9.495143 & -0.930195 & 1.997544 \\
\hline $\mathrm{H}$ & 10.083442 & -1.791976 & 2.332907 \\
\hline $\mathrm{H}$ & 10.132291 & -0.333613 & 1.334107 \\
\hline $\mathrm{H}$ & 9.262906 & -0.326560 & 2.882483 \\
\hline C & 7.374225 & 3.832976 & 2.328726 \\
\hline $\mathrm{H}$ & 7.683262 & 4.864896 & 2.537513 \\
\hline $\mathrm{H}$ & 6.406938 & 3.662154 & 2.814898 \\
\hline $\mathrm{H}$ & 8.104940 & 3.164916 & 2.796382 \\
\hline C & 8.651217 & 3.865937 & 0.151694 \\
\hline $\mathrm{H}$ & 8.970763 & 4.897637 & 0.344477 \\
\hline $\mathrm{H}$ & 9.425771 & 3.199082 & 0.544430 \\
\hline $\mathrm{H}$ & 8.605627 & 3.721437 & -0.933911 \\
\hline C & 6.269764 & 4.616315 & 0.228815 \\
\hline $\mathrm{H}$ & 6.618045 & 5.634599 & 0.436458 \\
\hline $\mathrm{H}$ & 6.165396 & 4.517766 & -0.857791 \\
\hline $\mathrm{H}$ & 5.276293 & 4.508977 & 0.678459 \\
\hline C & -2.116402 & -2.058688 & 1.588098 \\
\hline C & -1.764472 & -1.205065 & 2.637975 \\
\hline C & -3.410107 & -2.613585 & 1.569641 \\
\hline C & -2.675981 & -0.872830 & 3.652565 \\
\hline $\mathrm{H}$ & -0.756561 & -0.810200 & 2.678822 \\
\hline
\end{tabular}




\begin{tabular}{|c|c|c|c|}
\hline C & -4.341856 & -2.318814 & 2.565725 \\
\hline $\mathrm{H}$ & -3.675538 & -3.273888 & 0.752969 \\
\hline C & -3.951286 & -1.438508 & 3.592177 \\
\hline $\mathrm{H}$ & -4.671293 & -1.198095 & 4.365358 \\
\hline C & -5.763836 & -2.913369 & 2.582335 \\
\hline C & -2.231647 & 0.077292 & 4.780964 \\
\hline C & -6.802893 & -1.770662 & 2.489423 \\
\hline $\mathrm{H}$ & -6.711998 & -1.066757 & 3.323403 \\
\hline $\mathrm{H}$ & -6.680082 & -1.203129 & 1.559839 \\
\hline $\mathrm{H}$ & -7.821190 & -2.178364 & 2.506949 \\
\hline C & -6.012957 & -3.883806 & 1.412293 \\
\hline $\mathrm{H}$ & -7.029224 & -4.288157 & 1.480036 \\
\hline $\mathrm{H}$ & -5.921086 & -3.387546 & 0.439850 \\
\hline $\mathrm{H}$ & -5.319030 & -4.732153 & 1.431054 \\
\hline C & -5.979160 & -3.695570 & 3.900505 \\
\hline $\mathrm{H}$ & -5.261124 & -4.518977 & 3.989278 \\
\hline $\mathrm{H}$ & -5.863113 & -3.055794 & 4.781289 \\
\hline $\mathrm{H}$ & -6.989751 & -4.121211 & 3.929665 \\
\hline C & -1.028472 & -0.543150 & 5.530451 \\
\hline $\mathrm{H}$ & -1.298722 & -1.508216 & 5.974564 \\
\hline $\mathrm{H}$ & -0.174090 & -0.706826 & 4.866100 \\
\hline $\mathrm{H}$ & -0.699084 & 0.122467 & 6.337736 \\
\hline C & -1.809198 & 1.435382 & 4.169631 \\
\hline $\mathrm{H}$ & -0.998288 & 1.326428 & 3.442027 \\
\hline $\mathrm{H}$ & -2.656039 & 1.911529 & 3.661197 \\
\hline $\mathrm{H}$ & -1.463616 & 2.114293 & 4.959384 \\
\hline C & -3.350458 & 0.339385 & 5.806685 \\
\hline $\mathrm{H}$ & -2.986597 & 1.025491 & 6.579812 \\
\hline $\mathrm{H}$ & -4.230197 & 0.801079 & 5.343823 \\
\hline $\mathrm{H}$ & -3.670060 & -0.581437 & 6.308469 \\
\hline
\end{tabular}

\section{C-Catalyzed reaction TS 143}

\section{Datum}

Value

\begin{tabular}{lc}
\hline B3LYP/6-31G(d) Energy & -3038.942577 \\
\hline B3LYP-D3(BJ)/def2-TZVPP-IEF-PCM(DCM) Energy & -3040.496849 \\
\hline B3LYP-D3(BJ)/def2-TZVPP-IEF-PCM(DCM)//B3LYP/6-31G(d) Free Energy (Quasiharmonic) & -3039.267574 \\
\hline
\end{tabular}

Number of Imaginary Frequencies

Frequencies (Top 3 out of 450)

$$
\begin{array}{lr}
\text { 1. } & -366.4355 \mathrm{~cm}^{-1} \\
\text { 2. } & 8.0738 \mathrm{~cm}^{-1} \\
\text { 3. } & 14.1111 \mathrm{~cm}^{-1}
\end{array}
$$




\begin{tabular}{|c|c|c|c|}
\hline C & 1.498430 & -0.754551 & 0.976800 \\
\hline $\mathrm{H}$ & 2.332520 & -0.055921 & 0.895630 \\
\hline $\mathrm{H}$ & 0.578620 & -0.162760 & 0.984210 \\
\hline C & -1.789370 & -0.288800 & -1.251010 \\
\hline 0 & -1.996360 & -0.532990 & -2.414130 \\
\hline 0 & -1.329050 & 0.929470 & -0.876710 \\
\hline H & -1.229510 & 1.072270 & 0.102920 \\
\hline 0 & 1.674649 & -1.394971 & 2.250470 \\
\hline $\mathrm{H}$ & 0.937319 & -2.015100 & 2.374890 \\
\hline C & -0.434170 & 4.062620 & -1.896790 \\
\hline C & 1.136280 & 3.046720 & -0.820150 \\
\hline C & -1.314790 & 4.477470 & 0.371560 \\
\hline C & -0.690520 & 4.907840 & -0.834670 \\
\hline $\mathrm{H}$ & 0.688120 & 2.111280 & -1.159160 \\
\hline $\mathrm{H}$ & -2.149730 & 3.791420 & 0.218980 \\
\hline $\mathrm{H}$ & -1.554460 & 5.271380 & 1.077850 \\
\hline C & -1.136850 & 1.997230 & 2.939320 \\
\hline C & -0.078820 & 2.970810 & 3.578700 \\
\hline C & -2.582750 & 2.488660 & 3.115400 \\
\hline $\mathrm{H}$ & -3.247480 & 1.891250 & 2.482460 \\
\hline $\mathrm{H}$ & -2.919500 & 2.379030 & 4.151840 \\
\hline $\mathrm{H}$ & -2.689240 & 3.539840 & 2.831990 \\
\hline C & -1.035520 & 0.546660 & 3.406910 \\
\hline $\mathrm{H}$ & -1.786560 & -0.066740 & 2.897320 \\
\hline $\mathrm{H}$ & -1.238620 & 0.486240 & 4.483000 \\
\hline $\mathrm{H}$ & -0.049680 & 0.116410 & 3.221620 \\
\hline C & 1.289510 & 2.305999 & 3.802490 \\
\hline $\mathrm{H}$ & 1.266760 & 1.586559 & 4.628170 \\
\hline $\mathrm{H}$ & 1.634850 & 1.786789 & 2.904500 \\
\hline $\mathrm{H}$ & 2.019130 & 3.085449 & 4.044560 \\
\hline C & -0.536500 & 3.653820 & 4.869080 \\
\hline $\mathrm{H}$ & -0.742290 & 2.919200 & 5.656570 \\
\hline $\mathrm{H}$ & 0.254600 & 4.320310 & 5.228370 \\
\hline $\mathrm{H}$ & -1.433700 & 4.256440 & 4.707930 \\
\hline 0 & -0.798480 & 2.082970 & 1.524280 \\
\hline 0 & 0.067590 & 3.976180 & 2.563650 \\
\hline B & -0.187620 & 3.393700 & 1.300140 \\
\hline 0 & 1.036280 & 3.336890 & 0.447010 \\
\hline C & 2.346940 & 3.508739 & -1.528600 \\
\hline C & 3.196910 & 4.469299 & -0.959700 \\
\hline C & 2.649410 & 2.982689 & -2.794680 \\
\hline C & 4.321580 & 4.905599 & -1.656150 \\
\hline $\mathrm{H}$ & 2.966850 & 4.856579 & 0.027510 \\
\hline C & 3.772020 & 3.425189 & -3.489750 \\
\hline $\mathrm{H}$ & 1.996070 & 2.232309 & -3.234420 \\
\hline C & 4.609320 & 4.390769 & -2.923390 \\
\hline $\mathrm{H}$ & 4.976570 & 5.649259 & -1.210270 \\
\hline $\mathrm{H}$ & 3.995860 & 3.017559 & -4.471580 \\
\hline $\mathrm{H}$ & 5.484820 & 4.736719 & -3.465980 \\
\hline $\mathrm{H}$ & -0.158600 & 5.860840 & -0.816600 \\
\hline
\end{tabular}




\begin{tabular}{|c|c|c|c|}
\hline $\mathrm{H}$ & -1.040920 & 3.171800 & -2.035540 \\
\hline H & 0.079770 & 4.434860 & -2.776820 \\
\hline C & -0.967921 & -2.158400 & 0.186980 \\
\hline C & -2.000181 & -1.280550 & -0.128940 \\
\hline C & -3.279571 & -1.357220 & 0.521170 \\
\hline C & -3.418841 & -2.238780 & 1.577440 \\
\hline H & -4.370441 & -2.290810 & 2.099540 \\
\hline C & 1.494469 & -1.696971 & -0.214270 \\
\hline C & 0.292249 & -2.262700 & -0.639040 \\
\hline C & 2.655289 & -2.758071 & -2.065270 \\
\hline C & 2.714969 & -1.977211 & -0.925780 \\
\hline $\mathrm{H}$ & 3.574499 & -2.994051 & -2.594990 \\
\hline C & 1.446719 & -3.309701 & -2.547610 \\
\hline C & 0.237749 & -3.080920 & -1.818410 \\
\hline C & -0.958741 & -3.682700 & -2.298930 \\
\hline C & -0.955651 & -4.457300 & -3.436930 \\
\hline C & 0.242499 & -4.677840 & -4.157180 \\
\hline C & 1.417609 & -4.114921 & -3.718760 \\
\hline $\mathrm{H}$ & -1.888551 & -3.512600 & -1.769730 \\
\hline $\mathrm{H}$ & -1.883611 & -4.899580 & -3.788850 \\
\hline $\mathrm{H}$ & 0.229479 & -5.291010 & -5.054280 \\
\hline $\mathrm{H}$ & 2.346719 & -4.278091 & -4.259920 \\
\hline C & -2.375951 & -3.101120 & 1.989090 \\
\hline C & -1.144601 & -3.101380 & 1.258860 \\
\hline C & -0.147261 & -4.050780 & 1.628700 \\
\hline C & -0.344301 & -4.912870 & 2.686570 \\
\hline C & -1.550801 & -4.884320 & 3.424840 \\
\hline C & -2.545381 & -4.000230 & 3.076680 \\
\hline $\mathrm{H}$ & 0.776639 & -4.095020 & 1.062920 \\
\hline $\mathrm{H}$ & 0.432429 & -5.624840 & 2.951330 \\
\hline $\mathrm{H}$ & -1.691581 & -5.567080 & 4.258080 \\
\hline $\mathrm{H}$ & -3.483771 & -3.979150 & 3.625610 \\
\hline C & 6.647860 & -0.817831 & 0.321820 \\
\hline C & 5.750399 & -1.298781 & 1.284840 \\
\hline C & 4.461149 & -1.650111 & 0.866330 \\
\hline C & 4.059569 & -1.511701 & -0.469720 \\
\hline C & 4.985870 & -1.014531 & -1.399180 \\
\hline C & 6.290950 & -0.670431 & -1.026780 \\
\hline $\mathrm{H}$ & 7.649380 & -0.549881 & 0.629270 \\
\hline $\mathrm{H}$ & 3.747439 & -2.039641 & 1.582920 \\
\hline $\mathrm{H}$ & 4.667330 & -0.892721 & -2.430490 \\
\hline C & 6.128579 & -1.459721 & 2.770360 \\
\hline C & 7.281360 & -0.158261 & -2.092040 \\
\hline C & 7.579000 & -1.030271 & 3.061480 \\
\hline $\mathrm{H}$ & 7.793110 & -1.158431 & 4.128780 \\
\hline $\mathrm{H}$ & 8.305559 & -1.633652 & 2.504740 \\
\hline H & 7.749290 & 0.024099 & 2.815510 \\
\hline C & 5.184070 & -0.589411 & 3.634690 \\
\hline $\mathrm{H}$ & 5.422940 & -0.711891 & 4.698820 \\
\hline H & 5.296080 & 0.472099 & 3.383930 \\
\hline $\mathrm{H}$ & 4.132970 & -0.857751 & 3.490320 \\
\hline C & 5.976149 & -2.943221 & 3.183230 \\
\hline $\mathrm{H}$ & 6.239919 & -3.073411 & 4.240300 \\
\hline H & 4.949279 & -3.297631 & 3.049800 \\
\hline
\end{tabular}




\begin{tabular}{|c|c|c|c|}
\hline $\mathrm{H}$ & 6.634009 & -3.586531 & 2.587090 \\
\hline C & 7.477200 & -1.247311 & -3.174150 \\
\hline $\mathrm{H}$ & 8.176320 & -0.899831 & -3.945070 \\
\hline $\mathrm{H}$ & 7.883329 & -2.166241 & -2.735960 \\
\hline $\mathrm{H}$ & 6.534329 & -1.502051 & -3.669420 \\
\hline C & 6.719430 & 1.121319 & -2.752500 \\
\hline $\mathrm{H}$ & 7.408370 & 1.486039 & -3.525130 \\
\hline $\mathrm{H}$ & 5.749250 & 0.943799 & -3.226980 \\
\hline $\mathrm{H}$ & 6.581620 & 1.918569 & -2.013950 \\
\hline C & 8.663600 & 0.178658 & -1.500820 \\
\hline $\mathrm{H}$ & 9.326540 & 0.538798 & -2.295900 \\
\hline $\mathrm{H}$ & 8.601390 & 0.966098 & -0.741150 \\
\hline $\mathrm{H}$ & 9.139490 & -0.697902 & -1.046730 \\
\hline C & -4.498640 & -0.645640 & 0.026660 \\
\hline C & -4.552610 & 0.724490 & -0.240550 \\
\hline C & -5.657541 & -1.420700 & -0.175510 \\
\hline C & -5.729870 & 1.332000 & -0.712820 \\
\hline $\mathrm{H}$ & -3.669860 & 1.331170 & -0.076990 \\
\hline C & -6.847490 & -0.855929 & -0.631650 \\
\hline $\mathrm{H}$ & -5.594441 & -2.486500 & 0.007980 \\
\hline C & -6.854960 & 0.525111 & -0.894830 \\
\hline $\mathrm{H}$ & -7.770480 & 0.975041 & -1.259070 \\
\hline C & -8.127211 & -1.683309 & -0.859210 \\
\hline C & -5.741860 & 2.843730 & -1.012490 \\
\hline C & -9.257010 & -1.138699 & 0.047180 \\
\hline $\mathrm{H}$ & -8.982770 & -1.218659 & 1.105450 \\
\hline $\mathrm{H}$ & -9.476720 & -0.086639 & -0.161980 \\
\hline $\mathrm{H}$ & -10.180461 & -1.709889 & -0.108840 \\
\hline C & -8.560391 & -1.572609 & -2.340550 \\
\hline $\mathrm{H}$ & -9.470671 & -2.159149 & -2.515820 \\
\hline $\mathrm{H}$ & -8.771610 & -0.537609 & -2.628780 \\
\hline $\mathrm{H}$ & -7.777331 & -1.951729 & -3.006840 \\
\hline C & -7.928721 & -3.175159 & -0.530350 \\
\hline $\mathrm{H}$ & -7.161351 & -3.634819 & -1.163400 \\
\hline $\mathrm{H}$ & -7.645141 & -3.328199 & 0.517280 \\
\hline $\mathrm{H}$ & -8.865441 & -3.717819 & -0.700390 \\
\hline C & -7.098230 & 3.328501 & -1.559810 \\
\hline $\mathrm{H}$ & -7.363800 & 2.826101 & -2.496840 \\
\hline $\mathrm{H}$ & -7.911380 & 3.170201 & -0.841990 \\
\hline $\mathrm{H}$ & -7.046990 & 4.403611 & -1.765730 \\
\hline C & -5.445320 & 3.627970 & 0.287170 \\
\hline $\mathrm{H}$ & -6.208870 & 3.427971 & 1.047960 \\
\hline $\mathrm{H}$ & -4.473870 & 3.359500 & 0.713000 \\
\hline $\mathrm{H}$ & -5.436420 & 4.706730 & 0.088870 \\
\hline C & -4.660080 & 3.162510 & -2.071710 \\
\hline $\mathrm{H}$ & -4.636130 & 4.239180 & -2.280240 \\
\hline $\mathrm{H}$ & -3.660880 & 2.861740 & -1.742610 \\
\hline $\mathrm{H}$ & -4.867940 & 2.637290 & -3.010760 \\
\hline
\end{tabular}


Frequencies (Top 3 out of 450)

$\begin{array}{rr}\text { 1. } & -547.2489 \mathrm{~cm}^{-1} \\ \text { 2. } & 7.0745 \mathrm{~cm}^{-1} \\ \text { 3. } & 10.9837 \mathrm{~cm}^{-1}\end{array}$

\section{B3LYP/6-31G(d) Molecular Geometry in Cartesian Coordinates}

C

$\mathrm{H}$

$\mathrm{H}$

C

0

0

$\mathrm{H}$

0

$\mathrm{H}$

C

C

C

C

$\mathrm{H}$

$\mathrm{H}$

$\mathrm{H}$

C

C

C

$\mathrm{H}$

$\mathrm{H}$

$\mathrm{H}$

C

$\mathrm{H}$

$\mathrm{H}$

$\mathrm{H}$

C

$\mathrm{H}$

$\mathrm{H}$

$\mathrm{H}$

C

$\mathrm{H}$

\section{$-2.889369$}

$-3.887679$

$-2.165741$

0.228599

$-0.789625$

1. 238744

1.989730

$-2.801851$

$-2.107058$

5.064972

3.478348

4.619561

5.189341

2.857618

5.207820

3.694623

4.248407

5. 205915

3. 332982

2. 719033

3.926892

2.657915

4.999644

4.275045

5.549217

5.710984

6.576887

6.483987

7.123419

7.176438

4.558053

4.466538
0.701711

0.645327

0.639574

1.198022

1.754078

1.004250

0.439207

1.977384

1.930041

$-2.991718$

$-3.206404$

$-0.638859$

$-1.597164$

$-2.803320$

0.290151

$-0.317018$

$-0.287024$

$-1.537368$

$-0.205322$

0.698965

$-0.171948$

$-1.062780$

1.043278

1.834266

1.325228

0.987558

$-1.301986$

$-1.048302$

$-0.501206$

$-2.215818$

$-2.784913$

$-2.696359$

\subsection{6}

1.652347

2.033780

$-0.961833$

$-1.344036$

$-1.798188$

$-1.451734$

0.576912

$-0.115523$

1.548057

1.007970

0.900728

1.820557

1.823463

0.879677

1.449346

$-2.650552$

$-2.631664$

$-3.874659$

$-3.835200$

$-4.795778$

$-3.924363$

$-2.473892$

$-2.254006$

$-3.378894$

$-1.643293$

- 3.272525

$-4.335289$

$-2.768305$

$-3.196578$

$-3.260467$

$-4.348730$ 


\begin{tabular}{|c|c|c|c|}
\hline $\mathrm{H}$ & 5.190821 & -3.653338 & -3.044478 \\
\hline $\mathrm{H}$ & 3.566725 & -2.968442 & -2.838582 \\
\hline 0 & 3.443779 & -0.500098 & -1.462900 \\
\hline 0 & 5.398982 & -1.757909 & -1.229531 \\
\hline B & 4.188043 & -1.342176 & -0.529319 \\
\hline 0 & 3.313494 & -2.539094 & -0.170267 \\
\hline C & 3.267271 & -4.698034 & 0.910553 \\
\hline C & 2.690358 & -5.393046 & 1.979971 \\
\hline C & 3.662855 & -5.400240 & -0.235258 \\
\hline C & 2.506396 & -6.774500 & 1.906236 \\
\hline $\mathrm{H}$ & 2.366443 & -4.847037 & 2.863556 \\
\hline C & 3.477564 & -6.779208 & -0.308498 \\
\hline $\mathrm{H}$ & 4.098821 & -4.852173 & -1.064548 \\
\hline C & 2.901601 & -7.469906 & 0.762518 \\
\hline $\mathrm{H}$ & 2.048172 & -7.304934 & 2.736596 \\
\hline $\mathrm{H}$ & 3.778979 & -7.317907 & -1.202930 \\
\hline $\mathrm{H}$ & 2.757250 & -8.545260 & 0.702096 \\
\hline $\mathrm{H}$ & 5.611583 & -1.269790 & 2.777333 \\
\hline $\mathrm{H}$ & 5.577732 & -3.228145 & 0.604649 \\
\hline $\mathrm{H}$ & 5.347218 & -3.668467 & 2.354810 \\
\hline C & -0.260214 & -0.422800 & 0.884351 \\
\hline C & 0.434440 & 0.698224 & 0.451320 \\
\hline C & 1.374762 & 1.377741 & 1.299344 \\
\hline C & 1.686933 & 0.809798 & 2.520332 \\
\hline $\mathrm{H}$ & 2.357263 & 1.336820 & 3.195503 \\
\hline C & -2.680080 & -0.466132 & 0.256498 \\
\hline C & -1.399802 & -0.992380 & 0.079149 \\
\hline C & -3.556875 & -2.086609 & -1.328551 \\
\hline C & -3.787619 & -1.025486 & -0.471923 \\
\hline $\mathrm{H}$ & -4.389241 & -2.504558 & -1.889021 \\
\hline C & -2.269764 & -2.633956 & -1.532543 \\
\hline C & -1.159232 & -2.078507 & -0.821765 \\
\hline C & 0.131729 & -2.632584 & -1.041044 \\
\hline C & 0.307450 & -3.687488 & -1.909265 \\
\hline C & -0.792722 & -4.238390 & -2.608899 \\
\hline C & -2.052941 & -3.718162 & -2.425596 \\
\hline H & 0.997698 & -2.222969 & -0.533471 \\
\hline $\mathrm{H}$ & 1.302913 & -4.097915 & -2.050072 \\
\hline $\mathrm{H}$ & -0.638396 & -5.069387 & -3.292347 \\
\hline $\mathrm{H}$ & -2.905996 & -4.128123 & -2.961480 \\
\hline C & 1.095113 & -0.404275 & 2.955845 \\
\hline C & 0.086800 & -1.019216 & 2.145196 \\
\hline C & -0.535326 & -2.204364 & 2.622608 \\
\hline C & -0.169114 & -2.762371 & 3.829058 \\
\hline C & 0.830475 & -2.155873 & 4.625989 \\
\hline C & 1.444761 & -0.999062 & 4.198183 \\
\hline $\mathrm{H}$ & -1.303699 & -2.671592 & 2.016921 \\
\hline $\mathrm{H}$ & -0.657108 & -3.669480 & 4.174684 \\
\hline H & 1.102112 & -2.597113 & 5.581397 \\
\hline $\mathrm{H}$ & 2.198453 & -0.513919 & 4.814837 \\
\hline C & -7.838317 & 0.328060 & -0.173905 \\
\hline C & -7.565326 & -1.047957 & -0.060130 \\
\hline C & -6.233486 & -1.456894 & -0.140793 \\
\hline C & -5.190533 & -0.531078 & -0.333340 \\
\hline
\end{tabular}




\begin{tabular}{|c|c|c|c|}
\hline C & -5.505699 & 0.825787 & -0.443216 \\
\hline C & -6.831665 & 1.278943 & -0.359995 \\
\hline $\mathrm{H}$ & -8.869088 & 0.657599 & -0.108971 \\
\hline $\mathrm{H}$ & -5.974834 & -2.504662 & -0.038039 \\
\hline $\mathrm{H}$ & -4.696319 & 1.533090 & -0.581329 \\
\hline C & -8.728312 & -2.037147 & 0.153093 \\
\hline C & -7.112795 & 2.789564 & -0.472382 \\
\hline C & -8.247886 & -3.497190 & 0.257781 \\
\hline $\mathrm{H}$ & -9.108883 & -4.159315 & 0.405480 \\
\hline $\mathrm{H}$ & -7.732759 & -3.823400 & -0.652880 \\
\hline $\mathrm{H}$ & -7.568927 & -3.643644 & 1.105470 \\
\hline C & -9.475858 & -1.685361 & 1.461228 \\
\hline $\mathrm{H}$ & -10.313821 & -2.375534 & 1.620571 \\
\hline $\mathrm{H}$ & -8.805883 & -1.758058 & 2.325678 \\
\hline $\mathrm{H}$ & -9.881085 & -0.668495 & 1.438499 \\
\hline C & -9.710850 & -1.942117 & -1.038409 \\
\hline $\mathrm{H}$ & -10.549521 & -2.635730 & -0.898896 \\
\hline $\mathrm{H}$ & -10.125776 & -0.934575 & -1.145609 \\
\hline $\mathrm{H}$ & -9.210285 & -2.198122 & -1.979280 \\
\hline C & -8.612128 & 3.122120 & -0.354421 \\
\hline $\mathrm{H}$ & -8.756565 & 4.205674 & -0.434366 \\
\hline $\mathrm{H}$ & -9.200119 & 2.651496 & -1.151213 \\
\hline $\mathrm{H}$ & -9.026451 & 2.805360 & 0.609887 \\
\hline C & -6.360393 & 3.532102 & 0.658870 \\
\hline $\mathrm{H}$ & -6.531038 & 4.613460 & 0.581384 \\
\hline $\mathrm{H}$ & -6.718287 & 3.203946 & 1.642241 \\
\hline $\mathrm{H}$ & -5.280958 & 3.355325 & 0.618810 \\
\hline C & -6.611879 & 3.305281 & -1.842583 \\
\hline $\mathrm{H}$ & -6.798898 & 4.382432 & -1.935346 \\
\hline $\mathrm{H}$ & -5.537336 & 3.141333 & -1.970018 \\
\hline $\mathrm{H}$ & -7.129077 & 2.798366 & -2.665755 \\
\hline C & 1.905457 & 2.723507 & 0.941036 \\
\hline C & 3.263218 & 3.037798 & 1.092589 \\
\hline C & 1.014633 & 3.718523 & 0.512915 \\
\hline C & 3.747576 & 4.319260 & 0.806713 \\
\hline $\mathrm{H}$ & 3.939573 & 2.259519 & 1.423629 \\
\hline C & 1.459649 & 5.008663 & 0.208051 \\
\hline $\mathrm{H}$ & -0.037212 & 3.475612 & 0.441621 \\
\hline C & 2.826094 & 5.279583 & 0.363786 \\
\hline $\mathrm{H}$ & 3.186885 & 6.277380 & 0.131718 \\
\hline C & 0.502231 & 6.111640 & -0.282136 \\
\hline C & 5.233974 & 4.699728 & 0.954637 \\
\hline C & 0.966141 & 6.608559 & -1.672185 \\
\hline $\mathrm{H}$ & 1.982373 & 7.016562 & -1.645063 \\
\hline $\mathrm{H}$ & 0.951000 & 5.792590 & -2.403271 \\
\hline $\mathrm{H}$ & 0.299141 & 7.400914 & -2.033120 \\
\hline C & -0.948115 & 5.607501 & -0.416125 \\
\hline $\mathrm{H}$ & -1.584080 & 6.418174 & -0.789527 \\
\hline $\mathrm{H}$ & -1.026342 & 4.771213 & -1.119101 \\
\hline $\mathrm{H}$ & -1.362355 & 5.280902 & 0.544258 \\
\hline C & 0.519309 & 7.292068 & 0.717447 \\
\hline $\mathrm{H}$ & 0.192144 & 6.968262 & 1.712108 \\
\hline $\mathrm{H}$ & 1.519119 & 7.728601 & 0.819197 \\
\hline $\mathrm{H}$ & -0.157729 & 8.086137 & 0.379672 \\
\hline
\end{tabular}




\begin{tabular}{|llll|} 
C & 5.788142 & 5.169690 & -0.411229 \\
H & 5.710466 & 4.373471 & -1.160235 \\
H & 5.245529 & 6.040392 & -0.792879 \\
H & 6.844925 & 5.449475 & -0.319188 \\
C & 5.371809 & 5.844751 & 1.986209 \\
$H$ & 4.815688 & 6.736750 & 1.680532 \\
$H$ & 4.993304 & 5.536390 & 2.967608 \\
$H$ & 6.424513 & 6.131133 & 2.101383 \\
C & 6.098654 & 3.518957 & 1.436101 \\
H & 7.142168 & 3.839791 & 1.530947 \\
H & 5.776548 & 3.149818 & 2.416983 \\
H & 6.074515 & 2.683936 & 0.725839 \\
\hline
\end{tabular}

\section{C-Catalyzed reaction TS 145}

\section{Datum}

Value

\begin{tabular}{lc}
\hline B3LYP/6-31G(d) Energy & -3038.911704 \\
\hline B3LYP-D3(BJ)/def2-TZVPP-IEF-PCM(DCM) Energy & -3040.469863 \\
\hline B3LYP-D3(BJ)/def2-TZVPP-IEF-PCM(DCM)//B3LYP/6-31G(d) Free Energy (Quasiharmonic) & -3039.242676 \\
\hline
\end{tabular}

Number of Imaginary Frequencies

Frequencies (Top 3 out of 450)

$\begin{array}{lr}\text { 1. } & -592.5003 \mathrm{~cm}^{-1} \\ \text { 2. } & 10.7926 \mathrm{~cm}^{-1} \\ \text { 3. } & 11.3652 \mathrm{~cm}^{-1}\end{array}$

\section{B3LYP/6-31G(d) Molecular Geometry in Cartesian Coordinates}

$\begin{array}{rrrr}\mathrm{C} & -1.530310 & 0.394730 & 0.666450 \\ \mathrm{H} & -2.236500 & -0.029140 & -0.045920 \\ \mathrm{H} & -0.543310 & 0.000440 & 0.410490 \\ \mathrm{C} & 1.800101 & 1.846350 & -0.721000 \\ \mathrm{O} & 2.047531 & 2.959870 & -1.137260 \\ \mathrm{O} & 1.435790 & 0.870880 & -1.554020 \\ \mathrm{H} & 1.263880 & -0.029980 & -1.160150 \\ \mathrm{O} & -1.942330 & -0.108680 & 1.953640 \\ \mathrm{H} & -1.393230 & 0.327270 & 2.626400 \\ \mathrm{C} & -0.354740 & -4.379800 & 1.815870 \\ \mathrm{C} & -1.462590 & -3.282120 & 1.092910 \\ \mathrm{C} & 1.237310 & -2.573490 & 1.241960 \\ \mathrm{C} & 0.788340 & -3.607380 & 2.153450 \\ \mathrm{H} & -1.574810 & -2.488520 & 1.844630\end{array}$




\begin{tabular}{|c|c|c|c|}
\hline $\mathrm{H}$ & 2.311440 & -2.373610 & 1.338810 \\
\hline $\mathrm{H}$ & 0.760940 & -1.663140 & 1.681400 \\
\hline C & 1.581860 & -2.358310 & -2.374960 \\
\hline C & 1.168910 & -3.875240 & -2.245060 \\
\hline C & 1.100630 & -1.665650 & -3.652800 \\
\hline $\mathrm{H}$ & 1.418840 & -0.618900 & -3.653750 \\
\hline $\mathrm{H}$ & 1.523660 & -2.151390 & -4.540630 \\
\hline $\mathrm{H}$ & 0.011180 & -1.685270 & -3.730240 \\
\hline C & 3.093440 & -2.143551 & -2.206890 \\
\hline $\mathrm{H}$ & 3.302450 & -1.074891 & -2.104810 \\
\hline $\mathrm{H}$ & 3.652720 & -2.515291 & -3.072860 \\
\hline $\mathrm{H}$ & 3.463580 & -2.651081 & -1.311100 \\
\hline C & 2.212559 & -4.868530 & -2.765000 \\
\hline $\mathrm{H}$ & 2.404689 & -4.722010 & -3.834500 \\
\hline $\mathrm{H}$ & 3.157259 & -4.777371 & -2.223890 \\
\hline $\mathrm{H}$ & 1.843609 & -5.890820 & -2.626360 \\
\hline C & -0.186680 & -4.178560 & -2.910400 \\
\hline $\mathrm{H}$ & -0.116940 & -4.154730 & -4.003860 \\
\hline $\mathrm{H}$ & -0.504551 & -5.184200 & -2.612730 \\
\hline $\mathrm{H}$ & -0.952880 & -3.467740 & -2.592530 \\
\hline 0 & 0.913760 & -1.752220 & -1.236300 \\
\hline 0 & 1.062640 & -4.045410 & -0.826190 \\
\hline B & 0.582040 & -2.786320 & -0.263640 \\
\hline 0 & -0.931360 & -2.834280 & -0.075250 \\
\hline C & -2.755840 & -4.043780 & 0.952390 \\
\hline C & -3.106821 & -4.657910 & -0.255740 \\
\hline C & -3.609990 & -4.165410 & 2.057610 \\
\hline C & -4.295251 & -5.380949 & -0.353910 \\
\hline $\mathrm{H}$ & -2.448541 & -4.547530 & -1.109750 \\
\hline C & -4.794101 & -4.895369 & 1.961250 \\
\hline $\mathrm{H}$ & -3.347960 & -3.678630 & 2.995010 \\
\hline C & -5.138221 & -5.507069 & 0.753620 \\
\hline H & -4.565921 & -5.846809 & -1.297800 \\
\hline $\mathrm{H}$ & -5.450361 & -4.979989 & 2.823220 \\
\hline $\mathrm{H}$ & -6.062051 & -6.073749 & 0.673950 \\
\hline $\mathrm{H}$ & 1.252180 & -3.723670 & 3.139270 \\
\hline H & -0.151051 & -4.952070 & 0.898920 \\
\hline $\mathrm{H}$ & -0.780291 & -4.996100 & 2.607950 \\
\hline C & 0.807621 & 1.897100 & 1.563750 \\
\hline C & 1.880030 & 1.523730 & 0.760830 \\
\hline C & 3.059540 & 0.920169 & 1.319150 \\
\hline C & 3.074640 & 0.653159 & 2.676720 \\
\hline $\mathrm{H}$ & 3.967570 & 0.221619 & 3.121210 \\
\hline C & -1.521209 & 1.909750 & 0.589240 \\
\hline C & -0.387439 & 2.614530 & 0.990840 \\
\hline C & -2.612189 & 3.999460 & 0.000210 \\
\hline C & -2.664119 & 2.621480 & 0.086530 \\
\hline $\mathrm{H}$ & -3.480139 & 4.539250 & -0.370050 \\
\hline C & -1.480439 & 4.742740 & 0.408610 \\
\hline C & -0.341269 & 4.046180 & 0.919370 \\
\hline C & 0.784641 & 4.809450 & 1.334880 \\
\hline C & 0.777241 & 6.183530 & 1.253980 \\
\hline C & -0.353579 & 6.869970 & 0.750060 \\
\hline C & -1.456829 & 6.161940 & 0.334920 \\
\hline
\end{tabular}




\begin{tabular}{|c|c|c|c|}
\hline $\mathrm{H}$ & 1.663531 & 4.296680 & 1.708470 \\
\hline $\mathrm{H}$ & 1.649611 & 6.747040 & 1.573620 \\
\hline $\mathrm{H}$ & -0.344519 & 7.954980 & 0.689630 \\
\hline $\mathrm{H}$ & -2.330979 & 6.676640 & -0.056970 \\
\hline C & 1.991880 & 0.978380 & 3.527130 \\
\hline C & 0.845690 & 1.634070 & 2.974080 \\
\hline C & -0.208489 & 2.009430 & 3.857520 \\
\hline C & -0.141420 & 1.723820 & 5.204860 \\
\hline C & 0.986570 & 1.060200 & 5.743800 \\
\hline C & 2.030680 & 0.703260 & 4.921680 \\
\hline $\mathrm{H}$ & -1.065349 & 2.543080 & 3.458840 \\
\hline $\mathrm{H}$ & -0.955349 & 2.021460 & 5.860110 \\
\hline $\mathrm{H}$ & 1.029230 & 0.844620 & 6.807880 \\
\hline $\mathrm{H}$ & 2.909400 & 0.208289 & 5.328970 \\
\hline C & -6.333490 & 0.815911 & -1.160900 \\
\hline C & -5.833680 & 0.505931 & 0.117190 \\
\hline C & -4.619210 & 1.075471 & 0.503850 \\
\hline C & -3.918219 & 1.941211 & -0.353780 \\
\hline C & -4.448849 & 2.222571 & -1.616160 \\
\hline C & -5.663100 & 1.664271 & -2.046060 \\
\hline $\mathrm{H}$ & -7.274410 & 0.373181 & -1.465760 \\
\hline $\mathrm{H}$ & -4.191770 & 0.858911 & 1.474740 \\
\hline $\mathrm{H}$ & -3.888689 & 2.878931 & -2.275770 \\
\hline C & -6.636120 & -0.434629 & 1.036600 \\
\hline C & -6.197959 & 1.994011 & -3.453400 \\
\hline C & -5.945550 & -0.651649 & 2.396670 \\
\hline $\mathrm{H}$ & -6.545380 & -1.336379 & 3.007720 \\
\hline $\mathrm{H}$ & -4.947440 & -1.088089 & 2.284860 \\
\hline $\mathrm{H}$ & -5.845410 & 0.286871 & 2.954220 \\
\hline C & -8.035590 & 0.170271 & 1.300910 \\
\hline $\mathrm{H}$ & -8.619360 & -0.490049 & 1.954480 \\
\hline $\mathrm{H}$ & -7.954420 & 1.147671 & 1.790400 \\
\hline $\mathrm{H}$ & -8.604000 & 0.307701 & 0.374860 \\
\hline C & -6.795960 & -1.812149 & 0.350950 \\
\hline $\mathrm{H}$ & -7.371850 & -2.492069 & 0.991230 \\
\hline $\mathrm{H}$ & -7.322230 & -1.731969 & -0.606340 \\
\hline $\mathrm{H}$ & -5.820760 & -2.272449 & 0.160810 \\
\hline C & -6.385509 & 3.523731 & -3.590630 \\
\hline $\mathrm{H}$ & -6.763629 & 3.773791 & -4.589670 \\
\hline $\mathrm{H}$ & -7.103749 & 3.898041 & -2.851970 \\
\hline $\mathrm{H}$ & -5.444769 & 4.065231 & -3.447170 \\
\hline C & -5.182510 & 1.506111 & -4.514060 \\
\hline $\mathrm{H}$ & -5.541400 & 1.740681 & -5.523970 \\
\hline $\mathrm{H}$ & -4.203599 & 1.979911 & -4.388610 \\
\hline $\mathrm{H}$ & -5.038600 & 0.421651 & -4.447400 \\
\hline C & -7.553170 & 1.321231 & -3.742160 \\
\hline $\mathrm{H}$ & -7.892790 & 1.592941 & -4.748160 \\
\hline $\mathrm{H}$ & -7.483740 & 0.228201 & -3.702010 \\
\hline $\mathrm{H}$ & -8.326550 & 1.641231 & -3.034350 \\
\hline C & 4.277920 & 0.613719 & 0.514110 \\
\hline C & 4.975020 & -0.591171 & 0.724960 \\
\hline C & 4.794240 & 1.539479 & -0.396930 \\
\hline C & 6.157020 & -0.879181 & 0.042150 \\
\hline $\mathrm{H}$ & 4.564540 & -1.306411 & 1.428670 \\
\hline
\end{tabular}




\begin{tabular}{|lrrr|} 
C & 5.972140 & 1.282299 & -1.116530 \\
H & 4.277021 & 2.480239 & -0.537320 \\
C & 6.632390 & 0.074869 & -0.877160 \\
H & 7.546340 & -0.141271 & -1.417920 \\
C & 6.476751 & 2.331739 & -2.124610 \\
C & 6.950810 & -2.181741 & 0.262930 \\
C & 6.757211 & 3.662609 & -1.386270 \\
H & 5.858901 & 4.056479 & -0.900530 \\
H & 7.527001 & 3.531379 & -0.616560 \\
H & 7.110731 & 4.421169 & -2.095300 \\
C & 7.773531 & 1.893799 & -2.831010 \\
H & 8.084931 & 2.668509 & -3.540690 \\
H & 8.597480 & 1.745559 & -2.122890 \\
H & 7.636860 & 0.965349 & -3.397390 \\
C & 5.389511 & 2.563689 & -3.202350 \\
H & 5.193180 & 1.641179 & -3.761020 \\
H & 4.441561 & 2.897039 & -2.768570 \\
H & 5.722181 & 3.327809 & -3.915950 \\
C & 8.349090 & -1.834861 & 0.827940 \\
H & 8.911880 & -1.187202 & 0.147710 \\
H & 8.265650 & -1.315801 & 1.789760 \\
H & 8.936880 & -2.748222 & 0.983030 \\
C & 6.256130 & -3.133091 & 1.256020 \\
H & 6.146890 & -2.680011 & 2.248250 \\
H & 5.262940 & -3.434821 & 0.903380 \\
H & 6.854520 & -4.043471 & 1.374660 \\
C & 7.115370 & -2.932811 & -1.079660 \\
H & 7.680560 & -3.860811 & -0.928990 \\
H & 6.140290 & -3.192731 & -1.506420 \\
H & 7.654470 & -2.333971 & -1.820700 \\
& & & \\
\hline & & & \\
\hline & & & \\
\hline
\end{tabular}




\subsection{Optimization with B3LYP-D3(BJ)/6-31G(d,p)}

\section{Benzaldehyde}

\begin{tabular}{|c|c|}
\hline Datum & \\
\hline B3LYP-D & /6-31G(d,p) Energy \\
\hline B3LYP-D & /def2-TZVPP/IEF-PC \\
\hline B3LYP-D & /def2-TZVPP/IEF-PC \\
\hline Number & naginary Frequenci \\
\hline Frequenci & op 3 out of 36) \\
\hline 1. & $124.8422 \mathrm{~cm}^{-1}$ \\
\hline 2. & $222.1015 \mathrm{~cm}^{-1}$ \\
\hline 3. & $240.0186 \mathrm{~cm}^{-1}$ \\
\hline
\end{tabular}

B3LYP-D3(BJ)/6-31G(d,p) Molecular Geometry in Cartesian Coordinates

\section{Allylboronic acid pinacol ester 1}

Datum

\subsection{0 \\ $-0.000001$}

2.269395

1.547211

0.000002

2.842947

$-0.396275$

$-0.000003$

0.532926

0.215442

$-0.000000$

$-0.361444$

1. 292138

0.000003

0.047271

$-1.100161$

$-0.000003$

$-1.735111$

1.059261

0.000004

0.024946

2. 308363

0.000005

$-1.323036$

$-1.331345$

$-0.000002$

0.763825

$-1.914741$

$-0.000005$

$-2.213908$

$-0.252072$

0.000001

1.892847

0.000007

$-1.703926$

$-2.347842$

$-0.000004$

$-0.435695$
Value $-345.607035$

$-345.740457$

$-345.649416$

\begin{tabular}{lc}
\hline B3LYP-D3(BJ)/6-31G(d,p) Energy & -528.664855 \\
\hline B3LYP-D3(BJ)/def2-TZVPP/IEF-PCM(DCM) Energy & -528.858872 \\
\hline B3LYP-D3(BJ)/def2-TZVPP/IEF-PCM(DCM)//B3LYP-D3(BJ)/6-31G(d,p) Free Energy (Quasiharmonic) & -528.632163 \\
\hline
\end{tabular}


Frequencies (Top 3 out of 81)

$\begin{array}{lr}\text { 1. } & 15.6800 \mathrm{~cm}^{-1} \\ \text { 2. } & 49.8949 \mathrm{~cm}^{-1} \\ \text { 3. } & 101.3381 \mathrm{~cm}^{-1}\end{array}$

B3LYP-D3(BJ)/6-31G(d,p) Molecular Geometry in Cartesian Coordinates

\begin{tabular}{|c|c|c|c|}
\hline C & -4.110055 & 0.130368 & 0.727673 \\
\hline C & -2.292495 & -0.771250 & -0.771570 \\
\hline C & -3.207540 & 0.299633 & -0.238701 \\
\hline $\mathrm{H}$ & -2.393731 & -0.829985 & -1.865608 \\
\hline $\mathrm{H}$ & -2.561061 & -1.751211 & -0.365028 \\
\hline C & 1.066198 & 0.836017 & -0.091596 \\
\hline C & 1.373428 & -0.672259 & 0.226481 \\
\hline C & 2.088852 & 1.528519 & -0.983191 \\
\hline $\mathrm{H}$ & 1.778267 & 2.560814 & -1.164633 \\
\hline $\mathrm{H}$ & 3.071127 & 1.548660 & -0.500502 \\
\hline $\mathrm{H}$ & 2.183275 & 1.028910 & -1.948187 \\
\hline C & 0.796592 & 1.673779 & 1.161577 \\
\hline $\mathrm{H}$ & 1.711048 & 1.846359 & 1.735803 \\
\hline $\mathrm{H}$ & 0.390102 & 2.641477 & 0.857505 \\
\hline $\mathrm{H}$ & 0.062737 & 1.187443 & 1.809589 \\
\hline C & 2.063316 & -0.922095 & 1.561487 \\
\hline $\mathrm{H}$ & 2.215408 & -1.995569 & 1.701273 \\
\hline $\mathrm{H}$ & 3.042445 & -0.433308 & 1.587374 \\
\hline $\mathrm{H}$ & 1.465630 & -0.553399 & 2.396007 \\
\hline C & 2.121654 & -1.386595 & -0.902170 \\
\hline $\mathrm{H}$ & 3.162258 & -1.056868 & -0.966993 \\
\hline $\mathrm{H}$ & 2.110297 & -2.461967 & -0.708184 \\
\hline $\mathrm{H}$ & 1.639625 & -1.210167 & -1.867372 \\
\hline 0 & -0.196379 & 0.748073 & -0.809694 \\
\hline 0 & 0.040130 & -1.255719 & 0.276380 \\
\hline B & -0.792489 & -0.432046 & -0.436519 \\
\hline $\mathrm{H}$ & -3.072765 & 1.290152 & -0.671996 \\
\hline H & -4.269670 & -0.838908 & 1.194016 \\
\hline $\mathrm{H}$ & -4.722244 & 0.952281 & 1.086100 \\
\hline
\end{tabular}

Allylboronic acid pinacol ester 2 


\begin{tabular}{lr}
\hline B3LYP-D3(BJ)/6-31G(d,p) Energy & -528.664745 \\
\hline B3LYP-D3(BJ)/def2-TZVPP/IEF-PCM(DCM) Energy & -528.858731 \\
\hline B3LYP-D3(BJ)/def2-TZVPP/IEF-PCM(DCM)//B3LYP-D3(BJ)/6-31G(d,p) Free Energy (Quasiharmonic) & -528.631976 \\
\hline
\end{tabular}

Number of Imaginary Frequencies

Frequencies (Top 3 out of 81)

$\begin{array}{lr}\text { 1. } & 14.7095 \mathrm{~cm}^{-1} \\ \text { 2. } & 49.6287 \mathrm{~cm}^{-1} \\ \text { 3. } & 100.4716 \mathrm{~cm}^{-1}\end{array}$

B3LYP-D3(BJ)/6-31G(d,p) Molecular Geometry in Cartesian Coordinates

\begin{tabular}{|c|c|c|c|}
\hline C & 4.052284 & -0.451130 & 0.619505 \\
\hline C & 2.307254 & 0.294510 & -1.044329 \\
\hline C & 3.176520 & 0.451348 & 0.176650 \\
\hline $\mathrm{H}$ & 2.613569 & -0.579780 & -1.626750 \\
\hline $\mathrm{H}$ & 2.411071 & 1.180231 & -1.686826 \\
\hline C & -1.249117 & 0.731985 & 0.204429 \\
\hline C & -1.175138 & -0.818607 & -0.041374 \\
\hline C & -1.789133 & 1.143313 & 1.568268 \\
\hline $\mathrm{H}$ & -1.783640 & 2.233302 & 1.651875 \\
\hline $\mathrm{H}$ & -2.819782 & 0.797395 & 1.696176 \\
\hline $\mathrm{H}$ & -1.180665 & 0.738648 & 2.377980 \\
\hline C & -1.983493 & 1.484883 & -0.908383 \\
\hline $\mathrm{H}$ & -3.060919 & 1.300712 & -0.876225 \\
\hline $\mathrm{H}$ & -1.811274 & 2.556667 & -0.782520 \\
\hline $\mathrm{H}$ & -1.609823 & 1.193983 & -1.893705 \\
\hline C & -2.349701 & -1.407262 & -0.811950 \\
\hline $\mathrm{H}$ & -2.196506 & -2.480638 & -0.951078 \\
\hline $\mathrm{H}$ & -3.283736 & -1.265730 & -0.258944 \\
\hline $\mathrm{H}$ & -2.453303 & -0.949714 & -1.796684 \\
\hline C & -0.919933 & -1.619565 & 1.238432 \\
\hline $\mathrm{H}$ & -1.799327 & -1.631924 & 1.888265 \\
\hline $\mathrm{H}$ & -0.674809 & -2.649282 & 0.966963 \\
\hline $\mathrm{H}$ & -0.076405 & -1.207216 & 1.798362 \\
\hline 0 & 0.149441 & 1.124455 & 0.120743 \\
\hline 0 & 0.027204 & -0.946098 & -0.853293 \\
\hline B & 0.802757 & 0.156756 & -0.602521 \\
\hline $\mathrm{H}$ & 3.027249 & 1.369105 & 0.744674 \\
\hline $\mathrm{H}$ & 4.225343 & -1.384275 & 0.088824 \\
\hline H & 4.628759 & -0.288972 & 1.525119 \\
\hline
\end{tabular}




\section{Allylboronic acid pinacol ester 3}

Datum

Value

\begin{tabular}{lr}
\hline B3LYP-D3(BJ)/6-31G(d,p) Energy & -528.663441 \\
\hline B3LYP-D3(BJ)/def2-TZVPP/IEF-PCM(DCM) Energy & -528.856642 \\
\hline B3LYP-D3(BJ)/def2-TZVPP/IEF-PCM(DCM)//B3LYP-D3(BJ)/6-31G(d,p) Free Energy (Quasiharmonic) & -528.62969 \\
\hline
\end{tabular}

Number of Imaginary Frequencies

Frequencies (Top 3 out of 81)

$\begin{array}{ll}\text { 1. } & 27.9723 \mathrm{~cm}^{-1} \\ \text { 2. } & 61.6337 \mathrm{~cm}^{-1} \\ \text { 3. } & 88.1865 \mathrm{~cm}^{-1}\end{array}$

\section{B3LYP-D3(BJ)/6-31G(d,p) Molecular Geometry in Cartesian Coordinates}

C

C

C

$\mathrm{H}$

$\mathrm{H}$

C

C

C

$\mathrm{H}$

$\mathrm{H}$

$\mathrm{H}$

C

$\mathrm{H}$

$\mathrm{H}$

$\mathrm{H}$

C

$\mathrm{H}$

$\mathrm{H}$

$\mathrm{H}$

C

$\mathrm{H}$

$\mathrm{H}$

$\mathrm{H}$

0

0

B

$\mathrm{H}$

$\mathrm{H}$

$\mathrm{H}$

-3.172887
-2.348805
-3.360598
-2.572459
-2.473863
1.114722
1.155004
1.843776
1.595064
2.928736
1.532249
1.565754
0.955788
2.612588
1.477123
0.901013
0.743450
1.748630
0.004493
2.398097
2.322748
2.512636
3.296320
-0.302281
-0.000174
-0.862547
-4.328586
-2.237611
-3.961393

- 3.172887

0.515975

$-0.355569$

$-0.173330$

0.363074

$-1.350869$

0.785659

$-0.755923$

1.168332

2.202032

1.089914

0.530940

1.679464

1.525074

1.484132

2.728445

$-1.089585$

$-2.166724$

$-0.805795$

$-0.584468$

$-1.479241$

$-2.544245$

$-1.377799$

$-1.086259$

1.016674

$-1.245032$

$-0.188381$

$-0.648518$

1.027302

0.607844
1.086277

$-1.154407$

$-0.040105$

$-1.956548$

$-1.596149$

$-0.082190$

0.219266

$-1.372965$

$-1.625524$

$-1.261121$

$-2.204655$

1.066007

1.957025

1. 319445

0.771426

1. 692120

1.787033

2. 321981

2.061173

$-0.282594$

$-0.047999$

$-1.362575$

0.204277

$-0.321272$

$-0.518227$

$-0.672026$

$-0.201365$

1. 294215

1.827029 


\section{Catalyst 2C 1}

\section{Datum}

Value

B3LYP-D3(BJ)/6-31G(d,p) Energy

$-2165.164801$

B3LYP-D3(BJ)/def2-TZVPP/IEF-PCM(DCM) Energy

$-2165.904874$

B3LYP-D3(BJ)/def2-TZVPP/IEF-PCM(DCM)//B3LYP-D3(BJ)/6-31G(d,p) Free Energy (Quasiharmonic)

$-2165.026764$

Number of Imaginary Frequencies

Frequencies (Top 3 out of 321)

$\begin{array}{lr}\text { 1. } & 9.6231 \mathrm{~cm}^{-1} \\ \text { 2. } & 12.1625 \mathrm{~cm}^{-1} \\ \text { 3. } & 15.1874 \mathrm{~cm}^{-1}\end{array}$

B3LYP-D3(BJ)/6-31G(d,p) Molecular Geometry in Cartesian Coordinates

$\begin{array}{lrrr}C & 1.505562 & -0.078429 & -1.287872 \\ \mathrm{H} & 2.464523 & 0.191662 & -1.735078 \\ \mathrm{C} & 0.914449 & -0.637732 & -2.017499 \\ \mathrm{C} & -1.575808 & -0.131163 & 1.272215 \\ \mathrm{O} & -2.260201 & -0.426747 & 2.223828 \\ \mathrm{H} & -0.711813 & 0.894848 & 1.316360 \\ \mathrm{O} & -0.213144 & 0.979050 & 0.469734 \\ \mathrm{H} & 0.791554 & 1.136651 & -0.931554 \\ \mathrm{C} & 0.434234 & 1.532552 & -1.737226 \\ \mathrm{C} & -0.617027 & -1.755734 & -0.374923 \\ \mathrm{C} & -1.659895 & -0.917249 & -0.020727 \\ \mathrm{C} & -2.888143 & -0.914387 & -0.758596 \\ \mathrm{H} & -3.024602 & -1.784706 & -1.820319 \\ \mathrm{C} & -3.938202 & -1.765740 & -2.407171 \\ \mathrm{C} & 1.708914 & -0.909622 & -0.046956 \\ \mathrm{C} & 0.671447 & -1.726710 & 0.385345 \\ \mathrm{C} & 3.107239 & -1.705993 & 1.765813 \\ \mathrm{H} & 2.945030 & -0.873158 & 0.675389 \\ \mathrm{C} & 4.042172 & -1.678572 & 2.317591 \\ \mathrm{C} & 2.075038 & -2.557746 & 2.221476 \\ \mathrm{C} & 0.821356 & -2.558779 & 1.533510 \\ \mathrm{C} & -0.225793 & -3.389456 & 2.014535 \\ \mathrm{C} & -0.033486 & -4.189484 & 3.116277 \\ \mathrm{C} & 1.211201 & -4.196957 & 3.791219 \\ \mathrm{H} & -1.185095 & -3.396625 & 3.355255 \\ \mathrm{H} & -0.843795 & -4.814267 & 1.511213 \\ & & & 3.478006\end{array}$




\begin{tabular}{|c|c|c|c|}
\hline $\mathrm{H}$ & 1.346568 & -4.832595 & 4.660839 \\
\hline $\mathrm{H}$ & 3.194864 & -3.388920 & 3.873923 \\
\hline C & -1.997104 & -2.686191 & -2.189719 \\
\hline C & -0.766548 & -2.673929 & -1.461772 \\
\hline C & 0.263540 & -3.573728 & -1.843755 \\
\hline C & 0.085122 & -4.440254 & -2.898750 \\
\hline C & -1.130402 & -4.448129 & -3.622634 \\
\hline C & -2.147110 & -3.588985 & -3.274809 \\
\hline $\mathrm{H}$ & 1.193531 & -3.571614 & -1.286537 \\
\hline $\mathrm{H}$ & 0.880623 & -5.124451 & -3.177018 \\
\hline $\mathrm{H}$ & -1.258200 & -5.136171 & -4.452537 \\
\hline $\mathrm{H}$ & -3.083951 & -3.589269 & -3.824831 \\
\hline C & 6.126202 & 1.764823 & -0.388229 \\
\hline C & 6.413350 & 0.404559 & -0.179952 \\
\hline C & 5.359863 & -0.444949 & 0.155851 \\
\hline C & 4.050420 & 0.043079 & 0.293552 \\
\hline C & 3.807671 & 1.403254 & 0.098060 \\
\hline C & 4.837504 & 2.283606 & -0.258549 \\
\hline $\mathrm{H}$ & 6.937345 & 2.427858 & -0.660772 \\
\hline $\mathrm{H}$ & 5.528887 & -1.504804 & 0.299847 \\
\hline $\mathrm{H}$ & 2.798190 & 1.773752 & 0.227102 \\
\hline C & 7.857538 & -0.091591 & -0.340553 \\
\hline C & 4.500233 & 3.762101 & -0.494106 \\
\hline C & 8.337676 & 0.187779 & -1.781168 \\
\hline $\mathrm{H}$ & 9.369071 & -0.156819 & -1.913166 \\
\hline $\mathrm{H}$ & 7.707052 & -0.333759 & -2.507993 \\
\hline $\mathrm{H}$ & 8.308505 & 1.254927 & -2.017736 \\
\hline C & 8.766573 & 0.657055 & 0.658116 \\
\hline $\mathrm{H}$ & 9.803362 & 0.318086 & 0.557750 \\
\hline $\mathrm{H}$ & 8.749861 & 1.737286 & 0.489839 \\
\hline $\mathrm{H}$ & 8.444503 & 0.473997 & 1.687909 \\
\hline C & 7.989586 & -1.599547 & -0.073159 \\
\hline $\mathrm{H}$ & 9.033646 & -1.904700 & -0.192242 \\
\hline $\mathrm{H}$ & 7.681825 & -1.858249 & 0.944765 \\
\hline $\mathrm{H}$ & 7.389677 & -2.189421 & -0.773130 \\
\hline C & 5.730282 & 4.585371 & -0.906498 \\
\hline $\mathrm{H}$ & 5.438614 & 5.626933 & -1.072201 \\
\hline $\mathrm{H}$ & 6.501404 & 4.576863 & -0.129741 \\
\hline $\mathrm{H}$ & 6.174475 & 4.212701 & -1.835007 \\
\hline C & 3.447029 & 3.861565 & -1.620492 \\
\hline $\mathrm{H}$ & 3.172600 & 4.907645 & -1.794883 \\
\hline $\mathrm{H}$ & 3.842834 & 3.452242 & -2.555864 \\
\hline $\mathrm{H}$ & 2.537236 & 3.309559 & -1.368839 \\
\hline C & 3.920738 & 4.366247 & 0.803047 \\
\hline $\mathrm{H}$ & 3.668033 & 5.421398 & 0.652117 \\
\hline $\mathrm{H}$ & 3.013338 & 3.846083 & 1.120990 \\
\hline $\mathrm{H}$ & 4.647291 & 4.301689 & 1.618925 \\
\hline C & -3.975296 & 0.038189 & -0.429277 \\
\hline C & -3.674640 & 1.382257 & -0.163621 \\
\hline C & -5.305689 & -0.378639 & -0.401081 \\
\hline C & -4.672860 & 2.296950 & 0.155762 \\
\hline $\mathrm{H}$ & -2.642154 & 1.699421 & -0.201013 \\
\hline C & -6.342391 & 0.517196 & -0.102729 \\
\hline $\mathrm{H}$ & -5.526207 & -1.425063 & -0.583217 \\
\hline
\end{tabular}




\begin{tabular}{|lrrr|} 
C & -6.002090 & 1.842302 & 0.174520 \\
H & -6.784662 & 2.547255 & 0.423489 \\
C & -7.790822 & 0.008990 & -0.070061 \\
C & -4.359322 & 3.756342 & 0.510896 \\
C & -8.795718 & 1.124474 & 0.259438 \\
H & -8.610482 & 1.557234 & 1.247388 \\
H & -8.763269 & 1.930574 & -0.480378 \\
H & -9.810896 & 0.715950 & 0.261786 \\
C & -8.156049 & -0.577019 & -1.450999 \\
H & -9.185419 & -0.951676 & -1.446593 \\
H & -8.072896 & 0.186308 & -2.231114 \\
H & -7.499308 & -1.407711 & -1.723791 \\
C & -7.918959 & -1.091156 & 1.005672 \\
H & -7.257591 & -1.937049 & 0.799866 \\
H & -7.659673 & -0.698743 & 1.993581 \\
H & -8.946602 & -1.468986 & 1.044018 \\
C & -4.805281 & 4.018193 & 1.966106 \\
H & -5.878009 & 3.848458 & 2.095061 \\
H & -4.274821 & 3.355502 & 2.656164 \\
H & -4.591968 & 5.054705 & 2.249665 \\
C & -2.856690 & 4.068142 & 0.405656 \\
H & -2.261510 & 3.436780 & 1.072048 \\
H & -2.489346 & 3.930783 & -0.617186 \\
H & -2.678120 & 5.111643 & 0.682842 \\
C & -5.121804 & 4.700777 & -0.441773 \\
H & -4.908881 & 5.746038 & -0.192762 \\
H & -4.823037 & 4.529245 & -1.480893 \\
H & -6.203912 & 4.556597 & -0.376884 \\
& & & \\
\hline & & & \\
\hline & & & \\
\hline
\end{tabular}

\section{Catalyst 2C 2}

Datum

Value

\begin{tabular}{|c|c|}
\hline B3LYP-D3(BJ)/6-31G(d,p) Energy & -2165.164107 \\
\hline B3LYP-D3(BJ)/def2-TZVPP/IEF-PCM(DCM) Energy & -2165.904672 \\
\hline B3LYP-D3(BJ)/def2-TZVPP/IEF-PCM(DCM)//B3LYP-D3(BJ)/6-31G(d,p) Free Energy (Quasiharmonic) & -2165.026758 \\
\hline Number of Imaginary Frequencies & 0 \\
\hline \multicolumn{2}{|l|}{ Frequencies (Top 3 out of 321) } \\
\hline $9.5714 \mathrm{~cm}^{-1}$ & \\
\hline 2. $12.3681 \mathrm{~cm}^{-1}$ & \\
\hline 3. $15.2296 \mathrm{~cm}^{-1}$ & \\
\hline
\end{tabular}




\begin{tabular}{|c|c|c|c|}
\hline C & 1.492725 & -0.070655 & -1.286309 \\
\hline $\mathrm{H}$ & 2.450866 & 0.203225 & -1.733541 \\
\hline $\mathrm{H}$ & 0.905152 & -0.633811 & -2.016023 \\
\hline C & -1.585475 & -0.128218 & 1.276281 \\
\hline 0 & -2.271149 & -0.423687 & 2.227123 \\
\hline 0 & -0.722726 & 0.898473 & 1.320575 \\
\hline $\mathrm{H}$ & -0.223244 & 0.983026 & 0.474122 \\
\hline 0 & 0.773283 & 1.139849 & -0.929145 \\
\hline $\mathrm{H}$ & 0.407185 & 1.531412 & -1.732881 \\
\hline C & -0.622627 & -1.754012 & -0.367500 \\
\hline C & -1.667118 & -0.916646 & -0.015513 \\
\hline C & -2.894814 & -0.917311 & -0.754297 \\
\hline C & -3.029528 & -1.790677 & -1.813741 \\
\hline H & -3.942692 & -1.774325 & -2.401355 \\
\hline C & 1.700493 & -0.899919 & -0.045020 \\
\hline C & 0.666428 & -1.718897 & 0.391387 \\
\hline C & 3.104004 & -1.684079 & 1.768689 \\
\hline C & 2.937298 & -0.856800 & 0.674793 \\
\hline $\mathrm{H}$ & 4.039558 & -1.650456 & 2.319078 \\
\hline C & 2.075401 & -2.538034 & 2.228786 \\
\hline C & 0.820981 & -2.546460 & 1.542181 \\
\hline C & -0.222508 & -3.379252 & 2.027403 \\
\hline C & -0.025997 & -4.174431 & 3.131934 \\
\hline C & 1.219393 & -4.174589 & 3.805572 \\
\hline C & 2.245137 & -3.371945 & 3.365536 \\
\hline H & -1.182407 & -3.365290 & 1.525010 \\
\hline $\mathrm{H}$ & -0.833540 & -4.800924 & 3.496881 \\
\hline $\mathrm{H}$ & 1.358077 & -4.806377 & 4.677474 \\
\hline $\mathrm{H}$ & 3.200024 & -3.358589 & 3.883260 \\
\hline C & -2.000585 & -2.691738 & -2.180154 \\
\hline C & -0.770190 & -2.675279 & -1.452011 \\
\hline C & 0.261674 & -3.574087 & -1.831528 \\
\hline C & 0.085049 & -4.443766 & -2.884230 \\
\hline C & -1.130369 & -4.455887 & -3.608234 \\
\hline C & -2.148731 & -3.597712 & -3.262849 \\
\hline H & 1.191589 & -3.568682 & -1.274208 \\
\hline $\mathrm{H}$ & 0.881900 & -5.127163 & -3.160600 \\
\hline H & -1.256755 & -5.146379 & -4.436318 \\
\hline $\mathrm{H}$ & -3.085470 & -3.601186 & -3.813037 \\
\hline C & 6.116008 & 1.783197 & -0.389697 \\
\hline C & 6.401536 & 0.429822 & -0.202398 \\
\hline C & 5.340402 & -0.423463 & 0.134573 \\
\hline C & 4.040361 & 0.059996 & 0.286551 \\
\hline C & 3.793520 & 1.430543 & 0.104501 \\
\hline C & 4.819829 & 2.305773 & -0.243208 \\
\hline H & 6.917419 & 2.459037 & -0.659059 \\
\hline $\mathrm{H}$ & 5.516086 & -1.486128 & 0.266218 \\
\hline H & 2.784345 & 1.792395 & 0.243631 \\
\hline C & 7.814319 & -0.150009 & -0.361607 \\
\hline C & 4.572565 & 3.802412 & -0.477570 \\
\hline C & 8.852337 & 0.925898 & -0.718863 \\
\hline $\mathrm{H}$ & 9.840551 & 0.465992 & -0.815101 \\
\hline
\end{tabular}




\begin{tabular}{|c|c|c|c|}
\hline $\mathrm{H}$ & 8.619678 & 1.413517 & -1.670740 \\
\hline $\mathrm{H}$ & 8.918759 & 1.697149 & 0.054849 \\
\hline C & 8.243684 & -0.813859 & 0.964639 \\
\hline $\mathrm{H}$ & 9.249223 & -1.238095 & 0.870966 \\
\hline $\mathrm{H}$ & 8.254967 & -0.082358 & 1.778586 \\
\hline $\mathrm{H}$ & 7.564991 & -1.622094 & 1.250496 \\
\hline C & 7.807134 & -1.207653 & -1.486386 \\
\hline $\mathrm{H}$ & 8.806626 & -1.637966 & -1.612000 \\
\hline $\mathrm{H}$ & 7.115377 & -2.025715 & -1.267687 \\
\hline $\mathrm{H}$ & 7.505582 & -0.759282 & -2.438232 \\
\hline C & 4.944233 & 4.150985 & -1.935199 \\
\hline $\mathrm{H}$ & 4.780529 & 5.217031 & -2.126746 \\
\hline $\mathrm{H}$ & 5.993303 & 3.928616 & -2.149548 \\
\hline $\mathrm{H}$ & 4.330824 & 3.578936 & -2.638741 \\
\hline C & 3.102125 & 4.189359 & -0.247637 \\
\hline $\mathrm{H}$ & 2.966293 & 5.256458 & -0.448563 \\
\hline $\mathrm{H}$ & 2.427132 & 3.633612 & -0.905574 \\
\hline $\mathrm{H}$ & 2.792329 & 4.002604 & 0.785062 \\
\hline C & 5.448147 & 4.629401 & 0.487697 \\
\hline $\mathrm{H}$ & 5.280657 & 5.700531 & 0.331261 \\
\hline $\mathrm{H}$ & 5.205767 & 4.393729 & 1.528448 \\
\hline $\mathrm{H}$ & 6.513540 & 4.432722 & 0.338830 \\
\hline C & -3.982616 & 0.035976 & -0.429309 \\
\hline C & -3.682162 & 1.380796 & -0.167222 \\
\hline C & -5.313162 & -0.380414 & -0.402509 \\
\hline C & -4.680738 & 2.296770 & 0.147272 \\
\hline $\mathrm{H}$ & -2.649520 & 1.697559 & -0.203538 \\
\hline C & -6.350223 & 0.516671 & -0.109172 \\
\hline $\mathrm{H}$ & -5.533615 & -1.427326 & -0.581906 \\
\hline C & -6.010142 & 1.842558 & 0.164661 \\
\hline $\mathrm{H}$ & -6.793046 & 2.548524 & 0.409704 \\
\hline C & -7.798888 & 0.009013 & -0.078212 \\
\hline C & -4.367366 & 3.757176 & 0.498365 \\
\hline C & -7.929874 & -1.087611 & 1.000765 \\
\hline $\mathrm{H}$ & -7.268261 & -1.934340 & 0.799243 \\
\hline $\mathrm{H}$ & -7.672789 & -0.692082 & 1.988006 \\
\hline $\mathrm{H}$ & -8.957714 & -1.465028 & 1.037918 \\
\hline C & -8.804208 & 1.125863 & 0.245298 \\
\hline $\mathrm{H}$ & -9.819525 & 0.717676 & 0.246523 \\
\hline $\mathrm{H}$ & -8.621201 & 1.561740 & 1.232292 \\
\hline $\mathrm{H}$ & -8.769712 & 1.929571 & -0.497025 \\
\hline C & -8.161084 & -0.581365 & -1.458094 \\
\hline $\mathrm{H}$ & -8.075793 & 0.179381 & -2.240496 \\
\hline $\mathrm{H}$ & -7.504030 & -1.413197 & -1.726625 \\
\hline $\mathrm{H}$ & -9.190608 & -0.955615 & -1.454900 \\
\hline C & -4.816129 & 4.023830 & 1.951838 \\
\hline $\mathrm{H}$ & -5.889161 & 3.854802 & 2.079211 \\
\hline $\mathrm{H}$ & -4.287213 & 3.363216 & 2.645063 \\
\hline $\mathrm{H}$ & -4.603075 & 5.061188 & 2.232484 \\
\hline C & -2.864406 & 4.068119 & 0.395105 \\
\hline $\mathrm{H}$ & -2.270639 & 3.438656 & 1.064531 \\
\hline $\mathrm{H}$ & -2.495096 & 3.927503 & -0.626589 \\
\hline $\mathrm{H}$ & -2.685968 & 5.112410 & 0.669365 \\
\hline$C$ & -5.127650 & 4.698815 & -0.458818 \\
\hline
\end{tabular}




$\begin{array}{llll}\mathrm{H} & -4.914825 & 5.744796 & -0.212767 \\ \mathrm{H} & -4.826915 & 4.523831 & -1.496797 \\ \mathrm{H} & -6.209938 & 4.555230 & -0.395590\end{array}$

\section{Catalyst 2C 3}

Datum

Value

B3LYP-D3(BJ)/6-31G(d,p) Energy

$-2165.164252$

B3LYP-D3(BJ)/def2-TZVPP/IEF-PCM(DCM) Energy

$-2165.90467$

B3LYP-D3(BJ)/def2-TZVPP/IEF-PCM(DCM)//B3LYP-D3(BJ)/6-31G(d,p) Free Energy (Quasiharmonic) - 2165.026668

Number of Imaginary Frequencies

Frequencies (Top 3 out of 321)

$\begin{array}{ll}\text { 1. } & 10.2694 \mathrm{~cm}^{-1} \\ \text { 2. } & 12.4554 \mathrm{~cm}^{-1} \\ \text { 3. } & 14.8528 \mathrm{~cm}^{-1}\end{array}$

\section{B3LYP-D3(BJ)/6-31G(d,p) Molecular Geometry in Cartesian Coordinates}

C

$\mathrm{H}$

$\mathrm{H}$

C

0

0

$\mathrm{H}$

0

$\mathrm{H}$

C

C

C

C

$\mathrm{H}$

C

C

C

C

$\mathrm{H}$

C

C

C

C
1.494688

2.453493

0.910087

$-1.582069$

$-2.258495$

$-0.724476$

$-0.227343$

0.772026

0.406937

$-0.622682$

$-1.668281$

$-2.897170$

$-3.030150$

$-3.943893$

1. 700813

0.666395

3. 102857

2.936952

4.037850

2.074183

0.820282

$-0.223170$

$-0.027134$
$-0.078377$

0.194986

$-0.646088$

$-0.128828$

$-0.422905$

0.903688

0.983027

1.132827

1.518020

$-1.755946$

$-0.920242$

$-0.922573$

$-1.795842$

$-1.782396$

$-0.901477$

$-1.718759$

$-1.678258$

$-0.855273$

$-1.642169$

$-2.530888$

$-2.542167$

$-3.373819$

$-4.165087$
$-1.291533$

$-1.737590$

$-2.020080$

1.276402

2. 233895

1. 308785

0.460341

$-0.942827$

$-1.750119$

$-0.365867$

$-0.012861$

$-0.749633$

$-1.809826$

$-2.396488$

$-0.045910$

0.392961

1.772077

0.674819

2. 323257

2. 234388

1.546850

2.034126

3.141525 


\begin{tabular}{|c|c|c|c|}
\hline C & 1.217681 & -4.162271 & 3.816224 \\
\hline C & 2.243391 & -3.360692 & 3.374220 \\
\hline $\mathrm{H}$ & -1.182658 & -3.362177 & 1.530922 \\
\hline $\mathrm{H}$ & -0.834645 & -4.790766 & 3.507930 \\
\hline $\mathrm{H}$ & 1.355941 & -4.790944 & 4.690439 \\
\hline H & 3.197866 & -3.345078 & 3.892636 \\
\hline C & -1.999745 & -2.694181 & -2.178158 \\
\hline C & -0.769165 & -2.676932 & -1.450488 \\
\hline C & 0.263614 & -3.574391 & -1.830762 \\
\hline C & 0.087786 & -4.443199 & -2.884284 \\
\hline C & -1.127779 & -4.455927 & -3.608148 \\
\hline C & -2.147099 & -3.599386 & -3.261701 \\
\hline $\mathrm{H}$ & 1.193541 & -3.568524 & -1.273442 \\
\hline $\mathrm{H}$ & 0.885293 & -5.125520 & -3.161417 \\
\hline $\mathrm{H}$ & -1.253516 & -5.145791 & -4.436851 \\
\hline $\mathrm{H}$ & -3.084060 & -3.603653 & -3.811493 \\
\hline C & 6.115785 & 1.782026 & -0.395804 \\
\hline C & 6.401774 & 0.429591 & -0.202559 \\
\hline C & 5.340580 & -0.422899 & 0.136214 \\
\hline C & 4.040046 & 0.060457 & 0.284241 \\
\hline C & 3.792707 & 1.430099 & 0.096113 \\
\hline C & 4.819080 & 2.304472 & -0.253561 \\
\hline $\mathrm{H}$ & 6.917253 & 2.457233 & -0.666568 \\
\hline $\mathrm{H}$ & 5.516645 & -1.484919 & 0.272460 \\
\hline $\mathrm{H}$ & 2.783116 & 1.791968 & 0.232302 \\
\hline C & 7.815122 & -0.150086 & -0.357232 \\
\hline C & 4.571511 & 3.800047 & -0.494267 \\
\hline C & 8.853149 & 0.925019 & -0.716880 \\
\hline $\mathrm{H}$ & 9.841774 & 0.465293 & -0.809678 \\
\hline $\mathrm{H}$ & 8.621845 & 1.408843 & -1.671019 \\
\hline $\mathrm{H}$ & 8.917840 & 1.699273 & 0.053975 \\
\hline C & 8.242686 & -0.808658 & 0.972220 \\
\hline $\mathrm{H}$ & 9.248618 & -1.232659 & 0.881789 \\
\hline $\mathrm{H}$ & 8.252226 & -0.074078 & 1.783412 \\
\hline $\mathrm{H}$ & 7.564006 & -1.616209 & 1.260033 \\
\hline C & 7.810308 & -1.211993 & -1.478001 \\
\hline $\mathrm{H}$ & 8.810221 & -1.642263 & -1.600354 \\
\hline $\mathrm{H}$ & 7.118611 & -2.029574 & -1.257318 \\
\hline $\mathrm{H}$ & 7.510081 & -0.767392 & -2.432031 \\
\hline C & 4.946306 & 4.143302 & -1.952355 \\
\hline $\mathrm{H}$ & 4.782499 & 5.208531 & -2.148290 \\
\hline $\mathrm{H}$ & 5.995972 & 3.920688 & -2.163484 \\
\hline $\mathrm{H}$ & 4.334797 & 3.568289 & -2.655135 \\
\hline C & 3.100339 & 4.187011 & -0.269170 \\
\hline $\mathrm{H}$ & 2.964328 & 5.253207 & -0.474713 \\
\hline $\mathrm{H}$ & 2.427228 & 3.628156 & -0.926428 \\
\hline $\mathrm{H}$ & 2.788405 & 4.004239 & 0.763603 \\
\hline C & 5.444421 & 4.631178 & 0.469870 \\
\hline $\mathrm{H}$ & 5.276688 & 5.701617 & 0.309029 \\
\hline $\mathrm{H}$ & 5.199828 & 4.399276 & 1.510948 \\
\hline $\mathrm{H}$ & 6.510252 & 4.434527 & 0.324137 \\
\hline C & -3.990220 & 0.024565 & -0.422981 \\
\hline C & -3.706916 & 1.360767 & -0.143210 \\
\hline$\sigma$ & -5.325920 & -0.406124 & -0.416153 \\
\hline
\end{tabular}




\begin{tabular}{|c|c|c|c|}
\hline C & -4.720032 & 2.273971 & 0.170975 \\
\hline $\mathrm{H}$ & -2.676547 & 1.694017 & -0.159254 \\
\hline C & -6.364326 & 0.477277 & -0.127117 \\
\hline $\mathrm{H}$ & -5.529248 & -1.451515 & -0.610265 \\
\hline C & -6.036511 & 1.812265 & 0.166285 \\
\hline $\mathrm{H}$ & -6.837419 & 2.499946 & 0.405565 \\
\hline C & -7.836279 & 0.041733 & -0.097778 \\
\hline C & -4.337143 & 3.717014 & 0.524595 \\
\hline C & -8.635436 & 0.875757 & -1.122288 \\
\hline $\mathrm{H}$ & -8.582817 & 1.945612 & -0.902830 \\
\hline $\mathrm{H}$ & -8.247870 & 0.722388 & -2.134427 \\
\hline $\mathrm{H}$ & -9.691010 & 0.583004 & -1.111679 \\
\hline C & -8.011844 & -1.444905 & -0.446949 \\
\hline $\mathrm{H}$ & -9.074288 & -1.705963 & -0.422453 \\
\hline H & -7.636880 & -1.671768 & -1.450061 \\
\hline $\mathrm{H}$ & -7.494345 & -2.092242 & 0.267598 \\
\hline C & -8.409741 & 0.276843 & 1.316391 \\
\hline $\mathrm{H}$ & -7.853516 & -0.301207 & 2.060511 \\
\hline $\mathrm{H}$ & -8.356609 & 1.330345 & 1.604189 \\
\hline $\mathrm{H}$ & -9.460690 & -0.029813 & 1.357512 \\
\hline C & -5.565028 & 4.588003 & 0.833125 \\
\hline $\mathrm{H}$ & -6.243179 & 4.652606 & -0.024103 \\
\hline $\mathrm{H}$ & -6.128258 & 4.202553 & 1.688590 \\
\hline $\mathrm{H}$ & -5.243022 & 5.604285 & 1.079963 \\
\hline C & -3.423555 & 3.697575 & 1.770740 \\
\hline $\mathrm{H}$ & -3.947188 & 3.258116 & 2.624954 \\
\hline $\mathrm{H}$ & -2.516715 & 3.109792 & 1.606696 \\
\hline $\mathrm{H}$ & -3.125628 & 4.717661 & 2.037959 \\
\hline C & -3.578892 & 4.353413 & -0.659997 \\
\hline $\mathrm{H}$ & -3.295157 & 5.383841 & -0.420250 \\
\hline $\mathrm{H}$ & -2.663516 & 3.803316 & -0.895556 \\
\hline H & -4.202694 & 4.369936 & -1.559514 \\
\hline
\end{tabular}

\section{Catalyst 2C 4}

Datum

Value

B3LYP-D3(BJ)/6-31G(d,p) Energy

$-2165.164947$

B3LYP-D3(BJ)/def2-TZVPP/IEF-PCM(DCM) Energy

$-2165.904868$

B3LYP-D3(BJ)/def2-TZVPP/IEF-PCM(DCM)//B3LYP-D3(BJ)/6-31G(d,p) Free Energy (Quasiharmonic)

$-2165.026667$

Number of Imaginary Frequencies

Frequencies (Top 3 out of 321)

$\begin{array}{ll}\text { 1. } & 10.4074 \mathrm{~cm}^{-1} \\ \text { 2. } & 12.3131 \mathrm{~cm}^{-1} \\ \text { 3. } & 14.8474 \mathrm{~cm}^{-1}\end{array}$




\begin{tabular}{|c|c|c|c|}
\hline C & 1.506882 & -0.086396 & -1.292648 \\
\hline $\mathrm{H}$ & 2.466387 & 0.183330 & -1.738848 \\
\hline $\mathrm{H}$ & 0.918511 & -0.650032 & -2.021134 \\
\hline C & -1.572442 & -0.133122 & 1.272986 \\
\hline 0 & -2.247682 & -0.427628 & 2.231074 \\
\hline 0 & -0.713565 & 0.898666 & 1.305670 \\
\hline $\mathrm{H}$ & -0.217367 & 0.978062 & 0.457026 \\
\hline 0 & 0.789759 & 1.129272 & -0.944186 \\
\hline $\mathrm{H}$ & 0.433689 & 1.519450 & -1.753221 \\
\hline C & -0.617174 & -1.758719 & -0.373036 \\
\hline C & -1.661054 & -0.921857 & -0.017644 \\
\hline C & -2.890501 & -0.920400 & -0.753497 \\
\hline C & -3.025465 & -1.790582 & -1.815959 \\
\hline $\mathrm{H}$ & -3.939669 & -1.774327 & -2.401828 \\
\hline C & 1.708993 & -0.911829 & -0.047721 \\
\hline C & 0.671471 & -1.727662 & 0.386934 \\
\hline C & 3.106469 & -1.701220 & 1.768731 \\
\hline C & 2.944579 & -0.872230 & 0.675334 \\
\hline $\mathrm{H}$ & 4.040959 & -1.671321 & 2.321126 \\
\hline C & 2.074516 & -2.552132 & 2.226457 \\
\hline C & 0.821187 & -2.556021 & 1.537831 \\
\hline C & -0.225625 & -3.386006 & 2.020795 \\
\hline C & -0.033344 & -4.182573 & 3.125029 \\
\hline C & 1.210936 & -4.187082 & 3.800750 \\
\hline C & 2.239849 & -3.387375 & 3.362930 \\
\hline $\mathrm{H}$ & -1.184638 & -3.368966 & 1.516868 \\
\hline $\mathrm{H}$ & -0.843392 & -4.806894 & 3.488134 \\
\hline $\mathrm{H}$ & 1.346238 & -4.819964 & 4.672385 \\
\hline $\mathrm{H}$ & 3.193989 & -3.377419 & 3.882088 \\
\hline C & -1.996650 & -2.689480 & -2.187371 \\
\hline C & -0.765808 & -2.676534 & -1.460058 \\
\hline C & 0.265104 & -3.575004 & -1.842994 \\
\hline C & 0.087287 & -4.440611 & -2.898815 \\
\hline C & -1.128493 & -4.449009 & -3.622375 \\
\hline C & -2.146067 & -3.591456 & -3.273316 \\
\hline $\mathrm{H}$ & 1.195192 & -3.572493 & -1.285915 \\
\hline $\mathrm{H}$ & 0.883375 & -5.123752 & -3.177992 \\
\hline $\mathrm{H}$ & -1.255798 & -5.136384 & -4.452904 \\
\hline $\mathrm{H}$ & -3.083204 & -3.592455 & -3.822819 \\
\hline C & 6.124521 & 1.764779 & -0.394197 \\
\hline C & 6.412687 & 0.405523 & -0.180818 \\
\hline C & 5.359605 & -0.443770 & 0.156785 \\
\hline C & 4.049572 & 0.043539 & 0.291325 \\
\hline C & 3.805779 & 1.402783 & 0.090710 \\
\hline C & 4.835199 & 2.282833 & -0.267838 \\
\hline $\mathrm{H}$ & 6.935375 & 2.427605 & -0.668096 \\
\hline $\mathrm{H}$ & 5.529447 & -1.502952 & 0.304705 \\
\hline $\mathrm{H}$ & 2.795809 & 1.772802 & 0.217416 \\
\hline
\end{tabular}




\begin{tabular}{|c|c|c|c|}
\hline C & 7.857530 & -0.089827 & -0.337928 \\
\hline C & 4.496881 & 3.760184 & -0.508974 \\
\hline C & 8.764528 & 0.662899 & 0.659527 \\
\hline $\mathrm{H}$ & 9.801776 & 0.324625 & 0.561602 \\
\hline $\mathrm{H}$ & 8.746974 & 1.742568 & 0.487767 \\
\hline H & 8.441288 & 0.482824 & 1.689479 \\
\hline C & 7.990594 & -1.596788 & -0.065479 \\
\hline $\mathrm{H}$ & 9.035076 & -1.901381 & -0.182260 \\
\hline $\mathrm{H}$ & 7.681797 & -1.852475 & 0.952892 \\
\hline $\mathrm{H}$ & 7.392099 & -2.189470 & -0.764289 \\
\hline C & 8.339348 & 0.185304 & -1.778799 \\
\hline $\mathrm{H}$ & 9.371205 & -0.158856 & -1.908286 \\
\hline $\mathrm{H}$ & 7.710146 & -0.339118 & -2.504780 \\
\hline H & 8.309619 & 1.251655 & -2.018866 \\
\hline C & 5.726687 & 4.583148 & -0.922706 \\
\hline $\mathrm{H}$ & 5.434262 & 5.623849 & -1.092429 \\
\hline $\mathrm{H}$ & 6.496824 & 4.578084 & -0.144945 \\
\hline $\mathrm{H}$ & 6.172403 & 4.207644 & -1.849340 \\
\hline C & 3.445029 & 3.854718 & -1.637047 \\
\hline $\mathrm{H}$ & 3.169667 & 4.899898 & -1.815316 \\
\hline $\mathrm{H}$ & 3.842506 & 3.442719 & -2.570530 \\
\hline $\mathrm{H}$ & 2.535530 & 3.302480 & -1.384798 \\
\hline C & 3.915270 & 4.368328 & 0.785361 \\
\hline $\mathrm{H}$ & 3.661763 & 5.422709 & 0.630447 \\
\hline $\mathrm{H}$ & 3.008015 & 3.848410 & 1.104127 \\
\hline $\mathrm{H}$ & 4.640915 & 4.307322 & 1.602316 \\
\hline C & -3.982486 & 0.026532 & -0.422646 \\
\hline C & -3.698234 & 1.361869 & -0.139779 \\
\hline C & -5.318284 & -0.403842 & -0.414210 \\
\hline C & -4.710473 & 2.274440 & 0.179087 \\
\hline $\mathrm{H}$ & -2.667813 & 1.694907 & -0.157067 \\
\hline C & -6.355851 & 0.478982 & -0.120444 \\
\hline $\mathrm{H}$ & -5.522264 & -1.448650 & -0.610760 \\
\hline C & -6.027067 & 1.813064 & 0.175956 \\
\hline $\mathrm{H}$ & -6.827281 & 2.500263 & 0.418916 \\
\hline C & -7.827820 & 0.043656 & -0.088892 \\
\hline C & -4.326520 & 3.716407 & 0.535924 \\
\hline C & -8.004510 & -1.441944 & -0.441895 \\
\hline $\mathrm{H}$ & -7.631922 & -1.666026 & -1.446516 \\
\hline $\mathrm{H}$ & -7.485503 & -2.091425 & 0.269606 \\
\hline $\mathrm{H}$ & -9.066949 & -1.702850 & -0.415677 \\
\hline C & -8.397861 & 0.274819 & 1.327314 \\
\hline $\mathrm{H}$ & -9.448768 & -0.031756 & 1.370055 \\
\hline $\mathrm{H}$ & -7.839982 & -0.305471 & 2.068448 \\
\hline $\mathrm{H}$ & -8.343841 & 1.327480 & 1.618010 \\
\hline C & -8.629242 & 0.880769 & -1.109098 \\
\hline $\mathrm{H}$ & -8.575841 & 1.949984 & -0.886732 \\
\hline $\mathrm{H}$ & -8.244163 & 0.730182 & -2.122602 \\
\hline $\mathrm{H}$ & -9.684855 & 0.588233 & -1.096761 \\
\hline C & -3.570998 & 4.356033 & -0.648672 \\
\hline $\mathrm{H}$ & -2.656444 & 3.806292 & -0.888230 \\
\hline $\mathrm{H}$ & -4.197036 & 4.375402 & -1.546570 \\
\hline $\mathrm{H}$ & -3.286345 & 5.385676 & -0.406626 \\
\hline$c$ & -5.553501 & 4.586731 & 0.849871 \\
\hline
\end{tabular}




$\begin{array}{|lrrr|}\text { H } & -6.233651 & 4.653933 & -0.005565 \\ \text { H } & -6.114777 & 4.198924 & 1.705553 \\ \text { H } & -5.230707 & 5.602234 & 1.098871 \\ \text { C } & -3.410031 & 3.693253 & 1.779875 \\ \text { H } & -3.111243 & 4.712505 & 2.049308 \\ \text { H } & -3.931750 & 3.251482 & 2.634065 \\ \text { H } & -2.503731 & 3.105687 & 1.612087\end{array}$

\section{Catalyst 2C 5}

\section{Datum}

Value

B3LYP-D3(BJ)/6-31G(d,p) Energy

$-2165.16408$

B3LYP-D3(BJ)/def2-TZVPP/IEF-PCM(DCM) Energy

$-2165.904555$

B3LYP-D3(BJ)/def2-TZVPP/IEF-PCM(DCM)//B3LYP-D3(BJ)/6-31G(d,p) Free Energy (Quasiharmonic) -2165.026618

Number of Imaginary Frequencies

0

Frequencies (Top 3 out of 321)

$\begin{array}{lr}\text { 1. } & 9.5668 \mathrm{~cm}^{-1} \\ \text { 2. } & 12.2424 \mathrm{~cm}^{-1} \\ \text { 3. } & 15.3600 \mathrm{~cm}^{-1}\end{array}$

\section{B3LYP-D3(BJ)/6-31G(d,p) Molecular Geometry in Cartesian Coordinates}

$\begin{array}{lrrr}\mathrm{C} & 1.492004 & -0.093750 & -1.286396 \\ \mathrm{H} & 2.450294 & 0.180081 & -1.733256 \\ \mathrm{H} & 0.904566 & -0.657009 & -2.016100 \\ \mathrm{C} & -1.585512 & -0.153064 & 1.274004 \\ \mathrm{O} & -2.270165 & -0.450511 & 2.224935 \\ \mathrm{O} & -0.723428 & 0.874291 & 1.319435 \\ \mathrm{H} & -0.223842 & 0.959691 & 0.473113 \\ \mathrm{O} & 0.772311 & 1.117112 & -0.930238 \\ \mathrm{H} & 0.403674 & 1.506121 & -1.734056 \\ \mathrm{C} & -0.623015 & -1.777827 & -0.370401 \\ \mathrm{C} & -1.667078 & -0.939679 & -0.018969 \\ \mathrm{C} & -2.894143 & -0.938372 & -0.759068 \\ \mathrm{C} & -3.029004 & -1.812504 & -1.818014 \\ \mathrm{H} & -3.941774 & -1.796013 & -2.406192 \\ \mathrm{C} & 1.699484 & -0.923103 & -0.045135 \\ \mathrm{C} & 0.665640 & -1.743562 & 0.389238 \\ \mathrm{C} & 3.102349 & -1.710119 & 1.767707 \\ \mathrm{C} & 2.935805 & -0.879865 & 0.675876 \\ \mathrm{H} & 4.037484 & -1.677442 & 2.318832\end{array}$




\begin{tabular}{|c|c|c|c|}
\hline C & 2.074204 & -2.565829 & 2.225324 \\
\hline C & 0.819971 & -2.573293 & 1.538400 \\
\hline C & -0.223339 & -3.407569 & 2.021467 \\
\hline C & -0.026766 & -4.205228 & 3.124182 \\
\hline C & 1.218516 & -4.206468 & 3.798079 \\
\hline C & 2.244011 & -3.402375 & 3.360175 \\
\hline $\mathrm{H}$ & -1.183094 & -3.392807 & 1.518810 \\
\hline $\mathrm{H}$ & -0.834118 & -4.832924 & 3.487490 \\
\hline $\mathrm{H}$ & 1.357288 & -4.840297 & 4.668489 \\
\hline $\mathrm{H}$ & 3.198794 & -3.389897 & 3.878113 \\
\hline C & -2.000719 & -2.714597 & -2.183573 \\
\hline C & -0.770567 & -2.698904 & -1.455030 \\
\hline C & 0.260835 & -3.598464 & -1.833982 \\
\hline C & 0.083952 & -4.468312 & -2.886505 \\
\hline C & -1.131295 & -4.479841 & -3.610828 \\
\hline C & -2.149170 & -3.620875 & -3.266003 \\
\hline $\mathrm{H}$ & 1.190548 & -3.593563 & -1.276311 \\
\hline $\mathrm{H}$ & 0.880415 & -5.152339 & -3.162442 \\
\hline $\mathrm{H}$ & -1.257946 & -5.170550 & -4.438695 \\
\hline $\mathrm{H}$ & -3.085789 & -3.623958 & -3.816398 \\
\hline C & 6.107536 & 1.764938 & -0.377290 \\
\hline C & 4.815974 & 2.286594 & -0.237087 \\
\hline C & 3.788417 & 1.406171 & 0.108652 \\
\hline C & 4.037750 & 0.040028 & 0.291867 \\
\hline C & 5.343046 & -0.445824 & 0.145877 \\
\hline C & 6.398461 & 0.408350 & -0.187141 \\
\hline $\mathrm{H}$ & 6.914929 & 2.437861 & -0.645792 \\
\hline $\mathrm{H}$ & 2.778313 & 1.766778 & 0.244082 \\
\hline $\mathrm{H}$ & 5.513417 & -1.506688 & 0.280580 \\
\hline C & 4.564687 & 3.782294 & -0.474159 \\
\hline C & 7.841747 & -0.087193 & -0.358844 \\
\hline C & 4.937524 & 4.129045 & -1.931839 \\
\hline $\mathrm{H}$ & 4.771441 & 5.194267 & -2.125911 \\
\hline $\mathrm{H}$ & 5.987478 & 3.908670 & -2.144047 \\
\hline $\mathrm{H}$ & 4.326874 & 3.553910 & -2.635242 \\
\hline C & 3.092798 & 4.165387 & -0.246803 \\
\hline $\mathrm{H}$ & 2.954418 & 5.231893 & -0.449118 \\
\hline $\mathrm{H}$ & 2.420216 & 3.607120 & -0.905043 \\
\hline $\mathrm{H}$ & 2.782001 & 3.978962 & 0.785660 \\
\hline C & 5.436701 & 4.612979 & 0.491011 \\
\hline $\mathrm{H}$ & 5.266793 & 5.683471 & 0.332864 \\
\hline $\mathrm{H}$ & 5.193785 & 4.378124 & 1.531805 \\
\hline $\mathrm{H}$ & 6.502793 & 4.418674 & 0.343877 \\
\hline C & 8.755240 & 0.650248 & 0.643946 \\
\hline $\mathrm{H}$ & 9.791474 & 0.311946 & 0.535921 \\
\hline $\mathrm{H}$ & 8.738214 & 1.732229 & 0.487199 \\
\hline $\mathrm{H}$ & 8.437184 & 0.456720 & 1.673057 \\
\hline C & 7.973558 & -1.598062 & -0.108114 \\
\hline $\mathrm{H}$ & 9.016754 & -1.902834 & -0.235419 \\
\hline $\mathrm{H}$ & 7.670474 & -1.867382 & 0.908453 \\
\hline $\mathrm{H}$ & 7.369762 & -2.179929 & -0.811427 \\
\hline C & 8.315562 & 0.207386 & -1.798421 \\
\hline $\mathrm{H}$ & 9.346200 & -0.136076 & -1.938999 \\
\hline $\mathrm{H}$ & 7.681344 & -0.305724 & -2.528089 \\
\hline
\end{tabular}




\begin{tabular}{|c|c|c|c|}
\hline $\mathrm{H}$ & 8.285594 & 1.277089 & -2.023184 \\
\hline C & -3.980481 & 0.017146 & -0.435844 \\
\hline C & -3.678481 & 1.357136 & -0.170317 \\
\hline C & -5.315612 & -0.401821 & -0.415424 \\
\hline C & -4.678160 & 2.277176 & 0.145183 \\
\hline $\mathrm{H}$ & -2.645503 & 1.673082 & -0.203075 \\
\hline C & -6.346913 & 0.494829 & -0.123197 \\
\hline $\mathrm{H}$ & -5.530028 & -1.446850 & -0.599500 \\
\hline C & -6.002179 & 1.823160 & 0.156552 \\
\hline $\mathrm{H}$ & -6.791500 & 2.525322 & 0.402733 \\
\hline C & -7.822181 & 0.071426 & -0.077678 \\
\hline C & -4.361581 & 3.735438 & 0.502929 \\
\hline C & -8.012873 & -1.415829 & -0.416257 \\
\hline $\mathrm{H}$ & -7.648400 & -1.651871 & -1.421109 \\
\hline $\mathrm{H}$ & -7.494707 & -2.063148 & 0.297809 \\
\hline $\mathrm{H}$ & -9.077183 & -1.667874 & -0.381141 \\
\hline C & -8.380191 & 0.319754 & 1.340338 \\
\hline $\mathrm{H}$ & -9.433109 & 0.021837 & 1.393519 \\
\hline $\mathrm{H}$ & -7.821388 & -0.257896 & 2.082806 \\
\hline $\mathrm{H}$ & -8.315788 & 1.374593 & 1.620980 \\
\hline C & -8.623632 & 0.905993 & -1.099811 \\
\hline $\mathrm{H}$ & -8.559474 & 1.976665 & -0.887405 \\
\hline $\mathrm{H}$ & -8.246790 & 0.743402 & -2.114542 \\
\hline $\mathrm{H}$ & -9.681577 & 0.622608 & -1.077747 \\
\hline C & -2.857965 & 4.043735 & 0.400693 \\
\hline $\mathrm{H}$ & -2.265283 & 3.410760 & 1.067748 \\
\hline $\mathrm{H}$ & -2.488988 & 3.906225 & -0.621550 \\
\hline $\mathrm{H}$ & -2.677544 & 5.086656 & 0.678819 \\
\hline C & -5.120269 & 4.682851 & -0.449648 \\
\hline $\mathrm{H}$ & -4.820290 & 4.512153 & -1.488541 \\
\hline $\mathrm{H}$ & -6.202803 & 4.540764 & -0.386892 \\
\hline $\mathrm{H}$ & -4.905684 & 5.727327 & -0.198821 \\
\hline C & -4.809625 & 3.995588 & 1.957726 \\
\hline $\mathrm{H}$ & -4.594655 & 5.031053 & 2.243855 \\
\hline $\mathrm{H}$ & -5.882979 & 3.827659 & 2.084111 \\
\hline $\mathrm{H}$ & -4.282166 & 3.330302 & 2.647568 \\
\hline
\end{tabular}

\section{Catalyst 2C 6}

\section{Datum}

Value

B3LYP-D3(BJ)/6-31G(d,p) Energy

$-2165.163977$

B3LYP-D3(BJ)/def2-TZVPP/IEF-PCM(DCM) Energy

$-2165.904335$

B3LYP-D3(BJ)/def2-TZVPP/IEF-PCM(DCM)//B3LYP-D3(BJ)/6-31G(d,p) Free Energy (Quasiharmonic)

$-2165.026478$

Number of Imaginary Frequencies

Frequencies (Top 3 out of 321) 

1. $9.6931 \mathrm{~cm}^{-1}$
2. $\quad 12.0465 \mathrm{~cm}^{-1}$
3. $14.6124 \mathrm{~cm}^{-1}$

B3LYP-D3(BJ)/6-31G(d,p) Molecular Geometry in Cartesian Coordinates

\begin{tabular}{|c|c|c|c|}
\hline C & -1.487620 & -0.093571 & -1.291318 \\
\hline $\mathrm{H}$ & -0.896756 & -0.654560 & -2.019985 \\
\hline $\mathrm{H}$ & -2.449347 & 0.169454 & -1.737209 \\
\hline C & 1.589218 & -0.111870 & 1.276914 \\
\hline 0 & 2.269614 & -0.398321 & 2.233921 \\
\hline 0 & 0.719898 & 0.910660 & 1.309783 \\
\hline $\mathrm{H}$ & 0.222133 & 0.985003 & 0.461259 \\
\hline 0 & -0.778116 & 1.125466 & -0.942428 \\
\hline $\mathrm{H}$ & -0.415141 & 1.513383 & -1.749374 \\
\hline C & 0.648054 & -1.748068 & -0.367515 \\
\hline C & 1.684400 & -0.901684 & -0.012657 \\
\hline C & 2.913832 & -0.890538 & -0.748189 \\
\hline C & 3.056619 & -1.759519 & -1.810483 \\
\hline $\mathrm{H}$ & 3.970506 & -1.734655 & -2.396599 \\
\hline C & -1.684811 & -0.919429 & -0.046098 \\
\hline C & -0.641479 & -1.726281 & 0.391173 \\
\hline C & -3.077547 & -1.714480 & 1.770989 \\
\hline C & -2.921066 & -0.886959 & 0.675579 \\
\hline $\mathrm{H}$ & -4.012507 & -1.689759 & 2.322811 \\
\hline C & -2.039796 & -2.556999 & 2.231261 \\
\hline C & -0.785928 & -2.553309 & 1.543623 \\
\hline C & 0.266620 & -3.374406 & 2.029281 \\
\hline C & 0.079366 & -4.169856 & 3.135188 \\
\hline C & -1.165394 & -4.181988 & 3.809936 \\
\hline C & -2.199829 & -3.390864 & 3.369506 \\
\hline $\mathrm{H}$ & 1.225881 & -3.351397 & 1.526035 \\
\hline $\mathrm{H}$ & 0.893720 & -4.787322 & 3.500379 \\
\hline $\mathrm{H}$ & -1.296711 & -4.813841 & 4.682928 \\
\hline $\mathrm{H}$ & -3.154404 & -3.386743 & 3.887951 \\
\hline C & 2.035992 & -2.668105 & -2.181069 \\
\hline C & 0.805186 & -2.665670 & -1.453540 \\
\hline C & -0.217590 & -3.573771 & -1.835603 \\
\hline C & -0.032063 & -4.438643 & -2.890696 \\
\hline C & 1.183594 & -4.436571 & -3.614505 \\
\hline C & 2.193383 & -3.569478 & -3.266365 \\
\hline $\mathrm{H}$ & -1.147565 & -3.579188 & -1.278355 \\
\hline $\mathrm{H}$ & -0.821932 & -5.129269 & -3.169127 \\
\hline $\mathrm{H}$ & 1.316980 & -5.123424 & -4.444511 \\
\hline $\mathrm{H}$ & 3.130343 & -3.562320 & -3.816134 \\
\hline C & -6.123371 & 1.718114 & -0.384041 \\
\hline C & -6.399153 & 0.359436 & -0.186500 \\
\hline C & -5.333770 & -0.481577 & 0.148219 \\
\hline C & -4.033495 & 0.019090 & 0.288954 \\
\hline c & -3.799393 & 1.386942 & 0.098389 \\
\hline
\end{tabular}




\begin{tabular}{|c|c|c|c|}
\hline C & -4.837215 & 2.254416 & -0.249446 \\
\hline $\mathrm{H}$ & -6.938612 & 2.380957 & -0.653909 \\
\hline $\mathrm{H}$ & -5.492378 & -1.543538 & 0.288473 \\
\hline $\mathrm{H}$ & -2.793022 & 1.759261 & 0.230069 \\
\hline C & -7.837390 & -0.152531 & -0.352125 \\
\hline C & -4.602918 & 3.751616 & -0.494283 \\
\hline C & -8.318013 & 0.129808 & -1.791903 \\
\hline $\mathrm{H}$ & -9.345178 & -0.225603 & -1.928100 \\
\hline $\mathrm{H}$ & -8.300335 & 1.198657 & -2.021973 \\
\hline $\mathrm{H}$ & -7.680080 & -0.379931 & -2.520696 \\
\hline C & -7.952232 & -1.663491 & -0.093676 \\
\hline $\mathrm{H}$ & -8.992385 & -1.980105 & -0.216874 \\
\hline $\mathrm{H}$ & -7.343915 & -2.242257 & -0.795655 \\
\hline $\mathrm{H}$ & -7.643773 & -1.924519 & 0.923439 \\
\hline C & -8.756296 & 0.579901 & 0.649400 \\
\hline $\mathrm{H}$ & -9.789051 & 0.229805 & 0.545733 \\
\hline $\mathrm{H}$ & -8.433536 & 0.394940 & 1.678622 \\
\hline $\mathrm{H}$ & -8.751471 & 1.661215 & 0.487287 \\
\hline C & -4.984182 & 4.087547 & -1.952319 \\
\hline $\mathrm{H}$ & -4.830592 & 5.153648 & -2.151753 \\
\hline $\mathrm{H}$ & -4.369408 & 3.516038 & -2.655088 \\
\hline $\mathrm{H}$ & -6.032282 & 3.854558 & -2.160156 \\
\hline C & -5.480862 & 4.577166 & 0.469927 \\
\hline $\mathrm{H}$ & -5.323285 & 5.648715 & 0.306289 \\
\hline $\mathrm{H}$ & -6.545206 & 4.370407 & 0.327187 \\
\hline $\mathrm{H}$ & -5.232005 & 4.349830 & 1.510984 \\
\hline C & -3.134583 & 4.151709 & -0.273450 \\
\hline $\mathrm{H}$ & -3.008386 & 5.218640 & -0.481390 \\
\hline $\mathrm{H}$ & -2.818610 & 3.973701 & 0.758930 \\
\hline $\mathrm{H}$ & -2.458113 & 3.597501 & -0.931170 \\
\hline C & 3.997670 & 0.065828 & -0.417558 \\
\hline C & 5.333409 & -0.347629 & -0.404609 \\
\hline C & 3.701073 & 1.402944 & -0.138135 \\
\hline C & 6.367612 & 0.546714 & -0.111753 \\
\hline $\mathrm{H}$ & 5.554354 & -1.392201 & -0.596526 \\
\hline C & 4.702642 & 2.322336 & 0.178073 \\
\hline $\mathrm{H}$ & 2.667389 & 1.725733 & -0.157501 \\
\hline C & 6.029370 & 1.874691 & 0.178982 \\
\hline $\mathrm{H}$ & 6.816180 & 2.574187 & 0.419849 \\
\hline C & 4.306773 & 3.762944 & 0.529590 \\
\hline C & 7.818468 & 0.043054 & -0.097105 \\
\hline C & 5.525915 & 4.645280 & 0.841292 \\
\hline $\mathrm{H}$ & 6.090245 & 4.265896 & 1.698700 \\
\hline H & 6.206193 & 4.715856 & -0.013728 \\
\hline $\mathrm{H}$ & 5.193524 & 5.658650 & 1.086458 \\
\hline C & 3.546638 & 4.392494 & -0.657499 \\
\hline $\mathrm{H}$ & 3.254182 & 5.420914 & -0.419486 \\
\hline $\mathrm{H}$ & 4.172606 & 4.413145 & -1.555434 \\
\hline H & 2.636323 & 3.834878 & -0.894958 \\
\hline C & 3.389656 & 3.737099 & 1.773068 \\
\hline $\mathrm{H}$ & 2.488259 & 3.141662 & 1.606635 \\
\hline $\mathrm{H}$ & 3.914218 & 3.302467 & 2.629191 \\
\hline $\mathrm{H}$ & 3.082593 & 4.754898 & 2.038776 \\
\hline$C$ & 8.171331 & -0.535415 & -1.484461 \\
\hline
\end{tabular}




\begin{tabular}{|rrrr|}
$H$ & 7.515052 & -1.367583 & -1.753834 \\
$H$ & 8.076648 & 0.230928 & -2.260304 \\
$H$ & 9.202243 & -0.905819 & -1.492864 \\
$\mathrm{C}$ & 8.824037 & 1.159759 & 0.226857 \\
$\mathrm{H}$ & 8.781773 & 1.969399 & -0.508540 \\
$\mathrm{H}$ & 8.648786 & 1.587535 & 1.218747 \\
$\mathrm{H}$ & 9.840141 & 0.753594 & 0.216104 \\
$\mathrm{C}$ & 7.961896 & -1.061793 & 0.971902 \\
$\mathrm{H}$ & 8.991243 & -1.436108 & 0.997910 \\
$\mathrm{H}$ & 7.711434 & -0.675144 & 1.964362 \\
$\mathrm{H}$ & 7.301326 & -1.908951 & 0.768716 \\
& & & \\
\hline
\end{tabular}

\section{Catalyst 2C 7}

\section{Datum}

Value

\begin{tabular}{lc}
\hline B3LYP-D3(BJ)/6-31G(d,p) Energy & -2165.164634 \\
\hline B3LYP-D3(BJ)/def2-TZVPP/IEF-PCM(DCM) Energy & -2165.904645 \\
\hline B3LYP-D3(BJ)/def2-TZVPP/IEF-PCM(DCM)//B3LYP-D3(BJ)/6-31G(d,p) Free Energy (Quasiharmonic) & -2165.02646 \\
\hline
\end{tabular}

Number of Imaginary Frequencies

Frequencies (Top 3 out of 321)

$\begin{array}{ll}\text { 1. } & 10.1665 \mathrm{~cm}^{-1} \\ \text { 2. } & 12.6220 \mathrm{~cm}^{-1} \\ \text { 3. } & 15.4364 \mathrm{~cm}^{-1}\end{array}$

\section{B3LYP-D3(BJ)/6-31G(d,p) Molecular Geometry in Cartesian Coordinates}

$\begin{array}{lrrr}\mathrm{C} & 1.512679 & -0.063049 & -1.288011 \\ \mathrm{H} & 2.468687 & 0.216962 & -1.735528 \\ \mathrm{H} & 0.927571 & -0.629039 & -2.017349 \\ \mathrm{C} & -1.568656 & -0.147627 & 1.271159 \\ \mathrm{O} & -2.249157 & -0.450715 & 2.223186 \\ \mathrm{O} & -0.716398 & 0.888297 & 1.314822 \\ \mathrm{H} & -0.218279 & 0.977378 & 0.468371 \\ \mathrm{O} & 0.785951 & 1.144417 & -0.931994 \\ \mathrm{H} & 0.426915 & 1.537951 & -1.738059 \\ \mathrm{C} & -0.591458 & -1.763165 & -0.373373 \\ \mathrm{C} & -1.643573 & -0.935376 & -0.021374 \\ \mathrm{C} & -2.871027 & -0.946067 & -0.760753 \\ \mathrm{C} & -2.997379 & -1.820704 & -1.820329 \\ \mathrm{H} & -3.910609 & -1.813200 & -2.407965 \\ \mathrm{C} & 1.724831 & -0.891199 & -0.046513\end{array}$




\begin{tabular}{|c|c|c|c|}
\hline C & 0.696305 & -1.718603 & 0.387320 \\
\hline C & 3.131967 & -1.668681 & 1.767795 \\
\hline C & 2.960592 & -0.840750 & 0.675224 \\
\hline $\mathrm{H}$ & 4.066711 & -1.629661 & 2.319237 \\
\hline C & 2.108856 & -2.530439 & 2.225426 \\
\hline C & 0.855411 & -2.546708 & 1.537180 \\
\hline C & -0.182636 & -3.387823 & 2.019740 \\
\hline C & 0.018199 & -4.183296 & 3.123273 \\
\hline C & 1.262670 & -4.175546 & 3.798558 \\
\hline C & 2.283168 & -3.364870 & 3.361099 \\
\hline $\mathrm{H}$ & -1.141962 & -3.380041 & 1.516152 \\
\hline $\mathrm{H}$ & -0.785262 & -4.816204 & 3.486149 \\
\hline $\mathrm{H}$ & 1.404781 & -4.807706 & 4.669634 \\
\hline $\mathrm{H}$ & 3.237289 & -3.345467 & 3.880033 \\
\hline C & -1.960100 & -2.711919 & -2.187117 \\
\hline C & -0.730069 & -2.684794 & -1.458724 \\
\hline C & 0.310010 & -3.573941 & -1.838550 \\
\hline C & 0.141570 & -4.444439 & -2.891916 \\
\hline C & -1.073559 & -4.467159 & -3.616161 \\
\hline C & -2.099782 & -3.618580 & -3.270391 \\
\hline $\mathrm{H}$ & 1.239721 & -3.560502 & -1.281026 \\
\hline $\mathrm{H}$ & 0.944691 & -5.120322 & -3.168605 \\
\hline $\mathrm{H}$ & -1.193490 & -5.158242 & -4.444712 \\
\hline $\mathrm{H}$ & -3.036392 & -3.630328 & -3.820673 \\
\hline C & 6.117733 & 1.828517 & -0.400330 \\
\hline C & 4.818786 & 2.333574 & -0.263645 \\
\hline C & 3.800674 & 1.446637 & 0.095317 \\
\hline C & 4.056667 & 0.084983 & 0.289383 \\
\hline C & 5.366196 & -0.385694 & 0.144924 \\
\hline C & 6.415377 & 0.474902 & -0.195339 \\
\hline $\mathrm{H}$ & 6.915064 & 2.503704 & -0.674293 \\
\hline $\mathrm{H}$ & 2.787927 & 1.806687 & 0.228528 \\
\hline $\mathrm{H}$ & 5.552686 & -1.445387 & 0.285527 \\
\hline C & 4.468473 & 3.810010 & -0.496484 \\
\hline C & 7.836204 & -0.089222 & -0.344456 \\
\hline C & 5.689621 & 4.644763 & -0.912781 \\
\hline $\mathrm{H}$ & 5.387566 & 5.683758 & -1.076146 \\
\hline $\mathrm{H}$ & 6.463973 & 4.642697 & -0.139251 \\
\hline $\mathrm{H}$ & 6.133742 & 4.277452 & -1.843417 \\
\hline C & 3.410202 & 3.902179 & -1.618785 \\
\hline $\mathrm{H}$ & 3.126526 & 4.946152 & -1.791149 \\
\hline $\mathrm{H}$ & 3.805736 & 3.497014 & -2.556092 \\
\hline $\mathrm{H}$ & 2.506017 & 3.342338 & -1.364248 \\
\hline C & 3.888816 & 4.408429 & 0.803290 \\
\hline $\mathrm{H}$ & 3.627613 & 5.461845 & 0.654528 \\
\hline $\mathrm{H}$ & 2.986544 & 3.880896 & 1.123665 \\
\hline $\mathrm{H}$ & 4.618557 & 4.348462 & 1.616679 \\
\hline C & 8.863259 & 0.995434 & -0.707568 \\
\hline $\mathrm{H}$ & 9.857005 & 0.545783 & -0.795222 \\
\hline $\mathrm{H}$ & 8.629490 & 1.471533 & -1.664940 \\
\hline $\mathrm{H}$ & 8.917730 & 1.774722 & 0.058947 \\
\hline C & 8.269037 & -0.736165 & 0.989021 \\
\hline $\mathrm{H}$ & 9.280197 & -1.148509 & 0.902759 \\
\hline $\mathrm{H}$ & 8.268084 & 0.002356 & 1.796693 \\
\hline
\end{tabular}




\begin{tabular}{|c|c|c|c|}
\hline $\mathrm{H}$ & 7.599352 & -1.550285 & 1.279359 \\
\hline C & 7.846384 & -1.156877 & -1.459785 \\
\hline $\mathrm{H}$ & 8.851717 & -1.575456 & -1.578494 \\
\hline $\mathrm{H}$ & 7.164356 & -1.981681 & -1.235912 \\
\hline $\mathrm{H}$ & 7.542203 & -0.720956 & -2.416572 \\
\hline C & -3.967572 & -0.002947 & -0.435528 \\
\hline C & -5.297796 & -0.437225 & -0.413822 \\
\hline C & -3.680580 & 1.340120 & -0.168934 \\
\hline C & -6.338937 & 0.447193 & -0.119267 \\
\hline $\mathrm{H}$ & -5.500455 & -1.484452 & -0.598716 \\
\hline C & -4.690374 & 2.248260 & 0.148894 \\
\hline $\mathrm{H}$ & -2.651350 & 1.667981 & -0.202857 \\
\hline C & -6.009065 & 1.779064 & 0.161507 \\
\hline $\mathrm{H}$ & -6.806024 & 2.471860 & 0.409540 \\
\hline C & -4.390234 & 3.709669 & 0.508000 \\
\hline C & -7.809180 & 0.006854 & -0.072129 \\
\hline C & -2.890466 & 4.035511 & 0.403984 \\
\hline $\mathrm{H}$ & -2.289637 & 3.408664 & 1.069549 \\
\hline $\mathrm{H}$ & -2.521406 & 3.903410 & -0.618938 \\
\hline $\mathrm{H}$ & -2.721788 & 5.080134 & 0.683104 \\
\hline C & -5.161207 & 4.649425 & -0.442293 \\
\hline $\mathrm{H}$ & -4.958287 & 5.695992 & -0.190489 \\
\hline $\mathrm{H}$ & -4.860830 & 4.483455 & -1.481833 \\
\hline $\mathrm{H}$ & -6.241942 & 4.494842 & -0.378101 \\
\hline C & -4.839141 & 3.962763 & 1.963775 \\
\hline $\mathrm{H}$ & -5.910291 & 3.782265 & 2.091495 \\
\hline $\mathrm{H}$ & -4.303026 & 3.302742 & 2.652001 \\
\hline $\mathrm{H}$ & -4.635761 & 5.000278 & 2.250935 \\
\hline C & -8.621734 & 0.833583 & -1.091858 \\
\hline $\mathrm{H}$ & -8.569576 & 1.904627 & -0.878051 \\
\hline $\mathrm{H}$ & -8.244625 & 0.676745 & -2.107392 \\
\hline $\mathrm{H}$ & -9.676313 & 0.538030 & -1.068557 \\
\hline C & -7.983332 & -1.482027 & -0.412488 \\
\hline $\mathrm{H}$ & -7.617682 & -1.712508 & -1.418203 \\
\hline $\mathrm{H}$ & -7.456734 & -2.124352 & 0.299918 \\
\hline $\mathrm{H}$ & -9.044631 & -1.746292 & -0.376129 \\
\hline C & -8.367798 & 0.246798 & 1.347090 \\
\hline $\mathrm{H}$ & -9.417152 & -0.063223 & 1.401456 \\
\hline $\mathrm{H}$ & -7.801282 & -0.325454 & 2.087892 \\
\hline $\mathrm{H}$ & -8.315040 & 1.301911 & 1.629122 \\
\hline
\end{tabular}

\section{Catalyst 2C 8}

Datum

Value

B3LYP-D3(BJ)/6-31G(d,p) Energy

$-2165.164532$

B3LYP-D3(BJ)/def2-TZVPP/IEF-PCM(DCM) Energy

$-2165.90443$

B3LYP-D3(BJ)/def2-TZVPP/IEF-PCM(DCM)//B3LYP-D3(BJ)/6-31G(d,p) Free Energy (Quasiharmonic) - 
Frequencies (Top 3 out of 321)

$\begin{array}{ll}\text { 1. } & 10.4451 \mathrm{~cm}^{-1} \\ \text { 2. } & 12.3864 \mathrm{~cm}^{-1} \\ \text { 3. } & 14.6418 \mathrm{~cm}^{-1}\end{array}$

B3LYP-D3(BJ)/6-31G(d,p) Molecular Geometry in Cartesian Coordinates

\begin{tabular}{|c|c|c|c|}
\hline C & 1.507747 & -0.063168 & -1.291665 \\
\hline $\mathrm{H}$ & 2.467135 & 0.206281 & -1.738368 \\
\hline $\mathrm{H}$ & 0.918961 & -0.626516 & -2.020080 \\
\hline$C$ & -1.572776 & -0.107918 & 1.275334 \\
\hline 0 & -2.249301 & -0.400336 & 2.233188 \\
\hline 0 & -0.713388 & 0.923314 & 1.306925 \\
\hline $\mathrm{H}$ & -0.216945 & 1.001716 & 0.458343 \\
\hline 0 & 0.791332 & 1.152370 & -0.942079 \\
\hline $\mathrm{H}$ & 0.438442 & 1.545595 & -1.751032 \\
\hline C & -0.616642 & -1.734931 & -0.369466 \\
\hline C & -1.661025 & -0.898755 & -0.013972 \\
\hline$C$ & -2.890909 & -0.899473 & -0.748821 \\
\hline C & -3.025484 & -1.769140 & -1.811604 \\
\hline $\mathrm{H}$ & -3.939935 & -1.753142 & -2.397139 \\
\hline C & 1.710056 & -0.888579 & -0.046739 \\
\hline C & 0.672286 & -1.702882 & 0.389939 \\
\hline$C$ & 3.108253 & -1.675341 & 1.770452 \\
\hline C & 2.946163 & -0.849259 & 0.675067 \\
\hline $\mathrm{H}$ & 4.043224 & -1.644614 & 2.322022 \\
\hline C & 2.075770 & -2.524384 & 2.230703 \\
\hline C & 0.822208 & -2.529080 & 1.542463 \\
\hline C & -0.224849 & -3.357462 & 2.027627 \\
\hline C & -0.032664 & -4.151568 & 3.133665 \\
\hline C & 1.211764 & -4.155148 & 3.809055 \\
\hline C & 2.240998 & -3.357018 & 3.369051 \\
\hline $\mathrm{H}$ & -1.184036 & -3.341097 & 1.524018 \\
\hline $\mathrm{H}$ & -0.842958 & -4.774581 & 3.498452 \\
\hline $\mathrm{H}$ & 1.346955 & -4.786005 & 4.682169 \\
\hline $\mathrm{H}$ & 3.195273 & -3.346304 & 3.887946 \\
\hline C & -1.995944 & -2.667107 & -2.183389 \\
\hline C & -0.765036 & -2.653216 & -1.456177 \\
\hline C & 0.266418 & -3.551041 & -1.839210 \\
\hline C & 0.089097 & -4.416749 & -2.895025 \\
\hline $\mathrm{C}$ & -1.126682 & -4.425914 & -3.618558 \\
\hline C & -2.144811 & -3.569050 & -3.269408 \\
\hline $\mathrm{H}$ & 1.196582 & -3.547897 & -1.282274 \\
\hline $\mathrm{H}$ & 0.885630 & -5.099340 & -3.174267 \\
\hline
\end{tabular}




\begin{tabular}{|c|c|c|c|}
\hline $\mathrm{H}$ & -1.253533 & -5.113285 & -4.449154 \\
\hline $\mathrm{H}$ & -3.081937 & -3.570562 & -3.818928 \\
\hline C & 6.132211 & 1.782708 & -0.406845 \\
\hline C & 4.838570 & 2.301964 & -0.273040 \\
\hline C & 3.810807 & 1.427051 & 0.087933 \\
\hline C & 4.052233 & 0.063409 & 0.286705 \\
\hline C & 5.356799 & -0.421620 & 0.144861 \\
\hline C & 6.415356 & 0.426706 & -0.197165 \\
\hline $\mathrm{H}$ & 6.936867 & 2.448563 & -0.682214 \\
\hline $\mathrm{H}$ & 2.801831 & 1.798261 & 0.219319 \\
\hline $\mathrm{H}$ & 5.531924 & -1.482770 & 0.289034 \\
\hline C & 4.504269 & 3.781319 & -0.510840 \\
\hline C & 7.830255 & -0.152875 & -0.343246 \\
\hline C & 3.930746 & 4.390163 & 0.786807 \\
\hline $\mathrm{H}$ & 3.680789 & 5.445799 & 0.634522 \\
\hline $\mathrm{H}$ & 3.022894 & 3.873269 & 1.108754 \\
\hline $\mathrm{H}$ & 4.659655 & 4.325132 & 1.600548 \\
\hline C & 5.734502 & 4.601479 & -0.929431 \\
\hline $\mathrm{H}$ & 5.443692 & 5.643088 & -1.096508 \\
\hline $\mathrm{H}$ & 6.508442 & 4.593793 & -0.155527 \\
\hline $\mathrm{H}$ & 6.175063 & 4.226208 & -1.858584 \\
\hline C & 3.447488 & 3.881295 & -1.633874 \\
\hline $\mathrm{H}$ & 3.175090 & 4.927697 & -1.809640 \\
\hline $\mathrm{H}$ & 3.839024 & 3.468999 & -2.569748 \\
\hline $\mathrm{H}$ & 2.537228 & 3.331971 & -1.378050 \\
\hline C & 7.830428 & -1.222601 & -1.456629 \\
\hline $\mathrm{H}$ & 8.831350 & -1.652196 & -1.573227 \\
\hline $\mathrm{H}$ & 7.139239 & -2.039578 & -1.232145 \\
\hline $\mathrm{H}$ & 7.532226 & -0.785172 & -2.414609 \\
\hline C & 8.869362 & 0.920070 & -0.706886 \\
\hline $\mathrm{H}$ & 9.858435 & 0.459735 & -0.791873 \\
\hline $\mathrm{H}$ & 8.642343 & 1.396568 & -1.665681 \\
\hline $\mathrm{H}$ & 8.930768 & 1.700377 & 0.058069 \\
\hline C & 8.254410 & -0.801988 & 0.991966 \\
\hline $\mathrm{H}$ & 9.261290 & -1.225084 & 0.907797 \\
\hline $\mathrm{H}$ & 8.260140 & -0.062088 & 1.798353 \\
\hline $\mathrm{H}$ & 7.575872 & -1.608554 & 1.282831 \\
\hline C & -3.984514 & 0.045157 & -0.416628 \\
\hline C & -3.702152 & 1.385376 & -0.137376 \\
\hline C & -5.315620 & -0.382909 & -0.401952 \\
\hline C & -4.713363 & 2.293740 & 0.180074 \\
\hline $\mathrm{H}$ & -2.672071 & 1.719430 & -0.158149 \\
\hline C & -6.359169 & 0.500002 & -0.107619 \\
\hline $\mathrm{H}$ & -5.525372 & -1.429813 & -0.593696 \\
\hline C & -6.035111 & 1.831602 & 0.182692 \\
\hline $\mathrm{H}$ & -6.829213 & 2.522471 & 0.424561 \\
\hline C & -7.804365 & -0.019592 & -0.090936 \\
\hline C & -4.332959 & 3.738635 & 0.531081 \\
\hline C & -8.821697 & 1.086037 & 0.234238 \\
\hline $\mathrm{H}$ & -8.649841 & 1.515800 & 1.225860 \\
\hline $\mathrm{H}$ & -8.789271 & 1.896015 & -0.501283 \\
\hline $\mathrm{H}$ & -9.833289 & 0.668737 & 0.224868 \\
\hline C & -8.152732 & -0.602170 & -1.477708 \\
\hline $\mathrm{H}$ & -9.179555 & -0.983787 & -1.484653 \\
\hline
\end{tabular}




\begin{tabular}{|lrrr|}
$H$ & -8.067442 & 0.164988 & -2.253832 \\
$H$ & -7.487803 & -1.427211 & -1.747801 \\
$\mathrm{C}$ & -7.934129 & -1.125772 & 0.978445 \\
$\mathrm{H}$ & -7.264455 & -1.965584 & 0.774527 \\
$\mathrm{H}$ & -7.686680 & -0.736182 & 1.970512 \\
$\mathrm{H}$ & -8.959219 & -1.511491 & 1.005868 \\
$\mathrm{C}$ & -3.414904 & 3.722944 & 1.774023 \\
$\mathrm{H}$ & -3.934188 & 3.282741 & 2.630513 \\
$\mathrm{H}$ & -2.507227 & 3.137266 & 1.607086 \\
$\mathrm{H}$ & -3.118741 & 4.744041 & 2.039445 \\
$\mathrm{C}$ & -3.580459 & 4.376212 & -0.656586 \\
$\mathrm{H}$ & -2.664365 & 3.828438 & -0.894729 \\
$\mathrm{H}$ & -4.207223 & 4.390008 & -1.554088 \\
$\mathrm{H}$ & -3.298993 & 5.407765 & -0.418877 \\
$\mathrm{C}$ & -5.561415 & 4.607745 & 0.843359 \\
$\mathrm{H}$ & -5.239937 & 5.624793 & 1.087838 \\
$\mathrm{H}$ & -6.243063 & 4.670513 & -0.011177 \\
$\mathrm{H}$ & -6.120913 & 4.222596 & 1.701357 \\
\hline
\end{tabular}

\section{Catalyst 2C 9}

\section{Datum}

Value

\begin{tabular}{lr} 
B3LYP-D3(BJ)/6-31G(d,p) Energy & -2165.168298 \\
\hline B3LYP-D3(BJ)/def2-TZVPP/IEF-PCM(DCM) Energy & -2165.903072 \\
\hline B3LYP-D3(BJ)/def2-TZVPP/IEF-PCM(DCM)//B3LYP-D3(BJ)/6-31G(d,p) Free Energy (Quasiharmonic) & -2165.024724
\end{tabular}

Number of Imaginary Frequencies

Frequencies (Top 3 out of 321)

$\begin{array}{lr}\text { 1. } & 6.0717 \mathrm{~cm}^{-1} \\ \text { 2. } & 12.2256 \mathrm{~cm}^{-1} \\ \text { 3. } & 15.3600 \mathrm{~cm}^{-1}\end{array}$

\section{B3LYP-D3(BJ)/6-31G(d,p) Molecular Geometry in Cartesian Coordinates}

C

C

C

C

C

C

$\mathrm{H}$

-0.084674
-0.265116
0.782703
2.032017
2.183767
1.150168
-0.894165

$-4.353648$

$-3.486563$

$-2.623276$

$-2.669257$

$-3.572014$

$-4.397280$

$-5.008608$
3.013978

1.960513

1.545711

2. 240607

3. 325238

3. 703240

3. 320248 


\begin{tabular}{|c|c|c|c|}
\hline $\mathrm{H}$ & -1.210692 & -3.450531 & 1.432225 \\
\hline C & 0.628166 & -1.715426 & 0.451654 \\
\hline C & 3.076944 & -1.798557 & 1.845060 \\
\hline $\mathrm{H}$ & 3.133423 & -3.597693 & 3.851886 \\
\hline $\mathrm{H}$ & 1.278513 & -5.084878 & 4.533422 \\
\hline C & 2.934520 & -0.926473 & 0.787539 \\
\hline C & 1.693583 & -0.916554 & 0.074352 \\
\hline $\mathrm{H}$ & 4.002020 & -1.795913 & 2.413715 \\
\hline C & -1.208129 & -4.320633 & -3.598267 \\
\hline C & -2.222309 & -3.474660 & -3.215252 \\
\hline C & -2.054567 & -2.588694 & -2.118112 \\
\hline C & -0.812785 & -2.583388 & -1.411107 \\
\hline C & 0.216533 & -3.468106 & -1.832225 \\
\hline C & 0.022622 & -4.315438 & -2.899047 \\
\hline $\mathrm{H}$ & -4.012129 & -1.700414 & -2.260742 \\
\hline $\mathrm{H}$ & -1.347045 & -4.993988 & -4.438482 \\
\hline $\mathrm{H}$ & -3.169703 & -3.469316 & -3.746995 \\
\hline C & -3.077882 & -1.704814 & -1.707134 \\
\hline C & -0.660437 & -1.687518 & -0.309316 \\
\hline $\mathrm{H}$ & 1.163378 & -3.464624 & -1.304595 \\
\hline $\mathrm{H}$ & 0.819296 & -4.984566 & -3.209065 \\
\hline C & -1.678427 & -0.822928 & 0.072486 \\
\hline C & -2.918040 & -0.825853 & -0.652124 \\
\hline C & -1.443944 & 0.120139 & 1.238751 \\
\hline $\mathrm{H}$ & -2.386188 & 0.314814 & 1.755708 \\
\hline $\mathrm{H}$ & -0.763966 & -0.355020 & 1.956162 \\
\hline C & 1.561195 & -0.087516 & -1.164195 \\
\hline 0 & 0.787014 & 0.841414 & -1.328791 \\
\hline 0 & 2.394442 & -0.493929 & -2.134654 \\
\hline $\mathrm{H}$ & 2.257128 & 0.107236 & -2.887301 \\
\hline 0 & -0.931769 & 1.389967 & 0.839804 \\
\hline $\mathrm{H}$ & -0.303277 & 1.246691 & 0.107665 \\
\hline C & -4.050270 & 0.071029 & -0.298755 \\
\hline C & -5.350714 & -0.454356 & -0.203116 \\
\hline C & -3.853277 & 1.436596 & -0.093163 \\
\hline C & -6.438900 & 0.363325 & 0.096205 \\
\hline $\mathrm{H}$ & -5.485532 & -1.519170 & -0.346590 \\
\hline C & -4.921476 & 2.285487 & 0.225833 \\
\hline $\mathrm{H}$ & -2.847278 & 1.830427 & -0.163411 \\
\hline C & -6.199114 & 1.731976 & 0.309743 \\
\hline $\mathrm{H}$ & -7.037754 & 2.371727 & 0.554078 \\
\hline C & 4.011828 & 0.030284 & 0.440146 \\
\hline C & 3.698014 & 1.384926 & 0.246570 \\
\hline C & 5.335186 & -0.390186 & 0.317456 \\
\hline C & 4.682032 & 2.308984 & -0.094757 \\
\hline $\mathrm{H}$ & 2.673306 & 1.703481 & 0.382701 \\
\hline C & 6.356374 & 0.514835 & -0.006685 \\
\hline $\mathrm{H}$ & 5.560296 & -1.442933 & 0.452095 \\
\hline C & 6.004986 & 1.850410 & -0.212917 \\
\hline $\mathrm{H}$ & 6.775832 & 2.563672 & -0.474490 \\
\hline C & 7.799525 & 0.007437 & -0.137971 \\
\hline C & 4.361118 & 3.788570 & -0.345603 \\
\hline C & 8.238950 & -0.625702 & 1.199911 \\
\hline $\mathrm{H}$ & 7.597280 & -1.465714 & 1.479715 \\
\hline
\end{tabular}




\begin{tabular}{|c|c|c|c|}
\hline $\mathrm{H}$ & 8.199506 & 0.110248 & 2.009002 \\
\hline H & 9.266036 & -0.999027 & 1.125921 \\
\hline C & 8.787269 & 1.132970 & -0.484687 \\
\hline $\mathrm{H}$ & 9.799202 & 0.723593 & -0.560036 \\
\hline $\mathrm{H}$ & 8.800258 & 1.911660 & 0.284324 \\
\hline $\mathrm{H}$ & 8.547345 & 1.601671 & -1.444178 \\
\hline C & 7.865271 & -1.055705 & -1.255748 \\
\hline $\mathrm{H}$ & 7.212758 & -1.906979 & -1.043580 \\
\hline $\mathrm{H}$ & 8.887635 & -1.434371 & -1.361985 \\
\hline H & 7.556670 & -0.629013 & -2.215154 \\
\hline C & 4.779224 & 4.157284 & -1.785699 \\
\hline $\mathrm{H}$ & 5.848384 & 3.998818 & -1.952265 \\
\hline $\mathrm{H}$ & 4.232483 & 3.550973 & -2.514837 \\
\hline $\mathrm{H}$ & 4.560858 & 5.211528 & -1.986622 \\
\hline C & 5.145164 & 4.661915 & 0.656699 \\
\hline H & 6.225325 & 4.520308 & 0.558966 \\
\hline $\mathrm{H}$ & 4.929197 & 5.722401 & 0.488140 \\
\hline $\mathrm{H}$ & 4.866111 & 4.415741 & 1.685821 \\
\hline C & 2.861296 & 4.090672 & -0.188164 \\
\hline $\mathrm{H}$ & 2.251368 & 3.499006 & -0.877805 \\
\hline $\mathrm{H}$ & 2.511640 & 3.891439 & 0.829437 \\
\hline $\mathrm{H}$ & 2.675383 & 5.147920 & -0.400031 \\
\hline C & -4.631498 & 3.771211 & 0.477146 \\
\hline C & -7.872078 & -0.175643 & 0.212868 \\
\hline C & -7.951412 & -1.687287 & -0.054658 \\
\hline H & -7.603506 & -1.938272 & -1.061614 \\
\hline $\mathrm{H}$ & -7.356381 & -2.257672 & 0.665313 \\
\hline $\mathrm{H}$ & -8.989364 & -2.023227 & 0.032111 \\
\hline C & -8.772178 & 0.543395 & -0.815145 \\
\hline $\mathrm{H}$ & -8.791065 & 1.624079 & -0.650243 \\
\hline $\mathrm{H}$ & -8.413594 & 0.367139 & -1.834110 \\
\hline $\mathrm{H}$ & -9.801670 & 0.175146 & -0.745263 \\
\hline C & -8.405569 & 0.091058 & 1.636985 \\
\hline $\mathrm{H}$ & -7.781892 & -0.409717 & 2.384054 \\
\hline $\mathrm{H}$ & -8.414978 & 1.158948 & 1.871523 \\
\hline $\mathrm{H}$ & -9.429978 & -0.284113 & 1.738135 \\
\hline C & -5.898508 & 4.561783 & 0.838359 \\
\hline $\mathrm{H}$ & -6.638853 & 4.533196 & 0.032143 \\
\hline H & -6.368558 & 4.177742 & 1.749414 \\
\hline $\mathrm{H}$ & -5.640856 & 5.610621 & 1.015025 \\
\hline C & -4.016298 & 4.391633 & -0.795771 \\
\hline $\mathrm{H}$ & -3.796371 & 5.452625 & -0.633583 \\
\hline H & -3.083399 & 3.894357 & -1.073534 \\
\hline H & -4.706992 & 4.310459 & -1.641357 \\
\hline C & -3.624978 & 3.894522 & 1.643315 \\
\hline $\mathrm{H}$ & -2.689596 & 3.370055 & 1.431219 \\
\hline $\mathrm{H}$ & -3.391293 & 4.948591 & 1.831244 \\
\hline H & -4.047286 & 3.472048 & 2.561102 \\
\hline
\end{tabular}


Frequencies (Top 3 out of 321)

$\begin{array}{lr}\text { 1. } & 5.8422 \mathrm{~cm}^{-1} \\ \text { 2. } & 12.2597 \mathrm{~cm}^{-1} \\ \text { 3. } & 15.5304 \mathrm{~cm}^{-1}\end{array}$

\section{B3LYP-D3(BJ)/6-31G(d,p) Molecular Geometry in Cartesian Coordinates}

C

C

$\mathrm{C}$

C

C

C

$\mathrm{H}$

$\mathrm{H}$

C

C

$\mathrm{H}$

$\mathrm{H}$

C

C

$\mathrm{H}$

C

C

C

C

C

C

$\mathrm{H}$

$\mathrm{H}$

$\mathrm{H}$

C

C

$\mathrm{H}$

$\mathrm{H}$

C

C

C

$\mathrm{H}$

\subsection{1}

0.283106

$-0.768297$

$-2.018038$

$-2.166732$

$-1.129776$

0.917805

1. 229037

$-0.617175$

$-3.066019$

$-3.116775$

$-1.255846$

$-2.926343$

$-1.685949$

$-3.990930$

1. 246772

2. 253616

2.076912

0.833801

$-0.187881$

0.014644

4.027438

1. 392535

3. 201922

3.092348

0.672377

$-1.135705$

$-0.776198$

1.682807

2.923553

1.438985

0.762659
$-4.377382$

$-3.508231$

$-2.649397$

$-2.701684$

$-3.606542$

$-4.427562$

$-5.028927$

$-3.467258$

$-1.740295$

$-1.834192$

$-3.637180$

$-5.116786$

$-0.959633$

$-0.945990$

$-1.835570$

$-4.340015$

$-3.485339$

$-2.598360$

$-2.601353$

$-3.494819$

$-4.342890$

$-1.694487$

$-5.014087$

$-3.473748$

$-1.705373$

$-1.704610$

$-3.497447$

$-5.018856$

$-0.831220$

$-0.825489$

0.111407

$-0.368939$

\section{010281}

1.958019

1.543062

2. 236789

3. 320120

3.698150

3. 316655

1.430721

0.449584

1.842314

3.845802

4.527336

0.786406

0.072223

2.411219

$-3.591704$

$-3.208639$

$-2.113719$

$-1.409042$

$-1.830241$

$-2.894870$

$-2.254952$

$-4.430185$

$-3.738655$

$-1.702861$

$-0.309360$

$-1.304336$

$-3.204990$

0.072609

$-0.649993$

1. 237369

1.954770 


\begin{tabular}{|c|c|c|c|}
\hline $\mathrm{H}$ & 2.379070 & 0.314981 & 1.754862 \\
\hline C & -1.558584 & -0.119292 & -1.168464 \\
\hline 0 & -0.788829 & 0.812663 & -1.337019 \\
\hline 0 & -2.390414 & -0.532825 & -2.137223 \\
\hline $\mathrm{H}$ & -2.256060 & 0.066551 & -2.891829 \\
\hline 0 & 0.915495 & 1.376170 & 0.836704 \\
\hline $\mathrm{H}$ & 0.293250 & 1.227090 & 0.100357 \\
\hline C & 4.047462 & 0.081927 & -0.297042 \\
\hline C & 3.838029 & 1.446116 & -0.094645 \\
\hline C & 5.352428 & -0.431593 & -0.198709 \\
\hline C & 4.898180 & 2.305262 & 0.223655 \\
\hline $\mathrm{H}$ & 2.828656 & 1.830834 & -0.166816 \\
\hline C & 6.432891 & 0.396473 & 0.100091 \\
\hline H & 5.496946 & -1.495469 & -0.339697 \\
\hline C & 6.180618 & 1.763408 & 0.310258 \\
\hline $\mathrm{H}$ & 7.013202 & 2.411222 & 0.554060 \\
\hline C & -4.003999 & -0.002042 & 0.442814 \\
\hline C & -5.331615 & -0.426781 & 0.323970 \\
\hline C & -3.689950 & 1.349013 & 0.250946 \\
\hline C & -6.348159 & 0.477770 & 0.004492 \\
\hline $\mathrm{H}$ & -5.549714 & -1.478596 & 0.459759 \\
\hline C & -4.676112 & 2.277227 & -0.087984 \\
\hline $\mathrm{H}$ & -2.665020 & 1.667564 & 0.385366 \\
\hline C & -5.993422 & 1.817335 & -0.201830 \\
\hline $\mathrm{H}$ & -6.771432 & 2.527107 & -0.461772 \\
\hline C & -4.353409 & 3.756626 & -0.338573 \\
\hline C & -7.816708 & 0.053783 & -0.141541 \\
\hline C & -4.775849 & 4.127128 & -1.776847 \\
\hline $\mathrm{H}$ & -4.232805 & 3.520453 & -2.508411 \\
\hline $\mathrm{H}$ & -5.845836 & 3.970276 & -1.939773 \\
\hline $\mathrm{H}$ & -4.556641 & 5.181179 & -1.977856 \\
\hline C & -2.852469 & 4.055872 & -0.185928 \\
\hline $\mathrm{H}$ & -2.665445 & 5.112934 & -0.397724 \\
\hline $\mathrm{H}$ & -2.499597 & 3.855391 & 0.830310 \\
\hline $\mathrm{H}$ & -2.245916 & 3.463612 & -0.878021 \\
\hline C & -5.132797 & 4.629830 & 0.667338 \\
\hline $\mathrm{H}$ & -4.850762 & 4.382086 & 1.695256 \\
\hline $\mathrm{H}$ & -4.916283 & 5.690293 & 0.499350 \\
\hline $\mathrm{H}$ & -6.213451 & 4.489321 & 0.573136 \\
\hline C & -8.676849 & 0.844046 & 0.868166 \\
\hline $\mathrm{H}$ & -8.354414 & 0.642104 & 1.894332 \\
\hline $\mathrm{H}$ & -8.607530 & 1.922598 & 0.702573 \\
\hline $\mathrm{H}$ & -9.730256 & 0.557923 & 0.777258 \\
\hline C & -8.019600 & -1.446602 & 0.124038 \\
\hline $\mathrm{H}$ & -7.713115 & -1.722943 & 1.137776 \\
\hline $\mathrm{H}$ & -9.078772 & -1.699512 & 0.016872 \\
\hline $\mathrm{H}$ & -7.457992 & -2.063267 & -0.584487 \\
\hline C & -8.296304 & 0.360362 & -1.576717 \\
\hline $\mathrm{H}$ & -8.217004 & 1.425519 & -1.810831 \\
\hline $\mathrm{H}$ & -7.698765 & -0.189130 & -2.310724 \\
\hline $\mathrm{H}$ & -9.344558 & 0.067167 & -1.699473 \\
\hline C & 7.870702 & -0.129350 & 0.219617 \\
\hline C & 4.594547 & 3.788879 & 0.471267 \\
\hline$c$ & 3.585822 & 3.905749 & 1.636200 \\
\hline
\end{tabular}




\begin{tabular}{|c|c|c|c|}
\hline $\mathrm{H}$ & 2.655478 & 3.372232 & 1.424490 \\
\hline $\mathrm{H}$ & 4.011099 & 3.489330 & 2.555382 \\
\hline $\mathrm{H}$ & 3.342349 & 4.958087 & 1.821402 \\
\hline C & 3.974967 & 4.400792 & -0.803649 \\
\hline $\mathrm{H}$ & 4.667103 & 4.323805 & -1.648445 \\
\hline $\mathrm{H}$ & 3.046783 & 3.894575 & -1.081071 \\
\hline $\mathrm{H}$ & 3.745435 & 5.460158 & -0.644154 \\
\hline C & 5.854014 & 4.591707 & 0.831801 \\
\hline $\mathrm{H}$ & 6.326669 & 4.214050 & 1.744175 \\
\hline $\mathrm{H}$ & 6.595336 & 4.567916 & 0.026328 \\
\hline $\mathrm{H}$ & 5.586739 & 5.638584 & 1.005777 \\
\hline C & 7.963854 & -1.640856 & -0.044218 \\
\hline $\mathrm{H}$ & 7.373096 & -2.214826 & 0.676424 \\
\hline $\mathrm{H}$ & 7.619391 & -1.897335 & -1.050972 \\
\hline $\mathrm{H}$ & 9.004660 & -1.967307 & 0.044546 \\
\hline C & 8.400206 & 0.145494 & 1.643677 \\
\hline $\mathrm{H}$ & 9.427811 & -0.220274 & 1.746844 \\
\hline $\mathrm{H}$ & 8.399831 & 1.213983 & 1.875674 \\
\hline $\mathrm{H}$ & 7.780193 & -0.359048 & 2.391262 \\
\hline C & 8.765473 & 0.595292 & -0.809114 \\
\hline $\mathrm{H}$ & 8.774535 & 1.676484 & -0.646715 \\
\hline $\mathrm{H}$ & 9.798133 & 0.236412 & -0.737270 \\
\hline $\mathrm{H}$ & 8.409588 & 0.413473 & -1.828047 \\
\hline
\end{tabular}

\section{Catalyst 2C 11}

\section{Datum}

Value

\begin{tabular}{lr}
\hline B3LYP-D3(BJ)/6-31G(d,p) Energy & -2165.168092 \\
\hline B3LYP-D3(BJ)/def2-TZVPP/IEF-PCM(DCM) Energy & -2165.902949 \\
\hline B3LYP-D3(BJ)/def2-TZVPP/IEF-PCM(DCM)//B3LYP-D3(BJ)/6-31G(d,p) Free Energy (Quasiharmonic) & -2165.024609
\end{tabular}

Number of Imaginary Frequencies

Frequencies (Top 3 out of 321)
1. $7.5435 \mathrm{~cm}^{-1}$
2. $12.3201 \mathrm{~cm}^{-1}$
3. $15.1521 \mathrm{~cm}^{-1}$

\section{B3LYP-D3(BJ)/6-31G(d,p) Molecular Geometry in Cartesian Coordinates}

$\begin{array}{lrrr}C & -0.112072 & -4.346709 & 2.980131 \\ C & -0.281572 & -3.470638 & 1.932353 \\ C & 0.773731 & -2.610647 & 1.529688\end{array}$




\begin{tabular}{|c|c|c|c|}
\hline C & 2.019344 & -2.670470 & 2.230032 \\
\hline C & 2.159843 & -3.582467 & 3.308561 \\
\hline C & 1.118986 & -4.403555 & 3.675312 \\
\hline $\mathrm{H}$ & -0.927287 & -4.998744 & 3.277335 \\
\hline $\mathrm{H}$ & -1.224192 & -3.424539 & 1.399597 \\
\hline C & 0.630712 & -1.692691 & 0.442717 \\
\hline C & 3.072832 & -1.806071 & 1.844433 \\
\hline $\mathrm{H}$ & 3.106862 & -3.618686 & 3.839315 \\
\hline $\mathrm{H}$ & 1.238658 & -5.098246 & 4.500869 \\
\hline C & 2.942200 & -0.924028 & 0.793219 \\
\hline C & 1.703149 & -0.897642 & 0.076941 \\
\hline $\mathrm{H}$ & 3.996366 & -1.817758 & 2.415389 \\
\hline C & -1.174927 & -4.239595 & -3.657607 \\
\hline C & -2.193161 & -3.401251 & -3.268512 \\
\hline C & -2.034042 & -2.531332 & -2.157440 \\
\hline C & -0.796629 & -2.533635 & -1.442663 \\
\hline C & 0.236789 & -3.410521 & -1.870104 \\
\hline C & 0.051203 & -4.242471 & -2.950453 \\
\hline $\mathrm{H}$ & -3.994363 & -1.646511 & -2.296748 \\
\hline $\mathrm{H}$ & -1.307204 & -4.900703 & -4.508550 \\
\hline $\mathrm{H}$ & -3.137250 & -3.389840 & -3.806016 \\
\hline C & -3.062530 & -1.656649 & -1.739072 \\
\hline C & -0.652699 & -1.653549 & -0.326699 \\
\hline $\mathrm{H}$ & 1.180267 & -3.413369 & -1.336548 \\
\hline $\mathrm{H}$ & 0.851093 & -4.905614 & -3.265016 \\
\hline C & -1.675359 & -0.797531 & 0.061291 \\
\hline C & -2.910514 & -0.794040 & -0.669887 \\
\hline C & -1.452928 & 0.132191 & 1.240351 \\
\hline $\mathrm{H}$ & -2.399977 & 0.315522 & 1.752920 \\
\hline $\mathrm{H}$ & -0.775971 & -0.348937 & 1.956969 \\
\hline C & 1.575970 & -0.050687 & -1.149950 \\
\hline 0 & 0.811474 & 0.889850 & -1.294921 \\
\hline 0 & 2.399758 & -0.450335 & -2.130506 \\
\hline $\mathrm{H}$ & 2.268171 & 0.166592 & -2.871429 \\
\hline 0 & -0.945818 & 1.408516 & 0.858833 \\
\hline $\mathrm{H}$ & -0.301828 & 1.274225 & 0.139017 \\
\hline C & -4.049221 & 0.090566 & -0.305467 \\
\hline C & -5.334777 & -0.444372 & -0.193951 \\
\hline C & -3.861625 & 1.467470 & -0.107058 \\
\hline C & -6.437955 & 0.363172 & 0.116706 \\
\hline $\mathrm{H}$ & -5.465529 & -1.512773 & -0.332084 \\
\hline C & -4.933202 & 2.297952 & 0.213749 \\
\hline $\mathrm{H}$ & -2.859222 & 1.862971 & -0.188050 \\
\hline C & -6.212041 & 1.725908 & 0.317958 \\
\hline $\mathrm{H}$ & -7.046305 & 2.367966 & 0.570147 \\
\hline C & 4.034151 & 0.019113 & 0.453802 \\
\hline C & 3.749182 & 1.369045 & 0.245852 \\
\hline C & 5.360142 & -0.427706 & 0.351940 \\
\hline C & 4.755509 & 2.281888 & -0.095103 \\
\hline $\mathrm{H}$ & 2.728231 & 1.713377 & 0.362007 \\
\hline C & 6.391086 & 0.455300 & 0.035145 \\
\hline $\mathrm{H}$ & 5.559588 & -1.481543 & 0.499859 \\
\hline C & 6.062750 & 1.803721 & -0.190873 \\
\hline $\mathrm{H}$ & 6.857473 & 2.491267 & -0.450245 \\
\hline
\end{tabular}




\begin{tabular}{|c|c|c|c|}
\hline C & 7.854195 & 0.007484 & -0.090766 \\
\hline C & 4.374830 & 3.744205 & -0.361628 \\
\hline C & 8.031159 & -1.493000 & 0.192052 \\
\hline H & 7.709321 & -1.754230 & 1.205045 \\
\hline $\mathrm{H}$ & 7.467922 & -2.108185 & -0.516477 \\
\hline $\mathrm{H}$ & 9.087423 & -1.763159 & 0.099229 \\
\hline C & 8.715726 & 0.794626 & 0.920336 \\
\hline $\mathrm{H}$ & 9.765403 & 0.491152 & 0.843596 \\
\hline $\mathrm{H}$ & 8.665218 & 1.872376 & 0.743254 \\
\hline H & 8.379287 & 0.607861 & 1.944896 \\
\hline C & 8.354497 & 0.291836 & -1.523554 \\
\hline $\mathrm{H}$ & 8.295383 & 1.355661 & -1.769289 \\
\hline $\mathrm{H}$ & 9.399153 & -0.019553 & -1.631287 \\
\hline $\mathrm{H}$ & 7.756411 & -0.255662 & -2.258619 \\
\hline C & 5.595482 & 4.612614 & -0.703990 \\
\hline $\mathrm{H}$ & 6.321605 & 4.630896 & 0.115027 \\
\hline H & 6.104500 & 4.259517 & -1.606467 \\
\hline $\mathrm{H}$ & 5.274724 & 5.642607 & -0.886604 \\
\hline C & 3.692774 & 4.336303 & 0.890352 \\
\hline $\mathrm{H}$ & 4.366620 & 4.303229 & 1.752212 \\
\hline $\mathrm{H}$ & 3.415498 & 5.380713 & 0.712202 \\
\hline $\mathrm{H}$ & 2.782576 & 3.791485 & 1.154041 \\
\hline C & 3.391485 & 3.791152 & -1.552888 \\
\hline $\mathrm{H}$ & 3.863514 & 3.391256 & -2.456643 \\
\hline $\mathrm{H}$ & 2.487185 & 3.207713 & -1.359448 \\
\hline $\mathrm{H}$ & 3.088861 & 4.823987 & -1.756302 \\
\hline C & -4.746512 & 3.798572 & 0.477691 \\
\hline C & -7.830017 & -0.274198 & 0.235625 \\
\hline C & -8.195895 & -0.952932 & -1.102014 \\
\hline $\mathrm{H}$ & -8.214306 & -0.220921 & -1.915478 \\
\hline H & -7.476799 & -1.732087 & -1.369452 \\
\hline $\mathrm{H}$ & -9.185611 & -1.418221 & -1.036846 \\
\hline C & -8.920763 & 0.757871 & 0.564104 \\
\hline $\mathrm{H}$ & -8.735378 & 1.251729 & 1.523025 \\
\hline $\mathrm{H}$ & -8.994801 & 1.528445 & -0.209614 \\
\hline $\mathrm{H}$ & -9.892348 & 0.258544 & 0.630565 \\
\hline C & -7.811949 & -1.332488 & 1.359579 \\
\hline $\mathrm{H}$ & -7.081357 & -2.121086 & 1.159704 \\
\hline H & -7.554406 & -0.873513 & 2.319189 \\
\hline $\mathrm{H}$ & -8.796368 & -1.803322 & 1.457741 \\
\hline C & -5.123001 & 4.095440 & 1.945636 \\
\hline $\mathrm{H}$ & -6.159465 & 3.818529 & 2.160023 \\
\hline $\mathrm{H}$ & -4.477257 & 3.536171 & 2.629626 \\
\hline $\mathrm{H}$ & -5.007478 & 5.163319 & 2.161772 \\
\hline C & -5.662208 & 4.611787 & -0.461476 \\
\hline $\mathrm{H}$ & -5.532682 & 5.684814 & -0.282876 \\
\hline $\mathrm{H}$ & -5.420605 & 4.408551 & -1.509473 \\
\hline $\mathrm{H}$ & -6.718959 & 4.374387 & -0.309107 \\
\hline C & -3.294135 & 4.249840 & 0.248105 \\
\hline $\mathrm{H}$ & -2.988403 & 4.095724 & -0.791704 \\
\hline $\mathrm{H}$ & -3.204063 & 5.318729 & 0.466840 \\
\hline $\mathrm{H}$ & -2.589170 & 3.710543 & 0.886652 \\
\hline
\end{tabular}




\section{Catalyst 2C 12}

\section{Datum}

Value

B3LYP-D3(BJ)/6-31G(d,p) Energy

$-2165.167579$

B3LYP-D3(BJ)/def2-TZVPP/IEF-PCM(DCM) Energy

$-2165.902658$

B3LYP-D3(BJ)/def2-TZVPP/IEF-PCM(DCM)//B3LYP-D3(BJ)/6-31G(d,p) Free Energy (Quasiharmonic)

$-2165.024568$

Number of Imaginary Frequencies

0

Frequencies (Top 3 out of 321)

$\begin{array}{lr}\text { 1. } & 5.3527 \mathrm{~cm}^{-1} \\ \text { 2. } & 12.0413 \mathrm{~cm}^{-1} \\ \text { 3. } & 15.2808 \mathrm{~cm}^{-1}\end{array}$

B3LYP-D3(BJ)/6-31G(d,p) Molecular Geometry in Cartesian Coordinates

C

C

C

C

C

C

$\mathrm{H}$

$\mathrm{H}$

C

C

$\mathrm{H}$

$\mathrm{H}$

C

C

$\mathrm{H}$

C

C

C

C

C

C

$\mathrm{H}$

$\mathrm{H}$

$\mathrm{H}$

C

C

$\mathrm{H}$

$\mathrm{H}$

C
0.114975

0.288080

$-0.766311$

$-2.014291$

$-2.158420$

$-1.118745$

0.929418

1.232759

$-0.619917$

$-3.064692$

$-3.107157$

$-1.241368$

$-2.929364$

$-1.691627$

$-3.987506$

1. 260926

2. 263211

2.080833

0.836755

$-0.180080$

0.027900

4.028004

1.411016

3. 212092

3.091560

0.669152

$-1.128581$

$-0.759317$

1.674747
$-4.385137$

$-3.512504$

$-2.655115$

$-2.712177$

$-3.620481$

$-4.440244$

$-5.035603$

$-3.467764$

$-1.742922$

$-1.844940$

$-3.654733$

$-5.132160$

$-0.967344$

$-0.950648$

$-1.848546$

$-4.327285$

$-3.468350$

$-2.583833$

$-2.593845$

$-3.491502$

$-4.337039$

$-1.669724$

$-4.999359$

$-3.451383$

$-1.686447$

$-1.699739$

$-3.499249$

$-5.016255$

$-0.822037$

\subsection{8}

1.936418

1.526086

2. 222621

3. 303665

3. 676968

3. 289248

1.407200

0.434470

1.833941

3.831483

4.504428

0.780069

0.061327

2.406243

$-3.610241$

$-3.224559$

$-2.128672$

$-1.425614$

$-1.849515$

$-2.915136$

$-2.264722$

$-4.449563$

- 3.753413

$-1.714971$

$-0.324697$

$-1.324829$

$-3.227378$

0.059606 


\begin{tabular}{|c|c|c|c|}
\hline C & 2.915878 & -0.809533 & -0.661081 \\
\hline C & 1.427301 & 0.119268 & 1.224626 \\
\hline $\mathrm{H}$ & 0.755925 & -0.366158 & 1.943617 \\
\hline H & 2.367783 & 0.327316 & 1.740009 \\
\hline C & -1.571191 & -0.123380 & -1.179701 \\
\hline 0 & -0.801985 & 0.807936 & -1.353309 \\
\hline 0 & -2.409331 & -0.536741 & -2.143378 \\
\hline H & -2.279560 & 0.062957 & -2.898516 \\
\hline 0 & 0.895721 & 1.380285 & 0.825010 \\
\hline H & 0.277903 & 1.227549 & 0.085978 \\
\hline C & 4.034977 & 0.101738 & -0.302097 \\
\hline C & 5.329733 & -0.405906 & -0.171868 \\
\hline C & 3.818851 & 1.477607 & -0.127194 \\
\hline C & 6.414060 & 0.428109 & 0.135321 \\
\hline $\mathrm{H}$ & 5.482698 & -1.473511 & -0.292292 \\
\hline C & 4.870956 & 2.334180 & 0.189169 \\
\hline $\mathrm{H}$ & 2.809669 & 1.852059 & -0.222210 \\
\hline C & 6.159773 & 1.789135 & 0.313311 \\
\hline $\mathrm{H}$ & 6.979072 & 2.451262 & 0.562670 \\
\hline C & -4.007137 & -0.007031 & 0.444810 \\
\hline C & -3.690830 & 1.344160 & 0.257506 \\
\hline C & -5.336188 & -0.428323 & 0.330357 \\
\hline C & -4.676389 & 2.276115 & -0.072676 \\
\hline $\mathrm{H}$ & -2.664560 & 1.659711 & 0.388909 \\
\hline C & -6.352119 & 0.480015 & 0.019725 \\
\hline H & -5.556056 & -1.480222 & 0.462651 \\
\hline C & -5.995281 & 1.819700 & -0.182324 \\
\hline $\mathrm{H}$ & -6.772918 & 2.532412 & -0.435287 \\
\hline C & -7.822361 & 0.060103 & -0.120995 \\
\hline C & -4.351301 & 3.755911 & -0.317749 \\
\hline C & -8.675664 & 0.847736 & 0.896547 \\
\hline $\mathrm{H}$ & -8.348639 & 0.640352 & 1.920172 \\
\hline $\mathrm{H}$ & -8.604612 & 1.926868 & 0.735538 \\
\hline H & -9.730182 & 0.564488 & 0.809547 \\
\hline C & -8.027468 & -1.441002 & 0.138756 \\
\hline $\mathrm{H}$ & -9.087743 & -1.690967 & 0.035633 \\
\hline $\mathrm{H}$ & -7.470760 & -2.055722 & -0.575304 \\
\hline $\mathrm{H}$ & -7.716672 & -1.722671 & 1.149711 \\
\hline C & -8.308356 & 0.374329 & -1.552352 \\
\hline $\mathrm{H}$ & -7.715771 & -0.173217 & -2.291809 \\
\hline $\mathrm{H}$ & -9.357903 & 0.084161 & -1.671218 \\
\hline $\mathrm{H}$ & -8.227683 & 1.440349 & -1.782017 \\
\hline C & -5.122765 & 4.625899 & 0.697021 \\
\hline $\mathrm{H}$ & -6.204281 & 4.488338 & 0.608407 \\
\hline H & -4.835338 & 4.372337 & 1.722024 \\
\hline $\mathrm{H}$ & -4.904711 & 5.686683 & 0.533091 \\
\hline C & -4.781018 & 4.134783 & -1.751673 \\
\hline $\mathrm{H}$ & -5.852332 & 3.981572 & -1.909297 \\
\hline $\mathrm{H}$ & -4.560187 & 5.189272 & -1.948556 \\
\hline $\mathrm{H}$ & -4.243718 & 3.530458 & -2.489393 \\
\hline C & -2.848720 & 4.050543 & -0.172237 \\
\hline $\mathrm{H}$ & -2.490437 & 3.844294 & 0.840935 \\
\hline H & -2.247606 & 3.460078 & -0.870559 \\
\hline$H$ & -2.660126 & 5.108117 & -0.38003 \\
\hline
\end{tabular}




\begin{tabular}{|lrrr|} 
C & 4.651836 & 3.834691 & 0.427605 \\
C & 7.817304 & -0.179727 & 0.275520 \\
H & 7.810027 & -1.222696 & 1.413841 \\
H & 7.535157 & -0.755812 & 2.364792 \\
H & 7.096822 & -2.028233 & 1.218740 \\
C & 8.802576 & -1.672664 & 1.526891 \\
H & 8.884382 & 0.878223 & 0.599267 \\
H & 8.950802 & 1.638984 & -0.184777 \\
H & 8.679988 & 1.381946 & 1.549155 \\
C & 9.864865 & 0.399148 & 0.682060 \\
H & 8.208556 & -0.869363 & -1.049312 \\
H & 7.507326 & -1.666182 & -1.312128 \\
H & 8.219835 & -0.148359 & -1.872677 \\
C & 9.206620 & -1.314020 & -0.969071 \\
H & 5.006064 & 4.161570 & 1.894690 \\
H & 4.364324 & 3.599706 & 2.580307 \\
H & 6.045542 & 3.909268 & 2.124331 \\
C & 4.866441 & 5.230046 & 2.092938 \\
H & 3.193362 & 4.253246 & 0.175423 \\
H & 3.079857 & 5.323511 & 0.375776 \\
H & 2.902073 & 4.076511 & -0.864952 \\
C & 2.492183 & 3.710400 & 0.815140 \\
H & 5.561470 & 4.651594 & -0.514228 \\
H & 5.335762 & 4.427261 & -1.561467 \\
H & 5.408341 & 5.724408 & -0.353958 \\
& 6.621041 & 4.438218 & -0.346652 \\
\hline & & & \\
\hline & & & \\
\hline & & & \\
\hline
\end{tabular}

\section{Catalyst 2C 13}

Datum

Value

B3LYP-D3(BJ)/6-31G(d,p) Energy

$-2165.168622$

B3LYP-D3(BJ)/def2-TZVPP/IEF-PCM(DCM) Energy

$-2165.903092$

B3LYP-D3(BJ)/def2-TZVPP/IEF-PCM(DCM)//B3LYP-D3(BJ)/6-31G(d,p) Free Energy (Quasiharmonic) -2165.024499

Number of Imaginary Frequencies

Frequencies (Top 3 out of 321)

$\begin{array}{lr}\text { 1. } & 7.7790 \mathrm{~cm}^{-1} \\ \text { 2. } & 12.4931 \mathrm{~cm}^{-1} \\ \text { 3. } & 15.3377 \mathrm{~cm}^{-1}\end{array}$

B3LYP-D3(BJ)/6-31G(d,p) Molecular Geometry in Cartesian Coordinates 


\begin{tabular}{|c|c|c|c|}
\hline C & 0.127237 & -4.337615 & 2.996855 \\
\hline C & 0.297162 & -3.463692 & 1.947357 \\
\hline C & -0.759377 & -2.607508 & 1.539839 \\
\hline C & -2.006666 & -2.668930 & 2.237059 \\
\hline C & -2.147583 & -3.578660 & 3.317441 \\
\hline C & -1.105495 & -4.396045 & 3.688934 \\
\hline $\mathrm{H}$ & 0.943410 & -4.986709 & 3.297825 \\
\hline $\mathrm{H}$ & 1.241069 & -3.416407 & 1.416993 \\
\hline C & -0.615948 & -1.691825 & 0.451020 \\
\hline C & -3.061461 & -1.808354 & 1.846526 \\
\hline $\mathrm{H}$ & -3.095890 & -3.616114 & 3.845797 \\
\hline $\mathrm{H}$ & -1.225473 & -5.089016 & 4.515886 \\
\hline C & -2.930408 & -0.928423 & 0.793591 \\
\hline C & -1.689460 & -0.900322 & 0.080656 \\
\hline $\mathrm{H}$ & -3.986527 & -1.821253 & 2.414965 \\
\hline C & 1.202622 & -4.244701 & -3.639335 \\
\hline C & 2.218188 & -3.402919 & -3.250789 \\
\hline C & 2.055287 & -2.530826 & -2.141928 \\
\hline C & 0.816865 & -2.534493 & -1.428979 \\
\hline C & -0.213755 & -3.414991 & -1.855792 \\
\hline C & -0.024515 & -4.249013 & -2.933890 \\
\hline $\mathrm{H}$ & 4.013103 & -1.641584 & -2.281178 \\
\hline $\mathrm{H}$ & 1.337757 & -4.907479 & -4.488524 \\
\hline $\mathrm{H}$ & 3.163019 & -3.390401 & -3.786951 \\
\hline C & 3.080892 & -1.652678 & -1.724189 \\
\hline C & 0.669136 & -1.651792 & -0.315630 \\
\hline $\mathrm{H}$ & -1.157988 & -3.418994 & -1.323597 \\
\hline $\mathrm{H}$ & -0.822267 & -4.914929 & -3.248013 \\
\hline C & 1.689015 & -0.792097 & 0.071913 \\
\hline C & 2.925734 & -0.787724 & -0.657225 \\
\hline C & 1.460291 & 0.139936 & 1.248055 \\
\hline $\mathrm{H}$ & 0.781221 & -0.340039 & 1.963132 \\
\hline $\mathrm{H}$ & 2.404353 & 0.327929 & 1.764093 \\
\hline C & -1.561195 & -0.055597 & -1.147611 \\
\hline 0 & -0.798883 & 0.886918 & -1.292295 \\
\hline 0 & -2.380916 & -0.459297 & -2.129702 \\
\hline H & -2.249000 & 0.156498 & -2.871518 \\
\hline 0 & 0.951117 & 1.414497 & 0.861191 \\
\hline $\mathrm{H}$ & 0.308722 & 1.277161 & 0.140220 \\
\hline C & 4.061433 & 0.101360 & -0.295077 \\
\hline C & 5.356112 & -0.424927 & -0.201630 \\
\hline C & 3.870194 & 1.469633 & -0.078217 \\
\hline C & 6.452691 & 0.386730 & 0.104701 \\
\hline $\mathrm{H}$ & 5.493651 & -1.490856 & -0.351740 \\
\hline C & 4.939691 & 2.308450 & 0.246540 \\
\hline $\mathrm{H}$ & 2.865340 & 1.866932 & -0.145508 \\
\hline C & 6.221150 & 1.750503 & 0.327463 \\
\hline $\mathrm{H}$ & 7.055891 & 2.389848 & 0.575356 \\
\hline C & -4.024167 & 0.010707 & 0.448884 \\
\hline C & -5.348505 & -0.440357 & 0.344236 \\
\hline C & -3.742703 & 1.361020 & 0.238680 \\
\hline C & -6.381216 & 0.438800 & 0.022519 \\
\hline $\mathrm{H}$ & -5.545192 & -1.494456 & 0.493960 \\
\hline
\end{tabular}




\begin{tabular}{|c|c|c|c|}
\hline C & -4.750827 & 2.270056 & -0.107117 \\
\hline $\mathrm{H}$ & -2.723147 & 1.708731 & 0.356996 \\
\hline C & -6.056329 & 1.787715 & -0.205533 \\
\hline $\mathrm{H}$ & -6.852389 & 2.472286 & -0.468642 \\
\hline C & -4.373857 & 3.732950 & -0.375744 \\
\hline C & -7.842585 & -0.013743 & -0.106597 \\
\hline C & -3.696714 & 4.329702 & 0.876699 \\
\hline $\mathrm{H}$ & -4.372615 & 4.296418 & 1.736932 \\
\hline $\mathrm{H}$ & -2.785574 & 3.788166 & 1.143899 \\
\hline $\mathrm{H}$ & -3.422122 & 5.374569 & 0.697074 \\
\hline C & -5.596214 & 4.597009 & -0.722966 \\
\hline $\mathrm{H}$ & -5.278078 & 5.627587 & -0.906849 \\
\hline $\mathrm{H}$ & -6.101878 & 4.240572 & -1.626011 \\
\hline $\mathrm{H}$ & -6.324447 & 4.614752 & 0.094180 \\
\hline C & -3.387697 & 3.780314 & -1.564655 \\
\hline $\mathrm{H}$ & -3.856299 & 3.377224 & -2.468770 \\
\hline $\mathrm{H}$ & -3.087513 & 4.813590 & -1.769447 \\
\hline $\mathrm{H}$ & -2.482247 & 3.199804 & -1.367753 \\
\hline C & -8.339625 & 0.265968 & -1.541435 \\
\hline $\mathrm{H}$ & -8.283065 & 1.329432 & -1.789324 \\
\hline $\mathrm{H}$ & -7.737769 & -0.281298 & -2.273590 \\
\hline $\mathrm{H}$ & -9.383008 & -0.048853 & -1.651474 \\
\hline C & -8.709389 & 0.772973 & 0.900320 \\
\hline $\mathrm{H}$ & -8.661693 & 1.850481 & 0.721006 \\
\hline $\mathrm{H}$ & -9.757903 & 0.466104 & 0.821268 \\
\hline $\mathrm{H}$ & -8.375298 & 0.589490 & 1.926239 \\
\hline C & -8.015790 & -1.514143 & 0.178986 \\
\hline $\mathrm{H}$ & -7.448615 & -2.129151 & -0.526550 \\
\hline $\mathrm{H}$ & -7.696137 & -1.772181 & 1.193489 \\
\hline $\mathrm{H}$ & -9.070947 & -1.787729 & 0.083669 \\
\hline C & 4.657643 & 3.794269 & 0.509392 \\
\hline C & 7.852170 & -0.239555 & 0.200570 \\
\hline C & 8.938617 & 0.798316 & 0.525703 \\
\hline $\mathrm{H}$ & 8.996961 & 1.577166 & -0.240999 \\
\hline $\mathrm{H}$ & 8.761752 & 1.281288 & 1.491696 \\
\hline $\mathrm{H}$ & 9.914841 & 0.306020 & 0.574874 \\
\hline C & 8.208063 & -0.902455 & -1.147689 \\
\hline $\mathrm{H}$ & 7.493071 & -1.685916 & -1.413528 \\
\hline H & 8.209488 & -0.162912 & -1.954532 \\
\hline $\mathrm{H}$ & 9.202898 & -1.358830 & -1.099065 \\
\hline C & 7.857386 & -1.308707 & 1.314405 \\
\hline $\mathrm{H}$ & 7.606988 & -0.861397 & 2.281393 \\
\hline $\mathrm{H}$ & 7.131673 & -2.102043 & 1.115457 \\
\hline H & 8.847219 & -1.771275 & 1.396527 \\
\hline C & 3.652440 & 3.914156 & 1.677085 \\
\hline $\mathrm{H}$ & 2.714563 & 3.395508 & 1.461809 \\
\hline $\mathrm{H}$ & 4.073154 & 3.482876 & 2.591507 \\
\hline $\mathrm{H}$ & 3.423915 & 4.967928 & 1.873005 \\
\hline C & 4.045076 & 4.428045 & -0.758233 \\
\hline $\mathrm{H}$ & 3.831289 & 5.489043 & -0.587898 \\
\hline $\mathrm{H}$ & 4.734674 & 4.349417 & -1.604961 \\
\hline $\mathrm{H}$ & 3.109295 & 3.938019 & -1.039137 \\
\hline C & 5.928432 & 4.576486 & 0.876174 \\
\hline H & 6.668533 & 4.551188 & 0.069674 \\
\hline
\end{tabular}




\section{Catalyst 2C 14}

\section{Datum}

Value

\begin{tabular}{lr}
\hline B3LYP-D3(BJ)/6-31G(d,p) Energy & -2165.168108 \\
\hline B3LYP-D3(BJ)/def2-TZVPP/IEF-PCM(DCM) Energy & -2165.902826 \\
\hline B3LYP-D3(BJ)/def2-TZVPP/IEF-PCM(DCM)//B3LYP-D3(BJ)/6-31G(d,p) Free Energy (Quasiharmonic) & -2165.024462 \\
\hline
\end{tabular}

Number of Imaginary Frequencies

Frequencies (Top 3 out of 321)
1.
$6.1061 \mathrm{~cm}^{-1}$
2. $\quad 12.1578 \mathrm{~cm}^{-1}$
3. $15.3958 \mathrm{~cm}^{-1}$

\section{B3LYP-D3(BJ)/6-31G(d,p) Molecular Geometry in Cartesian Coordinates}

\begin{tabular}{|c|c|c|c|}
\hline C & -0.133494 & -4.367114 & 3.006108 \\
\hline C & -0.306065 & -3.496000 & 1.954652 \\
\hline C & 0.750249 & -2.642887 & 1.540349 \\
\hline C & 1.999734 & -2.703051 & 2.233899 \\
\hline C & 2.143303 & -3.609753 & 3.316379 \\
\hline C & 1.101648 & -4.425091 & 3.693782 \\
\hline $\mathrm{H}$ & -0.949447 & -5.014179 & 3.312007 \\
\hline $\mathrm{H}$ & -1.251824 & -3.449057 & 1.427552 \\
\hline C & 0.604356 & -1.731947 & 0.447691 \\
\hline C & 3.052655 & -1.841239 & 1.840156 \\
\hline $\mathrm{H}$ & 3.093208 & -3.646373 & 3.841923 \\
\hline $\mathrm{H}$ & 1.223806 & -5.115786 & 4.522326 \\
\hline $\mathrm{C}$ & 2.917977 & -0.964874 & 0.785087 \\
\hline C & 1.677657 & -0.943464 & 0.070959 \\
\hline $\mathrm{H}$ & 3.977554 & -1.848448 & 2.409032 \\
\hline C & -1.273673 & -4.314132 & -3.598588 \\
\hline C & -2.276127 & -3.455148 & -3.213583 \\
\hline C & -2.094780 & -2.571478 & -2.116798 \\
\hline C & -0.851581 & -2.582165 & -1.412302 \\
\hline C & 0.165442 & -3.479990 & -1.835486 \\
\hline C & -0.041545 & -4.324779 & -2.901875 \\
\hline $\mathrm{H}$ & -4.041205 & -1.657401 & -2.255091 \\
\hline $\mathrm{H}$ & -1.422930 & -4.985618 & -4.438524 \\
\hline $\mathrm{H}$ & -3.224389 & -3.437529 & -3.743514 \\
\hline
\end{tabular}




\begin{tabular}{|c|c|c|c|}
\hline C & -3.105806 & -1.674434 & -1.703627 \\
\hline C & -0.685097 & -1.688264 & -0.310983 \\
\hline $\mathrm{H}$ & 1.113306 & -3.488553 & -1.309720 \\
\hline $\mathrm{H}$ & 0.745804 & -5.004120 & -3.213501 \\
\hline C & -1.691004 & -0.810586 & 0.072874 \\
\hline C & -2.932011 & -0.798191 & -0.648725 \\
\hline C & -1.442259 & 0.130579 & 1.237788 \\
\hline $\mathrm{H}$ & -2.381098 & 0.338691 & 1.755776 \\
\hline $\mathrm{H}$ & -0.768021 & -0.353120 & 1.954945 \\
\hline C & 1.554816 & -0.114861 & -1.168894 \\
\hline 0 & 0.789821 & 0.821146 & -1.336592 \\
\hline 0 & 2.384668 & -0.531670 & -2.137954 \\
\hline $\mathrm{H}$ & 2.253528 & 0.069167 & -2.891963 \\
\hline 0 & -0.913061 & 1.392686 & 0.836594 \\
\hline $\mathrm{H}$ & -0.290947 & 1.240441 & 0.100803 \\
\hline C & -4.051971 & 0.112649 & -0.291966 \\
\hline C & -3.837645 & 1.480090 & -0.092129 \\
\hline C & -5.354557 & -0.391343 & -0.186633 \\
\hline C & -4.891802 & 2.340031 & 0.227281 \\
\hline $\mathrm{H}$ & -2.826888 & 1.860418 & -0.168275 \\
\hline C & -6.436369 & 0.441636 & 0.114976 \\
\hline $\mathrm{H}$ & -5.510154 & -1.456565 & -0.323574 \\
\hline C & -6.181765 & 1.804032 & 0.320488 \\
\hline $\mathrm{H}$ & -7.004869 & 2.459798 & 0.564437 \\
\hline C & 4.001102 & -0.013091 & 0.442564 \\
\hline C & 3.694765 & 1.339876 & 0.251702 \\
\hline C & 5.326364 & -0.445199 & 0.323992 \\
\hline C & 4.686314 & 2.262820 & -0.085897 \\
\hline $\mathrm{H}$ & 2.671590 & 1.664115 & 0.385913 \\
\hline C & 6.348150 & 0.453886 & 0.005818 \\
\hline $\mathrm{H}$ & 5.538477 & -1.498341 & 0.458984 \\
\hline C & 6.001065 & 1.795608 & -0.199496 \\
\hline $\mathrm{H}$ & 6.783190 & 2.501219 & -0.458401 \\
\hline C & 7.814372 & 0.021773 & -0.139750 \\
\hline C & 4.372093 & 3.744265 & -0.335188 \\
\hline C & 8.008638 & -1.479980 & 0.124543 \\
\hline $\mathrm{H}$ & 7.700038 & -1.755529 & 1.137856 \\
\hline $\mathrm{H}$ & 7.443951 & -2.092805 & -0.584866 \\
\hline $\mathrm{H}$ & 9.066421 & -1.738778 & 0.017730 \\
\hline C & 8.678332 & 0.806231 & 0.871226 \\
\hline $\mathrm{H}$ & 9.730152 & 0.514196 & 0.780755 \\
\hline $\mathrm{H}$ & 8.615266 & 1.885306 & 0.706546 \\
\hline $\mathrm{H}$ & 8.354073 & 0.605218 & 1.896999 \\
\hline C & 8.296573 & 0.326988 & -1.574343 \\
\hline $\mathrm{H}$ & 8.223481 & 1.392801 & -1.807486 \\
\hline $\mathrm{H}$ & 9.343218 & 0.027949 & -1.696735 \\
\hline $\mathrm{H}$ & 7.696371 & -0.218398 & -2.309239 \\
\hline C & 2.872783 & 4.051813 & -0.183066 \\
\hline $\mathrm{H}$ & 2.263267 & 3.463652 & -0.876053 \\
\hline $\mathrm{H}$ & 2.518230 & 3.852351 & 0.832785 \\
\hline $\mathrm{H}$ & 2.691825 & 5.110112 & -0.393937 \\
\hline C & 4.797446 & 4.113831 & -1.772842 \\
\hline $\mathrm{H}$ & 4.251343 & 3.511037 & -2.505337 \\
\hline $\mathrm{H}$ & 4.584397 & 5.169328 & -1.972876 \\
\hline
\end{tabular}




\begin{tabular}{|c|c|c|c|}
\hline $\mathrm{H}$ & 5.866607 & 3.951014 & -1.935341 \\
\hline C & 5.155817 & 4.612038 & 0.672059 \\
\hline $\mathrm{H}$ & 4.871911 & 4.364736 & 1.699567 \\
\hline $\mathrm{H}$ & 6.235725 & 4.465659 & 0.578225 \\
\hline $\mathrm{H}$ & 4.945253 & 5.673865 & 0.505128 \\
\hline C & -7.845361 & -0.160655 & 0.224265 \\
\hline C & -4.584193 & 3.824030 & 0.471073 \\
\hline C & -3.572019 & 3.941251 & 1.633021 \\
\hline $\mathrm{H}$ & -3.995758 & 3.527921 & 2.554314 \\
\hline $\mathrm{H}$ & -2.643714 & 3.404717 & 1.420038 \\
\hline $\mathrm{H}$ & -3.325395 & 4.993397 & 1.815285 \\
\hline C & -5.840357 & 4.631431 & 0.833569 \\
\hline $\mathrm{H}$ & -6.584220 & 4.608585 & 0.030460 \\
\hline $\mathrm{H}$ & -6.311772 & 4.257463 & 1.748060 \\
\hline $\mathrm{H}$ & -5.569019 & 5.677782 & 1.004576 \\
\hline C & -3.966687 & 4.432380 & -0.806590 \\
\hline $\mathrm{H}$ & -3.040073 & 3.923936 & -1.085164 \\
\hline $\mathrm{H}$ & -4.660934 & 4.354713 & -1.649600 \\
\hline $\mathrm{H}$ & -3.735057 & 5.491754 & -0.650012 \\
\hline C & -8.913051 & 0.898099 & 0.544068 \\
\hline $\mathrm{H}$ & -8.722918 & 1.388416 & 1.503823 \\
\hline $\mathrm{H}$ & -8.963081 & 1.669557 & -0.230647 \\
\hline $\mathrm{H}$ & -9.896810 & 0.422254 & 0.603818 \\
\hline C & -7.862406 & -1.218188 & 1.349036 \\
\hline $\mathrm{H}$ & -8.859170 & -1.663806 & 1.440529 \\
\hline $\mathrm{H}$ & -7.150588 & -2.025189 & 1.154884 \\
\hline $\mathrm{H}$ & -7.600161 & -0.765178 & 2.310215 \\
\hline C & -8.218750 & -0.831404 & -1.115359 \\
\hline $\mathrm{H}$ & -7.517890 & -1.629093 & -1.376502 \\
\hline $\mathrm{H}$ & -9.220632 & -1.270959 & -1.057273 \\
\hline $\mathrm{H}$ & -8.212118 & -0.100228 & -1.929766 \\
\hline
\end{tabular}

\section{Catalyst 2C 15}

Datum

Value

\begin{tabular}{lr}
\hline B3LYP-D3(BJ)/6-31G(d,p) Energy & -2165.167842 \\
\hline B3LYP-D3(BJ)/def2-TZVPP/IEF-PCM(DCM) Energy & -2165.902636 \\
\hline
\end{tabular}

B3LYP-D3(BJ)/def2-TZVPP/IEF-PCM(DCM)//B3LYP-D3(BJ)/6-31G(d,p) Free Energy (Quasiharmonic) -

Number of Imaginary Frequencies

Frequencies (Top 3 out of 321)

$\begin{array}{lr}\text { 1. } & 7.7945 \mathrm{~cm}^{-1} \\ \text { 2. } & 12.6379 \mathrm{~cm}^{-1} \\ \text { 3. } & 15.2053 \mathrm{~cm}^{-1}\end{array}$




\begin{tabular}{|c|c|c|c|}
\hline C & 0.065921 & -4.331726 & 2.988593 \\
\hline C & 0.242507 & -3.459518 & 1.938763 \\
\hline C & -0.804862 & -2.589967 & 1.535908 \\
\hline C & -2.050089 & -2.636443 & 2.237925 \\
\hline C & -2.197953 & -3.544657 & 3.318633 \\
\hline C & -1.164596 & -4.375037 & 3.685699 \\
\hline H & 0.875106 & -4.991165 & 3.285941 \\
\hline $\mathrm{H}$ & 1.184756 & -3.423704 & 1.404561 \\
\hline C & -0.653965 & -1.674919 & 0.447540 \\
\hline C & -3.096234 & -1.763818 & 1.850781 \\
\hline $\mathrm{H}$ & -3.144549 & -3.570565 & 3.850749 \\
\hline $\mathrm{H}$ & -1.289832 & -5.066753 & 4.512926 \\
\hline C & -2.958542 & -0.885827 & 0.797163 \\
\hline C & -1.718992 & -0.870037 & 0.081590 \\
\hline $\mathrm{H}$ & -4.019913 & -1.766336 & 2.421653 \\
\hline C & 1.108525 & -4.242210 & -3.657997 \\
\hline C & 2.138176 & -3.416791 & -3.271416 \\
\hline C & 1.992967 & -2.545269 & -2.159650 \\
\hline C & 0.757610 & -2.532316 & -1.441499 \\
\hline C & -0.287794 & -3.396200 & -1.866336 \\
\hline C & -0.115590 & -4.230030 & -2.947441 \\
\hline $\mathrm{H}$ & 3.963679 & -1.685815 & -2.304314 \\
\hline $\mathrm{H}$ & 1.230220 & -4.904667 & -4.509471 \\
\hline $\mathrm{H}$ & 3.080920 & -3.417070 & -3.811392 \\
\hline C & 3.033470 & -1.683811 & -1.743896 \\
\hline C & 0.628100 & -1.650849 & -0.324822 \\
\hline $\mathrm{H}$ & -1.229777 & -3.387638 & -1.330239 \\
\hline $\mathrm{H}$ & -0.924562 & -4.883045 & -3.259961 \\
\hline C & 1.662543 & -0.807896 & 0.060639 \\
\hline C & 2.895776 & -0.819222 & -0.674201 \\
\hline C & 1.454586 & 0.123276 & 1.241124 \\
\hline $\mathrm{H}$ & 0.772101 & -0.349258 & 1.958175 \\
\hline $\mathrm{H}$ & 2.404829 & 0.293049 & 1.752368 \\
\hline C & -1.582870 & -0.022252 & -1.143749 \\
\hline 0 & -0.809961 & 0.911840 & -1.285364 \\
\hline 0 & -2.409653 & -0.411908 & -2.125711 \\
\hline $\mathrm{H}$ & -2.272319 & 0.205662 & -2.865053 \\
\hline 0 & 0.964670 & 1.407024 & 0.861986 \\
\hline $\mathrm{H}$ & 0.313991 & 1.282055 & 0.146541 \\
\hline C & 4.046805 & 0.050804 & -0.313353 \\
\hline C & 3.877141 & 1.425324 & -0.108085 \\
\hline C & 5.329544 & -0.505918 & -0.212603 \\
\hline C & 4.962874 & 2.243877 & 0.211989 \\
\hline $\mathrm{H}$ & 2.879733 & 1.834629 & -0.182305 \\
\hline C & 6.439202 & 0.285359 & 0.095552 \\
\hline $\mathrm{H}$ & 5.439047 & -1.573393 & -0.358031 \\
\hline C & 6.228597 & 1.653868 & 0.305712 \\
\hline $\mathrm{H}$ & 7.078579 & 2.279102 & 0.558301 \\
\hline C & -4.045396 & 0.061550 & 0.452786 \\
\hline C & -5.369100 & -0.374709 & 0.345573 \\
\hline
\end{tabular}




\begin{tabular}{|c|c|c|c|}
\hline C & -3.753964 & 1.413424 & 0.242553 \\
\hline C & -6.400033 & 0.513253 & 0.022252 \\
\hline $\mathrm{H}$ & -5.580724 & -1.428563 & 0.492715 \\
\hline C & -4.753094 & 2.326640 & -0.102924 \\
\hline $\mathrm{H}$ & -2.731632 & 1.752828 & 0.361321 \\
\hline C & -6.068027 & 1.855743 & -0.204103 \\
\hline $\mathrm{H}$ & -6.852071 & 2.550471 & -0.466819 \\
\hline C & -4.366778 & 3.787812 & -0.370654 \\
\hline C & -7.839335 & -0.010675 & -0.090942 \\
\hline C & -3.687097 & 4.380170 & 0.882530 \\
\hline $\mathrm{H}$ & -4.363829 & 4.350511 & 1.742254 \\
\hline $\mathrm{H}$ & -2.779493 & 3.832932 & 1.150105 \\
\hline $\mathrm{H}$ & -3.406135 & 5.423495 & 0.703644 \\
\hline C & -5.582852 & 4.660555 & -0.718908 \\
\hline $\mathrm{H}$ & -5.256923 & 5.688884 & -0.901904 \\
\hline $\mathrm{H}$ & -6.090170 & 4.308429 & -1.622675 \\
\hline $\mathrm{H}$ & -6.312051 & 4.683369 & 0.097205 \\
\hline C & -3.379058 & 3.829847 & -1.558530 \\
\hline $\mathrm{H}$ & -3.848863 & 3.429332 & -2.463187 \\
\hline $\mathrm{H}$ & -3.072920 & 4.861442 & -1.763111 \\
\hline $\mathrm{H}$ & -2.477113 & 3.244275 & -1.360547 \\
\hline C & -7.904472 & -1.084446 & -1.198596 \\
\hline $\mathrm{H}$ & -7.608925 & -0.663358 & -2.164568 \\
\hline $\mathrm{H}$ & -7.241258 & -1.926989 & -0.984725 \\
\hline $\mathrm{H}$ & -8.923835 & -1.474457 & -1.292178 \\
\hline C & -8.843056 & 1.100686 & -0.438139 \\
\hline $\mathrm{H}$ & -8.618612 & 1.562909 & -1.404451 \\
\hline $\mathrm{H}$ & -9.850986 & 0.679141 & -0.499044 \\
\hline $\mathrm{H}$ & -8.857069 & 1.886676 & 0.323345 \\
\hline C & -8.259808 & -0.636668 & 1.256401 \\
\hline $\mathrm{H}$ & -7.607106 & -1.467836 & 1.537100 \\
\hline $\mathrm{H}$ & -8.220022 & 0.106463 & 2.058892 \\
\hline $\mathrm{H}$ & -9.283804 & -1.020758 & 1.195477 \\
\hline C & 7.857430 & -0.290200 & 0.221661 \\
\hline C & 4.794910 & 3.745238 & 0.484910 \\
\hline C & 3.347825 & 4.215672 & 0.260236 \\
\hline $\mathrm{H}$ & 3.038340 & 4.070518 & -0.779754 \\
\hline $\mathrm{H}$ & 2.637180 & 3.682241 & 0.897377 \\
\hline $\mathrm{H}$ & 3.271631 & 5.284511 & 0.484384 \\
\hline C & 5.719186 & 4.552306 & -0.450990 \\
\hline $\mathrm{H}$ & 6.773076 & 4.300484 & -0.301873 \\
\hline $\mathrm{H}$ & 5.473520 & 4.358283 & -1.499768 \\
\hline $\mathrm{H}$ & 5.603744 & 5.625826 & -0.265893 \\
\hline C & 5.177523 & 4.028210 & 1.953945 \\
\hline $\mathrm{H}$ & 4.526297 & 3.472570 & 2.635676 \\
\hline $\mathrm{H}$ & 6.210819 & 3.736790 & 2.164516 \\
\hline $\mathrm{H}$ & 5.075724 & 5.096016 & 2.177206 \\
\hline C & 8.782574 & 0.406550 & -0.799293 \\
\hline $\mathrm{H}$ & 8.426918 & 0.240218 & -1.820933 \\
\hline $\mathrm{H}$ & 8.827099 & 1.486353 & -0.633424 \\
\hline $\mathrm{H}$ & 9.802025 & 0.012656 & -0.722513 \\
\hline C & 7.899786 & -1.803207 & -0.046628 \\
\hline $\mathrm{H}$ & 8.928129 & -2.165708 & 0.047308 \\
\hline $\mathrm{H}$ & 7.285171 & -2.358661 & 0.668539 \\
\hline
\end{tabular}




\section{Catalyst 2C 16}

\section{Datum}

Value

\begin{tabular}{lc}
\hline B3LYP-D3(BJ)/6-31G(d,p) Energy & -2165.163716 \\
\hline B3LYP-D3(BJ)/def2-TZVPP/IEF-PCM(DCM) Energy & -2165.902013 \\
\hline B3LYP-D3(BJ)/def2-TZVPP/IEF-PCM(DCM)//B3LYP-D3(BJ)/6-31G(d,p) Free Energy (Quasiharmonic) & -2165.023805
\end{tabular}

Number of Imaginary Frequencies

Frequencies (Top 3 out of 321)
1. $9.6839 \mathrm{~cm}^{-1}$
2. $12.8416 \mathrm{~cm}^{-1}$
3. $13.9655 \mathrm{~cm}^{-1}$

\section{B3LYP-D3(BJ)/6-31G(d,p) Molecular Geometry in Cartesian Coordinates}

$\begin{array}{rrrr}\mathrm{C} & -1.498175 & 0.245032 & 1.265248 \\ \mathrm{H} & -2.445453 & 0.667387 & 1.607473 \\ \mathrm{H} & -0.959926 & -0.166058 & 2.122880 \\ \mathrm{C} & 1.477045 & -0.603435 & -1.208195 \\ \mathrm{O} & 1.955175 & -1.243970 & -2.121287 \\ \mathrm{O} & 0.809784 & 0.530673 & -1.425012 \\ \mathrm{H} & 0.371725 & 0.849935 & -0.600352 \\ \mathrm{O} & -0.699541 & 1.304639 & 0.672126 \\ \mathrm{H} & -0.367125 & 1.869354 & 1.382115 \\ \mathrm{C} & 0.522057 & -1.723779 & 0.798207 \\ \mathrm{C} & 1.599080 & -1.063660 & 0.230916 \\ \mathrm{C} & 2.813552 & -0.864341 & 0.964393 \\ \mathrm{C} & 2.893823 & -1.368728 & 2.248098 \\ \mathrm{H} & 3.809298 & -1.223226 & 2.813775 \\ \mathrm{C} & -1.744338 & -0.841422 & 0.247950 \\ \mathrm{C} & -0.771008 & -1.814641 & 0.051208 \\ \mathrm{C} & -3.201810 & -1.967875 & -1.329154 \\ \mathrm{C} & -2.975288 & -0.900180 & -0.481967 \\ \mathrm{H} & -4.132912 & -2.012317 & -1.886295 \\ \mathrm{C} & -2.238886 & -2.982074 & -1.534506 \\ \mathrm{C} & -0.986730 & -2.898608 & -0.849231\end{array}$




\begin{tabular}{|c|c|c|c|}
\hline C & -0.007253 & -3.900200 & -1.083375 \\
\hline C & -0.263273 & -4.940788 & -1.944729 \\
\hline C & -1.506807 & -5.029077 & -2.616432 \\
\hline C & -2.470781 & -4.069376 & -2.418165 \\
\hline $\mathrm{H}$ & 0.951680 & -3.822089 & -0.585225 \\
\hline $\mathrm{H}$ & 0.495507 & -5.696858 & -2.119389 \\
\hline $\mathrm{H}$ & -1.692603 & -5.855334 & -3.295688 \\
\hline $\mathrm{H}$ & -3.423352 & -4.124435 & -2.937372 \\
\hline C & 1.834549 & -2.093638 & 2.844169 \\
\hline C & 0.622314 & -2.280139 & 2.110174 \\
\hline C & -0.441747 & -3.000954 & 2.714810 \\
\hline C & -0.309296 & -3.514113 & 3.985255 \\
\hline C & 0.891080 & -3.329878 & 4.712141 \\
\hline C & 1.938309 & -2.635436 & 4.152562 \\
\hline $\mathrm{H}$ & -1.359153 & -3.146760 & 2.155503 \\
\hline $\mathrm{H}$ & -1.128607 & -4.067775 & 4.433130 \\
\hline $\mathrm{H}$ & 0.982316 & -3.740702 & 5.712821 \\
\hline $\mathrm{H}$ & 2.863578 & -2.490977 & 4.703180 \\
\hline C & -5.944070 & 2.149364 & -0.178109 \\
\hline C & -6.334730 & 0.806205 & -0.038865 \\
\hline C & -5.349894 & -0.176656 & -0.130016 \\
\hline C & -4.007388 & 0.161775 & -0.364262 \\
\hline C & -3.660670 & 1.504932 & -0.515728 \\
\hline C & -4.619806 & 2.520999 & -0.412732 \\
\hline $\mathrm{H}$ & -6.701689 & 2.918359 & -0.097251 \\
\hline $\mathrm{H}$ & -5.599493 & -1.222740 & -0.003456 \\
\hline $\mathrm{H}$ & -2.625627 & 1.753403 & -0.715718 \\
\hline C & -7.812113 & 0.476288 & 0.218291 \\
\hline C & -4.171040 & 3.981747 & -0.553543 \\
\hline C & -8.265980 & 1.149526 & 1.531543 \\
\hline $\mathrm{H}$ & -9.320238 & 0.927563 & 1.729546 \\
\hline $\mathrm{H}$ & -7.674969 & 0.785886 & 2.377971 \\
\hline $\mathrm{H}$ & -8.155244 & 2.236482 & 1.488513 \\
\hline C & -8.664029 & 1.009980 & -0.953693 \\
\hline $\mathrm{H}$ & -9.723454 & 0.786541 & -0.787486 \\
\hline $\mathrm{H}$ & -8.564051 & 2.092887 & -1.066855 \\
\hline $\mathrm{H}$ & -8.359911 & 0.545704 & -1.896917 \\
\hline C & -8.059271 & -1.035788 & 0.342402 \\
\hline $\mathrm{H}$ & -9.123211 & -1.221928 & 0.517840 \\
\hline $\mathrm{H}$ & -7.774897 & -1.568396 & -0.570427 \\
\hline $\mathrm{H}$ & -7.504031 & -1.469874 & 1.179709 \\
\hline C & -5.335562 & 4.970986 & -0.391511 \\
\hline $\mathrm{H}$ & -4.965466 & 5.995959 & -0.490721 \\
\hline $\mathrm{H}$ & -6.103410 & 4.820401 & -1.156886 \\
\hline $\mathrm{H}$ & -5.808948 & 4.882233 & 0.591528 \\
\hline C & -3.116852 & 4.287605 & 0.534081 \\
\hline $\mathrm{H}$ & -2.761758 & 5.319790 & 0.441857 \\
\hline $\mathrm{H}$ & -3.546742 & 4.162698 & 1.533494 \\
\hline $\mathrm{H}$ & -2.252706 & 3.622564 & 0.452740 \\
\hline C & -3.543430 & 4.191057 & -1.948370 \\
\hline $\mathrm{H}$ & -3.211870 & 5.228560 & -2.064972 \\
\hline $\mathrm{H}$ & -2.677217 & 3.542632 & -2.103716 \\
\hline $\mathrm{H}$ & -4.270500 & 3.973529 & -2.736886 \\
\hline C & 3.949370 & -0.071943 & 0.422194 \\
\hline
\end{tabular}




\begin{tabular}{|c|c|c|c|}
\hline C & 4.483505 & 0.971963 & 1.197744 \\
\hline C & 4.510031 & -0.350623 & -0.823454 \\
\hline C & 5.553333 & 1.736869 & 0.737671 \\
\hline $\mathrm{H}$ & 4.028535 & 1.180971 & 2.158075 \\
\hline C & 5.574941 & 0.409959 & -1.325760 \\
\hline $\mathrm{H}$ & 4.099683 & -1.161351 & -1.408683 \\
\hline C & 6.079208 & 1.439570 & -0.531500 \\
\hline $\mathrm{H}$ & 6.901615 & 2.037786 & -0.902723 \\
\hline C & 6.112594 & 0.092887 & -2.727327 \\
\hline C & 6.160195 & 2.887781 & 1.552855 \\
\hline C & 7.297578 & 0.990652 & -3.116297 \\
\hline $\mathrm{H}$ & 7.016175 & 2.048349 & -3.133022 \\
\hline $\mathrm{H}$ & 8.141412 & 0.870164 & -2.428822 \\
\hline $\mathrm{H}$ & 7.645503 & 0.726175 & -4.119503 \\
\hline C & 6.581647 & -1.376950 & -2.778865 \\
\hline $\mathrm{H}$ & 6.966968 & -1.616170 & -3.775897 \\
\hline $\mathrm{H}$ & 7.379533 & -1.557456 & -2.051172 \\
\hline $\mathrm{H}$ & 5.764641 & -2.069975 & -2.562906 \\
\hline C & 4.975210 & 0.307156 & -3.751498 \\
\hline $\mathrm{H}$ & 4.100006 & -0.307171 & -3.525564 \\
\hline $\mathrm{H}$ & 4.653017 & 1.353433 & -3.753054 \\
\hline $\mathrm{H}$ & 5.321454 & 0.055463 & -4.760193 \\
\hline C & 6.023442 & 4.205093 & 0.759260 \\
\hline $\mathrm{H}$ & 6.538555 & 4.152144 & -0.203615 \\
\hline $\mathrm{H}$ & 4.970842 & 4.431342 & 0.562439 \\
\hline $\mathrm{H}$ & 6.453908 & 5.038741 & 1.324955 \\
\hline $\mathrm{C}$ & 5.465252 & 3.070701 & 2.911715 \\
\hline $\mathrm{H}$ & 4.404255 & 3.312860 & 2.794302 \\
\hline H & 5.545208 & 2.171832 & 3.531119 \\
\hline $\mathrm{H}$ & 5.934807 & 3.894219 & 3.458428 \\
\hline $\mathrm{C}$ & 7.654331 & 2.596639 & 1.811096 \\
\hline $\mathrm{H}$ & 8.108074 & 3.410225 & 2.387642 \\
\hline H & 7.776076 & 1.667025 & 2.375817 \\
\hline $\mathrm{H}$ & 8.213549 & 2.495486 & 0.877115 \\
\hline
\end{tabular}

\section{Catalyst 2C 17}

Datum

Value

\begin{tabular}{lr}
\hline B3LYP-D3(BJ)/6-31G(d,p) Energy & -2165.163096 \\
\hline B3LYP-D3(BJ)/def2-TZVPP/IEF-PCM(DCM) Energy & -2165.901836 \\
\hline B3LYP-D3(BJ)/def2-TZVPP/IEF-PCM(DCM)//B3LYP-D3(BJ)/6-31G(d,p) Free Energy (Quasiharmonic) & -2165.023784 \\
\hline Number of Imaginary Frequencies & 0
\end{tabular}

Frequencies (Top 3 out of 321) 

1. $9.5109 \mathrm{~cm}^{-1}$
2. $\quad 12.7447 \mathrm{~cm}^{-1}$
3. $13.9059 \mathrm{~cm}^{-1}$

B3LYP-D3(BJ)/6-31G(d,p) Molecular Geometry in Cartesian Coordinates

\begin{tabular}{|c|c|c|c|}
\hline C & 1.509143 & 0.236141 & 1.265337 \\
\hline $\mathrm{H}$ & 0.974069 & -0.180062 & 2.122485 \\
\hline H & 2.456547 & 0.658650 & 1.607058 \\
\hline C & -1.471159 & -0.608080 & -1.207216 \\
\hline 0 & -1.947806 & -1.244988 & -2.122845 \\
\hline 0 & -0.807704 & 0.530162 & -1.418812 \\
\hline $\mathrm{H}$ & -0.368927 & 0.845764 & -0.593379 \\
\hline 0 & 0.706853 & 1.296918 & 0.679424 \\
\hline $\mathrm{H}$ & 0.375800 & 1.857776 & 1.393090 \\
\hline C & -0.508998 & -1.733878 & 0.793223 \\
\hline C & -1.588895 & -1.074799 & 0.230263 \\
\hline C & -2.802105 & -0.881130 & 0.967168 \\
\hline C & -2.878266 & -1.388617 & 2.249819 \\
\hline $\mathrm{H}$ & -3.792955 & -1.246759 & 2.817715 \\
\hline C & 1.754776 & -0.845077 & 0.242338 \\
\hline C & 0.782671 & -1.819023 & 0.043153 \\
\hline C & 3.210793 & -1.961723 & -1.343112 \\
\hline C & 2.984201 & -0.898307 & -0.490588 \\
\hline $\mathrm{H}$ & 4.140748 & -2.001964 & -1.902489 \\
\hline C & 2.249169 & -2.976619 & -1.551083 \\
\hline C & 0.998284 & -2.898369 & -0.862877 \\
\hline C & 0.019889 & -3.900346 & -1.099852 \\
\hline C & 0.275817 & -4.936470 & -1.966592 \\
\hline C & 1.518183 & -5.019717 & -2.641084 \\
\hline C & 2.481042 & -4.059433 & -2.440239 \\
\hline $\mathrm{H}$ & -0.938277 & -3.825921 & -0.599695 \\
\hline $\mathrm{H}$ & -0.482223 & -5.692768 & -2.143447 \\
\hline $\mathrm{H}$ & 1.703925 & -5.842494 & -3.324573 \\
\hline $\mathrm{H}$ & 3.432667 & -4.110547 & -2.961588 \\
\hline C & -1.815665 & -2.112382 & 2.841508 \\
\hline C & -0.604937 & -2.294281 & 2.103848 \\
\hline C & 0.462126 & -3.014587 & 2.703792 \\
\hline C & 0.334106 & -3.531379 & 3.973229 \\
\hline C & -0.864688 & -3.351507 & 4.703780 \\
\hline C & -1.914829 & -2.657797 & 4.148735 \\
\hline $\mathrm{H}$ & 1.378353 & -3.157029 & 2.141688 \\
\hline H & 1.155734 & -4.084558 & 4.417448 \\
\hline H & -0.952443 & -3.765134 & 5.703620 \\
\hline $\mathrm{H}$ & -2.838981 & -2.516777 & 4.702125 \\
\hline C & 5.947661 & 2.155766 & -0.180029 \\
\hline C & 6.341415 & 0.812757 & -0.048248 \\
\hline C & 5.358336 & -0.171671 & -0.141458 \\
\hline C & 4.014524 & 0.165107 & -0.370539 \\
\hline C & 3.664689 & 1.508266 & -0.514706 \\
\hline
\end{tabular}




\begin{tabular}{|c|c|c|c|}
\hline C & 4.622021 & 2.525785 & -0.409373 \\
\hline $\mathrm{H}$ & 6.703916 & 2.925921 & -0.097471 \\
\hline $\mathrm{H}$ & 5.610392 & -1.217832 & -0.020526 \\
\hline $\mathrm{H}$ & 2.628593 & 1.755533 & -0.710732 \\
\hline C & 7.820142 & 0.484644 & 0.203457 \\
\hline C & 4.169885 & 3.986270 & -0.541922 \\
\hline C & 8.070729 & -1.027504 & 0.319564 \\
\hline $\mathrm{H}$ & 9.135515 & -1.212302 & 0.491256 \\
\hline $\mathrm{H}$ & 7.518619 & -1.466783 & 1.156232 \\
\hline $\mathrm{H}$ & 7.784987 & -1.556260 & -0.595074 \\
\hline C & 8.667917 & 1.025791 & -0.968109 \\
\hline $\mathrm{H}$ & 9.728235 & 0.803776 & -0.805718 \\
\hline $\mathrm{H}$ & 8.362329 & 0.565449 & -1.912786 \\
\hline $\mathrm{H}$ & 8.565382 & 2.109022 & -1.075763 \\
\hline C & 8.276043 & 1.152418 & 1.518794 \\
\hline $\mathrm{H}$ & 9.331251 & 0.931624 & 1.713023 \\
\hline $\mathrm{H}$ & 8.163013 & 2.239344 & 1.481323 \\
\hline $\mathrm{H}$ & 7.687932 & 0.783485 & 2.364952 \\
\hline C & 3.537923 & 4.200992 & -1.933956 \\
\hline $\mathrm{H}$ & 3.203894 & 5.238357 & -2.044629 \\
\hline $\mathrm{H}$ & 4.263226 & 3.988771 & -2.725541 \\
\hline $\mathrm{H}$ & 2.672628 & 3.551509 & -2.089989 \\
\hline C & 3.118135 & 4.284723 & 0.550110 \\
\hline $\mathrm{H}$ & 2.760660 & 5.316607 & 0.463855 \\
\hline $\mathrm{H}$ & 2.255153 & 3.618270 & 0.467986 \\
\hline $\mathrm{H}$ & 3.551097 & 4.155898 & 1.547698 \\
\hline C & 5.332830 & 4.977120 & -0.378411 \\
\hline $\mathrm{H}$ & 4.960374 & 6.001798 & -0.471699 \\
\hline $\mathrm{H}$ & 5.809141 & 4.884651 & 0.602871 \\
\hline $\mathrm{H}$ & 6.098849 & 4.831756 & -1.146624 \\
\hline C & -3.941014 & -0.090840 & 0.428498 \\
\hline C & -4.465334 & 0.957448 & 1.197723 \\
\hline C & -4.511279 & -0.384370 & -0.814941 \\
\hline C & -5.540308 & 1.722594 & 0.738756 \\
\hline $\mathrm{H}$ & -4.002752 & 1.174453 & 2.152659 \\
\hline C & -5.576216 & 0.371101 & -1.311782 \\
\hline $\mathrm{H}$ & -4.102923 & -1.201988 & -1.388421 \\
\hline C & -6.072115 & 1.412138 & -0.518950 \\
\hline $\mathrm{H}$ & -6.897573 & 2.006375 & -0.896478 \\
\hline C & -6.187690 & 0.103864 & -2.694045 \\
\hline C & -6.137735 & 2.882351 & 1.548627 \\
\hline C & -7.692872 & -0.201700 & -2.544496 \\
\hline $\mathrm{H}$ & -7.848829 & -1.087059 & -1.920098 \\
\hline $\mathrm{H}$ & -8.233884 & 0.631111 & -2.085755 \\
\hline $\mathrm{H}$ & -8.141812 & -0.392009 & -3.525399 \\
\hline C & -6.001347 & 1.360251 & -3.572548 \\
\hline $\mathrm{H}$ & -6.432005 & 1.196637 & -4.566444 \\
\hline $\mathrm{H}$ & -6.489362 & 2.236370 & -3.135523 \\
\hline $\mathrm{H}$ & -4.938855 & 1.591794 & -3.693306 \\
\hline C & -5.515081 & -1.083202 & -3.403493 \\
\hline $\mathrm{H}$ & -4.441443 & -0.923365 & -3.536593 \\
\hline $\mathrm{H}$ & -5.651944 & -2.015045 & -2.845566 \\
\hline $\mathrm{H}$ & -5.963383 & -1.220350 & -4.392540 \\
\hline C & -5.431150 & 3.075488 & 2.900088 \\
\hline
\end{tabular}




\begin{tabular}{|llll|}
$H$ & -5.508122 & 2.182360 & 3.528111 \\
$H$ & -4.370601 & 3.314073 & 2.771858 \\
$H$ & -5.894266 & 3.904943 & 3.443287 \\
$\mathrm{C}$ & -6.003888 & 4.191856 & 0.741886 \\
$\mathrm{H}$ & -4.952379 & 4.413393 & 0.534271 \\
$\mathrm{H}$ & -6.526784 & 4.131204 & -0.216362 \\
$\mathrm{H}$ & -6.427581 & 5.031981 & 1.303086 \\
$\mathrm{C}$ & -7.630340 & 2.597134 & 1.821358 \\
$\mathrm{H}$ & -8.077834 & 3.416927 & 2.393970 \\
$\mathrm{H}$ & -8.197008 & 2.488607 & 0.892678 \\
$\mathrm{H}$ & -7.749869 & 1.672907 & 2.395291 \\
& & & \\
\hline
\end{tabular}

\section{Catalyst 2C 18}

\section{Datum}

Value

B3LYP-D3(BJ)/6-31G(d,p) Energy $-2165.162326$

B3LYP-D3(BJ)/def2-TZVPP/IEF-PCM(DCM) Energy $-2165.901585$

B3LYP-D3(BJ)/def2-TZVPP/IEF-PCM(DCM)//B3LYP-D3(BJ)/6-31G(d,p) Free Energy (Quasiharmonic) $-2165.023781$

Number of Imaginary Frequencies

Frequencies (Top 3 out of 321)
1. $9.0521 \mathrm{~cm}^{-1}$
2. $\quad 13.1541 \mathrm{~cm}^{-1}$
3. $13.8649 \mathrm{~cm}^{-1}$

\section{B3LYP-D3(BJ)/6-31G(d,p) Molecular Geometry in Cartesian Coordinates}

$\begin{array}{rrrr}\mathrm{C} & -1.484426 & 0.230338 & 1.258984 \\ \mathrm{H} & -2.433364 & 0.649310 & 1.601453 \\ \mathrm{H} & -0.945774 & -0.179998 & 2.116863 \\ \mathrm{C} & 1.493975 & -0.607700 & -1.217927 \\ \mathrm{O} & 1.977282 & -1.244265 & -2.130394 \\ \mathrm{O} & 0.819899 & 0.522968 & -1.434660 \\ \mathrm{H} & 0.378821 & 0.839591 & -0.610473 \\ \mathrm{O} & -0.689130 & 1.290848 & 0.665066 \\ \mathrm{H} & -0.349332 & 1.851749 & 1.374463 \\ \mathrm{C} & 0.541391 & -1.731738 & 0.788666 \\ \mathrm{C} & 1.616146 & -1.068074 & 0.221312 \\ \mathrm{C} & 2.829559 & -0.865493 & 0.955248 \\ \mathrm{C} & 2.912376 & -1.369708 & 2.238656 \\ \mathrm{H} & 3.827787 & -1.220989 & 2.803662 \\ \mathrm{C} & -1.728081 & -0.856220 & 0.241460\end{array}$




\begin{tabular}{|c|c|c|c|}
\hline C & -0.751679 & -1.825705 & 0.042176 \\
\hline C & -3.182529 & -1.983381 & -1.337372 \\
\hline C & -2.959053 & -0.916908 & -0.487713 \\
\hline $\mathrm{H}$ & -4.113642 & -2.029234 & -1.894354 \\
\hline C & -2.216577 & -2.994233 & -1.545296 \\
\hline C & -0.964323 & -2.908386 & -0.860499 \\
\hline C & 0.018349 & -3.906124 & -1.097646 \\
\hline C & -0.234794 & -4.945551 & -1.961258 \\
\hline C & -1.478505 & -5.036423 & -2.632277 \\
\hline C & -2.445477 & -4.080300 & -2.431249 \\
\hline $\mathrm{H}$ & 0.977482 & -3.825799 & -0.600231 \\
\hline $\mathrm{H}$ & 0.526461 & -5.698562 & -2.138334 \\
\hline $\mathrm{H}$ & -1.662032 & -5.861724 & -3.313321 \\
\hline $\mathrm{H}$ & -3.398175 & -4.137270 & -2.950035 \\
\hline C & 1.855392 & -2.098267 & 2.834660 \\
\hline C & 0.643780 & -2.288302 & 2.100501 \\
\hline C & -0.417837 & -3.012929 & 2.704894 \\
\hline C & -0.283766 & -3.526065 & 3.975197 \\
\hline C & 0.915922 & -3.338150 & 4.702219 \\
\hline C & 1.960920 & -2.640136 & 4.142829 \\
\hline $\mathrm{H}$ & -1.334725 & -3.161570 & 2.145482 \\
\hline $\mathrm{H}$ & -1.101286 & -4.082581 & 4.422824 \\
\hline $\mathrm{H}$ & 1.008491 & -3.749040 & 5.702753 \\
\hline $\mathrm{H}$ & 2.885780 & -2.492975 & 4.693441 \\
\hline C & -5.941521 & 2.112385 & -0.190478 \\
\hline C & -6.325235 & 0.774337 & -0.037319 \\
\hline C & -5.331120 & -0.204554 & -0.125889 \\
\hline C & -3.995539 & 0.140769 & -0.367049 \\
\hline C & -3.652383 & 1.489079 & -0.529438 \\
\hline C & -4.617036 & 2.494642 & -0.434688 \\
\hline $\mathrm{H}$ & -6.700837 & 2.883426 & -0.114837 \\
\hline H & -5.573990 & -1.251281 & 0.008231 \\
\hline $\mathrm{H}$ & -2.619508 & 1.734910 & -0.733146 \\
\hline C & -7.799196 & 0.435741 & 0.228937 \\
\hline C & -4.263964 & 3.981691 & -0.577407 \\
\hline C & -8.252095 & 1.117407 & 1.538063 \\
\hline $\mathrm{H}$ & -9.303943 & 0.890155 & 1.742759 \\
\hline $\mathrm{H}$ & -7.654887 & 0.765655 & 2.385148 \\
\hline $\mathrm{H}$ & -8.148791 & 2.204670 & 1.484788 \\
\hline C & -8.659617 & 0.952815 & -0.944171 \\
\hline $\mathrm{H}$ & -9.716770 & 0.723270 & -0.771931 \\
\hline $\mathrm{H}$ & -8.567732 & 2.035377 & -1.067246 \\
\hline $\mathrm{H}$ & -8.355765 & 0.482713 & -1.884577 \\
\hline C & -8.034484 & -1.077008 & 0.367503 \\
\hline $\mathrm{H}$ & -9.096137 & -1.269383 & 0.549915 \\
\hline $\mathrm{H}$ & -7.750808 & -1.615575 & -0.542037 \\
\hline $\mathrm{H}$ & -7.471889 & -1.499666 & 1.205743 \\
\hline C & -5.073985 & 4.596875 & -1.738177 \\
\hline $\mathrm{H}$ & -4.831490 & 5.659050 & -1.851390 \\
\hline $\mathrm{H}$ & -4.844528 & 4.091229 & -2.681077 \\
\hline $\mathrm{H}$ & -6.151349 & 4.516417 & -1.568451 \\
\hline C & -4.616233 & 4.709772 & 0.737872 \\
\hline $\mathrm{H}$ & -4.377232 & 5.775991 & 0.660457 \\
\hline H & -5.679537 & 4.620561 & 0.977477 \\
\hline
\end{tabular}




\begin{tabular}{|c|c|c|c|}
\hline $\mathrm{H}$ & -4.049000 & 4.292063 & 1.575886 \\
\hline C & -2.768541 & 4.196734 & -0.863987 \\
\hline $\mathrm{H}$ & -2.557114 & 5.268201 & -0.933065 \\
\hline $\mathrm{H}$ & -2.138315 & 3.778568 & -0.073270 \\
\hline $\mathrm{H}$ & -2.468366 & 3.736159 & -1.810038 \\
\hline C & 3.961484 & -0.067453 & 0.413211 \\
\hline C & 4.476011 & 0.986853 & 1.171551 \\
\hline C & 4.537323 & -0.362433 & -0.831415 \\
\hline C & 5.547499 & 1.763519 & 0.709588 \\
\hline $\mathrm{H}$ & 4.014181 & 1.210627 & 2.127898 \\
\hline C & 5.592881 & 0.398820 & -1.329053 \\
\hline $\mathrm{H}$ & 4.135753 & -1.186454 & -1.400506 \\
\hline C & 6.082939 & 1.453628 & -0.541600 \\
\hline $\mathrm{H}$ & 6.901920 & 2.047254 & -0.927012 \\
\hline C & 6.209800 & 0.130054 & -2.708448 \\
\hline C & 6.073228 & 2.916733 & 1.576488 \\
\hline C & 7.717076 & -0.163084 & -2.554176 \\
\hline $\mathrm{H}$ & 8.250712 & 0.675979 & -2.098248 \\
\hline $\mathrm{H}$ & 7.878391 & -1.044477 & -1.925509 \\
\hline $\mathrm{H}$ & 8.169503 & -0.354454 & -3.533283 \\
\hline C & 5.548319 & -1.065572 & -3.413901 \\
\hline $\mathrm{H}$ & 5.999776 & -1.203703 & -4.401388 \\
\hline $\mathrm{H}$ & 5.691436 & -1.993686 & -2.851355 \\
\hline $\mathrm{H}$ & 4.473719 & -0.914830 & -3.549879 \\
\hline C & 6.015967 & 1.380536 & -3.593869 \\
\hline $\mathrm{H}$ & 4.951987 & 1.603147 & -3.718373 \\
\hline $\mathrm{H}$ & 6.496430 & 2.262664 & -3.160645 \\
\hline $\mathrm{H}$ & 6.450200 & 1.214834 & -4.585868 \\
\hline C & 7.250964 & 3.653743 & 0.918889 \\
\hline $\mathrm{H}$ & 8.099097 & 2.984813 & 0.742952 \\
\hline $\mathrm{H}$ & 6.964510 & 4.102897 & -0.037092 \\
\hline $\mathrm{H}$ & 7.593662 & 4.459791 & 1.574993 \\
\hline C & 4.937682 & 3.935742 & 1.813001 \\
\hline $\mathrm{H}$ & 4.579655 & 4.343928 & 0.862765 \\
\hline $\mathrm{H}$ & 4.085317 & 3.478390 & 2.323247 \\
\hline $\mathrm{H}$ & 5.292313 & 4.767190 & 2.432016 \\
\hline C & 6.551145 & 2.356811 & 2.933605 \\
\hline $\mathrm{H}$ & 6.925740 & 3.165405 & 3.570816 \\
\hline $\mathrm{H}$ & 5.741794 & 1.854613 & 3.470914 \\
\hline $\mathrm{H}$ & 7.358185 & 1.631523 & 2.790690 \\
\hline
\end{tabular}

\section{Catalyst 2C 19}

Datum

Value

B3LYP-D3(BJ)/6-31G(d,p) Energy

$-2165.162984$

B3LYP-D3(BJ)/def2-TZVPP/IEF-PCM(DCM) Energy

$-2165.901777$

B3LYP-D3(BJ)/def2-TZVPP/IEF-PCM(DCM)//B3LYP-D3(BJ)/6-31G(d,p) Free Energy (Quasiharmonic) - 
Frequencies (Top 3 out of 321)

$\begin{array}{lr}\text { 1. } & 9.2920 \mathrm{~cm}^{-1} \\ \text { 2. } & 13.0667 \mathrm{~cm}^{-1} \\ \text { 3. } & 14.0115 \mathrm{~cm}^{-1}\end{array}$

B3LYP-D3(BJ)/6-31G(d,p) Molecular Geometry in Cartesian Coordinates

\begin{tabular}{|c|c|c|c|}
\hline C & -1.489844 & 0.255629 & 1.263226 \\
\hline $\mathrm{H}$ & -2.437172 & 0.679635 & 1.603957 \\
\hline $\mathrm{H}$ & -0.955294 & -0.157902 & 2.122132 \\
\hline C & 1.487016 & -0.594291 & -1.210416 \\
\hline 0 & 1.968373 & -1.233091 & -2.123151 \\
\hline 0 & 0.818033 & 0.538494 & -1.427450 \\
\hline $\mathrm{H}$ & 0.377623 & 0.857025 & -0.603419 \\
\hline 0 & -0.687652 & 1.311975 & 0.671205 \\
\hline $\mathrm{H}$ & -0.345477 & 1.870276 & 1.381505 \\
\hline C & 0.527863 & -1.715296 & 0.793656 \\
\hline C & 1.606756 & -1.056469 & 0.228330 \\
\hline C & 2.820829 & -0.860640 & 0.963440 \\
\hline C & 2.898502 & -1.366484 & 2.246749 \\
\hline $\mathrm{H}$ & 3.813606 & -1.223410 & 2.813650 \\
\hline C & -1.736921 & -0.829058 & 0.244518 \\
\hline C & -0.764538 & -1.802481 & 0.045132 \\
\hline C & -3.193541 & -1.946162 & -1.339555 \\
\hline C & -2.966964 & -0.883270 & -0.486408 \\
\hline $\mathrm{H}$ & -4.123769 & -1.986144 & -1.898487 \\
\hline C & -2.231787 & -2.961058 & -1.547676 \\
\hline C & -0.980747 & -2.882691 & -0.859739 \\
\hline C & -0.002672 & -3.885096 & -1.096152 \\
\hline C & -0.258987 & -4.921702 & -1.962226 \\
\hline C & -1.501353 & -5.004887 & -2.636688 \\
\hline C & -2.463939 & -4.044189 & -2.436325 \\
\hline $\mathrm{H}$ & 0.955465 & -3.810771 & -0.595886 \\
\hline $\mathrm{H}$ & 0.498718 & -5.678474 & -2.138512 \\
\hline $\mathrm{H}$ & -1.687359 & -5.827964 & -3.319740 \\
\hline $\mathrm{H}$ & -3.415589 & -4.095320 & -2.957630 \\
\hline C & 1.837105 & -2.089913 & 2.840815 \\
\hline C & 0.625463 & -2.273380 & 2.105087 \\
\hline C & -0.440660 & -2.992914 & 2.707639 \\
\hline C & -0.310824 & -3.507530 & 3.977762 \\
\hline C & 0.888943 & -3.326201 & 4.706394 \\
\hline C & 1.938179 & -2.633184 & 4.148815 \\
\hline $\mathrm{H}$ & -1.357613 & -3.136462 & 2.147004 \\
\hline $\mathrm{H}$ & -1.131746 & -4.060093 & 4.424047 \\
\hline
\end{tabular}




\begin{tabular}{|c|c|c|c|}
\hline $\mathrm{H}$ & 0.978117 & -3.738137 & 5.706805 \\
\hline $\mathrm{H}$ & 2.863022 & -2.490995 & 4.700747 \\
\hline C & -5.942823 & 2.158740 & -0.180042 \\
\hline C & -6.327982 & 0.825678 & -0.029257 \\
\hline C & -5.332649 & -0.158421 & -0.121216 \\
\hline C & -4.000028 & 0.177417 & -0.362734 \\
\hline C & -3.652030 & 1.528522 & -0.523604 \\
\hline C & -4.611136 & 2.533983 & -0.426069 \\
\hline $\mathrm{H}$ & -6.692263 & 2.935982 & -0.104313 \\
\hline $\mathrm{H}$ & -5.586543 & -1.204837 & 0.012967 \\
\hline $\mathrm{H}$ & -2.618268 & 1.770182 & -0.727885 \\
\hline C & -7.780395 & 0.406770 & 0.239995 \\
\hline C & -4.254328 & 4.020101 & -0.567558 \\
\hline C & -7.853706 & -0.341677 & 1.588426 \\
\hline H & -8.882904 & -0.654114 & 1.795933 \\
\hline H & -7.225617 & -1.236691 & 1.588959 \\
\hline $\mathrm{H}$ & -7.519868 & 0.302405 & 2.408009 \\
\hline C & -8.734910 & 1.609832 & 0.304748 \\
\hline $\mathrm{H}$ & -9.754598 & 1.260516 & 0.493351 \\
\hline H & -8.465600 & 2.298015 & 1.112137 \\
\hline $\mathrm{H}$ & -8.744426 & 2.170276 & -0.635281 \\
\hline C & -8.257274 & -0.527976 & -0.892614 \\
\hline H & -9.291779 & -0.842758 & -0.717678 \\
\hline $\mathrm{H}$ & -8.213156 & -0.018451 & -1.860169 \\
\hline $\mathrm{H}$ & -7.640387 & -1.428263 & -0.960137 \\
\hline C & -5.062220 & 4.638446 & -1.728263 \\
\hline $\mathrm{H}$ & -4.816561 & 5.700008 & -1.840439 \\
\hline H & -4.833490 & 4.132961 & -2.671444 \\
\hline $\mathrm{H}$ & -6.139875 & 4.561036 & -1.559125 \\
\hline C & -4.605011 & 4.748679 & 0.747959 \\
\hline $\mathrm{H}$ & -4.362991 & 5.814230 & 0.670747 \\
\hline $\mathrm{H}$ & -5.668591 & 4.662629 & 0.987363 \\
\hline $\mathrm{H}$ & -4.038879 & 4.329237 & 1.585871 \\
\hline C & -2.758305 & 4.231600 & -0.853450 \\
\hline $\mathrm{H}$ & -2.543984 & 5.302577 & -0.921264 \\
\hline H & -2.129512 & 3.810841 & -0.062942 \\
\hline $\mathrm{H}$ & -2.459073 & 3.771294 & -1.799927 \\
\hline C & 3.959387 & -0.070487 & 0.423636 \\
\hline C & 4.494435 & 0.971937 & 1.200546 \\
\hline C & 4.522115 & -0.350396 & -0.820787 \\
\hline C & 5.567249 & 1.734189 & 0.742998 \\
\hline $\mathrm{H}$ & 4.037919 & 1.181874 & 2.159948 \\
\hline C & 5.590237 & 0.407385 & -1.320478 \\
\hline H & 4.110691 & -1.159768 & -1.407121 \\
\hline C & 6.095353 & 1.435595 & -0.524941 \\
\hline $\mathrm{H}$ & 6.920229 & 2.031655 & -0.894162 \\
\hline C & 6.130390 & 0.088904 & -2.720761 \\
\hline C & 6.175172 & 2.883493 & 1.559666 \\
\hline C & 7.318961 & 0.983216 & -3.106734 \\
\hline H & 7.040707 & 2.041737 & -3.124070 \\
\hline $\mathrm{H}$ & 8.160738 & 0.860206 & -2.417182 \\
\hline $\mathrm{H}$ & 7.668589 & 0.717793 & -4.109102 \\
\hline C & 6.595280 & -1.382296 & -2.771306 \\
\hline $\mathrm{H}$ & 6.982397 & -1.622534 & -3.767400 \\
\hline
\end{tabular}




\begin{tabular}{|lrrr|} 
H & 7.390817 & -1.565214 & -2.041641 \\
$\mathrm{H}$ & 5.775719 & -2.072955 & -2.557464 \\
$\mathrm{C}$ & 4.996139 & 0.306593 & -3.747682 \\
$\mathrm{H}$ & 4.118451 & -0.304894 & -3.523696 \\
$\mathrm{H}$ & 4.677259 & 1.353883 & -3.750159 \\
$\mathrm{H}$ & 5.343994 & 0.053648 & -4.755514 \\
$\mathrm{C}$ & 6.044086 & 4.201136 & 0.765658 \\
$\mathrm{H}$ & 6.561562 & 4.146732 & -0.195866 \\
$\mathrm{H}$ & 4.992629 & 4.430249 & 0.566061 \\
$\mathrm{H}$ & 6.475360 & 5.033636 & 1.332434 \\
$\mathrm{C}$ & 5.477234 & 3.068435 & 2.916720 \\
$\mathrm{H}$ & 4.417187 & 3.313388 & 2.796520 \\
$\mathrm{H}$ & 5.553204 & 2.169445 & 3.536452 \\
$\mathrm{H}$ & 5.947562 & 3.890784 & 3.464533 \\
$\mathrm{C}$ & 7.667834 & 2.588276 & 1.821785 \\
$\mathrm{H}$ & 8.122354 & 3.400689 & 2.399379 \\
$\mathrm{H}$ & 7.785569 & 1.658406 & 2.386938 \\
$\mathrm{H}$ & 8.229148 & 2.485439 & 0.889244 \\
& & & \\
\hline
\end{tabular}

\section{Catalyst 2C 20}

\section{Datum}

Value

B3LYP-D3(BJ)/6-31G(d,p) Energy

$-2165.163496$

B3LYP-D3(BJ)/def2-TZVPP/IEF-PCM(DCM) Energy

$-2165.901786$

B3LYP-D3(BJ)/def2-TZVPP/IEF-PCM(DCM)//B3LYP-D3(BJ)/6-31G(d,p) Free Energy (Quasiharmonic)

$-2165.023619$

Number of Imaginary Frequencies

Frequencies (Top 3 out of 321)

$\begin{array}{lr}\text { 1. } & 9.5378 \mathrm{~cm}^{-1} \\ \text { 2. } & 12.8924 \mathrm{~cm}^{-1} \\ \text { 3. } & 13.8822 \mathrm{~cm}^{-1}\end{array}$

B3LYP-D3(BJ)/6-31G(d,p) Molecular Geometry in Cartesian Coordinates

$\begin{array}{rrrr}C & 1.486386 & 0.253532 & 1.256737 \\ H & 0.944801 & -0.149431 & 2.116146 \\ H & 2.433509 & 0.676824 & 1.598255 \\ C & -1.485992 & -0.609517 & -1.215227 \\ 0 & -1.965451 & -1.256072 & -2.123328 \\ 0 & -0.815740 & 0.521111 & -1.440569 \\ H & -0.378153 & 0.846355 & -0.617996 \\ 0 & 0.691647 & 1.309624 & 0.652337\end{array}$




\begin{tabular}{|c|c|c|c|}
\hline $\mathrm{H}$ & 0.360493 & 1.882840 & 1.356088 \\
\hline C & -0.535605 & -1.716184 & 0.801431 \\
\hline C & -1.610318 & -1.058309 & 0.227303 \\
\hline C & -2.825517 & -0.851562 & 0.957237 \\
\hline C & -2.909994 & -1.347184 & 2.243939 \\
\hline $\mathrm{H}$ & -3.826612 & -1.196057 & 2.806315 \\
\hline C & 1.733276 & -0.841627 & 0.248993 \\
\hline C & 0.758757 & -1.814737 & 0.057697 \\
\hline C & 3.192275 & -1.982826 & -1.316041 \\
\hline C & 2.965797 & -0.908236 & -0.477599 \\
\hline $\mathrm{H}$ & 4.124581 & -2.033257 & -1.870649 \\
\hline C & 2.227941 & -2.996782 & -1.515895 \\
\hline C & 0.974451 & -2.905887 & -0.834021 \\
\hline C & -0.006394 & -3.907378 & -1.062843 \\
\hline C & 0.249573 & -4.954835 & -1.915849 \\
\hline C & 1.494434 & -5.050473 & -2.584078 \\
\hline C & 2.459769 & -4.091111 & -2.390849 \\
\hline $\mathrm{H}$ & -0.966326 & -3.823733 & -0.567522 \\
\hline $\mathrm{H}$ & -0.510259 & -5.710739 & -2.086594 \\
\hline $\mathrm{H}$ & 1.680174 & -5.882129 & -3.256726 \\
\hline $\mathrm{H}$ & 3.413375 & -4.151814 & -2.907516 \\
\hline C & -1.853194 & -2.069887 & 2.847199 \\
\hline C & -0.639634 & -2.263130 & 2.117122 \\
\hline C & 0.422003 & -2.981382 & 2.729017 \\
\hline C & 0.285975 & -3.485657 & 4.002644 \\
\hline C & -0.915713 & -3.294759 & 4.725594 \\
\hline C & -1.960700 & -2.602626 & 4.158967 \\
\hline $\mathrm{H}$ & 1.340416 & -3.132359 & 2.172752 \\
\hline $\mathrm{H}$ & 1.103485 & -4.037436 & 4.456096 \\
\hline $\mathrm{H}$ & -1.009777 & -3.698667 & 5.728821 \\
\hline $\mathrm{H}$ & -2.887009 & -2.453143 & 4.706490 \\
\hline C & 5.940362 & 2.137267 & -0.189961 \\
\hline C & 6.327741 & 0.794381 & -0.039386 \\
\hline C & 5.340985 & -0.187014 & -0.125329 \\
\hline C & 3.999798 & 0.152489 & -0.365522 \\
\hline C & 3.656391 & 1.495174 & -0.528256 \\
\hline C & 4.617489 & 2.509934 & -0.430710 \\
\hline $\mathrm{H}$ & 6.699471 & 2.905214 & -0.113203 \\
\hline $\mathrm{H}$ & 5.587993 & -1.232622 & 0.009940 \\
\hline $\mathrm{H}$ & 2.622405 & 1.744316 & -0.732798 \\
\hline C & 7.803790 & 0.463236 & 0.223768 \\
\hline C & 4.172269 & 3.970504 & -0.584054 \\
\hline C & 8.047318 & -1.048364 & 0.360218 \\
\hline $\mathrm{H}$ & 9.110429 & -1.235462 & 0.539617 \\
\hline $\mathrm{H}$ & 7.489155 & -1.474716 & 1.199552 \\
\hline $\mathrm{H}$ & 7.763925 & -1.587430 & -0.549119 \\
\hline C & 8.659552 & 0.985887 & -0.950390 \\
\hline H & 9.718090 & 0.761373 & -0.780044 \\
\hline $\mathrm{H}$ & 8.356511 & 0.514973 & -1.890665 \\
\hline $\mathrm{H}$ & 8.562275 & 2.068099 & -1.072194 \\
\hline C & 8.256151 & 1.145703 & 1.532764 \\
\hline $\mathrm{H}$ & 9.309484 & 0.923048 & 1.734865 \\
\hline $\mathrm{H}$ & 8.147841 & 2.232523 & 1.481032 \\
\hline $\mathrm{H}$ & 7.662468 & 0.789919 & 2.380658 \\
\hline
\end{tabular}




\begin{tabular}{|c|c|c|c|}
\hline C & 3.549502 & 4.170421 & -1.982427 \\
\hline $\mathrm{H}$ & 3.220482 & 5.207681 & -2.108032 \\
\hline $\mathrm{H}$ & 4.278590 & 3.945332 & -2.766947 \\
\hline $\mathrm{H}$ & 2.682430 & 3.522606 & -2.135509 \\
\hline C & 3.115302 & 4.286934 & 0.497831 \\
\hline H & 2.762787 & 5.319156 & 0.396642 \\
\hline $\mathrm{H}$ & 2.249912 & 3.623241 & 0.418740 \\
\hline $\mathrm{H}$ & 3.541711 & 4.168667 & 1.499540 \\
\hline C & 5.338376 & 4.958496 & -0.425878 \\
\hline $\mathrm{H}$ & 4.970743 & 5.983465 & -0.533884 \\
\hline $\mathrm{H}$ & 5.808581 & 4.876104 & 0.559240 \\
\hline $\mathrm{H}$ & 6.108228 & 4.800594 & -1.187756 \\
\hline C & -3.957637 & -0.058788 & 0.407901 \\
\hline C & -4.486787 & 0.992432 & 1.167532 \\
\hline C & -4.521750 & -0.346990 & -0.838006 \\
\hline C & -5.556115 & 1.762101 & 0.700498 \\
\hline $\mathrm{H}$ & -4.034044 & 1.214596 & 2.128580 \\
\hline C & -5.579988 & 0.412571 & -1.345301 \\
\hline $\mathrm{H}$ & -4.114465 & -1.165211 & -1.414942 \\
\hline C & -6.083204 & 1.457260 & -0.561175 \\
\hline $\mathrm{H}$ & -6.901344 & 2.049815 & -0.943591 \\
\hline C & -6.121046 & 0.085540 & -2.743860 \\
\hline C & -6.097595 & 2.908542 & 1.567658 \\
\hline C & -6.595236 & -1.383058 & -2.783013 \\
\hline $\mathrm{H}$ & -5.780193 & -2.077102 & -2.563008 \\
\hline H & -7.392355 & -1.555131 & -2.052432 \\
\hline $\mathrm{H}$ & -6.983287 & -1.628901 & -3.777397 \\
\hline C & -7.303730 & 0.983595 & -3.139711 \\
\hline $\mathrm{H}$ & -7.653494 & 0.710609 & -4.140025 \\
\hline $\mathrm{H}$ & -8.147280 & 0.872406 & -2.450377 \\
\hline $\mathrm{H}$ & -7.019366 & 2.040243 & -3.166905 \\
\hline C & -4.984433 & 0.287326 & -3.771461 \\
\hline H & -4.658357 & 1.332359 & -3.782002 \\
\hline $\mathrm{H}$ & -4.111218 & -0.328276 & -3.541364 \\
\hline $\mathrm{H}$ & -5.332880 & 0.028741 & -4.777683 \\
\hline C & -4.971697 & 3.935127 & 1.817384 \\
\hline $\mathrm{H}$ & -4.120396 & 3.482153 & 2.333273 \\
\hline $\mathrm{H}$ & -4.608558 & 4.349230 & 0.871643 \\
\hline $\mathrm{H}$ & -5.337642 & 4.761924 & 2.436093 \\
\hline C & -7.275574 & 3.640038 & 0.904019 \\
\hline $\mathrm{H}$ & -6.985292 & 4.095859 & -0.047598 \\
\hline $\mathrm{H}$ & -8.117597 & 2.966114 & 0.718283 \\
\hline $\mathrm{H}$ & -7.629017 & 4.440485 & 1.561326 \\
\hline C & -6.582480 & 2.340408 & 2.918915 \\
\hline $\mathrm{H}$ & -6.969006 & 3.143824 & 3.555590 \\
\hline $\mathrm{H}$ & -7.382403 & 1.609109 & 2.766870 \\
\hline H & -5.773645 & 1.842927 & 3.461345 \\
\hline
\end{tabular}


Frequencies (Top 3 out of 321)

$\begin{array}{lr}\text { 1. } & 9.4976 \mathrm{~cm}^{-1} \\ \text { 2. } & 12.8356 \mathrm{~cm}^{-1} \\ \text { 3. } & 13.8309 \mathrm{~cm}^{-1}\end{array}$

\section{B3LYP-D3(BJ)/6-31G(d,p) Molecular Geometry in Cartesian Coordinates}

\begin{tabular}{|c|c|c|c|}
\hline C & -1.490639 & 0.278423 & 1.251820 \\
\hline $\mathrm{H}$ & -2.436076 & 0.707636 & 1.590689 \\
\hline $\mathrm{H}$ & -0.952596 & -0.124648 & 2.113416 \\
\hline C & 1.482284 & -0.606854 & -1.214338 \\
\hline 0 & 1.959727 & -1.258029 & -2.120193 \\
\hline 0 & 0.817924 & 0.526510 & -1.443418 \\
\hline $\mathrm{H}$ & 0.380744 & 0.856017 & -0.622360 \\
\hline 0 & -0.689642 & 1.328163 & 0.644947 \\
\hline $\mathrm{H}$ & -0.359174 & 1.904076 & 1.346815 \\
\hline C & 0.522639 & -1.702472 & 0.803880 \\
\hline C & 1.602086 & -1.052603 & 0.229498 \\
\hline C & 2.817398 & -0.850946 & 0.960657 \\
\hline C & 2.896774 & -1.342815 & 2.249122 \\
\hline $\mathrm{H}$ & 3.813403 & -1.195424 & 2.812468 \\
\hline C & -1.741373 & -0.818349 & 0.246762 \\
\hline C & -0.770993 & -1.796047 & 0.058229 \\
\hline C & -3.202955 & -1.954367 & -1.319781 \\
\hline C & -2.973145 & -0.880760 & -0.481175 \\
\hline $\mathrm{H}$ & -4.134543 & -2.000674 & -1.875980 \\
\hline C & -2.242766 & -2.972820 & -1.517141 \\
\hline C & -0.990306 & -2.887233 & -0.832674 \\
\hline C & -0.013824 & -3.893648 & -1.058471 \\
\hline C & -0.272983 & -4.940633 & -1.911104 \\
\hline C & -1.516778 & -5.030859 & -2.582012 \\
\hline C & -2.477929 & -4.066699 & -2.391712 \\
\hline $\mathrm{H}$ & 0.945442 & -3.814164 & -0.561195 \\
\hline $\mathrm{H}$ & 0.483577 & -5.700333 & -2.079502 \\
\hline $\mathrm{H}$ & -1.705016 & -5.862163 & -3.254396 \\
\hline $\mathrm{H}$ & -3.430667 & -4.123235 & -2.910450 \\
\hline C & 1.834696 & -2.057216 & 2.852991 \\
\hline C & 0.621291 & -2.245865 & 2.121452 \\
\hline 0 & -0.445450 & -2.956100 & 2.733834 \\
\hline
\end{tabular}




\begin{tabular}{|c|c|c|c|}
\hline C & -0.314528 & -3.456912 & 4.009358 \\
\hline C & 0.886966 & -3.270463 & 4.733789 \\
\hline C & 1.936878 & -2.586218 & 4.166696 \\
\hline $\mathrm{H}$ & -1.363759 & -3.103665 & 2.176484 \\
\hline $\mathrm{H}$ & -1.135972 & -4.002491 & 4.463199 \\
\hline H & 0.976966 & -3.671546 & 5.738519 \\
\hline H & 2.863088 & -2.440236 & 4.715328 \\
\hline C & -5.940861 & 2.177928 & -0.193275 \\
\hline C & -4.611085 & 2.543060 & -0.437289 \\
\hline C & -3.655669 & 1.528191 & -0.534740 \\
\hline C & -4.003768 & 0.183291 & -0.369170 \\
\hline C & -5.341793 & -0.146012 & -0.127187 \\
\hline C & -6.329783 & 0.840671 & -0.040804 \\
\hline $\mathrm{H}$ & -6.690146 & 2.952294 & -0.118608 \\
\hline $\mathrm{H}$ & -2.620812 & 1.772445 & -0.740946 \\
\hline $\mathrm{H}$ & -5.599654 & -1.190872 & 0.011644 \\
\hline C & -4.162395 & 4.002763 & -0.594092 \\
\hline C & -7.785340 & 0.430051 & 0.227335 \\
\hline C & -3.542415 & 4.199093 & -1.994252 \\
\hline $\mathrm{H}$ & -3.211601 & 5.235475 & -2.122581 \\
\hline $\mathrm{H}$ & -2.677095 & 3.549110 & -2.148035 \\
\hline H & -4.273695 & 3.974033 & -2.776747 \\
\hline C & -5.324971 & 4.994970 & -0.434811 \\
\hline $\mathrm{H}$ & -4.953721 & 6.018414 & -0.545152 \\
\hline $\mathrm{H}$ & -6.097148 & 4.838991 & -1.194675 \\
\hline $\mathrm{H}$ & -5.793499 & 4.915946 & 0.551344 \\
\hline C & -3.101902 & 4.318599 & 0.484553 \\
\hline H & -2.747302 & 5.349894 & 0.380994 \\
\hline $\mathrm{H}$ & -3.525886 & 4.202761 & 1.487586 \\
\hline $\mathrm{H}$ & -2.238278 & 3.652772 & 0.404175 \\
\hline C & -7.865623 & -0.311749 & 1.579082 \\
\hline $\mathrm{H}$ & -8.896965 & -0.617463 & 1.786097 \\
\hline $\mathrm{H}$ & -7.242460 & -1.210174 & 1.584832 \\
\hline $\mathrm{H}$ & -7.529725 & 0.334159 & 2.396387 \\
\hline C & -8.735007 & 1.637527 & 0.284397 \\
\hline $\mathrm{H}$ & -9.756578 & 1.292871 & 0.471517 \\
\hline $\mathrm{H}$ & -8.465557 & 2.328104 & 1.089633 \\
\hline H & -8.739764 & 2.194259 & -0.657833 \\
\hline C & -8.264628 & -0.507782 & -0.901741 \\
\hline $\mathrm{H}$ & -9.301261 & -0.816145 & -0.727826 \\
\hline $\mathrm{H}$ & -8.215434 & -0.003431 & -1.871764 \\
\hline $\mathrm{H}$ & -7.652335 & -1.411621 & -0.963242 \\
\hline C & 3.955603 & -0.067641 & 0.410324 \\
\hline C & 4.490686 & 0.982795 & 1.166870 \\
\hline C & 4.519916 & -0.364577 & -0.833438 \\
\hline C & 5.566278 & 1.743102 & 0.698853 \\
\hline $\mathrm{H}$ & 4.037775 & 1.211863 & 2.126217 \\
\hline C & 5.584428 & 0.385512 & -1.341665 \\
\hline $\mathrm{H}$ & 4.107883 & -1.182094 & -1.407985 \\
\hline C & 6.093570 & 1.429633 & -0.560624 \\
\hline $\mathrm{H}$ & 6.916648 & 2.014813 & -0.943798 \\
\hline C & 6.125777 & 0.049266 & -2.737930 \\
\hline C & 6.114180 & 2.889161 & 1.562482 \\
\hline C & 4.992646 & 0.255491 & -3.768485 \\
\hline
\end{tabular}




\begin{tabular}{|c|c|c|c|}
\hline $\mathrm{H}$ & 4.114480 & -0.352767 & -3.537704 \\
\hline $\mathrm{H}$ & 4.674310 & 1.302850 & -3.783638 \\
\hline $\mathrm{H}$ & 5.341088 & -0.009499 & -4.773041 \\
\hline C & 7.315769 & 0.937152 & -3.134821 \\
\hline $\mathrm{H}$ & 7.665503 & 0.657802 & -4.133387 \\
\hline $\mathrm{H}$ & 7.039182 & 1.995731 & -3.166605 \\
\hline $\mathrm{H}$ & 8.157115 & 0.822440 & -2.443377 \\
\hline C & 6.589283 & -1.422893 & -2.770665 \\
\hline $\mathrm{H}$ & 7.383581 & -1.598092 & -2.037761 \\
\hline $\mathrm{H}$ & 5.768718 & -2.110140 & -2.549854 \\
\hline $\mathrm{H}$ & 6.977606 & -1.675247 & -3.763308 \\
\hline C & 7.299156 & 3.609037 & 0.898610 \\
\hline $\mathrm{H}$ & 8.136481 & 2.928010 & 0.717597 \\
\hline $\mathrm{H}$ & 7.014520 & 4.063128 & -0.055532 \\
\hline $\mathrm{H}$ & 7.657118 & 4.409496 & 1.553452 \\
\hline C & 4.995308 & 3.925088 & 1.805085 \\
\hline $\mathrm{H}$ & 4.637439 & 4.337733 & 0.856706 \\
\hline $\mathrm{H}$ & 4.139488 & 3.480614 & 2.320893 \\
\hline $\mathrm{H}$ & 5.365886 & 4.751851 & 2.421077 \\
\hline C & 6.591600 & 2.323270 & 2.917334 \\
\hline $\mathrm{H}$ & 6.982601 & 3.126541 & 3.551452 \\
\hline $\mathrm{H}$ & 5.777799 & 1.834214 & 3.459993 \\
\hline $\mathrm{H}$ & 7.386401 & 1.585374 & 2.770387 \\
\hline
\end{tabular}

\section{Catalyst 2C 22}

\section{Datum}

Value

\begin{tabular}{lr}
\hline B3LYP-D3(BJ)/6-31G(d,p) Energy & -2165.16555 \\
\hline B3LYP-D3(BJ)/def2-TZVPP/IEF-PCM(DCM) Energy & -2165.900349 \\
\hline B3LYP-D3(BJ)/def2-TZVPP/IEF-PCM(DCM)//B3LYP-D3(BJ)/6-31G(d,p) Free Energy (Quasiharmonic) & -2165.022115
\end{tabular}

Number of Imaginary Frequencies

Frequencies (Top 3 out of 321)
1. $10.4686 \mathrm{~cm}^{-1}$
2. $11.2434 \mathrm{~cm}^{-1}$
3. $13.2872 \mathrm{~cm}^{-1}$

\section{B3LYP-D3(BJ)/6-31G(d,p) Molecular Geometry in Cartesian Coordinates}

$\begin{array}{rrrr}C & 0.288974 & -3.542320 & 3.931142 \\ C & 0.417712 & -2.973100 & 2.684824 \\ C & -0.657070 & -2.247862 & 2.107004\end{array}$




\begin{tabular}{|c|c|c|c|}
\hline C & -1.875086 & -2.109840 & 2.843746 \\
\hline C & -1.974229 & -2.708720 & 4.127156 \\
\hline C & -0.917212 & -3.409489 & 4.659454 \\
\hline $\mathrm{H}$ & 1.116915 & -4.097529 & 4.360537 \\
\hline $\mathrm{H}$ & 1.340944 & -3.072213 & 2.125575 \\
\hline C & -0.556154 & -1.639092 & 0.818558 \\
\hline C & -2.948645 & -1.381539 & 2.275876 \\
\hline $\mathrm{H}$ & -2.902240 & -2.601188 & 4.681353 \\
\hline $\mathrm{H}$ & -1.004288 & -3.862620 & 5.642065 \\
\hline C & -2.863899 & -0.815065 & 1.020763 \\
\hline C & -1.643375 & -0.964648 & 0.293246 \\
\hline $\mathrm{H}$ & -3.873024 & -1.285584 & 2.837515 \\
\hline C & 1.453820 & -4.968378 & -2.565371 \\
\hline C & 2.401985 & -3.984450 & -2.411854 \\
\hline C & 2.175035 & -2.887447 & -1.538793 \\
\hline C & 0.942670 & -2.810778 & -0.821718 \\
\hline C & -0.017507 & -3.841583 & -1.003202 \\
\hline C & 0.233124 & -4.894841 & -1.852317 \\
\hline $\mathrm{H}$ & 4.063852 & -1.922373 & -1.915116 \\
\hline $\mathrm{H}$ & 1.637541 & -5.804160 & -3.233517 \\
\hline $\mathrm{H}$ & 3.342052 & -4.030933 & -2.954499 \\
\hline C & 3.134111 & -1.865447 & -1.356805 \\
\hline C & 0.729071 & -1.701256 & 0.053359 \\
\hline $\mathrm{H}$ & -0.957213 & -3.784577 & -0.466369 \\
\hline $\mathrm{H}$ & -0.510949 & -5.674995 & -1.979860 \\
\hline C & 1.688401 & -0.707305 & 0.218590 \\
\hline C & 2.923849 & -0.792177 & -0.511531 \\
\hline C & 1.438562 & 0.426573 & 1.202549 \\
\hline $\mathrm{H}$ & 0.770463 & 0.074091 & 1.996534 \\
\hline $\mathrm{H}$ & 2.384360 & 0.709723 & 1.669341 \\
\hline C & -1.467432 & -0.285189 & -1.032109 \\
\hline 0 & -1.155141 & 0.881555 & -1.175355 \\
\hline 0 & -1.656711 & -1.108004 & -2.080663 \\
\hline $\mathrm{H}$ & -1.467330 & -0.585915 & -2.880373 \\
\hline 0 & 0.929476 & 1.619176 & 0.614084 \\
\hline $\mathrm{H}$ & 0.115395 & 1.413758 & 0.121579 \\
\hline C & 4.011314 & 0.212553 & -0.367010 \\
\hline C & 3.755162 & 1.581192 & -0.448942 \\
\hline C & 5.334338 & -0.225130 & -0.177669 \\
\hline C & 4.786663 & 2.522418 & -0.327793 \\
\hline $\mathrm{H}$ & 2.732476 & 1.908179 & -0.584043 \\
\hline C & 6.386506 & 0.681947 & -0.069447 \\
\hline $\mathrm{H}$ & 5.515376 & -1.289526 & -0.095454 \\
\hline C & 6.087585 & 2.053421 & -0.145682 \\
\hline $\mathrm{H}$ & 6.898192 & 2.765005 & -0.051702 \\
\hline C & -4.007708 & -0.055851 & 0.456852 \\
\hline C & -4.496200 & -0.340508 & -0.818096 \\
\hline C & -4.613023 & 0.957760 & 1.215883 \\
\hline C & -5.564768 & 0.383526 & -1.364588 \\
\hline $\mathrm{H}$ & -4.043693 & -1.145680 & -1.383806 \\
\hline C & -5.684611 & 1.690844 & 0.709455 \\
\hline $\mathrm{H}$ & -4.207448 & 1.174548 & 2.195946 \\
\hline C & -6.139315 & 1.387934 & -0.585645 \\
\hline $\mathrm{H}$ & -6.963786 & 1.961259 & -0.989279 \\
\hline
\end{tabular}




\begin{tabular}{|c|c|c|c|}
\hline C & -6.364740 & 2.816419 & 1.501351 \\
\hline C & -6.050926 & 0.044303 & -2.780272 \\
\hline C & -6.223121 & 4.143205 & 0.724336 \\
\hline $\mathrm{H}$ & -5.168571 & 4.398788 & 0.583054 \\
\hline $\mathrm{H}$ & -6.688912 & 4.085542 & -0.263315 \\
\hline $\mathrm{H}$ & -6.703528 & 4.959574 & 1.274424 \\
\hline C & -5.744229 & 3.005546 & 2.894882 \\
\hline $\mathrm{H}$ & -6.264378 & 3.810383 & 3.423031 \\
\hline $\mathrm{H}$ & -5.830841 & 2.099325 & 3.502645 \\
\hline $\mathrm{H}$ & -4.686301 & 3.278397 & 2.833790 \\
\hline C & -7.861422 & 2.481537 & 1.679695 \\
\hline $\mathrm{H}$ & -7.986735 & 1.543371 & 2.229342 \\
\hline $\mathrm{H}$ & -8.364859 & 3.276631 & 2.240135 \\
\hline $\mathrm{H}$ & -8.371034 & 2.376426 & 0.717984 \\
\hline C & -7.193624 & 0.961957 & -3.242703 \\
\hline $\mathrm{H}$ & -8.073103 & 0.869817 & -2.597768 \\
\hline $\mathrm{H}$ & -6.887540 & 2.012669 & -3.258296 \\
\hline $\mathrm{H}$ & -7.497564 & 0.688878 & -4.257758 \\
\hline C & -6.555238 & -1.414511 & -2.809553 \\
\hline $\mathrm{H}$ & -7.390525 & -1.550047 & -2.115496 \\
\hline $\mathrm{H}$ & -6.899020 & -1.679309 & -3.815356 \\
\hline $\mathrm{H}$ & -5.767972 & -2.119101 & -2.527767 \\
\hline C & -4.873925 & 0.200129 & -3.768440 \\
\hline $\mathrm{H}$ & -4.492401 & 1.225901 & -3.758120 \\
\hline $\mathrm{H}$ & -4.047240 & -0.469374 & -3.515524 \\
\hline $\mathrm{H}$ & -5.197665 & -0.036418 & -4.787705 \\
\hline C & 7.842041 & 0.240238 & 0.141401 \\
\hline C & 4.433180 & 4.014633 & -0.382982 \\
\hline C & 5.669886 & 4.915133 & -0.239325 \\
\hline $\mathrm{H}$ & 6.177814 & 4.755591 & 0.717205 \\
\hline $\mathrm{H}$ & 6.392457 & 4.744294 & -1.044151 \\
\hline $\mathrm{H}$ & 5.367401 & 5.966000 & -0.282041 \\
\hline C & 3.452729 & 4.336612 & 0.767441 \\
\hline $\mathrm{H}$ & 2.542565 & 3.734290 & 0.707025 \\
\hline $\mathrm{H}$ & 3.922084 & 4.139653 & 1.737059 \\
\hline $\mathrm{H}$ & 3.167812 & 5.394424 & 0.736992 \\
\hline C & 3.756881 & 4.330020 & -1.734486 \\
\hline $\mathrm{H}$ & 4.427892 & 4.100396 & -2.568619 \\
\hline $\mathrm{H}$ & 2.839818 & 3.750986 & -1.869675 \\
\hline $\mathrm{H}$ & 3.493905 & 5.392152 & -1.788848 \\
\hline C & 8.367440 & 0.824465 & 1.470478 \\
\hline $\mathrm{H}$ & 8.331404 & 1.917248 & 1.473805 \\
\hline $\mathrm{H}$ & 7.767961 & 0.467768 & 2.313838 \\
\hline $\mathrm{H}$ & 9.407366 & 0.522226 & 1.636645 \\
\hline C & 8.707047 & 0.762368 & -1.026109 \\
\hline $\mathrm{H}$ & 9.752075 & 0.462085 & -0.891599 \\
\hline $\mathrm{H}$ & 8.354505 & 0.358250 & -1.980243 \\
\hline H & 8.678002 & 1.853186 & -1.094555 \\
\hline C & 7.986035 & -1.289090 & 0.198602 \\
\hline $\mathrm{H}$ & 7.646363 & -1.761925 & -0.728238 \\
\hline $\mathrm{H}$ & 9.037811 & -1.555462 & 0.341924 \\
\hline $\mathrm{H}$ & 7.418391 & -1.718395 & 1.030010 \\
\hline
\end{tabular}




\section{Catalyst 2C 23}

Datum

Value

B3LYP-D3(BJ)/6-31G(d,p) Energy

$-2165.165045$

B3LYP-D3(BJ)/def2-TZVPP/IEF-PCM(DCM) Energy

$-2165.900193$

B3LYP-D3(BJ)/def2-TZVPP/IEF-PCM(DCM)//B3LYP-D3(BJ)/6-31G(d,p) Free Energy (Quasiharmonic) $\quad-2165.022043$

Number of Imaginary Frequencies

Frequencies (Top 3 out of 321)

$\begin{array}{ll}\text { 1. } & 10.4836 \mathrm{~cm}^{-1} \\ \text { 2. } & 11.4353 \mathrm{~cm}^{-1} \\ \text { 3. } & 13.2126 \mathrm{~cm}^{-1}\end{array}$

B3LYP-D3(BJ)/6-31G(d,p) Molecular Geometry in Cartesian Coordinates

C

C

C

C

C

C

$\mathrm{H}$

$\mathrm{H}$

C

C

$\mathrm{H}$

$\mathrm{H}$

C

C

$\mathrm{H}$

C

C

C

C

C

C

$\mathrm{H}$

$\mathrm{H}$

$\mathrm{H}$

C

C

$\mathrm{H}$

$\mathrm{H}$

C
$-0.305964$

$-0.435065$

0.641497

1.861533

1.961117

0.902396

$-1.135273$

$-1.359914$

0.541086

2.936435

2.890755

0.989758

2.851746

1.629649

3.862357

$-1.493833$

$-2.436579$

$-2.201836$

$-0.967357$

$-0.012832$

$-0.270954$

$-4.086560$

$-1.683496$

$-3.378228$

$-3.155172$

$-0.745905$

0.928603

0.468902

$-1.699754$
$-3.598785$

$-3.013627$

$-2.285764$

$-2.161690$

$-2.777022$

$-3.480026$

$-4.155824$

$-3.102059$

$-1.661452$

$-1.430210$

$-2.680116$

$-3.945711$

$-0.846241$

$-0.984121$

$-1.345472$

$-4.949969$

$-3.962612$

$-2.874490$

$-2.810467$

$-3.844386$

$-4.888848$

$-1.896405$

$-5.778917$

$-3.999556$

$-1.849193$

$-1.709912$

$-3.796679$

$-5.671499$

$-0.712608$
3.883821

2. 644934

2.073787

2.809475

4.085075

4. 610984

4. 308178

2. 086575

0.792817

2. 248581

4.638512

5.587686

1.001201

0.273780

2. 809401

$-2.616153$

$-2.451631$

$-1.569559$

$-0.854907$

$-1.047980$

$-1.905671$

$-1.932681$

$-3.291120$

$-2.992269$

$-1.376207$

0.029502

$-0.513293$

$-2.042100$

0.205992 


\begin{tabular}{|c|c|c|c|}
\hline C & -2.937362 & -0.784610 & -0.521922 \\
\hline C & -1.442445 & 0.410452 & 1.200416 \\
\hline $\mathrm{H}$ & -2.385953 & 0.693192 & 1.672077 \\
\hline $\mathrm{H}$ & -0.774221 & 0.047030 & 1.989355 \\
\hline C & 1.452760 & -0.292610 & -1.044978 \\
\hline 0 & 1.147977 & 0.877392 & -1.178538 \\
\hline 0 & 1.631993 & -1.109361 & -2.099567 \\
\hline $\mathrm{H}$ & 1.436759 & -0.582956 & -2.894894 \\
\hline 0 & -0.929299 & 1.606658 & 0.622881 \\
\hline $\mathrm{H}$ & -0.116916 & 1.402859 & 0.126951 \\
\hline C & -4.019381 & 0.224245 & -0.365631 \\
\hline C & -3.756522 & 1.592274 & -0.435650 \\
\hline C & -5.344143 & -0.208474 & -0.177003 \\
\hline C & -4.782947 & 2.537539 & -0.303352 \\
\hline $\mathrm{H}$ & -2.732513 & 1.915357 & -0.570080 \\
\hline C & -6.391438 & 0.702865 & -0.057839 \\
\hline $\mathrm{H}$ & -5.530351 & -1.272652 & -0.104031 \\
\hline C & -6.085776 & 2.073456 & -0.122295 \\
\hline $\mathrm{H}$ & -6.892546 & 2.788213 & -0.019726 \\
\hline C & 3.997802 & -0.081577 & 0.449317 \\
\hline C & 4.480837 & -0.344356 & -0.837933 \\
\hline C & 4.611517 & 0.906093 & 1.229001 \\
\hline C & 5.554191 & 0.378775 & -1.364287 \\
\hline $\mathrm{H}$ & 4.016786 & -1.131770 & -1.415243 \\
\hline C & 5.692814 & 1.642423 & 0.738341 \\
\hline $\mathrm{H}$ & 4.209382 & 1.104992 & 2.214231 \\
\hline C & 6.139738 & 1.362075 & -0.558842 \\
\hline $\mathrm{H}$ & 6.971481 & 1.933266 & -0.956883 \\
\hline C & 6.383739 & 2.742757 & 1.556234 \\
\hline C & 6.092596 & 0.135153 & -2.780944 \\
\hline C & 6.254342 & 4.088359 & 0.810266 \\
\hline $\mathrm{H}$ & 6.719060 & 4.048765 & -0.178809 \\
\hline $\mathrm{H}$ & 5.202244 & 4.357059 & 0.675283 \\
\hline $\mathrm{H}$ & 6.742656 & 4.887399 & 1.378536 \\
\hline C & 7.877086 & 2.389380 & 1.725846 \\
\hline $\mathrm{H}$ & 8.388588 & 3.166160 & 2.304367 \\
\hline $\mathrm{H}$ & 7.993611 & 1.437341 & 2.253089 \\
\hline $\mathrm{H}$ & 8.385123 & 2.301693 & 0.761536 \\
\hline C & 5.765238 & 2.904961 & 2.954099 \\
\hline $\mathrm{H}$ & 5.843439 & 1.984014 & 3.540492 \\
\hline $\mathrm{H}$ & 6.293060 & 3.692277 & 3.500714 \\
\hline $\mathrm{H}$ & 4.709918 & 3.189146 & 2.899928 \\
\hline C & 5.336736 & -0.991836 & -3.503538 \\
\hline $\mathrm{H}$ & 5.437828 & -1.947872 & -2.980626 \\
\hline $\mathrm{H}$ & 4.269526 & -0.768794 & -3.597704 \\
\hline $\mathrm{H}$ & 5.741393 & -1.119559 & -4.512079 \\
\hline C & 7.584864 & -0.252144 & -2.707754 \\
\hline $\mathrm{H}$ & 7.720127 & -1.167117 & -2.122706 \\
\hline $\mathrm{H}$ & 7.983335 & -0.426260 & -3.713096 \\
\hline $\mathrm{H}$ & 8.185085 & 0.534376 & -2.242169 \\
\hline C & 5.935996 & 1.430358 & -3.606974 \\
\hline $\mathrm{H}$ & 4.883768 & 1.724583 & -3.670725 \\
\hline $\mathrm{H}$ & 6.487954 & 2.262669 & -3.161756 \\
\hline $\mathrm{H}$ & 6.314146 & 1.282365 & -4.624344 \\
\hline
\end{tabular}




\begin{tabular}{|llll|} 
C & -7.848644 & 0.266603 & 0.152795 \\
C & -4.422041 & 4.028396 & -0.345640 \\
H & -3.746929 & 4.352765 & -1.695612 \\
H & -2.833047 & 3.770436 & -1.837992 \\
H & -4.420774 & 4.134135 & -2.530416 \\
C & -3.478747 & 5.414024 & -1.740800 \\
H & -3.437615 & 4.334873 & 0.805622 \\
H & -3.906089 & 4.131719 & 1.774387 \\
H & -2.530790 & 3.728292 & 0.737975 \\
C & -3.147150 & 5.391397 & 0.784073 \\
H & -5.653882 & 4.933760 & -0.191156 \\
H & -6.378974 & 4.773945 & -0.995979 \\
H & -6.160634 & 4.768015 & 0.764941 \\
C & -5.346163 & 5.983434 & -0.224874 \\
H & -8.714062 & 0.803854 & -1.007526 \\
H & -8.679643 & 1.895098 & -1.065989 \\
H & -8.366113 & 0.406784 & -1.966293 \\
C & -9.760247 & 0.507638 & -0.872976 \\
H & -8.367564 & 0.841240 & 1.488586 \\
H & -9.408580 & 0.542771 & 1.654752 \\
H & -7.767704 & 0.473771 & 2.327035 \\
C & -8.325937 & 1.933748 & 1.501843 \\
H & -8.000272 & -1.262430 & 0.196334 \\
H & -7.432645 & -1.702219 & 1.022254 \\
H & -9.053013 & -1.524773 & 0.340012 \\
& -7.665438 & -1.728445 & -0.735705 \\
\hline & & & \\
\hline & & & \\
\hline
\end{tabular}

\section{Catalyst 2C 24}

Datum

Value

B3LYP-D3(BJ)/6-31G(d,p) Energy

$-2165.165041$

B3LYP-D3(BJ)/def2-TZVPP/IEF-PCM(DCM) Energy

$-2165.900231$

B3LYP-D3(BJ)/def2-TZVPP/IEF-PCM(DCM)//B3LYP-D3(BJ)/6-31G(d,p) Free Energy (Quasiharmonic) -2165.022035

Number of Imaginary Frequencies

Frequencies (Top 3 out of 321)

$\begin{array}{ll}\text { 1. } & 10.7520 \mathrm{~cm}^{-1} \\ \text { 2. } & 11.4833 \mathrm{~cm}^{-1} \\ \text { 3. } & 13.2789 \mathrm{~cm}^{-1}\end{array}$

B3LYP-D3(BJ)/6-31G(d,p) Molecular Geometry in Cartesian Coordinates 


\begin{tabular}{|c|c|c|c|}
\hline C & 0.281455 & -3.566092 & 3.904797 \\
\hline C & 0.415058 & -2.990574 & 2.661871 \\
\hline C & -0.658426 & -2.264728 & 2.082444 \\
\hline C & -1.880129 & -2.132610 & 2.813967 \\
\hline C & -1.984428 & -2.738151 & 4.093835 \\
\hline C & -0.928554 & -3.439372 & 4.627832 \\
\hline $\mathrm{H}$ & 1.108466 & -4.121654 & 4.335526 \\
\hline $\mathrm{H}$ & 1.341157 & -3.085191 & 2.106609 \\
\hline C & -0.553333 & -1.650585 & 0.796906 \\
\hline C & -2.952041 & -1.403132 & 2.244656 \\
\hline$H$ & -2.915372 & -2.635263 & 4.643990 \\
\hline $\mathrm{H}$ & -1.019489 & -3.897566 & 5.607743 \\
\hline C & -2.862369 & -0.828543 & 0.993378 \\
\hline C & -1.639022 & -0.974884 & 0.269886 \\
\hline $\mathrm{H}$ & -3.879579 & -1.312379 & 2.801884 \\
\hline C & 1.480856 & -4.970703 & -2.581687 \\
\hline C & 2.425704 & -3.984439 & -2.422766 \\
\hline C & 2.191728 & -2.888887 & -1.549736 \\
\hline C & 0.955854 & -2.816249 & -0.838331 \\
\hline C & -0.000838 & -3.849258 & -1.025476 \\
\hline C & 0.256562 & -4.901002 & -1.874440 \\
\hline $\mathrm{H}$ & 4.079610 & -1.918274 & -1.916414 \\
\hline $\mathrm{H}$ & 1.669924 & -5.805351 & -3.249762 \\
\hline $\mathrm{H}$ & 3.368436 & -4.027918 & -2.961023 \\
\hline C & 3.147143 & -1.864421 & -1.362359 \\
\hline C & 0.735222 & -1.708235 & 0.036914 \\
\hline $\mathrm{H}$ & -0.943372 & -3.795018 & -0.493355 \\
\hline $\mathrm{H}$ & -0.484965 & -5.682826 & -2.006454 \\
\hline C & 1.691061 & -0.711829 & 0.207568 \\
\hline C & 2.930076 & -0.792570 & -0.517014 \\
\hline C & 1.434134 & 0.419786 & 1.192339 \\
\hline $\mathrm{H}$ & 0.763331 & 0.064242 & 1.982693 \\
\hline $\mathrm{H}$ & 2.377223 & 0.704180 & 1.663842 \\
\hline C & -1.458008 & -0.294472 & -1.054064 \\
\hline 0 & -1.150658 & 0.873829 & -1.196266 \\
\hline 0 & -1.637502 & -1.119227 & -2.102255 \\
\hline $\mathrm{H}$ & -1.440730 & -0.599519 & -2.901595 \\
\hline 0 & 0.925041 & 1.612458 & 0.604046 \\
\hline $\mathrm{H}$ & 0.113187 & 1.406684 & 0.108075 \\
\hline C & 4.014195 & 0.214882 & -0.366419 \\
\hline C & 3.754797 & 1.582944 & -0.447776 \\
\hline C & 5.337534 & -0.219532 & -0.171774 \\
\hline C & 4.783263 & 2.526742 & -0.320941 \\
\hline $\mathrm{H}$ & 2.731860 & 1.907430 & -0.586904 \\
\hline C & 6.386814 & 0.690180 & -0.057814 \\
\hline $\mathrm{H}$ & 5.521025 & -1.283545 & -0.090046 \\
\hline C & 6.084608 & 2.060954 & -0.133689 \\
\hline $\mathrm{H}$ & 6.892913 & 2.774553 & -0.035254 \\
\hline C & -4.005226 & -0.065173 & 0.433140 \\
\hline C & -4.489599 & -0.340218 & -0.855045 \\
\hline C & -4.617218 & 0.929297 & 1.196340 \\
\hline C & -5.558475 & 0.378175 & -1.387105 \\
\hline $\mathrm{H}$ & -4.026210 & -1.134553 & -1.423345 \\
\hline
\end{tabular}




\begin{tabular}{|c|c|c|c|}
\hline C & -5.700843 & 1.665934 & 0.697793 \\
\hline $\mathrm{H}$ & -4.218815 & 1.143544 & 2.182285 \\
\hline C & -6.147647 & 1.375181 & -0.592167 \\
\hline $\mathrm{H}$ & -6.976841 & 1.937407 & -1.001589 \\
\hline C & -6.334010 & 2.759884 & 1.568960 \\
\hline C & -6.098205 & 0.121125 & -2.800713 \\
\hline C & -5.269482 & 3.830698 & 1.891784 \\
\hline $\mathrm{H}$ & -4.417566 & 3.405693 & 2.429216 \\
\hline $\mathrm{H}$ & -4.889419 & 4.289050 & 0.973711 \\
\hline $\mathrm{H}$ & -5.701077 & 4.620164 & 2.516564 \\
\hline C & -6.846193 & 2.131014 & 2.882746 \\
\hline $\mathrm{H}$ & -7.297481 & 2.897941 & 3.521344 \\
\hline $\mathrm{H}$ & -7.602757 & 1.366827 & 2.678612 \\
\hline $\mathrm{H}$ & -6.037453 & 1.659665 & 3.447901 \\
\hline C & -7.517717 & 3.450389 & 0.872779 \\
\hline $\mathrm{H}$ & -8.316564 & 2.741597 & 0.632646 \\
\hline $\mathrm{H}$ & -7.938622 & 4.213523 & 1.534359 \\
\hline $\mathrm{H}$ & -7.209754 & 3.947834 & -0.052163 \\
\hline C & -5.942207 & 1.408079 & -3.639801 \\
\hline $\mathrm{H}$ & -6.493643 & 2.244979 & -3.202683 \\
\hline $\mathrm{H}$ & -4.889920 & 1.701210 & -3.707730 \\
\hline $\mathrm{H}$ & -6.321436 & 1.249750 & -4.655211 \\
\hline C & -7.590595 & -0.265158 & -2.722759 \\
\hline $\mathrm{H}$ & -8.190536 & 0.525945 & -2.264691 \\
\hline $\mathrm{H}$ & -7.989531 & -0.449065 & -3.726179 \\
\hline $\mathrm{H}$ & -7.725477 & -1.174491 & -2.128868 \\
\hline C & -5.343309 & -1.012917 & -3.513134 \\
\hline $\mathrm{H}$ & -4.276027 & -0.791229 & -3.609774 \\
\hline $\mathrm{H}$ & -5.444662 & -1.963947 & -2.981230 \\
\hline $\mathrm{H}$ & -5.748460 & -1.149890 & -4.520268 \\
\hline C & 7.842569 & 0.252049 & 0.158891 \\
\hline C & 4.426081 & 4.018088 & -0.375783 \\
\hline C & 3.440038 & 4.336066 & 0.770967 \\
\hline $\mathrm{H}$ & 2.531765 & 3.731354 & 0.706118 \\
\hline $\mathrm{H}$ & 3.905950 & 4.139240 & 1.742271 \\
\hline $\mathrm{H}$ & 3.152374 & 5.393135 & 0.740583 \\
\hline C & 3.754552 & 4.333377 & -1.729686 \\
\hline $\mathrm{H}$ & 4.429596 & 4.106517 & -2.561321 \\
\hline $\mathrm{H}$ & 2.839552 & 3.752149 & -1.869356 \\
\hline $\mathrm{H}$ & 3.489042 & 5.394887 & -1.783835 \\
\hline C & 5.659812 & 4.921654 & -0.225915 \\
\hline $\mathrm{H}$ & 6.164187 & 4.762281 & 0.732520 \\
\hline $\mathrm{H}$ & 6.386168 & 4.753694 & -1.027937 \\
\hline $\mathrm{H}$ & 5.354737 & 5.971773 & -0.268608 \\
\hline C & 7.990345 & -1.276961 & 0.214924 \\
\hline $\mathrm{H}$ & 7.420194 & -1.708743 & 1.043329 \\
\hline $\mathrm{H}$ & 7.655992 & -1.749589 & -0.713953 \\
\hline $\mathrm{H}$ & 9.042181 & -1.540731 & 0.362548 \\
\hline C & 8.360614 & 0.836074 & 1.490939 \\
\hline $\mathrm{H}$ & 9.400593 & 0.536363 & 1.661296 \\
\hline $\mathrm{H}$ & 8.321696 & 1.928754 & 1.495391 \\
\hline $\mathrm{H}$ & 7.758394 & 0.476809 & 2.331250 \\
\hline C & 8.711295 & 0.777846 & -1.004203 \\
\hline $\mathrm{H}$ & 8.679676 & 1.868664 & -1.071479 \\
\hline
\end{tabular}




\section{Catalyst 2C 25}

\section{Datum}

Value

\begin{tabular}{lr}
\hline B3LYP-D3(BJ)/6-31G(d,p) Energy & -2165.164802 \\
\hline B3LYP-D3(BJ)/def2-TZVPP/IEF-PCM(DCM) Energy & -2165.900046 \\
\hline B3LYP-D3(BJ)/def2-TZVPP/IEF-PCM(DCM)//B3LYP-D3(BJ)/6-31G(d,p) Free Energy (Quasiharmonic) & -2165.021997 \\
\hline
\end{tabular}

Number of Imaginary Frequencies

Frequencies (Top 3 out of 321)

$\begin{array}{ll}\text { 1. } & 10.3172 \mathrm{~cm}^{-1} \\ \text { 2. } & 11.4351 \mathrm{~cm}^{-1} \\ \text { 3. } & 13.2122 \mathrm{~cm}^{-1}\end{array}$

\section{B3LYP-D3(BJ)/6-31G(d,p) Molecular Geometry in Cartesian Coordinates}

\begin{tabular}{|c|c|c|c|}
\hline C & 0.295781 & -3.581993 & 3.885590 \\
\hline C & 0.421119 & -2.998247 & 2.645661 \\
\hline C & -0.655751 & -2.267678 & 2.078550 \\
\hline C & -1.872288 & -2.139580 & 2.819503 \\
\hline C & -1.967950 & -2.753414 & 4.096099 \\
\hline C & -0.908979 & -3.459045 & 4.617987 \\
\hline $\mathrm{H}$ & 1.125329 & -4.141084 & 4.306774 \\
\hline $\mathrm{H}$ & 1.343273 & -3.089738 & 2.083342 \\
\hline C & -0.558381 & -1.643928 & 0.797004 \\
\hline C & -2.947846 & -1.405871 & 2.262507 \\
\hline $\mathrm{H}$ & -2.894871 & -2.653371 & 4.653520 \\
\hline $\mathrm{H}$ & -0.993396 & -3.923596 & 5.595486 \\
\hline $\mathrm{C}$ & -2.866369 & -0.824509 & 1.013998 \\
\hline C & -1.647333 & -0.964326 & 0.282048 \\
\hline $\mathrm{H}$ & -3.871129 & -1.317595 & 2.827202 \\
\hline C & 1.454921 & -4.934353 & -2.623723 \\
\hline C & 2.401067 & -3.949806 & -2.461429 \\
\hline C & 2.172541 & -2.861994 & -1.577487 \\
\hline C & 0.940708 & -2.795148 & -0.858368 \\
\hline C & -0.017361 & -3.826167 & -1.049235 \\
\hline C & 0.234776 & -4.870494 & -1.908921 \\
\hline $\mathrm{H}$ & 4.059386 & -1.887604 & -1.945604 \\
\hline $\mathrm{H}$ & 1.639854 & -5.763082 & -3.300268 \\
\hline $\mathrm{H}$ & 3.340638 & -3.988787 & -3.005535 \\
\hline
\end{tabular}




\begin{tabular}{|c|c|c|c|}
\hline C & 3.129576 & -1.839353 & -1.386468 \\
\hline C & 0.725351 & -1.695272 & 0.028613 \\
\hline $\mathrm{H}$ & -0.956673 & -3.776475 & -0.510977 \\
\hline $\mathrm{H}$ & -0.507744 & -5.650951 & -2.043483 \\
\hline C & 1.682716 & -0.701241 & 0.202767 \\
\hline C & 2.916572 & -0.775837 & -0.530159 \\
\hline C & 1.435779 & 0.421911 & 1.199812 \\
\hline $\mathrm{H}$ & 0.771115 & 0.059369 & 1.992484 \\
\hline $\mathrm{H}$ & 2.383898 & 0.698322 & 1.666392 \\
\hline C & -1.474968 & -0.268948 & -1.035522 \\
\hline 0 & -1.167321 & 0.900412 & -1.165699 \\
\hline 0 & -1.662108 & -1.080763 & -2.093243 \\
\hline $\mathrm{H}$ & -1.475068 & -0.548900 & -2.887033 \\
\hline 0 & 0.925531 & 1.620867 & 0.627237 \\
\hline $\mathrm{H}$ & 0.110477 & 1.420122 & 0.134812 \\
\hline C & 4.001255 & 0.230363 & -0.374230 \\
\hline C & 3.739820 & 1.605610 & -0.474850 \\
\hline C & 5.313498 & -0.198977 & -0.159245 \\
\hline C & 4.764530 & 2.541116 & -0.347245 \\
\hline $\mathrm{H}$ & 2.718058 & 1.920541 & -0.628132 \\
\hline C & 6.370823 & 0.713369 & -0.037865 \\
\hline $\mathrm{H}$ & 5.501613 & -1.263530 & -0.064761 \\
\hline C & 6.071680 & 2.073498 & -0.133011 \\
\hline $\mathrm{H}$ & 6.869483 & 2.798074 & -0.031273 \\
\hline C & -4.012169 & -0.059986 & 0.461499 \\
\hline C & -4.501948 & -0.328987 & -0.816345 \\
\hline C & -4.617992 & 0.942870 & 1.234261 \\
\hline C & -5.572227 & 0.400470 & -1.352150 \\
\hline $\mathrm{H}$ & -4.049003 & -1.126182 & -1.392919 \\
\hline C & -5.691349 & 1.680776 & 0.738712 \\
\hline $\mathrm{H}$ & -4.211344 & 1.147728 & 2.216436 \\
\hline C & -6.147275 & 1.393896 & -0.559604 \\
\hline $\mathrm{H}$ & -6.973058 & 1.971192 & -0.954810 \\
\hline C & -6.371997 & 2.795142 & 1.545860 \\
\hline C & -6.059614 & 0.078830 & -2.771502 \\
\hline C & -7.868130 & 2.456294 & 1.721267 \\
\hline $\mathrm{H}$ & -8.378573 & 2.363184 & 0.758762 \\
\hline $\mathrm{H}$ & -7.991870 & 1.510886 & 2.258731 \\
\hline $\mathrm{H}$ & -8.371890 & 3.243446 & 2.292526 \\
\hline C & -6.232605 & 4.132100 & 0.786081 \\
\hline $\mathrm{H}$ & -6.713384 & 4.940701 & 1.347217 \\
\hline $\mathrm{H}$ & -5.178478 & 4.390666 & 0.647103 \\
\hline $\mathrm{H}$ & -6.699300 & 4.086729 & -0.201783 \\
\hline C & -5.750350 & 2.966755 & 2.941162 \\
\hline $\mathrm{H}$ & -4.692825 & 3.241722 & 2.882620 \\
\hline $\mathrm{H}$ & -6.270978 & 3.763942 & 3.480334 \\
\hline $\mathrm{H}$ & -5.835220 & 2.052521 & 3.537057 \\
\hline C & -7.203625 & 1.001267 & -3.220992 \\
\hline $\mathrm{H}$ & -8.082363 & 0.900078 & -2.576400 \\
\hline $\mathrm{H}$ & -6.898499 & 2.052370 & -3.223424 \\
\hline $\mathrm{H}$ & -7.508367 & 0.740924 & -4.239150 \\
\hline C & -6.562642 & -1.379955 & -2.818748 \\
\hline $\mathrm{H}$ & -7.397142 & -1.525028 & -2.125671 \\
\hline H & -6.907144 & -1.632345 & -3.827495 \\
\hline
\end{tabular}




\begin{tabular}{|c|c|c|c|}
\hline $\mathrm{H}$ & -5.774443 & -2.087292 & -2.546615 \\
\hline C & -4.883836 & 0.248258 & -3.758893 \\
\hline $\mathrm{H}$ & -4.503148 & 1.274129 & -3.735881 \\
\hline $\mathrm{H}$ & -4.056288 & -0.423683 & -3.515451 \\
\hline $\mathrm{H}$ & -5.208526 & 0.024447 & -4.780732 \\
\hline C & 7.794422 & 0.191070 & 0.204768 \\
\hline C & 4.497941 & 4.051541 & -0.409653 \\
\hline C & 4.869049 & 4.679206 & 0.951674 \\
\hline $\mathrm{H}$ & 4.261048 & 4.248245 & 1.753010 \\
\hline $\mathrm{H}$ & 5.921131 & 4.511649 & 1.200520 \\
\hline $\mathrm{H}$ & 4.695151 & 5.760786 & 0.933680 \\
\hline C & 3.021031 & 4.366286 & -0.701217 \\
\hline $\mathrm{H}$ & 2.715430 & 3.978058 & -1.678110 \\
\hline $\mathrm{H}$ & 2.353950 & 3.938835 & 0.052250 \\
\hline $\mathrm{H}$ & 2.873258 & 5.450932 & -0.713398 \\
\hline C & 5.360571 & 4.687544 & -1.520129 \\
\hline $\mathrm{H}$ & 6.429761 & 4.542016 & -1.341041 \\
\hline $\mathrm{H}$ & 5.121310 & 4.250399 & -2.494649 \\
\hline $\mathrm{H}$ & 5.175333 & 5.765879 & -1.574700 \\
\hline C & 7.827219 & -0.606617 & 1.526217 \\
\hline $\mathrm{H}$ & 7.539400 & 0.029208 & 2.369261 \\
\hline $\mathrm{H}$ & 7.142055 & -1.458381 & 1.501090 \\
\hline $\mathrm{H}$ & 8.835252 & -0.991831 & 1.715599 \\
\hline C & 8.826029 & 1.326403 & 0.303002 \\
\hline $\mathrm{H}$ & 9.822468 & 0.905399 & 0.469118 \\
\hline $\mathrm{H}$ & 8.864339 & 1.919110 & -0.616305 \\
\hline $\mathrm{H}$ & 8.607516 & 2.000861 & 1.136760 \\
\hline C & 8.203889 & -0.732909 & -0.962420 \\
\hline $\mathrm{H}$ & 8.188219 & -0.188060 & -1.911537 \\
\hline $\mathrm{H}$ & 9.216513 & -1.120907 & -0.806403 \\
\hline $\mathrm{H}$ & 7.528562 & -1.587806 & -1.056295 \\
\hline
\end{tabular}

\section{Catalyst 2C 26}

\section{Datum}

Value

B3LYP-D3(BJ)/6-31G(d,p) Energy

$-2165.164295$

B3LYP-D3(BJ)/def2-TZVPP/IEF-PCM(DCM) Energy

$-2165.899927$

B3LYP-D3(BJ)/def2-TZVPP/IEF-PCM(DCM)//B3LYP-D3(BJ)/6-31G(d,p) Free Energy (Quasiharmonic) -2165.021907

Number of Imaginary Frequencies

0

Frequencies (Top 3 out of 321)

$\begin{array}{ll}\text { 1. } & 10.6546 \mathrm{~cm}^{-1} \\ \text { 2. } & 11.7581 \mathrm{~cm}^{-1} \\ \text { 3. } & 13.2065 \mathrm{~cm}^{-1}\end{array}$




\begin{tabular}{|c|c|c|c|}
\hline C & 0.287689 & -3.605795 & 3.858742 \\
\hline C & 0.418097 & -3.015592 & 2.622380 \\
\hline C & -0.657318 & -2.284136 & 2.053690 \\
\hline C & -1.877595 & -2.161826 & 2.789272 \\
\hline C & -1.978624 & -2.782485 & 4.062147 \\
\hline C & -0.920948 & -3.488840 & 4.585707 \\
\hline $\mathrm{H}$ & 1.116183 & -4.165460 & 4.281237 \\
\hline $\mathrm{H}$ & 1.343158 & -3.102679 & 2.064164 \\
\hline C & -0.555569 & -1.654865 & 0.775210 \\
\hline C & -2.951374 & -1.426727 & 2.230840 \\
\hline $\mathrm{H}$ & -2.908521 & -2.687003 & 4.615405 \\
\hline $\mathrm{H}$ & -1.009389 & -3.958589 & 5.560364 \\
\hline C & -2.864792 & -0.837134 & 0.986335 \\
\hline C & -1.642860 & -0.973769 & 0.258558 \\
\hline $\mathrm{H}$ & -3.877885 & -1.343635 & 2.790966 \\
\hline C & 1.481553 & -4.935925 & -2.640540 \\
\hline C & 2.424540 & -3.949289 & -2.472611 \\
\hline C & 2.189143 & -2.863060 & -1.588522 \\
\hline C & 0.953791 & -2.800114 & -0.875141 \\
\hline C & -0.000950 & -3.833088 & -1.071895 \\
\hline C & 0.257805 & -4.875780 & -1.931598 \\
\hline $\mathrm{H}$ & 4.075196 & -1.883448 & -1.946748 \\
\hline $\mathrm{H}$ & 1.671710 & -5.763414 & -3.317157 \\
\hline $\mathrm{H}$ & 3.366781 & -3.985359 & -3.012287 \\
\hline C & 3.142669 & -1.838189 & -1.391903 \\
\hline C & 0.731540 & -1.701856 & 0.012150 \\
\hline $\mathrm{H}$ & -0.943094 & -3.786065 & -0.538389 \\
\hline $\mathrm{H}$ & -0.482295 & -5.657711 & -2.070809 \\
\hline C & 1.685558 & -0.705605 & 0.191931 \\
\hline C & 2.922997 & -0.776200 & -0.535391 \\
\hline C & 1.431659 & 0.415140 & 1.189973 \\
\hline $\mathrm{H}$ & 0.764254 & 0.049504 & 1.978932 \\
\hline $\mathrm{H}$ & 2.377124 & 0.692564 & 1.661322 \\
\hline C & -1.465217 & -0.277280 & -1.057484 \\
\hline 0 & -1.162489 & 0.893622 & -1.186393 \\
\hline 0 & -1.642410 & -1.090870 & -2.114993 \\
\hline $\mathrm{H}$ & -1.447900 & -0.561270 & -2.908365 \\
\hline 0 & 0.921507 & 1.614321 & 0.617809 \\
\hline $\mathrm{H}$ & 0.108671 & 1.413439 & 0.121837 \\
\hline C & 4.004419 & 0.232524 & -0.373269 \\
\hline C & 3.739928 & 1.607246 & -0.473081 \\
\hline C & 5.316827 & -0.193775 & -0.153245 \\
\hline C & 4.761697 & 2.545176 & -0.339828 \\
\hline $\mathrm{H}$ & 2.718043 & 1.919829 & -0.630247 \\
\hline C & 6.371309 & 0.721080 & -0.026165 \\
\hline $\mathrm{H}$ & 5.507237 & -1.257978 & -0.059401 \\
\hline C & 6.069115 & 2.080575 & -0.120727 \\
\hline $\mathrm{H}$ & 6.864646 & 2.807027 & -0.014679 \\
\hline C & -4.009653 & -0.068561 & 0.437481 \\
\hline C & -4.495256 & -0.327878 & -0.853494 \\
\hline
\end{tabular}




\begin{tabular}{|c|c|c|c|}
\hline C & -4.622384 & 0.914936 & 1.214171 \\
\hline C & -5.566104 & 0.395353 & -1.374902 \\
\hline $\mathrm{H}$ & -4.031156 & -1.113986 & -1.432568 \\
\hline C & -5.708043 & 1.655902 & 0.726585 \\
\hline $\mathrm{H}$ & -4.222925 & 1.117312 & 2.202195 \\
\hline C & -6.156084 & 1.380916 & -0.566399 \\
\hline $\mathrm{H}$ & -6.986843 & 1.946853 & -0.967454 \\
\hline C & -6.341954 & 2.737450 & 1.612565 \\
\hline C & -6.107061 & 0.155678 & -2.791079 \\
\hline C & -5.279025 & 3.806235 & 1.947175 \\
\hline $\mathrm{H}$ & -4.425372 & 3.376133 & 2.477751 \\
\hline $\mathrm{H}$ & -4.901405 & 4.276936 & 1.034362 \\
\hline $\mathrm{H}$ & -5.711147 & 4.586839 & 2.582643 \\
\hline C & -6.850699 & 2.091022 & 2.919139 \\
\hline $\mathrm{H}$ & -7.302407 & 2.848936 & 3.568122 \\
\hline $\mathrm{H}$ & -7.606115 & 1.327985 & 2.706621 \\
\hline $\mathrm{H}$ & -6.040100 & 1.614171 & 3.476965 \\
\hline C & -7.528211 & 3.434324 & 0.927142 \\
\hline $\mathrm{H}$ & -8.326017 & 2.726996 & 0.679339 \\
\hline $\mathrm{H}$ & -7.949566 & 4.188146 & 1.599038 \\
\hline $\mathrm{H}$ & -7.222805 & 3.944098 & 0.008085 \\
\hline C & -5.954193 & 1.453653 & -3.613608 \\
\hline $\mathrm{H}$ & -6.506591 & 2.283856 & -3.165079 \\
\hline $\mathrm{H}$ & -4.902491 & 1.749464 & -3.678858 \\
\hline $\mathrm{H}$ & -6.334290 & 1.307820 & -4.630568 \\
\hline C & -7.598711 & -0.234096 & -2.716468 \\
\hline $\mathrm{H}$ & -8.199437 & 0.549995 & -2.247505 \\
\hline $\mathrm{H}$ & -7.998484 & -0.405629 & -3.721746 \\
\hline $\mathrm{H}$ & -7.731414 & -1.151283 & -2.134277 \\
\hline C & -5.351089 & -0.967771 & -3.518973 \\
\hline $\mathrm{H}$ & -4.284292 & -0.743053 & -3.613932 \\
\hline $\mathrm{H}$ & -5.450222 & -1.925779 & -2.999308 \\
\hline $\mathrm{H}$ & -5.757171 & -1.092385 & -4.527340 \\
\hline C & 7.795210 & 0.202068 & 0.221695 \\
\hline C & 4.491540 & 4.055003 & -0.401356 \\
\hline C & 3.015028 & 4.366397 & -0.698477 \\
\hline $\mathrm{H}$ & 2.714391 & 3.978741 & -1.677138 \\
\hline $\mathrm{H}$ & 2.345983 & 3.936222 & 0.051689 \\
\hline $\mathrm{H}$ & 2.864535 & 5.450676 & -0.709791 \\
\hline C & 5.357049 & 4.694595 & -1.507524 \\
\hline $\mathrm{H}$ & 6.425867 & 4.551496 & -1.324303 \\
\hline $\mathrm{H}$ & 5.122819 & 4.258114 & -2.483565 \\
\hline $\mathrm{H}$ & 5.169332 & 5.772533 & -1.561463 \\
\hline C & 4.855564 & 4.681874 & 0.962250 \\
\hline $\mathrm{H}$ & 4.245443 & 4.248359 & 1.760590 \\
\hline $\mathrm{H}$ & 5.907063 & 4.516699 & 1.215121 \\
\hline $\mathrm{H}$ & 4.678965 & 5.763026 & 0.944912 \\
\hline C & 8.211891 & -0.719269 & -0.945027 \\
\hline $\mathrm{H}$ & 7.539191 & -1.575795 & -1.042844 \\
\hline $\mathrm{H}$ & 8.198721 & -0.173176 & -1.893467 \\
\hline $\mathrm{H}$ & 9.224870 & -1.104842 & -0.785349 \\
\hline C & 7.824562 & -0.597324 & 1.542190 \\
\hline $\mathrm{H}$ & 8.832770 & -0.980264 & 1.735212 \\
\hline $\mathrm{H}$ & 7.531670 & 0.036625 & 2.384899 \\
\hline
\end{tabular}




\section{Catalyst 2C 27}

\section{Datum}

Value

\begin{tabular}{lr}
\hline B3LYP-D3(BJ)/6-31G(d,p) Energy & -2165.164292 \\
\hline B3LYP-D3(BJ)/def2-TZVPP/IEF-PCM(DCM) Energy & -2165.899824 \\
\hline B3LYP-D3(BJ)/def2-TZVPP/IEF-PCM(DCM)//B3LYP-D3(BJ)/6-31G(d,p) Free Energy (Quasiharmonic) & -2165.021897 \\
\hline
\end{tabular}

Number of Imaginary Frequencies

Frequencies (Top 3 out of 321)
1. $\quad 10.2700 \mathrm{~cm}^{-1}$
2. $11.5834 \mathrm{~cm}^{-1}$
3. $13.2027 \mathrm{~cm}^{-1}$

\section{B3LYP-D3(BJ)/6-31G(d,p) Molecular Geometry in Cartesian Coordinates}

$\begin{array}{lrrr}\text { C } & 0.285452 & -3.645676 & 3.844302 \\ \text { C } & 0.415865 & -3.047692 & 2.611690 \\ \text { C } & -0.658156 & -2.309244 & 2.049426 \\ \text { C } & -1.876993 & -2.188052 & 2.787560 \\ \text { C } & -1.978006 & -2.816745 & 4.056522 \\ \text { C } & -0.921760 & -3.529783 & 4.573832 \\ \text { H } & 1.112818 & -4.210706 & 4.261842 \\ \text { H } & 1.339836 & -3.133902 & 2.051529 \\ \text { C } & -0.556298 & -1.671570 & 0.775165 \\ \text { C } & -2.949370 & -1.446187 & 2.235558 \\ \text { H } & -2.906749 & -2.721978 & 4.611837 \\ \text { H } & -1.010206 & -4.005643 & 5.545523 \\ \text { C } & -2.863257 & -0.848901 & 0.994587 \\ \text { C } & -1.642334 & -0.983815 & 0.264629 \\ \text { H } & -3.874568 & -1.364056 & 2.797961 \\ \text { C } & 1.465958 & -4.937360 & -2.663452 \\ \text { C } & 2.412092 & -3.954576 & -2.490899 \\ \text { C } & 2.181170 & -2.872964 & -1.599930 \\ \text { C } & 0.947058 & -2.810721 & -0.884412 \\ \text { C } & -0.011046 & -3.839648 & -1.086046 \\ \text { C } & 0.243367 & -4.877870 & -1.952415\end{array}$




\begin{tabular}{|c|c|c|c|}
\hline H & 4.069224 & -1.897163 & -1.955447 \\
\hline $\mathrm{H}$ & 1.652730 & -5.761342 & -3.345278 \\
\hline $\mathrm{H}$ & 3.353513 & -3.990238 & -3.032030 \\
\hline C & 3.137860 & -1.852086 & -1.398686 \\
\hline C & 0.729619 & -1.717323 & 0.009967 \\
\hline H & -0.952250 & -3.793123 & -0.550842 \\
\hline $\mathrm{H}$ & -0.499243 & -5.656760 & -2.095277 \\
\hline C & 1.686902 & -0.724979 & 0.194493 \\
\hline C & 2.922978 & -0.794168 & -0.535698 \\
\hline C & 1.437769 & 0.388928 & 1.201320 \\
\hline $\mathrm{H}$ & 0.770293 & 0.019335 & 1.988328 \\
\hline H & 2.384786 & 0.659832 & 1.673307 \\
\hline C & -1.463514 & -0.278342 & -1.046471 \\
\hline 0 & -1.157314 & 0.892543 & -1.167304 \\
\hline 0 & -1.643013 & -1.083847 & -2.109858 \\
\hline $\mathrm{H}$ & -1.446158 & -0.549277 & -2.899306 \\
\hline 0 & 0.930291 & 1.594226 & 0.639447 \\
\hline $\mathrm{H}$ & 0.116515 & 1.399427 & 0.142565 \\
\hline C & 4.007324 & 0.210954 & -0.370814 \\
\hline C & 3.745837 & 1.583542 & -0.455806 \\
\hline C & 5.323516 & -0.225977 & -0.162277 \\
\hline C & 4.772808 & 2.520869 & -0.319336 \\
\hline $\mathrm{H}$ & 2.724222 & 1.901147 & -0.604618 \\
\hline C & 6.376148 & 0.683534 & -0.033279 \\
\hline $\mathrm{H}$ & 5.504359 & -1.290520 & -0.080983 \\
\hline C & 6.074056 & 2.048678 & -0.113260 \\
\hline $\mathrm{H}$ & 6.878968 & 2.767843 & -0.002926 \\
\hline C & -4.006912 & -0.074272 & 0.451765 \\
\hline C & -4.490420 & -0.320236 & -0.838624 \\
\hline C & -4.618043 & 0.905709 & 1.243071 \\
\hline C & -5.561532 & 0.412211 & -1.356563 \\
\hline $\mathrm{H}$ & -4.028465 & -1.102016 & -1.425209 \\
\hline C & -5.697173 & 1.650876 & 0.761062 \\
\hline $\mathrm{H}$ & -4.215518 & 1.091681 & 2.230658 \\
\hline C & -6.144542 & 1.387415 & -0.539508 \\
\hline $\mathrm{H}$ & -6.974529 & 1.965748 & -0.930861 \\
\hline C & -6.385163 & 2.743270 & 1.591963 \\
\hline C & -6.100044 & 0.187229 & -2.776253 \\
\hline C & -7.879755 & 2.392677 & 1.756274 \\
\hline $\mathrm{H}$ & -8.387461 & 2.318558 & 0.790653 \\
\hline $\mathrm{H}$ & -7.999694 & 1.434576 & 2.271639 \\
\hline $\mathrm{H}$ & -8.389117 & 3.163908 & 2.344037 \\
\hline C & -6.250897 & 4.097505 & 0.862687 \\
\hline $\mathrm{H}$ & -6.737047 & 4.891094 & 1.440378 \\
\hline $\mathrm{H}$ & -5.197842 & 4.364418 & 0.731695 \\
\hline $\mathrm{H}$ & -6.715028 & 4.071491 & -0.127111 \\
\hline C & -5.767115 & 2.886249 & 2.992138 \\
\hline $\mathrm{H}$ & -4.710905 & 3.167879 & 2.942142 \\
\hline $\mathrm{H}$ & -6.292902 & 3.668338 & 3.548147 \\
\hline $\mathrm{H}$ & -5.848480 & 1.958333 & 3.567005 \\
\hline C & -5.347258 & -0.933289 & -3.512007 \\
\hline $\mathrm{H}$ & -4.279349 & -0.712339 & -3.603083 \\
\hline $\mathrm{H}$ & -5.451403 & -1.895213 & -3.000617 \\
\hline H & -5.751883 & -1.047721 & -4.522159 \\
\hline
\end{tabular}




\begin{tabular}{|c|c|c|c|}
\hline C & -5.939126 & 1.491784 & -3.586590 \\
\hline $\mathrm{H}$ & -4.885992 & 1.783599 & -3.646260 \\
\hline $\mathrm{H}$ & -6.317192 & 1.357143 & -4.605848 \\
\hline $\mathrm{H}$ & -6.488837 & 2.320329 & -3.131668 \\
\hline C & -7.593509 & -0.196383 & -2.708390 \\
\hline $\mathrm{H}$ & -7.731832 & -1.117940 & -2.134495 \\
\hline $\mathrm{H}$ & -8.191551 & 0.586284 & -2.233599 \\
\hline $\mathrm{H}$ & -7.992034 & -0.357125 & -3.715937 \\
\hline C & 7.828043 & 0.241960 & 0.202427 \\
\hline C & 4.504685 & 4.031810 & -0.363815 \\
\hline C & 3.027853 & 4.348642 & -0.653706 \\
\hline $\mathrm{H}$ & 2.723853 & 3.971362 & -1.635384 \\
\hline $\mathrm{H}$ & 2.359987 & 3.912221 & 0.093881 \\
\hline $\mathrm{H}$ & 2.879330 & 5.433248 & -0.653727 \\
\hline C & 5.368379 & 4.681889 & -1.465134 \\
\hline $\mathrm{H}$ & 6.437407 & 4.534769 & -1.286272 \\
\hline $\mathrm{H}$ & 5.131068 & 4.256467 & -2.445283 \\
\hline $\mathrm{H}$ & 5.182624 & 5.760696 & -1.506861 \\
\hline C & 4.873441 & 4.642531 & 1.005750 \\
\hline $\mathrm{H}$ & 4.264982 & 4.200878 & 1.800874 \\
\hline $\mathrm{H}$ & 5.925338 & 4.472306 & 1.253696 \\
\hline $\mathrm{H}$ & 4.698767 & 5.724127 & 1.001437 \\
\hline C & 8.713320 & 0.767491 & -0.948112 \\
\hline $\mathrm{H}$ & 8.378130 & 0.365708 & -1.909440 \\
\hline H & 8.684400 & 1.858465 & -1.014409 \\
\hline $\mathrm{H}$ & 9.756059 & 0.467745 & -0.795859 \\
\hline $\mathrm{C}$ & 7.971570 & -1.287481 & 0.258569 \\
\hline $\mathrm{H}$ & 9.020708 & -1.553739 & 0.420200 \\
\hline H & 7.389247 & -1.719018 & 1.078582 \\
\hline $\mathrm{H}$ & 7.648917 & -1.758289 & -0.675348 \\
\hline $\mathrm{C}$ & 8.328673 & 0.823445 & 1.542131 \\
\hline $\mathrm{H}$ & 7.714165 & 0.464633 & 2.373672 \\
\hline H & 9.365648 & 0.521750 & 1.726660 \\
\hline $\mathrm{H}$ & 8.291517 & 1.916217 & 1.547172 \\
\hline
\end{tabular}

\section{Catalyst 2C 28}

Datum

Value

\begin{tabular}{lr} 
B3LYP-D3(BJ)/6-31G(d,p) Energy & -2165.165329 \\
\hline B3LYP-D3(BJ)/def2-TZVPP/IEF-PCM(DCM) Energy & -2165.900116 \\
\hline B3LYP-D3(BJ)/def2-TZVPP/IEF-PCM(DCM)//B3LYP-D3(BJ)/6-31G(d,p) Free Energy (Quasiharmonic) & -2165.021868 \\
\hline
\end{tabular}

Number of Imaginary Frequencies

Frequencies (Top 3 out of 321) 

1.
$10.7663 \mathrm{~cm}^{-1}$
2.
$11.2597 \mathrm{~cm}^{-1}$
3.
$13.3553 \mathrm{~cm}^{-1}$

\section{B3LYP-D3(BJ)/6-31G(d,p) Molecular Geometry in Cartesian Coordinates}

C

C

C

C

C

C

$\mathrm{H}$

$\mathrm{H}$

C

C

$\mathrm{H}$

$\mathrm{H}$

C

C

$\mathrm{H}$

C

C

C

C

C

C

$\mathrm{H}$

$\mathrm{H}$

$\mathrm{H}$

C

C

$\mathrm{H}$

$\mathrm{H}$

C

C

C

$\mathrm{H}$

$\mathrm{H}$

C

0

0

$\mathrm{H}$

0

$\mathrm{H}$

C

C

C

C

$\mathrm{H}$

$$
-0.264325
$$

$-0.397644$

0.674052

1.893814

1.997753

0.943587

$-1.089962$

$-1.322184$

0.568377

2. 964371

2.927124

1.034299

2.874563

1.652708

3.890408

$-1.441404$

$-2.391484$

$-2.165137$

$-0.931403$

0.030752

$-0.219318$

$-4.056863$

$-1.624658$

$-3.332611$

$-3.126087$

$-0.718459$

0.971537

0.526280

$-1.679606$

$-2.916423$

$-1.430009$

$-2.375372$

$-0.759471$

1.472533

1.157726

1.661940

1.471084

$-0.924697$

$-0.111282$

$-4.005768$

$-3.752717$

$-5.327510$

$-4.786029$

$-2.731002$
$-3.509796$

$-2.950298$

$-2.227260$

$-2.081289$

$-2.670293$

$-3.369101$

$-4.063387$

$-3.055513$

$-1.628748$

$-1.355155$

$-2.556854$

$-3.814674$

$-0.798105$

$-0.956095$

$-1.253279$

$-4.989234$

$-4.006289$

$-2.901946$

$-2.816871$

$-3.846866$

$-4.907317$

$-1.944041$

$-5.830644$

$-4.059152$

$-1.880652$

$-1.699981$

$-3.783478$

$-5.686727$

$-0.706841$

$-0.800214$

0.435407

0.720332

0.090652

$-0.287885$

0.877076

$-1.118742$

$-0.603429$

1.624316

1.416790

0.203282

1.571783

$-0.235737$

2. 511717

1.899895
3.952209

2.701969

2.115745

2. 848064

4.135625

4.676140

4.388078

2.146005

0.822849

2. 271666

4.686357

5.661871

1.012790

0.289431

2.829544

$-2.530170$

$-2.382295$

$-1.518382$

$-0.804621$

$-0.980140$

$-1.820420$

$-1.898313$

$-3.191341$

$-2.922511$

$-1.342431$

0.061185

$-0.445919$

$-1.943516$

0.220572

$-0.506181$

1.194864

1.661458

1.990200

$-1.041063$

$-1.192991$

$-2.083125$

$-2.886869$

0.595809

0.103098

$-0.367440$

$-0.460578$

$-0.172305$

$-0.344953$

$-0.600217$ 


\begin{tabular}{|c|c|c|c|}
\hline C & -6.381445 & 0.669898 & -0.069365 \\
\hline $\mathrm{H}$ & -5.506125 & -1.299838 & -0.081436 \\
\hline C & -6.085611 & 2.041371 & -0.156855 \\
\hline H & -6.897576 & 2.751940 & -0.067042 \\
\hline C & 4.015025 & -0.040216 & 0.440396 \\
\hline C & 4.504177 & -0.336468 & -0.835590 \\
\hline C & 4.618910 & 0.979505 & 1.182771 \\
\hline C & 5.567628 & 0.382992 & -1.388233 \\
\hline $\mathrm{H}$ & 4.052253 & -1.148407 & -1.392051 \\
\hline C & 5.692316 & 1.713084 & 0.668185 \\
\hline $\mathrm{H}$ & 4.217394 & 1.211295 & 2.163512 \\
\hline C & 6.145830 & 1.400488 & -0.619918 \\
\hline $\mathrm{H}$ & 6.967379 & 1.965151 & -1.035194 \\
\hline C & 6.315328 & 2.833597 & 1.513443 \\
\hline C & 6.054773 & 0.031125 & -2.801048 \\
\hline C & 6.833334 & 2.241106 & 2.841811 \\
\hline $\mathrm{H}$ & 6.028894 & 1.776138 & 3.418254 \\
\hline H & 7.596722 & 1.479048 & 2.655887 \\
\hline $\mathrm{H}$ & 7.277840 & 3.027105 & 3.461744 \\
\hline C & 5.240815 & 3.901800 & 1.811110 \\
\hline $\mathrm{H}$ & 5.665071 & 4.710397 & 2.416186 \\
\hline $\mathrm{H}$ & 4.855802 & 4.334090 & 0.882509 \\
\hline $\mathrm{H}$ & 4.393322 & 3.481644 & 2.359233 \\
\hline C & 7.492788 & 3.519336 & 0.801800 \\
\hline $\mathrm{H}$ & 7.180839 & 3.992916 & -0.134217 \\
\hline H & 7.905923 & 4.301173 & 1.446256 \\
\hline $\mathrm{H}$ & 8.298627 & 2.813204 & 0.577885 \\
\hline C & 6.564556 & -1.426048 & -2.815896 \\
\hline H & 7.400065 & -1.551699 & -2.120233 \\
\hline $\mathrm{H}$ & 5.779716 & -2.130579 & -2.527287 \\
\hline $\mathrm{H}$ & 6.909774 & -1.699570 & -3.818899 \\
\hline C & 7.194000 & 0.947847 & -3.274284 \\
\hline $\mathrm{H}$ & 8.074867 & 0.865299 & -2.630015 \\
\hline H & 7.497177 & 0.665194 & -4.286972 \\
\hline $\mathrm{H}$ & 6.884808 & 1.997411 & -3.300286 \\
\hline C & 4.877052 & 0.172317 & -3.790610 \\
\hline H & 4.053028 & -0.497807 & -3.530743 \\
\hline $\mathrm{H}$ & 4.491487 & 1.196642 & -3.790604 \\
\hline $\mathrm{H}$ & 5.201513 & -0.073181 & -4.807549 \\
\hline C & -7.835674 & 0.226715 & 0.147325 \\
\hline C & -4.435889 & 4.004211 & -0.412546 \\
\hline C & -3.762824 & 4.310409 & -1.767770 \\
\hline H & -2.844758 & 3.732316 & -1.900143 \\
\hline $\mathrm{H}$ & -4.434910 & 4.072785 & -2.598793 \\
\hline H & -3.502258 & 5.372645 & -1.830978 \\
\hline C & -3.453991 & 4.337366 & 0.733448 \\
\hline $\mathrm{H}$ & -3.921022 & 4.146813 & 1.705466 \\
\hline $\mathrm{H}$ & -2.542519 & 3.736747 & 0.675919 \\
\hline $\mathrm{H}$ & -3.171614 & 5.395567 & 0.694265 \\
\hline C & -5.674288 & 4.903134 & -0.273642 \\
\hline $\mathrm{H}$ & -6.397995 & 4.724387 & -1.075726 \\
\hline H & -6.180068 & 4.750051 & 0.685081 \\
\hline H & -5.374183 & 5.954286 & -0.325222 \\
\hline c & -8.703670 & 0.737800 & -1.022851 \\
\hline
\end{tabular}




\begin{tabular}{|llll|}
$H$ & -8.677149 & 1.828108 & -1.099880 \\
$H$ & -8.351736 & 0.326997 & -1.974351 \\
$H$ & -9.747819 & 0.436287 & -0.884335 \\
$\mathrm{C}$ & -8.360216 & 0.820184 & 1.472634 \\
$\mathrm{H}$ & -9.399209 & 0.516990 & 1.642844 \\
$\mathrm{H}$ & -7.758602 & 0.471398 & 2.317781 \\
$\mathrm{H}$ & -8.326574 & 1.913035 & 1.467357 \\
$\mathrm{C}$ & -7.976254 & -1.302432 & 0.216712 \\
$\mathrm{H}$ & -7.406355 & -1.724008 & 1.050530 \\
$\mathrm{H}$ & -9.027217 & -1.569946 & 0.363806 \\
$\mathrm{H}$ & -7.637041 & -1.781758 & -0.706956 \\
& & & \\
\hline
\end{tabular}

\section{Catalyst 2C 29}

\section{Datum}

Value

B3LYP-D3(BJ)/6-31G(d,p) Energy $-2165.164831$

B3LYP-D3(BJ)/def2-TZVPP/IEF-PCM(DCM) Energy $-2165.899976$

B3LYP-D3(BJ)/def2-TZVPP/IEF-PCM(DCM)//B3LYP-D3(BJ)/6-31G(d,p) Free Energy (Quasiharmonic) $-2165.021798$

Number of Imaginary Frequencies

Frequencies (Top 3 out of 321)

$\begin{array}{ll}\text { 1. } & 10.7354 \mathrm{~cm}^{-1} \\ \text { 2. } & 11.4765 \mathrm{~cm}^{-1} \\ \text { 3. } & 13.0937 \mathrm{~cm}^{-1}\end{array}$

B3LYP-D3(BJ)/6-31G(d,p) Molecular Geometry in Cartesian Coordinates

C

C

C

C

C

C

$\mathrm{H}$

$\mathrm{H}$

C

C

$\mathrm{H}$

$\mathrm{H}$

C

C

$\mathrm{H}$
0.333340

0.457661

$-0.623521$

$-1.843210$

$-1.937813$

$-0.874666$

1.166190

1.382248

$-0.528162$

$-2.922672$

$-2.867203$

$-0.958233$

$-2.842753$

$-1.620988$

$-3.848202$
$-3.585761$

$-3.000462$

$-2.278591$

$-2.160678$

$-2.775995$

$-3.473130$

$-4.138143$

$-3.084173$

$-1.654237$

$-1.435000$

$-2.683777$

$-3.938833$

$-0.851065$

$-0.982887$

$-1.354752$

\subsection{2}

2.641905

2.071881

2.809163

4.085149

4.609936

4.304717

2.082389

0.790543

2. 249495

4.639801

5.586959

1.001789

0.272703

2.811618 


\begin{tabular}{|c|c|c|c|}
\hline C & 1.521440 & -4.929535 & -2.622903 \\
\hline C & 2.458923 & -3.937095 & -2.458710 \\
\hline C & 2.219195 & -2.851022 & -1.575511 \\
\hline C & 0.985133 & -2.794279 & -0.859453 \\
\hline C & 0.036142 & -3.833320 & -1.052221 \\
\hline C & 0.299086 & -4.875718 & -1.910969 \\
\hline $\mathrm{H}$ & 4.098554 & -1.861997 & -1.939337 \\
\hline $\mathrm{H}$ & 1.714888 & -5.756883 & -3.298755 \\
\hline $\mathrm{H}$ & 3.400103 & -3.968385 & -3.000521 \\
\hline C & 3.167296 & -1.820761 & -1.382115 \\
\hline C & 0.758289 & -1.695529 & 0.025914 \\
\hline $\mathrm{H}$ & -0.904938 & -3.791178 & -0.516443 \\
\hline $\mathrm{H}$ & -0.436575 & -5.662358 & -2.047127 \\
\hline C & 1.706907 & -0.693303 & 0.202237 \\
\hline C & 2.944259 & -0.758666 & -0.526324 \\
\hline C & 1.444558 & 0.428989 & 1.196245 \\
\hline $\mathrm{H}$ & 0.779121 & 0.062521 & 1.986158 \\
\hline $\mathrm{H}$ & 2.386931 & 0.717201 & 1.666897 \\
\hline C & -1.449621 & -0.290929 & -1.046551 \\
\hline 0 & -1.150712 & 0.880476 & -1.180957 \\
\hline 0 & -1.626667 & -1.108881 & -2.100587 \\
\hline $\mathrm{H}$ & -1.435464 & -0.581750 & -2.896413 \\
\hline 0 & 0.924544 & 1.621738 & 0.617930 \\
\hline $\mathrm{H}$ & 0.112664 & 1.413144 & 0.123182 \\
\hline C & 4.021476 & 0.255072 & -0.368185 \\
\hline C & 3.752290 & 1.625280 & -0.442152 \\
\hline C & 5.343863 & -0.165682 & -0.174149 \\
\hline C & 4.771599 & 2.572391 & -0.308465 \\
\hline $\mathrm{H}$ & 2.726823 & 1.942187 & -0.580138 \\
\hline C & 6.391260 & 0.752213 & -0.052538 \\
\hline $\mathrm{H}$ & 5.542231 & -1.229752 & -0.096982 \\
\hline C & 6.081974 & 2.116996 & -0.120796 \\
\hline $\mathrm{H}$ & 6.878018 & 2.840044 & -0.018542 \\
\hline C & -3.993365 & -0.092179 & 0.451394 \\
\hline C & -4.610231 & 0.893180 & 1.231517 \\
\hline C & -4.477416 & -0.358027 & -0.834840 \\
\hline C & -5.695752 & 1.624218 & 0.742274 \\
\hline $\mathrm{H}$ & -4.207262 & 1.094601 & 2.215895 \\
\hline C & -5.555059 & 0.359769 & -1.359741 \\
\hline $\mathrm{H}$ & -4.010702 & -1.143625 & -1.412482 \\
\hline C & -6.143702 & 1.340897 & -0.553907 \\
\hline $\mathrm{H}$ & -6.978784 & 1.907969 & -0.950841 \\
\hline C & -6.094905 & 0.112693 & -2.775252 \\
\hline C & -6.390215 & 2.721998 & 1.560602 \\
\hline C & -5.945839 & 1.408050 & -3.602435 \\
\hline $\mathrm{H}$ & -6.500901 & 2.238074 & -3.156804 \\
\hline $\mathrm{H}$ & -4.895115 & 1.707154 & -3.668256 \\
\hline $\mathrm{H}$ & -6.325093 & 1.257595 & -4.619033 \\
\hline C & -7.585220 & -0.281489 & -2.699058 \\
\hline $\mathrm{H}$ & -7.984727 & -0.458141 & -3.703545 \\
\hline $\mathrm{H}$ & -7.715155 & -1.196682 & -2.113147 \\
\hline $\mathrm{H}$ & -8.188231 & 0.502549 & -2.232895 \\
\hline C & -5.335140 & -1.011269 & -3.498462 \\
\hline $\mathrm{H}$ & -5.430854 & -1.967399 & -2.974709 \\
\hline
\end{tabular}




\begin{tabular}{|c|c|c|c|}
\hline $\mathrm{H}$ & -5.741038 & -1.141561 & -4.506174 \\
\hline $\mathrm{H}$ & -4.269151 & -0.783341 & -3.594733 \\
\hline C & -7.881587 & 2.361844 & 1.733312 \\
\hline $\mathrm{H}$ & -8.391005 & 2.270964 & 0.770027 \\
\hline $\mathrm{H}$ & -7.992711 & 1.409733 & 2.261591 \\
\hline $\mathrm{H}$ & -8.395605 & 3.136746 & 2.312120 \\
\hline C & -6.268453 & 4.067588 & 0.813322 \\
\hline $\mathrm{H}$ & -6.734851 & 4.025082 & -0.174842 \\
\hline $\mathrm{H}$ & -6.759377 & 4.864812 & 1.381893 \\
\hline $\mathrm{H}$ & -5.217867 & 4.341044 & 0.676139 \\
\hline C & -5.769833 & 2.888194 & 2.957161 \\
\hline $\mathrm{H}$ & -5.842681 & 1.967376 & 3.544446 \\
\hline $\mathrm{H}$ & -4.715941 & 3.177203 & 2.900770 \\
\hline $\mathrm{H}$ & -6.300255 & 3.673512 & 3.504133 \\
\hline C & 7.823256 & 0.239778 & 0.163886 \\
\hline C & 4.405241 & 4.062340 & -0.355160 \\
\hline C & 5.632872 & 4.973625 & -0.200486 \\
\hline $\mathrm{H}$ & 6.138496 & 4.813167 & 0.757066 \\
\hline $\mathrm{H}$ & 6.360558 & 4.815164 & -1.003188 \\
\hline $\mathrm{H}$ & 5.320183 & 6.021737 & -0.237792 \\
\hline C & 3.417348 & 4.368145 & 0.793349 \\
\hline $\mathrm{H}$ & 2.513170 & 3.757671 & 0.725438 \\
\hline $\mathrm{H}$ & 3.884590 & 4.169174 & 1.763588 \\
\hline $\mathrm{H}$ & 3.122830 & 5.423509 & 0.768693 \\
\hline C & 3.731579 & 4.381430 & -1.707149 \\
\hline $\mathrm{H}$ & 4.407561 & 4.162766 & -2.540226 \\
\hline $\mathrm{H}$ & 2.819797 & 3.795883 & -1.849739 \\
\hline $\mathrm{H}$ & 3.460255 & 5.441775 & -1.755560 \\
\hline C & 8.846512 & 1.382630 & 0.264722 \\
\hline $\mathrm{H}$ & 8.867760 & 1.988907 & -0.646218 \\
\hline $\mathrm{H}$ & 8.633985 & 2.043326 & 1.110896 \\
\hline $\mathrm{H}$ & 9.848503 & 0.967534 & 0.411371 \\
\hline C & 8.226066 & -0.663921 & -1.021392 \\
\hline $\mathrm{H}$ & 9.244573 & -1.043750 & -0.884540 \\
\hline $\mathrm{H}$ & 7.557997 & -1.524202 & -1.117937 \\
\hline $\mathrm{H}$ & 8.191986 & -0.106376 & -1.962628 \\
\hline C & 7.881327 & -0.576169 & 1.473312 \\
\hline $\mathrm{H}$ & 7.204046 & -1.434118 & 1.444885 \\
\hline $\mathrm{H}$ & 8.895459 & -0.954089 & 1.644136 \\
\hline $\mathrm{H}$ & 7.598465 & 0.044760 & 2.329047 \\
\hline
\end{tabular}

\section{Catalyst 2C 30}

Datum

Value

B3LYP-D3(BJ)/6-31G(d,p) Energy

$-2165.164574$

B3LYP-D3(BJ)/def2-TZVPP/IEF-PCM(DCM) Energy

$-2165.899749$

B3LYP-D3(BJ)/def2-TZVPP/IEF-PCM(DCM)//B3LYP-D3(BJ)/6-31G(d,p) Free Energy (Quasiharmonic) -2165.021741 
Frequencies (Top 3 out of 321)

$\begin{array}{ll}\text { 1. } & 10.3869 \mathrm{~cm}^{-1} \\ \text { 2. } & 11.3904 \mathrm{~cm}^{-1} \\ \text { 3. } & 13.2934 \mathrm{~cm}^{-1}\end{array}$

B3LYP-D3(BJ)/6-31G(d,p) Molecular Geometry in Cartesian Coordinates

\begin{tabular}{|c|c|c|c|}
\hline C & 0.243711 & -3.559240 & 3.912272 \\
\hline C & 0.378386 & -2.986279 & 2.668287 \\
\hline C & -0.690679 & -2.252146 & 2.091127 \\
\hline C & -1.909189 & -2.109195 & 2.826104 \\
\hline C & -2.014601 & -2.712222 & 4.107044 \\
\hline C & -0.963004 & -3.421558 & 4.638776 \\
\hline $\mathrm{H}$ & 1.067336 & -4.121209 & 4.341183 \\
\hline $\mathrm{H}$ & 1.302011 & -3.089161 & 2.110366 \\
\hline C & -0.583503 & -1.639614 & 0.804932 \\
\hline C & -2.977121 & -1.372133 & 2.258794 \\
\hline $\mathrm{H}$ & -2.943038 & -2.601024 & 4.659813 \\
\hline $\mathrm{H}$ & -1.054833 & -3.877805 & 5.619513 \\
\hline C & -2.885848 & -0.801128 & 1.006285 \\
\hline C & -1.665220 & -0.956038 & 0.280175 \\
\hline $\mathrm{H}$ & -3.902395 & -1.272944 & 2.818424 \\
\hline C & 1.413757 & -4.976663 & -2.579239 \\
\hline C & 2.367163 & -3.998193 & -2.423000 \\
\hline C & 2.144585 & -2.900509 & -1.549747 \\
\hline C & 0.911225 & -2.817467 & -0.835023 \\
\hline C & -0.054448 & -3.842564 & -1.019461 \\
\hline C & 0.191965 & -4.896630 & -1.868843 \\
\hline $\mathrm{H}$ & 4.039554 & -1.944567 & -1.921853 \\
\hline $\mathrm{H}$ & 1.594164 & -5.812996 & -3.247601 \\
\hline $\mathrm{H}$ & 3.308055 & -4.049586 & -2.963778 \\
\hline C & 3.108819 & -1.883535 & -1.365571 \\
\hline C & 0.702242 & -1.707899 & 0.041250 \\
\hline $\mathrm{H}$ & -0.994989 & -3.780549 & -0.484640 \\
\hline $\mathrm{H}$ & -0.556334 & -5.672361 & -1.998637 \\
\hline C & 1.666777 & -0.719642 & 0.209060 \\
\hline C & 2.902027 & -0.809915 & -0.520158 \\
\hline $\mathrm{C}$ & 1.425371 & 0.413171 & 1.196458 \\
\hline $\mathrm{H}$ & 0.755705 & 0.061773 & 1.989922 \\
\hline $\mathrm{H}$ & 2.374222 & 0.686092 & 1.663565 \\
\hline C & -1.483042 & -0.273184 & -1.042610 \\
\hline 0 & -1.166873 & 0.892922 & -1.181341 \\
\hline 0 & -1.672548 & -1.092497 & -2.093990 \\
\hline $\mathrm{H}$ & -1.479881 & -0.568601 & -2.891720 \\
\hline
\end{tabular}




\begin{tabular}{|c|c|c|c|}
\hline 0 & 0.925601 & 1.611663 & 0.613572 \\
\hline $\mathrm{H}$ & 0.110808 & 1.413347 & 0.119728 \\
\hline C & 3.993566 & 0.190073 & -0.372587 \\
\hline C & 3.741660 & 1.562849 & -0.480377 \\
\hline C & 5.306809 & -0.252584 & -0.157660 \\
\hline C & 4.775278 & 2.495083 & -0.360161 \\
\hline $\mathrm{H}$ & 2.722215 & 1.885063 & -0.634118 \\
\hline C & 6.365898 & 0.651523 & -0.044542 \\
\hline $\mathrm{H}$ & 5.480263 & -1.316873 & -0.058813 \\
\hline C & 6.073316 & 2.017249 & -0.146987 \\
\hline $\mathrm{H}$ & 6.883303 & 2.732499 & -0.049099 \\
\hline C & -4.023811 & -0.032724 & 0.443097 \\
\hline C & -4.513331 & -0.311684 & -0.836643 \\
\hline C & -4.625101 & 0.979397 & 1.197860 \\
\hline C & -5.574420 & 0.417680 & -1.380737 \\
\hline $\mathrm{H}$ & -4.063537 & -1.117983 & -1.402963 \\
\hline C & -5.696283 & 1.722300 & 0.692087 \\
\hline $\mathrm{H}$ & -4.223237 & 1.197911 & 2.181505 \\
\hline C & -6.150130 & 1.427095 & -0.599995 \\
\hline H & -6.969897 & 1.999196 & -1.008547 \\
\hline C & -6.316526 & 2.833907 & 1.551021 \\
\hline C & -6.061812 & 0.084944 & -2.798088 \\
\hline C & -6.836067 & 2.226513 & 2.872034 \\
\hline $\mathrm{H}$ & -7.601382 & 1.468729 & 2.676793 \\
\hline $\mathrm{H}$ & -6.032825 & 1.752476 & 3.442732 \\
\hline $\mathrm{H}$ & -7.278587 & 3.005993 & 3.501549 \\
\hline C & -7.492250 & 3.531268 & 0.847847 \\
\hline H & -7.903555 & 4.306087 & 1.501886 \\
\hline $\mathrm{H}$ & -7.179076 & 4.015622 & -0.082230 \\
\hline $\mathrm{H}$ & -8.299781 & 2.829917 & 0.615156 \\
\hline C & -5.239359 & 3.895689 & 1.861783 \\
\hline $\mathrm{H}$ & -4.853302 & 4.338453 & 0.938569 \\
\hline $\mathrm{H}$ & -5.661592 & 4.697792 & 2.476845 \\
\hline $\mathrm{H}$ & -4.392902 & 3.466651 & 2.404603 \\
\hline C & -4.882990 & 0.234506 & -3.785112 \\
\hline H & -4.493971 & 1.257435 & -3.772081 \\
\hline $\mathrm{H}$ & -4.061367 & -0.441543 & -3.533120 \\
\hline $\mathrm{H}$ & -5.207637 & 0.002784 & -4.805224 \\
\hline C & -7.197708 & 1.011224 & -3.260601 \\
\hline H & -6.885046 & 2.060005 & -3.273314 \\
\hline $\mathrm{H}$ & -7.501125 & 0.742204 & -4.276926 \\
\hline $\mathrm{H}$ & -8.079283 & 0.923561 & -2.617974 \\
\hline C & -6.576354 & -1.370249 & -2.831304 \\
\hline H & -5.794048 & -2.080819 & -2.550732 \\
\hline $\mathrm{H}$ & -7.412876 & -1.501728 & -2.137936 \\
\hline $\mathrm{H}$ & -6.921591 & -1.630270 & -3.837890 \\
\hline C & 7.814885 & 0.203693 & 0.197184 \\
\hline C & 4.517675 & 4.006902 & -0.429679 \\
\hline C & 3.043046 & 4.329149 & -0.724745 \\
\hline $\mathrm{H}$ & 2.736234 & 3.937593 & -1.699934 \\
\hline $\mathrm{H}$ & 2.372243 & 3.910035 & 0.030073 \\
\hline $\mathrm{H}$ & 2.902117 & 5.414613 & -0.742931 \\
\hline C & 5.385690 & 4.632686 & -1.541627 \\
\hline $\mathrm{H}$ & 6.453713 & 4.481231 & -1.360395 \\
\hline
\end{tabular}




\begin{tabular}{|lrrr|}
$H$ & 5.145323 & 4.192761 & -2.514601 \\
$H$ & 5.207349 & 5.711907 & -1.601179 \\
$\mathrm{C}$ & 4.890891 & 4.637590 & 0.929594 \\
$\mathrm{H}$ & 4.279521 & 4.213361 & 1.731944 \\
$\mathrm{H}$ & 5.941626 & 4.464158 & 1.180237 \\
$\mathrm{H}$ & 4.723712 & 5.720150 & 0.907423 \\
$\mathrm{C}$ & 8.702598 & 0.703719 & -0.962806 \\
$\mathrm{H}$ & 8.363506 & 0.288356 & -1.916968 \\
$\mathrm{H}$ & 8.681316 & 1.793610 & -1.047254 \\
$\mathrm{H}$ & 9.743358 & 0.399174 & -0.806611 \\
$\mathrm{C}$ & 7.947688 & -1.325595 & 0.278585 \\
$\mathrm{H}$ & 8.995088 & -1.596548 & 0.443641 \\
$\mathrm{H}$ & 7.363194 & -1.739369 & 1.106178 \\
$\mathrm{H}$ & 7.620765 & -1.809534 & -0.647100 \\
$\mathrm{C}$ & 8.321015 & 0.803791 & 1.526562 \\
$\mathrm{H}$ & 7.704854 & 0.463179 & 2.364510 \\
$\mathrm{H}$ & 9.356025 & 0.497867 & 1.715108 \\
$\mathrm{H}$ & 8.291595 & 1.896731 & 1.513494 \\
& & & \\
\hline
\end{tabular}

\section{Catalyst 2C 31}

\section{Datum}

Value

B3LYP-D3(BJ)/6-31G(d,p) Energy

$-2165.163758$

B3LYP-D3(BJ)/def2-TZVPP/IEF-PCM(DCM) Energy

$-2165.899698$

B3LYP-D3(BJ)/def2-TZVPP/IEF-PCM(DCM)//B3LYP-D3(BJ)/6-31G(d,p) Free Energy (Quasiharmonic)

$-2165.021596$

Number of Imaginary Frequencies

Frequencies (Top 3 out of 321)

$\begin{array}{ll}\text { 1. } & 10.0121 \mathrm{~cm}^{-1} \\ \text { 2. } & 12.5981 \mathrm{~cm}^{-1} \\ \text { 3. } & 14.8725 \mathrm{~cm}^{-1}\end{array}$

B3LYP-D3(BJ)/6-31G(d,p) Molecular Geometry in Cartesian Coordinates

C

C

C

C

C

C

$\mathrm{H}$

$\mathrm{H}$
0.235871

0.361755

$-0.720417$

$-1.949301$

$-2.044794$

$-0.977198$

1.071949

1.290725
$-4.117410$

$-3.274200$

$-2.449665$

$-2.517087$

$-3.394093$

$-4.177550$

$-4.741790$

$-3.228427$

\subsection{8}

2.002742

1.596567

2.324880

3. 436839

3.808777

3. 381992

1. 446547 


\begin{tabular}{|c|c|c|c|}
\hline C & -0.622703 & -1.562337 & 0.478213 \\
\hline C & -3.035359 & -1.699967 & 1.927351 \\
\hline $\mathrm{H}$ & -2.979797 & -3.434682 & 3.988195 \\
\hline $\mathrm{H}$ & -1.062485 & -4.845848 & 4.659968 \\
\hline C & -2.950478 & -0.853698 & 0.842776 \\
\hline C & -1.722890 & -0.806576 & 0.108531 \\
\hline $\mathrm{H}$ & -3.951103 & -1.724443 & 2.510331 \\
\hline C & 0.986017 & -4.035015 & -3.745952 \\
\hline C & 2.042599 & -3.244425 & -3.360402 \\
\hline C & 1.942082 & -2.403907 & -2.220130 \\
\hline C & 0.723884 & -2.383606 & -1.472997 \\
\hline C & -0.349548 & -3.212631 & -1.897693 \\
\hline C & -0.220457 & -4.017089 & -3.006133 \\
\hline $\mathrm{H}$ & 3.931575 & -1.593406 & -2.382520 \\
\hline $\mathrm{H}$ & 1.072385 & -4.673777 & -4.619524 \\
\hline $\mathrm{H}$ & 2.972823 & -3.249473 & -3.921614 \\
\hline C & 3.013934 & -1.583638 & -1.801770 \\
\hline C & 0.640392 & -1.533454 & -0.326711 \\
\hline $\mathrm{H}$ & -1.280662 & -3.196772 & -1.344035 \\
\hline $\mathrm{H}$ & -1.052034 & -4.640241 & -3.319598 \\
\hline C & 1.707957 & -0.731825 & 0.061517 \\
\hline C & 2.924702 & -0.752704 & -0.700770 \\
\hline C & 1.559412 & 0.163745 & 1.277090 \\
\hline $\mathrm{H}$ & 0.876451 & -0.301527 & 1.996119 \\
\hline $\mathrm{H}$ & 2.524960 & 0.291957 & 1.769342 \\
\hline C & -1.685930 & -0.057434 & -1.188952 \\
\hline 0 & -2.311718 & -0.349195 & -2.177931 \\
\hline 0 & -0.841002 & 1.016321 & -1.161539 \\
\hline $\mathrm{H}$ & -0.834552 & 1.392151 & -2.058736 \\
\hline 0 & 1.108696 & 1.480097 & 0.948366 \\
\hline $\mathrm{H}$ & 0.369225 & 1.388597 & 0.327952 \\
\hline C & 4.111215 & 0.067033 & -0.335833 \\
\hline C & 3.997419 & 1.431459 & -0.068242 \\
\hline C & 5.381039 & -0.534511 & -0.290279 \\
\hline C & 5.118687 & 2.203882 & 0.263737 \\
\hline $\mathrm{H}$ & 3.014492 & 1.883919 & -0.100076 \\
\hline C & 6.520025 & 0.206073 & 0.020457 \\
\hline $\mathrm{H}$ & 5.451226 & -1.597906 & -0.482085 \\
\hline C & 6.363494 & 1.575385 & 0.296947 \\
\hline $\mathrm{H}$ & 7.242026 & 2.154940 & 0.550741 \\
\hline C & -4.088846 & 0.021441 & 0.475009 \\
\hline C & -3.877727 & 1.377349 & 0.225651 \\
\hline C & -5.388333 & -0.499365 & 0.387653 \\
\hline C & -4.931802 & 2.223694 & -0.140216 \\
\hline $\mathrm{H}$ & -2.876174 & 1.778718 & 0.329950 \\
\hline C & -6.465295 & 0.315519 & 0.042757 \\
\hline $\mathrm{H}$ & -5.529850 & -1.557682 & 0.566481 \\
\hline C & -6.210818 & 1.672365 & -0.222211 \\
\hline $\mathrm{H}$ & -7.041162 & 2.306840 & -0.504020 \\
\hline C & -7.900549 & -0.216412 & -0.074757 \\
\hline C & -4.635780 & 3.700360 & -0.434439 \\
\hline C & -7.995326 & -1.715283 & 0.252789 \\
\hline $\mathrm{H}$ & -7.394992 & -2.318796 & -0.434893 \\
\hline $\mathrm{H}$ & -7.665007 & -1.927746 & 1.274487 \\
\hline
\end{tabular}




\begin{tabular}{|c|c|c|c|}
\hline H & -9.034501 & -2.046341 & 0.164164 \\
\hline $\mathrm{C}$ & -8.406966 & -0.003891 & -1.517819 \\
\hline $\mathrm{H}$ & -9.431233 & -0.378320 & -1.620913 \\
\hline $\mathrm{H}$ & -8.407805 & 1.053907 & -1.794719 \\
\hline $\mathrm{H}$ & -7.773017 & -0.536603 & -2.233181 \\
\hline C & -8.810386 & 0.551429 & 0.908434 \\
\hline H & -8.818964 & 1.624577 & 0.699080 \\
\hline $\mathrm{H}$ & -9.841132 & 0.187861 & 0.836301 \\
\hline $\mathrm{H}$ & -8.470436 & 0.414399 & 1.939738 \\
\hline C & -3.588422 & 3.787349 & -1.566914 \\
\hline $\mathrm{H}$ & -2.651187 & 3.298804 & -1.286162 \\
\hline $\mathrm{H}$ & -3.959721 & 3.304363 & -2.476099 \\
\hline $\mathrm{H}$ & -3.364362 & 4.834072 & -1.798948 \\
\hline C & -4.073266 & 4.370871 & 0.837219 \\
\hline $\mathrm{H}$ & -3.153245 & 3.886626 & 1.175786 \\
\hline $\mathrm{H}$ & -3.846257 & 5.424943 & 0.644163 \\
\hline $\mathrm{H}$ & -4.798733 & 4.321130 & 1.655155 \\
\hline C & -5.889511 & 4.471210 & -0.876562 \\
\hline $\mathrm{H}$ & -6.324759 & 4.047259 & -1.786976 \\
\hline $\mathrm{H}$ & -6.658361 & 4.474668 & -0.097562 \\
\hline $\mathrm{H}$ & -5.626912 & 5.512455 & -1.086634 \\
\hline C & 7.921535 & -0.418246 & 0.084745 \\
\hline C & 4.921652 & 3.691355 & 0.585666 \\
\hline C & 3.949022 & 3.820926 & 1.779445 \\
\hline $\mathrm{H}$ & 2.980159 & 3.361165 & 1.567733 \\
\hline H & 4.364819 & 3.334062 & 2.667714 \\
\hline $\mathrm{H}$ & 3.781539 & 4.877186 & 2.018473 \\
\hline C & 4.318855 & 4.403125 & -0.644525 \\
\hline $\mathrm{H}$ & 4.986175 & 4.319872 & -1.508403 \\
\hline $\mathrm{H}$ & 3.352830 & 3.973556 & -0.922413 \\
\hline H & 4.164904 & 5.466691 & -0.431519 \\
\hline C & 6.239938 & 4.390938 & 0.951652 \\
\hline $\mathrm{H}$ & 6.704722 & 3.940765 & 1.834610 \\
\hline $\mathrm{H}$ & 6.961110 & 4.355390 & 0.128581 \\
\hline $\mathrm{H}$ & 6.047699 & 5.444024 & 1.178955 \\
\hline C & 7.908272 & -1.918885 & -0.247978 \\
\hline $\mathrm{H}$ & 7.294363 & -2.485064 & 0.459334 \\
\hline $\mathrm{H}$ & 7.528471 & -2.105902 & -1.257301 \\
\hline H & 8.926381 & -2.316974 & -0.196696 \\
\hline C & 8.494697 & -0.244140 & 1.507748 \\
\hline $\mathrm{H}$ & 9.497570 & -0.680467 & 1.572120 \\
\hline H & 8.569531 & 0.810317 & 1.787044 \\
\hline $\mathrm{H}$ & 7.856950 & -0.740944 & 2.245526 \\
\hline C & 8.842874 & 0.292437 & -0.930164 \\
\hline $\mathrm{H}$ & 8.927211 & 1.362129 & -0.720135 \\
\hline $\mathrm{H}$ & 9.850400 & -0.136503 & -0.896871 \\
\hline H & 8.456619 & 0.181103 & -1.948172 \\
\hline
\end{tabular}


Frequencies (Top 3 out of 321)

$\begin{array}{ll}\text { 1. } & 10.4632 \mathrm{~cm}^{-1} \\ \text { 2. } & 12.6675 \mathrm{~cm}^{-1} \\ \text { 3. } & 14.6137 \mathrm{~cm}^{-1}\end{array}$

\section{B3LYP-D3(BJ)/6-31G(d,p) Molecular Geometry in Cartesian Coordinates}

C

C

$\mathrm{C}$

C

C

C

$\mathrm{H}$

$\mathrm{H}$

C

C

$\mathrm{H}$

$\mathrm{H}$

C

C

$\mathrm{H}$

C

C

C

C

C

C

$\mathrm{H}$

$\mathrm{H}$

$\mathrm{H}$

C

C

$\mathrm{H}$

$\mathrm{H}$

C

C

C

$\mathrm{H}$

$$
-0.235134
$$

$-0.363594$

0.716759

1.946424

2.044557

0.978699

$-1.069804$

$-1.293242$

0.616238

3.030640

2.980157

1.065943

2.944600

1.715148

3.946485

$-0.996428$

$-2.054206$

$-1.953116$

$-0.733036$

0.341540

0.211865

$-3.945294$

$-1.083277$

$-2.985874$

$-3.026176$

$-0.648952$

1.273971

1.044349

$-1.717729$

$-2.936345$

$-1.568483$

$-2.533095$
$-4.104951$

$-3.262730$

$-2.436620$

$-2.501555$

$-3.377293$

$-4.162334$

$-4.730617$

$-3.218964$

$-1.549117$

$-1.683152$

$-3.415708$

$-4.829747$

$-0.839026$

$-0.792389$

$-1.704527$

$-4.017011$

$-3.230229$

$-2.391913$

$-2.369790$

$-3.195031$

$-3.997393$

$-1.586637$

$-4.654086$

$-3.236669$

$-1.575554$

$-1.521839$

$-3.178076$

$-4.617588$

$-0.724026$

$-0.746690$

0.170021

0.294513
3.072974

1.991969

1.584114

2. 311109

3.423830

3. 797422

3. 372940

1.436772

0.466142

1.911543

3.974336

4.649111

0.825173

0.093919

2.494427

$-3.759434$

$-3.369389$

$-2.227544$

$-1.483483$

$-1.912743$

$-3.022640$

$-2.383073$

$-4.634188$

$-3.928191$

$-1.804681$

$-0.335614$

$-1.361329$

$-3.339543$

0.057042

$-0.702168$

1. 273703

1.768740 


\begin{tabular}{|c|c|c|c|}
\hline $\mathrm{H}$ & -0.882279 & -0.294371 & 1.990186 \\
\hline C & 1.672950 & -0.041198 & -1.201779 \\
\hline 0 & 2.304442 & -0.323661 & -2.190055 \\
\hline 0 & 0.816840 & 1.022876 & -1.173011 \\
\hline $\mathrm{H}$ & 0.803087 & 1.397930 & -2.070317 \\
\hline 0 & -1.122759 & 1.488237 & 0.945997 \\
\hline $\mathrm{H}$ & -0.382501 & 1.400093 & 0.326035 \\
\hline C & -4.124066 & 0.069248 & -0.332613 \\
\hline C & -4.013122 & 1.433549 & -0.063188 \\
\hline C & -5.392152 & -0.535743 & -0.284488 \\
\hline C & -5.135487 & 2.202468 & 0.273173 \\
\hline $\mathrm{H}$ & -3.031462 & 1.888615 & -0.096983 \\
\hline C & -6.532214 & 0.201334 & 0.030653 \\
\hline $\mathrm{H}$ & -5.460058 & -1.599012 & -0.477829 \\
\hline C & -6.378525 & 1.570617 & 0.308878 \\
\hline $\mathrm{H}$ & -7.257865 & 2.147448 & 0.566062 \\
\hline C & 4.085026 & 0.033794 & 0.457774 \\
\hline C & 3.872559 & 1.397664 & 0.203917 \\
\hline C & 5.378415 & -0.481218 & 0.382934 \\
\hline C & 4.927396 & 2.237673 & -0.142313 \\
\hline $\mathrm{H}$ & 2.869203 & 1.791341 & 0.297408 \\
\hline C & 6.468119 & 0.336329 & 0.050734 \\
\hline $\mathrm{H}$ & 5.525732 & -1.541203 & 0.560460 \\
\hline C & 6.217126 & 1.684419 & -0.209992 \\
\hline $\mathrm{H}$ & 7.041889 & 2.331839 & -0.478168 \\
\hline C & 7.873214 & -0.276678 & -0.031243 \\
\hline C & 4.721849 & 3.725388 & -0.457663 \\
\hline C & 8.946732 & 0.764746 & -0.385789 \\
\hline $\mathrm{H}$ & 8.763425 & 1.218568 & -1.364668 \\
\hline $\mathrm{H}$ & 8.997503 & 1.564429 & 0.359890 \\
\hline $\mathrm{H}$ & 9.928277 & 0.282593 & -0.423226 \\
\hline C & 7.885238 & -1.374073 & -1.117307 \\
\hline $\mathrm{H}$ & 8.879995 & -1.827014 & -1.190227 \\
\hline $\mathrm{H}$ & 7.169353 & -2.170166 & -0.895689 \\
\hline $\mathrm{H}$ & 7.626096 & -0.955186 & -2.094422 \\
\hline C & 8.237294 & -0.899745 & 1.333698 \\
\hline $\mathrm{H}$ & 7.531420 & -1.683584 & 1.621822 \\
\hline $\mathrm{H}$ & 9.236176 & -1.347413 & 1.293918 \\
\hline $\mathrm{H}$ & 8.233929 & -0.139481 & 2.121059 \\
\hline C & 5.173003 & 3.996672 & -1.909372 \\
\hline $\mathrm{H}$ & 6.228613 & 3.752599 & -2.056976 \\
\hline $\mathrm{H}$ & 4.589927 & 3.396057 & -2.614396 \\
\hline $\mathrm{H}$ & 5.034447 & 5.053854 & -2.160578 \\
\hline C & 5.563297 & 4.581410 & 0.512568 \\
\hline $\mathrm{H}$ & 6.630066 & 4.355401 & 0.430817 \\
\hline $\mathrm{H}$ & 5.429218 & 5.646624 & 0.295462 \\
\hline $\mathrm{H}$ & 5.261173 & 4.402891 & 1.549182 \\
\hline C & 3.249904 & 4.149162 & -0.323380 \\
\hline $\mathrm{H}$ & 2.601404 & 3.581933 & -0.998456 \\
\hline $\mathrm{H}$ & 2.879459 & 4.012077 & 0.697206 \\
\hline $\mathrm{H}$ & 3.147013 & 5.209098 & -0.574590 \\
\hline C & -7.931892 & -0.426770 & 0.097917 \\
\hline C & -4.941464 & 3.689934 & 0.596966 \\
\hline C & -6.260549 & 4.385423 & 0.967850 \\
\hline
\end{tabular}




\begin{tabular}{|c|c|c|c|}
\hline $\mathrm{H}$ & -6.983977 & 4.349316 & 0.146783 \\
\hline $\mathrm{H}$ & -6.721625 & 3.932580 & 1.851389 \\
\hline $\mathrm{H}$ & -6.070441 & 5.438641 & 1.196332 \\
\hline C & -4.344063 & 4.405299 & -0.633768 \\
\hline H & -3.377645 & 3.978813 & -0.915009 \\
\hline $\mathrm{H}$ & -5.013582 & 4.321606 & -1.495903 \\
\hline H & -4.192396 & 5.468936 & -0.419491 \\
\hline C & -3.965768 & 3.820148 & 1.788175 \\
\hline $\mathrm{H}$ & -4.377762 & 3.330766 & 2.676837 \\
\hline H & -2.996292 & 3.363308 & 1.572985 \\
\hline $\mathrm{H}$ & -3.800411 & 4.876467 & 2.028421 \\
\hline C & -8.501497 & -0.256375 & 1.522797 \\
\hline H & -7.860348 & -0.752603 & 2.258008 \\
\hline $\mathrm{H}$ & -8.578354 & 0.797449 & 1.803928 \\
\hline $\mathrm{H}$ & -9.503021 & -0.695473 & 1.589324 \\
\hline C & -7.915622 & -1.926853 & -0.237168 \\
\hline $\mathrm{H}$ & -8.932531 & -2.327704 & -0.183623 \\
\hline $\mathrm{H}$ & -7.538187 & -2.111313 & -1.247848 \\
\hline $\mathrm{H}$ & -7.298222 & -2.492499 & 0.467527 \\
\hline C & -8.857960 & 0.283053 & -0.913281 \\
\hline H & -8.474281 & 0.174326 & -1.932544 \\
\hline $\mathrm{H}$ & -9.864257 & -0.148594 & -0.877827 \\
\hline H & -8.944518 & 1.352190 & -0.701339 \\
\hline
\end{tabular}

\section{Catalyst 2C 33}

\section{Datum}

Value

\begin{tabular}{lr}
\hline B3LYP-D3(BJ)/6-31G(d,p) Energy & -2165.16312 \\
\hline B3LYP-D3(BJ)/def2-TZVPP/IEF-PCM(DCM) Energy & -2165.899398 \\
\hline B3LYP-D3(BJ)/def2-TZVPP/IEF-PCM(DCM)//B3LYP-D3(BJ)/6-31G(d,p) Free Energy (Quasiharmonic) & -2165.021491
\end{tabular}

Number of Imaginary Frequencies

Frequencies (Top 3 out of 321)
1. $9.8575 \mathrm{~cm}^{-1}$
2. $12.5767 \mathrm{~cm}^{-1}$
3. $14.7721 \mathrm{~cm}^{-1}$

\section{B3LYP-D3(BJ)/6-31G(d,p) Molecular Geometry in Cartesian Coordinates}

$\begin{array}{rrrr}C & 0.236746 & -4.128002 & 3.061581 \\ C & 0.361211 & -3.278009 & 1.986223 \\ C & -0.722206 & -2.452566 & 1.585252\end{array}$




\begin{tabular}{|c|c|c|c|}
\hline C & -1.950896 & -2.526255 & 2.313297 \\
\hline C & -2.044931 & -3.410220 & 3.419866 \\
\hline C & -0.976140 & -4.194377 & 3.786860 \\
\hline $\mathrm{H}$ & 1.073794 & -4.752953 & 3.356382 \\
\hline $\mathrm{H}$ & 1.290019 & -3.227429 & 1.430164 \\
\hline C & -0.626017 & -1.558392 & 0.472256 \\
\hline C & -3.038251 & -1.708387 & 1.920862 \\
\hline $\mathrm{H}$ & -2.979807 & -3.455585 & 3.971071 \\
\hline $\mathrm{H}$ & -1.060334 & -4.868018 & 4.633941 \\
\hline C & -2.954688 & -0.855303 & 0.841534 \\
\hline C & -1.727315 & -0.801908 & 0.107431 \\
\hline $\mathrm{H}$ & -3.953953 & -1.737890 & 2.503682 \\
\hline C & 0.983649 & -4.004270 & -3.767529 \\
\hline C & 2.039376 & -3.214379 & -3.378066 \\
\hline C & 1.938359 & -2.380573 & -2.233008 \\
\hline C & 0.720604 & -2.366240 & -1.484924 \\
\hline C & -0.351918 & -3.194238 & -1.913835 \\
\hline C & -0.222370 & -3.992192 & -3.026944 \\
\hline $\mathrm{H}$ & 3.926869 & -1.564758 & -2.391820 \\
\hline $\mathrm{H}$ & 1.070368 & -4.637844 & -4.644837 \\
\hline $\mathrm{H}$ & 2.969211 & -3.214770 & -3.939958 \\
\hline C & 3.009303 & -1.560740 & -1.810803 \\
\hline C & 0.636421 & -1.523092 & -0.333343 \\
\hline $\mathrm{H}$ & -1.282757 & -3.182755 & -1.359605 \\
\hline $\mathrm{H}$ & -1.053316 & -4.614622 & -3.343508 \\
\hline C & 1.703219 & -0.722640 & 0.058777 \\
\hline C & 2.918606 & -0.736986 & -0.704842 \\
\hline C & 1.557706 & 0.165512 & 1.280183 \\
\hline $\mathrm{H}$ & 0.879714 & -0.306921 & 1.999611 \\
\hline $\mathrm{H}$ & 2.525781 & 0.291673 & 1.768393 \\
\hline C & -1.691459 & -0.044375 & -1.185280 \\
\hline 0 & -2.316563 & -0.331076 & -2.176219 \\
\hline 0 & -0.848566 & 1.030466 & -1.150407 \\
\hline $\mathrm{H}$ & -0.842304 & 1.412680 & -2.044888 \\
\hline 0 & 1.103133 & 1.482377 & 0.962041 \\
\hline $\mathrm{H}$ & 0.362367 & 1.392839 & 0.343094 \\
\hline C & 4.103684 & 0.082929 & -0.335102 \\
\hline C & 3.986305 & 1.459764 & -0.087661 \\
\hline C & 5.364330 & -0.514922 & -0.267880 \\
\hline C & 5.102237 & 2.227927 & 0.237620 \\
\hline $\mathrm{H}$ & 3.002647 & 1.904461 & -0.134676 \\
\hline C & 6.510687 & 0.228872 & 0.045838 \\
\hline $\mathrm{H}$ & 5.441023 & -1.582910 & -0.444103 \\
\hline C & 6.354017 & 1.593078 & 0.296203 \\
\hline $\mathrm{H}$ & 7.222769 & 2.186185 & 0.551493 \\
\hline C & -4.094304 & 0.020449 & 0.479091 \\
\hline C & -3.885126 & 1.378319 & 0.238952 \\
\hline C & -5.392883 & -0.501845 & 0.387347 \\
\hline C & -4.940312 & 2.225438 & -0.121888 \\
\hline $\mathrm{H}$ & -2.884242 & 1.780496 & 0.346492 \\
\hline C & -6.470879 & 0.313607 & 0.047024 \\
\hline $\mathrm{H}$ & -5.532810 & -1.561563 & 0.559002 \\
\hline C & -6.218378 & 1.672618 & -0.208570 \\
\hline $\mathrm{H}$ & -7.049518 & 2.307651 & -0.486754 \\
\hline
\end{tabular}




\begin{tabular}{|c|c|c|c|}
\hline C & -7.905149 & -0.219866 & -0.075430 \\
\hline C & -4.646432 & 3.704533 & -0.405873 \\
\hline C & - 7.997731 & -1.721110 & 0.241703 \\
\hline $\mathrm{H}$ & -7.395708 & -2.318854 & -0.449529 \\
\hline $\mathrm{H}$ & -7.668038 & -1.940050 & 1.262237 \\
\hline $\mathrm{H}$ & -9.036262 & -2.053290 & 0.149786 \\
\hline C & -8.410601 & 0.001730 & -1.517473 \\
\hline $\mathrm{H}$ & -9.434149 & -0.373686 & -1.624096 \\
\hline $\mathrm{H}$ & -8.412942 & 1.061404 & -1.787092 \\
\hline $\mathrm{H}$ & -7.775099 & -0.524988 & -2.235889 \\
\hline C & -8.817175 & 0.539695 & 0.912151 \\
\hline $\mathrm{H}$ & -8.827435 & 1.614224 & 0.710078 \\
\hline $\mathrm{H}$ & -9.847227 & 0.174840 & 0.836649 \\
\hline $\mathrm{H}$ & -8.477877 & 0.396253 & 1.942799 \\
\hline C & -3.598779 & 3.800864 & -1.537320 \\
\hline $\mathrm{H}$ & -2.660913 & 3.311843 & -1.259558 \\
\hline $\mathrm{H}$ & -3.969038 & 3.323587 & -2.449943 \\
\hline $\mathrm{H}$ & -3.376202 & 4.849491 & -1.762064 \\
\hline C & -4.085367 & 4.367145 & 0.870561 \\
\hline $\mathrm{H}$ & -3.164679 & 3.882036 & 1.206051 \\
\hline $\mathrm{H}$ & -3.859927 & 5.422866 & 0.684826 \\
\hline $\mathrm{H}$ & -4.811021 & 4.310644 & 1.687899 \\
\hline C & -5.901179 & 4.476496 & -0.843131 \\
\hline $\mathrm{H}$ & -6.335395 & 4.058234 & -1.756666 \\
\hline $\mathrm{H}$ & -6.670354 & 4.473320 & -0.064446 \\
\hline $\mathrm{H}$ & -5.640111 & 5.519586 & -1.045828 \\
\hline C & 7.872814 & -0.476870 & 0.114338 \\
\hline C & 4.994211 & 3.726152 & 0.553897 \\
\hline C & 3.560732 & 4.253727 & 0.374261 \\
\hline $\mathrm{H}$ & 3.224837 & 4.149145 & -0.662567 \\
\hline $\mathrm{H}$ & 2.845807 & 3.728077 & 1.013178 \\
\hline $\mathrm{H}$ & 3.527416 & 5.317835 & 0.628972 \\
\hline C & 5.926594 & 4.525550 & -0.380718 \\
\hline $\mathrm{H}$ & 6.973518 & 4.232322 & -0.262101 \\
\hline $\mathrm{H}$ & 5.651639 & 4.369437 & -1.428605 \\
\hline $\mathrm{H}$ & 5.854071 & 5.596951 & -0.164286 \\
\hline C & 5.417154 & 3.956708 & 2.021120 \\
\hline $\mathrm{H}$ & 4.761481 & 3.405844 & 2.702476 \\
\hline $\mathrm{H}$ & 6.443891 & 3.625181 & 2.201668 \\
\hline $\mathrm{H}$ & 5.357205 & 5.021093 & 2.273757 \\
\hline C & 7.825718 & -1.570902 & 1.202677 \\
\hline $\mathrm{H}$ & 7.608635 & -1.133468 & 2.182151 \\
\hline $\mathrm{H}$ & 7.054956 & -2.317202 & 0.991735 \\
\hline $\mathrm{H}$ & 8.788335 & -2.090310 & 1.264744 \\
\hline C & 9.017657 & 0.491009 & 0.454010 \\
\hline $\mathrm{H}$ & 9.965352 & -0.055468 & 0.483364 \\
\hline $\mathrm{H}$ & 9.113501 & 1.283165 & -0.295143 \\
\hline H & 8.874892 & 0.959934 & 1.432559 \\
\hline C & 8.179713 & -1.125829 & -1.252597 \\
\hline $\mathrm{H}$ & 8.217524 & -0.368077 & -2.041458 \\
\hline $\mathrm{H}$ & 9.147095 & -1.639133 & -1.223617 \\
\hline $\mathrm{H}$ & 7.419253 & -1.860684 & -1.530737 \\
\hline
\end{tabular}




\section{Catalyst 2C 34}

Datum

Value

B3LYP-D3(BJ)/6-31G(d,p) Energy

$-2165.162658$

B3LYP-D3(BJ)/def2-TZVPP/IEF-PCM(DCM) Energy

$-2165.899275$

B3LYP-D3(BJ)/def2-TZVPP/IEF-PCM(DCM)//B3LYP-D3(BJ)/6-31G(d,p) Free Energy (Quasiharmonic) $\quad-2165.021437$

Number of Imaginary Frequencies

Frequencies (Top 3 out of 321)

$\begin{array}{ll}\text { 1. } & 10.0277 \mathrm{~cm}^{-1} \\ \text { 2. } & 12.8524 \mathrm{~cm}^{-1} \\ \text { 3. } & 14.6669 \mathrm{~cm}^{-1}\end{array}$

B3LYP-D3(BJ)/6-31G(d,p) Molecular Geometry in Cartesian Coordinates

C

C

C

C

C

C

$\mathrm{H}$

$\mathrm{H}$

C

C

$\mathrm{H}$

$\mathrm{H}$

C

C

$\mathrm{H}$

C

C

C

C

C

C

$\mathrm{H}$

$\mathrm{H}$

$\mathrm{H}$

C

C

$\mathrm{H}$

$\mathrm{H}$

C
$-0.260050$

$-0.382788$

0.702650

1.931432

2.023659

0.952983

$-1.098590$

$-1.311748$

0.608438

3.020661

2.958693

1.035776

2.940321

1.711963

3.935952

$-1.012962$

$-2.066440$

$-1.961947$

$-0.742979$

0.327109

0.194194

$-3.949061$

$-1.102361$

$-2.997163$

$-3.030572$

$-0.655227$

1. 258760

1.023323

$-1.719828$
$-4.128850$

$-3.277773$

$-2.454934$

$-2.532478$

$-3.417289$

$-4.198860$

$-4.751828$

$-3.224364$

$-1.558981$

$-1.717422$

$-3.465482$

$-4.873266$

$-0.863964$

$-0.805759$

$-1.749161$

$-3.990413$

- 3.199391

$-2.368872$

$-2.358993$

$-3.188174$

$-3.982873$

$-1.548629$

$-4.621453$

$-3.196384$

$-1.548035$

$-1.519048$

$-3.180318$

$-4.606257$

$-0.717595$
3.050169

1.975465

1.574571

2. 301833

3.407911

3. 774889

3. 344903

1.419944

0.462894

1.909269

3.958610

4.621502

0.829634

0.097289

2.492529

$-3.780920$

$-3.387678$

$-2.240538$

$-1.494312$

$-1.927087$

- 3.042129

$-2.394033$

$-4.659785$

$-3.948095$

$-1.814458$

$-0.340667$

$-1.374154$

$-3.361568$

0.055319 


\begin{tabular}{|c|c|c|c|}
\hline C & -2.936438 & -0.727483 & -0.706398 \\
\hline C & -1.570593 & 0.167369 & 1.278637 \\
\hline $\mathrm{H}$ & -2.537615 & 0.293925 & 1.768837 \\
\hline $\mathrm{H}$ & -0.892205 & -0.307962 & 1.995753 \\
\hline C & 1.674702 & -0.046049 & -1.193737 \\
\hline 0 & 2.302320 & -0.327792 & -2.184717 \\
\hline 0 & 0.827057 & 1.024384 & -1.157099 \\
\hline $\mathrm{H}$ & 0.814244 & 1.405024 & -2.052051 \\
\hline 0 & -1.114538 & 1.484251 & 0.963004 \\
\hline $\mathrm{H}$ & -0.372941 & 1.395089 & 0.344992 \\
\hline C & -4.119254 & 0.093901 & -0.332631 \\
\hline C & -3.998646 & 1.469855 & -0.081856 \\
\hline C & -5.381007 & -0.501544 & -0.264823 \\
\hline C & -5.112430 & 2.239429 & 0.247418 \\
\hline $\mathrm{H}$ & -3.014152 & 1.912644 & -0.129374 \\
\hline C & -6.525300 & 0.243775 & 0.052805 \\
\hline $\mathrm{H}$ & -5.460183 & -1.568912 & -0.443686 \\
\hline C & -6.365407 & 1.606992 & 0.306506 \\
\hline $\mathrm{H}$ & -7.232493 & 2.201185 & 0.564918 \\
\hline C & 4.085314 & 0.005999 & 0.469829 \\
\hline C & 3.880076 & 1.368654 & 0.223833 \\
\hline C & 5.379387 & -0.520730 & 0.394660 \\
\hline C & 4.941741 & 2.207759 & -0.118636 \\
\hline $\mathrm{H}$ & 2.879071 & 1.767933 & 0.318556 \\
\hline C & 6.468523 & 0.291430 & 0.067469 \\
\hline $\mathrm{H}$ & 5.513792 & -1.580759 & 0.568631 \\
\hline C & 6.222116 & 1.646213 & -0.187576 \\
\hline $\mathrm{H}$ & 7.056905 & 2.285564 & -0.453798 \\
\hline C & 7.901700 & -0.249319 & -0.036854 \\
\hline C & 4.742958 & 3.698000 & -0.427082 \\
\hline C & 7.982501 & -1.751371 & 0.279820 \\
\hline $\mathrm{H}$ & 7.639111 & -1.969699 & 1.295957 \\
\hline $\mathrm{H}$ & 7.386037 & -2.345334 & -0.419443 \\
\hline H & 9.020368 & -2.088802 & 0.200424 \\
\hline C & 8.804479 & 0.504789 & 0.963172 \\
\hline $\mathrm{H}$ & 9.833723 & 0.135223 & 0.900710 \\
\hline $\mathrm{H}$ & 8.822274 & 1.579481 & 0.762371 \\
\hline $\mathrm{H}$ & 8.451167 & 0.362223 & 1.989206 \\
\hline C & 8.426363 & -0.028647 & -1.472068 \\
\hline $\mathrm{H}$ & 8.436782 & 1.031360 & -1.740284 \\
\hline $\mathrm{H}$ & 9.449424 & -0.408632 & -1.566442 \\
\hline $\mathrm{H}$ & 7.797458 & -0.551323 & -2.199171 \\
\hline C & 3.273203 & 4.128267 & -0.289166 \\
\hline $\mathrm{H}$ & 2.621111 & 3.567737 & -0.966334 \\
\hline H & 2.903055 & 3.987938 & 0.731082 \\
\hline $\mathrm{H}$ & 3.175331 & 5.189940 & -0.534959 \\
\hline C & 5.193998 & 3.973236 & -1.877991 \\
\hline $\mathrm{H}$ & 4.607413 & 3.378479 & -2.585046 \\
\hline $\mathrm{H}$ & 5.060666 & 5.032141 & -2.124732 \\
\hline $\mathrm{H}$ & 6.248200 & 3.724185 & -2.027557 \\
\hline C & 5.589758 & 4.545071 & 0.546206 \\
\hline $\mathrm{H}$ & 5.287952 & 4.363243 & 1.582324 \\
\hline H & 6.655212 & 4.313562 & 0.462365 \\
\hline $\mathrm{H}$ & 5.461257 & 5.612012 & 0.334298 \\
\hline
\end{tabular}




\begin{tabular}{|lrrr|} 
C & -7.888755 & -0.459353 & 0.121788 \\
C & -5.000699 & 3.736535 & 0.567693 \\
C & -3.566512 & 4.261700 & 0.386621 \\
H & -2.851314 & 3.732814 & 1.022548 \\
H & -3.232944 & 4.159364 & -0.651183 \\
H & -3.530514 & 5.325019 & 0.644248 \\
C & -5.420158 & 3.963724 & 2.036443 \\
H & -6.447199 & 3.633765 & 2.218143 \\
H & -4.764207 & 3.409552 & 2.714842 \\
H & -5.357507 & 5.027251 & 2.292027 \\
C & -5.933377 & 4.540519 & -0.362679 \\
H & -5.660917 & 4.386864 & -1.411581 \\
H & -6.980659 & 4.249112 & -0.242731 \\
H & -5.858197 & 5.611142 & -0.143322 \\
C & -8.199347 & -1.104024 & -1.246340 \\
H & -8.236969 & -0.344086 & -2.033104 \\
H & -7.440868 & -1.839677 & -1.527757 \\
H & -9.167726 & -1.615430 & -1.217061 \\
C & -9.031020 & 0.509954 & 0.466035 \\
H & -9.979783 & -0.034656 & 0.495578 \\
H & -8.885590 & 0.975964 & 1.445583 \\
H & -9.126533 & 1.304310 & -0.280827 \\
C & -7.842029 & -1.556389 & 1.207115 \\
H & -7.622339 & -1.122021 & 2.187373 \\
H & -8.805608 & -2.073977 & 1.269475 \\
H & -7.073176 & -2.303707 & 0.992840 \\
& & & \\
\hline & & & \\
\hline
\end{tabular}

\section{Catalyst 2C 35}

Datum

Value

B3LYP-D3(BJ)/6-31G(d,p) Energy

$-2165.163093$

B3LYP-D3(BJ)/def2-TZVPP/IEF-PCM(DCM) Energy

$-2165.899362$

B3LYP-D3(BJ)/def2-TZVPP/IEF-PCM(DCM)//B3LYP-D3(BJ)/6-31G(d,p) Free Energy (Quasiharmonic) -2165.021353

Number of Imaginary Frequencies

0

Frequencies (Top 3 out of 321)

$\begin{array}{ll}\text { 1. } & 10.0265 \mathrm{~cm}^{-1} \\ \text { 2. } & 12.6462 \mathrm{~cm}^{-1} \\ \text { 3. } & 14.6001 \mathrm{~cm}^{-1}\end{array}$

B3LYP-D3(BJ)/6-31G(d,p) Molecular Geometry in Cartesian Coordinates 


\begin{tabular}{|c|c|c|c|}
\hline C & 0.278416 & -4.109264 & 3.073325 \\
\hline C & 0.399994 & -3.263617 & 1.994211 \\
\hline C & -0.687407 & -2.446228 & 1.587519 \\
\hline C & -1.916896 & -2.523580 & 2.313615 \\
\hline C & -2.007900 & -3.402715 & 3.424304 \\
\hline C & -0.935338 & -4.179045 & 3.796851 \\
\hline $\mathrm{H}$ & 1.118457 & -4.728084 & 3.372492 \\
\hline $\mathrm{H}$ & 1.329540 & -3.210414 & 1.439661 \\
\hline C & -0.594436 & -1.555851 & 0.471260 \\
\hline C & -3.008037 & -1.713862 & 1.915366 \\
\hline $\mathrm{H}$ & -2.943488 & -3.450760 & 3.974066 \\
\hline $\mathrm{H}$ & -1.017180 & -4.849118 & 4.646986 \\
\hline C & -2.928901 & -0.865916 & 0.831315 \\
\hline C & -1.699751 & -0.807886 & 0.100254 \\
\hline $\mathrm{H}$ & -3.923940 & -1.745203 & 2.497679 \\
\hline C & 1.038493 & -4.000322 & -3.760394 \\
\hline C & 2.089238 & -3.204613 & -3.369401 \\
\hline C & 1.981306 & -2.370068 & -2.225464 \\
\hline C & 0.761682 & -2.360922 & -1.480367 \\
\hline C & -0.305518 & -3.195111 & -1.910685 \\
\hline C & -0.169261 & -3.993699 & -3.022519 \\
\hline $\mathrm{H}$ & 3.965987 & -1.545540 & -2.380442 \\
\hline $\mathrm{H}$ & 1.130499 & -4.634473 & -4.636742 \\
\hline $\mathrm{H}$ & 3.020419 & -3.200979 & -3.929043 \\
\hline C & 3.047208 & -1.544911 & -1.801371 \\
\hline C & 0.670378 & -1.516222 & -0.330576 \\
\hline $\mathrm{H}$ & -1.237612 & -3.188037 & -1.358497 \\
\hline $\mathrm{H}$ & -0.996181 & -4.620921 & -3.340154 \\
\hline C & 1.732224 & -0.709914 & 0.063372 \\
\hline C & 2.950434 & -0.720343 & -0.696366 \\
\hline C & 1.576096 & 0.180909 & 1.281559 \\
\hline $\mathrm{H}$ & 0.894967 & -0.290886 & 1.998088 \\
\hline $\mathrm{H}$ & 2.540022 & 0.313490 & 1.775894 \\
\hline C & -1.663220 & -0.054984 & -1.194706 \\
\hline 0 & -2.289119 & -0.343416 & -2.184797 \\
\hline 0 & -0.818261 & 1.017996 & -1.163228 \\
\hline H & -0.805832 & 1.393456 & -2.060386 \\
\hline 0 & 1.118123 & 1.495372 & 0.956010 \\
\hline $\mathrm{H}$ & 0.378115 & 1.401407 & 0.336610 \\
\hline C & 4.131429 & 0.104468 & -0.324901 \\
\hline C & 4.009611 & 1.471434 & -0.055117 \\
\hline C & 5.401300 & -0.484498 & -0.275114 \\
\hline C & 5.123217 & 2.246835 & 0.279640 \\
\hline $\mathrm{H}$ & 3.023807 & 1.917469 & -0.089014 \\
\hline C & 6.540455 & 0.262926 & 0.039230 \\
\hline $\mathrm{H}$ & 5.485119 & -1.549319 & -0.466571 \\
\hline C & 6.377792 & 1.626664 & 0.315826 \\
\hline $\mathrm{H}$ & 7.246159 & 2.216262 & 0.570940 \\
\hline C & -4.076027 & -0.001282 & 0.465510 \\
\hline C & -5.368428 & -0.532266 & 0.391549 \\
\hline C & -3.874569 & 1.360651 & 0.212516 \\
\hline C & -6.459584 & 0.274889 & 0.058752 \\
\hline $\mathrm{H}$ & -5.499904 & -1.591753 & 0.570960 \\
\hline
\end{tabular}




\begin{tabular}{|c|c|c|c|}
\hline C & -4.938295 & 2.194743 & -0.135746 \\
\hline $\mathrm{H}$ & -2.874896 & 1.763455 & 0.306395 \\
\hline C & -6.216908 & 1.629024 & -0.203218 \\
\hline $\mathrm{H}$ & -7.053284 & 2.264449 & -0.473831 \\
\hline C & -4.743586 & 3.683877 & -0.452005 \\
\hline C & -7.891016 & -0.270691 & -0.044401 \\
\hline C & -5.594001 & 4.533689 & 0.515716 \\
\hline $\mathrm{H}$ & -6.658631 & 4.298380 & 0.432077 \\
\hline $\mathrm{H}$ & -5.292705 & 4.358556 & 1.553133 \\
\hline $\mathrm{H}$ & -5.468614 & 5.599832 & 0.298015 \\
\hline C & -5.193703 & 3.949939 & -1.904906 \\
\hline $\mathrm{H}$ & -5.063021 & 5.007848 & -2.157260 \\
\hline $\mathrm{H}$ & -4.604660 & 3.352965 & -2.608037 \\
\hline $\mathrm{H}$ & -6.247034 & 3.697168 & -2.054322 \\
\hline C & -3.275269 & 4.119249 & -0.314739 \\
\hline $\mathrm{H}$ & -2.620796 & 3.556997 & -0.988189 \\
\hline $\mathrm{H}$ & -3.180286 & 5.179866 & -0.566157 \\
\hline $\mathrm{H}$ & -2.905900 & 3.985526 & 0.706682 \\
\hline C & -7.967737 & -1.771243 & 0.280289 \\
\hline $\mathrm{H}$ & -7.368682 & -2.367199 & -0.415052 \\
\hline $\mathrm{H}$ & -7.624932 & -1.983063 & 1.297999 \\
\hline $\mathrm{H}$ & -9.004501 & -2.112177 & 0.201481 \\
\hline C & -8.414518 & -0.059326 & -1.481430 \\
\hline $\mathrm{H}$ & -7.783195 & -0.584107 & -2.204912 \\
\hline $\mathrm{H}$ & -9.436355 & -0.442775 & -1.575015 \\
\hline $\mathrm{H}$ & -8.427670 & 0.999178 & -1.755394 \\
\hline C & -8.797260 & 0.486115 & 0.950437 \\
\hline $\mathrm{H}$ & -8.444805 & 0.350130 & 1.977657 \\
\hline $\mathrm{H}$ & -8.818004 & 1.559650 & 0.743823 \\
\hline $\mathrm{H}$ & -9.825321 & 0.113159 & 0.888704 \\
\hline C & 7.910112 & -0.431197 & 0.085119 \\
\hline C & 4.918482 & 3.733515 & 0.602991 \\
\hline C & 4.314674 & 4.444248 & -0.627347 \\
\hline $\mathrm{H}$ & 4.983671 & 4.364742 & -1.490295 \\
\hline $\mathrm{H}$ & 3.350955 & 4.010800 & -0.907190 \\
\hline $\mathrm{H}$ & 4.155921 & 5.506973 & -0.413524 \\
\hline C & 6.232250 & 4.439958 & 0.972639 \\
\hline $\mathrm{H}$ & 6.697987 & 3.991524 & 1.855937 \\
\hline $\mathrm{H}$ & 6.955489 & 4.409890 & 0.151210 \\
\hline $\mathrm{H}$ & 6.033264 & 5.491606 & 1.200927 \\
\hline C & 3.942844 & 3.857305 & 1.794975 \\
\hline $\mathrm{H}$ & 2.976716 & 3.393003 & 1.580748 \\
\hline $\mathrm{H}$ & 4.359085 & 3.371389 & 2.683568 \\
\hline $\mathrm{H}$ & 3.769831 & 4.912491 & 2.034936 \\
\hline C & 8.209904 & -1.059569 & -1.292996 \\
\hline $\mathrm{H}$ & 7.453991 & -1.798366 & -1.573053 \\
\hline $\mathrm{H}$ & 8.232311 & -0.291523 & -2.072444 \\
\hline $\mathrm{H}$ & 9.182600 & -1.563463 & -1.280217 \\
\hline C & 7.884081 & -1.539862 & 1.159319 \\
\hline $\mathrm{H}$ & 8.852184 & -2.050733 & 1.205501 \\
\hline $\mathrm{H}$ & 7.672195 & -1.117490 & 2.146517 \\
\hline $\mathrm{H}$ & 7.118373 & -2.290613 & 0.945753 \\
\hline C & 9.050179 & 0.542049 & 0.426237 \\
\hline $\mathrm{H}$ & 8.914091 & 0.996483 & 1.412490 \\
\hline
\end{tabular}


$\mathrm{H}$

$\mathrm{H}$

\section{Catalyst 2C 36}

\section{Datum}

Value

\begin{tabular}{lr}
\hline B3LYP-D3(BJ)/6-31G(d,p) Energy & -2165.162644 \\
\hline B3LYP-D3(BJ)/def2-TZVPP/IEF-PCM(DCM) Energy & -2165.899209 \\
\hline B3LYP-D3(BJ)/def2-TZVPP/IEF-PCM(DCM)//B3LYP-D3(BJ)/6-31G(d,p) Free Energy (Quasiharmonic) & -2165.021353 \\
\hline
\end{tabular}

Number of Imaginary Frequencies

Frequencies (Top 3 out of 321)

$\begin{array}{ll}\text { 1. } & 10.2181 \mathrm{~cm}^{-1} \\ \text { 2. } & 13.0424 \mathrm{~cm}^{-1} \\ \text { 3. } & 14.9377 \mathrm{~cm}^{-1}\end{array}$

\section{B3LYP-D3(BJ)/6-31G(d,p) Molecular Geometry in Cartesian Coordinates}

\begin{tabular}{|c|c|c|c|}
\hline C & 0.240591 & -4.137485 & 3.049812 \\
\hline $\mathrm{C}$ & 0.365965 & -3.287948 & 1.974189 \\
\hline C & -0.716248 & -2.460690 & 1.573686 \\
\hline C & -1.944566 & -2.532085 & 2.302341 \\
\hline C & -2.039532 & -3.415420 & 3.409365 \\
\hline C & -0.971949 & -4.201414 & 3.775913 \\
\hline $\mathrm{H}$ & 1.076647 & -4.763963 & 3.344191 \\
\hline $\mathrm{H}$ & 1.294533 & -3.239197 & 1.417586 \\
\hline C & -0.619213 & -1.566213 & 0.461047 \\
\hline C & -3.030570 & -1.712552 & 1.910169 \\
\hline $\mathrm{H}$ & -2.974174 & -3.458930 & 3.961120 \\
\hline $\mathrm{H}$ & -1.056832 & -4.874665 & 4.623241 \\
\hline $\mathrm{C}$ & -2.947637 & -0.860591 & 0.829551 \\
\hline C & -1.719822 & -0.808577 & 0.095796 \\
\hline $\mathrm{H}$ & -3.945323 & -1.739578 & 2.494508 \\
\hline C & 0.989814 & -4.012599 & -3.778664 \\
\hline C & 2.046833 & -3.225385 & -3.387353 \\
\hline C & 1.946254 & -2.391847 & -2.242010 \\
\hline C & 0.727520 & -2.375105 & -1.495554 \\
\hline C & -0.346320 & -3.200498 & -1.926296 \\
\hline C & -0.217178 & -3.998143 & -3.039665 \\
\hline $\mathrm{H}$ & 3.936675 & -1.581026 & -2.397991 \\
\hline $\mathrm{H}$ & 1.076273 & -4.645974 & -4.656146 \\
\hline $\mathrm{H}$ & 2.977467 & -3.227769 & -3.947918 \\
\hline
\end{tabular}




\begin{tabular}{|c|c|c|c|}
\hline C & 3.018444 & -1.574675 & -1.818077 \\
\hline C & 0.643972 & -1.532557 & -0.343582 \\
\hline $\mathrm{H}$ & -1.277789 & -3.187367 & -1.373149 \\
\hline $\mathrm{H}$ & -1.049111 & -4.618556 & -3.357601 \\
\hline C & 1.712049 & -0.734609 & 0.050308 \\
\hline C & 2.928397 & -0.750640 & -0.712119 \\
\hline C & 1.566638 & 0.152205 & 1.272722 \\
\hline $\mathrm{H}$ & 0.886042 & -0.319392 & 1.990170 \\
\hline $\mathrm{H}$ & 2.534200 & 0.274920 & 1.762768 \\
\hline C & -1.680691 & -0.050706 & -1.196244 \\
\hline 0 & -2.310663 & -0.331028 & -2.186135 \\
\hline 0 & -0.828372 & 1.016118 & -1.162086 \\
\hline $\mathrm{H}$ & -0.814954 & 1.395359 & -2.057622 \\
\hline 0 & 1.116313 & 1.470929 & 0.956147 \\
\hline $\mathrm{H}$ & 0.374381 & 1.384464 & 0.338147 \\
\hline C & 4.114417 & 0.067414 & -0.341239 \\
\hline C & 3.998359 & 1.439847 & -0.089581 \\
\hline C & 5.377320 & -0.537854 & -0.277125 \\
\hline C & 5.117387 & 2.208207 & 0.240464 \\
\hline $\mathrm{H}$ & 3.015709 & 1.886891 & -0.135534 \\
\hline C & 6.519248 & 0.202379 & 0.040151 \\
\hline $\mathrm{H}$ & 5.445539 & -1.603191 & -0.458774 \\
\hline C & 6.361710 & 1.570021 & 0.296871 \\
\hline $\mathrm{H}$ & 7.237296 & 2.155459 & 0.557031 \\
\hline C & -4.089161 & 0.014116 & 0.470246 \\
\hline C & -5.385774 & -0.506739 & 0.397856 \\
\hline C & -3.878081 & 1.375494 & 0.222132 \\
\hline C & -6.471702 & 0.310008 & 0.071443 \\
\hline $\mathrm{H}$ & -5.524784 & -1.565908 & 0.573440 \\
\hline C & -4.936424 & 2.219022 & -0.119755 \\
\hline $\mathrm{H}$ & -2.875119 & 1.770313 & 0.314786 \\
\hline C & -6.219485 & 1.663303 & -0.185831 \\
\hline $\mathrm{H}$ & -7.051765 & 2.306138 & -0.451507 \\
\hline C & -4.731236 & 3.707897 & -0.430614 \\
\hline C & -7.907550 & -0.224227 & -0.029617 \\
\hline C & -5.572689 & 4.560290 & 0.542663 \\
\hline $\mathrm{H}$ & -6.639340 & 4.333710 & 0.460615 \\
\hline $\mathrm{H}$ & -5.270302 & 4.378479 & 1.578614 \\
\hline $\mathrm{H}$ & -5.439456 & 5.626319 & 0.329090 \\
\hline C & -5.183119 & 3.983102 & -1.881266 \\
\hline $\mathrm{H}$ & -5.045302 & 5.041025 & -2.129752 \\
\hline $\mathrm{H}$ & -4.600303 & 3.384627 & -2.588301 \\
\hline $\mathrm{H}$ & -6.238668 & 3.738658 & -2.028924 \\
\hline C & -3.259313 & 4.131576 & -0.295449 \\
\hline $\mathrm{H}$ & -2.610801 & 3.567070 & -0.972750 \\
\hline $\mathrm{H}$ & -3.156902 & 5.192435 & -0.542910 \\
\hline $\mathrm{H}$ & -2.888335 & 3.990996 & 0.724464 \\
\hline C & -8.433749 & -0.003109 & -1.464197 \\
\hline $\mathrm{H}$ & -8.439742 & 1.056563 & -1.733872 \\
\hline $\mathrm{H}$ & -7.808577 & -0.529703 & -2.191691 \\
\hline $\mathrm{H}$ & -9.458727 & -0.378481 & -1.556216 \\
\hline C & -8.805035 & 0.535441 & 0.970966 \\
\hline $\mathrm{H}$ & -8.818138 & 1.609936 & 0.768754 \\
\hline $\mathrm{H}$ & -9.836109 & 0.170626 & 0.910795 \\
\hline
\end{tabular}




\begin{tabular}{|c|c|c|c|}
\hline $\mathrm{H}$ & -8.450602 & 0.392590 & 1.996573 \\
\hline$C$ & -7.994760 & -1.725451 & 0.289293 \\
\hline $\mathrm{H}$ & -7.402316 & -2.323157 & -0.410198 \\
\hline $\mathrm{H}$ & -7.650565 & -1.943960 & 1.305119 \\
\hline $\mathrm{H}$ & -9.034323 & -2.058167 & 0.212224 \\
\hline C & 7.916908 & -0.427992 & 0.128109 \\
\hline C & 5.009443 & 3.705144 & 0.563491 \\
\hline C & 5.947884 & 4.507749 & -0.362094 \\
\hline $\mathrm{H}$ & 6.993745 & 4.212388 & -0.239257 \\
\hline $\mathrm{H}$ & 5.678643 & 4.357073 & -1.412238 \\
\hline $\mathrm{H}$ & 5.875816 & 5.578190 & -0.140869 \\
\hline C & 5.424935 & 3.927195 & 2.034076 \\
\hline $\mathrm{H}$ & 4.765278 & 3.373240 & 2.709028 \\
\hline $\mathrm{H}$ & 6.450298 & 3.593116 & 2.217897 \\
\hline $\mathrm{H}$ & 5.364985 & 4.990205 & 2.292446 \\
\hline C & 3.577541 & 4.235360 & 0.378748 \\
\hline $\mathrm{H}$ & 3.246635 & 4.135111 & -0.660121 \\
\hline $\mathrm{H}$ & 2.858613 & 3.708527 & 1.012146 \\
\hline $\mathrm{H}$ & 3.544556 & 5.298568 & 0.637214 \\
\hline C & 8.853922 & 0.267642 & -0.882718 \\
\hline $\mathrm{H}$ & 8.479683 & 0.147513 & -1.904191 \\
\hline $\mathrm{H}$ & 8.940336 & 1.339154 & -0.682973 \\
\hline $\mathrm{H}$ & 9.859023 & -0.165319 & -0.832580 \\
\hline C & 7.900132 & -1.932019 & -0.188924 \\
\hline $\mathrm{H}$ & 8.915520 & -2.334487 & -0.120759 \\
\hline $\mathrm{H}$ & 7.274680 & -2.487702 & 0.516587 \\
\hline $\mathrm{H}$ & 7.531990 & -2.127907 & -1.200874 \\
\hline C & 8.472852 & -0.241377 & 1.556265 \\
\hline $\mathrm{H}$ & 7.823344 & -0.726896 & 2.291267 \\
\hline $\mathrm{H}$ & 9.472687 & -0.681691 & 1.638197 \\
\hline H & 8.549160 & 0.815729 & 1.825085 \\
\hline
\end{tabular}

\section{Catalyst 2C 37}

Datum

Value

\begin{tabular}{lr}
\hline B3LYP-D3(BJ)/6-31G(d,p) Energy & -2165.16333 \\
\hline B3LYP-D3(BJ)/def2-TZVPP/IEF-PCM(DCM) Energy & -2165.899239 \\
\hline
\end{tabular}

B3LYP-D3(BJ)/def2-TZVPP/IEF-PCM(DCM)//B3LYP-D3(BJ)/6-31G(d,p) Free Energy (Quasiharmonic) -2165.021209

Number of Imaginary Frequencies

Frequencies (Top 3 out of 321)

$\begin{array}{ll}\text { 1. } & 10.0741 \mathrm{~cm}^{-1} \\ \text { 2. } & 12.2389 \mathrm{~cm}^{-1} \\ \text { 3. } & 14.4620 \mathrm{~cm}^{-1}\end{array}$




\begin{tabular}{|c|c|c|c|}
\hline C & 0.230744 & -4.095997 & 3.086006 \\
\hline C & 0.358433 & -3.253352 & 2.005248 \\
\hline C & -0.723032 & -2.428994 & 1.596842 \\
\hline C & -1.953134 & -2.496101 & 2.323153 \\
\hline C & -2.050483 & -3.372450 & 3.435429 \\
\hline C & -0.983498 & -4.155723 & 3.809553 \\
\hline $\mathrm{H}$ & 1.066337 & -4.720189 & 3.386466 \\
\hline $\mathrm{H}$ & 1.288360 & -3.207838 & 1.450638 \\
\hline C & -0.623214 & -1.541879 & 0.478459 \\
\hline C & -3.038605 & -1.679249 & 1.923265 \\
\hline $\mathrm{H}$ & -2.986359 & -3.412644 & 3.985332 \\
\hline $\mathrm{H}$ & -1.070168 & -4.823503 & 4.661007 \\
\hline C & -2.951719 & -0.833955 & 0.838235 \\
\hline C & -1.722683 & -0.786160 & 0.106642 \\
\hline $\mathrm{H}$ & -3.955452 & -1.703025 & 2.504570 \\
\hline C & 0.989801 & -4.010831 & -3.746328 \\
\hline C & 2.046259 & -3.221086 & -3.358644 \\
\hline C & 1.944806 & -2.382047 & -2.217412 \\
\hline C & 0.725816 & -2.362166 & -1.471531 \\
\hline C & -0.347424 & -3.190294 & -1.898446 \\
\hline C & -0.217416 & -3.993417 & -3.007760 \\
\hline $\mathrm{H}$ & 3.934899 & -1.571470 & -2.376533 \\
\hline $\mathrm{H}$ & 1.076848 & -4.648460 & -4.620654 \\
\hline $\mathrm{H}$ & 2.977043 & -3.225648 & -3.918930 \\
\hline C & 3.016569 & -1.562688 & -1.796821 \\
\hline C & 0.641176 & -1.513169 & -0.324378 \\
\hline $\mathrm{H}$ & -1.279150 & -3.174842 & -1.345815 \\
\hline $\mathrm{H}$ & -1.048896 & -4.615848 & -3.322900 \\
\hline C & 1.708659 & -0.712599 & 0.066045 \\
\hline C & 2.926176 & -0.733695 & -0.694606 \\
\hline C & 1.559515 & 0.183002 & 1.281566 \\
\hline $\mathrm{H}$ & 0.876095 & -0.282184 & 2.000263 \\
\hline $\mathrm{H}$ & 2.524765 & 0.311037 & 1.774504 \\
\hline C & -1.683641 & -0.035357 & -1.189785 \\
\hline 0 & -2.309353 & -0.324931 & -2.179461 \\
\hline 0 & -0.838064 & 1.037653 & -1.159847 \\
\hline $\mathrm{H}$ & -0.831467 & 1.415839 & -2.056053 \\
\hline 0 & 1.109416 & 1.499300 & 0.952253 \\
\hline $\mathrm{H}$ & 0.370097 & 1.407995 & 0.331641 \\
\hline C & 4.112912 & 0.084483 & -0.326836 \\
\hline C & 5.378829 & -0.512811 & -0.275686 \\
\hline C & 4.000419 & 1.453210 & -0.062008 \\
\hline C & 6.523172 & 0.228110 & 0.035194 \\
\hline $\mathrm{H}$ & 5.455381 & -1.578868 & -0.463284 \\
\hline C & 5.119437 & 2.222396 & 0.269032 \\
\hline $\mathrm{H}$ & 3.017597 & 1.905698 & -0.096900 \\
\hline C & 6.369837 & 1.593941 & 0.306746 \\
\hline $\mathrm{H}$ & 7.242308 & 2.178669 & 0.559043 \\
\hline C & -4.089892 & 0.040219 & 0.467443 \\
\hline C & -5.385224 & -0.475834 & 0.372404 \\
\hline
\end{tabular}




\begin{tabular}{|c|c|c|c|}
\hline C & -3.878674 & 1.400451 & 0.221707 \\
\hline C & -6.466370 & 0.340614 & 0.025403 \\
\hline $\mathrm{H}$ & -5.533830 & -1.536409 & 0.545554 \\
\hline C & -4.929561 & 2.244307 & -0.144416 \\
\hline $\mathrm{H}$ & -2.877361 & 1.801175 & 0.331020 \\
\hline C & -6.214164 & 1.694144 & -0.233631 \\
\hline $\mathrm{H}$ & -7.037358 & 2.334042 & -0.514992 \\
\hline C & -4.634790 & 3.722908 & -0.432806 \\
\hline C & -7.870939 & -0.271341 & -0.079734 \\
\hline C & -4.079638 & 4.390793 & 0.843506 \\
\hline $\mathrm{H}$ & -4.808611 & 4.336657 & 1.658051 \\
\hline $\mathrm{H}$ & -3.160065 & 3.907456 & 1.184562 \\
\hline $\mathrm{H}$ & -3.854322 & 5.446075 & 0.654985 \\
\hline C & -5.887271 & 4.493814 & -0.878900 \\
\hline $\mathrm{H}$ & -5.624217 & 5.535778 & -1.084937 \\
\hline $\mathrm{H}$ & -6.318008 & 4.072170 & -1.792489 \\
\hline $\mathrm{H}$ & -6.660015 & 4.494912 & -0.103812 \\
\hline C & -3.582043 & 3.815610 & -1.559872 \\
\hline $\mathrm{H}$ & -3.947917 & 3.334712 & -2.472373 \\
\hline $\mathrm{H}$ & -3.358925 & 4.863487 & -1.787658 \\
\hline $\mathrm{H}$ & -2.645236 & 3.327998 & -1.276070 \\
\hline C & -8.939278 & 0.771245 & -0.446992 \\
\hline $\mathrm{H}$ & -9.000749 & 1.569268 & 0.299623 \\
\hline $\mathrm{H}$ & -8.742670 & 1.227489 & -1.422113 \\
\hline $\mathrm{H}$ & -9.920113 & 0.288967 & -0.499259 \\
\hline C & -8.256224 & -0.898724 & 1.277410 \\
\hline $\mathrm{H}$ & -8.264014 & -0.141212 & 2.067398 \\
\hline $\mathrm{H}$ & -9.254855 & -1.345222 & 1.221088 \\
\hline $\mathrm{H}$ & -7.555478 & -1.684198 & 1.573429 \\
\hline C & -7.867453 & -1.365324 & -1.169366 \\
\hline $\mathrm{H}$ & -7.592541 & -0.943743 & -2.141004 \\
\hline $\mathrm{H}$ & -7.155907 & -2.162819 & -0.939014 \\
\hline $\mathrm{H}$ & -8.861489 & -1.816844 & -1.259336 \\
\hline C & 4.924981 & 3.711696 & 0.586648 \\
\hline C & 7.888158 & -0.475036 & 0.082912 \\
\hline C & 7.855262 & -1.579463 & 1.161288 \\
\hline $\mathrm{H}$ & 7.646808 & -1.151970 & 2.147012 \\
\hline $\mathrm{H}$ & 7.084372 & -2.325814 & 0.950962 \\
\hline $\mathrm{H}$ & 8.819915 & -2.096692 & 1.208856 \\
\hline C & 9.034935 & 0.491794 & 0.419749 \\
\hline $\mathrm{H}$ & 9.121442 & 1.291608 & -0.322333 \\
\hline $\mathrm{H}$ & 8.902473 & 0.950830 & 1.404364 \\
\hline $\mathrm{H}$ & 9.983739 & -0.053440 & 0.433175 \\
\hline C & 8.182916 & -1.110574 & -1.293001 \\
\hline $\mathrm{H}$ & 7.421902 & -1.845330 & -1.569863 \\
\hline $\mathrm{H}$ & 8.210026 & -0.345627 & -2.075342 \\
\hline $\mathrm{H}$ & 9.152214 & -1.620934 & -1.278871 \\
\hline C & 6.243715 & 4.410657 & 0.952846 \\
\hline $\mathrm{H}$ & 6.706895 & 3.962527 & 1.837643 \\
\hline $\mathrm{H}$ & 6.966277 & 4.372502 & 0.131158 \\
\hline $\mathrm{H}$ & 6.051982 & 5.464512 & 1.177130 \\
\hline C & 3.950855 & 3.846829 & 1.778623 \\
\hline $\mathrm{H}$ & 3.784974 & 4.904102 & 2.014382 \\
\hline $\mathrm{H}$ & 2.981550 & 3.388071 & 1.566811 \\
\hline
\end{tabular}




\section{Catalyst 2C 38}

\section{Datum}

Value

B3LYP-D3(BJ)/6-31G(d,p) Energy $-2165.162883$

B3LYP-D3(BJ)/def2-TZVPP/IEF-PCM(DCM) Energy $-2165.899096$

B3LYP-D3(BJ)/def2-TZVPP/IEF-PCM(DCM)//B3LYP-D3(BJ)/6-31G(d,p) Free Energy (Quasiharmonic) $-2165.021208$

Number of Imaginary Frequencies

Frequencies (Top 3 out of 321)

$\begin{array}{ll}\text { 1. } & 10.2419 \mathrm{~cm}^{-1} \\ \text { 2. } & 12.5728 \mathrm{~cm}^{-1} \\ \text { 3. } & 14.9568 \mathrm{~cm}^{-1}\end{array}$

B3LYP-D3(BJ)/6-31G(d,p) Molecular Geometry in Cartesian Coordinates

$\begin{array}{lrrr}\text { C } & -0.191873 & -4.124289 & 3.063321 \\ \text { C } & -0.323662 & -3.277823 & 1.986043 \\ \text { C } & 0.752403 & -2.443555 & 1.583569 \\ \text { C } & 1.981440 & -2.504580 & 2.312193 \\ \text { C } & 2.083064 & -3.385063 & 3.420819 \\ \text { C } & 1.021261 & -4.178048 & 3.789194 \\ \text { H } & -1.023320 & -4.756171 & 3.359194 \\ \text { H } & -1.252689 & -3.236842 & 1.429565 \\ \text { C } & 0.648210 & -1.552439 & 0.468766 \\ \text { C } & 3.061550 & -1.677832 & 1.918148 \\ \text { H } & 3.018069 & -3.420633 & 3.972529 \\ \text { H } & 1.111212 & -4.848954 & 4.637851 \\ \text { C } & 2.970532 & -0.828606 & 0.836538 \\ \text { C } & 1.742764 & -0.787000 & 0.102457 \\ H & 3.977326 & -1.697152 & 2.501312 \\ \text { C } & -0.941140 & -4.023775 & -3.763563 \\ \text { C } & -2.003823 & -3.242577 & -3.375543 \\ \text { C } & -1.909665 & -2.404443 & -2.233021 \\ \text { C } & -0.691556 & -2.376812 & -1.485914 \\ \text { C } & 0.388276 & -3.196083 & -1.913273 \\ \text { C } & 0.265326 & -3.998366 & -3.024009\end{array}$




\begin{tabular}{|c|c|c|c|}
\hline $\mathrm{H}$ & -3.905406 & -1.607621 & -2.393109 \\
\hline $\mathrm{H}$ & -1.022685 & -4.660708 & -4.638934 \\
\hline $\mathrm{H}$ & -2.934065 & -3.253275 & -3.936654 \\
\hline C & -2.987643 & -1.593068 & -1.812608 \\
\hline C & -0.614609 & -1.529883 & -0.336682 \\
\hline $\mathrm{H}$ & 1.319382 & -3.174447 & -1.359787 \\
\hline $\mathrm{H}$ & 1.101778 & -4.613944 & -3.339468 \\
\hline C & -1.688268 & -0.737717 & 0.053702 \\
\hline C & -2.903909 & -0.764499 & -0.709559 \\
\hline C & -1.549795 & 0.153884 & 1.273401 \\
\hline $\mathrm{H}$ & -2.518614 & 0.272039 & 1.762076 \\
\hline $\mathrm{H}$ & -0.866910 & -0.311099 & 1.993024 \\
\hline C & 1.700910 & -0.031363 & -1.191118 \\
\hline 0 & 2.330938 & -0.312655 & -2.180499 \\
\hline 0 & 0.847501 & 1.035105 & -1.158695 \\
\hline $\mathrm{H}$ & 0.839743 & 1.416983 & -2.053305 \\
\hline 0 & -1.107360 & 1.474408 & 0.953011 \\
\hline $\mathrm{H}$ & -0.366266 & 1.390595 & 0.333652 \\
\hline C & -4.095528 & 0.047122 & -0.342585 \\
\hline C & -5.354510 & -0.566106 & -0.277123 \\
\hline C & -3.988596 & 1.421266 & -0.096305 \\
\hline C & -6.501513 & 0.167976 & 0.036113 \\
\hline $\mathrm{H}$ & -5.415642 & -1.632584 & -0.454568 \\
\hline C & -5.112924 & 2.183714 & 0.229411 \\
\hline $\mathrm{H}$ & -3.008825 & 1.874486 & -0.143138 \\
\hline C & -6.353121 & 1.537677 & 0.287253 \\
\hline $\mathrm{H}$ & -7.232773 & 2.118505 & 0.544003 \\
\hline C & 4.102738 & 0.055943 & 0.472137 \\
\hline C & 5.402255 & -0.449586 & 0.377707 \\
\hline C & 3.881528 & 1.415632 & 0.232265 \\
\hline C & 6.477809 & 0.376837 & 0.037012 \\
\hline $\mathrm{H}$ & 5.558718 & -1.509763 & 0.546328 \\
\hline C & 4.926714 & 2.269372 & -0.127201 \\
\hline $\mathrm{H}$ & 2.876875 & 1.808048 & 0.341020 \\
\hline C & 6.215752 & 1.729628 & -0.216081 \\
\hline $\mathrm{H}$ & 7.034619 & 2.377252 & -0.492389 \\
\hline C & 4.621068 & 3.747041 & -0.408993 \\
\hline C & 7.887295 & -0.223773 & -0.067737 \\
\hline C & 4.055997 & 4.403818 & 0.868747 \\
\hline $\mathrm{H}$ & 3.139032 & 3.911486 & 1.203905 \\
\hline $\mathrm{H}$ & 4.782428 & 4.351217 & 1.685667 \\
\hline $\mathrm{H}$ & 3.822980 & 5.458247 & 0.684865 \\
\hline C & 3.571530 & 3.837051 & -1.539260 \\
\hline $\mathrm{H}$ & 3.340475 & 4.884246 & -1.762235 \\
\hline $\mathrm{H}$ & 3.944584 & 3.364117 & -2.453018 \\
\hline $\mathrm{H}$ & 2.637864 & 3.340141 & -1.261314 \\
\hline C & 5.868856 & 4.530319 & -0.846554 \\
\hline $\mathrm{H}$ & 6.306074 & 4.117122 & -1.760924 \\
\hline $\mathrm{H}$ & 5.598206 & 5.571266 & -1.047841 \\
\hline $\mathrm{H}$ & 6.638890 & 4.533277 & -0.068774 \\
\hline C & 8.274691 & -0.853560 & 1.287692 \\
\hline $\mathrm{H}$ & 8.275066 & -0.099148 & 2.080682 \\
\hline $\mathrm{H}$ & 7.579375 & -1.645538 & 1.579160 \\
\hline $\mathrm{H}$ & 9.276836 & -1.292162 & 1.231652 \\
\hline
\end{tabular}




\begin{tabular}{lrrr} 
C & 8.948223 & 0.828519 & -0.428712 \\
H & 9.002271 & 1.623834 & 0.321356 \\
H & 9.932824 & 0.354010 & -0.481289 \\
H & 8.749739 & 1.287351 & -1.402238 \\
H & 7.894539 & -1.313418 & -1.161695 \\
H & 7.188670 & -2.117285 & -0.936054 \\
H & 7.618463 & -0.890107 & -2.132250 \\
C & 8.892211 & -1.756926 & -1.251311 \\
C & -5.015130 & 3.682748 & 0.545868 \\
C & -7.895144 & -0.471060 & 0.125340 \\
H & -7.868263 & -1.976333 & -0.185030 \\
H & -7.497850 & -2.174292 & -1.195748 \\
H & -7.239925 & -2.524828 & 0.523538 \\
C & -8.881080 & -2.385093 & -0.116085 \\
H & -8.835481 & 0.214028 & -0.889595 \\
H & -8.928927 & 1.285852 & -0.694754 \\
H & -8.459364 & 0.091709 & -1.910118 \\
C & -9.837852 & -0.225112 & -0.838583 \\
H & -8.453898 & -0.281769 & 1.552045 \\
H & -7.802072 & -0.759795 & 2.289902 \\
H & -8.537413 & 0.775987 & 1.816122 \\
C & -9.450930 & -0.728233 & 1.634814 \\
H & -5.434399 & 3.908880 & 2.014751 \\
H & -6.457882 & 3.569064 & 2.198515 \\
H & -4.772275 & 3.362325 & 2.693312 \\
C & -5.381717 & 4.973436 & 2.268284 \\
H & -5.957214 & 4.475025 & -0.384910 \\
H & -5.892354 & 5.546922 & -0.168563 \\
H & -5.685340 & 4.321187 & -1.433916 \\
C & -7.001353 & 4.173529 & -0.262328 \\
H & -3.586381 & 4.221323 & 0.360949 \\
H & -3.253208 & 4.118579 & -0.676952 \\
H & -3.560606 & 5.285856 & 0.614724 \\
& -2.865135 & 3.701921 & 0.997849 \\
\hline
\end{tabular}

\section{Catalyst 2C 39}

Datum

Value

B3LYP-D3(BJ)/6-31G(d,p) Energy $-2165.162885$

B3LYP-D3(BJ)/def2-TZVPP/IEF-PCM(DCM) Energy $-2165.898604$

B3LYP-D3(BJ)/def2-TZVPP/IEF-PCM(DCM)//B3LYP-D3(BJ)/6-31G(d,p) Free Energy (Quasiharmonic) -

Number of Imaginary Frequencies

Frequencies (Top 3 out of 321) 


$\begin{array}{ll}\text { 1. } & 11.5553 \mathrm{~cm}^{-1} \\ \text { 2. } & 13.0267 \mathrm{~cm}^{-1} \\ \text { 3. } & 13.4662 \mathrm{~cm}^{-1}\end{array}$

B3LYP-D3(BJ)/6-31G(d,p) Molecular Geometry in Cartesian Coordinates

C

C

$\mathrm{C}$

C

C

C

$\mathrm{H}$

$\mathrm{H}$

C

$\mathrm{C}$

$\mathrm{H}$

$\mathrm{H}$

C

C

$\mathrm{H}$

C

C

C

C

C

C

$\mathrm{H}$

$\mathrm{H}$

$\mathrm{H}$

C

C

$\mathrm{H}$

$\mathrm{H}$

C

C

C

$\mathrm{H}$

$\mathrm{H}$

C

0

0

$\mathrm{H}$

0

$\mathrm{H}$

C

C

C

C

$\mathrm{H}$

$$
-0.331568
$$

$-0.445678$

0.639908

1.854836

1.939178

0.870899

$-1.168083$

$-1.365721$

0.553013

2. 943271

2.865280

0.946673

2.871394

1.649671

3.870087

$-1.371665$

$-2.345549$

$-2.143997$

$-0.911824$

0.076263

$-0.149767$

$-4.057170$

$-1.534985$

$-3.285750$

$-3.129035$

$-0.726225$

1.018610

0.616403

$-1.711688$

$-2.944087$

$-1.495790$

$-2.451472$

$-0.836877$

1.521579

1.518521

1.386263

1. 306871

$-0.987114$

$-0.121818$

$-4.052253$

$-3.824599$

$-5.365812$

$-4.874215$

$-2.809560$
$-3.439274$

$-2.880353$

$-2.170533$

$-2.040668$

$-2.629040$

$-3.312631$

$-3.982357$

$-2.976218$

$-1.572148$

$-1.336514$

$-2.527805$

$-3.757623$

$-0.776471$

$-0.909423$

$-1.260384$

$-4.914911$

$-3.958161$

$-2.860711$

$-2.755591$

$-3.756594$

$-4.811242$

$-1.941564$

$-5.750975$

$-4.026088$

$-1.864810$

$-1.648594$

$-3.674777$

$-5.567600$

$-0.682640$

$-0.791514$

0.447456

0.724220

0.106103

$-0.295106$

$-0.883775$

1.058480

1. 381249

1.648220

1.459129

0.189896

1.563897

$-0.273953$

2.484363

1.911945

\subsection{5}

2.686078

2.108992

2.852353

4.141616

4.673808

4.366792

2. 121185

0.813819

2. 282521

4.700183

5.661066

1.023933

0.292361

2.843176

$-2.599153$

$-2.436193$

$-1.558027$

$-0.844827$

$-1.038597$

$-1.892501$

$-1.927555$

$-3.272210$

$-2.976275$

$-1.368909$

0.039593

$-0.510129$

$-2.031400$

0.213516

$-0.518695$

1. 206835

1.655038

2. 012351

$-1.069498$

$-2.124664$

$-0.993770$

$-1.908575$

0.624024

0.229968

$-0.371228$

$-0.451595$

$-0.179963$

$-0.327077$

$-0.591651$ 


\begin{tabular}{|c|c|c|c|}
\hline C & -6.435927 & 0.611643 & -0.068507 \\
\hline $\mathrm{H}$ & -5.525039 & -1.341944 & -0.099860 \\
\hline C & -6.165011 & 1.988950 & -0.142794 \\
\hline $\mathrm{H}$ & -6.989607 & 2.683777 & -0.045961 \\
\hline C & 4.037063 & -0.062049 & 0.447843 \\
\hline C & 4.709080 & 0.903683 & 1.197301 \\
\hline C & 4.479558 & -0.355491 & -0.851592 \\
\hline C & 5.811669 & 1.593422 & 0.673433 \\
\hline $\mathrm{H}$ & 4.343968 & 1.134006 & 2.192571 \\
\hline C & 5.560849 & 0.321525 & -1.411257 \\
\hline $\mathrm{H}$ & 3.970597 & -1.127566 & -1.411396 \\
\hline C & 6.212209 & 1.289763 & -0.629041 \\
\hline $\mathrm{H}$ & 7.053572 & 1.818214 & -1.058332 \\
\hline C & 6.044151 & 0.048733 & -2.842241 \\
\hline C & 6.514731 & 2.654450 & 1.531961 \\
\hline C & 5.212591 & -1.042549 & -3.536803 \\
\hline $\mathrm{H}$ & 5.294589 & -2.003124 & -3.018616 \\
\hline $\mathrm{H}$ & 4.152609 & -0.776791 & -3.591528 \\
\hline $\mathrm{H}$ & 5.575891 & -1.186182 & -4.558967 \\
\hline C & 7.518907 & -0.405401 & -2.811957 \\
\hline $\mathrm{H}$ & 7.877887 & -0.600942 & -3.828140 \\
\hline $\mathrm{H}$ & 8.168693 & 0.354181 & -2.368200 \\
\hline $\mathrm{H}$ & 7.629577 & -1.324189 & -2.227730 \\
\hline C & 5.923297 & 1.347817 & -3.668445 \\
\hline $\mathrm{H}$ & 6.523074 & 2.157034 & -3.242547 \\
\hline $\mathrm{H}$ & 6.266370 & 1.179565 & -4.694913 \\
\hline $\mathrm{H}$ & 4.883060 & 1.686112 & -3.707425 \\
\hline C & 5.507730 & 3.769744 & 1.887018 \\
\hline $\mathrm{H}$ & 5.126016 & 4.250339 & 0.980924 \\
\hline $\mathrm{H}$ & 4.651448 & 3.379879 & 2.443921 \\
\hline $\mathrm{H}$ & 5.989410 & 4.535697 & 2.504406 \\
\hline C & 7.709503 & 3.295810 & 0.808169 \\
\hline $\mathrm{H}$ & 7.400431 & 3.810776 & -0.106782 \\
\hline $\mathrm{H}$ & 8.180491 & 4.036403 & 1.461579 \\
\hline $\mathrm{H}$ & 8.469683 & 2.553668 & 0.544789 \\
\hline C & 7.032380 & 1.997151 & 2.829590 \\
\hline $\mathrm{H}$ & 6.218451 & 1.558885 & 3.413617 \\
\hline H & 7.748660 & 1.201326 & 2.602632 \\
\hline $\mathrm{H}$ & 7.533646 & 2.740043 & 3.459308 \\
\hline C & -7.881924 & 0.140365 & 0.143803 \\
\hline C & -4.552115 & 3.983838 & -0.381656 \\
\hline C & -5.806939 & 4.858507 & -0.234944 \\
\hline $\mathrm{H}$ & -6.527488 & 4.672911 & -1.038203 \\
\hline $\mathrm{H}$ & -6.309226 & 4.687855 & 0.722560 \\
\hline $\mathrm{H}$ & -5.526573 & 5.915496 & -0.277770 \\
\hline C & -3.885855 & 4.314758 & -1.734388 \\
\hline $\mathrm{H}$ & -2.958114 & 3.753555 & -1.872912 \\
\hline $\mathrm{H}$ & -4.553914 & 4.072471 & -2.567158 \\
\hline $\mathrm{H}$ & -3.644040 & 5.381949 & -1.788312 \\
\hline C & -3.576878 & 4.326161 & 0.767220 \\
\hline $\mathrm{H}$ & -4.040159 & 4.118674 & 1.737460 \\
\hline $\mathrm{H}$ & -2.654343 & 3.743044 & 0.705649 \\
\hline $\mathrm{H}$ & -3.314228 & 5.389747 & 0.737197 \\
\hline c & -8.416787 & 0.711774 & 1.474675 \\
\hline
\end{tabular}




\begin{tabular}{|lrrr|}
$H$ & -7.809144 & 0.365628 & 2.316594 \\
$H$ & -8.402713 & 1.805071 & 1.479900 \\
$H$ & -9.450179 & 0.388503 & 1.641766 \\
$\mathrm{C}$ & -7.994932 & -1.391654 & 0.198570 \\
$\mathrm{H}$ & -9.040894 & -1.679336 & 0.343089 \\
$\mathrm{H}$ & -7.647363 & -1.856014 & -0.729590 \\
$\mathrm{H}$ & -7.417500 & -1.810895 & 1.028362 \\
$\mathrm{C}$ & -8.758945 & 0.647008 & -1.021580 \\
$\mathrm{H}$ & -8.399666 & 0.251875 & -1.976937 \\
$\mathrm{H}$ & -9.797387 & 0.325211 & -0.886116 \\
$\mathrm{H}$ & -8.752391 & 1.738337 & -1.088009 \\
\hline
\end{tabular}

\section{Catalyst 2C 40}

\section{Datum}

Value

B3LYP-D3(BJ)/6-31G(d,p) Energy $-2165.162875$

B3LYP-D3(BJ)/def2-TZVPP/IEF-PCM(DCM) Energy $-2165.898495$

B3LYP-D3(BJ)/def2-TZVPP/IEF-PCM(DCM)//B3LYP-D3(BJ)/6-31G(d,p) Free Energy (Quasiharmonic) $-2165.020511$

Number of Imaginary Frequencies

Frequencies (Top 3 out of 321)

$\begin{array}{ll}\text { 1. } & 11.2827 \mathrm{~cm}^{-1} \\ \text { 2. } & 13.1276 \mathrm{~cm}^{-1} \\ \text { 3. } & 13.7852 \mathrm{~cm}^{-1}\end{array}$

B3LYP-D3(BJ)/6-31G(d,p) Molecular Geometry in Cartesian Coordinates

C

C

C

$\mathrm{C}$

C

C

$\mathrm{H}$

$\mathrm{H}$

H

C

C

$\mathrm{H}$

$\mathrm{H}$

C

C

$\mathrm{H}$

\subsection{8}

0.422764

$-0.659589$

$-1.875087$

$-1.963232$

$-0.898118$

1.138961

1. 343207

$-0.569121$

$-2.960135$

$-2.889670$

$-0.976803$

$-2.885223$

$-1.662412$

$-3.886960$
$-3.536230$

$-2.947621$

$-2.220321$

$-2.104031$

$-2.723078$

$-3.423109$

$-4.092459$

$-3.033021$

$-1.591352$

$-1.382414$

$-2.631961$

$-3.891513$

$-0.792032$

$-0.911466$

$-1.316932$

\subsection{3}

2.632014

2.070868

2.815484

4.090113

4.606980

4.286568

2.066073

0.790680

2. 261585

4.649861

5.583119

1.016884

0.284359

2.823508 


\begin{tabular}{|c|c|c|c|}
\hline C & 1.345416 & -4.870825 & -2.688641 \\
\hline C & 2.321685 & -3.919706 & -2.507708 \\
\hline C & 2.123323 & -2.839069 & -1.608286 \\
\hline C & 0.891883 & -2.744941 & -0.892246 \\
\hline C & -0.098781 & -3.739484 & -1.105044 \\
\hline C & 0.124182 & -4.777930 & -1.979417 \\
\hline $\mathrm{H}$ & 4.038177 & -1.915839 & -1.961940 \\
\hline $\mathrm{H}$ & 1.506370 & -5.694050 & -3.377904 \\
\hline $\mathrm{H}$ & 3.261363 & -3.979334 & -3.049684 \\
\hline C & 3.110620 & -1.848815 & -1.401033 \\
\hline C & 0.709652 & -1.655535 & 0.014625 \\
\hline $\mathrm{H}$ & -1.040556 & -3.665760 & -0.574354 \\
\hline $\mathrm{H}$ & -0.643878 & -5.529644 & -2.132441 \\
\hline C & 1.697776 & -0.696081 & 0.207151 \\
\hline C & 2.928129 & -0.792322 & -0.529770 \\
\hline C & 1.489896 & 0.411586 & 1.227309 \\
\hline $\mathrm{H}$ & 0.833616 & 0.052319 & 2.027490 \\
\hline $\mathrm{H}$ & 2.449291 & 0.672218 & 1.677685 \\
\hline C & -1.529630 & -0.267483 & -1.063317 \\
\hline 0 & -1.528055 & -0.835303 & -2.130499 \\
\hline 0 & -1.389716 & 1.082773 & -0.960122 \\
\hline $\mathrm{H}$ & -1.312024 & 1.424703 & -1.868143 \\
\hline 0 & 0.984267 & 1.628199 & 0.677282 \\
\hline $\mathrm{H}$ & 0.119911 & 1.450228 & 0.276409 \\
\hline C & 4.037006 & 0.185495 & -0.364547 \\
\hline C & 3.807138 & 1.564339 & -0.442672 \\
\hline C & 5.343632 & -0.281409 & -0.162544 \\
\hline C & 4.855010 & 2.478014 & -0.305653 \\
\hline $\mathrm{H}$ & 2.793092 & 1.905838 & -0.590144 \\
\hline C & 6.417125 & 0.603600 & -0.033680 \\
\hline $\mathrm{H}$ & 5.500503 & -1.350140 & -0.087242 \\
\hline C & 6.145930 & 1.975592 & -0.106055 \\
\hline $\mathrm{H}$ & 6.967328 & 2.675756 & 0.004644 \\
\hline C & -4.048016 & -0.060015 & 0.457257 \\
\hline C & -4.476585 & -0.295837 & -0.849299 \\
\hline C & -4.732831 & 0.877648 & 1.246387 \\
\hline C & -5.556922 & 0.407798 & -1.398673 \\
\hline $\mathrm{H}$ & -3.964484 & -1.042192 & -1.443817 \\
\hline C & -5.824119 & 1.581411 & 0.740494 \\
\hline $\mathrm{H}$ & -4.374535 & 1.060937 & 2.251433 \\
\hline C & -6.213091 & 1.333876 & -0.587686 \\
\hline $\mathrm{H}$ & -7.050868 & 1.887737 & -0.991345 \\
\hline C & -6.595001 & 2.619338 & 1.568395 \\
\hline C & -5.952244 & 0.143601 & -2.857701 \\
\hline C & -6.039214 & 2.756833 & 2.994944 \\
\hline $\mathrm{H}$ & -6.096452 & 1.811714 & 3.544049 \\
\hline $\mathrm{H}$ & -4.997012 & 3.090612 & 2.993657 \\
\hline $\mathrm{H}$ & -6.624023 & 3.498100 & 3.547949 \\
\hline C & -8.076153 & 2.194134 & 1.665789 \\
\hline $\mathrm{H}$ & -8.644286 & 2.925277 & 2.250966 \\
\hline $\mathrm{H}$ & -8.542476 & 2.120056 & 0.679589 \\
\hline $\mathrm{H}$ & -8.168621 & 1.218849 & 2.153787 \\
\hline C & -6.499759 & 3.996768 & 0.877308 \\
\hline $\mathrm{H}$ & -6.922387 & 3.973750 & -0.130911 \\
\hline
\end{tabular}




\begin{tabular}{|c|c|c|c|}
\hline $\mathrm{H}$ & -7.047236 & 4.750701 & 1.453234 \\
\hline $\mathrm{H}$ & -5.456841 & 4.318402 & 0.796442 \\
\hline C & -4.753792 & 0.494636 & -3.767996 \\
\hline $\mathrm{H}$ & -4.496367 & 1.554679 & -3.670909 \\
\hline $\mathrm{H}$ & -3.865313 & -0.091571 & -3.518155 \\
\hline $\mathrm{H}$ & -5.002824 & 0.300436 & -4.816893 \\
\hline C & -7.160173 & 0.985441 & -3.297869 \\
\hline $\mathrm{H}$ & -6.954631 & 2.058133 & -3.225833 \\
\hline $\mathrm{H}$ & -7.402031 & 0.764305 & -4.341808 \\
\hline $\mathrm{H}$ & -8.048281 & 0.764800 & -2.697054 \\
\hline C & -6.310683 & -1.347684 & -3.032561 \\
\hline $\mathrm{H}$ & -5.468974 & -1.998980 & -2.783068 \\
\hline $\mathrm{H}$ & -7.152112 & -1.623149 & -2.388992 \\
\hline $\mathrm{H}$ & -6.592885 & -1.550597 & -4.071184 \\
\hline C & 7.859588 & 0.128193 & 0.193896 \\
\hline C & 4.621940 & 3.994964 & -0.343232 \\
\hline C & 3.151736 & 4.347543 & -0.625523 \\
\hline $\mathrm{H}$ & 2.835534 & 3.980472 & -1.607407 \\
\hline $\mathrm{H}$ & 2.477723 & 3.924962 & 0.124670 \\
\hline $\mathrm{H}$ & 3.028734 & 5.435316 & -0.621765 \\
\hline C & 5.495369 & 4.629196 & -1.446097 \\
\hline $\mathrm{H}$ & 6.561261 & 4.456071 & -1.272561 \\
\hline $\mathrm{H}$ & 5.244057 & 4.213181 & -2.426786 \\
\hline $\mathrm{H}$ & 5.335092 & 5.712275 & -1.482766 \\
\hline C & 5.010160 & 4.592051 & 1.026922 \\
\hline $\mathrm{H}$ & 4.395328 & 4.161391 & 1.823206 \\
\hline $\mathrm{H}$ & 6.058785 & 4.396487 & 1.269506 \\
\hline $\mathrm{H}$ & 4.860749 & 5.677432 & 1.027492 \\
\hline C & 8.751647 & 0.639047 & -0.958031 \\
\hline $\mathrm{H}$ & 8.403397 & 0.249592 & -1.919763 \\
\hline $\mathrm{H}$ & 8.747469 & 1.730724 & -1.019044 \\
\hline $\mathrm{H}$ & 9.787854 & 0.314867 & -0.811635 \\
\hline C & 7.968624 & -1.404359 & 0.242323 \\
\hline $\mathrm{H}$ & 9.012117 & -1.694972 & 0.398302 \\
\hline $\mathrm{H}$ & 7.380132 & -1.826551 & 1.062789 \\
\hline $\mathrm{H}$ & 7.631639 & -1.863355 & -0.692378 \\
\hline C & 8.378639 & 0.692004 & 1.534141 \\
\hline $\mathrm{H}$ & 7.759781 & 0.343119 & 2.366687 \\
\hline $\mathrm{H}$ & 9.409280 & 0.366163 & 1.712778 \\
\hline $\mathrm{H}$ & 8.366241 & 1.785317 & 1.544449 \\
\hline
\end{tabular}

\section{Catalyst 2C 41}

Datum

Value

B3LYP-D3(BJ)/6-31G(d,p) Energy

$-2165.162276$

B3LYP-D3(BJ)/def2-TZVPP/IEF-PCM(DCM) Energy

$-2165.898309$

B3LYP-D3(BJ)/def2-TZVPP/IEF-PCM(DCM)//B3LYP-D3(BJ)/6-31G(d,p) Free Energy (Quasiharmonic) - 
Frequencies (Top 3 out of 321)

$\begin{array}{ll}\text { 1. } & 11.2912 \mathrm{~cm}^{-1} \\ \text { 2. } & 13.2189 \mathrm{~cm}^{-1} \\ \text { 3. } & 13.3608 \mathrm{~cm}^{-1}\end{array}$

B3LYP-D3(BJ)/6-31G(d,p) Molecular Geometry in Cartesian Coordinates

\begin{tabular}{|c|c|c|c|}
\hline C & 0.353907 & -3.521818 & 3.870372 \\
\hline C & 0.463008 & -2.935053 & 2.630282 \\
\hline C & -0.625644 & -2.214586 & 2.072507 \\
\hline C & -1.838504 & -2.103529 & 2.822243 \\
\hline C & -1.917623 & -2.720448 & 4.098473 \\
\hline C & -0.846456 & -3.413653 & 4.612057 \\
\hline $\mathrm{H}$ & 1.192741 & -4.072705 & 4.283888 \\
\hline $\mathrm{H}$ & 1.381425 & -3.016588 & 2.060487 \\
\hline C & -0.543813 & -1.586918 & 0.791016 \\
\hline C & -2.930176 & -1.389501 & 2.271350 \\
\hline $\mathrm{H}$ & -2.842093 & -2.633283 & 4.662096 \\
\hline $\mathrm{H}$ & -0.918289 & -3.880476 & 5.589483 \\
\hline C & -2.863729 & -0.801785 & 1.025010 \\
\hline C & -1.643299 & -0.914763 & 0.287815 \\
\hline $\mathrm{H}$ & -3.855287 & -1.328137 & 2.836579 \\
\hline C & 1.376167 & -4.848244 & -2.702948 \\
\hline C & 2.348381 & -3.892866 & -2.522461 \\
\hline C & 2.147531 & -2.815651 & -1.619551 \\
\hline C & 0.917833 & -2.729390 & -0.899441 \\
\hline C & -0.068610 & -3.728176 & -1.111889 \\
\hline C & 0.156706 & -4.763164 & -1.989753 \\
\hline $\mathrm{H}$ & 4.056844 & -1.881408 & -1.976715 \\
\hline $\mathrm{H}$ & 1.538957 & -5.668780 & -3.394978 \\
\hline $\mathrm{H}$ & 3. 286617 & -3.946404 & -3.067570 \\
\hline C & 3.130658 & -1.821042 & -1.412741 \\
\hline C & 0.732844 & -1.643272 & 0.010901 \\
\hline $\mathrm{H}$ & -1.009144 & -3.660351 & -0.578237 \\
\hline $\mathrm{H}$ & -0.608236 & -5.518087 & -2.142554 \\
\hline C & 1.716875 & -0.679581 & 0.202848 \\
\hline C & 2.945246 & -0.768218 & -0.537884 \\
\hline C & 1.506948 & 0.425873 & 1.225034 \\
\hline $\mathrm{H}$ & 0.856385 & 0.062034 & 2.027823 \\
\hline $\mathrm{H}$ & 2.466688 & 0.691840 & 1.671575 \\
\hline C & -1.518655 & -0.267599 & -1.059199 \\
\hline 0 & -1.517215 & -0.830671 & -2.128350 \\
\hline 0 & -1.383056 & 1.083533 & -0.950863 \\
\hline $\mathrm{H}$ & -1.303278 & 1.428525 & -1.857471 \\
\hline
\end{tabular}




\begin{tabular}{|c|c|c|c|}
\hline 0 & 0.991730 & 1.639637 & 0.677831 \\
\hline $\mathrm{H}$ & 0.126572 & 1.456509 & 0.280923 \\
\hline C & 4.049585 & 0.214767 & -0.372866 \\
\hline C & 3.812396 & 1.595984 & -0.452865 \\
\hline C & 5.355160 & -0.239558 & -0.170620 \\
\hline C & 4.853322 & 2.512159 & -0.317651 \\
\hline $\mathrm{H}$ & 2.796116 & 1.930936 & -0.599931 \\
\hline C & 6.428957 & 0.652689 & -0.042867 \\
\hline $\mathrm{H}$ & 5.525057 & -1.308436 & -0.092500 \\
\hline C & 6.153260 & 2.018999 & -0.117022 \\
\hline $\mathrm{H}$ & 6.964145 & 2.728056 & -0.009855 \\
\hline C & -4.033342 & -0.079603 & 0.466930 \\
\hline C & -4.473437 & -0.342809 & -0.835859 \\
\hline C & -4.709957 & 0.866419 & 1.246237 \\
\hline C & -5.560450 & 0.344507 & -1.380900 \\
\hline $\mathrm{H}$ & -3.961929 & -1.098607 & -1.415179 \\
\hline C & -5.811645 & 1.560749 & 0.739488 \\
\hline $\mathrm{H}$ & -4.341881 & 1.067782 & 2.244304 \\
\hline C & -6.210580 & 1.286008 & -0.574797 \\
\hline H & -7.056041 & 1.827381 & -0.985598 \\
\hline C & -6.575558 & 2.611310 & 1.558174 \\
\hline C & -6.041275 & 0.105163 & -2.818840 \\
\hline C & -6.491453 & 3.976340 & 0.841672 \\
\hline $\mathrm{H}$ & -5.450323 & 4.298766 & 0.743061 \\
\hline $\mathrm{H}$ & -6.925214 & 3.934176 & -0.161229 \\
\hline $\mathrm{H}$ & -7.034187 & 4.739382 & 1.410043 \\
\hline C & -6.003499 & 2.774970 & 2.975513 \\
\hline H & -6.583467 & 3.524733 & 3.522111 \\
\hline $\mathrm{H}$ & -6.052385 & 1.839606 & 3.541844 \\
\hline $\mathrm{H}$ & -4.962102 & 3.110664 & 2.956293 \\
\hline C & -8.054307 & 2.184367 & 1.680025 \\
\hline H & -8.138786 & 1.217473 & 2.185812 \\
\hline $\mathrm{H}$ & -8.617799 & 2.924205 & 2.258716 \\
\hline $\mathrm{H}$ & -8.531489 & 2.091746 & 0.700586 \\
\hline C & -5.923507 & 1.424537 & -3.612543 \\
\hline H & -6.526242 & 2.221071 & -3.167268 \\
\hline $\mathrm{H}$ & -4.884377 & 1.767126 & -3.641718 \\
\hline $\mathrm{H}$ & -6.264868 & 1.280840 & -4.643308 \\
\hline C & -7.514564 & -0.354065 & -2.801011 \\
\hline H & -8.166865 & 0.392445 & -2.339055 \\
\hline $\mathrm{H}$ & -7.872378 & -0.525636 & -3.821919 \\
\hline $\mathrm{H}$ & -7.623044 & -1.287132 & -2.239469 \\
\hline C & -5.205463 & -0.966275 & -3.538780 \\
\hline H & -4.146294 & -0.695927 & -3.586034 \\
\hline $\mathrm{H}$ & -5.284807 & -1.939410 & -3.044140 \\
\hline $\mathrm{H}$ & -5.567406 & -1.086240 & -4.564467 \\
\hline C & 7.844601 & 0.102455 & 0.183792 \\
\hline C & 4.613663 & 4.027856 & -0.358117 \\
\hline C & 5.003655 & 4.630688 & 1.009095 \\
\hline H & 4.393051 & 4.199774 & 1.808505 \\
\hline $\mathrm{H}$ & 6.053909 & 4.440858 & 1.249035 \\
\hline $\mathrm{H}$ & 4.849351 & 5.715384 & 1.006727 \\
\hline C & 3.141033 & 4.373371 & -0.636294 \\
\hline $\mathrm{H}$ & 2.823416 & 4.003307 & -1.616594 \\
\hline
\end{tabular}




\begin{tabular}{|llll|}
$H$ & 2.471455 & 3.948762 & 0.116741 \\
$H$ & 3.012992 & 5.460574 & -0.633824 \\
$\mathrm{C}$ & 5.480540 & 4.663378 & -1.465536 \\
$\mathrm{H}$ & 6.547799 & 4.495760 & -1.295150 \\
$\mathrm{H}$ & 5.227651 & 4.243776 & -2.444311 \\
$\mathrm{H}$ & 5.314968 & 5.745598 & -1.504325 \\
$\mathrm{C}$ & 8.896773 & 1.218133 & 0.288983 \\
$\mathrm{H}$ & 8.939141 & 1.821724 & -0.623046 \\
$\mathrm{H}$ & 8.696340 & 1.885606 & 1.132873 \\
$\mathrm{H}$ & 9.886699 & 0.777504 & 0.442462 \\
$\mathrm{C}$ & 8.229964 & -0.814073 & -0.997408 \\
$\mathrm{H}$ & 9.236523 & -1.221672 & -0.852790 \\
$\mathrm{H}$ & 7.539147 & -1.655755 & -1.097334 \\
$\mathrm{H}$ & 8.217907 & -0.257385 & -1.939668 \\
$\mathrm{C}$ & 7.871453 & -0.711983 & 1.495117 \\
$\mathrm{H}$ & 7.171128 & -1.551087 & 1.463848 \\
$\mathrm{H}$ & 8.873630 & -1.117295 & 1.673087 \\
$\mathrm{H}$ & 7.600367 & -0.081717 & 2.347844 \\
& & & \\
\hline
\end{tabular}

\section{Catalyst 2C 42}

\section{Datum}

Value

B3LYP-D3(BJ)/6-31G(d,p) Energy

$-2165.162349$

B3LYP-D3(BJ)/def2-TZVPP/IEF-PCM(DCM) Energy

$-2165.898197$

B3LYP-D3(BJ)/def2-TZVPP/IEF-PCM(DCM)//B3LYP-D3(BJ)/6-31G(d,p) Free Energy (Quasiharmonic)

$-2165.020468$

Number of Imaginary Frequencies

Frequencies (Top 3 out of 321)

$\begin{array}{ll}\text { 1. } & 10.8685 \mathrm{~cm}^{-1} \\ \text { 2. } & 13.0047 \mathrm{~cm}^{-1} \\ \text { 3. } & 13.5728 \mathrm{~cm}^{-1}\end{array}$

B3LYP-D3(BJ)/6-31G(d,p) Molecular Geometry in Cartesian Coordinates

C

C

C

C

C

C

$\mathrm{H}$

$\mathrm{H}$

-0.103091
-0.244933
0.803875
2.019730
2.131679
1.094456
-0.913219
-1.157863

$-4.915743$

$-3.745223$

$-2.784343$

$-3.067121$

$-4.284529$

$-5.187384$

$-5.637709$

$-3.542310$
$-1.270419$

$-0.558695$

$-0.525327$

$-1.224875$

$-1.947182$

$-1.973626$

$-1.288882$

$-0.010554$ 


\begin{tabular}{|c|c|c|c|}
\hline C & 0.684446 & -1.551091 & 0.187394 \\
\hline C & 3.072666 & -2.122533 & -1.189653 \\
\hline $\mathrm{H}$ & 3.055184 & -4.488185 & -2.481551 \\
\hline $\mathrm{H}$ & 1.192157 & -6.113346 & -2.531434 \\
\hline C & 2.965162 & -0.934854 & -0.494392 \\
\hline C & 1.748256 & -0.665906 & 0.206904 \\
\hline $\mathrm{H}$ & 3.979217 & -2.327890 & -1.750817 \\
\hline C & -0.796472 & -2.196420 & 5.085942 \\
\hline C & -1.877993 & -1.640445 & 4.444363 \\
\hline C & -1.819186 & -1.324231 & 3.061016 \\
\hline C & -0.616951 & -1.592113 & 2.335414 \\
\hline C & 0.481848 & -2.170874 & 3.026581 \\
\hline C & 0.394031 & -2.463436 & 4.368138 \\
\hline $\mathrm{H}$ & -3.842901 & -0.596689 & 2.924155 \\
\hline $\mathrm{H}$ & -0.851827 & -2.433188 & 6.144044 \\
\hline $\mathrm{H}$ & -2.796995 & -1.433475 & 4.985614 \\
\hline C & -2.921213 & -0.765693 & 2.375145 \\
\hline C & -0.571573 & -1.260571 & 0.946924 \\
\hline $\mathrm{H}$ & 1.398198 & -2.373302 & 2.484625 \\
\hline $\mathrm{H}$ & 1.243634 & -2.902885 & 4.881528 \\
\hline C & -1.660551 & -0.692174 & 0.299880 \\
\hline C & -2.874293 & -0.459242 & 1.028155 \\
\hline C & -1.529478 & -0.290617 & -1.157581 \\
\hline $\mathrm{H}$ & -2.207138 & 0.534255 & -1.376512 \\
\hline $\mathrm{H}$ & -0.509300 & 0.058184 & -1.344545 \\
\hline C & 1.593921 & 0.598541 & 0.998798 \\
\hline 0 & 0.837103 & 1.505561 & 0.720470 \\
\hline 0 & 2.393090 & 0.615627 & 2.084290 \\
\hline $\mathrm{H}$ & 2.240568 & 1.471503 & 2.520801 \\
\hline 0 & -1.885605 & -1.333420 & -2.074337 \\
\hline $\mathrm{H}$ & -1.243600 & -2.049384 & -1.969310 \\
\hline C & -4.087545 & 0.105987 & 0.382270 \\
\hline C & -4.565078 & -0.411883 & -0.822399 \\
\hline C & -4.789100 & 1.153332 & 1.002886 \\
\hline C & -5.716181 & 0.105561 & -1.431277 \\
\hline $\mathrm{H}$ & -4.016168 & -1.217711 & -1.293532 \\
\hline C & -5.946477 & 1.682018 & 0.434083 \\
\hline $\mathrm{H}$ & -4.394203 & 1.557842 & 1.926339 \\
\hline C & -6.389870 & 1.142967 & -0.786053 \\
\hline $\mathrm{H}$ & -7.281797 & 1.556222 & -1.239690 \\
\hline C & 4.076386 & 0.047530 & -0.510282 \\
\hline C & 5.396614 & -0.375587 & -0.291788 \\
\hline C & 3.823531 & 1.397925 & -0.751881 \\
\hline C & 6.452567 & 0.534079 & -0.300357 \\
\hline $\mathrm{H}$ & 5.572460 & -1.424257 & -0.087961 \\
\hline C & 4.856166 & 2.344417 & -0.746176 \\
\hline $\mathrm{H}$ & 2.806746 & 1.714935 & -0.949563 \\
\hline C & 6.156271 & 1.890196 & -0.522866 \\
\hline $\mathrm{H}$ & 6.970040 & 2.603881 & -0.515610 \\
\hline C & 4.510480 & 3.823353 & -0.964776 \\
\hline C & 7.909078 & 0.113008 & -0.058608 \\
\hline C & 3.827102 & 3.994787 & -2.338278 \\
\hline $\mathrm{H}$ & 4.491129 & 3.670808 & -3.145817 \\
\hline $\mathrm{H}$ & 2.904591 & 3.412478 & -2.407494 \\
\hline
\end{tabular}




\begin{tabular}{|c|c|c|c|}
\hline $\mathrm{H}$ & 3.571254 & 5.046411 & -2.506160 \\
\hline C & 5.753735 & 4.725781 & -0.927142 \\
\hline $\mathrm{H}$ & 5.457437 & 5.767453 & -1.083103 \\
\hline $\mathrm{H}$ & 6.267263 & 4.668366 & 0.037841 \\
\hline $\mathrm{H}$ & 6.469030 & 4.462782 & -1.712986 \\
\hline C & 3.542723 & 4.277787 & 0.151231 \\
\hline $\mathrm{H}$ & 4.015581 & 4.175837 & 1.133809 \\
\hline $\mathrm{H}$ & 3.269887 & 5.329614 & 0.013075 \\
\hline $\mathrm{H}$ & 2.621050 & 3.689443 & 0.158173 \\
\hline C & 8.762605 & 0.505668 & -1.283908 \\
\hline $\mathrm{H}$ & 8.398910 & 0.005050 & -2.186656 \\
\hline $\mathrm{H}$ & 8.738006 & 1.583411 & -1.466463 \\
\hline $\mathrm{H}$ & 9.807484 & 0.215910 & -1.128724 \\
\hline C & 8.049703 & -1.402075 & 0.159606 \\
\hline $\mathrm{H}$ & 7.698362 & -1.969797 & -0.707748 \\
\hline $\mathrm{H}$ & 9.102427 & -1.655118 & 0.317935 \\
\hline $\mathrm{H}$ & 7.491873 & -1.738456 & 1.039012 \\
\hline C & 8.447735 & 0.834359 & 1.195762 \\
\hline $\mathrm{H}$ & 8.415801 & 1.921419 & 1.082261 \\
\hline $\mathrm{H}$ & 7.855309 & 0.572089 & 2.077781 \\
\hline $\mathrm{H}$ & 9.488037 & 0.547237 & 1.383497 \\
\hline C & -6.735649 & 2.830378 & 1.079369 \\
\hline C & -6.167496 & -0.477292 & -2.777254 \\
\hline C & -7.442214 & 0.196320 & -3.308999 \\
\hline $\mathrm{H}$ & -8.286170 & 0.066604 & -2.623624 \\
\hline $\mathrm{H}$ & -7.294899 & 1.268559 & -3.472738 \\
\hline $\mathrm{H}$ & -7.721825 & -0.249708 & -4.268433 \\
\hline C & -6.448764 & -1.986271 & -2.612598 \\
\hline $\mathrm{H}$ & -5.558757 & -2.527149 & -2.280742 \\
\hline $\mathrm{H}$ & -7.241043 & -2.156574 & -1.876502 \\
\hline $\mathrm{H}$ & -6.768482 & -2.419736 & -3.566607 \\
\hline C & -5.037533 & -0.276398 & -3.812125 \\
\hline $\mathrm{H}$ & -4.835427 & 0.789978 & -3.956869 \\
\hline $\mathrm{H}$ & -4.106026 & -0.753605 & -3.496729 \\
\hline $\mathrm{H}$ & -5.329694 & -0.700931 & -4.779299 \\
\hline C & -8.179703 & 2.361811 & 1.360537 \\
\hline $\mathrm{H}$ & -8.692297 & 2.057923 & 0.443809 \\
\hline $\mathrm{H}$ & -8.183669 & 1.507416 & 2.044620 \\
\hline $\mathrm{H}$ & -8.761121 & 3.169931 & 1.817877 \\
\hline C & -6.765740 & 4.035750 & 0.114983 \\
\hline $\mathrm{H}$ & -7.328208 & 4.865338 & 0.557444 \\
\hline $\mathrm{H}$ & -5.751036 & 4.385299 & -0.099294 \\
\hline $\mathrm{H}$ & -7.239054 & 3.779953 & -0.836925 \\
\hline C & -6.113626 & 3.292332 & 2.407014 \\
\hline $\mathrm{H}$ & -5.095271 & 3.668078 & 2.267983 \\
\hline $\mathrm{H}$ & -6.712267 & 4.104018 & 2.831879 \\
\hline $\mathrm{H}$ & -6.082416 & 2.481992 & 3.142126 \\
\hline
\end{tabular}


Frequencies (Top 3 out of 321)

$\begin{array}{ll}\text { 1. } & 11.6546 \mathrm{~cm}^{-1} \\ \text { 2. } & 13.0261 \mathrm{~cm}^{-1} \\ \text { 3. } & 13.3281 \mathrm{~cm}^{-1}\end{array}$

\section{B3LYP-D3(BJ)/6-31G(d,p) Molecular Geometry in Cartesian Coordinates}

C

C

C

C

C

C

$\mathrm{H}$

$\mathrm{H}$

C

C

$\mathrm{H}$

$\mathrm{H}$

C

C

$\mathrm{H}$

C

C

C

c

C

C

$\mathrm{H}$

$\mathrm{H}$

$\mathrm{H}$

C

C

$\mathrm{H}$

$\mathrm{H}$

C

C

C

$\mathrm{H}$

\subsection{7}

0.464639

$-0.625392$

$-1.841285$

$-1.922164$

$-0.849712$

1.193649

1.385447

$-0.542414$

$-2.933804$

$-2.848942$

$-0.922862$

$-2.865943$

$-1.642903$

$-3.860529$

1.400780

2. 368549

2. 160941

0.928972

$-0.052699$

0.179216

4.068142

1.568720

3. 308438

3. 139955

0.736845

$-0.994812$

$-0.582034$

1.716404

2.948926

1.494533

0.837938
$-3.468328$

$-2.894263$

$-2.184901$

$-2.071219$

$-2.674978$

$-3.357874$

$-4.010901$

$-2.977660$

$-1.571358$

$-1.367058$

$-2.585927$

$-3.814669$

$-0.791705$

$-0.908810$

$-1.303170$

$-4.867775$

$-3.906272$

$-2.819169$

$-2.729222$

$-3.734762$

$-4.779336$

$-1.883734$

$-5.695909$

$-3.962506$

$-1.819038$

$-1.632165$

$-3.664548$

$-5.539441$

$-0.661975$

$-0.756357$

0.457662

0.105137

\subsection{9}

2.662798

2.093577

2.837976

4. 120388

4.644937

4. 330942

2.097175

0.805381

2. 276365

4.679897

5.626985

1.024264

0.291705

2. 838631

$-2.642198$

$-2.470831$

$-1.581332$

$-0.865692$

$-1.068275$

$-1.932943$

$-1.942997$

- 3.323861

$-3.012797$

$-1.382871$

0.029776

$-0.537719$

$-2.078312$

0.212694

$-0.520966$

1. 216511

2.019145 


\begin{tabular}{|c|c|c|c|}
\hline $\mathrm{H}$ & 2.448795 & 0.735751 & 1.666979 \\
\hline C & -1.518525 & -0.281183 & -1.064381 \\
\hline 0 & -1.513171 & -0.862637 & -2.124129 \\
\hline 0 & -1.391841 & 1.071808 & -0.979184 \\
\hline $\mathrm{H}$ & -1.320348 & 1.402346 & -1.891935 \\
\hline 0 & 0.979013 & 1.660666 & 0.644648 \\
\hline $\mathrm{H}$ & 0.114757 & 1.470425 & 0.248992 \\
\hline C & 4.051938 & 0.229130 & -0.362229 \\
\hline C & 3.817406 & 1.606129 & -0.432755 \\
\hline C & 5.363778 & -0.224006 & -0.171154 \\
\hline C & 4.859716 & 2.527762 & -0.298753 \\
\hline $\mathrm{H}$ & 2.800543 & 1.949060 & -0.572226 \\
\hline C & 6.433984 & 0.667407 & -0.049964 \\
\hline $\mathrm{H}$ & 5.535647 & -1.292852 & -0.097304 \\
\hline C & 6.158699 & 2.039557 & -0.114288 \\
\hline $\mathrm{H}$ & 6.972660 & 2.742279 & -0.012097 \\
\hline C & -4.035951 & -0.076923 & 0.457410 \\
\hline C & -4.727059 & 0.865038 & 1.235874 \\
\hline C & -4.464764 & -0.332771 & -0.845289 \\
\hline C & -5.824877 & 1.553468 & 0.723059 \\
\hline $\mathrm{H}$ & -4.368537 & 1.063993 & 2.237865 \\
\hline C & -5.551725 & 0.355078 & -1.401587 \\
\hline $\mathrm{H}$ & -3.947620 & -1.082415 & -1.431258 \\
\hline C & -6.214002 & 1.286012 & -0.601200 \\
\hline $\mathrm{H}$ & -7.056888 & 1.827987 & -1.010298 \\
\hline C & -5.947389 & 0.069201 & -2.856428 \\
\hline C & -6.602848 & 2.595459 & 1.539132 \\
\hline C & -4.753373 & 0.418239 & -3.773300 \\
\hline $\mathrm{H}$ & -4.504329 & 1.481469 & -3.690174 \\
\hline $\mathrm{H}$ & -3.859771 & -0.157607 & -3.517696 \\
\hline $\mathrm{H}$ & -5.002639 & 0.208731 & -4.819184 \\
\hline C & -7.162780 & 0.895669 & -3.305099 \\
\hline $\mathrm{H}$ & -7.404623 & 0.659331 & -4.345704 \\
\hline $\mathrm{H}$ & -8.048089 & 0.675629 & -2.699951 \\
\hline $\mathrm{H}$ & -6.965723 & 1.970802 & -3.247099 \\
\hline C & -6.294111 & -1.427022 & -3.011622 \\
\hline $\mathrm{H}$ & -7.132137 & -1.701014 & -2.363009 \\
\hline $\mathrm{H}$ & -6.576512 & -1.645434 & -4.047039 \\
\hline $\mathrm{H}$ & -5.446740 & -2.068299 & -2.755423 \\
\hline C & -6.520432 & 3.964693 & 0.830315 \\
\hline $\mathrm{H}$ & -5.480403 & 4.294010 & 0.743397 \\
\hline $\mathrm{H}$ & -6.944674 & 3.925321 & -0.176726 \\
\hline $\mathrm{H}$ & -7.073169 & 4.721295 & 1.397647 \\
\hline C & -6.045602 & 2.755826 & 2.962706 \\
\hline $\mathrm{H}$ & -5.006203 & 3.098141 & 2.955129 \\
\hline $\mathrm{H}$ & -6.635508 & 3.499268 & 3.507304 \\
\hline $\mathrm{H}$ & -6.094036 & 1.817388 & 3.523979 \\
\hline C & -8.080209 & 2.159173 & 1.644821 \\
\hline $\mathrm{H}$ & -8.547670 & 2.068590 & 0.660541 \\
\hline $\mathrm{H}$ & -8.163640 & 1.189477 & 2.145423 \\
\hline $\mathrm{H}$ & -8.653371 & 2.892988 & 2.221686 \\
\hline C & 7.853290 & 0.119096 & 0.161765 \\
\hline C & 4.531442 & 4.026800 & -0.342551 \\
\hline C & 3.553042 & 4.356538 & 0.807365 \\
\hline
\end{tabular}




\begin{tabular}{|c|c|c|c|}
\hline $\mathrm{H}$ & 2.633255 & 3.769738 & 0.739996 \\
\hline $\mathrm{H}$ & 4.015662 & 4.143713 & 1.776775 \\
\hline $\mathrm{H}$ & 3.285845 & 5.419210 & 0.785040 \\
\hline C & 3.865734 & 4.365641 & -1.693630 \\
\hline H & 4.535687 & 4.132001 & -2.527359 \\
\hline $\mathrm{H}$ & 2.940253 & 3.802091 & -1.837587 \\
\hline H & 3.620166 & 5.432359 & -1.739945 \\
\hline C & 5.781870 & 4.906550 & -0.187351 \\
\hline $\mathrm{H}$ & 6.283773 & 4.731624 & 0.769543 \\
\hline $\mathrm{H}$ & 6.504708 & 4.730869 & -0.990737 \\
\hline $\mathrm{H}$ & 5.495951 & 5.962353 & -0.222765 \\
\hline C & 7.894577 & -0.698418 & 1.470850 \\
\hline $\mathrm{H}$ & 7.630060 & -0.070866 & 2.327638 \\
\hline $\mathrm{H}$ & 7.195823 & -1.539000 & 1.444086 \\
\hline $\mathrm{H}$ & 8.899343 & -1.101818 & 1.638486 \\
\hline C & 8.905264 & 1.235845 & 0.259486 \\
\hline $\mathrm{H}$ & 9.897029 & 0.795566 & 0.401911 \\
\hline $\mathrm{H}$ & 8.938220 & 1.842030 & -0.651177 \\
\hline $\mathrm{H}$ & 8.712899 & 1.901003 & 1.107016 \\
\hline C & 8.229339 & -0.794143 & -1.025019 \\
\hline $\mathrm{H}$ & 8.206497 & -0.235709 & -1.966055 \\
\hline $\mathrm{H}$ & 9.238340 & -1.199493 & -0.891293 \\
\hline $\mathrm{H}$ & 7.539627 & -1.637329 & -1.119742 \\
\hline
\end{tabular}

\section{Catalyst 2C 44}

Datum

Value

\begin{tabular}{lr}
\hline B3LYP-D3(BJ)/6-31G(d,p) Energy & -2165.162244 \\
\hline B3LYP-D3(BJ)/def2-TZVPP/IEF-PCM(DCM) Energy & -2165.898282 \\
\hline B3LYP-D3(BJ)/def2-TZVPP/IEF-PCM(DCM)//B3LYP-D3(BJ)/6-31G(d,p) Free Energy (Quasiharmonic) & -2165.020413 \\
\hline
\end{tabular}

Number of Imaginary Frequencies

Frequencies (Top 3 out of 321)
1. $11.4192 \mathrm{~cm}^{-1}$
2. $13.2330 \mathrm{~cm}^{-1}$
3. $13.5144 \mathrm{~cm}^{-1}$

\section{B3LYP-D3(BJ)/6-31G(d,p) Molecular Geometry in Cartesian Coordinates}

$\begin{array}{rrrr}C & 0.306517 & -3.500844 & 3.895188 \\ C & 0.423841 & -2.925378 & 2.650554 \\ C & -0.657987 & -2.202324 & 2.082946\end{array}$




\begin{tabular}{|c|c|c|c|}
\hline C & -1.872559 & -2.076652 & 2.827596 \\
\hline C & -1.960282 & -2.682229 & 4.108649 \\
\hline C & -0.895593 & -3.378318 & 4.631764 \\
\hline $\mathrm{H}$ & 1.140164 & -4.053895 & 4.316238 \\
\hline $\mathrm{H}$ & 1.343572 & -3.018027 & 2.084597 \\
\hline C & -0.567568 & -1.586600 & 0.796209 \\
\hline C & -2.957352 & -1.359581 & 2.266989 \\
\hline $\mathrm{H}$ & -2.886090 & -2.584109 & 4.668265 \\
\hline $\mathrm{H}$ & -0.973951 & -3.836419 & 5.612809 \\
\hline C & -2.882127 & -0.782648 & 1.016239 \\
\hline C & -1.660646 & -0.911225 & 0.283536 \\
\hline $\mathrm{H}$ & -3.884138 & -1.286894 & 2.828155 \\
\hline C & 1.343977 & -4.896702 & -2.655936 \\
\hline C & 2.321365 & -3.945358 & -2.482369 \\
\hline C & 2.123936 & -2.857105 & -1.591973 \\
\hline C & 0.892315 & -2.755653 & -0.877207 \\
\hline C & -0.099522 & -3.750640 & -1.082329 \\
\hline C & 0.122513 & -4.796443 & -1.948115 \\
\hline $\mathrm{H}$ & 4.039783 & -1.938725 & -1.952925 \\
\hline $\mathrm{H}$ & 1.504195 & -5.725735 & -3.338376 \\
\hline $\mathrm{H}$ & 3.261194 & -4.010582 & -3.023440 \\
\hline C & 3.112201 & -1.866164 & -1.392742 \\
\hline C & 0.711117 & -1.658764 & 0.020762 \\
\hline $\mathrm{H}$ & -1.041508 & -3.671303 & -0.552826 \\
\hline $\mathrm{H}$ & -0.646504 & -5.548299 & -2.095490 \\
\hline C & 1.700183 & -0.698745 & 0.205543 \\
\hline C & 2.930450 & -0.802169 & -0.530504 \\
\hline C & 1.493294 & 0.417613 & 1.216415 \\
\hline $\mathrm{H}$ & 0.837492 & 0.065326 & 2.020075 \\
\hline $\mathrm{H}$ & 2.453049 & 0.681773 & 1.663945 \\
\hline C & -1.528284 & -0.278199 & -1.069398 \\
\hline 0 & -1.526290 & -0.852069 & -2.132742 \\
\hline 0 & -1.387124 & 1.073345 & -0.974073 \\
\hline $\mathrm{H}$ & -1.303723 & 1.409086 & -1.883823 \\
\hline 0 & 0.987557 & 1.629630 & 0.656440 \\
\hline $\mathrm{H}$ & 0.122589 & 1.448525 & 0.258195 \\
\hline C & 4.039706 & 0.176631 & -0.373926 \\
\hline C & 3.810073 & 1.554763 & -0.464358 \\
\hline C & 5.346241 & -0.288679 & -0.167796 \\
\hline C & 4.858068 & 2.469468 & -0.335511 \\
\hline $\mathrm{H}$ & 2.796062 & 1.895065 & -0.614816 \\
\hline C & 6.419870 & 0.597296 & -0.046781 \\
\hline $\mathrm{H}$ & 5.502936 & -1.356729 & -0.083029 \\
\hline C & 6.148902 & 1.968634 & -0.131396 \\
\hline $\mathrm{H}$ & 6.970393 & 2.669643 & -0.026926 \\
\hline C & -4.044327 & -0.055623 & 0.449004 \\
\hline C & -4.486736 & -0.329765 & -0.854673 \\
\hline C & -4.713319 & 0.902477 & 1.210855 \\
\hline C & -5.564967 & 0.358815 & -1.406047 \\
\hline $\mathrm{H}$ & -3.980129 & -1.096108 & -1.424411 \\
\hline C & -5.812828 & 1.603397 & 0.695410 \\
\hline $\mathrm{H}$ & -4.348216 & 1.117979 & 2.209443 \\
\hline C & -6.213390 & 1.318827 & -0.611361 \\
\hline $\mathrm{H}$ & -7.052386 & 1.856151 & -1.034215 \\
\hline
\end{tabular}




\begin{tabular}{|c|c|c|c|}
\hline C & -6.512565 & 2.655532 & 1.567492 \\
\hline C & -6.048034 & 0.107235 & -2.840981 \\
\hline C & -7.033244 & 1.983139 & 2.856138 \\
\hline H & -7.752161 & 1.192854 & 2.618409 \\
\hline $\mathrm{H}$ & -6.221194 & 1.534440 & 3.434832 \\
\hline $\mathrm{H}$ & -7.532291 & 2.719474 & 3.495251 \\
\hline C & -7.704669 & 3.310597 & 0.851620 \\
\hline $\mathrm{H}$ & -8.173437 & 4.044160 & 1.514495 \\
\hline $\mathrm{H}$ & -7.393278 & 3.836513 & -0.056289 \\
\hline $\mathrm{H}$ & -8.467298 & 2.574675 & 0.578047 \\
\hline C & -5.501749 & 3.762445 & 1.937596 \\
\hline $\mathrm{H}$ & -5.117748 & 4.253376 & 1.038036 \\
\hline $\mathrm{H}$ & -5.980989 & 4.522057 & 2.564652 \\
\hline $\mathrm{H}$ & -4.647233 & 3.362187 & 2.489818 \\
\hline C & -5.219988 & -0.977688 & -3.549553 \\
\hline $\mathrm{H}$ & -4.158952 & -0.715268 & -3.599682 \\
\hline H & -5.306133 & -1.944887 & -3.044526 \\
\hline $\mathrm{H}$ & -5.582867 & -1.106050 & -4.573899 \\
\hline C & -5.921575 & 1.416887 & -3.649473 \\
\hline $\mathrm{H}$ & -4.880034 & 1.751710 & -3.683103 \\
\hline $\mathrm{H}$ & -6.264484 & 1.263796 & -4.678371 \\
\hline $\mathrm{H}$ & -6.518624 & 2.222559 & -3.213143 \\
\hline C & -7.524519 & -0.341687 & -2.818079 \\
\hline $\mathrm{H}$ & -7.639167 & -1.267874 & -2.246435 \\
\hline $\mathrm{H}$ & -8.171846 & 0.414265 & -2.364628 \\
\hline $\mathrm{H}$ & -7.883326 & -0.522099 & -3.837121 \\
\hline C & 7.862259 & 0.123718 & 0.185062 \\
\hline C & 4.625210 & 3.986045 & -0.386741 \\
\hline C & 3.155059 & 4.336254 & -0.672245 \\
\hline $\mathrm{H}$ & 2.838879 & 3.960414 & -1.650816 \\
\hline H & 2.480962 & 3.920486 & 0.081670 \\
\hline $\mathrm{H}$ & 3.032186 & 5.424030 & -0.678260 \\
\hline C & 5.498758 & 4.610204 & -1.495247 \\
\hline $\mathrm{H}$ & 6.564625 & 4.438558 & -1.320097 \\
\hline $\mathrm{H}$ & 5.247466 & 4.185356 & -2.472149 \\
\hline $\mathrm{H}$ & 5.338581 & 5.692924 & -1.541719 \\
\hline C & 5.013464 & 4.595417 & 0.977985 \\
\hline $\mathrm{H}$ & 4.398522 & 4.172067 & 1.778096 \\
\hline H & 6.062046 & 4.401875 & 1.222369 \\
\hline $\mathrm{H}$ & 4.864218 & 5.680783 & 0.968738 \\
\hline C & 8.754407 & 0.624076 & -0.971392 \\
\hline $\mathrm{H}$ & 8.406077 & 0.226071 & -1.929588 \\
\hline $\mathrm{H}$ & 8.750423 & 1.715161 & -1.042191 \\
\hline $\mathrm{H}$ & 9.790559 & 0.301040 & -0.822103 \\
\hline C & 7.971042 & -1.408359 & 0.247206 \\
\hline $\mathrm{H}$ & 9.014485 & -1.697740 & 0.405789 \\
\hline H & 7.382470 & -1.823100 & 1.071406 \\
\hline $\mathrm{H}$ & 7.633988 & -1.875631 & -0.683359 \\
\hline C & 8.381396 & 0.699405 & 1.520216 \\
\hline $\mathrm{H}$ & 7.762420 & 0.358133 & 2.355824 \\
\hline $\mathrm{H}$ & 9.411955 & 0.374934 & 1.701805 \\
\hline H & 8.369264 & 1.792771 & 1.520734 \\
\hline
\end{tabular}




\section{Catalyst 2C 45}

\section{Datum}

Value

B3LYP-D3(BJ)/6-31G(d,p) Energy

$-2165.161708$

B3LYP-D3(BJ)/def2-TZVPP/IEF-PCM(DCM) Energy

$-2165.897953$

B3LYP-D3(BJ)/def2-TZVPP/IEF-PCM(DCM)//B3LYP-D3(BJ)/6-31G(d,p) Free Energy (Quasiharmonic)

$-2165.020411$

Number of Imaginary Frequencies

Frequencies (Top 3 out of 321)

$\begin{array}{ll}\text { 1. } & 10.5272 \mathrm{~cm}^{-1} \\ \text { 2. } & 13.0543 \mathrm{~cm}^{-1} \\ \text { 3. } & 13.3712 \mathrm{~cm}^{-1}\end{array}$

B3LYP-D3(BJ)/6-31G(d,p) Molecular Geometry in Cartesian Coordinates

C

$\mathrm{C}$

$\mathrm{C}$

C

C

C

$\mathrm{H}$

$\mathrm{H}$

C

C

$\mathrm{H}$

$\mathrm{H}$

C

C

$\mathrm{H}$

C

C

C

C

C

C

$\mathrm{H}$

$\mathrm{H}$

$\mathrm{H}$

C

C

$\mathrm{H}$

$\mathrm{H}$

C
0.123617

0.264160

$-0.787555$

$-2.004749$

$-2.115284$

$-1.075397$

0.935936

1.178216

$-0.669873$

$-3.060122$

$-3.039855$

$-1.172100$

$-2.953652$

$-1.736122$

$-3.967530$

0.825030

1.903263

1.840021

0.636800

$-0.458581$

$-0.366519$

3.861113

0.883789

2.823000

2.938544

0.586899

$-1.375692$

$-1.213526$

1.672334
$-4.894761$

$-3.737832$

$-2.781198$

$-3.054049$

$-4.257517$

$-5.156487$

$-5.613644$

$-3.542763$

$-1.562117$

$-2.113091$

$-4.453608$

$-6.071756$

$-0.938872$

$-0.680536$

$-2.309321$

$-2.314662$

$-1.740685$

$-1.392713$

$-1.647502$

$-2.245295$

$-2.568547$

$-0.655818$

$-2.575712$

$-1.543486$

$-0.814941$

$-1.284272$

$-2.437745$

$-3.022283$

$-0.697421$
$-1.365409$

$-0.631541$

$-0.575933$

$-1.277116$

$-2.022583$

$-2.069828$

$-1.400551$

$-0.082431$

0.161183

$-1.220887$

$-2.557947$

$-2.645192$

$-0.503074$

0.200832

$-1.783955$

5.039919

4.408721

3.033216

2. 304566

2.984866

4. 319082

2. 908209

6.092112

4.952375

2. 357730

0.924204

2.440566

4.824278

0.287862 


\begin{tabular}{|c|c|c|c|}
\hline C & 2.887157 & -0.477409 & 1.018345 \\
\hline C & 1.536154 & -0.263015 & -1.159607 \\
\hline $\mathrm{H}$ & 0.514525 & 0.087010 & -1.335908 \\
\hline $\mathrm{H}$ & 2.210859 & 0.568552 & -1.361598 \\
\hline C & -1.585427 & 0.565347 & 1.022185 \\
\hline 0 & -0.828941 & 1.479685 & 0.770256 \\
\hline 0 & -2.387059 & 0.552477 & 2.106892 \\
\hline $\mathrm{H}$ & -2.231928 & 1.394124 & 2.569219 \\
\hline 0 & 1.892698 & -1.283871 & -2.100678 \\
\hline $\mathrm{H}$ & 1.253231 & -2.004025 & -2.009727 \\
\hline C & 4.096845 & 0.106966 & 0.382892 \\
\hline C & 4.572861 & -0.380289 & -0.835070 \\
\hline C & 4.796531 & 1.141581 & 1.026526 \\
\hline C & 5.720525 & 0.155619 & -1.434437 \\
\hline $\mathrm{H}$ & 4.025433 & -1.176562 & -1.323874 \\
\hline C & 5.950556 & 1.687756 & 0.467551 \\
\hline $\mathrm{H}$ & 4.402623 & 1.522451 & 1.960391 \\
\hline C & 6.392458 & 1.179595 & -0.766312 \\
\hline $\mathrm{H}$ & 7.281697 & 1.606779 & -1.212270 \\
\hline C & -4.065789 & 0.042568 & -0.500138 \\
\hline C & -3.809240 & 1.398286 & -0.736991 \\
\hline C & -5.380551 & -0.381659 & -0.275600 \\
\hline C & -4.840318 & 2.339656 & -0.723993 \\
\hline $\mathrm{H}$ & -2.793256 & 1.707814 & -0.939574 \\
\hline C & -6.439844 & 0.529897 & -0.271910 \\
\hline $\mathrm{H}$ & -5.555458 & -1.431449 & -0.076668 \\
\hline C & -6.142264 & 1.880733 & -0.491965 \\
\hline $\mathrm{H}$ & -6.953254 & 2.600983 & -0.478638 \\
\hline C & -7.894376 & 0.106852 & -0.020771 \\
\hline C & -4.582184 & 3.837229 & -0.939888 \\
\hline C & -8.032284 & -1.409220 & 0.192506 \\
\hline $\mathrm{H}$ & -7.687765 & -1.973326 & -0.679924 \\
\hline $\mathrm{H}$ & -7.467124 & -1.748808 & 1.065970 \\
\hline $\mathrm{H}$ & -9.083546 & -1.663486 & 0.358378 \\
\hline C & -8.757336 & 0.503986 & -1.237881 \\
\hline $\mathrm{H}$ & -9.800930 & 0.213210 & -1.076122 \\
\hline $\mathrm{H}$ & -8.734383 & 1.582485 & -1.416274 \\
\hline $\mathrm{H}$ & -8.400221 & 0.007429 & -2.145469 \\
\hline C & -8.423234 & 0.823054 & 1.240595 \\
\hline $\mathrm{H}$ & -8.392092 & 1.910570 & 1.131001 \\
\hline $\mathrm{H}$ & -9.461991 & 0.535173 & 1.435576 \\
\hline $\mathrm{H}$ & -7.823744 & 0.557687 & 2.116880 \\
\hline C & -5.030486 & 4.608908 & 0.320298 \\
\hline $\mathrm{H}$ & -6.093614 & 4.460748 & 0.529777 \\
\hline $\mathrm{H}$ & -4.466831 & 4.277229 & 1.197997 \\
\hline $\mathrm{H}$ & -4.859680 & 5.682969 & 0.190371 \\
\hline C & -5.389755 & 4.330240 & -2.159116 \\
\hline $\mathrm{H}$ & -6.464613 & 4.182893 & -2.020664 \\
\hline $\mathrm{H}$ & -5.216776 & 5.399232 & -2.323520 \\
\hline $\mathrm{H}$ & -5.091702 & 3.792605 & -3.064524 \\
\hline C & -3.094598 & 4.139431 & -1.186573 \\
\hline $\mathrm{H}$ & -2.466196 & 3.814978 & -0.351596 \\
\hline $\mathrm{H}$ & -2.728894 & 3.650550 & -2.094933 \\
\hline $\mathrm{H}$ & -2.954961 & 5.217452 & -1.312109 \\
\hline
\end{tabular}




\begin{tabular}{|rrrr|} 
C & 6.737387 & 2.823220 & 1.137983 \\
C & 6.169903 & -0.393115 & -2.795305 \\
H & 7.440759 & 0.297529 & -3.314293 \\
H & 7.289290 & 1.372872 & -3.451813 \\
H & 8.287109 & 0.154371 & -2.634561 \\
C & 7.719161 & -0.124349 & -4.284937 \\
H & 5.036238 & -0.171252 & -3.821820 \\
H & 4.107348 & -0.659346 & -3.515497 \\
H & 4.829938 & 0.897586 & -3.940067 \\
C & 5.327097 & -0.571145 & -4.799828 \\
H & 6.456770 & -1.904646 & -2.667911 \\
H & 7.252182 & -2.089874 & -1.938829 \\
H & 5.569736 & -2.456386 & -2.346124 \\
C & 6.774619 & -2.313965 & -3.633146 \\
H & 6.117493 & 3.250931 & 2.478038 \\
H & 5.097399 & 3.626173 & 2.350883 \\
H & 6.091255 & 2.423084 & 3.193582 \\
C & 6.714428 & 4.054372 & 2.920611 \\
H & 6.760252 & 4.051419 & 0.202622 \\
H & 7.320815 & 4.872363 & 0.663265 \\
H & 7.231888 & 3.820204 & -0.756380 \\
C & 5.743632 & 4.402061 & -0.000504 \\
H & 8.183971 & 2.353512 & 1.403799 \\
H & 8.695021 & 2.073620 & 0.478606 \\
H & 8.763704 & 3.152643 & 1.878739 \\
& 8.193096 & 1.482999 & 2.067203 \\
\hline & & & \\
\hline & & & \\
\hline & & & \\
\hline
\end{tabular}

\section{Catalyst 2C 46}

Datum

Value

B3LYP-D3(BJ)/6-31G(d,p) Energy

$-2165.162695$

B3LYP-D3(BJ)/def2-TZVPP/IEF-PCM(DCM) Energy

$-2165.89835$

B3LYP-D3(BJ)/def2-TZVPP/IEF-PCM(DCM)//B3LYP-D3(BJ)/6-31G(d,p) Free Energy (Quasiharmonic) -2165.020379

Number of Imaginary Frequencies

0

Frequencies (Top 3 out of 321)

$\begin{array}{ll}\text { 1. } & 11.5706 \mathrm{~cm}^{-1} \\ \text { 2. } & 13.0039 \mathrm{~cm}^{-1} \\ \text { 3. } & 13.1329 \mathrm{~cm}^{-1}\end{array}$

B3LYP-D3(BJ)/6-31G(d,p) Molecular Geometry in Cartesian Coordinates 


\begin{tabular}{|c|c|c|c|}
\hline C & -0.379300 & -3.456291 & 3.916738 \\
\hline C & -0.485012 & -2.887125 & 2.668181 \\
\hline C & 0.607394 & -2.180175 & 2.100512 \\
\hline C & 1.820426 & -2.064098 & 2.849224 \\
\hline C & 1.895984 & -2.662729 & 4.134340 \\
\hline C & 0.821233 & -3.343184 & 4.657395 \\
\hline $\mathrm{H}$ & -1.220998 & -3.997003 & 4.337786 \\
\hline $\mathrm{H}$ & -1.403601 & -2.972506 & 2.099250 \\
\hline C & 0.529251 & -1.570894 & 0.809905 \\
\hline C & 2.915773 & -1.363346 & 2.288669 \\
\hline $\mathrm{H}$ & 2.820610 & -2.571826 & 4.697111 \\
\hline $\mathrm{H}$ & 0.890348 & -3.796066 & 5.641549 \\
\hline C & 2.852842 & -0.793601 & 1.033847 \\
\hline C & 1.632303 & -0.911614 & 0.297568 \\
\hline $\mathrm{H}$ & 3.840820 & -1.298021 & 2.853554 \\
\hline C & -1.402337 & -4.868064 & -2.643596 \\
\hline C & -2.371237 & -3.907277 & -2.474652 \\
\hline C & -2.166720 & -2.820117 & -1.584506 \\
\hline C & -0.936716 & -2.729415 & -0.865548 \\
\hline C & 0.046178 & -3.734259 & -1.065674 \\
\hline C & -0.182701 & -4.778834 & -1.931135 \\
\hline $\mathrm{H}$ & -4.073410 & -1.885704 & -1.951515 \\
\hline $\mathrm{H}$ & -1.567880 & -5.696212 & -3.325832 \\
\hline $\mathrm{H}$ & -3.309654 & -3.964090 & -3.019108 \\
\hline C & -3.146850 & -1.820555 & -1.388745 \\
\hline C & -0.747753 & -1.632396 & 0.030650 \\
\hline$H$ & 0.986877 & -3.663407 & -0.532722 \\
\hline $\mathrm{H}$ & 0.579540 & -5.538280 & -2.074713 \\
\hline C & -1.728396 & -0.662778 & 0.210845 \\
\hline C & -2.958703 & -0.757714 & -0.526405 \\
\hline C & -1.509814 & 0.457302 & 1.214887 \\
\hline $\mathrm{H}$ & -2.465557 & 0.735945 & 1.661842 \\
\hline $\mathrm{H}$ & -0.856166 & 0.105100 & 2.020038 \\
\hline C & 1.512065 & -0.284029 & -1.058994 \\
\hline 0 & 1.509561 & -0.862667 & -2.119744 \\
\hline 0 & 1.382399 & 1.069381 & -0.971112 \\
\hline H & 1.306749 & 1.400836 & -1.883125 \\
\hline 0 & -0.991796 & 1.659711 & 0.643993 \\
\hline $\mathrm{H}$ & -0.126220 & 1.468482 & 0.251578 \\
\hline C & -4.062308 & 0.227680 & -0.371296 \\
\hline C & -3.827584 & 1.604659 & -0.441682 \\
\hline C & -5.374695 & -0.225386 & -0.183956 \\
\hline C & -4.870289 & 2.526339 & -0.311194 \\
\hline $\mathrm{H}$ & -2.810285 & 1.947521 & -0.578204 \\
\hline C & -6.445262 & 0.666077 & -0.066291 \\
\hline $\mathrm{H}$ & -5.546771 & -1.294206 & -0.110206 \\
\hline C & -6.169817 & 2.038205 & -0.130383 \\
\hline $\mathrm{H}$ & -6.984090 & 2.740960 & -0.030908 \\
\hline C & 4.026077 & -0.084798 & 0.466276 \\
\hline C & 4.706001 & 0.869677 & 1.232294 \\
\hline C & 4.466229 & -0.368956 & -0.832082 \\
\hline C & 5.811112 & 1.551797 & 0.716486 \\
\hline $\mathrm{H}$ & 4.337874 & 1.087225 & 2.226949 \\
\hline
\end{tabular}




\begin{tabular}{|c|c|c|c|}
\hline C & 5.556628 & 0.305704 & -1.386109 \\
\hline $\mathrm{H}$ & 3.952123 & -1.131043 & -1.400751 \\
\hline C & 6.210031 & 1.256154 & -0.593261 \\
\hline H & 7.058166 & 1.787872 & -1.011100 \\
\hline C & 6.037687 & 0.043236 & -2.819936 \\
\hline C & 6.578913 & 2.610818 & 1.520495 \\
\hline C & 7.508996 & -0.421909 & -2.793985 \\
\hline $\mathrm{H}$ & 8.164038 & 0.328548 & -2.342429 \\
\hline $\mathrm{H}$ & 7.613033 & -1.347076 & -2.218703 \\
\hline $\mathrm{H}$ & 7.866974 & -0.609964 & -3.811925 \\
\hline C & 5.926202 & 1.351331 & -3.632980 \\
\hline H & 6.267774 & 1.191136 & -4.661236 \\
\hline $\mathrm{H}$ & 4.888564 & 1.697875 & -3.668083 \\
\hline $\mathrm{H}$ & 6.531955 & 2.151725 & -3.198864 \\
\hline C & 5.197953 & -1.035106 & -3.524861 \\
\hline H & 4.139984 & -0.760994 & -3.577109 \\
\hline $\mathrm{H}$ & 5.560286 & -1.171632 & -4.548334 \\
\hline $\mathrm{H}$ & 5.272731 & -2.001210 & -3.015935 \\
\hline C & 8.055469 & 2.178725 & 1.650586 \\
\hline $\mathrm{H}$ & 8.134734 & 1.219084 & 2.170822 \\
\hline $\mathrm{H}$ & 8.533366 & 2.069253 & 0.673238 \\
\hline $\mathrm{H}$ & 8.621776 & 2.924423 & 2.218914 \\
\hline C & 6.005884 & 2.798208 & 2.934485 \\
\hline $\mathrm{H}$ & 6.049829 & 1.871193 & 3.514772 \\
\hline $\mathrm{H}$ & 6.588610 & 3.553390 & 3.470578 \\
\hline $\mathrm{H}$ & 4.966055 & 3.138303 & 2.908936 \\
\hline C & 6.502194 & 3.965473 & 0.783737 \\
\hline H & 6.937077 & 3.906533 & -0.217834 \\
\hline $\mathrm{H}$ & 5.462730 & 4.291312 & 0.678941 \\
\hline $\mathrm{H}$ & 7.047754 & 4.734271 & 1.341541 \\
\hline C & -7.865164 & 0.117825 & 0.141549 \\
\hline C & -4.541878 & 4.025356 & -0.354686 \\
\hline C & -3.566891 & 4.355615 & 0.797976 \\
\hline $\mathrm{H}$ & -4.032383 & 4.143220 & 1.766104 \\
\hline $\mathrm{H}$ & -2.646910 & 3.768783 & 0.733602 \\
\hline H & -3.299633 & 5.418281 & 0.775967 \\
\hline C & -5.792753 & 4.905184 & -0.203628 \\
\hline $\mathrm{H}$ & -6.513193 & 4.729103 & -1.009077 \\
\hline H & -6.297499 & 4.730714 & 0.751851 \\
\hline H & -5.506734 & 5.960973 & -0.238704 \\
\hline C & -3.872148 & 4.363580 & -1.703932 \\
\hline $\mathrm{H}$ & -2.946250 & 3.799947 & -1.844868 \\
\hline $\mathrm{H}$ & -4.539616 & 4.129560 & -2.539545 \\
\hline H & -3.626430 & 5.430275 & -1.750009 \\
\hline C & -7.910237 & -0.699032 & 1.450921 \\
\hline $\mathrm{H}$ & -7.211360 & -1.539588 & 1.426626 \\
\hline $\mathrm{H}$ & -7.648252 & -0.071028 & 2.308157 \\
\hline $\mathrm{H}$ & -8.915468 & -1.102399 & 1.615829 \\
\hline C & -8.237713 & -0.796032 & -1.045861 \\
\hline H & -9.247079 & -1.201368 & -0.914868 \\
\hline $\mathrm{H}$ & -8.212157 & -0.238073 & -1.987110 \\
\hline $\mathrm{H}$ & -7.547687 & -1.639230 & -1.138150 \\
\hline C & -8.917457 & 1.234580 & 0.235643 \\
\hline $\mathrm{H}$ & -8.947775 & 1.840307 & -0.675417 \\
\hline
\end{tabular}



H

\section{Catalyst 2C 47}

\section{Datum}

Value

\begin{tabular}{lr}
\hline B3LYP-D3(BJ)/6-31G(d,p) Energy & -2165.161741 \\
\hline B3LYP-D3(BJ)/def2-TZVPP/IEF-PCM(DCM) Energy & -2165.897925 \\
\hline B3LYP-D3(BJ)/def2-TZVPP/IEF-PCM(DCM)//B3LYP-D3(BJ)/6-31G(d,p) Free Energy (Quasiharmonic) & -2165.02033 \\
\hline
\end{tabular}

Number of Imaginary Frequencies

Frequencies (Top 3 out of 321)

$\begin{array}{ll}\text { 1. } & 10.9447 \mathrm{~cm}^{-1} \\ \text { 2. } & 12.9430 \mathrm{~cm}^{-1} \\ \text { 3. } & 13.8444 \mathrm{~cm}^{-1}\end{array}$

B3LYP-D3(BJ)/6-31G(d,p) Molecular Geometry in Cartesian Coordinates

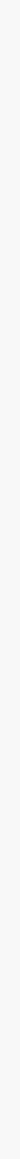

$\begin{array}{r}-0.100582 \\ -0.243020 \\ 0.805314 \\ 2.021306 \\ 2.133924 \\ 1.097150 \\ -0.910487 \\ -1.156245 \\ 0.685276 \\ 3.073663 \\ 3.057534 \\ 1.195306 \\ 2.965540 \\ 1.748612 \\ 3.980230 \\ -0.795259 \\ -1.876827 \\ -1.818363 \\ -0.616433 \\ 0.482326 \\ 0.394861 \\ -3.842657 \\ -0.850389 \\ -2.795637 \\ \hline\end{array}$

-4.929618
-3.755760
-2.794243
-3.079764
-4.300627
-5.204112
-5.651896
-3.550511
-1.557631
-2.134366
-4.506391
-6.132710
-0.943380
-0.671774
-2.341880
-2.176156
-1.623520
-1.315324
-1.587798
-2.162973
-2.447736
-0.587666
-2.406837
-1.413252

$-1.252543$

$-0.546504$

$-0.517745$

$-1.215928$

$-1.932261$

$-1.954314$

$-1.267790$

0.000268

0.189009

$-1.185468$

$-2.465642$

$-2.507646$

$-0.496002$

0.204237

$-1.745817$

5.091609

4.447131

3.062035

2. 337510

3.031699

4.374979

2.920460

6.151066

4.987448 


\begin{tabular}{|c|c|c|c|}
\hline C & -2.920597 & -0.760404 & 2.373141 \\
\hline C & -0.570834 & -1.263992 & 0.947077 \\
\hline $\mathrm{H}$ & 1.398407 & -2.368910 & 2.490602 \\
\hline $\mathrm{H}$ & 1.244477 & -2.884551 & 4.890593 \\
\hline C & -1.659732 & -0.698899 & 0.297336 \\
\hline C & -2.872722 & -0.461932 & 1.024671 \\
\hline C & -1.530949 & -0.302868 & -1.162010 \\
\hline $\mathrm{H}$ & -2.212164 & 0.518903 & -1.382397 \\
\hline $\mathrm{H}$ & -0.512182 & 0.050525 & -1.349430 \\
\hline C & 1.594132 & 0.595968 & 0.990792 \\
\hline 0 & 0.836282 & 1.501277 & 0.709695 \\
\hline 0 & 2.394642 & 0.618310 & 2.075259 \\
\hline $\mathrm{H}$ & 2.242107 & 1.476024 & 2.508124 \\
\hline 0 & -1.882920 & -1.349176 & -2.075232 \\
\hline $\mathrm{H}$ & -1.242230 & -2.065016 & -1.962163 \\
\hline C & -4.084912 & 0.100497 & 0.374208 \\
\hline C & -4.576423 & -0.449993 & -0.820425 \\
\hline C & -4.769666 & 1.162963 & 0.966944 \\
\hline C & -5.722677 & 0.058972 & -1.426573 \\
\hline $\mathrm{H}$ & -4.031628 & -1.269329 & -1.267832 \\
\hline C & -5.933336 & 1.693127 & 0.391558 \\
\hline H & -4.369185 & 1.592615 & 1.879288 \\
\hline C & -6.386513 & 1.128062 & -0.801639 \\
\hline $\mathrm{H}$ & -7.276334 & 1.527867 & -1.270817 \\
\hline C & 4.076016 & 0.039746 & -0.517126 \\
\hline C & 5.396696 & -0.381232 & -0.297255 \\
\hline C & 3.821960 & 1.388765 & -0.765086 \\
\hline C & 6.451902 & 0.529243 & -0.310734 \\
\hline $\mathrm{H}$ & 5.573495 & -1.428766 & -0.088472 \\
\hline C & 4.853811 & 2.336122 & -0.764397 \\
\hline $\mathrm{H}$ & 2.804814 & 1.703987 & -0.963788 \\
\hline C & 6.154399 & 1.884043 & -0.539571 \\
\hline $\mathrm{H}$ & 6.967595 & 2.598411 & -0.536099 \\
\hline C & 4.506787 & 3.813724 & -0.989792 \\
\hline C & 7.908882 & 0.110537 & -0.067719 \\
\hline C & 3.822485 & 3.978113 & -2.363693 \\
\hline $\mathrm{H}$ & 4.486300 & 3.650825 & -3.170072 \\
\hline $\mathrm{H}$ & 2.900404 & 3.394746 & -2.429595 \\
\hline $\mathrm{H}$ & 3.565703 & 5.028727 & -2.536409 \\
\hline C & 5.749318 & 4.717343 & -0.957113 \\
\hline $\mathrm{H}$ & 5.452068 & 5.758028 & -1.117793 \\
\hline $\mathrm{H}$ & 6.263432 & 4.664875 & 0.007838 \\
\hline $\mathrm{H}$ & 6.464397 & 4.451246 & -1.742111 \\
\hline C & 3.539290 & 4.272627 & 0.124610 \\
\hline $\mathrm{H}$ & 4.012839 & 4.175801 & 1.107374 \\
\hline $\mathrm{H}$ & 3.265414 & 5.323536 & -0.018397 \\
\hline $\mathrm{H}$ & 2.618159 & 3.683485 & 0.134930 \\
\hline C & 8.761437 & 0.497958 & -1.295361 \\
\hline $\mathrm{H}$ & 8.397660 & -0.007315 & -2.195478 \\
\hline $\mathrm{H}$ & 8.735851 & 1.574785 & -1.483114 \\
\hline $\mathrm{H}$ & 9.806637 & 0.209805 & -1.139346 \\
\hline C & 8.050871 & -1.403355 & 0.157753 \\
\hline $\mathrm{H}$ & 7.699590 & -1.975559 & -0.706672 \\
\hline $\mathrm{H}$ & 9.103881 & -1.654759 & 0.316803 \\
\hline
\end{tabular}




\begin{tabular}{|c|c|c|c|}
\hline $\mathrm{H}$ & 7.493728 & -1.735937 & 1.039036 \\
\hline C & 8.447610 & 0.838392 & 1.182855 \\
\hline $\mathrm{H}$ & 8.414627 & 1.924863 & 1.064146 \\
\hline $\mathrm{H}$ & 7.855920 & 0.579837 & 2.066463 \\
\hline $\mathrm{H}$ & 9.488277 & 0.553109 & 1.371376 \\
\hline C & -6.646301 & 2.867422 & 1.077526 \\
\hline C & -6.257333 & -0.496141 & -2.754063 \\
\hline C & -5.429181 & -1.690280 & -3.257518 \\
\hline $\mathrm{H}$ & -4.378454 & -1.426845 & -3.407112 \\
\hline $\mathrm{H}$ & -5.468994 & -2.528577 & -2.554458 \\
\hline $\mathrm{H}$ & -5.832774 & -2.038799 & -4.213559 \\
\hline C & -6.198979 & 0.619149 & -3.820583 \\
\hline $\mathrm{H}$ & -6.796912 & 1.487716 & -3.529654 \\
\hline $\mathrm{H}$ & -5.168525 & 0.955623 & -3.970582 \\
\hline $\mathrm{H}$ & -6.582457 & 0.251809 & -4.778904 \\
\hline C & -7.718728 & -0.958601 & -2.574150 \\
\hline $\mathrm{H}$ & -7.785754 & -1.744685 & -1.815468 \\
\hline $\mathrm{H}$ & -8.371450 & -0.137609 & -2.264117 \\
\hline $\mathrm{H}$ & -8.109868 & -1.358362 & -3.516122 \\
\hline C & -7.063635 & 2.446967 & 2.503330 \\
\hline $\mathrm{H}$ & -7.748004 & 1.593394 & 2.470997 \\
\hline $\mathrm{H}$ & -6.199490 & 2.160283 & 3.108887 \\
\hline $\mathrm{H}$ & -7.570568 & 3.273822 & 3.012793 \\
\hline C & -7.907670 & 3.310767 & 0.319245 \\
\hline $\mathrm{H}$ & -8.382812 & 4.142015 & 0.849280 \\
\hline $\mathrm{H}$ & -7.671925 & 3.654312 & -0.692791 \\
\hline $\mathrm{H}$ & -8.640634 & 2.501325 & 0.244655 \\
\hline C & -5.682888 & 4.071141 & 1.158791 \\
\hline $\mathrm{H}$ & -5.373376 & 4.387521 & 0.157868 \\
\hline $\mathrm{H}$ & -6.171701 & 4.919266 & 1.650861 \\
\hline $\mathrm{H}$ & -4.780105 & 3.828750 & 1.725886 \\
\hline
\end{tabular}

\section{Catalyst 2C 48}

Datum

Value

$\begin{array}{ll}\text { B3LYP-D3(BJ)/6-31G(d,p) Energy } & -2165.162654\end{array}$

$\begin{array}{lr}\text { B3LYP-D3(BJ)/def2-TZVPP/IEF-PCM(DCM) Energy } & -2165.898317\end{array}$

B3LYP-D3(BJ)/def2-TZVPP/IEF-PCM(DCM)//B3LYP-D3(BJ)/6-31G(d,p) Free Energy (Quasiharmonic) -2165.020318

Number of Imaginary Frequencies

Frequencies (Top 3 out of 321)

$\begin{array}{ll}\text { 1. } & 11.5126 \mathrm{~cm}^{-1} \\ \text { 2. } & 13.0688 \mathrm{~cm}^{-1} \\ \text { 3. } & 13.4584 \mathrm{~cm}^{-1}\end{array}$




\begin{tabular}{|c|c|c|c|}
\hline C & 0.305243 & -3.506326 & 3.880760 \\
\hline C & 0.423776 & -2.923989 & 2.639458 \\
\hline C & -0.659716 & -2.203216 & 2.072122 \\
\hline C & -1.877079 & -2.086718 & 2.813687 \\
\hline C & -1.966009 & -2.699309 & 4.091338 \\
\hline C & -0.899759 & -3.393202 & 4.614147 \\
\hline $\mathrm{H}$ & 1.140160 & -4.057566 & 4.301657 \\
\hline $\mathrm{H}$ & 1.345691 & -3.009466 & 2.075940 \\
\hline C & -0.568655 & -1.581345 & 0.788478 \\
\hline C & -2.962993 & -1.370821 & 2.253943 \\
\hline $\mathrm{H}$ & -2.893933 & -2.608213 & 4.648623 \\
\hline $\mathrm{H}$ & -0.979028 & -3.856699 & 5.592575 \\
\hline C & -2.886761 & -0.786520 & 1.006594 \\
\hline C & -1.662914 & -0.907499 & 0.276275 \\
\hline $\mathrm{H}$ & -3.891252 & -1.304589 & 2.813451 \\
\hline C & 1.369343 & -4.874029 & -2.665978 \\
\hline C & 2.340825 & -3.917308 & -2.488647 \\
\hline C & 2.135372 & -2.832132 & -1.596376 \\
\hline C & 0.901780 & -2.739259 & -0.883824 \\
\hline C & -0.083812 & -3.739598 & -1.092829 \\
\hline C & 0.146022 & -4.782393 & -1.960235 \\
\hline $\mathrm{H}$ & 4.047045 & -1.901710 & -1.950944 \\
\hline $\mathrm{H}$ & 1.535688 & -5.700695 & -3.349813 \\
\hline $\mathrm{H}$ & 3.281995 & -3.975883 & -3.028145 \\
\hline C & 3.117693 & -1.836036 & -1.392810 \\
\hline C & 0.712125 & -1.645080 & 0.015781 \\
\hline $\mathrm{H}$ & -1.027215 & -3.666849 & -0.564888 \\
\hline $\mathrm{H}$ & -0.618221 & -5.538542 & -2.110464 \\
\hline C & 1.695306 & -0.679939 & 0.204693 \\
\hline C & 2.927853 & -0.775545 & -0.528229 \\
\hline C & 1.480290 & 0.434066 & 1.216503 \\
\hline $\mathrm{H}$ & 0.824171 & 0.077445 & 2.018046 \\
\hline $\mathrm{H}$ & 2.437565 & 0.702305 & 1.666979 \\
\hline C & -1.530944 & -0.272177 & -1.075552 \\
\hline 0 & -1.524475 & -0.847599 & -2.138591 \\
\hline 0 & -1.399280 & 1.079503 & -0.981393 \\
\hline $\mathrm{H}$ & -1.322549 & 1.415935 & -1.891544 \\
\hline 0 & 0.970394 & 1.644249 & 0.656605 \\
\hline $\mathrm{H}$ & 0.107198 & 1.460034 & 0.256100 \\
\hline C & 4.031885 & 0.208100 & -0.365115 \\
\hline C & 3.796233 & 1.588733 & -0.458405 \\
\hline C & 5.335867 & -0.245420 & -0.151083 \\
\hline C & 4.837016 & 2.505301 & -0.324734 \\
\hline $\mathrm{H}$ & 2.781144 & 1.923138 & -0.614527 \\
\hline C & 6.409540 & 0.647128 & -0.024450 \\
\hline $\mathrm{H}$ & 5.504484 & -1.313709 & -0.062773 \\
\hline C & 6.135329 & 2.012949 & -0.111997 \\
\hline $\mathrm{H}$ & 6.946082 & 2.722334 & -0.005988 \\
\hline C & -4.049499 & -0.058139 & 0.442082 \\
\hline C & -4.480488 & -0.304880 & -0.865462 \\
\hline
\end{tabular}




\begin{tabular}{|c|c|c|c|}
\hline C & -4.732662 & 0.884372 & 1.217599 \\
\hline C & -5.558365 & 0.392808 & -1.417540 \\
\hline $\mathrm{H}$ & -3.968068 & -1.056234 & -1.453483 \\
\hline C & -5.828058 & 1.587474 & 0.706895 \\
\hline $\mathrm{H}$ & -4.377011 & 1.082156 & 2.223232 \\
\hline C & -6.218599 & 1.330098 & -0.613934 \\
\hline $\mathrm{H}$ & -7.054799 & 1.874720 & -1.026925 \\
\hline C & -6.542209 & 2.619121 & 1.592575 \\
\hline C & -5.956714 & 0.116779 & -2.874103 \\
\hline C & -7.732397 & 3.280906 & 0.879366 \\
\hline $\mathrm{H}$ & -7.417027 & 3.826888 & -0.015179 \\
\hline $\mathrm{H}$ & -8.488151 & 2.546134 & 0.584570 \\
\hline $\mathrm{H}$ & -8.211258 & 3.998154 & 1.552841 \\
\hline C & -5.541660 & 3.725084 & 1.992391 \\
\hline $\mathrm{H}$ & -6.031262 & 4.470531 & 2.628426 \\
\hline $\mathrm{H}$ & -4.690105 & 3.319615 & 2.545388 \\
\hline $\mathrm{H}$ & -5.151979 & 4.234441 & 1.105604 \\
\hline C & -7.070042 & 1.919868 & 2.863940 \\
\hline $\mathrm{H}$ & -6.260193 & 1.465988 & 3.441672 \\
\hline $\mathrm{H}$ & -7.579880 & 2.640996 & 3.511847 \\
\hline $\mathrm{H}$ & -7.781402 & 1.129299 & 2.605312 \\
\hline C & -6.314649 & -1.376029 & -3.036883 \\
\hline $\mathrm{H}$ & -5.471873 & -2.024803 & -2.784466 \\
\hline $\mathrm{H}$ & -7.154261 & -1.647208 & -2.389126 \\
\hline $\mathrm{H}$ & -6.599375 & -1.587058 & -4.073221 \\
\hline C & -4.760326 & 0.461195 & -3.789689 \\
\hline $\mathrm{H}$ & -3.871146 & -0.122644 & -3.536819 \\
\hline $\mathrm{H}$ & -5.011295 & 0.258753 & -4.836588 \\
\hline $\mathrm{H}$ & -4.503059 & 1.522058 & -3.701443 \\
\hline C & -7.166049 & 0.954047 & -3.319616 \\
\hline $\mathrm{H}$ & -8.053325 & 0.738044 & -2.715959 \\
\hline $\mathrm{H}$ & -6.961595 & 2.027514 & -3.257250 \\
\hline H & -7.408949 & 0.723597 & -4.361326 \\
\hline C & 7.823355 & 0.097860 & 0.215573 \\
\hline C & 4.598763 & 4.020755 & -0.379753 \\
\hline C & 5.472454 & 4.645942 & -1.487705 \\
\hline H & 6.538592 & 4.479031 & -1.309756 \\
\hline $\mathrm{H}$ & 5.224831 & 4.218005 & -2.464214 \\
\hline H & 5.307950 & 5.727903 & -1.536879 \\
\hline C & 4.981428 & 4.635237 & 0.984350 \\
\hline H & 4.365942 & 4.211795 & 1.784011 \\
\hline $\mathrm{H}$ & 6.030156 & 4.446790 & 1.231940 \\
\hline $\mathrm{H}$ & 4.827944 & 5.719975 & 0.971588 \\
\hline C & 3.128014 & 4.364888 & -0.669324 \\
\hline $\mathrm{H}$ & 2.815715 & 3.986593 & -1.648187 \\
\hline $\mathrm{H}$ & 2.453841 & 3.947276 & 0.083520 \\
\hline $\mathrm{H}$ & 3.000782 & 5.452156 & -0.676977 \\
\hline C & 8.875697 & 1.213661 & 0.317652 \\
\hline H & 8.924053 & 1.809417 & -0.599216 \\
\hline $\mathrm{H}$ & 8.670631 & 1.888444 & 1.154585 \\
\hline $\mathrm{H}$ & 9.864343 & 0.773676 & 0.480908 \\
\hline C & 8.215240 & -0.829036 & -0.955347 \\
\hline $\mathrm{H}$ & 9.220578 & -1.236103 & -0.801067 \\
\hline H & 7.524401 & -1.671052 & -1.052298 \\
\hline
\end{tabular}




\section{Catalyst 2C 49}

\section{Datum}

Value

\begin{tabular}{lr}
\hline B3LYP-D3(BJ)/6-31G(d,p) Energy & -2165.161069 \\
\hline B3LYP-D3(BJ)/def2-TZVPP/IEF-PCM(DCM) Energy & -2165.897625 \\
\hline B3LYP-D3(BJ)/def2-TZVPP/IEF-PCM(DCM)//B3LYP-D3(BJ)/6-31G(d,p) Free Energy (Quasiharmonic) & -2165.020297 \\
\hline
\end{tabular}

Number of Imaginary Frequencies

Frequencies (Top 3 out of 321)
1. $10.4599 \mathrm{~cm}^{-1}$
2. $13.0588 \mathrm{~cm}^{-1}$
3. $13.5155 \mathrm{~cm}^{-1}$

\section{B3LYP-D3(BJ)/6-31G(d,p) Molecular Geometry in Cartesian Coordinates}

\begin{tabular}{|c|c|c|c|}
\hline C & -0.088302 & -4.878318 & -1.396778 \\
\hline C & -0.233089 & -3.727350 & -0.654444 \\
\hline C & 0.813390 & -2.765218 & -0.595324 \\
\hline C & 2.029944 & -3.026463 & -1.301982 \\
\hline C & 2.144961 & -4.223968 & -2.056257 \\
\hline C & 1.109962 & -5.128402 & -2.106646 \\
\hline $\mathrm{H}$ & -0.896795 & -5.601350 & -1.434662 \\
\hline $\mathrm{H}$ & -1.146675 & -3.541144 & -0.101518 \\
\hline C & 0.690814 & -1.551685 & 0.150213 \\
\hline C & 3.080328 & -2.080122 & -1.242012 \\
\hline $\mathrm{H}$ & 3.068941 & -4.411042 & -2.595859 \\
\hline $\mathrm{H}$ & 1.209960 & -6.039017 & -2.688793 \\
\hline C & 2.969498 & -0.912071 & -0.515056 \\
\hline C & 1.752325 & -0.664520 & 0.193154 \\
\hline $\mathrm{H}$ & 3.987189 & -2.266875 & -1.809204 \\
\hline C & -0.783181 & -2.338089 & 5.030081 \\
\hline C & -1.867176 & -1.767972 & 4.405231 \\
\hline C & -1.810931 & -1.413250 & 3.031184 \\
\hline C & -0.608636 & -1.657022 & 2.297179 \\
\hline C & 0.492743 & -2.251189 & 2.970942 \\
\hline C & 0.407370 & -2.581201 & 4.303966 \\
\hline
\end{tabular}




\begin{tabular}{|c|c|c|c|}
\hline H & -3.837662 & -0.689024 & 2.915823 \\
\hline $\mathrm{H}$ & -0.836664 & -2.604364 & 6.081247 \\
\hline $\mathrm{H}$ & -2.786258 & -1.579121 & 4.952949 \\
\hline C & -2.915636 & -0.839663 & 2.362012 \\
\hline C & -0.565388 & -1.286383 & 0.918487 \\
\hline H & 1.409150 & -2.435466 & 2.422634 \\
\hline H & 1.258956 & -3.031983 & 4.804085 \\
\hline C & -1.656578 & -0.703233 & 0.288826 \\
\hline C & -2.870435 & -0.495827 & 1.024133 \\
\hline C & -1.528326 & -0.256361 & -1.155731 \\
\hline $\mathrm{H}$ & -2.211467 & 0.570892 & -1.347644 \\
\hline H & -0.510387 & 0.105724 & -1.330341 \\
\hline C & 1.597336 & 0.575658 & 1.022156 \\
\hline 0 & 0.832210 & 1.485440 & 0.780143 \\
\hline 0 & 2.407037 & 0.563654 & 2.100900 \\
\hline $\mathrm{H}$ & 2.249457 & 1.402121 & 2.568131 \\
\hline 0 & -1.877271 & -1.271086 & -2.105278 \\
\hline $\mathrm{H}$ & -1.235400 & -1.989253 & -2.016201 \\
\hline C & -4.085752 & 0.082734 & 0.394003 \\
\hline C & -4.570727 & -0.422591 & -0.818827 \\
\hline C & -4.777870 & 1.120374 & 1.032399 \\
\hline C & -5.722057 & 0.103870 & -1.408799 \\
\hline $\mathrm{H}$ & -4.021549 & -1.221057 & -1.297543 \\
\hline C & -5.939536 & 1.662972 & 0.476470 \\
\hline $\mathrm{H}$ & -4.376782 & 1.510391 & 1.959442 \\
\hline C & -6.387461 & 1.139966 & -0.742968 \\
\hline $\mathrm{H}$ & -7.280841 & 1.559885 & -1.193142 \\
\hline C & 4.077298 & 0.074294 & -0.507746 \\
\hline C & 5.390819 & -0.339436 & -0.287816 \\
\hline C & 3.814050 & 1.433502 & -0.738843 \\
\hline C & 6.451037 & 0.578310 & -0.283244 \\
\hline $\mathrm{H}$ & 5.578064 & -1.389803 & -0.091104 \\
\hline C & 4.838918 & 2.376162 & -0.724746 \\
\hline $\mathrm{H}$ & 2.795868 & 1.738410 & -0.937563 \\
\hline C & 6.149859 & 1.924476 & -0.497517 \\
\hline $\mathrm{H}$ & 6.951044 & 2.652078 & -0.486953 \\
\hline C & 4.575046 & 3.873326 & -0.935364 \\
\hline C & 7.880068 & 0.078205 & -0.028275 \\
\hline C & 5.025344 & 4.643365 & 0.325216 \\
\hline $\mathrm{H}$ & 4.465911 & 4.307517 & 1.204050 \\
\hline $\mathrm{H}$ & 6.089763 & 4.498729 & 0.530409 \\
\hline H & 4.850142 & 5.717107 & 0.198514 \\
\hline C & 3.085593 & 4.171307 & -1.175604 \\
\hline $\mathrm{H}$ & 2.941730 & 5.249248 & -1.297061 \\
\hline $\mathrm{H}$ & 2.718237 & 3.684103 & -2.084192 \\
\hline $\mathrm{H}$ & 2.461469 & 3.841891 & -0.339349 \\
\hline C & 5.376220 & 4.372939 & -2.156240 \\
\hline $\mathrm{H}$ & 5.076413 & 3.837003 & -3.062093 \\
\hline $\mathrm{H}$ & 5.198530 & 5.441774 & -2.316643 \\
\hline H & 6.452145 & 4.229360 & -2.022431 \\
\hline C & 8.253456 & -0.965119 & -1.103459 \\
\hline $\mathrm{H}$ & 7.575818 & -1.823170 & -1.087140 \\
\hline $\mathrm{H}$ & 8.211244 & -0.523553 & -2.104003 \\
\hline H & 9.269641 & -1.338100 & -0.936076 \\
\hline
\end{tabular}




\begin{tabular}{|c|c|c|c|}
\hline C & 7.947623 & -0.576573 & 1.368371 \\
\hline $\mathrm{H}$ & 7.260067 & -1.422580 & 1.451970 \\
\hline $\mathrm{H}$ & 8.959703 & -0.944906 & 1.568346 \\
\hline $\mathrm{H}$ & 7.685331 & 0.145343 & 2.147939 \\
\hline C & 8.915696 & 1.212870 & -0.079645 \\
\hline $\mathrm{H}$ & 8.926462 & 1.707424 & -1.055992 \\
\hline $\mathrm{H}$ & 8.725515 & 1.970358 & 0.687081 \\
\hline $\mathrm{H}$ & 9.915826 & 0.806060 & 0.097974 \\
\hline C & -6.719758 & 2.806128 & 1.141865 \\
\hline C & -6.249984 & -0.401766 & -2.758681 \\
\hline C & -6.190651 & 0.754025 & -3.781029 \\
\hline $\mathrm{H}$ & -6.792099 & 1.609036 & -3.458762 \\
\hline $\mathrm{H}$ & -5.160653 & 1.099080 & -3.913571 \\
\hline $\mathrm{H}$ & -6.569243 & 0.423229 & -4.754488 \\
\hline C & -7.710576 & -0.874776 & -2.602143 \\
\hline $\mathrm{H}$ & -7.778318 & -1.689115 & -1.873961 \\
\hline $\mathrm{H}$ & -8.366774 & -0.067898 & -2.263640 \\
\hline $\mathrm{H}$ & -8.097151 & -1.239553 & -3.560049 \\
\hline C & -5.416321 & -1.573456 & -3.304197 \\
\hline $\mathrm{H}$ & -4.366044 & -1.301372 & -3.440787 \\
\hline $\mathrm{H}$ & -5.455345 & -2.437776 & -2.633332 \\
\hline $\mathrm{H}$ & -5.816109 & -1.886970 & -4.273852 \\
\hline C & -6.749776 & 4.024086 & 0.193554 \\
\hline $\mathrm{H}$ & -5.734751 & 4.371568 & -0.022474 \\
\hline $\mathrm{H}$ & -7.229887 & 3.782756 & -0.758766 \\
\hline $\mathrm{H}$ & -7.305638 & 4.850561 & 0.649962 \\
\hline $\mathrm{C}$ & -6.087629 & 3.247379 & 2.471815 \\
\hline $\mathrm{H}$ & -6.679986 & 4.056009 & 2.911053 \\
\hline $\mathrm{H}$ & -6.055819 & 2.427175 & 3.195871 \\
\hline $\mathrm{H}$ & -5.068384 & 3.620305 & 2.331768 \\
\hline C & -8.164111 & 2.340072 & 1.425159 \\
\hline $\mathrm{H}$ & -8.167982 & 1.476597 & 2.097723 \\
\hline $\mathrm{H}$ & -8.739579 & 3.144420 & 1.896446 \\
\hline $\mathrm{H}$ & -8.683008 & 2.050318 & 0.507374 \\
\hline
\end{tabular}

\section{Catalyst 2C 50}

Datum

Value

\begin{tabular}{lr}
\hline B3LYP-D3(BJ)/6-31G(d,p) Energy & -2165.161099 \\
\hline B3LYP-D3(BJ)/def2-TZVPP/IEF-PCM(DCM) Energy & -2165.897679 \\
\hline B3LYP-D3(BJ)/def2-TZVPP/IEF-PCM(DCM)//B3LYP-D3(BJ)/6-31G(d,p) Free Energy (Quasiharmonic) & -2165.020295 \\
\hline Number of Imaginary Frequencies & 0 \\
\hline Frequencies (Top 3 out of 321)
\end{tabular}



1.
$10.6294 \mathrm{~cm}^{-1}$
2 .
$12.9683 \mathrm{~cm}^{-1}$
3.
$13.5206 \mathrm{~cm}^{-1}$

\section{B3LYP-D3(BJ)/6-31G(d,p) Molecular Geometry in Cartesian Coordinates}

C

C

C

C

C

C

$\mathrm{H}$

$\mathrm{H}$

C

C

$\mathrm{H}$

$\mathrm{H}$

C

C

$\mathrm{H}$

C

C

C

C

C

C

$\mathrm{H}$

$\mathrm{H}$

$\mathrm{H}$

C

C

$\mathrm{H}$

$\mathrm{H}$

C

C

C

$\mathrm{H}$

$\mathrm{H}$

C

0

0

$\mathrm{H}$

0

$\mathrm{H}$

C

C

C

C

$\mathrm{H}$

\subsection{1}

0.263324

$-0.788209$

$-2.005296$

$-2.115941$

$-1.076211$

0.935040

1.177486

$-0.670455$

$-3.060443$

$-3.040427$

$-1.172934$

$-2.953946$

$-1.736570$

$-3.967696$

0.822557

1.900926

1.838466

0.635885

$-0.459548$

$-0.368261$

3.860078

0.880762

2.820219

2.937352

0.586170

$-1.376133$

$-1.215348$

1.671666

2. 885461

1.538213

0.518067

2. 216677

$-1.586262$

$-0.828945$

$-2.389473$

$-2.234650$

1.890636

1.252163

4.094407

4.584932

4.777189

5.728364

4.041577
$-4.910388$

$-3.748539$

$-2.791356$

$-3.068738$

$-4.277254$

$-5.176713$

$-5.629477$

$-3.549759$

$-1.567277$

$-2.127156$

$-4.476793$

$-6.095844$

$-0.948112$

$-0.685247$

$-2.326993$

$-2.280205$

$-1.710910$

$-1.374584$

$-1.636190$

$-2.228949$

$-2.540891$

$-0.637518$

$-2.532405$

$-1.508746$

$-0.802001$

$-1.284269$

$-2.426612$

$-2.990936$

$-0.702197$

$-0.476109$

$-0.276863$

0.077517

0.551028

0.565713

1.478127

0.560054

1.404599

$-1.303639$

$-2.023347$

0.103459

$-0.420704$

1.155586

0.104152

$-1.232274$
$-1.337962$

$-0.611964$

$-0.563112$

$-1.262690$

$-1.999917$

$-2.040786$

$-1.368278$

$-0.064370$

0.165644

$-1.213219$

$-2.534156$

$-2.609962$

$-0.503371$

0.199135

$-1.775269$

5.051411

4.416118

3.037756

2. 310333

2.994912

4. 331894

2.906757

6.105792

4.958709

2. 358018

0.926906

2.451586

4.840251

0.286621

1.016058

$-1.163980$

$-1.341711$

$-1.369380$

1.012784

0.756393

2.096446

2.553547

$-2.099022$

$-1.998671$

0.374659

$-0.832159$

0.987750

$-1.430050$

$-1.295230$ 


\begin{tabular}{|c|c|c|c|}
\hline C & 5.938030 & 1.701071 & 0.421033 \\
\hline $\mathrm{H}$ & 4.377254 & 1.565044 & 1.909576 \\
\hline C & 6.390358 & 1.162082 & -0.784482 \\
\hline $\mathrm{H}$ & 7.278014 & 1.574177 & -1.247089 \\
\hline C & -4.065842 & 0.033589 & -0.507559 \\
\hline C & -3.808783 & 1.387716 & -0.752825 \\
\hline C & -5.380895 & -0.388837 & -0.281326 \\
\hline C & -4.839598 & 2.329436 & -0.746490 \\
\hline $\mathrm{H}$ & -2.792567 & 1.695682 & -0.956641 \\
\hline C & -6.439953 & 0.522994 & -0.284261 \\
\hline $\mathrm{H}$ & -5.556237 & -1.437288 & -0.075832 \\
\hline C & -6.141852 & 1.872344 & -0.512578 \\
\hline H & -6.952663 & 2.592878 & -0.504383 \\
\hline C & -7.894793 & 0.101920 & -0.031607 \\
\hline C & -4.580858 & 3.825574 & -0.971441 \\
\hline C & -8.033298 & -1.412757 & 0.191002 \\
\hline $\mathrm{H}$ & -7.688274 & -1.982382 & -0.677634 \\
\hline $\mathrm{H}$ & -7.468894 & -1.747060 & 1.066989 \\
\hline H & -9.084760 & -1.665692 & 0.357655 \\
\hline C & -8.756713 & 0.491695 & -1.251829 \\
\hline $\mathrm{H}$ & -9.800509 & 0.202196 & -1.089074 \\
\hline $\mathrm{H}$ & -8.733339 & 1.569059 & -1.436910 \\
\hline $\mathrm{H}$ & -8.399022 & -0.010587 & -2.156033 \\
\hline C & -8.424414 & 0.826115 & 1.224863 \\
\hline $\mathrm{H}$ & -8.392775 & 1.912919 & 1.108547 \\
\hline $\mathrm{H}$ & -9.463432 & 0.539823 & 1.420795 \\
\hline H & -7.825722 & 0.565975 & 2.103260 \\
\hline C & -5.029739 & 4.605115 & 0.283691 \\
\hline H & -6.093060 & 4.458614 & 0.493353 \\
\hline $\mathrm{H}$ & -4.466797 & 4.278606 & 1.163784 \\
\hline $\mathrm{H}$ & -4.858456 & 5.678301 & 0.147318 \\
\hline C & -5.387476 & 4.311349 & -2.194197 \\
\hline $\mathrm{H}$ & -6.462464 & 4.165113 & -2.055579 \\
\hline $\mathrm{H}$ & -5.214114 & 5.379277 & -2.365003 \\
\hline $\mathrm{H}$ & -5.088949 & 3.768118 & -3.096102 \\
\hline C & -3.093020 & 4.125816 & -1.218993 \\
\hline $\mathrm{H}$ & -2.465265 & 3.806368 & -0.381605 \\
\hline $\mathrm{H}$ & -2.726855 & 3.631204 & -2.124059 \\
\hline H & -2.952981 & 5.202998 & -1.351112 \\
\hline C & 6.648645 & 2.863439 & 1.129358 \\
\hline C & 6.261896 & -0.421700 & -2.769815 \\
\hline C & 7.724802 & -0.884016 & -2.602243 \\
\hline H & 8.375987 & -0.067939 & -2.276401 \\
\hline $\mathrm{H}$ & 7.795240 & -1.685505 & -1.860166 \\
\hline $\mathrm{H}$ & 8.115198 & -1.263117 & -3.553025 \\
\hline C & 6.198677 & 0.715430 & -3.812747 \\
\hline $\mathrm{H}$ & 5.167079 & 1.052285 & -3.953765 \\
\hline $\mathrm{H}$ & 6.794947 & 1.579304 & -3.504922 \\
\hline $\mathrm{H}$ & 6.581254 & 0.369131 & -4.779231 \\
\hline C & 5.435843 & -1.607195 & -3.296625 \\
\hline H & 5.479131 & -2.459940 & -2.611375 \\
\hline $\mathrm{H}$ & 4.384157 & -1.343425 & -3.438715 \\
\hline H & 5.838498 & -1.934546 & -4.260511 \\
\hline C & 5.681983 & 4.062617 & 1.235386 \\
\hline
\end{tabular}




\begin{tabular}{|llll|} 
H & 5.370558 & 4.397869 & 0.241220 \\
$\mathrm{H}$ & 4.780474 & 3.806406 & 1.798412 \\
$\mathrm{H}$ & 6.168900 & 4.902220 & 1.743685 \\
$\mathrm{C}$ & 7.907930 & 3.325153 & 0.378574 \\
$\mathrm{H}$ & 8.381536 & 4.146937 & 0.924504 \\
$\mathrm{H}$ & 8.642916 & 2.519291 & 0.286972 \\
$\mathrm{H}$ & 7.670024 & 3.688173 & -0.626134 \\
$\mathrm{C}$ & 7.068829 & 2.416136 & 2.546120 \\
$\mathrm{H}$ & 7.755500 & 1.565262 & 2.496185 \\
$\mathrm{H}$ & 7.574136 & 3.234193 & 3.071167 \\
$\mathrm{H}$ & 6.206228 & 2.115219 & 3.146953 \\
& & & \\
\hline
\end{tabular}

\section{Catalyst 2C 51}

\section{Datum}

Value

B3LYP-D3(BJ)/6-31G(d,p) Energy $-2165.162638$

B3LYP-D3(BJ)/def2-TZVPP/IEF-PCM(DCM) Energy $-2165.898253$

B3LYP-D3(BJ)/def2-TZVPP/IEF-PCM(DCM)//B3LYP-D3(BJ)/6-31G(d,p) Free Energy (Quasiharmonic) $-2165.02026$

Number of Imaginary Frequencies

Frequencies (Top 3 out of 321)

$\begin{array}{ll}\text { 1. } & 11.4281 \mathrm{~cm}^{-1} \\ \text { 2. } & 13.0647 \mathrm{~cm}^{-1} \\ \text { 3. } & 13.6883 \mathrm{~cm}^{-1}\end{array}$

B3LYP-D3(BJ)/6-31G(d,p) Molecular Geometry in Cartesian Coordinates

$\begin{array}{lrrl}\text { C } & -0.281795 & -3.507129 & 3.890699 \\ \text { C } & -0.403901 & -2.928401 & 2.648054 \\ \text { C } & 0.675654 & -2.203542 & 2.078426 \\ \text { C } & 1.892812 & -2.079173 & 2.819042 \\ \text { C } & 1.985476 & -2.688192 & 4.098135 \\ \text { C } & 0.922991 & -3.386156 & 4.623190 \\ \text { H } & -1.113689 & -4.061596 & 4.313344 \\ \text { H } & -1.325638 & -3.019912 & 2.085189 \\ \text { C } & 0.580760 & -1.585206 & 0.793350 \\ \text { C } & 2.974910 & -1.359397 & 2.256880 \\ \text { H } & 2.913229 & -2.591105 & 4.654695 \\ \text { H } & 1.005097 & -3.846883 & 5.602693 \\ \text { C } & 2.895121 & -0.778602 & 1.008112 \\ \text { C } & 1.671395 & -0.907226 & 0.278893 \\ \text { H } & 3.903200 & -1.287368 & 2.815620\end{array}$




\begin{tabular}{|c|c|c|c|}
\hline C & -1.340396 & -4.897864 & -2.650923 \\
\hline C & -2.316992 & -3.945968 & -2.475908 \\
\hline C & -2.117058 & -2.857028 & -1.586913 \\
\hline C & -0.883702 & -2.755402 & -0.875204 \\
\hline C & 0.107259 & -3.750963 & -1.081746 \\
\hline C & -0.117245 & -4.797492 & -1.946029 \\
\hline $\mathrm{H}$ & -4.033651 & -1.938575 & -1.943632 \\
\hline $\mathrm{H}$ & -1.502558 & -5.727451 & -3.332225 \\
\hline $\mathrm{H}$ & -3.258119 & -4.011312 & -3.014699 \\
\hline C & -3.104588 & -1.865698 & -1.385956 \\
\hline C & -0.699914 & -1.657688 & 0.021222 \\
\hline $\mathrm{H}$ & 1.050477 & -3.671583 & -0.554431 \\
\hline $\mathrm{H}$ & 0.651046 & -5.549895 & -2.094416 \\
\hline C & -1.688228 & -0.697232 & 0.207695 \\
\hline C & -2.920524 & -0.801120 & -0.524923 \\
\hline C & -1.478481 & 0.419738 & 1.217292 \\
\hline $\mathrm{H}$ & -2.436862 & 0.683547 & 1.667975 \\
\hline $\mathrm{H}$ & -0.819712 & 0.068168 & 2.018856 \\
\hline C & 1.535417 & -0.275825 & -1.074375 \\
\hline 0 & 1.531896 & -0.853688 & -2.136098 \\
\hline 0 & 1.396105 & 1.075334 & -0.983341 \\
\hline $\mathrm{H}$ & 1.317221 & 1.409115 & -1.894287 \\
\hline 0 & -0.975484 & 1.631875 & 0.655136 \\
\hline $\mathrm{H}$ & -0.111898 & 1.451459 & 0.253719 \\
\hline C & -4.029628 & 0.177412 & -0.365581 \\
\hline C & -3.800871 & 1.555618 & -0.457023 \\
\hline C & -5.335369 & -0.288433 & -0.155579 \\
\hline C & -4.848913 & 2.469878 & -0.325344 \\
\hline $\mathrm{H}$ & -2.787480 & 1.896363 & -0.610557 \\
\hline C & -6.409047 & 0.597073 & -0.031710 \\
\hline $\mathrm{H}$ & -5.491345 & -1.356528 & -0.070063 \\
\hline C & -6.138925 & 1.968512 & -0.117399 \\
\hline $\mathrm{H}$ & -6.960420 & 2.669180 & -0.010706 \\
\hline C & 4.054228 & -0.046526 & 0.440913 \\
\hline C & 4.485388 & -0.294831 & -0.866285 \\
\hline C & 4.734066 & 0.900723 & 1.213583 \\
\hline C & 5.560122 & 0.405761 & -1.420806 \\
\hline $\mathrm{H}$ & 3.975686 & -1.049785 & -1.452041 \\
\hline C & 5.826339 & 1.606883 & 0.700416 \\
\hline $\mathrm{H}$ & 4.378286 & 1.099593 & 2.218959 \\
\hline C & 6.217109 & 1.347712 & -0.619998 \\
\hline $\mathrm{H}$ & 7.050908 & 1.894577 & -1.034879 \\
\hline C & 6.536988 & 2.643544 & 1.583061 \\
\hline C & 5.958730 & 0.127543 & -2.876884 \\
\hline C & 7.068196 & 1.949606 & 2.855932 \\
\hline $\mathrm{H}$ & 6.260411 & 1.494103 & 3.435275 \\
\hline $\mathrm{H}$ & 7.782456 & 1.161117 & 2.598951 \\
\hline $\mathrm{H}$ & 7.575601 & 2.674365 & 3.501696 \\
\hline C & 5.532417 & 3.746674 & 1.980607 \\
\hline $\mathrm{H}$ & 6.019487 & 4.495579 & 2.614522 \\
\hline $\mathrm{H}$ & 5.140352 & 4.252314 & 1.092743 \\
\hline $\mathrm{H}$ & 4.682700 & 3.339332 & 2.535054 \\
\hline C & 7.724244 & 3.308065 & 0.867511 \\
\hline $\mathrm{H}$ & 7.406304 & 3.850556 & -0.028247 \\
\hline
\end{tabular}




\begin{tabular}{|c|c|c|c|}
\hline $\mathrm{H}$ & 8.200720 & 4.028851 & 1.538899 \\
\hline $\mathrm{H}$ & 8.482643 & 2.575440 & 0.574168 \\
\hline C & 7.164549 & 0.968358 & -3.325236 \\
\hline $\mathrm{H}$ & 6.955959 & 2.041180 & -3.265511 \\
\hline $\mathrm{H}$ & 8.052996 & 0.757355 & -2.721532 \\
\hline $\mathrm{H}$ & 7.407757 & 0.736177 & -4.366490 \\
\hline C & 4.760486 & 0.464926 & -3.792658 \\
\hline $\mathrm{H}$ & 4.499192 & 1.525023 & -3.707068 \\
\hline $\mathrm{H}$ & 5.011613 & 0.260670 & -4.839169 \\
\hline $\mathrm{H}$ & 3.873717 & -0.121654 & -3.537683 \\
\hline C & 6.322389 & -1.364275 & -3.036040 \\
\hline $\mathrm{H}$ & 7.163373 & -1.630537 & -2.388022 \\
\hline $\mathrm{H}$ & 5.482268 & -2.015679 & -2.781556 \\
\hline $\mathrm{H}$ & 6.607421 & -1.576830 & -4.071983 \\
\hline C & -7.850548 & 0.122886 & 0.204364 \\
\hline C & -4.616907 & 3.986551 & -0.377588 \\
\hline C & -5.001711 & 4.596084 & 0.988047 \\
\hline $\mathrm{H}$ & -6.049535 & 4.402144 & 1.235354 \\
\hline $\mathrm{H}$ & -4.384391 & 4.173225 & 1.786585 \\
\hline $\mathrm{H}$ & -4.852978 & 5.681513 & 0.978113 \\
\hline C & -5.493798 & 4.610008 & -1.483850 \\
\hline $\mathrm{H}$ & -5.244972 & 4.185052 & -2.461334 \\
\hline $\mathrm{H}$ & -6.559098 & 4.437881 & -1.305736 \\
\hline $\mathrm{H}$ & -5.334278 & 5.692793 & -1.531018 \\
\hline C & -3.147716 & 4.337385 & -0.667212 \\
\hline $\mathrm{H}$ & -2.471356 & 3.922127 & 0.084954 \\
\hline $\mathrm{H}$ & -2.834030 & 3.961466 & -1.646552 \\
\hline $\mathrm{H}$ & -3.025379 & 5.425215 & -0.673818 \\
\hline C & -8.746279 & 0.622771 & -0.949522 \\
\hline $\mathrm{H}$ & -8.743000 & 1.713852 & -1.020402 \\
\hline $\mathrm{H}$ & -8.400562 & 0.224862 & -1.908704 \\
\hline $\mathrm{H}$ & -9.781844 & 0.299278 & -0.797194 \\
\hline C & -8.366035 & 0.698450 & 1.540984 \\
\hline $\mathrm{H}$ & -9.395927 & 0.373568 & 1.725589 \\
\hline $\mathrm{H}$ & -7.744494 & 0.357488 & 2.374813 \\
\hline $\mathrm{H}$ & -8.354345 & 1.791820 & 1.541388 \\
\hline C & -7.958485 & -1.409235 & 0.266924 \\
\hline $\mathrm{H}$ & -7.367398 & -1.823669 & 1.089478 \\
\hline $\mathrm{H}$ & -9.001346 & -1.699058 & 0.428494 \\
\hline $\mathrm{H}$ & -7.623878 & -1.876422 & -0.664567 \\
\hline
\end{tabular}

\section{Catalyst 2C 52}

Datum

Value

B3LYP-D3(BJ)/6-31G(d,p) Energy

$-2165.163074$

B3LYP-D3(BJ)/def2-TZVPP/IEF-PCM(DCM) Energy

$-2165.898357$

B3LYP-D3(BJ)/def2-TZVPP/IEF-PCM(DCM)//B3LYP-D3(BJ)/6-31G(d,p) Free Energy (Quasiharmonic) - 
Frequencies (Top 3 out of 321)

$\begin{array}{ll}\text { 1. } & 11.8410 \mathrm{~cm}^{-1} \\ \text { 2. } & 12.9333 \mathrm{~cm}^{-1} \\ \text { 3. } & 13.3006 \mathrm{~cm}^{-1}\end{array}$

B3LYP-D3(BJ)/6-31G(d,p) Molecular Geometry in Cartesian Coordinates

\begin{tabular}{|c|c|c|c|}
\hline C & -0.331741 & -3.438781 & 3.928019 \\
\hline C & -0.446566 & -2.874742 & 2.677950 \\
\hline C & 0.640961 & -2.168234 & 2.100451 \\
\hline C & 1.858445 & -2.046748 & 2.841029 \\
\hline C & 1.943484 & -2.640321 & 4.127880 \\
\hline C & 0.873379 & -3.320741 & 4.660424 \\
\hline $\mathrm{H}$ & -1.169737 & -3.979274 & 4.356654 \\
\hline $\mathrm{H}$ & -1.368612 & -2.964053 & 2.115263 \\
\hline C & 0.553929 & -1.565493 & 0.807392 \\
\hline C & 2.948282 & -1.344775 & 2.271376 \\
\hline $\mathrm{H}$ & 2.871525 & -2.545502 & 4.684342 \\
\hline $\mathrm{H}$ & 0.949671 & -3.769739 & 5.645821 \\
\hline C & 2.875900 & -0.779139 & 1.015237 \\
\hline C & 1.652041 & -0.905283 & 0.285771 \\
\hline $\mathrm{H}$ & 3.876381 & -1.274445 & 2.830641 \\
\hline C & -1.396579 & -4.894717 & -2.604579 \\
\hline C & -2.364563 & -3.932379 & -2.439191 \\
\hline C & -2.155131 & -2.836985 & -1.560361 \\
\hline C & -0.921118 & -2.739630 & -0.849216 \\
\hline C & 0.060735 & -3.746239 & -1.045485 \\
\hline C & -0.172960 & -4.798871 & -1.899839 \\
\hline $\mathrm{H}$ & -4.064090 & -1.906225 & -1.924930 \\
\hline $\mathrm{H}$ & -1.565918 & -5.729204 & -3.278097 \\
\hline $\mathrm{H}$ & -3.306002 & -3.994269 & -2.977838 \\
\hline C & -3.134262 & -1.835718 & -1.368236 \\
\hline C & -0.727193 & -1.634313 & 0.035594 \\
\hline $\mathrm{H}$ & 1.004404 & -3.670399 & -0.518471 \\
\hline $\mathrm{H}$ & 0.588441 & -5.559701 & -2.040538 \\
\hline C & -1.706797 & -0.663016 & 0.212324 \\
\hline C & -2.941379 & -0.764947 & -0.516824 \\
\hline C & -1.482804 & 0.466001 & 1.205112 \\
\hline $\mathrm{H}$ & -2.435949 & 0.747504 & 1.655824 \\
\hline $\mathrm{H}$ & -0.823595 & 0.121360 & 2.009036 \\
\hline C & 1.524888 & -0.290634 & -1.075959 \\
\hline 0 & 1.518135 & -0.882342 & -2.129961 \\
\hline 0 & 1.398559 & 1.063088 & -1.003388 \\
\hline $\mathrm{H}$ & 1.326205 & 1.385178 & -1.919085 \\
\hline
\end{tabular}




\begin{tabular}{|c|c|c|c|}
\hline 0 & -0.969957 & 1.664104 & 0.620730 \\
\hline $\mathrm{H}$ & -0.106527 & 1.471212 & 0.224576 \\
\hline C & -4.044516 & 0.221317 & -0.363896 \\
\hline C & -3.811072 & 1.597712 & -0.448371 \\
\hline C & -5.355413 & -0.230763 & -0.164092 \\
\hline C & -4.853503 & 2.519973 & -0.319741 \\
\hline $\mathrm{H}$ & -2.794887 & 1.939874 & -0.594469 \\
\hline C & -6.425744 & 0.661147 & -0.047738 \\
\hline $\mathrm{H}$ & -5.526370 & -1.298956 & -0.079466 \\
\hline C & -6.151546 & 2.032782 & -0.126186 \\
\hline $\mathrm{H}$ & -6.965604 & 2.735967 & -0.027967 \\
\hline C & 4.042403 & -0.064294 & 0.441230 \\
\hline C & 4.474265 & -0.332464 & -0.861798 \\
\hline C & 4.728180 & 0.887135 & 1.203446 \\
\hline C & 5.555684 & 0.352603 & -1.422735 \\
\hline $\mathrm{H}$ & 3.959812 & -1.090672 & -1.439152 \\
\hline C & 5.827021 & 1.578195 & 0.683810 \\
\hline $\mathrm{H}$ & 4.371906 & 1.101422 & 2.205470 \\
\hline C & 6.218394 & 1.299396 & -0.632414 \\
\hline $\mathrm{H}$ & 7.057285 & 1.834525 & -1.052298 \\
\hline C & 6.544016 & 2.620200 & 1.554947 \\
\hline C & 5.955177 & 0.052858 & -2.874289 \\
\hline C & 7.737490 & 3.266905 & 0.833437 \\
\hline $\mathrm{H}$ & 8.490885 & 2.525059 & 0.550575 \\
\hline $\mathrm{H}$ & 7.425201 & 3.800660 & -0.069527 \\
\hline $\mathrm{H}$ & 8.218174 & 3.992334 & 1.496769 \\
\hline C & 7.067773 & 1.937865 & 2.837146 \\
\hline $\mathrm{H}$ & 7.579444 & 2.666631 & 3.474983 \\
\hline $\mathrm{H}$ & 6.255589 & 1.495476 & 3.420487 \\
\hline $\mathrm{H}$ & 7.776570 & 1.141009 & 2.591086 \\
\hline C & 5.547206 & 3.735719 & 1.937181 \\
\hline $\mathrm{H}$ & 4.693371 & 3.341726 & 2.494937 \\
\hline $\mathrm{H}$ & 6.038889 & 4.488502 & 2.562875 \\
\hline $\mathrm{H}$ & 5.160627 & 4.233553 & 1.042523 \\
\hline C & 6.307530 & -1.443646 & -3.013653 \\
\hline $\mathrm{H}$ & 7.144963 & -1.708182 & -2.360356 \\
\hline $\mathrm{H}$ & 5.461787 & -2.085204 & -2.752845 \\
\hline $\mathrm{H}$ & 6.593185 & -1.671582 & -4.046145 \\
\hline C & 7.168435 & 0.878530 & -3.330629 \\
\hline $\mathrm{H}$ & 8.053959 & 0.668365 & -2.722357 \\
\hline $\mathrm{H}$ & 7.412018 & 0.631252 & -4.368312 \\
\hline $\mathrm{H}$ & 6.968068 & 1.953609 & -3.285005 \\
\hline C & 4.761557 & 0.387868 & -3.796951 \\
\hline $\mathrm{H}$ & 3.869685 & -0.188503 & -3.536541 \\
\hline $\mathrm{H}$ & 4.508386 & 1.450980 & -3.725468 \\
\hline $\mathrm{H}$ & 5.013300 & 0.168340 & -4.840211 \\
\hline C & -7.843939 & 0.114056 & 0.174324 \\
\hline C & -4.526323 & 4.018718 & -0.379009 \\
\hline C & -5.776853 & 4.899158 & -0.228631 \\
\hline $\mathrm{H}$ & -6.502004 & 4.715143 & -1.028058 \\
\hline $\mathrm{H}$ & -6.275736 & 4.733326 & 0.731456 \\
\hline $\mathrm{H}$ & -5.491742 & 5.954743 & -0.275264 \\
\hline C & -3.864837 & 4.344919 & -1.735261 \\
\hline $\mathrm{H}$ & -2.939404 & 3.780611 & -1.876537 \\
\hline
\end{tabular}




\begin{tabular}{|c|c|c|c|}
\hline $\mathrm{H}$ & -4.537104 & 4.102831 & -2.564705 \\
\hline $\mathrm{H}$ & -3.620115 & 5.411295 & -1.792584 \\
\hline C & -3.544748 & 4.360133 & 0.764775 \\
\hline $\mathrm{H}$ & -4.004361 & 4.156310 & 1.737544 \\
\hline H & -2.624769 & 3.773313 & 0.700319 \\
\hline $\mathrm{H}$ & -3.278345 & 5.422713 & 0.731459 \\
\hline C & -8.896277 & 1.231053 & 0.264884 \\
\hline $\mathrm{H}$ & -8.701366 & 1.904510 & 1.105247 \\
\hline $\mathrm{H}$ & -8.932786 & 1.828353 & -0.651494 \\
\hline $\mathrm{H}$ & -9.887258 & 0.791585 & 0.415047 \\
\hline C & -7.880023 & -0.690646 & 1.491483 \\
\hline $\mathrm{H}$ & -8.883933 & -1.092961 & 1.666680 \\
\hline H & -7.180852 & -1.531049 & 1.470437 \\
\hline $\mathrm{H}$ & -7.612815 & -0.054578 & 2.341129 \\
\hline C & -8.223585 & -0.810981 & -1.002130 \\
\hline $\mathrm{H}$ & -7.533655 & -1.654621 & -1.091063 \\
\hline $\mathrm{H}$ & -9.231832 & -1.215646 & -0.860857 \\
\hline H & -8.204466 & -0.261764 & -1.948657 \\
\hline
\end{tabular}

\section{Catalyst 2C 53}

\section{Datum}

Value

B3LYP-D3(BJ)/6-31G(d,p) Energy

$-2165.161136$

B3LYP-D3(BJ)/def2-TZVPP/IEF-PCM(DCM) Energy

$-2165.8978$

B3LYP-D3(BJ)/def2-TZVPP/IEF-PCM(DCM)//B3LYP-D3(BJ)/6-31G(d,p) Free Energy (Quasiharmonic)

$-2165.020184$

Number of Imaginary Frequencies

Frequencies (Top 3 out of 321)

$\begin{array}{ll}\text { 1. } & 10.9722 \mathrm{~cm}^{-1} \\ \text { 2. } & 12.7238 \mathrm{~cm}^{-1} \\ \text { 3. } & 14.1739 \mathrm{~cm}^{-1}\end{array}$

B3LYP-D3(BJ)/6-31G(d,p) Molecular Geometry in Cartesian Coordinates

C

C

C

C

$\mathrm{C}$

C

$\mathrm{H}$

$\mathrm{H}$

-0.189893
-0.312341
0.747366
1.946559
2.041121
0.997012
-1.007030
-1.217512

3. 237149

2.081030

1.642359

2.420350

3.602352

4.005090

3. 559849

1.486882

\subsection{7}

3. 268675

2. 427397

2.365395

3. 146301

3.946167

4.644412

3. 322925 


\begin{tabular}{|c|c|c|c|}
\hline C & 0.645616 & 0.460823 & 1.628937 \\
\hline C & 3.006154 & 1.990326 & 1.531199 \\
\hline $\mathrm{H}$ & 2.955191 & 4.186947 & 3.095088 \\
\hline $\mathrm{H}$ & 1.080053 & 4.913877 & 4.533810 \\
\hline C & 2.912875 & 0.846805 & 0.764357 \\
\hline C & 1.705435 & 0.084484 & 0.823257 \\
\hline $\mathrm{H}$ & 3.924873 & 2.568725 & 1.510928 \\
\hline C & -1.183788 & -3.483314 & 4.409281 \\
\hline C & -2.183343 & -3.165247 & 3.520194 \\
\hline C & -2.015423 & -2.108259 & 2.586580 \\
\hline C & -0.789802 & -1.374813 & 2.578443 \\
\hline C & 0.223450 & -1.727749 & 3.508559 \\
\hline C & 0.030780 & -2.756101 & 4.401980 \\
\hline $\mathrm{H}$ & -3.974461 & -2.289010 & 1.705066 \\
\hline $\mathrm{H}$ & -1.322372 & -4.292539 & 5.119673 \\
\hline H & -3.119674 & -3.716286 & 3.518639 \\
\hline C & -3.033000 & -1.748409 & 1.673200 \\
\hline C & -0.632630 & -0.318157 & 1.630723 \\
\hline $\mathrm{H}$ & 1.157877 & -1.178667 & 3.499871 \\
\hline $\mathrm{H}$ & 0.815296 & -3.014446 & 5.106496 \\
\hline C & -1.645036 & 0.014237 & 0.738701 \\
\hline C & -2.884599 & -0.709938 & 0.773965 \\
\hline C & -1.425652 & 1.142757 & -0.253043 \\
\hline $\mathrm{H}$ & -0.383299 & 1.151857 & -0.584561 \\
\hline $\mathrm{H}$ & -2.049727 & 0.992207 & -1.133389 \\
\hline C & 1.490223 & -1.049141 & -0.137568 \\
\hline 0 & 1.280325 & -0.904757 & -1.320487 \\
\hline 0 & 1.523341 & -2.263119 & 0.460739 \\
\hline $\mathrm{H}$ & 1.312790 & -2.912700 & -0.232557 \\
\hline 0 & -1.801156 & 2.423971 & 0.269607 \\
\hline H & -1.217241 & 2.618284 & 1.016255 \\
\hline C & -4.022017 & -0.378130 & -0.123948 \\
\hline C & -4.473458 & 0.936003 & -0.252823 \\
\hline C & -4.681146 & -1.400621 & -0.826709 \\
\hline C & -5.555109 & 1.254920 & -1.084575 \\
\hline $\mathrm{H}$ & -3.959043 & 1.716285 & 0.294336 \\
\hline C & -5.770422 & -1.122337 & -1.650715 \\
\hline $\mathrm{H}$ & -4.307521 & -2.412304 & -0.729314 \\
\hline C & -6.187728 & 0.214892 & -1.766273 \\
\hline $\mathrm{H}$ & -7.025832 & 0.440194 & -2.413497 \\
\hline C & 4.045778 & 0.415012 & -0.088888 \\
\hline C & 4.480604 & -0.910063 & -0.067290 \\
\hline C & 4.696783 & 1.339377 & -0.919968 \\
\hline C & 5.537450 & -1.341883 & -0.879191 \\
\hline $\mathrm{H}$ & 3.991422 & -1.610513 & 0.599282 \\
\hline C & 5.760984 & 0.949441 & -1.730921 \\
\hline $\mathrm{H}$ & 4.331497 & 2.358297 & -0.938762 \\
\hline C & 6.159852 & -0.398020 & -1.696476 \\
\hline $\mathrm{H}$ & 6.977200 & -0.709665 & -2.333876 \\
\hline C & 6.490709 & 1.925695 & -2.664296 \\
\hline C & 5.956245 & -2.817651 & -0.837066 \\
\hline C & 5.928856 & 3.353302 & -2.568911 \\
\hline $\mathrm{H}$ & 4.874464 & 3.393936 & -2.858790 \\
\hline $\mathrm{H}$ & 6.022670 & 3.758073 & -1.556226 \\
\hline
\end{tabular}




\begin{tabular}{|c|c|c|c|}
\hline $\mathrm{H}$ & 6.483814 & 4.012970 & -3.242840 \\
\hline C & 6.341420 & 1.443547 & -4.123507 \\
\hline $\mathrm{H}$ & 6.856680 & 2.128551 & -4.805569 \\
\hline $\mathrm{H}$ & 6.767107 & 0.446424 & -4.265682 \\
\hline $\mathrm{H}$ & 5.286592 & 1.401347 & -4.411420 \\
\hline C & 7.987295 & 1.969174 & -2.287018 \\
\hline $\mathrm{H}$ & 8.456732 & 0.985700 & -2.376349 \\
\hline $\mathrm{H}$ & 8.526027 & 2.658454 & -2.946153 \\
\hline $\mathrm{H}$ & 8.117939 & 2.309958 & -1.255134 \\
\hline C & 4.738382 & -3.696845 & -1.198194 \\
\hline $\mathrm{H}$ & 3.914554 & -3.550607 & -0.494313 \\
\hline $\mathrm{H}$ & 4.370537 & -3.456106 & -2.200403 \\
\hline $\mathrm{H}$ & 5.014193 & -4.756720 & -1.177517 \\
\hline C & 6.438842 & -3.169583 & 0.586464 \\
\hline $\mathrm{H}$ & 5.654991 & -3.002662 & 1.330341 \\
\hline $\mathrm{H}$ & 6.733872 & -4.223192 & 0.639850 \\
\hline $\mathrm{H}$ & 7.302187 & -2.557645 & 0.865850 \\
\hline C & 7.089559 & -3.132989 & -1.825842 \\
\hline $\mathrm{H}$ & 6.797133 & -2.916523 & -2.858115 \\
\hline $\mathrm{H}$ & 7.995865 & -2.562812 & -1.598793 \\
\hline $\mathrm{H}$ & 7.344085 & -4.195625 & -1.767884 \\
\hline C & -6.512254 & -2.211855 & -2.438457 \\
\hline C & -5.979028 & 2.723875 & -1.216680 \\
\hline C & -4.785751 & 3.541552 & -1.760349 \\
\hline $\mathrm{H}$ & -3.911229 & 3.469863 & -1.108439 \\
\hline $\mathrm{H}$ & -4.494503 & 3.182081 & -2.752731 \\
\hline $\mathrm{H}$ & -5.060250 & 4.598752 & -1.849123 \\
\hline C & -6.380915 & 3.266014 & 0.171796 \\
\hline $\mathrm{H}$ & -7.221176 & 2.697570 & 0.583360 \\
\hline $\mathrm{H}$ & -5.552465 & 3.204549 & 0.882229 \\
\hline $\mathrm{H}$ & -6.681840 & 4.316776 & 0.097203 \\
\hline C & -7.170363 & 2.905630 & -2.170068 \\
\hline $\mathrm{H}$ & -6.935069 & 2.560198 & -3.181782 \\
\hline $\mathrm{H}$ & -8.056302 & 2.365561 & -1.820430 \\
\hline $\mathrm{H}$ & -7.432130 & 3.966231 & -2.234729 \\
\hline C & -5.926574 & -3.611555 & -2.192039 \\
\hline $\mathrm{H}$ & -4.879879 & -3.673193 & -2.505595 \\
\hline $\mathrm{H}$ & -5.985416 & -3.894185 & -1.136252 \\
\hline $\mathrm{H}$ & -6.489725 & -4.353310 & -2.766787 \\
\hline C & -6.415417 & -1.907162 & -3.948974 \\
\hline $\mathrm{H}$ & -6.942628 & -2.673414 & -4.527953 \\
\hline $\mathrm{H}$ & -6.857667 & -0.937938 & -4.195019 \\
\hline $\mathrm{H}$ & -5.370602 & -1.890164 & -4.274257 \\
\hline C & -7.996101 & -2.228056 & -2.011984 \\
\hline $\mathrm{H}$ & -8.482116 & -1.267234 & -2.201882 \\
\hline $\mathrm{H}$ & -8.544578 & -2.997036 & -2.567032 \\
\hline $\mathrm{H}$ & -8.089814 & -2.444568 & -0.943192 \\
\hline
\end{tabular}


Frequencies (Top 3 out of 321)

$\begin{array}{ll}\text { 1. } & 10.5701 \mathrm{~cm}^{-1} \\ \text { 2. } & 12.1082 \mathrm{~cm}^{-1} \\ \text { 3. } & 13.7984 \mathrm{~cm}^{-1}\end{array}$

\section{B3LYP-D3(BJ)/6-31G(d,p) Molecular Geometry in Cartesian Coordinates}

C

C

$\mathrm{C}$

C

$\mathrm{C}$

C

$\mathrm{H}$

$\mathrm{H}$

C

C

$\mathrm{H}$

$\mathrm{H}$

C

C

$\mathrm{H}$

C

C

C

C

C

C

$\mathrm{H}$

$\mathrm{H}$

$\mathrm{H}$

C

C

$\mathrm{H}$

$\mathrm{H}$

C

C

C

$\mathrm{H}$

\subsection{8}

0.294576

$-0.761772$

$-1.963720$

$-2.064740$

$-1.023814$

0.980817

1. 202092

$-0.654673$

$-3.019507$

$-2.981082$

$-1.111655$

$-2.920074$

$-1.711131$

$-3.940751$

1.184968

2. 184767

2.014470

0.786195

$-0.227230$

$-0.032231$

3.975930

1. 325390

3. 123117

3.032306

0.626238

$-1.163787$

$-0.817012$

1.638793

2.881056

1.417818

2.047424

\section{267314}

2. 104574

1.653509

2. 426175

3.615150

4.029762

3. 599605

1.514764

0.463757

1.984122

4. 195494

4.943807

0.834435

0.075572

2.558165

$-3.436258$

$-3.126403$

$-2.082593$

$-1.353811$

$-1.698079$

$-2.713715$

$-2.267347$

$-4.235437$

$-3.673964$

$-1.731058$

$-0.310764$

$-1.152872$

$-2.965648$

0.013261

$-0.705178$

1.126450

0.965693

\subsection{3}

3. 258007

2. 419101

2. 347053

3. 116452

3.914404

4.620340

3. 319639

1.633621

1.514453

3. 057852

4.493135

0.757415

0.828963

1.487181

4.469471

3.577724

2.629867

2.610077

3.543209

4.450573

1. 753561

5.190797

3. 584964

1.713355

1.647776

3. 525466

5.157108

0.752956

0.800416

$-0.255727$

$-1.130474$ 


\begin{tabular}{|c|c|c|c|}
\hline $\mathrm{H}$ & 0.377039 & 1.123449 & -0.592885 \\
\hline C & -1.491375 & -1.071229 & -0.114586 \\
\hline 0 & -1.287816 & -0.945153 & -1.300868 \\
\hline 0 & -1.514309 & -2.274567 & 0.504177 \\
\hline $\mathrm{H}$ & -1.298042 & -2.934528 & -0.177294 \\
\hline 0 & 1.782775 & 2.417163 & 0.249107 \\
\hline $\mathrm{H}$ & 1.200271 & 2.613918 & 0.996154 \\
\hline C & 4.018393 & -0.380188 & -0.100296 \\
\hline C & 4.475695 & 0.937545 & -0.226874 \\
\hline C & 4.668485 & -1.404247 & -0.801420 \\
\hline C & 5.556816 & 1.247208 & -1.055229 \\
\hline $\mathrm{H}$ & 3.960932 & 1.713704 & 0.321928 \\
\hline C & 5.760263 & -1.131919 & -1.630367 \\
\hline $\mathrm{H}$ & 4.290229 & -2.414113 & -0.702784 \\
\hline C & 6.181078 & 0.199026 & -1.741736 \\
\hline $\mathrm{H}$ & 7.019380 & 0.427393 & -2.391420 \\
\hline C & -4.050091 & 0.395833 & -0.096377 \\
\hline C & -4.700788 & 1.307823 & -0.926997 \\
\hline C & -4.485330 & -0.938121 & -0.064944 \\
\hline C & -5.775427 & 0.915214 & -1.737260 \\
\hline $\mathrm{H}$ & -4.339097 & 2.330029 & -0.958783 \\
\hline C & -5.542027 & -1.367129 & -0.864245 \\
\hline $\mathrm{H}$ & -3.989911 & -1.628646 & 0.604010 \\
\hline C & -6.172563 & -0.422064 & -1.690650 \\
\hline $\mathrm{H}$ & -6.993110 & -0.750027 & -2.315510 \\
\hline C & -6.020736 & -2.825146 & -0.878443 \\
\hline C & -6.452535 & 1.950727 & -2.646084 \\
\hline C & -5.229728 & -3.705170 & 0.103263 \\
\hline $\mathrm{H}$ & -4.160430 & -3.712985 & -0.129092 \\
\hline $\mathrm{H}$ & -5.348666 & -3.366036 & 1.137015 \\
\hline $\mathrm{H}$ & -5.591530 & -4.736348 & 0.047585 \\
\hline C & -5.835533 & -3.398529 & -2.300294 \\
\hline $\mathrm{H}$ & -6.168504 & -4.441501 & -2.337674 \\
\hline H & -6.410889 & -2.835934 & -3.040678 \\
\hline $\mathrm{H}$ & -4.783007 & -3.362264 & -2.597900 \\
\hline C & -7.512905 & -2.885341 & -0.488589 \\
\hline $\mathrm{H}$ & -8.136266 & -2.312557 & -1.180688 \\
\hline $\mathrm{H}$ & -7.867793 & -3.921579 & -0.498348 \\
\hline $\mathrm{H}$ & -7.668139 & -2.479982 & 0.516112 \\
\hline C & -5.413374 & 2.507123 & -3.643386 \\
\hline $\mathrm{H}$ & -4.576400 & 2.987673 & -3.129768 \\
\hline $\mathrm{H}$ & -5.005769 & 1.705164 & -4.266340 \\
\hline $\mathrm{H}$ & -5.876566 & 3.251217 & -4.300462 \\
\hline C & -7.003946 & 3.104303 & -1.780608 \\
\hline $\mathrm{H}$ & -6.210207 & 3.601806 & -1.216573 \\
\hline $\mathrm{H}$ & -7.487489 & 3.857153 & -2.412396 \\
\hline $\mathrm{H}$ & -7.743025 & 2.733134 & -1.063648 \\
\hline C & -7.618814 & 1.351851 & -3.448482 \\
\hline $\mathrm{H}$ & -7.283853 & 0.548117 & -4.111487 \\
\hline $\mathrm{H}$ & -8.399791 & 0.953845 & -2.792872 \\
\hline $\mathrm{H}$ & -8.072376 & 2.127980 & -4.072324 \\
\hline C & 6.493005 & -2.225857 & -2.420760 \\
\hline C & 6.050357 & 2.688301 & -1.245579 \\
\hline$c$ & 7.547287 & 2.778435 & -0.881151 \\
\hline
\end{tabular}




\begin{tabular}{|llll|} 
H & 7.710571 & 2.488791 & 0.161647 \\
$\mathrm{H}$ & 8.160436 & 2.126238 & -1.509577 \\
$\mathrm{H}$ & 7.909423 & 3.804010 & -1.011829 \\
$\mathrm{C}$ & 5.276693 & 3.685981 & -0.367212 \\
$\mathrm{H}$ & 4.204055 & 3.674698 & -0.579551 \\
$\mathrm{H}$ & 5.410861 & 3.468649 & 0.697365 \\
$\mathrm{H}$ & 5.648983 & 4.699165 & -0.549245 \\
$\mathrm{C}$ & 5.856100 & 3.089607 & -2.724002 \\
$\mathrm{H}$ & 6.410807 & 2.429695 & -3.397413 \\
$\mathrm{H}$ & 4.798679 & 3.040246 & -3.001417 \\
$\mathrm{H}$ & 6.206798 & 4.113986 & -2.891251 \\
$\mathrm{C}$ & 5.898450 & -3.621770 & -2.173900 \\
$\mathrm{H}$ & 5.958277 & -3.905814 & -1.118554 \\
$\mathrm{H}$ & 4.850510 & -3.676077 & -2.484631 \\
$\mathrm{H}$ & 6.455004 & -4.366776 & -2.750849 \\
$\mathrm{C}$ & 7.977634 & -2.251949 & -1.997871 \\
$\mathrm{H}$ & 8.520073 & -3.023774 & -2.554890 \\
$\mathrm{H}$ & 8.469049 & -1.293870 & -2.187857 \\
$\mathrm{H}$ & 8.072639 & -2.469861 & -0.929493 \\
$\mathrm{C}$ & 6.394149 & -1.918955 & -3.930619 \\
$\mathrm{H}$ & 6.842188 & -0.952364 & -4.176680 \\
$\mathrm{H}$ & 6.914628 & -2.687989 & -4.511981 \\
$\mathrm{H}$ & 5.348649 & -1.894335 & -4.253152 \\
& & & \\
\hline & & & \\
\hline
\end{tabular}

\section{Catalyst 2C 55}

Datum

Value

\begin{tabular}{lr}
\hline B3LYP-D3(BJ)/6-31G(d,p) Energy & -2165.16111 \\
\hline B3LYP-D3(BJ)/def2-TZVPP/IEF-PCM(DCM) Energy & -2165.897738 \\
\hline B3LYP-D3(BJ)/def2-TZVPP/IEF-PCM(DCM)//B3LYP-D3(BJ)/6-31G(d,p) Free Energy (Quasiharmonic) & -2165.020095
\end{tabular}

Number of Imaginary Frequencies

Frequencies (Top 3 out of 321)

$\begin{array}{ll}\text { 1. } & 11.1269 \mathrm{~cm}^{-1} \\ \text { 2. } & 12.6563 \mathrm{~cm}^{-1} \\ \text { 3. } & 14.1548 \mathrm{~cm}^{-1}\end{array}$

B3LYP-D3(BJ)/6-31G(d,p) Molecular Geometry in Cartesian Coordinates

$\begin{array}{lrrr}C & -0.124832 & -4.909779 & -1.212577 \\ C & -0.253655 & -3.734034 & -0.506387 \\ C & 0.799216 & -2.776955 & -0.490148\end{array}$




\begin{tabular}{|c|c|c|c|}
\hline C & 2.007902 & -3.072588 & -1.196252 \\
\hline C & 2.106255 & -4.294093 & -1.913382 \\
\hline C & 1.063783 & -5.191274 & -1.926538 \\
\hline $\mathrm{H}$ & -0.938051 & -5.628506 & -1.216777 \\
\hline $\mathrm{H}$ & -1.158771 & -3.526309 & 0.052817 \\
\hline C & 0.692279 & -1.537781 & 0.215876 \\
\hline C & 3.068301 & -2.135763 & -1.172372 \\
\hline $\mathrm{H}$ & 3.024303 & -4.506075 & -2.453874 \\
\hline $\mathrm{H}$ & 1.151367 & -6.120682 & -2.480241 \\
\hline C & 2.975650 & -0.947717 & -0.476976 \\
\hline C & 1.765714 & -0.663050 & 0.229795 \\
\hline $\mathrm{H}$ & 3.969370 & -2.349158 & -1.739346 \\
\hline C & -0.776227 & -2.053332 & 5.135260 \\
\hline C & -1.862785 & -1.523615 & 4.480561 \\
\hline C & -1.807093 & -1.244852 & 3.089019 \\
\hline C & -0.603664 & -1.523770 & 2.369480 \\
\hline C & 0.500944 & -2.073018 & 3.075173 \\
\hline C & 0.416200 & -2.328999 & 4.423973 \\
\hline $\mathrm{H}$ & -3.833420 & -0.528428 & 2.937095 \\
\hline $\mathrm{H}$ & -0.828140 & -2.260861 & 6.199622 \\
\hline $\mathrm{H}$ & -2.782889 & -1.308976 & 5.016911 \\
\hline C & -2.912906 & -0.710568 & 2.390276 \\
\hline C & -0.563471 & -1.234066 & 0.971321 \\
\hline $\mathrm{H}$ & 1.420659 & -2.277203 & 2.540015 \\
\hline $\mathrm{H}$ & 1.271234 & -2.742959 & 4.948997 \\
\hline C & -1.660302 & -0.696811 & 0.308879 \\
\hline C & -2.871688 & -0.443227 & 1.034539 \\
\hline C & -1.542084 & -0.349229 & -1.163951 \\
\hline $\mathrm{H}$ & -2.209833 & 0.478735 & -1.402932 \\
\hline $\mathrm{H}$ & -0.518526 & -0.026992 & -1.379316 \\
\hline C & 1.695637 & 0.548504 & 1.112880 \\
\hline 0 & 2.343504 & 0.698505 & 2.122209 \\
\hline 0 & 0.805488 & 1.470382 & 0.666580 \\
\hline H & 0.793582 & 2.177701 & 1.334618 \\
\hline 0 & -1.927057 & -1.414836 & -2.040959 \\
\hline $\mathrm{H}$ & -1.294595 & -2.137373 & -1.921737 \\
\hline c & -4.087495 & 0.108524 & 0.380757 \\
\hline C & -4.577324 & -0.438079 & -0.806100 \\
\hline C & -4.779746 & 1.175514 & 0.978714 \\
\hline C & -5.730602 & 0.069348 & -1.419564 \\
\hline $\mathrm{H}$ & -4.036855 & -1.259335 & -1.259620 \\
\hline C & -5.939075 & 1.694974 & 0.405447 \\
\hline $\mathrm{H}$ & -4.377627 & 1.600734 & 1.889767 \\
\hline C & -6.394222 & 1.126811 & -0.797012 \\
\hline $\mathrm{H}$ & -7.287845 & 1.532195 & -1.254352 \\
\hline C & 4.091882 & 0.027741 & -0.503716 \\
\hline C & 3.842763 & 1.373902 & -0.769219 \\
\hline C & 5.409747 & -0.396247 & -0.277226 \\
\hline C & 4.878148 & 2.316637 & -0.783355 \\
\hline $\mathrm{H}$ & 2.825613 & 1.687481 & -0.974124 \\
\hline C & 6.468287 & 0.510308 & -0.299617 \\
\hline $\mathrm{H}$ & 5.581400 & -1.441743 & -0.054514 \\
\hline C & 6.176421 & 1.862352 & -0.549398 \\
\hline H & 6.992446 & 2.573405 & -0.553934 \\
\hline
\end{tabular}




\begin{tabular}{|c|c|c|c|}
\hline C & 7.922613 & 0.090336 & -0.043919 \\
\hline C & 4.541769 & 3.790373 & -1.047173 \\
\hline C & 8.782655 & 0.456981 & -1.272688 \\
\hline $\mathrm{H}$ & 8.422097 & -0.060330 & -2.167316 \\
\hline $\mathrm{H}$ & 8.761648 & 1.531062 & -1.476303 \\
\hline $\mathrm{H}$ & 9.826118 & 0.167930 & -1.106968 \\
\hline C & 8.057991 & -1.420622 & 0.204201 \\
\hline $\mathrm{H}$ & 9.109306 & -1.673286 & 0.372301 \\
\hline $\mathrm{H}$ & 7.495151 & -1.738037 & 1.087369 \\
\hline $\mathrm{H}$ & 7.709151 & -2.004223 & -0.653643 \\
\hline C & 8.456998 & 0.834422 & 1.198966 \\
\hline $\mathrm{H}$ & 7.858356 & 0.592332 & 2.082370 \\
\hline $\mathrm{H}$ & 9.495233 & 0.547214 & 1.397892 \\
\hline $\mathrm{H}$ & 8.429733 & 1.919116 & 1.063681 \\
\hline C & 3.519026 & 4.269264 & 0.007133 \\
\hline $\mathrm{H}$ & 2.591371 & 3.692357 & -0.041351 \\
\hline $\mathrm{H}$ & 3.925945 & 4.164583 & 1.017551 \\
\hline $\mathrm{H}$ & 3.268174 & 5.322811 & -0.156968 \\
\hline C & 3.928336 & 3.928458 & -2.457036 \\
\hline $\mathrm{H}$ & 3.017293 & 3.332648 & -2.560371 \\
\hline $\mathrm{H}$ & 3.671129 & 4.973605 & -2.660707 \\
\hline $\mathrm{H}$ & 4.635810 & 3.594609 & -3.222479 \\
\hline C & 5.780086 & 4.696640 & -0.965198 \\
\hline $\mathrm{H}$ & 6.250254 & 4.650160 & 0.022107 \\
\hline $\mathrm{H}$ & 6.529732 & 4.427071 & -1.715790 \\
\hline $\mathrm{H}$ & 5.489082 & 5.735702 & -1.146817 \\
\hline C & -6.719334 & 2.861924 & 1.027883 \\
\hline C & -6.195903 & -0.547116 & -2.745657 \\
\hline C & -5.073206 & -0.380931 & -3.794481 \\
\hline $\mathrm{H}$ & -4.866485 & 0.679957 & -3.970045 \\
\hline $\mathrm{H}$ & -4.141954 & -0.854849 & -3.473436 \\
\hline $\mathrm{H}$ & -5.375472 & -0.830002 & -4.747323 \\
\hline C & -7.471145 & 0.119077 & -3.285408 \\
\hline $\mathrm{H}$ & -8.310367 & 0.012455 & -2.590329 \\
\hline $\mathrm{H}$ & -7.319479 & 1.185712 & -3.479159 \\
\hline $\mathrm{H}$ & -7.760714 & -0.351019 & -4.230254 \\
\hline C & -6.483997 & -2.049464 & -2.537753 \\
\hline $\mathrm{H}$ & -5.594186 & -2.586269 & -2.198859 \\
\hline $\mathrm{H}$ & -7.270887 & -2.195278 & -1.790733 \\
\hline $\mathrm{H}$ & -6.814197 & -2.506650 & -3.476968 \\
\hline C & -8.161683 & 2.404189 & 1.334636 \\
\hline H & -8.683332 & 2.077668 & 0.430920 \\
\hline $\mathrm{H}$ & -8.160877 & 1.567886 & 2.040675 \\
\hline $\mathrm{H}$ & -8.737155 & 3.225217 & 1.776171 \\
\hline C & -6.756096 & 4.042072 & 0.033048 \\
\hline $\mathrm{H}$ & -7.312887 & 4.883962 & 0.459046 \\
\hline $\mathrm{H}$ & -5.742805 & 4.384121 & -0.199613 \\
\hline $\mathrm{H}$ & -7.238540 & 3.762969 & -0.907618 \\
\hline C & -6.084125 & 3.356632 & 2.337361 \\
\hline $\mathrm{H}$ & -5.066107 & 3.726018 & 2.179306 \\
\hline $\mathrm{H}$ & -6.676629 & 4.180854 & 2.746290 \\
\hline $\mathrm{H}$ & -6.048093 & 2.565852 & 3.093193 \\
\hline
\end{tabular}




\section{Catalyst 2C 56}

Datum

Value

B3LYP-D3(BJ)/6-31G(d,p) Energy

$-2165.160638$

B3LYP-D3(BJ)/def2-TZVPP/IEF-PCM(DCM) Energy

$-2165.897645$

B3LYP-D3(BJ)/def2-TZVPP/IEF-PCM(DCM)//B3LYP-D3(BJ)/6-31G(d,p) Free Energy (Quasiharmonic) $\quad-2165.020086$

Number of Imaginary Frequencies

0

Frequencies (Top 3 out of 321)

$\begin{array}{ll}\text { 1. } & 10.9089 \mathrm{~cm}^{-1} \\ \text { 2. } & 12.7646 \mathrm{~cm}^{-1} \\ \text { 3. } & 14.0737 \mathrm{~cm}^{-1}\end{array}$

B3LYP-D3(BJ)/6-31G(d,p) Molecular Geometry in Cartesian Coordinates

C

C

C

C

C

C

$\mathrm{H}$

$\mathrm{H}$

C

C

$\mathrm{H}$

$\mathrm{H}$

C

C

$\mathrm{H}$

C

C

C

C

C

C

$\mathrm{H}$

$\mathrm{H}$

$\mathrm{H}$

C

C

$\mathrm{H}$

$\mathrm{H}$

C
$-0.122881$

$-0.255187$

0.795285

2.005211

2.107129

1.066885

$-0.934280$

$-1.161292$

0.684436

3.063426

3.026097

1.157109

2.968754

1.756103

3.965151

$-0.789721$

$-1.877510$

$-1.820750$

$-0.614926$

0.490903

0.405085

$-3.849771$

$-0.842498$

$-2.799453$

$-2.927652$

$-0.573778$

1.412346

1. 261063

$-1.671768$
$-4.894966$

$-3.719349$

$-2.759617$

$-3.052619$

$-4.273864$

$-5.173612$

$-5.615743$

$-3.513752$

$-1.519690$

$-2.113535$

$-4.483595$

$-6.102854$

$-0.926068$

$-0.642487$

- 2.324461

$-2.029259$

$-1.505920$

$-1.230157$

$-1.505479$

$-2.048221$

$-2.301343$

$-0.522556$

$-2.234508$

$-1.294096$

$-0.702130$

$-1.218964$

$-2.249938$

$-2.710322$

$-0.687871$
$-1.204570$

$-0.498792$

$-0.482080$

$-1.186895$

$-1.903974$

$-1.917749$

$-1.209127$

0.059560

0.222005

$-1.161612$

$-2.443788$

$-2.471309$

$-0.465117$

0.237445

$-1.728397$

5. 140212

4.482433

3.090331

2. 373382

3.082266

4.431546

2. 933556

6.204972

5.016744

2. 388603

0.974602

2. 549136

4.958931

0.309160 


\begin{tabular}{|c|c|c|c|}
\hline C & -2.885295 & -0.437481 & 1.032350 \\
\hline C & -1.552259 & -0.342500 & -1.164098 \\
\hline H & -2.221926 & 0.483238 & -1.405442 \\
\hline $\mathrm{H}$ & -0.529319 & -0.017701 & -1.378409 \\
\hline C & 1.679177 & 0.572077 & 1.115137 \\
\hline 0 & 2.332526 & 0.734744 & 2.119187 \\
\hline 0 & 0.776891 & 1.481308 & 0.669126 \\
\hline $\mathrm{H}$ & 0.758122 & 2.190984 & 1.334323 \\
\hline 0 & -1.932977 & -1.410603 & -2.039983 \\
\hline $\mathrm{H}$ & -1.298354 & -2.130952 & -1.919074 \\
\hline C & -4.102034 & 0.108816 & 0.375723 \\
\hline C & -4.587726 & -0.440762 & -0.811461 \\
\hline C & -4.799297 & 1.173900 & 0.971299 \\
\hline C & -5.741670 & 0.061922 & -1.427585 \\
\hline $\mathrm{H}$ & -4.043494 & -1.260510 & -1.263186 \\
\hline C & -5.959477 & 1.688525 & 0.395429 \\
\hline $\mathrm{H}$ & -4.400387 & 1.601596 & 1.882601 \\
\hline C & -6.410318 & 1.117551 & -0.807315 \\
\hline $\mathrm{H}$ & -7.304535 & 1.519268 & -1.266721 \\
\hline C & 4.087767 & 0.046537 & -0.490654 \\
\hline C & 5.397834 & -0.378168 & -0.272991 \\
\hline C & 3.839720 & 1.401408 & -0.759175 \\
\hline C & 6.470078 & 0.524711 & -0.307739 \\
\hline $\mathrm{H}$ & 5.573141 & -1.424358 & -0.045754 \\
\hline C & 4.877227 & 2.329223 & -0.789542 \\
\hline $\mathrm{H}$ & 2.822250 & 1.712772 & -0.954666 \\
\hline C & 6.184490 & 1.866768 & -0.562985 \\
\hline $\mathrm{H}$ & 6.995307 & 2.583275 & -0.584292 \\
\hline C & 4.634764 & 3.822922 & -1.044597 \\
\hline C & 7.894659 & 0.014040 & -0.049778 \\
\hline C & 5.438369 & 4.275621 & -2.282088 \\
\hline $\mathrm{H}$ & 6.512270 & 4.119685 & -2.147498 \\
\hline $\mathrm{H}$ & 5.127692 & 3.718745 & -3.171581 \\
\hline $\mathrm{H}$ & 5.276690 & 5.342188 & -2.472118 \\
\hline C & 5.097775 & 4.622583 & 0.192683 \\
\hline $\mathrm{H}$ & 4.932903 & 5.694452 & 0.037843 \\
\hline $\mathrm{H}$ & 4.541527 & 4.313430 & 1.082993 \\
\hline $\mathrm{H}$ & 6.161827 & 4.472623 & 0.395310 \\
\hline C & 3.149988 & 4.135674 & -1.292745 \\
\hline $\mathrm{H}$ & 2.526768 & 3.849178 & -0.439977 \\
\hline $\mathrm{H}$ & 3.021715 & 5.210312 & -1.453909 \\
\hline $\mathrm{H}$ & 2.769863 & 3.618530 & -2.179222 \\
\hline C & 8.245342 & -1.066230 & -1.095694 \\
\hline $\mathrm{H}$ & 7.556700 & -1.914306 & -1.047780 \\
\hline $\mathrm{H}$ & 8.200703 & -0.654782 & -2.108953 \\
\hline $\mathrm{H}$ & 9.257881 & -1.447581 & -0.925043 \\
\hline C & 7.965661 & -0.598318 & 1.365804 \\
\hline $\mathrm{H}$ & 7.269287 & -1.433333 & 1.480479 \\
\hline $\mathrm{H}$ & 8.975160 & -0.971952 & 1.569144 \\
\hline $\mathrm{H}$ & 7.716863 & 0.149767 & 2.124635 \\
\hline C & 8.944099 & 1.133151 & -0.144484 \\
\hline $\mathrm{H}$ & 8.953180 & 1.597348 & -1.135719 \\
\hline $\mathrm{H}$ & 8.769907 & 1.916026 & 0.600175 \\
\hline $\mathrm{H}$ & 9.940413 & 0.718992 & 0.037586 \\
\hline
\end{tabular}




\begin{tabular}{|rrrr|} 
C & -6.745142 & 2.853220 & 1.015298 \\
C & -6.202160 & -0.557363 & -2.754051 \\
H & -7.478725 & 0.103780 & -3.296870 \\
H & -8.318941 & -0.005300 & -2.603375 \\
H & -7.330520 & 1.170798 & -3.491193 \\
C & -7.764724 & -0.368120 & -4.241906 \\
H & -6.485239 & -2.060562 & -2.545441 \\
H & -5.594148 & -2.593871 & -2.204401 \\
H & -7.273030 & -2.208587 & -1.799806 \\
C & -6.811993 & -2.519713 & -3.484904 \\
H & -5.078008 & -0.388002 & -3.800813 \\
H & -4.874727 & 0.673478 & -3.976809 \\
H & -4.145708 & -0.858350 & -3.477567 \\
C & -5.376812 & -0.838893 & -4.753889 \\
H & -6.784319 & 4.032299 & 0.019291 \\
H & -5.771841 & 4.377791 & -0.211812 \\
H & -7.263990 & 3.750570 & -0.922009 \\
C & -7.344949 & 4.872565 & 0.443458 \\
H & -6.114210 & 3.351460 & 2.325502 \\
H & -6.710507 & 4.173868 & 2.732570 \\
H & -6.076670 & 2.561508 & 3.082126 \\
C & -5.097271 & 3.724462 & 2.168997 \\
H & -8.186388 & 2.390527 & 1.319788 \\
H & -8.183858 & 1.554901 & 2.026625 \\
H & -8.765675 & 3.209870 & 1.759462 \\
& -8.705151 & 2.061252 & 0.415412 \\
\hline & & & \\
\hline
\end{tabular}

\section{Catalyst 2C 57}

Datum

Value

B3LYP-D3(BJ)/6-31G(d,p) Energy

$-2165.160494$

B3LYP-D3(BJ)/def2-TZVPP/IEF-PCM(DCM) Energy

$-2165.897446$

B3LYP-D3(BJ)/def2-TZVPP/IEF-PCM(DCM)//B3LYP-D3(BJ)/6-31G(d,p) Free Energy (Quasiharmonic) -2165.020085

Number of Imaginary Frequencies

Frequencies (Top 3 out of 321)

$\begin{array}{ll}\text { 1. } & 10.7176 \mathrm{~cm}^{-1} \\ \text { 2. } & 12.5317 \mathrm{~cm}^{-1} \\ \text { 3. } & 13.8996 \mathrm{~cm}^{-1}\end{array}$

B3LYP-D3(BJ)/6-31G(d,p) Molecular Geometry in Cartesian Coordinates 


\begin{tabular}{|c|c|c|c|}
\hline C & 0.172787 & 3.307993 & 3.960803 \\
\hline C & 0.296535 & 2.139423 & 3.242425 \\
\hline C & -0.760773 & 1.687580 & 2.405152 \\
\hline C & -1.958944 & 2.465547 & 2.325883 \\
\hline C & -2.054949 & 3.660700 & 3.086320 \\
\hline C & -1.013135 & 4.075908 & 3.882791 \\
\hline $\mathrm{H}$ & 0.988188 & 3.640763 & 4.595066 \\
\hline $\mathrm{H}$ & 1.201007 & 1.545535 & 3.309603 \\
\hline C & -0.657578 & 0.492493 & 1.627326 \\
\hline C & -3.016121 & 2.022460 & 1.495476 \\
\hline $\mathrm{H}$ & -2.968238 & 4.245237 & 3.022094 \\
\hline $\mathrm{H}$ & -1.097214 & 4.994622 & 4.454642 \\
\hline C & -2.921485 & 0.865919 & 0.748583 \\
\hline C & -1.715122 & 0.103420 & 0.824687 \\
\hline $\mathrm{H}$ & -3.934138 & 2.601375 & 1.462196 \\
\hline C & 1.158413 & -3.406308 & 4.479898 \\
\hline C & 2.160832 & -3.104981 & 3.588175 \\
\hline C & 1.997284 & -2.063525 & 2.636548 \\
\hline C & 0.772997 & -1.328147 & 2.612846 \\
\hline C & -0.243230 & -1.663579 & 3.546176 \\
\hline C & -0.054752 & -2.677118 & 4.457264 \\
\hline $\mathrm{H}$ & 3.958596 & -2.262110 & 1.762977 \\
\hline $\mathrm{H}$ & 1.293699 & -4.203747 & 5.204123 \\
\hline $\mathrm{H}$ & 3.096163 & -3.657636 & 3.598317 \\
\hline C & 3.018003 & -1.720738 & 1.719953 \\
\hline C & 0.619800 & -0.287633 & 1.646699 \\
\hline$H$ & -1.176617 & -1.113033 & 3.525826 \\
\hline $\mathrm{H}$ & -0.841544 & -2.922149 & 5.164000 \\
\hline C & 1.635082 & 0.027745 & 0.751883 \\
\hline C & 2.873330 & -0.697266 & 0.803238 \\
\hline C & 1.420892 & 1.137975 & -0.261527 \\
\hline $\mathrm{H}$ & 2.050002 & 0.969908 & -1.135265 \\
\hline $\mathrm{H}$ & 0.380275 & 1.139243 & -0.599174 \\
\hline C & -1.498337 & -1.047321 & -0.115199 \\
\hline 0 & -1.285038 & -0.924524 & -1.299957 \\
\hline 0 & -1.534549 & -2.250401 & 0.504636 \\
\hline $\mathrm{H}$ & -1.322755 & -2.912455 & -0.176366 \\
\hline 0 & 1.792879 & 2.428746 & 0.237967 \\
\hline $\mathrm{H}$ & 1.210113 & 2.632717 & 0.982870 \\
\hline C & 4.013179 & -0.381949 & -0.097731 \\
\hline C & 4.477933 & 0.932721 & -0.228851 \\
\hline C & 4.657924 & -1.412214 & -0.794694 \\
\hline C & 5.561298 & 1.233261 & -1.057636 \\
\hline $\mathrm{H}$ & 3.967130 & 1.713765 & 0.316704 \\
\hline C & 5.751686 & -1.149058 & -1.623986 \\
\hline $\mathrm{H}$ & 4.273891 & -2.419555 & -0.692642 \\
\hline C & 6.180060 & 0.179082 & -1.739926 \\
\hline $\mathrm{H}$ & 7.020020 & 0.400363 & -2.389921 \\
\hline C & -4.051987 & 0.420925 & -0.101072 \\
\hline C & -4.488544 & -0.903070 & -0.058463 \\
\hline C & -4.698965 & 1.331758 & -0.950046 \\
\hline C & -5.543014 & -1.347441 & -0.866677 \\
\hline $\mathrm{H}$ & -4.002561 & -1.592626 & 0.621668 \\
\hline
\end{tabular}




\begin{tabular}{|c|c|c|c|}
\hline C & -5.760826 & 0.929326 & -1.757958 \\
\hline $\mathrm{H}$ & -4.332335 & 2.349777 & -0.984882 \\
\hline C & -6.161418 & -0.416902 & -1.702073 \\
\hline $\mathrm{H}$ & -6.976889 & -0.738382 & -2.336987 \\
\hline C & -6.486158 & 1.890460 & -2.710256 \\
\hline C & -5.963512 & -2.821870 & -0.801173 \\
\hline C & -5.922694 & 3.318753 & -2.637355 \\
\hline $\mathrm{H}$ & -6.019418 & 3.740909 & -1.632071 \\
\hline $\mathrm{H}$ & -4.867255 & 3.353012 & -2.924222 \\
\hline $\mathrm{H}$ & -6.474434 & 3.967525 & -3.324382 \\
\hline C & -7.983910 & 1.942289 & -2.338717 \\
\hline H & -8.519575 & 2.620893 & -3.011296 \\
\hline $\mathrm{H}$ & -8.454340 & 0.958018 & -2.412757 \\
\hline $\mathrm{H}$ & -8.117453 & 2.300830 & -1.313242 \\
\hline C & -6.332741 & 1.383345 & -4.160548 \\
\hline $\mathrm{H}$ & -6.759359 & 0.384523 & -4.287148 \\
\hline $\mathrm{H}$ & -6.844790 & 2.057338 & -4.855872 \\
\hline $\mathrm{H}$ & -5.277030 & 1.334821 & -4.444204 \\
\hline C & -6.451237 & -3.149430 & 0.626409 \\
\hline $\mathrm{H}$ & -5.669765 & -2.970697 & 1.370042 \\
\hline $\mathrm{H}$ & -7.314970 & -2.532152 & 0.892537 \\
\hline $\mathrm{H}$ & -6.747399 & -4.201745 & 0.696483 \\
\hline C & -4.745318 & -3.708160 & -1.143394 \\
\hline $\mathrm{H}$ & -3.923795 & -3.551002 & -0.439176 \\
\hline $\mathrm{H}$ & -5.022294 & -4.767276 & -1.106036 \\
\hline $\mathrm{H}$ & -4.373742 & -3.484473 & -2.148172 \\
\hline C & -7.093814 & -3.152662 & -1.788345 \\
\hline H & -8.000247 & -2.577777 & -1.574030 \\
\hline $\mathrm{H}$ & -6.797668 & -2.953948 & -2.823126 \\
\hline $\mathrm{H}$ & -7.349717 & -4.213902 & -1.713339 \\
\hline C & 6.478597 & -2.249959 & -2.410079 \\
\hline C & 6.063081 & 2.670842 & -1.252938 \\
\hline C & 7.560229 & 2.753861 & -0.887715 \\
\hline $\mathrm{H}$ & 7.721098 & 2.467183 & 0.156278 \\
\hline $\mathrm{H}$ & 8.170129 & 2.095853 & -1.513241 \\
\hline $\mathrm{H}$ & 7.928272 & 3.776873 & -1.021936 \\
\hline C & 5.294401 & 3.676090 & -0.378839 \\
\hline $\mathrm{H}$ & 4.221874 & 3.670045 & -0.591956 \\
\hline $\mathrm{H}$ & 5.426560 & 3.461923 & 0.686630 \\
\hline $\mathrm{H}$ & 5.672525 & 4.686487 & -0.564309 \\
\hline C & 5.872182 & 3.067791 & -2.732975 \\
\hline $\mathrm{H}$ & 6.423704 & 2.402333 & -3.403539 \\
\hline $\mathrm{H}$ & 4.814710 & 3.023312 & -3.011018 \\
\hline $\mathrm{H}$ & 6.228719 & 4.089577 & -2.903707 \\
\hline C & 7.962899 & -2.282847 & -1.986531 \\
\hline $\mathrm{H}$ & 8.459779 & -1.328265 & -2.179883 \\
\hline $\mathrm{H}$ & 8.056296 & -2.497335 & -0.917320 \\
\hline $\mathrm{H}$ & 8.501174 & -3.059774 & -2.540488 \\
\hline C & 6.382010 & -1.947989 & -3.921082 \\
\hline $\mathrm{H}$ & 6.898378 & -2.722031 & -4.499457 \\
\hline $\mathrm{H}$ & 5.336779 & -1.918681 & -4.244092 \\
\hline $\mathrm{H}$ & 6.835546 & -0.984817 & -4.170469 \\
\hline C & 5.876115 & -3.641605 & -2.158381 \\
\hline H & 4.828015 & -3.691185 & -2.469358 \\
\hline
\end{tabular}


$\mathrm{H}$

$\mathrm{H}$

\section{Catalyst 2C 58}

Datum

Value

\begin{tabular}{lr}
\hline B3LYP-D3(BJ)/6-31G(d,p) Energy & -2165.161489 \\
\hline B3LYP-D3(BJ)/def2-TZVPP/IEF-PCM(DCM) Energy & -2165.897594 \\
\hline B3LYP-D3(BJ)/def2-TZVPP/IEF-PCM(DCM)//B3LYP-D3(BJ)/6-31G(d,p) Free Energy (Quasiharmonic) & -2165.020077 \\
\hline
\end{tabular}

Number of Imaginary Frequencies

Frequencies (Top 3 out of 321)

$\begin{array}{ll}\text { 1. } & 10.8080 \mathrm{~cm}^{-1} \\ \text { 2. } & 13.1142 \mathrm{~cm}^{-1} \\ \text { 3. } & 13.7735 \mathrm{~cm}^{-1}\end{array}$

\section{B3LYP-D3(BJ)/6-31G(d,p) Molecular Geometry in Cartesian Coordinates}

\begin{tabular}{|c|c|c|c|}
\hline C & 0.067014 & -4.899743 & -1.306518 \\
\hline C & 0.213322 & -3.735771 & -0.585058 \\
\hline C & -0.830086 & -2.769220 & -0.547187 \\
\hline C & -2.045345 & -3.039562 & -1.252633 \\
\hline C & -2.162004 & -4.250419 & -1.985066 \\
\hline C & -1.129842 & -5.158941 & -2.015539 \\
\hline $\mathrm{H}$ & 0.873188 & -5.626012 & -1.328463 \\
\hline $\mathrm{H}$ & 1.125829 & -3.542339 & -0.032828 \\
\hline C & -0.705526 & -1.542137 & 0.175348 \\
\hline C & -3.093048 & -2.089303 & -1.212871 \\
\hline $\mathrm{H}$ & -3.084945 & -4.444438 & -2.523985 \\
\hline $\mathrm{H}$ & -1.231015 & -6.079794 & -2.581133 \\
\hline C & -2.980912 & -0.908331 & -0.507214 \\
\hline C & -1.764391 & -0.651114 & 0.198966 \\
\hline $\mathrm{H}$ & -3.999027 & -2.284437 & -1.778628 \\
\hline C & 0.755907 & -2.227821 & 5.074399 \\
\hline C & 1.842940 & -1.674657 & 4.439687 \\
\hline C & 1.790692 & -1.349776 & 3.058124 \\
\hline C & 0.589203 & -1.605815 & 2.326953 \\
\hline C & -0.515338 & -2.182048 & 3.011044 \\
\hline C & -0.433784 & -2.483226 & 4.351107 \\
\hline $\mathrm{H}$ & 3.819884 & -0.634343 & 2.931556 \\
\hline $\mathrm{H}$ & 0.806323 & -2.471283 & 6.131230 \\
\hline $\mathrm{H}$ & 2.761417 & -1.476655 & 4.985175 \\
\hline
\end{tabular}




\begin{tabular}{|c|c|c|c|}
\hline C & 2.898619 & -0.794281 & 2.379087 \\
\hline C & 0.550048 & -1.264988 & 0.940452 \\
\hline $\mathrm{H}$ & -1.431118 & -2.375659 & 2.464908 \\
\hline $\mathrm{H}$ & -1.287768 & -2.920547 & 4.859019 \\
\hline C & 1.644516 & -0.699223 & 0.300605 \\
\hline C & 2.857447 & -0.479656 & 1.033919 \\
\hline C & 1.520950 & -0.283324 & -1.153643 \\
\hline $\mathrm{H}$ & 0.504402 & 0.077638 & -1.338486 \\
\hline $\mathrm{H}$ & 2.206822 & 0.537803 & -1.361523 \\
\hline C & -1.605577 & 0.606900 & 1.000039 \\
\hline 0 & -0.840248 & 1.510000 & 0.732574 \\
\hline 0 & -2.412469 & 0.623349 & 2.079855 \\
\hline $\mathrm{H}$ & -2.257317 & 1.475668 & 2.522338 \\
\hline 0 & 1.869447 & -1.318969 & -2.080407 \\
\hline H & 1.225230 & -2.033239 & -1.977958 \\
\hline C & 4.075958 & 0.081031 & 0.393880 \\
\hline C & 4.562049 & -0.452945 & -0.806161 \\
\hline C & 4.769854 & 1.130693 & 1.010329 \\
\hline C & 5.716197 & 0.056664 & -1.405314 \\
\hline $\mathrm{H}$ & 4.011487 & -1.260308 & -1.268037 \\
\hline C & 5.934359 & 1.657127 & 0.444852 \\
\hline $\mathrm{H}$ & 4.368081 & 1.542762 & 1.927489 \\
\hline C & 6.383284 & 1.105519 & -0.761544 \\
\hline $\mathrm{H}$ & 7.278875 & 1.512519 & -1.219118 \\
\hline C & -4.087315 & 0.079601 & -0.518157 \\
\hline C & -3.826858 & 1.433491 & -0.752675 \\
\hline C & -5.406523 & -0.332343 & -0.305062 \\
\hline C & -4.852453 & 2.381984 & -0.745086 \\
\hline $\mathrm{H}$ & -2.807568 & 1.745332 & -0.945918 \\
\hline C & -6.462769 & 0.584087 & -0.312408 \\
\hline $\mathrm{H}$ & -5.595375 & -1.381666 & -0.104329 \\
\hline C & -6.161674 & 1.935415 & -0.527618 \\
\hline $\mathrm{H}$ & -6.965261 & 2.656980 & -0.522747 \\
\hline C & -7.895014 & 0.086994 & -0.066600 \\
\hline C & -4.500048 & 3.860974 & -0.956158 \\
\hline C & -8.260872 & -0.962422 & -1.138473 \\
\hline $\mathrm{H}$ & -8.209159 & -0.527247 & -2.141373 \\
\hline $\mathrm{H}$ & -7.584820 & -1.821428 & -1.110889 \\
\hline $\mathrm{H}$ & -9.279146 & -1.332681 & -0.977730 \\
\hline C & -8.929712 & 1.221923 & -0.133544 \\
\hline $\mathrm{H}$ & -9.931511 & 0.815979 & 0.036667 \\
\hline $\mathrm{H}$ & -8.747019 & 1.983403 & 0.630983 \\
\hline $\mathrm{H}$ & -8.931294 & 1.711652 & -1.112344 \\
\hline C & -7.975516 & -0.559385 & 1.333307 \\
\hline $\mathrm{H}$ & -7.718247 & 0.166586 & 2.110789 \\
\hline $\mathrm{H}$ & -8.989919 & -0.924654 & 1.527194 \\
\hline $\mathrm{H}$ & -7.290153 & -1.406067 & 1.427418 \\
\hline C & -3.533486 & 4.306546 & 0.164495 \\
\hline $\mathrm{H}$ & -4.009227 & 4.201794 & 1.145393 \\
\hline $\mathrm{H}$ & -2.614259 & 3.714384 & 0.171018 \\
\hline $\mathrm{H}$ & -3.256147 & 5.357965 & 0.032121 \\
\hline C & -5.739118 & 4.769376 & -0.917997 \\
\hline $\mathrm{H}$ & -6.453510 & 4.513988 & -1.707115 \\
\hline H & -5.436840 & 5.810205 & -1.068219 \\
\hline
\end{tabular}




\begin{tabular}{|c|c|c|c|}
\hline $\mathrm{H}$ & -6.256034 & 4.710316 & 0.045036 \\
\hline C & -3.811999 & 4.036193 & -2.326884 \\
\hline $\mathrm{H}$ & -2.891475 & 3.450734 & -2.395893 \\
\hline $\mathrm{H}$ & -4.474752 & 3.718170 & -3.137837 \\
\hline $\mathrm{H}$ & -3.551878 & 5.087636 & -2.489433 \\
\hline C & 6.716810 & 2.812485 & 1.086158 \\
\hline C & 6.245440 & -0.480878 & -2.742321 \\
\hline C & 7.704333 & -0.954407 & -2.572060 \\
\hline $\mathrm{H}$ & 8.362167 & -0.142023 & -2.250317 \\
\hline $\mathrm{H}$ & 7.768222 & -1.752412 & -1.825677 \\
\hline $\mathrm{H}$ & 8.091840 & -1.341659 & -3.520722 \\
\hline C & 6.191594 & 0.651704 & -3.790606 \\
\hline $\mathrm{H}$ & 5.162883 & 0.996588 & -3.933202 \\
\hline $\mathrm{H}$ & 6.794803 & 1.512059 & -3.486360 \\
\hline $\mathrm{H}$ & 6.571334 & 0.297960 & -4.755510 \\
\hline C & 5.409484 & -1.662197 & -3.262954 \\
\hline $\mathrm{H}$ & 5.444668 & -2.511222 & -2.572625 \\
\hline $\mathrm{H}$ & 4.360283 & -1.390235 & -3.407810 \\
\hline $\mathrm{H}$ & 5.810297 & -1.998699 & -4.224445 \\
\hline C & 8.158923 & 2.348143 & 1.383350 \\
\hline $\mathrm{H}$ & 8.158276 & 1.500083 & 2.075256 \\
\hline $\mathrm{H}$ & 8.678980 & 2.035967 & 0.473610 \\
\hline $\mathrm{H}$ & 8.736022 & 3.161003 & 1.837732 \\
\hline C & 6.083077 & 3.285651 & 2.404329 \\
\hline $\mathrm{H}$ & 6.677043 & 4.102107 & 2.826586 \\
\hline $\mathrm{H}$ & 5.065377 & 3.658580 & 2.253480 \\
\hline $\mathrm{H}$ & 6.046966 & 2.482124 & 3.146648 \\
\hline C & 6.753075 & 4.008692 & 0.110783 \\
\hline $\mathrm{H}$ & 5.739736 & 4.354706 & -0.115278 \\
\hline $\mathrm{H}$ & 7.310793 & 4.843310 & 0.549759 \\
\hline $\mathrm{H}$ & 7.234396 & 3.744434 & -0.834815 \\
\hline
\end{tabular}

\section{Catalyst 2C 59}

\section{Datum}

Value

\begin{tabular}{lr}
\hline B3LYP-D3(BJ)/6-31G(d,p) Energy & -2165.160899 \\
\hline B3LYP-D3(BJ)/def2-TZVPP/IEF-PCM(DCM) Energy & -2165.897555 \\
\hline
\end{tabular}

B3LYP-D3(BJ)/def2-TZVPP/IEF-PCM(DCM)//B3LYP-D3(BJ)/6-31G(d,p) Free Energy (Quasiharmonic) - -2165.020028

Number of Imaginary Frequencies

0

Frequencies (Top 3 out of 321)

$\begin{array}{ll}\text { 1. } & 10.7099 \mathrm{~cm}^{-1} \\ \text { 2. } & 12.5626 \mathrm{~cm}^{-1} \\ \text { 3. } & 14.0698 \mathrm{~cm}^{-1}\end{array}$




\begin{tabular}{|c|c|c|c|}
\hline C & 0.162336 & 3.049780 & 4.143007 \\
\hline C & 0.291118 & 1.928688 & 3.353358 \\
\hline C & -0.765183 & 1.523878 & 2.491057 \\
\hline C & -1.967407 & 2.299124 & 2.461591 \\
\hline C & -2.068575 & 3.444157 & 3.294927 \\
\hline C & -1.027738 & 3.814665 & 4.114406 \\
\hline $\mathrm{H}$ & 0.976940 & 3.346846 & 4.795760 \\
\hline $\mathrm{H}$ & 1.198814 & 1.336652 & 3.382656 \\
\hline C & -0.657313 & 0.379323 & 1.641087 \\
\hline C & -3.023436 & 1.903018 & 1.606301 \\
\hline $\mathrm{H}$ & -2.985078 & 4.026578 & 3.268298 \\
\hline $\mathrm{H}$ & -1.115810 & 4.695547 & 4.742418 \\
\hline C & -2.923428 & 0.796071 & 0.788515 \\
\hline C & -1.713804 & 0.035618 & 0.816710 \\
\hline $\mathrm{H}$ & -3.944734 & 2.477668 & 1.609990 \\
\hline C & 1.178431 & -3.672553 & 4.256801 \\
\hline C & 2.179422 & -3.313524 & 3.385092 \\
\hline C & 2.010127 & -2.218846 & 2.496231 \\
\hline C & 0.781712 & -1.490322 & 2.515167 \\
\hline C & -0.233032 & -1.886071 & 3.426221 \\
\hline C & -0.039028 & -2.950414 & 4.276137 \\
\hline $\mathrm{H}$ & 3.972365 & -2.354995 & 1.613901 \\
\hline $\mathrm{H}$ & 1.318055 & -4.510420 & 4.932956 \\
\hline $\mathrm{H}$ & 3.117916 & -3.860439 & 3.363346 \\
\hline C & 3.028920 & -1.817063 & 1.601895 \\
\hline C & 0.623414 & -0.395039 & 1.612571 \\
\hline $\mathrm{H}$ & -1.169658 & -1.340803 & 3.437538 \\
\hline $\mathrm{H}$ & -0.824720 & -3.241305 & 4.966511 \\
\hline C & 1.636938 & -0.021771 & 0.738152 \\
\hline C & 2.879075 & -0.742380 & 0.746495 \\
\hline C & 1.416011 & 1.147056 & -0.205324 \\
\hline $\mathrm{H}$ & 2.042752 & 1.036321 & -1.089675 \\
\hline $\mathrm{H}$ & 0.374453 & 1.166619 & -0.538848 \\
\hline C & -1.493556 & -1.054458 & -0.192116 \\
\hline 0 & -1.283993 & -0.858077 & -1.367582 \\
\hline 0 & -1.522761 & -2.293213 & 0.353103 \\
\hline $\mathrm{H}$ & -1.310335 & -2.911623 & -0.367584 \\
\hline 0 & 1.785558 & 2.406166 & 0.372423 \\
\hline $\mathrm{H}$ & 1.199818 & 2.565722 & 1.125841 \\
\hline C & 4.017624 & -0.369372 & -0.133617 \\
\hline C & 4.464122 & 0.950708 & -0.207161 \\
\hline C & 4.682775 & -1.359579 & -0.875861 \\
\hline C & 5.546691 & 1.307708 & -1.022113 \\
\hline $\mathrm{H}$ & 3.945163 & 1.705843 & 0.370160 \\
\hline C & 5.773160 & -1.043467 & -1.684579 \\
\hline $\mathrm{H}$ & 4.313018 & -2.375871 & -0.821172 \\
\hline C & 6.185375 & 0.298992 & -1.743986 \\
\hline $\mathrm{H}$ & 7.024244 & 0.553916 & -2.379078 \\
\hline C & -4.052458 & 0.400212 & -0.087016 \\
\hline C & -4.703601 & 1.352490 & -0.876879 \\
\hline
\end{tabular}




\begin{tabular}{|c|c|c|c|}
\hline C & -4.485805 & -0.928733 & -0.125386 \\
\hline C & -5.769540 & 0.997371 & -1.709227 \\
\hline $\mathrm{H}$ & -4.344292 & 2.375840 & -0.855524 \\
\hline C & -5.537668 & -1.324592 & -0.955289 \\
\hline $\mathrm{H}$ & -3.995867 & -1.656209 & 0.511056 \\
\hline C & -6.165721 & -0.346123 & -1.735446 \\
\hline $\mathrm{H}$ & -6.979926 & -0.637593 & -2.382293 \\
\hline C & -5.955924 & -2.801467 & -0.978597 \\
\hline C & -6.446444 & 2.074276 & -2.569693 \\
\hline C & -6.442515 & -3.215239 & 0.426902 \\
\hline $\mathrm{H}$ & -5.660400 & -3.081471 & 1.179268 \\
\hline $\mathrm{H}$ & -7.306206 & -2.615531 & 0.730705 \\
\hline $\mathrm{H}$ & -6.738418 & -4.269967 & 0.433390 \\
\hline C & -4.736856 & -3.664157 & -1.373837 \\
\hline $\mathrm{H}$ & -5.012310 & -4.724041 & -1.399375 \\
\hline $\mathrm{H}$ & -4.366397 & -3.380666 & -2.363838 \\
\hline $\mathrm{H}$ & -3.914931 & -3.547967 & -0.662158 \\
\hline C & -7.086331 & -3.075076 & -1.983238 \\
\hline $\mathrm{H}$ & -6.791864 & -2.813781 & -3.004460 \\
\hline $\mathrm{H}$ & -7.339147 & -4.139664 & -1.971893 \\
\hline $\mathrm{H}$ & -7.994231 & -2.516790 & -1.734289 \\
\hline C & -7.007862 & 3.181080 & -1.651114 \\
\hline $\mathrm{H}$ & -7.749427 & 2.771661 & -0.957920 \\
\hline $\mathrm{H}$ & -6.219494 & 3.652880 & -1.058185 \\
\hline $\mathrm{H}$ & -7.491107 & 3.962561 & -2.247400 \\
\hline C & -7.605928 & 1.512230 & -3.408065 \\
\hline $\mathrm{H}$ & -8.389369 & 1.079093 & -2.778211 \\
\hline $\mathrm{H}$ & -8.058648 & 2.317423 & -3.994619 \\
\hline $\mathrm{H}$ & -7.264557 & 0.744064 & -4.108827 \\
\hline C & -5.404485 & 2.684415 & -3.532104 \\
\hline $\mathrm{H}$ & -4.573195 & 3.143255 & -2.990186 \\
\hline $\mathrm{H}$ & -4.988851 & 1.916226 & -4.191258 \\
\hline $\mathrm{H}$ & -5.867743 & 3.457600 & -4.154670 \\
\hline C & 6.521456 & -2.096737 & -2.514389 \\
\hline C & 5.964727 & 2.782541 & -1.093145 \\
\hline C & 4.769436 & 3.616597 & -1.606659 \\
\hline $\mathrm{H}$ & 4.482582 & 3.296598 & -2.613724 \\
\hline $\mathrm{H}$ & 3.893423 & 3.514714 & -0.960792 \\
\hline $\mathrm{H}$ & 5.039552 & 4.677694 & -1.651463 \\
\hline C & 7.157865 & 3.007982 & -2.034901 \\
\hline $\mathrm{H}$ & 8.045222 & 2.457857 & -1.705099 \\
\hline $\mathrm{H}$ & 6.926783 & 2.703198 & -3.060549 \\
\hline $\mathrm{H}$ & 7.415228 & 4.071440 & -2.055457 \\
\hline C & 6.360450 & 3.269562 & 0.317361 \\
\hline $\mathrm{H}$ & 5.530188 & 3.176351 & 1.022190 \\
\hline $\mathrm{H}$ & 7.201693 & 2.688096 & 0.708196 \\
\hline $\mathrm{H}$ & 6.657529 & 4.323593 & 0.286197 \\
\hline C & 8.004166 & -2.124822 & -2.084635 \\
\hline $\mathrm{H}$ & 8.486729 & -1.155016 & -2.233122 \\
\hline $\mathrm{H}$ & 8.095887 & -2.385125 & -1.025482 \\
\hline $\mathrm{H}$ & 8.557277 & -2.867914 & -2.669538 \\
\hline C & 6.427630 & -1.730276 & -4.011337 \\
\hline $\mathrm{H}$ & 6.959173 & -2.470130 & -4.619935 \\
\hline $\mathrm{H}$ & 5.383658 & -1.703514 & -4.338638 \\
\hline
\end{tabular}




\section{Catalyst 2C 60}

\section{Datum}

Value

\begin{tabular}{lr}
\hline B3LYP-D3(BJ)/6-31G(d,p) Energy & -2165.161918 \\
\hline B3LYP-D3(BJ)/def2-TZVPP/IEF-PCM(DCM) Energy & -2165.897742 \\
\hline B3LYP-D3(BJ)/def2-TZVPP/IEF-PCM(DCM)//B3LYP-D3(BJ)/6-31G(d,p) Free Energy (Quasiharmonic) & -2165.01999 \\
\hline
\end{tabular}

Number of Imaginary Frequencies

Frequencies (Top 3 out of 321)

$\begin{array}{ll}\text { 1. } & 11.0725 \mathrm{~cm}^{-1} \\ \text { 2. } & 13.1866 \mathrm{~cm}^{-1} \\ \text { 3. } & 13.4929 \mathrm{~cm}^{-1}\end{array}$

\section{B3LYP-D3(BJ)/6-31G(d,p) Molecular Geometry in Cartesian Coordinates}

\begin{tabular}{lrrr} 
C & 0.087885 & -4.917930 & -1.227536 \\
C & 0.234997 & -3.740815 & -0.527862 \\
C & -0.810509 & -2.776095 & -0.502232 \\
C & -2.028570 & -3.061846 & -1.196711 \\
C & -2.145951 & -4.285967 & -1.906639 \\
C & -1.111818 & -5.192556 & -1.925864 \\
H & 0.895569 & -5.642748 & -1.240252 \\
H & 1.149713 & -3.535446 & 0.016353 \\
C & -0.685305 & -1.535861 & 0.197365 \\
C & -3.078229 & -2.113308 & -1.169090 \\
H & -3.071050 & -4.491748 & -2.437424 \\
H & -1.213606 & -6.123683 & -2.474258 \\
C & -2.965364 & -0.919464 & -0.485557 \\
C & -1.746064 & -0.646884 & 0.210000 \\
H & -3.986439 & -2.320610 & -1.726878 \\
C & 0.805114 & -2.136835 & 5.098746 \\
C & 1.887357 & -1.592004 & 4.448836 \\
C & 1.826229 & -1.289281 & 3.062614 \\
C & 0.621016 & -1.559336 & 2.342735 \\
C & -0.478458 & -2.126339 & 3.042524 \\
C & -0.388408 & -2.405763 & 4.386741 \\
\hline & & &
\end{tabular}




\begin{tabular}{|c|c|c|c|}
\hline H & 3.852841 & -0.571330 & 2.912878 \\
\hline $\mathrm{H}$ & 0.862239 & -2.363307 & 6.159001 \\
\hline $\mathrm{H}$ & 2.808691 & -1.383710 & 4.985597 \\
\hline C & 2.928742 & -0.741949 & 2.368392 \\
\hline C & 0.573439 & -1.241917 & 0.950982 \\
\hline $\mathrm{H}$ & -1.397108 & -2.330270 & 2.505033 \\
\hline $\mathrm{H}$ & -1.238589 & -2.836271 & 4.906704 \\
\hline C & 1.662908 & -0.684236 & 0.295644 \\
\hline C & 2.878864 & -0.448224 & 1.018804 \\
\hline C & 1.530968 & -0.297732 & -1.165837 \\
\hline $\mathrm{H}$ & 0.512100 & 0.054014 & -1.354601 \\
\hline $\mathrm{H}$ & 2.212053 & 0.521572 & -1.394954 \\
\hline C & -1.586067 & 0.625876 & 0.987194 \\
\hline 0 & -0.823863 & 1.525364 & 0.699474 \\
\hline 0 & -2.387704 & 0.660406 & 2.070469 \\
\hline $\mathrm{H}$ & -2.232424 & 1.521085 & 2.496428 \\
\hline 0 & 1.880554 & -1.352088 & -2.071683 \\
\hline $\mathrm{H}$ & 1.235110 & -2.063636 & -1.958168 \\
\hline C & 4.092046 & 0.107273 & 0.364361 \\
\hline C & 4.568928 & -0.428373 & -0.836943 \\
\hline C & 4.796308 & 1.157525 & 0.965071 \\
\hline C & 5.717772 & 0.077440 & -1.451056 \\
\hline $\mathrm{H}$ & 4.016764 & -1.238660 & -1.296520 \\
\hline C & 5.958907 & 1.678577 & 0.388594 \\
\hline $\mathrm{H}$ & 4.407411 & 1.581054 & 1.885247 \\
\hline C & 6.399971 & 1.125109 & -0.820486 \\
\hline $\mathrm{H}$ & 7.290641 & 1.522966 & -1.284385 \\
\hline C & -4.073795 & 0.065957 & -0.509459 \\
\hline C & -3.816985 & 1.415765 & -0.770090 \\
\hline C & -5.391220 & -0.344464 & -0.282836 \\
\hline C & -4.844351 & 2.362347 & -0.775311 \\
\hline $\mathrm{H}$ & -2.799214 & 1.725896 & -0.973807 \\
\hline C & -6.449254 & 0.569737 & -0.302327 \\
\hline $\mathrm{H}$ & -5.577147 & -1.390267 & -0.061988 \\
\hline C & -6.151699 & 1.917424 & -0.543719 \\
\hline $\mathrm{H}$ & -6.956637 & 2.637473 & -0.548500 \\
\hline C & -7.879475 & 0.074630 & -0.041213 \\
\hline C & -4.495749 & 3.837851 & -1.015134 \\
\hline C & -8.248123 & -0.994499 & -1.092451 \\
\hline $\mathrm{H}$ & -8.201652 & -0.577284 & -2.103207 \\
\hline $\mathrm{H}$ & -7.570354 & -1.851667 & -1.052484 \\
\hline $\mathrm{H}$ & -9.264984 & -1.363629 & -0.920559 \\
\hline C & -8.916564 & 1.206346 & -0.123956 \\
\hline $\mathrm{H}$ & -9.916840 & 0.801728 & 0.057961 \\
\hline $\mathrm{H}$ & -8.731958 & 1.981799 & 0.625930 \\
\hline $\mathrm{H}$ & -8.923402 & 1.678380 & -1.111390 \\
\hline C & -7.952653 & -0.546625 & 1.370416 \\
\hline $\mathrm{H}$ & -7.693210 & 0.193611 & 2.133593 \\
\hline $\mathrm{H}$ & -8.965555 & -0.910025 & 1.575330 \\
\hline $\mathrm{H}$ & -7.265435 & -1.390355 & 1.476682 \\
\hline C & -3.525041 & 4.305723 & 0.092778 \\
\hline $\mathrm{H}$ & -3.996279 & 4.218402 & 1.077551 \\
\hline $\mathrm{H}$ & -2.604754 & 3.715322 & 0.106177 \\
\hline $\mathrm{H}$ & -3.250098 & 5.354959 & -0.060274 \\
\hline
\end{tabular}




\begin{tabular}{|c|c|c|c|}
\hline C & -5.736386 & 4.744510 & -0.988060 \\
\hline $\mathrm{H}$ & -6.453780 & 4.473467 & -1.769193 \\
\hline $\mathrm{H}$ & -5.436743 & 5.782964 & -1.158587 \\
\hline $\mathrm{H}$ & -6.248915 & 4.702088 & -0.021812 \\
\hline C & -3.814160 & 3.989077 & -2.391935 \\
\hline $\mathrm{H}$ & -2.892748 & 3.404267 & -2.454256 \\
\hline $\mathrm{H}$ & -4.479854 & 3.654843 & -3.193910 \\
\hline $\mathrm{H}$ & -3.556903 & 5.037842 & -2.575009 \\
\hline C & 6.692838 & 2.835347 & 1.083427 \\
\hline C & 6.168080 & -0.523463 & -2.790045 \\
\hline C & 6.445323 & -2.031109 & -2.607063 \\
\hline $\mathrm{H}$ & 7.236416 & -2.194633 & -1.868150 \\
\hline $\mathrm{H}$ & 5.553578 & -2.565552 & -2.269509 \\
\hline $\mathrm{H}$ & 6.764883 & -2.476950 & -3.555436 \\
\hline C & 7.444796 & 0.139473 & -3.330767 \\
\hline $\mathrm{H}$ & 7.301135 & 1.209982 & -3.508259 \\
\hline $\mathrm{H}$ & 8.288891 & 0.015919 & -2.644481 \\
\hline $\mathrm{H}$ & 7.722291 & -0.319802 & -4.284584 \\
\hline C & 5.038980 & -0.332518 & -3.827783 \\
\hline $\mathrm{H}$ & 4.106174 & -0.803186 & -3.506477 \\
\hline $\mathrm{H}$ & 4.839610 & 0.732492 & -3.985832 \\
\hline $\mathrm{H}$ & 5.330148 & -0.769768 & -4.789610 \\
\hline C & 7.113683 & 2.393401 & 2.501710 \\
\hline $\mathrm{H}$ & 6.249555 & 2.112404 & 3.109948 \\
\hline $\mathrm{H}$ & 7.785479 & 1.530521 & 2.455464 \\
\hline $\mathrm{H}$ & 7.635890 & 3.207230 & 3.016752 \\
\hline $\mathrm{C}$ & 5.746887 & 4.051385 & 1.184815 \\
\hline $\mathrm{H}$ & 6.250793 & 4.887348 & 1.682514 \\
\hline H & 5.434634 & 4.382992 & 0.189682 \\
\hline $\mathrm{H}$ & 4.844875 & 3.815009 & 1.755679 \\
\hline $\mathrm{C}$ & 7.955661 & 3.270663 & 0.322599 \\
\hline H & 7.718957 & 3.629895 & -0.683699 \\
\hline H & 8.445123 & 4.088793 & 0.860026 \\
\hline $\mathrm{H}$ & 8.677207 & 2.452511 & 0.233519 \\
\hline
\end{tabular}

\section{Catalyst 2C 61}

Datum

Value

\begin{tabular}{lr}
\hline B3LYP-D3(BJ)/6-31G(d,p) Energy & -2165.160771 \\
\hline B3LYP-D3(BJ)/def2-TZVPP/IEF-PCM(DCM) Energy & -2165.897476 \\
\hline B3LYP-D3(BJ)/def2-TZVPP/IEF-PCM(DCM)//B3LYP-D3(BJ)/6-31G(d,p) Free Energy (Quasiharmonic) & -2165.01999 \\
\hline Number of Imaginary Frequencies & 0
\end{tabular}

Frequencies (Top 3 out of 321) 


$\begin{array}{ll}\text { 1. } & 10.7283 \mathrm{~cm}^{-1} \\ \text { 2. } & 12.4850 \mathrm{~cm}^{-1} \\ \text { 3. } & 14.0094 \mathrm{~cm}^{-1}\end{array}$

B3LYP-D3(BJ)/6-31G(d,p) Molecular Geometry in Cartesian Coordinates

C

C

C

C

C

C

$\mathrm{H}$

$\mathrm{H}$

C

$\mathrm{C}$

$\mathrm{H}$

$\mathrm{H}$

C

C

$\mathrm{H}$

C

C

C

C

C

C

$\mathrm{H}$

$\mathrm{H}$

$\mathrm{H}$

C

C

$\mathrm{H}$

$\mathrm{H}$

C

C

C

$\mathrm{H}$

$\mathrm{H}$

C

0

0

$\mathrm{H}$

0

$\mathrm{H}$

C

C

C

C

$\mathrm{H}$
0.232800

0.340453

$-0.725239$

$-1.917555$

$-1.996488$

$-0.945266$

1.054151

1.237335

$-0.637465$

$-2.986856$

$-2.904935$

$-1.016533$

$-2.911245$

$-1.708526$

$-3.900482$

1.121133

2.137553

1.989676

0.768015

$-0.264051$

$-0.090851$

3.958405

1.243337

3.070935

3.022354

0.634160

$-1.197855$

$-0.890226$

1.662059

2.893844

1.465312

2.084448

0.421399

$-1.548408$

$-1.568832$

$-1.354366$

$-1.269374$

1.866720

1.292886

4.041724

4.511658

4.691838

5.602305

4.004886
3.311626

2.133282

1.683630

2.470528

3.676215

4.091333

3.642178

1. 528492

0.480061

2.025161

4. 268240

5.017809

0.855318

0.086279

2.611066

$-3.501575$

$-3.180896$

$-2.116265$

$-1.376856$

$-1.734587$

$-2.770680$

$-2.306665$

$-4.317708$

$-3.736775$

$-1.757284$

$-0.309039$

$-1.185360$

$-3.034386$

0.023691

$-0.713065$

1.160072

0.997760

1.192100

$-1.148450$

$-2.288681$

$-0.837017$

$-1.683740$

2.434716

2.639772

$-0.394048$

0.913737

$-1.425586$

1.217636

1. 701174
3.903781

3. 198614

2. 370543

2. 292409

3.038312

3.822248

4.531498

3.271206

1.604602

1.479130

2.973907

4.383179

0.750416

0.823395

1.447346

4.373730

3.505214

2. 577242

2. 554732

3.461623

4.349615

1.720725

5.079200

3.514469

1.680983

1.616510

3.437759

5.035068

0.741974

0.785357

$-0.245322$

$-1.127650$

$-0.571802$

$-0.015821$

0.392640

$-1.316547$

$-1.788254$

0.272635

1.024390

$-0.104259$

$-0.231833$

$-0.802829$

$-1.057628$

0.311937 


\begin{tabular}{|c|c|c|c|}
\hline C & 5.789928 & -1.162581 & -1.620119 \\
\hline $\mathrm{H}$ & 4.304767 & -2.432274 & -0.706097 \\
\hline C & 6.225395 & 0.168966 & -1.734755 \\
\hline $\mathrm{H}$ & 7.070584 & 0.382556 & -2.376748 \\
\hline C & -4.056165 & 0.409385 & -0.080261 \\
\hline C & -4.506818 & -0.918197 & -0.010373 \\
\hline C & -4.701993 & 1.306917 & -0.930882 \\
\hline C & -5.570236 & -1.357170 & -0.795630 \\
\hline $\mathrm{H}$ & -4.015951 & -1.597403 & 0.672338 \\
\hline C & -5.786590 & 0.905407 & -1.723414 \\
\hline $\mathrm{H}$ & -4.329836 & 2.324187 & -0.990947 \\
\hline C & -6.196067 & -0.426861 & -1.642088 \\
\hline $\mathrm{H}$ & -7.023729 & -0.762052 & -2.253681 \\
\hline C & -6.461100 & 1.925508 & -2.651666 \\
\hline C & -6.058924 & -2.811821 & -0.769759 \\
\hline C & -7.640394 & 1.319511 & -3.429306 \\
\hline $\mathrm{H}$ & -7.319666 & 0.496727 & -4.075826 \\
\hline $\mathrm{H}$ & -8.419059 & 0.945026 & -2.757413 \\
\hline $\mathrm{H}$ & -8.092022 & 2.085059 & -4.067462 \\
\hline C & -5.425626 & 2.447398 & -3.671176 \\
\hline $\mathrm{H}$ & -5.886735 & 3.181004 & -4.341347 \\
\hline $\mathrm{H}$ & -4.578757 & 2.930714 & -3.176606 \\
\hline $\mathrm{H}$ & -5.033098 & 1.626737 & -4.279548 \\
\hline C & -6.993220 & 3.105434 & -1.809944 \\
\hline $\mathrm{H}$ & -6.189489 & 3.608609 & -1.265444 \\
\hline $\mathrm{H}$ & -7.475374 & 3.847354 & -2.455570 \\
\hline $\mathrm{H}$ & -7.729013 & 2.759124 & -1.077429 \\
\hline C & -5.900769 & -3.418326 & -2.181157 \\
\hline $\mathrm{H}$ & -6.479390 & -2.866409 & -2.927235 \\
\hline $\mathrm{H}$ & -4.851609 & -3.404211 & -2.492643 \\
\hline $\mathrm{H}$ & -6.245777 & -4.457842 & -2.189860 \\
\hline C & -7.545679 & -2.850523 & -0.357459 \\
\hline H & -8.175111 & -2.289122 & -1.053756 \\
\hline $\mathrm{H}$ & -7.908329 & -3.883872 & -0.336977 \\
\hline $\mathrm{H}$ & -7.682594 & -2.420529 & 0.639599 \\
\hline C & -5.256377 & -3.675163 & 0.217935 \\
\hline H & -4.189263 & -3.688555 & -0.023213 \\
\hline $\mathrm{H}$ & -5.366264 & -3.318295 & 1.246812 \\
\hline H & -5.621569 & -4.706211 & 0.184841 \\
\hline C & 6.523170 & -2.262943 & -2.400885 \\
\hline C & 6.046728 & 2.680721 & -1.187926 \\
\hline C & 6.446586 & 3.218652 & 0.202762 \\
\hline H & 5.612688 & 3.168912 & 0.907697 \\
\hline H & 7.276270 & 2.639224 & 0.620351 \\
\hline $\mathrm{H}$ & 6.762215 & 4.265187 & 0.129396 \\
\hline C & 4.868183 & 3.513960 & -1.740030 \\
\hline $\mathrm{H}$ & 4.579391 & 3.158136 & -2.734516 \\
\hline $\mathrm{H}$ & 3.988327 & 3.453718 & -1.094168 \\
\hline H & 5.157092 & 4.567462 & -1.827120 \\
\hline C & 7.246693 & 2.845935 & -2.133469 \\
\hline $\mathrm{H}$ & 8.122964 & 2.294374 & -1.777537 \\
\hline $\mathrm{H}$ & 7.013651 & 2.502800 & -3.146504 \\
\hline $\mathrm{H}$ & 7.523017 & 3.902903 & -2.197137 \\
\hline C & 5.917938 & -3.654624 & -2.156348 \\
\hline
\end{tabular}




$\begin{array}{|llll|}\mathrm{H} & 5.965671 & -3.936238 & -1.099809 \\ \mathrm{H} & 4.872632 & -3.703703 & -2.476741 \\ \mathrm{H} & 6.475449 & -4.404522 & -2.725998 \\ \mathrm{C} & 8.003631 & -2.297428 & -1.963841 \\ \mathrm{H} & 8.546024 & -3.074681 & -2.513308 \\ \mathrm{H} & 8.503369 & -1.343368 & -2.152229 \\ \mathrm{H} & 8.086808 & -2.512826 & -0.893991 \\ \mathrm{C} & 6.441358 & -1.959660 & -3.912574 \\ \mathrm{H} & 6.897257 & -0.996364 & -4.157029 \\ \mathrm{H} & 6.963205 & -2.733359 & -4.486470 \\ \mathrm{H} & 5.399212 & -1.930470 & -4.245710 \\ & & \end{array}$

\section{Catalyst 2C 62}

\section{Datum}

Value

\begin{tabular}{lr}
\hline B3LYP-D3(BJ)/6-31G(d,p) Energy & -2165.160494 \\
\hline B3LYP-D3(BJ)/def2-TZVPP/IEF-PCM(DCM) Energy & -2165.897468 \\
\hline B3LYP-D3(BJ)/def2-TZVPP/IEF-PCM(DCM)//B3LYP-D3(BJ)/6-31G(d,p) Free Energy (Quasiharmonic) & -2165.019952 \\
\hline
\end{tabular}

Number of Imaginary Frequencies

Frequencies (Top 3 out of 321)

$\begin{array}{ll}\text { 1. } & 11.1802 \mathrm{~cm}^{-1} \\ \text { 2. } & 12.6184 \mathrm{~cm}^{-1} \\ \text { 3. } & 14.3899 \mathrm{~cm}^{-1}\end{array}$

B3LYP-D3(BJ)/6-31G(d,p) Molecular Geometry in Cartesian Coordinates

$\begin{array}{lrrr}\text { C } & -0.121833 & -4.921178 & -1.205109 \\ \text { C } & -0.251530 & -3.743568 & -0.502250 \\ \text { C } & 0.800841 & -2.785937 & -0.488259 \\ \text { C } & 2.009894 & -3.082824 & -1.193201 \\ \text { C } & 2.109213 & -4.306314 & -1.906790 \\ \text { C } & 1.067232 & -5.204093 & -1.917747 \\ \text { H } & -0.934819 & -5.640164 & -1.207880 \\ \text { H } & -1.157140 & -3.534650 & 0.055703 \\ \text { C } & 0.693063 & -1.544905 & 0.214354 \\ \text { C } & 3.069639 & -2.145197 & -1.171840 \\ \text { H } & 3.027557 & -4.519295 & -2.446392 \\ \text { H } & 1.155498 & -6.135001 & -2.468821 \\ \text { C } & 2.976011 & -0.955151 & -0.480019 \\ \text { C } & 1.765839 & -0.669344 & 0.225874 \\ \text { H } & 3.970947 & -2.359602 & -1.738060\end{array}$




\begin{tabular}{|c|c|c|c|}
\hline C & -0.776101 & -2.047623 & 5.135304 \\
\hline C & -1.862816 & -1.520111 & 4.478991 \\
\hline C & -1.807150 & -1.245362 & 3.086721 \\
\hline C & -0.603598 & -1.526032 & 2.367949 \\
\hline C & 0.501077 & -2.072952 & 3.075305 \\
\hline C & 0.416378 & -2.325016 & 4.424869 \\
\hline $\mathrm{H}$ & -3.834427 & -0.529694 & 2.931497 \\
\hline $\mathrm{H}$ & -0.828026 & -2.252117 & 6.200253 \\
\hline $\mathrm{H}$ & -2.783047 & -1.304304 & 5.014668 \\
\hline C & -2.913239 & -0.713384 & 2.386235 \\
\hline C & -0.562894 & -1.240082 & 0.968902 \\
\hline $\mathrm{H}$ & 1.420847 & -2.278453 & 2.540738 \\
\hline $\mathrm{H}$ & 1.271516 & -2.737226 & 4.951103 \\
\hline C & -1.659638 & -0.704689 & 0.305146 \\
\hline C & -2.870700 & -0.449920 & 1.030051 \\
\hline C & -1.543265 & -0.358358 & -1.168279 \\
\hline $\mathrm{H}$ & -2.214373 & 0.467365 & -1.406356 \\
\hline $\mathrm{H}$ & -0.520928 & -0.031120 & -1.383038 \\
\hline C & 1.695013 & 0.544592 & 1.105606 \\
\hline 0 & 2.342604 & 0.697695 & 2.114660 \\
\hline 0 & 0.804600 & 1.464959 & 0.656655 \\
\hline $\mathrm{H}$ & 0.792753 & 2.174290 & 1.322552 \\
\hline 0 & -1.924110 & -1.424838 & -2.044829 \\
\hline $\mathrm{H}$ & -1.293178 & -2.147559 & -1.919267 \\
\hline C & -4.085338 & 0.100777 & 0.373061 \\
\hline C & -4.588553 & -0.473822 & -0.805194 \\
\hline C & -4.761348 & 1.179893 & 0.946207 \\
\hline C & -5.736542 & 0.027560 & -1.414688 \\
\hline $\mathrm{H}$ & -4.051828 & -1.306372 & -1.237325 \\
\hline C & -5.926397 & 1.703129 & 0.367400 \\
\hline $\mathrm{H}$ & -4.354318 & 1.626464 & 1.847574 \\
\hline C & -6.390450 & 1.114151 & -0.809931 \\
\hline $\mathrm{H}$ & -7.281522 & 1.507823 & -1.281863 \\
\hline C & 4.091400 & 0.021192 & -0.509775 \\
\hline C & 3.841075 & 1.366250 & -0.779716 \\
\hline C & 5.409644 & -0.400850 & -0.281869 \\
\hline C & 4.875615 & 2.309865 & -0.796997 \\
\hline $\mathrm{H}$ & 2.823636 & 1.678197 & -0.985678 \\
\hline C & 6.467368 & 0.506579 & -0.307278 \\
\hline $\mathrm{H}$ & 5.582228 & -1.445457 & -0.055731 \\
\hline C & 6.174298 & 1.857526 & -0.561550 \\
\hline $\mathrm{H}$ & 6.989699 & 2.569277 & -0.568468 \\
\hline C & 7.922085 & 0.088776 & -0.050260 \\
\hline C & 4.537933 & 3.782407 & -1.065782 \\
\hline C & 8.781671 & 0.451894 & -1.280397 \\
\hline $\mathrm{H}$ & 8.421465 & -0.068857 & -2.173170 \\
\hline $\mathrm{H}$ & 8.759673 & 1.525239 & -1.487758 \\
\hline $\mathrm{H}$ & 9.825412 & 0.164350 & -1.113795 \\
\hline C & 8.058818 & -1.421188 & 0.203111 \\
\hline $\mathrm{H}$ & 9.110362 & -1.672328 & 0.372074 \\
\hline $\mathrm{H}$ & 7.496260 & -1.736026 & 1.087379 \\
\hline $\mathrm{H}$ & 7.710490 & -2.008086 & -0.652688 \\
\hline C & 8.455959 & 0.837647 & 1.189968 \\
\hline $\mathrm{H}$ & 7.857666 & 0.598057 & 2.074289 \\
\hline
\end{tabular}




\begin{tabular}{|c|c|c|c|}
\hline $\mathrm{H}$ & 9.494488 & 0.552089 & 1.389737 \\
\hline $\mathrm{H}$ & 8.427671 & 1.921843 & 1.050944 \\
\hline C & 3.514675 & 4.263938 & -0.013183 \\
\hline $\mathrm{H}$ & 2.587580 & 3.685973 & -0.059726 \\
\hline $\mathrm{H}$ & 3.921657 & 4.163130 & 0.997606 \\
\hline $\mathrm{H}$ & 3.262812 & 5.316673 & -0.180911 \\
\hline C & 3.924495 & 3.915198 & -2.476151 \\
\hline $\mathrm{H}$ & 3.013989 & 3.318237 & -2.577525 \\
\hline $\mathrm{H}$ & 3.666393 & 4.959426 & -2.683379 \\
\hline $\mathrm{H}$ & 4.632321 & 3.579369 & -3.240401 \\
\hline C & 5.775427 & 4.690063 & -0.986756 \\
\hline $\mathrm{H}$ & 6.245585 & 4.647274 & 0.000720 \\
\hline $\mathrm{H}$ & 6.525358 & 4.418689 & -1.736412 \\
\hline $\mathrm{H}$ & 5.483490 & 5.728255 & -1.171833 \\
\hline C & -6.630279 & 2.894442 & 1.033259 \\
\hline C & -6.283896 & -0.555271 & -2.725054 \\
\hline C & -6.225928 & 0.534271 & -3.817895 \\
\hline $\mathrm{H}$ & -6.816331 & 1.413322 & -3.543482 \\
\hline $\mathrm{H}$ & -5.194407 & 0.860344 & -3.982997 \\
\hline $\mathrm{H}$ & -6.618391 & 0.147007 & -4.764620 \\
\hline C & -7.746921 & -1.003879 & -2.524388 \\
\hline $\mathrm{H}$ & -7.813723 & -1.771281 & -1.746826 \\
\hline $\mathrm{H}$ & -8.392490 & -0.171634 & -2.229783 \\
\hline $\mathrm{H}$ & -8.146848 & -1.423402 & -3.453955 \\
\hline C & -5.466879 & -1.766322 & -3.205740 \\
\hline $\mathrm{H}$ & -4.415604 & -1.513383 & -3.368858 \\
\hline $\mathrm{H}$ & -5.506989 & -2.587430 & -2.482725 \\
\hline $\mathrm{H}$ & -5.879455 & -2.134783 & -4.150371 \\
\hline C & -7.044270 & 2.503535 & 2.468418 \\
\hline $\mathrm{H}$ & -7.733151 & 1.653164 & 2.454825 \\
\hline $\mathrm{H}$ & -6.179347 & 2.223597 & 3.076057 \\
\hline $\mathrm{H}$ & -7.544908 & 3.342539 & 2.964005 \\
\hline C & -7.892033 & 3.330187 & 0.271234 \\
\hline $\mathrm{H}$ & -8.360183 & 4.174601 & 0.786429 \\
\hline $\mathrm{H}$ & -7.658479 & 3.651903 & -0.748467 \\
\hline $\mathrm{H}$ & -8.630012 & 2.523842 & 0.215891 \\
\hline C & -5.660523 & 4.094548 & 1.088073 \\
\hline $\mathrm{H}$ & -5.352352 & 4.389701 & 0.080209 \\
\hline $\mathrm{H}$ & -6.143364 & 4.954731 & 1.564751 \\
\hline $\mathrm{H}$ & -4.757709 & 3.858624 & 1.658038 \\
\hline
\end{tabular}

\section{Catalyst 2C 63}

Datum

Value

B3LYP-D3(BJ)/6-31G(d,p) Energy

$-2165.15999$

B3LYP-D3(BJ)/def2-TZVPP/IEF-PCM(DCM) Energy

$-2165.897247$

B3LYP-D3(BJ)/def2-TZVPP/IEF-PCM(DCM)//B3LYP-D3(BJ)/6-31G(d,p) Free Energy (Quasiharmonic) - 
Frequencies (Top 3 out of 321)

$\begin{array}{ll}\text { 1. } & 10.6751 \mathrm{~cm}^{-1} \\ \text { 2. } & 12.4456 \mathrm{~cm}^{-1} \\ \text { 3. } & 14.1427 \mathrm{~cm}^{-1}\end{array}$

B3LYP-D3(BJ)/6-31G(d,p) Molecular Geometry in Cartesian Coordinates

\begin{tabular}{|c|c|c|c|}
\hline C & 0.138659 & -4.904413 & -1.224285 \\
\hline C & 0.265268 & -3.731505 & -0.513028 \\
\hline C & -0.788691 & -2.775667 & -0.494185 \\
\hline C & -1.996116 & -3.069815 & -1.202784 \\
\hline C & -2.092207 & -4.288306 & -1.925382 \\
\hline C & -1.048707 & -5.184219 & -1.941017 \\
\hline $\mathrm{H}$ & 0.952783 & -5.622089 & -1.230601 \\
\hline $\mathrm{H}$ & 1.169595 & -3.525074 & 0.047890 \\
\hline C & -0.684091 & -1.538779 & 0.216102 \\
\hline C & -3.057692 & -2.134672 & -1.175644 \\
\hline $\mathrm{H}$ & -3.009377 & -4.499010 & -2.467871 \\
\hline $\mathrm{H}$ & -1.134477 & -6.111346 & -2.498824 \\
\hline C & -2.968935 & -0.949674 & -0.473984 \\
\hline C & -1.759071 & -0.665626 & 0.233394 \\
\hline $\mathrm{H}$ & -3.957385 & -2.346785 & -1.745181 \\
\hline C & 0.780321 & -2.060178 & 5.136485 \\
\hline C & 1.867578 & -1.530081 & 4.483202 \\
\hline C & 1.813396 & -1.250383 & 3.091816 \\
\hline C & 0.610603 & -1.528560 & 2.370792 \\
\hline C & -0.494635 & -2.078455 & 3.075043 \\
\hline C & -0.411316 & -2.335412 & 4.423765 \\
\hline $\mathrm{H}$ & 3.840613 & -0.534567 & 2.941743 \\
\hline $\mathrm{H}$ & 0.831164 & -2.268450 & 6.200758 \\
\hline $\mathrm{H}$ & 2.787189 & -1.315970 & 5.020625 \\
\hline C & 2.920247 & -0.716161 & 2.394439 \\
\hline C & 0.571346 & -1.237081 & 0.972901 \\
\hline $\mathrm{H}$ & -1.413729 & -2.282557 & 2.538775 \\
\hline $\mathrm{H}$ & -1.266864 & -2.749882 & 4.947553 \\
\hline C & 1.668896 & -0.699473 & 0.312146 \\
\hline C & 2.879803 & -0.448215 & 1.038923 \\
\hline C & 1.552308 & -0.345267 & -1.159401 \\
\hline $\mathrm{H}$ & 0.529498 & -0.018507 & -1.372441 \\
\hline $\mathrm{H}$ & 2.222168 & 0.482877 & -1.392480 \\
\hline C & -1.688575 & 0.543645 & 1.118974 \\
\hline 0 & -2.342933 & 0.696708 & 2.123885 \\
\hline 0 & -0.789896 & 1.459990 & 0.679929 \\
\hline $\mathrm{H}$ & -0.774415 & 2.164665 & 1.350507 \\
\hline
\end{tabular}




\begin{tabular}{|c|c|c|c|}
\hline 0 & 1.934947 & -1.405872 & -2.042452 \\
\hline H & 1.305206 & -2.130372 & -1.921237 \\
\hline C & 4.096013 & 0.102882 & 0.385184 \\
\hline C & 4.595350 & -0.463579 & -0.794293 \\
\hline C & 4.775248 & 1.179788 & 0.970965 \\
\hline C & 5.747227 & 0.039808 & -1.403296 \\
\hline $\mathrm{H}$ & 4.057081 & -1.291893 & -1.232572 \\
\hline C & 5.937090 & 1.701081 & 0.395388 \\
\hline $\mathrm{H}$ & 4.365605 & 1.615126 & 1.873893 \\
\hline C & 6.398784 & 1.116494 & -0.790465 \\
\hline $\mathrm{H}$ & 7.292581 & 1.518420 & -1.255906 \\
\hline C & -4.090891 & 0.019530 & -0.497938 \\
\hline C & -5.403683 & -0.416977 & -0.287868 \\
\hline C & -3.846862 & 1.373776 & -0.753859 \\
\hline C & -6.474004 & 0.480935 & -0.318042 \\
\hline $\mathrm{H}$ & -5.568795 & -1.464931 & -0.072019 \\
\hline C & -4.889639 & 2.301399 & -0.779482 \\
\hline H & -2.830214 & 1.691184 & -0.943752 \\
\hline C & -6.189566 & 1.830321 & -0.560923 \\
\hline $\mathrm{H}$ & -7.009437 & 2.540348 & -0.574813 \\
\hline C & -4.650267 & 3.798221 & -1.019697 \\
\hline C & -7.926675 & 0.044867 & -0.079401 \\
\hline C & -5.453926 & 4.260894 & -2.253340 \\
\hline $\mathrm{H}$ & -5.141427 & 3.713326 & -3.147940 \\
\hline $\mathrm{H}$ & -6.527527 & 4.100811 & -2.121069 \\
\hline H & -5.294917 & 5.329647 & -2.433033 \\
\hline C & -3.165950 & 4.116685 & -1.263517 \\
\hline $\mathrm{H}$ & -3.040146 & 5.193056 & -1.414732 \\
\hline $\mathrm{H}$ & -2.542761 & 3.823950 & -0.412865 \\
\hline H & -2.783742 & 3.608561 & -2.154307 \\
\hline C & -5.116315 & 4.584137 & 0.225125 \\
\hline $\mathrm{H}$ & -4.560332 & 4.267243 & 1.112858 \\
\hline $\mathrm{H}$ & -4.954153 & 5.657988 & 0.081531 \\
\hline H & -6.180158 & 4.429095 & 0.425190 \\
\hline C & -8.048814 & -1.468211 & 0.162792 \\
\hline $\mathrm{H}$ & -7.685043 & -2.045535 & -0.693091 \\
\hline $\mathrm{H}$ & -7.491961 & -1.782552 & 1.050840 \\
\hline $\mathrm{H}$ & -9.099144 & -1.732258 & 0.319105 \\
\hline C & -8.776879 & 0.406446 & -1.316365 \\
\hline $\mathrm{H}$ & -8.401309 & -0.104330 & -2.208552 \\
\hline $\mathrm{H}$ & -9.819185 & 0.106553 & -1.163093 \\
\hline H & -8.764182 & 1.481299 & -1.516666 \\
\hline C & -8.481735 & 0.779709 & 1.159810 \\
\hline $\mathrm{H}$ & -7.889956 & 0.541367 & 2.048828 \\
\hline $\mathrm{H}$ & -8.463785 & 1.865028 & 1.027773 \\
\hline $\mathrm{H}$ & -9.519065 & 0.481601 & 1.347078 \\
\hline C & 6.704493 & 2.883560 & 1.004731 \\
\hline C & 6.290711 & -0.535212 & -2.718875 \\
\hline C & 5.472195 & -1.743340 & -3.204463 \\
\hline $\mathrm{H}$ & 5.513691 & -2.568486 & -2.486123 \\
\hline $\mathrm{H}$ & 4.420636 & -1.489308 & -3.363905 \\
\hline $\mathrm{H}$ & 5.882648 & -2.106594 & -4.152026 \\
\hline C & 7.754234 & -0.984862 & -2.525090 \\
\hline $\mathrm{H}$ & 8.400455 & -0.154313 & -2.227067 \\
\hline
\end{tabular}




\begin{tabular}{|lrrr|}
$H$ & 7.823468 & -1.756880 & -1.752349 \\
$H$ & 8.151764 & -1.398548 & -3.458283 \\
$\mathrm{C}$ & 6.229327 & 0.561366 & -3.804369 \\
$\mathrm{H}$ & 5.197351 & 0.888606 & -3.964146 \\
$\mathrm{H}$ & 6.820323 & 1.438671 & -3.525554 \\
$\mathrm{H}$ & 6.618982 & 0.180591 & -4.754882 \\
$\mathrm{C}$ & 8.148211 & 2.441555 & 1.327245 \\
$\mathrm{H}$ & 8.149354 & 1.616190 & 2.046016 \\
$\mathrm{H}$ & 8.677561 & 2.104729 & 0.431797 \\
$\mathrm{H}$ & 8.715050 & 3.273508 & 1.759386 \\
$\mathrm{C}$ & 6.057058 & 3.393239 & 2.302472 \\
$\mathrm{H}$ & 6.640413 & 4.228429 & 2.702183 \\
$\mathrm{H}$ & 5.037151 & 3.751936 & 2.132444 \\
$\mathrm{H}$ & 6.022564 & 2.614038 & 3.070297 \\
$\mathrm{C}$ & 6.738159 & 4.048265 & -0.008122 \\
$\mathrm{H}$ & 5.723751 & 4.378583 & -0.252494 \\
$\mathrm{H}$ & 7.285735 & 4.901147 & 0.407822 \\
$\mathrm{H}$ & 7.228481 & 3.758164 & -0.941393 \\
& & & \\
\hline
\end{tabular}

\section{Catalyst 2C 64}

\section{Datum}

Value

\begin{tabular}{lr}
\hline B3LYP-D3(BJ)/6-31G(d,p) Energy & -2165.160021 \\
\hline B3LYP-D3(BJ)/def2-TZVPP/IEF-PCM(DCM) Energy & -2165.897375 \\
\hline B3LYP-D3(BJ)/def2-TZVPP/IEF-PCM(DCM)//B3LYP-D3(BJ)/6-31G(d,p) Free Energy (Quasiharmonic) & -2165.019931 \\
\hline
\end{tabular}

Number of Imaginary Frequencies

Frequencies (Top 3 out of 321)

$\begin{array}{ll}\text { 1. } & 11.0022 \mathrm{~cm}^{-1} \\ \text { 2. } & 12.7442 \mathrm{~cm}^{-1} \\ \text { 3. } & 14.3357 \mathrm{~cm}^{-1}\end{array}$

\section{B3LYP-D3(BJ)/6-31G(d,p) Molecular Geometry in Cartesian Coordinates}

\begin{tabular}{|c|c|c|c|}
\hline C & -0.119384 & -4.906291 & -1.198738 \\
\hline C & -0.252708 & -3.729083 & -0.495862 \\
\hline C & 0.797192 & -2.768731 & -0.480956 \\
\hline C & 2.007559 & -3.062624 & -1.184633 \\
\hline C & 2.110595 & -4.285579 & -1.898615 \\
\hline C & 1.070912 & -5.185999 & -1.910605 \\
\hline $\mathrm{H}$ & -0.930496 & -5.627385 & -1.202199 \\
\hline $\mathrm{H}$ & -1.159364 & -3.522576 & 0.061255 \\
\hline
\end{tabular}




\begin{tabular}{|c|c|c|c|}
\hline C & 0.685350 & -1.527231 & 0.220180 \\
\hline C & 3.065025 & -2.122638 & -1.161485 \\
\hline $\mathrm{H}$ & 3.029914 & -4.496036 & -2.437551 \\
\hline $\mathrm{H}$ & 1.161932 & -6.116527 & -2.461873 \\
\hline C & 2.969214 & -0.933424 & -0.468149 \\
\hline C & 1.756279 & -0.649109 & 0.233615 \\
\hline $\mathrm{H}$ & 3.967024 & -2.334262 & -1.727579 \\
\hline C & -0.790041 & -2.026121 & 5.139542 \\
\hline C & -1.877929 & -1.504765 & 4.480256 \\
\hline C & -1.821031 & -1.232415 & 3.087560 \\
\hline C & -0.614984 & -1.509125 & 2.371400 \\
\hline C & 0.490849 & -2.049775 & 3.081834 \\
\hline C & 0.404918 & -2.299561 & 4.431753 \\
\hline $\mathrm{H}$ & -3.850985 & -0.525535 & 2.927566 \\
\hline $\mathrm{H}$ & -0.842955 & -2.228795 & 6.204790 \\
\hline $\mathrm{H}$ & -2.800073 & -1.292050 & 5.013879 \\
\hline C & -2.928127 & -0.706417 & 2.384197 \\
\hline C & -0.573167 & -1.225777 & 0.971868 \\
\hline $\mathrm{H}$ & 1.412422 & -2.252528 & 2.549312 \\
\hline $\mathrm{H}$ & 1.260952 & -2.706966 & 4.960266 \\
\hline C & -1.671003 & -0.696307 & 0.305214 \\
\hline C & -2.884285 & -0.445040 & 1.027625 \\
\hline C & -1.553185 & -0.351635 & -1.168497 \\
\hline $\mathrm{H}$ & -2.226132 & 0.471986 & -1.408694 \\
\hline $\mathrm{H}$ & -0.531445 & -0.021830 & -1.382009 \\
\hline C & 1.678480 & 0.567458 & 1.108432 \\
\hline 0 & 2.331570 & 0.732888 & 2.112213 \\
\hline 0 & 0.775804 & 1.475249 & 0.660211 \\
\hline $\mathrm{H}$ & 0.757025 & 2.186647 & 1.323561 \\
\hline 0 & -1.929774 & -1.420263 & -2.044327 \\
\hline $\mathrm{H}$ & -1.296760 & -2.140890 & -1.917258 \\
\hline C & -4.099734 & 0.100626 & 0.367937 \\
\hline C & -4.598657 & -0.476279 & -0.811014 \\
\hline C & -4.780694 & 1.177642 & 0.939226 \\
\hline C & -5.747148 & 0.020890 & -1.423032 \\
\hline $\mathrm{H}$ & -4.058249 & -1.307173 & -1.241723 \\
\hline C & -5.946432 & 1.696516 & 0.357928 \\
\hline $\mathrm{H}$ & -4.377002 & 1.626132 & 1.841137 \\
\hline C & -6.406068 & 1.105445 & -0.820081 \\
\hline $\mathrm{H}$ & -7.297526 & 1.495856 & -1.293985 \\
\hline C & 4.087208 & 0.040284 & -0.496452 \\
\hline C & 5.397763 & -0.382291 & -0.277606 \\
\hline C & 3.837643 & 1.394059 & -0.769084 \\
\hline C & 6.469007 & 0.521664 & -0.315274 \\
\hline $\mathrm{H}$ & 5.574243 & -1.427599 & -0.047230 \\
\hline C & 4.874121 & 2.322921 & -0.802447 \\
\hline $\mathrm{H}$ & 2.819807 & 1.703666 & -0.965451 \\
\hline C & 6.181920 & 1.862614 & -0.574627 \\
\hline $\mathrm{H}$ & 6.991955 & 2.579931 & -0.598302 \\
\hline C & 4.629983 & 3.815539 & -1.062203 \\
\hline C & 7.894193 & 0.013364 & -0.055964 \\
\hline C & 5.432908 & 4.265171 & -2.301254 \\
\hline $\mathrm{H}$ & 6.506996 & 4.110811 & -2.166339 \\
\hline $\mathrm{H}$ & 5.122694 & 3.705126 & -3.188917 \\
\hline
\end{tabular}




\begin{tabular}{|c|c|c|c|}
\hline $\mathrm{H}$ & 5.270051 & 5.330954 & -2.494658 \\
\hline C & 5.092300 & 4.619658 & 0.172441 \\
\hline $\mathrm{H}$ & 4.926221 & 5.690847 & 0.014209 \\
\hline H & 4.536524 & 4.312737 & 1.063818 \\
\hline $\mathrm{H}$ & 6.156545 & 4.471516 & 0.375384 \\
\hline C & 3.144831 & 4.125881 & -1.311125 \\
\hline $\mathrm{H}$ & 2.522033 & 3.841368 & -0.457387 \\
\hline $\mathrm{H}$ & 3.015349 & 5.199866 & -1.475643 \\
\hline $\mathrm{H}$ & 2.765160 & 3.605534 & -2.195920 \\
\hline C & 8.245868 & -1.069844 & -1.098502 \\
\hline H & 7.558157 & -1.918509 & -1.047774 \\
\hline H & 8.200599 & -0.661664 & -2.113055 \\
\hline $\mathrm{H}$ & 9.258852 & -1.449549 & -0.926825 \\
\hline C & 7.966118 & -0.594411 & 1.361544 \\
\hline $\mathrm{H}$ & 7.270660 & -1.429801 & 1.478992 \\
\hline $\mathrm{H}$ & 8.976057 & -0.966304 & 1.565892 \\
\hline $\mathrm{H}$ & 7.716637 & 0.155814 & 2.118034 \\
\hline C & 8.942409 & 1.133301 & -0.154439 \\
\hline $\mathrm{H}$ & 8.950766 & 1.594366 & -1.147141 \\
\hline H & 8.767550 & 1.918346 & 0.587775 \\
\hline $\mathrm{H}$ & 9.939209 & 0.720788 & 0.028711 \\
\hline C & -6.655862 & 2.885566 & 1.021957 \\
\hline C & -6.289713 & -0.564249 & -2.734368 \\
\hline C & -5.467738 & -1.772866 & -3.212728 \\
\hline $\mathrm{H}$ & -4.416923 & -1.516630 & -3.373629 \\
\hline $\mathrm{H}$ & -5.506806 & -2.593782 & -2.489437 \\
\hline $\mathrm{H}$ & -5.877052 & -2.143054 & -4.158104 \\
\hline C & -6.232759 & 0.525062 & -3.827496 \\
\hline $\mathrm{H}$ & -6.826587 & 1.402321 & -3.554736 \\
\hline $\mathrm{H}$ & -5.201916 & 0.854375 & -3.990384 \\
\hline $\mathrm{H}$ & -6.621843 & 0.136200 & -4.774962 \\
\hline C & -7.751743 & -1.017461 & -2.536850 \\
\hline $\mathrm{H}$ & -7.817869 & -1.784741 & -1.759110 \\
\hline $\mathrm{H}$ & -8.400667 & -0.187175 & -2.244096 \\
\hline $\mathrm{H}$ & -8.148187 & -1.438664 & -3.467150 \\
\hline C & -7.917410 & 3.316817 & 0.257045 \\
\hline $\mathrm{H}$ & -7.682710 & 3.639048 & -0.762228 \\
\hline $\mathrm{H}$ & -8.652519 & 2.507952 & 0.200306 \\
\hline $\mathrm{H}$ & -8.389559 & 4.159769 & 0.770984 \\
\hline C & -5.690380 & 4.089025 & 1.078690 \\
\hline $\mathrm{H}$ & -6.177280 & 4.947646 & 1.554054 \\
\hline $\mathrm{H}$ & -4.788066 & 3.856366 & 1.650788 \\
\hline $\mathrm{H}$ & -5.380912 & 4.385002 & 0.071468 \\
\hline C & -7.071674 & 2.493510 & 2.456277 \\
\hline $\mathrm{H}$ & -6.207123 & 2.216619 & 3.065845 \\
\hline $\mathrm{H}$ & -7.576253 & 3.330885 & 2.950623 \\
\hline $\mathrm{H}$ & -7.757615 & 1.640788 & 2.441334 \\
\hline
\end{tabular}


Frequencies (Top 3 out of 321)

$\begin{array}{ll}\text { 1. } & 11.0482 \mathrm{~cm}^{-1} \\ \text { 2. } & 12.3615 \mathrm{~cm}^{-1} \\ \text { 3. } & 14.3999 \mathrm{~cm}^{-1}\end{array}$

B3LYP-D3(BJ)/6-31G(d,p) Molecular Geometry in Cartesian Coordinates

C

C

C

C

C

C

$\mathrm{H}$

$\mathrm{H}$

C

C

$\mathrm{H}$

$\mathrm{H}$

C

C

$\mathrm{H}$

C

C

C

C

C

C

$\mathrm{H}$

$\mathrm{H}$

$\mathrm{H}$

C

C

$\mathrm{H}$

$\mathrm{H}$

C

C

C

$\mathrm{H}$
$-0.192936$

$-0.320992$

0.738430

1.943027

2.043421

0.999523

$-1.009936$

$-1.230457$

0.631961

3.001955

2.961738

1.086996

2.903155

1.691481

3.925043

$-1.236363$

$-2.230411$

$-2.051120$

$-0.819995$

0.187363

$-0.016226$

$-4.008405$

$-1.383597$

$-3.170943$

$-3.062589$

$-0.651372$

1.126128

0.763987

$-1.657972$

$-2.902422$

$-1.428491$

$-0.385378$

\section{358944}

2. 180891

1.708616

2.476105

3.681405

4. 116352

3.707277

1.595010

0.502373

2. 012891

4. 257764

5.042619

0.847194

0.093708

2. 583550

$-3.329137$

$-3.035171$

$-2.013181$

$-1.289562$

$-1.617135$

$-2.611883$

$-2.210385$

$-4.111681$

$-3.578824$

$-1.678510$

$-0.267915$

$-1.076033$

$-2.851286$

0.040125

$-0.673980$

1.133116

1.124031
3. 901181

3.199183

2. 375957

2. 293414

3.037075

3.820189

4.524946

3. 269013

1.615974

1.476438

2.970661

4.379198

0.744197

0.825628

1.441645

4.525560

3. 622085

2.652373

2.623041

3.568660

4.497030

1.769567

5. 263565

3.636526

1. 722631

1.639501

3.543736

5.212913

0.732399

0.787795

$-0.296203$

$-0.625427$ 


\begin{tabular}{|c|c|c|c|}
\hline $\mathrm{H}$ & -2.051640 & 0.957658 & -1.172606 \\
\hline C & 1.472764 & -1.071985 & -0.094746 \\
\hline 0 & 1.275382 & -0.969857 & -1.284347 \\
\hline 0 & 1.488622 & -2.262220 & 0.549054 \\
\hline $\mathrm{H}$ & 1.273808 & -2.935555 & -0.119683 \\
\hline 0 & -1.796363 & 2.433391 & 0.182755 \\
\hline $\mathrm{H}$ & -1.213534 & 2.647939 & 0.924701 \\
\hline C & -4.033088 & -0.366802 & -0.127289 \\
\hline C & -4.479582 & 0.947624 & -0.301382 \\
\hline C & -4.692716 & -1.401840 & -0.800285 \\
\hline C & -5.552567 & 1.242720 & -1.146615 \\
\hline $\mathrm{H}$ & -3.963718 & 1.742179 & 0.223470 \\
\hline C & -5.780223 & -1.146707 & -1.641730 \\
\hline $\mathrm{H}$ & -4.328220 & -2.416019 & -0.673149 \\
\hline C & -6.191054 & 0.182918 & -1.802389 \\
\hline $\mathrm{H}$ & -7.022797 & 0.398113 & -2.457153 \\
\hline C & 4.036388 & 0.386507 & -0.093531 \\
\hline C & 4.466936 & -0.947854 & -0.030531 \\
\hline C & 4.694691 & 1.278045 & -0.940206 \\
\hline C & 5.526646 & -1.397641 & -0.814268 \\
\hline $\mathrm{H}$ & 3.965504 & -1.621966 & 0.650577 \\
\hline C & 5.772518 & 0.864351 & -1.735613 \\
\hline $\mathrm{H}$ & 4.336524 & 2.300447 & -0.996324 \\
\hline C & 6.164914 & -0.472866 & -1.657513 \\
\hline $\mathrm{H}$ & 6.987910 & -0.816996 & -2.270320 \\
\hline C & 6.458146 & 1.877463 & -2.663091 \\
\hline C & 6.000542 & -2.857148 & -0.793910 \\
\hline C & 7.007889 & 3.048398 & -1.820161 \\
\hline $\mathrm{H}$ & 6.212284 & 3.560710 & -1.272245 \\
\hline $\mathrm{H}$ & 7.741479 & 2.691155 & -1.090601 \\
\hline $\mathrm{H}$ & 7.497534 & 3.785549 & -2.465602 \\
\hline C & 5.426611 & 2.414557 & -3.678721 \\
\hline $\mathrm{H}$ & 5.895963 & 3.142487 & -4.349393 \\
\hline $\mathrm{H}$ & 5.020295 & 1.600124 & -4.286137 \\
\hline $\mathrm{H}$ & 4.588065 & 2.908878 & -3.180969 \\
\hline C & 7.627333 & 1.257530 & -3.444990 \\
\hline $\mathrm{H}$ & 7.293834 & 0.440418 & -4.092188 \\
\hline $\mathrm{H}$ & 8.087108 & 2.018346 & -4.082978 \\
\hline $\mathrm{H}$ & 8.403080 & 0.871694 & -2.776028 \\
\hline C & 7.490383 & -2.913587 & -0.394710 \\
\hline $\mathrm{H}$ & 8.119391 & -2.358245 & -1.095852 \\
\hline $\mathrm{H}$ & 7.641580 & -2.486670 & 0.601644 \\
\hline $\mathrm{H}$ & 7.841836 & -3.950927 & -0.379697 \\
\hline C & 5.821034 & -3.460943 & -2.203857 \\
\hline $\mathrm{H}$ & 6.402497 & -2.916761 & -2.953180 \\
\hline $\mathrm{H}$ & 6.150383 & -4.505655 & -2.216531 \\
\hline $\mathrm{H}$ & 4.770304 & -3.427522 & -2.508065 \\
\hline C & 5.201329 & -3.712846 & 0.202553 \\
\hline $\mathrm{H}$ & 5.315720 & -3.351387 & 1.229234 \\
\hline $\mathrm{H}$ & 4.133290 & -3.722434 & -0.035454 \\
\hline $\mathrm{H}$ & 5.560066 & -4.746130 & 0.171585 \\
\hline C & -6.467545 & -2.316169 & -2.362493 \\
\hline C & -5.970904 & 2.708550 & -1.328167 \\
\hline C & -4.772291 & 3.504547 & -1.891971 \\
\hline
\end{tabular}




\begin{tabular}{|c|c|c|c|}
\hline $\mathrm{H}$ & -3.901145 & 3.450615 & -1.233870 \\
\hline $\mathrm{H}$ & -4.477490 & 3.112484 & -2.870881 \\
\hline $\mathrm{H}$ & -5.042567 & 4.559311 & -2.016000 \\
\hline C & -6.377655 & 3.296828 & 0.040018 \\
\hline H & -7.221581 & 2.744542 & 0.465858 \\
\hline $\mathrm{H}$ & -5.552728 & 3.255911 & 0.756028 \\
\hline H & -6.675044 & 4.345511 & -0.070012 \\
\hline C & -7.157070 & 2.864614 & -2.292710 \\
\hline $\mathrm{H}$ & -6.918814 & 2.486373 & -3.291883 \\
\hline $\mathrm{H}$ & -8.047110 & 2.339782 & -1.930615 \\
\hline $\mathrm{H}$ & -7.413775 & 3.923789 & -2.392141 \\
\hline C & -7.003856 & -3.315575 & -1.314859 \\
\hline $\mathrm{H}$ & -6.200611 & -3.714122 & -0.689002 \\
\hline $\mathrm{H}$ & -7.733037 & -2.832982 & -0.656551 \\
\hline $\mathrm{H}$ & -7.494929 & -4.161125 & -1.808967 \\
\hline C & -5.442993 & -3.027556 & -3.272409 \\
\hline $\mathrm{H}$ & -5.913506 & -3.868819 & -3.793259 \\
\hline $\mathrm{H}$ & -5.047420 & -2.337217 & -4.023839 \\
\hline $\mathrm{H}$ & -4.597171 & -3.418022 & -2.700004 \\
\hline C & -7.647101 & -1.856458 & -3.234531 \\
\hline $\mathrm{H}$ & -7.324882 & -1.167918 & -4.021797 \\
\hline $\mathrm{H}$ & -8.105743 & -2.723626 & -3.719683 \\
\hline $\mathrm{H}$ & -8.420759 & -1.359986 & -2.640503 \\
\hline
\end{tabular}

\section{Catalyst 2C 66}

\section{Datum}

Value

\begin{tabular}{lr}
\hline B3LYP-D3(BJ)/6-31G(d,p) Energy & -2165.160936 \\
\hline B3LYP-D3(BJ)/def2-TZVPP/IEF-PCM(DCM) Energy & -2165.897603 \\
\hline B3LYP-D3(BJ)/def2-TZVPP/IEF-PCM(DCM)//B3LYP-D3(BJ)/6-31G(d,p) Free Energy (Quasiharmonic) & -2165.019911
\end{tabular}

Number of Imaginary Frequencies

Frequencies (Top 3 out of 321)

$\begin{array}{ll}\text { 1. } & 11.0496 \mathrm{~cm}^{-1} \\ \text { 2. } & 12.8159 \mathrm{~cm}^{-1} \\ \text { 3. } & 14.5436 \mathrm{~cm}^{-1}\end{array}$

\section{B3LYP-D3(BJ)/6-31G(d,p) Molecular Geometry in Cartesian Coordinates}

$\begin{array}{rrrr}C & 0.199527 & 3.395826 & 3.880019 \\ C & 0.322854 & 2.212675 & 3.185796 \\ C & -0.737547 & 1.740353 & 2.363862\end{array}$




\begin{tabular}{|c|c|c|c|}
\hline C & -1.938381 & 2.513128 & 2.274565 \\
\hline C & -2.033771 & 3.723830 & 3.010081 \\
\hline C & -0.988980 & 4.158662 & 3.792055 \\
\hline $\mathrm{H}$ & 1.017209 & 3.744087 & 4.502931 \\
\hline $\mathrm{H}$ & 1.229294 & 1.622734 & 3.260785 \\
\hline C & -0.634980 & 0.529529 & 1.610709 \\
\hline C & -2.998663 & 2.049684 & 1.459345 \\
\hline $\mathrm{H}$ & -2.949046 & 4.304372 & 2.938395 \\
\hline $\mathrm{H}$ & -1.072703 & 5.089013 & 4.344816 \\
\hline C & -2.904622 & 0.877922 & 0.736485 \\
\hline C & -1.695583 & 0.120701 & 0.822072 \\
\hline $\mathrm{H}$ & -3.918487 & 2.625247 & 1.418735 \\
\hline C & 1.210169 & -3.302844 & 4.533823 \\
\hline C & 2.206691 & -3.016764 & 3.630548 \\
\hline C & 2.033994 & -1.996425 & 2.657905 \\
\hline C & 0.806853 & -1.266169 & 2.625341 \\
\hline C & -0.203163 & -1.585590 & 3.570938 \\
\hline C & -0.005946 & -2.578915 & 4.502210 \\
\hline $\mathrm{H}$ & 3.990950 & -2.206916 & 1.777471 \\
\hline $\mathrm{H}$ & 1.352389 & -4.084180 & 5.274084 \\
\hline $\mathrm{H}$ & 3.144206 & -3.565533 & 3.647390 \\
\hline C & 3.048178 & -1.669867 & 1.728256 \\
\hline C & 0.644840 & -0.246326 & 1.638819 \\
\hline $\mathrm{H}$ & -1.138765 & -1.039117 & 3.543888 \\
\hline $\mathrm{H}$ & -0.788057 & -2.811932 & 5.218138 \\
\hline C & 1.654016 & 0.053718 & 0.731907 \\
\hline C & 2.894431 & -0.667062 & 0.790480 \\
\hline C & 1.431181 & 1.144575 & -0.300424 \\
\hline $\mathrm{H}$ & 2.053675 & 0.962561 & -1.175958 \\
\hline $\mathrm{H}$ & 0.388172 & 1.140139 & -0.630038 \\
\hline C & -1.479867 & -1.048208 & -0.095390 \\
\hline 0 & -1.272422 & -0.948456 & -1.283325 \\
\hline 0 & -1.509459 & -2.238683 & 0.548638 \\
\hline $\mathrm{H}$ & -1.299206 & -2.913667 & -0.120043 \\
\hline 0 & 1.806239 & 2.444357 & 0.174250 \\
\hline $\mathrm{H}$ & 1.223369 & 2.665433 & 0.914244 \\
\hline C & 4.027346 & -0.368975 & -0.124815 \\
\hline C & 4.681348 & -1.409699 & -0.794533 \\
\hline C & 4.481215 & 0.942408 & -0.302694 \\
\hline C & 5.770406 & -1.163131 & -1.636524 \\
\hline $\mathrm{H}$ & 4.311100 & -2.421429 & -0.664537 \\
\hline C & 5.556027 & 1.228992 & -1.148528 \\
\hline $\mathrm{H}$ & 3.969563 & 1.741401 & 0.219534 \\
\hline C & 6.188699 & 0.163696 & -1.801025 \\
\hline $\mathrm{H}$ & 7.021719 & 0.372294 & -2.456302 \\
\hline C & -4.038096 & 0.411842 & -0.097752 \\
\hline C & -4.470384 & -0.912238 & -0.024905 \\
\hline C & -4.691856 & 1.302553 & -0.962711 \\
\hline C & -5.527365 & -1.376807 & -0.818352 \\
\hline $\mathrm{H}$ & -3.978883 & -1.585710 & 0.667264 \\
\hline C & -5.756331 & 0.879882 & -1.756742 \\
\hline $\mathrm{H}$ & -4.328401 & 2.320649 & -1.021032 \\
\hline C & -6.152573 & -0.466013 & -1.670273 \\
\hline $\mathrm{H}$ & -6.970063 & -0.803270 & -2.294293 \\
\hline
\end{tabular}




\begin{tabular}{|c|c|c|c|}
\hline C & -6.488953 & 1.818316 & -2.725922 \\
\hline C & -5.942768 & -2.850830 & -0.719851 \\
\hline C & -7.984942 & 1.874401 & -2.347982 \\
\hline $\mathrm{H}$ & -8.453131 & 0.887556 & -2.398221 \\
\hline $\mathrm{H}$ & -8.114256 & 2.254824 & -1.329876 \\
\hline $\mathrm{H}$ & -8.525777 & 2.536871 & -3.032399 \\
\hline C & -6.341499 & 1.280261 & -4.165662 \\
\hline $\mathrm{H}$ & -6.858601 & 1.937900 & -4.872802 \\
\hline $\mathrm{H}$ & -5.287101 & 1.228013 & -4.453516 \\
\hline $\mathrm{H}$ & -6.766365 & 0.277973 & -4.268541 \\
\hline C & -5.928799 & 3.249216 & -2.686878 \\
\hline $\mathrm{H}$ & -4.874804 & 3.279701 & -2.979407 \\
\hline $\mathrm{H}$ & -6.485382 & 3.881477 & -3.385293 \\
\hline $\mathrm{H}$ & -6.021892 & 3.693014 & -1.690611 \\
\hline C & -6.422482 & -3.149972 & 0.716648 \\
\hline $\mathrm{H}$ & -5.638058 & -2.953119 & 1.452557 \\
\hline $\mathrm{H}$ & -7.287038 & -2.530133 & 0.974006 \\
\hline $\mathrm{H}$ & -6.714715 & -4.201557 & 0.810202 \\
\hline C & -4.723315 & -3.740198 & -1.049427 \\
\hline $\mathrm{H}$ & -3.898865 & -3.565565 & -0.352791 \\
\hline $\mathrm{H}$ & -4.996576 & -4.799175 & -0.988396 \\
\hline $\mathrm{H}$ & -4.357460 & -3.536523 & -2.060541 \\
\hline C & -7.076733 & -3.205849 & -1.694328 \\
\hline $\mathrm{H}$ & -7.983976 & -2.629401 & -1.487773 \\
\hline $\mathrm{H}$ & -6.786206 & -3.028103 & -2.734501 \\
\hline $\mathrm{H}$ & -7.328866 & -4.266062 & -1.595746 \\
\hline C & 5.982495 & 2.691919 & -1.334458 \\
\hline C & 6.451245 & -2.338503 & -2.353818 \\
\hline C & 7.633213 & -1.887845 & -3.227317 \\
\hline $\mathrm{H}$ & 7.314687 & -1.199815 & -4.016530 \\
\hline $\mathrm{H}$ & 8.409686 & -1.393939 & -2.634827 \\
\hline $\mathrm{H}$ & 8.086995 & -2.758926 & -3.710017 \\
\hline C & 5.422758 & -3.046991 & -3.261552 \\
\hline $\mathrm{H}$ & 4.574834 & -3.431097 & -2.687965 \\
\hline $\mathrm{H}$ & 5.030941 & -2.356752 & -4.015039 \\
\hline $\mathrm{H}$ & 5.888629 & -3.892377 & -3.779891 \\
\hline C & 6.982180 & -3.337690 & -1.303248 \\
\hline $\mathrm{H}$ & 7.713985 & -2.857110 & -0.646381 \\
\hline $\mathrm{H}$ & 6.176812 & -3.730044 & -0.676209 \\
\hline $\mathrm{H}$ & 7.468661 & -4.187329 & -1.794878 \\
\hline C & 4.788549 & 3.492668 & -1.901435 \\
\hline $\mathrm{H}$ & 4.492185 & 3.099158 & -2.879293 \\
\hline $\mathrm{H}$ & 3.916743 & 3.445520 & -1.243685 \\
\hline $\mathrm{H}$ & 5.064637 & 4.545553 & -2.028599 \\
\hline C & 7.170000 & 2.838438 & -2.298846 \\
\hline $\mathrm{H}$ & 7.432605 & 3.895865 & -2.401420 \\
\hline $\mathrm{H}$ & 8.056938 & 2.309809 & -1.934666 \\
\hline $\mathrm{H}$ & 6.930158 & 2.458430 & -3.296967 \\
\hline C & 6.391737 & 3.282302 & 0.032073 \\
\hline $\mathrm{H}$ & 7.232389 & 2.726751 & 0.460135 \\
\hline $\mathrm{H}$ & 6.694944 & 4.328977 & -0.081137 \\
\hline $\mathrm{H}$ & 5.566216 & 3.248212 & 0.747758 \\
\hline
\end{tabular}




\section{Catalyst 2C 67}

\section{Datum}

Value

B3LYP-D3(BJ)/6-31G(d,p) Energy

$-2165.160774$

B3LYP-D3(BJ)/def2-TZVPP/IEF-PCM(DCM) Energy

$-2165.897356$

B3LYP-D3(BJ)/def2-TZVPP/IEF-PCM(DCM)//B3LYP-D3(BJ)/6-31G(d,p) Free Energy (Quasiharmonic)

$-2165.019905$

Number of Imaginary Frequencies

Frequencies (Top 3 out of 321)

$\begin{array}{ll}\text { 1. } & 10.4754 \mathrm{~cm}^{-1} \\ \text { 2. } & 12.7632 \mathrm{~cm}^{-1} \\ \text { 3. } & 13.9059 \mathrm{~cm}^{-1}\end{array}$

B3LYP-D3(BJ)/6-31G(d,p) Molecular Geometry in Cartesian Coordinates

C

C

C

C

C

C

$\mathrm{H}$

$\mathrm{H}$

C

C

$\mathrm{H}$

$\mathrm{H}$

C

C

$\mathrm{H}$

C

C

C

C

C

C

$\mathrm{H}$

$\mathrm{H}$

$\mathrm{H}$

C

C

$\mathrm{H}$

$\mathrm{H}$

C

-0.214343
-0.324056
0.740201
1.932954
2.014018
0.964383
-1.034621
-1.221540
0.651016
3.000068
2.922735
1.037257
2.923260
1.720296
3.912963
-1.101777
-2.119000
-1.973141
-0.752522
0.280313
0.109000
-3.942602
-1.222514
-3.051557
-3.007024
-0.620144
1.213171
0.908911
-1.648945

3. 508869

2. 298382

1.808672

2.589902

3.829754

4.282611

3.869946

1.698810

0.569654

2.106026

4.417360

5.234729

0.902666

0.138493

2.690570

$-3.269798$

$-2.994280$

$-1.976949$

$-1.237874$

$-1.548305$

$-2.539085$

$-2.211638$

$-4.049939$

$-3.550367$

$-1.664125$

$-0.218485$

$-0.999345$

$-2.766897$

0.068721
3.749360

3.101368

2. 294502

2.176820

2.864284

3. 629164

4. 361454

3. 203606

1. 587769

1.383147

2.770160

4. 145153

0.710923

0.824004

1. 321105

4. 554784

3.671793

2. 691931

2. 631915

3.555197

4.493863

1.847175

5. 300101

3. 709194

1. 779731

1.640982

3. 503722

5.191464

0.751640 


\begin{tabular}{|c|c|c|c|}
\hline C & -2.880018 & -0.665480 & 0.833494 \\
\hline C & -1.454745 & 1.152729 & -0.293465 \\
\hline $\mathrm{H}$ & -0.412039 & 1.164902 & -0.625781 \\
\hline $\mathrm{H}$ & -2.077950 & 0.944741 & -1.163412 \\
\hline C & 1.559124 & -1.138042 & 0.050234 \\
\hline 0 & 1.587139 & -2.255119 & 0.519390 \\
\hline 0 & 1.357689 & -0.898022 & -1.263625 \\
\hline $\mathrm{H}$ & 1.278441 & -1.769315 & -1.689501 \\
\hline 0 & -1.851889 & 2.452800 & 0.158657 \\
\hline $\mathrm{H}$ & -1.279951 & 2.691327 & 0.901860 \\
\hline C & -4.028720 & -0.391172 & -0.070040 \\
\hline C & -4.664683 & -1.451694 & -0.729291 \\
\hline C & -4.510419 & 0.912558 & -0.242883 \\
\hline C & -5.765879 & -1.229710 & -1.560774 \\
\hline $\mathrm{H}$ & -4.268501 & -2.450482 & -0.594728 \\
\hline C & -5.601292 & 1.172191 & -1.075815 \\
\hline $\mathrm{H}$ & -4.007054 & 1.717465 & 0.273901 \\
\hline C & -6.210656 & 0.088642 & -1.719358 \\
\hline $\mathrm{H}$ & -7.056714 & 0.277993 & -2.371564 \\
\hline C & 4.065402 & 0.419684 & -0.102826 \\
\hline C & 4.505737 & -0.899842 & 0.003631 \\
\hline C & 4.719494 & 1.293185 & -0.986147 \\
\hline C & 5.567511 & -1.377568 & -0.776098 \\
\hline $\mathrm{H}$ & 4.015039 & -1.562289 & 0.705741 \\
\hline C & 5.792114 & 0.858837 & -1.762484 \\
\hline $\mathrm{H}$ & 4.351300 & 2.307747 & -1.070929 \\
\hline C & 6.193919 & -0.483292 & -1.644352 \\
\hline $\mathrm{H}$ & 7.017160 & -0.830583 & -2.255310 \\
\hline C & 6.528788 & 1.780301 & -2.744971 \\
\hline C & 5.973798 & -2.852359 & -0.655306 \\
\hline C & 6.398583 & 1.211373 & -4.174448 \\
\hline $\mathrm{H}$ & 6.828081 & 0.208778 & -4.251707 \\
\hline H & 5.347385 & 1.149446 & -4.472502 \\
\hline $\mathrm{H}$ & 6.920506 & 1.855775 & -4.890154 \\
\hline C & 8.020781 & 1.852197 & -2.354064 \\
\hline $\mathrm{H}$ & 8.565002 & 2.502957 & -3.046999 \\
\hline H & 8.137996 & 2.254565 & -1.343011 \\
\hline $\mathrm{H}$ & 8.494203 & 0.866918 & -2.378703 \\
\hline C & 5.961300 & 3.208836 & -2.741920 \\
\hline $\mathrm{H}$ & 6.042850 & 3.674234 & -1.754595 \\
\hline $\mathrm{H}$ & 6.521173 & 3.828919 & -3.448571 \\
\hline $\mathrm{H}$ & 4.909829 & 3.227907 & -3.044610 \\
\hline C & 4.768796 & -3.732941 & -1.056471 \\
\hline H & 3.894411 & -3.540157 & -0.428997 \\
\hline $\mathrm{H}$ & 4.484889 & -3.542543 & -2.096912 \\
\hline $\mathrm{H}$ & 5.025244 & -4.793861 & -0.963107 \\
\hline C & 6.371334 & -3.159843 & 0.804279 \\
\hline $\mathrm{H}$ & 5.545955 & -2.975068 & 1.496729 \\
\hline $\mathrm{H}$ & 6.661650 & -4.211141 & 0.904627 \\
\hline $\mathrm{H}$ & 7.217867 & -2.539672 & 1.115557 \\
\hline C & 7.160681 & -3.208199 & -1.564222 \\
\hline $\mathrm{H}$ & 6.926855 & -3.041102 & -2.620409 \\
\hline $\mathrm{H}$ & 8.052370 & -2.624701 & -1.313394 \\
\hline $\mathrm{H}$ & 7.411578 & -4.266475 & -1.444504 \\
\hline
\end{tabular}




\begin{tabular}{|llll|} 
C & -6.122058 & 2.596267 & -1.315699 \\
C & -6.484508 & -2.365361 & -2.303953 \\
H & -7.965947 & -2.400092 & -1.870561 \\
H & -8.050596 & -2.577655 & -0.793917 \\
H & -8.475020 & -1.458692 & -2.094433 \\
C & -8.498290 & -3.202277 & -2.393363 \\
H & -5.865036 & -3.740667 & -2.007684 \\
H & -4.818132 & -3.789825 & -2.322779 \\
H & -5.913534 & -3.984837 & -0.941908 \\
C & -6.412358 & -4.516508 & -2.551878 \\
H & -6.400206 & -2.114970 & -3.825061 \\
H & -6.865397 & -1.165779 & -4.104791 \\
H & -5.357222 & -2.086021 & -4.155537 \\
C & -6.911557 & -2.913946 & -4.373174 \\
H & -5.361453 & 3.639359 & -0.479727 \\
H & -5.485547 & 3.458964 & 0.592915 \\
H & -4.290012 & 3.639202 & -0.698330 \\
C & -5.753046 & 4.638356 & -0.696411 \\
H & -7.618160 & 2.672882 & -0.944808 \\
H & -7.999765 & 3.686262 & -1.110564 \\
H & -8.223077 & 1.986991 & -1.544677 \\
C & -7.769741 & 2.418889 & 0.108968 \\
H & -5.944261 & 2.946402 & -2.809109 \\
H & -6.490889 & 2.252238 & -3.454106 \\
H & -6.314592 & 3.957434 & -3.011548 \\
& -4.887770 & 2.906054 & -3.091652 \\
\hline & & & \\
\hline
\end{tabular}

\section{Catalyst 2C 68}

Datum

Value

B3LYP-D3(BJ)/6-31G(d,p) Energy

$-2165.160278$

B3LYP-D3(BJ)/def2-TZVPP/IEF-PCM(DCM) Energy

$-2165.897267$

B3LYP-D3(BJ)/def2-TZVPP/IEF-PCM(DCM)//B3LYP-D3(BJ)/6-31G(d,p) Free Energy (Quasiharmonic)

$-2165.019903$

Number of Imaginary Frequencies

Frequencies (Top 3 out of 321)

$\begin{array}{ll}\text { 1. } & 10.6294 \mathrm{~cm}^{-1} \\ \text { 2. } & 12.3459 \mathrm{~cm}^{-1} \\ \text { 3. } & 14.0561 \mathrm{~cm}^{-1}\end{array}$

B3LYP-D3(BJ)/6-31G(d,p) Molecular Geometry in Cartesian Coordinates 


\begin{tabular}{|c|c|c|c|}
\hline C & -0.155783 & 3.271222 & 3.988237 \\
\hline C & -0.286139 & 2.110926 & 3.257730 \\
\hline C & 0.768695 & 1.661755 & 2.415922 \\
\hline C & 1.971230 & 2.433769 & 2.344723 \\
\hline C & 2.074070 & 3.620251 & 3.117678 \\
\hline C & 1.034550 & 4.033043 & 3.918418 \\
\hline $\mathrm{H}$ & -0.969378 & 3.602071 & 4.625812 \\
\hline $\mathrm{H}$ & -1.194034 & 1.521604 & 3.318645 \\
\hline C & 0.658958 & 0.475007 & 1.626249 \\
\hline C & 3.025822 & 1.993450 & 1.509477 \\
\hline $\mathrm{H}$ & 2.990742 & 4.200128 & 3.059701 \\
\hline $\mathrm{H}$ & 1.123858 & 4.945174 & 4.499928 \\
\hline C & 2.924123 & 0.845704 & 0.750252 \\
\hline C & 1.714145 & 0.088424 & 0.819374 \\
\hline $\mathrm{H}$ & 3.947377 & 2.567066 & 1.482123 \\
\hline C & -1.179319 & -3.436182 & 4.447459 \\
\hline C & -2.179751 & -3.122490 & 3.557737 \\
\hline C & -2.009985 & -2.074855 & 2.614072 \\
\hline C & -0.781665 & -1.346047 & 2.596409 \\
\hline C & 0.232372 & -1.694283 & 3.527410 \\
\hline C & 0.037919 & -2.713698 & 4.430671 \\
\hline $\mathrm{H}$ & -3.971988 & -2.256047 & 1.737464 \\
\hline $\mathrm{H}$ & -1.319325 & -4.238296 & 5.165592 \\
\hline $\mathrm{H}$ & -3.118179 & -3.669930 & 3.563429 \\
\hline C & -3.028259 & -1.719760 & 1.699321 \\
\hline C & -0.622332 & -0.298755 & 1.638548 \\
\hline$H$ & 1.168842 & -1.148848 & 3.511550 \\
\hline $\mathrm{H}$ & 0.823119 & -2.968548 & 5.135697 \\
\hline C & -1.635256 & 0.028700 & 0.745497 \\
\hline C & -2.876794 & -0.690727 & 0.790143 \\
\hline C & -1.416033 & 1.146773 & -0.258181 \\
\hline $\mathrm{H}$ & -0.375684 & 1.145808 & -0.596717 \\
\hline $\mathrm{H}$ & -2.046548 & 0.989940 & -1.133015 \\
\hline C & 1.492337 & -1.052575 & -0.131087 \\
\hline 0 & 1.282600 & -0.917903 & -1.315182 \\
\hline 0 & 1.520674 & -2.261223 & 0.478100 \\
\hline H & 1.307727 & -2.916140 & -0.209411 \\
\hline 0 & -1.781244 & 2.434564 & 0.253625 \\
\hline $\mathrm{H}$ & -1.196918 & 2.628648 & 0.999938 \\
\hline C & -4.013293 & -0.363901 & -0.110960 \\
\hline C & -4.475940 & 0.956506 & -0.230840 \\
\hline C & -4.659137 & -1.379035 & -0.818819 \\
\hline C & -5.554005 & 1.266221 & -1.056931 \\
\hline $\mathrm{H}$ & -3.963466 & 1.730151 & 0.323631 \\
\hline C & -5.754414 & -1.106032 & -1.650864 \\
\hline $\mathrm{H}$ & -4.282202 & -2.392766 & -0.730533 \\
\hline C & -6.179279 & 0.219361 & -1.755133 \\
\hline $\mathrm{H}$ & -7.015488 & 0.456266 & -2.400340 \\
\hline C & 4.051518 & 0.404105 & -0.105301 \\
\hline C & 4.483986 & -0.925328 & -0.074850 \\
\hline C & 4.701276 & 1.313122 & -0.945640 \\
\hline C & 5.533188 & -1.365204 & -0.885721 \\
\hline $\mathrm{H}$ & 3.995079 & -1.617943 & 0.600106 \\
\hline
\end{tabular}




\begin{tabular}{|c|c|c|c|}
\hline C & 5.764890 & 0.913895 & -1.760837 \\
\hline $\mathrm{H}$ & 4.342455 & 2.336383 & -0.977244 \\
\hline C & 6.159876 & -0.429530 & -1.717729 \\
\hline $\mathrm{H}$ & 6.972020 & -0.755201 & -2.350697 \\
\hline C & 6.440220 & 1.943416 & -2.678632 \\
\hline C & 5.949392 & -2.841910 & -0.833375 \\
\hline C & 7.003079 & 3.097782 & -1.821566 \\
\hline $\mathrm{H}$ & 6.215634 & 3.600986 & -1.253799 \\
\hline $\mathrm{H}$ & 7.745513 & 2.725992 & -1.108391 \\
\hline $\mathrm{H}$ & 7.485638 & 3.846011 & -2.459598 \\
\hline C & 5.396482 & 2.501107 & -3.670481 \\
\hline H & 5.858398 & 3.240238 & -4.334067 \\
\hline $\mathrm{H}$ & 4.980137 & 1.698767 & -4.287139 \\
\hline $\mathrm{H}$ & 4.565823 & 2.987773 & -3.152398 \\
\hline C & 7.598321 & 1.337438 & -3.487815 \\
\hline $\mathrm{H}$ & 7.255737 & 0.533357 & -4.146423 \\
\hline $\mathrm{H}$ & 8.050316 & 2.110299 & -4.116875 \\
\hline $\mathrm{H}$ & 8.382584 & 0.937999 & -2.837062 \\
\hline C & 7.076277 & -3.169224 & -1.825839 \\
\hline $\mathrm{H}$ & 6.779043 & -2.960845 & -2.858371 \\
\hline $\mathrm{H}$ & 7.985818 & -2.600293 & -1.609025 \\
\hline H & 7.327548 & -4.232201 & -1.759934 \\
\hline C & 4.727777 & -3.721929 & -1.179706 \\
\hline $\mathrm{H}$ & 4.354197 & -3.488874 & -2.181615 \\
\hline $\mathrm{H}$ & 5.001720 & -4.782150 & -1.151901 \\
\hline $\mathrm{H}$ & 3.908536 & -3.568194 & -0.472074 \\
\hline C & 6.439623 & -3.182896 & 0.590245 \\
\hline H & 7.305449 & -2.569992 & 0.859697 \\
\hline $\mathrm{H}$ & 5.660189 & -3.008390 & 1.337016 \\
\hline $\mathrm{H}$ & 6.733447 & -4.236485 & 0.650805 \\
\hline C & -6.426719 & -2.256194 & -2.414382 \\
\hline C & -6.053369 & 2.706249 & -1.238808 \\
\hline C & -5.281705 & 3.702298 & -0.356913 \\
\hline $\mathrm{H}$ & -5.413559 & 3.479501 & 0.706813 \\
\hline $\mathrm{H}$ & -4.209356 & 3.695351 & -0.570955 \\
\hline $\mathrm{H}$ & -5.657468 & 4.715159 & -0.533568 \\
\hline C & -7.549936 & 2.788969 & -0.870706 \\
\hline H & -8.162133 & 2.137985 & -1.501280 \\
\hline $\mathrm{H}$ & -7.709738 & 2.493369 & 0.170978 \\
\hline $\mathrm{H}$ & -7.915919 & 3.813966 & -0.995130 \\
\hline C & -5.864005 & 3.116686 & -2.715429 \\
\hline H & -4.806931 & 3.073450 & -2.995229 \\
\hline $\mathrm{H}$ & -6.417549 & 2.458655 & -3.391579 \\
\hline $\mathrm{H}$ & -6.219329 & 4.140560 & -2.875860 \\
\hline C & -6.949729 & -3.300123 & -1.404113 \\
\hline $\mathrm{H}$ & -6.141394 & -3.710246 & -0.792427 \\
\hline $\mathrm{H}$ & -7.685681 & -2.851695 & -0.729394 \\
\hline $\mathrm{H}$ & -7.429003 & -4.133731 & -1.929073 \\
\hline C & -5.393393 & -2.920200 & -3.349780 \\
\hline H & -5.853343 & -3.747672 & -3.901176 \\
\hline $\mathrm{H}$ & -5.007113 & -2.197637 & -4.075295 \\
\hline $\mathrm{H}$ & -4.542339 & -3.320716 & -2.792291 \\
\hline C & -7.612074 & -1.778599 & -3.268497 \\
\hline H & -7.298258 & -1.056853 & -4.029019 \\
\hline
\end{tabular}




$\begin{array}{llll}H & -8.060362 & -2.632202 & -3.786224 \\ \mathrm{H} & -8.391374 & -1.314463 & -2.655943\end{array}$

\section{Catalyst 2C 69}

Datum

Value

\begin{tabular}{lr}
\hline B3LYP-D3(BJ)/6-31G(d,p) Energy & -2165.160168 \\
\hline B3LYP-D3(BJ)/def2-TZVPP/IEF-PCM(DCM) Energy & -2165.897242 \\
\hline B3LYP-D3(BJ)/def2-TZVPP/IEF-PCM(DCM)//B3LYP-D3(BJ)/6-31G(d,p) Free Energy (Quasiharmonic) & -2165.019872 \\
\hline
\end{tabular}

Number of Imaginary Frequencies

Frequencies (Top 3 out of 321)

$\begin{array}{ll}\text { 1. } & 10.7206 \mathrm{~cm}^{-1} \\ \text { 2. } & 12.5568 \mathrm{~cm}^{-1} \\ \text { 3. } & 14.0216 \mathrm{~cm}^{-1}\end{array}$

\section{B3LYP-D3(BJ)/6-31G(d,p) Molecular Geometry in Cartesian Coordinates}

$\begin{array}{lrrr}\text { C } & 0.225129 & 3.468302 & 3.782106 \\ \mathrm{C} & 0.335596 & 2.264675 & 3.121548 \\ \mathrm{C} & -0.728546 & 1.782375 & 2.310070 \\ \mathrm{C} & -1.922110 & 2.563692 & 2.201296 \\ \mathrm{C} & -2.004003 & 3.796197 & 2.901637 \\ \mathrm{C} & -0.954332 & 4.242153 & 3.670565 \\ \mathrm{H} & 1.045423 & 3.823757 & 4.397459 \\ \mathrm{H} & 1.233722 & 1.664967 & 3.217097 \\ \mathrm{C} & -0.638150 & 0.550910 & 1.590165 \\ \mathrm{C} & -2.989639 & 2.086429 & 1.403972 \\ \mathrm{H} & -2.913429 & 4.383762 & 2.814293 \\ \mathrm{H} & -1.027830 & 5.188788 & 4.196460 \\ \mathrm{C} & -2.911230 & 0.890395 & 0.719398 \\ \mathrm{C} & -1.707492 & 0.126428 & 0.822743 \\ \mathrm{H} & -3.904216 & 2.669156 & 1.349375 \\ \mathrm{C} & 1.125503 & -3.316793 & 4.513944 \\ \mathrm{C} & 2.141544 & -3.029289 & 3.633381 \\ \mathrm{C} & 1.992490 & -2.002027 & 2.664486 \\ \mathrm{C} & 0.770067 & -1.265257 & 2.613174 \\ \mathrm{C} & -0.261478 & -1.588227 & 3.533558 \\ \mathrm{C} & -0.087168 & -2.588627 & 4.461414 \\ \mathrm{H} & 3.961784 & -2.223356 & 1.814746 \\ \mathrm{H} & 1.248639 & -4.104540 & 5.250820 \\ \mathrm{H} & 3.075530 & -3.583378 & 3.664334\end{array}$




\begin{tabular}{|c|c|c|c|}
\hline C & 3.024757 & -1.677447 & 1.754418 \\
\hline C & 0.634500 & -0.235340 & 1.633575 \\
\hline $\mathrm{H}$ & -1.195808 & -1.041224 & 3.488357 \\
\hline $\mathrm{H}$ & -0.886214 & -2.826081 & 5.156780 \\
\hline C & 1.661697 & 0.063405 & 0.746327 \\
\hline C & 2.893790 & -0.669695 & 0.818658 \\
\hline C & 1.465536 & 1.158909 & -0.286400 \\
\hline $\mathrm{H}$ & 2.088787 & 0.961860 & -1.158895 \\
\hline $\mathrm{H}$ & 0.422732 & 1.173206 & -0.618438 \\
\hline C & -1.544496 & -1.138874 & 0.031078 \\
\hline 0 & -1.559709 & -2.262679 & 0.483044 \\
\hline 0 & -1.354458 & -0.877020 & -1.281142 \\
\hline $\mathrm{H}$ & -1.267760 & -1.740966 & -1.720155 \\
\hline 0 & 1.860970 & 2.454166 & 0.180567 \\
\hline $\mathrm{H}$ & 1.288789 & 2.683359 & 0.926512 \\
\hline C & 4.039553 & -0.384849 & -0.085333 \\
\hline C & 4.523826 & 0.923839 & -0.242626 \\
\hline C & 4.671985 & -1.430369 & -0.760936 \\
\hline C & 5.609466 & 1.192048 & -1.073464 \\
\hline $\mathrm{H}$ & 4.021999 & 1.721473 & 0.286773 \\
\hline C & 5.774368 & -1.199365 & -1.596298 \\
\hline $\mathrm{H}$ & 4.279192 & -2.435096 & -0.643339 \\
\hline C & 6.220486 & 0.115548 & -1.738293 \\
\hline $\mathrm{H}$ & 7.062754 & 0.320104 & -2.386707 \\
\hline C & -4.054642 & 0.412149 & -0.095233 \\
\hline C & -4.503041 & -0.912782 & 0.022736 \\
\hline C & -4.701605 & 1.276927 & -0.978294 \\
\hline C & -5.565394 & -1.381901 & -0.746382 \\
\hline $\mathrm{H}$ & -4.011416 & -1.565763 & 0.730086 \\
\hline C & -5.785168 & 0.844980 & -1.756086 \\
\hline $\mathrm{H}$ & -4.331113 & 2.291957 & -1.075175 \\
\hline C & -6.192444 & -0.484122 & -1.626399 \\
\hline $\mathrm{H}$ & -7.019347 & -0.842764 & -2.225563 \\
\hline C & -6.461063 & 1.829516 & -2.721002 \\
\hline C & -6.051955 & -2.835359 & -0.667382 \\
\hline C & -7.638901 & 1.193602 & -3.476627 \\
\hline $\mathrm{H}$ & -7.316427 & 0.348452 & -4.092717 \\
\hline $\mathrm{H}$ & -8.417230 & 0.842375 & -2.791900 \\
\hline $\mathrm{H}$ & -8.091610 & 1.934655 & -4.142342 \\
\hline C & -5.426064 & 2.316039 & -3.758307 \\
\hline $\mathrm{H}$ & -5.888252 & 3.023761 & -4.455052 \\
\hline $\mathrm{H}$ & -4.580518 & 2.818865 & -3.281226 \\
\hline $\mathrm{H}$ & -5.031509 & 1.474540 & -4.336129 \\
\hline C & -6.995686 & 3.038188 & -1.922757 \\
\hline $\mathrm{H}$ & -6.193093 & 3.562131 & -1.396467 \\
\hline $\mathrm{H}$ & -7.478797 & 3.755429 & -2.594992 \\
\hline $\mathrm{H}$ & -7.731239 & 2.717337 & -1.178499 \\
\hline C & -5.248345 & -3.660753 & 0.351362 \\
\hline $\mathrm{H}$ & -4.181230 & -3.681795 & 0.110738 \\
\hline $\mathrm{H}$ & -5.358518 & -3.266211 & 1.366349 \\
\hline $\mathrm{H}$ & -5.612312 & -4.692752 & 0.356412 \\
\hline C & -5.892780 & -3.492992 & -2.055575 \\
\hline $\mathrm{H}$ & -4.843618 & -3.488773 & -2.367354 \\
\hline H & -6.236255 & -4.532626 & -2.026057 \\
\hline
\end{tabular}




\begin{tabular}{|c|c|c|c|}
\hline $\mathrm{H}$ & -6.472175 & -2.969719 & -2.821435 \\
\hline C & -7.538715 & -2.861067 & -0.254049 \\
\hline $\mathrm{H}$ & -7.676341 & -2.395104 & 0.726615 \\
\hline $\mathrm{H}$ & -8.168847 & -2.326371 & -0.970441 \\
\hline $\mathrm{H}$ & -7.899942 & -3.893480 & -0.195828 \\
\hline C & 6.431937 & -2.382178 & -2.321919 \\
\hline C & 6.132861 & 2.617974 & -1.294979 \\
\hline C & 5.373697 & 3.651676 & -0.446197 \\
\hline $\mathrm{H}$ & 4.302227 & 3.655263 & -0.664699 \\
\hline $\mathrm{H}$ & 5.497705 & 3.458016 & 0.624133 \\
\hline $\mathrm{H}$ & 5.766361 & 4.652835 & -0.650658 \\
\hline C & 5.956745 & 2.988231 & -2.783803 \\
\hline $\mathrm{H}$ & 6.502524 & 2.301991 & -3.437880 \\
\hline $\mathrm{H}$ & 4.900294 & 2.953942 & -3.067349 \\
\hline $\mathrm{H}$ & 6.329247 & 4.001160 & -2.972301 \\
\hline C & 7.628975 & 2.687320 & -0.922290 \\
\hline $\mathrm{H}$ & 7.779332 & 2.419762 & 0.128319 \\
\hline $\mathrm{H}$ & 8.233157 & 2.008241 & -1.530561 \\
\hline $\mathrm{H}$ & 8.012225 & 3.702171 & -1.074804 \\
\hline C & 6.938407 & -3.401064 & -1.278233 \\
\hline $\mathrm{H}$ & 7.678632 & -2.941559 & -0.615794 \\
\hline $\mathrm{H}$ & 6.123185 & -3.781034 & -0.656387 \\
\hline $\mathrm{H}$ & 7.407805 & -4.256886 & -1.775650 \\
\hline C & 7.625275 & -1.948044 & -3.188025 \\
\hline $\mathrm{H}$ & 8.061921 & -2.823340 & -3.678759 \\
\hline $\mathrm{H}$ & 7.323128 & -1.245118 & -3.970639 \\
\hline $\mathrm{H}$ & 8.410368 & -1.476903 & -2.588399 \\
\hline C & 5.391440 & -3.061093 & -3.238447 \\
\hline $\mathrm{H}$ & 5.017148 & -2.356408 & -3.987614 \\
\hline $\mathrm{H}$ & 5.840904 & -3.911850 & -3.762334 \\
\hline $\mathrm{H}$ & 4.534015 & -3.432300 & -2.670513 \\
\hline
\end{tabular}

\section{Catalyst 2C 70}

\section{Datum}

Value

B3LYP-D3(BJ)/6-31G(d,p) Energy

$-2165.160439$

B3LYP-D3(BJ)/def2-TZVPP/IEF-PCM(DCM) Energy

$-2165.897409$

B3LYP-D3(BJ)/def2-TZVPP/IEF-PCM(DCM)//B3LYP-D3(BJ)/6-31G(d,p) Free Energy (Quasiharmonic)

$-2165.01987$

Number of Imaginary Frequencies

0

Frequencies (Top 3 out of 321)

$\begin{array}{ll}\text { 1. } & 10.9877 \mathrm{~cm}^{-1} \\ \text { 2. } & 12.4920 \mathrm{~cm}^{-1} \\ \text { 3. } & 14.0288 \mathrm{~cm}^{-1}\end{array}$




\begin{tabular}{|c|c|c|c|}
\hline C & -0.155023 & -4.922722 & -1.154597 \\
\hline C & -0.284016 & -3.737703 & -0.464121 \\
\hline C & 0.771151 & -2.783034 & -0.455664 \\
\hline C & 1.982111 & -3.090678 & -1.152434 \\
\hline C & 2.080590 & -4.321477 & -1.853536 \\
\hline C & 1.035977 & -5.216215 & -1.859745 \\
\hline $\mathrm{H}$ & -0.969990 & -5.639475 & -1.153135 \\
\hline $\mathrm{H}$ & -1.191111 & -3.520660 & 0.088276 \\
\hline C & 0.664277 & -1.534067 & 0.232752 \\
\hline C & 3.044719 & -2.156437 & -1.135425 \\
\hline $\mathrm{H}$ & 3.000482 & -4.542578 & -2.387199 \\
\hline $\mathrm{H}$ & 1.123631 & -6.152851 & -2.401124 \\
\hline C & 2.953336 & -0.959218 & -0.455170 \\
\hline C & 1.739910 & -0.661669 & 0.240387 \\
\hline $\mathrm{H}$ & 3.947443 & -2.379472 & -1.695922 \\
\hline C & -0.827810 & -1.985611 & 5.151318 \\
\hline C & -1.910722 & -1.463001 & 4.484936 \\
\hline C & -1.848042 & -1.201285 & 3.090413 \\
\hline C & -0.641514 & -1.490209 & 2.380011 \\
\hline C & 0.459174 & -2.031697 & 3.097758 \\
\hline C & 0.367742 & -2.270978 & 4.449198 \\
\hline $\mathrm{H}$ & -3.872869 & -0.483811 & 2.919730 \\
\hline $\mathrm{H}$ & -0.885054 & -2.180104 & 6.217864 \\
\hline $\mathrm{H}$ & -2.833222 & -1.241065 & 5.014160 \\
\hline C & -2.949783 & -0.673924 & 2.379959 \\
\hline C & -0.594365 & -1.218277 & 0.978445 \\
\hline $\mathrm{H}$ & 1.381188 & -2.243488 & 2.569553 \\
\hline $\mathrm{H}$ & 1.219858 & -2.679248 & 4.983344 \\
\hline C & -1.687288 & -0.687797 & 0.304370 \\
\hline C & -2.901026 & -0.422650 & 1.021514 \\
\hline C & -1.562901 & -0.359992 & -1.172489 \\
\hline $\mathrm{H}$ & -2.228133 & 0.465984 & -1.425109 \\
\hline $\mathrm{H}$ & -0.538006 & -0.042417 & -1.388307 \\
\hline C & 1.665184 & 0.563517 & 1.103545 \\
\hline 0 & 2.311638 & 0.733238 & 2.110855 \\
\hline 0 & 0.772207 & 1.473604 & 0.640506 \\
\hline $\mathrm{H}$ & 0.753119 & 2.190856 & 1.297523 \\
\hline 0 & -1.946828 & -1.436742 & -2.036182 \\
\hline $\mathrm{H}$ & -1.315735 & -2.158462 & -1.905347 \\
\hline C & -4.111822 & 0.125828 & 0.355750 \\
\hline C & -4.601901 & -0.436498 & -0.827863 \\
\hline C & -4.801109 & 1.200606 & 0.930691 \\
\hline C & -5.747900 & 0.066467 & -1.449895 \\
\hline $\mathrm{H}$ & -4.062694 & -1.266197 & -1.267345 \\
\hline C & -5.960469 & 1.719852 & 0.346232 \\
\hline $\mathrm{H}$ & -4.404329 & 1.642986 & 1.838659 \\
\hline C & -6.414142 & 1.139374 & -0.845375 \\
\hline $\mathrm{H}$ & -7.302418 & 1.535049 & -1.315648 \\
\hline C & 4.076478 & 0.008306 & -0.489414 \\
\hline C & 3.835490 & 1.357771 & -0.772018 \\
\hline
\end{tabular}




\begin{tabular}{|c|c|c|c|}
\hline C & 5.387316 & -0.425407 & -0.262129 \\
\hline C & 4.879335 & 2.283882 & -0.807598 \\
\hline $\mathrm{H}$ & 2.820493 & 1.672442 & -0.974806 \\
\hline C & 6.458667 & 0.470931 & -0.301360 \\
\hline $\mathrm{H}$ & 5.549877 & -1.469340 & -0.025813 \\
\hline C & 6.177226 & 1.815820 & -0.571231 \\
\hline H & 6.997903 & 2.524739 & -0.592558 \\
\hline C & 7.909198 & 0.038259 & -0.044330 \\
\hline C & 4.643031 & 3.776210 & -1.077002 \\
\hline C & 8.028183 & -1.470058 & 0.227344 \\
\hline $\mathrm{H}$ & 7.464609 & -1.767071 & 1.117113 \\
\hline $\mathrm{H}$ & 7.670116 & -2.063236 & -0.620061 \\
\hline $\mathrm{H}$ & 9.077110 & -1.731912 & 0.396253 \\
\hline C & 8.456271 & 0.795966 & 1.184623 \\
\hline $\mathrm{H}$ & 9.492038 & 0.500735 & 1.384648 \\
\hline H & 8.440123 & 1.878615 & 1.032059 \\
\hline $\mathrm{H}$ & 7.858186 & 0.574815 & 2.073862 \\
\hline C & 8.768364 & 0.375655 & -1.281939 \\
\hline $\mathrm{H}$ & 8.757920 & 1.446513 & -1.502736 \\
\hline H & 9.809320 & 0.078008 & -1.115670 \\
\hline $\mathrm{H}$ & 8.398645 & -0.151741 & -2.166872 \\
\hline C & 3.160881 & 4.091179 & -1.337942 \\
\hline $\mathrm{H}$ & 2.785009 & 3.566993 & -2.222099 \\
\hline H & 2.530980 & 3.814677 & -0.486805 \\
\hline H & 3.037148 & 5.164660 & -1.509936 \\
\hline C & 5.456445 & 4.215760 & -2.312699 \\
\hline $\mathrm{H}$ & 5.150299 & 3.652214 & -3.199538 \\
\hline H & 5.299671 & 5.281166 & -2.513022 \\
\hline $\mathrm{H}$ & 6.528881 & 4.057403 & -2.169372 \\
\hline C & 5.100191 & 4.584388 & 0.156818 \\
\hline $\mathrm{H}$ & 4.537227 & 4.284050 & 1.045910 \\
\hline H & 6.162362 & 4.432384 & 0.367772 \\
\hline H & 4.939894 & 5.655534 & -0.007530 \\
\hline C & -6.678536 & 2.902103 & 1.014378 \\
\hline C & -6.213163 & -0.566237 & -2.768972 \\
\hline C & -7.484394 & 0.097359 & -3.321646 \\
\hline $\mathrm{H}$ & -8.326217 & 0.003820 & -2.627882 \\
\hline $\mathrm{H}$ & -7.328414 & 1.160561 & -3.530096 \\
\hline $\mathrm{H}$ & -7.772839 & -0.385055 & -4.260645 \\
\hline C & -6.508276 & -2.064450 & -2.541708 \\
\hline $\mathrm{H}$ & -5.621392 & -2.600478 & -2.194038 \\
\hline H & -7.297049 & -2.196926 & -1.794179 \\
\hline $\mathrm{H}$ & -6.838922 & -2.532732 & -3.475316 \\
\hline C & -5.087504 & -0.419384 & -3.817552 \\
\hline $\mathrm{H}$ & -4.875501 & 0.638065 & -4.007049 \\
\hline $\mathrm{H}$ & -4.159139 & -0.892932 & -3.487740 \\
\hline $\mathrm{H}$ & -5.389589 & -0.879993 & -4.764963 \\
\hline C & -7.101872 & 2.499194 & 2.443509 \\
\hline H & -7.784121 & 1.643733 & 2.418805 \\
\hline H & -6.240040 & 2.221929 & 3.056735 \\
\hline $\mathrm{H}$ & -7.613125 & 3.331244 & 2.940047 \\
\hline C & -7.937259 & 3.334941 & 0.245393 \\
\hline $\mathrm{H}$ & -8.415029 & 4.172266 & 0.763384 \\
\hline H & -7.698231 & 3.666013 & -0.76998 \\
\hline
\end{tabular}




\section{Catalyst 2C 71}

\section{Datum}

Value

\begin{tabular}{lc}
\hline B3LYP-D3(BJ)/6-31G(d,p) Energy & -2165.160893 \\
\hline B3LYP-D3(BJ)/def2-TZVPP/IEF-PCM(DCM) Energy & -2165.897497 \\
\hline B3LYP-D3(BJ)/def2-TZVPP/IEF-PCM(DCM)//B3LYP-D3(BJ)/6-31G(d,p) Free Energy (Quasiharmonic) & -2165.019838
\end{tabular}

Number of Imaginary Frequencies

Frequencies (Top 3 out of 321)
1. $11.2395 \mathrm{~cm}^{-1}$
2. $12.9087 \mathrm{~cm}^{-1}$
3. $14.1738 \mathrm{~cm}^{-1}$

\section{B3LYP-D3(BJ)/6-31G(d,p) Molecular Geometry in Cartesian Coordinates}

$\begin{array}{lrrr}\text { C } & 0.100211 & -4.897238 & -1.216909 \\ \text { C } & 0.233699 & -3.722938 & -0.509172 \\ \text { C } & -0.814928 & -2.761216 & -0.492564 \\ \text { C } & -2.024342 & -3.050709 & -1.199951 \\ \text { C } & -2.127521 & -4.270776 & -1.918792 \\ \text { C } & -1.089015 & -5.172557 & -1.932233 \\ \text { H } & 0.910238 & -5.619557 & -1.221338 \\ \text { H } & 1.139314 & -3.519891 & 0.050929 \\ \text { C } & -0.702759 & -1.523125 & 0.214658 \\ \text { C } & -3.080674 & -2.109259 & -1.175554 \\ \text { H } & -3.046016 & -4.477935 & -2.460397 \\ \text { H } & -1.180228 & -6.100830 & -2.487247 \\ \text { C } & -2.983310 & -0.922948 & -0.478031 \\ \text { C } & -1.772364 & -0.643736 & 0.229028 \\ \text { H } & -3.982236 & -2.317448 & -1.743706 \\ \text { C } & 0.761579 & -2.047808 & 5.134262 \\ \text { C } & 1.851006 & -1.523392 & 4.480060 \\ \text { C } & 1.797095 & -1.243713 & 3.088632 \\ \text { C } & 0.592447 & -1.516178 & 2.368656 \\ \text { C } & -0.515141 & -2.060072 & 3.073842 \\ \text { C } & -0.432070 & -2.317036 & 4.422562\end{array}$




\begin{tabular}{|c|c|c|c|}
\hline H & 3.827071 & -0.537571 & 2.937576 \\
\hline $\mathrm{H}$ & 0.812156 & -2.256058 & 6.198545 \\
\hline $\mathrm{H}$ & 2.772061 & -1.313704 & 5.016735 \\
\hline C & 2.905786 & -0.714727 & 2.390427 \\
\hline C & 0.554158 & -1.225676 & 0.970614 \\
\hline H & -1.435766 & -2.259365 & 2.538401 \\
\hline H & -1.289367 & -2.726792 & 4.947191 \\
\hline C & 1.653798 & -0.693499 & 0.308732 \\
\hline C & 2.866262 & -0.446383 & 1.034830 \\
\hline C & 1.537545 & -0.344224 & -1.163868 \\
\hline $\mathrm{H}$ & 0.515585 & -0.016908 & -1.379169 \\
\hline H & 2.209274 & 0.480758 & -1.402017 \\
\hline C & -1.697726 & 0.567607 & 1.111928 \\
\hline 0 & -2.348040 & 0.721675 & 2.119082 \\
\hline 0 & -0.801521 & 1.484387 & 0.667566 \\
\hline $\mathrm{H}$ & -0.788162 & 2.192430 & 1.334808 \\
\hline 0 & 1.917615 & -1.410885 & -2.041725 \\
\hline $\mathrm{H}$ & 1.281634 & -2.130465 & -1.923396 \\
\hline C & 4.084977 & 0.099711 & 0.381719 \\
\hline C & 4.572132 & -0.448211 & -0.805636 \\
\hline C & 4.782675 & 1.162502 & 0.980837 \\
\hline C & 5.728058 & 0.053911 & -1.418481 \\
\hline $\mathrm{H}$ & 4.027547 & -1.266244 & -1.260047 \\
\hline C & 5.944759 & 1.676517 & 0.408250 \\
\hline $\mathrm{H}$ & 4.382648 & 1.588918 & 1.892250 \\
\hline C & 6.397081 & 1.107271 & -0.794761 \\
\hline $\mathrm{H}$ & 7.292824 & 1.508525 & -1.251593 \\
\hline C & -4.095944 & 0.056671 & -0.503903 \\
\hline C & -3.841243 & 1.405107 & -0.771431 \\
\hline C & -5.411994 & -0.356361 & -0.278133 \\
\hline C & -4.870634 & 2.348701 & -0.787633 \\
\hline $\mathrm{H}$ & -2.822408 & 1.713454 & -0.976072 \\
\hline C & -6.471908 & 0.555663 & -0.303268 \\
\hline $\mathrm{H}$ & -5.595122 & -1.401419 & -0.051659 \\
\hline C & -6.177114 & 1.901898 & -0.554399 \\
\hline $\mathrm{H}$ & -6.983767 & 2.619900 & -0.564440 \\
\hline C & -7.900774 & 0.059727 & -0.037277 \\
\hline C & -4.529648 & 3.821421 & -1.054227 \\
\hline C & -7.970377 & -0.551855 & 1.378770 \\
\hline $\mathrm{H}$ & -7.282353 & -1.394332 & 1.489201 \\
\hline $\mathrm{H}$ & -7.708893 & 0.193389 & 2.136137 \\
\hline H & -8.982711 & -0.914405 & 1.588155 \\
\hline C & -8.268849 & -1.017064 & -1.080844 \\
\hline $\mathrm{H}$ & -9.284512 & -1.387429 & -0.904532 \\
\hline $\mathrm{H}$ & -8.225371 & -0.606516 & -2.094529 \\
\hline $\mathrm{H}$ & -7.589098 & -1.872450 & -1.036412 \\
\hline C & -8.939973 & 1.189035 & -0.126180 \\
\hline $\mathrm{H}$ & -8.949405 & 1.654340 & -1.116865 \\
\hline $\mathrm{H}$ & -9.939213 & 0.783837 & 0.060120 \\
\hline H & -8.755430 & 1.969678 & 0.618263 \\
\hline C & -5.764320 & 4.732912 & -0.972221 \\
\hline $\mathrm{H}$ & -6.515729 & 4.466346 & -1.722064 \\
\hline $\mathrm{H}$ & -6.234271 & 4.689424 & 0.015291 \\
\hline H & -5.468716 & 5.770529 & -1.154842 \\
\hline
\end{tabular}




\begin{tabular}{|c|c|c|c|}
\hline C & -3.917454 & 3.955538 & -2.465053 \\
\hline $\mathrm{H}$ & -4.626605 & 3.622133 & -3.229150 \\
\hline $\mathrm{H}$ & -3.657751 & 4.999648 & -2.671016 \\
\hline $\mathrm{H}$ & -3.008188 & 3.356986 & -2.568168 \\
\hline C & -3.503938 & 4.298897 & -0.002112 \\
\hline $\mathrm{H}$ & -3.909802 & 4.197187 & 1.009042 \\
\hline $\mathrm{H}$ & -2.578209 & 3.718914 & -0.050828 \\
\hline $\mathrm{H}$ & -3.249934 & 5.351398 & -0.168219 \\
\hline C & 6.730986 & 2.838763 & 1.031982 \\
\hline C & 6.190255 & -0.563494 & -2.745228 \\
\hline C & 6.470617 & -2.067527 & -2.538967 \\
\hline $\mathrm{H}$ & 7.256676 & -2.218198 & -1.792036 \\
\hline $\mathrm{H}$ & 5.578033 & -2.600141 & -2.200755 \\
\hline $\mathrm{H}$ & 6.798564 & -2.525357 & -3.478658 \\
\hline C & 7.468924 & 0.096737 & -3.284198 \\
\hline $\mathrm{H}$ & 7.322748 & 1.164354 & -3.476752 \\
\hline $\mathrm{H}$ & 8.307576 & -0.014980 & -2.589233 \\
\hline $\mathrm{H}$ & 7.756093 & -0.373775 & -4.229570 \\
\hline C & 5.068482 & -0.390367 & -3.793922 \\
\hline $\mathrm{H}$ & 4.134795 & -0.859871 & -3.473466 \\
\hline $\mathrm{H}$ & 4.867221 & 0.671766 & -3.968297 \\
\hline $\mathrm{H}$ & 5.368497 & -0.839897 & -4.747258 \\
\hline C & 8.170957 & 2.373290 & 1.338268 \\
\hline $\mathrm{H}$ & 8.165833 & 1.536239 & 2.043402 \\
\hline $\mathrm{H}$ & 8.690959 & 2.045075 & 0.434217 \\
\hline $\mathrm{H}$ & 8.750624 & 3.190872 & 1.780707 \\
\hline C & 6.098297 & 3.335281 & 2.341993 \\
\hline $\mathrm{H}$ & 6.695054 & 4.155955 & 2.751875 \\
\hline $\mathrm{H}$ & 5.082221 & 3.710135 & 2.184320 \\
\hline $\mathrm{H}$ & 6.058127 & 2.543846 & 3.096932 \\
\hline C & 6.773811 & 4.019793 & 0.038442 \\
\hline $\mathrm{H}$ & 5.762289 & 4.367278 & -0.193861 \\
\hline $\mathrm{H}$ & 7.334895 & 4.858355 & 0.465370 \\
\hline $\mathrm{H}$ & 7.254835 & 3.739254 & -0.902523 \\
\hline
\end{tabular}

\section{Catalyst 2C 72}

Datum

Value

\begin{tabular}{lr}
\hline B3LYP-D3(BJ)/6-31G(d,p) Energy & -2165.160147 \\
\hline B3LYP-D3(BJ)/def2-TZVPP/IEF-PCM(DCM) Energy & -2165.897117 \\
\hline B3LYP-D3(BJ)/def2-TZVPP/IEF-PCM(DCM)//B3LYP-D3(BJ)/6-31G(d,p) Free Energy (Quasiharmonic) & -2165.019816 \\
\hline Number of Imaginary Frequencies & 0
\end{tabular}

Frequencies (Top 3 out of 321) 


$\begin{array}{ll}\text { 1. } & 10.6038 \mathrm{~cm}^{-1} \\ \text { 2. } & 12.7013 \mathrm{~cm}^{-1} \\ \text { 3. } & 13.9324 \mathrm{~cm}^{-1}\end{array}$

B3LYP-D3(BJ)/6-31G(d,p) Molecular Geometry in Cartesian Coordinates

C

C

C

C

C

C

$\mathrm{H}$

$\mathrm{H}$

C

C

$\mathrm{H}$

$\mathrm{H}$

C

C

$\mathrm{H}$

C

C

C

C

C

C

$\mathrm{H}$

$\mathrm{H}$

$\mathrm{H}$

C

C

$\mathrm{H}$

$\mathrm{H}$

C

C

C

$\mathrm{H}$

$\mathrm{H}$

C

0

0

$\mathrm{H}$

0

$\mathrm{H}$

C

C

C

C

$\mathrm{H}$
0.242887

0.346475

$-0.720540$

$-1.909861$

$-1.984602$

$-0.932255$

1.065283

1. 241268

$-0.637094$

$-2.980462$

$-2.890743$

$-1.000308$

$-2.909616$

$-1.709408$

$-3.891502$

1.104134

2.122385

1.979519

0.760907

$-0.273045$

$-0.104697$

3.948596

1.222539

3.053459

3.014475

0.631567

$-1.204481$

$-0.905527$

1.661244

2.890304

1.469782

2.091890

0.426913

$-1.552901$

$-1.578617$

$-1.354778$

$-1.271960$

1.871026

1.300166

4.039997

4.525026

4.673732

5.617061

4.023309
3.492189

2. 283062

1.800626

2.587609

3.825878

4. 271784

3.847624

1.679091

0.563618

2.110172

4.417852

5.222780

0.908099

0.139058

2.697978

$-3.299040$

$-3.020864$

$-1.997273$

$-1.254569$

$-1.567861$

$-2.564798$

$-2.232161$

$-4.084024$

$-3.579634$

$-1.681806$

$-0.228839$

$-1.016242$

$-2.794834$

0.060653

$-0.677363$

1.150547

0.944885

1.167343

$-1.132427$

$-2.253335$

$-0.880344$

$-1.747785$

2.447307

2.684096

$-0.400901$

0.902559

$-1.459329$

1.164082

1.705837

\subsection{4}

3.108205

2. 300575

2. 186395

2.877376

3.642637

4. 372341

3.208053

1. 589411

1. 393405

2. 785775

4.161352

0.718436

0.826192

1. 334711

4.533025

3.652059

2.678295

2.622390

3.543452

4.476104

1.832718

5.273621

3.686379

1.768238

1.637615

3.494933

5.171913

0.750025

0.827779

$-0.289489$

$-1.160801$

$-0.621129$

0.043099

0.501802

$-1.269866$

$-1.702702$

0.168462

0.913050

$-0.073841$

$-0.239242$

$-0.738603$

$-1.070056$

0.281643 


\begin{tabular}{|c|c|c|c|}
\hline C & 5.775990 & -1.235487 & -1.568173 \\
\hline $\mathrm{H}$ & 4.275022 & -2.457865 & -0.609762 \\
\hline C & 6.224153 & 0.082606 & -1.719200 \\
\hline $\mathrm{H}$ & 7.071127 & 0.273443 & -2.369785 \\
\hline C & -4.056513 & 0.429144 & -0.090827 \\
\hline C & -4.508736 & -0.889818 & 0.035756 \\
\hline C & -4.701199 & 1.297540 & -0.979945 \\
\hline C & -5.576502 & -1.361285 & -0.731173 \\
\hline $\mathrm{H}$ & -4.019725 & -1.541216 & 0.746320 \\
\hline C & -5.783249 & 0.864909 & -1.750882 \\
\hline $\mathrm{H}$ & -4.323027 & 2.307303 & -1.077517 \\
\hline C & -6.195637 & -0.466489 & -1.611532 \\
\hline $\mathrm{H}$ & -7.026184 & -0.819382 & -2.213462 \\
\hline C & -6.511525 & 1.782805 & -2.743184 \\
\hline C & -6.067315 & -2.812960 & -0.643040 \\
\hline C & -5.927296 & 3.204526 & -2.759028 \\
\hline $\mathrm{H}$ & -6.001474 & 3.683254 & -1.777518 \\
\hline $\mathrm{H}$ & -4.876277 & 3.207479 & -3.063841 \\
\hline $\mathrm{H}$ & -6.481269 & 3.822194 & -3.472400 \\
\hline C & -8.001574 & 1.876722 & -2.349903 \\
\hline $\mathrm{H}$ & -8.540142 & 2.524944 & -3.049593 \\
\hline $\mathrm{H}$ & -8.486155 & 0.896606 & -2.361015 \\
\hline $\mathrm{H}$ & -8.111844 & 2.292751 & -1.343624 \\
\hline C & -6.390886 & 1.194368 & -4.165490 \\
\hline $\mathrm{H}$ & -6.831780 & 0.195719 & -4.228835 \\
\hline $\mathrm{H}$ & -6.907003 & 1.835315 & -4.888467 \\
\hline $\mathrm{H}$ & -5.341144 & 1.116456 & -4.464874 \\
\hline C & -5.266197 & -3.634222 & 0.381044 \\
\hline $\mathrm{H}$ & -4.199156 & -3.660243 & 0.140626 \\
\hline $\mathrm{H}$ & -5.375119 & -3.232815 & 1.393474 \\
\hline $\mathrm{H}$ & -5.633462 & -4.664993 & 0.392742 \\
\hline C & -5.909743 & -3.479324 & -2.027153 \\
\hline $\mathrm{H}$ & -4.860562 & -3.479923 & -2.338840 \\
\hline $\mathrm{H}$ & -6.256317 & -4.517744 & -1.991769 \\
\hline $\mathrm{H}$ & -6.487280 & -2.958598 & -2.796187 \\
\hline C & -7.554126 & -2.831328 & -0.229850 \\
\hline $\mathrm{H}$ & -7.690579 & -2.358737 & 0.747785 \\
\hline $\mathrm{H}$ & -8.182231 & -2.298794 & -0.949667 \\
\hline $\mathrm{H}$ & -7.918926 & -3.862105 & -0.165373 \\
\hline C & 6.492330 & -2.368804 & -2.317102 \\
\hline C & 6.141604 & 2.588154 & -1.301593 \\
\hline C & 5.382949 & 3.628489 & -0.460426 \\
\hline $\mathrm{H}$ & 4.311670 & 3.632137 & -0.679777 \\
\hline $\mathrm{H}$ & 5.505821 & 3.441875 & 0.611292 \\
\hline $\mathrm{H}$ & 5.777153 & 4.627703 & -0.671313 \\
\hline C & 5.965933 & 2.947086 & -2.793163 \\
\hline $\mathrm{H}$ & 6.511322 & 2.255149 & -3.441593 \\
\hline $\mathrm{H}$ & 4.909573 & 2.911012 & -3.076773 \\
\hline $\mathrm{H}$ & 6.339006 & 3.958286 & -2.989647 \\
\hline C & 7.637588 & 2.658891 & -0.929067 \\
\hline $\mathrm{H}$ & 7.787639 & 2.398626 & 0.123397 \\
\hline $\mathrm{H}$ & 8.241249 & 1.974809 & -1.532261 \\
\hline H & 8.021916 & 3.672205 & -1.088847 \\
\hline$C$ & 6.409839 & -2.109797 & -3.836868 \\
\hline
\end{tabular}




$\begin{array}{|llll|}\mathrm{H} & 5.367186 & -2.076413 & -4.167974 \\ \mathrm{H} & 6.877608 & -1.160240 & -4.111000 \\ \mathrm{H} & 6.919620 & -2.907008 & -4.389004 \\ \mathrm{C} & 5.869172 & -3.744166 & -2.028933 \\ \mathrm{H} & 6.414930 & -4.518358 & -2.577036 \\ \mathrm{H} & 5.916264 & -3.994369 & -0.964496 \\ \mathrm{H} & 4.822372 & -3.788911 & -2.345036 \\ \mathrm{C} & 7.973341 & -2.409661 & -1.882799 \\ \mathrm{H} & 8.056727 & -2.593378 & -0.807089 \\ \mathrm{H} & 8.504079 & -3.210274 & -2.409631 \\ \mathrm{H} & 8.484920 & -1.468307 & -2.101082 \\ & & \end{array}$

\section{Catalyst 2C 73}

\section{Datum}

Value

\begin{tabular}{lr}
\hline B3LYP-D3(BJ)/6-31G $(d, p)$ Energy & -2165.15807 \\
\hline B3LYP-D3(BJ)/def2-TZVPP/IEF-PCM(DCM) Energy & -2165.896262 \\
\hline B3LYP-D3(BJ)/def2-TZVPP/IEF-PCM(DCM)//B3LYP-D3(BJ)/6-31G(d,p) Free Energy (Quasiharmonic) & -2165.019803 \\
\hline
\end{tabular}

Number of Imaginary Frequencies

Frequencies (Top 3 out of 321)
1. $5.5373 \mathrm{~cm}^{-1}$
2. $10.9979 \mathrm{~cm}^{-1}$
3. $14.3004 \mathrm{~cm}^{-1}$

\section{B3LYP-D3(BJ)/6-31G(d,p) Molecular Geometry in Cartesian Coordinates}

$\begin{array}{llll}\text { C } & -0.515449 & -5.124438 & -2.562547 \\ \text { C } & -0.135556 & -4.140444 & -1.678968 \\ \text { C } & -1.033532 & -3.098790 & -1.327701 \\ \text { C } & -2.338729 & -3.081609 & -1.912608 \\ \text { C } & -2.698432 & -4.111803 & -2.820983 \\ \text { C } & -1.808904 & -5.112050 & -3.137158 \\ \text { H } & 0.181180 & -5.914247 & -2.825136 \\ \text { H } & 0.856299 & -4.142707 & -1.241925 \\ \text { C } & -0.672635 & -2.066159 & -0.409924 \\ \text { C } & -3.228680 & -2.029593 & -1.586816 \\ H & -3.690201 & -4.092617 & -3.263774 \\ H & -2.095087 & -5.894647 & -3.833027 \\ \text { C } & -2.877680 & -1.020998 & -0.712903 \\ C & -1.579956 & -1.062372 & -0.113241 \\ \text { H } & -4.202769 & -2.000398 & -2.065987\end{array}$




\begin{tabular}{|c|c|c|c|}
\hline C & 1.335279 & -4.604891 & 3.622108 \\
\hline C & 2.317622 & -3.737596 & 3.202846 \\
\hline C & 2.103137 & -2.877512 & 2.094963 \\
\hline C & 0.850839 & -2.923153 & 1.406930 \\
\hline C & -0.148303 & -3.817602 & 1.874260 \\
\hline C & 0.090051 & -4.640230 & 2.952243 \\
\hline $\mathrm{H}$ & 3.991727 & -1.835989 & 2.255088 \\
\hline $\mathrm{H}$ & 1.509829 & -5.258911 & 4.470887 \\
\hline $\mathrm{H}$ & 3.273465 & -3.693378 & 3.717627 \\
\hline C & 3.074501 & -1.933082 & 1.680596 \\
\hline C & 0.657916 & -2.073670 & 0.270645 \\
\hline $\mathrm{H}$ & -1.106290 & -3.847084 & 1.368421 \\
\hline $\mathrm{H}$ & -0.684512 & -5.320003 & 3.293726 \\
\hline C & 1.652645 & -1.210604 & -0.155919 \\
\hline C & 2.856179 & -1.094263 & 0.610563 \\
\hline C & 1.488562 & -0.406162 & -1.439632 \\
\hline $\mathrm{H}$ & 0.626525 & -0.782534 & -1.991007 \\
\hline $\mathrm{H}$ & 1.277769 & 0.642917 & -1.203125 \\
\hline C & -1.201132 & -0.026764 & 0.907446 \\
\hline 0 & -0.403985 & 0.877432 & 0.746811 \\
\hline 0 & -1.858857 & -0.211605 & 2.067920 \\
\hline $\mathrm{H}$ & -1.579710 & 0.511014 & 2.656642 \\
\hline 0 & 2.598303 & -0.539226 & -2.318603 \\
\hline $\mathrm{H}$ & 3.339194 & -0.062672 & -1.919813 \\
\hline C & 3.826314 & -0.003221 & 0.322365 \\
\hline C & 5.163820 & -0.263622 & 0.027612 \\
\hline C & 3.375959 & 1.327388 & 0.376310 \\
\hline C & 6.065691 & 0.781401 & -0.232155 \\
\hline $\mathrm{H}$ & 5.491280 & -1.296966 & -0.018290 \\
\hline C & 4.239907 & 2.388104 & 0.115930 \\
\hline $\mathrm{H}$ & 2.339469 & 1.503412 & 0.634545 \\
\hline C & 5.580224 & 2.089290 & -0.187398 \\
\hline $\mathrm{H}$ & 6.256017 & 2.908688 & -0.395195 \\
\hline C & -3.811823 & 0.096416 & -0.431206 \\
\hline C & -5.159064 & -0.158961 & -0.132198 \\
\hline C & -3.360384 & 1.416047 & -0.470068 \\
\hline C & -6.044283 & 0.883419 & 0.137657 \\
\hline H & -5.488516 & -1.189429 & -0.089903 \\
\hline C & -4.214757 & 2.487964 & -0.181650 \\
\hline $\mathrm{H}$ & -2.327329 & 1.609045 & -0.730786 \\
\hline C & -5.546950 & 2.198497 & 0.114534 \\
\hline $\mathrm{H}$ & -6.226282 & 3.010963 & 0.338576 \\
\hline C & -3.645081 & 3.913006 & -0.185893 \\
\hline C & -7.523308 & 0.645929 & 0.474634 \\
\hline C & -3.052687 & 4.227449 & -1.576307 \\
\hline $\mathrm{H}$ & -2.247227 & 3.536819 & -1.839374 \\
\hline $\mathrm{H}$ & -3.821982 & 4.157979 & -2.351755 \\
\hline $\mathrm{H}$ & -2.640612 & 5.242179 & -1.592309 \\
\hline C & -2.529165 & 4.005486 & 0.879609 \\
\hline $\mathrm{H}$ & -2.090303 & 5.009306 & 0.884542 \\
\hline $\mathrm{H}$ & -2.934705 & 3.807641 & 1.877539 \\
\hline $\mathrm{H}$ & -1.726485 & 3.286250 & 0.695510 \\
\hline C & -4.713138 & 4.970150 & 0.134826 \\
\hline $\mathrm{H}$ & -5.149334 & 4.817269 & 1.127046 \\
\hline
\end{tabular}




\begin{tabular}{|c|c|c|c|}
\hline $\mathrm{H}$ & -4.261005 & 5.966513 & 0.121984 \\
\hline $\mathrm{H}$ & -5.523503 & 4.963132 & -0.601151 \\
\hline C & -7.819878 & 1.193539 & 1.887458 \\
\hline $\mathrm{H}$ & -7.204049 & 0.686222 & 2.636542 \\
\hline $\mathrm{H}$ & -7.616047 & 2.265574 & 1.956880 \\
\hline $\mathrm{H}$ & -8.872802 & 1.035046 & 2.144762 \\
\hline C & -7.895356 & -0.845294 & 0.448583 \\
\hline $\mathrm{H}$ & -7.325045 & -1.418222 & 1.186228 \\
\hline $\mathrm{H}$ & -8.957154 & -0.964118 & 0.685009 \\
\hline $\mathrm{H}$ & -7.722889 & -1.287748 & -0.537549 \\
\hline C & -8.405133 & 1.381529 & -0.557516 \\
\hline $\mathrm{H}$ & -8.216622 & 2.458542 & -0.557421 \\
\hline $\mathrm{H}$ & -8.212538 & 1.008340 & -1.568193 \\
\hline $\mathrm{H}$ & -9.465725 & 1.226887 & -0.331410 \\
\hline C & 3.772298 & 3.849732 & 0.139658 \\
\hline C & 7.526583 & 0.449704 & -0.567072 \\
\hline C & 8.152632 & -0.331936 & 0.608013 \\
\hline $\mathrm{H}$ & 8.132681 & 0.264720 & 1.525374 \\
\hline $\mathrm{H}$ & 7.617754 & -1.265260 & 0.803659 \\
\hline $\mathrm{H}$ & 9.195165 & -0.583663 & 0.385519 \\
\hline C & 8.372288 & 1.709641 & -0.811945 \\
\hline $\mathrm{H}$ & 7.996273 & 2.291939 & -1.658939 \\
\hline $\mathrm{H}$ & 8.395899 & 2.359484 & 0.068512 \\
\hline $\mathrm{H}$ & 9.403017 & 1.421658 & -1.039885 \\
\hline C & 7.569725 & -0.418257 & -1.843432 \\
\hline $\mathrm{H}$ & 7.018703 & -1.353866 & -1.715877 \\
\hline $\mathrm{H}$ & 7.129222 & 0.116027 & -2.690994 \\
\hline $\mathrm{H}$ & 8.604867 & -0.670126 & -2.098211 \\
\hline C & 3.979620 & 4.465958 & -1.260947 \\
\hline $\mathrm{H}$ & 5.030292 & 4.439949 & -1.563412 \\
\hline $\mathrm{H}$ & 3.400490 & 3.921194 & -2.013061 \\
\hline $\mathrm{H}$ & 3.653670 & 5.511690 & -1.269133 \\
\hline C & 4.599523 & 4.638427 & 1.177063 \\
\hline $\mathrm{H}$ & 4.279578 & 5.685708 & 1.202000 \\
\hline $\mathrm{H}$ & 4.468769 & 4.216928 & 2.178585 \\
\hline $\mathrm{H}$ & 5.667633 & 4.620927 & 0.943011 \\
\hline C & 2.284508 & 3.977879 & 0.508129 \\
\hline $\mathrm{H}$ & 2.077078 & 3.576087 & 1.504490 \\
\hline $\mathrm{H}$ & 1.996518 & 5.033690 & 0.507944 \\
\hline $\mathrm{H}$ & 1.639697 & 3.456759 & -0.205809 \\
\hline
\end{tabular}

\section{Catalyst 2C 74}

Datum

Value

B3LYP-D3(BJ)/6-31G(d,p) Energy

$-2165.160622$

B3LYP-D3(BJ)/def2-TZVPP/IEF-PCM(DCM) Energy

$-2165.897289$

B3LYP-D3(BJ)/def2-TZVPP/IEF-PCM(DCM)//B3LYP-D3(BJ)/6-31G(d,p) Free Energy (Quasiharmonic) - 
Frequencies (Top 3 out of 321)

$\begin{array}{ll}\text { 1. } & 10.8389 \mathrm{~cm}^{-1} \\ \text { 2. } & 13.1772 \mathrm{~cm}^{-1} \\ \text { 3. } & 14.5983 \mathrm{~cm}^{-1}\end{array}$

B3LYP-D3(BJ)/6-31G(d,p) Molecular Geometry in Cartesian Coordinates

\begin{tabular}{|c|c|c|c|}
\hline C & -0.265013 & 3.557683 & 3.697937 \\
\hline C & -0.369732 & 2.338812 & 3.064921 \\
\hline C & 0.699147 & 1.840473 & 2.269498 \\
\hline C & 1.891483 & 2.621801 & 2.148022 \\
\hline C & 1.967242 & 3.870511 & 2.819843 \\
\hline C & 0.913110 & 4.331657 & 3.573539 \\
\hline $\mathrm{H}$ & -1.088730 & 3.925192 & 4.301534 \\
\hline $\mathrm{H}$ & -1.266724 & 1.739082 & 3.170565 \\
\hline C & 0.614696 & 0.592883 & 1.577294 \\
\hline C & 2.963814 & 2.128670 & 1.367035 \\
\hline $\mathrm{H}$ & 2.875634 & 4.458153 & 2.722851 \\
\hline $\mathrm{H}$ & 0.982044 & 5.290440 & 4.077588 \\
\hline C & 2.891895 & 0.916423 & 0.710606 \\
\hline C & 1.688759 & 0.153032 & 0.825311 \\
\hline $\mathrm{H}$ & 3.876920 & 2.712680 & 1.302851 \\
\hline C & -1.156080 & -3.215027 & 4.574159 \\
\hline C & -2.168387 & -2.948210 & 3.682886 \\
\hline C & -2.016340 & -1.941704 & 2.692854 \\
\hline C & -0.794906 & -1.204082 & 2.631572 \\
\hline C & 0.232840 & -1.505556 & 3.563449 \\
\hline C & 0.055711 & -2.486137 & 4.511687 \\
\hline $\mathrm{H}$ & -3.980698 & -2.185307 & 1.838820 \\
\hline $\mathrm{H}$ & -1.281458 & -3.986967 & 5.327204 \\
\hline $\mathrm{H}$ & -3.101628 & -3.503077 & 3.721326 \\
\hline C & -3.044587 & -1.638722 & 1.771042 \\
\hline C & -0.656776 & -0.194683 & 1.631255 \\
\hline $\mathrm{H}$ & 1.166490 & -0.958066 & 3.510929 \\
\hline $\mathrm{H}$ & 0.851832 & -2.707305 & 5.215719 \\
\hline C & -1.680385 & 0.083581 & 0.733128 \\
\hline C & -2.911341 & -0.650915 & 0.814417 \\
\hline C & -1.480803 & 1.159506 & -0.319242 \\
\hline $\mathrm{H}$ & -0.435681 & 1.173241 & -0.643258 \\
\hline $\mathrm{H}$ & -2.096385 & 0.944546 & -1.192778 \\
\hline C & 1.530745 & -1.129763 & 0.061124 \\
\hline 0 & 1.551517 & -2.243593 & 0.536979 \\
\hline 0 & 1.336993 & -0.896848 & -1.255970 \\
\hline $\mathrm{H}$ & 1.252193 & -1.770318 & -1.676124 \\
\hline
\end{tabular}




\begin{tabular}{|c|c|c|c|}
\hline 0 & -1.885623 & 2.461807 & 0.121144 \\
\hline $\mathrm{H}$ & -1.314470 & 2.711844 & 0.861240 \\
\hline C & -4.053477 & -0.388644 & -0.100763 \\
\hline C & -4.693847 & -1.454409 & -0.744413 \\
\hline C & -4.529063 & 0.910869 & -0.306911 \\
\hline C & -5.790014 & -1.244338 & -1.587057 \\
\hline $\mathrm{H}$ & -4.307649 & -2.457035 & -0.592093 \\
\hline C & -5.611528 & 1.161147 & -1.154674 \\
\hline $\mathrm{H}$ & -4.028400 & 1.729547 & 0.194844 \\
\hline C & -6.229847 & 0.071651 & -1.780299 \\
\hline $\mathrm{H}$ & -7.068727 & 0.252079 & -2.436471 \\
\hline C & 4.040303 & 0.421199 & -0.086616 \\
\hline C & 4.487814 & -0.897001 & 0.062578 \\
\hline C & 4.690963 & 1.273371 & -0.986999 \\
\hline C & 5.556935 & -1.383878 & -0.692759 \\
\hline $\mathrm{H}$ & 3.994077 & -1.535474 & 0.781571 \\
\hline C & 5.774350 & 0.825146 & -1.747068 \\
\hline $\mathrm{H}$ & 4.316396 & 2.282639 & -1.101950 \\
\hline C & 6.182014 & -0.505140 & -1.585007 \\
\hline $\mathrm{H}$ & 7.013561 & -0.870214 & -2.178220 \\
\hline C & 6.508905 & 1.724765 & -2.751402 \\
\hline C & 6.043108 & -2.835383 & -0.579541 \\
\hline C & 6.390205 & 1.114511 & -4.164645 \\
\hline $\mathrm{H}$ & 6.827619 & 0.113402 & -4.210968 \\
\hline $\mathrm{H}$ & 5.341051 & 1.035697 & -4.465838 \\
\hline $\mathrm{H}$ & 6.910750 & 1.742127 & -4.896088 \\
\hline C & 7.998140 & 1.819484 & -2.355257 \\
\hline $\mathrm{H}$ & 8.541013 & 2.454839 & -3.063364 \\
\hline $\mathrm{H}$ & 8.107025 & 2.250721 & -1.355248 \\
\hline $\mathrm{H}$ & 8.479285 & 0.837629 & -2.349744 \\
\hline C & 5.929934 & 3.148179 & -2.791208 \\
\hline $\mathrm{H}$ & 6.002747 & 3.641811 & -1.817002 \\
\hline $\mathrm{H}$ & 6.488449 & 3.752678 & -3.512272 \\
\hline $\mathrm{H}$ & 4.879917 & 3.150263 & -3.099481 \\
\hline C & 5.888611 & -3.523518 & -1.953305 \\
\hline $\mathrm{H}$ & 4.840552 & -3.526280 & -2.268725 \\
\hline $\mathrm{H}$ & 6.470374 & -3.016893 & -2.728547 \\
\hline $\mathrm{H}$ & 6.232155 & -4.562168 & -1.899868 \\
\hline C & 5.235840 & -3.637692 & 0.454656 \\
\hline $\mathrm{H}$ & 4.169661 & -3.664697 & 0.210545 \\
\hline $\mathrm{H}$ & 5.600176 & -4.669131 & 0.484551 \\
\hline $\mathrm{H}$ & 5.342046 & -3.220102 & 1.460807 \\
\hline C & 7.528343 & -2.851425 & -0.160614 \\
\hline $\mathrm{H}$ & 8.160662 & -2.332325 & -0.886529 \\
\hline $\mathrm{H}$ & 7.662631 & -2.363655 & 0.809840 \\
\hline $\mathrm{H}$ & 7.889836 & -3.882098 & -0.078309 \\
\hline C & -6.062082 & 2.612548 & -1.371653 \\
\hline C & -6.455668 & -2.446433 & -2.273763 \\
\hline C & -6.968181 & -3.428198 & -1.197942 \\
\hline $\mathrm{H}$ & -6.154976 & -3.794034 & -0.565105 \\
\hline $\mathrm{H}$ & -7.704418 & -2.942750 & -0.549703 \\
\hline $\mathrm{H}$ & -7.444205 & -4.296001 & -1.667584 \\
\hline C & -5.419892 & -3.161229 & -3.168183 \\
\hline $\mathrm{H}$ & -5.041189 & -2.483326 & -3.939524 \\
\hline
\end{tabular}




\begin{tabular}{|llll|}
$H$ & -4.564743 & -3.519329 & -2.588513 \\
$H$ & -5.874862 & -4.025464 & -3.664619 \\
$\mathrm{C}$ & -7.646976 & -2.034189 & -3.153542 \\
$\mathrm{H}$ & -8.428970 & -1.538381 & -2.570017 \\
$\mathrm{H}$ & -7.341461 & -1.359923 & -3.959645 \\
$\mathrm{H}$ & -8.089062 & -2.923007 & -3.614237 \\
$\mathrm{C}$ & -7.256790 & 2.718441 & -2.332452 \\
$\mathrm{H}$ & -7.016268 & 2.319529 & -3.323021 \\
$\mathrm{H}$ & -8.133322 & 2.184397 & -1.951420 \\
$\mathrm{H}$ & -7.536620 & 3.769013 & -2.457574 \\
$\mathrm{C}$ & -4.883970 & 3.418729 & -1.963479 \\
$\mathrm{H}$ & -5.176869 & 4.464224 & -2.112237 \\
$\mathrm{H}$ & -4.007794 & 3.399245 & -1.310140 \\
$\mathrm{H}$ & -4.587523 & 3.008228 & -2.934382 \\
$\mathrm{C}$ & -6.473393 & 3.227868 & -0.016786 \\
$\mathrm{H}$ & -5.643597 & 3.223676 & 0.694734 \\
$\mathrm{H}$ & -6.794217 & 4.266599 & -0.152415 \\
$\mathrm{H}$ & -7.302512 & 2.668884 & 0.428867 \\
\hline
\end{tabular}

\section{Catalyst 2C 75}

\section{Datum}

Value

B3LYP-D3(BJ)/6-31G(d,p) Energy

$-2165.160699$

B3LYP-D3(BJ)/def2-TZVPP/IEF-PCM(DCM) Energy

$-2165.897298$

B3LYP-D3(BJ)/def2-TZVPP/IEF-PCM(DCM)//B3LYP-D3(BJ)/6-31G(d,p) Free Energy (Quasiharmonic)

$-2165.019604$

Number of Imaginary Frequencies

Frequencies (Top 3 out of 321)

$\begin{array}{ll}\text { 1. } & 11.3956 \mathrm{~cm}^{-1} \\ \text { 2. } & 12.9174 \mathrm{~cm}^{-1} \\ \text { 3. } & 14.1955 \mathrm{~cm}^{-1}\end{array}$

B3LYP-D3(BJ)/6-31G(d,p) Molecular Geometry in Cartesian Coordinates

C

C

C

C

C

C

$\mathrm{H}$

$\mathrm{H}$

-0.107316
-0.242272
0.806759
2.018016
2.122700
1.083829
-0.917684
-1.149423

$-4.912604$

$-3.730798$

$-2.769425$

$-3.066889$

$-4.294612$

$-5.196049$

$-5.634552$

$-3.521406$
$-1.173972$

$-0.479135$

$-0.469781$

$-1.170686$

$-1.876145$

$-1.882900$

$-1.173080$

0.076126 


\begin{tabular}{|c|c|c|c|}
\hline C & 0.693198 & -1.523831 & 0.223878 \\
\hline C & 3.074614 & -2.125568 & -1.153491 \\
\hline $\mathrm{H}$ & 3.042620 & -4.507912 & -2.412924 \\
\hline $\mathrm{H}$ & 1.176192 & -6.130226 & -2.427724 \\
\hline C & 2.975685 & -0.931687 & -0.469224 \\
\hline C & 1.762921 & -0.644536 & 0.231486 \\
\hline $\mathrm{H}$ & 3.977694 & -2.340187 & -1.716817 \\
\hline C & -0.785275 & -2.001636 & 5.144100 \\
\hline C & -1.872632 & -1.482844 & 4.481982 \\
\hline C & -1.814508 & -1.215767 & 3.088286 \\
\hline C & -0.607921 & -1.495286 & 2.374254 \\
\hline C & 0.497444 & -2.032992 & 3.087603 \\
\hline C & 0.410360 & -2.277599 & 4.438374 \\
\hline $\mathrm{H}$ & -3.843839 & -0.509474 & 2.924527 \\
\hline $\mathrm{H}$ & -0.839041 & -2.200248 & 6.210065 \\
\hline $\mathrm{H}$ & -2.795202 & -1.268065 & 5.014025 \\
\hline C & -2.920825 & -0.692230 & 2.382100 \\
\hline C & -0.565436 & -1.218025 & 0.973592 \\
\hline $\mathrm{H}$ & 1.419562 & -2.237392 & 2.556673 \\
\hline $\mathrm{H}$ & 1.266011 & -2.682760 & 4.969221 \\
\hline C & -1.662754 & -0.691131 & 0.303822 \\
\hline C & -2.876499 & -0.435731 & 1.024492 \\
\hline C & -1.543517 & -0.357186 & -1.172096 \\
\hline $\mathrm{H}$ & -2.214628 & 0.465342 & -1.420358 \\
\hline $\mathrm{H}$ & -0.521117 & -0.032159 & -1.388833 \\
\hline C & 1.685964 & 0.576511 & 1.100744 \\
\hline 0 & 2.332410 & 0.741106 & 2.108713 \\
\hline 0 & 0.792339 & 1.489086 & 0.642718 \\
\hline $\mathrm{H}$ & 0.777313 & 2.204794 & 1.301696 \\
\hline 0 & -1.922122 & -1.433196 & -2.038980 \\
\hline $\mathrm{H}$ & -1.286218 & -2.151366 & -1.912001 \\
\hline C & -4.092006 & 0.107618 & 0.363134 \\
\hline C & -4.786634 & 1.176266 & 0.943030 \\
\hline C & -4.581132 & -0.453894 & -0.821265 \\
\hline C & -5.950398 & 1.690257 & 0.362678 \\
\hline $\mathrm{H}$ & -4.390645 & 1.618135 & 1.851592 \\
\hline C & -5.731495 & 0.044025 & -1.439278 \\
\hline $\mathrm{H}$ & -4.037815 & -1.278883 & -1.264537 \\
\hline C & -6.403008 & 1.110862 & -0.829864 \\
\hline $\mathrm{H}$ & -7.294688 & 1.502602 & -1.296980 \\
\hline C & 4.088656 & 0.047329 & -0.502684 \\
\hline C & 3.835069 & 1.392739 & -0.786060 \\
\hline C & 5.403918 & -0.363455 & -0.268383 \\
\hline C & 4.864755 & 2.335863 & -0.809793 \\
\hline $\mathrm{H}$ & 2.816932 & 1.698947 & -0.997254 \\
\hline C & 6.464133 & 0.548002 & -0.300591 \\
\hline $\mathrm{H}$ & 5.586100 & -1.405965 & -0.029724 \\
\hline C & 6.170409 & 1.891399 & -0.567661 \\
\hline $\mathrm{H}$ & 6.977272 & 2.609055 & -0.583347 \\
\hline C & 7.892108 & 0.054766 & -0.024969 \\
\hline C & 4.525017 & 3.805571 & -1.094036 \\
\hline C & 8.263067 & -1.033287 & -1.055753 \\
\hline $\mathrm{H}$ & 7.583003 & -1.888019 & -1.004104 \\
\hline $\mathrm{H}$ & 8.222666 & -0.633695 & -2.073933 \\
\hline
\end{tabular}




\begin{tabular}{|c|c|c|c|}
\hline $\mathrm{H}$ & 9.278127 & -1.401906 & -0.872449 \\
\hline C & 7.957414 & -0.541485 & 1.397804 \\
\hline $\mathrm{H}$ & 8.969077 & -0.901831 & 1.614108 \\
\hline H & 7.693758 & 0.211920 & 2.146292 \\
\hline $\mathrm{H}$ & 7.268980 & -1.382668 & 1.515296 \\
\hline C & 8.931784 & 1.182887 & -0.122988 \\
\hline $\mathrm{H}$ & 8.745137 & 1.971626 & 0.612343 \\
\hline $\mathrm{H}$ & 9.930373 & 0.779576 & 0.070746 \\
\hline $\mathrm{H}$ & 8.944339 & 1.637375 & -1.118647 \\
\hline C & 5.759664 & 4.717646 & -1.018310 \\
\hline $\mathrm{H}$ & 6.226486 & 4.685054 & -0.028897 \\
\hline H & 6.513367 & 4.442603 & -1.762769 \\
\hline $\mathrm{H}$ & 5.464887 & 5.753224 & -1.213405 \\
\hline C & 3.496061 & 4.295145 & -0.050691 \\
\hline $\mathrm{H}$ & 3.898657 & 4.204816 & 0.962851 \\
\hline $\mathrm{H}$ & 3.242860 & 5.345750 & -0.229510 \\
\hline $\mathrm{H}$ & 2.570333 & 3.714875 & -0.095826 \\
\hline C & 3.917413 & 3.923907 & -2.508253 \\
\hline $\mathrm{H}$ & 4.628915 & 3.581681 & -3.266240 \\
\hline H & 3.008302 & 3.324475 & -2.607506 \\
\hline $\mathrm{H}$ & 3.658699 & 4.965692 & -2.726843 \\
\hline C & -6.195644 & -0.587236 & -2.759440 \\
\hline C & -6.674287 & 2.865927 & 1.036126 \\
\hline C & -7.092081 & 2.455877 & 2.464859 \\
\hline $\mathrm{H}$ & -7.769185 & 1.596374 & 2.438876 \\
\hline $\mathrm{H}$ & -6.227262 & 2.181906 & 3.075354 \\
\hline $\mathrm{H}$ & -7.607302 & 3.283217 & 2.965148 \\
\hline C & -7.937299 & 3.293457 & 0.271219 \\
\hline $\mathrm{H}$ & -7.702522 & 3.629227 & -0.743608 \\
\hline $\mathrm{H}$ & -8.664637 & 2.478525 & 0.202848 \\
\hline $\mathrm{H}$ & -8.419076 & 4.126171 & 0.792914 \\
\hline C & -5.720893 & 4.078180 & 1.108983 \\
\hline $\mathrm{H}$ & -4.818858 & 3.848446 & 1.682711 \\
\hline $\mathrm{H}$ & -5.409879 & 4.387044 & 0.106108 \\
\hline $\mathrm{H}$ & -6.218117 & 4.927437 & 1.590516 \\
\hline C & -5.073139 & -0.430009 & -3.809899 \\
\hline $\mathrm{H}$ & -4.868077 & 0.629345 & -3.996374 \\
\hline $\mathrm{H}$ & -4.141169 & -0.898893 & -3.483611 \\
\hline $\mathrm{H}$ & -5.374385 & -0.889363 & -4.758184 \\
\hline C & -7.472099 & 0.070316 & -3.307282 \\
\hline $\mathrm{H}$ & -7.759542 & -0.410806 & -4.247249 \\
\hline $\mathrm{H}$ & -8.311872 & -0.030666 & -2.612080 \\
\hline $\mathrm{H}$ & -7.323100 & 1.135129 & -3.512585 \\
\hline C & -6.481058 & -2.087969 & -2.536456 \\
\hline $\mathrm{H}$ & -7.267385 & -2.227721 & -1.787677 \\
\hline $\mathrm{H}$ & -6.810845 & -2.555227 & -3.470878 \\
\hline $\mathrm{H}$ & -5.590156 & -2.619677 & -2.192444 \\
\hline
\end{tabular}


Frequencies (Top 3 out of 321)

$\begin{array}{lr}\text { 1. } & 4.0791 \mathrm{~cm}^{-1} \\ \text { 2. } & 11.1951 \mathrm{~cm}^{-1} \\ \text { 3. } & 14.0891 \mathrm{~cm}^{-1}\end{array}$

\section{B3LYP-D3(BJ)/6-31G(d,p) Molecular Geometry in Cartesian Coordinates}

C

C

C

C

C

C

$\mathrm{H}$

$\mathrm{H}$

C

C

$\mathrm{H}$

$\mathrm{H}$

C

C

$\mathrm{H}$

C

C

C

C

C

C

$\mathrm{H}$

$\mathrm{H}$

$\mathrm{H}$

C

C

$\mathrm{H}$

$\mathrm{H}$

C

C

C

$\mathrm{H}$
$-0.575753$

$-0.187245$

$-1.074928$

$-2.378808$

$-2.747523$

$-1.867863$

0.113055

0.803656

$-0.704608$

$-3.258578$

$-3.738204$

$-2.160863$

$-2.898955$

$-1.602259$

$-4.231454$

1. 268072

2. 262863

2.060075

0.806497

$-0.205387$

0.021746

3.964879

1.433667

3. 219865

3.045151

0.625419

$-1.164244$

$-0.762525$

1.632142

2.838604

1.473532

0.614057
$-5.094201$

$-4.118973$

$-3.069856$

$-3.036140$

$-4.057451$

$-5.065199$

$-5.889893$

$-4.134107$

$-2.045633$

$-1.977028$

$-4.025485$

$-5.840979$

$-0.977772$

$-1.034614$

$-1.934647$

$-4.624288$

$-3.770421$

$-2.903334$

$-2.927710$

$-3.808937$

$-4.638731$

$-1.892720$

$-5.283777$

$-3.742416$

$-1.972856$

$-2.071460$

$-3.822359$

$-5.308047$

$-1.221299$

$-1.126170$

$-0.408424$

$-0.782658$
$-2.582183$

$-1.692627$

$-1.337374$

$-1.924438$

$-2.839146$

$-3.159153$

$-2.847627$

$-1.253707$

$-0.413763$

$-1.593948$

$-3.283635$

$-3.859799$

$-0.713028$

$-0.112437$

$-2.074629$

3. 609197

3. 191822

2.087146

1.400504

1.865624

2.940511

2.247303

4.455535

3. 705586

1.674214

0. 267522

1. 360732

3. 280494

$-0.157238$

0.607751

$-1.436705$

$-1.993645$ 


\begin{tabular}{|c|c|c|c|}
\hline $\mathrm{H}$ & 1.258759 & 0.638410 & -1.193431 \\
\hline C & -1.214618 & -0.007552 & 0.913446 \\
\hline 0 & -0.404895 & 0.886522 & 0.759581 \\
\hline 0 & -1.881184 & -0.186925 & 2.069782 \\
\hline $\mathrm{H}$ & -1.596346 & 0.530659 & 2.661911 \\
\hline 0 & 2.585881 & -0.532482 & -2.313213 \\
\hline $\mathrm{H}$ & 3.322397 & -0.052180 & -1.911054 \\
\hline C & 3.827993 & -0.051731 & 0.322737 \\
\hline C & 5.166592 & -0.342124 & 0.037307 \\
\hline C & 3.402479 & 1.283325 & 0.370621 \\
\hline C & 6.084503 & 0.683111 & -0.218507 \\
\hline $\mathrm{H}$ & 5.468100 & -1.381330 & -0.001680 \\
\hline C & 4.289103 & 2.330802 & 0.112068 \\
\hline $\mathrm{H}$ & 2.368191 & 1.480244 & 0.622532 \\
\hline C & 5.619583 & 2.003636 & -0.180534 \\
\hline $\mathrm{H}$ & 6.318582 & 2.806494 & -0.388870 \\
\hline C & -3.823606 & 0.146067 & -0.425569 \\
\hline C & -3.360002 & 1.465413 & -0.458578 \\
\hline C & -5.168786 & -0.093938 & -0.128488 \\
\hline C & -4.203736 & 2.540140 & -0.167778 \\
\hline $\mathrm{H}$ & -2.324808 & 1.649435 & -0.717549 \\
\hline C & -6.049794 & 0.957149 & 0.145247 \\
\hline $\mathrm{H}$ & -5.514251 & -1.121560 & -0.087579 \\
\hline C & -5.544348 & 2.264128 & 0.127372 \\
\hline $\mathrm{H}$ & -6.209887 & 3.084980 & 0.350669 \\
\hline C & -7.517810 & 0.645294 & 0.472016 \\
\hline C & -3.623175 & 3.961338 & -0.166767 \\
\hline C & -8.153128 & -0.112322 & -0.713858 \\
\hline $\mathrm{H}$ & -8.118552 & 0.493549 & -1.624675 \\
\hline $\mathrm{H}$ & -7.633787 & -1.052749 & -0.917524 \\
\hline $\mathrm{H}$ & -9.200970 & -0.348129 & -0.498636 \\
\hline C & -8.344881 & 1.915562 & 0.728167 \\
\hline $\mathrm{H}$ & -9.381493 & 1.641089 & 0.946180 \\
\hline $\mathrm{H}$ & -7.965161 & 2.481245 & 1.584678 \\
\hline $\mathrm{H}$ & -8.352605 & 2.577021 & -0.143795 \\
\hline C & -7.581381 & -0.236418 & 1.737917 \\
\hline $\mathrm{H}$ & -7.134584 & 0.280357 & 2.592942 \\
\hline H & -8.621737 & -0.473557 & 1.985928 \\
\hline $\mathrm{H}$ & -7.045621 & -1.179601 & 1.601041 \\
\hline C & -2.508679 & 4.042382 & 0.901173 \\
\hline $\mathrm{H}$ & -2.917511 & 3.844338 & 1.897725 \\
\hline $\mathrm{H}$ & -1.711151 & 3.317657 & 0.716151 \\
\hline $\mathrm{H}$ & -2.062347 & 5.042901 & 0.910247 \\
\hline C & -4.683129 & 5.026557 & 0.154855 \\
\hline $\mathrm{H}$ & -5.122717 & 4.875015 & 1.145741 \\
\hline $\mathrm{H}$ & -4.222286 & 6.018993 & 0.145562 \\
\hline $\mathrm{H}$ & -5.492165 & 5.028728 & -0.582561 \\
\hline C & -3.025446 & 4.275726 & -1.554951 \\
\hline $\mathrm{H}$ & -2.224338 & 3.580137 & -1.818213 \\
\hline $\mathrm{H}$ & -3.793454 & 4.213835 & -2.332321 \\
\hline $\mathrm{H}$ & -2.606270 & 5.287622 & -1.567220 \\
\hline C & 3.847081 & 3.800644 & 0.128412 \\
\hline C & 7.558369 & 0.409133 & -0.550632 \\
\hline C & 7.888891 & -1.092050 & -0.538018 \\
\hline
\end{tabular}




\begin{tabular}{|c|c|c|c|}
\hline $\mathrm{H}$ & 7.701484 & -1.539332 & 0.443161 \\
\hline $\mathrm{H}$ & 7.305400 & -1.641836 & -1.282825 \\
\hline $\mathrm{H}$ & 8.947720 & -1.237498 & -0.772730 \\
\hline C & 8.454907 & 1.110699 & 0.492250 \\
\hline $\mathrm{H}$ & 8.295369 & 2.192385 & 0.501873 \\
\hline $\mathrm{H}$ & 8.248097 & 0.733208 & 1.498513 \\
\hline $\mathrm{H}$ & 9.511869 & 0.929732 & 0.268944 \\
\hline C & 7.874260 & 0.962566 & -1.956984 \\
\hline $\mathrm{H}$ & 7.248063 & 0.479485 & -2.713464 \\
\hline $\mathrm{H}$ & 7.699184 & 2.040307 & -2.016420 \\
\hline $\mathrm{H}$ & 8.923403 & 0.778483 & -2.212454 \\
\hline C & 4.682826 & 4.577831 & 1.167538 \\
\hline $\mathrm{H}$ & 4.539339 & 4.162252 & 2.169771 \\
\hline $\mathrm{H}$ & 5.751671 & 4.539838 & 0.939270 \\
\hline $\mathrm{H}$ & 4.382008 & 5.630868 & 1.187248 \\
\hline C & 2.359916 & 3.956219 & 0.488655 \\
\hline $\mathrm{H}$ & 2.090853 & 5.016985 & 0.484268 \\
\hline $\mathrm{H}$ & 1.709725 & 3.445051 & -0.227619 \\
\hline $\mathrm{H}$ & 2.139959 & 3.560820 & 1.484885 \\
\hline C & 4.072725 & 4.407913 & -1.273192 \\
\hline $\mathrm{H}$ & 3.488610 & 3.870403 & -2.026664 \\
\hline $\mathrm{H}$ & 3.765075 & 5.459107 & -1.287224 \\
\hline $\mathrm{H}$ & 5.124473 & 4.362273 & -1.569607 \\
\hline
\end{tabular}

\section{Catalyst 2C 77}

\section{Datum}

Value

B3LYP-D3(BJ)/6-31G(d,p) Energy

$-2165.161$

B3LYP-D3(BJ)/def2-TZVPP/IEF-PCM(DCM) Energy

$-2165.897264$

B3LYP-D3(BJ)/def2-TZVPP/IEF-PCM(DCM)//B3LYP-D3(BJ)/6-31G(d,p) Free Energy (Quasiharmonic)

$-2165.019554$

Number of Imaginary Frequencies

Frequencies (Top 3 out of 321)
1. $10.7574 \mathrm{~cm}^{-1}$
2. $12.7772 \mathrm{~cm}^{-1}$
3. $14.4231 \mathrm{~cm}^{-1}$

\section{B3LYP-D3(BJ)/6-31G(d,p) Molecular Geometry in Cartesian Coordinates}

$\begin{array}{lrrr}C & -0.212085 & 3.449104 & 3.794609 \\ C & -0.327710 & 2.249882 & 3.126946 \\ C & 0.735457 & 1.766657 & 2.314730\end{array}$




\begin{tabular}{|c|c|c|c|}
\hline C & 1.933282 & 2.542307 & 2.212398 \\
\hline C & 2.020394 & 3.770311 & 2.920044 \\
\hline C & 0.971695 & 4.217319 & 3.689667 \\
\hline H & -1.031518 & 3.805305 & 4.410686 \\
\hline $\mathrm{H}$ & -1.229005 & 1.654158 & 3.217630 \\
\hline C & 0.640296 & 0.539674 & 1.588068 \\
\hline C & 2.999232 & 2.064688 & 1.413275 \\
\hline $\mathrm{H}$ & 2.932999 & 4.353638 & 2.837655 \\
\hline H & 1.049238 & 5.160476 & 4.221189 \\
\hline C & 2.915907 & 0.873338 & 0.720935 \\
\hline C & 1.708421 & 0.114462 & 0.819391 \\
\hline H & 3.916272 & 2.643889 & 1.362977 \\
\hline C & -1.145841 & -3.331885 & 4.492811 \\
\hline C & -2.158255 & -3.037559 & 3.610356 \\
\hline C & -2.002639 & -2.007344 & 2.645593 \\
\hline C & -0.777401 & -1.274893 & 2.600492 \\
\hline C & 0.250346 & -1.604933 & 3.522636 \\
\hline C & 0.069648 & -2.607958 & 4.446417 \\
\hline $\mathrm{H}$ & -3.969821 & -2.219002 & 1.789505 \\
\hline H & -1.273991 & -4.121771 & 5.226526 \\
\hline $\mathrm{H}$ & -3.094325 & -3.588348 & 3.636610 \\
\hline C & -3.030935 & -1.675842 & 1.733688 \\
\hline C & -0.635620 & -0.241643 & 1.625379 \\
\hline $\mathrm{H}$ & 1.186740 & -1.061081 & 3.482198 \\
\hline $\mathrm{H}$ & 0.865709 & -2.850717 & 5.143374 \\
\hline C & -1.659459 & 0.064739 & 0.736720 \\
\hline C & -2.894237 & -0.664910 & 0.802043 \\
\hline C & -1.456484 & 1.166375 & -0.287988 \\
\hline $\mathrm{H}$ & -0.412246 & 1.182662 & -0.614705 \\
\hline H & -2.075792 & 0.977945 & -1.164977 \\
\hline C & 1.541453 & -1.148472 & 0.024780 \\
\hline 0 & 1.558839 & -2.272897 & 0.476458 \\
\hline 0 & 1.348611 & -0.885885 & -1.285987 \\
\hline H & 1.265313 & -1.749458 & -1.726573 \\
\hline 0 & -1.852389 & 2.458982 & 0.187713 \\
\hline H & -1.277699 & 2.686114 & 0.932433 \\
\hline C & -4.036696 & -0.373411 & -0.103840 \\
\hline C & -4.683715 & -1.419062 & -0.773266 \\
\hline C & -4.505958 & 0.933375 & -0.275652 \\
\hline C & -5.780377 & -1.181934 & -1.608052 \\
\hline $\mathrm{H}$ & -4.302394 & -2.427204 & -0.647400 \\
\hline C & -5.588705 & 1.210782 & -1.114585 \\
\hline $\mathrm{H}$ & -4.000294 & 1.736316 & 0.246118 \\
\hline C & -6.213776 & 0.140800 & -1.766659 \\
\hline $\mathrm{H}$ & -7.052988 & 0.342263 & -2.416261 \\
\hline C & 4.056513 & 0.396983 & -0.098883 \\
\hline C & 4.491198 & -0.929532 & -0.010846 \\
\hline C & 4.716410 & 1.273380 & -0.966792 \\
\hline C & 5.549356 & -1.399429 & -0.793359 \\
\hline $\mathrm{H}$ & 3.994890 & -1.598791 & 0.680839 \\
\hline C & 5.792479 & 0.845318 & -1.750159 \\
\hline H & 4.357212 & 2.294179 & -1.043435 \\
\hline C & 6.187166 & -0.495573 & -1.651528 \\
\hline H & 7.008389 & -0.844348 & -2.260101 \\
\hline
\end{tabular}




\begin{tabular}{|c|c|c|c|}
\hline C & 6.481975 & 1.840956 & -2.694696 \\
\hline C & 5.949518 & -2.877995 & -0.693374 \\
\hline C & 5.455053 & 2.359760 & -3.724433 \\
\hline $\mathrm{H}$ & 5.051658 & 1.534396 & -4.319189 \\
\hline $\mathrm{H}$ & 4.614529 & 2.863200 & -3.239182 \\
\hline $\mathrm{H}$ & 5.927041 & 3.075248 & -4.406562 \\
\hline C & 7.654366 & 1.207220 & -3.460878 \\
\hline $\mathrm{H}$ & 8.116783 & 1.956941 & -4.110050 \\
\hline $\mathrm{H}$ & 8.427231 & 0.833021 & -2.782214 \\
\hline $\mathrm{H}$ & 7.324164 & 0.378773 & -4.095212 \\
\hline C & 7.028917 & 3.027777 & -1.872325 \\
\hline $\mathrm{H}$ & 7.758671 & 2.684081 & -1.132564 \\
\hline $\mathrm{H}$ & 7.522299 & 3.751927 & -2.529570 \\
\hline $\mathrm{H}$ & 6.231230 & 3.551048 & -1.337984 \\
\hline C & 4.742201 & -3.747715 & -1.111062 \\
\hline $\mathrm{H}$ & 3.866923 & -3.560679 & -0.483093 \\
\hline H & 4.461818 & -3.540549 & -2.149263 \\
\hline $\mathrm{H}$ & 4.994089 & -4.810991 & -1.033084 \\
\hline C & 6.341467 & -3.209241 & 0.762559 \\
\hline $\mathrm{H}$ & 5.514575 & -3.032014 & 1.455172 \\
\hline $\mathrm{H}$ & 6.627834 & -4.262976 & 0.847800 \\
\hline $\mathrm{H}$ & 7.189182 & -2.596904 & 1.085920 \\
\hline C & 7.137698 & -3.226262 & -1.603780 \\
\hline $\mathrm{H}$ & 6.908339 & -3.043102 & -2.658245 \\
\hline $\mathrm{H}$ & 8.031526 & -2.650948 & -1.342050 \\
\hline $\mathrm{H}$ & 7.382962 & -4.287398 & -1.498302 \\
\hline C & -6.032398 & 2.669525 & -1.293305 \\
\hline C & -6.453737 & -2.362819 & -2.323441 \\
\hline C & -7.643918 & -1.922206 & -3.190894 \\
\hline $\mathrm{H}$ & -8.422412 & -1.436853 & -2.594050 \\
\hline H & -7.335749 & -1.229707 & -3.980364 \\
\hline $\mathrm{H}$ & -8.091514 & -2.796825 & -3.672961 \\
\hline C & -6.970263 & -3.367939 & -1.271334 \\
\hline $\mathrm{H}$ & -6.158253 & -3.753488 & -0.648727 \\
\hline $\mathrm{H}$ & -7.703094 & -2.894622 & -0.610386 \\
\hline $\mathrm{H}$ & -7.451494 & -4.221443 & -1.761429 \\
\hline C & -5.423329 & -3.061194 & -3.236820 \\
\hline $\mathrm{H}$ & -5.041758 & -2.366645 & -3.991765 \\
\hline H & -4.569475 & -3.438505 & -2.667513 \\
\hline $\mathrm{H}$ & -5.884005 & -3.910242 & -3.753765 \\
\hline C & -6.438912 & 3.251519 & 0.077626 \\
\hline $\mathrm{H}$ & -7.270363 & 2.685377 & 0.509697 \\
\hline $\mathrm{H}$ & -5.608292 & 3.224543 & 0.787672 \\
\hline $\mathrm{H}$ & -6.754550 & 4.295059 & -0.030488 \\
\hline C & -7.227853 & 2.806000 & -2.249313 \\
\hline $\mathrm{H}$ & -7.502685 & 3.860791 & -2.346923 \\
\hline H & -6.990666 & 2.431553 & -3.250185 \\
\hline $\mathrm{H}$ & -8.106469 & 2.266610 & -1.880828 \\
\hline C & -4.851144 & 3.485041 & -1.865778 \\
\hline $\mathrm{H}$ & -4.557674 & 3.098135 & -2.847212 \\
\hline $\mathrm{H}$ & -5.139264 & 4.535363 & -1.987366 \\
\hline $\mathrm{H}$ & -3.974345 & 3.444773 & -1.214232 \\
\hline
\end{tabular}




\section{Catalyst 2C 78}

Datum

Value

B3LYP-D3(BJ)/6-31G(d,p) Energy

$-2165.157842$

B3LYP-D3(BJ)/def2-TZVPP/IEF-PCM(DCM) Energy

$-2165.896024$

B3LYP-D3(BJ)/def2-TZVPP/IEF-PCM(DCM)//B3LYP-D3(BJ)/6-31G(d,p) Free Energy (Quasiharmonic)

$-2165.019509$

Number of Imaginary Frequencies

Frequencies (Top 3 out of 321)

$\begin{array}{lr}\text { 1. } & 5.6124 \mathrm{~cm}^{-1} \\ \text { 2. } & 11.1043 \mathrm{~cm}^{-1} \\ \text { 3. } & 14.1181 \mathrm{~cm}^{-1}\end{array}$

B3LYP-D3(BJ)/6-31G(d,p) Molecular Geometry in Cartesian Coordinates

C

C

C

C

C

C

$\mathrm{H}$

$\mathrm{H}$

C

C

$\mathrm{H}$

$\mathrm{H}$

C

C

$\mathrm{H}$

C

C

C

C

C

C

$\mathrm{H}$

$\mathrm{H}$

$\mathrm{H}$

C

C

$\mathrm{H}$

$\mathrm{H}$

C
0.548019

0.162852

1.054771

2. 359351

2. 724553

1.840863

$-0.144012$

$-0.828642$

0.688018

3.243196

3.715775

2.131198

2.886855

1.589574

4.216591

$-1.303113$

$-2.291417$

$-2.082657$

$-0.829820$

0.175417

$-0.057469$

$-3.978892$

$-1.473304$

$-3.247718$

$-3.060645$

$-0.642512$

1.133744

0.721749

$-1.643116$
$-5.103144$

$-4.123219$

$-3.076836$

$-3.050652$

$-4.076709$

$-5.081741$

$-5.896655$

$-4.132354$

$-2.047978$

$-1.993940$

$-4.050521$

$-5.861164$

$-0.989919$

$-1.039499$

$-1.957242$

$-4.604381$

$-3.743620$

$-2.880911$

$-2.916953$

$-3.804902$

$-4.630291$

$-1.853401$

$-5.260460$

$-3.706685$

$-1.943161$

$-2.064878$

$-3.827173$

$-5.304952$

$-1.208376$
$-2.568106$

$-1.682284$

$-1.329617$

$-1.915553$

$-2.826360$

$-3.143782$

$-2.831686$

$-1.244534$

$-0.409840$

$-1.588205$

$-3.270018$

$-3.841488$

$-0.711373$

$-0.111445$

$-2.068308$

3. 619162

3. 200450

2.093516

1.405890

1.872573

2. 949638

2. 253647

4.467235

3. 714952

1.679596

0.270625

1. 366997

3. 290668

$-0.155385$ 


\begin{tabular}{|c|c|c|c|}
\hline C & -2.847911 & -1.101703 & 0.610507 \\
\hline C & -1.483970 & -0.401026 & -1.437866 \\
\hline $\mathrm{H}$ & -1.281328 & 0.649288 & -1.199734 \\
\hline $\mathrm{H}$ & -0.618597 & -0.770063 & -1.988967 \\
\hline C & 1.205277 & -0.006818 & 0.910070 \\
\hline 0 & 0.399337 & 0.889920 & 0.751825 \\
\hline 0 & 1.870013 & -0.184234 & 2.067723 \\
\hline $\mathrm{H}$ & 1.587690 & 0.536992 & 2.656625 \\
\hline 0 & -2.591880 & -0.541284 & -2.318027 \\
\hline $\mathrm{H}$ & -3.336701 & -0.070863 & -1.919268 \\
\hline C & -3.826180 & -0.017858 & 0.322619 \\
\hline C & -5.161378 & -0.288358 & 0.026462 \\
\hline C & -3.386228 & 1.316146 & 0.378248 \\
\hline C & -6.071129 & 0.749884 & -0.233033 \\
\hline $\mathrm{H}$ & -5.480753 & -1.324173 & -0.020700 \\
\hline C & -4.258155 & 2.370376 & 0.118097 \\
\hline $\mathrm{H}$ & -2.351374 & 1.499957 & 0.637617 \\
\hline C & -5.595848 & 2.061452 & -0.186644 \\
\hline $\mathrm{H}$ & -6.277783 & 2.875796 & -0.394245 \\
\hline C & 3.815266 & 0.131740 & -0.427484 \\
\hline C & 5.159314 & -0.111623 & -0.128063 \\
\hline C & 3.356052 & 1.452491 & -0.465752 \\
\hline C & 6.043387 & 0.937571 & 0.143125 \\
\hline $\mathrm{H}$ & 5.501389 & -1.140216 & -0.083174 \\
\hline C & 4.202826 & 2.525469 & -0.177415 \\
\hline $\mathrm{H}$ & 2.321809 & 1.639013 & -0.726684 \\
\hline C & 5.542169 & 2.246110 & 0.120335 \\
\hline $\mathrm{H}$ & 6.210061 & 3.065542 & 0.341806 \\
\hline C & 3.626694 & 3.948473 & -0.181640 \\
\hline C & 7.510040 & 0.622141 & 0.472595 \\
\hline C & 3.031912 & 4.260414 & -1.571637 \\
\hline $\mathrm{H}$ & 3.800811 & 4.193742 & -2.347730 \\
\hline $\mathrm{H}$ & 2.229013 & 3.566500 & -1.833865 \\
\hline $\mathrm{H}$ & 2.615881 & 5.273554 & -1.587620 \\
\hline C & 4.689517 & 5.011348 & 0.138244 \\
\hline $\mathrm{H}$ & 4.231800 & 6.005187 & 0.125257 \\
\hline $\mathrm{H}$ & 5.127209 & 4.861454 & 1.130220 \\
\hline H & 5.499609 & 5.008725 & -0.598011 \\
\hline C & 2.510891 & 4.036323 & 0.884421 \\
\hline $\mathrm{H}$ & 2.917631 & 3.840034 & 1.882182 \\
\hline $\mathrm{H}$ & 2.067748 & 5.038284 & 0.889790 \\
\hline $\mathrm{H}$ & 1.711294 & 3.313608 & 0.700478 \\
\hline C & 8.340714 & 1.890590 & 0.726109 \\
\hline $\mathrm{H}$ & 8.351650 & 2.549470 & -0.147768 \\
\hline $\mathrm{H}$ & 7.961626 & 2.459953 & 1.580459 \\
\hline $\mathrm{H}$ & 9.376184 & 1.613548 & 0.946289 \\
\hline C & 8.144409 & -0.140824 & -0.710349 \\
\hline $\mathrm{H}$ & 8.112793 & 0.462553 & -1.622928 \\
\hline $\mathrm{H}$ & 9.191257 & -0.379282 & -0.493212 \\
\hline $\mathrm{H}$ & 7.622358 & -1.080195 & -0.911949 \\
\hline C & 7.569430 & -0.256106 & 1.741106 \\
\hline $\mathrm{H}$ & 7.123144 & 0.264460 & 2.594095 \\
\hline $\mathrm{H}$ & 7.031046 & -1.198092 & 1.606312 \\
\hline H & 8.608774 & -0.495620 & 1.991065 \\
\hline
\end{tabular}




\begin{tabular}{|c|c|c|c|}
\hline C & -3.801939 & 3.835574 & 0.143479 \\
\hline C & -7.529179 & 0.407245 & -0.569329 \\
\hline C & -8.384325 & 1.660860 & -0.813895 \\
\hline $\mathrm{H}$ & -8.413654 & 2.309796 & 0.067060 \\
\hline $\mathrm{H}$ & -8.012086 & 2.246711 & -1.660107 \\
\hline $\mathrm{H}$ & -9.412630 & 1.365174 & -1.042921 \\
\hline C & -8.150093 & -0.380044 & 0.604710 \\
\hline $\mathrm{H}$ & -7.608208 & -1.309376 & 0.800059 \\
\hline $\mathrm{H}$ & -8.135397 & 0.216064 & 1.522526 \\
\hline $\mathrm{H}$ & -9.190500 & -0.639592 & 0.381253 \\
\hline C & -7.564760 & -0.460052 & -1.846370 \\
\hline $\mathrm{H}$ & -7.127732 & 0.078207 & -2.693215 \\
\hline $\mathrm{H}$ & -7.006735 & -1.391539 & -1.719114 \\
\hline $\mathrm{H}$ & -8.597771 & -0.719597 & -2.102090 \\
\hline C & -4.012709 & 4.451308 & -1.256831 \\
\hline $\mathrm{H}$ & -3.428729 & 3.911609 & -2.008846 \\
\hline $\mathrm{H}$ & -5.062875 & 4.417478 & -1.560279 \\
\hline $\mathrm{H}$ & -3.694779 & 5.499513 & -1.263883 \\
\hline C & -2.315561 & 3.974968 & 0.513489 \\
\hline $\mathrm{H}$ & -2.035779 & 5.032981 & 0.514477 \\
\hline $\mathrm{H}$ & -2.105982 & 3.573959 & 1.509717 \\
\hline $\mathrm{H}$ & -1.666051 & 3.459507 & -0.200299 \\
\hline C & -4.636250 & 4.616972 & 1.180727 \\
\hline $\mathrm{H}$ & -4.503157 & 4.195699 & 2.182036 \\
\hline $\mathrm{H}$ & -4.324479 & 5.666686 & 1.206797 \\
\hline $\mathrm{H}$ & -5.703968 & 4.591351 & 0.945648 \\
\hline
\end{tabular}

\section{Catalyst 2C 79}

Datum

Value

B3LYP-D3(BJ)/6-31G(d,p) Energy

$-2165.157517$

B3LYP-D3(BJ)/def2-TZVPP/IEF-PCM(DCM) Energy

$-2165.895581$

B3LYP-D3(BJ)/def2-TZVPP/IEF-PCM(DCM)//B3LYP-D3(BJ)/6-31G(d,p) Free Energy (Quasiharmonic) -2165.019117

Number of Imaginary Frequencies

Frequencies (Top 3 out of 321)

$\begin{array}{lr}\text { 1. } & 8.8374 \mathrm{~cm}^{-1} \\ \text { 2. } & 11.4801 \mathrm{~cm}^{-1} \\ \text { 3. } & 15.2081 \mathrm{~cm}^{-1}\end{array}$

B3LYP-D3(BJ)/6-31G(d,p) Molecular Geometry in Cartesian Coordinates 


\begin{tabular}{|c|c|c|c|}
\hline C & 0.598882 & -5.193764 & -2.595460 \\
\hline C & 0.191904 & -4.224832 & -1.707225 \\
\hline C & 1.065986 & -3.168637 & -1.339511 \\
\hline C & 2.375082 & -3.119430 & -1.914218 \\
\hline C & 2.762660 & -4.134452 & -2.828116 \\
\hline C & 1.896338 & -5.150164 & -3.159368 \\
\hline $\mathrm{H}$ & -0.079156 & -5.995684 & -2.869745 \\
\hline $\mathrm{H}$ & -0.802712 & -4.251408 & -1.277157 \\
\hline C & 0.676922 & -2.152395 & -0.415098 \\
\hline C & 3.238704 & -2.048872 & -1.577238 \\
\hline $\mathrm{H}$ & 3.757058 & -4.091205 & -3.263245 \\
\hline $\mathrm{H}$ & 2.204046 & -5.920996 & -3.859168 \\
\hline C & 2.860661 & -1.056944 & -0.695808 \\
\hline C & 1.563196 & -1.135531 & -0.099213 \\
\hline $\mathrm{H}$ & 4.212179 & -1.990578 & -2.055058 \\
\hline C & -1.418926 & -4.727360 & 3.556761 \\
\hline C & -2.385458 & -3.845211 & 3.131703 \\
\hline C & -2.145295 & -2.976663 & 2.035642 \\
\hline C & -0.883328 & -3.030592 & 1.365745 \\
\hline C & 0.099623 & -3.939367 & 1.839545 \\
\hline C & -0.163873 & -4.769808 & 2.905599 \\
\hline $\mathrm{H}$ & -4.014766 & -1.901612 & 2.190451 \\
\hline $\mathrm{H}$ & -1.613339 & -5.387684 & 4.396299 \\
\hline $\mathrm{H}$ & -3.348107 & -3.795302 & 3.633120 \\
\hline C & -3.096110 & -2.011956 & 1.620651 \\
\hline C & -0.665925 & -2.174381 & 0.240163 \\
\hline $\mathrm{H}$ & 1.064650 & -3.973898 & 1.347350 \\
\hline $\mathrm{H}$ & 0.597985 & -5.461255 & 3.252215 \\
\hline C & -1.646655 & -1.300196 & -0.197008 \\
\hline C & -2.853355 & -1.165424 & 0.561238 \\
\hline C & -1.447561 & -0.496170 & -1.477967 \\
\hline $\mathrm{H}$ & -1.146561 & 0.529488 & -1.234807 \\
\hline $\mathrm{H}$ & -0.628430 & -0.935885 & -2.048116 \\
\hline C & 1.169340 & -0.131337 & 0.947936 \\
\hline 0 & 0.356745 & 0.763788 & 0.820566 \\
\hline 0 & 1.837418 & -0.338979 & 2.099489 \\
\hline H & 1.546282 & 0.358958 & 2.711613 \\
\hline 0 & -2.576133 & -0.529248 & -2.340312 \\
\hline $\mathrm{H}$ & -3.265818 & 0.010478 & -1.930922 \\
\hline C & -3.788294 & -0.040375 & 0.290247 \\
\hline C & -3.283438 & 1.267698 & 0.324044 \\
\hline C & -5.147551 & -0.251391 & 0.035661 \\
\hline C & -4.110570 & 2.365181 & 0.077090 \\
\hline $\mathrm{H}$ & -2.234871 & 1.403756 & 0.555606 \\
\hline C & -6.008039 & 0.826298 & -0.203639 \\
\hline $\mathrm{H}$ & -5.511357 & -1.270767 & 0.005876 \\
\hline C & -5.464659 & 2.117166 & -0.183501 \\
\hline $\mathrm{H}$ & -6.119183 & 2.959382 & -0.380522 \\
\hline C & 3.757095 & 0.089568 & -0.409889 \\
\hline C & 5.108573 & -0.114985 & -0.109381 \\
\hline C & 3.250469 & 1.394351 & -0.450812 \\
\hline C & 5.955423 & 0.963246 & 0.162763 \\
\hline $\mathrm{H}$ & 5.477701 & -1.131909 & -0.066083 \\
\hline
\end{tabular}




\begin{tabular}{|c|c|c|c|}
\hline C & 4.060163 & 2.493444 & -0.159757 \\
\hline $\mathrm{H}$ & 2.213523 & 1.539898 & -0.717404 \\
\hline C & 5.406234 & 2.252091 & 0.138839 \\
\hline $\mathrm{H}$ & 6.049418 & 3.096028 & 0.364407 \\
\hline C & 3.511901 & 3.927367 & -0.145529 \\
\hline C & 7.442108 & 0.781677 & 0.501645 \\
\hline C & 4.275553 & 4.782403 & -1.178372 \\
\hline $\mathrm{H}$ & 5.347048 & 4.817819 & -0.961475 \\
\hline $\mathrm{H}$ & 4.151192 & 4.375034 & -2.186456 \\
\hline $\mathrm{H}$ & 3.898727 & 5.810939 & -1.175984 \\
\hline C & 3.709717 & 4.524983 & 1.264480 \\
\hline $\mathrm{H}$ & 3.329017 & 5.551413 & 1.300148 \\
\hline H & 3.171804 & 3.935098 & 2.013295 \\
\hline $\mathrm{H}$ & 4.764791 & 4.548337 & 1.551224 \\
\hline C & 2.010940 & 3.978190 & -0.481303 \\
\hline $\mathrm{H}$ & 1.414980 & 3.385145 & 0.218979 \\
\hline $\mathrm{H}$ & 1.658443 & 5.013026 & -0.430658 \\
\hline $\mathrm{H}$ & 1.810660 & 3.610380 & -1.492566 \\
\hline C & 8.296550 & 1.551332 & -0.528574 \\
\hline $\mathrm{H}$ & 8.119163 & 1.172676 & -1.539982 \\
\hline H & 8.067309 & 2.620433 & -0.527325 \\
\hline $\mathrm{H}$ & 9.362027 & 1.436693 & -0.301633 \\
\hline C & 7.870504 & -0.694384 & 0.474559 \\
\hline $\mathrm{H}$ & 7.716045 & -1.142174 & -0.512148 \\
\hline $\mathrm{H}$ & 8.935808 & -0.772867 & 0.711961 \\
\hline $\mathrm{H}$ & 7.321783 & -1.289346 & 1.211127 \\
\hline C & 7.715448 & 1.338616 & 1.915419 \\
\hline $\mathrm{H}$ & 7.471084 & 2.402137 & 1.985364 \\
\hline H & 7.117814 & 0.807937 & 2.663034 \\
\hline $\mathrm{H}$ & 8.773126 & 1.219701 & 2.174525 \\
\hline C & -7.502988 & 0.639830 & -0.500631 \\
\hline C & -3.578632 & 3.805110 & 0.075476 \\
\hline C & -2.071217 & 3.868444 & 0.376781 \\
\hline $\mathrm{H}$ & -1.835261 & 3.458950 & 1.363476 \\
\hline $\mathrm{H}$ & -1.482555 & 3.318779 & -0.363768 \\
\hline $\mathrm{H}$ & -1.737611 & 4.910644 & 0.359695 \\
\hline C & -4.325138 & 4.628850 & 1.146148 \\
\hline $\mathrm{H}$ & -5.402522 & 4.653795 & 0.959611 \\
\hline H & -4.167415 & 4.203556 & 2.142152 \\
\hline $\mathrm{H}$ & -3.962171 & 5.662316 & 1.153646 \\
\hline C & -3.819862 & 4.429057 & -1.316114 \\
\hline $\mathrm{H}$ & -3.299946 & 3.858131 & -2.091730 \\
\hline H & -4.882889 & 4.450980 & -1.572111 \\
\hline $\mathrm{H}$ & -3.447344 & 5.458818 & -1.341565 \\
\hline C & -7.818526 & 1.207292 & -1.901451 \\
\hline $\mathrm{H}$ & -7.581515 & 2.272618 & -1.969273 \\
\hline H & -7.239918 & 0.686001 & -2.670346 \\
\hline $\mathrm{H}$ & -8.882450 & 1.084974 & -2.131627 \\
\hline C & -8.331647 & 1.396225 & 0.559947 \\
\hline $\mathrm{H}$ & -9.402462 & 1.277147 & 0.362437 \\
\hline H & -8.123490 & 1.010293 & 1.562727 \\
\hline $\mathrm{H}$ & -8.108635 & 2.466665 & 0.560960 \\
\hline C & -7.922162 & -0.838885 & -0.474302 \\
\hline $\mathrm{H}$ & -7.738861 & -1.293986 & 0.504059 \\
\hline
\end{tabular}




\section{Catalyst 2C 80}

\section{Datum}

Value

\begin{tabular}{lr}
\hline B3LYP-D3(BJ)/6-31G(d,p) Energy & -2165.157854 \\
\hline B3LYP-D3(BJ)/def2-TZVPP/IEF-PCM(DCM) Energy & -2165.895393 \\
\hline B3LYP-D3(BJ)/def2-TZVPP/IEF-PCM(DCM)//B3LYP-D3(BJ)/6-31G(d,p) Free Energy (Quasiharmonic) & -2165.018737 \\
\hline
\end{tabular}

Number of Imaginary Frequencies

Frequencies (Top 3 out of 321)

$\begin{array}{lr}\text { 1. } & 8.9019 \mathrm{~cm}^{-1} \\ \text { 2. } & 12.1632 \mathrm{~cm}^{-1} \\ \text { 3. } & 15.2944 \mathrm{~cm}^{-1}\end{array}$

\section{B3LYP-D3(BJ)/6-31G(d,p) Molecular Geometry in Cartesian Coordinates}

\begin{tabular}{|c|c|c|c|}
\hline C & 0.669858 & -5.244051 & -2.575846 \\
\hline C & 0.234703 & -4.278364 & -1.697502 \\
\hline C & 1.083281 & -3.201910 & -1.328652 \\
\hline C & 2.395809 & -3.128323 & -1.892878 \\
\hline C & 2.812719 & -4.140597 & -2.796843 \\
\hline C & 1.971027 & -5.176587 & -3.128778 \\
\hline $\mathrm{H}$ & 0.011325 & -6.061840 & -2.850782 \\
\hline $\mathrm{H}$ & -0.762755 & -4.323476 & -1.275627 \\
\hline C & 0.664614 & -2.188640 & -0.414023 \\
\hline C & 3.232960 & -2.036683 & -1.556678 \\
\hline $\mathrm{H}$ & 3.809585 & -4.078824 & -3.224005 \\
\hline $\mathrm{H}$ & 2.301021 & -5.945112 & -3.820907 \\
\hline C & 2.826194 & -1.047646 & -0.684956 \\
\hline C & 1.526124 & -1.150262 & -0.098240 \\
\hline $\mathrm{H}$ & 4.208648 & -1.959616 & -2.027294 \\
\hline C & -1.473386 & -4.792175 & 3.523937 \\
\hline C & -2.435089 & -3.907670 & 3.092560 \\
\hline C & -2.183196 & -3.034076 & 2.003276 \\
\hline C & -0.914012 & -3.086181 & 1.346273 \\
\hline C & 0.063948 & -3.996328 & 1.827311 \\
\hline C & -0.211209 & -4.831647 & 2.886674 \\
\hline $\mathrm{H}$ & -4.045402 & -1.944812 & 2.156021 \\
\hline $\mathrm{H}$ & -1.677117 & -5.456302 & 4.358244 \\
\hline $\mathrm{H}$ & -3.402795 & -3.859465 & 3.584311 \\
\hline
\end{tabular}




\begin{tabular}{|c|c|c|c|}
\hline C & -3.126358 & -2.061263 & 1.587968 \\
\hline C & -0.685362 & -2.227071 & 0.225117 \\
\hline $\mathrm{H}$ & 1.034236 & -4.028717 & 1.345338 \\
\hline $\mathrm{H}$ & 0.546852 & -5.524561 & 3.238652 \\
\hline C & -1.663259 & -1.354184 & -0.220227 \\
\hline C & -2.870803 & -1.211351 & 0.534869 \\
\hline C & -1.459080 & -0.556460 & -1.505150 \\
\hline $\mathrm{H}$ & -1.134875 & 0.463409 & -1.269040 \\
\hline $\mathrm{H}$ & -0.654933 & -1.016141 & -2.081252 \\
\hline C & 1.107419 & -0.148367 & 0.942462 \\
\hline 0 & 0.295218 & 0.745812 & 0.802599 \\
\hline 0 & 1.755588 & -0.354971 & 2.104759 \\
\hline $\mathrm{H}$ & 1.451241 & 0.340397 & 2.713493 \\
\hline 0 & -2.596072 & -0.568521 & -2.356453 \\
\hline $\mathrm{H}$ & -3.266441 & -0.004964 & -1.947140 \\
\hline C & -3.771247 & -0.055400 & 0.278057 \\
\hline C & -5.137590 & -0.208916 & 0.002972 \\
\hline C & -3.221797 & 1.227613 & 0.341792 \\
\hline C & -5.948160 & 0.904521 & -0.228030 \\
\hline $\mathrm{H}$ & -5.540511 & -1.212584 & -0.049294 \\
\hline C & -4.001275 & 2.365886 & 0.102597 \\
\hline $\mathrm{H}$ & -2.169791 & 1.324405 & 0.585524 \\
\hline C & -5.356095 & 2.179186 & -0.179978 \\
\hline $\mathrm{H}$ & -5.978382 & 3.043735 & -0.372742 \\
\hline C & 3.695473 & 0.120054 & -0.400283 \\
\hline C & 5.042792 & -0.047932 & -0.082149 \\
\hline C & 3.161540 & 1.417004 & -0.463684 \\
\hline C & 5.868621 & 1.053690 & 0.185235 \\
\hline $\mathrm{H}$ & 5.439503 & -1.055748 & -0.018825 \\
\hline C & 3.943947 & 2.532996 & -0.179750 \\
\hline $\mathrm{H}$ & 2.124796 & 1.536441 & -0.743409 \\
\hline C & 5.297013 & 2.327081 & 0.137586 \\
\hline $\mathrm{H}$ & 5.913323 & 3.190086 & 0.354393 \\
\hline C & 3.367965 & 3.955932 & -0.194472 \\
\hline C & 7.345277 & 0.820513 & 0.535552 \\
\hline C & 3.552335 & 4.586992 & 1.202817 \\
\hline $\mathrm{H}$ & 3.025767 & 4.002198 & 1.963622 \\
\hline H & 4.606269 & 4.638866 & 1.489805 \\
\hline $\mathrm{H}$ & 3.150397 & 5.605818 & 1.216281 \\
\hline C & 1.866457 & 3.970600 & -0.531162 \\
\hline $\mathrm{H}$ & 1.492804 & 4.998765 & -0.499530 \\
\hline $\mathrm{H}$ & 1.672252 & 3.580159 & -1.534989 \\
\hline $\mathrm{H}$ & 1.283538 & 3.378746 & 0.180881 \\
\hline C & 4.115867 & 4.804074 & -1.244505 \\
\hline $\mathrm{H}$ & 3.999476 & 4.373885 & -2.244035 \\
\hline $\mathrm{H}$ & 3.719649 & 5.825151 & -1.263159 \\
\hline $\mathrm{H}$ & 5.186369 & 4.863941 & -1.028027 \\
\hline C & 8.104156 & 2.134558 & 0.780558 \\
\hline $\mathrm{H}$ & 8.090262 & 2.780941 & -0.102591 \\
\hline $\mathrm{H}$ & 7.684677 & 2.694145 & 1.622451 \\
\hline $\mathrm{H}$ & 9.150163 & 1.916460 & 1.016442 \\
\hline C & 8.032682 & 0.076255 & -0.629557 \\
\hline $\mathrm{H}$ & 7.980947 & 0.665300 & -1.550534 \\
\hline H & 9.087724 & -0.104458 & -0.397060 \\
\hline
\end{tabular}




\begin{tabular}{|c|c|c|c|}
\hline $\mathrm{H}$ & 7.562800 & -0.891677 & -0.824379 \\
\hline C & 7.434859 & -0.036467 & 1.816755 \\
\hline $\mathrm{H}$ & 6.952395 & 0.471800 & 2.657382 \\
\hline $\mathrm{H}$ & 6.947436 & -1.006816 & 1.689302 \\
\hline $\mathrm{H}$ & 8.482133 & -0.218576 & 2.081430 \\
\hline C & -3.332224 & 3.745912 & 0.158582 \\
\hline C & -7.445092 & 0.783926 & -0.547696 \\
\hline C & -8.257580 & 1.555548 & 0.514556 \\
\hline $\mathrm{H}$ & -8.079040 & 1.144904 & 1.513244 \\
\hline $\mathrm{H}$ & -7.992229 & 2.616027 & 0.536658 \\
\hline $\mathrm{H}$ & -9.329433 & 1.482677 & 0.300802 \\
\hline C & -7.924095 & -0.676704 & -0.552165 \\
\hline $\mathrm{H}$ & -7.406151 & -1.270746 & -1.311314 \\
\hline $\mathrm{H}$ & -7.772327 & -1.155085 & 0.420475 \\
\hline $\mathrm{H}$ & -8.994525 & -0.712065 & -0.776478 \\
\hline C & -7.718674 & 1.386688 & -1.942713 \\
\hline $\mathrm{H}$ & -7.439095 & 2.442766 & -1.989401 \\
\hline $\mathrm{H}$ & -7.150713 & 0.855327 & -2.712692 \\
\hline $\mathrm{H}$ & -8.783377 & 1.310338 & -2.188679 \\
\hline C & -2.765285 & 3.976874 & 1.576207 \\
\hline $\mathrm{H}$ & -3.562920 & 3.944337 & 2.325117 \\
\hline $\mathrm{H}$ & -2.023405 & 3.217815 & 1.838778 \\
\hline $\mathrm{H}$ & -2.279080 & 4.956819 & 1.635398 \\
\hline C & -4.312246 & 4.883074 & -0.168369 \\
\hline $\mathrm{H}$ & -3.790589 & 5.843929 & -0.123912 \\
\hline $\mathrm{H}$ & -4.731422 & 4.778778 & -1.174221 \\
\hline $\mathrm{H}$ & -5.140586 & 4.922298 & 0.546243 \\
\hline C & -2.172630 & 3.787869 & -0.862064 \\
\hline $\mathrm{H}$ & -2.538919 & 3.602470 & -1.876803 \\
\hline $\mathrm{H}$ & -1.689612 & 4.771051 & -0.847230 \\
\hline $\mathrm{H}$ & -1.408441 & 3.039278 & -0.636354 \\
\hline
\end{tabular}

\section{Catalyst 2C 81}

Datum

Value

\begin{tabular}{lr}
\hline B3LYP-D3(BJ)/6-31G(d,p) Energy & -2165.157721 \\
\hline B3LYP-D3(BJ)/def2-TZVPP/IEF-PCM(DCM) Energy & -2165.895269 \\
\hline B3LYP-D3(BJ)/def2-TZVPP/IEF-PCM(DCM)//B3LYP-D3(BJ)/6-31G(d,p) Free Energy (Quasiharmonic) & -2165.018704 \\
\hline
\end{tabular}

Number of Imaginary Frequencies

Frequencies (Top 3 out of 321)

$\begin{array}{lr}\text { 1. } & 8.4383 \mathrm{~cm}^{-1} \\ \text { 2. } & 12.2342 \mathrm{~cm}^{-1} \\ \text { 3. } & 15.2593 \mathrm{~cm}^{-1}\end{array}$




\begin{tabular}{|c|c|c|c|}
\hline C & -0.581017 & -5.260002 & -2.557519 \\
\hline C & -0.162369 & -4.284113 & -1.682459 \\
\hline C & -1.028652 & -3.220241 & -1.318317 \\
\hline C & -2.341719 & -3.170014 & -1.883830 \\
\hline C & -2.741403 & -4.192439 & -2.784209 \\
\hline C & -1.882714 & -5.215816 & -3.111613 \\
\hline $\mathrm{H}$ & 0.091004 & -6.067904 & -2.829031 \\
\hline $\mathrm{H}$ & 0.835447 & -4.311259 & -1.259891 \\
\hline C & -0.627652 & -2.197045 & -0.406948 \\
\hline C & -3.196710 & -2.090812 & -1.552644 \\
\hline $\mathrm{H}$ & -3.738828 & -4.148544 & -3.212274 \\
\hline $\mathrm{H}$ & -2.199615 & -5.992220 & -3.801066 \\
\hline C & -2.806761 & -1.091185 & -0.685104 \\
\hline C & -1.505967 & -1.171259 & -0.096327 \\
\hline $\mathrm{H}$ & -4.173182 & -2.032023 & -2.024231 \\
\hline C & 1.540108 & -4.753573 & 3.545257 \\
\hline C & 2.489320 & -3.855941 & 3.113265 \\
\hline C & 2.227079 & -2.990316 & 2.020125 \\
\hline C & 0.960680 & -3.064172 & 1.359818 \\
\hline C & -0.004470 & -3.987515 & 1.841541 \\
\hline C & 0.280518 & -4.814707 & 2.904690 \\
\hline $\mathrm{H}$ & 4.073207 & -1.873236 & 2.172623 \\
\hline $\mathrm{H}$ & 1.751692 & -5.411462 & 4.382545 \\
\hline H & 3.454798 & -3.791080 & 3.607476 \\
\hline C & 3.156933 & -2.005155 & 1.603468 \\
\hline C & 0.721356 & -2.212242 & 0.235177 \\
\hline $\mathrm{H}$ & -0.972812 & -4.036488 & 1.357100 \\
\hline $\mathrm{H}$ & -0.467816 & -5.517902 & 3.257086 \\
\hline C & 1.686821 & -1.325731 & -0.210028 \\
\hline C & 2.891049 & -1.163778 & 0.546402 \\
\hline C & 1.477384 & -0.533404 & -1.496922 \\
\hline $\mathrm{H}$ & 0.667175 & -0.990653 & -2.066195 \\
\hline $\mathrm{H}$ & 1.161646 & 0.489768 & -1.264041 \\
\hline C & -1.103282 & -0.159422 & 0.941021 \\
\hline 0 & -0.305696 & 0.747303 & 0.797645 \\
\hline 0 & -1.746215 & -0.373532 & 2.104906 \\
\hline $\mathrm{H}$ & -1.451898 & 0.328066 & 2.711418 \\
\hline 0 & 2.610150 & -0.557152 & -2.354191 \\
\hline $\mathrm{H}$ & 3.288526 & 0.000193 & -1.949422 \\
\hline C & 3.776024 & 0.002789 & 0.283346 \\
\hline C & 3.210264 & 1.282344 & 0.353095 \\
\hline C & 5.136596 & -0.129174 & -0.005514 \\
\hline C & 3.971640 & 2.426702 & 0.107526 \\
\hline $\mathrm{H}$ & 2.159290 & 1.364642 & 0.606607 \\
\hline C & 5.935250 & 0.997081 & -0.244717 \\
\hline $\mathrm{H}$ & 5.558596 & -1.126977 & -0.065052 \\
\hline C & 5.331332 & 2.260518 & -0.189744 \\
\hline $\mathrm{H}$ & 5.933583 & 3.135603 & -0.385116 \\
\hline C & -3.694578 & 0.063970 & -0.406624 \\
\hline C & -3.181627 & 1.365432 & -0.470059 \\
\hline
\end{tabular}




\begin{tabular}{|c|c|c|c|}
\hline C & -5.044920 & -0.129286 & $-0.09344 e$ \\
\hline C & -3.983912 & 2.472865 & -0.189919 \\
\hline $\mathrm{H}$ & -2.146046 & 1.501811 & -0.746238 \\
\hline C & -5.884501 & 0.957159 & 0.168249 \\
\hline $\mathrm{H}$ & -5.418883 & -1.143572 & -0.032965 \\
\hline C & -5.329128 & 2.242756 & 0.120983 \\
\hline H & -5.966830 & 3.093233 & 0.337362 \\
\hline C & -7.369781 & 0.788277 & 0.519586 \\
\hline C & -3.429085 & 3.904349 & -0.203724 \\
\hline C & -7.805524 & -0.685873 & 0.518196 \\
\hline $\mathrm{H}$ & -7.659875 & -1.149603 & -0.462471 \\
\hline H & -7.254801 & -1.272119 & 1.260244 \\
\hline $\mathrm{H}$ & -8.869582 & -0.755390 & 0.763800 \\
\hline C & -8.227175 & 1.546140 & -0.516913 \\
\hline $\mathrm{H}$ & -9.291684 & 1.440329 & -0.281332 \\
\hline $\mathrm{H}$ & -7.992741 & 2.613983 & -0.533725 \\
\hline $\mathrm{H}$ & -8.058258 & 1.150997 & -1.523452 \\
\hline C & -7.631122 & 1.368445 & 1.926286 \\
\hline $\mathrm{H}$ & -7.381170 & 2.431708 & 1.978131 \\
\hline $\mathrm{H}$ & -8.687639 & 1.258748 & 2.194044 \\
\hline $\mathrm{H}$ & -7.031195 & 0.846471 & 2.678189 \\
\hline C & -1.926654 & 3.941125 & -0.534675 \\
\hline $\mathrm{H}$ & -1.337575 & 3.358607 & 0.179977 \\
\hline $\mathrm{H}$ & -1.722740 & 3.552887 & -1.537425 \\
\hline $\mathrm{H}$ & -1.568566 & 4.974786 & -0.502451 \\
\hline C & -3.628495 & 4.533503 & $1.19226 e$ \\
\hline $\mathrm{H}$ & -3.096517 & 3.957159 & 1.955717 \\
\hline H & -3.242051 & 5.558286 & 1.206757 \\
\hline $\mathrm{H}$ & -4.684278 & 4.569529 & 1.474944 \\
\hline C & -4.185777 & 4.739876 & -1.257451 \\
\hline $\mathrm{H}$ & -4.059206 & 4.310545 & -2.256096 \\
\hline $\mathrm{H}$ & -5.257927 & 4.783221 & -1.045083 \\
\hline $\mathrm{H}$ & -3.805375 & 5.766952 & -1.275733 \\
\hline C & 7.423221 & 0.808367 & -0.574435 \\
\hline C & 3.287630 & 3.799635 & 0.171686 \\
\hline C & 4.249468 & 4.948464 & -0.168950 \\
\hline $\mathrm{H}$ & 5.086325 & 4.999292 & 0.534866 \\
\hline H & 4.657190 & 4.848452 & -1.179896 \\
\hline $\mathrm{H}$ & 3.716225 & 5.902686 & -0.118783 \\
\hline C & 2.737439 & 4.025281 & 1.596760 \\
\hline $\mathrm{H}$ & 2.007852 & 3.257951 & 1.869517 \\
\hline H & 3.545426 & 4.001859 & 2.334849 \\
\hline $\mathrm{H}$ & 2.241252 & 4.999830 & 1.662399 \\
\hline C & 2.113845 & 3.828264 & -0.833161 \\
\hline $\mathrm{H}$ & 2.468597 & 3.647173 & -1.852766 \\
\hline $\mathrm{H}$ & 1.361486 & 3.070803 & -0.597588 \\
\hline $\mathrm{H}$ & 1.619847 & 4.805888 & -0.811740 \\
\hline C & 7.554467 & -0.030790 & -1.864063 \\
\hline $\mathrm{H}$ & 7.091996 & -1.015415 & -1.755020 \\
\hline H & 7.070648 & 0.473913 & -2.706138 \\
\hline $\mathrm{H}$ & 8.609993 & -0.181019 & -2.115656 \\
\hline C & 8.113011 & 0.068847 & 0.592294 \\
\hline $\mathrm{H}$ & 9.175773 & -0.080913 & 0.373299 \\
\hline H & 8.032395 & 0.645565 & 1.519053 \\
\hline
\end{tabular}




\section{Catalyst 2C 82}

\section{Datum}

Value

\begin{tabular}{lr}
\hline B3LYP-D3(BJ)/6-31G(d,p) Energy & -2165.158261 \\
\hline B3LYP-D3(BJ)/def2-TZVPP/IEF-PCM(DCM) Energy & -2165.895143 \\
\hline B3LYP-D3(BJ)/def2-TZVPP/IEF-PCM(DCM)//B3LYP-D3(BJ)/6-31G(d,p) Free Energy (Quasiharmonic) & -2165.018519 \\
\hline
\end{tabular}

Number of Imaginary Frequencies

Frequencies (Top 3 out of 321)
1.
$6.3923 \mathrm{~cm}^{-1}$
2.
$9.7797 \mathrm{~cm}^{-1}$
3. $15.0156 \mathrm{~cm}^{-1}$

\section{B3LYP-D3(BJ)/6-31G(d,p) Molecular Geometry in Cartesian Coordinates}

$\begin{array}{lrll}\text { C } & -0.713662 & 5.302017 & -2.557135 \\ \text { C } & -0.263306 & 4.335217 & -1.687767 \\ \text { C } & -1.099634 & 3.249350 & -1.318543 \\ \text { C } & -2.415680 & 3.166794 & -1.873691 \\ \text { C } & -2.848346 & 4.180788 & -2.768360 \\ \text { C } & -2.018459 & 5.226179 & -3.100453 \\ \text { H } & -0.064511 & 6.127166 & -2.832384 \\ \text { H } & 0.736827 & 4.386777 & -1.272960 \\ \text { C } & -0.665648 & 2.235497 & -0.412039 \\ \text { C } & -3.240075 & 2.064745 & -1.539599 \\ \text { H } & -3.847768 & 4.112608 & -3.188498 \\ \text { H } & -2.360551 & 5.995756 & -3.785495 \\ \text { C } & -2.817317 & 1.073257 & -0.678252 \\ \text { C } & -1.515824 & 1.187349 & -0.097416 \\ \text { H } & -4.218112 & 1.981837 & -2.004311 \\ \text { C } & 1.510938 & 4.839766 & 3.508935 \\ \text { C } & 2.463978 & 3.947204 & 3.074714 \\ \text { C } & 2.200622 & 3.073517 & 1.988311 \\ \text { C } & 0.928782 & 3.134466 & 1.336823 \\ \text { C } & -0.040363 & 4.051969 & 1.821386 \\ \text { C } & 0.246002 & 4.887224 & 2.877897\end{array}$




\begin{tabular}{|c|c|c|c|}
\hline $\mathrm{H}$ & 4.050438 & 1.962661 & 2.142297 \\
\hline $\mathrm{H}$ & 1.723640 & 5.503911 & 4.340982 \\
\hline $\mathrm{H}$ & 3.433437 & 3.892481 & 3.562308 \\
\hline C & 3.133027 & 2.089501 & 1.573796 \\
\hline C & 0.688874 & 2.276026 & 0.217340 \\
\hline H & -1.012675 & 4.090566 & 1.343972 \\
\hline $\mathrm{H}$ & -0.505326 & 5.586185 & 3.232341 \\
\hline C & 1.659810 & 1.397251 & -0.231530 \\
\hline C & 2.866021 & 1.240675 & 0.523047 \\
\hline C & 1.452395 & 0.605700 & -1.519845 \\
\hline $\mathrm{H}$ & 1.124400 & -0.414141 & -1.289704 \\
\hline $\mathrm{H}$ & 0.651610 & 1.072068 & -2.095335 \\
\hline C & -1.084266 & 0.184465 & 0.937123 \\
\hline 0 & -0.282323 & -0.717168 & 0.781878 \\
\hline 0 & -1.706869 & 0.396549 & 2.111169 \\
\hline $\mathrm{H}$ & -1.397218 & -0.301659 & 2.714143 \\
\hline 0 & 2.591765 & 0.617207 & -2.368089 \\
\hline $\mathrm{H}$ & 3.260170 & 0.051869 & -1.957763 \\
\hline C & 3.739856 & 0.062573 & 0.273319 \\
\hline C & 3.156146 & -1.204045 & 0.356177 \\
\hline C & 5.107520 & 0.175685 & -0.013892 \\
\hline C & 3.901164 & -2.366121 & 0.124405 \\
\hline $\mathrm{H}$ & 2.103814 & -1.268907 & 0.607706 \\
\hline C & 5.884926 & -0.962998 & -0.238040 \\
\hline $\mathrm{H}$ & 5.537966 & 1.167037 & -0.080573 \\
\hline C & 5.258436 & -2.220361 & -0.170535 \\
\hline $\mathrm{H}$ & 5.855376 & -3.103852 & -0.357844 \\
\hline C & -3.668587 & -0.109102 & -0.398564 \\
\hline c & -5.024615 & 0.042041 & -0.070857 \\
\hline C & -3.123581 & -1.391599 & -0.473568 \\
\hline C & -5.825899 & -1.067953 & 0.192868 \\
\hline $\mathrm{H}$ & -5.427576 & 1.044698 & -0.002879 \\
\hline C & -3.891059 & -2.528907 & -0.192440 \\
\hline H & -2.084853 & -1.503101 & -0.756563 \\
\hline C & -5.234726 & -2.342773 & 0.133713 \\
\hline $\mathrm{H}$ & -5.849152 & -3.206755 & 0.352516 \\
\hline c & -3.216358 & -3.907203 & -0.232410 \\
\hline C & -7.311847 & -0.946222 & 0.559852 \\
\hline C & -2.591393 & -4.139517 & -1.624792 \\
\hline $\mathrm{H}$ & -3.355777 & -4.099970 & -2.407155 \\
\hline $\mathrm{H}$ & -1.830670 & -3.390348 & -1.859163 \\
\hline $\mathrm{H}$ & -2.111352 & -5.123215 & -1.664123 \\
\hline C & -4.204906 & -5.049171 & 0.050083 \\
\hline $\mathrm{H}$ & -3.681136 & -6.008997 & 0.008334 \\
\hline $\mathrm{H}$ & -4.653398 & -4.960572 & 1.044617 \\
\hline $\mathrm{H}$ & -5.011969 & -5.077595 & -0.689086 \\
\hline C & -2.102548 & -3.944813 & 0.838785 \\
\hline $\mathrm{H}$ & -2.527378 & -3.813796 & 1.839654 \\
\hline $\mathrm{H}$ & -1.582993 & -4.909125 & 0.813810 \\
\hline $\mathrm{H}$ & -1.361478 & -3.156191 & 0.684174 \\
\hline C & -8.156694 & -1.722239 & -0.473644 \\
\hline $\mathrm{H}$ & -8.009781 & -1.315081 & -1.478861 \\
\hline $\mathrm{H}$ & -7.890933 & -2.782488 & -0.500377 \\
\hline $\mathrm{H}$ & -9.221438 & -1.649684 & -0.226766 \\
\hline
\end{tabular}




\begin{tabular}{|c|c|c|c|}
\hline C & -7.791556 & 0.514097 & 0.573408 \\
\hline $\mathrm{H}$ & -7.670211 & 0.988541 & -0.405436 \\
\hline $\mathrm{H}$ & -8.854566 & 0.550075 & 0.830488 \\
\hline $\mathrm{H}$ & -7.250698 & 1.111536 & 1.313796 \\
\hline C & -7.542183 & -1.543668 & 1.964848 \\
\hline $\mathrm{H}$ & -7.260518 & -2.599384 & 2.006890 \\
\hline $\mathrm{H}$ & -6.950951 & -1.008994 & 2.714740 \\
\hline H & -8.598898 & -1.467192 & 2.243168 \\
\hline C & 7.382173 & -0.887789 & -0.570247 \\
\hline C & 3.192074 & -3.725090 & 0.202129 \\
\hline C & 4.134649 & -4.893830 & -0.122988 \\
\hline $\mathrm{H}$ & 4.546273 & -4.811775 & -1.134021 \\
\hline $\mathrm{H}$ & 4.968898 & -4.950089 & 0.583578 \\
\hline $\mathrm{H}$ & 3.585756 & -5.838560 & -0.063314 \\
\hline C & 2.019678 & -3.743315 & -0.804844 \\
\hline H & 1.280791 & -2.970183 & -0.577274 \\
\hline $\mathrm{H}$ & 2.378976 & -3.579179 & -1.825733 \\
\hline $\mathrm{H}$ & 1.508446 & -4.711773 & -0.773767 \\
\hline C & 2.632974 & -3.923761 & 1.627836 \\
\hline $\mathrm{H}$ & 3.438442 & -3.905964 & 2.368829 \\
\hline $\mathrm{H}$ & 1.915480 & -3.140827 & 1.888014 \\
\hline $\mathrm{H}$ & 2.119161 & -4.888456 & 1.702792 \\
\hline C & 7.901100 & 0.558873 & -0.597446 \\
\hline $\mathrm{H}$ & 7.393509 & 1.157225 & -1.360209 \\
\hline $\mathrm{H}$ & 7.770513 & 1.053677 & 0.370029 \\
\hline $\mathrm{H}$ & 8.970231 & 0.561827 & -0.830540 \\
\hline C & 7.628487 & -1.515815 & -1.959202 \\
\hline $\mathrm{H}$ & 8.693013 & -1.472276 & -2.213778 \\
\hline $\mathrm{H}$ & 7.319397 & -2.564217 & -1.990193 \\
\hline $\mathrm{H}$ & 7.069727 & -0.978779 & -2.731985 \\
\hline C & 8.181179 & -1.667783 & 0.496119 \\
\hline $\mathrm{H}$ & 7.886736 & -2.720108 & 0.534061 \\
\hline $\mathrm{H}$ & 9.252992 & -1.627413 & 0.273706 \\
\hline $\mathrm{H}$ & 8.021598 & -1.239471 & 1.490584 \\
\hline
\end{tabular}

\section{Uncatalyzed reaction TS 1 (Chair, Equatorial)}

Datum

Value

\begin{tabular}{lr}
\hline B3LYP-D3(BJ)/6-31G(d,p) Energy & -874.27248 \\
\hline B3LYP-D3(BJ)/def2-TZVPP/IEF-PCM(DCM) Energy & -874.588402 \\
\hline B3LYP-D3(BJ)/def2-TZVPP/IEF-PCM(DCM)//B3LYP-D3(BJ)/6-31G(d,p) Free Energy (Quasiharmonic) & -874.254195 \\
\hline Number of Imaginary Frequencies & 1 \\
Frequencies (Top 3 out of 123) &
\end{tabular}


1. $-285.7296 \mathrm{~cm}^{-1}$

2. $38.9894 \mathrm{~cm}^{-1}$

3. $46.3826 \mathrm{~cm}^{-1}$

B3LYP-D3(BJ)/6-31G(d,p) Molecular Geometry in Cartesian Coordinates

\begin{tabular}{|c|c|c|c|}
\hline C & -1.540853 & 2.398574 & -0.252676 \\
\hline C & -1.243883 & 0.304790 & 0.319081 \\
\hline C & 0.703252 & 2.062936 & -1.231797 \\
\hline $\mathrm{C}$ & -0.185114 & 2.648930 & -0.280015 \\
\hline $\mathrm{H}$ & -0.861137 & 0.545397 & 1.311543 \\
\hline $\mathrm{H}$ & 1.701635 & 2.496620 & -1.268603 \\
\hline $\mathrm{H}$ & 0.289357 & 1.888375 & -2.225111 \\
\hline C & 2.810161 & -0.051683 & 0.820373 \\
\hline C & 2.758509 & -0.995259 & -0.435768 \\
\hline C & 3.849210 & 1.067809 & 0.672302 \\
\hline $\mathrm{H}$ & 3.673617 & 1.814724 & 1.451502 \\
\hline $\mathrm{H}$ & 4.872447 & 0.694250 & 0.775944 \\
\hline $\mathrm{H}$ & 3.757936 & 1.558053 & -0.300105 \\
\hline C & 3.005656 & -0.768957 & 2.152900 \\
\hline $\mathrm{H}$ & 3.954667 & -1.315141 & 2.169427 \\
\hline $\mathrm{H}$ & 3.021440 & -0.038073 & 2.966917 \\
\hline $\mathrm{H}$ & 2.193661 & -1.471841 & 2.345836 \\
\hline C & 2.077334 & -2.337918 & -0.134387 \\
\hline $\mathrm{H}$ & 1.876299 & -2.846608 & -1.080806 \\
\hline $\mathrm{H}$ & 2.707343 & -2.986685 & 0.481834 \\
\hline $\mathrm{H}$ & 1.123441 & -2.188439 & 0.376825 \\
\hline C & 4.104242 & -1.235121 & -1.112268 \\
\hline $\mathrm{H}$ & 4.809223 & -1.713733 & -0.424290 \\
\hline $\mathrm{H}$ & 3.970119 & -1.894517 & -1.974464 \\
\hline $\mathrm{H}$ & 4.540110 & -0.300407 & -1.468235 \\
\hline 0 & 1.506341 & 0.553687 & 0.808876 \\
\hline 0 & 1.916132 & -0.257937 & -1.339527 \\
\hline B & 1.009715 & 0.474619 & -0.535932 \\
\hline 0 & -0.393540 & -0.109276 & -0.561839 \\
\hline C & -2.639992 & -0.147703 & 0.212362 \\
\hline C & -3.508711 & 0.027403 & 1.298150 \\
\hline C & -3.106463 & -0.763928 & -0.957728 \\
\hline C & -4.829479 & -0.404306 & 1.215289 \\
\hline $\mathrm{H}$ & -3.142789 & 0.501975 & 2.204645 \\
\hline C & -4.427294 & -1.193551 & -1.036612 \\
\hline $\mathrm{H}$ & -2.418877 & -0.904143 & -1.784278 \\
\hline C & -5.291601 & -1.013720 & 0.046876 \\
\hline $\mathrm{H}$ & -5.497462 & -0.268850 & 2.059995 \\
\hline $\mathrm{H}$ & -4.785285 & -1.673401 & -1.942124 \\
\hline $\mathrm{H}$ & -6.321348 & -1.351013 & -0.018181 \\
\hline $\mathrm{H}$ & 0.267561 & 3.086507 & 0.608720 \\
\hline $\mathrm{H}$ & -2.057206 & 2.111307 & -1.162743 \\
\hline $\mathrm{H}$ & -2.164629 & 2.800986 & 0.538842 \\
\hline
\end{tabular}




\section{Uncatalyzed reaction TS 2 (Chair, Axial)}

Datum

Value

\begin{tabular}{lc} 
B3LYP-D3(BJ)/6-31G(d,p) Energy & -874.265627 \\
\hline B3LYP-D3(BJ)/def2-TZVPP/IEF-PCM(DCM) Energy & -874.580757 \\
\hline B3LYP-D3(BJ)/def2-TZVPP/IEF-PCM(DCM)//B3LYP-D3(BJ)/6-31G(d,p) Free Energy (Quasiharmonic) & -874.246802 \\
\hline
\end{tabular}

Number of Imaginary Frequencies

Frequencies (Top 3 out of 123)

$\begin{array}{rr}\text { 1. } & -277.3015 \mathrm{~cm}^{-1} \\ \text { 2. } & 32.7294 \mathrm{~cm}^{-1} \\ \text { 3. } & 42.4451 \mathrm{~cm}^{-1}\end{array}$

B3LYP-D3(BJ)/6-31G(d,p) Molecular Geometry in Cartesian Coordinates

\begin{tabular}{|c|c|c|c|}
\hline C & 1.821837 & 2.709669 & -0.513237 \\
\hline C & 1.501046 & 1.103923 & 0.971149 \\
\hline C & -0.552830 & 2.265988 & -0.990814 \\
\hline C & 0.834102 & 2.206301 & -1.326464 \\
\hline $\mathrm{H}$ & -1.235401 & 2.084105 & -1.820485 \\
\hline $\mathrm{H}$ & -0.845866 & 3.133257 & -0.397385 \\
\hline C & -2.238580 & -0.900723 & -0.598854 \\
\hline C & -2.801540 & -0.213451 & 0.693945 \\
\hline C & -2.998150 & -0.485975 & -1.865436 \\
\hline $\mathrm{H}$ & -2.416888 & -0.790877 & -2.740049 \\
\hline $\mathrm{H}$ & -3.983714 & -0.957646 & -1.923468 \\
\hline $\mathrm{H}$ & -3.132339 & 0.597840 & -1.901749 \\
\hline C & -2.136018 & -2.421391 & -0.527778 \\
\hline $\mathrm{H}$ & -3.122258 & -2.873700 & -0.380369 \\
\hline $\mathrm{H}$ & -1.723420 & -2.808604 & -1.464373 \\
\hline $\mathrm{H}$ & -1.482377 & -2.736786 & 0.287232 \\
\hline C & -2.357023 & -0.927008 & 1.978786 \\
\hline $\mathrm{H}$ & -2.588125 & -0.284027 & 2.831926 \\
\hline $\mathrm{H}$ & -2.871879 & -1.883081 & 2.114430 \\
\hline $\mathrm{H}$ & -1.279166 & -1.106810 & 1.974876 \\
\hline C & -4.312814 & -0.009246 & 0.706182 \\
\hline $\mathrm{H}$ & -4.838609 & -0.967922 & 0.644416 \\
\hline $\mathrm{H}$ & -4.609678 & 0.481504 & 1.637357 \\
\hline $\mathrm{H}$ & -4.634024 & 0.621997 & -0.123799 \\
\hline 0 & -0.910662 & -0.352003 & -0.680640 \\
\hline 0 & -2.162928 & 1.072757 & 0.652394 \\
\hline B & -0.900802 & 0.892082 & 0.033161 \\
\hline 0 & 0.221173 & 1.015206 & 1.078698 \\
\hline
\end{tabular}




$\begin{array}{|lrrr|}H & 1.128604 & 1.527275 & -2.126022 \\ H & 1.582700 & 3.501549 & 0.190593 \\ H & 2.869054 & 2.584755 & -0.764217 \\ \mathrm{C} & 2.406317 & 0.041753 & 0.480700 \\ \mathrm{C} & 3.776393 & 0.191718 & 0.753084 \\ \mathrm{C} & 1.959914 & -1.104795 & -0.192334 \\ \mathrm{C} & 4.689737 & -0.776128 & 0.350170 \\ \mathrm{H} & 4.122053 & 1.076054 & 1.282879 \\ \mathrm{C} & 2.880816 & -2.070462 & -0.593437 \\ \mathrm{H} & 0.902547 & -1.216450 & -0.402877 \\ \mathrm{C} & 4.242116 & -1.912550 & -0.327689 \\ \mathrm{H} & 5.745783 & -0.648977 & 0.566797 \\ \mathrm{H} & 2.530559 & -2.956260 & -1.114660 \\ \mathrm{H} & 4.951324 & -2.672162 & -0.641991 \\ \mathrm{H} & 1.962538 & 1.761137 & 1.713692 \\ \end{array}$

\section{Uncatalyzed reaction TS 3 (Boat, Equatorial)}

Datum

Value

\begin{tabular}{ll}
\hline B3LYP-D3(BJ)/6-31G(d,p) Energy & -874.262029 \\
\hline B3LYP-D3(BJ)/def2-TZVPP/IEF-PCM(DCM) Energy & -874.577481 \\
\hline B3LYP-D3(BJ)/def2-TZVPP/IEF-PCM(DCM)//B3LYP-D3(BJ)/6-31G(d,p) Free Energy (Quasiharmonic) & -874.243856
\end{tabular}

Number of Imaginary Frequencies

Frequencies (Top 3 out of 123)

$\begin{array}{lr}\text { 1. } & -368.9929 \mathrm{~cm}^{-1} \\ \text { 2. } & 31.5673 \mathrm{~cm}^{-1} \\ \text { 3. } & 45.1175 \mathrm{~cm}^{-1}\end{array}$

B3LYP-D3(BJ)/6-31G(d,p) Molecular Geometry in Cartesian Coordinates

$$
\text { C }
$$

C

1.291222

1.940047

$-0.060789$

2.327910

2.473467

2.763803

2.506878

$-0.220096$

$-0.749290$

0.997488

1.451340

0.720406

1.313488
0.445866
-0.190269
0.163930
-1.140292
0.610592
-0.705799
0.692279
-0.588466
-1.577181
-0.224859




\begin{tabular}{|c|c|c|c|}
\hline $\mathrm{H}$ & -3.609028 & 1.747807 & 0.087080 \\
\hline C & -3.744087 & -1.275916 & -1.599913 \\
\hline $\mathrm{H}$ & -4.637327 & -1.697871 & -1.127134 \\
\hline $\mathrm{H}$ & -4.044240 & -0.821336 & -2.548292 \\
\hline H & -3.046528 & -2.085060 & -1.820658 \\
\hline C & -2.072308 & -2.182914 & 0.620294 \\
\hline $\mathrm{H}$ & -1.563080 & -2.411827 & 1.560782 \\
\hline H & -2.870327 & -2.917022 & 0.474429 \\
\hline $\mathrm{H}$ & -1.350555 & -2.284962 & -0.193793 \\
\hline C & -3.642925 & -0.634090 & 1.813749 \\
\hline $\mathrm{H}$ & -4.538196 & -1.222199 & 1.586515 \\
\hline $\mathrm{H}$ & -3.215638 & -1.014177 & 2.746313 \\
\hline H & -3.937229 & 0.403966 & 1.976002 \\
\hline 0 & -1.871950 & 0.208587 & -1.319360 \\
\hline 0 & -1.513352 & 0.130969 & 0.983497 \\
\hline B & -0.964567 & 0.513856 & -0.283449 \\
\hline 0 & 0.394451 & -0.042223 & -0.533817 \\
\hline $\mathrm{H}$ & 1.438495 & 2.747583 & -0.625975 \\
\hline $\mathrm{H}$ & 0.647221 & 1.726561 & 2.162045 \\
\hline $\mathrm{H}$ & 2.347116 & 2.063863 & 1.523692 \\
\hline C & 2.665272 & -0.297630 & 0.113005 \\
\hline C & 3.565867 & -0.662707 & 1.124035 \\
\hline C & 3.124053 & -0.163774 & -1.205259 \\
\hline C & 4.906113 & -0.886738 & 0.823334 \\
\hline $\mathrm{H}$ & 3.208812 & -0.768656 & 2.145005 \\
\hline C & 4.465274 & -0.387161 & -1.501837 \\
\hline $\mathrm{H}$ & 2.414727 & 0.104822 & -1.979957 \\
\hline C & 5.359446 & -0.747202 & -0.490441 \\
\hline $\mathrm{H}$ & 5.596978 & -1.171933 & 1.610508 \\
\hline $\mathrm{H}$ & 4.815701 & -0.284104 & -2.524163 \\
\hline $\mathrm{H}$ & 6.404780 & -0.921637 & -0.725475 \\
\hline H & 0.884535 & -0.407825 & 1.411534 \\
\hline
\end{tabular}

\section{Uncatalyzed reaction TS 4 (Boat, Axial)}

\section{Datum}

Value

B3LYP-D3(BJ)/6-31G(d,p) Energy

$-874.259195$

B3LYP-D3(BJ)/def2-TZVPP/IEF-PCM(DCM) Energy

$-874.574583$

B3LYP-D3(BJ)/def2-TZVPP/IEF-PCM(DCM)//B3LYP-D3(BJ)/6-31G(d,p) Free Energy (Quasiharmonic)

$-874.240925$

Number of Imaginary Frequencies

Frequencies (Top 3 out of 123)

$\begin{array}{lr}\text { 1. } & -330.2541 \mathrm{~cm}^{-1} \\ \text { 2. } & 31.2429 \mathrm{~cm}^{-1} \\ \text { 3. } & 51.3975 \mathrm{~cm}^{-1}\end{array}$




\begin{tabular}{|c|c|c|c|}
\hline C & -1.848276 & 2.614187 & -0.531659 \\
\hline C & -1.523602 & 1.046362 & 0.965810 \\
\hline C & 0.417555 & 1.967318 & -1.224935 \\
\hline C & -0.499246 & 2.882487 & -0.641058 \\
\hline $\mathrm{H}$ & 1.398059 & 2.371266 & -1.469845 \\
\hline $\mathrm{H}$ & 0.001800 & 1.351037 & -2.023134 \\
\hline C & 3.048966 & 0.012422 & 0.270343 \\
\hline C & 2.133376 & -1.191066 & -0.148400 \\
\hline C & 3.863784 & 0.565051 & -0.907438 \\
\hline $\mathrm{H}$ & 4.284706 & 1.531916 & -0.618272 \\
\hline $\mathrm{H}$ & 4.686659 & -0.100831 & -1.184699 \\
\hline $\mathrm{H}$ & 3.229935 & 0.714579 & -1.785158 \\
\hline C & 3.970119 & -0.266679 & 1.454416 \\
\hline $\mathrm{H}$ & 4.659859 & -1.087520 & 1.230700 \\
\hline $\mathrm{H}$ & 4.563905 & 0.624600 & 1.676810 \\
\hline $\mathrm{H}$ & 3.397561 & -0.520679 & 2.347634 \\
\hline C & 1.648608 & -2.007860 & 1.057273 \\
\hline $\mathrm{H}$ & 0.856632 & -2.687426 & 0.729651 \\
\hline $\mathrm{H}$ & 2.454434 & -2.606039 & 1.493038 \\
\hline $\mathrm{H}$ & 1.238381 & -1.355601 & 1.831663 \\
\hline C & 2.723439 & -2.116312 & -1.206519 \\
\hline $\mathrm{H}$ & 3.647060 & -2.581804 & -0.846974 \\
\hline $\mathrm{H}$ & 2.011564 & -2.913741 & -1.439620 \\
\hline $\mathrm{H}$ & 2.938958 & -1.576183 & -2.129654 \\
\hline 0 & 2.089829 & 1.006557 & 0.659552 \\
\hline 0 & 0.998151 & -0.508846 & -0.714721 \\
\hline B & 0.869590 & 0.731138 & -0.008871 \\
\hline 0 & -0.257414 & 0.801616 & 1.005849 \\
\hline $\mathrm{H}$ & -0.087446 & 3.691465 & -0.036891 \\
\hline $\mathrm{H}$ & -2.316659 & 1.948391 & -1.247132 \\
\hline $\mathrm{H}$ & -2.511985 & 3.307567 & -0.024923 \\
\hline C & -2.498406 & 0.059302 & 0.463760 \\
\hline C & -3.855194 & 0.229592 & 0.780477 \\
\hline C & -2.096076 & -1.041876 & -0.308042 \\
\hline C & -4.802357 & -0.683643 & 0.330133 \\
\hline $\mathrm{H}$ & -4.161643 & 1.082877 & 1.380330 \\
\hline C & -3.051805 & -1.949438 & -0.758709 \\
\hline $\mathrm{H}$ & -1.044831 & -1.169361 & -0.546329 \\
\hline C & -4.401297 & -1.775918 & -0.443668 \\
\hline $\mathrm{H}$ & -5.849498 & -0.548644 & 0.582351 \\
\hline $\mathrm{H}$ & -2.740888 & -2.801296 & -1.355789 \\
\hline $\mathrm{H}$ & -5.138828 & -2.490630 & -0.795777 \\
\hline $\mathrm{H}$ & -1.887739 & 1.702173 & 1.760243 \\
\hline
\end{tabular}




\begin{tabular}{lr}
\hline B3LYP-D3(BJ)/6-31G(d,p) Energy & -3039.493781 \\
\hline B3LYP-D3(BJ)/def2-TZVPP/IEF-PCM(DCM) Energy & -3040.532948 \\
\hline B3LYP-D3(BJ)/def2-TZVPP/IEF-PCM(DCM)//B3LYP-D3(BJ)/6-31G(d,p) Free Energy (Quasiharmonic) & -3039.301929 \\
\hline
\end{tabular}

Number of Imaginary Frequencies

Frequencies (Top 3 out of 450)

$\begin{array}{rr}\text { 1. } & -298.8983 \mathrm{~cm}^{-1} \\ \text { 2. } & 8.9052 \mathrm{~cm}^{-1} \\ \text { 3. } & 18.1481 \mathrm{~cm}^{-1}\end{array}$

B3LYP-D3(BJ)/6-31G(d,p) Molecular Geometry in Cartesian Coordinates

\begin{tabular}{|c|c|c|c|}
\hline C & 1.352020 & 0.814050 & 0.874260 \\
\hline $\mathrm{H}$ & 2.250580 & 0.234000 & 1.082750 \\
\hline $\mathrm{H}$ & 0.867320 & 1.051780 & 1.828040 \\
\hline C & -1.480780 & 1.975300 & -1.697670 \\
\hline 0 & -2.139540 & 2.606270 & -2.493820 \\
\hline 0 & -0.555100 & 1.089170 & -2.080120 \\
\hline $\mathrm{H}$ & -0.127500 & 0.665690 & -1.284690 \\
\hline 0 & 0.456130 & 0.004650 & 0.094290 \\
\hline $\mathrm{H}$ & 0.828950 & -0.919640 & 0.055430 \\
\hline C & -0.701970 & -2.103180 & 3.429360 \\
\hline C & -1.326630 & -2.421040 & 1.429190 \\
\hline C & 1.269500 & -3.396710 & 2.701260 \\
\hline C & 0.645920 & -2.190110 & 3.126410 \\
\hline $\mathrm{H}$ & -0.991360 & -1.442710 & 1.083200 \\
\hline $\mathrm{H}$ & 2.357420 & -3.378940 & 2.668560 \\
\hline $\mathrm{H}$ & 0.877780 & -4.315420 & 3.137980 \\
\hline C & 2.363270 & -2.919680 & -0.703210 \\
\hline C & 1.862000 & -4.394230 & -0.870590 \\
\hline C & 2.339140 & -2.070940 & -1.969930 \\
\hline $\mathrm{H}$ & 2.708830 & -1.064880 & -1.753710 \\
\hline $\mathrm{H}$ & 2.988260 & -2.507450 & -2.735850 \\
\hline $\mathrm{H}$ & 1.330250 & -1.986450 & -2.377610 \\
\hline $\mathrm{C}$ & 3.732930 & -2.830940 & -0.028630 \\
\hline $\mathrm{H}$ & 4.536450 & -3.137730 & -0.701260 \\
\hline $\mathrm{H}$ & 3.921690 & -1.798140 & 0.267900 \\
\hline $\mathrm{H}$ & 3.771010 & -3.464940 & 0.859950 \\
\hline C & 2.960310 & -5.397350 & -1.205270 \\
\hline $\mathrm{H}$ & 3.448140 & -5.141430 & -2.151650 \\
\hline $\mathrm{H}$ & 3.715480 & -5.434640 & -0.418760 \\
\hline $\mathrm{H}$ & 2.526599 & -6.395970 & -1.305600 \\
\hline C & 0.712150 & -4.510420 & -1.879010 \\
\hline $\mathrm{H}$ & 1.057850 & -4.375540 & -2.908260 \\
\hline
\end{tabular}




\begin{tabular}{|c|c|c|c|}
\hline $\mathrm{H}$ & 0.267940 & -5.504920 & -1.789120 \\
\hline $\mathrm{H}$ & -0.066790 & -3.773960 & -1.674260 \\
\hline 0 & 1.386260 & -2.387410 & 0.227310 \\
\hline 0 & 1.347700 & -4.697910 & 0.438980 \\
\hline B & 0.833610 & -3.500860 & 0.988400 \\
\hline 0 & -0.655080 & -3.473960 & 1.045600 \\
\hline C & -2.776830 & -2.588430 & 1.633830 \\
\hline C & -3.367060 & -3.860080 & 1.616580 \\
\hline C & -3.576240 & -1.452230 & 1.812840 \\
\hline C & -4.746330 & -3.984220 & 1.760730 \\
\hline $\mathrm{H}$ & -2.737580 & -4.729910 & 1.466720 \\
\hline C & -4.954160 & -1.581030 & 1.950850 \\
\hline $\mathrm{H}$ & -3.115720 & -0.471040 & 1.830140 \\
\hline C & -5.542160 & -2.846230 & 1.920710 \\
\hline $\mathrm{H}$ & -5.204590 & -4.967900 & 1.733770 \\
\hline $\mathrm{H}$ & -5.567880 & -0.693270 & 2.051880 \\
\hline $\mathrm{H}$ & -6.619180 & -2.945320 & 2.013520 \\
\hline $\mathrm{H}$ & 1.184770 & -1.259510 & 2.951170 \\
\hline $\mathrm{H}$ & -1.223440 & -2.979420 & 3.800740 \\
\hline $\mathrm{H}$ & -1.147770 & -1.145650 & 3.678340 \\
\hline C & -0.672190 & 2.780990 & 0.534450 \\
\hline C & -1.673030 & 2.179490 & -0.207270 \\
\hline C & -2.938010 & 1.862030 & 0.383090 \\
\hline C & -3.164320 & 2.222620 & 1.697150 \\
\hline $\mathrm{H}$ & -4.106650 & 1.956320 & 2.167280 \\
\hline C & 1.689930 & 2.093060 & 0.136190 \\
\hline C & 0.676030 & 3.022360 & -0.068210 \\
\hline C & 3.190630 & 3.437320 & -1.216460 \\
\hline C & 2.978210 & 2.307850 & -0.447670 \\
\hline $\mathrm{H}$ & 4.173280 & 3.605850 & -1.647260 \\
\hline C & 2.177150 & 4.394970 & -1.443730 \\
\hline C & 0.889390 & 4.189760 & -0.857990 \\
\hline C & -0.130940 & 5.150270 & -1.092760 \\
\hline C & 0.118660 & 6.262200 & -1.862180 \\
\hline C & 1.397190 & 6.469990 & -2.434200 \\
\hline C & 2.403140 & 5.555780 & -2.229810 \\
\hline $\mathrm{H}$ & -1.115880 & 4.984380 & -0.673050 \\
\hline $\mathrm{H}$ & -0.672230 & 6.983840 & -2.039980 \\
\hline $\mathrm{H}$ & 1.577450 & 7.352270 & -3.040580 \\
\hline $\mathrm{H}$ & 3.385550 & 5.704190 & -2.669200 \\
\hline C & -2.185990 & 2.889840 & 2.473240 \\
\hline C & -0.909750 & 3.166060 & 1.891310 \\
\hline C & 0.076800 & 3.810280 & 2.683710 \\
\hline C & -0.185070 & 4.157280 & 3.990260 \\
\hline C & -1.446220 & 3.877960 & 4.567410 \\
\hline C & -2.423530 & 3.258020 & 3.823390 \\
\hline $\mathrm{H}$ & 1.041630 & 4.028460 & 2.239980 \\
\hline $\mathrm{H}$ & 0.579060 & 4.651390 & 4.582290 \\
\hline $\mathrm{H}$ & -1.640330 & 4.156560 & 5.598550 \\
\hline $\mathrm{H}$ & -3.395180 & 3.040550 & 4.258280 \\
\hline C & 6.316850 & -0.303190 & 0.035050 \\
\hline C & 5.899710 & 0.032780 & -1.257940 \\
\hline C & 4.785160 & 0.867370 & -1.387230 \\
\hline C & 4.113460 & 1.363950 & -0.263310 \\
\hline
\end{tabular}




\begin{tabular}{|c|c|c|c|}
\hline C & 4.567850 & 1.011640 & 1.014430 \\
\hline C & 5.668430 & 0.166980 & 1.184530 \\
\hline $\mathrm{H}$ & 7.173970 & -0.957340 & 0.152600 \\
\hline $\mathrm{H}$ & 4.413330 & 1.145230 & -2.365110 \\
\hline $\mathrm{H}$ & 4.061280 & 1.430850 & 1.874810 \\
\hline C & 6.670360 & -0.506050 & -2.472390 \\
\hline C & 6.181490 & -0.249780 & 2.570500 \\
\hline C & 6.070850 & -0.023220 & -3.803030 \\
\hline $\mathrm{H}$ & 6.650560 & -0.430680 & -4.636750 \\
\hline $\mathrm{H}$ & 5.035380 & -0.355800 & -3.924590 \\
\hline $\mathrm{H}$ & 6.092360 & 1.067710 & -3.885740 \\
\hline C & 8.133980 & -0.020050 & -2.394050 \\
\hline $\mathrm{H}$ & 8.703090 & -0.385420 & -3.255560 \\
\hline $\mathrm{H}$ & 8.179870 & 1.073350 & -2.391940 \\
\hline $\mathrm{H}$ & 8.631410 & -0.377330 & -1.488120 \\
\hline C & 6.641960 & -2.049000 & -2.466800 \\
\hline $\mathrm{H}$ & 7.228810 & -2.442720 & -3.303520 \\
\hline $\mathrm{H}$ & 7.056790 & -2.461300 & -1.542860 \\
\hline $\mathrm{H}$ & 5.616820 & -2.416660 & -2.568890 \\
\hline C & 5.345090 & 0.357850 & 3.708250 \\
\hline $\mathrm{H}$ & 5.740060 & 0.026100 & 4.673120 \\
\hline $\mathrm{H}$ & 5.375090 & 1.451730 & 3.695870 \\
\hline $\mathrm{H}$ & 4.298110 & 0.042400 & 3.652920 \\
\hline C & 6.118130 & -1.787280 & 2.698040 \\
\hline $\mathrm{H}$ & 6.505440 & -2.104020 & 3.672320 \\
\hline $\mathrm{H}$ & 5.087330 & -2.142170 & 2.607530 \\
\hline $\mathrm{H}$ & 6.709250 & -2.284110 & 1.924090 \\
\hline C & 7.642310 & 0.220920 & 2.736640 \\
\hline $\mathrm{H}$ & 8.026360 & -0.067370 & 3.721030 \\
\hline $\mathrm{H}$ & 8.297250 & -0.219320 & 1.979790 \\
\hline $\mathrm{H}$ & 7.711750 & 1.309410 & 2.647020 \\
\hline C & -3.938940 & 1.041620 & -0.339100 \\
\hline C & -3.511110 & -0.068990 & -1.074790 \\
\hline C & -5.310250 & 1.286280 & -0.204160 \\
\hline C & -4.419590 & -0.928620 & -1.689980 \\
\hline $\mathrm{H}$ & -2.452610 & -0.270970 & -1.140280 \\
\hline C & -6.251920 & 0.435340 & -0.789630 \\
\hline $\mathrm{H}$ & -5.627340 & 2.158700 & 0.353190 \\
\hline C & -5.782370 & -0.657590 & -1.529810 \\
\hline $\mathrm{H}$ & -6.503700 & -1.318900 & -1.997000 \\
\hline C & -7.764910 & 0.651730 & -0.644600 \\
\hline C & -3.959500 & -2.123490 & -2.534180 \\
\hline C & -8.397730 & 0.817080 & -2.042890 \\
\hline $\mathrm{H}$ & -9.478600 & 0.973400 & -1.956820 \\
\hline $\mathrm{H}$ & -7.966360 & 1.677800 & -2.562730 \\
\hline $\mathrm{H}$ & -8.235790 & -0.065430 & -2.667580 \\
\hline C & -8.384690 & -0.575660 & 0.058850 \\
\hline $\mathrm{H}$ & -9.469250 & -0.454940 & 0.154820 \\
\hline $\mathrm{H}$ & -8.195370 & -1.498320 & -0.496160 \\
\hline $\mathrm{H}$ & -7.965950 & -0.699190 & 1.062960 \\
\hline C & -8.099740 & 1.901250 & 0.185490 \\
\hline $\mathrm{H}$ & -7.699730 & 1.831530 & 1.202230 \\
\hline $\mathrm{H}$ & -7.704310 & 2.811000 & -0.276310 \\
\hline $\mathrm{H}$ & -9.185590 & 2.012210 & 0.263810 \\
\hline
\end{tabular}




\begin{tabular}{|llll|} 
C & -4.322990 & -1.844120 & -4.008630 \\
$H$ & -5.401420 & -1.707090 & -4.133190 \\
$H$ & -3.825790 & -0.936580 & -4.363820 \\
$H$ & -4.011760 & -2.680410 & -4.644440 \\
$\mathrm{C}$ & -2.439940 & -2.346680 & -2.438380 \\
$\mathrm{H}$ & -1.873150 & -1.480230 & -2.792130 \\
$\mathrm{H}$ & -2.134250 & -2.565430 & -1.410500 \\
$\mathrm{H}$ & -2.157490 & -3.204870 & -3.055610 \\
$\mathrm{C}$ & -4.663190 & -3.411840 & -2.060810 \\
$\mathrm{H}$ & -4.328060 & -4.264820 & -2.660770 \\
$\mathrm{H}$ & -4.434150 & -3.616740 & -1.012230 \\
$\mathrm{H}$ & -5.750230 & -3.345300 & -2.161160 \\
\hline
\end{tabular}

\section{C-Catalyzed reaction TS 2}

\section{Datum}

Value

\begin{tabular}{lc} 
B3LYP-D3(BJ)/6-31G(d,p) Energy & -3039.493513 \\
\hline B3LYP-D3(BJ)/def2-TZVPP/IEF-PCM(DCM) Energy & -3040.532545 \\
\hline B3LYP-D3(BJ)/def2-TZVPP/IEF-PCM(DCM)//B3LYP-D3(BJ)/6-31G(d,p) Free Energy (Quasiharmonic) & -3039.301508
\end{tabular}

Number of Imaginary Frequencies

Frequencies (Top 3 out of 450)

$\begin{array}{lr}\text { 1. } & -312.7716 \mathrm{~cm}^{-1} \\ \text { 2. } & 11.6284 \mathrm{~cm}^{-1} \\ \text { 3. } & 16.4640 \mathrm{~cm}^{-1}\end{array}$

\section{B3LYP-D3(BJ)/6-31G(d,p) Molecular Geometry in Cartesian Coordinates}

C

$\mathrm{H}$

$\mathrm{H}$

C

0

0

$\mathrm{H}$

0

$\mathrm{H}$

C

C

C

C

$\mathrm{H}$
$-1.329890$

$-2.227500$

$-0.840530$

1.501840

2.162110

0.568590

0.140920

$-0.440580$

$-0.830660$

0.483870

1.199010

$-1.450190$

$-0.852190$

0.878940
0.792470

0.210100

1.012610

1.964620

2.600550

1.088660

0.660490

$-0.010570$

$-0.926830$

$-2.224650$

$-2.478360$

$-3.501090$

$-2.307770$

$-1.493620$
0.864221

1.065991

1.819801

$-1.706879$

$-2.497389$

$-2.096839$

$-1.303699$

0.069771

0.011561

3.429281

1.462511

2. 579551

3.070701

1.121461 


\begin{tabular}{|c|c|c|c|}
\hline $\mathrm{H}$ & -2.535260 & -3.487160 & 2.497661 \\
\hline $\mathrm{H}$ & -1.071330 & -4.433770 & 2.997291 \\
\hline C & -2.350940 & -2.861460 & -0.871939 \\
\hline C & -1.845510 & -4.330270 & -1.073239 \\
\hline C & -2.233260 & -1.954900 & -2.092859 \\
\hline $\mathrm{H}$ & -2.618470 & -0.958560 & -1.858629 \\
\hline $\mathrm{H}$ & -2.823310 & -2.351890 & -2.924149 \\
\hline $\mathrm{H}$ & -1.197090 & -1.853500 & -2.420459 \\
\hline C & -3.765540 & -2.791960 & -0.293149 \\
\hline $\mathrm{H}$ & -4.520110 & -3.057931 & -1.038109 \\
\hline H & -3.967600 & -1.773480 & 0.039871 \\
\hline $\mathrm{H}$ & -3.872200 & -3.468560 & 0.558201 \\
\hline C & -2.921210 & -5.308970 & -1.530619 \\
\hline $\mathrm{H}$ & -3.340230 & -5.006090 & -2.495839 \\
\hline $\mathrm{H}$ & -3.729740 & -5.377820 & -0.801269 \\
\hline $\mathrm{H}$ & -2.485080 & -6.304790 & -1.646859 \\
\hline C & -0.629060 & -4.406950 & -2.004999 \\
\hline $\mathrm{H}$ & -0.903480 & -4.224160 & -3.048149 \\
\hline $\mathrm{H}$ & -0.194210 & -5.406790 & -1.929599 \\
\hline $\mathrm{H}$ & 0.137140 & -3.685530 & -1.715549 \\
\hline 0 & -1.440560 & -2.380080 & 0.150121 \\
\hline 0 & -1.426110 & -4.698770 & 0.253581 \\
\hline B & -0.932020 & -3.534060 & 0.884361 \\
\hline 0 & 0.549450 & -3.522870 & 1.018371 \\
\hline C & 2.641830 & -2.640870 & 1.713051 \\
\hline C & 3.232140 & -3.911550 & 1.761101 \\
\hline C & 3.438060 & -1.496740 & 1.841271 \\
\hline C & 4.609970 & -4.027350 & 1.917791 \\
\hline $\mathrm{H}$ & 2.604690 & -4.787970 & 1.643561 \\
\hline C & 4.816230 & -1.616660 & 1.992001 \\
\hline $\mathrm{H}$ & 2.977400 & -0.516120 & 1.801741 \\
\hline C & 5.404270 & -2.881320 & 2.027101 \\
\hline $\mathrm{H}$ & 5.069420 & -5.010640 & 1.942281 \\
\hline $\mathrm{H}$ & 5.429870 & -0.725380 & 2.052771 \\
\hline $\mathrm{H}$ & 6.480670 & -2.975930 & 2.133831 \\
\hline $\mathrm{H}$ & -1.391220 & -1.375760 & 2.905101 \\
\hline $\mathrm{H}$ & 0.991660 & -3.109440 & 3.799631 \\
\hline $\mathrm{H}$ & 0.912210 & -1.274100 & 3.730751 \\
\hline C & 0.702890 & 2.748180 & 0.534541 \\
\hline C & 1.698500 & 2.146960 & -0.214579 \\
\hline C & 2.959120 & 1.806850 & 0.372661 \\
\hline C & 3.186370 & 2.146010 & 1.692301 \\
\hline $\mathrm{H}$ & 4.123430 & 1.860520 & 2.161451 \\
\hline C & -1.667010 & 2.084600 & 0.148031 \\
\hline C & -0.646480 & 3.006120 & -0.059129 \\
\hline C & -3.176260 & 3.466860 & -1.156849 \\
\hline C & -2.964270 & 2.324680 & -0.407019 \\
\hline $\mathrm{H}$ & -4.165370 & 3.655930 & -1.563749 \\
\hline C & -2.153900 & 4.412960 & -1.392739 \\
\hline C & -0.858740 & 4.185000 & -0.831809 \\
\hline C & 0.169150 & 5.135970 & -1.072169 \\
\hline C & -0.079571 & 6.259710 & -1.824559 \\
\hline C & -1.364951 & 6.489860 & -2.372149 \\
\hline C & -2.378721 & 5.585850 & -2.161029 \\
\hline
\end{tabular}




\begin{tabular}{|c|c|c|c|}
\hline $\mathrm{H}$ & 1.159010 & 4.952860 & -0.671609 \\
\hline $\mathrm{H}$ & 0.717139 & 6.973560 & -2.007639 \\
\hline $\mathrm{H}$ & -1.544491 & 7.381400 & -2.965039 \\
\hline $\mathrm{H}$ & -3.366631 & 5.751810 & -2.581379 \\
\hline C & 2.215230 & 2.814130 & 2.476081 \\
\hline C & 0.943280 & 3.113370 & 1.896511 \\
\hline C & -0.036360 & 3.758690 & 2.696741 \\
\hline C & 0.228270 & 4.084670 & 4.008121 \\
\hline C & 1.485100 & 3.781910 & 4.582951 \\
\hline C & 2.455630 & 3.160440 & 3.831531 \\
\hline H & -0.997920 & 3.995100 & 2.255371 \\
\hline $\mathrm{H}$ & -0.530390 & 4.580330 & 4.605881 \\
\hline $\mathrm{H}$ & 1.681190 & 4.043830 & 5.618081 \\
\hline H & 3.423900 & 2.924710 & 4.264401 \\
\hline C & -6.297990 & -0.295731 & 0.142911 \\
\hline C & -5.581430 & 0.126229 & 1.270351 \\
\hline C & -4.499140 & 0.989359 & 1.076171 \\
\hline C & -4.105740 & 1.393010 & -0.205189 \\
\hline C & -4.838560 & 0.939049 & -1.307609 \\
\hline C & -5.948430 & 0.101059 & -1.153469 \\
\hline H & -7.138950 & -0.959251 & 0.277441 \\
\hline H & -3.952240 & 1.372030 & 1.931581 \\
\hline $\mathrm{H}$ & -4.519780 & 1.248319 & -2.297259 \\
\hline C & -5.927280 & -0.336411 & 2.692941 \\
\hline C & -6.734480 & -0.347321 & -2.394279 \\
\hline C & -4.696920 & -1.046241 & 3.301641 \\
\hline $\mathrm{H}$ & -4.919150 & -1.397771 & 4.314711 \\
\hline $\mathrm{H}$ & -3.834000 & -0.376910 & 3.363471 \\
\hline $\mathrm{H}$ & -4.409480 & -1.909551 & 2.694201 \\
\hline C & -7.107070 & -1.321211 & 2.717441 \\
\hline $\mathrm{H}$ & -7.306780 & -1.630501 & 3.747781 \\
\hline $\mathrm{H}$ & -6.892680 & -2.222661 & 2.134931 \\
\hline H & -8.022330 & -0.867761 & 2.324601 \\
\hline C & -6.294930 & 0.888079 & 3.557431 \\
\hline $\mathrm{H}$ & -6.533510 & 0.575949 & 4.579871 \\
\hline $\mathrm{H}$ & -7.166590 & 1.405789 & 3.145321 \\
\hline H & -5.472890 & 1.607279 & 3.609891 \\
\hline C & -5.792690 & -1.083001 & -3.371339 \\
\hline $\mathrm{H}$ & -6.339780 & -1.381131 & -4.272009 \\
\hline $\mathrm{H}$ & -5.380540 & -1.984491 & -2.909119 \\
\hline $\mathrm{H}$ & -4.954090 & -0.454651 & -3.681739 \\
\hline C & -7.319740 & 0.900349 & -3.091419 \\
\hline $\mathrm{H}$ & -7.888420 & 0.609229 & -3.981109 \\
\hline $\mathrm{H}$ & -6.532520 & 1.590509 & -3.407119 \\
\hline $\mathrm{H}$ & -7.990860 & 1.442269 & -2.417769 \\
\hline C & -7.893270 & -1.294011 & -2.042519 \\
\hline $\mathrm{H}$ & -8.417860 & -1.588011 & -2.956569 \\
\hline $\mathrm{H}$ & -8.622680 & -0.815761 & -1.381469 \\
\hline H & -7.535990 & -2.207351 & -1.556309 \\
\hline C & 3.957010 & 0.990510 & -0.359219 \\
\hline C & 3.533150 & -0.090090 & -1.138889 \\
\hline C & 5.328730 & 1.212570 & -0.202569 \\
\hline C & 4.442500 & -0.932370 & -1.778829 \\
\hline $\mathrm{H}$ & 2.473060 & -0.284190 & -1.233799 \\
\hline
\end{tabular}




$\begin{array}{lrrr}\text { C } & 6.272350 & 0.371760 & -0.801529 \\ \text { H } & 5.654030 & 2.063790 & 0.386261 \\ \mathrm{C} & 5.807650 & -0.690670 & -1.587889 \\ \mathrm{H} & 6.524850 & -1.341650 & -2.065499 \\ \mathrm{C} & 7.768580 & 0.637320 & -0.578919 \\ \mathrm{C} & 3.907260 & -2.073350 & -2.654149 \\ \mathrm{C} & 8.119790 & 2.055050 & -1.078239 \\ \mathrm{H} & 9.185080 & 2.260421 & -0.925959 \\ \mathrm{H} & 7.551150 & 2.824050 & -0.548699 \\ \mathrm{H} & 7.899940 & 2.154300 & -2.145439 \\ \mathrm{C} & 8.660540 & -0.369819 & -1.322579 \\ \mathrm{H} & 9.713310 & -0.140759 & -1.130669 \\ \mathrm{H} & 8.502910 & -0.327449 & -2.404569 \\ \mathrm{H} & 8.477630 & -1.396319 & -0.989579 \\ \mathrm{C} & 8.080400 & 0.531260 & 0.929871 \\ \mathrm{H} & 7.848430 & -0.472150 & 1.302061 \\ \mathrm{H} & 7.498860 & 1.250610 & 1.513591 \\ \mathrm{H} & 9.141610 & 0.727001 & 1.118441 \\ \mathrm{C} & 3.028400 & -1.473530 & -3.774469 \\ \mathrm{H} & 3.612830 & -0.797610 & -4.406119 \\ \mathrm{H} & 2.187090 & -0.901810 & -3.373949 \\ \mathrm{H} & 2.626230 & -2.273110 & -4.406649 \\ \mathrm{C} & 3.056750 & -3.026070 & -1.789529 \\ \mathrm{H} & 2.211640 & -2.507730 & -1.329919 \\ \mathrm{H} & 3.652220 & -3.463750 & -0.984409 \\ \mathrm{H} & 2.653200 & -3.838890 & -2.402739 \\ \mathrm{C} & 5.036290 & -2.890400 & -3.301319 \\ \mathrm{H} & 4.607200 & -3.683650 & -3.921059 \\ \mathrm{H} & 5.674390 & -3.365720 & -2.549169 \\ \mathrm{H} & 5.666990 & -2.269960 & -3.945799\end{array}$

\section{C-Catalyzed reaction TS 3 (TS-2.2)}

\section{Datum}

Value

B3LYP-D3(BJ)/6-31G(d,p) Energy

$-3039.492527$

B3LYP-D3(BJ)/def2-TZVPP/IEF-PCM(DCM) Energy

$-3040.531638$

B3LYP-D3(BJ)/def2-TZVPP/IEF-PCM(DCM)//B3LYP-D3(BJ)/6-31G(d,p) Free Energy (Quasiharmonic)

$-3039.300804$

Number of Imaginary Frequencies

Frequencies (Top 3 out of 450)

$\begin{array}{lr}\text { 1. } & -249.0671 \mathrm{~cm}^{-1} \\ \text { 2. } & 9.4362 \mathrm{~cm}^{-1} \\ \text { 3. } & 11.4719 \mathrm{~cm}^{-1}\end{array}$




\begin{tabular}{|c|c|c|c|}
\hline C & -0.847980 & 0.262110 & -0.216670 \\
\hline $\mathrm{H}$ & -0.409630 & 0.225540 & 0.780650 \\
\hline $\mathrm{H}$ & -1.795850 & -0.271350 & -0.179050 \\
\hline C & 2.384850 & 1.999690 & -1.606220 \\
\hline 0 & 3.163570 & 2.827750 & -2.023400 \\
\hline 0 & 1.644840 & 1.244400 & -2.424250 \\
\hline $\mathrm{H}$ & 1.066690 & 0.610960 & -1.917570 \\
\hline 0 & 0.035320 & -0.424070 & -1.126890 \\
\hline $\mathrm{H}$ & 0.397820 & -1.169870 & -0.596380 \\
\hline C & -3.995630 & -2.722440 & 0.748110 \\
\hline C & -2.809940 & -1.953980 & 2.376600 \\
\hline C & -1.772290 & -3.309000 & -0.139990 \\
\hline C & -3.036240 & -3.660660 & 0.422890 \\
\hline $\mathrm{H}$ & -2.898950 & -2.857030 & 2.983110 \\
\hline $\mathrm{H}$ & -1.785150 & -2.448880 & -0.809220 \\
\hline $\mathrm{H}$ & -1.194771 & -4.139380 & -0.544240 \\
\hline C & 1.490140 & -3.097670 & 1.393850 \\
\hline C & 0.757550 & -3.734820 & 2.626240 \\
\hline C & 1.896669 & -4.135450 & 0.342650 \\
\hline $\mathrm{H}$ & 2.177540 & -3.616600 & -0.578110 \\
\hline $\mathrm{H}$ & 2.754159 & -4.728970 & 0.672610 \\
\hline $\mathrm{H}$ & 1.070789 & -4.814620 & 0.117780 \\
\hline C & 2.676310 & -2.210780 & 1.747420 \\
\hline $\mathrm{H}$ & 3.434470 & -2.782010 & 2.293440 \\
\hline $\mathrm{H}$ & 3.143780 & -1.820250 & 0.840610 \\
\hline $\mathrm{H}$ & 2.366540 & -1.361850 & 2.356170 \\
\hline C & 0.715290 & -2.783930 & 3.831840 \\
\hline $\mathrm{H}$ & 1.694900 & -2.693120 & 4.309680 \\
\hline $\mathrm{H}$ & 0.379130 & -1.785960 & 3.541300 \\
\hline $\mathrm{H}$ & 0.010390 & -3.183660 & 4.566260 \\
\hline C & 1.294329 & -5.097050 & 3.049050 \\
\hline $\mathrm{H}$ & 2.348299 & -5.029480 & 3.337290 \\
\hline $\mathrm{H}$ & 0.729809 & -5.464070 & 3.910920 \\
\hline $\mathrm{H}$ & 1.195859 & -5.827660 & 2.244690 \\
\hline 0 & 0.445240 & -2.272910 & 0.823870 \\
\hline 0 & -0.585710 & -3.906750 & 2.129570 \\
\hline B & -0.821340 & -2.819860 & 1.239870 \\
\hline 0 & -1.660690 & -1.710360 & 1.830210 \\
\hline C & -3.651790 & -0.799100 & 2.742750 \\
\hline C & -3.300570 & 0.498320 & 2.343700 \\
\hline C & -4.814070 & -0.997200 & 3.502250 \\
\hline C & -4.107830 & 1.576950 & 2.690980 \\
\hline $\mathrm{H}$ & -2.390000 & 0.654860 & 1.784660 \\
\hline C & -5.620500 & 0.083730 & 3.845840 \\
\hline $\mathrm{H}$ & -5.085190 & -2.001830 & 3.815380 \\
\hline C & -5.270460 & 1.373860 & 3.437990 \\
\hline $\mathrm{H}$ & -3.827390 & 2.577400 & 2.376570 \\
\hline $\mathrm{H}$ & -6.520090 & -0.076969 & 4.431120 \\
\hline $\mathrm{H}$ & -5.899990 & 2.216630 & 3.705440 \\
\hline $\mathrm{H}$ & -3.137151 & -4.663480 & 0.836940 \\
\hline
\end{tabular}




\begin{tabular}{|c|c|c|c|}
\hline $\mathrm{H}$ & -4.028590 & -1.776690 & 0.217390 \\
\hline $\mathrm{H}$ & -4.919310 & -3.024420 & 1.230330 \\
\hline C & 1.050020 & 2.231040 & 0.500140 \\
\hline C & 2.208830 & 1.796160 & -0.114380 \\
\hline C & 3.302460 & 1.285960 & 0.655950 \\
\hline C & 3.198530 & 1.270320 & 2.031010 \\
\hline H & 4.005520 & 0.842700 & 2.618510 \\
\hline C & -1.083440 & 1.688950 & -0.628870 \\
\hline C & -0.141010 & 2.651940 & -0.298890 \\
\hline C & -2.532280 & 3.397860 & -1.555000 \\
\hline C & -2.300350 & 2.065560 & -1.275250 \\
\hline H & -3.461180 & 3.686770 & -2.038480 \\
\hline C & -1.574320 & 4.398040 & -1.261040 \\
\hline C & -0.346360 & 4.021890 & -0.629240 \\
\hline C & 0.617440 & 5.024790 & -0.342980 \\
\hline C & 0.373160 & 6.340190 & -0.661560 \\
\hline C & -0.844900 & 6.715340 & -1.278690 \\
\hline C & -1.794840 & 5.765630 & -1.573310 \\
\hline $\mathrm{H}$ & 1.557750 & 4.731410 & 0.109750 \\
\hline H & 1.121380 & 7.096660 & -0.447230 \\
\hline $\mathrm{H}$ & -1.022660 & 7.757540 & -1.525670 \\
\hline $\mathrm{H}$ & -2.727490 & 6.046110 & -2.054820 \\
\hline C & 2.032200 & 1.715480 & 2.699440 \\
\hline C & 0.933280 & 2.202550 & 1.926580 \\
\hline C & -0.252220 & 2.598710 & 2.600370 \\
\hline C & -0.355010 & 2.494720 & 3.969400 \\
\hline C & 0.729940 & 2.004160 & 4.733030 \\
\hline C & 1.898800 & 1.630040 & 4.110340 \\
\hline $\mathrm{H}$ & -1.081540 & 2.981580 & 2.016800 \\
\hline $\mathrm{H}$ & -1.275510 & 2.786170 & 4.465420 \\
\hline H & 0.637180 & 1.924420 & 5.811690 \\
\hline $\mathrm{H}$ & 2.736700 & 1.251430 & 4.689090 \\
\hline C & -5.256820 & -0.857870 & -2.205270 \\
\hline C & -5.599630 & 0.199870 & -1.353630 \\
\hline C & -4.616710 & 1.153560 & -1.070180 \\
\hline C & -3.329510 & 1.044790 & -1.607370 \\
\hline C & -3.018420 & -0.032510 & -2.446560 \\
\hline C & -3.980190 & -0.994750 & -2.767960 \\
\hline H & -6.010560 & -1.601040 & -2.440030 \\
\hline $\mathrm{H}$ & -4.826000 & 1.982860 & -0.406960 \\
\hline H & -2.011520 & -0.103580 & -2.836820 \\
\hline C & -7.008770 & 0.264781 & -0.746170 \\
\hline C & -3.695270 & -2.135850 & -3.756040 \\
\hline C & -8.064500 & 0.203521 & -1.870260 \\
\hline $\mathrm{H}$ & -9.072730 & 0.262011 & -1.446420 \\
\hline $\mathrm{H}$ & -7.937640 & 1.037301 & -2.567660 \\
\hline $\mathrm{H}$ & -7.999560 & -0.724949 & -2.443690 \\
\hline C & -7.199580 & -0.934859 & 0.207440 \\
\hline $\mathrm{H}$ & -8.212250 & -0.933429 & 0.626000 \\
\hline $\mathrm{H}$ & -7.049570 & -1.886229 & -0.311450 \\
\hline $\mathrm{H}$ & -6.489090 & -0.883609 & 1.037640 \\
\hline C & -7.237050 & 1.556531 & 0.055850 \\
\hline $\mathrm{H}$ & -8.258700 & 1.569011 & 0.448380 \\
\hline $\mathrm{H}$ & -6.554830 & 1.629021 & 0.907070 \\
\hline
\end{tabular}




\begin{tabular}{|c|c|c|c|}
\hline H & -7.108520 & 2.446211 & -0.568710 \\
\hline $\mathrm{C}$ & -4.496440 & -1.864040 & -5.048750 \\
\hline $\mathrm{H}$ & -4.314250 & -2.653820 & -5.785880 \\
\hline $\mathrm{H}$ & -5.571680 & -1.826970 & -4.849500 \\
\hline $\mathrm{H}$ & -4.204320 & -0.907140 & -5.491980 \\
\hline C & -2.202500 & -2.229480 & -4.116270 \\
\hline H & -2.038470 & -3.082930 & -4.781260 \\
\hline $\mathrm{H}$ & -1.853840 & -1.334230 & -4.639050 \\
\hline $\mathrm{H}$ & -1.575950 & -2.366440 & -3.229940 \\
\hline C & -4.135340 & -3.491390 & -3.165660 \\
\hline $\mathrm{H}$ & -3.942121 & -4.292570 & -3.886780 \\
\hline $\mathrm{H}$ & -3.586050 & -3.716980 & -2.249090 \\
\hline $\mathrm{H}$ & -5.203130 & -3.511470 & -2.931310 \\
\hline C & 4.467710 & 0.651840 & -0.003960 \\
\hline C & 5.772420 & 0.875550 & 0.447070 \\
\hline C & 4.248230 & -0.253160 & -1.048810 \\
\hline C & 6.856750 & 0.211219 & -0.133920 \\
\hline H & 5.926020 & 1.592489 & 1.243680 \\
\hline C & 5.304660 & -0.928830 & -1.658400 \\
\hline $\mathrm{H}$ & 3.234260 & -0.429580 & -1.375840 \\
\hline C & 6.597070 & -0.683841 & -1.180080 \\
\hline $\mathrm{H}$ & 7.430640 & -1.203481 & -1.640380 \\
\hline C & 5.077540 & -1.917690 & -2.809130 \\
\hline C & 8.304840 & 0.435589 & 0.324880 \\
\hline C & 5.538790 & -3.323040 & -2.368270 \\
\hline H & 6.598800 & -3.333231 & -2.098170 \\
\hline $\mathrm{H}$ & 4.968120 & -3.661920 & -1.497450 \\
\hline $\mathrm{H}$ & 5.390229 & -4.046540 & -3.177390 \\
\hline C & 3.597110 & -1.995690 & -3.220940 \\
\hline $\mathrm{H}$ & 3.486800 & -2.682510 & -4.065710 \\
\hline $\mathrm{H}$ & 2.970390 & -2.374650 & -2.406750 \\
\hline $\mathrm{H}$ & 3.204980 & -1.020160 & -3.523030 \\
\hline C & 5.894600 & -1.464311 & -4.037790 \\
\hline H & 5.582080 & -0.467250 & -4.361780 \\
\hline $\mathrm{H}$ & 6.965990 & -1.427041 & -3.821680 \\
\hline $\mathrm{H}$ & 5.745300 & -2.159650 & -4.870940 \\
\hline C & 8.902070 & -0.905851 & 0.801940 \\
\hline $\mathrm{H}$ & 8.327890 & -1.309151 & 1.642110 \\
\hline $\mathrm{H}$ & 8.900730 & -1.655691 & 0.006110 \\
\hline $\mathrm{H}$ & 9.938020 & -0.767121 & 1.130070 \\
\hline C & 9.135850 & 0.977659 & -0.858050 \\
\hline $\mathrm{H}$ & 9.143590 & 0.279649 & -1.699660 \\
\hline $\mathrm{H}$ & 8.726160 & 1.927089 & -1.215720 \\
\hline $\mathrm{H}$ & 10.174020 & 1.144709 & -0.550460 \\
\hline C & 8.400860 & 1.442979 & 1.481990 \\
\hline $\mathrm{H}$ & 9.447490 & 1.566339 & 1.776890 \\
\hline $\mathrm{H}$ & 8.018300 & 2.426979 & 1.194380 \\
\hline $\mathrm{H}$ & 7.845690 & 1.102649 & 2.361890 \\
\hline
\end{tabular}


Frequencies (Top 3 out of 450)

$\begin{array}{lr}\text { 1. } & -300.8391 \mathrm{~cm}^{-1} \\ \text { 2. } & 9.0435 \mathrm{~cm}^{-1} \\ \text { 3. } & 13.8900 \mathrm{~cm}^{-1}\end{array}$

\section{B3LYP-D3(BJ)/6-31G(d,p) Molecular Geometry in Cartesian Coordinates}

\begin{tabular}{|c|c|c|c|}
\hline C & 1.706130 & 0.722929 & 0.865120 \\
\hline $\mathrm{H}$ & 2.561640 & 0.089899 & 1.102840 \\
\hline $\mathrm{H}$ & 1.108500 & 0.845549 & 1.774420 \\
\hline C & -0.907680 & 2.507100 & -1.458600 \\
\hline 0 & -1.532040 & 3.320780 & -2.103720 \\
\hline 0 & -0.031260 & 1.674079 & -2.020350 \\
\hline $\mathrm{H}$ & 0.358180 & 1.057549 & -1.340190 \\
\hline 0 & 0.925260 & 0.112199 & -0.167280 \\
\hline $\mathrm{H}$ & 0.712029 & -0.841851 & 0.025880 \\
\hline C & -2.360471 & -1.094530 & 2.708420 \\
\hline C & -2.475111 & -2.489130 & 1.162760 \\
\hline C & -0.003721 & -1.823171 & 2.779840 \\
\hline C & -1.013531 & -0.858550 & 2.489200 \\
\hline $\mathrm{H}$ & -2.296141 & -1.780290 & 0.354200 \\
\hline $\mathrm{H}$ & 1.022159 & -1.460291 & 2.731210 \\
\hline $\mathrm{H}$ & -0.181821 & -2.432601 & 3.666330 \\
\hline C & 1.472629 & -3.240961 & -0.286460 \\
\hline C & 1.260489 & -4.576711 & 0.508110 \\
\hline C & 1.356649 & -3.358791 & -1.801450 \\
\hline H & 1.481699 & -2.372431 & -2.257990 \\
\hline $\mathrm{H}$ & 2.136779 & -4.017951 & -2.195730 \\
\hline $\mathrm{H}$ & 0.384169 & -3.749391 & -2.101230 \\
\hline C & 2.786409 & -2.547551 & 0.082400 \\
\hline $\mathrm{H}$ & 3.649339 & -3.119411 & -0.263830 \\
\hline $\mathrm{H}$ & 2.837859 & -1.565511 & -0.389580 \\
\hline $\mathrm{H}$ & 2.870829 & -2.426111 & 1.165000 \\
\hline C & 2.542848 & -5.351291 & 0.795900 \\
\hline $\mathrm{H}$ & 3.050758 & -5.630281 & -0.133100 \\
\hline $\mathrm{H}$ & 3.228869 & -4.766451 & 1.410350 \\
\hline $\mathrm{H}$ & 2.300228 & -6.268361 & 1.339620 \\
\hline C & 0.231418 & -5.502001 & -0.158180 \\
\hline & 0.629028 & -5.958051 & -1.069730 \\
\hline
\end{tabular}




\begin{tabular}{|c|c|c|c|}
\hline $\mathrm{H}$ & -0.025632 & -6.298501 & 0.544670 \\
\hline $\mathrm{H}$ & -0.686051 & -4.964590 & -0.406960 \\
\hline 0 & 0.365419 & -2.422981 & 0.196160 \\
\hline 0 & 0.722199 & -4.112541 & 1.756510 \\
\hline B & -0.088501 & -2.989821 & 1.465420 \\
\hline 0 & -1.537441 & -3.353270 & 1.442670 \\
\hline C & -3.871171 & -2.943710 & 1.336610 \\
\hline C & -4.140721 & -4.163210 & 1.973470 \\
\hline C & -4.927911 & -2.162690 & 0.846840 \\
\hline C & -5.455171 & -4.598740 & 2.112600 \\
\hline $\mathrm{H}$ & -3.310771 & -4.758790 & 2.336070 \\
\hline C & -6.239571 & -2.607649 & 0.985250 \\
\hline $\mathrm{H}$ & -4.721761 & -1.218820 & 0.355140 \\
\hline C & -6.506541 & -3.823269 & 1.618390 \\
\hline $\mathrm{H}$ & -5.660602 & -5.546590 & 2.600350 \\
\hline $\mathrm{H}$ & -7.052421 & -2.008689 & 0.591300 \\
\hline $\mathrm{H}$ & -7.531011 & -4.167299 & 1.722640 \\
\hline $\mathrm{H}$ & -0.762901 & -0.026590 & 1.834440 \\
\hline $\mathrm{H}$ & -2.665371 & -1.792590 & 3.481850 \\
\hline $\mathrm{H}$ & -3.090231 & -0.347030 & 2.421810 \\
\hline C & -0.125540 & 2.892879 & 0.903470 \\
\hline C & -1.111420 & 2.438150 & 0.045830 \\
\hline C & -2.404800 & 2.074620 & 0.541730 \\
\hline C & -2.692810 & 2.281400 & 1.874020 \\
\hline $\mathrm{H}$ & -3.668770 & 1.999900 & 2.259670 \\
\hline C & 2.158840 & 2.076099 & 0.373270 \\
\hline C & 1.256680 & 3.131179 & 0.382300 \\
\hline C & 3.801200 & 3.465609 & -0.743370 \\
\hline C & 3.447070 & 2.239349 & -0.220410 \\
\hline $\mathrm{H}$ & 4.781440 & 3.588579 & -1.195050 \\
\hline C & 2.917280 & 4.571369 & -0.714770 \\
\hline C & 1.612500 & 4.403219 & -0.152870 \\
\hline C & 0.713041 & 5.502269 & -0.162210 \\
\hline C & 1.095471 & 6.715129 & -0.685590 \\
\hline C & 2.391811 & 6.886629 & -1.228200 \\
\hline C & 3.280491 & 5.837349 & -1.244580 \\
\hline $\mathrm{H}$ & -0.285339 & 5.365349 & 0.237110 \\
\hline $\mathrm{H}$ & 0.397301 & 7.546119 & -0.690710 \\
\hline $\mathrm{H}$ & 2.678701 & 7.849549 & -1.639470 \\
\hline $\mathrm{H}$ & 4.273351 & 5.958969 & -1.668400 \\
\hline C & -1.729180 & 2.801570 & 2.773380 \\
\hline C & -0.413990 & 3.090189 & 2.290400 \\
\hline C & 0.561770 & 3.564419 & 3.205720 \\
\hline C & 0.251240 & 3.743679 & 4.535100 \\
\hline C & -1.048770 & 3.455610 & 5.012890 \\
\hline C & -2.015730 & 2.994040 & 4.149590 \\
\hline $\mathrm{H}$ & 1.556930 & 3.786729 & 2.837140 \\
\hline $\mathrm{H}$ & 1.007470 & 4.108939 & 5.222910 \\
\hline $\mathrm{H}$ & -1.280170 & 3.599150 & 6.063740 \\
\hline $\mathrm{H}$ & -3.015140 & 2.766190 & 4.509620 \\
\hline C & 6.048299 & -1.115222 & -0.605140 \\
\hline C & 5.438089 & -0.595532 & -1.752100 \\
\hline C & 4.600040 & 0.514319 & -1.599620 \\
\hline C & 4.378850 & 1.081989 & -0.341060 \\
\hline
\end{tabular}




\begin{tabular}{|c|c|c|c|}
\hline C & 5.008290 & 0.537218 & 0.783700 \\
\hline C & 5.850069 & -0.573252 & 0.670970 \\
\hline $\mathrm{H}$ & 6.697229 & -1.978392 & -0.707290 \\
\hline $\mathrm{H}$ & 4.086950 & 0.945379 & -2.449810 \\
\hline H & 4.826320 & 0.996849 & 1.747590 \\
\hline C & 5.692359 & -1.258132 & -3.113510 \\
\hline C & 6.553079 & -1.206382 & 1.880350 \\
\hline C & 5.234579 & -2.731612 & -3.058020 \\
\hline $\mathrm{H}$ & 5.421489 & -3.224722 & -4.018060 \\
\hline $\mathrm{H}$ & 5.765839 & -3.293252 & -2.284430 \\
\hline $\mathrm{H}$ & 4.163979 & -2.794681 & -2.844250 \\
\hline C & 4.927699 & -0.562122 & -4.250870 \\
\hline H & 5.137549 & -1.067382 & -5.198510 \\
\hline $\mathrm{H}$ & 3.845779 & -0.593491 & -4.089750 \\
\hline $\mathrm{H}$ & 5.226980 & 0.484808 & -4.359330 \\
\hline C & 7.201399 & -1.199502 & -3.433620 \\
\hline $\mathrm{H}$ & 7.404419 & -1.670022 & -4.401710 \\
\hline $\mathrm{H}$ & 7.548329 & -0.162462 & -3.477760 \\
\hline H & 7.796109 & -1.719142 & -2.677130 \\
\hline C & 8.081609 & -1.119052 & 1.681320 \\
\hline H & 8.604089 & -1.567262 & 2.533380 \\
\hline $\mathrm{H}$ & 8.401299 & -1.644832 & 0.777350 \\
\hline H & 8.402059 & -0.076362 & 1.592670 \\
\hline C & 6.195169 & -0.499462 & 3.197510 \\
\hline $\mathrm{H}$ & 6.710789 & -0.987992 & 4.029790 \\
\hline $\mathrm{H}$ & 6.499460 & 0.551688 & 3.189610 \\
\hline H & 5.120499 & -0.544312 & 3.400780 \\
\hline C & 6.134889 & -2.687872 & 2.001820 \\
\hline H & 6.643789 & -3.158142 & 2.850010 \\
\hline $\mathrm{H}$ & 5.056009 & -2.775322 & 2.159560 \\
\hline $\mathrm{H}$ & 6.387719 & -3.256792 & 1.103010 \\
\hline C & -3.367710 & 1.382290 & -0.347240 \\
\hline C & -2.906360 & 0.341870 & -1.164460 \\
\hline C & -4.721690 & 1.729880 & -0.376300 \\
\hline C & -3.764461 & -0.338210 & -2.029580 \\
\hline $\mathrm{H}$ & -1.858610 & 0.080580 & -1.123640 \\
\hline C & -5.614650 & 1.067170 & -1.225110 \\
\hline $\mathrm{H}$ & -5.054640 & 2.554710 & 0.240630 \\
\hline C & -5.114111 & 0.037010 & -2.033670 \\
\hline $\mathrm{H}$ & -5.795551 & -0.480959 & -2.699730 \\
\hline C & -7.096540 & 1.458631 & -1.326890 \\
\hline C & -3.262391 & -1.443250 & -2.968880 \\
\hline C & -7.417720 & 1.852441 & -2.785180 \\
\hline $\mathrm{H}$ & -8.467180 & 2.153021 & -2.874810 \\
\hline $\mathrm{H}$ & -6.791260 & 2.689841 & -3.106220 \\
\hline $\mathrm{H}$ & -7.247210 & 1.023301 & -3.477030 \\
\hline C & -7.976520 & 0.261121 & -0.911830 \\
\hline $\mathrm{H}$ & -9.037400 & 0.504771 & -1.033540 \\
\hline $\mathrm{H}$ & -7.763931 & -0.626729 & -1.514090 \\
\hline $\mathrm{H}$ & -7.806801 & 0.008341 & 0.139620 \\
\hline C & -7.449410 & 2.648861 & -0.420160 \\
\hline $\mathrm{H}$ & -7.264620 & 2.423601 & 0.635000 \\
\hline H & -6.877440 & 3.542781 & -0.686160 \\
\hline $\mathrm{H}$ & -8.511640 & 2.889311 & -0.525970 \\
\hline
\end{tabular}




\begin{tabular}{|llll|} 
C & -3.568871 & -1.030540 & -4.425230 \\
$H$ & -4.642011 & -0.904310 & -4.594620 \\
$H$ & -3.075611 & -0.085340 & -4.670170 \\
$H$ & -3.208441 & -1.797530 & -5.119100 \\
C & -1.744591 & -1.662140 & -2.843930 \\
$H$ & -1.184411 & -0.749550 & -3.069290 \\
$H$ & -1.450861 & -2.002120 & -1.846420 \\
$H$ & -1.429641 & -2.432410 & -3.554810 \\
C & -3.980521 & -2.770570 & -2.647060 \\
$H$ & -3.655711 & -3.553410 & -3.340790 \\
$H$ & -3.758531 & -3.104270 & -1.629720 \\
$H$ & -5.066891 & -2.675510 & -2.733120 \\
\hline
\end{tabular}

\section{C-Catalyzed reaction TS 5}

\section{Datum}

Value

\begin{tabular}{lc} 
B3LYP-D3(BJ)/6-31G(d,p) Energy & -3039.491294 \\
\hline B3LYP-D3(BJ)/def2-TZVPP/IEF-PCM(DCM) Energy & -3040.530291 \\
\hline B3LYP-D3(BJ)/def2-TZVPP/IEF-PCM(DCM)//B3LYP-D3(BJ)/6-31G(d,p) Free Energy (Quasiharmonic) & -3039.299557
\end{tabular}

Number of Imaginary Frequencies

Frequencies (Top 3 out of 450)

$\begin{array}{lr}\text { 1. } & -293.4056 \mathrm{~cm}^{-1} \\ \text { 2. } & 9.7042 \mathrm{~cm}^{-1} \\ \text { 3. } & 10.4898 \mathrm{~cm}^{-1}\end{array}$

B3LYP-D3(BJ)/6-31G(d,p) Molecular Geometry in Cartesian Coordinates

\begin{tabular}{|c|c|c|c|}
\hline C & 1.382520 & 0.346821 & -0.069602 \\
\hline $\mathrm{H}$ & 2.304935 & -0.036051 & 0.377040 \\
\hline $\mathrm{H}$ & 0.736122 & 0.704867 & 0.734996 \\
\hline C & -1.542974 & 1.095586 & -2.507852 \\
\hline 0 & -2.249985 & 1.608918 & -3.347456 \\
\hline 0 & -0.701608 & 0.104336 & -2.795471 \\
\hline $\mathrm{H}$ & -0.189949 & -0.201459 & -1.991515 \\
\hline 0 & 0.714340 & -0.716985 & -0.768928 \\
\hline $\mathrm{H}$ & 0.280258 & -1.312479 & -0.103948 \\
\hline C & -2.273890 & 0.283973 & 4.063516 \\
\hline C & -0.282836 & -0.421874 & 4.054059 \\
\hline C & -2.481566 & -1.250251 & 2.135692 \\
\hline C & -2.775600 & -0.867742 & 3.477884 \\
\hline $\mathrm{H}$ & -0.446104 & -1.254241 & 4.739496 \\
\hline
\end{tabular}




\begin{tabular}{|c|c|c|c|}
\hline $\mathrm{H}$ & -2.392378 & -0.430558 & 1.426236 \\
\hline H & -3.071247 & -2.076995 & 1.742596 \\
\hline C & 0.434846 & -3.531054 & 1.260506 \\
\hline C & -0.288891 & -4.166003 & 2.501468 \\
\hline C & 0.441401 & -4.397105 & 0.008019 \\
\hline $\mathrm{H}$ & 0.916171 & -3.854885 & -0.812454 \\
\hline H & 1.012729 & -5.314888 & 0.179148 \\
\hline $\mathrm{H}$ & -0.568051 & -4.664405 & -0.302722 \\
\hline C & 1.856531 & -3.047059 & 1.574005 \\
\hline $\mathrm{H}$ & 2.537399 & -3.886708 & 1.736541 \\
\hline $\mathrm{H}$ & 2.230838 & -2.465956 & 0.727240 \\
\hline $\mathrm{H}$ & 1.872576 & -2.408401 & 2.459458 \\
\hline C & 0.639185 & -4.930863 & 3.441383 \\
\hline $\mathrm{H}$ & 1.119775 & -5.767868 & 2.924381 \\
\hline $\mathrm{H}$ & 1.413193 & -4.279623 & 3.850415 \\
\hline $\mathrm{H}$ & 0.060807 & -5.335915 & 4.276488 \\
\hline C & -1.479238 & -5.048864 & 2.108910 \\
\hline $\mathrm{H}$ & -1.159970 & -5.988668 & 1.648976 \\
\hline $\mathrm{H}$ & -2.049622 & -5.283291 & 3.011632 \\
\hline $\mathrm{H}$ & -2.140170 & -4.528639 & 1.413575 \\
\hline 0 & -0.389733 & -2.359456 & 1.006217 \\
\hline 0 & -0.807775 & -3.021813 & 3.198662 \\
\hline B & -0.877666 & -1.920692 & 2.289533 \\
\hline 0 & -0.097449 & -0.735930 & 2.800447 \\
\hline C & 0.426811 & 0.745419 & 4.603624 \\
\hline C & 0.563685 & 0.854652 & 5.995443 \\
\hline C & 0.991981 & 1.720711 & 3.772863 \\
\hline C & 1.265430 & 1.920061 & 6.550483 \\
\hline $\mathrm{H}$ & 0.123508 & 0.095771 & 6.636758 \\
\hline C & 1.702199 & 2.778168 & 4.331854 \\
\hline $\mathrm{H}$ & 0.870301 & 1.647004 & 2.699643 \\
\hline C & 1.840966 & 2.881963 & 5.717340 \\
\hline $\mathrm{H}$ & 1.371242 & 1.996813 & 7.627943 \\
\hline $\mathrm{H}$ & 2.141780 & 3.525937 & 3.682106 \\
\hline $\mathrm{H}$ & 2.396162 & 3.709925 & 6.147158 \\
\hline $\mathrm{H}$ & -3.182274 & -1.634918 & 4.135969 \\
\hline $\mathrm{H}$ & -2.034275 & 1.141618 & 3.443788 \\
\hline $\mathrm{H}$ & -2.477347 & 0.497084 & 5.108250 \\
\hline C & -0.573952 & 2.384940 & -0.579030 \\
\hline C & -1.604890 & 1.609380 & -1.078999 \\
\hline C & -2.835454 & 1.473523 & -0.357807 \\
\hline C & -2.999831 & 2.167722 & 0.822807 \\
\hline $\mathrm{H}$ & -3.914245 & 2.036142 & 1.393808 \\
\hline C & 1.698409 & 1.473692 & -1.024564 \\
\hline C & 0.745609 & 2.451139 & -1.277510 \\
\hline C & 3.278391 & 2.626726 & -2.461555 \\
\hline C & 2.977621 & 1.545237 & -1.658923 \\
\hline H & 4.254991 & 2.681535 & -2.934012 \\
\hline C & 2.329515 & 3.641178 & -2.732521 \\
\hline C & 1.026027 & 3.541956 & -2.153321 \\
\hline C & 0.059583 & 4.535845 & -2.462453 \\
\hline C & 0.376238 & 5.586745 & -3.291462 \\
\hline C & 1.671818 & 5.694338 & -3.852012 \\
\hline C & 2.624716 & 4.740573 & -3.581168 \\
\hline
\end{tabular}




\begin{tabular}{|c|c|c|c|}
\hline H & -0.938178 & 4.442680 & -2.049392 \\
\hline H & -0.373974 & 6.335331 & -3.525770 \\
\hline $\mathrm{H}$ & 1.906934 & 6.529024 & -4.505289 \\
\hline $\mathrm{H}$ & 3.616958 & 4.808915 & -4.018278 \\
\hline C & -1.997631 & 3.032413 & 1.326837 \\
\hline C & -0.758929 & 3.143066 & 0.621800 \\
\hline C & 0.232207 & 4.029535 & 1.120830 \\
\hline C & 0.006431 & 4.768785 & 2.260546 \\
\hline C & -1.205873 & 4.634946 & 2.975578 \\
\hline C & -2.183970 & 3.782278 & 2.518392 \\
\hline $\mathrm{H}$ & 1.165174 & 4.129554 & 0.578611 \\
\hline $\mathrm{H}$ & 0.765839 & 5.457298 & 2.617578 \\
\hline $\mathrm{H}$ & -1.362955 & 5.209804 & 3.882670 \\
\hline $\mathrm{H}$ & -3.123535 & 3.680056 & 3.054381 \\
\hline C & 5.856038 & -1.571907 & -1.290670 \\
\hline C & 6.193325 & -0.295719 & -0.824033 \\
\hline C & 5.237413 & 0.718437 & -0.945903 \\
\hline C & 3.978675 & 0.456764 & -1.496452 \\
\hline C & 3.673198 & -0.832611 & -1.947789 \\
\hline C & 4.612147 & -1.863607 & -1.864428 \\
\hline $\mathrm{H}$ & 6.590457 & -2.366306 & -1.209517 \\
\hline $\mathrm{H}$ & 5.448459 & 1.723936 & -0.603108 \\
\hline $\mathrm{H}$ & 2.693050 & -1.005770 & -2.369106 \\
\hline C & 7.577604 & -0.058691 & -0.203061 \\
\hline C & 4.325779 & -3.276680 & -2.392435 \\
\hline C & 8.669240 & -0.393307 & -1.242208 \\
\hline $\mathrm{H}$ & 9.665203 & -0.233168 & -0.814939 \\
\hline $\mathrm{H}$ & 8.609465 & -1.434310 & -1.571141 \\
\hline $\mathrm{H}$ & 8.569765 & 0.242773 & -2.127223 \\
\hline C & 7.773001 & 1.398051 & 0.247130 \\
\hline $\mathrm{H}$ & 8.770470 & 1.519216 & 0.680703 \\
\hline $\mathrm{H}$ & 7.688368 & 2.095552 & -0.591973 \\
\hline $\mathrm{H}$ & 7.042074 & 1.687699 & 1.008491 \\
\hline C & 7.744762 & -0.972091 & 1.030465 \\
\hline $\mathrm{H}$ & 8.728426 & -0.820327 & 1.488282 \\
\hline $\mathrm{H}$ & 6.979576 & -0.752368 & 1.781595 \\
\hline $\mathrm{H}$ & 7.657999 & -2.029256 & 0.764914 \\
\hline C & 5.386726 & -3.653456 & -3.448872 \\
\hline $\mathrm{H}$ & 5.186870 & -4.654006 & -3.847550 \\
\hline $\mathrm{H}$ & 5.372794 & -2.944187 & -4.282052 \\
\hline $\mathrm{H}$ & 6.396302 & -3.657176 & -3.028481 \\
\hline C & 4.389560 & -4.283368 & -1.224221 \\
\hline $\mathrm{H}$ & 4.207441 & -5.300956 & -1.587066 \\
\hline H & 5.368091 & -4.271024 & -0.735315 \\
\hline $\mathrm{H}$ & 3.635204 & -4.049919 & -0.468871 \\
\hline C & 2.939854 & -3.373564 & -3.050628 \\
\hline H & 2.756577 & -4.401183 & -3.379170 \\
\hline H & 2.140887 & -3.093147 & -2.359648 \\
\hline $\mathrm{H}$ & 2.865357 & -2.723351 & -3.927547 \\
\hline C & -3.882833 & 0.534979 & -0.822936 \\
\hline C & -5.237090 & 0.899088 & -0.806379 \\
\hline C & -3.524599 & -0.745647 & -1.241760 \\
\hline C & -6.224189 & 0.003521 & -1.214213 \\
\hline H & -5.494843 & 1.904117 & -0.497320 \\
\hline
\end{tabular}




\begin{tabular}{|c|c|c|c|}
\hline C & -4.486054 & -1.668145 & -1.669542 \\
\hline $\mathrm{H}$ & -2.479285 & -1.027849 & -1.226928 \\
\hline C & -5.823935 & -1.273800 & -1.644281 \\
\hline $\mathrm{H}$ & -6.584912 & -1.970219 & -1.972601 \\
\hline C & -4.024304 & -3.052568 & -2.139874 \\
\hline C & -7.715922 & 0.366041 & -1.225404 \\
\hline C & -5.194973 & -3.936897 & -2.595639 \\
\hline $\mathrm{H}$ & -5.908058 & -4.117085 & -1.784475 \\
\hline $\mathrm{H}$ & -5.735498 & -3.488855 & -3.435257 \\
\hline $\mathrm{H}$ & -4.815629 & -4.908799 & -2.925991 \\
\hline C & -3.047245 & -2.883548 & -3.324435 \\
\hline $\mathrm{H}$ & -2.682597 & -3.862109 & -3.656254 \\
\hline $\mathrm{H}$ & -3.546933 & -2.399378 & -4.168863 \\
\hline $\mathrm{H}$ & -2.182860 & -2.268464 & -3.062938 \\
\hline C & -3.303452 & -3.760730 & -0.973223 \\
\hline $\mathrm{H}$ & -2.442039 & -3.189031 & -0.619480 \\
\hline $\mathrm{H}$ & -3.985801 & -3.901601 & -0.127686 \\
\hline $\mathrm{H}$ & -2.948048 & -4.748536 & -1.288153 \\
\hline C & -8.256634 & 0.256997 & -2.667482 \\
\hline $\mathrm{H}$ & -7.720493 & 0.939170 & -3.334057 \\
\hline $\mathrm{H}$ & -8.144788 & -0.755185 & -3.065592 \\
\hline $\mathrm{H}$ & -9.321433 & 0.513324 & -2.696104 \\
\hline C & -8.485404 & -0.612350 & -0.311845 \\
\hline $\mathrm{H}$ & -8.375231 & -1.648250 & -0.644024 \\
\hline $\mathrm{H}$ & -8.119600 & -0.550543 & 0.718058 \\
\hline H & -9.554362 & -0.372624 & -0.310644 \\
\hline C & -7.972286 & 1.795783 & -0.722483 \\
\hline $\mathrm{H}$ & -9.046072 & 2.006136 & -0.741718 \\
\hline $\mathrm{H}$ & -7.625492 & 1.930573 & 0.307053 \\
\hline $\mathrm{H}$ & -7.476282 & 2.540779 & -1.351975 \\
\hline
\end{tabular}

\section{C-Catalyzed reaction TS 6}

\section{Datum}

Value

B3LYP-D3(BJ)/6-31G(d,p) Energy

$-3039.492216$

B3LYP-D3(BJ)/def2-TZVPP/IEF-PCM(DCM) Energy

$-3040.530219$

B3LYP-D3(BJ)/def2-TZVPP/IEF-PCM(DCM)//B3LYP-D3(BJ)/6-31G(d,p) Free Energy (Quasiharmonic)

$-3039.299272$

Number of Imaginary Frequencies

Frequencies (Top 3 out of 450)

$\begin{array}{lr}\text { 1. } & -273.3177 \mathrm{~cm}^{-1} \\ \text { 2. } & 11.7751 \mathrm{~cm}^{-1} \\ \text { 3. } & 12.9569 \mathrm{~cm}^{-1}\end{array}$




\begin{tabular}{|c|c|c|c|}
\hline C & -0.963690 & 0.167950 & -0.445790 \\
\hline $\mathrm{H}$ & -0.535680 & 0.168490 & 0.556110 \\
\hline $\mathrm{H}$ & -1.935460 & -0.314270 & -0.381680 \\
\hline C & 2.439170 & 1.683370 & -1.712790 \\
\hline 0 & 3.234780 & 2.486400 & -2.145200 \\
\hline 0 & 1.757080 & 0.858190 & -2.516720 \\
\hline $\mathrm{H}$ & 1.121600 & 0.281750 & -2.014330 \\
\hline 0 & -0.127630 & -0.607780 & -1.323340 \\
\hline $\mathrm{H}$ & -0.103470 & -1.492010 & -0.898330 \\
\hline C & -4.420200 & -2.583100 & 2.440000 \\
\hline C & -2.707950 & -1.336390 & 2.818150 \\
\hline C & -2.833630 & -3.723710 & 0.934720 \\
\hline C & -3.648650 & -3.680310 & 2.106850 \\
\hline $\mathrm{H}$ & -2.390020 & -1.875380 & 3.711640 \\
\hline $\mathrm{H}$ & -3.226850 & -3.180550 & 0.074850 \\
\hline $\mathrm{H}$ & -2.442540 & -4.704590 & 0.667210 \\
\hline C & 0.804830 & -3.348240 & 0.870590 \\
\hline C & 0.626520 & -3.295250 & 2.426700 \\
\hline C & 0.829150 & -4.777070 & 0.320340 \\
\hline $\mathrm{H}$ & 0.711440 & -4.738260 & -0.765950 \\
\hline $\mathrm{H}$ & 1.772300 & -5.282450 & 0.547020 \\
\hline $\mathrm{H}$ & 0.009120 & -5.368990 & 0.734140 \\
\hline C & 1.994430 & -2.554800 & 0.341180 \\
\hline $\mathrm{H}$ & 2.934090 & -2.938050 & 0.749920 \\
\hline $\mathrm{H}$ & 2.048880 & -2.645720 & -0.747400 \\
\hline $\mathrm{H}$ & 1.919750 & -1.496310 & 0.589840 \\
\hline C & 1.007950 & -1.928610 & 3.012040 \\
\hline $\mathrm{H}$ & 2.088520 & -1.764160 & 2.992460 \\
\hline $\mathrm{H}$ & 0.533390 & -1.110050 & 2.468740 \\
\hline $\mathrm{H}$ & 0.672030 & -1.882690 & 4.051610 \\
\hline C & 1.339050 & -4.408810 & 3.184980 \\
\hline $\mathrm{H}$ & 2.420080 & -4.364530 & 3.017990 \\
\hline $\mathrm{H}$ & 1.157230 & -4.297730 & 4.257720 \\
\hline $\mathrm{H}$ & 0.975820 & -5.391540 & 2.880440 \\
\hline 0 & -0.416930 & -2.722420 & 0.400220 \\
\hline 0 & -0.796330 & -3.479180 & 2.572070 \\
\hline B & -1.402410 & -2.849150 & 1.444030 \\
\hline 0 & -1.995460 & -1.498000 & 1.747210 \\
\hline C & -3.370300 & -0.034210 & 3.006620 \\
\hline C & -3.597790 & 0.824200 & 1.920910 \\
\hline C & -3.737040 & 0.369380 & 4.296990 \\
\hline C & -4.166100 & 2.075690 & 2.131660 \\
\hline $\mathrm{H}$ & -3.327350 & 0.511040 & 0.921720 \\
\hline C & -4.313650 & 1.620620 & 4.503260 \\
\hline $\mathrm{H}$ & -3.556580 & -0.294740 & 5.137750 \\
\hline C & -4.525130 & 2.478190 & 3.421440 \\
\hline $\mathrm{H}$ & -4.318420 & 2.741340 & 1.288570 \\
\hline $\mathrm{H}$ & -4.590670 & 1.929840 & 5.506020 \\
\hline $\mathrm{H}$ & -4.966890 & 3.456520 & 3.582160 \\
\hline $\mathrm{H}$ & -3.446930 & -4.420550 & 2.879880 \\
\hline
\end{tabular}




\begin{tabular}{|c|c|c|c|}
\hline H & -4.784850 & -1.930420 & 1.654030 \\
\hline H & -4.974590 & -2.558400 & 3.372630 \\
\hline C & 0.987130 & 2.066940 & 0.283710 \\
\hline C & 2.173700 & 1.572590 & -0.225710 \\
\hline C & 3.199280 & 1.072750 & 0.642440 \\
\hline C & 2.976790 & 1.089550 & 2.004840 \\
\hline H & 3.726560 & 0.669850 & 2.668850 \\
\hline C & -1.098510 & 1.575340 & -0.954890 \\
\hline C & -0.122400 & 2.501490 & -0.622480 \\
\hline C & -2.362490 & 3.284070 & -2.122730 \\
\hline C & -2.237960 & 1.968780 & -1.725930 \\
\hline $\mathrm{H}$ & -3.232840 & 3.590040 & -2.696750 \\
\hline C & -1.365550 & 4.246140 & -1.825880 \\
\hline C & -0.216280 & 3.851890 & -1.067920 \\
\hline C & 0.783530 & 4.817910 & -0.780110 \\
\hline C & 0.648720 & 6.115680 & -1.215410 \\
\hline C & -0.491200 & 6.509470 & -1.956810 \\
\hline C & -1.473540 & 5.594760 & -2.256490 \\
\hline $\mathrm{H}$ & 1.666010 & 4.508640 & -0.231890 \\
\hline $\mathrm{H}$ & 1.424770 & 6.842530 & -0.997550 \\
\hline $\mathrm{H}$ & -0.583030 & 7.537020 & -2.295220 \\
\hline $\mathrm{H}$ & -2.346320 & 5.888870 & -2.832860 \\
\hline C & 1.775900 & 1.585930 & 2.566440 \\
\hline C & 0.759970 & 2.089990 & 1.697470 \\
\hline C & -0.461650 & 2.539540 & 2.264460 \\
\hline C & -0.675750 & 2.475040 & 3.622750 \\
\hline C & 0.329610 & 1.973090 & 4.481830 \\
\hline C & 1.530600 & 1.545050 & 3.964260 \\
\hline $\mathrm{H}$ & -1.236990 & 2.917920 & 1.609910 \\
\hline $\mathrm{H}$ & -1.625600 & 2.800380 & 4.033090 \\
\hline $\mathrm{H}$ & 0.149780 & 1.925830 & 5.551610 \\
\hline $\mathrm{H}$ & 2.306840 & 1.154260 & 4.616170 \\
\hline C & -5.184880 & -1.069260 & -2.159500 \\
\hline C & -3.914920 & -1.285760 & -2.704330 \\
\hline C & -2.985510 & -0.241600 & -2.627430 \\
\hline C & -3.300080 & 0.965100 & -2.000330 \\
\hline C & -4.596610 & 1.164710 & -1.506160 \\
\hline C & -5.555100 & 0.152050 & -1.571840 \\
\hline $\mathrm{H}$ & -5.912420 & -1.870400 & -2.185660 \\
\hline $\mathrm{H}$ & -1.978040 & -0.376290 & -2.999390 \\
\hline $\mathrm{H}$ & -4.821900 & 2.109520 & -1.027460 \\
\hline C & -3.519820 & -2.599810 & -3.394570 \\
\hline C & -6.959220 & 0.310520 & -0.970330 \\
\hline C & -3.482440 & -2.349010 & -4.918210 \\
\hline $\mathrm{H}$ & -3.197790 & -3.262790 & -5.451540 \\
\hline $\mathrm{H}$ & -4.462920 & -2.028470 & -5.284500 \\
\hline H & -2.758120 & -1.568810 & -5.169370 \\
\hline C & -2.122330 & -3.063890 & -2.927580 \\
\hline $\mathrm{H}$ & -1.865670 & -4.009370 & -3.417200 \\
\hline H & -1.341960 & -2.341310 & -3.173780 \\
\hline $\mathrm{H}$ & -2.087260 & -3.221500 & -1.846790 \\
\hline C & -4.520750 & -3.730610 & -3.103530 \\
\hline $\mathrm{H}$ & -4.177660 & -4.656190 & -3.575530 \\
\hline H & -4.614880 & -3.917900 & -2.028670 \\
\hline
\end{tabular}




\begin{tabular}{|c|c|c|c|}
\hline H & -5.516080 & -3.511140 & -3.501790 \\
\hline C & -7.087520 & -0.632290 & 0.246100 \\
\hline $\mathrm{H}$ & -8.090820 & -0.566750 & 0.681440 \\
\hline $\mathrm{H}$ & -6.907500 & -1.674760 & -0.032230 \\
\hline $\mathrm{H}$ & -6.363750 & -0.355350 & 1.019300 \\
\hline C & -7.230500 & 1.746640 & -0.491990 \\
\hline H & -8.254250 & 1.820690 & -0.112560 \\
\hline $\mathrm{H}$ & -6.558190 & 2.036320 & 0.320840 \\
\hline $\mathrm{H}$ & -7.121680 & 2.470140 & -1.305960 \\
\hline C & -8.026310 & -0.054110 & -2.023700 \\
\hline $\mathrm{H}$ & -9.030500 & 0.063000 & -1.602480 \\
\hline $\mathrm{H}$ & -7.944570 & 0.596770 & -2.899660 \\
\hline $\mathrm{H}$ & -7.928380 & -1.087870 & -2.365040 \\
\hline C & 4.451080 & 0.481460 & 0.109720 \\
\hline C & 5.679000 & 0.758970 & 0.722560 \\
\hline C & 4.414580 & -0.390900 & -0.983780 \\
\hline C & 6.864090 & 0.182570 & 0.258130 \\
\hline H & 5.694370 & 1.455770 & 1.550890 \\
\hline C & 5.578670 & -0.973310 & -1.485540 \\
\hline $\mathrm{H}$ & 3.463210 & -0.610000 & -1.443940 \\
\hline C & 6.787280 & -0.676710 & -0.845120 \\
\hline $\mathrm{H}$ & 7.701210 & -1.121090 & -1.224740 \\
\hline C & 5.565300 & -1.895580 & -2.712040 \\
\hline C & 8.229780 & 0.470959 & 0.898120 \\
\hline C & 6.152630 & -3.270750 & -2.331140 \\
\hline H & 7.182900 & -3.187900 & -1.973430 \\
\hline $\mathrm{H}$ & 5.559760 & -3.739040 & -1.538730 \\
\hline H & 6.152410 & -3.939500 & -3.198790 \\
\hline C & 4.145310 & -2.110360 & -3.262880 \\
\hline $\mathrm{H}$ & 4.189830 & -2.746900 & -4.152060 \\
\hline $\mathrm{H}$ & 3.503530 & -2.611750 & -2.531170 \\
\hline H & 3.667420 & -1.168250 & -3.546310 \\
\hline C & 6.422910 & -1.255460 & -3.825180 \\
\hline H & 6.018970 & -0.278930 & -4.107910 \\
\hline $\mathrm{H}$ & 7.458380 & -1.110450 & -3.504010 \\
\hline $\mathrm{H}$ & 6.432790 & -1.895380 & -4.714370 \\
\hline C & 8.850530 & -0.853211 & 1.392840 \\
\hline $\mathrm{H}$ & 8.208180 & -1.326691 & 2.142140 \\
\hline H & 8.989980 & -1.565221 & 0.574850 \\
\hline $\mathrm{H}$ & 9.829860 & -0.669331 & 1.848140 \\
\hline C & 9.159870 & 1.114379 & -0.152900 \\
\hline H & 9.312900 & 0.457029 & -1.013140 \\
\hline $\mathrm{H}$ & 8.735680 & 2.053129 & -0.521740 \\
\hline $\mathrm{H}$ & 10.141020 & 1.328469 & 0.285140 \\
\hline C & 8.122510 & 1.428340 & 2.096000 \\
\hline $\mathrm{H}$ & 9.115970 & 1.597009 & 2.522890 \\
\hline H & 7.717930 & 2.401420 & 1.801370 \\
\hline $\mathrm{H}$ & 7.486370 & 1.017380 & 2.886410 \\
\hline
\end{tabular}


Frequencies (Top 3 out of 450)

$\begin{array}{lr}\text { 1. } & -251.5994 \mathrm{~cm}^{-1} \\ \text { 2. } & 11.3612 \mathrm{~cm}^{-1} \\ \text { 3. } & 16.7526 \mathrm{~cm}^{-1}\end{array}$

\section{B3LYP-D3(BJ)/6-31G(d,p) Molecular Geometry in Cartesian Coordinates}

C

$\mathrm{H}$

$\mathrm{H}$

C

0

0

$\mathrm{H}$

0

$\mathrm{H}$

C

C

C

C

$\mathrm{H}$

$\mathrm{H}$

$\mathrm{H}$

C

C

C

$\mathrm{H}$

$\mathrm{H}$

$\mathrm{H}$

C

$\mathrm{H}$

$\mathrm{H}$

$\mathrm{H}$

C

$\mathrm{H}$

$\mathrm{H}$

$\mathrm{H}$

C

$\mathrm{H}$

\section{$-1.290717$}

$-0.964166$

$-2.241266$

1.996743

2. 727134

1. 219962

0.639277

$-0.317639$

$-0.793576$

0.155371

0.860879

$-2.031939$

$-1.070384$

0.888533

$-3.011820$

$-2.085719$

$-1.883669$

$-2.797944$

$-0.591313$

0.084948

$-0.781226$

$-0.092253$

$-2.587184$

$-3.387735$

$-3.013019$

$-1.874171$

$-4.236498$

$-4.793905$

$-4.258462$

$-4.746452$

$-2.799884$

$-3.168937$
1.267194

1.835378

0.796015

1.620179

1.984045

0.538963

0.437685

0.231507

$-0.643795$

$-1.934510$

$-2.727702$

$-2.582020$

$-1.605619$

$-1.773715$

$-2.182054$

$-3.476536$

$-2.654621$

$-3.836703$

$-3.118024$

$-2.261685$

$-3.503044$

- 3.893151

$-1.605663$

$-1.118491$

$-2.063409$

$-0.840860$

$-3.384770$

$-3.194459$

$-2.478272$

$-4.178503$

$-5.039092$

$-4.761750$
1.097613

1.971762

1.334762

$-1.082724$

$-1.979481$

$-1.193902$

$-0.373813$

0.862230

0.802919

$-2.707283$

$-0.869081$

$-1.754175$

$-2.158179$

$-0.347950$

$-1.504303$

$-2.376070$

2.024388

1.550082

2.707496

2. 788264

3. 713364

2. 122349

2.878668

2. 319654

3. 777055

3. 200145

1. 269303

2.190829

0.660920

0.717507

2.489817

3.483049 


\begin{tabular}{|c|c|c|c|}
\hline $\mathrm{H}$ & -3.456544 & -5.815610 & 2.087979 \\
\hline $\mathrm{H}$ & -1.799446 & -5.462558 & 2.590051 \\
\hline 0 & -1.526249 & -2.055431 & 0.754793 \\
\hline 0 & -2.202799 & -4.230969 & 0.306241 \\
\hline B & -1.451327 & -3.146581 & -0.213197 \\
\hline 0 & -0.053964 & -3.589328 & -0.521374 \\
\hline C & 2.163268 & -3.255770 & -1.312798 \\
\hline C & 2.320188 & -4.623131 & -1.583544 \\
\hline C & 3.258367 & -2.387234 & -1.430742 \\
\hline C & 3.562847 & -5.116845 & -1.967269 \\
\hline $\mathrm{H}$ & 1.465257 & -5.280265 & -1.471269 \\
\hline C & 4.496665 & -2.889685 & -1.820205 \\
\hline $\mathrm{H}$ & 3.138439 & -1.330113 & -1.216659 \\
\hline C & 4.652786 & -4.250731 & -2.089165 \\
\hline $\mathrm{H}$ & 3.684649 & -6.176764 & -2.167873 \\
\hline $\mathrm{H}$ & 5.340328 & -2.216771 & -1.900265 \\
\hline $\mathrm{H}$ & 5.622439 & -4.636086 & -2.389496 \\
\hline $\mathrm{H}$ & -1.193103 & -0.589889 & -1.784538 \\
\hline $\mathrm{H}$ & 0.267643 & -2.874247 & -3.239428 \\
\hline $\mathrm{H}$ & 0.877598 & -1.155226 & -2.917672 \\
\hline C & 0.791636 & 3.112196 & 0.520259 \\
\hline C & 1.906110 & 2.343692 & 0.239318 \\
\hline C & 2.953822 & 2.177221 & 1.193969 \\
\hline C & 2.878801 & 2.861251 & 2.387547 \\
\hline $\mathrm{H}$ & 3.659881 & 2.723092 & 3.129554 \\
\hline C & -1.431446 & 2.186672 & -0.101054 \\
\hline C & -0.390487 & 3.062877 & -0.396800 \\
\hline C & -2.566444 & 2.852661 & -2.141426 \\
\hline C & -2.552889 & 2.090682 & -0.986968 \\
\hline $\mathrm{H}$ & -3.423124 & 2.789614 & -2.806054 \\
\hline C & -1.517114 & 3.739517 & -2.471304 \\
\hline C & -0.405804 & 3.857960 & -1.579582 \\
\hline C & 0.646173 & 4.753386 & -1.910660 \\
\hline C & 0.594141 & 5.498090 & -3.065276 \\
\hline C & -0.509483 & 5.385133 & -3.945478 \\
\hline C & -1.541269 & 4.524793 & -3.654398 \\
\hline $\mathrm{H}$ & 1.499214 & 4.827653 & -1.246622 \\
\hline $\mathrm{H}$ & 1.407581 & 6.173946 & -3.309207 \\
\hline $\mathrm{H}$ & -0.534711 & 5.978611 & -4.854180 \\
\hline $\mathrm{H}$ & -2.390397 & 4.429833 & -4.325330 \\
\hline C & 1.805010 & 3.741389 & 2.674304 \\
\hline C & 0.735398 & 3.866484 & 1.732259 \\
\hline C & -0.354244 & 4.722459 & 2.042170 \\
\hline C & -0.381637 & 5.426066 & 3.225008 \\
\hline C & 0.676836 & 5.304078 & 4.156169 \\
\hline C & 1.744276 & 4.479487 & 3.885200 \\
\hline $\mathrm{H}$ & -1.162634 & 4.817243 & 1.325524 \\
\hline $\mathrm{H}$ & -1.218114 & 6.081993 & 3.445549 \\
\hline $\mathrm{H}$ & 0.643130 & 5.864168 & 5.085522 \\
\hline $\mathrm{H}$ & 2.559670 & 4.379361 & 4.596041 \\
\hline C & -5.996617 & -0.324755 & -0.264322 \\
\hline C & -5.333620 & -0.444401 & -1.491673 \\
\hline C & -4.188439 & 0.334341 & -1.692613 \\
\hline C & -3.727099 & 1.217891 & -0.708877 \\
\hline
\end{tabular}




\begin{tabular}{|c|c|c|c|}
\hline C & -4.434422 & 1.325294 & 0.496253 \\
\hline C & -5.573326 & 0.553480 & 0.741741 \\
\hline $\mathrm{H}$ & -6.885919 & -0.921222 & -0.093731 \\
\hline H & -3.633531 & 0.266077 & -2.619620 \\
\hline $\mathrm{H}$ & -4.097507 & 2.048665 & 1.228009 \\
\hline C & -5.902475 & -1.377056 & -2.572725 \\
\hline C & -6.397915 & 0.689420 & 2.030795 \\
\hline C & -5.044620 & -1.384690 & -3.848959 \\
\hline $\mathrm{H}$ & -5.492084 & -2.058633 & -4.585705 \\
\hline $\mathrm{H}$ & -4.987300 & -0.390392 & -4.302860 \\
\hline $\mathrm{H}$ & -4.026740 & -1.733445 & -3.655386 \\
\hline C & -5.991086 & -2.821533 & -2.035272 \\
\hline $\mathrm{H}$ & -6.431191 & -3.477967 & -2.793453 \\
\hline $\mathrm{H}$ & -5.003008 & -3.212829 & -1.780443 \\
\hline $\mathrm{H}$ & -6.613859 & -2.883095 & -1.138694 \\
\hline C & -7.318731 & -0.887337 & -2.948818 \\
\hline $\mathrm{H}$ & -7.747692 & -1.527267 & -3.727357 \\
\hline $\mathrm{H}$ & -7.994586 & -0.905946 & -2.089314 \\
\hline $\mathrm{H}$ & -7.286968 & 0.138950 & -3.327814 \\
\hline C & -6.584246 & -0.691189 & 2.694051 \\
\hline $\mathrm{H}$ & -7.063561 & -1.408758 & 2.023339 \\
\hline $\mathrm{H}$ & -5.621693 & -1.109865 & 2.998956 \\
\hline $\mathrm{H}$ & -7.212459 & -0.597670 & 3.586202 \\
\hline C & -5.727927 & 1.620510 & 3.054654 \\
\hline $\mathrm{H}$ & -6.333188 & 1.661879 & 3.965213 \\
\hline $\mathrm{H}$ & -4.731209 & 1.263163 & 3.332847 \\
\hline $\mathrm{H}$ & -5.633266 & 2.642788 & 2.676210 \\
\hline C & -7.781805 & 1.272780 & 1.669905 \\
\hline $\mathrm{H}$ & -8.394247 & 1.390995 & 2.570417 \\
\hline $\mathrm{H}$ & -7.677437 & 2.253029 & 1.194613 \\
\hline $\mathrm{H}$ & -8.322109 & 0.621175 & 0.977294 \\
\hline C & 4.019109 & 1.165746 & 0.953749 \\
\hline C & 4.956742 & 1.323973 & -0.067901 \\
\hline C & 4.040098 & 0.005142 & 1.734180 \\
\hline C & 5.924793 & 0.345622 & -0.314167 \\
\hline $\mathrm{H}$ & 4.902375 & 2.211396 & -0.684994 \\
\hline C & 4.979535 & -1.005179 & 1.504138 \\
\hline $\mathrm{H}$ & 3.285410 & -0.109576 & 2.505155 \\
\hline C & 5.920468 & -0.804440 & 0.485523 \\
\hline $\mathrm{H}$ & 6.655497 & -1.573229 & 0.297607 \\
\hline C & 4.942706 & -2.280997 & 2.358996 \\
\hline C & 6.921996 & 0.561912 & -1.461638 \\
\hline C & 5.251713 & -1.908802 & 3.825013 \\
\hline $\mathrm{H}$ & 5.227297 & -2.800903 & 4.460545 \\
\hline $\mathrm{H}$ & 6.244072 & -1.454571 & 3.909775 \\
\hline $\mathrm{H}$ & 4.522214 & -1.194831 & 4.218263 \\
\hline C & 3.540018 & -2.923774 & 2.278405 \\
\hline $\mathrm{H}$ & 3.499619 & -3.820004 & 2.906927 \\
\hline $\mathrm{H}$ & 2.757270 & -2.242683 & 2.623326 \\
\hline $\mathrm{H}$ & 3.304743 & -3.216036 & 1.252198 \\
\hline C & 5.967914 & -3.326616 & 1.890901 \\
\hline $\mathrm{H}$ & 5.786380 & -3.627862 & 0.854755 \\
\hline $\mathrm{H}$ & 6.994571 & -2.955403 & 1.970763 \\
\hline $\mathrm{H}$ & 5.891386 & -4.220825 & 2.517185 \\
\hline
\end{tabular}




\begin{tabular}{|llll|} 
C & 7.683535 & 1.885810 & -1.233768 \\
$H$ & 8.231472 & 1.861550 & -0.286388 \\
$H$ & 7.005529 & 2.742551 & -1.208152 \\
$H$ & 8.403449 & 2.053512 & -2.042104 \\
C & 7.958525 & -0.568868 & -1.563323 \\
$H$ & 7.493839 & -1.538616 & -1.767864 \\
$H$ & 8.547344 & -0.661850 & -0.645145 \\
$H$ & 8.650766 & -0.359600 & -2.384546 \\
$\mathrm{C}$ & 6.143208 & 0.643844 & -2.794136 \\
$\mathrm{H}$ & 6.828589 & 0.858385 & -3.621647 \\
$\mathrm{H}$ & 5.375875 & 1.421068 & -2.770194 \\
$\mathrm{H}$ & 5.637121 & -0.301687 & -3.011728 \\
\hline
\end{tabular}

\section{C-Catalyzed reaction TS 8}

\section{Datum}

Value

\begin{tabular}{lc} 
B3LYP-D3(BJ)/6-31G(d,p) Energy & -3039.489684 \\
\hline B3LYP-D3(BJ)/def2-TZVPP/IEF-PCM(DCM) Energy & -3040.529557 \\
\hline B3LYP-D3(BJ)/def2-TZVPP/IEF-PCM(DCM)//B3LYP-D3(BJ)/6-31G(d,p) Free Energy (Quasiharmonic) & -3039.298809
\end{tabular}

Number of Imaginary Frequencies

Frequencies (Top 3 out of 450)

$\begin{array}{lr}\text { 1. } & -266.5651 \mathrm{~cm}^{-1} \\ \text { 2. } & 8.7122 \mathrm{~cm}^{-1} \\ \text { 3. } & 10.3946 \mathrm{~cm}^{-1}\end{array}$

B3LYP-D3(BJ)/6-31G(d,p) Molecular Geometry in Cartesian Coordinates

\begin{tabular}{|lrll|}
\hline C & & & \\
$H$ & 1.044837 & 0.577191 & 0.795680 \\
$H$ & 2.049623 & 0.166144 & 0.902440 \\
C & 0.530556 & 0.467755 & 1.754586 \\
O & -2.173260 & 1.810302 & -1.305107 \\
O & -2.930329 & 2.525041 & -1.922429 \\
$H$ & -1.188951 & 1.130463 & -1.905158 \\
O & -0.626670 & 0.641354 & -1.241153 \\
$H$ & 0.345627 & -0.173186 & -0.213454 \\
C & -0.032357 & -0.993980 & 0.205897 \\
$C$ & 1.578405 & -3.107914 & -2.405420 \\
$C$ & 2.260189 & -2.848111 & -0.390912 \\
$C$ & -0.353266 & -4.134081 & -1.269242 \\
$H$ & 0.244648 & -3.095668 & -2.043118 \\
& 1.922269 & -1.813267 & -0.353574 \\
\hline
\end{tabular}




\begin{tabular}{|c|c|c|c|}
\hline $\mathrm{H}$ & -1.440005 & -4.121661 & -1.222335 \\
\hline $\mathrm{H}$ & 0.042128 & -5.135585 & -1.440961 \\
\hline C & -1.317813 & -2.681850 & 1.919903 \\
\hline C & -0.807629 & -4.061138 & 2.461908 \\
\hline C & -1.196117 & -1.517728 & 2.893608 \\
\hline $\mathrm{H}$ & -1.535726 & -0.599353 & 2.413622 \\
\hline $\mathrm{H}$ & -1.824624 & -1.677725 & 3.774930 \\
\hline $\mathrm{H}$ & -0.166123 & -1.375791 & 3.224901 \\
\hline C & -2.738805 & -2.748816 & 1.353977 \\
\hline $\mathrm{H}$ & -3.483441 & -2.840273 & 2.149789 \\
\hline $\mathrm{H}$ & -2.953295 & -1.834058 & 0.798758 \\
\hline $\mathrm{H}$ & -2.852100 & -3.598022 & 0.676372 \\
\hline C & -1.880392 & -4.908604 & 3.136858 \\
\hline $\mathrm{H}$ & -2.300795 & -4.389946 & 4.004721 \\
\hline $\mathrm{H}$ & -2.688337 & -5.147608 & 2.443889 \\
\hline $\mathrm{H}$ & -1.441304 & -5.848718 & 3.481826 \\
\hline C & 0.408891 & -3.915133 & 3.387394 \\
\hline $\mathrm{H}$ & 0.134626 & -3.491623 & 4.358188 \\
\hline $\mathrm{H}$ & 0.841622 & -4.905400 & 3.550273 \\
\hline $\mathrm{H}$ & 1.176447 & -3.283576 & 2.933554 \\
\hline 0 & -0.409495 & -2.451792 & 0.812099 \\
\hline 0 & -0.383298 & -4.730596 & 1.262870 \\
\hline B & 0.106426 & -3.743678 & 0.376346 \\
\hline 0 & 1.602810 & -3.743578 & 0.286495 \\
\hline C & 3.699942 & -3.088779 & -0.591163 \\
\hline C & 4.253766 & -4.342930 & -0.291228 \\
\hline C & 4.521181 & -2.060825 & -1.072966 \\
\hline C & 5.613394 & -4.566825 & -0.479041 \\
\hline $\mathrm{H}$ & 3.605336 & -5.120289 & 0.096351 \\
\hline C & 5.879365 & -2.297075 & -1.269452 \\
\hline $\mathrm{H}$ & 4.103561 & -1.080571 & -1.283634 \\
\hline C & 6.428385 & -3.546083 & -0.975599 \\
\hline $\mathrm{H}$ & 6.039670 & -5.535828 & -0.239060 \\
\hline $\mathrm{H}$ & 6.509649 & -1.499063 & -1.639018 \\
\hline $\mathrm{H}$ & 7.488837 & -3.721479 & -1.128099 \\
\hline $\mathrm{H}$ & -0.290597 & -2.150030 & -2.123461 \\
\hline $\mathrm{H}$ & 2.094025 & -4.055824 & -2.520647 \\
\hline $\mathrm{H}$ & 2.015064 & -2.261702 & -2.925392 \\
\hline C & -1.331940 & 2.169685 & 1.026195 \\
\hline C & -2.308137 & 1.646773 & 0.195779 \\
\hline C & -3.518136 & 1.097617 & 0.732558 \\
\hline C & -3.706877 & 1.123842 & 2.099566 \\
\hline $\mathrm{H}$ & -4.601588 & 0.675235 & 2.520345 \\
\hline C & 1.091985 & 2.035975 & 0.400402 \\
\hline C & -0.086294 & 2.772919 & 0.455804 \\
\hline C & 2.235260 & 3.947779 & -0.562270 \\
\hline C & 2.283833 & 2.640793 & -0.111859 \\
\hline $\mathrm{H}$ & 3.144623 & 4.412733 & -0.931022 \\
\hline C & 1.049752 & 4.713684 & -0.532203 \\
\hline C & -0.140439 & 4.121813 & -0.007671 \\
\hline C & -1.330333 & 4.898394 & 0.027568 \\
\hline C & -1.334144 & 6.195022 & -0.429924 \\
\hline C & -0.152440 & 6.782314 & -0.943022 \\
\hline C & 1.013510 & 6.056514 & -0.992903 \\
\hline
\end{tabular}




\begin{tabular}{|c|c|c|c|}
\hline $\mathrm{H}$ & -2.242715 & 4.449530 & 0.400238 \\
\hline $\mathrm{H}$ & -2.252867 & 6.772210 & -0.404958 \\
\hline $\mathrm{H}$ & -0.173066 & 7.806858 & -1.301466 \\
\hline $\mathrm{H}$ & 1.925099 & 6.496252 & -1.387647 \\
\hline $\mathrm{C}$ & -2.747013 & 1.676661 & 2.980496 \\
\hline C & -1.531790 & 2.204478 & 2.442871 \\
\hline C & -0.566066 & 2.738536 & 3.336402 \\
\hline C & -0.784335 & 2.732195 & 4.695738 \\
\hline C & -1.981328 & 2.197641 & 5.227708 \\
\hline C & -2.940897 & 1.683918 & 4.386513 \\
\hline $\mathrm{H}$ & 0.349884 & 3.151845 & 2.929813 \\
\hline H & -0.034686 & 3.141774 & 5.365616 \\
\hline $\mathrm{H}$ & -2.140507 & 2.197150 & 6.301550 \\
\hline $\mathrm{H}$ & -3.863957 & 1.272757 & 4.785439 \\
\hline C & 6.173676 & 0.878112 & -0.272506 \\
\hline C & 5.641706 & 1.471376 & -1.423726 \\
\hline C & 4.340326 & 1.980684 & -1.357230 \\
\hline C & 3.599518 & 1.948085 & -0.169623 \\
\hline C & 4.168371 & 1.345738 & 0.959059 \\
\hline C & 5.445583 & 0.778070 & 0.919690 \\
\hline $\mathrm{H}$ & 7.173576 & 0.472251 & -0.306620 \\
\hline $\mathrm{H}$ & 3.883114 & 2.427780 & -2.233174 \\
\hline $\mathrm{H}$ & 3.609590 & 1.348540 & 1.888709 \\
\hline C & 6.423065 & 1.572861 & -2.742615 \\
\hline C & 5.996450 & 0.074129 & 2.168402 \\
\hline C & 5.698181 & 0.746219 & -3.827375 \\
\hline $\mathrm{H}$ & 6.231224 & 0.817140 & -4.781602 \\
\hline H & 5.642886 & -0.310149 & -3.546961 \\
\hline $\mathrm{H}$ & 4.676859 & 1.103077 & -3.985943 \\
\hline C & 6.487422 & 3.050989 & -3.186035 \\
\hline $\mathrm{H}$ & 7.046529 & 3.142161 & -4.123291 \\
\hline $\mathrm{H}$ & 5.491423 & 3.469756 & -3.350940 \\
\hline $\mathrm{H}$ & 6.987689 & 3.661899 & -2.428193 \\
\hline C & 7.866493 & 1.058363 & -2.614714 \\
\hline $\mathrm{H}$ & 8.385844 & 1.183764 & -3.569454 \\
\hline H & 8.426164 & 1.612053 & -1.854605 \\
\hline $\mathrm{H}$ & 7.908491 & -0.005155 & -2.360144 \\
\hline C & 6.103025 & 1.090728 & 3.324796 \\
\hline $\mathrm{H}$ & 6.487956 & 0.602836 & 4.226745 \\
\hline $\mathrm{H}$ & 6.781088 & 1.908770 & 3.062043 \\
\hline H & 5.131016 & 1.527740 & 3.570144 \\
\hline C & 5.034422 & -1.066706 & 2.570148 \\
\hline $\mathrm{H}$ & 5.404739 & -1.576031 & 3.466275 \\
\hline H & 4.031316 & -0.691356 & 2.792557 \\
\hline $\mathrm{H}$ & 4.947901 & -1.804284 & 1.767786 \\
\hline C & 7.385577 & -0.539234 & 1.928652 \\
\hline $\mathrm{H}$ & 7.727912 & -1.037251 & 2.840838 \\
\hline $\mathrm{H}$ & 7.360812 & -1.287418 & 1.130778 \\
\hline $\mathrm{H}$ & 8.128263 & 0.222215 & 1.670107 \\
\hline C & -4.528555 & 0.457314 & -0.142734 \\
\hline C & -4.128329 & -0.371185 & -1.189960 \\
\hline C & -5.899468 & 0.647112 & 0.090021 \\
\hline C & -5.059025 & -0.997538 & -2.025897 \\
\hline $\mathrm{H}$ & -3.070566 & -0.526223 & -1.361393 \\
\hline
\end{tabular}




\begin{tabular}{|c|c|c|c|}
\hline C & -6.857388 & 0.026269 & -0.709589 \\
\hline $\mathrm{H}$ & -6.197753 & 1.313442 & 0.889222 \\
\hline C & -6.413208 & -0.790330 & -1.764262 \\
\hline $\mathrm{H}$ & -7.151781 & -1.265510 & -2.397217 \\
\hline C & -8.365161 & 0.216520 & -0.491573 \\
\hline C & -4.546194 & -1.853152 & -3.190516 \\
\hline C & -8.671106 & 1.133293 & 0.703761 \\
\hline $\mathrm{H}$ & -9.754295 & 1.231720 & 0.824621 \\
\hline $\mathrm{H}$ & -8.267951 & 0.728436 & 1.637491 \\
\hline $\mathrm{H}$ & -8.260493 & 2.137283 & 0.559303 \\
\hline C & -8.988071 & 0.847115 & -1.755813 \\
\hline $\mathrm{H}$ & -10.065517 & 0.990999 & -1.619141 \\
\hline $\mathrm{H}$ & -8.535035 & 1.820727 & -1.965878 \\
\hline $\mathrm{H}$ & -8.843427 & 0.214813 & -2.636034 \\
\hline C & -9.018722 & -1.156860 & -0.225999 \\
\hline $\mathrm{H}$ & -8.870172 & -1.844602 & -1.062912 \\
\hline $\mathrm{H}$ & -8.593528 & -1.622306 & 0.668829 \\
\hline $\mathrm{H}$ & -10.097489 & -1.042729 & -0.073244 \\
\hline C & -5.687340 & -2.497995 & -3.991501 \\
\hline $\mathrm{H}$ & -6.299759 & -3.156173 & -3.366530 \\
\hline $\mathrm{H}$ & -6.341330 & -1.743934 & -4.440333 \\
\hline $\mathrm{H}$ & -5.272175 & -3.101746 & -4.804304 \\
\hline C & -3.721431 & -0.954965 & -4.139866 \\
\hline $\mathrm{H}$ & -4.342203 & -0.150638 & -4.545577 \\
\hline $\mathrm{H}$ & -2.875132 & -0.487631 & -3.629414 \\
\hline $\mathrm{H}$ & -3.332483 & -1.545292 & -4.977209 \\
\hline C & -3.643505 & -2.974787 & -2.636557 \\
\hline $\mathrm{H}$ & -4.201914 & -3.630500 & -1.960423 \\
\hline $\mathrm{H}$ & -3.240792 & -3.583779 & -3.453001 \\
\hline $\mathrm{H}$ & -2.799114 & -2.563609 & -2.079297 \\
\hline
\end{tabular}

\section{C-Catalyzed reaction TS 9}

\section{Datum}

Value

B3LYP-D3(BJ)/6-31G(d,p) Energy

$-3039.489686$

B3LYP-D3(BJ)/def2-TZVPP/IEF-PCM(DCM) Energy

$-3040.529817$

B3LYP-D3(BJ)/def2-TZVPP/IEF-PCM(DCM)//B3LYP-D3(BJ)/6-31G(d,p) Free Energy (Quasiharmonic)

$-3039.298768$

Number of Imaginary Frequencies

Frequencies (Top 3 out of 450)

$\begin{array}{lr}\text { 1. } & -251.0492 \mathrm{~cm}^{-1} \\ \text { 2. } & 7.4336 \mathrm{~cm}^{-1} \\ \text { 3. } & 11.8550 \mathrm{~cm}^{-1}\end{array}$




\begin{tabular}{|c|c|c|c|}
\hline C & 1.036749 & 0.678530 & 0.713990 \\
\hline $\mathrm{H}$ & 2.026409 & 0.277370 & 0.941450 \\
\hline $\mathrm{H}$ & 0.442409 & 0.659741 & 1.632810 \\
\hline C & -2.200480 & 1.760441 & -1.436750 \\
\hline 0 & -2.986450 & 2.393421 & -2.106080 \\
\hline 0 & -1.222370 & 1.035701 & -1.990380 \\
\hline $\mathrm{H}$ & -0.636181 & 0.609731 & -1.305250 \\
\hline 0 & 0.416769 & -0.145509 & -0.288390 \\
\hline $\mathrm{H}$ & 0.036999 & -0.962359 & 0.136290 \\
\hline C & 1.861519 & -3.120090 & -2.232960 \\
\hline C & 2.300379 & -2.959420 & -0.114830 \\
\hline C & -0.227931 & -4.086559 & -1.352450 \\
\hline C & 0.498429 & -3.056689 & -2.027630 \\
\hline $\mathrm{H}$ & 2.003689 & -1.911590 & -0.093960 \\
\hline $\mathrm{H}$ & -1.309831 & -4.045879 & -1.456950 \\
\hline $\mathrm{H}$ & 0.156809 & -5.097269 & -1.492890 \\
\hline C & -1.045021 & -2.526279 & 2.014380 \\
\hline C & -1.590701 & -3.991889 & 1.975280 \\
\hline C & 0.086889 & -2.345379 & 3.033770 \\
\hline $\mathrm{H}$ & 0.570959 & -1.378510 & 2.868770 \\
\hline $\mathrm{H}$ & -0.295501 & -2.357409 & 4.058050 \\
\hline $\mathrm{H}$ & 0.840089 & -3.129280 & 2.931160 \\
\hline C & -2.094931 & -1.441069 & 2.189330 \\
\hline $\mathrm{H}$ & -2.613591 & -1.554419 & 3.146360 \\
\hline $\mathrm{H}$ & -1.624101 & -0.455549 & 2.188030 \\
\hline $\mathrm{H}$ & -2.833201 & -1.460709 & 1.388080 \\
\hline C & -2.941411 & -4.085399 & 1.252390 \\
\hline $\mathrm{H}$ & -3.759661 & -3.689259 & 1.860690 \\
\hline $\mathrm{H}$ & -2.920671 & -3.533319 & 0.310150 \\
\hline $\mathrm{H}$ & -3.148041 & -5.135909 & 1.032190 \\
\hline C & -1.677051 & -4.670899 & 3.338430 \\
\hline $\mathrm{H}$ & -2.349831 & -4.123579 & 4.006940 \\
\hline $\mathrm{H}$ & -2.068061 & -5.684919 & 3.217920 \\
\hline $\mathrm{H}$ & -0.694101 & -4.742579 & 3.806370 \\
\hline 0 & -0.482301 & -2.410789 & 0.679940 \\
\hline 0 & -0.603371 & -4.675759 & 1.186990 \\
\hline B & 0.023819 & -3.734489 & 0.334180 \\
\hline 0 & 1.522369 & -3.836800 & 0.441110 \\
\hline C & 3.739109 & -3.264170 & -0.156700 \\
\hline C & 4.198499 & -4.553920 & 0.151220 \\
\hline C & 4.654299 & -2.260920 & -0.502300 \\
\hline C & 5.559499 & -4.836700 & 0.107650 \\
\hline $\mathrm{H}$ & 3.476269 & -5.312990 & 0.429200 \\
\hline C & 6.014839 & -2.554490 & -0.550380 \\
\hline $\mathrm{H}$ & 4.306059 & -1.258430 & -0.731180 \\
\hline C & 6.470609 & -3.838730 & -0.247960 \\
\hline $\mathrm{H}$ & 5.912119 & -5.833890 & 0.351560 \\
\hline $\mathrm{H}$ & 6.716619 & -1.774910 & -0.816470 \\
\hline $\mathrm{H}$ & 7.532759 & -4.059840 & -0.286120 \\
\hline $\mathrm{H}$ & 0.011449 & -2.089989 & -2.148510 \\
\hline
\end{tabular}




\begin{tabular}{|c|c|c|c|}
\hline $\mathrm{H}$ & 2.351369 & -4.085500 & -2.305870 \\
\hline $\mathrm{H}$ & 2.395409 & -2.278950 & -2.662710 \\
\hline C & -1.283820 & 2.365891 & 0.815730 \\
\hline C & -2.292550 & 1.773631 & 0.076190 \\
\hline C & -3.502360 & 1.329001 & 0.703910 \\
\hline C & -3.664760 & 1.541891 & 2.056620 \\
\hline H & -4.560030 & 1.173201 & 2.548010 \\
\hline C & 1.124920 & 2.092010 & 0.185900 \\
\hline C & -0.033830 & 2.859491 & 0.156980 \\
\hline C & 2.308740 & 3.835490 & -1.016480 \\
\hline C & 2.328480 & 2.599960 & -0.396750 \\
\hline $\mathrm{H}$ & 3.227490 & 4.227120 & -1.442940 \\
\hline C & 1.140290 & 4.626460 & -1.082360 \\
\hline C & -0.057580 & 4.140391 & -0.472740 \\
\hline C & -1.226750 & 4.946431 & -0.526740 \\
\hline C & -1.204750 & 6.171161 & -1.151690 \\
\hline C & -0.016270 & 6.653621 & -1.750610 \\
\hline C & 1.130770 & 5.897030 & -1.716050 \\
\hline $\mathrm{H}$ & -2.144680 & 4.574801 & -0.088360 \\
\hline H & -2.108290 & 6.770891 & -1.193240 \\
\hline $\mathrm{H}$ & -0.016280 & 7.622001 & -2.241350 \\
\hline $\mathrm{H}$ & 2.047530 & 6.257150 & -2.174340 \\
\hline C & -2.668830 & 2.167811 & 2.843570 \\
\hline C & -1.449060 & 2.580261 & 2.222280 \\
\hline C & -0.449840 & 3.193921 & 3.023340 \\
\hline C & -0.642180 & 3.374451 & 4.374790 \\
\hline C & -1.844360 & 2.954601 & 4.990720 \\
\hline C & -2.834770 & 2.365691 & 4.239220 \\
\hline $\mathrm{H}$ & 0.470070 & 3.520971 & 2.552560 \\
\hline $\mathrm{H}$ & 0.132260 & 3.844631 & 4.972870 \\
\hline $\mathrm{H}$ & -1.983200 & 3.101381 & 6.057340 \\
\hline $\mathrm{H}$ & -3.762300 & 2.041511 & 4.702730 \\
\hline C & 6.202720 & 0.815070 & -0.201330 \\
\hline C & 5.684830 & 1.182520 & -1.449570 \\
\hline C & 4.386820 & 1.703920 & -1.493680 \\
\hline C & 3.632640 & 1.887430 & -0.329480 \\
\hline C & 4.179210 & 1.489610 & 0.896410 \\
\hline C & 5.457990 & 0.930660 & 0.979570 \\
\hline $\mathrm{H}$ & 7.205749 & 0.418820 & -0.146850 \\
\hline $\mathrm{H}$ & 3.943140 & 1.989250 & -2.441300 \\
\hline $\mathrm{H}$ & 3.601530 & 1.653070 & 1.800030 \\
\hline C & 6.473840 & 1.014260 & -2.756660 \\
\hline C & 5.990309 & 0.469330 & 2.343990 \\
\hline C & 6.534590 & 2.365530 & -3.501510 \\
\hline $\mathrm{H}$ & 7.097590 & 2.258720 & -4.434700 \\
\hline $\mathrm{H}$ & 5.538480 & 2.736420 & -3.755780 \\
\hline $\mathrm{H}$ & 7.029700 & 3.124980 & -2.888310 \\
\hline C & 7.917939 & 0.543179 & -2.518110 \\
\hline $\mathrm{H}$ & 8.444179 & 0.477459 & -3.475030 \\
\hline H & 8.470430 & 1.240099 & -1.880300 \\
\hline $\mathrm{H}$ & 7.959189 & -0.448551 & -2.057580 \\
\hline C & 5.756429 & -0.028520 & -3.642330 \\
\hline $\mathrm{H}$ & 6.292659 & -0.160990 & -4.588220 \\
\hline $\mathrm{H}$ & 5.705449 & -0.999410 & -3.139950 \\
\hline
\end{tabular}




\begin{tabular}{|c|c|c|c|}
\hline $\mathrm{H}$ & 4.734229 & 0.282300 & -3.875930 \\
\hline C & 7.386829 & -0.165550 & 2.244220 \\
\hline $\mathrm{H}$ & 7.717399 & -0.480370 & 3.238600 \\
\hline $\mathrm{H}$ & 7.380769 & -1.050870 & 1.601550 \\
\hline $\mathrm{H}$ & 8.128919 & 0.540429 & 1.858200 \\
\hline C & 6.071110 & 1.679180 & 3.298960 \\
\hline $\mathrm{H}$ & 6.439650 & 1.365120 & 4.281410 \\
\hline $\mathrm{H}$ & 6.751890 & 2.440400 & 2.905180 \\
\hline $\mathrm{H}$ & 5.093280 & 2.147170 & 3.442520 \\
\hline C & 5.025159 & -0.586970 & 2.927790 \\
\hline $\mathrm{H}$ & 5.386109 & -0.933170 & 3.902170 \\
\hline $\mathrm{H}$ & 4.019579 & -0.181260 & 3.071210 \\
\hline $\mathrm{H}$ & 4.947969 & -1.452100 & 2.263080 \\
\hline C & -4.520821 & 0.564031 & -0.051880 \\
\hline C & -4.112431 & -0.416489 & -0.962350 \\
\hline C & -5.887831 & 0.772921 & 0.162450 \\
\hline C & -5.039811 & -1.166929 & -1.684730 \\
\hline $\mathrm{H}$ & -3.054191 & -0.580929 & -1.105310 \\
\hline C & -6.846661 & 0.020032 & -0.520870 \\
\hline $\mathrm{H}$ & -6.188890 & 1.552441 & 0.850700 \\
\hline C & -6.398241 & -0.939159 & -1.438240 \\
\hline $\mathrm{H}$ & -7.132171 & -1.520228 & -1.986360 \\
\hline C & -8.355081 & 0.221752 & -0.316490 \\
\hline C & -4.605251 & -2.202429 & -2.729330 \\
\hline C & -8.984931 & -1.102798 & 0.165650 \\
\hline $\mathrm{H}$ & -10.063721 & -0.981228 & 0.312390 \\
\hline $\mathrm{H}$ & -8.834251 & -1.909338 & -0.557070 \\
\hline $\mathrm{H}$ & -8.543561 & -1.419038 & 1.116080 \\
\hline C & -8.662420 & 1.307952 & 0.726900 \\
\hline $\mathrm{H}$ & -9.745620 & 1.409182 & 0.845090 \\
\hline $\mathrm{H}$ & -8.243050 & 1.057692 & 1.706570 \\
\hline $\mathrm{H}$ & -8.268860 & 2.283042 & 0.424000 \\
\hline C & -8.999681 & 0.641262 & -1.655240 \\
\hline $\mathrm{H}$ & -8.562910 & 1.576332 & -2.018720 \\
\hline $\mathrm{H}$ & -8.854171 & -0.117568 & -2.428900 \\
\hline $\mathrm{H}$ & -10.077740 & 0.790682 & -1.529740 \\
\hline C & -3.077671 & -2.227769 & -2.899350 \\
\hline $\mathrm{H}$ & -2.689491 & -1.260199 & -3.231440 \\
\hline $\mathrm{H}$ & -2.578591 & -2.484339 & -1.961240 \\
\hline $\mathrm{H}$ & -2.797881 & -2.981669 & -3.641660 \\
\hline C & -5.079631 & -3.604279 & -2.292700 \\
\hline $\mathrm{H}$ & -4.638091 & -3.883449 & -1.331150 \\
\hline $\mathrm{H}$ & -6.167491 & -3.644009 & -2.184440 \\
\hline $\mathrm{H}$ & -4.787521 & -4.355789 & -3.034390 \\
\hline C & -5.234281 & -1.844179 & -4.092640 \\
\hline $\mathrm{H}$ & -4.920721 & -2.564869 & -4.855750 \\
\hline $\mathrm{H}$ & -6.327031 & -1.856079 & -4.051540 \\
\hline $\mathrm{H}$ & -4.920121 & -0.846039 & -4.411570 \\
\hline
\end{tabular}


Frequencies (Top 3 out of 450)

$\begin{array}{lr}\text { 1. } & -277.9547 \mathrm{~cm}^{-1} \\ \text { 2. } & 6.6273 \mathrm{~cm}^{-1} \\ \text { 3. } & 15.7435 \mathrm{~cm}^{-1}\end{array}$

\section{B3LYP-D3(BJ)/6-31G(d,p) Molecular Geometry in Cartesian Coordinates}

C

$\mathrm{H}$

$\mathrm{H}$

C

0

0

$\mathrm{H}$

0

$\mathrm{H}$

C

C

C

C

$\mathrm{H}$

$\mathrm{H}$

$\mathrm{H}$

C

C

C

$\mathrm{H}$

$\mathrm{H}$

$\mathrm{H}$

C

$\mathrm{H}$

$\mathrm{H}$

$\mathrm{H}$

C

$\mathrm{H}$

$\mathrm{H}$

$\mathrm{H}$

C

$\mathrm{H}$

\section{$-1.349465$}

$-1.067765$

$-2.301299$

1.996930

2. 765901

1.172522

0.593042

$-0.349656$

$-0.803383$

0.016263

0.738025

$-2.165405$

$-1.179361$

0.788075

$-3.116207$

$-2.282123$

$-1.817445$

$-2.857146$

$-0.533970$

0.223743

$-0.699714$

$-0.145524$

$-2.363891$

$-3.171289$

$-2.744009$

$-1.570485$

$-4.254456$

$-4.749615$

$-4.209667$

$-4.866834$

$-2.940117$

$-3.244963$
1. 258060

1.842118

0.773007

1. 511461

1.807867

0.462483

0.398343

0.239305

$-0.648440$

$-1.963645$

$-2.795832$

$-2.524755$

$-1.587601$

$-1.857569$

$-2.087358$

$-3.410572$

$-2.691295$

$-3.768700$

$-3.296158$

$-2.509890$

$-3.700363$

$-4.090647$

$-1.617599$

$-1.060764$

$-2.064090$

$-0.913679$

- 3.169165

$-2.939169$

$-2.254672$

$-3.896496$

$-4.988252$

$-4.701998$
1.090436

1.970611

1.295338

$-1.082510$

$-1.970961$

$-1.176905$

$-0.352749$

0.902468

0.863683

$-2.692749$

$-0.898848$

$-1.673114$

$-2.104411$

$-0.351645$

$-1.379765$

$-2.298778$

2.098370

1.641031

2.680028

2.747876

3.682660

2.040027

3.029844

2. 554640

3.953925

3.297460

1.427369

2. 376412

0.832151

0.888494

2. 553473

3. 565743 


\begin{tabular}{|c|c|c|c|}
\hline $\mathrm{H}$ & -3.681419 & -5.689543 & 2.160589 \\
\hline $\mathrm{H}$ & -1.981307 & -5.506113 & 2.606220 \\
\hline 0 & -1.492686 & -2.076785 & 0.826561 \\
\hline 0 & -2.344778 & -4.186799 & 0.366454 \\
\hline B & -1.542310 & -3.145589 & -0.168382 \\
\hline 0 & -0.187102 & -3.650840 & -0.551135 \\
\hline C & 2.032246 & -3.326998 & -1.361886 \\
\hline C & 3.125096 & -2.455505 & -1.482410 \\
\hline C & 2.190276 & -4.692530 & -1.637083 \\
\hline C & 4.364927 & -2.952062 & -1.872881 \\
\hline $\mathrm{H}$ & 3.000320 & -1.399146 & -1.267994 \\
\hline C & 3.434212 & -5.181581 & -2.024653 \\
\hline $\mathrm{H}$ & 1.337708 & -5.352624 & -1.523325 \\
\hline C & 4.522401 & -4.313037 & -2.144338 \\
\hline $\mathrm{H}$ & 5.206109 & -2.276319 & -1.954180 \\
\hline $\mathrm{H}$ & 3.558415 & -6.240677 & -2.228345 \\
\hline $\mathrm{H}$ & 5.492468 & -4.696676 & -2.445767 \\
\hline $\mathrm{H}$ & -1.247598 & -0.569550 & -1.723824 \\
\hline $\mathrm{H}$ & 0.071149 & -2.900525 & -3.239012 \\
\hline $\mathrm{H}$ & 0.759311 & -1.210410 & -2.924374 \\
\hline C & 0.789429 & 3.044407 & 0.487171 \\
\hline C & 1.907611 & 2.276890 & 0.214650 \\
\hline C & 2.969789 & 2.148135 & 1.158106 \\
\hline C & 2.900827 & 2.855971 & 2.337409 \\
\hline $\mathrm{H}$ & 3.692797 & 2.744650 & 3.072326 \\
\hline C & -1.459843 & 2.161030 & -0.123908 \\
\hline C & -0.391198 & 2.998846 & -0.432656 \\
\hline C & -2.571957 & 2.827178 & -2.177018 \\
\hline C & -2.583542 & 2.086260 & -1.008975 \\
\hline $\mathrm{H}$ & -3.429111 & 2.778442 & -2.842249 \\
\hline C & -1.494164 & 3.672726 & -2.521818 \\
\hline C & -0.380198 & 3.771663 & -1.630773 \\
\hline C & 0.699387 & 4.627830 & -1.977842 \\
\hline C & 0.671939 & 5.351229 & -3.146743 \\
\hline C & -0.433682 & 5.256414 & -4.026516 \\
\hline C & -1.492445 & 4.435318 & -3.719977 \\
\hline $\mathrm{H}$ & 1.553791 & 4.689624 & -1.314488 \\
\hline $\mathrm{H}$ & 1.506602 & 5.996318 & -3.402016 \\
\hline $\mathrm{H}$ & -0.439247 & 5.832678 & -4.946553 \\
\hline $\mathrm{H}$ & -2.343952 & 4.354472 & -4.389741 \\
\hline C & 1.817617 & 3.726392 & 2.618926 \\
\hline C & 0.738440 & 3.822804 & 1.684306 \\
\hline C & -0.354043 & 4.678447 & 1.986529 \\
\hline C & -0.377536 & 5.403957 & 3.156014 \\
\hline C & 0.688959 & 5.307468 & 4.080983 \\
\hline C & 1.760766 & 4.486693 & 3.816195 \\
\hline $\mathrm{H}$ & -1.168580 & 4.754624 & 1.274716 \\
\hline $\mathrm{H}$ & -1.217063 & 6.058023 & 3.370500 \\
\hline $\mathrm{H}$ & 0.658343 & 5.884672 & 4.999912 \\
\hline $\mathrm{H}$ & 2.583308 & 4.407058 & 4.521373 \\
\hline C & -6.081533 & -0.251914 & -0.265107 \\
\hline C & -5.655238 & 0.639392 & 0.721452 \\
\hline C & -4.496510 & 1.388361 & 0.470557 \\
\hline C & -3.782413 & 1.250053 & -0.722246 \\
\hline
\end{tabular}




\begin{tabular}{|c|c|c|c|}
\hline C & -4.254311 & 0.355500 & -1.696390 \\
\hline C & -5.406397 & -0.403682 & -1.487323 \\
\hline $\mathrm{H}$ & -6.972770 & -0.840586 & -0.093311 \\
\hline $\mathrm{H}$ & -4.156413 & 2.117706 & 1.198599 \\
\hline $\mathrm{H}$ & -3.693256 & 0.261865 & -2.617380 \\
\hline C & -6.433720 & 0.860022 & 2.027082 \\
\hline C & -5.972950 & -1.363370 & -2.545002 \\
\hline C & -5.513115 & 0.609665 & 3.239335 \\
\hline $\mathrm{H}$ & -6.048158 & 0.819901 & 4.171540 \\
\hline $\mathrm{H}$ & -5.183350 & -0.432520 & 3.266154 \\
\hline $\mathrm{H}$ & -4.622834 & 1.244115 & 3.216459 \\
\hline C & -6.940421 & 2.318618 & 2.061901 \\
\hline $\mathrm{H}$ & -7.509609 & 2.502881 & 2.979455 \\
\hline $\mathrm{H}$ & -6.113278 & 3.033247 & 2.030383 \\
\hline $\mathrm{H}$ & -7.592182 & 2.523145 & 1.206926 \\
\hline C & -7.645456 & -0.077863 & 2.150199 \\
\hline $\mathrm{H}$ & -8.154435 & 0.102639 & 3.101813 \\
\hline $\mathrm{H}$ & -8.372683 & 0.089402 & 1.349776 \\
\hline $\mathrm{H}$ & -7.344331 & -1.130008 & 2.127435 \\
\hline C & -5.110249 & -1.406756 & -3.817125 \\
\hline $\mathrm{H}$ & -5.048174 & -0.424406 & -4.295868 \\
\hline $\mathrm{H}$ & -4.094380 & -1.753373 & -3.610479 \\
\hline $\mathrm{H}$ & -5.557026 & -2.097936 & -4.538180 \\
\hline C & -6.064166 & -2.792178 & -1.966069 \\
\hline $\mathrm{H}$ & -6.494770 & -3.471693 & -2.709254 \\
\hline $\mathrm{H}$ & -5.078379 & -3.173768 & -1.688454 \\
\hline $\mathrm{H}$ & -6.697454 & -2.828057 & -1.075409 \\
\hline C & -7.387582 & -0.884534 & -2.940469 \\
\hline $\mathrm{H}$ & -7.813875 & -1.547545 & -3.700946 \\
\hline $\mathrm{H}$ & -8.066893 & -0.877058 & -2.083566 \\
\hline $\mathrm{H}$ & -7.353789 & 0.129748 & -3.350441 \\
\hline C & 4.055533 & 1.155806 & 0.918673 \\
\hline C & 5.053652 & 1.390932 & -0.034280 \\
\hline C & 4.045798 & -0.043505 & 1.630161 \\
\hline C & 6.048123 & 0.443821 & -0.272792 \\
\hline $\mathrm{H}$ & 5.018185 & 2.314800 & -0.593277 \\
\hline C & 5.016122 & -1.030656 & 1.401702 \\
\hline H & 3.253986 & -0.206999 & 2.353483 \\
\hline C & 6.005857 & -0.758660 & 0.454381 \\
\hline $\mathrm{H}$ & 6.770253 & -1.501804 & 0.266057 \\
\hline C & 4.977871 & -2.333626 & 2.214712 \\
\hline C & 7.181804 & 0.689361 & -1.279637 \\
\hline C & 5.304995 & -1.998868 & 3.686465 \\
\hline $\mathrm{H}$ & 5.286914 & -2.906492 & 4.299973 \\
\hline $\mathrm{H}$ & 6.298510 & -1.547128 & 3.770737 \\
\hline $\mathrm{H}$ & 4.580180 & -1.293697 & 4.103934 \\
\hline C & 3.575115 & -2.974772 & 2.138700 \\
\hline $\mathrm{H}$ & 3.545986 & -3.884653 & 2.747659 \\
\hline $\mathrm{H}$ & 2.796133 & -2.305003 & 2.512777 \\
\hline $\mathrm{H}$ & 3.324080 & -3.244650 & 1.110770 \\
\hline C & 5.995991 & -3.367378 & 1.706150 \\
\hline $\mathrm{H}$ & 5.813218 & -3.623710 & 0.658270 \\
\hline $\mathrm{H}$ & 7.025632 & -3.009121 & 1.803449 \\
\hline H & 5.911566 & -4.285730 & 2.295360 \\
\hline
\end{tabular}




$\begin{array}{lrrr}\mathrm{C} & 8.527326 & 0.708979 & -0.522720 \\ \mathrm{H} & 8.714485 & -0.239325 & -0.010452 \\ \mathrm{H} & 8.536173 & 1.503295 & 0.230089 \\ \mathrm{H} & 9.355453 & 0.886200 & -1.217817 \\ \mathrm{C} & 7.206991 & -0.436530 & -2.334172 \\ \mathrm{H} & 6.266407 & -0.464610 & -2.892866 \\ \mathrm{H} & 7.366827 & -1.418322 & -1.878763 \\ \mathrm{H} & 8.020061 & -0.269148 & -3.048698 \\ \mathrm{C} & 7.015310 & 2.026484 & -2.020369 \\ \mathrm{H} & 7.827559 & 2.150028 & -2.743440 \\ \mathrm{H} & 7.052967 & 2.876738 & -1.332523 \\ \mathrm{H} & 6.067179 & 2.067973 & -2.564774\end{array}$

\section{C-Catalyzed reaction TS 11}

\section{Datum}

Value

\begin{tabular}{lc} 
B3LYP-D3(BJ)/6-31G(d,p) Energy & -3039.49662 \\
\hline B3LYP-D3(BJ)/def2-TZVPP/IEF-PCM(DCM) Energy & -3040.529838 \\
\hline B3LYP-D3(BJ)/def2-TZVPP/IEF-PCM(DCM)//B3LYP-D3(BJ)/6-31G(d,p) Free Energy (Quasiharmonic) & -3039.298263 \\
\hline
\end{tabular}

Number of Imaginary Frequencies

Frequencies (Top 3 out of 450)

$\begin{array}{lr}\text { 1. } & -265.6563 \mathrm{~cm}^{-1} \\ \text { 2. } & 11.0651 \mathrm{~cm}^{-1} \\ \text { 3. } & 17.8511 \mathrm{~cm}^{-1}\end{array}$

B3LYP-D3(BJ)/6-31G(d,p) Molecular Geometry in Cartesian Coordinates

$\begin{array}{rrrr}\mathrm{C} & -1.303326 & 1.280837 & 1.094316 \\ \mathrm{H} & -0.971330 & 1.847940 & 1.967208 \\ \mathrm{H} & -2.249019 & 0.803696 & 1.338589 \\ \mathrm{C} & 1.983397 & 1.617275 & -1.099162 \\ \mathrm{O} & 2.774824 & 1.938801 & -1.959691 \\ \mathrm{O} & 1.145717 & 0.588151 & -1.255091 \\ \mathrm{H} & 0.570510 & 0.479518 & -0.432252 \\ \mathrm{O} & -0.326184 & 0.253086 & 0.842775 \\ \mathrm{H} & -0.792717 & -0.628272 & 0.793448 \\ \mathrm{C} & 0.082570 & -1.887887 & -2.752052 \\ \mathrm{C} & 0.869494 & -2.646483 & -0.942349 \\ \mathrm{C} & -2.053595 & -2.604126 & -1.738612 \\ \mathrm{C} & -1.138533 & -1.597631 & -2.169947 \\ \mathrm{H} & 0.883065 & -1.691677 & -0.422901\end{array}$




\begin{tabular}{|c|c|c|c|}
\hline $\mathrm{H}$ & -3.038291 & -2.238054 & -1.458218 \\
\hline $\mathrm{H}$ & -2.094065 & -3.502781 & -2.355277 \\
\hline C & -1.826849 & -2.649803 & 2.029821 \\
\hline C & -2.712185 & -3.858215 & 1.569871 \\
\hline C & -0.517720 & -3.074747 & 2.705326 \\
\hline H & 0.135428 & -2.199997 & 2.777330 \\
\hline $\mathrm{H}$ & -0.688551 & -3.459836 & 3.714566 \\
\hline $\mathrm{H}$ & -0.003652 & -3.839625 & 2.119330 \\
\hline C & -2.551382 & -1.613473 & 2.881900 \\
\hline $\mathrm{H}$ & -3.364951 & -1.148046 & 2.323199 \\
\hline $\mathrm{H}$ & -2.963080 & -2.076393 & 3.784216 \\
\hline $\mathrm{H}$ & -1.855548 & -0.830422 & 3.197006 \\
\hline C & -4.162037 & -3.443302 & 1.286256 \\
\hline H & -4.723880 & -3.261921 & 2.207004 \\
\hline $\mathrm{H}$ & -4.207248 & -2.540245 & 0.673857 \\
\hline $\mathrm{H}$ & -4.652195 & -4.252344 & 0.738664 \\
\hline C & -2.684364 & -5.050461 & 2.521737 \\
\hline $\mathrm{H}$ & -3.058006 & -4.771505 & 3.512764 \\
\hline $\mathrm{H}$ & -3.323227 & -5.846423 & 2.129367 \\
\hline $\mathrm{H}$ & -1.673974 & -5.449075 & 2.624151 \\
\hline 0 & -1.494678 & -2.050754 & 0.753449 \\
\hline 0 & -2.106341 & -4.250319 & 0.330677 \\
\hline B & -1.403412 & -3.144432 & -0.211073 \\
\hline 0 & -0.003777 & -3.538650 & -0.559218 \\
\hline C & 2.173960 & -3.127727 & -1.432443 \\
\hline C & 3.218241 & -2.212052 & -1.631861 \\
\hline C & 2.380149 & -4.491850 & -1.685523 \\
\hline C & 4.451839 & -2.663559 & -2.090237 \\
\hline $\mathrm{H}$ & 3.060856 & -1.156990 & -1.434976 \\
\hline C & 3.620627 & -4.934598 & -2.134541 \\
\hline $\mathrm{H}$ & 1.565486 & -5.185781 & -1.511762 \\
\hline C & 4.656683 & -4.020657 & -2.343216 \\
\hline $\mathrm{H}$ & 5.256463 & -1.954168 & -2.234989 \\
\hline $\mathrm{H}$ & 3.781481 & -5.991944 & -2.320782 \\
\hline H & 5.623574 & -4.366897 & -2.695668 \\
\hline $\mathrm{H}$ & -1.284528 & -0.586398 & -1.792530 \\
\hline $\mathrm{H}$ & 0.208351 & -2.823319 & -3.288757 \\
\hline $\mathrm{H}$ & 0.771896 & -1.085942 & -2.985606 \\
\hline C & 0.774659 & 3.117759 & 0.499997 \\
\hline C & 1.882911 & 2.337791 & 0.222451 \\
\hline C & 2.925447 & 2.161719 & 1.180134 \\
\hline C & 2.858727 & 2.853460 & 2.369347 \\
\hline H & 3.634062 & 2.705029 & 3.115337 \\
\hline C & -1.456401 & 2.201317 & -0.101777 \\
\hline C & -0.414205 & 3.072158 & -0.409046 \\
\hline C & -2.604337 & 2.860947 & -2.136543 \\
\hline C & -2.583436 & 2.102001 & -0.980118 \\
\hline H & -3.464959 & 2.796114 & -2.795896 \\
\hline C & -1.555221 & 3.744296 & -2.476943 \\
\hline C & -0.435901 & 3.861743 & -1.594895 \\
\hline C & 0.618134 & 4.749886 & -1.938883 \\
\hline C & 0.560496 & 5.489410 & -3.096667 \\
\hline C & -0.551313 & 5.378353 & -3.966744 \\
\hline C & -1.585185 & 4.524773 & -3.663054 \\
\hline
\end{tabular}




\begin{tabular}{|c|c|c|c|}
\hline $\mathrm{H}$ & 1.476564 & 4.823328 & -1.281506 \\
\hline $\mathrm{H}$ & 1.375341 & 6.159860 & -3.350620 \\
\hline $\mathrm{H}$ & -0.581209 & 5.967847 & -4.877890 \\
\hline $\mathrm{H}$ & -2.440215 & 4.431240 & -4.326658 \\
\hline C & 1.797380 & 3.751050 & 2.648923 \\
\hline C & 0.728249 & 3.880837 & 1.707015 \\
\hline C & -0.351791 & 4.750800 & 2.012158 \\
\hline C & -0.369885 & 5.463626 & 3.189599 \\
\hline C & 0.688389 & 5.337098 & 4.120362 \\
\hline C & 1.746210 & 4.498579 & 3.854376 \\
\hline $\mathrm{H}$ & -1.160107 & 4.849152 & 1.295958 \\
\hline $\mathrm{H}$ & -1.198890 & 6.130273 & 3.406191 \\
\hline $\mathrm{H}$ & 0.662051 & 5.904510 & 5.045509 \\
\hline $\mathrm{H}$ & 2.561122 & 4.394342 & 4.565175 \\
\hline C & -5.994345 & -0.352742 & -0.230641 \\
\hline C & -5.344041 & -0.458945 & -1.465923 \\
\hline C & -4.210896 & 0.334752 & -1.676487 \\
\hline C & -3.749793 & 1.220872 & -0.694705 \\
\hline C & -4.445411 & 1.315006 & 0.518430 \\
\hline C & -5.570487 & 0.526430 & 0.774294 \\
\hline $\mathrm{H}$ & -6.874435 & -0.960537 & -0.052725 \\
\hline $\mathrm{H}$ & -3.664918 & 0.275578 & -2.609384 \\
\hline $\mathrm{H}$ & -4.109606 & 2.039599 & 1.249479 \\
\hline C & -5.916494 & -1.390788 & -2.545783 \\
\hline C & -6.380081 & 0.643635 & 2.074603 \\
\hline C & -7.333106 & -0.897742 & -2.916513 \\
\hline $\mathrm{H}$ & -7.766528 & -1.535981 & -3.694000 \\
\hline $\mathrm{H}$ & -8.005269 & -0.915454 & -2.054035 \\
\hline $\mathrm{H}$ & -7.300412 & 0.128831 & -3.294629 \\
\hline C & -5.062037 & -1.398713 & -3.824254 \\
\hline $\mathrm{H}$ & -5.509816 & -2.074933 & -4.558724 \\
\hline $\mathrm{H}$ & -5.008364 & -0.405231 & -4.280322 \\
\hline $\mathrm{H}$ & -4.042811 & -1.744430 & -3.632263 \\
\hline C & -6.006925 & -2.835905 & -2.010403 \\
\hline $\mathrm{H}$ & -6.450893 & -3.489803 & -2.768468 \\
\hline $\mathrm{H}$ & -5.018881 & -3.230108 & -1.760128 \\
\hline $\mathrm{H}$ & -6.627049 & -2.898578 & -1.112106 \\
\hline C & -5.704643 & 1.570383 & 3.098822 \\
\hline $\mathrm{H}$ & -4.701097 & 1.219217 & 3.359818 \\
\hline $\mathrm{H}$ & -5.624192 & 2.597250 & 2.729666 \\
\hline $\mathrm{H}$ & -6.297876 & 1.597248 & 4.017793 \\
\hline C & -7.773040 & 1.218851 & 1.736285 \\
\hline $\mathrm{H}$ & -8.375702 & 1.322767 & 2.645127 \\
\hline $\mathrm{H}$ & -7.682607 & 2.204696 & 1.269786 \\
\hline $\mathrm{H}$ & -8.315917 & 0.569588 & 1.043447 \\
\hline C & -6.547066 & -0.744778 & 2.726875 \\
\hline $\mathrm{H}$ & -7.161848 & -0.664099 & 3.629554 \\
\hline $\mathrm{H}$ & -7.032513 & -1.458833 & 2.056838 \\
\hline $\mathrm{H}$ & -5.577325 & -1.160424 & 3.012522 \\
\hline C & 3.970992 & 1.123870 & 0.956620 \\
\hline C & 3.900689 & -0.067923 & 1.693979 \\
\hline C & 4.980882 & 1.293610 & 0.014496 \\
\hline C & 4.835711 & -1.085742 & 1.505078 \\
\hline H & 3.086740 & -0.181733 & 2.398949 \\
\hline
\end{tabular}




$\begin{array}{lrrr}\text { C } & 5.942003 & 0.295526 & -0.199406 \\ \text { H } & 4.997653 & 2.207188 & -0.566023 \\ \text { C } & 5.852158 & -0.874532 & 0.557782 \\ \text { H } & 6.583988 & -1.657476 & 0.405963 \\ \text { C } & 7.043311 & 0.538728 & -1.241129 \\ \text { C } & 4.803976 & -2.392483 & 2.312250 \\ \text { C } & 6.400206 & 0.818928 & -2.617637 \\ \text { H } & 7.175248 & 1.054005 & -3.355337 \\ \text { H } & 5.843329 & -0.050268 & -2.979546 \\ \text { H } & 5.698258 & 1.654794 & -2.579874 \\ \text { C } & 7.874246 & 1.767746 & -0.811006 \\ \text { H } & 8.671587 & 1.962446 & -1.536587 \\ \text { H } & 7.255077 & 2.666172 & -0.743317 \\ \text { H } & 8.334381 & 1.602085 & 0.168313 \\ \text { C } & 7.996839 & -0.658999 & -1.379097 \\ \text { H } & 8.520154 & -0.870231 & -0.441171 \\ \text { H } & 7.470689 & -1.568491 & -1.685980 \\ \text { H } & 8.752889 & -0.441925 & -2.139790 \\ \text { C } & 3.551261 & -2.494388 & 3.198304 \\ \text { H } & 2.634717 & -2.454677 & 2.601382 \\ \text { H } & 3.510544 & -1.694832 & 3.944440 \\ \text { H } & 3.556923 & -3.447665 & 3.735638 \\ \text { C } & 4.812609 & -3.611932 & 1.367100 \\ \text { H } & 5.684573 & -3.617284 & 0.708995 \\ \text { H } & 3.922464 & -3.625432 & 0.734584 \\ \text { H } & 4.831638 & -4.537378 & 1.952940 \\ \text { C } & 6.053180 & -2.439261 & 3.219049 \\ \text { H } & 6.060373 & -3.358717 & 3.814855 \\ \text { H } & 6.068777 & -1.585795 & 3.904288 \\ \text { H } & 6.974445 & -2.411944 & 2.629814\end{array}$

\section{C-Catalyzed reaction TS 12}

\section{Datum}

Value

B3LYP-D3(BJ)/6-31G(d,p) Energy

$-3039.494815$

B3LYP-D3(BJ)/def2-TZVPP/IEF-PCM(DCM) Energy

$-3040.528218$

B3LYP-D3(BJ)/def2-TZVPP/IEF-PCM(DCM)//B3LYP-D3(BJ)/6-31G(d,p) Free Energy (Quasiharmonic)

$-3039.297544$

Number of Imaginary Frequencies

Frequencies (Top 3 out of 450)

$\begin{array}{lr}\text { 1. } & -178.4742 \mathrm{~cm}^{-1} \\ \text { 2. } & 11.0056 \mathrm{~cm}^{-1} \\ \text { 3. } & 18.0424 \mathrm{~cm}^{-1}\end{array}$




\begin{tabular}{|c|c|c|c|}
\hline C & -1.449310 & 1.365820 & -1.091120 \\
\hline $\mathrm{H}$ & -2.444740 & 1.089490 & -1.447860 \\
\hline $\mathrm{H}$ & -0.873730 & 1.750820 & -1.938910 \\
\hline C & 1.550510 & 1.833500 & 1.228550 \\
\hline 0 & 2.064740 & 2.364590 & 2.191550 \\
\hline 0 & 0.839260 & 0.712530 & 1.351700 \\
\hline $\mathrm{H}$ & 0.299660 & 0.507620 & 0.528550 \\
\hline 0 & -0.807330 & 0.210360 & -0.542400 \\
\hline $\mathrm{H}$ & -0.902230 & -0.588560 & -1.132400 \\
\hline C & 2.312240 & -2.043950 & 0.140230 \\
\hline C & 0.299220 & -2.655280 & 0.738580 \\
\hline C & 1.482521 & -2.852980 & -2.043850 \\
\hline C & 1.886410 & -1.807350 & -1.144700 \\
\hline $\mathrm{H}$ & -0.123850 & -1.658390 & 0.647740 \\
\hline $\mathrm{H}$ & 1.413880 & -2.555300 & -3.090490 \\
\hline $\mathrm{H}$ & 2.026951 & -3.791700 & -1.928910 \\
\hline C & -2.247880 & -2.509240 & -2.391210 \\
\hline C & -1.787269 & -3.803820 & -3.142380 \\
\hline C & -3.166250 & -2.799101 & -1.201490 \\
\hline $\mathrm{H}$ & -2.769939 & -3.615300 & -0.593430 \\
\hline $\mathrm{H}$ & -3.234770 & -1.904981 & -0.579740 \\
\hline $\mathrm{H}$ & -4.177319 & -3.063291 & -1.525520 \\
\hline C & -2.862750 & -1.434430 & -3.279840 \\
\hline $\mathrm{H}$ & -3.727540 & -1.832191 & -3.820320 \\
\hline $\mathrm{H}$ & -3.212790 & -0.596101 & -2.671290 \\
\hline $\mathrm{H}$ & -2.141400 & -1.057670 & -4.006860 \\
\hline C & -1.224809 & -3.500860 & -4.538480 \\
\hline $\mathrm{H}$ & -2.012809 & -3.237220 & -5.250420 \\
\hline $\mathrm{H}$ & -0.504060 & -2.680270 & -4.501960 \\
\hline $\mathrm{H}$ & -0.709549 & -4.391730 & -4.906410 \\
\hline C & -2.858489 & -4.886620 & -3.236760 \\
\hline $\mathrm{H}$ & -3.735919 & -4.525511 & -3.783790 \\
\hline $\mathrm{H}$ & -2.457499 & -5.752380 & -3.770940 \\
\hline $\mathrm{H}$ & -3.172009 & -5.218061 & -2.245880 \\
\hline 0 & -0.983690 & -2.021960 & -1.877030 \\
\hline 0 & -0.718409 & -4.287940 & -2.320010 \\
\hline B & -0.152339 & -3.196040 & -1.611230 \\
\hline 0 & -0.036769 & -3.545950 & -0.143370 \\
\hline C & 0.641951 & -3.102160 & 2.097430 \\
\hline C & 0.759901 & -4.466490 & 2.394910 \\
\hline C & 0.839900 & -2.143300 & 3.104080 \\
\hline C & 1.069571 & -4.867480 & 3.691430 \\
\hline $\mathrm{H}$ & 0.595111 & -5.191720 & 1.605950 \\
\hline C & 1.154990 & -2.552880 & 4.396040 \\
\hline $\mathrm{H}$ & 0.763200 & -1.087530 & 2.861540 \\
\hline C & 1.269681 & -3.913560 & 4.692510 \\
\hline $\mathrm{H}$ & 1.154041 & -5.924570 & 3.923790 \\
\hline $\mathrm{H}$ & 1.313280 & -1.809980 & 5.171150 \\
\hline $\mathrm{H}$ & 1.514011 & -4.230150 & 5.701890 \\
\hline $\mathrm{H}$ & 1.606990 & -0.787270 & -1.400330 \\
\hline
\end{tabular}




\begin{tabular}{|c|c|c|c|}
\hline $\mathrm{H}$ & 2.738151 & -3.007610 & 0.398760 \\
\hline $\mathrm{H}$ & 2.532710 & -1.220220 & 0.806750 \\
\hline C & 0.694220 & 3.241820 & -0.631780 \\
\hline C & 1.700440 & 2.410680 & -0.166590 \\
\hline C & 2.873830 & 2.156530 & -0.952310 \\
\hline C & 3.000580 & 2.816400 & -2.161070 \\
\hline H & 3.889080 & 2.648590 & -2.762030 \\
\hline C & -1.573930 & 2.423620 & -0.020130 \\
\hline C & -0.565990 & 3.359540 & 0.165210 \\
\hline C & -2.831750 & 3.387850 & 1.815780 \\
\hline C & -2.702820 & 2.410110 & 0.853890 \\
\hline $\mathrm{H}$ & -3.691650 & 3.372729 & 2.479360 \\
\hline C & -1.854950 & 4.400370 & 1.982270 \\
\hline C & -0.684350 & 4.374100 & 1.161890 \\
\hline C & 0.322240 & 5.353270 & 1.371180 \\
\hline C & 0.164850 & 6.325590 & 2.331080 \\
\hline C & -1.000950 & 6.361160 & 3.133120 \\
\hline C & -1.986230 & 5.416650 & 2.964320 \\
\hline $\mathrm{H}$ & 1.221180 & 5.317180 & 0.766850 \\
\hline H & 0.942170 & 7.068150 & 2.481050 \\
\hline $\mathrm{H}$ & -1.110220 & 7.133350 & 3.888390 \\
\hline $\mathrm{H}$ & -2.878590 & 5.430020 & 3.583750 \\
\hline C & 2.020720 & 3.715540 & -2.643440 \\
\hline C & 0.834390 & 3.926520 & -1.875120 \\
\hline C & -0.162040 & 4.805770 & -2.374370 \\
\hline C & 0.013750 & 5.453750 & -3.575940 \\
\hline C & 1.190960 & 5.249970 & -4.335330 \\
\hline C & 2.171060 & 4.399730 & -3.878610 \\
\hline $\mathrm{H}$ & -1.060610 & 4.962960 & -1.788030 \\
\hline $\mathrm{H}$ & -0.752770 & 6.128130 & -3.944820 \\
\hline $\mathrm{H}$ & 1.316650 & 5.767800 & -5.281140 \\
\hline $\mathrm{H}$ & 3.075730 & 4.236410 & -4.457470 \\
\hline C & -5.460280 & -0.849521 & 0.791610 \\
\hline C & -4.380520 & -0.877881 & 1.684820 \\
\hline C & -3.495280 & 0.204459 & 1.673150 \\
\hline C & -3.681810 & 1.284269 & 0.805420 \\
\hline C & -4.764610 & 1.278829 & -0.075390 \\
\hline C & -5.670150 & 0.209919 & -0.098900 \\
\hline $\mathrm{H}$ & -6.148780 & -1.681231 & 0.782410 \\
\hline $\mathrm{H}$ & -2.638460 & 0.226260 & 2.337040 \\
\hline $\mathrm{H}$ & -4.896820 & 2.125369 & -0.741910 \\
\hline C & -4.168380 & -2.026081 & 2.682870 \\
\hline C & -6.851940 & 0.240099 & -1.079220 \\
\hline C & -4.477990 & -1.498861 & 4.101320 \\
\hline $\mathrm{H}$ & -4.333910 & -2.290671 & 4.844660 \\
\hline $\mathrm{H}$ & -3.821990 & -0.663791 & 4.363830 \\
\hline H & -5.512620 & -1.147811 & 4.169280 \\
\hline C & -5.082829 & -3.227671 & 2.392620 \\
\hline H & -4.865389 & -4.032971 & 3.100730 \\
\hline $\mathrm{H}$ & -6.141809 & -2.973761 & 2.500840 \\
\hline $\mathrm{H}$ & -4.923589 & -3.617831 & 1.382380 \\
\hline C & -2.706760 & -2.515380 & 2.634960 \\
\hline $\mathrm{H}$ & -2.553689 & -3.329940 & 3.350100 \\
\hline $\mathrm{H}$ & -2.447669 & -2.887570 & 1.641030 \\
\hline
\end{tabular}




\begin{tabular}{|c|c|c|c|}
\hline H & -1.999220 & -1.725270 & 2.892970 \\
\hline C & -6.318460 & 0.343169 & -2.524030 \\
\hline $\mathrm{H}$ & -7.150080 & 0.386969 & -3.235640 \\
\hline H & -5.707730 & 1.238619 & -2.668770 \\
\hline $\mathrm{H}$ & -5.702340 & -0.525891 & -2.771700 \\
\hline C & -7.725870 & -1.020891 & -0.983060 \\
\hline H & -8.546180 & -0.955451 & -1.704320 \\
\hline $\mathrm{H}$ & -7.154910 & -1.926801 & -1.210140 \\
\hline $\mathrm{H}$ & -8.167400 & -1.134291 & 0.011800 \\
\hline C & -7.735630 & 1.467719 & -0.769590 \\
\hline $\mathrm{H}$ & -8.585690 & 1.510459 & -1.459220 \\
\hline $\mathrm{H}$ & -8.125350 & 1.416849 & 0.251820 \\
\hline $\mathrm{H}$ & -7.176450 & 2.402219 & -0.867840 \\
\hline C & 3.886880 & 1.137150 & -0.566840 \\
\hline C & 4.365550 & 1.012020 & 0.737770 \\
\hline C & 4.350710 & 0.240260 & -1.547320 \\
\hline C & 5.243250 & -0.021060 & 1.096380 \\
\hline H & 4.042190 & 1.720540 & 1.485520 \\
\hline C & 5.254910 & -0.772430 & -1.236080 \\
\hline $\mathrm{H}$ & 3.953610 & 0.327140 & -2.550300 \\
\hline C & 5.681150 & -0.890120 & 0.097520 \\
\hline $\mathrm{H}$ & 6.362710 & -1.690750 & 0.354360 \\
\hline C & 5.775990 & -1.766000 & -2.284080 \\
\hline C & 5.642780 & -0.167860 & 2.570650 \\
\hline C & 7.302470 & -1.583480 & -2.426650 \\
\hline H & 7.705030 & -2.286240 & -3.164390 \\
\hline $\mathrm{H}$ & 7.542850 & -0.567130 & -2.754220 \\
\hline H & 7.817410 & -1.759170 & -1.477890 \\
\hline C & 5.472421 & -3.211080 & -1.833090 \\
\hline $\mathrm{H}$ & 5.849161 & -3.924880 & -2.573480 \\
\hline $\mathrm{H}$ & 5.942851 & -3.447140 & -0.874950 \\
\hline H & 4.395531 & -3.364410 & -1.727230 \\
\hline C & 5.128220 & -1.552810 & -3.661720 \\
\hline $\mathrm{H}$ & 4.042970 & -1.687070 & -3.620100 \\
\hline $\mathrm{H}$ & 5.338120 & -0.554930 & -4.059330 \\
\hline $\mathrm{H}$ & 5.526850 & -2.282720 & -4.372840 \\
\hline C & 6.336220 & 1.125110 & 3.049610 \\
\hline $\mathrm{H}$ & 7.240760 & 1.321500 & 2.465000 \\
\hline H & 5.679100 & 1.993630 & 2.958030 \\
\hline H & 6.622550 & 1.033050 & 4.102820 \\
\hline C & 4.363450 & -0.404970 & 3.407090 \\
\hline H & 3.650220 & 0.417110 & 3.303770 \\
\hline $\mathrm{H}$ & 3.860820 & -1.326840 & 3.097350 \\
\hline H & 4.619090 & -0.501380 & 4.468330 \\
\hline C & 6.599950 & -1.347520 & 2.800730 \\
\hline $\mathrm{H}$ & 6.851720 & -1.415250 & 3.863430 \\
\hline H & 6.145940 & -2.299380 & 2.507100 \\
\hline $\mathrm{H}$ & 7.535330 & -1.226520 & 2.244600 \\
\hline
\end{tabular}


Frequencies (Top 3 out of 450)

$\begin{array}{rr}\text { 1. } & -277.1619 \mathrm{~cm}^{-1} \\ \text { 2. } & 13.2642 \mathrm{~cm}^{-1} \\ \text { 3. } & 19.0132 \mathrm{~cm}^{-1}\end{array}$

\section{B3LYP-D3(BJ)/6-31G(d,p) Molecular Geometry in Cartesian Coordinates}

\section{C}

$\mathrm{H}$

$\mathrm{H}$

C

0

0

$\mathrm{H}$

0

$\mathrm{H}$

C

C

C

C

$\mathrm{H}$

$\mathrm{H}$

$\mathrm{H}$

C

C

C

$\mathrm{H}$

$\mathrm{H}$

$\mathrm{H}$

C

$\mathrm{H}$

$\mathrm{H}$

$\mathrm{H}$

C

$\mathrm{H}$

$\mathrm{H}$

$\mathrm{H}$

C

$\mathrm{H}$

\section{$-1.329143$}

$-2.269104$

$-0.984447$

1.870036

2.735636

0.891783

0.383416

$-0.344122$

$-0.768899$

$-0.046010$

0.808951

$-1.719392$

$-1.325842$

0.339402

$-1.267184$

$-2.784227$

$-2.097717$

$-1.963694$

$-1.582947$

$-1.699772$

$-0.526458$

$-2.152229$

$-3.510858$

$-3.485417$

$-4.206665$

$-3.900483$

$-0.726165$

$-0.830940$

0.173390

$-0.595630$

$-3.206734$

$-3.433722$
1.511106

1.083218

2. 228722

1. 248897

1. 278403

0.341440

0.379094

0.467469

$-0.426633$

$-2.471650$

$-3.426293$

$-1.737676$

$-2.522905$

$-4.399876$

$-0.749039$

$-1.721950$

$-2.750573$

$-4.185771$

$-2.627535$

$-1.596084$

$-2.887928$

$-3.275392$

$-2.174840$

$-1.112731$

$-2.672395$

$-2.256177$

$-4.941120$

$-5.249230$

$-4.330691$

$-5.838593$

$-5.055743$

$-5.229471$
$-1.155708$

$-1.501038$

$-1.906783$

1.161937

2.012228

1. 178762

0.307518

$-1.074476$

$-1.174574$

3. 198897

1.517824

1. 550292

2.674414

1.673385

1.498877

1.336353

$-1.914064$

$-1.283826$

$-3.344722$

$-3.691308$

$-3.412276$

$-4.019068$

$-1.810554$

$-2.065982$

$-2.492005$

$-0.796701$

$-1.787801$

$-2.832337$

$-1.691645$

$-1.176872$

$-1.439474$

$-2.496481$ 


\begin{tabular}{|c|c|c|c|}
\hline $\mathrm{H}$ & -3.036211 & -6.026274 & -0.965217 \\
\hline $\mathrm{H}$ & -4.074705 & -4.595563 & -0.964984 \\
\hline 0 & -1.244267 & -1.965667 & -1.055586 \\
\hline 0 & -1.778762 & -3.901791 & 0.114038 \\
\hline B & -1.089680 & -2.651783 & 0.207152 \\
\hline 0 & 0.372302 & -2.720325 & 0.510804 \\
\hline C & 2.235683 & -3.303656 & 1.868399 \\
\hline C & 2.921379 & -2.104890 & 1.630070 \\
\hline C & 2.912941 & -4.393846 & 2.431678 \\
\hline C & 4.268279 & -1.994455 & 1.952733 \\
\hline $\mathrm{H}$ & 2.385301 & -1.271982 & 1.202987 \\
\hline C & 4.268801 & -4.286395 & 2.735240 \\
\hline H & 2.381789 & -5.323946 & 2.616309 \\
\hline C & 4.946051 & -3.087032 & 2.498774 \\
\hline $\mathrm{H}$ & 4.775916 & -1.054994 & 1.770668 \\
\hline H & 4.796434 & -5.136178 & 3.157115 \\
\hline $\mathrm{H}$ & 6.000923 & -3.004816 & 2.744058 \\
\hline $\mathrm{H}$ & -1.964056 & -3.362470 & 2.949733 \\
\hline $\mathrm{H}$ & 0.538759 & -1.566142 & 3.070380 \\
\hline H & 0.237995 & -3.118764 & 4.022662 \\
\hline C & 0.795081 & 3.132560 & -0.113225 \\
\hline C & 1.849594 & 2.249811 & 0.034243 \\
\hline C & 2.999435 & 2.321886 & -0.807292 \\
\hline C & 3.116126 & 3.366668 & -1.694060 \\
\hline $\mathrm{H}$ & 3.976986 & 3.413663 & -2.354906 \\
\hline C & -1.518243 & 2.220935 & 0.176138 \\
\hline C & -0.460039 & 2.968115 & 0.686941 \\
\hline C & -2.788259 & 2.658102 & 2.201571 \\
\hline C & -2.713554 & 2.067374 & 0.952824 \\
\hline H & -3.700708 & 2.552687 & 2.781014 \\
\hline C & -1.725406 & 3.411403 & 2.745729 \\
\hline C & -0.532834 & 3.573833 & 1.975831 \\
\hline C & 0.539597 & 4.326112 & 2.526980 \\
\hline C & 0.428879 & 4.893559 & 3.774761 \\
\hline C & -0.756847 & 4.739027 & 4.533379 \\
\hline C & -1.809909 & 4.013609 & 4.029180 \\
\hline H & 1.453352 & 4.434849 & 1.954861 \\
\hline H & 1.258365 & 5.460470 & 4.185402 \\
\hline $\mathrm{H}$ & -0.828408 & 5.192477 & 5.517219 \\
\hline $\mathrm{H}$ & -2.722099 & 3.885819 & 4.605286 \\
\hline C & 2.103060 & 4.353496 & -1.810553 \\
\hline C & 0.907634 & 4.221087 & -1.034670 \\
\hline C & -0.123127 & 5.183981 & -1.198016 \\
\hline C & 0.021834 & 6.228608 & -2.083070 \\
\hline C & 1.203717 & 6.359751 & -2.848822 \\
\hline C & 2.219181 & 5.440620 & -2.714683 \\
\hline $\mathrm{H}$ & -1.026427 & 5.086266 & -0.606318 \\
\hline $\mathrm{H}$ & -0.774017 & 6.958723 & -2.193467 \\
\hline $\mathrm{H}$ & 1.306423 & 7.187845 & -3.543352 \\
\hline H & 3.127996 & 5.531720 & -3.302939 \\
\hline $\mathrm{C}$ & -6.101458 & -0.222905 & -0.275828 \\
\hline C & -5.631904 & 0.777239 & -1.137235 \\
\hline C & -4.518845 & 1.521157 & -0.733289 \\
\hline C & -3.888212 & 1.275828 & 0.493475 \\
\hline
\end{tabular}




\begin{tabular}{|c|c|c|c|}
\hline C & -4.412172 & 0.291161 & 1.340195 \\
\hline C & -5.517894 & -0.479323 & 0.970354 \\
\hline $\mathrm{H}$ & -6.953853 & -0.817516 & -0.585260 \\
\hline H & -4.141580 & 2.321376 & -1.357454 \\
\hline $\mathrm{H}$ & -3.919276 & 0.125055 & 2.288320 \\
\hline C & -6.361913 & 1.039251 & -2.463234 \\
\hline C & -6.075574 & -1.595943 & 1.867092 \\
\hline C & -7.809100 & 1.483219 & -2.157702 \\
\hline $\mathrm{H}$ & -8.352823 & 1.680901 & -3.087862 \\
\hline $\mathrm{H}$ & -8.356790 & 0.715254 & -1.604388 \\
\hline $\mathrm{H}$ & -7.815313 & 2.397310 & -1.555957 \\
\hline C & -5.683095 & 2.139753 & -3.294777 \\
\hline $\mathrm{H}$ & -6.227624 & 2.280587 & -4.233298 \\
\hline $\mathrm{H}$ & -5.675471 & 3.099909 & -2.769881 \\
\hline $\mathrm{H}$ & -4.650389 & 1.877704 & -3.546219 \\
\hline C & -6.390986 & -0.251090 & -3.309995 \\
\hline $\mathrm{H}$ & -6.941083 & -0.079215 & -4.241337 \\
\hline $\mathrm{H}$ & -5.377693 & -0.572579 & -3.566137 \\
\hline $\mathrm{H}$ & -6.878677 & -1.074814 & -2.781822 \\
\hline C & -7.594901 & -1.400360 & 2.056647 \\
\hline $\mathrm{H}$ & -7.997338 & -2.179752 & 2.712205 \\
\hline $\mathrm{H}$ & -7.807858 & -0.427613 & 2.510652 \\
\hline $\mathrm{H}$ & -8.137143 & -1.453759 & 1.108935 \\
\hline C & -5.814835 & -2.964623 & 1.199063 \\
\hline $\mathrm{H}$ & -6.253786 & -3.769728 & 1.798484 \\
\hline $\mathrm{H}$ & -6.255111 & -3.010787 & 0.198588 \\
\hline $\mathrm{H}$ & -4.742505 & -3.163495 & 1.104363 \\
\hline C & -5.420922 & -1.605807 & 3.259047 \\
\hline $\mathrm{H}$ & -5.872156 & -2.393111 & 3.870494 \\
\hline $\mathrm{H}$ & -4.347122 & -1.805908 & 3.205856 \\
\hline $\mathrm{H}$ & -5.565759 & -0.653658 & 3.779007 \\
\hline C & 3.937272 & 1.165905 & -0.856269 \\
\hline C & 5.262440 & 1.246130 & -0.442393 \\
\hline C & 3.442322 & -0.035017 & -1.390000 \\
\hline C & 6.116262 & 0.136706 & -0.552259 \\
\hline H & 5.617776 & 2.175895 & -0.011495 \\
\hline C & 4.272640 & -1.143143 & -1.551609 \\
\hline $\mathrm{H}$ & 2.402604 & -0.067019 & -1.693000 \\
\hline C & 5.605372 & -1.031108 & -1.122500 \\
\hline $\mathrm{H}$ & 6.254793 & -1.888900 & -1.235665 \\
\hline C & 3.795920 & -2.429012 & -2.242746 \\
\hline C & 7.559225 & 0.241555 & -0.038440 \\
\hline C & 4.512401 & -2.530174 & -3.607984 \\
\hline H & 4.283121 & -1.660797 & -4.232290 \\
\hline $\mathrm{H}$ & 5.598665 & -2.577547 & -3.485272 \\
\hline $\mathrm{H}$ & 4.191709 & -3.431400 & -4.142632 \\
\hline C & 4.142033 & -3.673237 & -1.398567 \\
\hline $\mathrm{H}$ & 3.829399 & -4.578849 & -1.929942 \\
\hline $\mathrm{H}$ & 5.215482 & -3.757257 & -1.209210 \\
\hline $\mathrm{H}$ & 3.632950 & -3.648369 & -0.434216 \\
\hline C & 2.277154 & -2.422821 & -2.485255 \\
\hline $\mathrm{H}$ & 1.714050 & -2.315883 & -1.554884 \\
\hline $\mathrm{H}$ & 1.975322 & -1.617230 & -3.161622 \\
\hline $\mathrm{H}$ & 1.982460 & -3.368333 & -2.952306 \\
\hline
\end{tabular}




\begin{tabular}{|lrrr|} 
C & 7.537235 & 0.573392 & 1.469616 \\
H & 7.003779 & 1.505286 & 1.672995 \\
H & 7.041481 & -0.221941 & 2.035319 \\
H & 8.558540 & 0.676869 & 1.852497 \\
C & 8.344917 & -1.065786 & -0.228541 \\
H & 7.877619 & -1.897951 & 0.307638 \\
$H$ & 8.425803 & -1.341405 & -1.284688 \\
$H$ & 9.360101 & -0.945558 & 0.162150 \\
C & 8.293529 & 1.365368 & -0.800415 \\
H & 9.325134 & 1.458850 & -0.443715 \\
H & 8.320150 & 1.152160 & -1.873701 \\
$H$ & 7.802907 & 2.332813 & -0.663228 \\
\hline
\end{tabular}

\section{C-Catalyzed reaction TS 14}

\section{Datum}

Value

\begin{tabular}{lc} 
B3LYP-D3(BJ)/6-31G(d,p) Energy & -3039.496208 \\
\hline B3LYP-D3(BJ)/def2-TZVPP/IEF-PCM(DCM) Energy & -3040.528807 \\
\hline B3LYP-D3(BJ)/def2-TZVPP/IEF-PCM(DCM)//B3LYP-D3(BJ)/6-31G(d,p) Free Energy (Quasiharmonic) & -3039.297193
\end{tabular}

Number of Imaginary Frequencies

Frequencies (Top 3 out of 450)

$\begin{array}{lr}\text { 1. } & -352.0725 \mathrm{~cm}^{-1} \\ \text { 2. } & 9.6821 \mathrm{~cm}^{-1} \\ \text { 3. } & 16.9983 \mathrm{~cm}^{-1}\end{array}$

\section{B3LYP-D3(BJ)/6-31G(d,p) Molecular Geometry in Cartesian Coordinates}

C

$\mathrm{H}$

$\mathrm{H}$

C

0

0

$\mathrm{H}$

0

$\mathrm{H}$

C

C

C

C

$\mathrm{H}$

$$
\begin{array}{r}
2.142970 \\
3.054290 \\
1.625250 \\
-0.886210 \\
-1.487280 \\
-0.181570 \\
0.308840 \\
1.297100 \\
0.978900 \\
-1.409591 \\
-2.314890 \\
0.437409 \\
-0.624781 \\
-2.850531
\end{array}
$$

0.237529

$-0.338871$

0.359179

2.033030

2.860100

1.066880

0.481680

$-0.460131$

$-1.308241$

$-4.264620$

$-3.085220$

$-4.362270$

$-4.965150$

$-3.895580$
0.900640

1.078250

1.855970

$-0.956390$

$-1.614240$

$-1.543730$

$-0.894050$

$-0.025820$

0.383560

$-2.689800$

$-1.230450$

$-1.057640$

$-1.784160$

$-0.734400$ 


\begin{tabular}{|c|c|c|c|}
\hline $\mathrm{H}$ & 1.002690 & -3.595221 & -1.585700 \\
\hline $\mathrm{H}$ & 1.074209 & -5.041061 & -0.493680 \\
\hline C & 0.030060 & -2.790490 & 2.372060 \\
\hline C & -0.651251 & -4.203670 & 2.411790 \\
\hline C & -0.965270 & -1.638670 & 2.547250 \\
\hline $\mathrm{H}$ & -0.481650 & -0.694710 & 2.288140 \\
\hline $\mathrm{H}$ & -1.823320 & -1.761770 & 1.884780 \\
\hline $\mathrm{H}$ & -1.319660 & -1.562380 & 3.578730 \\
\hline C & 1.213820 & -2.619031 & 3.315920 \\
\hline $\mathrm{H}$ & 1.609630 & -1.602281 & 3.243290 \\
\hline H & 0.904700 & -2.781731 & 4.353190 \\
\hline $\mathrm{H}$ & 2.017600 & -3.315861 & 3.074270 \\
\hline C & -1.805291 & -4.316130 & 3.402770 \\
\hline $\mathrm{H}$ & -1.464311 & -4.127380 & 4.425980 \\
\hline $\mathrm{H}$ & -2.602800 & -3.611530 & 3.162190 \\
\hline $\mathrm{H}$ & -2.224321 & -5.325540 & 3.365270 \\
\hline C & 0.360859 & -5.332490 & 2.645880 \\
\hline $\mathrm{H}$ & 0.723209 & -5.351471 & 3.677900 \\
\hline $\mathrm{H}$ & -0.126491 & -6.287620 & 2.433340 \\
\hline $\mathrm{H}$ & 1.220799 & -5.229661 & 1.979230 \\
\hline 0 & 0.519530 & -2.751690 & 1.005940 \\
\hline 0 & -1.173111 & -4.348460 & 1.079760 \\
\hline B & -0.433570 & -3.489600 & 0.211880 \\
\hline 0 & -1.298760 & -2.576510 & -0.579630 \\
\hline C & -3.111820 & -2.168710 & -2.065320 \\
\hline C & -2.652510 & -0.871470 & -2.319920 \\
\hline C & -4.343520 & -2.588500 & -2.587640 \\
\hline C & -3.418580 & 0.001660 & -3.084130 \\
\hline $\mathrm{H}$ & -1.705440 & -0.547100 & -1.912150 \\
\hline C & -5.105600 & -1.714620 & -3.357370 \\
\hline $\mathrm{H}$ & -4.701000 & -3.595100 & -2.386460 \\
\hline C & -4.642900 & -0.419840 & -3.606380 \\
\hline $\mathrm{H}$ & -3.056800 & 1.010220 & -3.248790 \\
\hline $\mathrm{H}$ & -6.059700 & -2.039779 & -3.760340 \\
\hline $\mathrm{H}$ & -5.242170 & 0.259890 & -4.203660 \\
\hline $\mathrm{H}$ & -1.005071 & -5.916030 & -1.412270 \\
\hline $\mathrm{H}$ & -0.983890 & -3.418450 & -3.220140 \\
\hline $\mathrm{H}$ & -2.242781 & -4.754980 & -3.182660 \\
\hline C & 0.274580 & 2.360440 & 1.230610 \\
\hline C & -0.896870 & 2.046250 & 0.557040 \\
\hline C & -2.105740 & 1.764840 & 1.274930 \\
\hline C & -2.070910 & 1.789190 & 2.655370 \\
\hline $\mathrm{H}$ & -2.979210 & 1.565390 & 3.206530 \\
\hline C & 2.468180 & 1.591809 & 0.318240 \\
\hline C & 1.541650 & 2.615119 & 0.473730 \\
\hline C & 3.915780 & 3.081259 & -0.930420 \\
\hline C & 3.673810 & 1.818139 & -0.422410 \\
\hline $\mathrm{H}$ & 4.821541 & 3.257249 & -1.502980 \\
\hline C & 3.000481 & 4.146309 & -0.772780 \\
\hline C & 1.778301 & 3.913339 & -0.068120 \\
\hline C & 0.851161 & 4.981069 & 0.066680 \\
\hline C & 1.126141 & 6.219619 & -0.464050 \\
\hline C & 2.338541 & 6.451879 & -1.157410 \\
\hline C & 3.252831 & 5.436769 & -1.309800 \\
\hline
\end{tabular}




\begin{tabular}{|c|c|c|c|}
\hline $\mathrm{H}$ & -0.084209 & 4.802090 & 0.583500 \\
\hline $\mathrm{H}$ & 0.406181 & 7.024880 & -0.357380 \\
\hline $\mathrm{H}$ & 2.540301 & 7.434259 & -1.573260 \\
\hline $\mathrm{H}$ & 4.182551 & 5.603999 & -1.846370 \\
\hline C & -0.901930 & 2.120810 & 3.378530 \\
\hline C & 0.291330 & 2.433760 & 2.658370 \\
\hline C & 1.458490 & 2.782599 & 3.389530 \\
\hline C & 1.445580 & 2.805369 & 4.765620 \\
\hline C & 0.265510 & 2.484680 & 5.478330 \\
\hline C & -0.882630 & 2.152990 & 4.797860 \\
\hline $\mathrm{H}$ & 2.362510 & 3.028839 & 2.844510 \\
\hline $\mathrm{H}$ & 2.345540 & 3.073699 & 5.310160 \\
\hline $\mathrm{H}$ & 0.269770 & 2.505460 & 6.563750 \\
\hline $\mathrm{H}$ & -1.795150 & 1.910910 & 5.335430 \\
\hline C & 6.550950 & -1.265751 & -1.101510 \\
\hline C & 6.996410 & 0.011909 & -0.721390 \\
\hline C & 6.039910 & 1.000219 & -0.493790 \\
\hline C & 4.669340 & 0.740299 & -0.661280 \\
\hline C & 4.268020 & -0.534121 & -1.067540 \\
\hline C & 5.197850 & -1.561601 & -1.270210 \\
\hline $\mathrm{H}$ & 7.286860 & -2.043981 & -1.259280 \\
\hline $\mathrm{H}$ & 6.337460 & 1.986479 & -0.160570 \\
\hline $\mathrm{H}$ & 3.211900 & -0.724151 & -1.211410 \\
\hline C & 8.501940 & 0.263238 & -0.552720 \\
\hline C & 4.685080 & -2.969821 & -1.601870 \\
\hline C & 9.217050 & -0.014802 & -1.892580 \\
\hline $\mathrm{H}$ & 10.294690 & 0.155078 & -1.792580 \\
\hline $\mathrm{H}$ & 9.069820 & -1.046502 & -2.223360 \\
\hline $\mathrm{H}$ & 8.837570 & 0.645518 & -2.678550 \\
\hline C & 8.809370 & 1.710518 & -0.135850 \\
\hline $\mathrm{H}$ & 9.891110 & 1.843968 & -0.037740 \\
\hline $\mathrm{H}$ & 8.451430 & 2.429748 & -0.879080 \\
\hline $\mathrm{H}$ & 8.355540 & 1.959708 & 0.828360 \\
\hline C & 9.061100 & -0.681052 & 0.533340 \\
\hline $\mathrm{H}$ & 10.136360 & -0.517762 & 0.665030 \\
\hline $\mathrm{H}$ & 8.567400 & -0.502062 & 1.493630 \\
\hline $\mathrm{H}$ & 8.911640 & -1.732092 & 0.271450 \\
\hline C & 3.912850 & -3.501621 & -0.372570 \\
\hline $\mathrm{H}$ & 3.547189 & -4.517101 & -0.561800 \\
\hline $\mathrm{H}$ & 4.565110 & -3.533651 & 0.506280 \\
\hline $\mathrm{H}$ & 3.051650 & -2.874721 & -0.129280 \\
\hline C & 3.737530 & -2.918641 & -2.818750 \\
\hline $\mathrm{H}$ & 3.356519 & -3.920781 & -3.043660 \\
\hline $\mathrm{H}$ & 2.877960 & -2.267671 & -2.639420 \\
\hline $\mathrm{H}$ & 4.260100 & -2.544821 & -3.704840 \\
\hline C & 5.826409 & -3.948001 & -1.921970 \\
\hline $\mathrm{H}$ & 5.411939 & -4.928461 & -2.176260 \\
\hline $\mathrm{H}$ & 6.423710 & -3.605881 & -2.773270 \\
\hline $\mathrm{H}$ & 6.495559 & -4.085691 & -1.066950 \\
\hline C & -3.391230 & 1.402360 & 0.616640 \\
\hline C & -4.044260 & 0.224150 & 0.999740 \\
\hline C & -4.014250 & 2.260530 & -0.292940 \\
\hline C & -5.294710 & -0.118300 & 0.478280 \\
\hline $\mathrm{H}$ & -3.559480 & -0.418760 & 1.724330 \\
\hline
\end{tabular}




\begin{tabular}{|c|c|c|c|}
\hline C & -5.262300 & 1.949360 & -0.839490 \\
\hline $\mathrm{H}$ & -3.507749 & 3.175180 & -0.566160 \\
\hline C & -5.878400 & 0.754810 & -0.448740 \\
\hline $\mathrm{H}$ & -6.840830 & 0.502021 & -0.868330 \\
\hline C & -5.936170 & 2.958570 & -1.781880 \\
\hline C & -6.033700 & -1.358049 & 1.006930 \\
\hline C & -6.260009 & 4.233381 & -0.970830 \\
\hline $\mathrm{H}$ & -6.917919 & 4.000281 & -0.127530 \\
\hline $\mathrm{H}$ & -5.350779 & 4.690520 & -0.570920 \\
\hline $\mathrm{H}$ & -6.761289 & 4.973861 & -1.604110 \\
\hline C & -4.991849 & 3.330370 & -2.945130 \\
\hline $\mathrm{H}$ & -5.434819 & 4.132840 & -3.545030 \\
\hline $\mathrm{H}$ & -4.013589 & 3.667040 & -2.595460 \\
\hline $\mathrm{H}$ & -4.829150 & 2.473420 & -3.603420 \\
\hline C & -7.245180 & 2.417631 & -2.379180 \\
\hline $\mathrm{H}$ & -7.079490 & 1.488121 & -2.932990 \\
\hline $\mathrm{H}$ & -7.997670 & 2.225581 & -1.607820 \\
\hline $\mathrm{H}$ & -7.664939 & 3.151961 & -3.073680 \\
\hline C & -5.096710 & -2.579320 & 1.084780 \\
\hline $\mathrm{H}$ & -4.745340 & -2.853920 & 0.089610 \\
\hline $\mathrm{H}$ & -4.221250 & -2.397180 & 1.712260 \\
\hline $\mathrm{H}$ & -5.630930 & -3.436390 & 1.508390 \\
\hline C & -6.550800 & -1.031879 & 2.425960 \\
\hline $\mathrm{H}$ & -5.721920 & -0.798790 & 3.101390 \\
\hline $\mathrm{H}$ & -7.219440 & -0.165729 & 2.405610 \\
\hline $\mathrm{H}$ & -7.101600 & -1.882939 & 2.842530 \\
\hline C & -7.230360 & -1.742499 & 0.119660 \\
\hline $\mathrm{H}$ & -7.685140 & -2.665709 & 0.492560 \\
\hline $\mathrm{H}$ & -8.007470 & -0.972589 & 0.123380 \\
\hline $\mathrm{H}$ & -6.916600 & -1.909669 & -0.915150 \\
\hline
\end{tabular}

\section{C-Catalyzed reaction TS 15}

\section{Datum}

Value

B3LYP-D3(BJ)/6-31G(d,p) Energy

$-3039.487847$

B3LYP-D3(BJ)/def2-TZVPP/IEF-PCM(DCM) Energy

$-3040.527202$

B3LYP-D3(BJ)/def2-TZVPP/IEF-PCM(DCM)//B3LYP-D3(BJ)/6-31G(d,p) Free Energy (Quasiharmonic)

$-3039.296767$

Number of Imaginary Frequencies

Frequencies (Top 3 out of 450)

$\begin{array}{lr}\text { 1. } & -223.9906 \mathrm{~cm}^{-1} \\ \text { 2. } & 5.2075 \mathrm{~cm}^{-1} \\ \text { 3. } & 11.3500 \mathrm{~cm}^{-1}\end{array}$ 


\begin{tabular}{|c|c|c|c|}
\hline C & 1.067122 & 0.378763 & -0.238305 \\
\hline $\mathrm{H}$ & 1.995344 & -0.183439 & -0.339445 \\
\hline $\mathrm{H}$ & 0.639879 & 0.146661 & 0.739155 \\
\hline C & -2.151741 & 2.431337 & -1.035251 \\
\hline 0 & -2.767993 & 3.458988 & -1.248116 \\
\hline 0 & -1.620626 & 1.716250 & -2.014916 \\
\hline $\mathrm{H}$ & -1.026408 & 0.987537 & -1.676052 \\
\hline 0 & 0.164623 & -0.038853 & -1.274684 \\
\hline $\mathrm{H}$ & -0.018902 & -1.003421 & -1.154273 \\
\hline C & 0.203670 & -1.713411 & 3.680631 \\
\hline C & 1.671697 & -2.700605 & 2.380571 \\
\hline C & -1.192271 & -1.964398 & 1.659970 \\
\hline C & -0.846168 & -2.297247 & 3.010209 \\
\hline $\mathrm{H}$ & 1.449173 & -3.702027 & 2.751342 \\
\hline $\mathrm{H}$ & -1.051483 & -0.919430 & 1.389662 \\
\hline $\mathrm{H}$ & -2.165339 & -2.330252 & 1.336513 \\
\hline C & -0.708316 & -3.818929 & -1.338561 \\
\hline C & -0.215354 & -4.944226 & -0.361934 \\
\hline C & -2.236217 & -3.700314 & -1.384517 \\
\hline $\mathrm{H}$ & -2.506571 & -2.745242 & -1.843035 \\
\hline $\mathrm{H}$ & -2.686647 & -4.505918 & -1.971221 \\
\hline $\mathrm{H}$ & -2.662489 & -3.729567 & -0.380446 \\
\hline C & -0.147896 & -3.905674 & -2.751864 \\
\hline $\mathrm{H}$ & -0.455746 & -4.841746 & -3.228770 \\
\hline $\mathrm{H}$ & -0.530112 & -3.078511 & -3.356802 \\
\hline $\mathrm{H}$ & 0.939892 & -3.851989 & -2.754225 \\
\hline C & 1.251695 & -5.325980 & -0.599641 \\
\hline $\mathrm{H}$ & 1.383666 & -5.889673 & -1.527741 \\
\hline $\mathrm{H}$ & 1.886898 & -4.438382 & -0.638369 \\
\hline $\mathrm{H}$ & 1.588232 & -5.950885 & 0.232203 \\
\hline C & -1.089223 & -6.193483 & -0.346140 \\
\hline $\mathrm{H}$ & -1.118625 & -6.663075 & -1.334696 \\
\hline $\mathrm{H}$ & -0.680290 & -6.919657 & 0.362023 \\
\hline $\mathrm{H}$ & -2.109313 & -5.959305 & -0.037906 \\
\hline 0 & -0.185662 & -2.632533 & -0.697898 \\
\hline 0 & -0.313335 & -4.300434 & 0.922534 \\
\hline B & -0.088570 & -2.905876 & 0.714748 \\
\hline 0 & 1.256174 & -2.399951 & 1.192164 \\
\hline C & 2.873688 & -2.028520 & 2.895760 \\
\hline C & 3.535132 & -2.559159 & 4.012513 \\
\hline C & 3.369396 & -0.865964 & 2.286655 \\
\hline C & 4.675478 & -1.936717 & 4.512600 \\
\hline $\mathrm{H}$ & 3.151987 & -3.460036 & 4.484214 \\
\hline C & 4.507129 & -0.245873 & 2.789904 \\
\hline $\mathrm{H}$ & 2.864647 & -0.463472 & 1.420567 \\
\hline C & 5.163003 & -0.778157 & 3.903134 \\
\hline $\mathrm{H}$ & 5.185726 & -2.354855 & 5.374415 \\
\hline $\mathrm{H}$ & 4.885376 & 0.647839 & 2.306085 \\
\hline $\mathrm{H}$ & 6.053015 & -0.293538 & 4.292140 \\
\hline $\mathrm{H}$ & -1.269872 & -3.214429 & 3.418030 \\
\hline
\end{tabular}




\begin{tabular}{|c|c|c|c|}
\hline $\mathrm{H}$ & 0.549249 & -0.726007 & 3.398131 \\
\hline $\mathrm{H}$ & 0.480596 & -2.044923 & 4.675909 \\
\hline C & -0.748075 & 2.193045 & 1.007143 \\
\hline C & -1.960159 & 1.943350 & 0.388587 \\
\hline C & -3.040489 & 1.329929 & 1.106466 \\
\hline C & -2.871752 & 1.071294 & 2.452412 \\
\hline $\mathrm{H}$ & -3.687530 & 0.618573 & 3.007741 \\
\hline C & 1.325746 & 1.862268 & -0.332948 \\
\hline C & 0.412918 & 2.739205 & 0.237009 \\
\hline C & 2.729770 & 3.733750 & -0.981691 \\
\hline C & 2.513014 & 2.370263 & -0.953165 \\
\hline $\mathrm{H}$ & 3.631446 & 4.117687 & -1.450243 \\
\hline C & 1.796180 & 4.651704 & -0.446393 \\
\hline C & 0.608510 & 4.150874 & 0.173091 \\
\hline C & -0.330288 & 5.073992 & 0.705152 \\
\hline C & -0.098081 & 6.427715 & 0.633702 \\
\hline C & 1.081913 & 6.923972 & 0.028915 \\
\hline C & 2.006076 & 6.055008 & -0.501489 \\
\hline $\mathrm{H}$ & -1.245630 & 4.695680 & 1.144462 \\
\hline $\mathrm{H}$ & -0.828619 & 7.122970 & 1.034927 \\
\hline $\mathrm{H}$ & 1.250139 & 7.995393 & -0.021401 \\
\hline $\mathrm{H}$ & 2.909787 & 6.428073 & -0.975241 \\
\hline C & -1.669860 & 1.371444 & 3.136562 \\
\hline C & -0.571422 & 1.911280 & 2.399499 \\
\hline C & 0.655856 & 2.144458 & 3.076483 \\
\hline C & 0.777758 & 1.884378 & 4.424201 \\
\hline C & -0.320686 & 1.380177 & 5.159413 \\
\hline C & -1.514602 & 1.123001 & 4.525549 \\
\hline $\mathrm{H}$ & 1.495981 & 2.539177 & 2.517069 \\
\hline $\mathrm{H}$ & 1.722431 & 2.069113 & 4.925931 \\
\hline $\mathrm{H}$ & -0.213958 & 1.185125 & 6.221972 \\
\hline $\mathrm{H}$ & -2.357966 & 0.718026 & 5.077475 \\
\hline C & 5.430189 & -0.385238 & -2.392885 \\
\hline C & 5.838654 & 0.636264 & -1.533221 \\
\hline C & 4.867914 & 1.546410 & -1.088613 \\
\hline C & 3.537963 & 1.445502 & -1.500221 \\
\hline C & 3.172557 & 0.429435 & -2.395427 \\
\hline C & 4.102810 & -0.507799 & -2.837122 \\
\hline $\mathrm{H}$ & 6.154859 & -1.118002 & -2.722130 \\
\hline $\mathrm{H}$ & 5.134786 & 2.332673 & -0.389571 \\
\hline $\mathrm{H}$ & 2.138266 & 0.372952 & -2.705933 \\
\hline C & 7.290167 & 0.784481 & -1.054295 \\
\hline C & 3.687640 & -1.702549 & -3.705359 \\
\hline C & 7.334967 & 0.730141 & 0.488093 \\
\hline $\mathrm{H}$ & 8.367965 & 0.815128 & 0.842670 \\
\hline $\mathrm{H}$ & 6.763620 & 1.548115 & 0.936424 \\
\hline $\mathrm{H}$ & 6.920698 & -0.212563 & 0.858187 \\
\hline C & 8.202781 & -0.326487 & -1.598034 \\
\hline $\mathrm{H}$ & 9.223280 & -0.176481 & -1.232548 \\
\hline $\mathrm{H}$ & 7.873761 & -1.317301 & -1.269264 \\
\hline $\mathrm{H}$ & 8.238324 & -0.321984 & -2.691721 \\
\hline C & 7.841471 & 2.144497 & -1.534122 \\
\hline $\mathrm{H}$ & 8.874856 & 2.280302 & -1.196557 \\
\hline H & 7.827128 & 2.204202 & -2.626732 \\
\hline
\end{tabular}




\begin{tabular}{|c|c|c|c|}
\hline $\mathrm{H}$ & 7.248352 & 2.977485 & -1.146421 \\
\hline C & 4.660213 & -1.886899 & -4.887307 \\
\hline $\mathrm{H}$ & 4.345744 & -2.735112 & -5.504480 \\
\hline $\mathrm{H}$ & 4.677869 & -0.993638 & -5.519358 \\
\hline $\mathrm{H}$ & 5.682995 & -2.084414 & -4.555139 \\
\hline C & 3.713180 & -2.961098 & -2.809132 \\
\hline $\mathrm{H}$ & 3.411306 & -3.848262 & -3.377448 \\
\hline $\mathrm{H}$ & 4.713574 & -3.141887 & -2.404414 \\
\hline $\mathrm{H}$ & 3.026457 & -2.840843 & -1.965395 \\
\hline C & 2.267916 & -1.533778 & -4.272632 \\
\hline $\mathrm{H}$ & 2.014426 & -2.398054 & -4.894381 \\
\hline $\mathrm{H}$ & 1.517783 & -1.462965 & -3.481480 \\
\hline $\mathrm{H}$ & 2.191099 & -0.636860 & -4.895180 \\
\hline C & -4.278903 & 0.860128 & 0.429362 \\
\hline C & -4.691085 & -0.468240 & 0.639134 \\
\hline C & -5.037434 & 1.682626 & -0.402003 \\
\hline C & -5.827207 & -0.979315 & 0.016281 \\
\hline $\mathrm{H}$ & -4.087509 & -1.093723 & 1.283366 \\
\hline C & -6.173014 & 1.195010 & -1.063688 \\
\hline $\mathrm{H}$ & -4.727588 & 2.708079 & -0.542553 \\
\hline C & -6.547052 & -0.130314 & -0.842078 \\
\hline $\mathrm{H}$ & -7.418429 & -0.525146 & -1.348991 \\
\hline C & -6.929929 & 2.132601 & -2.013495 \\
\hline C & -6.295213 & -2.428419 & 0.212532 \\
\hline C & -5.966680 & 2.586365 & -3.133707 \\
\hline $\mathrm{H}$ & -5.080969 & 3.087150 & -2.734701 \\
\hline $\mathrm{H}$ & -5.625036 & 1.725903 & -3.717968 \\
\hline $\mathrm{H}$ & -6.475984 & 3.279370 & -3.812735 \\
\hline C & -8.146654 & 1.452435 & -2.660150 \\
\hline $\mathrm{H}$ & -8.649837 & 2.157299 & -3.329085 \\
\hline $\mathrm{H}$ & -7.854118 & 0.582309 & -3.256536 \\
\hline $\mathrm{H}$ & -8.876058 & 1.126308 & -1.911267 \\
\hline C & -7.423755 & 3.367047 & -1.228919 \\
\hline $\mathrm{H}$ & -8.100858 & 3.070292 & -0.421256 \\
\hline $\mathrm{H}$ & -6.592929 & 3.922252 & -0.786232 \\
\hline $\mathrm{H}$ & -7.963535 & 4.048449 & -1.895474 \\
\hline C & -5.436841 & -3.185586 & 1.238893 \\
\hline $\mathrm{H}$ & -4.394626 & -3.263709 & 0.916575 \\
\hline $\mathrm{H}$ & -5.456234 & -2.699135 & 2.219249 \\
\hline $\mathrm{H}$ & -5.819835 & -4.203295 & 1.362219 \\
\hline C & -7.755088 & -2.435711 & 0.714630 \\
\hline $\mathrm{H}$ & -7.838778 & -1.910250 & 1.671109 \\
\hline $\mathrm{H}$ & -8.430772 & -1.949318 & 0.006281 \\
\hline $\mathrm{H}$ & -8.103868 & -3.464459 & 0.856734 \\
\hline C & -6.211554 & -3.174799 & -1.136551 \\
\hline $\mathrm{H}$ & -6.543337 & -4.212855 & -1.022279 \\
\hline $\mathrm{H}$ & -6.840658 & -2.701933 & -1.895760 \\
\hline$H$ & -5.183729 & -3.182071 & -1.511515 \\
\hline
\end{tabular}


Frequencies (Top 3 out of 450)

$\begin{array}{lr}\text { 1. } & -314.8806 \mathrm{~cm}^{-1} \\ \text { 2. } & 7.9185 \mathrm{~cm}^{-1} \\ \text { 3. } & 16.2060 \mathrm{~cm}^{-1}\end{array}$

\section{B3LYP-D3(BJ)/6-31G(d,p) Molecular Geometry in Cartesian Coordinates}

C

$\mathrm{H}$

$\mathrm{H}$

C

0

0

$\mathrm{H}$

0

$\mathrm{H}$

C

C

C

C

$\mathrm{H}$

$\mathrm{H}$

$\mathrm{H}$

C

C

C

$\mathrm{H}$

$\mathrm{H}$

$\mathrm{H}$

C

$\mathrm{H}$

$\mathrm{H}$

$\mathrm{H}$

C

$\mathrm{H}$

$\mathrm{H}$

$\mathrm{H}$

C

$\mathrm{H}$

\section{204340}

3.136400

1.842350

$-0.656901$

$-1.054581$

$-0.000370$

0.381880

1. 226040

0.904970

$-0.943428$

$-1.951319$

0.667612

$-0.336638$

$-2.568269$

1. 352781

1.178282

0.410071

$-1.052069$

0.587891

1.638901

$-0.000640$

0.281671

1.442391

2.440801

1.405071

1.279662

$-2.096649$

$-2.172799$

$-1.856189$

$-3.069369$

$-1.377099$

$-1.266219$
0.219190

$-0.351650$

0.265520

2. 128919

3.078639

1.121720

0.489440

$-0.444610$

$-1.233380$

$-4.680261$

$-3.258141$

$-4.639410$

$-5.282701$

$-3.981751$

$-4.000650$

$-5.270370$

$-2.899900$

$-3.461861$

$-1.596080$

$-1.294990$

$-0.789600$

$-1.716440$

$-3.933510$

$-3.575600$

$-4.084660$

$-4.896220$

$-2.339861$

$-1.781331$

$-1.636001$

$-2.788211$

$-4.397261$

$-3.883531$
1.051070

1.043680

2.080830

$-0.847370$

$-1.490000$

$-1.428450$

$-0.750030$

0.239360

0.741070

$-2.015320$

$-0.831210$

$-0.146440$

$-0.924430$

$-0.295930$

$-0.700860$

0.579090

3.009000

3.007450

3. 776580

3.772800

3.340630

4.820150

3.469620

3. 204720

4.552340

2.979640

2.920890

3.858160

2.121370

2.703840

4.165570

5.126010 


\begin{tabular}{|c|c|c|c|}
\hline H & -2.412218 & -4.740281 & 4.081960 \\
\hline $\mathrm{H}$ & -0.728858 & -5.274881 & 4.158850 \\
\hline 0 & 0.636151 & -2.657730 & 1.598320 \\
\hline 0 & -1.084439 & -4.220811 & 1.782130 \\
\hline B & -0.248119 & -3.524971 & 0.858170 \\
\hline 0 & -0.982749 & -2.704111 & -0.153470 \\
\hline C & -2.615159 & -2.442661 & -1.863960 \\
\hline C & -2.178280 & -1.136951 & -2.115570 \\
\hline C & -3.714179 & -2.956082 & -2.568090 \\
\hline C & -2.834640 & -0.347911 & -3.054540 \\
\hline $\mathrm{H}$ & -1.342810 & -0.737181 & -1.561690 \\
\hline C & -4.356569 & -2.171882 & -3.520510 \\
\hline $\mathrm{H}$ & -4.060009 & -3.966762 & -2.367990 \\
\hline C & -3.920490 & -0.865662 & -3.761470 \\
\hline $\mathrm{H}$ & -2.499060 & 0.670929 & -3.214710 \\
\hline $\mathrm{H}$ & -5.202269 & -2.573302 & -4.070050 \\
\hline $\mathrm{H}$ & -4.434250 & -0.252262 & -4.493340 \\
\hline $\mathrm{H}$ & -0.824708 & -6.154881 & -0.489970 \\
\hline $\mathrm{H}$ & -0.403169 & -3.926451 & -2.579600 \\
\hline $\mathrm{H}$ & -1.738028 & -5.190481 & -2.549410 \\
\hline C & 0.218989 & 2.244160 & 1.477840 \\
\hline C & -0.865051 & 1.995529 & 0.648750 \\
\hline C & -2.132110 & 1.593319 & 1.184400 \\
\hline C & -2.235600 & 1.424059 & 2.553190 \\
\hline $\mathrm{H}$ & -3.189130 & 1.121309 & 2.974540 \\
\hline C & 2.453980 & 1.627010 & 0.559680 \\
\hline C & 1.531019 & 2.619410 & 0.860030 \\
\hline C & 3.905369 & 3.268811 & -0.481140 \\
\hline C & 3.632850 & 1.947711 & -0.186370 \\
\hline $\mathrm{H}$ & 4.797259 & 3.513331 & -1.050990 \\
\hline C & 3.018509 & 4.311140 & -0.123440 \\
\hline C & 1.786139 & 3.982600 & 0.525180 \\
\hline C & 0.869729 & 5.025670 & 0.823290 \\
\hline C & 1.167328 & 6.331590 & 0.511640 \\
\hline C & 2.391398 & 6.658520 & -0.120240 \\
\hline C & 3.293388 & 5.669461 & -0.433620 \\
\hline $\mathrm{H}$ & -0.072821 & 4.775069 & 1.295840 \\
\hline H & 0.456858 & 7.118460 & 0.744760 \\
\hline $\mathrm{H}$ & 2.611218 & 7.693570 & -0.363310 \\
\hline $\mathrm{H}$ & 4.229538 & 5.910321 & -0.929490 \\
\hline C & -1.153920 & 1.663929 & 3.431260 \\
\hline C & 0.094339 & 2.104480 & 2.891730 \\
\hline C & 1.176239 & 2.348640 & 3.778720 \\
\hline C & 1.033469 & 2.148950 & 5.132860 \\
\hline C & -0.201360 & 1.706889 & 5.666970 \\
\hline C & -1.271500 & 1.476869 & 4.834510 \\
\hline $\mathrm{H}$ & 2.119839 & 2.690100 & 3.367150 \\
\hline $\mathrm{H}$ & 1.870389 & 2.334710 & 5.798750 \\
\hline $\mathrm{H}$ & -0.300500 & 1.554829 & 6.737310 \\
\hline $\mathrm{H}$ & -2.224040 & 1.142489 & 5.236080 \\
\hline C & 6.063770 & -1.205699 & -1.699090 \\
\hline C & 6.696340 & -0.136168 & -1.051260 \\
\hline C & 5.895480 & 0.897181 & -0.552580 \\
\hline C & 4.505970 & 0.868201 & -0.711730 \\
\hline
\end{tabular}




\begin{tabular}{|c|c|c|c|}
\hline C & 3.914010 & -0.198639 & -1.398970 \\
\hline C & 4.676581 & -1.260849 & -1.882880 \\
\hline $\mathrm{H}$ & 6.672561 & -2.025468 & -2.065580 \\
\hline $\mathrm{H}$ & 6.335550 & 1.726941 & -0.013120 \\
\hline $\mathrm{H}$ & 2.841950 & -0.188810 & -1.529570 \\
\hline C & 8.224130 & -0.147188 & -0.893930 \\
\hline C & 4.025251 & -2.484939 & -2.541480 \\
\hline C & 8.645741 & -1.388548 & -0.078430 \\
\hline $\mathrm{H}$ & 9.734201 & -1.414647 & 0.043190 \\
\hline $\mathrm{H}$ & 8.190221 & -1.371228 & 0.916630 \\
\hline $\mathrm{H}$ & 8.341201 & -2.317078 & -0.569070 \\
\hline C & 8.879610 & -0.203158 & -2.290690 \\
\hline $\mathrm{H}$ & 9.971300 & -0.215997 & -2.200030 \\
\hline $\mathrm{H}$ & 8.578630 & -1.097318 & -2.843340 \\
\hline $\mathrm{H}$ & 8.595520 & 0.669312 & -2.887190 \\
\hline C & 8.745350 & 1.104162 & -0.169040 \\
\hline $\mathrm{H}$ & 9.835860 & 1.057173 & -0.090540 \\
\hline $\mathrm{H}$ & 8.489819 & 2.020432 & -0.710280 \\
\hline $\mathrm{H}$ & 8.342820 & 1.182062 & 0.845680 \\
\hline C & 4.677191 & -2.765679 & -3.910470 \\
\hline $\mathrm{H}$ & 4.214081 & -3.640489 & -4.379680 \\
\hline H & 4.551401 & -1.911029 & -4.582250 \\
\hline $\mathrm{H}$ & 5.748331 & -2.966519 & -3.820050 \\
\hline C & 4.231931 & -3.700289 & -1.610640 \\
\hline $\mathrm{H}$ & 3.769402 & -4.596959 & -2.038300 \\
\hline $\mathrm{H}$ & 5.294591 & -3.908919 & -1.455240 \\
\hline $\mathrm{H}$ & 3.780841 & -3.515639 & -0.630520 \\
\hline C & 2.513371 & -2.289080 & -2.756750 \\
\hline $\mathrm{H}$ & 2.090801 & -3.189960 & -3.214420 \\
\hline $\mathrm{H}$ & 1.985641 & -2.103790 & -1.817030 \\
\hline $\mathrm{H}$ & 2.307121 & -1.447450 & -3.425260 \\
\hline C & -3.336840 & 1.351458 & 0.345020 \\
\hline C & -3.752521 & 2.299108 & -0.599960 \\
\hline C & -4.124990 & 0.215278 & 0.567070 \\
\hline C & -4.923341 & 2.114358 & -1.334350 \\
\hline $\mathrm{H}$ & -3.145301 & 3.178319 & -0.745870 \\
\hline C & -5.321360 & 0.012108 & -0.131120 \\
\hline H & -3.788340 & -0.510082 & 1.296700 \\
\hline C & -5.689310 & 0.967908 & -1.083230 \\
\hline $\mathrm{H}$ & -6.606320 & 0.826147 & -1.641670 \\
\hline C & -6.257720 & -1.155942 & 0.216370 \\
\hline C & -5.396261 & 3.137238 & -2.377680 \\
\hline C & -7.221850 & -0.669473 & 1.321970 \\
\hline $\mathrm{H}$ & -6.666810 & -0.360253 & 2.213210 \\
\hline $\mathrm{H}$ & -7.806910 & 0.188817 & 0.977620 \\
\hline $\mathrm{H}$ & -7.916869 & -1.466953 & 1.609120 \\
\hline C & -7.086049 & -1.609463 & -1.000980 \\
\hline $\mathrm{H}$ & -7.685959 & -2.485623 & -0.734260 \\
\hline $\mathrm{H}$ & -7.781460 & -0.836053 & -1.338150 \\
\hline $\mathrm{H}$ & -6.437969 & -1.876342 & -1.838970 \\
\hline C & -5.479319 & -2.375842 & 0.742410 \\
\hline $\mathrm{H}$ & -4.725169 & -2.699692 & 0.021790 \\
\hline $\mathrm{H}$ & -4.978369 & -2.164772 & 1.690010 \\
\hline H & -6.167639 & -3.208522 & 0.919070 \\
\hline
\end{tabular}




\begin{tabular}{|llll|} 
C & -6.797061 & 3.656037 & -1.988200 \\
$\mathrm{H}$ & -7.535261 & 2.849507 & -1.954800 \\
$\mathrm{H}$ & -6.773301 & 4.129417 & -1.001740 \\
$\mathrm{H}$ & -7.144471 & 4.398007 & -2.715440 \\
$\mathrm{C}$ & -4.442791 & 4.339248 & -2.482540 \\
$\mathrm{H}$ & -4.395441 & 4.897308 & -1.542270 \\
$\mathrm{H}$ & -3.426071 & 4.031918 & -2.742610 \\
$\mathrm{H}$ & -4.800501 & 5.024768 & -3.257330 \\
$\mathrm{C}$ & -5.469921 & 2.460978 & -3.762920 \\
$\mathrm{H}$ & -5.854921 & 3.163788 & -4.509780 \\
$\mathrm{H}$ & -4.475911 & 2.136478 & -4.084440 \\
$\mathrm{H}$ & -6.126080 & 1.585898 & -3.752780 \\
\hline
\end{tabular}

\section{C-Catalyzed reaction TS 17}

\section{Datum}

Value

\begin{tabular}{lr} 
B3LYP-D3(BJ)/6-31G(d,p) Energy & -3039.495797 \\
\hline B3LYP-D3(BJ)/def2-TZVPP/IEF-PCM(DCM) Energy & -3040.527542 \\
\hline B3LYP-D3(BJ)/def2-TZVPP/IEF-PCM(DCM)//B3LYP-D3(BJ)/6-31G(d,p) Free Energy (Quasiharmonic) & -3039.296259
\end{tabular}

Number of Imaginary Frequencies

Frequencies (Top 3 out of 450)

$\begin{array}{lr}\text { 1. } & -303.8390 \mathrm{~cm}^{-1} \\ \text { 2. } & 11.8440 \mathrm{~cm}^{-1} \\ \text { 3. } & 17.4546 \mathrm{~cm}^{-1}\end{array}$

B3LYP-D3(BJ)/6-31G(d,p) Molecular Geometry in Cartesian Coordinates

\begin{tabular}{|lrlr|}
\hline$C$ & 1.329092 & 1.396784 & -1.047199 \\
$H$ & 0.951992 & 2.063701 & -1.825332 \\
$H$ & 2.282130 & 0.992292 & -1.380907 \\
$C$ & -2.028399 & 1.429522 & 1.066509 \\
$O$ & -2.916157 & 1.551389 & 1.884317 \\
$O$ & -1.130894 & 0.446012 & 1.142729 \\
$H$ & -0.540998 & 0.443601 & 0.321436 \\
$O$ & 0.379616 & 0.319849 & -0.927313 \\
$H$ & 0.830303 & -0.563006 & -0.820267 \\
$C$ & -0.222532 & -1.900584 & 2.875731 \\
$C$ & -0.984971 & -2.674535 & 1.094815 \\
$C$ & 1.930536 & -2.535144 & 1.838335 \\
$C$ & 0.984496 & -1.565552 & 2.282978 \\
$H$ & -0.986652 & -1.735201 & 0.551210 \\
\hline
\end{tabular}




\begin{tabular}{|c|c|c|c|}
\hline $\mathrm{H}$ & 2.888173 & -2.131793 & 1.520915 \\
\hline $\mathrm{H}$ & 2.030694 & -3.424520 & 2.461762 \\
\hline C & 2.042026 & -2.627045 & -1.819724 \\
\hline C & 2.081556 & -4.171803 & -1.547511 \\
\hline C & 1.365146 & -2.216627 & -3.123682 \\
\hline $\mathrm{H}$ & 1.350066 & -1.126252 & -3.210025 \\
\hline $\mathrm{H}$ & 1.910001 & -2.617256 & -3.984488 \\
\hline $\mathrm{H}$ & 0.334354 & -2.569617 & -3.165343 \\
\hline C & 3.422162 & -1.976974 & -1.709623 \\
\hline $\mathrm{H}$ & 4.061534 & -2.239841 & -2.557555 \\
\hline $\mathrm{H}$ & 3.317468 & -0.890991 & -1.691862 \\
\hline $\mathrm{H}$ & 3.927513 & -2.273020 & -0.790743 \\
\hline C & 3.345927 & -4.866602 & -2.043130 \\
\hline $\mathrm{H}$ & 3.454980 & -4.759243 & -3.127420 \\
\hline $\mathrm{H}$ & 4.235229 & -4.462487 & -1.556975 \\
\hline $\mathrm{H}$ & 3.289820 & -5.933604 & -1.811113 \\
\hline C & 0.839343 & -4.897967 & -2.084069 \\
\hline $\mathrm{H}$ & 0.841134 & -4.953108 & -3.176923 \\
\hline $\mathrm{H}$ & 0.834183 & -5.915672 & -1.685530 \\
\hline $\mathrm{H}$ & -0.078941 & -4.405398 & -1.758542 \\
\hline 0 & 1.238544 & -2.144818 & -0.713574 \\
\hline 0 & 2.062975 & -4.235365 & -0.113459 \\
\hline B & 1.267048 & -3.154933 & 0.345896 \\
\hline 0 & -0.108377 & -3.580145 & 0.742127 \\
\hline C & -2.314160 & -3.140852 & 1.538963 \\
\hline C & -2.555493 & -4.503596 & 1.761874 \\
\hline C & -3.357991 & -2.212364 & 1.672587 \\
\hline C & -3.833016 & -4.934696 & 2.107654 \\
\hline $\mathrm{H}$ & -1.740426 & -5.207451 & 1.636210 \\
\hline C & -4.631141 & -2.652231 & 2.022152 \\
\hline $\mathrm{H}$ & -3.174132 & -1.156232 & 1.507585 \\
\hline C & -4.873193 & -4.010512 & 2.238232 \\
\hline $\mathrm{H}$ & -4.020898 & -5.991688 & 2.269387 \\
\hline $\mathrm{H}$ & -5.435141 & -1.932011 & 2.117818 \\
\hline $\mathrm{H}$ & -5.869877 & -4.348711 & 2.505318 \\
\hline $\mathrm{H}$ & 1.086368 & -0.547591 & 1.910333 \\
\hline $\mathrm{H}$ & -0.308362 & -2.835873 & 3.420952 \\
\hline $\mathrm{H}$ & -0.932782 & -1.120088 & 3.120431 \\
\hline C & -0.748530 & 3.170759 & -0.214790 \\
\hline C & -1.871501 & 2.372421 & -0.097965 \\
\hline C & -2.924618 & 2.421291 & -1.058851 \\
\hline C & -2.872393 & 3.360766 & -2.060607 \\
\hline $\mathrm{H}$ & -3.654079 & 3.386072 & -2.814300 \\
\hline C & 1.481355 & 2.154455 & 0.261567 \\
\hline C & 0.429591 & 2.962508 & 0.686638 \\
\hline C & 2.614919 & 2.522827 & 2.378964 \\
\hline C & 2.612371 & 1.952284 & 1.119424 \\
\hline $\mathrm{H}$ & 3.477124 & 2.377419 & 3.023219 \\
\hline C & 1.546881 & 3.321801 & 2.845566 \\
\hline C & 0.434676 & 3.562611 & 1.980008 \\
\hline C & -0.632632 & 4.375953 & 2.447042 \\
\hline C & -0.595921 & 4.924018 & 3.707834 \\
\hline C & 0.507971 & 4.688917 & 4.562760 \\
\hline C & 1.555732 & 3.905775 & 4.139877 \\
\hline
\end{tabular}




\begin{tabular}{|c|c|c|c|}
\hline $\mathrm{H}$ & -1.485140 & 4.545621 & 1.799958 \\
\hline $\mathrm{H}$ & -1.421444 & 5.537682 & 4.054019 \\
\hline $\mathrm{H}$ & 0.521541 & 5.127546 & 5.555706 \\
\hline $\mathrm{H}$ & 2.406083 & 3.719037 & 4.789615 \\
\hline C & -1.792496 & 4.277943 & -2.155614 \\
\hline C & -0.700121 & 4.170741 & -1.236429 \\
\hline C & 0.398166 & 5.060402 & -1.372369 \\
\hline C & 0.413869 & 6.013574 & -2.365642 \\
\hline C & -0.665864 & 6.120273 & -3.272995 \\
\hline C & -1.742593 & 5.269536 & -3.168999 \\
\hline $\mathrm{H}$ & 1.223101 & 4.980906 & -0.673159 \\
\hline $\mathrm{H}$ & 1.258096 & 6.690492 & -2.452871 \\
\hline $\mathrm{H}$ & -0.641728 & 6.875768 & -4.052195 \\
\hline $\mathrm{H}$ & -2.573634 & 5.342975 & -3.864780 \\
\hline C & 6.060212 & -0.272255 & -0.066646 \\
\hline C & 5.602970 & 0.748579 & -0.901514 \\
\hline C & 4.474624 & 1.471754 & -0.491959 \\
\hline C & 3.805444 & 1.169722 & 0.695834 \\
\hline C & 4.309996 & 0.150657 & 1.519760 \\
\hline C & 5.443003 & -0.579494 & 1.157335 \\
\hline H & 6.923720 & -0.850579 & -0.366923 \\
\hline $\mathrm{H}$ & 4.112663 & 2.296704 & -1.096672 \\
\hline $\mathrm{H}$ & 3.789932 & -0.062747 & 2.444819 \\
\hline C & 6.293531 & 1.113231 & -2.223382 \\
\hline C & 6.022732 & -1.703191 & 2.030869 \\
\hline C & 7.449608 & 0.158075 & -2.560011 \\
\hline H & 7.896379 & 0.443926 & -3.516994 \\
\hline $\mathrm{H}$ & 8.239461 & 0.192998 & -1.803404 \\
\hline $\mathrm{H}$ & 7.104039 & -0.876756 & -2.648901 \\
\hline C & 5.270389 & 1.053300 & -3.378332 \\
\hline $\mathrm{H}$ & 5.751235 & 1.323753 & -4.324333 \\
\hline $\mathrm{H}$ & 4.856773 & 0.046014 & -3.480957 \\
\hline H & 4.437064 & 1.743788 & -3.221720 \\
\hline C & 6.858243 & 2.546175 & -2.113848 \\
\hline $\mathrm{H}$ & 7.354511 & 2.833789 & -3.047055 \\
\hline $\mathrm{H}$ & 6.067917 & 3.275428 & -1.914724 \\
\hline H & 7.588831 & 2.614177 & -1.301860 \\
\hline C & 7.523475 & -1.435588 & 2.280002 \\
\hline $\mathrm{H}$ & 7.944092 & -2.219132 & 2.918900 \\
\hline $\mathrm{H}$ & 8.099088 & -1.422413 & 1.350813 \\
\hline H & 7.669185 & -0.472461 & 2.779120 \\
\hline C & 5.330500 & -1.795257 & 3.401336 \\
\hline $\mathrm{H}$ & 5.804249 & -2.581106 & 3.997336 \\
\hline $\mathrm{H}$ & 5.418139 & -0.856348 & 3.957452 \\
\hline $\mathrm{H}$ & 4.270661 & -2.045142 & 3.313940 \\
\hline C & 5.862245 & -3.056062 & 1.300686 \\
\hline $\mathrm{H}$ & 6.307994 & -3.861230 & 1.894693 \\
\hline $\mathrm{H}$ & 4.810159 & -3.305684 & 1.134677 \\
\hline H & 6.361101 & -3.043418 & 0.326913 \\
\hline C & -3.925750 & 1.315497 & -1.046987 \\
\hline C & -3.569776 & 0.119686 & -1.679212 \\
\hline C & -5.131743 & 1.401030 & -0.356174 \\
\hline C & -4.409492 & -0.997588 & -1.638693 \\
\hline $\mathrm{H}$ & -2.615643 & 0.076874 & -2.194053 \\
\hline
\end{tabular}




\begin{tabular}{|c|c|c|c|}
\hline C & -5.990062 & 0.298322 & -0.277600 \\
\hline $\mathrm{H}$ & -5.372055 & 2.325796 & 0.155217 \\
\hline C & -5.611087 & -0.883442 & -0.927138 \\
\hline $\mathrm{H}$ & -6.262640 & -1.742471 & -0.867775 \\
\hline C & -7.294238 & 0.420392 & 0.523327 \\
\hline C & -4.023921 & -2.268984 & -2.410406 \\
\hline C & -6.964359 & 0.812825 & 1.980329 \\
\hline $\mathrm{H}$ & -7.885736 & 0.915665 & 2.564013 \\
\hline $\mathrm{H}$ & -6.337877 & 0.055593 & 2.460782 \\
\hline $\mathrm{H}$ & -6.421228 & 1.759214 & 2.033553 \\
\hline C & -8.176562 & 1.516829 & -0.111913 \\
\hline $\mathrm{H}$ & -9.111767 & 1.627566 & 0.447906 \\
\hline $\mathrm{H}$ & -7.671056 & 2.486341 & -0.116110 \\
\hline $\mathrm{H}$ & -8.424993 & 1.263566 & -1.147447 \\
\hline C & -8.097078 & -0.890637 & 0.542987 \\
\hline $\mathrm{H}$ & -8.397337 & -1.197437 & -0.463842 \\
\hline $\mathrm{H}$ & -7.528197 & -1.710697 & 0.992040 \\
\hline $\mathrm{H}$ & -9.007864 & -0.755243 & 1.134304 \\
\hline C & -2.580584 & -2.701291 & -2.077558 \\
\hline $\mathrm{H}$ & -1.853978 & -1.896653 & -2.213707 \\
\hline $\mathrm{H}$ & -2.513577 & -3.046085 & -1.045172 \\
\hline $\mathrm{H}$ & -2.282074 & -3.532145 & -2.726066 \\
\hline C & -4.950869 & -3.451849 & -2.083563 \\
\hline $\mathrm{H}$ & -4.945625 & -3.678254 & -1.012782 \\
\hline $\mathrm{H}$ & -5.983116 & -3.260433 & -2.392978 \\
\hline $\mathrm{H}$ & -4.609018 & -4.344023 & -2.617464 \\
\hline C & -4.123742 & -1.958456 & -3.920439 \\
\hline $\mathrm{H}$ & -3.876676 & -2.847030 & -4.512117 \\
\hline $\mathrm{H}$ & -5.136526 & -1.639166 & -4.185942 \\
\hline $\mathrm{H}$ & -3.434511 & -1.158025 & -4.205519 \\
\hline
\end{tabular}

\section{C-Catalyzed reaction TS 18}

\section{Datum}

Value

B3LYP-D3(BJ)/6-31G(d,p) Energy

$-3039.49448$

B3LYP-D3(BJ)/def2-TZVPP/IEF-PCM(DCM) Energy

$-3040.52752$

B3LYP-D3(BJ)/def2-TZVPP/IEF-PCM(DCM)//B3LYP-D3(BJ)/6-31G(d,p) Free Energy (Quasiharmonic)

$-3039.296002$

Number of Imaginary Frequencies

Frequencies (Top 3 out of 450)

$\begin{array}{lr}\text { 1. } & -215.1033 \mathrm{~cm}^{-1} \\ \text { 2. } & 11.6114 \mathrm{~cm}^{-1} \\ \text { 3. } & 16.5938 \mathrm{~cm}^{-1}\end{array}$




\begin{tabular}{|c|c|c|c|}
\hline C & -1.602930 & 0.785570 & 1.085150 \\
\hline $\mathrm{H}$ & -1.003829 & 1.192480 & 1.903140 \\
\hline $\mathrm{H}$ & -2.490170 & 0.309760 & 1.508460 \\
\hline C & 1.180781 & 1.829340 & -1.456080 \\
\hline 0 & 1.606561 & 2.471790 & -2.394330 \\
\hline 0 & 0.532440 & 0.677920 & -1.638730 \\
\hline $\mathrm{H}$ & 0.068050 & 0.378290 & -0.797890 \\
\hline 0 & -0.831950 & -0.196870 & 0.370370 \\
\hline $\mathrm{H}$ & -0.284150 & -0.767390 & 0.977050 \\
\hline C & 2.254320 & -2.229881 & -1.993040 \\
\hline C & 0.345200 & -2.761740 & -1.137980 \\
\hline C & 2.967390 & -2.660981 & 0.335240 \\
\hline C & 2.708590 & -1.789671 & -0.768760 \\
\hline $\mathrm{H}$ & 0.069300 & -1.737390 & -0.918820 \\
\hline $\mathrm{H}$ & 3.513650 & -2.193331 & 1.151200 \\
\hline $\mathrm{H}$ & 3.378960 & -3.637221 & 0.073360 \\
\hline C & 0.694630 & -2.060830 & 3.057370 \\
\hline C & 0.751930 & -3.624500 & 3.162970 \\
\hline C & 1.912600 & -1.367301 & 3.676870 \\
\hline $\mathrm{H}$ & 1.936310 & -0.327281 & 3.337890 \\
\hline $\mathrm{H}$ & 1.873390 & -1.375581 & 4.769940 \\
\hline $\mathrm{H}$ & 2.837390 & -1.853701 & 3.361570 \\
\hline C & -0.589330 & -1.439080 & 3.599100 \\
\hline H & -0.735730 & -1.716180 & 4.647530 \\
\hline $\mathrm{H}$ & -0.532820 & -0.347550 & 3.553300 \\
\hline $\mathrm{H}$ & -1.461900 & -1.766050 & 3.030350 \\
\hline C & -0.619430 & -4.277760 & 2.947470 \\
\hline $\mathrm{H}$ & -1.295460 & -4.109870 & 3.791410 \\
\hline $\mathrm{H}$ & -1.090720 & -3.900260 & 2.039040 \\
\hline $\mathrm{H}$ & -0.474960 & -5.354600 & 2.828980 \\
\hline C & 1.385650 & -4.143690 & 4.449740 \\
\hline H & 0.819030 & -3.815690 & 5.327570 \\
\hline $\mathrm{H}$ & 1.391630 & -5.236980 & 4.438620 \\
\hline $\mathrm{H}$ & 2.417430 & -3.802691 & 4.548880 \\
\hline 0 & 0.758690 & -1.853610 & 1.627290 \\
\hline 0 & 1.594660 & -3.982480 & 2.060600 \\
\hline B & 1.423460 & -3.020290 & 1.033570 \\
\hline 0 & 0.642880 & -3.546680 & -0.145070 \\
\hline C & -0.281510 & -3.336420 & -2.338040 \\
\hline C & -0.747280 & -2.466360 & -3.335940 \\
\hline C & -0.442070 & -4.720010 & -2.482810 \\
\hline C & -1.365030 & -2.980270 & -4.471290 \\
\hline $\mathrm{H}$ & -0.614870 & -1.394490 & -3.212520 \\
\hline C & -1.068930 & -5.227030 & -3.618230 \\
\hline $\mathrm{H}$ & -0.084890 & -5.374930 & -1.695950 \\
\hline C & -1.528880 & -4.360760 & -4.613180 \\
\hline $\mathrm{H}$ & -1.725510 & -2.306440 & -5.241780 \\
\hline H & -1.202250 & -6.298800 & -3.727900 \\
\hline $\mathrm{H}$ & -2.016810 & -4.760480 & -5.496670 \\
\hline H & 2.629420 & -0.727171 & -0.559540 \\
\hline
\end{tabular}




\begin{tabular}{|c|c|c|c|}
\hline $\mathrm{H}$ & 2.459440 & -3.244631 & -2.319620 \\
\hline $\mathrm{H}$ & 1.989910 & -1.512041 & -2.760900 \\
\hline C & 0.246031 & 2.895680 & 0.576550 \\
\hline C & 1.344921 & 2.299660 & -0.021830 \\
\hline C & 2.594711 & 2.183529 & 0.678990 \\
\hline C & 2.674771 & 2.715409 & 1.953360 \\
\hline H & 3.615371 & 2.651629 & 2.491000 \\
\hline C & -1.993799 & 1.895380 & 0.137600 \\
\hline C & -1.081209 & 2.912450 & -0.116240 \\
\hline C & -3.607089 & 2.968800 & -1.319790 \\
\hline C & -3.277519 & 1.909550 & -0.495570 \\
\hline $\mathrm{H}$ & -4.580559 & 2.981050 & -1.801310 \\
\hline C & -2.701069 & 4.019210 & -1.592990 \\
\hline C & -1.403069 & 3.989280 & -0.993580 \\
\hline C & -0.486619 & 5.033810 & -1.287540 \\
\hline C & -0.843679 & 6.062370 & -2.127420 \\
\hline C & -2.131519 & 6.096730 & -2.715220 \\
\hline C & -3.037709 & 5.096100 & -2.455650 \\
\hline $\mathrm{H}$ & 0.505481 & 4.998220 & -0.853260 \\
\hline $\mathrm{H}$ & -0.131749 & 6.851180 & -2.348710 \\
\hline $\mathrm{H}$ & -2.397969 & 6.914650 & -3.377550 \\
\hline $\mathrm{H}$ & -4.025009 & 5.110950 & -2.908670 \\
\hline C & 1.588161 & 3.366870 & 2.580240 \\
\hline C & 0.345301 & 3.463590 & 1.884330 \\
\hline C & -0.747639 & 4.109460 & 2.521040 \\
\hline C & -0.611929 & 4.634510 & 3.786270 \\
\hline C & 0.620571 & 4.539040 & 4.475540 \\
\hline C & 1.696421 & 3.919680 & 3.883730 \\
\hline $\mathrm{H}$ & -1.690949 & 4.186440 & 1.992800 \\
\hline $\mathrm{H}$ & -1.454609 & 5.128800 & 4.259450 \\
\hline $\mathrm{H}$ & 0.713181 & 4.957840 & 5.472780 \\
\hline $\mathrm{H}$ & 2.646511 & 3.841679 & 4.404660 \\
\hline C & -6.130470 & -1.205179 & 0.112810 \\
\hline C & -6.530780 & 0.129531 & 0.295690 \\
\hline C & -5.582499 & 1.131941 & 0.095440 \\
\hline C & -4.266830 & 0.820440 & -0.283650 \\
\hline C & -3.911240 & -0.515410 & -0.474470 \\
\hline C & -4.833570 & -1.550640 & -0.268870 \\
\hline $\mathrm{H}$ & -6.857700 & -1.989949 & 0.277970 \\
\hline $\mathrm{H}$ & -5.837039 & 2.173041 & 0.250280 \\
\hline $\mathrm{H}$ & -2.897250 & -0.743030 & -0.780170 \\
\hline C & -7.976050 & 0.429911 & 0.718310 \\
\hline C & -4.384660 & -3.001540 & -0.487530 \\
\hline C & -8.239619 & 1.937081 & 0.865940 \\
\hline $\mathrm{H}$ & -9.280779 & 2.102121 & 1.159610 \\
\hline $\mathrm{H}$ & -7.602839 & 2.386401 & 1.634290 \\
\hline H & -8.070629 & 2.471001 & -0.074400 \\
\hline C & -8.944840 & -0.127079 & -0.347140 \\
\hline $\mathrm{H}$ & -9.982960 & 0.074831 & -0.061500 \\
\hline H & -8.760440 & 0.339011 & -1.320070 \\
\hline $\mathrm{H}$ & -8.835180 & -1.208139 & -0.468430 \\
\hline C & -8.262530 & -0.246249 & 2.076480 \\
\hline $\mathrm{H}$ & -9.291730 & -0.045439 & 2.393540 \\
\hline $\mathrm{H}$ & -8.133820 & -1.330769 & 2.023460 \\
\hline
\end{tabular}




\begin{tabular}{|c|c|c|c|}
\hline H & -7.586140 & 0.133671 & 2.848550 \\
\hline $\mathrm{C}$ & -5.471750 & -4.017839 & -0.104780 \\
\hline $\mathrm{H}$ & -5.092570 & -5.033670 & -0.252590 \\
\hline $\mathrm{H}$ & -5.765540 & -3.920579 & 0.945440 \\
\hline $\mathrm{H}$ & -6.367620 & -3.907139 & -0.723890 \\
\hline C & -4.037510 & -3.193460 & -1.978120 \\
\hline $\mathrm{H}$ & -3.671340 & -4.208290 & -2.163410 \\
\hline $\mathrm{H}$ & -4.919320 & -3.024040 & -2.604540 \\
\hline $\mathrm{H}$ & -3.259690 & -2.500090 & -2.300330 \\
\hline C & -3.136030 & -3.285260 & 0.375380 \\
\hline $\mathrm{H}$ & -2.761470 & -4.296270 & 0.184950 \\
\hline $\mathrm{H}$ & -2.323090 & -2.585530 & 0.170390 \\
\hline $\mathrm{H}$ & -3.380730 & -3.207380 & 1.439970 \\
\hline C & 3.778481 & 1.460349 & 0.139090 \\
\hline C & 4.518070 & 0.622149 & 0.998170 \\
\hline C & 4.193881 & 1.596849 & -1.186180 \\
\hline C & 5.646240 & -0.061861 & 0.549600 \\
\hline H & 4.177090 & 0.500759 & 2.018140 \\
\hline C & 5.285130 & 0.873789 & -1.686610 \\
\hline $\mathrm{H}$ & 3.655561 & 2.269949 & -1.835650 \\
\hline C & 5.998420 & 0.063049 & -0.805330 \\
\hline $\mathrm{H}$ & 6.856240 & -0.487111 & -1.169500 \\
\hline C & 5.626001 & 0.994119 & -3.177460 \\
\hline C & 6.530730 & -0.908011 & 1.478050 \\
\hline C & 5.926401 & 2.468199 & -3.522510 \\
\hline H & 5.069821 & 3.114339 & -3.314710 \\
\hline $\mathrm{H}$ & 6.778631 & 2.837989 & -2.943250 \\
\hline $\mathrm{H}$ & 6.167531 & 2.565809 & -4.586500 \\
\hline C & 6.845790 & 0.145129 & -3.567570 \\
\hline $\mathrm{H}$ & 7.044080 & 0.255869 & -4.637950 \\
\hline $\mathrm{H}$ & 7.746760 & 0.456819 & -3.029030 \\
\hline $\mathrm{H}$ & 6.678100 & -0.918461 & -3.370060 \\
\hline C & 4.408550 & 0.511629 & -3.998910 \\
\hline $\mathrm{H}$ & 4.185160 & -0.535681 & -3.770750 \\
\hline $\mathrm{H}$ & 3.513571 & 1.101479 & -3.783720 \\
\hline $\mathrm{H}$ & 4.619350 & 0.590499 & -5.071190 \\
\hline C & 7.913520 & -0.225811 & 1.580080 \\
\hline $\mathrm{H}$ & 8.392960 & -0.146441 & 0.600370 \\
\hline $\mathrm{H}$ & 7.818250 & 0.785169 & 1.988500 \\
\hline $\mathrm{H}$ & 8.576410 & -0.800781 & 2.236030 \\
\hline C & 5.947970 & -1.026551 & 2.895110 \\
\hline $\mathrm{H}$ & 5.843700 & -0.048861 & 3.375480 \\
\hline $\mathrm{H}$ & 4.967340 & -1.510701 & 2.891710 \\
\hline $\mathrm{H}$ & 6.613400 & -1.632081 & 3.517740 \\
\hline C & 6.709560 & -2.331421 & 0.908500 \\
\hline $\mathrm{H}$ & 7.351210 & -2.922681 & 1.570420 \\
\hline $\mathrm{H}$ & 5.748280 & -2.842941 & 0.818260 \\
\hline $\mathrm{H}$ & 7.176690 & -2.319521 & -0.079690 \\
\hline
\end{tabular}


Frequencies (Top 3 out of 450)

$\begin{array}{rr}\text { 1. } & -283.1370 \mathrm{~cm}^{-1} \\ \text { 2. } & 12.4862 \mathrm{~cm}^{-1} \\ \text { 3. } & 14.8527 \mathrm{~cm}^{-1}\end{array}$

\section{B3LYP-D3(BJ)/6-31G(d,p) Molecular Geometry in Cartesian Coordinates}

\section{C}

$\mathrm{H}$

$\mathrm{H}$

C

0

0

$\mathrm{H}$

0

$\mathrm{H}$

C

C

C

C

$\mathrm{H}$

$\mathrm{H}$

$\mathrm{H}$

C

C

C

$\mathrm{H}$

$\mathrm{H}$

$\mathrm{H}$

C

$\mathrm{H}$

$\mathrm{H}$

$\mathrm{H}$

C

$\mathrm{H}$

$\mathrm{H}$

$\mathrm{H}$

C

$\mathrm{H}$
$-0.776773$

$-0.430209$

$-1.752614$

2.574853

3.183260

2.095729

1.483726

0.117609

0.047079

$-4.922008$

- 3.245971

$-3.172939$

$-4.247884$

- 3.231644

$-2.845114$

$-3.265180$

0.422377

$-0.096530$

0.495575

0.636277

1.330110

$-0.431041$

1. 742729

2. 060774

1.663217

2. 528769

0.243786

$-0.031015$

$-0.315105$

1.309486

0.346514

1.437912
$-0.237695$

0.085990

0.211384

$-2.088918$

$-3.130100$

$-1.404909$

$-0.676427$

0.265839

1.235704

2.503979

1.884054

3. 402592

3. 575578

2.755377

4. 301556

2.540336

3.630941

4.110855

4.761361

4.325682

5.439932

5.340570

2. 867508

2.586638

1.956100

3. 495982

3.118224

2.095681

3. 397438

3.130910

5.515298

5.578856
0.334405

$-0.646314$

0.491377

0.908644

1.052267

1.945283

1.659880

1. 344109

1.233398

$-0.873124$

$-2.027642$

0.607931

$-0.312604$

$-2.684664$

1.128398

1.267952

$-0.110718$

$-1.511891$

0.919502

1.912456

0.720425

0.927255

$-0.158126$

0.850333

$-0.751627$

$-0.588929$

$-2.633782$

$-2.370231$

$-3.531285$

$-2.878268$

$-1.905217$

$-1.964804$ 


\begin{tabular}{|c|c|c|c|}
\hline $\mathrm{H}$ & -0.061295 & 5.767108 & -2.888263 \\
\hline $\mathrm{H}$ & -0.010504 & 6.258312 & -1.190540 \\
\hline 0 & -0.621480 & 2.723026 & 0.314330 \\
\hline 0 & -1.525175 & 4.119422 & -1.318522 \\
\hline B & -1.811269 & 3.035114 & -0.436742 \\
\hline 0 & -2.303725 & 1.799469 & -1.137992 \\
\hline C & -3.757093 & 0.619842 & -2.588829 \\
\hline C & -3.566718 & -0.590565 & -1.908987 \\
\hline C & -4.401270 & 0.620413 & -3.832751 \\
\hline C & -4.003344 & -1.784587 & -2.470879 \\
\hline $\mathrm{H}$ & -3.077435 & -0.588198 & -0.946694 \\
\hline C & -4.838467 & -0.577398 & -4.394412 \\
\hline $\mathrm{H}$ & -4.546154 & 1.557955 & -4.362665 \\
\hline C & -4.637600 & -1.782284 & -3.716179 \\
\hline $\mathrm{H}$ & -3.837082 & -2.716253 & -1.938723 \\
\hline $\mathrm{H}$ & -5.330382 & -0.572892 & -5.361831 \\
\hline H & -4.974185 & -2.715275 & -4.157071 \\
\hline $\mathrm{H}$ & -4.361968 & 4.555174 & -0.775304 \\
\hline $\mathrm{H}$ & -4.966161 & 1.567899 & -0.325401 \\
\hline $\mathrm{H}$ & -5.703128 & 2.664634 & -1.609294 \\
\hline C & 1.111073 & -1.789731 & -1.077621 \\
\hline C & 2.316329 & -1.502746 & -0.464713 \\
\hline C & 3.291236 & -0.669138 & -1.103183 \\
\hline C & 2.995652 & -0.145682 & -2.347057 \\
\hline H & 3.724106 & 0.494702 & -2.835698 \\
\hline C & -0.888128 & -1.737223 & 0.375558 \\
\hline C & 0.031997 & -2.503626 & -0.324597 \\
\hline C & -2.141416 & -3.732669 & 0.957084 \\
\hline C & -1.993451 & -2.362847 & 1.038447 \\
\hline $\mathrm{H}$ & -2.984551 & -4.209481 & 1.449440 \\
\hline C & -1.202141 & -4.542515 & 0.272170 \\
\hline C & -0.084678 & -3.922677 & -0.373402 \\
\hline C & 0.860267 & -4.738556 & -1.048849 \\
\hline C & 0.701351 & -6.103952 & -1.092253 \\
\hline C & -0.408215 & -6.718001 & -0.462578 \\
\hline C & -1.336090 & -5.954593 & 0.206410 \\
\hline $\mathrm{H}$ & 1.720602 & -4.266636 & -1.509025 \\
\hline $\mathrm{H}$ & 1.435254 & -6.718188 & -1.604287 \\
\hline $\mathrm{H}$ & -0.519058 & -7.797292 & -0.503123 \\
\hline $\mathrm{H}$ & -2.184107 & -6.420892 & 0.700275 \\
\hline C & 1.776226 & -0.421468 & -3.012086 \\
\hline C & 0.818514 & -1.271313 & -2.378206 \\
\hline C & -0.418788 & -1.513275 & -3.030160 \\
\hline C & -0.705301 & -0.928356 & -4.242611 \\
\hline C & 0.245202 & -0.090299 & -4.872510 \\
\hline C & 1.461360 & 0.148670 & -4.274124 \\
\hline $\mathrm{H}$ & -1.147282 & -2.154008 & -2.550301 \\
\hline $\mathrm{H}$ & -1.667687 & -1.107481 & -4.711483 \\
\hline $\mathrm{H}$ & 0.011523 & 0.364961 & -5.830080 \\
\hline $\mathrm{H}$ & 2.195169 & 0.792442 & -4.750932 \\
\hline C & -4.839044 & 0.306002 & 2.753294 \\
\hline $\mathrm{C}$ & -3.511575 & 0.351955 & 3.205824 \\
\hline C & -2.609898 & -0.579196 & 2.685051 \\
\hline C & -3.005475 & -1.515183 & 1.723330 \\
\hline
\end{tabular}




\begin{tabular}{|c|c|c|c|}
\hline C & -4.346189 & -1.561582 & 1.328117 \\
\hline C & -5.282952 & -0.648707 & 1.827436 \\
\hline $\mathrm{H}$ & -5.543510 & 1.031597 & 3.132720 \\
\hline $\mathrm{H}$ & -1.568406 & -0.558732 & 2.978774 \\
\hline $\mathrm{H}$ & -4.638803 & -2.293458 & 0.583946 \\
\hline C & -3.037255 & 1.353503 & 4.270597 \\
\hline C & -6.753483 & -0.740739 & 1.389023 \\
\hline C & -4.044175 & 2.497350 & 4.481064 \\
\hline $\mathrm{H}$ & -3.643535 & 3.209650 & 5.208631 \\
\hline $\mathrm{H}$ & -4.237236 & 3.040686 & 3.550549 \\
\hline $\mathrm{H}$ & -5.000284 & 2.136296 & 4.872079 \\
\hline C & -2.869596 & 0.588785 & 5.602353 \\
\hline $\mathrm{H}$ & -2.529449 & 1.265418 & 6.394148 \\
\hline $\mathrm{H}$ & -3.817845 & 0.141217 & 5.916719 \\
\hline $\mathrm{H}$ & -2.134827 & -0.215310 & 5.502542 \\
\hline C & -1.678844 & 1.975289 & 3.876682 \\
\hline $\mathrm{H}$ & -1.366798 & 2.691543 & 4.644134 \\
\hline $\mathrm{H}$ & -0.891301 & 1.224702 & 3.785647 \\
\hline $\mathrm{H}$ & -1.736074 & 2.503836 & 2.921939 \\
\hline C & -7.549873 & 0.521903 & 1.761527 \\
\hline $\mathrm{H}$ & -8.573744 & 0.435800 & 1.385108 \\
\hline $\mathrm{H}$ & -7.612206 & 0.663238 & 2.844376 \\
\hline $\mathrm{H}$ & -7.104264 & 1.421412 & 1.324301 \\
\hline C & -6.867191 & -0.938159 & -0.137506 \\
\hline $\mathrm{H}$ & -7.921851 & -0.991169 & -0.427086 \\
\hline $\mathrm{H}$ & -6.407232 & -0.111344 & -0.685051 \\
\hline $\mathrm{H}$ & -6.389022 & -1.859800 & -0.475948 \\
\hline C & -7.389094 & -1.956285 & 2.099456 \\
\hline $\mathrm{H}$ & -8.442555 & -2.061973 & 1.817040 \\
\hline $\mathrm{H}$ & -6.870002 & -2.881876 & 1.833096 \\
\hline $\mathrm{H}$ & -7.334203 & -1.842763 & 3.186503 \\
\hline C & 4.566655 & -0.301195 & -0.435295 \\
\hline C & 4.950806 & 1.040207 & -0.368750 \\
\hline C & 5.404017 & -1.282812 & 0.115466 \\
\hline C & 6.151192 & 1.425302 & 0.243862 \\
\hline $\mathrm{H}$ & 4.286639 & 1.790115 & -0.782629 \\
\hline C & 6.595593 & -0.934507 & 0.747626 \\
\hline H & 5.098064 & -2.315631 & 0.048818 \\
\hline C & 6.951114 & 0.423353 & 0.796104 \\
\hline $\mathrm{H}$ & 7.876347 & 0.696775 & 1.286950 \\
\hline C & 7.503840 & -1.982533 & 1.405014 \\
\hline C & 6.519454 & 2.914408 & 0.317175 \\
\hline C & 8.909052 & -1.923583 & 0.769968 \\
\hline $\mathrm{H}$ & 9.567660 & -2.666033 & 1.233758 \\
\hline $\mathrm{H}$ & 8.858898 & -2.134698 & -0.302916 \\
\hline $\mathrm{H}$ & 9.372522 & -0.940800 & 0.896750 \\
\hline C & 7.607129 & -1.674949 & 2.914992 \\
\hline $\mathrm{H}$ & 8.251762 & -2.409867 & 3.409520 \\
\hline $\mathrm{H}$ & 8.028137 & -0.682083 & 3.098542 \\
\hline $\mathrm{H}$ & 6.619473 & -1.714261 & 3.384032 \\
\hline C & 6.951902 & -3.408605 & 1.242018 \\
\hline $\mathrm{H}$ & 5.952053 & -3.510335 & 1.673156 \\
\hline $\mathrm{H}$ & 6.897353 & -3.700297 & 0.188285 \\
\hline H & 7.614536 & -4.117744 & 1.748188 \\
\hline
\end{tabular}




\begin{tabular}{|llll|} 
C & 6.505159 & 3.523425 & -1.101184 \\
H & 7.227465 & 3.016917 & -1.748927 \\
H & 5.520517 & 3.440290 & -1.569577 \\
H & 6.766576 & 4.586523 & -1.061927 \\
C & 5.483727 & 3.645239 & 1.198934 \\
H & 4.473577 & 3.549325 & 0.793485 \\
H & 5.476775 & 3.230172 & 2.211514 \\
H & 5.721836 & 4.712750 & 1.266933 \\
C & 7.914707 & 3.142962 & 0.920810 \\
H & 8.142635 & 4.213376 & 0.926917 \\
H & 7.972983 & 2.787518 & 1.954046 \\
H & 8.693181 & 2.638074 & 0.340200 \\
\hline
\end{tabular}

\section{C-Catalyzed reaction TS 20 (TS-2.4)}

\section{Datum}

Value

B3LYP-D3(BJ)/6-31G(d,p) Energy

$-3039.489451$

B3LYP-D3(BJ)/def2-TZVPP/IEF-PCM(DCM) Energy

$-3040.525791$

B3LYP-D3(BJ)/def2-TZVPP/IEF-PCM(DCM)//B3LYP-D3(BJ)/6-31G(d,p) Free Energy (Quasiharmonic)

$-3039.295339$

Number of Imaginary Frequencies

Frequencies (Top 3 out of 450)

$\begin{array}{lr}\text { 1. } & -284.7254 \mathrm{~cm}^{-1} \\ \text { 2. } & 6.1834 \mathrm{~cm}^{-1} \\ \text { 3. } & 13.2164 \mathrm{~cm}^{-1}\end{array}$

B3LYP-D3(BJ)/6-31G(d,p) Molecular Geometry in Cartesian Coordinates

\begin{tabular}{|rrrr}
\hline C & -2.886136 & 0.780951 & 1.317089 \\
H & -3.899670 & 0.740849 & 1.723230 \\
H & -2.192607 & 0.572855 & 2.141913 \\
C & 0.291748 & 1.294208 & -0.783030 \\
O & -0.692757 & 1.946331 & -1.094864 \\
O & 1.272294 & 1.095371 & -1.650565 \\
H & 1.980333 & 0.472316 & -1.329258 \\
O & -2.691926 & 2.104551 & 0.829471 \\
H & -1.981458 & 2.084271 & 0.155953 \\
C & 4.431139 & -3.303657 & 2.011751 \\
C & 3.594678 & -3.813936 & 0.120785 \\
C & 4.576034 & -1.074896 & 0.964263 \\
C & 5.172202 & -2.263573 & 1.479849 \\
H & 4.532104 & -4.125667 & -0.341620
\end{tabular}




\begin{tabular}{|c|c|c|c|}
\hline $\mathrm{H}$ & 5.270554 & -0.266159 & 0.747968 \\
\hline $\mathrm{H}$ & 3.678694 & -0.739378 & 1.478740 \\
\hline C & 4.154461 & -0.213234 & -2.531281 \\
\hline C & 4.975085 & -1.530418 & -2.738751 \\
\hline C & 3.247417 & 0.137368 & -3.707546 \\
\hline H & 2.762854 & 1.102085 & -3.550888 \\
\hline H & 3.839903 & 0.196668 & -4.626208 \\
\hline $\mathrm{H}$ & 2.465093 & -0.611168 & -3.841043 \\
\hline C & 5.024911 & 0.989595 & -2.154885 \\
\hline H & 5.572688 & 1.376979 & -3.018851 \\
\hline $\mathrm{H}$ & 5.746610 & 0.724653 & -1.378606 \\
\hline H & 4.380506 & 1.782533 & -1.767068 \\
\hline C & 6.334424 & -1.328664 & -3.398607 \\
\hline $\mathrm{H}$ & 6.224576 & -0.894028 & -4.397224 \\
\hline H & 6.971231 & -0.676065 & -2.799313 \\
\hline H & 6.839675 & -2.293111 & -3.502434 \\
\hline C & 4.175239 & -2.604985 & -3.489263 \\
\hline H & 4.071491 & -2.370091 & -4.552054 \\
\hline $\mathrm{H}$ & 4.702012 & -3.559010 & -3.397920 \\
\hline H & 3.176546 & -2.718879 & -3.062484 \\
\hline 0 & 3.346934 & -0.542860 & -1.363543 \\
\hline 0 & 5.184062 & -1.978725 & -1.389060 \\
\hline B & 4.045857 & -1.570024 & -0.628346 \\
\hline 0 & 3.093162 & -2.684120 & -0.292882 \\
\hline C & 2.670907 & -4.877034 & 0.546035 \\
\hline C & 1.376286 & -4.576865 & 0.989819 \\
\hline C & 3.097291 & -6.211483 & 0.494520 \\
\hline C & 0.516160 & -5.603260 & 1.361292 \\
\hline H & 1.054349 & -3.546145 & 1.039685 \\
\hline C & 2.232527 & -7.236549 & 0.868884 \\
\hline H & 4.102806 & -6.440828 & 0.152066 \\
\hline C & 0.939507 & -6.933460 & 1.300624 \\
\hline H & -0.485835 & -5.361569 & 1.698499 \\
\hline H & 2.563549 & -8.269018 & 0.820096 \\
\hline $\mathrm{H}$ & 0.263667 & -7.732423 & 1.589417 \\
\hline $\mathrm{H}$ & 6.206951 & -2.462676 & 1.204028 \\
\hline H & 3.462251 & -3.099059 & 2.457601 \\
\hline H & 4.918449 & -4.217659 & 2.335548 \\
\hline C & -0.315120 & -0.419753 & 0.907752 \\
\hline C & 0.484933 & 0.654393 & 0.566794 \\
\hline C & 1.534484 & 1.117136 & 1.424970 \\
\hline C & 1.807245 & 0.401395 & 2.571759 \\
\hline H & 2.584474 & 0.749764 & 3.245586 \\
\hline C & -2.717111 & -0.281951 & 0.245608 \\
\hline C & -1.459019 & -0.830636 & 0.033828 \\
\hline C & -3.614088 & -1.689146 & -1.521803 \\
\hline C & -3.820384 & -0.711727 & -0.565916 \\
\hline H & -4.450234 & -2.012899 & -2.134629 \\
\hline C & -2.340241 & -2.251907 & -1.764057 \\
\hline C & -1.229490 & -1.802689 & -0.985647 \\
\hline C & 0.056339 & -2.339178 & -1.255061 \\
\hline C & 0.228531 & -3.301202 & -2.223749 \\
\hline C & -0.873374 & -3.759436 & -2.983944 \\
\hline C & -2.127845 & -3.239643 & -2.762309 \\
\hline
\end{tabular}




\begin{tabular}{|c|c|c|c|}
\hline H & 0.913507 & -1.987018 & -0.698276 \\
\hline $\mathrm{H}$ & 1.217098 & -3.713548 & -2.396588 \\
\hline $\mathrm{H}$ & -0.724406 & -4.517645 & -3.746736 \\
\hline $\mathrm{H}$ & -2.978041 & -3.575713 & -3.349447 \\
\hline C & 1.076343 & -0.763808 & 2.914059 \\
\hline C & -0.028967 & -1.160845 & 2.097248 \\
\hline C & -0.802740 & -2.284507 & 2.490632 \\
\hline C & -0.475323 & -3.004709 & 3.617706 \\
\hline C & 0.641081 & -2.635992 & 4.404101 \\
\hline C & 1.393371 & -1.534443 & 4.063841 \\
\hline $\mathrm{H}$ & -1.652948 & -2.567913 & 1.880756 \\
\hline H & -1.074024 & -3.862894 & 3.905815 \\
\hline $\mathrm{H}$ & 0.895141 & -3.218111 & 5.284325 \\
\hline $\mathrm{H}$ & 2.239461 & -1.232751 & 4.674860 \\
\hline C & -7.758017 & 0.870127 & -0.149381 \\
\hline C & -7.590119 & -0.519445 & -0.199819 \\
\hline C & -6.289949 & -1.016146 & -0.324831 \\
\hline C & -5.188466 & -0.152753 & -0.402742 \\
\hline C & -5.396735 & 1.231156 & -0.361924 \\
\hline C & -6.681594 & 1.761577 & -0.226083 \\
\hline $\mathrm{H}$ & -8.760565 & 1.270180 & -0.039330 \\
\hline H & -6.108169 & -2.083617 & -0.342133 \\
\hline $\mathrm{H}$ & -4.534189 & 1.880154 & -0.415792 \\
\hline C & -8.820371 & -1.433808 & -0.104625 \\
\hline C & -6.928209 & 3.273894 & -0.131908 \\
\hline C & -9.541006 & -1.184712 & 1.237853 \\
\hline $\mathrm{H}$ & -10.424647 & -1.826924 & 1.323216 \\
\hline H & -8.876539 & -1.401999 & 2.079977 \\
\hline $\mathrm{H}$ & -9.870682 & -0.146513 & 1.332569 \\
\hline C & -9.782264 & -1.116858 & -1.269919 \\
\hline $\mathrm{H}$ & -10.670882 & -1.755684 & -1.217281 \\
\hline $\mathrm{H}$ & -10.115727 & -0.075865 & -1.246614 \\
\hline $\mathrm{H}$ & -9.292943 & -1.288842 & -2.233691 \\
\hline C & -8.448462 & -2.923396 & -0.181121 \\
\hline $\mathrm{H}$ & -9.354740 & -3.533686 & -0.117195 \\
\hline $\mathrm{H}$ & -7.948742 & -3.167135 & -1.123880 \\
\hline $\mathrm{H}$ & -7.790026 & -3.218920 & 0.641664 \\
\hline C & -5.633097 & 4.083471 & -0.315252 \\
\hline $\mathrm{H}$ & -5.853399 & 5.151784 & -0.221666 \\
\hline $\mathrm{H}$ & -4.875498 & 3.824966 & 0.429533 \\
\hline $\mathrm{H}$ & -5.196657 & 3.918857 & -1.305533 \\
\hline C & -7.931387 & 3.708564 & -1.220978 \\
\hline $\mathrm{H}$ & -8.109953 & 4.787891 & -1.163855 \\
\hline $\mathrm{H}$ & -7.543696 & 3.479047 & -2.218402 \\
\hline $\mathrm{H}$ & -8.896675 & 3.205619 & -1.112691 \\
\hline C & -7.511569 & 3.594909 & 1.261343 \\
\hline $\mathrm{H}$ & -7.706001 & 4.668975 & 1.356654 \\
\hline $\mathrm{H}$ & -8.452691 & 3.064928 & 1.436031 \\
\hline $\mathrm{H}$ & -6.810300 & 3.303029 & 2.049141 \\
\hline C & 2.259104 & 2.370335 & 1.102433 \\
\hline C & 1.528760 & 3.489169 & 0.683052 \\
\hline C & 3.651874 & 2.458574 & 1.214867 \\
\hline C & 2.167959 & 4.677220 & 0.328117 \\
\hline H & 0.451551 & 3.418431 & 0.639167 \\
\hline
\end{tabular}




$\begin{array}{lrrr}\text { C } & 4.323781 & 3.641921 & 0.893144 \\ \text { H } & 4.201982 & 1.584857 & 1.533928 \\ \text { C } & 3.561846 & 4.728445 & 0.444776 \\ \text { H } & 4.072373 & 5.647285 & 0.177170 \\ \text { C } & 5.847405 & 3.790252 & 1.016768 \\ \text { C } & 1.392290 & 5.892135 & -0.197497 \\ \text { C } & 6.527489 & 2.486441 & 1.463349 \\ \text { H } & 6.161555 & 2.147624 & 2.437472 \\ \text { H } & 6.368121 & 1.683489 & 0.737760 \\ \text { H } & 7.606746 & 2.644291 & 1.550681 \\ \text { C } & 6.445286 & 4.199185 & -0.346602 \\ \text { H } & 7.532049 & 4.310466 & -0.266029 \\ \text { H } & 6.234180 & 3.442001 & -1.107117 \\ \text { H } & 6.038842 & 5.150499 & -0.699748 \\ \text { C } & 6.159478 & 4.882769 & 2.062538 \\ \text { H } & 5.728519 & 5.846411 & 1.777417 \\ \text { H } & 5.751168 & 4.610976 & 3.040973 \\ \text { H } & 7.241982 & 5.014954 & 2.166795 \\ \text { C } & 1.885306 & 6.214016 & -1.625239 \\ \text { H } & 2.953573 & 6.449117 & -1.642635 \\ \text { H } & 1.716358 & 5.362881 & -2.291751 \\ \text { H } & 1.344827 & 7.077542 & -2.027581 \\ \text { C } & -0.121855 & 5.628067 & -0.260019 \\ \text { H } & -0.360844 & 4.779227 & -0.907329 \\ \text { H } & -0.540599 & 5.427648 & 0.731166 \\ \text { H } & -0.630536 & 6.510239 & -0.660672 \\ \text { C } & 1.644715 & 7.104709 & 0.722630 \\ \text { H } & 1.101819 & 7.981468 & 0.353602 \\ \text { H } & 1.303735 & 6.896190 & 1.741515 \\ \text { H } & 2.706341 & 7.364693 & 0.770610\end{array}$

\section{C-Catalyzed reaction TS 21}

\section{Datum}

Value

B3LYP-D3(BJ)/6-31G(d,p) Energy

$-3039.493534$

B3LYP-D3(BJ)/def2-TZVPP/IEF-PCM(DCM) Energy

$-3040.52672$

B3LYP-D3(BJ)/def2-TZVPP/IEF-PCM(DCM)//B3LYP-D3(BJ)/6-31G(d,p) Free Energy (Quasiharmonic)

$-3039.295154$

Number of Imaginary Frequencies

Frequencies (Top 3 out of 450)

$\begin{array}{rr}\text { 1. } & -285.3835 \mathrm{~cm}^{-1} \\ \text { 2. } & 12.1631 \mathrm{~cm}^{-1} \\ \text { 3. } & 15.3903 \mathrm{~cm}^{-1}\end{array}$




\begin{tabular}{|c|c|c|c|}
\hline C & 1.613480 & 0.353460 & 0.371340 \\
\hline $\mathrm{H}$ & 2.372650 & -0.289781 & 0.823210 \\
\hline $\mathrm{H}$ & 0.911150 & 0.665800 & 1.151380 \\
\hline C & -0.909030 & 2.152400 & -1.958150 \\
\hline 0 & -1.374690 & 2.991430 & -2.705700 \\
\hline 0 & -0.316020 & 1.061090 & -2.419400 \\
\hline $\mathrm{H}$ & 0.119470 & 0.525790 & -1.691350 \\
\hline 0 & 0.925970 & -0.351850 & -0.668760 \\
\hline $\mathrm{H}$ & 0.485439 & -1.207370 & -0.418860 \\
\hline C & 0.734659 & -2.183140 & 2.993800 \\
\hline C & -1.153731 & -1.567110 & 2.108500 \\
\hline C & 0.450159 & -4.095280 & 1.452340 \\
\hline C & 1.129989 & -2.922200 & 1.895590 \\
\hline $\mathrm{H}$ & -0.702510 & -0.863670 & 1.409480 \\
\hline $\mathrm{H}$ & 0.972789 & -4.684150 & 0.700180 \\
\hline $\mathrm{H}$ & 0.005099 & -4.712310 & 2.232650 \\
\hline C & -1.279711 & -2.786290 & -1.664430 \\
\hline C & -1.716251 & -4.277690 & -1.446320 \\
\hline C & -2.437211 & -1.794020 & -1.547730 \\
\hline $\mathrm{H}$ & -2.045410 & -0.776110 & -1.537130 \\
\hline $\mathrm{H}$ & -3.117491 & -1.871769 & -2.399170 \\
\hline $\mathrm{H}$ & -3.011401 & -1.951139 & -0.635730 \\
\hline C & -0.500511 & -2.529320 & -2.948300 \\
\hline $\mathrm{H}$ & -1.118561 & -2.787770 & -3.814090 \\
\hline $\mathrm{H}$ & -0.243001 & -1.471400 & -3.034050 \\
\hline $\mathrm{H}$ & 0.416569 & -3.116110 & -2.990060 \\
\hline C & -0.687941 & -5.280590 & -1.984630 \\
\hline $\mathrm{H}$ & -0.682411 & -5.314070 & -3.077900 \\
\hline $\mathrm{H}$ & 0.319209 & -5.031940 & -1.642700 \\
\hline $\mathrm{H}$ & -0.941932 & -6.274890 & -1.608340 \\
\hline C & -3.102491 & -4.604819 & -1.992940 \\
\hline $\mathrm{H}$ & -3.146231 & -4.438059 & -3.074180 \\
\hline $\mathrm{H}$ & -3.333902 & -5.655859 & -1.799740 \\
\hline $\mathrm{H}$ & -3.868401 & -3.995319 & -1.511720 \\
\hline 0 & -0.397611 & -2.589640 & -0.516670 \\
\hline 0 & -1.752731 & -4.408180 & -0.013830 \\
\hline B & -0.908021 & -3.429370 & 0.547130 \\
\hline 0 & -1.628521 & -2.674460 & 1.631930 \\
\hline C & -1.794510 & -1.005660 & 3.306290 \\
\hline C & -2.694801 & -1.773189 & 4.060760 \\
\hline C & -1.505420 & 0.309720 & 3.692680 \\
\hline C & -3.301891 & -1.222699 & 5.184660 \\
\hline $\mathrm{H}$ & -2.915551 & -2.785859 & 3.743950 \\
\hline C & -2.113150 & 0.854270 & 4.820320 \\
\hline $\mathrm{H}$ & -0.812990 & 0.906840 & 3.109350 \\
\hline C & -3.012460 & 0.090371 & 5.566780 \\
\hline $\mathrm{H}$ & -4.006631 & -1.813769 & 5.760700 \\
\hline $\mathrm{H}$ & -1.880940 & 1.872910 & 5.109110 \\
\hline $\mathrm{H}$ & -3.488570 & 0.516941 & 6.444110 \\
\hline $\mathrm{H}$ & 1.827839 & -2.460871 & 1.197910 \\
\hline
\end{tabular}




\begin{tabular}{|c|c|c|c|}
\hline $\mathrm{H}$ & 0.214499 & -2.671370 & 3.810780 \\
\hline $\mathrm{H}$ & 1.232689 & -1.250840 & 3.239470 \\
\hline C & 0.147890 & 2.809350 & 0.202330 \\
\hline C & -0.989390 & 2.374680 & -0.458170 \\
\hline C & -2.251510 & 2.302090 & 0.228290 \\
\hline C & -2.334670 & 2.835210 & 1.499530 \\
\hline $\mathrm{H}$ & -3.287140 & 2.810221 & 2.018890 \\
\hline C & 2.252860 & 1.560759 & -0.270390 \\
\hline C & 1.503590 & 2.720050 & -0.426860 \\
\hline C & 4.134990 & 2.609859 & -1.379010 \\
\hline C & 3.595720 & 1.496569 & -0.763400 \\
\hline H & 5.154050 & 2.566689 & -1.752060 \\
\hline C & 3.388981 & 3.795349 & -1.577040 \\
\hline C & 2.042401 & 3.855609 & -1.100590 \\
\hline C & 1.288211 & 5.039460 & -1.315540 \\
\hline C & 1.847431 & 6.116329 & -1.963200 \\
\hline C & 3.184791 & 6.062239 & -2.424810 \\
\hline C & 3.936801 & 4.926229 & -2.238370 \\
\hline $\mathrm{H}$ & 0.257061 & 5.071700 & -0.983370 \\
\hline H & 1.257891 & 7.012170 & -2.130110 \\
\hline $\mathrm{H}$ & 3.611921 & 6.920239 & -2.934770 \\
\hline $\mathrm{H}$ & 4.960411 & 4.873819 & -2.598410 \\
\hline C & -1.219530 & 3.400580 & 2.161050 \\
\hline C & 0.060640 & 3.335830 & 1.531970 \\
\hline C & 1.193791 & 3.815980 & 2.241160 \\
\hline C & 1.059581 & 4.355510 & 3.501190 \\
\hline C & -0.213339 & 4.445840 & 4.111680 \\
\hline C & -1.327909 & 3.978300 & 3.453290 \\
\hline $\mathrm{H}$ & 2.168641 & 3.761749 & 1.771210 \\
\hline $\mathrm{H}$ & 1.934981 & 4.722769 & 4.027500 \\
\hline $\mathrm{H}$ & -0.307459 & 4.886760 & 5.099300 \\
\hline $\mathrm{H}$ & -2.310769 & 4.042010 & 3.911520 \\
\hline C & 6.055129 & -1.948751 & -0.236500 \\
\hline C & 4.797389 & -2.114411 & -0.829050 \\
\hline C & 3.999250 & -0.980501 & -1.005820 \\
\hline C & 4.440590 & 0.283539 & -0.589300 \\
\hline C & 5.708200 & 0.410159 & -0.006800 \\
\hline C & 6.535970 & -0.701162 & 0.177210 \\
\hline $\mathrm{H}$ & 6.682149 & -2.822112 & -0.092340 \\
\hline $\mathrm{H}$ & 3.023629 & -1.056751 & -1.466220 \\
\hline $\mathrm{H}$ & 6.025140 & 1.393029 & 0.318820 \\
\hline C & 4.342739 & -3.515881 & -1.261560 \\
\hline C & 7.927230 & -0.600592 & 0.819170 \\
\hline C & 2.946609 & -3.501501 & -1.903010 \\
\hline $\mathrm{H}$ & 2.670139 & -4.518291 & -2.199480 \\
\hline $\mathrm{H}$ & 2.923109 & -2.877561 & -2.801540 \\
\hline $\mathrm{H}$ & 2.174439 & -3.133521 & -1.222260 \\
\hline C & 5.341829 & -4.081791 & -2.293490 \\
\hline $\mathrm{H}$ & 5.033169 & -5.082981 & -2.613190 \\
\hline $\mathrm{H}$ & 5.389309 & -3.439551 & -3.178210 \\
\hline $\mathrm{H}$ & 6.351639 & -4.158552 & -1.880860 \\
\hline C & 4.301659 & -4.443631 & -0.027980 \\
\hline $\mathrm{H}$ & 3.976869 & -5.448801 & -0.318120 \\
\hline $\mathrm{H}$ & 5.283469 & -4.532961 & 0.445300 \\
\hline
\end{tabular}




\begin{tabular}{|c|c|c|c|}
\hline H & 3.603229 & -4.065341 & 0.724410 \\
\hline C & 8.289160 & 0.842928 & 1.204060 \\
\hline $\mathrm{H}$ & 9.288500 & 0.865848 & 1.649230 \\
\hline H & 8.298600 & 1.503818 & 0.331770 \\
\hline $\mathrm{H}$ & 7.589420 & 1.254338 & 1.938160 \\
\hline C & 7.963459 & -1.465812 & 2.097330 \\
\hline H & 8.950809 & -1.410132 & 2.568460 \\
\hline $\mathrm{H}$ & 7.218909 & -1.119622 & 2.821070 \\
\hline $\mathrm{H}$ & 7.754879 & -2.517002 & 1.880120 \\
\hline C & 8.986789 & -1.114242 & -0.179490 \\
\hline $\mathrm{H}$ & 9.987669 & -1.053582 & 0.261500 \\
\hline H & 8.807279 & -2.155602 & -0.460410 \\
\hline $\mathrm{H}$ & 8.979060 & -0.515092 & -1.095270 \\
\hline C & -3.425310 & 1.555961 & -0.303270 \\
\hline C & -3.892690 & 1.689951 & -1.612440 \\
\hline C & -4.084380 & 0.667121 & 0.563690 \\
\hline C & -4.964350 & 0.921391 & -2.079430 \\
\hline H & -3.414850 & 2.401641 & -2.268770 \\
\hline C & -5.198940 & -0.066289 & 0.151370 \\
\hline $\mathrm{H}$ & -3.696150 & 0.544521 & 1.567600 \\
\hline C & -5.607920 & 0.061711 & -1.184400 \\
\hline $\mathrm{H}$ & -6.447940 & -0.522259 & -1.531780 \\
\hline C & -5.911220 & -1.019359 & 1.126030 \\
\hline C & -5.376750 & 1.056071 & -3.551530 \\
\hline C & -5.973170 & -0.401319 & 2.538340 \\
\hline H & -6.527111 & -1.065169 & 3.210500 \\
\hline $\mathrm{H}$ & -4.982710 & -0.257599 & 2.974050 \\
\hline H & -6.482670 & 0.567431 & 2.519680 \\
\hline C & -7.355361 & -1.313838 & 0.679350 \\
\hline $\mathrm{H}$ & -7.857601 & -1.923338 & 1.437110 \\
\hline $\mathrm{H}$ & -7.930340 & -0.391588 & 0.548780 \\
\hline H & -7.390281 & -1.873978 & -0.259350 \\
\hline C & -5.135061 & -2.351749 & 1.188800 \\
\hline $\mathrm{H}$ & -5.117901 & -2.838719 & 0.209110 \\
\hline $\mathrm{H}$ & -4.100351 & -2.202789 & 1.502350 \\
\hline $\mathrm{H}$ & -5.609421 & -3.040219 & 1.897690 \\
\hline C & -6.522290 & 0.103191 & -3.927090 \\
\hline $\mathrm{H}$ & -6.246540 & -0.943669 & -3.763400 \\
\hline $\mathrm{H}$ & -7.430990 & 0.312062 & -3.353260 \\
\hline H & -6.766150 & 0.220501 & -4.987390 \\
\hline C & -5.835790 & 2.504691 & -3.823420 \\
\hline H & -6.696330 & 2.763801 & -3.198130 \\
\hline $\mathrm{H}$ & -5.039020 & 3.223781 & -3.616370 \\
\hline $\mathrm{H}$ & -6.127650 & 2.620361 & -4.872970 \\
\hline C & -4.157160 & 0.729571 & -4.443160 \\
\hline $\mathrm{H}$ & -4.424800 & 0.828741 & -5.500930 \\
\hline $\mathrm{H}$ & -3.313660 & 1.395071 & -4.243270 \\
\hline $\mathrm{H}$ & -3.817780 & -0.297369 & -4.272800 \\
\hline
\end{tabular}


Frequencies (Top 3 out of 450)

$\begin{array}{rr}\text { 1. } & -264.0469 \mathrm{~cm}^{-1} \\ \text { 2. } & 11.1721 \mathrm{~cm}^{-1} \\ \text { 3. } & 17.4532 \mathrm{~cm}^{-1}\end{array}$

\section{B3LYP-D3(BJ)/6-31G(d,p) Molecular Geometry in Cartesian Coordinates}

\section{C}

$\mathrm{H}$

$\mathrm{H}$

C

0

0

$\mathrm{H}$

0

$\mathrm{H}$

C

C

C

C

$\mathrm{H}$

$\mathrm{H}$

$\mathrm{H}$

C

C

C

$\mathrm{H}$

$\mathrm{H}$

$\mathrm{H}$

C

$\mathrm{H}$

$\mathrm{H}$

$\mathrm{H}$

C

$\mathrm{H}$

$\mathrm{H}$

$\mathrm{H}$

C

$\mathrm{H}$

\section{$-1.563593$}

$-2.479852$

$-1.283843$

1.706607

2.448762

0.888094

0.327704

$-0.505002$

$-0.831513$

2.840570

1. 297082

1.404082

1.999035

0.724341

0.904855

2.027648

$-2.202246$

$-1.896703$

$-2.488543$

$-2.496116$

$-3.459840$

$-1.716584$

$-3.290023$

$-4.238248$

$-3.459062$

$-3.021266$

$-2.011113$

$-3.051573$

$-1.469813$

$-1.563473$

$-2.730926$

$-3.800493$
1. 271599

0.771044

1.978276

1.409091

1.604128

0.360357

0.361376

0.299444

$-0.637612$

$-1.930123$

$-2.748984$

$-2.713641$

$-1.677226$

$-1.832759$

$-2.381701$

$-3.592351$

$-2.991868$

$-4.166150$

$-3.468840$

$-2.599786$

$-3.964415$

$-4.160166$

$-2.031870$

$-2.558836$

$-1.259304$

$-1.551610$

$-3.735174$

$-3.597047$

$-2.802072$

$-4.511950$

$-5.421016$

$-5.209009$
$-0.975704$
$-1.280706$
$-1.761815$
1.303673
2. 246857
1. 272568
0.430052
$-0.880054$
$-0.949342$
$-0.285659$
0.884594
$-2.138769$
$-1.355130$
0.988865
$-3.048585$
$-2.308967$
$-0.984683$
$-1.974944$
0.444014
1.107978
0.517506
0.787753
$-1.447815$
$-1.587788$
$-0.696615$
$-2.391064$
$-3.443937$
$-3.753552$
$-3.620354$
$-4.069050$
$-1.732065$
$-1.836667$ 


\begin{tabular}{|c|c|c|c|}
\hline $\mathrm{H}$ & -2.462827 & -6.187384 & -2.464558 \\
\hline $\mathrm{H}$ & -2.546690 & -5.828055 & -0.736771 \\
\hline 0 & -0.931069 & -2.296339 & -0.968084 \\
\hline 0 & -0.523788 & -4.469189 & -1.703684 \\
\hline B & 0.098979 & -3.324620 & -1.139397 \\
\hline 0 & 0.800527 & -3.692267 & 0.126844 \\
\hline C & 2.089105 & -3.147800 & 2.062629 \\
\hline C & 2.564489 & -4.459121 & 2.202222 \\
\hline C & 2.365495 & -2.191858 & 3.052033 \\
\hline C & 3.309631 & -4.810207 & 3.323854 \\
\hline $\mathrm{H}$ & 2.332634 & -5.186287 & 1.431765 \\
\hline C & 3.110980 & -2.552331 & 4.171710 \\
\hline $\mathrm{H}$ & 2.011585 & -1.172761 & 2.930613 \\
\hline C & 3.585372 & -3.858343 & 4.309519 \\
\hline $\mathrm{H}$ & 3.674355 & -5.826990 & 3.433446 \\
\hline $\mathrm{H}$ & 3.327583 & -1.809602 & 4.932763 \\
\hline $\mathrm{H}$ & 4.168558 & -4.135099 & 5.182589 \\
\hline H & 1.575345 & -0.678833 & -1.438191 \\
\hline $\mathrm{H}$ & 3.426230 & -2.843820 & -0.272759 \\
\hline $\mathrm{H}$ & 3.227302 & -1.111355 & 0.308463 \\
\hline C & 0.463870 & 3.076512 & -0.057902 \\
\hline C & 1.628614 & 2.345325 & 0.118698 \\
\hline C & 2.720284 & 2.463199 & -0.801561 \\
\hline C & 2.617474 & 3.386528 & -1.823784 \\
\hline $\mathrm{H}$ & 3.435948 & 3.480167 & -2.531157 \\
\hline C & -1.756351 & 2.004725 & 0.342494 \\
\hline C & -0.732944 & 2.826793 & 0.807136 \\
\hline C & -2.969540 & 2.352930 & 2.418648 \\
\hline C & -2.910735 & 1.783560 & 1.160310 \\
\hline $\mathrm{H}$ & -3.852266 & 2.191368 & 3.030456 \\
\hline C & -1.933922 & 3.169892 & 2.923553 \\
\hline C & -0.794556 & 3.427447 & 2.099335 \\
\hline C & 0.237294 & 4.265055 & 2.602619 \\
\hline C & 0.141678 & 4.817832 & 3.857952 \\
\hline C & -0.988283 & 4.563774 & 4.672591 \\
\hline C & -2.003156 & 3.758039 & 4.214317 \\
\hline $\mathrm{H}$ & 1.110703 & 4.449527 & 1.988810 \\
\hline $\mathrm{H}$ & 0.940928 & 5.450096 & 4.231301 \\
\hline $\mathrm{H}$ & -1.047930 & 5.006284 & 5.662142 \\
\hline $\mathrm{H}$ & -2.873939 & 3.556924 & 4.831930 \\
\hline C & 1.482967 & 4.215734 & -1.984469 \\
\hline C & 0.376026 & 4.055234 & -1.095090 \\
\hline C & -0.772111 & 4.871525 & -1.278110 \\
\hline C & -0.819531 & 5.800771 & -2.292367 \\
\hline C & 0.277089 & 5.959053 & -3.173007 \\
\hline C & 1.402449 & 5.183375 & -3.020410 \\
\hline $\mathrm{H}$ & -1.609922 & 4.755163 & -0.600100 \\
\hline $\mathrm{H}$ & -1.701825 & 6.420929 & -2.416744 \\
\hline $\mathrm{H}$ & 0.226497 & 6.696047 & -3.968473 \\
\hline $\mathrm{H}$ & 2.248765 & 5.297552 & -3.691831 \\
\hline C & -6.316563 & -0.438792 & -0.119742 \\
\hline C & -5.842292 & 0.592388 & -0.940116 \\
\hline C & -4.724764 & 1.308589 & -0.506178 \\
\hline C & -4.078452 & 0.988646 & 0.695275 \\
\hline
\end{tabular}




\begin{tabular}{|c|c|c|c|}
\hline C & -4.576313 & -0.056797 & 1.482830 \\
\hline C & -5.706934 & -0.782984 & 1.091640 \\
\hline $\mathrm{H}$ & -7.190813 & -0.994766 & -0.439007 \\
\hline $\mathrm{H}$ & -4.345373 & 2.138569 & -1.089085 \\
\hline $\mathrm{H}$ & -4.054260 & -0.298398 & 2.400080 \\
\hline C & -6.547802 & 0.887855 & -2.270691 \\
\hline C & -6.304970 & -1.907330 & 1.949474 \\
\hline C & -5.889901 & 2.048894 & -3.033698 \\
\hline $\mathrm{H}$ & -6.420797 & 2.218165 & -3.975273 \\
\hline $\mathrm{H}$ & -5.923752 & 2.981282 & -2.461573 \\
\hline $\mathrm{H}$ & -4.844751 & 1.833022 & -3.276921 \\
\hline C & -6.484282 & -0.372304 & -3.161397 \\
\hline $\mathrm{H}$ & -6.982121 & -0.187762 & -4.119389 \\
\hline $\mathrm{H}$ & -5.445817 & -0.652762 & -3.362780 \\
\hline $\mathrm{H}$ & -6.974748 & -1.227227 & -2.687875 \\
\hline C & -8.022325 & 1.256614 & -2.002257 \\
\hline $\mathrm{H}$ & -8.540838 & 1.463949 & -2.944450 \\
\hline $\mathrm{H}$ & -8.557056 & 0.447224 & -1.497741 \\
\hline $\mathrm{H}$ & -8.090591 & 2.147734 & -1.370623 \\
\hline C & -6.516232 & -3.175518 & 1.095363 \\
\hline $\mathrm{H}$ & -6.921421 & -3.982828 & 1.714126 \\
\hline $\mathrm{H}$ & -7.218073 & -3.006628 & 0.274493 \\
\hline $\mathrm{H}$ & -5.571775 & -3.517784 & 0.664185 \\
\hline C & -5.400957 & -2.275566 & 3.137319 \\
\hline $\mathrm{H}$ & -5.853629 & -3.094878 & 3.703814 \\
\hline $\mathrm{H}$ & -4.412257 & -2.604879 & 2.804321 \\
\hline $\mathrm{H}$ & -5.269797 & -1.434806 & 3.825064 \\
\hline C & -7.668510 & -1.431212 & 2.496985 \\
\hline $\mathrm{H}$ & -8.129455 & -2.212975 & 3.110528 \\
\hline $\mathrm{H}$ & -7.547280 & -0.536700 & 3.115821 \\
\hline $\mathrm{H}$ & -8.359684 & -1.185425 & 1.685431 \\
\hline C & 3.878876 & 1.528175 & -0.795354 \\
\hline C & 4.169416 & 0.833239 & -1.983251 \\
\hline C & 4.668807 & 1.305409 & 0.332143 \\
\hline C & 5.216761 & -0.082735 & -2.048986 \\
\hline $\mathrm{H}$ & 3.530838 & 1.001061 & -2.840955 \\
\hline C & 5.726462 & 0.383852 & 0.304264 \\
\hline $\mathrm{H}$ & 4.438316 & 1.841675 & 1.241373 \\
\hline C & 5.983159 & -0.289738 & -0.890300 \\
\hline $\mathrm{H}$ & 6.792732 & -1.007218 & -0.927864 \\
\hline C & 6.519204 & 0.126112 & 1.592561 \\
\hline C & 5.542894 & -0.875147 & -3.322814 \\
\hline C & 5.557578 & -0.470150 & 2.644185 \\
\hline $\mathrm{H}$ & 4.706113 & 0.187944 & 2.835455 \\
\hline $\mathrm{H}$ & 5.162273 & -1.432191 & 2.306110 \\
\hline $\mathrm{H}$ & 6.083042 & -0.636807 & 3.591404 \\
\hline C & 7.678647 & -0.859555 & 1.379195 \\
\hline $\mathrm{H}$ & 8.216112 & -1.004016 & 2.321359 \\
\hline $\mathrm{H}$ & 7.320739 & -1.840488 & 1.051026 \\
\hline $\mathrm{H}$ & 8.395893 & -0.489430 & 0.639211 \\
\hline C & 7.102683 & 1.454564 & 2.118326 \\
\hline $\mathrm{H}$ & 7.776766 & 1.903376 & 1.381577 \\
\hline $\mathrm{H}$ & 6.317864 & 2.180815 & 2.344040 \\
\hline $\mathrm{H}$ & 7.669644 & 1.279929 & 3.039066 \\
\hline
\end{tabular}




$\begin{array}{llrl}\mathrm{C} & 6.964722 & -0.503311 & -3.795396 \\ \mathrm{H} & 7.031929 & 0.565998 & -4.019367 \\ \mathrm{H} & 7.714377 & -0.732602 & -3.032798 \\ \mathrm{H} & 7.224931 & -1.060782 & -4.701862 \\ \mathrm{C} & 5.479620 & -2.388806 & -3.024592 \\ \mathrm{H} & 6.181128 & -2.679023 & -2.237860 \\ \mathrm{H} & 4.474992 & -2.676436 & -2.703647 \\ \mathrm{H} & 5.730062 & -2.962383 & -3.923531 \\ \mathrm{C} & 4.555553 & -0.575975 & -4.462361 \\ \mathrm{H} & 4.823004 & -1.164490 & -5.345305 \\ \mathrm{H} & 3.530477 & -0.840048 & -4.184187 \\ \mathrm{H} & 4.574033 & 0.479921 & -4.749851\end{array}$

\section{C-Catalyzed reaction TS 23}

\section{Datum}

Value

\begin{tabular}{lc} 
B3LYP-D3(BJ)/6-31G(d,p) Energy & -3039.488603 \\
\hline B3LYP-D3(BJ)/def2-TZVPP/IEF-PCM(DCM) Energy & -3040.525528 \\
\hline B3LYP-D3(BJ)/def2-TZVPP/IEF-PCM(DCM)//B3LYP-D3(BJ)/6-31G(d,p) Free Energy (Quasiharmonic) & -3039.29458
\end{tabular}

Number of Imaginary Frequencies

Frequencies (Top 3 out of 450)

$\begin{array}{lr}\text { 1. } & -250.1260 \mathrm{~cm}^{-1} \\ \text { 2. } & 9.8724 \mathrm{~cm}^{-1} \\ \text { 3. } & 15.2524 \mathrm{~cm}^{-1}\end{array}$

B3LYP-D3(BJ)/6-31G(d,p) Molecular Geometry in Cartesian Coordinates

$\begin{array}{rrrr}\mathrm{C} & 1.531495 & 0.794416 & -0.972766 \\ \mathrm{H} & 0.944828 & 0.833454 & -1.896680 \\ \mathrm{H} & 2.521532 & 0.405774 & -1.213958 \\ \mathrm{C} & -1.244862 & 2.166245 & 1.371548 \\ \mathrm{O} & -1.783361 & 2.969416 & 2.097076 \\ \mathrm{O} & -0.334416 & 1.295175 & 1.841231 \\ \mathrm{H} & 0.052821 & 0.728311 & 1.123352 \\ \mathrm{O} & 0.889792 & -0.098131 & -0.046820 \\ \mathrm{H} & 1.246115 & -1.009098 & -0.199463 \\ \mathrm{C} & -1.796103 & -3.006915 & 0.832043 \\ \mathrm{C} & -1.210618 & -2.690843 & -1.198567 \\ \mathrm{C} & 0.386869 & -4.139220 & 0.947214 \\ \mathrm{C} & -0.489826 & -3.064031 & 1.278672 \\ \mathrm{H} & -0.841572 & -1.679022 & -1.018185\end{array}$




\begin{tabular}{|c|c|c|c|}
\hline $\mathrm{H}$ & 1.311672 & -4.185492 & 1.518770 \\
\hline $\mathrm{H}$ & -0.089790 & -5.114990 & 0.849917 \\
\hline C & 2.857951 & -2.826990 & -1.608390 \\
\hline C & 3.036225 & -4.376818 & -1.459151 \\
\hline C & 2.369377 & -2.416234 & -3.002236 \\
\hline $\mathrm{H}$ & 3.158871 & -2.525211 & -3.751022 \\
\hline $\mathrm{H}$ & 1.509216 & -3.014688 & -3.309967 \\
\hline $\mathrm{H}$ & 2.066046 & -1.365895 & -2.981417 \\
\hline C & 4.093184 & -2.028886 & -1.217336 \\
\hline $\mathrm{H}$ & 3.954031 & -0.959531 & -1.388306 \\
\hline $\mathrm{H}$ & 4.346169 & -2.160811 & -0.167619 \\
\hline $\mathrm{H}$ & 4.948540 & -2.346942 & -1.821800 \\
\hline C & 3.893648 & -4.757340 & -0.243207 \\
\hline $\mathrm{H}$ & 4.953696 & -4.538335 & -0.402182 \\
\hline $\mathrm{H}$ & 3.563042 & -4.229162 & 0.653870 \\
\hline $\mathrm{H}$ & 3.784699 & -5.830226 & -0.065531 \\
\hline C & 3.574474 & -5.068269 & -2.709042 \\
\hline $\mathrm{H}$ & 4.562239 & -4.680292 & -2.979290 \\
\hline $\mathrm{H}$ & 3.670882 & -6.140885 & -2.519840 \\
\hline $\mathrm{H}$ & 2.898504 & -4.935405 & -3.554876 \\
\hline 0 & 1.779225 & -2.573047 & -0.663978 \\
\hline 0 & 1.700132 & -4.833884 & -1.224664 \\
\hline B & 0.943374 & -3.769972 & -0.674687 \\
\hline 0 & -0.342969 & -3.630819 & -1.432173 \\
\hline C & -2.541911 & -2.830131 & -1.819121 \\
\hline C & -3.471085 & -1.791411 & -1.686537 \\
\hline C & -2.896612 & -3.996516 & -2.511372 \\
\hline C & -4.744049 & -1.913687 & -2.232404 \\
\hline $\mathrm{H}$ & -3.199392 & -0.901429 & -1.136709 \\
\hline C & -4.168693 & -4.110839 & -3.066133 \\
\hline $\mathrm{H}$ & -2.167377 & -4.792659 & -2.609517 \\
\hline C & -5.095690 & -3.074095 & -2.925903 \\
\hline $\mathrm{H}$ & -5.460212 & -1.108383 & -2.103790 \\
\hline $\mathrm{H}$ & -4.440556 & -5.011401 & -3.607961 \\
\hline $\mathrm{H}$ & -6.088732 & -3.172583 & -3.353644 \\
\hline $\mathrm{H}$ & -0.046264 & -2.149654 & 1.671501 \\
\hline $\mathrm{H}$ & -2.321188 & -3.928232 & 0.601178 \\
\hline $\mathrm{H}$ & -2.418710 & -2.143239 & 1.032865 \\
\hline C & -0.685310 & 2.618888 & -1.013787 \\
\hline C & -1.582735 & 2.082049 & -0.104722 \\
\hline C & -2.858022 & 1.583715 & -0.532702 \\
\hline C & -3.149944 & 1.635557 & -1.885372 \\
\hline $\mathrm{H}$ & -4.078222 & 1.208967 & -2.248788 \\
\hline C & 1.686017 & 2.193735 & -0.420185 \\
\hline C & 0.643333 & 3.099543 & -0.530163 \\
\hline C & 3.116342 & 3.927636 & 0.494145 \\
\hline C & 2.922132 & 2.601853 & 0.170170 \\
\hline $\mathrm{H}$ & 4.059194 & 4.234556 & 0.937952 \\
\hline C & 2.091339 & 4.890638 & 0.327494 \\
\hline C & 0.812788 & 4.464740 & -0.152014 \\
\hline C & -0.236227 & 5.416674 & -0.248615 \\
\hline C & -0.021944 & 6.732047 & 0.091777 \\
\hline C & 1.247573 & 7.157366 & 0.551210 \\
\hline & 2.278843 & 6.255262 & 0.670157 \\
\hline
\end{tabular}




\begin{tabular}{|c|c|c|c|}
\hline $\mathrm{H}$ & -1.212108 & 5.088396 & -0.587136 \\
\hline $\mathrm{H}$ & -0.832961 & 7.449332 & 0.015311 \\
\hline $\mathrm{H}$ & 1.401114 & 8.198361 & 0.818425 \\
\hline $\mathrm{H}$ & 3.251765 & 6.571327 & 1.035815 \\
\hline C & -2.267463 & 2.191006 & -2.838857 \\
\hline C & -1.009634 & 2.703468 & -2.400826 \\
\hline C & -0.121691 & 3.258096 & -3.359407 \\
\hline C & -0.464582 & 3.295137 & -4.692190 \\
\hline C & -1.707965 & 2.777938 & -5.128514 \\
\hline C & -2.588792 & 2.237085 & -4.221082 \\
\hline $\mathrm{H}$ & 0.830349 & 3.653308 & -3.023225 \\
\hline $\mathrm{H}$ & 0.222215 & 3.722814 & -5.415901 \\
\hline $\mathrm{H}$ & -1.963338 & 2.810254 & -6.183089 \\
\hline $\mathrm{H}$ & -3.544575 & 1.836930 & -4.547435 \\
\hline C & 5.796014 & -0.302775 & 1.371118 \\
\hline C & 4.519157 & -0.325180 & 1.938030 \\
\hline C & 3.598949 & 0.636619 & 1.506812 \\
\hline C & 3.942741 & 1.586870 & 0.543934 \\
\hline C & 5.231280 & 1.584576 & -0.004230 \\
\hline C & 6.174560 & 0.635496 & 0.397006 \\
\hline $\mathrm{H}$ & 6.522318 & -1.042958 & 1.681927 \\
\hline $\mathrm{H}$ & 2.599299 & 0.670135 & 1.920605 \\
\hline $\mathrm{H}$ & 5.473090 & 2.328132 & -0.753697 \\
\hline C & 4.112109 & -1.329301 & 3.025015 \\
\hline C & 7.589839 & 0.573420 & -0.194932 \\
\hline C & 5.076154 & -2.525878 & 3.097472 \\
\hline $\mathrm{H}$ & 4.720105 & -3.241316 & 3.844918 \\
\hline $\mathrm{H}$ & 5.144025 & -3.048662 & 2.138228 \\
\hline $\mathrm{H}$ & 6.084681 & -2.222260 & 3.393576 \\
\hline C & 4.116343 & -0.602673 & 4. 387357 \\
\hline $\mathrm{H}$ & 3.811766 & -1.285307 & 5.188559 \\
\hline $\mathrm{H}$ & 5.115248 & -0.221619 & 4.622251 \\
\hline $\mathrm{H}$ & 3.425281 & 0.245210 & 4.380932 \\
\hline C & 2.693106 & -1.868597 & 2.743028 \\
\hline $\mathrm{H}$ & 2.443652 & -2.659014 & 3.458828 \\
\hline $\mathrm{H}$ & 1.934575 & -1.087884 & 2.844287 \\
\hline $\mathrm{H}$ & 2.619445 & -2.273938 & 1.730877 \\
\hline C & 7.785693 & -0.787210 & -0.898425 \\
\hline $\mathrm{H}$ & 8.795732 & -0.859993 & -1.316257 \\
\hline $\mathrm{H}$ & 7.646250 & -1.623265 & -0.207667 \\
\hline $\mathrm{H}$ & 7.067718 & -0.905789 & -1.715788 \\
\hline C & 7.840634 & 1.687219 & -1.224101 \\
\hline $\mathrm{H}$ & 8.860014 & 1.606457 & -1.613676 \\
\hline $\mathrm{H}$ & 7.154394 & 1.615605 & -2.073644 \\
\hline $\mathrm{H}$ & 7.732356 & 2.681066 & -0.778781 \\
\hline C & 8.628066 & 0.720778 & 0.938147 \\
\hline $\mathrm{H}$ & 9.644283 & 0.678912 & 0.531480 \\
\hline $\mathrm{H}$ & 8.505055 & 1.677845 & 1.454489 \\
\hline $\mathrm{H}$ & 8.532968 & -0.074825 & 1.681994 \\
\hline C & -3.837961 & 0.962715 & 0.397921 \\
\hline C & -3.430288 & 0.267321 & 1.545119 \\
\hline C & -5.208380 & 1.013029 & 0.098681 \\
\hline C & -4.353615 & -0.337497 & 2.401447 \\
\hline $\mathrm{H}$ & -2.379837 & 0.195591 & 1.777766 \\
\hline
\end{tabular}




\begin{tabular}{|c|c|c|c|}
\hline C & -6.153499 & 0.357022 & 0.889056 \\
\hline $\mathrm{H}$ & -5.530711 & 1.588104 & -0.758600 \\
\hline C & -5.703733 & -0.303799 & 2.038480 \\
\hline $\mathrm{H}$ & -6.429411 & -0.794180 & 2.677988 \\
\hline C & -7.648243 & 0.335515 & 0.540397 \\
\hline C & -3.933113 & -0.993412 & 3.725129 \\
\hline C & -8.460762 & 0.956173 & 1.696590 \\
\hline $\mathrm{H}$ & -9.530763 & 0.939293 & 1.462849 \\
\hline $\mathrm{H}$ & -8.314723 & 0.410752 & 2.632772 \\
\hline $\mathrm{H}$ & -8.162801 & 1.995472 & 1.865335 \\
\hline C & -7.961839 & 1.121176 & -0.743110 \\
\hline $\mathrm{H}$ & -9.034935 & 1.072645 & -0.951117 \\
\hline $\mathrm{H}$ & -7.689025 & 2.176834 & -0.649929 \\
\hline $\mathrm{H}$ & -7.438580 & 0.707604 & -1.611593 \\
\hline C & -8.085743 & -1.130706 & 0.328964 \\
\hline $\mathrm{H}$ & -7.509794 & -1.595980 & -0.477429 \\
\hline $\mathrm{H}$ & -7.935481 & -1.730125 & 1.230939 \\
\hline $\mathrm{H}$ & -9.148081 & -1.178780 & 0.066184 \\
\hline C & -4.325157 & -2.485856 & 3.721544 \\
\hline $\mathrm{H}$ & -5.401184 & -2.621528 & 3.578583 \\
\hline $\mathrm{H}$ & -3.809020 & -3.025243 & 2.921916 \\
\hline $\mathrm{H}$ & -4.052873 & -2.951691 & 4.674677 \\
\hline C & -2.420376 & -0.878483 & 3.980593 \\
\hline $\mathrm{H}$ & -1.837934 & -1.428615 & 3.237108 \\
\hline $\mathrm{H}$ & -2.082023 & 0.161240 & 3.975318 \\
\hline $\mathrm{H}$ & -2.183720 & -1.306732 & 4.959450 \\
\hline C & -4.664070 & -0.272992 & 4.880125 \\
\hline $\mathrm{H}$ & -4.378412 & -0.713820 & 5.841211 \\
\hline $\mathrm{H}$ & -4.403006 & 0.789236 & 4.898159 \\
\hline $\mathrm{H}$ & -5.750781 & -0.351277 & 4.785443 \\
\hline
\end{tabular}

\section{C-Catalyzed reaction TS 24}

\section{Datum}

Value

B3LYP-D3(BJ)/6-31G(d,p) Energy

$-3039.487014$

B3LYP-D3(BJ)/def2-TZVPP/IEF-PCM(DCM) Energy

$-3040.525036$

B3LYP-D3(BJ)/def2-TZVPP/IEF-PCM(DCM)//B3LYP-D3(BJ)/6-31G(d,p) Free Energy (Quasiharmonic)

$-3039.294347$

Number of Imaginary Frequencies

Frequencies (Top 3 out of 450)

$\begin{array}{lr}\text { 1. } & -259.9588 \mathrm{~cm}^{-1} \\ \text { 2. } & 7.8264 \mathrm{~cm}^{-1} \\ \text { 3. } & 10.1334 \mathrm{~cm}^{-1}\end{array}$




\begin{tabular}{|c|c|c|c|}
\hline C & 0.941105 & 0.419043 & -0.009353 \\
\hline $\mathrm{H}$ & 1.923770 & -0.036768 & -0.105353 \\
\hline $\mathrm{H}$ & 0.491445 & 0.039409 & 0.907653 \\
\hline C & -2.420238 & 2.287619 & -0.785694 \\
\hline 0 & -3.079388 & 3.299945 & -0.927206 \\
\hline 0 & -1.768497 & 1.730580 & -1.798635 \\
\hline $\mathrm{H}$ & -1.133674 & 1.019147 & -1.501132 \\
\hline 0 & 0.136815 & 0.029709 & -1.135272 \\
\hline $\mathrm{H}$ & -0.048975 & -0.935602 & -1.020371 \\
\hline C & 1.815192 & -2.650856 & 3.460566 \\
\hline C & 2.716754 & -3.078614 & 1.541137 \\
\hline C & -0.234302 & -2.584719 & 2.091878 \\
\hline C & 0.604323 & -3.191278 & 3.076925 \\
\hline $\mathrm{H}$ & 2.671068 & -4.162623 & 1.654505 \\
\hline $\mathrm{H}$ & -0.228837 & -1.496563 & 2.094454 \\
\hline $\mathrm{H}$ & -1.246272 & -2.982950 & 2.033348 \\
\hline C & -0.777009 & -3.611538 & -1.303282 \\
\hline C & 0.105311 & -4.839304 & -0.887201 \\
\hline C & -2.225564 & -3.720251 & -0.819163 \\
\hline $\mathrm{H}$ & -2.702564 & -2.740969 & -0.909765 \\
\hline $\mathrm{H}$ & -2.798289 & -4.436496 & -1.414280 \\
\hline $\mathrm{H}$ & -2.270160 & -4.029460 & 0.227852 \\
\hline C & -0.751192 & -3.269106 & -2.788216 \\
\hline $\mathrm{H}$ & -1.159127 & -4.095736 & -3.378419 \\
\hline $\mathrm{H}$ & -1.368702 & -2.385918 & -2.977150 \\
\hline $\mathrm{H}$ & 0.259490 & -3.057262 & -3.135139 \\
\hline C & 1.410806 & -4.919465 & -1.689599 \\
\hline $\mathrm{H}$ & 1.236074 & -5.238522 & -2.721275 \\
\hline $\mathrm{H}$ & 1.923182 & -3.955686 & -1.706377 \\
\hline $\mathrm{H}$ & 2.070484 & -5.649168 & -1.211972 \\
\hline C & -0.620066 & -6.179406 & -0.922363 \\
\hline $\mathrm{H}$ & -0.971218 & -6.407299 & -1.933940 \\
\hline $\mathrm{H}$ & 0.062388 & -6.976007 & -0.612902 \\
\hline $\mathrm{H}$ & -1.474948 & -6.184440 & -0.244423 \\
\hline 0 & -0.147077 & -2.538171 & -0.566089 \\
\hline 0 & 0.431246 & -4.531026 & 0.482723 \\
\hline B & 0.490231 & -3.108667 & 0.592695 \\
\hline 0 & 1.885724 & -2.540416 & 0.700308 \\
\hline C & 4.020843 & -2.431682 & 1.753686 \\
\hline C & 5.065617 & -3.174884 & 2.322197 \\
\hline C & 4.239654 & -1.094110 & 1.393531 \\
\hline C & 6.310881 & -2.588542 & 2.529586 \\
\hline $\mathrm{H}$ & 4.896969 & -4.212770 & 2.596453 \\
\hline C & 5.483560 & -0.510934 & 1.604792 \\
\hline $\mathrm{H}$ & 3.439231 & -0.519739 & 0.947915 \\
\hline C & 6.520431 & -1.254046 & 2.174488 \\
\hline $\mathrm{H}$ & 7.116688 & -3.169934 & 2.966110 \\
\hline $\mathrm{H}$ & 5.638952 & 0.519949 & 1.310742 \\
\hline $\mathrm{H}$ & 7.490283 & -0.794737 & 2.338722 \\
\hline $\mathrm{H}$ & 0.404167 & -4.231654 & 3.330979 \\
\hline
\end{tabular}




\begin{tabular}{|c|c|c|c|}
\hline $\mathrm{H}$ & 1.972707 & -1.580736 & 3.371872 \\
\hline $\mathrm{H}$ & 2.457962 & -3.171917 & 4.162501 \\
\hline C & -1.120955 & 1.840970 & 1.279648 \\
\hline C & -2.287288 & 1.614292 & 0.568016 \\
\hline C & -3.335494 & 0.794627 & 1.107118 \\
\hline C & -3.144530 & 0.229569 & 2.354315 \\
\hline $\mathrm{H}$ & -3.930889 & -0.387495 & 2.777880 \\
\hline C & 1.040070 & 1.920725 & 0.053099 \\
\hline C & 0.003631 & 2.615744 & 0.663568 \\
\hline C & 2.179484 & 4.008631 & -0.408396 \\
\hline C & 2.160870 & 2.628187 & -0.493424 \\
\hline $\mathrm{H}$ & 3.015727 & 4.555292 & -0.834107 \\
\hline C & 1.129748 & 4.742931 & 0.188868 \\
\hline C & 0.013701 & 4.038885 & 0.740447 \\
\hline C & -1.038709 & 4.780881 & 1.338983 \\
\hline C & -0.984208 & 6.153812 & 1.392815 \\
\hline C & 0.122087 & 6.851723 & 0.850423 \\
\hline C & 1.153571 & 6.161372 & 0.259644 \\
\hline $\mathrm{H}$ & -1.896812 & 4.249110 & 1.732203 \\
\hline $\mathrm{H}$ & -1.799288 & 6.709430 & 1.845575 \\
\hline $\mathrm{H}$ & 0.148710 & 7.936078 & 0.897511 \\
\hline $\mathrm{H}$ & 2.002921 & 6.689528 & -0.164616 \\
\hline C & -1.969776 & 0.438673 & 3.112839 \\
\hline C & -0.938032 & 1.268514 & 2.577416 \\
\hline C & 0.253032 & 1.448475 & 3.331266 \\
\hline C & 0.417011 & 0.825591 & 4.548471 \\
\hline C & -0.606283 & 0.003036 & 5.077921 \\
\hline C & -1.775827 & -0.177648 & 4.377761 \\
\hline $\mathrm{H}$ & 1.036189 & 2.080318 & 2.928230 \\
\hline $\mathrm{H}$ & 1.334348 & 0.971043 & 5.110772 \\
\hline $\mathrm{H}$ & -0.463886 & -0.483084 & 6.037957 \\
\hline $\mathrm{H}$ & -2.566487 & -0.808173 & 4.774523 \\
\hline C & 5.522713 & 0.519168 & -2.066254 \\
\hline C & 5.745736 & 1.641588 & -1.261099 \\
\hline C & 4.626287 & 2.314590 & -0.756860 \\
\hline C & 3.323207 & 1.915302 & -1.086358 \\
\hline C & 3.145618 & 0.821256 & -1.945342 \\
\hline C & 4.236110 & 0.083025 & -2.406796 \\
\hline $\mathrm{H}$ & 6.374030 & -0.042606 & -2.430688 \\
\hline $\mathrm{H}$ & 4.749271 & 3.148610 & -0.077285 \\
\hline $\mathrm{H}$ & 2.138900 & 0.544287 & -2.226362 \\
\hline C & 7.178022 & 2.128655 & -0.989106 \\
\hline C & 4.054040 & -1.221470 & -3.193885 \\
\hline C & 7.648577 & 2.927400 & -2.225263 \\
\hline $\mathrm{H}$ & 8.672125 & 3.292706 & -2.084133 \\
\hline $\mathrm{H}$ & 7.627967 & 2.302828 & -3.123284 \\
\hline $\mathrm{H}$ & 6.997165 & 3.788799 & -2.401834 \\
\hline C & 7.254731 & 3.044812 & 0.245514 \\
\hline $\mathrm{H}$ & 8.294488 & 3.334368 & 0.425742 \\
\hline $\mathrm{H}$ & 6.680408 & 3.965338 & 0.110728 \\
\hline $\mathrm{H}$ & 6.885849 & 2.543789 & 1.146830 \\
\hline C & 8.141543 & 0.944833 & -0.763812 \\
\hline $\mathrm{H}$ & 9.136618 & 1.319529 & -0.503107 \\
\hline $\mathrm{H}$ & 7.795172 & 0.296342 & 0.045393 \\
\hline
\end{tabular}




\begin{tabular}{|c|c|c|c|}
\hline $\mathrm{H}$ & 8.254181 & 0.328430 & -1.659186 \\
\hline C & 4.886220 & -1.191831 & -4.491418 \\
\hline $\mathrm{H}$ & 4.753149 & -2.125520 & -5.048265 \\
\hline $\mathrm{H}$ & 4.573504 & -0.363394 & -5.134595 \\
\hline $\mathrm{H}$ & 5.955080 & -1.076602 & -4.291197 \\
\hline C & 4.528206 & -2.386559 & -2.295397 \\
\hline $\mathrm{H}$ & 4.416079 & -3.343344 & -2.817704 \\
\hline $\mathrm{H}$ & 5.578707 & -2.274120 & -2.011959 \\
\hline $\mathrm{H}$ & 3.937682 & -2.425151 & -1.375474 \\
\hline C & 2.583222 & -1.471145 & -3.564769 \\
\hline $\mathrm{H}$ & 2.501305 & -2.412946 & -4.116912 \\
\hline $\mathrm{H}$ & 1.947107 & -1.543658 & -2.679011 \\
\hline $\mathrm{H}$ & 2.184349 & -0.674807 & -4.200765 \\
\hline C & -4.595612 & 0.491661 & 0.375898 \\
\hline C & -5.089853 & -0.821698 & 0.359107 \\
\hline C & -5.340185 & 1.499877 & -0.243506 \\
\hline C & -6.305476 & -1.135163 & -0.257048 \\
\hline $\mathrm{H}$ & -4.504996 & -1.602146 & 0.835009 \\
\hline C & -6.537628 & 1.215703 & -0.905752 \\
\hline $\mathrm{H}$ & -4.968999 & 2.513543 & -0.209218 \\
\hline C & -7.005218 & -0.102547 & -0.895664 \\
\hline $\mathrm{H}$ & -7.935653 & -0.330684 & -1.394429 \\
\hline C & -7.274076 & 2.357071 & -1.620203 \\
\hline C & -6.849974 & -2.570748 & -0.188186 \\
\hline C & -6.346869 & 2.936395 & -2.712517 \\
\hline $\mathrm{H}$ & -5.401065 & 3.293890 & -2.297766 \\
\hline $\mathrm{H}$ & -6.113338 & 2.172984 & -3.461738 \\
\hline $\mathrm{H}$ & -6.838107 & 3.773699 & -3.220974 \\
\hline C & -8.578475 & 1.887810 & -2.283506 \\
\hline $\mathrm{H}$ & -9.061819 & 2.733278 & -2.782631 \\
\hline $\mathrm{H}$ & -8.393931 & 1.118810 & -3.040366 \\
\hline $\mathrm{H}$ & -9.286081 & 1.485675 & -1.550823 \\
\hline C & -7.619676 & 3.463862 & -0.600863 \\
\hline $\mathrm{H}$ & -8.265446 & 3.074876 & 0.193224 \\
\hline $\mathrm{H}$ & -6.721909 & 3.877220 & -0.134462 \\
\hline $\mathrm{H}$ & -8.145991 & 4.285696 & -1.098483 \\
\hline C & -5.804075 & -3.562007 & -0.736517 \\
\hline $\mathrm{H}$ & -5.537316 & -3.318133 & -1.769477 \\
\hline $\mathrm{H}$ & -4.888164 & -3.550299 & -0.142656 \\
\hline $\mathrm{H}$ & -6.199936 & -4.583380 & -0.715887 \\
\hline C & -7.148624 & -2.915380 & 1.287609 \\
\hline $\mathrm{H}$ & -6.247432 & -2.842747 & 1.903439 \\
\hline $\mathrm{H}$ & -7.893489 & -2.229438 & 1.702806 \\
\hline $\mathrm{H}$ & -7.536178 & -3.936758 & 1.373869 \\
\hline C & -8.145976 & -2.746225 & -0.996581 \\
\hline $\mathrm{H}$ & -8.486047 & -3.783968 & -0.924346 \\
\hline $\mathrm{H}$ & -8.950814 & -2.108141 & -0.619361 \\
\hline $\mathrm{H}$ & -7.995136 & -2.516995 & -2.056129 \\
\hline
\end{tabular}


Frequencies (Top 3 out of 450)

$\begin{array}{lr}\text { 1. } & -250.5215 \mathrm{~cm}^{-1} \\ \text { 2. } & 8.3087 \mathrm{~cm}^{-1} \\ \text { 3. } & 10.7733 \mathrm{~cm}^{-1}\end{array}$

\section{B3LYP-D3(BJ)/6-31G(d,p) Molecular Geometry in Cartesian Coordinates}

\begin{tabular}{|c|c|c|c|}
\hline C & 1.070093 & 0.490675 & 0.687246 \\
\hline $\mathrm{H}$ & 2.127843 & 0.215656 & 0.597258 \\
\hline $\mathrm{H}$ & 0.666646 & 0.012015 & 1.585519 \\
\hline C & -2.217408 & 2.193095 & -0.293315 \\
\hline 0 & -2.821190 & 3.233352 & -0.464924 \\
\hline 0 & -1.502723 & 1.631193 & -1.264986 \\
\hline $\mathrm{H}$ & -0.897082 & 0.914760 & -0.922736 \\
\hline 0 & 0.366562 & 0.012345 & -0.465189 \\
\hline $\mathrm{H}$ & 0.324386 & -0.979282 & -0.378230 \\
\hline C & 3.939449 & -1.948274 & -1.188413 \\
\hline C & 3.157627 & -2.775018 & 0.611351 \\
\hline C & 1.936837 & -3.039093 & -2.149376 \\
\hline C & 2.691057 & -1.883188 & -1.779724 \\
\hline $\mathrm{H}$ & 2.594449 & -1.891413 & 0.910800 \\
\hline $\mathrm{H}$ & 1.060363 & -2.842223 & -2.766284 \\
\hline $\mathrm{H}$ & 2.516143 & -3.887940 & -2.514255 \\
\hline C & -0.938190 & -3.129753 & 0.123684 \\
\hline C & -0.643790 & -4.671207 & 0.072634 \\
\hline C & -1.510640 & -2.619191 & 1.439881 \\
\hline $\mathrm{H}$ & -1.666104 & -1.540625 & 1.382876 \\
\hline $\mathrm{H}$ & -2.477685 & -3.084905 & 1.653744 \\
\hline $\mathrm{H}$ & -0.836302 & -2.822288 & 2.272839 \\
\hline C & -1.788640 & -2.643348 & -1.054859 \\
\hline $\mathrm{H}$ & -2.821359 & -2.987986 & -0.975710 \\
\hline $\mathrm{H}$ & -1.814776 & -1.550724 & -1.068310 \\
\hline $\mathrm{H}$ & -1.376175 & -2.995424 & -2.003495 \\
\hline C & -1.777664 & -5.510940 & -0.506240 \\
\hline $\mathrm{H}$ & -2.684512 & -5.418637 & 0.099726 \\
\hline $\mathrm{H}$ & -2.007822 & -5.215005 & -1.530682 \\
\hline $\mathrm{H}$ & -1.481987 & -6.563499 & -0.516709 \\
\hline C & -0.219622 & -5.234584 & 1.436312 \\
\hline & -1.057848 & -5.273385 & 2.138224 \\
\hline
\end{tabular}




\begin{tabular}{|c|c|c|c|}
\hline $\mathrm{H}$ & 0.158383 & -6.249520 & 1.289984 \\
\hline $\mathrm{H}$ & 0.581292 & -4.638811 & 1.879659 \\
\hline 0 & 0.390869 & -2.565662 & -0.029726 \\
\hline 0 & 0.481161 & -4.750815 & -0.819733 \\
\hline B & 1.247528 & -3.578778 & -0.639377 \\
\hline 0 & 2.477755 & -3.805845 & 0.202050 \\
\hline C & 4.426114 & -3.011378 & 1.322719 \\
\hline C & 5.052406 & -4.264645 & 1.285157 \\
\hline C & 5.009254 & -1.963360 & 2.047341 \\
\hline C & 6.251004 & -4.460448 & 1.964425 \\
\hline $\mathrm{H}$ & 4.582458 & -5.068838 & 0.730028 \\
\hline C & 6.209383 & -2.162984 & 2.723686 \\
\hline $\mathrm{H}$ & 4.523243 & -0.991942 & 2.068746 \\
\hline C & 6.833066 & -3.411612 & 2.681733 \\
\hline $\mathrm{H}$ & 6.733286 & -5.432537 & 1.937942 \\
\hline $\mathrm{H}$ & 6.658262 & -1.346753 & 3.280412 \\
\hline $\mathrm{H}$ & 7.768950 & -3.568308 & 3.208816 \\
\hline $\mathrm{H}$ & 2.163179 & -0.934633 & -1.722029 \\
\hline $\mathrm{H}$ & 4.580622 & -2.805626 & -1.369220 \\
\hline $\mathrm{H}$ & 4.434195 & -1.034152 & -0.881659 \\
\hline C & -1.231224 & 1.720957 & 1.945765 \\
\hline C & -2.259702 & 1.481025 & 1.048024 \\
\hline C & -3.370592 & 0.647190 & 1.405746 \\
\hline C & -3.400228 & 0.111010 & 2.679871 \\
\hline $\mathrm{H}$ & -4.240239 & -0.513460 & 2.968037 \\
\hline C & 0.980122 & 1.990184 & 0.830010 \\
\hline C & -0.076483 & 2.567804 & 1.516746 \\
\hline C & 2.034479 & 4.160051 & 0.575539 \\
\hline C & 2.017722 & 2.811637 & 0.293948 \\
\hline $\mathrm{H}$ & 2.826673 & 4.781306 & 0.167359 \\
\hline C & 1.005518 & 4.770036 & 1.334810 \\
\hline C & -0.094497 & 3.971103 & 1.779135 \\
\hline C & -1.160324 & 4.598925 & 2.475848 \\
\hline C & -1.126125 & 5.948251 & 2.740437 \\
\hline C & -0.029985 & 6.736200 & 2.315151 \\
\hline C & 1.009725 & 6.159616 & 1.623815 \\
\hline $\mathrm{H}$ & -2.006318 & 3.998497 & 2.788936 \\
\hline $\mathrm{H}$ & -1.948538 & 6.415732 & 3.272624 \\
\hline $\mathrm{H}$ & -0.018142 & 7.800540 & 2.528916 \\
\hline $\mathrm{H}$ & 1.847474 & 6.760249 & 1.280836 \\
\hline C & -2.373787 & 0.339624 & 3.625387 \\
\hline C & -1.261327 & 1.156707 & 3.256132 \\
\hline C & -0.223561 & 1.371745 & 4.201071 \\
\hline C & -0.284745 & 0.801259 & 5.452235 \\
\hline C & -1.386238 & -0.009072 & 5.818346 \\
\hline C & -2.407602 & -0.232343 & 4.924561 \\
\hline $\mathrm{H}$ & 0.616954 & 1.996757 & 3.920133 \\
\hline $\mathrm{H}$ & 0.514586 & 0.974573 & 6.166091 \\
\hline $\mathrm{H}$ & -1.421494 & -0.453216 & 6.808292 \\
\hline $\mathrm{H}$ & -3.256043 & -0.853577 & 5.197342 \\
\hline C & 4.761494 & 1.275834 & -2.588988 \\
\hline C & 3.409601 & 1.426684 & -2.930590 \\
\hline C & 2.536670 & 1.898424 & -1.946580 \\
\hline C & 2.998818 & 2.239873 & -0.670470 \\
\hline
\end{tabular}




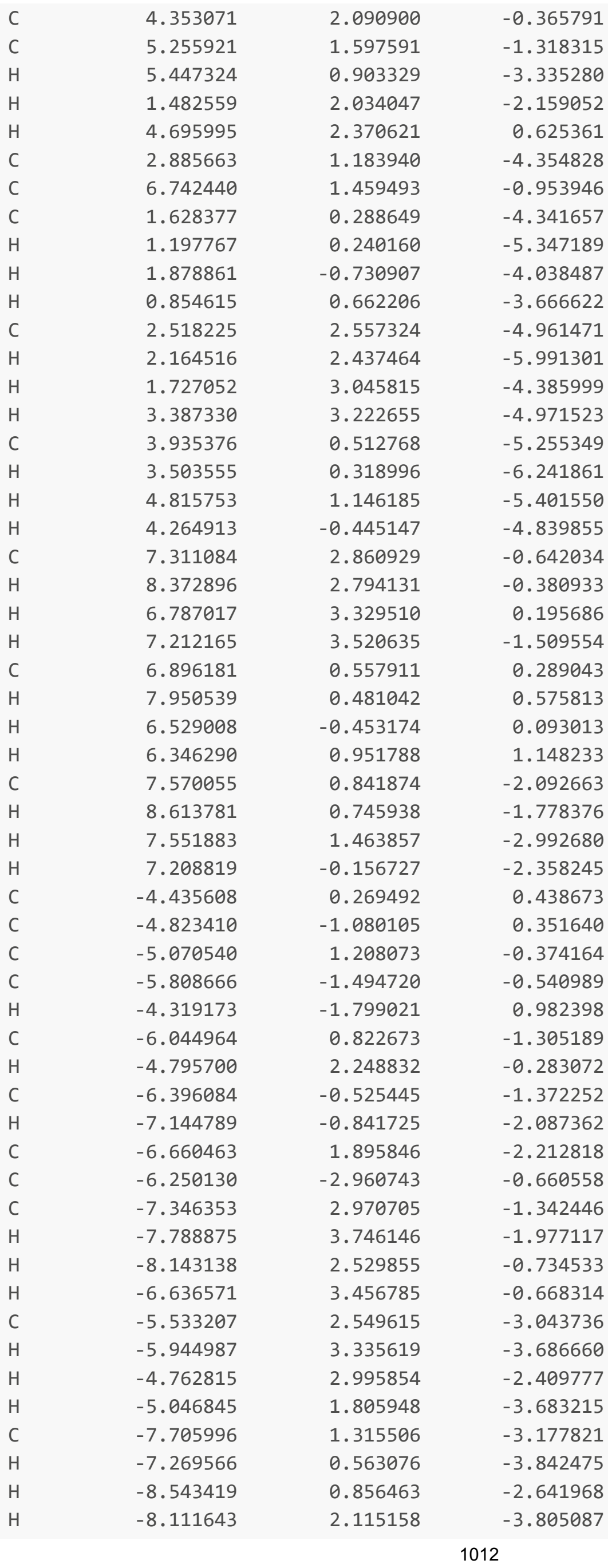




\begin{tabular}{|lrrr|} 
C & -5.890304 & -3.488062 & -2.066418 \\
$H$ & -6.379150 & -2.903128 & -2.850394 \\
$H$ & -4.810603 & -3.437042 & -2.237726 \\
$H$ & -6.205230 & -4.531564 & -2.177147 \\
C & -5.572902 & -3.862166 & 0.385104 \\
$H$ & -4.486493 & -3.883496 & 0.262425 \\
$H$ & -5.795065 & -3.533416 & 1.405257 \\
$H$ & -5.935277 & -4.889131 & 0.277481 \\
C & -7.776932 & -3.057346 & -0.452199 \\
H & -8.106663 & -4.098884 & -0.532498 \\
$H$ & -8.057438 & -2.684346 & 0.537776 \\
$H$ & -8.326673 & -2.475469 & -1.196510 \\
\hline
\end{tabular}

\section{C-Catalyzed reaction TS 26}

\section{Datum}

Value

\begin{tabular}{lc} 
B3LYP-D3(BJ)/6-31G(d,p) Energy & -3039.486074 \\
\hline B3LYP-D3(BJ)/def2-TZVPP/IEF-PCM(DCM) Energy & -3040.523369 \\
\hline B3LYP-D3(BJ)/def2-TZVPP/IEF-PCM(DCM)//B3LYP-D3(BJ)/6-31G(d,p) Free Energy (Quasiharmonic) & -3039.293164 \\
\hline
\end{tabular}

Number of Imaginary Frequencies

Frequencies (Top 3 out of 450)

$\begin{array}{lr}\text { 1. } & -293.8623 \mathrm{~cm}^{-1} \\ \text { 2. } & 5.5455 \mathrm{~cm}^{-1} \\ \text { 3. } & 12.3912 \mathrm{~cm}^{-1}\end{array}$

\section{B3LYP-D3(BJ)/6-31G(d,p) Molecular Geometry in Cartesian Coordinates}

$\begin{array}{lrrr}\mathrm{C} & 0.928840 & 1.530629 & 1.011090 \\ \mathrm{H} & 1.903080 & 1.248829 & 1.400520 \\ \mathrm{H} & 0.383301 & 2.057960 & 1.800510 \\ \mathrm{C} & -2.118390 & 1.140170 & -1.481900 \\ \mathrm{O} & -2.898550 & 1.272261 & -2.399190 \\ \mathrm{O} & -1.062610 & 0.324500 & -1.564910 \\ \mathrm{H} & -0.549800 & 0.304530 & -0.702920 \\ \mathrm{O} & 0.220150 & 0.324710 & 0.684150 \\ \mathrm{H} & 0.739390 & -0.429301 & 1.055480 \\ \mathrm{C} & 2.990399 & -3.877351 & -1.599400 \\ \mathrm{C} & 1.015569 & -3.748271 & -0.808360 \\ \mathrm{C} & 3.543449 & -2.488431 & 0.364420 \\ \mathrm{C} & 3.611339 & -3.704141 & -0.374610 \\ \mathrm{H} & 1.044799 & -4.712171 & -0.298190\end{array}$




\begin{tabular}{|c|c|c|c|}
\hline $\mathrm{H}$ & 3.507809 & -1.567741 & -0.217450 \\
\hline $\mathrm{H}$ & 4.218519 & -2.414702 & 1.214940 \\
\hline C & 1.045869 & -1.962771 & 3.153340 \\
\hline C & 1.665859 & -3.397501 & 3.312410 \\
\hline C & -0.466011 & -2.001060 & 2.904000 \\
\hline H & -1.011091 & -2.278630 & 3.810300 \\
\hline $\mathrm{H}$ & -0.809660 & -1.015200 & 2.582880 \\
\hline $\mathrm{H}$ & -0.713281 & -2.715890 & 2.117830 \\
\hline C & 1.372420 & -0.984081 & 4.274560 \\
\hline $\mathrm{H}$ & 0.911580 & -0.014691 & 4.063090 \\
\hline $\mathrm{H}$ & 0.975820 & -1.343331 & 5.229450 \\
\hline $\mathrm{H}$ & 2.448420 & -0.835341 & 4.376470 \\
\hline C & 0.752029 & -4.404461 & 4.005010 \\
\hline $\mathrm{H}$ & 0.506079 & -4.078030 & 5.020730 \\
\hline $\mathrm{H}$ & -0.174521 & -4.546540 & 3.447330 \\
\hline $\mathrm{H}$ & 1.258588 & -5.371331 & 4.073080 \\
\hline C & 3.035959 & -3.380111 & 4.002360 \\
\hline $\mathrm{H}$ & 2.951989 & -3.159011 & 5.070480 \\
\hline $\mathrm{H}$ & 3.495919 & -4.365461 & 3.889540 \\
\hline $\mathrm{H}$ & 3.699419 & -2.640751 & 3.549390 \\
\hline 0 & 1.698690 & -1.491841 & 1.941550 \\
\hline 0 & 1.858479 & -3.816571 & 1.950550 \\
\hline B & 1.965719 & -2.652901 & 1.132500 \\
\hline 0 & 1.061369 & -2.683051 & -0.058470 \\
\hline C & 0.271499 & -3.660390 & -2.074650 \\
\hline C & -0.040481 & -2.409780 & -2.623830 \\
\hline C & -0.118821 & -4.831130 & -2.739750 \\
\hline C & -0.743941 & -2.330190 & -3.820220 \\
\hline $\mathrm{H}$ & 0.242060 & -1.507560 & -2.100470 \\
\hline C & -0.810721 & -4.747740 & -3.945280 \\
\hline $\mathrm{H}$ & 0.120198 & -5.800350 & -2.309820 \\
\hline C & -1.122471 & -3.497880 & -4.487410 \\
\hline $\mathrm{H}$ & -1.014520 & -1.354320 & -4.209400 \\
\hline $\mathrm{H}$ & -1.114322 & -5.654910 & -4.458230 \\
\hline $\mathrm{H}$ & -1.672921 & -3.435620 & -5.420900 \\
\hline $\mathrm{H}$ & 3.923459 & -4.595191 & 0.168450 \\
\hline $\mathrm{H}$ & 2.838019 & -3.020971 & -2.246510 \\
\hline H & 3.006699 & -4.843261 & -2.093820 \\
\hline C & -1.374399 & 2.912100 & 0.120610 \\
\hline C & -2.285170 & 1.917090 & -0.191730 \\
\hline C & -3.446450 & 1.700371 & 0.617560 \\
\hline C & -3.676479 & 2.547411 & 1.682720 \\
\hline $\mathrm{H}$ & -4.531439 & 2.368771 & 2.327830 \\
\hline C & 1.046511 & 2.423029 & -0.208780 \\
\hline C & -0.105019 & 3.058990 & -0.660050 \\
\hline C & 2.270081 & 3.302359 & -2.112490 \\
\hline C & 2.262141 & 2.537689 & -0.959900 \\
\hline $\mathrm{H}$ & 3.196861 & 3.410729 & -2.668370 \\
\hline C & 1.115611 & 3.963019 & -2.588830 \\
\hline C & -0.104219 & 3.836330 & -1.854940 \\
\hline C & -1.269659 & 4.484410 & -2.344580 \\
\hline C & -1.222429 & 5.231390 & -3.498390 \\
\hline C & -0.010849 & 5.367420 & -4.218490 \\
\hline$\sigma$ & 1.131891 & 4.745889 & -3.773540 \\
\hline
\end{tabular}




\begin{tabular}{|c|c|c|c|}
\hline $\mathrm{H}$ & -2.201479 & 4.366880 & -1.804200 \\
\hline $\mathrm{H}$ & -2.121758 & 5.716050 & -3.864900 \\
\hline $\mathrm{H}$ & 0.010072 & 5.960380 & -5.127750 \\
\hline $\mathrm{H}$ & 2.064361 & 4.839779 & -4.323210 \\
\hline C & -2.807789 & 3.621011 & 1.991760 \\
\hline C & -1.626699 & 3.805560 & 1.208270 \\
\hline C & -0.745369 & 4.868340 & 1.540360 \\
\hline C & -1.019718 & 5.706510 & 2.597660 \\
\hline C & -2.186898 & 5.520960 & 3.375560 \\
\hline C & -3.059169 & 4.499551 & 3.078150 \\
\hline $\mathrm{H}$ & 0.147661 & 5.012650 & 0.942860 \\
\hline $\mathrm{H}$ & -0.337938 & 6.516950 & 2.836490 \\
\hline $\mathrm{H}$ & -2.391608 & 6.188170 & 4.207220 \\
\hline $\mathrm{H}$ & -3.957259 & 4.348941 & 3.670750 \\
\hline C & 5.854740 & 0.488008 & 0.120200 \\
\hline C & 5.416180 & 0.438868 & -1.203980 \\
\hline C & 4.220530 & 1.100148 & -1.522250 \\
\hline C & 3.515760 & 1.839099 & -0.568480 \\
\hline C & 4.029720 & 1.919029 & 0.735690 \\
\hline C & 5.173390 & 1.216978 & 1.108670 \\
\hline $\mathrm{H}$ & 6.749100 & -0.052482 & 0.400730 \\
\hline $\mathrm{H}$ & 3.818680 & 1.043709 & -2.528210 \\
\hline $\mathrm{H}$ & 3.519621 & 2.546129 & 1.454440 \\
\hline C & 6.203090 & -0.278512 & -2.310640 \\
\hline C & 5.660470 & 1.164518 & 2.562740 \\
\hline C & 5.315850 & -1.335142 & -2.999280 \\
\hline $\mathrm{H}$ & 5.857019 & -1.800752 & -3.830010 \\
\hline $\mathrm{H}$ & 5.036259 & -2.121122 & -2.294330 \\
\hline $\mathrm{H}$ & 4.398050 & -0.899442 & -3.403810 \\
\hline C & 6.649860 & 0.772128 & -3.351150 \\
\hline $\mathrm{H}$ & 7.226200 & 0.296028 & -4.151900 \\
\hline $\mathrm{H}$ & 5.791650 & 1.274208 & -3.806490 \\
\hline H & 7.277530 & 1.537958 & -2.884950 \\
\hline C & 7.454160 & -0.989522 & -1.770330 \\
\hline $\mathrm{H}$ & 7.976580 & -1.489713 & -2.591460 \\
\hline $\mathrm{H}$ & 8.155540 & -0.286043 & -1.311240 \\
\hline $\mathrm{H}$ & 7.194479 & -1.751042 & -1.028030 \\
\hline C & 5.455380 & -0.280372 & 3.070810 \\
\hline $\mathrm{H}$ & 5.743640 & -0.366512 & 4.124390 \\
\hline $\mathrm{H}$ & 4.404090 & -0.566352 & 2.970570 \\
\hline $\mathrm{H}$ & 6.054010 & -0.994522 & 2.497270 \\
\hline C & 7.152090 & 1.545348 & 2.650000 \\
\hline $\mathrm{H}$ & 7.497200 & 1.491438 & 3.687980 \\
\hline $\mathrm{H}$ & 7.781430 & 0.876347 & 2.057190 \\
\hline $\mathrm{H}$ & 7.314091 & 2.565798 & 2.288950 \\
\hline C & 4.867071 & 2.109698 & 3.480060 \\
\hline $\mathrm{H}$ & 5.262151 & 2.049938 & 4.498700 \\
\hline $\mathrm{H}$ & 4.944541 & 3.151308 & 3.152780 \\
\hline H & 3.807340 & 1.839849 & 3.522330 \\
\hline C & -4.343570 & 0.541191 & 0.391440 \\
\hline C & -5.732490 & 0.678661 & 0.532620 \\
\hline C & -3.810880 & -0.714309 & 0.092280 \\
\hline C & -6.582640 & -0.415718 & 0.381870 \\
\hline $\mathrm{H}$ & -6.132970 & 1.662851 & 0.739540 \\
\hline
\end{tabular}




\begin{tabular}{|c|c|c|c|}
\hline C & -4.633621 & -1.832119 & -0.087120 \\
\hline $\mathrm{H}$ & -2.738250 & -0.817770 & -0.001390 \\
\hline C & -6.010281 & -1.659999 & 0.071530 \\
\hline $\mathrm{H}$ & -6.666201 & -2.511488 & -0.058130 \\
\hline C & -4.022301 & -3.165679 & -0.537850 \\
\hline C & -8.107330 & -0.304988 & 0.525790 \\
\hline C & -3.739281 & -3.054409 & -2.051330 \\
\hline $\mathrm{H}$ & -4.669501 & -2.897929 & -2.606780 \\
\hline $\mathrm{H}$ & -3.078151 & -2.211579 & -2.262770 \\
\hline $\mathrm{H}$ & -3.259641 & -3.965139 & -2.424390 \\
\hline C & -2.698051 & -3.449240 & 0.200340 \\
\hline $\mathrm{H}$ & -2.300131 & -4.420090 & -0.113240 \\
\hline $\mathrm{H}$ & -1.930951 & -2.704670 & -0.021710 \\
\hline $\mathrm{H}$ & -2.849611 & -3.473809 & 1.284140 \\
\hline C & -4.967731 & -4.353439 & -0.289550 \\
\hline $\mathrm{H}$ & -5.249581 & -4.429519 & 0.765900 \\
\hline $\mathrm{H}$ & -5.883221 & -4.280279 & -0.883730 \\
\hline $\mathrm{H}$ & -4.470722 & -5.285709 & -0.575780 \\
\hline C & -8.559400 & 1.124302 & 0.865840 \\
\hline $\mathrm{H}$ & -8.130650 & 1.469412 & 1.812010 \\
\hline $\mathrm{H}$ & -8.277870 & 1.834712 & 0.082590 \\
\hline $\mathrm{H}$ & -9.649020 & 1.152652 & 0.964500 \\
\hline C & -8.777480 & -0.720008 & -0.801830 \\
\hline $\mathrm{H}$ & -8.451280 & -0.069798 & -1.619260 \\
\hline $\mathrm{H}$ & -8.529431 & -1.749328 & -1.074950 \\
\hline $\mathrm{H}$ & -9.867610 & -0.647867 & -0.718660 \\
\hline C & -8.581830 & -1.243048 & 1.656570 \\
\hline H & -9.669720 & -1.182908 & 1.771600 \\
\hline $\mathrm{H}$ & -8.323561 & -2.285728 & 1.452390 \\
\hline $\mathrm{H}$ & -8.120780 & -0.965088 & 2.609610 \\
\hline
\end{tabular}

\section{C-Catalyzed reaction TS 27}

\section{Datum}

Value

B3LYP-D3(BJ)/6-31G(d,p) Energy

$-3039.486341$

B3LYP-D3(BJ)/def2-TZVPP/IEF-PCM(DCM) Energy

$-3040.522883$

B3LYP-D3(BJ)/def2-TZVPP/IEF-PCM(DCM)//B3LYP-D3(BJ)/6-31G(d,p) Free Energy (Quasiharmonic)

$-3039.292067$

Number of Imaginary Frequencies

Frequencies (Top 3 out of 450)

$\begin{array}{lr}\text { 1. } & -211.5772 \mathrm{~cm}^{-1} \\ \text { 2. } & 6.9666 \mathrm{~cm}^{-1} \\ \text { 3. } & 9.5807 \mathrm{~cm}^{-1}\end{array}$




\begin{tabular}{|c|c|c|c|}
\hline C & 2.065700 & 0.278279 & -0.762210 \\
\hline $\mathrm{H}$ & 1.436350 & 0.488680 & -1.632440 \\
\hline $\mathrm{H}$ & 2.818380 & -0.459261 & -1.054190 \\
\hline C & -0.046780 & 2.536270 & 1.572270 \\
\hline 0 & -0.425709 & 3.462840 & 2.254400 \\
\hline 0 & 0.646330 & 1.515670 & 2.080590 \\
\hline H & 0.843630 & 0.827000 & 1.382040 \\
\hline 0 & 1.249920 & -0.269470 & 0.287320 \\
\hline $\mathrm{H}$ & 0.597800 & -0.878890 & -0.144310 \\
\hline C & -4.181460 & -0.113590 & -2.121490 \\
\hline C & -3.805790 & -1.527190 & -0.507920 \\
\hline C & -1.854640 & -0.690890 & -2.708870 \\
\hline C & -3.264240 & -0.708100 & -2.957760 \\
\hline $\mathrm{H}$ & -4.121590 & -0.756540 & 0.192840 \\
\hline $\mathrm{H}$ & -1.238000 & -0.946060 & -3.570950 \\
\hline $\mathrm{H}$ & -1.496040 & 0.199200 & -2.191770 \\
\hline C & 0.304680 & -3.307190 & -1.071490 \\
\hline C & -0.447260 & -3.972910 & -2.271960 \\
\hline C & 1.822920 & -3.266990 & -1.203070 \\
\hline $\mathrm{H}$ & 2.129840 & -2.747761 & -2.112130 \\
\hline $\mathrm{H}$ & 2.260520 & -2.744631 & -0.350100 \\
\hline $\mathrm{H}$ & 2.233170 & -4.281521 & -1.226920 \\
\hline C & -0.104220 & -3.895700 & 0.283460 \\
\hline $\mathrm{H}$ & 0.269880 & -3.249190 & 1.080070 \\
\hline $\mathrm{H}$ & -1.191430 & -3.952050 & 0.373810 \\
\hline $\mathrm{H}$ & 0.313330 & -4.895600 & 0.431450 \\
\hline C & -0.689060 & -5.471000 & -2.114950 \\
\hline $\mathrm{H}$ & 0.258000 & -6.014200 & -2.033700 \\
\hline $\mathrm{H}$ & -1.222830 & -5.851010 & -2.990850 \\
\hline $\mathrm{H}$ & -1.291790 & -5.684590 & -1.230740 \\
\hline C & 0.225940 & -3.688070 & -3.620590 \\
\hline $\mathrm{H}$ & 0.466280 & -2.626990 & -3.722470 \\
\hline $\mathrm{H}$ & -0.466340 & -3.961990 & -4.421070 \\
\hline $\mathrm{H}$ & 1.146950 & -4.264480 & -3.747360 \\
\hline 0 & -0.204500 & -1.951290 & -1.137480 \\
\hline 0 & -1.715550 & -3.297340 & -2.257610 \\
\hline B & -1.556950 & -2.016860 & -1.636510 \\
\hline 0 & -2.523170 & -1.719430 & -0.508300 \\
\hline C & -4.826860 & -2.567530 & -0.744210 \\
\hline C & -4.565110 & -3.806270 & -1.347080 \\
\hline C & -6.121720 & -2.284330 & -0.276050 \\
\hline C & -5.594930 & -4.733830 & -1.487210 \\
\hline $\mathrm{H}$ & -3.565330 & -4.018830 & -1.706770 \\
\hline C & -7.144070 & -3.215770 & -0.419330 \\
\hline $\mathrm{H}$ & -6.315320 & -1.330080 & 0.205580 \\
\hline C & -6.882040 & -4.445120 & -1.028460 \\
\hline $\mathrm{H}$ & -5.390040 & -5.692200 & -1.954680 \\
\hline $\mathrm{H}$ & -8.139990 & -2.987899 & -0.052580 \\
\hline $\mathrm{H}$ & -7.676610 & -5.176609 & -1.139600 \\
\hline H & -3.631260 & -1.419920 & -3.696590 \\
\hline
\end{tabular}




\begin{tabular}{|c|c|c|c|}
\hline $\mathrm{H}$ & -3.884310 & 0.713850 & -1.490020 \\
\hline $\mathrm{H}$ & -5.244980 & -0.228760 & -2.299300 \\
\hline C & 0.652760 & 2.778960 & -0.829960 \\
\hline C & -0.355750 & 2.528830 & 0.083890 \\
\hline C & -1.726550 & 2.469450 & -0.333040 \\
\hline C & -2.033910 & 2.765800 & -1.644970 \\
\hline $\mathrm{H}$ & -3.064020 & 2.711970 & -1.982250 \\
\hline C & 2.750650 & 1.547119 & -0.312690 \\
\hline C & 2.081870 & 2.759849 & -0.392740 \\
\hline C & 4.756390 & 2.683379 & 0.443100 \\
\hline C & 4.094960 & 1.504219 & 0.169210 \\
\hline $\mathrm{H}$ & 5.780520 & 2.647629 & 0.803630 \\
\hline C & 4.114661 & 3.939779 & 0.327140 \\
\hline C & 2.739251 & 3.982349 & -0.063660 \\
\hline C & 2.080401 & 5.239339 & -0.119330 \\
\hline C & 2.757341 & 6.399329 & 0.177270 \\
\hline C & 4.122911 & 6.359159 & 0.548430 \\
\hline C & 4.784051 & 5.155849 & 0.625350 \\
\hline $\mathrm{H}$ & 1.030871 & 5.269210 & -0.388410 \\
\hline $\mathrm{H}$ & 2.240531 & 7.352879 & 0.134460 \\
\hline $\mathrm{H}$ & 4.643881 & 7.282809 & 0.781250 \\
\hline $\mathrm{H}$ & 5.828141 & 5.115569 & 0.922900 \\
\hline C & -1.034200 & 3.071890 & -2.600220 \\
\hline C & 0.336980 & 3.051830 & -2.198830 \\
\hline C & 1.337651 & 3.307050 & -3.172330 \\
\hline C & 0.995701 & 3.569710 & -4.480200 \\
\hline C & -0.361899 & 3.588070 & -4.877070 \\
\hline C & -1.354009 & 3.342810 & -3.955660 \\
\hline $\mathrm{H}$ & 2.377901 & 3.295019 & -2.867710 \\
\hline $\mathrm{H}$ & 1.772281 & 3.763910 & -5.213440 \\
\hline $\mathrm{H}$ & -0.617719 & 3.794470 & -5.911730 \\
\hline $\mathrm{H}$ & -2.399079 & 3.347570 & -4.252540 \\
\hline C & 6.001310 & -2.192491 & 1.101760 \\
\hline C & 4.829990 & -1.842221 & 1.776070 \\
\hline C & 4. 210070 & -0.635461 & 1.425560 \\
\hline C & 4.757460 & 0.203079 & 0.453860 \\
\hline C & 5.943310 & -0.170341 & -0.193450 \\
\hline C & 6.575740 & -1.376241 & 0.112360 \\
\hline $\mathrm{H}$ & 6.493770 & -3.123911 & 1.350660 \\
\hline $\mathrm{H}$ & 3.297950 & -0.323141 & 1.919280 \\
\hline $\mathrm{H}$ & 6.348180 & 0.495559 & -0.945610 \\
\hline C & 4.224820 & -2.694421 & 2.900920 \\
\hline C & 7.861650 & -1.837861 & -0.588260 \\
\hline C & 5.021860 & -3.983831 & 3.152920 \\
\hline $\mathrm{H}$ & 4.546570 & -4.560261 & 3.952550 \\
\hline $\mathrm{H}$ & 5.056680 & -4.618411 & 2.261440 \\
\hline $\mathrm{H}$ & 6.049800 & -3.771331 & 3.463330 \\
\hline C & 4.216150 & -1.864441 & 4.203550 \\
\hline $\mathrm{H}$ & 3.800570 & -2.454611 & 5.027610 \\
\hline $\mathrm{H}$ & 5.231520 & -1.560801 & 4.476990 \\
\hline $\mathrm{H}$ & 3.611570 & -0.959691 & 4.100210 \\
\hline C & 2.777100 & -3.082901 & 2.535270 \\
\hline $\mathrm{H}$ & 2.303690 & -3.618171 & 3.365660 \\
\hline $\mathrm{H}$ & 2.167470 & -2.205641 & 2.304120 \\
\hline
\end{tabular}




\begin{tabular}{|c|c|c|c|}
\hline $\mathrm{H}$ & 2.764900 & -3.738921 & 1.660300 \\
\hline C & 7.595680 & -3.178641 & -1.306440 \\
\hline $\mathrm{H}$ & 8.502360 & -3.527901 & -1.812570 \\
\hline $\mathrm{H}$ & 7.281890 & -3.956941 & -0.605400 \\
\hline $\mathrm{H}$ & 6.806440 & -3.066061 & -2.056420 \\
\hline C & 8.353880 & -0.822451 & -1.632040 \\
\hline $\mathrm{H}$ & 8.581440 & 0.146679 & -1.177340 \\
\hline $\mathrm{H}$ & 7.614350 & -0.664371 & -2.423150 \\
\hline $\mathrm{H}$ & 9.270110 & -1.192161 & -2.102610 \\
\hline C & 8.977620 & -2.030011 & 0.461260 \\
\hline $\mathrm{H}$ & 9.902620 & -2.363231 & -0.021940 \\
\hline $\mathrm{H}$ & 9.183470 & -1.090861 & 0.984120 \\
\hline $\mathrm{H}$ & 8.704170 & -2.776931 & 1.211440 \\
\hline C & -2.774110 & 1.975800 & 0.593010 \\
\hline C & -2.480010 & 0.900280 & 1.439380 \\
\hline C & -4.073840 & 2.497840 & 0.581940 \\
\hline C & -3.436710 & 0.364190 & 2.301690 \\
\hline $\mathrm{H}$ & -1.494710 & 0.465840 & 1.401260 \\
\hline C & -5.071400 & 1.963190 & 1.403100 \\
\hline $\mathrm{H}$ & -4.287779 & 3.338800 & -0.065270 \\
\hline C & -4.726480 & 0.908870 & 2.261620 \\
\hline $\mathrm{H}$ & -5.485770 & 0.502070 & 2.920800 \\
\hline C & -6.513650 & 2.489860 & 1.397650 \\
\hline C & -3.094980 & -0.769220 & 3.279410 \\
\hline C & -6.882080 & 2.997690 & 2.808000 \\
\hline $\mathrm{H}$ & -7.909779 & 3.376561 & 2.820740 \\
\hline $\mathrm{H}$ & -6.808290 & 2.204140 & 3.556500 \\
\hline $\mathrm{H}$ & -6.213389 & 3.807910 & 3.113390 \\
\hline C & -6.709939 & 3.644350 & 0.402040 \\
\hline $\mathrm{H}$ & -7.751149 & 3.979791 & 0.427370 \\
\hline $\mathrm{H}$ & -6.078119 & 4.502680 & 0.649130 \\
\hline $\mathrm{H}$ & -6.485059 & 3.336630 & -0.624210 \\
\hline C & -7.467240 & 1.342530 & 0.997860 \\
\hline $\mathrm{H}$ & -7.228090 & 0.972080 & -0.004820 \\
\hline $\mathrm{H}$ & -7.401580 & 0.501470 & 1.694190 \\
\hline $\mathrm{H}$ & -8.505790 & 1.690471 & 0.991520 \\
\hline C & -4.081750 & -1.944070 & 3.114020 \\
\hline $\mathrm{H}$ & -5.121200 & -1.633980 & 3.254450 \\
\hline $\mathrm{H}$ & -3.996880 & -2.397460 & 2.122710 \\
\hline $\mathrm{H}$ & -3.865820 & -2.720420 & 3.855410 \\
\hline C & -1.668530 & -1.305100 & 3.060500 \\
\hline $\mathrm{H}$ & -1.544470 & -1.698200 & 2.047240 \\
\hline $\mathrm{H}$ & -0.913130 & -0.531580 & 3.226630 \\
\hline $\mathrm{H}$ & -1.472940 & -2.119950 & 3.764540 \\
\hline C & -3.198620 & -0.209410 & 4.714980 \\
\hline $\mathrm{H}$ & -2.947590 & -0.986000 & 5.445650 \\
\hline $\mathrm{H}$ & -2.509190 & 0.628740 & 4.851970 \\
\hline $\mathrm{H}$ & -4.209910 & 0.146830 & 4.934060 \\
\hline
\end{tabular}


Frequencies (Top 3 out of 450)

$\begin{array}{lr}\text { 1. } & -196.4939 \mathrm{~cm}^{-1} \\ \text { 2. } & 8.5995 \mathrm{~cm}^{-1} \\ \text { 3. } & 12.1460 \mathrm{~cm}^{-1}\end{array}$

\section{B3LYP-D3(BJ)/6-31G(d,p) Molecular Geometry in Cartesian Coordinates}

\begin{tabular}{|c|c|c|c|}
\hline C & -2.048530 & 0.256530 & 0.808070 \\
\hline $\mathrm{H}$ & -1.434650 & 0.516080 & 1.676490 \\
\hline $\mathrm{H}$ & -2.771950 & -0.506180 & 1.113560 \\
\hline C & -0.069550 & 2.516570 & -1.618910 \\
\hline 0 & 0.274730 & 3.440470 & -2.322740 \\
\hline 0 & -0.744910 & 1.470440 & -2.097220 \\
\hline $\mathrm{H}$ & -0.897510 & 0.789970 & -1.381870 \\
\hline 0 & -1.204830 & -0.276970 & -0.223790 \\
\hline $\mathrm{H}$ & -0.525730 & -0.854390 & 0.209430 \\
\hline C & 4.262600 & 0.139420 & 2.163710 \\
\hline C & 3.951680 & -1.331770 & 0.571960 \\
\hline C & 1.975640 & -0.566390 & 2.773400 \\
\hline C & 3.388890 & -0.499090 & 3.011270 \\
\hline $\mathrm{H}$ & 4.242410 & -0.570610 & -0.150120 \\
\hline $\mathrm{H}$ & 1.382790 & -0.841570 & 3.646350 \\
\hline $\mathrm{H}$ & 1.566840 & 0.303680 & 2.258670 \\
\hline C & -0.286510 & -3.109180 & 1.568070 \\
\hline C & 0.925360 & -4.064100 & 1.822590 \\
\hline C & -1.068690 & -2.800780 & 2.849410 \\
\hline $\mathrm{H}$ & -1.745980 & -1.963460 & 2.666820 \\
\hline $\mathrm{H}$ & -1.667820 & -3.658290 & 3.168480 \\
\hline $\mathrm{H}$ & -0.393750 & -2.527040 & 3.663340 \\
\hline C & -1.237350 & -3.556070 & 0.464470 \\
\hline $\mathrm{H}$ & -2.054020 & -2.838920 & 0.350210 \\
\hline $\mathrm{H}$ & -0.724370 & -3.636390 & -0.494820 \\
\hline $\mathrm{H}$ & -1.673900 & -4.529420 & 0.709790 \\
\hline C & 1.474830 & -4.671280 & 0.523580 \\
\hline $\mathrm{H}$ & 1.635480 & -3.903510 & -0.236440 \\
\hline $\mathrm{H}$ & 2.436620 & -5.147670 & 0.730940 \\
\hline $\mathrm{H}$ & 0.799250 & -5.429120 & 0.116780 \\
\hline C & 0.670020 & -5.162040 & 2.848360 \\
\hline & -0.150230 & -5.812700 & 2.528450 \\
\hline
\end{tabular}




\begin{tabular}{|c|c|c|c|}
\hline $\mathrm{H}$ & 1.566390 & -5.779120 & 2.958700 \\
\hline $\mathrm{H}$ & 0.426340 & -4.742370 & 3.825420 \\
\hline 0 & 0.385370 & -1.897820 & 1.145810 \\
\hline 0 & 1.900570 & -3.152750 & 2.362230 \\
\hline B & 1.721010 & -1.894500 & 1.705180 \\
\hline 0 & 2.679310 & -1.566710 & 0.574750 \\
\hline C & 5.016040 & -2.317960 & 0.851030 \\
\hline C & 4.852300 & -3.468270 & 1.637190 \\
\hline C & 6.258900 & -2.064580 & 0.246870 \\
\hline C & 5.922820 & -4.341390 & 1.810540 \\
\hline $\mathrm{H}$ & 3.893440 & -3.656670 & 2.105410 \\
\hline C & 7.323530 & -2.943030 & 0.423080 \\
\hline $\mathrm{H}$ & 6.379360 & -1.177860 & -0.368100 \\
\hline C & 7.157140 & -4.085470 & 1.207550 \\
\hline $\mathrm{H}$ & 5.793390 & -5.230410 & 2.420370 \\
\hline $\mathrm{H}$ & 8.277760 & -2.739430 & -0.052550 \\
\hline $\mathrm{H}$ & 7.984530 & -4.774400 & 1.347190 \\
\hline $\mathrm{H}$ & 3.803710 & -1.174060 & 3.759390 \\
\hline $\mathrm{H}$ & 3.910860 & 0.929410 & 1.512600 \\
\hline $\mathrm{H}$ & 5.332780 & 0.089580 & 2.331820 \\
\hline C & -0.733870 & 2.803340 & 0.791100 \\
\hline C & 0.261850 & 2.549560 & -0.135010 \\
\hline C & 1.640740 & 2.526160 & 0.256340 \\
\hline C & 1.970780 & 2.881500 & 1.547240 \\
\hline $\mathrm{H}$ & 3.009060 & 2.865210 & 1.862860 \\
\hline C & -2.789990 & 1.484040 & 0.329960 \\
\hline C & -2.168640 & 2.723880 & 0.380270 \\
\hline C & -4.840410 & 2.527470 & -0.438450 \\
\hline C & -4.134570 & 1.378060 & -0.144620 \\
\hline $\mathrm{H}$ & -5.861950 & 2.445390 & -0.798430 \\
\hline C & -4.248920 & 3.809750 & -0.348590 \\
\hline C & -2.874360 & 3.913510 & 0.032540 \\
\hline C & -2.264010 & 5.195650 & 0.058300 \\
\hline C & -2.987320 & 6.322430 & -0.256400 \\
\hline C & -4.352530 & 6.221960 & -0.617620 \\
\hline C & -4.966870 & 4.992730 & -0.666750 \\
\hline $\mathrm{H}$ & -1.214870 & 5.271290 & 0.319690 \\
\hline $\mathrm{H}$ & -2.507730 & 7.295950 & -0.235970 \\
\hline $\mathrm{H}$ & -4.910260 & 7.119930 & -0.865260 \\
\hline $\mathrm{H}$ & -6.010150 & 4.906060 & -0.957140 \\
\hline C & 0.983350 & 3.201300 & 2.511770 \\
\hline C & -0.395710 & 3.129650 & 2.142730 \\
\hline C & -1.381660 & 3.391470 & 3.129280 \\
\hline C & -1.018970 & 3.711650 & 4.418670 \\
\hline C & 0.345880 & 3.783130 & 4.783060 \\
\hline C & 1.324340 & 3.531550 & 3.848670 \\
\hline $\mathrm{H}$ & -2.427550 & 3.339520 & 2.848790 \\
\hline $\mathrm{H}$ & -1.784560 & 3.910670 & 5.162100 \\
\hline $\mathrm{H}$ & 0.618350 & 4.034860 & 5.803330 \\
\hline $\mathrm{H}$ & 2.375000 & 3.575790 & 4.121610 \\
\hline C & -5.949610 & -2.372870 & -1.047190 \\
\hline C & -4.707150 & -2.055220 & -1.623050 \\
\hline C & -4.121440 & -0.837410 & -1.285790 \\
\hline C & -4.756890 & 0.053450 & -0.405370 \\
\hline
\end{tabular}




\begin{tabular}{|c|c|c|c|}
\hline C & -5.990360 & -0.291620 & 0.144700 \\
\hline C & -6.606080 & -1.514810 & -0.163090 \\
\hline $\mathrm{H}$ & -6.413030 & -3.315410 & -1.308440 \\
\hline $\mathrm{H}$ & -3.170640 & -0.546320 & -1.708130 \\
\hline $\mathrm{H}$ & -6.466050 & 0.404390 & 0.828250 \\
\hline C & -4.062600 & -3.017480 & -2.631370 \\
\hline C & -7.959240 & -1.859500 & 0.475510 \\
\hline C & -3.935190 & -4.424940 & -2.011180 \\
\hline $\mathrm{H}$ & -3.471430 & -5.112110 & -2.726990 \\
\hline $\mathrm{H}$ & -3.316570 & -4.401630 & -1.110840 \\
\hline $\mathrm{H}$ & -4.909420 & -4.839930 & -1.738100 \\
\hline C & -4.962180 & -3.097190 & -3.884200 \\
\hline $\mathrm{H}$ & -4.526340 & -3.775770 & -4.625840 \\
\hline $\mathrm{H}$ & -5.962350 & -3.465000 & -3.636400 \\
\hline $\mathrm{H}$ & -5.071730 & -2.110810 & -4.345090 \\
\hline C & -2.663250 & -2.546550 & -3.066260 \\
\hline $\mathrm{H}$ & -2.218640 & -3.287150 & -3.738430 \\
\hline $\mathrm{H}$ & -2.706550 & -1.595950 & -3.606050 \\
\hline $\mathrm{H}$ & -1.992660 & -2.415140 & -2.213110 \\
\hline C & -8.996210 & -0.784010 & 0.085940 \\
\hline $\mathrm{H}$ & -9.968880 & -1.010230 & 0.536320 \\
\hline $\mathrm{H}$ & -8.693870 & 0.210620 & 0.424760 \\
\hline $\mathrm{H}$ & -9.122730 & -0.743460 & -1.000400 \\
\hline C & -8.491460 & -3.227580 & 0.019700 \\
\hline $\mathrm{H}$ & -9.454160 & -3.426870 & 0.500380 \\
\hline $\mathrm{H}$ & -8.647690 & -3.260990 & -1.063050 \\
\hline $\mathrm{H}$ & -7.809530 & -4.038890 & 0.293040 \\
\hline C & -7.803020 & -1.889230 & 2.011310 \\
\hline $\mathrm{H}$ & -8.759780 & -2.128310 & 2.488450 \\
\hline $\mathrm{H}$ & -7.071990 & -2.646520 & 2.311650 \\
\hline $\mathrm{H}$ & -7.465250 & -0.925260 & 2.401430 \\
\hline C & 2.663240 & 1.992740 & -0.675210 \\
\hline C & 2.347940 & 0.855570 & -1.427770 \\
\hline C & 3.949910 & 2.536610 & -0.767490 \\
\hline C & 3.266550 & 0.276020 & -2.301460 \\
\hline $\mathrm{H}$ & 1.376520 & 0.407710 & -1.302310 \\
\hline C & 4.910660 & 1.967660 & -1.610510 \\
\hline $\mathrm{H}$ & 4.179700 & 3.423000 & -0.189840 \\
\hline C & 4.542900 & 0.849390 & -2.374460 \\
\hline $\mathrm{H}$ & 5.273580 & 0.413540 & -3.047550 \\
\hline C & 6.333910 & 2.531020 & -1.734940 \\
\hline C & 2.897180 & -0.937570 & -3.166740 \\
\hline C & 6.585990 & 2.966450 & -3.194370 \\
\hline $\mathrm{H}$ & 7.597720 & 3.372210 & -3.302600 \\
\hline $\mathrm{H}$ & 6.484270 & 2.128710 & -3.889580 \\
\hline $\mathrm{H}$ & 5.871850 & 3.738540 & -3.495520 \\
\hline C & 6.562670 & 3.746070 & -0.821640 \\
\hline $\mathrm{H}$ & 7.589980 & 4.104950 & -0.936350 \\
\hline $\mathrm{H}$ & 5.892060 & 4.572980 & -1.073360 \\
\hline $\mathrm{H}$ & 6.414850 & 3.493050 & 0.233190 \\
\hline C & 7.350410 & 1.437110 & -1.341690 \\
\hline $\mathrm{H}$ & 7.194400 & 1.116940 & -0.305960 \\
\hline H & 7.266240 & 0.556660 & -1.985410 \\
\hline $\mathrm{H}$ & 8.374480 & 1.815790 & -1.428240 \\
\hline
\end{tabular}




\begin{tabular}{|llll|} 
C & 3.895670 & -2.090810 & -2.933220 \\
$H$ & 4.929410 & -1.786100 & -3.120960 \\
$H$ & 3.834320 & -2.464650 & -1.907980 \\
$H$ & 3.668860 & -2.924140 & -3.606500 \\
C & 1.480450 & -1.455990 & -2.856050 \\
$H$ & 1.386890 & -1.761010 & -1.809280 \\
$H$ & 0.717560 & -0.700150 & -3.063910 \\
$H$ & 1.265780 & -2.327260 & -3.482840 \\
C & 2.948820 & -0.505180 & -4.648360 \\
H & 2.675180 & -1.342890 & -5.298960 \\
$H$ & 2.251820 & 0.317220 & -4.833900 \\
$H$ & 3.950290 & -0.169250 & -4.934300 \\
\hline
\end{tabular}

\section{C-Catalyzed reaction TS 29}

\section{Datum}

Value

B3LYP-D3(BJ)/6-31G(d,p) Energy

$-3039.48308$

B3LYP-D3(BJ)/def2-TZVPP/IEF-PCM(DCM) Energy

$-3040.522075$

B3LYP-D3(BJ)/def2-TZVPP/IEF-PCM(DCM)//B3LYP-D3(BJ)/6-31G(d,p) Free Energy (Quasiharmonic)

$-3039.291572$

Number of Imaginary Frequencies

Frequencies (Top 3 out of 450)

$\begin{array}{lr}\text { 1. } & -237.3357 \mathrm{~cm}^{-1} \\ \text { 2. } & 10.1237 \mathrm{~cm}^{-1} \\ \text { 3. } & 12.7291 \mathrm{~cm}^{-1}\end{array}$

B3LYP-D3(BJ)/6-31G(d,p) Molecular Geometry in Cartesian Coordinates

\begin{tabular}{|c|c|c|c|}
\hline C & -0.584840 & 0.904200 & 0.561540 \\
\hline $\mathrm{H}$ & -0.035710 & 0.770140 & 1.498480 \\
\hline $\mathrm{H}$ & -1.590220 & 0.509630 & 0.698740 \\
\hline C & 2.740250 & 1.886280 & -1.363530 \\
\hline 0 & 3.666490 & 2.470830 & -1.882300 \\
\hline 0 & 1.719170 & 1.396740 & -2.067240 \\
\hline $\mathrm{H}$ & 1.067890 & 0.931830 & -1.465500 \\
\hline 0 & 0.057490 & 0.169640 & -0.489690 \\
\hline $\mathrm{H}$ & 0.268570 & -0.723250 & -0.123090 \\
\hline C & -2.697600 & -2.604570 & -2.570790 \\
\hline C & -3.135100 & -1.897180 & -0.612040 \\
\hline C & -0.721680 & -3.558260 & -1.467490 \\
\hline C & -2.008430 & -3.690010 & -2.067890 \\
\hline $\mathrm{H}$ & -3.403570 & -0.924870 & -1.031930 \\
\hline
\end{tabular}




\begin{tabular}{|c|c|c|c|}
\hline $\mathrm{H}$ & -0.184040 & -4.491270 & -1.301690 \\
\hline $\mathrm{H}$ & -0.093490 & -2.799070 & -1.934030 \\
\hline C & 0.832890 & -2.805240 & 1.749140 \\
\hline C & -0.419150 & -3.558850 & 2.325060 \\
\hline $\mathrm{C}$ & 1.975460 & -3.745090 & 1.356370 \\
\hline $\mathrm{H}$ & 2.726180 & -3.177260 & 0.802180 \\
\hline $\mathrm{H}$ & 2.456140 & -4.182190 & 2.236170 \\
\hline $\mathrm{H}$ & 1.614460 & -4.554500 & 0.717030 \\
\hline C & 1.370570 & -1.675960 & 2.621770 \\
\hline $\mathrm{H}$ & 2.193330 & -1.172220 & 2.109140 \\
\hline $\mathrm{H}$ & 0.605620 & -0.930640 & 2.842320 \\
\hline H & 1.752990 & -2.067380 & 3.569870 \\
\hline C & -1.340550 & -2.634180 & 3.133710 \\
\hline $\mathrm{H}$ & -0.888780 & -2.349440 & 4.088050 \\
\hline $\mathrm{H}$ & -1.580650 & -1.726230 & 2.576710 \\
\hline $\mathrm{H}$ & -2.277420 & -3.157910 & 3.342500 \\
\hline C & -0.093080 & -4.809430 & 3.132310 \\
\hline $\mathrm{H}$ & 0.519990 & -4.561900 & 4.004810 \\
\hline $\mathrm{H}$ & -1.019150 & -5.269710 & 3.488500 \\
\hline $\mathrm{H}$ & 0.438670 & -5.543500 & 2.525350 \\
\hline 0 & 0.298310 & -2.235830 & 0.532430 \\
\hline 0 & -1.114890 & -3.962800 & 1.126030 \\
\hline B & -0.903330 & -2.940470 & 0.156040 \\
\hline 0 & -1.995990 & -1.897140 & 0.009060 \\
\hline C & -4.293910 & -2.724820 & -0.199880 \\
\hline C & -5.543870 & -2.418520 & -0.758320 \\
\hline C & -4.192050 & -3.765240 & 0.734220 \\
\hline C & -6.677960 & -3.135870 & -0.393430 \\
\hline $\mathrm{H}$ & -5.618810 & -1.606250 & -1.472130 \\
\hline C & -5.333450 & -4.477040 & 1.100370 \\
\hline $\mathrm{H}$ & -3.223230 & -4.010740 & 1.154420 \\
\hline C & -6.575310 & -4.167410 & 0.543260 \\
\hline $\mathrm{H}$ & -7.639950 & -2.887390 & -0.830990 \\
\hline $\mathrm{H}$ & -5.251540 & -5.278540 & 1.828100 \\
\hline $\mathrm{H}$ & -7.459150 & -4.725260 & 0.836560 \\
\hline H & -2.560180 & -4.616160 & -1.911590 \\
\hline $\mathrm{H}$ & -2.147350 & -1.718170 & -2.873520 \\
\hline $\mathrm{H}$ & -3.686620 & -2.729060 & -2.996850 \\
\hline C & 1.779000 & 2.416780 & 0.894800 \\
\hline C & 2.705790 & 1.716870 & 0.143970 \\
\hline C & 3.740590 & 0.955520 & 0.776760 \\
\hline C & 3.859180 & 1.001290 & 2.149530 \\
\hline $\mathrm{H}$ & 4.620410 & 0.400090 & 2.638350 \\
\hline C & -0.594380 & 2.362130 & 0.174200 \\
\hline C & 0.598360 & 3.071290 & 0.246960 \\
\hline C & -1.667910 & 4.249980 & -0.904730 \\
\hline C & -1.755540 & 2.968960 & -0.395270 \\
\hline $\mathrm{H}$ & -2.553590 & 4.716990 & -1.326330 \\
\hline C & -0.460720 & 4.987170 & -0.866930 \\
\hline C & 0.697320 & 4.394700 & -0.270430 \\
\hline C & 1.907060 & 5.137910 & -0.231030 \\
\hline C & 1.963550 & 6.409950 & -0.750610 \\
\hline C & 0.815460 & 7.000970 & -1.331400 \\
\hline C & -0.368770 & 6.304520 & -1.388550 \\
\hline
\end{tabular}




\begin{tabular}{|c|c|c|c|}
\hline $\mathrm{H}$ & 2.791270 & 4.676800 & 0.193480 \\
\hline $\mathrm{H}$ & 2.896560 & 6.963740 & -0.723110 \\
\hline $\mathrm{H}$ & 0.876200 & 8.005910 & -1.737810 \\
\hline H & -1.252380 & 6.750030 & -1.837060 \\
\hline $\mathrm{C}$ & 2.973500 & 1.763340 & 2.950240 \\
\hline C & 1.900640 & 2.468810 & 2.320600 \\
\hline C & 0.989660 & 3.191540 & 3.135440 \\
\hline C & 1.131490 & 3.212370 & 4.505030 \\
\hline C & 2.190070 & 2.510230 & 5.127130 \\
\hline C & 3.089590 & 1.801830 & 4.364050 \\
\hline $\mathrm{H}$ & 0.179330 & 3.732240 & 2.660010 \\
\hline H & 0.427740 & 3.772630 & 5.112940 \\
\hline $\mathrm{H}$ & 2.290350 & 2.533010 & 6.207920 \\
\hline $\mathrm{H}$ & 3.904620 & 1.257400 & 4.832840 \\
\hline C & -5.565710 & 1.027760 & -0.423630 \\
\hline C & -4.888120 & 1.176490 & 0.794650 \\
\hline C & -3.644090 & 1.811860 & 0.776580 \\
\hline C & -3.066940 & 2.266300 & -0.415310 \\
\hline C & -3.763850 & 2.084650 & -1.613000 \\
\hline C & -5.025940 & 1.475850 & -1.636440 \\
\hline $\mathrm{H}$ & -6.535450 & 0.552710 & -0.423680 \\
\hline $\mathrm{H}$ & -3.106470 & 1.976730 & 1.704360 \\
\hline $\mathrm{H}$ & -3.301710 & 2.427710 & -2.532580 \\
\hline C & -5.462150 & 0.686680 & 2.132520 \\
\hline C & -5.762170 & 1.321010 & -2.976300 \\
\hline C & -6.823860 & -0.007240 & 1.968950 \\
\hline $\mathrm{H}$ & -7.186240 & -0.335080 & 2.948080 \\
\hline H & -7.575240 & 0.669370 & 1.549110 \\
\hline $\mathrm{H}$ & -6.754020 & -0.890520 & 1.328810 \\
\hline C & -4.479550 & -0.320480 & 2.770040 \\
\hline $\mathrm{H}$ & -4.865960 & -0.667960 & 3.734270 \\
\hline $\mathrm{H}$ & -4.340170 & -1.192650 & 2.126800 \\
\hline $\mathrm{H}$ & -3.497950 & 0.127580 & 2.947310 \\
\hline C & -5.642960 & 1.894700 & 3.077020 \\
\hline $\mathrm{H}$ & -6.048800 & 1.566410 & 4.039970 \\
\hline H & -4.694760 & 2.404080 & 3.269590 \\
\hline $\mathrm{H}$ & -6.334130 & 2.625470 & 2.645440 \\
\hline C & -4.944240 & 0.395820 & -3.903350 \\
\hline $\mathrm{H}$ & -5.434050 & 0.297330 & -4.878000 \\
\hline $\mathrm{H}$ & -3.935490 & 0.783040 & -4.070010 \\
\hline $\mathrm{H}$ & -4.850080 & -0.605760 & -3.471710 \\
\hline C & -7.170540 & 0.725850 & -2.811010 \\
\hline $\mathrm{H}$ & -7.658140 & 0.660060 & -3.788250 \\
\hline $\mathrm{H}$ & -7.147710 & -0.283900 & -2.389470 \\
\hline $\mathrm{H}$ & -7.797620 & 1.348290 & -2.165600 \\
\hline C & -5.909340 & 2.706770 & -3.641980 \\
\hline $\mathrm{H}$ & -6.439700 & 2.615960 & -4.595880 \\
\hline $\mathrm{H}$ & -6.475840 & 3.386770 & -2.998410 \\
\hline $\mathrm{H}$ & -4.938380 & 3.166330 & -3.842880 \\
\hline C & 4.587820 & 0.035270 & -0.018350 \\
\hline C & 3.991390 & -0.788980 & -0.972810 \\
\hline C & 5.970020 & -0.056070 & 0.199120 \\
\hline C & 4.747760 & -1.676330 & -1.747730 \\
\hline $\mathrm{H}$ & 2.920210 & -0.728080 & -1.117790 \\
\hline
\end{tabular}




$\begin{array}{lrrr}\text { C } & 6.752720 & -0.945910 & -0.535210 \\ \text { H } & 6.419300 & 0.602520 & 0.931700 \\ \mathrm{C} & 6.120410 & -1.741590 & -1.506980 \\ \mathrm{H} & 6.726980 & -2.420910 & -2.092310 \\ \mathrm{C} & 8.269890 & -1.070790 & -0.335490 \\ \mathrm{C} & 4.041870 & -2.499780 & -2.833230 \\ \mathrm{C} & 8.792400 & -0.133540 & 0.765120 \\ \mathrm{H} & 9.873240 & -0.263560 & 0.877340 \\ \mathrm{H} & 8.607370 & 0.917320 & 0.522750 \\ \mathrm{H} & 8.329110 & -0.349150 & 1.733170 \\ \mathrm{C} & 8.613600 & -2.522250 & 0.063370 \\ \mathrm{H} & 9.694150 & -2.633180 & 0.206010 \\ \mathrm{H} & 8.115010 & -2.795830 & 0.998680 \\ \mathrm{H} & 8.301880 & -3.237280 & -0.702880 \\ \mathrm{C} & 8.989710 & -0.715300 & -1.654330 \\ \mathrm{H} & 8.693070 & -1.381540 & -2.468980 \\ \mathrm{H} & 8.755360 & 0.309250 & -1.958610 \\ \mathrm{H} & 10.075210 & -0.798260 & -1.530980 \\ \mathrm{C} & 3.389530 & -1.529310 & -3.843710 \\ \mathrm{H} & 2.671970 & -0.857500 & -3.366040 \\ \mathrm{H} & 4.149330 & -0.904320 & -4.322530 \\ \mathrm{H} & 2.865830 & -2.092430 & -4.624620 \\ \mathrm{C} & 5.011240 & -3.417040 & -3.594510 \\ \mathrm{H} & 5.791460 & -2.844510 & -4.105650 \\ \mathrm{H} & 5.495020 & -4.138450 & -2.927840 \\ \mathrm{H} & 4.463310 & -3.982400 & -4.354750 \\ \mathrm{C} & 2.950520 & -3.377480 & -2.186040 \\ \mathrm{H} & 2.399380 & -3.929190 & -2.955860 \\ \mathrm{H} & 3.392900 & -4.104420 & -1.497370 \\ \mathrm{H} & 2.231910 & -2.782120 & -1.618550\end{array}$

\section{C-Catalyzed reaction TS 30 (TS-2.5)}

\section{Datum}

Value

B3LYP-D3(BJ)/6-31G(d,p) Energy

$-3039.485572$

B3LYP-D3(BJ)/def2-TZVPP/IEF-PCM(DCM) Energy

$-3040.521121$

B3LYP-D3(BJ)/def2-TZVPP/IEF-PCM(DCM)//B3LYP-D3(BJ)/6-31G(d,p) Free Energy (Quasiharmonic)

$-3039.291427$

Number of Imaginary Frequencies

Frequencies (Top 3 out of 450)

$\begin{array}{rr}\text { 1. } & -250.0792 \mathrm{~cm}^{-1} \\ \text { 2. } & 5.9064 \mathrm{~cm}^{-1} \\ \text { 3. } & 9.7755 \mathrm{~cm}^{-1}\end{array}$




\begin{tabular}{|c|c|c|c|}
\hline C & 3.085210 & -1.256240 & -1.683410 \\
\hline $\mathrm{H}$ & 4.150000 & -1.406480 & -1.879090 \\
\hline $\mathrm{H}$ & 2.555930 & -2.148870 & -2.040190 \\
\hline C & -0.326650 & 0.051510 & -0.614900 \\
\hline 0 & 0.433670 & 0.826630 & -1.179410 \\
\hline 0 & -1.132430 & 0.513061 & 0.323010 \\
\hline H & -1.704360 & -0.109579 & 0.875700 \\
\hline 0 & 2.698470 & -0.113880 & -2.433860 \\
\hline $\mathrm{H}$ & 1.830540 & 0.189160 & -2.102480 \\
\hline C & -0.711100 & 2.697660 & 3.174650 \\
\hline C & -2.521050 & 2.606241 & 2.029060 \\
\hline C & -1.677730 & 0.699371 & 4.243590 \\
\hline C & -0.694120 & 1.339390 & 3.429550 \\
\hline $\mathrm{H}$ & -2.029270 & 2.131531 & 1.185270 \\
\hline $\mathrm{H}$ & -2.065140 & 1.299161 & 5.068070 \\
\hline $\mathrm{H}$ & -1.432920 & -0.315649 & 4.546880 \\
\hline C & -3.893100 & -1.226429 & 1.839110 \\
\hline C & -4.502780 & -1.318879 & 3.278120 \\
\hline C & -4.779320 & -0.437269 & 0.869750 \\
\hline $\mathrm{H}$ & -4.210470 & -0.198799 & -0.030680 \\
\hline $\mathrm{H}$ & -5.654900 & -1.018899 & 0.569960 \\
\hline $\mathrm{H}$ & -5.117650 & 0.496161 & 1.324420 \\
\hline C & -3.481740 & -2.560959 & 1.231180 \\
\hline $\mathrm{H}$ & -3.033120 & -2.409149 & 0.248740 \\
\hline $\mathrm{H}$ & -4.355361 & -3.208409 & 1.103540 \\
\hline $\mathrm{H}$ & -2.757211 & -3.080819 & 1.859590 \\
\hline C & -3.841070 & -2.411409 & 4.128470 \\
\hline $\mathrm{H}$ & -4.143721 & -3.416469 & 3.819860 \\
\hline $\mathrm{H}$ & -2.753310 & -2.340449 & 4.065870 \\
\hline $\mathrm{H}$ & -4.134060 & -2.265119 & 5.171130 \\
\hline C & -6.019800 & -1.486509 & 3.304530 \\
\hline $\mathrm{H}$ & -6.322420 & -2.407519 & 2.794920 \\
\hline $\mathrm{H}$ & -6.363390 & -1.542889 & 4.341060 \\
\hline $\mathrm{H}$ & -6.518400 & -0.640179 & 2.830270 \\
\hline 0 & -2.698060 & -0.440619 & 2.089530 \\
\hline 0 & -4.171440 & -0.048429 & 3.848300 \\
\hline B & -3.037860 & 0.484591 & 3.180850 \\
\hline 0 & -3.333470 & 1.893751 & 2.756120 \\
\hline C & -2.807069 & 4.045301 & 1.899630 \\
\hline C & -3.736079 & 4.679591 & 2.735680 \\
\hline C & -2.145629 & 4.782761 & 0.906980 \\
\hline C & -3.998419 & 6.036951 & 2.575980 \\
\hline $\mathrm{H}$ & -4.247389 & 4.092531 & 3.490450 \\
\hline C & -2.411639 & 6.139971 & 0.751060 \\
\hline $\mathrm{H}$ & -1.427459 & 4.285441 & 0.261910 \\
\hline C & -3.337579 & 6.769441 & 1.585980 \\
\hline $\mathrm{H}$ & -4.721839 & 6.526421 & 3.220660 \\
\hline $\mathrm{H}$ & -1.900329 & 6.705781 & -0.021240 \\
\hline $\mathrm{H}$ & -3.546249 & 7.827811 & 1.464000 \\
\hline H & -0.077310 & 0.705210 & 2.796120 \\
\hline
\end{tabular}




\begin{tabular}{|c|c|c|c|}
\hline $\mathrm{H}$ & -1.146450 & 3.374211 & 3.902990 \\
\hline $\mathrm{H}$ & 0.001300 & 3.135250 & 2.483160 \\
\hline C & 0.631050 & -2.221340 & -0.472720 \\
\hline C & -0.385380 & -1.414540 & -0.957970 \\
\hline C & -1.439610 & -1.942039 & -1.776060 \\
\hline C & -1.420201 & -3.294949 & -2.059520 \\
\hline $\mathrm{H}$ & -2.186421 & -3.711039 & -2.705770 \\
\hline C & 2.870570 & -1.128450 & -0.182560 \\
\hline C & 1.706650 & -1.631200 & 0.386500 \\
\hline C & 3.661620 & -0.494560 & 2.027750 \\
\hline C & 3.867590 & -0.530730 & 0.661380 \\
\hline $\mathrm{H}$ & 4.411110 & -0.031360 & 2.662520 \\
\hline C & 2.507040 & -1.039030 & 2.633520 \\
\hline C & 1.501730 & -1.621240 & 1.801270 \\
\hline C & 0.360590 & -2.202820 & 2.417900 \\
\hline C & 0.236790 & -2.224590 & 3.788300 \\
\hline C & 1.222750 & -1.629350 & 4.610670 \\
\hline C & 2.327680 & -1.040390 & 4.042630 \\
\hline $\mathrm{H}$ & -0.407100 & -2.646940 & 1.796550 \\
\hline $\mathrm{H}$ & -0.624890 & -2.701170 & 4.243720 \\
\hline $\mathrm{H}$ & 1.101670 & -1.639040 & 5.689280 \\
\hline $\mathrm{H}$ & 3.091770 & -0.581760 & 4.663840 \\
\hline C & -0.422621 & -4.161420 & -1.555750 \\
\hline C & 0.621989 & -3.623350 & -0.742600 \\
\hline C & 1.618949 & -4.497950 & -0.236650 \\
\hline C & 1.582649 & -5.843100 & -0.525120 \\
\hline C & 0.550769 & -6.376140 & -1.334270 \\
\hline C & -0.428191 & -5.552800 & -1.839250 \\
\hline $\mathrm{H}$ & 2.409709 & -4.086710 & 0.380190 \\
\hline $\mathrm{H}$ & 2.350509 & -6.501720 & -0.131760 \\
\hline $\mathrm{H}$ & 0.535909 & -7.438840 & -1.555270 \\
\hline $\mathrm{H}$ & -1.222731 & -5.954249 & -2.461750 \\
\hline C & 7.519570 & 1.103759 & -0.812000 \\
\hline C & 7.561770 & 0.224589 & 0.277440 \\
\hline C & 6.353080 & -0.307511 & 0.733340 \\
\hline C & 5.135850 & 0.030250 & 0.124410 \\
\hline C & 5.132310 & 0.920670 & -0.955590 \\
\hline C & 6.323530 & 1.462549 & -1.444050 \\
\hline $\mathrm{H}$ & 8.451310 & 1.515749 & -1.185350 \\
\hline $\mathrm{H}$ & 6.338550 & -1.011911 & 1.555700 \\
\hline $\mathrm{H}$ & 4.186080 & 1.156990 & -1.419950 \\
\hline C & 8.914300 & -0.127701 & 0.913880 \\
\hline C & 6.349150 & 2.405539 & -2.655130 \\
\hline C & 9.585770 & 1.162889 & 1.430710 \\
\hline $\mathrm{H}$ & 10.556290 & 0.933759 & 1.884510 \\
\hline $\mathrm{H}$ & 9.754200 & 1.881809 & 0.624330 \\
\hline $\mathrm{H}$ & 8.960510 & 1.649119 & 2.186080 \\
\hline C & 8.768040 & -1.099181 & 2.096150 \\
\hline $\mathrm{H}$ & 9.753490 & -1.314751 & 2.520570 \\
\hline $\mathrm{H}$ & 8.148110 & -0.675911 & 2.892740 \\
\hline $\mathrm{H}$ & 8.324610 & -2.050491 & 1.786100 \\
\hline C & 9.821480 & -0.789611 & -0.145600 \\
\hline $\mathrm{H}$ & 10.793960 & -1.045751 & 0.289260 \\
\hline $\mathrm{H}$ & 9.364300 & -1.707671 & -0.527640 \\
\hline
\end{tabular}




\begin{tabular}{|c|c|c|c|}
\hline $\mathrm{H}$ & 9.999010 & -0.126301 & -0.996520 \\
\hline C & 7.041431 & 3.729879 & -2.270110 \\
\hline $\mathrm{H}$ & 7.062331 & 4.411159 & -3.127740 \\
\hline $\mathrm{H}$ & 6.505091 & 4.224449 & -1.454170 \\
\hline $\mathrm{H}$ & 8.073901 & 3.572279 & -1.945040 \\
\hline C & 7.137080 & 1.726619 & -3.796480 \\
\hline $\mathrm{H}$ & 7.178580 & 2.380819 & -4.674390 \\
\hline $\mathrm{H}$ & 8.164500 & 1.498579 & -3.497450 \\
\hline $\mathrm{H}$ & 6.656670 & 0.788639 & -4.091190 \\
\hline C & 4.934650 & 2.727310 & -3.166580 \\
\hline $\mathrm{H}$ & 5.002080 & 3.382740 & -4.040890 \\
\hline $\mathrm{H}$ & 4.387290 & 1.826160 & -3.455910 \\
\hline $\mathrm{H}$ & 4.344050 & 3.247290 & -2.405430 \\
\hline C & -2.544370 & -1.094889 & -2.295280 \\
\hline C & -2.323840 & 0.214291 & -2.725200 \\
\hline C & -3.851520 & -1.612409 & -2.351120 \\
\hline C & -3.377250 & 1.031401 & -3.155170 \\
\hline $\mathrm{H}$ & -1.317560 & 0.607901 & -2.731190 \\
\hline C & -4.918210 & -0.843099 & -2.810140 \\
\hline $\mathrm{H}$ & -4.023450 & -2.617689 & -1.990820 \\
\hline C & -4.658360 & 0.482011 & -3.199250 \\
\hline $\mathrm{H}$ & -5.484070 & 1.093781 & -3.538120 \\
\hline C & -6.349620 & -1.390799 & -2.902840 \\
\hline C & -3.076880 & 2.490641 & -3.521400 \\
\hline C & -6.476890 & -2.793199 & -2.285530 \\
\hline $\mathrm{H}$ & -5.853231 & -3.525739 & -2.807080 \\
\hline $\mathrm{H}$ & -6.195620 & -2.795369 & -1.227530 \\
\hline $\mathrm{H}$ & -7.514091 & -3.134288 & -2.356190 \\
\hline C & -7.328970 & -0.452138 & -2.165770 \\
\hline $\mathrm{H}$ & -8.347560 & -0.849998 & -2.224900 \\
\hline $\mathrm{H}$ & -7.060570 & -0.354628 & -1.110280 \\
\hline $\mathrm{H}$ & -7.340960 & 0.550392 & -2.600890 \\
\hline C & -6.748260 & -1.472929 & -4.392690 \\
\hline $\mathrm{H}$ & -6.703390 & -0.490519 & -4.871400 \\
\hline $\mathrm{H}$ & -6.075520 & -2.141909 & -4.938300 \\
\hline $\mathrm{H}$ & -7.770210 & -1.853858 & -4.496210 \\
\hline C & -4.334870 & 3.250151 & -3.970340 \\
\hline $\mathrm{H}$ & -4.783070 & 2.800801 & -4.862430 \\
\hline $\mathrm{H}$ & -5.093180 & 3.279551 & -3.181520 \\
\hline $\mathrm{H}$ & -4.072489 & 4.283661 & -4.215640 \\
\hline C & -2.497870 & 3.197701 & -2.274470 \\
\hline $\mathrm{H}$ & -3.213500 & 3.170151 & -1.447010 \\
\hline $\mathrm{H}$ & -1.571130 & 2.726281 & -1.935340 \\
\hline $\mathrm{H}$ & -2.282219 & 4.248091 & -2.499700 \\
\hline C & -2.042900 & 2.537391 & -4.666220 \\
\hline $\mathrm{H}$ & -1.817989 & 3.576221 & -4.930370 \\
\hline $\mathrm{H}$ & -1.102910 & 2.055511 & -4.385090 \\
\hline $\mathrm{H}$ & -2.427070 & 2.033181 & -5.558580 \\
\hline
\end{tabular}


Frequencies (Top 3 out of 450)

$\begin{array}{lr}\text { 1. } & -250.1081 \mathrm{~cm}^{-1} \\ \text { 2. } & 5.9133 \mathrm{~cm}^{-1} \\ \text { 3. } & 9.7732 \mathrm{~cm}^{-1}\end{array}$

\section{B3LYP-D3(BJ)/6-31G(d,p) Molecular Geometry in Cartesian Coordinates}

\begin{tabular}{|c|c|c|c|}
\hline C & -3.085231 & -1.256453 & -1.683280 \\
\hline $\mathrm{H}$ & -2.555923 & -2.149095 & -2.039978 \\
\hline H & -4.150014 & -1.406736 & -1.878942 \\
\hline C & 0.326649 & 0.051393 & -0.614925 \\
\hline 0 & -0.433660 & 0.826429 & -1.179556 \\
\hline 0 & 1.132397 & 0.513085 & 0.322942 \\
\hline $\mathrm{H}$ & 1.704346 & -0.109453 & 0.875718 \\
\hline 0 & -2.698528 & -0.114146 & -2.433833 \\
\hline $\mathrm{H}$ & -1.830591 & 0.188922 & -2.102507 \\
\hline C & 0.710989 & 2.698113 & 3.174246 \\
\hline C & 2.520870 & 2.606612 & 2.028627 \\
\hline C & 1.677773 & 0.700082 & 4.243530 \\
\hline C & 0.694094 & 1.339879 & 3.429410 \\
\hline $\mathrm{H}$ & 2.029100 & 2.131728 & 1.184925 \\
\hline $\mathrm{H}$ & 1.433063 & -0.314903 & 4.547013 \\
\hline $\mathrm{H}$ & 2.065185 & 1.300057 & 5.067879 \\
\hline C & 3.893057 & -1.226073 & 1.839318 \\
\hline C & 4.502832 & -1.318202 & 3.278318 \\
\hline C & 3.481717 & -2.560737 & 1.231686 \\
\hline $\mathrm{H}$ & 3.033032 & -2.409140 & 0.249249 \\
\hline H & 4.355351 & -3.208174 & 1.104133 \\
\hline $\mathrm{H}$ & 2.757246 & -3.080497 & 1.860248 \\
\hline C & 4.779187 & -0.437060 & 0.869745 \\
\hline $\mathrm{H}$ & 5.654776 & -1.018715 & 0.570020 \\
\hline $\mathrm{H}$ & 4.210284 & -0.198804 & -0.030703 \\
\hline $\mathrm{H}$ & 5.117496 & 0.496471 & 1.324215 \\
\hline C & 6.019857 & -1.485788 & 3.304668 \\
\hline $\mathrm{H}$ & 6.363516 & -1.541961 & 4.341188 \\
\hline H & 6.322476 & -2.406887 & 2.795207 \\
\hline $\mathrm{H}$ & 6.518407 & -0.639531 & 2.830212 \\
\hline C & 3.841204 & -2.410584 & 4.128930 \\
\hline $\mathrm{H}$ & 4.143862 & -3.415693 & 3.820497 \\
\hline
\end{tabular}




\begin{tabular}{|c|c|c|c|}
\hline $\mathrm{H}$ & 4.134254 & -2.264075 & 5.171536 \\
\hline $\mathrm{H}$ & 2.753439 & -2.339662 & 4.066380 \\
\hline 0 & 2.698009 & -0.440250 & 2.089658 \\
\hline 0 & 4.171491 & -0.047655 & 3.848255 \\
\hline B & 3.037856 & 0.485194 & 3.180759 \\
\hline 0 & 3.333380 & 1.894274 & 2.755763 \\
\hline C & 2.806826 & 4.045665 & 1.898929 \\
\hline C & 2.145262 & 4.782941 & 0.906232 \\
\hline C & 3.735905 & 4.680115 & 2.734773 \\
\hline C & 2.411198 & 6.140139 & 0.750076 \\
\hline $\mathrm{H}$ & 1.427043 & 4.285493 & 0.261305 \\
\hline C & 3.998186 & 6.037458 & 2.574833 \\
\hline $\mathrm{H}$ & 4.247314 & 4.093185 & 3.489587 \\
\hline C & 3.337210 & 6.769771 & 1.584800 \\
\hline $\mathrm{H}$ & 1.899787 & 6.705810 & -0.022257 \\
\hline $\mathrm{H}$ & 4.721658 & 6.527047 & 3.219367 \\
\hline $\mathrm{H}$ & 3.545828 & 7.828131 & 1.462630 \\
\hline $\mathrm{H}$ & 0.077292 & 0.705543 & 2.796127 \\
\hline $\mathrm{H}$ & 1.146294 & 3.374830 & 3.902465 \\
\hline $\mathrm{H}$ & -0.001477 & 3.135526 & 2.482708 \\
\hline C & -0.631059 & -2.221427 & -0.472470 \\
\hline C & 0.385397 & -1.414701 & -0.957803 \\
\hline C & 1.439636 & -1.942319 & -1.775796 \\
\hline C & 1.420191 & -3.295259 & -2.059105 \\
\hline $\mathrm{H}$ & 2.186416 & -3.711446 & -2.705298 \\
\hline C & -2.870589 & -1.128516 & -0.182441 \\
\hline C & -1.706661 & -1.631181 & 0.386674 \\
\hline C & -3.661668 & -0.494405 & 2.027793 \\
\hline C & -3.867622 & -0.530727 & 0.661429 \\
\hline $\mathrm{H}$ & -4.411181 & -0.031159 & 2.662506 \\
\hline C & -2.507065 & -1.038755 & 2.633627 \\
\hline C & -1.501733 & -1.621029 & 1.801441 \\
\hline C & -0.360565 & -2.202481 & 2.418143 \\
\hline C & -0.236743 & -2.224056 & 3.788544 \\
\hline C & -1.222719 & -1.628754 & 4.610847 \\
\hline C & -2.327692 & -1.039919 & 4.042737 \\
\hline $\mathrm{H}$ & 0.407145 & -2.646635 & 1.796841 \\
\hline $\mathrm{H}$ & 0.624970 & -2.700526 & 4. 244012 \\
\hline $\mathrm{H}$ & -1.101626 & -1.638284 & 5.689455 \\
\hline $\mathrm{H}$ & -3.091791 & -0.581244 & 4.663887 \\
\hline C & 0.422587 & -4.161650 & -1.555257 \\
\hline C & -0.622016 & -3.623471 & -0.742182 \\
\hline C & -1.618992 & -4.497993 & -0.236128 \\
\hline C & -1.582717 & -5.843180 & -0.524441 \\
\hline C & -0.550840 & -6.376328 & -1.333528 \\
\hline C & 0.428133 & -5.553063 & -1.838599 \\
\hline $\mathrm{H}$ & -2.409754 & -4.086678 & 0.380662 \\
\hline $\mathrm{H}$ & -2.350579 & -6.501746 & -0.130997 \\
\hline $\mathrm{H}$ & -0.535993 & -7.439055 & -1.554405 \\
\hline $\mathrm{H}$ & 1.222669 & -5.954602 & -2.461052 \\
\hline C & -7.519560 & 1.103682 & -0.812126 \\
\hline C & -6.323496 & 1.462494 & -1.444117 \\
\hline C & -5.132295 & 0.920616 & -0.955607 \\
\hline C & -5.135877 & 0.030194 & 0.124384 \\
\hline
\end{tabular}




\begin{tabular}{|c|c|c|c|}
\hline C & -6.353131 & -0.307607 & 0.733245 \\
\hline C & -7.561809 & 0.224483 & 0.277289 \\
\hline $\mathrm{H}$ & -8.451290 & 1.515678 & -1.185505 \\
\hline $\mathrm{H}$ & -4.186051 & 1.156942 & -1.419923 \\
\hline $\mathrm{H}$ & -6.338624 & -1.012017 & 1.555590 \\
\hline C & -6.349061 & 2.405462 & -2.655213 \\
\hline C & -8.914359 & -0.127848 & 0.913645 \\
\hline C & -4.934537 & 2.727274 & -3.166570 \\
\hline $\mathrm{H}$ & -5.001926 & 3.382689 & -4.040890 \\
\hline $\mathrm{H}$ & -4.344007 & 3.247282 & -2.405379 \\
\hline $\mathrm{H}$ & -4.387132 & 1.826136 & -3.455849 \\
\hline C & -7.136887 & 1.726480 & -3.796613 \\
\hline $\mathrm{H}$ & -7.178335 & 2.380652 & -4.674539 \\
\hline $\mathrm{H}$ & -6.656415 & 0.788512 & -4.091248 \\
\hline $\mathrm{H}$ & -8.164314 & 1.498421 & -3.497647 \\
\hline C & -7.041423 & 3.729787 & -2.270282 \\
\hline $\mathrm{H}$ & -7.062267 & 4.411048 & -3.127929 \\
\hline $\mathrm{H}$ & -8.073913 & 3.572165 & -1.945305 \\
\hline $\mathrm{H}$ & -6.505172 & 4.224393 & -1.454305 \\
\hline C & -9.585795 & 1.162692 & 1.430635 \\
\hline $\mathrm{H}$ & -10.556337 & 0.933532 & 1.884384 \\
\hline $\mathrm{H}$ & -8.960540 & 1.648799 & 2.186087 \\
\hline $\mathrm{H}$ & -9.754175 & 1.881721 & 0.624350 \\
\hline C & -9.821553 & -0.789593 & -0.145927 \\
\hline $\mathrm{H}$ & -10.794029 & -1.045782 & 0.288891 \\
\hline $\mathrm{H}$ & -9.999077 & -0.126169 & -0.996754 \\
\hline $\mathrm{H}$ & -9.364385 & -1.707610 & -0.528096 \\
\hline C & -8.768149 & -1.099496 & 2.095791 \\
\hline $\mathrm{H}$ & -9.753615 & -1.315125 & 2.520139 \\
\hline $\mathrm{H}$ & -8.324719 & -2.050766 & 1.785619 \\
\hline $\mathrm{H}$ & -8.148249 & -0.676346 & 2.892465 \\
\hline C & 2.544443 & -1.095271 & -2.295079 \\
\hline C & 3.851570 & -1.612852 & -2.350823 \\
\hline C & 2.323982 & 0.213875 & -2.725136 \\
\hline C & 4.918305 & -0.843647 & -2.809890 \\
\hline $\mathrm{H}$ & 4.023443 & -2.618100 & -1.990391 \\
\hline C & 3.377447 & 1.030890 & -3.155158 \\
\hline $\mathrm{H}$ & 1.317718 & 0.607535 & -2.731201 \\
\hline C & 4.658526 & 0.481431 & -3.199164 \\
\hline $\mathrm{H}$ & 5.484277 & 1.093120 & -3.538085 \\
\hline C & 3.077161 & 2.490107 & -3.521546 \\
\hline C & 6.349691 & -1.391430 & -2.902497 \\
\hline C & 2.043203 & 2.536798 & -4.666392 \\
\hline $\mathrm{H}$ & 2.427373 & 2.032500 & -5.558703 \\
\hline $\mathrm{H}$ & 1.103193 & 2.054988 & -4.385237 \\
\hline $\mathrm{H}$ & 1.818348 & 3.575620 & -4.930636 \\
\hline C & 2.498152 & 3.197316 & -2.274696 \\
\hline $\mathrm{H}$ & 2.282554 & 4.247695 & -2.500037 \\
\hline $\mathrm{H}$ & 1.571389 & 2.725971 & -1.935541 \\
\hline $\mathrm{H}$ & 3.213754 & 3.169822 & -1.447217 \\
\hline C & 4.335200 & 3.249515 & -3.970531 \\
\hline $\mathrm{H}$ & 5.093492 & 3.278935 & -3.181701 \\
\hline $\mathrm{H}$ & 4.783387 & 2.800058 & -4.862569 \\
\hline $\mathrm{H}$ & 4.072880 & 4.283009 & -4.215922 \\
\hline
\end{tabular}




\begin{tabular}{|llrl|} 
C & 7.329109 & -0.452680 & -2.165620 \\
$H$ & 7.341146 & 0.549767 & -2.600922 \\
$H$ & 7.060731 & -0.354964 & -1.110139 \\
$H$ & 8.347671 & -0.850614 & -2.224695 \\
C & 6.476918 & -2.793720 & -2.284917 \\
$H$ & 6.195693 & -2.795668 & -1.226900 \\
$H$ & 5.853190 & -3.526326 & -2.806292 \\
$H$ & 7.514091 & -3.134882 & -2.355554 \\
C & 6.748320 & -1.473868 & -4.392327 \\
H & 7.770249 & -1.854854 & -4.495787 \\
$H$ & 6.075549 & -2.142941 & -4.937806 \\
$H$ & 6.703476 & -0.491558 & -4.871239 \\
\hline
\end{tabular}

\section{C-Catalyzed reaction TS 32}

\section{Datum}

Value

\begin{tabular}{lr} 
B3LYP-D3(BJ)/6-31G(d,p) Energy & -3039.488652 \\
\hline B3LYP-D3(BJ)/def2-TZVPP/IEF-PCM(DCM) Energy & -3040.521127 \\
\hline B3LYP-D3(BJ)/def2-TZVPP/IEF-PCM(DCM)//B3LYP-D3(BJ)/6-31G(d,p) Free Energy (Quasiharmonic) & -3039.290173 \\
\hline
\end{tabular}

Number of Imaginary Frequencies

Frequencies (Top 3 out of 450)

$\begin{array}{lr}\text { 1. } & -256.3416 \mathrm{~cm}^{-1} \\ \text { 2. } & 11.6893 \mathrm{~cm}^{-1} \\ \text { 3. } & 14.3661 \mathrm{~cm}^{-1}\end{array}$

B3LYP-D3(BJ)/6-31G(d,p) Molecular Geometry in Cartesian Coordinates

$\begin{array}{rrrr}C & -1.218260 & -0.210580 & 1.149760 \\ H & -1.879140 & -0.853160 & 0.571320 \\ H & -0.204860 & -0.384660 & 0.785980 \\ \mathrm{C} & 1.608380 & 1.574300 & -0.624940 \\ \mathrm{O} & 1.366850 & 2.599040 & -1.231420 \\ \mathrm{O} & 1.720470 & 0.409570 & -1.264240 \\ \mathrm{H} & 1.792720 & -0.377150 & -0.661030 \\ \mathrm{O} & -1.330810 & -0.664170 & 2.509950 \\ \mathrm{H} & -0.764910 & -0.100090 & 3.056070 \\ \mathrm{C} & -0.104540 & -1.969570 & -4.409320 \\ \mathrm{C} & -1.275080 & -1.758570 & -2.554240 \\ \mathrm{C} & 1.696071 & -2.450590 & -2.803040 \\ \mathrm{C} & 0.777421 & -2.848810 & -3.823990 \\ \mathrm{H} & -1.745039 & -2.736080 & -2.676950\end{array}$




\begin{tabular}{|c|c|c|c|}
\hline $\mathrm{H}$ & 2.046980 & -1.421250 & -2.875300 \\
\hline $\mathrm{H}$ & 2.502981 & -3.155900 & -2.607320 \\
\hline C & 1.761191 & -3.034350 & 0.721950 \\
\hline C & 0.658531 & -4.105620 & 0.377420 \\
\hline C & 3.182591 & -3.516070 & 0.411160 \\
\hline $\mathrm{H}$ & 3.847851 & -2.650470 & 0.415710 \\
\hline $\mathrm{H}$ & 3.538581 & -4.238710 & 1.151320 \\
\hline $\mathrm{H}$ & 3.237231 & -3.980970 & -0.576050 \\
\hline C & 1.692521 & -2.474570 & 2.139210 \\
\hline $\mathrm{H}$ & 1.880111 & -3.266030 & 2.872280 \\
\hline $\mathrm{H}$ & 0.720781 & -2.026820 & 2.351450 \\
\hline $\mathrm{H}$ & 2.458860 & -1.705030 & 2.268100 \\
\hline C & -0.665189 & -3.862650 & 1.109170 \\
\hline $\mathrm{H}$ & -0.592169 & -4.087830 & 2.176840 \\
\hline $\mathrm{H}$ & -0.997429 & -2.831210 & 1.006040 \\
\hline $\mathrm{H}$ & -1.426729 & -4.513680 & 0.671510 \\
\hline C & 1.105801 & -5.549540 & 0.588350 \\
\hline $\mathrm{H}$ & 1.370951 & -5.727240 & 1.635720 \\
\hline $\mathrm{H}$ & 0.288331 & -6.227090 & 0.326540 \\
\hline $\mathrm{H}$ & 1.963421 & -5.797860 & -0.038680 \\
\hline 0 & 1.452830 & -1.973800 & -0.207990 \\
\hline 0 & 0.439241 & -3.900400 & -1.032240 \\
\hline B & 0.754901 & -2.549050 & -1.335340 \\
\hline 0 & -0.386530 & -1.615050 & -1.630690 \\
\hline C & -2.029820 & -0.558301 & -2.946580 \\
\hline C & -3.174930 & -0.674261 & -3.746280 \\
\hline C & -1.573350 & 0.710680 & -2.558000 \\
\hline C & -3.848130 & 0.469179 & -4.164240 \\
\hline $\mathrm{H}$ & -3.524260 & -1.657741 & -4.049030 \\
\hline C & -2.262750 & 1.849549 & -2.960090 \\
\hline $\mathrm{H}$ & -0.675330 & 0.792510 & -1.961090 \\
\hline C & -3.395400 & 1.731779 & -3.769230 \\
\hline $\mathrm{H}$ & -4.727530 & 0.378199 & -4.793240 \\
\hline $\mathrm{H}$ & -1.907900 & 2.823850 & -2.642090 \\
\hline $\mathrm{H}$ & -3.926690 & 2.621359 & -4.093670 \\
\hline $\mathrm{H}$ & 0.605571 & -3.917190 & -3.953390 \\
\hline $\mathrm{H}$ & 0.126710 & -0.910260 & -4.429380 \\
\hline $\mathrm{H}$ & -0.846899 & -2.311220 & -5.123290 \\
\hline C & 0.706930 & 1.852270 & 1.666400 \\
\hline C & 1.794860 & 1.533280 & 0.871000 \\
\hline C & 3.053900 & 1.149110 & 1.441590 \\
\hline C & 3.162740 & 1.098840 & 2.817370 \\
\hline $\mathrm{H}$ & 4.116860 & 0.840940 & 3.266220 \\
\hline C & -1.605590 & 1.238610 & 0.946360 \\
\hline C & -0.630240 & 2.218400 & 1.093670 \\
\hline C & -3.213770 & 2.938189 & 0.304680 \\
\hline C & -2.943030 & 1.609789 & 0.572710 \\
\hline $\mathrm{H}$ & -4.225480 & 3.230029 & 0.040110 \\
\hline C & -2.227050 & 3.946089 & 0.396370 \\
\hline C & -0.909280 & 3.590340 & 0.818230 \\
\hline C & 0.068690 & 4.611560 & 0.940720 \\
\hline C & -0.242860 & 5.920260 & 0.654860 \\
\hline C & -1.547730 & 6.273100 & 0.233920 \\
\hline C & -2.517280 & 5.306509 & 0.108080 \\
\hline
\end{tabular}




\begin{tabular}{|c|c|c|c|}
\hline $\mathrm{H}$ & 1.073120 & 4.340710 & 1.242370 \\
\hline $\mathrm{H}$ & 0.517960 & 6.689230 & 0.745230 \\
\hline $\mathrm{H}$ & -1.778850 & 7.310060 & 0.009940 \\
\hline $\mathrm{H}$ & -3.522450 & 5.567149 & -0.211710 \\
\hline C & 2.075690 & 1.400360 & 3.669070 \\
\hline C & 0.826310 & 1.791690 & 3.092650 \\
\hline C & -0.256740 & 2.102680 & 3.962500 \\
\hline C & -0.109650 & 2.015890 & 5.329160 \\
\hline C & 1.125860 & 1.623530 & 5.895780 \\
\hline C & 2.194310 & 1.327870 & 5.082060 \\
\hline $\mathrm{H}$ & -1.201920 & 2.412640 & 3.533090 \\
\hline $\mathrm{H}$ & -0.946630 & 2.255560 & 5.977180 \\
\hline $\mathrm{H}$ & 1.227150 & 1.558740 & 6.974510 \\
\hline $\mathrm{H}$ & 3.147670 & 1.028220 & 5.507810 \\
\hline C & -6.177230 & -1.176311 & 0.373660 \\
\hline C & -5.247260 & -1.298501 & 1.405890 \\
\hline C & -4.202500 & -0.367801 & 1.464670 \\
\hline C & -4.052940 & 0.623729 & 0.491830 \\
\hline C & -5.008650 & 0.714899 & -0.535400 \\
\hline C & -6.092550 & -0.160461 & -0.593570 \\
\hline $\mathrm{H}$ & -6.995280 & -1.882241 & 0.313370 \\
\hline $\mathrm{H}$ & -3.486180 & -0.415211 & 2.274580 \\
\hline $\mathrm{H}$ & -4.874420 & 1.471969 & -1.296090 \\
\hline C & -5.320949 & -2.397721 & 2.474460 \\
\hline C & -7.168870 & -0.071601 & -1.686150 \\
\hline C & -4.008919 & -3.213211 & 2.449340 \\
\hline $\mathrm{H}$ & -4.042249 & -4.008921 & 3.202140 \\
\hline $\mathrm{H}$ & -3.863339 & -3.679471 & 1.469640 \\
\hline $\mathrm{H}$ & -3.137469 & -2.587331 & 2.655820 \\
\hline C & -5.488840 & -1.746081 & 3.863880 \\
\hline $\mathrm{H}$ & -5.537029 & -2.515621 & 4.642310 \\
\hline $\mathrm{H}$ & -4.651570 & -1.083611 & 4.098620 \\
\hline $\mathrm{H}$ & -6.409720 & -1.155721 & 3.908170 \\
\hline C & -6.497309 & -3.359071 & 2.243770 \\
\hline $\mathrm{H}$ & -6.501719 & -4.129541 & 3.020950 \\
\hline $\mathrm{H}$ & -7.460619 & -2.840481 & 2.285370 \\
\hline $\mathrm{H}$ & -6.421629 & -3.864431 & 1.275550 \\
\hline C & -8.574790 & -0.123801 & -1.050340 \\
\hline $\mathrm{H}$ & -9.341990 & -0.032461 & -1.826780 \\
\hline $\mathrm{H}$ & -8.754850 & -1.061771 & -0.519200 \\
\hline $\mathrm{H}$ & -8.709840 & 0.696259 & -0.338270 \\
\hline C & -7.072580 & 1.235869 & -2.489940 \\
\hline $\mathrm{H}$ & -7.878680 & 1.274779 & -3.229590 \\
\hline $\mathrm{H}$ & -6.125430 & 1.316219 & -3.025930 \\
\hline $\mathrm{H}$ & -7.171170 & 2.110209 & -1.838570 \\
\hline C & -6.999720 & -1.265381 & -2.650310 \\
\hline $\mathrm{H}$ & -7.753280 & -1.231971 & -3.445480 \\
\hline $\mathrm{H}$ & -6.008210 & -1.247711 & -3.111030 \\
\hline $\mathrm{H}$ & -7.106419 & -2.217551 & -2.122010 \\
\hline C & 4.222220 & 0.805690 & 0.593020 \\
\hline C & 4.499580 & 1.520350 & -0.571800 \\
\hline C & 5.064680 & -0.260600 & 0.953260 \\
\hline C & 5.552910 & 1.157480 & -1.419370 \\
\hline H & 3.875330 & 2.364500 & -0.835080 \\
\hline
\end{tabular}




\begin{tabular}{|c|c|c|c|}
\hline C & 6.150930 & -0.622850 & 0.158620 \\
\hline $\mathrm{H}$ & 4.836910 & -0.817890 & 1.852650 \\
\hline C & 6.365090 & 0.091140 & -1.033170 \\
\hline $\mathrm{H}$ & 7.187330 & -0.204610 & -1.671970 \\
\hline C & 7.096650 & -1.776030 & 0.525160 \\
\hline C & 5.735160 & 1.919500 & -2.738000 \\
\hline C & 8.541940 & -1.239020 & 0.610480 \\
\hline $\mathrm{H}$ & 8.872560 & -0.809620 & -0.338990 \\
\hline $\mathrm{H}$ & 8.622150 & -0.460590 & 1.375700 \\
\hline $\mathrm{H}$ & 9.233121 & -2.047940 & 0.870760 \\
\hline C & 6.745081 & -2.413780 & 1.879370 \\
\hline $\mathrm{H}$ & 7.459631 & -3.210990 & 2.105910 \\
\hline $\mathrm{H}$ & 6.793480 & -1.683040 & 2.692990 \\
\hline $\mathrm{H}$ & 5.745931 & -2.858000 & 1.875090 \\
\hline C & 7.019041 & -2.868380 & -0.563570 \\
\hline $\mathrm{H}$ & 6.003941 & -3.268080 & -0.642820 \\
\hline $\mathrm{H}$ & 7.303151 & -2.478990 & -1.545110 \\
\hline $\mathrm{H}$ & 7.695211 & -3.695700 & -0.321970 \\
\hline C & 4.451850 & 1.750570 & -3.583890 \\
\hline $\mathrm{H}$ & 4.271600 & 0.693050 & -3.801940 \\
\hline $\mathrm{H}$ & 3.566930 & 2.137080 & -3.071870 \\
\hline $\mathrm{H}$ & 4.554250 & 2.284060 & -4.535400 \\
\hline C & 5.964200 & 3.417120 & -2.443130 \\
\hline $\mathrm{H}$ & 5.120380 & 3.858020 & -1.906020 \\
\hline $\mathrm{H}$ & 6.863980 & 3.561950 & -1.836560 \\
\hline $\mathrm{H}$ & 6.089070 & 3.972230 & -3.378990 \\
\hline C & 6.929290 & 1.397900 & -3.552210 \\
\hline $\mathrm{H}$ & 7.017010 & 1.967100 & -4.482550 \\
\hline $\mathrm{H}$ & 7.871970 & 1.506580 & -3.005900 \\
\hline $\mathrm{H}$ & 6.806690 & 0.343590 & -3.819820 \\
\hline
\end{tabular}

\section{C-Catalyzed reaction TS 33}

\section{Datum}

Value

B3LYP-D3(BJ)/6-31G(d,p) Energy

$-3039.484173$

B3LYP-D3(BJ)/def2-TZVPP/IEF-PCM(DCM) Energy

$-3040.52096$

B3LYP-D3(BJ)/def2-TZVPP/IEF-PCM(DCM)//B3LYP-D3(BJ)/6-31G(d,p) Free Energy (Quasiharmonic)

$-3039.290142$

Number of Imaginary Frequencies

Frequencies (Top 3 out of 450)

$\begin{array}{lr}\text { 1. } & -212.7460 \mathrm{~cm}^{-1} \\ \text { 2. } & 11.2371 \mathrm{~cm}^{-1} \\ \text { 3. } & 14.1266 \mathrm{~cm}^{-1}\end{array}$




\begin{tabular}{|c|c|c|c|}
\hline C & -0.730040 & 0.924210 & 0.627990 \\
\hline $\mathrm{H}$ & -0.144360 & 0.852300 & 1.548950 \\
\hline $\mathrm{H}$ & -1.712280 & 0.497930 & 0.824010 \\
\hline C & 2.535860 & 1.980290 & -1.384670 \\
\hline 0 & 3.426460 & 2.584180 & -1.941940 \\
\hline 0 & 1.536220 & 1.404620 & -2.053970 \\
\hline $\mathrm{H}$ & 0.905880 & 0.948660 & -1.427110 \\
\hline 0 & -0.092370 & 0.173220 & -0.416360 \\
\hline $\mathrm{H}$ & 0.252040 & -0.669450 & -0.027380 \\
\hline C & -2.821640 & -1.718140 & -2.058990 \\
\hline C & -3.022140 & -1.941630 & 0.037860 \\
\hline C & -0.734660 & -2.956600 & -1.685400 \\
\hline C & -2.093410 & -2.888820 & -2.120400 \\
\hline $\mathrm{H}$ & -3.421860 & -0.926110 & 0.057310 \\
\hline $\mathrm{H}$ & -0.213080 & -3.877030 & -1.945380 \\
\hline $\mathrm{H}$ & -0.157850 & -2.070240 & -1.949070 \\
\hline C & 1.111310 & -2.854950 & 1.599820 \\
\hline C & 0.647840 & -4.339590 & 1.391170 \\
\hline C & 2.619020 & -2.644040 & 1.648200 \\
\hline $\mathrm{H}$ & 2.842450 & -1.582040 & 1.778270 \\
\hline $\mathrm{H}$ & 3.055420 & -3.188780 & 2.491750 \\
\hline $\mathrm{H}$ & 3.104510 & -2.971870 & 0.729330 \\
\hline C & 0.449460 & -2.183050 & 2.809520 \\
\hline $\mathrm{H}$ & 0.670680 & -1.112020 & 2.784560 \\
\hline $\mathrm{H}$ & -0.635020 & -2.310560 & 2.791840 \\
\hline $\mathrm{H}$ & 0.836630 & -2.584030 & 3.750320 \\
\hline C & 0.407131 & -5.113460 & 2.683140 \\
\hline $\mathrm{H}$ & 1.324511 & -5.177870 & 3.277250 \\
\hline $\mathrm{H}$ & -0.369969 & -4.641970 & 3.286930 \\
\hline $\mathrm{H}$ & 0.084091 & -6.131520 & 2.447570 \\
\hline C & 1.591811 & -5.124700 & 0.472620 \\
\hline $\mathrm{H}$ & 2.539951 & -5.358610 & 0.965640 \\
\hline $\mathrm{H}$ & 1.107241 & -6.063850 & 0.192580 \\
\hline $\mathrm{H}$ & 1.806191 & -4.565290 & -0.440580 \\
\hline 0 & 0.592020 & -2.239380 & 0.399370 \\
\hline 0 & -0.611280 & -4.190910 & 0.706460 \\
\hline B & -0.640740 & -2.920250 & 0.060400 \\
\hline 0 & -1.803320 & -2.037150 & 0.475680 \\
\hline C & -4.035540 & -3.005450 & 0.235270 \\
\hline C & -3.709040 & -4.263050 & 0.761090 \\
\hline C & -5.370410 & -2.713230 & -0.086670 \\
\hline C & -4.713039 & -5.213190 & 0.945140 \\
\hline $\mathrm{H}$ & -2.678949 & -4.485420 & 1.017700 \\
\hline C & -6.365790 & -3.666300 & 0.099020 \\
\hline $\mathrm{H}$ & -5.616710 & -1.733940 & -0.480860 \\
\hline C & -6.037769 & -4.922590 & 0.615590 \\
\hline $\mathrm{H}$ & -4.457929 & -6.186160 & 1.353800 \\
\hline $\mathrm{H}$ & -7.394550 & -3.430760 & -0.155830 \\
\hline $\mathrm{H}$ & -6.812539 & -5.668430 & 0.764250 \\
\hline $\mathrm{H}$ & -2.636620 & -3.818100 & -2.287810 \\
\hline
\end{tabular}




\begin{tabular}{|c|c|c|c|}
\hline $\mathrm{H}$ & -2.300320 & -0.766720 & -2.002590 \\
\hline $\mathrm{H}$ & -3.855020 & -1.688430 & -2.382440 \\
\hline C & 1.560020 & 2.560630 & 0.856110 \\
\hline C & 2.521620 & 1.882090 & 0.129150 \\
\hline C & 3.602590 & 1.211690 & 0.786790 \\
\hline C & 3.711170 & 1.305890 & 2.158400 \\
\hline $\mathrm{H}$ & 4.507120 & 0.768910 & 2.666220 \\
\hline C & -0.814870 & 2.358760 & 0.169120 \\
\hline C & 0.344720 & 3.124600 & 0.188650 \\
\hline C & -1.977510 & 4.127670 & -1.013310 \\
\hline C & -2.004670 & 2.875970 & -0.428610 \\
\hline $\mathrm{H}$ & -2.884930 & 4.527160 & -1.457300 \\
\hline C & -0.806860 & 4.921880 & -1.025330 \\
\hline C & 0.379760 & 4.419460 & -0.403460 \\
\hline C & 1.553390 & 5.219450 & -0.414290 \\
\hline C & 1.547780 & 6.460600 & -1.006380 \\
\hline C & 0.371090 & 6.962710 & -1.613120 \\
\hline C & -0.779280 & 6.209660 & -1.622670 \\
\hline $\mathrm{H}$ & 2.459780 & 4.825990 & 0.030810 \\
\hline $\mathrm{H}$ & 2.453630 & 7.058260 & -1.016420 \\
\hline $\mathrm{H}$ & 0.383100 & 7.944210 & -2.077030 \\
\hline $\mathrm{H}$ & -1.684500 & 6.586640 & -2.090430 \\
\hline C & 2.786150 & 2.048760 & 2.932350 \\
\hline C & 1.681550 & 2.678370 & 2.278020 \\
\hline C & 0.740550 & 3.393380 & 3.065360 \\
\hline C & 0.884430 & 3.478670 & 4.432140 \\
\hline C & 1.974500 & 2.850950 & 5.079010 \\
\hline C & 2.903070 & 2.151580 & 4.343050 \\
\hline $\mathrm{H}$ & -0.093820 & 3.877550 & 2.570980 \\
\hline $\mathrm{H}$ & 0.158190 & 4.032920 & 5.018720 \\
\hline $\mathrm{H}$ & 2.076140 & 2.924160 & 6.157400 \\
\hline $\mathrm{H}$ & 3.743120 & 1.665220 & 4.831060 \\
\hline C & -5.777740 & 0.876480 & -0.341950 \\
\hline C & -5.228120 & 1.229850 & -1.585730 \\
\hline C & -3.976210 & 1.851170 & -1.596320 \\
\hline C & -3.290450 & 2.125400 & -0.403970 \\
\hline C & -3.865520 & 1.744740 & 0.811640 \\
\hline C & -5.113190 & 1.109620 & 0.866150 \\
\hline $\mathrm{H}$ & -6.757010 & 0.417120 & -0.319120 \\
\hline $\mathrm{H}$ & -3.507810 & 2.139550 & -2.528840 \\
\hline $\mathrm{H}$ & -3.334500 & 1.975980 & 1.728950 \\
\hline C & -6.037890 & 1.006340 & -2.872930 \\
\hline C & -5.701710 & 0.719870 & 2.230330 \\
\hline C & -6.677040 & -0.397670 & -2.897570 \\
\hline $\mathrm{H}$ & -7.251990 & -0.528410 & -3.819740 \\
\hline $\mathrm{H}$ & -5.917620 & -1.184480 & -2.864890 \\
\hline $\mathrm{H}$ & -7.363460 & -0.558050 & -2.062050 \\
\hline C & -7.161080 & 2.066580 & -2.917700 \\
\hline $\mathrm{H}$ & -7.763820 & 1.951290 & -3.825240 \\
\hline $\mathrm{H}$ & -7.825120 & 1.974600 & -2.052990 \\
\hline $\mathrm{H}$ & -6.740110 & 3.076510 & -2.910990 \\
\hline C & -5.169570 & 1.151730 & -4.134560 \\
\hline $\mathrm{H}$ & -5.770020 & 0.933100 & -5.022660 \\
\hline $\mathrm{H}$ & -4.778700 & 2.166610 & -4.247980 \\
\hline
\end{tabular}




\begin{tabular}{|c|c|c|c|}
\hline H & -4.321750 & 0.459370 & -4.122000 \\
\hline $\mathrm{C}$ & -4.732540 & -0.241690 & 2.953110 \\
\hline $\mathrm{H}$ & -5.118780 & -0.489140 & 3.947520 \\
\hline $\mathrm{H}$ & -4.618130 & -1.175420 & 2.395670 \\
\hline $\mathrm{H}$ & -3.740510 & 0.199950 & 3.081400 \\
\hline C & -5.887600 & 1.995970 & 3.079690 \\
\hline $\mathrm{H}$ & -6.309570 & 1.742680 & 4.058030 \\
\hline H & -4.938540 & 2.512080 & 3.248070 \\
\hline $\mathrm{H}$ & -6.566870 & 2.696850 & 2.584480 \\
\hline C & -7.064610 & 0.019860 & 2.104180 \\
\hline $\mathrm{H}$ & -6.990030 & -0.911760 & 1.535730 \\
\hline $\mathrm{H}$ & -7.809530 & 0.663210 & 1.625270 \\
\hline $\mathrm{H}$ & -7.438830 & -0.232480 & 3.100840 \\
\hline C & 4.518940 & 0.328990 & 0.026840 \\
\hline C & 3.980550 & -0.587430 & -0.882480 \\
\hline C & 5.899360 & 0.348710 & 0.249660 \\
\hline C & 4.796230 & -1.474720 & -1.583830 \\
\hline H & 2.909360 & -0.610900 & -1.019070 \\
\hline C & 6.748350 & -0.528980 & -0.430960 \\
\hline $\mathrm{H}$ & 6.298440 & 1.077060 & 0.944280 \\
\hline C & 6.173720 & -1.427080 & -1.339720 \\
\hline $\mathrm{H}$ & 6.822270 & -2.109080 & -1.879480 \\
\hline C & 8.270120 & -0.532570 & -0.225950 \\
\hline C & 4.223750 & -2.474410 & -2.597070 \\
\hline C & 8.722150 & 0.513220 & 0.806000 \\
\hline H & 9.809560 & 0.470380 & 0.921840 \\
\hline $\mathrm{H}$ & 8.460650 & 1.528720 & 0.493470 \\
\hline $\mathrm{H}$ & 8.275790 & 0.331020 & 1.788760 \\
\hline C & 8.714160 & -1.925090 & 0.271520 \\
\hline $\mathrm{H}$ & 9.799420 & -1.949760 & 0.419420 \\
\hline $\mathrm{H}$ & 8.233170 & -2.168130 & 1.224310 \\
\hline $\mathrm{H}$ & 8.455650 & -2.711400 & -0.442990 \\
\hline C & 8.966150 & -0.219200 & -1.567890 \\
\hline H & 8.718120 & -0.959010 & -2.333890 \\
\hline $\mathrm{H}$ & 8.661240 & 0.762970 & -1.941480 \\
\hline $\mathrm{H}$ & 10.054570 & -0.217400 & -1.442370 \\
\hline C & 4.828150 & -2.183620 & -3.987140 \\
\hline $\mathrm{H}$ & 4.584920 & -1.166140 & -4.307030 \\
\hline $\mathrm{H}$ & 5.917490 & -2.283010 & -3.982730 \\
\hline $\mathrm{H}$ & 4.429650 & -2.883370 & -4.730130 \\
\hline C & 4.587080 & -3.911340 & -2.166910 \\
\hline $\mathrm{H}$ & 5.669740 & -4.056320 & -2.110710 \\
\hline $\mathrm{H}$ & 4.167850 & -4.142400 & -1.182720 \\
\hline $\mathrm{H}$ & 4.188591 & -4.636790 & -2.884700 \\
\hline C & 2.692890 & -2.369230 & -2.700420 \\
\hline $\mathrm{H}$ & 2.321150 & -3.100390 & -3.425450 \\
\hline $\mathrm{H}$ & 2.208580 & -2.569830 & -1.740850 \\
\hline $\mathrm{H}$ & 2.378950 & -1.375290 & -3.034810 \\
\hline
\end{tabular}


Frequencies (Top 3 out of 450)

$\begin{array}{rr}\text { 1. } & -196.8452 \mathrm{~cm}^{-1} \\ \text { 2. } & 12.3600 \mathrm{~cm}^{-1} \\ \text { 3. } & 15.1401 \mathrm{~cm}^{-1}\end{array}$

\section{B3LYP-D3(BJ)/6-31G(d,p) Molecular Geometry in Cartesian Coordinates}

C

$\mathrm{H}$

$\mathrm{H}$

C

0

0

$\mathrm{H}$

0

$\mathrm{H}$

C

C

C

C

$\mathrm{H}$

$\mathrm{H}$

$\mathrm{H}$

C

C

C

$\mathrm{H}$

$\mathrm{H}$

$\mathrm{H}$

C

$\mathrm{H}$

$\mathrm{H}$

$\mathrm{H}$

C

$\mathrm{H}$

$\mathrm{H}$

$\mathrm{H}$

C

$\mathrm{H}$

\subsection{0}

1.775600

3.147530

$-0.498000$

$-0.894930$

$-0.045140$

0.431140

1.412090

0.874620

$-1.908770$

$-2.979710$

$-0.426030$

$-1.431840$

$-3.078210$

0.325470

0.025280

$-0.050920$

$-1.420750$

1.144170

2.022840

1.375270

0.958420

0.221990

0.246610

1.195050

$-0.536920$

$-2.528020$

$-2.386230$

$-2.567250$

$-3.491950$

$-1.363580$

$-1.040510$

\subsection{0}

0.490620

$-0.442230$

2.026720

2.864910

0.848260

0.328710

$-0.544970$

$-1.204620$

$-1.486150$

$-1.266430$

$-2.852310$

$-2.714960$

$-0.200440$

$-2.063550$

$-3.842330$

$-3.335490$

$-4.086400$

$-4.191900$

$-3.549210$

$-4.966250$

$-4.672630$

$-2.706080$

$-3.468790$

$-2.204970$

$-1.964380$

$-3.403940$

$-3.537200$

$-2.335760$

$-3.840710$

$-5.573890$

$-5.735290$
0.933250

1.844410

1.214300

$-1.119470$

$-1.906170$

$-1.537690$

$-0.818540$

0.080740

0.600210

$-2.918140$

$-1.074650$

$-1.505130$

$-2.512700$

$-1.282190$

$-1.524650$

$-1.447740$

2.028870

1.904900

1.596390

1.491970

2.333350

0.633750

3.391680

4.176460

3. 381550

3. 643530

2. 716410

3.792890

2.495480

2.443160

2.232420

3. 266050 


\begin{tabular}{|c|c|c|c|}
\hline H & -2.357850 & -6.014460 & 2.116230 \\
\hline $\mathrm{H}$ & -0.680930 & -6.100390 & 1.563650 \\
\hline 0 & -0.222130 & -2.276620 & 1.069300 \\
\hline 0 & -1.721800 & -3.939190 & 0.505640 \\
\hline B & -1.182010 & -2.689290 & 0.059290 \\
\hline 0 & -2.120500 & -1.529720 & -0.134920 \\
\hline C & -4.212780 & -2.051360 & -1.325210 \\
\hline C & -5.187930 & -1.470119 & -2.150430 \\
\hline C & -4.452430 & -3.314390 & -0.766470 \\
\hline C & -6.379930 & -2.133849 & -2.417990 \\
\hline $\mathrm{H}$ & -5.006580 & -0.488279 & -2.571930 \\
\hline C & -5.652710 & -3.972629 & -1.032020 \\
\hline $\mathrm{H}$ & -3.694460 & -3.772620 & -0.141970 \\
\hline C & -6.618170 & -3.389929 & -1.854210 \\
\hline $\mathrm{H}$ & -7.125160 & -1.669949 & -3.056830 \\
\hline $\mathrm{H}$ & -5.834250 & -4.948159 & -0.591250 \\
\hline $\mathrm{H}$ & -7.550660 & -3.908609 & -2.054150 \\
\hline $\mathrm{H}$ & -1.997210 & -3.602830 & -2.794500 \\
\hline $\mathrm{H}$ & -1.298990 & -0.598150 & -2.778540 \\
\hline $\mathrm{H}$ & -2.708030 & -1.426360 & -3.648870 \\
\hline C & 0.720860 & 2.578630 & 0.974230 \\
\hline C & -0.491670 & 2.275720 & 0.375520 \\
\hline C & -1.707550 & 2.257510 & 1.140120 \\
\hline C & -1.632870 & 2.556630 & 2.487680 \\
\hline $\mathrm{H}$ & -2.545170 & 2.561410 & 3.075810 \\
\hline C & 2.783790 & 1.412620 & 0.185580 \\
\hline C & 1.992490 & 2.554170 & 0.184500 \\
\hline C & 4.427200 & 2.542110 & -1.194420 \\
\hline C & 4.019750 & 1.396510 & -0.537430 \\
\hline $\mathrm{H}$ & 5.361740 & 2.530290 & -1.747600 \\
\hline C & 3.645220 & 3.719590 & -1.209600 \\
\hline C & 2.393890 & 3.729410 & -0.518360 \\
\hline C & 1.601560 & 4.907750 & -0.552530 \\
\hline C & 2.033290 & 6.022910 & -1.231720 \\
\hline C & 3.275580 & 6.015520 & -1.910860 \\
\hline C & 4.061640 & 4.887750 & -1.901650 \\
\hline $\mathrm{H}$ & 0.642190 & 4.909460 & -0.049170 \\
\hline $\mathrm{H}$ & 1.414630 & 6.914480 & -1.254560 \\
\hline $\mathrm{H}$ & 3.601840 & 6.903020 & -2.444390 \\
\hline $\mathrm{H}$ & 5.012800 & 4.870390 & -2.426320 \\
\hline C & -0.419360 & 2.894230 & 3.128970 \\
\hline C & 0.784740 & 2.914920 & 2.362390 \\
\hline C & 2.000900 & 3.261760 & 3.008950 \\
\hline C & 2.020150 & 3.569430 & 4.350580 \\
\hline C & 0.826360 & 3.546670 & 5.110280 \\
\hline C & -0.366720 & 3.217690 & 4.510490 \\
\hline $\mathrm{H}$ & 2.915690 & 3.282960 & 2.428130 \\
\hline $\mathrm{H}$ & 2.956940 & 3.835020 & 4.830540 \\
\hline $\mathrm{H}$ & 0.856190 & 3.792030 & 6.167390 \\
\hline $\mathrm{H}$ & -1.289020 & 3.199780 & 5.084360 \\
\hline C & 6.531230 & -2.048680 & -0.762910 \\
\hline C & 5.163550 & -2.188360 & -1.053280 \\
\hline C & 4.353380 & -1.057450 & -0.975120 \\
\hline C & 4.883980 & 0.188000 & -0.600310 \\
\hline
\end{tabular}




\begin{tabular}{|c|c|c|c|}
\hline C & 6.247610 & 0.289190 & -0.319890 \\
\hline C & 7.095430 & -0.825170 & -0.399210 \\
\hline $\mathrm{H}$ & 7.163730 & -2.925110 & -0.821940 \\
\hline $\mathrm{H}$ & 3.296910 & -1.116890 & -1.196530 \\
\hline $\mathrm{H}$ & 6.643040 & 1.253400 & -0.017510 \\
\hline C & 4.605830 & -3.568640 & -1.428170 \\
\hline C & 8.587690 & -0.662270 & -0.076310 \\
\hline C & 5.346320 & -4.110110 & -2.669050 \\
\hline $\mathrm{H}$ & 4.954430 & -5.094730 & -2.946620 \\
\hline $\mathrm{H}$ & 5.215280 & -3.437060 & -3.521950 \\
\hline $\mathrm{H}$ & 6.419580 & -4.217050 & -2.488290 \\
\hline C & 4.816850 & -4.534090 & -0.241460 \\
\hline $\mathrm{H}$ & 4.425890 & -5.528560 & -0.483130 \\
\hline $\mathrm{H}$ & 5.876050 & -4.640790 & 0.009370 \\
\hline $\mathrm{H}$ & 4.296640 & -4.171000 & 0.650340 \\
\hline C & 3.103020 & -3.515680 & -1.744960 \\
\hline $\mathrm{H}$ & 2.739560 & -4.520940 & -1.981200 \\
\hline $\mathrm{H}$ & 2.524050 & -3.139740 & -0.898900 \\
\hline $\mathrm{H}$ & 2.892890 & -2.874570 & -2.606460 \\
\hline C & 8.743420 & -0.170660 & 1.378950 \\
\hline $\mathrm{H}$ & 9.802590 & -0.042480 & 1.627750 \\
\hline $\mathrm{H}$ & 8.243480 & 0.788850 & 1.536730 \\
\hline $\mathrm{H}$ & 8.311890 & -0.892100 & 2.079860 \\
\hline C & 9.366700 & -1.978890 & -0.225740 \\
\hline $\mathrm{H}$ & 10.422430 & -1.810470 & 0.007950 \\
\hline $\mathrm{H}$ & 8.994880 & -2.749480 & 0.456830 \\
\hline $\mathrm{H}$ & 9.310260 & -2.368770 & -1.246860 \\
\hline C & 9.205680 & 0.375990 & -1.037580 \\
\hline $\mathrm{H}$ & 10.270960 & 0.510940 & -0.820700 \\
\hline $\mathrm{H}$ & 9.106430 & 0.048790 & -2.077250 \\
\hline $\mathrm{H}$ & 8.719300 & 1.350980 & -0.945950 \\
\hline C & -3.040280 & 1.905540 & 0.577760 \\
\hline C & -3.887650 & 1.054250 & 1.304840 \\
\hline C & -3.508850 & 2.453820 & -0.620210 \\
\hline C & -5.181280 & 0.758791 & 0.867620 \\
\hline $\mathrm{H}$ & -3.512750 & 0.617400 & 2.222820 \\
\hline C & -4.780070 & 2.140631 & -1.114840 \\
\hline $\mathrm{H}$ & -2.869800 & 3.133080 & -1.164040 \\
\hline C & -5.602410 & 1.304201 & -0.353720 \\
\hline $\mathrm{H}$ & -6.588890 & 1.062121 & -0.720170 \\
\hline C & -5.211810 & 2.737841 & -2.462090 \\
\hline C & -6.118820 & -0.083339 & 1.747540 \\
\hline C & -5.218640 & 4.278251 & -2.354320 \\
\hline $\mathrm{H}$ & -5.913800 & 4.609591 & -1.576350 \\
\hline $\mathrm{H}$ & -4.227200 & 4.667480 & -2.109530 \\
\hline $\mathrm{H}$ & -5.529310 & 4.724821 & -3.305130 \\
\hline C & -4.203700 & 2.311870 & -3.553900 \\
\hline $\mathrm{H}$ & -4.468050 & 2.770720 & -4.512880 \\
\hline $\mathrm{H}$ & -3.180840 & 2.604510 & -3.304900 \\
\hline $\mathrm{H}$ & -4.212680 & 1.225070 & -3.693190 \\
\hline C & -6.616370 & 2.277841 & -2.884300 \\
\hline $\mathrm{H}$ & -6.677670 & 1.189271 & -2.984560 \\
\hline $\mathrm{H}$ & -7.380090 & 2.595331 & -2.167320 \\
\hline $\mathrm{H}$ & -6.870330 & 2.713961 & -3.855190 \\
\hline
\end{tabular}




\begin{tabular}{|lrrr|} 
C & -6.565310 & 0.793461 & 2.938740 \\
H & -7.239340 & 0.234161 & 3.597360 \\
$H$ & -5.705020 & 1.121081 & 3.530460 \\
H & -7.090880 & 1.687501 & 2.588650 \\
C & -7.369720 & -0.553959 & 0.986060 \\
$H$ & -7.993080 & 0.285561 & 0.663430 \\
$H$ & -7.104840 & -1.147039 & 0.106940 \\
$H$ & -7.982820 & -1.178879 & 1.642920 \\
C & -5.393450 & -1.331729 & 2.284030 \\
H & -6.080870 & -1.933309 & 2.888300 \\
H & -5.026180 & -1.952239 & 1.464600 \\
$H$ & -4.544160 & -1.072559 & 2.920210 \\
\hline
\end{tabular}

\section{C-Catalyzed reaction TS 35}

\section{Datum}

Value

\begin{tabular}{lc} 
B3LYP-D3(BJ)/6-31G(d,p) Energy & -3039.481178 \\
\hline B3LYP-D3(BJ)/def2-TZVPP/IEF-PCM(DCM) Energy & -3040.519749 \\
\hline B3LYP-D3(BJ)/def2-TZVPP/IEF-PCM(DCM)//B3LYP-D3(BJ)/6-31G(d,p) Free Energy (Quasiharmonic) & -3039.289681
\end{tabular}

Number of Imaginary Frequencies

Frequencies (Top 3 out of 450)

$\begin{array}{lr}\text { 1. } & -248.2949 \mathrm{~cm}^{-1} \\ \text { 2. } & 9.4034 \mathrm{~cm}^{-1} \\ \text { 3. } & 12.0600 \mathrm{~cm}^{-1}\end{array}$

\section{B3LYP-D3(BJ)/6-31G(d,p) Molecular Geometry in Cartesian Coordinates}

C

$\mathrm{H}$

$\mathrm{H}$

C

0

0

$\mathrm{H}$

0

$\mathrm{H}$

C

C

C

C

$\mathrm{H}$
1.932310

2.717950

1. 204180

$-0.474530$

$-0.991670$

0.303320

0.641860

1. 292730

0.762891

$-2.687110$

$-3.269049$

$-0.627409$

$-1.348670$

$-3.458270$
0.344760

$-0.304640$

0.530440

2. 308500

3.137540

1.341510

0.727290

$-0.296710$

$-1.067320$

$-0.270130$

$-1.110920$

$-1.555020$

$-0.325190$

$-0.131300$
0.491690

0.890550

1.289170

$-1.913720$

$-2.631740$

$-2.392690$

$-1.679210$

$-0.618080$

$-0.282400$

3.202730

1.304030

2.831700

2.876960

0.872070 


\begin{tabular}{|c|c|c|c|}
\hline $\mathrm{H}$ & 0.455571 & -1.452480 & 2.769130 \\
\hline $\mathrm{H}$ & -0.926819 & -2.275910 & 3.593930 \\
\hline C & 0.587481 & -3.672610 & 0.189440 \\
\hline C & -0.551049 & -4.618880 & 0.710930 \\
\hline C & 0.969541 & -3.863880 & -1.272930 \\
\hline $\mathrm{H}$ & 1.703601 & -3.105590 & -1.552290 \\
\hline $\mathrm{H}$ & 1.418491 & -4.848530 & -1.435490 \\
\hline $\mathrm{H}$ & 0.107791 & -3.754810 & -1.931080 \\
\hline C & 1.843541 & -3.693860 & 1.068800 \\
\hline $\mathrm{H}$ & 2.400301 & -4.628300 & 0.956840 \\
\hline $\mathrm{H}$ & 2.499821 & -2.870880 & 0.770550 \\
\hline H & 1.588241 & -3.568920 & 2.123830 \\
\hline C & -0.052529 & -5.900680 & 1.369920 \\
\hline $\mathrm{H}$ & 0.522211 & -6.506600 & 0.661940 \\
\hline H & 0.573451 & -5.683400 & 2.236650 \\
\hline $\mathrm{H}$ & -0.905809 & -6.494550 & 1.709340 \\
\hline C & -1.586679 & -4.951560 & -0.370000 \\
\hline $\mathrm{H}$ & -1.182059 & -5.630150 & -1.126700 \\
\hline $\mathrm{H}$ & -2.443679 & -5.438870 & 0.103000 \\
\hline H & -1.944129 & -4.048630 & -0.866330 \\
\hline 0 & -0.026159 & -2.372580 & 0.326500 \\
\hline 0 & -1.190509 & -3.809470 & 1.713000 \\
\hline B & -1.040579 & -2.434710 & 1.354560 \\
\hline 0 & -2.242199 & -1.730390 & 0.805330 \\
\hline C & -4.466899 & -1.855480 & 1.725480 \\
\hline C & -4.418109 & -3.223900 & 2.032510 \\
\hline C & -5.688009 & -1.167281 & 1.772080 \\
\hline C & -5.589859 & -3.885820 & 2.389290 \\
\hline $\mathrm{H}$ & -3.467649 & -3.746230 & 1.984510 \\
\hline C & -6.854759 & -1.837291 & 2.124850 \\
\hline H & -5.712620 & -0.112951 & 1.513120 \\
\hline C & -6.806169 & -3.198531 & 2.436000 \\
\hline $\mathrm{H}$ & -5.558169 & -4.944900 & 2.625900 \\
\hline $\mathrm{H}$ & -7.800159 & -1.304221 & 2.151550 \\
\hline $\mathrm{H}$ & -7.715849 & -3.724441 & 2.709100 \\
\hline H & -0.909780 & 0.551250 & 2.405010 \\
\hline $\mathrm{H}$ & -3.119729 & -1.054720 & 3.814150 \\
\hline $\mathrm{H}$ & -3.215950 & 0.675870 & 3.190410 \\
\hline C & 0.313790 & 2.743060 & 0.439150 \\
\hline C & -0.713100 & 2.387240 & -0.416050 \\
\hline C & -2.066610 & 2.316190 & 0.055340 \\
\hline C & -2.344730 & 2.707160 & 1.347480 \\
\hline $\mathrm{H}$ & -3.364290 & 2.640600 & 1.715760 \\
\hline C & 2.527370 & 1.654090 & 0.033370 \\
\hline C & 1.734270 & 2.791420 & -0.026240 \\
\hline C & 4.443680 & 2.933720 & -0.726430 \\
\hline C & 3.901730 & 1.722190 & -0.350420 \\
\hline $\mathrm{H}$ & 5.492230 & 2.982101 & -1.005790 \\
\hline C & 3.660060 & 4.109730 & -0.801000 \\
\hline C & 2.272490 & 4.039200 & -0.463720 \\
\hline C & 1.480870 & 5.213940 & -0.570570 \\
\hline C & 2.041300 & 6.402030 & -0.978300 \\
\hline C & 3.418180 & 6.474830 & -1.298690 \\
\hline C & 4.208040 & 5.352430 & -1.214460 \\
\hline
\end{tabular}




\begin{tabular}{|c|c|c|c|}
\hline H & 0.422450 & 5.156660 & -0.344820 \\
\hline $\mathrm{H}$ & 1.422660 & 7.289790 & -1.063320 \\
\hline $\mathrm{H}$ & 3.846140 & 7.419540 & -1.619710 \\
\hline $\mathrm{H}$ & 5.263180 & 5.396741 & -1.469270 \\
\hline C & -1.328810 & 3.129220 & 2.239740 \\
\hline C & 0.029770 & 3.114890 & 1.795290 \\
\hline C & 1.049830 & 3.474650 & 2.714350 \\
\hline C & 0.739970 & 3.827320 & 4.009280 \\
\hline C & -0.604310 & 3.839920 & 4.447550 \\
\hline C & -1.615700 & 3.497540 & 3.579470 \\
\hline H & 2.080660 & 3.468270 & 2.380220 \\
\hline $\mathrm{H}$ & 1.532600 & 4.098090 & 4.699670 \\
\hline $\mathrm{H}$ & -0.835200 & 4.119270 & 5.470790 \\
\hline H & -2.651380 & 3.500240 & 3.908130 \\
\hline C & 6.437841 & -1.703859 & -0.449390 \\
\hline C & 5.366361 & -1.626809 & -1.356250 \\
\hline C & 4.521300 & -0.520050 & -1.283150 \\
\hline C & 4.765090 & 0.508700 & -0.359650 \\
\hline C & 5.850830 & 0.411531 & 0.508630 \\
\hline C & 6.699400 & -0.705999 & 0.489630 \\
\hline $\mathrm{H}$ & 7.085521 & -2.569869 & -0.491560 \\
\hline $\mathrm{H}$ & 3.668780 & -0.422030 & -1.941870 \\
\hline H & 6.022140 & 1.218461 & 1.213830 \\
\hline C & 5.177511 & -2.743400 & -2.393250 \\
\hline C & 7.866760 & -0.785819 & 1.483300 \\
\hline C & 4.856961 & -4.065480 & -1.663500 \\
\hline $\mathrm{H}$ & 4.718751 & -4.877950 & -2.385440 \\
\hline $\mathrm{H}$ & 5.664771 & -4.352969 & -0.984050 \\
\hline $\mathrm{H}$ & 3.941811 & -3.972890 & -1.073790 \\
\hline C & 4.042931 & -2.429490 & -3.381900 \\
\hline $\mathrm{H}$ & 3.937441 & -3.251950 & -4.096030 \\
\hline $\mathrm{H}$ & 3.080961 & -2.300900 & -2.880510 \\
\hline H & 4.249381 & -1.516900 & -3.949270 \\
\hline C & 6.478201 & -2.914719 & -3.208200 \\
\hline $\mathrm{H}$ & 6.350361 & -3.697799 & -3.963330 \\
\hline $\mathrm{H}$ & 6.739241 & -1.984259 & -3.721630 \\
\hline $\mathrm{H}$ & 7.323901 & -3.197789 & -2.575740 \\
\hline C & 8.805440 & 0.420451 & 1.265510 \\
\hline $\mathrm{H}$ & 9.643630 & 0.384141 & 1.969890 \\
\hline $\mathrm{H}$ & 9.211630 & 0.416631 & 0.249250 \\
\hline H & 8.284430 & 1.370201 & 1.413930 \\
\hline C & 7.310460 & -0.750119 & 2.923210 \\
\hline $\mathrm{H}$ & 8.128280 & -0.801019 & 3.650310 \\
\hline $\mathrm{H}$ & 6.747170 & 0.167051 & 3.115680 \\
\hline $\mathrm{H}$ & 6.641091 & -1.597699 & 3.101010 \\
\hline C & 8.690531 & -2.072859 & 1.317630 \\
\hline $\mathrm{H}$ & 9.506321 & -2.084249 & 2.046910 \\
\hline $\mathrm{H}$ & 8.082391 & -2.967229 & 1.485700 \\
\hline H & 9.135851 & -2.143189 & 0.320330 \\
\hline C & -3.130130 & 1.693720 & -0.767070 \\
\hline C & -2.827900 & 0.526810 & -1.485300 \\
\hline C & -4.450990 & 2.144160 & -0.726530 \\
\hline C & -3.819190 & -0.199980 & -2.135550 \\
\hline $\mathrm{H}$ & -1.811630 & 0.163880 & -1.478170 \\
\hline
\end{tabular}




\begin{tabular}{|c|c|c|c|}
\hline C & -5.477250 & 1.442840 & -1.377010 \\
\hline $\mathrm{H}$ & -4.672850 & 3.062510 & -0.193180 \\
\hline C & -5.139400 & 0.273880 & -2.063460 \\
\hline $\mathrm{H}$ & -5.917420 & -0.291201 & -2.559650 \\
\hline C & -6.913390 & 1.985469 & -1.326380 \\
\hline C & -3.508309 & -1.505460 & -2.877410 \\
\hline C & -6.943540 & 3.387989 & -1.971440 \\
\hline $\mathrm{H}$ & -7.960830 & 3.794079 & -1.954200 \\
\hline $\mathrm{H}$ & -6.291920 & 4.089689 & -1.443930 \\
\hline $\mathrm{H}$ & -6.609030 & 3.341779 & -3.012020 \\
\hline C & -7.906050 & 1.084599 & -2.078570 \\
\hline $\mathrm{H}$ & -8.912790 & 1.507789 & -2.008670 \\
\hline $\mathrm{H}$ & -7.653610 & 1.002529 & -3.140010 \\
\hline $\mathrm{H}$ & -7.938410 & 0.076079 & -1.654040 \\
\hline C & -7.379800 & 2.086769 & 0.141780 \\
\hline $\mathrm{H}$ & -7.413520 & 1.095489 & 0.605090 \\
\hline $\mathrm{H}$ & -6.715240 & 2.718279 & 0.738010 \\
\hline $\mathrm{H}$ & -8.385510 & 2.517409 & 0.194340 \\
\hline C & -3.973929 & -1.384600 & -4.343510 \\
\hline $\mathrm{H}$ & -5.049279 & -1.197630 & -4.415750 \\
\hline $\mathrm{H}$ & -3.454560 & -0.564230 & -4.847880 \\
\hline $\mathrm{H}$ & -3.759349 & -2.311680 & -4.885980 \\
\hline C & -2.002499 & -1.821900 & -2.870230 \\
\hline $\mathrm{H}$ & -1.418909 & -1.027590 & -3.346040 \\
\hline $\mathrm{H}$ & -1.622679 & -1.959660 & -1.854580 \\
\hline $\mathrm{H}$ & -1.823059 & -2.748190 & -3.425910 \\
\hline C & -4.260099 & -2.664310 & -2.186830 \\
\hline $\mathrm{H}$ & -4.051899 & -3.610900 & -2.697660 \\
\hline $\mathrm{H}$ & -3.948139 & -2.762200 & -1.144000 \\
\hline $\mathrm{H}$ & -5.342819 & -2.507850 & -2.199810 \\
\hline
\end{tabular}

\section{C-Catalyzed reaction TS 36}

\section{Datum}

Value

B3LYP-D3(BJ)/6-31G(d,p) Energy

$-3039.489978$

B3LYP-D3(BJ)/def2-TZVPP/IEF-PCM(DCM) Energy

$-3040.521288$

B3LYP-D3(BJ)/def2-TZVPP/IEF-PCM(DCM)//B3LYP-D3(BJ)/6-31G(d,p) Free Energy (Quasiharmonic)

$-3039.28962$

Number of Imaginary Frequencies

Frequencies (Top 3 out of 450)

$\begin{array}{lr}\text { 1. } & -197.3477 \mathrm{~cm}^{-1} \\ \text { 2. } & 12.4589 \mathrm{~cm}^{-1} \\ \text { 3. } & 15.2432 \mathrm{~cm}^{-1}\end{array}$




\begin{tabular}{|c|c|c|c|}
\hline C & 2.298740 & 0.213070 & 0.937270 \\
\hline $\mathrm{H}$ & 3.148160 & -0.414910 & 1.220580 \\
\hline $\mathrm{H}$ & 1.768730 & 0.509540 & 1.846950 \\
\hline C & -0.509311 & 2.029350 & -1.119260 \\
\hline 0 & -0.910171 & 2.864760 & -1.906910 \\
\hline 0 & -0.048850 & 0.853420 & -1.536350 \\
\hline $\mathrm{H}$ & 0.429760 & 0.337060 & -0.816480 \\
\hline 0 & 1.415530 & -0.530430 & 0.083910 \\
\hline $\mathrm{H}$ & 0.881560 & -1.193010 & 0.603200 \\
\hline C & -1.897170 & -1.492860 & -2.917530 \\
\hline C & -2.970900 & -1.277081 & -1.076180 \\
\hline C & -0.408220 & -2.849690 & -1.502130 \\
\hline C & -1.413720 & -2.718770 & -2.510740 \\
\hline $\mathrm{H}$ & -3.075150 & -0.211791 & -1.284530 \\
\hline $\mathrm{H}$ & 0.048260 & -3.837210 & -1.443010 \\
\hline $\mathrm{H}$ & 0.338940 & -2.056820 & -1.521440 \\
\hline C & -0.035360 & -3.327250 & 2.033230 \\
\hline C & -1.401260 & -4.085050 & 1.908230 \\
\hline C & 1.164500 & -4.178380 & 1.603570 \\
\hline $\mathrm{H}$ & 2.040240 & -3.531610 & 1.499830 \\
\hline $\mathrm{H}$ & 1.398170 & -4.950780 & 2.341740 \\
\hline $\mathrm{H}$ & 0.982510 & -4.661030 & 0.641180 \\
\hline C & 0.232470 & -2.694750 & 3.395610 \\
\hline $\mathrm{H}$ & 0.259710 & -3.456320 & 4.181410 \\
\hline $\mathrm{H}$ & 1.203050 & -2.188850 & 3.386240 \\
\hline $\mathrm{H}$ & -0.530460 & -1.956480 & 3.645390 \\
\hline C & -2.513140 & -3.406871 & 2.717020 \\
\hline $\mathrm{H}$ & -2.372340 & -3.537820 & 3.793910 \\
\hline $\mathrm{H}$ & -2.557330 & -2.339231 & 2.494420 \\
\hline $\mathrm{H}$ & -3.474470 & -3.848821 & 2.442950 \\
\hline C & -1.337389 & -5.571790 & 2.237950 \\
\hline $\mathrm{H}$ & -1.015149 & -5.730170 & 3.272310 \\
\hline $\mathrm{H}$ & -2.329339 & -6.017320 & 2.120850 \\
\hline $\mathrm{H}$ & -0.651179 & -6.095930 & 1.570970 \\
\hline 0 & -0.210360 & -2.270450 & 1.072020 \\
\hline 0 & -1.700780 & -3.941300 & 0.508280 \\
\hline B & -1.167090 & -2.688960 & 0.061420 \\
\hline 0 & -2.111300 & -1.534710 & -0.135090 \\
\hline C & -4.199600 & -2.068801 & -1.327140 \\
\hline C & -4.432900 & -3.332891 & -0.768090 \\
\hline C & -5.177250 & -1.493161 & -2.153310 \\
\hline C & -5.629420 & -3.997691 & -1.034290 \\
\hline $\mathrm{H}$ & -3.673000 & -3.786771 & -0.142750 \\
\hline C & -6.365470 & -2.163391 & -2.421500 \\
\hline $\mathrm{H}$ & -5.000870 & -0.510521 & -2.575040 \\
\hline C & -6.597390 & -3.420511 & -1.857420 \\
\hline $\mathrm{H}$ & -5.806040 & -4.974001 & -0.593250 \\
\hline $\mathrm{H}$ & -7.112700 & -1.703731 & -3.061070 \\
\hline $\mathrm{H}$ & -7.526930 & -3.944281 & -2.057870 \\
\hline $\mathrm{H}$ & -1.973930 & -3.609970 & -2.792310 \\
\hline
\end{tabular}




\begin{tabular}{|c|c|c|c|}
\hline $\mathrm{H}$ & -1.292270 & -0.601470 & -2.778350 \\
\hline H & -2.695900 & -1.438171 & -3.649240 \\
\hline C & 0.703269 & 2.589760 & 0.975800 \\
\hline C & -0.506571 & 2.279050 & 0.375650 \\
\hline C & -1.723361 & 2.253840 & 1.138630 \\
\hline C & -1.652281 & 2.553950 & 2.486160 \\
\hline H & -2.565391 & 2.553519 & 3.073060 \\
\hline C & 2.774379 & 1.436460 & 0.189440 \\
\hline C & 1.976069 & 2.573190 & 0.187720 \\
\hline C & 4.412799 & 2.577031 & -1.187210 \\
\hline C & 4.011459 & 1.428010 & -0.532200 \\
\hline $\mathrm{H}$ & 5.348369 & 2.571831 & -1.738740 \\
\hline C & 3.623649 & 3.749620 & -1.202930 \\
\hline C & 2.371049 & 3.751240 & -0.513970 \\
\hline C & 1.571389 & 4.924610 & -0.548900 \\
\hline C & 1.997339 & 6.042840 & -1.226690 \\
\hline C & 3.240929 & 6.043640 & -1.903510 \\
\hline C & 4.034019 & 4.920840 & -1.893500 \\
\hline $\mathrm{H}$ & 0.611129 & 4.920020 & -0.047270 \\
\hline $\mathrm{H}$ & 1.373129 & 6.930520 & -1.250150 \\
\hline $\mathrm{H}$ & 3.562619 & 6.933510 & -2.435870 \\
\hline $\mathrm{H}$ & 4.986279 & 4.909791 & -2.416330 \\
\hline C & -0.441731 & 2.899300 & 3.128910 \\
\hline C & 0.763209 & 2.927140 & 2.363880 \\
\hline C & 1.976329 & 3.281980 & 3.011810 \\
\hline C & 1.991889 & 3.590510 & 4.353290 \\
\hline C & 0.797279 & 3.560630 & 5.111470 \\
\hline C & -0.392921 & 3.223800 & 4.510320 \\
\hline $\mathrm{H}$ & 2.891699 & 3.308660 & 2.432140 \\
\hline $\mathrm{H}$ & 2.926349 & 3.862320 & 4.834310 \\
\hline $\mathrm{H}$ & 0.824189 & 3.806770 & 6.168480 \\
\hline $\mathrm{H}$ & -1.315841 & 3.200410 & 5.083000 \\
\hline C & 6.539880 & -1.998589 & -0.755650 \\
\hline C & 5.178600 & -2.150759 & -1.044490 \\
\hline C & 4.360210 & -1.021119 & -0.967250 \\
\hline C & 4.882710 & 0.224611 & -0.595900 \\
\hline C & 6.250600 & 0.339181 & -0.315970 \\
\hline C & 7.100600 & -0.767649 & -0.393970 \\
\hline $\mathrm{H}$ & 7.185040 & -2.869109 & -0.810200 \\
\hline $\mathrm{H}$ & 3.304070 & -1.088320 & -1.188000 \\
\hline $\mathrm{H}$ & 6.632919 & 1.307301 & -0.017410 \\
\hline C & 4.627650 & -3.534979 & -1.415440 \\
\hline C & 8.603280 & -0.680859 & -0.089390 \\
\hline C & 3.124860 & -3.490110 & -1.733710 \\
\hline $\mathrm{H}$ & 2.766570 & -4.497760 & -1.967580 \\
\hline $\mathrm{H}$ & 2.543180 & -3.114780 & -0.889260 \\
\hline H & 2.912400 & -2.852340 & -2.597130 \\
\hline C & 5.371970 & -4.076719 & -2.653790 \\
\hline $\mathrm{H}$ & 4.985370 & -5.064169 & -2.928700 \\
\hline H & 5.238760 & -3.407019 & -3.508960 \\
\hline $\mathrm{H}$ & 6.445580 & -4.177649 & -2.471520 \\
\hline C & 4.842500 & -4.495059 & -0.225170 \\
\hline $\mathrm{H}$ & 4.456581 & -5.492369 & -0.463270 \\
\hline H & 5.902100 & -4.595379 & 0.026680 \\
\hline
\end{tabular}




\begin{tabular}{|c|c|c|c|}
\hline $\mathrm{H}$ & 4.320210 & -4.131249 & 0.665100 \\
\hline C & 9.041750 & 0.744271 & 0.284330 \\
\hline $\mathrm{H}$ & 10.117360 & 0.758101 & 0.485380 \\
\hline $\mathrm{H}$ & 8.845809 & 1.453221 & -0.526090 \\
\hline $\mathrm{H}$ & 8.531269 & 1.102521 & 1.183660 \\
\hline C & 8.940360 & -1.615779 & 1.092090 \\
\hline $\mathrm{H}$ & 10.010490 & -1.570549 & 1.322140 \\
\hline $\mathrm{H}$ & 8.384760 & -1.323139 & 1.988510 \\
\hline $\mathrm{H}$ & 8.689510 & -2.656319 & 0.868420 \\
\hline C & 9.401450 & -1.120099 & -1.335890 \\
\hline $\mathrm{H}$ & 10.477660 & -1.068319 & -1.137930 \\
\hline $\mathrm{H}$ & 9.163490 & -2.146619 & -1.627720 \\
\hline $\mathrm{H}$ & 9.178100 & -0.470449 & -2.187850 \\
\hline C & -3.053361 & 1.894229 & 0.574620 \\
\hline C & -3.523341 & 2.439669 & -0.624090 \\
\hline C & -3.897121 & 1.038689 & 1.300910 \\
\hline C & -4.792311 & 2.119689 & -1.120170 \\
\hline $\mathrm{H}$ & -2.887231 & 3.122159 & -1.167310 \\
\hline C & -5.188710 & 0.736429 & 0.862270 \\
\hline $\mathrm{H}$ & -3.521110 & 0.604109 & 2.219510 \\
\hline C & -5.611201 & 1.279259 & -0.359750 \\
\hline $\mathrm{H}$ & -6.596001 & 1.032029 & -0.727280 \\
\hline C & -6.122990 & -0.110061 & 1.741470 \\
\hline C & -5.225591 & 2.714349 & -2.468050 \\
\hline C & -5.392220 & -1.354981 & 2.278680 \\
\hline $\mathrm{H}$ & -6.077230 & -1.959541 & 2.882720 \\
\hline $\mathrm{H}$ & -5.021620 & -1.974031 & 1.459640 \\
\hline $\mathrm{H}$ & -4.544510 & -1.091731 & 2.915270 \\
\hline C & -6.574570 & 0.764719 & 2.932250 \\
\hline $\mathrm{H}$ & -7.246540 & 0.202309 & 3.590320 \\
\hline $\mathrm{H}$ & -5.716341 & 1.096389 & 3.524690 \\
\hline $\mathrm{H}$ & -7.104021 & 1.656269 & 2.581660 \\
\hline C & -7.371070 & -0.586581 & 0.979040 \\
\hline $\mathrm{H}$ & -7.998060 & 0.249999 & 0.655790 \\
\hline $\mathrm{H}$ & -7.102700 & -1.178531 & 0.100220 \\
\hline $\mathrm{H}$ & -7.981780 & -1.214271 & 1.635480 \\
\hline C & -5.240801 & 4.254719 & -2.360510 \\
\hline $\mathrm{H}$ & -5.938671 & 4.582439 & -1.583450 \\
\hline $\mathrm{H}$ & -4.251741 & 4.649249 & -2.114540 \\
\hline $\mathrm{H}$ & -5.552671 & 4.699489 & -3.311770 \\
\hline C & -4.213901 & 2.293639 & -3.558590 \\
\hline $\mathrm{H}$ & -3.192921 & 2.591759 & -3.308390 \\
\hline $\mathrm{H}$ & -4.216941 & 1.206789 & -3.697800 \\
\hline $\mathrm{H}$ & -4.479551 & 2.750979 & -4.517930 \\
\hline C & -6.627151 & 2.246749 & -2.891890 \\
\hline $\mathrm{H}$ & -6.882231 & 2.681249 & -3.863210 \\
\hline $\mathrm{H}$ & -6.682481 & 1.157829 & -2.991940 \\
\hline$H$ & -7.393451 & 2.560319 & -2.175950 \\
\hline
\end{tabular}


Frequencies (Top 3 out of 450)

$\begin{array}{lr}\text { 1. } & -274.5230 \mathrm{~cm}^{-1} \\ \text { 2. } & 5.3075 \mathrm{~cm}^{-1} \\ \text { 3. } & 10.3120 \mathrm{~cm}^{-1}\end{array}$

B3LYP-D3(BJ)/6-31G(d,p) Molecular Geometry in Cartesian Coordinates

\begin{tabular}{|c|c|c|c|}
\hline C & -3.017991 & 0.241260 & -2.023654 \\
\hline $\mathrm{H}$ & -2.469279 & -0.167022 & -2.882613 \\
\hline $\mathrm{H}$ & -4.078192 & 0.255795 & -2.290718 \\
\hline C & 0.372768 & 0.515967 & -0.360362 \\
\hline 0 & -0.417031 & 1.444139 & -0.238960 \\
\hline 0 & 1.250838 & 0.281757 & 0.594217 \\
\hline $\mathrm{H}$ & 1.780450 & -0.568168 & 0.512143 \\
\hline 0 & -2.645609 & 1.590490 & -1.791479 \\
\hline $\mathrm{H}$ & -1.787844 & 1.590470 & -1.320439 \\
\hline C & 1.559817 & -0.654316 & 5.136190 \\
\hline C & 3.233540 & -0.004669 & 3.995634 \\
\hline C & 1.265749 & -2.203084 & 3.240149 \\
\hline C & 1.582401 & -1.929868 & 4.601885 \\
\hline $\mathrm{H}$ & 3.969288 & -0.595640 & 4.545170 \\
\hline $\mathrm{H}$ & 0.503429 & -1.566702 & 2.789986 \\
\hline $\mathrm{H}$ & 1.122156 & -3.251732 & 2.992660 \\
\hline C & 3.830024 & -2.387818 & 0.446406 \\
\hline C & 4. 310297 & -3.331572 & 1.603793 \\
\hline C & 3.551984 & -3.071138 & -0.886165 \\
\hline $\mathrm{H}$ & 3.172481 & -2.341935 & -1.606356 \\
\hline $\mathrm{H}$ & 4.467836 & -3.510114 & -1.294800 \\
\hline $\mathrm{H}$ & 2.805024 & -3.859729 & -0.782513 \\
\hline C & 4.760070 & -1.185345 & 0.253417 \\
\hline $\mathrm{H}$ & 5.691867 & -1.482218 & -0.236450 \\
\hline $\mathrm{H}$ & 4.268011 & -0.441538 & -0.374486 \\
\hline $\mathrm{H}$ & 5.002808 & -0.716541 & 1.208773 \\
\hline C & 5.823837 & -3.499866 & 1.692150 \\
\hline H & 6.224116 & -3.937243 & 0.771553 \\
\hline $\mathrm{H}$ & 6.318654 & -2.544356 & 1.871464 \\
\hline $\mathrm{H}$ & 6.070514 & -4.169707 & 2.520721 \\
\hline C & 3.629577 & -4.704695 & 1.558834 \\
\hline $\mathrm{H}$ & 4.009499 & -5.324992 & 0.741630 \\
\hline
\end{tabular}




\begin{tabular}{|c|c|c|c|}
\hline $\mathrm{H}$ & 3.819748 & -5.221599 & 2.503091 \\
\hline $\mathrm{H}$ & 2.550267 & -4.593213 & 1.439547 \\
\hline 0 & 2.568662 & -1.908700 & 0.980860 \\
\hline 0 & 3.851437 & -2.647567 & 2.783340 \\
\hline B & 2.755732 & -1.810319 & 2.415545 \\
\hline 0 & 2.946857 & -0.376237 & 2.785255 \\
\hline C & 3.164281 & 1.440031 & 4.279741 \\
\hline C & 3.805730 & 1.959652 & 5.411269 \\
\hline C & 2.449515 & 2.292623 & 3.423074 \\
\hline C & 3.736291 & 3.322431 & 5.689338 \\
\hline $\mathrm{H}$ & 4.358616 & 1.293801 & 6.068790 \\
\hline C & 2.379881 & 3.652267 & 3.711157 \\
\hline $\mathrm{H}$ & 1.962485 & 1.878165 & 2.545915 \\
\hline C & 3.020532 & 4.169324 & 4.841033 \\
\hline $\mathrm{H}$ & 4.238559 & 3.723285 & 6.564090 \\
\hline $\mathrm{H}$ & 1.825809 & 4.311159 & 3.051295 \\
\hline $\mathrm{H}$ & 2.962388 & 5.231274 & 5.058933 \\
\hline $\mathrm{H}$ & 2.111807 & -2.705897 & 5.154249 \\
\hline $\mathrm{H}$ & 0.897215 & 0.090423 & 4.707701 \\
\hline $\mathrm{H}$ & 1.867649 & -0.478807 & 6.162081 \\
\hline C & -0.578710 & -1.313954 & -1.765660 \\
\hline C & 0.415103 & -0.364669 & -1.587622 \\
\hline C & 1.476475 & -0.209483 & -2.541429 \\
\hline C & 1.508671 & -1.058970 & -3.629967 \\
\hline $\mathrm{H}$ & 2.287401 & -0.933768 & -4.375857 \\
\hline C & -2.827596 & -0.699923 & -0.841233 \\
\hline C & -1.675920 & -1.473731 & -0.759458 \\
\hline C & -3.708529 & -1.827642 & 1.125278 \\
\hline C & -3.858699 & -0.864091 & 0.146004 \\
\hline $\mathrm{H}$ & -4.487256 & -1.947134 & 1.872717 \\
\hline C & -2.580483 & -2.676761 & 1.184070 \\
\hline C & -1.530995 & -2.495577 & 0.231384 \\
\hline C & -0.421882 & -3.383619 & 0.264474 \\
\hline C & -0.377743 & -4.418188 & 1.171126 \\
\hline C & -1.409244 & -4.586584 & 2.125035 \\
\hline C & -2.478843 & -3.722722 & 2.139446 \\
\hline H & 0.388817 & -3.245014 & -0.439093 \\
\hline $\mathrm{H}$ & 0.458416 & -5.109896 & 1.158639 \\
\hline $\mathrm{H}$ & -1.352440 & -5.398952 & 2.843120 \\
\hline $\mathrm{H}$ & -3.277820 & -3.841695 & 2.865962 \\
\hline C & 0.536342 & -2.065999 & -3.830346 \\
\hline C & -0.532051 & -2.195779 & -2.891035 \\
\hline C & -1.515095 & -3.197077 & -3.108205 \\
\hline C & -1.439087 & -4.031040 & -4.200390 \\
\hline C & -0.379039 & -3.903378 & -5.129171 \\
\hline C & 0.585293 & -2.939807 & -4.948294 \\
\hline $\mathrm{H}$ & -2.328008 & -3.293999 & -2.398356 \\
\hline $\mathrm{H}$ & -2.197968 & -4.791958 & -4.353088 \\
\hline $\mathrm{H}$ & -0.331855 & -4.567573 & -5.986577 \\
\hline $\mathrm{H}$ & 1.400053 & -2.830932 & -5.658506 \\
\hline C & -7.453790 & 1.428847 & 0.174419 \\
\hline C & -6.234997 & 2.096351 & 0.008290 \\
\hline C & -5.063512 & 1.335791 & 0.000469 \\
\hline C & -5.107147 & -0.056751 & 0.134232 \\
\hline
\end{tabular}




\begin{tabular}{|c|c|c|c|}
\hline C & -6.346674 & -0.692701 & 0.295701 \\
\hline C & -7.536817 & 0.038461 & 0.321838 \\
\hline $\mathrm{H}$ & -8.370582 & 2.009260 & 0.179585 \\
\hline $\mathrm{H}$ & -4.101372 & 1.805175 & -0.141063 \\
\hline $\mathrm{H}$ & -6.363781 & -1.772340 & 0.377152 \\
\hline C & -6.214432 & 3.619000 & -0.186093 \\
\hline C & -8.912184 & -0.623884 & 0.490150 \\
\hline C & -6.969941 & 3.963607 & -1.488029 \\
\hline $\mathrm{H}$ & -6.977887 & 5.047218 & -1.650033 \\
\hline $\mathrm{H}$ & -6.486885 & 3.492357 & -2.349394 \\
\hline H & -8.008298 & 3.620338 & -1.455293 \\
\hline C & -6.909121 & 4.306350 & 1.008140 \\
\hline $\mathrm{H}$ & -6.897687 & 5.394335 & 0.880036 \\
\hline $\mathrm{H}$ & -7.952578 & 3.993880 & 1.109277 \\
\hline $\mathrm{H}$ & -6.395526 & 4.067481 & 1.944729 \\
\hline C & -4.781674 & 4.167261 & -0.297933 \\
\hline $\mathrm{H}$ & -4.815723 & 5.249539 & -0.459886 \\
\hline $\mathrm{H}$ & -4.212206 & 3.985678 & 0.619266 \\
\hline $\mathrm{H}$ & -4.232929 & 3.713140 & -1.127407 \\
\hline C & -9.782408 & -0.318509 & -0.747980 \\
\hline $\mathrm{H}$ & -10.770514 & -0.780734 & -0.645290 \\
\hline $\mathrm{H}$ & -9.927586 & 0.756639 & -0.884085 \\
\hline $\mathrm{H}$ & -9.313650 & -0.709577 & -1.656352 \\
\hline C & -8.810874 & -2.150237 & 0.641797 \\
\hline $\mathrm{H}$ & -9.811482 & -2.575843 & 0.766211 \\
\hline $\mathrm{H}$ & -8.359111 & -2.615534 & -0.239752 \\
\hline $\mathrm{H}$ & -8.218446 & -2.430712 & 1.518365 \\
\hline C & -9.598766 & -0.058713 & 1.752198 \\
\hline $\mathrm{H}$ & -10.585904 & -0.514485 & 1.887515 \\
\hline $\mathrm{H}$ & -9.000042 & -0.266352 & 2.644605 \\
\hline $\mathrm{H}$ & -9.735426 & 1.024124 & 1.687120 \\
\hline C & 2.520236 & 0.836564 & -2.398638 \\
\hline C & 2.197119 & 2.129450 & -1.986817 \\
\hline C & 3.859698 & 0.534350 & -2.699857 \\
\hline C & 3.182513 & 3.111241 & -1.822145 \\
\hline $\mathrm{H}$ & 1.162921 & 2.377238 & -1.791342 \\
\hline C & 4.864284 & 1.490357 & -2.567847 \\
\hline $\mathrm{H}$ & 4.103418 & -0.474549 & -3.005933 \\
\hline C & 4.502496 & 2.771839 & -2.118337 \\
\hline $\mathrm{H}$ & 5.279685 & 3.515035 & -1.994813 \\
\hline C & 6.338837 & 1.183921 & -2.864823 \\
\hline C & 2.768591 & 4.492495 & -1.299093 \\
\hline C & 7.172836 & 1.397091 & -1.582666 \\
\hline $\mathrm{H}$ & 7.100369 & 2.426197 & -1.220624 \\
\hline $\mathrm{H}$ & 6.829814 & 0.736317 & -0.781181 \\
\hline $\mathrm{H}$ & 8.229716 & 1.183171 & -1.775964 \\
\hline C & 6.549815 & -0.262043 & -3.342589 \\
\hline $\mathrm{H}$ & 7.611418 & -0.430876 & -3.547188 \\
\hline $\mathrm{H}$ & 6.237641 & -0.988888 & -2.586722 \\
\hline $\mathrm{H}$ & 5.996966 & -0.467779 & -4.264626 \\
\hline C & 6.843938 & 2.135852 & -3.970336 \\
\hline $\mathrm{H}$ & 6.267660 & 2.001569 & -4.891069 \\
\hline $\mathrm{H}$ & 6.757424 & 3.184125 & -3.671897 \\
\hline $\mathrm{H}$ & 7.897764 & 1.936416 & -4.193179 \\
\hline
\end{tabular}




\begin{tabular}{|llll|} 
C & 2.147983 & 4.317800 & 0.105449 \\
$\mathrm{H}$ & 1.271721 & 3.664036 & 0.087678 \\
$\mathrm{H}$ & 2.877500 & 3.881090 & 0.793852 \\
$\mathrm{H}$ & 1.838923 & 5.290995 & 0.504278 \\
$\mathrm{C}$ & 3.960558 & 5.455802 & -1.187785 \\
$\mathrm{H}$ & 4.719753 & 5.081956 & -0.493426 \\
$\mathrm{H}$ & 4.436410 & 5.626349 & -2.158976 \\
$\mathrm{H}$ & 3.616827 & 6.424241 & -0.811878 \\
$\mathrm{C}$ & 1.725458 & 5.115800 & -2.250722 \\
$\mathrm{H}$ & 1.420205 & 6.101538 & -1.883626 \\
$\mathrm{H}$ & 2.140086 & 5.238583 & -3.256353 \\
$\mathrm{H}$ & 0.827050 & 4.498391 & -2.329623 \\
& & & \\
\hline
\end{tabular}

\section{C-Catalyzed reaction TS 38}

\section{Datum}

Value

B3LYP-D3(BJ)/6-31G(d,p) Energy

$-3039.483751$

B3LYP-D3(BJ)/def2-TZVPP/IEF-PCM(DCM) Energy

$-3040.519133$

B3LYP-D3(BJ)/def2-TZVPP/IEF-PCM(DCM)//B3LYP-D3(BJ)/6-31G(d,p) Free Energy (Quasiharmonic)

$-3039.289373$

Number of Imaginary Frequencies

Frequencies (Top 3 out of 450)

$\begin{array}{lr}\text { 1. } & -290.2767 \mathrm{~cm}^{-1} \\ \text { 2. } & 7.5884 \mathrm{~cm}^{-1} \\ \text { 3. } & 10.9175 \mathrm{~cm}^{-1}\end{array}$

B3LYP-D3(BJ)/6-31G(d,p) Molecular Geometry in Cartesian Coordinates

C

$\mathrm{H}$

$\mathrm{H}$

C

0

0

$\mathrm{H}$

0

$\mathrm{H}$

C

C

c

C

$\mathrm{H}$

\section{355875}

1.377963

3.084657

$-0.766925$

0.199824

$-1.720960$

$-2.357899$

2. 761706

2.109473

$-1.349853$

$-0.907675$

$-3.669407$

$-2.565409$

$-0.827947$
$-2.075388$

$-2.398662$

$-2.299238$

$-0.582754$

$-0.856736$

0.220147

0.614825

$-2.843185$

$-2.688857$

3.190832

3. 259763

3.193052

2.560267

2.183701
0.644278

0.283748

$-0.134090$

$-0.698678$

$-1.383826$

$-1.175167$

$-0.509633$

1. 787641

2.485442

$-3.040055$

$-0.964532$

$-2.200617$

$-2.842894$

$-0.849962$ 


\begin{tabular}{|c|c|c|c|}
\hline $\mathrm{H}$ & -4.612473 & 2.652626 & -2.260594 \\
\hline H & -3.792811 & 4.256200 & -2.410681 \\
\hline C & -4.401122 & 1.713987 & 0.980420 \\
\hline C & -4.711543 & 3.206389 & 1.353580 \\
\hline C & -5.512484 & 1.059839 & 0.152036 \\
\hline H & -5.136132 & 0.128539 & -0.277120 \\
\hline $\mathrm{H}$ & -6.385651 & 0.825575 & 0.768013 \\
\hline $\mathrm{H}$ & -5.835351 & 1.712467 & -0.661571 \\
\hline C & -4.043888 & 0.824376 & 2.165786 \\
\hline $\mathrm{H}$ & -4.884248 & 0.763483 & 2.864866 \\
\hline $\mathrm{H}$ & -3.815952 & -0.185983 & 1.824311 \\
\hline H & -3.174442 & 1.200370 & 2.706255 \\
\hline C & -3.862081 & 3.705427 & 2.527504 \\
\hline $\mathrm{H}$ & -4.166182 & 3.262533 & 3.480513 \\
\hline $\mathrm{H}$ & -2.810880 & 3.478296 & 2.350551 \\
\hline $\mathrm{H}$ & -3.967831 & 4.790920 & 2.597211 \\
\hline C & -6.186787 & 3.493846 & 1.616836 \\
\hline $\mathrm{H}$ & -6.559474 & 2.898016 & 2.456666 \\
\hline $\mathrm{H}$ & -6.313345 & 4.550573 & 1.867436 \\
\hline $\mathrm{H}$ & -6.795751 & 3.281961 & 0.736760 \\
\hline 0 & -3.237203 & 1.854736 & 0.131569 \\
\hline 0 & -4.306388 & 3.922114 & 0.180756 \\
\hline B & -3.286608 & 3.189919 & -0.477114 \\
\hline 0 & -1.949079 & 3.854152 & -0.447229 \\
\hline C & 0.367376 & 3.989750 & -1.042642 \\
\hline C & 0.440224 & 5.368321 & -0.806435 \\
\hline C & 1.527707 & 3.272569 & -1.365677 \\
\hline C & 1.665692 & 6.021207 & -0.896369 \\
\hline $\mathrm{H}$ & -0.465156 & 5.904031 & -0.543334 \\
\hline C & 2.748671 & 3.932214 & -1.464192 \\
\hline $\mathrm{H}$ & 1.467032 & 2.199242 & -1.524626 \\
\hline C & 2.820115 & 5.306698 & -1.229556 \\
\hline H & 1.724394 & 7.088484 & -0.706129 \\
\hline $\mathrm{H}$ & 3.645321 & 3.372967 & -1.711908 \\
\hline $\mathrm{H}$ & 3.773743 & 5.820701 & -1.302679 \\
\hline $\mathrm{H}$ & -2.583821 & 1.477649 & -2.936152 \\
\hline $\mathrm{H}$ & -1.311455 & 4.270778 & -3.142505 \\
\hline $\mathrm{H}$ & -0.514624 & 2.642269 & -3.461982 \\
\hline C & -0.034753 & -0.846620 & 1.663243 \\
\hline C & -0.970452 & -1.144183 & 0.683841 \\
\hline C & -2.077836 & -2.018420 & 0.948587 \\
\hline C & -2.219842 & -2.531519 & 2.222645 \\
\hline H & -3.032397 & -3.221068 & 2.429261 \\
\hline C & 2.336504 & -0.587470 & 0.931091 \\
\hline C & 1.168368 & 0.006219 & 1.393220 \\
\hline C & 3.479932 & 1.549989 & 1.089135 \\
\hline C & 3.517084 & 0.209016 & 0.761485 \\
\hline $\mathrm{H}$ & 4.380783 & 2.146718 & 0.982864 \\
\hline C & 2.312552 & 2.173280 & 1.579503 \\
\hline C & 1.119442 & 1.397898 & 1.716291 \\
\hline C & -0.056026 & 2.043807 & 2.186539 \\
\hline C & -0.037578 & 3.379955 & 2.516512 \\
\hline C & 1.147597 & 4.141053 & 2.388930 \\
\hline C & 2.297041 & 3.548356 & 1.928754 \\
\hline
\end{tabular}




\begin{tabular}{|c|c|c|c|}
\hline H & -0.974196 & 1.474146 & 2.267712 \\
\hline $\mathrm{H}$ & -0.938727 & 3.860987 & 2.877076 \\
\hline $\mathrm{H}$ & 1.138888 & 5.196063 & 2.642265 \\
\hline $\mathrm{H}$ & 3.207176 & 4.125935 & 1.802020 \\
\hline C & -1.313636 & -2.222896 & 3.262668 \\
\hline C & -0.197688 & -1.372819 & 2.984382 \\
\hline C & 0.725148 & -1.094745 & 4.031063 \\
\hline C & 0.549097 & -1.633782 & 5.285890 \\
\hline C & -0.557507 & -2.472186 & 5.559213 \\
\hline C & -1.466992 & -2.759023 & 4.568475 \\
\hline $\mathrm{H}$ & 1.568381 & -0.445389 & 3.827736 \\
\hline $\mathrm{H}$ & 1.262761 & -1.411977 & 6.072924 \\
\hline $\mathrm{H}$ & -0.684116 & -2.889359 & 6.553233 \\
\hline $\mathrm{H}$ & -2.316965 & -3.405326 & 4.767851 \\
\hline C & 7.186564 & -1.315617 & -0.795381 \\
\hline C & 6.641877 & -0.141474 & -1.343680 \\
\hline C & 5.437511 & 0.325603 & -0.822372 \\
\hline C & 4.786669 & -0.346872 & 0.224989 \\
\hline C & 5.363974 & -1.500794 & 0.754605 \\
\hline C & 6.566619 & -2.009423 & 0.244803 \\
\hline $\mathrm{H}$ & 8.115879 & -1.693326 & -1.202742 \\
\hline $\mathrm{H}$ & 4.963980 & 1.208801 & -1.231987 \\
\hline $\mathrm{H}$ & 4.855237 & -2.008731 & 1.564752 \\
\hline C & 7.372566 & 0.567284 & -2.492896 \\
\hline C & 7.133590 & -3.303553 & 0.843736 \\
\hline C & 8.788146 & 0.970331 & -2.026915 \\
\hline $\mathrm{H}$ & 9.327800 & 1.474548 & -2.836101 \\
\hline $\mathrm{H}$ & 8.735400 & 1.652731 & -1.172761 \\
\hline $\mathrm{H}$ & 9.376693 & 0.100427 & -1.723204 \\
\hline C & 7.481445 & -0.391595 & -3.697927 \\
\hline $\mathrm{H}$ & 8.003978 & 0.095261 & -4.528762 \\
\hline $\mathrm{H}$ & 8.032840 & -1.300213 & -3.441181 \\
\hline $\mathrm{H}$ & 6.487774 & -0.690589 & -4.045614 \\
\hline C & 6.639363 & 1.838062 & -2.953839 \\
\hline $\mathrm{H}$ & 7.198907 & 2.313424 & -3.765308 \\
\hline $\mathrm{H}$ & 5.636403 & 1.612998 & -3.329987 \\
\hline $\mathrm{H}$ & 6.547061 & 2.567038 & -2.142057 \\
\hline C & 7.421452 & -3.085944 & 2.344832 \\
\hline $\mathrm{H}$ & 7.821786 & -4.002286 & 2.792395 \\
\hline $\mathrm{H}$ & 8.154943 & -2.285970 & 2.488304 \\
\hline $\mathrm{H}$ & 6.514991 & -2.813823 & 2.891797 \\
\hline C & 6.089441 & -4.430947 & 0.680713 \\
\hline $\mathrm{H}$ & 6.467572 & -5.363116 & 1.115289 \\
\hline $\mathrm{H}$ & 5.144218 & -4.185663 & 1.171978 \\
\hline $\mathrm{H}$ & 5.880113 & -4.610517 & -0.378884 \\
\hline C & 8.437188 & -3.743668 & 0.159532 \\
\hline $\mathrm{H}$ & 8.797201 & -4.671570 & 0.614485 \\
\hline $\mathrm{H}$ & 8.289029 & -3.933509 & -0.908321 \\
\hline $\mathrm{H}$ & 9.226614 & -2.992838 & 0.268275 \\
\hline C & -3.047418 & -2.398153 & -0.110669 \\
\hline C & -4.419934 & -2.471437 & 0.182425 \\
\hline C & -2.618426 & -2.689653 & -1.405241 \\
\hline C & -5.352234 & -2.800980 & -0.800316 \\
\hline $\mathrm{H}$ & -4.747346 & -2.237324 & 1.186850 \\
\hline
\end{tabular}




\begin{tabular}{|c|c|c|c|}
\hline C & -3.526528 & -2.988857 & -2.427374 \\
\hline $\mathrm{H}$ & -1.559280 & -2.671081 & -1.627252 \\
\hline C & -4.882320 & -3.043296 & -2.102454 \\
\hline $\mathrm{H}$ & -5.601926 & -3.276282 & -2.876715 \\
\hline C & -2.993059 & -3.202536 & -3.849645 \\
\hline C & -6.857847 & -2.891657 & -0.513087 \\
\hline C & -2.283103 & -1.907500 & -4.307878 \\
\hline $\mathrm{H}$ & -2.985402 & -1.067408 & -4.316504 \\
\hline $\mathrm{H}$ & -1.452132 & -1.639662 & -3.650126 \\
\hline $\mathrm{H}$ & -1.887502 & -2.031867 & -5.321911 \\
\hline C & -1.984550 & -4.371019 & -3.854202 \\
\hline $\mathrm{H}$ & -1.592913 & -4.528838 & -4.864722 \\
\hline $\mathrm{H}$ & -1.134185 & -4.176014 & -3.195619 \\
\hline $\mathrm{H}$ & -2.461937 & -5.298889 & -3.523405 \\
\hline C & -4.113460 & -3.525569 & -4.850287 \\
\hline $\mathrm{H}$ & -4.643870 & -4.445152 & -4.582393 \\
\hline $\mathrm{H}$ & -4.844697 & -2.713625 & -4.915628 \\
\hline $\mathrm{H}$ & -3.686540 & -3.666943 & -5.847708 \\
\hline C & -7.191757 & -2.578330 & 0.954521 \\
\hline $\mathrm{H}$ & -6.704030 & -3.279610 & 1.638970 \\
\hline $\mathrm{H}$ & -6.891059 & -1.563423 & 1.230883 \\
\hline H & -8.271616 & -2.659189 & 1.111313 \\
\hline C & -7.617089 & -1.887194 & -1.406945 \\
\hline $\mathrm{H}$ & -7.293668 & -0.862276 & -1.203962 \\
\hline $\mathrm{H}$ & -7.453047 & -2.086239 & -2.469394 \\
\hline $\mathrm{H}$ & -8.694139 & -1.949925 & -1.217714 \\
\hline C & -7.343905 & -4.323832 & -0.824439 \\
\hline $\mathrm{H}$ & -8.418249 & -4.412669 & -0.630112 \\
\hline $\mathrm{H}$ & -7.168997 & -4.591001 & -1.870194 \\
\hline $\mathrm{H}$ & -6.821103 & -5.055379 & -0.200402 \\
\hline
\end{tabular}

\section{C-Catalyzed reaction TS 39}

\section{Datum}

Value

B3LYP-D3(BJ)/6-31G(d,p) Energy

$-3039.482991$

B3LYP-D3(BJ)/def2-TZVPP/IEF-PCM(DCM) Energy

$-3040.519392$

B3LYP-D3(BJ)/def2-TZVPP/IEF-PCM(DCM)//B3LYP-D3(BJ)/6-31G(d,p) Free Energy (Quasiharmonic)

$-3039.289201$

Number of Imaginary Frequencies

Frequencies (Top 3 out of 450)

$\begin{array}{lr}\text { 1. } & -286.5931 \mathrm{~cm}^{-1} \\ \text { 2. } & 6.9500 \mathrm{~cm}^{-1} \\ \text { 3. } & 10.4898 \mathrm{~cm}^{-1}\end{array}$




\begin{tabular}{|c|c|c|c|}
\hline C & -3.104105 & 0.467396 & -1.726161 \\
\hline $\mathrm{H}$ & -2.552464 & 0.194446 & -2.634004 \\
\hline $\mathrm{H}$ & -4.155762 & 0.576563 & -2.001303 \\
\hline C & 0.314242 & 0.406162 & -0.037994 \\
\hline 0 & -0.461085 & 1.268328 & 0.351669 \\
\hline 0 & 1.250272 & -0.050516 & 0.777532 \\
\hline $\mathrm{H}$ & 1.775811 & -0.822167 & 0.439268 \\
\hline 0 & -2.677944 & 1.740035 & -1.250813 \\
\hline $\mathrm{H}$ & -1.864439 & 1.616507 & -0.721477 \\
\hline C & 3.075157 & -1.676103 & 4.987558 \\
\hline C & 3.984153 & -0.516194 & 3.451878 \\
\hline C & 2.435271 & -3.115219 & 3.090314 \\
\hline C & 3.177278 & -2.860481 & 4.279604 \\
\hline $\mathrm{H}$ & 4.982466 & -0.935905 & 3.589657 \\
\hline $\mathrm{H}$ & 1.436910 & -2.681376 & 3.042245 \\
\hline $\mathrm{H}$ & 2.457348 & -4.144949 & 2.738253 \\
\hline C & 3.610585 & -3.028605 & -0.315791 \\
\hline C & 5.008735 & -2.803005 & 0.354067 \\
\hline C & 3.184944 & -4.500695 & -0.301890 \\
\hline $\mathrm{H}$ & 2.133411 & -4.575707 & -0.588504 \\
\hline $\mathrm{H}$ & 3.770971 & -5.089576 & -1.013055 \\
\hline $\mathrm{H}$ & 3.309432 & -4.938437 & 0.691088 \\
\hline C & 3.472585 & -2.460820 & -1.719126 \\
\hline $\mathrm{H}$ & 4.200462 & -2.921546 & -2.395065 \\
\hline $\mathrm{H}$ & 2.473455 & -2.659436 & -2.114734 \\
\hline $\mathrm{H}$ & 3.627231 & -1.384075 & -1.718555 \\
\hline C & 5.597730 & -1.426352 & 0.012181 \\
\hline $\mathrm{H}$ & 5.972482 & -1.393168 & -1.014552 \\
\hline $\mathrm{H}$ & 4.858354 & -0.631695 & 0.136125 \\
\hline $\mathrm{H}$ & 6.434311 & -1.226650 & 0.687530 \\
\hline C & 6.034068 & -3.896377 & 0.077969 \\
\hline $\mathrm{H}$ & 6.234204 & -3.984542 & -0.994826 \\
\hline $\mathrm{H}$ & 6.975175 & -3.653090 & 0.579243 \\
\hline $\mathrm{H}$ & 5.692015 & -4.862110 & 0.453183 \\
\hline 0 & 2.733252 & -2.292540 & 0.574034 \\
\hline 0 & 4.677032 & -2.830196 & 1.754273 \\
\hline B & 3.371819 & -2.264000 & 1.874756 \\
\hline 0 & 3.335221 & -0.863608 & 2.381828 \\
\hline C & 3.724362 & 0.831536 & 3.986626 \\
\hline C & 4.606619 & 1.387586 & 4.922804 \\
\hline C & 2.594121 & 1.553310 & 3.571460 \\
\hline C & 4.362395 & 2.653839 & 5.446941 \\
\hline $\mathrm{H}$ & 5.481681 & 0.824371 & 5.236619 \\
\hline C & 2.353281 & 2.815732 & 4.106207 \\
\hline $\mathrm{H}$ & 1.929474 & 1.123706 & 2.829183 \\
\hline C & 3.232245 & 3.367507 & 5.042214 \\
\hline $\mathrm{H}$ & 5.050028 & 3.083725 & 6.168400 \\
\hline $\mathrm{H}$ & 1.479799 & 3.373452 & 3.785673 \\
\hline $\mathrm{H}$ & 3.038447 & 4.353608 & 5.452827 \\
\hline $\mathrm{H}$ & 4.035968 & -3.501490 & 4.476728 \\
\hline
\end{tabular}




\begin{tabular}{|c|c|c|c|}
\hline $\mathrm{H}$ & 2.149989 & -1.110403 & 4.950211 \\
\hline $\mathrm{H}$ & 3.705030 & -1.494128 & 5.852325 \\
\hline C & -0.716227 & -1.125852 & -1.686143 \\
\hline C & 0.270265 & -0.191500 & -1.420513 \\
\hline C & 1.244553 & 0.176477 & -2.405564 \\
\hline C & 1.217897 & -0.476285 & -3.621499 \\
\hline $\mathrm{H}$ & 1.926226 & -0.190388 & -4.392933 \\
\hline C & -2.958112 & -0.645266 & -0.701305 \\
\hline C & -1.792795 & -1.403482 & -0.681600 \\
\hline C & -3.833995 & -1.963547 & 1.143535 \\
\hline C & -4.000737 & -0.926183 & 0.245347 \\
\hline $\mathrm{H}$ & -4.620287 & -2.170364 & 1.863497 \\
\hline C & -2.659439 & -2.747518 & 1.183142 \\
\hline C & -1.607678 & -2.461573 & 0.258766 \\
\hline C & -0.423197 & -3.242086 & 0.311585 \\
\hline C & -0.293643 & -4.262856 & 1.224937 \\
\hline C & -1.337236 & -4.549876 & 2.136628 \\
\hline C & -2.492390 & -3.804026 & 2.117295 \\
\hline $\mathrm{H}$ & 0.392573 & -3.012012 & -0.360981 \\
\hline $\mathrm{H}$ & 0.619493 & -4.846827 & 1.250360 \\
\hline $\mathrm{H}$ & -1.219548 & -5.356607 & 2.853710 \\
\hline $\mathrm{H}$ & -3.297429 & -4.010571 & 2.817053 \\
\hline C & 0.279442 & -1.496042 & -3.907896 \\
\hline C & -0.713762 & -1.824660 & -2.932857 \\
\hline C & -1.667259 & -2.831329 & -3.237078 \\
\hline C & -1.632444 & -3.488346 & -4.445949 \\
\hline C & -0.648509 & -3.165061 & -5.410476 \\
\hline C & 0.283614 & -2.188559 & -5.147497 \\
\hline $\mathrm{H}$ & -2.421216 & -3.075919 & -2.497972 \\
\hline $\mathrm{H}$ & -2.364940 & -4.259167 & -4.663882 \\
\hline $\mathrm{H}$ & -0.634080 & -3.689891 & -6.360678 \\
\hline $\mathrm{H}$ & 1.038926 & -1.931093 & -5.884594 \\
\hline C & -7.661210 & 1.266125 & 0.418928 \\
\hline C & -6.460994 & 1.972180 & 0.336918 \\
\hline C & -5.266629 & 1.241509 & 0.283051 \\
\hline C & -5.270586 & -0.153302 & 0.285890 \\
\hline C & -6.498402 & -0.833492 & 0.365472 \\
\hline C & -7.704533 & -0.138927 & 0.437371 \\
\hline $\mathrm{H}$ & -8.594835 & 1.812804 & 0.462545 \\
\hline $\mathrm{H}$ & -4.316240 & 1.754925 & 0.211881 \\
\hline $\mathrm{H}$ & -6.486935 & -1.916188 & 0.347618 \\
\hline C & -6.388874 & 3.504135 & 0.288353 \\
\hline C & -9.064426 & -0.847467 & 0.520567 \\
\hline C & -5.691808 & 3.930069 & -1.023574 \\
\hline $\mathrm{H}$ & -5.612086 & 5.022078 & -1.072096 \\
\hline $\mathrm{H}$ & -4.686258 & 3.509289 & -1.105800 \\
\hline $\mathrm{H}$ & -6.269146 & 3.593490 & -1.891133 \\
\hline C & -7.778641 & 4.157479 & 0.339222 \\
\hline $\mathrm{H}$ & -7.676091 & 5.246222 & 0.295689 \\
\hline $\mathrm{H}$ & -8.401508 & 3.849124 & -0.506626 \\
\hline $\mathrm{H}$ & -8.310066 & 3.910745 & 1.264304 \\
\hline C & -5.566862 & 4.013553 & 1.491849 \\
\hline $\mathrm{H}$ & -5.499670 & 5.106958 & 1.470643 \\
\hline $\mathrm{H}$ & -6.034954 & 3.717467 & 2.436305 \\
\hline
\end{tabular}




\begin{tabular}{|c|c|c|c|}
\hline H & -4.549407 & 3.614599 & 1.480107 \\
\hline C & -9.785153 & -0.417085 & 1.816424 \\
\hline $\mathrm{H}$ & -10.761166 & -0.909099 & 1.892682 \\
\hline $\mathrm{H}$ & -9.194388 & -0.689287 & 2.696688 \\
\hline $\mathrm{H}$ & -9.951141 & 0.663162 & 1.847025 \\
\hline C & -9.923654 & -0.452119 & -0.699752 \\
\hline $\mathrm{H}$ & -10.900952 & -0.945603 & -0.655993 \\
\hline H & -10.094445 & 0.627058 & -0.740723 \\
\hline $\mathrm{H}$ & -9.431578 & -0.748052 & -1.631493 \\
\hline C & -8.925877 & -2.378323 & 0.534794 \\
\hline $\mathrm{H}$ & -9.916864 & -2.837886 & 0.602765 \\
\hline $\mathrm{H}$ & -8.449073 & -2.750098 & -0.377443 \\
\hline $\mathrm{H}$ & -8.339509 & -2.722935 & 1.392342 \\
\hline C & 2.240582 & 1.248051 & -2.153808 \\
\hline C & 1.870025 & 2.426874 & -1.505559 \\
\hline C & 3.567474 & 1.104731 & -2.596214 \\
\hline C & 2.799752 & 3.442349 & -1.246783 \\
\hline H & 0.839309 & 2.561060 & -1.207561 \\
\hline C & 4.517325 & 2.097553 & -2.366375 \\
\hline $\mathrm{H}$ & 3.844987 & 0.195458 & -3.111726 \\
\hline C & 4.112453 & 3.254313 & -1.678491 \\
\hline $\mathrm{H}$ & 4.848270 & 4.023553 & -1.482606 \\
\hline C & 5.978132 & 1.962891 & -2.818671 \\
\hline C & 2.336924 & 4.694371 & -0.490964 \\
\hline C & 6.897001 & 1.983408 & -1.577774 \\
\hline H & 6.789183 & 2.913551 & -1.013035 \\
\hline $\mathrm{H}$ & 6.659292 & 1.154241 & -0.905117 \\
\hline $\mathrm{H}$ & 7.946605 & 1.892300 & -1.878261 \\
\hline C & 6.232859 & 0.657266 & -3.589161 \\
\hline $\mathrm{H}$ & 7.282527 & 0.608148 & -3.894229 \\
\hline $\mathrm{H}$ & 6.024737 & -0.224099 & -2.975516 \\
\hline $\mathrm{H}$ & 5.619923 & 0.596426 & -4.494034 \\
\hline C & 6.338948 & 3.144163 & -3.745015 \\
\hline H & 5.697625 & 3.151876 & -4.631854 \\
\hline $\mathrm{H}$ & 6.222007 & 4.106526 & -3.239728 \\
\hline $\mathrm{H}$ & 7.380208 & 3.064151 & -4.075588 \\
\hline C & 3.467372 & 5.719979 & -0.313243 \\
\hline $\mathrm{H}$ & 4.300656 & 5.308075 & 0.264942 \\
\hline $\mathrm{H}$ & 3.855043 & 6.067874 & -1.276292 \\
\hline $\mathrm{H}$ & 3.089652 & 6.592874 & 0.227795 \\
\hline C & 1.184878 & 5.368912 & -1.265871 \\
\hline $\mathrm{H}$ & 1.511609 & 5.669752 & -2.266416 \\
\hline $\mathrm{H}$ & 0.325306 & 4.703274 & -1.378153 \\
\hline $\mathrm{H}$ & 0.845035 & 6.263660 & -0.733362 \\
\hline C & 1.840507 & 4.267100 & 0.907663 \\
\hline $\mathrm{H}$ & 1.493759 & 5.141764 & 1.469958 \\
\hline $\mathrm{H}$ & 1.015494 & 3.551857 & 0.848163 \\
\hline $\mathrm{H}$ & 2.648887 & 3.796121 & 1.473503 \\
\hline
\end{tabular}


Frequencies (Top 3 out of 450)

$\begin{array}{lrl}\text { 1. } & -254.2066 \mathrm{~cm}^{-1} \\ \text { 2. } & 9.5571 \mathrm{~cm}^{-1} \\ \text { 3. } & 12.4813 \mathrm{~cm}^{-1}\end{array}$

\section{B3LYP-D3(BJ)/6-31G(d,p) Molecular Geometry in Cartesian Coordinates}

C

$\mathrm{H}$

$\mathrm{H}$

C

0

0

$\mathrm{H}$

0

$\mathrm{H}$

C

C

C

C

$\mathrm{H}$

$\mathrm{H}$

$\mathrm{H}$

C

C

C

$\mathrm{H}$

$\mathrm{H}$

$\mathrm{H}$

C

$\mathrm{H}$

$\mathrm{H}$

$\mathrm{H}$

C

$\mathrm{H}$

$\mathrm{H}$

$\mathrm{H}$

C

$\mathrm{H}$

\subsection{0}

4.023110

2.373650

$-0.339820$

0.521730

$-1.273430$

$-1.911720$

2.613660

1.860650

$-3.688960$

$-3.286810$

$-4.341740$

$-4.654100$

$-4.255570$

$-3.412360$

$-5.159030$

$-4.078290$

$-5.520390$

$-4.023860$

$-2.984280$

$-4.531850$

$-4.491250$

$-3.297730$

$-3.733760$

- 3.282670

$-2.270180$

$-6.200100$

$-6.309310$

$-5.636010$

$-7.198520$

$-6.437450$

$-6.761960$

\section{$-1.764160$}

$-2.078300$

$-2.194560$

$-0.347780$

$-0.615290$

0.557070

0.688110

$-2.298250$

$-1.775950$

2. 167971

3.503740

0.629931

1.484011

4.004771

0.073480

$-0.003629$

1.505181

1.878601

0.422691

0.198290

0.755961

$-0.499639$

2. 738020

3.161041

3.508920

2.453220

2.998131

2. 731551

3. 929191

3.175481

0.652391

0. 322941
0.943650

1.119190

1.739920

$-0.435280$

$-1.258030$

$-0.709760$

0.038700

$-0.323610$

$-0.663480$

$-3.400560$

$-1.744650$

$-1.586920$

$-2.690410$

$-1.797550$

$-1.689510$

$-1.245890$

2.065000

1.564190

3.135430

3.387390

4.046160

2.792180

2.532650

3.441620

1.759410

2.762540

2.346000

3.402420

2. 273260

1.936220

1.493940

2.485650 


\begin{tabular}{|c|c|c|c|}
\hline $\mathrm{H}$ & -7.324270 & 0.908331 & 0.908040 \\
\hline $\mathrm{H}$ & -5.925360 & -0.176339 & 1.002380 \\
\hline 0 & -3.477900 & 0.990900 & 0.850230 \\
\hline 0 & -5.286260 & 2.334831 & 0.222300 \\
\hline B & -4.090300 & 1.713711 & -0.243150 \\
\hline 0 & -3.075780 & 2.688620 & -0.763120 \\
\hline C & -2.117020 & 4.218240 & -2.280270 \\
\hline C & -2.297550 & 5.365400 & -3.065150 \\
\hline C & -0.820830 & 3.768570 & -1.981840 \\
\hline C & -1.192140 & 6.061490 & -3.546110 \\
\hline $\mathrm{H}$ & -3.303360 & 5.710030 & -3.291000 \\
\hline C & 0.279190 & 4.471550 & -2.462070 \\
\hline $\mathrm{H}$ & -0.693210 & 2.878860 & -1.377240 \\
\hline C & 0.096960 & 5.615490 & -3.243910 \\
\hline $\mathrm{H}$ & -1.333429 & 6.951850 & -4.150640 \\
\hline $\mathrm{H}$ & 1.278280 & 4.128350 & -2.216020 \\
\hline $\mathrm{H}$ & 0.958610 & 6.160680 & -3.616960 \\
\hline $\mathrm{H}$ & -5.692710 & 1.792111 & -2.812990 \\
\hline $\mathrm{H}$ & -2.669950 & 1.794820 & -3.413240 \\
\hline $\mathrm{H}$ & -3.962700 & 2.846351 & -4.202090 \\
\hline C & 0.560690 & -0.538020 & 1.839140 \\
\hline C & -0.410280 & -0.946580 & 0.940610 \\
\hline C & -1.480820 & -1.823710 & 1.315850 \\
\hline C & -1.536960 & -2.235110 & 2.633040 \\
\hline $\mathrm{H}$ & -2.308030 & -2.933740 & 2.941410 \\
\hline C & 2.889180 & -0.251310 & 0.979300 \\
\hline C & 1.692180 & 0.331770 & 1.376570 \\
\hline C & 3.798120 & 1.950730 & 0.498780 \\
\hline C & 3.970420 & 0.579850 & 0.532210 \\
\hline $\mathrm{H}$ & 4.612300 & 2.576760 & 0.145590 \\
\hline C & 2.575980 & 2.564980 & 0.852040 \\
\hline C & 1.497290 & 1.744510 & 1.305610 \\
\hline C & 0.262580 & 2.365430 & 1.628830 \\
\hline C & 0.096030 & 3.724430 & 1.496610 \\
\hline C & 1.165900 & 4.536110 & 1.050410 \\
\hline C & 2.379470 & 3.967660 & 0.741700 \\
\hline $\mathrm{H}$ & -0.560490 & 1.744850 & 1.959320 \\
\hline $\mathrm{H}$ & -0.863060 & 4.175300 & 1.726020 \\
\hline $\mathrm{H}$ & 1.020800 & 5.606440 & 0.944110 \\
\hline $\mathrm{H}$ & 3.206530 & 4.581770 & 0.395920 \\
\hline C & -0.606200 & -1.792280 & 3.603480 \\
\hline C & 0.466730 & -0.932660 & 3.208060 \\
\hline C & 1.399750 & -0.501940 & 4.186660 \\
\hline C & 1.275280 & -0.901280 & 5.497960 \\
\hline C & 0.215740 & -1.753530 & 5.890390 \\
\hline C & -0.701590 & -2.190360 & 4.963220 \\
\hline $\mathrm{H}$ & 2.211580 & 0.148300 & 3.881320 \\
\hline $\mathrm{H}$ & 1.993920 & -0.562580 & 6.237570 \\
\hline $\mathrm{H}$ & 0.130340 & -2.062290 & 6.927630 \\
\hline $\mathrm{H}$ & -1.516020 & -2.846350 & 5.257200 \\
\hline C & 7.746930 & -0.958800 & -0.732380 \\
\hline C & 7.715070 & 0.091670 & 0.201260 \\
\hline C & 6.470670 & 0.568580 & 0.610110 \\
\hline C & 5.280170 & 0.022290 & 0.100760 \\
\hline
\end{tabular}




\begin{tabular}{|c|c|c|c|}
\hline C & 5.351110 & -1.009710 & -0.834930 \\
\hline C & 6.584320 & -1.523820 & -1.256980 \\
\hline $\mathrm{H}$ & 8.709680 & -1.341810 & -1.046750 \\
\hline $\mathrm{H}$ & 6.397860 & 1.359020 & 1.346870 \\
\hline $\mathrm{H}$ & 4.426550 & -1.423000 & -1.218480 \\
\hline C & 9.036810 & 0.659870 & 0.738270 \\
\hline C & 6.592720 & -2.681940 & -2.263480 \\
\hline C & 9.869520 & 1.211830 & -0.439000 \\
\hline $\mathrm{H}$ & 10.820550 & 1.618350 & -0.077440 \\
\hline $\mathrm{H}$ & 10.095010 & 0.434370 & -1.173940 \\
\hline $\mathrm{H}$ & 9.328640 & 2.011730 & -0.954360 \\
\hline C & 8.816230 & 1.799530 & 1.745940 \\
\hline $\mathrm{H}$ & 9.782930 & 2.175810 & 2.094840 \\
\hline $\mathrm{H}$ & 8.275570 & 2.638050 & 1.295880 \\
\hline $\mathrm{H}$ & 8.255020 & 1.461480 & 2.622570 \\
\hline C & 9.828010 & -0.462100 & 1.444570 \\
\hline $\mathrm{H}$ & 10.777340 & -0.075560 & 1.831670 \\
\hline $\mathrm{H}$ & 9.256000 & -0.869410 & 2.284080 \\
\hline $\mathrm{H}$ & 10.054710 & -1.286940 & 0.763620 \\
\hline C & 5.913130 & -2.221960 & -3.571290 \\
\hline $\mathrm{H}$ & 5.902760 & -3.038100 & -4.302210 \\
\hline $\mathrm{H}$ & 4.879090 & -1.913110 & -3.398120 \\
\hline H & 6.449430 & -1.374920 & -4.011330 \\
\hline C & 8.015340 & -3.157450 & -2.596620 \\
\hline $\mathrm{H}$ & 7.969500 & -3.987840 & -3.307980 \\
\hline $\mathrm{H}$ & 8.541900 & -3.512830 & -1.704990 \\
\hline $\mathrm{H}$ & 8.613130 & -2.362020 & -3.053630 \\
\hline C & 5.803670 & -3.869890 & -1.668320 \\
\hline $\mathrm{H}$ & 5.784860 & -4.704670 & -2.378020 \\
\hline $\mathrm{H}$ & 6.275960 & -4.220460 & -0.744620 \\
\hline H & 4.770890 & -3.597520 & -1.435600 \\
\hline C & -2.476220 & -2.307510 & 0.325360 \\
\hline C & -3.804960 & -2.564219 & 0.709670 \\
\hline C & -2.108930 & -2.540900 & -1.001010 \\
\hline C & -4.744340 & -3.038299 & -0.203600 \\
\hline $\mathrm{H}$ & -4.091160 & -2.379399 & 1.735610 \\
\hline C & -3.041970 & -2.945530 & -1.964370 \\
\hline $\mathrm{H}$ & -1.077260 & -2.413170 & -1.296520 \\
\hline C & -4.347130 & -3.193799 & -1.543700 \\
\hline $\mathrm{H}$ & -5.081020 & -3.527579 & -2.265710 \\
\hline C & -2.586750 & -3.085780 & -3.422820 \\
\hline C & -6.177420 & -3.419899 & 0.197400 \\
\hline C & -2.065970 & -1.716320 & -3.916740 \\
\hline $\mathrm{H}$ & -2.861760 & -0.965540 & -3.880610 \\
\hline $\mathrm{H}$ & -1.231520 & -1.352270 & -3.311420 \\
\hline $\mathrm{H}$ & -1.720460 & -1.796280 & -4.953310 \\
\hline C & -1.451410 & -4.128040 & -3.510270 \\
\hline $\mathrm{H}$ & -1.118280 & -4.238060 & -4.547780 \\
\hline $\mathrm{H}$ & -0.584050 & -3.835170 & -2.912980 \\
\hline $\mathrm{H}$ & -1.791820 & -5.105600 & -3.154290 \\
\hline C & -3.729680 & -3.532319 & -4.347590 \\
\hline $\mathrm{H}$ & -4.123440 & -4.513329 & -4.062480 \\
\hline $\mathrm{H}$ & -4.556600 & -2.814929 & -4.342580 \\
\hline H & -3.362850 & -3.608190 & -5.375610 \\
\hline
\end{tabular}




$\begin{array}{|llll|}\text { C } & -7.193010 & -2.576939 & -0.603200 \\ \text { H } & -7.072810 & -1.511009 & -0.392510 \\ \text { H } & -7.076640 & -2.721429 & -1.680760 \\ \text { H } & -8.216660 & -2.861609 & -0.336830 \\ \text { C } & -6.397440 & -4.915659 & -0.122380 \\ H & -6.269280 & -5.124989 & -1.187730 \\ H & -5.685960 & -5.538339 & 0.428740 \\ H & -7.411020 & -5.219899 & 0.160620 \\ \text { C } & -6.441800 & -3.214799 & 1.697940 \\ \text { H } & -7.466730 & -3.516159 & 1.934850 \\ \text { H } & -5.767510 & -3.821639 & 2.310360 \\ \text { H } & -6.328840 & -2.169499 & 1.995560 \\ \end{array}$

\section{C-Catalyzed reaction TS 41}

\section{Datum}

Value

B3LYP-D3(BJ)/6-31G(d,p) Energy

$-3039.485$

B3LYP-D3(BJ)/def2-TZVPP/IEF-PCM(DCM) Energy

$-3040.519414$

B3LYP-D3(BJ)/def2-TZVPP/IEF-PCM(DCM)//B3LYP-D3(BJ)/6-31G(d,p) Free Energy (Quasiharmonic)

$-3039.288723$

Number of Imaginary Frequencies

Frequencies (Top 3 out of 450)

$\begin{array}{lr}\text { 1. } & -278.2041 \mathrm{~cm}^{-1} \\ \text { 2. } & 10.2927 \mathrm{~cm}^{-1} \\ \text { 3. } & 12.7288 \mathrm{~cm}^{-1}\end{array}$

B3LYP-D3(BJ)/6-31G(d,p) Molecular Geometry in Cartesian Coordinates

C

$\mathrm{H}$

$\mathrm{H}$

C

0

0

$\mathrm{H}$

0

$\mathrm{H}$

C

C

c

C

$\mathrm{H}$
2.982928

4.014264

2.434804

$-0.326897$

0.555895

$-1.458754$

$-2.171448$

2.437945

1.797837

$-4.123804$

$-3.668881$

$-5.306276$

$-4.245276$

$-2.672013$
0.389219

0.632828

0.202608

$-0.009233$

0.546328

$-0.331379$

$-0.809362$

1.533914

1. 212182

1.614586

$-0.374853$

0.568304

1.354595

$-0.348323$
$-1.492420$

$-1.758932$

$-2.423383$

0.208281

0.847228

0.814745

0.285890

$-0.832158$

$-0.162640$

2.430509

2.925706

0.539507

1.075134

2.493012 


\begin{tabular}{|c|c|c|c|}
\hline $\mathrm{H}$ & -5.392107 & 0.588611 & -0.544065 \\
\hline $\mathrm{H}$ & -6.269736 & 0.661159 & 1.041995 \\
\hline C & -4.002042 & -2.530035 & -0.829070 \\
\hline C & -5.225152 & -3.146885 & -0.072842 \\
\hline C & -4.397059 & -1.936760 & -2.183609 \\
\hline $\mathrm{H}$ & -3.559898 & -1.357709 & -2.574014 \\
\hline $\mathrm{H}$ & -4.631211 & -2.722915 & -2.906930 \\
\hline $\mathrm{H}$ & -5.268237 & -1.285608 & -2.085323 \\
\hline C & -2.805569 & -3.456892 & -0.997265 \\
\hline $\mathrm{H}$ & -3.088969 & -4.360145 & -1.546437 \\
\hline H & -2.394316 & -3.748710 & -0.029855 \\
\hline $\mathrm{H}$ & -2.024064 & -2.951525 & -1.572138 \\
\hline C & -4.795357 & -4.081177 & 1.067863 \\
\hline $\mathrm{H}$ & -4.398801 & -5.027650 & 0.687986 \\
\hline $\mathrm{H}$ & -4.038769 & -3.612750 & 1.700966 \\
\hline $\mathrm{H}$ & -5.668142 & -4.295578 & 1.689592 \\
\hline C & -6.237323 & -3.851345 & -0.969908 \\
\hline $\mathrm{H}$ & -5.773436 & -4.684113 & -1.508737 \\
\hline $\mathrm{H}$ & -7.049043 & -4.253295 & -0.357544 \\
\hline $\mathrm{H}$ & -6.672424 & -3.160819 & -1.693798 \\
\hline 0 & -3.628525 & -1.443538 & 0.057715 \\
\hline 0 & -5.848077 & -1.982699 & 0.489929 \\
\hline B & -4.812979 & -1.082884 & 0.848516 \\
\hline 0 & -4.586359 & -1.085529 & 2.323781 \\
\hline C & -3.743854 & -0.291086 & 4. 397214 \\
\hline C & -4.912491 & -0.646149 & 5.084353 \\
\hline C & -2.620739 & 0.146290 & 5.112573 \\
\hline C & -4.953587 & -0.560391 & 6.472647 \\
\hline $\mathrm{H}$ & -5.767839 & -0.995586 & 4.516741 \\
\hline C & -2.666888 & 0.231536 & 6.501482 \\
\hline $\mathrm{H}$ & -1.715540 & 0.414314 & 4.574293 \\
\hline C & -3.833772 & -0.119767 & 7.183404 \\
\hline $\mathrm{H}$ & -5.857849 & -0.840098 & 7.004311 \\
\hline $\mathrm{H}$ & -1.793802 & 0.567240 & 7.051997 \\
\hline $\mathrm{H}$ & -3.869639 & -0.054422 & 8.266461 \\
\hline $\mathrm{H}$ & -3.377394 & 1.527680 & 0.444919 \\
\hline $\mathrm{H}$ & -5.014170 & 1.650595 & 3.050419 \\
\hline $\mathrm{H}$ & -3.263850 & 2.160569 & 2.804195 \\
\hline C & 0.722684 & -1.347019 & -1.610749 \\
\hline C & -0.227600 & -0.408507 & -1.240269 \\
\hline C & -1.161178 & 0.125005 & -2.191174 \\
\hline C & -1.212657 & -0.439028 & -3.447960 \\
\hline $\mathrm{H}$ & -1.883245 & -0.021534 & -4.192909 \\
\hline C & 2.958083 & -0.865942 & -0.636037 \\
\hline C & 1.849883 & -1.705299 & -0.691599 \\
\hline C & 3.985242 & -2.347822 & 0.993829 \\
\hline C & 4.049067 & -1.191834 & 0.237705 \\
\hline $\mathrm{H}$ & 4.804545 & -2.585165 & 1.665895 \\
\hline C & 2.873742 & -3.218521 & 0.949487 \\
\hline C & 1.780206 & -2.896796 & 0.088240 \\
\hline C & 0.668057 & -3.777407 & 0.040638 \\
\hline C & 0.633211 & -4.914529 & 0.813873 \\
\hline C & 1.712310 & -5.227827 & 1.675216 \\
\hline C & 2.807251 & -4.398128 & 1.738134 \\
\hline
\end{tabular}




\begin{tabular}{|c|c|c|c|}
\hline $\mathrm{H}$ & -0.154946 & -3.542355 & -0.620072 \\
\hline $\mathrm{H}$ & -0.226045 & -5.576423 & 0.763486 \\
\hline $\mathrm{H}$ & 1.672664 & -6.126147 & 2.283517 \\
\hline $\mathrm{H}$ & 3.641199 & -4.631554 & 2.394158 \\
\hline C & -0.359597 & -1.506321 & -3.822979 \\
\hline C & 0.654770 & -1.940076 & -2.912095 \\
\hline C & 1.559857 & -2.951418 & -3.327764 \\
\hline C & 1.449770 & -3.523392 & -4.575114 \\
\hline C & 0.438729 & -3.102937 & -5.470644 \\
\hline C & -0.441865 & -2.111641 & -5.103656 \\
\hline $\mathrm{H}$ & 2.334425 & -3.273047 & -2.641877 \\
\hline $\mathrm{H}$ & 2.144353 & -4.301216 & -4.876081 \\
\hline $\mathrm{H}$ & 0.364026 & -3.561701 & -6.451650 \\
\hline $\mathrm{H}$ & -1.213302 & -1.774322 & -5.789956 \\
\hline C & 7.539140 & 1.240558 & 0.618673 \\
\hline C & 6.287106 & 1.853962 & 0.662117 \\
\hline C & 5.150754 & 1.043795 & 0.540790 \\
\hline C & 5.258577 & -0.334340 & 0.354855 \\
\hline C & 6.536027 & -0.919432 & 0.312764 \\
\hline C & 7.688327 & -0.146921 & 0.447916 \\
\hline $\mathrm{H}$ & 8.429982 & 1.848876 & 0.711915 \\
\hline $\mathrm{H}$ & 4.164433 & 1.488257 & 0.566584 \\
\hline $\mathrm{H}$ & 6.605483 & -1.987719 & 0.149828 \\
\hline C & 6.096705 & 3.368216 & 0.822066 \\
\hline C & 9.099589 & -0.750737 & 0.402276 \\
\hline C & 7.433934 & 4.118968 & 0.916538 \\
\hline $\mathrm{H}$ & 7.247884 & 5.192366 & 1.021059 \\
\hline $\mathrm{H}$ & 8.019748 & 3.797665 & 1.784015 \\
\hline $\mathrm{H}$ & 8.043137 & 3.973884 & 0.018611 \\
\hline C & 5.314889 & 3.905949 & -0.397882 \\
\hline $\mathrm{H}$ & 5.151794 & 4.984961 & -0.296875 \\
\hline $\mathrm{H}$ & 5.877755 & 3.733069 & -1.321154 \\
\hline $\mathrm{H}$ & 4.340595 & 3.421459 & -0.503146 \\
\hline C & 5.289189 & 3.646730 & 2.108166 \\
\hline $\mathrm{H}$ & 5.138634 & 4.724368 & 2.236537 \\
\hline $\mathrm{H}$ & 4.305065 & 3.172253 & 2.074617 \\
\hline $\mathrm{H}$ & 5.816873 & 3.267300 & 2.989169 \\
\hline C & 9.830617 & -0.438619 & 1.725946 \\
\hline $\mathrm{H}$ & 10.842886 & -0.857484 & 1.711430 \\
\hline $\mathrm{H}$ & 9.915991 & 0.637934 & 1.896713 \\
\hline $\mathrm{H}$ & 9.293307 & -0.870223 & 2.576215 \\
\hline C & 9.076575 & -2.276332 & 0.215404 \\
\hline $\mathrm{H}$ & 10.100923 & -2.661256 & 0.197451 \\
\hline $\mathrm{H}$ & 8.547714 & -2.775586 & 1.033338 \\
\hline $\mathrm{H}$ & 8.597974 & -2.561813 & -0.726502 \\
\hline C & 9.883582 & -0.131974 & -0.775133 \\
\hline $\mathrm{H}$ & 10.895622 & -0.549088 & -0.822222 \\
\hline $\mathrm{H}$ & 9.382493 & -0.340180 & -1.725610 \\
\hline $\mathrm{H}$ & 9.972627 & 0.953211 & -0.675337 \\
\hline C & -1.913240 & 1.366140 & -1.884289 \\
\hline C & -3.218095 & 1.593080 & -2.352444 \\
\hline C & -1.257932 & 2.381295 & -1.183944 \\
\hline C & -3.861517 & 2.809098 & -2.120712 \\
\hline $\mathrm{H}$ & -3.721878 & 0.803388 & -2.891960 \\
\hline
\end{tabular}




\begin{tabular}{|c|c|c|c|}
\hline C & -1.886427 & 3.597545 & -0.894785 \\
\hline $\mathrm{H}$ & -0.226375 & 2.233534 & -0.896174 \\
\hline C & -3.182414 & 3.790326 & -1.375371 \\
\hline $\mathrm{H}$ & -3.682553 & 4.730250 & -1.181461 \\
\hline C & -1.127654 & 4.633339 & -0.056305 \\
\hline C & -5.257848 & 3.123496 & -2.678910 \\
\hline C & -1.907009 & 5.949756 & 0.084480 \\
\hline $\mathrm{H}$ & -2.096791 & 6.412763 & -0.889362 \\
\hline $\mathrm{H}$ & -2.868014 & 5.801263 & 0.587475 \\
\hline $\mathrm{H}$ & -1.327755 & 6.658818 & 0.683389 \\
\hline C & -0.899403 & 4.040941 & 1.353273 \\
\hline $\mathrm{H}$ & -0.336137 & 4.745169 & 1.975171 \\
\hline $\mathrm{H}$ & -1.858459 & 3.846005 & 1.845209 \\
\hline $\mathrm{H}$ & -0.342522 & 3.100829 & 1.314009 \\
\hline C & 0.238395 & 4.938923 & -0.710068 \\
\hline $\mathrm{H}$ & 0.875648 & 4.053396 & -0.779795 \\
\hline $\mathrm{H}$ & 0.104154 & 5.338355 & -1.720414 \\
\hline $\mathrm{H}$ & 0.775850 & 5.686674 & -0.117269 \\
\hline C & -6.197792 & 3.594252 & -1.548462 \\
\hline $\mathrm{H}$ & -6.312975 & 2.821638 & -0.784464 \\
\hline $\mathrm{H}$ & -5.825237 & 4.497426 & -1.058173 \\
\hline $\mathrm{H}$ & -7.187988 & 3.824113 & -1.955801 \\
\hline C & -5.118646 & 4.251682 & -3.725428 \\
\hline $\mathrm{H}$ & -4.697537 & 5.158218 & -3.281719 \\
\hline $\mathrm{H}$ & -4.460848 & 3.943207 & -4.543955 \\
\hline $\mathrm{H}$ & -6.097418 & 4.504495 & -4.147500 \\
\hline C & -5.900937 & 1.906993 & -3.365056 \\
\hline $\mathrm{H}$ & -6.892532 & 2.178057 & -3.739500 \\
\hline $\mathrm{H}$ & -5.309402 & 1.561493 & -4.218380 \\
\hline $\mathrm{H}$ & -6.026663 & 1.067326 & -2.674663 \\
\hline
\end{tabular}

\section{C-Catalyzed reaction TS 42}

\section{Datum}

Value

B3LYP-D3(BJ)/6-31G(d,p) Energy

$-3039.482142$

B3LYP-D3(BJ)/def2-TZVPP/IEF-PCM(DCM) Energy

$-3040.519236$

B3LYP-D3(BJ)/def2-TZVPP/IEF-PCM(DCM)//B3LYP-D3(BJ)/6-31G(d,p) Free Energy (Quasiharmonic)

$-3039.288427$

Number of Imaginary Frequencies

Frequencies (Top 3 out of 450)

$\begin{array}{lr}\text { 1. } & -220.6648 \mathrm{~cm}^{-1} \\ \text { 2. } & 10.4315 \mathrm{~cm}^{-1} \\ \text { 3. } & 13.6866 \mathrm{~cm}^{-1}\end{array}$ 


\begin{tabular}{|c|c|c|c|}
\hline C & -0.667530 & 0.897050 & 0.610410 \\
\hline $\mathrm{H}$ & -0.098670 & 0.836080 & 1.542360 \\
\hline $\mathrm{H}$ & -1.650260 & 0.468740 & 0.792710 \\
\hline C & 2.608360 & 1.885450 & -1.426020 \\
\hline 0 & 3.505800 & 2.473060 & -1.989140 \\
\hline 0 & 1.599770 & 1.315710 & -2.087870 \\
\hline $\mathrm{H}$ & 0.971440 & 0.872970 & -1.449960 \\
\hline 0 & -0.010430 & 0.128340 & -0.409720 \\
\hline $\mathrm{H}$ & 0.294090 & -0.716920 & 0.005820 \\
\hline C & -2.442710 & -1.735830 & -2.328980 \\
\hline C & -2.983660 & -1.845910 & -0.289920 \\
\hline C & -0.558730 & -3.128120 & -1.589160 \\
\hline C & -1.826590 & -2.966750 & -2.227400 \\
\hline $\mathrm{H}$ & -3.326490 & -0.812310 & -0.370980 \\
\hline $\mathrm{H}$ & 0.142890 & -2.329930 & -1.833160 \\
\hline $\mathrm{H}$ & -0.106680 & -4.112390 & -1.705500 \\
\hline C & 0.897800 & -2.795490 & 1.859940 \\
\hline C & 0.363030 & -4.270020 & 1.748040 \\
\hline C & 2.407160 & -2.656350 & 2.021950 \\
\hline $\mathrm{H}$ & 2.739200 & -3.119370 & 2.956880 \\
\hline $\mathrm{H}$ & 2.944990 & -3.113770 & 1.191750 \\
\hline $\mathrm{H}$ & 2.679250 & -1.597420 & 2.051860 \\
\hline C & 0.176990 & -1.978000 & 2.939170 \\
\hline $\mathrm{H}$ & -0.907470 & -2.030960 & 2.818300 \\
\hline $\mathrm{H}$ & 0.437080 & -2.323700 & 3.943340 \\
\hline $\mathrm{H}$ & 0.482720 & -0.931390 & 2.856720 \\
\hline C & -0.073870 & -4.883630 & 3.074870 \\
\hline $\mathrm{H}$ & 0.763340 & -4.931130 & 3.778880 \\
\hline $\mathrm{H}$ & -0.883040 & -4.309610 & 3.529060 \\
\hline $\mathrm{H}$ & -0.433210 & -5.902850 & 2.906850 \\
\hline C & 1.347720 & -5.204800 & 1.037100 \\
\hline $\mathrm{H}$ & 2.221220 & -5.425720 & 1.657570 \\
\hline $\mathrm{H}$ & 0.836590 & -6.144920 & 0.812980 \\
\hline $\mathrm{H}$ & 1.692060 & -4.770170 & 0.097430 \\
\hline 0 & 0.527280 & -2.268150 & 0.567270 \\
\hline 0 & -0.798800 & -4.132630 & 0.907170 \\
\hline B & -0.689200 & -2.933080 & 0.147150 \\
\hline 0 & -1.855760 & -1.981530 & 0.340290 \\
\hline C & -4.067780 & -2.854000 & -0.209680 \\
\hline C & -5.314090 & -2.537470 & -0.770700 \\
\hline C & -3.891880 & -4.083100 & 0.439960 \\
\hline C & -6.369980 & -3.438380 & -0.697910 \\
\hline $\mathrm{H}$ & -5.448530 & -1.573190 & -1.246970 \\
\hline C & -4.956750 & -4.980910 & 0.512550 \\
\hline $\mathrm{H}$ & -2.930890 & -4.323360 & 0.882250 \\
\hline C & -6.192590 & -4.666240 & -0.054210 \\
\hline $\mathrm{H}$ & -7.330560 & -3.184290 & -1.135910 \\
\hline $\mathrm{H}$ & -4.820090 & -5.930930 & 1.019950 \\
\hline $\mathrm{H}$ & -7.016120 & -5.370840 & 0.008660 \\
\hline $\mathrm{H}$ & -2.425640 & -3.852310 & -2.435550 \\
\hline
\end{tabular}




\begin{tabular}{|c|c|c|c|}
\hline $\mathrm{H}$ & -1.848390 & -0.832140 & -2.222190 \\
\hline $\mathrm{H}$ & -3.403430 & -1.636540 & -2.819100 \\
\hline C & 1.637750 & 2.502450 & 0.806310 \\
\hline C & 2.589010 & 1.801000 & 0.088270 \\
\hline C & 3.650070 & 1.108850 & 0.754060 \\
\hline C & 3.756200 & 1.205930 & 2.125060 \\
\hline $\mathrm{H}$ & 4.539040 & 0.654420 & 2.637900 \\
\hline C & -0.742820 & 2.328190 & 0.139930 \\
\hline C & 0.428310 & 3.076630 & 0.135890 \\
\hline C & -1.904580 & 4.114260 & -1.016680 \\
\hline C & -1.941060 & 2.866600 & -0.423320 \\
\hline $\mathrm{H}$ & -2.817550 & 4.533910 & -1.429610 \\
\hline C & -0.717920 & 4.882890 & -1.069360 \\
\hline C & 0.473500 & 4.365050 & -0.468930 \\
\hline C & 1.660250 & 5.144130 & -0.511490 \\
\hline C & 1.663950 & 6.379390 & -1.115960 \\
\hline C & 0.483480 & 6.896630 & -1.702250 \\
\hline C & -0.680220 & 6.164530 & -1.679270 \\
\hline $\mathrm{H}$ & 2.569030 & 4.738200 & -0.082670 \\
\hline $\mathrm{H}$ & 2.579850 & 6.960520 & -1.151480 \\
\hline $\mathrm{H}$ & 0.502810 & 7.873320 & -2.175960 \\
\hline $\mathrm{H}$ & -1.588710 & 6.553930 & -2.130270 \\
\hline C & 2.843070 & 1.971820 & 2.891190 \\
\hline C & 1.755510 & 2.622870 & 2.228640 \\
\hline C & 0.824730 & 3.358460 & 3.009490 \\
\hline C & 0.961880 & 3.442920 & 4.376940 \\
\hline C & 2.035020 & 2.794430 & 5.031580 \\
\hline C & 2.953690 & 2.075300 & 4.302370 \\
\hline $\mathrm{H}$ & 0.003170 & 3.858640 & 2.509730 \\
\hline $\mathrm{H}$ & 0.243230 & 4.012560 & 4.958060 \\
\hline $\mathrm{H}$ & 2.131400 & 2.867220 & 6.110480 \\
\hline $\mathrm{H}$ & 3.780580 & 1.572610 & 4.796250 \\
\hline C & -5.728360 & 0.916140 & -0.099100 \\
\hline C & -5.300210 & 1.338140 & -1.359410 \\
\hline C & -4.033990 & 1.940460 & -1.457070 \\
\hline C & -3.240170 & 2.147580 & -0.328820 \\
\hline C & -3.717570 & 1.726030 & 0.924940 \\
\hline C & -4.951510 & 1.094670 & 1.060020 \\
\hline $\mathrm{H}$ & -6.695770 & 0.441380 & -0.000530 \\
\hline $\mathrm{H}$ & -3.655760 & 2.266750 & -2.420280 \\
\hline $\mathrm{H}$ & -3.106110 & 1.924680 & 1.795670 \\
\hline C & -6.173750 & 1.206540 & -2.617280 \\
\hline C & -5.490070 & 0.621910 & 2.418270 \\
\hline C & -5.452490 & 0.350780 & -3.679960 \\
\hline $\mathrm{H}$ & -6.032520 & 0.329610 & -4.608510 \\
\hline $\mathrm{H}$ & -4.459050 & 0.742150 & -3.913830 \\
\hline $\mathrm{H}$ & -5.338200 & -0.682480 & -3.337800 \\
\hline C & -7.536490 & 0.558540 & -2.321650 \\
\hline $\mathrm{H}$ & -8.118820 & 0.491060 & -3.245400 \\
\hline $\mathrm{H}$ & -7.431330 & -0.456180 & -1.924370 \\
\hline $\mathrm{H}$ & -8.117110 & 1.146470 & -1.604480 \\
\hline C & -6.428550 & 2.618120 & -3.191400 \\
\hline $\mathrm{H}$ & -7.066650 & 2.558600 & -4.079510 \\
\hline $\mathrm{H}$ & -6.927340 & 3.252090 & -2.452010 \\
\hline
\end{tabular}




\begin{tabular}{|c|c|c|c|}
\hline H & -5.496380 & 3.110370 & -3.480830 \\
\hline $\mathrm{C}$ & -4.504310 & 0.906540 & 3.563300 \\
\hline $\mathrm{H}$ & -4.923830 & 0.544160 & 4.506510 \\
\hline $\mathrm{H}$ & -3.547890 & 0.397090 & 3.408550 \\
\hline $\mathrm{H}$ & -4.309410 & 1.977430 & 3.677170 \\
\hline C & -6.813840 & 1.356670 & 2.721100 \\
\hline $\mathrm{H}$ & -7.211740 & 1.038440 & 3.690560 \\
\hline H & -6.659270 & 2.439670 & 2.752810 \\
\hline $\mathrm{H}$ & -7.574460 & 1.148550 & 1.963390 \\
\hline C & -5.744930 & -0.899530 & 2.374920 \\
\hline $\mathrm{H}$ & -6.158310 & -1.238560 & 3.330810 \\
\hline $\mathrm{H}$ & -6.449820 & -1.177390 & 1.587730 \\
\hline $\mathrm{H}$ & -4.817240 & -1.448920 & 2.192650 \\
\hline C & 4.549530 & 0.209150 & -0.006380 \\
\hline C & 5.934250 & 0.238640 & 0.173300 \\
\hline C & 3.996320 & -0.717220 & -0.894740 \\
\hline C & 6.772850 & -0.630090 & -0.533210 \\
\hline H & 6.352930 & 0.972530 & 0.853980 \\
\hline C & 4.800810 & -1.587890 & -1.631330 \\
\hline $\mathrm{H}$ & 2.919410 & -0.754690 & -1.002270 \\
\hline C & 6.185730 & -1.529880 & -1.432810 \\
\hline $\mathrm{H}$ & 6.821920 & -2.197450 & -1.995330 \\
\hline C & 4.134570 & -2.556870 & -2.617040 \\
\hline C & 8.291180 & -0.555180 & -0.315100 \\
\hline C & 3.309830 & -1.747140 & -3.642100 \\
\hline H & 2.554230 & -1.119910 & -3.162720 \\
\hline $\mathrm{H}$ & 3.959160 & -1.083360 & -4.220820 \\
\hline $\mathrm{H}$ & 2.801690 & -2.424060 & -4.338130 \\
\hline C & 5.157140 & -3.410150 & -3.383290 \\
\hline $\mathrm{H}$ & 5.841970 & -2.789320 & -3.969310 \\
\hline $\mathrm{H}$ & 5.751720 & -4.035090 & -2.709060 \\
\hline $\mathrm{H}$ & 4.634870 & -4.075660 & -4.077700 \\
\hline C & 3.199430 & -3.502990 & -1.834680 \\
\hline H & 3.772190 & -4.109550 & -1.125010 \\
\hline $\mathrm{H}$ & 2.445030 & -2.951090 & -1.269430 \\
\hline $\mathrm{H}$ & 2.680640 & -4.182640 & -2.520450 \\
\hline C & 8.791820 & 0.852430 & -0.704850 \\
\hline $\mathrm{H}$ & 8.314200 & 1.631380 & -0.104640 \\
\hline $\mathrm{H}$ & 8.574280 & 1.063090 & -1.756260 \\
\hline $\mathrm{H}$ & 9.874630 & 0.927649 & -0.555090 \\
\hline C & 8.605370 & -0.818400 & 1.173510 \\
\hline H & 8.259090 & -1.812640 & 1.473290 \\
\hline $\mathrm{H}$ & 8.121010 & -0.085040 & 1.824070 \\
\hline $\mathrm{H}$ & 9.684910 & -0.763271 & 1.351850 \\
\hline C & 9.057260 & -1.588600 & -1.156690 \\
\hline $\mathrm{H}$ & 10.130170 & -1.500381 & -0.959710 \\
\hline $\mathrm{H}$ & 8.903170 & -1.432130 & -2.228760 \\
\hline $\mathrm{H}$ & 8.757330 & -2.612680 & -0.912810 \\
\hline
\end{tabular}


Frequencies (Top 3 out of 450)

$\begin{array}{rr}\text { 1. } & -222.1033 \mathrm{~cm}^{-1} \\ \text { 2. } & 11.0422 \mathrm{~cm}^{-1} \\ \text { 3. } & 14.8098 \mathrm{~cm}^{-1}\end{array}$

\section{B3LYP-D3(BJ)/6-31G(d,p) Molecular Geometry in Cartesian Coordinates}

C

$\mathrm{H}$

$\mathrm{H}$

C

0

0

$\mathrm{H}$

0

$\mathrm{H}$

C

C

C

C

$\mathrm{H}$

$\mathrm{H}$

$\mathrm{H}$

C

C

C

$\mathrm{H}$

$\mathrm{H}$

$\mathrm{H}$

C

$\mathrm{H}$

$\mathrm{H}$

$\mathrm{H}$

C

$\mathrm{H}$

$\mathrm{H}$

$\mathrm{H}$

C

$\mathrm{H}$

\subsection{0}

3.175840

1.799600

$-0.508630$

$-0.965780$

$-0.008300$

0.478190

1.446380

0.880600

$-1.789070$

$-2.927620$

$-0.342370$

$-1.304950$

$-3.038580$

0.402770

0.108230

$-0.236750$

$-1.606390$

0.970910

1.874060

1.106880

0.864410

$-0.089590$

$-0.167560$

0.892080

$-0.850400$

$-2.779210$

$-2.761800$

$-2.765080$

$-3.717750$

$-1.627340$

$-1.419290$

\subsection{0}

$-0.402580$

0.547520

1.981460

2.785670

0.820660

0.318730

$-0.541260$

$-1.167090$

$-1.514370$

$-1.252930$

$-2.834320$

$-2.730870$

$-0.190080$

$-2.039840$

$-3.819700$

$-3.187970$

$-3.918510$

$-4.096250$

$-3.481620$

$-4.826390$

$-4.632590$

$-2.474210$

$-3.182390$

$-1.993870$

$-1.701910$

$-3.153950$

$-3.205410$

$-2.105490$

$-3.593880$

$-5.377440$

$-5.466680$

\subsection{0}

1.237780

1.831100

$-1.143690$

$-1.933440$

$-1.556360$

$-0.832240$

0.097380

0.627730

$-3.010870$

$-1.251790$

$-1.517440$

$-2.567100$

$-1.470970$

$-1.517280$

$-1.404870$

2.095040

1.878300

1.839660

1.783030

2.642560

0.894270

3.435270

4.266370

3.495750

3.556090

2.507910

3.600440

2.202570

2. 160290

2. 317340

3. 388540 


\begin{tabular}{|c|c|c|c|}
\hline $\mathrm{H}$ & -2.616060 & -5.805050 & 2.128220 \\
\hline $\mathrm{H}$ & -0.893170 & -5.966540 & 1.765620 \\
\hline 0 & -0.279210 & -2.192610 & 1.055900 \\
\hline 0 & -1.745680 & -3.868060 & 0.448050 \\
\hline B & -1.181740 & -2.627660 & 0.003190 \\
\hline 0 & -2.105490 & -1.479750 & -0.266180 \\
\hline C & -4.149450 & -2.051150 & -1.527810 \\
\hline C & -4.357590 & -3.354610 & -1.055600 \\
\hline C & -5.145590 & -1.437040 & -2.301060 \\
\hline C & -5.546800 & -4.017510 & -1.353420 \\
\hline $\mathrm{H}$ & -3.591720 & -3.834670 & -0.457870 \\
\hline C & -6.329860 & -2.103540 & -2.598060 \\
\hline $\mathrm{H}$ & -4.988560 & -0.426310 & -2.657580 \\
\hline C & -6.536010 & -3.399130 & -2.120350 \\
\hline $\mathrm{H}$ & -5.703400 & -5.024610 & -0.978820 \\
\hline $\mathrm{H}$ & -7.091510 & -1.611560 & -3.195370 \\
\hline $\mathrm{H}$ & -7.460490 & -3.922370 & -2.344440 \\
\hline $\mathrm{H}$ & -1.845090 & -3.632200 & -2.855330 \\
\hline $\mathrm{H}$ & -1.191350 & -0.617940 & -2.872570 \\
\hline $\mathrm{H}$ & -2.552280 & -1.484070 & -3.781240 \\
\hline C & 0.750220 & 2.596280 & 0.909650 \\
\hline C & -0.470510 & 2.260180 & 0.345170 \\
\hline C & -1.667890 & 2.238700 & 1.138390 \\
\hline C & -1.567320 & 2.570730 & 2.476760 \\
\hline $\mathrm{H}$ & -2.465460 & 2.571310 & 3.086160 \\
\hline C & 2.810320 & 1.416930 & 0.144180 \\
\hline C & 2.011080 & 2.552410 & 0.103410 \\
\hline C & 4.435050 & 2.499700 & -1.293120 \\
\hline C & 4.041600 & 1.378640 & -0.586500 \\
\hline $\mathrm{H}$ & 5.365760 & 2.472830 & -1.852180 \\
\hline C & 3.643560 & 3.669480 & -1.351560 \\
\hline C & 2.397830 & 3.699900 & -0.650410 \\
\hline C & 1.596200 & 4.869950 & -0.726790 \\
\hline C & 2.013410 & 5.958390 & -1.456530 \\
\hline C & 3.250010 & 5.931130 & -2.145630 \\
\hline C & 4.044930 & 4.810650 & -2.095710 \\
\hline $\mathrm{H}$ & 0.641140 & 4.885500 & -0.215330 \\
\hline $\mathrm{H}$ & 1.387810 & 6.843670 & -1.511680 \\
\hline $\mathrm{H}$ & 3.564960 & 6.797640 & -2.718970 \\
\hline $\mathrm{H}$ & 4.991830 & 4.778460 & -2.627340 \\
\hline C & -0.347150 & 2.949640 & 3.080800 \\
\hline C & 0.838480 & 2.972760 & 2.286450 \\
\hline C & 2.061840 & 3.359640 & 2.895910 \\
\hline C & 2.104860 & 3.703970 & 4.228040 \\
\hline C & 0.929110 & 3.678670 & 5.015300 \\
\hline C & -0.270220 & 3.310670 & 4.451850 \\
\hline $\mathrm{H}$ & 2.963080 & 3.382530 & 2.294480 \\
\hline $\mathrm{H}$ & 3.046700 & 4.000540 & 4.679100 \\
\hline $\mathrm{H}$ & 0.977720 & 3.952870 & 6.064600 \\
\hline $\mathrm{H}$ & -1.178990 & 3.290110 & 5.046820 \\
\hline C & 6.568640 & -2.056910 & -0.636240 \\
\hline C & 7.134330 & -0.805740 & -0.361740 \\
\hline C & 6.285790 & 0.304900 & -0.342830 \\
\hline C & 4.914750 & 0.174950 & -0.600030 \\
\hline
\end{tabular}




\begin{tabular}{|c|c|c|c|}
\hline C & 4.388910 & -1.090780 & -0.890400 \\
\hline C & 5.204230 & -2.224920 & -0.900120 \\
\hline $\mathrm{H}$ & 7.212070 & -2.930400 & -0.639030 \\
\hline $\mathrm{H}$ & 6.671770 & 1.288610 & -0.106470 \\
\hline $\mathrm{H}$ & 3.331230 & -1.170870 & -1.098400 \\
\hline C & 8.640500 & -0.701930 & -0.080570 \\
\hline C & 4.643930 & -3.629930 & -1.163180 \\
\hline C & 9.423520 & -1.222120 & -1.305330 \\
\hline $\mathrm{H}$ & 10.502050 & -1.160380 & -1.123460 \\
\hline $\mathrm{H}$ & 9.180370 & -2.264670 & -1.527930 \\
\hline $\mathrm{H}$ & 9.191390 & -0.627930 & -2.194610 \\
\hline C & 9.084620 & 0.743630 & 0.195270 \\
\hline $\mathrm{H}$ & 10.162710 & 0.768730 & 0.381420 \\
\hline $\mathrm{H}$ & 8.878850 & 1.399370 & -0.656470 \\
\hline $\mathrm{H}$ & 8.585920 & 1.159650 & 1.076150 \\
\hline C & 8.990560 & -1.559090 & 1.154880 \\
\hline $\mathrm{H}$ & 10.063290 & -1.500530 & 1.369260 \\
\hline $\mathrm{H}$ & 8.445470 & -1.208370 & 2.036790 \\
\hline $\mathrm{H}$ & 8.736580 & -2.611590 & 1.001860 \\
\hline C & 3.139530 & -3.601500 & -1.476150 \\
\hline $\mathrm{H}$ & 2.777050 & -4.622120 & -1.635830 \\
\hline $\mathrm{H}$ & 2.562770 & -3.164810 & -0.657690 \\
\hline $\mathrm{H}$ & 2.924570 & -3.026240 & -2.381790 \\
\hline C & 5.378650 & -4.270050 & -2.359420 \\
\hline $\mathrm{H}$ & 4.984400 & -5.273010 & -2.556150 \\
\hline $\mathrm{H}$ & 5.245630 & -3.666970 & -3.262860 \\
\hline $\mathrm{H}$ & 6.452440 & -4.364300 & -2.174820 \\
\hline C & 4.858160 & -4.496390 & 0.097090 \\
\hline $\mathrm{H}$ & 4.468860 & -5.507880 & -0.062980 \\
\hline $\mathrm{H}$ & 5.917840 & -4.579810 & 0.354760 \\
\hline $\mathrm{H}$ & 4.337770 & -4.063010 & 0.957130 \\
\hline C & -3.009460 & 1.852690 & 0.620960 \\
\hline C & -3.821780 & 1.005990 & 1.398520 \\
\hline C & -3.530310 & 2.384580 & -0.559300 \\
\hline C & -5.129510 & 0.705560 & 1.023460 \\
\hline $\mathrm{H}$ & -3.405790 & 0.592450 & 2.307930 \\
\hline c & -4.821460 & 2.057160 & -0.998990 \\
\hline $\mathrm{H}$ & -2.922680 & 3.070660 & -1.130150 \\
\hline C & -5.599350 & 1.220770 & -0.197970 \\
\hline $\mathrm{H}$ & -6.601510 & 0.963730 & -0.515720 \\
\hline C & -5.328490 & 2.678480 & -2.309060 \\
\hline C & -6.070760 & -0.129530 & 1.905880 \\
\hline C & -5.394750 & 4.212180 & -2.135110 \\
\hline $\mathrm{H}$ & -6.067770 & 4.480270 & -1.314550 \\
\hline H & -4.409960 & 4.632070 & -1.914770 \\
\hline $\mathrm{H}$ & -5.765040 & 4.684160 & -3.051770 \\
\hline C & -4.351370 & 2.346820 & -3.459680 \\
\hline $\mathrm{H}$ & -4.668440 & 2.850260 & -4.379370 \\
\hline $\mathrm{H}$ & -3.328610 & 2.658350 & -3.235290 \\
\hline $\mathrm{H}$ & -4.330340 & 1.271130 & -3.664210 \\
\hline C & -6.730250 & 2.177060 & -2.691920 \\
\hline $\mathrm{H}$ & -6.757610 & 1.089420 & -2.811550 \\
\hline $\mathrm{H}$ & -7.477940 & 2.453230 & -1.941800 \\
\hline $\mathrm{H}$ & -7.034510 & 2.623930 & -3.643280 \\
\hline
\end{tabular}




\begin{tabular}{|llll|} 
C & -7.368750 & 0.670030 & 2.155780 \\
H & -7.150590 & 1.627300 & 2.639650 \\
H & -7.905720 & 0.878890 & 1.226860 \\
H & -8.041380 & 0.101750 & 2.807220 \\
C & -6.414770 & -1.455730 & 1.200460 \\
H & -6.863720 & -1.289370 & 0.218280 \\
$H$ & -5.517980 & -2.059660 & 1.050970 \\
H & -7.122870 & -2.034980 & 1.803660 \\
C & -5.449230 & -0.452460 & 3.274770 \\
H & -6.166460 & -1.017110 & 3.878490 \\
H & -4.547990 & -1.063070 & 3.180520 \\
H & -5.192030 & 0.458140 & 3.825480 \\
& & & \\
\hline
\end{tabular}

\section{C-Catalyzed reaction TS 44}

\section{Datum}

Value

B3LYP-D3(BJ)/6-31G(d,p) Energy

$-3039.484559$

B3LYP-D3(BJ)/def2-TZVPP/IEF-PCM(DCM) Energy

$-3040.51904$

B3LYP-D3(BJ)/def2-TZVPP/IEF-PCM(DCM)//B3LYP-D3(BJ)/6-31G(d,p) Free Energy (Quasiharmonic)

$-3039.288419$

Number of Imaginary Frequencies

Frequencies (Top 3 out of 450)

$\begin{array}{lr}\text { 1. } & -264.3116 \mathrm{~cm}^{-1} \\ \text { 2. } & 7.9944 \mathrm{~cm}^{-1} \\ \text { 3. } & 13.3132 \mathrm{~cm}^{-1}\end{array}$

B3LYP-D3(BJ)/6-31G(d,p) Molecular Geometry in Cartesian Coordinates

C

$\mathrm{H}$

$\mathrm{H}$

C

0

0

$\mathrm{H}$

0

$\mathrm{H}$

C

C

C

C

$\mathrm{H}$

\section{983221}

4.020081

2. 366261

$-0.339880$

0.509170

$-1.250530$

$-1.832240$

2.638441

1.870451

$-3.887000$

$-3.264050$

$-4.433780$

$-4.811920$

$-4.208480$
$-1.756839$

$-2.026979$

$-2.139469$

$-0.515740$

$-0.859979$

0.397190

0.618120

$-2.402279$

$-1.939129$

1.868170

3. 311720

0.538980

1.303470

3.860590
1.042860

1.254740

1.864200

$-0.476440$

$-1.282540$

$-0.801220$

$-0.031910$

$-0.181230$

$-0.568880$

$-3.349090$

$-1.905570$

$-1.344630$

$-2.489990$

$-1.902360$ 


\begin{tabular}{|c|c|c|c|}
\hline H & -3.549710 & -0.081620 & -1.478740 \\
\hline $\mathrm{H}$ & -5.243220 & -0.002220 & -0.858910 \\
\hline C & -3.959860 & 1.449300 & 2.171280 \\
\hline C & -4.838780 & 2.685170 & 1.746400 \\
\hline C & -4.804450 & 0.232980 & 2.554680 \\
\hline H & -4.136730 & -0.617050 & 2.697520 \\
\hline H & -5.355030 & 0.398540 & 3.484870 \\
\hline $\mathrm{H}$ & -5.515180 & -0.011840 & 1.762390 \\
\hline C & -2.950180 & 1.731040 & 3.279420 \\
\hline $\mathrm{H}$ & -2.280020 & 2.552140 & 3.025890 \\
\hline $\mathrm{H}$ & -2.345110 & 0.838810 & 3.464350 \\
\hline $\mathrm{H}$ & -3.466700 & 1.985840 & 4.210580 \\
\hline C & -4.070450 & 4.011840 & 1.827060 \\
\hline $\mathrm{H}$ & -4.663260 & 4.788260 & 1.335810 \\
\hline $\mathrm{H}$ & -3.889240 & 4.318720 & 2.861180 \\
\hline $\mathrm{H}$ & -3.113400 & 3.937860 & 1.307830 \\
\hline C & -6.163140 & 2.806370 & 2.492900 \\
\hline $\mathrm{H}$ & -5.999460 & 2.918390 & 3.569670 \\
\hline $\mathrm{H}$ & -6.703640 & 3.688410 & 2.138130 \\
\hline $\mathrm{H}$ & -6.793900 & 1.932940 & 2.321020 \\
\hline 0 & -3.256890 & 1.130710 & 0.947240 \\
\hline 0 & -5.122710 & 2.409590 & 0.362900 \\
\hline B & -3.983120 & 1.728510 & -0.150350 \\
\hline 0 & -2.982900 & 2.588860 & -0.867980 \\
\hline C & -2.123910 & 3.924790 & -2.609770 \\
\hline C & -0.819580 & 3.465830 & -2.367140 \\
\hline C & -2.338170 & 4.990780 & -3.493850 \\
\hline C & 0.255520 & 4.080851 & -3.000410 \\
\hline $\mathrm{H}$ & -0.665910 & 2.639630 & -1.683940 \\
\hline C & -1.258180 & 5.597300 & -4.129340 \\
\hline $\mathrm{H}$ & -3.349940 & 5.344230 & -3.675390 \\
\hline C & 0.039830 & 5.143710 & -3.881610 \\
\hline $\mathrm{H}$ & 1.262750 & 3.732361 & -2.797610 \\
\hline $\mathrm{H}$ & -1.425730 & 6.425130 & -4.811120 \\
\hline $\mathrm{H}$ & 0.882040 & 5.620261 & -4.373950 \\
\hline $\mathrm{H}$ & -5.841950 & 1.656360 & -2.545630 \\
\hline $\mathrm{H}$ & -2.897090 & 1.430100 & -3.427760 \\
\hline $\mathrm{H}$ & -4.209440 & 2.477120 & -4.187490 \\
\hline C & 0.512110 & -0.528539 & 1.818770 \\
\hline C & -0.430460 & -1.024120 & 0.934680 \\
\hline C & -1.497449 & -1.887560 & 1.351650 \\
\hline C & -1.568799 & -2.204150 & 2.694990 \\
\hline $\mathrm{H}$ & -2.333349 & -2.891590 & 3.042510 \\
\hline C & 2.840310 & -0.249109 & 0.957410 \\
\hline C & 1.628000 & 0.333841 & 1.306070 \\
\hline C & 3.694260 & 1.928881 & 0.300850 \\
\hline C & 3.902510 & 0.570881 & 0.448830 \\
\hline $\mathrm{H}$ & 4.493190 & 2.544561 & -0.102000 \\
\hline C & 2.457060 & 2.539301 & 0.605280 \\
\hline C & 1.398980 & 1.732851 & 1.128060 \\
\hline C & 0.152340 & 2.349991 & 1.412710 \\
\hline C & -0.040150 & 3.692250 & 1.180330 \\
\hline C & 1.006340 & 4.489231 & 0.659320 \\
\hline C & 2.228780 & 3.923681 & 0.383070 \\
\hline
\end{tabular}




\begin{tabular}{|c|c|c|c|}
\hline $\mathrm{H}$ & -0.659420 & 1.746450 & 1.794860 \\
\hline $\mathrm{H}$ & -1.002850 & 4.143930 & 1.392070 \\
\hline $\mathrm{H}$ & 0.837030 & 5.544701 & 0.471470 \\
\hline $\mathrm{H}$ & 3.039370 & 4.525191 & -0.019090 \\
\hline $\mathrm{C}$ & -0.662889 & -1.676370 & 3.646840 \\
\hline C & 0.399820 & -0.823919 & 3.210410 \\
\hline C & 1.302230 & -0.300039 & 4.171780 \\
\hline C & 1.159010 & -0.604639 & 5.506370 \\
\hline C & 0.110740 & -1.451009 & 5.939840 \\
\hline C & -0.777279 & -1.976300 & 5.030180 \\
\hline $\mathrm{H}$ & 2.105040 & 0.345481 & 3.834100 \\
\hline H & 1.853790 & -0.195309 & 6.232920 \\
\hline $\mathrm{H}$ & 0.010441 & -1.684780 & 6.995190 \\
\hline $\mathrm{H}$ & -1.583289 & -2.627390 & 5.356540 \\
\hline C & 7.740260 & -0.960919 & -0.644660 \\
\hline C & 6.594251 & -1.598919 & -1.134490 \\
\hline C & 5.344630 & -1.088759 & -0.772520 \\
\hline C & 5.231770 & 0.016351 & 0.076930 \\
\hline C & 6.398020 & 0.630041 & 0.550500 \\
\hline C & 7.665540 & 0.156951 & 0.196590 \\
\hline $\mathrm{H}$ & 8.711630 & -1.345599 & -0.919430 \\
\hline $\mathrm{H}$ & 4.436500 & -1.557149 & -1.131120 \\
\hline $\mathrm{H}$ & 6.300650 & 1.477281 & 1.221770 \\
\hline C & 6.650171 & -2.835769 & -2.041830 \\
\hline C & 8.914950 & 0.861401 & 0.746760 \\
\hline C & 5.977621 & -2.505699 & -3.391870 \\
\hline $\mathrm{H}$ & 6.001801 & -3.379159 & -4.052900 \\
\hline H & 4.932651 & -2.214529 & -3.258040 \\
\hline $\mathrm{H}$ & 6.495981 & -1.682109 & -3.893640 \\
\hline C & 8.090081 & -3.296449 & -2.317210 \\
\hline $\mathrm{H}$ & 8.076801 & -4.183869 & -2.957580 \\
\hline $\mathrm{H}$ & 8.672661 & -2.525199 & -2.831630 \\
\hline H & 8.613551 & -3.561889 & -1.393100 \\
\hline C & 5.886051 & -3.993309 & -1.360420 \\
\hline $\mathrm{H}$ & 5.901271 & -4.884119 & -1.998540 \\
\hline H & 6.354031 & -4.252549 & -0.404920 \\
\hline $\mathrm{H}$ & 4.842761 & -3.732779 & -1.163970 \\
\hline C & 10.219080 & 0.220062 & 0.245640 \\
\hline $\mathrm{H}$ & 11.077050 & 0.759902 & 0.658340 \\
\hline $\mathrm{H}$ & 10.302930 & -0.825268 & 0.558610 \\
\hline H & 10.295610 & 0.257902 & -0.845480 \\
\hline C & 8.904060 & 2.340011 & 0.302190 \\
\hline $\mathrm{H}$ & 9.786480 & 2.862492 & 0.687950 \\
\hline H & 8.910980 & 2.416121 & -0.789660 \\
\hline $\mathrm{H}$ & 8.017380 & 2.864871 & 0.668300 \\
\hline C & 8.903850 & 0.790141 & 2.288990 \\
\hline $\mathrm{H}$ & 9.786040 & 1.291892 & 2.701980 \\
\hline $\mathrm{H}$ & 8.016500 & 1.271861 & 2.708620 \\
\hline $\mathrm{H}$ & 8.911230 & -0.250369 & 2.628300 \\
\hline C & -2.494199 & -2.420940 & 0.386380 \\
\hline C & -3.818809 & -2.662600 & 0.792470 \\
\hline C & -2.153179 & -2.678500 & -0.943250 \\
\hline C & -4.788249 & -3.103190 & -0.104150 \\
\hline $\mathrm{H}$ & -4.088889 & -2.475510 & 1.820540 \\
\hline
\end{tabular}




\begin{tabular}{|c|c|c|c|}
\hline C & -3.110449 & -3.071890 & -1.887610 \\
\hline $\mathrm{H}$ & -1.125889 & -2.571340 & -1.259490 \\
\hline C & -4.418349 & -3.273580 & -1.449400 \\
\hline $\mathrm{H}$ & -5.175309 & -3.574120 & -2.162080 \\
\hline C & -2.682059 & -3.230630 & -3.352400 \\
\hline C & -6.240069 & -3.372940 & 0.319010 \\
\hline C & -3.842989 & -3.682890 & -4.251760 \\
\hline $\mathrm{H}$ & -4.242439 & -4.653870 & -3.941390 \\
\hline $\mathrm{H}$ & -4.662599 & -2.957150 & -4.249730 \\
\hline $\mathrm{H}$ & -3.491999 & -3.782030 & -5.283310 \\
\hline C & -2.166989 & -1.867060 & -3.868150 \\
\hline $\mathrm{H}$ & -1.316030 & -1.503700 & -3.286020 \\
\hline $\mathrm{H}$ & -2.959310 & -1.113340 & -3.815710 \\
\hline $\mathrm{H}$ & -1.848719 & -1.954180 & -4.912870 \\
\hline C & -1.551929 & -4.277230 & -3.451340 \\
\hline $\mathrm{H}$ & -0.671809 & -3.981400 & -2.874660 \\
\hline $\mathrm{H}$ & -1.888269 & -5.250260 & -3.079430 \\
\hline $\mathrm{H}$ & -1.239639 & -4.398300 & -4.494050 \\
\hline C & -6.649739 & -4.794410 & -0.123560 \\
\hline $\mathrm{H}$ & -6.596789 & -4.917350 & -1.208370 \\
\hline $\mathrm{H}$ & -5.996009 & -5.545300 & 0.330770 \\
\hline $\mathrm{H}$ & -7.679329 & -5.004730 & 0.185030 \\
\hline C & -6.434029 & -3.277540 & 1.841460 \\
\hline $\mathrm{H}$ & -5.789529 & -3.984800 & 2.373080 \\
\hline $\mathrm{H}$ & -6.225999 & -2.272700 & 2.218610 \\
\hline $\mathrm{H}$ & -7.471539 & -3.515680 & 2.095150 \\
\hline C & -7.166789 & -2.339170 & -0.356180 \\
\hline $\mathrm{H}$ & -8.213919 & -2.539700 & -0.104210 \\
\hline $\mathrm{H}$ & -6.926840 & -1.325130 & -0.020760 \\
\hline $\mathrm{H}$ & -7.070109 & -2.363850 & -1.445310 \\
\hline
\end{tabular}

\section{C-Catalyzed reaction TS 45}

\section{Datum}

Value

B3LYP-D3(BJ)/6-31G(d,p) Energy $-3039.484052$

B3LYP-D3(BJ)/def2-TZVPP/IEF-PCM(DCM) Energy $-3040.518841$

B3LYP-D3(BJ)/def2-TZVPP/IEF-PCM(DCM)//B3LYP-D3(BJ)/6-31G(d,p) Free Energy (Quasiharmonic) $-3039.288381$

Frequencies (Top 3 out of 450)

$\begin{array}{lr}\text { 1. } & -265.7764 \mathrm{~cm}^{-1} \\ \text { 2. } & 7.9737 \mathrm{~cm}^{-1} \\ \text { 3. } & 13.5920 \mathrm{~cm}^{-1}\end{array}$




\begin{tabular}{|c|c|c|c|}
\hline C & 2.989860 & -1.742880 & 1.056240 \\
\hline $\mathrm{H}$ & 4.026620 & -2.010630 & 1.271540 \\
\hline $\mathrm{H}$ & 2.371720 & -2.122000 & 1.878290 \\
\hline C & -0.333520 & -0.515700 & -0.473160 \\
\hline 0 & 0.522390 & -0.855210 & -1.273200 \\
\hline 0 & -1.250280 & 0.389710 & -0.805260 \\
\hline $\mathrm{H}$ & -1.831570 & 0.612870 & -0.036840 \\
\hline 0 & 2.648020 & -2.395220 & -0.165060 \\
\hline $\mathrm{H}$ & 1.881020 & -1.933960 & -0.557150 \\
\hline C & -3.887639 & 1.852320 & -3.348090 \\
\hline C & -3.268059 & 3.302580 & -1.912390 \\
\hline C & -4.433650 & 0.530740 & -1.338680 \\
\hline C & -4.812170 & 1.289910 & -2.486940 \\
\hline $\mathrm{H}$ & -4.213559 & 3.849640 & -1.910080 \\
\hline $\mathrm{H}$ & -3.549850 & -0.091020 & -1.470070 \\
\hline $\mathrm{H}$ & -5.242800 & -0.008170 & -0.850280 \\
\hline C & -3.957200 & 1.459780 & 2.173150 \\
\hline C & -4.838749 & 2.691910 & 1.742360 \\
\hline C & -4.799360 & 0.244830 & 2.566150 \\
\hline $\mathrm{H}$ & -4.130010 & -0.603030 & 2.714480 \\
\hline $\mathrm{H}$ & -5.349200 & 0.416080 & 3.495740 \\
\hline $\mathrm{H}$ & -5.510600 & -0.006660 & 1.776420 \\
\hline C & -2.945860 & 1.749880 & 3.277680 \\
\hline $\mathrm{H}$ & -2.277219 & 2.570180 & 3.017750 \\
\hline $\mathrm{H}$ & -2.339220 & 0.859660 & 3.467030 \\
\hline $\mathrm{H}$ & -3.461019 & 2.009830 & 4.208200 \\
\hline C & -4.072889 & 4.020440 & 1.815450 \\
\hline $\mathrm{H}$ & -4.667799 & 4.793250 & 1.321060 \\
\hline $\mathrm{H}$ & -3.891019 & 4.332820 & 2.847810 \\
\hline $\mathrm{H}$ & -3.116309 & 3.945780 & 1.295480 \\
\hline C & -6.162799 & 2.814730 & 2.489170 \\
\hline $\mathrm{H}$ & -5.998579 & 2.932830 & 3.565210 \\
\hline $\mathrm{H}$ & -6.705119 & 3.693900 & 2.130060 \\
\hline $\mathrm{H}$ & -6.792159 & 1.939300 & 2.322420 \\
\hline 0 & -3.255980 & 1.134280 & 0.949980 \\
\hline 0 & -5.123319 & 2.408620 & 0.360530 \\
\hline B & -3.982930 & 1.726550 & -0.149640 \\
\hline 0 & -2.984209 & 2.584610 & -0.871870 \\
\hline C & -2.129779 & 3.916350 & -2.619270 \\
\hline C & -0.823909 & 3.463390 & -2.373810 \\
\hline C & -2.347499 & 4.977160 & -3.508740 \\
\hline C & 0.249281 & 4.079140 & -3.009700 \\
\hline $\mathrm{H}$ & -0.667429 & 2.641530 & -1.686020 \\
\hline C & -1.269449 & 5.584360 & -4.146840 \\
\hline $\mathrm{H}$ & -3.360459 & 5.326090 & -3.692400 \\
\hline C & 0.030111 & 5.136730 & -3.896330 \\
\hline $\mathrm{H}$ & 1.257661 & 3.735460 & -2.804460 \\
\hline $\mathrm{H}$ & -1.439709 & 6.408180 & -4.832800 \\
\hline $\mathrm{H}$ & 0.870801 & 5.613920 & -4.390660 \\
\hline $\mathrm{H}$ & -5.842400 & 1.641790 & -2.544360 \\
\hline
\end{tabular}




\begin{tabular}{|c|c|c|c|}
\hline $\mathrm{H}$ & -2.896460 & 1.416240 & -3.423730 \\
\hline $\mathrm{H}$ & -4.210249 & 2.455820 & -4.190300 \\
\hline C & 0.516460 & -0.515480 & 1.822650 \\
\hline C & -0.424980 & -1.017250 & 0.940550 \\
\hline C & -1.490810 & -1.879970 & 1.362470 \\
\hline C & -1.561370 & -2.190150 & 2.707420 \\
\hline $\mathrm{H}$ & -2.324430 & -2.877720 & 3.058020 \\
\hline C & 2.845310 & -0.235780 & 0.962960 \\
\hline C & 1.631380 & 0.346490 & 1.307120 \\
\hline C & 3.696501 & 1.941100 & 0.299650 \\
\hline C & 3.907040 & 0.583800 & 0.452150 \\
\hline $\mathrm{H}$ & 4.494841 & 2.557069 & -0.103860 \\
\hline C & 2.457881 & 2.550550 & 0.599770 \\
\hline C & 1.399960 & 1.744220 & 1.123030 \\
\hline C & 0.151481 & 2.360150 & 1.402350 \\
\hline C & -0.042599 & 3.701330 & 1.165100 \\
\hline C & 1.003941 & 4.498380 & 0.644260 \\
\hline C & 2.227891 & 3.933890 & 0.372640 \\
\hline $\mathrm{H}$ & -0.660430 & 1.756570 & 1.784130 \\
\hline $\mathrm{H}$ & -1.006649 & 4.152070 & 1.372680 \\
\hline $\mathrm{H}$ & 0.833421 & 5.553010 & 0.452730 \\
\hline $\mathrm{H}$ & 3.038491 & 4.535420 & -0.029480 \\
\hline C & -0.657040 & -1.655490 & 3.656720 \\
\hline C & 0.403890 & -0.803110 & 3.215930 \\
\hline C & 1.304740 & -0.271960 & 4.174830 \\
\hline C & 1.161630 & -0.569480 & 5.511020 \\
\hline C & 0.115130 & -1.415880 & 5.948760 \\
\hline C & -0.771260 & -1.948220 & 5.041640 \\
\hline $\mathrm{H}$ & 2.106250 & 0.373490 & 3.833960 \\
\hline $\mathrm{H}$ & 1.855140 & -0.154510 & 6.235580 \\
\hline $\mathrm{H}$ & 0.014930 & -1.644060 & 7.005350 \\
\hline $\mathrm{H}$ & -1.575950 & -2.599350 & 5.371220 \\
\hline C & 7.743620 & -0.946331 & -0.630010 \\
\hline C & 6.605550 & -1.591981 & -1.113760 \\
\hline C & 5.351780 & -1.079541 & -0.754680 \\
\hline C & 5.237310 & 0.030089 & 0.082720 \\
\hline C & 6.404260 & 0.656729 & 0.552910 \\
\hline C & 7.667760 & 0.183529 & 0.202940 \\
\hline $\mathrm{H}$ & 8.721510 & -1.327041 & -0.896810 \\
\hline $\mathrm{H}$ & 4.445340 & -1.553881 & -1.109710 \\
\hline $\mathrm{H}$ & 6.297900 & 1.508009 & 1.213820 \\
\hline C & 6.662660 & -2.836981 & -2.008870 \\
\hline C & 8.964430 & 0.838309 & 0.701070 \\
\hline C & 5.988640 & -2.520531 & -3.361410 \\
\hline $\mathrm{H}$ & 6.013160 & -3.400381 & -4.013850 \\
\hline $\mathrm{H}$ & 4.943440 & -2.229251 & -3.229660 \\
\hline $\mathrm{H}$ & 6.505850 & -1.701381 & -3.871520 \\
\hline C & 8.103340 & -3.297171 & -2.280100 \\
\hline $\mathrm{H}$ & 8.092280 & -4.190581 & -2.912080 \\
\hline $\mathrm{H}$ & 8.683930 & -2.529221 & -2.801740 \\
\hline $\mathrm{H}$ & 8.627210 & -3.552561 & -1.353340 \\
\hline C & 5.900620 & -3.988851 & -1.315700 \\
\hline $\mathrm{H}$ & 5.917340 & -4.885861 & -1.944990 \\
\hline $\mathrm{H}$ & 6.369240 & -4.237741 & -0.357770 \\
\hline
\end{tabular}




\begin{tabular}{|c|c|c|c|}
\hline H & 4.856820 & -3.728311 & -1.121970 \\
\hline C & 9.801630 & 1.298979 & -0.511700 \\
\hline $\mathrm{H}$ & 10.734770 & 1.765579 & -0.177080 \\
\hline $\mathrm{H}$ & 10.061250 & 0.461899 & -1.165370 \\
\hline $\mathrm{H}$ & 9.247911 & 2.030029 & -1.109140 \\
\hline C & 8.696421 & 2.062469 & 1.591300 \\
\hline $\mathrm{H}$ & 9.646711 & 2.498939 & 1.914240 \\
\hline $\mathrm{H}$ & 8.139151 & 2.837349 & 1.055570 \\
\hline $\mathrm{H}$ & 8.131800 & 1.794109 & 2.489580 \\
\hline C & 9.773620 & -0.188251 & 1.522750 \\
\hline $\mathrm{H}$ & 10.706160 & 0.259749 & 1.883550 \\
\hline $\mathrm{H}$ & 9.199150 & -0.529011 & 2.389770 \\
\hline $\mathrm{H}$ & 10.032530 & -1.067951 & 0.927320 \\
\hline C & -2.488670 & -2.417890 & 0.401120 \\
\hline C & -3.810590 & -2.651530 & 0.807670 \\
\hline C & -2.142710 & -2.684790 & -0.930610 \\
\hline C & -4.784720 & -3.094460 & -0.088430 \\
\hline $\mathrm{H}$ & -4.081160 & -2.456330 & 1.834070 \\
\hline C & -3.099760 & -3.076760 & -1.868900 \\
\hline $\mathrm{H}$ & -1.113460 & -2.586780 & -1.237440 \\
\hline C & -4.412820 & -3.269110 & -1.427100 \\
\hline $\mathrm{H}$ & -5.167940 & -3.567200 & -2.146160 \\
\hline C & -2.753780 & -3.263810 & -3.352460 \\
\hline C & -6.237140 & -3.357900 & 0.337450 \\
\hline C & -3.138140 & -4.688660 & -3.802860 \\
\hline H & -2.589730 & -5.439030 & -3.225030 \\
\hline $\mathrm{H}$ & -4.207010 & -4.882670 & -3.675390 \\
\hline H & -2.897250 & -4.830170 & -4.861850 \\
\hline C & -3.552050 & -2.229310 & -4.176470 \\
\hline $\mathrm{H}$ & -3.287290 & -1.209660 & -3.881610 \\
\hline H & -4.630790 & -2.346080 & -4.037200 \\
\hline $\mathrm{H}$ & -3.335180 & -2.344540 & -5.244010 \\
\hline C & -1.256260 & -3.051840 & -3.631810 \\
\hline H & -0.920280 & -2.050500 & -3.347750 \\
\hline H & -0.638880 & -3.779160 & -3.095220 \\
\hline H & -1.062740 & -3.179810 & -4.701300 \\
\hline C & -6.426370 & -3.266680 & 1.860830 \\
\hline H & -5.782210 & -3.977400 & 2.388260 \\
\hline H & -6.214590 & -2.263690 & 2.240700 \\
\hline H & -7.463800 & -3.502729 & 2.116750 \\
\hline C & -7.160330 & -2.317579 & -0.332250 \\
\hline H & -6.916360 & -1.305880 & 0.007180 \\
\hline H & -7.064330 & -2.338010 & -1.421580 \\
\hline H & -8.208050 & -2.514959 & -0.080300 \\
\hline C & -6.654850 & -4.775860 & -0.108560 \\
\hline $\mathrm{H}$ & -7.684450 & -4.982429 & 0.202480 \\
\hline H & -6.605920 & -4.895410 & -1.193950 \\
\hline H & -6.003180 & -5.531420 & 0.340890 \\
\hline
\end{tabular}


Frequencies (Top 3 out of 450)

$\begin{array}{lr}\text { 1. } & -283.1243 \mathrm{~cm}^{-1} \\ \text { 2. } & 9.4813 \mathrm{~cm}^{-1} \\ \text { 3. } & 12.6014 \mathrm{~cm}^{-1}\end{array}$

\section{B3LYP-D3(BJ)/6-31G(d,p) Molecular Geometry in Cartesian Coordinates}

C

$\mathrm{H}$

$\mathrm{H}$

C

0

0

$\mathrm{H}$

0

$\mathrm{H}$

C

C

C

C

$\mathrm{H}$

$\mathrm{H}$

$\mathrm{H}$

C

C

C

$\mathrm{H}$

$\mathrm{H}$

$\mathrm{H}$

C

$\mathrm{H}$

$\mathrm{H}$

$\mathrm{H}$

C

$\mathrm{H}$

$\mathrm{H}$

$\mathrm{H}$

C

$\mathrm{H}$

\section{$-3.142810$}

$-2.595090$

$-4.199260$

0.295400

$-0.457760$

1.100650

1.649860

$-2.743960$

$-1.882930$

2.430739

3. 291609

2. 268910

2.816219

4. 325889

1.220470

2. 511320

3.768410

5.009100

3.651880

2.667080

4.407800

3.768750

3.663670

4.516150

2.750800

3.643510

5.296200

5.743170

4.387860

5.994650

6.275250

6.593970
$-1.516120$

$-2.451590$

$-1.716451$

$-0.012290$

0.648410

0.608260

0.052990

$-0.532580$

$-0.164780$

4.123890

3.333580

1.735610

3.041850

3.453381

1.688710

1.022520

$-1.110370$

$-0.167309$

$-1.724580$

$-2.186810$

$-2.498889$

$-0.965190$

$-2.196590$

$-2.880149$

$-2.782260$

$-1.774220$

0.189761

$-0.645469$

0.484121

1.030841

$-0.667439$

$-1.625919$
$-1.328120$

$-1.491350$

$-1.520520$

$-0.499250$

$-1.203180$

0.340880

0.957450

$-2.276310$

$-1.994610$

3. 549810

1.768690

4.153790

4. 321640

2.096350

3.860260

4.940410

2.513570

2. 372690

3.912420

4.018730

4.071000

4.688590

1.456430

1.523410

1.599970

0.455310

0.906320

0.364020

0.377030

0.880370

3.056030

2.633350 


\begin{tabular}{|c|c|c|c|}
\hline $\mathrm{H}$ & 7.083900 & 0.053831 & 2.907500 \\
\hline $\mathrm{H}$ & 6.123880 & -0.788599 & 4.129950 \\
\hline 0 & 2.673200 & -0.175040 & 2.338150 \\
\hline 0 & 4.552250 & 1.016001 & 3.057900 \\
\hline B & 3.157520 & 1.124310 & 2.770450 \\
\hline 0 & 2.816880 & 2.126900 & 1.724710 \\
\hline C & 2.772629 & 4.284410 & 0.770690 \\
\hline C & 3.477009 & 5.465580 & 0.504080 \\
\hline C & 1.600979 & 3.988490 & 0.055980 \\
\hline C & 3.018589 & 6.346060 & -0.472370 \\
\hline $\mathrm{H}$ & 4.387499 & 5.684741 & 1.055580 \\
\hline C & 1.147869 & 4.874470 & -0.916200 \\
\hline $\mathrm{H}$ & 1.083759 & 3.053870 & 0.243250 \\
\hline C & 1.852549 & 6.051700 & -1.182670 \\
\hline $\mathrm{H}$ & 3.570209 & 7.256890 & -0.682670 \\
\hline $\mathrm{H}$ & 0.249449 & 4.637790 & -1.476700 \\
\hline $\mathrm{H}$ & 1.496239 & 6.736520 & -1.946000 \\
\hline $\mathrm{H}$ & 3.744899 & 3.123120 & 4.885700 \\
\hline $\mathrm{H}$ & 1.432739 & 4.148350 & 3.124240 \\
\hline $\mathrm{H}$ & 2.917629 & 5.086590 & 3.668100 \\
\hline C & -0.714860 & -2.171760 & 0.085640 \\
\hline C & 0.326360 & -1.518370 & -0.555150 \\
\hline C & 1.345240 & -2.237370 & -1.268950 \\
\hline C & 1.242770 & -3.616550 & -1.305330 \\
\hline $\mathrm{H}$ & 1.962220 & -4.187900 & -1.882190 \\
\hline C & -2.962220 & -1.072590 & 0.114350 \\
\hline C & -1.783530 & -1.384510 & 0.782350 \\
\hline C & -3.775160 & 0.066459 & 2.101820 \\
\hline C & -3.982010 & -0.322341 & 0.791920 \\
\hline $\mathrm{H}$ & -4.542010 & 0.645329 & 2.608100 \\
\hline C & -2.583310 & -0.230870 & 2.799470 \\
\hline C & -1.560100 & -0.973940 & 2.132930 \\
\hline C & -0.363350 & -1.264810 & 2.839600 \\
\hline C & -0.186590 & -0.835590 & 4.134640 \\
\hline C & -1.199080 & -0.097990 & 4.792550 \\
\hline C & -2.371600 & 0.196540 & 4.136900 \\
\hline $\mathrm{H}$ & 0.424700 & -1.816780 & 2.343250 \\
\hline $\mathrm{H}$ & 0.742090 & -1.053560 & 4.649690 \\
\hline $\mathrm{H}$ & -1.044800 & 0.236350 & 5.814000 \\
\hline $\mathrm{H}$ & -3.155620 & 0.764020 & 4.630600 \\
\hline C & 0.225110 & -4.325670 & -0.627850 \\
\hline C & -0.767040 & -3.598080 & 0.097920 \\
\hline C & -1.779990 & -4.316030 & 0.786080 \\
\hline C & -1.808929 & -5.691540 & 0.753200 \\
\hline C & -0.830929 & -6.413240 & 0.027380 \\
\hline C & 0.161981 & -5.744470 & -0.649120 \\
\hline $\mathrm{H}$ & -2.530820 & -3.760810 & 1.336280 \\
\hline $\mathrm{H}$ & -2.587589 & -6.229150 & 1.285080 \\
\hline $\mathrm{H}$ & -0.868419 & -7.498000 & 0.007110 \\
\hline $\mathrm{H}$ & 0.916301 & -6.291060 & -1.208080 \\
\hline C & -7.693590 & 0.768189 & -1.016590 \\
\hline C & -6.512500 & 0.996239 & -1.722960 \\
\hline C & -5.300490 & 0.636049 & -1.118960 \\
\hline C & -5.269550 & 0.043399 & 0.143260 \\
\hline
\end{tabular}




\begin{tabular}{|c|c|c|c|}
\hline C & -6.478630 & -0.175371 & 0.826350 \\
\hline C & -7.701210 & 0.184849 & 0.262430 \\
\hline $\mathrm{H}$ & -8.640180 & 1.040779 & -1.466130 \\
\hline $\mathrm{H}$ & -4.363480 & 0.791029 & -1.638730 \\
\hline $\mathrm{H}$ & -6.439410 & -0.652501 & 1.797720 \\
\hline C & -6.479780 & 1.606869 & -3.130280 \\
\hline C & -9.041540 & -0.043881 & 0.976160 \\
\hline C & -5.807360 & 0.603579 & -4.094450 \\
\hline $\mathrm{H}$ & -5.755860 & 1.025999 & -5.104440 \\
\hline $\mathrm{H}$ & -4.792310 & 0.351139 & -3.776800 \\
\hline $\mathrm{H}$ & -6.384870 & -0.325511 & -4.144570 \\
\hline C & -7.884990 & 1.930019 & -3.661180 \\
\hline $\mathrm{H}$ & -7.810471 & 2.355969 & -4.666570 \\
\hline $\mathrm{H}$ & -8.508540 & 1.032589 & -3.726790 \\
\hline $\mathrm{H}$ & -8.400501 & 2.660159 & -3.028540 \\
\hline C & -5.658381 & 2.913719 & -3.098970 \\
\hline $\mathrm{H}$ & -5.618371 & 3.361719 & -4.098070 \\
\hline $\mathrm{H}$ & -6.109311 & 3.640579 & -2.415450 \\
\hline $\mathrm{H}$ & -4.631621 & 2.733589 & -2.770580 \\
\hline C & -8.863760 & -0.686521 & 2.361220 \\
\hline $\mathrm{H}$ & -9.841880 & -0.823161 & 2.832920 \\
\hline $\mathrm{H}$ & -8.387400 & -1.669471 & 2.293210 \\
\hline $\mathrm{H}$ & -8.259590 & -0.058561 & 3.023490 \\
\hline C & -9.758120 & 1.311009 & 1.162450 \\
\hline $\mathrm{H}$ & -10.720190 & 1.168578 & 1.667040 \\
\hline $\mathrm{H}$ & -9.150430 & 1.990199 & 1.768580 \\
\hline $\mathrm{H}$ & -9.951440 & 1.801219 & 0.204450 \\
\hline C & -9.924770 & -0.977831 & 0.120930 \\
\hline $\mathrm{H}$ & -10.888380 & -1.150302 & 0.613140 \\
\hline $\mathrm{H}$ & -10.123990 & -0.552821 & -0.866570 \\
\hline $\mathrm{H}$ & -9.436010 & -1.946221 & -0.024630 \\
\hline C & 2.508070 & -1.567830 & -1.912780 \\
\hline C & 2.429470 & -0.265160 & -2.427650 \\
\hline C & 3.742660 & -2.228370 & -1.970050 \\
\hline C & 3.559640 & 0.401580 & -2.898980 \\
\hline $\mathrm{H}$ & 1.473210 & 0.230340 & -2.463840 \\
\hline C & 4.888340 & -1.614599 & -2.488080 \\
\hline $\mathrm{H}$ & 3.818330 & -3.229590 & -1.563870 \\
\hline C & 4.780020 & -0.290009 & -2.919580 \\
\hline $\mathrm{H}$ & 5.661710 & 0.224281 & -3.277820 \\
\hline C & 6.196050 & -2.415579 & -2.589920 \\
\hline C & 3.504520 & 1.879560 & -3.309460 \\
\hline C & 7.380130 & -1.542689 & -3.036190 \\
\hline $\mathrm{H}$ & 7.221480 & -1.121009 & -4.033200 \\
\hline $\mathrm{H}$ & 7.556690 & -0.717809 & -2.338510 \\
\hline $\mathrm{H}$ & 8.290540 & -2.148489 & -3.075620 \\
\hline C & 6.559510 & -3.051869 & -1.231690 \\
\hline $\mathrm{H}$ & 7.466240 & -3.657679 & -1.332380 \\
\hline $\mathrm{H}$ & 6.750450 & -2.284469 & -0.476080 \\
\hline $\mathrm{H}$ & 5.767260 & -3.703779 & -0.855100 \\
\hline C & 5.992860 & -3.537209 & -3.632900 \\
\hline $\mathrm{H}$ & 5.186630 & -4.214399 & -3.335980 \\
\hline $\mathrm{H}$ & 5.732770 & -3.116139 & -4.608840 \\
\hline $\mathrm{H}$ & 6.908410 & -4.128279 & -3.745770 \\
\hline
\end{tabular}




$\begin{array}{llll}\mathrm{C} & 4.226039 & 2.693531 & -2.211410 \\ \mathrm{H} & 3.753379 & 2.525680 & -1.241130 \\ \mathrm{H} & 5.278799 & 2.404891 & -2.130580 \\ \mathrm{H} & 4.178389 & 3.765951 & -2.429090 \\ \mathrm{C} & 4.206510 & 2.097901 & -4.664690 \\ \mathrm{H} & 5.262880 & 1.816841 & -4.634310 \\ \mathrm{H} & 3.724110 & 1.511730 & -5.453220 \\ \mathrm{H} & 4.156269 & 3.154801 & -4.946750 \\ \mathrm{C} & 2.059489 & 2.393220 & -3.429460 \\ \mathrm{H} & 2.070569 & 3.447090 & -3.723400 \\ \mathrm{H} & 1.498500 & 1.836940 & -4.187410 \\ \mathrm{H} & 1.516109 & 2.323920 & -2.484610\end{array}$

\section{C-Catalyzed reaction TS 47}

\section{Datum}

Value

\begin{tabular}{lc} 
B3LYP-D3(BJ)/6-31G(d,p) Energy & -3039.481113 \\
\hline B3LYP-D3(BJ)/def2-TZVPP/IEF-PCM(DCM) Energy & -3040.516893 \\
\hline B3LYP-D3(BJ)/def2-TZVPP/IEF-PCM(DCM)//B3LYP-D3(BJ)/6-31G(d,p) Free Energy (Quasiharmonic) & -3039.286725
\end{tabular}

Number of Imaginary Frequencies

Frequencies (Top 3 out of 450)

$\begin{array}{rr}\text { 1. } & -247.5304 \mathrm{~cm}^{-1} \\ \text { 2. } & 8.0704 \mathrm{~cm}^{-1} \\ \text { 3. } & 10.2684 \mathrm{~cm}^{-1}\end{array}$

B3LYP-D3(BJ)/6-31G(d,p) Molecular Geometry in Cartesian Coordinates

$\begin{array}{lrrr}C & -3.191390 & -1.487550 & -1.389710 \\ H & -2.675240 & -2.435700 & -1.580230 \\ H & -4.253790 & -1.645810 & -1.588960 \\ \mathrm{C} & 0.256220 & -0.079230 & -0.460710 \\ \mathrm{O} & -0.501230 & 0.625330 & -1.113440 \\ \mathrm{O} & 1.059680 & 0.489620 & 0.419830 \\ \mathrm{H} & 1.708130 & -0.047630 & 0.968690 \\ \mathrm{O} & -2.755830 & -0.489900 & -2.306330 \\ \mathrm{H} & -1.898750 & -0.140000 & -1.993000 \\ \mathrm{C} & 0.994250 & 3.401100 & 2.710490 \\ \mathrm{C} & 2.530440 & 2.926330 & 1.316280 \\ \mathrm{C} & 2.075400 & 1.622180 & 4.029190 \\ \mathrm{C} & 0.988730 & 2.129670 & 3.252900 \\ \mathrm{H} & 1.877640 & 2.295040 & 0.722050\end{array}$




\begin{tabular}{|c|c|c|c|}
\hline $\mathrm{H}$ & 1.859000 & 0.702550 & 4.569730 \\
\hline $\mathrm{H}$ & 2.587980 & 2.358149 & 4.650240 \\
\hline C & 3.817450 & -1.122431 & 2.414570 \\
\hline C & 5.078460 & -0.304571 & 2.846760 \\
\hline C & 3.252570 & -1.967941 & 3.559510 \\
\hline H & 2.280530 & -2.370510 & 3.267490 \\
\hline $\mathrm{H}$ & 3.908750 & -2.812011 & 3.789120 \\
\hline $\mathrm{H}$ & 3.130120 & -1.368981 & 4.464540 \\
\hline C & 4.005930 & -1.979351 & 1.174920 \\
\hline $\mathrm{H}$ & 4.789650 & -2.725511 & 1.338110 \\
\hline $\mathrm{H}$ & 3.081180 & -2.513071 & 0.935750 \\
\hline $\mathrm{H}$ & 4.276930 & -1.374911 & 0.312130 \\
\hline C & 5.871840 & 0.221999 & 1.640380 \\
\hline $\mathrm{H}$ & 6.433040 & -0.577201 & 1.146770 \\
\hline $\mathrm{H}$ & 5.215840 & 0.691569 & 0.904210 \\
\hline $\mathrm{H}$ & 6.579840 & 0.976059 & 1.993300 \\
\hline C & 6.013260 & -1.033521 & 3.806030 \\
\hline $\mathrm{H}$ & 6.398030 & -1.954991 & 3.356660 \\
\hline $\mathrm{H}$ & 6.865010 & -0.390671 & 4.044590 \\
\hline $\mathrm{H}$ & 5.506720 & -1.280381 & 4.740310 \\
\hline 0 & 2.872280 & -0.063471 & 2.112070 \\
\hline 0 & 4.491320 & 0.806059 & 3.537790 \\
\hline B & 3.280400 & 1.128089 & 2.871570 \\
\hline 0 & 3.461590 & 2.358539 & 2.029770 \\
\hline C & 2.821960 & 4.263909 & 0.768540 \\
\hline C & 1.977570 & 4.793430 & -0.217560 \\
\hline C & 3.952040 & 4.983789 & 1.177430 \\
\hline C & 2.263150 & 6.027520 & -0.793350 \\
\hline $\mathrm{H}$ & 1.106080 & 4.228230 & -0.534430 \\
\hline C & 4.234710 & 6.217479 & 0.597970 \\
\hline $\mathrm{H}$ & 4.602790 & 4.552489 & 1.929800 \\
\hline C & 3.393371 & 6.741279 & -0.387060 \\
\hline $\mathrm{H}$ & 1.609560 & 6.430760 & -1.560330 \\
\hline $\mathrm{H}$ & 5.115471 & 6.770689 & 0.908970 \\
\hline $\mathrm{H}$ & 3.619401 & 7.702129 & -0.838970 \\
\hline $\mathrm{H}$ & 0.263720 & 1.412970 & 2.872580 \\
\hline $\mathrm{H}$ & 1.571290 & 4.186830 & 3.188110 \\
\hline $\mathrm{H}$ & 0.180120 & 3.728590 & 2.072400 \\
\hline C & -0.777720 & -2.248390 & 0.001050 \\
\hline C & 0.278720 & -1.577930 & -0.597620 \\
\hline C & 1.295230 & -2.270370 & -1.339940 \\
\hline C & 1.177710 & -3.645930 & -1.440580 \\
\hline $\mathrm{H}$ & 1.893600 & -4.197550 & -2.040460 \\
\hline C & -2.999700 & -1.095070 & 0.065990 \\
\hline C & -1.836270 & -1.472660 & 0.726920 \\
\hline C & -3.770430 & 0.025670 & 2.080810 \\
\hline C & -3.990570 & -0.322760 & 0.761570 \\
\hline $\mathrm{H}$ & -4.512990 & 0.624470 & 2.599750 \\
\hline C & -2.599620 & -0.349640 & 2.776160 \\
\hline C & -1.609020 & -1.121340 & 2.093300 \\
\hline C & -0.444490 & -1.512690 & 2.806690 \\
\hline C & -0.271600 & -1.159630 & 4.125530 \\
\hline C & -1.244960 & -0.379910 & 4.794460 \\
\hline C & -2.381930 & 0.017320 & 4.131050 \\
\hline
\end{tabular}




\begin{tabular}{|c|c|c|c|}
\hline $\mathrm{H}$ & 0.308130 & -2.102510 & 2.297020 \\
\hline $\mathrm{H}$ & 0.619130 & -1.476770 & 4.655680 \\
\hline $\mathrm{H}$ & -1.089090 & -0.097820 & 5.830960 \\
\hline H & -3.137200 & 0.614010 & 4.634730 \\
\hline C & 0.145450 & -4.374520 & -0.806830 \\
\hline C & -0.847490 & -3.671890 & -0.057360 \\
\hline C & -1.876520 & -4.409660 & 0.584180 \\
\hline C & -1.920730 & -5.781430 & 0.484020 \\
\hline C & -0.942320 & -6.478380 & -0.265250 \\
\hline C & 0.066150 & -5.789810 & -0.897440 \\
\hline $\mathrm{H}$ & -2.627110 & -3.872370 & 1.152480 \\
\hline $\mathrm{H}$ & -2.711460 & -6.335150 & 0.980270 \\
\hline $\mathrm{H}$ & -0.991890 & -7.560300 & -0.338620 \\
\hline $\mathrm{H}$ & 0.820450 & -6.317980 & -1.473720 \\
\hline C & -7.659030 & 0.949820 & -1.014140 \\
\hline C & -6.470830 & 1.154440 & -1.715670 \\
\hline C & -5.272330 & 0.734770 & -1.123050 \\
\hline C & -5.262920 & 0.108020 & 0.122870 \\
\hline C & -6.478790 & -0.085170 & 0.801750 \\
\hline C & -7.687740 & 0.333190 & 0.248860 \\
\hline $\mathrm{H}$ & -8.595260 & 1.268010 & -1.455220 \\
\hline $\mathrm{H}$ & -4.330670 & 0.869830 & -1.639790 \\
\hline $\mathrm{H}$ & -6.456760 & -0.589270 & 1.759930 \\
\hline C & -6.417030 & 1.801460 & -3.105960 \\
\hline C & -9.035390 & 0.133180 & 0.957310 \\
\hline C & -5.781180 & 0.801560 & -4.098040 \\
\hline $\mathrm{H}$ & -5.715500 & 1.249230 & -5.096180 \\
\hline $\mathrm{H}$ & -4.775340 & 0.505200 & -3.789190 \\
\hline $\mathrm{H}$ & -6.391000 & -0.104960 & -4.172510 \\
\hline C & -7.810140 & 2.188560 & -3.625850 \\
\hline $\mathrm{H}$ & -7.720920 & 2.638580 & -4.619430 \\
\hline $\mathrm{H}$ & -8.465260 & 1.316010 & -3.714870 \\
\hline $\mathrm{H}$ & -8.299020 & 2.919290 & -2.973100 \\
\hline C & -5.549720 & 3.077040 & -3.040320 \\
\hline $\mathrm{H}$ & -5.494980 & 3.550520 & -4.026820 \\
\hline $\mathrm{H}$ & -5.973950 & 3.800420 & -2.336370 \\
\hline $\mathrm{H}$ & -4.529520 & 2.851930 & -2.719190 \\
\hline C & -9.950970 & -0.745450 & 0.077780 \\
\hline $\mathrm{H}$ & -10.920080 & -0.896750 & 0.565990 \\
\hline $\mathrm{H}$ & -10.134940 & -0.287090 & -0.897680 \\
\hline $\mathrm{H}$ & -9.496980 & -1.726330 & -0.094260 \\
\hline C & -8.880670 & -0.552380 & 2.324430 \\
\hline $\mathrm{H}$ & -9.863050 & -0.666720 & $2.79306 e$ \\
\hline $\mathrm{H}$ & -8.439750 & -1.549570 & $2.22976 e$ \\
\hline $\mathrm{H}$ & -8.254810 & 0.035730 & 3.002996 \\
\hline C & -9.703280 & 1.507250 & $1.18037 e$ \\
\hline $\mathrm{H}$ & -10.669750 & 1.385470 & $1.68185 e$ \\
\hline $\mathrm{H}$ & -9.072020 & 2.148080 & 1.803910 \\
\hline $\mathrm{H}$ & -9.879310 & 2.029430 & $0.23603 e$ \\
\hline C & 2.458100 & -1.578420 & $-1.95958 e$ \\
\hline C & 3.682890 & -2.246551 & $-2.07831 e$ \\
\hline C & 2.386100 & -0.245010 & $-2.39360 e$ \\
\hline C & 4.824540 & -1.615761 & $-2.58704 e$ \\
\hline $\mathrm{H}$ & 3.761370 & -3.265031 & -1.717310 \\
\hline
\end{tabular}




\begin{tabular}{|c|c|c|c|}
\hline C & 3.514920 & 0.437069 & -2.843880 \\
\hline $\mathrm{H}$ & 1.435480 & 0.262360 & -2.386650 \\
\hline C & 4.723950 & -0.268061 & -2.938110 \\
\hline $\mathrm{H}$ & 5.603050 & 0.255589 & -3.289760 \\
\hline C & 3.478920 & 1.935209 & -3.174900 \\
\hline C & 6.132740 & -2.410251 & -2.718430 \\
\hline C & 4.382270 & 2.670859 & -2.158830 \\
\hline $\mathrm{H}$ & 4.057200 & 2.472549 & -1.134270 \\
\hline $\mathrm{H}$ & 5.424690 & 2.350309 & -2.244990 \\
\hline $\mathrm{H}$ & 4.343940 & 3.753209 & -2.319790 \\
\hline C & 4.003920 & 2.180189 & -4.604370 \\
\hline $\mathrm{H}$ & 3.988080 & 3.250799 & -4.834690 \\
\hline $\mathrm{H}$ & 5.032430 & 1.829709 & -4.727890 \\
\hline $\mathrm{H}$ & 3.381380 & 1.663229 & -5.341300 \\
\hline C & 2.058390 & 2.515410 & -3.076750 \\
\hline $\mathrm{H}$ & 1.380580 & 2.048650 & -3.798330 \\
\hline $\mathrm{H}$ & 1.628440 & 2.383030 & -2.080820 \\
\hline $\mathrm{H}$ & 2.086060 & 3.588620 & -3.287230 \\
\hline C & 6.566630 & -2.938841 & -1.334930 \\
\hline $\mathrm{H}$ & 6.740560 & -2.111281 & -0.640770 \\
\hline $\mathrm{H}$ & 5.810110 & -3.592621 & -0.893180 \\
\hline $\mathrm{H}$ & 7.495220 & -3.513051 & -1.422270 \\
\hline C & 5.899680 & -3.602641 & -3.671400 \\
\hline $\mathrm{H}$ & 5.120200 & -4.271531 & -3.295810 \\
\hline $\mathrm{H}$ & 5.591920 & -3.253541 & -4.661840 \\
\hline $\mathrm{H}$ & 6.818970 & -4.187571 & -3.783800 \\
\hline C & 7.278880 & -1.556581 & -3.284590 \\
\hline $\mathrm{H}$ & 8.183110 & -2.166531 & -3.371790 \\
\hline $\mathrm{H}$ & 7.041950 & -1.169251 & -4.280300 \\
\hline $\mathrm{H}$ & 7.512380 & -0.709111 & -2.632510 \\
\hline
\end{tabular}

\section{C-Catalyzed reaction TS 48 (TS-2.6)}

\section{Datum}

Value

B3LYP-D3(BJ)/6-31G(d,p) Energy

$-3039.487577$

B3LYP-D3(BJ)/def2-TZVPP/IEF-PCM(DCM) Energy

$-3040.515908$

B3LYP-D3(BJ)/def2-TZVPP/IEF-PCM(DCM)//B3LYP-D3(BJ)/6-31G(d,p) Free Energy (Quasiharmonic)

$-3039.284630$

Number of Imaginary Frequencies

Frequencies (Top 3 out of 450)

$\begin{array}{lr}\text { 1. } & -360.7243 \mathrm{~cm}^{-1} \\ \text { 2. } & 17.7681 \mathrm{~cm}^{-1} \\ \text { 3. } & 18.4182 \mathrm{~cm}^{-1}\end{array}$




\begin{tabular}{|c|c|c|c|}
\hline C & 2.005133 & 0.938067 & 1.026000 \\
\hline C & -0.374794 & 2.306967 & -1.388656 \\
\hline 0 & -0.549105 & 0.900768 & -1.479649 \\
\hline $\mathrm{H}$ & 0.054147 & 0.493046 & -0.831908 \\
\hline 0 & 1.352554 & -0.014840 & 0.359271 \\
\hline $\mathrm{H}$ & 1.041341 & -0.752574 & 0.990203 \\
\hline C & -0.274297 & -2.331574 & -2.581876 \\
\hline C & -1.721460 & -1.789775 & -1.233203 \\
\hline C & 0.848674 & -3.334642 & -0.631814 \\
\hline C & 0.119785 & -3.442683 & -1.847322 \\
\hline $\mathrm{H}$ & -1.810489 & -0.798523 & -1.678491 \\
\hline $\mathrm{H}$ & 1.217735 & -4.271878 & -0.217754 \\
\hline $\mathrm{H}$ & 1.606675 & -2.550119 & -0.625407 \\
\hline C & -0.701654 & -3.659656 & 2.739228 \\
\hline C & -0.068064 & -2.227894 & 2.889897 \\
\hline C & -2.002859 & -3.848136 & 3.513983 \\
\hline $\mathrm{H}$ & -2.382141 & -4.861443 & 3.354057 \\
\hline $\mathrm{H}$ & -1.841782 & -3.711523 & 4.588372 \\
\hline $\mathrm{H}$ & -2.767734 & -3.145292 & 3.180700 \\
\hline C & 0.283005 & -4.783778 & 3.077238 \\
\hline $\mathrm{H}$ & 0.489377 & -4.837532 & 4.150243 \\
\hline $\mathrm{H}$ & -0.152879 & -5.735393 & 2.761336 \\
\hline $\mathrm{H}$ & 1.228355 & -4.649099 & 2.547565 \\
\hline C & 0.991505 & -2.083577 & 3.974839 \\
\hline $\mathrm{H}$ & 0.549648 & -2.232545 & 4.965278 \\
\hline $\mathrm{H}$ & 1.803565 & -2.798925 & 3.843566 \\
\hline $\mathrm{H}$ & 1.411048 & -1.075200 & 3.929285 \\
\hline C & -1.105843 & -1.106642 & 3.025616 \\
\hline $\mathrm{H}$ & -1.602356 & -1.141119 & 3.999372 \\
\hline $\mathrm{H}$ & -0.597630 & -0.142535 & 2.934563 \\
\hline $\mathrm{H}$ & -1.859960 & -1.168260 & 2.239941 \\
\hline 0 & -0.995120 & -3.748787 & 1.330176 \\
\hline 0 & 0.576454 & -2.081510 & 1.601203 \\
\hline B & -0.262235 & -2.753523 & 0.624835 \\
\hline 0 & -1.087663 & -1.740438 & -0.087143 \\
\hline C & -2.869474 & -2.689965 & -1.518544 \\
\hline C & -3.083708 & -3.930183 & -0.900482 \\
\hline C & -3.792135 & -2.227683 & -2.466799 \\
\hline C & -4.203139 & -4.686306 & -1.239272 \\
\hline $\mathrm{H}$ & -2.402238 & -4.276596 & -0.132425 \\
\hline C & -4.911551 & -2.985672 & -2.800772 \\
\hline $\mathrm{H}$ & -3.636088 & -1.258291 & -2.927445 \\
\hline C & -5.118422 & -4.221820 & -2.187507 \\
\hline $\mathrm{H}$ & -4.369128 & -5.641642 & -0.750702 \\
\hline $\mathrm{H}$ & -5.619794 & -2.609797 & -3.532759 \\
\hline $\mathrm{H}$ & -5.990285 & -4.817217 & -2.440777 \\
\hline $\mathrm{H}$ & -0.359247 & -4.393348 & -2.078348 \\
\hline $\mathrm{H}$ & 0.284963 & -1.403848 & -2.496849 \\
\hline $\mathrm{H}$ & -0.839822 & -2.463952 & -3.498344 \\
\hline C & 0.630778 & 3.355851 & 0.671560 \\
\hline
\end{tabular}




\begin{tabular}{|c|c|c|c|}
\hline C & -0.493267 & 2.844964 & 0.029254 \\
\hline C & -1.739448 & 2.777119 & 0.738177 \\
\hline C & -1.816901 & 3.304254 & 2.012667 \\
\hline $\mathrm{H}$ & -2.764695 & 3.266445 & 2.541653 \\
\hline C & 2.599035 & 1.979190 & 0.113755 \\
\hline C & 1.969475 & 3.206594 & 0.018468 \\
\hline C & 4.291440 & 2.657341 & -1.468010 \\
\hline C & 3.749653 & 1.667433 & -0.673764 \\
\hline $\mathrm{H}$ & 5.169914 & 2.436839 & -2.067627 \\
\hline C & 3.730896 & 3.957897 & -1.525354 \\
\hline C & 2.536337 & 4.237285 & -0.787716 \\
\hline C & 1.945082 & 5.523109 & -0.894422 \\
\hline C & 2.522110 & 6.497608 & -1.676461 \\
\hline C & 3.710405 & 6.227349 & -2.396607 \\
\hline C & 4.298316 & 4.985651 & -2.323870 \\
\hline $\mathrm{H}$ & 1.035721 & 5.724979 & -0.338896 \\
\hline $\mathrm{H}$ & 2.065689 & 7.480311 & -1.742936 \\
\hline $\mathrm{H}$ & 4.154968 & 7.004587 & -3.010569 \\
\hline $\mathrm{H}$ & 5.206481 & 4.770868 & -2.880088 \\
\hline C & -0.705803 & 3.896142 & 2.658517 \\
\hline C & 0.552872 & 3.905532 & 1.986800 \\
\hline C & 1.681844 & 4.446362 & 2.654651 \\
\hline C & 1.561133 & 4.977512 & 3.918365 \\
\hline C & 0.309242 & 4.983072 & 4.578206 \\
\hline C & -0.798985 & 4.449961 & 3.961844 \\
\hline $\mathrm{H}$ & 2.643353 & 4.430847 & 2.154555 \\
\hline $\mathrm{H}$ & 2.432494 & 5.390519 & 4.416945 \\
\hline $\mathrm{H}$ & 0.228872 & 5.403788 & 5.575881 \\
\hline $\mathrm{H}$ & -1.762073 & 4.439879 & 4.464650 \\
\hline C & 4.890120 & -2.424719 & -0.823307 \\
\hline C & 4.670020 & -1.739061 & -2.024334 \\
\hline C & 4.346984 & -0.381280 & -1.950216 \\
\hline C & 4.251328 & 0.270620 & -0.715035 \\
\hline C & 4.512134 & -0.438154 & 0.462229 \\
\hline C & 4.814975 & -1.800634 & 0.427784 \\
\hline $\mathrm{H}$ & 5.111930 & -3.485795 & -0.862654 \\
\hline $\mathrm{H}$ & 4.114139 & 0.181596 & -2.845313 \\
\hline $\mathrm{H}$ & 4.440053 & 0.081914 & 1.407357 \\
\hline C & 4.745970 & -2.500012 & -3.355750 \\
\hline C & 4.996201 & -2.629968 & 1.705678 \\
\hline C & 3.685382 & -3.622239 & -3.362008 \\
\hline $\mathrm{H}$ & 3.733615 & -4.187171 & -4.299557 \\
\hline $\mathrm{H}$ & 3.835394 & -4.325366 & -2.538147 \\
\hline $\mathrm{H}$ & 2.678582 & -3.205737 & -3.263662 \\
\hline C & 4.484498 & -1.586818 & -4.564287 \\
\hline $\mathrm{H}$ & 4.562371 & -2.167479 & -5.488608 \\
\hline $\mathrm{H}$ & 3.481947 & -1.149178 & -4.531128 \\
\hline $\mathrm{H}$ & 5.212763 & -0.771738 & -4.620435 \\
\hline C & 6.151829 & -3.118269 & -3.511115 \\
\hline $\mathrm{H}$ & 6.224023 & -3.669850 & -4.454823 \\
\hline $\mathrm{H}$ & 6.920505 & -2.339156 & -3.510119 \\
\hline $\mathrm{H}$ & 6.380233 & -3.813483 & -2.698680 \\
\hline C & 6.375346 & -3.319908 & 1.695649 \\
\hline $\mathrm{H}$ & 6.504298 & -3.925167 & 2.599486 \\
\hline
\end{tabular}




\begin{tabular}{|c|c|c|c|}
\hline H & 6.493561 & -3.980823 & 0.832171 \\
\hline H & 7.180409 & -2.578985 & 1.663265 \\
\hline C & 4.888006 & -1.767933 & 2.974250 \\
\hline H & 5.006176 & -2.401146 & 3.859146 \\
\hline $\mathrm{H}$ & 5.668857 & -1.001427 & 3.008206 \\
\hline $\mathrm{H}$ & 3.917363 & -1.269891 & 3.045322 \\
\hline C & 3.877792 & -3.694908 & 1.750562 \\
\hline $\mathrm{H}$ & 3.954498 & -4.290322 & 2.667537 \\
\hline $\mathrm{H}$ & 2.896194 & -3.214551 & 1.726457 \\
\hline H & 3.940230 & -4.381267 & 0.900641 \\
\hline C & -2.945922 & 2.084153 & 0.202206 \\
\hline C & -3.557013 & 1.113568 & 1.004724 \\
\hline C & -3.533444 & 2.414233 & -1.025243 \\
\hline C & -4.730063 & 0.461834 & 0.609166 \\
\hline $\mathrm{H}$ & -3.095686 & 0.880570 & 1.954918 \\
\hline C & -4.680856 & 1.758113 & -1.475620 \\
\hline $\mathrm{H}$ & -3.103032 & 3.214751 & -1.610753 \\
\hline C & -5.250008 & 0.776717 & -0.650655 \\
\hline $\mathrm{H}$ & -6.141721 & 0.265241 & -0.992656 \\
\hline C & -5.363934 & 2.114385 & -2.805547 \\
\hline C & -5.423221 & -0.567627 & 1.517621 \\
\hline C & -5.418913 & 0.868666 & -3.714566 \\
\hline $\mathrm{H}$ & -5.962644 & 1.093606 & -4.638583 \\
\hline $\mathrm{H}$ & -4.408871 & 0.547763 & -3.988694 \\
\hline $\mathrm{H}$ & -5.920113 & 0.030001 & -3.223959 \\
\hline C & -6.801702 & 2.601489 & -2.521433 \\
\hline $\mathrm{H}$ & -7.305024 & 2.874318 & -3.455531 \\
\hline H & -7.400057 & 1.829476 & -2.030121 \\
\hline $\mathrm{H}$ & -6.789731 & 3.479959 & -1.869026 \\
\hline C & -4.621331 & 3.224853 & -3.566144 \\
\hline $\mathrm{H}$ & -4.590753 & 4.157058 & -2.993855 \\
\hline $\mathrm{H}$ & -3.593204 & 2.933864 & -3.803666 \\
\hline $\mathrm{H}$ & -5.134963 & 3.432391 & -4.509982 \\
\hline C & -6.956891 & -0.436061 & 1.415498 \\
\hline $\mathrm{H}$ & -7.284117 & 0.580021 & 1.657526 \\
\hline $\mathrm{H}$ & -7.327645 & -0.682867 & 0.417196 \\
\hline $\mathrm{H}$ & -7.435930 & -1.126361 & 2.117404 \\
\hline C & -5.009704 & -1.986860 & 1.083364 \\
\hline H & -5.302542 & -2.185321 & 0.049908 \\
\hline H & -3.927361 & -2.119553 & 1.151049 \\
\hline $\mathrm{H}$ & -5.485797 & -2.739021 & 1.723045 \\
\hline C & -5.035803 & -0.370013 & 2.995399 \\
\hline $\mathrm{H}$ & -5.608219 & -1.062869 & 3.620129 \\
\hline H & -3.977412 & -0.572403 & 3.174502 \\
\hline $\mathrm{H}$ & -5.250594 & 0.648797 & 3.333579 \\
\hline $\mathrm{H}$ & 0.589296 & 2.607241 & -1.810386 \\
\hline $\mathrm{H}$ & -1.146040 & 2.747055 & -2.022750 \\
\hline 0 & 2.114128 & 0.965657 & 2.237436 \\
\hline
\end{tabular}


Frequencies (Top 3 out of 168)

$\begin{array}{ll}\text { 1. } & 22.5659 \mathrm{~cm}^{-1} \\ \text { 2. } & 26.0700 \mathrm{~cm}^{-1} \\ \text { 3. } & 34.1318 \mathrm{~cm}^{-1}\end{array}$

\section{B3LYP-D3(BJ)/6-31G(d,p) Molecular Geometry in Cartesian Coordinates}

C

C

C

C

$\mathrm{H}$

C

C

C

$\mathrm{C}$

$\mathrm{H}$

0

$P$

0

c

C

C

C

C

C

$\mathrm{H}$

$\mathrm{H}$

$\mathrm{C}$

C

C

C

C

C

$\mathrm{H}$

$\mathrm{H}$

C

C

C
$-0.688279$

$-1.497225$

$-2.899146$

$-3.475884$

$-4.548791$

3. 566912

0.777053

1.490235

2.896542

4.645158

$-0.904343$

$-0.094112$

0.798927

$-5.221220$

$-4.326239$

$-3.568155$

$-3.695146$

$-4.596975$

$-5.355004$

$-4.216721$

$-6.042210$

5.085598

5. 314855

4.589510

3. 623871

3. 397453

4.124859

6.050787

3.941086

1.475920

2.894420

3.598889
1.171201

0.139538

0.081742

1.146352

1.149137

0.895768

1.104724

$-0.012769$

$-0.149644$

0.836462

$-0.930610$

$-2.024137$

$-1.073519$

$-3.374839$

$-2.833468$

$-1.711124$

$-1.107638$

$-1.661324$

$-2.783856$

- 3.285981

$-3.201748$

$-3.619196$

$-3.053811$

$-1.935713$

$-1.363822$

$-1.943568$

- 3.059821

$-3.489303$

$-3.493613$

2. 147954

2.039999

3.077017
0.082499

$-0.354851$

$-0.129665$

0.533386

0.699779

$-0.530343$

$-0.169703$

0.230966

0.074731

$-0.640816$

$-1.027449$

$-0.135181$

0.828043

$-1.181829$

$-2.106447$

$-1.779978$

$-0.518532$

0.403705

0.074933

$-3.087090$

0.804331

1.334879

0.080864

$-0.324423$

0.516777

1.774570

2.178702

$-0.588053$

3.156900

$-0.862180$

$-1.021258$

$-1.688962$ 


\begin{tabular}{|lrrr|} 
C & 2.933153 & 4.161397 & -2.208600 \\
C & 1.526332 & 4.247995 & -2.089522 \\
H & 0.815995 & 3.267925 & -1.434755 \\
H & 4.676621 & 2.987714 & -1.791062 \\
H & 3.481120 & 4.945151 & -2.722006 \\
H & 1.001199 & 5.092926 & -2.523816 \\
C & -0.261507 & 3.340629 & -1.358169 \\
C & -1.288136 & 2.236748 & 0.832793 \\
C & -2.707398 & 2.228678 & 1.026455 \\
C & -3.315887 & 3.290658 & 1.746949 \\
C & -2.558602 & 4.303003 & 2.286304 \\
C & -1.152462 & 4.287147 & 2.135791 \\
H & -0.533023 & 3.281105 & 1.429835 \\
H & -4.394625 & 3.278162 & 1.874137 \\
H & -3.033972 & 5.106789 & 2.839514 \\
H & -0.554948 & 5.073660 & 2.585949 \\
O & 0.544675 & 3.276575 & 1.328894 \\
O & 0.554753 & -3.037066 & -0.980443 \\
H & -1.114816 & -2.542095 & 0.992398 \\
H & -1.640508 & -3.285765 & 0.663360 \\
H & 4.749630 & -1.513366 & -1.311254 \\
H & 2.654314 & -1.513932 & 2.435554 \\
H & 5.646825 & -4.493274 & 1.650602 \\
H & -2.873444 & -1.299485 & -2.501755 \\
H & -5.806512 & -4.252511 & -1.437986 \\
& -4.679549 & -1.219992 & 1.391764 \\
\hline & & & \\
\hline & & & \\
\hline
\end{tabular}

\section{Simple BPA 2}

Datum

Value

B3LYP-D3(BJ)/6-31G(d,p) Energy

$-1874.620096$

B3LYP-D3(BJ)/def2-TZVPP/IEF-PCM(DCM) Energy

$-1875.235359$

B3LYP-D3(BJ)/def2-TZVPP/IEF-PCM(DCM)//B3LYP-D3(BJ)/6-31G(d,p) Free Energy (Quasiharmonic) -1874.827463

Number of Imaginary Frequencies

Frequencies (Top 3 out of 168)

$\begin{array}{ll}\text { 1. } & 23.0974 \mathrm{~cm}^{-1} \\ \text { 2. } & 29.0049 \mathrm{~cm}^{-1} \\ \text { 3. } & 36.1402 \mathrm{~cm}^{-1}\end{array}$

B3LYP-D3(BJ)/6-31G(d,p) Molecular Geometry in Cartesian Coordinates 


\begin{tabular}{|c|c|c|c|}
\hline C & 0.619376 & 1.162048 & -0.050418 \\
\hline C & 1.531515 & 0.202036 & 0.339359 \\
\hline C & 2.918225 & 0.249282 & 0.034746 \\
\hline C & 3.371418 & 1.350635 & -0.662570 \\
\hline $\mathrm{H}$ & 4.429497 & 1.435668 & -0.890660 \\
\hline C & -3.598670 & 0.696912 & 0.649676 \\
\hline C & -0.820090 & 0.990755 & 0.285910 \\
\hline C & -1.487389 & -0.170860 & -0.071528 \\
\hline C & -2.893013 & -0.339121 & 0.069130 \\
\hline $\mathrm{H}$ & -4.672369 & 0.594626 & 0.772186 \\
\hline 0 & 1.059554 & -0.910527 & 1.041640 \\
\hline $\mathrm{P}$ & 0.298366 & -2.056160 & 0.179799 \\
\hline 0 & -0.772951 & -1.206413 & -0.686280 \\
\hline C & 5.551273 & -3.015220 & 0.958615 \\
\hline C & 4.676286 & -2.533303 & 1.933847 \\
\hline C & 3.818146 & -1.473405 & 1.649112 \\
\hline C & 3.823334 & -0.873641 & 0.379614 \\
\hline C & 4.705966 & -1.367441 & -0.593913 \\
\hline C & 5.564020 & -2.427802 & -0.306870 \\
\hline $\mathrm{H}$ & 4.660511 & -2.983827 & 2.921336 \\
\hline $\mathrm{H}$ & 6.235036 & -2.800294 & -1.074895 \\
\hline C & -5.082638 & -3.743513 & -1.361501 \\
\hline C & -3.972856 & -3.931822 & -0.536786 \\
\hline C & -3.240052 & -2.840828 & -0.073528 \\
\hline C & -3.613532 & -1.537583 & -0.432668 \\
\hline C & -4.732269 & -1.356620 & -1.260228 \\
\hline C & -5.461475 & -2.450446 & -1.722067 \\
\hline $\mathrm{H}$ & -3.673807 & -4.934249 & -0.246097 \\
\hline $\mathrm{H}$ & -6.319849 & -2.292026 & -2.367984 \\
\hline C & -1.551315 & 2.013790 & 0.976143 \\
\hline C & -2.964307 & 1.862918 & 1.139154 \\
\hline C & -3.699225 & 2.877985 & 1.807589 \\
\hline C & -3.066762 & 3.986548 & 2.317719 \\
\hline C & -1.664343 & 4.119714 & 2.188722 \\
\hline C & -0.925624 & 3.159731 & 1.535761 \\
\hline $\mathrm{H}$ & -4.772676 & 2.752988 & 1.916856 \\
\hline $\mathrm{H}$ & -3.637235 & 4.754136 & 2.831025 \\
\hline $\mathrm{H}$ & -1.165168 & 4.984262 & 2.614931 \\
\hline $\mathrm{H}$ & 0.148530 & 3.267575 & 1.451599 \\
\hline C & 1.090603 & 2.265185 & -0.840133 \\
\hline C & 2.493149 & 2.365691 & -1.114117 \\
\hline C & 2.976349 & 3.465063 & -1.872365 \\
\hline C & 2.113903 & 4.410632 & -2.373451 \\
\hline C & 0.724000 & 4.285896 & -2.145359 \\
\hline C & 0.224879 & 3.241644 & -1.400497 \\
\hline $\mathrm{H}$ & 4.044057 & 3.534679 & -2.059744 \\
\hline $\mathrm{H}$ & 2.493703 & 5.243818 & -2.956214 \\
\hline $\mathrm{H}$ & 0.042464 & 5.018254 & -2.566527 \\
\hline $\mathrm{H}$ & -0.841964 & 3.154561 & -1.241853 \\
\hline 0 & -0.173918 & -3.149320 & 1.047471 \\
\hline 0 & 1.273677 & -2.454956 & -1.031276 \\
\hline $\mathrm{H}$ & 1.891756 & -3.149896 & -0.760830 \\
\hline $\mathrm{H}$ & -2.389181 & -3.006318 & 0.576204 \\
\hline
\end{tabular}




$\begin{array}{rrrr}H & -5.015426 & -0.350899 & -1.555552 \\ \mathrm{H} & -5.647527 & -4.598094 & -1.721509 \\ \mathrm{H} & 3.139606 & -1.107732 & 2.410089 \\ \mathrm{H} & 6.214842 & -3.844660 & 1.182544 \\ \mathrm{H} & 4.695519 & -0.929610 & -1.586934\end{array}$

\section{Simple BHCA 1}

\section{Datum}

Value

B3LYP-D3(BJ)/6-31G(d,p) Energy $-1535.993087$

B3LYP-D3(BJ)/def2-TZVPP/IEF-PCM(DCM) Energy $-1536.545421$

B3LYP-D3(BJ)/def2-TZVPP/IEF-PCM(DCM)//B3LYP-D3(BJ)/6-31G(d,p) Free Energy (Quasiharmonic) $-1536.099056$

Number of Imaginary Frequencies

Frequencies (Top 3 out of 177)

$\begin{array}{ll}\text { 1. } & 20.0793 \mathrm{~cm}^{-1} \\ \text { 2. } & 27.1652 \mathrm{~cm}^{-1} \\ \text { 3. } & 35.5871 \mathrm{~cm}^{-1}\end{array}$

B3LYP-D3(BJ)/6-31G(d,p) Molecular Geometry in Cartesian Coordinates

$\begin{array}{lrrr}C & -1.383886 & -0.927203 & -1.538867 \\ \mathrm{H} & -2.285068 & -1.164185 & -2.109478 \\ \mathrm{H} & -0.696320 & -0.365864 & -2.176975 \\ \mathrm{C} & 1.404883 & -0.957139 & 1.371347 \\ \mathrm{O} & 1.973169 & -0.695680 & 2.406010 \\ \mathrm{O} & 0.543252 & -1.979827 & 1.276471 \\ \mathrm{H} & 0.138438 & -2.029967 & 0.378876 \\ \mathrm{O} & -0.747189 & -2.160246 & -1.115806 \\ \mathrm{H} & -0.344262 & -2.580653 & -1.887026 \\ \mathrm{C} & 0.635948 & 0.700482 & -0.338053 \\ \mathrm{C} & 1.640673 & -0.133618 & 0.123053 \\ \mathrm{C} & 2.950520 & -0.102016 & -0.455625 \\ \mathrm{C} & 3.195976 & 0.784904 & -1.484514 \\ \mathrm{H} & 4.173133 & 0.791124 & -1.957985 \\ \mathrm{C} & -1.722936 & -0.123986 & -0.311049 \\ \mathrm{C} & -0.730677 & 0.655382 & 0.270859 \\ \mathrm{C} & -3.307730 & 0.620724 & 1.363513 \\ \mathrm{C} & -3.034824 & -0.164561 & 0.259573 \\ \mathrm{H} & -4.299912 & 0.589005 & 1.803656 \\ \mathrm{C} & -2.322237 & 1.430320 & 1.972994 \\ \mathrm{C} & -0.999499 & 1.441605 & 1.429995\end{array}$




\begin{tabular}{|c|c|c|c|}
\hline C & -0.004940 & 2.235231 & 2.061732 \\
\hline C & -0.311574 & 2.988055 & 3.170813 \\
\hline C & -1.623492 & 2.983998 & 3.703185 \\
\hline C & -2.605447 & 2.219832 & 3.118790 \\
\hline $\mathrm{H}$ & 1.004685 & 2.224505 & 1.669080 \\
\hline $\mathrm{H}$ & 0.459456 & 3.583887 & 3.648543 \\
\hline $\mathrm{H}$ & -1.848719 & 3.581892 & 4.580854 \\
\hline $\mathrm{H}$ & -3.612126 & 2.203684 & 3.526550 \\
\hline C & 2.205954 & 1.676037 & -1.963379 \\
\hline C & 0.899988 & 1.640488 & -1.383554 \\
\hline C & -0.087826 & 2.538697 & -1.867689 \\
\hline C & 0.202290 & 3.422682 & -2.882561 \\
\hline C & 1.492608 & 3.451434 & -3.462183 \\
\hline C & 2.471185 & 2.596085 & -3.011401 \\
\hline $\mathrm{H}$ & -1.075658 & 2.520873 & -1.421546 \\
\hline $\mathrm{H}$ & -0.562553 & 4.104877 & -3.240297 \\
\hline $\mathrm{H}$ & 1.707895 & 4.152986 & -4.262100 \\
\hline $\mathrm{H}$ & 3.465398 & 2.613253 & -3.448852 \\
\hline C & -6.217313 & -2.628581 & -1.250534 \\
\hline C & -6.422399 & -1.266551 & -1.028575 \\
\hline C & -5.381497 & -0.470944 & -0.553774 \\
\hline C & -4.117183 & -1.021109 & -0.292910 \\
\hline C & -3.922483 & -2.393594 & -0.518342 \\
\hline C & -4.964569 & -3.187366 & -0.994020 \\
\hline $\mathrm{H}$ & -7.027339 & -3.249696 & -1.620256 \\
\hline $\mathrm{H}$ & -5.537789 & 0.591627 & -0.394874 \\
\hline $\mathrm{H}$ & -2.952863 & -2.833738 & -0.311880 \\
\hline C & 4.026953 & -1.019843 & -0.007605 \\
\hline C & 3.777966 & -2.382303 & 0.215090 \\
\hline C & 5.335433 & -0.542426 & 0.157634 \\
\hline C & 4.807402 & -3.240714 & 0.591658 \\
\hline $\mathrm{H}$ & 2.773150 & -2.772594 & 0.095154 \\
\hline C & 6.365630 & -1.401426 & 0.533051 \\
\hline $\mathrm{H}$ & 5.535306 & 0.514880 & 0.015535 \\
\hline C & 6.105641 & -2.754770 & 0.749985 \\
\hline $\mathrm{H}$ & 6.906978 & -3.424363 & 1.046616 \\
\hline $\mathrm{H}$ & -4.799141 & -4.248337 & -1.155873 \\
\hline $\mathrm{H}$ & -7.392000 & -0.821252 & -1.230131 \\
\hline $\mathrm{H}$ & 4.594270 & -4.291709 & 0.760477 \\
\hline $\mathrm{H}$ & 7.369951 & -1.011048 & 0.666696 \\
\hline
\end{tabular}

\section{Simple BHCA 2}

Datum

Value

\begin{tabular}{lr}
\hline B3LYP-D3(BJ)/6-31G(d,p) Energy & -1535.993622 \\
\hline B3LYP-D3(BJ)/def2-TZVPP/IEF-PCM(DCM) Energy & -1536.544582 \\
\hline B3LYP-D3(BJ)/def2-TZVPP/IEF-PCM(DCM)//B3LYP-D3(BJ)/6-31G(d,p) Free Energy (Quasiharmonic) & -1536.098428 \\
\hline
\end{tabular}


Frequencies (Top 3 out of 177)

$\begin{array}{ll}\text { 1. } & 16.6214 \mathrm{~cm}^{-1} \\ \text { 2. } & 25.1320 \mathrm{~cm}^{-1} \\ \text { 3. } & 30.6507 \mathrm{~cm}^{-1}\end{array}$

B3LYP-D3(BJ)/6-31G(d,p) Molecular Geometry in Cartesian Coordinates

\begin{tabular}{|c|c|c|c|}
\hline C & -1.431682 & -0.780265 & -1.602376 \\
\hline $\mathrm{H}$ & -2.319370 & -0.838793 & -2.238386 \\
\hline $\mathrm{H}$ & -0.629567 & -0.314497 & -2.173204 \\
\hline C & 1.228211 & -1.043422 & 1.280426 \\
\hline 0 & 1.722660 & -0.861732 & 2.368337 \\
\hline 0 & 0.351958 & -2.036774 & 1.062315 \\
\hline $\mathrm{H}$ & 0.005122 & -2.033309 & 0.136703 \\
\hline 0 & -0.966437 & -2.119294 & -1.312559 \\
\hline $\mathrm{H}$ & -1.746747 & -2.636057 & -1.059947 \\
\hline C & 0.649169 & 0.778105 & -0.335039 \\
\hline C & 1.575147 & -0.153343 & 0.105142 \\
\hline C & 2.907413 & -0.172259 & -0.419177 \\
\hline C & 3.252416 & 0.759118 & -1.377980 \\
\hline $\mathrm{H}$ & 4.248768 & 0.731898 & -1.808738 \\
\hline C & -1.727868 & 0.018833 & -0.353746 \\
\hline C & -0.733204 & 0.784338 & 0.235736 \\
\hline C & -3.318161 & 0.740492 & 1.333740 \\
\hline C & -3.027998 & -0.037717 & 0.235326 \\
\hline $\mathrm{H}$ & -4.310329 & 0.698298 & 1.774043 \\
\hline C & -2.335370 & 1.565988 & 1.933507 \\
\hline C & -1.009785 & 1.570010 & 1.395637 \\
\hline C & -0.014945 & 2.358426 & 2.032452 \\
\hline C & -0.324133 & 3.118964 & 3.135902 \\
\hline C & -1.639191 & 3.126067 & 3.658939 \\
\hline C & -2.621529 & 2.363349 & 3.072056 \\
\hline $\mathrm{H}$ & 0.996028 & 2.341505 & 1.643088 \\
\hline $\mathrm{H}$ & 0.447139 & 3.713218 & 3.615319 \\
\hline $\mathrm{H}$ & -1.867213 & 3.730232 & 4.531547 \\
\hline $\mathrm{H}$ & -3.630402 & 2.353819 & 3.474690 \\
\hline C & 2.340475 & 1.737458 & -1.841350 \\
\hline C & 1.012200 & 1.753955 & -1.313906 \\
\hline C & 0.100909 & 2.736809 & -1.782377 \\
\hline C & 0.486041 & 3.656268 & -2.731890 \\
\hline C & 1.799138 & 3.636139 & -3.258599 \\
\hline C & 2.704444 & 2.696588 & -2.822465 \\
\hline $\mathrm{H}$ & -0.904465 & 2.754045 & -1.376846 \\
\hline $\mathrm{H}$ & -0.220629 & 4.403324 & -3.079671 \\
\hline
\end{tabular}




\begin{tabular}{|lrrr|}
$H$ & 2.089586 & 4.366157 & -4.007690 \\
$H$ & 3.714578 & 2.674705 & -3.221478 \\
C & -5.902588 & -2.838364 & -1.305960 \\
C & -4.841682 & -3.278227 & -0.513036 \\
C & -3.918164 & -2.360250 & -0.012045 \\
C & -4.041321 & -0.990913 & -0.300129 \\
C & -5.116903 & -0.559747 & -1.084843 \\
C & -6.040757 & -1.478277 & -1.585062 \\
$H$ & -6.621430 & -3.550783 & -1.698108 \\
$H$ & -3.115952 & -2.689168 & 0.644383 \\
$H$ & -5.219598 & 0.497950 & -1.306729 \\
C & 3.906902 & -1.177289 & 0.019852 \\
C & 3.576303 & -2.536046 & 0.129621 \\
C & 5.224901 & -0.783091 & 0.293709 \\
C & 4.537214 & -3.471857 & 0.503702 \\
$H$ & 2.564242 & -2.862950 & -0.081476 \\
C & 6.185951 & -1.719692 & 0.666776 \\
$H$ & 5.485316 & 0.269121 & 0.237338 \\
C & 5.845553 & -3.068530 & 0.772094 \\
$H$ & 6.592787 & -3.799086 & 1.066245 \\
$H$ & -6.868364 & -1.130930 & -2.196015 \\
$H$ & -4.738806 & -4.332584 & -0.275288 \\
$H$ & 4.262716 & -4.519239 & 0.582382 \\
$H$ & 7.198519 & -1.394192 & 0.885276 \\
& & & \\
\hline
\end{tabular}

\section{Simple BHCA 3}

\section{Datum}

Value

B3LYP-D3(BJ)/6-31G(d,p) Energy

$-1535.996861$

B3LYP-D3(BJ)/def2-TZVPP/IEF-PCM(DCM) Energy

$-1536.543786$

B3LYP-D3(BJ)/def2-TZVPP/IEF-PCM(DCM)//B3LYP-D3(BJ)/6-31G(d,p) Free Energy (Quasiharmonic)

$-1536.097061$

Number of Imaginary Frequencies

Frequencies (Top 3 out of 177)
1. $\quad 18.0230 \mathrm{~cm}^{-1}$
2. $26.3373 \mathrm{~cm}^{-1}$
3. $33.6630 \mathrm{~cm}^{-1}$

\section{B3LYP-D3(BJ)/6-31G(d,p) Molecular Geometry in Cartesian Coordinates}

$\begin{array}{llll}\mathrm{C} & -1.389161 & -1.040563 & -1.519925 \\ \mathrm{H} & -2.267276 & -1.143134 & -2.161633\end{array}$




\begin{tabular}{|c|c|c|c|}
\hline $\mathrm{H}$ & -0.604750 & -0.550763 & -2.109844 \\
\hline C & 1.388248 & -1.089923 & 1.194006 \\
\hline 0 & 0.604709 & -2.023772 & 1.172265 \\
\hline 0 & 2.101446 & -0.785559 & 2.290096 \\
\hline $\mathrm{H}$ & 1.877221 & -1.452918 & 2.961787 \\
\hline 0 & -0.995961 & -2.360745 & -1.158455 \\
\hline $\mathrm{H}$ & -0.400806 & -2.293603 & -0.389142 \\
\hline C & 0.632411 & 0.671117 & -0.369138 \\
\hline C & 1.660024 & -0.150762 & 0.062684 \\
\hline C & 2.975513 & -0.069558 & -0.494046 \\
\hline C & 3.219152 & 0.878940 & -1.464763 \\
\hline $\mathrm{H}$ & 4.203989 & 0.932630 & -1.918814 \\
\hline C & -1.722019 & -0.185730 & -0.312485 \\
\hline C & -0.726540 & 0.598564 & 0.255558 \\
\hline C & -3.270618 & 0.554861 & 1.405443 \\
\hline C & -3.029599 & -0.209061 & 0.278564 \\
\hline $\mathrm{H}$ & -4.255626 & 0.527788 & 1.861719 \\
\hline C & -2.270994 & 1.350377 & 2.009706 \\
\hline C & -0.966962 & 1.381015 & 1.426705 \\
\hline C & 0.033453 & 2.185634 & 2.036297 \\
\hline C & -0.245947 & 2.919176 & 3.166632 \\
\hline C & -1.537517 & 2.886045 & 3.744504 \\
\hline C & -2.526563 & 2.117561 & 3.177011 \\
\hline $\mathrm{H}$ & 1.026587 & 2.211809 & 1.603241 \\
\hline $\mathrm{H}$ & 0.530113 & 3.527502 & 3.620697 \\
\hline H & -1.743341 & 3.468967 & 4.636932 \\
\hline $\mathrm{H}$ & -3.521146 & 2.084415 & 3.612884 \\
\hline C & 2.209968 & 1.763253 & -1.916857 \\
\hline C & 0.893404 & 1.659938 & -1.368412 \\
\hline C & -0.113092 & 2.548227 & -1.830650 \\
\hline C & 0.170095 & 3.489571 & -2.793767 \\
\hline C & 1.471452 & 3.587782 & -3.340493 \\
\hline C & 2.468003 & 2.742376 & -2.911538 \\
\hline H & -1.109751 & 2.470642 & -1.412413 \\
\hline $\mathrm{H}$ & -0.609164 & 4.161891 & -3.138383 \\
\hline H & 1.680350 & 4.333881 & -4.100807 \\
\hline H & 3.469091 & 2.810714 & -3.327396 \\
\hline C & -6.369577 & -2.441911 & -1.257271 \\
\hline C & -5.130570 & -3.064659 & -1.104251 \\
\hline C & -4.032386 & -2.352406 & -0.624797 \\
\hline C & -4.159129 & -0.994714 & -0.288988 \\
\hline C & -5.410418 & -0.377106 & -0.451355 \\
\hline C & -6.505428 & -1.092224 & -0.930890 \\
\hline $\mathrm{H}$ & -7.221149 & -3.001666 & -1.632268 \\
\hline $\mathrm{H}$ & -3.068146 & -2.837189 & -0.518601 \\
\hline $\mathrm{H}$ & -5.514136 & 0.677603 & -0.215254 \\
\hline C & 4.048193 & -1.008877 & -0.084704 \\
\hline C & 3.811228 & -2.391077 & -0.030083 \\
\hline C & 5.330213 & -0.531358 & 0.221993 \\
\hline C & 4.830381 & -3.270053 & 0.330181 \\
\hline $\mathrm{H}$ & 2.830873 & -2.778296 & -0.288009 \\
\hline C & 6.348761 & -1.411679 & 0.580443 \\
\hline $\mathrm{H}$ & 5.516336 & 0.537820 & 0.199118 \\
\hline & 6.101847 & -2.784008 & 0.637240 \\
\hline
\end{tabular}




$\begin{array}{rrrr}\mathrm{H} & 6.895127 & -3.469879 & 0.917836 \\ \mathrm{H} & -7.461966 & -0.593440 & -1.056036 \\ \mathrm{H} & -5.015234 & -4.114611 & -1.356442 \\ \mathrm{H} & 4.632473 & -4.337072 & 0.360876 \\ \mathrm{H} & 7.333851 & -1.025095 & 0.823378\end{array}$

\section{Simple BHCA 4}

\section{Datum}

Value

B3LYP-D3(BJ)/6-31G(d,p) Energy $-1535.991936$

B3LYP-D3(BJ)/def2-TZVPP/IEF-PCM(DCM) Energy $-1536.542879$

B3LYP-D3(BJ)/def2-TZVPP/IEF-PCM(DCM)//B3LYP-D3(BJ)/6-31G(d,p) Free Energy (Quasiharmonic) $-1536.096608$

Number of Imaginary Frequencies

Frequencies (Top 3 out of 177)

$\begin{array}{ll}\text { 1. } & 18.4217 \mathrm{~cm}^{-1} \\ \text { 2. } & 27.0294 \mathrm{~cm}^{-1} \\ \text { 3. } & 33.9615 \mathrm{~cm}^{-1}\end{array}$

B3LYP-D3(BJ)/6-31G(d,p) Molecular Geometry in Cartesian Coordinates

$\begin{array}{lrrr}\mathrm{C} & 1.303764 & -1.119170 & 1.402707 \\ \mathrm{H} & 2.190945 & -1.461615 & 1.941024 \\ \mathrm{H} & 0.634925 & -0.616608 & 2.106092 \\ \mathrm{C} & -1.342619 & -0.663527 & -1.544911 \\ \mathrm{O} & -1.736225 & -0.181815 & -2.585639 \\ \mathrm{O} & -0.618321 & -1.783815 & -1.522567 \\ \mathrm{H} & -0.280766 & -1.978097 & -0.616181 \\ \mathrm{O} & 0.622890 & -2.265553 & 0.831290 \\ \mathrm{H} & 0.223080 & -2.774521 & 1.548830 \\ \mathrm{C} & -0.633732 & 0.728362 & 0.394658 \\ \mathrm{C} & -1.638042 & -0.008273 & -0.211754 \\ \mathrm{C} & -2.943862 & -0.094581 & 0.370166 \\ \mathrm{C} & -3.184356 & 0.588694 & 1.546542 \\ \mathrm{H} & -4.170230 & 0.528701 & 1.997835 \\ \mathrm{C} & 1.690843 & -0.174963 & 0.293757 \\ \mathrm{C} & 0.747220 & 0.728402 & -0.181939 \\ \mathrm{C} & 3.342640 & 0.733040 & -1.229901 \\ \mathrm{C} & 3.011012 & -0.192993 & -0.259011 \\ \mathrm{H} & 4.341180 & 0.718419 & -1.656358 \\ \mathrm{C} & 2.409346 & 1.672662 & -1.723519 \\ \mathrm{C} & 1.077036 & 1.665339 & -1.204880\end{array}$




\begin{tabular}{lrrr} 
C & 0.133817 & 2.592764 & -1.722377 \\
C & 0.498753 & 3.490452 & -2.697724 \\
C & 1.820452 & 3.503420 & -3.205702 \\
C & 2.753270 & 2.612194 & -2.731352 \\
H & -0.883122 & 2.570232 & -1.349680 \\
H & -0.233105 & 4.189725 & -3.089163 \\
H & 2.091895 & 4.216456 & -3.977990 \\
H & 3.766729 & 2.609232 & -3.122275 \\
C & -2.199894 & 1.391280 & 2.170621 \\
C & -0.897747 & 1.470077 & 1.587123 \\
C & 0.088106 & 2.274895 & 2.218030 \\
C & -0.203493 & 2.966998 & 3.371632 \\
C & -1.492584 & 2.887434 & 3.950356 \\
C & -2.467855 & 2.116860 & 3.361268 \\
H & 1.074083 & 2.340717 & 1.771945 \\
H & 0.558296 & 3.582166 & 3.840279 \\
H & -1.708952 & 3.439153 & 4.859850 \\
H & -3.460748 & 2.052258 & 3.797276 \\
C & 6.045101 & -3.005765 & 0.907853 \\
C & 4.772324 & -3.458208 & 0.557469 \\
C & 3.777885 & -2.552707 & 0.192179 \\
C & 4.041453 & -1.173361 & 0.173813 \\
C & 5.325244 & -0.730577 & 0.527581 \\
C & 6.318390 & -1.637724 & 0.891731 \\
H & 6.817922 & -3.713720 & 1.191025 \\
H & 2.792407 & -2.909045 & -0.087483 \\
H & 5.534471 & 0.334769 & 0.529095 \\
C & -4.024031 & -0.949655 & -0.193719 \\
C & -4.470400 & -0.821450 & -1.517676 \\
C & -4.653662 & -1.885666 & 0.642584 \\
C & -5.515624 & -1.615193 & -1.986802 \\
H & -3.993114 & -0.104466 & -2.172773 \\
C & -5.696686 & -2.678831 & 0.169848 \\
H & -4.307288 & -1.996685 & 1.665837 \\
C & -6.131164 & -2.545496 & -1.149182 \\
H & -6.942457 & -3.163505 & -1.521679 \\
H & 7.304012 & -1.275336 & 1.167921 \\
H & 4.554012 & -4.521957 & 0.559029 \\
& -5.850834 & -1.502071 & -3.013201 \\
\hline & -6.165531 & -3.403122 & 0.829248 \\
\hline
\end{tabular}

\section{Simple BHCA 5}

Datum

Value

\begin{tabular}{lr}
\hline B3LYP-D3(BJ)/6-31G(d,p) Energy & -1535.992951 \\
\hline B3LYP-D3(BJ)/def2-TZVPP/IEF-PCM(DCM) Energy & -1536.542097 \\
\hline B3LYP-D3(BJ)/def2-TZVPP/IEF-PCM(DCM)//B3LYP-D3(BJ)/6-31G(d,p) Free Energy (Quasiharmonic) & -1536.095911 \\
\hline
\end{tabular}


Frequencies (Top 3 out of 177)

$\begin{array}{ll}\text { 1. } & 14.3856 \mathrm{~cm}^{-1} \\ \text { 2. } & 25.9566 \mathrm{~cm}^{-1} \\ \text { 3. } & 30.8612 \mathrm{~cm}^{-1}\end{array}$

B3LYP-D3(BJ)/6-31G(d,p) Molecular Geometry in Cartesian Coordinates

\begin{tabular}{|c|c|c|c|}
\hline C & -1.304625 & -0.835319 & -1.595024 \\
\hline $\mathrm{H}$ & -2.174468 & -0.950744 & -2.248443 \\
\hline $\mathrm{H}$ & -0.516468 & -0.340345 & -2.160417 \\
\hline C & 1.127157 & -0.878276 & 1.389665 \\
\hline 0 & 1.378302 & -0.552364 & 2.529546 \\
\hline 0 & 0.452183 & -1.998950 & 1.115698 \\
\hline $\mathrm{H}$ & 0.199666 & -2.053950 & 0.161195 \\
\hline 0 & -0.779374 & -2.144117 & -1.277842 \\
\hline $\mathrm{H}$ & -1.535980 & -2.693108 & -1.021171 \\
\hline C & 0.648578 & 0.869474 & -0.318439 \\
\hline C & 1.559090 & -0.030301 & 0.210460 \\
\hline C & 2.891452 & -0.119561 & -0.303741 \\
\hline C & 3.253050 & 0.722026 & -1.338093 \\
\hline $\mathrm{H}$ & 4.262195 & 0.665058 & -1.735069 \\
\hline C & -1.678388 & -0.009872 & -0.381680 \\
\hline C & -0.758387 & 0.859526 & 0.186520 \\
\hline C & -3.375408 & 0.723812 & 1.194273 \\
\hline C & -2.992743 & -0.112586 & 0.169147 \\
\hline $\mathrm{H}$ & -4.377818 & 0.644113 & 1.605273 \\
\hline C & -2.473686 & 1.658682 & 1.759963 \\
\hline C & -1.130188 & 1.708356 & 1.272022 \\
\hline C & -0.212755 & 2.605184 & 1.880006 \\
\hline C & -0.613579 & 3.427413 & 2.907024 \\
\hline C & -1.947002 & 3.388664 & 3.380225 \\
\hline C & -2.855126 & 2.520318 & 2.821169 \\
\hline $\mathrm{H}$ & 0.811813 & 2.623431 & 1.527678 \\
\hline $\mathrm{H}$ & 0.098415 & 4.106748 & 3.364761 \\
\hline $\mathrm{H}$ & -2.247770 & 4.042139 & 4.193239 \\
\hline $\mathrm{H}$ & -3.876835 & 2.475560 & 3.187428 \\
\hline C & 2.362334 & 1.677968 & -1.882213 \\
\hline C & 1.032022 & 1.759701 & -1.365876 \\
\hline C & 0.137126 & 2.712909 & -1.919793 \\
\hline C & 0.543789 & 3.549839 & -2.934072 \\
\hline C & 1.861773 & 3.471283 & -3.444126 \\
\hline C & 2.749585 & 2.555570 & -2.928930 \\
\hline $\mathrm{H}$ & -0.871098 & 2.774685 & -1.525335 \\
\hline $\mathrm{H}$ & -0.148520 & 4.277625 & -3.345752 \\
\hline
\end{tabular}




\begin{tabular}{|lrrr|} 
H & 2.169181 & 4.137597 & -4.244096 \\
H & 3.762573 & 2.489065 & -3.315571 \\
C & -5.610079 & -3.226615 & -1.219024 \\
C & -4.512546 & -3.531588 & -0.412407 \\
C & -3.672361 & -2.512439 & 0.036754 \\
C & -3.916649 & -1.174949 & -0.318150 \\
C & -5.028516 & -0.879389 & -1.114853 \\
C & -5.868718 & -1.899484 & -1.563249 \\
H & -6.264117 & -4.018125 & -1.570892 \\
H & -2.843103 & -2.735173 & 0.704119 \\
H & -5.224802 & 0.152895 & -1.387604 \\
C & 3.873370 & -1.120643 & 0.192031 \\
C & 4.201089 & -1.232614 & 1.551789 \\
C & 4.527467 & -1.951604 & -0.731363 \\
C & 5.156985 & -2.156455 & 1.970448 \\
H & 3.704965 & -0.597000 & 2.274201 \\
C & 5.481112 & -2.874895 & -0.309027 \\
H & 4.267250 & -1.880187 & -1.783041 \\
C & 5.799087 & -2.979922 & 1.045463 \\
H & 6.540438 & -3.700336 & 1.377636 \\
H & -6.725190 & -1.657078 & -2.184992 \\
H & -4.316967 & -4.559612 & -0.123086 \\
H & 5.401737 & -2.229140 & 3.025688 \\
H & 5.970153 & -3.515963 & -1.036365 \\
& & & \\
\hline
\end{tabular}

\section{Simple BHCA 6}

\section{Datum}

Value

B3LYP-D3(BJ)/6-31G(d,p) Energy

$-1535.991798$

B3LYP-D3(BJ)/def2-TZVPP/IEF-PCM(DCM) Energy

$-1536.541229$

B3LYP-D3(BJ)/def2-TZVPP/IEF-PCM(DCM)//B3LYP-D3(BJ)/6-31G(d,p) Free Energy (Quasiharmonic)

$-1536.094774$

Number of Imaginary Frequencies

Frequencies (Top 3 out of 177)

$\begin{array}{ll}\text { 1. } & 16.8710 \mathrm{~cm}^{-1} \\ \text { 2. } & 24.3699 \mathrm{~cm}^{-1} \\ \text { 3. } & 33.8344 \mathrm{~cm}^{-1}\end{array}$

B3LYP-D3(BJ)/6-31G(d,p) Molecular Geometry in Cartesian Coordinates

\section{C}

$\mathrm{H}$ 


\begin{tabular}{|c|c|c|c|}
\hline $\mathrm{H}$ & -0.648677 & -0.508549 & -2.137633 \\
\hline C & 1.371619 & -1.162602 & 1.148462 \\
\hline 0 & 0.541834 & -2.046939 & 1.098305 \\
\hline 0 & 2.098833 & -1.004193 & 2.271254 \\
\hline $\mathrm{H}$ & 2.735285 & -0.284301 & 2.148466 \\
\hline 0 & -1.039085 & -2.336732 & -1.222618 \\
\hline $\mathrm{H}$ & -0.448085 & -2.290241 & -0.447303 \\
\hline C & 0.626030 & 0.652704 & -0.375953 \\
\hline C & 1.650284 & -0.185076 & 0.039840 \\
\hline C & 2.968485 & -0.086375 & -0.513723 \\
\hline C & 3.214251 & 0.880845 & -1.466245 \\
\hline $\mathrm{H}$ & 4.200189 & 0.943169 & -1.916738 \\
\hline C & -1.739377 & -0.177657 & -0.318059 \\
\hline C & -0.730291 & 0.584710 & 0.254435 \\
\hline C & -3.257504 & 0.534275 & 1.438454 \\
\hline C & -3.038641 & -0.204309 & 0.290380 \\
\hline $\mathrm{H}$ & -4.234966 & 0.501028 & 1.910092 \\
\hline C & -2.244880 & 1.312470 & 2.043352 \\
\hline C & -0.950523 & 1.350289 & 1.440161 \\
\hline C & 0.058038 & 2.148489 & 2.044697 \\
\hline C & -0.200596 & 2.862850 & 3.192051 \\
\hline C & -1.480425 & 2.815380 & 3.794167 \\
\hline C & -2.478933 & 2.057441 & 3.229329 \\
\hline $\mathrm{H}$ & 1.039032 & 2.196387 & 1.585172 \\
\hline $\mathrm{H}$ & 0.581097 & 3.468705 & 3.639931 \\
\hline $\mathrm{H}$ & -1.670127 & 3.380583 & 4.701289 \\
\hline $\mathrm{H}$ & -3.465546 & 2.015861 & 3.682077 \\
\hline C & 2.206188 & 1.772551 & -1.903602 \\
\hline C & 0.890764 & 1.661776 & -1.353756 \\
\hline C & -0.113356 & 2.563718 & -1.795242 \\
\hline C & 0.170997 & 3.522294 & -2.740589 \\
\hline C & 1.471238 & 3.626562 & -3.289423 \\
\hline C & 2.465964 & 2.769830 & -2.879781 \\
\hline $\mathrm{H}$ & -1.109140 & 2.481152 & -1.376205 \\
\hline $\mathrm{H}$ & -0.606723 & 4.203819 & -3.070123 \\
\hline $\mathrm{H}$ & 1.680341 & 4.386329 & -4.035946 \\
\hline $\mathrm{H}$ & 3.466245 & 2.842668 & -3.296723 \\
\hline C & -6.414828 & -2.378657 & -1.248667 \\
\hline C & -5.178256 & -3.013105 & -1.127216 \\
\hline C & -4.068500 & -2.319471 & -0.647169 \\
\hline C & -4.181424 & -0.969033 & -0.278867 \\
\hline C & -5.430401 & -0.339366 & -0.409257 \\
\hline C & -6.536898 & -1.035941 & -0.889688 \\
\hline $\mathrm{H}$ & -7.275465 & -2.924088 & -1.624083 \\
\hline $\mathrm{H}$ & -3.106404 & -2.813105 & -0.564927 \\
\hline $\mathrm{H}$ & -5.523548 & 0.710210 & -0.147157 \\
\hline C & 4.053382 & -1.014532 & -0.103703 \\
\hline C & 3.838124 & -2.400850 & -0.051061 \\
\hline C & 5.325281 & -0.515615 & 0.216752 \\
\hline C & 4.868554 & -3.262078 & 0.316528 \\
\hline $\mathrm{H}$ & 2.864101 & -2.803294 & -0.308866 \\
\hline C & 6.355526 & -1.379710 & 0.583405 \\
\hline $\mathrm{H}$ & 5.496648 & 0.556379 & 0.190819 \\
\hline C & 6.129503 & -2.755385 & 0.635072 \\
\hline
\end{tabular}




$\begin{array}{rrrr}H & 6.930436 & -3.428826 & 0.923388 \\ \mathrm{H} & -7.491688 & -0.528218 & -0.989700 \\ \mathrm{H} & -5.073897 & -4.057883 & -1.404318 \\ \mathrm{H} & 4.686666 & -4.331539 & 0.349189 \\ \mathrm{H} & 7.332019 & -0.977608 & 0.835204\end{array}$

\section{Simple BHCA 7}

\section{Datum}

Value

B3LYP-D3(BJ)/6-31G(d,p) Energy $-1535.993915$

B3LYP-D3(BJ)/def2-TZVPP/IEF-PCM(DCM) Energy $-1536.541031$

B3LYP-D3(BJ)/def2-TZVPP/IEF-PCM(DCM)//B3LYP-D3(BJ)/6-31G(d,p) Free Energy (Quasiharmonic) $-1536.094662$

Number of Imaginary Frequencies

Frequencies (Top 3 out of 177)

$\begin{array}{ll}\text { 1. } & 18.5262 \mathrm{~cm}^{-1} \\ \text { 2. } & 26.3413 \mathrm{~cm}^{-1} \\ \text { 3. } & 29.7147 \mathrm{~cm}^{-1}\end{array}$

B3LYP-D3(BJ)/6-31G(d,p) Molecular Geometry in Cartesian Coordinates

$\begin{array}{lrrr}C & -1.281181 & -1.139547 & 1.442559 \\ \mathrm{H} & -0.495840 & -0.663747 & 2.041206 \\ \mathrm{H} & -2.148863 & -1.278673 & 2.091574 \\ \mathrm{C} & 1.317560 & -0.985363 & -1.268057 \\ \mathrm{O} & 0.944760 & -2.134572 & -1.129961 \\ \mathrm{O} & 1.432871 & -0.410545 & -2.478688 \\ \mathrm{H} & 1.145618 & -1.074633 & -3.130283 \\ \mathrm{O} & -0.886618 & -2.444621 & 1.037267 \\ \mathrm{H} & -0.156287 & -2.369438 & 0.398237 \\ \mathrm{C} & 0.652004 & 0.735303 & 0.369116 \\ \mathrm{C} & 1.663534 & -0.057159 & -0.142597 \\ \mathrm{C} & 2.971757 & -0.076337 & 0.430263 \\ \mathrm{C} & 3.220154 & 0.739833 & 1.514960 \\ \mathrm{H} & 4.212093 & 0.744709 & 1.956649 \\ \mathrm{C} & -1.659202 & -0.224593 & 0.288748 \\ \mathrm{C} & -0.723267 & 0.670251 & -0.219398 \\ \mathrm{C} & -3.292691 & 0.601465 & -1.310253 \\ \mathrm{C} & -2.980810 & -0.257929 & -0.273169 \\ \mathrm{H} & -4.287649 & 0.566355 & -1.743942 \\ \mathrm{C} & -2.358353 & 1.517726 & -1.844055 \\ \mathrm{C} & -1.043414 & 1.562190 & -1.289323\end{array}$




\begin{tabular}{|c|c|c|c|}
\hline C & -0.110466 & 2.492363 & -1.820417 \\
\hline C & -0.464528 & 3.333656 & -2.849987 \\
\hline C & -1.766809 & 3.285178 & -3.402779 \\
\hline C & -2.691686 & 2.394970 & -2.910072 \\
\hline $\mathrm{H}$ & 0.890619 & 2.527129 & -1.406914 \\
\hline $\mathrm{H}$ & 0.260158 & 4.039099 & -3.244582 \\
\hline $\mathrm{H}$ & -2.031639 & 3.953409 & -4.216391 \\
\hline $\mathrm{H}$ & -3.694155 & 2.349747 & -3.326379 \\
\hline C & 2.228089 & 1.594620 & 2.053681 \\
\hline C & 0.920934 & 1.599860 & 1.473979 \\
\hline C & -0.070620 & 2.455149 & 2.021959 \\
\hline C & 0.221506 & 3.271767 & 3.090432 \\
\hline C & 1.515680 & 3.269060 & 3.663229 \\
\hline C & 2.495917 & 2.448567 & 3.155773 \\
\hline $\mathrm{H}$ & -1.061443 & 2.454563 & 1.582513 \\
\hline $\mathrm{H}$ & -0.544510 & 3.923607 & 3.498384 \\
\hline $\mathrm{H}$ & 1.731427 & 3.917607 & 4.506555 \\
\hline $\mathrm{H}$ & 3.491020 & 2.440246 & 3.591146 \\
\hline C & -6.178915 & -2.776596 & 1.117332 \\
\hline C & -4.920290 & -3.326462 & 0.873135 \\
\hline C & -3.866579 & -2.523423 & 0.439385 \\
\hline C & -4.058377 & -1.146541 & 0.242234 \\
\hline C & -5.329050 & -0.603071 & 0.496123 \\
\hline C & -6.379424 & -1.408645 & 0.929636 \\
\hline $\mathrm{H}$ & -6.995502 & -3.406974 & 1.456366 \\
\hline $\mathrm{H}$ & -2.886009 & -2.951816 & 0.267058 \\
\hline $\mathrm{H}$ & -5.482904 & 0.464145 & 0.368605 \\
\hline C & 4.041970 & -0.963389 & -0.093326 \\
\hline C & 4.404587 & -0.955326 & -1.448506 \\
\hline C & 4.733148 & -1.810730 & 0.785364 \\
\hline C & 5.428493 & -1.780200 & -1.910891 \\
\hline $\mathrm{H}$ & 3.894303 & -0.289207 & -2.134796 \\
\hline C & 5.756084 & -2.634427 & 0.321079 \\
\hline $\mathrm{H}$ & 4.446212 & -1.833621 & 1.831915 \\
\hline C & 6.106266 & -2.622739 & -1.029594 \\
\hline $\mathrm{H}$ & 6.901774 & -3.266058 & -1.392555 \\
\hline $\mathrm{H}$ & -7.351455 & -0.966593 & 1.127495 \\
\hline $\mathrm{H}$ & -4.754197 & -4.389766 & 1.018196 \\
\hline $\mathrm{H}$ & 5.702766 & -1.757630 & -2.961242 \\
\hline $\mathrm{H}$ & 6.274217 & -3.291440 & 1.012848 \\
\hline
\end{tabular}

\section{Simple BHCA 8}

Datum

Value

\begin{tabular}{lr}
\hline B3LYP-D3(BJ)/6-31G(d,p) Energy & -1535.991718 \\
\hline B3LYP-D3(BJ)/def2-TZVPP/IEF-PCM(DCM) Energy & -1536.539448 \\
\hline B3LYP-D3(BJ)/def2-TZVPP/IEF-PCM(DCM)//B3LYP-D3(BJ)/6-31G(d,p) Free Energy (Quasiharmonic) & -1536.093313 \\
\hline
\end{tabular}


Frequencies (Top 3 out of 177)

$\begin{array}{ll}\text { 1. } & 19.0140 \mathrm{~cm}^{-1} \\ \text { 2. } & 25.8005 \mathrm{~cm}^{-1} \\ \text { 3. } & 29.5223 \mathrm{~cm}^{-1}\end{array}$

B3LYP-D3(BJ)/6-31G(d,p) Molecular Geometry in Cartesian Coordinates

\begin{tabular}{|c|c|c|c|}
\hline C & 1.334110 & -1.088006 & -1.472498 \\
\hline $\mathrm{H}$ & 0.565528 & -0.597062 & -2.079370 \\
\hline $\mathrm{H}$ & 2.218303 & -1.214781 & -2.100054 \\
\hline C & -1.370717 & -0.975654 & 1.288026 \\
\hline 0 & -1.254737 & -0.643663 & 2.443344 \\
\hline 0 & -1.226445 & -2.268797 & 0.884802 \\
\hline $\mathrm{H}$ & -1.034643 & -2.790187 & 1.684148 \\
\hline 0 & 0.924442 & -2.407047 & -1.115058 \\
\hline $\mathrm{H}$ & 0.126923 & -2.340600 & -0.567990 \\
\hline C & -0.644373 & 0.723089 & -0.355605 \\
\hline C & -1.668151 & -0.070128 & 0.131027 \\
\hline C & -2.973761 & -0.060289 & -0.450691 \\
\hline C & -3.204391 & 0.775792 & -1.523812 \\
\hline $\mathrm{H}$ & -4.195425 & 0.808854 & -1.966307 \\
\hline C & 1.679809 & -0.211846 & -0.280867 \\
\hline C & 0.724542 & 0.650242 & 0.247085 \\
\hline C & 3.288649 & 0.593078 & 1.353200 \\
\hline C & 2.996730 & -0.243437 & 0.292627 \\
\hline $\mathrm{H}$ & 4.280648 & 0.561147 & 1.793910 \\
\hline C & 2.335922 & 1.480073 & 1.904028 \\
\hline C & 1.023060 & 1.515323 & 1.344764 \\
\hline C & 0.069744 & 2.410059 & 1.898305 \\
\hline C & 0.404525 & 3.230916 & 2.950258 \\
\hline C & 1.706055 & 3.194468 & 3.505012 \\
\hline C & 2.649494 & 2.335157 & 2.993346 \\
\hline $\mathrm{H}$ & -0.932117 & 2.432231 & 1.486432 \\
\hline $\mathrm{H}$ & -0.336183 & 3.908291 & 3.363607 \\
\hline $\mathrm{H}$ & 1.954883 & 3.845475 & 4.337404 \\
\hline $\mathrm{H}$ & 3.650728 & 2.296851 & 3.413216 \\
\hline C & -2.195796 & 1.622215 & -2.044529 \\
\hline C & -0.894542 & 1.605507 & -1.451705 \\
\hline C & 0.110406 & 2.459168 & -1.977742 \\
\hline C & -0.162346 & 3.292679 & -3.038314 \\
\hline C & -1.450211 & 3.310348 & -3.624630 \\
\hline C & -2.443780 & 2.493519 & -3.137618 \\
\hline $\mathrm{H}$ & 1.096118 & 2.444348 & -1.527378 \\
\hline $\mathrm{H}$ & 0.614125 & 3.942822 & -3.428812 \\
\hline
\end{tabular}




\begin{tabular}{|lrrr|}
$H$ & -1.650831 & 3.972057 & -4.461440 \\
$H$ & -3.434738 & 2.501735 & -3.582346 \\
C & 6.228530 & -2.701639 & -1.129591 \\
C & 4.973413 & -3.267917 & -0.905472 \\
C & 3.910420 & -2.483070 & -0.461473 \\
C & 4.087457 & -1.108861 & -0.233892 \\
C & 5.354779 & -0.549293 & -0.467739 \\
C & 6.415193 & -1.336361 & -0.910966 \\
$H$ & 7.052957 & -3.317262 & -1.476525 \\
$H$ & 2.933768 & -2.924873 & -0.302163 \\
$H$ & 5.498075 & 0.516140 & -0.315850 \\
C & -4.065674 & -0.917857 & 0.074636 \\
C & -4.380213 & -0.940931 & 1.442205 \\
C & -4.824803 & -1.702701 & -0.805983 \\
C & -5.423592 & -1.735580 & 1.913653 \\
$H$ & -3.816130 & -0.325178 & 2.133842 \\
C & -5.868026 & -2.495166 & -0.332583 \\
$H$ & -4.575618 & -1.703170 & -1.862483 \\
C & -6.170024 & -2.515272 & 1.029754 \\
$H$ & -6.981572 & -3.134261 & 1.399554 \\
$H$ & 7.384609 & -0.881704 & -1.092286 \\
$H$ & 4.818127 & -4.329410 & -1.074090 \\
$H$ & -5.658317 & -1.737684 & 2.973666 \\
$H$ & -6.439925 & -3.103460 & -1.026646 \\
& & & \\
\hline
\end{tabular}

\section{Simple BHCA 9}

\section{Datum}

Value

B3LYP-D3(BJ)/6-31G(d,p) Energy

$-1535.991718$

B3LYP-D3(BJ)/def2-TZVPP/IEF-PCM(DCM) Energy

$-1536.539448$

B3LYP-D3(BJ)/def2-TZVPP/IEF-PCM(DCM)//B3LYP-D3(BJ)/6-31G(d,p) Free Energy (Quasiharmonic)

$-1536.093313$

Number of Imaginary Frequencies

Frequencies (Top 3 out of 177)
1. $\quad 18.9961 \mathrm{~cm}^{-1}$
2. $\quad 25.7991 \mathrm{~cm}^{-1}$
3. $29.5059 \mathrm{~cm}^{-1}$

\section{B3LYP-D3(BJ)/6-31G(d,p) Molecular Geometry in Cartesian Coordinates}

$\begin{array}{llll}\mathrm{C} & -1.334523 & -1.088028 & -1.472375 \\ \mathrm{H} & -2.218878 & -1.214886 & -2.099683\end{array}$




\begin{tabular}{|c|c|c|c|}
\hline $\mathrm{H}$ & -0.566139 & -0.597059 & -2.079478 \\
\hline C & 1.370808 & -0.975557 & 1.288083 \\
\hline 0 & 1.255341 & -0.643432 & 2.443416 \\
\hline 0 & 1.225835 & -2.268666 & 0.885014 \\
\hline $\mathrm{H}$ & 1.033976 & -2.789892 & 1.684448 \\
\hline 0 & -0.924681 & -2.407025 & -1.114982 \\
\hline $\mathrm{H}$ & -0.127224 & -2.340489 & -0.567845 \\
\hline C & 0.644322 & 0.722867 & -0.355796 \\
\hline C & 1.668171 & -0.070227 & 0.130913 \\
\hline C & 2.973723 & -0.060477 & -0.450965 \\
\hline C & 3.204164 & 0.775319 & -1.524352 \\
\hline $\mathrm{H}$ & 4.195139 & 0.808303 & -1.966979 \\
\hline C & -1.679962 & -0.211817 & -0.280706 \\
\hline C & -0.724532 & 0.650200 & 0.247063 \\
\hline C & -3.288491 & 0.593399 & 1.353523 \\
\hline C & -2.996796 & -0.243253 & 0.292995 \\
\hline $\mathrm{H}$ & -4.280419 & 0.561580 & 1.794396 \\
\hline C & -2.335612 & 1.480371 & 1.904121 \\
\hline C & -1.022815 & 1.515419 & 1.344696 \\
\hline C & -0.069335 & 2.410120 & 1.898015 \\
\hline C & -0.403902 & 3.231127 & 2.949916 \\
\hline C & -1.705367 & 3.194875 & 3.504835 \\
\hline C & -2.648959 & 2.335608 & 2.993383 \\
\hline $\mathrm{H}$ & 0.932473 & 2.432144 & 1.486008 \\
\hline $\mathrm{H}$ & 0.336924 & 3.908474 & 3.363097 \\
\hline $\mathrm{H}$ & -1.954023 & 3.846001 & 4.337185 \\
\hline $\mathrm{H}$ & -3.650145 & 2.297455 & 3.413379 \\
\hline C & 2.195492 & 1.621589 & -2.045155 \\
\hline C & 0.894335 & 1.605041 & -1.452127 \\
\hline C & -0.110690 & 2.458583 & -1.978211 \\
\hline C & 0.161901 & 3.291816 & -3.039041 \\
\hline C & 1.449671 & 3.309319 & -3.625569 \\
\hline C & 2.443312 & 2.492609 & -3.138506 \\
\hline $\mathrm{H}$ & -1.096326 & 2.443881 & -1.527680 \\
\hline $\mathrm{H}$ & -0.614624 & 3.941868 & -3.429580 \\
\hline $\mathrm{H}$ & 1.650161 & 3.970809 & -4.462581 \\
\hline $\mathrm{H}$ & 3.434200 & 2.500705 & -3.583390 \\
\hline C & -6.228895 & -2.701562 & -1.128347 \\
\hline C & -4.973705 & -3.267801 & -0.904540 \\
\hline C & -3.910626 & -2.482920 & -0.460813 \\
\hline C & -4.087645 & -1.108714 & -0.233205 \\
\hline C & -5.355043 & -0.549186 & -0.466732 \\
\hline C & -6.415545 & -1.336290 & -0.909680 \\
\hline $\mathrm{H}$ & -7.053389 & -3.317210 & -1.475071 \\
\hline $\mathrm{H}$ & -2.933929 & -2.924701 & -0.301720 \\
\hline $\mathrm{H}$ & -5.498327 & 0.516245 & -0.314829 \\
\hline C & 4.065837 & -0.917772 & 0.074408 \\
\hline C & 4.380557 & -0.940583 & 1.441937 \\
\hline C & 4.825011 & -1.702603 & -0.806185 \\
\hline C & 5.424137 & -1.734968 & 1.913374 \\
\hline $\mathrm{H}$ & 3.816457 & -0.324823 & 2.133550 \\
\hline C & 5.868437 & -2.494807 & -0.332799 \\
\hline $\mathrm{H}$ & 4.575695 & -1.703279 & -1.862653 \\
\hline C & 6.170602 & -2.514659 & 1.029502 \\
\hline
\end{tabular}




$\begin{array}{rrrr}\mathrm{H} & 6.982303 & -3.133447 & 1.399297 \\ \mathrm{H} & -7.385021 & -0.881665 & -1.090755 \\ \mathrm{H} & -4.818431 & -4.329289 & -1.073187 \\ \mathrm{H} & 5.658990 & -1.736871 & 2.973358 \\ \mathrm{H} & 6.440362 & -3.103096 & -1.026843\end{array}$

\section{Simple BHCA 10}

\section{Datum}

Value

B3LYP-D3(BJ)/6-31G(d,p) Energy $-1535.990594$

B3LYP-D3(BJ)/def2-TZVPP/IEF-PCM(DCM) Energy $-1536.538938$

B3LYP-D3(BJ)/def2-TZVPP/IEF-PCM(DCM)//B3LYP-D3(BJ)/6-31G(d,p) Free Energy (Quasiharmonic) $-1536.093208$

Number of Imaginary Frequencies

Frequencies (Top 3 out of 177)

$\begin{array}{ll}\text { 1. } & 19.2168 \mathrm{~cm}^{-1} \\ \text { 2. } & 26.9688 \mathrm{~cm}^{-1} \\ \text { 3. } & 28.6630 \mathrm{~cm}^{-1}\end{array}$

B3LYP-D3(BJ)/6-31G(d,p) Molecular Geometry in Cartesian Coordinates

$\begin{array}{lrrr}\mathrm{C} & -1.420954 & 0.035540 & -1.799729 \\ \mathrm{H} & -0.390769 & -0.284571 & -1.984540 \\ \mathrm{H} & -2.078952 & -0.641061 & -2.345345 \\ \mathrm{C} & 1.410167 & -1.677105 & 0.268477 \\ \mathrm{O} & 0.651664 & -2.393057 & -0.349431 \\ \mathrm{O} & 2.101948 & -2.094736 & 1.349159 \\ \mathrm{H} & 1.875565 & -3.032834 & 1.471567 \\ \mathrm{O} & -1.660002 & 1.335371 & -2.350810 \\ \mathrm{H} & -1.031115 & 1.945210 & -1.940497 \\ \mathrm{C} & 0.647564 & 0.667070 & 0.107011 \\ \mathrm{C} & 1.683964 & -0.237830 & -0.047994 \\ \mathrm{C} & 2.981521 & 0.172855 & -0.484392 \\ \mathrm{C} & 3.195376 & 1.512828 & -0.738672 \\ \mathrm{H} & 4.166609 & 1.835622 & -1.101138 \\ \mathrm{C} & -1.711620 & -0.048345 & -0.313727 \\ \mathrm{C} & -0.696464 & 0.223809 & 0.593312 \\ \mathrm{C} & -3.211061 & -0.551497 & 1.529404 \\ \mathrm{C} & -3.007413 & -0.428501 & 0.167528 \\ \mathrm{H} & -4.195555 & -0.828169 & 1.894896 \\ \mathrm{C} & -2.188681 & -0.299008 & 2.471586 \\ \mathrm{C} & -0.901143 & 0.106581 & 2.001873\end{array}$




\begin{tabular}{|c|c|c|c|}
\hline C & 0.119468 & 0.371889 & 2.954794 \\
\hline C & -0.124261 & 0.238073 & 4.302322 \\
\hline C & -1.399879 & -0.163617 & 4.766220 \\
\hline C & -2.408457 & -0.424761 & 3.869019 \\
\hline $\mathrm{H}$ & 1.100003 & 0.675155 & 2.607010 \\
\hline $\mathrm{H}$ & 0.667156 & 0.442185 & 5.016806 \\
\hline $\mathrm{H}$ & -1.578040 & -0.264002 & 5.832431 \\
\hline $\mathrm{H}$ & -3.391441 & -0.732414 & 4.214557 \\
\hline C & 2.173266 & 2.477806 & -0.577105 \\
\hline C & 0.876873 & 2.055334 & -0.143905 \\
\hline C & -0.140339 & 3.035145 & 0.028373 \\
\hline C & 0.110186 & 4.362465 & -0.240090 \\
\hline C & 1.388692 & 4.776397 & -0.683346 \\
\hline C & 2.396491 & 3.854396 & -0.844090 \\
\hline $\mathrm{H}$ & -1.116402 & 2.720188 & 0.379309 \\
\hline $\mathrm{H}$ & -0.676772 & 5.097820 & -0.106703 \\
\hline $\mathrm{H}$ & 1.571529 & 5.825592 & -0.892618 \\
\hline $\mathrm{H}$ & 3.381495 & 4.165352 & -1.180098 \\
\hline C & -6.381240 & -1.231414 & -2.372877 \\
\hline C & -5.614283 & -0.083602 & -2.575875 \\
\hline C & -4.507311 & 0.178891 & -1.770826 \\
\hline C & -4.148079 & -0.709489 & -0.744139 \\
\hline C & -4.924896 & -1.863078 & -0.551760 \\
\hline C & -6.030996 & -2.122573 & -1.358057 \\
\hline $\mathrm{H}$ & -7.241953 & -1.432738 & -3.003466 \\
\hline $\mathrm{H}$ & -3.909022 & 1.067710 & -1.937858 \\
\hline $\mathrm{H}$ & -4.641942 & -2.567274 & 0.224538 \\
\hline C & 4.076185 & -0.805083 & -0.703197 \\
\hline $\mathrm{C}$ & 3.862446 & -1.977897 & -1.443570 \\
\hline C & 5.359882 & -0.549908 & -0.199838 \\
\hline C & 4.906291 & -2.873067 & -1.667280 \\
\hline $\mathrm{H}$ & 2.879948 & -2.181558 & -1.855952 \\
\hline C & 6.402778 & -1.445712 & -0.425106 \\
\hline $\mathrm{H}$ & 5.528993 & 0.345919 & 0.389439 \\
\hline C & 6.179201 & -2.611121 & -1.159206 \\
\hline $\mathrm{H}$ & 6.991198 & -3.310102 & -1.334074 \\
\hline $\mathrm{H}$ & -6.614248 & -3.024607 & -1.198524 \\
\hline $\mathrm{H}$ & -5.879050 & 0.615113 & -3.363848 \\
\hline $\mathrm{H}$ & 4.725683 & -3.772737 & -2.247529 \\
\hline $\mathrm{H}$ & 7.388543 & -1.237244 & -0.020537 \\
\hline
\end{tabular}

\section{Simple BHCA 11}

Datum

Value

\begin{tabular}{lr}
\hline B3LYP-D3(BJ)/6-31G(d,p) Energy & -1535.985141 \\
\hline B3LYP-D3(BJ)/def2-TZVPP/IEF-PCM(DCM) Energy & -1536.537487 \\
\hline B3LYP-D3(BJ)/def2-TZVPP/IEF-PCM(DCM)//B3LYP-D3(BJ)/6-31G(d,p) Free Energy (Quasiharmonic) & -1536.09304 \\
\hline
\end{tabular}


Frequencies (Top 3 out of 177)

$\begin{array}{ll}\text { 1. } & 12.5381 \mathrm{~cm}^{-1} \\ \text { 2. } & 25.6172 \mathrm{~cm}^{-1} \\ \text { 3. } & 26.2844 \mathrm{~cm}^{-1}\end{array}$

B3LYP-D3(BJ)/6-31G(d,p) Molecular Geometry in Cartesian Coordinates

\begin{tabular}{|c|c|c|c|}
\hline C & -1.278769 & -1.021234 & -1.471008 \\
\hline $\mathrm{H}$ & -0.267472 & -0.796883 & -1.807136 \\
\hline $\mathrm{H}$ & -1.296673 & -2.090300 & -1.222222 \\
\hline C & 1.242849 & -1.184173 & 1.155358 \\
\hline 0 & 1.030616 & -0.985763 & 2.330909 \\
\hline 0 & 1.112051 & -2.404440 & 0.583445 \\
\hline $\mathrm{H}$ & 0.876341 & -3.012950 & 1.305127 \\
\hline 0 & -2.130473 & -0.731961 & -2.573559 \\
\hline $\mathrm{H}$ & -3.009802 & -1.070237 & -2.355605 \\
\hline C & 0.700555 & 0.766777 & -0.257117 \\
\hline C & 1.655717 & -0.144509 & 0.156385 \\
\hline C & 2.984073 & -0.131008 & -0.369120 \\
\hline C & 3.304417 & 0.824759 & -1.312495 \\
\hline $\mathrm{H}$ & 4.317093 & 0.866223 & -1.702336 \\
\hline C & -1.611721 & -0.185627 & -0.243587 \\
\hline C & -0.687058 & 0.694652 & 0.295737 \\
\hline C & -3.280512 & 0.591142 & 1.349716 \\
\hline C & -2.929286 & -0.250779 & 0.318117 \\
\hline $\mathrm{H}$ & -4.283271 & 0.536515 & 1.764493 \\
\hline C & -2.358109 & 1.508317 & 1.910204 \\
\hline C & -1.029508 & 1.551676 & 1.389472 \\
\hline C & -0.099491 & 2.450964 & 1.972456 \\
\hline C & -0.473010 & 3.275308 & 3.009573 \\
\hline C & -1.791995 & 3.237286 & 3.518697 \\
\hline C & -2.713168 & 2.368462 & 2.980949 \\
\hline $\mathrm{H}$ & 0.914925 & 2.476403 & 1.592452 \\
\hline $\mathrm{H}$ & 0.251046 & 3.956812 & 3.445137 \\
\hline $\mathrm{H}$ & -2.072348 & 3.891616 & 4.338341 \\
\hline $\mathrm{H}$ & -3.726703 & 2.324983 & 3.369827 \\
\hline C & 2.359553 & 1.775301 & -1.768427 \\
\hline $\mathrm{C}$ & 1.031986 & 1.750895 & -1.236880 \\
\hline C & 0.083786 & 2.694604 & -1.711328 \\
\hline C & 0.439271 & 3.629611 & -2.656013 \\
\hline C & 1.756374 & 3.663182 & -3.173036 \\
\hline C & 2.694130 & 2.755343 & -2.739671 \\
\hline $\mathrm{H}$ & -0.926573 & 2.661227 & -1.321100 \\
\hline $\mathrm{H}$ & -0.295061 & 4.345561 & -3.011268 \\
\hline
\end{tabular}




\begin{tabular}{|lrrr|}
$H$ & 2.022368 & 4.406733 & -3.917961 \\
$H$ & 3.705216 & 2.771391 & -3.136465 \\
C & -5.888161 & -3.012370 & -1.141479 \\
C & -4.697303 & -3.489490 & -0.591224 \\
C & -3.734594 & -2.597364 & -0.122999 \\
C & -3.944133 & -1.211337 & -0.199571 \\
C & -5.148494 & -0.744227 & -0.747956 \\
C & -6.112185 & -1.637484 & -1.216169 \\
$H$ & -6.636132 & -3.707892 & -1.508926 \\
$H$ & -2.818274 & -2.970511 & 0.322931 \\
$H$ & -5.313893 & 0.326429 & -0.815698 \\
C & 4.011398 & -1.099941 & 0.087192 \\
C & 4.234503 & -1.334943 & 1.452840 \\
C & 4.803494 & -1.777958 & -0.851231 \\
C & 5.220246 & -2.229790 & 1.864600 \\
$H$ & 3.643482 & -0.805812 & 2.192257 \\
C & 5.789546 & -2.670740 & -0.437540 \\
$H$ & 4.624317 & -1.615975 & -1.909495 \\
C & 6.000671 & -2.900754 & 0.922605 \\
$H$ & 6.767709 & -3.597829 & 1.245681 \\
$H$ & -7.035436 & -1.258285 & -1.643420 \\
$H$ & -4.520426 & -4.558329 & -0.520463 \\
$H$ & 5.383904 & -2.395541 & 2.925091 \\
$H$ & 6.387667 & -3.193342 & -1.177898 \\
& & & \\
\hline
\end{tabular}

\section{Simple BHCA 12}

\section{Datum}

Value

B3LYP-D3(BJ)/6-31G(d,p) Energy

$-1535.986927$

B3LYP-D3(BJ)/def2-TZVPP/IEF-PCM(DCM) Energy

$-1536.537467$

B3LYP-D3(BJ)/def2-TZVPP/IEF-PCM(DCM)//B3LYP-D3(BJ)/6-31G(d,p) Free Energy (Quasiharmonic)

$-1536.09286$

Number of Imaginary Frequencies

Frequencies (Top 3 out of 177)
1. $\quad 12.7809 \mathrm{~cm}^{-1}$
2. $\quad 23.7740 \mathrm{~cm}^{-1}$
3. $29.8228 \mathrm{~cm}^{-1}$

B3LYP-D3(BJ)/6-31G(d,p) Molecular Geometry in Cartesian Coordinates

$\begin{array}{llll}C & -1.392859 & -0.929586 & -1.555069 \\ H & -0.404297 & -0.681551 & -1.940515\end{array}$




\begin{tabular}{|c|c|c|c|}
\hline $\mathrm{H}$ & -1.373790 & -1.998272 & -1.310129 \\
\hline C & 1.202768 & -1.280247 & 1.057029 \\
\hline 0 & 0.401528 & -2.166522 & 0.841110 \\
\hline 0 & 1.813379 & -1.124527 & 2.249756 \\
\hline $\mathrm{H}$ & 1.500656 & -1.853769 & 2.812499 \\
\hline 0 & -2.305862 & -0.644331 & -2.608587 \\
\hline $\mathrm{H}$ & -3.158561 & -1.027805 & -2.363049 \\
\hline C & 0.695959 & 0.710193 & -0.326542 \\
\hline C & 1.628493 & -0.232845 & 0.072450 \\
\hline C & 2.976771 & -0.200514 & -0.402775 \\
\hline C & 3.344289 & 0.810800 & -1.267085 \\
\hline $\mathrm{H}$ & 4.358082 & 0.833895 & -1.655457 \\
\hline C & -1.667426 & -0.114222 & -0.301154 \\
\hline C & -0.684992 & 0.692099 & 0.247349 \\
\hline C & -3.246387 & 0.664509 & 1.378850 \\
\hline C & -2.964723 & -0.149710 & 0.303769 \\
\hline $\mathrm{H}$ & -4.234929 & 0.630412 & 1.828502 \\
\hline C & -2.266739 & 1.515013 & 1.945808 \\
\hline C & -0.951256 & 1.515792 & 1.387893 \\
\hline C & 0.037854 & 2.342784 & 1.984105 \\
\hline C & -0.265619 & 3.134927 & 3.068296 \\
\hline C & -1.570757 & 3.138699 & 3.613732 \\
\hline C & -2.548369 & 2.342491 & 3.063582 \\
\hline $\mathrm{H}$ & 1.041417 & 2.341522 & 1.575261 \\
\hline $\mathrm{H}$ & 0.503187 & 3.760629 & 3.511020 \\
\hline $\mathrm{H}$ & -1.795907 & 3.768016 & 4.469201 \\
\hline $\mathrm{H}$ & -3.552415 & 2.331674 & 3.478620 \\
\hline C & 2.426712 & 1.798332 & -1.699141 \\
\hline C & 1.076537 & 1.748014 & -1.231063 \\
\hline C & 0.155821 & 2.729899 & -1.681858 \\
\hline C & 0.555865 & 3.717939 & -2.551876 \\
\hline C & 1.893243 & 3.771136 & -3.011925 \\
\hline C & 2.806756 & 2.830664 & -2.596508 \\
\hline $\mathrm{H}$ & -0.870115 & 2.680688 & -1.336421 \\
\hline $\mathrm{H}$ & -0.158887 & 4.460210 & -2.892700 \\
\hline $\mathrm{H}$ & 2.194497 & 4.556299 & -3.698443 \\
\hline $\mathrm{H}$ & 3.833331 & 2.861527 & -2.950302 \\
\hline C & -6.031373 & -2.835789 & -1.062453 \\
\hline C & -4.817207 & -3.337714 & -0.590409 \\
\hline C & -3.819940 & -2.467114 & -0.154443 \\
\hline C & -4.020908 & -1.077645 & -0.188141 \\
\hline C & -5.247664 & -0.584608 & -0.656737 \\
\hline C & -6.244905 & -1.457324 & -1.091961 \\
\hline $\mathrm{H}$ & -6.805927 & -3.514972 & -1.404745 \\
\hline $\mathrm{H}$ & -2.883097 & -2.856293 & 0.231538 \\
\hline $\mathrm{H}$ & -5.404574 & 0.488950 & -0.691038 \\
\hline C & 3.964397 & -1.237935 & -0.014423 \\
\hline C & 3.647654 & -2.603134 & -0.088576 \\
\hline C & 5.251188 & -0.867037 & 0.401722 \\
\hline C & 4.592919 & -3.568691 & 0.251137 \\
\hline $\mathrm{H}$ & 2.662621 & -2.907613 & -0.425925 \\
\hline C & 6.195844 & -1.833633 & 0.739576 \\
\hline $\mathrm{H}$ & 5.498342 & 0.187052 & 0.479760 \\
\hline C & 5.869613 & -3.188412 & 0.666383 \\
\hline
\end{tabular}




$\begin{array}{rrrr}\mathrm{H} & 6.604917 & -3.942029 & 0.930891 \\ \mathrm{H} & -7.186163 & -1.059620 & -1.458980 \\ \mathrm{H} & -4.648203 & -4.409595 & -0.555095 \\ \mathrm{H} & 4.333347 & -4.620712 & 0.182067 \\ \mathrm{H} & 7.184776 & -1.528395 & 1.067950\end{array}$

\section{Simple BHCA 13}

\section{Datum}

Value

B3LYP-D3(BJ)/6-31G(d,p) Energy $-1535.989703$

B3LYP-D3(BJ)/def2-TZVPP/IEF-PCM(DCM) Energy $-1536.538461$

B3LYP-D3(BJ)/def2-TZVPP/IEF-PCM(DCM)//B3LYP-D3(BJ)/6-31G(d,p) Free Energy (Quasiharmonic) $-1536.092819$

Number of Imaginary Frequencies

Frequencies (Top 3 out of 177)

$\begin{array}{ll}\text { 1. } & 19.1368 \mathrm{~cm}^{-1} \\ \text { 2. } & 26.5101 \mathrm{~cm}^{-1} \\ \text { 3. } & 28.5704 \mathrm{~cm}^{-1}\end{array}$

B3LYP-D3(BJ)/6-31G(d,p) Molecular Geometry in Cartesian Coordinates

$\begin{array}{lrrr}\mathrm{C} & -1.295051 & -0.463510 & -1.723699 \\ \mathrm{H} & -0.248737 & -0.778510 & -1.785319 \\ \mathrm{H} & -1.909776 & -1.288464 & -2.085002 \\ \mathrm{C} & 1.375241 & -1.522943 & 0.754839 \\ \mathrm{O} & 1.226454 & -1.709742 & 1.941683 \\ \mathrm{O} & 1.257951 & -2.504744 & -0.166820 \\ \mathrm{H} & 1.065821 & -3.320536 & 0.327503 \\ \mathrm{O} & -1.547842 & 0.624816 & -2.619026 \\ \mathrm{H} & -0.976523 & 1.358665 & -2.351664 \\ \mathrm{C} & 0.670163 & 0.727470 & 0.019361 \\ \mathrm{C} & 1.688673 & -0.203798 & 0.111803 \\ \mathrm{C} & 2.984508 & 0.040768 & -0.437891 \\ \mathrm{C} & 3.208089 & 1.241858 & -1.080998 \\ \mathrm{H} & 4.192841 & 1.450777 & -1.487848 \\ \mathrm{C} & -1.653652 & -0.138853 & -0.285614 \\ \mathrm{C} & -0.696406 & 0.424939 & 0.549609 \\ \mathrm{C} & -3.259412 & -0.083124 & 1.536448 \\ \mathrm{C} & -2.972978 & -0.390730 & 0.219995 \\ \mathrm{H} & -4.261733 & -0.260576 & 1.915041 \\ \mathrm{C} & -2.302389 & 0.490297 & 2.404727 \\ \mathrm{C} & -0.990681 & 0.755782 & 1.906923\end{array}$




\begin{tabular}{|c|c|c|c|}
\hline C & -0.033162 & 1.336016 & 2.779773 \\
\hline C & -0.362806 & 1.642717 & 4.079547 \\
\hline C & -1.664254 & 1.382743 & 4.571720 \\
\hline C & -2.611825 & 0.817609 & 3.751344 \\
\hline $\mathrm{H}$ & 0.967678 & 1.525661 & 2.410267 \\
\hline $\mathrm{H}$ & 0.380811 & 2.083734 & 4.735978 \\
\hline $\mathrm{H}$ & -1.909787 & 1.629168 & 5.600117 \\
\hline $\mathrm{H}$ & -3.612896 & 0.612024 & 4.119886 \\
\hline C & 2.198489 & 2.226384 & -1.200423 \\
\hline C & 0.909136 & 1.975792 & -0.633830 \\
\hline C & -0.097236 & 2.974308 & -0.750473 \\
\hline C & 0.160502 & 4.157383 & -1.406958 \\
\hline C & 1.435263 & 4.402564 & -1.970322 \\
\hline C & 2.431107 & 3.459767 & -1.864397 \\
\hline $\mathrm{H}$ & -1.069282 & 2.792013 & -0.306240 \\
\hline $\mathrm{H}$ & -0.616957 & 4.910283 & -1.488031 \\
\hline $\mathrm{H}$ & 1.624353 & 5.339313 & -2.484873 \\
\hline $\mathrm{H}$ & 3.412777 & 3.642745 & -2.291822 \\
\hline C & -6.176781 & -2.073463 & -2.102620 \\
\hline C & -5.432138 & -1.009465 & -2.612921 \\
\hline C & -4.379539 & -0.465908 & -1.878618 \\
\hline C & -4.052750 & -0.983234 & -0.614467 \\
\hline C & -4.807487 & -2.055518 & -0.112399 \\
\hline C & -5.859466 & -2.596392 & -0.848650 \\
\hline $\mathrm{H}$ & -6.995269 & -2.494343 & -2.678743 \\
\hline $\mathrm{H}$ & -3.799539 & 0.356991 & -2.281471 \\
\hline $\mathrm{H}$ & -4.551029 & -2.474111 & 0.856032 \\
\hline C & 4.077264 & -0.956591 & -0.322930 \\
\hline C & 4.382488 & -1.560590 & 0.906644 \\
\hline C & 4.847383 & -1.288285 & -1.447438 \\
\hline C & 5.428528 & -2.476169 & 1.003474 \\
\hline $\mathrm{H}$ & 3.807243 & -1.303323 & 1.789137 \\
\hline C & 5.893139 & -2.203040 & -1.348040 \\
\hline $\mathrm{H}$ & 4.604499 & -0.840664 & -2.406037 \\
\hline C & 6.186819 & -2.800897 & -0.121725 \\
\hline $\mathrm{H}$ & 7.000519 & -3.515317 & -0.044060 \\
\hline $\mathrm{H}$ & -6.426542 & -3.430227 & -0.445560 \\
\hline $\mathrm{H}$ & -5.672691 & -0.595511 & -3.587604 \\
\hline $\mathrm{H}$ & 5.655610 & -2.930243 & 1.963144 \\
\hline $\mathrm{H}$ & 6.473411 & -2.455510 & -2.230299 \\
\hline
\end{tabular}

\section{Simple BHCA 14}

Datum

Value

B3LYP-D3(BJ)/6-31G(d,p) Energy

$-1535.99$

B3LYP-D3(BJ)/def2-TZVPP/IEF-PCM(DCM) Energy

$-1536.53883$

B3LYP-D3(BJ)/def2-TZVPP/IEF-PCM(DCM)//B3LYP-D3(BJ)/6-31G(d,p) Free Energy (Quasiharmonic) - 
Frequencies (Top 3 out of 177)

$\begin{array}{ll}\text { 1. } & 20.0939 \mathrm{~cm}^{-1} \\ \text { 2. } & 28.3753 \mathrm{~cm}^{-1} \\ \text { 3. } & 31.9300 \mathrm{~cm}^{-1}\end{array}$

B3LYP-D3(BJ)/6-31G(d,p) Molecular Geometry in Cartesian Coordinates

\begin{tabular}{|c|c|c|c|}
\hline C & -1.299879 & -1.040951 & -1.538546 \\
\hline $\mathrm{H}$ & -2.170101 & -1.132116 & -2.192784 \\
\hline $\mathrm{H}$ & -0.508815 & -0.541438 & -2.109831 \\
\hline C & 1.230008 & -1.132970 & 1.140812 \\
\hline 0 & 1.093278 & -2.305677 & 0.868954 \\
\hline 0 & 1.018255 & -0.702317 & 2.400655 \\
\hline $\mathrm{H}$ & 1.039839 & 0.268812 & 2.431004 \\
\hline 0 & -0.925843 & -2.371617 & -1.208129 \\
\hline $\mathrm{H}$ & -0.147521 & -2.357525 & -0.624774 \\
\hline C & 0.656164 & 0.758229 & -0.360754 \\
\hline C & 1.632172 & -0.096804 & 0.122842 \\
\hline C & 2.961519 & -0.093953 & -0.407162 \\
\hline C & 3.260750 & 0.810910 & -1.406360 \\
\hline $\mathrm{H}$ & 4.271660 & 0.847347 & -1.800701 \\
\hline C & -1.668207 & -0.183843 & -0.336808 \\
\hline C & -0.728263 & 0.686002 & 0.201856 \\
\hline C & -3.275768 & 0.545712 & 1.335312 \\
\hline C & -2.982910 & -0.246153 & 0.240691 \\
\hline $\mathrm{H}$ & -4.260972 & 0.478806 & 1.786650 \\
\hline C & -2.330435 & 1.426137 & 1.909729 \\
\hline C & -1.032345 & 1.516587 & 1.324060 \\
\hline C & -0.085637 & 2.411235 & 1.896678 \\
\hline C & -0.406697 & 3.160542 & 3.008209 \\
\hline C & -1.690247 & 3.059897 & 3.594039 \\
\hline C & -2.630903 & 2.215436 & 3.050749 \\
\hline $\mathrm{H}$ & 0.889379 & 2.517942 & 1.431541 \\
\hline $\mathrm{H}$ & 0.326033 & 3.840298 & 3.431892 \\
\hline $\mathrm{H}$ & -1.930517 & 3.654187 & 4.469793 \\
\hline $\mathrm{H}$ & -3.620465 & 2.134935 & 3.491539 \\
\hline C & 2.299884 & 1.716532 & -1.917704 \\
\hline C & 0.971675 & 1.696579 & -1.389335 \\
\hline C & 0.010120 & 2.602485 & -1.908016 \\
\hline C & 0.351716 & 3.493607 & -2.899689 \\
\hline C & 1.667709 & 3.518032 & -3.419916 \\
\hline C & 2.619521 & 2.649007 & -2.939285 \\
\hline $\mathrm{H}$ & -0.997531 & 2.580078 & -1.508762 \\
\hline $\mathrm{H}$ & -0.391384 & 4.183324 & -3.287132 \\
\hline
\end{tabular}




\begin{tabular}{|lrrr|}
$H$ & 1.922731 & 4.225619 & -4.202576 \\
$H$ & 3.630583 & 2.661012 & -3.335979 \\
C & -6.210397 & -2.659385 & -1.259169 \\
C & -4.955045 & -3.232439 & -1.054735 \\
C & -3.891128 & -2.463200 & -0.586100 \\
C & -4.070797 & -1.097889 & -0.312958 \\
C & -5.338522 & -0.530448 & -0.525419 \\
C & -6.398566 & -1.302356 & -0.994952 \\
$H$ & -7.034693 & -3.263649 & -1.625846 \\
$H$ & -2.914023 & -2.909086 & -0.441854 \\
$H$ & -5.482462 & 0.529174 & -0.337080 \\
C & 4.008874 & -1.020347 & 0.089487 \\
C & 4.228118 & -1.214972 & 1.461144 \\
C & 4.828844 & -1.699528 & -0.824172 \\
C & 5.236794 & -2.066438 & 1.905343 \\
$H$ & 3.613171 & -0.694169 & 2.186795 \\
C & 5.838494 & -2.549515 & -0.379481 \\
$H$ & 4.651249 & -1.576538 & -1.887778 \\
C & 6.046388 & -2.735751 & 0.987544 \\
$H$ & 6.830320 & -3.401321 & 1.335000 \\
$H$ & -7.368405 & -0.842630 & -1.160306 \\
$H$ & -4.799454 & -4.287695 & -1.257818 \\
$H$ & 5.391781 & -2.203399 & 2.970936 \\
$H$ & 6.456050 & -3.074996 & -1.101374 \\
& & & \\
\hline
\end{tabular}

\section{Simple BHCA 15}

\section{Datum}

Value

B3LYP-D3(BJ)/6-31G(d,p) Energy

$-1535.9834$

B3LYP-D3(BJ)/def2-TZVPP/IEF-PCM(DCM) Energy

$-1536.536108$

B3LYP-D3(BJ)/def2-TZVPP/IEF-PCM(DCM)//B3LYP-D3(BJ)/6-31G(d,p) Free Energy (Quasiharmonic)

$-1536.091655$

Number of Imaginary Frequencies

Frequencies (Top 3 out of 177)
1. $\quad 15.1301 \mathrm{~cm}^{-1}$
2. $23.7848 \mathrm{~cm}^{-1}$
3. $\quad 26.2957 \mathrm{~cm}^{-1}$

\section{B3LYP-D3(BJ)/6-31G(d,p) Molecular Geometry in Cartesian Coordinates}

$\begin{array}{llll}\mathrm{C} & -1.512727 & 0.783119 & -1.607027 \\ \mathrm{H} & -1.971881 & 1.784045 & -1.615011\end{array}$




\begin{tabular}{|c|c|c|c|}
\hline $\mathrm{H}$ & -0.444913 & 0.930206 & -1.766209 \\
\hline C & 1.500385 & -1.614555 & -0.185511 \\
\hline 0 & 0.701509 & -2.163059 & -0.911142 \\
\hline 0 & 2.304480 & -2.289720 & 0.667958 \\
\hline $\mathrm{H}$ & 2.110014 & -3.232454 & 0.529697 \\
\hline 0 & -1.957304 & 0.002040 & -2.702754 \\
\hline $\mathrm{H}$ & -2.922800 & -0.019628 & -2.677552 \\
\hline C & 0.652602 & 0.652573 & 0.331137 \\
\hline C & 1.713816 & -0.133975 & -0.087689 \\
\hline C & 2.990480 & 0.433015 & -0.393258 \\
\hline C & 3.162622 & 1.792743 & -0.231615 \\
\hline $\mathrm{H}$ & 4.116983 & 2.241213 & -0.490676 \\
\hline C & -1.723997 & 0.128477 & -0.246568 \\
\hline C & -0.673346 & 0.039994 & 0.654361 \\
\hline C & -3.145067 & -1.104893 & 1.296036 \\
\hline C & -2.994827 & -0.431590 & 0.102295 \\
\hline $\mathrm{H}$ & -4.112597 & -1.529809 & 1.548361 \\
\hline C & -2.084776 & -1.225674 & 2.224386 \\
\hline C & -0.826520 & -0.624466 & 1.912714 \\
\hline C & 0.223171 & -0.719236 & 2.865907 \\
\hline C & 0.037179 & -1.385230 & 4.056181 \\
\hline C & -1.206770 & -1.987216 & 4.357659 \\
\hline C & -2.245311 & -1.905451 & 3.459590 \\
\hline $\mathrm{H}$ & 1.180976 & -0.264581 & 2.643718 \\
\hline $\mathrm{H}$ & 0.851856 & -1.448218 & 4.770907 \\
\hline $\mathrm{H}$ & -1.339072 & -2.509571 & 5.300119 \\
\hline $\mathrm{H}$ & -3.207356 & -2.358558 & 3.682246 \\
\hline C & 2.117770 & 2.630845 & 0.224589 \\
\hline C & 0.838162 & 2.058802 & 0.509552 \\
\hline C & -0.202693 & 2.909436 & 0.968595 \\
\hline C & 0.009547 & 4.260044 & 1.125120 \\
\hline C & 1.272603 & 4.826829 & 0.831763 \\
\hline C & 2.302494 & 4.028584 & 0.392262 \\
\hline $\mathrm{H}$ & -1.168592 & 2.474458 & 1.197526 \\
\hline $\mathrm{H}$ & -0.795752 & 4.896866 & 1.477692 \\
\hline $\mathrm{H}$ & 1.425857 & 5.894133 & 0.957560 \\
\hline $\mathrm{H}$ & 3.275808 & 4.455043 & 0.166980 \\
\hline C & -6.465129 & -0.085803 & -2.417377 \\
\hline C & -5.809398 & 1.062024 & -1.970075 \\
\hline C & -4.679920 & 0.953202 & -1.159897 \\
\hline C & -4.182540 & -0.306429 & -0.787811 \\
\hline C & -4.855045 & -1.452363 & -1.238835 \\
\hline C & -5.985583 & -1.342873 & -2.047062 \\
\hline $\mathrm{H}$ & -7.342483 & -0.000876 & -3.050810 \\
\hline $\mathrm{H}$ & -4.184172 & 1.848441 & -0.798364 \\
\hline $\mathrm{H}$ & -4.467863 & -2.428794 & -0.966281 \\
\hline C & 4.102806 & -0.392841 & -0.925178 \\
\hline C & 3.892528 & -1.290745 & -1.983002 \\
\hline C & 5.396693 & -0.258218 & -0.402336 \\
\hline C & 4.949828 & -2.037586 & -2.497658 \\
\hline $\mathrm{H}$ & 2.900645 & -1.393474 & -2.410949 \\
\hline C & 6.453418 & -1.005118 & -0.918736 \\
\hline $\mathrm{H}$ & 5.564044 & 0.420397 & 0.428261 \\
\hline C & 6.233396 & -1.898169 & -1.968004 \\
\hline
\end{tabular}




$\begin{array}{rrrr}\mathrm{H} & 7.056202 & -2.481227 & -2.370061 \\ \mathrm{H} & -6.487361 & -2.240754 & -2.394564 \\ \mathrm{H} & -6.180854 & 2.044210 & -2.245846 \\ \mathrm{H} & 4.771154 & -2.723403 & -3.320166 \\ \mathrm{H} & 7.447578 & -0.895288 & -0.496116\end{array}$

\section{Simple BHCA 16}

\section{Datum}

Value

B3LYP-D3(BJ)/6-31G(d,p) Energy $-1535.987262$

B3LYP-D3(BJ)/def2-TZVPP/IEF-PCM(DCM) Energy $-1536.536302$

B3LYP-D3(BJ)/def2-TZVPP/IEF-PCM(DCM)//B3LYP-D3(BJ)/6-31G(d,p) Free Energy (Quasiharmonic) $-1536.091217$

Number of Imaginary Frequencies

Frequencies (Top 3 out of 177)

$\begin{array}{ll}\text { 1. } & 19.0985 \mathrm{~cm}^{-1} \\ \text { 2. } & 25.2570 \mathrm{~cm}^{-1} \\ \text { 3. } & 27.9402 \mathrm{~cm}^{-1}\end{array}$

B3LYP-D3(BJ)/6-31G(d,p) Molecular Geometry in Cartesian Coordinates

$\begin{array}{lrrr}\mathrm{C} & -1.451319 & -0.303397 & -1.774075 \\ \mathrm{H} & -0.417885 & -0.639898 & -1.900900 \\ \mathrm{H} & -2.112088 & -1.086603 & -2.162156 \\ \mathrm{C} & 1.343635 & -1.603353 & 0.532025 \\ \mathrm{O} & 0.571098 & -2.401059 & 0.042807 \\ \mathrm{O} & 2.012714 & -1.840961 & 1.679651 \\ \mathrm{H} & 1.759873 & -2.737213 & 1.960611 \\ \mathrm{O} & -1.670349 & 0.938936 & -2.460643 \\ \mathrm{H} & -1.377955 & 0.821827 & -3.374030 \\ \mathrm{C} & 0.648829 & 0.692363 & -0.067341 \\ \mathrm{C} & 1.660784 & -0.253495 & -0.034284 \\ \mathrm{C} & 2.978913 & 0.040848 & -0.502280 \\ \mathrm{C} & 3.238332 & 1.312250 & -0.972775 \\ \mathrm{H} & 4.228721 & 1.545452 & -1.352313 \\ \mathrm{C} & -1.722868 & -0.085261 & -0.307510 \\ \mathrm{C} & -0.698267 & 0.377816 & 0.504594 \\ \mathrm{C} & -3.199273 & -0.203330 & 1.614356 \\ \mathrm{C} & -3.009544 & -0.365148 & 0.254696 \\ \mathrm{H} & -4.177512 & -0.403720 & 2.041167 \\ \mathrm{C} & -2.169019 & 0.248693 & 2.468887 \\ \mathrm{C} & -0.889707 & 0.555022 & 1.908949\end{array}$




\begin{tabular}{|c|c|c|c|}
\hline C & 0.138858 & 1.018553 & 2.773809 \\
\hline C & -0.089709 & 1.168049 & 4.122086 \\
\hline C & -1.357173 & 0.864771 & 4.674989 \\
\hline C & -2.372960 & 0.416298 & 3.864529 \\
\hline $\mathrm{H}$ & 1.112378 & 1.249427 & 2.357959 \\
\hline $\mathrm{H}$ & 0.707339 & 1.521840 & 4.768619 \\
\hline $\mathrm{H}$ & -1.523285 & 0.988507 & 5.740732 \\
\hline $\mathrm{H}$ & -3.349717 & 0.181938 & 4.278502 \\
\hline C & 2.240850 & 2.315347 & -1.011562 \\
\hline C & 0.918808 & 2.004627 & -0.562512 \\
\hline C & -0.076709 & 3.015874 & -0.610307 \\
\hline C & 0.224578 & 4.273484 & -1.078376 \\
\hline C & 1.532507 & 4.581104 & -1.524303 \\
\hline C & 2.517103 & 3.621951 & -1.494653 \\
\hline $\mathrm{H}$ & -1.081441 & 2.773858 & -0.288359 \\
\hline $\mathrm{H}$ & -0.547058 & 5.036063 & -1.112509 \\
\hline H & 1.754619 & 5.578145 & -1.892264 \\
\hline $\mathrm{H}$ & 3.522713 & 3.848509 & -1.837483 \\
\hline C & -6.396384 & -1.701720 & -2.032613 \\
\hline C & -5.647763 & -0.608509 & -2.470467 \\
\hline C & -4.537409 & -0.176569 & -1.747296 \\
\hline C & -4.155443 & -0.836511 & -0.567784 \\
\hline C & -4.914239 & -1.936969 & -0.139075 \\
\hline C & -6.023985 & -2.366421 & -0.863893 \\
\hline $\mathrm{H}$ & -7.260714 & -2.035033 & -2.599011 \\
\hline $\mathrm{H}$ & -3.954468 & 0.670619 & -2.092046 \\
\hline $\mathrm{H}$ & -4.614537 & -2.466670 & 0.759866 \\
\hline C & 4.048637 & -0.987756 & -0.521198 \\
\hline C & 3.814525 & -2.268075 & -1.046213 \\
\hline C & 5.331240 & -0.681031 & -0.044084 \\
\hline C & 4.835497 & -3.215238 & -1.086112 \\
\hline $\mathrm{H}$ & 2.832814 & -2.517350 & -1.434847 \\
\hline $\mathrm{C}$ & 6.351868 & -1.628358 & -0.086229 \\
\hline $\mathrm{H}$ & 5.516278 & 0.300229 & 0.381540 \\
\hline C & 6.107402 & -2.899707 & -0.606833 \\
\hline $\mathrm{H}$ & 6.901857 & -3.638916 & -0.638281 \\
\hline $\mathrm{H}$ & -6.593345 & -3.224528 & -0.519283 \\
\hline $\mathrm{H}$ & -5.932574 & -0.083729 & -3.377737 \\
\hline $\mathrm{H}$ & 4.638155 & -4.199142 & -1.500753 \\
\hline $\mathrm{H}$ & 7.336567 & -1.376152 & 0.295576 \\
\hline
\end{tabular}

\section{Simple BHCA 17}

Datum

Value

\begin{tabular}{lr}
\hline B3LYP-D3(BJ)/6-31G(d,p) Energy & -1535.985995 \\
\hline B3LYP-D3(BJ)/def2-TZVPP/IEF-PCM(DCM) Energy & -1536.536954 \\
\hline B3LYP-D3(BJ)/def2-TZVPP/IEF-PCM(DCM)//B3LYP-D3(BJ)/6-31G(d,p) Free Energy (Quasiharmonic) & -1536.09115 \\
\hline
\end{tabular}


Frequencies (Top 3 out of 177)

$\begin{array}{ll}\text { 1. } & 19.4574 \mathrm{~cm}^{-1} \\ \text { 2. } & 29.2048 \mathrm{~cm}^{-1} \\ \text { 3. } & 32.1745 \mathrm{~cm}^{-1}\end{array}$

B3LYP-D3(BJ)/6-31G(d,p) Molecular Geometry in Cartesian Coordinates

\begin{tabular}{|c|c|c|c|}
\hline C & -1.328303 & -0.202806 & -1.795692 \\
\hline $\mathrm{H}$ & -0.292520 & -0.538057 & -1.909271 \\
\hline $\mathrm{H}$ & -1.967846 & -0.947546 & -2.268892 \\
\hline C & 1.304192 & -1.689628 & 0.297199 \\
\hline 0 & 1.306565 & -2.527096 & -0.570378 \\
\hline 0 & 0.972136 & -2.013455 & 1.569655 \\
\hline $\mathrm{H}$ & 0.920288 & -1.214807 & 2.120021 \\
\hline 0 & -1.553326 & 1.015826 & -2.511887 \\
\hline $\mathrm{H}$ & -0.930717 & 1.673085 & -2.170670 \\
\hline C & 0.668099 & 0.711191 & 0.084503 \\
\hline C & 1.666108 & -0.248412 & 0.029017 \\
\hline C & 2.978909 & 0.069922 & -0.445121 \\
\hline C & 3.233538 & 1.364649 & -0.851522 \\
\hline $\mathrm{H}$ & 4.232879 & 1.628612 & -1.183626 \\
\hline C & -1.672140 & -0.082655 & -0.322304 \\
\hline C & -0.702610 & 0.356089 & 0.568643 \\
\hline C & -3.240045 & -0.328298 & 1.518239 \\
\hline C & -2.982861 & -0.410115 & 0.162951 \\
\hline $\mathrm{H}$ & -4.231389 & -0.576568 & 1.885087 \\
\hline C & -2.264329 & 0.100073 & 2.447648 \\
\hline C & -0.972820 & 0.474146 & 1.966347 \\
\hline C & 0.001472 & 0.922085 & 2.901709 \\
\hline C & -0.287107 & 0.975304 & 4.248494 \\
\hline C & -1.564667 & 0.597328 & 4.723300 \\
\hline C & -2.531639 & 0.176316 & 3.839543 \\
\hline $\mathrm{H}$ & 0.973043 & 1.242784 & 2.539635 \\
\hline $\mathrm{H}$ & 0.466777 & 1.319968 & 4.949344 \\
\hline $\mathrm{H}$ & -1.779768 & 0.645060 & 5.785989 \\
\hline $\mathrm{H}$ & -3.516999 & -0.110840 & 4.195332 \\
\hline C & 2.239734 & 2.372047 & -0.826115 \\
\hline C & 0.933745 & 2.049104 & -0.341919 \\
\hline C & -0.058645 & 3.067288 & -0.311539 \\
\hline C & 0.230086 & 4.341533 & -0.747800 \\
\hline C & 1.522936 & 4.660219 & -1.226161 \\
\hline C & 2.505062 & 3.697446 & -1.259857 \\
\hline $\mathrm{H}$ & -1.046510 & 2.825833 & 0.064442 \\
\hline $\mathrm{H}$ & -0.537293 & 5.108477 & -0.720397 \\
\hline
\end{tabular}




\begin{tabular}{|lrrr|}
$H$ & 1.736670 & 5.668472 & -1.566311 \\
$H$ & 3.499898 & 3.935579 & -1.625015 \\
C & -6.234577 & -1.652532 & -2.355808 \\
C & -5.484439 & -0.525094 & -2.692330 \\
C & -4.415542 & -0.123285 & -1.893548 \\
C & -4.080376 & -0.848953 & -0.738892 \\
C & -4.839882 & -1.982989 & -0.411181 \\
C & -5.907123 & -2.382371 & -1.212702 \\
$H$ & -7.064885 & -1.963471 & -2.982494 \\
$H$ & -3.829549 & 0.748711 & -2.162575 \\
$H$ & -4.573459 & -2.563130 & 0.466930 \\
C & 4.064987 & -0.939751 & -0.479562 \\
C & 4.295711 & -1.798129 & 0.605535 \\
C & 4.912440 & -1.023316 & -1.593939 \\
C & 5.343894 & -2.714020 & 0.576003 \\
$H$ & 3.655025 & -1.747250 & 1.479531 \\
C & 5.961447 & -1.939135 & -1.622972 \\
$H$ & 4.726501 & -0.383415 & -2.450641 \\
C & 6.181699 & -2.787075 & -0.537253 \\
$H$ & 6.996842 & -3.503544 & -0.560608 \\
$H$ & -6.477271 & -3.267907 & -0.948564 \\
$H$ & -5.732208 & 0.047915 & -3.580724 \\
$H$ & 5.507302 & -3.369613 & 1.425578 \\
$H$ & 6.600485 & -1.997613 & -2.498697 \\
& & & \\
\hline
\end{tabular}

\section{Simple BHCA 18}

\section{Datum}

Value

B3LYP-D3(BJ)/6-31G(d,p) Energy

$-1535.984908$

B3LYP-D3(BJ)/def2-TZVPP/IEF-PCM(DCM) Energy

$-1536.536209$

B3LYP-D3(BJ)/def2-TZVPP/IEF-PCM(DCM)//B3LYP-D3(BJ)/6-31G(d,p) Free Energy (Quasiharmonic)

$-1536.090884$

Number of Imaginary Frequencies

Frequencies (Top 3 out of 177)
1. $18.4944 \mathrm{~cm}^{-1}$
2. $22.4170 \mathrm{~cm}^{-1}$
3. $29.5807 \mathrm{~cm}^{-1}$

\section{B3LYP-D3(BJ)/6-31G(d,p) Molecular Geometry in Cartesian Coordinates}

$\begin{array}{llll}\mathrm{C} & -1.455767 & -0.070102 & -1.803536 \\ \mathrm{H} & -0.429771 & -0.405953 & -1.982068\end{array}$




\begin{tabular}{|c|c|c|c|}
\hline $\mathrm{H}$ & -2.123091 & -0.774999 & -2.299302 \\
\hline C & 1.367686 & -1.691150 & 0.278589 \\
\hline 0 & 0.569310 & -2.374596 & -0.315368 \\
\hline 0 & 2.048872 & -2.191730 & 1.335994 \\
\hline $\mathrm{H}$ & 2.654749 & -1.518672 & 1.679158 \\
\hline 0 & -1.698640 & 1.197166 & -2.425626 \\
\hline $\mathrm{H}$ & -1.056548 & 1.824946 & -2.066482 \\
\hline C & 0.642493 & 0.668755 & 0.036259 \\
\hline C & 1.668440 & -0.256940 & -0.075914 \\
\hline C & 2.974046 & 0.126262 & -0.519707 \\
\hline C & 3.201539 & 1.451266 & -0.836838 \\
\hline $\mathrm{H}$ & 4.178492 & 1.747965 & -1.206142 \\
\hline C & -1.726277 & -0.062776 & -0.311726 \\
\hline C & -0.699867 & 0.267937 & 0.561339 \\
\hline C & -3.195113 & -0.458678 & 1.581376 \\
\hline C & -3.013068 & -0.415955 & 0.211602 \\
\hline $\mathrm{H}$ & -4.171503 & -0.720407 & 1.978082 \\
\hline C & -2.161125 & -0.141165 & 2.490563 \\
\hline C & -0.885054 & 0.245826 & 1.976905 \\
\hline C & 0.142377 & 0.589582 & 2.896962 \\
\hline C & -0.079948 & 0.540270 & 4.253916 \\
\hline C & -1.341902 & 0.148010 & 4.760898 \\
\hline C & -2.358793 & -0.181500 & 3.896291 \\
\hline $\mathrm{H}$ & 1.108179 & 0.902277 & 2.515593 \\
\hline $\mathrm{H}$ & 0.715621 & 0.807236 & 4.942790 \\
\hline $\mathrm{H}$ & -1.503544 & 0.111754 & 5.833684 \\
\hline $\mathrm{H}$ & -3.332393 & -0.479385 & 4.275111 \\
\hline C & 2.186469 & 2.430561 & -0.731932 \\
\hline c & 0.886088 & 2.040610 & -0.280172 \\
\hline C & -0.123147 & 3.036366 & -0.158615 \\
\hline C & 0.138843 & 4.345984 & -0.493419 \\
\hline C & 1.421573 & 4.726858 & -0.954697 \\
\hline C & 2.422133 & 3.790191 & -1.067204 \\
\hline H & -1.102364 & 2.746678 & 0.204889 \\
\hline $\mathrm{H}$ & -0.642290 & 5.093515 & -0.399627 \\
\hline $\mathrm{H}$ & 1.612947 & 5.762650 & -1.216370 \\
\hline H & 3.410091 & 4.075837 & -1.416646 \\
\hline C & -6.418743 & -1.376637 & -2.228065 \\
\hline C & -5.660614 & -0.239028 & -2.507426 \\
\hline C & -4.543394 & 0.073158 & -1.734983 \\
\hline C & -4.165499 & -0.753983 & -0.664824 \\
\hline C & -4.933282 & -1.898003 & -0.395452 \\
\hline C & -6.049466 & -2.207356 & -1.169633 \\
\hline $\mathrm{H}$ & -7.287356 & -1.617181 & -2.833577 \\
\hline H & -3.951716 & 0.953372 & -1.960993 \\
\hline $\mathrm{H}$ & -4.634868 & -2.556168 & 0.414645 \\
\hline C & 4.073350 & -0.863914 & -0.656694 \\
\hline C & 3.875957 & -2.085011 & -1.320405 \\
\hline C & 5.345632 & -0.574483 & -0.139690 \\
\hline C & 4.924481 & -2.990905 & -1.458557 \\
\hline $\mathrm{H}$ & 2.901638 & -2.320864 & -1.734456 \\
\hline C & 6.393514 & -1.482379 & -0.279666 \\
\hline H & 5.503315 & 0.361466 & 0.387816 \\
\hline$c$ & 6.185474 & -2.693801 & -0.939573 \\
\hline
\end{tabular}




$\begin{array}{rrrr}H & 7.000112 & -3.403099 & -1.046840 \\ \mathrm{H} & -6.625544 & -3.101539 & -0.951014 \\ \mathrm{H} & -5.940157 & 0.412443 & -3.330007 \\ \mathrm{H} & 4.755743 & -3.929514 & -1.976746 \\ \mathrm{H} & 7.369697 & -1.246297 & 0.132668\end{array}$

\section{Simple BHCA 19}

\section{Datum}

Value

B3LYP-D3(BJ)/6-31G(d,p) Energy

$-1535.985883$

B3LYP-D3(BJ)/def2-TZVPP/IEF-PCM(DCM) Energy

$-1536.535791$

B3LYP-D3(BJ)/def2-TZVPP/IEF-PCM(DCM)//B3LYP-D3(BJ)/6-31G(d,p) Free Energy (Quasiharmonic)

$-1536.090803$

Number of Imaginary Frequencies

Frequencies (Top 3 out of 177)

$\begin{array}{ll}\text { 1. } & 18.8672 \mathrm{~cm}^{-1} \\ \text { 2. } & 26.0250 \mathrm{~cm}^{-1} \\ \text { 3. } & 26.9647 \mathrm{~cm}^{-1}\end{array}$

B3LYP-D3(BJ)/6-31G(d,p) Molecular Geometry in Cartesian Coordinates

$\begin{array}{lrrr}\text { C } & 1.316040 & -0.637790 & -1.668353 \\ \text { H } & 1.932400 & -1.508858 & -1.918846 \\ \text { H } & 0.266725 & -0.945993 & -1.700476 \\ \text { C } & -1.298902 & -1.447039 & 0.869623 \\ \text { O } & -1.107593 & -1.516518 & 2.063128 \\ \text { O } & -1.186117 & -2.515720 & 0.045882 \\ \text { H } & -0.964644 & -3.272500 & 0.615961 \\ \text { O } & 1.551236 & 0.436975 & -2.591394 \\ \text { H } & 1.207227 & 0.162196 & -3.451543 \\ \text { C } & -0.673416 & 0.739192 & -0.099109 \\ \text { C } & -1.662883 & -0.206049 & 0.110097 \\ \text { C } & -2.979195 & -0.046550 & -0.421493 \\ \text { C } & -3.255031 & 1.089725 & -1.155502 \\ \text { H } & -4.259121 & 1.241564 & -1.540351 \\ \text { C } & 1.661861 & -0.144089 & -0.285422 \\ \text { C } & 0.699299 & 0.525978 & 0.457777 \\ \text { C } & 3.253462 & 0.129548 & 1.526319 \\ \text { C } & 2.974574 & -0.338463 & 0.256549 \\ \text { H } & 4.251417 & -0.004482 & 1.933138 \\ \text { C } & 2.292644 & 0.814349 & 2.304693 \\ \text { C } & 0.986677 & 1.022168 & 1.765207\end{array}$




$\begin{array}{lrrr}\text { C } & 0.024961 & 1.710602 & 2.550760 \\ \mathrm{C} & 0.345428 & 2.176051 & 3.804697 \\ \mathrm{C} & 1.641354 & 1.973800 & 4.337158 \\ \mathrm{C} & 2.592547 & 1.306328 & 3.602611 \\ \mathrm{H} & -0.970726 & 1.859170 & 2.150146 \\ \mathrm{H} & -0.400915 & 2.699702 & 4.393873 \\ \mathrm{H} & 1.879773 & 2.346014 & 5.328780 \\ \mathrm{H} & 3.589270 & 1.143824 & 4.003109 \\ \mathrm{C} & -2.276114 & 2.083484 & -1.395001 \\ \mathrm{C} & -0.959365 & 1.910262 & -0.864156 \\ \mathrm{C} & 0.019258 & 2.907299 & -1.116107 \\ \mathrm{C} & -0.296494 & 4.029177 & -1.846260 \\ \mathrm{C} & -1.602136 & 4.206113 & -2.364399 \\ \mathrm{C} & -2.568870 & 3.253281 & -2.145396 \\ \mathrm{H} & 1.021915 & 2.762171 & -0.733899 \\ \mathrm{H} & 0.461090 & 4.784038 & -2.031644 \\ \mathrm{H} & -1.836297 & 5.097144 & -2.938883 \\ \mathrm{H} & -3.572223 & 3.379932 & -2.542118 \\ \mathrm{C} & 6.178042 & -2.319060 & -1.820383 \\ \mathrm{C} & 5.450879 & -1.314007 & -2.459494 \\ \mathrm{C} & 4.399495 & -0.675588 & -1.804044 \\ \mathrm{C} & 4.055380 & -1.036204 & -0.490861 \\ \mathrm{C} & 4.793039 & -2.049882 & 0.140976 \\ \mathrm{C} & 5.844120 & -2.686023 & -0.516499 \\ \mathrm{H} & 6.996590 & -2.813517 & -2.334616 \\ \mathrm{H} & 3.834619 & 0.104173 & -2.303400 \\ \mathrm{H} & 4.524055 & -2.347272 & 1.149919 \\ \mathrm{C} & -4.042020 & -1.055004 & -0.184484 \\ \mathrm{C} & -4.296047 & -1.556692 & 1.101570 \\ \mathrm{C} & -4.837832 & -1.501083 & -1.250152 \\ \mathrm{C} & -5.314769 & -2.484332 & 1.310537 \\ \mathrm{H} & -3.702565 & -1.209565 & 1.940123 \\ \mathrm{C} & -5.857095 & -2.426802 & -1.038972 \\ \mathrm{H} & -4.636367 & -1.131562 & -2.250767 \\ \mathrm{C} & -6.098426 & -2.923003 & 0.242823 \\ \mathrm{H} & -6.891272 & -3.646008 & 0.408001 \\ \mathrm{H} & 6.398070 & -3.472198 & -0.012135 \\ \mathrm{H} & 5.707287 & -1.019158 & -3.472894 \\ \mathrm{H} & -5.501437 & -2.857870 & 2.312784 \\ & -6.457862 & -2.767015 & -1.877057\end{array}$

\section{Simple BHCA 20}

Datum

Value

\begin{tabular}{lr}
\hline B3LYP-D3(BJ)/6-31G(d,p) Energy & -1535.981994 \\
\hline B3LYP-D3(BJ)/def2-TZVPP/IEF-PCM(DCM) Energy & -1536.534839 \\
\hline B3LYP-D3(BJ)/def2-TZVPP/IEF-PCM(DCM)//B3LYP-D3(BJ)/6-31G(d,p) Free Energy (Quasiharmonic) & -1536.090443 \\
\hline
\end{tabular}


Frequencies (Top 3 out of 177)

$\begin{array}{ll}\text { 1. } & 13.7105 \mathrm{~cm}^{-1} \\ \text { 2. } & 21.2713 \mathrm{~cm}^{-1} \\ \text { 3. } & 30.1885 \mathrm{~cm}^{-1}\end{array}$

B3LYP-D3(BJ)/6-31G(d,p) Molecular Geometry in Cartesian Coordinates

\begin{tabular}{|c|c|c|c|}
\hline C & -1.429388 & -0.913349 & -1.566155 \\
\hline $\mathrm{H}$ & -0.434115 & -0.688878 & -1.949073 \\
\hline $\mathrm{H}$ & -1.443326 & -1.985051 & -1.338106 \\
\hline C & 1.175807 & -1.345490 & 1.001606 \\
\hline 0 & 0.326278 & -2.168032 & 0.751276 \\
\hline 0 & 1.794491 & -1.352859 & 2.205794 \\
\hline $\mathrm{H}$ & 2.460119 & -0.649713 & 2.230720 \\
\hline 0 & -2.336128 & -0.581970 & -2.612499 \\
\hline $\mathrm{H}$ & -3.198750 & -0.945741 & -2.370889 \\
\hline C & 0.688956 & 0.695878 & -0.335666 \\
\hline C & 1.615892 & -0.261431 & 0.050304 \\
\hline C & 2.968858 & -0.216800 & -0.417799 \\
\hline C & 3.342518 & 0.808835 & -1.263399 \\
\hline $\mathrm{H}$ & 4.358606 & 0.837211 & -1.645308 \\
\hline C & -1.682047 & -0.108844 & -0.301548 \\
\hline C & -0.689907 & 0.686982 & 0.242859 \\
\hline C & -3.238934 & 0.662413 & 1.401888 \\
\hline C & -2.972766 & -0.142452 & 0.316326 \\
\hline $\mathrm{H}$ & -4.221660 & 0.626949 & 1.863795 \\
\hline C & -2.249621 & 1.505462 & 1.963275 \\
\hline C & -0.941167 & 1.507521 & 1.389015 \\
\hline C & 0.053904 & 2.334271 & 1.976174 \\
\hline C & -0.234493 & 3.119881 & 3.069324 \\
\hline C & -1.531221 & 3.117487 & 3.634011 \\
\hline C & -2.515548 & 2.325050 & 3.090531 \\
\hline $\mathrm{H}$ & 1.049177 & 2.346878 & 1.546605 \\
\hline $\mathrm{H}$ & 0.538571 & 3.747147 & 3.502584 \\
\hline $\mathrm{H}$ & -1.744932 & 3.740140 & 4.497132 \\
\hline $\mathrm{H}$ & -3.513862 & 2.310981 & 3.518906 \\
\hline C & 2.427950 & 1.802232 & -1.685935 \\
\hline C & 1.076683 & 1.747173 & -1.221266 \\
\hline C & 0.159406 & 2.738588 & -1.659142 \\
\hline C & 0.563959 & 3.738224 & -2.513351 \\
\hline C & 1.902769 & 3.795701 & -2.969431 \\
\hline C & 2.813283 & 2.847259 & -2.566378 \\
\hline $\mathrm{H}$ & -0.867629 & 2.685511 & -1.317987 \\
\hline $\mathrm{H}$ & -0.148448 & 4.486611 & -2.845435 \\
\hline
\end{tabular}




\begin{tabular}{|lrrr|}
$H$ & 2.207038 & 4.590378 & -3.643484 \\
$H$ & 3.840726 & 2.880964 & -2.917260 \\
C & -6.050189 & -2.816753 & -1.045081 \\
C & -6.256593 & -1.437508 & -1.083670 \\
C & -5.256318 & -0.567522 & -0.649195 \\
C & -4.034532 & -1.065329 & -0.173265 \\
C & -3.840020 & -2.455358 & -0.130752 \\
C & -4.840616 & -3.322697 & -0.565231 \\
$H$ & -6.827209 & -3.493810 & -1.386067 \\
$H$ & -5.407790 & 0.506721 & -0.688560 \\
$H$ & -2.906265 & -2.846241 & 0.261157 \\
C & 3.964190 & -1.248193 & -0.025963 \\
C & 3.662127 & -2.617039 & -0.101871 \\
C & 5.243060 & -0.863074 & 0.405188 \\
C & 4.614268 & -3.571298 & 0.247154 \\
$H$ & 2.681363 & -2.932206 & -0.441388 \\
C & 6.194908 & -1.819758 & 0.752942 \\
$H$ & 5.480812 & 0.193754 & 0.480660 \\
C & 5.882863 & -3.177246 & 0.675228 \\
$H$ & 6.622376 & -3.923357 & 0.948605 \\
$H$ & -4.677854 & -4.395249 & -0.522670 \\
$H$ & -7.194558 & -1.037077 & -1.456139 \\
$H$ & 4.365046 & -4.625595 & 0.180138 \\
$H$ & 7.177204 & -1.504318 & 1.091137 \\
& & & \\
\hline
\end{tabular}

\section{Simple BHCA 21}

\section{Datum}

Value

B3LYP-D3(BJ)/6-31G(d,p) Energy

$-1535.978461$

B3LYP-D3(BJ)/def2-TZVPP/IEF-PCM(DCM) Energy

$-1536.534595$

B3LYP-D3(BJ)/def2-TZVPP/IEF-PCM(DCM)//B3LYP-D3(BJ)/6-31G(d,p) Free Energy (Quasiharmonic)

$-1536.090316$

Number of Imaginary Frequencies

Frequencies (Top 3 out of 177)

$\begin{array}{ll}\text { 1. } & 11.9413 \mathrm{~cm}^{-1} \\ \text { 2. } & 24.0465 \mathrm{~cm}^{-1} \\ \text { 3. } & 27.5893 \mathrm{~cm}^{-1}\end{array}$

B3LYP-D3(BJ)/6-31G(d,p) Molecular Geometry in Cartesian Coordinates

$\begin{array}{llll}\mathrm{C} & 1.418748 & -0.860202 & -1.599799 \\ \mathrm{H} & 0.363842 & -0.771808 & -1.857596\end{array}$




\begin{tabular}{|c|c|c|c|}
\hline $\mathrm{H}$ & 1.988837 & -0.415679 & -2.429038 \\
\hline C & -1.270412 & -1.407107 & 0.888791 \\
\hline 0 & -1.349564 & -2.532711 & 0.469287 \\
\hline 0 & -0.851869 & -1.170325 & 2.157998 \\
\hline $\mathrm{H}$ & -0.784789 & -0.215744 & 2.320448 \\
\hline 0 & 1.673894 & -2.249135 & -1.481302 \\
\hline $\mathrm{H}$ & 2.629502 & -2.367495 & -1.396209 \\
\hline C & -0.680148 & 0.729739 & -0.285325 \\
\hline C & -1.648325 & -0.201217 & 0.060200 \\
\hline C & -2.973860 & -0.122304 & -0.477857 \\
\hline C & -3.268273 & 0.899382 & -1.357729 \\
\hline $\mathrm{H}$ & -4.278732 & 0.989548 & -1.744646 \\
\hline C & 1.695750 & -0.086553 & -0.318233 \\
\hline C & 0.696690 & 0.649801 & 0.296640 \\
\hline C & 3.230837 & 0.485479 & 1.484267 \\
\hline C & 2.995506 & -0.148774 & 0.284889 \\
\hline $\mathrm{H}$ & 4.216070 & 0.420437 & 1.937132 \\
\hline C & 2.228319 & 1.245581 & 2.134549 \\
\hline C & 0.943334 & 1.352498 & 1.520835 \\
\hline C & -0.056783 & 2.128077 & 2.170475 \\
\hline C & 0.202912 & 2.750264 & 3.373284 \\
\hline C & 1.474234 & 2.636768 & 3.980019 \\
\hline C & 2.465617 & 1.903536 & 3.368052 \\
\hline $\mathrm{H}$ & -1.026888 & 2.239899 & 1.698125 \\
\hline $\mathrm{H}$ & -0.571871 & 3.339388 & 3.853829 \\
\hline $\mathrm{H}$ & 1.666118 & 3.131840 & 4.926552 \\
\hline $\mathrm{H}$ & 3.447232 & 1.813438 & 3.824534 \\
\hline C & -2.304176 & 1.858511 & -1.749692 \\
\hline C & -0.986397 & 1.782969 & -1.201753 \\
\hline C & -0.026739 & 2.754944 & -1.589676 \\
\hline C & -0.357861 & 3.754704 & -2.475877 \\
\hline C & -1.663334 & 3.830462 & -3.016548 \\
\hline C & -2.614385 & 2.903066 & -2.659470 \\
\hline $\mathrm{H}$ & 0.973329 & 2.697219 & -1.175354 \\
\hline $\mathrm{H}$ & 0.385528 & 4.491993 & -2.762406 \\
\hline $\mathrm{H}$ & -1.911152 & 4.623868 & -3.714745 \\
\hline $\mathrm{H}$ & -3.618849 & 2.954289 & -3.069745 \\
\hline C & 6.243625 & -2.321851 & -1.510824 \\
\hline C & 5.742501 & -2.705801 & -0.266636 \\
\hline C & 4.685404 & -2.002489 & 0.310376 \\
\hline C & 4.110903 & -0.906311 & -0.350784 \\
\hline C & 4.627935 & -0.525006 & -1.598672 \\
\hline C & 5.684776 & -1.228345 & -2.174127 \\
\hline $\mathrm{H}$ & 7.063645 & -2.871708 & -1.961757 \\
\hline $\mathrm{H}$ & 4.281385 & -2.308233 & 1.270143 \\
\hline $\mathrm{H}$ & 4.206816 & 0.335313 & -2.108992 \\
\hline C & -4.042262 & -1.071664 & -0.078781 \\
\hline C & -4.255685 & -1.400891 & 1.267766 \\
\hline C & -4.895572 & -1.618945 & -1.047252 \\
\hline C & -5.292749 & -2.253974 & 1.634425 \\
\hline $\mathrm{H}$ & -3.607967 & -0.985299 & 2.032792 \\
\hline C & -5.933491 & -2.472682 & -0.680444 \\
\hline $\mathrm{H}$ & -4.723098 & -1.391685 & -2.094461 \\
\hline C & -6.136703 & -2.792076 & 0.662112 \\
\hline
\end{tabular}




$\begin{array}{rrrr}H & -6.943212 & -3.459873 & 0.948593 \\ H & 6.075199 & -0.918788 & -3.138629 \\ H & 6.169633 & -3.557383 & 0.253740 \\ H & -5.442602 & -2.497155 & 2.681726 \\ H & -6.577271 & -2.896543 & -1.445120\end{array}$

\section{Simple BHCA 22}

\section{Datum}

Value

B3LYP-D3(BJ)/6-31G(d,p) Energy

$-1535.982573$

B3LYP-D3(BJ)/def2-TZVPP/IEF-PCM(DCM) Energy

$-1536.535014$

B3LYP-D3(BJ)/def2-TZVPP/IEF-PCM(DCM)//B3LYP-D3(BJ)/6-31G(d,p) Free Energy (Quasiharmonic)

$-1536.090165$

Number of Imaginary Frequencies

Frequencies (Top 3 out of 177)

$\begin{array}{ll}\text { 1. } & 13.7192 \mathrm{~cm}^{-1} \\ \text { 2. } & 27.8808 \mathrm{~cm}^{-1} \\ \text { 3. } & 30.5074 \mathrm{~cm}^{-1}\end{array}$

B3LYP-D3(BJ)/6-31G(d,p) Molecular Geometry in Cartesian Coordinates

$\begin{array}{lrrr}\mathrm{C} & 1.225745 & -0.883165 & -1.609001 \\ \mathrm{H} & 1.070726 & -1.931305 & -1.325361 \\ \mathrm{H} & 0.275113 & -0.527631 & -2.006123 \\ \mathrm{C} & -0.987613 & -1.294658 & 0.967920 \\ \mathrm{O} & -0.844196 & -2.421394 & 0.560890 \\ \mathrm{O} & -0.608915 & -0.967260 & 2.229509 \\ \mathrm{H} & -0.692051 & -0.009162 & 2.358609 \\ \mathrm{O} & 2.163295 & -0.752913 & -2.668858 \\ \mathrm{H} & 2.946237 & -1.266134 & -2.429265 \\ \mathrm{C} & -0.732623 & 0.833823 & -0.289285 \\ \mathrm{C} & -1.577898 & -0.177784 & 0.141037 \\ \mathrm{C} & -2.942462 & -0.239889 & -0.282838 \\ \mathrm{C} & -3.407012 & 0.746677 & -1.130696 \\ \mathrm{H} & -4.449442 & 0.735220 & -1.434225 \\ \mathrm{C} & 1.600124 & -0.053046 & -0.386004 \\ \mathrm{C} & 0.689171 & 0.828636 & 0.173376 \\ \mathrm{C} & 3.287860 & 0.684058 & 1.205193 \\ \mathrm{C} & 2.915304 & -0.151296 & 0.175163 \\ \mathrm{H} & 4.285059 & 0.596226 & 1.627032 \\ \mathrm{C} & 2.387429 & 1.619022 & 1.771955 \\ \mathrm{C} & 1.053415 & 1.680759 & 1.264942\end{array}$




\begin{tabular}{lrrr} 
C & 0.134285 & 2.576497 & 1.876564 \\
C & 0.523466 & 3.376229 & 2.929617 \\
C & 1.847528 & 3.320961 & 3.421470 \\
C & 2.758057 & 2.459615 & 2.852347 \\
H & -0.877751 & 2.638381 & 1.491542 \\
H & -0.190366 & 4.057415 & 3.382221 \\
H & 2.141561 & 3.956600 & 4.250651 \\
H & 3.775408 & 2.403566 & 3.229139 \\
C & -2.576429 & 1.792875 & -1.599883 \\
C & -1.211587 & 1.842999 & -1.176950 \\
C & -0.376803 & 2.882796 & -1.662927 \\
C & -0.876816 & 3.840006 & -2.515750 \\
C & -2.231397 & 3.799817 & -2.924359 \\
C & -3.061370 & 2.798237 & -2.477163 \\
H & 0.661957 & 2.906478 & -1.354063 \\
H & -0.229148 & 4.630438 & -2.881418 \\
H & -2.611960 & 4.561612 & -3.597584 \\
H & -4.100103 & 2.758049 & -2.792247 \\
C & 5.726488 & -3.128952 & -1.128596 \\
C & 4.474763 & -3.509655 & -0.641789 \\
C & 3.559625 & -2.543656 & -0.226451 \\
C & 3.883367 & -1.178597 & -0.297899 \\
C & 5.146878 & -0.807554 & -0.780155 \\
C & 6.060994 & -1.775838 & -1.193931 \\
H & 6.436655 & -3.882406 & -1.454533 \\
H & 2.594826 & -2.841430 & 0.172232 \\
H & 5.397336 & 0.246608 & -0.845231 \\
C & -3.862199 & -1.306408 & 0.183502 \\
C & -3.918389 & -1.684853 & 1.533040 \\
C & -4.727480 & -1.933125 & -0.725440 \\
C & -4.813210 & -2.662570 & 1.959999 \\
H & -3.263666 & -1.208667 & 2.255045 \\
C & -5.623465 & -2.910094 & -0.297907 \\
H & -4.674506 & -1.667176 & -1.776379 \\
C & -5.670130 & -3.277921 & 1.047068 \\
H & -6.365604 & -4.041784 & 1.380593 \\
H & 7.031958 & -1.472538 & -1.573372 \\
H & 4.211490 & -4.560832 & -0.577650 \\
H & -4.842631 & -2.941076 & 3.008791 \\
H & -6.278265 & -3.391598 & -1.017761 \\
& & & \\
\hline & & & \\
\hline
\end{tabular}

\section{Simple BHCA 23}

Datum

Value

\begin{tabular}{lr}
\hline B3LYP-D3(BJ)/6-31G(d,p) Energy & -1535.980443 \\
\hline B3LYP-D3(BJ)/def2-TZVPP/IEF-PCM(DCM) Energy & -1536.534555 \\
\hline B3LYP-D3(BJ)/def2-TZVPP/IEF-PCM(DCM)//B3LYP-D3(BJ)/6-31G(d,p) Free Energy (Quasiharmonic) & -1536.090031 \\
\hline
\end{tabular}


Frequencies (Top 3 out of 177)

$\begin{array}{ll}\text { 1. } & 14.8619 \mathrm{~cm}^{-1} \\ \text { 2. } & 26.3344 \mathrm{~cm}^{-1} \\ \text { 3. } & 27.7787 \mathrm{~cm}^{-1}\end{array}$

B3LYP-D3(BJ)/6-31G(d,p) Molecular Geometry in Cartesian Coordinates

\begin{tabular}{|c|c|c|c|}
\hline C & -1.204616 & -1.052780 & -1.446585 \\
\hline $\mathrm{H}$ & -0.206765 & -0.783573 & -1.793695 \\
\hline $\mathrm{H}$ & -1.160371 & -2.101447 & -1.121660 \\
\hline C & 1.137947 & -1.216890 & 1.128586 \\
\hline 0 & 0.736289 & -1.018748 & 2.247388 \\
\hline 0 & 1.138586 & -2.475850 & 0.612201 \\
\hline $\mathrm{H}$ & 1.542922 & -2.461305 & -0.268149 \\
\hline 0 & -2.054608 & -0.897257 & -2.574697 \\
\hline $\mathrm{H}$ & -2.917562 & -1.265127 & -2.339359 \\
\hline C & 0.710860 & 0.808529 & -0.234988 \\
\hline C & 1.625879 & -0.142961 & 0.189086 \\
\hline C & 2.976313 & -0.142219 & -0.284170 \\
\hline C & 3.360104 & 0.836110 & -1.180173 \\
\hline $\mathrm{H}$ & 4.390577 & 0.868688 & -1.521042 \\
\hline C & -1.594773 & -0.157692 & -0.278302 \\
\hline C & -0.701240 & 0.758046 & 0.253661 \\
\hline C & -3.331327 & 0.666030 & 1.213505 \\
\hline C & -2.927800 & -0.223459 & 0.243414 \\
\hline $\mathrm{H}$ & -4.345612 & 0.610437 & 1.598813 \\
\hline C & -2.445276 & 1.628277 & 1.756644 \\
\hline C & -1.096371 & 1.661914 & 1.290401 \\
\hline C & -0.200480 & 2.597302 & 1.870300 \\
\hline C & -0.626170 & 3.467713 & 2.848062 \\
\hline C & -1.966206 & 3.441857 & 3.299297 \\
\hline C & -2.854637 & 2.536971 & 2.766078 \\
\hline $\mathrm{H}$ & 0.830185 & 2.615637 & 1.536358 \\
\hline $\mathrm{H}$ & 0.072847 & 4.176135 & 3.281606 \\
\hline $\mathrm{H}$ & -2.288185 & 4.132693 & 4.072329 \\
\hline $\mathrm{H}$ & -3.883384 & 2.500161 & 3.113334 \\
\hline C & 2.457045 & 1.820475 & -1.646524 \\
\hline C & 1.106939 & 1.809203 & -1.173802 \\
\hline C & 0.200412 & 2.785514 & -1.665238 \\
\hline C & 0.617076 & 3.737752 & -2.566398 \\
\hline C & 1.956930 & 3.758288 & -3.022619 \\
\hline C & 2.855604 & 2.819485 & -2.573529 \\
\hline $\mathrm{H}$ & -0.826292 & 2.765355 & -1.319984 \\
\hline $\mathrm{H}$ & -0.086000 & 4.478566 & -2.933568 \\
\hline
\end{tabular}




\begin{tabular}{|lrrr|}
$H$ & 2.271113 & 4.516475 & -3.733144 \\
$H$ & 3.883807 & 2.824700 & -2.923755 \\
C & -5.738878 & -3.191088 & -1.093231 \\
C & -4.546598 & -3.579997 & -0.479967 \\
C & -3.631780 & -2.619520 & -0.052289 \\
C & -3.893856 & -1.251986 & -0.234643 \\
C & -5.099054 & -0.872602 & -0.844833 \\
C & -6.013950 & -1.835093 & -1.272064 \\
$H$ & -6.449236 & -3.940390 & -1.428271 \\
$H$ & -2.715831 & -2.921842 & 0.445545 \\
$H$ & -5.303510 & 0.183118 & -0.993113 \\
C & 3.960837 & -1.155265 & 0.175262 \\
C & 4.099750 & -1.471072 & 1.536221 \\
C & 4.788975 & -1.802678 & -0.755023 \\
C & 5.040211 & -2.410686 & 1.951079 \\
$H$ & 3.474444 & -0.973803 & 2.269799 \\
C & 5.730426 & -2.741307 & -0.337317 \\
$H$ & 4.677387 & -1.576737 & -1.811197 \\
C & 5.858443 & -3.048456 & 1.017590 \\
$H$ & 6.589151 & -3.781918 & 1.343816 \\
$H$ & -6.938990 & -1.524924 & -1.748370 \\
$H$ & -4.331483 & -4.633043 & -0.326797 \\
$H$ & 5.136194 & -2.641300 & 3.007369 \\
$H$ & 6.358025 & -3.237985 & -1.070938 \\
& & & \\
\hline
\end{tabular}

\section{Simple BHCA 24}

\section{Datum}

Value

B3LYP-D3(BJ)/6-31G(d,p) Energy

$-1535.983728$

B3LYP-D3(BJ)/def2-TZVPP/IEF-PCM(DCM) Energy

$-1536.53536$

B3LYP-D3(BJ)/def2-TZVPP/IEF-PCM(DCM)//B3LYP-D3(BJ)/6-31G(d,p) Free Energy (Quasiharmonic)

$-1536.08993$

Number of Imaginary Frequencies

Frequencies (Top 3 out of 177)
1. $\quad 19.2238 \mathrm{~cm}^{-1}$
2. $27.1078 \mathrm{~cm}^{-1}$
3. $28.6596 \mathrm{~cm}^{-1}$

\section{B3LYP-D3(BJ)/6-31G(d,p) Molecular Geometry in Cartesian Coordinates}

$\begin{array}{llll}\mathrm{C} & 1.270848 & -0.971538 & -1.496235 \\ \mathrm{H} & 1.892629 & -1.858170 & -1.621295\end{array}$




\begin{tabular}{|c|c|c|c|}
\hline $\mathrm{H}$ & 0.229090 & -1.305699 & -1.430714 \\
\hline C & -1.380962 & -1.306005 & 1.137864 \\
\hline 0 & -1.022480 & -1.152161 & 2.278354 \\
\hline 0 & -1.498600 & -2.554075 & 0.615926 \\
\hline H & -1.861282 & -2.500085 & -0.280794 \\
\hline 0 & 1.470580 & -0.190097 & -2.677454 \\
\hline $\mathrm{H}$ & 0.939567 & 0.613882 & -2.587554 \\
\hline C & -0.664964 & 0.691286 & -0.146738 \\
\hline C & -1.690971 & -0.180089 & 0.183698 \\
\hline C & -2.989722 & -0.060610 & -0.407118 \\
\hline C & -3.207766 & 0.942309 & -1.330777 \\
\hline $\mathrm{H}$ & -4.195863 & 1.058451 & -1.765548 \\
\hline C & 1.653496 & -0.240452 & -0.224407 \\
\hline C & 0.707916 & 0.538486 & 0.430731 \\
\hline C & 3.293582 & 0.343255 & 1.468451 \\
\hline C & 2.984282 & -0.332984 & 0.304159 \\
\hline $\mathrm{H}$ & 4.304170 & 0.284640 & 1.861635 \\
\hline C & 2.348426 & 1.141588 & 2.152685 \\
\hline C & 1.025616 & 1.248910 & 1.626877 \\
\hline C & 0.080493 & 2.055176 & 2.313335 \\
\hline C & 0.432444 & 2.727086 & 3.460711 \\
\hline C & 1.745332 & 2.624024 & 3.979736 \\
\hline C & 2.681153 & 1.846577 & 3.339469 \\
\hline $\mathrm{H}$ & -0.929398 & 2.126100 & 1.926681 \\
\hline $\mathrm{H}$ & -0.302087 & 3.337357 & 3.976864 \\
\hline $\mathrm{H}$ & 2.008627 & 3.158613 & 4.887182 \\
\hline $\mathrm{H}$ & 3.690422 & 1.758056 & 3.731467 \\
\hline C & -2.188294 & 1.848893 & -1.703989 \\
\hline C & -0.896979 & 1.731788 & -1.099615 \\
\hline C & 0.120363 & 2.652575 & -1.476714 \\
\hline C & -0.129886 & 3.631215 & -2.412648 \\
\hline C & -1.407422 & 3.743455 & -3.010993 \\
\hline C & -2.413688 & 2.874293 & -2.659946 \\
\hline $\mathrm{H}$ & 1.094387 & 2.577316 & -1.007184 \\
\hline $\mathrm{H}$ & 0.656044 & 4.326562 & -2.689366 \\
\hline $\mathrm{H}$ & -1.590133 & 4.520134 & -3.746726 \\
\hline $\mathrm{H}$ & -3.397665 & 2.955980 & -3.112566 \\
\hline C & 6.153011 & -2.606330 & -1.505972 \\
\hline C & 5.392852 & -1.731926 & -2.283470 \\
\hline C & 4.351301 & -1.002068 & -1.713248 \\
\hline C & 4.051596 & -1.138143 & -0.347916 \\
\hline C & 4.821380 & -2.023054 & 0.423785 \\
\hline C & 5.862273 & -2.750802 & -0.149046 \\
\hline $\mathrm{H}$ & 6.962822 & -3.173806 & -1.954460 \\
\hline $\mathrm{H}$ & 3.759337 & -0.328160 & -2.322677 \\
\hline $\mathrm{H}$ & 4.584082 & -2.149908 & 1.475524 \\
\hline C & -4.096471 & -0.981238 & -0.040404 \\
\hline C & -4.380859 & -1.276103 & 1.302504 \\
\hline C & -4.893929 & -1.557185 & -1.041645 \\
\hline C & -5.434200 & -2.125340 & 1.631204 \\
\hline H & -3.778648 & -0.834014 & 2.089022 \\
\hline C & -5.948020 & -2.406312 & -0.710048 \\
\hline H & -4.670761 & -1.348300 & -2.083560 \\
\hline C & -6.221033 & -2.692728 & 0.627735 \\
\hline
\end{tabular}




$\begin{array}{rrrr}H & -7.039739 & -3.356604 & 0.886935 \\ H & 6.441020 & -3.435961 & 0.463190 \\ H & 5.612654 & -1.612582 & -3.340213 \\ H & -5.642113 & -2.341346 & 2.674314 \\ H & -6.550367 & -2.849826 & -1.496858\end{array}$

\section{Simple BHCA 25}

Datum

Value

\begin{tabular}{lc} 
B3LYP-D3(BJ)/6-31G(d,p) Energy & -1535.982974 \\
\hline B3LYP-D3(BJ)/def2-TZVPP/IEF-PCM(DCM) Energy & -1536.534566 \\
\hline B3LYP-D3(BJ)/def2-TZVPP/IEF-PCM(DCM)//B3LYP-D3(BJ)/6-31G(d,p) Free Energy (Quasiharmonic) & -1536.089329
\end{tabular}

Number of Imaginary Frequencies

Frequencies (Top 3 out of 177)
1. $19.3916 \mathrm{~cm}^{-1}$
2. $26.6764 \mathrm{~cm}^{-1}$
3. $31.4647 \mathrm{~cm}^{-1}$

\section{B3LYP-D3(BJ)/6-31G(d,p) Molecular Geometry in Cartesian Coordinates}

$\begin{array}{lrrr}\mathrm{C} & 1.335122 & -0.607016 & -1.702529 \\ \mathrm{H} & 1.979380 & -1.448794 & -1.978190 \\ \mathrm{H} & 0.298065 & -0.955424 & -1.730088 \\ \mathrm{C} & -1.233451 & -1.584082 & 0.631618 \\ \mathrm{O} & -1.199576 & -2.589927 & -0.033841 \\ \mathrm{O} & -0.895421 & -1.614773 & 1.943919 \\ \mathrm{H} & -0.880758 & -0.713827 & 2.306159 \\ \mathrm{O} & 1.522815 & 0.500324 & -2.596640 \\ \mathrm{H} & 1.182589 & 0.235341 & -3.461316 \\ \mathrm{C} & -0.671964 & 0.727453 & -0.107750 \\ \mathrm{C} & -1.642986 & -0.246950 & 0.065079 \\ \mathrm{C} & -2.974032 & -0.066876 & -0.428830 \\ \mathrm{C} & -3.277586 & 1.115909 & -1.073283 \\ \mathrm{H} & -4.293823 & 1.286158 & -1.415832 \\ \mathrm{C} & 1.675626 & -0.134672 & -0.310207 \\ \mathrm{C} & 0.704896 & 0.514144 & 0.438047 \\ \mathrm{C} & 3.240162 & 0.067883 & 1.535520 \\ \mathrm{C} & 2.983136 & -0.340256 & 0.240646 \\ \mathrm{H} & 4.230400 & -0.085705 & 1.953509 \\ \mathrm{C} & 2.263866 & 0.712691 & 2.328891 \\ \mathrm{C} & 0.974013 & 0.962272 & 1.767738\end{array}$




$\begin{array}{lrrr}\text { C } & -0.002426 & 1.620530 & 2.566771 \\ \text { C } & 0.283787 & 1.996510 & 3.861245 \\ \text { C } & 1.560686 & 1.744836 & 4.415588 \\ \text { C } & 2.528873 & 1.122624 & 3.661935 \\ \text { H } & -0.973429 & 1.842219 & 2.136603 \\ \text { H } & -0.471535 & 2.499101 & 4.457247 \\ \text { H } & 1.773874 & 2.047912 & 5.435728 \\ \text { H } & 3.513207 & 0.928948 & 4.078568 \\ \text { C } & -2.313709 & 2.131661 & -1.280012 \\ \text { C } & -0.982532 & 1.938938 & -0.796117 \\ \text { C } & -0.017110 & 2.955958 & -1.015452 \\ \text { C } & -0.360201 & 4.116743 & -1.668932 \\ \text { C } & -1.681376 & 4.314315 & -2.137466 \\ \text { C } & -2.635603 & 3.342194 & -1.948910 \\ \text { H } & 0.997838 & 2.795139 & -0.673635 \\ \text { H } & 0.387567 & 4.886392 & -1.831970 \\ \text { H } & -1.937312 & 5.235947 & -2.651044 \\ \text { H } & -3.650286 & 3.483861 & -2.309997 \\ \text { C } & 6.230041 & -2.164995 & -1.903559 \\ \text { C } & 5.486725 & -1.145600 & -2.500026 \\ \text { C } & 4.419946 & -0.559296 & -1.821619 \\ \text { C } & 4.079162 & -0.987353 & -0.528111 \\ \text { C } & 4.831773 & -2.015026 & 0.061002 \\ \text { C } & 5.897233 & -2.599733 & -0.620399 \\ \text { H } & 7.059732 & -2.619840 & -2.436035 \\ \text { H } & 3.840120 & 0.229660 & -2.288730 \\ \text { H } & 4.561551 & -2.367432 & 1.051609 \\ \text { C } & -4.029740 & -1.091032 & -0.233850 \\ \text { C } & -4.214506 & -1.724855 & 1.003863 \\ \text { C } & -4.896912 & -1.414609 & -1.287882 \\ \text { C } & -5.235016 & -2.655494 & 1.180775 \\ \text { H } & -3.560040 & -1.486106 & 1.835322 \\ \text { C } & -5.918993 & -2.344110 & -1.110468 \\ \text { H } & -4.748182 & -0.949623 & -2.257281 \\ \text { C } & -6.092363 & -2.967771 & 0.125369 \\ \text { H } & -6.886274 & -3.695083 & 0.263734 \\ \text { H } & 6.462287 & -3.399721 & -0.151650 \\ \text { H } & 5.741174 & -0.800260 & -3.497722 \\ \text { H } & -5.362016 & -3.134610 & 2.146564 \\ \text { H } & -6.573933 & -2.588858 & -1.941157\end{array}$

\section{Simple BHCA 26}

Datum

Value

\begin{tabular}{lr}
\hline B3LYP-D3(BJ)/6-31G(d,p) Energy & -1535.983661 \\
\hline B3LYP-D3(BJ)/def2-TZVPP/IEF-PCM(DCM) Energy & -1536.534835 \\
\hline B3LYP-D3(BJ)/def2-TZVPP/IEF-PCM(DCM)//B3LYP-D3(BJ)/6-31G(d,p) Free Energy (Quasiharmonic) & -1536.08909 \\
\hline
\end{tabular}


Frequencies (Top 3 out of 177)

$\begin{array}{ll}\text { 1. } & 19.8546 \mathrm{~cm}^{-1} \\ \text { 2. } & 28.2883 \mathrm{~cm}^{-1} \\ \text { 3. } & 32.3379 \mathrm{~cm}^{-1}\end{array}$

B3LYP-D3(BJ)/6-31G(d,p) Molecular Geometry in Cartesian Coordinates

\begin{tabular}{|c|c|c|c|}
\hline C & -1.417058 & 0.950387 & -1.525215 \\
\hline $\mathrm{H}$ & -0.365296 & 0.833041 & -1.814830 \\
\hline $\mathrm{H}$ & -2.032401 & 0.582139 & -2.347520 \\
\hline C & 1.586490 & -1.747432 & -0.114836 \\
\hline 0 & 2.390801 & -2.479900 & 0.405744 \\
\hline 0 & 0.534434 & -2.258275 & -0.797124 \\
\hline $\mathrm{H}$ & -0.097426 & -1.554462 & -1.013645 \\
\hline 0 & -1.742160 & 2.333802 & -1.394975 \\
\hline $\mathrm{H}$ & -1.142200 & 2.715869 & -0.738041 \\
\hline C & 0.665757 & 0.529912 & 0.438493 \\
\hline C & 1.713650 & -0.243316 & -0.043858 \\
\hline C & 2.986526 & 0.346038 & -0.341246 \\
\hline C & 3.165468 & 1.692182 & -0.097407 \\
\hline $\mathrm{H}$ & 4.110382 & 2.156373 & -0.362346 \\
\hline C & -1.699514 & 0.138887 & -0.270764 \\
\hline C & -0.690874 & -0.059312 & 0.671131 \\
\hline C & -3.213873 & -1.184269 & 1.091921 \\
\hline C & -2.997096 & -0.436505 & -0.049694 \\
\hline $\mathrm{H}$ & -4.196919 & -1.612460 & 1.263096 \\
\hline C & -2.209673 & -1.393060 & 2.064874 \\
\hline C & -0.917878 & -0.819324 & 1.859357 \\
\hline C & 0.087347 & -1.027046 & 2.841043 \\
\hline C & -0.177195 & -1.770852 & 3.967914 \\
\hline C & -1.457027 & -2.339296 & 4.170536 \\
\hline C & -2.451324 & -2.152885 & 3.239186 \\
\hline $\mathrm{H}$ & 1.071937 & -0.600765 & 2.689569 \\
\hline $\mathrm{H}$ & 0.602533 & -1.926025 & 4.706542 \\
\hline $\mathrm{H}$ & -1.649746 & -2.924864 & 5.063959 \\
\hline $\mathrm{H}$ & -3.436737 & -2.585649 & 3.385609 \\
\hline C & 2.146226 & 2.498156 & 0.462519 \\
\hline C & 0.873042 & 1.912219 & 0.740311 \\
\hline C & -0.139764 & 2.723151 & 1.324866 \\
\hline C & 0.090210 & 4.056503 & 1.586464 \\
\hline C & 1.342294 & 4.641052 & 1.283078 \\
\hline C & 2.347461 & 3.876009 & 0.738360 \\
\hline $\mathrm{H}$ & -1.093216 & 2.272816 & 1.577150 \\
\hline $\mathrm{H}$ & -0.691804 & 4.661123 & 2.034434 \\
\hline
\end{tabular}




\begin{tabular}{|c|c|c|c|}
\hline $\mathrm{H}$ & 1.508717 & 5.693409 & 1.489589 \\
\hline $\mathrm{H}$ & 3.314715 & 4.314975 & 0.511737 \\
\hline C & -6.308275 & -0.071515 & -2.767335 \\
\hline C & -5.599341 & 1.066285 & -2.380856 \\
\hline C & -4.512919 & 0.962527 & -1.513842 \\
\hline C & -4.117022 & -0.290175 & -1.018128 \\
\hline C & -4.836496 & -1.428859 & -1.415812 \\
\hline C & -5.921680 & -1.321480 & -2.282718 \\
\hline $\mathrm{H}$ & -7.152902 & 0.014178 & -3.444130 \\
\hline $\mathrm{H}$ & -3.960531 & 1.848430 & -1.222373 \\
\hline H & -4.526102 & -2.404124 & -1.053504 \\
\hline C & 4.084088 & -0.426102 & -0.974805 \\
\hline C & 3.840236 & -1.246067 & -2.086048 \\
\hline C & 5.399116 & -0.298037 & -0.508115 \\
\hline C & 4.883651 & -1.922033 & -2.711230 \\
\hline $\mathrm{H}$ & 2.828230 & -1.349487 & -2.465172 \\
\hline C & 6.443821 & -0.976134 & -1.132876 \\
\hline $\mathrm{H}$ & 5.594703 & 0.316916 & 0.364725 \\
\hline C & 6.189714 & -1.789363 & -2.237095 \\
\hline $\mathrm{H}$ & 7.002763 & -2.319434 & -2.723277 \\
\hline $\mathrm{H}$ & -6.460238 & -2.214662 & -2.584688 \\
\hline $\mathrm{H}$ & -5.893882 & 2.043010 & -2.752681 \\
\hline $\mathrm{H}$ & 4.677047 & -2.551440 & -3.571156 \\
\hline $\mathrm{H}$ & 7.455252 & -0.875414 & -0.751262 \\
\hline
\end{tabular}

\section{Simple BHCA 27}

\section{Datum}

Value

B3LYP-D3(BJ)/6-31G(d,p) Energy

$-1535.982153$

B3LYP-D3(BJ)/def2-TZVPP/IEF-PCM(DCM) Energy

$-1536.533728$

B3LYP-D3(BJ)/def2-TZVPP/IEF-PCM(DCM)//B3LYP-D3(BJ)/6-31G(d,p) Free Energy (Quasiharmonic)

$-1536.089001$

Number of Imaginary Frequencies

Frequencies (Top 3 out of 177)
1. $18.0981 \mathrm{~cm}^{-1}$
2. $20.3378 \mathrm{~cm}^{-1}$
3. $29.3191 \mathrm{~cm}^{-1}$

\section{B3LYP-D3(BJ)/6-31G(d,p) Molecular Geometry in Cartesian Coordinates}

$\begin{array}{llll}\mathrm{C} & -1.497561 & -0.235110 & -1.789793 \\ \mathrm{H} & -0.469758 & -0.575541 & -1.947266\end{array}$




\begin{tabular}{|c|c|c|c|}
\hline H & -2.171403 & -0.998887 & -2.193020 \\
\hline C & 1.295694 & -1.656998 & 0.383083 \\
\hline 0 & 0.514642 & -2.385945 & -0.181129 \\
\hline 0 & 1.914867 & -2.062606 & 1.516419 \\
\hline $\mathrm{H}$ & 2.501319 & -1.358981 & 1.830858 \\
\hline 0 & -1.719990 & 1.033973 & -2.425397 \\
\hline H & -1.464283 & 0.942452 & -3.352654 \\
\hline C & 0.642534 & 0.690937 & -0.086218 \\
\hline C & 1.642616 & -0.270067 & -0.093091 \\
\hline C & 2.969431 & 0.036848 & -0.532734 \\
\hline C & 3.243536 & 1.325713 & -0.946798 \\
\hline $\mathrm{H}$ & 4.240281 & 1.567058 & -1.303894 \\
\hline C & -1.739926 & -0.066955 & -0.311786 \\
\hline C & -0.699660 & 0.371009 & 0.492910 \\
\hline C & -3.171565 & -0.259795 & 1.637565 \\
\hline C & -3.013181 & -0.369044 & 0.268702 \\
\hline $\mathrm{H}$ & -4.137974 & -0.483158 & 2.079457 \\
\hline C & -2.124425 & 0.168179 & 2.483879 \\
\hline C & -0.861899 & 0.506203 & 1.905410 \\
\hline C & 0.178657 & 0.958768 & 2.762071 \\
\hline C & -0.018557 & 1.059004 & 4.119945 \\
\hline C & -1.266895 & 0.714762 & 4.691832 \\
\hline C & -2.295912 & 0.283011 & 3.889168 \\
\hline $\mathrm{H}$ & 1.132663 & 1.238187 & 2.329123 \\
\hline $\mathrm{H}$ & 0.786125 & 1.409095 & 4.759239 \\
\hline $\mathrm{H}$ & -1.408567 & 0.796967 & 5.764939 \\
\hline $\mathrm{H}$ & -3.259313 & 0.021142 & 4.317484 \\
\hline C & 2.253664 & 2.336265 & -0.956947 \\
\hline C & 0.927044 & 2.019174 & -0.525360 \\
\hline C & -0.060160 & 3.039904 & -0.536993 \\
\hline C & 0.253638 & 4.311757 & -0.955267 \\
\hline C & 1.566289 & 4.625796 & -1.383414 \\
\hline C & 2.543189 & 3.658568 & -1.387140 \\
\hline $\mathrm{H}$ & -1.068412 & 2.792751 & -0.230443 \\
\hline $\mathrm{H}$ & -0.511637 & 5.081373 & -0.964073 \\
\hline $\mathrm{H}$ & 1.797695 & 5.634444 & -1.711744 \\
\hline $\mathrm{H}$ & 3.552009 & 3.890101 & -1.716862 \\
\hline C & -6.446677 & -1.621209 & -1.994890 \\
\hline C & -5.709335 & -0.509681 & -2.404961 \\
\hline C & -4.583982 & -0.104924 & -1.689366 \\
\hline C & -4.176095 & -0.810864 & -0.545772 \\
\hline C & -4.923377 & -1.929329 & -0.144817 \\
\hline C & -6.047963 & -2.331653 & -0.862308 \\
\hline $\mathrm{H}$ & -7.322613 & -1.933568 & -2.555356 \\
\hline $\mathrm{H}$ & -4.009250 & 0.756343 & -2.012544 \\
\hline $\mathrm{H}$ & -4.602616 & -2.494700 & 0.724591 \\
\hline C & 4.042646 & -0.990434 & -0.554228 \\
\hline C & 3.825721 & -2.261605 & -1.108993 \\
\hline C & 5.312116 & -0.689685 & -0.036368 \\
\hline C & 4.850923 & -3.203690 & -1.140576 \\
\hline $\mathrm{H}$ & 2.853716 & -2.509104 & -1.521356 \\
\hline C & 6.337078 & -1.633078 & -0.070537 \\
\hline $\mathrm{H}$ & 5.485016 & 0.285431 & 0.408931 \\
\hline C & 6.109190 & -2.893888 & -0.622699 \\
\hline
\end{tabular}




$\begin{array}{rrrr}H & 6.905724 & -3.630976 & -0.646844 \\ \mathrm{H} & -6.608143 & -3.204522 & -0.540623 \\ \mathrm{H} & -6.014509 & 0.050206 & -3.284183 \\ \mathrm{H} & 4.666137 & -4.181101 & -1.575009 \\ \mathrm{H} & 7.310872 & -1.385726 & 0.341044\end{array}$

\section{Simple BHCA 28}

\section{Datum}

Value

B3LYP-D3(BJ)/6-31G(d,p) Energy $-1535.979261$

B3LYP-D3(BJ)/def2-TZVPP/IEF-PCM(DCM) Energy $-1536.533324$

B3LYP-D3(BJ)/def2-TZVPP/IEF-PCM(DCM)//B3LYP-D3(BJ)/6-31G(d,p) Free Energy (Quasiharmonic) $-1536.088606$

Number of Imaginary Frequencies

Frequencies (Top 3 out of 177)

$\begin{array}{ll}\text { 1. } & 18.4822 \mathrm{~cm}^{-1} \\ \text { 2. } & 25.9434 \mathrm{~cm}^{-1} \\ \text { 3. } & 31.7294 \mathrm{~cm}^{-1}\end{array}$

B3LYP-D3(BJ)/6-31G(d,p) Molecular Geometry in Cartesian Coordinates

$\begin{array}{lrrr}\mathrm{C} & -1.618832 & 0.964543 & -1.486650 \\ \mathrm{H} & -0.596373 & 1.321947 & -1.605468 \\ \mathrm{H} & -1.883416 & 0.413852 & -2.399101 \\ \mathrm{C} & 1.647136 & -1.687559 & -0.162900 \\ \mathrm{O} & 2.439178 & -2.410489 & 0.388507 \\ \mathrm{O} & 0.632292 & -2.215361 & -0.893114 \\ \mathrm{H} & 0.013875 & -1.512305 & -1.144591 \\ \mathrm{O} & -2.428478 & 2.127966 & -1.348943 \\ \mathrm{H} & -3.351432 & 1.839052 & -1.391722 \\ \mathrm{C} & 0.655140 & 0.568598 & 0.351704 \\ \mathrm{C} & 1.736512 & -0.181960 & -0.092887 \\ \mathrm{C} & 3.007143 & 0.433280 & -0.344010 \\ \mathrm{C} & 3.147605 & 1.781738 & -0.092812 \\ \mathrm{H} & 4.095782 & 2.263842 & -0.310642 \\ \mathrm{C} & -1.722209 & 0.057892 & -0.270407 \\ \mathrm{C} & -0.665884 & -0.084522 & 0.621602 \\ \mathrm{C} & -3.131513 & -1.314304 & 1.162260 \\ \mathrm{C} & -2.974598 & -0.581815 & 0.008083 \\ \mathrm{H} & -4.084532 & -1.795281 & 1.363683 \\ \mathrm{C} & -2.075184 & -1.472481 & 2.092669 \\ \mathrm{C} & -0.816073 & -0.852967 & 1.822338\end{array}$




\begin{tabular}{|c|c|c|c|}
\hline C & 0.233454 & -1.009791 & 2.766029 \\
\hline C & 0.044473 & -1.748703 & 3.911782 \\
\hline C & -1.200056 & -2.366183 & 4.173194 \\
\hline C & -2.237983 & -2.229187 & 3.280969 \\
\hline $\mathrm{H}$ & 1.193457 & -0.547568 & 2.572344 \\
\hline $\mathrm{H}$ & 0.859881 & -1.862241 & 4.618767 \\
\hline $\mathrm{H}$ & -1.332478 & -2.949387 & 5.079045 \\
\hline $\mathrm{H}$ & -3.198601 & -2.699203 & 3.471264 \\
\hline C & 2.083918 & 2.572313 & 0.403985 \\
\hline C & 0.807194 & 1.966382 & 0.619075 \\
\hline C & -0.260158 & 2.772270 & 1.095803 \\
\hline C & -0.068616 & 4.111442 & 1.348348 \\
\hline C & 1.195297 & 4.709807 & 1.137510 \\
\hline C & 2.247391 & 3.956047 & 0.671757 \\
\hline $\mathrm{H}$ & -1.236377 & 2.326181 & 1.235308 \\
\hline H & -0.896121 & 4.715707 & 1.705889 \\
\hline $\mathrm{H}$ & 1.330692 & 5.767653 & 1.340311 \\
\hline $\mathrm{H}$ & 3.220478 & 4.407491 & 0.501161 \\
\hline C & -6.320992 & -0.172835 & -2.664085 \\
\hline C & -6.352011 & 0.420468 & -1.402326 \\
\hline C & -5.263819 & 0.285919 & -0.538643 \\
\hline C & -4.128380 & -0.441230 & -0.926742 \\
\hline C & -4.112365 & -1.038799 & -2.195869 \\
\hline C & -5.199597 & -0.905617 & -3.057199 \\
\hline $\mathrm{H}$ & -7.165983 & -0.067320 & -3.337087 \\
\hline $\mathrm{H}$ & -5.282097 & 0.748347 & 0.443550 \\
\hline $\mathrm{H}$ & -3.248394 & -1.623478 & -2.496620 \\
\hline C & 4.146224 & -0.317810 & -0.927982 \\
\hline C & 3.967289 & -1.147109 & -2.044687 \\
\hline C & 5.437940 & -0.161481 & -0.407538 \\
\hline C & 5.049721 & -1.803753 & -2.622560 \\
\hline H & 2.974284 & -1.273317 & -2.464969 \\
\hline C & 6.522035 & -0.819127 & -0.985327 \\
\hline $\mathrm{H}$ & 5.583858 & 0.460595 & 0.469943 \\
\hline C & 6.331913 & -1.641777 & -2.095453 \\
\hline $\mathrm{H}$ & 7.175582 & -2.156340 & -2.544842 \\
\hline $\mathrm{H}$ & -5.173731 & -1.378585 & -4.033978 \\
\hline $\mathrm{H}$ & -7.221607 & 0.989568 & -1.088516 \\
\hline $\mathrm{H}$ & 4.892333 & -2.440898 & -3.487319 \\
\hline $\mathrm{H}$ & 7.514298 & -0.695368 & -0.562213 \\
\hline
\end{tabular}

\section{Simple BHCA 29}

Datum

Value

\begin{tabular}{lr}
\hline B3LYP-D3(BJ)/6-31G(d,p) Energy & -1535.979797 \\
\hline B3LYP-D3(BJ)/def2-TZVPP/IEF-PCM(DCM) Energy & -1536.532717 \\
\hline B3LYP-D3(BJ)/def2-TZVPP/IEF-PCM(DCM)//B3LYP-D3(BJ)/6-31G(d,p) Free Energy (Quasiharmonic) & -1536.087898 \\
\hline
\end{tabular}


Frequencies (Top 3 out of 177)

$\begin{array}{ll}\text { 1. } & 19.0506 \mathrm{~cm}^{-1} \\ \text { 2. } & 25.7614 \mathrm{~cm}^{-1} \\ \text { 3. } & 27.4193 \mathrm{~cm}^{-1}\end{array}$

B3LYP-D3(BJ)/6-31G(d,p) Molecular Geometry in Cartesian Coordinates

\begin{tabular}{|c|c|c|c|}
\hline C & 1. 312792 & -0.919176 & -1.534736 \\
\hline $\mathrm{H}$ & 1.946332 & -1.803189 & -1.665937 \\
\hline $\mathrm{H}$ & 0.270304 & -1.254844 & -1.497108 \\
\hline C & -1.316817 & -1.395941 & 1.014117 \\
\hline 0 & -0.866327 & -1.314341 & 2.129300 \\
\hline 0 & -1.498868 & -2.614955 & 0.437995 \\
\hline $\mathrm{H}$ & -1.953128 & -2.507190 & -0.410860 \\
\hline 0 & 1.497026 & 0.010018 & -2.612569 \\
\hline $\mathrm{H}$ & 1.250172 & -0.439950 & -3.431593 \\
\hline C & -0.664918 & 0.688591 & -0.166201 \\
\hline C & -1.669109 & -0.217875 & 0.142644 \\
\hline C & -2.987562 & -0.078132 & -0.398918 \\
\hline C & -3.248123 & 0.981691 & -1.244114 \\
\hline $\mathrm{H}$ & -4.254372 & 1.118861 & -1.628767 \\
\hline C & 1.669327 & -0.219911 & -0.247293 \\
\hline C & 0.710935 & 0.542043 & 0.405155 \\
\hline C & 3.283603 & 0.323502 & 1.481156 \\
\hline C & 2.991655 & -0.322761 & 0.296158 \\
\hline $\mathrm{H}$ & 4.288138 & 0.256359 & 1.888185 \\
\hline C & 2.326374 & 1.105913 & 2.166696 \\
\hline C & 1.012188 & 1.225328 & 1.621659 \\
\hline C & 0.056241 & 2.015431 & 2.312342 \\
\hline C & 0.389358 & 2.660393 & 3.480562 \\
\hline C & 1.693608 & 2.545016 & 4.018330 \\
\hline C & 2.639661 & 1.782910 & 3.374928 \\
\hline $\mathrm{H}$ & -0.946260 & 2.098855 & 1.909594 \\
\hline $\mathrm{H}$ & -0.353302 & 3.259642 & 3.998059 \\
\hline $\mathrm{H}$ & 1.942343 & 3.058498 & 4.941983 \\
\hline $\mathrm{H}$ & 3.642700 & 1.685395 & 3.780636 \\
\hline C & -2.250463 & 1.921000 & -1.594828 \\
\hline C & -0.934497 & 1.774874 & -1.054264 \\
\hline C & 0.062669 & 2.718337 & -1.417900 \\
\hline C & -0.234847 & 3.761996 & -2.262410 \\
\hline C & -1.540298 & 3.912039 & -2.789319 \\
\hline C & -2.525307 & 3.009857 & -2.464060 \\
\hline $\mathrm{H}$ & 1.064591 & 2.594575 & -1.027150 \\
\hline $\mathrm{H}$ & 0.537348 & 4.475646 & -2.531476 \\
\hline
\end{tabular}




\begin{tabular}{|lrrr|}
$H$ & -1.759727 & 4.741675 & -3.454323 \\
$H$ & -3.529021 & 3.115280 & -2.865907 \\
C & 6.187341 & -2.537332 & -1.541024 \\
C & 5.446976 & -1.627345 & -2.296833 \\
C & 4.397747 & -0.916122 & -1.717289 \\
C & 4.069515 & -1.106459 & -0.364950 \\
C & 4.820096 & -2.025614 & 0.384658 \\
C & 5.868944 & -2.735026 & -0.197178 \\
$H$ & 7.004167 & -3.089401 & -1.995946 \\
$H$ & 3.822195 & -0.209704 & -2.305724 \\
$H$ & 4.561849 & -2.192472 & 1.425775 \\
C & -4.077359 & -1.025951 & -0.048930 \\
C & -4.317097 & -1.398062 & 1.283736 \\
C & -4.905179 & -1.550477 & -1.053908 \\
C & -5.354937 & -2.271090 & 1.598514 \\
$H$ & -3.691126 & -0.996505 & 2.073692 \\
C & -5.944626 & -2.423211 & -0.736502 \\
$H$ & -4.718301 & -1.279880 & -2.088783 \\
C & -6.172251 & -2.786253 & 0.590995 \\
$H$ & -6.979317 & -3.468413 & 0.839109 \\
$H$ & 6.433012 & -3.446927 & 0.397997 \\
$H$ & 5.691707 & -1.463957 & -3.342381 \\
$H$ & -5.527469 & -2.546523 & 2.634104 \\
$H$ & -6.571330 & -2.824695 & -1.526957 \\
& & & \\
\hline
\end{tabular}

\section{Simple BHCA 30}

\section{Datum}

Value

B3LYP-D3(BJ)/6-31G(d,p) Energy

$-1535.979384$

B3LYP-D3(BJ)/def2-TZVPP/IEF-PCM(DCM) Energy

$-1536.531809$

B3LYP-D3(BJ)/def2-TZVPP/IEF-PCM(DCM)//B3LYP-D3(BJ)/6-31G(d,p) Free Energy (Quasiharmonic)

$-1536.086886$

Number of Imaginary Frequencies

Frequencies (Top 3 out of 177)
1. $\quad 19.8159 \mathrm{~cm}^{-1}$
2. $\quad 25.0631 \mathrm{~cm}^{-1}$
3. $\quad 31.4777 \mathrm{~cm}^{-1}$

B3LYP-D3(BJ)/6-31G(d,p) Molecular Geometry in Cartesian Coordinates

$\begin{array}{llll}C & -1.477627 & 0.754473 & -1.636139 \\ H & -0.419334 & 0.652447 & -1.904209\end{array}$




\begin{tabular}{|c|c|c|c|}
\hline $\mathrm{H}$ & -2.076737 & 0.231113 & -2.391332 \\
\hline C & 1.521099 & -1.724675 & 0.164472 \\
\hline 0 & 2.312281 & -2.379546 & 0.796957 \\
\hline 0 & 0.448722 & -2.321108 & -0.411817 \\
\hline $\mathrm{H}$ & -0.169384 & -1.648823 & -0.738908 \\
\hline 0 & -1.838411 & 2.139668 & -1.575277 \\
\hline $\mathrm{H}$ & -1.546381 & 2.557502 & -2.396126 \\
\hline C & 0.666360 & 0.645621 & 0.306820 \\
\hline C & 1.687679 & -0.235675 & -0.030375 \\
\hline C & 2.980080 & 0.251844 & -0.415443 \\
\hline C & 3.207498 & 1.611846 & -0.390083 \\
\hline $\mathrm{H}$ & 4.171814 & 1.992312 & -0.713114 \\
\hline C & -1.719384 & 0.142115 & -0.276395 \\
\hline C & -0.692258 & 0.126257 & 0.665021 \\
\hline C & -3.187008 & -0.970073 & 1.304414 \\
\hline C & -2.998557 & -0.419463 & 0.051507 \\
\hline $\mathrm{H}$ & -4.156228 & -1.389527 & 1.556604 \\
\hline C & -2.169795 & -0.982482 & 2.285809 \\
\hline C & -0.893313 & -0.425210 & 1.967727 \\
\hline C & 0.125738 & -0.441528 & 2.957271 \\
\hline C & -0.111495 & -0.985163 & 4.198464 \\
\hline C & -1.375905 & -1.537612 & 4.512098 \\
\hline C & -2.382760 & -1.535031 & 3.575865 \\
\hline $\mathrm{H}$ & 1.097865 & -0.025337 & 2.721941 \\
\hline $\mathrm{H}$ & 0.677987 & -0.993650 & 4.942927 \\
\hline $\mathrm{H}$ & -1.546822 & -1.965048 & 5.495151 \\
\hline $\mathrm{H}$ & -3.356403 & -1.957337 & 3.807023 \\
\hline C & 2.216298 & 2.535447 & 0.018436 \\
\hline C & 0.915511 & 2.052731 & 0.362093 \\
\hline C & -0.072643 & 2.985188 & 0.774819 \\
\hline C & 0.214581 & 4.329160 & 0.838520 \\
\hline C & 1.498320 & 4.807692 & 0.484781 \\
\hline C & 2.476374 & 3.929442 & 0.080178 \\
\hline $\mathrm{H}$ & -1.060590 & 2.624384 & 1.029079 \\
\hline $\mathrm{H}$ & -0.550607 & 5.029480 & 1.157912 \\
\hline $\mathrm{H}$ & 1.708974 & 5.871528 & 0.536014 \\
\hline $\mathrm{H}$ & 3.465736 & 4.286804 & -0.190607 \\
\hline C & -6.325409 & -0.627697 & -2.665288 \\
\hline C & -5.668371 & 0.586910 & -2.465761 \\
\hline C & -4.578357 & 0.668046 & -1.600701 \\
\hline C & -4.125824 & -0.472837 & -0.918661 \\
\hline C & -4.794008 & -1.690028 & -1.128292 \\
\hline C & -5.883037 & -1.767801 & -1.993776 \\
\hline $\mathrm{H}$ & -7.173908 & -0.686092 & -3.340120 \\
\hline $\mathrm{H}$ & -4.067969 & 1.612305 & -1.449246 \\
\hline $\mathrm{H}$ & -4.440671 & -2.581248 & -0.618824 \\
\hline C & 4.049696 & -0.651258 & -0.908685 \\
\hline C & 3.776434 & -1.634679 & -1.870532 \\
\hline C & 5.369716 & -0.493665 & -0.465585 \\
\hline C & 4.794539 & -2.439686 & -2.372584 \\
\hline $\mathrm{H}$ & 2.760059 & -1.765743 & -2.229092 \\
\hline C & 6.389524 & -1.299768 & -0.967456 \\
\hline $\mathrm{H}$ & 5.588106 & 0.249194 & 0.295120 \\
\hline C & 6.105774 & -2.274818 & -1.923482 \\
\hline
\end{tabular}




$\begin{array}{rrrr}H & 6.899251 & -2.904900 & -2.313212 \\ \mathrm{H} & -6.381597 & -2.720003 & -2.147781 \\ \mathrm{H} & -6.009762 & 1.479910 & -2.980982 \\ \mathrm{H} & 4.564304 & -3.195481 & -3.117104 \\ \mathrm{H} & 7.404746 & -1.171633 & -0.604372\end{array}$

\section{Simple BHCA 31}

\section{Datum}

Value

B3LYP-D3(BJ)/6-31G(d,p) Energy $-1535.976596$

B3LYP-D3(BJ)/def2-TZVPP/IEF-PCM(DCM) Energy $-1536.529778$

B3LYP-D3(BJ)/def2-TZVPP/IEF-PCM(DCM)//B3LYP-D3(BJ)/6-31G(d,p) Free Energy (Quasiharmonic) $-1536.085122$

Number of Imaginary Frequencies

Frequencies (Top 3 out of 177)

$\begin{array}{lr}\text { 1. } & 7.8642 \mathrm{~cm}^{-1} \\ \text { 2. } & 23.7021 \mathrm{~cm}^{-1} \\ \text { 3. } & 31.4614 \mathrm{~cm}^{-1}\end{array}$

B3LYP-D3(BJ)/6-31G(d,p) Molecular Geometry in Cartesian Coordinates

$\begin{array}{lrrr}\mathrm{C} & -1.236089 & -0.983970 & -1.518384 \\ \mathrm{H} & -2.119905 & -1.258246 & -2.105572 \\ \mathrm{H} & -0.571792 & -0.400694 & -2.167512 \\ \mathrm{C} & 1.323867 & -0.736239 & 1.536801 \\ \mathrm{O} & 0.301877 & -1.320580 & 1.790757 \\ \mathrm{O} & 2.287114 & -0.623203 & 2.492006 \\ \mathrm{H} & 3.073290 & -0.190039 & 2.128553 \\ \mathrm{O} & -0.557622 & -2.156669 & -1.047610 \\ \mathrm{H} & -0.362878 & -2.696598 & -1.825458 \\ \mathrm{C} & 0.638994 & 0.794175 & -0.307821 \\ \mathrm{C} & 1.623142 & -0.015700 & 0.241057 \\ \mathrm{C} & 2.924517 & -0.083808 & -0.358236 \\ \mathrm{C} & 3.221594 & 0.742224 & -1.422054 \\ \mathrm{H} & 4.194417 & 0.666557 & -1.898767 \\ \mathrm{C} & -1.664264 & -0.130437 & -0.347553 \\ \mathrm{C} & -0.760618 & 0.759735 & 0.213363 \\ \mathrm{C} & -3.372241 & 0.638983 & 1.196222 \\ \mathrm{C} & -2.992569 & -0.214944 & 0.177551 \\ \mathrm{H} & -4.376044 & 0.565502 & 1.603842 \\ \mathrm{C} & -2.480422 & 1.579935 & 1.758300 \\ \mathrm{C} & -1.139593 & 1.639027 & 1.267753\end{array}$




$\begin{array}{lrrr}\text { C } & -0.240544 & 2.574887 & 1.845372 \\ \text { C } & -0.650303 & 3.412322 & 2.856929 \\ \text { C } & -1.978615 & 3.352784 & 3.343222 \\ \text { C } & -2.871328 & 2.455904 & 2.805488 \\ \text { H } & 0.778093 & 2.621314 & 1.476133 \\ \text { H } & 0.048108 & 4.122512 & 3.288635 \\ \text { H } & -2.288153 & 4.016820 & 4.144365 \\ \text { H } & -3.891176 & 2.400740 & 3.175784 \\ \text { C } & 2.273342 & 1.652299 & -1.945736 \\ \text { C } & 0.953827 & 1.667431 & -1.395675 \\ \text { C } & -0.005833 & 2.557264 & -1.948639 \\ \text { C } & 0.327933 & 3.392425 & -2.990260 \\ \text { C } & 1.634198 & 3.374862 & -3.534518 \\ \text { C } & 2.584271 & 2.521118 & -3.024187 \\ \text { H } & -1.005683 & 2.569865 & -1.530885 \\ \text { H } & -0.414744 & 4.070343 & -3.398913 \\ \text { H } & 1.883044 & 4.037538 & -4.357539 \\ \text { H } & 3.588082 & 2.498339 & -3.438753 \\ \text { C } & -5.901835 & -3.072558 & -1.179284 \\ \text { C } & -6.236116 & -1.722081 & -1.074895 \\ \text { C } & -5.283536 & -0.797338 & -0.651041 \\ \text { C } & -3.980868 & -1.204845 & -0.325382 \\ \text { C } & -3.654227 & -2.566764 & -0.433877 \\ \text { C } & -4.608898 & -3.489029 & -0.858273 \\ \text { H } & -6.642923 & -3.794549 & -1.508749 \\ \text { H } & -5.540300 & 0.255525 & -0.583457 \\ \text { H } & -2.649279 & -2.888768 & -0.182350 \\ \text { C } & 3.920065 & -1.094208 & 0.083526 \\ \text { C } & 3.529482 & -2.431112 & 0.268066 \\ \text { C } & 5.262706 & -0.744706 & 0.289932 \\ \text { C } & 4.461520 & -3.390366 & 0.651709 \\ \text { H } & 2.492672 & -2.707514 & 0.100937 \\ \text { C } & 6.194265 & -1.708431 & 0.674969 \\ \text { H } & 5.568734 & 0.289296 & 0.161139 \\ \text { C } & 5.795993 & -3.032653 & 0.856734 \\ \text { H } & 6.520243 & -3.782245 & 1.159780 \\ \text { H } & -4.343447 & -4.539751 & -0.930194 \\ \text { H } & -7.237490 & -1.386895 & -1.328428 \\ & 4.146598 & -4.419870 & 0.789828 \\ \text { H } & 7.228672 & -1.422687 & 0.839279\end{array}$

\section{Catalyst 2A 1}

Datum

Value

B3LYP-D3(BJ)/6-31G(d,p) Energy $-1998.173911$

B3LYP-D3(BJ)/def2-TZVPP/IEF-PCM(DCM) Energy $-1998.880215$

B3LYP-D3(BJ)/def2-TZVPP/IEF-PCM(DCM)//B3LYP-D3(BJ)/6-31G(d,p) Free Energy (Quasiharmonic) -1998.280528 
Frequencies (Top 3 out of 237)

$\begin{array}{ll}\text { 1. } & 10.0895 \mathrm{~cm}^{-1} \\ \text { 2. } & 15.2785 \mathrm{~cm}^{-1} \\ \text { 3. } & 22.5520 \mathrm{~cm}^{-1}\end{array}$

B3LYP-D3(BJ)/6-31G(d,p) Molecular Geometry in Cartesian Coordinates

\begin{tabular}{|c|c|c|c|}
\hline C & -1.593547 & -0.103763 & -1.210580 \\
\hline $\mathrm{H}$ & -2.575542 & 0.154591 & -1.614213 \\
\hline $\mathrm{H}$ & -1.027689 & -0.645609 & -1.972960 \\
\hline C & 1.640528 & -0.180781 & 1.190577 \\
\hline 0 & 2.370245 & -0.479409 & 2.107205 \\
\hline 0 & 0.784896 & 0.848015 & 1.274237 \\
\hline $\mathrm{H}$ & 0.236841 & 0.930248 & 0.458716 \\
\hline 0 & -0.884459 & 1.112603 & -0.860881 \\
\hline $\mathrm{H}$ & -0.615340 & 1.558859 & -1.674741 \\
\hline C & 0.584346 & -1.777238 & -0.420082 \\
\hline C & 1.658409 & -0.961420 & -0.106494 \\
\hline C & 2.853259 & -0.976633 & -0.896824 \\
\hline C & 2.915731 & -1.829302 & -1.981022 \\
\hline $\mathrm{H}$ & 3.799371 & -1.821626 & -2.612006 \\
\hline C & -1.728997 & -0.946493 & 0.030044 \\
\hline C & -0.660705 & -1.749285 & 0.410334 \\
\hline C & -3.017720 & -1.744606 & 1.920511 \\
\hline C & -2.926331 & -0.920974 & 0.814201 \\
\hline $\mathrm{H}$ & -3.921214 & -1.724635 & 2.522545 \\
\hline C & -1.951888 & -2.579039 & 2.327397 \\
\hline C & -0.738758 & -2.575219 & 1.570422 \\
\hline C & 0.340253 & -3.393846 & 1.998784 \\
\hline C & 0.216672 & -4.184828 & 3.116808 \\
\hline C & -0.987645 & -4.195690 & 3.861177 \\
\hline C & -2.046569 & -3.408072 & 3.476410 \\
\hline $\mathrm{H}$ & 1.270018 & -3.372254 & 1.443158 \\
\hline $\mathrm{H}$ & 1.051340 & -4.799685 & 3.437740 \\
\hline $\mathrm{H}$ & -1.068290 & -4.823825 & 4.742901 \\
\hline $\mathrm{H}$ & -2.970605 & -3.403290 & 4.047428 \\
\hline C & 1.852692 & -2.701472 & -2.316168 \\
\hline C & 0.662342 & -2.682426 & -1.525086 \\
\hline C & -0.399538 & -3.561579 & -1.865993 \\
\hline C & -0.290282 & -4.411639 & -2.943541 \\
\hline C & 0.884588 & -4.423756 & -3.731740 \\
\hline C & 1.931526 & -3.586319 & -3.423474 \\
\hline $\mathrm{H}$ & -1.298565 & -3.556577 & -1.260259 \\
\hline $\mathrm{H}$ & -1.109508 & -5.079719 & -3.190259 \\
\hline
\end{tabular}




\begin{tabular}{|c|c|c|c|}
\hline $\mathrm{H}$ & 0.957439 & -5.098445 & -4.579008 \\
\hline $\mathrm{H}$ & 2.838331 & -3.591018 & -4.021629 \\
\hline C & -6.310601 & 1.622454 & -0.059493 \\
\hline C & -6.470658 & 0.246297 & 0.164219 \\
\hline C & -5.375530 & -0.568652 & 0.429233 \\
\hline C & -4.076318 & -0.041508 & 0.481649 \\
\hline C & -3.913252 & 1.335751 & 0.261404 \\
\hline C & -5.010094 & 2.147970 & -0.005585 \\
\hline $\mathrm{H}$ & -5.521962 & -1.631890 & 0.592878 \\
\hline $\mathrm{H}$ & -2.918714 & 1.767018 & 0.298817 \\
\hline C & 3.996104 & -0.078906 & -0.605006 \\
\hline C & 5.312384 & -0.554720 & -0.696434 \\
\hline C & 3.805547 & 1.271448 & -0.276591 \\
\hline C & 6.395695 & 0.285675 & -0.468545 \\
\hline H & 5.483159 & -1.604542 & -0.912477 \\
\hline C & 4.888733 & 2.110910 & -0.049064 \\
\hline H & 2.800653 & 1.673118 & -0.206013 \\
\hline C & 6.206636 & 1.637094 & -0.140880 \\
\hline $\mathrm{H}$ & -7.466844 & -0.184235 & 0.149853 \\
\hline H & -4.855657 & 3.206017 & -0.192187 \\
\hline $\mathrm{H}$ & 4.712735 & 3.157609 & 0.176558 \\
\hline H & 7.402454 & -0.117747 & -0.507184 \\
\hline C & -7.475430 & 2.491456 & -0.344089 \\
\hline C & -7.537920 & 3.799922 & 0.161207 \\
\hline C & -8.545638 & 2.028325 & -1.126000 \\
\hline C & -8.633073 & 4.617790 & -0.106995 \\
\hline $\mathrm{H}$ & -6.733239 & 4.166061 & 0.790852 \\
\hline C & -9.641677 & 2.845398 & -1.393117 \\
\hline $\mathrm{H}$ & -8.503063 & 1.029053 & -1.547692 \\
\hline C & -9.690157 & 4.144177 & -0.885169 \\
\hline H & -8.665336 & 5.623693 & 0.300625 \\
\hline $\mathrm{H}$ & -10.455248 & 2.470197 & -2.006654 \\
\hline $\mathrm{H}$ & -10.544023 & 4.781291 & -1.093561 \\
\hline C & 7.359361 & 2.532655 & 0.106060 \\
\hline C & 8.538872 & 2.412579 & -0.645901 \\
\hline C & 7.300705 & 3.523643 & 1.098940 \\
\hline C & 9.623664 & 3.254779 & -0.413169 \\
\hline $\mathrm{H}$ & 8.592893 & 1.669169 & -1.435007 \\
\hline C & 8.384714 & 4.367100 & 1.330682 \\
\hline $\mathrm{H}$ & 6.408252 & 3.611463 & 1.710258 \\
\hline C & 9.551175 & 4.236506 & 0.575887 \\
\hline $\mathrm{H}$ & 10.523970 & 3.150066 & -1.011258 \\
\hline $\mathrm{H}$ & 8.321935 & 5.121165 & 2.109483 \\
\hline H & 10.396345 & 4.893246 & 0.757383 \\
\hline
\end{tabular}

\section{Catalyst 2A 2}


Frequencies (Top 3 out of 237)

$\begin{array}{ll}\text { 1. } & 10.1232 \mathrm{~cm}^{-1} \\ \text { 2. } & 15.4821 \mathrm{~cm}^{-1} \\ \text { 3. } & 22.7832 \mathrm{~cm}^{-1}\end{array}$

B3LYP-D3(BJ)/6-31G(d,p) Molecular Geometry in Cartesian Coordinates

\begin{tabular}{|c|c|c|c|}
\hline C & 1.586571 & -0.068278 & 1.219360 \\
\hline $\mathrm{H}$ & 1.017161 & -0.589144 & 1.993591 \\
\hline $\mathrm{H}$ & 2.566483 & 0.201600 & 1.620445 \\
\hline C & -1.637339 & -0.213778 & -1.192326 \\
\hline 0 & -2.363999 & -0.537920 & -2.102698 \\
\hline 0 & -0.781093 & 0.812037 & -1.301932 \\
\hline $\mathrm{H}$ & -0.235865 & 0.917124 & -0.487130 \\
\hline & 0.878680 & 1.137734 & 0.832876 \\
\hline & 0.599820 & 1.602534 & 1.632945 \\
\hline$c$ & -0.586725 & -1.764018 & 0.466276 \\
\hline$\zeta$ & -1.659916 & -0.957797 & 0.126015 \\
\hline C & -2.857734 & -0.951563 & 0.912006 \\
\hline r & -2.923984 & -1.773909 & 2.019174 \\
\hline $\mathrm{H}$ & -3.809939 & -1.749063 & 2.646452 \\
\hline & 1.728184 & -0.944927 & 0.003189 \\
\hline$C$ & 0.661714 & -1.758278 & -0.359478 \\
\hline C & 3.025147 & -1.794598 & -1.858858 \\
\hline C & 2.929084 & -0.940886 & -0.775951 \\
\hline $\mathrm{H}$ & 3.931318 & -1.790963 & -2.457170 \\
\hline C & 1.961247 & -2.640089 & -2.247399 \\
\hline C & 0.744892 & -2.615834 & -1.496016 \\
\hline C & -0.332128 & -3.446218 & -1.906389 \\
\hline C & -0.203588 & -4.267522 & -3.001761 \\
\hline C & 1.003902 & -4.298445 & -3.740405 \\
\hline C & 2.061010 & -3.500256 & -3.372850 \\
\hline $\mathrm{H}$ & -1.264312 & -3.409673 & -1.355624 \\
\hline $\mathrm{H}$ & -1.036766 & -4.891183 & -3.309290 \\
\hline $\mathrm{H}$ & 1.088413 & -4.950483 & -4.604232 \\
\hline $\mathrm{H}$ & 2.987484 & -3.510832 & -3.939825 \\
\hline & -1.862002 & -2.636114 & 2.382295 \\
\hline & -0.668620 & -2.638359 & 1.595569 \\
\hline C & 0.392320 & -3.507145 & 1.964793 \\
\hline$C$ & 0.279201 & -4.327214 & 3.064948 \\
\hline & -0.898753 & -4.318225 & 3.848571 \\
\hline
\end{tabular}




\begin{tabular}{|c|c|c|c|}
\hline C & -1.944811 & -3.490124 & 3.513269 \\
\hline $\mathrm{H}$ & 1.293765 & -3.518354 & 1.362752 \\
\hline $\mathrm{H}$ & 1.097771 & -4.987737 & 3.333232 \\
\hline $\mathrm{H}$ & -0.974643 & -4.969349 & 4.713818 \\
\hline $\mathrm{H}$ & -2.853940 & -3.478785 & 4.107799 \\
\hline C & 6.312386 & 1.623173 & 0.039917 \\
\hline C & 6.472463 & 0.242022 & -0.151194 \\
\hline c & 5.377766 & -0.578717 & -0.399130 \\
\hline C & 4.078198 & -0.053362 & -0.462965 \\
\hline c & 3.914936 & 1.328518 & -0.273473 \\
\hline C & 5.011766 & 2.147174 & -0.026912 \\
\hline $\mathrm{H}$ & 5.522502 & -1.647828 & -0.520368 \\
\hline $\mathrm{H}$ & 2.922421 & 1.761215 & -0.337973 \\
\hline C & -3.999683 & -0.062584 & 0.591312 \\
\hline C & -5.316260 & -0.535647 & 0.692382 \\
\hline C & -3.808142 & 1.277874 & 0.225044 \\
\hline C & -6.398872 & 0.297848 & 0.437439 \\
\hline $\mathrm{H}$ & -5.487737 & -1.578895 & 0.937724 \\
\hline C & -4.890620 & 2.110414 & -0.029611 \\
\hline $\mathrm{H}$ & -2.803057 & 1.677486 & 0.145949 \\
\hline C & -6.208805 & 1.639348 & 0.071810 \\
\hline $\mathrm{H}$ & 7.462208 & -0.196227 & -0.071986 \\
\hline $\mathrm{H}$ & 4.863245 & 3.216977 & 0.080606 \\
\hline $\mathrm{H}$ & -4.713930 & 3.150221 & -0.284654 \\
\hline $\mathrm{H}$ & -7.405754 & -0.104360 & 0.484603 \\
\hline C & 7.477173 & 2.498389 & 0.304428 \\
\hline C & 8.710352 & 2.265954 & -0.325203 \\
\hline C & 7.377070 & 3.582247 & 1.191200 \\
\hline C & 9.806439 & 3.088447 & -0.075732 \\
\hline $\mathrm{H}$ & 8.799510 & 1.450066 & -1.035439 \\
\hline C & 8.472326 & 4.406187 & 1.439603 \\
\hline $\mathrm{H}$ & 6.439416 & 3.762190 & 1.707493 \\
\hline C & 9.692351 & 4.162638 & 0.807631 \\
\hline $\mathrm{H}$ & 10.748775 & 2.895999 & -0.579722 \\
\hline $\mathrm{H}$ & 8.375371 & 5.234641 & 2.134837 \\
\hline $\mathrm{H}$ & 10.546215 & 4.804352 & 1.001357 \\
\hline C & -7.360719 & 2.527401 & -0.204299 \\
\hline C & -8.542517 & 2.429083 & 0.547224 \\
\hline C & -7.298951 & 3.489281 & -1.225217 \\
\hline C & -9.626521 & 3.264212 & 0.286949 \\
\hline $\mathrm{H}$ & -8.599027 & 1.708757 & 1.357293 \\
\hline C & -8.382179 & 4.325677 & -1.484547 \\
\hline $\mathrm{H}$ & -6.404624 & 3.559398 & -1.836081 \\
\hline C & -9.550941 & 4.216939 & -0.729847 \\
\hline $\mathrm{H}$ & -10.528659 & 3.176818 & 0.885059 \\
\hline $\mathrm{H}$ & -8.316962 & 5.056913 & -2.284625 \\
\hline $\mathrm{H}$ & -10.395504 & 4.868146 & -0.932830 \\
\hline
\end{tabular}


Frequencies (Top 3 out of 237)

$\begin{array}{lr}\text { 1. } & 8.4980 \mathrm{~cm}^{-1} \\ \text { 2. } & 14.5750 \mathrm{~cm}^{-1} \\ \text { 3. } & 19.0052 \mathrm{~cm}^{-1}\end{array}$

\section{B3LYP-D3(BJ)/6-31G(d,p) Molecular Geometry in Cartesian Coordinates}

\begin{tabular}{|c|c|c|c|}
\hline C & -1.688285 & -0.345259 & -1.235690 \\
\hline $\mathrm{H}$ & -1.008372 & -0.789247 & -1.961490 \\
\hline $\mathrm{H}$ & -2.675428 & -0.254621 & -1.697420 \\
\hline C & 1.449571 & -0.219750 & 1.126295 \\
\hline 0 & 2.130476 & -0.450921 & 2.097928 \\
\hline 0 & 0.560217 & 0.785761 & 1.113216 \\
\hline $\mathrm{H}$ & 0.051352 & 0.824890 & 0.266524 \\
\hline 0 & -1.161532 & 0.977654 & -0.978944 \\
\hline $\mathrm{H}$ & -1.878111 & 1.489107 & -0.573358 \\
\hline C & 0.568109 & -1.966184 & -0.433953 \\
\hline C & 1.568596 & -1.057950 & -0.129593 \\
\hline C & 2.784692 & -1.020773 & -0.885199 \\
\hline C & 2.939793 & -1.910871 & -1.929410 \\
\hline $\mathrm{H}$ & 3.841563 & -1.868016 & -2.532697 \\
\hline C & -1.763877 & -1.197588 & 0.010640 \\
\hline $\mathrm{C}$ & -0.688552 & -1.992928 & 0.376523 \\
\hline C & -3.033531 & -1.991309 & 1.922628 \\
\hline C & -2.936224 & -1.163428 & 0.826377 \\
\hline $\mathrm{H}$ & -3.929764 & -1.966000 & 2.535953 \\
\hline C & -1.968755 & -2.847180 & 2.297831 \\
\hline C & -0.761238 & -2.830315 & 1.530900 \\
\hline C & 0.322692 & -3.650444 & 1.942337 \\
\hline C & 0.207839 & -4.460489 & 3.047900 \\
\hline C & -0.992119 & -4.487919 & 3.797806 \\
\hline C & -2.055083 & -3.695763 & 3.432048 \\
\hline $\mathrm{H}$ & 1.247540 & -3.618516 & 1.378774 \\
\hline $\mathrm{H}$ & 1.045818 & -5.078677 & 3.353628 \\
\hline $\mathrm{H}$ & -1.066791 & -5.131304 & 4.668972 \\
\hline $\mathrm{H}$ & -2.975197 & -3.701876 & 4.009440 \\
\hline C & 1.948262 & -2.864805 & -2.260877 \\
\hline C & 0.737086 & -2.899683 & -1.502832 \\
\hline C & -0.255067 & -3.857676 & -1.839819 \\
\hline
\end{tabular}




\begin{tabular}{|c|c|c|c|}
\hline C & -0.058515 & -4.735965 & -2.881635 \\
\hline C & 1.137870 & -4.697458 & -3.636108 \\
\hline C & 2.117848 & -3.781237 & -3.331624 \\
\hline $\mathrm{H}$ & -1.170712 & -3.889007 & -1.260060 \\
\hline $\mathrm{H}$ & -0.824944 & -5.464486 & -3.127104 \\
\hline $\mathrm{H}$ & 1.279680 & -5.394912 & -4.455782 \\
\hline H & 3.039341 & -3.745447 & -3.905752 \\
\hline C & -6.020616 & 1.736364 & -0.051976 \\
\hline C & -6.209233 & 0.377592 & -0.349061 \\
\hline C & -5.225047 & -0.566136 & -0.065808 \\
\hline C & -4.016492 & -0.182524 & 0.525838 \\
\hline C & -3.829248 & 1.172948 & 0.841013 \\
\hline C & -4.812699 & 2.115957 & 0.554203 \\
\hline $\mathrm{H}$ & -5.390684 & -1.612518 & -0.302897 \\
\hline H & -2.914537 & 1.474951 & 1.345717 \\
\hline C & 3.857945 & -0.041206 & -0.592269 \\
\hline C & 5.201972 & -0.442452 & -0.599052 \\
\hline C & 3.574081 & 1.309768 & -0.344018 \\
\hline C & 6.223419 & 0.470609 & -0.365260 \\
\hline $\mathrm{H}$ & 5.441981 & -1.489607 & -0.753709 \\
\hline C & 4.596068 & 2.221648 & -0.110802 \\
\hline $\mathrm{H}$ & 2.546237 & 1.654748 & -0.346042 \\
\hline C & 5.941591 & 1.822713 & -0.116585 \\
\hline $\mathrm{H}$ & -7.148162 & 0.055674 & -0.787944 \\
\hline $\mathrm{H}$ & -4.638636 & 3.161051 & 0.789357 \\
\hline H & 4.350257 & 3.266335 & 0.049450 \\
\hline $\mathrm{H}$ & 7.252270 & 0.126304 & -0.337183 \\
\hline C & -7.066677 & 2.738271 & -0.362690 \\
\hline C & -7.830690 & 2.638507 & -1.536014 \\
\hline C & -7.317647 & 3.810366 & 0.507947 \\
\hline C & -8.814338 & 3.580142 & -1.829243 \\
\hline $\mathrm{H}$ & -7.632061 & 1.831497 & -2.234242 \\
\hline C & -8.300416 & 4.752777 & 0.214246 \\
\hline $\mathrm{H}$ & -6.756189 & 3.888327 & 1.433596 \\
\hline C & -9.053088 & 4.641549 & -0.955472 \\
\hline $\mathrm{H}$ & -9.388838 & 3.489973 & -2.746114 \\
\hline $\mathrm{H}$ & -8.484861 & 5.570133 & 0.904674 \\
\hline $\mathrm{H}$ & -9.819153 & 5.375803 & -1.184130 \\
\hline C & 7.027971 & 2.796152 & 0.136648 \\
\hline C & 8.248078 & 2.709784 & -0.552572 \\
\hline C & 6.864209 & 3.829577 & 1.072885 \\
\hline C & 9.270292 & 3.625408 & -0.313878 \\
\hline $\mathrm{H}$ & 8.383088 & 1.933217 & -1.298865 \\
\hline C & 7.885529 & 4.746456 & 1.310455 \\
\hline $\mathrm{H}$ & 5.938901 & 3.894270 & 1.636362 \\
\hline C & 9.093365 & 4.648560 & 0.618396 \\
\hline H & 10.203404 & 3.545228 & -0.863550 \\
\hline $\mathrm{H}$ & 7.741386 & 5.533021 & 2.045093 \\
\hline $\mathrm{H}$ & 9.889680 & 5.362634 & 0.804365 \\
\hline
\end{tabular}


Frequencies (Top 3 out of 237)

$\begin{array}{lr}\text { 1. } & 8.5833 \mathrm{~cm}^{-1} \\ \text { 2. } & 15.0586 \mathrm{~cm}^{-1} \\ \text { 3. } & 19.0623 \mathrm{~cm}^{-1}\end{array}$

B3LYP-D3(BJ)/6-31G(d,p) Molecular Geometry in Cartesian Coordinates

C

$\mathrm{H}$

$\mathrm{H}$

C

0

0

$\mathrm{H}$

0

$\mathrm{H}$

C

C

C

C

$\mathrm{H}$

C

C

C

C

$\mathrm{H}$

C

C

C

C

C

C

$\mathrm{H}$

$\mathrm{H}$

$\mathrm{H}$

$\mathrm{H}$

C

C

C

\section{$-1.686851$}

$-1.004904$

$-2.672084$

1.445976

2.123754

0.560670

0.053471

$-1.158939$

$-1.874991$

0.561442

1. 564634

2. 782581

2.936689

3.839990

$-1.768646$

$-0.697251$

$-3.046718$

$-2.943289$

$-3.944726$

$-1.985847$

$-0.776123$

0.303800

0.183046

$-1.019081$

$-2.078267$

1.230355

1.018039

$-1.098392$

$-2.999982$

1.942352

0.729331

$-0.265674$
$-0.316748$

$-0.748763$

$-0.216170$

$-0.244313$

$-0.494667$

0.764876

0.820388

1.000457

1.502916

$-1.960558$

$-1.061443$

$-1.016065$

$-1.888902$

$-1.839263$

$-1.190778$

$-1.996345$

$-2.013703$

$-1.166888$

$-1.996144$

$-2.879521$

$-2.853503$

$-3.684458$

$-4.513210$

$-4.549488$

$-3.747373$

$-3.645934$

$-5.139531$

$-5.207634$

$-3.760247$

$-2.833328$

$-2.876434$

$-3.824760$
$-1.243219$

$-1.974291$

$-1.707026$

1.126953

2.095998

1. 129111

0.282364

$-0.960692$

$-0.542872$

$-0.464699$

$-0.142758$

$-0.894953$

$-1.953773$

$-2.554250$

$-0.012419$

0. 342261

1.881548

0.800466

2. 492547

2. 244866

1.481672

1.881735

2. 972707

3. 718735

3. 363908

1. 321391

3. 269936

4.578388

3.938631

$-2.303558$

$-1.548898$

$-1.904316$ 


\begin{tabular}{|c|c|c|c|}
\hline C & -0.070040 & -4.686012 & -2.960412 \\
\hline C & 1.128216 & -4.639309 & -3.711444 \\
\hline C & 2.110938 & -3.732128 & -3.389305 \\
\hline $\mathrm{H}$ & -1.182801 & -3.862390 & -1.327285 \\
\hline $\mathrm{H}$ & -0.838692 & -5.407271 & -3.219964 \\
\hline $\mathrm{H}$ & 1.269244 & -5.323297 & -4.542520 \\
\hline $\mathrm{H}$ & 3.033868 & -3.690119 & -3.960699 \\
\hline C & -6.013777 & 1.759095 & -0.039183 \\
\hline C & -6.207727 & 0.405718 & -0.356392 \\
\hline C & -5.229437 & -0.546974 & -0.082547 \\
\hline C & -4.019143 & -0.177052 & 0.514058 \\
\hline C & -3.825543 & 1.173439 & 0.847332 \\
\hline C & -4.805354 & 2.124432 & 0.575135 \\
\hline $\mathrm{H}$ & -5.393073 & -1.586418 & -0.349572 \\
\hline H & -2.917807 & 1.460849 & 1.372795 \\
\hline C & 3.858899 & -0.045732 & -0.583105 \\
\hline C & 5.201438 & -0.451828 & -0.594304 \\
\hline C & 3.579610 & 1.301818 & -0.312093 \\
\hline C & 6.225827 & 0.453215 & -0.342877 \\
\hline $\mathrm{H}$ & 5.437844 & -1.497051 & -0.766561 \\
\hline C & 4.604521 & 2.205671 & -0.061197 \\
\hline $\mathrm{H}$ & 2.553063 & 1.650641 & -0.310016 \\
\hline C & 5.948556 & 1.801854 & -0.071354 \\
\hline $\mathrm{H}$ & -7.125560 & 0.102293 & -0.849703 \\
\hline $\mathrm{H}$ & -4.649139 & 3.156106 & 0.873509 \\
\hline $\mathrm{H}$ & 4.362311 & 3.248344 & 0.116699 \\
\hline $\mathrm{H}$ & 7.253339 & 0.104627 & -0.318841 \\
\hline C & -7.053940 & 2.770803 & -0.336853 \\
\hline C & -8.416643 & 2.465301 & -0.195403 \\
\hline C & -6.700175 & 4.059100 & -0.767504 \\
\hline C & -9.394019 & 3.417041 & -0.476616 \\
\hline $\mathrm{H}$ & -8.708285 & 1.483374 & 0.163636 \\
\hline C & -7.677325 & 5.011465 & -1.047413 \\
\hline $\mathrm{H}$ & -5.652367 & 4.305077 & -0.908366 \\
\hline C & -9.028516 & 4.694252 & -0.903568 \\
\hline $\mathrm{H}$ & -10.442518 & 3.163748 & -0.352852 \\
\hline $\mathrm{H}$ & -7.383210 & 5.999875 & -1.387004 \\
\hline $\mathrm{H}$ & -9.790168 & 5.436166 & -1.122000 \\
\hline C & 7.038036 & 2.766674 & 0.200816 \\
\hline C & 8.259029 & 2.687963 & -0.487745 \\
\hline C & 6.876400 & 3.784023 & 1.154857 \\
\hline C & 9.284154 & 3.595465 & -0.231198 \\
\hline $\mathrm{H}$ & 8.392523 & 1.924158 & -1.247368 \\
\hline C & 7.900649 & 4.692787 & 1.410312 \\
\hline $\mathrm{H}$ & 5.950352 & 3.842154 & 1.717842 \\
\hline C & 9.109332 & 4.602656 & 0.718683 \\
\hline $\mathrm{H}$ & 10.217943 & 3.521543 & -0.780601 \\
\hline $\mathrm{H}$ & 7.758103 & 5.466772 & 2.158499 \\
\hline $\mathrm{H}$ & 9.907936 & 5.310381 & 0.918586 \\
\hline
\end{tabular}


Frequencies (Top 3 out of 237)

$\begin{array}{lr}\text { 1. } & 9.1309 \mathrm{~cm}^{-1} \\ \text { 2. } & 14.7614 \mathrm{~cm}^{-1} \\ \text { 3. } & 22.0093 \mathrm{~cm}^{-1}\end{array}$

B3LYP-D3(BJ)/6-31G(d,p) Molecular Geometry in Cartesian Coordinates

\begin{tabular}{|c|c|c|c|}
\hline C & 1.538891 & -0.109556 & 1.181783 \\
\hline $\mathrm{H}$ & 2.502589 & -0.268352 & 1.671267 \\
\hline $\mathrm{H}$ & 0.865892 & 0.345567 & 1.918687 \\
\hline C & -1.623171 & 0.076293 & -1.059496 \\
\hline 0 & -0.869082 & -0.867127 & -1.226565 \\
\hline 0 & -2.488867 & 0.470847 & -2.006923 \\
\hline $\mathrm{H}$ & -2.386727 & -0.146936 & -2.751720 \\
\hline 0 & 1.064857 & -1.395600 & 0.795107 \\
\hline $\mathrm{H}$ & 0.359122 & -1.266035 & 0.134986 \\
\hline C & -0.592953 & 1.709321 & 0.485194 \\
\hline C & -1.693828 & 0.933056 & 0.163937 \\
\hline C & -2.905099 & 0.990490 & 0.923734 \\
\hline C & -2.973305 & 1.871693 & 1.982486 \\
\hline $\mathrm{H}$ & -3.874118 & 1.905048 & 2.587763 \\
\hline C & 1.701673 & 0.826524 & -0.000015 \\
\hline C & 0.650639 & 1.664874 & -0.347734 \\
\hline C & 2.984360 & 1.673096 & -1.880890 \\
\hline C & 2.901117 & 0.829223 & -0.788564 \\
\hline $\mathrm{H}$ & 3.885554 & 1.666478 & -2.486664 \\
\hline C & 1.923973 & 2.525718 & -2.262357 \\
\hline C & 0.726699 & 2.529748 & -1.482637 \\
\hline C & -0.335574 & 3.390994 & -1.869228 \\
\hline C & -0.215836 & 4.203841 & -2.973239 \\
\hline C & 0.969905 & 4.197311 & -3.745995 \\
\hline C & 2.015324 & 3.375205 & -3.396636 \\
\hline $\mathrm{H}$ & -1.249106 & 3.397495 & -1.286170 \\
\hline $\mathrm{H}$ & -1.037412 & 4.854726 & -3.255798 \\
\hline $\mathrm{H}$ & 1.049910 & 4.843013 & -4.615037 \\
\hline $\mathrm{H}$ & 2.929526 & 3.361728 & -3.983428 \\
\hline C & -1.886148 & 2.709273 & 2.330343 \\
\hline C & -0.672755 & 2.628000 & 1.577996 \\
\hline 0 & 0.413874 & 3.468098 & 1.937239 \\
\hline
\end{tabular}




\begin{tabular}{|c|c|c|c|}
\hline C & 0.305138 & 4.342702 & 2.994242 \\
\hline C & -0.893632 & 4.419021 & 3.742028 \\
\hline C & -1.964253 & 3.619021 & 3.417087 \\
\hline $\mathrm{H}$ & 1.332010 & 3.407341 & 1.364860 \\
\hline H & 1.144039 & 4.978730 & 3.258272 \\
\hline H & -0.964934 & 5.112228 & 4.574342 \\
\hline $\mathrm{H}$ & -2.887244 & 3.670799 & 3.987333 \\
\hline C & 6.397078 & -1.575124 & 0.047241 \\
\hline C & 5.115873 & -2.147775 & 0.054159 \\
\hline C & 3.978359 & -1.386999 & -0.194697 \\
\hline C & 4.082734 & -0.012671 & -0.463450 \\
\hline C & 5.363864 & 0.563319 & -0.469014 \\
\hline C & 6.497624 & -0.200567 & -0.217771 \\
\hline H & 2.998980 & -1.852383 & -0.180103 \\
\hline $\mathrm{H}$ & 5.464342 & 1.630134 & -0.643995 \\
\hline C & -4.048172 & 0.096072 & 0.624932 \\
\hline C & -5.355237 & 0.600211 & 0.572481 \\
\hline C & -3.858545 & -1.279504 & 0.422189 \\
\hline C & -6.434081 & -0.238823 & 0.318248 \\
\hline $\mathrm{H}$ & -5.518073 & 1.665822 & 0.699917 \\
\hline C & -4.939063 & -2.116850 & 0.169439 \\
\hline H & -2.860919 & -1.700760 & 0.491222 \\
\hline C & -6.248715 & -1.614839 & 0.110409 \\
\hline H & 5.010494 & -3.213959 & 0.228314 \\
\hline H & 7.471014 & 0.279060 & -0.191678 \\
\hline H & -4.770380 & -3.182328 & 0.051780 \\
\hline H & -7.431951 & 0.181404 & 0.244801 \\
\hline C & 7.602780 & -2.391349 & 0.316814 \\
\hline C & 8.806740 & -2.145250 & -0.362482 \\
\hline C & 7.572474 & -3.431988 & 1.259014 \\
\hline C & 9.941505 & -2.911940 & -0.107929 \\
\hline $\mathrm{H}$ & 8.842226 & -1.363039 & -1.114181 \\
\hline C & 8.706318 & -4.200383 & 1.512415 \\
\hline H & 6.658542 & -3.620322 & 1.813228 \\
\hline C & 9.896510 & -3.943643 & 0.830595 \\
\hline $\mathrm{H}$ & 10.860132 & -2.709522 & -0.650582 \\
\hline H & 8.662715 & -4.995478 & 2.250766 \\
\hline H & 10.780461 & -4.542011 & 1.028588 \\
\hline C & -7.397776 & -2.508158 & -0.161883 \\
\hline C & -7.278037 & -3.581909 & -1.058463 \\
\hline C & -8.634673 & -2.303500 & 0.470053 \\
\hline C & -8.358371 & -4.423088 & -1.314135 \\
\hline H & -6.337522 & -3.740486 & -1.576496 \\
\hline C & -9.715760 & -3.143381 & 0.213320 \\
\hline $\mathrm{H}$ & -8.738545 & -1.495878 & 1.187712 \\
\hline C & -9.582375 & -4.207246 & -0.679770 \\
\hline $\mathrm{H}$ & -8.246593 & -5.243331 & -2.016758 \\
\hline H & -10.661300 & -2.972574 & 0.719076 \\
\hline $\mathrm{H}$ & -10.424542 & -4.862501 & -0.879219 \\
\hline
\end{tabular}


Frequencies (Top 3 out of 237)

$\begin{array}{lr}\text { 1. } & 9.0751 \mathrm{~cm}^{-1} \\ \text { 2. } & 14.5090 \mathrm{~cm}^{-1} \\ \text { 3. } & 21.9109 \mathrm{~cm}^{-1}\end{array}$

B3LYP-D3(BJ)/6-31G(d,p) Molecular Geometry in Cartesian Coordinates

\begin{tabular}{|c|c|c|c|}
\hline C & -1.543116 & -0.073652 & -1.181980 \\
\hline $\mathrm{H}$ & -2.508541 & -0.218885 & -1.672278 \\
\hline $\mathrm{H}$ & -0.873248 & 0.403251 & -1.907872 \\
\hline C & 1.626309 & 0.046417 & 1.054225 \\
\hline 0 & 0.872570 & -0.901380 & 1.196338 \\
\hline 0 & 2.495298 & 0.413122 & 2.009791 \\
\hline $\mathrm{H}$ & 2.395515 & -0.226015 & 2.736670 \\
\hline 0 & -1.066710 & -1.370052 & -0.834701 \\
\hline $\mathrm{H}$ & -0.359407 & -1.259362 & -0.172815 \\
\hline C & 0.591162 & 1.723698 & -0.438916 \\
\hline C & 1.693014 & 0.938427 & -0.143983 \\
\hline C & 2.901775 & 1.017909 & -0.905799 \\
\hline C & 2.966541 & 1.929418 & -1.938792 \\
\hline $\mathrm{H}$ & 3.865338 & 1.980285 & -2.545843 \\
\hline C & -1.701922 & 0.827101 & 0.027466 \\
\hline $\mathrm{C}$ & -0.649718 & 1.654826 & 0.396402 \\
\hline C & -2.978030 & 1.616827 & 1.937330 \\
\hline C & -2.898551 & 0.805988 & 0.819960 \\
\hline $\mathrm{H}$ & -3.876905 & 1.591929 & 2.546052 \\
\hline C & -1.916332 & 2.457871 & 2.340297 \\
\hline C & -0.721829 & 2.485466 & 1.556820 \\
\hline C & 0.341736 & 3.334997 & 1.965170 \\
\hline C & 0.225871 & 4.114457 & 3.093399 \\
\hline C & -0.957114 & 4.084566 & 3.869831 \\
\hline C & -2.003710 & 3.273075 & 3.499783 \\
\hline $\mathrm{H}$ & 1.253158 & 3.359080 & 1.379271 \\
\hline $\mathrm{H}$ & 1.048378 & 4.756790 & 3.392352 \\
\hline $\mathrm{H}$ & -1.034093 & 4.703989 & 4.758065 \\
\hline $\mathrm{H}$ & -2.915814 & 3.241908 & 4.089163 \\
\hline C & 1.878340 & 2.776772 & -2.258554 \\
\hline C & 0.667484 & 2.673761 & -1.504785 \\
\hline C & -0.420196 & 3.524043 & -1.835749 \\
\hline
\end{tabular}




\begin{tabular}{|c|c|c|c|}
\hline C & -0.314925 & 4.428921 & -2.867304 \\
\hline C & 0.881284 & 4.526781 & -3.616687 \\
\hline C & 1.952874 & 3.717632 & -3.318738 \\
\hline $\mathrm{H}$ & -1.336323 & 3.446739 & -1.262145 \\
\hline $\mathrm{H}$ & -1.154584 & 5.072452 & -3.109884 \\
\hline $\mathrm{H}$ & 0.949847 & 5.243802 & -4.428812 \\
\hline H & 2.873916 & 3.785856 & -3.890405 \\
\hline C & -6.396840 & -1.574468 & -0.072435 \\
\hline C & -5.115785 & -2.146898 & -0.098119 \\
\hline C & -3.977557 & -1.393515 & 0.169601 \\
\hline C & -4.081078 & -0.026574 & 0.474100 \\
\hline C & -5.361785 & 0.549635 & 0.496255 \\
\hline C & -6.496451 & -0.207455 & 0.228815 \\
\hline $\mathrm{H}$ & -2.997062 & -1.854872 & 0.124967 \\
\hline H & -5.463166 & 1.607783 & 0.717356 \\
\hline C & 4.045840 & 0.115344 & -0.636744 \\
\hline C & 5.353048 & 0.617965 & -0.574208 \\
\hline C & 3.856942 & -1.265502 & -0.472892 \\
\hline C & 6.432763 & -0.227831 & -0.347571 \\
\hline $\mathrm{H}$ & 5.515399 & 1.686810 & -0.671692 \\
\hline C & 4.938308 & -2.109559 & -0.247595 \\
\hline $\mathrm{H}$ & 2.859138 & -1.684773 & -0.550852 \\
\hline C & 6.248103 & -1.609223 & -0.178404 \\
\hline $\mathrm{H}$ & -5.006669 & -3.195900 & -0.354822 \\
\hline $\mathrm{H}$ & -7.474809 & 0.260683 & 0.272566 \\
\hline H & 4.770091 & -3.177995 & -0.159837 \\
\hline $\mathrm{H}$ & 7.430869 & 0.190231 & -0.265437 \\
\hline C & -7.603487 & -2.383776 & -0.358323 \\
\hline C & -8.686382 & -1.839148 & -1.067064 \\
\hline C & -7.695079 & -3.716987 & 0.072716 \\
\hline C & -9.821976 & -2.600256 & -1.334333 \\
\hline $\mathrm{H}$ & -8.622902 & -0.819270 & -1.433030 \\
\hline C & -8.829601 & -4.479136 & -0.195935 \\
\hline $\mathrm{H}$ & -6.879049 & -4.147625 & 0.644169 \\
\hline C & -9.898873 & -3.924221 & -0.900252 \\
\hline $\mathrm{H}$ & -10.644523 & -2.161471 & -1.891288 \\
\hline $\mathrm{H}$ & -8.882332 & -5.506028 & 0.153299 \\
\hline $\mathrm{H}$ & -10.783354 & -4.518014 & -1.109309 \\
\hline C & 7.398031 & -2.509753 & 0.064906 \\
\hline C & 8.632850 & -2.287464 & -0.565126 \\
\hline C & 7.281191 & -3.608113 & 0.931554 \\
\hline C & 9.714734 & -3.134141 & -0.335472 \\
\hline $\mathrm{H}$ & 8.734399 & -1.460093 & -1.260273 \\
\hline C & 8.362324 & -4.456032 & 1.160140 \\
\hline $\mathrm{H}$ & 6.342345 & -3.781123 & 1.447998 \\
\hline C & 9.584250 & -4.222502 & 0.528039 \\
\hline H & 10.658615 & -2.949250 & -0.839371 \\
\hline $\mathrm{H}$ & 8.252821 & -5.295565 & 1.839970 \\
\hline $\mathrm{H}$ & 10.427048 & -4.883015 & 0.706364 \\
\hline
\end{tabular}


Frequencies (Top 3 out of 237)

$\begin{array}{lr}\text { 1. } & 9.1981 \mathrm{~cm}^{-1} \\ \text { 2. } & 14.9102 \mathrm{~cm}^{-1} \\ \text { 3. } & 21.9656 \mathrm{~cm}^{-1}\end{array}$

B3LYP-D3(BJ)/6-31G(d,p) Molecular Geometry in Cartesian Coordinates

\begin{tabular}{|c|c|c|c|}
\hline C & -1.546026 & -0.143110 & 1.172471 \\
\hline $\mathrm{H}$ & -0.875568 & 0.291034 & 1.924184 \\
\hline $\mathrm{H}$ & -2.511963 & -0.313812 & 1.653461 \\
\hline C & 1.627326 & 0.099908 & -1.048837 \\
\hline 0 & 0.871417 & -0.836603 & -1.244306 \\
\hline 0 & 2.500208 & 0.515290 & -1.980579 \\
\hline $\mathrm{H}$ & 2.402145 & -0.084427 & -2.740576 \\
\hline 0 & -1.071906 & -1.418845 & 0.753080 \\
\hline $\mathrm{H}$ & -0.363312 & -1.272107 & 0.099645 \\
\hline C & 0.590724 & 1.691611 & 0.534296 \\
\hline C & 1.693151 & 0.924306 & 0.196877 \\
\hline C & 2.901003 & 0.960930 & 0.963592 \\
\hline C & 2.963757 & 1.812289 & 2.046900 \\
\hline $\mathrm{H}$ & 3.861482 & 1.828996 & 2.657408 \\
\hline C & -1.702676 & 0.824619 & 0.015612 \\
\hline C & -0.649111 & 1.670500 & -0.305164 \\
\hline C & -2.976164 & 1.722523 & -1.847607 \\
\hline C & -2.898732 & 0.849898 & -0.777671 \\
\hline $\mathrm{H}$ & -3.874672 & 1.733164 & -2.457294 \\
\hline C & -1.913092 & 2.583543 & -2.201709 \\
\hline C & -0.719250 & 2.565385 & -1.416947 \\
\hline C & 0.345574 & 3.435457 & -1.775774 \\
\hline C & 0.231575 & 4.277512 & -2.858299 \\
\hline C & -0.950698 & 4.292836 & -3.636220 \\
\hline C & -1.998507 & 3.462951 & -3.313423 \\
\hline $\mathrm{H}$ & 1.256450 & 3.425511 & -1.188627 \\
\hline $\mathrm{H}$ & 1.055023 & 4.934820 & -3.119766 \\
\hline $\mathrm{H}$ & -1.026177 & 4.961470 & -4.488157 \\
\hline $\mathrm{H}$ & -2.910115 & 3.466118 & -3.904378 \\
\hline C & 1.874843 & 2.639839 & 2.412659 \\
\hline C & 0.665244 & 2.579881 & 1.652297 \\
\hline C & -0.423007 & 3.410100 & 2.029254 \\
\hline
\end{tabular}




\begin{tabular}{|c|c|c|c|}
\hline C & -0.319429 & 4.254945 & 3.110693 \\
\hline C & 0.875573 & 4.309991 & 3.866364 \\
\hline C & 1.947663 & 3.518987 & 3.524683 \\
\hline $\mathrm{H}$ & -1.338269 & 3.365624 & 1.450811 \\
\hline $\mathrm{H}$ & -1.159508 & 4.883702 & 3.388055 \\
\hline $\mathrm{H}$ & 0.942832 & 4.979756 & 4.717985 \\
\hline $\mathrm{H}$ & 2.867805 & 3.554597 & 4.100744 \\
\hline C & -6.401191 & -1.571271 & -0.021359 \\
\hline C & -5.120706 & -2.145557 & -0.024041 \\
\hline C & -3.981202 & -1.379906 & -0.247571 \\
\hline C & -4.082767 & 0.001219 & -0.480153 \\
\hline C & -5.363183 & 0.578813 & -0.475940 \\
\hline C & -6.498944 & -0.190016 & -0.250110 \\
\hline $\mathrm{H}$ & -3.002431 & -1.846782 & -0.241069 \\
\hline $\mathrm{H}$ & -5.461643 & 1.650037 & -0.622857 \\
\hline C & 4.046636 & 0.076405 & 0.645029 \\
\hline C & 5.352914 & 0.583996 & 0.611121 \\
\hline C & 3.860380 & -1.293408 & 0.404331 \\
\hline C & 6.434460 & -0.246206 & 0.339901 \\
\hline $\mathrm{H}$ & 5.515322 & 1.642849 & 0.786487 \\
\hline C & 4.943125 & -2.121160 & 0.130131 \\
\hline $\mathrm{H}$ & 2.861261 & -1.715204 & 0.440520 \\
\hline C & 6.252073 & -1.615762 & 0.091375 \\
\hline $\mathrm{H}$ & -5.017366 & -3.216134 & 0.122138 \\
\hline $\mathrm{H}$ & -7.471885 & 0.289993 & -0.215572 \\
\hline $\mathrm{H}$ & 4.770894 & -3.173198 & -0.072720 \\
\hline H & 7.438004 & 0.166893 & 0.336914 \\
\hline C & -7.609037 & -2.392810 & 0.221100 \\
\hline C & -7.584250 & -3.457923 & 1.135708 \\
\hline C & -8.809584 & -2.127396 & -0.456965 \\
\hline C & -8.720129 & -4.231277 & 1.363640 \\
\hline $\mathrm{H}$ & -6.673063 & -3.661958 & 1.688886 \\
\hline C & -9.946382 & -2.899089 & -0.227855 \\
\hline $\mathrm{H}$ & -8.840724 & -1.325607 & -1.187945 \\
\hline C & -9.906887 & -3.955179 & 0.683400 \\
\hline $\mathrm{H}$ & -8.680820 & -5.045588 & 2.080993 \\
\hline $\mathrm{H}$ & -10.862273 & -2.681305 & -0.769181 \\
\hline $\mathrm{H}$ & -10.792424 & -4.557428 & 0.861539 \\
\hline C & 7.403952 & -2.499002 & -0.201775 \\
\hline C & 7.441505 & -3.817037 & 0.280504 \\
\hline C & 8.486220 & -2.039668 & -0.969175 \\
\hline C & 8.524693 & -4.647993 & 0.004225 \\
\hline $\mathrm{H}$ & 6.626888 & -4.181325 & 0.898315 \\
\hline C & 9.570170 & -2.870016 & -1.244433 \\
\hline $\mathrm{H}$ & 8.462478 & -1.032769 & -1.373827 \\
\hline C & 9.594115 & -4.178130 & -0.759201 \\
\hline $\mathrm{H}$ & 8.537672 & -5.661472 & 0.393647 \\
\hline $\mathrm{H}$ & 10.393336 & -2.497744 & -1.846802 \\
\hline $\mathrm{H}$ & 10.438502 & -4.825597 & -0.974124 \\
\hline
\end{tabular}


Frequencies (Top 3 out of 237)

$\begin{array}{lr}\text { 1. } & 9.0981 \mathrm{~cm}^{-1} \\ \text { 2. } & 14.6523 \mathrm{~cm}^{-1} \\ \text { 3. } & 21.7634 \mathrm{~cm}^{-1}\end{array}$

B3LYP-D3(BJ)/6-31G(d,p) Molecular Geometry in Cartesian Coordinates

C

$\mathrm{H}$

$\mathrm{H}$

C

0

0

$\mathrm{H}$

0

$\mathrm{H}$

C

C

C

C

$\mathrm{H}$

C

C

C

C

$\mathrm{H}$

C

C

C

C

C

C

$\mathrm{H}$

$\mathrm{H}$

$\mathrm{H}$

$\mathrm{H}$

C

C

C
$-1.549683$

$-0.882000$

$-2.517093$

1.630520

0.875099

2. 506665

2.411068

$-1.073344$

$-0.363266$

0.588729

1.692260

2.897660

2.956981

3.852730

$-1.702944$

$-0.648542$

$-2.970812$

$-2.896489$

$-3.867297$

$-1.906732$

$-0.715353$

0.350465

0.239733

$-0.940116$

$-1.988808$

1.259509

1.063896

$-1.013054$

$-2.898559$

1.866953

0.659757

$-0.429650$
$-0.108855$

0.346739

$-0.266656$

0.071900

$-0.869658$

0.460911

$-0.159851$

$-1.395558$

$-1.266883$

1.707344

0.930967

0.989366

1.871048

1.905043

0.825534

1.662117

1.669070

0.827629

1.661954

2.519805

2.524619

3. 384247

4.194733

4.187330

3. 366825

3. 391404

4.844428

4.831127

3. 352748

2. 708585

2.626978

3.467505
1.171840

1.913253

1.654277

$-1.046361$

$-1.218099$

$-1.986408$

$-2.729626$

0.790087

0.134462

0.488040

0.175899

0.945117

2.004089

2.616775

$-0.012539$

$-0.354239$

$-1.904806$

$-0.809946$

$-2.517506$

$-2.280193$

$-1.491498$

$-1.871912$

$-2.978603$

$-3.760320$

$-3.417032$

$-1.281879$

$-3.256343$

$-4.631397$

$-4.010684$

2. 342768

1.580615

1.930399 


\begin{tabular}{|c|c|c|c|}
\hline C & -0.329462 & 4.342721 & 2.987735 \\
\hline C & 0.863134 & 4.419247 & 3.745332 \\
\hline C & 1.936272 & 3.618924 & 3.429637 \\
\hline $\mathrm{H}$ & -1.343040 & 3.406539 & 1.350502 \\
\hline H & -1.170368 & 4.979096 & 3.244459 \\
\hline H & 0.927716 & 5.112908 & 4.577819 \\
\hline $\mathrm{H}$ & 2.854579 & 3.670913 & 4.007376 \\
\hline c & -6.400259 & -1.572883 & 0.003800 \\
\hline C & -5.119889 & -2.147261 & 0.017612 \\
\hline C & -3.980014 & -1.387953 & -0.225212 \\
\hline C & -4.081121 & -0.012909 & -0.491667 \\
\hline C & -5.361139 & 0.565142 & -0.501884 \\
\hline C & -6.497461 & -0.197871 & -0.259443 \\
\hline H & -3.000111 & -1.851388 & -0.190258 \\
\hline $\mathrm{H}$ & -5.460696 & 1.629172 & -0.693625 \\
\hline c & 4.044226 & 0.096127 & 0.655575 \\
\hline C & 3.858561 & -1.279944 & 0.453175 \\
\hline C & 5.350712 & 0.602448 & 0.611898 \\
\hline C & 4.942137 & -2.115256 & 0.206573 \\
\hline $\mathrm{H}$ & 2.859242 & -1.700468 & 0.497831 \\
\hline C & 6.433079 & -0.235227 & 0.368336 \\
\hline H & 5.512687 & 1.665846 & 0.757673 \\
\hline C & 6.251325 & -1.611296 & 0.158303 \\
\hline H & -5.012711 & -3.203159 & 0.245205 \\
\hline $\mathrm{H}$ & -7.475183 & 0.272404 & -0.293306 \\
\hline H & 7.436717 & 0.177516 & 0.357238 \\
\hline H & 4.770453 & -3.172636 & 0.033175 \\
\hline C & -7.608663 & -2.388488 & 0.263151 \\
\hline C & -8.693239 & -1.862638 & 0.983417 \\
\hline C & -7.700262 & -3.709058 & -0.205196 \\
\hline C & -9.830453 & -2.629645 & 1.225802 \\
\hline H & -8.629888 & -0.853425 & 1.377865 \\
\hline C & -8.836407 & -4.477147 & 0.038549 \\
\hline H & -6.882865 & -4.124495 & -0.785873 \\
\hline C & -9.907331 & -3.940909 & 0.754732 \\
\hline $\mathrm{H}$ & -10.654310 & -2.205641 & 1.792187 \\
\hline H & -8.889086 & -5.493838 & -0.339362 \\
\hline H & -10.793074 & -4.539320 & 0.944379 \\
\hline C & 7.404128 & -2.502691 & -0.105010 \\
\hline C & 7.439546 & -3.806335 & 0.415066 \\
\hline C & 8.489486 & -2.065653 & -0.881021 \\
\hline C & 8.523676 & -4.644945 & 0.167050 \\
\hline H & 6.622458 & -4.152654 & 1.039906 \\
\hline C & 9.574373 & -2.903629 & -1.128035 \\
\hline $\mathrm{H}$ & 8.467516 & -1.070771 & -1.314472 \\
\hline C & 9.596185 & -4.197264 & -0.605327 \\
\hline $\mathrm{H}$ & 8.534960 & -5.646808 & 0.585499 \\
\hline H & 10.399980 & -2.548857 & -1.737583 \\
\hline $\mathrm{H}$ & 10.441319 & -4.850674 & -0.798199 \\
\hline
\end{tabular}


Frequencies (Top 3 out of 237)

$\begin{array}{lr}\text { 1. } & 8.6341 \mathrm{~cm}^{-1} \\ \text { 2. } & 16.8596 \mathrm{~cm}^{-1} \\ \text { 3. } & 21.7855 \mathrm{~cm}^{-1}\end{array}$

B3LYP-D3(BJ)/6-31G(d,p) Molecular Geometry in Cartesian Coordinates

\begin{tabular}{|c|c|c|c|}
\hline C & 1.558735 & 0.111403 & 1.207439 \\
\hline $\mathrm{H}$ & 2.535916 & -0.176369 & 1.603119 \\
\hline $\mathrm{H}$ & 1.002775 & 0.639133 & 1.986542 \\
\hline C & -1.568248 & 0.335242 & -1.253702 \\
\hline 0 & -2.148634 & 0.745129 & -2.236246 \\
\hline 0 & -0.818292 & -0.767441 & -1.294160 \\
\hline $\mathrm{H}$ & -0.323726 & -0.900318 & -0.450969 \\
\hline 0 & 0.829818 & -1.083423 & 0.826283 \\
\hline $\mathrm{H}$ & 0.583082 & -1.562054 & 1.628711 \\
\hline C & -0.579670 & 1.855698 & 0.452794 \\
\hline C & -1.650306 & 1.063813 & 0.071593 \\
\hline C & -2.833274 & 0.985271 & 0.875651 \\
\hline C & -2.887570 & 1.731785 & 2.037152 \\
\hline $\mathrm{H}$ & -3.778785 & 1.679208 & 2.655353 \\
\hline C & 1.718989 & 0.996082 & -0.002018 \\
\hline C & 0.680484 & 1.850420 & -0.354343 \\
\hline C & 3.055670 & 1.849533 & -1.835329 \\
\hline C & 2.924469 & 0.973568 & -0.774058 \\
\hline $\mathrm{H}$ & 3.965529 & 1.831140 & -2.427755 \\
\hline C & 2.023077 & 2.739325 & -2.208593 \\
\hline C & 0.800422 & 2.733473 & -1.467464 \\
\hline C & -0.245862 & 3.609166 & -1.862254 \\
\hline C & -0.081797 & 4.456778 & -2.932331 \\
\hline C & 1.132016 & 4.468660 & -3.661446 \\
\hline C & 2.159589 & 3.626364 & -3.309191 \\
\hline $\mathrm{H}$ & -1.182474 & 3.586585 & -1.318354 \\
\hline $\mathrm{H}$ & -0.891269 & 5.116596 & -3.227636 \\
\hline $\mathrm{H}$ & 1.244619 & 5.141647 & -4.505796 \\
\hline $\mathrm{H}$ & 3.090446 & 3.622480 & -3.869023 \\
\hline C & -1.835061 & 2.589568 & 2.435736 \\
\hline C & -0.656375 & 2.660951 & 1.630801 \\
\hline 0 & 0.398397 & 3.521877 & 2.035444 \\
\hline
\end{tabular}




\begin{tabular}{|c|c|c|c|}
\hline C & 0.289347 & 4.274651 & 3.182782 \\
\hline C & -0.877265 & 4.202541 & 3.980768 \\
\hline C & -1.915492 & 3.378418 & 3.613546 \\
\hline $\mathrm{H}$ & 1.290010 & 3.581830 & 1.421523 \\
\hline H & 1.101364 & 4.932132 & 3.477262 \\
\hline H & -0.950097 & 4.802354 & 4.882596 \\
\hline $\mathrm{H}$ & -2.815656 & 3.319049 & 4.218762 \\
\hline C & 6.214347 & -1.721243 & -0.000660 \\
\hline C & 6.421907 & -0.341534 & -0.151927 \\
\hline C & 5.357000 & 0.521857 & -0.384758 \\
\hline C & 4.041878 & 0.041449 & -0.475913 \\
\hline C & 3.831220 & -1.338963 & -0.327694 \\
\hline C & 4.897931 & -2.199751 & -0.092831 \\
\hline H & 5.539943 & 1.586593 & -0.492473 \\
\hline $\mathrm{H}$ & 2.823341 & -1.733814 & -0.395393 \\
\hline C & -3.967021 & 0.077512 & 0.555956 \\
\hline C & -4.430540 & -0.808777 & 1.541399 \\
\hline C & -4.631181 & 0.100583 & -0.679705 \\
\hline C & -5.510971 & -1.649597 & 1.300651 \\
\hline $\mathrm{H}$ & -3.916791 & -0.852853 & 2.497097 \\
\hline C & -5.714317 & -0.739375 & -0.914924 \\
\hline H & -4.298170 & 0.782505 & -1.451038 \\
\hline C & -6.175209 & -1.632263 & 0.064587 \\
\hline H & 7.431508 & 0.054220 & -0.106472 \\
\hline H & 4.706889 & -3.260204 & 0.037862 \\
\hline H & -6.230808 & -0.681412 & -1.867560 \\
\hline H & -5.824521 & -2.352022 & 2.066382 \\
\hline C & 7.347019 & -2.642050 & 0.248664 \\
\hline C & 8.424142 & -2.255531 & 1.062082 \\
\hline C & 7.371405 & -3.924318 & -0.322760 \\
\hline C & 9.490038 & -3.121307 & 1.295691 \\
\hline $\mathrm{H}$ & 8.410343 & -1.278248 & 1.534224 \\
\hline C & 8.436362 & -4.790920 & -0.088102 \\
\hline H & 6.561370 & -4.230697 & -0.976964 \\
\hline C & 9.500770 & -4.393283 & 0.721971 \\
\hline $\mathrm{H}$ & 10.309452 & -2.805336 & 1.934310 \\
\hline H & 8.439495 & -5.775159 & -0.546687 \\
\hline $\mathrm{H}$ & 10.331139 & -5.068349 & 0.904201 \\
\hline C & -7.323796 & -2.529316 & -0.197921 \\
\hline C & -7.510068 & -3.107350 & -1.463783 \\
\hline C & -8.253882 & -2.823993 & 0.811646 \\
\hline C & -8.590571 & -3.950748 & -1.711755 \\
\hline H & -6.786307 & -2.912539 & -2.248698 \\
\hline C & -9.333596 & -3.668649 & 0.564477 \\
\hline $\mathrm{H}$ & -8.141419 & -2.364683 & 1.788714 \\
\hline C & -9.507068 & -4.235639 & -0.698735 \\
\hline $\mathrm{H}$ & -8.711766 & -4.393597 & -2.695773 \\
\hline H & -10.046130 & -3.876872 & 1.357066 \\
\hline $\mathrm{H}$ & -10.348879 & -4.893352 & -0.891968 \\
\hline
\end{tabular}


Frequencies (Top 3 out of 237)

$\begin{array}{lr}\text { 1. } & 8.6397 \mathrm{~cm}^{-1} \\ \text { 2. } & 16.8281 \mathrm{~cm}^{-1} \\ \text { 3. } & 21.8465 \mathrm{~cm}^{-1}\end{array}$

B3LYP-D3(BJ)/6-31G(d,p) Molecular Geometry in Cartesian Coordinates

\begin{tabular}{|c|c|c|c|}
\hline C & 1.553169 & 0.075650 & 1.216849 \\
\hline $\mathrm{H}$ & 2.528463 & -0.223569 & 1.608604 \\
\hline $\mathrm{H}$ & 0.994212 & 0.582245 & 2.007709 \\
\hline C & -1.564026 & 0.368987 & -1.250565 \\
\hline 0 & -2.141275 & 0.805839 & -2.223288 \\
\hline 0 & -0.813006 & -0.731568 & -1.319263 \\
\hline $\mathrm{H}$ & -0.321403 & -0.887846 & -0.478337 \\
\hline 0 & 0.825084 & -1.107861 & 0.800009 \\
\hline $\mathrm{H}$ & 0.568185 & -1.604056 & 1.588441 \\
\hline C & -0.581569 & 1.840318 & 0.501852 \\
\hline C & -1.651144 & 1.060172 & 0.094276 \\
\hline C & -2.837363 & 0.959886 & 0.891114 \\
\hline C & -2.895718 & 1.673353 & 2.073024 \\
\hline $\mathrm{H}$ & -3.789408 & 1.604053 & 2.685980 \\
\hline C & 1.718847 & 0.993029 & 0.032789 \\
\hline C & 0.681748 & 1.856829 & -0.300198 \\
\hline C & 3.062235 & 1.895448 & -1.771835 \\
\hline C & 2.927348 & 0.991327 & -0.734867 \\
\hline $\mathrm{H}$ & 3.974307 & 1.892792 & -2.361125 \\
\hline C & 2.031138 & 2.795075 & -2.124925 \\
\hline C & 0.805861 & 2.769625 & -1.388576 \\
\hline C & -0.238829 & 3.655936 & -1.763389 \\
\hline C & -0.070827 & 4.531848 & -2.809801 \\
\hline C & 1.145517 & 4.562885 & -3.534122 \\
\hline C & 2.171685 & 3.711188 & -3.200927 \\
\hline $\mathrm{H}$ & -1.177348 & 3.619093 & -1.223577 \\
\hline $\mathrm{H}$ & -0.879117 & 5.199592 & -3.090142 \\
\hline $\mathrm{H}$ & 1.261177 & 5.258179 & -4.359774 \\
\hline $\mathrm{H}$ & 3.104507 & 3.722019 & -3.757385 \\
\hline C & -1.844219 & 2.518683 & 2.499864 \\
\hline C & -0.662378 & 2.612021 & 1.701847 \\
\hline 0 & 0.391416 & 3.460244 & 2.134899 \\
\hline
\end{tabular}




\begin{tabular}{|c|c|c|c|}
\hline C & 0.278482 & 4.180060 & 3.302830 \\
\hline C & -0.891274 & 4.086195 & 4.093918 \\
\hline C & -1.928673 & 3.273756 & 3.699339 \\
\hline $\mathrm{H}$ & 1.285497 & 3.536870 & 1.526443 \\
\hline $\mathrm{H}$ & 1.089851 & 4.828150 & 3.619089 \\
\hline $\mathrm{H}$ & -0.967160 & 4.660103 & 5.012200 \\
\hline $\mathrm{H}$ & -2.831224 & 3.197928 & 4.299141 \\
\hline c & 6.217821 & -1.719934 & -0.023163 \\
\hline c & 4.901527 & -2.196761 & -0.126628 \\
\hline c & 3.834316 & -1.330997 & -0.339963 \\
\hline C & 4.044420 & 0.052497 & -0.457878 \\
\hline c & 5.359680 & 0.530964 & -0.357160 \\
\hline C & 6.424658 & -0.336908 & -0.142549 \\
\hline $\mathrm{H}$ & 2.828710 & -1.726343 & -0.433503 \\
\hline H & 5.540242 & 1.599492 & -0.422902 \\
\hline C & -3.970621 & 0.062567 & 0.541565 \\
\hline C & -4.438940 & -0.850507 & 1.499926 \\
\hline C & -4.629948 & 0.121093 & -0.695510 \\
\hline C & -5.519337 & -1.682958 & 1.231506 \\
\hline $\mathrm{H}$ & -3.929032 & -0.921948 & 2.456034 \\
\hline C & -5.713061 & -0.710641 & -0.958392 \\
\hline H & -4.293207 & 0.823992 & -1.446102 \\
\hline C & -6.178744 & -1.630131 & -0.006132 \\
\hline $\mathrm{H}$ & 4.717244 & -3.265155 & -0.074761 \\
\hline $\mathrm{H}$ & 7.427371 & 0.063826 & -0.033372 \\
\hline H & -6.225758 & -0.625320 & -1.911019 \\
\hline $\mathrm{H}$ & -5.836652 & -2.406224 & 1.975989 \\
\hline c & 7.350987 & -2.645324 & 0.205540 \\
\hline C & 7.207276 & -3.768797 & 1.035167 \\
\hline C & 8.596997 & -2.422156 & -0.401795 \\
\hline C & 8.272697 & -4.639977 & 1.249836 \\
\hline $\mathrm{H}$ & 6.259361 & -3.943632 & 1.534178 \\
\hline C & 9.663303 & -3.291966 & -0.185978 \\
\hline H & 8.719373 & -1.574774 & -1.068894 \\
\hline C & 9.505943 & -4.405150 & 0.640633 \\
\hline $\mathrm{H}$ & 8.142203 & -5.498964 & 1.901145 \\
\hline $\mathrm{H}$ & 10.616123 & -3.105483 & -0.672194 \\
\hline H & 10.336566 & -5.083720 & 0.808041 \\
\hline C & -7.327304 & -2.518029 & -0.298181 \\
\hline C & -8.261576 & -2.839920 & 0.699139 \\
\hline C & -7.509379 & -3.059911 & -1.580539 \\
\hline C & -9.341286 & -3.675941 & 0.424164 \\
\hline $\mathrm{H}$ & -8.152356 & -2.408472 & 1.689192 \\
\hline c & -8.589876 & -3.894650 & -1.856282 \\
\hline $\mathrm{H}$ & -6.782403 & -2.843956 & -2.356901 \\
\hline C & -9.510563 & -4.206868 & -0.855186 \\
\hline $\mathrm{H}$ & -10.057082 & -3.905542 & 1.207859 \\
\hline H & -8.707797 & -4.309431 & -2.852848 \\
\hline $\mathrm{H}$ & -10.352366 & -4.857832 & -1.070094 \\
\hline
\end{tabular}


Frequencies (Top 3 out of 237)

$\begin{array}{lr}\text { 1. } & 8.7211 \mathrm{~cm}^{-1} \\ \text { 2. } & 16.8305 \mathrm{~cm}^{-1} \\ \text { 3. } & 21.7386 \mathrm{~cm}^{-1}\end{array}$

B3LYP-D3(BJ)/6-31G(d,p) Molecular Geometry in Cartesian Coordinates

\begin{tabular}{|c|c|c|c|}
\hline C & -1.560891 & -0.053821 & 1.211836 \\
\hline $\mathrm{H}$ & -2.538480 & 0.249021 & 1.594996 \\
\hline $\mathrm{H}$ & -1.002372 & -0.543187 & 2.013770 \\
\hline C & 1.565384 & -0.382226 & -1.241263 \\
\hline 0 & 2.147915 & -0.835051 & -2.203445 \\
\hline 0 & 0.809174 & 0.713052 & -1.333616 \\
\hline $\mathrm{H}$ & 0.314563 & 0.883425 & -0.497270 \\
\hline 0 & -0.836037 & 1.124373 & 0.774696 \\
\hline $\mathrm{H}$ & -0.582661 & 1.635847 & 1.554443 \\
\hline C & 0.583048 & -1.822950 & 0.536264 \\
\hline C & 1.651164 & -1.046993 & 0.116869 \\
\hline C & 2.834912 & -0.927778 & 0.914743 \\
\hline C & 2.892152 & -1.618405 & 2.110261 \\
\hline $\mathrm{H}$ & 3.783924 & -1.534881 & 2.724222 \\
\hline C & -1.719447 & -0.994010 & 0.044854 \\
\hline C & -0.677803 & -1.859475 & -0.269042 \\
\hline C & -3.053786 & -1.935298 & -1.746554 \\
\hline C & -2.925675 & -1.011601 & -0.726126 \\
\hline $\mathrm{H}$ & -3.964109 & -1.947337 & -2.338425 \\
\hline C & -2.017918 & -2.836939 & -2.079978 \\
\hline C & -0.794951 & -2.792785 & -1.340673 \\
\hline C & 0.254464 & -3.681535 & -1.696018 \\
\hline C & 0.093200 & -4.577241 & -2.726607 \\
\hline C & -1.120816 & -4.626662 & -3.453800 \\
\hline C & -2.151474 & -3.773282 & -3.139326 \\
\hline $\mathrm{H}$ & 1.191198 & -3.630856 & -1.154235 \\
\hline $\mathrm{H}$ & 0.905064 & -5.246656 & -2.992253 \\
\hline $\mathrm{H}$ & -1.231126 & -5.337474 & -4.266874 \\
\hline $\mathrm{H}$ & -3.082566 & -3.798226 & -3.698218 \\
\hline C & 1.842131 & -2.458741 & 2.550335 \\
\hline C & 0.662893 & -2.571314 & 1.750997 \\
\hline 0 & -0.389335 & -3.414634 & 2.197276 \\
\hline
\end{tabular}




\begin{tabular}{|c|c|c|c|}
\hline C & -0.277418 & -4.111452 & 3.379161 \\
\hline C & 0.889750 & -3.998457 & 4.171585 \\
\hline C & 1.925621 & -3.190330 & 3.764362 \\
\hline H & -1.281407 & -3.505920 & 1.587894 \\
\hline $\mathrm{H}$ & -1.087559 & -4.756048 & 3.705550 \\
\hline $\mathrm{H}$ & 0.964871 & -4.554303 & 5.100975 \\
\hline $\mathrm{H}$ & 2.826181 & -3.099964 & 4.365137 \\
\hline C & -6.229380 & 1.698807 & -0.075265 \\
\hline C & -4.914801 & 2.179130 & -0.184224 \\
\hline C & -3.843383 & 1.313955 & -0.377960 \\
\hline C & -4.047394 & -0.072412 & -0.469907 \\
\hline C & -5.360907 & -0.554433 & -0.363597 \\
\hline C & -6.430102 & 0.312883 & -0.168593 \\
\hline $\mathrm{H}$ & -2.839162 & 1.711651 & -0.476339 \\
\hline $\mathrm{H}$ & -5.536800 & -1.624778 & -0.409396 \\
\hline C & 3.967588 & -0.034874 & 0.551408 \\
\hline C & 4.427935 & 0.900423 & 1.491912 \\
\hline C & 4.635485 & -0.120367 & -0.679443 \\
\hline C & 5.511302 & 1.725692 & 1.213573 \\
\hline $\mathrm{H}$ & 3.922633 & 0.980214 & 2.449820 \\
\hline C & 5.719666 & 0.706628 & -0.953485 \\
\hline $\mathrm{H}$ & 4.293278 & -0.827867 & -1.423262 \\
\hline C & 6.179940 & 1.645363 & -0.017578 \\
\hline $\mathrm{H}$ & -4.735146 & 3.249096 & -0.152435 \\
\hline $\mathrm{H}$ & -7.431448 & -0.089911 & -0.054539 \\
\hline $\mathrm{H}$ & 6.204451 & 0.638262 & -1.921948 \\
\hline $\mathrm{H}$ & 5.858421 & 2.426640 & 1.966079 \\
\hline C & -7.367069 & 2.623631 & 0.132306 \\
\hline C & -7.230530 & 3.763487 & 0.940517 \\
\hline C & -8.610312 & 2.383557 & -0.474252 \\
\hline C & -8.300187 & 4.634173 & 1.135201 \\
\hline $\mathrm{H}$ & -6.284855 & 3.951890 & 1.438841 \\
\hline C & -9.680852 & 3.252885 & -0.278393 \\
\hline $\mathrm{H}$ & -8.727164 & 1.522973 & -1.125246 \\
\hline C & -9.530605 & 4.382467 & 0.527023 \\
\hline $\mathrm{H}$ & -8.175215 & 5.506104 & 1.770190 \\
\hline $\mathrm{H}$ & -10.631418 & 3.053061 & -0.763721 \\
\hline H & -10.364533 & 5.060649 & 0.678845 \\
\hline C & 7.334088 & 2.523397 & -0.317775 \\
\hline C & 8.429309 & 2.045850 & -1.055101 \\
\hline C & 7.362314 & 3.854386 & 0.128130 \\
\hline C & 9.515480 & 2.870988 & -1.337023 \\
\hline $\mathrm{H}$ & 8.436928 & 1.012464 & -1.386689 \\
\hline C & 8.448978 & 4.679508 & -0.152220 \\
\hline $\mathrm{H}$ & 6.512098 & 4.250029 & 0.674677 \\
\hline C & 9.530694 & 4.191574 & -0.886346 \\
\hline $\mathrm{H}$ & 10.355524 & 2.479239 & -1.902697 \\
\hline H & 8.446492 & 5.708517 & 0.194697 \\
\hline $\mathrm{H}$ & 10.377234 & 4.834721 & -1.106041 \\
\hline
\end{tabular}


Frequencies (Top 3 out of 237)

$\begin{array}{lr}\text { 1. } & 8.7020 \mathrm{~cm}^{-1} \\ \text { 2. } & 16.9902 \mathrm{~cm}^{-1} \\ \text { 3. } & 21.8364 \mathrm{~cm}^{-1}\end{array}$

B3LYP-D3(BJ)/6-31G(d,p) Molecular Geometry in Cartesian Coordinates

\begin{tabular}{|c|c|c|c|}
\hline C & -1.566741 & -0.088857 & 1.203088 \\
\hline $\mathrm{H}$ & -2.546272 & 0.202648 & 1.590132 \\
\hline $\mathrm{H}$ & -1.010988 & -0.598914 & 1.994019 \\
\hline C & 1.569346 & -0.348980 & -1.244471 \\
\hline 0 & 2.155066 & -0.775450 & -2.216710 \\
\hline 0 & 0.814055 & 0.748910 & -1.309255 \\
\hline $\mathrm{H}$ & 0.316500 & 0.896372 & -0.470329 \\
\hline 0 & -0.841466 & 1.101213 & 0.800624 \\
\hline $\mathrm{H}$ & -0.598541 & 1.596057 & 1.594324 \\
\hline C & 0.581124 & -1.838781 & 0.488948 \\
\hline C & 1.650205 & -1.050853 & 0.095180 \\
\hline C & 2.830771 & -0.952806 & 0.900598 \\
\hline C & 2.884103 & -1.676104 & 2.076808 \\
\hline $\mathrm{H}$ & 3.773458 & -1.608852 & 2.696251 \\
\hline C & -1.719705 & -0.997683 & 0.010753 \\
\hline C & -0.676575 & -1.854245 & -0.321914 \\
\hline C & -3.047339 & -1.892002 & -1.809586 \\
\hline C & -2.922937 & -0.995188 & -0.765067 \\
\hline $\mathrm{H}$ & -3.955486 & -1.888870 & -2.404911 \\
\hline C & -2.009892 & -2.784413 & -2.162592 \\
\hline C & -0.789512 & -2.759129 & -1.418118 \\
\hline C & 0.261560 & -3.637888 & -1.792877 \\
\hline C & 0.104270 & -4.506564 & -2.846955 \\
\hline C & -1.107238 & -4.537535 & -3.579325 \\
\hline C & -2.139369 & -3.692986 & -3.246343 \\
\hline $\mathrm{H}$ & 1.196401 & -3.600948 & -1.246725 \\
\hline $\mathrm{H}$ & 0.917364 & -5.168526 & -3.127131 \\
\hline $\mathrm{H}$ & -1.214458 & -5.227031 & -4.410960 \\
\hline $\mathrm{H}$ & -3.068518 & -3.703743 & -3.808912 \\
\hline C & 1.833139 & -2.529085 & 2.489471 \\
\hline C & 0.657010 & -2.620321 & 1.682853 \\
\hline 0 & -0.396122 & -3.476545 & 2.101551 \\
\hline
\end{tabular}




\begin{tabular}{|c|c|c|c|}
\hline C & -0.287971 & -4.205921 & 3.263982 \\
\hline C & 0.876094 & -4.114059 & 4.063669 \\
\hline C & 1.912725 & -3.294041 & 3.683014 \\
\hline $\mathrm{H}$ & -1.285769 & -3.551631 & 1.486439 \\
\hline $\mathrm{H}$ & -1.098706 & -4.860070 & 3.569220 \\
\hline $\mathrm{H}$ & 0.948249 & -4.695501 & 4.977504 \\
\hline $\mathrm{H}$ & 2.810934 & -3.219657 & 4.289475 \\
\hline C & -6.225811 & 1.700919 & -0.054347 \\
\hline C & -6.427337 & 0.317579 & -0.178066 \\
\hline C & -5.358311 & -0.545929 & -0.390749 \\
\hline C & -4.044964 & -0.061991 & -0.488379 \\
\hline C & -3.840304 & 1.321985 & -0.367590 \\
\hline C & -4.911132 & 2.182926 & -0.152903 \\
\hline $\mathrm{H}$ & -5.536592 & -1.613375 & -0.477425 \\
\hline $\mathrm{H}$ & -2.833848 & 1.719490 & -0.440748 \\
\hline C & 3.963892 & -0.049192 & 0.566297 \\
\hline C & 4.419389 & 0.860589 & 1.533785 \\
\hline C & 4.636649 & -0.100305 & -0.663814 \\
\hline C & 5.502790 & 1.694389 & 1.282303 \\
\hline $\mathrm{H}$ & 3.910207 & 0.913639 & 2.491486 \\
\hline C & 5.720877 & 0.735076 & -0.910934 \\
\hline $\mathrm{H}$ & 4.298197 & -0.787636 & -1.427997 \\
\hline C & 6.176314 & 1.648443 & 0.052047 \\
\hline $\mathrm{H}$ & -7.435431 & -0.081338 & -0.127126 \\
\hline $\mathrm{H}$ & -4.724802 & 3.246605 & -0.043312 \\
\hline $\mathrm{H}$ & 6.209529 & 0.693719 & -1.878987 \\
\hline $\mathrm{H}$ & 5.846087 & 2.374963 & 2.054992 \\
\hline C & -7.362894 & 2.621918 & 0.173227 \\
\hline C & -8.440572 & 2.247662 & 0.991630 \\
\hline C & -7.391042 & 3.892094 & -0.424431 \\
\hline C & -9.510640 & 3.113593 & 1.204697 \\
\hline $\mathrm{H}$ & -8.424029 & 1.280310 & 1.483717 \\
\hline C & -8.460178 & 4.758873 & -0.210333 \\
\hline $\mathrm{H}$ & -6.580560 & 4.188358 & -1.082729 \\
\hline C & -9.525087 & 4.373486 & 0.604986 \\
\hline $\mathrm{H}$ & -10.330449 & 2.807351 & 1.847535 \\
\hline $\mathrm{H}$ & -8.466162 & 5.733471 & -0.689039 \\
\hline $\mathrm{H}$ & -10.358725 & 5.048664 & 0.771176 \\
\hline C & 7.330515 & 2.535688 & -0.219508 \\
\hline C & 8.429258 & 2.079732 & -0.965218 \\
\hline C & 7.355278 & 3.854040 & 0.262668 \\
\hline C & 9.515494 & 2.913523 & -1.220150 \\
\hline $\mathrm{H}$ & 8.439504 & 1.055793 & -1.324858 \\
\hline C & 8.442003 & 4.687769 & 0.009316 \\
\hline $\mathrm{H}$ & 6.502399 & 4.233622 & 0.816405 \\
\hline C & 9.527250 & 4.221320 & -0.733515 \\
\hline $\mathrm{H}$ & 10.358278 & 2.538340 & -1.792935 \\
\hline $\mathrm{H}$ & 8.436837 & 5.706909 & 0.384208 \\
\hline $\mathrm{H}$ & 10.373851 & 4.871202 & -0.932150 \\
\hline
\end{tabular}


Frequencies (Top 3 out of 237)

$\begin{array}{lr}\text { 1. } & 7.2550 \mathrm{~cm}^{-1} \\ \text { 2. } & 15.6451 \mathrm{~cm}^{-1} \\ \text { 3. } & 20.9100 \mathrm{~cm}^{-1}\end{array}$

B3LYP-D3(BJ)/6-31G(d,p) Molecular Geometry in Cartesian Coordinates

\begin{tabular}{|c|c|c|c|}
\hline C & -1.630600 & 0.439043 & 1.282506 \\
\hline $\mathrm{H}$ & -0.964202 & 0.918257 & 1.998186 \\
\hline $\mathrm{H}$ & -2.614581 & 0.332253 & 1.748596 \\
\hline C & 1.332894 & 0.374648 & -1.169600 \\
\hline 0 & 1.802742 & 0.706438 & -2.236323 \\
\hline 0 & 0.602782 & -0.737724 & -1.043159 \\
\hline $\mathrm{H}$ & 0.168628 & -0.795473 & -0.156447 \\
\hline 0 & -1.070302 & -0.875485 & 1.064395 \\
\hline $\mathrm{H}$ & -1.770685 & -1.415063 & 0.667196 \\
\hline C & 0.562914 & 2.117225 & 0.433900 \\
\hline C & 1.542820 & 1.204526 & 0.080834 \\
\hline C & 2.747771 & 1.083873 & 0.843569 \\
\hline C & 2.915106 & 1.908079 & 1.940088 \\
\hline $\mathrm{H}$ & 3.826549 & 1.828075 & 2.524964 \\
\hline C & -1.744808 & 1.280178 & 0.028222 \\
\hline C & -0.718248 & 2.139626 & -0.335896 \\
\hline C & -3.089628 & 2.064573 & -1.836866 \\
\hline C & -2.927268 & 1.207385 & -0.770930 \\
\hline $\mathrm{H}$ & -3.993116 & 2.007803 & -2.437359 \\
\hline C & -2.080854 & 2.990493 & -2.200172 \\
\hline C & -0.857541 & 3.008752 & -1.459386 \\
\hline C & 0.174783 & 3.896154 & -1.862371 \\
\hline C & -0.005334 & 4.739277 & -2.933916 \\
\hline C & -1.221498 & 4.731690 & -3.658030 \\
\hline C & -2.234630 & 3.873047 & -3.300895 \\
\hline $\mathrm{H}$ & 1.111248 & 3.890734 & -1.317188 \\
\hline $\mathrm{H}$ & 0.792763 & 5.411241 & -3.233026 \\
\hline $\mathrm{H}$ & -1.347844 & 5.401414 & -4.503008 \\
\hline $\mathrm{H}$ & -3.165954 & 3.852148 & -3.859675 \\
\hline C & 1.952145 & 2.875848 & 2.312988 \\
\hline C & 0.750109 & 2.989383 & 1.547926 \\
\hline 0 & -0.218813 & 3.954677 & 1.929314 \\
\hline
\end{tabular}




\begin{tabular}{|c|c|c|c|}
\hline C & -0.003550 & 4.773301 & 3.014757 \\
\hline C & 1.187688 & 4.663379 & 3.771233 \\
\hline C & 2.142770 & 3.735234 & 3.426992 \\
\hline $\mathrm{H}$ & -1.129235 & 4.040428 & 1.346463 \\
\hline $\mathrm{H}$ & -0.750009 & 5.510670 & 3.293173 \\
\hline $\mathrm{H}$ & 1.344504 & 5.315506 & 4.624789 \\
\hline H & 3.059275 & 3.644737 & 4.003087 \\
\hline C & -5.824102 & -1.898987 & 0.031255 \\
\hline C & -6.117560 & -0.556101 & 0.314904 \\
\hline C & -5.193995 & 0.454132 & 0.057949 \\
\hline C & -3.942735 & 0.155016 & -0.492042 \\
\hline C & -3.650429 & -1.185058 & -0.795423 \\
\hline C & -4.573729 & -2.194046 & -0.535052 \\
\hline $\mathrm{H}$ & -5.440478 & 1.486890 & 0.284341 \\
\hline $\mathrm{H}$ & -2.701684 & -1.421623 & -1.271471 \\
\hline C & 3.788833 & 0.069730 & 0.534608 \\
\hline C & 4.253684 & -0.773176 & 1.556332 \\
\hline C & 4.362927 & -0.052468 & -0.739858 \\
\hline C & 5.250520 & -1.711003 & 1.313698 \\
\hline $\mathrm{H}$ & 3.803210 & -0.708038 & 2.542016 \\
\hline C & 5.362623 & -0.989603 & -0.977111 \\
\hline $\mathrm{H}$ & 4.029223 & 0.594909 & -1.540758 \\
\hline C & 5.825449 & -1.838662 & 0.039742 \\
\hline $\mathrm{H}$ & -7.090318 & -0.300093 & 0.722383 \\
\hline $\mathrm{H}$ & -4.319520 & -3.224722 & -0.760692 \\
\hline $\mathrm{H}$ & 5.813249 & -1.044272 & -1.962819 \\
\hline $\mathrm{H}$ & 5.564386 & -2.377194 & 2.110988 \\
\hline C & -6.806158 & -2.971376 & 0.314208 \\
\hline C & -6.952147 & -4.061937 & -0.557479 \\
\hline C & -7.613180 & -2.921686 & 1.461675 \\
\hline C & -7.874733 & -5.070597 & -0.289822 \\
\hline $\mathrm{H}$ & -6.356731 & -4.103520 & -1.464048 \\
\hline C & -8.536730 & -3.929619 & 1.728792 \\
\hline $\mathrm{H}$ & -7.495016 & -2.099991 & 2.161023 \\
\hline C & -8.671125 & -5.008635 & 0.854312 \\
\hline $\mathrm{H}$ & -7.978162 & -5.901640 & -0.980805 \\
\hline $\mathrm{H}$ & -9.145735 & -3.876939 & 2.626118 \\
\hline $\mathrm{H}$ & -9.390300 & -5.794549 & 1.062666 \\
\hline C & 6.884398 & -2.839760 & -0.223427 \\
\hline C & 7.858917 & -3.130030 & 0.744677 \\
\hline C & 6.939249 & -3.523646 & -1.448416 \\
\hline C & 8.854268 & -4.072508 & 0.497046 \\
\hline $\mathrm{H}$ & 7.848547 & -2.591924 & 1.687312 \\
\hline C & 7.935496 & -4.464876 & -1.697099 \\
\hline $\mathrm{H}$ & 6.178358 & -3.332279 & -2.198283 \\
\hline C & 8.897398 & -4.744047 & -0.725427 \\
\hline H & 9.603208 & -4.276051 & 1.256610 \\
\hline $\mathrm{H}$ & 7.954983 & -4.988122 & -2.648412 \\
\hline $\mathrm{H}$ & 9.673459 & -5.478101 & -0.919080 \\
\hline
\end{tabular}


Frequencies (Top 3 out of 237)

$\begin{array}{lr}\text { 1. } & 8.8687 \mathrm{~cm}^{-1} \\ \text { 2. } & 13.9043 \mathrm{~cm}^{-1} \\ \text { 3. } & 22.1548 \mathrm{~cm}^{-1}\end{array}$

B3LYP-D3(BJ)/6-31G(d,p) Molecular Geometry in Cartesian Coordinates

\begin{tabular}{|c|c|c|c|}
\hline C & -1.583893 & -0.095644 & 1.213362 \\
\hline $\mathrm{H}$ & -2.556558 & -0.228156 & 1.693296 \\
\hline $\mathrm{H}$ & -0.916678 & 0.373846 & 1.947306 \\
\hline C & 1.603475 & -0.029833 & -1.012352 \\
\hline 0 & 0.798630 & -0.928191 & -1.148289 \\
\hline 0 & 2.490808 & 0.213947 & -1.996105 \\
\hline $\mathrm{H}$ & 3.082361 & 0.933611 & -1.730390 \\
\hline 0 & -1.118318 & -1.395330 & 0.869842 \\
\hline $\mathrm{H}$ & -0.413660 & -1.290169 & 0.202724 \\
\hline C & 0.586175 & 1.669647 & 0.487619 \\
\hline C & 1.682743 & 0.875832 & 0.185865 \\
\hline C & 2.896771 & 0.957522 & 0.943688 \\
\hline C & 2.967472 & 1.864854 & 1.980954 \\
\hline $\mathrm{H}$ & 3.869521 & 1.912192 & 2.583399 \\
\hline C & -1.719256 & 0.809575 & 0.004252 \\
\hline C & -0.653173 & 1.624370 & -0.351261 \\
\hline C & -2.962332 & 1.617422 & -1.919501 \\
\hline C & -2.906476 & 0.804545 & -0.802209 \\
\hline $\mathrm{H}$ & -3.852484 & 1.601333 & -2.541067 \\
\hline C & -1.888258 & 2.450744 & -2.304779 \\
\hline C & -0.705124 & 2.466077 & -1.504358 \\
\hline C & 0.364806 & 3.318568 & -1.889191 \\
\hline C & 0.270203 & 4.106173 & -3.013660 \\
\hline C & -0.898508 & 4.081421 & -3.811318 \\
\hline C & -1.953375 & 3.271890 & -3.461451 \\
\hline $\mathrm{H}$ & 1.260639 & 3.349249 & -1.279023 \\
\hline $\mathrm{H}$ & 1.096619 & 4.752716 & -3.292398 \\
\hline $\mathrm{H}$ & -0.958686 & 4.704744 & -4.697964 \\
\hline $\mathrm{H}$ & -2.855937 & 3.246948 & -4.065498 \\
\hline C & 1.882112 & 2.711019 & 2.309796 \\
\hline C & 0.670742 & 2.616541 & 1.555658 \\
\hline 0 & -0.411954 & 3.472293 & 1.890266 \\
\hline
\end{tabular}




\begin{tabular}{|c|c|c|c|}
\hline C & -0.301551 & 4.371311 & 2.926152 \\
\hline C & 0.895123 & 4.459280 & 3.676453 \\
\hline C & 1.962487 & 3.646339 & 3.374624 \\
\hline $\mathrm{H}$ & -1.328572 & 3.402138 & 1.316765 \\
\hline H & -1.137827 & 5.017880 & 3.172098 \\
\hline H & 0.967021 & 5.171757 & 4.492216 \\
\hline $\mathrm{H}$ & 2.884031 & 3.706991 & 3.946273 \\
\hline C & -6.441843 & -1.536845 & 0.044155 \\
\hline C & -5.167420 & -2.122813 & 0.088726 \\
\hline C & -4.017471 & -1.381908 & -0.162863 \\
\hline C & -4.102374 & -0.014882 & -0.472531 \\
\hline C & -5.376638 & 0.574545 & -0.516042 \\
\hline C & -6.522814 & -0.169510 & -0.261771 \\
\hline H & -3.043768 & -1.857385 & -0.119548 \\
\hline $\mathrm{H}$ & -5.462366 & 1.636816 & -0.723648 \\
\hline c & 4.054023 & 0.076081 & 0.650905 \\
\hline C & 5.351700 & 0.605626 & 0.583323 \\
\hline C & 3.889424 & -1.304683 & 0.457814 \\
\hline C & 6.445052 & -0.214168 & 0.325475 \\
\hline $\mathrm{H}$ & 5.496636 & 1.674544 & 0.708232 \\
\hline C & 4.983839 & -2.121285 & 0.202303 \\
\hline H & 2.899804 & -1.743700 & 0.528500 \\
\hline C & 6.283437 & -1.594399 & 0.128458 \\
\hline H & -5.076813 & -3.184688 & 0.294530 \\
\hline $\mathrm{H}$ & -7.491120 & 0.320974 & -0.265849 \\
\hline H & 4.834236 & -3.189638 & 0.087617 \\
\hline H & 7.434150 & 0.224242 & 0.241583 \\
\hline C & -7.660842 & -2.332067 & 0.316683 \\
\hline C & -8.850731 & -2.092572 & -0.389242 \\
\hline C & -7.657490 & -3.345739 & 1.288293 \\
\hline C & -9.997956 & -2.839567 & -0.132017 \\
\hline H & -8.865166 & -1.331908 & -1.163412 \\
\hline C & -8.803797 & -4.094475 & 1.544434 \\
\hline H & -6.754765 & -3.528153 & 1.862492 \\
\hline C & -9.979776 & -3.844544 & 0.835946 \\
\hline $\mathrm{H}$ & -10.905241 & -2.643010 & -0.695507 \\
\hline H & -8.781038 & -4.868805 & 2.305445 \\
\hline H & -10.873408 & -4.427675 & 1.035948 \\
\hline C & 7.446106 & -2.467118 & -0.151874 \\
\hline C & 8.683530 & -2.239579 & 0.470863 \\
\hline C & 7.337464 & -3.542291 & -1.048042 \\
\hline C & 9.777294 & -3.060208 & 0.205809 \\
\hline H & 8.778366 & -1.430259 & 1.187958 \\
\hline C & 8.431045 & -4.363370 & -1.312484 \\
\hline $\mathrm{H}$ & 6.396240 & -3.716795 & -1.559513 \\
\hline C & 9.655600 & -4.125748 & -0.686974 \\
\hline $\mathrm{H}$ & 10.723493 & -2.873054 & 0.704404 \\
\hline H & 8.328844 & -5.184809 & -2.015024 \\
\hline $\mathrm{H}$ & 10.507773 & -4.765749 & -0.893243 \\
\hline
\end{tabular}


Frequencies (Top 3 out of 237)

$\begin{array}{lr}\text { 1. } & 8.8744 \mathrm{~cm}^{-1} \\ \text { 2. } & 13.8248 \mathrm{~cm}^{-1} \\ \text { 3. } & 22.0819 \mathrm{~cm}^{-1}\end{array}$

B3LYP-D3(BJ)/6-31G(d,p) Molecular Geometry in Cartesian Coordinates

\begin{tabular}{|c|c|c|c|}
\hline C & -1.588029 & -0.056382 & -1.214427 \\
\hline $\mathrm{H}$ & -0.923944 & 0.435917 & -1.936159 \\
\hline $\mathrm{H}$ & -2.562309 & -0.175223 & -1.694676 \\
\hline C & 1.606010 & -0.058879 & 1.003668 \\
\hline 0 & 0.801080 & -0.960540 & 1.115104 \\
\hline 0 & 2.496790 & 0.154539 & 1.991362 \\
\hline $\mathrm{H}$ & 3.087988 & 0.881534 & 1.745551 \\
\hline 0 & -1.119939 & -1.365434 & -0.912327 \\
\hline $\mathrm{H}$ & -0.414130 & -1.280135 & -0.243581 \\
\hline C & 0.584433 & 1.685482 & -0.440931 \\
\hline C & 1.681739 & 0.882703 & -0.166776 \\
\hline C & 2.893517 & 0.987372 & -0.925374 \\
\hline C & 2.961336 & 1.925833 & -1.934750 \\
\hline $\mathrm{H}$ & 3.861611 & 1.991361 & -2.538144 \\
\hline C & -1.719694 & 0.811173 & 0.022373 \\
\hline $\mathrm{C}$ & -0.652497 & 1.614599 & 0.399743 \\
\hline C & -2.956772 & 1.558617 & 1.974223 \\
\hline C & -2.904289 & 0.780805 & 0.832054 \\
\hline $\mathrm{H}$ & -3.844790 & 1.523053 & 2.598017 \\
\hline C & -1.881482 & 2.379711 & 2.381782 \\
\hline C & -0.700825 & 2.420032 & 1.578582 \\
\hline C & 0.370381 & 3.260112 & 1.986458 \\
\hline C & 0.279333 & 4.012325 & 3.135188 \\
\hline C & -0.886935 & 3.962740 & 3.935268 \\
\hline C & -1.942958 & 3.164484 & 3.563644 \\
\hline $\mathrm{H}$ & 1.264347 & 3.309666 & 1.374788 \\
\hline $\mathrm{H}$ & 1.106669 & 4.649868 & 3.431382 \\
\hline $\mathrm{H}$ & -0.944334 & 4.558161 & 4.841070 \\
\hline $\mathrm{H}$ & -2.843641 & 3.120756 & 4.169425 \\
\hline C & 1.875197 & 2.781795 & -2.234360 \\
\hline C & 0.666097 & 2.664549 & -1.479776 \\
\hline C & -0.417306 & 3.530434 & -1.784666 \\
\hline
\end{tabular}




\begin{tabular}{|c|c|c|c|}
\hline C & -0.309801 & 4.460668 & -2.792923 \\
\hline C & 0.884554 & 4.571192 & -3.543937 \\
\hline C & 1.952579 & 3.749164 & -3.270388 \\
\hline $\mathrm{H}$ & -1.332095 & 3.443036 & -1.210614 \\
\hline $\mathrm{H}$ & -1.146576 & 5.114725 & -3.016355 \\
\hline $\mathrm{H}$ & 0.954159 & 5.308221 & -4.337792 \\
\hline H & 2.872367 & 3.827037 & -3.842775 \\
\hline C & -6.440781 & -1.537388 & -0.071198 \\
\hline C & -5.166294 & -2.121387 & -0.135179 \\
\hline C & -4.016020 & -1.387542 & 0.135193 \\
\hline C & -4.100739 & -0.029224 & 0.481192 \\
\hline C & -5.374796 & 0.558817 & 0.541672 \\
\hline C & -6.521470 & -0.178858 & 0.271273 \\
\hline $\mathrm{H}$ & -3.041229 & -1.857242 & 0.061523 \\
\hline H & -5.461652 & 1.610885 & 0.795637 \\
\hline C & 4.051508 & 0.097422 & -0.662800 \\
\hline C & 3.887334 & -1.288593 & -0.511517 \\
\hline C & 5.349355 & 0.624741 & -0.582312 \\
\hline C & 4.982320 & -2.112519 & -0.283459 \\
\hline $\mathrm{H}$ & 2.897547 & -1.725297 & -0.593324 \\
\hline C & 6.443299 & -0.202456 & -0.352064 \\
\hline $\mathrm{H}$ & 5.493980 & 1.696977 & -0.675046 \\
\hline C & 6.282077 & -1.588016 & -0.196552 \\
\hline $\mathrm{H}$ & -5.072233 & -3.163574 & -0.423557 \\
\hline $\mathrm{H}$ & -7.494145 & 0.297247 & 0.345330 \\
\hline H & 7.432622 & 0.233220 & -0.257253 \\
\hline $\mathrm{H}$ & 4.832994 & -3.183877 & -0.200907 \\
\hline C & -7.660274 & -2.326349 & -0.359448 \\
\hline C & -8.749438 & -1.751079 & -1.033510 \\
\hline C & -7.758067 & -3.670441 & 0.034888 \\
\hline C & -9.897115 & -2.493137 & -1.302758 \\
\hline $\mathrm{H}$ & -8.681806 & -0.721721 & -1.371117 \\
\hline C & -8.904720 & -4.413482 & -0.235766 \\
\hline H & -6.936943 & -4.125158 & 0.579745 \\
\hline C & -9.980092 & -3.828214 & -0.905383 \\
\hline $\mathrm{H}$ & -10.724534 & -2.030575 & -1.832611 \\
\hline $\mathrm{H}$ & -8.961983 & -5.449384 & 0.084961 \\
\hline $\mathrm{H}$ & -10.874031 & -4.407141 & -1.115926 \\
\hline C & 7.445292 & -2.468762 & 0.054914 \\
\hline C & 7.338532 & -3.570061 & 0.919003 \\
\hline C & 8.681331 & -2.222897 & -0.563579 \\
\hline C & 8.432599 & -4.398685 & 1.156521 \\
\hline $\mathrm{H}$ & 6.398421 & -3.759598 & 1.427163 \\
\hline C & 9.775578 & -3.051116 & -0.325462 \\
\hline $\mathrm{H}$ & 8.774659 & -1.392625 & -1.256513 \\
\hline C & 9.655774 & -4.142666 & 0.535589 \\
\hline H & 8.331876 & -5.240604 & 1.834607 \\
\hline H & 10.720676 & -2.849311 & -0.820420 \\
\hline $\mathrm{H}$ & 10.508335 & -4.788549 & 0.720848 \\
\hline
\end{tabular}


Frequencies (Top 3 out of 237)

$\begin{array}{lr}\text { 1. } & 8.7634 \mathrm{~cm}^{-1} \\ \text { 2. } & 13.8516 \mathrm{~cm}^{-1} \\ \text { 3. } & 22.1009 \mathrm{~cm}^{-1}\end{array}$

B3LYP-D3(BJ)/6-31G(d,p) Molecular Geometry in Cartesian Coordinates

C

$\mathrm{H}$

$\mathrm{H}$

C

0

0

$\mathrm{H}$

0

$\mathrm{H}$

C

C

C

C

$\mathrm{H}$

C

C

C

C

$\mathrm{H}$

C

C

C

C

C

C

$\mathrm{H}$

$\mathrm{H}$

$\mathrm{H}$

$\mathrm{H}$

C

C

C
$-1.584411$

$-0.917922$

$-2.557209$

1.606106

0.800722

2.498115

3.089421

$-1.117518$

$-0.413166$

0.583660

1.681565

2.893588

2.960983

3.861079

$-1.719816$

$-0.654344$

$-2.962210$

$-2.906410$

$-3.851887$

$-1.888654$

$-0.706259$

0.362999

0.268501

$-0.899425$

$-1.953651$

1.258216

1.094386

$-0.959524$

$-2.855606$

1.874407

0.664948

$-0.419091$
$-0.139639$

0.303352

$-0.290345$

0.010460

$-0.881238$

0.288499

0.997045

$-1.425585$

$-1.295442$

1.653921

0.872119

0.927125

1.796941

1.822964

0.808818

1.637110

1.684322

0.831774

1.689845

2.532040

2.519826

3. 386913

4. 214516

4. 217195

3. 394328

3. 396475

4.871688

4.872041

3. 390161

2.630530

2.562324

3.405065

\subsection{1}

1.947547

1.670949

$-1.019424$

$-1.191206$

$-1.989731$

$-1.695191$

0.806304

0.143273

0.538269

0.210964

0.974445

2.043652

2.650277

0.021203

$-0.303641$

$-1.873141$

$-0.785827$

$-2.495571$

$-2.227203$

$-1.425632$

$-1.778335$

$-2.873708$

$-3.672894$

$-3.353538$

$-1.166567$

$-3.128110$

$-4.536528$

$-3.958987$

2. 399443

1.639478

2.001684 


\begin{tabular}{|c|c|c|c|}
\hline C & -0.311649 & 4.266888 & 3.069015 \\
\hline C & 0.883197 & 4.328792 & 3.824820 \\
\hline C & 1.951721 & 3.527540 & 3.496967 \\
\hline $\mathrm{H}$ & -1.334350 & 3.354857 & 1.423938 \\
\hline $\mathrm{H}$ & -1.148878 & 4.903928 & 3.335711 \\
\hline H & 0.952777 & 5.011967 & 4.665472 \\
\hline $\mathrm{H}$ & 2.871855 & 3.568296 & 4.072638 \\
\hline C & -6.440450 & -1.540956 & -0.025765 \\
\hline C & -5.165704 & -2.127457 & -0.002556 \\
\hline C & -4.016175 & -1.377216 & -0.226905 \\
\hline c & -4.101871 & 0.000131 & -0.486557 \\
\hline C & -5.376451 & 0.590035 & -0.508687 \\
\hline C & -6.522207 & -0.163441 & -0.281734 \\
\hline H & -3.042172 & -1.853328 & -0.200789 \\
\hline $\mathrm{H}$ & -5.462736 & 1.659110 & -0.677522 \\
\hline C & 4.051985 & 0.056050 & 0.655336 \\
\hline C & 5.349720 & 0.587654 & 0.609581 \\
\hline C & 3.888659 & -1.317898 & 0.417949 \\
\hline C & 6.444873 & -0.224198 & 0.334857 \\
\hline $\mathrm{H}$ & 5.495898 & 1.649057 & 0.786374 \\
\hline C & 4.984441 & -2.125846 & 0.141008 \\
\hline H & 2.896722 & -1.756037 & 0.452383 \\
\hline C & 6.284594 & -1.597502 & 0.093692 \\
\hline $\mathrm{H}$ & -5.074512 & -3.196075 & 0.164423 \\
\hline $\mathrm{H}$ & -7.490805 & 0.326296 & -0.268020 \\
\hline H & 4.829141 & -3.180197 & -0.061843 \\
\hline H & 7.440833 & 0.206521 & 0.324966 \\
\hline C & -7.659005 & -2.346194 & 0.217804 \\
\hline C & -8.849052 & -2.081982 & -0.478975 \\
\hline C & -7.655095 & -3.394276 & 1.152189 \\
\hline C & -9.995889 & -2.838347 & -0.248823 \\
\hline $\mathrm{H}$ & -8.863909 & -1.293883 & -1.225191 \\
\hline C & -8.801018 & -4.152329 & 1.381208 \\
\hline H & -6.752240 & -3.596848 & 1.719381 \\
\hline C & -9.977165 & -3.877578 & 0.682257 \\
\hline $\mathrm{H}$ & -10.903301 & -2.622043 & -0.804826 \\
\hline H & -8.777830 & -4.953597 & 2.113791 \\
\hline H & -10.870502 & -4.467980 & 0.861132 \\
\hline C & 7.449999 & -2.461699 & -0.201773 \\
\hline C & 7.512037 & -3.776459 & 0.286533 \\
\hline C & 8.520165 & -1.986650 & -0.976312 \\
\hline C & 8.608535 & -4.589368 & 0.009462 \\
\hline H & 6.706332 & -4.152430 & 0.908960 \\
\hline C & 9.616975 & -2.799317 & -1.252948 \\
\hline $\mathrm{H}$ & 8.476670 & -0.982724 & -1.386650 \\
\hline C & 9.665878 & -4.104274 & -0.761193 \\
\hline $\mathrm{H}$ & 8.640923 & -5.600697 & 0.403109 \\
\hline H & 10.430305 & -2.416089 & -1.861651 \\
\hline $\mathrm{H}$ & 10.520243 & -4.738031 & -0.977358 \\
\hline
\end{tabular}


Frequencies (Top 3 out of 237)

$\begin{array}{lr}\text { 1. } & 9.5307 \mathrm{~cm}^{-1} \\ \text { 2. } & 16.4099 \mathrm{~cm}^{-1} \\ \text { 3. } & 21.2128 \mathrm{~cm}^{-1}\end{array}$

B3LYP-D3(BJ)/6-31G(d,p) Molecular Geometry in Cartesian Coordinates

\begin{tabular}{|c|c|c|c|}
\hline C & 1.560277 & -0.040945 & 1.210661 \\
\hline $\mathrm{H}$ & 2.542029 & -0.161746 & 1.672594 \\
\hline $\mathrm{H}$ & 0.904517 & 0.434623 & 1.948023 \\
\hline C & -1.588342 & 0.068133 & -1.028137 \\
\hline 0 & -1.682258 & 0.412893 & -2.181753 \\
\hline 0 & -1.360993 & -1.227052 & -0.672379 \\
\hline $\mathrm{H}$ & -1.307364 & -1.737952 & -1.499236 \\
\hline 0 & 1.108917 & -1.361991 & 0.916289 \\
\hline $\mathrm{H}$ & 0.227215 & -1.300972 & 0.517943 \\
\hline C & -0.600277 & 1.758260 & 0.481952 \\
\hline C & -1.686149 & 0.958313 & 0.173980 \\
\hline C & -2.868309 & 0.946088 & 0.977819 \\
\hline C & -2.912759 & 1.767462 & 2.085962 \\
\hline $\mathrm{H}$ & -3.810053 & 1.784276 & 2.697200 \\
\hline C & 1.682280 & 0.850243 & -0.013312 \\
\hline C & 0.641222 & 1.707257 & -0.353809 \\
\hline C & 2.970394 & 1.691034 & -1.895266 \\
\hline C & 2.877794 & 0.839222 & -0.810627 \\
\hline $\mathrm{H}$ & 3.868872 & 1.674616 & -2.504993 \\
\hline C & 1.927513 & 2.573186 & -2.258124 \\
\hline C & 0.733365 & 2.587280 & -1.476089 \\
\hline C & -0.311087 & 3.476564 & -1.841987 \\
\hline C & -0.174651 & 4.312419 & -2.926105 \\
\hline C & 1.009159 & 4. 297091 & -3.701618 \\
\hline C & 2.036154 & 3.443405 & -3.374860 \\
\hline $\mathrm{H}$ & -1.225018 & 3.482565 & -1.260139 \\
\hline $\mathrm{H}$ & -0.983082 & 4.985375 & -3.194223 \\
\hline $\mathrm{H}$ & 1.101213 & 4.959760 & -4.556591 \\
\hline $\mathrm{H}$ & 2.948050 & 3.421160 & -3.964918 \\
\hline C & -1.836280 & 2.620018 & 2.430610 \\
\hline C & -0.660768 & 2.625779 & 1.616201 \\
\hline 0 & 0.412927 & 3.485756 & 1.966652 \\
\hline
\end{tabular}




\begin{tabular}{|c|c|c|c|}
\hline C & 0.324298 & 4.304395 & 3.069381 \\
\hline C & -0.839217 & 4.299995 & 3.874918 \\
\hline C & -1.895120 & 3.476369 & 3.561447 \\
\hline $\mathrm{H}$ & 1.303212 & 3.487878 & 1.348535 \\
\hline $\mathrm{H}$ & 1.151135 & 4.959693 & 3.324307 \\
\hline $\mathrm{H}$ & -0.894848 & 4.950154 & 4.742409 \\
\hline $\mathrm{H}$ & -2.791461 & 3.467675 & 4.174909 \\
\hline C & 6.349404 & -1.613110 & -0.006795 \\
\hline C & 5.061978 & -2.171462 & -0.002087 \\
\hline C & 3.932950 & -1.395059 & -0.240981 \\
\hline C & 4.050561 & -0.019475 & -0.496499 \\
\hline C & 5.338403 & 0.541797 & -0.499142 \\
\hline C & 6.464174 & -0.237189 & -0.258662 \\
\hline $\mathrm{H}$ & 2.949477 & -1.849949 & -0.226383 \\
\hline $\mathrm{H}$ & 5.450278 & 1.609034 & -0.663829 \\
\hline C & -4.026474 & 0.082238 & 0.643737 \\
\hline C & -4.572904 & 0.053193 & -0.648528 \\
\hline C & -4.624241 & -0.710066 & 1.634848 \\
\hline C & -5.673711 & -0.746469 & -0.936007 \\
\hline $\mathrm{H}$ & -4.149245 & 0.678587 & -1.426348 \\
\hline C & -5.723439 & -1.509558 & 1.343862 \\
\hline $\mathrm{H}$ & -4.198210 & -0.720127 & 2.633178 \\
\hline C & -6.270600 & -1.545517 & 0.051455 \\
\hline $\mathrm{H}$ & 4.945160 & -3.237971 & 0.162523 \\
\hline $\mathrm{H}$ & 7.442867 & 0.231380 & -0.230622 \\
\hline $\mathrm{H}$ & -6.143770 & -2.142564 & 2.118673 \\
\hline $\mathrm{H}$ & -6.099600 & -0.725379 & -1.933912 \\
\hline C & 7.546603 & -2.445249 & 0.251644 \\
\hline C & 7.507390 & -3.494068 & 1.184378 \\
\hline C & 8.751113 & -2.206368 & -0.429222 \\
\hline C & 8.633266 & -4.277486 & 1.427097 \\
\hline $\mathrm{H}$ & 6.593199 & -3.677019 & 1.740008 \\
\hline C & 9.877899 & -2.988138 & -0.185348 \\
\hline $\mathrm{H}$ & 8.793162 & -1.417642 & -1.173736 \\
\hline C & 9.824120 & -4.027886 & 0.743792 \\
\hline $\mathrm{H}$ & 8.583077 & -5.078710 & 2.158362 \\
\hline $\mathrm{H}$ & 10.797081 & -2.791052 & -0.728983 \\
\hline $\mathrm{H}$ & 10.701878 & -4.637923 & 0.933479 \\
\hline C & -7.439434 & -2.399589 & -0.260020 \\
\hline C & -7.545921 & -3.049075 & -1.500143 \\
\hline C & -8.469182 & -2.580814 & 0.676916 \\
\hline C & -8.645356 & -3.852217 & -1.793911 \\
\hline $\mathrm{H}$ & -6.746915 & -2.942564 & -2.226982 \\
\hline C & -9.567971 & -3.385214 & 0.384170 \\
\hline $\mathrm{H}$ & -8.418050 & -2.064889 & 1.630489 \\
\hline C & -9.661159 & -4.024329 & -0.852789 \\
\hline $\mathrm{H}$ & -8.704365 & -4.351938 & -2.756088 \\
\hline $\mathrm{H}$ & -10.357412 & -3.505669 & 1.119893 \\
\hline $\mathrm{H}$ & -10.517730 & -4.650799 & -1.081301 \\
\hline
\end{tabular}


Frequencies (Top 3 out of 237)

$\begin{array}{lr}\text { 1. } & 9.5302 \mathrm{~cm}^{-1} \\ \text { 2. } & 16.4093 \mathrm{~cm}^{-1} \\ \text { 3. } & 21.2129 \mathrm{~cm}^{-1}\end{array}$

B3LYP-D3(BJ)/6-31G(d,p) Molecular Geometry in Cartesian Coordinates

\begin{tabular}{|c|c|c|c|}
\hline C & 1.560298 & -0.040987 & 1.210647 \\
\hline $\mathrm{H}$ & 0.904525 & 0.434561 & 1.948013 \\
\hline $\mathrm{H}$ & 2.542049 & -0.161783 & 1.672583 \\
\hline C & -1.588313 & 0.068063 & -1.028119 \\
\hline 0 & -1.682229 & 0.412773 & -2.181750 \\
\hline 0 & -1.360971 & -1.227114 & -0.672311 \\
\hline $\mathrm{H}$ & -1.307318 & -1.738040 & -1.499151 \\
\hline 0 & 1.108954 & -1.362032 & 0.916243 \\
\hline $\mathrm{H}$ & 0.227247 & -1.301010 & 0.517902 \\
\hline C & -0.600270 & 1.758219 & 0.481952 \\
\hline C & -1.686142 & 0.958277 & 0.173968 \\
\hline C & -2.868320 & 0.946076 & 0.977783 \\
\hline C & -2.912779 & 1.767458 & 2.085921 \\
\hline $\mathrm{H}$ & -3.810084 & 1.784284 & 2.697143 \\
\hline C & 1.682296 & 0.850217 & -0.013314 \\
\hline C & 0.641233 & 1.707225 & -0.353805 \\
\hline C & 2.970403 & 1.691015 & -1.895268 \\
\hline C & 2.877809 & 0.839203 & -0.810630 \\
\hline $\mathrm{H}$ & 3.868881 & 1.674604 & -2.504995 \\
\hline C & 1.927517 & 2.573163 & -2.258123 \\
\hline C & 0.733371 & 2.587252 & -1.476084 \\
\hline C & -0.311084 & 3.476537 & -1.841976 \\
\hline C & -0.174653 & 4.312393 & -2.926092 \\
\hline C & 1.009154 & 4.297068 & -3.701611 \\
\hline C & 2.036151 & 3.443385 & -3.374857 \\
\hline $\mathrm{H}$ & -1.225012 & 3.482536 & -1.260124 \\
\hline $\mathrm{H}$ & -0.983085 & 4.985350 & -3.194205 \\
\hline $\mathrm{H}$ & 1.101204 & 4.959739 & -4.556582 \\
\hline $\mathrm{H}$ & 2.948045 & 3.421143 & -3.964919 \\
\hline C & -1.836297 & 2.620003 & 2.430584 \\
\hline C & -0.660773 & 2.625751 & 1.616192 \\
\hline 0 & 0.412921 & 3.485725 & 1.966651 \\
\hline
\end{tabular}




\begin{tabular}{|c|c|c|c|}
\hline C & 0.324281 & 4.304371 & 3.069373 \\
\hline C & -0.839244 & 4.299983 & 3.874894 \\
\hline C & -1.895148 & 3.476362 & 3.561414 \\
\hline $\mathrm{H}$ & 1.303214 & 3.487838 & 1.348547 \\
\hline $\mathrm{H}$ & 1.151119 & 4.959666 & 3.324306 \\
\hline $\mathrm{H}$ & -0.894884 & 4.950149 & 4.742380 \\
\hline H & -2.791498 & 3.467678 & 4.174863 \\
\hline C & 6.349446 & -1.613085 & -0.006787 \\
\hline C & 5.062029 & -2.171457 & -0.002093 \\
\hline C & 3.932990 & -1.395068 & -0.240988 \\
\hline C & 4.050584 & -0.019480 & -0.496496 \\
\hline C & 5.338417 & 0.541811 & -0.499126 \\
\hline C & 6.464197 & -0.237160 & -0.258643 \\
\hline H & 2.949523 & -1.849971 & -0.226401 \\
\hline H & 5.450276 & 1.609051 & -0.663805 \\
\hline C & -4.026491 & 0.082233 & 0.643702 \\
\hline C & -4.624302 & -0.710007 & 1.634838 \\
\hline C & -4.572894 & 0.053142 & -0.648573 \\
\hline C & -5.723515 & -1.509485 & 1.343867 \\
\hline $\mathrm{H}$ & -4.198293 & -0.720029 & 2.633178 \\
\hline C & -5.673716 & -0.746505 & -0.936038 \\
\hline $\mathrm{H}$ & -4.149204 & 0.678492 & -1.426411 \\
\hline C & -6.270646 & -1.545491 & 0.051449 \\
\hline $\mathrm{H}$ & 4.945226 & -3.237969 & 0.162506 \\
\hline $\mathrm{H}$ & 7.442883 & 0.231424 & -0.230590 \\
\hline H & -6.099584 & -0.725449 & -1.933952 \\
\hline $\mathrm{H}$ & -6.143878 & -2.142443 & 2.118698 \\
\hline C & 7.546655 & -2.445207 & 0.251653 \\
\hline C & 7.507447 & -3.494046 & 1.184366 \\
\hline C & 8.751171 & -2.206292 & -0.429190 \\
\hline C & 8.633333 & -4.277449 & 1.427086 \\
\hline $\mathrm{H}$ & 6.593251 & -3.677023 & 1.739980 \\
\hline C & 9.877967 & -2.988047 & -0.185315 \\
\hline H & 8.793217 & -1.417550 & -1.173688 \\
\hline C & 9.824193 & -4.027814 & 0.743803 \\
\hline $\mathrm{H}$ & 8.583147 & -5.078688 & 2.158334 \\
\hline $\mathrm{H}$ & 10.797154 & -2.790933 & -0.728933 \\
\hline H & 10.701959 & -4.637839 & 0.933491 \\
\hline C & -7.439496 & -2.399546 & -0.260011 \\
\hline C & -8.469262 & -2.580712 & 0.676915 \\
\hline C & -7.545979 & -3.049077 & -1.500112 \\
\hline C & -9.568065 & -3.385098 & 0.384183 \\
\hline $\mathrm{H}$ & -8.418133 & -2.064751 & 1.630469 \\
\hline C & -8.645429 & -3.852205 & -1.793866 \\
\hline $\mathrm{H}$ & -6.746959 & -2.942614 & -2.226942 \\
\hline C & -9.661249 & -4.024258 & -0.852753 \\
\hline H & -10.357521 & -3.505506 & 1.119898 \\
\hline $\mathrm{H}$ & -8.704434 & -4.351961 & -2.756025 \\
\hline $\mathrm{H}$ & -10.517832 & -4.650716 & -1.081255 \\
\hline
\end{tabular}


Frequencies (Top 3 out of 237)

$\begin{array}{lr}\text { 1. } & 9.4536 \mathrm{~cm}^{-1} \\ \text { 2. } & 16.0750 \mathrm{~cm}^{-1} \\ \text { 3. } & 20.9568 \mathrm{~cm}^{-1}\end{array}$

B3LYP-D3(BJ)/6-31G(d,p) Molecular Geometry in Cartesian Coordinates

\begin{tabular}{|c|c|c|c|}
\hline C & 1.564399 & -0.005843 & 1.204684 \\
\hline $\mathrm{H}$ & 2.547637 & -0.113532 & 1.666700 \\
\hline $\mathrm{H}$ & 0.911423 & 0.491826 & 1.929819 \\
\hline C & -1.593901 & 0.038239 & -1.025312 \\
\hline 0 & -1.693208 & 0.348452 & -2.188258 \\
\hline 0 & -1.365430 & -1.245872 & -0.632179 \\
\hline $\mathrm{H}$ & -1.316247 & -1.781260 & -1.443665 \\
\hline 0 & 1.111353 & -1.334744 & 0.951128 \\
\hline $\mathrm{H}$ & 0.228499 & -1.285000 & 0.553801 \\
\hline C & -0.597903 & 1.771819 & 0.429486 \\
\hline C & -1.685648 & 0.963963 & 0.150139 \\
\hline C & -2.864306 & 0.976627 & 0.959107 \\
\hline C & -2.903472 & 1.830716 & 2.042418 \\
\hline $\mathrm{H}$ & -3.798033 & 1.866270 & 2.656848 \\
\hline C & 1.682481 & 0.848057 & -0.045952 \\
\hline C & 0.640366 & 1.694464 & -0.409065 \\
\hline C & 2.964227 & 1.630067 & -1.957390 \\
\hline C & 2.875234 & 0.812265 & -0.846577 \\
\hline $\mathrm{H}$ & 3.860543 & 1.594775 & -2.569486 \\
\hline C & 1.920048 & 2.500648 & -2.343791 \\
\hline C & 0.728678 & 2.539301 & -1.558346 \\
\hline C & -0.316960 & 3.417060 & -1.947928 \\
\hline C & -0.184298 & 4.218831 & -3.057937 \\
\hline C & 0.996752 & 4.179156 & -3.836797 \\
\hline C & 2.024817 & 3.335801 & -3.487363 \\
\hline $\mathrm{H}$ & -1.228835 & 3.441199 & -1.363331 \\
\hline $\mathrm{H}$ & -0.993613 & 4.883300 & -3.343962 \\
\hline $\mathrm{H}$ & 1.085857 & 4.814953 & -4.712245 \\
\hline $\mathrm{H}$ & 2.934608 & 3.295084 & -4.079681 \\
\hline C & -1.825088 & 2.692566 & 2.356648 \\
\hline C & -0.653126 & 2.673131 & 1.537372 \\
\hline 0 & 0.422406 & 3.542743 & 1.857042 \\
\hline
\end{tabular}




\begin{tabular}{|c|c|c|c|}
\hline C & 0.338935 & 4.394258 & 2.934993 \\
\hline C & -0.821003 & 4.414655 & 3.745423 \\
\hline C & -1.878598 & 3.582539 & 3.461490 \\
\hline $\mathrm{H}$ & 1.309878 & 3.525830 & 1.235128 \\
\hline H & 1.167083 & 5.056597 & 3.166391 \\
\hline H & -0.872573 & 5.090638 & 4.593203 \\
\hline $\mathrm{H}$ & -2.772241 & 3.592794 & 4.078847 \\
\hline C & 6.349635 & -1.614068 & 0.021433 \\
\hline C & 5.062732 & -2.173237 & 0.042906 \\
\hline C & 3.932833 & -1.404950 & -0.217569 \\
\hline C & 4.049085 & -0.036533 & -0.509646 \\
\hline C & 5.336130 & 0.525922 & -0.527239 \\
\hline C & 6.462949 & -0.245526 & -0.267831 \\
\hline H & 2.948530 & -1.856378 & -0.173865 \\
\hline $\mathrm{H}$ & 5.448369 & 1.584836 & -0.738847 \\
\hline C & -4.024351 & 0.103866 & 0.656095 \\
\hline C & -4.617454 & -0.658866 & 1.672868 \\
\hline C & -4.577066 & 0.037278 & -0.632093 \\
\hline C & -5.718278 & -1.466064 & 1.410742 \\
\hline $\mathrm{H}$ & -4.186518 & -0.639896 & 2.668960 \\
\hline C & -5.679523 & -0.769939 & -0.890683 \\
\hline H & -4.156945 & 0.639432 & -1.429919 \\
\hline C & -6.271800 & -1.539502 & 0.122640 \\
\hline H & 4.942765 & -3.223162 & 0.290852 \\
\hline H & 7.446059 & 0.212819 & -0.308205 \\
\hline H & -6.110311 & -0.777846 & -1.886675 \\
\hline H & -6.134992 & -2.075982 & 2.205759 \\
\hline C & 7.547897 & -2.438271 & 0.299836 \\
\hline C & 7.625776 & -3.768434 & -0.143075 \\
\hline C & 8.636282 & -1.911035 & 1.013272 \\
\hline C & 8.752396 & -4.544572 & 0.118671 \\
\hline H & 6.805493 & -4.185530 & -0.718504 \\
\hline C & 9.763973 & -2.686132 & 1.273668 \\
\hline H & 8.583358 & -0.893820 & 1.388202 \\
\hline C & 9.827248 & -4.006910 & 0.827843 \\
\hline $\mathrm{H}$ & 8.794710 & -5.568749 & -0.239803 \\
\hline H & 10.591011 & -2.260769 & 1.834334 \\
\hline H & 10.705611 & -4.611544 & 1.031478 \\
\hline C & -7.442472 & -2.401724 & -0.157959 \\
\hline C & -8.467528 & -2.555122 & 0.789039 \\
\hline C & -7.555441 & -3.086959 & -1.378106 \\
\hline C & -9.568027 & -3.367201 & 0.525381 \\
\hline H & -8.411394 & -2.011689 & 1.726921 \\
\hline C & -8.656595 & -3.897805 & -1.642823 \\
\hline $\mathrm{H}$ & -6.760091 & -3.001989 & -2.111762 \\
\hline C & -9.667669 & -4.042001 & -0.691966 \\
\hline $\mathrm{H}$ & -10.353772 & -3.465831 & 1.268276 \\
\hline H & -8.720638 & -4.425281 & -2.589742 \\
\hline $\mathrm{H}$ & -10.525581 & -4.674466 & -0.897814 \\
\hline
\end{tabular}


Frequencies (Top 3 out of 237)

$\begin{array}{lr}\text { 1. } & 9.4592 \mathrm{~cm}^{-1} \\ \text { 2. } & 16.0787 \mathrm{~cm}^{-1} \\ \text { 3. } & 20.9625 \mathrm{~cm}^{-1}\end{array}$

B3LYP-D3(BJ)/6-31G(d,p) Molecular Geometry in Cartesian Coordinates

C

$\mathrm{H}$

$\mathrm{H}$

C

0

0

$\mathrm{H}$

0

$\mathrm{H}$

C

C

C

C

$\mathrm{H}$

C

C

C

C

$\mathrm{H}$

C

C

C

C

C

C

$\mathrm{H}$

$\mathrm{H}$

$\mathrm{H}$

$\mathrm{H}$

C

C

C

\section{$-1.564433$}

$-0.911493$

$-2.547664$

1.593459

1.692602

1. 364848

1. 315175

$-1.111179$

$-0.228035$

0.597897

1.685549

2.864328

2.903678

3.798326

$-1.682569$

$-0.640436$

$-2.964301$

$-2.875312$

$-3.860628$

$-1.920101$

$-0.728740$

0.316901

0.184260

$-0.996775$

$-2.024854$

1.228768

0.993581

$-1.085862$

$-2.934636$

1.825397

0.653336

$-0.422068$
$-0.005755$

0.491899

$-0.113567$

0.037896

0.347850

$-1.246079$

$-1.781556$

$-1.334554$

$-1.284693$

1.771914

0.963895

0.976660

1.830937

1.866553

0.848209

1.694580

1.630100

0.812372

1.594782

2.500627

2.539329

3.417058

4. 218732

4. 178992

3. 335673

3. 441272

4.883174

4.814703

3. 294902

2. 692908

2.673435

3. 543219
1.204738

1.929905

1.666748

$-1.025068$

$-2.188102$

$-0.631603$

$-1.443003$

0.950972

0.554325

0.429468

0.150142

0.958918

2.042083

2.656383

$-0.045839$

$-0.408989$

$-1.957341$

$-0.846470$

$-2.569420$

$-2.343799$

$-1.558343$

$-1.947989$

$-3.058080$

$-3.836954$

$-3.487449$

$-1.363382$

$-3.344148$

$-4.712466$

$-4.079775$

2. 356308

1. 537175

1.856814 


\begin{tabular}{|c|c|c|c|}
\hline C & -0.338387 & 4.394917 & 2.934611 \\
\hline C & 0.821643 & 4.415338 & 3.744906 \\
\hline C & 1.879126 & 3.583065 & 3.460991 \\
\hline $\mathrm{H}$ & -1.309612 & 3.526298 & 1.235005 \\
\hline $\mathrm{H}$ & -1.166441 & 5.057382 & 3.165985 \\
\hline $\mathrm{H}$ & 0.873378 & 5.091463 & 4.592562 \\
\hline H & 2.772844 & 3.593343 & 4.078238 \\
\hline C & -6.349613 & -1.614062 & 0.021608 \\
\hline C & -5.062689 & -2.173193 & 0.043031 \\
\hline C & -3.932821 & -1.404861 & -0.217461 \\
\hline C & -4.049139 & -0.036436 & -0.509488 \\
\hline C & -5.336199 & 0.525977 & -0.527055 \\
\hline C & -6.462988 & -0.245519 & -0.267626 \\
\hline $\mathrm{H}$ & -2.948501 & -1.856260 & -0.173813 \\
\hline H & -5.448469 & 1.584887 & -0.738669 \\
\hline C & 4.024319 & 0.103824 & 0.655943 \\
\hline C & 4.617510 & -0.658724 & 1.672807 \\
\hline C & 4.576933 & 0.037009 & -0.632279 \\
\hline C & 5.718313 & -1.465972 & 1.410738 \\
\hline $\mathrm{H}$ & 4.186668 & -0.639562 & 2.668936 \\
\hline C & 5.679373 & -0.770257 & -0.890810 \\
\hline $\mathrm{H}$ & 4.156753 & 0.639029 & -1.430172 \\
\hline C & 6.271728 & -1.539647 & 0.122600 \\
\hline $\mathrm{H}$ & -4.942686 & -3.223124 & 0.290928 \\
\hline $\mathrm{H}$ & -7.446115 & 0.212790 & -0.307958 \\
\hline H & 6.110079 & -0.778339 & -1.886836 \\
\hline $\mathrm{H}$ & 6.135100 & -2.075733 & 2.205836 \\
\hline C & -7.547842 & -2.438322 & 0.300005 \\
\hline C & -7.625743 & -3.768422 & -0.143092 \\
\hline C & -8.636172 & -1.911197 & 1.013609 \\
\hline C & -8.752337 & -4.544611 & 0.118636 \\
\hline $\mathrm{H}$ & -6.805502 & -4.185430 & -0.718643 \\
\hline C & -9.763836 & -2.686345 & 1.273990 \\
\hline H & -8.583230 & -0.894033 & 1.388674 \\
\hline C & -9.827137 & -4.007060 & 0.827976 \\
\hline $\mathrm{H}$ & -8.794672 & -5.568736 & -0.239984 \\
\hline $\mathrm{H}$ & -10.590831 & -2.261071 & 1.834785 \\
\hline $\mathrm{H}$ & -10.705476 & -4.611731 & 1.031595 \\
\hline C & 7.442380 & -2.401920 & -0.157939 \\
\hline C & 8.467562 & -2.555069 & 0.788964 \\
\hline C & 7.555206 & -3.087450 & -1.377936 \\
\hline C & 9.568047 & -3.367192 & 0.525362 \\
\hline $\mathrm{H}$ & 8.411537 & -2.011417 & 1.726724 \\
\hline C & 8.656346 & -3.898339 & -1.642599 \\
\hline $\mathrm{H}$ & 6.759765 & -3.002669 & -2.111514 \\
\hline C & 9.667547 & -4.042285 & -0.691836 \\
\hline H & 10.353889 & -3.465627 & 1.268180 \\
\hline $\mathrm{H}$ & 8.720278 & -4.426042 & -2.589399 \\
\hline $\mathrm{H}$ & 10.525444 & -4.674782 & -0.897642 \\
\hline
\end{tabular}


Frequencies (Top 3 out of 237)

$\begin{array}{lr}\text { 1. } & 9.5449 \mathrm{~cm}^{-1} \\ \text { 2. } & 16.4571 \mathrm{~cm}^{-1} \\ \text { 3. } & 21.1350 \mathrm{~cm}^{-1}\end{array}$

B3LYP-D3(BJ)/6-31G(d,p) Molecular Geometry in Cartesian Coordinates

\begin{tabular}{|c|c|c|c|}
\hline C & -1.559840 & -0.066852 & 1.202555 \\
\hline $\mathrm{H}$ & -0.905869 & 0.389662 & 1.953438 \\
\hline $\mathrm{H}$ & -2.542649 & -0.199610 & 1.658935 \\
\hline C & 1.590474 & 0.096300 & -1.022264 \\
\hline 0 & 1.680660 & 0.471034 & -2.166797 \\
\hline 0 & 1.370038 & -1.208658 & -0.699385 \\
\hline $\mathrm{H}$ & 1.319661 & -1.698773 & -1.538975 \\
\hline 0 & -1.107671 & -1.379962 & 0.875546 \\
\hline $\mathrm{H}$ & -0.224656 & -1.309262 & 0.481747 \\
\hline C & 0.601697 & 1.750984 & 0.525953 \\
\hline C & 1.686667 & 0.956174 & 0.201764 \\
\hline C & 2.866501 & 0.921855 & 1.008517 \\
\hline C & 2.909815 & 1.716866 & 2.135821 \\
\hline $\mathrm{H}$ & 3.805431 & 1.717438 & 2.749735 \\
\hline C & -1.679283 & 0.855770 & 0.001837 \\
\hline $\mathrm{C}$ & -0.637974 & 1.722015 & -0.313531 \\
\hline C & -2.963728 & 1.745619 & -1.859960 \\
\hline C & -2.873082 & 0.865387 & -0.798090 \\
\hline $\mathrm{H}$ & -3.860820 & 1.744951 & -2.471942 \\
\hline C & -1.920571 & 2.637689 & -2.196869 \\
\hline C & -0.728187 & 2.631584 & -1.412098 \\
\hline C & 0.316577 & 3.530817 & -1.751774 \\
\hline C & 0.182060 & 4.395266 & -2.813476 \\
\hline C & -1.000013 & 4.399984 & -3.591776 \\
\hline C & -2.027221 & 3.537317 & -3.290244 \\
\hline $\mathrm{H}$ & 1.229160 & 3.521716 & -1.167836 \\
\hline $\mathrm{H}$ & 0.990655 & 5.075615 & -3.061720 \\
\hline $\mathrm{H}$ & -1.090558 & 5.085174 & -4.428974 \\
\hline $\mathrm{H}$ & -2.937759 & 3.530317 & -3.882768 \\
\hline C & 1.834187 & 2.563268 & 2.497836 \\
\hline C & 0.660683 & 2.590668 & 1.680983 \\
\hline C & -0.412516 & 3.443698 & 2.049523 \\
\hline
\end{tabular}




\begin{tabular}{|c|c|c|c|}
\hline C & -0.325176 & 4.235482 & 3.171784 \\
\hline C & 0.836456 & 4.209839 & 3.979656 \\
\hline C & 1.891760 & 3.392182 & 3.649011 \\
\hline $\mathrm{H}$ & -1.301356 & 3.462149 & 1.429599 \\
\hline H & -1.151582 & 4.885799 & 3.440492 \\
\hline H & 0.891081 & 4.838885 & 4.862642 \\
\hline $\mathrm{H}$ & 2.786574 & 3.367181 & 4.264251 \\
\hline C & -6.345609 & -1.608668 & -0.068181 \\
\hline C & -5.057942 & -2.166435 & -0.074737 \\
\hline C & -3.928626 & -1.383559 & -0.289994 \\
\hline C & -4.046177 & -0.001758 & -0.509463 \\
\hline C & -5.334267 & 0.558896 & -0.500540 \\
\hline C & -6.460323 & -0.226597 & -0.283778 \\
\hline H & -2.945016 & -1.838345 & -0.284834 \\
\hline $\mathrm{H}$ & -5.446181 & 1.630079 & -0.637157 \\
\hline C & 4.023327 & 0.062598 & 0.657803 \\
\hline C & 4.613700 & -0.756401 & 1.631375 \\
\hline C & 4.575170 & 0.063422 & -0.632385 \\
\hline C & 5.712074 & -1.551536 & 1.325747 \\
\hline $\mathrm{H}$ & 4.194647 & -0.775613 & 2.632532 \\
\hline C & 5.672501 & -0.735310 & -0.935725 \\
\hline H & 4.146426 & 0.696386 & -1.401270 \\
\hline C & 6.263018 & -1.559494 & 0.034566 \\
\hline H & -4.941089 & -3.236888 & 0.061857 \\
\hline H & -7.439303 & 0.240692 & -0.245963 \\
\hline H & 6.068070 & -0.733421 & -1.946268 \\
\hline H & 6.161590 & -2.163419 & 2.101173 \\
\hline C & -7.543127 & -2.447848 & 0.164720 \\
\hline C & -8.745764 & -2.191586 & -0.513133 \\
\hline C & -7.506115 & -3.520920 & 1.069535 \\
\hline C & -9.872856 & -2.980021 & -0.293312 \\
\hline H & -8.786033 & -1.383483 & -1.236670 \\
\hline C & -8.632298 & -4.310963 & 1.288171 \\
\hline H & -6.593449 & -3.718100 & 1.622804 \\
\hline C & -9.821274 & -4.043922 & 0.608199 \\
\hline $\mathrm{H}$ & -10.790536 & -2.769050 & -0.834267 \\
\hline H & -8.583836 & -5.131208 & 1.998157 \\
\hline H & -10.699270 & -4.659144 & 0.779108 \\
\hline C & 7.429549 & -2.410968 & -0.292838 \\
\hline C & 7.564403 & -3.692276 & 0.265036 \\
\hline C & 8.428416 & -1.957761 & -1.169196 \\
\hline C & 8.661653 & -4.493352 & -0.042421 \\
\hline H & 6.788461 & -4.070765 & 0.922888 \\
\hline C & 9.525090 & -2.759124 & -1.477808 \\
\hline $\mathrm{H}$ & 8.355283 & -0.959611 & -1.589178 \\
\hline C & 9.646831 & -4.030457 & -0.915534 \\
\hline $\mathrm{H}$ & 8.742606 & -5.484578 & 0.393390 \\
\hline H & 10.290589 & -2.386397 & -2.151664 \\
\hline $\mathrm{H}$ & 10.501680 & -4.654900 & -1.155761 \\
\hline
\end{tabular}


Frequencies (Top 3 out of 237)

$\begin{array}{lr}\text { 1. } & 9.5438 \mathrm{~cm}^{-1} \\ \text { 2. } & 16.4572 \mathrm{~cm}^{-1} \\ \text { 3. } & 21.1346 \mathrm{~cm}^{-1}\end{array}$

B3LYP-D3(BJ)/6-31G(d,p) Molecular Geometry in Cartesian Coordinates

\begin{tabular}{|c|c|c|c|}
\hline C & 1.559892 & -0.066820 & 1.202586 \\
\hline $\mathrm{H}$ & 2.542707 & -0.199547 & 1.658962 \\
\hline $\mathrm{H}$ & 0.905918 & 0.389706 & 1.953459 \\
\hline C & -1.590520 & 0.096295 & -1.022282 \\
\hline 0 & -1.680977 & 0.471031 & -2.166794 \\
\hline 0 & -1.369794 & -1.208625 & -0.699460 \\
\hline $\mathrm{H}$ & -1.319470 & -1.698720 & -1.539066 \\
\hline 0 & 1.107743 & -1.379947 & 0.875617 \\
\hline $\mathrm{H}$ & 0.224745 & -1.309257 & 0.481776 \\
\hline C & -0.601688 & 1.750950 & 0.525943 \\
\hline C & -1.686667 & 0.956146 & 0.201766 \\
\hline C & -2.866502 & 0.921853 & 1.008517 \\
\hline C & -2.909808 & 1.716875 & 2.135812 \\
\hline $\mathrm{H}$ & -3.805422 & 1.717457 & 2.749730 \\
\hline C & 1.679305 & 0.855762 & 0.001834 \\
\hline C & 0.637972 & 1.721971 & -0.313555 \\
\hline C & 2.963697 & 1.745541 & -1.860032 \\
\hline C & 2.873088 & 0.865361 & -0.798115 \\
\hline $\mathrm{H}$ & 3.860777 & 1.744860 & -2.472031 \\
\hline C & 1.920516 & 2.637574 & -2.196965 \\
\hline C & 0.728147 & 2.631487 & -1.412171 \\
\hline C & -0.316642 & 3.530682 & -1.751874 \\
\hline C & -0.182162 & 4.395079 & -2.813623 \\
\hline C & 0.999898 & 4. 399781 & -3.591945 \\
\hline C & 2.027128 & 3.537150 & -3.290387 \\
\hline $\mathrm{H}$ & -1.229214 & 3.521593 & -1.167918 \\
\hline $\mathrm{H}$ & -0.990775 & 5.075399 & -3.061887 \\
\hline $\mathrm{H}$ & 1.090414 & 5.084930 & -4.429180 \\
\hline $\mathrm{H}$ & 2.937656 & 3.530138 & -3.882927 \\
\hline C & -1.834171 & 2.563271 & 2.497817 \\
\hline C & -0.660668 & 2.590652 & 1.680961 \\
\hline 0 & 0.412536 & 3.443683 & 2.049485 \\
\hline
\end{tabular}




\begin{tabular}{|c|c|c|c|}
\hline C & 0.325204 & 4.235482 & 3.171736 \\
\hline C & -0.836425 & 4.209855 & 3.979612 \\
\hline C & -1.891735 & 3.392200 & 3.648982 \\
\hline $\mathrm{H}$ & 1.301373 & 3.462120 & 1.429557 \\
\hline $\mathrm{H}$ & 1.151614 & 4.885798 & 3.440433 \\
\hline H & -0.891044 & 4.838914 & 4.862590 \\
\hline $\mathrm{H}$ & -2.786548 & 3.367212 & 4.264224 \\
\hline C & 6.345654 & -1.608619 & -0.068139 \\
\hline C & 5.057998 & -2.166410 & -0.074704 \\
\hline C & 3.928670 & -1.383557 & -0.289984 \\
\hline C & 4.046200 & -0.001758 & -0.509469 \\
\hline C & 5.334278 & 0.558922 & -0.500537 \\
\hline C & 6.460346 & -0.226548 & -0.283752 \\
\hline $\mathrm{H}$ & 2.945068 & -1.838360 & -0.284830 \\
\hline $\mathrm{H}$ & 5.446173 & 1.630105 & -0.637169 \\
\hline C & -4.023338 & 0.062601 & 0.657810 \\
\hline C & -4.613676 & -0.756427 & 1.631377 \\
\hline C & -4.575215 & 0.063457 & -0.632363 \\
\hline C & -5.712058 & -1.551556 & 1.325761 \\
\hline $\mathrm{H}$ & -4.194595 & -0.775659 & 2.632523 \\
\hline C & -5.672554 & -0.735269 & -0.935691 \\
\hline $\mathrm{H}$ & -4.146481 & 0.696430 & -1.401247 \\
\hline C & -6.263041 & -1.559479 & 0.034597 \\
\hline $\mathrm{H}$ & 4.941163 & -3.236864 & 0.061904 \\
\hline $\mathrm{H}$ & 7.439317 & 0.240758 & -0.245934 \\
\hline $\mathrm{H}$ & -6.068152 & -0.733357 & -1.946223 \\
\hline $\mathrm{H}$ & -6.161551 & -2.163461 & 2.101184 \\
\hline C & 7.543186 & -2.447774 & 0.164784 \\
\hline C & 8.745824 & -2.191499 & -0.513063 \\
\hline C & 7.506184 & -3.520835 & 1.069612 \\
\hline C & 9.872927 & -2.979911 & -0.293221 \\
\hline $\mathrm{H}$ & 8.786084 & -1.383404 & -1.236609 \\
\hline C & 8.632379 & -4.310855 & 1.288269 \\
\hline $\mathrm{H}$ & 6.593516 & -3.718023 & 1.622874 \\
\hline C & 9.821356 & -4.043801 & 0.608303 \\
\hline $\mathrm{H}$ & 10.790609 & -2.768931 & -0.834171 \\
\hline $\mathrm{H}$ & 8.583926 & -5.131091 & 1.998265 \\
\hline $\mathrm{H}$ & 10.699362 & -4.659005 & 0.779228 \\
\hline C & -7.429582 & -2.410944 & -0.292795 \\
\hline C & -8.428476 & -1.957712 & -1.169109 \\
\hline C & -7.564419 & -3.692267 & 0.265048 \\
\hline C & -9.525160 & -2.759066 & -1.477709 \\
\hline $\mathrm{H}$ & -8.355354 & -0.959550 & -1.589066 \\
\hline C & -8.661679 & -4.493334 & -0.042397 \\
\hline $\mathrm{H}$ & -6.788456 & -4.070774 & 0.922866 \\
\hline C & -9.646884 & -4.030414 & -0.915466 \\
\hline $\mathrm{H}$ & -10.290680 & -2.386319 & -2.151531 \\
\hline $\mathrm{H}$ & -8.742619 & -5.484572 & 0.393389 \\
\hline $\mathrm{H}$ & -10.501741 & -4.654850 & -1.155683 \\
\hline
\end{tabular}


Frequencies (Top 3 out of 237)

$\begin{array}{lr}\text { 1. } & 9.4835 \mathrm{~cm}^{-1} \\ \text { 2. } & 16.2540 \mathrm{~cm}^{-1} \\ \text { 3. } & 21.0451 \mathrm{~cm}^{-1}\end{array}$

B3LYP-D3(BJ)/6-31G(d,p) Molecular Geometry in Cartesian Coordinates

\begin{tabular}{|c|c|c|c|}
\hline C & 1.563481 & -0.034566 & 1.196174 \\
\hline $\mathrm{H}$ & 2.547571 & -0.155042 & 1.653196 \\
\hline $\mathrm{H}$ & 0.911911 & 0.443002 & 1.935962 \\
\hline C & -1.595711 & 0.067263 & -1.020732 \\
\hline 0 & -1.690040 & 0.409254 & -2.175159 \\
\hline 0 & -1.375465 & -1.228192 & -0.661562 \\
\hline $\mathrm{H}$ & -1.329412 & -1.742187 & -1.486990 \\
\hline 0 & 1.109879 & -1.356049 & 0.907137 \\
\hline $\mathrm{H}$ & 0.225457 & -1.295975 & 0.514770 \\
\hline C & -0.599232 & 1.764897 & 0.475178 \\
\hline C & -1.686087 & 0.961945 & 0.178523 \\
\hline C & -2.862539 & 0.951756 & 0.990882 \\
\hline C & -2.900672 & 1.779074 & 2.094881 \\
\hline $\mathrm{H}$ & -3.793697 & 1.797999 & 2.712258 \\
\hline C & 1.679496 & 0.853678 & -0.030523 \\
\hline C & 0.637292 & 1.710593 & -0.367701 \\
\hline C & 2.958278 & 1.688657 & -1.921442 \\
\hline C & 2.870830 & 0.839902 & -0.833980 \\
\hline $\mathrm{H}$ & 3.853469 & 1.670211 & -2.535916 \\
\hline C & 1.913920 & 2.570399 & -2.281163 \\
\hline C & 0.724031 & 2.587502 & -1.492766 \\
\hline C & -0.321838 & 3.476414 & -1.855439 \\
\hline C & -0.190705 & 4.309269 & -2.942512 \\
\hline C & 0.988928 & 4.291043 & -3.724313 \\
\hline C & 2.017111 & 3.437536 & -3.400813 \\
\hline $\mathrm{H}$ & -1.232642 & 3.484400 & -1.268711 \\
\hline $\mathrm{H}$ & -1.000132 & 4.982018 & -3.208149 \\
\hline $\mathrm{H}$ & 1.076834 & 4.951329 & -4.581565 \\
\hline $\mathrm{H}$ & 2.925776 & 3.413078 & -3.995744 \\
\hline C & -1.823115 & 2.634925 & 2.427710 \\
\hline C & -0.652988 & 2.637789 & 1.605608 \\
\hline 0 & 0.422133 & 3.500546 & 1.944699 \\
\hline
\end{tabular}




\begin{tabular}{|c|c|c|c|}
\hline C & 0.339810 & 4.324784 & 3.043732 \\
\hline C & -0.818467 & 4.323360 & 3.856807 \\
\hline C & -1.875511 & 3.497034 & 3.554493 \\
\hline $\mathrm{H}$ & 1.308336 & 3.500377 & 1.320751 \\
\hline $\mathrm{H}$ & 1.167587 & 4.982240 & 3.289913 \\
\hline $\mathrm{H}$ & -0.869156 & 4.977910 & 4.721297 \\
\hline $\mathrm{H}$ & -2.767782 & 3.490507 & 4.173882 \\
\hline c & 6.345973 & -1.611049 & -0.041344 \\
\hline c & 5.058899 & -2.170179 & -0.033060 \\
\hline C & 3.928762 & -1.394492 & -0.269480 \\
\hline C & 4.044939 & -0.018422 & -0.523115 \\
\hline C & 5.332151 & 0.543910 & -0.527231 \\
\hline C & 6.459199 & -0.234902 & -0.291966 \\
\hline $\mathrm{H}$ & 2.944400 & -1.846694 & -0.236595 \\
\hline H & 5.444352 & 1.608345 & -0.709038 \\
\hline C & -4.021306 & 0.083419 & 0.670250 \\
\hline C & -4.608097 & -0.706177 & 1.669945 \\
\hline C & -4.578592 & 0.046730 & -0.617072 \\
\hline C & -5.708292 & -1.509051 & 1.392457 \\
\hline $\mathrm{H}$ & -4.184806 & -0.696329 & 2.669453 \\
\hline C & -5.677702 & -0.759750 & -0.892193 \\
\hline H & -4.152775 & 0.656562 & -1.406021 \\
\hline C & -6.264698 & -1.554551 & 0.104397 \\
\hline $\mathrm{H}$ & 4.939043 & -3.226661 & 0.185325 \\
\hline $\mathrm{H}$ & 7.442382 & 0.224108 & -0.321383 \\
\hline H & -6.077532 & -0.787255 & -1.900685 \\
\hline H & -6.154952 & -2.097467 & 2.187442 \\
\hline C & 7.544512 & -2.443209 & 0.210998 \\
\hline C & 8.634654 & -1.936808 & 0.936741 \\
\hline C & 7.620935 & -3.760264 & -0.269728 \\
\hline C & 9.762634 & -2.719342 & 1.172501 \\
\hline H & 8.582949 & -0.930640 & 1.340540 \\
\hline c & 8.747846 & -4.543875 & -0.032648 \\
\hline H & 6.799256 & -4.160552 & -0.855015 \\
\hline C & 9.824452 & -4.026926 & 0.689156 \\
\hline $\mathrm{H}$ & 10.591065 & -2.310335 & 1.743190 \\
\hline $\mathrm{H}$ & 8.789012 & -5.557443 & -0.420229 \\
\hline H & 10.703051 & -4.637363 & 0.873551 \\
\hline C & -7.433256 & -2.414289 & -0.192794 \\
\hline C & -7.567043 & -3.678162 & 0.403784 \\
\hline c & -8.435246 & -1.986524 & -1.078328 \\
\hline C & -8.666292 & -4.486930 & 0.124938 \\
\hline H & -6.788811 & -4.037561 & 1.069589 \\
\hline C & -9.533909 & -2.795631 & -1.358351 \\
\hline $\mathrm{H}$ & -8.362924 & -1.001475 & -1.528309 \\
\hline C & -9.654574 & -4.049388 & -0.757689 \\
\hline $\mathrm{H}$ & -8.746414 & -5.464537 & 0.590638 \\
\hline H & -10.301797 & -2.442463 & -2.039979 \\
\hline $\mathrm{H}$ & -10.510998 & -4.679832 & -0.975596 \\
\hline
\end{tabular}


Frequencies (Top 3 out of 237)

$\begin{array}{lr}\text { 1. } & 9.4752 \mathrm{~cm}^{-1} \\ \text { 2. } & 16.2455 \mathrm{~cm}^{-1} \\ \text { 3. } & 21.0326 \mathrm{~cm}^{-1}\end{array}$

B3LYP-D3(BJ)/6-31G(d,p) Molecular Geometry in Cartesian Coordinates

\begin{tabular}{|c|c|c|c|}
\hline C & 1.563547 & -0.034873 & 1.196079 \\
\hline $\mathrm{H}$ & 2.547629 & -0.155323 & 1.653132 \\
\hline $\mathrm{H}$ & 0.911911 & 0.442572 & 1.935891 \\
\hline C & -1.595866 & 0.067128 & -1.020652 \\
\hline 0 & -1.691090 & 0.408995 & -2.175035 \\
\hline 0 & -1.374709 & -1.228193 & -0.661534 \\
\hline $\mathrm{H}$ & -1.328868 & -1.742228 & -1.486955 \\
\hline 0 & 1.110046 & -1.356344 & 0.906866 \\
\hline $\mathrm{H}$ & 0.225625 & -1.296254 & 0.514466 \\
\hline C & -0.599209 & 1.764678 & 0.475284 \\
\hline C & -1.686102 & 0.961801 & 0.178612 \\
\hline C & -2.862606 & 0.951763 & 0.990890 \\
\hline C & -2.900751 & 1.779121 & 2.094849 \\
\hline $\mathrm{H}$ & -3.793804 & 1.798088 & 2.712189 \\
\hline C & 1.679526 & 0.853467 & -0.030540 \\
\hline C & 0.637286 & 1.710357 & -0.367642 \\
\hline C & 2.958119 & 1.688394 & -1.921607 \\
\hline C & 2.870804 & 0.839705 & -0.834087 \\
\hline $\mathrm{H}$ & 3.853257 & 1.669944 & -2.536159 \\
\hline C & 1.913701 & 2.570075 & -2.281296 \\
\hline C & 0.723894 & 2.587211 & -1.492769 \\
\hline C & -0.322024 & 3.476088 & -1.855399 \\
\hline C & -0.191022 & 4.308854 & -2.942550 \\
\hline C & 0.988525 & 4.290588 & -3.724480 \\
\hline C & 2.016756 & 3.437130 & -3.401023 \\
\hline $\mathrm{H}$ & -1.232763 & 3.484122 & -1.268572 \\
\hline $\mathrm{H}$ & -1.000490 & 4.981570 & -3.208148 \\
\hline $\mathrm{H}$ & 1.076328 & 4.950809 & -4.581794 \\
\hline $\mathrm{H}$ & 2.925360 & 3.412642 & -3.996047 \\
\hline C & -1.823138 & 2.634894 & 2.427712 \\
\hline C & -0.652959 & 2.637623 & 1.605675 \\
\hline 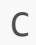 & 0.422209 & 3.500312 & 1.944793 \\
\hline
\end{tabular}




\begin{tabular}{|c|c|c|c|}
\hline C & 0.339893 & 4.324594 & 3.043788 \\
\hline C & -0.818429 & 4.323297 & 3.856800 \\
\hline C & -1.875522 & 3.497051 & 3.554460 \\
\hline $\mathrm{H}$ & 1.308450 & 3.500048 & 1.320897 \\
\hline $\mathrm{H}$ & 1.167713 & 4.981989 & 3.289990 \\
\hline $\mathrm{H}$ & -0.869112 & 4.977885 & 4.721262 \\
\hline H & -2.767829 & 3.490624 & 4.173798 \\
\hline C & 6.346240 & -1.610833 & -0.041387 \\
\hline C & 5.059216 & -2.170072 & -0.032889 \\
\hline C & 3.928986 & -1.394532 & -0.269345 \\
\hline C & 4.045012 & -0.018499 & -0.523234 \\
\hline C & 5.332176 & 0.543944 & -0.527573 \\
\hline C & 6.459314 & -0.234722 & -0.292273 \\
\hline $\mathrm{H}$ & 2.944673 & -1.846843 & -0.236387 \\
\hline H & 5.444269 & 1.608360 & -0.709557 \\
\hline C & -4.021402 & 0.083450 & 0.670262 \\
\hline C & -4.608031 & -0.706307 & 1.669916 \\
\hline C & -4.578850 & 0.046975 & -0.616992 \\
\hline C & -5.708256 & -1.509150 & 1.392448 \\
\hline $\mathrm{H}$ & -4.184614 & -0.696563 & 2.669373 \\
\hline C & -5.677992 & -0.759467 & -0.892085 \\
\hline $\mathrm{H}$ & -4.153072 & 0.656887 & -1.405900 \\
\hline C & -6.264838 & -1.554437 & 0.104460 \\
\hline $\mathrm{H}$ & 4.939469 & -3.226526 & 0.185691 \\
\hline $\mathrm{H}$ & 7.442451 & 0.224377 & -0.321866 \\
\hline H & -6.077956 & -0.786822 & -1.900528 \\
\hline $\mathrm{H}$ & -6.154806 & -2.097692 & 2.187402 \\
\hline C & 7.544880 & -2.442841 & 0.211002 \\
\hline C & 7.621358 & -3.759994 & -0.269440 \\
\hline C & 8.635063 & -1.936192 & 0.936507 \\
\hline C & 8.748359 & -4.543459 & -0.032315 \\
\hline $\mathrm{H}$ & 6.799649 & -4.160484 & -0.854548 \\
\hline C & 9.763132 & -2.718579 & 1.172310 \\
\hline H & 8.583325 & -0.929940 & 1.340093 \\
\hline C & 9.825004 & -4.026263 & 0.689250 \\
\hline $\mathrm{H}$ & 8.789562 & -5.557111 & -0.419675 \\
\hline $\mathrm{H}$ & 10.591596 & -2.309376 & 1.742813 \\
\hline $\mathrm{H}$ & 10.703674 & -4.636588 & 0.873681 \\
\hline C & -7.433435 & -2.414137 & -0.192711 \\
\hline C & -8.435543 & -1.986241 & -1.078045 \\
\hline C & -7.567139 & -3.678102 & 0.403686 \\
\hline C & -9.534237 & -2.795309 & -1.358049 \\
\hline $\mathrm{H}$ & -8.363289 & -1.001122 & -1.527882 \\
\hline C & -8.666419 & -4.486829 & 0.124861 \\
\hline $\mathrm{H}$ & -6.788818 & -4.037605 & 1.069331 \\
\hline C & -9.654819 & -4.049158 & -0.757567 \\
\hline H & -10.302216 & -2.442037 & -2.039523 \\
\hline $\mathrm{H}$ & -8.746477 & -5.464509 & 0.590422 \\
\hline $\mathrm{H}$ & -10.511270 & -4.679572 & -0.975459 \\
\hline
\end{tabular}


Frequencies (Top 3 out of 237)

$\begin{array}{lr}\text { 1. } & 9.3285 \mathrm{~cm}^{-1} \\ \text { 2. } & 16.4102 \mathrm{~cm}^{-1} \\ \text { 3. } & 22.0803 \mathrm{~cm}^{-1}\end{array}$

B3LYP-D3(BJ)/6-31G(d,p) Molecular Geometry in Cartesian Coordinates

\begin{tabular}{|c|c|c|c|}
\hline C & 1.574261 & -0.103874 & -1.128856 \\
\hline $\mathrm{H}$ & 2.202731 & -0.988738 & -1.025832 \\
\hline $\mathrm{H}$ & 0.539632 & -0.444440 & -1.235359 \\
\hline C & -1.644097 & -0.068201 & 1.083259 \\
\hline 0 & -0.910060 & -1.028819 & 1.173504 \\
\hline 0 & -2.493994 & 0.304396 & 2.062984 \\
\hline $\mathrm{H}$ & -2.391372 & -0.349415 & 2.775856 \\
\hline 0 & 2.022293 & 0.552224 & -2.320128 \\
\hline $\mathrm{H}$ & 1.429372 & 1.299427 & -2.481085 \\
\hline C & -0.601412 & 1.630788 & -0.375102 \\
\hline C & -1.712989 & 0.853913 & -0.096367 \\
\hline C & -2.899507 & 0.926900 & -0.890304 \\
\hline C & -2.927371 & 1.812522 & -1.949397 \\
\hline $\mathrm{H}$ & -3.809274 & 1.856338 & -2.581202 \\
\hline C & 1.691344 & 0.759601 & 0.112304 \\
\hline $\mathrm{C}$ & 0.621820 & 1.562633 & 0.484216 \\
\hline C & 2.913727 & 1.493215 & 2.078968 \\
\hline C & 2.875336 & 0.738646 & 0.920860 \\
\hline $\mathrm{H}$ & 3.814491 & 1.487175 & 2.685546 \\
\hline C & 1.830556 & 2.302376 & 2.489227 \\
\hline C & 0.657178 & 2.351176 & 1.674463 \\
\hline C & -0.423648 & 3.177745 & 2.085037 \\
\hline C & -0.345247 & 3.913673 & 3.244987 \\
\hline C & 0.817345 & 3.864419 & 4.051017 \\
\hline C & 1.880869 & 3.076535 & 3.678831 \\
\hline $\mathrm{H}$ & -1.318980 & 3.217023 & 1.475777 \\
\hline $\mathrm{H}$ & -1.180961 & 4.537938 & 3.545542 \\
\hline $\mathrm{H}$ & 0.865477 & 4.451337 & 4.963052 \\
\hline $\mathrm{H}$ & 2.778318 & 3.032673 & 4.289527 \\
\hline C & -1.823706 & 2.640582 & -2.262116 \\
\hline C & -0.639409 & 2.555990 & -1.463925 \\
\hline C & 0.460008 & 3.402533 & -1.778397 \\
\hline
\end{tabular}




\begin{tabular}{|c|c|c|c|}
\hline C & 0.395853 & 4.269827 & -2.846312 \\
\hline C & -0.770489 & 4.340740 & -3.644420 \\
\hline C & -1.855323 & 3.546361 & -3.355192 \\
\hline $\mathrm{H}$ & 1.349073 & 3.357380 & -1.159879 \\
\hline $\mathrm{H}$ & 1.243800 & 4.907239 & -3.075462 \\
\hline $\mathrm{H}$ & -0.806533 & 5.027698 & -4.483947 \\
\hline $\mathrm{H}$ & -2.755458 & 3.598456 & -3.960877 \\
\hline C & 6.411064 & -1.562099 & -0.021278 \\
\hline c & 5.778921 & -0.781629 & -1.001712 \\
\hline c & 4.630247 & -0.051444 & -0.716657 \\
\hline C & 4.067925 & -0.074554 & 0.569939 \\
\hline C & 4.697460 & -0.855731 & 1.551961 \\
\hline C & 5.844663 & -1.585639 & 1.262671 \\
\hline $\mathrm{H}$ & 4.160770 & 0.543642 & -1.492037 \\
\hline H & 4.260460 & -0.912075 & 2.544106 \\
\hline C & -4.069016 & 0.055151 & -0.625253 \\
\hline C & -5.364908 & 0.590430 & -0.613823 \\
\hline C & -3.919342 & -1.324425 & -0.417040 \\
\hline C & -6.471536 & -0.221758 & -0.394220 \\
\hline $\mathrm{H}$ & -5.498166 & 1.659647 & -0.745446 \\
\hline C & -5.027678 & -2.134882 & -0.199771 \\
\hline H & -2.930614 & -1.769569 & -0.449257 \\
\hline C & -6.326137 & -1.601726 & -0.181998 \\
\hline $\mathrm{H}$ & 6.211034 & -0.722663 & -1.995627 \\
\hline $\mathrm{H}$ & 6.288888 & -2.210637 & 2.030895 \\
\hline H & -4.888605 & -3.203997 & -0.076279 \\
\hline H & -7.460607 & 0.222833 & -0.351665 \\
\hline C & 7.631913 & -2.340207 & -0.332199 \\
\hline C & 8.658745 & -2.479528 & 0.615249 \\
\hline C & 7.793163 & -2.958519 & -1.582490 \\
\hline C & 9.806714 & -3.212531 & 0.323422 \\
\hline H & 8.564227 & -1.986321 & 1.577518 \\
\hline C & 8.941868 & -3.689815 & -1.875613 \\
\hline $\mathrm{H}$ & 6.998505 & -2.884849 & -2.318029 \\
\hline C & 9.953931 & -3.820798 & -0.923820 \\
\hline $\mathrm{H}$ & 10.592074 & -3.300990 & 1.068184 \\
\hline $\mathrm{H}$ & 9.042525 & -4.166398 & -2.846158 \\
\hline H & 10.848852 & -4.391501 & -1.151877 \\
\hline C & -7.504342 & -2.466995 & 0.053775 \\
\hline C & -8.715370 & -2.233372 & -0.617030 \\
\hline c & -7.438947 & -3.542327 & 0.954076 \\
\hline C & -9.823951 & -3.046745 & -0.394385 \\
\hline $\mathrm{H}$ & -8.777051 & -1.424044 & -1.337641 \\
\hline C & -8.546777 & -4.356991 & 1.175723 \\
\hline $\mathrm{H}$ & -6.519449 & -3.722606 & 1.501863 \\
\hline C & -9.744556 & -4.112535 & 0.502837 \\
\hline $\mathrm{H}$ & -10.748684 & -2.853727 & -0.929772 \\
\hline H & -8.477120 & -5.178783 & 1.881980 \\
\hline $\mathrm{H}$ & -10.608181 & -4.747061 & 0.675828 \\
\hline
\end{tabular}


Frequencies (Top 3 out of 237)

$\begin{array}{lr}\text { 1. } & 9.3137 \mathrm{~cm}^{-1} \\ \text { 2. } & 16.4178 \mathrm{~cm}^{-1} \\ \text { 3. } & 21.9004 \mathrm{~cm}^{-1}\end{array}$

B3LYP-D3(BJ)/6-31G(d,p) Molecular Geometry in Cartesian Coordinates

\begin{tabular}{|c|c|c|c|}
\hline C & 1.577479 & 0.069719 & -1.128081 \\
\hline $\mathrm{H}$ & 0.542747 & 0.405102 & -1.249052 \\
\hline $\mathrm{H}$ & 2.204374 & 0.958365 & -1.051236 \\
\hline C & -1.647855 & 0.099809 & 1.074088 \\
\hline 0 & -0.915291 & 1.063782 & 1.136364 \\
\hline 0 & -2.500465 & -0.242811 & 2.062369 \\
\hline $\mathrm{H}$ & -2.400957 & 0.433304 & 2.754578 \\
\hline 0 & 2.030762 & -0.623543 & -2.296054 \\
\hline $\mathrm{H}$ & 1.438961 & -1.375893 & -2.435729 \\
\hline C & -0.598574 & -1.643178 & -0.326555 \\
\hline C & -1.711893 & -0.859171 & -0.076047 \\
\hline C & -2.895784 & -0.958662 & -0.871049 \\
\hline C & -2.919285 & -1.877376 & -1.901682 \\
\hline $\mathrm{H}$ & -3.799064 & -1.942138 & -2.534648 \\
\hline C & 1.691404 & -0.753652 & 0.140274 \\
\hline $\mathrm{C}$ & 0.621910 & -1.546306 & 0.533936 \\
\hline C & 2.908546 & -1.422742 & 2.133043 \\
\hline C & 2.872590 & -0.705057 & 0.951630 \\
\hline $\mathrm{H}$ & 3.807177 & -1.396072 & 2.742213 \\
\hline C & 1.825381 & -2.220591 & 2.564978 \\
\hline C & 0.654676 & -2.296955 & 1.748502 \\
\hline C & -0.426178 & -3.111994 & 2.181443 \\
\hline C & -0.350341 & -3.810993 & 3.364191 \\
\hline C & 0.809600 & -3.734466 & 4.171912 \\
\hline C & 1.873083 & -2.956887 & 3.778498 \\
\hline $\mathrm{H}$ & -1.319455 & -3.171865 & 1.570838 \\
\hline $\mathrm{H}$ & -1.186018 & -4.426920 & 3.681587 \\
\hline H & 0.855746 & -4.292342 & 5.102097 \\
\hline $\mathrm{H}$ & 2.768494 & -2.892352 & 4.390351 \\
\hline C & -1.813783 & -2.713712 & -2.184453 \\
\hline C & -0.632158 & -2.602510 & -1.385564 \\
\hline C & 0.469080 & -3.457460 & -1.669381 \\
\hline
\end{tabular}




\begin{tabular}{|c|c|c|c|}
\hline C & 0.409213 & -4.358369 & -2.709349 \\
\hline C & -0.754432 & -4.455830 & -3.508599 \\
\hline C & -1.840960 & -3.653788 & -3.248301 \\
\hline $\mathrm{H}$ & 1.355999 & -3.391723 & -1.049627 \\
\hline $\mathrm{H}$ & 1.258470 & -5.001917 & -2.915345 \\
\hline $\mathrm{H}$ & -0.787066 & -5.169160 & -4.325982 \\
\hline $\mathrm{H}$ & -2.739057 & -3.726042 & -3.854942 \\
\hline C & 6.407548 & 1.571828 & -0.049319 \\
\hline C & 5.783879 & 0.755510 & -1.005672 \\
\hline C & 4.636002 & 0.031448 & -0.701917 \\
\hline C & 4.064750 & 0.099452 & 0.579159 \\
\hline C & 4.684504 & 0.918235 & 1.536220 \\
\hline C & 5.832404 & 1.639731 & 1.229005 \\
\hline $\mathrm{H}$ & 4.163879 & -0.579475 & -1.463346 \\
\hline $\mathrm{H}$ & 4.250754 & 0.994760 & 2.528451 \\
\hline C & -4.067042 & -0.080316 & -0.637356 \\
\hline C & -5.362433 & -0.616389 & -0.613204 \\
\hline C & -3.919406 & 1.305277 & -0.472025 \\
\hline C & -6.470564 & 0.201055 & -0.422512 \\
\hline $\mathrm{H}$ & -5.494212 & -1.689351 & -0.711678 \\
\hline C & -5.029206 & 2.120894 & -0.283490 \\
\hline $\mathrm{H}$ & -2.931045 & 1.750300 & -0.515293 \\
\hline C & -6.327172 & 1.587112 & -0.252930 \\
\hline $\mathrm{H}$ & 6.191645 & 0.705342 & -2.010317 \\
\hline $\mathrm{H}$ & 6.301573 & 2.250047 & 1.994220 \\
\hline $\mathrm{H}$ & -4.891622 & 3.193505 & -0.193026 \\
\hline $\mathrm{H}$ & -7.459337 & -0.243043 & -0.369139 \\
\hline C & 7.629707 & 2.340201 & -0.378960 \\
\hline C & 8.613735 & 1.800750 & -1.222901 \\
\hline C & 7.835723 & 3.626938 & 0.143926 \\
\hline C & 9.763604 & 2.522873 & -1.533627 \\
\hline $\mathrm{H}$ & 8.484444 & 0.797388 & -1.616010 \\
\hline C & 8.986315 & 4.348751 & -0.164974 \\
\hline $\mathrm{H}$ & 7.073463 & 4.072292 & 0.775341 \\
\hline C & 9.955706 & 3.800211 & -1.005591 \\
\hline $\mathrm{H}$ & 10.515435 & 2.083452 & -2.182404 \\
\hline $\mathrm{H}$ & 9.121491 & 5.345678 & 0.243833 \\
\hline $\mathrm{H}$ & 10.852055 & 4.362992 & -1.247296 \\
\hline C & -7.506877 & 2.458051 & -0.047482 \\
\hline C & -8.715558 & 2.202929 & -0.714667 \\
\hline C & -7.445246 & 3.560395 & 0.819809 \\
\hline C & -9.825507 & 3.021719 & -0.520455 \\
\hline H & -8.774257 & 1.371908 & -1.410410 \\
\hline C & -8.554449 & 4.380420 & 1.013010 \\
\hline $\mathrm{H}$ & -6.527625 & 3.758104 & 1.364728 \\
\hline C & -9.749865 & 4.114451 & 0.344091 \\
\hline $\mathrm{H}$ & -10.748365 & 2.811626 & -1.052643 \\
\hline $\mathrm{H}$ & -8.487728 & 5.223422 & 1.694107 \\
\hline $\mathrm{H}$ & -10.614564 & 4.753163 & 0.494900 \\
\hline
\end{tabular}


Frequencies (Top 3 out of 237)

$\begin{array}{lr}\text { 1. } & 9.3419 \mathrm{~cm}^{-1} \\ \text { 2. } & 16.2731 \mathrm{~cm}^{-1} \\ \text { 3. } & 22.2641 \mathrm{~cm}^{-1}\end{array}$

B3LYP-D3(BJ)/6-31G(d,p) Molecular Geometry in Cartesian Coordinates

\begin{tabular}{|c|c|c|c|}
\hline C & -1.579688 & -0.146763 & 1.118115 \\
\hline $\mathrm{H}$ & -2.208220 & -1.026753 & 0.979704 \\
\hline $\mathrm{H}$ & -0.545649 & -0.491698 & 1.215968 \\
\hline C & 1.648143 & -0.031821 & -1.077685 \\
\hline 0 & 0.911110 & -0.985757 & -1.206536 \\
\hline 0 & 2.506193 & 0.371461 & -2.037881 \\
\hline $\mathrm{H}$ & 2.407564 & -0.257785 & -2.773156 \\
\hline 0 & -2.032232 & 0.464690 & 2.331279 \\
\hline $\mathrm{H}$ & -1.440117 & 1.205511 & 2.522032 \\
\hline C & 0.600005 & 1.613086 & 0.437804 \\
\hline C & 1.712748 & 0.846953 & 0.134786 \\
\hline C & 2.896122 & 0.890374 & 0.935727 \\
\hline C & 2.919364 & 1.735692 & 2.027417 \\
\hline $\mathrm{H}$ & 3.798472 & 1.755859 & 2.664283 \\
\hline C & -1.691331 & 0.762266 & -0.090621 \\
\hline C & -0.619731 & 1.577474 & -0.428408 \\
\hline C & -2.905251 & 1.568902 & -2.033782 \\
\hline C & -2.872079 & 0.772144 & -0.904136 \\
\hline $\mathrm{H}$ & -3.803556 & 1.586000 & -2.643787 \\
\hline C & -1.819847 & 2.391682 & -2.409594 \\
\hline C & -0.649708 & 2.409404 & -1.588900 \\
\hline C & 0.433291 & 3.249717 & -1.964373 \\
\hline C & 0.360048 & 4.028006 & -3.096685 \\
\hline C & -0.799308 & 4.009448 & -3.908641 \\
\hline C & -1.864839 & 3.209237 & -3.570016 \\
\hline $\mathrm{H}$ & 1.326129 & 3.265903 & -1.350414 \\
\hline $\mathrm{H}$ & 1.197356 & 4.662301 & -3.370644 \\
\hline $\mathrm{H}$ & -0.843381 & 4.629624 & -4.798608 \\
\hline $\mathrm{H}$ & -2.759859 & 3.188619 & -4.185487 \\
\hline C & 1.814351 & 2.551578 & 2.366221 \\
\hline C & 0.633518 & 2.497230 & 1.560333 \\
\hline 0 & -0.467109 & 3.331852 & 1.901371 \\
\hline
\end{tabular}




\begin{tabular}{|c|c|c|c|}
\hline C & -0.407538 & 4.158546 & 3.001266 \\
\hline C & 0.755318 & 4.199140 & 3.806573 \\
\hline C & 1.841312 & 3.415837 & 3.492554 \\
\hline H & -1.353402 & 3.310129 & 1.277635 \\
\hline $\mathrm{H}$ & -1.256367 & 4.787241 & 3.250374 \\
\hline $\mathrm{H}$ & 0.787793 & 4.854180 & 4.671380 \\
\hline $\mathrm{H}$ & 2.738826 & 3.444977 & 4.103647 \\
\hline C & -6.413635 & -1.557933 & -0.061215 \\
\hline C & -5.784515 & -0.814929 & 0.949786 \\
\hline C & -4.634013 & -0.076038 & 0.696289 \\
\hline C & -4.066720 & -0.052284 & -0.588102 \\
\hline C & -4.693125 & -0.796094 & -1.600670 \\
\hline C & -5.842197 & -1.534839 & -1.342936 \\
\hline H & -4.166937 & 0.489478 & 1.494894 \\
\hline $\mathrm{H}$ & -4.252242 & -0.816395 & -2.592489 \\
\hline C & 4.067328 & 0.029612 & 0.642948 \\
\hline C & 3.919881 & -1.341832 & 0.385713 \\
\hline C & 5.362469 & 0.566476 & 0.653564 \\
\hline C & 5.029618 & -2.142351 & 0.140228 \\
\hline H & 2.930068 & -1.785749 & 0.382005 \\
\hline C & 6.470799 & -0.236441 & 0.409735 \\
\hline H & 5.495520 & 1.627241 & 0.841775 \\
\hline C & 6.327417 & -1.607650 & 0.146217 \\
\hline $\mathrm{H}$ & -6.220476 & -0.792160 & 1.943513 \\
\hline $\mathrm{H}$ & -6.284043 & -2.130604 & -2.135379 \\
\hline $\mathrm{H}$ & 7.464142 & 0.199541 & 0.440708 \\
\hline $\mathrm{H}$ & 4.887806 & -3.196112 & -0.076878 \\
\hline C & -7.636673 & -2.345312 & 0.215990 \\
\hline C & -8.660024 & -2.447814 & -0.739879 \\
\hline C & -7.803611 & -3.009331 & 1.441852 \\
\hline C & -9.810148 & -3.189348 & -0.479827 \\
\hline $\mathrm{H}$ & -8.561078 & -1.919389 & -1.682813 \\
\hline C & -8.954471 & -3.749224 & 1.703254 \\
\hline $\mathrm{H}$ & -7.011705 & -2.964193 & 2.182652 \\
\hline C & -9.963040 & -3.843230 & 0.743417 \\
\hline $\mathrm{H}$ & -10.592751 & -3.248887 & -1.230346 \\
\hline $\mathrm{H}$ & -9.059552 & -4.261326 & 2.655061 \\
\hline H & -10.859645 & -4.420601 & 0.946746 \\
\hline C & 7.507545 & -2.462392 & -0.117441 \\
\hline C & 8.602178 & -1.972847 & -0.847741 \\
\hline C & 7.560299 & -3.782992 & 0.356316 \\
\hline C & 9.712722 & -2.776364 & -1.095207 \\
\hline $\mathrm{H}$ & 8.567796 & -0.963697 & -1.245939 \\
\hline C & 8.670115 & -4.587154 & 0.107810 \\
\hline $\mathrm{H}$ & 6.735434 & -4.170354 & 0.945859 \\
\hline C & 9.751567 & -4.087352 & -0.618766 \\
\hline $\mathrm{H}$ & 10.545294 & -2.380942 & -1.669242 \\
\hline H & 8.694184 & -5.603129 & 0.490177 \\
\hline $\mathrm{H}$ & 10.616701 & -4.713917 & -0.812082 \\
\hline
\end{tabular}


Frequencies (Top 3 out of 237)

$\begin{array}{lr}\text { 1. } & 9.3009 \mathrm{~cm}^{-1} \\ \text { 2. } & 16.2415 \mathrm{~cm}^{-1} \\ \text { 3. } & 21.9651 \mathrm{~cm}^{-1}\end{array}$

B3LYP-D3(BJ)/6-31G(d,p) Molecular Geometry in Cartesian Coordinates

\begin{tabular}{|c|c|c|c|}
\hline C & -1.583254 & 0.111583 & -1.118066 \\
\hline $\mathrm{H}$ & -2.210309 & 0.996412 & -1.006317 \\
\hline $\mathrm{H}$ & -0.549198 & 0.451859 & -1.230907 \\
\hline C & 1.652019 & 0.063171 & 1.071111 \\
\hline 0 & 0.916098 & 1.021250 & 1.172689 \\
\hline 0 & 2.513533 & -0.310280 & 2.040257 \\
\hline $\mathrm{H}$ & 2.418190 & 0.341940 & 2.755666 \\
\hline 0 & -2.040882 & -0.538787 & -2.308824 \\
\hline $\mathrm{H}$ & -1.449987 & -1.285890 & -2.477569 \\
\hline C & 0.596939 & -1.627718 & -0.387968 \\
\hline C & 1.711456 & -0.853550 & -0.113182 \\
\hline C & 2.892038 & -0.923633 & -0.916352 \\
\hline C & 2.910764 & -1.803219 & -1.980707 \\
\hline $\mathrm{H}$ & 3.787622 & -1.844459 & -2.619653 \\
\hline C & -1.691676 & -0.757531 & 0.119918 \\
\hline $\mathrm{C}$ & -0.620032 & -1.562885 & 0.480450 \\
\hline C & -2.900596 & -1.499666 & 2.091716 \\
\hline C & -2.869782 & -0.739545 & 0.937019 \\
\hline $\mathrm{H}$ & -3.796902 & -1.495954 & 2.704873 \\
\hline C & -1.815080 & -2.311555 & 2.490290 \\
\hline C & -0.647434 & -2.357063 & 1.667127 \\
\hline C & 0.435780 & -3.186167 & 2.066164 \\
\hline C & 0.365071 & -3.927654 & 3.223064 \\
\hline C & -0.791846 & -3.881668 & 4.037410 \\
\hline C & -1.857493 & -3.091420 & 3.676475 \\
\hline $\mathrm{H}$ & 1.326778 & -3.222992 & 1.450414 \\
\hline $\mathrm{H}$ & 1.202497 & -4.553833 & 3.514766 \\
\hline H & -0.833949 & -4.472926 & 4.946940 \\
\hline $\mathrm{H}$ & -2.750610 & -3.049964 & 4.293656 \\
\hline C & 1.803906 & -2.628428 & -2.289567 \\
\hline C & 0.625884 & -2.547278 & -1.481820 \\
\hline C & -0.476538 & -3.391423 & -1.792263 \\
\hline
\end{tabular}




\begin{tabular}{|c|c|c|c|}
\hline C & -0.421339 & -4.252930 & -2.865340 \\
\hline C & 0.738676 & -4.320214 & -3.672945 \\
\hline C & 1.826323 & -3.528210 & -3.387831 \\
\hline $\mathrm{H}$ & -1.360659 & -3.349043 & -1.166509 \\
\hline $\mathrm{H}$ & -1.271464 & -4.888586 & -3.091297 \\
\hline $\mathrm{H}$ & 0.767672 & -5.002564 & -4.516498 \\
\hline H & 2.721693 & -3.577621 & -4.000763 \\
\hline C & -6.410075 & 1.569130 & 0.032330 \\
\hline C & -5.789099 & 0.788870 & -0.955360 \\
\hline C & -4.639681 & 0.055155 & -0.682221 \\
\hline C & -4.064015 & 0.076791 & 0.598491 \\
\hline C & -4.681128 & 0.859390 & 1.587023 \\
\hline C & -5.830631 & 1.590634 & 1.310338 \\
\hline $\mathrm{H}$ & -4.169743 & -0.527070 & -1.467115 \\
\hline $\mathrm{H}$ & -4.244001 & 0.899949 & 2.579896 \\
\hline C & 4.065015 & -0.055165 & -0.655118 \\
\hline C & 3.919699 & 1.323905 & -0.441067 \\
\hline C & 5.359633 & -0.593382 & -0.653134 \\
\hline C & 5.030999 & 2.130698 & -0.224975 \\
\hline $\mathrm{H}$ & 2.930311 & 1.768741 & -0.448117 \\
\hline C & 6.469530 & 0.215763 & -0.438810 \\
\hline $\mathrm{H}$ & 5.491086 & -1.659727 & -0.807953 \\
\hline C & 6.328301 & 1.594791 & -0.218488 \\
\hline $\mathrm{H}$ & -6.200221 & 0.775180 & -1.959796 \\
\hline $\mathrm{H}$ & -6.297598 & 2.172121 & 2.098994 \\
\hline $\mathrm{H}$ & 7.462366 & -0.221983 & -0.459399 \\
\hline $\mathrm{H}$ & 4.890888 & 3.190970 & -0.040993 \\
\hline C & -7.633813 & 2.348132 & -0.264974 \\
\hline C & -8.619934 & 1.839500 & -1.125453 \\
\hline C & -7.839237 & 3.614476 & 0.305750 \\
\hline C & -9.771171 & 2.571723 & -1.405976 \\
\hline $\mathrm{H}$ & -8.491143 & 0.851467 & -1.555785 \\
\hline C & -8.991213 & 4.346307 & 0.027075 \\
\hline $\mathrm{H}$ & -7.075462 & 4.036805 & 0.950999 \\
\hline C & -9.962639 & 3.828498 & -0.830508 \\
\hline $\mathrm{H}$ & -10.524559 & 2.156008 & -2.068430 \\
\hline $\mathrm{H}$ & -9.125888 & 5.327314 & 0.472892 \\
\hline $\mathrm{H}$ & -10.860064 & 4. 399127 & -1.048679 \\
\hline C & 7.510080 & 2.456345 & 0.013745 \\
\hline C & 8.606872 & 1.989244 & 0.755416 \\
\hline C & 7.562268 & 3.761154 & -0.501968 \\
\hline C & 9.718951 & 2.799179 & 0.973313 \\
\hline $\mathrm{H}$ & 8.573062 & 0.993293 & 1.185613 \\
\hline C & 8.673621 & 4.571767 & -0.283051 \\
\hline $\mathrm{H}$ & 6.735666 & 4.130329 & -1.100674 \\
\hline C & 9.757209 & 4.094311 & 0.455278 \\
\hline H & 10.553214 & 2.421428 & 1.556717 \\
\hline $\mathrm{H}$ & 8.697196 & 5.575034 & -0.697644 \\
\hline $\mathrm{H}$ & 10.623543 & 4.725890 & 0.625531 \\
\hline
\end{tabular}


Frequencies (Top 3 out of 237)

$\begin{array}{lr}\text { 1. } & 6.8651 \mathrm{~cm}^{-1} \\ \text { 2. } & 15.4506 \mathrm{~cm}^{-1} \\ \text { 3. } & 19.4105 \mathrm{~cm}^{-1}\end{array}$

B3LYP-D3(BJ)/6-31G(d,p) Molecular Geometry in Cartesian Coordinates

C

$\mathrm{H}$

$\mathrm{H}$

C

0

0

$\mathrm{H}$

0

$\mathrm{H}$

C

C

C

C

$\mathrm{H}$

C

C

C

C

$\mathrm{H}$

C

C

C

C

C

C

$\mathrm{H}$

$\mathrm{H}$

$\mathrm{H}$

$\mathrm{H}$

C

C

C

\subsection{5}

0.599127

1.486510

$-1.408791$

$-1.407970$

$-1.171373$

$-1.061018$

2. 571719

3. 391334

$-0.639247$

$-1.646640$

$-2.861858$

$-3.015946$

$-3.944184$

1.637260

0.629837

2. 995461

2.834005

3.908004

1.987433

0.771482

$-0.248906$

$-0.066007$

1.142615

2.146117

$-1.180499$

$-0.857095$

1. 272529

3.075185

$-2.011324$

$-0.798749$

0.212080

\subsection{5}

0.396977

$-0.900672$

0.006271

0.212841

$-1.216301$

$-1.818647$

0.435634

0.088714

1.952259

1.036833

1.036536

1.982450

2.013666

1.011469

1.892918

1.808825

0.954740

1.760450

2. 728994

2. 761785

3. 662901

4.499312

4.471924

3.601394

3.680380

5.182171

5.135706

3. 565919

2.936539

2.926922

3.874788

\subsection{5}

1.706563

0.931440

$-0.953427$

$-2.146598$

$-0.421973$

$-1.178053$

2. 116272

1.738298

0.356340

0.109973

0.861497

1.855628

2.418305

$-0.073467$

$-0.432538$

$-1.930035$

$-0.861522$

$-2.517799$

$-2.308182$

$-1.560793$

$-1.961062$

$-3.038899$

$-3.772966$

$-3.415534$

$-1.407942$

$-3.332661$

$-4.622123$

$-3.977615$

2. 145880

1. 387402

1.694543 


\begin{tabular}{|c|c|c|c|}
\hline C & 0.023501 & 4.799648 & 2.695655 \\
\hline C & -1.181208 & 4.818437 & 3.438297 \\
\hline C & -2.174922 & 3.906407 & 3.169802 \\
\hline $\mathrm{H}$ & 1.137215 & 3.852950 & 1.130738 \\
\hline H & 0.804283 & 5.519002 & 2.921332 \\
\hline H & -1.315864 & 5.554055 & 4.225224 \\
\hline $\mathrm{H}$ & -3.099761 & 3.911264 & 3.739572 \\
\hline C & 6.023158 & -1.835601 & 0.012348 \\
\hline C & 6.244398 & -0.450010 & 0.034411 \\
\hline C & 5.215063 & 0.445914 & -0.239787 \\
\hline C & 3.923705 & -0.009541 & -0.545808 \\
\hline C & 3.703534 & -1.395503 & -0.577905 \\
\hline C & 4.732211 & -2.289740 & -0.301765 \\
\hline H & 5.403093 & 1.514654 & -0.213339 \\
\hline $\mathrm{H}$ & 2.717001 & -1.770570 & -0.830347 \\
\hline C & -3.946939 & 0.064919 & 0.584844 \\
\hline C & -4.571981 & -0.616922 & 1.639252 \\
\hline C & -4.399561 & -0.176977 & -0.721402 \\
\hline C & -5.606833 & -1.512969 & 1.397676 \\
\hline $\mathrm{H}$ & -4.216288 & -0.463872 & 2.653286 \\
\hline C & -5.435643 & -1.073115 & -0.960031 \\
\hline H & -3.953207 & 0.357263 & -1.552840 \\
\hline C & -6.059994 & -1.760738 & 0.092280 \\
\hline $\mathrm{H}$ & 7.238903 & -0.072481 & 0.249290 \\
\hline $\mathrm{H}$ & 4.530233 & -3.355941 & -0.310735 \\
\hline H & -5.790759 & -1.216689 & -1.975448 \\
\hline H & -6.047834 & -2.056170 & 2.227320 \\
\hline C & 7.116671 & -2.788842 & 0.310786 \\
\hline C & 7.225186 & -4.001379 & -0.388271 \\
\hline C & 8.070517 & -2.502049 & 1.300031 \\
\hline C & 8.253441 & -4.897930 & -0.107101 \\
\hline $\mathrm{H}$ & 6.512851 & -4.227940 & -1.175281 \\
\hline C & 9.099581 & -3.397914 & 1.580525 \\
\hline H & 7.986486 & -1.582192 & 1.870056 \\
\hline C & 9.195382 & -4.599965 & 0.878374 \\
\hline $\mathrm{H}$ & 8.324436 & -5.826780 & -0.664913 \\
\hline H & 9.822266 & -3.161017 & 2.355460 \\
\hline H & 9.996880 & -5.298527 & 1.097421 \\
\hline C & -7.160327 & -2.717322 & -0.166178 \\
\hline C & -7.148335 & -3.537698 & -1.305518 \\
\hline C & -8.242640 & -2.827570 & 0.721336 \\
\hline C & -8.183579 & -4.437021 & -1.549701 \\
\hline H & -6.307097 & -3.485718 & -1.989380 \\
\hline C & -9.277135 & -3.728161 & 0.478634 \\
\hline $\mathrm{H}$ & -8.283549 & -2.182283 & 1.593121 \\
\hline C & -9.252567 & -4.536819 & -0.658334 \\
\hline H & -8.150819 & -5.067599 & -2.433097 \\
\hline $\mathrm{H}$ & -10.108830 & -3.791733 & 1.173860 \\
\hline $\mathrm{H}$ & -10.059156 & -5.238149 & -0.848090 \\
\hline
\end{tabular}




\begin{tabular}{lr}
\hline B3LYP-D3(BJ)/6-31G(d,p) Energy & -1998.165722 \\
\hline B3LYP-D3(BJ)/def2-TZVPP/IEF-PCM(DCM) Energy & -1998.872142 \\
\hline B3LYP-D3(BJ)/def2-TZVPP/IEF-PCM(DCM)//B3LYP-D3(BJ)/6-31G(d,p) Free Energy (Quasiharmonic) & -1998.27434 \\
\hline
\end{tabular}

Number of Imaginary Frequencies

Frequencies (Top 3 out of 237)
1. $6.9459 \mathrm{~cm}^{-1}$
2. $15.6474 \mathrm{~cm}^{-1}$
3. $19.6067 \mathrm{~cm}^{-1}$

B3LYP-D3(BJ)/6-31G(d,p) Molecular Geometry in Cartesian Coordinates

\begin{tabular}{|c|c|c|c|}
\hline C & 1.523623 & 0.140682 & 1.200577 \\
\hline $\mathrm{H}$ & 0.594867 & 0.366737 & 1.722489 \\
\hline $\mathrm{H}$ & 1.475755 & -0.920401 & 0.923191 \\
\hline C & -1.405886 & 0.030444 & -0.959228 \\
\hline 0 & -1.401568 & 0.263972 & -2.147400 \\
\hline 0 & -1.170999 & -1.204120 & -0.455033 \\
\hline $\mathrm{H}$ & -1.058710 & -1.789034 & -1.224420 \\
\hline 0 & 2.566407 & 0.385500 & 2.136697 \\
\hline $\mathrm{H}$ & 3.385605 & 0.044470 & 1.752508 \\
\hline C & -0.637909 & 1.944970 & 0.396956 \\
\hline C & -1.645910 & 1.037071 & 0.126389 \\
\hline C & -2.864025 & 1.022242 & 0.873049 \\
\hline C & -3.020293 & 1.946070 & 1.887424 \\
\hline $\mathrm{H}$ & -3.950607 & 1.966382 & 2.447167 \\
\hline C & 1.639169 & 1.010568 & -0.043238 \\
\hline C & 0.634151 & 1.900404 & -0.388110 \\
\hline C & 3.004444 & 1.843177 & -1.878943 \\
\hline $\mathrm{C}$ & 2.838568 & 0.967810 & -0.828354 \\
\hline $\mathrm{H}$ & 3.918660 & 1.804958 & -2.464844 \\
\hline C & 1.999159 & 2.772353 & -2.241798 \\
\hline C & 0.780754 & 2.791797 & -1.497996 \\
\hline C & -0.237008 & 3.702314 & -1.883328 \\
\hline C & -0.049265 & 4.560101 & -2.943398 \\
\hline C & 1.161757 & 4.545755 & -3.673874 \\
\hline C & 2.162804 & 3.666668 & -3.330798 \\
\hline $\mathrm{H}$ & -1.170460 & 3.709955 & -1.333131 \\
\hline $\mathrm{H}$ & -0.838383 & 5.249947 & -3.225913 \\
\hline $\mathrm{H}$ & 1.295427 & 5.226353 & -4.509017 \\
\hline $\mathrm{H}$ & 3.093674 & 3.641113 & -3.890431 \\
\hline C & -2.015199 & 2.891724 & 2.202622 \\
\hline C & -0.799729 & 2.896815 & 1.448735 \\
\hline $\mathrm{C}$ & 0.211589 & 3.835728 & 1.780737 \\
\hline
\end{tabular}




\begin{tabular}{|c|c|c|c|}
\hline C & 0.020786 & 4.738397 & 2.801494 \\
\hline C & -1.186746 & 4.742853 & 3.539764 \\
\hline C & -2.181035 & 3.838862 & 3.247248 \\
\hline $\mathrm{H}$ & 1.138886 & 3.824681 & 1.220166 \\
\hline $\mathrm{H}$ & 0.801982 & 5.451055 & 3.046166 \\
\hline $\mathrm{H}$ & -1.323114 & 5.460990 & 4.342385 \\
\hline H & -3.108036 & 3.832728 & 3.813482 \\
\hline C & 6.022666 & -1.843586 & -0.004251 \\
\hline C & 4.727818 & -2.289627 & -0.314510 \\
\hline C & 3.700342 & -1.388930 & -0.572827 \\
\hline C & 3.926154 & -0.003912 & -0.529764 \\
\hline C & 5.221972 & 0.443333 & -0.230220 \\
\hline C & 6.249258 & -0.459095 & 0.029826 \\
\hline $\mathrm{H}$ & 2.715290 & -1.757989 & -0.839372 \\
\hline H & 5.410498 & 1.510992 & -0.177547 \\
\hline C & -3.949628 & 0.058883 & 0.570727 \\
\hline C & -4.579603 & -0.645441 & 1.607270 \\
\hline C & -4.397819 & -0.152898 & -0.742262 \\
\hline C & -5.614916 & -1.534192 & 1.341862 \\
\hline $\mathrm{H}$ & -4.227440 & -0.515724 & 2.625781 \\
\hline C & -5.434400 & -1.041767 & -1.004719 \\
\hline $\mathrm{H}$ & -3.947596 & 0.399137 & -1.559865 \\
\hline C & -6.063645 & -1.751882 & 0.029585 \\
\hline $\mathrm{H}$ & 4.534734 & -3.355174 & -0.385225 \\
\hline $\mathrm{H}$ & 7.233652 & -0.086516 & 0.294156 \\
\hline H & -5.786009 & -1.161885 & -2.024387 \\
\hline $\mathrm{H}$ & -6.059801 & -2.095157 & 2.157495 \\
\hline C & 7.113511 & -2.804508 & 0.278216 \\
\hline C & 6.861414 & -3.990685 & 0.985410 \\
\hline C & 8.425007 & -2.552029 & -0.154095 \\
\hline C & 7.886910 & -4.895162 & 1.250951 \\
\hline $\mathrm{H}$ & 5.859233 & -4.189378 & 1.351884 \\
\hline C & 9.450941 & -3.455626 & 0.112594 \\
\hline $\mathrm{H}$ & 8.633419 & -1.653246 & -0.725836 \\
\hline C & 9.186269 & -4.631407 & 0.815963 \\
\hline $\mathrm{H}$ & 7.672639 & -5.802852 & 1.806925 \\
\hline $\mathrm{H}$ & 10.457038 & -3.245515 & -0.237515 \\
\hline $\mathrm{H}$ & 9.985682 & -5.335854 & 1.023494 \\
\hline C & -7.164422 & -2.700706 & -0.254339 \\
\hline C & -8.249993 & -2.829463 & 0.626683 \\
\hline C & -7.149618 & -3.495000 & -1.411990 \\
\hline C & -9.284929 & -3.722734 & 0.359983 \\
\hline $\mathrm{H}$ & -8.293047 & -2.204076 & 1.512749 \\
\hline C & -8.185307 & -4.386954 & -1.680150 \\
\hline $\mathrm{H}$ & -6.305928 & -3.428800 & -2.091587 \\
\hline C & -9.257552 & -4.505359 & -0.794998 \\
\hline H & -10.119153 & -3.800818 & 1.050685 \\
\hline $\mathrm{H}$ & -8.150370 & -4.997321 & -2.577546 \\
\hline $\mathrm{H}$ & -10.064490 & -5.200953 & -1.003460 \\
\hline
\end{tabular}


Frequencies (Top 3 out of 237)

$\begin{array}{lr}\text { 1. } & 6.8451 \mathrm{~cm}^{-1} \\ \text { 2. } & 15.5234 \mathrm{~cm}^{-1} \\ \text { 3. } & 19.4692 \mathrm{~cm}^{-1}\end{array}$

B3LYP-D3(BJ)/6-31G(d,p) Molecular Geometry in Cartesian Coordinates

C

$\mathrm{H}$

$\mathrm{H}$

C

0

0

$\mathrm{H}$

0

$\mathrm{H}$

C

C

C

C

$\mathrm{H}$

C

C

C

C

$\mathrm{H}$

C

C

C

C

C

C

$\mathrm{H}$

$\mathrm{H}$

$\mathrm{H}$

$\mathrm{H}$

C

C

C

\subsection{9}

1.472970

0.597775

$-1.406991$

$-1.397140$

$-1.181659$

$-1.072825$

2.570639

3.387802

$-0.638400$

$-1.645403$

$-2.861629$

- 3.017224

$-3.946266$

1.637387

0.632028

2.999050

2.834938

3.911869

1.993550

0.776734

$-0.241438$

$-0.055495$

1.154039

2.155370

$-1.173788$

$-0.844904$

1.286317

3.085064

$-2.013040$

$-0.799195$

0.211444
$-0.119210$

0.935453

$-0.335851$

$-0.050706$

$-0.308848$

1.195493

1.765510

$-0.340924$

$-0.006717$

$-1.941377$

$-1.036387$

$-1.005979$

$-1.911872$

$-1.921071$

$-1.015827$

$-1.913265$

$-1.887091$

$-0.989296$

$-1.860918$

$-2.824488$

$-2.828349$

$-3.747321$

$-4.627763$

$-4.628785$

$-3.742071$

$-3.743428$

$-5.323851$

$-5.327230$

- 3.728194

$-2.853584$

$-2.873878$

$-3.808288$
1.196245

0.895817

1.726209

$-0.952966$

$-2.136026$

$-0.473763$

$-1.254771$

2.134031

1.739658

0.436187

0.151973

0.901464

1.932042

2.494162

$-0.028724$

$-0.352226$

$-1.849135$

$-0.817397$

$-2.437864$

$-2.189526$

$-1.442921$

$-1.806486$

$-2.848136$

$-3.581206$

$-3.259267$

$-1.254385$

$-3.114061$

$-4.401709$

$-3.821260$

2. 261504

1.505306

1.851877 


\begin{tabular}{|c|c|c|c|}
\hline C & 0.021391 & -4.692516 & 2.888771 \\
\hline C & -1.184665 & -4.682124 & 3.629410 \\
\hline C & -2.178183 & -3.781907 & 3.323012 \\
\hline $\mathrm{H}$ & 1.137570 & -3.808673 & 1.289273 \\
\hline $\mathrm{H}$ & 0.802028 & -5.401929 & 3.144410 \\
\hline $\mathrm{H}$ & -1.320477 & -5.385777 & 4.444853 \\
\hline $\mathrm{H}$ & -3.103991 & -3.764314 & 3.890954 \\
\hline c & 6.018518 & 1.842668 & -0.064936 \\
\hline c & 4.722313 & 2.280458 & -0.381200 \\
\hline C & 3.694972 & 1.373251 & -0.616287 \\
\hline C & 3.922335 & -0.010250 & -0.543052 \\
\hline c & 5.219440 & -0.449482 & -0.237285 \\
\hline C & 6.246558 & 0.459493 & -0.000471 \\
\hline $\mathrm{H}$ & 2.708813 & 1.735351 & -0.888234 \\
\hline $\mathrm{H}$ & 5.409191 & -1.515518 & -0.161319 \\
\hline c & -3.946282 & -0.045673 & 0.585597 \\
\hline C & -4.570276 & 0.678987 & 1.611556 \\
\hline C & -4.399067 & 0.143564 & -0.729163 \\
\hline C & -5.605354 & 1.564400 & 1.334355 \\
\hline $\mathrm{H}$ & -4.226802 & 0.552967 & 2.633505 \\
\hline C & -5.432743 & 1.032183 & -1.003978 \\
\hline $\mathrm{H}$ & -3.941656 & -0.411195 & -1.540950 \\
\hline C & -6.057094 & 1.761211 & 0.019919 \\
\hline $\mathrm{H}$ & 4.527963 & 3.343972 & -0.475234 \\
\hline $\mathrm{H}$ & 7.232093 & 0.093840 & 0.269228 \\
\hline H & -5.750295 & 1.176751 & -2.031633 \\
\hline $\mathrm{H}$ & -6.083488 & 2.099729 & 2.148421 \\
\hline c & 7.109351 & 2.810640 & 0.192372 \\
\hline C & 6.858416 & 4.012285 & 0.873375 \\
\hline C & 8.419698 & 2.549596 & -0.238337 \\
\hline C & 7.883897 & 4.923437 & 1.115073 \\
\hline H & 5.857216 & 4.218349 & 1.238457 \\
\hline c & 9.445624 & 3.459889 & 0.004532 \\
\hline H & 8.627125 & 1.638307 & -0.790297 \\
\hline C & 9.182103 & 4.651054 & 0.681966 \\
\hline $\mathrm{H}$ & 7.670550 & 5.843298 & 1.651042 \\
\hline $\mathrm{H}$ & 10.450787 & 3.242793 & -0.343997 \\
\hline H & 9.981507 & 5.360725 & 0.870899 \\
\hline C & -7.156292 & 2.708232 & -0.276488 \\
\hline C & -8.104708 & 2.422260 & -1.271513 \\
\hline c & -7.276892 & 3.915496 & 0.430120 \\
\hline C & -9.138123 & 3.313397 & -1.550943 \\
\hline $\mathrm{H}$ & -8.043945 & 1.481764 & -1.809834 \\
\hline C & -8.311183 & 4.806113 & 0.152109 \\
\hline $\mathrm{H}$ & -6.537466 & 4.166821 & 1.183909 \\
\hline C & -9.246414 & 4.509313 & -0.840083 \\
\hline $\mathrm{H}$ & -9.865672 & 3.069006 & -2.319072 \\
\hline H & -8.381393 & 5.738025 & 0.705033 \\
\hline $\mathrm{H}$ & -10.052155 & 5.203595 & -1.057381 \\
\hline
\end{tabular}




\begin{tabular}{lc}
\hline B3LYP-D3(BJ)/6-31G(d,p) Energy & -1998.170468 \\
\hline B3LYP-D3(BJ)/def2-TZVPP/IEF-PCM(DCM) Energy & -1998.873295 \\
\hline B3LYP-D3(BJ)/def2-TZVPP/IEF-PCM(DCM)//B3LYP-D3(BJ)/6-31G(d,p) Free Energy (Quasiharmonic) & -1998.274283
\end{tabular}

Frequencies (Top 3 out of 237)

$\begin{array}{lr}\text { 1. } & 8.5259 \mathrm{~cm}^{-1} \\ \text { 2. } & 18.9899 \mathrm{~cm}^{-1} \\ \text { 3. } & 21.5440 \mathrm{~cm}^{-1}\end{array}$

\section{B3LYP-D3(BJ)/6-31G(d,p) Molecular Geometry in Cartesian Coordinates}

\begin{tabular}{|c|c|c|c|}
\hline C & -1.519764 & -0.038328 & 1.128743 \\
\hline $\mathrm{H}$ & -2.126909 & -0.937958 & 1.023116 \\
\hline $\mathrm{H}$ & -0.478863 & -0.354090 & 1.249066 \\
\hline C & 1.588192 & -0.043933 & -1.047898 \\
\hline 0 & 1.654845 & 0.252770 & -2.219741 \\
\hline 0 & 1.379540 & -1.308327 & -0.617832 \\
\hline $\mathrm{H}$ & 1.325168 & -1.862145 & -1.416026 \\
\hline 0 & -1.991742 & 0.606727 & 2.316618 \\
\hline $\mathrm{H}$ & -1.437406 & 1.386351 & 2.462794 \\
\hline C & 0.622405 & 1.735042 & 0.367383 \\
\hline C & 1.702433 & 0.912629 & 0.102723 \\
\hline C & 2.869377 & 0.913412 & 0.927425 \\
\hline C & 2.900222 & 1.758939 & 2.018801 \\
\hline $\mathrm{H}$ & 3.786957 & 1.785241 & 2.644828 \\
\hline C & -1.657084 & 0.829625 & -0.108131 \\
\hline C & -0.613977 & 1.672148 & -0.473875 \\
\hline C & -2.938473 & 1.605717 & -2.020878 \\
\hline C & -2.855177 & 0.803542 & -0.898344 \\
\hline $\mathrm{H}$ & -3.850438 & 1.597239 & -2.610549 \\
\hline C & -1.890358 & 2.469344 & -2.413053 \\
\hline C & -0.699841 & 2.511427 & -1.625670 \\
\hline C & 0.349092 & 3.383267 & -2.019213 \\
\hline C & 0.220393 & 4.177875 & -3.134666 \\
\hline C & -0.961370 & 4.138817 & -3.912818 \\
\hline C & -1.992795 & 3.301339 & -3.559192 \\
\hline $\mathrm{H}$ & 1.260144 & 3.407281 & -1.433058 \\
\hline $\mathrm{H}$ & 1.032116 & 4.837665 & -3.424699 \\
\hline $\mathrm{H}$ & -1.048388 & 4.770950 & -4.791128 \\
\hline $\mathrm{H}$ & -2.903065 & 3.262183 & -4.150882 \\
\hline C & 1.820606 & 2.619083 & 2.330308 \\
\hline C & 0.664192 & 2.620549 & 1.488269 \\
\hline C & -0.412296 & 3.495500 & 1.802738 \\
\hline
\end{tabular}




\begin{tabular}{|c|c|c|c|}
\hline C & -0.348619 & 4.318922 & 2.905071 \\
\hline C & 0.794131 & 4.313559 & 3.739695 \\
\hline C & 1.854924 & 3.485795 & 3.454570 \\
\hline H & -1.281434 & 3.509807 & 1.154780 \\
\hline $\mathrm{H}$ & -1.177511 & 4.981503 & 3.132626 \\
\hline H & 0.830708 & 4.967613 & 4.605070 \\
\hline $\mathrm{H}$ & 2.736485 & 3.479093 & 4.089110 \\
\hline C & -6.300210 & -1.655353 & -0.015822 \\
\hline C & -5.694227 & -0.882017 & 0.986580 \\
\hline C & -4.574858 & -0.100391 & 0.721629 \\
\hline C & -4.016155 & -0.062902 & -0.566269 \\
\hline C & -4.620679 & -0.835522 & -1.570666 \\
\hline C & -5.738698 & -1.616854 & -1.301490 \\
\hline H & -4.126710 & 0.489992 & 1.513069 \\
\hline $\mathrm{H}$ & -4.187737 & -0.841482 & -2.566297 \\
\hline C & 4.029835 & 0.040411 & 0.629676 \\
\hline C & 4.637386 & -0.703283 & 1.651979 \\
\hline C & 4.570095 & -0.043060 & -0.662864 \\
\hline C & 5.741043 & -1.507037 & 1.391557 \\
\hline H & 4.215275 & -0.673937 & 2.651583 \\
\hline C & 5.675487 & -0.846440 & -0.919744 \\
\hline H & 4.136035 & 0.541478 & -1.466408 \\
\hline C & 6.283150 & -1.595936 & 0.099573 \\
\hline $\mathrm{H}$ & -6.125108 & -0.869487 & 1.982712 \\
\hline $\mathrm{H}$ & -6.163072 & -2.232538 & -2.088261 \\
\hline $\mathrm{H}$ & 6.096046 & -0.867434 & -1.919895 \\
\hline $\mathrm{H}$ & 6.168961 & -2.102631 & 2.191474 \\
\hline C & -7.489473 & -2.488615 & 0.274085 \\
\hline C & -8.513869 & -2.637785 & -0.674499 \\
\hline C & -7.622239 & -3.150835 & 1.505080 \\
\hline C & -9.631863 & -3.422868 & -0.402443 \\
\hline $\mathrm{H}$ & -8.442172 & -2.111684 & -1.621188 \\
\hline C & -8.741046 & -3.934262 & 1.778496 \\
\hline $\mathrm{H}$ & -6.828337 & -3.069267 & 2.240622 \\
\hline C & -9.750944 & -4.074527 & 0.825740 \\
\hline $\mathrm{H}$ & -10.416190 & -3.518191 & -1.147429 \\
\hline $\mathrm{H}$ & -8.819840 & -4.443864 & 2.734167 \\
\hline H & -10.622586 & -4.685742 & 1.038412 \\
\hline C & 7.457283 & -2.454031 & -0.179016 \\
\hline C & 8.492097 & -2.586097 & 0.760584 \\
\hline C & 7.564038 & -3.156592 & -1.389864 \\
\hline C & 9.595993 & -3.394174 & 0.498817 \\
\hline H & 8.440613 & -2.029151 & 1.690780 \\
\hline C & 8.668569 & -3.963437 & -1.652713 \\
\hline $\mathrm{H}$ & 6.761391 & -3.088227 & -2.117265 \\
\hline C & 9.689375 & -4.086287 & -0.709272 \\
\hline $\mathrm{H}$ & 10.389228 & -3.476124 & 1.235768 \\
\hline H & 8.727734 & -4.504466 & -2.592281 \\
\hline $\mathrm{H}$ & 10.549946 & -4.715598 & -0.913709 \\
\hline
\end{tabular}


Frequencies (Top 3 out of 237)

$\begin{array}{lr}\text { 1. } & 6.8909 \mathrm{~cm}^{-1} \\ \text { 2. } & 15.4239 \mathrm{~cm}^{-1} \\ \text { 3. } & 19.3345 \mathrm{~cm}^{-1}\end{array}$

B3LYP-D3(BJ)/6-31G(d,p) Molecular Geometry in Cartesian Coordinates

\begin{tabular}{|c|c|c|c|}
\hline C & -1.528555 & -0.145319 & 1.184147 \\
\hline $\mathrm{H}$ & -0.602130 & -0.367554 & 1.711826 \\
\hline $\mathrm{H}$ & -1.482335 & 0.915221 & 0.904329 \\
\hline C & 1.409596 & -0.028660 & -0.948218 \\
\hline 0 & 1.403153 & -0.261234 & -2.136605 \\
\hline 0 & 1.181805 & 1.206661 & -0.442777 \\
\hline $\mathrm{H}$ & 1.074865 & 1.793541 & -1.211424 \\
\hline 0 & -2.576183 & -0.390482 & 2.114935 \\
\hline $\mathrm{H}$ & -3.393323 & -0.049632 & 1.726263 \\
\hline C & 0.639795 & -1.950475 & 0.396895 \\
\hline C & 1.645982 & -1.037586 & 0.135963 \\
\hline C & 2.859262 & -1.020729 & 0.890648 \\
\hline C & 3.012898 & -1.948165 & 1.902165 \\
\hline $\mathrm{H}$ & 3.939828 & -1.967504 & 2.467500 \\
\hline C & -1.635402 & -1.018523 & -0.058051 \\
\hline C & -0.627768 & -1.908260 & -0.395465 \\
\hline C & -2.990208 & -1.855912 & -1.899430 \\
\hline C & -2.830272 & -0.978263 & -0.849972 \\
\hline $\mathrm{H}$ & -3.901328 & -1.819753 & -2.490264 \\
\hline C & -1.982198 & -2.785167 & -2.254669 \\
\hline C & -0.767748 & -2.801959 & -1.504353 \\
\hline C & 0.252893 & -3.712280 & -1.882543 \\
\hline C & 0.071530 & -4.572506 & -2.941737 \\
\hline C & -1.135707 & -4.560923 & -3.678493 \\
\hline C & -2.139327 & -3.682022 & -3.342532 \\
\hline $\mathrm{H}$ & 1.183476 & -3.717893 & -1.327476 \\
\hline $\mathrm{H}$ & 0.862781 & -5.262181 & -3.218668 \\
\hline $\mathrm{H}$ & -1.264433 & -5.243459 & -4.512834 \\
\hline $\mathrm{H}$ & -3.067278 & -3.658535 & -3.907076 \\
\hline C & 2.009459 & -2.898723 & 2.207558 \\
\hline C & 0.798520 & -2.905274 & 1.446488 \\
\hline 0 & -0.211392 & -3.848996 & 1.769140 \\
\hline
\end{tabular}




\begin{tabular}{|c|c|c|c|}
\hline C & -0.023340 & -4.754854 & 2.787565 \\
\hline C & 1.179882 & -4.757841 & 3.532860 \\
\hline C & 2.172599 & -3.849210 & 3.249581 \\
\hline H & -1.135354 & -3.839317 & 1.203085 \\
\hline $\mathrm{H}$ & -0.803380 & -5.471250 & 3.024915 \\
\hline H & 1.314159 & -5.478535 & 4.333542 \\
\hline $\mathrm{H}$ & 3.096228 & -3.841872 & 3.821278 \\
\hline C & -6.017370 & 1.836347 & -0.049674 \\
\hline C & -6.241044 & 0.451984 & 0.003416 \\
\hline C & -5.212413 & -0.451675 & -0.246984 \\
\hline C & -3.919327 & -0.005453 & -0.559248 \\
\hline C & -3.696633 & 1.379060 & -0.622216 \\
\hline C & -4.724652 & 2.281069 & -0.369897 \\
\hline $\mathrm{H}$ & -5.402370 & -1.519215 & -0.196691 \\
\hline $\mathrm{H}$ & -2.708685 & 1.746643 & -0.880080 \\
\hline C & 3.943023 & -0.051593 & 0.599710 \\
\hline C & 4.561504 & 0.652045 & 1.643487 \\
\hline C & 4.400375 & 0.166979 & -0.708901 \\
\hline C & 5.595631 & 1.545472 & 1.389458 \\
\hline H & 4.214395 & 0.503279 & 2.661137 \\
\hline C & 5.433092 & 1.063565 & -0.960487 \\
\hline H & 3.947271 & -0.371098 & -1.534231 \\
\hline C & 6.051902 & 1.771640 & 0.081326 \\
\hline $\mathrm{H}$ & -7.236869 & 0.081116 & 0.223723 \\
\hline $\mathrm{H}$ & -4.520827 & 3.346452 & -0.402671 \\
\hline $\mathrm{H}$ & 5.754223 & 1.231001 & -1.983549 \\
\hline H & 6.069441 & 2.064131 & 2.216731 \\
\hline C & -7.110242 & 2.797949 & 0.223164 \\
\hline C & -7.214460 & 3.994240 & -0.503981 \\
\hline C & -8.067816 & 2.535522 & 1.215570 \\
\hline C & -8.242155 & 4.898643 & -0.246937 \\
\hline H & -6.499191 & 4.201484 & -1.293647 \\
\hline C & -9.096317 & 3.439225 & 1.471951 \\
\hline $\mathrm{H}$ & -7.987206 & 1.628885 & 1.806876 \\
\hline C & -9.187824 & 4.624942 & 0.742001 \\
\hline $\mathrm{H}$ & -8.309799 & 5.814496 & -0.826243 \\
\hline $\mathrm{H}$ & -9.821952 & 3.221355 & 2.249708 \\
\hline H & -9.988898 & 5.329614 & 0.942219 \\
\hline C & 7.150047 & 2.727279 & -0.190268 \\
\hline C & 7.265245 & 3.919160 & 0.542869 \\
\hline C & 8.102854 & 2.465089 & -1.187649 \\
\hline C & 8.298556 & 4.817866 & 0.288224 \\
\hline H & 6.522435 & 4.152409 & 1.299134 \\
\hline C & 9.135293 & 3.364348 & -1.443705 \\
\hline $\mathrm{H}$ & 8.046257 & 1.536391 & -1.746515 \\
\hline C & 9.238193 & 4.544761 & -0.706610 \\
\hline $\mathrm{H}$ & 8.364576 & 5.737673 & 0.861557 \\
\hline H & 9.866290 & 3.138300 & -2.214175 \\
\hline $\mathrm{H}$ & 10.043178 & 5.245357 & -0.905672 \\
\hline
\end{tabular}


Frequencies (Top 3 out of 237)

$\begin{array}{lr}\text { 1. } & 8.4858 \mathrm{~cm}^{-1} \\ \text { 2. } & 18.8045 \mathrm{~cm}^{-1} \\ \text { 3. } & 21.0378 \mathrm{~cm}^{-1}\end{array}$

B3LYP-D3(BJ)/6-31G(d,p) Molecular Geometry in Cartesian Coordinates

\begin{tabular}{|c|c|c|c|}
\hline C & 1.523742 & -0.005522 & -1.125762 \\
\hline $\mathrm{H}$ & 2.129251 & -0.908955 & -1.046400 \\
\hline $\mathrm{H}$ & 0.482936 & -0.315786 & -1.260372 \\
\hline C & -1.591139 & -0.076901 & 1.037754 \\
\hline 0 & -1.658784 & 0.181906 & 2.218483 \\
\hline 0 & -1.385345 & -1.327395 & 0.567431 \\
\hline $\mathrm{H}$ & -1.334306 & -1.906784 & 1.347476 \\
\hline 0 & 2.001501 & 0.676362 & -2.290506 \\
\hline H & 1.448555 & 1.460815 & -2.414385 \\
\hline C & -0.619268 & 1.745841 & -0.316397 \\
\hline$C$ & -1.700879 & 0.916473 & -0.081654 \\
\hline$C$ & -2.865126 & 0.944799 & -0.909697 \\
\hline C & -2.891493 & 1.824481 & -1.973840 \\
\hline $\mathrm{H}$ & -3.776151 & 1.871705 & -2.601576 \\
\hline$C$ & 1.657348 & 0.822748 & 0.138404 \\
\hline$C$ & 0.614185 & 1.655081 & 0.526597 \\
\hline C & 2.933162 & 1.536885 & 2.078827 \\
\hline$c$ & 2.852523 & 0.770085 & 0.931632 \\
\hline$\Pi$ & 3.842917 & 1.508693 & 2.671284 \\
\hline C & 1.885009 & 2.389758 & 2.493848 \\
\hline C & 0.697303 & 2.458160 & 1.704100 \\
\hline C & -0.351613 & 3. 319211 & 2.120769 \\
\hline$c$ & -0.225596 & 4.078694 & 3.260730 \\
\hline$C$ & 0.953343 & 4.013506 & 4.041408 \\
\hline C & 1.984699 & 3.185660 & 3.665585 \\
\hline $\mathrm{H}$ & -1.260484 & 3.363048 & 1.532367 \\
\hline $\mathrm{H}$ & -1.037256 & 4.730627 & 3.568192 \\
\hline $\mathrm{H}$ & 1.038278 & 4.617972 & 4.939185 \\
\hline & 2.892808 & 3.126672 & 4.258948 \\
\hline C & -1.809947 & 2.692825 & -2.254395 \\
\hline C & -0.656472 & 2.666504 & -1.408762 \\
\hline & 0.421840 & 3.549953 & -1.691577 \\
\hline
\end{tabular}




\begin{tabular}{|c|c|c|c|}
\hline C & 0.362718 & 4.407937 & -2.767485 \\
\hline C & -0.777062 & 4.430028 & -3.605878 \\
\hline C & -1.839560 & 3.594684 & -3.350796 \\
\hline $\mathrm{H}$ & 1.288607 & 3.542916 & -1.040330 \\
\hline $\mathrm{H}$ & 1.192921 & 5.076665 & -2.971030 \\
\hline $\mathrm{H}$ & -0.810035 & 5.111150 & -4.450264 \\
\hline $\mathrm{H}$ & -2.718876 & 3.608926 & -3.988320 \\
\hline C & 6.297547 & -1.665715 & -0.012694 \\
\hline c & 5.699504 & -0.857642 & -0.992163 \\
\hline C & 4.580818 & -0.081802 & -0.707552 \\
\hline C & 4.013223 & -0.088040 & 0.576983 \\
\hline c & 4.608327 & -0.897304 & 1.557680 \\
\hline C & 5.727464 & -1.670319 & 1.269645 \\
\hline $\mathrm{H}$ & 4.129492 & 0.524076 & -1.485449 \\
\hline $\mathrm{H}$ & 4.179251 & -0.922804 & 2.554690 \\
\hline C & -4.027759 & 0.064703 & -0.643008 \\
\hline C & -4.633637 & -0.645370 & -1.689895 \\
\hline C & -4.571785 & -0.058495 & 0.644771 \\
\hline C & -5.739472 & -1.454843 & -1.457981 \\
\hline $\mathrm{H}$ & -4.208576 & -0.585412 & -2.686876 \\
\hline C & -5.679328 & -0.867488 & 0.873161 \\
\hline H & -4.138971 & 0.499657 & 1.467533 \\
\hline C & -6.285487 & -1.583279 & -0.170974 \\
\hline $\mathrm{H}$ & 6.104201 & -0.854102 & -1.999302 \\
\hline $\mathrm{H}$ & 6.178809 & -2.271152 & 2.052899 \\
\hline $\mathrm{H}$ & -6.102787 & -0.919166 & 1.870973 \\
\hline $\mathrm{H}$ & -6.166189 & -2.024147 & -2.277444 \\
\hline c & 7.488595 & -2.489319 & -0.322440 \\
\hline C & 7.650303 & -3.764699 & 0.242160 \\
\hline C & 8.486620 & -2.015049 & -1.188784 \\
\hline C & 8.771496 & -4.538607 & -0.048146 \\
\hline H & 6.876275 & -4.160183 & 0.892218 \\
\hline C & 9.607051 & -2.789296 & -1.480945 \\
\hline H & 8.392254 & -1.021126 & -1.614435 \\
\hline C & 9.755147 & -4.054656 & -0.911526 \\
\hline $\mathrm{H}$ & 8.872457 & -5.525641 & 0.393244 \\
\hline $\mathrm{H}$ & 10.370568 & -2.399822 & -2.147707 \\
\hline H & 10.628607 & -4.657982 & -1.138735 \\
\hline C & -7.462264 & -2.447104 & 0.077080 \\
\hline C & -8.494637 & -2.547021 & -0.869148 \\
\hline C & -7.574173 & -3.187209 & 1.264876 \\
\hline C & -9.601173 & -3.360264 & -0.636111 \\
\hline $\mathrm{H}$ & -8.439134 & -1.961169 & -1.781179 \\
\hline c & -8.681340 & -3.999266 & 1.499028 \\
\hline $\mathrm{H}$ & -6.773524 & -3.143701 & 1.996386 \\
\hline C & -9.699690 & -4.089854 & 0.549303 \\
\hline $\mathrm{H}$ & -10.392446 & -3.417027 & -1.377528 \\
\hline $\mathrm{H}$ & -8.744513 & -4.569468 & 2.420919 \\
\hline $\mathrm{H}$ & -10.562326 & -4.723192 & 0.731380 \\
\hline
\end{tabular}


Frequencies (Top 3 out of 237)

$\begin{array}{lr}\text { 1. } & 8.5356 \mathrm{~cm}^{-1} \\ \text { 2. } & 19.1355 \mathrm{~cm}^{-1} \\ \text { 3. } & 21.7469 \mathrm{~cm}^{-1}\end{array}$

B3LYP-D3(BJ)/6-31G(d,p) Molecular Geometry in Cartesian Coordinates

C

$\mathrm{H}$

$\mathrm{H}$

C

0

0

$\mathrm{H}$

0

$\mathrm{H}$

C

C

C

C

$\mathrm{H}$

C

C

C

C

$\mathrm{H}$

C

C

C

C

C

C

$\mathrm{H}$

$\mathrm{H}$

$\mathrm{H}$

$\mathrm{H}$

C

C

C
$-1.521179$

$-2.129013$

$-0.480802$

1.592807

1.655104

1. 394117

1. 344381

$-1.994843$

$-1.441106$

0.622968

1.703303

2.867674

2.895829

3. 780725

$-1.655173$

$-0.610982$

$-2.931823$

$-2.851427$

$-3.842403$

$-1.882533$

$-0.693938$

0.356093

0.230211

$-0.949671$

$-1.982053$

1. 265751

1.042728

$-1.034469$

$-2.890878$

1.815688

0.661535

$-0.415881$
0.076084

0.970975

0.396832

0.010423

$-0.326612$

1. 290204

1.817100

$-0.528873$

$-1.303496$

$-1.722090$

$-0.907604$

$-0.881352$

$-1.692529$

$-1.699007$

$-0.833407$

$-1.687526$

$-1.674719$

$-0.834780$

$-1.686709$

$-2.550871$

$-2.565705$

$-3.450215$

$-4.282614$

$-4.270503$

$-3.421624$

$-3.453803$

$-4.951768$

$-4.932353$

- 3.402927

$-2.542942$

$-2.571228$

$-3.435696$
1.117950

0.980767

1.229537

$-1.046025$

$-2.207178$

$-0.659038$

$-1.475585$

2. 326099

2. 498775

0.423162

0.135853

0.963976

2.081252

2. 710371

$-0.089060$

$-0.423318$

$-1.977227$

$-0.882480$

$-2.568971$

$-2.337172$

$-1.545937$

$-1.906881$

$-2.994741$

$-3.776629$

$-3.454410$

$-1.318084$

$-3.260063$

$-4.632994$

$-4.049309$

2. 416581

1.571941

1. 911327 


\begin{tabular}{|c|c|c|c|}
\hline C & -0.355073 & -4.223759 & 3.039345 \\
\hline C & 0.785625 & -4.192078 & 3.876207 \\
\hline C & 1.847168 & -3.373706 & 3.567755 \\
\hline $\mathrm{H}$ & -1.283475 & -3.470455 & 1.262086 \\
\hline $\mathrm{H}$ & -1.184657 & -4.878691 & 3.285744 \\
\hline $\mathrm{H}$ & 0.819972 & -4.818422 & 4.761938 \\
\hline H & 2.727083 & -3.346810 & 4.204044 \\
\hline C & -6.300849 & 1.648784 & -0.091141 \\
\hline C & -5.696434 & 0.910253 & 0.938095 \\
\hline C & -4.575568 & 0.121509 & 0.702161 \\
\hline C & -4.013736 & 0.041488 & -0.582433 \\
\hline C & -4.616502 & 0.779472 & -1.613564 \\
\hline C & -5.736030 & 1.568061 & -1.373396 \\
\hline $\mathrm{H}$ & -4.128688 & -0.441520 & 1.513995 \\
\hline $\mathrm{H}$ & -4.181084 & 0.752618 & -2.607766 \\
\hline C & 4.028447 & -0.016662 & 0.643363 \\
\hline C & 4.630224 & 0.760111 & 1.644142 \\
\hline C & 4.574407 & 0.026704 & -0.648668 \\
\hline C & 5.734903 & 1.555675 & 1.363729 \\
\hline $\mathrm{H}$ & 4.214933 & 0.747603 & 2.646959 \\
\hline C & 5.678185 & 0.825409 & -0.926611 \\
\hline $\mathrm{H}$ & 4.134148 & -0.570253 & -1.439642 \\
\hline C & 6.281148 & 1.606320 & 0.071535 \\
\hline $\mathrm{H}$ & -6.129785 & 0.930562 & 1.933029 \\
\hline $\mathrm{H}$ & -6.159154 & 2.156563 & -2.181356 \\
\hline H & 6.068837 & 0.858567 & -1.938514 \\
\hline $\mathrm{H}$ & 6.193018 & 2.134205 & 2.159462 \\
\hline C & -7.492109 & 2.489413 & 0.167643 \\
\hline C & -7.629167 & 3.192344 & 1.375368 \\
\hline C & -8.514291 & 2.604864 & -0.788006 \\
\hline C & -8.749991 & 3.982543 & 1.619478 \\
\hline $\mathrm{H}$ & -6.837064 & 3.136867 & 2.115274 \\
\hline C & -9.634303 & 3.396657 & -0.545303 \\
\hline H & -8.439240 & 2.047399 & -1.716307 \\
\hline C & -9.757658 & 4.088961 & 0.660003 \\
\hline $\mathrm{H}$ & -8.832128 & 4.523792 & 2.557305 \\
\hline $\mathrm{H}$ & -10.416857 & 3.465519 & -1.295056 \\
\hline $\mathrm{H}$ & -10.630879 & 4.705421 & 0.849836 \\
\hline C & 7.455107 & 2.457532 & -0.228630 \\
\hline C & 8.445031 & 2.028013 & -1.126832 \\
\hline C & 7.606440 & 3.714866 & 0.377538 \\
\hline C & 9.548874 & 2.828995 & -1.409788 \\
\hline $\mathrm{H}$ & 8.359146 & 1.047697 & -1.584682 \\
\hline C & 8.710888 & 4.515489 & 0.095780 \\
\hline $\mathrm{H}$ & 6.837604 & 4.075939 & 1.053274 \\
\hline C & 9.687009 & 4.076260 & -0.799457 \\
\hline H & 10.307160 & 2.474464 & -2.101397 \\
\hline $\mathrm{H}$ & 8.804544 & 5.488235 & 0.569096 \\
\hline $\mathrm{H}$ & 10.547467 & 4.700388 & -1.019670 \\
\hline
\end{tabular}




\begin{tabular}{lc}
\hline B3LYP-D3(BJ)/6-31G(d,p) Energy & -1998.170374 \\
\hline B3LYP-D3(BJ)/def2-TZVPP/IEF-PCM(DCM) Energy & -1998.873196 \\
\hline B3LYP-D3(BJ)/def2-TZVPP/IEF-PCM(DCM)//B3LYP-D3(BJ)/6-31G(d,p) Free Energy (Quasiharmonic) & -1998.274191 \\
\hline
\end{tabular}

Frequencies (Top 3 out of 237)

$\begin{array}{lr}\text { 1. } & 8.5032 \mathrm{~cm}^{-1} \\ \text { 2. } & 18.8189 \mathrm{~cm}^{-1} \\ \text { 3. } & 21.5914 \mathrm{~cm}^{-1}\end{array}$

B3LYP-D3(BJ)/6-31G(d,p) Molecular Geometry in Cartesian Coordinates

\begin{tabular}{|c|c|c|c|}
\hline C & -1.524346 & 0.044360 & 1.114592 \\
\hline $\mathrm{H}$ & -0.484032 & 0.360283 & 1.239702 \\
\hline $\mathrm{H}$ & -2.130676 & 0.943805 & 1.002865 \\
\hline C & 1.596415 & 0.040908 & -1.038796 \\
\hline 0 & 1.660536 & -0.260312 & -2.209655 \\
\hline 0 & 1.400083 & 1.308718 & -0.613047 \\
\hline $\mathrm{H}$ & 1.354075 & 1.860596 & -1.413133 \\
\hline 0 & -2.002971 & -0.596870 & 2.301872 \\
\hline $\mathrm{H}$ & -1.450606 & -1.377002 & 2.452722 \\
\hline $\mathrm{C}$ & 0.619746 & -1.735132 & 0.372746 \\
\hline C & 1.701851 & -0.913310 & 0.114482 \\
\hline C & 2.863231 & -0.913700 & 0.947227 \\
\hline C & 2.886536 & -1.758699 & 2.039245 \\
\hline $\mathrm{H}$ & 3.769125 & -1.785407 & 2.671065 \\
\hline C & -1.655279 & -0.827803 & -0.119978 \\
\hline C & -0.611275 & -1.673141 & -0.476436 \\
\hline C & -2.927230 & -1.610257 & -2.036448 \\
\hline C & -2.848957 & -0.803500 & -0.916851 \\
\hline $\mathrm{H}$ & -3.835892 & -1.603277 & -2.631203 \\
\hline C & -1.878057 & -2.476904 & -2.419117 \\
\hline C & -0.691916 & -2.517135 & -1.625105 \\
\hline C & 0.358041 & -3.391950 & -2.009158 \\
\hline C & 0.234390 & -4.191259 & -3.121810 \\
\hline C & -0.943081 & -4.154058 & -3.906540 \\
\hline C & -1.975312 & -3.313707 & -3.562208 \\
\hline $\mathrm{H}$ & 1.265869 & -3.414465 & -1.417947 \\
\hline H & 1.046819 & -4.853337 & -3.404582 \\
\hline H & -1.026155 & -4.789865 & -4.782581 \\
\hline H & -2.882256 & -3.275900 & -4.159066 \\
\hline C & 1.804390 & -2.617871 & 2.344432 \\
\hline C & 0.653365 & -2.619164 & 1.495084 \\
\hline C & -0.426001 & -3.492650 & 1.803759 \\
\hline
\end{tabular}




\begin{tabular}{|c|c|c|c|}
\hline C & -0.370033 & -4.315025 & 2.907269 \\
\hline C & 0.767525 & -4.310020 & 3.748964 \\
\hline C & 1.830874 & -3.483547 & 3.469712 \\
\hline H & -1.291106 & -3.506680 & 1.150438 \\
\hline $\mathrm{H}$ & -1.201017 & -4.976511 & 3.130345 \\
\hline $\mathrm{H}$ & 0.798061 & -4.963248 & 4.615200 \\
\hline $\mathrm{H}$ & 2.708409 & -3.476994 & 4.109807 \\
\hline C & -6.297258 & 1.661086 & -0.063632 \\
\hline C & -5.700047 & 0.888282 & 0.944398 \\
\hline C & -4.580357 & 0.103877 & 0.688733 \\
\hline C & -4.010794 & 0.065682 & -0.594380 \\
\hline C & -4.605041 & 0.839637 & -1.603677 \\
\hline C & -5.725245 & 1.621279 & -1.344506 \\
\hline $\mathrm{H}$ & -4.129756 & -0.474112 & 1.487986 \\
\hline $\mathrm{H}$ & -4.174471 & 0.830619 & -2.600326 \\
\hline C & 4.026126 & -0.041121 & 0.657196 \\
\hline C & 4.625337 & 0.704030 & 1.683228 \\
\hline C & 4.576603 & 0.040886 & -0.631038 \\
\hline C & 5.731969 & 1.506276 & 1.430972 \\
\hline H & 4.206494 & 0.661600 & 2.683741 \\
\hline C & 5.682317 & 0.846208 & -0.880740 \\
\hline H & 4.138354 & -0.531120 & -1.441345 \\
\hline C & 6.282794 & 1.595513 & 0.142817 \\
\hline $\mathrm{H}$ & -6.106251 & 0.919627 & 1.950449 \\
\hline $\mathrm{H}$ & -6.175912 & 2.193914 & -2.148981 \\
\hline $\mathrm{H}$ & 6.076543 & 0.909632 & -1.889809 \\
\hline $\mathrm{H}$ & 6.188026 & 2.059662 & 2.245544 \\
\hline C & -7.489461 & 2.493979 & 0.215328 \\
\hline C & -8.488070 & 2.049759 & 1.096802 \\
\hline C & -7.651761 & 3.748341 & -0.394395 \\
\hline C & -9.609615 & 2.832738 & 1.359981 \\
\hline H & -8.393272 & 1.071660 & 1.557562 \\
\hline C & -8.774084 & 4.530899 & -0.133090 \\
\hline $\mathrm{H}$ & -6.877364 & 4.121278 & -1.057215 \\
\hline C & -9.758295 & 4.076910 & 0.745789 \\
\hline H & -10.373550 & 2.466389 & 2.039255 \\
\hline $\mathrm{H}$ & -8.875506 & 5.501517 & -0.609399 \\
\hline $\mathrm{H}$ & -10.632640 & 4.687009 & 0.950385 \\
\hline C & 7.458957 & 2.453772 & -0.127153 \\
\hline C & 8.451365 & 2.050480 & -1.034728 \\
\hline C & 7.610022 & 3.691675 & 0.517830 \\
\hline $\mathrm{C}$ & 9.557368 & 2.858024 & -1.289173 \\
\hline H & 8.365650 & 1.084800 & -1.522720 \\
\hline C & 8.716638 & 4.498812 & 0.264595 \\
\hline H & 6.839390 & 4.033054 & 1.201706 \\
\hline C & 9.695228 & 4.085731 & -0.640341 \\
\hline $\mathrm{H}$ & 10.317540 & 2.523682 & -1.988719 \\
\hline $\mathrm{H}$ & 8.810092 & 5.456405 & 0.767899 \\
\hline H & 10.557388 & 4.714948 & -0.838292 \\
\hline
\end{tabular}




B3LYP-D3(BJ)/6-31G(d,p) Energy
B3LYP-D3(BJ)/def2-TZVPP/IEF-PCM(DCM) Energy
B3LYP-D3(BJ)/def2-TZVPP/IEF-PCM(DCM)//B3LYP-D3(BJ)/6-31G(d,p) Free Energy (Quasiharmonic)

$-1998.167493$

Number of Imaginary Frequencies

$-1998.872134$

Frequencies (Top 3 out of 237)

$\begin{array}{lr}\text { 1. } & 7.1196 \mathrm{~cm}^{-1} \\ \text { 2. } & 15.1230 \mathrm{~cm}^{-1} \\ \text { 3. } & 18.6212 \mathrm{~cm}^{-1}\end{array}$

B3LYP-D3(BJ)/6-31G(d,p) Molecular Geometry in Cartesian Coordinates

\begin{tabular}{|c|c|c|c|}
\hline C & -1.565010 & -0.195399 & -1.275123 \\
\hline $\mathrm{H}$ & -1.471890 & 0.860651 & -0.994824 \\
\hline $\mathrm{H}$ & -0.654840 & -0.453992 & -1.815569 \\
\hline C & 1.389616 & -0.003192 & 0.938806 \\
\hline 0 & 0.589072 & 0.907790 & 0.880681 \\
\hline 0 & 2.162441 & -0.226034 & 2.021762 \\
\hline $\mathrm{H}$ & 1.952449 & 0.481085 & 2.655935 \\
\hline 0 & -2.634811 & -0.408717 & -2.188478 \\
\hline $\mathrm{H}$ & -3.427706 & -0.013644 & -1.801732 \\
\hline C & 0.638625 & -1.922585 & -0.433551 \\
\hline C & 1.640714 & -1.012682 & -0.140997 \\
\hline C & 2.903105 & -1.046966 & -0.812258 \\
\hline C & 3.116031 & -2.027760 & -1.760070 \\
\hline $\mathrm{H}$ & 4.060178 & -2.051036 & -2.295973 \\
\hline C & -1.674017 & -1.056849 & -0.026372 \\
\hline C & -0.642131 & -1.904338 & 0.338507 \\
\hline C & -3.008188 & -1.878932 & 1.835476 \\
\hline C & -2.867233 & -1.022810 & 0.764885 \\
\hline $\mathrm{H}$ & -3.918460 & -1.845588 & 2.427798 \\
\hline C & -1.977742 & -2.771763 & 2.216483 \\
\hline C & -0.758355 & -2.771949 & 1.471584 \\
\hline C & 0.286865 & -3.642007 & 1.881539 \\
\hline C & 0.125763 & -4.475664 & 2.965136 \\
\hline C & -1.085416 & -4.479927 & 3.695852 \\
\hline C & -2.113154 & -3.642510 & 3.328677 \\
\hline $\mathrm{H}$ & 1.219731 & -3.640715 & 1.330124 \\
\hline $\mathrm{H}$ & 0.935540 & -5.134034 & 3.264072 \\
\hline $\mathrm{H}$ & -1.198423 & -5.142244 & 4.548558 \\
\hline $\mathrm{H}$ & -3.045136 & -3.631710 & 3.887039 \\
\hline C & 2.122979 & -2.981375 & -2.088667 \\
\hline C & 0.858042 & -2.928243 & -1.423917 \\
\hline C & -0.140847 & -3.875274 & -1.770483 \\
\hline
\end{tabular}




\begin{tabular}{|c|c|c|c|}
\hline C & 0.104066 & -4.832848 & -2.727762 \\
\hline C & 1.357267 & -4.888828 & -3.383027 \\
\hline C & 2.343027 & -3.981656 & -3.071779 \\
\hline $\mathrm{H}$ & -1.103001 & -3.823753 & -1.274598 \\
\hline $\mathrm{H}$ & -0.669610 & -5.548531 & -2.986996 \\
\hline $\mathrm{H}$ & 1.536122 & -5.649603 & -4.136525 \\
\hline H & 3.305130 & -4.014739 & -3.575005 \\
\hline C & -6.052040 & 1.793065 & -0.024037 \\
\hline C & -4.748749 & 2.233166 & 0.258860 \\
\hline C & -3.721594 & 1.328276 & 0.504650 \\
\hline C & -3.959920 & -0.055349 & 0.474136 \\
\hline C & -5.262991 & -0.497710 & 0.203376 \\
\hline C & -6.288863 & 0.409866 & -0.043836 \\
\hline $\mathrm{H}$ & -2.727825 & 1.689062 & 0.750101 \\
\hline H & -5.459194 & -1.564494 & 0.161183 \\
\hline C & 3.958923 & -0.043772 & -0.535198 \\
\hline C & 5.289359 & -0.444927 & -0.347259 \\
\hline C & 3.668122 & 1.328298 & -0.488908 \\
\hline C & 6.291927 & 0.489547 & -0.114597 \\
\hline $\mathrm{H}$ & 5.529027 & -1.503587 & -0.351706 \\
\hline C & 4.672486 & 2.261143 & -0.256996 \\
\hline $\mathrm{H}$ & 2.652184 & 1.668210 & -0.659111 \\
\hline C & 6.004459 & 1.862619 & -0.063706 \\
\hline $\mathrm{H}$ & -4.547493 & 3.297896 & 0.318377 \\
\hline $\mathrm{H}$ & -7.280531 & 0.042433 & -0.287512 \\
\hline H & 4.427689 & 3.318416 & -0.260656 \\
\hline $\mathrm{H}$ & 7.307039 & 0.150480 & 0.065509 \\
\hline C & -7.141804 & 2.758987 & -0.293717 \\
\hline C & -8.447205 & 2.517896 & 0.162971 \\
\hline C & -6.894992 & 3.938861 & -1.013291 \\
\hline C & -9.472150 & 3.425962 & -0.092159 \\
\hline $\mathrm{H}$ & -8.651119 & 1.624288 & 0.744375 \\
\hline C & -7.919391 & 4.847930 & -1.267166 \\
\hline $\mathrm{H}$ & -5.898057 & 4.128946 & -1.398111 \\
\hline C & -9.212662 & 4.595304 & -0.808076 \\
\hline $\mathrm{H}$ & -10.473306 & 3.224414 & 0.276753 \\
\hline $\mathrm{H}$ & -7.709132 & 5.750553 & -1.832880 \\
\hline $\mathrm{H}$ & -10.011254 & 5.303302 & -1.006609 \\
\hline C & 7.071447 & 2.858329 & 0.186518 \\
\hline C & 8.360197 & 2.681565 & -0.341784 \\
\hline C & 6.820420 & 4.004011 & 0.958299 \\
\hline C & 9.364113 & 3.617785 & -0.105558 \\
\hline $\mathrm{H}$ & 8.565932 & 1.816341 & -0.963991 \\
\hline C & 7.823447 & 4.941522 & 1.193140 \\
\hline $\mathrm{H}$ & 5.837813 & 4.144745 & 1.397333 \\
\hline C & 9.100200 & 4.752388 & 0.662722 \\
\hline H & 10.351799 & 3.465801 & -0.530362 \\
\hline $\mathrm{H}$ & 7.610034 & 5.816869 & 1.799195 \\
\hline $\mathrm{H}$ & 9.882230 & 5.482607 & 0.846171 \\
\hline
\end{tabular}


Frequencies (Top 3 out of 237)

$\begin{array}{lr}\text { 1. } & 7.0521 \mathrm{~cm}^{-1} \\ \text { 2. } & 14.8349 \mathrm{~cm}^{-1} \\ \text { 3. } & 18.4402 \mathrm{~cm}^{-1}\end{array}$

B3LYP-D3(BJ)/6-31G(d,p) Molecular Geometry in Cartesian Coordinates

\begin{tabular}{|c|c|c|c|}
\hline C & -1.569503 & -0.221810 & -1.264047 \\
\hline $\mathrm{H}$ & -1.478806 & 0.839759 & -1.004637 \\
\hline $\mathrm{H}$ & -0.660371 & -0.488142 & -1.802485 \\
\hline C & 1.394067 & 0.016859 & 0.933266 \\
\hline 0 & 0.591231 & 0.924846 & 0.861576 \\
\hline 0 & 2.173054 & -0.183038 & 2.016344 \\
\hline $\mathrm{H}$ & 1.964982 & 0.536130 & 2.637448 \\
\hline 0 & -2.642098 & -0.455945 & -2.169162 \\
\hline $\mathrm{H}$ & -3.433722 & -0.052091 & -1.788978 \\
\hline C & 0.639928 & -1.930117 & -0.398061 \\
\hline C & 1.641866 & -1.013203 & -0.127700 \\
\hline C & 2.901540 & -1.059008 & -0.803384 \\
\hline C & 3.112177 & -2.058195 & -1.732286 \\
\hline $\mathrm{H}$ & 4.054181 & -2.090739 & -2.271465 \\
\hline C & -1.672151 & -1.059365 & 0.001292 \\
\hline $\mathrm{C}$ & -0.637827 & -1.898438 & 0.378511 \\
\hline C & -2.998491 & -1.846999 & 1.883543 \\
\hline C & -2.862386 & -1.011589 & 0.796142 \\
\hline $\mathrm{H}$ & -3.906690 & -1.803567 & 2.478393 \\
\hline C & -1.965432 & -2.731084 & 2.277788 \\
\hline C & -0.748790 & -2.744173 & 1.528507 \\
\hline C & 0.299016 & -3.604987 & 1.951205 \\
\hline C & 0.142984 & -4.417850 & 3.051209 \\
\hline C & -1.065498 & -4.409489 & 3.786350 \\
\hline C & -2.095644 & -3.580507 & 3.406970 \\
\hline $\mathrm{H}$ & 1.229789 & -3.613208 & 1.396325 \\
\hline $\mathrm{H}$ & 0.954672 & -5.069419 & 3.359715 \\
\hline H & -1.174563 & -5.055463 & 4.652012 \\
\hline $\mathrm{H}$ & -3.025598 & -3.560152 & 3.968439 \\
\hline C & 2.119296 & -3.019666 & -2.037681 \\
\hline C & 0.856934 & -2.955013 & -1.369073 \\
\hline C & -0.141858 & -3.910271 & -1.692574 \\
\hline
\end{tabular}




\begin{tabular}{|c|c|c|c|}
\hline C & 0.100753 & -4.886593 & -2.631323 \\
\hline C & 1.351429 & -4.953921 & -3.290327 \\
\hline C & 2.336987 & -4.039195 & -3.001353 \\
\hline $\mathrm{H}$ & -1.102103 & -3.850193 & -1.193963 \\
\hline $\mathrm{H}$ & -0.672816 & -5.608526 & -2.872963 \\
\hline $\mathrm{H}$ & 1.528499 & -5.729467 & -4.029044 \\
\hline $\mathrm{H}$ & 3.297140 & -4.080980 & -3.507649 \\
\hline C & -6.053157 & 1.784872 & -0.036016 \\
\hline C & -6.286037 & 0.400861 & -0.039525 \\
\hline C & -5.257776 & -0.500690 & 0.219413 \\
\hline C & -3.957262 & -0.051044 & 0.490199 \\
\hline c & -3.723141 & 1.333330 & 0.505582 \\
\hline C & -4.751888 & 2.232225 & 0.244063 \\
\hline $\mathrm{H}$ & -5.454336 & -1.568180 & 0.206584 \\
\hline $\mathrm{H}$ & -2.726925 & 1.699553 & 0.732427 \\
\hline C & 3.956802 & -0.048799 & -0.550788 \\
\hline C & 5.288595 & -0.443999 & -0.359926 \\
\hline C & 3.663879 & 1.323454 & -0.531068 \\
\hline C & 6.290470 & 0.496562 & -0.149888 \\
\hline $\mathrm{H}$ & 5.530003 & -1.502156 & -0.344027 \\
\hline C & 4.667550 & 2.262375 & -0.321770 \\
\hline H & 2.646740 & 1.658232 & -0.704291 \\
\hline C & 6.000893 & 1.869916 & -0.125481 \\
\hline $\mathrm{H}$ & -7.288250 & 0.029600 & -0.228045 \\
\hline $\mathrm{H}$ & -4.541569 & 3.296847 & 0.238178 \\
\hline $\mathrm{H}$ & 4.420981 & 3.318970 & -0.345817 \\
\hline $\mathrm{H}$ & 7.306815 & 0.162805 & 0.033160 \\
\hline c & -7.146102 & 2.743647 & -0.318737 \\
\hline C & -8.125640 & 2.454326 & -1.281866 \\
\hline C & -7.228615 & 3.964477 & 0.369441 \\
\hline C & -9.154192 & 3.355342 & -1.547409 \\
\hline H & -8.062346 & 1.527892 & -1.843867 \\
\hline C & -8.256236 & 4.866280 & 0.103103 \\
\hline H & -6.495625 & 4.193784 & 1.136405 \\
\hline C & -9.223896 & 4.565546 & -0.856275 \\
\hline $\mathrm{H}$ & -9.896966 & 3.116018 & -2.302392 \\
\hline $\mathrm{H}$ & -8.306398 & 5.801678 & 0.652223 \\
\hline H & -10.024898 & 5.268230 & -1.063766 \\
\hline C & 7.067127 & 2.872137 & 0.100822 \\
\hline C & 6.816953 & 4.032689 & 0.850343 \\
\hline C & 8.354293 & 2.686834 & -0.428412 \\
\hline C & 7.819261 & 4.976295 & 1.062766 \\
\hline $\mathrm{H}$ & 5.835657 & 4.180674 & 1.289926 \\
\hline C & 9.357503 & 3.629175 & -0.214576 \\
\hline $\mathrm{H}$ & 8.559250 & 1.809630 & -1.033876 \\
\hline C & 9.094449 & 4.778551 & 0.531725 \\
\hline $\mathrm{H}$ & 7.606543 & 5.863308 & 1.651868 \\
\hline H & 10.343931 & 3.470247 & -0.639762 \\
\hline $\mathrm{H}$ & 9.875926 & 5.513527 & 0.697702 \\
\hline
\end{tabular}


Frequencies (Top 3 out of 237)

$\begin{array}{lr}\text { 1. } & 7.1666 \mathrm{~cm}^{-1} \\ \text { 2. } & 15.1643 \mathrm{~cm}^{-1} \\ \text { 3. } & 18.5766 \mathrm{~cm}^{-1}\end{array}$

B3LYP-D3(BJ)/6-31G(d,p) Molecular Geometry in Cartesian Coordinates

C

$\mathrm{H}$

$\mathrm{H}$

C

0

0

$\mathrm{H}$

0

$\mathrm{H}$

C

C

C

C

$\mathrm{H}$

C

C

C

C

$\mathrm{H}$

C

C

C

C

C

C

$\mathrm{H}$

$\mathrm{H}$

$\mathrm{H}$

$\mathrm{H}$

C

C

C

\section{$-1.571455$}

$-0.664193$

$-1.475613$

1. 392772

0.588007

2.175952

1.970085

$-2.645767$

$-3.436342$

0.635147

1.639031

2.898627

3.106788

4.048415

$-1.675694$

$-0.642562$

$-3.002559$

$-2.865581$

$-3.910302$

$-1.971007$

$-0.754614$

0.291814

0.134664

$-1.073548$

$-2.102342$

1. 222439

0.945288

$-1.183449$

$-3.032060$

2. 111768

0.849823

$-0.151044$
$-0.171265$

$-0.420735$

0.879130

$-0.020098$

0.888299

$-0.256204$

0.441438

$-0.365847$

0.022699

$-1.914381$

$-1.010572$

$-1.033022$

$-1.995854$

$-2.009535$

$-1.056006$

$-1.910282$

$-1.912230$

$-1.036274$

$-1.889500$

$-2.812491$

$-2.799053$

$-3.677146$

$-4.531051$

$-4.548591$

$-3.703982$

$-3.665956$

$-5.195342$

$-5.226816$

$-3.703316$

$-2.942582$

$-2.901478$

$-3.841438$
1. 272369

1.821941

0.972474

$-0.931767$

$-0.894664$

$-2.004377$

$-2.650326$

2. 184622

1.786685

0.471986

0.166508

0.843738

1.810872

2. 351495

0.039601

$-0.305310$

$-1.812059$

$-0.757120$

$-2.408743$

$-2.171953$

$-1.422285$

$-1.811556$

$-2.879862$

$-3.615269$

$-3.268083$

$-1.256463$

$-3.162998$

$-4.455790$

$-3.830307$

2.153050

1.481854

1.841804 


\begin{tabular}{|c|c|c|c|}
\hline C & 0.089176 & -4.780865 & 2.818055 \\
\hline C & 1.339459 & -4.824950 & 3.479787 \\
\hline C & 2.327023 & -3.924216 & 3.155832 \\
\hline $\mathrm{H}$ & -1.110985 & -3.798930 & 1.340812 \\
\hline $\mathrm{H}$ & -0.685976 & -5.491223 & 3.087325 \\
\hline $\mathrm{H}$ & 1.514638 & -5.571437 & 4.248291 \\
\hline H & 3.286890 & -3.948185 & 3.663819 \\
\hline C & -6.051156 & 1.796952 & -0.036535 \\
\hline C & -4.746190 & 2.230362 & -0.322039 \\
\hline C & -3.718819 & 1.319989 & -0.545705 \\
\hline C & -3.958653 & -0.062580 & -0.489576 \\
\hline C & -5.263319 & -0.498495 & -0.216083 \\
\hline C & -6.289392 & 0.414600 & 0.008990 \\
\hline $\mathrm{H}$ & -2.723625 & 1.675073 & -0.793702 \\
\hline H & -5.460753 & -1.564091 & -0.154184 \\
\hline C & 3.956614 & -0.035851 & 0.552711 \\
\hline C & 5.286758 & -0.441990 & 0.374451 \\
\hline C & 3.668032 & 1.335630 & 0.483305 \\
\hline C & 6.291610 & 0.487185 & 0.130592 \\
\hline $\mathrm{H}$ & 5.527897 & -1.499553 & 0.415221 \\
\hline C & 4.674071 & 2.262919 & 0.236540 \\
\hline $\mathrm{H}$ & 2.649064 & 1.678526 & 0.627579 \\
\hline C & 6.006028 & 1.859396 & 0.054721 \\
\hline $\mathrm{H}$ & -4.543635 & 3.293559 & -0.401292 \\
\hline $\mathrm{H}$ & -7.282488 & 0.052823 & 0.255296 \\
\hline H & 4.421629 & 3.316108 & 0.166717 \\
\hline $\mathrm{H}$ & 7.316146 & 0.148616 & 0.014094 \\
\hline C & -7.141224 & 2.768859 & 0.209362 \\
\hline C & -8.444785 & 2.520009 & -0.248426 \\
\hline C & -6.896558 & 3.962374 & 0.906833 \\
\hline C & -9.470005 & 3.433782 & -0.015761 \\
\hline $\mathrm{H}$ & -8.646927 & 1.615391 & -0.813180 \\
\hline C & -7.921220 & 4.877131 & 1.138218 \\
\hline H & -5.901180 & 4.159102 & 1.292344 \\
\hline C & -9.212645 & 4.616694 & 0.678298 \\
\hline $\mathrm{H}$ & -10.469671 & 3.225922 & -0.385210 \\
\hline $\mathrm{H}$ & -7.712650 & 5.790486 & 1.687083 \\
\hline $\mathrm{H}$ & -10.011446 & 5.329144 & 0.859286 \\
\hline C & 7.075426 & 2.849207 & -0.208888 \\
\hline C & 7.065093 & 4.104911 & 0.419164 \\
\hline C & 8.125832 & 2.556353 & -1.093310 \\
\hline C & 8.070517 & 5.036360 & 0.170808 \\
\hline $\mathrm{H}$ & 6.276310 & 4.339311 & 1.126912 \\
\hline C & 9.132330 & 3.486970 & -1.340630 \\
\hline $\mathrm{H}$ & 8.136197 & 1.601521 & -1.609370 \\
\hline C & 9.109074 & 4.731521 & -0.709951 \\
\hline H & 8.047845 & 5.998642 & 0.673370 \\
\hline H & 9.931278 & 3.243215 & -2.034439 \\
\hline $\mathrm{H}$ & 9.893038 & 5.457167 & -0.903059 \\
\hline
\end{tabular}


Frequencies (Top 3 out of 237)

$\begin{array}{lr}\text { 1. } & 7.1582 \mathrm{~cm}^{-1} \\ \text { 2. } & 14.9418 \mathrm{~cm}^{-1} \\ \text { 3. } & 18.5061 \mathrm{~cm}^{-1}\end{array}$

B3LYP-D3(BJ)/6-31G(d,p) Molecular Geometry in Cartesian Coordinates

\begin{tabular}{|c|c|c|c|}
\hline C & 1.576519 & 0.197073 & -1.260927 \\
\hline $\mathrm{H}$ & 1.483926 & -0.859271 & -0.981665 \\
\hline $\mathrm{H}$ & 0.670079 & 0.453680 & -1.808556 \\
\hline C & -1.397891 & 0.000750 & 0.927460 \\
\hline 0 & -0.590860 & -0.905033 & 0.878009 \\
\hline 0 & -2.187807 & 0.214890 & 1.999808 \\
\hline $\mathrm{H}$ & -1.984228 & -0.494533 & 2.633524 \\
\hline 0 & 2.653387 & 0.413190 & -2.165474 \\
\hline $\mathrm{H}$ & 3.443061 & 0.016539 & -1.773815 \\
\hline C & -0.636109 & 1.922055 & -0.436857 \\
\hline C & -1.640171 & 1.011348 & -0.153220 \\
\hline C & -2.896680 & 1.045076 & -0.835723 \\
\hline C & -3.101838 & 2.025984 & -1.785150 \\
\hline $\mathrm{H}$ & -4.040996 & 2.048718 & -2.329748 \\
\hline C & 1.673976 & 1.058453 & -0.011295 \\
\hline C & 0.638289 & 1.904641 & 0.345661 \\
\hline C & 2.992463 & 1.880905 & 1.861571 \\
\hline C & 2.860673 & 1.025193 & 0.789547 \\
\hline $\mathrm{H}$ & 3.897985 & 1.848253 & 2.461169 \\
\hline C & 1.958127 & 2.772710 & 2.234475 \\
\hline C & 0.744705 & 2.771997 & 1.479855 \\
\hline C & -0.304485 & 3.641133 & 1.881621 \\
\hline C & -0.152782 & 4.474709 & 2.966629 \\
\hline C & 1.052510 & 4.479817 & 3.707014 \\
\hline C & 2.083869 & 3.643363 & 3.347881 \\
\hline $\mathrm{H}$ & -1.232848 & 3.639271 & 1.322641 \\
\hline $\mathrm{H}$ & -0.965444 & 5.132414 & 3.259152 \\
\hline $\mathrm{H}$ & 1.158171 & 5.142066 & 4.560719 \\
\hline $\mathrm{H}$ & 3.011385 & 3.633284 & 3.913642 \\
\hline C & -2.106655 & 2.980492 & -2.104482 \\
\hline C & -0.847665 & 2.928107 & -1.428535 \\
\hline C & 0.153444 & 3.876198 & -1.765767 \\
\hline
\end{tabular}




\begin{tabular}{|c|c|c|c|}
\hline C & -0.083774 & 4.834042 & -2.724702 \\
\hline C & -1.331141 & 4.889228 & -3.391083 \\
\hline C & -2.318828 & 3.981037 & -3.089066 \\
\hline $\mathrm{H}$ & 1.111176 & 3.825292 & -1.261347 \\
\hline $\mathrm{H}$ & 0.691526 & 5.550575 & -2.976643 \\
\hline $\mathrm{H}$ & -1.503982 & 5.650223 & -4.145765 \\
\hline H & -3.276450 & 4.013521 & -3.600803 \\
\hline C & 6.052431 & -1.789416 & 0.025260 \\
\hline C & 4.749570 & -2.230157 & 0.308414 \\
\hline C & 3.720559 & -1.325490 & 0.548065 \\
\hline C & 3.956071 & 0.058127 & 0.506883 \\
\hline C & 5.258137 & 0.501353 & 0.233015 \\
\hline C & 6.286635 & -0.405934 & -0.004055 \\
\hline H & 2.723031 & -1.686378 & 0.777707 \\
\hline H & 5.455764 & 1.568228 & 0.200398 \\
\hline C & -3.954418 & 0.040917 & -0.568828 \\
\hline C & -3.663937 & -1.331202 & -0.524616 \\
\hline C & -5.285977 & 0.441542 & -0.388720 \\
\hline C & -4.669555 & -2.264602 & -0.300181 \\
\hline $\mathrm{H}$ & -2.643796 & -1.669695 & -0.671030 \\
\hline C & -6.290394 & -0.493683 & -0.167219 \\
\hline $\mathrm{H}$ & -5.528628 & 1.499329 & -0.410209 \\
\hline C & -6.002932 & -1.866660 & -0.116457 \\
\hline $\mathrm{H}$ & 4.538280 & -3.294512 & 0.322295 \\
\hline $\mathrm{H}$ & 7.289993 & -0.039265 & -0.195464 \\
\hline H & -7.315977 & -0.158985 & -0.048738 \\
\hline $\mathrm{H}$ & -4.415729 & -3.318557 & -0.249578 \\
\hline C & 7.145662 & -2.754410 & -0.234240 \\
\hline C & 8.129602 & -2.484523 & -1.198526 \\
\hline C & 7.224071 & -3.961852 & 0.477635 \\
\hline C & 9.158439 & -3.391356 & -1.442263 \\
\hline $\mathrm{H}$ & 8.069595 & -1.569012 & -1.778501 \\
\hline C & 8.251973 & -4.869492 & 0.233106 \\
\hline H & 6.487597 & -4.175745 & 1.245714 \\
\hline C & 9.224033 & -4.588105 & -0.727695 \\
\hline $\mathrm{H}$ & 9.904678 & -3.167223 & -2.198493 \\
\hline $\mathrm{H}$ & 8.298897 & -5.794192 & 0.800333 \\
\hline $\mathrm{H}$ & 10.025260 & -5.295338 & -0.918170 \\
\hline C & -7.071869 & -2.862954 & 0.123567 \\
\hline C & -8.126543 & -2.588578 & 1.008835 \\
\hline C & -7.056805 & -4.106469 & -0.528197 \\
\hline C & -9.132601 & -3.525261 & 1.234051 \\
\hline $\mathrm{H}$ & -8.140648 & -1.643745 & 1.542894 \\
\hline C & -8.061792 & -5.044005 & -0.301950 \\
\hline $\mathrm{H}$ & -6.264613 & -4.326174 & -1.236850 \\
\hline C & -9.104622 & -4.757556 & 0.579934 \\
\hline H & -9.934924 & -3.295963 & 1.928896 \\
\hline $\mathrm{H}$ & -8.035408 & -5.996515 & -0.822619 \\
\hline $\mathrm{H}$ & -9.888243 & -5.487940 & 0.755810 \\
\hline
\end{tabular}




\begin{tabular}{lr}
\hline B3LYP-D3(BJ)/6-31G(d,p) Energy & -1998.16395 \\
\hline B3LYP-D3(BJ)/def2-TZVPP/IEF-PCM(DCM) Energy & -1998.870768 \\
\hline B3LYP-D3(BJ)/def2-TZVPP/IEF-PCM(DCM)//B3LYP-D3(BJ)/6-31G(d,p) Free Energy (Quasiharmonic) & -1998.272916 \\
\hline
\end{tabular}

Number of Imaginary Frequencies

Frequencies (Top 3 out of 237)

$\begin{array}{lr}\text { 1. } & 7.6807 \mathrm{~cm}^{-1} \\ \text { 2. } & 15.2823 \mathrm{~cm}^{-1} \\ \text { 3. } & 18.6356 \mathrm{~cm}^{-1}\end{array}$

B3LYP-D3(BJ)/6-31G(d,p) Molecular Geometry in Cartesian Coordinates

C

$\mathrm{H}$

$\mathrm{H}$

C

0

0

$\mathrm{H}$

0

$\mathrm{H}$

C

C

C

C

$\mathrm{H}$

C

C

C

C

$\mathrm{H}$

C

C

C

C

C

C

$\mathrm{H}$

$\mathrm{H}$

$\mathrm{H}$

$\mathrm{H}$

C

C

C
1.673374

0.652808

2.288066

$-1.750657$

$-1.009250$

$-2.698913$

$-2.649345$

2.039872

2.986958

$-0.582739$

$-1.731872$

$-2.885707$

$-2.849012$

$-3.705378$

1.707688

0.611367

2.831671

2.853100

3.704360

1.719625

0.591478

$-0.507248$

$-0.491499$

0.622913

1.705773

$-1.367186$

$-1.341231$

0.621413

2. 570154

$-1.709968$

$-0.552863$

0.584419
0.122844

0.159336

$-0.325769$

$-0.094363$

0.823290

$-0.551215$

0.017848

1.471021

1.490604

$-1.587606$

$-0.879829$

$-0.913236$

$-1.700031$

$-1.709418$

$-0.762201$

$-1.546230$

$-1.497165$

$-0.766814$

$-1.490077$

$-2.291205$

$-2.338191$

$-3.166075$

$-3.896140$

$-3.840215$

$-3.055113$

$-3.213821$

$-4.521665$

$-4.421009$

$-3.008435$

$-2.465784$

$-2.410888$

$-3.184557$
1.140852

1.520658

1.936838

$-1.117147$

$-1.388978$

$-1.967472$

$-2.754512$

0.903001

0.713564

0.473845

0.159531

1.004107

2.137707

2.805216

$-0.099878$

$-0.426356$

$-2.129079$

$-0.959590$

$-2.776137$

$-2.494060$

$-1.618356$

$-1.974275$

$-3.141204$

$-4.010470$

$-3.689847$

$-1.317244$

$-3.396484$

$-4.927672$

$-4.346400$

2. 481275

1. 642224

1.996550 


\begin{tabular}{|c|c|c|c|}
\hline C & 0.580333 & -3.963188 & 3.131219 \\
\hline C & -0.560867 & -4.007855 & 3.966856 \\
\hline C & -1.680088 & -3.275622 & 3.647161 \\
\hline $\mathrm{H}$ & 1.456700 & -3.153605 & 1.354102 \\
\hline $\mathrm{H}$ & 1.457046 & -4.549456 & 3.388128 \\
\hline H & -0.549954 & -4.624536 & 4.860179 \\
\hline $\mathrm{H}$ & -2.561089 & -3.305813 & 4.281882 \\
\hline C & 6.477622 & 1.414458 & -0.051277 \\
\hline c & 5.968180 & 0.458818 & 0.841852 \\
\hline C & 4.799074 & -0.239690 & 0.556575 \\
\hline C & 4.088116 & -0.003898 & -0.631114 \\
\hline c & 4.600126 & 0.946008 & -1.527630 \\
\hline C & 5.771056 & 1.640929 & -1.242877 \\
\hline $\mathrm{H}$ & 4.432387 & -0.981442 & 1.258860 \\
\hline $\mathrm{H}$ & 4.060808 & 1.143587 & -2.448364 \\
\hline C & -4.084114 & -0.088549 & 0.718117 \\
\hline C & -5.368818 & -0.641114 & 0.813896 \\
\hline C & -3.969340 & 1.269679 & 0.385023 \\
\hline C & -6.499209 & 0.133574 & 0.579337 \\
\hline $\mathrm{H}$ & -5.477020 & -1.692493 & 1.061453 \\
\hline C & -5.100732 & 2.041855 & 0.148841 \\
\hline H & -2.986996 & 1.725120 & 0.314158 \\
\hline C & -6.388710 & 1.491065 & 0.240269 \\
\hline $\mathrm{H}$ & 6.484917 & 0.275170 & 1.778297 \\
\hline $\mathrm{H}$ & 6.154513 & 2.358467 & -1.961092 \\
\hline H & -4.984698 & 3.084687 & -0.128199 \\
\hline H & -7.483457 & -0.313269 & 0.676824 \\
\hline C & 7.719542 & 2.160935 & 0.254813 \\
\hline C & 8.795534 & 1.533460 & 0.902369 \\
\hline C & 7.849880 & 3.514150 & -0.095365 \\
\hline C & 9.963200 & 2.235994 & 1.190786 \\
\hline $\mathrm{H}$ & 8.723439 & 0.480624 & 1.156365 \\
\hline c & 9.018005 & 4.216491 & 0.191729 \\
\hline $\mathrm{H}$ & 7.017941 & 4.023534 & -0.571256 \\
\hline C & 10.079731 & 3.580613 & 0.836362 \\
\hline $\mathrm{H}$ & 10.787003 & 1.729952 & 1.685030 \\
\hline $\mathrm{H}$ & 9.095321 & 5.264906 & -0.079894 \\
\hline H & 10.989792 & 4.128162 & 1.060813 \\
\hline C & -7.592266 & 2.315665 & -0.013387 \\
\hline C & -7.643846 & 3.658693 & 0.392817 \\
\hline c & -8.711271 & 1.774869 & -0.666631 \\
\hline C & -8.775533 & 4.434668 & 0.153428 \\
\hline H & -6.799200 & 4.086936 & 0.923067 \\
\hline C & -9.843810 & 2.550076 & -0.904628 \\
\hline H & -8.679541 & 0.746668 & -1.012921 \\
\hline C & -9.881007 & 3.883814 & -0.495996 \\
\hline $\mathrm{H}$ & -8.797465 & 5.469079 & 0.482953 \\
\hline H & -10.695272 & 2.114379 & -1.418754 \\
\hline $\mathrm{H}$ & -10.763231 & 4.488396 & -0.682118 \\
\hline
\end{tabular}




\begin{tabular}{lc}
\hline B3LYP-D3(BJ)/6-31G(d,p) Energy & -1998.168022 \\
\hline B3LYP-D3(BJ)/def2-TZVPP/IEF-PCM(DCM) Energy & -1998.871125 \\
\hline B3LYP-D3(BJ)/def2-TZVPP/IEF-PCM(DCM)//B3LYP-D3(BJ)/6-31G(d,p) Free Energy (Quasiharmonic) & -1998.272685
\end{tabular}

Frequencies (Top 3 out of 237)

$\begin{array}{lr}\text { 1. } & 9.3337 \mathrm{~cm}^{-1} \\ \text { 2. } & 15.5295 \mathrm{~cm}^{-1} \\ \text { 3. } & 21.0968 \mathrm{~cm}^{-1}\end{array}$

B3LYP-D3(BJ)/6-31G(d,p) Molecular Geometry in Cartesian Coordinates

\begin{tabular}{|c|c|c|c|}
\hline C & 1.596254 & 0.005530 & -1.161707 \\
\hline $\mathrm{H}$ & 2.218494 & 0.904986 & -1.093970 \\
\hline $\mathrm{H}$ & 0.559213 & 0.322772 & -1.306875 \\
\hline C & -1.583856 & 0.145216 & 1.001443 \\
\hline 0 & -0.836791 & 1.101478 & 0.998343 \\
\hline 0 & -2.412738 & -0.133329 & 2.029319 \\
\hline $\mathrm{H}$ & -2.284635 & 0.576741 & 2.681631 \\
\hline 0 & 2.027342 & -0.836089 & -2.242752 \\
\hline $\mathrm{H}$ & 1.847003 & -0.365636 & -3.067200 \\
\hline C & -0.599208 & -1.677368 & -0.347184 \\
\hline C & -1.693779 & -0.865129 & -0.098838 \\
\hline C & -2.906581 & -0.990886 & -0.845233 \\
\hline C & -2.979071 & -1.968837 & -1.816996 \\
\hline $\mathrm{H}$ & -3.885247 & -2.063028 & -2.407982 \\
\hline C & 1.696631 & -0.783445 & 0.118685 \\
\hline$C$ & 0.626167 & -1.575325 & 0.506560 \\
\hline C & 2.893636 & -1.405826 & 2.135849 \\
\hline C & 2.866419 & -0.710026 & 0.941412 \\
\hline $\mathrm{H}$ & 3.784134 & -1.361663 & 2.755793 \\
\hline C & 1.811007 & -2.205374 & 2.565142 \\
\hline C & 0.651032 & -2.303617 & 1.735151 \\
\hline$C$ & -0.430223 & -3.117667 & 2.169709 \\
\hline C & -0.364172 & -3.796271 & 3.364554 \\
\hline C & 0.785383 & -3.698559 & 4.184975 \\
\hline C & 1.848460 & -2.920772 & 3.791676 \\
\hline $\mathrm{H}$ & -1.314628 & -3.194456 & 1.548509 \\
\hline $\mathrm{H}$ & -1.199529 & -4.412567 & 3.682033 \\
\hline $\mathrm{H}$ & 0.823749 & -4.240623 & 5.124860 \\
\hline $\mathrm{H}$ & 2.735843 & -2.839919 & 4.413247 \\
\hline C & -1.892249 & -2.831065 & -2.095647 \\
\hline C & -0.674700 & -2.681883 & -1.359818 \\
\hline & 0.412430 & -3.547521 & -1.650545 \\
\hline
\end{tabular}




\begin{tabular}{|c|c|c|c|}
\hline C & 0.297100 & -4.514618 & -2.621624 \\
\hline C & -0.907166 & -4.661826 & -3.351142 \\
\hline C & -1.976197 & -3.835828 & -3.095846 \\
\hline $\mathrm{H}$ & 1.339518 & -3.421016 & -1.106298 \\
\hline $\mathrm{H}$ & 1.137337 & -5.167429 & -2.835952 \\
\hline H & -0.982440 & -5.429169 & -4.115549 \\
\hline $\mathrm{H}$ & -2.903024 & -3.939922 & -3.652849 \\
\hline C & 6.390941 & 1.583008 & -0.063286 \\
\hline C & 5.792705 & 0.733760 & -1.007256 \\
\hline C & 4.649096 & 0.003510 & -0.702194 \\
\hline C & 4.055904 & 0.097996 & 0.567247 \\
\hline C & 4.650661 & 0.948989 & 1.511664 \\
\hline C & 5.794511 & 1.676783 & 1.203471 \\
\hline $\mathrm{H}$ & 4.197384 & -0.637134 & -1.451863 \\
\hline $\mathrm{H}$ & 4.200393 & 1.045020 & 2.494755 \\
\hline C & -4.058629 & -0.085204 & -0.620082 \\
\hline C & -3.889280 & 1.304928 & -0.529841 \\
\hline C & -5.359862 & -0.599785 & -0.527915 \\
\hline C & -4.981772 & 2.145241 & -0.348393 \\
\hline $\mathrm{H}$ & -2.896932 & 1.732809 & -0.624658 \\
\hline C & -6.451029 & 0.242122 & -0.346000 \\
\hline $\mathrm{H}$ & -5.508363 & -1.674338 & -0.566667 \\
\hline C & -6.284988 & 1.632780 & -0.251568 \\
\hline $\mathrm{H}$ & 6.220035 & 0.660556 & -2.002516 \\
\hline $\mathrm{H}$ & 6.244228 & 2.312357 & 1.959747 \\
\hline $\mathrm{H}$ & -7.443255 & -0.184592 & -0.239748 \\
\hline $\mathrm{H}$ & -4.827483 & 3.219000 & -0.315832 \\
\hline C & 7.608942 & 2.357503 & -0.394039 \\
\hline C & 8.613927 & 1.807471 & -1.205855 \\
\hline C & 7.789735 & 3.660995 & 0.095623 \\
\hline C & 9.759613 & 2.535702 & -1.517883 \\
\hline $\mathrm{H}$ & 8.504576 & 0.791453 & -1.571640 \\
\hline C & 8.936127 & 4.388935 & -0.214537 \\
\hline $\mathrm{H}$ & 7.011591 & 4.113560 & 0.702070 \\
\hline C & 9.926423 & 3.829850 & -1.023241 \\
\hline $\mathrm{H}$ & 10.528118 & 2.087974 & -2.140928 \\
\hline $\mathrm{H}$ & 9.051918 & 5.398481 & 0.168414 \\
\hline $\mathrm{H}$ & 10.819619 & 4.397276 & -1.265696 \\
\hline C & -7.446436 & 2.529915 & -0.055029 \\
\hline C & -8.678566 & 2.259048 & -0.671257 \\
\hline C & -7.344055 & 3.673852 & 0.752564 \\
\hline C & -9.771378 & 3.102504 & -0.485496 \\
\hline $\mathrm{H}$ & -8.769502 & 1.394606 & -1.321312 \\
\hline C & -8.435993 & 4.518713 & 0.937010 \\
\hline $\mathrm{H}$ & -6.407545 & 3.885381 & 1.258801 \\
\hline C & -9.654989 & 4.236582 & 0.319210 \\
\hline $\mathrm{H}$ & -10.713004 & 2.878847 & -0.977857 \\
\hline $\mathrm{H}$ & -8.337294 & 5.394063 & 1.571913 \\
\hline $\mathrm{H}$ & -10.506335 & 4.894509 & 0.463393 \\
\hline
\end{tabular}




\begin{tabular}{lc} 
B3LYP-D3(BJ)/6-31G(d,p) Energy & -1998.167978 \\
\hline B3LYP-D3(BJ)/def2-TZVPP/IEF-PCM(DCM) Energy & -1998.871095 \\
\hline B3LYP-D3(BJ)/def2-TZVPP/IEF-PCM(DCM)//B3LYP-D3(BJ)/6-31G(d,p) Free Energy (Quasiharmonic) & -1998.272659
\end{tabular}

Frequencies (Top 3 out of 237)

$\begin{array}{lr}\text { 1. } & 9.3728 \mathrm{~cm}^{-1} \\ \text { 2. } & 15.5557 \mathrm{~cm}^{-1} \\ \text { 3. } & 21.1963 \mathrm{~cm}^{-1}\end{array}$

B3LYP-D3(BJ)/6-31G(d,p) Molecular Geometry in Cartesian Coordinates

\begin{tabular}{|c|c|c|c|}
\hline C & -1.590920 & -0.034879 & -1.166719 \\
\hline $\mathrm{H}$ & -0.553840 & -0.357500 & -1.298869 \\
\hline $\mathrm{H}$ & -2.215080 & -0.931269 & -1.079009 \\
\hline C & 1.580610 & -0.124000 & 1.009871 \\
\hline 0 & 0.832500 & -1.079270 & 1.027661 \\
\hline 0 & 2.405700 & 0.179370 & 2.033731 \\
\hline $\mathrm{H}$ & 2.274230 & -0.514010 & 2.703111 \\
\hline 0 & -2.015420 & 0.779691 & -2.270859 \\
\hline $\mathrm{H}$ & -1.823370 & 0.292261 & -3.082659 \\
\hline C & 0.603310 & 1.665440 & -0.387499 \\
\hline C & 1.695930 & 0.858300 & -0.114989 \\
\hline C & 2.911410 & 0.963520 & -0.860209 \\
\hline C & 2.988380 & 1.916300 & -1.856329 \\
\hline $\mathrm{H}$ & 3.896640 & 1.994320 & -2.446459 \\
\hline C & -1.695470 & 0.786661 & 0.092731 \\
\hline C & -0.625160 & 1.586610 & 0.464271 \\
\hline C & -2.899060 & 1.462911 & 2.088441 \\
\hline C & -2.868640 & 0.736411 & 0.912451 \\
\hline $\mathrm{H}$ & -3.792200 & 1.436441 & 2.705581 \\
\hline C & -1.816600 & 2.271111 & 2.501511 \\
\hline C & -0.653280 & 2.346010 & 1.673771 \\
\hline C & 0.427870 & 3.168900 & 2.091621 \\
\hline C & 0.358530 & 3.877870 & 3.268511 \\
\hline C & -0.794380 & 3.803320 & 4.086661 \\
\hline C & -1.857430 & 3.017711 & 3.709201 \\
\hline $\mathrm{H}$ & 1.314850 & 3.228060 & 1.472171 \\
\hline $\mathrm{H}$ & 1.193880 & 4.500480 & 3.573441 \\
\hline $\mathrm{H}$ & -0.835290 & 4.369280 & 5.012241 \\
\hline $\mathrm{H}$ & -2.747370 & 2.954371 & 4.329151 \\
\hline C & 1.903670 & 2.772560 & -2.160449 \\
\hline C & 0.683550 & 2.643870 & -1.425019 \\
\hline & -0.401370 & 3.503340 & -1.741269 \\
\hline
\end{tabular}




\begin{tabular}{|c|c|c|c|}
\hline C & -0.281500 & 4.445160 & -2.736349 \\
\hline C & 0.925310 & 4.572020 & -3.465489 \\
\hline C & 1.992300 & 3.751340 & -3.185709 \\
\hline $\mathrm{H}$ & -1.330400 & 3.392151 & -1.196989 \\
\hline $\mathrm{H}$ & -1.120090 & 5.093460 & -2.969969 \\
\hline $\mathrm{H}$ & 1.004150 & 5.319490 & -4.248979 \\
\hline H & 2.921090 & 3.839920 & -3.742139 \\
\hline C & -6.396080 & -1.572659 & -0.044929 \\
\hline C & -5.787350 & -0.756159 & -1.010899 \\
\hline C & -4.641970 & -0.022459 & -0.721239 \\
\hline C & -4.058570 & -0.078259 & 0.555051 \\
\hline C & -4.664630 & -0.895609 & 1.521991 \\
\hline C & -5.808820 & -1.628779 & 1.228471 \\
\hline $\mathrm{H}$ & -4.192130 & 0.605361 & -1.482709 \\
\hline H & -4.211650 & -0.976329 & 2.505191 \\
\hline C & 4.061770 & 0.062820 & -0.608039 \\
\hline C & 5.363220 & 0.578480 & -0.525349 \\
\hline C & 3.890850 & -1.324360 & -0.482269 \\
\hline C & 6.453060 & -0.259360 & -0.318439 \\
\hline $\mathrm{H}$ & 5.512860 & 1.651540 & -0.591459 \\
\hline C & 4.982030 & -2.160620 & -0.275829 \\
\hline $\mathrm{H}$ & 2.898420 & -1.753740 & -0.568929 \\
\hline C & 6.285440 & -1.646960 & -0.188499 \\
\hline $\mathrm{H}$ & -6.238490 & -0.669879 & -1.994519 \\
\hline $\mathrm{H}$ & -6.234740 & -2.280819 & 1.984481 \\
\hline H & 4.826630 & -3.233040 & -0.215899 \\
\hline $\mathrm{H}$ & 7.445360 & 0.169120 & -0.220309 \\
\hline C & -7.614940 & -2.352409 & -0.359539 \\
\hline C & -8.624800 & -2.529499 & 0.599791 \\
\hline C & -7.791740 & -2.934449 & -1.625009 \\
\hline C & -9.770980 & -3.263949 & 0.304701 \\
\hline $\mathrm{H}$ & -8.518800 & -2.064379 & 1.574741 \\
\hline C & -8.938730 & -3.667079 & -1.921519 \\
\hline $\mathrm{H}$ & -7.010090 & -2.831469 & -2.370959 \\
\hline C & -9.933670 & -3.835839 & -0.957739 \\
\hline $\mathrm{H}$ & -10.543100 & -3.381829 & 1.059131 \\
\hline $\mathrm{H}$ & -9.051440 & -4.115179 & -2.904239 \\
\hline $\mathrm{H}$ & -10.827290 & -4.407539 & -1.188269 \\
\hline C & 7.445500 & -2.539670 & 0.034621 \\
\hline C & 8.679680 & -2.285830 & -0.584739 \\
\hline C & 7.339720 & -3.662240 & 0.871241 \\
\hline C & 9.771220 & -3.125030 & -0.373919 \\
\hline $\mathrm{H}$ & 8.773290 & -1.438590 & -1.256699 \\
\hline C & 8.430400 & -4.502870 & 1.080791 \\
\hline $\mathrm{H}$ & 6.401540 & -3.859880 & 1.379981 \\
\hline C & 9.651470 & -4.237770 & 0.459561 \\
\hline H & 10.714500 & -2.914930 & -0.869089 \\
\hline $\mathrm{H}$ & 8.329060 & -5.361400 & 1.737851 \\
\hline $\mathrm{H}$ & 10.501830 & -4.892390 & 0.623311 \\
\hline
\end{tabular}


Frequencies (Top 3 out of 237)

$\begin{array}{lr}\text { 1. } & 9.3063 \mathrm{~cm}^{-1} \\ \text { 2. } & 15.5557 \mathrm{~cm}^{-1} \\ \text { 3. } & 21.0070 \mathrm{~cm}^{-1}\end{array}$

B3LYP-D3(BJ)/6-31G(d,p) Molecular Geometry in Cartesian Coordinates

\begin{tabular}{|c|c|c|c|}
\hline C & -1.603253 & 0.043317 & -1.155287 \\
\hline $\mathrm{H}$ & -2.225135 & 0.940056 & -1.055636 \\
\hline $\mathrm{H}$ & -0.566920 & 0.365159 & -1.295230 \\
\hline C & 1.587079 & 0.115385 & 0.997643 \\
\hline 0 & 0.836710 & 1.068577 & 1.028962 \\
\hline 0 & 2.424596 & -0.191055 & 2.010408 \\
\hline $\mathrm{H}$ & 2.300541 & 0.499687 & 2.683960 \\
\hline 0 & -2.039641 & -0.763092 & -2.260794 \\
\hline $\mathrm{H}$ & -1.863547 & -0.266178 & -3.070501 \\
\hline C & 0.596805 & -1.663376 & -0.404554 \\
\hline C & 1.692643 & -0.859733 & -0.134328 \\
\hline C & 2.902373 & -0.961320 & -0.889485 \\
\hline C & 2.970259 & -1.906679 & -1.893343 \\
\hline $\mathrm{H}$ & 3.873726 & -1.981662 & -2.491170 \\
\hline C & -1.697519 & -0.786366 & 0.099589 \\
\hline C & -0.624900 & -1.589415 & 0.457303 \\
\hline C & -2.885393 & -1.473765 & 2.100997 \\
\hline C & -2.863714 & -0.740043 & 0.929370 \\
\hline $\mathrm{H}$ & -3.773177 & -1.449965 & 2.725931 \\
\hline C & -1.800462 & -2.285917 & 2.499743 \\
\hline C & -0.644001 & -2.356620 & 1.662063 \\
\hline C & 0.439621 & -3.183336 & 2.065716 \\
\hline C & 0.379119 & -3.899941 & 3.238467 \\
\hline C & -0.766966 & -3.829469 & 4.066496 \\
\hline C & -1.832197 & -3.040271 & 3.702868 \\
\hline $\mathrm{H}$ & 1.321389 & -3.239473 & 1.438581 \\
\hline $\mathrm{H}$ & 1.216207 & -4.525476 & 3.532466 \\
\hline $\mathrm{H}$ & -0.800961 & -4.401388 & 4.988691 \\
\hline $\mathrm{H}$ & -2.716940 & -2.979986 & 4.330506 \\
\hline C & 1.881976 & -2.759061 & -2.195522 \\
\hline C & 0.667763 & -2.634328 & -1.449713 \\
\hline 0 & -0.420634 & -3.490213 & -1.763785 \\
\hline
\end{tabular}




\begin{tabular}{|c|c|c|c|}
\hline C & -0.309688 & -4.424790 & -2.766687 \\
\hline C & 0.891227 & -4.547614 & -3.506177 \\
\hline C & 1.961394 & -3.730299 & -3.228673 \\
\hline H & -1.345241 & -3.381944 & -1.211451 \\
\hline $\mathrm{H}$ & -1.150843 & -5.070398 & -2.998522 \\
\hline $\mathrm{H}$ & 0.963048 & -5.289338 & -4.295791 \\
\hline $\mathrm{H}$ & 2.885700 & -3.815850 & -3.792973 \\
\hline C & -6.392742 & 1.583268 & 0.013985 \\
\hline C & -5.798854 & 0.764406 & -0.959131 \\
\hline C & -4.653960 & 0.024905 & -0.682543 \\
\hline C & -4.055047 & 0.079104 & 0.586569 \\
\hline C & -4.645539 & 0.899560 & 1.560238 \\
\hline C & -5.790681 & 1.636749 & 1.280422 \\
\hline $\mathrm{H}$ & -4.205583 & -0.591472 & -1.454221 \\
\hline $\mathrm{H}$ & -4.190854 & 0.964332 & 2.543852 \\
\hline C & 4.056308 & -0.064410 & -0.639215 \\
\hline C & 5.356717 & -0.583684 & -0.565125 \\
\hline C & 3.889499 & 1.322526 & -0.506346 \\
\hline C & 6.449840 & 0.250617 & -0.361167 \\
\hline H & 5.505294 & -1.655136 & -0.655460 \\
\hline C & 4.983603 & 2.154909 & -0.299377 \\
\hline H & 2.895651 & 1.753014 & -0.568161 \\
\hline C & 6.286144 & 1.637716 & -0.222196 \\
\hline $\mathrm{H}$ & -6.230620 & 0.722863 & -1.954302 \\
\hline $\mathrm{H}$ & -6.236921 & 2.247995 & 2.058501 \\
\hline H & 4.825277 & 3.221695 & -0.177948 \\
\hline $\mathrm{H}$ & 7.447725 & -0.174676 & -0.325529 \\
\hline C & -7.611982 & 2.367991 & -0.286741 \\
\hline C & -8.620501 & 1.844174 & -1.111421 \\
\hline C & -7.790380 & 3.655306 & 0.244813 \\
\hline C & -9.767239 & 2.582144 & -1.395438 \\
\hline H & -8.512983 & 0.840261 & -1.509725 \\
\hline C & -8.937823 & 4.392925 & -0.037338 \\
\hline $\mathrm{H}$ & -7.009586 & 4.088276 & 0.862061 \\
\hline C & -9.931610 & 3.859977 & -0.859299 \\
\hline $\mathrm{H}$ & -10.538446 & 2.154547 & -2.029194 \\
\hline $\mathrm{H}$ & -9.051719 & 5.389823 & 0.377950 \\
\hline H & -10.825622 & 4.434971 & -1.079941 \\
\hline C & 7.449767 & 2.526327 & -0.000468 \\
\hline C & 7.503558 & 3.801928 & -0.584664 \\
\hline C & 8.527840 & 2.115115 & 0.799540 \\
\hline C & 8.597738 & 4.638128 & -0.375603 \\
\hline H & 6.692210 & 4.126959 & -1.228179 \\
\hline C & 9.622986 & 2.950382 & 1.007427 \\
\hline $\mathrm{H}$ & 8.491874 & 1.143561 & 1.282129 \\
\hline C & 9.662811 & 4.216008 & 0.421074 \\
\hline $\mathrm{H}$ & 8.622776 & 5.617744 & -0.843370 \\
\hline H & 10.442563 & 2.615853 & 1.636309 \\
\hline $\mathrm{H}$ & 10.515885 & 4.867398 & 0.583580 \\
\hline
\end{tabular}




\begin{tabular}{lc} 
B3LYP-D3(BJ)/6-31G(d,p) Energy & -1998.16796 \\
\hline B3LYP-D3(BJ)/def2-TZVPP/IEF-PCM(DCM) Energy & -1998.871036 \\
\hline B3LYP-D3(BJ)/def2-TZVPP/IEF-PCM(DCM)//B3LYP-D3(BJ)/6-31G(d,p) Free Energy (Quasiharmonic) & -1998.272614
\end{tabular}

Number of Imaginary Frequencies

Frequencies (Top 3 out of 237)

$\begin{array}{lr}\text { 1. } & 9.3117 \mathrm{~cm}^{-1} \\ \text { 2. } & 15.5010 \mathrm{~cm}^{-1} \\ \text { 3. } & 21.0308 \mathrm{~cm}^{-1}\end{array}$

B3LYP-D3(BJ)/6-31G(d,p) Molecular Geometry in Cartesian Coordinates

\begin{tabular}{|c|c|c|c|}
\hline C & -1.597999 & -0.074694 & -1.159475 \\
\hline $\mathrm{H}$ & -0.561618 & -0.401846 & -1.285926 \\
\hline $\mathrm{H}$ & -2.221872 & -0.967458 & -1.038831 \\
\hline C & 1.584858 & -0.091989 & 1.006199 \\
\hline 0 & 0.833519 & -1.043366 & 1.060591 \\
\hline 0 & 2.418978 & 0.241168 & 2.013309 \\
\hline $\mathrm{H}$ & 2.291847 & -0.430925 & 2.704917 \\
\hline 0 & -2.027875 & 0.702592 & -2.288192 \\
\hline $\mathrm{H}$ & -1.840309 & 0.188013 & -3.084123 \\
\hline C & 0.600957 & 1.648886 & -0.446913 \\
\hline C & 1.695206 & 0.852008 & -0.151403 \\
\hline C & 2.907544 & 0.932179 & -0.904966 \\
\hline C & 2.979560 & 1.850051 & -1.933747 \\
\hline $\mathrm{H}$ & 3.885044 & 1.908162 & -2.530399 \\
\hline C & -1.696292 & 0.788388 & 0.072404 \\
\hline C & -0.623737 & 1.599215 & 0.412449 \\
\hline C & -2.890469 & 1.531455 & 2.049929 \\
\hline C & -2.865785 & 0.766257 & 0.898621 \\
\hline $\mathrm{H}$ & -3.780834 & 1.526174 & 2.671606 \\
\hline C & -1.805627 & 2.352041 & 2.431071 \\
\hline C & -0.645917 & 2.398353 & 1.596193 \\
\hline C & 0.437644 & 3.233760 & 1.981702 \\
\hline C & 0.374005 & 3.981566 & 3.134636 \\
\hline C & -0.775328 & 3.935254 & 3.959876 \\
\hline C & -1.840590 & 3.138423 & 3.613419 \\
\hline $\mathrm{H}$ & 1.321894 & 3.271553 & 1.356702 \\
\hline $\mathrm{H}$ & 1.211118 & 4.613288 & 3.415015 \\
\hline $\mathrm{H}$ & -0.811761 & 4.531710 & 4.866299 \\
\hline $\mathrm{H}$ & -2.727816 & 3.096449 & 4.239041 \\
\hline C & 1.893108 & 2.694955 & -2.262351 \\
\hline C & 0.676312 & 2.591393 & -1.517514 \\
\hline 0 & -0.410190 & 3.439536 & -1.858085 \\
\hline
\end{tabular}




\begin{tabular}{|c|c|c|c|}
\hline C & -0.294977 & 4.346776 & -2.885316 \\
\hline C & 0.908507 & 4.448606 & -3.623823 \\
\hline C & 1.976923 & 3.638039 & -3.320930 \\
\hline $\mathrm{H}$ & -1.336752 & 3.346951 & -1.306183 \\
\hline $\mathrm{H}$ & -1.134709 & 4.986707 & -3.137168 \\
\hline $\mathrm{H}$ & 0.983700 & 5.168837 & -4.432782 \\
\hline H & 2.903192 & 3.707587 & -3.884209 \\
\hline C & -6.398384 & -1.571435 & 0.034098 \\
\hline C & -5.793767 & -0.787585 & -0.961055 \\
\hline C & -4.646879 & -0.045231 & -0.700948 \\
\hline C & -4.057744 & -0.059056 & 0.573846 \\
\hline C & -4.659723 & -0.843684 & 1.570018 \\
\hline C & -5.805449 & -1.585645 & 1.306028 \\
\hline $\mathrm{H}$ & -4.200259 & 0.556830 & -1.484781 \\
\hline $\mathrm{H}$ & -4.202346 & -0.892097 & 2.553304 \\
\hline C & 4.059888 & 0.041492 & -0.626754 \\
\hline C & 5.360596 & 0.561470 & -0.563542 \\
\hline C & 3.891429 & -1.341097 & -0.455987 \\
\hline C & 6.452380 & -0.267821 & -0.333678 \\
\hline $\mathrm{H}$ & 5.510455 & 1.629881 & -0.683029 \\
\hline C & 4.984192 & -2.168385 & -0.223101 \\
\hline $\mathrm{H}$ & 2.897345 & -1.772285 & -0.508467 \\
\hline C & 6.287019 & -1.650398 & -0.156803 \\
\hline $\mathrm{H}$ & -6.249298 & -0.733675 & -1.944955 \\
\hline $\mathrm{H}$ & -6.228159 & -2.212150 & 2.085083 \\
\hline H & 4.824517 & -3.231253 & -0.072570 \\
\hline $\mathrm{H}$ & 7.450559 & 0.157440 & -0.307186 \\
\hline C & -7.618849 & -2.360666 & -0.249130 \\
\hline C & -7.801511 & -2.984039 & -1.493920 \\
\hline C & -8.624407 & -2.505642 & 0.720051 \\
\hline C & -8.950007 & -3.725579 & -1.760950 \\
\hline $\mathrm{H}$ & -7.023205 & -2.906003 & -2.246368 \\
\hline C & -9.772099 & -3.248964 & 0.454494 \\
\hline H & -8.513892 & -2.008680 & 1.678640 \\
\hline C & -9.940626 & -3.862096 & -0.787655 \\
\hline $\mathrm{H}$ & -9.067270 & -4.205777 & -2.727855 \\
\hline $\mathrm{H}$ & -10.540824 & -3.341608 & 1.215881 \\
\hline $\mathrm{H}$ & -10.835420 & -4.440728 & -0.995198 \\
\hline C & 7.449253 & -2.533483 & 0.092553 \\
\hline C & 7.503475 & -3.824824 & -0.455927 \\
\hline C & 8.525569 & -2.101111 & 0.883721 \\
\hline C & 8.596386 & -4.655770 & -0.220883 \\
\hline $\mathrm{H}$ & 6.693530 & -4.166932 & -1.092318 \\
\hline C & 9.619445 & -2.931157 & 1.117573 \\
\hline $\mathrm{H}$ & 8.489162 & -1.116540 & 1.339130 \\
\hline C & 9.659725 & -4.212573 & 0.566612 \\
\hline H & 8.621808 & -5.647994 & -0.661249 \\
\hline H & 10.437649 & -2.579981 & 1.739122 \\
\hline $\mathrm{H}$ & 10.511804 & -4.859882 & 0.749369 \\
\hline
\end{tabular}




\begin{tabular}{lc} 
B3LYP-D3(BJ)/6-31G(d,p) Energy & -1998.166609 \\
\hline B3LYP-D3(BJ)/def2-TZVPP/IEF-PCM(DCM) Energy & -1998.870568 \\
\hline B3LYP-D3(BJ)/def2-TZVPP/IEF-PCM(DCM)//B3LYP-D3(BJ)/6-31G(d,p) Free Energy (Quasiharmonic) & -1998.272193 \\
\hline
\end{tabular}

Frequencies (Top 3 out of 237)

$\begin{array}{lr}\text { 1. } & 8.4997 \mathrm{~cm}^{-1} \\ \text { 2. } & 18.8795 \mathrm{~cm}^{-1} \\ \text { 3. } & 21.0504 \mathrm{~cm}^{-1}\end{array}$

B3LYP-D3(BJ)/6-31G(d,p) Molecular Geometry in Cartesian Coordinates

\begin{tabular}{|c|c|c|c|}
\hline C & 1.531516 & 0.042900 & -1.165289 \\
\hline $\mathrm{H}$ & 2.131636 & -0.870266 & -1.078348 \\
\hline $\mathrm{H}$ & 0.488154 & -0.251651 & -1.311858 \\
\hline C & -1.517509 & -0.088324 & 0.980605 \\
\hline 0 & -1.540358 & 0.139246 & 2.169534 \\
\hline 0 & -1.315370 & -1.328263 & 0.475531 \\
\hline $\mathrm{H}$ & -1.232089 & -1.921146 & 1.242550 \\
\hline 0 & 1.983818 & 0.848772 & -2.264665 \\
\hline $\mathrm{H}$ & 1.772748 & 0.374206 & -3.079484 \\
\hline C & -0.624199 & 1.782319 & -0.364915 \\
\hline C & -1.682394 & 0.926863 & -0.111124 \\
\hline C & -2.877586 & 0.956791 & -0.893374 \\
\hline C & -2.962829 & 1.873637 & -1.922395 \\
\hline $\mathrm{H}$ & -3.876345 & 1.931283 & -2.506794 \\
\hline C & 1.658997 & 0.863688 & 0.094065 \\
\hline C & 0.618957 & 1.705433 & 0.464890 \\
\hline C & 2.924407 & 1.567764 & 2.042155 \\
\hline C & 2.846068 & 0.800136 & 0.895582 \\
\hline $\mathrm{H}$ & 3.828435 & 1.532304 & 2.642845 \\
\hline C & 1.880762 & 2.432498 & 2.442849 \\
\hline C & 0.701043 & 2.509819 & 1.641537 \\
\hline C & -0.344393 & 3.380388 & 2.047678 \\
\hline C & -0.221988 & 4.141360 & 3.186858 \\
\hline C & 0.949301 & 4.067679 & 3.978364 \\
\hline C & 1.976729 & 3.230263 & 3.613754 \\
\hline $\mathrm{H}$ & -1.246163 & 3.432795 & 1.449325 \\
\hline $\mathrm{H}$ & -1.030296 & 4.801698 & 3.485084 \\
\hline $\mathrm{H}$ & 1.031493 & 4.673626 & 4.875441 \\
\hline $\mathrm{H}$ & 2.878864 & 3.164719 & 4.215509 \\
\hline C & -1.906939 & 2.767991 & -2.219125 \\
\hline C & -0.712510 & 2.725279 & -1.433392 \\
\hline C & 0.345224 & 3.620098 & -1.742487 \\
\hline
\end{tabular}




\begin{tabular}{|c|c|c|c|}
\hline C & 0.219090 & 4.524008 & -2.771366 \\
\hline C & -0.966288 & 4.573433 & -3.543946 \\
\hline C & -2.004425 & 3.713026 & -3.274922 \\
\hline $\mathrm{H}$ & 1.257745 & 3.569276 & -1.161811 \\
\hline $\mathrm{H}$ & 1.035364 & 5.202800 & -2.997269 \\
\hline $\mathrm{H}$ & -1.051040 & 5.292567 & -4.352921 \\
\hline $\mathrm{H}$ & -2.916114 & 3.742473 & -3.864911 \\
\hline C & 6.272458 & -1.672960 & -0.023528 \\
\hline c & 5.691870 & -0.856272 & -1.006580 \\
\hline C & 4.579310 & -0.068914 & -0.729900 \\
\hline C & 4.001107 & -0.069200 & 0.550046 \\
\hline C & 4.580250 & -0.885259 & 1.534559 \\
\hline C & 5.691865 & -1.671966 & 1.254098 \\
\hline H & 4.152479 & 0.560030 & -1.503635 \\
\hline $\mathrm{H}$ & 4.132497 & -0.919607 & 2.522984 \\
\hline C & -4.015295 & 0.049183 & -0.610368 \\
\hline C & -4.652522 & -0.634704 & -1.656224 \\
\hline C & -4.506807 & -0.127303 & 0.692369 \\
\hline C & -5.736230 & -1.469584 & -1.409906 \\
\hline $\mathrm{H}$ & -4.269500 & -0.531875 & -2.666686 \\
\hline C & -5.591474 & -0.962558 & 0.935890 \\
\hline $\mathrm{H}$ & -4.051302 & 0.410503 & 1.516416 \\
\hline C & -6.228117 & -1.652037 & -0.107779 \\
\hline $\mathrm{H}$ & 6.140660 & -0.812756 & -1.994092 \\
\hline $\mathrm{H}$ & 6.096505 & -2.320268 & 2.024900 \\
\hline $\mathrm{H}$ & -5.974070 & -1.055218 & 1.947224 \\
\hline H & -6.187322 & -2.016443 & -2.231711 \\
\hline C & 7.455862 & -2.510519 & -0.324767 \\
\hline C & 8.463669 & -2.705403 & 0.633265 \\
\hline C & 7.599950 & -3.131223 & -1.575927 \\
\hline C & 9.576194 & -3.494525 & 0.350756 \\
\hline $\mathrm{H}$ & 8.383795 & -2.211553 & 1.596493 \\
\hline c & 8.713348 & -3.918594 & -1.859892 \\
\hline $\mathrm{H}$ & 6.818594 & -3.014217 & -2.320140 \\
\hline C & 9.706563 & -4.104539 & -0.897506 \\
\hline $\mathrm{H}$ & 10.347671 & -3.625456 & 1.103671 \\
\hline $\mathrm{H}$ & 8.800941 & -4.395594 & -2.831503 \\
\hline H & 10.574017 & -4.718804 & -1.118240 \\
\hline C & -7.380624 & -2.543481 & 0.156038 \\
\hline C & -8.448621 & -2.628202 & -0.751413 \\
\hline c & -7.433717 & -3.325987 & 1.320569 \\
\hline C & -9.532226 & -3.467582 & -0.503638 \\
\hline H & -8.438848 & -2.010183 & -1.643639 \\
\hline C & -8.518042 & -4.164013 & 1.569749 \\
\hline H & -6.605260 & -3.293989 & 2.021054 \\
\hline C & -9.572109 & -4.239128 & 0.658410 \\
\hline $\mathrm{H}$ & -10.351634 & -3.511933 & -1.214771 \\
\hline H & -8.535525 & -4.766376 & 2.473034 \\
\hline $\mathrm{H}$ & -10.416975 & -4.892670 & 0.852150 \\
\hline
\end{tabular}


Frequencies (Top 3 out of 237)

$\begin{array}{lr}\text { 1. } & 8.5108 \mathrm{~cm}^{-1} \\ \text { 2. } & 18.8743 \mathrm{~cm}^{-1} \\ \text { 3. } & 21.1172 \mathrm{~cm}^{-1}\end{array}$

B3LYP-D3(BJ)/6-31G(d,p) Molecular Geometry in Cartesian Coordinates

\begin{tabular}{|c|c|c|c|}
\hline C & -1.536674 & 0.064932 & -1.155172 \\
\hline $\mathrm{H}$ & -2.135344 & -0.850749 & -1.086572 \\
\hline $\mathrm{H}$ & -0.493508 & -0.224842 & -1.312546 \\
\hline C & 1.522464 & -0.109914 & 0.973662 \\
\hline 0 & 1.548681 & 0.089640 & 2.167555 \\
\hline 0 & 1.322773 & -1.338372 & 0.440290 \\
\hline $\mathrm{H}$ & 1.244600 & -1.949353 & 1.193508 \\
\hline 0 & -1.994334 & 0.894863 & -2.234184 \\
\hline $\mathrm{H}$ & -1.793837 & 0.434958 & -3.060052 \\
\hline C & 0.619217 & 1.790204 & -0.323152 \\
\hline C & 1.680267 & 0.931225 & -0.094374 \\
\hline C & 2.871992 & 0.982424 & -0.880840 \\
\hline C & 2.950837 & 1.923709 & -1.888080 \\
\hline $\mathrm{H}$ & 3.861668 & 1.997265 & -2.474877 \\
\hline C & -1.660357 & 0.856488 & 0.123140 \\
\hline $\mathrm{C}$ & -0.620313 & 1.691062 & 0.509713 \\
\hline C & -2.919367 & 1.511668 & 2.092416 \\
\hline C & -2.844217 & 0.772038 & 0.927418 \\
\hline $\mathrm{H}$ & -3.820888 & 1.460361 & 2.695731 \\
\hline C & -1.875453 & 2.368370 & 2.509497 \\
\hline C & -0.699137 & 2.467041 & 1.705526 \\
\hline C & 0.346446 & 3.329533 & 2.128182 \\
\hline C & 0.227443 & 4.062713 & 3.285804 \\
\hline C & -0.940452 & 3.967884 & 4.080059 \\
\hline C & -1.967944 & 3.137596 & 3.699624 \\
\hline $\mathrm{H}$ & 1.245676 & 3. 397814 & 1.527610 \\
\hline $\mathrm{H}$ & 1.035838 & 4.717160 & 3.596521 \\
\hline $\mathrm{H}$ & -1.020007 & 4.551936 & 4.991777 \\
\hline $\mathrm{H}$ & -2.867471 & 3.055981 & 4.303314 \\
\hline C & 1.891864 & 2.822649 & -2.158779 \\
\hline C & 0.701016 & 2.758674 & -1.369095 \\
\hline C & -0.359670 & 3.658681 & -1.651846 \\
\hline
\end{tabular}




\begin{tabular}{|c|c|c|c|}
\hline C & -0.239793 & 4.587305 & -2.659238 \\
\hline C & 0.941974 & 4.657508 & -3.435721 \\
\hline C & 1.982881 & 3.792912 & -3.192028 \\
\hline $\mathrm{H}$ & -1.269384 & 3.592340 & -1.068337 \\
\hline $\mathrm{H}$ & -1.058255 & 5.269838 & -2.865071 \\
\hline H & 1.021775 & 5.396004 & -4.227576 \\
\hline $\mathrm{H}$ & 2.891868 & 3.838286 & -3.785166 \\
\hline C & -6.269776 & -1.683238 & -0.040695 \\
\hline C & -5.698174 & -0.836305 & -1.003130 \\
\hline c & -4.586715 & -0.053152 & -0.710459 \\
\hline C & -3.999233 & -0.090539 & 0.564662 \\
\hline C & -4.568314 & -0.938013 & 1.528050 \\
\hline C & -5.680486 & -1.718476 & 1.232341 \\
\hline H & -4.156557 & 0.587100 & -1.473060 \\
\hline $\mathrm{H}$ & -4.124292 & -0.986843 & 2.517574 \\
\hline C & 4.012944 & 0.070979 & -0.624426 \\
\hline C & 4.647343 & -0.586054 & -1.689044 \\
\hline C & 4.510345 & -0.135576 & 0.671646 \\
\hline C & 5.734044 & -1.423950 & -1.467366 \\
\hline $\mathrm{H}$ & 4.259809 & -0.459950 & -2.695139 \\
\hline C & 5.597968 & -0.973805 & 0.890527 \\
\hline $\mathrm{H}$ & 4.057130 & 0.381238 & 1.510270 \\
\hline C & 6.231853 & -1.636416 & -0.172058 \\
\hline $\mathrm{H}$ & -6.120822 & -0.806151 & -2.002618 \\
\hline $\mathrm{H}$ & -6.111767 & -2.349296 & 2.003222 \\
\hline H & 5.985071 & -1.089777 & 1.897736 \\
\hline H & 6.182946 & -1.949810 & -2.303941 \\
\hline C & -7.453499 & -2.514200 & -0.358729 \\
\hline C & -8.472092 & -2.027260 & -1.193563 \\
\hline C & -7.587291 & -3.809608 & 0.166199 \\
\hline C & -9.585353 & -2.808754 & -1.493858 \\
\hline $\mathrm{H}$ & -8.399798 & -1.018255 & -1.586891 \\
\hline C & -8.701306 & -4.590794 & -0.132196 \\
\hline $\mathrm{H}$ & -6.797564 & -4.213871 & 0.791524 \\
\hline C & -9.705496 & -4.094189 & -0.964196 \\
\hline $\mathrm{H}$ & -10.365321 & -2.409352 & -2.135213 \\
\hline $\mathrm{H}$ & -8.780814 & -5.592960 & 0.278377 \\
\hline $\mathrm{H}$ & -10.573469 & -4.703061 & -1.197485 \\
\hline C & 7.387668 & -2.530963 & 0.065423 \\
\hline C & 7.447714 & -3.341074 & 1.210586 \\
\hline C & 8.452001 & -2.591071 & -0.848279 \\
\hline C & 8.535215 & -4.181983 & 1.435005 \\
\hline H & 6.622176 & -3.328097 & 1.915122 \\
\hline C & 9.538779 & -3.433300 & -0.625294 \\
\hline $\mathrm{H}$ & 8.436859 & -1.951811 & -1.725333 \\
\hline C & 9.585580 & -4.232420 & 0.517710 \\
\hline $\mathrm{H}$ & 8.558077 & -4.805814 & 2.323480 \\
\hline H & 10.355255 & -3.458393 & -1.340725 \\
\hline $\mathrm{H}$ & 10.432922 & -4.888190 & 0.692143 \\
\hline
\end{tabular}




\begin{tabular}{lc} 
B3LYP-D3(BJ)/6-31G(d,p) Energy & -1998.166576 \\
\hline B3LYP-D3(BJ)/def2-TZVPP/IEF-PCM(DCM) Energy & -1998.870545 \\
\hline B3LYP-D3(BJ)/def2-TZVPP/IEF-PCM(DCM)//B3LYP-D3(BJ)/6-31G(d,p) Free Energy (Quasiharmonic) & -1998.272165 \\
\hline
\end{tabular}

Frequencies (Top 3 out of 237)

$\begin{array}{lr}\text { 1. } & 8.4659 \mathrm{~cm}^{-1} \\ \text { 2. } & 18.7838 \mathrm{~cm}^{-1} \\ \text { 3. } & 21.3312 \mathrm{~cm}^{-1}\end{array}$

\section{B3LYP-D3(BJ)/6-31G(d,p) Molecular Geometry in Cartesian Coordinates}

C

$\mathrm{H}$

$\mathrm{H}$

C

0

0

$\mathrm{H}$

0

$\mathrm{H}$

C

C

C

C

$\mathrm{H}$

C

C

C

C

$\mathrm{H}$

C

C

C

C

C

C

$\mathrm{H}$

$\mathrm{H}$

$\mathrm{H}$

$\mathrm{H}$

C

C

C
1.535215

0.492599

2.136159

$-1.521125$

$-1.539227$

$-1.329464$

$-1.251002$

1.989709

1.782656

$-0.623724$

$-1.682188$

$-2.874749$

$-2.957175$

$-3.868864$

1.658142

0.616621

2. 917314

2. 842782

3.819504

1.872179

0.694932

$-0.351870$

$-0.233062$

0.935779

1.964410

$-1.251769$

$-1.042342$

1.015142

2.864686

$-1.900693$

$-0.708703$

0.349999
0.005725

$-0.294633$

$-0.903450$

$-0.060078$

0.206205

$-1.317193$

$-1.885806$

0.776894

0.275877

1.769110

0.920669

0.926289

1.812303

1.852467

0.866343

1.719069

1.633651

0.829357

1.618025

2.510555

2.561348

3. 444452

4. 242351

4. 194849

3. 346157

3.477062

4.911956

4.829827

3. 300499

2.697899

2.679130

3.564252
1.157881

1.298045

1.040290

$-0.978074$

$-2.159051$

$-0.513074$

$-1.298786$

2. 281029

3.080911

0.420801

0.145128

0.932162

1.988110

2.576784

$-0.075042$

$-0.415250$

$-2.003195$

$-0.881815$

$-2.607475$

$-2.372341$

$-1.565315$

$-1.939574$

$-3.053587$

$-3.850698$

$-3.516849$

$-1.337010$

$-3.327544$

$-4.727727$

$-4.123205$

2. 308029

1.517723

1.850575 


\begin{tabular}{|c|c|c|c|}
\hline C & 0.226865 & 4.436856 & 2.906483 \\
\hline C & -0.956332 & 4.463036 & 3.683542 \\
\hline C & -1.995192 & 3.610900 & 3.391914 \\
\hline H & 1.260924 & 3.530727 & 1.266154 \\
\hline $\mathrm{H}$ & 1.043848 & 5.108526 & 3.150390 \\
\hline H & -1.038783 & 5.157591 & 4.513950 \\
\hline H & -2.905114 & 3.622438 & 3.985242 \\
\hline C & 6.273664 & -1.669023 & -0.052352 \\
\hline C & 5.695646 & -0.884310 & 0.957889 \\
\hline C & 4.581666 & -0.089482 & 0.709754 \\
\hline C & 3.999417 & -0.049720 & -0.567738 \\
\hline C & 4.575966 & -0.833757 & -1.579413 \\
\hline C & 5.688993 & -1.628086 & -1.327461 \\
\hline H & 4.156824 & 0.514356 & 1.504311 \\
\hline H & 4.125115 & -0.837129 & -2.567020 \\
\hline C & -4.013234 & 0.026891 & 0.626195 \\
\hline C & -4.645189 & -0.689530 & 1.653179 \\
\hline C & -4.510556 & -0.109666 & -0.679042 \\
\hline C & -5.730592 & -1.515890 & 1.386392 \\
\hline H & -4.269228 & -0.603329 & 2.667852 \\
\hline C & -5.594445 & -0.939584 & -0.943734 \\
\hline H & -4.048038 & 0.440002 & -1.491309 \\
\hline C & -6.226968 & -1.659947 & 0.081253 \\
\hline $\mathrm{H}$ & 6.147565 & -0.871679 & 1.944853 \\
\hline $\mathrm{H}$ & 6.091630 & -2.251355 & -2.119660 \\
\hline H & -5.946335 & -1.044636 & -1.964973 \\
\hline $\mathrm{H}$ & -6.213227 & -2.044974 & 2.201909 \\
\hline C & 7.458673 & -2.514686 & 0.218455 \\
\hline C & 7.607406 & -3.174314 & 1.448984 \\
\hline C & 8.463409 & -2.678510 & -0.748576 \\
\hline C & 8.722310 & -3.969314 & 1.704336 \\
\hline H & 6.828473 & -3.081400 & 2.199110 \\
\hline C & 9.577427 & -3.475232 & -0.494745 \\
\hline H & 8.379955 & -2.154661 & -1.695511 \\
\hline C & 9.712415 & -4.124106 & 0.733267 \\
\hline H & 8.813513 & -4.476553 & 2.660172 \\
\hline $\mathrm{H}$ & 10.346464 & -3.581783 & -1.253974 \\
\hline H & 10.581040 & -4.744304 & 0.931674 \\
\hline C & -7.380404 & -2.543629 & -0.204396 \\
\hline C & -7.543111 & -3.759204 & 0.479134 \\
\hline C & -8.339647 & -2.187575 & -1.165859 \\
\hline C & -8.628540 & -4.590088 & 0.211306 \\
\hline $\mathrm{H}$ & -6.797201 & -4.064707 & 1.206052 \\
\hline C & -9.424261 & -3.018980 & -1.435164 \\
\hline $\mathrm{H}$ & -8.245650 & -1.239249 & -1.685319 \\
\hline C & -9.573993 & -4.223932 & -0.747460 \\
\hline H & -8.731048 & -5.529802 & 0.745673 \\
\hline $\mathrm{H}$ & -10.158922 & -2.720777 & -2.177061 \\
\hline H & -10.419580 & -4.871675 & -0.956897 \\
\hline
\end{tabular}


Frequencies (Top 3 out of 237)

$\begin{array}{lr}\text { 1. } & 8.4637 \mathrm{~cm}^{-1} \\ \text { 2. } & 18.8148 \mathrm{~cm}^{-1} \\ \text { 3. } & 21.1822 \mathrm{~cm}^{-1}\end{array}$

B3LYP-D3(BJ)/6-31G(d,p) Molecular Geometry in Cartesian Coordinates

\begin{tabular}{|c|c|c|c|}
\hline C & 1.539981 & -0.030865 & -1.149159 \\
\hline $\mathrm{H}$ & 0.497543 & 0.264579 & -1.300846 \\
\hline $\mathrm{H}$ & 2.139212 & 0.881718 & -1.050747 \\
\hline C & -1.525447 & 0.081217 & 0.971336 \\
\hline 0 & -1.547486 & -0.155935 & 2.158446 \\
\hline 0 & -1.335052 & 1.327045 & 0.476346 \\
\hline H & -1.261438 & 1.914926 & 1.248212 \\
\hline 0 & 2.000244 & -0.827825 & -2.251719 \\
\hline $\mathrm{H}$ & 1.802781 & -0.342610 & -3.063719 \\
\hline C & -0.618976 & -1.780415 & -0.378131 \\
\hline C & -1.679936 & -0.927194 & -0.128005 \\
\hline$C$ & -2.868997 & -0.954078 & -0.919884 \\
\hline 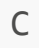 & -2.945358 & -1.865730 & -1.954225 \\
\hline$\Pi$ & -3.854318 & -1.921705 & -2.545826 \\
\hline C & 1.659171 & -0.861492 & 0.104532 \\
\hline C & 0.617826 & -1.707535 & 0.461471 \\
\hline C & 2.912059 & -1.578575 & 2.056071 \\
\hline$c$ & 2.840514 & -0.802576 & 0.914782 \\
\hline П & 3.811706 & -1.546380 & 2.663487 \\
\hline C & 1.866896 & -2.448025 & 2.442552 \\
\hline C & 0.693036 & -2.521023 & 1.632291 \\
\hline C & -0.353754 & -3.396491 & 2.024122 \\
\hline$c$ & -0.238145 & -4.166251 & 3.158093 \\
\hline & 0.927337 & -4.096794 & 3.958496 \\
\hline C & 1.955860 & -3.254762 & 3.607845 \\
\hline $\mathrm{H}$ & -1.251144 & -3.445646 & 1.418934 \\
\hline $\mathrm{H}$ & -1.047387 & -4.830315 & 3.445333 \\
\hline $\mathrm{H}$ & 1.004216 & -4.709623 & 4.851363 \\
\hline & 2.853543 & -3.192389 & 4.216553 \\
\hline C & -1.886147 & -2.757226 & -2.247636 \\
\hline C & -0.697788 & -2.717302 & -1.452668 \\
\hline$\Omega$ & 0.363501 & -3.608952 & -1.758747 \\
\hline
\end{tabular}




\begin{tabular}{|c|c|c|c|}
\hline C & 0.246353 & -4.507394 & -2.793449 \\
\hline C & -0.933153 & -4.554203 & -3.575126 \\
\hline C & -1.974445 & -3.696639 & -3.309243 \\
\hline $\mathrm{H}$ & 1.271552 & -3.560049 & -1.170940 \\
\hline $\mathrm{H}$ & 1.065245 & -5.183826 & -3.016957 \\
\hline $\mathrm{H}$ & -1.010864 & -5.269006 & -4.388638 \\
\hline $\mathrm{H}$ & -2.881604 & -3.724008 & -3.906269 \\
\hline C & 6.269110 & 1.681212 & 0.034282 \\
\hline c & 5.700493 & 0.864710 & -0.955824 \\
\hline C & 4.588162 & 0.072918 & -0.691073 \\
\hline C & 3.996750 & 0.070675 & 0.582778 \\
\hline c & 4.562892 & 0.887609 & 1.573869 \\
\hline C & 5.675963 & 1.676790 & 1.306004 \\
\hline $\mathrm{H}$ & 4.160348 & -0.543310 & -1.474508 \\
\hline H & 4.115832 & 0.905609 & 2.563067 \\
\hline C & -4.010240 & -0.049402 & -0.640930 \\
\hline C & -4.638579 & 0.640966 & -1.687771 \\
\hline C & -4.513636 & 0.117897 & 0.658385 \\
\hline C & -5.726321 & 1.471910 & -1.445896 \\
\hline $\mathrm{H}$ & -4.257905 & 0.530810 & -2.698363 \\
\hline C & -5.599876 & 0.952312 & 0.898082 \\
\hline $\mathrm{H}$ & -4.053977 & -0.411259 & 1.485766 \\
\hline C & -6.228758 & 1.646740 & -0.146853 \\
\hline $\mathrm{H}$ & 6.126218 & 0.865668 & -1.954460 \\
\hline $\mathrm{H}$ & 6.104889 & 2.283259 & 2.097466 \\
\hline $\mathrm{H}$ & -5.956544 & 1.081488 & 1.914891 \\
\hline H & -6.206018 & 1.980409 & -2.276114 \\
\hline C & 7.453743 & 2.521669 & -0.254069 \\
\hline C & 7.585910 & 3.799975 & 0.311613 \\
\hline C & 8.474846 & 2.061100 & -1.100713 \\
\hline C & 8.700782 & 4.590072 & 0.041211 \\
\hline $\mathrm{H}$ & 6.794312 & 4.184433 & 0.946981 \\
\hline c & 9.588966 & 2.851571 & -1.373017 \\
\hline $\mathrm{H}$ & 8.403773 & 1.064929 & -1.525704 \\
\hline C & 9.707478 & 4.119751 & -0.802940 \\
\hline $\mathrm{H}$ & 8.779028 & 5.578856 & 0.483270 \\
\hline $\mathrm{H}$ & 10.370871 & 2.472437 & -2.024242 \\
\hline H & 10.576135 & 4.735582 & -1.014403 \\
\hline C & -7.384653 & 2.535391 & 0.112196 \\
\hline C & -7.545867 & 3.733896 & -0.601172 \\
\hline c & -8.347762 & 2.201255 & 1.077655 \\
\hline C & -8.633593 & 4.569441 & -0.358332 \\
\hline H & -6.797081 & 4.022832 & -1.331899 \\
\hline C & -9.434680 & 3.037356 & 1.321960 \\
\hline $\mathrm{H}$ & -8.254852 & 1.265860 & 1.620246 \\
\hline C & -9.582888 & 4.225161 & 0.604731 \\
\hline $\mathrm{H}$ & -8.734933 & 5.495839 & -0.915679 \\
\hline H & -10.172301 & 2.756073 & 2.067521 \\
\hline $\mathrm{H}$ & -10.430280 & 4.876550 & 0.794666 \\
\hline
\end{tabular}




\begin{tabular}{lc} 
B3LYP-D3(BJ)/6-31G(d,p) Energy & -2872.49919 \\
\hline B3LYP-D3(BJ)/def2-TZVPP/IEF-PCM(DCM) Energy & -2873.503886 \\
\hline B3LYP-D3(BJ)/def2-TZVPP/IEF-PCM(DCM)//B3LYP-D3(BJ)/6-31G(d,p) Free Energy (Quasiharmonic) & -2872.552023 \\
\hline
\end{tabular}

Number of Imaginary Frequencies

Frequencies (Top 3 out of 366)

$\begin{array}{lr}\text { 1. } & -265.0052 \mathrm{~cm}^{-1} \\ \text { 2. } & 10.7393 \mathrm{~cm}^{-1} \\ \text { 3. } & 13.3274 \mathrm{~cm}^{-1}\end{array}$

B3LYP-D3(BJ)/6-31G(d,p) Molecular Geometry in Cartesian Coordinates

C

$\mathrm{H}$

$\mathrm{H}$

C

0

0

$\mathrm{H}$

0

$\mathrm{H}$

C

C

C

C

$\mathrm{H}$

$\mathrm{H}$

$\mathrm{H}$

C

C

C

$\mathrm{H}$

$\mathrm{H}$

$\mathrm{H}$

C

$\mathrm{H}$

$\mathrm{H}$

$\mathrm{H}$

C

$\mathrm{H}$

$\mathrm{H}$

$\mathrm{H}$

C

$\mathrm{H}$

\subsection{7}

2.114750

0.518115

$-1.951532$

$-2.761006$

$-0.932515$

$-0.359595$

0.546736

0.039384

0.746524

1.657518

$-1.268743$

$-0.502166$

1.580579

$-2.304642$

$-1.171993$

$-1.050236$

$-1.801492$

0.283607

0.849010

0.129101

0.884555

$-1.880949$

$-2.195712$

$-1.296444$

$-2.765967$

$-3.283015$

$-3.869707$

$-3.416705$

$-3.680637$

$-1.666735$

$-2.077508$

\subsection{2}

0.914208

1.487159

1.816700

2.238856

1.036444

0.787453

0.261725

$-0.394739$

$-2.934307$

$-2.737449$

$-3.345202$

$-2.549938$

$-1.650694$

$-3.040053$

$-4.423941$

$-1.628376$

$-2.993855$

$-1.590344$

$-0.705686$

$-1.528937$

$-2.475495$

$-0.394328$

$-0.403156$

0.515840

$-0.336626$

$-2.899863$

$-2.368042$

$-2.389679$

$-3.913598$

$-3.621858$

$-2.962763$
0.838975

1.199993

1.705831

$-1.603977$

$-2.399720$

$-1.974316$

$-1.193540$

0.024169

0.581561

$-2.077653$

$-0.150558$

$-0.719382$

$-1.625652$

$-0.149238$

$-0.584328$

$-0.846195$

2.650317

2.813166

3.406328

3.098090

4.487014

3.187100

2.969493

4.017786

2.808348

2. 337995

2.424216

3.179318

1.468480

2. 329680

4.197930

4.970053 


\begin{tabular}{|c|c|c|c|}
\hline $\mathrm{H}$ & -2.220849 & -4.564000 & 4.225797 \\
\hline $\mathrm{H}$ & -0.623871 & -3.838028 & 4.434624 \\
\hline 0 & -0.760387 & -1.636343 & 1.227306 \\
\hline 0 & -1.135523 & -3.840251 & 1.865571 \\
\hline B & -0.562046 & -3.036829 & 0.849907 \\
\hline 0 & 0.871177 & -3.415682 & 0.636926 \\
\hline C & 2.983227 & -3.307725 & -0.442703 \\
\hline C & 3.280312 & -4.642038 & -0.130476 \\
\hline C & 3.963163 & -2.492940 & -1.027177 \\
\hline C & 4.547452 & -5.150674 & -0.399730 \\
\hline $\mathrm{H}$ & 2.515054 & -5.255097 & 0.332388 \\
\hline C & 5.225582 & -3.010010 & -1.302392 \\
\hline $\mathrm{H}$ & 3.735608 & -1.456340 & -1.256898 \\
\hline C & 5.520741 & -4.337632 & -0.986705 \\
\hline $\mathrm{H}$ & 4.779469 & -6.181161 & -0.148765 \\
\hline $\mathrm{H}$ & 5.986232 & -2.378232 & -1.744677 \\
\hline $\mathrm{H}$ & 6.511450 & -4.731866 & -1.189582 \\
\hline $\mathrm{H}$ & -0.795440 & -1.511506 & -1.775590 \\
\hline $\mathrm{H}$ & 0.995914 & -3.988760 & -2.138585 \\
\hline $\mathrm{H}$ & 1.329904 & -2.270167 & -2.706563 \\
\hline C & -1.110943 & 2.975198 & 0.470526 \\
\hline C & -2.070181 & 2.182665 & -0.136952 \\
\hline C & -3.287726 & 1.856074 & 0.542401 \\
\hline C & -3.524876 & 2.394499 & 1.788530 \\
\hline $\mathrm{H}$ & -4.432582 & 2.124827 & 2.320355 \\
\hline C & 1.301621 & 2.525503 & -0.000357 \\
\hline C & 0.172353 & 3.303056 & -0.227077 \\
\hline C & 2.565184 & 3.904496 & -1.546856 \\
\hline C & 2.522630 & 2.826942 & -0.682737 \\
\hline $\mathrm{H}$ & 3.495846 & 4.139751 & -2.055087 \\
\hline C & 1.436435 & 4.721219 & -1.785058 \\
\hline C & 0.209550 & 4.417036 & -1.117169 \\
\hline C & -0.928966 & 5.227243 & -1.373400 \\
\hline C & -0.849293 & 6.291943 & -2.240214 \\
\hline C & 0.368943 & 6.599764 & -2.892465 \\
\hline C & 1.486244 & 5.830242 & -2.670188 \\
\hline H & -1.867373 & 4.984084 & -0.889573 \\
\hline $\mathrm{H}$ & -1.728726 & 6.898155 & -2.432280 \\
\hline $\mathrm{H}$ & 0.414983 & 7.443048 & -3.574551 \\
\hline $\mathrm{H}$ & 2.423383 & 6.055165 & -3.171457 \\
\hline C & -2.586235 & 3.239566 & 2.428137 \\
\hline C & -1.349056 & 3.525707 & 1.770893 \\
\hline C & -0.402738 & 4.354003 & 2.430316 \\
\hline C & -0.664467 & 4.864952 & 3.681882 \\
\hline C & -1.885280 & 4.574395 & 4.334489 \\
\hline C & -2.824118 & 3.779120 & 3.718923 \\
\hline $\mathrm{H}$ & 0.531709 & 4.581067 & 1.930121 \\
\hline $\mathrm{H}$ & 0.069499 & 5.497081 & 4.171932 \\
\hline $\mathrm{H}$ & -2.078879 & 4.982304 & 5.321682 \\
\hline $\mathrm{H}$ & -3.764370 & 3.549911 & 4.212388 \\
\hline C & 6.075708 & 0.390043 & -0.309710 \\
\hline C & 5.497015 & 0.657509 & -1.559801 \\
\hline C & 4.350336 & 1.438423 & -1.667678 \\
\hline C & 3.744083 & 1.993527 & -0.531256 \\
\hline
\end{tabular}




$\begin{array}{lrrr}\text { C } & 4.344840 & 1.758262 & 0.714826 \\ \text { C } & 5.482646 & 0.966090 & 0.824322 \\ \text { H } & 3.895529 & 1.601861 & -2.639524 \\ \text { H } & 3.926689 & 2.220432 & 1.603799 \\ \text { C } & -4.236191 & 0.872711 & -0.028724 \\ \text { C } & -3.767769 & -0.356508 & -0.516857 \\ \text { C } & -5.615964 & 1.111699 & -0.041222 \\ \text { C } & -4.651800 & -1.307073 & -1.012159 \\ \text { H } & -2.708140 & -0.579954 & -0.464360 \\ \text { C } & -6.499100 & 0.157850 & -0.535370 \\ \text { H } & -5.992217 & 2.067935 & 0.308529 \\ \text { C } & -6.035156 & -1.069978 & -1.032386 \\ \text { H } & 5.923715 & 0.211134 & -2.452519 \\ \text { H } & 5.935097 & 0.810669 & 1.798447 \\ \text { H } & -7.560907 & 0.379469 & -0.570679 \\ \text { H } & -4.271076 & -2.260987 & -1.362195 \\ \mathrm{C} & 7.248193 & -0.505282 & -0.192729 \\ \mathrm{C} & 8.260132 & -0.504589 & -1.165486 \\ \mathrm{C} & 7.342607 & -1.418914 & 0.868697 \\ \mathrm{C} & 9.328291 & -1.395593 & -1.083912 \\ \mathrm{H} & 8.215799 & 0.211588 & -1.980000 \\ \mathrm{C} & 8.411342 & -2.307624 & 0.951930 \\ \mathrm{H} & 6.547480 & -1.460754 & 1.605597 \\ \mathrm{C} & 9.407344 & -2.302146 & -0.025669 \\ \mathrm{H} & 10.103775 & -1.376657 & -1.843619 \\ \mathrm{H} & 8.457839 & -3.016451 & 1.772942 \\ \mathrm{H} & 10.238295 & -2.997868 & 0.036538 \\ \mathrm{C} & -6.973112 & -2.086383 & -1.560294 \\ \mathrm{C} & -8.227472 & -2.288741 & -0.963244 \\ \mathrm{C} & -6.628715 & -2.874853 & -2.669751 \\ \mathrm{C} & -9.108493 & -3.247037 & -1.458914 \\ \mathrm{H} & -8.499820 & -1.706927 & -0.088293 \\ \mathrm{C} & -7.508707 & -3.834570 & -3.164388 \\ \mathrm{H} & -5.675352 & -2.712046 & -3.162255 \\ \mathrm{C} & -8.752708 & -4.024950 & -2.561430 \\ \mathrm{H} & -10.070861 & -3.393068 & -0.977509 \\ \mathrm{H} & -7.226192 & -4.428064 & -4.028705 \\ & -9.438867 & -4.772421 & -2.947488\end{array}$

\section{A-Catalyzed reaction TS 2}

\section{Datum}

Value

B3LYP-D3(BJ)/6-31G(d,p) Energy

$-2872.496869$

B3LYP-D3(BJ)/def2-TZVPP/IEF-PCM(DCM) Energy

$-2873.502701$

B3LYP-D3(BJ)/def2-TZVPP/IEF-PCM(DCM)//B3LYP-D3(BJ)/6-31G(d,p) Free Energy (Quasiharmonic)

$-2872.55124$

Number of Imaginary Frequencies 
$\begin{array}{lr}\text { 1. } & -257.7322 \mathrm{~cm}^{-1} \\ \text { 2. } & 9.4996 \mathrm{~cm}^{-1} \\ \text { 3. } & 10.7080 \mathrm{~cm}^{-1}\end{array}$

B3LYP-D3(BJ)/6-31G(d,p) Molecular Geometry in Cartesian Coordinates

C

$\mathrm{H}$

$\mathrm{H}$

C

0

0

$\mathrm{H}$

0

$\mathrm{H}$

C

C

C

C

$\mathrm{H}$

$\mathrm{H}$

$\mathrm{H}$

C

C

C

$\mathrm{H}$

$\mathrm{H}$

$\mathrm{H}$

C

$\mathrm{H}$

$\mathrm{H}$

$\mathrm{H}$

C

$\mathrm{H}$

$\mathrm{H}$

$\mathrm{H}$

C

$\mathrm{H}$

$\mathrm{H}$

$\mathrm{H}$

0

0

B

0

C

C

C

C
1.466984

2.331312

0.880962

$-1.434547$

$-2.165966$

$-0.654826$

$-0.144281$

0.656859

0.048155

$-2.023729$

$-0.174292$

$-2.681088$

$-2.771528$

$-0.422188$

$-2.491622$

$-3.467466$

$-0.532870$

$-1.359469$

$-0.859038$

$-0.256799$

$-0.630697$

$-1.911235$

0.979534

1.358043

1.494243

1.224105

$-0.726997$

$-0.608483$

0.249278

$-1.370553$

$-2.809173$

$-2.875204$

$-3.393042$

$-3.258165$

$-0.962304$

$-1.371683$

$-1.257716$

$-0.198250$

0.765325

1.179521

1.259380

2.089938
0.481365

$-0.074386$

0.812000

1.802368

2.452780

0.832773

0.386605

$-0.378187$

$-0.912012$

0.003620

$-1.000342$

$-0.866956$

$-0.848576$

$-1.937315$

0.098527

$-1.419387$

$-3.299351$

$-4.071859$

$-3.642174$

$-3.021553$

$-4.691536$

$-3.460302$

$-3.386260$

$-4.387729$

$-2.675375$

$-3.143727$

$-5.377333$

$-6.077857$

$-5.202305$

$-5.847366$

$-4.317369$

$-5.106309$

$-4.625116$

$-3.410134$

$-1.929370$

$-3.146101$

$-1.824624$

$-1.004457$

$-0.100144$

$-0.415986$

1.053137

0.401993
-0.166366
0.206947
0.693114
-2.433054
-3.148546
-2.900776
-2.166801
-0.985129
-0.406831

4.282091

3.776368

2.068324

3.494469

4.276715

1.607634

1.556469

0.135662

1.218094

$-1.312321$

$-1.981929$

$-1.523598$

$-1.536357$

0.378592

0.156479

$-0.271511$

1.415131

1.688100

0.855164

2.142467

2.437089

0.777249

0.022281

1.648694

0.365723

0.377157

2. 319112

1.785926

2.477635

4.461052

5.762604

3.834708

6.426226 


\begin{tabular}{|c|c|c|c|}
\hline $\mathrm{H}$ & 0.792056 & -1.308761 & 6.245838 \\
\hline C & 2.169933 & 1.865231 & 4.500701 \\
\hline $\mathrm{H}$ & 0.918737 & 1.312472 & 2.839693 \\
\hline C & 2.588844 & 1.542408 & 5.794286 \\
\hline $\mathrm{H}$ & 2.412815 & 0.149027 & 7.431039 \\
\hline H & 2.546108 & 2.755036 & 4.009218 \\
\hline $\mathrm{H}$ & 3.300831 & 2.179943 & 6.309145 \\
\hline $\mathrm{H}$ & -3.246686 & -1.702717 & 3.975594 \\
\hline $\mathrm{H}$ & -1.675071 & 0.947918 & 3.875884 \\
\hline $\mathrm{H}$ & -2.066817 & -0.061368 & 5.364595 \\
\hline C & -0.270433 & 2.712117 & -0.392702 \\
\hline C & -1.389735 & 2.118249 & -0.948703 \\
\hline C & -2.597961 & 1.982071 & -0.192783 \\
\hline C & -2.658667 & 2.520681 & 1.076120 \\
\hline $\mathrm{H}$ & -3.558804 & 2.386586 & 1.668838 \\
\hline C & 1.902208 & 1.667582 & -0.992408 \\
\hline C & 1.032388 & 2.741760 & -1.126911 \\
\hline C & 3.527830 & 2.800808 & -2.390053 \\
\hline C & 3.167458 & 1.682779 & -1.660780 \\
\hline $\mathrm{H}$ & 4.485524 & 2.814484 & -2.902129 \\
\hline C & 2.664775 & 3.911182 & -2.538279 \\
\hline C & 1.380576 & 3.879991 & -1.910175 \\
\hline C & 0.500427 & 4.980277 & -2.087751 \\
\hline C & 0.881498 & 6.066001 & -2.841302 \\
\hline C & 2.157713 & 6.103386 & -3.453580 \\
\hline C & 3.027341 & 5.048317 & -3.307877 \\
\hline $\mathrm{H}$ & -0.484243 & 4.942182 & -1.636010 \\
\hline $\mathrm{H}$ & 0.196977 & 6.897399 & -2.976261 \\
\hline $\mathrm{H}$ & 2.443452 & 6.966502 & -4.046899 \\
\hline $\mathrm{H}$ & 4.003582 & 5.065156 & -3.784191 \\
\hline C & -1.554149 & 3.189261 & 1.657931 \\
\hline C & -0.332565 & 3.279163 & 0.920974 \\
\hline C & 0.775357 & 3.939132 & 1.516323 \\
\hline C & 0.678405 & 4.478327 & 2.780301 \\
\hline C & -0.521764 & 4.364877 & 3.519631 \\
\hline C & -1.613372 & 3.734111 & 2.967886 \\
\hline $\mathrm{H}$ & 1.697654 & 4.023009 & 0.953355 \\
\hline H & 1.528817 & 4.995176 & 3.214188 \\
\hline $\mathrm{H}$ & -0.579760 & 4.780809 & 4.520428 \\
\hline $\mathrm{H}$ & -2.542750 & 3.648960 & 3.524171 \\
\hline C & 5.927663 & -1.641064 & -1.522876 \\
\hline C & 6.349298 & -0.332793 & -1.240529 \\
\hline C & 5.454736 & 0.731861 & -1.276108 \\
\hline C & 4.103926 & 0.531416 & -1.595737 \\
\hline C & 3.680777 & -0.775628 & -1.887773 \\
\hline C & 4.576180 & -1.838370 & -1.847764 \\
\hline $\mathrm{H}$ & 5.800797 & 1.734741 & -1.044899 \\
\hline $\mathrm{H}$ & 2.640957 & -0.954528 & -2.137458 \\
\hline C & -3.718581 & 1.150143 & -0.689328 \\
\hline C & -5.051434 & 1.555749 & -0.545264 \\
\hline C & -3.462975 & -0.125496 & -1.216478 \\
\hline C & -6.095695 & 0.715232 & -0.915495 \\
\hline $\mathrm{H}$ & -5.266403 & 2.549185 & -0.164065 \\
\hline C & -4.507920 & -0.965560 & -1.579545 \\
\hline
\end{tabular}




\begin{tabular}{|c|c|c|c|}
\hline $\mathrm{H}$ & -2.443528 & -0.482605 & -1.293597 \\
\hline C & -5.845627 & -0.564418 & -1.435758 \\
\hline $\mathrm{H}$ & -7.119642 & 1.064578 & -0.829937 \\
\hline $\mathrm{H}$ & -4.285117 & -1.961234 & -1.950012 \\
\hline $\mathrm{H}$ & 7.393342 & -0.147020 & -1.008952 \\
\hline $\mathrm{H}$ & 4.218105 & -2.842436 & -2.052436 \\
\hline C & 6.876473 & -2.777031 & -1.477706 \\
\hline C & 6.781514 & -3.831951 & -2.399498 \\
\hline C & 7.893939 & -2.828434 & -0.511516 \\
\hline C & 7.672691 & -4.901603 & -2.356176 \\
\hline $\mathrm{H}$ & 6.018834 & -3.796733 & -3.170903 \\
\hline C & 8.786618 & -3.896908 & -0.469041 \\
\hline $\mathrm{H}$ & 7.966351 & -2.035883 & 0.226575 \\
\hline C & 8.679832 & -4.938955 & -1.390980 \\
\hline $\mathrm{H}$ & 7.586394 & -5.702876 & -3.083974 \\
\hline $\mathrm{H}$ & 9.561460 & -3.920348 & 0.291438 \\
\hline $\mathrm{H}$ & 9.375032 & -5.772099 & -1.357783 \\
\hline C & -6.955829 & -1.465846 & -1.818451 \\
\hline C & -8.141908 & -1.507587 & -1.068366 \\
\hline C & -6.847440 & -2.304579 & -2.939252 \\
\hline C & -9.184770 & -2.358676 & -1.426634 \\
\hline $\mathrm{H}$ & -8.233465 & -0.885020 & -0.183880 \\
\hline C & -7.889279 & -3.157250 & -3.296615 \\
\hline $\mathrm{H}$ & -5.950035 & -2.264066 & -3.548267 \\
\hline C & -9.062803 & -3.188254 & -2.542089 \\
\hline $\mathrm{H}$ & -10.090916 & -2.380558 & -0.828542 \\
\hline $\mathrm{H}$ & -7.788454 & -3.791164 & -4.172467 \\
\hline $\mathrm{H}$ & -9.875263 & -3.851911 & -2.821466 \\
\hline
\end{tabular}

\section{A-Catalyzed reaction TS 3 (TS-2.2')}

Datum

Value

\begin{tabular}{|c|c|}
\hline B3LYP-D3(BJ)/6-31G(d,p) Energy & -2872.50313 \\
\hline B3LYP-D3(BJ)/def2-TZVPP/IEF-PCM(DCM) Energy & -2873.502758 \\
\hline B3LYP-D3(BJ)/def2-TZVPP/IEF-PCM(DCM)//B3LYP-D3(BJ)/6-31G(d,p) Free Energy (Quasiharmonic) & -2872.550693 \\
\hline Number of Imaginary Frequencies & 1 \\
\hline \multicolumn{2}{|l|}{ Frequencies (Top 3 out of 366) } \\
\hline 1. $\quad-272.4959 \mathrm{~cm}^{-1}$ & \\
\hline 2. $\quad 6.8013 \mathrm{~cm}^{-1}$ & \\
\hline $8.0656 \mathrm{~cm}^{-1}$ & \\
\hline
\end{tabular}




\begin{tabular}{|c|c|c|c|}
\hline C & -1.351604 & 1.540334 & 1.085761 \\
\hline $\mathrm{H}$ & -0.972785 & 2.087113 & 1.952493 \\
\hline $\mathrm{H}$ & -2.322670 & 1.126034 & 1.345931 \\
\hline C & 1.912439 & 1.808757 & -1.111025 \\
\hline 0 & 2.642695 & 2.150902 & -2.016629 \\
\hline 0 & 1.086036 & 0.766729 & -1.227022 \\
\hline $\mathrm{H}$ & 0.510954 & 0.663780 & -0.403589 \\
\hline 0 & -0.446326 & 0.452636 & 0.825973 \\
\hline $\mathrm{H}$ & -0.957965 & -0.404551 & 0.780661 \\
\hline C & 0.058542 & -1.821975 & -2.627536 \\
\hline C & 0.756759 & -2.509221 & -0.753903 \\
\hline C & -2.124363 & -2.531062 & -1.712560 \\
\hline C & -1.198404 & -1.528375 & -2.129065 \\
\hline H & 0.747751 & -1.531881 & -0.277218 \\
\hline $\mathrm{H}$ & -3.136406 & -2.179565 & -1.520066 \\
\hline $\mathrm{H}$ & -2.101656 & -3.459025 & -2.284865 \\
\hline C & -2.082918 & -2.351304 & 2.067127 \\
\hline C & -2.945373 & -3.585299 & 1.636058 \\
\hline C & -0.803658 & -2.736125 & 2.819174 \\
\hline $\mathrm{H}$ & -0.150721 & -1.859418 & 2.864726 \\
\hline $\mathrm{H}$ & -1.018692 & -3.060092 & 3.841453 \\
\hline H & -0.269793 & -3.535694 & 2.301419 \\
\hline C & -2.846883 & -1.279060 & 2.835697 \\
\hline $\mathrm{H}$ & -3.642752 & -0.846496 & 2.227195 \\
\hline $\mathrm{H}$ & -3.290419 & -1.702912 & 3.742121 \\
\hline H & -2.167975 & -0.478184 & 3.143051 \\
\hline C & -4.374655 & -3.185314 & 1.251674 \\
\hline $\mathrm{H}$ & -4.984999 & -2.928314 & 2.122452 \\
\hline $\mathrm{H}$ & -4.370725 & -2.329637 & 0.574441 \\
\hline $\mathrm{H}$ & -4.848159 & -4.026465 & 0.739928 \\
\hline C & -2.968996 & -4.719507 & 2.656085 \\
\hline $\mathrm{H}$ & -3.394895 & -4.383775 & 3.607607 \\
\hline H & -3.587378 & -5.538016 & 2.277497 \\
\hline $\mathrm{H}$ & -1.966069 & -5.109859 & 2.835518 \\
\hline 0 & -1.696682 & -1.820578 & 0.773701 \\
\hline 0 & -2.277821 & -4.048291 & 0.453799 \\
\hline B & -1.554616 & -2.971531 & -0.118583 \\
\hline 0 & -0.138503 & -3.380006 & -0.373425 \\
\hline C & 2.081240 & -3.014678 & -1.158285 \\
\hline C & 3.138287 & -2.109787 & -1.341977 \\
\hline C & 2.292844 & -4.386506 & -1.355120 \\
\hline C & 4.390427 & -2.577761 & -1.726810 \\
\hline H & 2.972333 & -1.047709 & -1.194269 \\
\hline C & 3.552986 & -4.847588 & -1.725999 \\
\hline $\mathrm{H}$ & 1.467289 & -5.071942 & -1.199284 \\
\hline C & 4.602690 & -3.945282 & -1.914527 \\
\hline H & 5.204035 & -1.876997 & -1.866267 \\
\hline $\mathrm{H}$ & 3.718259 & -5.911538 & -1.866219 \\
\hline H & 5.588018 & -4.303696 & -2.194667 \\
\hline $\mathrm{H}$ & -1.375823 & -0.507090 & -1.794148 \\
\hline H & 0.225749 & -2.772644 & -3.124466 \\
\hline $\mathrm{H}$ & 0.750962 & -1.019309 & -2.850104 \\
\hline
\end{tabular}




\begin{tabular}{|c|c|c|c|}
\hline C & 0.792561 & 3.329272 & 0.519289 \\
\hline C & 1.880718 & 2.529106 & 0.216431 \\
\hline C & 2.961381 & 2.368092 & 1.137844 \\
\hline C & 2.928002 & 3.074043 & 2.323067 \\
\hline $\mathrm{H}$ & 3.736517 & 2.948164 & 3.037228 \\
\hline C & -1.462032 & 2.473156 & -0.105362 \\
\hline C & -0.400697 & 3.328563 & -0.385264 \\
\hline C & -2.578455 & 3.201330 & -2.136271 \\
\hline C & -2.575216 & 2.408189 & -1.003658 \\
\hline H & -3.428866 & 3.158161 & -2.810611 \\
\hline C & -1.519006 & 4.086672 & -2.437020 \\
\hline C & -0.402564 & 4.155582 & -1.546765 \\
\hline C & 0.666952 & 5.037580 & -1.856841 \\
\hline C & 0.624840 & 5.818598 & -2.987690 \\
\hline C & -0.484856 & 5.755850 & -3.865080 \\
\hline C & -1.532521 & 4.907767 & -3.595546 \\
\hline H & 1.524136 & 5.075073 & -1.195117 \\
\hline H & 1.450992 & 6.484750 & -3.214936 \\
\hline $\mathrm{H}$ & -0.501926 & 6.377428 & -4.754933 \\
\hline H & -2.385729 & 4.849679 & -4.265423 \\
\hline C & 1.874754 & 3.968370 & 2.634079 \\
\hline C & 0.781208 & 4.099092 & 1.722069 \\
\hline C & -0.283350 & 4.979664 & 2.049884 \\
\hline C & -0.264078 & 5.698998 & 3.223333 \\
\hline C & 0.818176 & 5.570033 & 4.126035 \\
\hline C & 1.862823 & 4.723224 & 3.836285 \\
\hline $\mathrm{H}$ & -1.109852 & 5.080324 & 1.355175 \\
\hline H & -1.081901 & 6.373058 & 3.458498 \\
\hline $\mathrm{H}$ & 0.820814 & 6.143010 & 5.048060 \\
\hline $\mathrm{H}$ & 2.696900 & 4.618397 & 4.524344 \\
\hline C & -5.956006 & -0.237457 & -0.441117 \\
\hline C & -5.590679 & 0.682700 & 0.555360 \\
\hline C & -4.505683 & 1.536080 & 0.384681 \\
\hline C & -3.728692 & 1.494954 & -0.783091 \\
\hline C & -4.100127 & 0.585401 & -1.783162 \\
\hline C & -5.191557 & -0.261782 & -1.617413 \\
\hline $\mathrm{H}$ & -4.262333 & 2.252700 & 1.163108 \\
\hline $\mathrm{H}$ & -3.519451 & 0.541765 & -2.698911 \\
\hline C & 4.036948 & 1.366178 & 0.908237 \\
\hline C & 4.899245 & 1.420412 & -0.196307 \\
\hline C & 4.203492 & 0.324889 & 1.833763 \\
\hline C & 5.892070 & 0.460239 & -0.362950 \\
\hline $\mathrm{H}$ & 4.771181 & 2.203200 & -0.932931 \\
\hline C & 5.189957 & -0.639249 & 1.657421 \\
\hline $\mathrm{H}$ & 3.537374 & 0.268178 & 2.689119 \\
\hline C & 6.053689 & -0.590843 & 0.553573 \\
\hline $\mathrm{H}$ & -6.155391 & 0.718431 & 1.481250 \\
\hline H & -5.465434 & -0.939753 & -2.419085 \\
\hline H & 6.534323 & 0.509038 & -1.236734 \\
\hline H & 5.302062 & -1.432364 & 2.389550 \\
\hline C & 7.081390 & -1.635344 & 0.340918 \\
\hline C & 8.339532 & -1.314567 & -0.192400 \\
\hline C & 6.808064 & -2.979108 & 0.643292 \\
\hline C & 9.293588 & -2.304719 & -0.416931 \\
\hline
\end{tabular}




\begin{tabular}{|lrrr|}
$H$ & 8.575964 & -0.277966 & -0.410927 \\
$\mathrm{C}$ & 7.762008 & -3.969019 & 0.419460 \\
$\mathrm{H}$ & 5.826629 & -3.250412 & 1.016489 \\
$\mathrm{C}$ & 9.008884 & -3.636520 & -0.112203 \\
$\mathrm{H}$ & 10.263446 & -2.034700 & -0.823773 \\
$\mathrm{H}$ & 7.525916 & -5.003589 & 0.649789 \\
$\mathrm{H}$ & 9.751822 & -4.408288 & -0.288334 \\
$\mathrm{C}$ & -7.107356 & -1.150119 & -0.256764 \\
$\mathrm{C}$ & -8.249076 & -0.737804 & 0.448378 \\
$\mathrm{C}$ & -7.083371 & -2.452982 & -0.780140 \\
$\mathrm{C}$ & -9.327810 & -1.600294 & 0.629162 \\
$\mathrm{H}$ & -8.300929 & 0.275197 & 0.834502 \\
$\mathrm{C}$ & -8.161811 & -3.315366 & -0.599949 \\
$\mathrm{H}$ & -6.199870 & -2.802027 & -1.303591 \\
$\mathrm{C}$ & -9.288691 & -2.893483 & 0.106655 \\
$\mathrm{H}$ & -10.204118 & -1.258485 & 1.171574 \\
$\mathrm{H}$ & -8.117114 & -4.322178 & -1.003788 \\
$\mathrm{H}$ & -10.128789 & -3.566147 & 0.248409 \\
& & & \\
\hline
\end{tabular}

\section{A-Catalyzed reaction TS 4}

\section{Datum}

Value

B3LYP-D3(BJ)/6-31G(d,p) Energy

$-2872.50313$

B3LYP-D3(BJ)/def2-TZVPP/IEF-PCM(DCM) Energy

$-2873.502758$

B3LYP-D3(BJ)/def2-TZVPP/IEF-PCM(DCM)//B3LYP-D3(BJ)/6-31G(d,p) Free Energy (Quasiharmonic)

$-2872.550693$

Number of Imaginary Frequencies

Frequencies (Top 3 out of 366)

$\begin{array}{lr}\text { 1. } & -272.4977 \mathrm{~cm}^{-1} \\ \text { 2. } & 6.8018 \mathrm{~cm}^{-1} \\ \text { 3. } & 8.0663 \mathrm{~cm}^{-1}\end{array}$

\section{B3LYP-D3(BJ)/6-31G(d,p) Molecular Geometry in Cartesian Coordinates}

C

$\mathrm{H}$

$\mathrm{H}$

C

0

0

$\mathrm{H}$

0
$-1.351628$

$-0.972830$

$-2.322699$

1.912460

2.642735

1.086059

0.510961

$-0.446341$
1.540291

2.087037

1.125979

1.808803

2.150982

0.766780

0.663798

0.452607
1.085792

1.952554

1. 345925

$-1.110917$

$-2.016493$

$-1.226972$

$-0.403554$

0.825982 


\begin{tabular}{|c|c|c|c|}
\hline $\mathrm{H}$ & -0.957976 & -0.404581 & 0.780627 \\
\hline C & 0.058593 & -1.821870 & -2.627610 \\
\hline C & 0.756777 & -2.509188 & -0.753990 \\
\hline C & -2.124328 & -2.530996 & -1.712699 \\
\hline C & -1.198363 & -1.528291 & -2.129149 \\
\hline $\mathrm{H}$ & 0.747760 & -1.531866 & -0.277269 \\
\hline H & -3.136374 & -2.179509 & -1.520210 \\
\hline $\mathrm{H}$ & -2.101609 & -3.458937 & -2.285040 \\
\hline C & -2.082948 & -2.351385 & 2.066996 \\
\hline C & -2.945392 & -3.585366 & 1.635866 \\
\hline C & -0.803698 & -2.736230 & 2.819048 \\
\hline H & -0.150766 & -1.859522 & 2.864644 \\
\hline $\mathrm{H}$ & -1.018747 & -3.060237 & 3.841312 \\
\hline $\mathrm{H}$ & -0.269823 & -3.535777 & 2.301271 \\
\hline C & -2.846929 & -1.279173 & 2.835595 \\
\hline $\mathrm{H}$ & -3.642790 & -0.846588 & 2.227097 \\
\hline $\mathrm{H}$ & -3.290478 & -1.703060 & 3.741996 \\
\hline $\mathrm{H}$ & -2.168028 & -0.478306 & 3.142990 \\
\hline C & -4.374671 & -3.185371 & 1.251477 \\
\hline H & -4.985028 & -2.928407 & 2.122255 \\
\hline $\mathrm{H}$ & -4.370733 & -2.329668 & 0.574277 \\
\hline $\mathrm{H}$ & -4.848164 & -4.026504 & 0.739690 \\
\hline C & -2.969027 & -4.719614 & 2.655848 \\
\hline $\mathrm{H}$ & -3.394941 & -4.383921 & 3.607377 \\
\hline $\mathrm{H}$ & -3.587399 & -5.538111 & 2.277219 \\
\hline $\mathrm{H}$ & -1.966101 & -5.109970 & 2.835280 \\
\hline 0 & -1.696693 & -1.820607 & 0.773596 \\
\hline 0 & -2.277821 & -4.048309 & 0.453598 \\
\hline B & -1.554608 & -2.971526 & -0.118730 \\
\hline 0 & -0.138491 & -3.379989 & -0.373562 \\
\hline C & 2.081265 & -3.014628 & -1.158370 \\
\hline C & 2.292874 & -4.386447 & -1.355256 \\
\hline C & 3.138316 & -2.109728 & -1.342007 \\
\hline C & 3.553023 & -4.847514 & -1.726132 \\
\hline $\mathrm{H}$ & 1.467317 & -5.071890 & -1.199461 \\
\hline C & 4.390463 & -2.577687 & -1.726838 \\
\hline $\mathrm{H}$ & 2.972358 & -1.047656 & -1.194260 \\
\hline C & 4.602730 & -3.945199 & -1.914606 \\
\hline H & 3.718299 & -5.911458 & -1.866392 \\
\hline $\mathrm{H}$ & 5.204072 & -1.876916 & -1.866253 \\
\hline $\mathrm{H}$ & 5.588062 & -4.303602 & -2.194744 \\
\hline $\mathrm{H}$ & -1.375790 & -0.507020 & -1.794196 \\
\hline $\mathrm{H}$ & 0.225809 & -2.772520 & -3.124574 \\
\hline H & 0.751015 & -1.019195 & -2.850135 \\
\hline C & 0.792548 & 3.329253 & 0.519433 \\
\hline C & 1.880711 & 2.529100 & 0.216565 \\
\hline C & 2.961356 & 2.368051 & 1.137995 \\
\hline C & 2.927952 & 3.073955 & 2.323244 \\
\hline $\mathrm{H}$ & 3.736452 & 2.948049 & 3.037417 \\
\hline C & -1.462033 & 2.473160 & -0.105297 \\
\hline C & -0.400693 & 3.328579 & -0.385144 \\
\hline C & -2.578416 & 3.201410 & -2.136200 \\
\hline C & -2.575199 & 2.408227 & -1.003617 \\
\hline $\mathrm{H}$ & -3.428813 & 3.158266 & -2.810559 \\
\hline
\end{tabular}




\begin{tabular}{|c|c|c|c|}
\hline C & -1.518961 & 4.086764 & -2.436894 \\
\hline C & -0.402537 & 4.155642 & -1.546614 \\
\hline C & 0.666984 & 5.037652 & -1.856635 \\
\hline C & 0.624894 & 5.818714 & -2.987455 \\
\hline C & -0.484785 & 5.755998 & -3.864869 \\
\hline C & -1.532454 & 4.907904 & -3.595389 \\
\hline H & 1.524155 & 5.075120 & -1.194893 \\
\hline H & 1.451050 & 6.484875 & -3.214660 \\
\hline $\mathrm{H}$ & -0.501837 & 6.377610 & -4.754699 \\
\hline $\mathrm{H}$ & -2.385649 & 4.849841 & -4.265286 \\
\hline C & 1.874695 & 3.968269 & 2.634270 \\
\hline C & 0.781169 & 4.099026 & 1.722243 \\
\hline C & -0.283397 & 4.979584 & 2.050071 \\
\hline C & -0.264150 & 5.698871 & 3.223549 \\
\hline C & 0.818085 & 5.569871 & 4.126268 \\
\hline C & 1.862740 & 4.723075 & 3.836506 \\
\hline H & -1.109885 & 5.080270 & 1.355349 \\
\hline $\mathrm{H}$ & -1.081980 & 6.372920 & 3.458724 \\
\hline H & 0.820704 & 6.142812 & 5.048316 \\
\hline $\mathrm{H}$ & 2.696802 & 4.618222 & 4.524578 \\
\hline C & -5.955997 & -0.237444 & -0.441239 \\
\hline C & -5.191525 & -0.261725 & -1.617521 \\
\hline C & -4.100094 & 0.585466 & -1.783218 \\
\hline C & -3.728679 & 1.494982 & -0.783107 \\
\hline C & -4.505691 & 1.536064 & 0.384652 \\
\hline C & -5.590690 & 0.682677 & 0.555279 \\
\hline $\mathrm{H}$ & -3.519400 & 0.541865 & -2.698958 \\
\hline $\mathrm{H}$ & -4.262357 & 2.252656 & 1.163111 \\
\hline C & 4.036927 & 1.366146 & 0.908370 \\
\hline C & 4.899248 & 1.420425 & -0.196152 \\
\hline C & 4.203450 & 0.324819 & 1.833858 \\
\hline C & 5.892076 & 0.460257 & -0.362813 \\
\hline H & 4.771201 & 2.203243 & -0.932747 \\
\hline C & 5.189917 & -0.639314 & 1.657498 \\
\hline H & 3.537313 & 0.268073 & 2.689196 \\
\hline C & 6.053673 & -0.590862 & 0.553671 \\
\hline H & 6.534349 & 0.509091 & -1.236580 \\
\hline H & 5.302004 & -1.432458 & 2.389596 \\
\hline H & -6.155419 & 0.718373 & 1.481160 \\
\hline $\mathrm{H}$ & -5.465387 & -0.939667 & -2.419223 \\
\hline C & 7.081378 & -1.635357 & 0.340995 \\
\hline C & 8.339533 & -1.314558 & -0.192281 \\
\hline C & 6.808044 & -2.979132 & 0.643308 \\
\hline C & 9.293592 & -2.304702 & -0.416830 \\
\hline H & 8.575971 & -0.277948 & -0.410759 \\
\hline C & 7.761992 & -3.969035 & 0.419458 \\
\hline H & 5.826600 & -3.250451 & 1.016472 \\
\hline C & 9.008881 & -3.636515 & -0.112163 \\
\hline H & 10.263461 & -2.034667 & -0.823639 \\
\hline H & 7.525894 & -5.003614 & 0.649739 \\
\hline H & 9.751822 & -4.408277 & -0.288308 \\
\hline C & -7.107349 & -1.150115 & -0.256941 \\
\hline C & -8.249083 & -0.737827 & 0.448194 \\
\hline C & -7.083352 & -2.452959 & -0.780364 \\
\hline
\end{tabular}




\begin{tabular}{|lrrr|} 
C & -9.327819 & -1.600325 & 0.628925 \\
$H$ & -8.300945 & 0.275160 & 0.834353 \\
C & -8.161794 & -3.315351 & -0.600225 \\
H & -6.199840 & -2.801983 & -1.303811 \\
C & -9.288688 & -2.893495 & 0.106373 \\
$H$ & -10.204138 & -1.258537 & 1.171332 \\
$H$ & -8.117087 & -4.322149 & -1.004100 \\
$H$ & -10.128788 & -3.566165 & 0.248085 \\
\hline
\end{tabular}

\section{A-Catalyzed reaction TS 5}

Datum

Value

\begin{tabular}{lr}
\hline B3LYP-D3(BJ)/6-31G(d,p) Energy & -2872.496828 \\
\hline B3LYP-D3(BJ)/def2-TZVPP/IEF-PCM(DCM) Energy & -2873.502163 \\
\hline B3LYP-D3(BJ)/def2-TZVPP/IEF-PCM(DCM)//B3LYP-D3(BJ)/6-31G(d,p) Free Energy (Quasiharmonic) & -2872.550541 \\
\hline
\end{tabular}

Number of Imaginary Frequencies

Frequencies (Top 3 out of 366)

$\begin{array}{lr}\text { 1. } & -278.0877 \mathrm{~cm}^{-1} \\ \text { 2. } & 9.1331 \mathrm{~cm}^{-1} \\ \text { 3. } & 10.4701 \mathrm{~cm}^{-1}\end{array}$

\section{B3LYP-D3(BJ)/6-31G(d,p) Molecular Geometry in Cartesian Coordinates}

\begin{tabular}{rrrr}
\hline C & 1.929569 & 1.065824 & 0.691780 \\
H & 2.845437 & 0.538663 & 0.969324 \\
H & 1.365918 & 1.264512 & 1.610253 \\
$\mathrm{C}$ & -0.753852 & 2.551597 & -1.696609 \\
O & -1.330076 & 3.321332 & -2.432423 \\
O & 0.078487 & 1.613857 & -2.154318 \\
H & 0.458444 & 1.069127 & -1.408956 \\
O & 1.155566 & 0.264502 & -0.202317 \\
H & 0.990585 & -0.642419 & 0.178346 \\
$\mathrm{C}$ & -2.061172 & -0.637306 & 3.000599 \\
$\mathrm{C}$ & -2.117239 & -2.079800 & 1.467926 \\
$\mathrm{C}$ & 0.263649 & -1.434435 & 3.233335 \\
$\mathrm{C}$ & -0.698658 & -0.450629 & 2.854382 \\
$\mathrm{H}$ & -1.845426 & -1.395609 & 0.661429 \\
$\mathrm{H}$ & 1.300480 & -1.099684 & 3.225588 \\
$\mathrm{H}$ & 0.019753 & -1.999365 & 4.133775 \\
$\mathrm{C}$ & 1.926387 & -3.013904 & 0.381225 \\
$\mathrm{C}$ & 1.594460 & -4.317257 & 1.184450
\end{tabular}




\begin{tabular}{|c|c|c|c|}
\hline C & 1.970132 & -3.164667 & -1.134585 \\
\hline $\mathrm{H}$ & 2.146385 & -2.186888 & -1.589827 \\
\hline $\mathrm{H}$ & 2.786843 & -3.829434 & -1.433614 \\
\hline $\mathrm{H}$ & 1.031069 & -3.560176 & -1.524163 \\
\hline C & 3.208023 & -2.340850 & 0.880422 \\
\hline $\mathrm{H}$ & 4.090591 & -2.930495 & 0.621170 \\
\hline $\mathrm{H}$ & 3.320295 & -1.367669 & 0.403991 \\
\hline $\mathrm{H}$ & 3.181792 & -2.210669 & 1.965101 \\
\hline C & 2.815993 & -5.125288 & 1.610931 \\
\hline $\mathrm{H}$ & 3.402507 & -5.439955 & 0.741648 \\
\hline $\mathrm{H}$ & 3.457996 & -4.547700 & 2.277305 \\
\hline $\mathrm{H}$ & 2.492021 & -6.021646 & 2.146891 \\
\hline C & 0.599004 & -5.226377 & 0.449289 \\
\hline H & 1.063532 & -5.723805 & -0.407348 \\
\hline $\mathrm{H}$ & 0.247451 & -5.991445 & 1.146219 \\
\hline $\mathrm{H}$ & -0.269915 & -4.664813 & 0.099535 \\
\hline 0 & 0.793599 & -2.161382 & 0.717574 \\
\hline 0 & 0.958097 & -3.795658 & 2.363254 \\
\hline B & 0.224204 & -2.651732 & 1.967419 \\
\hline 0 & -1.236917 & -2.961617 & 1.845861 \\
\hline C & -3.539331 & -2.478242 & 1.540725 \\
\hline C & -3.900593 & -3.716631 & 2.089770 \\
\hline C & -4.529974 & -1.627937 & 1.030734 \\
\hline C & -5.238639 & -4.101614 & 2.109898 \\
\hline $\mathrm{H}$ & -3.123500 & -4.366901 & 2.474749 \\
\hline C & -5.864912 & -2.019796 & 1.048777 \\
\hline $\mathrm{H}$ & -4.253707 & -0.664617 & 0.623010 \\
\hline C & -6.221918 & -3.258233 & 1.586626 \\
\hline $\mathrm{H}$ & -5.515114 & -5.065326 & 2.526338 \\
\hline $\mathrm{H}$ & -6.620298 & -1.362244 & 0.632729 \\
\hline $\mathrm{H}$ & -7.262873 & -3.566263 & 1.594948 \\
\hline $\mathrm{H}$ & -0.383096 & 0.354406 & 2.193160 \\
\hline $\mathrm{H}$ & -2.434015 & -1.314130 & 3.763014 \\
\hline H & -2.750308 & 0.122238 & 2.652794 \\
\hline C & 0.006214 & 3.175131 & 0.614460 \\
\hline C & -0.975888 & 2.630980 & -0.196525 \\
\hline C & -2.265313 & 2.303403 & 0.331397 \\
\hline C & -2.527787 & 2.581131 & 1.659120 \\
\hline $\mathrm{H}$ & -3.494388 & 2.317490 & 2.078363 \\
\hline C & 2.279380 & 2.377755 & 0.032893 \\
\hline C & 1.368708 & 3.421925 & 0.050092 \\
\hline C & 3.886152 & 3.759172 & -1.145177 \\
\hline C & 3.533911 & 2.531053 & -0.630543 \\
\hline $\mathrm{H}$ & 4.842138 & 3.876500 & -1.647532 \\
\hline c & 3.003616 & 4.866802 & -1.082184 \\
\hline C & 1.704583 & 4.690427 & -0.507825 \\
\hline C & 0.797998 & 5.783130 & -0.509693 \\
\hline C & 1.168481 & 6.999514 & -1.033984 \\
\hline C & 2.458879 & 7.179823 & -1.587078 \\
\hline C & 3.353434 & 6.135432 & -1.613881 \\
\hline $\mathrm{H}$ & -0.195142 & 5.639791 & -0.099524 \\
\hline $\mathrm{H}$ & 0.465330 & 7.826324 & -1.031502 \\
\hline $\mathrm{H}$ & 2.736476 & 8.145005 & -1.999292 \\
\hline $\mathrm{H}$ & 4.340309 & 6.263139 & -2.049654 \\
\hline
\end{tabular}




\begin{tabular}{|c|c|c|c|}
\hline C & -1.559714 & 3.161691 & 2.512925 \\
\hline C & -0.263296 & 3.457828 & 1.988905 \\
\hline C & 0.716045 & 4.009804 & 2.855693 \\
\hline C & 0.425944 & 4.250918 & 4.179694 \\
\hline C & -0.855534 & 3.951207 & 4.699894 \\
\hline C & -1.825802 & 3.418223 & 3.883284 \\
\hline H & 1.697158 & 4.238927 & 2.455150 \\
\hline $\mathrm{H}$ & 1.184286 & 4.673187 & 4.831501 \\
\hline $\mathrm{H}$ & -1.069501 & 4.142947 & 5.746661 \\
\hline $\mathrm{H}$ & -2.811028 & 3.182447 & 4.275651 \\
\hline C & 5.824840 & -1.079076 & -1.208035 \\
\hline C & 4.708119 & -0.781063 & -2.004047 \\
\hline C & 3.992383 & 0.396087 & -1.819814 \\
\hline C & 4.383757 & 1.326117 & -0.845728 \\
\hline C & 5.523582 & 1.056538 & -0.080940 \\
\hline C & 6.229945 & -0.132030 & -0.255874 \\
\hline H & 3.101543 & 0.592850 & -2.406496 \\
\hline $\mathrm{H}$ & 5.844433 & 1.773855 & 0.668483 \\
\hline C & -3.286529 & 1.599973 & -0.481383 \\
\hline C & -2.936360 & 0.539063 & -1.333371 \\
\hline C & -4.647499 & 1.916068 & -0.351115 \\
\hline C & -3.911246 & -0.192684 & -2.001567 \\
\hline $\mathrm{H}$ & -1.894003 & 0.276510 & -1.468579 \\
\hline C & -5.621371 & 1.181481 & -1.018912 \\
\hline $\mathrm{H}$ & -4.941670 & 2.748660 & 0.279579 \\
\hline C & -5.274084 & 0.100412 & -1.843492 \\
\hline $\mathrm{H}$ & -3.613307 & -1.007920 & -2.652419 \\
\hline $\mathrm{H}$ & -6.668914 & 1.428400 & -0.877695 \\
\hline $\mathrm{H}$ & 4.366393 & -1.505941 & -2.735338 \\
\hline $\mathrm{H}$ & 7.109792 & -0.327352 & 0.348969 \\
\hline C & 6.512748 & -2.383922 & -1.336170 \\
\hline C & 6.696820 & -2.986944 & -2.590194 \\
\hline C & 6.965694 & -3.066192 & -0.195352 \\
\hline C & 7.313721 & -4.231065 & -2.699991 \\
\hline $\mathrm{H}$ & 6.372673 & -2.464040 & -3.484433 \\
\hline C & 7.582359 & -4.310767 & -0.304965 \\
\hline $\mathrm{H}$ & 6.806473 & -2.626513 & 0.784177 \\
\hline C & 7.758647 & -4.898763 & -1.558005 \\
\hline $\mathrm{H}$ & 7.453542 & -4.677087 & -3.680058 \\
\hline $\mathrm{H}$ & 7.916718 & -4.826015 & 0.590426 \\
\hline $\mathrm{H}$ & 8.237594 & -5.869119 & -1.644039 \\
\hline C & -6.316021 & -0.732001 & -2.484341 \\
\hline C & -6.181308 & -2.129032 & -2.525561 \\
\hline C & -7.468840 & -0.152600 & -3.036353 \\
\hline C & -7.168958 & -2.921700 & -3.105690 \\
\hline $\mathrm{H}$ & -5.313256 & -2.592335 & -2.068478 \\
\hline C & -8.455831 & -0.946108 & -3.616737 \\
\hline $\mathrm{H}$ & -7.575557 & 0.927713 & -3.031917 \\
\hline C & -8.309634 & -2.333642 & -3.653924 \\
\hline $\mathrm{H}$ & -7.051815 & -4.001052 & -3.120484 \\
\hline $\mathrm{H}$ & -9.336292 & -0.479820 & -4.048382 \\
\hline H & -9.079131 & -2.951578 & -4.106199 \\
\hline
\end{tabular}




\section{A-Catalyzed reaction TS 6 (TS-2.3')}

Datum

Value

B3LYP-D3(BJ)/6-31G(d,p) Energy

$-2872.495924$

B3LYP-D3(BJ)/def2-TZVPP/IEF-PCM(DCM) Energy

$-2873.502065$

B3LYP-D3(BJ)/def2-TZVPP/IEF-PCM(DCM)//B3LYP-D3(BJ)/6-31G(d,p) Free Energy (Quasiharmonic) $\quad-2872.550514$

Number of Imaginary Frequencies

Frequencies (Top 3 out of 366)

$\begin{array}{rrr}\text { 1. } & -267.5984 \mathrm{~cm}^{-1} \\ \text { 2. } & 4.3220 \mathrm{~cm}^{-1} \\ \text { 3. } & 10.7422 \mathrm{~cm}^{-1}\end{array}$

B3LYP-D3(BJ)/6-31G(d,p) Molecular Geometry in Cartesian Coordinates

\begin{tabular}{|c|c|c|}
\hline-0.794135 & 0.391892 & -0.578334 \\
\hline-0.404359 & 0.223839 & 0.424219 \\
\hline-1.782652 & -0.056384 & -0.611038 \\
\hline 2.739132 & 1.934294 & -1.437872 \\
\hline 3.591586 & 2.750408 & -1.708000 \\
\hline 2.070907 & 1.264141 & -2.382511 \\
\hline 1.378489 & 0.658854 & -2.003584 \\
\hline 0.046539 & -0.272949 & -1.538598 \\
\hline 0.061813 & -1.202035 & -1.217869 \\
\hline-4.662081 & -2.360350 & 1.093621 \\
\hline-3.005578 & -1.505621 & 2.153624 \\
\hline-2.846687 & -3.369232 & -0.243326 \\
\hline-3.923719 & -3.457992 & 0.691436 \\
\hline-2.937976 & -2.284702 & 2.914859 \\
\hline-2.977092 & -2.627006 & -1.032080 \\
\hline-2.478596 & -4.321645 & -0.623200 \\
\hline 0.748945 & -3.300132 & 0.465572 \\
\hline 0.267220 & -3.576058 & 1.932299 \\
\hline 0.859559 & -4.577457 & -0.372041 \\
\hline 0.963229 & -4.302400 & -1.425192 \\
\hline 1.729706 & -5.175282 & -0.085948 \\
\hline-0.037008 & -5.192711 & -0.263856 \\
\hline 2.027686 & -2.476546 & 0.354652 \\
\hline 2.864217 & -2.983301 & 0.845588 \\
\hline 2.298671 & -2.342161 & -0.696546 \\
\hline 1.911741 & -1.489701 & 0.802332 \\
\hline 0.555311 & -2.396085 & 2.872800 \\
\hline 1.622144 & -2.308871 & 3.096347 \\
\hline 0.219189 & -1.447823 & 2.450410 \\
\hline
\end{tabular}




\begin{tabular}{|c|c|c|c|}
\hline $\mathrm{H}$ & 0.020566 & -2.556553 & 3.813196 \\
\hline C & 0.794028 & -4.870036 & 2.541482 \\
\hline $\mathrm{H}$ & 1.887505 & -4.860218 & 2.593646 \\
\hline H & 0.407255 & -4.981505 & 3.558426 \\
\hline $\mathrm{H}$ & 0.477045 & -5.738628 & 1.962390 \\
\hline 0 & -0.346294 & -2.519984 & -0.072636 \\
\hline 0 & -1.160650 & -3.699776 & 1.762169 \\
\hline B & -1.516025 & -2.785290 & 0.725868 \\
\hline 0 & -2.078114 & -1.484949 & 1.247541 \\
\hline C & -3.590845 & -0.214689 & 2.558674 \\
\hline C & -3.572389 & 0.889342 & 1.694564 \\
\hline C & -4.130498 & -0.078117 & 3.843892 \\
\hline C & -4.077836 & 2.113079 & 2.116642 \\
\hline $\mathrm{H}$ & -3.164757 & 0.782911 & 0.699244 \\
\hline C & -4.632312 & 1.151398 & 4.266339 \\
\hline $\mathrm{H}$ & -4.140985 & -0.932561 & 4.514956 \\
\hline C & -4.604888 & 2.249733 & 3.404368 \\
\hline $\mathrm{H}$ & -4.051857 & 2.963149 & 1.442444 \\
\hline $\mathrm{H}$ & -5.040270 & 1.253927 & 5.266833 \\
\hline $\mathrm{H}$ & -4.993911 & 3.208060 & 3.733855 \\
\hline $\mathrm{H}$ & -3.989809 & -4.365330 & 1.290912 \\
\hline $\mathrm{H}$ & -4.756287 & -1.508173 & 0.427725 \\
\hline $\mathrm{H}$ & -5.435450 & -2.462429 & 1.848086 \\
\hline C & 1.178927 & 2.072896 & 0.506676 \\
\hline C & 2.384903 & 1.624459 & 0.000317 \\
\hline C & 3.342959 & 0.972166 & 0.842275 \\
\hline C & 3.029879 & 0.781848 & 2.174018 \\
\hline $\mathrm{H}$ & 3.723811 & 0.240135 & 2.809653 \\
\hline C & -0.861504 & 1.861135 & -0.878132 \\
\hline C & 0.141803 & 2.681702 & -0.385913 \\
\hline C & -1.985651 & 3.777802 & -1.848310 \\
\hline C & -1.954056 & 2.416308 & -1.617854 \\
\hline $\mathrm{H}$ & -2.814629 & 4.207607 & -2.403789 \\
\hline C & -0.949455 & 4.633090 & -1.401597 \\
\hline C & 0.139619 & 4.081135 & -0.653411 \\
\hline C & 1.173575 & 4.944145 & -0.204097 \\
\hline C & 1.128960 & 6.291091 & -0.478840 \\
\hline C & 0.049235 & 6.839651 & -1.212117 \\
\hline C & -0.964894 & 6.028465 & -1.664282 \\
\hline $\mathrm{H}$ & 2.011823 & 4.519235 & 0.335732 \\
\hline $\mathrm{H}$ & 1.931302 & 6.938599 & -0.139858 \\
\hline $\mathrm{H}$ & 0.029253 & 7.904503 & -1.422787 \\
\hline $\mathrm{H}$ & -1.792257 & 6.442030 & -2.234202 \\
\hline C & 1.801325 & 1.213367 & 2.728663 \\
\hline C & 0.857567 & 1.881211 & 1.889407 \\
\hline C & -0.392582 & 2.265716 & 2.442106 \\
\hline C & -0.704710 & 1.979793 & 3.752010 \\
\hline C & 0.230440 & 1.317541 & 4.581314 \\
\hline C & 1.458914 & 0.951071 & 4.081511 \\
\hline $\mathrm{H}$ & -1.114002 & 2.768288 & 1.810208 \\
\hline $\mathrm{H}$ & -1.678149 & 2.254696 & 4.144719 \\
\hline $\mathrm{H}$ & -0.025525 & 1.097145 & 5.613129 \\
\hline $\mathrm{H}$ & 2.181622 & 0.438186 & 4.709862 \\
\hline C & -5.246860 & -0.247721 & -2.440316 \\
\hline
\end{tabular}




\begin{tabular}{|c|c|c|c|}
\hline C & -3.955147 & -0.540215 & -2.909436 \\
\hline C & -2.897854 & 0.339502 & -2.706551 \\
\hline C & -3.092166 & 1.549097 & -2.019256 \\
\hline C & -4.393822 & 1.871745 & -1.608846 \\
\hline C & -5.450962 & 0.989200 & -1.810217 \\
\hline $\mathrm{H}$ & -1.897589 & 0.066679 & -3.025937 \\
\hline $\mathrm{H}$ & -4.566476 & 2.807173 & -1.085531 \\
\hline C & 4.619239 & 0.432181 & 0.315223 \\
\hline C & 5.804354 & 0.573507 & 1.051862 \\
\hline C & 4.674608 & -0.284306 & -0.889882 \\
\hline C & 6.996891 & 0.015706 & 0.606165 \\
\hline $\mathrm{H}$ & 5.788811 & 1.139255 & 1.978049 \\
\hline C & 5.867256 & -0.841705 & -1.334255 \\
\hline $\mathrm{H}$ & 3.780093 & -0.402002 & -1.489982 \\
\hline C & 7.052797 & -0.706858 & -0.595493 \\
\hline $\mathrm{H}$ & 7.895160 & 0.125321 & 1.205421 \\
\hline $\mathrm{H}$ & 5.885753 & -1.369869 & -2.281974 \\
\hline $\mathrm{H}$ & -6.446215 & 1.258639 & -1.471525 \\
\hline $\mathrm{H}$ & -3.769397 & -1.490636 & -3.399861 \\
\hline C & 8.321548 & -1.304748 & -1.069560 \\
\hline C & 8.332711 & -2.550455 & -1.717264 \\
\hline C & 9.545095 & -0.641951 & -0.882452 \\
\hline C & 9.526004 & -3.114276 & -2.162563 \\
\hline $\mathrm{H}$ & 7.399692 & -3.089196 & -1.849001 \\
\hline C & 10.738861 & -1.206269 & -1.325889 \\
\hline $\mathrm{H}$ & 9.553212 & 0.335620 & -0.411068 \\
\hline C & 10.735016 & -2.445131 & -1.968142 \\
\hline $\mathrm{H}$ & 9.512615 & -4.082067 & -2.654983 \\
\hline $\mathrm{H}$ & 11.672948 & -0.672322 & -1.178965 \\
\hline $\mathrm{H}$ & 11.665242 & -2.884329 & -2.315088 \\
\hline C & -6.346205 & -1.230134 & -2.574814 \\
\hline C & -6.463935 & -2.034849 & -3.718746 \\
\hline C & -7.285832 & -1.395331 & -1.544377 \\
\hline C & -7.486980 & -2.973815 & -3.828539 \\
\hline $\mathrm{H}$ & -5.761418 & -1.904466 & -4.535731 \\
\hline C & -8.309380 & -2.333580 & -1.654244 \\
\hline $\mathrm{H}$ & -7.195254 & -0.799462 & -0.641569 \\
\hline C & -8.414030 & -3.127718 & -2.796834 \\
\hline $\mathrm{H}$ & -7.565126 & -3.581059 & -4.725238 \\
\hline $\mathrm{H}$ & -9.021023 & -2.451059 & -0.842509 \\
\hline $\mathrm{H}$ & -9.210763 & -3.859986 & -2.882666 \\
\hline
\end{tabular}

\section{A-Catalyzed reaction TS 7}

\section{Datum}

Value 
Frequencies (Top 3 out of 366)

$\begin{array}{rr}\text { 1. } & -277.6535 \mathrm{~cm}^{-1} \\ \text { 2. } & 7.8127 \mathrm{~cm}^{-1} \\ \text { 3. } & 11.0881 \mathrm{~cm}^{-1}\end{array}$

B3LYP-D3(BJ)/6-31G(d,p) Molecular Geometry in Cartesian Coordinates

\begin{tabular}{|lrrr}
\hline & & & \\
C & -0.796523 & 0.393048 & -0.559054 \\
H & -0.398915 & 0.200800 & 0.435931 \\
H & -1.781146 & -0.063023 & -0.597788 \\
C & 2.721689 & 1.985970 & -1.397894 \\
O & 3.567752 & 2.814494 & -1.649711 \\
O & 2.051781 & 1.338701 & -2.357172 \\
H & 1.365042 & 0.719079 & -1.991312 \\
O & 0.044100 & -0.237305 & -1.542663 \\
H & 0.078256 & -1.172742 & -1.243144 \\
C & -4.616965 & -2.438050 & 1.040572 \\
C & -2.972985 & -1.603890 & 2.125383 \\
C & -2.794274 & -3.388171 & -0.330942 \\
C & -3.869778 & -3.515732 & 0.600116 \\
H & -2.898790 & -2.403387 & 2.864550 \\
H & -2.920386 & -2.615460 & -1.090726 \\
H & -2.414290 & -4.322712 & -0.741739 \\
C & 0.798795 & -3.318338 & 0.379847 \\
C & 0.322366 & -3.646164 & 1.837700 \\
C & 0.913035 & -4.565762 & -0.501143 \\
H & 1.011741 & -4.254601 & -1.544669 \\
H & 1.787344 & -5.168131 & -0.238345 \\
H & 0.020360 & -5.189543 & -0.410882 \\
C & 2.072627 & -2.484622 & 0.293105 \\
H & 2.913612 & -3.003232 & 0.763625 \\
H & 2.339183 & -2.312748 & -0.753721 \\
H & 1.952646 & -1.514280 & 0.774518 \\
C & 0.606112 & -2.496761 & 2.816413 \\
H & 1.672959 & -2.411376 & 3.040598 \\
H & 0.264108 & -1.536775 & 2.426349 \\
H & 0.074154 & -2.691423 & 3.751895 \\
C & 0.857037 & -4.957273 & 2.401341 \\
H & 1.950547 & -4.943441 & 2.451408 \\
H & 0.473272 & -5.105307 & 3.414733 \\
H & 0.543295 & -5.807336 & 1.793680 \\
O & -0.301808 & -2.525241 & -0.128002 \\
B & -1.105920 & -3.770992 & 1.667052 \\
& -1.466794 & -2.823565 & 0.663869 \\
\hline
\end{tabular}




\begin{tabular}{|c|c|c|c|}
\hline 0 & -2.042210 & -1.548768 & 1.222590 \\
\hline C & -3.565889 & -0.328683 & 2.567799 \\
\hline C & -3.568151 & 0.796103 & 1.730618 \\
\hline C & -4.091672 & -0.228844 & 3.862022 \\
\hline C & -4.079144 & 2.004382 & 2.188596 \\
\hline H & -3.173524 & 0.717897 & 0.727382 \\
\hline C & -4.599047 & 0.985426 & 4.320520 \\
\hline $\mathrm{H}$ & -4.086812 & -1.099523 & 4.511911 \\
\hline C & -4.591469 & 2.104662 & 3.485621 \\
\hline $\mathrm{H}$ & -4.070032 & 2.870406 & 1.534641 \\
\hline $\mathrm{H}$ & -4.996273 & 1.059549 & 5.327798 \\
\hline $\mathrm{H}$ & -4.985291 & 3.050944 & 3.843022 \\
\hline $\mathrm{H}$ & -3.927096 & -4.443287 & 1.168707 \\
\hline $\mathrm{H}$ & -4.725994 & -1.565825 & 0.403191 \\
\hline $\mathrm{H}$ & -5.388437 & -2.574325 & 1.791474 \\
\hline C & 1.170817 & 2.057314 & 0.557635 \\
\hline C & 2.377761 & 1.633345 & 0.032885 \\
\hline C & 3.345594 & 0.965808 & 0.851369 \\
\hline C & 3.040900 & 0.735322 & 2.178728 \\
\hline $\mathrm{H}$ & 3.742475 & 0.181617 & 2.795357 \\
\hline C & -0.878134 & 1.869128 & -0.818519 \\
\hline C & 0.124183 & 2.682610 & -0.312194 \\
\hline C & -2.017538 & 3.803855 & -1.732511 \\
\hline C & -1.979490 & 2.436942 & -1.535462 \\
\hline $\mathrm{H}$ & -2.851741 & 4.243373 & -2.272293 \\
\hline C & -0.982689 & 4.652975 & -1.271918 \\
\hline C & 0.113428 & 4.088276 & -0.543947 \\
\hline C & 1.145681 & 4.945212 & -0.079286 \\
\hline C & 1.092853 & 6.298223 & -0.320680 \\
\hline C & 0.006321 & 6.859188 & -1.034321 \\
\hline C & -1.006424 & 6.054278 & -1.500457 \\
\hline $\mathrm{H}$ & 1.989144 & 4.511599 & 0.445248 \\
\hline $\mathrm{H}$ & 1.893922 & 6.941261 & 0.029626 \\
\hline $\mathrm{H}$ & -0.019985 & 7.928760 & -1.218738 \\
\hline $\mathrm{H}$ & -1.839128 & 6.477474 & -2.055325 \\
\hline C & 1.811749 & 1.140779 & 2.751411 \\
\hline C & 0.858353 & 1.824501 & 1.936135 \\
\hline C & -0.391783 & 2.183377 & 2.505879 \\
\hline C & -0.694795 & 1.857894 & 3.808681 \\
\hline C & 0.249728 & 1.179602 & 4.614060 \\
\hline C & 1.478426 & 0.837320 & 4.097931 \\
\hline $\mathrm{H}$ & -1.120553 & 2.697839 & 1.892242 \\
\hline H & -1.668243 & 2.113836 & 4.213921 \\
\hline $\mathrm{H}$ & 0.000845 & 0.927894 & 5.640432 \\
\hline $\mathrm{H}$ & 2.208437 & 0.312665 & 4.707864 \\
\hline C & -5.290697 & -0.188613 & -2.416128 \\
\hline C & -5.487609 & 1.042519 & -1.772825 \\
\hline C & -4.422955 & 1.910817 & -1.549368 \\
\hline C & -3.120146 & 1.579600 & -1.950091 \\
\hline C & -2.931902 & 0.374698 & -2.648117 \\
\hline C & -3.996367 & -0.490854 & -2.872467 \\
\hline $\mathrm{H}$ & -4.588595 & 2.833369 & -1.001567 \\
\hline $\mathrm{H}$ & -1.934925 & 0.104744 & -2.980204 \\
\hline C & 4.623443 & 0.451289 & 0.303143 \\
\hline
\end{tabular}




\begin{tabular}{|c|c|c|c|}
\hline C & 5.810897 & 0.580574 & 1.038231 \\
\hline C & 4.678634 & -0.229831 & -0.922317 \\
\hline C & 7.005504 & 0.044854 & 0.571491 \\
\hline $\mathrm{H}$ & 5.795437 & 1.119511 & 1.980270 \\
\hline C & 5.873341 & -0.765215 & -1.387724 \\
\hline $\mathrm{H}$ & 3.782281 & -0.336902 & -1.521707 \\
\hline C & 7.061227 & -0.642664 & -0.650560 \\
\hline $\mathrm{H}$ & 7.905692 & 0.144143 & 1.169660 \\
\hline $\mathrm{H}$ & 5.891468 & -1.265731 & -2.350337 \\
\hline $\mathrm{H}$ & -3.827230 & -1.413736 & -3.418232 \\
\hline $\mathrm{H}$ & -6.473138 & 1.296352 & -1.395624 \\
\hline C & -6.403448 & -1.151437 & -2.580245 \\
\hline C & -7.704761 & -0.712449 & -2.870032 \\
\hline C & -6.182403 & -2.529912 & -2.427710 \\
\hline C & -8.751562 & -1.621789 & -3.003311 \\
\hline $\mathrm{H}$ & -7.888378 & 0.347404 & -3.015896 \\
\hline C & -7.229326 & -3.438923 & -2.561330 \\
\hline $\mathrm{H}$ & -5.189513 & -2.886728 & -2.175157 \\
\hline C & -8.518480 & -2.989138 & -2.849354 \\
\hline $\mathrm{H}$ & -9.749411 & -1.262783 & -3.236639 \\
\hline $\mathrm{H}$ & -7.039151 & -4.500070 & -2.431139 \\
\hline $\mathrm{H}$ & -9.334356 & -3.697621 & -2.953527 \\
\hline C & 8.332087 & -1.217368 & -1.147087 \\
\hline C & 8.349178 & -2.444013 & -1.830080 \\
\hline C & 9.551694 & -0.551256 & -0.946553 \\
\hline C & 9.544401 & -2.986225 & -2.296534 \\
\hline $\mathrm{H}$ & 7.419424 & -2.985546 & -1.973018 \\
\hline C & 10.747400 & -1.094006 & -1.411183 \\
\hline $\mathrm{H}$ & 9.555007 & 0.412534 & -0.447548 \\
\hline C & 10.749467 & -2.314081 & -2.088458 \\
\hline $\mathrm{H}$ & 9.535670 & -3.939660 & -2.816301 \\
\hline $\mathrm{H}$ & 11.678307 & -0.557660 & -1.253235 \\
\hline $\mathrm{H}$ & 11.681203 & -2.736414 & -2.451892 \\
\hline
\end{tabular}

\section{A-Catalyzed reaction TS 8}

Datum

Value

\begin{tabular}{lc}
\hline B3LYP-D3(BJ)/6-31G(d,p) Energy & -2872.496747 \\
\hline B3LYP-D3(BJ)/def2-TZVPP/IEF-PCM(DCM) Energy & -2873.502166 \\
\hline B3LYP-D3(BJ)/def2-TZVPP/IEF-PCM(DCM)//B3LYP-D3(BJ)/6-31G(d,p) Free Energy (Quasiharmonic) & -2872.550391
\end{tabular}

Number of Imaginary Frequencies

Frequencies (Top 3 out of 366)

1. $-316.7343 \mathrm{~cm}^{-1}$

2. $9.4315 \mathrm{~cm}^{-1}$ 


\section{B3LYP-D3(BJ)/6-31G(d,p) Molecular Geometry in Cartesian Coordinates}

C

$\mathrm{H}$

$\mathrm{H}$

C

0

0

$\mathrm{H}$

0

$\mathrm{H}$

C

C

C

C

$\mathrm{H}$

$\mathrm{H}$

$\mathrm{H}$

C

C

C

$\mathrm{H}$

$\mathrm{H}$

$\mathrm{H}$

C

$\mathrm{H}$

$\mathrm{H}$

$\mathrm{H}$

C

$\mathrm{H}$

$\mathrm{H}$

$\mathrm{H}$

C

$\mathrm{H}$

$\mathrm{H}$

$\mathrm{H}$

0

0

B

0

C

C

C

C

$\mathrm{H}$

C

$\mathrm{H}$

C

$\mathrm{H}$
1.573443

2.457890

1.194625

$-1.367474$

$-2.035703$

$-0.491003$

$-0.051353$

0.563856

0.924965

$-0.488415$

$-1.289724$

1. 218544

0.809988

$-0.839061$

2.291053

0.728667

2.061658

1. 314211

2.004366

2.535575

2.492573

0.979871

3.506265

4.157439

3.884789

3.565534

2. 187964

2. 586900

3.020094

1.591416

0.045763

0.280751

$-0.535528$

$-0.577720$

1. 312584

0.921259

0.643481

$-0.815512$

$-2.733218$

$-3.510296$

$-3.339536$

$-4.881749$

$-3.029027$

$-4.710214$

$-2.738876$

$-5.484888$

$-5.487369$
1.081222

0.475002

1.448130

2.034715

2. 582842

1.066312

0.750794

0.246269

$-0.677244$

$-2.336669$

$-2.115273$

$-3.683277$

$-2.527176$

$-1.138263$

$-3.796364$

$-4.616825$

$-2.607103$

$-3.928868$

$-1.501737$

$-0.616896$

$-1.829228$

$-1.211261$

$-2.843496$

$-3.068392$

$-1.941483$

$-3.672935$

$-4.963825$

$-4.567548$

$-5.272909$

$-5.850407$

$-3.664186$

$-3.346562$

$-4.588382$

$-2.898269$

$-2.182540$

$-4.453311$

$-3.350894$

$-3.139155$

$-2.121789$

$-3.267435$

$-0.948304$

$-3.228555$

$-4.164471$

$-0.915674$

$-0.056328$

$-2.052609$

$-4.110369$
0.900890

1.094484

1.860708

$-1.660727$

$-2.507977$

$-1.948959$

$-1.109200$

0.312538

0.179854

3.398777

1.467996

2. 232823

2. 952767

1. 291741

2.085412

2. 509301

$-1.140248$

$-1.527861$

$-2.189249$

$-1.831013$

$-3.112530$

$-2.424729$

$-0.690940$

$-1.540425$

$-0.205703$

0.017547

$-2.227576$

$-3.167086$

$-1.593201$

$-2.458815$

$-2.351078$

$-3.370894$

$-2.399575$

$-1.883954$

0.029395

$-0.246606$

0.592632

0.806159

1.769952

1.551003

2. 237557

1. 785456

1.177898

2.471530

2. 387791

2. 239414

1.601657 


\begin{tabular}{|c|c|c|c|}
\hline $\mathrm{H}$ & -5.179439 & 0.004133 & 2.802407 \\
\hline $\mathrm{H}$ & -6.558147 & -2.020504 & 2.395871 \\
\hline $\mathrm{H}$ & 1.462989 & -1.655575 & 2.909360 \\
\hline $\mathrm{H}$ & -1.100531 & -3.200384 & 3.638191 \\
\hline $\mathrm{H}$ & -0.768734 & -1.410499 & 3.889689 \\
\hline C & -0.421505 & 3.057565 & 0.421190 \\
\hline C & -1.483765 & 2.424734 & -0.201628 \\
\hline C & -2.724802 & 2.221397 & 0.479851 \\
\hline C & -2.861153 & 2.717720 & 1.762787 \\
\hline $\mathrm{H}$ & -3.782607 & 2.536801 & 2.308307 \\
\hline C & 1.903227 & 2.253779 & -0.004122 \\
\hline C & 0.902034 & 3.182812 & -0.268440 \\
\hline C & 3.370301 & 3.396307 & -1.566609 \\
\hline C & 3.167325 & 2.365772 & -0.667040 \\
\hline $\mathrm{H}$ & 4.334358 & 3.487322 & -2.058576 \\
\hline C & 2.370110 & 4.351034 & -1.854493 \\
\hline C & 1.105939 & 4.247448 & -1.194656 \\
\hline C & 0.098997 & 5.205113 & -1.491270 \\
\hline C & 0.338867 & 6.217268 & -2.390474 \\
\hline C & 1.594054 & 6.324264 & -3.037096 \\
\hline C & 2.586806 & 5.410602 & -2.774898 \\
\hline $\mathrm{H}$ & -0.868917 & 5.115884 & -1.012979 \\
\hline $\mathrm{H}$ & -0.442307 & 6.936932 & -2.613330 \\
\hline $\mathrm{H}$ & 1.766588 & 7.128394 & -3.745703 \\
\hline $\mathrm{H}$ & 3.551229 & 5.481629 & -3.269845 \\
\hline C & -1.820635 & 3.422813 & 2.412613 \\
\hline C & -0.571505 & 3.592561 & 1.738498 \\
\hline C & 0.474970 & 4.283861 & 2.404740 \\
\hline C & 0.295640 & 4.775393 & 3.678240 \\
\hline C & -0.938630 & 4.600289 & 4.347678 \\
\hline C & -1.972301 & 3.938406 & 3.726720 \\
\hline $\mathrm{H}$ & 1.418953 & 4.423167 & 1.890121 \\
\hline $\mathrm{H}$ & 1.104456 & 5.304381 & 4.172697 \\
\hline $\mathrm{H}$ & -1.067218 & 4.992670 & 5.351671 \\
\hline $\mathrm{H}$ & -2.924119 & 3.801238 & 4.232429 \\
\hline C & 6.381615 & -0.467995 & -0.077051 \\
\hline C & 5.905313 & -0.157312 & -1.359424 \\
\hline C & 4.871964 & 0.755924 & -1.538886 \\
\hline C & 4.273464 & 1.398373 & -0.444520 \\
\hline C & 4.773930 & 1.114559 & 0.835546 \\
\hline C & 5.802918 & 0.196090 & 1.016101 \\
\hline $\mathrm{H}$ & 4.504975 & 0.967265 & -2.538125 \\
\hline $\mathrm{H}$ & 4.354575 & 1.625104 & 1.697251 \\
\hline C & -3.813282 & 1.396235 & -0.094843 \\
\hline C & -3.533868 & 0.189479 & -0.754209 \\
\hline C & -5.157500 & 1.730972 & 0.120594 \\
\hline C & -4.555772 & -0.660744 & -1.152716 \\
\hline $\mathrm{H}$ & -2.506240 & -0.101421 & -0.936550 \\
\hline C & -6.180142 & 0.876095 & -0.273433 \\
\hline $\mathrm{H}$ & -5.400657 & 2.670393 & 0.607079 \\
\hline C & -5.900078 & -0.345634 & -0.905404 \\
\hline $\mathrm{H}$ & 6.149016 & -0.028723 & 2.019871 \\
\hline $\mathrm{H}$ & 6.353338 & -0.635178 & -2.224565 \\
\hline $\mathrm{H}$ & -4.306554 & -1.585649 & -1.661273 \\
\hline
\end{tabular}




\begin{tabular}{|rrrr|} 
H & -7.210366 & 1.144753 & -0.062700 \\
C & 7.446186 & -1.477746 & 0.119372 \\
C & 8.436309 & -1.303594 & 1.098943 \\
C & 7.481724 & -2.640950 & -0.665949 \\
H & 9.430025 & -2.261421 & 1.287096 \\
C & 8.439478 & -0.398228 & 1.697790 \\
H & 8.475616 & -3.598590 & -0.478209 \\
C & 6.707583 & -2.805167 & -1.408448 \\
H & 9.454018 & -3.413057 & 0.499298 \\
H & 10.192136 & -2.104113 & 2.044341 \\
H & 8.480269 & -4.495766 & -1.089755 \\
C & 10.228285 & -4.159567 & 0.646217 \\
C & -6.985291 & -1.279703 & -1.279497 \\
C & -6.792456 & -2.668809 & -1.199944 \\
C & -8.234278 & -0.805987 & -1.711914 \\
H & -7.814804 & -3.552459 & -1.537268 \\
C & -5.843194 & -3.052063 & -0.843065 \\
H & -9.257128 & -1.689868 & -2.047949 \\
C & -8.393249 & 0.263055 & -1.811022 \\
H & -9.052800 & -3.067615 & -1.961320 \\
H & -7.646370 & -4.622801 & -1.462402 \\
H & -10.212493 & -1.301783 & -2.388342 \\
& -9.849644 & -3.756400 & -2.224405 \\
\hline & & & \\
\hline
\end{tabular}

\section{A-Catalyzed reaction TS 9}

\section{Datum}

Value

B3LYP-D3(BJ)/6-31G(d,p) Energy
B3LYP-D3(BJ)/def2-TZVPP/IEF-PCM(DCM) Energy
B3LYP-D3(BJ)/def2-TZVPP/IEF-PCM(DCM)//B3LYP-D3(BJ)/6-31G(d,p) Free Energy (Quasiharmonic)

$-2872.496747$

$-2873.502166$

Number of Imaginary Frequencies

Frequencies (Top 3 out of 366)

$$
\begin{array}{lr}
\text { 1. } & -316.7340 \mathrm{~cm}^{-1} \\
\text { 2. } & 9.4315 \mathrm{~cm}^{-1} \\
\text { 3. } & 10.6806 \mathrm{~cm}^{-1}
\end{array}
$$

\section{B3LYP-D3(BJ)/6-31G(d,p) Molecular Geometry in Cartesian Coordinates}

$\begin{array}{llll}\mathrm{C} & -1.573442 & 1.081224 & 0.900892 \\ \mathrm{H} & -2.457890 & 0.475005 & 1.094487 \\ \mathrm{H} & -1.194623 & 1.448134 & 1.860709\end{array}$




\begin{tabular}{|c|c|c|c|}
\hline C & 1.367476 & 2.034714 & -1.660726 \\
\hline 0 & 2.035704 & 2.582840 & -2.507976 \\
\hline 0 & 0.491005 & 1.066311 & -1.948957 \\
\hline $\mathrm{H}$ & 0.051355 & 0.750794 & -1.109198 \\
\hline 0 & -0.563856 & 0.246270 & 0.312541 \\
\hline $\mathrm{H}$ & -0.924966 & -0.677241 & 0.179852 \\
\hline C & 0.488412 & -2.336675 & 3. 398771 \\
\hline C & 1.289722 & -2.115275 & 1.467992 \\
\hline C & -1.218548 & -3.683278 & 2.232814 \\
\hline C & -0.809990 & -2.527179 & 2.952760 \\
\hline H & 0.839060 & -1.138265 & 1.291738 \\
\hline $\mathrm{H}$ & -2.291056 & -3.796363 & 2.085401 \\
\hline $\mathrm{H}$ & -0.728672 & -4.616828 & 2.509289 \\
\hline C & -2.061660 & -2.607100 & -1.140254 \\
\hline C & -1.314213 & -3.928864 & -1.527871 \\
\hline C & -2.004371 & -1.501733 & -2.189254 \\
\hline $\mathrm{H}$ & -2.535581 & -0.616893 & -1.831015 \\
\hline $\mathrm{H}$ & -2.492579 & -1.829223 & -3.112535 \\
\hline $\mathrm{H}$ & -0.979878 & -1.211255 & -2.424736 \\
\hline C & -3.506266 & -2.843494 & -0.690943 \\
\hline $\mathrm{H}$ & -4.157442 & -3.068385 & -1.540428 \\
\hline $\mathrm{H}$ & -3.884788 & -1.941483 & -0.205700 \\
\hline $\mathrm{H}$ & -3.565533 & -3.672937 & 0.017539 \\
\hline C & -2.187966 & -4.963821 & -2.227586 \\
\hline $\mathrm{H}$ & -2.586892 & -4.567547 & -3.167102 \\
\hline $\mathrm{H}$ & -3.020102 & -5.272898 & -1.593217 \\
\hline $\mathrm{H}$ & -1.591419 & -5.850406 & -2.458817 \\
\hline C & -0.045766 & -3.664180 & -2.351088 \\
\hline $\mathrm{H}$ & -0.280755 & -3.346549 & -3.370902 \\
\hline $\mathrm{H}$ & 0.535524 & -4.588376 & -2.399591 \\
\hline $\mathrm{H}$ & 0.577718 & -2.898266 & -1.883961 \\
\hline 0 & -1.312583 & -2.182538 & 0.029387 \\
\hline 0 & -0.921260 & -4.453309 & -0.246617 \\
\hline B & -0.643482 & -3.350893 & 0.592623 \\
\hline 0 & 0.815511 & -3.139156 & 0.806153 \\
\hline C & 2.733216 & -2.121792 & 1.769949 \\
\hline C & 3.510295 & -3.267438 & 1.551000 \\
\hline C & 3.339534 & -0.948307 & 2.237555 \\
\hline C & 4.881747 & -3.228559 & 1.785453 \\
\hline $\mathrm{H}$ & 3.029025 & -4.164474 & 1.177893 \\
\hline C & 4.710212 & -0.915678 & 2.471529 \\
\hline $\mathrm{H}$ & 2.738874 & -0.056332 & 2.387789 \\
\hline C & 5.484886 & -2.052613 & 2.239413 \\
\hline $\mathrm{H}$ & 5.487367 & -4.110372 & 1.601654 \\
\hline $\mathrm{H}$ & 5.179438 & 0.004129 & 2.802406 \\
\hline $\mathrm{H}$ & 6.558145 & -2.020508 & 2.395870 \\
\hline $\mathrm{H}$ & -1.462990 & -1.655577 & 2.909354 \\
\hline $\mathrm{H}$ & 1.100527 & -3.200390 & 3.638184 \\
\hline $\mathrm{H}$ & 0.768731 & -1.410506 & 3.889685 \\
\hline C & 0.421505 & 3.057567 & 0.421190 \\
\hline C & 1.483766 & 2.424734 & -0.201627 \\
\hline C & 2.724802 & 2.221398 & 0.479853 \\
\hline C & 2.861153 & 2.717723 & 1.762788 \\
\hline $\mathrm{H}$ & 3.782607 & 2.536805 & 2.308309 \\
\hline
\end{tabular}




\begin{tabular}{|c|c|c|c|}
\hline C & -1.903227 & 2.253780 & -0.004122 \\
\hline C & -0.902034 & 3.182813 & -0.268441 \\
\hline C & -3.370301 & 3.396306 & -1.566611 \\
\hline C & -3.167325 & 2.365772 & -0.667041 \\
\hline $\mathrm{H}$ & -4.334357 & 3.487322 & -2.058577 \\
\hline C & -2.370110 & 4.351033 & -1.854495 \\
\hline C & -1.105939 & 4.247448 & -1.194659 \\
\hline C & -0.098996 & 5.205112 & -1.491274 \\
\hline C & -0.338866 & 6.217267 & -2.390478 \\
\hline C & -1.594053 & 6.324262 & -3.037101 \\
\hline C & -2.586805 & 5.410600 & -2.774901 \\
\hline $\mathrm{H}$ & 0.868918 & 5.115883 & -1.012983 \\
\hline $\mathrm{H}$ & 0.442308 & 6.936930 & -2.613335 \\
\hline $\mathrm{H}$ & -1.766587 & 7.128391 & -3.745708 \\
\hline $\mathrm{H}$ & -3.551228 & 5.481628 & -3.269848 \\
\hline C & 1.820634 & 3.422818 & 2.412612 \\
\hline C & 0.571505 & 3.592565 & 1.738496 \\
\hline C & -0.474970 & 4.283866 & 2.404737 \\
\hline C & -0.295641 & 4.775401 & 3.678236 \\
\hline C & 0.938630 & 4.600297 & 4.347675 \\
\hline C & 1.972300 & 3.938413 & 3.726718 \\
\hline $\mathrm{H}$ & -1.418953 & 4.423172 & 1.890117 \\
\hline $\mathrm{H}$ & -1.104456 & 5.304390 & 4.172692 \\
\hline $\mathrm{H}$ & 1.067217 & 4.992681 & 5.351667 \\
\hline $\mathrm{H}$ & 2.924118 & 3.801246 & 4.232428 \\
\hline C & -6.381615 & -0.467994 & -0.077049 \\
\hline C & -5.802916 & 0.196090 & 1.016103 \\
\hline C & -4.773928 & 1.114560 & 0.835547 \\
\hline C & -4.273464 & 1.398374 & -0.444520 \\
\hline C & -4.871967 & 0.755926 & -1.538885 \\
\hline C & -5.905316 & -0.157310 & -1.359422 \\
\hline $\mathrm{H}$ & -4.354574 & 1.625105 & 1.697252 \\
\hline $\mathrm{H}$ & -4.504980 & 0.967268 & -2.538124 \\
\hline C & 3.813282 & 1.396235 & -0.094840 \\
\hline C & 3.533868 & 0.189478 & -0.754204 \\
\hline C & 5.157501 & 1.730972 & 0.120598 \\
\hline C & 4.555772 & -0.660745 & -1.152710 \\
\hline $\mathrm{H}$ & 2.506241 & -0.101422 & -0.936547 \\
\hline C & 6.180142 & 0.876094 & -0.273428 \\
\hline $\mathrm{H}$ & 5.400657 & 2.670393 & 0.607082 \\
\hline C & 5.900078 & -0.345635 & -0.905398 \\
\hline $\mathrm{H}$ & -6.353343 & -0.635172 & -2.224564 \\
\hline $\mathrm{H}$ & -6.149012 & -0.028725 & 2.019874 \\
\hline $\mathrm{H}$ & 4.306554 & -1.585651 & -1.661266 \\
\hline $\mathrm{H}$ & 7.210367 & 1.144752 & -0.062695 \\
\hline C & 6.985291 & -1.279705 & -1.279489 \\
\hline C & 6.792455 & -2.668811 & -1.199935 \\
\hline C & 8.234279 & -0.805990 & -1.711906 \\
\hline C & 7.814803 & -3.552461 & -1.537258 \\
\hline $\mathrm{H}$ & 5.843193 & -3.052065 & -0.843057 \\
\hline C & 9.257129 & -1.689871 & -2.047939 \\
\hline $\mathrm{H}$ & 8.393250 & 0.263052 & -1.811014 \\
\hline C & 9.052800 & -3.067619 & -1.961309 \\
\hline $\mathrm{H}$ & 7.646369 & -4.622804 & -1.462392 \\
\hline
\end{tabular}




$\begin{array}{|lrrr|}H & 10.212494 & -1.301787 & -2.388333 \\ H & 9.849644 & -3.756404 & -2.224393 \\ \mathrm{C} & -7.446182 & -1.477748 & 0.119378 \\ \mathrm{C} & -8.436295 & -1.303603 & 1.098961 \\ \mathrm{C} & -7.481730 & -2.640948 & -0.665949 \\ \mathrm{C} & -9.430009 & -2.261431 & 1.287120 \\ \mathrm{H} & -8.439458 & -0.398240 & 1.697813 \\ \mathrm{C} & -8.475621 & -3.598588 & -0.478204 \\ \mathrm{H} & -6.707598 & -2.805161 & -1.408459 \\ \mathrm{C} & -9.454011 & -3.413062 & 0.499315 \\ \mathrm{H} & -10.192111 & -2.104127 & 2.044375 \\ \mathrm{H} & -8.480280 & -4.495760 & -1.089756 \\ \mathrm{H} & -10.228276 & -4.159573 & 0.646239 \\ \end{array}$

\section{A-Catalyzed reaction TS 10}

\section{Datum}

Value

\begin{tabular}{lr}
\hline B3LYP-D3(BJ)/6-31G(d,p) Energy & -2872.501395 \\
\hline B3LYP-D3(BJ)/def2-TZVPP/IEF-PCM(DCM) Energy & -2873.501855 \\
\hline B3LYP-D3(BJ)/def2-TZVPP/IEF-PCM(DCM)//B3LYP-D3(BJ)/6-31G(d,p) Free Energy (Quasiharmonic) & -2872.549934 \\
\hline
\end{tabular}

Number of Imaginary Frequencies

Frequencies (Top 3 out of 366)

$\begin{array}{lr}\text { 1. } & -282.6893 \mathrm{~cm}^{-1} \\ \text { 2. } & 5.6145 \mathrm{~cm}^{-1} \\ \text { 3. } & 9.3350 \mathrm{~cm}^{-1}\end{array}$

B3LYP-D3(BJ)/6-31G(d,p) Molecular Geometry in Cartesian Coordinates

\begin{tabular}{|lrrr|}
\hline C & -1.352121 & 1.489340 & 1.094667 \\
$H$ & -0.952609 & 2.018340 & 1.962987 \\
$H$ & -2.341905 & 1.118881 & 1.351068 \\
C & 1.948542 & 1.659048 & -1.108535 \\
O & 2.674226 & 1.967284 & -2.029329 \\
O & 1.090466 & 0.638579 & -1.200703 \\
H & 0.512774 & 0.559041 & -0.379039 \\
O & -0.495032 & 0.362981 & 0.836492 \\
$H$ & -1.050146 & -0.465243 & 0.779786 \\
C & 0.004274 & -1.942164 & -2.562060 \\
C & 0.579027 & -2.634186 & -0.655418 \\
C & -2.251057 & -2.535148 & -1.742310 \\
$C$ & -1.259481 & -1.583047 & -2.125476
\end{tabular}




\begin{tabular}{|c|c|c|c|}
\hline $\mathrm{H}$ & 0.570583 & -1.657069 & -0.178695 \\
\hline $\mathrm{H}$ & -3.250978 & -2.132134 & -1.591571 \\
\hline $\mathrm{H}$ & -2.252562 & -3.466653 & -2.309276 \\
\hline C & -2.331119 & -2.352917 & 2.040919 \\
\hline C & -3.238529 & -3.545129 & 1.583947 \\
\hline C & -1.094297 & -2.797912 & 2.830005 \\
\hline $\mathrm{H}$ & -0.402325 & -1.953027 & 2.896106 \\
\hline $\mathrm{H}$ & -1.355000 & -3.111033 & 3.845023 \\
\hline $\mathrm{H}$ & -0.583198 & -3.621864 & 2.327881 \\
\hline C & -3.066224 & -1.246891 & 2.789220 \\
\hline $\mathrm{H}$ & -3.821763 & -0.775987 & 2.158482 \\
\hline $\mathrm{H}$ & -3.556421 & -1.650841 & 3.680601 \\
\hline $\mathrm{H}$ & -2.359760 & -0.479764 & 3.119819 \\
\hline C & -4.636310 & -3.078893 & 1.161547 \\
\hline $\mathrm{H}$ & -5.257215 & -2.790302 & 2.014729 \\
\hline $\mathrm{H}$ & -4.572281 & -2.226667 & 0.483123 \\
\hline $\mathrm{H}$ & -5.135112 & -3.898530 & 0.638904 \\
\hline C & -3.342599 & -4.677543 & 2.601040 \\
\hline $\mathrm{H}$ & -3.778347 & -4.322826 & 3.541168 \\
\hline $\mathrm{H}$ & -3.987699 & -5.466281 & 2.204273 \\
\hline $\mathrm{H}$ & -2.364182 & -5.114135 & 2.807120 \\
\hline 0 & -1.882866 & -1.840728 & 0.759907 \\
\hline 0 & -2.562974 & -4.038347 & 0.418604 \\
\hline B & -1.766324 & -3.000000 & -0.125930 \\
\hline 0 & -0.362676 & -3.476085 & -0.324007 \\
\hline C & 1.913484 & -3.173418 & -0.969694 \\
\hline C & 3.003960 & -2.294184 & -1.036801 \\
\hline C & 2.107588 & -4.545797 & -1.181055 \\
\hline C & 4.275041 & -2.786411 & -1.312934 \\
\hline $\mathrm{H}$ & 2.850287 & -1.231741 & -0.880519 \\
\hline C & 3.384333 & -5.031371 & -1.449483 \\
\hline $\mathrm{H}$ & 1.255307 & -5.212981 & -1.114617 \\
\hline C & 4.469960 & -4.153419 & -1.516110 \\
\hline $\mathrm{H}$ & 5.112156 & -2.103966 & -1.359205 \\
\hline $\mathrm{H}$ & 3.535244 & -6.095716 & -1.602131 \\
\hline $\mathrm{H}$ & 5.468002 & -4.529860 & -1.719068 \\
\hline $\mathrm{H}$ & -1.401536 & -0.549925 & -1.809981 \\
\hline $\mathrm{H}$ & 0.148329 & -2.906238 & -3.040346 \\
\hline $\mathrm{H}$ & 0.741699 & -1.174998 & -2.764292 \\
\hline C & 0.871752 & 3.195391 & 0.533918 \\
\hline C & 1.952909 & 2.391797 & 0.211816 \\
\hline C & 3.057927 & 2.241190 & 1.106132 \\
\hline C & 3.038299 & 2.934425 & 2.299446 \\
\hline $\mathrm{H}$ & 3.865438 & 2.816133 & 2.993246 \\
\hline C & -1.416725 & 2.424151 & -0.097551 \\
\hline C & -0.319135 & 3.233371 & -0.373231 \\
\hline C & -2.486672 & 3.185624 & -2.141132 \\
\hline C & -2.525111 & 2.400836 & -1.003549 \\
\hline $\mathrm{H}$ & -3.333212 & 3.173750 & -2.821556 \\
\hline C & -1.390195 & 4.025779 & -2.437948 \\
\hline C & -0.279648 & 4.056031 & -1.538325 \\
\hline C & 0.823726 & 4.897420 & -1.843152 \\
\hline C & 0.820393 & 5.673945 & -2.977863 \\
\hline C & -0.282478 & 5.647546 & -3.865416 \\
\hline
\end{tabular}




\begin{tabular}{|c|c|c|c|}
\hline C & -1.363111 & 4.840438 & -3.600752 \\
\hline $\mathrm{H}$ & 1.675822 & 4.908587 & -1.174199 \\
\hline $\mathrm{H}$ & 1.672360 & 6.308551 & -3.200020 \\
\hline $\mathrm{H}$ & -0.268587 & 6.264637 & -4.758431 \\
\hline $\mathrm{H}$ & -2.212422 & 4.810688 & -4.277392 \\
\hline C & 1.982418 & 3.814436 & 2.639603 \\
\hline C & 0.878164 & 3.956053 & 1.742429 \\
\hline C & -0.178874 & 4.838032 & 2.090920 \\
\hline C & -0.144935 & 5.543280 & 3.272426 \\
\hline C & 0.946268 & 5.400340 & 4.162276 \\
\hline C & 1.985728 & 4.555083 & 3.850786 \\
\hline $\mathrm{H}$ & -1.012406 & 4.949361 & 1.406377 \\
\hline $\mathrm{H}$ & -0.957949 & 6.217242 & 3.523954 \\
\hline $\mathrm{H}$ & 0.960320 & 5.962343 & 5.090910 \\
\hline $\mathrm{H}$ & 2.827947 & 4.441426 & 4.527406 \\
\hline C & -6.055001 & -0.045444 & -0.458288 \\
\hline C & -5.275583 & -0.129662 & -1.622110 \\
\hline C & -4.132707 & 0.648253 & -1.779903 \\
\hline C & -3.722916 & 1.546357 & -0.784420 \\
\hline C & -4.509719 & 1.643472 & 0.373093 \\
\hline C & -5.646633 & 0.858847 & 0.535958 \\
\hline $\mathrm{H}$ & -3.543632 & 0.561245 & -2.687191 \\
\hline $\mathrm{H}$ & -4.233591 & 2.351344 & 1.148633 \\
\hline C & 4.172567 & 1.293742 & 0.833065 \\
\hline C & 4.423008 & 0.248499 & 1.734486 \\
\hline C & 5.012040 & 1.425060 & -0.282727 \\
\hline C & 5.471166 & -0.641307 & 1.525748 \\
\hline $\mathrm{H}$ & 3.765690 & 0.118954 & 2.588733 \\
\hline C & 6.070702 & 0.544466 & -0.478122 \\
\hline $\mathrm{H}$ & 4.827936 & 2.218799 & -0.995150 \\
\hline C & 6.321980 & -0.507418 & 0.417427 \\
\hline $\mathrm{H}$ & 6.726979 & 0.689974 & -1.329907 \\
\hline $\mathrm{H}$ & 5.609026 & -1.471989 & 2.209747 \\
\hline H & -5.578758 & -0.797757 & -2.421517 \\
\hline $\mathrm{H}$ & -6.220119 & 0.937360 & 1.453744 \\
\hline C & -7.266132 & -0.879630 & -0.284818 \\
\hline c & -8.386063 & -0.389720 & 0.405670 \\
\hline C & -7.323740 & -2.182737 & -0.804981 \\
\hline C & -9.522059 & -1.177522 & 0.576064 \\
\hline $\mathrm{H}$ & -8.374955 & 0.625825 & 0.788340 \\
\hline C & -8.459468 & -2.970463 & -0.635258 \\
\hline $\mathrm{H}$ & -6.460057 & -2.591847 & -1.317649 \\
\hline C & -9.563593 & -2.472130 & 0.057292 \\
\hline $\mathrm{H}$ & -10.379698 & -0.776013 & 1.107299 \\
\hline H & -8.477620 & -3.979195 & -1.036366 \\
\hline $\mathrm{H}$ & -10.448352 & -3.086692 & 0.190912 \\
\hline C & 7.425260 & -1.468652 & 0.184803 \\
\hline C & 8.110348 & -2.056179 & 1.260375 \\
\hline C & 7.802970 & -1.834189 & -1.118255 \\
\hline C & 9.127767 & -2.982018 & 1.042541 \\
\hline $\mathrm{H}$ & 7.856942 & -1.766233 & 2.274886 \\
\hline C & 8.819827 & -2.760857 & -1.337195 \\
\hline H & 7.280876 & -1.405079 & -1.967455 \\
\hline$c$ & 9.485947 & -3.341336 & -0.257342 \\
\hline
\end{tabular}




$\begin{array}{rrrr}H & 9.647061 & -3.418602 & 1.890350 \\ H & 9.087633 & -3.033704 & -2.353499 \\ H & 10.277615 & -4.064401 & -0.427013\end{array}$

\section{A-Catalyzed reaction TS 11}

Datum

Value

\begin{tabular}{lr}
\hline B3LYP-D3(BJ)/6-31G(d,p) Energy & -2872.502295 \\
\hline B3LYP-D3(BJ)/def2-TZVPP/IEF-PCM(DCM) Energy & -2873.502029 \\
\hline B3LYP-D3(BJ)/def2-TZVPP/IEF-PCM(DCM)//B3LYP-D3(BJ)/6-31G(d,p) Free Energy (Quasiharmonic) & -2872.549852
\end{tabular}

Number of Imaginary Frequencies

Frequencies (Top 3 out of 366)

$\begin{array}{rr}\text { 1. } & -374.3981 \mathrm{~cm}^{-1} \\ \text { 2. } & 10.4235 \mathrm{~cm}^{-1} \\ \text { 3. } & 11.6553 \mathrm{~cm}^{-1}\end{array}$

\section{B3LYP-D3(BJ)/6-31G(d,p) Molecular Geometry in Cartesian Coordinates}

C

$\mathrm{H}$

$\mathrm{H}$

C

0

0

$\mathrm{H}$

0

$\mathrm{H}$

C

C

C

C

$\mathrm{H}$

$\mathrm{H}$

$\mathrm{H}$

C

C

C

$\mathrm{H}$

$\mathrm{H}$

$\mathrm{H}$

C
1. 391899

2. 364089

0.902975

$-1.757184$

$-2.662425$

$-0.747675$

$-0.203523$

0.575822

1.137018

0.203543

$-0.814354$

2.187194

1. 401435

-0. 796961

2.195677

3.170963

2. 317245

1. 344530

2. 174181

2. 915085

1. 181868

2. 356283

3. 781415
1.754301

1.412052

2.290496

1.844277

2.021506

1.016508

0.844679

0.611347

$-0.206389$

$-3.866412$

$-3.220867$

$-3.053823$

$-4.097913$

$-4.172149$

$-2.108058$

$-3.338592$

$-2.214921$

$-3.431812$

$-1.149991$

$-0.360346$

$-0.698024$

$-1.585941$

$-2.638219$
1.161587

1.514096

1.977650

$-1.073637$

$-1.864018$

$-1.332061$

$-0.503443$

0.849316

0.767327

$-2.718399$

$-1.031580$

$-1.494094$

$-2.054502$

$-0.497513$

$-2.034451$

$-1.126440$

2.027280

2.173611

3. 109342

2.952875

3. 101288

4.096499

1.898805 


\begin{tabular}{|c|c|c|c|}
\hline $\mathrm{H}$ & 4.366639 & -1.784774 & 1.554703 \\
\hline $\mathrm{H}$ & 4.187438 & -2.974175 & 2.857299 \\
\hline $\mathrm{H}$ & 3.896393 & -3.447423 & 1.174900 \\
\hline C & -0.047415 & -3.008137 & 2.667313 \\
\hline $\mathrm{H}$ & -0.038345 & -2.746003 & 3.729180 \\
\hline H & -0.426325 & -2.152991 & 2.102877 \\
\hline $\mathrm{H}$ & -0.738259 & -3.844245 & 2.527304 \\
\hline C & 1.880636 & -4.575950 & 3.025737 \\
\hline $\mathrm{H}$ & 2.078636 & -4.242646 & 4.049565 \\
\hline $\mathrm{H}$ & 1.140895 & -5.380428 & 3.068545 \\
\hline $\mathrm{H}$ & 2.800593 & -4.984118 & 2.604566 \\
\hline 0 & 1.905230 & -1.654261 & 0.749808 \\
\hline 0 & 1.236511 & -3.887064 & 0.812652 \\
\hline B & 1.288648 & -2.717745 & -0.010704 \\
\hline 0 & -0.032566 & -2.282570 & -0.545710 \\
\hline C & -2.106392 & -2.821558 & -1.615621 \\
\hline C & -2.294675 & -1.539892 & -2.147605 \\
\hline C & -3.174486 & -3.731776 & -1.591166 \\
\hline C & -3.546092 & -1.162015 & -2.623603 \\
\hline $\mathrm{H}$ & -1.480346 & -0.827398 & -2.143010 \\
\hline C & -4.424110 & -3.352427 & -2.073546 \\
\hline $\mathrm{H}$ & -3.029524 & -4.722633 & -1.168232 \\
\hline C & -4.609213 & -2.066918 & -2.587666 \\
\hline $\mathrm{H}$ & -3.683804 & -0.146736 & -2.979381 \\
\hline $\mathrm{H}$ & -5.260524 & -4.040832 & -2.016241 \\
\hline $\mathrm{H}$ & -5.592056 & -1.767084 & -2.936389 \\
\hline $\mathrm{H}$ & 1.600227 & -5.109611 & -1.703195 \\
\hline $\mathrm{H}$ & 0.063784 & -2.931155 & -3.251432 \\
\hline $\mathrm{H}$ & -0.389750 & -4.701118 & -3.077536 \\
\hline C & -0.729618 & 3.529591 & 0.489730 \\
\hline C & -1.730873 & 2.607809 & 0.234613 \\
\hline C & -2.847939 & 2.474469 & 1.117414 \\
\hline C & -2.982548 & 3.355898 & 2.166993 \\
\hline $\mathrm{H}$ & -3.814634 & 3.242745 & 2.856133 \\
\hline C & 1.541261 & 2.668172 & -0.038335 \\
\hline C & 0.504391 & 3.536780 & -0.356312 \\
\hline C & 2.719773 & 3.331902 & -2.055126 \\
\hline C & 2.656622 & 2.543814 & -0.923350 \\
\hline $\mathrm{H}$ & 3.570722 & 3.235306 & -2.723435 \\
\hline C & 1.704302 & 4.264022 & -2.373578 \\
\hline C & 0.563584 & 4.361632 & -1.517335 \\
\hline C & -0.477640 & 5.264057 & -1.862383 \\
\hline C & -0.381924 & 6.045918 & -2.989923 \\
\hline C & 0.754498 & 5.959669 & -3.829897 \\
\hline C & 1.772913 & 5.086046 & -3.528991 \\
\hline $\mathrm{H}$ & -1.353138 & 5.321497 & -1.225885 \\
\hline $\mathrm{H}$ & -1.185207 & 6.730736 & -3.242656 \\
\hline $\mathrm{H}$ & 0.815069 & 6.582068 & -4.717370 \\
\hline $\mathrm{H}$ & 2.643963 & 5.007349 & -4.173459 \\
\hline C & -2.026213 & 4.374725 & 2.407889 \\
\hline C & -0.863480 & 4.447738 & 1.577382 \\
\hline C & 0.117193 & 5.435271 & 1.856764 \\
\hline C & -0.047712 & 6.314888 & 2.903149 \\
\hline C & -1.198515 & 6.244666 & 3.722699 \\
\hline
\end{tabular}




\begin{tabular}{|c|c|c|c|}
\hline C & -2.163312 & 5.293599 & 3.480343 \\
\hline $\mathrm{H}$ & 0.996757 & 5.489480 & 1.224874 \\
\hline $\mathrm{H}$ & 0.708354 & 7.068232 & 3.101608 \\
\hline $\mathrm{H}$ & -1.317033 & 6.943395 & 4.545122 \\
\hline $\mathrm{H}$ & -3.046844 & 5.230181 & 4.109326 \\
\hline C & 5.721472 & -0.453431 & -0.359203 \\
\hline C & 5.580016 & 0.602915 & 0.555722 \\
\hline C & 4.597521 & 1.573464 & 0.386058 \\
\hline C & 3.715682 & 1.521267 & -0.702071 \\
\hline C & 3.858040 & 0.470622 & -1.617984 \\
\hline C & 4.842909 & -0.497163 & -1.451384 \\
\hline $\mathrm{H}$ & 4.514663 & 2.386728 & 1.101019 \\
\hline $\mathrm{H}$ & 3.182356 & 0.419161 & -2.466047 \\
\hline C & -3.756582 & 1.312390 & 0.973736 \\
\hline C & -5.148495 & 1.451122 & 0.928185 \\
\hline C & -3.206745 & 0.022868 & 0.913239 \\
\hline C & -5.966885 & 0.333633 & 0.792721 \\
\hline $\mathrm{H}$ & -5.585933 & 2.443686 & 0.972523 \\
\hline C & -4.026336 & -1.090621 & 0.792257 \\
\hline $\mathrm{H}$ & -2.129122 & -0.101243 & 0.952447 \\
\hline C & -5.419937 & -0.956308 & 0.712583 \\
\hline $\mathrm{H}$ & 6.234716 & 0.651060 & 1.419985 \\
\hline $\mathrm{H}$ & 4.942793 & -1.289045 & -2.186261 \\
\hline $\mathrm{H}$ & -7.044473 & 0.460098 & 0.754366 \\
\hline $\mathrm{H}$ & -3.578226 & -2.072633 & 0.699541 \\
\hline C & -6.270714 & -2.146965 & 0.494286 \\
\hline C & -5.956076 & -3.378857 & 1.088266 \\
\hline C & -7.379566 & -2.087701 & -0.364088 \\
\hline C & -6.713852 & -4.516437 & 0.821474 \\
\hline $\mathrm{H}$ & -5.113830 & -3.438626 & 1.769986 \\
\hline C & -8.138954 & -3.224633 & -0.632218 \\
\hline $\mathrm{H}$ & -7.620696 & -1.149587 & -0.853692 \\
\hline C & -7.806963 & -4.446361 & -0.044176 \\
\hline $\mathrm{H}$ & -6.454036 & -5.459167 & 1.293993 \\
\hline $\mathrm{H}$ & -8.984765 & -3.159160 & -1.310225 \\
\hline $\mathrm{H}$ & -8.396258 & -5.333423 & -0.255085 \\
\hline C & 6.753576 & -1.497803 & -0.166346 \\
\hline C & 8.010176 & -1.182581 & 0.374029 \\
\hline C & 6.495335 & -2.834352 & -0.510992 \\
\hline C & 8.973599 & -2.170000 & 0.567130 \\
\hline $\mathrm{H}$ & 8.240276 & -0.150931 & 0.620514 \\
\hline C & 7.458326 & -3.821899 & -0.319571 \\
\hline $\mathrm{H}$ & 5.522027 & -3.102095 & -0.908136 \\
\hline C & 8.702010 & -3.494439 & 0.221929 \\
\hline $\mathrm{H}$ & 9.941778 & -1.902781 & 0.979734 \\
\hline $\mathrm{H}$ & 7.233633 & -4.850550 & -0.584988 \\
\hline $\mathrm{H}$ & 9.452682 & -4.263917 & 0.372768 \\
\hline
\end{tabular}


Frequencies (Top 3 out of 366)

$\begin{array}{lr}\text { 1. } & -264.8896 \mathrm{~cm}^{-1} \\ \text { 2. } & 9.1634 \mathrm{~cm}^{-1} \\ \text { 3. } & 11.3995 \mathrm{~cm}^{-1}\end{array}$

\section{B3LYP-D3(BJ)/6-31G(d,p) Molecular Geometry in Cartesian Coordinates}

C

$\mathrm{H}$

$\mathrm{H}$

C

0

0

$\mathrm{H}$

0

$\mathrm{H}$

C

C

C

C

$\mathrm{H}$

$\mathrm{H}$

$\mathrm{H}$

C

C

C

$\mathrm{H}$

$\mathrm{H}$

$\mathrm{H}$

C

$\mathrm{H}$

$\mathrm{H}$

$\mathrm{H}$

C

$\mathrm{H}$

$\mathrm{H}$

$\mathrm{H}$

C

$\mathrm{H}$

\subsection{0}

2.233002

0.727951

$-1.981639$

$-2.795780$

$-1.014795$

$-0.421547$

0.512904

0.053622

0.028943

1. 356774

$-1.607296$

$-1.081720$

1.276898

$-2.582483$

$-1.538830$

$-1.174513$

$-0.735314$

$-0.548989$

$-0.921968$

$-0.819777$

0.539927

$-2.695070$

$-3.113270$

$-2.943665$

$-3.169570$

$-1.620009$

$-1.619432$

$-2.647198$

$-1.241059$

0.736476

0.891670
1. 252553

0.856209

1.526764

1.746195

2.170418

0.894809

0.681347

0.241933

$-0.381182$

$-3.044530$

$-2.712170$

$-3.388234$

$-2.640190$

$-1.625703$

$-3.065926$

$-4.472241$

$-1.585939$

$-2.997366$

$-0.399737$

0.538051

$-0.412865$

$-0.409832$

$-1.421067$

$-1.330839$

$-0.515291$

$-2.269880$

$-3.570935$

$-2.922503$

$-3.696887$

$-4.552028$

$-3.028504$

$-2.496421$
0.778159

1.049795

1.696536

$-1.570684$

$-2.359588$

$-1.930923$

$-1.154241$

0.077779

0.708622

$-2.149230$

$-0.521726$

$-0.334951$

$-1.431935$

$-0.547599$

0.027118

$-0.432980$

2. 788642

3. 307695

3. 515348

3.092230

4.575642

3. 438479

2. 741777

3. 748494

2. 188049

2. 245341

4.409052

5.291433

4.064229

4.708035

3. 744537

4. 687969 


\begin{tabular}{|c|c|c|c|}
\hline $\mathrm{H}$ & 1.037226 & -4.070305 & 3.880443 \\
\hline $\mathrm{H}$ & 1.384970 & -2.588977 & 2.983098 \\
\hline 0 & -0.677730 & -1.612953 & 1.424432 \\
\hline 0 & -0.881468 & -3.814206 & 2.134635 \\
\hline B & -0.553953 & -3.012560 & 1.015005 \\
\hline 0 & 0.802839 & -3.350400 & 0.471295 \\
\hline C & 2.577987 & -3.295275 & -1.109273 \\
\hline C & 2.967380 & -4.609597 & -0.816544 \\
\hline C & 3.353298 & -2.516883 & -1.979293 \\
\hline C & 4.116887 & -5.138443 & -1.396050 \\
\hline $\mathrm{H}$ & 2.364413 & -5.193117 & -0.129771 \\
\hline C & 4.493793 & -3.055568 & -2.568311 \\
\hline $\mathrm{H}$ & 3.060358 & -1.492198 & -2.186129 \\
\hline C & 4.878127 & -4.367007 & -2.278370 \\
\hline $\mathrm{H}$ & 4.420623 & -6.154175 & -1.163002 \\
\hline $\mathrm{H}$ & 5.084870 & -2.454053 & -3.251520 \\
\hline $\mathrm{H}$ & 5.769249 & -4.784996 & -2.736434 \\
\hline $\mathrm{H}$ & -1.399865 & -1.606814 & -1.562098 \\
\hline $\mathrm{H}$ & 0.274039 & -4.099823 & -2.214142 \\
\hline $\mathrm{H}$ & 0.427917 & -2.413359 & -2.936309 \\
\hline C & -1.008373 & 2.942555 & 0.417555 \\
\hline C & -2.023558 & 2.166058 & -0.115127 \\
\hline C & -3.197065 & 1.864744 & 0.646765 \\
\hline C & -3.317475 & 2.379462 & 1.920644 \\
\hline $\mathrm{H}$ & -4.188256 & 2.123242 & 2.516968 \\
\hline C & 1.373003 & 2.451015 & -0.134172 \\
\hline C & 0.238461 & 3.220157 & -0.363855 \\
\hline C & 2.582121 & 3.706497 & -1.821466 \\
\hline C & 2.569778 & 2.702370 & -0.873618 \\
\hline $\mathrm{H}$ & 3.494253 & 3.900545 & -2.378597 \\
\hline C & 1.443385 & 4.505278 & -2.077564 \\
\hline C & 0.244780 & 4.265564 & -1.333660 \\
\hline C & -0.897417 & 5.068191 & -1.598163 \\
\hline C & -0.850248 & 6.062127 & -2.547160 \\
\hline C & 0.338252 & 6.304433 & -3.277308 \\
\hline C & 1.458919 & 5.542139 & -3.047470 \\
\hline $\mathrm{H}$ & -1.814191 & 4.873344 & -1.054657 \\
\hline $\mathrm{H}$ & -1.732883 & 6.662006 & -2.744360 \\
\hline $\mathrm{H}$ & 0.358834 & 7.092069 & -4.024124 \\
\hline $\mathrm{H}$ & 2.373652 & 5.718591 & -3.606269 \\
\hline C & -2.315710 & 3.201130 & 2.491931 \\
\hline C & -1.138365 & 3.491084 & 1.733510 \\
\hline C & -0.136692 & 4.310754 & 2.318431 \\
\hline C & -0.286495 & 4.804900 & 3.594628 \\
\hline C & -1.445720 & 4.506426 & 4.348572 \\
\hline C & -2.437722 & 3.722782 & 3.806454 \\
\hline $\mathrm{H}$ & 0.750844 & 4.543146 & 1.741236 \\
\hline $\mathrm{H}$ & 0.488737 & 5.430191 & 4.026442 \\
\hline $\mathrm{H}$ & -1.550465 & 4.900544 & 5.354592 \\
\hline $\mathrm{H}$ & -3.332877 & 3.490222 & 4.376293 \\
\hline C & 6.116391 & 0.320138 & -0.222969 \\
\hline C & 5.487173 & 0.356150 & -1.475922 \\
\hline C & 4.349914 & 1.131587 & -1.686941 \\
\hline C & 3.798953 & 1.895711 & -0.649987 \\
\hline
\end{tabular}




$\begin{array}{lrrr}\text { C } & 4.442751 & 1.881784 & 0.596228 \\ \text { C } & 5.579430 & 1.108959 & 0.806202 \\ \text { H } & 3.871681 & 1.143425 & -2.661903 \\ \text { H } & 4.041289 & 2.482598 & 1.407033 \\ \text { C } & -4.232929 & 0.943415 & 0.122960 \\ \text { C } & -3.866559 & -0.305717 & -0.396900 \\ \text { C } & -5.594288 & 1.263045 & 0.186658 \\ \text { C } & -4.826031 & -1.200885 & -0.849712 \\ \text { H } & -2.820987 & -0.587110 & -0.396608 \\ \text { C } & -6.556235 & 0.366832 & -0.268418 \\ \text { H } & -5.895054 & 2.235072 & 0.564878 \\ \text { C } & -6.192217 & -0.881101 & -0.797613 \\ \text { H } & -7.603579 & 0.650321 & -0.246507 \\ \text { H } & -4.517322 & -2.172876 & -1.220705 \\ \text { H } & 5.904469 & -0.221400 & -2.294093 \\ \text { H } & 6.044674 & 1.092341 & 1.786694 \\ \text { C } & -7.215262 & -1.833821 & -1.284971 \\ \text { C } & -8.448713 & -1.966682 & -0.627851 \\ \text { C } & -6.974601 & -2.629301 & -2.416567 \\ \text { C } & -9.409879 & -2.864267 & -1.086934 \\ \text { H } & -8.641924 & -1.379181 & 0.264194 \\ \text { C } & -7.934792 & -3.528319 & -2.874738 \\ \text { H } & -6.038403 & -2.518785 & -2.954398 \\ \text { C } & -9.156939 & -3.649789 & -2.212215 \\ \text { H } & -10.354539 & -2.957126 & -0.559478 \\ \text { H } & -7.731961 & -4.127974 & -3.757000 \\ \text { H } & -9.905659 & -4.349831 & -2.569873 \\ \text { C } & 7.304403 & -0.532315 & 0.011884 \\ \text { C } & 8.385747 & -0.069343 & 0.777143 \\ \text { C } & 7.365438 & -1.830121 & -0.518838 \\ \text { C } & 9.497800 & -0.878355 & 1.000650 \\ \text { H } & 8.361666 & 0.939783 & 1.176856 \\ \text { C } & 8.477205 & -2.638556 & -0.294093 \\ \text { H } & 6.520606 & -2.216584 & -1.077901 \\ \text { C } & 9.548631 & -2.166145 & 0.465040 \\ \text { H } & 10.329229 & -0.500017 & 1.587723 \\ \text { H } & 8.500463 & -3.644355 & -0.703142 \\ & 10.415037 & -2.796427 & 0.640153\end{array}$

\section{A-Catalyzed reaction TS 13}

Datum

Value

B3LYP-D3(BJ)/6-31G(d,p) Energy

$-2872.500286$

B3LYP-D3(BJ)/def2-TZVPP/IEF-PCM(DCM) Energy

$-2873.500649$

B3LYP-D3(BJ)/def2-TZVPP/IEF-PCM(DCM)//B3LYP-D3(BJ)/6-31G(d,p) Free Energy (Quasiharmonic)

$-2872.548168$

Number of Imaginary Frequencies 
$\begin{array}{lr}\text { 1. } & -368.2418 \mathrm{~cm}^{-1} \\ \text { 2. } & 9.0552 \mathrm{~cm}^{-1} \\ \text { 3. } & 12.6065 \mathrm{~cm}^{-1}\end{array}$

B3LYP-D3(BJ)/6-31G(d,p) Molecular Geometry in Cartesian Coordinates

C

$\mathrm{H}$

$\mathrm{H}$

C

0

0

$\mathrm{H}$

0

$\mathrm{H}$

C

C

C

C

$\mathrm{H}$

$\mathrm{H}$

$\mathrm{H}$

C

C

C

$\mathrm{H}$

$\mathrm{H}$

$\mathrm{H}$

C

$\mathrm{H}$

$\mathrm{H}$

$\mathrm{H}$

C

$\mathrm{H}$

$\mathrm{H}$

H

C

$\mathrm{H}$

$\mathrm{H}$

$\mathrm{H}$

0

0

B

0

C

C

C

C
2. 306781

3. 237481

1.806861

$-0.766609$

$-1.382689$

$-0.023699$

0.465381

1.464951

1.104361

$-1.868539$

$-2.509449$

0.037541

$-1.134959$

$-3.163759$

0.663771

0.610841

$-0.006799$

$-0.925009$

$-0.776349$

$-0.148739$

$-1.661939$

$-1.077849$

1. 238071

1.809241

0.961061

1.887231

$-2.038199$

$-1.628019$

$-2.711699$

$-2.624919$

$-0.124529$

0.268461

$-0.784259$

0.713891

0.406471

$-1.522819$

$-0.674439$

$-1.402379$

$-3.164059$

$-2.489179$

$-4.474549$

$-3.126069$
0.594790

0.090320

0.912110

1.888800

2.541460

0.850510

0.411460

$-0.303580$

$-1.014480$

$-4.396500$

$-2.682010$

$-4.263360$

$-4.895900$

$-3.183960$

$-3.765840$

$-4.819380$

$-1.864150$

$-3.085230$

$-0.544970$

0.225590

$-0.652920$

$-0.194830$

$-1.715970$

$-0.836270$

$-1.578620$

$-2.588860$

$-2.761550$

$-2.405150$

$-2.001950$

$-3.662560$

$-4.300140$

$-4.153150$

$-5.171770$

$-4.512420$

$-2.199620$

$-3.420690$

$-2.944630$

$-2.173590$

$-1.986850$

$-0.998050$

$-2.335650$

$-0.355360$
0.828770

1.103380

1.749310

$-1.304450$

$-2.125470$

$-1.672290$

$-0.911810$

0.094940

0.689480

$-1.523900$

$-0.532770$

0.031930

$-0.455910$

0.181710

$-0.708510$

0.770960

2.943610

3.303680

2.877010

2.427590

2. 251740

3.867820

3.809700

3.499640

4.859690

3.728600

4.295880

5.246380

3.895970

4.495830

3.790600

4.800890

3.800480

3.122800

1.593350

2.041850

0.996600

$-0.043560$

$-1.656460$

$-2.381930$

$-2.010150$

$-3.438810$ 


\begin{tabular}{|c|c|c|c|}
\hline $\mathrm{H}$ & -1.482519 & -0.717740 & -2.104420 \\
\hline C & -5.105069 & -1.701310 & -3.075450 \\
\hline $\mathrm{H}$ & -5.004569 & -3.093460 & -1.440690 \\
\hline C & -4.429999 & -0.710430 & -3.792150 \\
\hline $\mathrm{H}$ & -2.604779 & 0.440230 & -3.960650 \\
\hline H & -6.128779 & -1.959160 & -3.325260 \\
\hline $\mathrm{H}$ & -4.926909 & -0.205760 & -4.615290 \\
\hline $\mathrm{H}$ & -1.620529 & -5.614160 & 0.203440 \\
\hline $\mathrm{H}$ & -1.357209 & -3.843200 & -2.305160 \\
\hline $\mathrm{H}$ & -2.792329 & -4.879960 & -1.824380 \\
\hline C & 0.392341 & 2.739490 & 0.721610 \\
\hline C & -0.782449 & 2.249230 & 0.166820 \\
\hline C & -1.987709 & 2.171060 & 0.946500 \\
\hline C & -1.946839 & 2.621870 & 2.253000 \\
\hline $\mathrm{H}$ & -2.851139 & 2.577950 & 2.851720 \\
\hline C & 2.590071 & 1.801170 & -0.033810 \\
\hline C & 1.649111 & 2.821010 & -0.090990 \\
\hline C & 4.017671 & 3.019270 & -1.569530 \\
\hline C & 3.788341 & 1.885740 & -0.812180 \\
\hline $\mathrm{H}$ & 4.921971 & 3.083870 & -2.167370 \\
\hline C & 3.087151 & 4.080780 & -1.634320 \\
\hline C & 1.867601 & 3.980080 & -0.894970 \\
\hline C & 0.924001 & 5.038200 & -0.985300 \\
\hline C & 1.181970 & 6.143550 & -1.761850 \\
\hline C & 2.392760 & 6.246030 & -2.488300 \\
\hline C & 3.322381 & 5.235300 & -2.427290 \\
\hline $\mathrm{H}$ & -0.010979 & 4.956500 & -0.444150 \\
\hline $\mathrm{H}$ & 0.449030 & 6.941610 & -1.825070 \\
\hline $\mathrm{H}$ & 2.580900 & 7.123780 & -3.098900 \\
\hline $\mathrm{H}$ & 4.250781 & 5.301120 & -2.987590 \\
\hline C & -0.776509 & 3.146770 & 2.845610 \\
\hline C & 0.420971 & 3.210410 & 2.071200 \\
\hline C & 1.596051 & 3.735500 & 2.672350 \\
\hline C & 1.584341 & 4.165410 & 3.979920 \\
\hline C & 0.397871 & 4.096660 & 4.748430 \\
\hline C & -0.757219 & 3.600490 & 4.190810 \\
\hline $\mathrm{H}$ & 2.504511 & 3.792420 & 2.084040 \\
\hline $\mathrm{H}$ & 2.490401 & 4.563690 & 4.425830 \\
\hline $\mathrm{H}$ & 0.403011 & 4.439590 & 5.778430 \\
\hline $\mathrm{H}$ & -1.673979 & 3.546020 & 4.771190 \\
\hline C & 6.755341 & -1.252950 & -0.986830 \\
\hline C & 7.118571 & 0.080660 & -0.744530 \\
\hline C & 6.157111 & 1.082890 & -0.673470 \\
\hline C & 4.794681 & 0.793170 & -0.841860 \\
\hline C & 4.427981 & -0.540290 & -1.087440 \\
\hline C & 5.391691 & -1.540320 & -1.155440 \\
\hline $\mathrm{H}$ & 6.460141 & 2.106910 & -0.477470 \\
\hline $\mathrm{H}$ & 3.379701 & -0.788780 & -1.213880 \\
\hline C & -3.257049 & 1.548270 & 0.477610 \\
\hline C & -3.908149 & 0.630200 & 1.321610 \\
\hline C & -3.882909 & 1.871990 & -0.734640 \\
\hline C & -5.112429 & 0.040730 & 0.961550 \\
\hline $\mathrm{H}$ & -3.460049 & 0.374600 & 2.274470 \\
\hline C & -5.088519 & 1.278470 & -1.091660 \\
\hline
\end{tabular}




\begin{tabular}{|lrrr|} 
H & -3.411879 & 2.578220 & -1.403630 \\
C & -5.727299 & 0.346000 & -0.262030 \\
H & -5.519489 & 1.518200 & -2.056690 \\
H & -5.589089 & -0.651040 & 1.648490 \\
$H$ & 8.167341 & 0.336060 & -0.630450 \\
$H$ & 5.080851 & -2.566800 & -1.322660 \\
C & 7.776921 & -2.322510 & -1.059340 \\
C & 7.647791 & -3.381110 & -1.972720 \\
C & 8.899901 & -2.305340 & -0.216680 \\
C & 8.607851 & -4.388060 & -2.040580 \\
H & 6.800711 & -3.397260 & -2.651100 \\
C & 9.861271 & -3.311000 & -0.285510 \\
H & 9.003441 & -1.509950 & 0.514600 \\
C & 9.719481 & -4.357560 & -1.197560 \\
H & 8.492431 & -5.193160 & -2.760100 \\
H & 10.718151 & -3.282230 & 0.380960 \\
H & 10.468241 & -5.141810 & -1.250980 \\
C & -6.987539 & -0.310120 & -0.675620 \\
C & -7.900819 & 0.339080 & -1.521330 \\
C & -7.289739 & -1.618150 & -0.261660 \\
C & -9.061199 & -0.300300 & -1.951830 \\
H & -7.707349 & 1.360680 & -1.830680 \\
C & -8.450399 & -2.258140 & -0.688130 \\
H & -6.589569 & -2.149670 & 0.373920 \\
C & -9.341139 & -1.604020 & -1.540830 \\
H & -9.752519 & 0.224650 & -2.604280 \\
H & -8.655349 & -3.273540 & -0.361910 \\
H & -10.244369 & -2.103160 & -1.877600 \\
& & & \\
\hline & & & \\
\hline
\end{tabular}




\section{References}

(1) Mohamadi, F.; Richards, N. G. J.; Guida, W. C.; Liskamp, R.; et al. Macromodel - an integrated software system for modeling organic and bioorganic molecules using molecular mechanics. J. Comput. Chem. 1990, 11, 440-467.

(2) Macromodel, Version 11.3. Schrödinger, LLC: New York, NY 2016.

(3) Banks, J. L.; Beard, H. S.; Cao, Y.; Cho, A. E.; et al. Integrated Modeling Program, Applied Chemical Theory (IMPACT). J. Comput. Chem. 2005, 26, 1752-1780.

(4) Chang, G.; Guida, W. C.; Still, W. C. An internal-coordinate Monte Carlo method for searching conformational space. J. Am. Chem. Soc. 1989, 111, 4379-4386.

(5) Kolossvary, I.; Guida, W. C. Low-mode conformational search elucidated: Application to $\mathrm{C} 39 \mathrm{H} 80$ and flexible docking of 9-deazaguanine inhibitors into PNP. J. Comput. Chem. 1999, 20, 1671-1684.

(6) Kolossváry, I.; Guida, W. C. Low mode search. An efficient, automated computational method for conformational analysis: Application to cyclic and acyclic alkanes and cyclic peptides. J. Am. Chem. Soc. 1996, 118, 5011-5019.

(7) Hohenberg, P.; Kohn, W. Inhomogeneous Electron Gas. Phys. Rev. 1964, 136, B864-871.

(8) Kohn, W.; Sham, L. J. Self-Consistent Equations Including Exchange and Correlation Effects. Phys. Rev. 1965, 140, A1133-1138.

(9) Frisch, M. J. et al. Gaussian 9, Revision D.01. Gaussian, Inc.: Wallingford CT 2009.

(10) Becke, A. D. Density-functional thermochemistry. III. The role of exact exchange. J. Chem. Phys. 1993, 98, 56485652.

(11) Stephens, P. J.; Devlin, F. J.; Chabalowski, C. F.; Frisch, M. J. Ab Initio Calculation of Vibrational Absorption and Circular Dichroism Spectra Using Density Functional Force Fields. J. Phys. Chem. 1994, 98, 11623-11627.

(12) Hehre, W. J.; Ditchfield, R.; Pople, J. A. Self-consistent molecular orbital methods. XII. Further extensions of Gaussian-type basis sets for use in molecular orbital studies of organic molecules. J. Chem. Phys. 1972, 56, 2257-2261.

(13) Simón, L;; Goodman, J. M. How reliable are DFT transition structures? Comparison of GGA, hybrid-meta-GGA and meta-GGA functionals. Org. Biomol. Chem. 2011, 9, 689-700.

(14) Grimme, S.; Antony, J.; Ehrlich, S.; Krieg, H. A consistent and accurate ab initio parametrization of density functional dispersion correction (DFT-D) for the 94 elements H-Pu. J. Chem. Phys. 2010, 132, 154104.

(15) Becke, A. D.; Johnson, E. R. A density-functional model of the dispersion interaction. J. Chem. Phys. 2005, 123, 154101.

(16) Johnson, E. R.; Becke, A. D. A post-Hartree-Fock model of intermolecular interactions: Inclusion of higher-order corrections. J. Chem. Phys. 2006, 124, 174104.

(17) Grimme, S.; Ehrlich, S.; Goerigk, L. Effect of the damping function in dispersion corrected density functional theory. J. Comput. Chem. 2011, 32, 1456-1465.

(18) Weigend, F.; Ahlrichs, R. Balanced basis sets of split valence, triple zeta valence and quadruple zeta valence quality for $\mathrm{H}$ to Rn: Design and assessment of accuracy. Phys. Chem. Chem. Phys. 2005, 7, 3297.

(19) Mennucci, B.; Cammi, R.; Tomasi, J. Excited states and solvatochromic shifts within a nonequilibrium solvation approach: A new formulation of the integral equation formalism method at the self-consistent field, configuration interaction, and multiconfiguration self-consistent field level. J. Chem. Phys. 1998, 109, 2798-2807.

(20) Frisch, M. J. et al. Gaussian 16, Revision A.03. Gaussian, Inc.: Wallingford CT 2016. 
(21) Grimme, S. Supramolecular Binding Thermodynamics by Dispersion-Corrected Density. Chem. A Eur. J. 2012, 18, 9955-9964.

(22) Funes-Ardoiz, I.; Paton, R. S. (2018). GoodVibes, v2.0.3 http://doi.org/10.5281/zenodo.595246.

(23) Alecu, I. M.; Zheng, J.; Zhao, Y.; Truhlar, D. G. Computational Thermochemistry: Scale Factor Databases and Scale Factors for Vibrational Frequencies Obtained from Electronic Model Chemistries. J. Chem. Theory Comput. 2010, 6, 2872-2887.

(24) Yang, J. Six-Membered Transition States in Organic Synthesis; Wiley: Hoboken, NJ, 2008.

(25) Ota, Y.; Kawato, Y.; Egami, H.; Hamashima, Y. Enantioselective Allyl-, and Allenylboration of Aldehydes Catalyzed by Chiral Hydroxyl Carboxylic Acid. Synlett 2017, 28, 976-980.

(26) Grayson, M. N.; Pellegrinet, S. C.; Goodman, J. M. Mechanistic Insights into the BINOL-Derived Phosphoric AcidCatalyzed Asymmetric Allylboration of Aldehydes. J. Am. Chem. Soc. 2012, 134 (5), 2716-2722.

(27) Grayson, M. N.; Goodman, J. M. Understanding the Mechanism of the Asymmetric Propargylation of Aldehydes Promoted by 1,1'-Bi-2-Naphthol-Derived Catalysts. J. Am. Chem. Soc. 2013, 135 (16), 6142-6148.

(28) Pedregal, J. R.-G.; Gómez-Orellana, P.; Maréchal, J.-D. ESIgen: Electronic Supporting Information Generator for Computational Chemistry Publications. J. Chem. Inf. Model. 2018, 58, 561-564. 